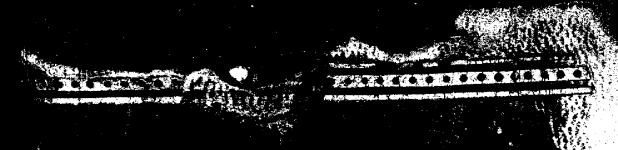

N. A

H

(

OOF BOTANY

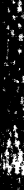

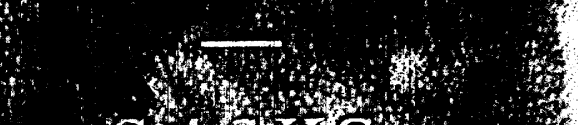

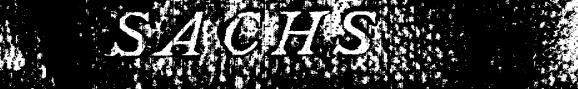

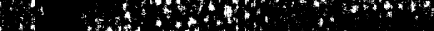

$19+4)$

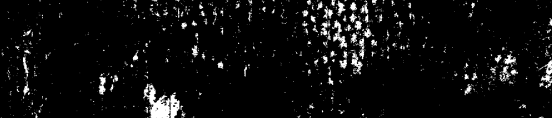

- $\quad \frac{4 K}{47}, 4$

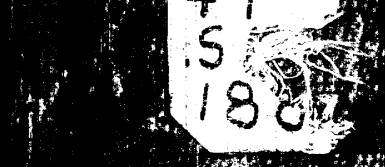

Secong to

* Secongerition.

,

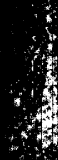

4
$4+2$

tis

i.

8.

4

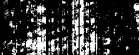

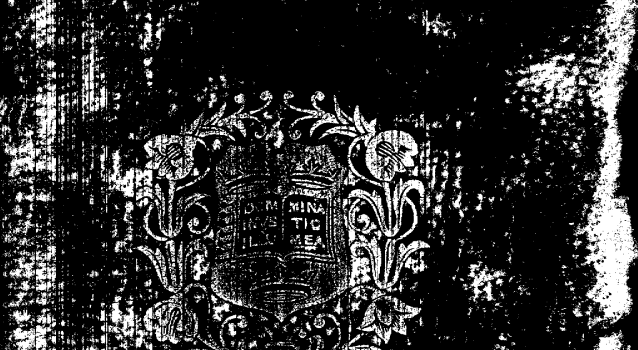

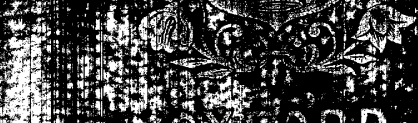

this

. RD

,

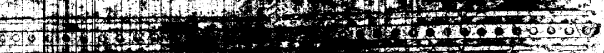

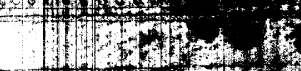




TEXT-B00K O'F BOTANY

$S A C H S$ 
Iondon

HENRY FROWDE

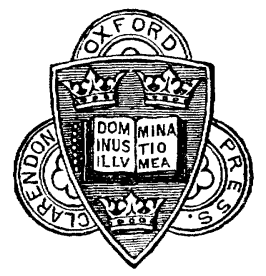

OXFORD UNIVERSITY PRESS WAREHOUSE

7 PATERNOSTER ROW 


\title{
TEXT-BOOK
}

\author{
OF \\ B O T A N Y
}

MORPHOLOGICAL AND PHYSIOLOGICAL

BY

\section{JULIUS SACHS}

PROFESSOR OF BOTANY IN THE UNIVERSITY OF WÜRZBURG

EDITED, WITH AN APPENDIX,

BY

SYDNEY H. VINES, M.A., D.Sc., F.L.S.

FELLOW AND LECTURER OF CHRIST'S COLLEGE, CAMBRIDGE

SECOND EDITION

(of * * ford

AT THE CLARENDON PRESS

M DCCC LXXXII

[ All rights reserved] 
<smiles>[CH]1CC1</smiles> 


\section{AUTHOR'S PREFACE.}

This Text-book of Botany is intended to introduce the student to the present state of our knowledge of botanical science. Its purpose is not only to describe the phenomena of plant-life which are already accurately known, but also to indicate those theories and problems in which botanical research is at present especially engaged; the arrangement of the material and the mode of treatment of the separate subjects are adapted to this purpose. Detailed discussions of questions of minor importance have been avoided, as these would only mar clearness of outline in the design; critical remarks have been introduced occasionally where they seemed necessary, in order to determine facts, or to justify the views taken on matters of fundamental importance.

The historical development of botanical views and theories does not seem to come within the scope of a Text-book of Botany, and would only interfere with the unity of design of the work. It would therefore be superfluous to quote scientific works which have only a historical interest. In the references which will be found in the work the chief object has been to introduce the student to those writings in which he will find a fuller discussion of those parts of the subject which have been only touched on briefly. In some cases the writings of others have been quoted because they represent views different from those of the author, and because it is desirable to place the student in a position to form a judgment for himself. Others again of the references are simply for the purpose of citing the authorities on which reliance is placed for statements that have not come within the range of the author's own observation. The reader of this work will at least learn the names and standing of those workers who have in recent times contributed most essentially to the science of which it treats.

By far the greater number of the illustrations are original, many of them the result of laborious investigation. Where they have been copied the name of the author from whom they are borrowed is in each case given in the description; illustrations from other sources are used only when the objects themselves have not been accessible, or when it seemed impossible to obtain better ones.

The Table of Contents will give sufficient indication of the plan of the work; the Index should be consulted for references to other parts of the book where an explanation of technical terms will be found when their meaning does not appear in any particular passage. 



\section{EDITOR'S PREFACE.}

WHEN I undertook, about a year ago, to prepare a new English edition of this important work, based upon the German edition of 1874 , I found that it would be necessary to make considerable alterations and additions if the work were to maintain in any degree its high reputation as adequately representing the attainments of Botanical Science. It is with the object of maintaining this reputation that $\mathrm{I}$ have ventured, not without considerable diffidence, to add to and to alter Professor Sachs' work; but I have been careful to distinguish my alterations and additions either by enclosing them in brackets or by quoting my authority, so that the reader will have no difficulty in recognising them. I cannot flatter myself, however, that $I$ have been altogether successful in my attempt. Complete success could only have been attained by rewriting a considerable portion of the work, but this did not come within my province.

I found also that nearly the whole of Book I. had already been for some time in print, and that consequently a number of important recent discoveries had not been noticed in it. In order to meet this difficulty I suggested to the Delegates of the Clarendon Press that the first thirty-two pages should be revised and reprinted, and that the additional notes necessary for the completion of Book I. should be incorporated in an Appendix, a suggestion which met with their approval. An opportunity was thus afforded me of adding some further notes and references on the remainder of the work. As it also contains the Corrigenda, the Appendix has come to be an important feature, and I therefore especially recommend it to the notice of the reader.

I have no doubt that many errors of omission and of commission will be detected; for these I would beg the reader's indulgence, in so far as I am responsible for them. They would have been much more numerous but for the valuable criticisms and suggestions of many friends, among whom I may especially mention Mr. W. T. Thiselton Dyer, Assistant Director of the Royal Gardens, Kew, Mr. D. H. Scott, Assistant Professor of Botany in University College, London, and Mr. F. O. Bower, Lecturer in Botany at the Normal School of Science, South Kensington. To my friend Mr. A. E. Shipley, Scholar of this College, I am much indebted for his kindness in assisting me in the serious labour of preparing the Index.

$$
\text { S. H. V. }
$$

Christ's College, Cambridge, August, $\mathbf{1 8 8 2 .}$ 



\title{
CONTENTS.
}

\author{
BOOK I. \\ GENERAL MORPHOLOGY. \\ CHAPTER I. \\ Morphology of the Cell.
}

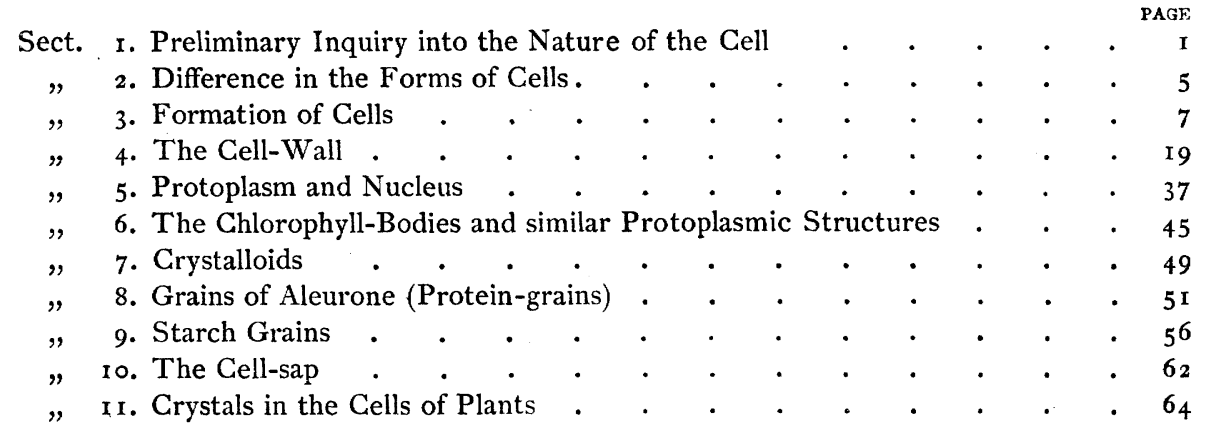

CHA P T E R II.

\section{Morphology of Tissues.}

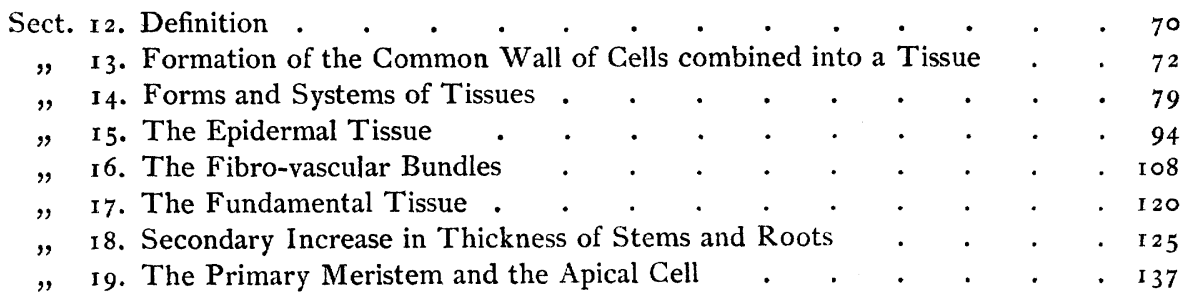


C. H A P T E R III.

\section{Morphology of the External Conformation of Plants.}

Sect. 20. Difference between Members and Organs. Metamorphosis . . $\quad$ I 49

" 2 r. Leaves and Leaf-forming Axes . . . . . . . . . . . . I53

" 22. Hairs (Trichomes) . . . . . . . . . . . . . 160

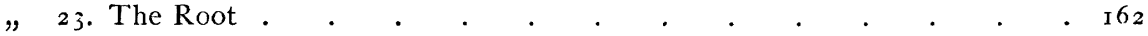

„24. Different Origin of Equivalent Members . . . . . . . . . $\quad$ I69

" 25. Different Capacity for Development of the Members of a Branch-system . ${ }_{17} 6$

" 26. The Relative Positions of Lateral Members on a Common Axis . $\quad$ - 187

"27. Directions of Growth . . . . . . . . . . . . . 202

,28. Characteristic Forms of Leaves and Shoots . . . . . . . 2 Ir

" 29. Reproduction; Sexual Organs; Alternation of Generations _ . . . 222

\section{BOOK II.}

\section{SPECIAL MORPHOLOGY}

AND OUTLINES OF CLASSIFICATION.

Group I. Thallophytes

Group II. Muscineæ .

Class I. Protophyta.

, 2. Zygosporeæ

, 3. Oosporeæ .

, 4. Carposporex

Class 5. Hepatic

, 6. Musci.

Croup III. Vascular Cryptogams .

Class 7. Equisetineæ

" 8. Filicineæ

Group IV. Phanerogams

9. Dichotomex

- 633 


\section{BOOK III.}

\section{P H Y I O LOG Y.}

\section{CHA P TER I. \\ Molecular Forces in the Plant.}

Sect. The Condition of Agregation of Organised Structures

2. Movement of Water in Plants . . . . . . . . . 674

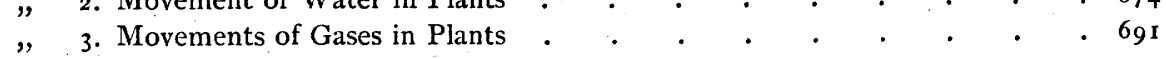

CHAPTER II.

\section{Chemical Processes in the Plant.}

Sect. 4. The Elementary Constituents of the Food of Plants . . . . . . 694

" 5. Assimilation and Metastasis . . . . . . . . . . 703

" 6. The Respiration of Plants . . . . . . . . . . . . . $\quad$. 72 I

\section{CHA P T ER III.}

\section{General Conditions of Plant-life.}

Sect. 7. The Influence of Temperature on Vegetation . . . . . . . $\quad$. 725

\# 8. Action of Light on Vegetation . . . . . . . . . . . . 737

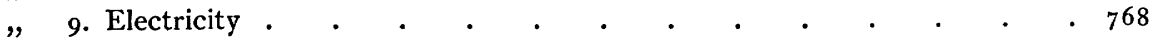

" Io. Action of Gravitation on the Processes of Vegetation . . . . . 770

\section{CHAPTER IV.}

\section{The Mechanics of Growth.}

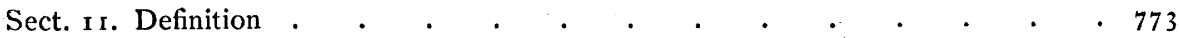

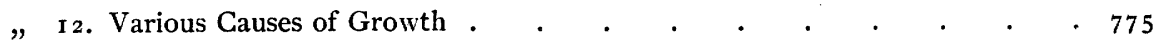

" 13. General Properties of the Growing Parts of Plants . . . . . $\quad$. 778

" r4. Causes of the Condition of Tension in Plants . . . . . . . $\quad$. 787

"15. Phenomena due to the Tension of Tissues in the Growing Parts of Plants . 794

" I6. Modification of Growth caused by Pressure and Traction . . . . 809

" $"$ 7. Course of the Growth in Length under constant External Conditions . . 815

" 18. Periodicity of Growth in Length caused by the alternation of Day and

Night . . . . . . . . . . . . .823

" 19. Effect of Temperature on Growth . . . . . . . . . $\quad$. 828

"20. Action of Light on Growth in Length.-Heliotropism . . . . 832

" 21. Action of Gravitation on Growth in Length.-Geotropism . . . . . . 839

"22. Unequal Growth . . . . . . . . . . . . . 854 
"24. The Twining of Climbing Plants . . . . . . . . 862

"25. The Twining of Tendrils . . . . . . . . . . . $86_{5}$

"26. Movements of growing Leaves and Floral Organs produced by Variations of Light and of Temperature . . . . . . . . 871

\section{CHA P TER V.}

\section{Periodic Movements of the Mature Parts of Plants and Movements dependent on Irritation.}

Sect. 27. Introduction

„28. Review of the Phenomena connected with Periodically Motile and Irritable Parts of Plants

„29. Mechanism of the Movements caused by Contact or Concussion

" 30. Mechanism of the Movements produced by Variations of Temperature and of Light .

" 3r. Mechanism of spontaneous periodic Movements . $\quad . \quad . \quad . \quad . \quad . \quad . \quad 895$

\section{CHA P TE R VI.}

\section{The Phenomena of Sexual Reproduction.}

Sect. 32. The Nature of Sexuality . . . . . . . . . . 896

" 33. Influence of the origin of the Reproductive Cells on the product of

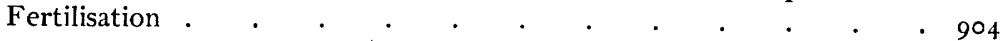

"34. Hybridisation . . . . . . . . . . . . . . $9 \mathrm{I}_{4}$

\section{CHAPTER VII. \\ The Origin of Species.}

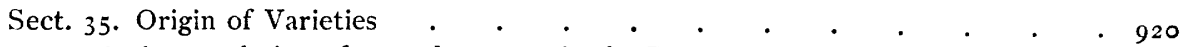

" 36. Accumulation of new characters in the Reproduction of Varieties . . $\quad 924$

" 37. Causes of the Progressive Development of Varieties . . . . . . $\quad 9^{28}$

"38. Relationship of the Morphological Nature of the Organ to its adaptation to the Conditions of Plant-life . . . . . . . . . 933

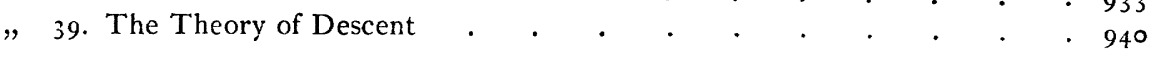

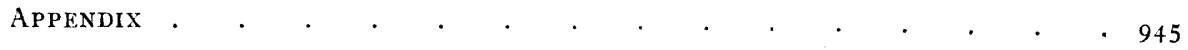

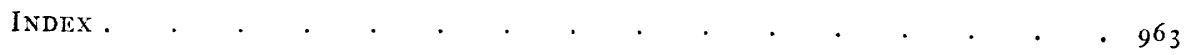




\title{
$\mathrm{BOOK} \mathrm{I}$.
}

\section{GENERA L MORPHOLOGY.}

\author{
CHAPTER I. \\ MORPHOLOGY OF THE CELL.
}

Sect. I. Preliminary Inquiry into the Nature of the Cell.-The substance of plants is not homogeneous, but is composed of small structures, generally indistinguishable by the naked eye. Each of these is, at least for a time, a whole complete in itself, being composed of solid, semi-solid, and fluid parts which differ in their chemical nature. These structures are termed Cells. For the most part, large numbers of them are in close contact and firmly united; and they then form a Cellular Tissue. But in every plant which completes its term of life there is at least one period in which certain cells or groups of cells separate at definite points from the union, and, after isolation, begin for themselves an independent course of life, as spores, pollen-grains, oospores, gemmæ, \&c.

Like the shape and size of the whole plant, the form, structure, and size of its individual cells are subject to regular changes; and the nature of these cannot be ascertained from the study of a single phase, but only from the entire series of changes which may be called the life-history of the cell. And as, moreover, each cell fulfils its own definite part in the economy of the plant, i.e. is specially intended for certain chemical or mechanical purposes, so also cells exhibit a diversity of form corresponding to their different functions. These differences, however, do not usually arise until the cells have passed through their earlier stages; the youngest cells of a plant differ only slightly from one another.

The general morphological laws which prevail in all cells are also more clearly evident in the young state; the more the developing cells adapt themselves to the special purposes which they have to fulfil, the more difficult it becomes to recognise in them these laws, which we will now expound more in detail.

By far the largest proportion of cells in the living succulent parts of plants, such as young roots, leaves, internodes, and fruits, are made up of three concentrically-disposed parts. First is an outer skin, firm and elastic, the Cell-wall, 
consisting of a peculiar substance, which we call Cellulose (Fig. $\mathbf{1}, B, C, h$ ). Close to the inner side of this membrane, which forms a closed envelope, is a second layer, also entirely continuous, the substance of which is soft and inelastic, and which always contains albuminous matter; to this substance H. v. Mohl gave the distinctive appellation of Protoplasm ${ }^{1}$. In the cells now under consideration it

$A$.
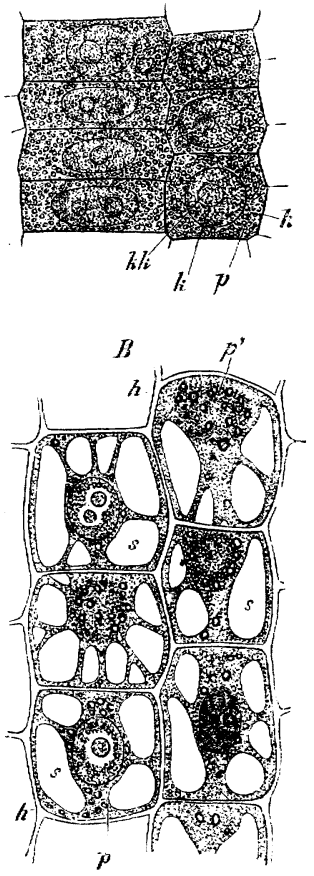

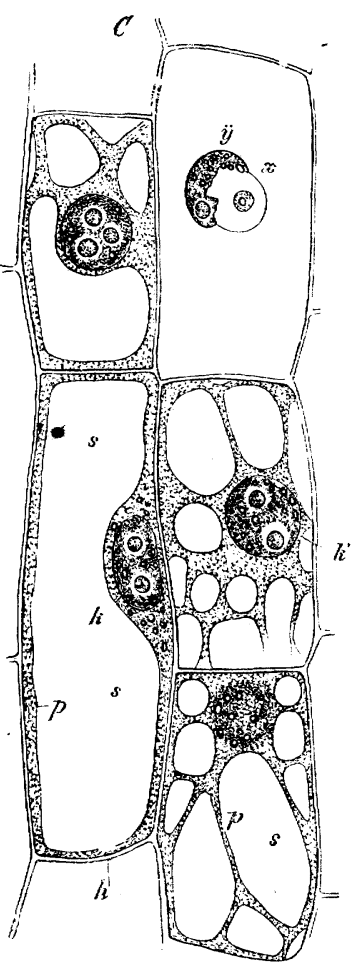

FIG. I.-Parenchymatous cells from the median cortical layer of the root of Fritillaria imperialis; longitudinal sections $(\times 550)$. A very young cells lying close to the apex of the root, still without cell-sap. $B$ cells of the same description about $2 \mathrm{~mm}$. from the apex of the root, the cell-sap $s$ forming separate drops in the protoplasm $p$; between the drops are plates of protoplasm; $C$ cells of the same protoplasm $p$; bescription about 7 in the cell to the right above is opened by the section; the nucleus shows, under the influence of the penetrating water, a peculiar appearance of swelling $(x, y)$. forms a sac enclosed by the cell-wall, in which sac other portions of protoplasm are also usually present in the form of plates and threads (Fig. I, $B, C, p$ ). Absent from some of the lowest organisms, but present in all the higher plants without exception, there lies imbedded in the protoplasm a roundish body, the substance of which is very similar to that of the protoplasm, the Nucleus (A, $C, k)$. The cavity enclosed by the protoplasm-sac is filled with a watery fluid, the Cell$\operatorname{sap}(B, C, s)$. In addition to these, there are also very commonly found in the interior of the cell granular bodies, which however may be passed over for the present.

Cells, in the stage of development now described, consist therefore of a firm membrane, semi-solid protoplasm (including the nucleus), and fluid cellsap. At first, however, the cellsap is wanting. If the same cells are examined in a very early stage of their development they are smaller $(A)$, their cell-wall thinner, and the protoplasm forms a continuous mass, in the middle of which lies the nucleus, at

1 H. v. Mohl, Ueber die Saftbewegungen im Inneren der Zellen, Bot. Zeitg. 1846, p. 73. The importance of this substance to the life of the cell was recognised at the same time by Nägeli, who, in conjunction with Schleiden, termed it 'Schleim.' (Zeitschr. für wissensch. Bot. von Schleiden u. Nägeli, Heft III, 1846, p. 53.) [The 'nucleus' was figured by F. Bauer in I 830 in the stigmatic cells of Bletia Tankervillice from a sketch made in 1802 . Meyen in the former year indicated it in his 'Phytotomie.' It was first described by Robert Brown (see Misc. Bot. Works, vol. I. p. $5^{12}$ ) in 1833 . Schwann in 1839 applied the term 'nucleolus' to the body previously discovered by Schleiden (Schwann and Schleiden's Researches, p. 3). Cohn in $185^{\circ}$ pointed out the analogy of the 'protoplasm' of botanists with the 'sarcode' of zoologists.] 
this time relatively very large $(k)$. The cell-sap first appears, when the cell is growing quickly $(B)$, in the form of drops (Vacuoles) in the interior of the protoplasm $(B, s)$; at a later period these drops usually coalesce, and form a single sap-cavity $(C, s)$, which is enclosed by the now sac-like hollow substance of the protoplasm.

In their earliest state the cells of the wood and cork of trees show conditions of development which correspond essentially to those represented in Fig. I. In these cells, however, a new condition follows very soon after the appearance of the cell-sap; the protoplasm containing the nucleus disappears, leaving the cell-cavity filled either with air or with water. Older wood and cork thus consist of a mere framework of cell-walls.

An important difference exists between the further behaviour of those cells which enclose protoplasm, and of those from which it has disappeared. The former only can grow, develope new chemical combinations, and, under certain conditions,
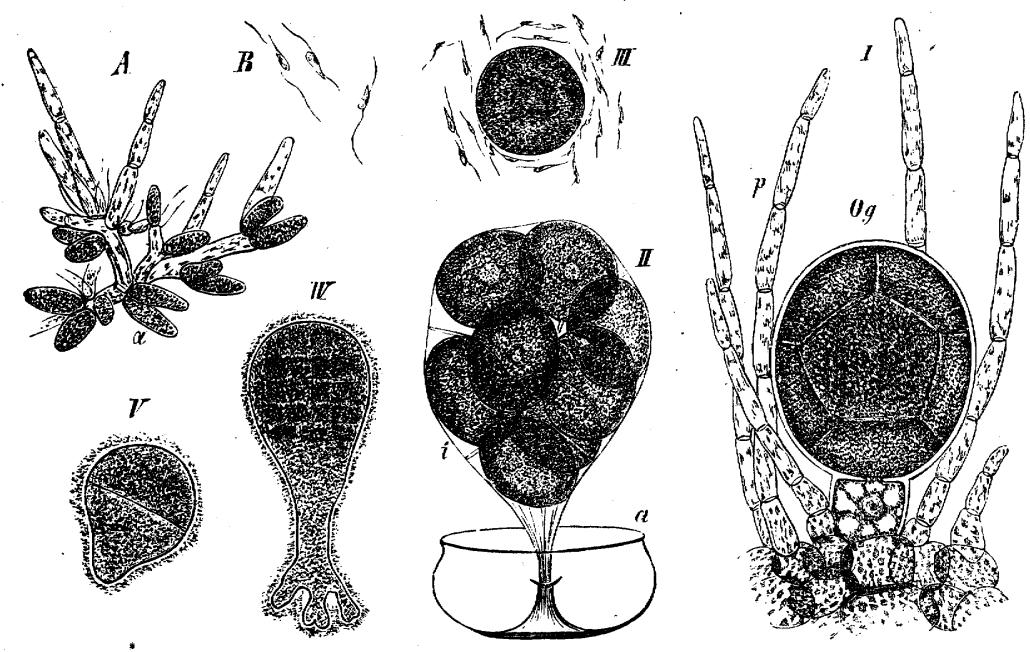

FIG. 2.-Sexual reproduction of Fucus vesiculosus; $A$ branched hair bearing antheridia; $B$ antherozoids; $I$ an oogonium $O g$ with paraphyses $p ; I I$ the exterior membrane $a$ of the oogonium is split, the inner membrane $i$ protrudes, containing the oospheres; III an oosphere escaped, with antherozoids swarming round it $V$ first division of the oospore or fertilised oosphere; $I V$ a young plant resulting from the growth of the oospore (after Thuret, Ann. des Sci. Nat. 1854, vol. II). ( $\beta \times 33^{\circ}$; all the rest $\times 160$.)

form new cells. The latter are never capable of further development; in the case of wood, they are of service to the plant only from their firmness, power of absorbing water, and peculiar form; in cork, by forming protecting envelopes which surround the living succulent cellular tissue.

Since then no further development can take place in cells which no longer contain protoplasm, it may be concluded that the latter is the proximate cause of growth. We shall see hereafter that the development of each cell begins with the formation of a protoplasm-mass, and that the cell-wall is also generated from it; but the relation of the protoplasm to cell-formation is still more striking when it exists for some time in the free state as a mass of definite form, which eventually clothes itself again with a fresh cell-wall, and takes up cell-sap. We have an excellent example of this in the reproduction of the Fucaceæ. On the fertile branches of these large marine Algæ, of which we may take Fucus resiculosus as 
an example, large cells, the oogonia (Fig. 2, $I, O g$ ), are formed in peculiar receptacles; the space enclosed by the cell-wall is densely filled with fine-grained protoplasm, which is at first homogeneous, but subsequently breaks up into eight portions (oospheres); these, completely filling up the cell-cavity of the oogonium, press against one another and become polygonal. The wall of the oogonium consists of two layers; the outer one splits, and the inner one protrudes in the form of a sac, which becomes distended by absorption of water; in this enlarged sac

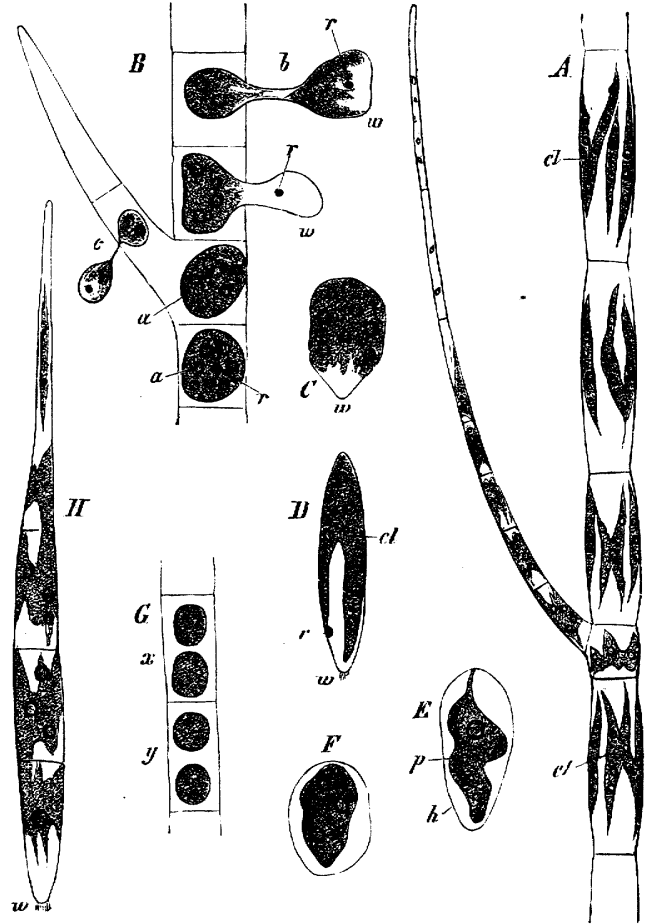

FIG. 3.-Stigeoclonium insigne (after Nägeli, Pflanzenphysiol. Untersuch ungen, Heft I); $A$ a filament of the Alga consisting of one row of cells, with a lateral branch; $c l$ green protoplasm-masses (chlorophyll-bodies), imbedded in the colourless protoplasm of each cell not shown in the drawing; $B$ the protoplasm of the cells contracting and protruding through openings in the cellwall; $C$ swarm-cells still without cell-wall; $D$ one come to rest; at $E$ and $F$ killed; the protoplasm $p$ is contracted and shows the newly-formed cell-wall $h$; $H$ a young plant grown from the swarm-cell; $G$ two cells of a flament in the $H$ a young plant grown from the swarm-cell; $G$ two cells of a filament in the
act of dividing; the protoplasm of each cell $(x, y)$ has split into two equal parts, and contracted by addition of a reagent.

the protoplasm shows, by its motions and changes of form, that it is soft and extensible; but, once freed, it assumes a definite form. Usually after some hours, the swarm-cell comes to rest; if killed by proper means, the protoplasm contracts $(E, F, p)$, and a delicate cell-wall may now be recognised, which it did not possess at the time of its exit, when it began to swim about. When once, at

1 [For the exact meaning of this term see Book II. Chap. I, the Introduction to Thallophytes. The term 'swarming' is applied to any apparently spontaneous motion imparted to a naked protoplasmic body by vibratile cilia.] the oospheres, now completely spherical, escape. By the fertilising action of other smaller protoplasm-masses, the antherozoids, these balls of protoplasm or oospheres are excited to further development; on the exterior of each fertilised oosphere or oospore a colourless substance makes its appearance, which hardens into a continuous cell-wall. The newlyformed cell now grows in two different directions in different modes, and produces, after further transformations (Fig. 2, $V$ and $I V)$, a young Fucus-plant.

Still more clearly does the independence of the protoplasm of a cell show itself in the formation of the swarm-cells ${ }^{1}$ (zoogonidia) of Algæ and of some Fungi. In many cases, as in Stigeoclonium insigne (Fig. $3, B, a$ ), the protoplasm of a cell filled with cell-sap contracts, expels the cell-sap, and forms a roundish ball, which, escaping through an opening in the cell-wall, swims about in the water $(C)$. While passing out,

- the oospheres become globular (Fig. 2, II); when this bursts, 
rest, it also changes its form, and increases in volume, cell-sap collecting in the interior. The cell formed in this way now grows in a manner dependent on the specific nature of the plant;-in our example it elongates itself (Fig. $3, D$ and $H$ ), - and new changes (in this case cell-divisions) begin.

These examples-and many more might be added-show us that the protoplasm constitutes the cell; the cell, in the sense defined above, is evidently only a further development of it; the formative forces proceed from it. It has hence become usual to consider a protoplasm-mass of this kind as a cell, and to designate it as a naked membraneless or Primordial Cell.

The development of a swarm-cell, like that of the oosphere of Fucus, shows,as does also the case of every other cell,-that the substance of the cell-wall was already contained in the protoplasm in some form or other which could not be recognised; the formation of the cell-wall must be regarded as a separation of matter already existing in the protoplasm. In the same manner the water of the cell-sap, although taken up from without, must nevertheless pass through the protoplasm; and, while it collects inside it as cell-sap, it takes up from it soluble substances; so far the formation of the cell-sap is also a separation of matter hitherto contained in the protoplasm. The nucleus is probably to be regarded as a differentiated portion of the protoplasm. Thus the mature cell, provided with cell-wall, nucleus, and cell-sap, is the result of a differentiation of matter already contained in the protoplasm. The essential point is this,- that this differentiation always leads to the formation of concentrically disposed layers, the outer of which, the cell-wall, is firm and elastic, the inner, the protoplasm-sac, soft and inelastic. If the cell, as is usually the case, is at first without any sap-cavity, the protoplasm is less firm and more watery in the centre, or a nucleus is in this case formed, which, at least in young cells, is always more watery than the surrounding protoplasm. When at last the cell-sap makes its appearance, the cavity of the cell is always filled with actual fluid, in which the nucleus often takes up a central position surrounded by protoplasm, or, more usually, approaches the circumference of the sap-cavity, and becomes parietal. So long as the phase of cell-development in which the cell appears as a sap-cavity bounded by a membrane - certainly the one most commonly seen-had alone been observed, it was correct enough to define the cell as a vesicle; but it is obvious that this view does not apply to many true cells, e.g. to young cells which form component parts of a tissue (as Fig. I, $A$ ), of the true nature of which we should get but an ill-defined conception were we to regard them as vesicles. The term applies still less to swarm-cells and to the oospheres of Fucus.

Sect. 2. Difference in the Form of Cells.-The development of the individual cell by no means always results in the forms just described; further changes of form usually take place in the separate parts of the cell. The volume of the entire cell generally increases for a considerable time, with corresponding increase of the cell-sap; not unfrequently it amounts to a hundred or even a thousandfold the original volume. During this increase, the form commonly changes; if it was at first roundish or polyhedral, it may become filiform, prismatic, or tabular, or branch into a number of arms. The cell-wall may increase very considerably in 
thickness; but this thickening is usually not uniform; particular spots remain thin, in others the thickened membrane projects internally or externally in ribbon-shaped prominences, spines, or knobs. In the substance of the cell-wall itself differences also manifest themselves, which give it greater firmness, elasticity, or hardness, or, on the other hand, greater softness or pliancy. The protoplasm may, in these processes, decrease more and more, until at last it forms an extremely thin membrane, applied so closely to the cell-wall that it does not become visible till contraction takes place; after the completion of the growth of the cell-wall it may even entirely disappear. But in many other cases the protoplasm increases with the increase in volume of the cell; it forms a thick-walled sac, the substance of which is endowed with constant motion, while filiform or ribbon-shaped strings of protoplasm often traverse the sap-cavity. In those cells which appear externally green, certain portions of the protoplasm become separated, and assume a green colour; these particles, the Chlorophyll-bodies, may have the form of bands, stars, or irregular masses; but they usually form numerous roundish granules, and are always parts of the collective protoplasm-mass of the cell. Sometimes, mixed with the green colouring-matter which tinges them, are pigments of other colours, red, blue, or yellow (as in the Florideæ, Oscillatorieæ, and Diatomaceæ); or the chlorophyll-granules assume, through changes in their colouring-matter, other tints, mostly yellow or red. Colouring-matters may also be dissolved in the cell-sap. The other chemical compounds, which are formed in extremely large numbers in the cell, are mostly dissolved in the cell-sap; but-many of them assume definite forms; thus arise granules of fat, drops of oil, and frequently true crystals or crystal-like bodies. One of the commonest granular compounds present in almost all plants, with the exception of Fungi and some Algæ and Lichens, is starch, the grains of which often accumulate in the cell in quantities greatly exceeding all other substances.

Cells of the most perfectly developed form are found in certain families of Algæ, the Conjugatæ, Siphonex, and Diatomacex. Since in these cases the same cell unites in itself all vegetative functions, and at the same time a many-sidedness presents itself in the vital phenomena, the whole cell attains a high degree of differentiation; the separate parts-the cell-wall, the protoplasm, and the bodies enclosed in it-show a variety of structure which does not occur elsewhere concurrently in the different parts of one and the same cell. In addition to this, the same cell has in these cases often to go through the most diverse metamorphoses, so that besides its manifold development as to size, it also undergoes a series of transitory changes of form. Hence these types of Algæ are of great importance for an accurate comprehension of the nature of the cell. (See Book II, Algæ.) But these cells are also remarkable in this respect,- that, after they have attained their highest grade of development, they are still able to divide and to multiply; sooner or later the protoplasm can abandon the cell-wall, contract, with all its contents (starch, oil, chlorophyll, \&c.), expel the water of the cell-sap, and form eventually a new cell.

We may pass over the innumerable intermediate forms, and turn our attention to the other extreme, namely, to those plants of which each usually consists of thousands or even millions of cells, as is the case with Vascular Cryptogams and Phanerogams, and in which the different parts of the plant undergo an entirely different morphological development, and are adapted to different functions for the support of the whole. Here we find that certain cells never attain their full development; they remain constantly in the immature condition which is represented in Fig. $\mathrm{I}, A$; these 
however assist the whole by continually giving rise to new cells by division, which then, on their part, undergo a further development. Such cells, which serve exclusively for the purpose of producing new ones, are found at the extremities of all roots and branches, and between the bark and wood of exogenous trees and shrubs. The cells produced in these positions undergo a different development according to their situation, and usually in such a way that aggregations of them into layers or strands follow simultaneously the same mode of development. Some grow quickly in all directions, their wall remains thin, the great bulk of their protoplasm becomes transformed into chlorophyll-granules, they are rich in cell-sap, and serve, as we shall see hereafter, for assimilation, i.e. the production of new organic substance which is formed out of the elements of the absorbed nutrient material. In other parts of the same plants the cells extend greatly in length, their diameter remains small, they form no chlorophyll; a certain number remain succulent and serve to convey assimilated substances; other cells of the same strand thicken their walis rapidly in many ways, their septa become absorbed, numerous cells in the same row combine into a long tube (vessel), from which the protoplasm and the cell-sap disappear; they serve then as organs of conduction for the plant. In their neighbourhood are formed the wood-cells; these are mostly prosenchymatous, extended in length, their wall greatly thickened, and its substance chemically changed (lignified); they form collectively a firm frame-work which supports the remaining tissues, lends firmness and elasticity to the whole, and is especially adapted for the rapid conduction of water through the plant. In the tissue of tubers, bulbs, and seeds most of the cells remain thin-walled; they become filled in the interior with albuminous substances, starch, oil, inulin, \&c., which afterwards, when new organs are being formed, serve. as material for the construction of new cells. In the same manner a considerable series of other forms of tissue could be named, cork, the testa of seeds, the stone of stone-fruit, \&c., which all alike attain their needful firmness and strength by a peculiar development of their cell-walls, in order to serve as protective envelopes for other masses of cells which are still capable of further development; their contents disappear as soon as the cellwall has assumed these properties, and their purpose has thus been fulfilled.

Each of the forms of cell just spoken of, occurring in the same plant, serves principally or even exclusively for one purpose only; in correlation with this, either the cell-wall, the protoplasm, the chlorophyll-granules, the cell-sap, or its granular deposits, is specially developed. Very commonly these specialised cells lose the power of reproduction and of multiplying by division; when they have fulfilled their function, they disappear, or their lignified cell-wall alone remains. The whole plant, of which these cells form a part, continues no less to live, since at special points it possesses cells, which, at the proper time, again produce new masses of cells capable of fulfilling in their turn the same functions.

Sect. 3: Formation of Cells ${ }^{1}$. - The formation of a new cell always commences with the re-arrangement of a protoplasm-mass round a new centre; the material required is always afforded by protoplasm already present, and the

1 H. von Mohl, Vermischte Schriften botanischen Inhalts, Tübingen $18_{45}$, pp. $67,8_{4}, 3^{62}$ [Anatomy and Physiology of the Vegetable Cell, translated by Henfrey, London I852]. - Schleiden in Müller's Archiv, I838, p. I37 [Taylor's Scient. Mem., vol. II. pp. 281-312, and Sydenham Society, I847].-Unger, Bot. Zeit. I844, p. 489 ; H. v. Mohl, Bot. Zeit. I844, p. 273.-Nägeli, Zeitschrift für wiss. Botanik, vol. I, I 844 , p. 34 , vols. III, IV, 1846 , p. 50.-A. Braun, Verjüngung in der Natur, Freiburg I850, p. I 29 et seq. [Ray Soc. Botanical and Physiological Memoirs, I 853 ].Hofmeister, Vergleichende Untersuchungen über die Embryobildung der Kryptog. u. Conif., Leipzig 1851 [Ray Soc. 1862]. - De Bary, Untersuchungen über die Familie der Conjugaten, Leipzig I858 Nägeli, Pflanzenphys. Untersuch. Heft I.-Pringsheim, Jabrb. fuir wiss. Botanik, vol. I, I858, pp. I, 284, vol. II. p. 1.-Hofmeister, Lehre von der. Pflanzenzelle, Leipzig 1867. [Strasburger, Ueber Zellbildung und Zelltheilung, Jena 1880.-id., Studien uiber Protoplasma, Jena I876.] 
newly constituted protoplasm-mass clothes itself, sooner or later, with a cell-wall. All processes of cell-formation agree in these points; but a description which goes more into detail requires a distinction to be drawn between different cases in which the process now varies in many different ways.

It must first be noted that the formation of new cells does not always result in an increase in the actual number of cells. Three types may be distinguished in this respect:-(I) The Renewal or Rejuvenescence of a cell; that is, the formation of a single new cell from the whole of the protoplasm of a cell already in existence; (2) The Conjugation or Coalescence of two (or more) protoplasm-masses in the formation of a new cell; (3) The Multiplication of a cell by the formation of two or more protoplasm-masses out of one. Each of these types shows a series of variations and transitions into the others. The greatest diversity is exhibited in the multiplication of cells; two cases must be distinguished, according as part only of the protoplasm of the mother-cell is applied to the formation of the new cells, or as the whole mass is converted into daughter-cells. The latter, by far the more common case, again exhibits variations, according as the protoplasmmasses, which collect around new centres, expel water, contract and become globular; according as the cell-wall is secreted during division or after its completion; and, finally, according to the way the cell-sap and nuclei make their appearance.

The different processes of cell-formation are in turn brought into play throughout the life of the plant;-Rejuvenescence, Conjugation, Free cell-formation, and Celldivision with contraction and rounding off, are the forms connected with reproduction; while in the growth or increase in volume of an organ by the formation of new cells, cell-division only comes into play, and almost invariably by division of one cell into two without any considerable contraction and rounding off of the new cells; the multiplication of cells in growing tissue may therefore be described as a bipartition of those already in existence. In the formation of reproductive cells, the tendency to isolation and rounding off is most strongly displayed; while in cell-formation accompanying growth, the mother-cells are divided by partitionwalls in such a manner that the resulting daughter-cells resemble their mothercells, or are able to develope into any required form.

The more important modes of cell-division must now be examined in a few examples.

\section{A. Cell-formation in relation to Reproduction.}

1. Cell-formation by Rejuvenescence.-A good example is afforded in the formation of the swarm-cells of Stigeoclonium insigne (Fig. 3, p. 4). The protoplasm of a cell of a filament contracts, and expels a portion of the water of the cell-sap; the arrangement of the differentiated protoplasm-mass is changed, and the chlorophyll-bodies become indistinct; its form alters as it escapes from its cell-wall; from almost cylindrical, the protoplasm-mass becomes ovoid, with a broad green and a narrower hyaline end; after its movement has ceased, the latter becomes the base, and the green end alone grows when the new cell clothes itself with a cell-wall. The pbservations of Pringsheim on $E$ dogonium also show that the direction of growth of the renewed cell is at right angles to the original direction of growth before the renewal; for the hyaline or radicular end of the swarm-cell, which afterwards becomes the point of attachment, is 
formed on the side (Fig. $4, A, E$ ), not at one end of the protoplasm-mass. The arrangement of the entire protoplasm of the cell is therefore entirely changed; the transverse becomes the longer diameter of the cell and of the plant arising from it. The material remains, as far as can be seen, the same, but its arrangement is different. This is the point of morphological importance, that every formation of a new cell depends essentially on a fresh arrangement of protoplasm already in existence. Hence the rejuvenescence of a cell not only may but must be regarded morphologically as the formation of a new one:

2. Cell-formation by Conjugation.-The protoplasm of two or more cells coalesces to form one common protoplasm-mass, which surrounds itself with a cell-wall and becomes endowed with the other properties of a cell. To study this process, which presents many variations, we may take one of our commonest filamentous Algæ, Spirogyra longata (Figs. 5, 6). Each filament (Fig. 5) consists of a row of similar cylindrical cells, each of which contains a protoplasm-sac; this encloses a relatively large quantity of cell-sap, in the midst of which hangs a nucleus, enveloped in a small mass of protoplasm, and attached to the sac by threads of the same substance; in the sac lies a spirally coiled chlorophyll-band, with thickenings (chlorophyll-granules) at intervals which contain starch-grains. The conjugation always takes place between opposite cells of two more or less parallel filaments. The first stage is the formation of lateral protuberances (Fig. 5, a), which continue to grow until they meet $(b)$. The protoplasm of each of the two cells concerned then contracts ${ }^{1}$, detaches itself from the surrounding cell-wall, rounds itself into an ellipsoidal form, and contracts still more by expulsion of the water of the cell-sap. This may occur simultaneously in the two conjugating cells. Next, the cell-wall opens between the two protuberances (Fig. 6, a), and one of the two ellipsoidal protoplasm-masses forces itself into the connecting channel thus formed, gliding slowly through it into the other cell-cavity; and. as soon as it touches the protoplasm-mass contained in it, they coalesce (Fig. $6, a$ ). After complete union (Fig. 6, b) the united body is again ellipsoidal, and scarcely larger than one of the two from which it was formed; during the union a contraction has evidently taken place, with expulsion of water. The coalescence gives the impression of a union of two drops of fluid; but

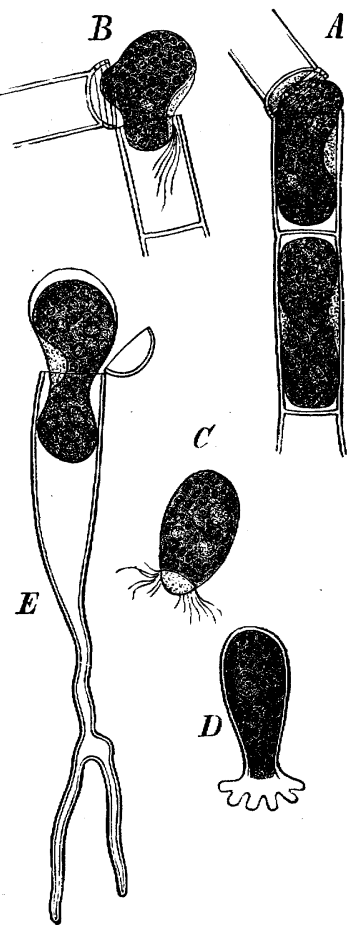

FIG. $4-A, B$ escape of the swarm-cells of an Edogonium; $C$ one free in motion; $D$ the same after it has become fixed and has formed the attaching disc; $E$ escape of the whole protoplasm of a young plant of $F$ dogonium in the foplasm swarm-cell $(\times 350)$. (After Pringsheim, Jahrb. fiir wiss. Bot. vol. I. pl, r.) the protoplasm is never fluid in the physical sense of the word. The conjugated protoplasm-mass clothes itself with a cell-wall, and forms a Zygospore, which germinates after a period of repose of some months, and then developes a new filament of cells. With greater or smaller deviations from this plan, conjugation takes place in a large group of Algæ, the Conjugatæ, among which the Diatoms must be included, and in some Fungi, the Zygomycetes. In the latter more considerable de- viations occur. In Spirogyra nitida it also happens (De Bary, Conjugaten, p. 6) that one

1 [Strasburger, Ueber Befruchtung und Zelltheilung, I878. The protoplasm of one cell, the one the protoplasm of which passes over into the other, usually contracts first.] 
cell conjugates with two others, and takes up both their protoplasm-masses; in these cases the zygospore is the product of three cells. In the Myxomycetes the swarm-cells (Myxo-amœbæ), which are endowed with a peculiar motion, coalesce gradually in great numbers, and finally form large, motile, naked protoplasm-masses, the Plasmodia, which only at a subsequent period are transformed into a number of cells.

In the cases hitherto considered the uniting protoplasm-masses are of equal size; the process of fertilisation in many Cryptogams differs from them only in the fact that the two protoplasm-masses which coalesce are of unequal size, and otherwise of different properties. In Book II. we shall treat in detail of the reproduction of Cryptogams; here we need only state that the male fertilising bodies (antherozoids) of Cryptogams are usually motile naked protoplasm-masses, that is, primordial cells; the female organ of these plants usually opens outwardly, and contains a primordial cell
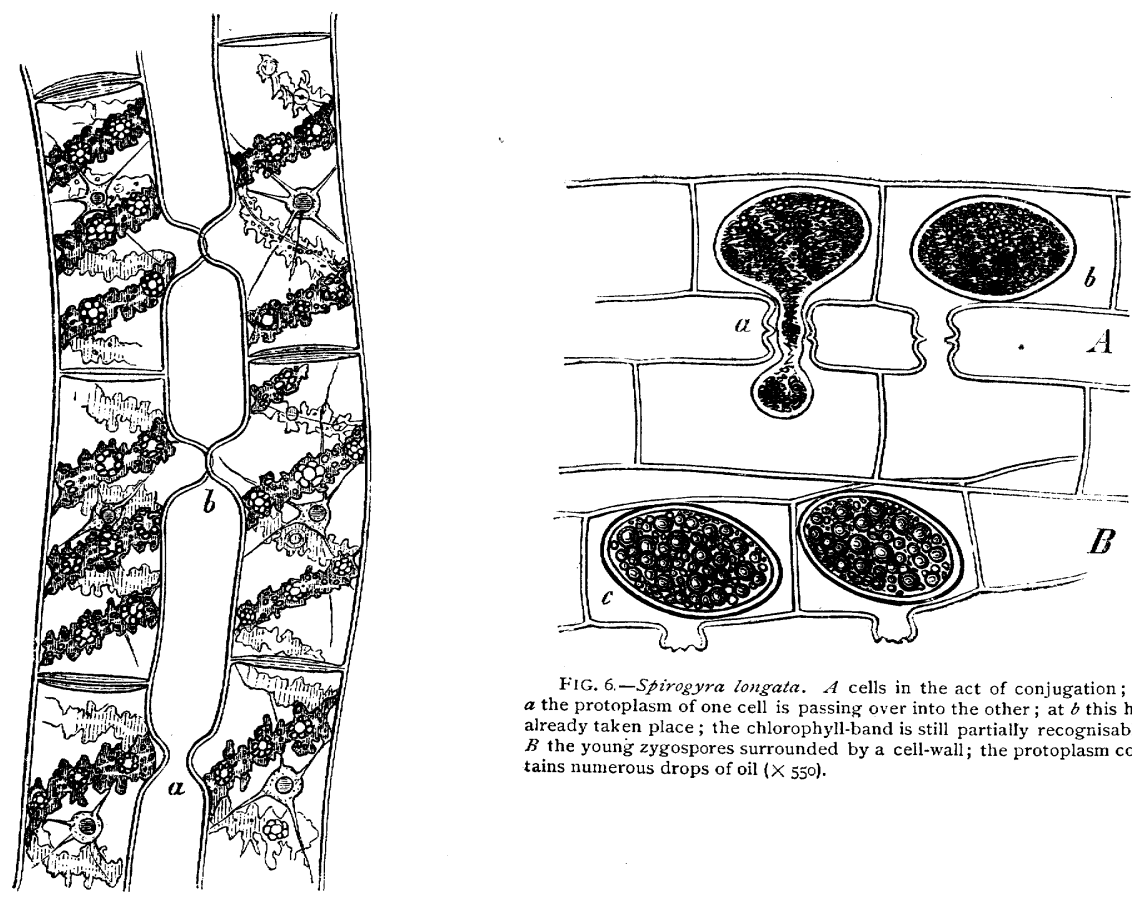

FIG. 6.-Spirogyra longata. A cells in the act of conjugation; at $a$ the protoplasm of one cell is passing over into the other; at $b$ this has already taken place; the chlorophyll-band is still partially recognisable. $B$ the young zygospores surrounded by a cell-wall; the protoplasm contains numerous drops of oil $(\times 550)$.

FIG. 5.-Spirogyra longata. Cells of two filaments in an early stage of conjugation, showing the spirally coiled chlorophyll-bands, in the chlorophyll-granules of which lie rings of starch-grains; small drops of oil are also distributed through them (see Sect. 6). This is the condition of the chlorophyll after the action of strong sunlight; the nuclei are also to be seen in the cells, each surrounded by protoplasin, threads of which reach the cell-wall in different places; $a$ and $b$ are the protuberances in two different stages $(\times 550)$.

(oosphere) which is fertilised by the antherozoids. In cases which have been accurately observed (Edogonium, Vaucheria) these coalesce with the former, and the new cell results from this coalescence. As with the Conjugatæ and some Fungi, the cell formed in this manner is always a reproductive cell; with it begins the growth of a new individual plant.

3. Free Cell-formation.-In the protoplasm of a cell new centres of formation arise, round each of which a portion of the protoplasm collects, and forms a cell. A portion of the protoplasm may remain and represent the protoplasm-mass of the mother-cell which persists for a longer or shorter time. [The new centres of formation are indicated by the previous appearance of nuclei formed by the repeated 
bipartition of the nucleus or nuclei of the cell.] Generally, the number of daughter-cells which arise in this manner is considerable; as an instance may be mentioned the formation of spores in Ascomycetes (Peziza ${ }^{1}$ ), (Fig. 7). The tubular mother-cells of the spores (asci) (a) are at first densely filled with protoplasm, and contain only one small nucleus. [This divides into two, and this process is repeated until eight nuclei are formed:] the protoplasm becomes frothy, and roundish drops of sap make their appearance in it $(b, c)$. The first stage in the formation of the spores is the condensation of the protoplasm in the upper part of the ascus, while it remains frothy in the lower part $(e, f)$. In this case eight spores are always formed in each ascus within the upper dense protoplasm; i.e. round each of the eight nuclei an ellipsoidal protoplasmmass collects $(d)$; each consists at first of coarse-grained protoplasm surrounded by a clear space; a portion of fine-grained protoplasm forms the matrix in which the spores are imbedded. Afterwards each spore becomes more sharply defined; the clear space disappears $(e)$; its substance becomes more fine-grained and clearer; and in one of its foci is formed a vacuole, i.e. a transparent drop of fluid. Finally, each spore surrounds itself with a firm membrane, the vacuole disappears, and in the centre is formed a large strongly refractive oil-drop, as well as numerous smaller ones.

An example is afforded by the formation of the oospheres of Acblya (Fig. 8) of a somewhat different mode of free cellformation. The protoplasm collects at the end of a hypha or of a branch of one; the larger end itself swells up into a globular form $(A, B)$, and, after the formation of a septum $(C)$, becomes an independent cell (the oogonium). [Numerous small nuclei are present in the protoplasm (as in $C$ ). The nuclei multiply by bipartition.] The whole protoplasm breaks up into two, three, four, or more parts, which very quickly round themselves off into a perfectly spherical form; [in each of these several nuclei are uniformly distributed.] The parts thus formed $(e, e$ in $D)$ contract greatly during their separation, and their

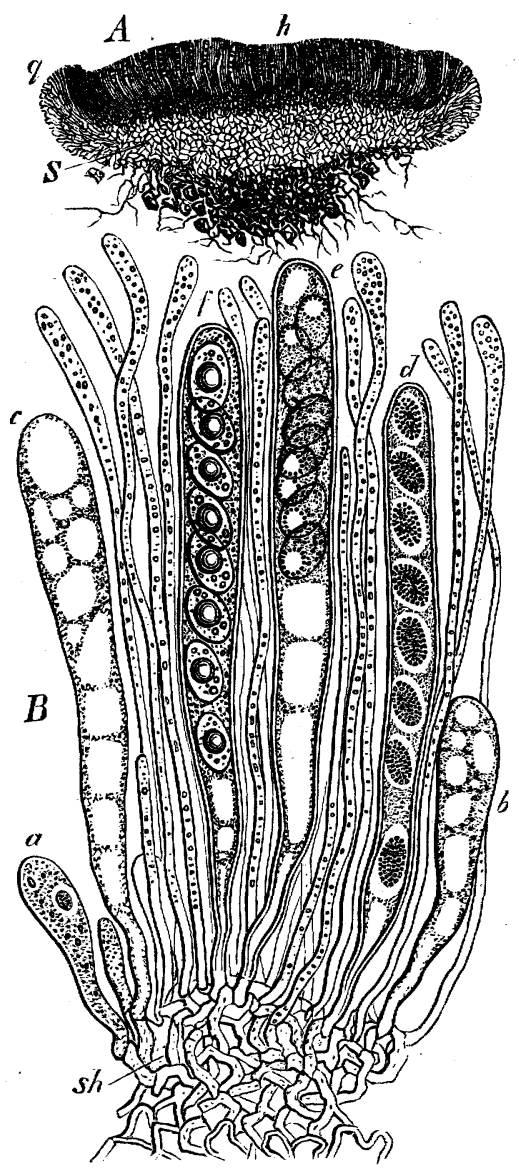

FIG. 7.-Peziza conzexula.'A vertical section of the whole plant (X about 20 ); $h$ hymeniun, $i . e$. the layer in which the spore-sacs (asci) lie; $S$ the tissue of the Fungus enveloping the hymenium at its edge $q$ in a cup-like manner; at the base of the tissue $S$ are delicate rhizoids, which grow between the particles of earth. $B$ a smaller portion of the hymenium $(\times 550)$; $s h$ sub-hymenial layer of densely interwoven cell-filaments (hyphæ); $a-f$ asci; among them thinner sacs, the paraphyses, in which lie red granules. protoplasm becomes denser by expulsion of water; after they have become fertilised by the antheridial tubes $(a, b$ in $D)$, they become invested with a cell-wall, [and the nuclei in each coalesce to constitute the nucleus of the oospore].

${ }^{1}$ [Strasburger, Zellbildung und Zelltheilung, 1880 ; Schmitz, Sitzber. d. niederrhein. Ges. zu Bonn, I879.] 
This form of free cell-formation is distinguished from the preceding by the circumstance that the whole protoplasm (and not a part only) collects round several centres. If the whole protoplasm, in its contraction, were to form only one mass, which sometimes happens, the case would be analogous to Rejuvenescence.

There occurs also in Acblya a variation of this process, when it forms its swarm-cells or zoogonidia (Fig. 9). The protoplasm in the club-shaped swollen end of a hypha [contains a number of small nuclei which multiply by bipartition]; it breaks up into a large number of small portions $(A)$, [each including a nucleus, ] which become completely rounded off $(a)$ after their escape from the cell in which they are produced

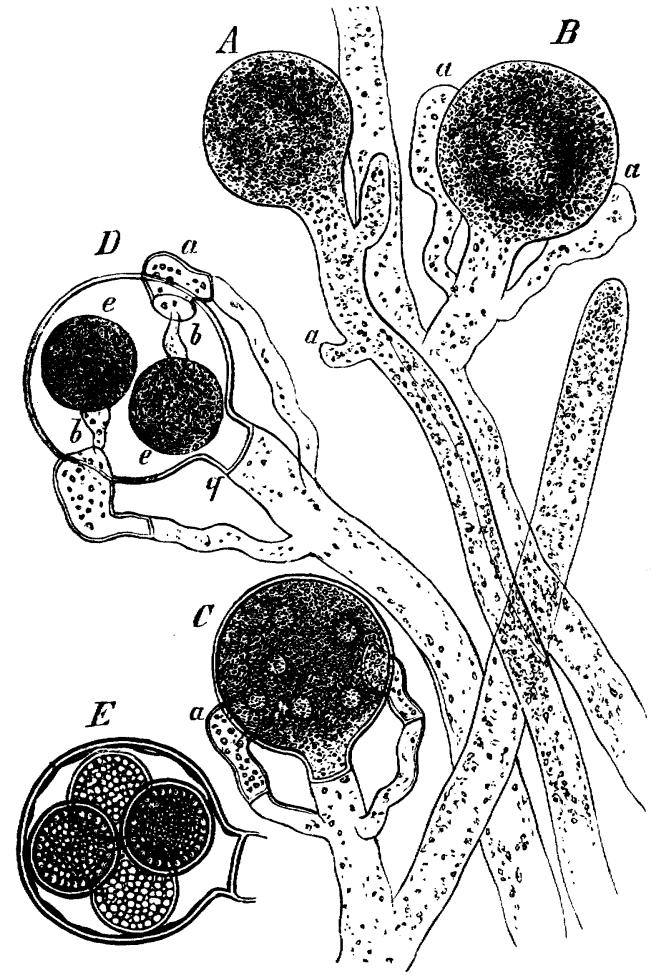

F1G. 8.-Oogonia and antheridia of Achlya lignicola, growing on wood in water; the course of development is indicated by the letters $A-F$. $a$ the antheridium, $b$ its tube penetrating into the oogonium $(\times 550)$.

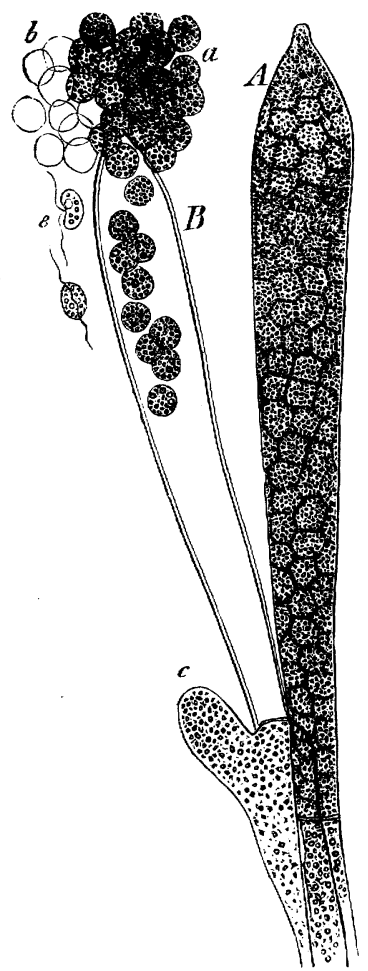

Fig. 9.-Cell in which are produced the swarm-cells or zongonidia of an Achlya $\left(\times 55^{\circ}\right) . A$ one still closed, $B$ one allowing the zongonidia to escape; beneath it a ?ateral shoot $c$; $a$ the zoogonidia just escaped; $b$ the abandoned membranes of the zoogonidia which have already swarmed; $e$ swarming zoogonidia.

$(B)$, and then clothe themselves with a delicate membrane, which they abandon $(b)$ when their movement commences $(e)$.

4. Formation of Reproductive Cells by Division of the Mother-Cell.-In the protoplasm of a cell new nuclei are formed by division; round each of these a portion of the protoplasm collects and becomes rounded off with a greater or less amount of contraction, in order to form a new cell; in this manner the entire protoplasm of the mother-cell is completely used up; its cell-wall alone remains, if it possess one, which is not always the case.

The formation of the spores of Mosses and Vascular Cryptogams, and of the pollengrains of Phanerogams, always takes place by the division of the mother-cell into four 
parts, either at once or by a repeated bipartition. In the special details of cell-division, however, some deviations occur.

[The first stage is, in all cases, the division of the nucleus of the mother-cell into two. This may be at once followed by the simultaneous formation of a cell-wall in the plane of the cell-plate (see infra, The Behaviour of the Nucleus during Division), as in the development of the pollen-grains of most Monocotyledons, and of the microspores of Isoëtes; a repetition of these processes in each of the two daughter-cells leads to the formation of the four pollen-grains or spores. More commonly the cell-plate between the two nuclei undergoes absorption; the two nuclei then divide, and the missing cell-plates are reconstituted; cellulose walls are now simultaneously formed in the cell-plates so as to divide the protoplasm into four parts, each containing a nucleus, which constitute the spores or the pollen-grains.

The division of the two secondary nuclei takes place in certain cases (pollen-grains of most Monocotyledons) in one plane, and this plane is at right angles to that of the division of the nucleus of the mother-cell. As the result of this, the four spores or pollen-grains formed lie in one plane and have a rounded form; they are said to be bilateral. In other cases (pollen-grains of Dicotyledons, spores of Equisetum) the division of each secondary nucleus takes place in a plane which is at right angles to that of the other and to that of the nucleus of the mother-cell; as a consequence the four spores or pollen-grains do not lie in one plane but are arranged tetrahedrally, and have moreover a somewhat tetrahedral form; they are said to be radial. In some plants the spores or pollen-grains are formed sometimes in one way and sometimes in the other, and are therefore either bilateral or radial. This is the case, for instance, in Liverworts and Mosses: amongst Ferns, whereas in the Hymenophyllaceæ and Cyatheaceæ only radial spores, have as yet been observed, radial spores have been found in some and bilateral spores in others of the genera of Polypodiacex, and this is probably also the case in the Schizæaceæ and Gleicheniacex: in the Marattiaceæ and in Ophioglossum radial and bilateral spores may be produced in the same sporangium: this last condition also obtains in Psilotum and in Lycopodium Selago and inundatum: finally, these two modes of the development of the pollen-grains have been observed by Strasburger in Allium Moly among Monocotyledons.

In the following paragraphs a detailed account is given of the course of development in certain cases illustrating the two modes above-mentioned.

a. No cell-avall is produced until after four nuclei bave been formed by division; the resulting cells are arranged tetrabedrally.]

Development of the spores of Equisetum. At first the mother-cells swim in the fluid which fills the cavity of the sporangium, in groups of two or four together (Fig. 10, $a, b)$. Each mother-cell consists at first of a large spherical nucleus (including nucleoli), surrounded by fine-grained protoplasm, with a sharply-defined outline, but is without a cell-wall ${ }^{1}$. [The first indication of division is the coalescence of the granules in the nucleus to form fibrillæ; it becomes elongated in form, assuming a spindle-shape. An aggregation of coarse granules now makes its appearance in the equatorial plane of the spindle, constituting the nuclear disc: this splits into two discoid halves, and each half then travels to one pole of the nucleus and there forms a new nucleus. A fresh aggregation of granules now appears in the equatorial plane of the spindle, constituting the cell-plate; this extends quite across the protoplasm of the cell. The fibrillæ connecting the two new nuclei with the cell-plate now disappear, and then the cell-plate also. During this time the two nuclei have begun to divide in the same way as the primary nucleus, in planes at right angles to each other and to that of the division of the primary nucleus. Six cell-plates are now formed between the four nuclei, and become connected with them by the formation of fibrillæ. In the cellplates cellulose walls are now formed and the division is complete. The protoplasm of each of the young spores rounds itself off, and its nucleus assumes a central position

1 [According to Strasburger (loc. cit.) a delicate cell-wall is present.] 
and a rounded form. The next step is the absorption of the cellulose walls and the setting free of the young spores as primordial cells, which soon become clothed with a cell-wall. This cell-wall subsequently becomes differentiated in a manner which is described in detail on pare 403 .

In other cases the wall of the mother-cell presents ingrowths at points corresponding to the lines along which the cellulose walls are formed, and this conveys the erroneous impression that the wall of the mother-cell grows inwards constricting the protoplasm and ultimately dividing it into four parts. A good example of this case is afforded by the development of the pollen-grains of Dicotyledons. Fig. I I, taken from Troprolum minus, will serve to illustrate it. In $a$ four nuclei are present, which have been formed in the manner described above with reference to Equisetum: they are connected by

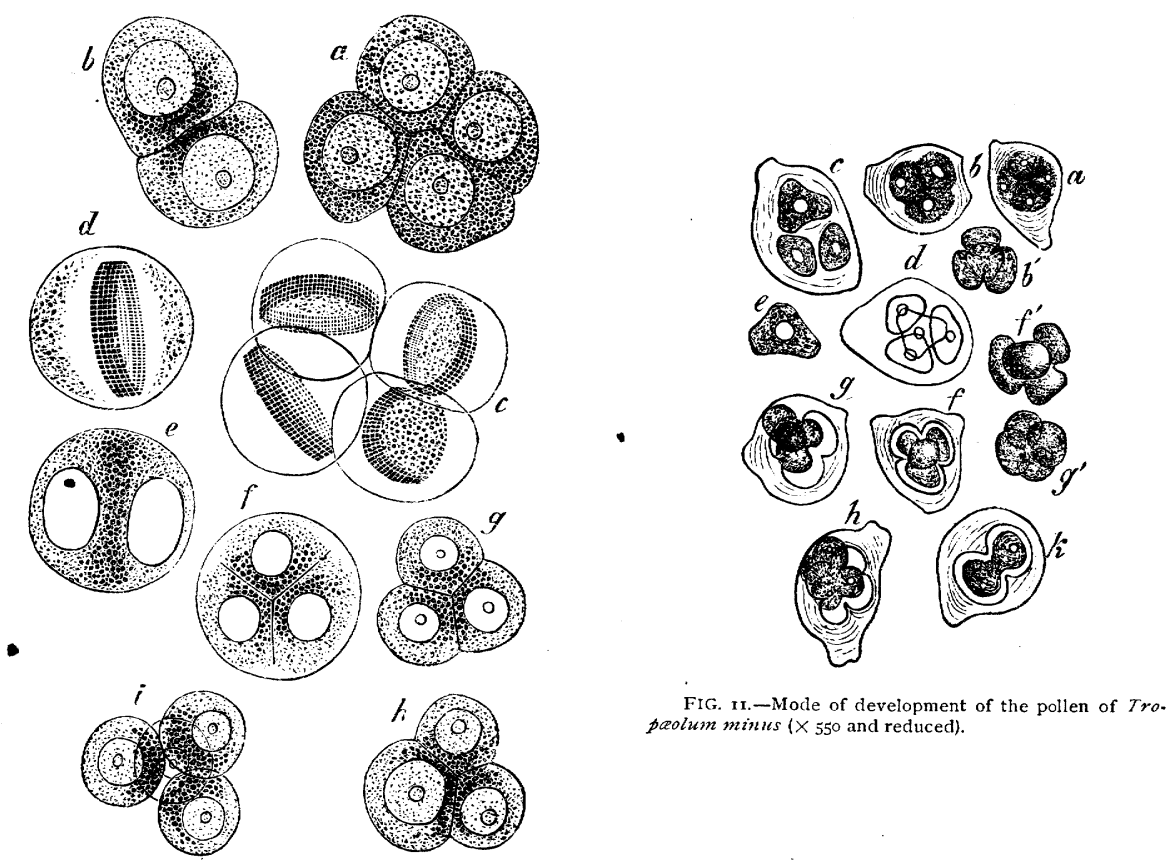

FIG. I0.-Mode of development of the spores of Equisetum limosum $(\times 550)$; $a$ group of four, $b$ group of two mother-cells ; $c$ and $d$ mother-cells preparing for division, showing the nuclear disc; $e$ one with two nuclei; $f, g$, and $i$ division into four spores $h$ abnormal formation of three spores from one mother-cell.

fibrillæ, and six cell-plates are present in the equatorial planes of the groups of fibrillæ which connect the nuclei. The wall of the mother-cell is thickened, especially at two parts of its surface, but it presents no ingrowths; but. now (b) ingrowths begin to be formed at points which correspond to the superficial ends of the six cell-plates $(f, g, b, k)$. Their growth does not, however, proceed so as to cause the constriction of the protoplasm into four masses: it is soon arrested; the cell-walls are formed simultaneously in the six cell-plates and are attached externally to the ingrowths of the cell-wall of the mother-cell. Later each mass of protoplasm forms a new proper wall around itself, which is the wall of the pollen-grain; the thick cell-walls surrounding them are absorbed, and the four pollen-grains are set free. (See also p. $55 \mathrm{I}$.)

b. A cell-wall is formed after each nuclear division; the resulting cells may be arranged either tetrabedrally or in one plane.

This mode of cell-division closely resembles that which takes place in a growing tissue. It may be very clearly observed in the formation of the pollen-grains of most Monocotyledons. Fig. I 2 shows the process in Funkia ovata. In $I$ two nuclei are 
present which have been formed by the division of the nucleus of the mother-cell. The next stage is that represented in $I I$, where a lamella of cellulose, which has been formed in the cell-plate between the two nuclei, completely divides the mother-cell. Thickening takes place at the junction of this partition-wall with the wall of the mother-cell, and the protoplasm of the two daughter-cells becomes rounded off. In $I V$ the nucleus of each daughter-cell is seen to have divided into two; sometimes this division does not take place in one of the cells $(V)$. A cell-wall is now formed simultaneously between each pair of nuclei : a proper cell-wall is subsequently formed round each mass of protoplasm, the thick investing cell-walls undergo absorption, and the pollen-grains are set free.]

The four cavities into which the mother-cell is divided in the development of spores and pollen-grains were at one time designated the 'Special mother-cells' of the pollen-grains, in which the grains themselves were formed. The term is however inaccurate, because the four protoplasmmasses are themselves the essential parts of the new cells, becoming subsequently enclosed in cell-walls. If the contents of the cavities are termed special-mothercells, they are identical with the daughtercells, i.e. the pollen-grains; if, on the other hand, the term is applied to the walls of the cavities, this is not in accordance with the present cell-theory. The term is in fact altogether superfluous.

c. Cell-formation by Budding and Abstriction. Among Fungi certain kinds of reproductive cells, conidia, stylospores, and basidiospores, are produced by small wartlike protuberances on a mother-cell, which then swell out and become rounded. The daughter-cell thus formed retains however at first its connection with the mothercell at its base, the contents of the two being connected by a channel through this narrow portion. A septum is eventually formed across this channel which splits into two lamellæ, and in this manner the

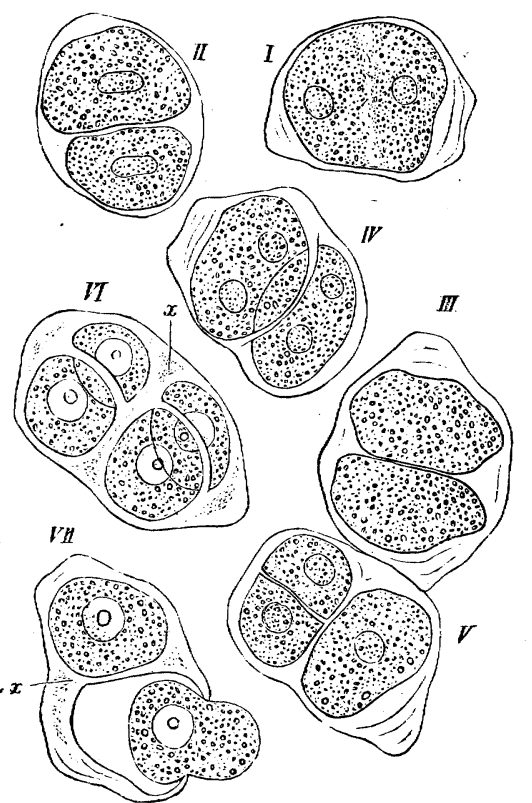

FIG. 12, $-I-V I I$ Successive stages in the formation of the pollen of Funkia ovata $(\times 550)$. In VII the wall of the daughter-cell has absorbed water till it has burst; its protoplasm is forcing itself through the cleft, and is becoming rounded off. separation of the spore from its mother-

cell is effected. The production of these spores therefore commences by a budding and is completed by division; and the whole process may be termed Abstriction. It occurs in a typical form in the reproduction of the yeast-fungus (Saccbaromyces). A wart-like out-growth from which several spores may be abstricted one after another is called a Sterigma; when the mother-cell bears several sterigmata, as in the Basidiomycetes, it is termed a Basidium. Intermediate processes between cell-formation by abstriction and ordinary cell-division occur when the protuberance is broad, and the daughter-cell therefore attached to the mother-cell by a broad base and separated from it by a broad septum, as in the branching of Cladophora; or, on the other hand, when the terminal portion of a hypha is divided by septa, and the resulting cells become rounded off and detached, as in the formation of the spores of Cystopus, Acidium, and other Fungi. [When the budding cell contains a single nucleus this probably divides, and one of the new nuclei goes to the bud; when the cell contains several nuclei some of these travel into the bud.] 


\section{B. Cell-formation in Growing Organs: Vegetative Cell-formation.}

This mode of cell-formation consists almost invariably in the bipartition of a mothercell; there is no or scarcely any perceptible rounding off or contraction of the dividing protoplasm; the two daughter-cells entirely occupy the place of the mother-cell.

I. The partition-wall is formed gradually. The protoplasm of the mother-cell projects in the plane of division as a circular protuberance, and a ridge of cellulose is formed in it which finally developes into a complete septum.

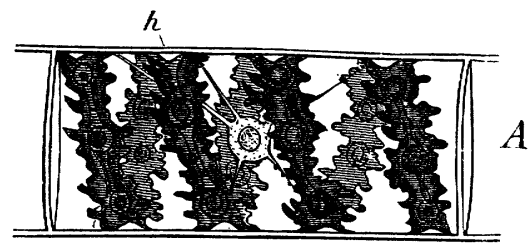

9
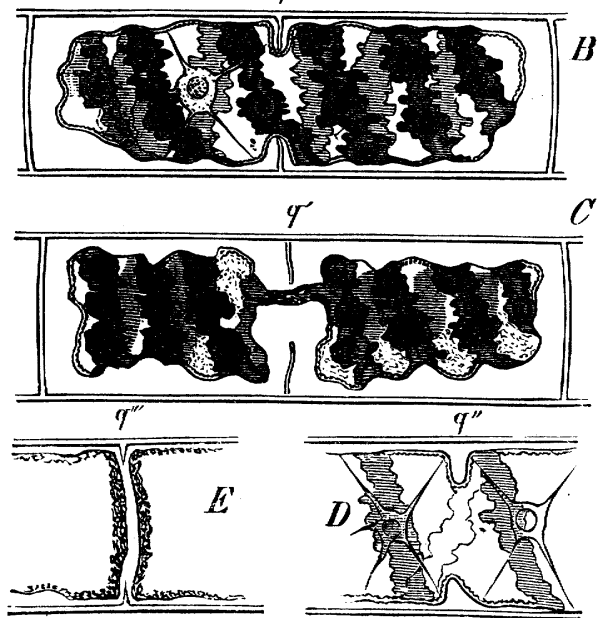

FIG. 13.-Spirogyra longata $(\times 550) . A$ a cell in the living state; $B, C$ cells laid in dilute alcohol during the division by night; $D, E$ central portion of cells in the act of division.
A clear and well-studied example is afforded in the stouter species of the genus Spirogyra ${ }^{1}$. In order to observe the divisions, it is necessary to place filaments that are in active growth in very dilute alcohol after midnight, that they may be examined by day, the divisions taking place only by night. Fig. I $3 A$ shows a living cell of a filament of $S$. longata by day; $B$ to $E$ the stages of division at night; the protoplasm of the cells is contracted by the alcohol.

[The phenomena of division fall into two groups, namely those which attend the division of the nucleus, and those which are connected with the ingrowth of the partition-wall. The nucleus of a cell which is about to divide becomes broader, assuming the form of a biconcave lens, and its nucleolus breaks up into irregular granules, which, together with its other granular contents, begin to form a nuclear disc in the equatorial plane. A delicate striation is now apparent in what is becoming the long axis of the nucleus, at right angles to the nuclear disc, and a characteristic nuclear spindle is gradually produced. The nuclear disc splits into two halves lying side by side, each of which travels to the corresponding pole of the nucleus; thus two new nuclei are constituted which are connected by fibrillæ.

The first indication of the formation of the partition-wall is the accumulation of protoplasm at about the middle of the length of the cell, so as to form a slight annular protuberance into the sap-cavity: this takes place about the same time as the first changes in the nucleus. Within this protuberance a rim of cellulose is formed which grows inwards, carrying before it the spiral chlorophyll-band (Fig. I3, $B$ and $C$ ); soon the chlorophyll-band is cut through, and margins of the ring of protoplasm coalesce in the middle so as to form a plate, to which the fibrillæ connecting the two nuclei, which have been formed in the meantime, become attached; in this plate the remainder of the cellulose wall is formed. The nucleus of each cell now travels to

1 This case was the first of all the processes of cell-formation that was accurately examined: H. von Mohl first described it in 1835 in Conferva glomerata. Mohl, Vermischte Schriften bot. Inhalts, Tübingen 1845 : [Strasburger, Zellbildung und Zelltheilung, I880.] 
its centre, and the protoplasm surrounding it throws out pseudopodia which attach themselves to the chlorophyll-granules in the green band and suspend the nucleus in the sap-cavity.]
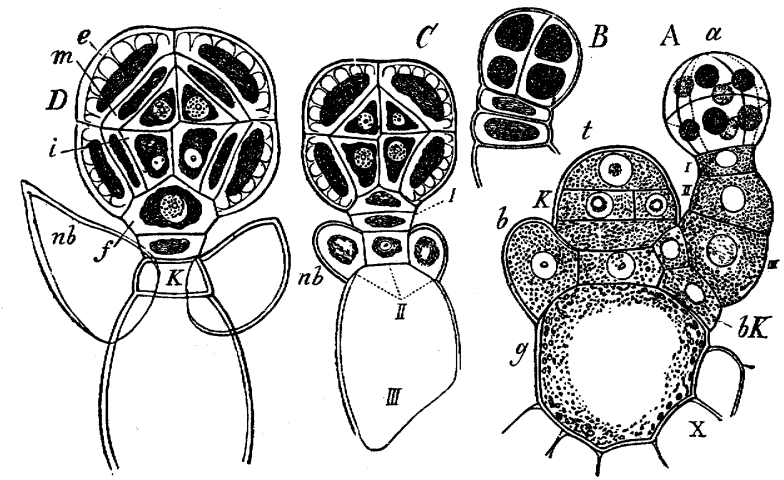

FIG. I4.-Formation of the antheridium of Nitella flexilis (see Book II). In $B$ the protoplasm has been made to contract by reagents.

2. The partition-rwall is formed all at once; that is, when first visible it appears as a thin membrane of cellulose stretching across the whole interior of the mother-cell; the protoplasm-masses of the two daughter-cells lie in the two cavities of the mother-

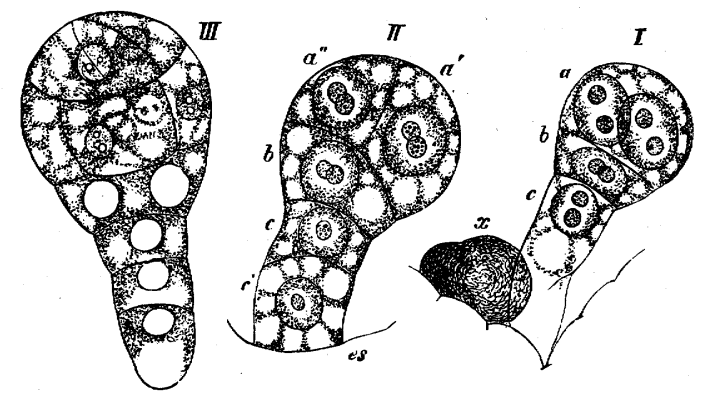

FIG. 15.-Embryos in the embryo-sac of Allium Cepa; the cells contain very large nuclei, each with two nucleoli. In $I$ the spherical apical cell contains two nuclei $\langle a\rangle$; in $I I$ it has already divided $\left(a\right.$ has split up into $\alpha^{\prime}$ and $\left.\alpha^{\prime \prime}\right)$; and in the same manner the cell $c$ (in $I$ ) has split up into $c$ and $c^{\prime}$.

cell thus produced. This mode of cell-division is usual and perhaps even universal in the formation of tissues, especially in the case of more highly organised plants.

[The nucleus divides, and between the two new nuclei a cell-plate is formed in which the cellulose wall is simultaneously produced.] (See Figs. $\mathrm{I}_{4}$ and $\mathrm{i}_{5}$.)

[The Bebaviour of the Nucleus during Division. The general rule is that the division of a cell is preceded by that of its nucleus, but to this there are certain exceptions. Strasburger has found that, in the mother-cells of the spores of Antboceros and of the macrospores of Isötes, the protoplasm divides into four parts before the nucleus shows any signs of division, and that in rare instances cells of Spirogyra had divided without any division of the nucleus having taken place.

In the process of division into two the nucleus usually goes through a series of changes which are designated by the term Karyokinesis : but in order that the description of the more complicated forms of karyokinesis may be intelligible, some account 
must be given of the structure of the nucleus in a state of rest ${ }^{1}$. The nucleus consists of a ground-substance which is homogenous, but sometimes presents, after careful preparation and staining, a finely punctuated appearance: it is denser towards the periphery, and thus constitutes a sort of membrane which gives to the nucleus its sharply-defined contour. In the ground-substance a more solid substance is present either in the form of granules, the larger of which are the nucleoli, or in the form of fibrillæ united into a network, the points of junction of the fibrillæ, the nodes, being somewhat thickened. Since, when the tissue is carefully stained with certain colouring-matters, the granules or the network become coloured more readily than the ground-substance of the nucleus, Flemming has designated the former chromatin and the latter acbromatin.

When nuclear division is about to take place the nucleus presents a coarsely granular appearance; the granules then coalesce to form convoluted threads, and these, together with the fibrillæ of the nuclear network which have become free, arrange themselves parallel to the long axis of the now elongated nucleus. At this time the nucleus has usually lost (Strasburger mentions Spirogyra nitida and the mather-cells of the spores of Equisetum as exceptions) its well-defined contour: it has assumed a spindle-shape, and an aggregation of protoplasm has been formed at each pole, in which a radial arrangement of the granules, with the pole as a centre, is usually evident. The fibrillæ of chromatin now contract so as to form a disc of greater or less thickness (equatorial plate of Flemming, nuclear disc of Strasburger) in the equatorial plane of the spindle, and faint striæ can now be detected between the nuclear disc and each pole, some of which apparently pass through the disc from one pole to the other. The nuclear disc now splits into two halves lying side by side, and each half travels to the corresponding pole along the fibrillæ which cause the striation of the spindle, and each then constitutes a new nucleus at the pole. The few fibrillæ which now connect the two nuclei are augmented by the formation of new ones, and the whole complex of fibrillæ assumes the form of a biconvex lens and extends across the cell. A row of granules now makes its appearance in the equatorial plane of the fibrillæ consisting, according to Strasburger, of starch or of a substance allied to starch and cellulose ${ }^{2}$; this is the cell-plate, and in this the cell-wall is simultaneously formed. If the complex of fibrillæ does not extend across the cell, so as to be in contact with both its lateral walls, the new wall is formed in contact with one lateral wall, and the complex gradually grows towards the other wall by the formation of new fibrillæ in which additions to the new cellwall are deposited. The new wall is clothed on each side with a layer of protoplasm, the nucleus assumes its normal position in the cells, and the process of division is complete.

The above description applies to the most complicated form of karyokinesis, as it occurs in relation to the development of the reproductive cells and to the reproductive processes of the higher plants. In the cell-division of the vegetative organs of these plants the process is usually simpler; thus, according to Schmitz, in the meristematic cells of Phanerogams the nucleus becomes elongated, the ends being swollen and the middle connecting part remaining narrow and presenting a longitudinal striation; the swollen ends become defined as new nuclei, and a cell-plate appears in the narrow portion in which the cell-wall is soon formed; the narrow portion gradually loses its striated appearance and assumes the appearance of the cell-protoplasm. In the celldivision of Spirogyra, as we have already seen, no cell-plate is formed. Strasburger draws attention to the fact that in those Thallophytes (excluding the Characex) in which karyokinesis has been observed, the cell-plate is not formed, as in the higher plants, in a complex of fibrillæ which connect the two new nuclei, but in a bridge of protoplasm which connects the two nuclei and extends across the cell in the plane of division.]

1 [This account is taken from the above-quoted work of Strasburger, and from those of Schmitz (Sitzber. d. niederrhein. Ges. in Bonn, 1880) and Flemming (Arch. f. micr. Anat. vol. I8) ]

2 [According to Schmitz (loc. cit.) the granules in question are microsomata, i.e. constituent elements of the protoplasm.] 
SECT. 4. The Cell-Wall ${ }^{1}$.-The substance of the cell-wall is secreted from the protoplasm. In what form it is previously contained in the protoplasm is not yet certainly known. The substance capable of forming cell-wall always consists of a combination of water, cellulose, and incombustible materials (ash-constituents), but may afterwards undergo further chemical changes.

By the continual secretion from the protoplasm of the substance out of which the cell-wall is formed, and its intercalation between the micellæ (see Bk. III. Sect. 1 ) of the cell-wall already in existence, this grows in both surface and thickness. The mode of both processes of growth is dependent on the specific nature of the cell, and on the function which it has to fulfil in the life of the plant; it therefore varies almost infinitely. Generally the surface-growth first preponderates, afterwards that in thickness. Neither the one nor the other is uniform over all points of a cell-wall; hence each cell during its growth also changes its form. The growth of a cell-wall continues only so long as it is in immediate contact on its inner side with protoplasm.

The want of uniformity in the surface-growth at different points causes cells which are at first, for example, spherical, ovoid, or polyhedral, to become subsequently cylindrical, conical, tubular, tabular, bounded by waved surfaces, \&c. The want of uniformity in the growth in thickness usually brings about a sculpture of the surface which is very characteristic. The thickened parts may project either outwardly or inwardly. The former occurs commonly in the surface of the cell-wall which is exposed, the latter in the partition-walls of adjoining cells. The thickenings which project outwards may take the form of knots, humps, spines, or ridges ; but those which project on the inside are much more various. In this case conical protuberances occur but seldom; annular ridges or spiral bands are much more common; these latter may be united in a reticulate manner, so that the thin interstices are polygonal; or the thickened part of the wall may be broader, and the thin parts then appear in the thick wall as fissures or roundish pits. If the wall is very thick, the latter become channels, which pass entirely or partially through the wall. Not unfrequently the thin portion of the wall which at first closes such a channel on the outside becomes absorbed, and the cell-wall is then perforated. But as, when contiguous cells are united into a tissue, the partitionwall usually becomes thickened in the same manner on both sides, the pits and pit-canals on the two sides meet, and the intermediate thin portion of membrane (sooner or later) becomes absorbed; a channel thus arises uniting two cell-cavities (Bordered Pits, perforated septa of vessels).

During the increase of the wall both in surface and thickness by the intercalation of new substance between the micellæ already in existence, a further internal structure usually becomes visible, which is termed Stratification and Striation. Both are the result of a different regularly alternating distribution of water and solid material in the cell-wall; at every point water is combined with cellulose,

I H. von Mohl, Vermischte Schriften bot. Inhalts, Tübingen I845 (numerous memoirs). Schacht, Lehrbuch der Anat. und Phys. der Gewächse, 1856.-Nägeli, Sitzungsberichte der Münch. Akademie, 1864, May and July.-Hofmeister, Die Lehre von der Pflanzenzelle, Leipzig 1867. Also numerous memoirs in the Botanische Zeitung. 
but in different proportions; portions less and more watery, denser and less dense, alternate. Thus, in every cell-wall sufficiently thick, a system of concentric layers becomes visible, of which the outermost and innermost are always denser, while between them alternate more and less watery layers. The stratification is visible on the transverse and longitudinal sections of the cell-wall, the striation on the surface as well; it is usually most evident there, but is in general less easily seen than the stratification; it depends on the presence of alternately more and less dense layers in the cell-wall, meeting its surface at an angle. Generally two such systems of layers may be recognised mutually intersecting one another. There are thus altogether three systems of layers present in a cell-wall, one concentric with the surface, and two vertical or oblique to it mutually intersecting, like the cleavageplanes of a crystal splitting in three directions (Nägeli); and just as this cleavage is sometimes more evident in one direction, sometimes in another, so is it also with the stratification and striation.

Independently of this internal structure, chemical changes arise in the cell-wall which never affect the whole mass uniformly, but usually mark out the thickened

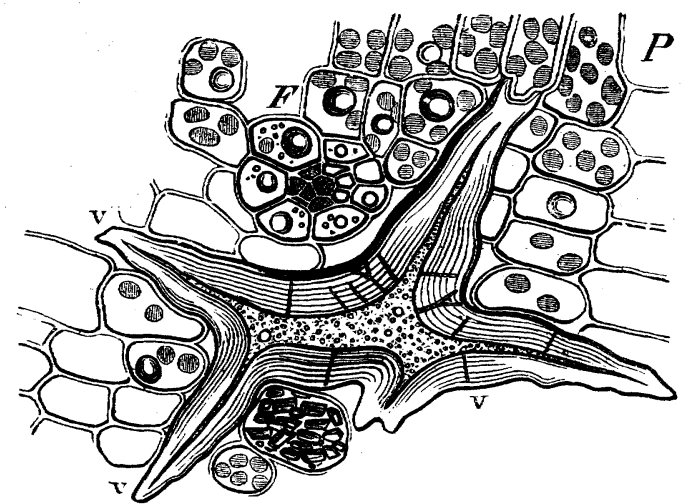

FIG. 16.-From the transverse section of a leaf of Camellia japonica; $P$ parenchymatous cells with chlorophyllgranules and drops of oil; $F$ a very thin fibro-vascular bundle; $v v$ a large, branched, thick-walled cell, which intrudes its branches between the parenchymatous cells.

cell-wall into concentric layers differing from one another chemically and physically. These chemical changes, which are always attended by an alteration of physical properties, are very various, but can conveniently be reduced to three categories;-Suberous or Cuticular change, Ligneous change, and Mucilaginous change. The first consists in the change of the outer layers of the cell-wall into an extensible very elastic substance, which water is unable, or nearly so, to penetrate or cause to swell, as the outer cell-wall-layer of the epidermis (cuticle) and of pollen-grains and spores, and cork. Lignification increases the hardness of the cell-wall, diminishes its extensibility, and renders it more easily permeable to water without considerable swelling. The conversion into mucilage renders the cell-wall capable of absorbing great quantities of water, so as to increase its volume and give it a gelatinous consistence. In the dry state such cell-walls are hard, brittle, or flexible like horn, as the cell-walls of many Algæ, the so-called 'intercellular substance' of the endosperm of Ceratonia Siliqua, linseed, and quince-mucilage. Several of these changes may occur simultaneously in a cell-wall, so that, for 
instance, the outer layers become woody and the inner mucilaginous, as in the wood-cells of the root of Phaseolus.

Besides these changes in the substance of the cell-wall, which are not unfrequently correlated with peculiar colourings, changes in its chemico-physical behaviour also arise from the interposition between its micellæ of considerable quantities of incombustible substances, especially lime and silica. If the deposition of these substances take place in sufficient .quantities, they remain behind, after the combustion of the organic groundwork of the cell-wall, as an ash-skeleton.

(a) The Surface-growth causes not only an increase in the size of the cell, but also changes in its form, when it takes place irregularly at different points of the circumference. Hence cells of originally dissimilar form may become similar by unequal growth; but it is much more common for cells originally alike in form to become entirely unlike. This is most usually the case in multicellular organs of the higher plants, such as leaves, stems, and roots; in their earliest state their cells are often scarcely distinguishable from one another; whereas in the completely developed organ the most various forms are juxtaposed (Fig. 16). It is only rarely, as in the growth of some spores and pollen-grains, that the surface-growth is so uniform that the original form is nearly retained even after considerable increase in size (e.g. pollen of Cucurbita and Althaa). But even in these cases the uniformity is only temporary; for the pollen-grains subsequently emit their pollen-tubes, or the spores germinate, in both cases by the local growth of the inner layer of the cell-wall. This also shows at the same time that the surface-growth of a cell-wall may be very different at different times; and this indeed is usually the case. From the infinite variety of the surface-growth of cell-walls, it is convenient, for the sake of arrangement, to reduce the different cases to classes, and to bestow names upon them ${ }^{1}$. Thus it is usual to distinguish between intercalary and apical growth of the cell-wall. Apical growth takes place when the surface-growth attains a maximum at any one spot (by interposition of new micellæ of cellulose), while its intensity decreases in all directions from it, and at a definite distance reaches a minimum, so that this portion of the cell-wall projects as a point, or appears as the rounded apex of an excrescence or of a cylindrical tube, as in hairs or filamentous Algx. If several points of apical growth occur in a cell which was originally round, it may become star-shaped; if new points of growth are formed behind the continuously growing end of a tubular cell, it branches, as in many filamentous Algæ, hyphæ of Fungi, Vaucberia, Bryopsis. Hofmeister ${ }^{2}$ distinguishes as a peculiar form of apical growth the case in which the maximum of growth is localised in a line instead of at a point; this may occur as the line of intersection of two curved surfaces. Intercalary growth of the cell-wall occurs in a typical form when the deposition of new substance is localised in a zone of the cell-wall; this zone extends, and a fresh piece of cell-wall is by degrees intercalated between the old ones. Very similar to this is the common occurrence of growth in the whole of the side-wall of a cubical, tabular, or cylindrical cell, as, for example, in the cells of Spirogyra, and the parenchymatous cells of growing roots and stems of Phanerogams (see Fig. r). Edogonium presents a peculiar case of intercalary surface-growth (Fig. 17). Below a septum an annular deposit of cellulose $(A, w)$ is formed; at this place the cell-wall splits, as if by a circular cut, into two pieces, and these separate from one another, but remain united by a zone of cell-wall $\left(B, w^{\prime}\right)$ formed by the extension of the annulus $w$. After the intercalation of this new zone, cell-division follows; and, since

1 A good classification of the processes of growth is, of course, still more important for the study of the mechanics of growth; but little has, however, yet been done in this direction, and we can only give a brief abstract.

${ }^{2}$ Handbuch der physiol. Botanik, vol. I. p. 162. 
this is repeated many times, the appearance is presented which is figured at $A, c$ (the formation of a so-called cap ${ }^{1}$ ).

(b) The Growth in Thickness of a Cell-wall is usually strictly localised, so that the thicker parts appear as very abrupt projections on the thinner parts of the cell-wall, either outside or inside. The general effect produced by the sculpture depends on whether the thick or thin portions occupy the greater extent of surface. If the thickening is strongest at certain points only, it takes the form of knobs or spines projecting outwardly (Fig. 19) or inwardly (Fig. I $8, C, D$ ); if it occurs most strongly in linear or ribbon-shaped portions of the cell-wall, projecting ridges or bands are formed on the inner or outer side. These ridge-like projections may form reticulate figures (Fig. I 8, B, Fig. 20, l), or rings, or spiral bands, an appearance especially frequent in certain thickened tissue-cells. If the rings or spiral bands

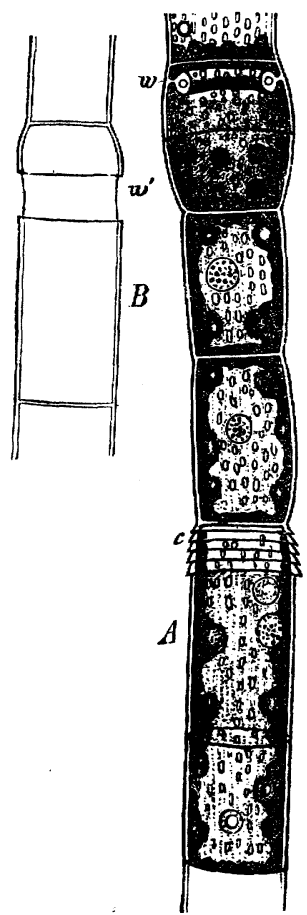

FIG. 17.-Intercalary surface-growth of Edogonium. which project inwards are thick and firm, and the intermediate portions of cell-wall thin and easily destructible, these thickenings may become detached even within the plant, and remain lying as isolated threads of cellulose in channels of the tissue, as in the annular vessels in the fibrovascular bundles of Equisetacex, Maize, \&c.; but the spiral thickenings may often also be drawn out to a considerable length as isolated fibres. Very striking examples are found in the rachis of the inflorescence of Ricinus communis and in the leaves of Agapantbus. If the thickening affects the whole surface of the cell-wall more completely, the small portions which remain thin will appear as Pits of very various outline, either roundish or fissure-like, or, when the thickening of the cell-wall is very considerable, as Canals, which perforate them. Thickenings of this kind most frequently affect the inner side of the cell-wall; the canals therefore run from the cavity of the cell outwards, and are there closed by a thin membrane ${ }^{2}$. When the cell loses its protoplasm and dies, this membrane is in many cases destroyed, and the pit or the canal then becomes open, as, for instance, in Spbagnum and in many wood-cells. The pits, especially in elongated cells, are generally arranged in spiral rows; but in other cases they are peculiarly grouped (Fig. $2 \mathrm{I}, A$ ). A remarkably striking form is the Sieve-like or Lattice-like marking which occurs in the Sieve-tubes of fibro-vascular bundles, generally in the septa, but also in the side-walls. In the simplest case the thin spots (pits) are densely crowded, only separated by thicker ridges, and polygonal in shape; they very often appear as sharply circumscribed groups of numerous dots.

In many cases the thin parts of such an area become absorbed, and the protoplasmic contents of adjoining cells enter into communication through these narrow channels. Sometimes the structure of these Sieve-plates (e.g. in Cucurbita Pepo) becomes, when old, very peculiar and complicated, from further thickening and swelling of the thickened portions ${ }^{3}$.

1 For further details of these somewhat complicated processes see Pringsheim, Jahrbuch für wissen. Bot. vol. I; Hofmeister, Handbuch der phys. Bot. vol. I. p. I54; and Nägeli und Schwendener, Das Mikroskop, vol. II. p. 549.

${ }^{2}$ Sometimes strongly thickened cell-walls with branched pit-canals have a very complicated structure, e.g. in the hard testa of Bertholletia. (See Millardet in Ann. des Sciences Nat., fifth series, vol. VI.)

${ }^{3}$ Compare Nägeli, Ueber die Siebröhren von Cucurbita, in the Sitzungsberichte der k. bayerischen 
One form of internal thickening which is extremely common in wood-cells and vessels, viz. the formation of Bordered Pits ${ }^{1}$, deserves a fuller exposition.

The formation of Bordered Pits takes place as follows. When the cell-wall begins to thicken, comparatively large spaces remain thin (Fig. 23, $t$; Fig. 24, $B, t$ ); but, as the thickening augments, it reaches even the thin spaces of the wall (Fig. 23, a-e; Fig. 24, $C-F)$. The outline of the thin spaces of the wall in the wood of Pinus sylvestris appears circular on a front view; the edge of the thickening-mass which arches over it grows also in a circular manner, and gradually contracts the opening; thus the front view of such a pit shows two concentric circles, the larger of which corresponds to the original thin space (Fig. $23, t$ ), and the smaller to the inner edge of the thickening

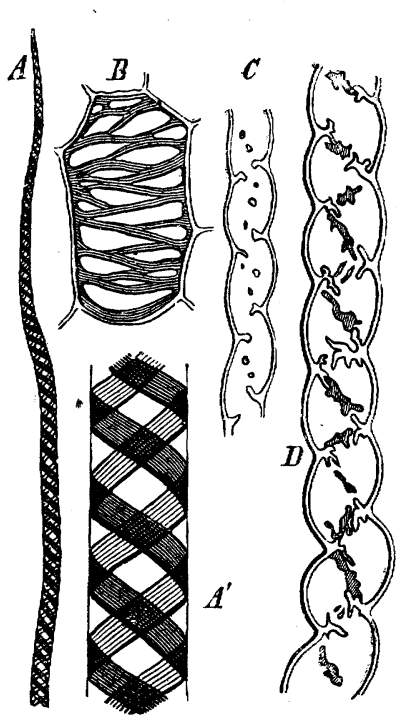

Fig. r8.-Cell-forms of Marchantia polymor. pha with thickenings projecting inwards; $A$ half of an elater from the sporogonium, with two spiral bands; $A^{\prime}$ a portion more strongly magnified; bands ; $A^{\prime}$ a portion more strongly magnified;
$B$ a parenchymatous cell from the centre of the $B$ a parenchymatous cell from the centre of the
thallus, with reticulate thickenings projecting inwards; $C$ a slender rhizoid with thickenings projecting inwards, arranged on a spiral constriction of the cell-wall ; $D$ a thicker rhizoid, with thicker branched projections, and the spiral arrangement still clearer.

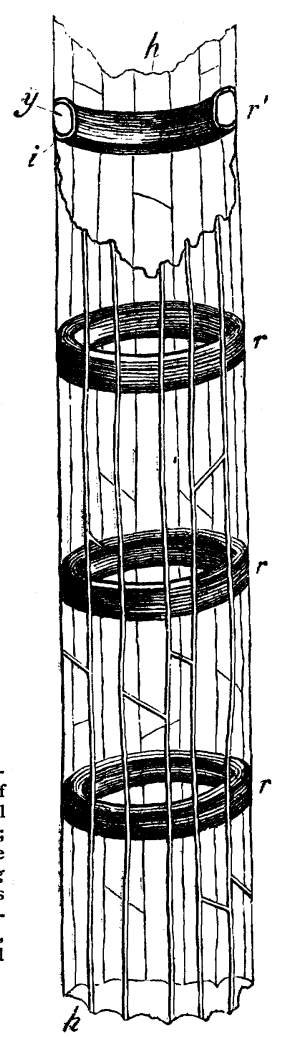

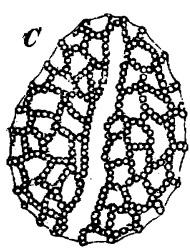

FIC. $B$ a young pollen-grain of Funkio ovata; the knob-like thickenings projecting outwards are still small ; in the older pollen-grain $C$ they are larger; they are arranged in lines united into a net-work.

\footnotetext{
FIG. I8 bis.-Piece of an annular vessel from the fibro-vascular bundle of the stem of Maize $(\times 550)$ $h h$ the thin cell-wall of the vessel, on which the boundary lines of the adjoining cells are clearly seen; $r r$ the annular thickenings of the wall of the vessel; $y$ the inner substance of one of the rings laid open; $i$ the denser layer which extends over the inner side of the ring projecting into the cavity of the cell.
}

(Fig. 23, a-e; Fig. $24, C, D$ ). Now since this process takes place on both sides 'of a partition-wall of two cells, a lenticular space is enclosed by the two overarchings, divided in the middle by the original thin cell-wall (Fig. $24, F, w)$, each half of this pit-cavity communicating with the cell-cavity by a circular opening. When the wood-

Akad. der Wissenschaften, München 1861. On the actual perforation of sieve-plates see Sachs in Flora, 1863 , p. 68, and Hanstein, Die Milchsaftgefässe, Berlin 1864, p. 23 et seq.

1 The development of these was first accurately recognised by Schacht, De maculis in plantarum vasis, \&c., Bonn 1860 . 
cells lose their protoplasm and become filled with air and water, the thin cell-wall disappears (as in Fig. 24, E), and the two pits form a single cavity, which is bounded by the over-arching thickening-masses, and is united with the adjoining cell-cavities by a circular opening (Fig. $24, A, D, E$ ). In Pinus sylvestris the pits are large and distant from one another, and the whole process may be easily traced step by step.

The process appears somewhat different when pits lie very near to one another, as in Pitted Vessels. In this case the thickening first appears in the form of a net-work, of which the thin parts of the cell-wall occupy the polygonal meshes, as may be very easily seen in young Maize-roots, for instance. Fig. 25, $A$, represents a portion of the side-wall of an already mature vessel ${ }^{1}$ of the root-tuber of the Dahlia. The ridges which first appear on the cell-wall are indicated at $a$, and are left white; they enclose elliptical spaces pointed at both ends. As the thickening continues, the free edge of each ridge, as it grows further inwards, spreads, and becomes arched over the thin parts of the cell-wall. In this case, however, the overarchings do not grow uniformly, but in such a manner that their edges form at length a narrow fissure $(c$, in $A$ and $B$ ).

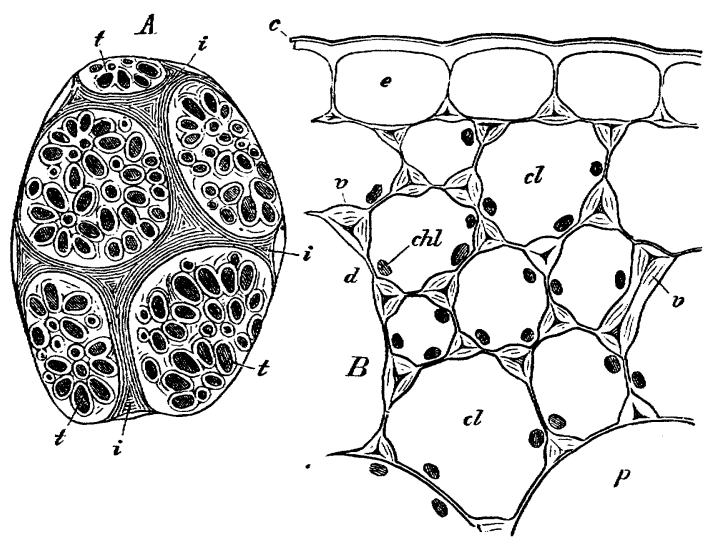

F1G. 21, $-A$ a parenchymatous cell from the cotyledon of Phaseolus multiflorus isolated by maceration; $i$ i the parts of the cell-wall where it is bounded by intercellular spaces; $t t$ cell-wall furnished with numerous simple pits, but not greatly thickened; the thinnest parts of the pits are figured dark. $B$ epidermis $(e)$ and collenchyma $(c l)$ of the leaf-stalk of a Begonia; the epidermal cells are thickened collenchyma $(c l)$ of the leaf-stalk of a Begronia; the epidermal cells are thickened
uniformly on the outer wall, but where they adjoin the collenchyma only at the angles where three cells meet; these thickenings have great capacity for swelling; $c h l$ chlorophyll-granules; $p$ parenchymatous cell $\left(\times 55^{\circ}\right)$.

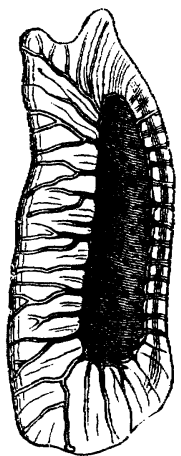

F16. 22.-Hypodermal cell of the underground stem of Pteris aquilina, isolated by boiling with potassium chlorate and nitric acid : it is more strongly thickened on the left side; the unthickened spaces appear as branched canals $\left(\times 55^{\circ}\right)$.

Here also, when two similar cells adjoin, the same process takes place on both sides of the partition-wall, and lenticular spaces are formed by the overarchings; these are at first bisected by the original thin cell-wall, which afterwards disappears, and the two cell-cavities are placed in communication at each bordered pit; the canal or bordered pit which unites them is wide in the middle, and opens into each cell by a narrow fissure (Fig. 25, B, C). If, on the other hand, a vessel of this kind adjoins a parenchymatous cell which remains always full of sap and closed, the thickening and overarching of the pit occur only on the side of the vessel (Fig. 26, V), the thin parts of the cell-wall remaining intact ${ }^{2}$, and the bordered pit remains closed; from

1 For the definition of a vessel see Chap. ii.

2 These thin pieces of cell-wall which close up bordered pits may, by rapid surface-growth, form bag-like protrusions, which grow through the pores of the pits into the vessels, spread themselves out there, become divided by septa, and thus form a thin-walled tissue, which not unfrequently fills up the whole of the cavity. These formations are known under the name of 'Tüllen' or 'Tyloses;' they are abundantly and easily seen, for instance, in old roots of Cucurbita, and in the 
the cell-cavity of the vessel a narrow fissure $(c)$ opens between the expanded thickeningmasses $(b)$ into a wider cavity, which is bounded on the sides by the narrow part of the thickening-masses $(a)$, on the outside of the primary cell-wall. These processes can only be seen in sections of extraordinary tenuity; but these are easily obtained if goodsized pieces of the parts to be studied are allowed to lie for months in plenty of absolute alcohol, taken out before the preparation is made, and the alcohol allowed to evaporate; in this manner pieces of sufficient hardness are obtained to cut extremely well with a very sharp knife.

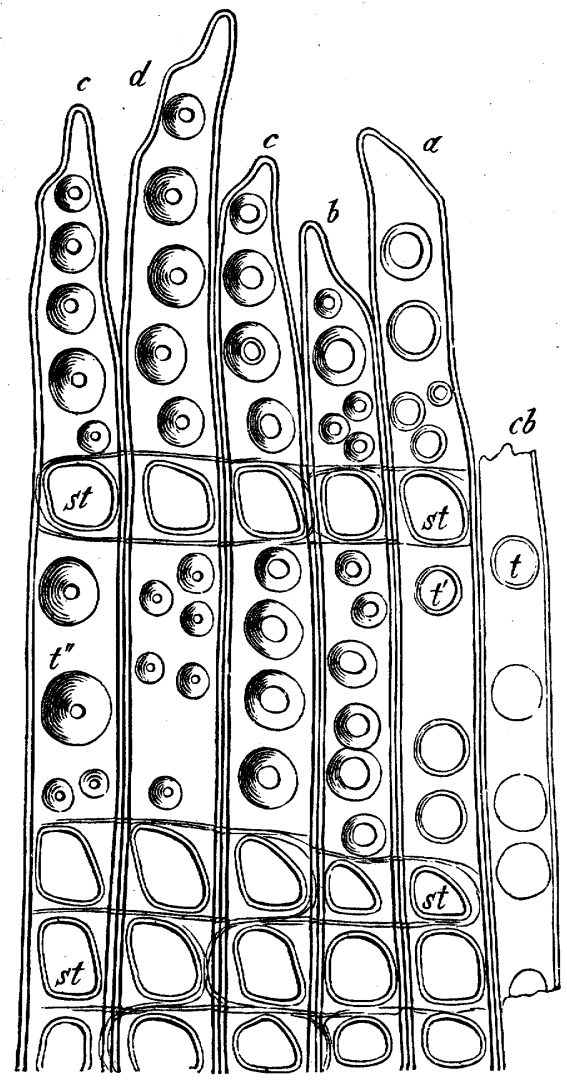

FIG. 23.-Pinus sylvestris; radial longitudinal section through the wood of a rapidly growing branch; $c b$ cambial wood-cells; $a$-e older wood-cells; $t t^{\prime} t^{\prime \prime}$ bordered pits of the wood-cells, enlarging with age; st large pits where cells of the medullary rays touch the wood-cells $(\times 550)$.
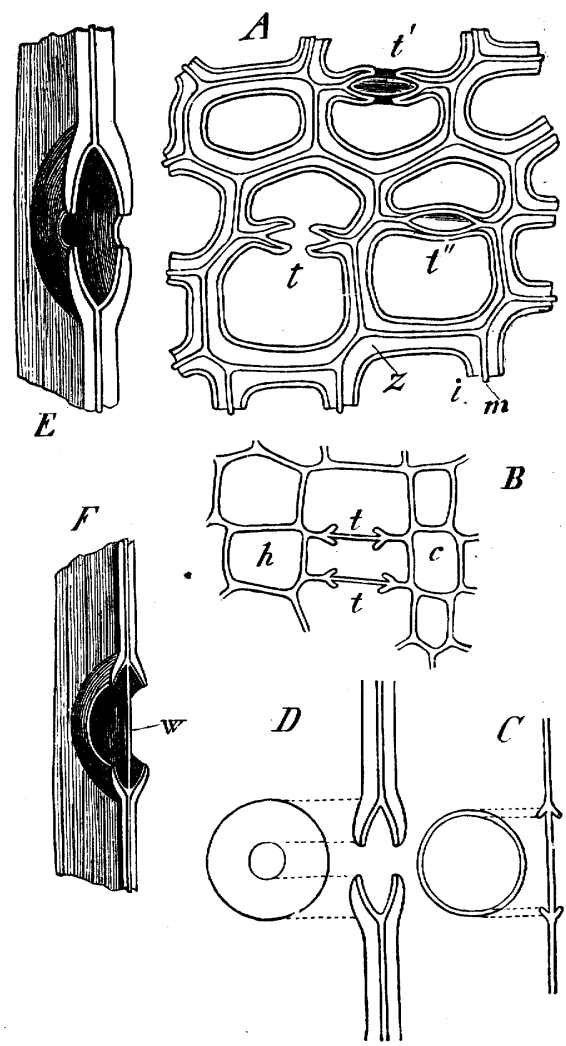

FIG 24 -Pinus sylvestris; $A$ transverse section of mature wood-cells $(\times 800) ; m$ middle lamella of the common partition; $i$ inner layer, clothing the cavity; $z$ intermediate layer of the cellwall; $t$ a mature pit cut through the middle; $t^{\prime}$ the same, but at a thicker part of the section, the part of the cavity of the pit lying beneath is seen in perspective; $t^{\prime \prime}$ a pit cut through beneath its inner opening: $B$ transwerse section through the cambium $(\times 800)$; $c$ opening: $B$ transverse wood-cells with pits $t t$ beginning to form; ( $C-F$ diagrammatic).

In the walls of scalariform vessels, which are developed with peculiar beauty in the higher Cryptogams, the bordered pits are fissure-like; they often stretch across the partition-wall of two adjoining cells, but are very narrow in the longitudinal direction. In Fig. 27, $A$, is shown the lower half of a vessel of this kind with fissurelike pits, separated by thickening-masses like rungs of a ladder; the larger white spaces

wood of Robinia pseudacacia, \&c. [See Journ. of Bot. 1872, pp. 321-323, t. 126; and Reess, Bot. Zeitg. I868, pp. I-I I, t. I ; also infra, Book iii. Sect. I6.] 
are the angles of contiguous cells. The formation of such a scalariform thickening begins by the growth, on the thin wall which separates two vessels $\left(C, s^{\prime}\right)$, of transverse ridges $(v)$, which unite on either side with the thickening which always lies at the angle of a cell-wall. $C$ shows this in front view, $D$ in vertical section. When completely developed, the thin cell-wall $\left(s^{\prime}\right)$ is absorbed $(c, c$, in $B)$, the thickening-ridges have overarched, so that only a narrow fissure $(B, d)$ remains between their margins; still further inwards the ridge again becomes narrower. The interior cavities of two adjoining vessels are thus united by a number of long narrow fissures $(B, s)$; the framework of the ladder is formed of peculiarly-shaped rungs, which may be seen in $B$ at $c c$ in section, at $e$ in front view. Where the wall of a vessel bounds a parenchymatous cell $(E)$, the scalariform thickening takes place only on one side of the vessel $(g)$, and is absent from the other side $(p)$. In this case also the thin original wall remains, closing externally the space of the fissure-like pit.

The variety in the formation of pits is by no means exhausted by these examples; but all the processes cannot be described here; we can only indicate a few.

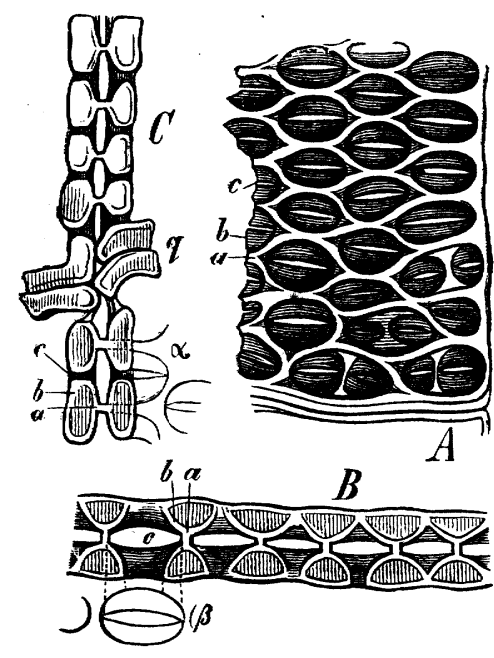

FIG. 25.-Wall of a vessel with bordered pits from the tuberous root of the Dahlia; $A$ front view of the wall of a vessel from. without ; $B$ transverse section of the same (horizontal, at right angles to the paper) ; $C$ longitudinal section (vertical, at right angles to the paper): $q$ septum; $a$ the (vertical, at right angles to the paper): $q$ septum; $a$ the
original thickening-ridges; $b$ the overarching part of the thickening-masses; $c$ the fissure through which the cavity of the pit communicates with the cell-cavity. At $\alpha$ and $\beta$ the corresponding front view is appended in order to make the transverse and longitudinal sections more clear $(\times 800)$.

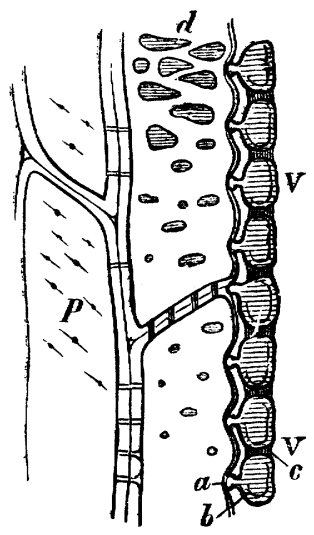

FIG. 26.-From the root-tuber of the Dahlia; $P$ parenchymatous woodcell; $V$ a piece of the wall of a vessel, where it adjoins a parenchymatous wood-cell; $a b$ the thickening-masses of the wall of the vessel cut through at right angles; $c$ fissure of the pit; $d$ simple pits in the parenchymatous wood-cell $(\times 800)$.

In the formation of vessels in the Dahlia (Fig. 25) the pit occupies at first a large round space, while the edges of the overarching thickening enclose a fissure. By a modification of this process of growth the fissure may attain a length much greater than the diameter of the pit, which then appears, on a front view, as a roundish opening crossed by a fissure (Fig. 26, $P$ ). It also sometimes happens that the pit-fissure changes its direction as the thickening increases; in this case, on a front view, two fissures appear as if they cross one another (Fig. 28, $A$ and $B, s t$ ). But in order to be certain that this takes place within the layers of the wall of a single cell, the cells must be isolated by maceration. Similar appearances are also often seen if the partition-wall of two cells is observed from the front. If the fissure inclines to the left in the one 
cell, the corresponding fissure may incline to the right in the other; viewed one over the other they then appear crossed ${ }^{1}$.

In cells which form a tissue, the partition-wall is always at first a very thin lamella; as the thickness increases, the thickening-masses project into the adjoining cell-cavities. Generally, as we have already seen, the thickenings on either side of a partition-wall correspond; and this is very evident in the formation of pits, inasmuch as the pitcanals of adjoining cells meet one another. But since a cell often adjoins cells of a very different character, different sides of the same cell may show different forms of thickening and different descriptions of pits. The total growth in thickness may also be very different on different sides; thus, for instance, epidermal cells are mostly strongly thickened on the outer exposed wall (cuticle); the inner wall, where they adjoin parenchymatous cells, being either very thin or corresponding in form to that of the adjoining cells.

The correspondence in the growth of the thickenings is less evident when they have a distinctly spiral structure, or when they occur in the form of strong spiral bands, as in spiral vessels; if, in this case, in each adjoining cell one or more spiral bands wind in the same direction, they must necessarily cross on the common partition=wall.

(c) Stratification and Striation of the Cell-zuall ${ }^{2}$. When the cell-walls have attained a certain thickness and extent of surface, stratification and striation become more or less evident. In consequence of stratification the cell-wall appears as if composed of very thin membranes enclosed one within another and fitting very closely together; the stratification is seen both in the transverse and the longitudinal section of the cell-wall. The striation is generally to be seen most plainly from the front; it may be observed in the form of two systems of lines (sometimes apparently more) marked on the surface. The one system, consisting of parallel strix, is always intersected by the other system which also consists of parallel strix. A closer investigation shows that the appearance of striation does not belong to the surface only or to one layer of the cell-wall, but that the striation rather penetrates the whole thickness of the cell-wall, and that the strix are due to layers which intersect the surface obliquely, and all other layers concentric with it. If the striation is very strongly marked, and nearly parallel to the longer axis of the cell, it may be recognised in a
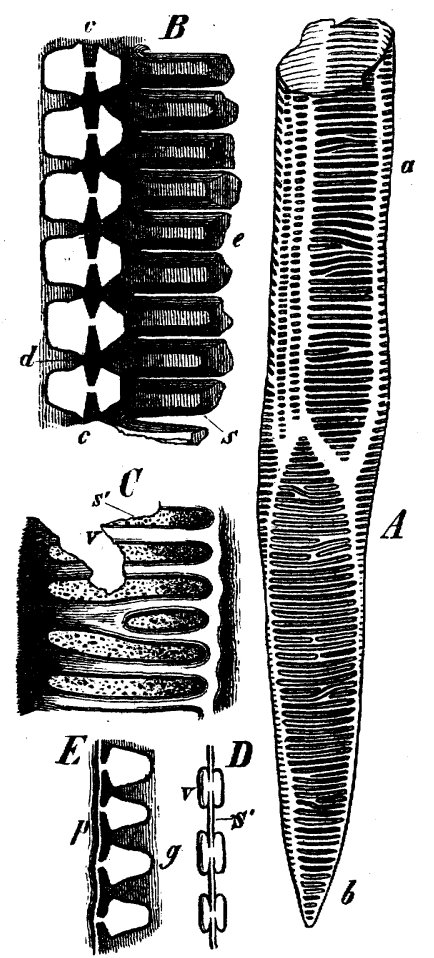

Fic. 27.-Vessel from the underground stem of Pteris aquilina thickened in a scalariform manner; $A$ half of a vessel, isolated by Schulse's maceration; $B-D$ sections obtained from pieces of the stem hardened in absolute alcohol; $B$ after a very clean section, represented in a partly diagrammatic manner; to the right, front view of grammatic manner; to the right, front view of
the wall of the vessels from within; $c c$ vertical the wall of the vessels from within; $c c$ vertical
section of the same; $C$ front view of the young wall of a vessel; $D$ its vertical section; $E$ place where a vessel adjoins a parenchymatous cell, in section vertical to the thickening-ridges of the vessel $(\times 800)$. transverse section in the form of lines crossing the concentric layers; in a longitudinal section only those systems of striation are easily seen which, viewed on the surface, cut the longer axis nearly at right angles.

1 A representation of a twisted pit-canal, whose outer and inner fissure (within the same cellwall) cross, may be seen in Nägeli, Berichte der Münchener Akademie, I867 (July 9), t. v. fig. 45.

${ }^{2} \mathrm{H}$. von Mohl, Bot. Zeitg. 1858, pp. I, 9.-Nägeli, Ueber den inneren Bau der vegetabilischen Zellenmembran, in the Sitzungsberichte der Münchener Akad. der Wissenschaften, I864, May and July.-Hofmeister, Lehre von der Pflanzenzelle, p. I97. 
Every system of stratification or striation consists of layers of visible thickness and of different refractive powers, so that a more strongly refractive layer or stria always alternates with a less strongly refractive one. This difference of refraction results from a different distribution of water and of solid particles in the cell-wall; the less strongly refractive layers contain more water and less cellulose, and are therefore less dense; the more strongly refractive and denser layers contain less water and more cellulose. Hence stratification and striation of the cell-wall disappear both when water is completely eliminated, and also when it absorbs much water; because, in the first case, the more watery layers are reduced to the condition of the less watery ones, in the latter case the less watery become similar to the others. On the other hand stratification and striation become most conspicuous when, from the particular proportion of water in the cell-wall, the difference between the dense and the watery layers is greatest. In many cases this may be brought about by addition of acids or alkalies
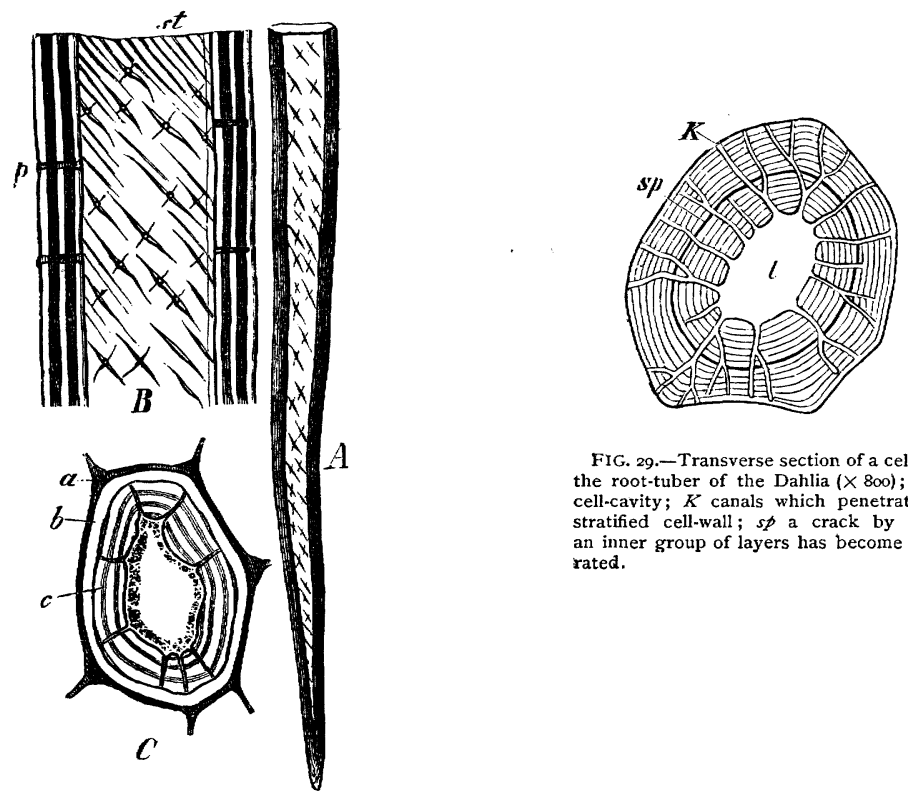

FIG. 29.-Transverse section of a cell from the root-tuber of the Dahlia $(\times 800)$; $l$ the cell-cavity; $K$ canals which penetrate the stratified cell-wall; $s p$ a crack by which an inner group of layers has become separated.

FIG. 28.-Sclerenchymatous cells of Pterts aquilina; $A$ half of a cell isolated and rendered colourless by Schulze's maceration; $B$ a piece more strongly magnified $(\times 550)$; the fissure-like pits cross; $i$.e. the fissure is twisted; at $p$ sideview of a fissure, appearing here as a simple canal. $C$ transverse section of the same.

which occasion a moderate swelling. But if the dense layers are very dense, and the others very watery, as is the case with some wood-cells (e.g. Pinus sylvestris), the striation becomes more evident through desiccation, because this brings out the dense layers and effaces the less dense ones.

The systems of striation and stratification of a cell-wall intersect one another, like the cleavage-planes of a crystal splitting in three directions. But since the striation and stratification are produced by layers of a measurable thickness, composed of alternately denser and less dense substance, the cell-wall appears to be composed of parallelopipedal pieces, distinguished from one another by the proportion of water contained in them. If we for a moment disregard the stratification, and assume that we have two intersecting systems of striation; then, where two dense striæ intersect, the densest or least watery places are always to be found; where two watery ones intersect, the least dense or most watery, and where places of greater and less density intersect, areolæ of inter- 
mediate density are found. The intersecting systems of striation must form prisms which stand vertically or obliquely upon the surface of the cell-wall. If the concentric stratification is very strongly developed, every one of these prisms must be cut up into more and less dense sections parallel with its base; if the concentric stratification is feebly developed, the prismatic structure may predominate. The peculiar internal structure of the epispore of Rhizocarpex (Fig. 33, p. 3I) ${ }^{1}$, and the yet more various structure of the extine of many pollen-grains, may perhaps be resolved into a further development of this kind of process; but our space does not permit us to pursue this question in detail. The layers which produce the external appearance of striation may possess the form of closed rings, i.e. may be similar to thin sections of the cell, or may run in a spiral manner round the axis of the cell. A distinction must accordingly be drawn between annular and spiral striation; it is often, however, very difficult to decide which of the two is present; sometimes both are developed at different parts of the same cell-wall. Sometimes one system of striation is very obscure, the other all the more strongly marked; or one system may be the better developed in one layer of the cell-wall, the other system in another layer; and this is genetically connected with the above-mentioned twisting of the pit-fissures. The striation is mostly clearest in cells with broad uniform thickening-surfaces, as Valonia utricularis, hairs of Opuntia, pith-cells of the root-tubers of the Dahlia (in the latter case remarkably plain); but it may also be recognised when the sculpture of the cell-wall is complicated; e.g. in the walls of very wide vessels of Cucurbita Pepo provided with densely crowded small bordered pits (after Schulze's maceration, especially in vessels of the root, where the crossed spiral striation is very clear). The striation may itself give occasion to differences of elevation; sometimes the denser layers project a little on the inner side of the cell-wall (Fig. $32 B$ ); or individual denser layers of one system of striation alone become prominent; thus, for instance, a fine spiral band makes its appearance on the inner sides of the wood-cells of the Yew, which is not unfrequently crossed by one running in the opposite direction. When elongated fissure-like pits are arranged in spiral lines on the cell-wall, a system of striation is generally found in a corresponding direction.

This slight sketch must suffice to explain the nature

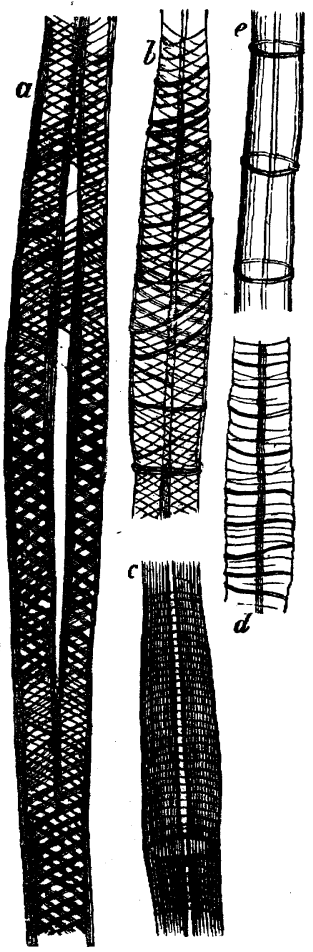

FIG. 30.-Cells from the leaf of Hoya car nosa $(\times 800)$, showing striation. The strix are not nearly so strongly marked in nature, but are quite as evident; $a$ optical longitudinal section of the crossed annular striation; $b$ front view where the annular striations cross; $c, d$ front view where they do not cross; $e$ a piece of cell-wall, where only a few annular striations are to be seen. of stratification and striation, and their relation to the sculpture of the cell-wall; further detail would exceed the limits of this work ${ }^{2}$.

(d) Intussusception the cause of growth of the Cell-rwall in surface and tbickness. The

${ }^{3}$ See also Book II, Rhizocarpeæ.

2 The striation may easily be seen, even with slight magnifying power, on the large pith-cells of the root-tubers of Dahlia, on the hairs of Opuntia, and on Valonia utricularis, but only with very high magnifying power on isolated wood-cells of Pinus, bast-fibres, \&c.; one of the examples longest known are the bast-cells of Apocynaceæ possessed of alternate dilatations and constrictions. (Mohl, Veget. Zelle, Fig. 27.) 
surface-growth of the cell-wall can be regarded only as an intercalation, between its already existing particles, of new particles which force the old ones asunder. It is very probable that the striation has a genetic connection with this process, similar to that which Nägeli has shown to exist between the stratification of starch-grains and their growth. It was long thought that the growth in thickness of the cell-wall arose from the repeated deposition of new concentric layers on its inner side; in which case the innermost layer would always be the youngest. This appeared to be an extremely simple explanation of the stratification of the cell-wall; and the chemical differentiation of thick cell-walls appeared entirely to support this idea. But the increased powers of the microscope revealed a fact quite fatal to the theory of apposition; the stratification of thickened cell-walls was shown, as we have seen, to be not a contiguity of similar, but an alternation of dissimilar, layers. For reasons which cannot here be discussed, it must

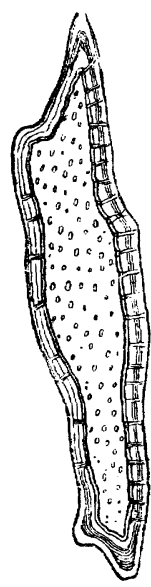

FIG. 3r.-Hypodermal cel
from the stem of Pteris aqui-
lina, isolated by Schulze's
maceration. The wall is seen
in optical longitudinal sec-
tion; it shows an innermost
very dense layer, a central
less dense layer (the dark
streak to the right below) en-
closed by two denser layers;
these layers are penetrated
by pit-canals, which are seen
on the hinder wall in trans-
verse section.

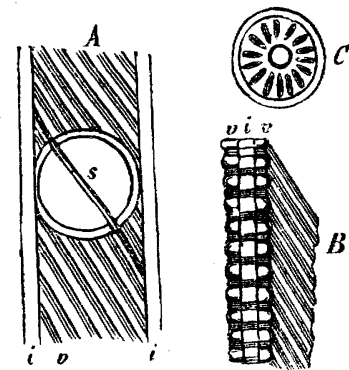

FIG. 32.-Striation of the wood-cells of Pinus Strobus: $A$ front view of a young cell; a fissure $s$ robus ; $A$ front view of a young cell; a fissure $s$ runs across the still young bordered pit, cor-
responding to the spiral striation; $B$ sectional view of the cell-wall with a part of the side view; $i$ the middle lamella of the wall common to two cells; $v v$ the thickening-layers in contact with it ; these are striated, the striation may be recognised as a formation of layers penetrating the whole thickness; the denser (white) layers project in the form of little knobs. $C$ front view of a pit; the striation here appears as a star-like arrangement of less dense spots $(\times 800)$.

be concluded that these alternate deposits of more and less watery layers must be the result not of an apposition, but of an internal differentiation of the cell-wall already formed. The fact is decisive, that on the inner side of every cell-wall there always lies a dense layer containing but little water; if growth in thickness took place by successive deposition of layers, the innermost and youngest layer would be alternately more and less dense, which is not the case. The growth also of such thickening-masses as project externally, like the crests and spines of pollen-grains, \&c., can only be explained by intussusception, not by apposition.

Growth by intussusception can be regarded only as the diffusion of an aqueous solution from the protoplasm between the micellæ of the cell-wall. What this solution is, cannot at present be said with certainty; probably it contains some carbo-hydrate which is easily transformed into cellulose. This substance then forms between the micellæ of the cell-wall new solid micellæ of cellulose. The actual process of growth, the internal structure of the cell-wall already described, and certain phenomena which it exhibits with polarised light, as well as the swelling of the cell-wall, lead to the conclusion 
that it consists of solid micellæ of definite form, each of which is surrounded by an envelope of water, and is thus separated from the adjoining micellæ; the more watery a cell-wall-layer, the smaller, according to the principles laid down by Nägeli ${ }^{1}$, are the solid micellæ, the more numerous and the thicker their aqueous envelopes. From this it follows that a certain quantity of water is as indispensable to the growth and to the internal organisation of the cell-wall as is cellulose itself. This water may be designated avater of organisation, in the same sense as we speak of 'water of crystallisation ;' and as the latter is indispensable to the formation of many crystals, so is the former to the structure of the cell-wall. It is moreover, as we shall see, a peculiarity of all organised structures to contain this water of organisation, at least as long as they continue to grow, because they all alike grow by intussusception.

From what has been said it will easily be seen that the concentric formation of layers of a cell-wall growing by intussusception differs essentially from the repeated

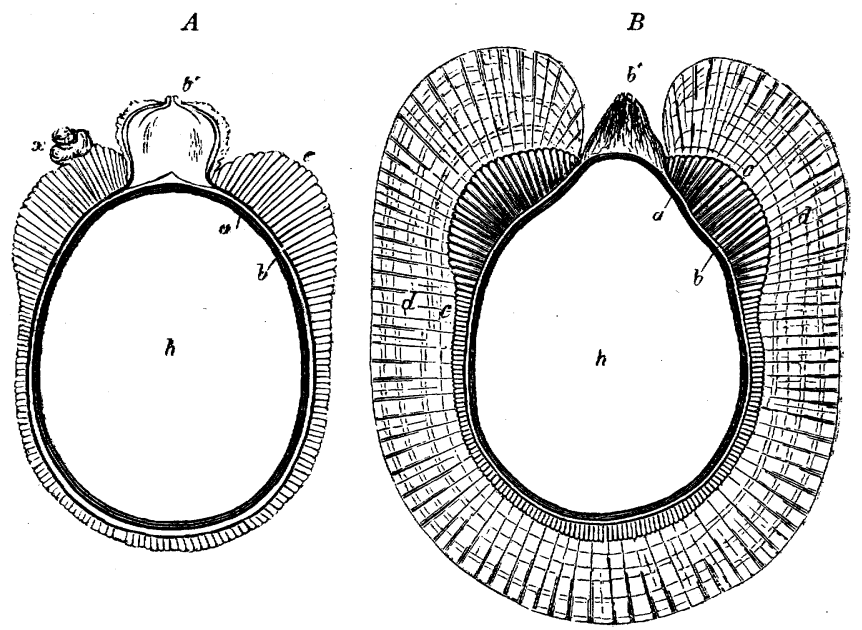

\begin{abstract}
FIG. 33--Macrospores of Pilularia globulifera, in optical longitudinal section; $A$ a still unripe spore, in which the outermost gelatinous layer of the cell-wall is still wanting, though present in the ripe spore $B$; the two outermost layers of the cell-wall of the latter $(c$ and $d)$ have assumed a prismatic structure, especially in $c$; in $d$ stratification is feebly indicated at the same time. Seen from the surface the prisms appear like areolæ. The bounding-surfaces of the prisms are, in the corresponding cell-wall layer of Marsilia Saivatrix, more solid and cuticularised, by which the appearance of a honey-comb is produced. (The layers $b, c$, and $d$ together form the epispore.) (See also Book II., Rhizocarpeæ.)
\end{abstract}

formation of a cell-wall round one and the same protoplasm-mass; cell-walls enclosed one within another may be produced in this manner; but these cannot be considered as layers of one cell-wall ${ }^{2}$. Such a process is very common in the formation of the pollengrains of Phanerogams; within each of the four cells formed by the division of the mother-cell the protoplasm forms round itself a new cell-wall, before the mother-cellwall has disappeared (Fig. 34).

But the renewal of a cell-wall may also be brought about by the internal layers of the cell-wall increasing by intussusception, while the external mass of layers undergoes no further growth. Thus the cell-wall of spores and pollen-grains originally grows as a

1 The theory of the growth of the cell-wall (as of all organised structures) by intussusception was first originated by Nägeli in his great work on Starch (1858). Compare also Sachs, Handbuch der Experimental-physiologie der Pflanzen, § I I4.

${ }^{2}$ [A striking instance of such a multilaminated cellulose-envelope is afforded in the remarkable organism described by Archer under the name of Chlamydomyxa, Quart. Jour. Micr. Sci. 1875, p. I 29.] 
whole, increasing by intussusception; by subsequent internal differentiation, masses (shells) of layers are formed, differing in their chemical and physical properties; the outer firm cuticularised shell (exospore, extine) remains unchanged, and is thrown off as an envelope, while an inner mass of layers (endospore, intine) begins a new growth with the germination of the spores or the development of the pollen-tubes. A similar process occurs with many filamentous Algæ (Rivularieæ and Scytonemeæ), where a large number of cell-wall-layers are successively formed one within another, while from time to time the older masses of layers cease to increase, and are broken through by the growing filament, which now forms new cell-wall-layers (see Nägeli und Schwendener, Das Mikroskop, 2nd ed., p. 547). It need scarcely be mentioned that these facts do not contradict the theory of growth by intussusception, but only correspond to particular modifications in the life of the cell.

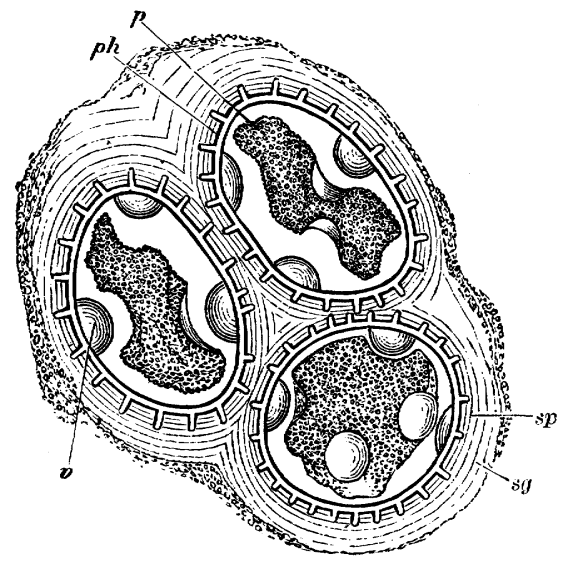

FIG. 34-- Pollen-mother-cell of Cucurbita Pepo; sg the externa common layers of the mother-cell undergoing absorption; $s p$ the so-called 'special mother-cells' consisting of masses of layers of the mother-cell-wall which surround the young pollen-grains; they also are afterwards absorbed; ph the wall of the pollen-grain; its spines grow out wards and penetrate the 'special mother-cell;' $v$ hemispherical deposits of cellulose on the cell-wall of the pollen-grain, from which the pollen-tubes are afterwards formed; $p$ the contracted protoplasm of the pollen-grain (the preparation was obtained by section of an anther which had lain for some months in absolute alcohol) ( $\left.\times 55^{\circ}\right)$.

(e) Differentiation of the Cell-wall into Systems of layers (Shells) with different chemical and physical properties.

Very young and thin cell-walls, while still in rapid growth, as also many older ones, are composed throughout their whole thickness of what has been termed pure cellulose; i.e. they are easily permeable by water, only slightly extensible or capable of swelling, very elastic, colourless, and soluble in sulphuric acid; with iodine and sulphuric acid they assume an intense blue colour, as also with Schultz's solution, rarely with solution of iodine alone (as the asci of Lichens). Together with these common properties, they may, according to the nature of the cell, possess many other peculiar reactions. Among older fully developed cells, succulent thin-walled parenchymatous cells of the higher plants behave for the most part in this manner, many thick-walled cells of Algæ, and-with the exception of the blue colour produced by iodine and sulphuric acid, and by Schultz's solution-most hyphæ of Fungi and Lichens.

With more strongly thickened cell-walls (rarely with moderately thin ones, as some cork-cells), whole masses of layers behave in a different manner chemically and physically; in consequence of this the cell-wall is at once seen to consist of two or more Shells ${ }^{1}$, each

1 It is desirable to employ the expression 'layers' (Schichten) only in the sense mentioned in paragraph (d), where it implies a regularly alternating difference in the proportion of water, 
of which may again exhibit numerous layers and the striation already described. In the case of exposed cells which require protection (as pollen-grains or spores), or of those which themselves serve as a protection to other tissues (as cork), an outermost shell (of greater or less thickness) of each cell-wall is transformed into cork or cuticle. When the cells are destined to form a firm frame-work (as in wood-cells), the outer masses of layers become lignified; in other cases, on the other hand, the outer layers, rarely the inner ones, are transformed into mucilage. Usually an inner layer of the cell-wall remains unchanged in all three cases, and gives the above-mentioned cellulose reactions, while the suberised and lignified shells of the cell-wall may, after previous treatment with alkalies or with nitric acid, also exhibit these reactions; the layers which are transformed into mucilage are the most refractory.

Some of the morphological relations here treated of find their explanation only when we study the formation of tissues; but I cannot here discuss the chemical behaviour of the cellwall ; the reactions must properly be regarded not as chemical tests, but only as the means of recognising a morphological differentiation. The description of some examples will be sufficient to guide the beginner.

The pollen of Thunbergia alata (Fig. 36) shows that the different development of two shells of a cell-wall may go so far that the cuticularised shell, the Extine, becomes actually separated from the non-cuticularised shell, the Intine, which still possesses the power of growth; by this means it becomes broken up in most cases by fissures previously formed into one or two spiral bands. This can be artificially induced by laying these pollen-grains in

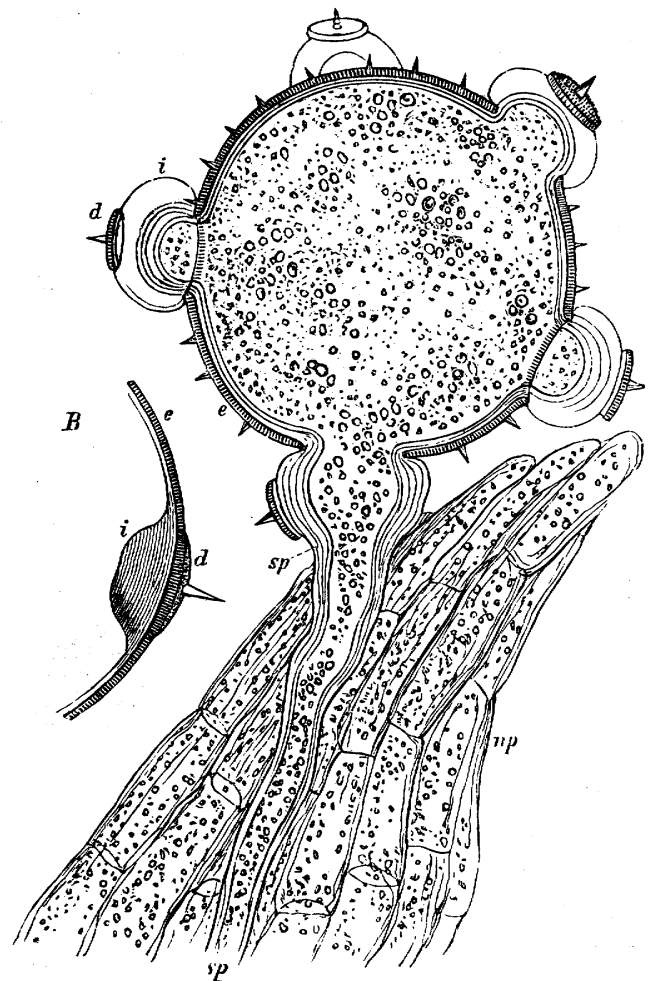

FIG. 35-A pollen-grain of Cucurbita Pepo, which has emitted a pollentube $(s p)$ into a papilla of the stigma. The cell-wall of the pollen-grain consists of a cuticularised extine $(e)$, and an intine capable of growth $(i) ;$ the latter is greatly thickened at certain places $(B i)$; on each thickening-mass the exţine forms a roundish lid $(d)$; when the pollen-grain is commencing to emit its pollen-tubes, the thick parts of the intine swell, and thus tilt up and lift off the pollen-tubes, the thick parts of the intine swell, and thus tilt up and lift off the
lid-like piece of the extine; one or two of these thickening-masses form pollentubes $(X 550)$.

concentrated sulphuric acid or potash solution; the extine then assumes a very beautiful red colour, while the intine in the first case dissolves, in the second case swells a little and remains colourless. In the germination also of many spores (e.g. Spirogyra, Mosses, \&c.) the cuticularised exospore becomes completely separated and stripped from the endospore, which still continues to develop; both shells, however-corresponding to the extine and intine of the pollen-grain-consist, in their actual development, of systems of layers of a single cell-wall possessing different chemicophysical properties.

as in the striæ; another term must be employed for the structures now under consideration, and the expression 'shells' (Schalen) appears to answer the purpose. 
In the epidermal cells, the cuticularisation either affects a shell of the outer wall, or it attacks the side-walls, as may be well seen, for instance, on the under-side of the leaf-veins of the holly. If a very thin transverse section (Fig. $37, A$ ) is treated with Schultz's solution, and submitted to a very high magnifying power (800), each cell-wall of the epidermis appears to be composed of two shells, of which the inner one, which is softer and more capable of swelling (c), becomes dark blue, while the outer shell does not. But this latter shows itself to be further composed of two chemically different layers, an inner $(b)$, which assumes a yellow colour and penetrates laterally between the cells $\left(b^{\prime}\right)$, and an outer one which remains colourless $(a)$, and extends continuously over the cells (the so-called true Cuticle). Between these two may be observed yet another boundary-zone, which, when the microscope is focussed to it, passes over the field of view like a shadow. The inner shell which assumes the blue colour, as well as the outer cuticularised substance, are each composed of a system of layers. In

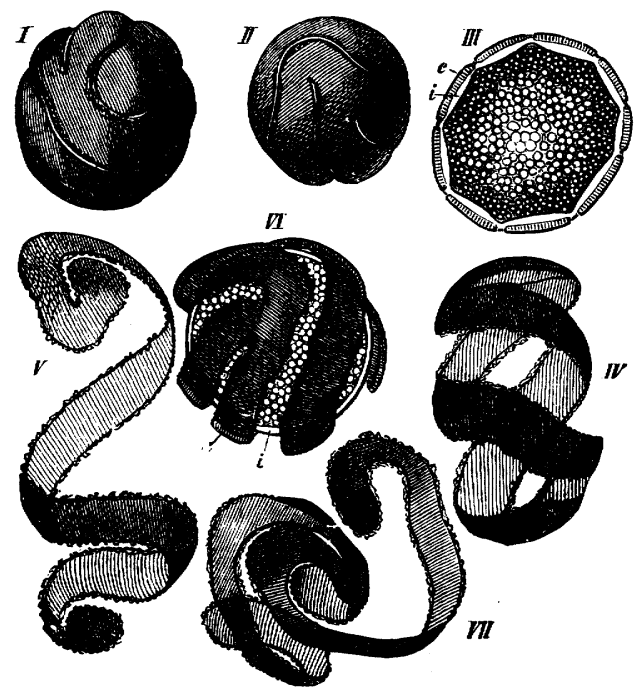

FIG. 36.-Pollen of Thunbergia alata $(\times 550)$. $I$ and $I I$ placed in concentrated sulphuric acid; $I V, V, V I I$ after solution of the intine; sometimes the fissures of the extine run so that isolated pieces of it fall off, corresponding to the lids of the extine of other pollen-grains, e.g. of Cucurbita; III in Schultz's solution, section; VI in strong solution of potash, $e$ extine, $i$ intine. The fissure s of the extine clearly arise from subsequent internal differentiation, in the same manner as the elaters are formed from the so-called 'special mother-cells' of the spores of Equise. tum. (See Book II., Equisetaceæ.) 28, p. 28); the two other shells of the cell-wall ( $b$ and $c$ ) remain unchanged by the maceration, except that they lose their colour; and hence the shell $c$ is shown to be composed of different layers, some more and some less watery (Fig. 28, $C, c$ ). The three shells also show a different behaviour on treatment with concentrated sulphuric acid; $a$ becomes a dark reddish brown and does not swell, or only slightly; $b$ swells in the radial direction and becomes thicker; while $c$ swells in the radial, tangential, and longitudinal directions (see Fig. $38, C, c$, and $D, c$ ); in transverse sections $c$ breaks away from $b$ and curves in a vermiform manner $(C)$; in longitudinal sections it is bent in a wavy manner $(D)$.

In true wood-cells, e.g. in Pinus sylvestris (Fig. 24, $A$, p. 25), three shells are likewise generally to be distinguished; one in the centre of the double cell-wall $(A, m)$, next a thicker one $(z)$, and then an innermost $(i)$; the two first turn yellow on treatment with solution of iodine or iodine and sulphuric acid, the innermost blue with the latter reagent $; \boldsymbol{z}$ and $i$ are dissolved by concentrated sulphuric acid, while the central lameila 
$m$ remains. Here also the possibility of isolating the cell depends on the circumstance that the central lamella $m$ may be dissolved by boiling in nitric acid with potassium chlorate; and thus the walls of the isolated cells consist only of the two inner shells. In many wood-cells (the 'Libriform Fibres' of Sanio) the inner thickening-layers form a shell of cartilaginous or gelatinous consistence, as in the wood of many Papilionaceæ.

When the outermost layers of the walls of cells which are combined into tissues become gelatinous or mucilaginous, their boundary-line disappears; and the cells, enclosed by the inner shell which is not mucilaginous, appear to be imbedded in a homogeneous jelly; this appearance gave rise to the theory of 'Intercellular Substance,' to which we shall recur. This behaviour occurs in the tissue of some Fucacex, and also in the endosperm of Ceratonia Siliqua (Fig. 39); $c c$ are the outer layers of the

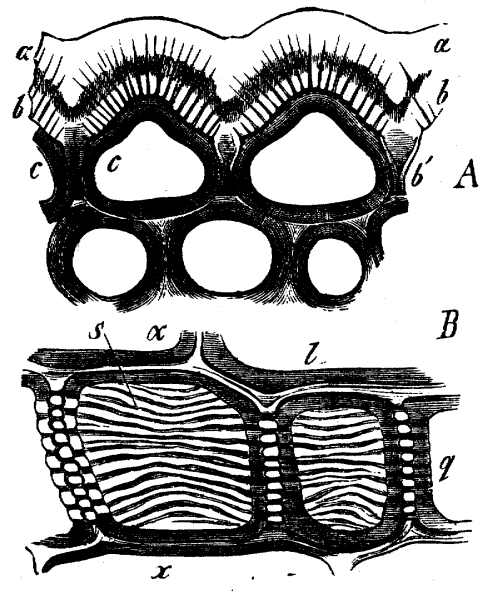

FIG. 37.-Epidermis of the central vein of the leaf of the holly; $A$ transverse section; $B$ superficial appearance (front view).

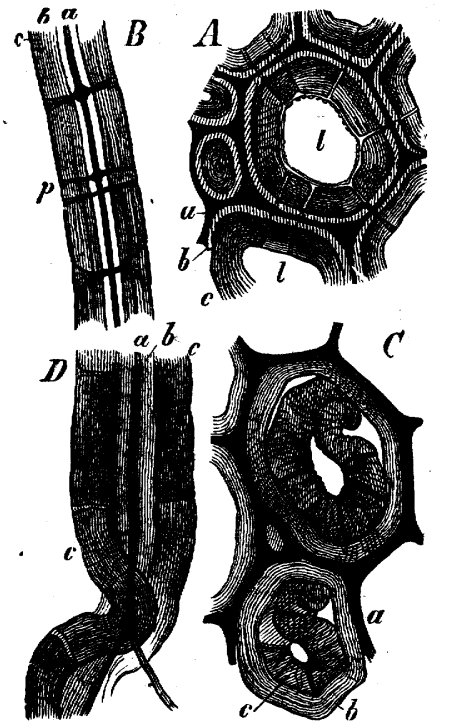

FIG. 38.-Structure of the sclerencinyma in the stem of Pteris aquilina ( $\mathrm{X} 550$ ). $A$ a fresh thin transverse section; $B$ the longitudinal wall between two cells, fresh (a curved pit-canal at the lower end); $C$ transverse section in concentrated sulphuric acid; $D$ longitudinal section of the wall in sulphuric acid; $a$ the central lamella of the wall ; $b$ second shell; $c$ third or innermost shell of the cell-wall ; $p$ pore-canals; $l$ cavity of the cell.

wall of the cells $a$, which have become entirely converted into mucilage and rendered indistinguishable, their innermost system of layers $(b)$ appearing as a strongly refractive shell. In the dry state the mucilaginous mass is almost horny; it swells up strongly in water with potash solution; with iodine and sulphuric acid it does not become coloured, but the sharply defined inner shell $b$ turns blue.

In isolated cells numerous cell-wall-layers may also form a mucilaginous shell which is most beautifully developed in the spores of Pilularia (Fig. $3 \dot{3}$, p. 32) and Marsilea. In the sporocarp of these plants are certain masses of parenchyma, the cell-walls of which become mucilaginous on the inner side; when dry the mucilaginous masses are firm and horny, but can absorb so much water that they increase in bulk several hundred-fold, and burst the wall of the sporocarp (Book II., Rhizocarpeæ). A similar transformation into mucilage of inner layers of cell-wall, while an outer, thin, and cuticularised shell resists, occurs with linseed and quince-seed. The inner thickening-masses of the epidermis of the seed, transformed into mucilage, absorb the surrounding water, swell up violently, and, bursting the cuticle which is incapable 
of swelling, appear, in the presence of a small quantity of water, as a hyaline layer enveloping the seed; with more copious addition of water they become more and more diluted into thin mucilage. A similar process occurs in some other seeds, as those of Teesdalia nudicaulis and Plantago Psyllium, in the seed-hairs of Ruellia, and the pericarp of Salvia. Gum-tragacanth consists of the cells of the pith and medullary rays of Astragalus creticus, $A$. Tragacantha, and other species, transformed into mucilage 1 . When the walls of these cells become mucilaginous, and swell up on copious addition of water, they force themselves through. cracks in the stem as viscid masses, and dry up on the outside into a horny substance capable of swelling in water. Vegetable mucilage can, however, arise in other ways ${ }^{2}$.

(f) Incombustible Deposits occur in every cell-wall. The presence of lime and silica can be directly proved, and it can scarcely be doubted that potash, soda, magnesia, iron, sulphuric acid, \&c., also occur in small quantities; the lime-salts and silica increase with age. The deposition may take place in two ways. Usually only extremely small particles of incombustible substance are deposited regularly between

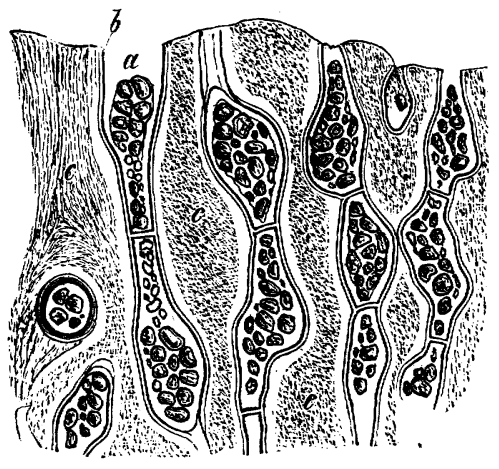

FIG. 39.-Section of the endosperm of Ceratonia Siliqua. the molecules of the organised substance of the cell-wall; and this may be recognised by the ash remaining behind after ignition in the form of the organised cell-wall (as a skeleton). But lime-salts may also be contained in the cell-wall in the form of numerous very small crystals; they then lie imbedded in the substance of the cellwall itself, sometimes in the form of growths which project into the cell-cavity (Cystoliths).

Skeletons composed of a substance ${ }^{3}$ soluble in weak acids (generally thought to be lime) are obtained by combustion of very thin layers of tissue on glass or platinum-foil; they occur so generally that it is unneces-

sary to adduce examples; from entire vascular cells I obtained, in the case of Cucurbita $P e p o$, beautiful lime-skeletons. Silica-skeletons are obtained most abundantly from epidermal cells and from Diatoms; but silicified cell-walls occur also in the interior of tissues, as in the leaves of Ficus Sycomorus, Fagus sylvatica, Quercus suber, Deutzia scabra, Phragmites communis, Ceratonia Siliqua, Magnolia grandiflora, \&c., according to Mohl ${ }^{4}$. The silicification does not generally affect the whole thickness of the cell-wall, but only an outer shell; as, for instance, in the case of epidermal cells, the cuticularised portion only. In order to obtain fine skeletons, it is necessary previously to soak the removed epidermis or thin sections of it in nitric or hydrochloric acid, and then to burn them on platinum-foil. I have found another method much more convenient. I place larger pieces of the tissue (e.g. of leaves of grass, stems of Equisetum, \&c.) on platinum-foil in a large drop of concentrated sulphuric acid, and heat over the flame;

' [H. von Mohl, Bot. Zeitg. 1857, p. 33; Pharmaceut. Journ. Jan. 1859.]

2 Compare further, Frank, Ueber die anatomische Bedeutung und die Entstehung der vegetabilischen Schleime, in Jahrb. für wissen. Bot. vol. V. I866.

8 The salts found in the ash are partly products of combustion. Carbonates may be produced by the combustion of salts of vegetable acids. Since a strong red heat is necessary for complete combustion, easily volatile chlorides (potassium or sodium chloride) may disappear from the ash.

${ }^{4} \mathrm{H}$. von Mohl, Ueber das Kieselskelet lebender Pflanzenzellen, in Bot. Zeitg. I86I, no. 30 et seq.-Rosanoff, Bot. Zeitg. I8 7 I, nos. 44, 45 . 
the mass immediately turns black, and a violent formation of gas follows; the heat must be continued until only the pure white ash remains. This is soon effected by this means, whereas otherwise the reduction to ash is generally very tedious, and often does not afford an entirely colourless skeleton ${ }^{1}$.

Sect. 5. Protoplasm and Nucleus ${ }^{2}$.- Now that the significance of protoplasm as the peculiar living essence of the cell has been sufficiently brought out, we need only add what is absolutely essential, both as respects its chemical and physical nature and its structure and movements. Protoplasm consists of a combination of (apparently different) albuminous substances (proteids) with water and small quantities of incombustible materials (ash). In most cases it also contains, as may be concluded on physiological grounds, considerable quantities of other organic compounds, belonging probably to the series of carbo-hydrates and oils. These admixtures are distributed through its mass in an invisible form; but it not unfrequently includes granules of starch and drops of oil, which at a subsequent period may either entirely disappear or may increase in bulk. Very commonly the rapidly increasing protoplasm, in itself colourless and hyaline, is rendered turbid by numerous small granules, consisting, probably, of minute drops of oil. The protoplasm, as it is generally met with, ought therefore to be considered as true protoplasm with varying admixtures of different formative materials (Metaplasm of Hanstein). The consistence of protoplasm varies greatly at different times and under different circumstances, even in the same protoplasm-mass. It commonly appears soft, plastic, tough, inelastic, and very extensible; in other cases it is more gelatinous, sometimes stiff, brittle (in the embryos of seeds before germination); but very commonly it gives the impression of being a fluid. All these properties depend essentially on the quantity of water it has absorbed. But, however great may be the quantity of water, and its consequent similarity to a fluid, the protoplasm is nevertheless never a fluid; even the mucilaginous or gelatinous conditions of other bodies can only be very superficially compared with it. For the living and life-giving protoplasm is endowed with internal forces, and, as the result of this, with an internal and external variability which is wanting in every other known structure; its active molecular forces cannot, in short, be compared with those of any other substance ${ }^{3}$. The capacity which protoplasm has, in consequence of the forces which become manifested in it, of assuming varying definite external forms, as well as its capacity of secreting substances of different chemical and physical properties according to definite laws, is the immediate cause of cell-formation and of every process of organic life.

1 On the crystals sometimes deposited in the cell-wall, see Sect. I I.

${ }^{2}$ H. von Mohl, Bot. Zeitg. I 844 , p. 273 , and I855, p. 689 ; [Ann. des Sci. Nat. I857, vol. VII. p. 253].-Unger, Anatomie und Physiologie der Pflanzen, p. 274, I85.-Nägeli, Pflanzenphysiol. Untersuchungen, Heft I. Zürich. - Brücke, Wiener akad. Berichte, p. 408 et seq., 186I.-Max Schultze, Ueber das Protoplasma der Rhizopoden und Pflanzenzellen, Leipzig I863.-De Bary, Die Mycetozoen, Leipzig 1864.-Hofmeister, Die Lehre von der Pflanzenzelle, Leipzig I867.Hanstein, Sitzungsberichte der niederrheinischen Gesellschaft in Bonn, Dec. I9, 1870.

3 For further details on this point, see Book III; also my Handbuch der ExperimentalPhysiologie der Pflanzen, § 116 , Leipzig $186_{5}$. 
The protoplasm of plants, when in a state of vital activity, is generally very watery; on one hand it exhibits an internal differentiation of its substance into layers and portions differing in their consistence and chemical nature; on the other hand it assumes definite outlines, and becomes bounded by surfaces of determinate, and mostly very variable, form.

The internal differentiation of protoplasm usually manifests itself by the formation of an external hyaline, firmer, but very thin layer, surrounding the inner mass, with which it remains in the most intimate contact. Every portion of a protoplasm-mass immediately surrounds itself, when it becomes isolated, with such a skin. In the interior a quantity of fluid sap, which permeates its substance throughout, becomes separated in the form of drops (Vacuoles); when the protoplasm is contained in a growing cell these vacuoles increase as the cell grows, and the protoplasm-mass becomes a sac filled with watery sap. One of the most common internal differentiations of the young protoplasmmass, while constituting itself a separate individual, is the formation of the Nucleus. The substance of the nucleus is at first indistinguishable from the rest of the protoplasm, and its formation is essentially the accumulation of certain particles of protoplasm round a centre, which is also usually the centre of the whole protoplasm-mass. Once formed, the nucleus-whose chemical nature, as far as observation goes, is altogether similar to that of the protoplasm -becomes more sharply defined; it may itself become enveloped in a skin, and vacuoles and granules (the Nucleoli) may become separated in it. But the nucleus always remains a part of the protoplasm-mass; it is always imbedded in it; very commonly it becomes again absorbed, after a short existence, in the protoplasm, e.g. in cells which divide frequently, see p. 14 ; in the elongated cells of the Characeæ the nucleus disappears altogether when the circulating motion of the protoplasm begins. Another very common differentiation of the substance of the protoplasm consists in portions of it becoming separated in a definite form, and assuming a green colour, forming the Chlorophyll-bodies, which, like the nucleus, not only arise out of the protoplasm, but always remain portions of the protoplasm-mass. But since these require more minute investigation, the next section will be devoted to them.

The external configuration of the protoplasm as a mass of definite form can be reduced to two cases:-either all its most minute particles group themselves concentrically round a common centre; or an internal motion takes place, which causes the protoplasm-mass to become elongated in some one direction, and disturbs the centripetal arrangement. The former occurs commonly in the formation of new cells, the latter during their growth.

The movements of the minute particles of protoplasm which determine its grouping and configuration during the formation and growth of cells, are generally so slow as not to be visible even when subjected to a very high magnifying power. Much quicker movements, even appearing rapid under a very high magnifying power, occur in cells either before their growth, as in swarm-cells, or when it is nearly completed.' Merely having regard to external appearance, the following kinds of movements of this nature may be distinguished:-

(A) Movements of naked protoplasm-masses. (I) Swimming of swarm-cells 
and antherozoids. This is characterised by the naked protoplasm-mass-swarm-cell or antherozoid-not changing its external form, while motile vibratile cilia, which are probably slender threads of protoplasm, cause rotation round the longer axis, and at the same time a progressive motion in the water. (2) Amoboid movement;consisting of rapid changes in the external shape of naked protoplasmic structures, Myxoamœbæ and plasmodia, which, while under water or in moist air on a firm support, creep about as if flowing, extending, and contracting; while within both the principal mass and the appendages which proceed from it a 'streaming' motion occurs.

(B) Movements of the protoplasm within the cell-wall. These commence after the protoplasm-mass of the cell has formed a larger sap-cavity, and continue commonly after the growth of the cell has ceased until the end of its life. (3) Those movements are distinguished as Circulation where threads and bands, proceeding from the parietal protoplasm, run to that portion which envelopes the nucleus, and often stretch completely across the sap-cavity. A distinction is drawn between movements of larger portions of protoplasm, and the 'streaming' movement of the substance of which they are composed; the former consist in the accumulation or diminution of the parietal layer, in movements in different directions of the mass which contains the nucleus, and, dependent on this, in different groupings of the threads. Within these structures themselves currents often occur, which are apparent from the movement of the enclosed granules, and are often in opposite directions within the same slender thread. In the cells of lower and higher plants which contain much protoplasm and sap but only a small quantity of granular contents, the circulation is a widely distributed phenomenon, especially visible in the hairs. (4) The term Rotation is applied to those cases where the whole mass of protoplasm enclosing a cell-cavity circulates as a thick current complete in itself, and carries along with it the grains and granules contained in it. This occurs in some water-plants, Characeæ, Vallisneria, root-hairs of Hydrocharis, \&c.

(a) The protoplasm exists in two conditions, which may be distinguished as the living and the dead; the former passes over into the latter by the most various chemical and mechanical processes; the reactions of living protoplasm towards chemical reagents are essentially different from those of dead protoplasm; but this of course can only be perceived when the reagents do not at the same moment cause death. Solutions of different colouring matters, as aqueous solutions of the colours of flowers and the juices of fruits, especially also weak acetic solution of carmine, have no power of colouring living protoplasm ${ }^{1}$; but if it has been previously killed, or if it has lost its vital properties by long-continued action of these reagents, it absorbs a relatively larger quantity of colouring material than of the solvent, and the whole substance assumes a much more intense colour than the reagent. Solutions of iodine in water, alcohol, potassium iodide, or glycerin, act in a similar manner; they all cause a yellow or brown colouring of the protoplasm, which is more intense than that of the solution itself. If protoplasm is first treated with nitric acid, the excess of acid removed by water, and potash solution added, it assumes a deep yellow colour; saturated with a solution of cupric sulphate and then treated with potash, it becomes

1 In consequence of this the protoplasm and nucleus are colourless even when the sap is coloured in living cells; in other cases, on the other hand, the protoplasm is tinged by a colouring matter soluble in water which is not present in the cell-sap, as in Florideæ and the flowers of Compositæ. 
of a beautiful dark violet. Protoplasm containing but little water treated with a large quantity of concentrated sulphuric acid assumes a beautiful rose-red colour, without at first changing its form; subsequently this colour and the form disappear together, the protoplasm dissolving. Dilute potash solution (sometimes also ammonia solution) dissolves protoplasm, or at least destroys its form, and makes it homogeneously transparent. If, on the other hand, cells with protoplasm of characteristic form are placed in concentrated potash solution, the form remains for weeks, but disappears immediately on addition of water. All these reactions are collectively characteristic of true albuminoids, as casein, fibrin, and albumen; and we are therefore justified in assuming that substances of this kind are always contained in protoplasm. If the protoplasm-sac in cells with much sap is very thin, it acquires a greater power of resistance to the solvents. In another respect also protoplasm behaves like albuminoids. By heating very watery protoplasm to above $50^{\circ} \mathrm{C}$, it is killed and becomes turbid and stiff, as if coagulated; alcohol and dilute mineral acids act in the same manner. The nucleus behaves towards all colouring substances, solvents, and coagulating agents, in the same manner as living watery protoplasm, or it shows itself even more sensitive, especially in young cells; in older cells however it is acted on less easily.

At the base of all protoplasmic structures there probably lies a substance which is colourless, homogeneous, and not visibly granular, to which alone the name Protoplasm ought perhaps to be applied, or which ought at all events to be distinguished as the basis of protoplasm. The fine granules which are so often mingled with it, and which some consider an essential ingredient, are probably finely divided assimilated food-materials, which undergo a further chemical metamorphosis into protoplasm; every intermediate form occurs from these more or less fine granules to the largest which may be clearly recognised as oil and starch. Homogeneous protoplasm destitute of granules is found in the cotyledons of dormant embryos of Helianthus, and in the first leaves of Phaseolus; out of it chlorophyll is subsequently formed, and it contains but very little water; but the extremely watery protoplasm which rotates in the cells of Vallisneria is also not itself granular; nothing but nucleus and chlorophyll-granules can be recognised in it. In the development of the spores of Equisetum (Fig. IO, p. 14) the finer granules separate repeatedly from the homogeneous protoplasm, and afterwards become again distributed through it. But in some cases the protoplasm is so loaded with granular and coloured materials, that the colourless hyaline basis can no longer be distinguished; as, for instance, in the oospheres of Fucus (Fig. 2, p. 3), the zygospores of Spirogyra (Fig. 6, p. Io), and in many spores and pollen-grains ${ }^{1}$. In the reservoirs for reserve-materials contained in dry seeds (e.g. the cotyledons of peas and beans), the protoplasm itself is often contracted into small roundish grains, among which lie the starch-grains.

(b) Skin, Vacuoles, Movement ${ }^{2}$. Naked protoplasm-bodies, as the plasmodia of the

\footnotetext{
${ }^{13}$ Hanstein gives to the substances mingled with the true protoplasm, and which undergo many transformations, the collective name of Metaplasm. (Bot. Zeitg. 1868, p. 7Io.)

${ }^{2}$ [The recent observations of Strasburger differ in some respects from what is stated in the text. Strasburger recognises a differentiation of protoplasm into two layers, which may be termed Ectoplasm and Endoplasm respectively. In vegetable cells the former is hyaline, while the chlorophyll-grains are imbedded in the latter. The ectoplasm does not however consist of the mere hyaline basis of the protoplasm, nor is it identical with the skin which is formed on a free surface when exposed, but is the result of a true process of differentiation. In numerous cases, though not universally, Strasburger has found that the ectoplasm presents the radial striation referred to in the text, and sometimes also a striation parallel with the surface. In the swarm-cells of Viaucheria treated with a one per cent. solution of osmic acid, he found that this striation depended on the presence in the ectoplasm of small rods of denser, imbedded in more watery protoplasm. Externally and internally these rods are in contact with a delicate continuous layer of protoplasm, and the cilia, which are more slender than the rods and about twice as long, arise from them. In the earliest stage the cilia are small processess of the ectoplasm corresponding in position to the rods
} 
Myxomycetes, some swarm-cells, e.g. of Vaucheria, allow the skin to be recognised, under sufficient magnifying power, as a hyaline edging; in the swarm-cells of Vaucheria it is evidently striated radially when seen in section, just as some cell-walls are; Hofmeister (Handbuch, vol. I. p. 25) found the same appearance in the plasmodia of Atthalium. Probably this skin is nothing but the pure basis of the protoplasm itself free from granules, of which the whole body is formed, but which is masked in the interior by grains and granules. It follows that in the amœboid movements of plasmodia the new extensions are always at first formed of the skin alone; it is only
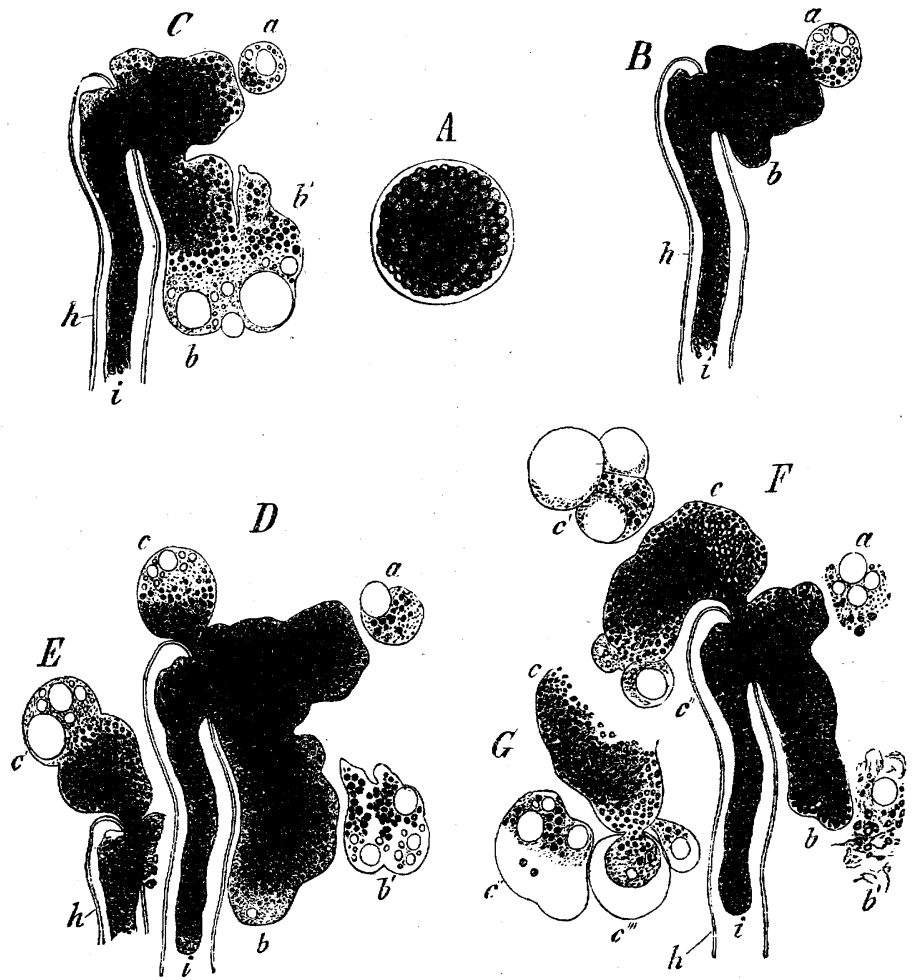

FIG. 40.-B-G protoplasm from an injured filament of Vaucheria terrestris, slowly emerging in water, in different successive conditions, at intervals of about five minutes; $h$ the wall of the ruptured filament; $i$ the protoplasm which still remains in the filament; $a$ in $B, C, D$, and $F$, a ball of protoplasm detaching itself, forming vacuoles, then dissolving (in $F$ ); $b$ a branchlet of the protoplasm from which the mass $b^{\prime}$ is detached, this mass isolated in $D$, dissolved in $F ; c$ and $c^{\prime}$ behave in a similar manner; $G$ shows the further changes of the part $c^{\prime \prime}$ in $F$. $A$ a freshly escaped mass of protoplasm, rounded off into a sphere, the chlorophyllgranules lying all together in the inside; a hyaline layer of protoplasm envelopes the whole as a skin.

when they increase in size that the interior granular substance makes its appearance in them. This is more clearly the case in the masses of protoplasm that escape into water from the injured filaments of Vaucheria, which often instantly become rounded into globular bodies, but not unfrequently show the amœboid movement of plasmodia

at a later stage; they are somewhat longer, the full extremity of each being terminated by a knob; as development proceeds the cilia become longer and the knobs become smaller in proportion to the increase in length, until the final hair-like form is reached. As long as the swarm-cell is in contact with the mother-cell-walls, the cilia are closely adpressed to the surface of the ectoplasm, with their apices directed forwards. The secretion of a cellulose envelope is closely and even inseparably connected with the presence of an ectoplasmic layer. See Strasburger, Studien uiber Protoplasma, Jena I876; Vines in Quart. Journ. Micr. Sci. 1877, pp. I 24-132.] 
for as much as half-an-hour or an hour (Fig. 40). This interpretation of the skin is not at all opposed to the fact that it is denser than the inner and more watery substance. That the cohesion in each protoplasm-mass decreases from without inwards is shown by the greater mobility of the inner portion, especially with plasmodia, and also by the formation of vacuoles, which clearly depends on the collection of part of the water present in the protoplasm round internal points in drops, presupposing that the cohesion is overcome at these points. The view that the hyaline homogeneous basis itself forms on each exposed surface of the protoplasm the skin destitute of granules, entirely agrees with the supposition that not only every vacuole in a protoplasm-mass, but also every thread of protoplasm which traverses the sap-cavity, and finally the sap-cavity itself, is also bounded by a skin, even if it be so thin that it cannot be seen when strongly magnified ${ }^{1}$.

If the protoplasm is not enclosed in a cell-wall, the vacuoles are usually small and not numerous. If, on the other hand, a cell-wall is formed, and if the cell grows

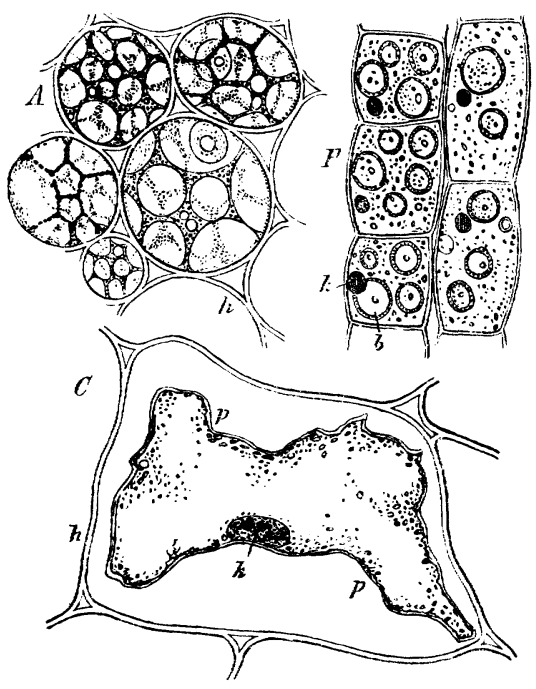

FIG. 41.-- Forms of the protoplasm contained in cells. $A$ and $B$ of maize; $A$ cells from the first leaf-sheath of a germinating plant; $B$ from its first internode; $C$ from the tuber of the Jerusalem artichoke, after action of iodine and dilute sulphuric acid; $h$ cell-wall; $k$ nucleus; $p$ protoplasm (primordial utricle). rapidly, this is always accompanied by an increase in number and size of the vacuoles (Fig. I, p. 2). This not unfrequently leads to a frothy condition of the protoplasm where the vacuoles are separated only by thin lamellæ of that substance (Fig. 4I, $A$ ); but in other cases the inner protoplasm-mass of a cell breaks up into smaller portions, each of which encloses a large vacuole surrounded by a thin skin of protoplasm (Fig. $4 \mathrm{I}, B, b$ ). These are the 'Sap-vesicles' which are so common, and which sometimes enclose granules of chlorophyll and other substances, and thus resemble cells; they are not uncommon in the flesh of berry-like fruits, and in tissues with mucilaginous juices. If the rapidly growing cell does not form new protoplasm as the size of the cell and the amount of sap increase, the quantity of protoplasm decreases; and not unfrequently it forms a thin sac not immediately visible, lying between the cell-wall which it lines and the cell-sap which it invests, and becoming visible only by means of reagents that remove the water and separate the protoplasm-sac-the Primordial Utricle of $\mathrm{Mohl}^{2}$-from the cell wall by contraction (Fig. $4 \mathrm{r}, C, p$ ). The significance of this thin protoplasm-sac, and its production owing to the increase in number and size of the vacuoles in an originally continuous protoplasm-mass, will no longer be doubtful to the reader after all that has been said in Sects. 1, 2, and 3, and by comparison of Fig. I with Fig: 4I.

In young cells, where the protoplasm still forms a thick layer or a net-work permeated by vacuoles, its substance-with the exception perhaps of the outermost layer lying on the cell-wall-appears to be always engaged in a 'streaming' movement, which is however usually very slow. In many mature and large cells, which do not serve for the storing up of assimilated materials, and where the protoplasm-mass is sufficiently nourished, and does not, as the cell increases in size, contract to a mere thin skin,

1 See Hanstein, Die Bewegungserscheinung des Zellkerns, u. s. w. in Sitzungsberichte der niederrheinischen Gesellschaft zu Bonn, Dec. I9, I870, p. 224.

${ }^{2}$ [H. von Mohl, Bot. Zeitg. I 844 , p. $273 \cdot$ ] 
this condition is permanent. If the whole protoplasm-mass withdraws to the cellwall, enclosing a single large vacuole (the Sap-cavity of the cell), all the particles of protoplasm, flowing in one direction, may form a continuous broad current encircling the cell (rotation), the direction of which is always such as to describe the longest course round the cell-cavity (Nägeli). Examples occur in Characeæ, and in many. other submerged water-plants, as Vallisneria, Ceratophyllum, Hydrilla, and root-hairs of Hydrocharis; the globular nucleus, when present (in Characeæ it soon disappears), is carried along with the current. The protoplasm-mass which encloses a large sap-cavity may, however, possess a net-work of ridge-like prominences, the substance of which flows in different directions; the nucleus may then either remain at rest and form the centre of movement, or be carried along with the current. Cases of this kind occur tolerably frequently in the hairs of land-plants, as in the stinging hairs of the

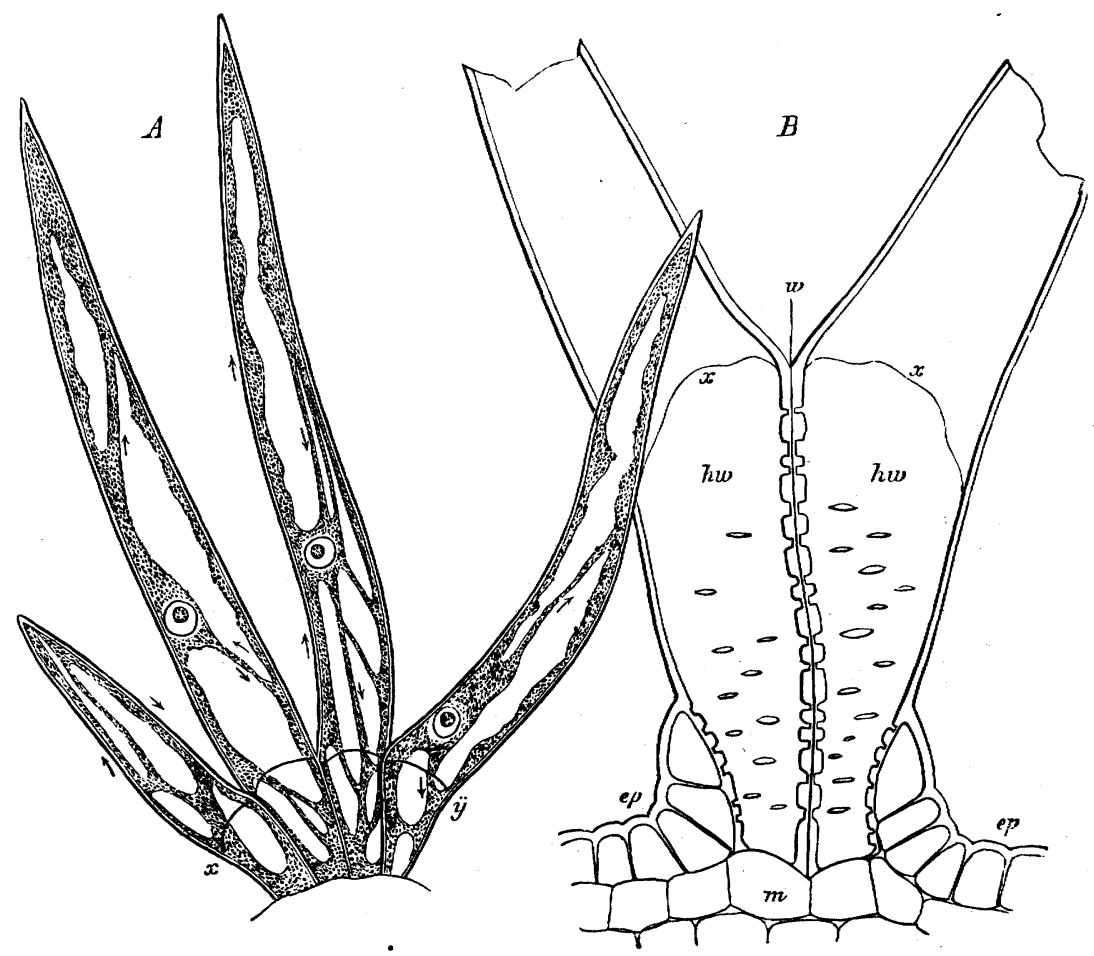

FIG. 42.- $A$ stellate hair on the calyx of the young flower-bud of the hollyhock; thicker ridges of protoplasm project into the sap-cavity of each cell; these are in 'streaming' motion (indicated by the arrows). $B$ epidermis (ep) with the basal portion of a mature stellate hair, showing the structure of the wall $\left(x_{550}\right)$.

stinging-nettle, and the stellate hairs of the hollyhock. But threads of protoplasm which exhibit these currents may also penetrate the sap-cavity of the cell; not unfrequently (e.g. Spirogyra, hairs of Cucurbita) the nucleus then lies in the centre, enveloped by a mass of protoplasm, the threads uniting it with the layer which clothes the cell-wall. These threads, stretching across the sap-cavity, arise from the thin lamellæ of protoplasm which in young quickly-growing cells separate adjoining vacuoles; when these finally flow together into a single sap-cavity, the thicker parts of the lamellæ (Fig. I, $B$, p. 2) remain as threads, forming a more or less irregular net-work, which at first corresponds to the coalescent vacuoles, but which undergoes further changes of form as the cell continues to grow, and in consequence of the internal movements of the whole protoplasm. New threads also make their appearance; ridgelike portions arise from the peripheral protoplasm, or even on the thicker threads, and 
finally become detached, leaving the two ends of the new thread united with the rest of the protoplasm; they do not grow up as branches with one free extremity. (Hanstein, l.c. p. $22 \mathrm{r}$.) Some of the threads also disappear, the two ends remaining in connexion with the rest of the protoplasm and coalescing with it. The threads form, with the central mass of protoplasm which contains the nucleus and the layer which clothes the cell-wall, a connected system, portions of which may change their position with respect to one another.

Besides these displacements-in consequence of which the parietal protoplasm accumulates or diminishes at any one spot, and the mass of protoplasm in the cell-cavity which contains the nucleus wanders about, and alters accordingly the grouping and form of the threads-under high magnifying power another form of movement comes into view, which is undoubtedly connected with the former, although the exact mode is unknown. In the parietal protoplasm and in the mass which contains the nucleus, but most distinctly in the threads, the minute granules-generally of chlorophyllare to be seen in a 'streaming' motion, which, under high magnifying power, may even appear very rapid. It must not, however, be overlooked that when the cell is magnified, say five hundred times, the rapidity of the motion is also apparently increased five hundred-fold. ' Within even a very slender thread, the granules near one another not unfrequently flow in opposite directions. Chlorophyll-granules often appear to be in motion on the surface of slender threads; it may nevertheless be assumed with certainty that they also are enclosed in the substance of the thread; but, being very prominent, are covered by only a very thin lamella of it.

Those movements of protoplasm which produce changes in the internal grouping of the protoplasm of the cell may be compared to the displacements of the mass which, in the case of naked Amœbæ, change the external contour, and cause its creeping motion. In the case of circulating protoplasm, the firm cell-wall hinders the change of contour, as well as the change of place of the whole mass; but the large sap-cavity allows of internal changes of position of larger or smaller portions. The 'streaming' movement, which is visible by means of the imbedded granules, occurs in the creeping naked protoplasm of the Amœbæ as well as in that enclosed in a cell-wall.

(c) The Nucleus. That the nucleus, which is never absent from Muscineæ and Vascular plants, but more often from Thallophytes, is a product of differentiation of the protoplasm, is sufficiently evident, not only from its chemical behaviour (vide supra, under $a$ ), but also from its participation in the processes of cell-formation (see Sect. 3). On the other hand, it must be borne in mind that, once formed, it constitutes a characteristically organised portion of the cell, which, to a certain extent, has a mode of development of its own. At first the nucleus is always a homogeneous roundish protoplasm-mass; subsequently its surface becomes firmer without taking the form of a special skin; in the interior there appear usually two or three (sometimes more) large granules, called Nucleoli, which, however, are often wanting. The nucleus has, at the time of its origin, generally already attained its permanent size, or nearly so ; its growth is never proportional to that of the cell; while in young tissue-cells (Fig. I, p. 2) it usually occupies a large portion of the cell-cavity, in mature cells its mass is progressively smaller in proportion to that of the whole cell. Its further development consists in its obtaining a firmer outer layer, and in the formation of small vacuoles and nucleoli; only rarely does it grow for a longer time; its substance may become frothy from the further formation of vacuoles, and sometimes it exhibits a circulation in the interior of the firmer enveloping layer, as in a cell ${ }^{1}$. The nucleus always remains enclosed in the substance of the protoplasm; if this latter forms vacuoles, or developes the circulation already described, the nucleus remains enveloped in a coating

1 In young hairs of Hyoscyamus niger, according to A. Weiss in the Sitzungsberichte der kais. Akademie der Wissenschaften zu Wien, vol. LIV, July $\mathbf{1} 866$. 
or mass of protoplasm which is connected with the parietal protoplasm-sac by the lamellæ lying between the vacuoles or by the threads. The nucleus apparently follows passively the movements of the protoplasm in which it is enveloped; it also undergoes changes of form under the pressure and traction of the moving mass beneath the eye of the observer. 'During the movement,' says Hanstein (l. c. p. 226), 'the bands of protoplasm are very tightly stretched, so that the envelope of the nucleus is drawn out into sharp angles. It looks as if the nucleus (with its envelope) were towed about like a ferry-boat by ropes. But since during this towing the bands alter their direction and form, it is evident that the envelope of the nucleus must also change its form. But not only the envelope, but also the nucleus itself, does this; this latter is never spherical or of any regular form during its movement, but is irregularly elongated, and usually in the direction of its motion at the time.' This change in the form of the nucleus may also be recognised from the displacement of the nucleoli within its mass.

Secr. 6. The Chlorophyll-bodies and similar Protoplasmic Structures'. -Chlorophyll, the green colouring matter so generally distributed through the vegetable kingdom, is always united to definite portions of the protoplasm-mass of the cells in which it is found; these green-coloured portions of protoplasm may, in contradistinction to the colouring matter itself by which they are tinged, be designated Chlorophyll-bodies. Every chlorophyll-body consists then of at least two substances, the colouring matter and its protoplasmic vehicle; if the former is removed by alcohol, ether, chloroform, benzin, or essential or fatty oils, the latter remains behind colourless. The colouring matter contained in each chlorophyllbody is itself only extremely small in quantity; after its removal the protoplasmic basis retains not only its form but also its previous volume. The latter is always a continuous soft substance containing extremely small vacuoles, in which the colouring matter is generally distributed universally, though not always uniformly.

Chlorophyll-bodies arise in the young cells by the separation of the protoplasm into portions which remain colourless and others which become green and sharply defined. This may be due to very small particles of a somewhat different nature either originally existing or being produced in the previously homogeneous protoplasm, and which collect to form distinct masses. The chlorophyll-bodies which arise in this manner always remain imbedded in the colourless protoplasm in a similar manner to the nucleus; they are never in immediate contact with the cell-sap. Their chemical and physical properties distinctly show that their colourless basis is a substance altogether similar to protoplasm. The chlorophyllbodies consequently always behave as integral parts of the protoplasm; and this is especially evident in the division of cells, conjugation, the formation of swarmcells, \&c. But the chlorophyll-bodies, when once formed, grow; and if they possess roundish forms they may increase by division. Both processes appear always to depend on the growth of the protoplasm-mass in which they are imbedded.

1 H. von Mohl, Bot. Zeitg. nos. 6 and 7, I855; [Ann. des Sci. Nat. vol. VI, I856, p. I 39.] -A. Gris, Ann. des Sci. Nat. 4th Ser. vol. VII. 1857, p. 179.-Sachs, Flora, 1862, p. I29; 1863, p. 193. Sachs, Handbuch der Exper. Physiol. der Pflanzen, § 87, Leipzig 1865.-Hofmeister, Die Lehre von der Pflanzenzelle, § 4I, Leipzig I867.-Kraus, Jahrb. für wissensch. Bot. VIII. 1871, p. 131. [Ditto, Zur Kenntniss der Chlorophyllfarbstoffe u. ihrer Verwandten. Stuttgart i872. -For Sorby's researches on chlorophyll see Book II. chap. 3. sect. 8.] 
It is only in the Algæ that the forms of the chlorophyll-bodies show much variety. In them it is frequently the case that the whole protoplasm-mass, with the exception of an outermost layer or a little more than this, either appears homogeneously green (as many swarm-cells, Palmellaceæ, gonidia of Lichens); or the chlorophyll-bodies assume stellate forms (e.g. Zygnema cruciatum, Fig. 43),

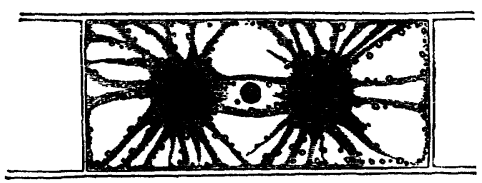

FIG. 43.-A cell of Zygnema cruciatum, with two stellate chlorophyll-bodies which are suspended in the interior of the cell; they are united by a colourless bridge of protoplasm in which lies a nucleus; the rays which form the union with the primordial utricle are nearly colourless in the middle. In each of the two chlorophyll-bodies lies a large starch-grain $(\times 550)$. or they form several lamellæ with a stellate transverse section when the cell is cut across (as in Closterium, \&c.), or straight or spiral bands (e.g. Spirogyra). But in most Algæ, and in all Muscineæ and Vascular plants, the chlorophyll-bodies are rounded or polygonal masses collected around a centre, and are termed Chlorophyll-granules. Generally a large number are contained in one cell; sometimes, however, only a few relatively large ones (e.g. Selaginella), and in one of the Hepaticæ of simplest structure (Anthoceros) only a single chlorophyll-granule exists in each cell, enclosing the nucleus; this therefore, when the cells divide, itself also divides in a corresponding manner.

With extremely few exceptions Starch-grains arise in the homogeneous substance of the chlorophyll-bodies, and, where these have special forms, are distributed in definite places (see, e.g., Fig. 5, p. ro); they are produced, in larger or smaller numbers, in the interior of ordinary chlorophyll-granules. They are at first visible as points, gradually increase in size, and finally may so completely fill up the space of the chlorophyll-granule that its green substance is represented only by a fine coating on the mature starch-grain; even this disappears under certain circumstances (as in old yellow leaves of Pisum sativum or Nicotiana), and the starch-contents then lie in the cell, which now contains no protoplasm, in the place of the chlorophyll-granules. Sometimes drops of oil also form in the interior of the chlorophyll-body ( $e . g$. in the bands of Spirogyra); and here and there granular contents of an unknown nature are observed. All these structures which arise in the chlorophyll-bodies are, however, not constant portions of them; their appearance and disappearance depend entirely on light, temperature, and other circumstances; the appearance of the chlorophyll-bodies themselves is also bound up with these conditions of life, to a description of which we shall recur in Book III, where it will be shown that chlorophyll is one of the most important elementary structures, and that its contents are the products of its assimilation. The consideration of these and other purely physiological properties of chlorophyll must be deferred till then. Sooner or later, in the normal course of things, the chlorophyll-bodies are again absorbed; this occurs in the most conspicuous manner at the time when the leaves of the higher plants are preparing for their fall; for instance, in the case of most of our native trees and shrubs, in the autumn. The whole protoplasm-mass - and with it the chlorophyll-granules from the cells of the leaves about to fall-is then absorbed and transferred to the perennial persistent structures. The phenomena which accompany this process vary greatly; but finally there remain in the cells filled with water and often with acicular crystals, a number of yellow glittering granules which bear no resemblance to chlorophyll; if the falling 
leaves are red, this depends on a substance dissolved in the sap; but in this case also the yellow granules are to be found.

The presence of chlorophyll in tissues is not always to be recognised by the naked eye. Sometimes the cells that possess chlorophyll contain a red sap; in other cases the green tissue of the leaves is covered by an epidermis with red sap, as in young plants of Atriplex hortensis; in this case, if the coloured epidermis be removed, the green tissue may be recognised. But in Algæ and Lichens we find that the chlorophyll-body of the cell itself contains, in addition to the green colouring matter, a red, blue, or yellow substance soluble in water; the fresh

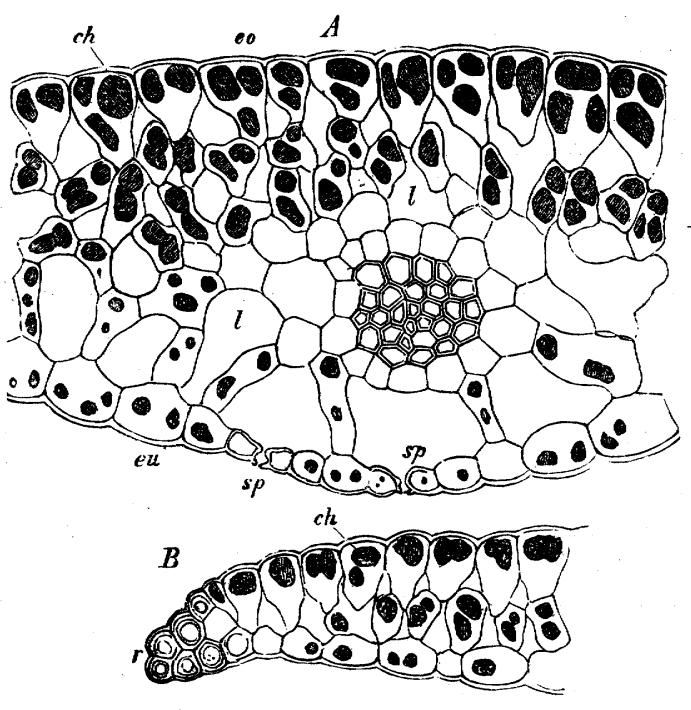

FIG. 44-Transverse section through a leaf of Selaginella inaqualifolia $\mathrm{X} 550$ ). $A$ in the middle, $B$ at the margin; $c h$ the chlorophyll-granules; visible in them are the small starch-grains; $e x$ the lower epidermis; eo the upper epıdermis; $l$ an air-conducting intercellular space; sp stomata.

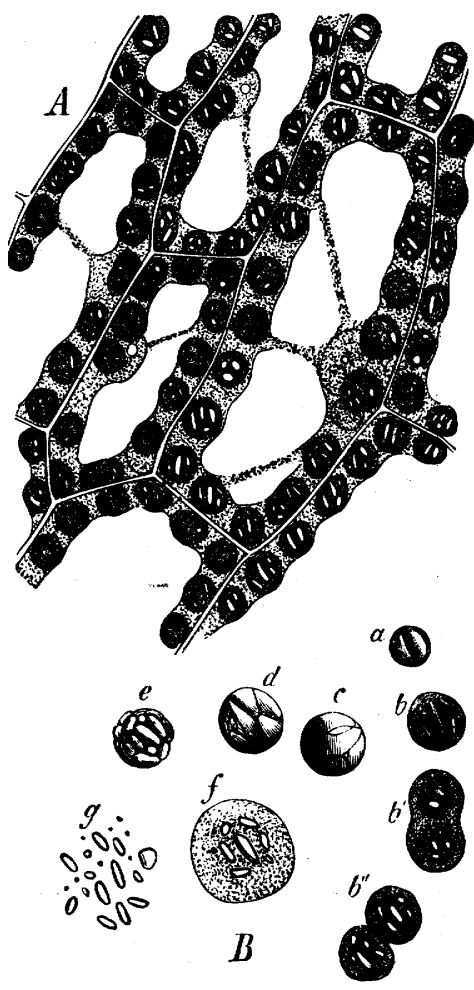

FIG. 45-Chlorophyll-granules of Funaria hygrometrica ( $\times 550)$. A cells of a mature leaf, seen from the surface; the chlorophyllgranules lie in a parietal layer of protoplasm, in which the nucleus is also imbedded; they contain starch-grains (left white). $B$ single chlorophyll-granule containing starch; $a$ a young one, $b$ an older one, $b^{\prime}$ and $b^{\prime \prime}$ granules in the act of division; $c, d, e$ old chlorophyllgranules, the starch-grains of which take up the space of the chlorophyll; $f$ a young chlorophyll-granule swollen up in water; $g$ the same after longer action of the water; the chlorophyll is destroyed, the starch-grains which it contained remaining behind.

chlorophyll-body appears then, by the admixture of the chlorophyll contained in it with these substances, verdigris-green (Oscillatoria, Peltigera canina, \&c.), a fine red (Florideæ), brown (Fucus, Laminaria saccharina), or buff (Diatomaceæ). (See Book II., Algæ.)

From this are to be distinguished those cases in which the originally green chlorophyll-granules assume a red or yellow colour from the alteration of their colouring material, a phenomenon which, from its physiological bearings, I have termed Degradation of chlorophyll. Thus the green bodies in the walls of the 
antheridia of Mosses and Characeæ become, at the time of fertilisation, of a beautiful red; in ripening fruits (Lycium barbarum, Solanum pseudo-capsicum, \&c.), the change of colour from green to yellow and red depends also on a similar loss of colour of the chlorophyll-granules, accompanied by a breaking up into angular forms with two or three points (Kraus, l.c.). Nearly related to the chlorophyllgranules are the vehicles of the yellow colouring materials to which many petals owe their colour (e.g. Cucurbita). The occasional blue (Tillandsia amona) or brown and violet bodies (Orchis Morio) are much further removed from this type, although they also have a basis similar to protoplasm, which is tinged by a colouring material, in these cases soluble in water.

(a) The Substance of the Chlorophyll-bodies is, irrespectively of the contents referred to, destitute of those fine granules which are so generally distributed through colourless motile protoplasm. In spite of their sharply defined form they are very soft and greasy when crushed; when they come into contact with pure water, vacuoles are formed, which at last burst through the green substance as hyaline bladders. Young chlorophyll-granules may thus become converted into delicate bladders, in which the starch-grains remain; old grains have much greater consistence. After extraction of the green colouring matter out of true chlorophyll-bodies, e. $g$. the bands of Spirogyra or granules of Allium Cepa, the remaining colourless basis possesses greater power of resistance, is coagulated, and shows all the reactions of protoplasm already mentioned.

(b) The Origin of the Cbloropbyll-bodies has at present only been directly observed in the granular forms; it can to some extent be compared with the process of free cell-formation. Round centres of formation within the protoplasm small portions of it collect in defined masses; if the centres of formation are at a considerable distance from one another, the chlorophyll-granules become round (as in the hairs of Cucurbita); but if they are large and lie close to one another, they are at first polygonal, as if flattened by pressure. The process then resembles the formation of numerous small swarm-cells in a single cell of Acblya (Fig. 9, $A$, p. 13); only that in this latter case colourless protoplasm always continues to lie between the green portion, as in the parietal chlorophyll of the leaves of Phanerogams. If a mass of protoplasm collects round the central nucleus during the formation of chlorophyll, the granules are often formed in its neighbourhood; they may then revolve with the protoplasm in the cell, or afterwards assume definite positions. In the filamentous Algæ with apical growth (e.g. Vaucheria, Bryopsis), they are produced in the colourless protoplasm-mass of the growing end of the filament, and then remain closely applied to the wall. In ripe spores of Osmunda regalis the chlorophyll surrounds the nucleus in the form of amorphous cloudy masses, which, however, separate on germination as ovoid granules, at first weakly defined, afterwards more sharply (Kny). In those cells of young leaves of Phanerogams which contain chlorophyll (cotyledons of the sunflower, primordial leaves of Phaseolus, buds of the tubers of the Jerusalem artichoke, \&c.) a definite layer of hyaline protoplasm devoid of granules is to be observed, close to the cell-wall, in which the chlorophyll-granules are subsequently formed; here the appearance is sometimes presented as if the mass were cut up into polyhedral pieces. The formation of the chlorophyll-granules is not always contemporaneous with that of its colouring matter; they may be at first colourless (as in Vaucheria or Bryopsis, according to Hofmeister) or yellow (in the case of leaves of Monocotylidons or Dicotyledons imperfectly exposed to light or in process of development), and become green at a subsequent period; in the cotyledons of Coniferæ the green colour appears contemporaneously with their origin even in the dark when the temperature is sufficiently high, as also in Ferns. The chlorophyll-granules, after assuming their green colour, grow by intussusception to many times their original size; if they are 
parietal, their growth in length and breadth is generally proportional to that of the cell-wall and protoplasm in which they lie. But if the growth of the cell is very considerable, the growing parietal chlorophyll-granules divide; this takes place by bipartition, in a direction at right angles to the longest diameter, into two secondary granules usually equal in size. If it contained small starch-grains before the division, these arrange themselves round the centres of the newly formed granules. These processes are inferred from the increase of the number of granules on the one hand, and from the frequent occurrence of constricted hour-glass-shaped forms on the other. After this bipartition had been discovered by Nägeli in Nitella, Bryopsis, Valonia, and in the prothallia of Ferns, it was subsequently noticed in all the families of Cryptogams which form chlorophyll; among Phanerogams also it appears widely distributed; it was discovered by Sanio in Peperomia and Ficaria, subsequently by Kny in Ceratophyllum, Myriophyllum, Anacharis, Utricularia, Sambucus, Impatiens, \&c. In cells of the prothallium of Osmunda exposed to feeble light and containing but little chlorophyll, Kny states that moniliform rows of chlorophyll-granules are produced by repeated bipartition, which, like the chains of cells of Nostoc, continue to elongate by intercalary divisions; branching takes place also, in a manner similar to that which occurs in Nostoc, some of the chlorophyll-granules increasing in size transversely, and producing branch-rows by division.

(c) With reference to the Internal Structure of the Cblorophyll-bodies, scarcely anything more can be said than that their outer layer often appears denser, and that the proportion of water increases towards the interior, the cohesion decreasing, as is apparent from the formation of vacuoles. A differentiation into intersecting layers of different density has only been once observed, by Rosanoff, in old chlorophyll-granules of Bryopsis plumosa.

Sect. 7. Crystalloids ${ }^{1}$.-A portion of the protoplasmic substance of a cell sometimes assumes crystal-like forms; bodies are produced which, bounded by plane surfaces and sharp edges and angles, possess an illusory resemblance to true crystals, even in their behaviour to polarised light; but they are essentially distinguished from crystals by the action of external influences, and at the same time present significant resemblances to organised parts of cells. It is therefore legitimate to distinguish them by the term Crystalloids ${ }^{2}$ proposed by Nägeli. They are usually colourless, but sometimes act as yehicles of colouring matters (not green), which may be removed from them. Their substance exhibits all the more essential reactions of protoplasm, its power of coagulation and of taking up colouring matters, the yellow reaction with potash after treatment by nitric acid, as well as that with iodine. The solubility of different crystalloids is very different, as is generally the case with proteids. They are capable of imbibing water, and swell up enormously under the influence of certain solutions; their outer layer possesses greater power of resistance than the inner more watery mass. Those crystalloids which have been

${ }^{1}$ Hartig, Bot. Zeitg. I856, p. 262.-Radlkofer, Ueber die Krystalle proteïnartiger Körper pflanzlichen und thierischen Ursprungs, Leipzig 1859.-Maschke, Bot. Zeitg. 1859, p. 409.-Cohn, Ueber Proteinkrystalle in den Kartoffein, in the thirty-seventh Jahresbericht der schlesischen Gesellschaft für vaterländ. Cultur, 1858 , Breslau.-Nägeli, Sitzungsberichte der k. bayer. Akademie der Wissenschaften, 1862 , p. 233.-Cramer, Das Rhodospermin, in the seventh volume of the Vierteljahrsschrift der naturforsch. Gesellschaft in Zürich.-J. Klein, Flora, 187I, No. I1.-Kraus, in Jahrb. für wissensch. Bot. vol. VIII. p. $4^{26 .}$

${ }^{2}$ [The term 'crystalloid' is, in another portion of this work, used in a different sense, to express any substance capable of crystallisation; see Book III. Chap. i. Sect. I.] 
most carefully examined consist of a mixture of two ingredients of different solubility; the two are so combined that when the more soluble is slowly removed, the less soluble remains as a skeleton (Nägeli).

Their form varies greatly in different plants; they appear as cubes, tetrahedra, octohedra, rhombohedra, and in other forms ; usually, however, their crystallographic characters cannot be exactly defined, a consequence of their small size and of the inconstancy of their angles.

In the rapidly growing organs of flowering plants they are known only in Lathrea squamaria ${ }^{1}$; more commonly they are produced in cells where large quantities of reserve-materials are collected which are only turned to use at a later period. The crystalloids themselves appear to be a form of protoplasmic structure especially adapted for a dormant condition (as in potato-tubers and many oily seeds); they are seldom found in cells which contain sap (potato-tubers), but more often in cells which do not contain it, and especially in oily seeds. Crystalloids containing colouring matters are found in petals and fruits. Sometimes they are formed only after the action of alcohol or a solution of sodium chloride on the plants externally or internally (Rhodospermin).

The crystalloids of potato-tubers are imbedded in the protoplasm; those that are widely distributed in the tissues of Lathrea squamaria are contained in great numbers in the interior of the nucleus; those found in oily seeds are generally enclosed in aleurone-grains.

The crystalloids discovered by Cohn in the tubers of the potato are convenient for observation; they are found very abundantly in some kinds, in others less frequently, in the parenchymatous cells which contain but little starch beneath the skin but tolerably deep in this tissue, lying enclosed in the protoplasm. Generally they are in the form of perfect cubes (less often of derivative forms, as tetrahedra). Those found by Radlkofer in the nucleus of the cells of Latbraa squamaria lie together in great quantities; they have the form of thin rectangular plates; sometimes they have rhombic or trapezoid forms; Radlkofer thinks it most probable that they belong to the rhombic system. In these cases they may be seen in sections without further preparation, and their relation to their surroundings is clear. The case is different with the crystalloids of oily seeds enclosed in aleurone-grains; I shall recur to their properties, and will only mention that from the brazil-nut they are obtained in quantities by washing the crushed oily parenchyma by oil or ether, the crystalloids settling down in the form of a fine powder; in sections but little can be made out. They were carefully investigated in the isolated state by Nägeli; according to him they appear rhombohedral, octohedral, or tabular; but it is uncertain whether they belong to the hexagonal or the klinorhombic system. Dried and then placed in water, they alter their angles about $2^{\circ}$ or $3^{\circ}$; in potash solution they swell strongly and alter their angles $15^{\circ}$ or $16^{\circ}$. By weak acids and dilute glycerin a substance is extracted, and a weak skeleton with firmer skin remains behind. The crystalloids in the cells of the endosperm of Ricinus communis are, like all crystalloids, insoluble in water, and are easily seen when thin sections of the tissue are laid in water, which destroys the substance surrounding the crystalloid, and sets it free. They frequently take the form of octohedra or tetrahedra, less often of rhombohedra; but the system is not certainly determined. The crystalloids which contain colouring matters were first detected by Nägeli in an imperfect form in the

1 [According to Prillieux, the brown colour of Neottia nidus-avis is due to brown crystalloids which assume a green colour when the plant is immersed in alcohol or boiling water; see Ann. des Sci. Nat., 5th ser., vol. XIX. p. I08.] 
petals of Viola tricolor and Orchis, and better developed in the dried fruits of Solanum americanum; in the latter case they form in the large parenchymatous cells clusters of a deep violet colour; the separate crystalloids are thin rhombic plates, often with truncated angles, \&c. According to Nägeli the crystalline form is the rhombic prism in a very abbreviated tabular shape; the six-sided plates are composed of six simple ones. In pure water they remain unchanged; alcohol extracts the colouring matter, as also do dilute acids; both leave, after long treatment, a very slight skeleton which is capable of swelling, while the whole crystalloid does not swell; Nägeli states that the crystalloid consists of a very small quantity of albuminous and a large quantity of another substance, with some colouring matter.

Crystalloids of albuminous substance have also been found in red marine Algæ (Florideæ) and in one Fungus. Cramer observed the first case of this kind; in specimens of Bornetia secundiffora which had lain a long while in solution of sodium chloride, as well as in specimens in alcohol of Callithamnion caudatum and seminudum, he found hexagonal plates and prisms with all the properties of crystalloids, and coloured red by the colouring matters of the Algx. They were found in the vegetative cells as well as in the spores. In sodium chloride preparations of Bornetia octohedral crystalloids were found also, apparently belonging to the klino-rhombic system; they were colourless. In living plants of the same Alga, Cohn also discovered colourless octohedral crystalloids which absorb the red colouring matter expelled from the pigment-grains. Within and without the cells of Ceramium rubrum preserved in sea-water with glycerin, klino-rhombic prisms formed, coloured red by the expelled pigment; they are clearly, like the hexagonal crystalloids observed by Cramer, produced only after death, while the colourless octohedra are to be found in the living cells. Finally, in dried specimens of other Florideæ, Griffitbsia barbata, G. neapolitana, Gongroceras pellucidum, and Callitbannion seminudum, Klein observed colourless crystalloids of a different form. These bodies may all be comprised in the name first given by Cramer,-Rbodospermin. In the sporangiophores of Pilobolus Klein also found colourless octohedra of tolerably regular structure with the properties of crystalloids.

Sect. 8. Alourone-Grains ${ }^{1}$.- The reservoirs for reserve material contained in ripe seeds, i.e. in the endosperm or the cotyledons of the embryo, always contain considerable quantities of proteids, together with starch or oily matter. If they contain much starch, as in Grasses, Phaseolus, Vicia, the oak, horse-chestnut, Spanish chestnut, \&c., the proteid, which only contains very little oily matter, occupies the interstices; it then consists of small or even minute granules, as shown in Fig. 46. In oily seeds, on the other hand, in place of the starch-grains are found granular roundish or angular structures (Fig. 47), sometimes not dissimilar

1 These structures were discovered by Hartig (Bot. Zeitg. I 855 , p. $88 \mathrm{I}$, and described in detail but imperfectly (ibid. 1856 , p. 257 ; [Ann. des Sci. Nat., 1856 , vol. VI. p. 325]); further researches were undertaken by Holle (Neues Jahrb. der Pharmacie, vol. X. 1858) and Maschke (Bot. Zeitg. I859). All these observations left undecided the relationship of the grains to the surrounding matrix ; it appeared to be assumed that in oily seeds the matrix consists of oil only. In the first and second editions of this book I opposed this view, and pointed out that the matrix in the cells of oily seeds consists of a mixture of oil and proteids, or rather, of a very oily protoplasm; on the other hand I fell into the error, partly in consequence of the use of diluted ether, of considering the aleurone-grains themselves as a compound of proteids and oil. This error has been refuted by Dr. Pfeffer's recent researches, commenced in the Würzburg laboratory, where I had the opportunity of seeing numerous preparations which were decisive as to the principal question. Dr. Pfeffer had the kindness to communicate to me a detailed account of his labours; what I have said above follows his views tolerably closely. See Pfeffer, in Pringsheim's Jahrb. für wissens. Bot. vol. VIII. p. 429 . 
to starch-grains in their appearance, and surrounded by a more or less homogeneous matrix, which, as closer investigation shows, consists, according to the oiliness of the seed, of more or less oil combined with proteids. The grains themselves, on the other hand, consist, independently of certain enclosed matters, of proteids.

In the aleurone-grains the proteid itself must be distinguished from the enclosed substances. The latter are either crystals of calcium oxalate, or noncrystalline, roundish, or clustered granules, known as Globoids. These are a double calcium and magnesium phosphate, in which the latter base is greatly in excess.

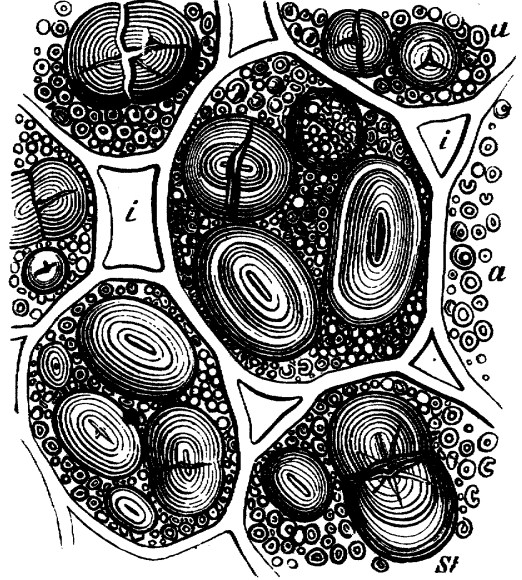

FIG. 46. - Cells shown in a very thin section through a cotyledon in a ripe seed of Pisum sativum; the large concentrically stratified grains st are starch-grains (cut through); the small granules $a$ are aleurone, consisting principally of legumin with a little oily matter; $i$ the intercellular spaces.

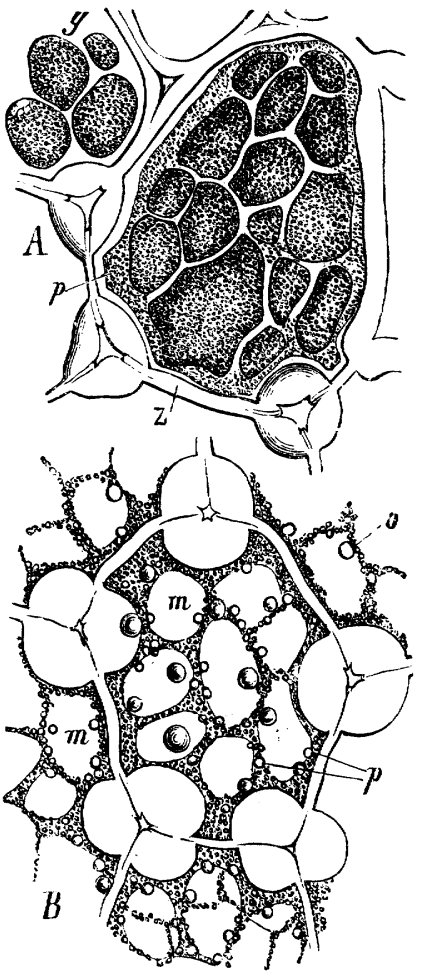

FIG. 47.-Cells from the cotyledon in a ripe seed of Lupinus varius; $A$ in an alcoholic solution of iodine; $B$ after destruction of the aleurone-grains by sulphuric acid; $z$ the cell-wall; $p$ the protoplasmic matrix, containing but little oily matter; $y$ the aleurone-grains; $a$ drops of oil expelled from the matrix by the action of the sulphuric acid; $m$ empty spaces from which the aleurone-grains have been dissolved $(\times 800)$.

The whole proteid is sometimes amorphous, and in that case is not doubly refractive; or the greater part, with the enclosed substances already named, is developed into a crystalloid (Sect. 7), and this, surrounded by a thin amorphous envelope, constitutes the aleurone-grain. (Fig. 48.)

The proteids are all insoluble in water; neither alcohol nor water extracts anything from them. The grains which contain no crystalloids dissolve in water entirely (Paonia), partially (Lupinus), or not at all (Cynoglossum). But all dissolve completely in water containing only a trace of potash. With careful treatment there always remains behind a membrane surrounding the grain, which behaves like 
coagulated albumen; but it may be a yet unknown proteid. With aleurone-grains containing crystalloids, after careful solution, a similar membrane remains, but the crystalloid itself also leaves behind one of the same kind; this occurs also in the solution of globoids in acetic or hydrochloric acid, and reminds one of the similar behaviour of true crystals of calcium oxalate.

The crystalline enclosures of calcium oxalate occur as clusters, distinct crystals, and needles, but are not commonly met. with. The globoids, on the other hand, are never absent from aleurone-grains; when they are accompanied by crystals, it is almost always the case that the grains of one cell contain only globoids, those of another only crystals, as in Silybum marianum, and all Umbelliferæ that have been examined. There occur however exceptions; in the grape-vine a globoid forms itself round a crystal or a cluster of crystals. The globoids are soluble in all inorganic acids, and in acetic; oxalic, and tartaric acid, but not in dilute potash.

The globoids, like the crystals, may occur in an aleurone-grain singly or many together; in the latter case they are small, and even too minute to be measured, but are then present in enormous numbers in one grain, e.g. Lu-

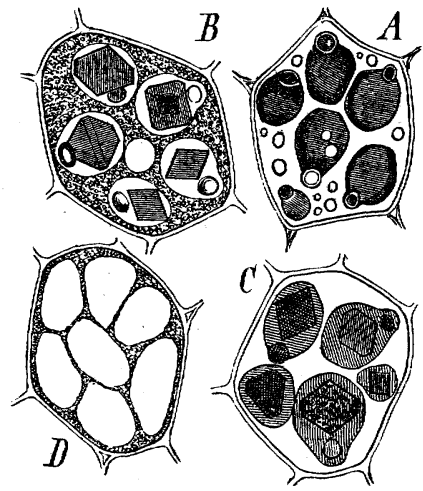

FIG. 48.-Cells from the endosperm of Ricinus commun is ( $\times 800)$. A fresh, in undiluted glycerin, $B$ in dilute glycerin, $C$ warmed in glycerin, $D$ after treatment with an alcoholic solution of iodine, the aleu* ment with an alcoholic solution of iodine, the aleu*
rone-grains are destroyed by sulphuric acid, the proteid remaining behind as a net-work. In the aleurone-grains the globoid may be recognised, and in $(B, C)$ the crystalloid.

pinus luteus, L. polyphyllus, Delphinium Requienii, \&c. Large globoids surrounding crystals occur singly, the largest in the grape-vine. Pfeffer found crystals accompanying crystalloids only in Ethusa Cynapium. The enclosed substances are usually absent from very small aleurone-grains.

In some seeds there is in each cell one aleurone-grain distinguished from the others by its size ('Solitär' of Hartig), both when crystalloids are present and when they are absent (Elaeis, Myristica, Vitis, Lupinus luteus); it may be distinguished by its enclosed substances. Thus in Lupinus luteus it contains a tabular crystalloid; the other surrounding grains only small and numerous globoids. In Silybum a cluster of crystals lies in one large grain, in the others a number of needle-shaped crystals. In other cases the enclosed substances are similar, as is the case with the globoids, which are merely larger in the large grain.

The crystalloids are tolerably widely distributed in aleurone-grains, although the greater number of seeds are destitute of them. They are not, however, characteristic of natural families, but may be present or absent in members of the same family; thus among palms, Sabal Adansonii is without, Elaeis guineensis has crystalloids; in the same manner all Umbelliferæ which have been investigated want them except AEthusa Cynatium. In other cases all seeds of the same family appear to contain crystalloids, as in the Euphorbiaceæ, among which Ricinus supplied the first example of fine crystalloids in the aleurone-grains.

The matrix which surrounds the grains of aleurone in oily seeds is, as has been mentioned, always a mixture of oily matter and proteids, but the proportion 
of the latter may be very small. Thus even in the castor-oil-plant and brazil-nut, where the matrix appears to consist entirely of oily matter, the albuminous constituent is quite discernible, as is shown in Fig. $48, D$; Pfeffer succeeded most readily by extracting with an alcoholic solution of corrosive sublimate, and then colouring with anilin-blue dissolved in water. The matrix may be considered as the protoplasm-mass of the cell, in which the water is replaced on drying by oil. But in addition it contains not only insoluble proteids, but other substances soluble in water rendered alkaline by potash. This composition of the matrix, together with the solubility of the amorphous mass of the aleurone-grains in water, are the cause of the complete loss of form which the cell-contents of oily seeds immediately undergo in water, as shown in sections under the microscope. In order to recognise their structure it is necessary to place fresh sections in undiluted glycerin, alcoholic solution of corrosive sublimate, oil, or concentrated sulphuric acid.

The oily matter may also separate from the matrix in crystals, as Pfeffer has observed in the brazil-nut, Elaeis guineensis, and the nutmeg.

To the above may be added, from Pfeffer's communication, some explanations concerning the more difficult points.

(a) The substance of the aleurone-grains always consists, to by far the greater extent, of proteids, with which very small quantities of other vegetable substances are usually or always mixed which are difficult of detection. All aleurone-grains are absolutely insoluble in alcohol, ether, benzol, or chloroform ${ }^{1}$; these reagents would dissolve oil (alcohol dissolves also glucose), if it were present, and would consequently alter the appearance of the grain. Some are insoluble in water (e.g.those of Cynoglossum officinale); those soluble in water ${ }^{2}$ yield with corrosive sublimate in absolute alcohol a mercurycompound insoluble in water. Gum, pectinaceous substances, cane-sugar, and dextrin do not, under this treatment, yield an insoluble compound. Of all widely distributed vegetable substances, only proteids behave in this manner towards corrosive sublimate. Boiling the mercury-compound with water reproduces a proteid insoluble in dilute acids and alkalies.

(b) In proving that the aleurone-grains of oily seeds contain no oil, we have already seen that it must be present in the matrix. The doubt which arises from the first glance at sections of oily seeds, whether the great mass of oily matter can find space in the interstices between the grains, can be settled by calculation; for if spheres (the grains may be so regarded) are placed in an equal number of cubes forming part of one great cube, $47^{\circ} 6$ p. c. of the cavity remains unoccupied; and if the spheres are distant from one another only about one-third of their radius, $69^{\circ} 7$ p. c. of the cavity is left, and this is more than is sufficient in oily seeds to take up the oily matter.

Immediate proof can be given of the existence of oil in seeds which contain it by the appearance presented by dry sections; if benzol is added, the matrix disappears, while small quantities of proteids remain. With alcoholic tincture of alkanet the matrix becomes of a deep blood-red colour if it contains a considerable amount of oil; but if the oily constituents of the seed are very small, the evidence cannot be obtained in this manner.

If the oil is extracted from the sections by alcohol, and the aleurone-grains then removed by potash solution, a net-work remains behind in which the grains are replaced

1 That I formerly considered them soluble in ether, was the result, as Pfeffer showed, of the containing a small quantity of water.

2 On the causes of the solubility in water see Pfeffer's treatise already cited. 
by cavities; with acetic acid and iodine the net-work assumes a yellow-brown colour (Fig. $47, B ; 4^{8} \mathrm{D}$ ). In most seeds this net-work is very beautiful and comparable to a parenchymatous tissue; in extremely oily seeds it often breaks up into fragments, the nucleus lying in it like a shrivelled ball. The threads of the net-work are composed of the insoluble proteids of the matrix and of the enveloping membranes of the aleuronegrains; although the net-work may exist without the latter if the grains have fallen from their places.

(c) The Crystalloids of the aleurone-grains are insoluble in water; they may therefore easily be isolated by treatment of sections with water, the amorphous portions of the grains dissolving, and any residue of cell-contents being destroyed; they then show all the reactions and the different forms of the crystalloids mentioned in Sect. 7. But that they consist of two proteids and grow by intussusception Pfeffer thinks very doubtful.

(d) If sections of the endosperm of the peony are treated with alcohol containing a small quantity of sulphuric acid, and if, after washing, they are placed in water, the substance of the aleurone-grains (not containing crystalloids) is seen to be distinctly stratified; but only a few firm and soft layers occur, the inner part of the mass being amorphous. Pfeffer's work should also be consulted here.

(e) The Development of the aleurone-grains is thus described by Pfeffer.-Their formation does not commence till the seeds have attained their last condition of ripeness and the funiculus begins to dry up; in the turbid emulsion which now fills the cells, the enclosed substances, especially the globoids, are already formed, but not fully developed. As the seed loses water, the formation of mucilaginous proteidmasses commences, mostly surrounding enclosed substances; these mucilaginous bodies, usually nearly globular, grow, their mutual distance thus decreases, and at last their differentiation is complete; the aleurone-grains, still consisting of mucilaginous substance, are distinctly separated from the still turbid matrix, which becomes clearer and clearer as the seed dries. The previously spherical or ellipsoidal grains become more or less polyhedral, especially in oily seeds which have but little matrix (e.g. Lupinus).

While the formation of the aleurone-grains is beginning, the protoplasm of the cell is only to be detected with difficulty in the turbid cell-contents; yet, on removing the oily matter by alcohol, it may be shown that it is present in the normal form; sometimes in the copious matrix of some seeds the dried threads of protoplasm may still be seen. In Lupinus luteus the crystal of calcium oxalate, which is afterwards enclosed by the largest grain, is already present before the formation of the aleurone-grains. Pfeffer was able to follow the development of the grains with remarkable ease in the peony; in this case the seed, even when it has attained its full size, is entirely filled with large starch-grains, which become changed into oil only when fully ripe. The starch is not always, however, completely changed into oily matter. If the starch-grains in the seeds of the peony were not completely transformed, and the intermediate mass, almost devoid of oily matter but very rich in proteids, formed very small aleurone-grains, we should have what does actually occur in Pbaseolus and in other seeds extremely rich in starch. There are, however, also seeds in which aleurone- and starch-grains occur in nearly equal quantities, but then always associated with oily matter.

No conclusion as to the manner of growth can be deduced from the turbid condition of the cell-contents and the softness of the growing aleurone-grains. Nevertheless it can mostly be affirmed with regard to ripe grains, that they are softer towards the inside, and that, consequently, on the application of very dilute reagents, they dissolve from within outwards. Different facts appear, nevertheless, to show that no growth takes place by intussusception, as is the case with starch-grains. The origin of aleurone-grains is simply a dissociation, which arises from loss of water by the seed; and, on germination, the original cell-contents are again more or less 
completely reconstituted by the union of the matrix with the substance of the aleuronegrains.

Pfeffer followed out the formation of the crystalloids in Ricinus and Euphorbia segetum; they arise nearly simultaneously with the globoids, at a rather early period, and both grow gradually, while the turbidity of the cell-contents at first somewhat increases. They lie, at an early stage, close to one another, and completely surrounded by the turbid mass; the vacuoles which Gris figures (Recherches sur la germination, Pl. I, Figs. IO-I3) are the result of the disorganisation of the cell-contents. The crystalloids are from the first sharp-edged; and, as soon as their size permits their shape to be recognised, it agrees with the mature form. The envelopment of crystalloid and globoid by an amorphous coating does not begin till the crystalloids are mature and the seed has begun to dry.

On germination the crystalloids dissolve as well from without as from within, till after the amorphous envelope has first disappeared; their external membranes for a time persist, but gradually become invisible. The globoids also dissolve (no doubt in consequence of the acid reaction which the tissue assumes), and in the case of old seeds from the outside inwards. The aleurone-grains destitute of a crystalloid swell up, and resume, on the germination of the seed, the form which they possessed in ripe but undried seeds; they begin to mix gradually with the substance of the matrix; their solution can sometimes be followed from without inwards; but they often coalesce as mucilaginous masses. These changes occur with the first signs of germination in the embryo; formation of starch takes place simultaneously in the contents of the cells.

SEct. 9. Starch-Grains. ${ }^{1}$.-Plants which grow under favourable circumstances produce by assimilation a larger quantity of formative organisable substance than they require or can employ at the time for the growth of the cells. These materials are stored up in some form or other in the cells themselves, and only come into use later. It has already been shown how this takes place with albuminous protoplasm-forming materials and with oily substances. Another substance, in the highest degree organisable, Starch, is formed beforehand and stored up in far larger quantities in anticipation of future use. Starch always appears in an organised form as solid grains having a concentrically stratified structure, which arise at first as minute dots in the protoplasm, and continue to grow while lying in it ; if, at a subsequent period, they reach the cell-sap and cease to remain in contact with the protoplasm which nourishes them, their growth ceases ${ }^{2}$. Every starch-grain consists of starch, water, and of very small quantities of mineral substances (ash). Starch itself is a carbo-hydrate of the same percentage composition as cellulose, to which it bears the greatest resemblance of all known substances in chemical and morphological properties. Starch, however, occurs in each grain in two modifications:-Granulose, more easily soluble, and assuming a beautiful blue colour with iodine in the presence of water, and Farinose, easily soluble, and more resembling

1 Nägeli, Die Stärkekörner, in Pflanzenphys. Untersuchungen, Heft II, and Sitzungsber. der $\mathrm{k}$. bayer. Akad. der Wissenschaften, 1863--Sachs, Handbuch der Exp. Physiol., Leipzig 1865, § 107. The account given here is chiefly derived from Nägeli's work.

2 According to Hofmeister, the starch-grains in the latex of Euphorbia appear to form an exception; nothing however is known about their development; the latex always contains protoplasm-forming substances, proteids, which perhaps here also take part in the production of the starch-grains. 
cellulose in its reactions ${ }^{1}$. At every point of a starch-grain both constituents occur together; if the granulose is extracted, the farinose remains behind as a skeleton; this skeleton presents the internal organisation of the whole grain, but is less dense or prorer in substance, and its weight amounts to only from 2 to 6 p.c. of the whole grain. Since the granulose greatly preponderates and is present at every point, the starch-grain shows the blue granulose-colouring with iodine throughout its whole extent.

The starch-grains have always rounded forms organised around an internal centre of formation; when young and small the grains appear to be always spherical; but since their growth is scarcely ever uniform, their form changes into ovoid, lenticular, rounded polyhedral, \&c.

The internal organisation of the starch-grain depends essentially on the different distribution of water in it (water of organisation). Every point of the grain contains water in addition to granulose and farinose. Most usually the amount of water increases from without inwards, and attains its maximum at a fixed point in the interior. With the increase in the proportion of water, the cohesion and density decrease, as also the index of refraction. This change in the proportion of water is not, however, constant, but intermittent. To the outermost least watery layer succeeds a sharply defined watery layer, to this again a less watery one, and so on, until the innermost less watery denser layer surrounds finally a very watery part, the nucleus. All the layers of a grain are disposed round this nucleus as their common centre, but every layer is not continuously developed round the whole nucleus; in small spherical grains with few layers this is always the case, but when their number increases, it does so most in the direction of most vigorous growth, which is continuous in a straight or curved line with the direction of least vigorous growth. This line is called the axis of the grain, and always passes through the nucleus.

The growth of the grains of starch is accomplished exclusively by Intussusception; new particles become intercalated between those already existing both in a radial and tangential direction, by which "means the proportion of water at particular places is at the same time changed. The youngest visible globular starch-grains consist of denser less watery substance; in this is formed subsequently the central watery nucleus; in the latter a central part may become denser; and in this, when the increase in size has advanced sufficiently, a softer nucleus may again arise. It may however also happen, after a softer nucleùs surrounded by a dense layer has arisen by differentiation of the originally dense grain, that in the dense layer a new soft one may arise, and it may thus become split into two dense layers, the inner of which encloses the soft nucleus. The layers increase in thickness and circumference by intercalation. When a layer

1 [The most recent researches seem to show that the supposed distinction between granulose and farinose is one of mechanical or molecular condition only. The coloration of starch by iodine appears not to depend on the formation of a definite chemical compound, but to be the consequence of the mechanical interposition of the iodine between the molecules of starch (see Miller's Chemistry, 3rd ed., vol. III. Sect. I57 I, p. 616 et seq.)] 
has attained a definite thickness, it becomes differentiated by further growth into three layers. If it is a dense layer, watery substance becomes intercalated in its middle, and the dense layer splits into two layers separated by a less dense one. But when a watery layer becomes sufficiently thick, its middle lamella may become denser, and a new dense layer be thus formed between two less dense layers. This process of splitting of the layers depends on their increase in thickness; and since this itself is the most vigorous where the layers are intersected by the longer branch of the axis of growth, the splittings, i.e. the new formations of layers, take place there most abundantly, and least so on the opposite side of the nucleus, where they may even entirely cease. The layers of the more quickly growing side of the grain become, as they pass to the slowly growing side, gradually thinner, and finally disappear. Lenticular grains $(e . g$. in the endosperm of wheat) have a lenticular nucleus; their layers grow most quickly in the direction of the radii of a great circle concentric with it, and commonly split, the nucleus remaining central. If, on the other hand, the growth takes place in one direction (e. $g$. in the ovoid grains of the potato-tuber) the nucleus becomes eccentric, is further and further removed from the centre of gravity of the grain, and is in this case globular. In some ellipsoidal (in the cotyledons of peas and beans) or elongated grains, the nucleus is extended in the direction of the longest axis.

It is very common for two nuclei to form in a small young grain; round each of them layers are formed, and the growth is strongest in the line of union. The distance of the nuclei from one another becomes continually greater; thus a tension arises in the few layers which are common to both; this leads to the formation of an inner fissure, which lies at right angles to the line of union of the two nuclei; it is continued towards the outside, and the grain breaks up into two half-grains which may nevertheless adhere to one another. If this division occurs more often, compound grains arise, consisting of numerous secondary grains, the number of which may amount even to thousands (e.g. in the endosperm of Spinacia and Avena).

Compound grains of from two to ten secondary grains, with a mulberry-like appearance, are extremely common in the parenchyma of quickly growing plants, e. $g$. in seedlings of Phaseolus and stem of Cucurbita. Grains of this description are different in their origin from compound grains of the kind which occur in chlorophyll; in this latter case a number of small grains exist from the first, which only touch and adhere to one another in consequence of increase of size. (See Fig. 45, p. 47.)

Partially compound starch-grains result when new nuclei with their surrounding masses of layers are formed after the grain has already formed several layers. The secondary grains appear therefore to be inclosed within the layers of the mother-grain. In this case also tension arises from the unequal growth of the common layers and of those belonging to each secondary grain, leading at length to the formation of fissures; but these do not usually extend to the outside; the secondary grains remain united.

(a) The growth of starch-grains by intussusception must be inferred from the following considerations:- Supposing that the formation of layers occurs by deposition, 
grains would be found the outermost layer of which would be a watery one; this, however, never occurs; the outermost layer is always the densest and least watery. According to this supposition the nucleus would also possess the properties of the youngest grains, whereas the nucleus is always soft, the youngest grains dense. The theory of deposition could only explain the formation of partially compound grains if we suppose that the common layers had been subsequently deposited round two or more previously isolated grains; but the common layers would have a different form, and the fissures in the interior of such grains remain unexplained. The theory of deposition, finally, is incompetent to explain why, in the secondary grains, the strongest growth always takes place in the line joining their nuclei (Fig. 49). The older hypothesis of a deposition of new layers from within presupposes that the starch-grains were at first hollow vesicles, which has never been observed; on this hypothesis, moreover, it cannot be explained how the phenomena arise which occur in the formation of secondary grains; and this hypothesis must moreover suppose growth by intussusception to explain the superficial extension of the layers. The hypothesis of growth by intussusception affords the simplest explanation of all the phenomena; and, after Nägeli's researches, may be considered as a fully established fact. The material which penetrates into the grain, and there becomes deposited in the form of new particles of starch, is, of course, in solution; but its chemical nature is not yet certainly known; dissolved starch can never be proved to exist in the plant, at least in those cells where active formation and growth of starch-

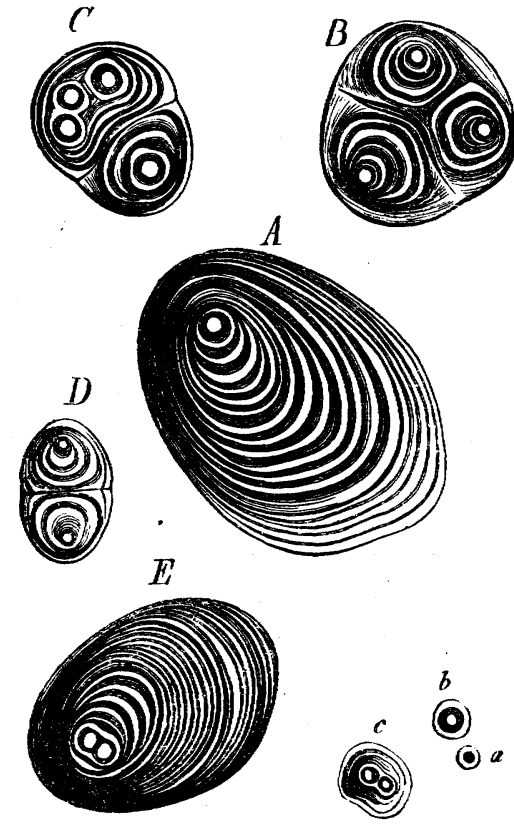

FIG. 49.-Starch-grains from the tuber of a potato ( $\times 800) . A$ an older simple grain; $B$ a partially compound grain; $C, D$ perfectly compound grains ; $E$ an older grain, the nucleus of which has divided; $a$ a very young grain, $b$ an older grain; $c$ a still older grain with divided nucleus. grains has been observed. It is, however, probable that a solution of sugar contained in the protoplasm is the material out of which particles of starch are formed by chemical and physical changes. The starch is easily changed into sugar by different agencies. From various facts (e.g. the production of radial fissures on drying), it must be concluded that the molecules of starch have not only a definite order of deposition in the direction of the radii, but are also arranged tangentially in a definite manner in each layer. A corresponding stratified structure with radial striation, and the consequent formation of areolæ, has, however, been observed only occasionally and imperfectly.

Growth by intussusception depends on the permeability of all parts of the grain to water and aqueous solutions. This can only be explained by supposing that the substance of starch is not continuous, but consists of distinct molecules, each of which possesses the power of attracting water, and surrounds itself with an aqueous envelope; the molecules of starch are separated from one another by these aqueous envelopes; the smaller the molecules in a given portion of a starch-grain, the thicker are these envelopes and the more watery the particular portion. From this it results, on purely mechanical principles, that, as the molecules increase in size, the aqueous envelopes become thinner, and the molecules approach nearer one another. The watery layers therefore consist of small molecules which are separated by thick aqueous envelopes, the denser less watery layers of larger molecules with thinner 
envelopes. The internal organisation depends, in these cases, on a definite relation of the water and the molecules of starch; the stratification of a starch-grain disappears, like that of a cell-wall, when the water is removed from it (e.g. by evaporation or action of absolute alcohol, \&c.), because the more watery layers then become similar to the less watery ones, and the difference of refractive power in the two ceases. In the same manner the stratification also disappears when the substance of the grain is rendered capable by chemical means (as dilute potash solution) of absorbing large quantities of water; the denser layers absorb relatively more water; they thus become similar to the more watery layers, and it is no longer possible to distinguish between them.

Besides the differentiation in the proportion of water which is recognised in the form of stratification, there is also in every grain an increase from without inwards in the amount of water. This is partly ascertained by the refraction, partly by the regular decrease of cohesion from without inwards. If the water is removed from fresh starch-grains, fissures are formed which cross the layers at right angles; in the interior a cavity is formed from which the fissures radiate; these become narrower outwardly, and are widest in the centre. From this it follows that on drying the greatest loss of water occurs in the interior, and that this regularly decreases towards the outside; but it also follows that the cohesion of the layers is less in the tangential direction (at right angles to the fissures) than in the radial. This points to the conclusion that within every layer the loss of water is greater in the tangential direction than in the radial.

If the water be removed from a fresh starch-grain or from one which has taken up as much as it can, it contracts; the molecules contained in it approach one another as their envelopes of water become thinner. A similar change takes place if the granulose is removed from a grain; the farinose-skeleton of the grain which remains is, although saturated with water, much smaller than the original grain. This possibly results from the fact that the molecules, now consisting only of farinose, possess less attraction for water, and, having thinner envelopes, approach nearer; the cause may however also be that the number of molecules has diminished.

(b) The Extraction of the Granulose of starch-grains, leaving behind a skeleton of farinose, can be brought about in very different ways:-1. By maceration in saliva at an elevated temperature; in the starch of Canna indica the extraction, according to $\mathrm{H}$. von Mohl, is slow at $35^{\circ}-40^{\circ} \mathrm{C}$., but is accomplished in a few hours at $50^{\circ}-55^{\circ} \mathrm{C}$.; a lower temperature suffices for wheat-starch, a higher is required for that of the potato; Nägeli gives in general $40^{\circ}-47^{\circ} \mathrm{C}$. 2. According to Melsens a similar extraction may also be effected by organic acids, diastase, and pepsin. 3. According to Nägeli it can be accomplished also by very slow action of hydrochloric or sulphuric acid which has been so diluted with water that it does not cause the starch-grains to swell. 4. According to Franz Schulze, the granulose is extracted in from two to four days by a saturated solution of sodium chloride containing I p. c. of hydrochloric acid, at a temperature of $60^{\circ} \mathrm{C}$; the residuum, which perfectly exhibits the organisation of the starch-grain, amounted, according to Dragendorff, to 5.7 p. c. in potato-starch, $2 * 3$ p.c. in wheatstarch. These skeletons are not coloured by iodine (Nägeli's preparation with sulphuric acid after fifteen months' extraction), or they become copper-red, and in places where the extraction was not perfect, bluish. They do not swell in boiling water, i.e. do not form paste. At $70^{\circ} \mathrm{C}$. the whole of the starch-grain, according to Mohl, is dissolved in saliva ; the skeleton produced at $40^{\circ}-55^{\circ} \mathrm{C}$. is, however, not affected by saliva at $70^{\circ}$.

Within the living cell the starch may be dissolved in very different ways; probably solution occurs mostly under the influence of protoplasm, or by the assistance of nitrogenous combinations in the cell-sap. Sometimes the solution begins, as in the extractions mentioned above, with the removal of the granulose, the farinose remaining behind; but this often takes place only partially; the extraction proceeds in places 
from without inwards; the extracted places are coloured copper-red by dilute iodine, the remaining mass blue; then the grain breaks up into pieces, $\cdot$ which are finally completely dissolved (as in the endosperm of germinating wheat, Fig. 50, B). In other cases the solution begins also in particular spots of the circumference; the whole substance, however, gradually dissolves; holes are formed, and finally the grain breaks up into pieces (as in the maize, Fig. 50, $A$ ). In the cotyledons of germinating beans, the solution of the ellipsoidal grains begins from within; but before they break up into pieces, the granulose is often so completely extracted that they assume with iodine a copper-red and in parts a bluish colour; afterwards the whole is dissolved. In germinating potatoes and the rhizome of Canna lanuginosa, on the other hand, the solution of the grains advances from without inwards, removing layer after layer. Probably this takes place when saliva is employed, whether the solvent acting slowly first extracts the granulose; or attacking it energetically dissolves the whole substance. Observations on embryos of the same species, germinating at different temperatures, would possibly show corresponding differences.

(c) Solubility, Swelling. If starch-grains are crushed in cold water, a small portion of the granulose is dissolved; addition of iodine occasions precipitation of finegrained blue pellicles ${ }^{1}$. Starch-grains ground with fine sand yield an actual solution of granulose to cold water. Other fluids, as dilute acids, do not cause a solution of the starch, but rather a transformation into other substances (dextrin, dextrose), which then dissolve.

Water of at least $55^{\circ}$ C. causes the swelling and conversion into paste of larger more watery starch-grains; in smaller denser ones this begins, according to Nägeli, at $65^{\circ}$. After heating in the dry state to about $200^{\circ} \mathrm{C}$., subsequent moisten-
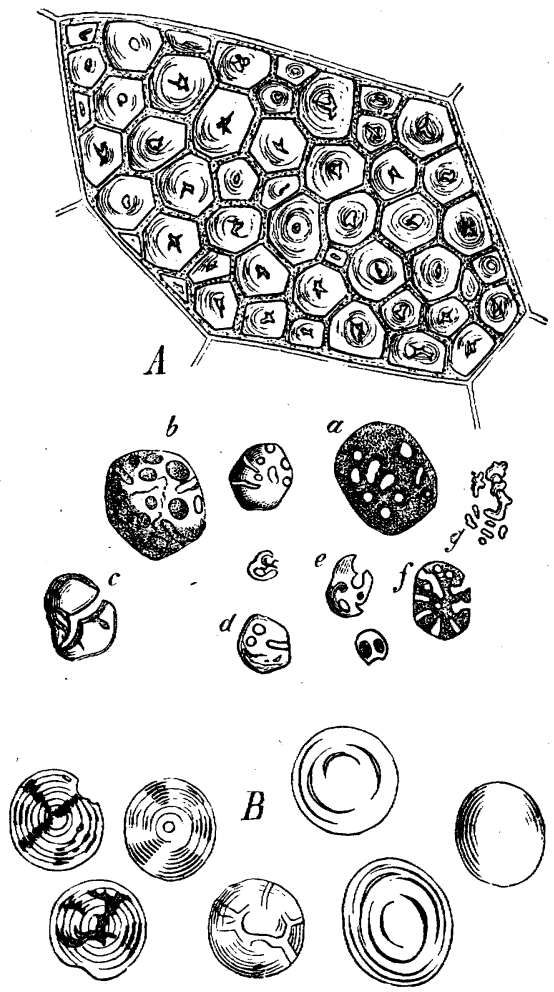

FIG. 50.- $A$ a cell of the endosperm of the maize, filled with crowded and therefore polyhedral starch-grains: between the grains lie thin plates of dried-up fine-grained protoplasm; small cavities and fissures are formed in the interior of the drying; $a-g$ starch-grains from the endor of the grains by drying; $a-g$ starch-grains from the endosperm of a germi-
nating seed of maize; $B$ lenticular starch-grains from the endosperm of a germinating seed of wheat; the commencing of the action of the solvent is shown by the nore evident ap-
pearance of stratification ( $\times 800)$.

ing causes swelling; but the substance is by this means chemically changed; it is transformed into dextrin. In the production of paste, the interior watery parts swell first, the outermost layer scarcely at all; it bursts and remains for a long time recognisable by iodine as a pellicle, even after the breaking up of the inner parts into small particles. A similar effect is caused by weak cold potash or soda solution; the volume of a grain may thus be increased one hundred and twenty-five fold, and so much fluid be absorbed that the swollen grain contains only from 2 to $0^{\circ} 5$ per cent. of solid starch.

1 On the actual solubility of starch, see the remarks in my Handbuch der Experimental Physiologie, p. 4 ro. 
SEct. I0. The Cell-sap.-The term Cell-sap may be understood in a wider or in a narrower sense. In the former it would express the collective mass of all the fluids by which the cell-wall, the protoplasm-body, and all other organised structures of the cell are saturated, and would also include the fluids contained in the vacuoles of the protoplasm; in a narrower sense the latter only is ordinarily designated as cell-sap. In any case there are grounds for considering the composition of the cell-sap as very variable, according as it has been imbibed by the protoplasm, the chlorophyll, the cell-wall, or the starch-grains of one and the same cell, or occurs as vacuole-fluid. The latter may in general represent the reservoir out of which the organised absorbent parts of the cell supply their needs, but in which, on the other hand, the superfluous soluble products of assimilation and metabolism ${ }^{1}$, and the food-materials that have been absorbed, also for a time collect. One constituent of the cell-sap, water, is always common to the vacuole-fluid and to the fluid which saturates the organised structures. The share taken by the water of the cell-sap in the entire building-up of the cell has already been entered into sufficiently in detail. Its function in the cell is a very manifold one; it is at once the general solvent and the agent of transport of the food-materials within the cell; the water itself enters in many ways into the chemical constitution of the substances produced in the plant; its elements are essential for the production of assimilated substances; for the formation of organised structures, the cell-wall, the protoplasmic structures, and the starch-grains, it is indispensable (water of organisation); the growth of the whole cell depends immediately on the absorption of water, and on the accumulation of the cell-sap as vacuole-fluid (see Figs. I, 4 I, 42, pp. 2, 42, 43). The increase in size of rapidly growing cells is nearly proportional to the accumulation of the sap in them. The hydrostatic pressure which the vacuole-fluid exercises on the primordial utricle and cell-wall is a factor in determining the form of the cell.

The substances dissolved in the water of the cell-sap-whether salts absorbed from without, or compounds produced in the plant itself by assimilation and metabolismare, as such, not immediately the subject of morphological observation, to which we are for the time confining ourselves. But the cell-sap sometimes contains substances the presence of which in the cell in characteristic forms can be proved by simple reactions, or which occur in nature in the form of definite structures, as drops or granules. Among the most important of the former is Inulin ${ }^{2}$. This substance, nearly related in composition to starch and sugar, occurs in the cell-sap of many Compositæ ${ }^{3}$. In sap

1 [In the first edition of this translation the term 'Stoffwechsel,' which includes as a general term any transformations which are effected in the products of assimilation, was translated 'metastasis.' In the literature of animal physiology the same idea has been rendered by the terms metabolism and metabolic (see Mayne's Expository Lexicon, 1860; Foster's Text-book of Physiology). For the sake of uniformity these terms will be adopted in the following pages. The products of metabolic transformations have been conveniently termed by Foster ' metabolites.']

${ }^{2}$ Sachs, Bot. Zeitg. p. 77, I864.-Prantl, Das Inulin, ein Beitrag zur Pflanzen-Physiologie; Preisschrift, Munich 1870.-Dragendorff, Materialien zu einer Monographie des Inulins, Petersburg I 870 .

3 [Kraus, Bot. Zeitg. 1875 , p. I 7 I, shows that, in addition to Compositæ, inulin is found also in the Campanulaceæ, Goodeniaceæ, Lobeliaceæ, and Stylideæ; and in these orders not only in the underground organs, but also in the stems and in the cells of the leaves which contain chlorophyll. Its solubility in water appears to vary.] 
obtained by pressure or boiling, it precipitates spontaneously after some time in the form of a white fine-grained precipitate. From solutions it crystallises in the form of socalled Sphere-crystals (Fig. 5 I $A$ ), consisting of crystalline elements disposed in a radiate manner. Within the cells it may be made visible as a finely granular precipitate by drying or by rapid removal of water by alcohol (Fig. $5 \mathrm{I}, F$ ). It is abundantly precipitated in the cells on dipping thin sections of the tissue into alcohol, in the form of smaller sphere-crystals which become readily visible on addition of water (Fig. $5 \mathrm{I}, B$ ). They are obtained much larger by laying entire specimens of Acetabularia or large pieces of tissue containing inulin (tubers and stems of the dahlia or Jerusalem artichoke) for a longer time in alcohol or glycerin; in the latter case a sphere-crystal very commonly includes several cells of the tissue (Fig. $5 \mathrm{r}, E$ ), a proof that the crystalline arrangement is not necessarily destroyed by the cell-walls. Similar forms (as in Fig. $5 \mathrm{I}, B$ ) are formed when tissues containing inulin freeze ; and they do not again become dissolved in the cellsap on thawing. Since the sphere - crystals consist of doubly refractive crystalline elements arranged radially, they show, with polarised light, the characteristic cross. They are not capable of swelling, are slowly dissolved in a large quantity of cold water, rapidly in a small quantity of warm water of from $50^{\circ}-55^{\circ}$ C. ; in nitric or hydrochloric acid or potash solution they dissolve easily, the solution always commencing from without; by boiling in very dilute sulphuric or hydrochloric acid the inulin is immediately transformed into glucose. Solutions of iodine in alcohol or water penetrate into the fine crevices of the sphere-crystals, but produce

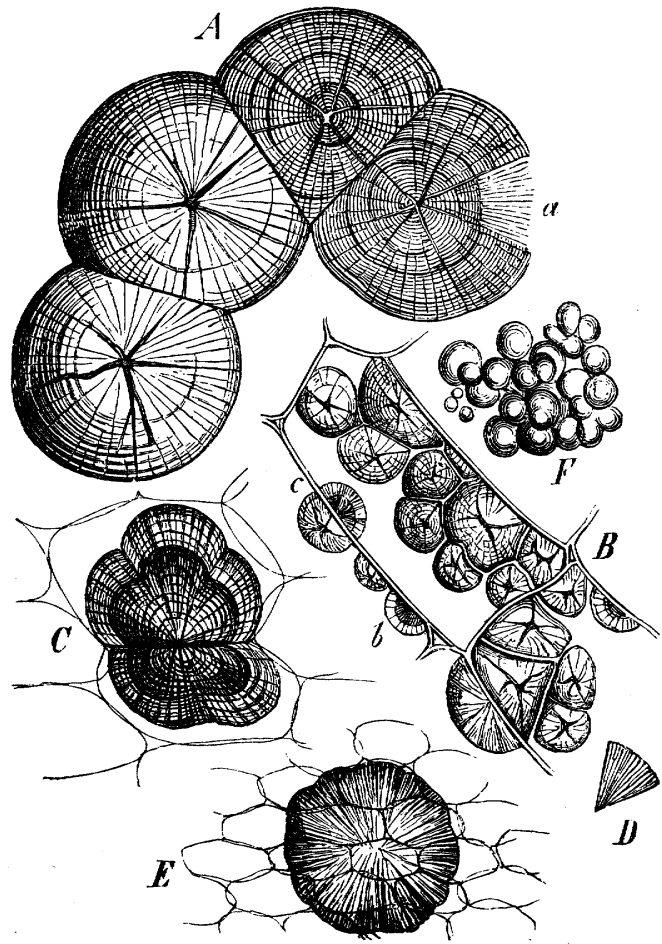

FIG. 5r.-Sphere-crystals of inulin. $A$ from an aqueous solution laid aside for $2 \frac{1}{2}$ months; at $a$ the action of nitric acid is commencing. $B$ cells of the root-tuber of the dahlia; a thin section was placed for 24 hours in alcohol of 90 p. c., and was then dipped in water. $C$ two cells with half sphere-crystals having their common centre in the middle of the separating cell-wall; from an internode $8 \mathrm{~mm}$. thick at the apex of an older plant of the Jerusalem artichoke which had remained for some time in alcohol. $D$ fragment of a sphere-crystal. $E$ a large sphere-crystal including several cells, from a larger piece of the stem-tuber of the artichoke, after lying for a longer time in alcohol. $F$ inulin after evaporation of the water from a thin section from the same $\left(X_{500} ; E\right.$ not so much).

no special colour. Inulin-structures are easily and certainly recognised by these reactions. If masses of tissue containing much inulin (tubers of Inula Helenium and Jerusalem artichoke, roots of dandelion and of other Compositæ) are examined in the air-dried state, the parenchymatous cells are found to be filled with angular, irregular, shining, colourless fragments, which are seen in polarised light to be crystalline, and may be recognised as inulin by the reactions above-named.

If the ovaries and unripe fruits of the orange or citron are laid for some time in alcohol, concretions are found in their tissues, which completely resemble in form the sphere-crystals of inulin; but the chemical reactions and the degree of solubility show that they do not consist of this substance. Pfeffer has examined these structures more 
minutely, and has ascertained (as Lebreton also thought, see Husemann, Die Pflanzenstoffe, p. 709) that they consist of Hesperidin. It is remarkable that only some individuals of the species named yield sphere-crystals, as for instance those in the botanical garden at Würzburg; a tree in the garden at Marburg yielded them in its unripe fruits in $187 \mathrm{r}$, but not since. Sphere-crystals of organic structure (combustible), but of otherwise unknown constitution, have also been described by Kraus and Russow; the former found them in the epidermis of the leaves and stem of Cocculus laurifolius, on treatment of the fresh cells with alcohol, glycerin, or even water. Russow ${ }^{1}$ found, in the living cells of the petiole and mesophyll of Marattia cicutafolia and Angiopteris erecta, sphere-crystals which enclosed a small crystal or other minute body as a central nucleus. Where the living cells did not contain these structures, he obtained them by treatment with alcohol; they left behind, on combustion, a considerable quantity of ash. Russow also found similar bodies in the cortex of Selaginella Martensii and in tropical orchids, when the plants had lain for some time in alcohol. He states that all these spherecrystals have the property of staining with carmine.

In the cell-sap of the Hepaticæ there occur vesicles or nodules of a peculiar appearance. In a letter Pfeffer states that they are formed in the very young leaves of Jungermannieæ (Alicularia scolaris, Radula complanata, \&c.) by the coalescence or grouping together of minute drops of oil, which are first formed in the cell-sap, not in the protoplasm, and must be regarded as products of excretion which have no further purpose in assisting growth; as is the case also with the fatty oils which are stored up as reserve-materials. A membrane-like envelope surrounds these drops of oil, whose substance consists, in addition to oil, of water and small quantities of proteids. Of a similar nature are the bodies found in the thallus of the Marchantiex, which, in the case of Lunularia, also contain tannin.

Among other organised constituents of the cell-sap must be mentioned the spherical drops or granules containing tannin and surrounded by a thin membrane which are found in particular cells of the cortex of many plants rich in tannin, as Salix, Betula, Alnus, Quercus, \&c. (see Nägeli u. Schwendener, Das Mikroskop, p. 492). They are still more conspicuous in the motile parts of the leaves of the sensitive plant, where Pfeffer ${ }^{2}$ has investigated them with care. Here they consist of a thin but tolerably firm membrane, enclosing a concentrated solution which contains a large quantity of tannin. The strongly refractive contents of the spherical bodies are coloured blue by solutions of iron, and form a reddish-brown mass with potassium bichromate. If the contents are extracted with water containing alkali, acid, or alcohol, the membrane is left, and is perhaps what has been termed a 'pellicle-precipitate,' consisting of a combination of tannin with a proteid ${ }^{3}$. Pfeffer states that spherical bodies of this nature are found in particular cells of the cortex of the stem and petiole of the sensitive plant, as well as in the petioles of Oxalis stricta and Acetosellä; according to Meyen they occur also in Desmodium gyrans; and, according to Unger, in Glycyrrbiza.

Sect. II. Crystals in the Cells of Plants ${ }^{4}$.- The crystal-like forms described in Sect. 7 , in which proteids are sometimes found, though always mixed with other organic compounds, are not common phenomena, and must not be placed in the same category as the very abundant true crystals of lime salts now

3 Untersuchungen über die Leitbündelkryptogamen, Petersburg, I872, p. I०9.

2 Physiol. Untersuch., pt. I, Ueber Reizbarkeit, Leipzig I873, p. I3 et seq.

3 See Book III. Chap. i. Sect. I, Traube's artificial cells.

4 Sanio, Monatsber, der Berl. Akad., April 1857, p. 254.-Hanstein, ibid. Nov. I7, I859.Holzner, Flora, 1864 , pp. $27.3,556$, and 1867 , p. 499.-Hilgers, Jahrbuch für wiss. Bot. vol. VI. 1867, p. 285 .-Rosanoff, Bot. Zeitg. 1865 and 1867 - - Solms-Laubach, Bot. Zeitg. 1871 , nos. 31-33. -Pfitzer, Flora, 1872 , p. 97 . 
to be described; both from a morphological and physiological point of view the difference is very great.

Calcium carbonate occurs, where it has hitherto been observed in plants, not in the form of large crystals with clearly defined faces, but in finely granular deposits whose crystalline nature is recognised only by their behaviour to polarised light (illuminating in a dark field of view by a crossed Nicol); while their solubility in weak acids with evolution of bubbles of gas characterises them as calcium carbonate. It occurs, according to De Bary, in the form of roundish grains in the plasmodium of Physarum. The calcium carbonate imbedded in the cell-walls of many marine Algæ, Acetabularia, Corallina, Melobesia, \&c., seems to be still more finely divided, their structure becoming in consequence stony and brittle. It occurs in an excessively fine state of division, in the form of molecules invisible even under a magnifying power of 800 , in the structures known as Cystoliths, club-shaped outgrowths of the walls of certain cells projecting into the cavity, found in Urticaceæ and Acanthaceæ (vide infra).

All other crystals found in plants and hitherto accurately examined are shown, by their form where this is recognisable, and by their reactions, especially by their insolubility in acetic acid, and their solubility in hydrochloric acid without evolution of bubbles, to consist of Calcium oxalate. This salt is widely distributed, especially in the tissue of the Crustaceous Lichens, most Fungi, and Phanerogams, and in the form of very small granules of crystalline structure, of clusters, of bundles of needles (Raphides), or often of large, heautiful individuals with perfectly formed crystalline faces.

In Fungi and Lichens the crystalline granules are commonly small, and are not deposited in the interior of the cells, but on the outside of the cell-walls, and frequently in such large numbers that the hyphal tissue becomes opaque and brittle in consequence. In some Lichens, as in Psorosma lentigerum, according to De Bary, minute granules of calcium oxalate are deposited in the cell-walls of the dense cortical tissue. It is only exceptionally that crystalline deposits occur in the interior of the cells of Fungi, as, for example, in the form of radiate spheres (sphere-crystals) in the swellings of some of the hyphæ of the mycelium of Phallus caninus.

Little or nothing is known of the occurrence of calcium oxalate in most Algæ, in Muscineæ, and in Vascular Cryptogams; but it is found very abundantly in the tissues of most Phanerogams. In Dicotyledons it often occurs in the form of large beautifully perfect Crystals in the cavities of cells $(e . g$. in the mesophyll and petiole of Begonia, and the stem and root of Phaseolus). Clusters of crystals are, however, much more common in this class, and are especially abundant in the bark of many trees, in the rhizome of Rheum, \&c. They are deposited in a protoplasmic nucleus (e.g. in the cotyledons of Cardiospermum Halicacabum), the separate crystals being completely formed only in the exposed part. Sometimes also (as in the hairs of Cucurbita) small and perfectly developed crystals are seen in the circulating protoplasm.

In Monocotyledons, especially those allied to the Liliaceæ and Aroideæ, the crystals of calcium oxalate occur mostly in the form of bundles of long very slender needles, forming the so-called Raphides, which lie parallel to one another, and 
usually more or less completely fill up the cells, which are mostly elongated. Needles of this kind are formed also in great quantities when the leaves of many woody plants change their colour and lose water by evaporation in the autumn, although absent during the period of growth.

Where the crystals lie in the cavity of the cell-and this is usually the case with Angiosperms - they are commonly, perhaps always, coated by a thin membrane, which remains after solution of the calcium oxalate, and must probably be considered as a coating of protoplasm. This is also the case, according to Payen, even with raphides, and, according to the accurate observations of others, also in the larger single crystals and clusters.

In Angiosperms calcium oxalate occurs apparently only rarely deposited in the substance of the cell-wall; Solms-Laubach $(l . c$.$) cites different species of Mesem-$

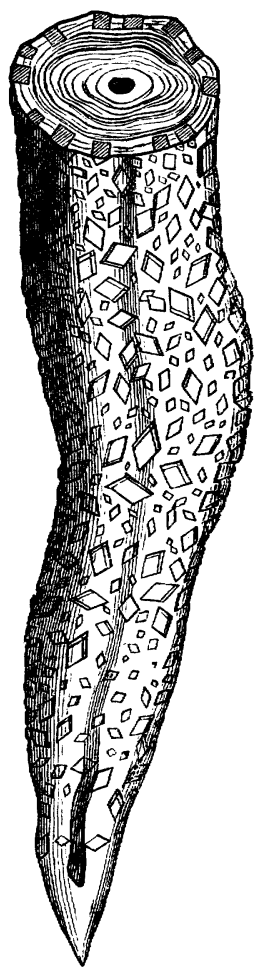

FIG. 52.-Half of a spicular cell of Weluitschia mirabilis, with a great number of crystals of calouter layer of the very thick cellwall. bryanthemum ( $M$. rhombeum, tigrinum lacerum, stramineum, Lemanni) and Sempervizum calcareum, in which fine granules or (in the last case) larger angular fragments of crystalline calcium oxalate are scattered through certain layers of the outer wall of the epidermal cells of leaves. Among Monocotyledons, Pfeffer has observed well-developed crystals in the thickened cuticle, and in cells which lie deeper in the tissue, of Draccena reflexa, arborea, Draco, and umbraculifera.

The occurrence of crystals of calcium oxalate in the substance of the cell-walls is, on the other hand, according to Solms-Laubach, of common occurrence in Gymnosperms. They generally consist of numerous small granules of unrecognisable shape; not unfrequently, however, they are well-developed crystals. In the bast-tissue of all parts of the stem deposits of this kind are found in the Cupressineæ, Podocarpus, Taxus, Cephalotaxus, and Ephedra; they are absent, on the other hand, from Phyllocladus trichomanoides, Salisburia adianiffolia, Dammara australis, and from all Abietineæ that have been examined. The small angular granules or larger individual crystals are usually deposited in the soft middle lamella of the walls between the bast-cells. Calcium oxalate occurs still more widely deposited in the cell-wall of the cortical parenchyma of the branches and leaves of Gymnosperms, with the possible exception of some Abietineæ; here also the middle lamella of a common cell-wall is the place where the crystals are formed, as also in the bundles of thick-walled hypodermal cells (e.g. Ephedra). The thick-walled often branched prosenchymatous cells abundantly scattered through the parenchymatous tissue of Gymnosperms, the socalled 'Spicular cells,' not unfrequently contain crystals deposited in the outer layers of their cell-walls; these occur in unusually large numbers and great perfection in Welwitschia mirabilis (Fig. 52). If the crystals are dissolved in hydrochloric acid, the empty cavities in the substance of the cell-wall retain completely the form of 
the crystals, so that the unpractised observer thinks that he still sees them. Fine granules are abundantly scattered through the thickened cuticle of Gymnosperms (Welwitschia, Taxus baccata, Ephedra, \&c.); or, in other cases, well-developed small crystals (Biota orientalis, Libocedrus Doniana, Cephalotaxus Fortunei, \&c.).

Closely related to these deposits in the cell-wall itself are the clusters of crystals discovered by Rosanoff (Bot. Zeitg. $186_{5}, \mathbf{1} 86_{7}$ ) in the pith of Kerria japonica and Ricinus communis, and in the petiole of some Aroideæ (Anthurium, Philodendron, and Pothos), which, lying in the cavity of the cell, are attached to the cell-wall by simple or branched threads of cellulose, and are even covered with a cellulose membrane. Pfeffer has shown that the large and beautifully developed crystals which occur in the leaves and branches of Citrus vulgaris, as well as in the bark of Salix aurita, Celtis australis, Rhamnus Frangula, Acer opulifolium, the Lombardy poplar, beech, and oriental plane, are also enclosed in a cellulose membrane which is often quite thick, and united in its growth, in one or more spots, with the cell-wall.

The Crystalline forms in which the calcium oxalate occurs in the cells of plants are extremely numerous, a result of the circumstance that this salt crystallises in two different systems, according as it is combined with six or with two equivalents of water. The calcium oxalate containing six equivalents of water of crystallisation $\left(\begin{array}{l}\mathrm{CaO} O \\ \mathrm{CaO}\end{array}\right\} \mathrm{C}_{4} \mathrm{O}_{6}+6$ aq.) crystallises in the quadratic system, the fundamental form being an obtuse quadrate-octahedron (the shape of a letter-envelope); combinations of the quadratic prism with the obtuse octahedron are met with in abundance. The raphides, however, belong, as respects their behaviour in polarised light, according to Holzner, to the klino-rhombic system, in which calcium oxalate crystallises with two equivalents of water of crystallisation $\left(\begin{array}{l}\mathrm{CaO} O \\ \mathrm{CaO}\end{array}\right\} \mathrm{C}_{4} \mathrm{O}_{6}+2$ aq. $)$. The fundamental form of the numerous combinations belonging to this class is a hendyohedron; it produces derivative forms which are very similar to calcspar (as, for instance, in the deposits in the cell-wall), and others very similar to calcium sulphate. The clusters of crystals (sphere-crystals) may consist of individuals of one or the other system ${ }^{1}$.

a. As respects its physiological significance, calcium oxalate is a metabolic product which is of no further use to the plant, an excretion similar to the volatile oils, resin, and other substances which are often contained in glands.

When the crystals remain so small that their volume appears inconsiderable in proportion to that of the cell itself, this latter may possess protoplasm capable of motion, nucleus, chlorophyll, and starch, as in the case of the hairs of Cucurbita or the mesophyll of Begonia. When, on the other hand, a crystal or a cluster or a bundle of raphides, or finally a mass of small crystals, nearly fills up a cell, no other organised constituent is usually present. The cells which contain raphides have loosened walls which easily swell, and the bundles of raphides are generally surrounded by a thick gummy mucilage. Such cells, which serve as receptacles for crystals, may be compared to simple glands which contain volatile oils and similar substances.

1 [Vesque has succeeded in reproducing artificially the crystalline forms in which calcium oxalate makes its appearance in vegetable tissues. Raphides are produced, according to his experiments, in the presence of glucose and also of dextrin. See Ann. des Sc. Nat., 5th ser., vol. XIX. p. 310.] 
b. The crystals of calcium oxalate which are imbedded in the middle lamella of a common cell-wall, such as those found in the soft bast of Coniferæ, have evidently been formed in the very spot where they occur. Pfitzer has shown, on the other hand, that the crystals found in Citrus vulgaris are formed in the cell-cavity, and become enclosed at a subsequent period by cellulose secreted from the surrounding protoplasm, which then coalesces in its growth, at one or more spots, with the cellwall. It is highly probable that the same is the case in other instances.

c. Cystoliths are at present known only in the Urticaceæ, Cannabineæ, Moraceæ, and Acanthacex (Justicia, Adbatoda). In the three first-named families they occur in isolated but numerous cells belonging to the epidermal system, especially of the leaves,

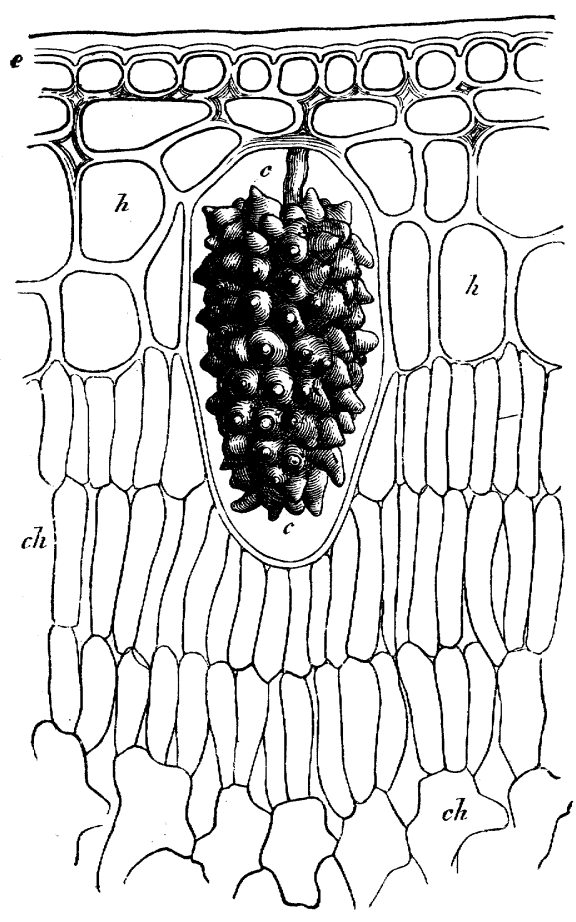

FIG. 53-Cystolith $c c$ in a hypodermal cell of Ficus elastica; $h h$ cells near the upper surface of the leaf; $e$ epidermis; $c h$ the inner tissue of the leaf containing chlorophyll. while the previously opaque substance of the concretion becomes gradually transparent from without inwards. Finally, there remains behind an insignificant skeleton of an organic matrix, in which the calcium carbonate was evidently deposited in the finest state of division. No cavities are to be seen out of which crystals can have disappeared; the matrix is perfectly homogeneous. Neither is there any reason for assuming that the lime was deposited between the layers of the matrix; since the outer portion of the mass, which contains an especially large quantity of lime, is quite unstratified. There is, on the other hand, a central nucleus, in direct connexion with the stalk, which is much denser than the external portion, and manifests an evident transverse stratification, as well as radiating fibres of a denser substance, which are obviously a faint indication of an intersecting striation. On addition of Schultz's solution the stratified and striated nucleus of the matrix assumes a beautiful dark blue colour, the outer portion only a light blue; the former consists of denser, the latter of more watery 
cellulose, between the molecules of which those of the lime have been deposited. The cystoliths originate (in Ficus elastica on Schacht's authority, in Broussonetia from my own observation) as wart-like outgrowths from the inner side of the cell-wall, which then swell up into a club-shaped form at their free end, and become impregnated with lime. After the lime has been dissolved and solution of iodine added, it is seen that the surface of the cystolith is coated with a thin protoplasmic membrane in which the original sculpture of the whole can still be perfectly made out.

[ $\mathrm{M}^{\mathrm{c}} \mathrm{Nab}$ gives (Journal of Botany, new series, vol. I. p. 33) for the composition of the potassium chlorate solution: three grains of potassium chlorate dissolved in two drachms of nitric acid of sp. gr. I'Io. The preparation of 'Schultz's solution' is thus described by Schacht (The Microscope and its application to vegetable anatomy and physiology, translated by F. Currey, p. 43) : Zinc is dissolved in hydrochloric acid; the solution is allowed to evaporate under contact with metallic zinc, until it attains the thickness of a syrup; the syrup is then saturated with potassium iodide, the iodine added, and the solution, when necessary, diluted with water. For the 'iodine-solution' the same authority recommends one grain of iodine and three grains of potassium iodide in one ounce of distilled water.] 
CHAPTER II.

\section{MORPHOLOGY OF TISSUES.}

SECT. I 2. Definition.-In the widest sense of the term, every aggregate of cells which obeys a common law of growth (usually however not uniform in its action) may be termed a Tissue. Aggregates of this kind may originate in different ways. The cells may be at first isolated; subsequently during their growth they may come into contact, and so completely coalesce at the surfaces of their walls that the boundary between them becomes indistinguishable. This happens, for

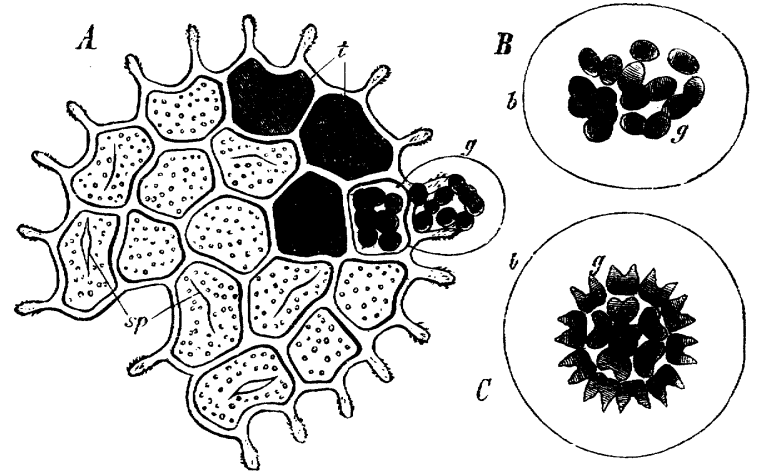

FIG. 54-Pediastrum granulatum (after A. Braun, $\times 400$ ). $A$ a plate consisting of united cells; at $g$ the innermost layer of a cell-wall is protruding; it encloses the daughter-cells resulting from division of the green protoplasm; at $t$ are various states of division of the cells; $s p$ the fissures in the already empty cell-walls; $B$ the inner lamella of the mother-cell-wall which has entirely escaped (greatly enlarged); $b$ contains the daughter-cells $g$, these are in active 'creeping' motion; $C$ the same family of cells $4 \frac{1}{2}$ hours after its birth, 4 hours after the small cells have come to rest; these have arranged themselves into a plate, which is already beginning to develop into one similar to $A$.

the wall of the embryo-sac itself, continuing then to develop as a continuous tissue (the endosperm) and to increase by division.

In Fungi and Lichens tissues originate by the juxtaposition and apical growth of slender filaments consisting of rows of cells (hyphæ), and of different orders of branchlets from them; each filament has its own growth, increasing the number of its cells by division, and branches copiously; but this takes place in such a manner that the different hyphæ undergo a similar development at definite 
spots on the whole body of the Fungus or Lichen, so as to form surfaces, threads, hollow structures, \&c., which possess a common growth, and yet consist of single elementary structures developing individually (Fig. 55).

With the exception, however, of the instances named, and of some similar ones, the formation in the vegetable kingdom of multicellular bodies obeying a common law of growth always arises from the cells which originate by bipartition from eommon mother-cells remaining in connexion; the cells are in these cases,
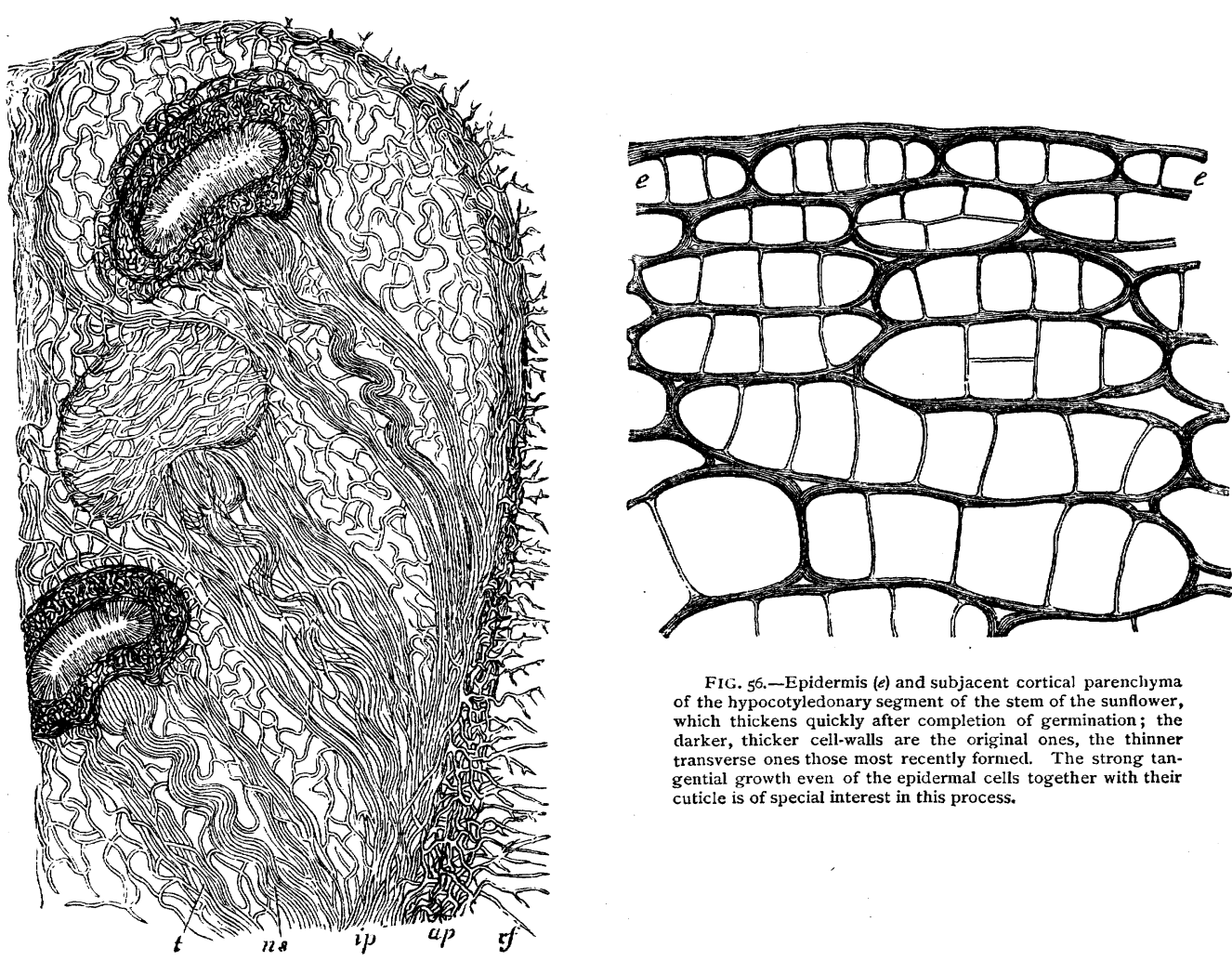

FIG. 56.-Epidermis (e) and subjacent cortical parenchyma of the hypocotyledonary segment of the stem of the sunflower, which thickens quickly after completion of germination; the darker, thicker cell-walls are the original ones, the thinner transverse ones those most recently formed. The strong tangential growth even of the epidermal cells together with their cuticle is of special interest in this process.

FIG. 55.-Part of a longitudinal section of a Gasteromycete (Crucibulum vulgare), showing the course of the hyphæ: their interstices are filled with a watery jelly, which has probably resulted from the conversion into mucilage of the outer cell-wall layers of the hyphx. (For further details of the internal organisation, see Book II, Fungi. The drawing is partially diagrammatic, inasmuch as the hyphæ are shown too thick for the small magnifying of the whole (about 25), and not so numerous as in nature.)

at least originally, so united that they appear like chambers in a mass which continues to grow as a whole (Fig. 56).

The two first-named kinds may be distinguished as false tissues from the latter or true form; but there is no sharp boundary-line between them. In many cases, for example, the endosperm is only in its rudimentary state a false tissue, due to the coalescence of isolated cells; in its further development by cell-division it becomes a true tissue (e.g. Ricinus, \&c.). The cortex of many Algæ and of the genus Cbara is formed by the coalescence of isolated filaments; but the result cannot be distinguished from true tissues. Nägeli and Schwendener (Das Mikroskop, vol. II. p. $5_{63}$ et seq.) may 
be consulted further on the growth of Acrochaticum pulvereum, Stypopodium atomarium, Delesseria Hypoglossum, and the leaves of Mosses ${ }^{1}$.

SeCT. 13. Formation of the Common Wall of Cells combined into a Tissue ${ }^{2}$. - If the cell-wall between two adjoining cells is thin, it appears, even

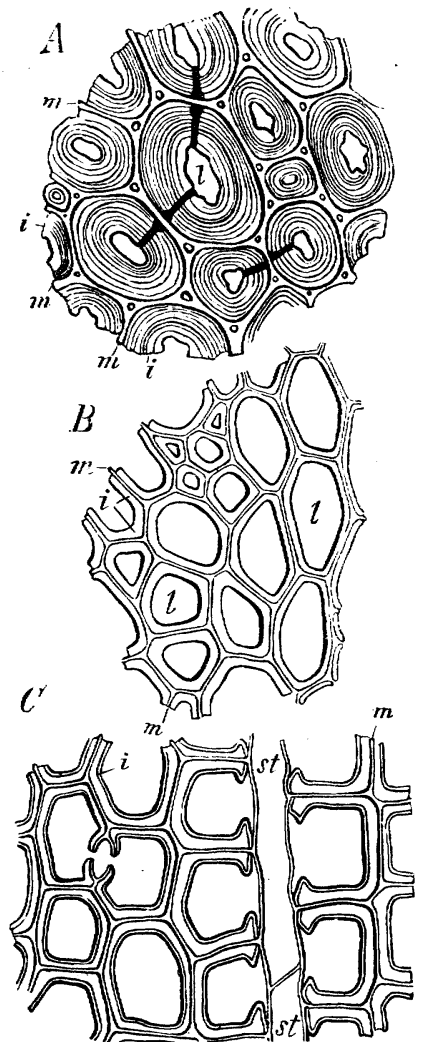

FIG. 57.-Transverse section through thickened cells with evident formation of middle lamellæ $(m)$; $i$ the whole of the cellulose superposed on this middle lamella; $l$ the cavity of the cell, from which the contents have been removed. $A$ from the cortical tissue of the stem of Lycopodium Chamacyparissus; $B$ wood-cells from the inner part of the wood of a young fibro-vascular inner part of the wood of a young fibro-vascular
bundle of the sunflower; $C$ wood of Pinus sylvestris, st a medullary ray $(x 800)$. condary,' or if it is differentiated into two shells, as 'Secondary' and 'Tertiary Cell-wall.'

1 On the formation of the cortex of Ceramiaceæ see Nägeli, Die neueren Algensysteme (Neuenburg 1847), and Nägeli und Cramer, Pflanzenphysiologische Untersuchungen.

${ }^{2}$ H. v. Mohl, Vermischte Schriften botanischen Inhalts. Tübingen 1845 , p. 3 I 1 et seq. Ditto, Die regetabilische Zelle, p. I96.-Wigand, Intercellularsubstanz und Cuticula. Braunschweig 1850. - Schacht, Lehrbuch der Anatomie und Physiologie der Gewächse, I 856 , vol. I. p. I08.Müller, Jahrb. für wiss. Bot. vol. V. 1867, p. 387.-Hofmeister, Lehre von der Pflanzenzelle. Leipzig I $867, \S 31$. times this is also the case when it has already attained a considerable thickness, as in succulent parenchymatous cells. Usually it is only when the wall has become moderately thick that it can be seen that the one side of the partition-wall belongs to one, the other to the adjoining cell. If stratification and differentiation into layers occur in a sufficiently thickened wall between two cells, a middle lamella always becomes discernible (Fig. $57, m$ ), on either side of which the cellulose is superposed in the form of layers and shells generally symmetrically distributed, so that those on one side appear to belong exclusively to the one adjoining cell, those on the other side to the other cell (Fig. 57, $i$ ). The impression may thus be given as if the layers which are concentrically deposited round each cell-cavity formed the wall belonging to it alone, while the middle lamella belonged to a common matrix in which the cells are imbedded; or as if it were excreted from the neighbouring cells. Both views were actually held for a considerable time, and the middle lamella was then termed 'Intercellular Substance.' If the older fragments of tissue represented in Fig. 57 are compared with the younger condition of the same, the thought at first suggests itself that the middle lamellæ may be the original thin walls, on which the thickening-layers have been deposited on both sides; this view has also found its defenders, by whom the middle lamella was distinguished as the 'Primary Cell-wall.' The remaining thickness is then correspondingly described as 'Sewhen very highly magnified, single; and some- 
In lignified tissues the middle lamella is generally thin but strongly refractive, and is formed of dense substance not capable of swelling. When the rest of the substance of the cell-wall has been dissolved in concentrated sulphuric acid, it remains (in fine transverse sections) as a delicate net-work; if, on the other hand, the cells are isolated by boiling in potash or nitric acid, this middle lamella is dissolved, while the rest of the cell-wall is preserved, as in all wood-cells and very many bast-cells. In other cases, as has already been mentioned in Sect. 4, the middle layers of the partition-wall of adjoining cells are, on the contrary, converted into mucilage; the layer of cellulose immediately surrounding each cell-cavity is dense, and the whole appears as if the cell-wall were imbedded in a mucilaginous refractive matrix (the so-called 'intercellular substance'); this occurs in many Fucaceæ and in the endosperm of Ceratonia Siliqua (Fig. 39, p. 36). On a fine transverse section through the cambial tissue of a branch of Pinus sylvestris, the two phenomena here described may be seen side by side; the woodcells show the thin dense middle lamella; the young bast-cells appear deposited in a soft mucilaginous substance, which is especially thick between the radial rows of cells, and is interspersed with fine strongly refractive granules (crystals); but both forms arise out of the same young tissue (the cambium), the walls of which are simple thin lamellæ, between which the cell-cavities themselves appear as so . many compartments. Objects of this kind are well adapted to prove the correctness of the supposition that in general the formation of denser or softer middle lamellæ depends only on a differentiation of the substance of the partition-walls during their thickening, a view which explains in a perfectly simple manner all the phenomena belonging to it, and altogether accords with growth by intussusception.

The thin perfectly homogeneous lamella of cellulose which bounds the young cells never exhibits a separation into two lamellæ; the boundary-line between the two cells is never marked by a fissure dividing the partition-wall. Nevertheless such a splitting of the still very thin lamella often takes place when the surfacegrowth is more rapid, as in the formation of the intercellular space, in the large-celled succulent tissue (parenchyma) of vascular plants, in the formation of stomata, \&c. Fig. $5^{8}$ shows some fully grown parenchymatous cells from the stem of the maize in transverse section; the cells were at first bounded by

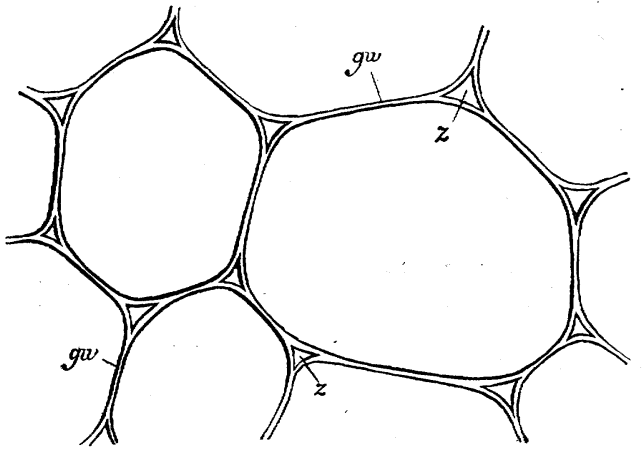

FIG. 58.-Transverse section through the succulent parenchyma of the stem of the maize; $g w$ common partition-wall of each pair of cells : $z$ intercellular space caused by the splitting of the wall $(x 550)$.

perfectly flat walls, which met nearly at right angles; as the size increased, a tendency arose towards a rounding off of the polyhedral forms, the unequal growth clearly leading to tensions which are only neutralised by the destruction of the cohesion in the substance of the cell-wall on the line where one wall meets 
the other. Thus a fissure arises which, in consequence of the mode of its origin, assumes the form of a triangular prism with concave sides (Fig. 58, z). It is filled with air, and becomes one of those intercellular spaces which very usually form in the parenchyma a continuous system of narrow channels. Not unfrequently the portions of the wall which bound the intercellular space grow rapidly, and thus it increases in size; the cells assume irregular star-shaped outlines in transverse section, touching one another only at small portions of the surface, as in the parenchyma on the under side of the leaves of many Dicotyledons, and the stem of Juncus effusus. In the faces of the cell, where no other wall intersects them, splittings of the homogeneous lamella may also occur locally; sometimes these are limited to narrowly circumscribed places, and produce flattened cavities in the homogeneous partition-wall. In other cases the splitting into two lamellæ takes place in such a manner that only isolated roundish places remain unaffected by it; the separated lamellæ continue to grow rapidly by intercalary

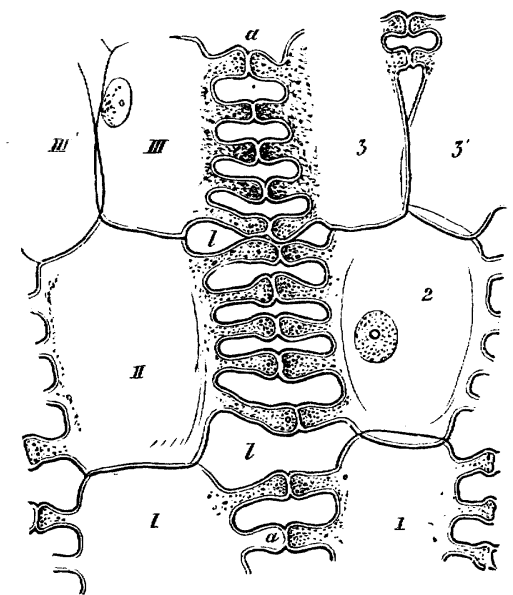

FIG. 59.-Two rows of cells running in a radial direc. tion $(I, I I, I I I$ and $\mathrm{r}, 2,3)$ from the cortical parenchyma of the root of Sagittaria sagittifolia in transverse section; $a$ the protrusions, $l$ the intercellular spaces between them (X about 350 ).

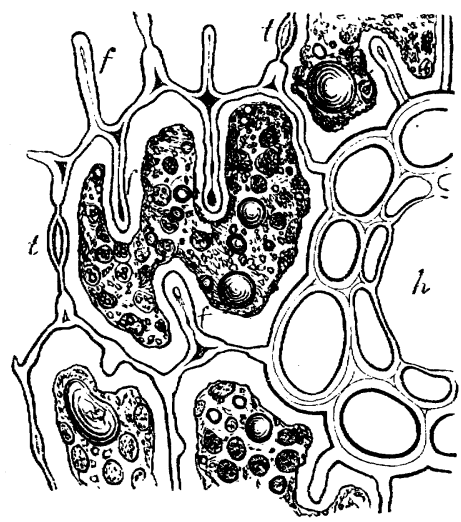

FIG. 60.-From a transverse section of the leaf of Pinus Pinaster; $h$ half of a resin-passage, to the left parenchymatous cells containing chlorophyll with folds $(f)$ of the cell-wall; $t$ pit-like formations (the contents of the cells contracted by glycerin, and containing drops of oil) ( $\times 800)$

growth, and bag-shaped protrusions of adjoining cells are formed which are separated by the fragments of the originally unsplit cell-wall (Fig. 59). In other cases there follows on the partial splitting of the partition-wall a local growth of one or both of the two lamellæ (or of only one), so that a fold arises which intrudes into the cell-cavity, as shown in Fig. $60, f$. Finally, in some species of the genus Spirogyra, the septum between each pair of cells splits into two lamellæ, each of which grows as a protrusion into the interior of the adjoining cell, and, when the adjacent cells separate, becomes turned inside out somewhat like the finger of a glove previously folded in. When the walls of cells forming a tissue split everywhere into two lamellæ (the separation proceeding always from the intercellular spaces) and become rounded off, a complete dissolution of the tissue takes place into a mere mass of isolated cells. This occurs in the flesh of many 
succulent fruits (e.g. Symphoricarpus in winter), and can sometimes be artificially brought about by continued boiling in water (as in potato-tubers).

The origin of the partition-walls in tissue-cells which increase by bipartition by no means requires the supposition that they were originally composed of two lamellæ. In this case one would be led, by a consideration of the properties of tissues where numerous divisions follow one another and intercellular spaces afterwards arise, to extremely complicated hypotheses, which, moreover, are not in harmony with growth by intussusception. Even where the union of the cells into a tissue arises from the amalgamation of originally separate cells (not sistercells), the union of the cell-walls is so intimate that no boundary-line can any longer be perceived; and the formation of a middle lamella proves also in such cases $^{1}$ (as does the formation of a middle lamella generally) that the hypothetical boundary-surface does not exist, and that the splitting of the homogeneous lamella is a consequence of different growth on its two sides. Both the manner in which the splittings of the thin homogeneous partition-walls arise, and also the formation of the middle lamella of thick walls, contradict the supposition of an originally double partition-wall in tissue-cells ${ }^{2}$.

The splitting of the partition-wall and the growth of its now separated lamellæ lead to a variety of configurations in the interior of tissues, which may be collectively included in the conception of the Intercellular Space. To this belong especially the large air-conducting channels in the tissue of many water and marshplants (Nymphæaceæ, Irideæ, Marsileaceæ, \&c.), and the formation of the cavity between the wall and the spore-sac in the sporogonium of Mosses ${ }^{3}$. Peculiar processes of growth of the adjoining cells are not unfrequently connected with the origin of intercellular spaces, of which the following are examples:- the formation of ordinary stomata, of the peculiar stomata of the Marchantieæ, and of resin and gum-passages (vide infra).

But the behaviour of the partition-wall ' of two cells contributes in quite a different manner to the production of air- or sap-conducting channels, which, like the air- or sap-conducting intercellular spaces, may form a continuous system throughout the plant. This happens by the partial or entire absorption of the partition-walls of adjoining cells, by which the cavities of long rows of cells of a tissue become connected. Unger has appropriately designated this a Coalescence of Cells. Vessels of this kind (Tracheïdes of Sanio) are formed in the xylem of the fibro-vascular bundles, from which the protoplasm and cell-sap have disappeared; they serve for conducting air. In the sieve-tubes in the bast-portion of the fibro-vascular bundles, on the other hand, the watery mucilaginous contents of the cells are not replaced by air; the communication established

1 For examples see Hofmeister, Handbuch, vol. I. pp. 262, 263.

${ }^{2}$ I may remind the reader of the cleavage of crystals as an analogous case; the cleavagesurfaces are determined by the molecular structure, but there is a wide difference between them and true fissures, however fine.

3 The wide air-canals in the stem of Equisetaceæ, Grasses, species of Allium, Umbelliferæ, and Compositæ, arise, on the other hand, from the cessation of the growth of inner masses of tissue and their drying and splitting, while the surrounding tissues continue to grow. 
between the cells of one row serves rather for a more rapid movement of the contents over greater distances. Laticiferous Vessels must also be regarded as composed of.coalesced cells; they are the result of very early and complete absorption of the partition-walls of adjoining cells belonging to straight or much branched rows in different systems of tissues.

Here however it is only necessary to point out the contrast between vessels produced by the coalescence of cells and intercellular spaces; a more minute consideration will come better in describing the systems of tissue.

(a) 'Intercellular Substance' and 'Primary Cell-avall.' The hypothesis implied by these terms could only be entertained so long as it was supposed that the original thin lamella between two adjoining tissue-cells was double, and so long as it was believed that the stratification of the cell-wall was brought about by the deposition of new layers. The expression that the original partition-wall betwe en two tissue-cells is double can only be understood in two senses:-either it means that the lamella consists of molecular layers, and that two of these contain between them the ideal boundary-surface of the two adjoining cells, or that there is an actual interruption of molecular connexion. The last supposition does not rest upon observation; it is besides contradicted by the detection of weak boundary-lines between layers which nevertheless are molecularly united, and have no cleft between them. Thus in the layers of thick cell-walls and of starch-grains there are no clefts, and yet the boundary-lines between the layers may be seen. If the first alternative is assumed to be correct, the question with reference to the intercellular substance depends on a mere verbal controversy; for if the original homogenous partition-wall is held together everywhere by molecular forces, and the supposed boundary-surface is no interruption of the molecular structure, then the deposition of a special intercellular substance at the same place is nothing but a process of ordinary growth by intussusception. The fact that the boundary-line between cells previously separate disappears by subsequent coalescence proves that the outer molecular layers of cellwalls may enter into molecular union. If in such cases a middle lamella is afterwards differentiated, this is the most striking evidence against the explanation of it as primary cell-wall. If an attempt is made to follow step by step the behaviour of developing woody tissue on the theory of the primary cell-wall, one is immediately involved in difficulties which do not arise on the supposition that the middle lamella is simply the result of subsequent differentiation.

(b) Examples of the formation of Intercellular Spaces. The origin of these spaces is very often connected, as has been mentioned, with a peculiar development of the separating cells, quite different from that of the rest of the tissue; so that the intercellular space together with its surrounding cells constitutes a peculiar form of tissue or an organ for a definite purpose. The observation of some cases of this kind is well calculated to show the beginner how, even in tissue-formation, processes which are morphologically similar or equivalent lead to entirely different physiological results. This subject will be treated in a more general and detailed manner in the third Chapter, and in Book III.

(I) The cleft of the Stomata of the epidermis belongs to the category of Intercellular Spaces, and its origin is peculiarly calculated to afford an insight into the mode of formation of an intercellular space. I have chosen the stomata on the leaves of Hyacinthus orientalis as an example. Figs. $6 \mathrm{I}-6_{4}$ are transverse sections perpendicular to the surface of the leaf; $e e$ in all of them are the epidermal cells, $p p$ the parenchyma of the leaf. The stoma, $S$, is formed of a rather small epidermal cell, which divides into two equal sister-cells by a wall vertical to the leaf; in Fig. $6 \mathrm{r}, S$, this has just 
taken place; the partition-wall is formed ${ }^{1}$, and appears as a very thin simple lamella, which soon thickens, and especially where it meets the wall of the mother-cell (Fig. 62, A). The thickening-mass appears at first quite homogeneous; afterwards an indication of stratification is to be observed, and the first trace of a separation into two (Fig. 62, B). In Fig. 63 , $t$, the splitting is already completed; the growth of the separated lamellæ now proceeds in a peculiar manner, so that a cleft arises
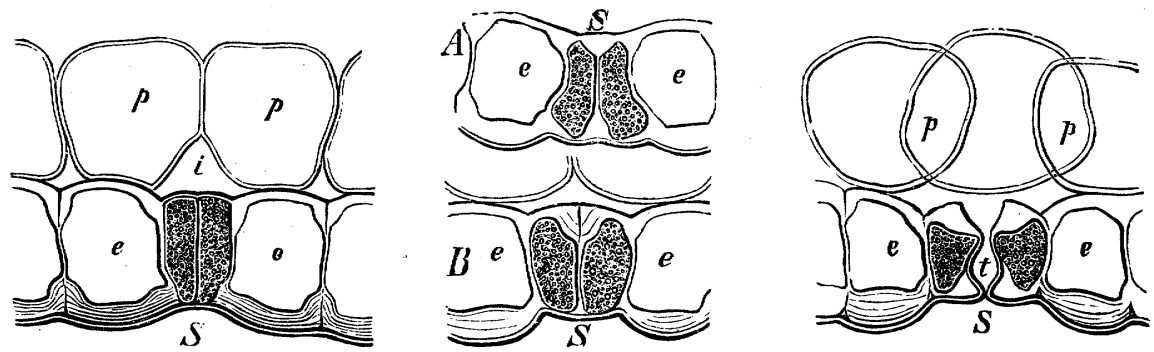

FIGS. 6x-63.-Development of the stomata in the leaf of Hyacinthus orientalis, seen in vertical section ( $x 800)$.

which is narrower in the middle, wider without and within, and which connects the intercellular space $i$ (the stoma) with the external air (Fig. 64). It is worth mention that before the division of the mother-cell an obvious cuticle has already overspread it together with the adjoining epidermal cells. This cuticle is easily recognised in the condition $B$, Fig. 62 , while still continuous; by the splitting of the partition-wall into two lamellæ it finally becomes ruptured (Fig. 63), and by the cuticularising of the outermost layer of the now separated lamellæ it is afterwards continued over the surfaces of the cleft (Fig. 64 ). If the process of the formation of the stoma is followed in a front view, it is seen that the splitting of the partition-wall does not extend throughout, but that a portion still remains undivided at each end where it adjoins the original mother-cell-well. The two cells which enclose the cleft, or Guardcells, are not only distinguished from the other epidermal cells by this peculiar mode

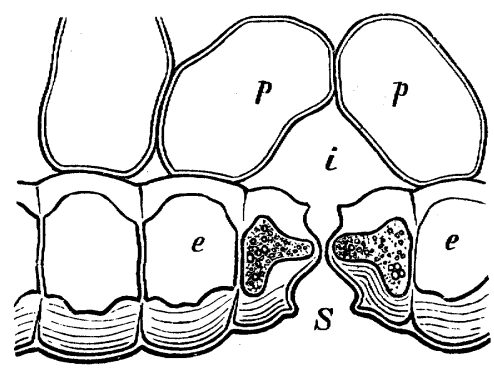

FIG. 64. of division and of growth; they also differ from them in containing chlorophyll and starch.

(2) In the family of Marchantiex belonging to the Hepaticx, the origin and structure of the stomata (Fig. $65, B, s p$ ) is much more complicated; of this we must speak hereafter. Here it need only be pointed out that even before the formation of the stoma the epidermal cells become detached from those lying beneath over rhomboidal areas which are marked off from one another by walls formed of cells which are not detached (Fig. $65, B, s s$ ). These large hypodermal chambers, each of which opens to the outside in its middle by a stoma, are destined to enclose the chlorophyll-containing tissue of these plants. The layer of cells which forms the

${ }^{1}$ I was unable to detect nuclei immediately before and for a considerable time after the division. [Strasburger has however succeeded with preparations preserved in alcohol in distinguishing, in the development of stomata in Iris pumila, the nucleus of the mother-cell and the successive stages of its division; see Ueber Zellbildung und Zelltheilung, p. I I5, t. v. figs. 38, 39; French translation, pp. II4-I I6.] 
bottom of each chamber, after repeated divisions vertical to the surface, sends out protrusions upwards into the cavity; these grow in a manner similar to many fila-
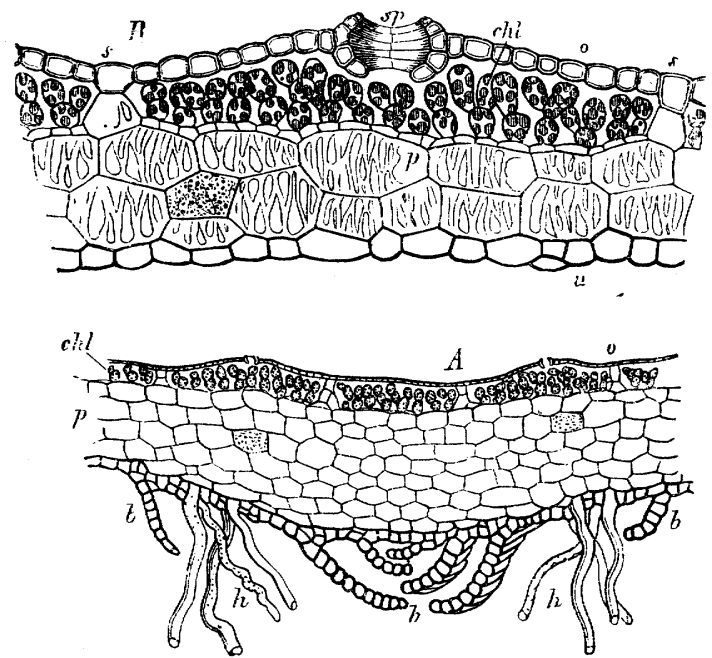

FIG. 65.-Transverse section through the horizontal thallus of Marchantia polymorpha; $A$ central part, furnished on the under side with the leaf-like appendages $b$, and the rhizoids $h\left(\mathbf{x}_{30}\right) ; B$ marginal part of the thallus, more highly magnified; $p$ colourless reticulately thickened parenchyma; $o$ epidermis of the upper side; chl the cells containing chlorophyll; $s p$ stoma; $s$ partition-walls between the hypodermal chambers; $u$ lower epidermis with dark-coloured cell-walls.
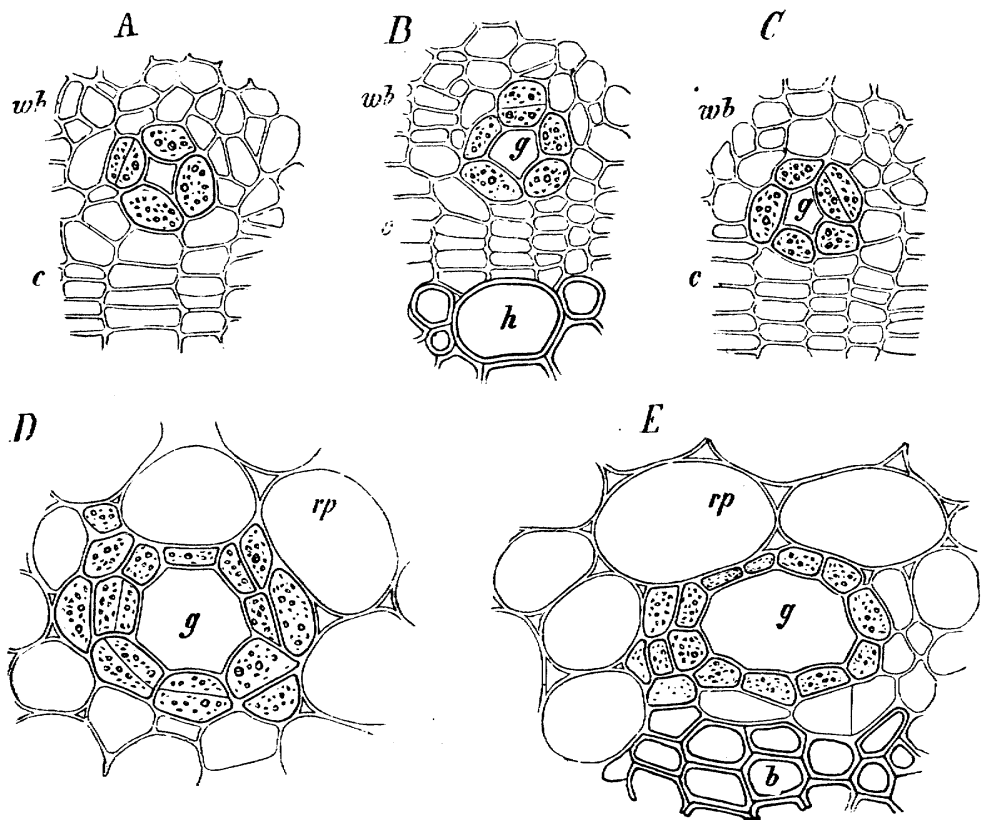

FIG. 66.-Sap.conducting intercellular passages in the young stem of ivy, in transverse section (X 80o); $A, B, C$ show young passages at $g$, placed at the boundary of the cambium $c$ and the soft bast $w b ; h$ the xylem; $g$ in $D$ and $E$ larger and older passages, lying at the boundary of the bast $b$ and the cortical parenchyma $r p$.

mentous Algæ, divide, branch, and form chlorophyll, while the whole of the rest of the tissue of these plants is devoid of it. 
(3) The origin of Resin-, Gum-, and Latex-passages depends also on the formation of intercellular spaces with a peculiar development of the cells which bound them. As I shall recur again to these structures, it is sufficient here to refer to one example. Fig. 66 represents passages of this kind in the transverse section of young portions of the stem of the ivy. Conditions such as $B, C$ show clearly that the intercellular space arises by the parting of four or five cells; and that these latter, distinguished by their turbid granular contents, increase by division. The formation of the much wider passages $D, E$ must also be referred to a subsequent increase and growth of the cells which surround the passage. By the growth of the cells which bound the intercellular passage, as well as by the manner of their division, by their contents, and by the circumstance that they excrete a peculiar sap into the passage, a structure of this kind becomes a differentiated part of the tissue, which is sharply marked off from its environment, and has a physiological function of its own.

Sect. r4. Forms and Systems of Tissues.-The entire mass of the celltissue which forms the body of a plant may be uniform or not; in the first case the cells are all similar to one another, and their modes of union everywhere the same. This case is rare in the vegetable kingdom; and it is only the simplest organisms that are constructed in this manner. Since in a homogeneous undifferentiated tissue all the cells are alike, their union into a whole is physiologically and morphologically of very subordinate importance, because each cell represents the character of the whole tissue. Hence it not unfrequently happens in these cases that the cells become actually isolated, and continue their life singly; and such individuals are termed Unicellular Plants. Only a little higher are those which consist of an unbranched row of perfectly uniform cells, or of an aggregation of such into a plate or mass. When numerous and densely crowded cells form a mass of tissue, then it is usually the case that different layers of tissue develop differently; the plant then consists of differentiated tissues. In general their arrangement is determined by the fact that the whole mass of tissue has a tendency to become definitely bounded on the outside, so that there arises a differentiation of outer layers of tissue from the inner mass. But in the interior of the mass enclosed by this Epidermal Tissue fresh differentiations arise in the higher plants; string-like arrangements of cells are formed, separated from one another and from the epidermis by Fundamental Tissue; these strings of tissue, the Vascular, Fibrous, or Fibro-Vascular Bundles, usually follow in their longitudinal course the direction of the most vigorous growth which immediately precedes their differentiation. Not only the epidermal layer, but also the vascular bundles and fundamental tissue, are however usually themselves differentiated; the epidermal tissue into layers of different nature; the bundles also exhibit differentiation and generally in a still higher degree. In this manner arise in the higher plants, instead of different layers, Systems of Tissues. We thus usually find an Epidermal System, a Fascicular System, and the System of Fundamental Tissue lying between them (Fig. 67). But whenever a differentiation of tissues of this kind occurs in a plant, it only takes place progressively; originally the whole mass of a growing portion of the plant (stem, leaf, or root) consists of a uniform tissue out of which, by diverse development of its layers, these tissue-systems have their origin. - This tissue of the youngest parts of plants 
which is not yet differentiated may be termed Primary Tissue, or, since its cells are always capable of division, Primary Meristem ${ }^{1}$.

In the present section a separate paragraph is devoted to each tissue-system, and a description will first be given of the various forms of cells and tissues which

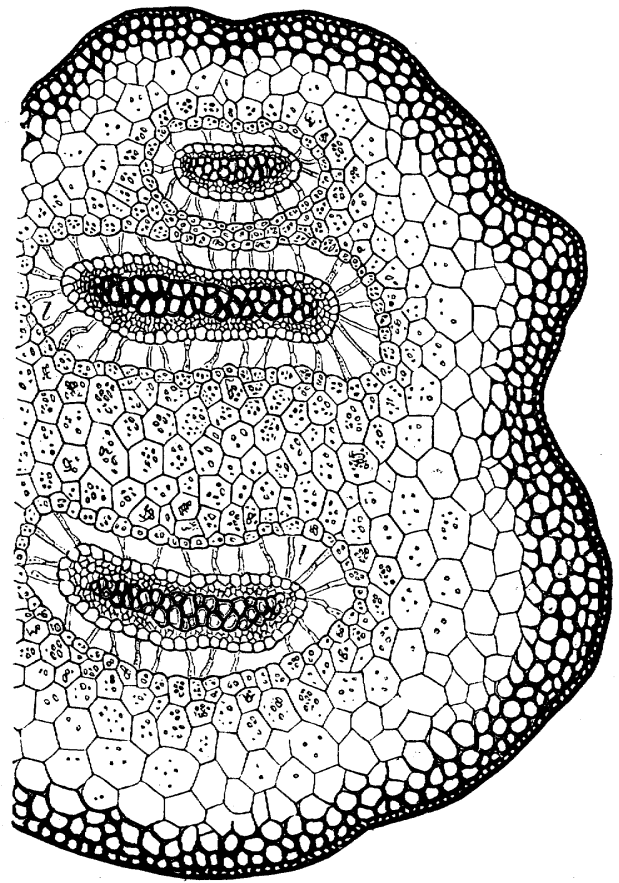

FIG. 67.-Transverse section of the stem of Selaginella inaquali: folia. The outer layers of cortical have thick dark-coloured cell-walls ; the thinner-walled fundamental tissue envelopes three fibro-vascular bund!es, separated from it by large intercellular spaces $l(\times 8 \infty)$. are constituents of all systems. Those forms which are peculiar to one or another system will be discussed in their proper place.

(a) In reference to external form, the following cell-combinations may be enumerated :-

(I) The term Tissue may be applied par excellence to aggregations of similar cells which, without any welldefined external form, consist, in whatever direction the section be made, of numbers of cells. Illustrations of such tissues occur in the greater number of the larger Fungi; the fundamental tissue of thick stems of Ferns and Monocotyledons is a parenchymatous tissue penetrated by other forms of tissue in the shape of strings. In Dicotyledons the pith especially comes under the same denomination; it may also be found in the purest form in the flesh of succulent fruits. In stonefruits, such as the peach, plum, cocoanut, \&c., the stone consists of a sclerenchymatous tissue. It may also occur that forms of tissue which differ morphologically may be aggregated into a mass of tissue uniform in its physiological characters, as in the secondary wood of trees and the succulent tissue of tubers, such as the potato, dahlia, \&c.

(2) A row or Filament of Cells is composed of similar cells placed singly side by side or in rows; but they are usually connected genetically. Isolated filaments of this character occur in the hyphæ of Fungi and in many Algæ, where they have arisen by transverse partition of an apical cell or by intercalary transverse division. In the higher plants we have abundant illustrations:-in the epidermal hairs, vessels, laticiferous vessels, sieve-tubes, tannin-receptacles (as in the phloëm of Phaseolus), \&c. which are found in the interior of tissues.

(3) A simple Layer of Cells results where similar cells are so united in one plane that the entire layer is only a single cell in thickness. Among Cryptogams it is not

1 It may not be superfluous to remark that the pith and cortex are neither forms nor systems of tissue, but are altogether indefinite and undefinable; we speak, for example, of cortex in Thallophytes in quite a different sense to what we do in Vascular Plants ; the cortex of Monocotyledons is something different from that of Conifers and Dicotyledons; in the latter the cortex has quite a different signification in young and in older paits of stems. The same is the case with the pith. 
rare for a whole organ to consist of a single layer of cells, as the leaves of Jungermannieæ, or even the entire plant, as among Algæ in Ulva and Rytipblaa. In the higher plants the epidermis usually consists of a single layer, and this is not unfrequently the composition of the vascular bundle-sheath (always in the case of young roots); in water and marshplants the fundamental tissue often resolves itself into simple layers which enclose large intercellular spaces, as in Nuphar, Fig. 68, Salvinia, Musa, \&c. It is not uncommon for masses of tissue to be composed of a number of simple layers of cells, as occurs frequently in the secondary wood of trees and the primary cortex of branches and roots; or several simple layers alike among themselves are in close juxtaposition, as in the epidermis of the leaves of Begonia and Ficus elastica.

(4) A String or Bundle of Cells is an elongated mass of tissue, the transverse section of which consists of a

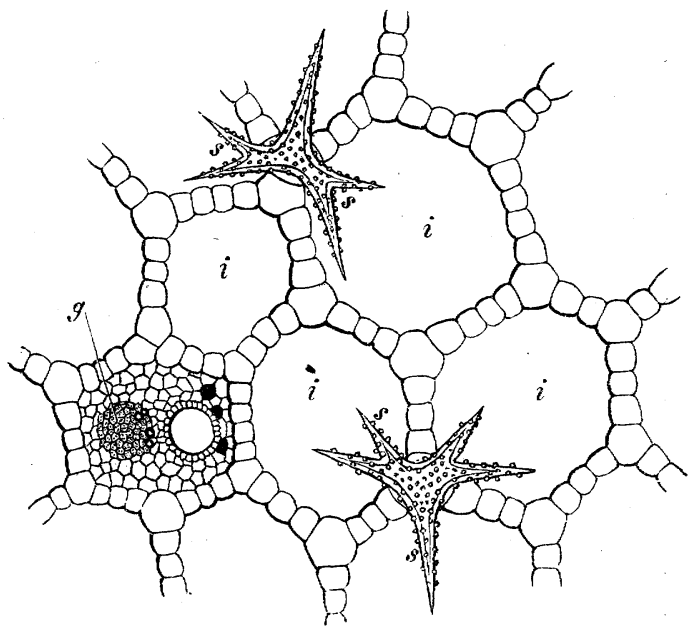

FIG. 68-Transverse section of a part of the petiole of Nuphar atiena; $i$ large intercellular spaces bounded by simple layers of cells; $s s$ stellate id oblasts; $g$ a fibro-vascular bundle. number of cells. Many of the lower plants, such as some simple Fungi and Floridex, consist of such strings of cells. The fundamental tissue of the higher plants is sometimes traversad by bundles of peculiar cells; such are the brown sclerenchymatous strings in the stem of Pteris aquilina and of Tree-ferns. The true bast of Dicotyledons not unfrequently forms bundles in the soft bast. In all vascular plants the bast-like and wood-forming elements are united into bundles, the Fibro-vascular Bundles, which form true tissue-systems, and traverse the fundamental tissue.

(5) Groups of Cells are roundish aggrègations of similar cells. In the lower Algæ, as the Chroococcacex, groups of this nature arise, each from a single mother-cell, and carry on an independent life as Cell-families. In the fundamental tissue of the higher plants groups of peculiar cells are often formed, strikingly different from those that surround them, as for example the groups of laticiferous cells illustrated in Fig. 69, or the groups of sclerenchymatous cells in

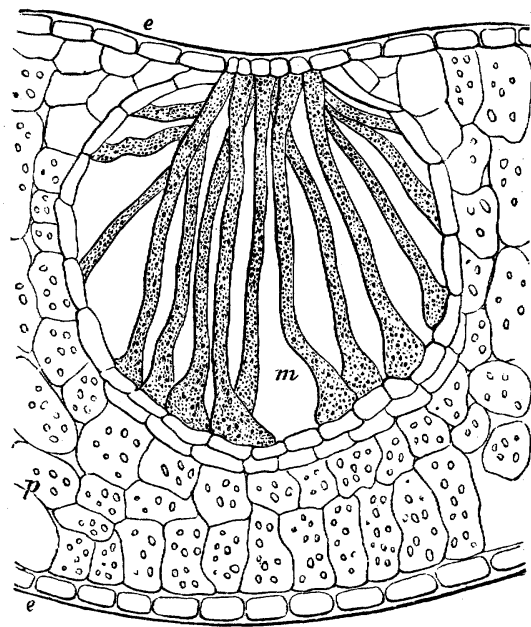

FIG. 69.-Vertical section of a leaf of Psoralea hirta $m$ a group of laticiferous cells imbedded in the chlorophyll. tissue $p ; e e$ the upper and under epidermis. the soft flesh of pears. True (compound) glands are formed by the dissolution of such groups of cells (vide infra).

(6) In the cases already named a number of similar cells are always united into a whole; but it also frequently happens that a single cell acquires a character 
different from that of the surrounding ones, owing to a difference either in its form or its contents; as for instance the stellate hair formed in the interior of the tissue of Nymphæaceæ (Fig. 68), and the Lithocysts in which the cystoliths (Fig. 53, p. 68) are formed, \&c. Cells marked by such a striking peculiarity I propose to call Idioblasts; the special forms will be described further on. The term Eremoblast, on the other hand, may be given to all those forms of cells which detach themselves altogether from their sister-cells, and continue an independent life; such as the spores of Cryptogams, antherozoids, pollen-grains, some gemmæ of Hepatic $x, \& c$. The entire individuals of some lower plants are eremoblasts, as most Desmidieæ, Bacillarieæ, and Siphonex (Unicellular Plants). There is this in common between idioblasts and eremoblasts, that the individual cells usually attain a high degree of development; but with this difference, that in the former the development is commonly in one direction only, while in the latter the individual cell may undergo the most varied differentiation, constituting in itself an organism endowed with independent life.

(b) Nägeli classifies all the various forms of tissue under two primary heads:Generating Tissue or Meristem, and Permanent Tissue. This classification is by no means parallel with one into younger and older cells; for, even when the former consists of younger, the latter of older cells, there are also other additional and important differences.

(1) Meristem consists of cells which increase slowly in size, and divide repeatedly in such a manner that some of the resulting cells continue to divide, while others pass over into permanent tissue. The cells which are still capable of division (merismatic) are mostly much smaller than the permanent cells which are developed from them, and are more or less similar to one another; they are easily recognised by their smooth thin walls, the quantity of protoplasm they contain, and the absence of any coarsely granular deposits. Meristem may be again divided into the two following kinds :-

(a) Primary Meristem comprises the whole cellular tissue of very young organs or parts of organs, as the apices of roots and stems, the youngest leaves, and the embryo; from it are subsequently developed the later forms of tissue. We shall hereafter discuss this form of tissue more in detail.

(b) Secondary Meristem occurs in those organs or parts of organs which have advanced beyond the condition of primary meristem, and therefore contain differentiated forms of permanent tissue, among which the secondary meristem is usually found in the form of thin layers, and furnishes, from its power of forming cells, the material for the production of new permanent tissue in addition to that already in existence. Various forms of secondary meristem will be hereafter described under the names of Cambium, Tbickening-ring, and Phellogen or Cork-cambium.

It not unfrequently happens that the large cells of a permanent tissue which have already attained a high degree of development, with moderately thick walls, a large quantity of cell-sap, but a relatively small proportion of protoplasm, and which contain chlorophyll-granules or other coarsely granular deposits, begin to grow and to divide afresh; as, for example, in the primary cortex of annual shoots and stems when they increase rapidly in diameter. The cortical cells are compelled, by the increase in size of the wood, to stretch in a tangential direction; each breaks up by repeated radial division into a number of segments (as shown in Fig. 56, p. 7I); and these, as soon as they are formed, behave like mature permanent cells. Among plants of very simple structure such a division of more mature cells takes place in Conjugatæ, especially in Spirogyra (Fig. 13, p. I7). A tissue in this condition has been termed Older Meristem, in contrast to Younger Meristem, in which all the dividing cells are still in their youngest condition.

(2) Permanent Tissue is the result of a further development of those cells of the 
primary and of the various forms of secondary meristem which no longer divide, but grow vigorously, and finally attain a definite development, in which they are of service to the plant by the rigidity or some other property of their cell-walls, or by the chemical activity of their contents. The most various forms of permanent tissue result from similar cells of the same primary or secondary meristem.

On attaining their permanent condition many forms of permanent tissue lose the whole of their living contents, especially their protoplasm, of which dry granular vestiges are sometimes left behind. Such forms of tissue may be termed Kenenchyma ${ }^{1}$, and include, for example, cork-tissue, and the tracheal elements of the wood-vessels and vessel-like wood-cells. In contrast to this is the Succulent Tissue, the cells of which, during the life of the organ, remain filled with chemical products of the vital activity of the plant, not with air or water. These succulent tissues may again be divided into two groups:- In the first the cells still contain protoplasm in an active vital condition, and are therefore able, under favourable conditions, to grow and divide afresh-i.e. to pass over into older meristem, phellogen, \&c., or, on being wounded to form a callus or cork-tissue-as, for example, in chlorophyll-containing tissue, the succulent parenchyma of cortex, or of tubers, \&c. In the second kind the cells pass into a condition of permanent quiescence, as in all those cases in which the protoplasm becomes unrecognisable, or remains behind as a doubtful residue; and where the cells are filled, not with chlorophyll, starch, sugar, inulin, fatty oil, aleurone, or other reserve-materials, but with excrementitious products of various kinds, as volatile oils, resin, gum, cystoliths, clusters of crystals, \&c. Cells of this description, and tissues composed of them, are apparently never capable of any further development; they are incapable, for example, of forming a healing cork-tissue over wounded surfaces. There is however no sharp line of demarcation between these different forms of tissue distinguished by their contents; it is only the extreme cases that can be thus characterised.

We also find the greatest variety of intermediate links, if we consider the various kinds of tissue in reference to the thickness and consistency of the cell-wall. Starting from ordinary succulent parenchyma with thin but firm and elastic walls composed of nearly pure cellulose, we see how, on the one hand, the thin cell-walls become converted into cork (periderm), while on the other hand the cell-walls of other kinds of tissue thicken and become lignified or converted into mucilage, or as hard as stone. The nature of lignification and conversion into mucilage has already been pointed out; the collenchymatous development of certain hypodermal tissues will be spoken of hereafter; here it is necessary only to refer to the fact that layers, strings, or groups of cells are frequently distinguished by the extraordinary hardness and thickness of their cell-walls. Such tissues, which may arise in all systems - as for instance the 'Stone-cells' (scleroblasts) in the flesh of pears and in the bark of many trees, the dark-brown strings in the stem of Tree-ferns, \&c. - may be included under the collective term Sclerenchyma.

If we now consider tissues in reference to the form and the mode of combination of their cells, it is of course evident that the latter must depend on the former; but that, on the other hand, the position of cells already existing must influence the growth, and therefore the form, of those which are developing. If we for the moment leave idioblasts out of account, and consider merely the mode of association of similar cells, we find that the old distinction into Prosencbyma and Parenchyma can still be maintained. By the former is meant a grouping together of elongated fusiform pointed and usually thick-walled cells, whose ends are dove-tailed between one another without intercellular spaces. A prosenchymatous arrangement of this kind is well seen in

${ }^{1}$ [This term, which has not hitherto been employed, is proposed as the equivalent of the German 'Leerzellengewebe.'] 
the fascicular elements of the wood and of true bast; but the fundamental tissue is also not unfrequently prosenchymatous. When, on the contrary, the cells are roundish or polyhedral, leave wide spaces between them, and touch one another by broad faces, the tissue is said to be parenchymatous. In elongated organs, like roots, internodes, \&c., the cells are usually elongated in the direction of the axis of growth; but are truncated at both ends, and arranged in parallel rows with broad septa. In green leaves, on the contrary, there are usually two kinds of parenchymatous tissue: - the so-called Palisade-parenchyma beneath the upper epidermis, with cells elongated in a direction vertical to the surface of the leaf, but densely crowded together side by side; and the Spongy Parencibyma of which the under half of the leaf consists, with roundish cells which either leave between them comparatively large intercellular spaces, or are furnished with outgrowths and branches with which they touch the adjoining cells, so that the tissue becomes still more spongy. The parenchyma attains the highest degree of looseness when the cells form a number of many-rayed stars which are in contact only by the ends of the rays, as in the stems of many rushes, the petiole of Musa, \&c. The tissue of the larger Fungi (Fig. 55, p. 7I) can be properly termed neither prosenchymatous nor parenchymatous; but consists of a number of Hypha, i.e. long slender branched filaments growing at the apex, and dividing transversely; it is best to term it a Hyphal Tissue. When the hyphæ are densely interwoven, and their cells short and broad, a parenchymatous appearance is presented in transverse or longitudinal sections, and such a tissue has been called $P$ seudo-parenchyma.

It has already been mentioned that it is not unusual for individual cells in a tissue otherwise homogeneous to become developed in a manner strikingly different from their neighbours; to such cells I have applied the term Idioblast. They may differ from the surrounding tissue in three different ways:-(I) Their form is the same, but they are distinguished by their contents; as, for example, by a coloured cell-sap, or by containing a volatile oil, resin, gum, or other similar substance, in which case they are termed Simple Glands; or they may contain groups of crystals, bundles of raphides, or cystoliths, in which case I have termed them Lithocysts. (2) In cells which contain a coloured sap, simple glands, and lithocysts, the cell-wall remains thin, while in other cases it becomes so thickened that the cavity of the cell is reduced to a narrow canal or a small central hollow; the thickened cell-wall manifests stratification and pore-canals, and is usually very hard. Idioblasts of this kind may be included in the general term Stone-cells or Scleroblasts. They are seldom isolated, but more often associated in groups or layers, and then form the tissue already described as Sclerenchyma. (3) In the two kinds of idioblasts already described a tendency is exhibited to attain a lärger size than that of the surrounding cells; scleroblasts are especially characterised by outgrowths. In the third group this takes place to a remarkable extent, this kind of idioblast being distinguished not only by its contents and the form of its cell-wall, but especially by its great increase in size and vigorous growth and branching. This is illustrated to a moderate extent in Fig. I6 (p. 2r), more strikingly in the Spicular Cells of Gymnosperms (Fig. 52, p. 66), which usually contain a number of crystals in their thick wall, are considerable in size, and generally branch extensively. To this class belong also the internal 'stellate hairs' of the petiole of $N_{u p h a r}$ (Fig. 68, p. 8I) ; and closely allied to these are the structures termed by Van Tieghem' 'poils' in the fundamental tissue of the Monsterineæ, a group of Aroideæ. Fig. 70 represents a longitudinal section through the petiole of Monstera deliciosa; a cell $s$ lying in the middle parenchymatous row has branched right and left and put out two arms extending upwards and downwards into the intercellular spaces of the parenchyma, one of which has again put out a small

1 Van Tieghem, Structure des Aroidées; in Ann. des Sci. Nat. 1866, vol. VI. See also Otto Buch, Ueber Sclerenchymzellen. Breslau 1870. 
lateral branch. The wall of this cell has become so thick that its cavity is reduced to a narrow canal. These cells are very abundant in the tissue of Monsterineæ, and present the appearance, when the petiole is broken across or cut with a blunt knife, of tough, slender hairs projecting out of the tissue. For idioblasts of this kind I propose the term Trichoblast, in order to express their resemblance to many epidermal trichomes. Those now referred to have such thick walls that their contents are of very little importance from a physiological point of view, if indeed they do not altogether disappear and become replaced by air. But in other cases they contain latex, which flows out in greater or less abundance when the plant is wounded. Such structures, the peculiarities of which were first recognised by David ${ }^{1}$ and compared by him to the trichoblasts in the petiole of Monsterineæ, and termed Laticiferous Cells, were previously confounded with true laticiferous vessels which result from a coalescence of cells (see p. 86). The receptacles for latex in Euphorbiaceæ, Morex, Apocynaceæ, and Asclepiadeæ are very long cells which are closed on all sides, often much branched at the ends, but not communicating with one another; they are formed at an early period, near the apex of the stem in the young fundamental tissue of the primary cortex, or of the pith when the side of the vascular bundles next the pith contains phloëm (Hoya carnosa); in other cases laticiferous cells belonging to the cortex put out branches through the ring of wood into the pith (Euphorbiacex, Morex). The laticiferous cells of the leaves are, in the case of Euphorbia, only prolongations of those of the internodes. The extraordinary length of the laticiferous cells, especially in Hoya carnosa, makes it difficult to recognise their true nature; but it is easily explained by their early formation near the apex of the stem, which necessitates their keeping pace with the growth in length of the stem as well as that of the whole surface of the leaf. These laticiferous trichoblasts are most easily seen in Euphorbia splendens, since they are readily isolated in consequence of their thick firm cellwall, and can be distinguished with certainty from any other form of tissue, by the peculiarity in the form of the starch-grains as well as the coagulated latex which they contain. The laticiferous cells represented in Fig. $7 \mathbf{r}$ (slightly magnified and reduced about $\frac{x}{4}$ in length) have been obtained by allowing the ends of branches of Euphorbia splendens to decay in water until the tissue has become very soft; lumps of the soft

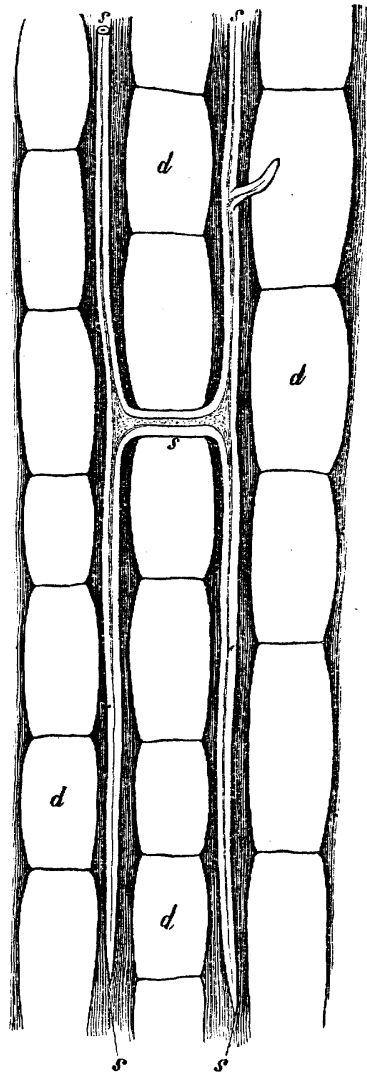

FIG. 70.-From a longitudinal section through the petiole of Monstera reli giosa: $d d$ parenchymatous cells; $s s$ a trichoblast. mass were then dissected with needles under the microscope, and the pulpy tissue washed away as completely as possible, until the long laticiferous cells were exposed, and allowed their closed ends to be fully examined. The structure of the laticiferous cells in the leaves can, according to David, be determined with much certainty by first extracting with alcohol, and then rendering them transparent by boiling in potash. In Ficus elastica the laticiferous cells are thin-walled and more

1. G. David, Ueber die Milchzellen der Euphorbiaceen, Morcen, Apocyneen, u. Asclepiadeen; Dissertation. Breslau 1872 . 
difficult to follow; still more so in the oleander, where also the contents are more limpid.

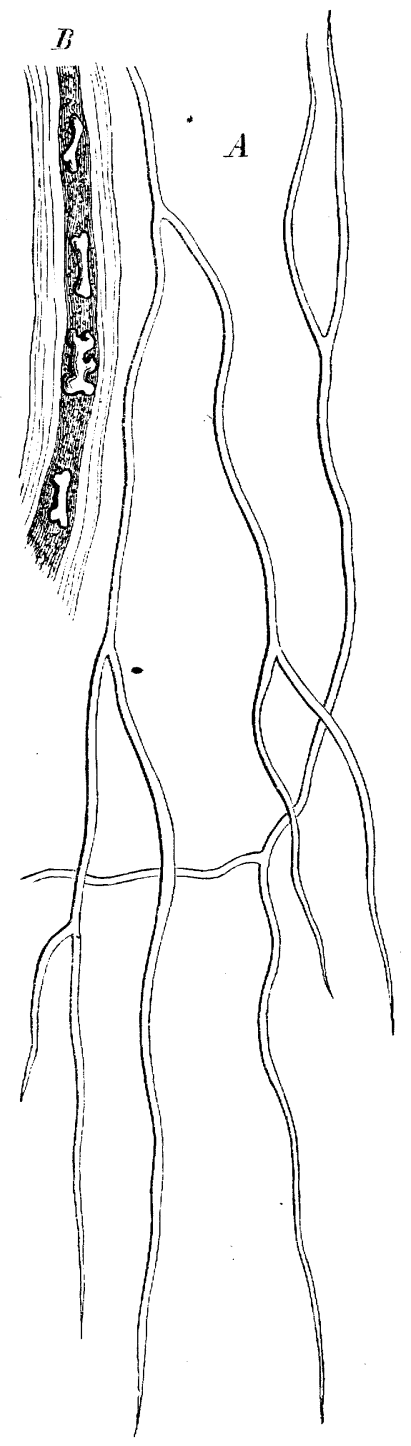

FIG. 71.--Laticiferous cells ${ }^{\top}$ from the end of a branch of Euphorbit splendens, exposed by maceration; $A$ the whole of one and part of another laticiferous cell (slightly magnified) ; $B$ a piece of one containing starch-grains of peculiar form (str ngly magnified).
A Coalescence of Cells arises from the tissue-like union of similar cells, until their contents completely coalesce, the partition-walls becoming partially or wholly absorbed. In this manner are formed those filiform aggregates of intercommunicating cells, constituting tubes filled with air or sap, which are known as Vessels, such as Wood-vessels, Bast-vessels or Sieve-tubes, and Laticiferous Vessels. But roundish groups of cells may also coalesce by the absorption of their walls and the formation of a single large cavity filled with sap; these are included under the general term Glands (or may be specially distinguished as Compound Glands). Just as the distinction between idioblasts and true tissue-cells is only a progressive one, depending on the augmentation of certain characters, so a coalescence is only an extreme case of the ordinary behaviour of adjacent cells; the contents of these mingle to a certain extent through their partition-walls by diffusion (osmose). Hence we frequently meet with tissues consisting of peculiar cells, which behave physiologically as if they had coalesced, although it is questionable whether the cavities of the cells are actually in communication one with another.

True Laticiferous Vessels are composed of coalescent cells containing latex and endowed with the same properties as we have already described as belonging to the laticiferous cells of Euphorbiaceæ, Moreæ, A pocynaceæ, and Asclepiadeæ. As far as can be judged from observations which are not yet brought to a conclusion, these vessels originate from rows of cells in the young tissue, and especially in the fibro-vascular bundles, coalescing at an early stage by the complete disappearance of their transverse septa; long tubes (as shown in Fig. 72) being thus formed filled with latex, which usually anastomose with one another laterally, and traverse the whole plant in the form of a continuous system of tubes ${ }^{1}$.

The Cichoriacex, Campanulaceæ, and Lobeliacex possess very perfectly developed laticiferous vessels belonging to the fibro-vascular bundles, which they accompany throughout the whole plant in the form of reticulately anastomosing tubes, imbedded, in the case of Cichoriace $x$ in the outer, in that of the two other orders in the inner phloëm-layer. Their form is best recognised by boiling sections of these plants for some minutes in dilute potash solution; the anastomosing tubes are then clearly recognised in the transparent tissue (Fig. 72 .), and it is easy to expose them entirely in large pieces. In

1 What follows is founded mainly on Hanstein's researches reported in his Preisschrift, Die Milchsaftgefässe und die verwandten Organe der Rinde. Berlin 1864. See also Dippel, Entstehung der Milchsaftgef ässe und deren Stellung in Gefässbündelsystem. Rotterdam $186_{\mathbf{5}}$ - - Vogel in Jahrb. fuir wiss. Bot. vol. V. p. 3 I. 
Papayacex (Carica and Vasconcella) the laticiferous vessels, on the other hand, run through the xylem-portion of the fibro-vascular bundles; they-i.e. the cells by the coalescence of which they are formed-are repeatedly produced in layers from the cambium with the other elements of the xylem; pitted and reticulately thickened vessels alternate with them. The branches of the laticiferous vessels envelope these in all directions, and are sometimes firmly fixed to them superficially; but horizontal branches of these tubes also penetrate the medullary rays, and terminate, towards the primary cortex, in scattered ramifications or recurrent knots, as also in the pith if the stem is hollow. As in the other families, a copious anastomosis of laticiferous vessels is developed in the horizontal partition-walls which the medullary tissue forms at the origin of each petiole in the hollow of the stem, penetrating the horizontal partition-wall in countless ramifications and in several layers one over another, and connecting the vessels belonging to the medullary rays with thcse of the whole wood-cylinder. In Papaveraceæ (Chelidonium, Papaver, Sanguinaria) the laticiferous vessels are also very perfectly developed; they are not here, however, as in the families just named, united into ribbon-shaped groups, but run mostly at a greater distance from one another, dispersed through the phloëm and the surrounding parenchyma; single ones appear also in the pith, but do not penetrate into the xylem. Lateral outgrowths and crossanastomoses are seldom found in the stem, but abundantly in the leaves, and especially in the carpels, in which closemeshed reticulations are formed, according to Unger, in the parenchymatous fundamental tissue; similarly also in the cortex of the root. In this family, especially in the parenchyma of the root of Sanguinaria canadensis, the origin of the laticiferous vessels from the coalescence of rows of cells may, according to Hanstein, be proved; owing to their imperfect union the re-

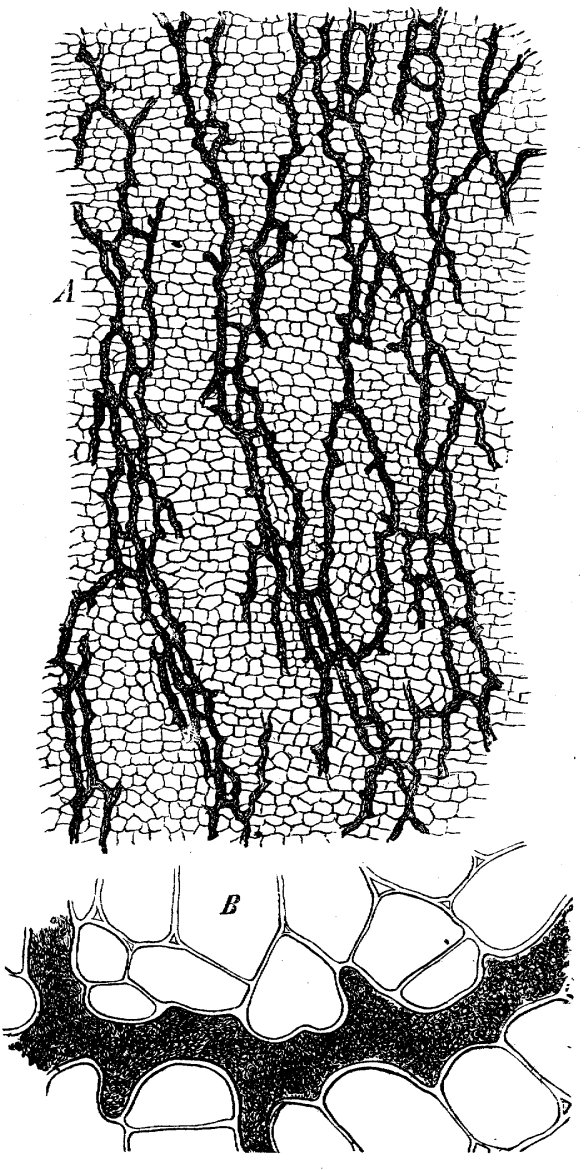

FIG. $72 .-A$ tangential longitudinal section through the phloëm of the root of Scorzonera hispanica; a number of laticiferous vessels, anastomosing laterally with one another, traverse the parenchymatous tissue; $B$ a small piece of a laticiferous vessel with the adjoining parenchymatous cells, more strongly magnified.

sulting tubes appear moniliform. In Ároideæ laticiferous vessels united into a net-work occur in the fibro-vascular bundles and the fundamental tissue; but some genera, as Caladium and Arum, also exhibit the peculiarity of laticiferous tubes running within the xylem, which, from their position, and to a certain extent also from their structure, must be regarded as metamorphosed spiral vessels. Simple broad tubes similar to these also traverse the fundamental tissue. In the genus Acer the sieve-tubes are transformed into laticiferous vessels, as may be seen from their position in the phloëm and the structure of their wall.

True laticiferous vessels scarcely occur among Monocotyledons. The peculiar and 
abundant latex in the bulb-scales of Allium Cepa is found in broad elongated rows of cells, whose broad septa exhibit sieve- or latticed structure, but whose actual perforation is not quite certain (Fig. 73). Where two tubes of this kind lie side by side, the longitudinal walls also show a pitted structure similar to that of immature sieve-tubes. These rows of cells traverse the bulb-scales, at whose base they anastomose, as well as the leaves and scapes, in long nearly parallel rows, which are 'generally separated from the epidermis by from one to three layers of cells. Similar rows are formed by the Utricular Vessels ${ }^{1}$ of Amaryllidex, as Narcissus, Leucojum, and Galantbus; they resemble, moreover, laticiferous vessels in this, that the septa

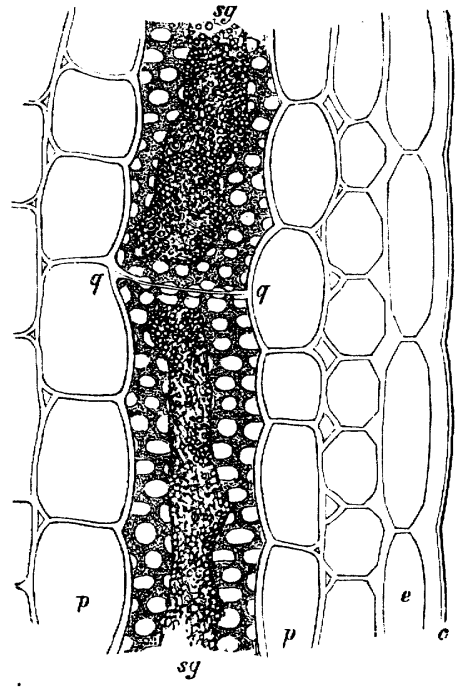

FIG. 73.-Longitudinal section through a bulbscale of Allium Cepa; $e$ the epidermis; $c$ the cuticle; $p$ parenchyma; $s g$ the latex of the utricular vessel coagulated by potash solution; $q q$ its septum; the longitudinal wall which separates the utricular vessel from one lying behind it exhibits a pitted structure. of the rows of cells become partially, sometimes entirely, absorbed; but their sap is not milky, and contains numerous needle-like crystals of calcium oxalate (raphides). Allied to these are numerous other structures in Monocotyledons which bear scarcely any other resemblance to laticiferous vessels. In some genera of Liliaceæ, as Scilla, Ornithogalum, and Muscari, the utricular vessels often consist of short interrupted rows of cells, and in the bulbs themselves larger isolated parenchymatous cells, resembling the former in containing raphides. That cells containing raphides may, however, unite into tubes, which resemble morphologically laticiferous vessels, is shown in Commelynaceæ. Here rows of cells which are early distinguished from those which surround them by containing raphides arise in the young parenchyma of the fundamental tissue of the internodes and leaves. While their neighbours continue to divide, they do not, but remain unaltered, and their septa are, according to Hanstein, absorbed as the entire organ grows and the cells in consequence elongate. In this manner long continuous tubes, filled with raphides of enormous length, are formed from the rows of cells of the fundamental tissue which contain crystals ${ }^{2}$.

As in Monocotyledons we find transitional forms between the imperfect laticiferous vessels of bulb-scales and simple lithocysts which do not contain latex but only raphides; so, on the other hand, Sieve-tubes result from a true coalescence of cells which usually, it is true, contain mucilaginous proteids, but sometimes also latex, as in Acer according to Hanstein, and in Convolvulaceæ according to Vogel, l.c. ${ }^{3}$

While true laticiferous vessels are confined to a few natural orders, Sieve-tubes, on the contrary, are apparently an essential constituent of the phloëm (bast-portion) of the fibro-vascular bundles. They occur nowhere else, and their morphological structure will therefore be spoken of under the fibro-vascular bundles; we must here speak

1 [These vessels were termed 'vesicular' in the Ist edition of this translation. The present rendering, corresponding to the French 'vaisseaux utriculeux,' is a more correct rendering of 'Schlauchgefässe,' and expresses the fact that they are composed of rows of cells (utricles) the cavities of which have not coalesced by the absorption of their septa.]

${ }^{2}$ See Hanstein, l.c.; also in Monatsber. der Berliner Akad. 1859.

$3 \mathrm{I}_{11}$ addition to the forms of cells already named, latex occurs also in the intercellular passages of miny plants, as Rhus, Alisma Plantago, \&c., and occasionally in the vessels of the wood, as in Carica, some Convolvulacex, \&c. On this subject see Trécul, Compt. rend. vol. LXI, IS65.Van Tieghem, Ann. des Sci. Nat., 5th ser. vol. VI, I866.-David, l.c. p. 57. 
of them as a special form of coalescence of cells. They make their appearance in the young phloëm in the form of long tubes arranged in rows, with thin walls and transverse or oblique septa, on which a net-work of thickening-ridges is soon observed, enclosing thinner areolæ. At a later period these latter appear to be actually perforated, while the thickening-ridges between them often swell up enormously. In this condition the septum, perforated by a number of pores, is termed a Sieve-plate; it is usually broader than the diameter of the tube, which therefore appears dilated at its septa, the sieve-plates, and hence acquires a very characteristic form (Fig. 74). Sieveplates of simpler structure are also usually formed in the side-walls where two sievetubes come into contact. In their early stage the sieve-tubes usually contain a tough albuminous mucilage very little affected by various solvents, which accumulates on both sides of the plate, and fills up the pores. The peculiar configuration of the sieve-plates, and the difficulty of obtaining longitudinal sections of them, render the observation of these characters extremely difficult; this is especially the case with the perforation of the sieve-plates, which can, however, be proved by a method first employed by myself ${ }^{1}$. It is sufficient to saturate thin longitudinal sections of the phloëm with iodine-solution until the contents of the sieve-tubes begin to turn brown, and then to add concentrated sulphuric acid; this dissolves the cell-walls and the substance of the sieve-plates, and nothing is left but the mucilaginous contents coloured a deep brown. The accumulations of protoplasm on each side of the sieve-plate are now seen to be united by slender threads of the same substance (Fig. $74, p$ ), which evidently previously filled the perforations or Sieve-Pores; and their continuity proves that the pores actually constituted a connection between two neighbouring tubes. Mohl gave to the sieve-tubes discovered by Hartig the term Latticed cells $^{2}$, since neither he nor subsequent observers were able to ascertain the actual perforation; but Hanstein succeeded in determining it by means of Schulze's solution. It is not even yet by any means certain whether all the rows of cells in the phloëm

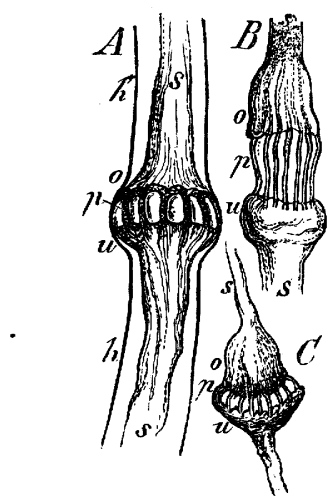

FIG. 74--Places where sieve-tules unite, showing the perforation of the septa after solution of the cell-wall by sulphuric acid. $A$ and $B$ from the petiole of Cucurbita; $C$ from the stem of the dahlia. In $A$ the cell-wall $h h^{\prime}$ is not yet completely absorbed; $s^{\prime}$ the protoplasmic mucilace 0 and $u$ accumulation of it on the upper mucilage, $o$ and $u$ accumulation of it on the uppe and under side of the septum (sieve-plate) ; $p$ the threads of protoplasm which unite these accumulations and pass through the pores of the sieveplates. which have in recent times been called sieve-tubes have perforated sieve-plates, and are therefore the result of actual coalescence. The cells of the parenchymatous fundamental tissue also not unfrequently exhibit a sieveplate-like structure on their walls (see Fig. $2 \mathrm{I}$, p: 24), as, for example, in the pinnæ of Cycadex, the bark of Ceropegia aphylla (Asclepiadex), \&c.; but with respect to the latter Borscow unhesitatingly asserts ${ }^{3}$ that they are not perforated, the pores being still closed by thin membranes. It is an interesting fact that the laticiferous cells of Ceropegia, as well as (according to David, l.c. p. 57) those of Euphorbiacex, are connected

${ }^{1}$ Mohl, Bot. Zeit. 1855 , p. 873 . [Ann. des Sci. Nat. $1_{5} 6$, vol. V. pp. I41-I59.]-Nägeli, Sitzungsber. der k. bayer. Akad. der Wissen. I861.-Sachs, Flora, I863, p. 68.-Hanstein, Die Milchsaftgefässe, Berlin I864, p. 23 et seq.

${ }^{2}$ [Hartig termed the sieve-tubes Siebröhren, which has been rendered 'cribriform vessels' by some English, and 'tubes cribreux' by French writers. Mohl preferred, for the reason stated in the text, to call them 'Gitterzellen,' which has been variously rendered 'cellulæ clathratæ,' 'cellules treillisées ' or 'grillagées,' ' latticed cells' or 'clathrate cells.']

${ }^{3}$ Borscow, Jahrb. für wiss. Bot. vol. VII. p. $34^{8}$. 
with the parenchymatous cells which adjoin them by such lattice plates, which, although they do not bring the cells into communication, must nevertheless facilitate the interchange of certain constituents by diffusion.

An illustration of the coalescence of cells is furnished by the vessels of the wood (mostly filled with air) especially those which have bordered pits, as well as the woodcells with bordered pits (Trache ides), of which sufficient has already been said (Figs. 2327, pp. 25-27). In the vessels of the secondary wood of Angiosperms formed from short cells with large cavities, the transverse or oblique septa commonly disappear altogether, so that the entire row of cells forms a completely continuous tube. But frequently,

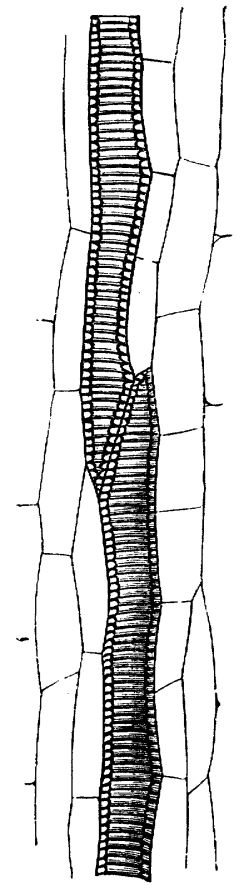

FiG. 75.-From a very young fibro-vascular bundle of a young petiole of Scrophularia aquatica; part of a spiral vessel surrounded by procambium; two spirally thickened cells are in prosenchymatous apposition; by the elongation of the petiole the coils of the spiral band, now lying close to one another, are drawn apart; the spiral band becomes detached from the thin wall which is common to the vessel and to the adjoining cells, and in this way a spiral band is formed capable of unwinding. as in Heliantbus, Sonchus, Cirsium, \&c. ${ }^{1}$, the septa are only partially absorbed, thick ridges remaining, which have a reticulate or latticed form, or, when the septa are very oblique, even scalariform. When true wood-vessels form air-conducting tubes in this manner, the separate parts having previously been closed cells, the tubes are also in communication with one another laterally through the open bordered pits already described. In tracheïdes which are arranged in a prosenchymatous manner, as those of Conifers (Fig. 23, p. 25) and Ferns (Fig. 27, p. 27), this lateral communication is the only one, since in the cells pointed at both ends there are no true septa which could be broken through. It is, on the other hand, doubtful whether the cells out of which annular and spiral vessels are formed (see Fig. I8, p. 23) are always in communication with one another, especially when the spiral cells remain short, as in the ultimate branches of the vascular bundles in the veins of leaves, where they are often considerably enlarged. Preparations in which the structure can be very easily observed may be obtained by boiling very young leaves for some time in potash solution and then placing them in glycerin. Where, however, the spiral vessels are formed at an early period in these organs, attaining subsequently a ccnsiderable length, so that the coils of the spiral thread which were at first very close become widely separated (Fig. 75), it may be assumed that the thin membrane which separates the contiguous ends of the spiral prosenchymatous cells becomes ruptured, and thus the cells are placed in communication with one another for considerable lengths. For these and other reasons, it is convenient not to limit the definition of a vessel to cases in which the component cells actually coalesce into a tube. Here, as elsewhere in the different forms of tissue, we find transitional structures; and the definition of terms must not be founded on a single characteristic selected arbitrarily, but on a general consideration of all the morphological and physiological characters.

The forms of coalescence now described possess the common physiological function of providing a means for the transport of food-materials, and of promoting and accelerating

1 See E. Tangl in Sitzungsber. der kais. Akad. der Wiss. Vienna, May r 8 ; 1. 
this transport to the more distant organs through the continuous tubes which they form. This is shown, among other evidence, by the course of these tubes, which is almost invariably in the direction of growth, and therefore enables them to place those organs in which food-material is produced in connection with those which require it. This is unquestionably the case with the sieve-tubes, which serve for the transport of the difficultly diffusible proteids, and secondarily also of the carbohydrates ${ }^{1}$. It is also true with respect to the laticiferous vessels, in so far as they contain proteids, cils, and carbohydrates. This function of the laticiferous vessels is not disproved by the fact ${ }^{2}$ that they usually also contain secretions that are not serviceable. The purpose, finally, of the wcod-vessels is to form channels filled with air within the close woody tissue, replacing the air-conducting intercellular spaces of the succulent parenchyma.

A totally different physiological function must, on the other hand, be assigned to the last form of coalescence of cells to be described, the Compound Glands. That these have no use connected with the transport of food-material is shown by their round form, which renders them quite unserviceable for placing different parts of the plant in communication. The same conclusion is indicated by the fact that the substances which accumulate in glands do not in any way contribute to growth, but must be regarded as excrementitious, or as secondary products of metabolism ${ }^{3}$.

The popular usage of the term Gland is extremely indefinite, including not only single cells with peculiar contents, but also certain external organs like the nectaries of flowers and the colleters or glandular hairs of many leaf-buds. In this extended signification it is impossible to give an exact definition to the term. In order to get a good definition, we must exclude in the first place the bodies hitherto known as Unicellular Glands, which must be associated with lithocysts and gum-cells under the designation of Idioblasts, as the term has already been defined (p. 84 ). We may now define a Gland ${ }^{4}$ as a group of cells sharply differentiated from those that surround them, whose intervening septa become absorbed, so that a single cavity is formed, which is often surrounded by special layers of tissue, and filled with excrementitious products, especially volatile oils. This definition excludes certain closely related forms of tissue, such as the nectaries and colleters ${ }^{5}$ already mentioned, which, however, in order to indicate their affinity, may be designated Gland-like bodies, in contradistinction to true glands.

Good examples of glands in this sense are furnished by the large receptacles for volatile oil which occur abundantly in the rind of various species of Citrus. They may be recognised, even in the young ovary of the flower, as roundish groups of cells, distinguished by containing a turbid protoplasm and small drops of oil. The walls of these cells soon begin to swell, and the individual cells can be separated by pressure. The walls then deliquesce, and a large globular cavity is formed,

1 See Sachs, Flora, I 863 , p. 50.-Briosi, Bot. Zeit. 1873, nos. 20-22. [Briosi detected the presence of extremely finely-divided starch in the sieve-tubes of a large number of plants. Experiments lead him to think that by compression the starch particles may be made to pass through the perforated partition from one cell to another.]

2 Sachs, Experimental-Physiologie, p. $3^{86 .}$

${ }^{3}$ [See also Meyen, Ueber die Secretionsorgane der Pflanzen, Berlin 1837.-J. B. Martinet, Organes de sécrétion des végétaux: Ann. des Sci. Nat. $5^{\text {th }}$ ser. vol. XIV, 1872 . It has been suggested that the contents of glands and similar secreting organs are not really excrementitious, but that they serve to protect the plant by preventing the consumption of the leaves \&c. by insects and other animals.]

4 [The term is here used in a somewhat more restricted sense than is usual in English botanical works, in consequence of the etymological meaning of the corresponding German term 'Druise,' in which the idea of something compound is implied.]

5 See Sect. I5, under the head of Epidermis, p. ror. 
filled with watery protoplasm with large drops of a volatile oil floating in it. The layers of cells which surround the cavity form an envelope, which differentiates it sharply from the surrounding tissue. The origin of two different forms of gland in Dictamnus Fraxinella is illustrated in the accompanying figures, taken from Rauter ${ }^{1}$. Fig. 76 represents the development of a gland on the upper side of the leaf, the contents of which are the source of the powerful odour of the plant. These leaf-glands of Dictamnus originate from only two cells, one of which belongs to the young epidermis, the other to the subjacent parenchymatous layer; the former divides again into two layers of cells, the outer of which $(d)$ forms a continuation of the epidermis, while the inner one $(c)$ contributes to the formation of the tissue of the gland, the principal

A
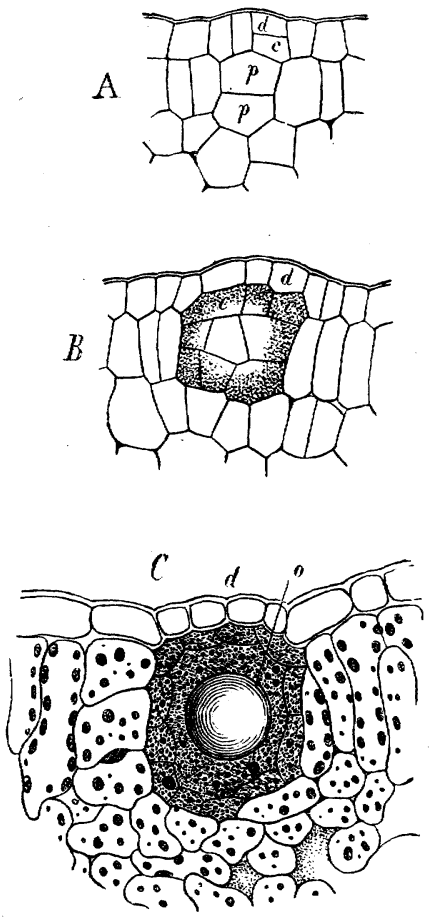

FIG. 76.-Gland from the upper side of the leaf of Dictam. nus Fraxinella (after Rauter). $A$ and $B$ early stages of development, $C$ mature gland; $d$ the covering layer, forming a continuation of the epidermis: $c$ and $p$ mother-cells of the gland-tissue; $o$ a large drop of volatile oil.

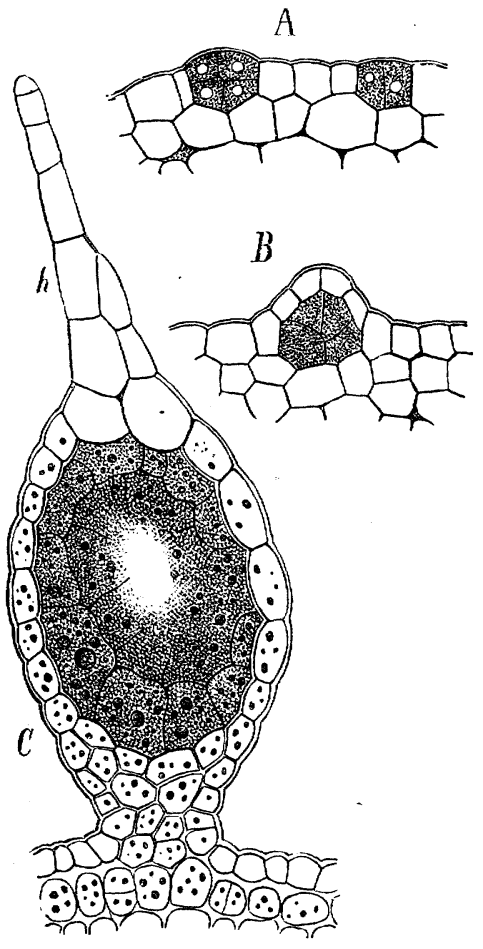

FIG. 77,-Gland and hair from the inflorescence of Dictamnus Fraxinella (after Rauter). $A$ and $B$ early stages of development; $C$ mature gland, with the hair $h$ at its apex.

- part of which originates by divisions of the two mother-cells of the gland $(p p)$; the enveloping layer of the gland is here but slightly developed, as is shown in Fig. 76, $C$. On the flower-stalks, bracts, and sepals of the same plant are formed large sessile or shortly-stalked glands of somewhat ovoid form, bearing at their apex a single hair (Fig. 77, b). These always arise, as Rauter has shown, from a single cell of the young epidermis, which divides first vertically, then tangentially (Fig. $77, A$ ); thus two layers are formed, the outer of which is a continuation of the epidermis, while the inner produces, by further divisions, the tissue of the gland $(B)$. In the further course of

1 Rauter, Zur Entwickelungsgeschichte einiger Trichomgebilde. Wien I 871 . 
development the whole substance of the gland now becomes, as it were, forced outwards above the surface of the organ $(C)$; and when, finally, the secreting tissue is absorbed, a cavity is formed filled with mucilage and drops of volatile oil, and surrounded only by the continuation of the epidermis. Similar to glands in their origin are the gum-passages and gummy swellings of diseased stone-fruit. Grégorieff found the seat of the formation of the gum in them to ba principally the soft bast of the fibro-vascular bundles which traverse the fruit-pulp; the cell-walls become absorbed after they have swelled up, and cavities with undefined boundaries filled with gum are thus formed, which sometimes exude their contents externally through the flesh of th? fruit when the production of gum is excessive.

While the origin of the structures now described is the coalescence of cells previously separated by partition-walls, the canal-like Receptacles for Secretions are formed, in many plants, by cells, previously in contact by their partition-walls, separating from one another and leaving an intercellular space (in the manner represented in Fig. 66, p. 78), into which the secretion flows from the surrounding cells. According to the nature of the secretion, we thus get Resin-passages, as in most Coniferæ and Terebinthacex, Gum-passages in Cycadex, passages with a mixture of gum and resin in Umbelliferæ and Araliacex; Latex-passages in Rbus and in Alisma Plantago beneath the epidermis of the petiole in front of the fibro-vascular bundles; and passages with different volatile oils, often coloured, as in the Compositæ. Similar Secretion-canals ${ }^{1}$ occur also in Clusiaceæ (the contents of the canals of Garcinia Morella yielding gamboge) and in Pittosporex; among Monocotyledons, in addition to Alisma Plantago, we find them in Aroidex. Among Ferns, they are stated to have been detected in Marattia and Angiopteris, and by Hegelmaier in some species of Lycopodium, as L. inundatum, alopecuroides, and annotinum. Plants which possess secretion-canals have, as a rule, no laticiferous vessels; but they both occur in some Cichoriaceæ as Scoiymus, Cynaraceæ as Cirsium and Lappa, and in some Aroidex as Pbilodendron. They are in that case distributed through different tissue-systems. Thus in Pbilodendron the laticiferous vessels are found in the phloëm of the fibro-vascular bundles, the secretion-canals in the fundamental tissue; while in Scolymus and Cirsium the laticiferous vessels run through the phloëm, the secretion-canals through the fundamental tissue of the cortex ${ }^{2}$. Where there are only secretion-canals, they may belong exclusively to the fundamental tissue of the primary cortex (Tagetes patula, according to Van Tieghem), or exclusively to the phloëm (the stem of Pittosporum Tobira, according to Van Tieghem), or to both systems (Umbelliferæ); in Coniferæ they occur in the pith and primary cortex, and also in the phlo:m and xylem. Where they' are found in the secondary phloëm formed out of the cambium, the secretion-canals may be produced repeatedly with other elements of the soft bast in concentric layers, as in Cussonia, Umbelliferæ, \&c.

The simplest forms of secretion-canals are produced by three, or more commonly four, longitudinal rows of secreting cells separating from one another far enough to form a narrow intercellular passage which they fill with their secretion, as, for example, in the roots of Compositæ, where definite groups of canals of this nature are formed in the double vascular bundle-sheath. If the tissue which surrounds the secretioncanal attains a vigorous development in breadth and thickness, the intercellular passages, which were at first narrow, become considerably broader (Fig. 66, p. 78), while the secreting cells which surround them also enlarge, divide in radial and tangential directions (in reference to the centre of the canal), and thus form round the canal

1 See especially Van Tieghem, Les canaux sécréteurs des plantes, Ann. des Sci. Nat., $5^{\text {th }}$ ser. vol. XVI, I872.-Müller in Jahrb. für wiss. Bot. vol. V. p. 387.-Thomas, ibid. vol. IV. pp. $4^{8-60 .}$

${ }^{2}$ Van Tieghem, in the French translation of the 3 rd ed. of this work, p. ${ }_{5} 8$. 
a mass of tissue of characteristic form one or more layers in thickness, the cells of which are distinguished by containing a turbid fluid with drops of oil and resin floating in it, and by their walls being not lignified and usually thin. If the tissue which surrounds the secretion-canal does not increase in size, the formation of the canal or intercellular passage is sometimes altogether suppressed; the secreting cells retain their secretion, and form gland-like groups of cells instead (Fig. $78, A, D$ ).

Secretion-canals resemble laticiferous vessels in this respect, that they follow, as a rule, the direction of growth of the organ, even when they do not lie in the fibrovascular bundles, and also that they penetrate into all parts of the plant, although particular organs, especially the fruit and flower, are destitute of them. When any part of the plant, especially the stem, which is rich in them, is wounded, and the

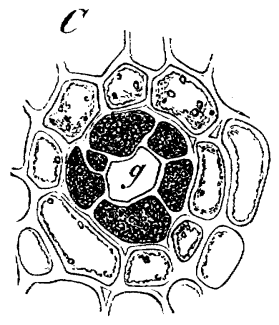

\section{$B$}

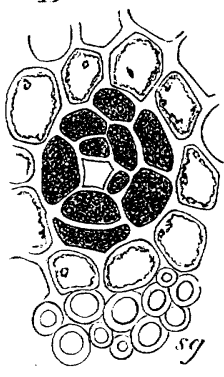

E

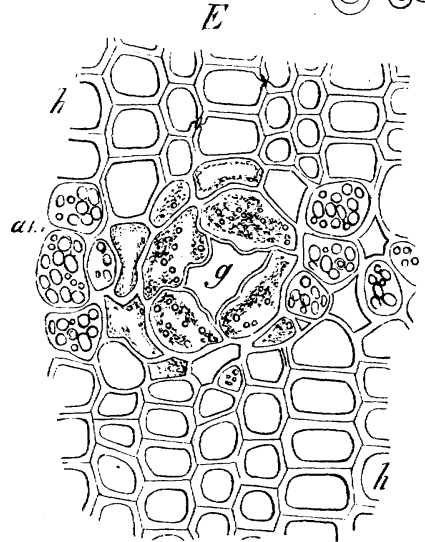

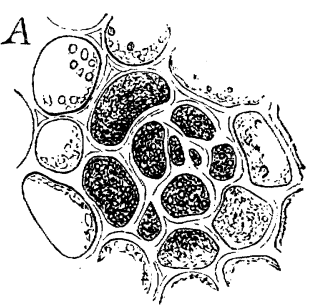

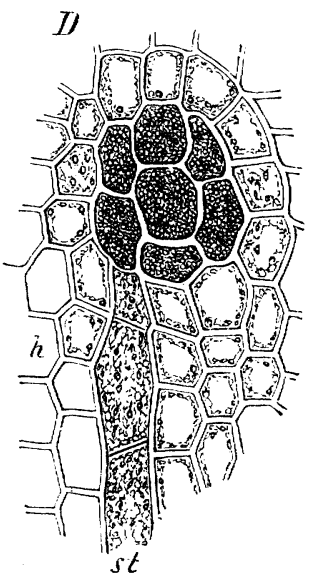

FIG. 78.-Transverse section of resin-passages $(g)$ at the base of a first year's branch of Pinus sylvestris (X 550$)$. A, $B, C$ passages lying in the periphery of the pith ( $s g$ spiral vessels of a fibro-vascular bundle); at $A$ the formation of a passage has not taken place, but the cells destined for its formation are there, their walls hiving become softer; $D$ wood-cells $(h)$ enclosing a group of resin-cells, not forming a passage ( $s t$ a medullary ray); $E$ part of the wood containing a resin-passage $(g)$; next it wood-cells containing starch $(a m)$, forming a zone passing from one passage to another.

canal therefore broken, the contents, forced by the pressure which masses of tissue exercise on one another, escape, in the same manner as latex, collect round the wound, and finally become hard. In this manner are obtained the various kinds of resin, as that of Coniferæ, mastic, sandarach, \&c., in the same way as dried latex such as euphorbium, lactucarium, opium, \&c.

Sect. 15. The Epidermal Tissue.-A differentiation into epidermal tissue and inner fundamental tissue can evidently only arise in those plants and parts of plants which consist of masses of tissue. In general the contrast of the two 
is the plainer the more the part of the plant is exposed to air and light, underground and submerged parts showing it in a smaller degree; in those destined to a longer term of life the formation of epidermis is usually also more perfect. The difference between epidermis and fundamental tissue can only be established by the outer layers of cells, whose morphological character is otherwise similar, becoming distinguished by the thickness and firmness of their cell-walls; and by having smaller cell-cavities than those which lie deeper. In this case there is usually no sharp boundary-line between the two tissues; the characteristics gradually increase the more nearly the cell-layers approach the surface. This is usually the case, among Algæ, with the Fucaceæ and larger Florideæ, with many Lichens and the fructifications of Fungi; even in the stem of Mosses the formation of epidermis is often indicated only in this manner. The contrast between epidermal and inner tissue becomes most marked when, besides a sharp boundary between the two, a different morphological development distinguishes the two kinds of tissue. In the sporogonia of Mosses and in all Vascular Plants at least one outer layer of cells may be distinguished in this sense as epidermal tissue, and is termed the Epidermis. In true roots and many root-like underground stems, as also in many submerged plants, the epidermis is only slightly different from the subjacent tissue; but in most parts of stems and leaves it shows an altogether peculiar development of its cells, giving rise to stomata and trichomes of the most various kinds. In many leaves and parts of stems, the epidermis, after it has already become a recognisable tissue (during or after the bud-condition) undergoes cell-division tolerably late, by which it becomes divided into two or more layers. From this epidermis formed of several layers of cells (Pfitzer, l.c. p. 53) those layers of tissue may be conveniently distinguished as Hypoderma which lie beneath the simple, rarely beneath the multilamellar epidermis, and perform the physiological function of strengthening the epidermal tissue, without however belonging to it genetically, while they are strikingly distinct from the deeper lying fundamental tissue, although genetically a part of it. This hypoderma consists chiefly of layers of thick-walled sclerenchymatous cells, sometimes even of bast-like fibres. In Phanerogams, especially Dicotyledons, the hypoderma is mostly developed as Collenchyma, the cell-walls being strongly thickened, and in a high degree capable of swelling at the longitudinal angles where three or four of them meet (Fig. $2 \mathrm{I}$, $B$, p. 24).

In those parts of plants which live long and which increase greatly in thickness, the epidermal system attains a further development in the production of Cork. This originates in the epidermis itself or in the subjacent layers of tissue by subsequent cell-division, occurring often very late, and by the suberisation of the newly-formed cells. The formation of cork often continues for a very long period, or is renewed after interruption; and when this occurs uniformly over the whole circumference, there arises a stratified cork-envelope, the Periderm, replacing the epidermis, which generally perishes, and surpassing it as a means of protection. But not unfrequently the formation of cork penetrates much deeper; lamellæ of cork arise deep within the stem as it increases in thickness; parts of the fundamental tissue and of the fibro-vascular bundles, or of the tissue which afterwards proceeds from them, become, as it were, cut out by lamellæ of cork. Since everything which 
lies outside such a structure dies and dries up, a peripheral layer of dried tissue collects, which is very various in its form and origin. This structure, abundant in Coniferæ and in many dicotyledonous trees, is the Bark, the most complicated epidermal structure in the vegetable kingdom.

(a) The Epidermal Formation of Thallophytes is chiefly confined to the cells of the fundamental tissue becoming smaller and firmer the nearer they lie to the surface; the cell-walls very generally become darker, as in the outer layers of the cortical tissue of many Lichens, and the outer layers of the peridia in Gasteromycetes and Pyrenomycetes; in the pileus of many Hymenomycetes the epidermal layer may be detached in large pieces (Fig. 79). From the small difference between cortex and fundamental tisswe in these Thallophytes, it may appear doubtful whether the
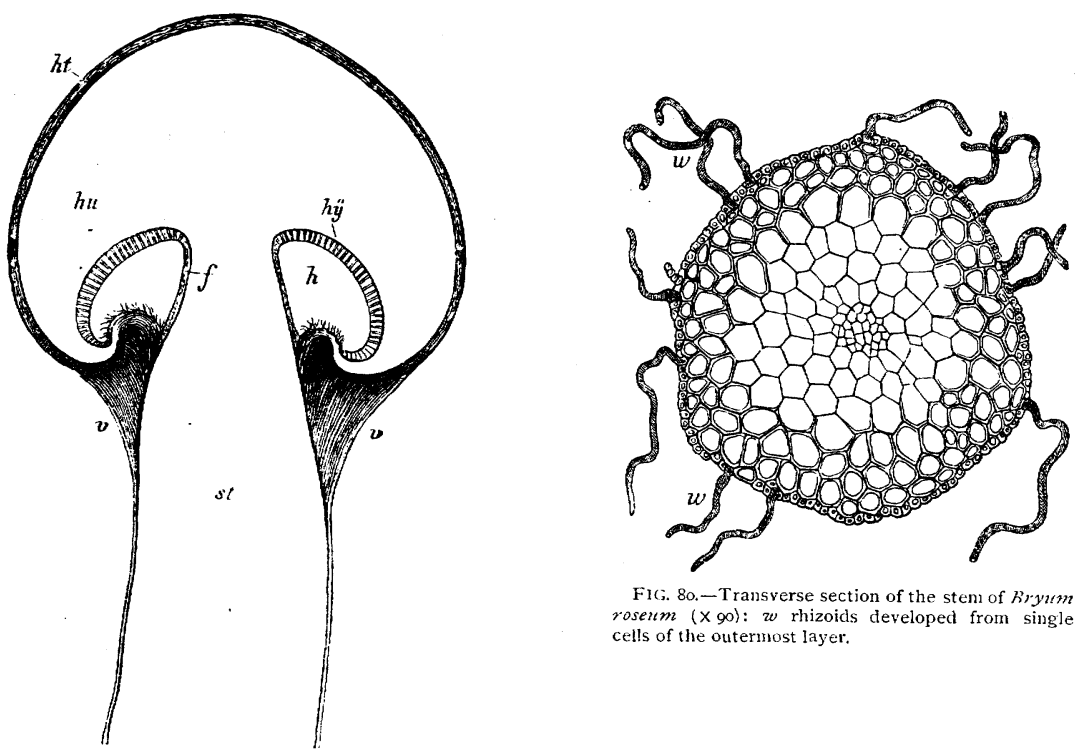

FIG. 80.-Transverse section of the stem of $h, y u m$ roserm $(X 90)$ : $w$ rhizoids developed from single cells of the outermost layer. FIG. 79.-Fructification of Boletus favidus in longitudinal section slightly magnified; st stipes; $h u$ pileus, $h y$ hymenium; $v$ velum ;
$h$ cavity beneath the hymenium ; f prolongation of the hymenial layer on the stipes; $h t$ the separable yellow epidermal layer of the
pileus.

outer layer should be termed cortex or epidermis; when the cortical tissue is moderately thick, this layer can usually be distinguished from it. With Thallophytes, as with higher plants, the outermost layer of cells displays a tendency to the formation of hairs.

The Muscineæ (Hepaticæ, Sphagnaceæ, Musci) exhibit a great variety with reference to the epidermal formation. While in many other Hepaticæ we have scarcely any indications of one, in the family of Marchantiex (Fig. 65, p. 78) an epidermis perfectly developed and provided with stomata suddenly makes its appearance. In Mosses the epidermal formation on the leafy stem is limited to this,- that the cells towards the surface become narrower, their walls becoming thicker and assuming a deeper red colour; the outermost layer often produces numerous long rhizoids (Fig. 80). In Bog-mosses (Sphagnaceæ), on the other hand, a single outermost layer of cells of the stem, or from two to four such, assume an entirely different character. These cells (Fig. 8I,e) have thin colourless walls; their cavities are much larger than those 
of the inner tissue; the walls sometimes show slender thickening-bands running in a spiral manner, and open externally by large orifices, being also in communication with one another by similar ones $(l)$. In the mature state they contain nothing but air or water, which rises in them by capillarity. Within this epidermal tissue the stem is similar to that of Mosses; the cells become towards the surface gradually narrower, thicker-walled, and of a darker colour. A similar epidermal layer, and with similar hygroscopic properties, occurs in the aërial roots of Orchids and of some Aroidex.

Like the other forms of tissue, the epidermis also attains a greater perfection in the sporogonia of Mosses; the variously differentiated internal tissue of the sporangium

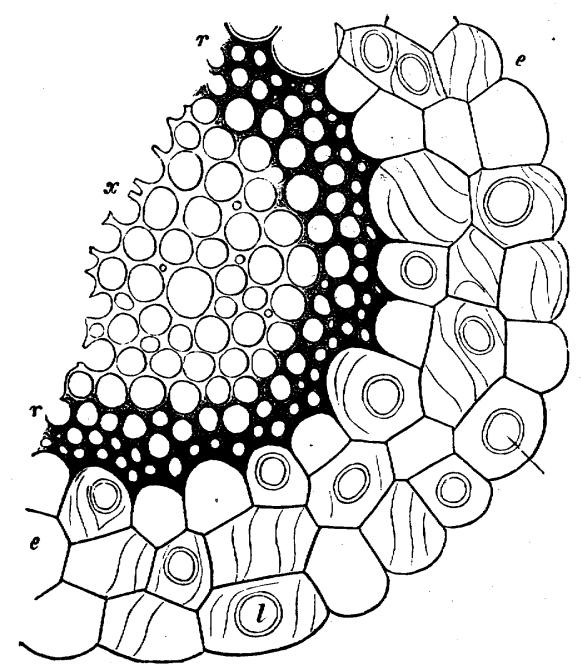

Fig. 8r.-Transverse section of the stem of Sphagnum cymbifolium ( $\mathrm{X} 900) ; x$ inner cells with colourless soft walls; cymbifolium (X goo); $x$ in $r$ cortical cells, becoming gradually narrower and thickerwalled towards the surface; $e e$ the epidermal layer;
$l$ orifices through which adjacent cells communicate with one another.

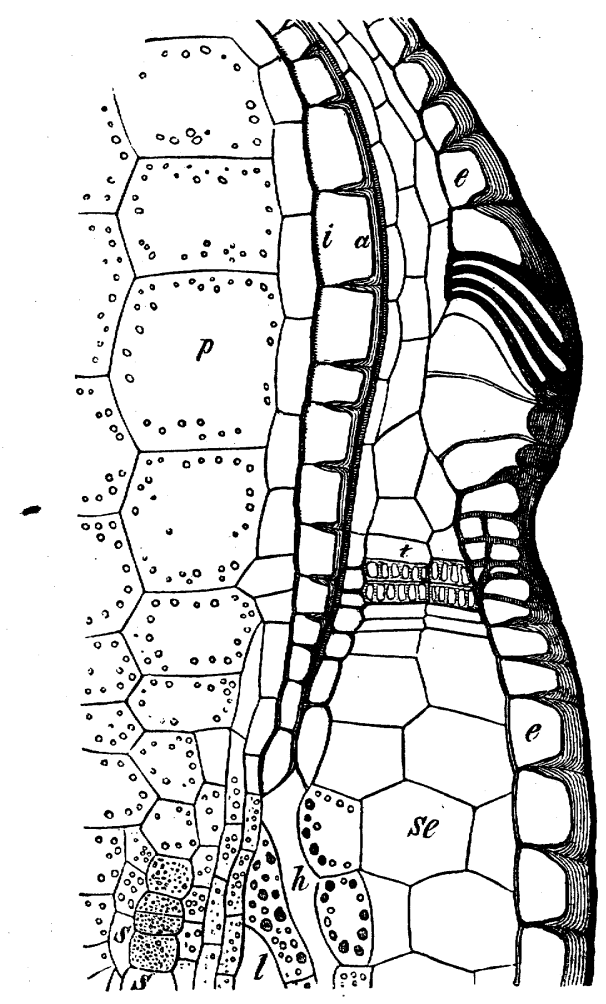

FIG. 82,-Part of a rangiuni of Funaria hygrometrica ( $\left.X_{300}\right) ; e$ epidermis; the thick (For further explanation of the Fig. see Book II.)

is surrounded by a highly developed true epidermis, sometimes provided with stomata (Fig. 82).

(b) The Epidermis ${ }^{1}$. In Vascular Plants the epidermal tissue consists usually only of a single superficial layer of cells, the true Epidermis. In its origin it always consists of a single layer; but this sometimes splits into two or more by divisions parallel to the

1 H. von Mohl, Vermischte Schriften bot. Inhalts. Tübingen 1845, p. 26o-F. Cohn, De Cuticula. Vratislaviæ 1850.- Leitgeb, Denkschriften der Wịener Akad. I865, vol. XXIV. p. 253.Nicolai, Schriften der phys.-ökonom. Gesells. Königsberg, i 865, p. 73.-Thomas, Jahrb. für wiss. Bot. vol. IV. p. 33.-Kraus, ibid., vol. IV. p. 305, and vol. V. p. 83 . - Pfitzer, ibid., vol. VII. p. 56I, and vol. VIII. p. 17.-De Bary, Bot. Zeitg. 1871, nos. 9-1 I and 34-37. 
surface, during or after the bud-condition of the organ in question. In such cases the outermost may be distinguished as the Epidermis proper from those which lie beneath, or the Strengthening-layers; these latter generally consist of large thin-walled cells with contents as clear as water, for which reason Pfitzer terms them Aqueous Tissue. Epidermis of this kind consisting of several layers occurs in the leaves of most species of Ficus, in the stems and leaves of many Piperaceæ, and in the leaves of Begonia. In the roots also of some species of Crinum the epidermis, at first simple, splits into several layers; but this is much more striking in the aërial roots of Orchids and Aroidex, where these cell-layers afterwards lose their succulent contents and surround the substance of the root as an air-containing envelope to the root (Velamen).

The Hypoderma is genetically distinct from the strengthening-layers which result by division from the originally simple epidermal layer, since it arises from the layers of the fundamental tissue which are covered by the true and simple epidermis. The cells of the hypoderma may also become developed as aqueous tissue like that mentioned above, and often to an enormous thickness; this occurs in many Bromeliaceæ and some species of Tradescantia. The hypoderma more often exists in the form of layers of very thick-walled often sclerenchymatous cells, whose origin has been proved to be from the fundamental tissue, not from the epidermis, at least in the case of Ephedra and E'egia, and is very probably so in other cases. While this sclerenchymatous hypoderma is especially frequent in Vascular Cryptogams (e.g. Equisetaceæ and Ferns) and in the leaves of Gymnosperms, a third form, the Collenchyma, occurs very abundantly in the petioles and succulent stems of Angiosperms, especially of Dicotyledons; its usually narrow but long cells are strikingly distinguished by the thickening-masses often forming longitudinal ridges at the angles projecting internally, and swelling greatly with water or more powerful reagents (Fig. 2 I, B, p. 24). That the collenchyma originates from the fundamental tissue, and thus not from the epidermis, has been actually observed only in Euonymus latifolius, Peperomia, Nerium, and liex, but is probable also in other cases.

When the term Epidermis is hereafter used without further remark, the ordinary simple layer, or the outermost when the epidermal tissue consists of several layers, is always to be understood.

The cells of the epidermis, as also those of the strengthening-layers and of the hypoderma, are in close contact on all sides; the only intercellular spaces are those between the guard-cells of the stomata, through which the large cavities in the fundamental tissue communicate with the external air. This close approximation of its cells is sometimes the only distinguishing mark of the epidermis, as in the submerged Hydrillex, Ceratophyllum, \&c.; in other cases the formation of hairs helps to distinguish it, as in most roots, where the cells of the epidermis are otherwise similar to those of the fundamental tissue in contents and in the nature of their wall. But usually in the stem and foliar organs the epidermis is destitute of chlorophyll, starch, and granular contents generally, while in Ferns and in the water-plants mentioned above, as well as in other cases, the epidermal cells contain chlorophyll-granules. Not unfrequently the otherwise colourless cell-sap is in them tinged by a red substance.

In organs which grow chiefly in length, as roots, long internodes, and the leaves of Monocotyledons, the epidermal cells are usually elongated longitudinally; in leaves with a broad surface they are mostly broadly tabular; in both cases the side-walls are often undulated, so that the adjoining cells interlock with one another.

The outermost lamella of the epidermal cells is always cuticularised, and usually to such an extent that cellulose cannot be detected in it, or only with difficulty. This true Cuticle extends uninterruptedly over the surface, and is strongly contrasted with the subjacent layers of the cell-wall. With preparations of iodine, with or without addition of sulphuric acid, the cuticle is coloured yellow or yellow-brown; it is insoluble in concentrated sulphuric acid, but soluble in boiling caustic potash. In submerged organs and roots it is very thin, difficult to be seen immediately, but 
rendered visible by iodine and sulphuric acid. The true cuticle is much thicker in aërial stems and leaves; it may be obtained in them even in large lamellæ by decay or solution of the subjacent cells in concentrated sulphuric acid. In many cases, and especially in stout leaves and internodes, the outer cell-wall layers of the epidermal cells which lie beneath the cuticle are strongly, often enormously, thickened; while the inner walls remain thin, the lateral walls are usually strongly thickened towards the surface, becoming suddenly thinner towards the inside. The thick portions of the wall are usually differentiated into at least two shells; an innermost thin shell, immediately surrounding the cell-cavity, shows the reactions of pure cellulose, while the layers of the cell-wall lying between it and the cuticle are more or less cuticularised, and the more so the nearer they lie to the cuticle. Not unfrequently these cuticularised layers extend downwards into the thick part of the lateral walls, in, which case the middle lamella sometimes behaves like the true cuticle, with which it is in contact on the outside. Like the cuticle of isolated cells (pollen-grains, spores), that of the epidermis has also a tendency to form projections, ridges, \&c., but they almost always remain very insignificant, and are best seen on a superficial view; as, for example, in many delicate petals (see Sect. 4 (e), p. 33 ).

According to the recent researches of De Bary, particles of Wax, which cannot be seen on section, but which exude in the form of drops when warmed to about $100^{\circ} \mathrm{C}$., are deposited in the substance of the cuticular layers of the epidermis. This deposit of wax (often associated with resin) is one of the contrivances which protect the aërial parts of plants from becoming moistened with water. But very frequently the wax extends in an unexplained manner over the cuticle, and becomes deposited there in different forms, constituting the so-called 'bloom' on fruits and some leaves, or as a continuous shining coating, which is again formed on young organs after being wiped off, and in ripe fruits of Benincasa cerifera (the wax-cucumber) appears again long after maturity. De Bary distinguishes four principal forms of this wax-coating. The bloom or gloss which is easily wiped off consists of small particles of two forms:-(I) Quantities of delicate minute rods or needles, as in the white-dusted Eucalypti, Acaciæ, many Grasses, \&c.; or of granules collected into several layers, as in Kleinia ficoides and Ricinus communis; these are aggregated wax-coatings. (2) Simple granular coatings consisting of grains isolated or touching one another in one layer; this is the most common form, e.g. in Iris pallida, the onion and cabbage, \&c. (3) Coatings of minute rods consisting of long, slender, rod-shaped particles, bent above or even curl-shaped, and standing vertically upon the cuticle, e.g. Heliconia farinosa and other Musaceæ, Cannaceæ, Saccharum, Benincasa cerifera, leaves of Cotyledon orbicularis. (4) Membrane-like layers of wax or incrustations; (a) as a gritty glazing in various species of Sempervivum, Euphorbia Caput-Medusa, Thuja occidentalis; (b) as thin scales, in Cereus alatus, Opuntia, Portulaca oleracea, the yew; (c) as thick continuous incrustations of wax, which sometimes permit a finer internal structure to be recognised, similar to the striation and stratification of the cell-wall: Euphorbia canariensis, fruits of species of Myrica, stems of Panicum turgidum. On the stem of the Peruvian wax-palms, especially of Ceroxylon andicola, these incrustations attain a thickness of $5 \mathrm{~mm}$; those on the stem of Cbamcedorea Schiedeana are thinner, but of similar structure. According to Wiesner (Bot. Zeitg. 1871, p. 77I), these flakes of wax consist of doubly refractive four-sided prisms standing perpendicularly close to one another.

Hairs ${ }^{1}$ are products of the epidermis; they originate from the growth of single epidermal cells, and are present in most plants in large numbers; when they are wanting in any part of a plant, it is termed glabrous. Their form is subject to

1 A. Weiss, Die Pflanzenhaare, in vols. IV and V of the Bot. Untersuchungen aus dem phys. Laborat. by Karsten, I867.-J. Hanstein, Bot. Zeitg. I868, p. 697 et seq.-Rauter, Zur Entwickelungsgeschichte einiger Trichomgebilde. Wien $187 \mathrm{I}$. [See also J. B. Martinet: Organes de sécrétion des végétaux, Ann. des Sci. Nat., 5th series, vol. XIV, I871.] 
extraordinary variation. The first indication of the formation of hairs occurs in the papillose protuberances of the epidermis of many petals, to which their velvety appearance is due. Among the simplest forms are also the Root-bairs which grow from the epidermis of true roots or underground stems, as Pteris aquilina, Equisetaceæ, \&c.; they are thin-walled protuberances of the epidermal cells which lengthen by growth at the apex, or only branch exceptionally, as occurs sometimes in the turnip. In Vascular Cryptogams their wall readily acquires a brown-red colour; their length of life is usually short, and when they die all trace of them disappears. The structure is similar of the woolly hairs which appear on the leaves and internodes of vascular plants while still in the bud, especially Dicotyledons. On the unfolding of these organs they commonly fall off and disappear, as in the horse-chestnut, Rbododendron, and Aralia papyrifera, where they form a felt easily wiped off from the newly unfolded leaves; in other cases they remain as a woolly coating, especially on the under-sides of leaves. In Prickles the wall is mostly thicker, silicified, and hard; they are shorter than the woolly hairs, pointed at the apex, and are usually separated by a septum from the mother-cell. When the free outer wall of unicellular hairs exhibits greater apical and surface-growth at two or more spots, branched forms result with a continuous cavity. The papillose bulging of an epidermal cell may become separated by a septum; the hair then consists of a basal cell and a free haircell; as in Anemia fraxinifolia; but the separated papilla may also become segmented by the formation of more or less numerous septa when the hair grows considerably in length, and thus arise segmented hairs, as e.g. on the filaments of Tradescantia. Sometimes the segments form lateral shoots, and thus arise tree-like bianched structures with whoried or alternate branches, e.g. in Verbascum Thapsus and Nicandra physaloides. If longitudinal divisions occur in the segment-cells of the hair, or if the hair continues to grow by an apical cell which forms segments on two sides, flatly expanded hairs are the result. To this form belong, for example, the so-called Palea of Ferns which sometimes entirely cover the younger leaves. Finally, the divisions in the young hair may be so arranged that it forms at length a tissue, which on its part may again assume different forms, e.g. the pappus-like hairs of Hieracium aurantiacum and Azalea indica, the capitate hairs of Korrea and Ribes sanguineum.

The papilla which projects above the epidermis and is separated by a septum often becomes divided by vertical and radial walls, and expands in a disc-like manner, so that the head consists of a radially arranged disc of numerous cells; thus arise Peltate Hairs, such as those of Elaagnus, Hippuris, and Pinguicula. Tufts of hairs arise when the mother-cell of the hair which belongs to the epidermis divides into several cells lying close to one another; each of these then grows independently into a hair, as is shown in Fig. 83, which is supplemented by Fig. 42, p. 43.

Not unfrequently a luxuriant growth of the parenchyma takes place beneath the hair, and subsequently also in the epidermis; the hair itself is then borne on a conical prominence or protuberance of the leaf or stem, into which its lower part is often deeply implanted; as, for instance, in the stinging hairs of the stinging-nettle. Thus also the prickles (climbing hairs) on the six projecting angles of the stem of the hop are inserted at their base into a protuberant mass of tissue, while the upper part grows into two opposite sharp points. Such double-pointed unicellular hairs occur also on the under-side of the leaf of Malpigbia urens; they are from five to six $\mathrm{mm}$. long, fusiform, very thick-walled, and are attached to the epidermis (without any protuberance) by their central part. In this case they easily become detached, and remain sticking in the skin of the hand which touches the leaf. (For further details on the Morphology of Hairs, see Sect. 2r.)

It is very common for hairs to be secreting organs. Such are the Stinging Hairs already mentioned of Urticacex, many Loasaceæ, \&c., as well as the short hairs of some Urticaceæ which contain cystoliths. But the most remarkable examples are 
Glandular Hairs. These consist of a stalk and a terminal head which is either composed of a single cell filled with resin or a volatile oil, or constitutes a true Gland, made up of a number of cells which have coalesced, so that nothing remains but the external cuticularised cell-wall in the form of a hollow vesicle containing the secretion. The oily, viscid, odoriferous secretion not unfrequently penetrates through the cell-wall, and raises the cuticle in the form of a bladder, collecting beneath it as a clear fluid, while the cells which produce it partially or entirely disappear, as in Salvia, Cannabis, and Humulus, in the latter case on the perianth of the female flowers. We are indebted to a careful work by Hanstein ${ }^{1}$ for an accurate knowledge of the glandular hairs on the leaf-buds of many trees, shrubs, and herbs. The parts of the bud are coated by a gummy substance, or one composed of gum-mucilage and drops of balsam, which he calls Blastocolla, while the glandular hairs which produce them he terms Colleters.

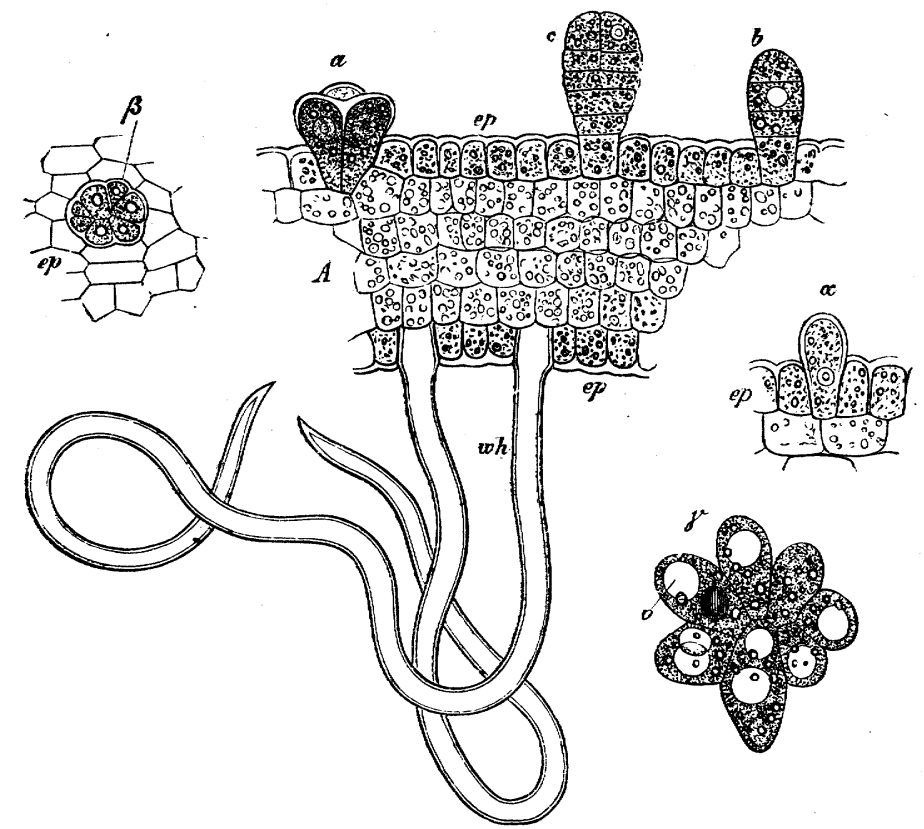

FIG. 83.-Development of the hairs on the calyx of a flower-bud of the hollyhock $\left(X_{300}\right) ; A$ wh woolly hairs on the inner surface; $b$ and $c$ glandular hairs in different stages of development; at $\alpha$ in $B$ rudiment of a glandular hair; ep always signifies the (still young) epidermis. The figures $\alpha$ in $A, \beta$ in $C$, and $\gamma$ in $D$ show the first stages of development of the stellate hairs (or rather tufts of hairs), the subsequent condition of which may be compared in Fig. 42 (p. 43 ); at $A \boldsymbol{a}$ is the hair in longitudinal section; $\beta$ and $\gamma$ show the appearance seen from above; the cells are rich in protoplasm; the formation of vacuoles $(v)$ in the protoplasm is beginning in $\gamma$.

These shortly-stalked multicellular hairs springing from an epidermal cell may expand towards the apex in a strap-shaped manner (Rumex), or may bear cells arranged in a fan-like manner on a kind of mid-rib (Cunonia, Coffea), or may form spherical or clubshaped knobs (Ribes sanguineum, Syringa vulgaris); in Platanuis acerifolia branched rows of cells occur, the roundish terminal cells of which are glandular. The colleters attain their full development at a very early period in the bud, when the foliar structures and portion of the stem out of which they spring are still very young and consist of tissue which is scarcely differentiated. They are borne especially on the en-

1 Ueber cie Organe der Harz- und Schleimabsonderung in den Laubknospen, Bot. Zeitg. I868, no. 43 et seq. The very instructive illustrations to this paper should be consulted.-See also Martinet, l.c. 
veloping scales of leaf-buds (Aesculus), on stipules which precede the leaves in development (Cunonia, Viola, Prunus), on ochreæ (Polygonaceæ), or on young leaves themselves (Ribes, Syringa). The secretion of the colleters is a watery mucilage in the Polygonacex; in the rest it is mixed with drops of balsam or resin. The gummucilage always arises from the conversion of a layer of cellulose lying beneath the cuticle of the colleter, the substance of which swells on addition of water, and raises the cuticle in p'aces into small bladders (Rumex), or detaches it continuously from the hair as a larger bladder; finally the cuticle bursts, and the mucilage escapes and flows over the bud; the uninjured inner layer of cell-wall can, on its part, form a cuticle, beneath which a layer of cellulose again separates, and the process is repeated. Where balsam is also excreted, it may be recognised even in the cells of the hair; but it appears outside the cell-wall in dro s as a deposit in the mucilage, or forms the basis of the secretion. Frequently also the young epidermis itself between the colleters participates in these processes (Polygonaceæ, Cunonia); and the blastocolla is even produced exclusively from the epidermis; thus arises, for instance, the greenish balsam on the bud-scales and foliage-leaves of poplars'.

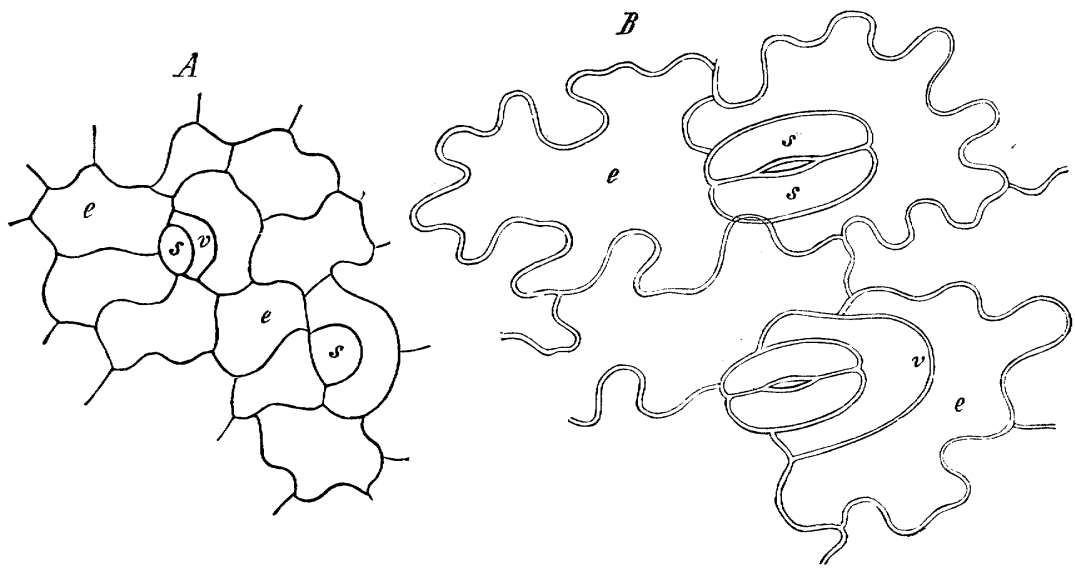

FIG. 84-Development of the stomata of Pteris flabellata (seen from the surface); $A$ very young epidermal cells ; $B$ nearly mature; $v$ cell formed by the preliminary division; $A s$ mother-cell of the two guard-cells $s s$ in $B$.

The Stomata ${ }^{2}$ are never found on the epidermis of true roots; on the other hand they are usually present on underground stems and leaves; according to Borodin they are occasionally found even on submerged parts; but they are formed in the largest numbers on the aërial internodes and foliage-leaves, though not altogether absent from the petals and carpels; they even occur in the interior of the cavity of

1 [Some reference should here be made to the remarkable discovery by F. Darwin (Quart. Journ. Micr. Sci. 1877 , p. 245 ) of the protrusion of protoplasmic filaments from the glands within the cup formed by the connate bases of the leaves of Dipsacus sylvestris, which he believes to have a function connected with the absorption of nitrogenous matter for the nutrition of the plant.]

${ }^{2}$ H. von Mohl, Verm. Schriften bot. Inhalts. Tübingen 1845 , pp. 245, 252.-Ditto, Bot. Zeitg. 1 856 , p. 7or.-A. Weiss, Jahrb. für wiss. Bot. vol. IV, 1865, p. I25.-Czech, Bot. Zeitg. I865, p. IoI.-Strasburger, Jahrb. für wiss. Bot. vol. V. 1866, p. 297.-E. Pfitzer, ibid., vol. VII, J870, p. 532.-Rauter, Mittheil. der naturwiss. Vereins für Steiermark, vol. II. Heft 2, I870.Borodin, Bot. Zeitg. I87o, p. 84r.-Hildebrand, ibid., p. I.-Ditto, Einige Beobachtungen aus dem Gebiete der Pflanzenanatomie. Bonn I861.-Prantl, Ergebnisse der neuern Untersuchungen über Spaltöffnungen, Flora 1872 . 
the ovary (e.g. in Ricinus). They are most numerous where an active interchange of gases takes place between the plant and the external air; for, considered physiologically, they are nothing but the mouths of the intercellular spaces of the inner tissue which open externally between the epidermal cells; this is however always preceded by a peculiar development in a young cell of the epidermis. Since the stomata do not arise till a late stage in the development of the internodes and leaves, or even after their expansion, their arrangement is partially dependent on the already elongated form of the epidermal cells; if these are greatly elongated in one direction and arranged in rows (as in Equisetum and the stem and leaves of many Monocotyledons and Pinus), the stomata are also arranged in longitudinal rows, the cleft lying in the direction of the axis of growth, the guard-cells right and left; if the epidermal cells are irregular on a superficial view, curved, \&c., the position of the stomata is more undefined and apparently irregular. The number of the stomata is generally extraordinarily great in the epidermis of organs containing chlorophyll. In 54 species A. Weiss counted on one square mm. from $\mathrm{r}$ to roo stomata, in 38 species $100-200$, in 39 species $200-300$, in 9 species $400-500$, and in 3 species $600-700$ stomata. The origin of stomata is always the formation of a mother-cell, first of all by division of a young epidermal cell, which is sometimes preceded by several preparatory divisions in it; this mother-cell becomes

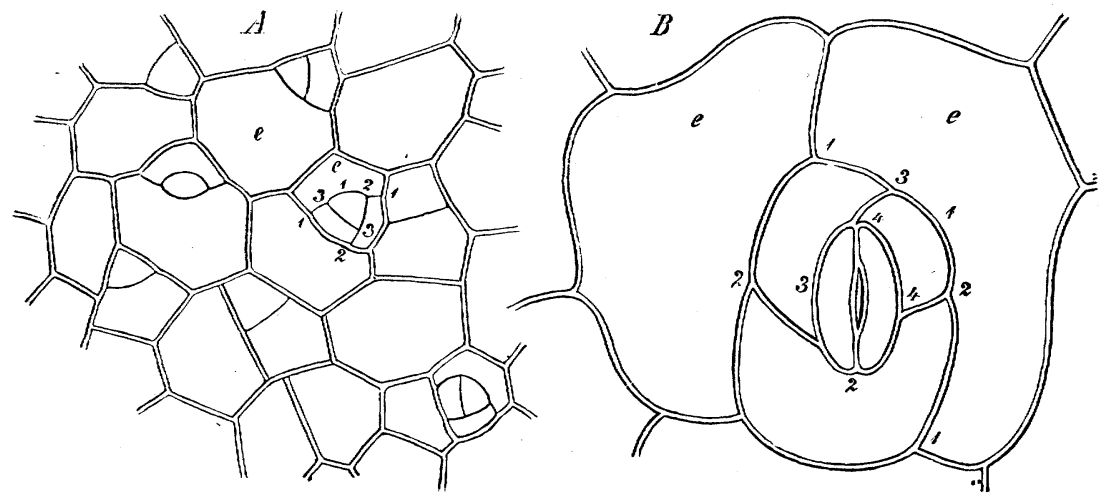

FIG. 85.-Development of the stomata in the leaf of Sea'um purpurascens. $A$ very young stomata; $B$ one nearly mature; $e$ epidermal cells; the numbers indicate the successive order of the preparatory divisiuns.

then more and more rounded off, and the Guard-cells of the stoma are produced from it by division. The variety of these processes up to the point when the cleft itself appears can hardly be explained in a few words; I prefer therefore to describe some examples more minutely. One of the simplest is afforded by the development of the stomata on the leaf of Hyacintbus orientalis, which has already been represented in vertical section in Figs. 6r-64 (p. 77). The preparation for the formation of the stoma is here very simple. A nearly cubical piece of a long epidermal cell is separated by a septum, and this is the mother-cell of the stoma. It is divided by a longitudinal wall (i.e. by one parallel to the axis of growth' of the leaf and at right angles to its surface) into two equal cells, which round themselves off as they grow. How the splitting of the partition-wall takes place has already been depicted in Figs، $61-64$, and can now easily be understood by the help of the surface-view in Figs. 84-86. In Equisetum limosum a similar appearance to that represented in Fig. 6I shows itself immediately after the first formation of the mother-cells of the stomata; but the mother-cell undergoes in these cases three divisions, first one obliquely to the right, then one obliquely to the left, finally the middle cell is bisected by a wall at right angles to the surface. Four cells thus arise in one plane, of which the two outer ones grow more rapidly, while the inner ones are forced downwards and beneath them; 
the stoma then appears, when perfect, as if it had been formed according to the Hyacintbus type, in which each guard-cell has been again divided into an upper and a lower cell. But, according to Strasburger, this is not the case; the two pairs of guard-cells lie originally in one plane, and, strictly speaking, it is only the middle cell, - which is divided by a vertical wall, and the splitting of which forms the cleft,that is to be considered as the mother-cell of the stoma; the two oblique divisions by which the two lateral cells are formed that afterwards lie uppermost must be regarded merely as a preparation for the formation of the mother-cell. Preparatory divisions of this kind occur in many Dicotyledons; one of the young epidermal cells becomes the primary mother-cell of the stoma, and is divided successively in different directions by walls at right angles to the surface; finally (Fig. 85) we have a cell surrounded by several cells formed in this manner, which afterwards forms the two guard-cells (as in Crassulaceæ, Begoniaceæ, Cruciferæ, Violaceæ, Asperifolieæ, Solanaceæ, Papilionaceæ). In other plants, on the contrary, especially Monocotyledons, after the formation of the mother-cell of the stoma which results from the division
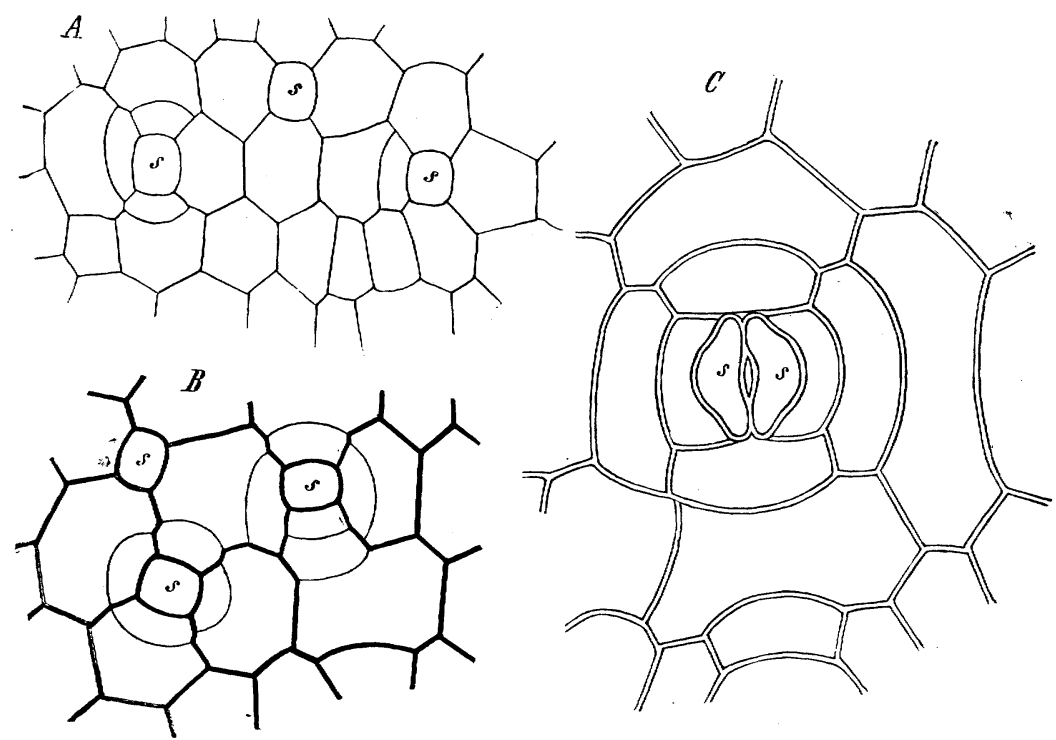

FIG. 86.-Development of the stomata in a leaf of Commelyna coelestis; $A$ very young stomata; $B$ nearly mature; $s s$ in $A$ and $B$ the mother-cells of the stoma in $C$; $s$ in $C$ the guard-cells; $A$ and $B$ show the formation of the neighbouring cells.

of a young epidermal cell, divisions also take place in the adjoining epidermal cells, so that the stoma is surrounded by a pair or by two decussate pairs or by some other arrangement of neighbouring cells (Fig. 86); as in Aloë socotrina, Gramineæ, Juncaceæ, Cyperaceæ, Alismaceæ, Marantaceæ, Proteaceæ, Coniferæ, Potbos crassinervia, Ficus elastica, Tradescantia zebrina. The origin of the mother-cell of the stoma in Plantagineæ, Enothereæ, Sileneæ, Centradenia, and many Ferns deserves special study in reference to the mode of cell-division. In these cases the mother-cell ${ }^{1}$ is cut out on one side from the young but already tolerably large epidermal cell by a wall bent in a $U$-shape, the convexity of which faces the cavity of the epidermal cell, while the ends

1 Strasburger calls them 'special mother-cells.' I think it, however, better entirely to abandon this expression, the more so as its first introduction in the formation of pollen depended on an obsolete view of the formation of the cell-wall (compare our description, pp. 33, 34). 
are applied to one of its side-walls (Fig. 84 ). Not unfrequently, especially with Ferns, e.g. Asplenium bulbiferum, Pteris cretica, Cibotium Scbiedei, \&c., preparatory cells are cut out in this manner from the epidermal cell before the formation of the mothercell, out of which the guard-cells are then formed by simple longitudinal division.

In consequence of the U-shape of the division-wall which separates the mothercell of the stoma from the epidermal cell, the former is half, or more than half, enclosed by the latter when looked at from above. In some Ferns and Sileneæ the wall of the mother-cell of the stoma is from the first so strongly curved that it touches the epidermal cell only in a narrow band; in Anemia villosa it touches it only at one spot, the partition-wall seen from above appearing like a circle. In Anemia densa and fraxinifolia the side-wall of the epidermal cell does not anywhere touch the wall of the mother-cell of the stoma ${ }^{1}$. When first formed this cell has the form of a hollow cylinder, or, more exactly, of a truncated cone, the base and truncated end of which are portions of the upper and lower wall of the epidermal cell; out of the latter a cell is thus cut out like a piece out of a cork by a corkborer; this piece is the mother-cell of the

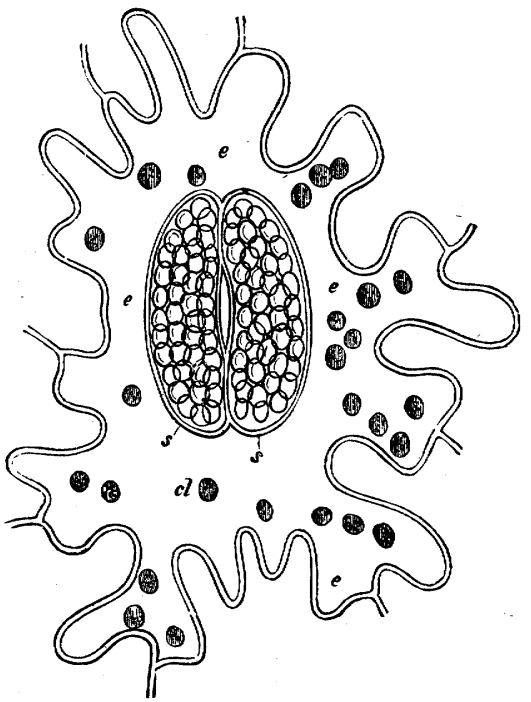

FIG. 87.-Superficial view of a stoma of Anemia fraxinifolia with the epidermal cell completely surrounding it; $e$ epidermis, ss guard-cells; $c i$ cblorophyll-granules.

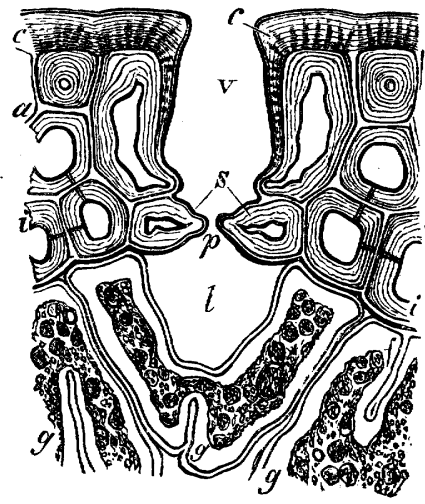

FIG, 88-Transverse section of a leaf of $p_{2 n u s}$ Pinaster $(\times 800) ; s$ guard-cells of the stoma; $p$ its cleft; $v$ entrance; $l$ air-cavity; $c$ cuticularised layers of the epidermis; $a$ middle lamella, $i$ inner thickening-layers of the cells beneath the epidermis $g$ parenchyma of the leaf containing chlorophyll.

stoma; and thus arises the remarkable arrangement represented in Fig. 87, where, as may be seen, the two guard-cells are entirely enclosed within a single epidermal cell. Similar, but more complicated, is, according to Rauter, the structure in Niphobolus Lingua.

By further growth of the guard-cells and of the epidermal cells which surround them, different relative positions of the former to the surface may be brought about; the guard-cells may, when mature, lie in one plane with those of the epidermis, or may be deeply depressed and apparently belong to a deeper layer of cells (Fig. 88); sometimes they are, on the contrary, elevated above the surface of the epidermis.

The stomata of Marchantiex may shortly be mentioned here in connexion with what has already been said on Fig. 65 , p. 78 . After the formation of the air-cavities, which are filled with outgrowths containing chlorophyll (Fig. 89, $A, c b l$ ), one cell of the

' Strasburger, in Jahrb. für wiss. Bot. VII. p. 393 ; also Rauter, l.c. 
epidermis lying above the centre is divided by several bipartitions into four, six (Marchantia, Fegatella), or several (Rebouillia) cells, which are arranged radially about a point where their walls unite. Here the cells separate from one another, and the cleft (Fig. $89, B$ and $C, p o$ ) is surrounded by four, six, or more guard-cells $(s l)$. Each of these cells is finally divided by walls parallel to the surface into from 4 to 8 cells lying one above another, and the stoma becomes a canal surrounded by 4,8 , or more rows of cells.

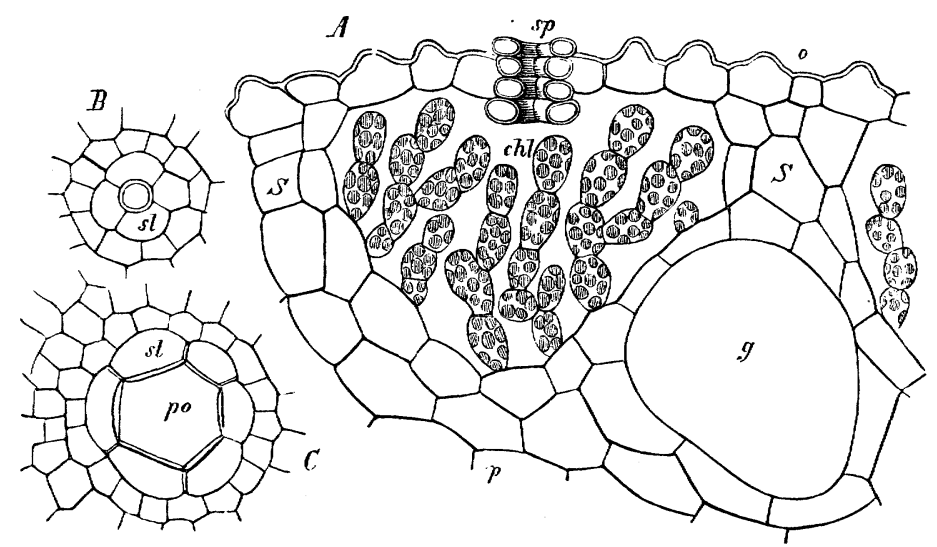

FIG.89.-Marchantia polymorpha. Part of a young fructification; $A$ vertical section, o epidermis, $S$ partitionwall between the air-cavities with their chlorophyll-cells $c h l ; g$ large parenchymatous cell; $s p$ stoma; $B$ and $C$ young stomata seen from above; po cleft; $s l$ guard-cells $(\times 550)$.

(c) Cork, and Epidermal Formations formed from it ${ }^{1}$ (Periderm, Lenticels, Bark). When succulent organs of the higher plants, no longer in the bud-condition, are injured, the wound generally becomes closed up by cork-tissue; i.e. new cells arise near the wounded surface by repeated division of those which are yet sound, and these, forming a firm skin, separate the inner living tissue from the outermost injured layers of cells. The walls of this tissue resist the most various agents; similar to the cuticular layers of the epidermis in their physical properties, flexible and elastic, permeable only with difficulty by air and water, they for the most part soon lose their contents and become filled with air. They are arranged in rows lying at right angles to the surface, of parallelopipedal form, and constitute a close tissue without intercellular spaces. These are the general distinguishing features of cork-tissue. It not merely forms on wounded surfaces, but arises in much greater mass where succulent organs require an effectual protection, as on potato-tubers, or where the epidermis is unable to keep up with the increase of circumference when growth in thickness continues for a long period. In these cases, which occur but seldom in Monocotyledons (e.g. stem of Dracena), but generally in stems and roots of Conifers and Dicotyledons when several years old, the cork-tissue is formed even before the destruction of the epidermis; and when this splits under the action of the weather and falls off, the new envelope formed by the cork is already present. The cork-tissue is the result of repeated bipartition of the cells by partition-walls, rarely in the epidermis itself, more often in the subjacent tissue. These partition-walls lie parallel to the surface of the organ; where the increase of the circumference necessitates it, vertical divisions also

1 H. von Mohl, Vermischte Schriften bot. Inhalts. Tübingen I 845, pp. 22 I, 233.-Hanstein, Untersuch. über den Bau u. die Entwickelung der Baumrinde. Berlin 1853.--Sanio, in Jahrb. für wiss. Bot., vol. II. p. 39.-Merklin, Mélanges biol. du Bulletin de l'Acad. Imp. des sciences de St. Pétersbourg, vol. IV. Feb. 26, 1864. 
take place, by which the number of the rows of cells is increased. Of the two newly formed cells of each radial row (i.e. vertical to the surface of the organ) one remains thin-walled, rich in protoplasm, and capable of division; the other becomes suberised and permanent. Thus arises, usually parallel to the surface of the organ, a layer of cells capable of division, which continues to form new cork-cells, the Cork-cambium or layer of Phellogen. In general this is the innermost layer of the whole cork-tissue, so that the cork increases centrifugally, and new layers of cork are constantly formed out of the phellogen inside those already in existence. But, according to Sanio, it also happens, when cork is beginning to be produced, that the formation of permanent cells proceeds centripetally, or an atternation of centripetal and centrifugal cellformation takes place in the young cork-tissue. But sooner or later the centrifugal formation of cork with phellogen on the inner side always commences, a result of the circumstance that the tissues lying on the outside of completely suberised layers of cells die sooner or later. Usually the formation of cork begins first at single places of the periphery of lignified branches; but the phellogen gradually forms a continuous layer, from which new layers of cork are developed centrifugally. When in this manner a layer of cork arises, increasing progressively from the inside, it is termed Periderm. The development and configuration of the corkcells may change periodically during the formation of periderm; alternate layers of narrow thick-walled and broad thin-walled cork-cells are formed; the periderm then appears stratified, like wood with annual rings, as in the periderm of the cork-oak, birch, \&c. In some cases the phellogen gives rise not only to cork-cells, by which the periderm increases in thickness, but parenchymatous cells are also formed containing chlorophyll ; but it is only daughtercells of the phellogen lying on the inner side (facing the wood) that undergo this metamorphosis. In this manner the green cortical tissue of some dicotyledonous plants becomes thickened by the layers of tissue proceeding from the phellogen, which Sanio terms the suberous cortical layer or

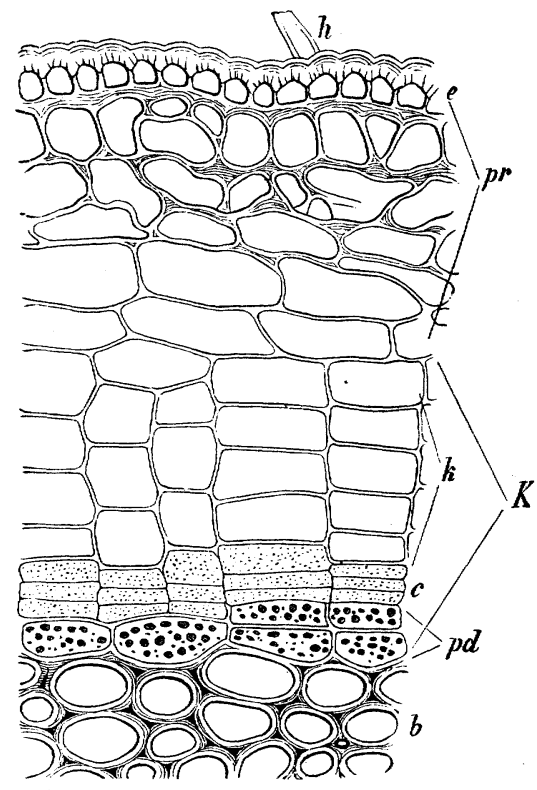

FIG. 90.-Formation of cork in a branch of Riles ugrum one year old; part of a transverse section; e epidermis, $h$ hair, $b$ bast-cells, $p r$ cortical parenchyma distorted by the increase in thickness of the branch; $K$ the total product of the phellogen $c ; k$ the cork-cells arranged radially in rows formed from $c$ in centrifugal order, $p d$ phelloderm (parenchyma containing chlorophyll formed centripetally from $c)(\times 550)$.

Pbelloderm. This occurs, for example, in

branches two years old or more of Salix purpurea and alba, the beech, \&c. In such cases the phellogen lies between the periderm and the phelloderm, the outer daughter-cells producing cork-cells; the inner phelloderm (Fig. 90). The layers of periderm which first undergo suberisation sometimes bear a very close resemblance to true epidermis, as, for instance, in branches one year old (August) of the Scotch fir, where, while the epidermis still remains, the cork-cambium is formed in the cortical parenchyma, and at first presents the appearance as if a second epidermis were formed with cells greatly thickened on the outside.

As the epidermis is at first replaced by the periderm, so this again is afterwards replaced by the formation of bark when the increase in thickness continues long and vigorous. In large trees, as oaks and poplars, the boughs are covered with epidermis in their first year, when several years old with periderm, the older branches and the stem 
with bark ${ }^{\mathrm{l}}$. The formation of bark depends on the repeated production of new layers of phellogen in the succulent cortical tissues of Conifers and Dicotyledons which continue to grow centrifugally. Layers of cells which can extend through the most different tissues of the cortex are changed into cork-cambium, which ceases to be active after the production of thicker or thinner layers of cork. These layers of cork cut out, so to speak, from the cortex, scaly or annular pieces of the surface; everything which lies outside them becomes dried up; and since this process is constantly repeated on the outside of the stem, and the new layers of cork continually intrench further on the growing cortical tissue, a mass of dried up portions of tissue, constantly increasing in thickness, becomes separated from the living part of the cortex; and this is the Bark. The process is very clear in the bark of the oriental plane which detaches itself in large scales, and almost as clear in old stems of the Scotch fir. Since the bark does not follow the increase in thickness of the stem, it splits in longitudinal crevices from the surface inwards, as in the oak, according to the direction of weakest cohesion; in other cases it peels off in the form of horizontal annular bands from the stem (ring-bark), as in the cherry.

Lenticels are a peculiarity of cork-forming Dicotyledons. They appear before the formation of periderm in branches during their first year, as long as the cortex is still covered with uninjured epidermis, and are visible as roundish bodies. At the end of the first or in the following summer, the epidermis splits above the lenticel in the direction of its length; the lenticel becomes changed into a more or less projecting wart, which is often divided by a central furrow into two lip-like ridges; its surface is generally brown, its substance to a certain depth dry, brittle, and cork-like. With the further increase in thickness of the branch, the lenticels become extended in a direction transverse to the branch, and present the appearance of transverse streaks; when afterwards cork or bark is formed, the splitting of the cortex commences with these, and they become indistinguishable (as in the silver poplar, apple, and birch); by the scaling off of the bark they are of course removed. According to Unger, the lenticels arise only at those portions of the cortex where stomata occur in the epidermis; according to Mohl the inner cortical parenchyma projects in a wart-like manner through the outer, and forms a cork-tissue, which, on the formation of periderm, coalesces with the cork of that tissue; as occurs, for example, in young potato-tubers. The formation of cork on the lenticel continues for a number of years, until the cortex which afterwards grows from within dies off on the outside, periderm or barkformations becoming interposed between the lenticels and the living part of the cortex. In many trees, as Cratagus, Pyrus, Salix, Populus, where the formation of periderm begins from single spots, and becomes further extended, the lenticels are, according to Mohl, the points of departure ${ }^{2}$.

SECT. 16. The Fibro-vascular Bundles ${ }^{3}$. - The tissue of the higher Cryptogams and of Phanerogams is traversed by filiform or string-like masses of

1 A considerable increase of thickness is not always associated with the formation of periderm, as, for example, in the sunflower and other annual stems. In Viscum the epidermis always remains capable of development, and its thick cuticular layers render the protection of periderm superfluous. The formation of bark is also not a necessary consequence of vigorous increase of thickness; the copper-beech and the cork-oak, for example, form nothing but periderm.

2 [For a detailed investigation of the development of lenticels, see E. Stahl, Entwickelungsgeschichte und Anatomie der Lenticellen, Bot. Zeitg., 1873.]

3 H. von Mohl, Vermischte Schriften, pp. 108, 1 29, 195, 268, 272, 285.-Ditto, Bot. Zeitg. 1855, p. 873.-Schacht, Lehrb. der Anat. u. Phys. der Gewächse, pp. 216 , 307-354.-Nägeli, Beiträge zur wiss. Bot. Leipzig 1858 , Heft I. - Sanio, Bot. Zeity. 1863 , no. 12 et seq-Nägeli, Das Dickenwachsthum des Stammes u. die Anordnung der Gefässtränge bei den Sapindaceen. München I864. - Caractère et formation du liège dans les dicotylédons, in Rauwenhoff's Archives Néerlandaises, vol. V. 1870. [For the recent literature see Vines in Quart. Jour. Micr. Sci. 1876, pp. 388-3y8.] 
tissue, which in some cases develop by increase in thickness in such a manner that they lose externally the form of strings and present that of large masses, retaining, however, internally their characteristic structure. These are the Vascular or Fibro-vascular Bundles. Very often they can be completely isolated with ease from the rest of the tissue of the plant. If, for instance, the petiole of Plantago major is broken across, they hang out from the parenchyma as tolerably thick, extensible, elastic threads. In Pteris aquilina it is possible, by scraping off the mucilaginous parenchyma, after removing the hard epidermal tissue of the underground stem, to expose them as strap-shaped or filiform very firm light yellowish bands (Fig. 9I). In older leaves of trees, dry pericarps (as Datura), stems of Cactus, \&c., the fibro-vascular bundles are left, through the decay of the parenchyma which surrounds them, as a skeleton retaining more or less the original form. Beautiful and instructive skeletons of this nature are afforded by the stems of Tree-ferns, Dracana, Yucca, maize, \&c., when their parenchyma has been destroyed by gradual decay, and only the epidermal tissue and the firm bundles in the interior remain; and the student would do well in any case to make for himself preparations of this kind, or to examine them in collections; they are extremely useful for a clear comprehension of structure. This is, however, the case only with lignified fibro-vascular bundles which run isolated between soft parenchyma; in some plants, on the contrary, the tissue of the bundles is even softer and more delicate than that which surrounds them (e.g. Ceratophyllum, Myriophyllum, Hydrillex, and other water-plants); and in these cases they cannot of course be isolated. But in the older lignified stems and roots of Conifers and Dicotyledons, the fibro-vascular bundles are so densely crowded, and so extended by the further development

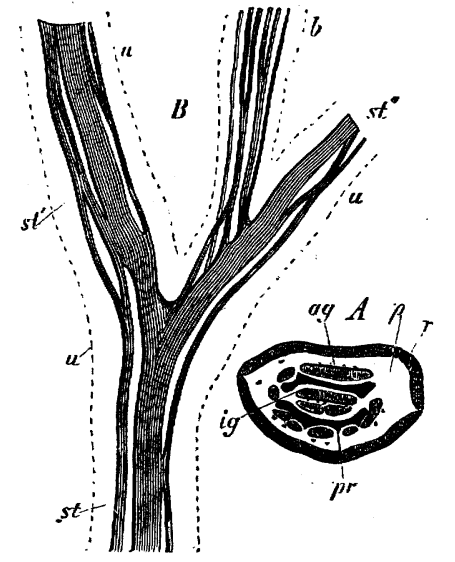

FIG. 9I.-Pteris aquilina. A transverse section

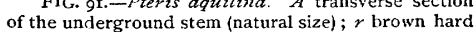
epidermal tissue; $p$ soft mucilag nous parenchyma containing starch; $p r$ dark-walled sclerenchyma, forming two broad bands traversing the stem; $a g$ fibrovascular trundles running outside these bands of sclerenchyma; ig others running within them. $B$ the fibro-vascular bundle represented in $A$, isolated by scraping off scraping off the parenchyma; it exhibits divisions and anastomoses; the dotted lines $u$ show the outline
of the stem $s^{*}$, of its branches $s t^{\prime}$ and $s t^{\prime \prime}$, and of a petiole $b$. of their tissue, that at last very little or even nothing is left of the original fundamental tissue which separated them, and such stems consist almost entirely of fibro-vascular masses.

Each separate fibro-vascular bundle consists, when it is sufficiently developed, of several different forms of tissue, and must therefore itself be considered as a tissuesystem; but different bundles, often in very large number, unite in most plants to form a system of a higher order. At present however we shall consider only the separate bundle.

The fibro-vascular bundle consists at first of similar cells fitting together without intercellular spaces ${ }^{1}$; this form of tissue in the young undifferentiated

1 The young cells of the fibro-vascular masses are not always elongated and prosenchymatous; in the roots of maize the young vascular cells which no longer divide, as well as the adjoining ones, are tabular or cubical. 
bundle may be termed Procambium ${ }^{1}$. As it grows older, some of its rows of cells change into permanent cells of definite form (vessels, bast-fibres, \&c.); and from these starting-points the transformation of the procambium-cells into permanent cells in the transverse section of the bundle advances until the cells have all undergone this change; or an inner layer of the bundle remains capable of

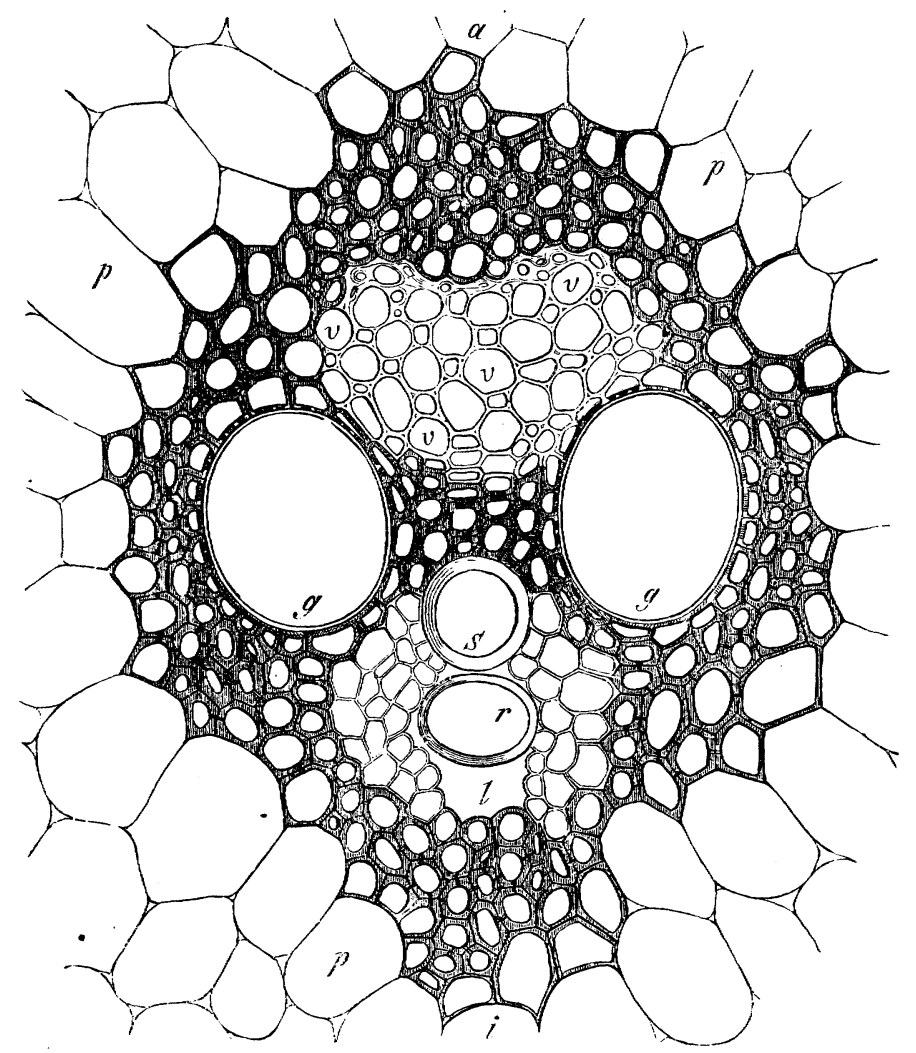

FIG 92.-Transverse section of a closed fibro-vascular bundle in the stem of maize $(\times 550)$; the fibro-vascular bunulle consists of the xylem-portion $g g, s, r, l$, and the phloëm-portion $v v v$; the thick-walled tissue on the outside is the bundlesheath belonging to the fundamental tissue; $p p$ the thin-walled parenchyma of the fundamental tissue; $a$ outer side, $i$ inner sheath belonging to the fundamental tissue; $\not p p$ the thin-walled parenchyma of the fundamental tissue; $a$ outer side, $i$ inner
side (facing the axis of the stem); $g g$ two large pitted vessels; $s$ spirally thickened vessel; $r$ isolated ring of an annular side (facing the axis of the stem); $g g$ two large pitted vessels; $s$ spirally thickened vessel; $r$ isolated ring of an annular
vessel; $l$ air-containing cavity, caused by splitting resulting from growth; $v v$ the cambiform or latticed cellular tissue which has last passed over into permanent tissue; between it and the vessel $s$ lie reticulately thickened vessels with bordered pits.

further development, and is then called Cambium. In advanced age there are thus bundles devoid of and bundles containing cambium; the former may be termed closed, the latter open ${ }^{2}$. As soon as a procambium-bundle has become transformed

${ }^{1}$ Nägeli calls the tissue of the young fibro-vascular bundles simply Cambium, and applies the same term to the tissue, capable of further development, of the bundles which increase in thickness, which nevertheless ought to be distinguished from them. Sanio terms the latter only Cambium, a restriction which I adopt. (Sanio in Bot. Zeitg. I 863, p. $3^{62 .)}$

2 This distinction was first made by Schleiden; but he incorrectly ascribed to Dicotyledons in general only open bundles; his distinction of simultaneous and successive cannot be sustained; all bundles become differentiated successively in transverse section. Schleiden's simultaneous bundles of the higher Cryptogams belong to the closed description. 
into a closed fibro-vascular bundle, all further growth ceases, as in Cryptogams, Monocotyledons, and some Dicotyledons. The open fibro-vascular bundle, on the other hand, continues to produce new layers of permanent tissue on both sides of its cambium, and thus the portion of the stem or root concerned continually increases in thickness, as occurs in woody Dicotyledons and Conifers; the foliar organs, however, of these plants possess closed bundles, or, if they are open, the activity of their cambium soon ceases.

The different forms of tissue of a differentiated fibro-vascular bundle may be classified into two groups, which Nägeli calls the Phlö̈m-(Bast) and Xylem- (Wood) portion of the bundle. They are separated by the cambium, if there is any. In each of the two constituents of the bundle, the phloëm and the xylem, three forms of tissue are especially to be distinguished:-(I) Vascular cell-unions (the wood-vessels of the xylem, the sieve-tubes of the phloëm); (2) Prosenchymatous Tissue (the wood-fibres in the xylem, the bast-fibres in the phloëm); and (3) Parenchymatous Tissue (the wood-parenchyma in the xylem, the bast-parenchyma in the phloëm). The phloëm consists of succulent, generally thin-walled cells; only the bast-cells, which are often absent, but very frequently massively developed, are usually greatly thickened (mostly however not lignified but flexible). The thin-walled succulent cells are either parenchymatous, or they are cambiform or latticed-cells, or finally sieve-tubes. The sylem-portion of the fibro-vascular bundle has mostly a strong tendency to thicken its cell-walls, which become hard and lignified; in vessels and wood-cells with bordered pits the contents disappear, and they henceforth contain air. Lignified parenchyma is also abundant, but in some cases no lignifying takes place; the whole bundle is then soft and succulent, sometimes traversed only by single thinner strings of lignified vessels and wood-cells, as in the roots of the radish, tubers of the potato, \&c. The elements of the fibro-vascular bundles, as far as they consist exclusively of procambium, are mainly prosenchymatous, or at least elongated in the direction of the axis of growth of the - bundle. In open bundles there arise also in the cambium, with the increase of their thickness, radial rows and layers of horizontally extended cells, by which the laterformed xylem- and phloëm-layers of the bundle become broken up in a fan-like manner. These horizontal elements mostly assurne the character of parenchymatous cells, and may be generally designated as rays; within the xylem they are called Xylem-rays, within the phloëm Phloëm-rays.

The position of the layers of phloëm and xylem in the transverse section of a bundle varies according to the class to which the plant belongs and the organ in which they are found; in the open bundle of the stem of Dicotyledons and Conifers the former lie towards the circumference ", the xylem facing the axis of the organ; between the two lies the cambium-layer (Fig. 93). But a layer of phloëm is sometimes found in addition on the axial side of the xylem, so that the bundle possesses two phloëm-layers, a peripheral, and an axial-layer, e.g. in Cucurbitaceæ, Solanaceæ, and Apocynaceæ. In the closed bundles of Dicotyledons there occur considerable deviations from the typical position of the tissues; among

${ }^{1}$ See, however, what is said in Book II., at the end of the section on Dicotyledons, on the formation of tissue in that class. 
Monocotyledons these are still more conspicuous, especially if the bundle-sheath of lignified prosenchyma, which often occurs in them, is taken into account (see Fig. 92). Among Ferns, Lycopodiaceæ (with isolated bundles ${ }^{1}$ ), and Rhizocarpeæ, the xylem lies in the centre of the bundle, while the phloëm forms a soft succulent sheath round it (Fig. 67, p. 80, and Fig. 94).

According to the relative position of the two principal constituents of fibrovascular bundles, they may be classified under two varieties:-(i) Collateral bundles, in which the phloëm and xylem run parallel to one another, like the two halves of a

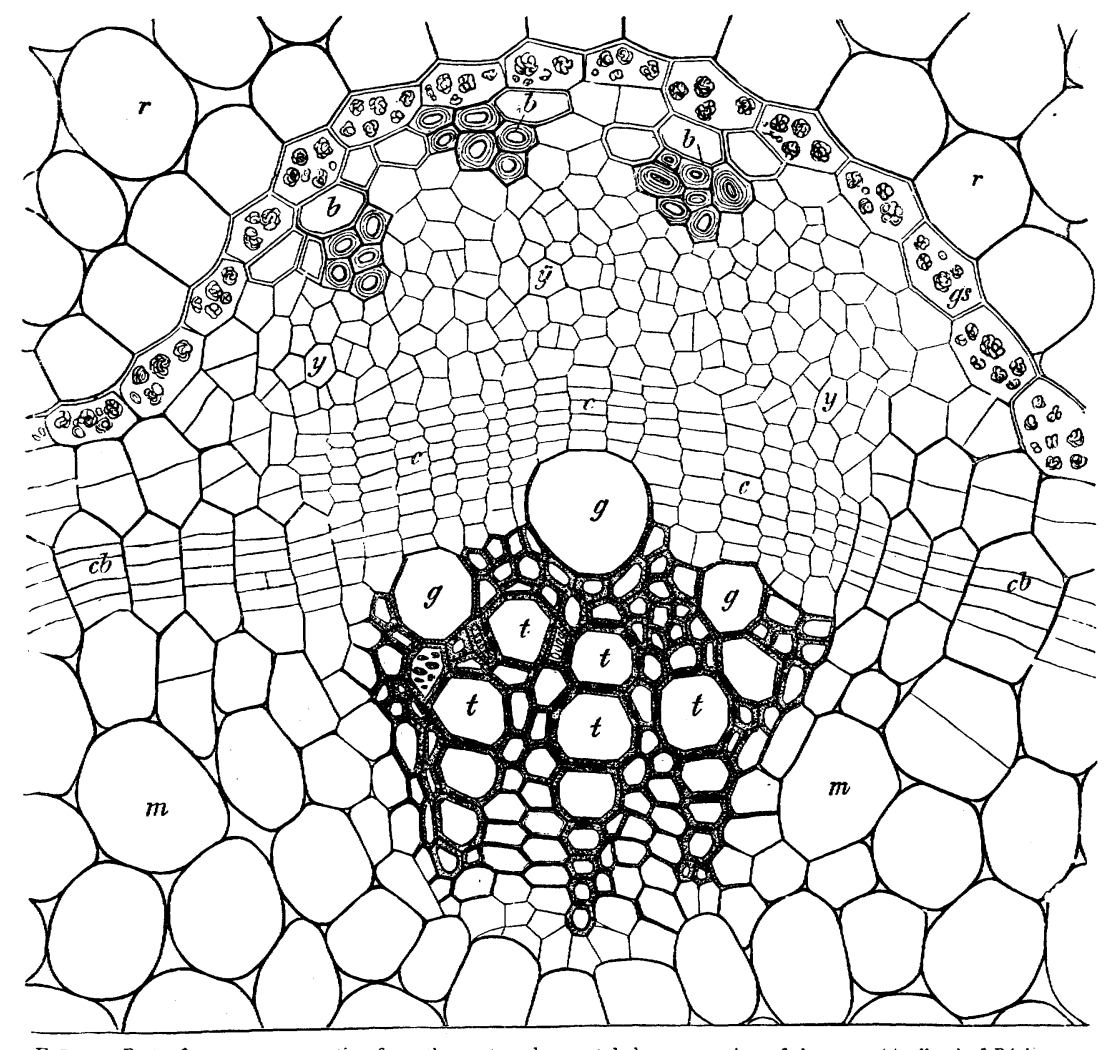

FIG. 93.-Part of a transverse section from the mature lypocotyledonary portion of the stem (tigellum) of $R$ icinus communis; $r$ parenchyma of the primary cortex, $m$ of the pith, both belonging to the fundamental tissue; between $r$ and $b$ is the mumis; $r$ parenchyma of the primary cortex, $m$ of the pith, both belonging to the fundamental tissue; between $r$ and $b$ is the of the phloëm $b, y$, the xylem $g, t$, and the cambiun $c c$, and is therefore an open bundle. The cainbium of the bundle of the phloëm $b, y$, the xylem $g, t$, and the cambiuin $c c$, and is therefore an open bundie. The cainbium of the bundle
$c c$ is also continued into the fundamental tissue that lies between it and the neighbouring bundles in the form of interfascicular cambium $c b$, formed by subsequent divisions of large parenchymatous cells (secondary meristem). In the phloëm are bastfibres $b b$, the soft-hast $y y$ (partly parenchyma and partly sieve-tubes); in the xylem narrow pitted vessels $t t$, broad pitted vessels $g g$, and between them wood-fibres.

rod bisected lengthwise, as occurs in Phanerogams and Equisetaceæ; and (2) Concentric, when the xylem is completely enclosed within a layer of phloëm, as in Ferns, the simple bundles of Lycopodiaceæ ${ }^{2}$, \&c. The latter kind are always

1 The bundle in the stem of Lycopodium Chamcecyparissus, \&c., is clearly a union of several fibro-vascular bundles, such as occurs in the axial cylinder of roots (see Book II., Lycopodiaceæ).

${ }^{2}$ See Russow, Vergleichende Untersuchungen über die Leitbündelkryptogamen. Petersburg 1872, p. 159 et seq. The terminology employed in this paper does not seem to me happy, with the exception of the term 'collateral bundles.' 
closed; while the former may be either closed or open. (Vide infra under the Fibro-vascular system of roots.)

Every one of its cell-forms may at one time or other be absent from a fibrovascular bundle; bundles may occur without wood-cells, without vessels (very rarely), without bast-fibres, \&c. ; it is only the soft bast (the succulent thin-walled cells of the phloëm) that is scarcely ever absent. All these variations may occur in the same fibro-vascular bundle in different parts of its length, when this is considerable. The terminations of the bundles which traverse the stem of Phanerogams are usually found in the leaves; there, as their thickness decreases, they lose all the elements of the xylem except one or two spiral vessels, and finally these also; the extreme ends of these bundles which traverse the mesophyll of the leaves often consist only of long narrow thinwalled cambiform cells.

If the fibro-vascular bundle is formed at the very earliest period within an organ which afterwards grows rapidly in length, then the elements which were formed before the increase in length (the innermost vessels and the outermost bastcells) are the longest, since they participate in the whole increase in length of the organ; the elements developed during the elongation are shorter; and those are shortest of all which arise after the increase in length of the whole organ has been completed; this occurs in particular with the open bundles of Dicotyledons and Conifers.

The development of the elements of a bundle always begins at single points in the transverse section, and extends from them in different directions; and thus the permanent

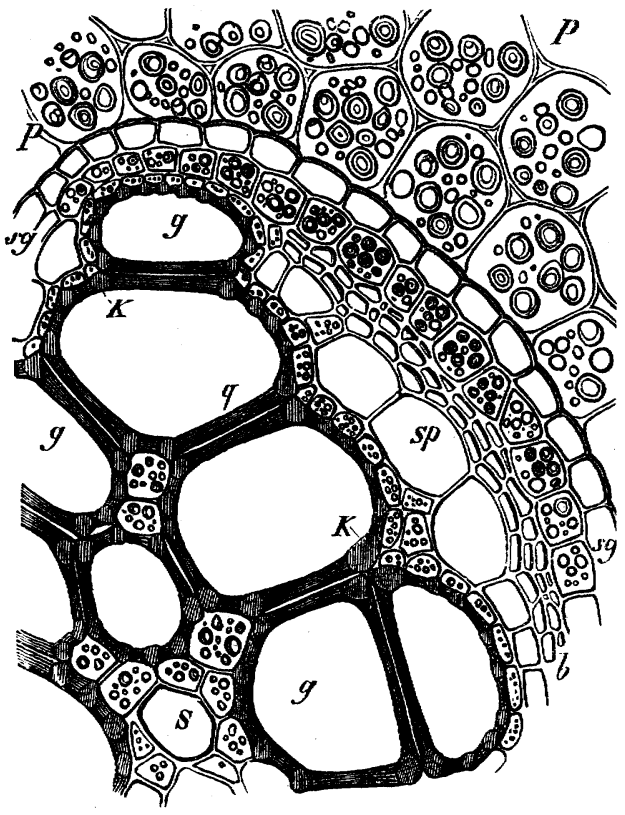

FIG. 94.-A fourth of a transverse section of one of the large fibrovascular bundles in the stem of Pteris aquilina, with a portion of the surrounding parenchyma, $P$, this is filled with starch (in winter); $s$ spiral vessel in the focus of the elliptical transverse section of the bundle, sur rounded by thin-walled wood-cells containing starch; $g g$ the vessels thickened in a scalariform manner, the structure of which is explained in Fig. 29 (p. 28); $s p$ wide sieve-tubes; between them and the xylem lies, in the winter, a layer of cells containing starch; $b$ bast-cells, with thick soft wall; $s g$ the bundle-sheath; between $b$ and $s g$ is a layer of cells containing starch.

cells which arise successively acquire different mature forms. In the open bundles in the stem of Dicotyledons and Gymnosperms the development usually begins with the thickening of single bast-cells on the peripheral side of the bundle; somewhat later single spiral vessels (or annular vessels) arise next the pith; and while the development of the phloëm proceeds centripetally-forming successively and often alternately bast-fibres, sieve-tubes and parenchyma-annular or spiral vessels with reticulate thickenings (or both forms), and eventually vessels with bordered pits alternating with wood-fibres and wood-parenchyma, arise centrifugally in the xylem (Fig. 95). In Coniferæ only prosenchymatous cells with bordered pits (together with xylem-rays) are subsequently produced, so long as 
the stem or root grows. In Dicotyledons, on the contrary, after the first year, a combination of vessels and wood-prosenchyma, often mixed with wood-parenchyma, is annually formed. In trees with annual rings in the wood a periodicity may be remarked in the development of the cells of the xylem; and on this depends its stratification into annual layers. Not unfrequently the phloëm-portion also shows a similar stratification. In the closed bundles of Monocotyledons the order of development in the first year is similar to that already described. In Fig. 92, for example, the annular vessel $r$ is first formed in the xylem-portion, then the spiral vessel $s$, then, advancing right and left, the pitted vessels $g g$, and in the middle (advancing radially) the narrow pitted vessels. It sometimes occurs $(e . g$. in Calodracon, according to Nägeli) that the formation of vessels advancing right and left encloses the procambium, which afterwards passes over into latticed cells.

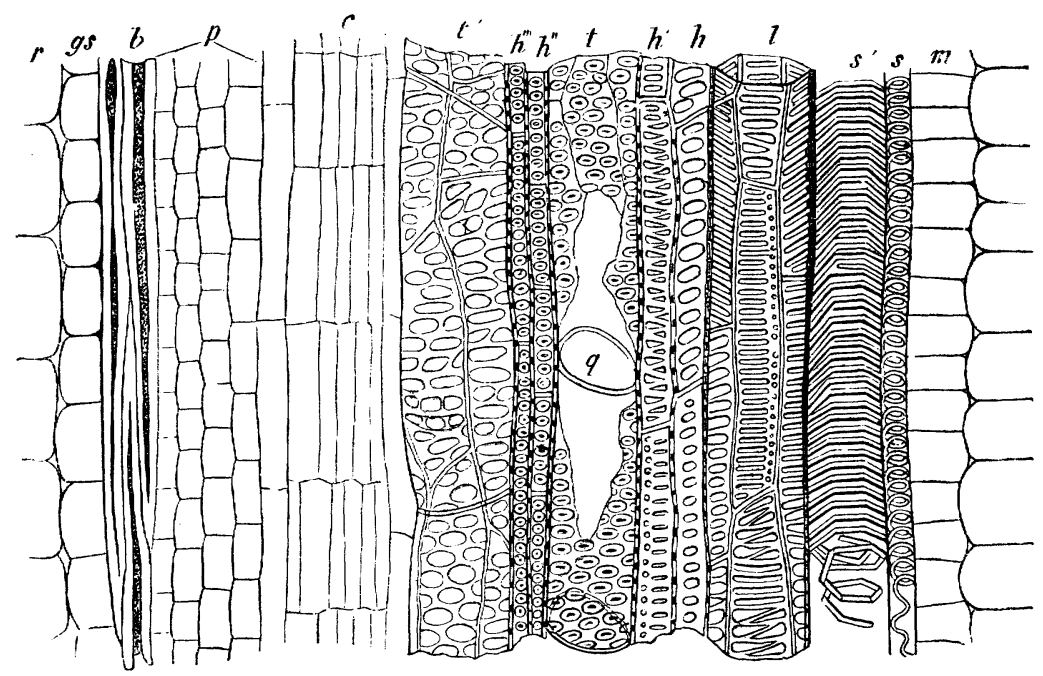

FIG. 95.-Longitudinal section of a fibro-vascular bundle of Ricinus, the transverse section being shown in Fig. 93 ; $r$ cortical parenchyma; $g s$ bundle-sheath; $m$ parenchyma of the pith : $b$ bast-fibres; $p$ phloëm-parenchyma; $c$ cambium, the row of cells between $c$ and $p$ develops afterwards into a sieve-tube. In the xylem-portion of the bundle the elements are developed from $s$ successively to $t^{\prime} ; s$ the first narrow and very long spiral vessel, $s^{\prime}$ wide spiral vessel, both with a spiral band which can be unrolled; $l$ vessel thickened partly in a scalariform, partly in a reticulate manner; $h h^{\prime}$ wood-cells; $t$ pitted vessel, at $q$ the absorbed septum; $h^{\prime \prime} h^{\prime \prime \prime}$ wood-cells; $t^{\prime}$ pitted vessel, still young; the pits at first show the outer border; afterwards the formation of the inner orifice commences; at $l t t^{\prime}$ in the wall of the vessel are observed the boundary-lines of the adjoining cells which have been removed.

In the petiole of Pteris aquilina the development of the xylem begins in the procambium-bundles, by the formation of some narrow spiral vessels in the foci of their elliptical section; scalariform vessels are then formed in the direction of the longer axis of the ellipse, first centrifugally then centripetally, until a compact woody mass is produced, elongated in transverse section; round this the procambium which is still left is transformed into latticed cells, sieve-tubes, and cambiform tissue, and partly (at the circumference) into bast-fibres (Figs. 94, 96). The same is the case with most concentric bundles of Cryptogams.

The Fibro-vascular System of Roots. Bundles of the kind now described traverse the stem and branches usually in large numbers (sometimes, as in Palms, the number is enormous), bending at their upper end into the leaves, where they ramify copiously in 
the lamina, constituting its venation. Every bundle is completely surrounded by fundamental tissue both in the stem and in the leaf, and is therefore isolated from the rest; the only connection between different bundies takes place at their lower ends within the stem. The arrangement of the fibro-vascular system in the root is strikingly different, if we compare with it in the case of stems only the original structures produced by differentiation from the primary meristem, and not the thickening-tissues which subsequently arise from the secondary meristem or cambium (see Sect. I8). The root is usually cylindrical and very slender; and its transverse section, both in Cryptogams and Phanerogams, shows, beneath the epidermis, a thick layer of parenchymatous fundamental tissue, surrounding a cylindrical bundle which traverses the whole length of the root. This bundle may be termed the axial cylinder or Plerome; it is always sharply separated from the cortical parenchyma by an innermost layer of the latter, the Bundle-sheath or Plerome-sheath. In most stems also a similar sheath separates the cortex from an internal cylinder of tissue ${ }^{1}$ containing the fibro-vascular bundles (Fig. 93 , p. I I 2 ). It may easily be recognised by the character of the longitudinal partitions of its cells in a radial section, which, in consequence of their peculiar folding, appear, on transverse section, as if marked with a black dot (Fig. $\left.9^{6}, s\right)^{2}$.

Within this bundlesheath is usually found in thick roots a large number of ribbon-shaped vascular bundles arranged in a ring. In each vascular bundle the oldest but smallest vessels lie on the outside next the sheath (Fig. $96, p, p)$; from them the formation of vessels advances centripetally, so that the later-formed

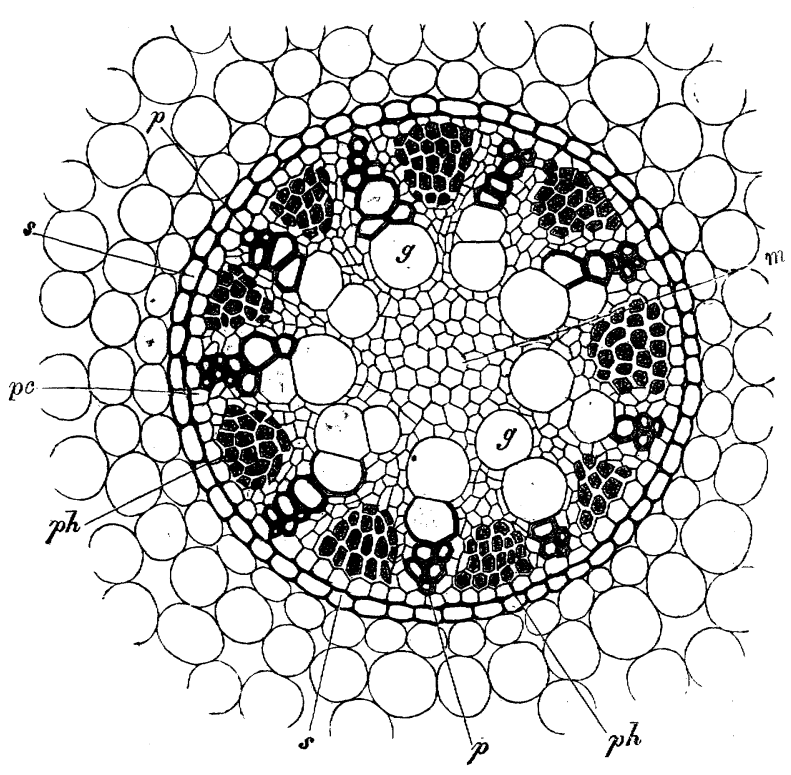

FIG. 96.-Transverse section of root of Acorus Calamus, showing the axial cylinder and the surrounding cortical tissue; $s$ bundle-sheath or plerome-sheath; $p p$ the oldest narrow peripheral vessels; $g$ large broader vessels which are always younger; $p h$ phloëm. bundle; $m$ central parenchymatous tissue or pith; $p c$ pericambium.

vessels which lie nearer the centre are always larger and broader. Between any two groups of vessels there always lies a bundle of phloëm $(p b)$, which not unfrequently has true bast-fibres on its outer margin. The rest of the axial cylinder consists of parenchymatous tissue.

In slenderer roots the number of xylem- and phloëm-bundles in the axial cylinder is commonly reduced to two or three, and in that case the former usually meet in the axis, so as to form either a broad band which divides the cylinder in two, or a three-rayed star of vessels. In thicker roots, on the contrary, the inner edges of the vascular bundles do not usually reach the centre of the cylinder, which in these cases consists of a mass of parenchymatous tissue or pith, $m$. Roots which, like most

${ }^{1}$ See also the end of Sect. 19, and Van Tieghem, Mémoire sur les canaux sécréteurs; Paris, I 872 ; foot-note, p. I 7 .

${ }^{2}$ [This is well shown by Van Tieghem, Recherches sur la symétrie de structure des plantes vasculaires, in Ann. des Sci. Nat. 5th ser, I871, vol. xiii. pl. 3-8.] 
primary roots of seedling Dicotyledons and Conifers, are thick in their upper part, gradually decreasing in thickness below as they lengthen, have no pith in their lower part, so that the vascular bundles, separated above by this pith, are in contact in the lower parts of the root, presenting a star-shaped appearance on transverse section.

The outermost portion of the axial cylinder consists (except, according to Nägeli and Leitgeb, in the case of Equisetum) of a simple layer of cells, which they have termed Pericambium. It must lie, as will be seen from what has been said, on the inside of the plerome- or bundle-sheath (Fig. $96, p c$ ). The importance of the pericambium in the branching of the root and in its increase in thickness will be discussed further on in Sects. 18 and 23 .

In the present state of our knowledge of tissues it is still doubtful whether the axial cylinder of roots should be regarded as a single fibro-vascular bundle, or as a coalescence of a number of such bundles, corresponding to the bundles of xylem and phloëm. Van Tieghem $(l . c$.$) adopts the latter vicw, laying special stress on the fact that in most stems$ also a plerome-sheath (which he terms 'membrane protectrice') separates all the fibrovascular bundles together with the pith from the cortex. On this view it is only necessary that the bundles in the stem should be closely crowded together, and their phloëm-portion placed at the side of instead of in front of the xylem-portion, in order to get an axial cylinder like that of roots. This view is supported by the fact that in some stems (as in those of Lycopodium, Fig. 100, B. p. 122, and some water-plants like Hippuris, Hydrilla, \&c.) there is an actual axial cylinder similar to that of roots. Further investigation is, however, required to show how far one is justified in regarding the entire axial cylinder of roots, like that of the stems just referred to, as the result of a longitudinal coalescence of true fibro-vascular bundies.

Forms of Cells. I have at present indicated only the relative positions of the separate forms of tissue in the fibro-vascular bundle; some remarks will follow on the forms of their cells; but here also, in consequence of the numerous special modes of development, reference must be made to the special morphology of separate classes of plants in Book II. The cell-forms of the fibro-vascular bundles attain their most perfect and varied development in Dicotyledons; the forms which occur in them may therefore be employed as a basis for the critical examination of those of other classes of plants.

The Xylem-portion of the fibro-vascular bundle of Dicotyledons is composed of numerous cell-forms, which may be referred, according to Sanio's careful researches, to three types. He distinguishes (I) Vascular, (2) Prosenchymatous, and (3) Parenchymatous.

To the Vascular forms belong the Ducts (pitted or dotted wood-vessels) and the vascular wood-cells or Trachëdes. This form of cells is characterised by their walls forming open orifices where two cells of the same kind meet, so that their contents soon disappear and air takes their place; the thickenings show a tendency towards the formation of spiral bands, reticulations, and bordered pits. True Vessels (Figs. 25, 94, pp. 26, I I $_{3}$ ) arise when the septa of similar cells arranged in longitudinal rows are entirely or partially absorbed; and thus long tubes originate, filled with air, and distinguished from the adjoining wood-cells principally by their greater breadth ${ }^{1}$. The septa may be horizontal or more or less oblique; and in general the mode of their perforation corresponds; horizontal walls are often entirely absorbed, or are pierced by large round openings. The more oblique the septum, the more do the perforations take the form of narrower or broader parallel fissures; and the thickening-bands of the septum which remain present more or less the appearance of rungs of a ladder, while they often combine into a network. These scalariform septa are found, according to Sanio, not only in reticulately thickened vessels and those with bordered pits, as was previously supposed, but also in spiral vessels (e.g. in Casuarina, Olea, Vitis), where coils of the spiral band pass imme- 
diately into the scalariform markings. The detachment of the spiral band of the firstformed spiral vessels in stems and petioles which are growing rapidly, appears to depend solely on the separation of the band from the thin quickly-growing wall common to the vessel and to the adjoining cells. If the band were unrolled owing to the absorption of this wall, the adjoining cells would necessarily be opened. If the septa of the separate vascular cells are very oblique, the cells assume a prosenchymatous appearance, and the more this is the case the more does the vessel appear completely continuous. In the xylem of Ferns this is often carried to so great an extent that, after the cells have been isolated by maceration, it would be easy to believe that we have not cells united into vessels, but fusiform prosenchyma (Fig. 27, p. 27); but in this case also all kinds of transitions occur to the typical scalariform septa ${ }^{1}$. Vessels with prosenchymatous constituents form the immediate passage to the vascular wood-cells (Tracheides). If the form of the cells is such that there is no difference between the Iongitudinal wall and the septum-which is possible only in decided prosenchyma-then the perforations between cells which lie above and cells which lie beside one another are no longer different in form; rows of cells no longer give rise to continuous tubes, but whole masses of cells (bundles, \&c.) are connected with one another by means of open bordered pits. This occurs in an especially marked manner in the tracheïdes in the wood of Coniferæ (vide Figs. 23, 24, p. 25). There

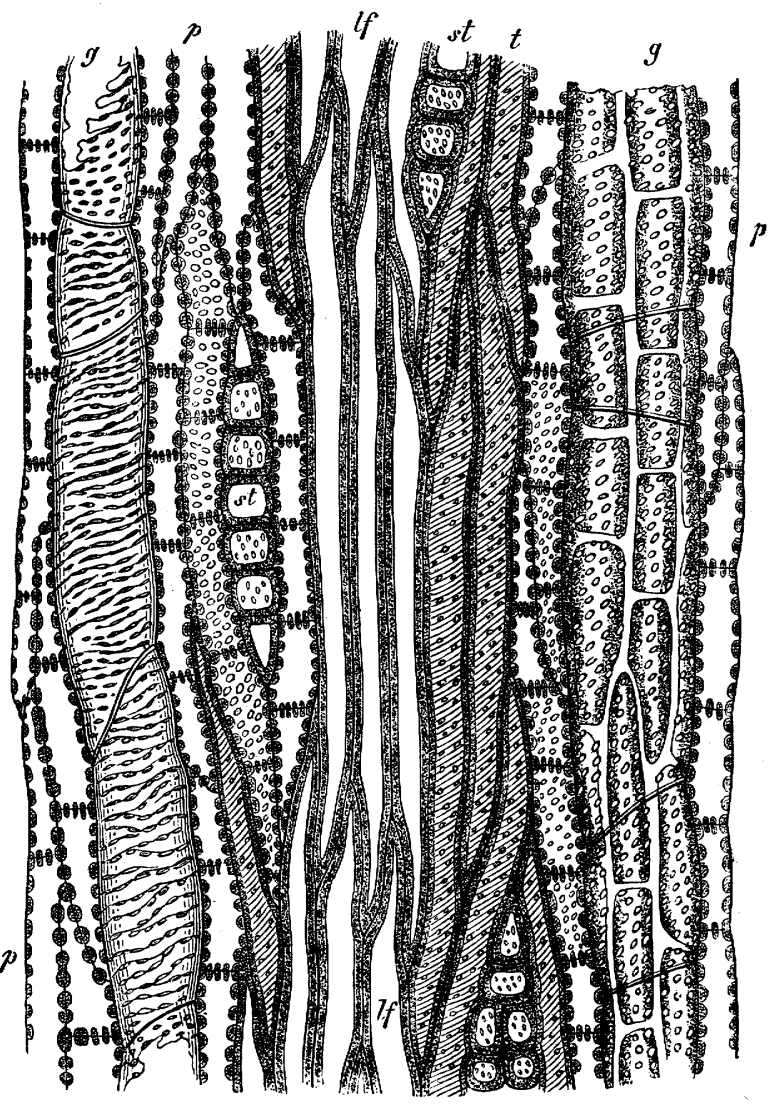

FIG. 97.-Tangential longitudinal section through the secondary wood of Ailantus glandulosa; $g$ vessels ; st xylem-rays cut through transversely ; $p$ woodparenchyma; $t$ tracheides; $l f$ libriform fibres.

is no other difference between these and true vessels; for vessels with open bordered

1 See Dippel in the Amtlichen Bericht der 39. Vers. der Naturforscher u. Aerzte. I865 (Giessen), P1. 3, Figs. 7-9. Dippel's observations on Cryptogams and the whole description of the formation of vessels here given, their passage into tracheides, and especially the fact that the air-conducting vascular forms have open bordered pits, and are thus in communication even when the parenchymatous constituents of a vessel are united not by large openings, but by narrow fissures, \&c. (and are hence not closed cells, as Caspary thinks), compel us to reject Caspary's hypotkesis of the absence of vessels in Cryptogams and many Phanerogams. (See Caspary, Monatsberichte der $\mathrm{k}$. Akademie der Wissenschaften in Berlin, 1862, p. $44^{8}$ [Nat. Hist. Rev., 1863, pp. $3^{6} 4-3^{6} 7$ ].) 
pits (ducts) behave in reference to the side-walls exactly like tracheïdes (Fig. 25, p. 26). The separate elements of the vessels of Ferns composed of prosenchymatous cells (Fig. 27, p. 27) may be correctly designated tracheïdes.

The Prosencbymatous cell-forms of the xylem are always fusiform, very thick-walled in comparison with their diameter, with usually simple, but sometimes bordered pits, the pits small, always without a spiral band; during the repose of vegetation they contain starch. Next to the middle lamella of their partition-walls there often lies an unlignified gelatinous thickening-mass which is coloured violet-red by Schultz's solution, and resembles many bast-fibres. These cells are generally much longer than the vascular forms. Sanio distinguishes two forms; - the simple (libriform) and

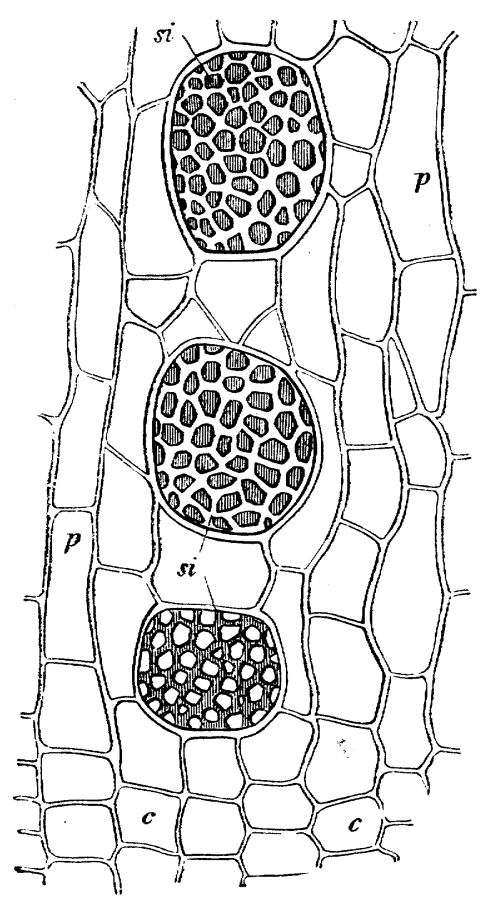

FIG. 99.-Transverse section through the phloëm of a fibro-vascular bundle in the stem of Cucurbita Pepo $\left(\times 55^{\circ}\right) ; s i$ the septa of the young sieve-tubes with areolæ, the sieve-pores being not yet developed; $p \not p$ phloëmparenchyna; $c c$ cambium. The bast-fibres are here wanting, the whole of the phloëm consisting of soft bast. (Respecting the sieve-tubes see Fig. 74.)

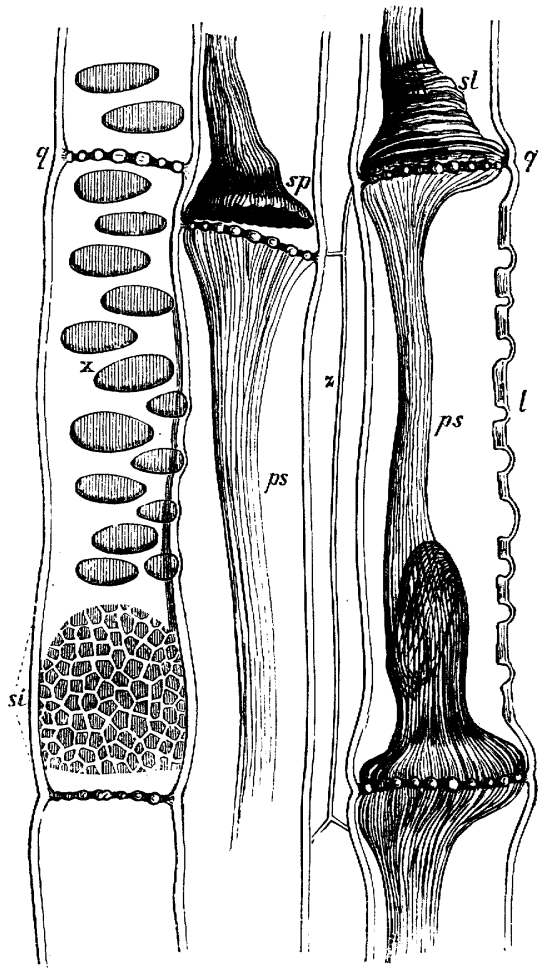

FIG. 99--Longitudinal section through the phloëm of a fibro-vascular bundle of Cucurbita Pepo; three sieve-tubes are visible, whose septa $q q$ are not yet perforated; the protoplasm $s l$ and $p s$ contained in the sieve-tubes is contracted; si a young sieve-plate in the side-wall; at $x$ and $l$ sieve-pores will be formed later; $z$ narrow parenchymatous cells between the sieve-tubes.

the septate fibres; the latter are distinguished from the former by their cavity being partitioned by several thin septa, while the common wall of the whole fibre is thick. These prosenchymatous cell-forms are found in the wood of dicotyledonous trees and shrubs in the most various intermixture with the vascular elements and the other forms to be named immediately. Whether libriform fibres occur in Cryptogams is at least doubtful.

The Parenchymatous cell-forms of the xylem are widely distributed, and especially abundant when the woody substance of the fibro-vascular bundles attains a considerable thickness. They arise by transverse division of the cambium-cells before their thickening commences. The sister-cells show this origin chiefly by the mode in which they are 
arranged; when completely developed they are thin-walled, with simple closed pits. Their contents in winter consist of starch, often associated with chlorophyll, tannin, and crystals of calcium oxalate. It also happens sometimes that the cambium-cells on the xylem-side of the bundle become transformed, without transverse division, into parenchymatous, thin-walled, simply pitted, elongated, succulent cells, which must also be considered as parenchymatous forms of wood-cells ${ }^{1}$. To this last type are also to be referred the parenchymatous elements in the xylem-portion of the closed fibrovascular bundles of Monocotyledons and Cryptogams; but these thin-walled, mostly elongated cells do not in this case originate in the cambium (since this, according to the terms in customary use, is absent from the closed bundles), but immediately from the procambium of the bundle (Fig. 95, p. Ir4, near $S$ ). Sometimes the woodparenchyma derived from the cambium of Dicotyledons (parenchyma of the xylem) is more strongly developed, while only a few vessels and tracheïdes are formed: this occurs in the thick napiform roots of the radish, carrot, beet, and dahlia, and in potato-tubers. The apparent pith of these organs corresponds, in its origin, to the wood of a dicotyledonous tree; but the elements of the xylem are not, or only slightly, lignified; the succulent contents and the thin soft cell-walls scarcely give this xylem the appearance of a homologue of the ordinary wood, although there can be no doubt about the homology.

The Phloëm-portion of the fibro-vascular bundles shows, when fully devalopcd, similar cell-forms to the xylem-portion; the sieve-tubes correspond to the vessels, the true bast-cells to the libriform fibres, the bast- or phloëm-parenchyma to the wood-parenchyma. When the bast-parenchyma consists of long, narrow, very thinwalled cells, it has been termed by Nägeli Cambiform tissue.

Parenchyma, cambiform tissue, and sieve-tubes may be included in the term Softbast, in opposition to the true bast which is sometimes entirely absent (as in Cucurbita), but in other cases is very abundantly developed (as in the Jerusalem artichoke, lime, \&c.), and consists of elongated, prosenchymatous, flexible, tough, firm cells, usually with strongly thickened walls. In Dicotyledons they are generally arranged in bundles, frequently forming layers alternating with soft-bast, as in the grape-vine; but sometimes, especially in the later portions of the phloëm, which are formed from the cambium, they occur also as separate fibres, as in the stem and tuber of the potato. The middle lamella of the partition-wall of two fibres is generally lignified or cuticularised (resistant and coloured yellow by iodine) when they are closely crowded; but in other cases it forms a mucilaginous 'intercellular substance' in which the cells (in transverse section) appear imbedded (e.g. the laburnum according to Sanio, Coniferæ and especially in Cryptogams, see Fig. 95, b, p. I 14). The true bast-fibres of the phloëm, like the libriform fibres of the xylem, may become partitioned by subsequent septa (as in the vine, occidental plane, horse-chestnut, Pelargonium roseum, Tamarix gallica, according to Sanio, l.c.p. III). As the libriform fibres of the xylem are often found branched after isolation by maceration, so also are the bast-fibres ${ }^{2}$.

The forms of cells now described are the ordinary and essential constituents of the fibro-vascular bundles; various other forms of tissue occur, however, occasionally, as

1 Sanio applies to these cells the term 'Ersatzzellen.'

2 It may not be superfluous to point out that many writers very inconveniently also designate certain cell-forms of the fundamental tissue as bast, when they are thick-walled, elongated, pointed at the ends, or even branched. In that case the libriform fibres of the xylem must also be called bast; and it is evident that the term would then have no exact scientific meaning. The term bast-cells has, till recently, been given to hypodermal prosenchyma, to the bundle-sheaths of Grasses, Aroider, and Palms, as well as to the cells which we have, in Sect. 14, called trichoblasts, even when they contain latex, as the laticiferous cells of Euphorbiaceæ. This practice is greatly to be deprecated, as it must import great uncertaintý into a correct interpretation of the different forms and systems of tissue. 
will have been seen from Sect. I4; and this happens especially in the phloëm. Thus lithocysts, cells containing pigments, receptacles for oil, and other idioblasts like the rows of cells in Phaseolus which contain tannin; the true laticiferous vessels of Cichoriaceæ, Campanulaceæ, and Lobeliaceæ, belong to the phloëm, while those of Papayaceæ and Aroideæ belong to the whole fibro-vascular bundle. In may even occur that vessels belonging to the xylem contain latex, as in Ipomaa, Argemone, Gomphocarpus, Euphorbia, Carica, Lactuca, and in Campanulacex (David, l.c.). In the same manner it has already been shown, in Sect. $\mathrm{I}_{4}$, that the secretion-canals-i.e. intercellular spaces containing oil, resin, or gum-may occur either in the phloëm, or in the xylem, or in both.

Sect. I7. The Fundamental Tissue.-By this name I designate the tissue of a plant or of an organ which still remains after the formation and development of the epidermal tissue and the fibro-vascular bundles. It consists very commonly of thin-walled succulent parenchyma filled with assimilated food-materials ; but not unfrequently it is thick-walled; sometimes portions assume the form of strings of strongly lignified prosenchymatous cells. The most various forms of calls may arise in the fundamental tissue, as in the epidermal system and the fibrovascular bundles; a portion may persist in a condition capable of division, while the surrounding portion passes over into permanent tissue; or special layers of the fundamental tissue, long after it has been transformed into permanent tissue, may again become subject to cell-division, and a generating tissue thus be produced, out of which originate, not only new fundamental tissue, but also fibrovascular bundles (e.g. in Aloineæ).

In Thallophytes and many Muscineæ the whole mass of tissue, with the exception of the outermost layer which is often developed as epidermal tissue, may be considered as fundamental; but in these cases, in consequence of the absence of fibro-vascular bundles, this distinction has but little practical value. In Mosses, which have string-like cell-groups in the stem, it may appear doubtful whether these are to be considered as peculiar forms of fundamental tissue, or as very rudimentary fibro-vascular bundles. In Vascular plants, on the other hand, the independence of the fundamental tissue, in contradistinction to the epidermal system and fibro-vascular bundles, is at once apparent; it fills up the interstices of the fibro-vascular bundles within the space enclosed by the epidermal tissues. Where the fibro-vascular bundles are closed and do not increase in thickness (as in many Ferns), it is frequently the one which occupies the greatest space; where, on the other hand, closely crowded fibro-vascular bundles produce large masses of xylem and phloëm by the development of cambium (as in the stems and roots of Conifers and Dicotyledons), the fundamental tissue becomes less important. The fibro-vascular bundles in stems are usually so arranged as to separate the fundamental tissue into an inner medullary portion the Pith, surrounded by the bundles, and an outer cortical layer or Cortex enveloping them. Since the bundles are not in contact laterally, or only partially so, there still remain between them portions of the fundamental tissue which connect the pith with the cortex, and which are termed Medullary Rays. If the fibro-vascular bundles of an organ form a solid axial cylinder, as occurs in some stems and in all roots, the fundamental tissue takes the form of cortex only. 
(a) The whole course of my description of tissue-systems necessitates the introduction of the idea of a Fundamental Tissue. It has, in fact, long been required; it was often necessary, in anatomical descriptions of tissues which are neither epidermal nor fibro-vascular, to distinguish them by some common term. Many writers employ the term Parenchyma in this sense, in opposition to the fibro-vascular bundles and the epidermis; but this usage is not scientific; the fibro-vascular bundles often contain parenchyma, and, vice vers $\hat{a}$, the fundamental tissue is not always parenchymatous but sometimes distinctly prosenchymatous. We have, moreover, to deal here not with forms of cells, but with the contrast of different systems of tissue, each of which may contain the most various cell-forms. I must compare somewhat more closely my description and use of terms with those of Nägeli. It might be supposed that Nägeli's Protenchyma is synonymous with my fundamental tissue; but this is not the case; the protenchyma of Nägeli is a much more comprehensive idea; everything which I call fundamental tissue is protenchyma, but all protenchyma is not fundamental tissue. Nägeli ${ }^{1}$ says, for example, that he would call the primary meristem and all tissues which arise immediately from it (i.e. only through the medium of secondary meristem, but not of cambium) Protenchyma (or Proten); the cambium, on the other hand, and everything which directly or indirectly originates from it, Epenchyma (or Epen). When Nägeli gave these definitions, he was treating of fibro-vascular bundles; and it is intelligible that he on this occasion included everything which does not belong to the fibro-vascular bundles under one common name (Proten). But our business is to give a uniform description of the various differentiations of plant-tissues; and there is no reason for bringing only into prominence the contrast between fibro-vascular and nonfibro-vascular tissues (Epenchyma and Protenchyma), and for considering all other differentiations as less important; the protenchyma of Nägeli therefore includes, according to my use of terms, three kinds of tissue each of equal value with his epenchyma. The primary meristem is not more opposed to the fibro-vascular (epenchyma) than to the epidermal and fundamental tissues; for the three systems of tissue equally arise by differentiation from it. The term Proten, after distinguishing from it the primary meristem, might be applied collectively to the epidermal and fundamental tissues; but I see no reason for bringing into prominence this contrast alone; the differentiation between epidermal and fundamental tissues is as essential as that between fibro-vascular bundles and fundamental tissue. From all this it follows that epidermal tissue, fibro-vascular bundles, and fundamental tissue are conceptions of equal value; in each we find the most various forms of cells; and secondary meristem may also arise in each. In the fibro-vascular bundles the cambium is of this nature; the whole of the young epidermis is a generating tissue in as accurate a sense as the cambium; if this latter forms vessels, xylem, phloëm, \&c., the former produces hairs, stomata, prickles, \&c.; the phellogen, belonging to the epidermal system, arises still more decidedly as a generating tissue; finally, even in the fundamental tissue a portion may persist for a considerable time as generating tissue, or may subsequently produce such a tissue, e.g. the meristem of the stems of Dracana, which brings about its increase in thickness, and thus forms new fibro-vascular bundles ${ }^{2}$.

(b) Examples. The relationship of the three systems of tissue may be observed very readily, and undisturbed by subsequent formations, in the foliage-leaves of Ferns and of most Phanerogams; in these the fundamental tissue is generally the prevailing system, and is developed into different cell-forms. Isolated fibro-vascular bundles, separated by the fundamental tissue, traverse the petiole, and are distributed through the

1 Beiträge zur wissenschaftlichen Botanik, Heft 1, p. 4.

2 Since the publication of the Ist edition of this work, the classification of tissues here proposed has been generally adopted, especially by younger botanists; as also in the main, with some deviations in particular points, by Russow, Unters. uiber die Leitbündelkryptogamen, Petersburg I $8_{7} 2$. 
lamina; in the petiole they are generally surrounded by a thin-walled parenchymatous fundamental tissue with wide elongated cells; this also forms sheath-like envelopes around the stronger bundles of the lamina, which are conspicuous on its under-side as the Veins; but the finer branches, and the finest of all, run through the socalled Mesophyll, i.e. a peculiar form of the fundamental tissue distinguished
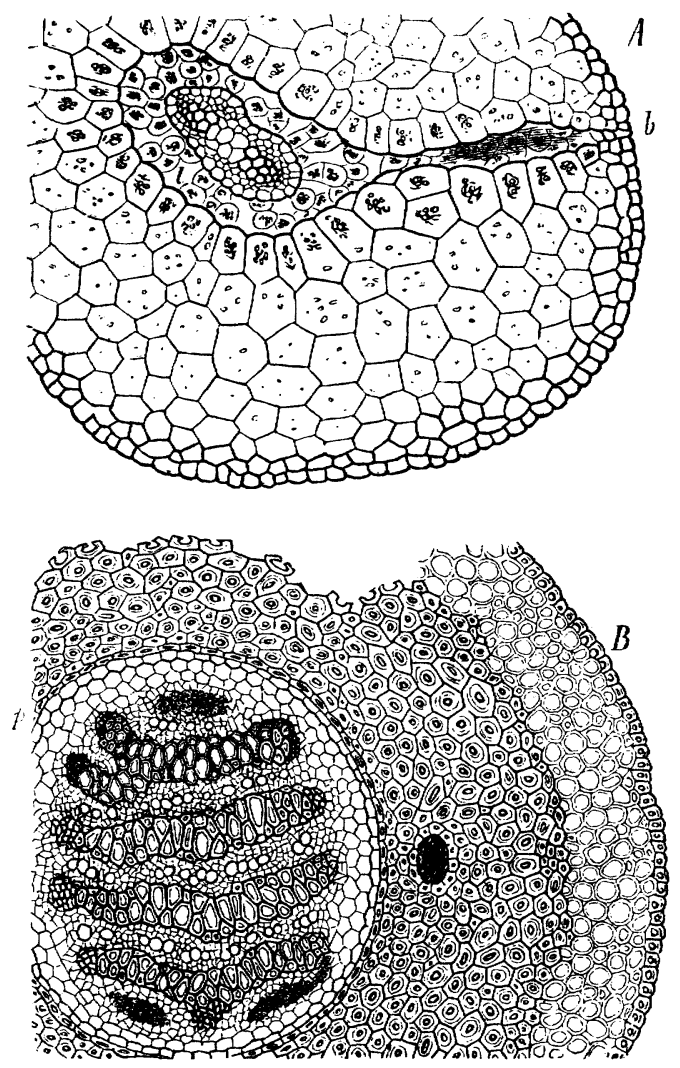

FIG. roo.- $A$ transverse section of the stem of Selaginella denticulata; the fibro-vascular bundle is not yet fully developed; the vessels are already lignified on both sides, but not yet in the centre; $l$ air-conducting intercellular spaces in the parenchyma enveloping the bundle; towards $b$ is the tissue corresponding to the bundle which bends outwards to the leaf. $B$ transverse corresponding to the bundle which bends outwards to the leaf. $B$ transverse der consists of densely crowded and coalescent fibro-vascular bundles; the four xylem-portions are quite separated, forming four bands on the transverse section, between and round which are found the narrower cells of the phloem. The phloëm portions of the four bundles have coalesced; between each pair of xylem-bundles is seen a row of wider cells, the latticed cells or sieve-tubes; the narrow cells lying on the right and left edge of each xylem-portion are spiral vessels (also in $A$ ). In the thick-walled prosenchymatous fundamental spiral vessels (also in $A$ ). In the thick-walled prosenchymatous fundamental
tissue which envelopes the axial cylinder is seen the dark transverse section of a slender fibro-vascular bundle which bends outwards to a leaf; it consists almost exclusively of long spiral vessels ( $x$ about 9 ).

common with the fibro-vascular bundles, but are only a peculiar form of the fundamental tissue, a prosenchymatous form of which often occurs also elsewhere in Cryptogams. The tendency to a prosenchymatous development of the cells of the fundamental tissue is also well seen in the stems of Lycopodiaceæ. In Selaginella denticulata (Fig. too $A$ ) the axial fibro-vascular bundle is surrounded by a very 
loose parenchyma with large intercellular spaces; this innermost portion of the fundamental tissue is enveloped by a thin-walled tissue without interstices, which is seen on longitudinal section to be prosenchymatous; the cells are pointed at both ends, and penetrate to a considerable distance between one another; towards the circumference they become gradually narrower and more pointed; the outermost are darkwalled, and form the epidermal system which gradually passes over into this fundamental tissue. In Lycopodium Cbamacyparissus $(B)$ the axial cylinder, which consists of several fibro-vascular bundles, is surrounded by a thick layer of greatly thickened prosenchyma; in the young stem the cells are similar to those of Selaginella; but here also the prosenchymatous cells of the fundamental tissue undergo an enormous thickening. This prosenchymatous fundamental tissue is in its turn enveloped by a layer of tissue, the cells of which are thin-walled and not prosenchymatous; this layer is a descending continuation of the fundamental tissue of the leaves, which envelopes the stem everywhere, and is itself covered by a clearly developed epidermis.

(c) The Forms of Cells and Tissues in the system of the fundamental tissue have not yet undergone a comparative and comprehensive investigation ${ }^{1}$, like those of the fibrovascular bundles. Out of the very scattered material I select the following for the information of the student.

Irrespectively of many altogether special phenomena, it is chiefly in connexion with the true epidermal tissue on the one hand and the fibrovascular bundles on the other hand that the differentiation of the fundamental tissue has to be considered. Certain forms of this tissue occur as strengthenings, or at least as accompaniments of the epidermal tissue, and have already been described as Hypoderma; other masses of tissue

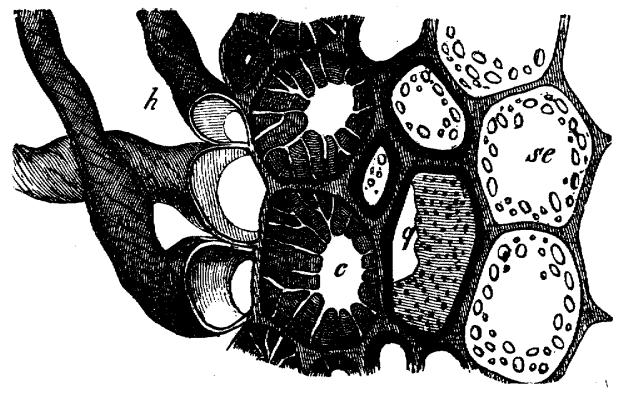

FIG. ror.-Transverse section through the underground stem of pteris aquilina; $h$ root-hairs; beneath the epidermis are strongly thick ened brown-walled cells; $q$ one lying deeper and less strongly thickened; a part of the wall is seen in front; se cells of the deeper layers containing starch, forming the passage to the inner colourless parenchyma of the starch, forming the accompany the separate fibro-vascular bundles as partially or entirely closed envelopes or sheaths; these I term generally Bundle-sheaths or Vascular Bundle-sheaths. In the same manner the whole remaining internal space of the organ concerned is commonly filled up by other forms of tissue, which do not, as for the most part the two former do, occur in the form of layers, but in masses; these I will designate simple Intermediate Tissue. Each of these combinations may be composed of very different forms of tissue.

(a). The Hypoderma appears sometimes as a thin-walled succulent watery tissue, as in the leaves of Tradescantia and Bromeliaceæ; in the stems and petioles of Dicotyledons it commonly consists of collenchyma, the cells of which are extended longitudinally, narrow, and thickened in the angles by a mass capable of great swelling; or the hypodermal fundamental tissue is developed in a sclerenchymatous manner, as in the stem of Pteris aquilina; or it occurs in the form of thick-walled but flexible fibres, forming either layers and bundles as in the stem of Equisetaceæ and leaves of Coniferæ (Fig. 102), or in long isolated fibres, similar to true bast-fibres, e.g. leaves of Cycadex. In all these cases the hypodermal cells are extended longitudinally; but when layers capable of great resistance are also required, the cells often extend in a direction vertical to the surface of the organ, and, increasing greatly in thickness, form layers of

${ }^{1}$ Since the publication of the $3^{\text {rd }}$ edition of this work, a close investigation of the fundamental tissue has been undertaken by Russow (l.c.); he has however occupied himself chiefly with the various forms of the bundle-sheaths or 'Critenchyma.' 
closely packed prisms, as in the pericarp of Marsilea and Pilularia, and the testa of the seeds of Papilionaceæ. Isolated cells of the same kind are sometimes found in the hypoderma, as accompaniments of the stomata and air-cavities, e.g. in leaves of Hakea.

$(\beta)$ The Bundle-sheaths consist, in many Monocotyledons (as Palms, Grasses, Aroideæ, \&c.) ${ }^{1}$, of elongated very thick-walled cells belonging to the fundamental tissue which is in close contact with each fibro-vascular bundle, either as a continuous sheath composed of several layers (Fig. 92, p. I10), or only as a partial investment. In Ferns and allied Cryptogams, on the other hand, a single layer formed of peculiar cells encloses each separate bundle as a cylinder (Fig. 95, p. I 14); and the same is the case in a few Phanerogams, as in the petiole of Menyantbes trifoliata, Hydrocleis Humboldti, \&c. A layer of fundamental tissue of similar structure envelopes, as we have already seen on p. I I 5 , the axial fibro-vascular cylinder of all roots and of many stems (Lycopodium, Hydrillex, Hippuris), and in most stems of Phanerogams with isolated vascular bundles (not forming an axial cylinder) separates the cortex from the internal tissue which encloses the vascular bundles and the pith. In Dicotyledons with the bundles arranged in a ring, this layer (Fig. 93, p. I I 2) surrounds the mass of tissue enclosed by the cortex in such a manner that the separate fibro-vascular bundles are only in contact with it at their phloëm-portion. If the axial mass of tissue of roots and stems is called plerome, then

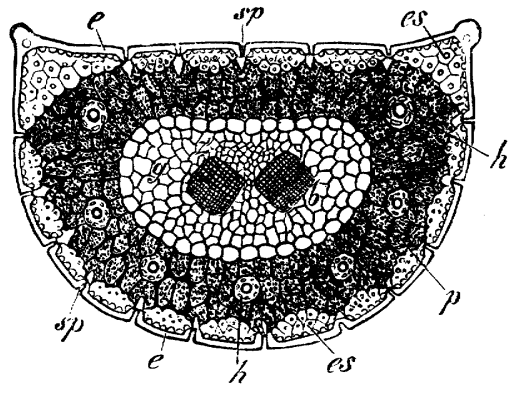

FIG. 102.-Transverse section of the acicular leaf of Pinus Pinaster ( $\mathbf{X}$ about 50 ); $e$ epidermis; es hypodermal bundles of prosenchyma: $s p$ stomata; $h$ resin-passages; $g b$ colourless inner tissue enclosing two fibro-vascular bundles.

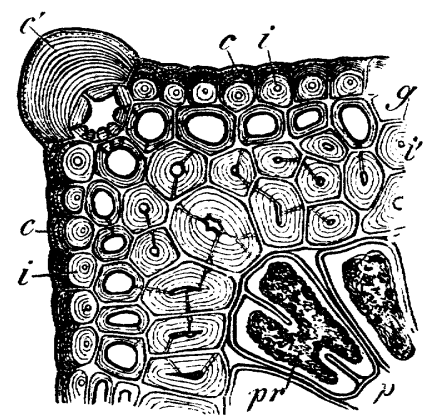

FIG. 103.-The left-iand corner of the previous figure $(x 800)$; $c$ outer cuticularised layer of the epidermal cells; $i$ inner mon-cuticularised layer; $c^{\prime}$ very strongly thickened outer wall of the epidermal cells situated at the corner; $g i^{\prime}$ hypodermal cells; $g$ central lamella; $i^{\prime}$ stratified thickening mass; $p$ parenchyma containing chlorophyll; $p r$ its contents contracted.

this layer, which separates it from the cortex, may conveniently be called the Pleromesheath. In the rhizome of many Monocotyledons, as Aroideæ, Zingiberacex, Iris, Veratrum, it may be seen with the naked eye. The unilamellar plerome- or bundlesheath consists of cells which usually become lignified at an early period, and strongly resist solution in sulphuric acid; the radial side-walls and the upper and under septa are distinguished by a peculiar folding, which, in transverse section, gives the appearance of a thickening of the walls, or of a black dot. The inner walls which face the bundle, as well as the radial side-walls, often become greatly thickened, especially in Ferns, where the thickened walls frequently assume also a deep brown-red colour.

$(\gamma)$ The Intermediate $\mathcal{T}$ issue usually consists of thin-walled succulent parenchyma with intercellular spaces which are absent from all other forms of tissue; in the stem, however, of Lycopodiaceæ and of some other Cryptogams it consists of prosenchyma, and this is then either thin-walled as in Selaginelleæ, or thick-walled as in Lycopodiex.

1 Caspary, Jahrb. für wiss. Bot. vols. I and IV, p. Ior et seq. - Sanio, Bot. Zeitg. 1865, p. I 76 et seq.-Pfitzer, Jahrb. fuir wiss. Bot. vol. IV. p. 297.-Van Tieghem, Canaux sécréteurs, in Ann. des Sci. Nat. I872, vol. XVI. 
When it is parenchymatous, it may be termed simply Fundamental Parenchyma. Two principal forms of this may be distinguished, which are nevertheless united by transitional forms, viz. the colourless parenchyma which occurs in the interior of large succulent stems and tubers, and in all roots and succulent fruits, and the parenchyma containing chlorophyll which forms the superficial layers beneath the epidermal tissues of stems and fruits. In foliage-leaves, when thin and delicate, it fills up the space between the upper. and lower epidermis; if they are very thick, as in Aloe, it forms only the superficial layers, while the inner mass of tissue consists of colourless parenchyma.

The hypodermal layers, bundle-sheaths, and intermediate tissue are the ordinary and essential constituents of the fundamental system; but in addition forms of cells and tissues developed in a peculiar manner occur, and much more frequently than in the fibro-vascular bundles. Of the former kind.are the majority of the cells described as idioblasts in Sect. I 4 , isolated cells containing a pigment, tannin, a volatile oil, clusters of crystals, \&c., or large utricular vessels, or isolated scleroblasts, or the branched cells comprised under the term trichoblasts, such as spicular cells, the hairs in the interior of Nuphar, in the root of Pilularia, in the petiole and stem of Monsterinex, \&c., or finally, the laticiferous cells of Euphorbiaceæ, Moreæ, Asclepiadeæ, and Apocynaceæ. True laticiferous vessels are, on the other hand, less often found in the fundamental tissue; but in the cortex of many Liliaceæ they are replaced by utricular vessels (see Sect. I4). Among the more complicated forms of tissue which occasionally enter into the composition of the fundamental tissue may be named true (compound) glands, or more frequently secretion-canals containing gum, resin, a volatile oil, or even latex, as in Alisma and Rhus. Of very common occurrence are, moreover, groups or layers of scleroblasts (especially in the cortex of many woody plants and the juicy flesh of pears), and layers, bundles, or bands of brown-walled sclerenchyma (in Pteris aquilina and Treeferns). Attention has already been called to the sclerenchyma of which the stone of stone-fruit (drupes) consists as a form of fundamental tissue; the natural contrast to this is the pulp or flesh of berries and of many. stone-fruits.

Sect. 18. The Secondary Increase in Thickness of Stems and Roots ${ }^{1}$.During the period when the younger portions of stems and branches are still increasing in length, they are also increasing in girth, the primary meristem becoming differentiated into other tissues which grow not only in the direction parallel to the axis of growth, but also in the radial and tangential directions. At an early period roots attain, immediately behind the growing point, the size which they retain until they have ceased growing in length.

This increase in diameter of stems, which accompanies, or even for a short time outlasts, the growth in length, is frequently occasioned mainly by the tangential extension of the outer layers of tissue, while that of the pith does not keep pace with it. The pith will then split and the stem become hollow; and this is often carried to such an extent that the substance of the cylinder itself

1 Nägeli, Ueber das Wachsthum des Stammes u. der Wurzel, in Beiträge zur wiss. Bot. Leipzig I8 8 , Heft 1.-Sanio, Bot. Zeitg. I 865, p. 165, et seq.-Millardet, Sur l'anatomie et le développement du corps ligneux dans les genres Yucca et Draccena, in Mém. de la Société Impér. des Sci. Nat. de Cherbourg, vol. XI, I865.-On abnormal formations of wood in Dicotyledons see Cruiger, Bot. Zeitg. $185^{\circ}$ and I 85 I. - Nägeli, Dickenwachsthum des Stengels u. s. w. bei den Sapindaceen. Munich I864.-Eichler, Ueber Menispermaceen, in Denkschrift der k. bayer. bot. Gesellschaft zu Regensburg, I 864, vol. V.-Sanio, Bot. Zeitg. I864, p. I93 et seq.-Askenasy, Botanische morphologische Studien, Dissertation, Frankfort-a-M. 1872.-On the increase in thickness of roots see Van Tieghem, Recherches sur la symétrie, \&c., Ann. des Sci. Nat., $5^{\text {th }}$ ser., vol. XIII. 
becomes very thin. In this mode are produced the hollow stems of Equisetaceæ, Grasses, many species of Allium, and many exogenous plants, such as Umbelliferæ, Dipsacus, Taraxacum, \&c. Septate hollow stems have a diaphragm of firm tissue, traversed by fibro-vascular bundles, at the insertion of each leaf.

In existing Cryptogams ${ }^{1}$ and in most Monocotyledons the root and every part of the stem retain the diameter which they had attained during their growth in length; even at a great age no further increase in thickness takes place. When, in these plants, the stems of older specimens greatly exceed in diameter those of seedlings, this is a consequence of the circumstance that the apex of the stem which is enclosed within the leaf-bud increases in diameter as it lengthens, so that thicker parts of the stem are constantly emerging from the bud, until a stationary condition is at length reached, when the stem no longer increases in girth. When this occurs, as in Palms, Ferns, thick-stemmed Grasses, Aroideæ, \&c., the stem may attain a very considerable thickness while still very young, in and below the bud, without having any power, at a later period, to increase further in size. In the same manner the roots, when they first emerge from the stem, are thicker the higher they stand on it; and in Pandanus it would appear as if roots as thick as the arm were formed without any secondary increase in thickness.

Very different processes are, on the other hand, the cause of the considerable diameters of the stems and roots of Gymnosperms, Dicotyledons, and arborescent Liliaceæ. After its growth in length is completed every part is slender, usually only a few millimetres in thickness, rarely so much as I or 2 centimetres; but in the course of months and years those parts become much thicker; the stem of a seedling Cistus only 2 or $3 \mathrm{~mm}$. in diameter may attain, after two or three months, a thickness of 2 or $3 \mathrm{cms}$. ; while in the case of the oak the increase from the same original thickness may amount to from 40 to $60 \mathrm{cms}$. Since the girth increases with the age of each section, the oldest, and therefore the lowermost sections of the erect stem are the broadest, the successive diameters decreasing gradually to the summit of the tree, as is well illustrated in the slender conical stem of pines. In them the stem of the mature plant is a cone standing on its base, while in Monocotyledons and Cryptogams the cone stands on its apex, as is well seen in the large climbing Aroideæ. In Dicotyledons and Gymnosperms every part of the stem is at first slender, always becoming thicker at a later period; in Monocotyledons and Cryptogams, on the contrary, every part retains the thickness which it had acquired at the close of its growth in length; the increase in thickness of the entire stem takes place at the upper end from the growth of the bud.

This increase in girth which commences only after the close of the growth of length of the organ, and which then generally lasts during the whole of its life, may be termed, in contradistinction to that arising from growth of the bud, the secondary increase in thickness. It is always the result of an inner layer of tissue remaining in a condition capable of division (merismatic), and continually producing

1 In the Lycopodiaceæ of the Carboniferous period Williamson has recently demonstrated a secondary increase in thickness. An indication of this is found in the stem of Isoëtes. (See Book II.) 
new layers concentrically one on another. The mode in which the meristem itself is formed, and in which the secondary layers of tissue are produced from it, differs greatly according to the nature of the plant. The numerous special modes of increase in thickness may be classified under three types, viz..-

I. Type of the Arborescent Liliaceæ. The innermost primary cortical layer produces a meristem, in which new closed fibro-vascular bundles continue to arise, which anastomose into a network, while the tissue between the bundles developes as secondary fundamental tissue.

2. Type of normal Gymnosperms and Dicotyledons. The vascular bundles of the stem are open and arranged in a ring; the generating tissue which lies between the phloëm and the xylem of each bundle is also continued through the medullary rays, $i . e$. those parts of the fundamental tissue which lie between any two adjoining bundles. Thus arises a continuous ring of meristem, which, according to the old use of terms, and to distinguish it from the ring of meristem in the preceding type, is commonly called the Cambium-ring. While in the preceding type new vascular bundles arise only in the ring of meristem, the meristem- or cambium-ring in this type crosses the primary vascular bundles, which have their phloëm lying on the outer side, their xylem on the inner side of the cambium. The increase in thickness consists in new secondary xylem being continually formed out of the cambium-ring on its inner side, new phloëm or secondary cortex on its outer side.

3. The type of Roots (of Gymnosperms and Dicotyledons). In the axial fibrovascular cylinder or plerome-bundle there lie, as has been mentioned, alternate groups of vessels (xylem) and phloëm-bundles side by side; on the inner side of each of the latter arises a cambium-layer, which produces secondary xylem on its inner side, phloëm on its outer side; on the outer side of the primary groups of xylem meristem is also formed, which either produces only secondary fundamental tissue, or combines with the cambium-layers already mentioned into a complete cambium-ring, out of which xylem is again formed on the inside, phloëm on the outside.

Further details may now be given regarding each of these three types, with an example; the nomenclature of the more important deviations, especially for the second type, will be deferred till the end of this section.

(1) The Type of Arborescent Liliacece is represented in the genera Draccena, Aletris (Calodracon), Yucca, Alö̈, Lomatophyllum, and Beaucarnea ${ }^{1}$. Specimens of the old stems of these plants are often found in botanical collections so decayed that within the thin layer of periderm the whole of the parenchymatous fundamental tissue has completely disappeared, while the fibro-vascular bundles are preserved entire. If one of these stems is split lengthwise, it is seen that completely isolated bundles run down the middle, as is the case in all Monocotyledons. Each bundle begins below at the periphery of the stem; higher up, it bends towards the centre of the stem, and then again outwards, finally entering a leaf at its upper end. The

1 A fourth type may be furnished by the mode of the increase in thickness in the primeval Lycopodiacex (vide supra); but scarcely anything certain is at present known about it. 
course of these bundles necessitates that they must cross one another, and form a loose mass consisting of slender isolated strings, surrounded by a more or less thick layer of denser woody substance. This woody substance forms a cylinder which, in decayed stems, is altogether separated from the layer of periderm, and loosely enveloped by it. The isolated strings in the interior are the primary vascular bundles which have been formed during the growth in length (properly only their lower ends or Leaf-traces, since the upper ends bend outwards into the leaves). The woody cylinder which envelopes them all consists, on the contrary, of

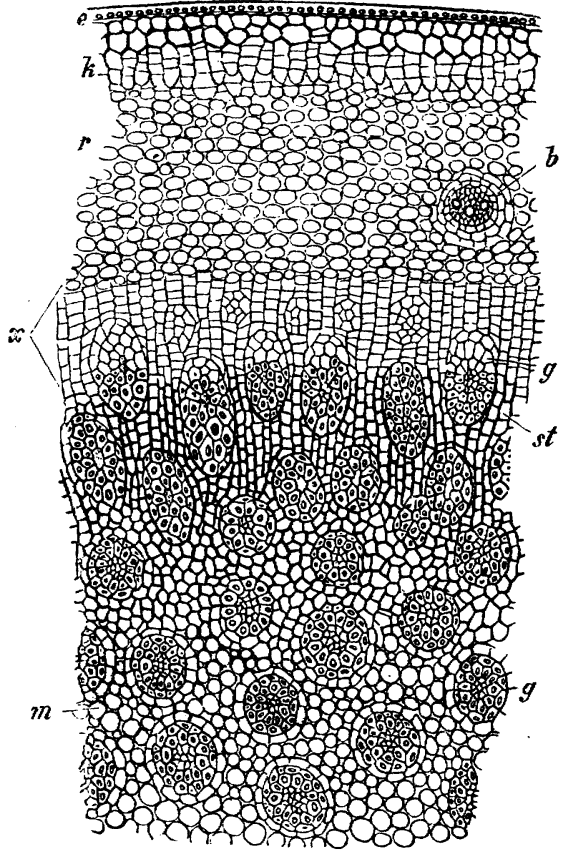

FIG. 104.-Part of the transverse section of a stem of Dracana (probably reflexa) about $13 \mathrm{~mm}$. thick and I metre high, about $20 \mathrm{~cm}$. below the summit. $e$ epidermis; $k$ cork (periderm); $r$ cortical portion of the fundamental tissue; $b$ transverse section of a fibro-vascular bundle, bending out to a leaf; $m$ the primary fundamental tissue (pith); $g$ the primary vascular bundles: $x$ the ring of meristem in which very young fibrovascular bundles are to be seen, while the older ones $g$ have already partially or entirely passed out of it, its lower part becoming transformed into fundamental tissue st arranged in radial rows. rows. In this meristem new fibro-vascular bundles are produced; one, two, or more adjoining cells (on the transverse section) dividing repeatedly by longitudinal walls in various positions. Out of the procambium-bundles which arise in this manner the fibro-vascular bundles proceed immediately, the procambium-cells being transformed into fibro-vascular tissue ${ }^{1}$; the intermediate meristem passes over likewise

1 It appears, however, that the thick-walled lignified cells on the outside of such a bundle do not belong to it, but to the secondary fundamental tissue, and therefore represent only a sclerenchymatous bundle-sheath, while the bundles enveloped by them are themselves very slender. 
into permanent tissue, and indeed into thick-walled parenchyma, which now forms the secondary fundamental tissue between the secondary fibro-vascular bundles. Since the cells of the thickening-ring which face inwards pass over in centrifugal succession into permanent tissue, while the outermost divide repeatedly, the whole ring continually moves centrifugally as it increases in diameter, and leaves behind new bundles and parenchymatous cells. In Yucca Millardet found the origin of the ring of meristem (thickening-ring) as little as $3 \mathrm{~mm}$. below the apex of the stem; in Calodracon (Cordyline) Jacquini, the meristem-ring is derived immediately, according to Nägeli, from the primary meristem of the apex of the stem, this layer remaining in a condition capable of division while the primary vascular bundles and fundamental tissue are being differentiated out of the primary meristem.

(2) The Type of normal Gymnosperms and Dicotyledons may be made clear by a reference to Fig. 105, which-with the exceptions of a few points of subordinate importance, such as the substitution of six fibro-vascular bundles for eight-represents in a simple diagrammatic manner the phenomena connected with the growth in thickness of the hypocotyledonary portion of the stem (tigellum) of Ricinus communis. We may commence with the period when, in the seedling stem, the fibro-vascular bundles-which are prolongations downwards of those bundles which bend outwards above into the first leaves or cotyledons-have become clearly differentiated. They lie, when seen in transverse section (Fig. ${ }_{105} A$ ), in a ring, and run parallel to one another and to the surface of the stem. The ring of fibrovascular bundles divides the primary fundamental tissue into pith $(M)$ and
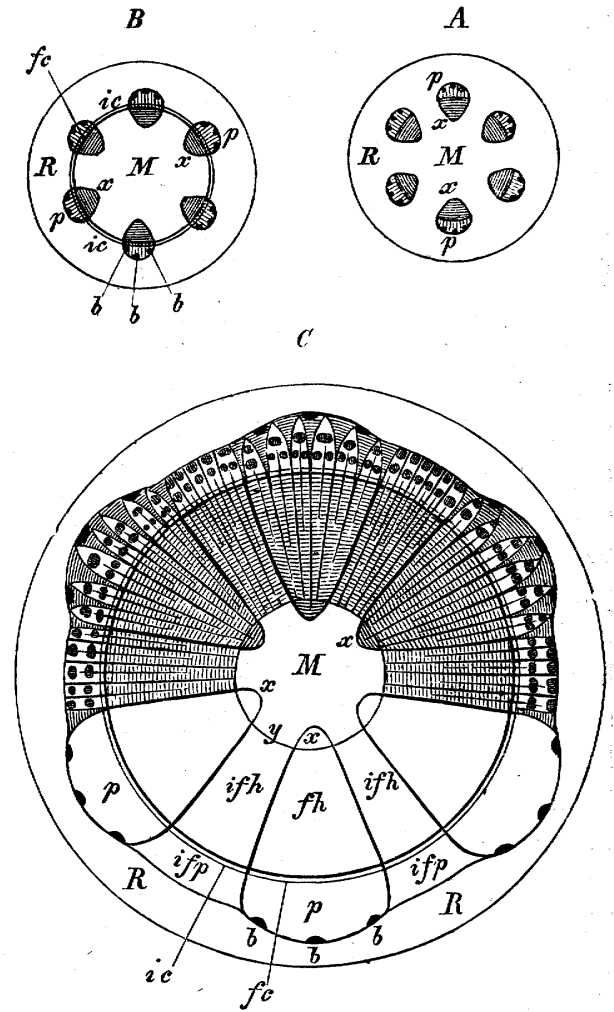

FIG. 105.-Diagramunatic representation of the secondary increase in thickness of a normal dicotyledonous stem. cortex $(R)$, which, however, still retain their connection by broad bands of fundamental tissue lying between the bundles, the Medullary Rays. Each of the bundles consists of an outer phloëm-portion $(p)$ and an inner xylem-portion $(x)$, between which lies a layer of cambium. The next change consists in the bands of cambium belonging to the bundles uniting into a continuous ring (Fig. ro5, $B$ ), meristem being formed between each pair of adjacent bundles by divisions in the corresponding layer of the medullary rays, as is more exactly shown in Fig. 93 (p. I I 2), which relates to this stage of development. Although there is no essential difference between this portion of the cambium-ring and that which lies in the 
bundles themselves, they may conveniently be distinguished, the latter as Fascicular Cambium $(B, f c)$, the former as Interfascicular Cambium $(B, i c)$. It may further be mentioned that three small groups of bast-fibres $b b b$ lie in the phloëm-portion of each bundle.

The activity of the whole continuous cambium-ring now commences. The ring consists of rows of cells arranged radially; in each of these rows the cells which lie on the inner side are the origin of the secondary xylem, those which lie on the outer side of the secondary phloëm; while a middle layer of the cambial cells always remains capable of division, and thus maintains the continued formation of secondary xylem and phloëm. In this manner the seedling stem increases considerably, in the course of a few weeks, both in diameter and rigidity, in consequence of the formation of a cylinder of secondary wood (xylem) surrounded by a layer of secondary cortex (phloëm), understanding by the latter term all the layers of phloëm developed out of the cambium-ring. Fig. 105, $C$ is the transverse section of the tigellum of the seedling plant which has already increased considerably in thickness; by the deposition of secondary phloëm, the primary phloëm $(R)$ has been compelled to grow tangentially; its cells, as well as those of the epidermis, stretch in that direction, and become divided by radial longitudinal walls. At this time it is still possible to refer back the various parts of the secondary tissue to their origin; the original xylem-portions of the bundles $(x)$, which were developed before the increase in thickness, can still be recognised; they appear as projections of the woody substance into the pith, and are collectively comprised under the term Medullary Sheath $x \times x$. It is somewhat more difficult to make out the original phloëm-portions of the bundles; but we are assisted by the position of the groups of true bast-fibres $b b b$ already mentioned; they still remain unchanged, but are pushed much further asunder, because the intermediate soft bast has extended in the tangential direction, in consequence of the pressure of the secondary tissue from within. The radial diameter of each phloëm-portion $p$ has also increased, secondary layers, formed out of the fascicular cambium, having been added to the primary phloëm from within. The primary xylem-portion $x$ of each bundle is separated from its phloëm-portion $p$ by a thick layer of secondary xylem $f h$, which has been developed from the fascicular cambium. These portions may be distinguished as Fascicular Xylem from the Interfascicular $X y l e m$ (Fig. 105, $C$, ifh) formed out of the interfascicular cambium; a layer of Interfascicular Phloëm ifp on the outside corresponds to each of these latter. The entire mass of secondary xylem consists therefore of fascicular and interfascicular xylem, the entire mass of secondary phloëm similarly of fascicular and interfascicular portions. The primary xylem is wanting on the inner or medullary side of the interfascicular xylem, the primary phloëm in the interfascicular phloëm.

With reference to the elementary constituents of the secondary or thickeningtissues, it is first to be noted that in this respect the fascicular and interfascicular formations are alike. The whole mass of secondary xylem, with the exception of the medullary rays or 'silver-grain,' consists, in Conifers of tracheides with bordered pits (Fig. 23, p. 25); in Dicotyledons of wood-prosenchyma, wood-parenchyma, and pitted vessels formed from short cells. In the secondary xylem no annular, spiral, or reticulated vessels are formed; these arise only in the original xylem-portions 
of the bundles, and are therefore subsequently found only in the medullary sheath (in Gymnosperms also); and since these were formed during the growth in length, they are considerably longer than the secondary elements. The secondary phloëm consists of phloëm-parenchyma, sieve-tubes, and sometimes of true bast-fibres; but the latter are often wanting. The mode in which these elements combine to form the secondary tissues varies greatly in different plants, and is, at present, of subordinate importance.

The elements of the secondary xylem of which we have now spoken, as well as those of the secondary phloëm, are, like those of the primary xylem and phloëm, elongated in the direction of the axis of growth. But elements also occur in the thickening tissue placed horizontally (i.e. at right angles to the axis of growth) and radially, out of which the radiate tissue is composed. In Fig. 105, $C$ (p. 129) the secondary xylem and phloëm are represented as crossed in a radial direction by dark lines, some of which pass through all the secondary layers, while others begin only in the secondary xylem and end in the secondary phloëm; the former are first formed, the latter subsequently, and constantly in increasing numbers. Each of these dark lines in the figure represents a ray of parenchymatous cells placed horizontally; each of the rays runs, as will be seen, uninterruptedly from the xylem through the cambium into the secondary phloëm; as long as it runs through the xylem it is called a Xylem-ray ${ }^{1}$; its continuation into the secondary cortex is a Phloëm-ray. These rays split up, as it were, the secondary tissue in the longitudinal and radial directions into sections which have a wedge-shaped form when cut through horizontally, and which increase in number as the cambium-ring increases in size. Each separate ray does not, however, by any means extend through the whole length (in the direction of growth) of the secondary tissue; but has generally only an inconsiderable height. If a thick stem is split longitudinally, the rays have the appearance, in many close woods, of glistening bands (the 'Silver-grain'), traversing the prosenchymatous woody tissue in a radial direction; in a tangential section they have the appearance of wedges driven into the mass of the wood. Each ray is sharp-edged above and below (i.e. in the direction of the axis of growth), thin, but usually thickened in the middle (in reference to its height), and sometimes composed of a number of layers of cells, as is shown in Fig. 97, p. II7. This and the position of the rays causes the elements of the secondary xylem and phloëm which are elongated longitudinally to be more or less bent in different directions. If the rays were imagined to be altogether removed, the entire thickening-tissue would then consist of bundles penetrated by empty meshes, and anastomosing tangentially above and below them. A very good idea of this structure may be obtained by examining a piece of ordinary lime-bast, or stems, such as the cabbage, in which the soft medullary rays have decayed.

Just as the elements of the secondary xylem and phloëm which are elongated

1 The term Medullary Ray should be avoided in reference to these; since most of the rays are neither connected with the pith, nor have any of its properties. [It is difficult however to substitute any other expression, although the term is used in a somewhat conventional sense. It is convenient to distinguish as Primary Medullary Rays those which are connected with the pith, and as Secondary Medullary Rays those which only commence in the secondary xylem.] 
longitudinally are formed out of longitudinally elongated cambial cells, so the rays are formed out of cambial cells lying in rows and elongated in the radial direction. In the xylem the cells of the rays are usually lignified, and sometimes have very hard walls, as in the copper-beech, where they alone remain after the decay of the wood, and then have completely the appearance of constituents of the wood. At other times they continue thin-walled, unlignified, and different from the true woody tissue. The phloëm-rays are usually thin-walled, parenchymatous, and at their outer end, where they pass through the primary phloëm, they are frequently compelled, by the increase in size of the stem, to extend tangentially, when they become divided by radial longitudinal walls; the phloëm-bundles which lie between them becoming thus pushed further and further apart (Fig. I05, C).

All the most essential points have now been spoken of in reference to the theory of the increase in thickness of the stems of Gymnosperms and Dicotyledons. But the formation of secondary xylem and phloëm from the cambium, which we have at present followed only in the early period of growth, continues, in perennial plants, throughout their whole growth; the wood and the secondary phloëm are therefore constantly increasing in thickness; but the increase of the xylem is generally considerably greater than that of the phloëm. At an earlier or later period in the thickening of the stem periderm is formed in the primary cortex, which sometimes, as in the beech, copper-beech, birch, and cork-oak, follows the increase in size of the stem, and surrounds it as a continuous envelope of cork. But usually Bark is formed; i.e. lamellæ of cork cut out flakes of the primary, and subsequently also of the secondary phloëm, which then dry up, and, accumulating on the surface, as in the pine, oak, \&c., form the bark, or fall off periodically, as in the plane. The whole of the primary cortex (phloëm) is then replaced by bark; with the exception of the pith and medullary sheath, the stem consists then entirely of masses of secondary tissue which have all originated in the cambium; but even of the secondary phloëm only the inner younger layer usually retains its vitality, the outer layers uniting sooner or later, in the production of bark.

In tropical woody plants when several years old, the additions to the wood formed in each successive year are not generally distinguishable on a transverse or longitudinal section; the entire mass of the wood is homogeneous. In woody plants, on the contrary, that grow in a climate in which the periods of growth are interrupted by a cold or wet season, as is the case with us, the annual additions to the wood may be recognised as sharply separated concentric layers, known as Annual Rings. Their sharp separation from one another is caused by the Vernal Wood being of a looser texture than the Autumnal Wood. Every annual ring consists, therefore, on the inside of looser, on the outside of denser wood which pass into one another insensibly without any sharp line of demarcation; while, on the contrary, the dense autumnal wood of the preceding ring is very sharply separated from the looser vernal wood of the succeeding ring. In Coniferæ the distinction between the looser vernal and the denser autumnal wood consists only in the tracheïdes of the former having larger transverse diameters, while in the later wood, and especially that formed at the end of the period of growth, they are narrower. The same mass of vernal wood includes therefore a larger amount of cell-cavity, and is consequently looser, than the autumnal wood. The walls 
of the later-formed wood-cells being thicker tends to the same result. In Dicotyledons, in addition to this difference, the vernal wood contains a larger number of large pitted vessels than that formed in the autumn, where, indeed, they may be altogether wanting.

The cause of the formation of annual rings lies, as I was the first to suggest, and as has been proved experimentally by de Vries, in the pressure, changing with the time of year, which the cortex, and especially the bark, exercises on the cambium and the young wood. This will be shown more in detail in Book III. Sect. I6.

In most woods, but not all, whether the annual rings are conspicuous or not, there is a distinction, when the stem has attained a sufficient thickness, between the so-called Duramen and Alburnum. The Duramen consists of the older and inner layers of wood, and is of a dark-brown, yellow, red, or even black colour, and of a firmer harder texture; the Alburnum, consisting of the last annual additions, forms a light-coloured or white, softer and more spongy tissue round this nucleus. The inner layers of alburnum are gradually transformed into duramen, the cambium depositing new layers of wood on the outside, and the cell-walls assuming a darker colour, from saturation with resin, colouring substances, \&c. The distinction between alburnum and duramen is very clear and well-marked in the oak, walnut, cherry, elm, 'acacia' (Robinia Pseud-Acacia), lignum-vitæ (Guaiacum officinale), logwood (Hematoxylon campechianum), brazil-wood (Casalpinia echinata), \&c. On the other hand no duramen is found in the silver fir, Scotch fir, lime, or Paulowenia imperialis, \&c.

(3) The Secondary Increase in Thickness of Roots in Gymnosperms and Dicotyledons differs from that which takes place in the stem only in the first production of the cambium-ring; and this difference consists merely in the fact that in roots the phloëm does not lie outside the xylem-bundles (in the radial direction), but is disposed alternately with it on the periphery of the axial fibro-vascular cylinder. When the cambium is once formed, it behaves in precisely the same manner as it does in the stem.

We may first investigate the process in a root which is from the first moderately thick, and has a true pith enclosed within its axial fibro-vascular cylinder or plerome, as in many primary roots, at least in their upper thicker portion. Fig. I06 represents a transverse section through the upper part of a root of the scarlet-runner (Phaseolus multiflorus) at the time when the cambium has been formed and has commenced its work; $s$ is the plerome- or bundle-sheath belonging to the fundamental tissue, which immediately encloses the pericambium or outermost layer of the plerome $p c$. Within this may be observed the four primary xylem-bundles, consisting of vessels of which the oldest and smallest $p$ lie on the outside, while the younger and larger $g g$ are next to the pith $\dot{M}$. In the interval between each pair of xylem-bundles lies a broad phloëm-bundle $b$, $b$, with true bast-fibres. The parts now described have been formed during the growth in length; this has however, at the time when the section was taken, ceased for three or four days in this part of the root and increase in thickness has taken its place. The cells lying on the inner side of each phloëm-bundle first divide repeatedly by tangential longitudinal walls; a layer of cambium $(c)$ is thus formed behind each 
phloëm-bundle, consisting of radial rows of cells. Some of the innermost cambial cells $g^{\prime} g^{\prime}$ have already enlarged considerably, to form the first vessels of the secondary xylem; these are arranged, in Phaseolus, in an annular zone continuous with the inner youngest vessels of the primary xylem-bundles, a structure which is seen nowhere else. Between and in front of these vessels other cambial cells develope into prosenchymatous and parenchymatous wood-cells. Since therefore the cambium-layer behind each phloëm-bundle is continually producing vessels, wood-parenchyma, and wood-prosenchyma on its inner side, a four-rayed cross of woody tissue is formed, as is shown in Fig. 107 (less strongly magnified). The four arms of this cross correspond in their position - as will be seen from what has been said, and from a comparison with Fig. 106-to the four primary phloëm -bundles $b, b, b, b$.

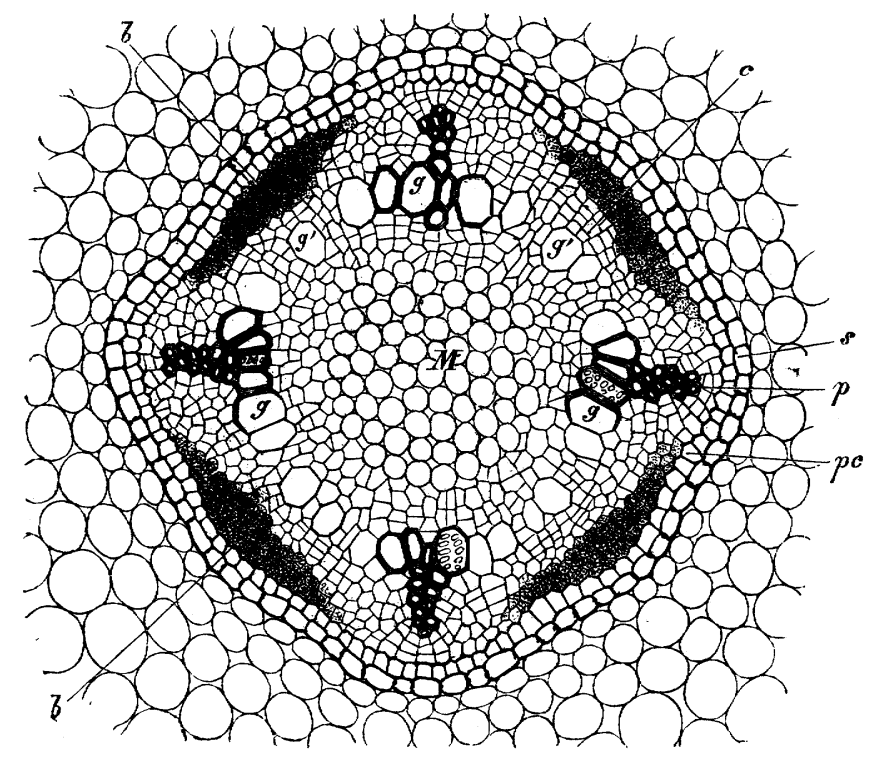

FIG. ro6.-Transverse section of the primary root of a seedling of Phaseolus multiflorus, from the upper thicker part, at the time when the first leaves of the seedling have emerged above the ground and the first lateral roots have already been formed. (The pericambium $p c$ has been drawn too thick-walled.)

On the outer side of each cambium-layer, but within the primary phloëm, a zone of new true bast-fibres (Fig. 107 $b^{\prime}$ ) has already been formed in the secondary phloëm. Between the four arms of the secondary xylem and secondary phloëm belonging to it, lie four broad rays of tissue, consisting of large parenchymatous cells elongated in the horizontal and radial directions. These four bands lie on the same radii as the four primary xylem-bundles (Fig. 106, $p$ ); in front of each of these latter a meristem-layer has been formed, which however has not produced xylem and phloëm like the cambium behind the phloëm-bundles, but only radiate parenchyma to the extent required by the growth of the adjacent masses of secondary xylem and phloëm. Fig. 1o7 further shows the tissue which surrounds the primary and secondary phloëm in the act of division; radial rows of cells have been produced here also, and form a layer of cork or periderm $k$ on the outside of the cortex. 
Precisely similar to the growth in thickness in Phaseolus is that of the primary roots containing pith of seedlings of Cucurbita Pepo, Convolvulus tricolor, Cereus, Clusia, \&c.; only that in these cases the secondary xylem does not coalesce with the primary xylem, but remains quite distinct, so that the alternation of the primary and secondary elements of the xylem is at once evident ${ }^{1}$. In slender roots which have no pith, and where the primary xylem-bundles meet in the centre, cambium is also formed on the inner side of the primary phloëm, and the secondary fibrovascular masses form, therefore, in this case also two, three, or more groups, which originate in the intervals between the primary xylem-bundles, as is very clearly seen in Tropacolum, but project much further outwardly. When there are two of these primary xylem-bundles, as in Beta, Tropcolum, Taxus, and Umbelliferæ, they form a vascular band dividing the axial cylinder in half; when three or more, as in Pisum, a three- or four-rayed star.

In the cases hitherto considered, the secondary fibro-vascular tissues (consisting of xylem and the phloëm belonging to it) remain separated into two, three, four, or more masses, nothing but parenchymatous fundamental tissue being formed between them and in front of the primary xylem, as in Phaseolus. In other cases, on the contrary, true cambium is formed in front of the primary xylem, producing xylem on the inside, phloëm on the outside; and thus is formed a compact cylinder of secondary xylem, surrounded by a continuous layer of secondary phloëm, as in Taxus, Pinus, Beta, \&c., and as is shown, in the case of the stem, in Fig. 105.

The secondary xylem of roots very frequently consists for the most part of succulent woodparenchyma, in which the few vessels, surrounded by a few lignified cells, are collected into isolated groups. This occurs especially in the cultivated

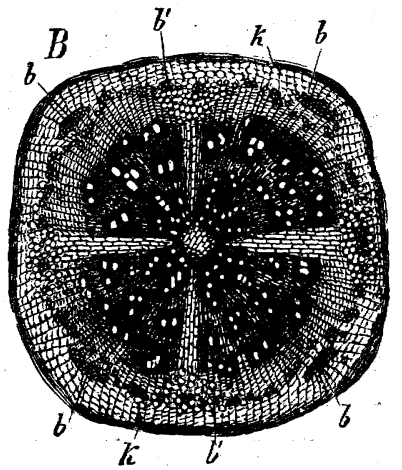

FIG, 107.- Transverse section of the upper part of the primary root of an older plant of Phaseolus multiflorus; $b \quad b \quad b \quad b$ primary phloëm. bundles; $b^{\prime} b^{\prime}$ bast-fibres in the secondary phloèm; $k$ pericambium. beet-root, the cultivated carrot, and in Althcea officinalis, Rheum rhaponticum, Atropa Belladonna, Cocculus palmatus, Inula Helenium, \&c. The secondary phloëm of roots also has a tendency towards an abundant formation of parenchyma, with a diminished development of bast. The secondary tissues of roots so completely resemble, on the other hand, those of stems in the formation of xylem-and phloëm-rays (medullary rays) and in other respects, that it is not always possible, especially when the wood of the root is not strongly lignified, to distinguish it from the wood of the stem without examining the central axis, when its real nature may always be determined from the primary xylem-bundles and the occasional absence of the pith.

From the great numbers of species belonging to the classes Gymnosperms and Dicotyledons, and from their extremely different adaptations to various vital conditions, it is not surprising that in the processes connected with the increase in size of the stem there should be a great variety of deviations from the normal types described

1 Very instructive figures of this are given by Van Tieghem, l.c. 
above. An exhaustive description of these deviations ${ }^{1}$ would far exceed our space, and would lead us to great difficulties, since there is at present no portion of the field of botany so unworked as the anatomy of plants properly so called. What follows will only serve to direct the attention of the student to some of the most striking deviations from the processes described under the head of the second type, to which some reference will again be made at the conclusion of the account of the structure of Dicotyledons in Book II.

(a) The variation is only slight when the increase in thickness is not uniformly concentric, but is altogether suspended on one side, as in the roots of Polygala Senega, or is much stronger on one side than elsewhere, as in the root of Ononis spinosa and in many stems.

(b) The cambium is sometimes formed only in the fibro-vascular bundles, which in consequence alone produce secondary xylem and phloëm, while the bridging over of the medullary rays by cambium does not take place, and interfascicular secondary fibrovascular masses are therefore not formed. The fundamental parenchyma which lies between the bundles increases like them slowly in thickness by the extension of its cells and their occasional division, as for example in the stem of Cucurbita, where the bundles are also singular in possessing a layer of phloëm on the inside of the xylem, a peculiarity belonging also to Solanaceæ and Apocynaceæ. But in old stems this ends in an over-bridging of the medullary rays by meristem.

(c) The processes deviate much more widely from the normal type in those cases where the activity of the original cambium soon ceases, and new bundles are produced out of a meristem in the surrounding cortex; these bundles first increasing in thickness by cambium, but then ceasing to grow, when a new ring of bundles is formed in a new meristem; so that at length the wood consists, not of a compact mass, but of concentric layers of isolated fibro-vascular bundles. This process presents a certain resemblance to what takes place in the Aloinex (Type I). The rather frequent instances of this mode may, according to Nägeli and Eichler, be classified into two groups, according as the ring of meristem which produces the new bundles is formed in the primary or secondary phloëm.

Each successive ring of bundles originates in the primary cortex in Gnetum (Gymnosperms), in Menispermaceæ, and in Rhynchosia scandens among Leguminosæ. Each of the secondary bundles has on its outer side a border of phloëm, which in Gnetum even forms true bast.

The new rings of fibro-vascular bundles originate successively in the secondary cortex, according to Eichler in Dilleniaceæ, many Leguminosæ (e.g. Baubinia, Caulotretus), some Polygalaceæ (Securidaca and Comesperma), Ampelideæ (Cissus according to Crüger), and Phytolaccaceæ.

(d) 'The stem of Malpighiacex and Bignoniacex' (Eichler l.c.) 'at a later period often agrees so far with the foregoing, that the wood appears as if broken up by layèrs of a parenchyma resembling cortical tissue into a number of isolated portions, of which only the outer ones are merismatic. The history of development is, however, quite different. It does not consist, as in the previous cases, of an actual new formation of vascular bundles outside those already in existence, but in a subsequent vigorous cell-multiplication within the medullary rays and the layers of woodparenchyma which intersect the fibro-vascular bundles. All the portions of wood which are by this means more or less driven apart and displaced are therefore only portions of the primary ring of fibro-vascular bundles; it is obvious that only the outer ones are capable of increase, since cambium is found in them only.' The wood however does not always break up longitudinally into pieces that are actually separated; sometimes it is only, as seen on transverse section, divided into lobes, or shows deep indentations, which appear from without as deep furrows, running usually in a spiral

1 [Oliver has collected the bibliography of the stem in Dicotyledons in the Nat. Hist. Review 1862 , pp. 298-329, and 1863 , pp. $251-25^{8}$.] 
direction. These lobes, separated by radial lamellæ of tissue resembling cortex, may again be divided in the same manner into smaller lobes; as occurs in some Malpighiaceæ like Heteropterys and Bannisteria. In some species of Bignonia the wood, as seen in transverse section, forms a four-armed cross, the arms of which are separated by very broad soft masses of tissue resembling medullary rays, which pass outwards into the cortex surrounding the stem (see Fig. 107). In this order Tecoma radicans has also the peculiarity, noticed by Sanio, that a new ring of wood is formed in the pith, altogether separated from the medullary sheath of the ordinary ring; this new ring has on its inner side a secondary layer of phloëm, and is increased by cambium.

(e) Some of the most peculiar modes in which the wood is formed are found in many climbing Sapindacex. The abnormalities here described are, in fact, met with chiefly among lianes, i.e. woody climbing and twining plants growing in tropical forests; although there are typical liane-stems which do not climb. In the abnormal Sapindacex, especially the genus Serjania, a transverse section shows three, four, five, or more portions of wood completely separated from one another, of which the central one is usually much the most strongly developed. Each of these masses of wood presents the structure of a normal dicotyledonous stem; medullary rays proceed from its central pith towards its circumference; each is surrounded by a narrow secondary cortex, by which they are, however, united into a whole. Looked at from without, the more slender outer portions of wood have the appearance of cushions, which project below out of the central larger one, and coalesce again with it above. The separate masses, which on a transverse section seems to be completely independent, are therefore only projections or outgrowths of the originally homogenous stem. According to Nägeli this abnormality is caused, at the very origin of the fibro-vascular bundles, by their not being arranged in a ring, so that a single cambium-ring cannot unite them all ${ }^{1}$.

(f) In the cases hitherto described the deviation from the typical processes is caused essentially by abnormalities in the origin of the cambium-ring, or by its subsequent behaviour, or finally by repeated fresh formation of fibro-vascular bundles in a secondary meristem. The case is different in Piperaceæ, Begoniaceæ, Nyctagineæ, and Amaranthacex. An abnormality is here brought about at a very early period in the growth of length of the stem, of such a nature that, in addition to the leaf-trace-bundles which bend above into the leaves, vascular bundles also arise in the stem which do not pass out into the leaves, i.e. 'cauline bundles.' On a transverse section even of a very young internode a number of fibro-vascular bundles are seen dispersed through the fundamental tissue; and the alternative now presents itself whether the leaf-trace- or the cauline bundles shall be united by cambium and hence become thicker, or whether the formation of cambium shall be altogether suppressed. Sanio states that the latter occurs among Piperacex, in the genus Peperomia, where the leaftrace-bundles which constitute an outer circle are not united by a ring of cambium, any more than the inner ones which do not bend out into the leaves. In Chavica, on the contrary, this takes place in the outer ring of leaf-trace-bundles, while in the pith which is enclosed by them the cauline bundles remain isolated and without any secondary increase in thickness. The structure is the same in Begoniacex. That of Nyctaginex, on the contrary, to a certain extent varies from this; the thick leaftrace-bundles, ascending through the middle of the fundamental tissue, remain isolated, while a wider outer ring of cauline bundles forms a ring of wood by the activity of its cambium.

Sect. 19. The Primary Meristem and the Apical Cell ${ }^{2}$.-At the growing ends of shoots, leaves, and roots, the forms of tissue hitherto described do not

1 Nägeli (l.c.) describes the very complicated processes more fully.

2 Nägeli, Die neueren Algensysteme. Neuenburg I847.-Cramer in Pflanzenphysiol. Untersuchungen, Heft III. p. 2r. Zürich.-Pringsheim, Jahrb. für wissen. Bot. vol. III. p. 484.-Kny, 
exist; here is found a homogenous tissue, the cells of which are all capable of division, rich in protoplasm, with thin and smooth walls, and containing no coarse granules. This tissue is termed Primary Meristem; it is a meristem because all the cells are capable of division, and primary because it presents the condition out of which the different permanent forms of tissue are successively formed by differentiation ('Proto-meristem' might perhaps, therefore, be a better term). If the general structure of the plant is simple, as in Algæ and Characeæ, the cell-forms arising from the primary meristem only differ slightly from one another. If the plant belongs to a higher type, as in Vascular Cryptogams and Phanerogams, layers of tissue of a different character first originate from the undifferentiated primary meristem which proceeds from the growing apex, and in these the different cell-forms of the epidermal and fundamental tissues, as well as of the fibro-vascular bundles, finally arise by further development, at a still greater distance from the primary meristem. The differentiation takes place so gradually, and at such different periods in the various layers of the tissue, that it is impossible at any one time to assign a definite lower limit to the primary meristem. As growth proceeds at the end of shoots, leaves, and roots, lower portions of the primary meristem become gradually transformed into permanent tissue; but the primary meristem is always again renewed by the production of new cells close to the apex. Nevertheless whole organs, the apical growth of which soon ceases, may at first consist entirely of primary meristem, which finally passes over altogether into permanent tissue, so that no primary meristem is left. Examples of this are furnished by the development of the sporogonium of Muscineæ, of the sporangia of Ferns, and even of most leaves and fruits of Phanerogams.

The terminal portion of an organ with permanent apical growth, consisting entirely of primary meristem, is termed the Growing Point or 'Punctum Vegetationis;' not unfrequently (but by no means always) it projects as a conical elongation, and is in this case distinguished as the Vegetative Cone or Cone of Growth.

The production and renewal of the primary meristem commence with the cells lying at the apex of the growing point; and, by the manner in which this happens, two extreme cases may be distinguished, which are however united by transitional forms. In the one case, the usual one with Cryptogams, though not without exception, the whole of the cells of the primary meristem trace their origin back to a single mother-cell lying at the apex of the growing point and called the Apical Cell. In some Cryptogams, on the other hand, and in Phanerogams, there is no single apical cell of this character; even when a cell lies at the

ditto, vol. IV. p. 64.-Hanstein, ditto, vol. IV. p. 238.-Geyler, ditto, vol. IV. p. 481.-Muiller, ditto, vol. V. p. 2+7.-Rees, ditto, vol. VI. p. 209.-Nägeli und Leitgeb, in Beiträge zur wissen. Bot., Heft IV. München I867.-J. Hanstein, Die Scheitelzellgruppe im Vegetationspunkt der Phanerogamen (in the Festschrift der niederrh. Ges. für Natur- und Heilkunde, Bonn; and Monatsiibersicht of the same Society, July 5, 1869).-Hofmeister, Bot. Zeitg. 1870, p. 441.Leitgeb, Sitzungsb. der Wiener Akad. 1868 and 1869 , and Bot. Zeitg. $187 \mathrm{I}$, nos. 3 and 34.Reinke in Hanstein's Botan. Untersuchungen, Bonn 1871, Heft III.-Russow, Vergleich. Untersuch. der Leitbuindelkryptogamen, in Mém. de l'Acad. Imp. de St. Petersbourg, 7th ser., vol. XIX. no. I, Petersburg 1872.-Warming, Récherches sur la ramification des Phanerogames, in Vidensk. Selsk., Skr. 5, Räkke Io, B. I, Copenhagen $187^{2}$ (Danish, with French abstract). 
apex, it is not, as in the former case, distinguished by its greater size; and, what is of more importance, it cannot be recognised as the single original mothercell of all the cells of the primary meristem, nor even of a definite layer. We may distinguish, therefore, between the Growing Point with and without an Apical Cell.

(a) Growing Point with an Apical Cell. The formation of the primary meristem out of the apical cell may be brought about, as will be shown presently, in different ways, but it generally results from the constant repeated division of the apical cell into two unequal daughter-cells. One of the two daughter-cells (the Apical Cell) remains from the first similar to the mother-cell, and includes the apex; it is immediately enlarged by growth till it equals the previous apical cell in size, and then again divides, and so on. This process produces the appearance as if the apical cell always remained intact; and this has been assumed in ordinary language, although the apical cell existing at any time is only a daughter-cell of the preceding one. The other daughter-cell, on the other hand, appears from the first like a piece cut off from the back or side of the apical cell, generally in the form of a disc or angular plate, and is hence called the Segment ${ }^{1}$. In the simplest case the segment may remain undivided; and then the whole tissue which is produced from the apical cell has the form of a simple row of cells, as in some Algæ, and in Fungus-hyphæ and hairs. But generally the segment divides into two cells, each of which again breaks up into two, and this process is mostly repeated many times in the daughter-cells, until a more or less extensive mass of tissue is produced from the segment. The aggregate of such masses of tissue constitutes the primary meristem. A very simple case of this kind is shown in Fig. Io8, where the apical cell $(s)$, here very large, growing straight out from its base, is divided by septa $\left(I^{a}, I^{b}\right)$, and thus forms the segments which lie in a row one over another. But each of these

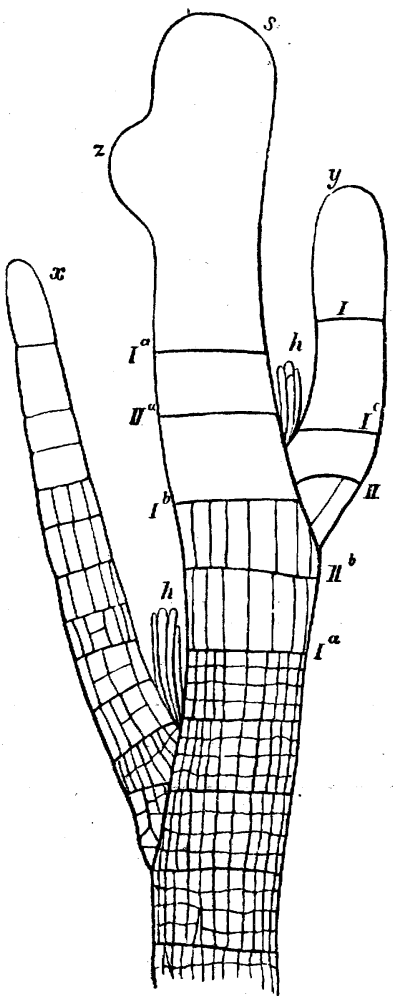

FIG. 108.-A branch of the thallome of Stypocaulon scoparium with two branchlets $x$ and $y$, and the rudiment of a third branchlet $z$ (after Geyler); all the lines indicate cell-walls.

1 The portions of wall which enclose a segment-cell differ in their nature and origin, and behave differently in their subsequent growth. Each segment possesses two walls which were originally division-walls of the apical cell; they are generally parallel to one another, and are called the 'Principal walls' of the segment; the older faces the base, the younger the apex of the organ. Another portion of the wall of the segment is a part of the outer wall of the apical cell; it may be termed the 'Outer wall' of the segment. Where the segments arise as transverse discs of an apical cell, as in Stypocaulon and Characeæ, the outer wall is cylindrical; when the segmentation takes place on two or three sides the process is very complicated; the segments have in this case, besides the two principal walls and the outer wall, side-walls which intersect at an acute angle. The side-walls are portions of the principal walls of older adjoining segments, and are successively cut off by the, youngest septum of the apical cell, which is at the same time the youngest principal wall. 
last is again immediately broken up by a septum $\left(I I^{a}, I I^{b}\right)$ into two disc-shaped cells, and in each of these numerous small cells arise by the formation of vertical and afterwards horizontal walls (as may be seen in the figure further back from the apex); and it is easily seen how the whole branch is built up of portions of tissue, each of which originated from a single segment. The same takes place

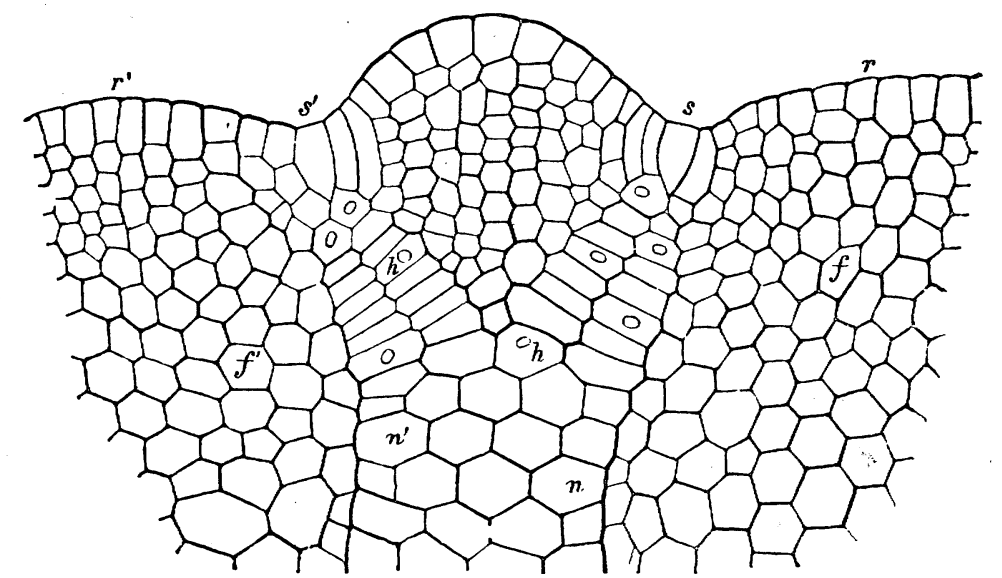

FIG. rog.-Apical region of a shoot of Metzgeria fircata in the act of bifurcation, looked at from the surface (after Kny). The shoots consist of a single layer of cells $\left(f f^{\prime}\right)$, which is however penetrated by a mid-rib $n n^{\prime}$, three to six layers in thickness.

in the lateral branchlets $x, y$, which in this case arise originally from lateral protuberances of the apical cell. These processes are remarkably clearly seen in Stypocaulon, in the first place because only one row of segments is formed lying one over another, and in the second place because the segments themselves are transformed into portions of tissue without at the same time growing, as is

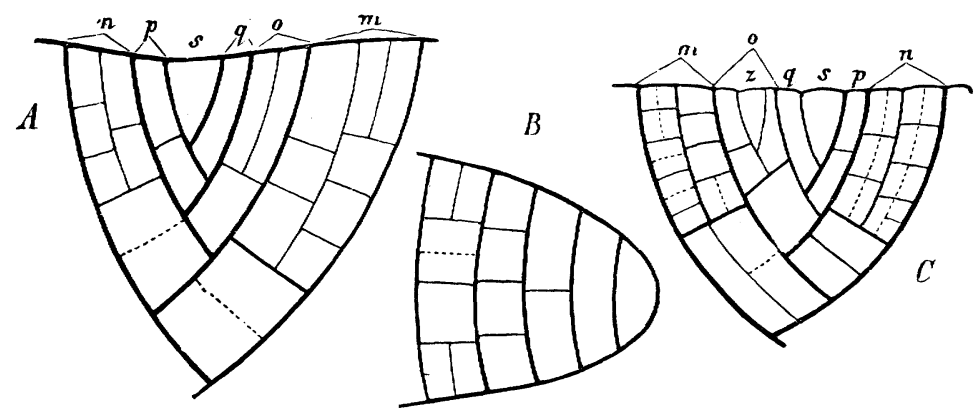

FIG. I10.-Diagrammatic representation of the segmentation of the apical cell, and of the first divisions in the segment of
Metzgeria furcata (after Kny). $A$ apex seen from the surface; $B$ the same in vertical longitudinal section; $C$ an apex in the Metzgeria furcata (after Kny). $A$ apex seen from the surface; $B$ the same
act of bifurcation; a new apical cell is formed in the third-youngest segment.

usually the case. Distortions often occur from the growth of the segments, which render difficult an investigation of the processes of division.

Figs. rog and r to show us a case in which the apical cell is divided alternately right and left by oblique walls, so as to produce two rows of segments attached to one another in a zigzag manner within and behind, but separated to some 
distance in front; in the angle which the two youngest segments enclose lies the apical cell s. Fig. Io9 shows the end of a shoot of Metzgeria furcata in the act of bifurcation; each fork ends in an apical cell $s$; the segments and the masses of tissue which are formed from them are drawn just as they appear to the eye under the microscope on a superficial view of the flat ribbon-shaped shoot. From the course of the cell-walls and the resulting grouping of cells round the apical cell, the diagram Fig. I ro, $A$ is deduced, in which the distortions of the cell-walls occasioned by growth are neglected, and hence the genetic relationships represented more clearly. For further elucidation Fig. I 10, $B$ is added, which also represents diagrammatically the longitudinal section of the apical region, at right angles to the broad surface of the ribbon-shaped shoot. This longitudinal section bisects, behind the apical cell, the mid-rib (Fig. I09, $n, n^{\prime}$ ), which consists of several layers of cells, while the lateral expansions of the shoot are only one layer in thickness. The origin of the tissue is now clear from the diagrammatic Fig. I $10, A$ and $B$, if it is observed in the first place that the portions of the surface indicated by $m, n, o, p$, and $q$ are the segments of the apical cell $s$ which were formed successively in the same order, so that $m$ represents the oldest, $q$ the youngest segment. From each segment a small piece is first cut off behind by a wall oblique to the axis of the shoot; from the zigzag row of these posterior sections arises the mid-rib of the shoot, which attains a thickness of several layers of cells, each division first of all splitting up by a wall parallel to the surface of the shoot into two cells lying one over another, and each of these cells on its part again dividing in the same manner. Divisions at right angles to the surface of the shoot (Fig. Iro, $B$ ) are then also formed in the uppermost and undermost of the cells produced in this way; an outer small-celled layer is formed on the mid-rib covering its upper and under side, and surrounding an inner bundle which consists of longer cells. While the posterior sections of the segment produce the tissue of the mid-rib, the tissue of the flat lateral portion (Fig. Io9, $f, f^{\prime}$ ) proceeds from the anterior sections which face the margin of the shoot; and this tissue is only one cell-layer in thickness, no division taking place in it parallel to the surface of the shoot. All the divisions in these marginal sections of the segment are, on the contrary, at right angles to the surface, and are produced by the marginal section first of all breaking up into two cells lying side by side (see Fig. Iro, $A, o$ ), each of which then forms several shorter cells by repeated bi-partition, and these may again undergo further division according to the activity of the growth. In general the first divisions only of the segment are constant; the further course of cell-multiplication is, according to the minute investigations of Kny, subject to many deviations. Since the tissue produced from the marginal sections becomes prominent during growth, it results that the apical cell, with the youngest segments, lies in a depression of the margin of the shoot; and we have here a simple example of the depression of the growing point in the tissue which grows more luxuriantly around it, such as often occurs to a much greater extent in Fucaceæ, Ferns, and Phanerogams. The differentiation of the tissue of the shoot of Metzgeria furcata does not attain a high degree; the cells of the margin and of the mid-rib, when mature, differ only slightly from one another; but this differentiation is brought about 
very early, even in the first division of the segment, so that the marginal tissue and the youngest extremity of the mid-rib can be traced close up to the apical cell. Fig. I ro, $C$, finally, affords an opportunity of learning the mode of formation of a new apical cell out of a cell of the meristem, a case which occurs often enough in Muscineæ and the higher Cryptogams. While the thallome of Stypocaulon (Fig. 108) shows how the apical cell of the lateral shoot grows immediately from that of the principal shoot as a lateral protuberance, which is then cut off by a wall, in Metzgeria furcata, as is shown by the researches of Hofmeister, Kny, and Müller, it appears that the origin of a new apical cell may be brought about in a different manner. Fig. I ro, $C$ shows the case described by Kny; in the third-youngest segment $o$, which is formed from the apical cell $s$, the customary separation into a mid-rib-cell and a margin-cell has first taken place; the latter then divides, as usual, into two cells lying side by side; but the new apical cell is constituted by the appearance of a curved wall in one of these margin-cells of the second order; and this wall intersects the dividing wall of the margin-cell previously formed, thus cutting out a wedge-shaped piece $z$, which assumes at once the function of the apical cell of a new shoot ${ }^{1}$.

In Equisetace $æ$ and many Ferns, the axis of the shoot terminates in a comparatively very large apical cell, which is bounded by four walls-an outer one, overarching the apex and spherically triangular, and three converging obliquely below and within, which form at the same time the upper principal walls of the youngest segments (Fig. III, $A, D$ ); the apical cell has hence the form of a segment of a sphere, or of a three-sided pyramid with its spherical base uppermost. The three plane principal walls of the apical cell are of different ages. The next division-wall arises in the apical cell, and is parallel to the oldest wall; a segment is formed bounded by two triangular principal walls, an arched outer wall, and two nearly oblong side-walls ${ }^{2}$; after the apical cell has again grown to its original size, a second division follows parallel to the next-younger principal wall, which is followed again, after fresh renewal of the apical cell, by a division parallel to the youngest principal wall. Three segments are now formed, placed somewhat like the steps of a winding staircase; each is in contact with a principal wall of the apical cell; and in this manner the divisions are repeated; and since each segment takes in a third of a circuit of the winding staircase, the segments out of which the stem is built up all lie in three rows parallel to the axis, each embracing a third of the diameter of the stem. In Fig. I I I, $B$ and $C$, the segments are numbered $I, I I, I I I$, \&c., according to the order of their formation, and are represented as they appear when the apex of the stem is seen from above (not in transverse section), or as if the arched surface of the apex were spread out flat. If the segments are followed according to the order of their numbering, the path described is an ascending spiral, because each segment lies higher than the older ones, as is shown in Fig. III, D, where, however, only two rows of

1 We shall recur, in Chap. III, to this case of spurious dichotomy.

2 These side-walls are pieces of the principal walls of the adjoining segments, as is seen in $B$ and $C$. 
segments are to be seen from without. The formation of tissue begins by each segment dividing into two, soon after its production, by a partition parallel to the principal walls; the new half-segments are indicated in $B, C$, and $D$ by $\mathbf{I}, \mathbf{I}$. Since in each of these two half-segments the further processes are almost exactly the same, it is necessary to keep in view only one of them. Each half-segment becomes divided first of all by a curved vertical wall, which meets internally a side-wall, externally the outer wall of the segment at its middle (Fig. I I r, $E$ ). Since three segments compose a transverse section of the stem, and each half-segment divides into two cells, the

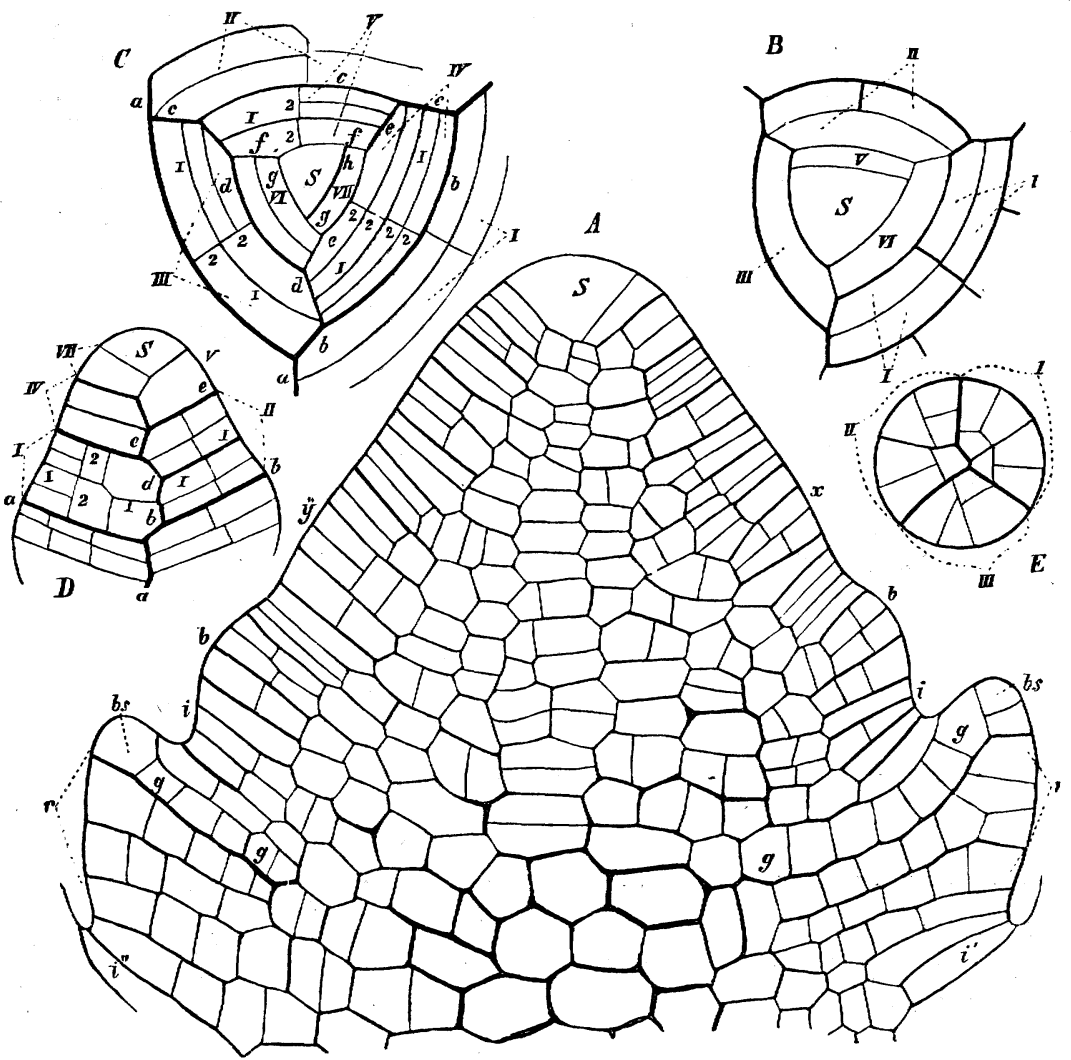

FIG F. maximum, in September $(\times 550) ; B$ view of the apex from above (both from nature); $C, D, E$ the same of $E$. arvense (after . maximic $C$ diagrammatic ground-plan of the apical cell and of the youngest segment; $D$ external view of the apex of Cramer). $C$ diagrammatic ground-plan of the apical cell and of the youngest segment; $D$ a the $I, I I, I I I, \&$ c. the segments; $\mathrm{x}, 2,3, \& \mathrm{c}$. the division-walls in the segments in the order of their formation; $x, y, b, b s$ in $A$ the first rudiments of leaves.

section of the stem now appears as if composed of six cells or sextants, whose walls are placed nearly radially, forming a six-rayed star, as is shown in the transverse section Fig. I I I $E$. Hence the walls by which this division is brought about are called sextant-walls; in $C$ and $D$ they are indicated by the figure 2 . Each of the sextant-cells is still further broken up by vertical walls into an outer larger and an inner smaller cell (Fig. III, E); and thus the foundation is laid of the two layers of tissue into which the primary meristem separates, viz. into an outer and an inner layer, as is clearly shown in Fig. III, $A$. In the outer layer divisions 
parallel to the principal walls and in the vertical radial direction at first preponderate; in the inner layer the divisions are less numerous, so that the cells become more uniform in diameter. This inner mass of tissue, arising from the inner sections of the sextants, is the pith which splits as the stem developes, dries up, and thus causes the hollowness of the stem; from the outer layer of the primary meristem are also formed further from the apex the cortex, the fibro-vascular bundles, and later the epidermis ${ }^{1}$. The external organs of Equisetum are also derived from the outermost layer of the primary meristem, as has already been shown in Fig. II I, $A$, where the protuberances $x, y, b, b s$ represent the rudiments of leaves. To these processes I shall recur hereafter; here it need only be mentioned that each set of three consecutive segments undergoes at an early period a small vertical displacement, so that at least their outer surfaces form a horizontal zone which then bulges out and is the origin of a leaf-sheath.

As a final example of the formation of the primary meristem from an apical cell, we may now consider the processes that take place at the growing end of a

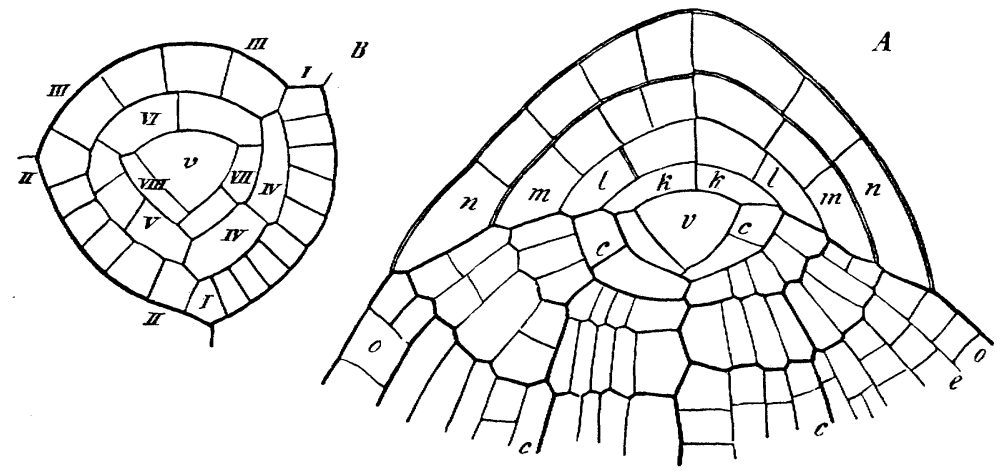

FIG. ri2.-Apical region of a Fern-root; $A$ longitudinal section through the end of the root of Pteris hastata $B$ transverse section through the apical cell and adjacent segments of the root of Asplenium Filix-famina (after Nägeli and Leitgeb).

Fern-root, with which the greater number of roots of Cryptogams agree in the main. Fig. I 1 2, $A$ represents an axial longitudinal section through a Fern-root, with the apex uppermost. From the apical cell $v$ arises not merely the tissue of the substance of the root $o, c$, but also the root-cap $k, l, m, n$, a mass of tissue which covers like a helmet the growing point of every root. The apical cell in this case resembles that of the stem of Equisetaceæ and of many other Cryptogams, in so far as it presents a three-sided pyramidal segment of a sphere; this form is sufficiently seen by comparing the longitudinal section $A$ with the transverse section $B$. Here also three straight rows of segments are formed by successive divisions of the apical cell, which are numbered according to their order in age, $I, I I, I I I, \& c$, in Fig. $B$; and here also a spiral is described by the line connecting the centres of the consecutive segments. The great difference between the apices of roots and the growing stem of Cryptogams is however, that in the former the apical cell not only produces these segments which build up the

${ }^{1}$ Compare Book II, Class Equisetacex, under the formation of their tissue. 
tissue of the root ${ }^{1}$, but other segments also which build up the Root-cap. These latter are cut off from the apical cell by septa in such a manner that they cover them like a cap; and every such segment belonging to a root-cap is hence termed simply a Cap-cell. According to the investigations of Nägeli and Leitgeb, it appears to be the rule that whenever three segments have been formed (from the substance of the root), a new cap-cell arises; but this rule is not always strictly adhered to.

The cap-cell increases quickly in breadth; and its transverse section, originally spherically triangular, becomes circular. It is simultaneously divided into two equal halves by a vertical wall (parallel to the axis of the root); in each of these halves a wall again arises at right angles to the former; and thus four quadrant-cells are formed. Each quadrant again breaks up into two cells (octants), the further divisions varying in different species. In the successive layers of the cap the quadrants are not superimposed but alternate; i.e. the quadrant-walls of one layer deviate from those of the preceding and following ones by about $45^{\circ}$.

The growth in length of the substance of the root, in so far as it is occasioned by divisions of the apical cell, proceeds, as has already been indicated, in such a manner that the septa which arise in spiral succession are parallel to the sides of the apical cell. Each segment-cell is bounded by five walls, as at the apex of the stem of Equisetum,-two principal triangular walls, two oblong side-walls, and one somewhat convex outer wall, in contact with a root-cap. The first wall which arises in each segment-cell stands at right angles to the principal walls, and is, with reference to the whole root, a radial longitudinal wall. Two cells arise in this manner side by side, unequal in form and size, the septum meeting internally a side-wall, but externally the middle of the outer wall. In this manner the transverse section of the root, composed at first of three segment-cells, breaks up into six cells or sextants (compare the processes described above in the stem of Equisetum); three of these sextants reach to the centre of the section; but the three which alternate with them do not. The sextant-walls are seen in Fig. Ir $2, B$, in the segments $I V, V, V I, V I I$, as lines dividing the outer wall in half; in a deeper transverse section they would form, together with the three side-walls of the three segments, a six-rayed star, similar to that in Fig. I I $1, E^{2}$. Each sextant-cell is next divided again by a wall parallel to the surface of the root into an inner and an outer cell ; in the transverse section of the root $(i . e$. in the corresponding transverse section beneath the apex), twelve cells can therefore be recognised at this stage, of which the six outer ones form a peripheral layer, and the six inner ones a central nucleus. The longitudinal section, Fig. I I 2, $A$, shows this wall at $c c$, and it may thus be seen how the mass of the substance of the root is broken up by it into an outer layer $0 c$ and an inner thick bundle $c c c c$. Out of the former arises by further division a tissue which becomes differentiated further backwards into epidermis $O$ and cortex (between $o$ and $c$ ); the axial bundle $c c c c$, on the other hand, which is the result of further longitudinal divisions of the inner sections of the sextants, forms the procambium-cylinder of the root, in which the vascular bundles arise. In this

1 They are bounded by thicker lines in the longitudinal section $A$.

${ }^{2}$ Compare Book II, Equisetaceæ, diagram of root, Fig. 284. 
case also the ultimate separation of the mass of tissue into two distinct portions is determined by the first divisions of the youngest segments; but a comparison of the corresponding process in the stem of Equisetum shows that the mass of tissue which is formed from the central portions of the sextant has quite a different signification from what it has there; and the same is the case with the peripheral layer. A further discussion of the origin of the forms of tissue of the root out of these portions of the primary meristem will be entered into when treating of Ferns and Equisetaceæ.

In conclusion, it may be remarked that the segments of the apical cell, where they arise in two or three rows, have at first a position oblique to the ideal axis of the organ, and enclose an angle open towards the apical cell; but, in consequence of growth, the position of the segments generally changes so that they come to lie gradually more transversely, and finally, at a certain distance from the apical cell, the principal walls lie at right angles to the axis of the organ. The process is not clearly shown in Figs. III and II 2 ; but more evidently in examples to be brought forward later (e.g. Fig. I 16, p. I53).

(b) Growing Point without an Apical Cell. This occurs universally in Phanerogams; the apical region of growing shoots, leaves, and roots consisting of a primary meristem, the cells of which are very small in proportion to the size of the entire growing point, and very numerous. It has not yet been demonstrated whether even the cells next the apex can be traced back to a single primary mothercell, although sometimes undoubtedly one cell lying at the apex is distinguished by a somewhat greater size and by its form. In many shoots the surface of the apex seen from above shows an arrangement of the superficial rows of cells which to a certain extent points to this one cell as their common primary mothercell; but even if this were the case, which is by no means proved, it is, on the other hand, altogether impossible to connect genetically the inner layers of cells also with this cell. The peculiar significance of the apical cell of Cryptogams lies in the fact that all the cells of the primary meristem furnish evidence of descent from it in different degrees.

But as in Cryptogams the first divisions of the segment-cells determine certain layers of the primary meristem which subsequently pass into the differentiated tissuesystems further backwards from the apex, so also in Phanerogams a definite arrangement of the cells is brought about in the primary meristem of the growing point, of such a kind that the various layers of the primary meristem, when followed further backwards, have a genetic relation with the epidermal tissue, the cortex, and the fibro-vascular bundles, and may be recognised as the first rudiments of them. The outermost layers run uninterruptedly over the apex of the growing point, overarching an inner mass of tissue of the primary meristem, which latter, on its part, sometimes runs out beneath the apex into a single cell (in Hippuris and Anacharis canadensis, according to Sanio), but usually terminates in a somewhat subordinate group of cells.

While in Cryptogams with an apical cell an evident cell of this kind is formed where a lateral outgrowth (shoot, leaf, or root) is about to be developed on the growing point, in Phanerogams, on the other hand, a whole group of cells, including inner and outer layers, makes its appearance at the spot in question, so 
that even at the first commencement of an organ no one predominating apical cell can be recognised (Fig. II3). After Sanio ${ }^{1}$ had investigated these processes in Phanerogams, Hanstein ${ }^{2}$ studied them in a more general and detailed manner, and has recently shown that even in the embyro of Phanerogams the first divisions take place in such a manner as to negative from the first the existence of an apical cell; while, on the other hand, a differentiation into an outer layer and an inner nucleus of tissue early manifests itself ${ }^{3}$.

The outermost layer of the primary meristem which covers the growing point together with its apex is the immediate continuation of the epidermis of the older part which lies further backwards; it may therefore be termed the Primordial Epidermis; Hanstein has however already applied to it the name Dermatogen. It is distinguished by the circumstance that divisions occur in it exclusively at right angles to the surface; it is only at a subsequent period that tangential divisions also sometimes occur, when the epidermis becomes divided into several layers.

Beneath the Primordial Epidermis one or more layers are generally found which also cover the apex continuously, and out of which the cortex originates further backwards from the apex (Fig. I 22, $r r$, p. I63); they represent therefore the Primordial Cortex; Hanstein calls this layer of the primary meristem the Periblem. Enclosed and overarched by this is a nucleus of tissue,

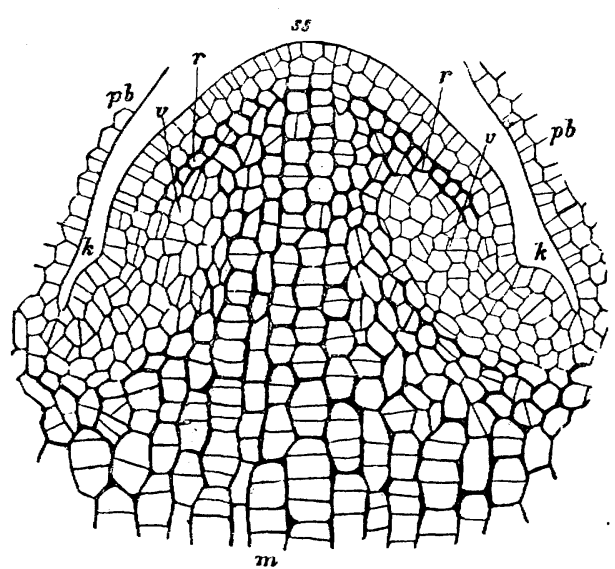

FIG. 113.-Longitudinal section through the apical region of the stem of an embryo of Phaseolus multiflorus; ss apex; pb parts of the two first leaves; $k k$ their axillary buds. which may be followed out as an immediate continuation of the fibro-vascular bundles, and of the pith enclosed or traversed by them. The layer of tissue in which the first fibro-vascular bundles originate, termed by Sanio the Thickening-ring, thus corresponds to the outer layer of this inner tissue-nucleus (which Hanstein terms Plerome), when a pith is formed ${ }^{4}$. If no pith is formed, as in many roots and some stems (e. $g$. Hippuris, Anacharis, \&c.), the whole of the plerome is developed into procambium, and this into an axial fibro-vascular cylinder, which is then traversed by two or more vascular bundles and bast-bundles.

The origin of the root-cap in Phanerogams may be considered, according to the recent investigations of Hanstein and Reinke, simply as a luxuriant growth of the primordial epidermis or dermatogen, localised at the apex in such a manner that

1 Sanio, in Bot. Zeitg. 1865 , p. 184 et seq.

2 Hanstein, Die Scheitelzellgruppe im Vegetationspunct der Phanerogamen. Bonn I 868.

3 Hansteir, Monatsber. der niederrh. Gesell., July 5, 1859. For further details see the general characteristics of Phanerogams in Book II.

4 Compare however Russow, l.c., pp. I77, 183 , from which it appears questionable whether this first differentiation of the primary meristem invariably takes place in this way. 
the part of the dermatogen which covers the apex of the root divides periodically by tangential walls. Thus the dermatogen splits at the apex into two layers of cells, the outermost of which developes into a many-celled cap, the Root-cap, while the inner layer at first again performs the functions of dermatogen, until a new splitting of the layer at the apex causes the formation of a new stratum, which again, on its part, as

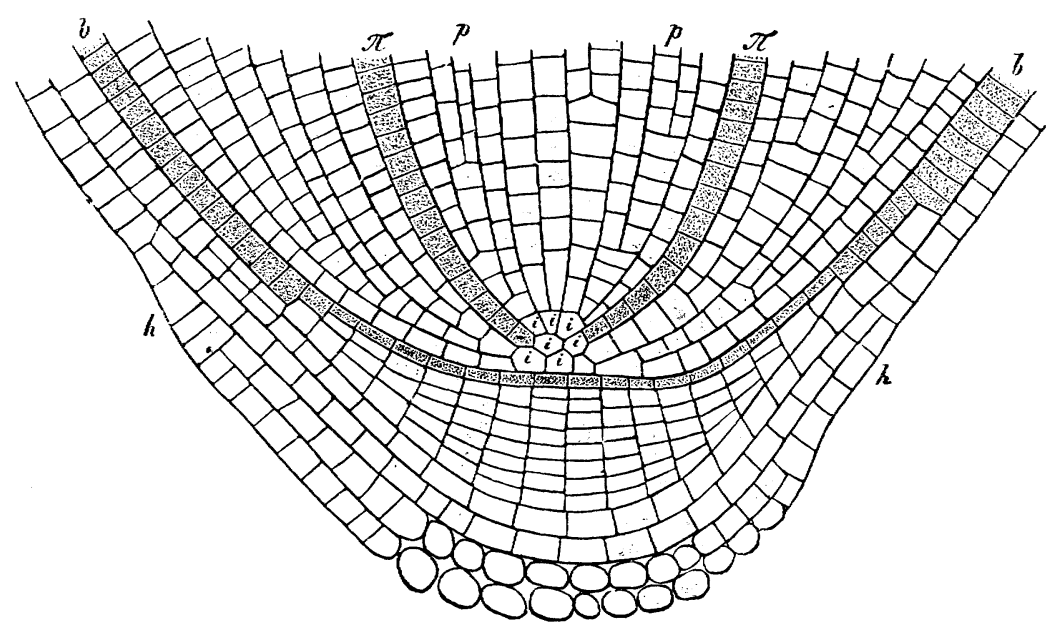

FIG. Ir4,-Longitudinal section through the apical region of the young root of the sunflower (after Reinke); $h h$ the root. cap; $b b$ (figured dark) the dermatogen; $\not p \not$ the plerome; its inner (dark) layer $\pi \pi$ the pericambium; between $\pi$ and $b$ lies the periblem; $i$ i the primary mother-cells, the source of the periblem and plerome.

in Cryptogams, becomes separated by tangential divisions into several layers, as is exemplified in Fig. I I 4 .

According to the description here given, which can only serve as an introduction to what follows for the student in a few examples, it might almost appear as if the processes in the growing point of Phanerogams were essentially different from those in Cryptogams, a hypothesis which I however do not accept. On the one hand the careful investigations of Nägeli and Leitgeb in Lycopodiacex (l.c.) on this point prove that in this family the significance of the apical cell in the production of the primary meristem is different from that in other Cryptogams, and approximates to what occurs in Phanerogams; and that, on the other hand, the apical cell of Cryptogams may, equally with the apical cellgroup of Phanerogams, be considered the starting-point of the first differentation of the layers of tissue. 


\section{MORPHOLOGY OF MEMBERS.}

SECT. 20. Distinction between Members and Organs ${ }^{1}$. Metamorphcsis. The parts of plants which are ordinarily termed their Organs-very various in their form, and serving different physiological purposes-may be considered scientifically from two different points of view. The question may be asked at the outset, How far are these parts adapted, by their form and structure, to perform their physiological work? In this case they are regarded from one side only, as instruments or organs, and this mode of regarding them belongs to physiology. In the other case these relationships may be completely put aside, and the question may be kept out of consideration what functions the parts of the plant have to fulfil, and the only point kept in view may be where and how they arise, that is in what manner the origin and growth of one member are related in space and time to those of another. This mode of regarding them is the morphological one. It is obvious that it is as one-sided as the physiological ; but investigation and description require, here as everywhere else in science, abstractions of this kind; and they are not only not hurtful, but even of the greatest assistance to investigation, if the investigator is only clearly conscious that they are abstractions.

In this chapter we shall concern ourselves exclusively with the morphological consideration of the parts of a plant. But before we proceed to a more minute investigation, it will be useful to get a somewhat more exact comprehension of the relationship between the physiological and the morphological view.

Morphological investigation has led to the result that the infinite variety of the parts of plants, which in their mature state are adapted to functions altogether different, may nevertheless be referred to a few Original forms, if regard is paid to their development, their mutual positions, the relative time of their formation, and their earliest states; that, for instance, the thick scales of a bulb, the membranous appendages of many tubers, the parts of the calyx and corolla, the stamens and carpels, many tendrils and spines, \&c., altogether resemble, in these respects,

1 Nägeli und Schwendener, Das Mikroskop. Leipzig I 867 , p. 599.-Hofmeister, Allgemeine Morphologie der Gewächse. Leipzig 1868, Sect. 1, 2.-Hanstein, Botanische Abhandlungen aus dem Gebiete der Morphologie u. Physiologie. Bonn 1870, Heft I. p. 85 . 
the green organs which have been termed simply leaves (foliage-leaves). All these structures are therefore also called Leaves (Phyllomes); and this designation is frequently justified by the fact that many of these organs actually become transformed, under peculiar conditions, into green leaves. Since the green organs which are termed leaves in popular language (foliage-leaves) may be considered as leaves par excellence, the other structures, which are also considered to be foliar, are termed metamorphosed leaves. The same is also the case with those parts to which the leaves are attached, and on which they grow as lateral appendages. They sometimes have the form of cylindrical or prismatic, slender, greatly elongated stems, sometimes of thick roundish tubers, or are often hard and lignified (trunks); in other cases they are soft and flexible, either embracing other firm bodies (bines), or firmly attached to them, as in the ivy; they may also occur as sharp spines or tendrils (grape-vine). All this is connected with the mode of life of the plant, and with the functions of the structures under consideration. But if the one characteristic only is kept in view, that they all bear leaves which arise below their growing apices, an agreement is found as important as complete, which may for the time be altogether abstracted from the physiological functions and the corresponding structure; and when once this abstraction is made, the agreement may be denoted by applying a common name to all those parts which bear leaves; they may be termed Stem-structures (Caulomes) or simply Axes. In the same sense therefore in which, for example, the tendril of a pea is a leaf, the tuber of a potato is also a stem or axial structure ; and just as the tendril of a pea is termed a metamorphosed leaf, so the tuber of a potato may also be called a metamorphosed stem.

The same is the case with hairs as with leaves and stems; the distinguishing characters of root-hairs, woolly hairs, prickles, glandular hairs, \&c., is that they all originate as outgrowths of epidermal cells. If we now go a step further, we may term all appendages of other parts which originate as outgrowths of epidermal cells, whatever their form and function, Hairs (Trichomes). Thus the so-called paleæ and the sporangia of Ferns are trichomes; or, if the ordinary filiform hairs are considered the original form, they are then metamorphosed hairs. It does not necessarily follow that hairs grow from a true epidermis; it is held sufficient if they arise from single superficial cells; and thus the number of the external appendages termed trichomes is still further increased.

As in the case of stems, leaves, and hairs, we may speak also of metamorphosed roots ; they are usually filiform, long, and slender, but sometimes thick and tuberous; usually they grow beneath the ground, but also sometimes above ground, and even in an upward direction. Nevertheless, under all circumstances roots maintain so striking a similarity to their typical forms, that the term metamorphosed is but seldom applied to them.

This mode of investigation, applied to Vascular Cryptogams and Phanerogams, has shown that all the organs of these plants may be referred to one of these morphological categories; every organ is either Stem (Axis), Root, Leaf, or Hair. The Muscineæ have no roots in a morphological sense, although they possess organs which completely fulfil the functions of roots; on the other hand most have leaves which grow on stems (axes). In Algæ, Fungi, and Lichens, the plant has 
generally appendages which may be termed hairs; but there are never any roots in the morphological sense, and the term leaf, as understood in higher plants, can no longer be rightly applied even in those cases where the external form of the mature parts is similar to the foliage-leaves of higher plants, e.g. Laminaria digitata, \&c. It is now agreed to apply to those vegetable structures in which the morphological distinction of stem and leaves cannot be carried out in the present state of our knowledge (and which have never any true roots), the morphological term Thallus or Thallome. In contradistinction to Thallophytes, all plants in which leaves can be morphologically distinguished might be termed Phyllophytes; the name Cormophytes has, however, been given in preference to them. From what has been said it will be seen that the thallophyte is only distinguished from a cormophyte by the lateral outgrowths which occur somewhere or other on it not presenting sufficient morphological distinctions from the part which bears them, to permit us to term them leaves in the same sense as in the more highly differentiated plants. But as the morphological distinctions of stem and leaf are not yet sufficiently established even in higher plants, it is impossible to draw a sharp boundary between Thallophytes and Cormophytes, and indeed it is certain that one does not exist.

If we now accept the terms Thallome, Stem (Caulome), Leaf (Phyllome), and Hair (Trichome) ${ }^{1}$, in the senses indicated, it can no longer be said that the leaf is the organ for this or that function; for leaves may undertake all possible functions; and the same remark applies also to the other parts. It is therefore on all accounts inexpedient simply to apply the term Organs to thallomes, stems, leaves, and hairs, for many of them have in fact no function at all. In order to avoid this mode of expression, which is confusing and foreign to morphology, it is obviously best to speak in this sense not of Organs, but of Members. The term Member is used when we speak of a part of a structure in reference to its form or position, and not to any special purpose it may serve. Thus, from a morphological point of view, stems, leaves, hairs, roots, thallus-branches, are simply members of the plant-form; but a particular leaf, a particular portion of the stem, \&c., may be an organ for this or that function, which it is the province of physiology to investigate.

The morphological nature of a member is best recognised in its earliest stages of development, and by its relative position in the series of processes of growth; the morphological definitions depend therefore essentially on the history of development.

The older a member becomes, the more obvious becomes its adaptation to a definite function, the more completely is its morphological character often lost. In their earliest states the members to which the same morphological term is applied (e.g. all the leaves of a plant) are extremely similar to one another; at a subsequent period all those distinctions arise which correspond to their different functions. We can now arrive at a definition of Metamorphosis which may be used in a scientific manner:-Metamorphosis is the varied development of members of the same morphological value resulting from their adaptation to definite functions.

${ }^{1}$ See Nägeli und Schwendener, Das Mikroskop, vol. II. p. 59I. 
(a) The conceptions of Stem, Leaf, Root, Trichome, as at present employed in botany, result from the examination of highly developed plants, the different members of which actually present considerable diversities, or display considerable differentiation; but if the attempt is made to apply these conceptions in the same manner to the less differentiated Hepaticæ, Algæ, Lichens and Fungi, many difficulties arise, depending principally on the fact that the members of the thallome sometimes display striking resemblances to leaves, hairs, stems, and even roots, while wanting others of their characteristics. Transitions occur from the members of Thallophytes which are but slightly differentiated morphologically to the highly differentiated members of Cormophytes. In the members which we term stem, leaf, root, hair, it is clear that those differences are only augmented which also occur, though in a lesser degree, in the more homogeneous ramifications of the thallome, especially of the higher Algæ; absolute distinctions between thallomes and leaf-bearing axes are not to be found. It is therefore a matter of convenience where the boundary-line is drawn.

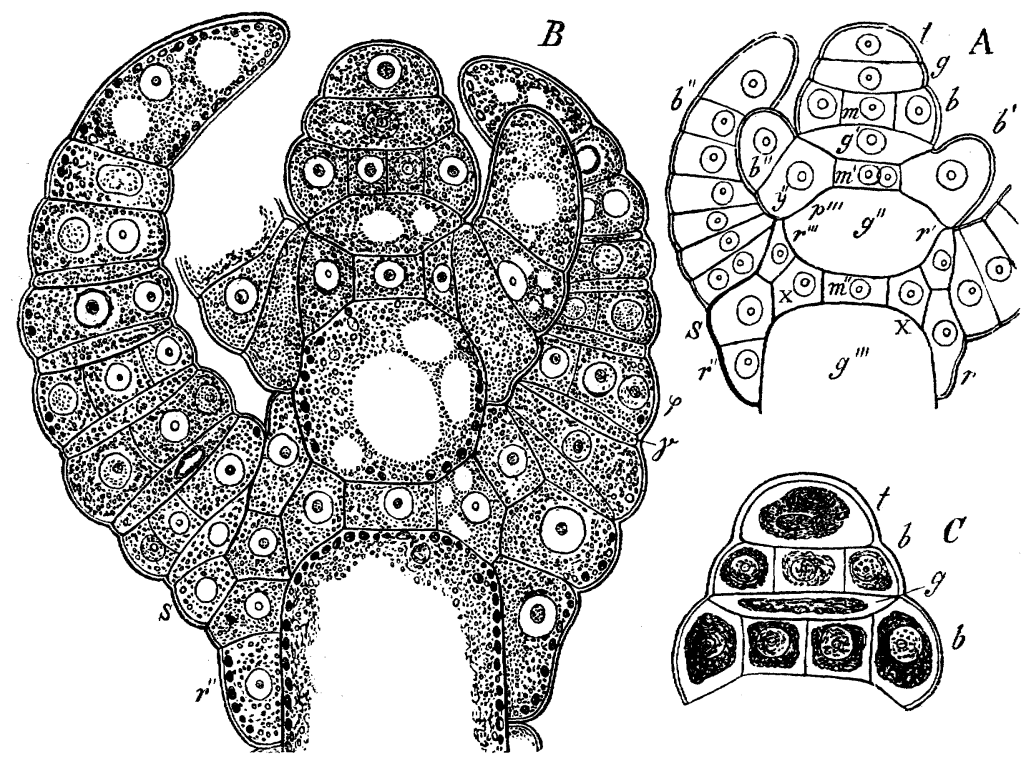

FIG. II5.-Longitudinal section through the apical region of three primary shoots of Charafragilis; $t$ the apical cell, in which segments are formed by septa; each segment being further divided by a curved septum into a lower cell which does not further divide and which developes into an internode $g^{\prime} g^{\prime \prime} g^{\prime \prime \prime}$, and an upper cell which produces a node $m m^{\prime}$ and the leaves. Each node cell produces a whorl of leaves of different ages. (For a more exact description, see Book II, Characeæ.),

(b) The expressions Thallome, Caulome, Phyllome, Trichome, Root, designate, as has been said, general ideas, from the definition of which are eliminated all those properties of the members which adapt them only for definite functions, while a few characters only, drawn from their origin and mutual position, are kept in view. Parts which are physiologically entirely different may therefore be morphologically equivalent, and, vice vers $\hat{a}$, physiologically equivalent organs may fall morphologically under quite different conceptions. The statement, for example, that the sporangia of Ferns are trichomes, means only that they originate, like all hairs, from epidermal cells; in this characteristic hairs and the sporangia of Ferns are morphologically equivalent. On the other hand the underground hairs of Mosses and the true roots of vascular plants are physiologically equivalent; both serve for the absorption of nourishment and the fixing of the plant in the ground, although the former are morphologically trichomes, the latter rcots. 
(c) General ideas, like those considered here and in the sequel, depend always on abstractions; they therefore necessarily want the practical clearness of the particular ideas from which they have been abstracted. How far the abstraction may be carried is more or less arbitrary; and the only correction for this lies in the scientific usefulness of the idea. Those ideas are the most useful which, from the greater precision of the definition, and from their greater clearness, include the greatest possible number of particular cases; for in this manner is that complete general comprehension of the phenomena most easily obtained which must precede a closer examination of them. The definitions in the following paragraphs are given from this point of view.

SeCT. 2r. Leaves and Leaf-bearing Axes ${ }^{1}$.-The members of the plant which are called Leaves (Phyllomes) in Characeæ, Muscineæ, Vascular Cryptogams, and Phanerogams, are related to the axis or stem from which they are derived in the manner described in the following paragraphs.

(I) The Leaves always originate below the growing apex of the stem as lateral outgrowths, either singly, or several at the same height, i.e. at an equal distance from the apex; in the latter case they form a whorl, the single leaves of which may differ in age, as in Chara and Salvinia, and in the whorls of many flowers.

(2) So long as the apex of the shoot continues growing in a straight line, and the portion of the shoot which produces leaves lengthens, the leaves arise in acropetal order; i.e. the nearer the leaves are to the apex, the

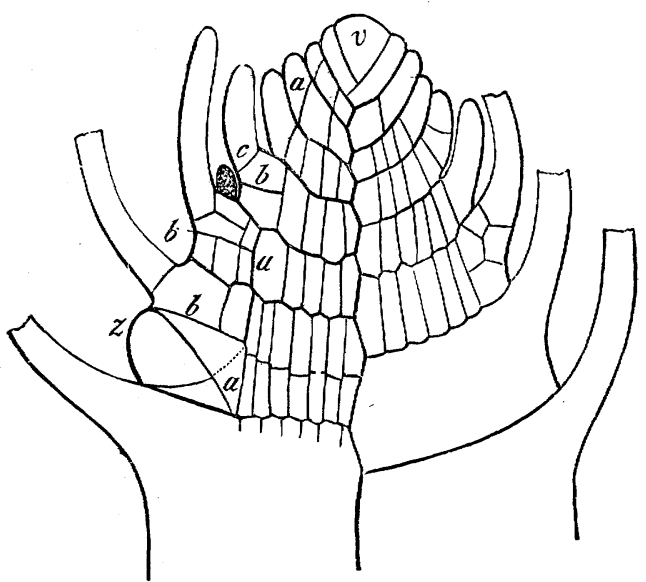

FIG. 116.-Longitudinal section through the apical region of a stem of Fontinalis antipyretica, an aquatic Moss (after Leitgeb); $v$ the apical cell of the shoot, producing three rows of segments which are at first oblique and afterwards placed transversely (distinguished by a stronger outline). Each segment is first of all divided by the septum $a$ into an inner and an outer cell; the former produces a part of the inner tissue of the stem, the latter the cortex of the stem and a leaf. Leaf-forming shoots arise beneath certain leaves, a triangular apical cell $z$ being formed from an outer cell of the segment, which then, like $v$, produces three rows of segments; and each segment here also forms a leaf. (For a more exact description see Book II, Mosses.)

younger they are. In this case leaves are never produced further from the apex than those already in existence. It is only when, as not unfrequently happens with the flowers of Phanerogams, the growth in length of the shoot ceases or becomes weaker at the apex, while, at the same time, active growth continues in a transverse zone or place beneath the apex, that new leaves can arise between those already in existence ${ }^{2}$.

(3) The Leaves always originate from the Primary Meristem of the Growing

1 Nägeli u. Schwendener, Das Mikroskop. Leipzig 1869, p. 599 et seq.-Hofmeister, Allgemeine Morphologie der Gewebe. Leipzig 1868, Sect. 2.-Pringsheim, Jahrb. für wissen. Bot. vol. III. p. 484.-Ditto on Utricularia, in Monatsber. der Berliner Akad., Feb. I869.-Hanstein, Bot. Abhandlungen, Bonn 1870, Heft I.-Leitgeb, Botan. Zeitg. 187 I, no. 3.-Warming, Recherches sur la ramification des Phanérogames. Copenhagen, I 872 , p. vi.

${ }^{2}$ Since phenomena of this kind are confined to the flowers and inflorescence of Phanerogams, their consideration may for the time be postponed. 
Point, never from those parts of the stem which already consist of fully differentiated tissues. In Characeæ, Muscineæ, \&c., before or during the first divisions of their segments, the leaves become visible close beneath the growing point as protuberances, the outer portion constituting an apical cell, from the segments of which a leaf is built up. In Vascular Cryptogams a many-celled cone of growth often overtops the youngest rudiment of a leaf, as in strong Equisetum buds, Salvinia, many Ferns and Selaginelleæ. In Phanerogams (Figs. I I 7, I 18,119 ) this is general ${ }^{1}$; in them the rudiment of the leaf does not begin with an apical cell projecting from the cone of growth, as in Cryptogams, but a rounded or broad cushion is formed, which from its very first origin consists of numerous small merismatic cells.

(4) The Leaves are always Exogenous Formations, i.e. the rudiment of the leaf never has its origin exclusively in the interior of the tissue of the stem, and is never covered by layers of tissue of the stem which take no part in its formation,

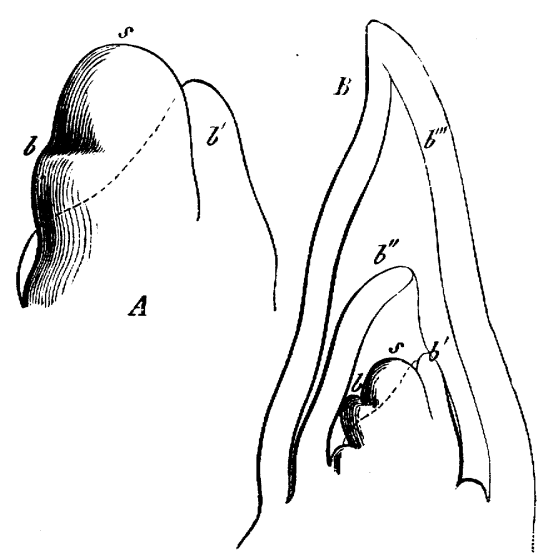

FIG. II7.-Terminal region of two primary shoots of maize. A pex of the very small-celled cone of growth, out of which the leaves $b, b^{\prime}, b^{\prime \prime}, b^{\prime \prime \prime}$ arise as multicellular protuberances, which soon embrace the stem, and envelope it and the younger leaves like a sheath. In the axil of the third youngest leaf $b^{\prime \prime}$ the young. est rudiment of a branchlet is visible as a roundish protuberance.

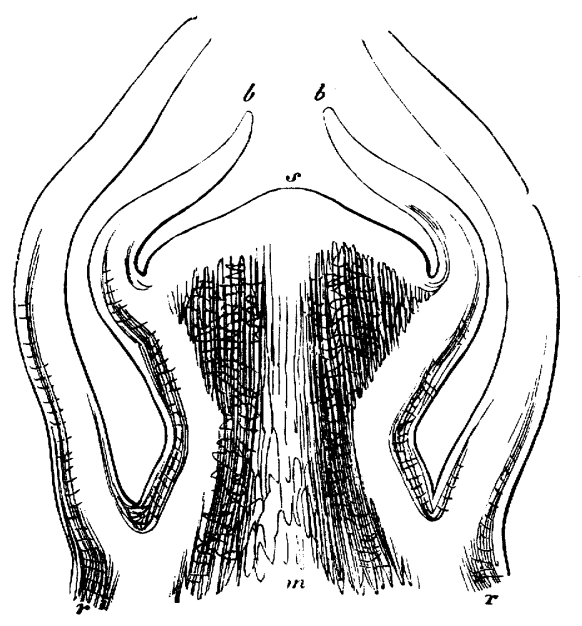

FIG. I18.-Longitudinal section through the apical region of the primary stem of the sunflower, immediately before the formation the flowers; $s$ apex of the broad growing point; $b b$ the youngest leaves ; $r$ cortex ; $m$ pith.

as is the case with roots and many endogenous shoots. In Cryptogams it is usually a single superficial cell (i.e. superficial before the differentiation of the epidermis) which forms the foliar protuberance. In Phanerogams a mass of tissue bulges out as the rudiment of the leaf, and consists of a luxuriant growth of the periblem covered by dermatogen (Sect. 19, Fig. I13, p. 147). By this means the leaf is at once distinguished from the hair even in its most rudimentary state. The hair is an outgrowth of the epidermis; but since in Phanerogams the primordial epidermis or dermatogen covers the whole of the growing point above the leaves, hairs may also spring up higher in position than the youngest leaves, from single cells

1 [Warming however remarks (Ramification des Phanérogames, p. iii) that the growing point may have the most various forms, from that of a rather acute cone, as in Gramineæ, Amaranthus, and Plantago, to that of a cup-shaped depression, e.g. Digitalis, and that the form may differ even in species belonging to the same genus; thus in Digitalis lutea it is convex, in D. parviflora concave.] 
belonging to the dermatogen, as in Utricularia according to Pringsheim. But in Cryptogams the dermatogen becomes differentiated only after the formation of the leaf; and hence the hairs are always at a greater distance from the apex than the youngest leaves (Fig. I16); the superficial cell of the stem, which in Cryptogams becomes the apical cell of a new leaf, is not an epidermal cell, since its origin dates long before the differentiation of the tissue into epidermis and periblem.

(5) The Tissue of the mature Leaf is continuous in its formation with that of the Stem. It is impossible, histologically, to find a boundary line between the stem and the base of the leaf, although such a boundary line must be assumed theoretically. If the surface of the stem is imagined to be continued through the base of the leaf, the transverse section thus caused is called the Insertion of the Leaf.

The continuity of the tissue is especially observable in vascular plants, where the well-developed leaves ${ }^{1}$ consist, like the stem, of epidermal and fundamental tissues and fibro-vascular bundles. The cortical layers of the stem bend out without interruption into the leaf, and constitute its fundamental tissue; in the same manner the epidermis passes over from the stem into the leaf; the fibro-vascular bundles of the leaves have, in Phanerogams and many Cryptogams, the appearance of being the upper ends of the 'common' bundles which ascend in the stem (Fig. I19); and where this is not the case, as in Lycopodiaceæ, the basal portions of the foliar bundles and the fibro. vascular mass of the stem are nevertheless in continuity.

The main cause of the continuity of tissue between stem and leaf is that the

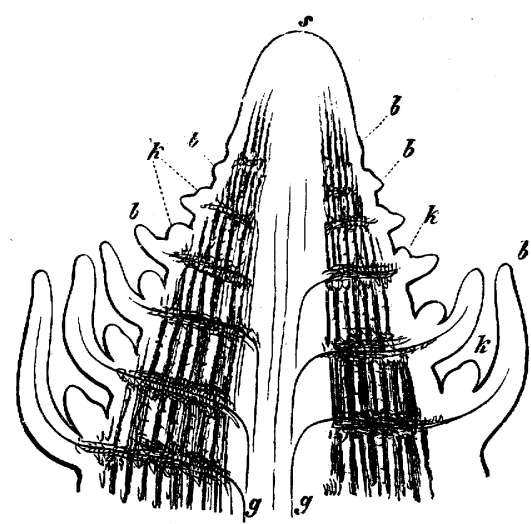

FIG. Ix.-Longitudinal section through the apical region of an upright shoot of Hippuris vulgaris; $s$ apex of the stem; $b, b, b$ the verticillate leaves; $k k$ the buds in their axils, which all develope into flowers; $g g$ the first vessels (the dark parts of the tissue indicate the inner cortex with its intercellular spaces).

leaf arises from the cone of growth of the stem, where it still consists entirely of primary meristem; in vascular plants the young leaf appears as a luxuriant development of its outer layers (the dermatogen and inner layers of periblem, see Sect. 19). And as vascular bundles (at first in the form of procambium) become differentiated in the central tissue of the stem or plerome, similar bundles also appear in the tissue of the growing leaf, in such a manner that the two are in connection with one another. This connection may be such that the foliar bundles appear as the upper prolongations of those of the stem; thus arise the 'common' bundles of Phanerogams, the portion that runs through the stem being termed the Leaf-trace (see Sect. 18). But in some Vascular Cryptogams, as Lycopodiaceæ and Equisetaceæ, the procambium bundles which are differentiated in the tissue of the young leaf are so connected with the young fibro-vascular

1 Leaves which wither early, or which persist as small scales, like all the leaves of Psilolum, and many small leaf-scales of Phanerogams, have no fibro-vascular bundles. 
bundles of the stem, that although they are actually continuous, their independent origin is still easily seen in radial longitudinal sections through the apices of young stems. In both cases the development of the first vessels usually commences in the region where the bundle that bends out into the leaf unites with that of the stem. In addition to the Common Bundles formed in this way, fibro-vascular bundles may also arise in the stem, as has already been described in Sect. 18, which belong to it alone, and are therefore termed Cauline Bundles. Cauline bundles may be formed at an early period of the growth of the stem, as in Piperaceæ, Nyctagineæ, \&c., or only as a consequence of increase in thickness, when the leaves have already long been developed, or have even fallen off, as in Aloïneæ, Menispermaceæ, \&c. (see Sect. I8).

(6) The Leaves usually grow more rapidly in length than the parent shoot above their insertion (Figs. $116, I_{7}, 118$ ). If the leaves are formed rapidly one after another, they envelope the end of the shoot, and thus form a Bud, in the centre of which lies the leaf-forming growing point. This production of a bud depends also on the more rapid growth of the outer or under side of the leaves in their young state, by which they become concave on the inner (afterwards the upper) side, and adpressed to the stem. It is only by the extension of their tissue that the leaves ultimately turn outwards in the order of their age, and thus escape from the bud. If the portion of the stem between the insertions of the leaves undergoes at the same time a considerable extension, the leaves then become placed at a distance from one another, and a shoot results with elongated internodes. In such cases the section of the stem in which the leaf-insertion lies usually developes in a different manner from the intermediate portions; these zones are termed the Nodes, the intermediate portions the Internodes, as in Characeæ, Equisetaceæ, and Grasses. If the stem remains entirely undeveloped between the nodes, it possesses no proper exposed surface, but is entirely covered by the leaf-insertions, as in Nephrodium Filix-mas; but more commonly this is only apparently so from the internodes being very short, as in many palm-stems. The internodes may be present immediately after the first formation of the leaves, when the consecutive leaves or leaf-whorls appear at considerable distances in height from one another, as in Chara ${ }^{1}$ and Zea (Fig. II 7 ); or they may originate only after further development of the stem-tissue, as in Mosses (Fig. I I6) and Equisetacex, where every segment of the apical cell of the stem forms a rudiment of a leaf, so that the leaf-rudiments follow immediately one after another; and it is only by further cell-formation, growth, and differentiation that the lower portions of the segment become developed into the exposed portions of the surface of the stem; as is clearly shown in Fig. I 6 . The formation of a bud in the way described above does not take place when on the one hand the leaves are developed very slowly one after another, or on the other hand when the stem grows rapidly in length between the youngest leaf-rudiments or even before the appearance of the youngest, so that there is always only one slightly developed leaf near the apex, as in the underground creeping shoots of Pteris aquilina (see Book II, Ferns).

${ }^{1}$ I consider in Chara, as in Muscinex, and in fact universally, that the cortex belongs originally to the stem, and not to the leaf. 
(7) Every Leaf assumes a form different to that of the Stem which produces it, and to that of its lateral Shoots. This is usually so conspicuous that no further description is needed. Nevertheless one point must be mentioned which often causes difficulty to the student. It not unfrequently occurs that lateral shoots of certain plants present a great similarity in form and physiological properties to the foliageleaves of other plants, as the flat lateral shoots (phylloclades) which bear the flowers in Ruscus, Xylophylla, Mühlenbeckia platyclada, \&c. ; but the course of development shows that these apparent leaves must, from their position, be lateral shoots, themselves producing leaves; and the leaves of these plants are usually of quite a different form from these leaf-like branches. The phrase 'leaf-like' has in these cases usually no distinct morphological, but only a popular meaning; and what will be said under paragraph (8) may be applied here. The branches or leaf-bearing lateral shoots arise in very different ways in different plants; but very commonly they have this in common with leaves,-that they originate also as lateral and exogenous outgrowths in the primary meristem of the growing point; that they are formed; like the leaves, in acropetal succession; and that the differentiation of their tissue proceeds continuously with that of the primary shoot. They are distinguished, however, from the leaves of the same plant by their place of origin, by their much slower growth-at least at first (later they may overtake the leaves), -and by their relations in point of symmetry, of which we shall speak hereafter. The leading fact, however, is that the lateral shoot repeats in itself, by the formation of leaves, all the relations hitherto named between leaf and stem, and is therefore a repetition of the primary shoot, although in other physiological characters it may differ greatly from it.

(8) The morphological conceptions of Stem and Leaf are correlative; one cannot be conceived without the other; Stem (Caulome) is merely that which bears Leaves ; Leaf (Phyllome) is only that which is produced on an axial structure in the manner described in paragraphs $(\mathbf{I}-7)^{\mathbf{1}}$. All the distinguishing characters which are applicable to the definition of Caulome and Phyllome express only mutual relationships of one to the other; nothing is implied as to the positive properties of either. If we compare together all the structures which we call leaves without reference to the stems to which they belong, we shall be unable to find a single characteristic which is common to them all and which is wanting in all stems ${ }^{2}$. But that which is common to all leaves is their relation to the stem. Hence the ideas Phyllome and Caulome cannot be obtained by comparing together the positive properties of leaves or the positive properties of stems, or by laying stress on the points which they have in common and on those wherein they differ; but these ideas

1 There are, for instance, thallomes strikingly similar to certain leaf-forms, as those of Laminaria, Delesseria, \&c.; they are, however, not leaves, since they are not formed on a stem as lateral structures.

${ }^{2}$ [Warming (Ramification des Phanérogames, p. xvii) remarks that while it is impossible to find constant characters for separating phyllomes from caulomes, they spring from the peripheral tissue at slightly different depths. Phyllomes originate in the superficial layers of the periblem, from the first to the third; feebly developed foliar organs, such as bracts, even in the first layer alone. Caulomes scarcely ever originate in the first layer, but usually in the third or fourth. Warming attributes this to the necessity for the largest structure to have the deepest origin.] 
are obtained by observing leaves exclusively in their relation to the stem which produces them, and stems in relation to the leaves produced from them. In other words, the expressions Stem and Leaf denote only certain relationships of the parts of a whole-the Shoot; the greater the differentiation, the more clearly are Stem and Leaf distinguished. The measure of the difference is usually arbitrary; but if we confine ourselves to those plants to which the term leaf is applied in ordinary language, the distinction of leaves from stem depends on the relationships named in paragraphs $(\mathbf{I}-7)$; and in this sense certain lateral outgrowths in some Algæ may be termed Leaves, and the axial structures which produce them Stems (e.g. Characeæ, Sargassum). But when the difference between the outgrowths and the axial structures which produce them is less, one or more of the relationships named in paragraphs $(\mathrm{I}-7)$ disappear, and it becomes doubtful whether the expressions Leaf and Stem ought still to be used; and when finally the similarity preponderates, the whole shoot is no longer called a Leafy Stem, but a Thallome. A branched thallome has the same relation to a leaf-bearing stem as a slightly differentiated to a highly differentiated whole.

The external differentiation of the members of the shoot into Stem and Leaf is to a certain extent independent of the internal differentiation which brings about the different forms of tissue and the cell-divisions, as is shown in the comparison of Muscineæ and Characeæ with Phanerogams. The internal segmentation may be reduced to a minimum of cell-divisions, or may altogether disappear; in the latter case the single cell represents a shoot, the lateral outgrowths of which behave as leaves and the axial part as stem, as, for example, in Caulerpa amongst Algæ. What has already been said as to the continuity of the tissue of stem and leaf and their common origin from the primary meristem, must here be understood in an extended sense. In place of the primary meristem we have the growing point of a single cell continuing its growth, and instead of the differentiation of tissue the development of the older part of the cell-wall and of its contents. Caulerpa consists of a single cell, which grows like a creeping stem and puts out lateral leaf-like protuberances and tubular hairs which even perform the function of roots, the whole enclosing a continuous cell-cavity without partition-walls ${ }^{1}$.

(a) The leaves, like the shoots, grow at first at the apex, i.e. at the end opposite the place of their origin. This apical growth continues indefinitely in many thallomes and leaf-bearing axes until checked by some external cause; this is especially the case in the primary shoots of Fucacex, pleurocarpous Mosses, Characex, the rhizomes of Equisetaceæ, Ferns, and the primary stems of Coniferæ and of many Angiosperms. If the primary shoots themselves bear organs of reproduction, the apical growth generally ceases with their development, as in many acrocarpous Mosses, the fertile stems of Equisetacex, the haulms of grasses which bear the inflorescence, and in all cases in Angiosperms where a primary shoot ends in a flower. The lateral shoots are usually of limited growth; the growth frequently ceases without any external cause, more especially when they bear reproductive organs, or become transformed into spines, or are very different in their shape from the primary shoot, as the horizontal lateral branchlets of many Coniferæ, the leaf-like shoots (phylloclades) of Phyllocladus, Xylophylla, Ruscus, \&c.

${ }^{1}$ See Nägeli, Zeitschrift für wissenschaftliche Botanik, and Neuere Algensysteme. 
In by far the greater number of leaves the apical growth ceases early, the apex itself becoming transformed into permanent tissue. In Ferns, however, the apical growth of the leaves usually continues, and in many genera is even unlimited, the apex of the leaf always remaining capable of development, and not becoming transformed into permanent tissue, as in Nephrolepis; in Gleichenia, Mertensia, Lygodium, and Guarea, the growth of the apex of the leaf is, as in many shoots, periodically interrupted, and again renewed in each period of growth.

(b) Besides the apical growth, there always exists, however, both in stems and in leaves, an interstitial growth, by which the parts produced by the apical growth increase in size and become further developed. The development of the internodes of the stem depends almost exclusively on this interstitial growth, as indeed is shown by the crowded position of the leaves and the shortness of the internodes in the bud; it is generally at first very rapid, and the increase in size occasioned by it is often very considerable; but it usually soon ceases, and the tissues become differentiated into unchanging permanent forms. Not unfrequently, however, a basal zone of the internodes (as in Grasses, Equisetum byemale, \&c.), and in many cases the base of the leaf also, remains for a long time in a condition capable of development, while the parts nearer to the apex, long since transformed into permanent tissue, have attained their full growth. In this manner a secondary basal increase in length, often continuing for a long time, is occasioned in parts which have long ceased to grow above; this occurs in a peculiarly marked manner in the long leaves of many Monocotyledons (Grasses, Liliaceæ, \&c.) which are sheath-like in their lower part, and to a smaller degree in many Dicotyledons (e.g. Umbelliferæ). Where, as in Ferns, and in a lower degree in many pinnate leaves of Dicotyledons, the apical growth long remains active, the basal interstitial growth usually soon ceases, and, vice vers $\hat{a}$, continues the longer the earlier the apical growth comes to an end. Two extreme cases may therefore be distinguished in leaves, although closely connected by intermediate forms; the predominantly apical and the predominantly basal growth.

If the interstitial growth continues at one part of the surface of the leaf, and attains there a maximum which then decreases, a bag-like projection of the surface of the leaf is formed, which is termed a Spur, such as occurs in many petals, as Aquilegia, Dicentra, \&c.

(c) Before the tissues which are differentiated from the condition of primary meristem assume their definite forms, a rapid growth usually takes place in their cells, which is no longer accompanied by cell-division; the size of the cells is not unfrequently increased by this means ten or even a hundred-fold and more. This process, which is mainly dependent on the rapid increase of the watery sap, may be termed Extension, in contradistinction to the growth of the younger cells which is connected with their divisions, and which always precedes the extension. On this extension depends the rapid unfolding of the parts of the bud, which had long before assumed their main outlines, but had remained small. The buds very often remain a long time in a condition of rest, until a rapid unfolding of the leaves and internodes already formed suddenly takes place; as, for instance, in the germination of many seeds, and in the persistent buds of many trees (horse-chestnut), bulbs (tulip), and corms (crocus, \&c.), formed in the summer and germinating in the spring after long rest in winter.

(d) The Axis of growth or of length of a member (as will further be shown in a special paragraph) is an imaginary line passing from the centre of the base to the apex. The entire growth both of leaves and of stems is usually most rapid in the direction of this line; they are therefore for the most part longer than they are broad or thick. In stems the growth is most often nearly equal along all diameters; they assume therefore cylindrical, prismatic, or bulbous rounded forms. It is, however, sometimes the case that the growth in length advances much more slowly than that in diameter; and then the stem becomes tabular or flat, as in many bulbs, the corm of the crocus, and especially in Isoëtes. It is only in the. lateral shoots of higher plants which have a very limited 
growth that the internodes expand in a plane which also includes the axis of length, and thus become leaf-like, as in Ruscus, Xylophylla, \&c.

In leaves the principal growth is usually in a plane which cuts the stem transversely, and is mostly symmetrical right and left of a plane which includes the axes of length both of the leaf and the stem; the common form of leaves is therefore that of thin plates symmetrically divided in half in the direction of their length. There occur, however, cylindrical and roundish tuber-like leaves, in which the growth has been nearly equally rapid in all diameters at right angles to the axis of the leaf, as in Mesembryanthemum ecbinatum ${ }^{1}$.

Sect. 22. Hair (Trichome) ${ }^{2}$ is the term given in the higher plants to those outgrowths which arise only from the epidermis, i.e. from the layer of cells which always remains the outermost in roots, stems, and leaves, whether these outgrowths assume the form of simple tubular protuberances, rows or plates of cells, or masses of tissue, or have the physiological character of woolly envelopes of the young leaves, root-like absorbing organs as in Muscineæ, glands, prickles, or sporangia as in Ferns ${ }^{3}$.

Hairs may originate from the primary meristem of the growing point, or from young leaves and lateral shoots, if an external layer of cells has already been differentiated as dermatogen, as in Phanerogams; but they may originate also in much older parts the tissue-systems of which have already become further differentiated, and which exhibit interstitial growth, because in such cases the epidermis produces new cells, for example stomata, and long remains capable of cell-division.

When hairs spring from the growing point, they are usually formed after the leaves, i.e. further from the apex than the youngest leaves; but it also occurs in Phanerogams that they are developed above the youngest leaves and nearer to the apex, the outermost layer of cells of the growing point having in this case already become differentiated as dermatogen, as in Utricularia according to Pringsheim. In Muscineæ and Vascular Cryptogams also, where the leaves become visible long before the differentiation of the external layers of tissue, the hairs do not appear on the surface of the stem till a later period and further from the apex.

If the hairs arise near the apex of a growing point or on a zone of interstitial basal growth, as do the sporangia of Hymenophyllaceæ, they may be arranged according to a definite law, which is not the case with hairs that spring from older organs, or at least not evidently so.

Hairs are always strikingly different in their form from the leaves and lateral shoots of the same plant, although they sometimes bear a certain resemblance to these organs in other plants. The development in size of a single hair is usually extremely small compared to that of the member which produces it; even the mass of all the hairs of a leaf, root, or stem is generally quite inconsiderable compared to the weight of the organ.

${ }^{1}$ [The leaf is also frequently unsymmetrical, i.e. the growth has not been equally vigorous of the two halves separated by the axial plane, as in the lime, Begonia, \&c.]

${ }^{2}$ Rauter, Zur Entwickelungsgeschichte einiger Trichomgebilde. Vienna I871, p. 33.-Compare also Sects. 15 and 19 (b).-Warming, Sur la différence entre les trichômes et les epiblastêmes, d'un ordre plus élevé (extract from the Videnskabelige Meddelelser de la société d'Hist. Nat. de Copenhague, nos. IO-1 2,1872 .

${ }^{3}$ [Hairs may develope into adventitious buds, as in Begonia; see Caruel, Trasformazione di peli in gemme, Nuov. Giorn. Bot. Ital., July 1875.$]$ 
(a) The woolly and glandular hairs on buds are distinguished by a remarkably rapid growth; they are often perfectly formed long before the parts of the bud unfold, but then they generally die off; the persistent hairs which remain during the life of the leaves are formed much more slowly, and are marked by a great variety of form. The root-hairs are formed at a considerable distance from the growing point of the root, often from I to $2 \mathrm{~cm}$. from the apex, and mostly die off after a few days or weeks, so that the older parts of the roots of even annual plants are destitute of living hairs. The existence of these hairs is connected with the activity of the roots in the ground.

The root-hairs which spring from the stems of Mosses are marked by a very long continued apical growth, and often by repeated branching. They consist of cells divided into rows by oblique septa, and, viewed physiologically, replace the root-system of vascular plants. These root-hairs of Muscineæ are remarkably endowed with generative power, and behave in many respects like the Protonema, a means of propagation peculiar to Muscineæ; like it, they produce gemmæ, which, when exposed to light, grow into leafy stems. If the root-hairs themselves are exposed to the air (e.g. by turning up a sod) they put out rows of cells containing chlorophyll, on which also gemmæ are produced.

(b) Thallophytes, when they consist of a mass of tissue, also form true hairs, like Cormophytes; but when the thallome consists only of one layer of cells, or, like Caulerpa and others, is unicellular, one can no longer speak of an external layer corresponding to the epidermis; and its hair-like outgrowths cannot therefore be considered as trichomes in the same sense as those of the higher plants. Nevertheless it is customary to speak in such cases also of hairs, when the outgrowths are long and slender, destitute of chlorophyll, and otherwise dissimilar to the thallus which produces them. On the other hand structures occur in highly organised plants which are closely analogous to many forms of hairs in their physiological, and partly also in their morphological properties, but which differ from true hairs in not originating from single epidermal cells, but consist of outgrowths of the tissue which lies beneath the epidermis, remaining however covered by a continuation of it. Examples of such structures, which may perhaps be distinguished by the term Emergences, are afforded, according to Rauter, by the prickles ${ }^{1}$ and glandular hairs of roses, and perhaps also of the various species of Rubus. Closely related to these are probably the warts, tubercles, and knobs on the surface of many fruits (according to Warming, for example, on the fruit of Datura Stramonium, and, according to my own observations, on that of Ricinus). To the same category belong the 'beards' of many petals (according to Warming, e.g. those of Menyantbes trifoliata); the 'tentacles' on the leaves of Drosera, the sharp hairs beneath the calyx of Agrimonia Eupatorium, the pappus of Compositæ, \&c. Larger emergences of this nature may even be penetrated by branches of the vascular bundles from the organs which produce them, as in Drosera, Datura, \&c. They resemble the leaves and branches of Phanerogams in their origin and mode of formation, while they agree with hairs in the late period at which they are produced, their occurrence on stems and leaves, and their frequently irregular distribution both as respects one another and the organ on which they grow. The classification adopted by Warming (l.c. p. 27), viz. including emergences under the term trichome, and dividing this class of structures into two sub-classes, hairs and emergences, seems to me, if not false, at all events inconvenient; because it becomes impossible to give any exact definition to the term trichome. The fact that emergences constitute a transition between trichomes, in the stricter sense of the term, and leaves or secondary axes, does not justify including them under the former term; they might as well be treated as branches of leaves or of stems. If the occurrence of transitional structures were held to prevent our distinguishing certain groups of members sharply from one another, then the distinction must be abandoned between phyllome and caulome, or

1 On spines, which must not be confounded with prickles, see Sect. 28. 
between caulome and root, since transitional structures occur also in these cases. The occurrence of these transitional structures in nature is in itself a reason for framing exact definitions. Definitions are not in themselves objects; but are means for arranging objects, and for enabling us to understand them.

Sect. 23. The term Root $^{1}$ is applied, in botanical morphology, in contrast to its use in popular language, only to such outgrowths of the substance of the plant as are clothed at their growing apex with the Root-cap already described in Sect. 19. Roots do not form leaves or other exogenous foliar structures; their epidermal cells, on the contrary, generally develope into long tubular appendages, the Ront-hairs. The apex of every root which is just beginning to be formed lies beneath the surface of the organ from which it proceeds ${ }^{2}$; it is then usually

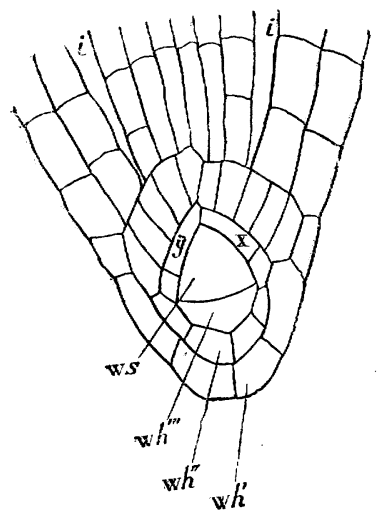

FIG. r20.-Longitudinal section through the young primary root of the embryo of Marsilea salvatrix; ws the apical cell, wh', wh', wh' the still simple layers of the root-cap; $x, y$ the last segments of the substance of the root; $i$ i intercellular $\mathbf{s}$ aces.

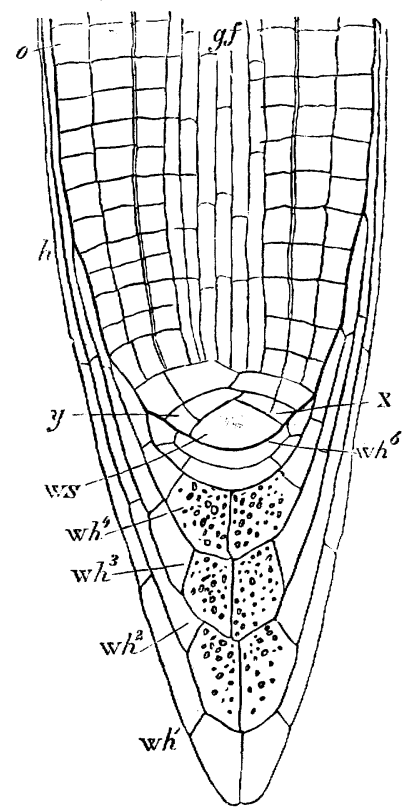

FIG. 12r.-Longitudinal section through a somewhat older primary root of Marsilea salvatrix; ws the apical cell; wh1 $w h^{2}$ the first, wh3 3 z $h^{4}$ the second, wh6 the third layer of the root-cap, each layer now consists of two divisions; $x y$ the youngest segments of the substance of the root; 0 epidermis; $g f$ fibro-vascular bundle; $h$ the part of the root-cap which extends furthest back.

covered with thick layers of tissue, which it breaks through in its further growth. Hence roots are always endogenous formations, by which character they are distinguished from all trichomes and leaves, and from most lateral shoots.

1 Nägeli und Leitgeb in Nägeli's Beiträgen zur wissen. Bot., Heft IV, 1867.-Hofmeister, Morphologie der Gewebe. Leipzig 1868, Sect. 5--Hanstein, Botan. Abhandlungen. Bonn 1870, Heft I.-Dödel, Jahrb. für wiss. Bot., vol. VII. p. I49 et seq.-Reinke, Wachsthumsgeschichte der Phanerogamenwurzel, in Hanstein's Botan. Untersuchungen, Heft III. Bonn I87I.-Van Tieghem, Recherches sur la symétrie de la structure des plantes vasculaires, Fasc. I, La racine, Paris I871 ; (also in Ann. des Sci. Nat., 5th ser., vol. XIII, I87I.)

2 I choose this expression because it appears also to fit the primary root of the embryo of Vascular Cryptogams. 
Roots occur only in those plants the tissue of which is traversed by fibrovascular bundles, and they themselves therefore always contain fibro-vascular bundles; but these latter differ from those of the stem and leaves in the first vessels being formed near the circumference of the bundle, while the later ones are always formed further inside, and hence centripetally in reference to the diameter of the root. The phloëm-bundles lie in the intervals between the

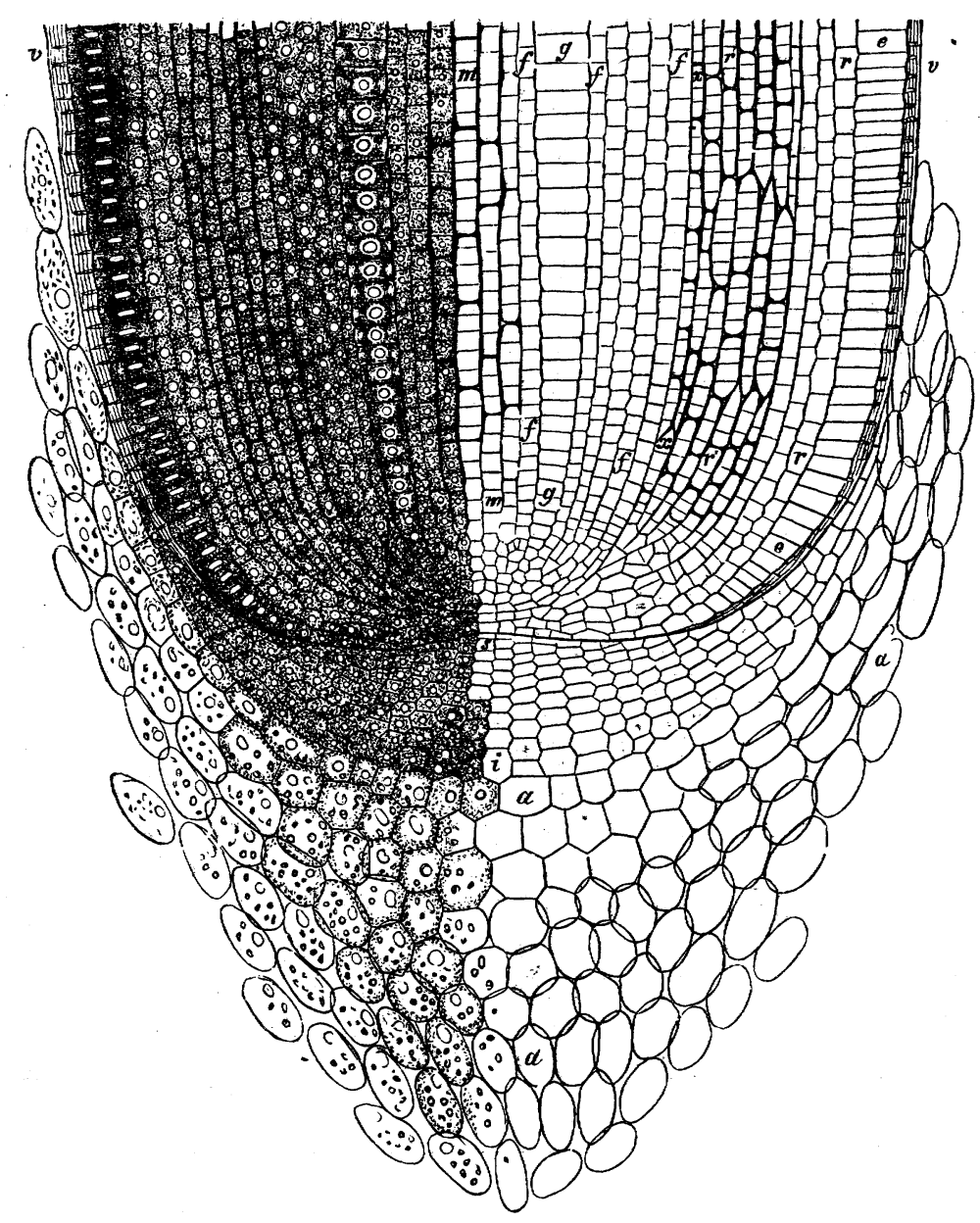

FIG. 122.-Longitudinal section through the apex of a root of maize; $a$ a outer and older layers of the root-cap; $i t$ intrer and younger layers; $s$ apex; $m g f$ the plerome; $m$ becomes the pith, $g$ a vessel, $f$ xylem; $x r$ the cortex which is producet from the periblem at the apex; $e$ e epidermis, continued into the dermatogen at the apex; $v v$ thickened outer wall of the epidermis (cuticle); (the origin of the root-cap from the dermatogen is not evident here; the figure was drawn long before this discovery).

primary vascular bundles at the circumference of the fibro-vascular cylinder (see Sects. $16,18$.

Although roots are commonly present in vascular plants, i. e. the higher Cryptogams and all Phanerogams, there occur even in these groups particular species from which they are entirely absent. Thus among Rhizocarpeæ the genus Salvinia, 
among Lycopodiaceæ the genus Psilotum, among Orchideæ Epipogum Gmelini and Corallorhiza innata, are destitute of roots; the little Lemna (Wolffa) arrhiza does not form roots, and is at the same time destitute of vascular bundles.

With reference to the place of their formation roots are remarkably variable. A root is usually present even in the young embryo which proceeds from the fertilised ovule (but not in Orchidex); it appears at the posterior end of the embryonal stem, and may be termed the Primary Root, whether it remains weakly and soon dies, as in Cryptogams and Monocotyledons ${ }^{1}$, or whether it continues to grow more vigorously, like the rest, as in many Dicotyledons. But besides the primary roots, there are usually formed in addition a large number of Secondary Roots, or simply Roots; since they are enormously more numerous than the primary roots, and of much greater importance to the plant, a special name is superfluous where the contrast to the primary root is not of importance. They arise in the interior of the primary or secondary roots, and on stems and petioles. The primary root with its secondary roots, or any root with its lateral roots, may be termed a Root-system. With the exception of many Dicotyledons with a persistent strongly developed primary root, the majority of roots spring from stems, especially when these latter creep, float, climb, or form bulbs or tubers. In Tree-ferns the stem is often densely covered throughout its whole length with a felt of delicate roots. In Ferns with densely crowded leaves in which no portion of the surface of the stem is left bare, the roots spring exclusively from the petioles, as, for example, in Nephrodium Filix-mas, Asplenium Filix-fomina, Ceratopteris thalictroides, \&c.; sometimes the fronds put out roots as in Mertensia ${ }^{2}$. When the stem possesses clearly developed nodes and internodes, the roots usually spring from the former; thus, for example, exclusively from the nodes in Equisetaceæ, and most commonly so in Grasses.

Roots owe their origin either to the primary meristem, or to partially differentiated masses of tissue, or finally to a secondary meristem enclosed between layers completely differentiated. The primary roots of embryos arise from quite undifferentiated primary meristem; the lateral roots of Cryptogams, as Nägeli and Leitgeb have shown, originate near the growing point of roots, where the differentiation of their tissues first begins; and with Phanerogams the same is the case. But stems may also produce roots near their growing point, where the differentiation of the primary meristem first commences; this occurs in the case of the creeping stems of Rhizo-

1 [On the primary root of Monocotyledons, which disappears at an early period, see Falkenberg, Vergleichende Untersuchungen über den Bau der vegetationsorgane der Monocotyledonen. Stuttgart 1876 .]

${ }^{2}$ A leaf of Phaseolus multiflorus cut off at the pulvinus and placed in water developed from the callus an abundant root-system, and remained living for some months. [The leaves of Ficus elastica behave in the same way.] According to Van Tieghem, the cotyledons of the sunflower, scarlet runner, Cucurbita maxima, Mirabilis Falappa, \&c., when laid on damp moss in a temperature of from $22^{\circ}$ to $25^{\circ} \mathrm{C}$., produce in a few days a number of roots; and this takes place even if the cotyledons are cut into small pieces, the roots then proceeding from the sections of the vascular bundles. I have myself seen a seedling of Cucurbita covered up too thickly with earth put forth long roots from its cotyledons. See further Dodel, Jahrb. für wiss. Bot. vol. VIII. p. I 77 . 
carps and in Pteris aquilina. Roots are formed out of a secondary meristem much further backwards from the growing point, where the tissue is already completely differentiated, in older portions of stems, and especially when mutilated, or when kept dark and damp.

The order of development of the secondary roots is, according to Nägeli and Leitgeb, distinctly acropetal in the primary roots of Cryptogams, where they arise near the apex; new roots are probably never formed in these plants between those already in existence. The same is probably always the case where roots are produced in the primary meristem or near the growing point of the stem, as in Pilularia, Marsilea, Cereus, \&c. But even where their origin is further from the apex, as with the lateral roots from the primary root of Phanerogams and from many stems, such as the maize, they generally appear in acropetal order; but owing to subsequent disturbance roots may arise adventitiously, i.e. in abnormal positions, especially on older primary roots of Dicotyledons.

Secondary roots usually make their appearance on the exterior of the fibro-vascular bundles; the fibro-vascular bundle of the secondary root is then placed at right angles, or nearly so, to those of the mother-root; the cortex is then only incompletely continuous with that of the latter, the epidermis not at all so. The case - is different in the primary roots of embryos, which are formed early and mostly so near the surface of the embryo that a complete continuity is possible in all the tissue-systems between stem and primary root; but in Grasses and some other Phanerogams the first root arises so deep in the interior of the substance of the embryo that it is covered, in the fully developed embryo of the ripe seed, by a thick layer of tissue (Fig. 1 24, ws), which is ruptured on germination (Fig. 123, ws), and is known by the name of Root-sheath or Coleorhiza. Similar formations occur also in the first lateral roots of seedlings of Allium Cepa, and occasionally elsewhere. But in other cases the secondary roots which are formed deeper in the tissue simply split
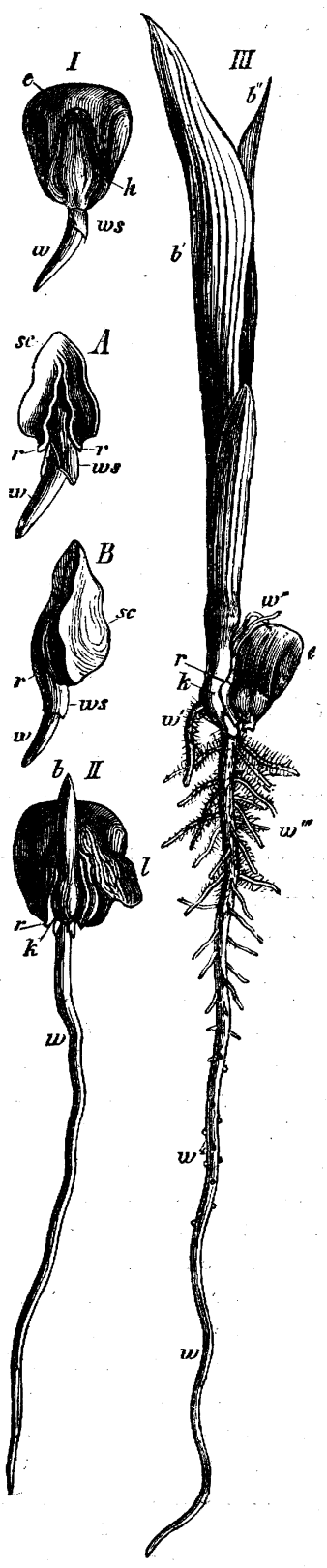

FIG. 123.-Germination of maize in the order $I, I I$, $I I I ; A$ and $B$ the embryo separated from $I$, in $A$ seen in front, in $B$ from the side; $w$ the primary root; ws its root-sheath; $w^{\prime}, w^{\prime \prime}, w^{\prime \prime \prime}$ secondary roots; $e$ the part of the seed filled with endosperm; $k$ the plumule; sc scutellum of the embryo; $r r$ its open margins; $b b^{\prime} b^{\prime \prime}$ the first leaves of the seedling (natural size). the layers of tissue which cover them, and project from a two-lipped chink. 
The typical form of roots is filiform and cylindrical; their section is usually circular when not altered by external pressure. It is only when roots undergo

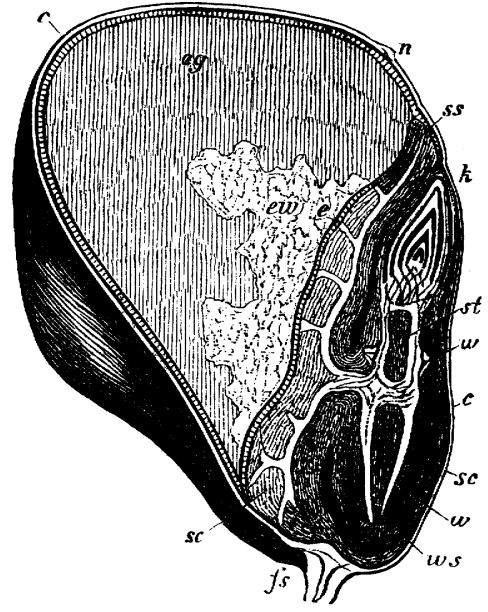

FIG. 124.-Longitudinal section through a grain of maize (X about 6 ); $c$ pericarp; $n$ remains of the stigma; $f s$ base of the grain ; eg hard yellowish part of the endosperm; ew whiter less dense part of the endosperm; sc scutellum of the embryo; $s s$ its point; $e$ its skin; $k$ plumule; $z$ (below) the primary root; ws its root-sheath; w (above) secondary roots springing from the first internode of the embryonal stem st. of the tissue commences immediately behind the terminal part of the root formed of primary meristem, an arrangement by which the elongation of the roots in the ground is essentially assisted.

(a) The primary root of the embryo of most Phanerogams gives the impression of being entirely exogenous, as if its apex were the actual posterior termination of the embryonal stem; but its first origin is endogenous; for the posterior end of the embryo is originally attached to the 'pro-embryo' or suspensor, in Phanerogams, and the primary root is, at its first origin, covered by this ${ }^{1}$. There was formerly some doubt as to the endogenous origin of the primary root of Ferns and Rhizocarps; but when' it is observed that the root is not constituted as such until the apical cell has thrown off the first layer of the root-cap, it is evident that in this case also the apex of the new root lies from the first inside the tissue of the embryo ${ }^{2}$.

(b) The origin of lateral roots in a mother-root is always on the outside of its axial fibro-vascular or plerome-cylinder; and the points where the new formation commences is-with a few exceptions among Phanerogams-on the outside of the vascular bundles, so that each bundle corresponds to a longitudinal row of secondary roots. There are however some differences between the phenomena in Cryptogams (Ferns, Marsileaceæ, and Equisetace ${ }^{3}$ ) and Phanerogams, viz., that in the former the roots originate from the innermost cortical layer or plerome-sheath which surrounds the

1 A more exact account of this, according to Hanstein's researches on the formation of the embryo, will be given in Book II, on the Characteristics of Phanerogams.

2 Compare the drawings of the embryos of Ferns and Rhizocarps in Book II.

3 In Lycopodiaceæ (and according to Van Tieghem, Ophioglossaceæ) no lateral roots are formed in the mother-roots, the roots branching dichotomously, and the growing point which is enveloped by the root-cap splitting into two growing points, each of which forms its own root-cap (see Fig. 138, p. 182). 
fibro-vascular bundle, while in Phanerogams they proceed from the pericambium which is enveloped by the plerome-sheath (see Sects. 16, 18). In the Cryptogams named above the new roots originate each from a single primary mother-cell, and there are always particular cells of the plerome-sheath which give rise to the rudiment of a root, while in Phanerogams, on the contrary, several of the pericambial cells take part in the production of each secondary root. Another difference consists in this, that the plane of symmetry of the secondary root is, in Cryptogams, at right angles to that of the mother-root, while in Phanerogams (according to Van Tieghem) the two coincide, at least when the mother-root and lateral roots each contain only two vascular bundles.

In Ferns, Marsileaceæ, and Equisetacex, where the root developes with an apical cell which becomes segmented on three sides, and contributes the capcells to form the rootcap (Sect. 19), the formation of the lateral roots commences with cell-divisions, by which a three-sided pyramidal cell is formed with its base outwards, which behaves as the mother-cell of the young root. These mother-cells of the lateral roots lie in the plerome-sheath of the axial bundle, in front of its groups of vessels, and are therefore separated from the outermost of these vessels by the pericambium. Further transformations take place subsequently in the pericambium, in consequence of which the fibro-vascular cylinder of the lateral root coalesces with that of the mother-root. This does not take place, however,
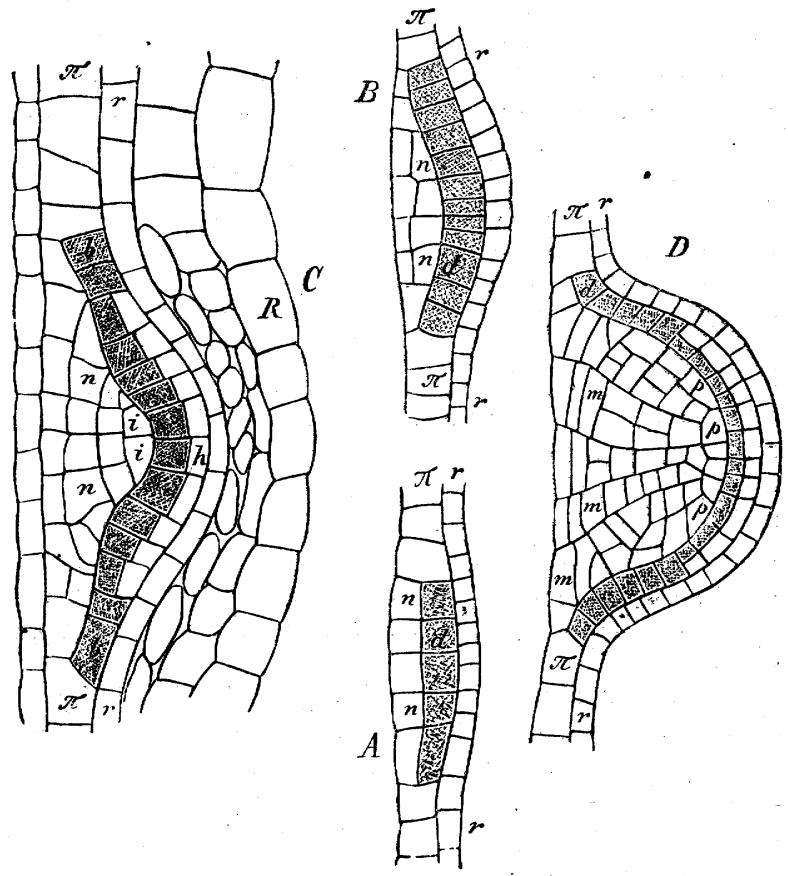

in Equisetace , where there is no pericambium.

In Phanerogams it is also the general rule, as has already been mentioned, for the lateral roots to originate outside the vascular bundles of the mother-root. An exception to this is however, according to Van Tieghem, afforded by Grasses, since these have no pericambium exterior to the vascular bundles; the new roots originate therefore on the outside of the phloëm-bundles which lie between the vascular bundles and exterior to which pericambium occurs. The phenomena are also different in Umbelliferæ and Araliaceæ; a secretion-canal lies here in the pericambium outside each fibrovascular bundle; and the lateral roots are therefore formed midway between each pair of bundles, and therefore outside the phloëm-bundle.

In Phanerogams, according to Reinke, the commencement of a lateral root is 
indicated by the splitting of several cells of the pericambium of the mother-root by tangential walls, so that it is divided into two layers (Fig. 125, $A$ ). The outer layer is immediately constituted into dermatogen $(d)$, which afterwards forms the layers of the root-cap by tangential divisions; since each outer layer of cells which results from the successive layers of the dermatogen constitutes a layer of the root-cap $(C b)$. The inner layer of cells $(A, n n)$, which faces the vessels of the vascular bundle of the mother-root, then also splits again into two layers $(B)$; and further longitudinal and transverse divisions follow, by which the primary meristem of the young root is formed. This soon divides into periblem and plerome, as may be clearly seen in $D$, where $p p$ is the periblem, and $m m$ the basal portion of the plerome, by which a union is effected with the fibro-vascular cylinder of the mother-root. While the young root lengthens somewhat obliquely to the axis of the mother-root and downwards, it compresses the cortical tissue $(D)$; the plerome-sheath $(A-D, r)$ resists disorganisation longest, and, at least at first, follows the growth of the young root, surrounding it with a sheath until it is destroyed. Finally the young root lengthens and its apex protrudes through the cortical tissue of the mother-root.

(c) In-stems lateral roots arise either from the interfascicular cambium (e.g. in Impatiens parvifora immediately above the soil in the primary stem), or from the outermost phloëm-layer of the fibro-vascular bundles, which is more commonly the case. These layers of tissue then behave like the pericambium of a primary root, as in Veronica Beccabunga, Lysimachia nummularia, or the ivy, according to Reinke.

(d) While the formation of the root-cap, as has already been shown in Sect. I9, is proceeding at the apex of the root, its outermost layers pass over into permanent tissue; the cells retain simple forms, but their walls become thicker, and in the outermost cell-layers of the cap swell up, become gelatinous, and thus cause the apex of the root to appear viscid; finally they die and become detached. In aërial and underground roots the root-cap is closely attached to the substance of the root by its oldest layers, which generally extend backwards; in the roots of Lemnacex, Stratiotes, and some other plants, which float on the water, it forms a loose sheath which envelopes the substance of the root high up, and is only fixed below to its apex.

(e) Roots are generally clearly distinguished, by the characteristics mentioned above, from leaf-bearing shoots; there occur, however, a few transitional forms which show that roots can become directly transformed into leafy shoots, as in Neottia Nidus-avis, where (according to Reichenbach, Irmisch, Prillieux, and Hofmeister) older lateral roots of the stem throw off their root-caps and form leaves beneath the apex. On the other hand, leaf-bearing shoots cease to produce leaves, as in many Hymenophyllaceæ, and, according to Mettenius, form root-hairs, and assume the habit of true roots (whether they actually form a root-cap is doubtful); in these species true roots are wanting. In Psilotum triquetrum Nägeli and Leitgeb have shown that the apparent roots are only underground shoots, on which more or less evident traces of leaf-formation may be recognised; they resemble true roots in function and in the mode of formation of their tissue, but have no root-cap, and, when they come above ground, grow in the manner of ordinary leafy shoots. In Selaginellex also, the same investigators have shown the presence of leafless shoots (rhizophores) which grow downwards, and do not form root-caps until they touch the ground (see Book II, Lycopodiaceæ).

We thus see that transitional structures between roots and leafy shoots are found even in highly differentiated plants. But even in Algæ the thallus is often fixed to its substratum by organs of attachment, which may be compared with roots in their habit and in many functional properties; and this occurs not only in the case of the large Fucaceæ and Laminarieæ, but even in the unicellular Vaucheria and Caulerpa.

In confirmation of the Theory of Descent referred to at the conclusion of this work (see Book III. Chap. 7), it is of great importance to know that members differing 
to the greatest extent morphologically and physiologically are connected by transitional forms, and that, especially in the branched thallomes of Algæ, the rudiments: are to be found of all the differentiations of the higher plants. Distinctions which, in the ramifications of the Alga-thallus, are only of a weak, undefined, and rudimentary character, increase more and more in the higher plants; points which can be sharply defined in the latter become indistinguishable when we are considering the more simple Thallophytes. The more the attempt is made to establish exact definitions for single forms, the more does one become convinced that all definition, all limitation, is arbitrary, and that Nature presents gradual transitions from the indistinguishable step by step to the distinct, and finally to the opposite.

SECT. 24. Different Origin of Equivalent Members ${ }^{1}$--(I) The different members of a plant spring out of one another; the members produced may therefore be similar (homogeneous), or dissimilar (heterogeneous) to the member which produced them. In the former case the formation of new members is ordinarily termed Branching; in the latter it is regarded as the production of a new member. A root, for instance, branches in the production of new roots, a stem in that of new stems, a thallome in that of new thallomes; in the same sense the production by a leaf of lateral leaf-structures must also be considered a case of branching. On the other hand the stem produces also leaves, roots, and hairs; leaves not unfrequently produce leaf-bearing shoots, sometimes roots, generally hairs; leaf-forming buds may also arise from roots. But since members which are morphologically dissimilarstem, leaf, root, trichome-do not differ absolutely, but only in degree, the difference between branching and the production of new members, between homogeneous and heterogeneous growth, must be regarded not as an opposition, but only as a gradually increasing differentiation of the members which grow out of one another.

(2) New members may originate either by Lateral Budding or by Dichotomy. Lateral budding occurs when the producing member, after its previous increase in length at the apex, forms outgrowths below it, which are from the very first weaker than the portion of the axial structure which lies above them. Dichotomy, on the other hand (rarely Polytomy), is caused by the cessation of the previous increase in length of a member at its apex, and by two (or more) new apices arising side by side at the apical surface, which, at least at first, are equally strong, and develope in diverging directions. Lateral budding may either form structures which are similar or dissimilar to the axial structure; and thus leaves, roots, hairs, or branches arise by lateral budding from the stem; leaflets, lobes, hairs, sometimes leaf-bearing shoots, or even roots, from the leaf. Dichotomy, on the contrary, never produces structures which are dissimilar to the producing structure; the divisions of a root produced by dichotomy are both roots, those of a leaf-bearing shoot both leafbearing shoots, those of a leaf both foliar structures; dichotomy hence always falls under the conception of branching in the above-named narrower sense.

1 Compare the literature mentioned in the previous sections, and in addition, $\mathrm{H}$. von Mohl, Linnæa, 1837 , p. 487.-Trécul in Ann. des Sci. Nat. 1847, vol. VIII.' p. 268.-Peter-Petershausen, Beiträge zur Entwickelungsgeschichte der Brutknospen. Hameln 1869.-Braun and Magnus, Verhandlungen des Bot. Vereins der Provinz Brandenburg, 187 I (on Calliopsis).-[Warming, Ramification des Phanérogames; Danish with French abstract. Copenhagen 1872.] 
Dichotomous branching is very common among Thallophytes, especially Algæ and the lower Hepaticæ; among Phanerogams it occurs only exceptionally; among Vascular Cryptogams it appears to occur in Ferns (e.g. the leaves of Platycerium alcicorne); but it is the only mode of branching in all shoots and roots of Selaginelleæ, Lycopodieæ, and in the roots of Isoëteæ ${ }^{1}$.

(3) The origin of lateral members, whether similar or dissimilar to the producing member, is either exogenous or endogenous. The former term is applied when they are formed by lateral outgrowth of a superficial cell or of a mass of cells which includes the outer layers of tissue, as in the case of all leaves and hairs and most normal leaf-bearing shoots. A member is of endogenous origin when it is covered, even when in a rudimentary condition, by a layer of the tissue of the producing member which does not take part in the new formation, as in all roots, all lateral shoots of Equisetaceæ, and in adventitious buds.

(4) Lateral members of any kind are almost always formed in considerable numbers on the axial structure which produces them, and even repeatedly one after another, because the producing structure continues to increase in length, and the conditions for similar equivalent outgrowths are repeated. Thus the stem, so long as it continues to grow at the apex, produces leaves, hairs, often even roots, and generally lateral shoots in great numbers, one after another; roots usually form in succession many lateral roots, branching leaves usually several segments. If the apical growth ceases early, the number of the lateral members is also limited; thus the short primary stem of Welwitschia mirabilis produces only two leaves. When the increase in length of the stem is very slow, the formation of lateral shoots from it is sometimes altogether suppressed, as in Isoëtes, Botrychium, and Ophioglossum.

(5) An axial structure may produce either several equivalent lateral members at the same level, or only one; in the second case the members formed in succession are termed solitary, in the first case a Whorl or Verticil. Leaves often occur in whorls, branches less frequently, roots occasionally (in the primary roots of Phanerogams). In the same whorl the members may arise either simultaneously, as the petals and stamens of many flowers, or the foliage-leaves of many Phanerogams; or successively, as in Characeæ and Salvinieæ. A whorl is a true one when the zone on which the lateral members are inserted is transverse from the first, as occurs in both the last-named plants and in many flowers; Spurious Whorls, on the other hand, are formed by displacement and unequal growth in the axis, as in Equisetaceæ, where the leaves, roots, and branches arise from transverse zones which are themselves formed by displacement of three segments of the stem ${ }^{2}$.

(6) Similar and equivalent lateral members usually arise on their common axial structure in acropetal or basifugal order, i.e. the younger a member is the

1 For further details of lateral branching and dichotomy see the conclusion of this section and Sect. 25 .

2 The three segments, which together form the periphery of the stem, stand at first at different heights, but arrange themselves, as Rees has shown, in a transverse zone, which developes externally a circular protuberance, the rudiment of the leaves (see Book II, Equisetaceæ). 
nearer it is to the apex. The lateral members which are formed from and sufficiently near the growing apex of an axial structure are apparently always acropetal; but the order is disturbed when lengthening at the apex ceases and new formations occur in the primary meristem below it, as in many flowers and in the abnormal inflorescence represented in Fig. 126. The lateral members formed at a greater distance from the growing apex of the axial structure are sometimes, but not always, acropetal. Since branching and the formation of lateral members out of the growing point occur in nearly all plants, and, by their regular repetition at definite points of the growing axis, determine the external form of the plant, they may be considered as normal, in opposition to the adventitious production of members which takes place at the older parts of the axial structure at a distance from the apex and without definite order. Such new formations are equally adventitious even when they are of great importance to the plant from a physiological point of view. Adventitious shoots are generally formed internally by the side of the fibro-vascular bundles of the branch, leaf, or root; but it does not follow from this that all endogenous shoots are adventitious. All the shoots of Equisetaceæ are endogenous in their origin; but they are not adventitious, since they are produced in the primary meristem below the apex of the mother-shoot, and in a perfectly definite order. It is equally incorrect to call all roots adventitious although they arise in the interior of the stem, leaves, or roots. They are adventitious

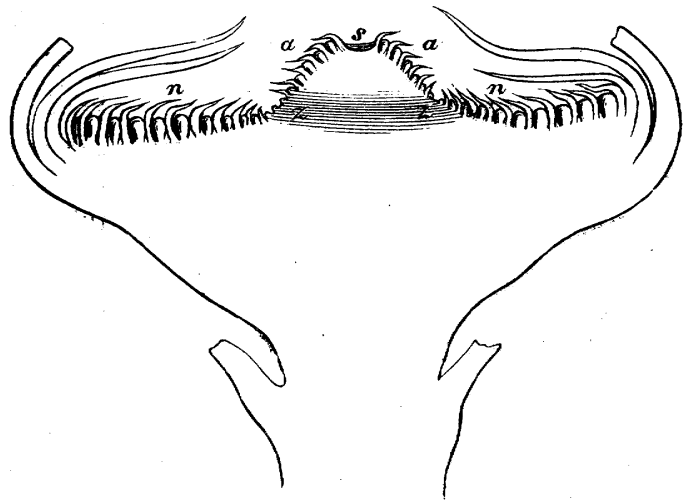

FIG. I25.-Median longitudinal section through a young inflorescence of the sunflower, the broad axis of which has been injured at the apex $s$, and has in consequence ceased growing. The zone $z z$ commenced instead an intercalary growth, and behaved as an apical region taking the place of the summit $a s a$; the bracts and flowers of this apical portion have in consequence been formed from above downwards in a centrifugal direction; while before the injury to the apex they were produced at $n n$ in the normal manner acropetally. The relative position of the bracts and flowers is also the reverse in the abnormal part of the inforescence of what we find normally.

only when they occur in older parts, and even then not always; when they arise close to the growing point of a mother-rost or a stem, they are arranged in strictly acropetal order, and are for that reason not adventitious. When a member has a basal zone of growth, and produces lateral members from it, they may be arranged in basipetal order, as the sporangia on the columella of Hymenophyllaceæ, according to Mettenius, or the segments of the leaves of Myriophyllum.

(7) When in the higher plants a new individual is formed destined for permanent and independent growth, a leaf-bearing axis is first constituted, i.e. a shoot on which roots, hairs, and lateral shoots subsequently arise. In all vascular plants this first shoot arises immediately out of the sexually-produced embryo; and the externally undifferentiated embryo must therefore be considered as itself a primary axis ${ }^{1}$. In Muscineæ, on the other hand, the sexually-produced embryo is

${ }^{1}$ Cómpare what.will be found under Rhizocarpeæ and Angiosperms in Book II. 
transformed into the sporogonium, a structure without leaves, roots, or branches, the sole function of which is the production of spores. A new Moss-plant is, on the contrary, constituted by the production of a leaf-bearing shoot from a branch of the alga-like Protonema, which branches, strikes root (by roothairs), and is independently nourished. The shoot first produced, from which are developed the rest, is termed the Primary Shoot; it is often more strongly developed than its lateral shoots, as in most Ferns, Cycadeæ, Coniferæ, Palms, and Amentiferæ. The primary shoot produces Lateral Shoots of the first order or Secondary Shoots, these again lateral shoots of the second order, and so on. Nevertheless it often happens that lateral shoots of any order take root and

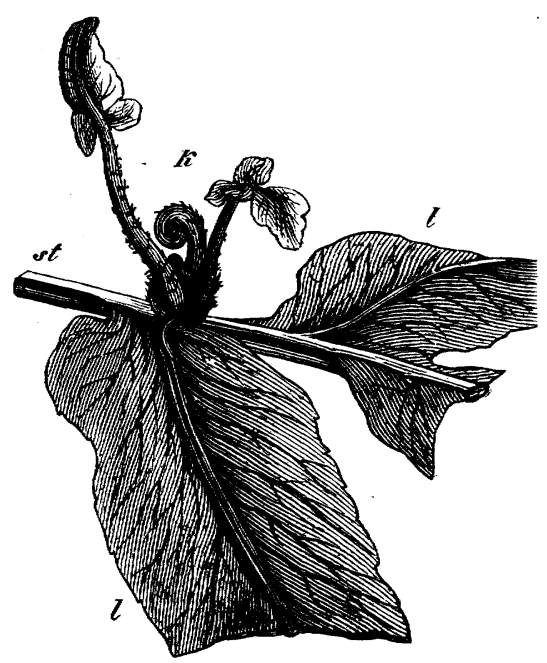

FIG. 127.-Asplenium decussatum; middle part of a mature leaf; its mid-rib st bears the pinnæ $l l$; at the base of one of these is formed the bud $K$, which has also already put out a root (natural size). become detached from the primary shoot; they then assume all its peculiarities, and may equally be considered as primary shoots. But it also happens that the primary shoot itself is arrested at an early period, while new orders of shoots proceed from it which gradually become more vigorous, as in many bulbous and tuberous plants. Shoots which become detached from the mother-plant when but slightly developed, continue to grow by independent nourishment, and repeat the peculiarities of the primary shoot, are called Gemme or Bulbils; they are often adventitious shoots; but bulbils may also be shoots of normal origin, as in many species of Allium.

Now that we have already spoken of the origin of leaves, hairs, and roots, and entered sufficiently into detail on the more important points (Sects. 20, 21,22 ), it only remains to go a little further into the various modes of origin of leaf-bearing shoots.

(a) In many Ferns leafy shoots arise from Leaves, and especially when the stem branches but little or not at all, as in Nephrodium Filix-mas, Asplenium Filix-fomina, Pteris aquilina, \&c. In these speoies the buds spring singly out of the lower parts of the petiole at a greater or less height above its insertion. In other species it is usually the lamina which produces numerous buds, generally in the axils of the pinnæ, as in Asplenium decussatum (Fig. I 27), A. Bellangeri, A. caudatum, Ceratopteris thalictroides, or on the surface of the leaf itself, as in Asplenium furcatum, \&c. In all these cases the buds produced on the leaves are exogenous in their origin, and those on the petioles of the first-named species arise, while the leaves are still very young, out of single superficial cells ${ }^{1}$. These shoots take root while still in connexion with the mother-leaf, but sooner or later become detached; in Nephrodium Filix-mas and Pteris aquilina often only after some years, when they have already acquired considerable strength, and the base of the mother-leaf has died off and decayed.

In Phanerogams buds also occur on leaves, although much more rarely. The best

${ }^{1}$ Hofmeister, Beiträge zur Kenntniss der Gefäss-Kryptogamen, vol. II. Leipzig 1857. 
known are those which are formed abundantly in the indentations of the leaves of Bryophyllum calycinum; according to Hofmeister ${ }^{1}$ they arise before the complete unfolding of the leaf as small masses of primitive parenchyma in the deepest parts of the incisions of the leaf. In the aquatic Utricularia vulgaris weak shoots arise, according - to Pringsheim ${ }^{2}$, mostly in the neighbourhood of the axils of the divisions of the leaf; in both cases these shoots are of exogenous origin. Nothing is known of the development of the buds produced on the leaves of Atherurus ternatus or Hyacintbus Pouzolsii (Döll, Flora von Baden, p. 348).

(b) Adventitious shoots springing from Roots are always endogenous; they arise, according to Hofmeister, in the neighbourhood of the fibro-vascular bundles or in the cambium, as in Opbioglossum, Epipactis microphylla, Linaria vulgaris, Cirsium arvense, the aspen, and apple.

(c) Adventitious Buds arise moreover in an endogenous manner under peculiar circumstances from older detached leaves or pieces of stem and root, especially when kept damp and in darkness. On this depends the propagation of many plants in gardens, as of Begonias from leaves, Marattias from their thick stipules, \&c. Adventitious buds also sometimes appear in considerable quantity in old stems of woody plants; this occurs on the callus formed between the bark and the wood, when the stem is cut off above the root. The branchlets which break out in old stems of Dicotyledons and Monocotyledons are, however, often not true adventitious shoots, but old dormant 'eyes' which have been left behind, having been formed at an earlier period as normal exogenous axillary buds, when the stem itself was still in the bud-condition; they had become enveloped by the bark as the stem increased in thickness, and carried on a feeble existence, until placed in a condition for active growth by a favourable accident, as the

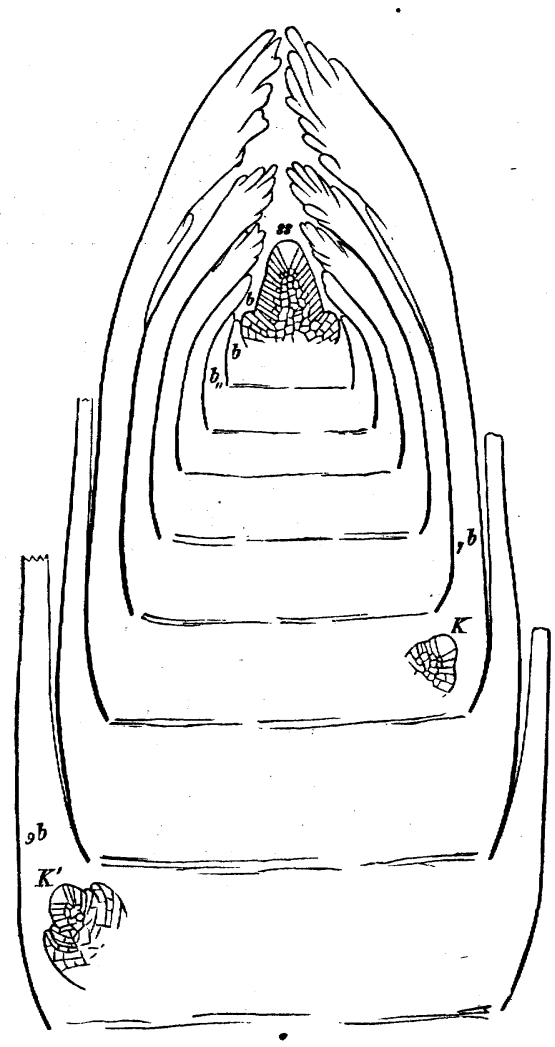

FIG. 128.-Equisetum arvense; longitudinal section through an underground bud in March ; ss the apical cell of the stem; $b \rightarrow b$ its leaves ; $K K^{\prime}$ two endogenous lateral buds exposed by the section. The youngest rudiments of buds are to be found, however, at $b^{\prime \prime}$, amd they have probably begun to be formed even at a greater height $(\times 50)$. removal of the stem above them (Hartig).

(d) In the genus Isoëtes the leaf-bearing shoot arises exclusively from the fertilised germ-cell or embryo, and forms neither normal lateral buds out of the stem nor any from the leaves or roots, nor any kind of adventitious buds.

(e) The Normal Formation of Lateral Shoots from the primary meristem of the growing point of the primary axis is endogenous in Equisetace ${ }^{3}$. With the exception of the primary axis which is developed out of the embryo, all the lateral shoots are here of

1 Hofmeister, Allgemeine Morphologie, p. 423.

2 Pringsheim, Zur Morphologie der Utricularien; in Monatsb. der k. Akad. der Wissen. Berlin 1869 .

s. [Some doubt is, however, now thrown on this exception ; see Book II, Equisetacex.] 
endogenous origin (Fig. $128, K K^{\prime}$ ); they are developed out of a cell in the interior of the tissue of the stem near to the growing point, and afterwards break through the base of the older leaf-sheaths. In some Jungermannieæ the normal terminal branching of: the stem takes place partially or entirely by endogenous formation of shoots ${ }^{1}$.

With these exceptions all normal lateral branches produced at the cone of growth of the bud or in its neighbourhood are, like the leaves, exogenous.

(f) The lateral branches which arise normally below the growing apex of a mothershoot are always produced in acropetal order, like the leaves, with which they exhibit various relationships as to position, age, and number.

(a) The numerical relationship of the lateral branches to the leaves formed on the same axis is variable. If the number is unequal, a greater number of leaves than of branchlets usually arises on the same axis; in Muscinex, Ferns, Rhizocarpex; Cycadeæ, and Coniferæ a much larger number. A branchlet may arise when a definite number of leaves has been formed, as in many Muscineæ and some Ferns, or

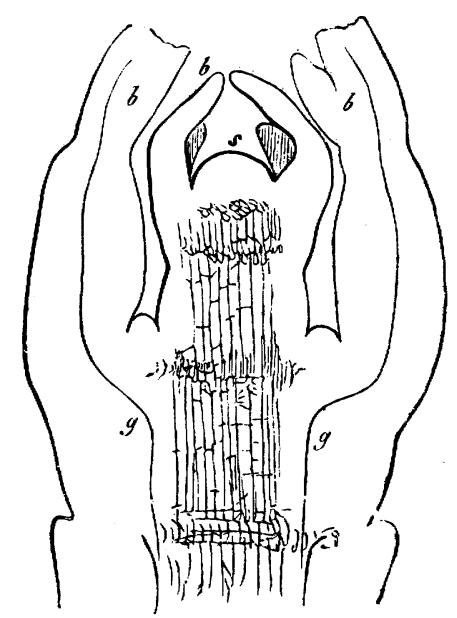

FIG. 129.-Longitudinal section through the apical region of a branch of Clematis apiifolia; $s$ apex of the stem; $b b$ leaves; $g g$ the first traces of spiral vessels, bending out uninterruptedly from the stem into the leaves.

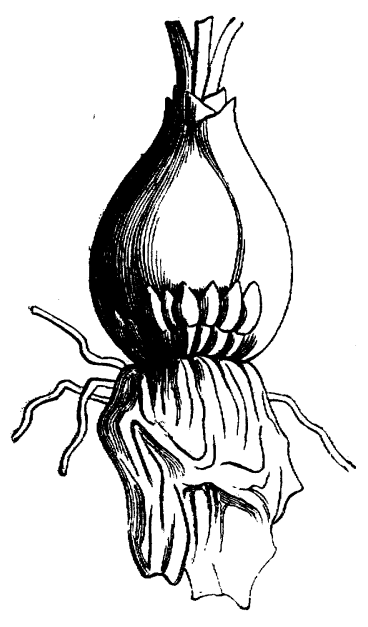

FIG. 130.-Bulb of Muscari botryoides; one of the lower bulb-scales is thrown back, in order to show the numerous buds standing side by side in its axil.

the formation of a branchlet results when the increase in length of the primary axis and the formation of its leaves ceases for a time and is subsequently renewed, as in Abies. When the leaves stand in whorls, the number of the lateral branches may be equal to that of the members of the whorl, as in Equisetacex, or smaller, as in Characex. It is unusual for the number of branchlets to be larger than that of the leaves, but this occurs in some Angiosperms, where two or more lateral buds often arise side by side above a leaf (Fig. 130), or one above another, as in Aristolochia Sipho, Gleditscbia, \&c. In most Angiosperms the number of the lateral branchlets (with the exception of the flower-shoots) is, at first, the same as that of the leaves; but usually only a much smaller number continue to develope.

$(\beta)$ The relationship in position and origin of leaves and branches is constant in each species and often in a whole class of plants. The lateral branches arise below the leaves (according to the acute investigations of Leitgeb ${ }^{2}$ ) probably in all Mosses, as well as in

1 [See Leitgeb, Bot. Zeit., I 872.$]$

2 Leitgeb, Beiträge zar Entwickelungsgeschichte der Pflanzenorgane, in Sitzungsber. der kais. 
the Hepaticæ Radula and Lejeunia; the branch springs (as shown in Fig. II6, z, p. 153) out of the lower part of a segment of the stem, the upper part of which has developed into a leaf. In Fontinalis this occurs below the median line of the leaf, in Spbagnum laterally below one half. According to the same observer, the lateral branches arise in place of a half-leaf beside the remaining half in many Jungermanniex, as Frullania, Madotheca, Mastigobryum, Jungermannia tricbophylla ${ }^{1}$. If each tooth in the leaf-sheath of an Equisetum be considered as a leaf, the buds originate at the side of the leaves and between them, for they break through the leaf-sheaths between the teeth. In Characeæ and Angiosperms the normal lateral branchlets spring from the acute angle which the leaf forms with the stem (Figs. 129, 131). Usually only one is formed above the middle of the insertion of the leaf, or two or three one above another; sometimes several are formed side by side, as in the bulbs of Muscari (Fig. 130), and the flowers in the axils of the bracts of Musa. Such branchlets are called Axillary Sboots; in Angiosperms the branching is, with a few doubtful exceptions, always axillary ${ }^{2}$.

The axillary shoot is usually so situated that it is attached both to the Subtending leaf in the axil of which it grows and to the primary axis, and is therefore in direct con-

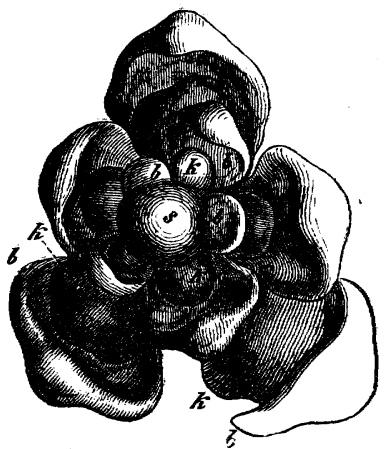

FIG. I31.-A pical region of a primary axis of Dictamnus Fraxinella, seen from above; $s$ apex of the primary axis; $b b b$ the young leaves; $k k$ their axillary buds, the two youngest leaves have not yet axillary buds.

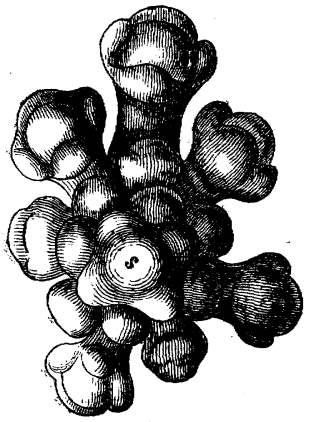

FIG. 132.-Young inflorescence of Isatis taurica, seen from above; $s$ apex of the axis of the inflorescence; the flower buds appear beneath it (in whorls of four); the youngest are still simple leafless elevations.

nection with both. But it is not unusual for the lateral shoot to advance along the primary axis and thus lose its connection with the subtending leaf ${ }^{3}$, or contrariwise to appear as if attached to the base of the leaf away from the axis. Examples of both are furnished by the sporangia of Lycopodium and Selaginella. The advance of the axillary shoot along the base of the subtending leaf is not uncommon in the inflorescence of Phanerogams, where the flower-bud springs from the base of the bract, as in Hippuris (Fig. I19, p. 155), Amorpha, Salix nigricans, Sedum Fabaria, \&c. But, on the other hand, the subtending leaf may advance on its axillary shoot, when it originates later than

Akad. der Wissen. zu Wien, vol. LVII, 1868, and vol. LIX, 1869; and Bot. Zeitg. 1871, no. 34. See also more in detail, Book II, Muscineæ.

1 Leitgẹb, Bot. Zeitg. I87 r, p. 563; see also Book II, Hepaticæ.

${ }^{2}$ [The relationship of leaf and lateral shoot is intelligible in the Muscinea, where the two struc. tures are derived from the same ultimate segment of the apical cell. In Phanerogams Warming considers ( $l . c$. p. xxiii) the leaf and its axillary bud (which are always united at their base) to no less form a whole, and to constitute a double organ whose parts have a different morphological value and are sometimes equally developed, while in some cases one is developed at the expense of the other.]

3 [This occurs not unfrequently in the flowering shoots or inflorescences of Phanerogams, when such shoots are termed extra-axillary.] 
the latter; in this manner is formed the bract on the flower-bud in Anthemis, Sisymbrium, and Umbelliferæ. When in addition to this it also happens that after the formation of the subtending leaf the basal portion common to it and to the bud lengthens, then the former is elevated on the axis of its axillary shoot, and the shoot appears to have no subtending leaf, because this latter is placed upon it, and constitutes its first leaf. This occurs in Thesium ebracteatum, Samolus Valerandi, Spircea, Ruta, Tilia (in this case the bract and the entire inflorescence), Borragineæ, Solanaceæ, and Crassulaceæ ${ }^{1}$.

$(\gamma)$ In reference to the relative time of production of the leaves and lateral branches on a primary axis, the general rule is that axillary buds are formed later than their subtending leaves. This is the case in Characeæ, Hepaticæ, Equisetaceæ, and, with the exception of some inflorescences, in Phanerogams. In the region of growth the subtending leaf attains a considerable size, even in the latter, before its axillary shoot is formed; and in the meantime new and younger leaves are produced above the youngest bud. In those inflorescences, on the contrary, where the formation of leaves is more or less suppressed, the bud is often formed immediately after the bract, or at the same time, or even earlier than it; and in the two last cases the bract has often the appearance of being a product of the bud (see conclusion of par. $\beta$ ). It may even happen that when the flower-buds are formed very rapidly, the production of bracts is altogether suppressed, as in most Cruciferæ (Fig. I 32$)^{2}$.

$(\delta)$ The fact that lateral shoots arise far most frequently at a greater distance from the apex of the stem than the youngest leaves, distinguishes them sufficiently from dichotomous branchings, which must always of necessity arise above the youngest leaf. But even when the leaves are visible only later than the corresponding buds, as in the inflorescence of Grasses, or is even completely suppressed, as in most Cruciferæ, it is still impossible to confound lateral with dichotomous branching, if, as in these cases, the cone of growth greatly overtops the youngest lateral bud, and continues to grow in a straight line (Figs. II7, I19, pp. 154, 155). Still more conspicuous is the distinction between lateral branching and dichotomy when the primary axis ends in a broad flat apical surface, as in the young capitula of Compositæ. Here the lateral shoots (the flowers) are so small in comparison to the mother-shoot, and are from the first placed at so great a distance from its apex, and so uniformly on all sides of it, that the mothershoot must be regarded as the independent centre of all new formations. The idea of dichotomy supposes, on the contrary, that the mother-shoot ceases as such, and that two branches, at first at least equally strong, continue to grow in length in diverging directions in its place.

If it is desired to include lateral branching from the growing point and dichotomy of the apex under one common term, in order to distinguish them from the adventitious formation of branches from older portions of the stem, leaves, or roots, the expression Terminal Brancbing commends itself.

SECT. 25. Different capacity for Development of the members of a Branch-system ${ }^{3}$.- Systems of similar members originate by branching; out of a root a root-system originates, out of a shoot a shoot-system; when a leaf branches, we get a pinnate, digitate, lobed, or incised leaf, \&c. We may therefore examine the more important relationships of form of such a system, taking into account for the time only the relative size and capacity for development of the

1 See Warming, Recherches sur la ramification des Phanérogames. Copenhagen I872, p. xx.

2 [On the suppression of the bracts in Cruciferæ, see Masters, Journ. Linn. Soc. 1875, vol. XIV. p. 39I.]

3 Nägeli und Schwendener, Das Mikroskop, p. 599.-Hofmeister, Allgemeine Morphologie der Gewebe, Sect. 7.-Kaufmann, Bot. Zeitg. 1869, p. 886.-Kraus, Medic.-Phys. Soc. in Erlangen, Dec. 5, I 870 .-Warming, $l$. $c$. 
branches of the various orders. We may here leave adventitious branchings entirely out of consideration; for it is evident that in respect to the phenomena now under consideration they play no essential part in the building up of the whole plant. We have therefore to do only with the branchings which arise at the end of a growing shoot, leaf, or root, i.e. with terminal branchings. These may be referred (as has already been shown in Sect. 24, div. 2) to two principal forms, dependent on the origin of the branching by dichotomy or by lateral branching; branch-systems of the first kind may be called simply Dichotomies, of the second kind Monopodia.

A Dichotomous Branch-system, according to the definition given in Sect. 24, is the result of the cessation of the growth at the apex in the original direction, and its continuation in two new directions at newly' constituted apical points, as is very clearly shown in Fig. I $333^{\mathbf{1}}$. We may term the newly formed branches Bifurcations, and the member which produces them the Base of the bifurcation. Every base can only bifurcate once; but every branch may again become the base of a new bifurcation ${ }^{2}$.

A Monopodium arises when the generating structure, following the direction of its previous growth, continues to grow at its apex, while lateral structures of a like kind are produced beneath it in acropetal succession, their longitudinal axes being placed obliquely or transversely to that of the generating member. The
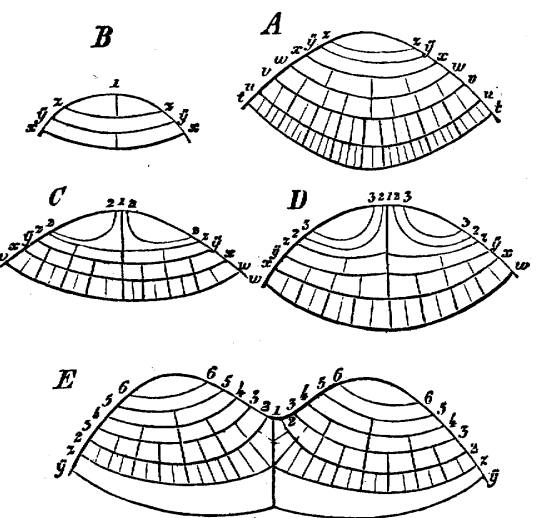

FIG. 133.-Dichotomy of the thallus of Dictyota dichotoma (after Nägeli); the order of development is according to the letters $A-E$; the letters $t-z$ indicate the segmentations of the apical cell before it dichotomises; $I$ is the division-wall by which the dichotomy commences; $2-6$ the segments of the new apical cells.

generating member, since it continues to grow during the branching, may form numerous lateral members; for all these it is the common base; hence the name Monopodium (Figs. 119, 123, 132). Every lateral branch may again branch in the same manner, and thus itself become a monopodium of the second order.

${ }^{1}$ Since we have to give here a narrower application to the term Direction of Growth; it will be necessary to compare with this Sect. 27 .

${ }^{2}$ In Cryptogams with apical cells it may be thought that dichotomy must necessarily be brought about by longitudinal division of the apical cell. When the segments arise by transverse division this is actually the case, as is shown in Fig. I33; but when the segmentation of the apical cell takes place in two or three rows, this would necessitate that the dichotomising wall should bisect its inferior angle, and thus have a position which is apparently universally avoided in cell-division. It is nevertheless possible that a true dichotomy may take place without this. Suppose the old apical cell, immediately after the formation of a new one by its side, were to change the direction of its longitudinal growth, so that both apices diverge from the previous direction of growth; the old apical cell then represents the apex of a new direction of growth. From this it seems to me that we are able to arrive at the distinction between a dichotomy and monopodium. Mutatis mutandis this is also true of Phanerogams which have no apical cell. It is necessary here again to point out that the occurrence of transitional forms between dichotomies and monopodia does not prevent our giving an exact definition of these terms; it is only, in fact, by this very means that transitional forms can be recognised as such. 
Just as the dichotomy may consist of numerous bifurcations, so may a monopodium consist of several orders of monopodial branching.

These definitions refer only to the bud-condition of the branch-system. Not unfrequently, both in dichotomous and in monopodial systems, the original character is maintained in their further growth; the two bifurcations develope, in the case of dichotomy, with equal strength and branch uniformly; in the case of a monopodium the primary axis continues to grow more strongly than all the secondary axes, and branches more copiously. But it is very commonly the case that in a dichotomous system some of the bifurcations are weaker, or that in a monopodial system some of the lateral axes, soon after their formation, grow more

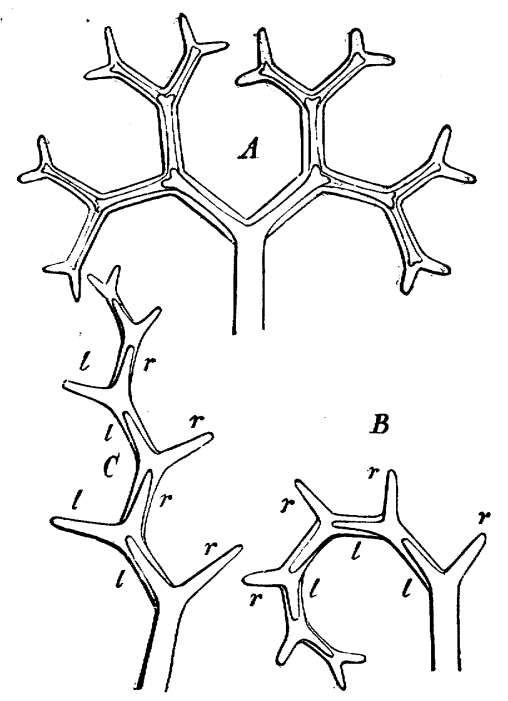

FIG. 134-Diagram of the various modes of development of a dichotomy; $A$ ane developed in a bifurcate manner; $B$ a helicoid; $C$ a scorpioid dichotomy. strongly and branch more copiously than the primary axis. In such cases the original character of the branch-system becomes less and less evident as it developes; and it may happen that systems originally dichotomous have subsequently the appearance of monopodia, and vice versá. It is hence impossible to infer from a mature system whether it originated in dichotomy or in lateral branching. It will therefore be desirable to make a simple classification of the most important changes which a branch-system undergoes during the development of its members.

(I) The Development of Dichotomous Systems may take place either in a bifurcate or a sympodial manner; I call a system bifurcate when at each fork the two branches develope with equal strength, as in Fig. 134, $A$. The dichotomous system is developed sympodially when at each bifurcation one branch developes more strongly than the other; in this case the base of each successive bifurcation forms apparently a primary shoot, on which the weaker branches appear as lateral shoots (Fig. 134, $B$, $C)$. The apparent primary shoot, which in fact consists of the bases of consecutive bifurcations, may on this account be termed a $P_{\text {seud-axis }}$ or Sympodium. Thus in $B$ (Fig. 134) the sympodium is composed of the left-hand branches $l, l, l$; in $C$ of the alternate left and right-hand branches $l r, l r$. Whether the case represented in $B$, which, on account of its similarity to certain monopodial systems, may be termed a Helicoid (bostrychoid) Dichotomy, actually occurs is doubtful (it probably does however in the leaf of Adiantum pedatum). On the other hand the development represented in Fig. 134, $C$ is common in shoots of Selaginellex, and, on account of its resemblance to some monopodial systems, may be termed a Scorpioid (cicinal) Dichotomy ${ }^{1}$.

(2) The development of Monopodial Systems may take place in a racemose

1 On Dichotomous Inflorescences see Book II, Phanerogams. 
or cymose manner; and the cymose development may be either apparently. dichotomous (or even apparently polytomous) or sympodial.

(a) A racemose system occurs when the monopodial mother-shoot continues to develope more strongly than all the lateral shoots, and when the lateral shoots of each successive order behave in the same manner in respect to their mothershoot. This occurs very clearly, for instance, in the stems of most Conifers (especially Pinus, Araucaria, \&c.) and in the compound leaves of Umbellifers.

(b) The cymose development of a monopodial system, or a Cyme, depends on the fact that each lateral shoot begins from an early period to grow more strongly, and in consequence of this, also branches more copiously than the mother-shoot; the growth of which then usually soon ceases. Two principal forms of Cyme may be distinguished, according as a pseud-axis (sympodium) is formed or not.

(a) When two, three, or more lateral shoots arise beneath the growing end of each shoot, which develope in different directions more strongly than their mothershoot, the growth of which soon ceases, a false Dichotomy (or Trichotomy, or Polytomy) arises. Fig. I 35 represents the formation of a false dichotomy; the shoot $I$ produces the shoots $I I$, $I I^{\prime \prime}$, originally weaker, but soon growing more strongly, while the growth of $I$ ceases; the same takes place with $I I I^{\prime}$, and $I I I^{\prime \prime}$. False

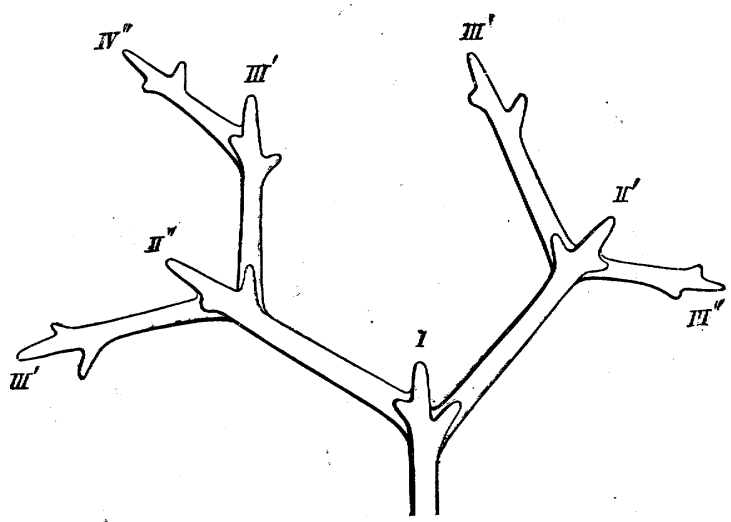

FIG. 135-Diagram of a false dichotomy (dichasium); the numerals indicate the order of development of the shoots of the system. dichotomies of this kind, which occur abundantly in the inflorescences of Phanerogams, are termed by Schimper Dichasia. But instead of two lateral branches growing out in opposite directions, three or more shoots standing in a true or spurious whorl may develope more strongly than their mother-shoot, and thus arises an umbellate system, such as is developed in a typical manner in the inflorescences of our native Euphorbias; a system of this kind may be called a Cymose Umbel.

$(\beta)$ The sympodial development of an originally monopodial system occurs when one lateral shoot always developes .with greater vigour than its mothershoot, as is shown in Fig. I $36, A$, where the lateral shoot 2,2 grows more strongly than the part 2, I of its mother-shoot, and so on. Usually the portions of all the shoots which lie below their lateral branches develope more strongly than the terminal portions, as is indicated in the figure by the thicker lines ; the terminal portions (indicated by thin lines) often die off early; the thicker basal portions of the different ramifications which proceed from one another then commonly place themselves in a straight line, and have the appearance of a connected whole; like a primary shoot to which the terminal portions of each separate order of shoots are attached as if they were lateral branches. The apparent primary 
shoot of the system is called the Sympodium or Pseud-axis. It consists, in Fig. ${ }_{3} 6, B$, of the pieces between $\mathrm{I}$ and 2,2 and 3,3 and 4,4 and 5 ; the weaker terminal portions of the respective branches I, 2, 3, \&c. are bent sideways. A comparison of Fig. I $36 C$ with $A$ shows that between a sympodially developed and a spurious dichotomous system the only point of difference is that in the latter each branch produces two stronger lateral branches. If in $C$ one of the branches is imagined to be suppressed alternately left and right, the form $A$ results, which is then easily transformed into $B$.

Sympodial systems occur in two different forms, according as the lateral shoots, the basal portions of which form the pseud-axis, arise always on the same side or on different sides of it.
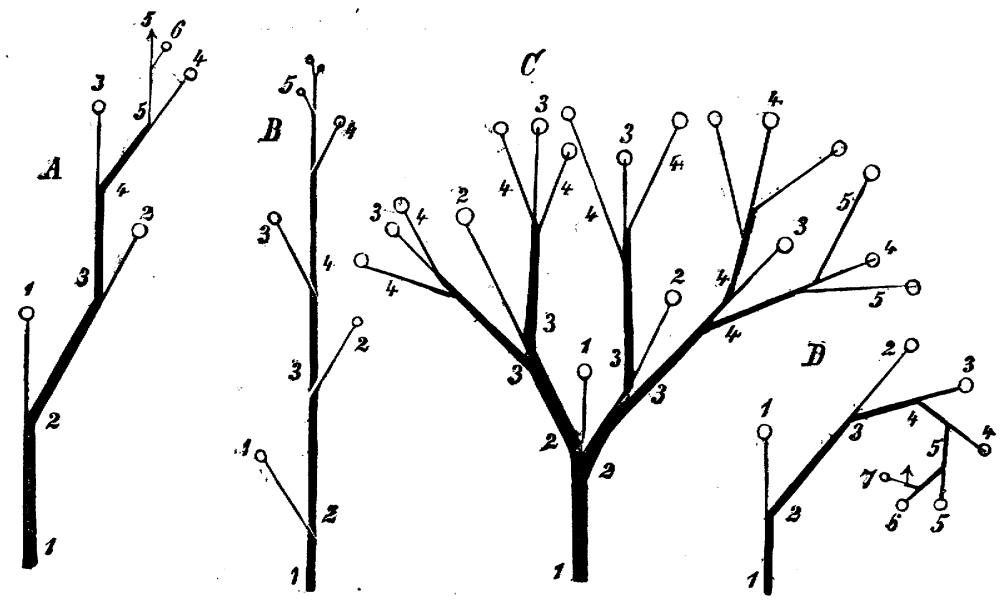

FIG. 136.-Cymose branchings represented diagrammatically; $A, B$ scorpioid (cicinal) cyme ; $C$ dichasium; $D$ helicoid (bostrychoid) cyme; the numerals indicate the order of succession of the lateral shoots which spring from one another.

If the sympodial ramification takes place always on the same side-e.g. always to the right, as in Fig. I $28, D$, or always to the left-the whole system is called a Helicoid Cyme ${ }^{1}$ or Bostryx; if, on the other hand, each branch which continues the system arises alternately right and left, as in Fig. $136, A, B$, the system is a Scorpioid Cyme or Cicinus. If in these cases we have to do with leafy shoots where the leaves are arranged spirally, a more exact definition of the terms right and left becomes needful. It is then necessary to imagine a median plane drawn through

1 [Some difficulty will perhaps be felt with regard to Fig. D, which stands for a helicoid cyme in the text, but which is also identical with the scorpioid cyme of descriptive botany, and corresponds to the specific name 'scorpioides' given by Linnæus to several plants in which it occurs. The term scorpioid was introduced by A. P. De Candolle (Organographie, vol. I. p. 415), to express a unilateral cyme the undeveloped portion of which is usually rolled up. This is the characteristic inflorescence of Borragineæ, amongst which Myosotis has long been distinguished as 'scorpion-grass' on this account. Bravais (Ann. des Sci. Nat. 2nd ser. vol. VII. p. I97) distinguished the helicoid cyme, which he defined as having the successive flowers ranged in a spiral round the pseud-axis. He amended De Candolle's definition of the scorpioid cyme by pointing out that the flowers are in two rows parallel to the pseud-axis.] 
the axis of growth of each shoot and through that of its immediate mother-shoot; then, in the helicoid cyme each following median plane always stands right or left of the preceding one, following the course of the leaf-spiral; in the scorpioid cyme, on the other hand, the consecutive median planes stand alternately right and left.

(a) In Thallophytes and the Thalloid Hepaticx, dichotomy is very common, but monopodial branchings also occur developed in the most various ways. The dichotomous branching is unusually clear and generally bifurcate among Algæ, especially in Dictyoteæ and species of Fucus (in particular F. serratus). In some there occurs a tendency towards a sympodial development of the bifurcations, but usually only at $\boldsymbol{x}$ late period; so that the dichotomous branching can be clearly recognised at the ends: of the branches even with the naked eye. The same is the case, among Hepaticæ, in Anthocerotex, Riccieæ, Marchantiex, and in Metzgeria (Fig... 137), where a flat expansion of the thallus or thalloid stem arises.between the young bifurcations, first of all as a protuberance $\left(f^{\prime} f^{\prime \prime}\right)$, which however cannot be considered as a continuation of the shoot, since it has no apical cell or mid-rib; subsequently: this protuberance disappears, as in $f^{\prime \prime \prime}$.

Distinctly monopodial:(1ateral) ramifications are particularly clear in filamentous Algæ, when the apical cell remains unbranched, and lateral branches grow only out of the individual cells (segments of the filament); as in Cladophora, Lejolisia, \&c. It occurs how-ever sometimes that lateral branches proceed out of the apical cell itself, as is espe-

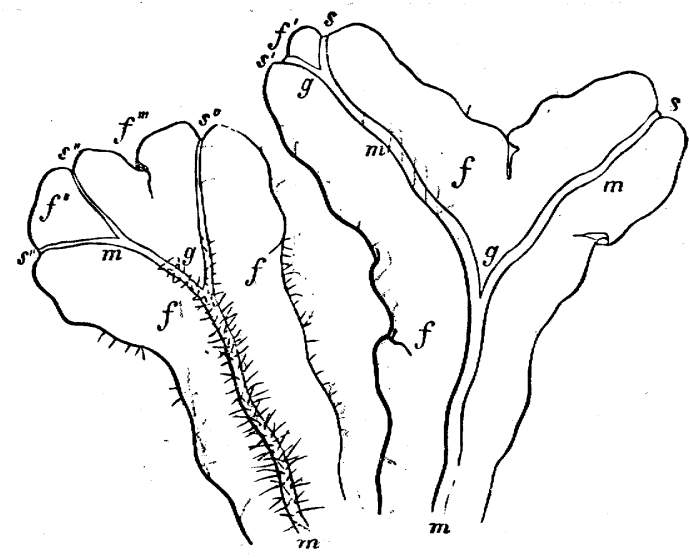

FIG. 137.-Flat thallus of AFetzgeria furcata branching dichotomously $(\mathrm{x}$ about $\mathrm{I5}$ ); $m \mathrm{~m}$ mid-rib consisting of several layers, branching dichotomously at $g$; $s s$ apical points of the branches; $f f$ the wing-like expansions of the thallus, consisting of one layex of cells ; $f^{\prime} f^{\prime \prime}$ the wings between the mid-ribs of younger branches. (The left-hand figure is seen from below, the right-hand one from*above.)

cially shown in Stypocaulon (Fig. 108, p. 139.). In other cases the branching of the apical cell is dichotomous, as in Coleochate soluta (see Böok II; Algæ).

(b) In the roots of Ferns, Equisetacex, and Rhizocarpex, as well as in those of Conifers, Monocotyledons; and Dicotyledons, the branching is always, as far as is known, at first monopodial, and even at a later period the primary root generally remains stronger than its lateral roots; these root-systems are therefore developed in a racemose manner (Fig. I 23 , p. 165 ); this is seen very beautifully in the root-systems which proceed from the primary roots of Dicotyledons when they are allowed to germinate and grow in water. Dichotomy of roots occurs only in Lycopodiaceæ, and probably in Cycadeæ, where they appear at a later period as systems of bifurcations. According to the most recent researches of Nägeli and Leitgeb, it is still altogether doubtful whether the branching depends, even in Lycopodiaceæ, on true dichotomy ${ }^{2}$; but the root-branches of Lycopo-

1 For the above-mentioned reasons I share Kny's view that the branching is in this case dichotomous. (See Hofmeister, Allgemeine Morphologie, p. 433.)

${ }^{2}$ Compare Nägeli's Beiträge zur wissen. Botanik, Heft IV, 1867. I would lay less stress on the relation of dichotomies to the apical cell, because the latter has scarcely the same decided signification in Lycopodiaceæ as in Ferns, Equisetaceæ, and other Cryptogams; and the apical growth apparently approaches nearer to that of Phanerogams. 
diaceæ always arise so near to the apex, and they assume at so early a period the character of dichotomies developed in a bifurcate manner, that, until further investigation proves the contrary, they must be considered as such. It is scarcely necessary to mention in conclusion that when roots branch dichotomously the bifurcations are at first covered by the original root-cap, as is shown in Fig. 138 .

(c) Leaves. Bifurcations resulting apparently from true dichotomy occur in the leaves of some Ferns, e.g. Platycerium alcicorne ${ }^{1}$; and, according to an older statement of Hofmeister, it appears that the branching of Fern-leaves generally commences dichotomously, although mature leaves mostly resemble a monopodium. On a mid-rib forming a continuation of the petiole are placed numerous secondary mid-ribs with secondary laciniæ (pinnæ). Since these branches are apparently always alternate and not opposite, and the terminal lobes of the leaves are frequently developed as equally strong bifurcations, leaves of this kind may be considered, according to Hofmeister's hypothesis, as dichotomies developed in a sympodial (and indeed a scorpioid) manner, the mid-rib representing the sympodium, and the apparent lateral branchlets the weaker

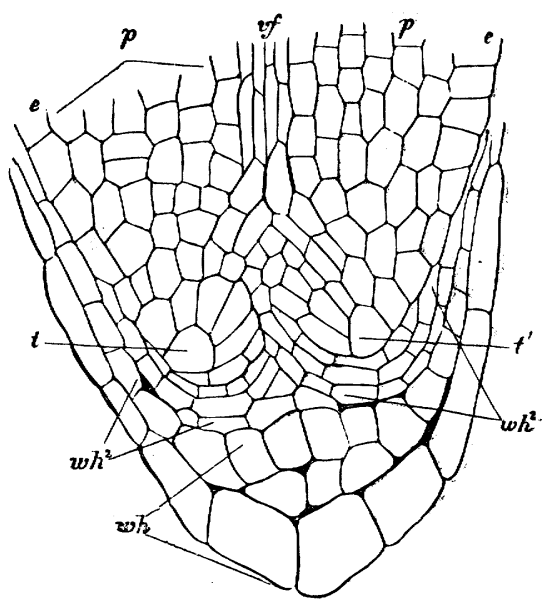

FIG. 138.-Dichotomy of the root of Isoëtes lacustris (after Hofmeister) $(x 400) ; t t^{\prime}$ the apical cells of the branches; wh the old root-cap formed before the bifurcation; zwh2 the two root-caps of the branches, sti.l covered by the former one; $e$ epiderinis; $p$ parenchyma; $v f$ fibro-vascular bundle of the root.

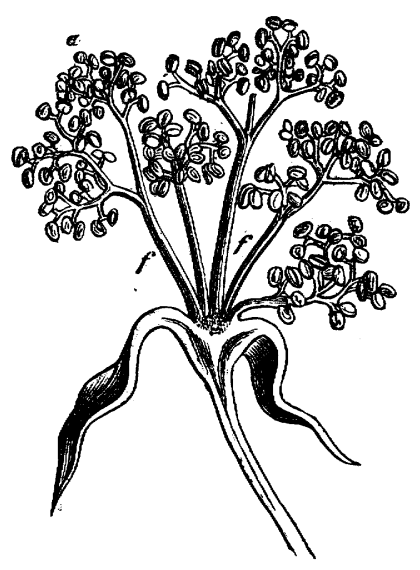

FIG. 139.-Part of a male flower of Ricinus communis cut through lengthways; $f f$ the basal portions of the compoundly branched stamens; $a$ their anthers.

branches (as in Fig. $\mathrm{I}_{34}, C$, p. $\mathrm{17} 7$ ); a process which is repeated in the segments of the leaf itself when the leaf is doubly or many times pinnate. A similar interpretation may perhaps be permitted of the simply pinnate leaves of Cycadex. The repeated branching of the stamens in the male flowers of Ricinus appears, according to Payer ${ }^{2}$, to proceed from dichotomy, and to a certain extent even from polytomy, commencing at an early period. The separate stamens appear as roundish protuberances on the floral axis, and each of these immediately forms two or more similar protuberances on its surface, and on these the same process is again repeated. When mature, the stamens (Fig. 139) appear as if divided dichotomously or trichotomously upon long stalks, the branches being developed somewhat irregularly.

1 The petiole of Adiantum pedatum divides above into two equally strong branches, each of which forms a helicoid cyme of ramifications arising probably by dichotomy; the weaker branches 'of the helicoid cyme stand upright and their numerous pinnæ form a scorpioid cyme produced by further dichotomy. This is one of the most beautiful forms of leaves, the history of the development of which would be of unusual interest.

${ }^{2}$ Payer, Organogénie de la fleur, pl. ro8, confirmed by Warming $l$. . 
On an originally monopodial branching depends, on the other hand, the form of the pinnate, lobed, divided, and toothed foliage-leaves of Angiosperms ${ }^{1}$. The leaf appears on the cone of growth as a roundish protuberance which quickly broadens into a shell-like form (Fig. 140, $A, b$ ), and grows vigorously at its apex. Beneath the apex protuberances arise at the right and left in acropetal order; these also grow in the same manner at their apex $(f)$, and produce again lateral protuberances of the second order $(\phi)$; which, according to the extent to which the surface of the leaf is developed, become lobes of a simple leaf or distinctly separated leaflets.

When two rows of lateral branches arise successively on the median axis of the leaf, they generally remain weaker than it, and their lateral branches are also less numerous and weaker; the development of such an originally monopodial branch-system of leaves

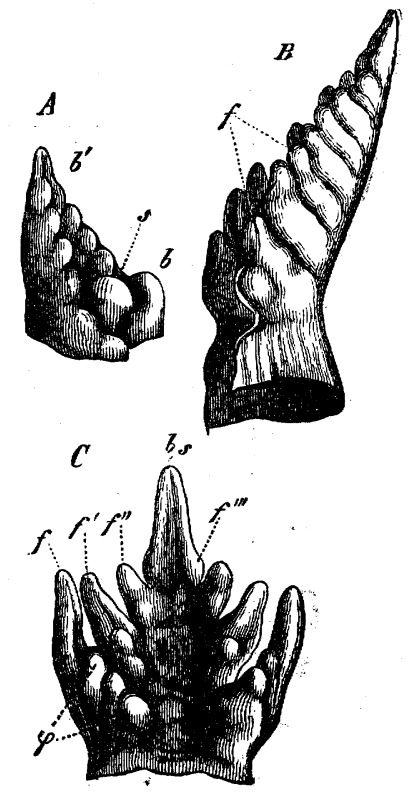

FIG. 140.-Developmentit of the pinnate leaves of Unbelliferæ; $A, B$ of Pastintaca sativa; $C$ of Levisticum officinale; $A$ apical region of the primary stem; its cone of gröwth is seen at $s$, its youngest leaf at $b ; b^{\prime}$ youngest leaf but one with the pinnation commencing; $c$, bs apex of the leaf; $f, f^{\prime}, f^{\prime \prime}$ leafcommencing; $C, b s$ apex of the leaf ; $f, f^{\prime}, f^{\prime \prime}$
branches of the first order; $\phi$ of the second order.

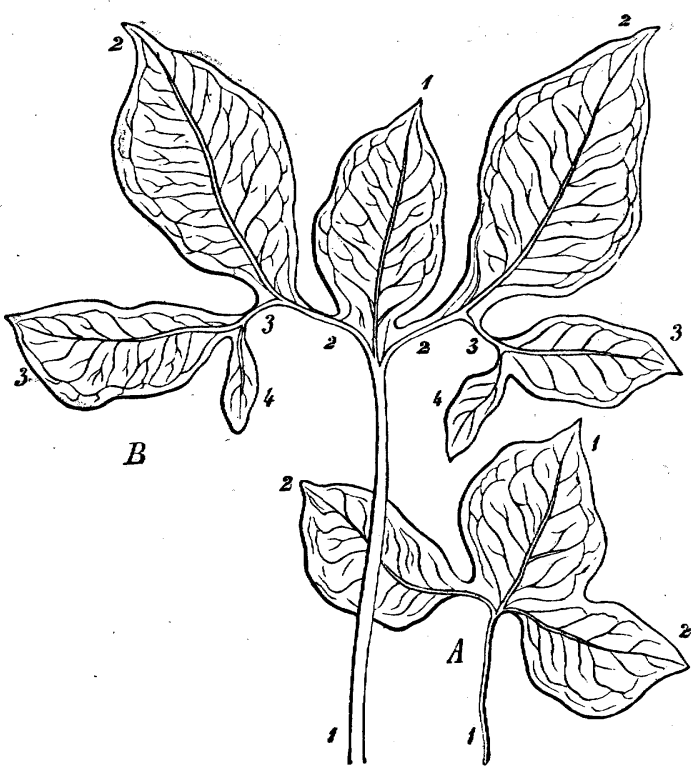

FIG. I4I.-Leaves of Amorphophallu bulbosus; $A$ with a simple, $B$ with a threefold branching of the lamina.

is therefore racemose. But the development may also be cymose, and may even lead to the formation of sympodia, especially when only one branch arises right and left on the primary leaf. This is the case, for instance, in the leaves of Helleborus, Rubus, and of several Aroidex, as Sauromatum and Amorphophallus. Fig. I4 I, $A$ represents a weakly leaf of the last-named plant with only one branch on each side; but when the leaves attain a more vigorous development, as shown at $B$, each lateral lobe, 22 , forms on its outer side again a lobe of the third order, 33 , which again produces a similar one of the fourth order, 4 4, and so on. According to the general definitions given above, the first branch of the leaf, 1 , forms with 22 a dichasium; but each branch of the dichasium

1 This was first shown in detail by Nägeli (Pflanzenphys. Untersuch. von Nägeli und Cramer, Ifeft II) in the leaves of Aralia spinosa.-See Eichler, Zur Entwickelungsgeschichte des Blattes. (Dissertation, Marburg, 1861). 
developes further only on one side, the new branches always arising either only on the left or only on the right side, 3 from 2 , and 4 from 3 ; every lateral branch thus produces a sympodial system, and in fact a helicoid cyme.

If now the basal pieces $2,3,4$, combined in a sympodial manner on both lateral shoots, are imagined to be much shortened, so that the bases of the lobes $2,3,4$ come close to the base of the lamina $\mathbf{r}$, then all the lobes of the leaf will appear to spring from one point, and the leaf is called digitate. It would appear, however, that such leaves may also arise by the formation from the broad end of the young leaf itself, first of a middle lobe, and then of new lateral lobes right and left from above downwards, as in Lupinus, according to Payer's drawings (Organogénie de la fleur, pl. 104). If the lobes remain completely united or have the appearance of a continuous lamella, we have a peltate leaf ${ }^{i}$. It is impossible to go more into the detail of these processes without numerous illustrations which cannot be given here. Fig. $\mathbf{I}_{4} 2$ will explain, in conclusion, the origin of the quadripartite lamina of the leaf of Marsilea Drummondi, according to
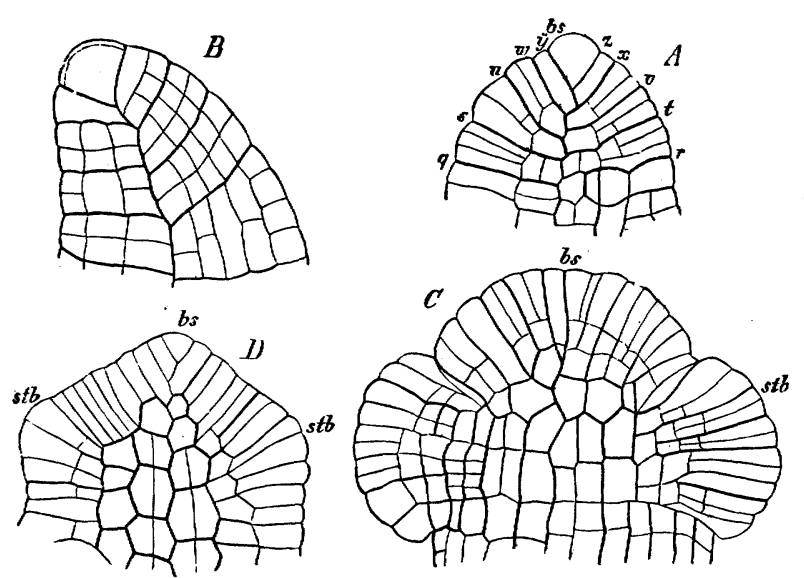

FIG. 142.-Development of the leaf of Marsilea Drummondi (after Hanstein). $A, C, D$ seen from the inner surface; $B$ longitudinal section vertical to $A$; bs apex of the leaf ; $q-z$ the segments of the apical cell; stb lateral lobes of the lanina in their earliest state.
Hanstein (Jahrb. für. wissen. Bot. vol. IV). The leaf has its origin in a cell of the cone of growth of the stem, which, becoming the apical cell of the leaf, produces two rows of segments from which the right and left halves are formed. Thus a broad cone first arises, growing at its apex, and bent towards the stem $(A, B)$; when this, which is the future petiole, has attained a certain height, it increases in breadth right and left. Beneath the still growing apex, $D, b s$,

a protuberance $(s t b)$ arises on both sides; and while the latter (destitute of an apical cell) becomes still more arched $(C, s t b)$, the apical growth of the leaf ceases $(C, b s)$, its apical cell disappears, and soon two equally strong outgrowths arise near the apical point, which, like the earlier lateral ones, increase vigorously and grow out into broad lobes of the leaf. Thus arises a quadripartite lamina at the end of the petiole, the lateral lobes of which have resulted from lateral branching, but the middle ones by dichotomy. The four lobes remain, as they grow, narrow at their base, becoming much broader at the free margin; and, since the part of the leaf from which they originated remains short and narrow, they appear, in the mature leaf, to spring from a single point, the end of the petiole.

(d) Branch-system of Leaf-bearing Shoots. The branching of the stem of Lycopodiaceæ is dichotomous. In Psilotum triquetrum all the branches develope uniformly; and this is the most regularly developed dichotomy found among vascular plants. In I.ycopodiex the development is much more irregular, but the bifurcation is always evident throughout; in Selaginellex, on the other hand, it is generally to be

1 Compare further Trécul, Formation des feuilles, in Ann. des Sci. Nat. vol. xx. I853; and Payer, l.c. p. 403 ; also Entwickelung der Blattgestalten, Jena 1846 . 
recognised only on the youngest branches, since the bifurcations are developed sympodially, and in fact as scorpioid cymes. This often happens (as in Selaginella fabellata) in such a manner that the entire outline of a branch consisting of numerous bifurcations assumes a form similar to that of a multipinnate Fern-leaf. The student who desires to obtain a clear idea of the different modes of development of a system produced from a dichotomous origin, and especially of the formation of sympodial forms out of dichotomies, could find no better object of study than the Selaginelleæ which are cultivated in all hot-houses. On the branching of the stem of Ferns and Rhizocarps, reference should be made to the description of the respective classes in Book II.

The branching always originates monopodially in the stems of Characex, Equisetaceæ, and Coniferæ, and here also its future, development is always racemose. The branch-systems of Mosses also always originate monopodially, but are sometime developed sympodially (as the 'innovations' of Acrocarpous Mosses beneath the sexual organs). It is often very irregular, but is sometimes of such a nature that much-branched systems of shoots develope racemosely and assume defined outlines, like those of multipinnate leaves, as in Hylocomium, Tbuidium, \&c.

The branching of Monocotyledons and Dicotyledons is always originally monopodial, but the mode of development of the system is extraordinarily variable; on the same plant, and even on the same branch-system, different forms, both racemose and cymose, may arise. The peculiarities of the different forms of development are usually very conspicuous in inflorescences, and are of many different kinds; and since the attention of botanists has been turned for a long time in this direction, they are not only copiously employed in the description of plants, but also furnished with names, which are here used in a more general sense. A more special description of those branch-systems which, in the case of Flowering Plants, are called Inflorescences, will follow in the general consideration of Angiosperms in Book II ; here it is only necessary to mention that the forms distinguished as spikes, racemes, and panicles are examples of the racemose development, while those termed dichasia, cymose umbels (in Euphorbia), and scorpioid and helicoid cymes, are examples of the cymose development of branchsystems which are at first monopodial.

Every other form of vegetative branching of Flowering Plants may be regarded from the same point of view. The formation of sympodia is not unfrequently brought about by arrest of the growth of the terminal portion or bud of the shoot, while the nearest lateral bud developes more vigorously, and appears like a continuation of the mother-shoot, as in Robinia, Corylus, Cercis, and many other plants; in the lime the primary stem itself is a sympodium formed in this manner. If the flower-bearing shoots above ground die annually, while the underground portions remain in a living condition, underground sympodia sometimes arise composed of the comparatively short but thick basal portions of numerous larger shoots which have long since died off. This is the case, for instance, in Polygonatum multiforum, the underground stem of which is known under the name of Solomon's Seal. In Fig. I43 is represented the anterior portion of one of these underground stems, those produced during eight previous years having been removed. The stem denoted by $b$ I 866 is the lower portion of the upright aërial shoot bearing leaves and lateral flowers, which was in existence in that year; but this shoot is itself only the terminal part, its much thicker basal portion is denoted in the diagram $B$ (as seen from above) by $n+2$; the slenderer terminal part dies off in the autumn, and at $b, b$, beneath the numbers $\mathrm{I} 86_{4}$ and $\mathbf{1} 86_{5}$, are shown the scars which remain behind after the death of the similar earlier terminal parts. The portion of the sympodium here represented thus consists of the three basal portions $n, n+1, n+2$, of three shoots, each of which unfolded its aërial portion bearing leaves and flowers in the year indicated. In the same manner the bud $n+3$ will now develope further; it springs from the axil of the leaf, the scar or insertion of which is denoted by $9^{\prime \prime}$. The basal portion of the shoot which proceeds from it will add a new piece to the sympodium, its terminal part 
will grow upwards, develope leaves and flowers, and then die off. Just as $n+3$ sprang from a leaf-axil as a lateral shoot of $n+2$, so did this also spring from $n+1$. Each of these shoots produced on its basal portion nine membranous colourless scale-like leaves ${ }^{1}$ which are still partially retained in $n+3$, while in $n, n+1$, and $n+2$, only their scars are to be seen; the numbers $\mathbf{I}-9$ indicate these in each year's growth. The new lateral shoot arises each year in the axil of the ninth and last scale-leaf, and the succeeding leaves are foliage-leaves on slender elongated internodes, while the internodes of the basal portion between the membranous scale-leaves are thick and short. The leaves are in two rows on the basal parts, alternately right and left, as may be seen by their scars; if the position of the ninth leaf of the segment $n$ is called left, then that of the segment $n+\mathrm{I}$ is right, that of the segment $n+2$ left; the shoots which continue the sympodium are thus again alternately right and left; and hence the sympodium is in this case a scorpioid cyme.

It is evident that the processes of growth would remain precisely the same, if, at the

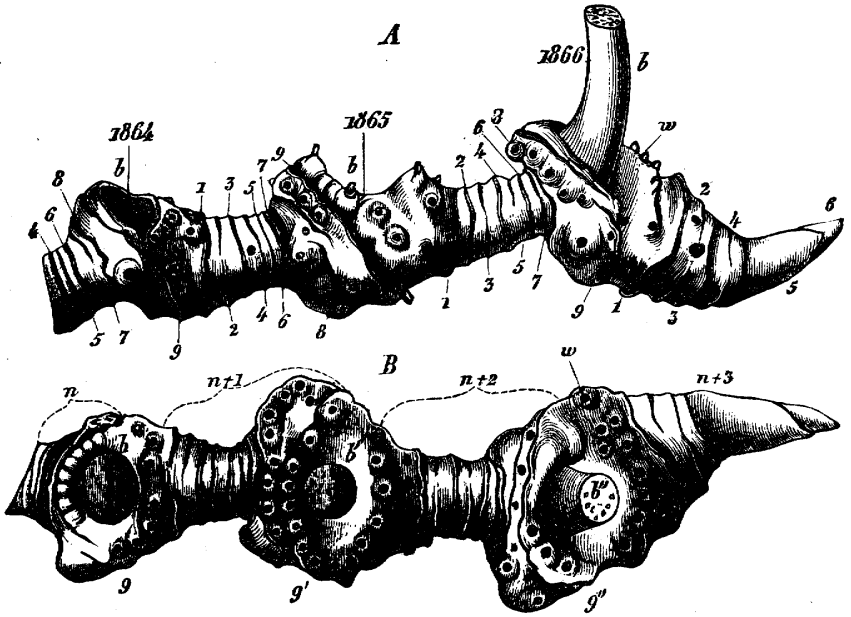

FIG. I43.-Polygonatum multifiorum; the anterior piece of a much longer rhizome, consisting of four annual growths. $A$ seen in profile, $B$ from above; all the adventitious roots have been cut off, their position being indicated by the roundish scars. The numbers $1864,1865,1866$ denote the years in which the respective pieces of the sympodium have been produced. close of each period of growth, after the bud for the next year had attained sufficient vigour, the whole shoot, including its basal portion, had died off and decayed; then, of course, no sympodium would be formed, but the development of the underground buds would nevertheless be sympodial. This occurs, for instance, in our native tuberous species of Opbrys, but with the difference that if a sympodium were actually formed, it would be a helicoid cyme. The processes in Colcbicum are similar, but somewhat more complicated.

The explanation of processes of growth of this nature requires much space, as is shown by the above example; I must refer therefore to the labours of Irmisch mentioned balow ${ }^{2}$. Where the leaves are clearly developed in Monocotyledons and Dicotyledons-and it is only in a few forms of inflorescence that this is not the case-it is almost always easy to understand the true nature of a branch-system, even without microscopic examination; because, with but few exceptions, the branching is axillary; the position of the leaves then makes it sufficiently clear which is mother-shoot and

${ }^{1}$ [Niederblätter or 'Cataphyllary leaves' of Henfrey; Braun's Rejuvenescence in Nature; in Ray Soc., Botanical and Physiological Memoirs, I $8_{53}$, p. 4.]

${ }^{2}$ Irmisch, Knollen und Zwiebelgewächse. Berlin 1850.-Ditto, Biologie und Morphologie dèr Orchideen. Leipzig 1853.-Ditto, Beiträge zur Morphologie der Pflanzen. Halle 1854, 1856.See also his papers in the Botanische Zeitung and the Regensburg 'Flora.' [Henfrey, Bot. Gaz. I 850 , I 85 I:] 
which lateral shoot when we have sympodial pseud-axes. Sometimes, however, distortions occur (e.g. in Solanaceæ) which might lead to erroneous conclusions if reference were not made to the earliest stages of development.

SECT. 26. The Relative Positions of Lateral Members on a Common Axis ${ }^{1}$.- In order to bring the facts which we have now to consider into a clear and simple arrangement, it is necessary, in the first place, to explain the use of a few technical expressions and geometrical modes of representation.

By the term Axial Structure or Axis is to be understood, in future, when the contrary is not expressly stated, any member that continues to grow at its apex and produces lateral members; for example, a mother-root with its lateral roots, a stem with its leaves ${ }^{2}$, the mid-rib of a leaf with its leaflets, pinnæ, or lobes, or a thallus-shoot with its lateral outgrowths.

If two or more similar lateral members proceed in different directions from the same transverse zone of an axis, they constitute a Whorl. A true whorl results when the zone of the axis which produces it is always at right angles to the axis (Fig. I 36 ); a Spurious or PseudoWhorl when the zone is the result of unequal development of the axis, or when lateral members which were formed at the same level have become so far separated by subsequent unequal elongation of the axis, that they appear, in the mature state, distributed into different zones. Simultaneous Whorls are those whose members are formed simultaneously (Fig. I44). Whorls are successive when the members at the same zone grow in succession either
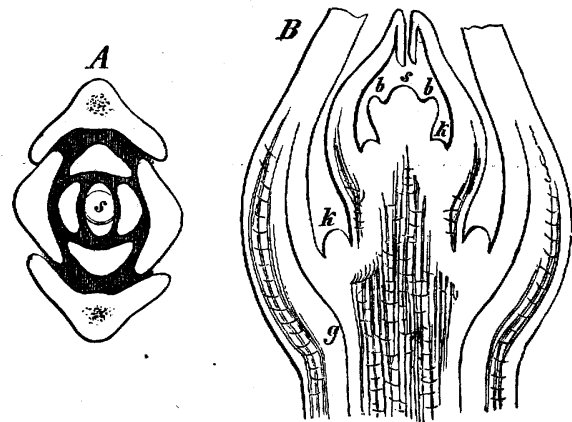

FIG. 144.-Apical region of a shoot of Coriaria murtifolia; $A$ in transverse section, $B$ in longitudinal section; $s$ apex of the stem; $; b b$ leaves in pairs, i.e. in decussate whorls of twos; $k$ axillary bud; $g$ youngest vessel.

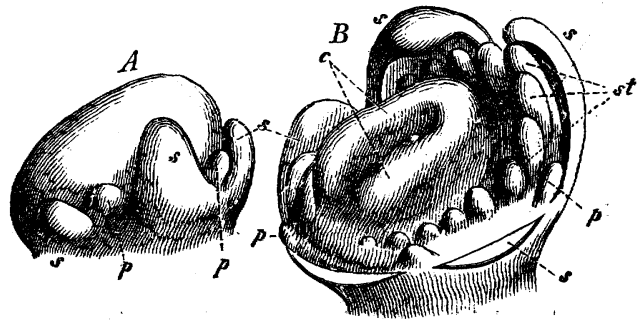

FIG 145-Development of the flower of the mignonette (after Payer); $A$ a younger, $B$ an older bud; from the latter the anterior sepals $s$ have been removed, the posterior ones left; $\not p p$ petals; st stamens, the posbeen removed, the posterior ones left ; $p p$ petals ; st stamens, the pos-
terior ones already large, the anterior ones not yet even in a rudimentary state; $c$ the rudiment of the pistil.

right and left, as is shown in Fig I45, and as occurs in the true leaf-whorls of

1 Röper, Iinnæa, I827, p. 84.-Schimper-Braun, Flora, i 835 , pp. 145, 737, 748.-Bravais, Ann. des Sci. Nat., vol. VII. 1837, pp. 42, I93.-Wichura, Flora, 1844, p. 161 - - Sendtner, Flora, I847, pp. 201, 21 7.-Brongniart, Flora, 1 849, p. 25.-Braun, Jahrb. für wissen. Bot. vol. I. I858, p. 307.-Irmisch, Flora, 185 I, pp. 81, 497.-Hanstein, Flora, I 857 , p. 407.-Schimper, ditto, p. 680.Buchenau, Flora, I860, p. 448.-Stenzel, Flora, I860, p. 45.-Numerous papers by Wydler, e. $g$. Linnæa, I 843 , p. I53; Flora, I $8_{44}, 18_{50}$, I $8_{5}$ I, I $8_{57}, 1859,1860,186_{3}$, and èlsewhere.-Hofmeister, Allgemeine Morphologie der Gewebe, $\$ 8$, 9. [Haughton, Manual of Geology.-Ellis, Mathematical Tracts.-A. Dickson, Trans. Royal Soc. Edinb. vol. XXVI. p. 505.-Chauncey Wright, Mem. Amer. Acad. vòl. IX. p. 379.-H. Airy, Proceedings Royal Society, vol. XXI. p. I76.Beal, American Naturalist, 1873 , vol. VII. p. 449.]

${ }^{2}$ [The term Phyllotaxis is used in works on descriptive botany to denote the mode of arrangement of leaves, and especially of the foliage-leaves on the stem.] 
Chara; or in a different order, as in the true leaf-whorls of Salvinia (vide infra), and in the three- or five-parted calyces of most Phanerogams.

The lateral members are, on the other hand, isolated or scattered when each member stands on a different zone of the axis. If the surface of an axial structure (which sometimes is quite imaginary, as in Nephrodium Filix-mas, \&c.) is supposed to be continued through the base of each lateral member, the section forms its Plane of Insertion. An imaginary point in this is considered its organic centre, but does not usually correspond to its geometrical centre; this point may be termed the Point of Insertion (see Sect. 27). A plane which bisects a lateral member symmetrically, or divides it into two similar halves, and contains the axis of growth of the lateral member as well as that of the axial member, passes through the point of insertion, and is called the Median Plane of the lateral member in question. If members are so arranged at different heights on an axis that their median planes coincide, they form a straight row or Orthostichy; generally there are two, three, or more orthostichies on an axial structure, and the members are then said to be recti-serial. If there are

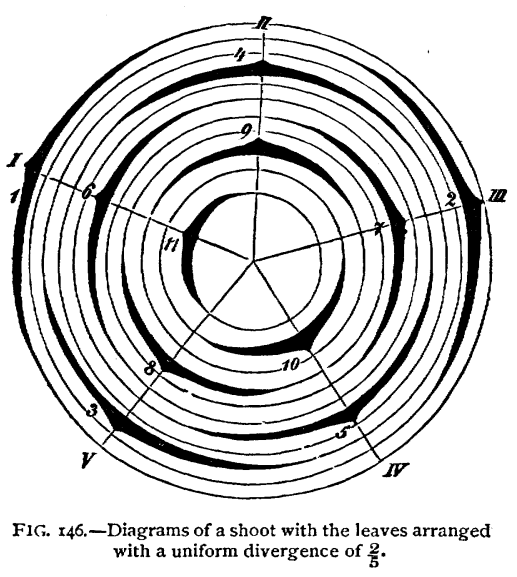

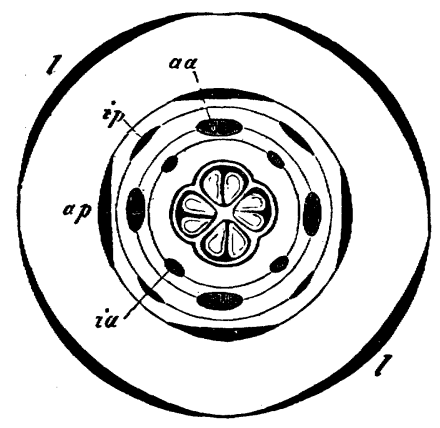

FiG. 147.-Diagram of the flower-stalk of Paris quadrifolia; $l l$ whorl of the large foliage-leaves beneath the flower; $a p$ outer, ip inner periantlr-whorl; $\alpha a$ outer, ia inner stamens; in the centre is the rudiment of the pistil consisting of four carpellary leaves.

no orthostichies, i.e. if the median planes of all the members intersect one another on an axis without coinciding, their arrangement is solitary.

The angle which the median planes of two members of the same axis enclose is their Divergence; it is expressed either in degrees or as a fraction of the circumference of the axis, which is then supposed to be a circle, although in fact this is not usually the case. In order to represent the divergences clearly, they may be drawn on a horizontal section of the vertical axial structure, in the manner represented in Figs. 146 and 147 . The transverse sections of the axial structure which bear the lateral members-in this case leaves-are denoted by concentric circles, the outermost circle corresponding to the lowest, the innermost to the highest transverse section. On these circles, which thus represent the relative ages from without inwards according to their succession in the acropetal development of the axis, the positions of the members are denoted by dots, or the forms of the planes of insertion themselves may be approximately indicated, as in the figures. On such a projection or diagram the median planes of the members 
appear as radial lines, indicated in Fig. 146 by $I-V$. Since in this case several members stand upon each median plane, they are arranged in orthostichies; and these again are so placed that they divide the circumference into five equal parts. But if the members are considered in reference to their age, as indicated by the figures $I-I I$, it is seen that the divergence between $I$ and 2 is $\frac{2}{5}$, as also is that between 2 and 3 , between 3 and 4 , and so on. The divergences are therefore all equal, or the members have in this case the constant divergence $\frac{2}{5}$. In Fig. 147 the members are arranged in a quaternary whorl; on each circle or section there stand in this case four similar members with the divergence $\frac{1}{4}$; but the successive whorls are so placed that the median planes of one whorl exactly bisect the angle of divergence of the preceding and following whorls; the whorls are here alternate, and all the members are arranged in eight orthostichies. If, on the other

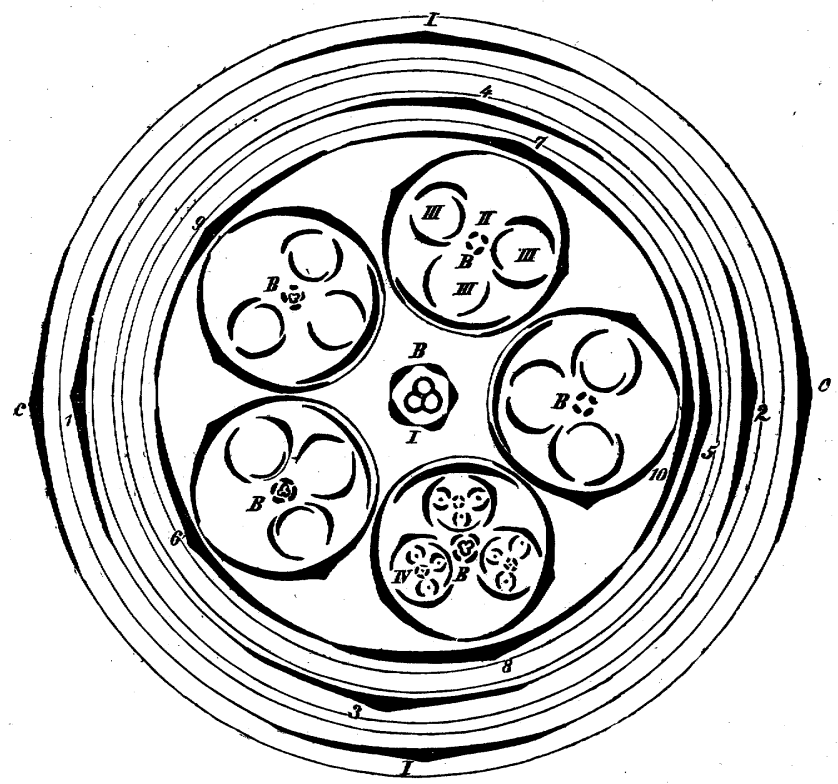

FiG. r48.-Diagram of a weakly plant of Euphorbia helioscopia ; $c c$ the cotyledons ; $I, I$ the first, I-to the later foliage: leaves; numbers 6 -ro form one whorl; at $B I$ in the centre is the terminal flower of the primary shoot, $B I I$ the terminal flower of one of the five axillaryshoots, $I I I, I I I, I I I$ the leaves of three axillary shoots of the second order.

hand, two whorls stand one over the other in such a manner that their members fall into the same median planes or cover one another, they are said to be superposed. Thus, for instance, the staminal whorl is superposed to that of the corolla in Primula; and in the primary roots of Phaseolus, Tropacolum, Cucurbita, and other Dicotyledons, superposed whorls of lateral roots not unfrequently occur. When alternate whorls have only two members, they are said to be decussate, as in Fig. 144, a very common arrangement with leaves.

If it is required to represent. by a horizontal projection not merely the divergences on an axis but those on an axial system, such as a system of leaf-bearing shoots, it may be done on the same principle, as is shown in Fig. 148. Each 
system of concentric circles comprises the members-in this case leaves-on an axis; the lateral axes-here secondary shoots-are interposed between the insertion of the respective leaves and their primary axis.

If the axial members are greatly shortened, the view (from above) of an axis, with its lateral members, often itself supplies the diagram; as, for instance, in the leaf-rosettes of Crassulaceæ, and in most flowers. In other cases a transverse section through the bud enables the observer to examine the divergence of the leaves; but in many other cases the relative positions are more obscure, and can only be ascertained by careful examination. In addition to the study of the history of development, particular methods, depending on geometrical principles, are often necessary in order to represent the relative positions correctly and at the same time clearly.

There are also circumstances in which it is desirable, instead of representing the relative positions on a horizontal projection, to project them on the unrolled surface of the axial structure, considered as a cylinder the surface of which is flattened out. The transverse sections of the axis lying one over another are denoted on this surface by straight horizontal lines on which the positions of the members are drawn.

Among the different arbitrary constructions which may be attempted on paper, for the purpose of comparing the relative positions of the members on an axis, or of reducing them to short geometrical or arithmetical expressions, the following has been employed. A line is imagined proceeding from any one of the older members in such a direction that, passing round the axis towards the right or the left, it includes the points of insertion of all the successive lateral members in the order of their age; the horizontal projection of this line is called the Genetic Spiral; in reality it is a spiral ${ }^{1}$ running round the stem more or less regularly. The importance of this construction has been very much overrated, and it has been employed where it is not only inapplicable to the elucidation of the history of development, but even where it has not even a geometrical meaning, and no longer assists a conception of the relative positions, but even makes it more difficult and complicated.

When we are dealing. with solitary leaves or shoots, standing out from the axis in three, four, five, eight, or more directions, and when the divergences are not too variable, the construction of the genetic spiral is of excellent service for a ready understanding of the position of the leaves (Fig. I49); and a more exact knowledge of the peculiar properties of this ideal line may, under these circumstances, be of great use in morphology. In some cases it may be applied with advantage even to the relative position of whorls; but in a large number other constructions appear much more natural, since they afford an easier explanation of the relative positions, and are more in accordance with the phenomena of growth. The construction of the desired genetic spiral is altogether impossible where the leaves are formed

1 If the spiral winds from right to left, the right edge of the leaves (as you ascend) is called the kathodic, the left edge the anodic; the reverse in the spiral of an opposite direction seen from without. 
in simultaneous whorls ${ }^{1}$, as the petals, stamens, and carpels of most flowers; or even in successive whorls where the members are formed in advancing order right and left, as in Characeæ and the flowers of Reseda (Fig. 145). In the successive whorls of Salvinia natans the construction of a genetic spiral would be equally impossible. Fig. I50, $B$ shows the diagram of the stem of this plant with three consecutive three-leaved whorls; in each of these the leaf $w$ is formed first, then the leaf $L_{1}$, and finally the leaf $L_{2}$. If an attempt be made to construct the spiral, it must pass from $w$ over $L_{2}$ across to $L_{1}$, then again in the same direction over $w$ across to $L_{2}$; the figure thus formed is a circle, in which the divergences of successive leaves vary greatly. If we now pass to the next whorl, the line proceeds in a spiral direction to the next

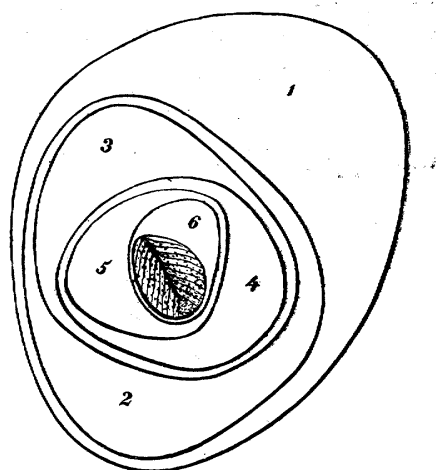

Fig. 149-Transverse section through the convolution of the leaf-sheaths $1-6$ of Sabal umbraculifera; in the centre is a young leaf-blade. The arrangement of the leaves is a 2 divergence. If the numbers $1-6$ are united by a line, the genetic spiral is obtained. leaf $w$; but then, to retain the genetic succession in the second whorl, the line must be continued in an opposite direction; and this is repeated with every new whorl. It is evident that no clear conception can be obtained in this forced manner, and the whole construction appears altogether superfluous, since it is required by no feature in the history of development. The stem of this plant is constructed, as Pringsheim has shown, of two rows of segments $(G$, $H, J, K$, \&c., in Fig. $\left.55^{\circ}, A\right)$, which arise alternately right and left from the apical cell. Even before the production of the leaves each segment undergoes various divisions, and in this manner the stem is built up of transverse disks which are in alternate succession nodes and internodes. Each nodal disk consists of the anterior half of an older segment and the posterior

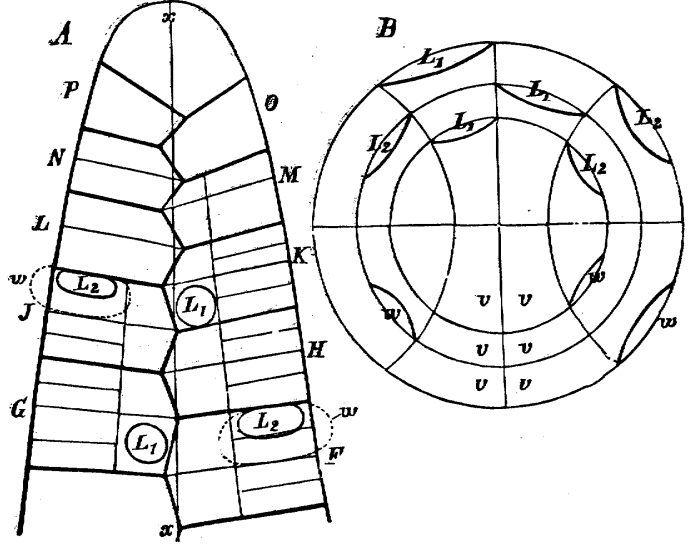

FIG. 150.-A the cone of growth of the stem of Salvinia natans, regarded diagrammatically and looked at from above; $x x$ projection of the plane which divides it vertically into a right and left half; the segments are indicated by stronger outlines, their divisions by thinner lines; the succession of the segments is denoted by the letters $F-P ; B$ diagram of the stem with three whorls of leaves, its ventral side indicated by $v v$; $w$ the first-formed floating leaf; $L_{1}$ the aërial leaf its ventral side indicated by $v v ; w$ the first-formed floating leaf; $L_{1}$ the aerial leaf
formed next; $L_{2}$ the second aèrial leaf of the same whorl formed last of all between the two first (after Pringsheim).

half of a segment next younger in age, as shown in the figure. An internode is formed of a whole segment of one row and of two half-segments of the other

1 Many writers employ even in such cases the conceptions borrowed from a spiral arrangement, considering arbitrarily as of successive origin the members of the whorl which arise simultaneously; but this is not in harmony with a true scientific method. 
row. Nodal cells occupying clearly-defined positions produce the leaves in the order stated. This development furnishes no evidence that the leaves are formed in spiral succession; the bilateral structure of the stem shows rather that a spiral construction is in this case altogether inadmissible. The same may be shown to be the case in Marsilea, where the creeping stem bears on its upper side two rows of leaves, while the under side forms roots; the leaves borne on the upper side may in this case be united in the order of their age by a zigzag

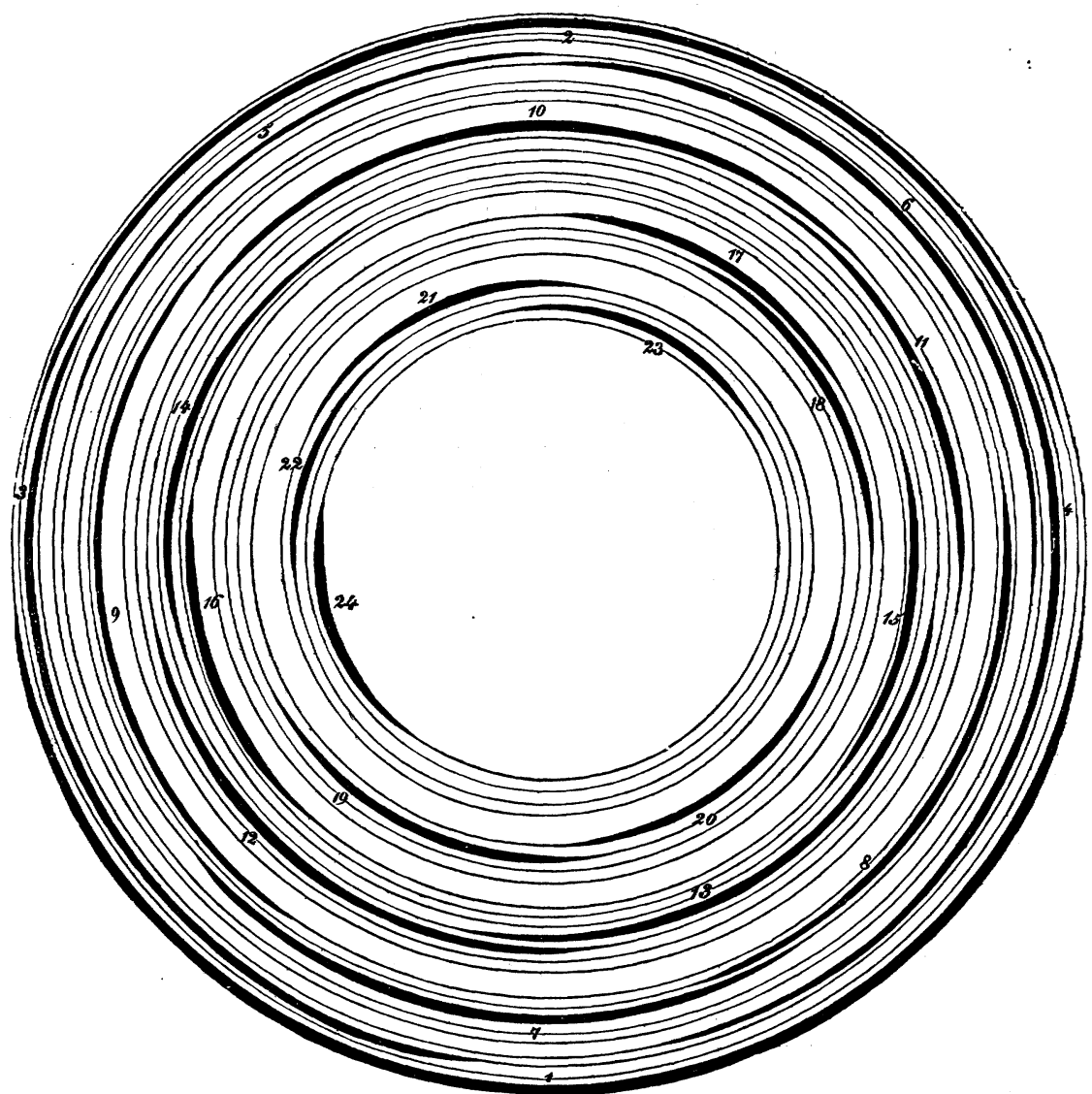

FIG. I5r.-Diagram of a flower-stalk of Fritillaria imperialis, showing the divergences of the first twenty-four foliage-leaves; the relative lengths of the internodes are indicated by the larger or smaller distances between the circles.

line broken right and left, which does not anywhere touch the leafless under side of the stem, and corresponds in its course to the bilateral structure of the stem. The spiral construction appears also to be meaningless in all those cases where it is indifferent whether the spiral be carried right or left. This is the case where the members are placed in two rows, with a constant divergence of $\frac{1}{2}$, and are thus arranged alternately in two orthostichies lying exactly opposite to one another, as is the case with the branchings of many thallomes (e.g. Stypocaulon, Fig. 108, p. r 39), the leaves of Grasses, the lateral shoots of the lime, elm, hazel, \&c. In all these 
cases of decidedly bilateral construction the genetic spiral might be imagined just as well, and with the same divergence, ascending right or left, by which of course it loses its importance for any morphological conclusion, as much as if one supposed it to change its direction from leaf to leaf.

It is principally in upright axes with solitary leaves arranged in three, four, five, or more directions, that the spiral construction appears conformable to nature, and agrees with the symmetrical relationships of plants, of which more will be said hereafter. The spiral construction proves to be opposed to nature in bilateral structures, especially in creeping or climbing stems, and in lateral branches.

In those cases in which the spiral construction may be employed naturally to elucidate the relative positions of the members, two cases may be distinguished, according as the divergences, on the one hand, are very unequal and change abruptly, or, on the other hand, are nearly or quite equal to one another or only change gradually. In the first case the members appear to be arranged irregularly and without order, as the foliage-leaves on the stem of Fritillaria imperialis (Fig. $\mathbf{1}_{5} \mathrm{I}$ ), the flowers on the rachis of the raceme of Triglochin palustre or of many Dicotyledons. When the change of divergence on the same axis is abrupt, it may also appear more natural to represent the phyllotaxis by two homodromal spirals instead of one, as in many species of aloe, where the shoots commence with leaves arranged in two rows, and then pass over into complicated divergences which lead finally to rosettes of leaves radiat-

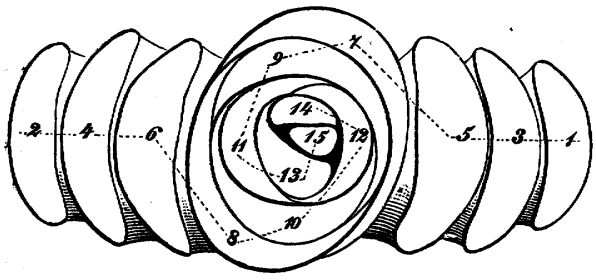

FIG. 152.-Transverse section of a shoat of Alö̈ Serra. ing on all sides. This occurs, e.g. in Alö̈ ciliaris, latifolia, brachyphylla, Lingua, nigricans, and Serra. Fig. I $5^{2}$ shows the transverse section of a shoot of the last-named species; the first six leaves are arranged alternately in two rows with a constant divergence $\frac{1}{2}$; at the $7^{\text {th }}$ leaf this arrangement is suddenly changed; instead of being placed over 5 , its position is between 5 and 6 ; but the 8th leaf exhibits the divergence $\frac{1}{2}$ from the $7^{\text {th }}$; the $9^{\text {th }}$ again changes the divergence, instead of being placed over 7 , it is between 7 and 6 ; the roth leaf again diverges about $\frac{1}{2}$ from the $9^{\text {th }}$; and so on. The leaves $7-15$ are evidently arranged in pairs, the pairs being 7,$8 ; 9, \mathbf{I 0} ; \mathbf{I} \mathbf{I}, \mathbf{I} 2 ; \mathbf{I}_{3}, \mathbf{I} 4$; each pair consists of two alternate (i.e. not opposite) leaves, the divergence of which is $\frac{1}{2}$; but the pairs themselves diverge from one another by smaller fractions. If it is desired to unite all the leaves from $\mathbf{I}$ to $\mathbf{I} 5$ by a genetic spiral, an abrupt alteration of the divergence would occur in it. The relative positions are shown, however, more simply and clearly if, keeping in view the bilateral origin of the shoot, two spirals are constructed, each of which commences from one of the original orthostichies, and, so to speak, continues it in a spiral curve; the one contains all the leaves with an even number, the other those with an uneven number; the two are homodromal, running in the same direction round the stem. The bilateral origin of the shoot 
may be followed in this manner up to the terminal rosette of leaves. Similar phyllotaxes appear to occur in Draccena and in some Aroideæ; and at first sight present the appearance as if the leaves were placed in two rows which have become changed into spirals by the torsion of the stem.

If we now turn to those cases which clearly gave rise to the erroneous hypothesis that the primary law of phyllotaxis is a universal spiral arrangement, we find the leaves placed singly, and their divergences almost or quite equal or gradually passing over into some other value, thus corresponding to the second case named above of spiral arrangement. In these cases the spiral construction affords a simple expression of the law of phyllotaxis; the only thing required is to name the constant angle of divergence;-according as this is $\frac{1}{3}, \frac{1}{4}, \frac{1}{5}, \frac{2}{5}, \frac{3}{8}$, \&c., the phyllotaxis is termed simply one of $\frac{1}{3}, \frac{1}{4}, \frac{1}{5}$, and so on. It is usual in such cases for the divergence not to remain constant for all the lateral members of an axis; shoots which form numerous leaves mostly begin with more simple arrangements, as $\frac{1}{2}$, and then pass over into more complicated ones, an arrange-

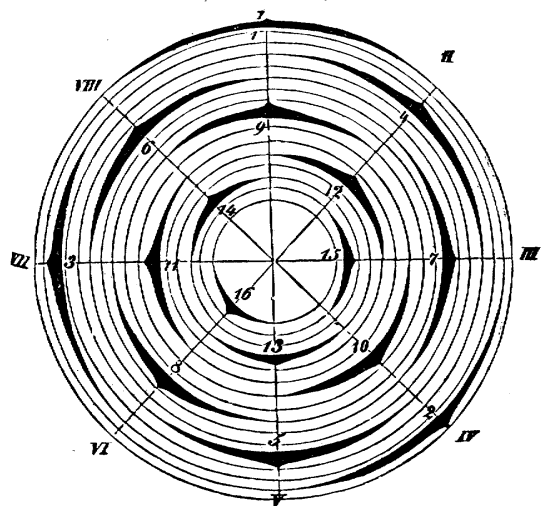

FIG. 153.-Diagram of a shoot in which the leaves have a constant phyllotaxis of $\frac{3}{8}$. ment being considered more complicated when the numerator and denominator of the fraction of divergence are larger. When the divergences between lateral members placed solitarily with a spiral arrangement are equal, they must also stand in straight rows, the number of which is expressed by the denominator of the angle of divergence. If, for instance, the divergence is a constant one of $\frac{3}{8}$, as in Fig. 153 , there are eight orthostichies, the 9 th member standing on the same median plane as the rst, the roth as the 2 nd, the IIth as the

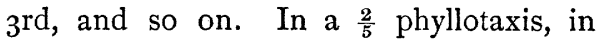
the same manner, the 6 th member stands over the ist, the 7 th over the 2 nd, and so on. In some cases the orthostichies are very obvious, as, for instance, in cacti with prominent angles to the stem, the angles corresponding to the orthostichies of the spirally arranged leaves, which, however, in this case mostly remain undeveloped. In verticillate leaves also the straight rows are mostly conspicuous if the shoot be looked at from above, as, for instance, in the decussate two-leaved whorls of $E u$ phorbia Lathyris, and the cactus-like $E$. canariensis.

When the members of a spiral phyllotaxis with a constant angle of divergence stand sufficiently close to one another, other spiral arrangements are easily seen, one of which may be followed to the right and the other to the left, and more or less completely concealing the genetic spiral. These rows are called Parastichies, and are particularly clear in fir-cones, the leaf-rosettes of Crassulaceæ, the flower-heads of the sunflower and other Compositæ, and the spadices of Aroideæ. They may be seen in every spiral phyllotaxis with a constant divergence, and can always be made clear in the diagram, or when the arrangement is represented on an unrolled cylindrical surface. The consideration of these constructions leads to definite 
geometrical rules, by means of which the genetic spiral can be easily deduced from the parastichies 1 .

It is evident that the constructions hitherto mentioned can only be more or less convenient aids to an understanding of the actual principles of the arrangement of leaves. But in order to obtain, with their assistance, a deeper insight into the processes of growth themselves of which these principles are the result, it is necessary to follow the development, and in every single case to ask the question; what circumstances are the cause of a new member being formed just in this place, and nowhere else. It may be well, therefore, to bring forward here some of the points which must be considered Areference to this view.

- (I) The first point is always to determine with certainty the order of succession in which the lateral members are formed.

(2) Attention must be paid not only to the lateral divergence, but also to the longitudinal distance at which a new member is formed at the growing point above the members last preceding it. The longitudinal distances of the youngest lateral structures of a growing point from one another are usually very small; there is often no space to be distinguished between them, $i_{\text {. }} e_{\text {c }}$ between the planes of insertion of the youngest members. This circumstance may, on the one hand, assist in the determination of the place where the next member must be produced; but, on the other hand, may give occasion, as the development of the axis proceeds with its crowded lateral members, to compression and distortion, by which the original arrangement is altered.

(3) By the increase in length of the common axis, members which were at first closely crowded become placed at a considerable distance from one another, while others, in consequence of slower growth, remain closely packed; so that a different distribution occurs in different parts of the stem, as in the leaf-rosettes and flower-stalks of Crassulaceæ, Agave, Aloe, \&c. In the same manner the angle of divergence frequently becomes changed by the more rapid increase in thickness of the axial structure on one side than on the other; and still more commonly by torsion round its own axis of growth. By such torsions lateral members, arranged at first exactly in straight rows, become displaced so that the orthostichies appear as if wound spirally round the axis. This occurs, for instance, according to Nägeli and Leitgeb, in the root-systems of Ferns, Equisetaceæ, and Rhizocarpeæ, as well as in the three-rowed phyllotaxis of the Moss Fontinalis antipyretica, according to Leitgeb. But the most striking example is furnished by the stem of the screw-pine, Pandanus utilis. In the bud, the numerous leaves, already strongly developed, stand, as is shown by the transverse section, in three perfectly straight lines with the phyllotaxis $\frac{1}{3}$; but, as the development of the stem advances, it undergoes so severe a torsion that the three orthostichies are transformed into three strongly curved spiral lines running round the stem (see Fig. 154). In these and similar cases the change in the relative positions caused by the torsion of the actual structure can be easily and certainly determined. But when the structures are so arranged at the apex of the axial structure that the angle of divergence cannot

1 As the treatment of the subject is only of value to those who are practically concerned with phyllotaxis, I must refer to the detailed description in Hofmeister's Allgemeine Morphologie, $\$ 9$. 
be accurately estimated by an apical view from above, it must remain uncertain whether the position of the mature members is unchanged, or has been altered by lateral displacement and torsion of the axis. A displacement, for instance, of about $9^{\circ}$ would be sufficient to alter the divergence from $\frac{2}{5}$ to $\frac{3}{8}$, a similar displacement of $1 \cdot 3^{\circ}$ would change the divergence from $\frac{5}{13}$ to $\frac{8}{21}$. When the phyllotaxis is very complicated and the number of the longitudinal rows very large, extremely smatl and almost inappreciable distortions are sufficient to destroy the original arrangement, and to bring into existence altogether different systems of

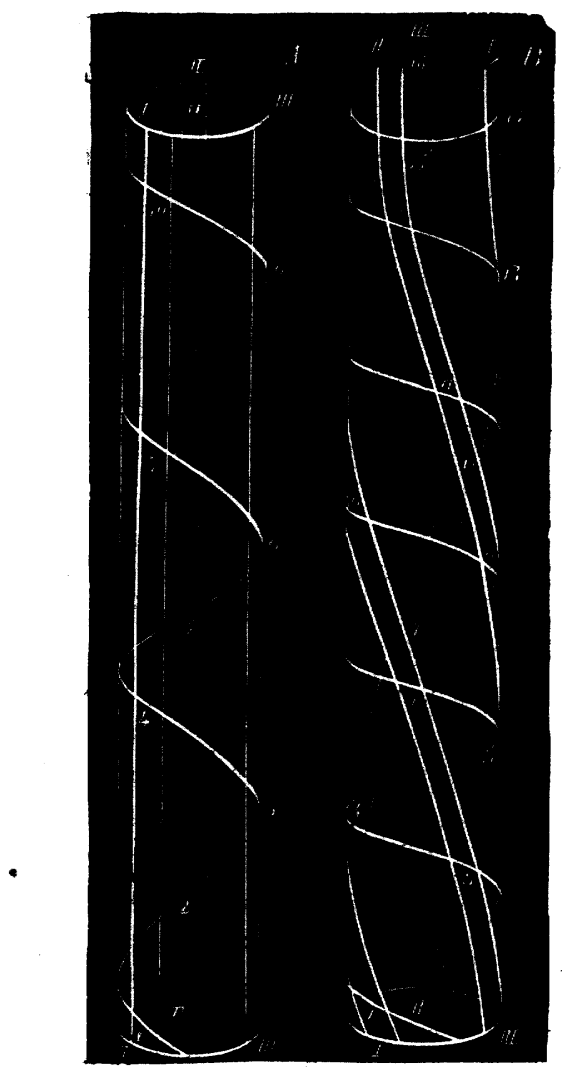

FrG. 154-Diagrammatic representation of the orthostichies of a $\frac{1}{d}$ phyllotaxis in Pandanus utilis; $A$ before, $B$ after the torsion of the stem. Each of the orthostichies $I, I I, I I I$ is indicated by a double line; the genetic spiral is simple; where it crosses the orthostichy, the leaf-insertions are indicated by figures.

leaves in the lateral buds before unfolding (see Fig. 155, p. 208).

(5) It must further be observed whether the first appearance of lateral

1 [See Airy, Proc. Royal Soc. l. c.]

2 Hofmeister (Allgemeine Morphologie, §§ 23, 24) has collected a series of facts which show relationships of this kind; but, both with reference to the individual facts and to the interpretation which he gives, I am deeidedly of a different opinion, the reasons for which would carry me too far. (Vide infra, Sect. 27.) terest so far as it makes it seem doubtful whether certain complicated phyllotaxes are always due to the original arrangement of the members ${ }^{1}$.

(4) It must be observed whether the position of newly-formed members or the subsequent change shows any relation to the direction of the force of gravitation, of the light which falls upon them, or of any pressure acting from without ${ }^{2}$. The effect of the force of gravitation is that primary shoots which are in the main upright put forth leaves spreading on all sides; while such as have a decidedly horizontal growth, in which a rooting under side is contrasted with an upper side, usually show an arrangement of leaves on the latter in two rows, or one which is divided into two equal halves by a plane cutting the stem longitudinally, as Salvinia, Marsitiea, Polypodium aureum, Pteris aquilina, \&c. When vertical primary shoots with leaves in several rows bear secondary horizontal branches with leaves in two rows, this relationship is less clear, as in the cherrylaurel, sweet chestnut, hazel, \&c., because an influence independent of gravitation must in these cases be presumed to be exercised by the primary upon the lateral axis, as is shown by the position of the

parastichies. This observation is of in- 
members is preceded by circumstances connected with their development which assist in determining their place of origin. Of this nature, for instance, is the connexion between the points of origin of lateral roots and the fibro-vascular bundles, the course of which determines the arrangement of the roots in rows; and this in turn determines the lateral roots being arranged spirally or in whorls. Here the arrangement in longitudinal rows is clearly the general and primary one; the divergences and longitudinal distances are a secondary effect determined by special accessory circumstances. The point of origin of a lateral shoot is, on the other hand, in general primarily determined by its relation to the nearest leaf, since it must be formed beneath, beside, or above its median plane; forces of secondary importance then determine whether lateral shoots are formed in connexion with each leaf or only with particular leaves of an axis, and so forth. The phyllotaxis of the lateral shoot may differ from that of its primary shoot, because the growth of the latter assists in influencing it; as, for instance, in the case of lateral shoots with a distichous phyllotaxis on primary shoots with an arrangement in several rows. Under this heading falls also the bilateral branching of leaves, whether the stem*itself be bilateral or multilateral. The dimensions of the growing point and the thickness of the axial structure derived from it may also determine the number of the rows of lateral structures; thus thick mother-roots usually produce three or more rows of seconcary roots, while more slender primary roots produce only two rows or at all events a smaller number. Thus, for instance, the roots of Cryptogams (according to Nägeli and Leitgeb), the thick primary roots of the maize, oak, pea, scarlet-runner, \&c., form three, four, five, six, or more orthostichies of lateral roots, which, on their part, are much slenderer and produce fewer orthostichies. The same is not unfrequently the case with the phyllotaxis of stems. When the size of the growing point increases, the leaves are arranged in a larger number of rows, as in the vigorous seedlings of many Dicotyledons, in Palms, Nephrodium Filix-mas, \&c. This is most strikingly exhibited in the many-rowed flower-heads of the sunflower on the four-rowed foliagestem, the size of the growing point undergoing a sudden and great increase at the period when the flower-head is being formed (Fig. 126, p. i7 I). But, vice versá, the number of the rows of leaves diminishes when the size of the growing end of the stem decreases in consequence of vigorous growth in length; this is seen, for instance, in the few-rowed long and slender peduncles which proceed from the

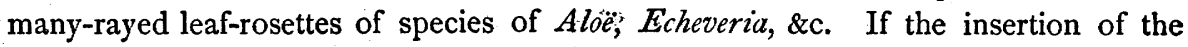
leaves or shoots takes up, at an early stage, a large part of the periphery at the growing point, only a few rows of leaves are formed; if the; insertion-planes are relatively small, the number of rows on the axis increases. This is illustrated by the many rows of small flowers in the spadices of Aroideæ or the racemes of Trifolium, while the leaves of the same plants are in few rows, their insertions embracing the stem or being even broader. Hofmeister ${ }^{1}$, to whom we owe the introduction of this point of view in the theory of phyllotaxis, states the general rule in the following

1 Allgemeine Morphologie, § 11, where particular cases are discussed in detail. This treatise is beyond question the most important that has hitherto been written on phyllotaxis; nevertheless, in my account, which necessary limits have confined almost to a mere sketch, I differ from Hofmeister's views even in some points of primary importance. 
words:-New lateral members have their origin above the centres of the widest gaps which are left at the circumference of the growing point between the insertions of the nearest older members of the same kind. The rule is illustrated by the case of alternating whorls (especially of pairs crossing one another at right angles, or 'decussating '), or by that of distichous leaves with a base which grows early in breadth, in Phanerogams where the growing point consists of small cells. Where, on the other hand, we have decidedly bilateral horizontal axes, as in Pteris aquilina, Salvinia, and Marsilea, or definite relations of the phyllotaxis to the segmentation of an apical cell as in Mosses, or distinctly successive formation of the members of the same whorl, as in Chara, Salvinia, the flowers of Reseda, \&c., the mechanical importance of the rule is, in my opinion, subordinate to the other causes which then have the greatest influence in determining the position of the new members. Independently of the points of view referred to in paragraphs $1-4$, the genetic relationships indicated in this paragraph show that it is scarcely possible to find a single rule which will govern all cases of phyllotaxis. Causes which belong to altogether different categories must, according to circumstances, exercise the greatest influence in determining the point at which a new member is formed.

(6) I consider it a circumstance of primary importance that the same or very similar kinds of phyllotaxis may be brought into existence by very different combinations of causes, and arrangements apparently very different by very similar combinations of causes. Among the causes here referred to I include the anterior development of the axis and of its lateral members, the influence of the primary on the secondary axes, the effect of pressure, gravitation, light, and similar conditions. This position becomes evident when it is observed that the same or similar divergences of leaves or lateral shoots may occur everywhere, in unicellular plants, in multicellular plants with a distinct apical cell, and in those in which the growing point consists of a small-celled tissue without any definite relation to the segmentation of an apical cell, as in Phanerogams. The mechanics of growth must undoubtedly be different when the lateral branches of the single cell of Vaucheria are formed in two rows, and the leaves of a Fissidens or of a Grass are produced in the same or a similar position, in which case the cell-walls of the primary meristem represent a multiplicity of causes of growth and of hindrances to it. The similar arrangement of the outgrowths under such different circumstances does not prove that the circumstances themselves are indifferent, but only that altogether different combinations of causes may lead to very similar relationships of position. In Muscinex and Vascular Cryptogams the relation of the formation of leaves to the segmentation of the apical cell is the more obvious the nearer the leaves originate to the apex. It is most obvious of all in Mosses, where each segment grows out into a leafforming protuberance as soon as it is formed, and before further cell-division takes place. Here the immediate controlling cause of the position of the leaves is that of the leaf-forming 'segments' themselves; when these latter are formed in two alternating longitudinal rows, as in Fissidens ${ }^{1}$, two rows or orthostichies of alternating leaves arise with the divergence $\frac{1}{2}$. When the segmentation of the apical cell is into

1 Lorentz, Moosstudien. Leipzig 1864. 
three rows, so that each new division-wall of the apical cell is parallel to the last division-wall but two, as in Fontinalis, three rows of leaves result, arranged spirally with the constant divergence $\frac{1}{3}$. When the apical cell is a three-sided pyramid, but the new walls which are formed in it are not parallel to those already in existence, but oblique, so that, for example, all the segments are broader on the anodic than on the kathodic side, then the segments no longer lie in three straight rows, but either three spirals or one only can be recognised encircling the axis; and since each 'segment' in this case (..$g$. in Polytrichum, Catharinea, and Sphagnum $\left.{ }^{1}\right)$ developes into a leaf, the leaves are formed in spiral phyllotaxes, with divergences depending on the obliquity of the principal walls of the segments to one another ${ }^{2}$. These phenomena show clearly that when each segment produces a leaf, the phyllotaxis depends on the manner in which the new principal walls of the segments arise; and since the direction taken by the segmentation of the apical cell depends again on causes of which we are at present ignorant, the phyllotaxis must also finally be referred to these unknown causes. In certain cases a reason may be given why, when the mode of segmentation of the apical cell is the same, the positions at which the leaves are formed are nevertheless variable. The segments of the apical cell, both in Fontinalis and in Equisetum, lie in three straight rows; but in Fontinalis the solitary leaves stand in straight rows and are arranged spirally with the constant divergence $\frac{1}{3}$, while in Equisetum, on the contrary, alternating whorls of leaves arise which have grown together in the form of a sheath; because here, as Rees has shown ${ }^{3}$, the three segments of each cycle, arranged originally in a spiral manner, are finally placed, in consequence of the growth not being uniform, on the same zone. From this a circular projection next grows out, on which the sheath-teeth are formed. From the want of uniformity in the growth of the segments, the causes of which are at present unknown, still further differences, as compared with Fontinalis, are introduced, in consequence of which the development of the whorls themselves becomes alternate instead of superposed, as might be the case. If the processes which take place in Marsilea, as Hanstein has described them ${ }^{4}$, are compared with this, it is seen that the segmentation of the apical cell of the stem agrees in the main with that of Fontinalis and Equiselum; it is in three rows with a divergence $\frac{1}{3}$. As in Fontinalis, the leaves originate by a curving outwards of the segment-cells; but the leaves are in this case not arranged in three rows as in Fontinalis, nor in whorls as in Equisetum, but in two rows. The immediate cause of this must be sought in the fact that the stem, together with the growing point, lies in a horizontal position; it has an upper and an under side. The segments of the apical cell form two rows on the upper and one on the under side; but the former produce leaves, the latter roots. The horizontal position of the stem and its bilateral development are here perhaps the cause why the upper side

1 Compare the admirable description by Leitgeb in the case of Sphagnum, in the Sitzungsber. der kais. Akad. der Wissenschaften, Wien, March 1869.

${ }^{2}$ See Hofmeister, Allg. Morph. p. 494; and Müller, Eine algemeine morphologische Studie, Bot. Zeitg. I869, t. IX. fig. 24. In such cases the behaviour of the apical cell may be represented by imagining it to rotate on its axis, as I expressed it in my first edition. The description there given does not however now appear to me suited to the beginner.

3 Rees, Jahrb. für wissen. Bot. vol: VI. p. 216.

4 Hanstein, in Jahrb. für wissen. Bot. vol. IV. p. 252. 
only produces leaves; and since its segments lie in two rows, there are two rows of leaves, which we may imagine united by a zigzag line. But a further cause of the difference, as compared with Fontinalis and Equisetum, arises from the fact that in Marsilea it is not every segment of the two rows on the upper side that forms a leaf; according to Hanstein, certain segments remain sterile, and these form the internodes which are at first wanting in Fontinalis and Equisetum, and are only formed at a later period by further differentiation and intercalary growth. In Pteris aquilina and in Salvinia the segments of the apical cell of the stem are also formed, as in Fissidens, in two rows; but the phyllotaxis is in all these cases very different. The effect of the difference of growth is first of all shown in the decidedly horizontal position of the stem of these plants, and also in the circumstance that the segments themselves grow vigorously in thickness and length, and divide before the formation of the leaves commences; it is not from the segment-cells which are already in existence that the leaves originate, but from certain cells resulting from their division at a distance from the apex of the stem. This is common to Pteris and Salvinia; but in the divisions of the segments and in the whole growth of the stem considerable differences between the two occur. Pteris aquilina forms on the upper side of its thick underground horizontal shoots two alternating rows of leaves, while Salvinia forms alternating whorls on its slender floating shoots, the members of the whorls showing a very peculiar order of succession corresponding to the bilateral arrangement and the horizontal growth of the axis.

The genetic forces which have an evident influence on the phyllotaxis of Cryptogams through the segmentation of the apical cell and the further behaviour of the segments, are wanting in Phanerogams, where the leaves spring from a small-celled cone of growth the tissue of which behaves like an almost homogeneous plastic mass. The immediate causes which determine the spot where a leaf or shoot is to arise can no longer be referred here, step by step, to the behaviour of an apical cell; they lie rather in the position of leaves already in existence, in their increase in breadth, in the form and size of the cone of growth, in its inclination to the vertical, in its relation to the size of the mother-shoot, \&c.- - conditions which, as has already been mentioned under paragraph 5 , have been treated in detail by Hofmeister. The rule there enunciated, that lateral shoots arise above the centres of the widest intervals between the youngest contiguous shoots, gives an efficient cause for the determination of the place of origin of new members, and may be applied also to the first leaves of lateral shoots, which generally show a definite relationship to the subtending leaf. In Monocotyledons, for instance, the first leaf of an axillary shoot usually stands on its posterior side, i.e. next the mother-axis; while in Dicotyledons the axillary shoot generally begins with two leaves, which stand right and left of the median plane of the subtending leaf, and thus fall in the space between it and the primary axis which is least exposed to pressure.

As has now been shown in this brief introduction, the investigations of phyllotaxis cannot at present do more than ascertain in each separate case the phenomena preceding and accompanying the origin of a member, as well as those forces which, from their direction, exercise an influence on the point of origin, and then lay down more general laws as the result of comparison in a sufficient number of cases. In these as in all other investigations into organisms, we are 
always however met at the very outset by a consideration of great importance which imposes itself upon us, I mean the tout ensemble of properties which define the character of the natural group, class, or order. By recognising a plant as a member of a particular class, e.g. Muscineæ, Filices, Equisetaceæ, Rhizocarpeæ, Phanerogamia, \&c., an aggregate of properties is ascribed to it, which must be taken into account as such. If we pay special regard to the point of view opened out by the Theory of Descent, we must recognise in the law of heredity and the physiological adaptation of organs, the difficulty or even impossibility of demonstrating the causes of any morphological phenomenon in any other manner than genetically. Organic forms are not the result of combinations of forces and materials given once for all, and always again reproduced in exactly the same manner, as in the case of a crystal which is first dissolved and then re-crystallised, but of combinations which repeat themselves hereditarily and which at the same time undergo change. To understand these it is necessary to refer to the past, and not merely to the immediate present.

Abundant opportunity will be afforded in the description of the various classes in Book II. for a more exact observation of particular relations of position; but what has now been said is sufficient as a preliminary. Some additional remarks on the Spiral Theory in the doctrine of phyllotaxis may however find a place here. It has already been shown that the construction required and employed in this theory is not in all cases possible, being sometimes arbitrary and without relation to development, and at other times simply meaningless; and that, finally, only those cases"admit of the application of this theory without violence, where the shoot forms three or more rows of leaves distributed singly and uniformly in all directions. The history of development often points to quite different constructions, even in those cases in which the spiral is still geometrically possible. But even where the connection of the leaves in order of succession in age by a spiral running round the stem in the same direction is possible and even apparently useful, there is not in the phenomena connected with development any sufficient reason for the hypothesis that the growth of the generating axis itself actually follows a spiral ${ }^{3}$.

Closely connected with the spiral theory, which must be carefully distinguished from the doctrine of phyllotaxis, is another very peculiar law connected with the angles of divergence. It was thought, namely, that a kind of natural law was found when it was discovered that some of the most commonly occurring constant divergences $\frac{1}{2}, \frac{1}{3}, \frac{2}{5}, \frac{a}{8}, \frac{5}{13}$, and some of the less common ones, as $\frac{8}{21}, \frac{13}{2} \frac{3}{4}, \frac{21}{45}, \frac{55}{114}, \&$ c. ${ }^{2}$, may be represented as successive convergents of the continued fraction

$$
\frac{I}{2+\frac{I}{I+\frac{I}{I}}}
$$

Were it possible to combine all kinds of phyllotaxis without exception in this manner into one single continued fraction, we should actually have a kind of natural law, in which there would be no relation of cause and effect, and which would hence stand out as an

${ }^{1}$ See on this point Hofmeister, Bot. Zeitg. 1867, nos. 5, 6, 7, and Allgemeine Morphologie, p. $4^{81}$.

${ }_{2}^{2}$ It must be observed in reference to this that it remains uncertain whether such complicated divergences are ever so formed originally, or whether they are not always consequences of complicated displacements, in consequence of which the direct observation of the growing point does not give in these cases a certain conclusion. 
inexplicable curiosity. This is not however exactly the case. There are many phyllotaxes which cannot be expressed by this continued fraction; and in order to carry out the method, new continued fractions have to be constructed, e.g.

$$
\frac{I}{3+\frac{I}{I+\frac{I}{I}} \ldots . .} \quad \text { or } \quad \frac{\mathrm{I}}{4+\frac{\mathrm{I}}{\mathrm{I}+\frac{\mathrm{I}}{\mathrm{I}}}} \quad \& \mathrm{c}_{\text {. }} \text {; }
$$

of which indeed only one or two convergents are for the most part met with as actual angles of divergence. And since it is possible immediately to construct a new continued fraction for every phyllotaxis which cannot be arranged under those already in existence, it is of course possible to represent by this method all varieties of phyllotaxis; but it follows at the same time that the method itself thus loses all deeper significance. If those divergences only occurred on one and the same axis or on one system of axes which can be represented by convergents of one and the same continued fraction, or if the different values of one particular continued fraction occurred exclusively in a genus, family, or order, the method would even in that case be of some value. But this is not the case. Since moreover no actual relationship of the method to the history of development, to the classification of plants, or to the mechanics of growth, has been established, in spite of numberless observations, it seems to me absolutely impossible to imagine what value the method can have for a deeper insight into the laws of phyllotaxis. But even as a mnemonic assistance it appears to me not only superfluous, but even disadvantageous, since the use of it diverts the attention from relationships which are of real importance ${ }^{1}$.

SECT. 27. Directions of Growth ${ }^{2}$.-(I) In every thallus, branch, stem, leaf, hair, and root, it is easy to distinguish between two opposite ends, the Base and the Apex. The base is the place where the member originated and began to grow; the apex is the extremity in the direction of the growth. The direction from the

1 [Chauncey Wright (Memoirs of Amer. Acad. vol. ix. p. $3^{89}$ ) has pointed out an interesting property of the series $\frac{1}{2}, \frac{2}{3}, \frac{2}{5}, \frac{3}{8} \ldots \ldots$ which includes all the more common arrangements of phyllotaxis. If the spiral line passing through successive leaves be traced the long way round, we obtain the complementary series $\frac{1}{2}, \frac{2}{3}, \frac{3}{5}, \frac{5}{8} \ldots .$. the terms of which are successive convergents of the continued fraction

$$
\overline{I+I} \frac{}{I+\frac{I}{I+8 c .}}
$$

If we put this $=K$

or

$$
\begin{aligned}
& \text { then } K=\frac{\mathrm{I}}{\mathrm{I}+K} \\
& K^{2}=\mathrm{I}-K \\
& \therefore \mathrm{I}: K=K: \mathrm{I}-K
\end{aligned}
$$

or $K$ is the ratio of the extreme and mean proportion; and generally

$$
K^{n}=K^{n-2}-K^{n-1} \text {. }
$$

$K$ is therefore the angular divergence which would effect ' the most thorough and rapid distribution of the leaves round the stem, each new or higher leaf falling over the angular space between the two older ones which are nearest in direction, so as to divide it in the same ratio, $K$, in which the first two or any two successive ones divide the circumference. Now $\frac{5}{3}$ and all successive fractions differ inappreciably from $K$,' Practically, therefore, all terms of the series above the third fulfil the condition of that leaf-distribution which is theoretically the most efficient by distributing the leaves most completely to the action of the surrounding atmosphere.]

${ }^{2}$ H. von Mohl, Ueber die Symmetrie der Pflanzen, in his Vermischte Schriften. 1846.Wichura, Flora, 1844, pp. I6 I et seq.-Hofmeister, Allgemeine Morphologie, §§ I, 23, 24.-Pfeffer, Arbeiten des botan. Instituts in Würzburg, 1871, p. 77 . 
base to the apex is the longitudinal direction of the member under consideration; and a section made in this direction is called a longitudinal section. The transverse direction and section of the member are at right angles to the longitudinal ones.

(2) In-every transverse section of a member there is a point about which the internal structure and external contour are so arranged that it must be considered as its Organic Centre. Every line drawn from this point towards any point of the circumference is a radius; every portion of the transverse section has one side facing the circumference and one facing the centre, these being usually developed in a different manner from the sides that face the radii, and hence easily distinguishable from them. These relationships are recognised with ease in the transverse section of woody stems and of all roots, but can be easily made out in other cases also, even in unicellular plants and hairs. The organic centre of the transverse section does not usually coincide with the geometrical centre, as is easily seen in the transverse sections of most petioles and horizontal branches with an 'eccentric' pith.

(3) If a line be imagined uniting the organic centres of all the transverse sections of a member, this is the Longitudinal Axis or Axis of Growth of the member. The axis of growth may be a straight or a crooked line; in the younger parts' (nearer the apex) it may be crooked, and again straight in those which are further developed (further from the apex), as in Salvinia and Utricularia; or the reverse. A plane which passes through the member in such a manner as to contain the axis is called an Axial Longitudinal Section. If the axis be curved in a plane, this plane coincides with the axial longitudinal section; if the axis is straight, the number of possible axial longitudinal sections is very large or even infinite.

Growth in the direction of the longitudinal axis is generally quicker, and also generally lasts longer, than in the transverse directions, as is clearly seen in most stems (haulms, flower-stalks, scapes, palm-stems), in long leaves, in all roots, and in most hairs and thallomes. This characteristic cannot however be used in the general definition; for there are cases in which it appears doubtful whether the growth in the direction of the longitudinal axis is more intense or more prolonged than in the radial directions; as, $e . g$. in the stem of Isoëtes, and the prothallium of some Polypodiacex. But the characteristic is superfluous for the determination of the longitudinal axis; its direction can always be recognised by the position of the base and apex of a member; and the point where it cuts the transverse section (the organic centre) can be found without anything else being known about the relationships of growth. It is always possible, without even knowing the duration or intensity of the growth, to decide which is the longitudinal and which the transverse section of a member; this can indeed be determined from a very small fraction of it; in a Mammilaria, a Melocactus, or a Cereus, it is just as easy to determine the longitudinal axis of growth in early youth, when these cacti are often as thick as they are long, as it is later when they are much longer than thick. This is also the case in the abbreviated axis of bulbs, in many tubers and corms (as the crocus), and in fruits, like those of many gourds, whose diameter is much greater than their length.

The growth of roots and stems in the direction of their longitudinal axis is generally unlimited, that of leaves and hairs mostly limited, although these rela- 
tionships are sometimes reversed. When the growth is unlimited, its products along the axis are usually constantly repeated, the segments formed one after another are similar, the lateral members that spring from them (branches, leaves, lateral roots, \&c.) are uniform, or they exhibit in their development a repeated alternation, as, e.g. in Moss-stems, rhizomes of Equisetum, primary stems of Conifers, \&c. When, on the contrary, the growth along the axis is limited and definite, the resulting segments are dissimilar, and their outgrowths exhibit progressive changes (metamorphosis). This occurs in most leaves, the basal portions of which are usually strikingly different in form from the parts nearer the apex; it occurs also in the stems of Angiosperms with terminal flowers, which commence, for instance, with the formation of radical leaves, proceed to that of foliage-leaves, and then, through the bracts, pass over into the production of floral leaves, closing with that of carpellary leaves.

Axial growth is always limited when true dichotomy occurs at the apex; on the other hand, bifurcations repeat and continue the mode of development of their common basal portion (as in Fuccus or Selaginella), although individual branches may terminate their growth without dichotomy by producing fruit.

(4) If an axial longitudinal section is imagined to pass through a member, the conformation right and left may be similar, like the right and left halves of the human body. If the two halves are so similar that the one is a reflected image of the other, they are symmetrical, and the dividing plane between them is called a plane of symmetry. In this strictest sense symmetry is very rarely found in plants (most nearly in many flowers and stems with decussating whorls); and accordingly the term is constantly employed in a laxer sense. Two, three, four, or a larger number of symmetrically dividing planes often pass through a member (a branch or root), all of which intersect in the axis of growth. Such members are called polysymmetrical; so-called 'regular' flowers, stems with alternating whorls, and most roots, are polysymmetrical. If, on the contrary, it is possible to imagine only one symmetrically dividing plane, as in the flowers of Labiatæ and Papilionaceæ ${ }^{1}$, in stems with opposite pairs of leaves, where the median plane of the two rows of leaves is at the same time the plane of symmetry, in the thalloid shoots of Marchantia, and in most leaves, the object is monosymmetrical, or simply symmetrical. Monosymmetry is however only a particular case of the ordinary bilateral structure, which consists in the processes of growth being similar to the right and left of an axial longitudinal section, although the two halves of the member do not lie exactly opposite to one another like reflected images. Thus, for example, the oblique leaves of Begonia are not symmetrical, although bilateral; the one half to the right of the mid-rib of the lamina is larger and of somewhat different shape to the other half to the left of the mid-rib; and the same is the case with the elm. A branch with alternating leaves in two rows is also simply bilateral without being monosymmetrical; if it is divided at right angles to the common median plane of all the leaves, the two halves bear each one row of leaves; but the one is not the reflected image

${ }^{1}$ A. Braun calls monosymmetrical flowers zygomorphic, an expression which is also elsewhere interchangeable with monosymmetrical. 
of the other, since the leaves of the two rows spring from different heights. Where a true monosymmetrical structure occurs, it may be considered a particular case of the bilateral; the latter, therefore, being the more common, is the more important phenomenon.

There is the same relationship between polysymmetry and multilateral arrangement as between monosymmetry and bilateral arrangement; polysymmetry must be considered only as a particular case of the multilateral structure. This latter always occurs where several pairs of halves can be produced by axial longitudinal sections, so that the two halves of each pair are very similar to one another, but not exactly alike, like an object and its reflected image. Thus the short stems of Sempervivum, the leaf-rosettes of Exonium, and fir-cones with their scales, can be easily halved by numerous longitudinal sections, but the halves thus formed are never symmetrical, because the leaves and scales are arranged spirally, and a spiral can never be divided symmetrically; but in so far as the spirally arranged leaves stand in three, four, five, eight, thirteen, \&c. orthostichies, the shoot itself may be said to be tri-, quadri-, quinqui- octo-, trideci-lateral, \&c.

The most common distinction is between bilateral and multilateral structures; in both cases the lateral arrangement may rise into symmetry, the former into monosymmetry, the latter into polysymmetry. The extremes are seen on the one side in roots with a circular transverse section, on the other side in most leaves and leaf-like shoots with only two symmetrical halves. If, however, in the case of roots regard is paid to the number of their fibro-vascular bundles, the apparently infinite number of their planes of symmetry may usually be reduced to two, three, four, or five.

To obtain a convenient mode of expression for relationships of this kind, each longitudinal section which produces two similar halves may be termed a principal section or principal plane; and if the two halves are symmetrical it is a symmetrical section or plane. Thus bilateral structures have one principal section, multilateral structures two or more principal sections.

(5) Lateral arrangement and relationships of symmetry may be looked at from two important points of view, according as the members of a plant are compared with one another, or are considered in reference to their relation with the external world, with gravitation, light, or the pressure of external objects.

If the members of a plant are compared with one another, it is seen, for example, that the principal sections of all the leaves, though on opposite sides of the stem, may lie in one plane, in which case the shoot itself is bilateral; or they may lie in two planes crossing one another at right angles, when the shoot is quadrilateral, as, for instance, when it bears decussate whorls of two members, a case which, in reference to other relationships, is very near to that of bilateral arrangements, and may be termed a double bilateral arrangement. In these cases the principal sections of the leaves are also at the same time principal sections of the stem. In Salvinia, Marsilea, Polypodium aureum, and Pteris aquilina, on the contrary, the principal sections of the leaves, forming two rectilineal series, lie right and left of the single principal section of the bilateral stem, an arrangement which is in these cases dependent on the horizontal growth.

The relationship of lateral arrangement and symmetry to the external envi- 
ronment of the plant is shown, for example, in the fact that multilateral shoots usually grow upright, while bilateral shoots generally lie horizontal, the principal section being then vertical. Many bilateral shoots cling on one side to a horizontal, oblique, or vertical support, as the Marchantieæ, Jungermannieæ, the ivy, \&c.; and in that case the principal section is at right angles to the support. Bilateral structures, leaves or whole shoots and systems of shoots, generally form their two sides to which the principal section is perpendicular very differently in respect to the external world; so that, in addition to a right and left half (right and left of the principal section), there is also a clear distinction between an upper and under side, an attached and free side, a dark and light side; and it is in this respect that the dependence of lateral arrangement on external conditions is most clearly evident.

In each special case it must, however, be left for more exact observation to settle how far the position of the principal sections of the members of a plant are determined by internal relationships of growth, or by external influences ${ }^{1}$, a question which can seldom be satisfactorily answered when not decided by experiment. To this end the researches on Marchantia polymorpha, begun by Mirbel in 1835 and carried on with great success by Dr. Pfeffer in $1870\left(l . c_{.}\right)$, are of peculiar interest. Dr. Pfeffer shows that the two flat sides of the gemmæ of this liverwort are identical; $i . e$. each of the two sides has the power of forming root-hairs when turned downwards. Bilateral arrangement and differentiation of the upper and under sides are first developed in the flat shoot which springs from the gemma. The side of the shoot exposed to light, whatever its position, becomes under all circumstances the upper side which forms stomata, the dark side becomes the under side which produces root-hairs and leaf-like processes. Even after the lateral shoots have been formed, the two sides of the gemma itself are still alike. Similar. relationships may also prevail in the germinating spores of creeping Jungermannieæ and in the formation of the prothallium of Ferns; but on this point more exact researches are still wanting. In Ferns only thus much is known, that (according to Wigand) when the light is stronger from one side, the principal section places itself in the direction of the strongest illumination, while the apex of the axis of growth is turned towards the shade.

What has now been said will serve as a definition only of the most important ideas, and an illustration of the points of view from which observations must be made. The results obtained by them cannot be given in detail; and since a definite theory has not yet been elaborated, a more detailed description must deal with numerous peculiarities and critical comparisons, for which we have no space. A few important facts may, nevertheless, be briefly mentioned.

(I) In reference to the Direction of the Axis of Growth, it appears to be the general rule that the origin of a new individual coincides with the commencement of a new direction of growth. This is very strikingly the case in the swarmspores of $E$ dogonium (Fig. 4, p. 9), the longitudinal axis of which is transverse to that of the filament which produces them, and becomes the longitudinal axis of the new plant; and the same is the case with the origin of new filaments of Nostoc and Rivularia (see Book II, Algæ). In many Cryptogams, researches have not yet been made on this point, or it would carry us too far to mention them. It may be mentioned, merely by way of example, that the

1 Compare Hofmeister, Allgemeine Morphologie, §§ 23, 24. 
axis of growth of the embryo of Ferns and Rhizocarps is distinctly transverse to the axis of the archegonium. In Phanerogams the direction of growth of the embryonal stem is opposed to that of the ovule; the apex of the young stem is formed in a direction different to that of the ovule, and it continues its growth in this direction. The formation of the sporocarp of Mosses forms an exception to this manner of growth, if it is considered as a new individual, but this appears very questionable; it grows in the same direction as the archegonium, and even in the direction of the axis of the stem when the archegonium is apical (i.e. in Acrocarpous Mosses).

A second remark relates to the fixation of the base of the axis of growth. In all lateral members and bifurcations the base is the fixed point at which the branching or new formation begins; and in the new formation of an axis of growth from swarmspores and fertilised oospheres, the growth in a definite direction does not begin until some one cell has become fixed. This occurs in all swarmspores, which do not begin to grow into tubes and filaments until their hyaline end, the anterior one in the swarming, has become fixed, even if only on the surface of the water. The germinating spore also of Ferns and Equisetaceæ puts out at an early stage a root-hair which fixes it to the support (the macrospore of Rhizocarps and Selaginelleæ does not require this in consequence of its weight). In a similar manner also the longitudinal growth of the embryo of Phanerogams does not begin until it has become attached at its posterior end to the apex of the embryo-sac. The embryo of Vascular Cryptogams produced by a sexual process fixes itself laterally by the portion called the foot into the tissue of the pro:hallium.

It is only in some Algæ of the simplest structure that there is no attachment of a point of the newly constituted plant to an external object (for which purpose any portion of the generating body may serve). In this case the opposition of base and apex disappears; growth may then produce a uniform arrangement in different and even opposite directions; simple threads result in which an anterior and posterior end can no longer be distinguished, as in some Desmidieæ and Diatoms, or spherical families of cells, like Glococapsa.

But when once a fixed point is established as a base, the increase of length takes place uniformly in one direction only from it; i.e. whatever grows in this direction is a member of a morphologically definite character. This does not however prevent the setting up of a new growth in the opposite direction; but the member which is formed in this direction is of a different nature morphologically; as occurs, for example, in the embryos of Phanerogams, in which, according to J. Hanstein's recent researches, the origin of the primary root is in fact such that its longitudinal axis must be considered as the prolongation of that of the stem in a posterior direction.

(2) With reference to tbe Relations of Symmetry, the most important point is that dichotomous branching is frequently repeated in one and the same plane in thallomes (as in Fucaceæ and Metzgeria), stems (Marchantia, Selaginella ${ }^{1}$ ), and leaves (in some Ferns). A different development then generally takes place on the two sides of the plane of dichotomy, one side of the shoot clinging closely to the ground or to upright bodies (as in Hepaticæ), or one side turning to the light, the other side to the shade (Selaginellex); in such cases the shoots have their greatest diameter in the plane of dichotomy. Where no such different development of the two sides occurs, as in Lycopodium (especially $L$. Selago according to Cramer), consecutive bifurcations may take place in different planes; and this is also the case with the roots of Lycopodiaceæ (see Nägeli and Leitgeb, and Pfeffer, l.c. p. 97).

As has already been mentioned, it is usually impossible, without experiment, to determine whether the position of the principal section of bilateral shoots and leaves is directly brought about by external conditions, such as pressure, gravitation, and light ${ }^{2}$. The position of the principal section usually shows a definite relation to the

1 In the roots of Selaginella, however, the successive bifurcations lie in planes at right angles.

2 This subject has been treated by Hofmeister (Allgemeine Morph. §§ 23, 24) from another 
mother-shoot, and at the same time to the direction of gravitation, light, and pressure (the latter in clinging or climbing plants, such as ivy, Jungermanniex, \&c.). It is therefore probable that internal and external causes generally cooperate to determine the direction of the longitudinal axis of a member when first formed, as well as of its lateral shoots ${ }^{1}$. Further development may show new relations to the original axis and to external influences. The horizontal lateral shoots of numerous woody Dicotyledons with alternate leaves in two rows have the principal section vertical, their rows of leaves right and left. The axillary buds of these leaves which remain dormant through

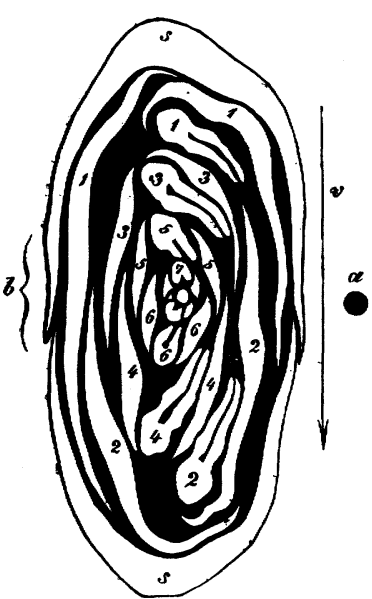

FIG. 155.- Lateral bud of a horizontal branch of Cercis canadensis (in December), in vertical transverse section ; $\mathrm{I} \rightarrow \mathrm{7}$ the consecutive leaves with their pairs of stipules indicated by the same numbers. The outer bud-scales have been renumbers. The outer bud-scales have been re-
moved, the two inner ones indicated by 3,3 . In moved, the two inner ones indicated by $3,3$. In
the centre is the growing point of the bud. $b$ the position of the leaf in whose axil the bud grows; $a$ axis of the mother-shoot; $v$ direction of gravitation.

$a$ lies above, the subtending leaf $b$ beneath the bud (the apparently terminal bud thus becoming axillary), and the vertical line $v$ becomes horizontal. This difference, which exists from the very first in the position of the lateral and terminal buds in horizontal bilateral mother-shoots, excludes the immediate influence of gravitation; but by a useful adaptation, even in the bud all the parts are so arranged that by a single twist of the axis during unfolding, they assume those positions which are most favourable for the functions of the leaves, and by which their faces are turned towards the light. In the terminal buds of such shoots this twisting is not necessary. Whether it is gravitation or the influence of light on growth which occasions this torsion of the axis of the bud is not

point of view; but on consulting the facts themselves I find much that is not in agreement with his statements, and in their interpretation $I$ arrive at essentially different conclusions, the reasons for which cannot be explained here in detail.

1 In order to determine this question experimentally, the apparatus which is described in Book III. Chap. 3, Sect. Io, should be used, a drum which holds the plant, and which rotates slowly round a horizontal axis.

${ }^{2}$ It is for our present purpose the same whether the bud at the end of the horizontal shoot be its true terminal bud, or a lateral bud the development of which is induced by the abortion of a terminal bud, as in Cercis and Corylus. In reference to the position of the terminal bud it is also indifferent that the position of the lateral buds is sometimes not quite horizontal, but a little oblique upwards and outwards, as in Corylus, Celtis, \&c. 
the question now under discussion. But the most important result is that the principal sections of the axillary buds of a bilateral mother-shoot may have greatly varying positions, and that, in consequence, the arrangement of the parts of the bud is independent of gravitation; on the other hand, there is a definite relationship to the mother-axis in the arrangement of the parts of the bud. The axillary bud of such a shoot may arise either laterally or on the under or upper side $^{1}$; in all cases its leaves turn their prominent mid-ribs outwards, away from the mother-shoot, so that the principal section of the bud is always at the same time an axial longitudinal section of the mother-shoot.

We are led to the same conclusion by the study of two- or three-year-old seedlings of Thuja and other Cupressinex. The leaves of the primary stem are arranged below in alternating whorls of fours, and consequently in eight longitudinal rows; higher up the whorls are alternate and of three leaves, and the leaves are in six rows. The axillary shoots, the number of which is very small in proportion to the leaves, appear, both in the eight-rowed and in the six-rowed region of the stem, to be generally in two rows, so that in reference to its branching the primary stem is bilateral (other positions of the branches occur, however, higher up, especially later). These lateral shoots of the first order begin at once with alternating whorls of two leaves, or a decussate arrangement; and the first pair always stands right and left of the subtending-leaf. Every such lateral shoot of the first order usually produces a bilateral branch-system, which spreads out in one plane. This plane is usually horizontal in seedlings of Thuja gigantea, T. Lobbii, \&c., and the principal section therefore vertical. But this is not without exception; lateral branch-systems are formed here and there spreading in a vertical plane, the principal section of which is therefore horizontal; and this is sometimes repeated in some of the lateral shoots of the second order. I found, however, on a strong seedling of Cupressus Lawsoniana seventeen lateral systems of shoots (standing in two opposite rows on the primary stem) all spreading in a vertical plane, while only one lower system spread horizontally. These differences in the position of the principal section of lateral branch-systems are not however brought about by torsions, which would easily be recognised from the phyllotaxis; they are original, and permanent. Where a lateral shoot of the first order branches horizontally, its branches are produced only from the axils of leaves that stand right and left; where it branches vertically, only from the axils of leaves that stand above and below. Now since the principal sections of these lateral branch-systems have altogether different inclinations to the horizon, it is scarcely possible to suppose that gravitation (or light) has any immediate influence on the origin of the lateral branches of the second order. The vertical position of the principal section of lateral shoots of the first order is much more constant in Araucaria excelsa; and here, as horticultural experience shows, we have a phenomenon inherent to lateral arrangement;--lateral shoots, planted vertically as cuttings, take root and continue to grow, vertically, but produce only lateral shoots in two rows; the branch which has once been produced as a lateral shoot does not change, when placed vertically, into a many-sided primary stem ${ }^{2}$.

I may add a few remarks on the species of the genus Begonia, which show that the relation of the bilateral arrangement of the parts to external influences may be entirely different in very closely allied forms, while it is constant for members of the same plant. The leaves of Begonias are alternate in two rows; on thick stems the two rows approximate on one side of the stem, and the other side of the stem appears naked; the shoot is thus not only bilateral, but it has a leaf-bearing anterior and a naked posterior side which are very unlike. The blade of the leaves is very unsymmetrical, one balf being much larger. The larger halves of all the leaves are turned towards the posterior side of the stem; and this can be used to distinguish the posterior and

1 Axillary shoots are formed on the upper side of the mother-shoot near its base in Cercis, and bear inflorescences.

${ }^{2}$ [This is not without exception; see Gœppert, Acta Acad. Nat. Cur. 1868, p. 34, t. I.] 
anterior sides even in slender-stemmed species, as B. undulata and incarnata, although in these cases the leaves are not approximated on the anterior side, but follow exactly the divergence $\frac{1}{2}$. It is well to remark at the outset that the leaf-stalks of Begonias are moderately heliotropic, while the axes of the shoot are scarcely so. In thick axes heliotropism appears to be entirely wanting; in slender axes (B. undulata and incarnata) it is always very slight. Some species with moderately thick stems, as B. Verschaffeltii and manicata, grow upright when the light comes from one side; very thick-stemmed species bend without reference to the direction of the light, while slender-stemmed species allow their weak branches to hang down without pointing in any one definite direction. If attention be now paid to the direction in which the stems bend, the plane of curvature is found always to coincide with the principal section of the shoot which divides it into two similar halves, each possessing a row of leaves. A definite relationship is also manifested between the tendency to bend and the relative thickness and length of the internodes. If the thickness of the internodes be represented by $\mathbf{I}$, then, in the upright stems of Begonia nitens, Möbringi, and sinuata, their respective lengths are $9,3^{*}$, and 2 ; in the slightly curved $B$. manicata it is $I$ or less; but in procumbent and strongly-curved stems as low as 0.7 (B. bydrocotylifolia), $0_{4}$ (B. pruinata), and $0_{2}$ (B. ricinifolia). In the slender-stemmed upright species the rows of leaves are diametrically opposite to one another; in the species with slightly-curved thicker stems they approach on the anterior side; in the very thick-stemmed species which are bent downwards the insertions of the leaves are placed entirely on the anterior side ${ }^{1}$. In the thick-stemmed species the stem curves downwards with the anterior side concave, or lies procumbent; but in this case it is always the leafless posterior side which faces downwards and puts out adventitious roots ( $B$. ricinifolia and macrophylla). In species on the other hand which have tall stems and slender internodes, the branches hang down, and in this case it is the posterior side which becomes convex and faces upwards (B. undulata and incarnata). Or, in other words, if we look at the buds, in the slender-stemmed forms all the larger halves of the leaves when first formed turn upwards, while in the thick-stemmed forms they turn downwards. The want of symmetry of the leaves is thus, when the position of the bud is inclined, opposed to gravitation, and when the stem is upright has no relationship with it. In species with short internodes and thick stems only a few lateral shoots are developed, in those with slender stems a great many. Such a relation constantly occurs in other cases (Cactaceæ, Palms, Ferns, and to an extreme extent in Isoëtes). The bilateral arrangement of the lateral branches is connected with that of the parent axis in the following way :-in all species the posterior side of the lateral branch, and hence the larger halves of the leaves, faces the parent axis ; the principal section of the former in the slenderstemmed species is therefore at right angles to that of the latter. In thick-stemmed species, where the axillary shoots are approximated in front, the principal section of the lateral branch makes an acute angle with that of the parent axis. As development progresses, the branches of slender-stemmed species retain nearly their original position; in thick-stemmed species where the anterior and posterior sides differ greatly, the lateral branch twists in such a manner that its posterior side faces in the same direction as that of the parent axis.

I have no precise information as to the mode of life of different species of Begonia, but suppose that those species in which the anterior and posterior sides are differentiated, and which do not cling to the ground, may have the power of climbing, like the ivy; although observations which I have had made for this purpose in the botanical garden at Würzburg have not yet led to any satisfactory result, partly because the plants were already too old, partly because the access of light was possibly too small on

1 The absolute measures of thickness run almost parallel to the above-named relative ones; the relatively thickest internodes are also usually absolutely the thickest, and these stems show the most decided tendency to a horizontal growth. 
the anterior side. The facts stated above do not negative the hypothesis that with stronger access of light from one side the stems of Begonias may possibly be apheliotropic. It appears moreover from Martius ('Flora brasiliensis', fasc. XXVII. p. 394) that at least some Begonias cling to rocks and the stems of trees ${ }^{1}$.

SECT. 28. Characteristic Forms of Leaves and Shoots.-The peculiarities of thallomes, leaves, axes, and roots which are common to whole classes, orders, or families, are the subject of special morphology and systematic botany; on the other hand it is the province of physiology to study the special organisation by which the members of a plant become adapted to perform definite functions. There are, however, some peculiarities of growth which recur in different divisions of the vegetable kingdom, or present themselves in striking contrast to the ordinary phenomena, and which are for this reason well adapted to bring into prominence the value of general morphological ideas. Peculiarities of this kind are termed characteristic, and they must be briefly mentioned here, chiefly in order to explain some scientific terms which will be used in Book II. We may limit our remarks to leaves and leaf-bearing shoots, since the forms of the thallus will be treated in sufficient detail in the chapter on Thallophytes, and those of roots present only slight characteristic differences, to which reference has already been made; the characteristic forms of hairs has already been sufficiently alluded to.

(1) Forms of Leaves. When fully developed, leaves are usually flatly extended plates of tissue, the extension being generally in directions right and left perpendicularly to the median plane or principal section, so that the surface of the leaf lies transversely (at right angles or obliquely) to the longitudinal axis of the stem. This is generally quite true for the base of flat leaves; but the upper part of the surface of the leaf is sometimes itself extended in the direction of the median plane, so that the plane of extension coincides with an axial longitudinal section of the stem, as in the genera Ixia, Iris, \&c. But sometimes the leaves are not flat, but conical or polyhedral; conical with almost circular transverse section in Characeæ and Pilularia, polyhedral in some species of Mesembryanthemum and Alö̈.

The outline of leaves is either simple or segmented; the former is the case when no definitely separated parts can be distinguished in the leaf; a leaf is segmented when it consists of pieces of various shapes, which are more or less separated from one another. Leaves which are not flat are usually simple, as also are those which are flat but small, their length and breadth being inconsiderable relatively to the stem, and not exceeding a few millimetres or centimetres in absolute measurement. Larger leaves are usually distinctly segmented, and in general the degree of segmentation increases with the increase of size; the small simple leaves of Mosses, for instance, may be contrasted with the large segmented leaves of Ferns, the small simple leaves of Lycopodiaceæ and Coniferæ with the large compound leaves of Cycadeæ, the small simple leaves of Linaceæ with the large much-divided leaves of the nearly-allied Geraniaceæ, \&c. The segmentation of leaves usually consists in the distinction of a basal portion which generally remains narrow, cylindrical, or prismatic, and of an upper portion which is flatly extended; the

1 On heliotropism see Book III. Chap. 3. Sect. 8. 
former being called the leaf-stalk or petiole, the latter the blade or lamina. Or the lower portion of the leaf has the form of a sheath enclosing the stem and younger leaves like a hollow cylinder. If the upper part is flatly expanded the leaf then consists of a sheath or vagina and a blade; it sometimes also happens that a stalk intervenes between the sheath-like basal portion and the lamina, as in Palms and some Aroideæ and Umbelliferæ. Segmentation into sheath, petiole, and blade may be distinguished as longitudinal, from lateral segmentation, which consists of actual branching, as in pinnate, deeply lobed, or compound leaves, or of a rudimentary branching, as in indented, toothed, and sinuate leaves. Leaves are termed compound in which the individual lateral pieces of the lamina are completely separated at their base; while those forms are termed lobed in which the lateral branches are only more or less projecting portions which unite at their base. If the individual branches of a branched leaf are sharply separated, each branch forms independently, so to speak, a leaf, and is hence distinguished as a leaflet. The pinnation, like the formation of lobes, may be repeated. If the branches are obviously arranged in two rows the leaf is said to be pinnate if it is a compound leaf; pinnately-lobed, pinnatisect, or pinnatifid if the divisions are incomplete; dentate, serrate, or crenate if the lateral projections are very small relatively to the lamina. If, on the contrary, the branches or lobes of the lamina are aggregated at the end of the petiole, and radiate from it, the leaf is said to be digitate, palmately lobed, \&c. It is termed peltate when the lamina is attached not by a portion of its margin, but at a point on its under surface (as in Tropaolum, Nelumbium, \&c.). These are only a few of the more important forms; the student will find in every text-book a number of other distinctions and terms employed in the special description of plants ${ }^{1}$.

As occasional appendages, which indicate a still further segmentation of leaves, must be mentioned stipules, ligular structures, and hood-like outgrowths.

Stipules may be considered as lateral branches of leaves which arise at their very point of insertion; they stand in pairs right and left of the base of the leaf, either entirely distinct from it ( $\mathrm{free}$ ) or united to it in growth (adnate); each single stipule is usually bilaterally unsymmetrical, and its shape is the reflected image of the other. Stipules are not formed until after the origin of the leaf, but then grow much more rapidly, and attain their final development at an earlier period; hence they play an important part in the position of the parts in the bud. In vernation they either extend by their inner margins (those facing the median plane of the leaf) over the back of the leaf and cover it outside either partially or entirely, or they extend in front of the leaf (on the side facing the stem) right and left, and thus cover the parts of the bud next youngest in age. In one or the other of these modes chambers are not unfrequently formed by the stipules, in which the formation of the leaves is completed, and from which they expand and unfold; the stipules then either also remain and unfold, or die and drop off.

The term ligule is applied to a membranous outgrowth on the inner side of the leaf of Grasses at the point where the flat lamina bends out at an angle from the sheath; it stands transversely to the median plane of the leaf. Similar. outgrowths are also found elsewhere, as on the petals of Lychnis and Narcissus (where

1 [Gray's Botanical Text-book, Part I, Structural Botany, I879, gives perhaps the best English exposition of the current terminology of Flowering plants.] 
they form the so-called Corona), on the leaves of Allium, \&c., and may be included in the general term of ligular structures. Outgrowths sometimes occur from the posterior (outer) side of leaves, as, for instance, the large hood-like appendages of the stamens in Asclepiadeæ.

It is only in some Muscineæ that the tissue of the leaf consists throughout of a single layer of cells. Usually, especially in large leaves; the tissue is composed of several layers, and, in vascular plants, is distinguished into epidermis, parenchymatous fundamental tissue, and fibro-vascular bundles. The fundamental tissue is termed mesophyll; the system of the fibro-vascular bundles running into the leaf forms the so-called venation. In the leaves of many Mosses which otherwise consist of only one layer, there runs in the middle from the base towards the apex a bundle of several layers, called the mid-rib; in leaves of more complicated structure there is also usually a mid-rib which runs from the base to the apex of the lamina, and divides it more or less symmetrically into two halves. The same occurs in every lateral leaflet or in every branch or lobe of the lamina; from the mid-rib spring the lateral veins which run to the margin of the leaf. In larger leaves, especially those of Dicotyledons, the fibro-vascular bundles which traverse the mid-rib and its stronger branches are enclosed in a thick parenchymatous layer of tissue, the cells of which differ from those of the mesophyll. Usually these veins project on the under side of the leaf, and the larger the whole lamina the more strongly are they constructed (especially the mid-rib). The finer veins, on the contrary, consist of single fibro-vascular bundles, often branching extensively, which run through the mesophyll of the lamina itself. The kind of venation varies in different classes of vascular plants, and is often very characteristic of large groups. This will be explained more in detail in the proper place.

In Characeæ, Muscineæ, and Vascular Cryptogams, all the leaves of a plant are usually similar, being either simple or segmented in the same manner, although the segmentation, especially in Ferns and Rhizocarps, is simpler in young than in the large leaves of mature plants. But it also happens, even in Cryptogams, that leaves of very different forms are found on the same plant. Thus some Muscineæ form colourless minute leaves on the underground creeping shoots, and in the neighbourhood of the organs of reproduction they often produce leaves of a different shape from those on the rest of the upright parts. In the same manner among Ferns the leaves on the underground shoots (stolons) of Struthiopteris germanica are represented by thin membranous scales, which are replaced on the upright end of the stolon by large green pinnate leaves. In Salvinia, among Rhizocarps, each whorl consists of two simple roundish leaves which rise into the air, and one that hangs down into the water and consists of filiform branches. Even in Coniferæ and Cycadeæ variation in the leaves of the individual plant is much more common; while in Monocotyledons and Dicotyledons the shapes of leaves become extraordinarily variable, not only on the same plant but often on the same axis.

The two most common forms of leaves are the scale- or 'cataphyllary' leaves ${ }^{1}$ and the foliage-leaves.

' [Henfrey, in his translation of Braun's 'Rejuvenescence in Nature' (Ray Soc., Botanical and Physiological Memoirs, 1853), first proposed to render the terms Hochblatt, Niederblatt, and 
The foliage-leaves ${ }^{1}$ are always distinguished by their green colour, owing to their containing chlorophyll (which however is sometimes concealed by red sap); and it is these which, in popular language, are exclusively called leaves. Usually they are the largest foliar organs of the plant, lasting the longest, and distinguished by the greater degree of segmentation of the outline, as well as by the more perfect development of their tissue. As the chief store-houses of chlorophyll they are the most important organs of assimilation, and are always destined to be expanded to the light even when they are formed on underground growing points (as in Sabal, Pteris aquilina, \&c.). When small they are usually produced in great numbers on a shoot; as they increase in size their number and the rapidity of their growth diminishes in proportion. In this respect the numerous small leaves of Mosses may be compared with the few large leaves of Ferns, the numerous small leaves of Conifers with the few large ones of Cycads, \&c.

Scale- or Cataphyllary leaves are usually produced on underground shoots, and remain buried in the earth, although they also frequently occur above ground, especially as an envelope to the winter-buds of woody plants (as the Horse-Chesnut, Oak, \&c.). In the genus Pinus the primary and strong lateral axes form leaves of this kind only; the acicular foliage-leaves appear in tufts on small axillary shoots; in Cycas scale-leaves alternate regularly on the stem with large foliageleaves. Seedlings (as of the Oak) and the lateral shoots from underground axes often begin with scales and only advance at a later period to the production of foliage-leaves (e.g. Struthiopteris, A gopodium, Orchis, Polygonatum, \&c.). In parasites and plants which live on decaying vegetable matter (saprophytes) and are destitute of chlorophyll (e.g. Monotropa, Neottia, Corallorhiza, Orobanche, \&c.) the scales are the only foliar structures of the vegetative parts, the foliage-leaves being absent. Even in those plants whose foliage-leaves are much segmented the scales remain simple; they are distinguished by a broad base, usually diminutive length, the absence of prominent veins, and by forming no chlorophyll or only very little. They are colourless, yellowish, reddish, or often brown; their texture is, according to circumstances, fleshy, succulent (as in some bulbs), membranous, or tough like leather.

In Phanerogams, especially in Monocotyledons and Dicotyledons, several other forms of leaves make their appearance as a preliminary to fertilisation-bracts, sepals, petals, stamens, and carpels. The thick seed-leaves or cotyledons will be spoken of in detail as a peculiarity of these classes.

From the point of view of the Theory of Descent we are justified in considering all other forms of leaves as subsequent metamorphoses of foliage-leaves; and these latter are therefore regarded as the original typical leaves. When they lost their original function-the assimilation of food-materials-and served other purposes, they assumed at the same time other forms and structure. The same is meant when certain tendrils and spines are said to be metamorphosed leaves. Leaf-tendrils

Laubblatt by 'hypsophyll,' 'cataphyll,' and 'euphyll.' The two first of these are useful additions to botanical terminology; the last, however, does not seem to be required, being precisely equivalent to the term foliage-leaf, which is already in general use (hypsophyll=bract)].

1 Compare the characteristics of the formations of leaves in A. Braun, Verjiingung in der Natur, Freiburg 1849-50, p. 66. [Ray Soc., Bot. and Phys. Mem. 1853, p. 62.] 
are leaves or parts of leaves which have become filiform, and possess the power of winding round slender bodies and thus of serving as climbing organs (as in Vicia, Gloriosa, Smilax aspera, \&c.). Leaf-spines are leaves which have developed into long, conical, pointed, woody bodies; they take the place of foliage-leaves (Berberis) or stipules (Xanthium spinosum, some species of Acacia). These two kinds of metamorphosis occur almost exclusively in Flowering plants (Angiosperms), the morphological and physiological perfection of which, in comparison with Cryptogams and Gymnosperms, is especially due to the capability of their leaves to assume the most various forms.

(2) Forms of Shoots. The axis of leaf-bearing shoots is, when sufficiently developed, usually columnar, with a cylindrical or prismatic surface. If the growth in length is very small in proportion to that in thickness, the short column forms a plate, as in the bulbs of Allium Cepa, and Isoëtes; if the growth in length is somewhat greater, with at the same time considerable increase of thickness, rounded or elongated masses are produced (as the tuber of the Potato and Artichoke, the aërial stems of Mammillaria and Euphorbia meloformis); when the growth in length greatly preponderates we have stems, scapes, and filiform structures of various kinds. Very commonly the same shoot shows differences of this kind in successive stages of its longitudinal growth; thus the stem of the Onion, which is at first broad and tabular, afterwards rises as a high naked scape, the end of which in its turn remains short, and thus produces the umbellate inflorescence; and in the same manner the thick tuber of the Potato is only the swollen end of a slender filiform shoot. Among the numerous deviations from the columnar form of the axis the conical is of peculiar interest. The conical stem is of two kinds; it may be slender at the base, increasing in thickness with further growth in length, so that each portion of the axis is thick in proportion to its youth, and the upright stem resembles a cone placed upon its point; the growing apex then lies on the surface which is turned uppermost, or rises above it as an upright cone. This form occurs in the stems of Tree-ferns, Palms, in Maize and in many Aroideæ; it depends on the absence of a secondary growth in thickness, while, with the age of the plant, the young tissue of the stem becomes constantly larger in circumference immediately beneath its apex; when this increase ceases, the circumference of the later increment of length remains the same, and the inverted conical stem continues to grow in the form of a cylinder. The second form of conical stem is caused by a long-continued secondary growth in thickness together with the small circumference of the shoot at the growing point; this occurs in Conifers and many dicotyledonous trees, the older stems of which are thick below but slender above, and thus resemble a slender cone placed on its base.

The habit of a leafy axis or of a segment of one is usually in close relation to the number, size, and formation of its leaves. If the internodes are very short but the leaves small and numerous, the surface of that portion of the axis is nowhere exposed, and the leaves only are seen, as in species of Thuja and Cupressus, and some Mosses (Thuidium); in such cases whole branch-systems frequently have the appearance of multipinnate leaves. If the closely packed leaves are large, they form a rosette enveloping the end of the stem, while the older parts of the stem are 
clothed with the remains of the leaves, or are naked, as in Tree-Ferns, procumbent species of Aspidium, Palms, species of Alö̈, \&c.

If a comparison is made between the amount of development in bulk which takes place in the leaves and in the axis of a shoot, we find as extremes on one side, for example, the Cacti (Cereus, Mammillaria, Echinocactus, \&c.) with gigantic axes and entirely abortive leaves, on the other side the Crassulaceæ with fleshy crowded leaves and comparatively weak stems; or on one side the underground tubers of the Potato with scarcely visible scales, and on the other side the bulbs of Liliaceæ with fleshy scales which entirely envelope the short stem.

In reference to the formation of leaves which appear on the shoots, it must first be noted whether the same axis always produces only similar leaves or such as gradually vary in form. The first is the case, for example, in most Muscineæ, Ferns, Lycopodiaceæ, Rhizocarps, all Equisetaceæ, and most Conifers; the latter, on the other hand, occurs commonly in shrubby Dicotyledons. In Monocotyledons and Dicotyledons (to a certain extent even in Conifers) it not unfrequently happens

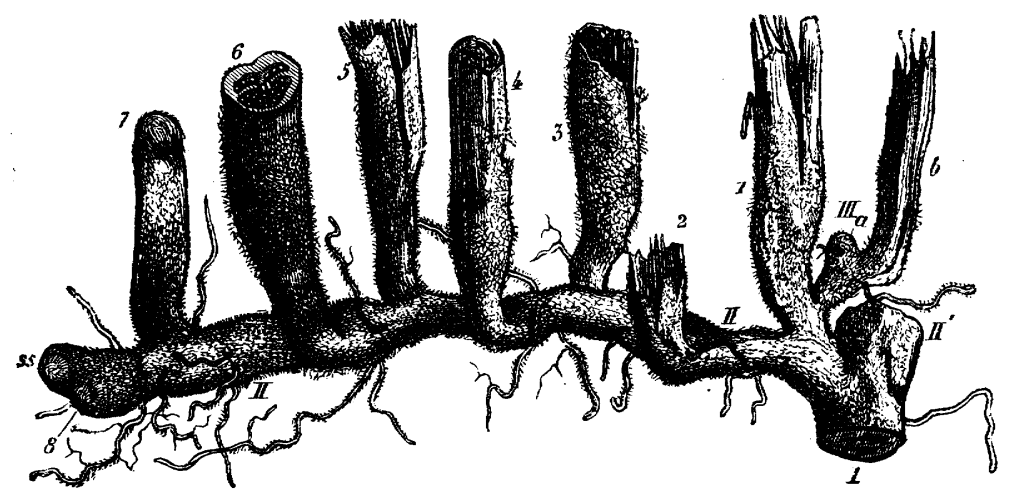

FIG. 156.-Rhizome of Pteris aquilina; $I, I I, I I I$ the underground creeping axes; ss the apex of one of them ; $1-6$ the basal parts of the leaf-stalks; 7 a young leaf; $b$ a decayed leaf-stalk, the basal portion of which is still living and bears a bud $I I I a$; the hairy threads are roots which arise behind the growing apex of the stern.

that the different forms of leaves are distributed over different generations of shoots; certain shoots produce, for example, little or nothing but foliage-leaves, others produce only bracts with or without flowers (e.g. Begonia). In such cases the shoots may be designated, according to their leaves, scaly shoots, leafy shoots, bract-axes, flowers, peduncles, \&c. On this point further details will be given in Book II.

It is of very common occurrence with Cryptogams and Angiosperms (not with Gymnosperms) for a persistent primary axis or branch-system to continue to grow underground, and to send up only at intervals long foliage-leaves or shoots, which subsequently disappear in their turn and are replaced by others. When such axes or branch-systems lie horizontally or obliquely in the ground, and produce lateral roots, they are called rhizomes (Fig. 156), (as in Iris, Polygonatum, Pteris aquilina and many other Ferns). Frequently they die at the posterior and continue to grow at the anterior end. Underground tubers and bulbs are more transitery structures, usually lasting only for one period of vegetation; the former 
are characterised by the preponderance of the axial mass with a very small amount of leaves, the latter, on the contrary, by the preponderance of leaves closely packed round a short stem. If the lower parts of a plant produce slender lateral shoots with small scales growing upon or beneath the earth, and after rooting at a considerable distance from the mother-stock produce foliage-shoots or shoots stronger than themselves, they are called stolons, as, for instance, in Asgopodium Podagraria, Fragaria, Struthiopteris germanica, and in Mnium and Catharinea among Mosses.

The greatest degree of variation from the ordinary forms of shoots is displayed by the flat leaf-like axes and branch-systems, and by the stem-tendrils and spiny shoots which occur frequently in Angiosperms. Leaf-like axes (phylloclades) are found in those Phanerogams in which large green foliage-leaves are wanting, and they replace them physiologically; their axial structure is of considerable superficial extent, and they produce and expose to the light large quantities of chlorophyll; they generally bear only very small membranous scale-leaves. Examples may be found in Phyllocladus among Conifers, Ruscus among Monocotyledons, and among Dicotyledons in Mühlenbeckia platyclada (Polygonaceæ), Xylophylla (Euphorbiaceæ), Carmichaelia (Papilionaceæ), and in Opuntia brasiliensis and Rhipsalis crispata (Cactacea), \&c.

Stem-tendrils, like leaf-tendrils, are long, slender, filiform structures, which have the power of winding spirally round slender bodies in a horizontal or oblique position with which they come laterally into contact, and thus serve as climbing organs; they spring laterally from shoots which have not the form of tendrils, and are distinguished by the absence of foliage-leaves, their power of forming leaves being mostly limited to very minute membranous scales. They are usually easily distinguished from leaftendrils by their origin, position, and by the production of leaves ; cases, however, occur where the morphological nature of a tendril is doubtful, as, for instance, in Cucurbitaceæ ${ }^{1}$. Evident examples of stem-tendrils are to be met with in Vitis, Ampelopsis, and Passiflora. Shoots which bear strongly developed foliageleaves on long slender internodes, and which have the power of winding in an ascending manner round upright supports, are not considered tendrils, but are called twining or climbing stems ${ }^{2}$; a distinction is drawn between tendrilclimbers (as Vitis) and stem-climbers (as Phaseolus, Humulus, Convolvulus, \&c.). In Cuscuta, where the primary shoot and all the lateral shoots, except the inflorescences, twine in the manner of tendrils and of climbing stems, and where foliage-leaves are also entirely suppressed, the peculiarities of tendrils and of climbing stems are to a certain extent united. A distinction similar to that between stem-tendrils and climbing stems is also possible in leaves; the foliage-leaves of Lygodium exhibit continuous growth in length, and behave completely like climbing stems, the rachis of the leaf corresponding to a climbing axis, and the leaflets to its foliage-leaves ${ }^{3}$.

The axial shoots of many Angiosperms have, like the leaves, the power of

1 According to Warming these are also metamorphosed branches.

2 Compare H. von Mohl, Ueber den Bau urd das Winden der Ranken und Schlingpflanzen. Tübingen 1827. [See also Darwin, On the Movements and Habits of Climbing Plants, London I875.]

${ }^{3}$ Compare Book II, Ferns, and Book III, on the Physiological Signification of Tendrils and Climbing Stems. 
forming spines, becoming transformed into conical, pointed, hardened bodies. This may take place either by the whole shoot or even a whole branch-system becoming spiny, with suppression of the foliage-leaves, as in the branched spines of Gleditschia ferox, or by the shoot first producing foliage-leaves, growing in the ordinary manner, and finally finishing its growth in length by a spiny point, as in the lower axillary shoots of Gleditschia triacanthos, Prunus spinosa, and many others.

Among Phanerogams, especially Monocotyledons and Dicotyledons, displacements of the leaves and lateral shoots (as well as roots), and adhesions of members, constantly occur, which, as development advances, are in apparent contradiction to the typical laws of growth and local position which are the ordinary ones in these classes; and it appears

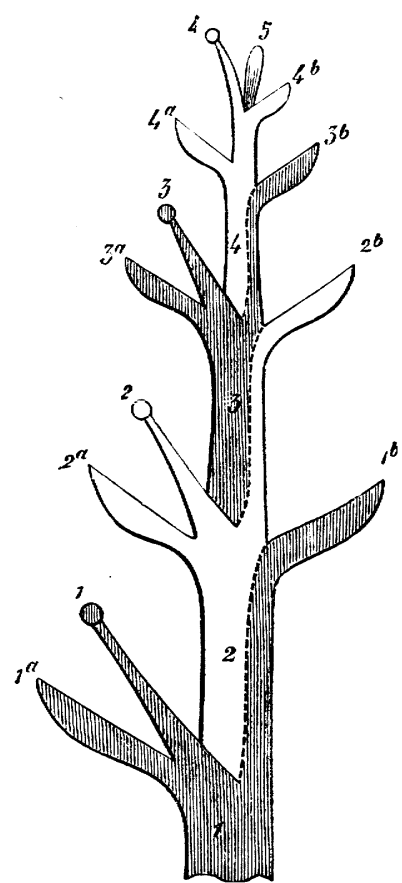

FIG. 157.-Diagram of the adhesion of leaves with the axial parts of their axillary shoots (after Nägeli and Schwendener; Das Mikroskop).

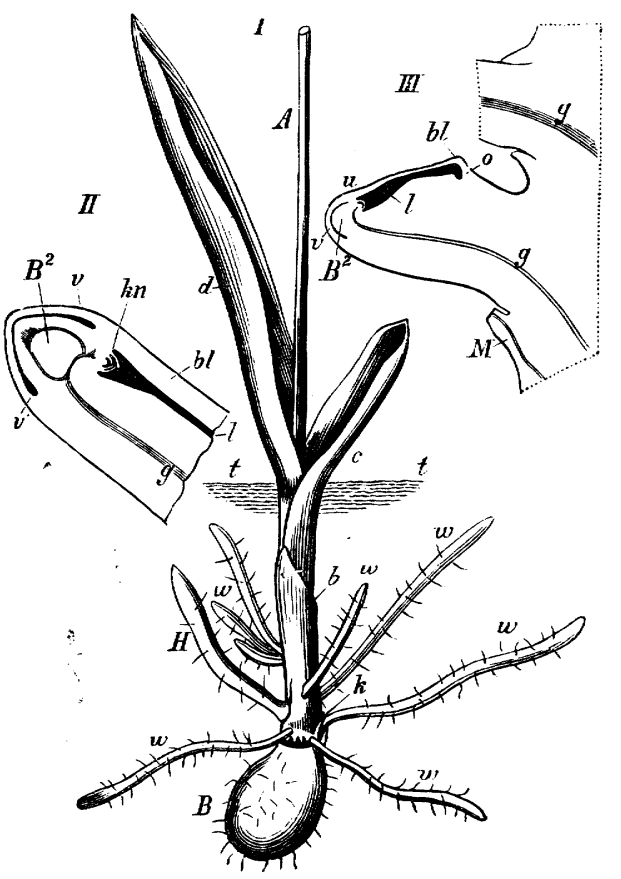

FIG. 158.-Herminium Monorchis (after T. Irnisch : Biologie und Morphologie der Orchideen. Leipzig 1853); $I$ the lower part of a flowering shoot (natural size); II, III the part containing the bud (magnified).

impossible to apply even the most general rules of growth which we have now been considering. It would be difficult for even a clever beginner to explain by the principles which have been regarded in this chapter as most universal, the structure, for instance, of the expanded flower of an Orchis, Rose, Lamium, Salvia, or of many other plants, of a partially or wholly ripe fig, or the phyllotaxis in the inflorescences of Borragineæ and Solanaceæ and many others. But the history of development shows that even such cases may be ranged under these laws, and that peculiarities of structures of this kind only arise at a later period, or in such a manner that they confirm general rules. The deviations from these laws are caused by the cessation of the growth of particular parts at an early period, while others undergo a great advance; or they are caused by the adhesion of parts originally distinct. Although it is quite impossible to give general rules for the explanation 
of abnormal formations, yet the causes which most commonly produce these results may be mentioned; these may be included under the heads displacement, adbesion, and abortion. Very commonly the two first act in unison, and in many flowers combine with abortion to produce complex organs difficult to explain. It belongs to the most beautiful problems of morphology to refer such apparent exceptions to more general laws of development; and the determination of natural affinity, the definition of the typical characters of whole classes, orders, and families, depends upon it. Since, however, these complicated phenomena belong almost exclusively to Angiosperms, and in them occur to much the largest extent in the flowers and inflorescences, the best place for a more detailed description will be when the characteristics of this class are under consideration. Some explanation may, however, be given here, by means of a few examples, of the use of the terms displacement, adhesion, and abortion.

Fig. 157 represents diagrammatically a branch-system developed sympodially and proceeding from an axillary shoot; I, I being the first shoot with its two leaves $\mathrm{I}^{\mathrm{a}}$ and $\mathrm{I}^{\mathrm{b}}$; in the axil of the leaf $\mathbf{I}^{\mathrm{b}}$ is developed the shoot 2 , 2, with its two leaves $2^{\mathrm{a}}, 2^{\mathrm{b}}$; in the axil of its leaf $\left(2^{b}\right)$ again arises the lateral shoot 3,3 , with its leaves $3^{a}, 3^{b}$, and so on. The parts of the stem of the shoots I, 2, 3, 4, which proceed from one another, form a straight pseud-axis or sympodium with the peculiarity that the mother-leaf in whose axis the lateral shoot developes adheres to it, and is carried up by it for some distance. If we call the globular ends I, 2, 3, 4 of the figure flowers, the whole is well adapted to represent diagrammatically the inflorescence of some Solanaceæ. If the leaves $I^{\mathrm{a}}, 2^{\mathrm{a}}, 3^{\mathrm{a}}$, $4^{2}$ are supposed to be removed, the diagram might stand for the primary branch of the inflorescence of Sedum. If, on the other hand, a lateral shoot is supposed to be formed in each case in the axil of the leaves $1^{a}, 2^{a}, 3^{a}, 4^{a}$ in the same manner as on the other side with displacement of the mother-leaf, this would repeat diagrammatically in a simple manner the branching and phyllotaxis of Datura ${ }^{1}$.

Still more complicated are the phenomena in Fig. ${ }_{5} 5$, where $I$ represents the lower part of a flowering plant of Herminium Monorchis. $t t$ is the surface of the ground, and what lies below this is therefore underground: $B$ is a swollen spherical root, above which rises the leaf-bearing shoot, which produces in its lower part slender lateral roots, $w, w, w$, as well as a sheath-like $\operatorname{scale}^{2} b$, and two foliage-leaves $c, d$, and continues higher as a slender scape $A$, bearing a raceme of flowers at its summit. Turning our attention exclusively to the structure $H$; we find it to be a shoot which contains the bud for the next year; for the whole plant $A, B$ in $I$ dies off after flowering, a similar plant being produced the next year from the bud contained in $H$. $H$ is therefore an axillary shoot of the scale $b$, an earlier condition being represented in Fig. $I I I$, where $M$ represents the base of the leaf $b$ cut through its median plane; $g$ is a fibro-vascular bundle running from the primary axis to the bud $u ; b l$ is the first leaf of this bud $u$ which is placed with its back to the mother-axis and forms a diminutive sheath enclosing the succeeding leaves of the bud $u ; B^{2}$ is the young tuberous root with its root-sheath $v$. In order to understand the displacement which has already taken place, the whole lower part between $M$ and $v$ must be imagined shortened to such an extent that $B^{2}$ would be somewhere near the letter $g$; and the bud $u$ must be supposed at the same time moved backwards towards $o$. By this means the normal position of the parts of $H$ under consideration is restored, and it is intelligible that the channel $l$, inclosed by the base of the leaf $b l$, is a consequence of the oblique direction outwards of the growth of the tissue lying between $o$ and $u$, that the root-sheath $v$ must be regarded as a part of the surface of the primary axis above $M$, and that in consequence $B^{2}$ has been formed in the tissue of the mother-axis beneath the bud $u$, and laterally on the fibro-vascular bundle $g$. In the normal position of the bud and root, the axis of growth of the latter would form almost a right angle with that of the bud, whereas by the displacement one forms a

1 [See Payer, Éléments de Botanique, p. II 7 .]

2 A first scale in the axil of which the bud $k$ stands is no longer to be seen. 
prolongation of the other. The growth of the mass of tissue lying between $g$ and $u$ further continues in the same direction, and the whole lateral shoot assumes the form represented in $H$ (Fig. $I$ ) ; the still further change of position of the parts which takes place in consequence is explained by Fig. $I I$, where $k n$ represents the bud $u$ in $I I I, b l$ the still more elongated sheath of the leaf $b l$ in $I I I$; the channel $l$ is the cavity inclosed by the leaf $b l$ increased in breadth, and which, were there no displacement, would be entirely filled up by the bud $u$ (or $k n)^{1}$.

In order to make the following displacement, which occurs very commonly, more intelligible, reference should be first made to Fig. I18, p. 154. This shows how the tissue beneath the apex expands laterally by early growth, so that the surface of the growing point, which would otherwise be elevated in a cone, becomes almost level; and the apical point thus comes to lie in the middle of a plane instead of at the point of a cone. In the Sunflower this state of things remains nearly unchanged as the capitulum

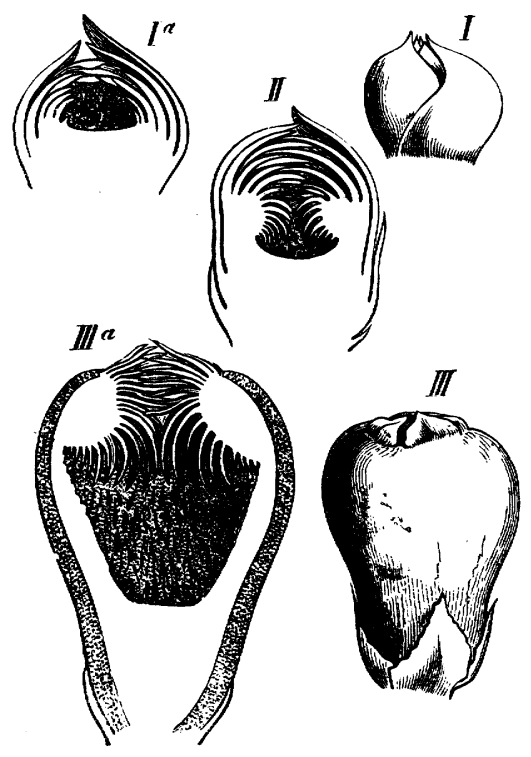

FIG. 159.-Development of the fig of Ficus carica (after Payer: Organogénie de la fleur).

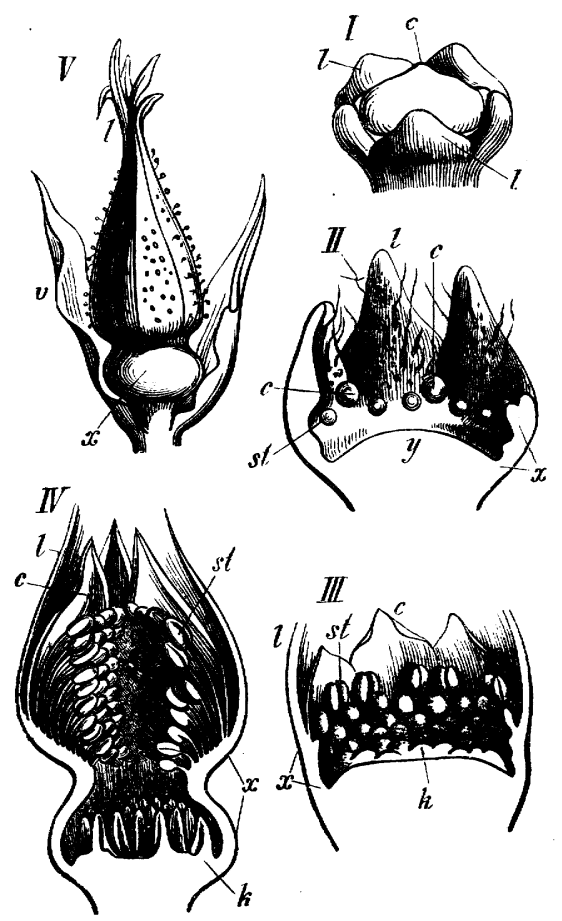

FIG. 160.-Development of the flower of Rosa alpina (after Payer Organogénie de la fleur).

developes; but the abnormal growth increases in many cases to such an extent that the apical point eventually lies at the base of a deep hollow, the walls of which result from older masses of tissue, which properly lie beneath the apex, growing upwards and overarching the apex itself. This occurs, for instance, in the development of the fig, which, as shown in Fig. I59, is a metamorphosed branch, the apex of which is at $I^{\text {a }}$ still nearly level, at $I I$ has already been outstripped by a circular leaf-bearing cushion, and at $I I I^{\mathrm{a}}$ is depressed in the form of an urn. The apical point of this shoot lies in this case in the deepest part of the hollow, the inner side of which is properly only the

1 [See also J. H. Fabre, De la germination des Ophrydées et de la nature de leurs tubercules, Ann. des sc. nat. 1856 , vol. V.] 
prolongation of the outside of the fig, and bears in consequence a large number of flowers (exogenous lateral shoots). In the nearly related genus Dorstenia the fig remains open; the margins of the tabular part of the axis which bears the small flowers do not arch over and unite.

On a process very similar to the formation of the common fig depends the origin of perigynous flowers and of inferior ovaries. Fig. 160 represents this in the perigynous flower of a Rose. I shows the very young shoot which is to develope into a flower, seen half from above and from the outside; the end of the shoot is thickly swollen; it has already produced the five sepals $l l$, and the five petals alternating with them are visible as little knobs, $c$, between which the apical region of the floral axis appears broad and flat. While the sepals grow quickly, the zone of the tissue of the axis out of which they spring becomes elevated in the form of a circular wall $x$ in $I I$, which afterwards contracts the opening above as seen in $I V$; an urn-shaped structure is thus formed which is known under the name of a hip, and is distinguished when ripe by its rëd or yellow colour and its sweet pulpy tissue. Here also the apical point lies in the middle of the bottom of the hollow, and the inner surface of the wall of the urn is a portion of the outer side of the floral axis which has been turned in. To this corresponds the acropetal succession of the leaves (which, however, is only adhered to in a general way). It is clear that if the apical point lies at $y$ (in $I I$ ), the order of succession of the leaves (in this case stamens st and carpels $k$ ) from above downwards must be termed acropetal.

If an. additional proof of what has just been said were wanted, it would be furnished by the history of development of the flowers of Geum, a genus very nearly related to the Rose (Fig. I6r). That part of the floral axis which bears the sepals $l$, the corolla $c$, and the stamens $a a$, is elevated in the form of a circular wall $y y$; but the apical region which in Rosa entirely ceases to elongate, becomes here again elevated as a conical body $x$, bearing at its summit the apical point of the floral axis. The order of succession of the foliar structures is again acropetal, and in consequence the stamens $\dot{a}$ are formed on the inner side of the axis $y y$ from above downwards, the carpels which succeed them on $x$ from below upwards. In Geum and other Dryadex the urn $y y$ spreads out at the time of fertilisation, its margin grows so vigorously in size that it expands in the form of a flat plate, and after the expansion its inner surface becomes the outer surface, in the middle of which the gynophore $x$ rises like a cone, and in Fragaria afterwards swells out, becomes fleshy, and forms the strawberry (a pseudocarp like the hip).

It will be seen that the formation of the fig, the hip, and that of the subsequently flat receptacle of Geum depends on a displacement which is caused by vigorous growth of masses of tissue that arise in the form of zones beneath the growing point. There is in these cases no such thing as

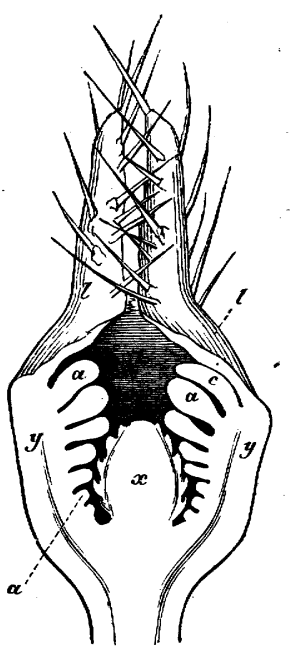

FIG. $16 \mathrm{r},-$ Longitudinal section of a young flower of Geum rivale. adhesion of foliar structures (as is usually stated in works on descriptive botany). The so-called coherent corolla and calyx of gamopetalous or sympetalous and gamosepalous or synsepalous flowers are also not the result of cohesion; the petals or sepals are on the contrary formed as a whorl of separate protuberances on the broad end of the young flower-stalk. That a gamopetalous corolla or gamosepalous calyx subsequently has the appearance of a bell having at its margin only as many teeth as the leaves of which it is considered to consist, does not depend on lateral cohesion of the margins of the leaves, but on the fact that the whole annular zone of the young receptacle which bears the corolla or calyx grows up; the bell-shaped part 
therefore never consisted of distinct leaves, but is the common basal piece which is developed from the floral axis as a whole, and which shows at its margin the original still separate leaves as teeth of the bell. The reverse is the case in the leaf-sheaths of Equisetum, where an annular wall originally projects round the axis, from which the separate leaf-teeth afterwards grow out. In this case also the sheath cannot be considered as formed by the cohesion of previously distinct pieces, but the separate teeth of the sheath must rather be considered as branches of a single annular rudimentary leaf. A similar explanation applies to the bundles of stamens which are generally termed coherent (monadelphous, polyadelphous, \&c.) stamens. As many protuberances are formed originally as there are bundles of filaments to be produced; and these protuberances must be considered as the original staminal leaves which subsequently produce by branching a larger or smaller number of stalked anthers (as e.g. in Hypericum, Callithamnus, \&c.). Cohesions of parts originally distinct are rare; examples are furnished by the connate inferior ovaries of two opposite flowers of an inflorescence in Lonicera alpigena, the fruits of Bentbamia fragifera which cohere into a large pseudoberry, and the cohesion of the two stigmata in the flower of Asclepias to each other and to the anthers. The anthers of Compositæ are not truly coherent, but only glued together by their sides.

Much more common than actual cohesion is the abortion of members already formed. Thus, for instance, the paripinnate leaves of some Leguminosæ ${ }^{1}$ originate as imparipinnate leaves; the terminal leaflet which is finally aborted is at first in the bud even larger than the lateral leaflets; but, as development progresses, it is so retarded that in the mature leaf it overtops the origin of the uppermost lateral leaflets only as a minute point. In the same manner the whole (branched) leaf-blades of many Acacias are also abortive, and are replaced by the petiole (phyllode), which is then expanded in its median plane. Still more complete is the abortion of the leaves from the axils of which spring the branches of the panicles of Grasses; and in this class whole flowers are often aborted. In diclinous Phanerogams the unisexuality of the flowers usually depends on the abortion of the stamens in the female, of the carpels in the male flowers. Sometimes only one of several stamens is aborted, as in Gesneraceæ (e. g. Columnea, where it is transformed into a small nectary); and the same occurs with the carpellary leaves (e.g. in Terebinthacex). In all these cases the structure which is afterwards abortive is actually present in the bud or even later, but its further growth ceases. The comparison, however, of nearly related plants shows that very commonly certain members are wanting in the flower the presence of which might be expected from the position and number of the others and from their presence in nearly related forms, although in such cases even the earliest condition of the bud does not exhibit a trace of the absent member. Since from the point of view of the Theory of Descent it must be assumed that nearly related plants are descended from a common ancestral form, the absent member may in such cases also be supposed to be aborted, only the arrest of development which has once taken place at an early period is so complete and has become so hereditary, that even its first rudiment is suppressed. The true theory of the structure of many flowers, and the reference of different forms of flowers to common types, often depends on the restoration of aborted members of this kind; but to this we shall recur in detail in Book II, when treating of Phanerogams.

SECT. 29. Reproduction; Sexual Organs; Alternation of Generations. Reproduction, or the production of new individuals, is generally brought about by particular portions of an individual becoming detached, which then have the power, first of all of producing new organs of nutrition, and afterwards of continuing

${ }^{1}$ Hofmeister, Allgemeine Morphologie, p. 546. 
their growth in such a manner that all the vital phenomena of the mother-plant are one by one reproduced in it. Since the same individual can form a number of reproductive organs, either simultaneously or successively, so there is also, in the reproductive process, at least the possibility of a multiplication of individuals, inasmuch as, under favourable vital conditions, a number of descendants of the same mother-plant actually come into existence. But since all those portions of the surface of the globe which it is possible for plants to inhabit are already covered with vegetation, it is in general only possible for such a proportion of these descendants to arrive at full maturity, that the number of individuals in existence remains the same from year to year. We shall see in the third book what an important bearing this fact has on the struggle for existence and on the consequent production of new vegetable forms. At present we have to consider only the most important morphological phenomena connected with the organs of reproduction.

The parts which become separated for the purpose of reproduction are very various in their nature. Among Cryptogams they consist most commonly of single cells,-spores, gonidia (or conidia), oospheres, antherozoids: less often they are bodies consisting of a small number of cells united into a tissue, like the gemma or bulbils of the Marchantieæ. In the more highly organised plants it frequently occurs that shoots, i.e. portions of the axis bearing leaves, become detached of their own accord in the bud-condition, then put out roots, and continue an independent growth; buds of this kind occur, for example, in some Mosses, in many Ferns, in Litium bulbiferum, several species of Allium, \&c. Very often almost any part of the plant, such as detached pieces of leaves, stems, roots, \&c., may become organs of reproduction, that is, they are able, under favourable conditions, to put out adventitious buds, and thus develope into new plants. In Phanerogams, finally, the normal reproductive bodies are the seeds, in which, even before separation from the mother-plant, a new individual has already advanced to a lower or higher stage of development, so that when the seed germinates nothing more is necessary than an increase in size of the parts that are already formed,-root, stem, and leaves.

In some cases the organs of reproduction appear, as it were, accidentally; but we will not here consider these cases, but rather turn our attention to those in which the formation of these organs is a necessary part of the life-history, and is essential to the complete development of the plant. These normal but still very various reproductive organs may be divided first of all into two groups, the sexual and the asexual.

Reproduction is said to be asexual when the part of the plant which becomes detached is able, without the assistance of any other organ, to produce a new individual. Of this nature are the spores of the Hymenomycetous Fungi and of Ferns, the gemmæ of Hepaticæ, and most zoogonidia of Algæ.

Reproduction is, on the contrary, sexual when two organs, developed expressly for this purpose, co-operate to produce a body out of which, either directly or after some further processes, one or more new individuals arise. Notwithstanding the great variety in the form of the organs of sexual reproduction in-the vegetable kingdom, and the complicated nature of the processes which often, especially in the higher plants, precede the act of sexual union, the essential feature of this 
act is always that two cells of the simplest possible kind combine, either completely coalescing with one another, or at least their contents becoming partially intermingled so as to afford a starting-point for a fresh development. It is the essential characteristic of sexual reproductive cells that each is incapable of further development by itself, this being the result of the combined action of two such cells.

It is only in the lowest forms of vegetable life, in some Algæ and Fungi, that the two cells which take part in the act of sexual union are alike or at least very similar in size, form, and physical properties. In this case their union is called conjugation, and the cell capable of germination which results from the union a zygospore. In all other cases the two uniting cells are strikingly different in size, form, and physical properties. In these cases one of the two cells, the male cell, conveys to the other only a very small quantity of material by means of which it produces an effect upon it; this other cell, the female cell, contains by far the largest proportion of the material which takes part in the development incited by the act of union. With the exception of a few complicated cases among Algæ and Fungi, which will be particularly described, the relationship between the two sexual reproductive cells is still more clearly indicated. by the fact that the male cell is motile, carrying to the other cell the fertilising material. The motion of the male cell is however of two kinds; it may either be spontaneous, as in most Cryptogams, when the cell is termed an antherozoid ${ }^{1}$; or, as in Phanerogams, the male cell, then called a pollen-grain, becomes detached from the parent-plant, and conveys the fertilising material to the female cell first of all by the aid of external forces, then by its own growth. The female cell, on the other hand, which is fertilised by means of the male cell, remains at rest at the place where it was formed, or at most, as in the Fucaceæ, is carried about passively; with the exception of the cases above referred to it is always a naked primordial cell, and is termed the oosphere, or germinal vesicle. After fertilisation, it secretes a cell-wall, and is then termed an oospore; from this, either at once, or after a period of rest (resting spore), the young plant is subsequently developed. Some not inconsiderable deviations from this plan will have to be pointed out in the class Carposporeæ of Thallophytes; but even in these cases the essential condition of fertilisation remains, that the male cell only incites to development, while the development itself proceeds entirely from the female organ.

Very considerable variety is shown in the morphological characters of the sexual organs, if we take a comparative view of the whole vegetable kingdom; but in the larger groups of plants we find the morphology of these organs to agree completely in all essential points, even when the anatomy of the vegetative organs, the habit and mode of life of the plants that compose them, vary greatly. When considering, in Book II, the distinguishing characteristics of the different classes of plants, our attention will be specially directed to the morphology of the sexual organs, and it will be sufficient here, as an introduction to what follows, to define the most general terms connected with these organs.

' [The term 'antherozoid' was first proposed by Derbès and Solier, Ann. des sc. nat. 1850, vol. XIV. p. 263.] 
In some plants we find only one kind of reproduction, either only the asexual, as in some of the simplest Algx and Fungi, or only the sexual, as in the Conjugatæ.

But in most plants reproductive organs of both kinds, sexual and asexual, are produced either simultaneously or successively. The two kinds of reproduction may then occur in the same individual, as in Vaucheria or Eurotium, or may be distributed on different individuals. In both cases the entire process of development may be divided into two sharply separated stages:-At the termination of one stage sexual organs are formed: by their union the second stage of development is rendered possible, and this closes with the production of asexual spores. Such a course of development is termed, from the analogy of certain processes in the animal kingdom, an Alternation of Generations, a term which is especially applicable in those cases in which, in one or both of the two stages of development, multiplication of the individual also takes place by gonidia or gemmæ, so that each of the two stages is complete in itself as a sexual or asexual generation.

Since we have here to do with phenomena which are foreign to ordinary life, and hence somewhat difficult to understand, we will illustrate the nature of alternation of generations by a few simple examples.

An alternation of generations is very evident in Ferns. The plant which we call in common language the Fern is merely the second stage in the process of development of the plant, or the asexual generation [sporophore]. It consists of a stem which forms true leaves and roots; on the leaves are produced small capsules or sporangia, in which the spores are produced without any sexual process. But each of these spores does not, on germination, again produce a Fern, but a minute plant of extremely simple structure which nourishes itself independently as a leaf-like thallus with root-hairs. This little plant, termed a prothallium, may, under certain circumstances, reproduce itself by gemmæ; and thus from a single spore an entire generation of prothallia will arise, which also behave as independent plants, although each prothallium is only the first stage of development, the sexual generation [oophore], of a Fern. For, finally, the prothallia produce sexual organs of reproduction; and from the oosphere of the female organ is produced an embryo which developes into a Fern with true roots and leaves. In this stage also the Fern is capable of immediate multiplication by the production of bulbils from which Fern-plants are directly developed, but the normal development closes with the production of spores. Precisely the same processes as in Ferns take place also in the Equisetaceæ and Ophioglossaceæ. In the Selaginelleæ the prothallium is formed inside the spore; and this class therefore establishes a transition to Phanerogams, where the prothallium is altogether rudimentary, and is found in a spore-like structure, the embryo-sac, within the ovule; so that the alternation of generations, so evident in Ferns, can be recognised here only by the most careful comparison with the most highly developed Cryptogams. This will be-explained more in detail in Book II.

In the Muscineæ the alternation of generations is no less clear than in Ferns, although it assumes an entirely different form. A Moss, in the state in which we ordinarily see it, consists of a stem provided with numerous leaves and root-hairs; 
but this leafy plant does not, as in the case of the Fern, produce spores, but can be reproduced by bulbils of different kinds. At length however, like the prothallium of Ferns, it produces sexual organs, and an embryo is the result of the fertilisation of the oosphere : this is not connected organically with the Moss-plant, but remains attached to it, deriving its nourishment from it, and finally developes into a capsule supported on a long stalk, the Sporogonium, in the interior of which are produced numbers of spores. A number of these stalked capsules, i.e. entire generations, may arise on the same Moss-plant either simultaneously or successively. The course of development of a Moss is therefore divided into two sharply separated stages, viz. the formation of a leafy stem which produces sexual organs (oophore), and the production of stalked capsules out of the fertilised oospheres of the female organs. In Muscineæ the second or asexual generation (sporophore), the sporogonium, has no power of directly producing its like from itself, as is possible in the case of Ferns, by bulbils; its only function is to produce spores $^{1}$; and when the spore germinates it gives rise first of all to a Protonema, which sometimes continues to grow for a long while, and can reproduce itself by gemmæ, until at lengh Moss-stems with true leaves again appear on it, which also are capable of multiplication by means of bulbils.

Even in Thallophytes we meet with various forms of an alternation of generations ${ }^{2}$. It is well shown in certain Fungi of the class Ascomycetes which have been closely investigated, as, for example, in the common mould, Penicillium glaucum, the ordinary form of which is only the first generation or stage of development in its life. During this stage, the first or sexual generation, the so-called Mycelium, developes on special branches a number of cells (conidia), by which the Fungus is continually propagated in this form. But when the excessive development of these conidia is prevented by exclusion of the air, sexual organs arise, as Brefeld has shown, on the luxuriant mycelium, and in consequence of their union a tuberous body is formed of a totally different nature, within which spores are finally produced in extremely numerous sacs (asci) of peculiar form; and these, when they germinate, again produce the mycelium with its penicillate conidiophores. The mycelium of this Fungus (and strictly speaking of all Fungi) corresponds therefore to the first stage of development, the prothallium, of Ferns, or to the leafy Moss-plant; and all three may be considered as the Sexual Generation [oophore], since their normal development ends with the formation of sexual organs. In all three cases, this sexual generation (prothallium, Moss-plant, mycelium) may propagate itself by gemmæ or by conidia before it produces the sexual organs. The small tubers which are the result of fertilisation in Penicillium correspond to the second stage of development, viz. the sporogonium of Mosses, and the mature Fern-plant ${ }^{3}$; in all three cases the product of this second generation [sporophore] is a large number of spores, by

${ }^{1}$ [The researches of Pringsheim and Stahl however have shown that this limitation can no longer be maintained. See Book II. Group 2.]

${ }_{2}^{2}$ [This explanation is now no longer generally accepted; see Journ. of Botany, I879.]

3 If these small tubers in Penicillium are termed the fructification, then in the same sense the sporogonium of Mosses is a fructification; and the Fern is also the fructification of the prothallium. 
which the whole process of development is again repeated. And as in Ferns, in addition to the spores, bulbils are also sometimes developed on the mature plant, so penicilliate conidiophores are also occasionally produced on the tuber of Penicillium. For the purpose of including this second stage of development in the various classes of plants under a common name, it may be termed the Sporeforming or Asexual Generation [sporophore], excluding from the idea of true spores the conidia of Penicillium, as well as the gonidia of Thallophytes.

If now, in the three examples cited of alternation of generations, we compare the organisation of the first or sexual with that of the second or spore-forming generation, it is seen that the latter, the result of an act of sexual union, is more highly and perfectly developed than the former, and therefore in this respect also represents the true close of the process of development. Thus in Penicillium we find the first generation developed in the form of a so-called mycelium, consisting of slender segmented branched filaments, while the second generation consists of a compact tissue of complicated structure. In Mosses again the first generation commences with a protonema, consisting, like a mycelium, of branched segmented filaments of cells; but here this generation advances to a higher development, since the protonema produces the leaf-bearing Moss-plant, the histological structure of which is still however very simple in comparison with the much more complete differentiation of the sporogonium. Still more strikingly are these characteristics seen in Ferns, where the first generation or prothallium consists of a plate of tissue which shows scarcely any external differentiation, while the second generation or true Fern is a very highly organised plant, differentiated externally into root, stem, and leaves, the tissue itself being also differentiated into three well-marked systems, the epidermal system, the fibrovascular bundles, and the fundamental tissue.

Starting then from Algæ and Fungi, and proceeding through the classes of Muscineæ, Filices, and Equisetaceæ to the Lycopodiaceæ, and finally to the Phanerogams, it is seen that in the alternation of generations, the first generation (oophore) continually recedes in importance and independence, while the development of the second generation (sporophore) continually advances; so that at length in Phanerogams the former is no longer a plant with independent power of growth, but takes the form of a special mass of tissue, the so-called Endosperm in the reproductive apparatus of the latter, filling up along with the embryo the cavity of the seed-coats. In contrast to this, at the starting-point of the series (Algæ and Fungi), the first or sexual generation is alone developed as a plant with independent growth; the second (asexual) generation appearing on it as its fructification or spore-fruit, represented, in its simplest form, by a single spore resulting from fertilisation, as will be illustrated in the introduction to the Thallophytes.

Designating the course of development which we have sketched out as alternation of generations, each of the two stages may be termed an Alternating Generation. Each may, as we have seen, be propagated directly by gemmæ, or by conidia, those developed by the first generation again producing individuals of the same kind; and in the same manner bodies of the same nature produced by the second generation will reproduce it. But this mode of reproduction may be wanting in either of the two alternating generations. 
If we now regard the two alternating generations as two stages of development of the same plant, each of which is necessary to supplement the other, it is seen that, in the first place, the entire course of development of a plant commences twice with a simple cell:-the first time the development begins with the spore to form the first (sexual) generation, the second time with the oosphere in the female organ to produce the second (spore-forming or asexual) generation. Secondly, we find that, in addition to these two beginnings from spore and oosphere, which are united to one another by the complete course of development, a subsidiary mode of development may also occur, each of the two generations having the power of propagating itself directly. For the purpose of distinguishing them from the true spores with which the development of the second generation closes, we term all those reproductive organs which immediately propagate the same generation either Gemme or Gonidia. A Spore, in our sense of the term, arises only from the second generation, and gives rise, on germination, to the first generation; a bulbil or gonidium, on the contrary, may arise from either of the alternating generations and reproduce it. The same facts may be expressed in the following manner:-Sexual cells (oospheres) and true spores indicate the turning-points in the alternation of generations; they are not organs for direct reproduction, for each of them always produces something different from that from which it immediately sprung; the spore of the Fern, for example, gives rise to a prothallium, the oosphere of the prothallium to a Fern; the spore of the tuber of Penicillium does not again give rise to a tuber, but to a filamentous mycelium, on which the tuber again arises as the result of fertilisation of the female cell. Bulbils and gonidia are, on the contrary, organs for direct reproduction, by means of which the same stage in the process of development is again repeated; the bulbil, for example, which arises on the leaf of a Fern, does not produce a prothallium, but a Fern; in the same manner the conidia formed on the branches of the mycelium of Penicillium do not, on development, give rise to the tuber, but to a mycelium like that on which they were borne.

The alternation of generations, as we have now described it in a few examples where it is peculiarly well exhibited, does not occur in those classes of Thallophytes which have the simplest structure; its first indications are met with where an act of sexual union is first detected, until at length, in the more highly developed plants, the alternation is manifested with perfect sharpness.

$a$. The idea of an alternation of generations is extended by some botanists considerably beyond the limits to which we have here confined it. It has been proposed, for instance, to apply the term to the case of Phanerogams in which lateral branches with foliage-leaves spring from a rhizome clothed only with scales, and from these other branches which develope into flowers; and to others of a like nature. It is clear, however, that the cases in question have a totally different significance in the history of development to that of the alternation of generations, using the term in the sense indicated above; they might be included, by way of distinction, under the common phrase Alternation of Axes. This phenomenon is one which is very inconstant even within limited groups of plants, while, on the other hand, true alternation of generations prevails over almost the entire vegetable kingdom; and the mode in which it runs through particular groups of plants is one of the weightiest arguments in favour of the natural system. This will be further elucidated in Book II. 
b. The problem which is met by the theory of alternation of generations in the form in which it has now been presented, is to refer the most important stages in the history of development of all plants to a single scheme which is illustrated most clearly in the cases of Muscinex and Filices, where Hofmeister first discovered this alternation in $185 \mathrm{I}$. The same botanist was also the first to explain the development of the seed in Gymnosperms by the alternation of generations in Lycopodiaceæ, and hence to compare it with the same phenomenon in Filices and Muscinex. At the present time our knowledge of the development of Thallophytes has made so much progress that it is possible to determine what are its main features, and to compare them with those of Muscineæ and vascular plants. This comparison, which has only been briefly indicated above, will be followed out more in detail in the sequel, and will lead to the result that Thallophytes may also be included in the scheme under which the other classes are comprised, the first stage of development closing with the formation of sexual organs, from which proceeds the second generation, essentially different from the first, and closing with the production of true spores. It will therefore show that the development of all plants which possess sexual organs may be divided into two stages which correspond in all essential points to the two generations in the lifehistory of a Fern; and that there is, therefore, in the whole vegetable kingdom, only one type of alternation of generations so far as it is brought about by sexual organs. 


\section{B OOK II.}

\section{SPECIAL MORPHOLOGY}

\section{AND \\ OUTLINES OF CLASSIFICATION: \\ GROUP I.}

\section{T H A L L O P H Y T E S.}

IN this group are comprised Algæ, Fungi, and Lichens, the term being applied to them because their vegetative body is usually a Thallus, i.e. exhibits no differentiation into stem, leaf, and root, or, if at all, only in a very rudimentary degree. There occur however in various groups of Thallophytes transitions from the simplest forms, which display no external differentiation, to others which show some indication of it; in the most highly developed representatives of some groups the external differentiation is carried so far that the terms leaf and stem are as applicable to them as to the higher plants. A true root, in the sense in which the term is applied to vascular plants, is however never found, though rootlike organs are commonly present which are termed Rhizoids; these are however always distinguishable by the absence of a root-cap and by the branching not being endogenous.

Like the external, the internal differentiation of Thallophytes also begins at the lowest stages, ascending by numberless transitional steps to a more perfect development of cells and tissues; but even in the most perfectly developed forms we do not meet with any sharp differentiation into those different systems which we know among the higher plants as epidermal tissue, fundamental tissue, and fibrovascular bundles. Even where the thallus consists of large masses of tissue, as in Fungi, it is still strikingly homogeneous.

Thallophytes nevertheless present a great variety of examples of the mode in which morphological differentiation proceeds from the simplest organic forms to 
others which are both externally and internally more and more complex. In the simplest stage the whole vegetative body consists of a single small cell of a roundish form, the cell-wall of which is thin and smooth, and within which protoplasm, chlorophyll, and cell-sap are only imperfectly separated. Advancing from this, progressive development is first displayed in the perfection of the single cell, which increases in size, and often attains dimensions unknown elsewhere in the vegetable kingdom, the differentiation being either chiefly in the contents, or in the external form, i.e. in branching. The growth of the cells may, on the other hand, be accompanied by cell-division, the thallus becoming multicellular, so that from a single cell there arises either a row of cells or a segmented filament, a simple plate of cells, or finally a massive tissue growing on all sides. Each of these processes further presents a great variety of modifications.

In the simpler Thallophytes a tendency prevails for a larger or smaller portion of their existence to be passed in the condition of motile primordial cells, which bear more or less resemblance to the simplest Infusoria, and were in fact until recently confounded with them. In some cases cells which are already clothed with a cell-wall, or assemblages of such cells, remain for a considerable time in a motile condition, swimming freely in the water. But these motile conditions are always interrupted by long periods of rest, during which growth and increase in size usually take place. In many of the more highly developed Thallophytes this power of motility is however limited to the male 'swarming' fertilising elements, the antherozoids; and in many cases is not displayed even here.

Like the structure of the vegetative body, the mode of reproduction of Thallophytes also exhibits great variety, commencing with the simplest kinds, and progressing finally to modes of reproduction almost as complicated as those which are met with in the highest plants. In the simplest cases reproduction appears to be coincident with ordinary cell-multiplication; the cell which constitutes the vegetative body grows and divides, each derivative celi then carrying on an independent life and repeating the process. In the more highly developed forms the unicellular or multicellular thallus continues to grow for a longer time, and becomes differentiated externally and internally, until at length at some one spot reproductive cells are produced. In most Thallophytes both kinds of reproduction, sexual and non-sexual, occur; and in the higher forms an evident alternation of generations is manifested. The reproductive organ which becomes separated from the mother-plant is almost always a single cell, which however varies greatly in its origin, significance, and capacity for development. The nomenclature which gives to all these- reproductive cells the name 'Spores' is a very unfortunate one, obscuring an insight into the course of development of the different forms, and rendering difficult the comparison of members of one group of Thallophytes with those of another. The erroneous theory of so-called 'Pleomorphy among Fungi was the result of a defective perception of the true nature of the different kinds of reproductive organs on which the common name of Spore had been bestowed. What we have now to say on this point for the purpose of counteracting the prevalent confusion of terms is founded on the view explained in Sect. 29 of Book I, on alternation of generations and on the relationship to it of the different reproductive organs. I begin by designating as Spores the reproductive cells which are produced in the 
sporangia of Ferns and capsules of Mosses. These are obviously the result of a vegetative process, excited by the act of sexual union, in consequence of which they arise on the second (non-sexual) generation [sporophore] which springs from the fertilised oosphere of the first (sexual) generation [oophore]. Let us now transfer these conceptions to the most highly developed Thallophytes which exhibit an evident alternation of generations, as the Ascomycetes. We have already seen, in the Section referred to, that the ascospores of Penicillium are the result of a vegetative process brought into action by the sexual organs of the mycelium, and which has for its result the formation of the tuberous fructification which constitutes the second generation. The ascospores of Penicillium therefore correspond to the spores of a Moss or a Fern. If now we suppose the result of the union of the sexual organs to be a very inconsiderable vegetative structure, and the second generation consequently to be merely rudimentary and a simple appendage to the first, the spores themselves would then seem to be an almost immediate result of fertilisation, as occurs for instance in the Nemalieæ (Fig. I64, $C$, p. 237). If we were further to imagine that the act of fertilisation did not result in the production of any vegetative structure, or the second generation to be altogether suppressed, the fertilised oosphere would then itself become a spore, as in the Coleochæteæ, Edogonieæ, and Vaucheria. In this case the spore is an equivalent for the whole of the second generation; it stands for the entire fructification of the Ascomycetes, the entire spore-capsule of a Moss, \&c. Precisely the same is true for the zygospore which results from conjugation. The zygospore (as for example in the Mucorini), or the oospore (as in Vaucheria), represents therefore in a morphological sense the entire second generation of these plants. This conclusion, which might easily be proved more in detail, may be briefly summed up in the statement that the Spore is either an immediate product of fertilisation (zygospore, oospore), or of a process of growth which is induced by fertilisation; and this vegetative growth may either be inconsiderable, as in the Nemalieæ and Erysipheæ, or it may be considerable, and it then gives rise to the second generation in which the spores are produced, as in Penicillium and other Ascomycetes. This explanation shows at once how in Thallophytes the second generation is a gradually increasing structure developed in consequence of the act of fertilisation. But for the purpose of a scientific nomenclature the term Spore (if used in the same sense as in Muscineæ and Vascular Cryptogams) must be applied in Thallophytes only to those reproductive cells which are the result of an act of impregnation, whether direct, or indirect through the production of a vegetative body which constitutes a second generation and closes the entire course of development of the plant. All other unicellular and non-sexual organs of reproduction we shall not term spores, but gonidia or conidia.

We may now proceed to a further description of the various kinds of sexual organs found among Thallophytes, and of the true spores which result from their union with or without an alternation of generations. The following three principal forms or types may be distinguished ${ }^{1}$.

1 More minute evidence of the statements here made will be found in the sequel in the description" of Algæ and Fungi. The facts stated are derived from the writings of Pringsheim, 
I. Conjugation and Production of Zygospores. Two cells of similar if not always of precisely the same nature coalesce, and produce a reproductive cell termed a $Z$ ygospore, which germinates after a shorter or longer period of rest, and then gives rise either to spores or at once to a plant of the same kind as that in which the conjugation took place. An alternation of generations is exhibited only in so far as the zygospore constitutes the entire second (non-sexual) generation.

The process of the formation of zygospores has a very different appearance according to the nature of the conjugating cells. The simplest case is presented

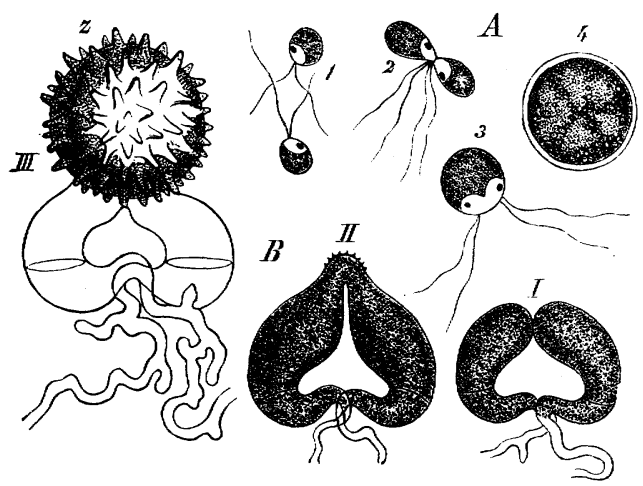

FIG. J62.-Various forms of conjugation and the production of zygospores $A$ conjugation of the zoogonidia of Pandorina; $B$ formation of zygospores in Piptocephalis (after Pringsheim and Brefeld). The numbers indicate in each case the successive stages of development. in the conjugation of zoogonidia discovered by Pringsheim (Fig. I62, $A$ ). These bodies during the process of swarming come into contact in pairs by their hyaline anterior ends, and then gradually coalesce into a primordial cell, which subsequently becomes invested with a cell-wall, and then grows, producing again motile cells, and each of these gives rise to a plant of the original kind. These Zoospores which result from the zygospore may be considered as true spores in the same sense as those of the

Muscineæ; for the zygospore is homologous with the spore-capsule of Mosses, and represents a rudimentary alternate generation. The conjugation of Spirogyra, as illustrated in Fig. 6, p. 10, is somewhat more complicated. The conjugating cells are here surrounded by a firm cell-wall; they put out protuberances opposite to one another, which unite to form a canal, through which the contents of one cell pass over into the other, and coalesce with its contents; the resulting protoplasmic body invests itself with a cell-wall, and becomes a zygospore, which again produces a Spirogyra filament by direct germination. The formation of zygospores in the Zygomycetes is represented in Fig. 162, $B$. Here the two cells which coalesce after having grown towards each other are perfectly alike and immotile ; and it is only a portion of the coalesced contents which becomes separated by a partition-wall, and produces the thick-walled zygospore which germinates after a period of rest.

2. The Formation of Oospores in Oogonia. The two reproductive cells are here essentially different; the female cell or Oosphere is always a naked iminotile primordial cell developed within an older cell which is termed the Oogonium. The male cells, the Antherozoids, the mother-cells of which are called Antheridia, are very small, and are endowed with motion by means of vibratile cilia; they swarm round the oosphere, and cause its impregnation by the coalescence of their

De Bary, Thuret, Nägeli, Janczewski, Brefeld, and others; though a different signification to that of the authors is sometimes applied to them. 
substance with it. The size of the antherozoids is so inconsiderable that they scarcely add appreciably to the mass of the oosphere, but yet produce a change in it, one consequence of which is that it becomes invested with a firm cell-wall, and then constitutes the Oospore.

The oospore may germinate immediately and give rise to a plant resembling the mother-plant, as in Fucus, or only after a certain period of rest like the zygospores, and this is the usual case. But here again the oospore may on germination give rise directly to a plant resembling the mother-plant, as in Vaucheria and some Saprolegnieæ; or it may, after remaining dormant, produce out of its contents a larger or smaller number of zoospores, each of which finally gives rise to a plant like the mother-plant, as in Spharoplea, EEdogonium, and Cystopus. In this process a rudimentary alternation of generations can again be detected:-an oospore which breaks up into zoospores may be compared to the sporogonium of a Moss in

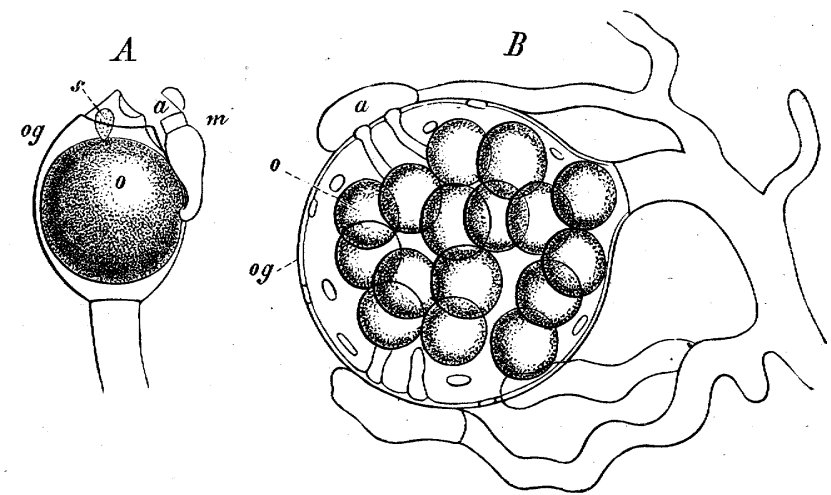

FIG. 163.-Examples of the production of oospores; $A$ in Edogonium; $B$ in Saprolegnin (after Pringsheim); og the oogonium; $o$ oosphere; $a$ antheridium; $m$ small male plant or dwarf male; $s$ antherozoid.

which all the parts except the spores are suppressed. If we were to imagine the fertilised oosphere in the archegonium of a Moss as itself producing the mothercells of the spores ${ }^{1}$, we should have something similar to one of these oospores. In this case therefore the oospore is properly a many-spored fructification in the same sense as the Moss-capsule; the zoospores produced from it are true spores in the sense of those of Muscineæ and Ferns, and we have consequently the first indication of the alternation of generations which attains its highest development in those classes. The new plants which result from the direct germination of the oospores, or through the medium of zoospores, have the power, in most cases, of propagating non-sexually by the formation of gonidia, until at length individuals arise which produce antheridia and oogonia. This non-sexual reproduction may be compared to that of the Marchantieæ by gemmæ produced on their vegetative body, until finally antheridia and archegonia are developed.

1 That such an analogy is not altogether fanciful is shown by Riccia, a genus of Hepaticæ, the extremely simple sporocarp of which may well be compared to the oospore of an EEdogonium. Pringsheim and De Bary have already pointed out this analogy (see De Bary, Die Familie der Conjugaten, Leipzig $185^{8}$, p. 60). 
But on the other hand the formation of oospores exhibits a certain resemblance to the process of conjugation. It is distinguished from that of the Pandorinex in this point of importance only, that the two coalescing sexual cells are not alike, so that the fertilisation of Vaucheria and $\mathbb{E}$ dogonium may be considered as a higher form of conjugation from a morphological point of view. But the mode of formation of many oospores displays also a greater or less resemblance to the mode of fertilisation which we shall describe as a third type; and in this respect the Saprolegnieæ in particular present a similarity to certain Ascomycetes.

3. Formation of Carpospores in Carpogonia. This type resembles the second in the fact that the two sexual organs contribute in very different degrees to the production of the fertilised body, the male organ only inciting to change, while the whole of the further development of the plant proceeds from the female organ, the result being the production of the Sporocarp.

The female organ, which may consist either of one cell or of more, may be designated by the general term Carpogonium. The male organs vary greatly according to the group to which the plant belongs; they may be swarming or passively motile antherozoids, or tubular Pollinodia; and fertilisation may be effected by the entrance of the antherozoids, as in the case of oospores, or by a kind of conjugation, the sexual cells coalescing by means of openings in the cellwalls of both, or finally by simple apposition and probably diffusion of a fertilising substance. The product of fertilisation is sometimes a single cell germinating directly or through the medium of zoospores; but more generally a multicellular body results, from which spores are finally produced. An alternation of generations may here also be recognised, rudimentary or more fully developed according as the structure of the fructification is simpler or more complicated. In the simplest cases the sporocarp appears only as an appendage of inconsiderable size to the plant; in the other extreme the fructification is able to continue an independent growth for a considerable time, and thus constitutes a second alternating generation. These phenomena will be described more in detail in the special description of the Carposporeæ. One essential difference between sporocarps and oospores consists in this, that in the production of the former certain cells also take part which were not immediately concerned in the act of impregnation; and that, with the exception of the simplest cases, the portion of the fructification which produces the spores is surrounded by a sterile envelope which serves merely for protection or also for further nourishment. Fig. 164 illustrates some of the most different forms of sporocarps.

In Coleochate $(A)$ the female organ or carpogonium (hitherto described as the oogonium) consists of a single cell $w$ which runs out upwards into a long narrow canal opening at the apex. Fertilisation is effected by small roundish swarming antherozoids $m$, and as a consequence the portion of the protoplasm (oosphere) which occupies the basal part of the cell becomes invested with a firm cell-wall. So far the phenomena are the same as in the formation of the oospores of Vaucheria or Edogonium, the only important difference consisting in the long canal formed by the cell-wall. A more essential deviation is now manifested in that the body which previously had the appearance of an oospore grows considerably after fertilisation, and in the fact that the effect of fertilisation shows itself also in the growth of the 
cells which adjoin the female organ, so that this latter becomes surrounded by an envelope $h$. A sporocarp is thus formed, the fertilised oosphere of which produces out of its contents after a certain period of rest a mass of tissue, all the cells of which produce zoospores, and each of these gives rise to a plant of

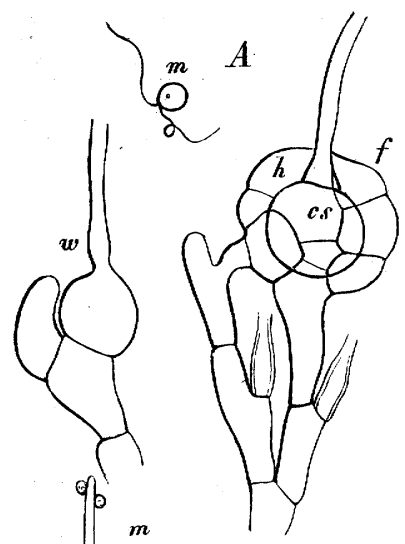

o
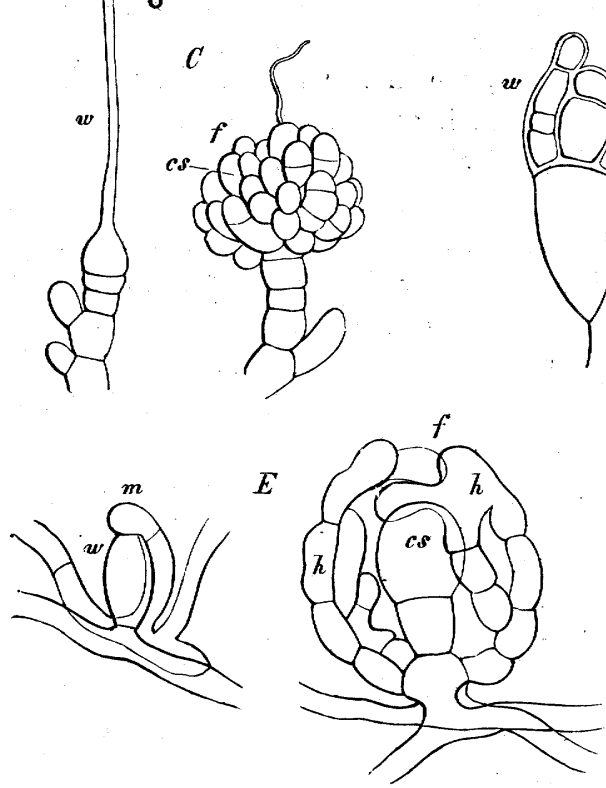
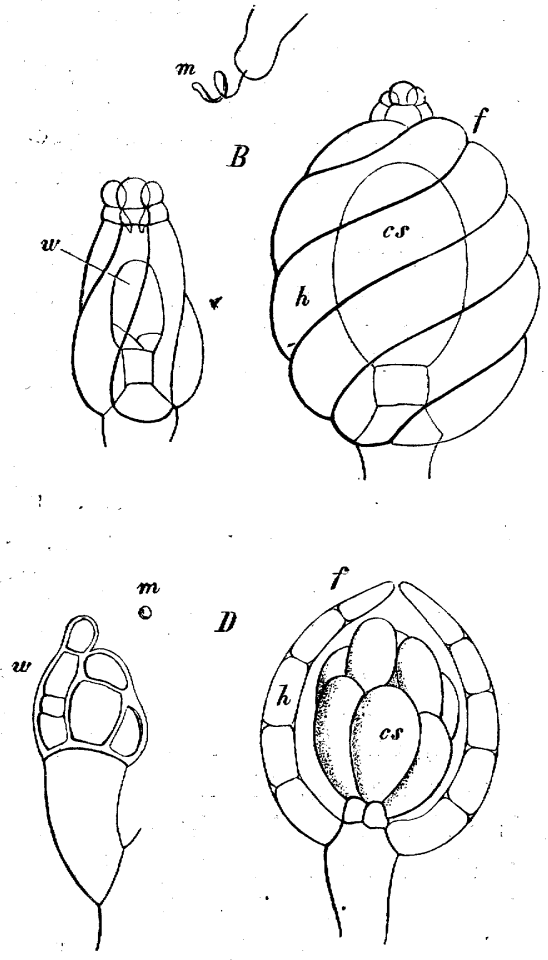

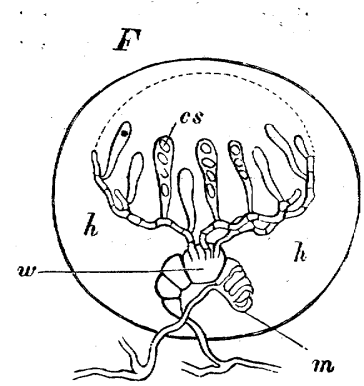

FIG, 164.-Various forms of carpogonia, and of sporocarps resulting from them; $w$ the female organ before fertilisation $m$ the male organ; $f$ the entire sporocarp; $h$ its envelope; cs the spores; $A$ Coleochate; $B$ Characea; $C$ Nemalion; $D$ Lejolisia; E Podosphara; F Ascobolus (after various authorities).

the same kind. The sporocarp of the Coleochæteæ combines the most essential characters of an oospore with those of the sporocarp of the Florideæ and of some Fungi. As respects an alternation of generations, the oosphere surrounded by its envelope, together with the tissue which subsequently fills it up and which produces 
the zoospores, must be regarded as the second generation, while the zoospores may be compared homologically with the spores of Mosses.

In Nemalion $(C)$ the carpogonium $w$ consists also of a single cell which is wide below and narrow and elongated above. This elongation, termed the Trichogyne, is a closed tube; the male fertilising cells attach themselves to it, empty their contents into it, and thus incite a further development of the basal part of the female cell, which now increases in size, and divides into a number of cells which grow out into densely crowded branches. A spore is formed at the end of each of these branches; and the whole assemblage of spores together with its short pedicel constitute the sporocarp, which in this case has no envelope.

In the true Florideæ, of which Nemalion may be considered the simplest form, the carpogonium $w$ consists, even before impregnation, of a number of cells $(D)$; a lateral row of cells bears at its apex a closed hair-like prolongation, the trichogyne, and is hence termed the Trichophore. The trichogyne receives the fertilising substance from the male cells which become attached to it; but neither the trichogyne itself nor the trichophore is thereby excited to any further development, the sporocarp resulting from the other cells of the carpogonium which lie beside the trichophore. The fertilisation therefore takes effect at a distance from the spot to which the male cell has attached itself. Certain cells of the carpogonium grow, divide, and finally produce the stalked spores, the pericarp or envelope of the fruit arising, as the result of branching beneath the carpogonium.

The sporocarp of the Characeæ $(B)$, which has hitherto been without any analogy, becomes intelligible if we compare it on the one hand with that of the Coleochæteæ, on the other with that of the Florideæ. The carpogonium w consists of a large ovoid cell which is borne on certain small round basal cells (Braun's 'Wendezellen'). These basal cells take no part in the development brought about by fertilisation, their behaviour being similar to that of the trichophore of the Florideæ. The large cell is fertilised by filiform antherozoids, and itself forms the single carpospore in the sporocarp, the envelope of which has been completely developed before fertilisation; and it behaves also in other respects in a similar manner to that of the Coleochæteæ, Florideæ, and Erysipheæ. That the large cell which becomes the carpospore does not possess any hair-like receptive organ or trichogyne is a point of very subordinate importance, since in the carpogonium of the Ascomycetes this organ is sometimes present, sometimes absent ${ }^{1}$.

One of the simplest cases of the formation of the fructification in the Ascomycetes is afforded by Podosphera $(E)$; and we here get the transition to an evident alternation of generations. The carpogonium $w$ consists of a single cell, and is fertilised by another tubular cell, the pollinodium. The result of fertilisation is that the female cell grows, and divides into two cells, of which the upper one forms in its interior several spores (Ascospores), and is hence termed the Ascus. Beneath the pedicel-cell of the ascus shoot out filaments which form the envelope of the fructification $f$.

The processes are somewhat more complicated in Ascobolus, another Asco-

${ }^{1}$ See De Bary, Beiträge zur Morphologie u. Physiologie der Pilze, vol. III. p. 88. 
mycete, of which a diagrammatic section is given in $F$. w is the carpogonium consisting of several cells, which is fertilised by the tubular branched pollinodium; the result is that a number of filaments shoot out from a central cell of the carpogonium, which then form sacs at the apices of their branches, and in these a number of carpospores. The envelope of the sporocarp is in this case very massive, and consists of cellular filaments which shoot out beneath the carpogonium; and it finally forms a compact pseudo-parenchyma in which the carpogonium is enclosed together with the ascogenous filaments and the asci which proceed from them. The mycelium which produces the carpogonia in both the Fungi now described is inconsiderable in comparison to the large sporocarp which results from the fertilisation of the carpogonium; the sporocarp itself in many cases continues to grow for some time independently of the mycelium, and therefore constitutes a second (non-sexual) generation of these Fungi. If the mycelium were large and vigorous, and the sporocarp which springs from it small, as is the case in the Florideæ and Characeæ, the sporocarp would in these cases also have the appearance of being only an appendage of the sexual generation, and an alternation of generations would scarcely be suggested, although this does actually occur, since the entire course of development of such a Fungus can be divided into two sharply-defined phases, of which the first is the mycelium with its sexual organs, while the second is the sporocarp with its independent power of growth.

Besides the true spores produced directly or indirectly by the act of fertilisation, which complete the course of development of the plant by a rudimentary or an evident alternation of generations, there is commonly among Thallophytes an extremely productive propagation by gonidia which are not brought into existence either directly or indirectly by any act of fertilisation, and which have therefore nothing to do with the alternation of generations.

The Gonidia ${ }^{1}$ often arise on the thallus by the whole of the contents of certain cells of the thallus dividing, and thus producing one or more gonidia which become detached from the plant. But in other cases special supports or receptacles are formed on the thallus, the sole function of which is to produce gonidia, either by the abstriction of the ends of special branches (Stylogonidia, as in Piptocephalis, Penicillium, \&c.), or by free cell-formation in the interior of large cells (Endogonidia, as in the Saprolegnieæ, Mucorini, and Vaucheria). In many cases, especially among Fungi, propagation is effected almost exclusively by such gonidia, the normal completion of the development by actual fertilisation being attained only under specially favourable conditions. This is the reason why the sexual organs of many Thallophytes are at present entirely unknown, while their gonidia are perfectly familiar. It is therefore often a very difficult matter to assert with respect to a Thallophyte, that it never produces sexual organs; since even in the case of the common Mould-fungus (Penicillium) and in many Algæ, the gonidia of which have long been known, it is only quite recently that the sexual organs and the alternate generation resulting from them have been discovered. Even in the majority of the large marine Algæ, the Phæosporeæ, and the numberless

1 Among Fungi the small gonidia which become detached in great numbers are also called Conidia, from the Greek kovia, dust. 
large Fungi (Basidiomycetes), the sexual organs are still unknown; although in the latter case the analogy of the Ascomycetes renders it very probable that the Fungi known as Hymenomycetes and Gasteromycetes are only the fructification which is the result of the union of sexual organs on the mycelium. The spores which are produced on these Fungi must therefore be treated as true spores in our sense of the term, and their mode of formation as something quite different from that of the gonidia of the Mould-fungi.

It is very common among Algæ, and occurs also in some Fungi which grow in water or on a moist substratum, for the gonidia when they escape from the mothercell to be naked, i.e. without any cell-wall, and motile; after their escape they have for some minutes or even hours the power of swimming about, at the same time rotating on their axis (swarming). The anterior end is hyaline, destitute of granules or colouring matter; and in some Algæ a minute red dot lies at one side behind the hyaline part ; the cause of the motion is the vibration of certain very fine threads, the Vibratile Cilia. Usually two of these cilia are attached to the hyaline anterior end, or one in front, the other at the side; but sometimes there is only one, while in others the hyaline anterior end is encircled by a dense circlet of numerous cilia; or finally the entire surface of the zoogonidium is covered with short cilia. During swarming a cell-wall of cellulose begins to be secreted; the zoogonidium then comes to rest, attaches itself to some solid body by its anterior end, the cilia disappear, and germination commences, the end which was posterior during swarming becoming the growing point and hence the anterior end of the young plant. It has already been mentioned that in some cases swarming cells conjugate, and these must then of course be regarded, not as gonidia, but as sexual organs which bear only a deceptive resemblance to zoogonidia; at any rate there are reasons for believing that the motile cells of some Algæ which have hitherto been regarded simply as gonidia, are capable of conjugation and are therefore sexual organs.

Motile cells of the kind now described may make their appearance at any stage in the course of development; it is not uncommon, as we have seen, for the entire contents of an oospore or even of a carpospore (as in Coleochate) to be transformed into motile cells which can then germinate; even in the so-called conidia of the Peronosporeæ the whole of the contents may break up into motile cells. In other cases again these bodies are produced in special branches of the thallus, and not unfrequently any vegetative cell of the thallus may allow its whole contents to escape in the form of motile cells. These motile cells have hitherto been all known as Swarm-spores or Zoospores; but, according to the definition of the term Spore which we have now adopted, we must term the asexually produced motile cells Zoogonidia, and designate the receptacles in which they sometimes arise in large numbers, not zoosporangia, but Zoogonidia-receptacles. It is moreover obviously of secondary importance whether the gonidia simply become detached, as in most Fungi, or whether they take the form of motile cells. The difference is evidently dependent on the mode of life of the plant; the presence or absence of the power of swarming is not one of morphological, but only of physiological importance; just as, in the seeds and fruits of Phanerogams, some have a power of transportation by means of a special floating-apparatus, while others simply fall 
off. In the genus Vaucheria we find all stages of transition from motile cells to gonidia which simply fall off.

The Classification of Thallophytes has been till quite recently based essentially on characteristics relating merely to the mode of life, according to which they have been divided into three classes,-Algæ, Fungi, and Lichens; the Characeæ have been sometimes included under Algæ, sometimes altogether separated from Thallophytes. But since more accurate investigations have enabled us not only to elucidate the morphological significance of the growth of these plants, but also to discover the sexual organs in the main divisions, and in many cases to follow the whole course of development, this classification can no longer be maintained, since it depends essentially only on differences in the external appearance and mode of life, while on the other hand it is seen that a totally different classification is necessitated by their morphological characteristics. The admirable labours of Schwendener, for example, have shown that Lichens, hitherto considered as a separate class, must not only be included among Fungi, but must be regarded as a section of a particular order, the Ascomycetes. Since we have become more accurately acquainted with the sexual organs of the Coleochæteæ and Florideæ, it can scarcely be doubted that these Algæ have a close affinity to Characeæ in the structure of their fructification. We can therefore now distinguish only two classes of Thallophytes,-Algæ and Fungi. But it has long been admitted that it is impossible to draw any satisfactory boundary-line between these two classes; several writers have indeed frequently pointed out that some families of Fungi must be closely associated with certain families of Algæ. It has been recognised more and more clearly that these two classes are separated only by a single distinguishing character; if the two old-established groups are to be retained, the only distinction between the two (and the one adopted in the earlier editions of this book) is to place under the head of Algæ all those Thallophytes which contain chlorophyll, under the head of Fungi all those which do not. But this separation is altogether artificial, and could only be tolerated so long as want of an accurate knowledge of the morphology of these plants compelled us to admit a classification having no foundation in morphology. In the present state of our knowledge, in which at least the morphological foundation for a scientific classification of Algæ and Fungi may be laid down, it is not only permissible but incumbent, in the interest of progress, at least to attempt a morphological classification of Thallophytes.

The first point to note is that the presence or absence of chlorophyll can be no sufficient reason for separating plants which are nearly related to one another morphologically, and which agree in their structure, their sexual organs, and their alternation of generations. In Phanerogams this principle is thoroughly admitted. If all Flowering-plants which do not contain chlorophyll were formed into one class in contradistinction to those which do contain it, the Rafflesiaceæ, Balanophoraceæ, Corallorhiza, Cuscuta, Orobanche, Monotropa, \&c. would have, in spite of the differences in their organic structure, to be combined into one class, and removed from their true relationship. No one however disputes that Cuscuta belongs to the Convolvulaceæ, Orobanche to the Labiatifloræ, Monotropa to the Pyrolaceæ, and Corallorhiza to the Orchideæ. These affinities are inferred, among Phanerogams, chiefly from the structure of the flowers and the embryo, and no one 
attaches the least importance to the fact that the want of chlorophyll and the peculiar mode of life of these plants gives them so different an appearance from that of their nearest allies. It is one of the most beautiful results of a truly scientific morphology and classification that, among Phanerogams, the remarkable habit of parasites and saprophytes is regarded as an altogether secondary matter. But the same principle should also be applied in determining the systematic relationships of Thallophytes;-habit and mode of life, the presence or absence of chlorophyll should also be treated as characters of altogether subordinate importance. All Thallophytes which are destitute of chlorophyll-i.e. all those which have hitherto been termed Fungi-must necessarily agree with one another more or less in their habit and mode of life, because they are all adapted to absorb organic carbonaceous nutriment from their environments. If they obtain it from living bodies, we have parasitism developed in its various forms; if they have the capacity of consuming dead organic remains, the habit and mode of life of the plant must vary accordingly. Algæ, in the sense in which the term has hitherto been used, are able themselves to produce carbonaceous food-materials out of carbon dioxide by assimilation; they are not therefore usually either parasites or saprophytes, but can maintain an independent life; they are however compelled, by the peculiarities of their organisation, to live in water or in damp places. Their dependence on assimilation requires that Algæ should inhabit localities where there is free access of light, while Fungi are not absolutely dependent on light for their supply of food.

But all these facts are of altogether secondary importance in determining: degrees of affinity in the compilation of a natural system of classification of Thallophytes. This object can be attained only by a comparison of such morphological characteristics as a thorough knowledge of development reveals. The determining considerations of a morphological nature are in Thallophytes, still more than in other groups of plants, dependent on the question whether they possess sexual organs, and, when this is the case, how these are formed, how the act of fertilisation is effected, and especially what is the nature of that structure which results either directly or indirectly from it, in one word, how the act of fertilisation affects the entire course of development.

We have already described the more important forms of the organs of fertilisation in Thallophytes, and the origin of an alternation of generations consequent upon it. If now the ${ }_{\text {plants }}$ which agree in these characters are compared with one another, it is seen that the remaining morphological facts also suggest a close affinity. The structural peculiarities connected with sexuality may therefore be regarded as the guiding characteristics, by which we are directed to relationships within the group. With our present still very imperfect knowledge of Thallophytes it is however not surprising if, in a classification founded on these principles, forms are nevertheless occasionally found placed near each other which appear to have but little affinity. This is unavoidable, because the intermediate transitional forms are unknown; and it must moreover be observed that in Thallophytes of a simple structure the morphological characteristics are more easily concealed by physiological adaptations and by changes in habit than in the higher plants. 
The classification here adopted does not therefore make any pretension to be one which will endure for all time; it rather claims to be in accordance with the present state of our knowledge, and to bring into proximity those forms which agree in the most important features of their development. In a certain sense this classification may still be called an artificial one; but it is natural in so far as it attempts to bring into prominence actual affinities and not merely differences and resemblances of external habit ${ }^{1}$.

It is, as every one knows, easier to make objections to a system than to lay down clear principles on which one should be established; and we will therefore only add a few explanatory remarks respecting the following table. In the present state of our knowledge, we seem compelled, first of all, to establish a class of Thallophytes in which not only is sexual reproduction unknown, but in which there is no near affinity to any sexual forms; this class will include only the simplest and most minute of all plants, and is therefore formed into the first class under the name Protophytes. But in a large number of Thallophytes in which we are unacquainted with the sexual organs, there is an obvious close affinity with well-known forms, with which therefore they may be associated in classification. Finally, there are other Thallophytes in which the sexual organs are still unknown, but in which no distinct affinity is exhibited with the Protophytes or with other well-known forms. These plants are altogether omitted from our classification, since it is not my purpose to present an index of all existing forms, but only to show the affinities of those that are best known ${ }^{2}$.

Each of the four classes here proposed starts with very simple forms, and attains, through diverging lines, very different degrees of development. The closest affinities are therefore found by comparing the simplest primary members of each class, especially those of the $2 \mathrm{nd}, 3^{\mathrm{rd}}$, and $4^{\text {th }}$ classes; and the widest differences by comparing the most perfect forms of the different classes. In this respect therefore these proposed classes resemble the recognised divisions in the groups of Muscineæ, Vascular Cryptogams, and Phanerogams.

In order not to depart too widely from the classification still current, and to facilitate a general view, I shall, as will be seen from the following table, treat separately the forms which contain chlorophyll (so-called Algæ) from those destitute of chlorophyll (so-called Fungi) within each class ${ }^{3}$.

${ }^{1}$ Cohn, who was the first to give up the division of Thallophytes into Algæ and Fungi, has not been, in my opinion, so happy in the classes which he has proposed. He does not start from any definite principle, but employs as his typical characters sometimes points of great, sometimes those of secondary morphological importance, as is shown by the names of the classes:Schizosporeæ, Zygosporeæ, Basidiosporeæ, Ascosporeæ, Tetrasporeæ, Zoosporeæ, and Oosporeæ (see Hedwigia, 1872, p. 18).

2 [Reference for some criticism and additional details on this classification of the Thallophytes may be made to Quart. Journ. Micr. Sc. 1875, pp. 295-326, and pp. 396-40r : see also de Bary, Bot. Zeitg. I88I. For a Classification of Fungi, see de Bary, Beitr. IV. I88I.]

3 Since this classification and the following account of Thallophytes has been ready for the press, I have had the opportunity of seeing a letter addressed to Dr. Brefeld by Prof. Fischer (Oct. 29th, 1873 ), in which the following classification is proposed:-

THALLOPHYTES.

$\overbrace{\text { Myxomycetes. }}^{\substack{\text { Fungi. } \\ \text { R } 2}}$ Algæ.




\section{THALLOPHYTES.}

\section{Class I. Protophyta.}

Containing Chlorophyll.

Cyanophycea.

Palmellaceæ (in part).
Not containing Chlorophyll.

Schizomycetes.

Saccharomycetes.

Class II. Zygosporeæ.

Conjugating cells motile.

Pandorineæ.

(Hydrodictyeæ).

Myxomycetes.

Conjugating cells stationary.

Conjugatæ (including Diatomaceæ). Zygomycetes.

Class III. Oosporeæ.

Sphæroplea.

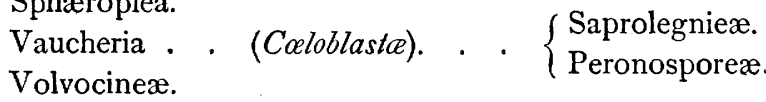

Edogonieæ.

Fucoideæ.

\section{Class IV. Carposporeæ.}

Coleochæteæ.

Florideæ.

Characeæ.
Ascomycetes (including Lichens). Acidiomycetes (Uredineæ). Basidiomycetes.

Class I. No sexual reproduction.

Saccharomyces.

Phycochromaceæ.

Class II. Reproduction by conjugation.

Zygomycetes.

Diatomaceæ, Conjugatæ.

Class III. Reproduction by oospores, the result of fertilisation.

Peronosporeæ.

Saprolegnieæ.
Palmellaceæ, Siphoneæ.

Confervaceæ, Fucaceæ, Coleochæteæ, Characeæ (?).

\section{Class IV. A componnd fructification resulting from fertilisation} (alternation of generations).

Ascomycetes.

Florideæ.

Basidiomycetes.

Prof. Fischer still treats Algæ and Fungi as two entirely distinct series developed in parallel rows; while I suppose that in each class Fungi have diverged as ramifications from various types of Algæ: there are other important differences between our systems in the position of the Myxomycetes, Coleochæteæ, and Characeæ. But, with the exception of these differences, the main point is the agreement of our views with respect to the establishment of four classes which serve equally for Fungi as for Algæ. 


\section{CLASS I.}

\section{PROTOPHYTA.}

In this class are comprised the most simple and minute of all plants, whether they contain chlorophyll, and therefore have been regarded as belonging to the Algæ, or whether they contain no chlorophyll, as the Yeast-fungi and the so-called Schizomycetes (Bacteria, etc.).

Those Protophytes which contain chlorophyll live chiefly in water, or at least in damp localities, sometimes as pseudo-parasites, and their green colouring matter is often mixed with a blue one which is soluble in water. Those which are destitute of chlorophyll are either true parasites or inhabit the moist surfaces of organic bodies, or are found in fluids which contain organic substances in solution from which they derive their nutriment, and which they decompose, causing putrefaction or fermentation.

The structure of Protophytes is always extremely simple, and in the simplest of all the cells are so small that they can be seen only under a high magnifying power. In the smallest a distinction can scarcely be detected between cell-wall and cell-contents; and when this can be done the contents are homogeneous, or minutely granular. The cell-wall has a tendency to deliquesce into a soft jelly in which the cells remain imbedded either regularly or irregularly; but sometimes it only swells up, and is then manifestly stratified.

In the simplest forms the cells are isolated; the two halves of a divided mothercell increase till they attain its size, and then again divide; the derivative cells separate, and carry on an independent life. In the more highly developed forms the derivative cells remain united, and the final result is either simple and often extremely slender rows of cells, thin lamellæ, cell-division taking place in one plane, or agglomerations in consequence of cell-division taking place in all directions. It is only in the most perfectly developed individual that the multicellular body has a determinate external form.

The species which contain chlorophyll are in general larger than those which do not, and the structure of their cells more perfect; the largest and most perfectly developed among the latter being Yeast-cells. Even in this, the lowest stage in the vegetable kingdom, the want of chlorophyll is seen to be usually associated with a degradation of structure. All the cells in the individual are usually exactly alike; it is only in the higher forms that a few larger cells of a different colour -termed Heterocysts-are intercalated among the otherwise similar cells of a filament.

In most cases there is no distinct base and apex, and therefore no definite direction of growth; but in a few of the highest members of the class a base 
and apex of growth can be distinguished, and a kind of branching makes its appearance.

Although zoogonidia, in the sense in which the term is used in the higher Thallophytes, do not occur (with the exception of some Palmellaceæ which perhaps do not belong to this class), many Protophytes are nevertheless endowed with a power of motion by means of which they swim about; spirally-wound multicellular filaments turn on their axis; or the filaments themselves bend backwards and forwards; or some other kind of motion occurs.

No sexual organs have yet been observed, and in most cases there are no non-sexual organs of reproduction, the multiplication of individuals being effected by the separation of the ordinary vegetative cells ${ }^{1}$. In other words, organs for nutrition and reproduction are not differentiated; it is only in the most highly developed forms that cells of peculiar form are produced for the sole purpose of reproduction.

The class has hitherto been divided into three groups distinguished by their colour; viz. I. those containing pure chlorophyll, Palmellaceæ; 2. those in which the chlorophyll is mixed with a blue pigment, and which therefore appear of a light green or bluish green colour, Cyanophyceæ; and 3. those in which there is no chlorophyll, Schizomycetes and Yeast. While limiting the class of Protophyta to these three groups, it is nevertheless possible that some of the forms included in it are not independent species, but merely stages in the development of other higher Thallophytes, which have a perpetual power of reproducing themselves. Thus it has already been determined ${ }^{2}$ that the genus Pleurococcus, hitherto placed among Palmellaceæ, is merely a stage of development of Chlamydomonas which belongs to Pandorineæ, a class of Zygosporeæ; and it is not improbable that the whole group of Palmellaceæ, and perhaps also some Chroococcaceæ, are of the same nature, and must at some time be eliminated from the class of Protophyta.

\section{FORMS CONTAINING GHLOROPHYLL.}

A. Cyanophycees. These organisms are of a bluish, emerald, or brownish green, or some similar colour, due to a mixture of true chlorophyll and phycocyanin; this pigment becomes diffused out of dead or ruptured cells, and thus produces the blue stain on the paper on which Oscillatoriex are dried. From crushed specimens treated with cold water phycocyanin is extracted as a beautiful blue solution, blood-red in reflected light ${ }^{3}$. When the crushed plants are treated with strong alcohol after the extraction of the blue pigment, a green solution is obtained which contains true chlorophyll, and probably a special yellow pigment, phycoxanthin ${ }^{4}$.

I. The Chroococcacem exist as isolated roundish cells or in roundish families, the cells of which are imbedded either in an amorphous mucilage or in the swollen walls of their mother-cells. They occur as gelatinous growths in damp places. Several genera are distinguished, with numerous species:-e.g. Cbroococcus and Gloocapsa

1 [Gonidia have been discovered in Gloocapsa by Bornet (Ann. sci. nat., sér. V. XVII; in Nostoc by Janczewski (ib. XIX), and in Bacillus by Cohn (Beit. zur Biol. d. Pflzn. I).]

${ }^{2}$ Cienkowski, Bot. Zeit. I865, no. 3 ; and Rostafinski, Bot. Zeit. 187 I, p. 786.

${ }^{3}$ Cohn, in Schulze's Archiv für mikrosk. Anatomie, vol. III. p. I2.-Askenasy, Bot. Zeit. 186 7 .

- Millardet and Kraus, Comptes Rendus, vol. LXVI. p. $5 \circ 5$. 
(Fig: 165), which divide in all directions, the latter imbedded in a stratified jelly; Gloeothece, imbedded in a stratified jelly but dividing in one direction only; and Mexismopedia, the cells of which divide cross-wise in a single plane.

2. The Nostocaceæ form lumps of mucilage or gelatinous pellicles which float in water or lie on damp earth or among moss. In the jelly are serpentine moniliform rows of roundish cells, a few larger cells, termed Heterocysts, the contents of which are of a different colour, being interposed at intervals. The filaments increase in length by the division of the individual cells, thus constantly adding to the coils which lie in the jelly that they excrete. New colonies are, according to Thuret ${ }^{1}$, formed in the following manner:-The jelly of the old colony becomes softened by water, the portions of the threads lying between the heterocysts become detached, separate from the jelly, and straighten themselves, while the heterocysts themselves remain in the jelly. After they have entered the water, the old portions of the threads become endowed with motion like the Oscillatoriex, and their exit is probably caused by this movement ${ }^{2}$. The roundish

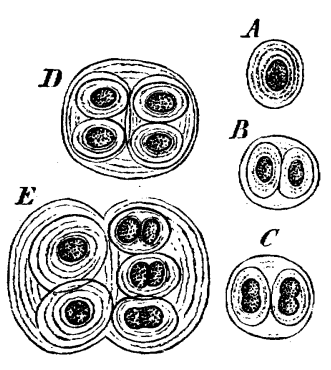

FIG. 165.-Mode of cell-division in Glococapsa. cells of the filaments grow transversely, i.e. at right angles to the axis of the filament, and then divide, the division-planes being parallel to the axis of the filament, which now consists of a series of short articulate threads, the axis of whose growth is at right angles to its own. The threads which are thus formed increase in length and join, placing their terminal cells in contact [alternately above and below in each successive thread], and thus unite into a single curved Nostoc-filament. Individual cells, apparently without any definite law, become heterocysts. In the meantime the gelatinous envelope is developed, and the new colony, which is at first microscopic, attains the size of a walnut ${ }^{3}$.

3. The Oscillatorieæ consist of rigid cylindrical filaments of various thickness, often extremely slender, divided into disc-like cells by very delicate transverse septa. The filaments are not straight, but somewhat coiled in the form of a very oblique spiral; they revolve on their axis, and become matted, when large numbers grow together (in water or on moist earth), into balls or pellicles. When a lump is placed in water or on wet paper, Nägeli has shown that it assumes a star-like arrangement in consequence of these movements.

4. The Rivularieæ ${ }^{4}$ form soft greenish blue lumps of jelly which swim about in stagnant water or grow attached; in the first case they are spherical, in the second hemispherical, the smallest about $\frac{1}{2}$ millimetre in diameter, the largest the size of a hazel-nut. A number of moniliform filaments consisting of roundish cells lie in the

1 Thuret, Observations sur la réproduction de quelques Nostochinées, Mem. de la soc. imp. des sci. nat. de Cherbourg, vol. V. I857. [Ann. and Mag. Nat. Hist. 1858, vol. II.]

2 These motile threads of Nostoc were seen by Janczewski to enter the young stomata on the lower side of the thallus of Anthoceros lavis, where they further develope into round balls. Such colonies of Nostoc have been known for a long time in cavities and in the tissues of different Hepaticæ (Blasia, Pellia, Diplolana, Aneura, Riccia), but have generally been considered endogenous gemmæ of these species, until Janczewski proved their true nature. Nostoc also establishes itself in the large porous cells of the leaves of Sphagnum. The entrance of Nostoc into the parenchyma of the stem of a dicotyledonous plant, Gunnera, is brought about, according to Reinke, in a different manner; the deeper-lying parenchymatous cells of the outer part of the stem, themselves covered by layers of parenchyma, are densely filled with colonies of the Alga. (Bot. Zeitg. 1872, pp. 59 and 74.) [See also Ann. des Sc. Nat. 1872, p. 306, and Quart. Journ. Micr. Sc. 1873, p. 369.]

3 [Archer has described the occurrence of 'spores' in Nostoc paludosum which were always placed singly between the heterocysts. Quart. Journ. Micr. Sc. 1872, p. 367.]

${ }^{4}$ De Bary, Flora, I863, p. 553 . 
jelly arranged radially; at the free end the filament runs out into a long hyaline hair, while at the central end is a large heterocyst which gives the whole filament the form of a riding-whip. The filament increases in length by the transverse division of its cells. Reproduction is effected by the cell which lies next to a basal heterocyst becoming thicker, increasing considerably in length and assuming a cylindrical form; its contents become denser, and invested by a firm membrane. When the whole of the rest of the colony perishes these Resting-spores only remain. They subsequently germinate, the contents dividing into from 4 to 12 shorter cylindrical pieces; each of which again divides repeatedly, until more than roo cells are formed which become rounded off and the filament moniliform. During this lengthening the membrane of the germinating cell ruptures, the upper end of the filament projects, the lower portion subsequently creeps out of the sheath, and the terminal cells become pointed. The filament, now free, breaks up into several pieces, which become closely packed together into a tuft or ball. Each filament now lengthens at one end into a segmented hair, while the cell at the other end becomes a heterocyst. Such a tuft which springs from a germinating cell forms a young mass of Rivularia, the filaments becoming enveloped in jelly. The multiplication of the filaments of a growing mass takes place by a kind of branching; i.e. one of the lower cells becomes a new heterocyst; the portion of the filament that lies between it and the old heterocyst developes into an independent filament alongside the parent-filament.

5. The Scytonemem form branched filaments enclosed in thick gelatinous envelopes, which-at all events in their older portions-consist also of several rows of cells. To this family belong Scytonema, Sirosiphon, \&c. ${ }^{1}$

B. The Palmellace e contain pure chlorophyll. The cells live singly or remain in families imbedded in mucilage; they resemble the Cyanophyceæ in many ways. Thus Gloocystis, belonging to this family, has the appearance of a pure-green Gloocapsa; Tetraspora forms Nostoc-like lumps of jelly, but is propagated by zoogonidia; in Oocardium the filaments are arranged radially in jelly, as in Rivularia. The delicate green growths on damp walls, stems of trees, \&c. consist of cells either isolated or grouped into families which are known under the names Protococcus, Palmella, Cystococcus, \&c.; Palmella cruenta forming blood-red incrustations. Probably, as has already been said, all these forms are only stages in the development of higher Algæ, which attain their further normal development only under favourable conditions of growth.

\section{FORMS NOT CONTAINING CLOROPHYLL.}

C. The SCHizom ycetes ${ }^{2}$ (Fig. I66) live in fluids which contain organic substances (albuminoids) liable to putrefaction, from which they obtain their nutriment, and of the putrefaction of which they are the cause. The greater number consist of extremely small cells without any differentiation into cell-wall and cell-contents, so that in some cases their organic nature can only be determined by indirect methods ${ }^{3}$. Where they occur, an enormous number of individuals are usually imbedded in a gelatinous

1 Various forms of the Algæ described under A. and B. occur again in the bodies described as gonidia of Lichens. (See Fig. 22 2.)

${ }^{2}$ Cohn, Untersuchungen über Bacterien, Beiträge zur Biologie, I872, Hft. 2. p. I 27 ; I876, Bd. ii. Hft. 2. [Quart. Journ. Micr. Sc. 1873 , p. I56; 1877 , p. 81 ; 1879, pp. 356-404.]

3 Since the smaller Schizomycetes, usually called Bacteria, are found also on the slimy surface of living bodies, on wounds, \&c., they have recently, from a medical point of view, been regarded as the cause of diseases. A copious literature, generally deficient in even an elementary acquaintance with scientific botany, treats of the Schizomycetes from this point of view; but it can hardly be doubted that observers have frequently mistaken the mere products of decomposition of organic substances, and every crystalline precipitates of an inorganic character, for Bacteria. 
mucilage, and this is especially true of the most minute forms, the investigation of which is hence rendered extremely difficult.

The forms and vital phenomena of the Schizomycetes, as far as they are accurately known, recall various species of Chroococcaceæ and Oscillatoriex; but they are in general much smaller than the corresponding forms belonging to the chlorophyllaceous series. Thus, for example, Sarcina (Fig. $166, \mathrm{I})$, which grows in the human stomach, corresponds to Merismopedia, the small cells dividing cross-wise and remaining for a time united into tetrads. In the remaining Schizomycetes, which are commonly known as Bacteria, growth takes place only in the direction of length, and the cells formed by repeated transverse division either separate or remain united into filaments. Cohn divides them into four groups:-I. Sphærobacteria, with extremely small roundish cells which become detached, corresponding to the most minute forms of the Chroococ-

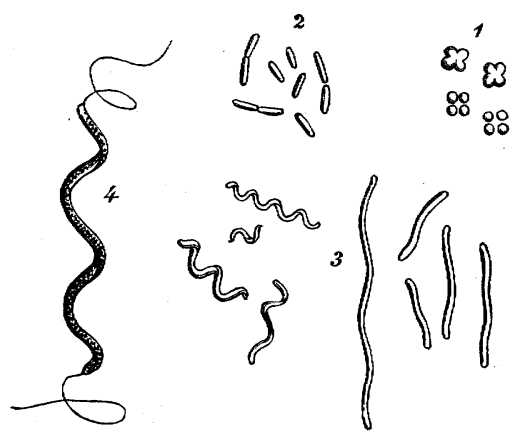

FIG. 166.-Schizomycetes: I Sarcina; 2 Bacterium; 3 Vibrio; 4 Spirillum (after Cohn). caceæ and Palmellaceæ. They grow on the surface of moist dead organic bodies forming gelatinous growths, often of an intense yellow-green, blue, or violet colour; these pigments which are contained in the protoplasm are sometimes soluble, sometimes insoluble in water. 2. Bacteria proper, in which the cells, when separate, are long, rod-like, very minute, and able to swim about in the fluid. Fluids that contain albuminoids putrefy and become milk-white from the multiplication of these ordinary Bacteria; they correspond in form to the genus Synechococcus among Chroococcacex which forms bluish-green coatings on rocks ${ }^{1}$. 3. Filobacteria, in which the slender cells remain united into threads, which are either straight, forming the genus Bacillus of Cohn, or curved and bent, when they are Vibrio (Fig. 166, 3). They do not excrete a gelatinous envelope and resemble small Oscillatoriex. If the Schizomycetes are constituted into a distinct group, then Beggiatoa, with contractile filaments, which has been hitherto referred to the Oscillatorieæ, must be included in it. 4. Spirobacteria, which form spirally curved filaments sometimes of considerable size in comparison to the preceding ones. Cohn distinguishes the genera Spirillum (Fig. 166, 4) and Spirocbate, which recall Spirulina among the Oscillatorieæ.

D. The SACCHAROMyCETES, of which the genus Saccbaromyces is the only one that is accurately known, consist of small round cells which live isolated, and resemble in form some Chroococcaceæ and Palmellaceæ; their organisation is nevertheless capable of a more accurate investigation than that of the Schizomycetes, which they also usually greatly exceed in size. The genus Saccharomyces, which causes the alcoholic fermentation in saccharine fluids, consists of separate cells of an ellipsoidal form with smooth and thin walls, the protoplasm in which can be clearly recognised as such and encloses one or more vacuoles. When growing in a solution capable of fermentation these cells multiply very rapidly; not however by the ordinary mode of division, but by budding and abstriction. At some point or other of the yeast-cell a small protuberance makes its appearance, which increases to the size of the mother-cell; the very narrow point of union then gives way, and the two cells then carry on an independent life, and again repeat the process. Whether under some circumstances the cells can also grow out

${ }^{1}$ [E. R. Lankester (Quart. Journ. Micr. Sc. I873, p. 408) believes, from the investigation of a peach-coloured Bacterium, that the series of forms distinguished by Cohn cannot be maintained as distinct. Lister (ibid. 1873, p. 393) believes that he has demonstrated the origin of Bacteria from a Fungus, a species of Dematium.] 
into tubes and assume hypha-like forms, as asserted by Cienkowski ${ }^{1}$, appears to be uncertain. Reess ${ }^{2}$, however, has discovered that when yeast-cells are grown on the surface of cut pieces of potato, turnips, \&c., they attaip a larger size, and the protoplasm contained in them breaks up into from two to four roundish endogenous gonidia, which, when placed in a saccharine fluid, at once again produce yeast-cells by budding and abstriction. Reess considers these endogonidia of Saccharomyces to be ascospores, and the yeast-fungus therefore to be an Ascomycete. Brefeld, however, makes the forcible objection to this view, - that if a yeast-cell which forms gonidia is considered an ascus, and the gonidia ascospores, it must be shown that the supposed ascus is developed from an ascogonium, i.e. from a female sexual organ, as in the Ascomycetes. Such an origin has, however, never been proved, and is extremely improbable.

The view advocated by Pasteur ${ }^{3}$, and since his time very popular, but never entertained by me, that the yeast-fungus can live in fluids which do not contain any oxygen diffused through them, and that they obtain the oxygen necessary for their respiration by the decomposition of chemical compounds, and especially by that of sugar into alcohol, carbon dioxide, and other products, has been shown to be altogether without foundation by the recent researches of Brefeld ${ }^{4}$ carried out in the botanical institute of Würzburg. Yeast-cells, like all other vegetable cells, require for their growth oxygen either free or diffused through the fluid ${ }^{5}$. The Fungi of fermentation afford no exception to this general law, and are only distinguished by the fact that they are able to make use of even the most minute quantities of oxygen diffused in the fluid. If the saccharine fluid is altogether free from diffused oxygen and from other nutrient substances, fermentation still takes place, but the yeast-cells do not grow, but pass into a dormant condition, perishing after a time; dead yeast-cells do not cause fermentation. It follows from this that the decomposition of the sugar into alcohol and carbon dioxide is caused by the living yeast-fungus, but has nothing to do with its respiration, growth, or nutrition ${ }^{6}$.

\section{CLASS II.}

\section{ZYGOSPORE $Æ$.}

AcCording to the principles laid down in the Introduction to the group, this class comprises plants which have hitherto been placcd among Algæ on the one hand and among Fungi on the other hand,-all those, in fact, in which sexual

1 [Bull. Acad. imp. St. Petersb. 1872, vol. XVII ; Quart. Journ. Micr. Sc. 1875, pp. 145-149.]

${ }^{2}$ Reess, Botanische Untersuchungen über des Alkoholgährugspilze, Leipzig I870. [Quart. Journ. Micr. Sc. 1875 , pp. I 42 , I 43.]

3 [Comptes rendus, 1872 , pp. 784-790; Quart. Journ. Micr. Sc. 1873, p. 351.-See also Mayer, Lebrbuch der Gahrungs Chemie, I876 ; Schützenberger, Les Fermentations, I 876 .]

4 Vortrag, July 26th, 1873, in the Physik.-medic. Gesellschaft of Würzburg.

5 The nature and the necessity of respiration in plants was first pointed out by me in my Handbuch der Experimental-physiologie, pp. 263-264; (see infra, Book III. Chap. 2. Sect. 6).

${ }^{6}$ On other Fermentation-fungi see Van Tieghem, Ann. Scientif. de l'école normale, vol. I. I864, and Ann. des Sci. Nat. $5^{\text {e }}$ sér. vol. VIII. I868, as well as the French translation of this work, p. 352 . 
reproduction takes place by means of conjugation, the essential characteristics of this process being that the two cells which take part in it are alike, and produce, by the coalescence of their protoplasmic contents, a cell of peculiar form, the Zygospore, which usually remains for a time dormant, and is then termed a resting-spore. Conjugation is the simplest form of sexual reproduction, and the morphological characters of the plants belonging to this class are, as might be expected, much simpler than of those which constitute the succeeding classes. The mere fact of sexuality does nevertheless show an advance on the mode of reproduction of the Protophyta, and the Zygosporeæ manifest in consequence a higher degree of organisation, and present the transition from the non-sexually propagated Protophytes to those forms of Thallophytes in which reproduction is sexual in the strict sense of the term.

Like that of the vegetative organs, the form of the organs of conjugation varies greatly in the different sections of this class; the zygospore is sometimes produced by the conjugation of naked zoogonidia, sometimes of highly developed cells belonging to the thallus, sometimes of special branches which do not occur elsewhere in the thallus. One of the most remarkable phenomena connected with sexuality is the formation of Auxospores in the Diatomaceæ, which, as far as our present knowledge goes, takes place in some cases by actual conjugation, but in others, as Schmitz has shown, by the simple approximation of two cells without any coalescence or actual contact, an interchange of substance taking place probably by diffusion.

The plants comprised in this class differ greatly in the structure of their vegetative body; and we are at present acquainted with but few intermediate transitional forms connecting the various sections belonging to it. This evidently arises from the fact that it is only of late years that the process of conjugation, previously known only in the Conjugatæ, has been studied in the Pandorineæ, Zygomycetes, and other families. It may be expected that the further investigation of the zoogonidia of a large number of Algæ will show them to be conjugating sexual organs; and it is even possible that among plants which are very nearly allied some produce zoogonidia which actually conjugate, while in others the corresponding cells do not usually conjugate, but proceed to a further development, or, in other words, are propagated parthenogenetically. Some such phenomenon is indicated in the formation of the auxospores of the Diatomaceæ already mentioned; and, on the other hand, we find forms, like the Hydrodictye ${ }^{1}$, in which no conjugation of the zoogonidia has hitherto been observed, although they are nearly connected, in their morphological characters, with the Pandorineæ in which this mode of reproduction does occur. I do not therefore hesitate in assigning the Hydrodictyeæ a place among the Zygosporeæ. The question is more difficult whether, in addition to the Zygomycetes, the Chytridineæ and Myxomycetes should also be included in this class. In the Chytridineæ it is probable that some of the zoogonidia conjugate, although this has not hitherto been observed ${ }^{2}$. In the

${ }^{1}$ [The conjugation of microzoogonidia was observed by Suppanetz in I873. Rostafinski, Mém. Soc. Sc. Nat. de Cherbourg, I875, vol. XIX. p. I5 2.]

${ }^{2}$ [Novakowski has discovered sexual reproduction in Polyphagus Euglenc. Cohn, Beitr. 3. Biol.

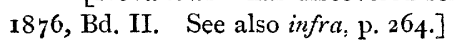


Myxomycetes a conjugation of zoogonidia does take place if the term is used in its most extended sense; for there is absolutely no reason why the coalescence of the Myxoamœbæ ${ }^{1}$ should not be regarded as a form of conjugation, and the production of plasmodia as analogous to that of zygospores. What has hitherto prevented botanists from recognising this analogy is merely the habit of life of the Myxomycetes, especially the peculiarity of not forming cells, and the circumstance that conjugation takes place between thousands of zoogonidia. If the coalescence of the myxoamobæ into a plasmodium is compared to the conjugation of the zoogonidia of the Pandorineæ, then the fructification which results from the plasmodium must be regarded as a large zygospore, the contents of which break up into a number of spores, like the large resting-spore of Synchitrium. They have at first, it is true, no power of motion, but this is quite a secondary consideration if regard is had to the corresponding processes in the Pandorineæ.

With reference to the structure of the thallus, all the plants included in this class may be termed unicellular, the thallus consisting of a single independent cell, an eremoblast; or, when it is multicellular, the separate cells are nevertheless essentially similar, so that their union is scarcely necessary physiologically for the existence of the whole. Such unions of equivalent cells, the product of a single mothercell, sometimes take place only after the individual cells have passed through a kind of swarming phase; when they come to rest, a number of cells unite to form the so-called Conobium, and continue their development as a body with definite form. This formation of cœnobia, as it occurs in Hydrodictyon and Pediastrum, exhibits a certain analogy, not only with the process of conjugation generally, but with the formation of plasmodia in the Myxomycetes in particular, except that a plasmodium, if regarded as a cœnobium consisting of masses of naked protop!asm; no longer manifests any independence of the separate parts.

The formation of a tissue in the ordinary sense of the term occurs only in a few Algæ, as Ulothrix and some Conjugatæ, in so far that the divisions take place.in one direction only, and the cells thus produced remain more or less firmly united, forming filaments in which all the cells are still perfectly equivalent, each one of them representing therefore the entire plant. The predominant unicellular character is manifested also in individual cells being capable of a high development, especially in the Conjugatæ, Diatomaceæ, and Zygomycetes. This tendency is manifested in the former in the contents of the cells and in the peculiar sculpture of the cell-wall, in the Zygomycetes in the extremely complicated branching, while the cell-contents remain simple.

The greater number of forms belonging to the class, especially those which contain chlorophyll, the Pandorineæ, Hydrodictyeæ, Desmidieæ, and Diatomaceæ, exhibit in their thallus no distinction between base and apex; they form either spherical or tabular cœnobia or rows of cells, all of which multiply by division without any distinction between base and apex; but in some Diatomaceæ and at all events in the germination of the zygospores of the Mesocarpeæ and Zygnemeæ adhesion takes place to a substratum, and hence a kind of contrast arises between

\footnotetext{
1 See Brefeld, Ueber Dictyostelium mucoroides in Abhandl. des Senkenb. Gesellsch., vol. VII. I 869, p. 20.
} 
base and apex. This occurs in a much higher degree in the Zygomycetes, and even in the Myxomycetes, which, growing on a substratum, send up their gonidiophores and sporocarps into the air.

A non-sexual reproduction takes place universally, but in different ways. In the Pandorineæ, Desmidieæ, and Diatomaceæ every cell-division may be regarded as a vegetative multiplication, since every single cell constitutes an individual; but in the Hydrodictyeæ and in Ulothrix peculiar zoogonidia are formed, different from the ordinary vegetative cells. Propagation by gonidia takes place however in the most perfect form in the Zygomycetes, where, before the formation of the zygospores, receptacles are produced on long stalks which develope endogonidia in vesicular swellings, the so-called sporangia, or conidia (stylogonidia) on branched stalks. In many Zygomycetes the much-branched cellular filament which forms the mycelium may break up under unfavourable vital conditions into a number of spherical cells or gonidia, each of which may subsequently reproduce the plant.

Many Zygosporeæ recall the Protophyta in this respect, that they have also conditions, during their true vegetative period, in which they have the power of motion; this occurs to an especially high degree in the Pandorineæ and Myxomycetes, much less so in some Conjugatæ and Diatomaceæ.

\section{FORMS GONTAINING CHLOROPHYLL.}

\section{A. Conjugation takes place between motile cells (Zoosporea).}

I. The PANDORINEA ${ }^{1}$ consist of cells which are either isolated or united into cœnobia by gelatinous envelopes; the cœnobia are either spherical, as in Stephanosphara, ellipsoid, as in Pandorina, or are square plates, as in Gonium. In these states, although surrounded by a cell-wall, they have still a power of motion, each cell possessing two long cilia which protrude through the cell-wall. The isolated cells of Cblamydomonas and Cblamydococcus swim about in this manner like ordinary zoogonidia; in the conobia, on the contrary, the cilia of all the individual cells project through the common envelope, and, by their united action, set the whole cœnobium in a twisting and rolling motion. The course of life of these plants may be illustrated in two examples.

The genus Chlamydomonas consists of isolated zoogonidia which multiply in the vegetative state by bi- or quadri-partition. But in the sexual reproduction the zoogonidia divide into eight motile daughter-cells each, provided with four cilia, differing from one another in size but smaller than the mother-cells. According to Rostafinski these cells conjugate in precisely the same way as Pringsheim has described in the case of Pandorina (vide infra); the zygospores thus formed come to rest and continue to grow for some weeks; if then dried and again placed in water they divide repeatedly, and form resting motionless families of cells identical with the genus Pleurococcus formerly placed under Palmellaceæ.

1 Cohn, Ueber Chlamydococcus und Chlamydomonas, Berichte der schles. Ges. ${ }^{8}{ }_{5} 6$. [Ray Soc., Bot. and Physiol. Mem. I852.]-Cohn und Wichura, Ueber Stephanosphara pluvialis, Nova Acta Acad. nat. curios. vol. XXVI. p. I. [Quart. Journ. Micr. Sc. I858, p. I 31 ; Archer, ib., I $86_{5}$, pp. I 16-I 85.]-Pringsheim, Ueber Paarung der Schwärmsporen, Monatsber. der Berliner Akad., Oct. I869. [Ann. des Sc. Nat. 1869, vol. XII.]-De Bary, Bot. Zeitg. I858, p. 73.-Rostafinski, Bot. Zeit. I $87 \mathrm{r}, \mathrm{p} .787$. 
In Pandorina Morum (Fig. I67) the complete course of development was followed by Pringsheim, this being the first instance that had been observed of the conjugation of zoogonidia. Pandorina is one of the commonest of the Pandorinex. The sixteen cells of a cœnobium (Fig. $167, I$ ) are closely crowded together, and surrounded by a thin gelatinous envelope out of which the long cilia protrude. The non-sexual multiplication results from each of the sixteen cells breaking up into sixteen smaller cells; the sixteen daughter-families $(I I)$ become free by the absorption of the gelatinous envelope of the mother-family ; each daughter-family, again surrounded by a gelatinous envelope, grows to the original size of the mother-family. The sexual reproduction

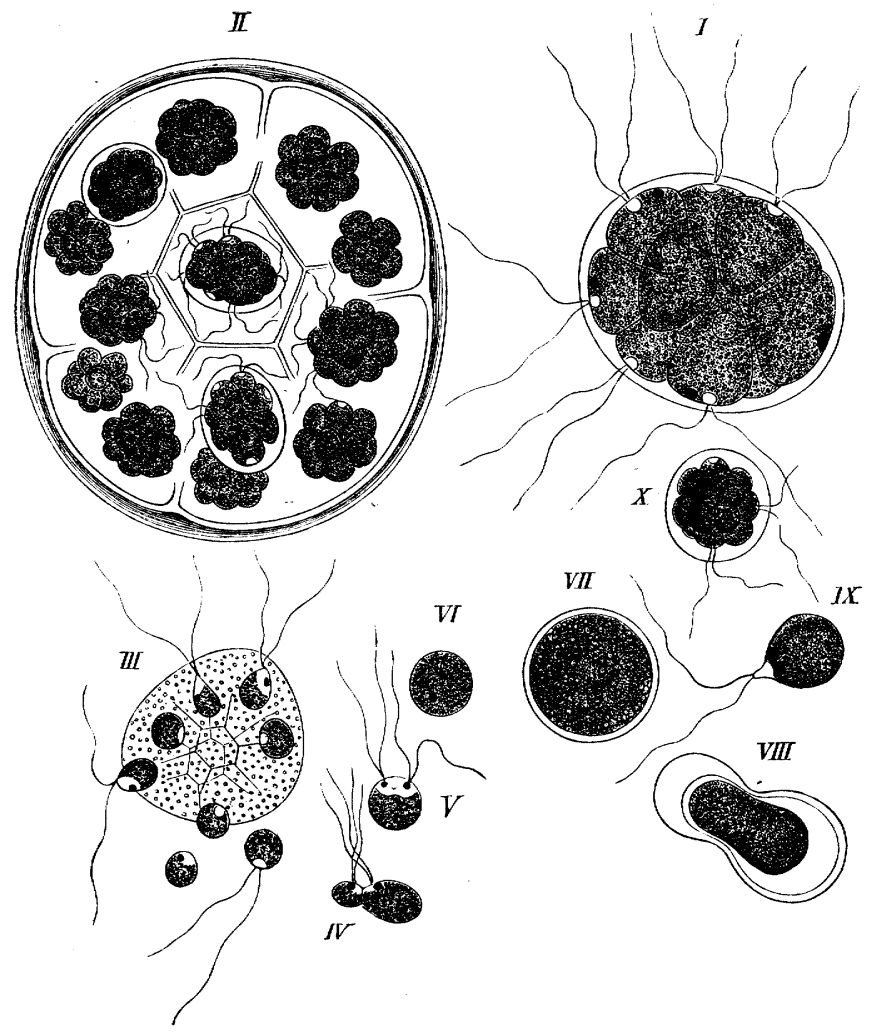

FIG. 167.-Development of Pandorina Morum (after Pringsheim) ; $I$ a swarming family ; $I I$ one divided into sixteen daughter-families; $I I I$ a sexual family, the separate cells emerging from the gelatinous envelope; $I V, V$ conjugation of the zoogonidia; $V I$ a zygospore just formed; $V I I$ a mature zygospore; $V I I I$ transformation of the contents of a zygospore into a large zoospore; $I X$ free zoospore; $X$ young family produced from the last.

is brought about in exactly the same way, but the gelatinous envelopes of the young families become softened, and the separate cells are thus freed and each swims about by itself $(I I I)$; these free zoogonidia are of very variable size, rounded and green at the posterior end, pointed, hyaline, and furnished with a red corpuscle in front, where they bear the two cilia. Among the crowd of these zoogonidia may be seen some which approach in pairs as if they were seeking one another. When they meet, their points come in contact, and they coalesce into a body at first hour-glass-shaped (IV), but gradually contracting into a ball $(V)$; in this ball the two corpuscles and the four cilia at the enlarged hyaline spot are still to be seen for a time; but these all soon 
disappear. Some minutes after the commencement of conjugation the resulting zygospore is a spherical cell $(V I)$, which remains at rest for some time enclosed in its cell-wall, its green colour passing over into a brick-red. If the dried-up zygospores, which have now greatly increased in size, are placed in water, germination begins after twenty-four hours; the outer shell of the cell-wall breaks up, an inner membrane protrudes and now contains one, two, or three large zoospores which finally escape $(V I I I, I X)$, surround themselves, after a short period of swarming, with a gelatinous envelope, and break up by successive divisions into sixteen primordial cells which now again form a cœnobium like Fig. $\mathrm{r}$.

A further illustration of the course of development in the Pandorinex is furnished by Stephanosphara pluvialis, one of the rarest and most beautiful of the family (Fig. 168), occurring rarely in the rain-water which collects in the hollow of large stones. The process of vegetative reproduction is the same as in Pandorina; but, according to Cohn and Wichura, the succession of generations of this kind is interrupted by the cells belonging to a family dividing repeatedly (Fig. I68, XII) into zoogonidia which ultimately become free $(X I I I)$, and probably produce resting zygospores by

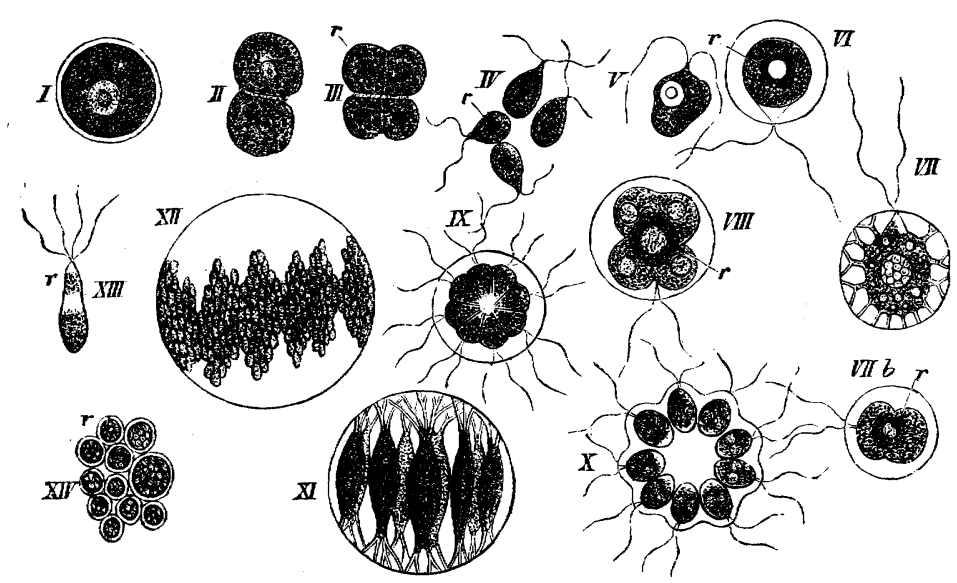

FIG. 168.-Successive stages in the reproduction of Stephanosphara pluvialis (after Cohn and Wichura).

conjugation after the manner of Pandorina. It is stated by the observers above-named that stationary immotile balls $(I)$ accumulate at the bottom of the water which, as they grow, assume a red colour. After these resting-cells, which are probably zygospores, have lain for some time dry and then again been moistened, they germinate, the contents breaking up into from 4 to 8 zoospores $(I I-V)$ which invest themselves with a cellwall, and each gives rise, in a single day by successive division $(V I I-I X)$, to an eight-cell conobium $(X, X I)$, which again in the next night gives birth to eight motile families ${ }^{1}$.

2. The nearest allies to the Pandorinex are probably the Hydrodictyex, as is shown by their formation of cœnobia, and still more by the whole course of their development, which Pringsheim ${ }^{2}$ first described in detail. Although the conjugation of the zoogonidia has in this case also not been actually observed, they offer a striking

1 [Archer has described, $l . c .$, pp. 7, 8, a remarkable amœboid phase which the primordial cells of Stephanosphara undergo.]

2 Pringsheim, Mon. der königl. Akad. der Wiss, zu Berlin. Dec. 13, r860. [Ann. des Sc. Nat. 1860, vol. XIV; Quart. Journ. Micr. Sc. I862, pp. 54, 104. See also A. Braun, Verjungung, - p. 146; Ray Soc. Bot. and Physiol. Mem. 1853, pp. 137, I90, see ante, p. 25 I.] 
resemblance in their mode of origin and subsequent development to those of the Pandorineæ. When the cœnobia multiply, a large number of zoogonidia are formed in each mother-cell, within which they move about for some time; when they come to rest they congregate in some definite arrangement, and then continue their development unitedly, eventually again multiplying in the same way, as for instance in Pediastrum (Fig. 169). In Hydrodictyon utriculatum, which occurs occasionally in ditches, the mature

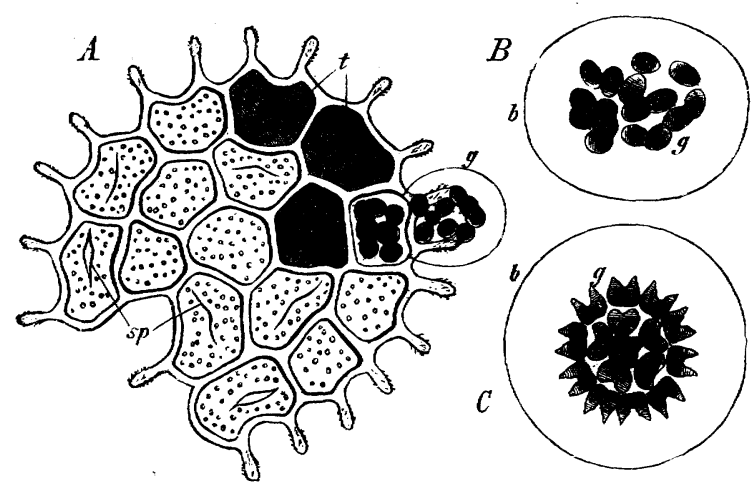

FIG. I69.-Pediastrum granulatum (after A. Braun, $\times 400$ ). A a plate consisting of united cells; at $g$ the innermost layer of a cell-wall is protruding; it encloses the daughter-cells resulting from division of the green protoplasm; at $t$ are various states of division of the cells; sp the fissures in the already empty of the mother-cell-wall which has entirely escaped (greatly enlarged) $b$ contains the of the mother-cell-wall which has entirely escaped (greatly enlarged); $b$ contains the
zoogidia $g$, these are in active 'creeping' motion; $C$ the same family of cells $4 \frac{1}{2}$ hours after its birth, 4 hours after the zoogonidia have come to rest; these have arranged themselves into a plate, which is already beginning to develope into one similar to $A$.

plant or cœnobium consists of a sac-like net several centimetres long, which is composed of a great number of cylindrical cells united at their ends so as to form a four- or sixcornered mesh. The ordinary mode of reproduction consists in the green contents of one of the cells of the net breaking up into from 7,000 to 20,000 zoogonidia which move about with a trembling motion within the wall of the mother-cell, come to rest in the course of half-an-hour, and then arrange themselves in such a way that, by their elongation, they again form a net of the original kind which is set free by the absorption of the wall of the mother-cell, and attains, in the course of three or four weeks, the size of the mother-plant. In other cells of the mature net the green contents break up into from 30,000 to $x 00,000$ microzoogonidia which at once leave the mother-cell and swarm about for some hours. The hypothesis has not yet however been confirmed that conjugation then takes place between these zoogonidia ${ }^{1}$; but when they come to rest they are spherical, invested with a firm cell-wall, and may retain their vitality for months when dried up if protected from light. After remaining several months at rest these resting-spores begin to grow slowly, and after they have attained a considerable size their contents break up into two or four large zoospores which come to rest after a few minutes, and assume a peculiar angular form when they have reached a considerable size, putting out horn-like appendages. In each of these so-called polybedra the green parietal protoplasm again breaks up into zoogonidia which move about for 20 or 40 minutes within a sac which protrudes out of the polyhedron. When come to rest they arrange themselves into a sac-like net consisting of from 200 to 300 cells, but in other respects resembling one of the ordinary ones. In some of the polyhedra smaller and more numerous zoogonidia are formed, but these also unite into a net.

1 [See note supra, p. $\left.25^{1 .}\right]$ 
3. Ulothrichaceze. Differing in many respects from the Pandorineæ and Hydrodictyex is the genus Ulotbrix, an Alga consisting of segmented filaments composed of cells which are all alike. It is mentioned in this connection only because it is characterised by the conjugation of equivalent zoogonidia. Cramer states ${ }^{1}$ that the contents of some of the individual cells of a filament break up into two, four, or eight zoogonidia which immediately germinate and produce new filaments. Other of the cells, on the other hand, give birth to 16 or 32 microzoogonidia which, after escaping, conjugate exactly like those of Pandorina. Nothing is known of the development of the zygospores. It is questionable whether Ulotbrix is not more nearly related to Spbaroplea among the Oosporex, and whether the conjugation of the zoogonidia is not to be regarded as a simpler case of the formation of oospores which occurs in the latter ${ }^{2}$.

\section{B. Conjugation takes place between stationary cells.}

(a) The Conjugate ${ }^{3}$ consist of cells with a limited power of growth, which multiply to an unlimited extent by bipartition; the cells thus produced either live entirely independently, or remain united in filaments. The chlorophyll-bodies form either parietal bands, axile plates, or radiate bodies arranged in pairs. Conjugation takes place between ordinary vegetative cells, the contents coalescing in a variety of ways, the resulting zygospore becoming invested with a cell-wall, germinating after a period of repose, and presenting essential differences in form from that of the vegetative cells. There are no distinct gonidia, the ordinary vegetative cells performing the functions of reproductive organs. De Bary divides the Conjugatæ into three families:-

(I) The Mesocarpeæ consist of cylindrical segmented filaments with an axile plate of chlorophyll ; filaments which lie parallel to one another put out conjugative processes, or two cells of contiguous filaments come into contact by knee-like projections; the parts of the walls which are in contact become absorbed and a broad canal is formed in which the protoplasm of the two conjugating cells collects; the canal then becomes shut off by two or four transverse septa and constitutes the zygospore. This mode of production of zygospores is clearly analogous to similar processes in the Zygonycetes. On germination the zygospore produces at once a segmented filament, the end that remains in the spore forming its base and the exposed end its apex. This contrast is not, however, permanent ; all the cells subsequently multiply by transverse division. To this family belong the genera Mesocarpus, Craterospermum, and Staurospermum.

(2) The Zygnemeæ consist also of cylindrical segmented filaments with the chlorophyll arranged in straight or spiral parietal bands or in stars placed in pairs. Conjugation takes place between two parallel filaments; the individual cells put out opposite conjugative protuberances (see Figs. 5 and 6, p. 10) which eventually touch one another, when the absorption of the cell-walls at the point of contact forms a narrow canal. Since a number of cells of two filaments usually conjugate at the same time, the whole forms a ladder-like structure in which the rungs are represented by the canals. After the formation of the conjugating canal the proto-

${ }^{1}$ Cramer, Naturfor. Gesellsch. in Zürich, March 21 st, 1870.

${ }^{2}$ [A. Dodel (Jahrb. für wiss. Bot. 1876 , vol. X. Heft 4 ) has described in detail the germination of the zygospores of Ulothrix. The whole course of development presents a striking analogy to that of Hydrodictyon. Dodel has, like Areschoug, observed occasional conjugation between the microzoogonidia; if these do not conjugate, they then propagate themselves non-sexually like the macrozoogonidia.]

${ }^{3}$ De Bary, Untersuchungen über die Familie der Conjugaten, 1858. [See also Hassall, Hist. Brit. Freshwater Algæ, 1845; Wittrock on Mesocarpeæ; Quart. Journ. Micr. Sc. 1873, p. I 23. Besides the modes of conjugation described in the text, contiguous cells of the same filament conjugate by lateral processes both in Mesocartea and Zygnemea.] 
plasmic bodies of the two cells contract, one of them passes through the canal into the other, and the two coalesce into a round zygospore invested with a thick cell-wall consisting of several layers which lies within the much larger mother-cell, and which germinates only after a period of rest (Fig. I70). In the Zygnemeæ there is also at first a contrast in the young plant between base and apex, which afterwards disappears, all the cells behaving exactly alike during growth. Among the genera are Zygnema, Spirogyra, Mougeotia, Sirogonium, and Zygogonium.

(3) The Desmidieæ ${ }^{1}$ consist of cells which live isolated or less often in rows which easily break up and are imbedded in mucilage. The cells are either cylindrical or fusiform, sometimes furnished with horn-like appendages (Scenedesmus); in other cases they have a circular or elliptical outline, and are divided by a deep constriction into two symmetrical halves. Even where there is no such constriction, the chlorophyll-body is divided symmetrically in the interior of the cell, or the symmetry is indicated by the so-called chlorophyll-vesicles [amylum-bodies] ${ }^{2}$ and the distribution of the starch-grains. In accordance with this symmetrical structure the vegetative propagation of the cells (individuals) is effected by the formation of a septum in the plane of symmetry or within the constriction which splits into two lamellæ and divides the cell into two halves. Growth at the point of separation then produces a segment which restores the symmetry. The made of formation of the zygospores is similar to that in the Zygnemex; but in the

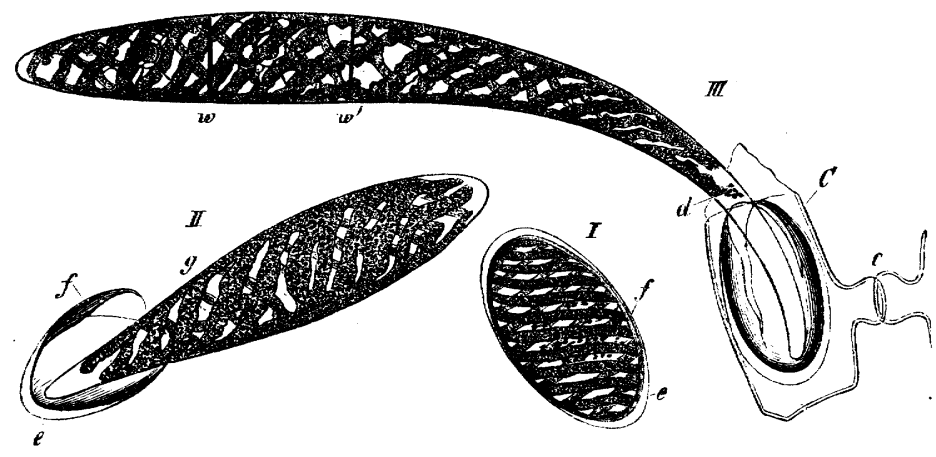

FIG. I70.-Germination of Spirogyra jugalis (after Pringsheim, Flora, 1852, no. $30 ; I$ a resting zygospore ; II commencement of its germination; $I I /$ the young plant further developed from a zygospore, which had been enclosed in the cell $C$, the conjugating canal $c$ being still visible; $e$ outer cell-wall of the spore; $f$ yellowish brown layer of the cell-wall; $g$ the third and innermost layer of the cell-wall of the spore, which forms the germinating filament; $w w^{\prime}$ the first septa of the germinating filament, the posterior end $d$ growing into a narrow appendage.

simplest forms, as Cylindrocystis and Mesotanium, where the conjugating individuals are of very simple form, conjugation appears to be nothing but a coalescence, altogether similar to that of the zoogonidia of Pandorina. The zygospore either germinates directly or divides into two or four daughter-cells, each of which repeats the vegetative mode of reproduction already described. In the last-named case we may consider, as in Pandorina, the zygospore to be a fructification which produces several spores in the sense in which the term is used in the Muscinex; and we may here again have an indication of an alternation of generations.

These processes may now be explained more in detail in the case of Cosmarium Botrytis (Fig. I7I), as described by De Bary (l.c.). The cells live isolated, and are symmetrically bisected by a deep constriction (Fig. I $7 \mathbf{1}, X$ ), and are also compressed

1 [See also Ralfs, British Desmidiex, 1848.-Archer in Pritchard's Infusoria.]

2 [Strasburger has shown (Zelltheilung und Zellbildung, $2^{\text {to }}$ Aufl. p. 83) that the structure existing in the chlorophyll-bodies of Zygnema and Spirogyra are true chlorophyll-granules in which numerous starch-grains are placed concentrically so as to form a layer at the surface. These structures are identical with those mentioned above as chlorophyll-vesicles and amylum-bodies.] 
at right angles to the plane of constriction $(I, a)$; in each half-cell are two amylumbodies and eight chlorophyll-plates, which curve and, running from two centres to the wall, converge in pairs. The multiplication of the cells by division is brought about by the narrowest part of the constriction elongating a little, when the thicker outer layer of the cell-wall opens by a circular fissure; the two halves of the cell hence appear separated from one another and united by a short canal, the wall of which is a continuation of the inner layer of the walls of the half-cells. A septum soon appears in the canal which unites them, by which the cell is divided into two daughter-cells, each of which is a half of the mother-cell. The septum, at first simple, splits into two lamellæ, which immediately become convex towards one another $(I X, b)$, and each daughter-cell now possesses a small rounded outgrowth which grows gradually and assumes the form of a half-cell, so that each daughter-cell now again consists of two symmetrical halves $(X)$. While the wall is undergoing this growth, the chlorophyllbody of the old half-cell grows into the newly-formed half. The two amylum-bodies in the old half-cells elongate, become constricted, and each divides into two; of these four, two pass over into the new growing half-cell, and all again arrange them-

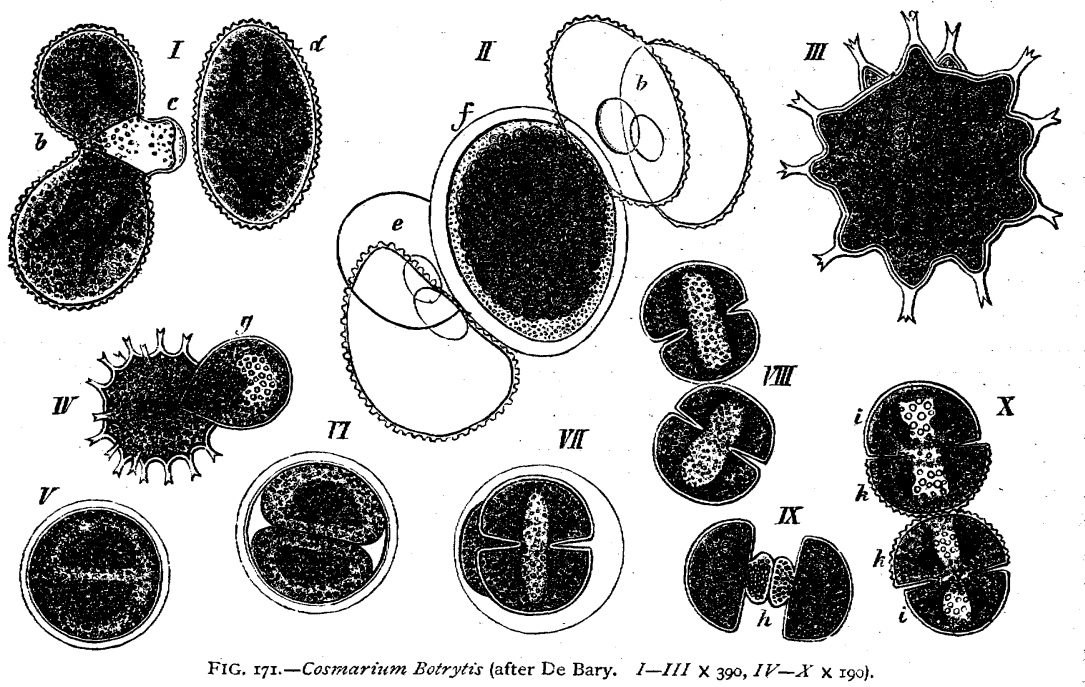

selves in the original symmetrical manner. Conjugation takes place between cells lying in pairs in a crossed position enclosed in soft jelly (Fig. $17 \mathrm{I}, I$ ). Each of the two cells puts out from its centre a conjugating protuberance $(I, c)$ which meets the other; these protuberances are formed of a delicate membrane which is a continuation of the inner layer of the cell, the firm outer layer of which is split $(I, c)$. Each of the two protuberances swells up into a hemispherical bladder, they come into contact, the separating wall disappears, and the contents unite in the broad canal thus formed; the protoplasm becomes loosened from the cell-wall, and contracts into a spherical form. The united protoplasmic body appears as if surrounded by a delicate gelatinous wall $(I I, f)$ by the side of which lie the empty cell-walls $(I I, e, b)$. The zygospore now becomes rounded into a ball; its wall forms, as it matures, three layers, an outer and an inner colourless layer of cellulose, and a middle firmer brown layer, This stratified cell-wall grows out at several points into spiny protuberances which are at first hollow and afterwards solid, each of them again producing at its end a few: smaller teeth (III). The starch-grains of the conjugating cells become transformed into oil in the zygospore. Germination commences by the protrusion of the colourless inner layer through a wide split in the outer layer $(I V)$, the thin-walled ball thus set free 
considerably exceeding the zygospore itself in size. In the contents of this sphere $(V)$ may be recognised two chlorophyll-masses surrounded by oily protoplasm, which might have been distinguished even before their escape from the external layer of the zygospore: The contents now contract and become surrounded by a new wall $(V)$ from which the older wall detaches itself as a delicate vesicle. After some time the protoplasm becomes constricted by a circular furrow, and splits into two hemispheres, each of which contains one of the two chlorophyll-masses $(V I)$. Each hemisphere remains for a time naked, and again constricts itself; but this time the constriction does not advance to the centre; the hemisphere changes its form in other respects also, and each assumes the form of a symmetrically divided Cosmarium-cell ( $\left.V^{r} I I\right)$, which surrounds itself with a wall of its own. The planes of constriction of the two cells derived from the zygospore cut the dividing plane of the zygospore itself at right angles ; they themselves also lie crossed in the mother-cell at right angles to one another. The contents in each now arrange themselves in the manner above described; the mothercell-wall is absorbed and the new cells separate from one another. All these processes of germination are completed in one or two days. The new cells, whose outer wall is smooth, now divide in the usual manner, but the newly-grown halves increase in size and become rough on the outside $(V I I I-X)$; the two daughter-cells of each of the two cells produced from the zygospore have dissimilar halves, and the four cells produced by their further bipartition are therefore of two different forms: two have their halves equal and two unequal; the latter constantly produce by division one with equal and one with unequal halves.

(b) The Diatomace $\mathbb{E}^{1}$ (Bacillariex), a group extremely rich in species, follow naturally after the Desmidiex; in particular they are allied to the Conjugatæ by processes of development which correspond to the conjugation of the latter, or at least bear a certain resemblance to it ${ }^{2}$. They bear a special resemblance to the Desmidiex in the form of their cells, in the manner of division, and in the mode of completion of the daughter-cells. Like the Desmidieæ, the similar cells of the Diatomaceæ may be united into rows, or may live entirely isolated. The tendency of Diatoms to secrete a soft jelly in which they live socially is found also in Desmids, although less strongly displayed. In the same manner the movements of Diatoms are not altogether dissimilar to those of Desmids, and even the silicification of the cell-wall, which is very strong in the former, is found, though to a smaller extent, in Closterium and other Desmids; and the fine sculpturing of the silicious shell also finds an analogue, although in a coarser form, in the cell-wall of some Desmids. The Diatoms are the only Algx, except the Conjugatæ, in which chlorophyll occurs in plates and bands, but in some forms it is also found in granules, and the green colouring matter is concealed, as in the chlorophyll-granules of the Fucacex, by a buff-coloured substance, Diatomin or Phycoxanthin $^{3}$. One of the most prominent peculiarities of Diatoms consists in their silicified cell-wall being composed of two separated halves or valves of unequal age, of which the older one partially envelopes the younger like the lid of a box. When the cell begins to divide, the valves separate from one another, and after the division of the contents into two daughter-cells, each of them forms a new layer at the plane of division which is adjusted by its turned-in margin (the girdle) to the girdle of the

1 Lüders, Ueber Organisation, Theilung und Copulation der Diatomeen, Bot. Zeitg. 1862.Millardet and Kraus discuss their colouring-matter in Compt. rend. vol. LXVI. p. 505-Askenasy in Bot. Zeit. I869, p. 799.-Pfitzer, in Hanstein's Bot. Abhandl., Heft II, 187 , gives the most important contribution to our knowledge of the Bacillarieæ. [Quart. Journ. Micr. Sc., I872, 1873.] Borscow, die Süswasser-bacillariaceen Russlands, 1873 .

2 [Thwaites first discovered the conjugation of the Diatomaceæ, Ann. Nat. Hist. I 847 , vol. XX; see also Carter, Ann. and Mag. Nat. Hist. 1856.-Schmitz, Quart. Journ. Micr. Sc., 1873, p. 145.-Smith, Synopsis of British Diatomaceæ.]

3 [Pinnularia viridis and Synedia splendens are green. Navicula fusiformis var. ostrearia is cobalt blue and communicates a green colour to the oysters which feed on it; Nature, vol. XVI. p. 397.] 
old valve of the mother-cell; this latter extends, like the lid of a box, over the newlyformed valve; and the two valves of the two daughter-cells lie next one another. Since, according to Pfitzer, the silicious valves, which also contain some organic matter, do not grow, it is clear that the new cells must constantly become smaller from generation to generation. When they have thus attained a certain minimum size, large cells, the Auxospores, are suddenly formed; the contents of the small cells, leaving the silicious valves which fall apart, increase either simply by growth or by both growth and conjugation. After this the auxospores surround themselves with new valves. Since the large auxospores are of somewhat different shape to their smaller mother-cells and primary mother-cells, the first result of their division must necessarily be cells of a different form and with unequal halves, as in the Desmidiex. The formation of the auxospores has been more exactly followed out only in a few cases. It would appear that they are formed in very different ways, from two or from one mother-cell, singly or in pairs, and with or without conjugation; they are alike only in so far that their size greatly exceeds that of the mother-cell. It has already been mentioned that, according to the recent researches of Schmitz in the case of Cocconema Cistula, the auxospores are produced by two cells laying themselves parallel side by side and allowing their contents to escape by the opening of the two valves; the cell-contents then commence growing rapidly without coming actually into contact, and a pair of auxospores is thus formed. Diatoms are found in enormous numbers at the bottom both of the sea and of fresh water, and attached to the submerged parts of other plants. Besides the ordinary rotation of protoplasm in their interior, they also exhibit a creeping motion by means of which they crawl over hard bodies or push small granules along their surface. This occurs only in a line drawn along the length of the cell-wall, in which Schultze ${ }^{1}$ supposes crevices or holes through which the protoplasm protrudes; and this, although not yet actually seen, probably occasions the creeping motion.

\section{FORMS NOT CONTAINING CHLOROPHYLL.}

\section{A. Conjugation takes place between motile cells (myxoamœbæ).}

The MyXonycetes ${ }^{2}$ differ so greatly in external appearance from the rest of the Thallophytes that they have been by some altogether separated by the vegetable kingdom. But if attention be directed not so much to the peculiarities of their external appearance as to those points in each stage of their development which are important from a morphological point of view, and especially to the fact that the formation of a cell-wall is a point only of secondary importance in the lower Thallophytes generally, it becomes clear that the Myxomycetes are allied in all essential points to the Pandorinex; and even if their mode of life is so remarkably different from that of this group of plants, the chief cause of this is that they do not, like them, live in water, but in the interstices of moist substrata, in consequence of which their motion is necessarily of a very different kind from that of the Pandorinex. In place of swimming and rotating, we have in the Myxomycetes a creeping of amoboid masses of protoplasm such as alone is practicable on the substratum on which they grow. It is impossible, in the space we have here at command, to go into these relationships more in detail; it must suffice to trace the main points in the history of their development, and to point out, in passing, the most important analogies.

${ }^{1}$ [See Pop. Sci. Rev. I866, p. 395-Engelmann, Bot. Zeit. I879, p. 49.]

2 De Bary, Die Mycetozoen, I864. [Ann. Nat. Hist. 1860, vol. V. p. 233.]-Cienkowski in Jahrb. für wiss. Bot. vol. III. pp. 325, 400.-Brefeld, Ueber Dictyostelium mucoroides, T869, Abhandl. der Senkenberg. Gesellsch., vol. VII.-Rostafinsky, Versuch eines Systems der Mycetozoen, 1873.Brefeld, Ueber Mucor racemosus und Hefe, Flora ${ }^{8} 873$ (latter part of the article). 
The Myxomycetes generally first make themselves visible at the time when they emerge from their porous substratum, and form their comparatively large fructification. The largest of these are the sulphur-yellow bodies which appear in summer on tanheaps, and which are known under the name of 'Flowers of Tan' (Etbalium scpticum); those of Lycogala, which are found on the stumps of trees, are of the size and form of hazel-nuts. In most other Myxomycetes the fructification is a small stalked capsule, containing, like those already mentioned, an enormous number of minute roundish, thick-walled spores. When these capsules burst, other structures often make their appearance, to which the term Capillitium has been given:-capillary tubes or threads, often united together in a reticulate or lattice-like manner, to the origin of which we shall recur presently (Fig. 172, C). In the simplest form at present known, Dictyostelium mucoroides discovered by Brefeld, not only is the capillitium wanting, but also the outer wall of the fructification, which consists only of a stalk composed of parenchymatous

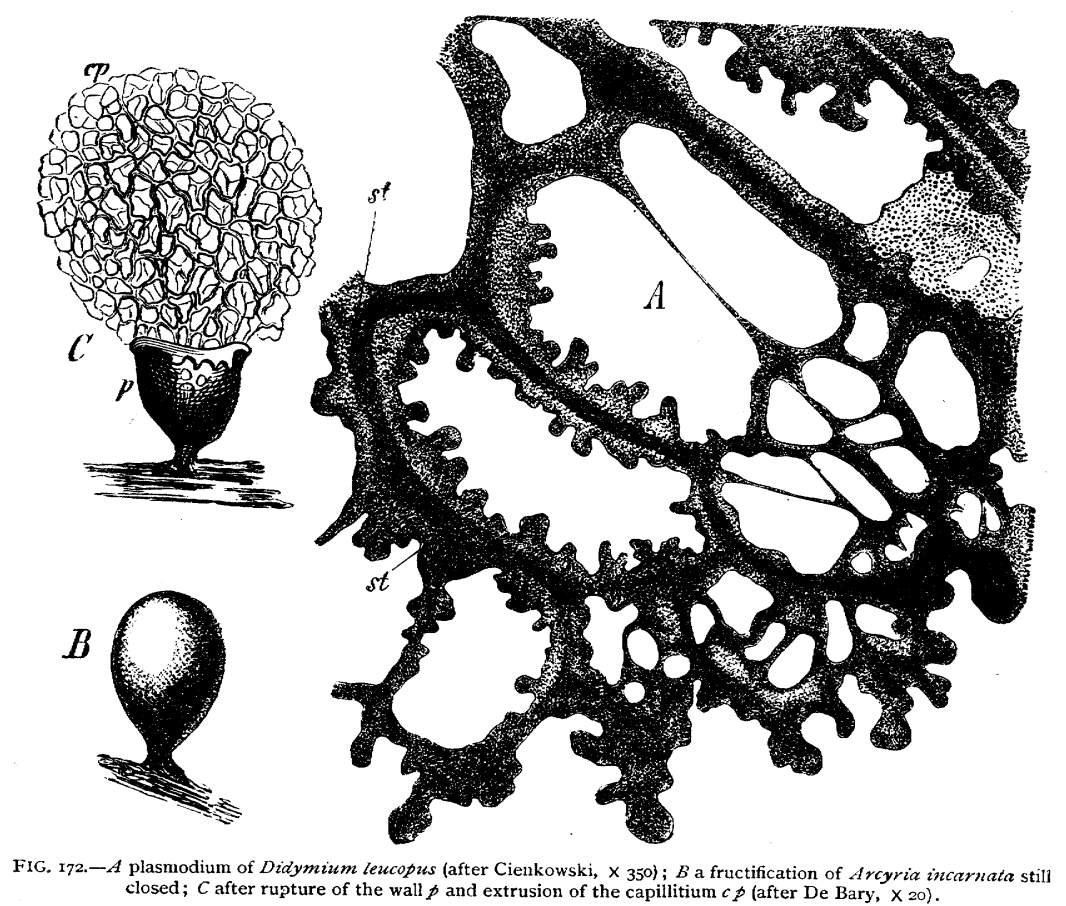

cells and of a roundish mass of spores. It is instructive to dwell on this simplest form of the Myxomycetes in order to arrive at an understanding of their systematic position. The spores of Dictyostelium will develope on the stage of the microscope in an aqueous decoction of rabbit's dung, and produce ripe fructifications after some days beneath the eye of the observer. When the spores germinate, the entire protoplasm of each of them escapes from the ruptured wall, creeps about with an amœboid motion, absorbs nutriment, and grows. It cannot be doubted that these amœboid bodies must be regarded as zoogonidia, only differing from ordinary ones in their mode of motion. After they have increased considerably in size in the course of several days, they divide, and multiply repeatedly in this way, a process which may unquestionably be compared directly with the vegetative propagation of Cklamydomonas, and indirectly also with that of Pandorina. The nucleus of these bodies subsequently disappears, their motion becomes more sluggish, and conjugation commences. They 
creep about in troops, come into close contact with one another, and finally coalesce into large lumps. As soon as one of these lumps is formed, the rest collect from all sides round it as a centre, coalesce with it, and increase in this manner the mass of protoplasm which then becomes more and more rounded off. There is every reason to believe that this collective union of zoogonidia is a conjugation and therefore a sexual act, in the same sense as the conjugation of the zoogonidia of the Pandorineæ; and the large mass of protoplasm formed in this way, which is called a Plasmodium, must therefore be treated as the analogue of the zygospore. The only difference is that in this case the zygospore does not become invested with a cell-wall nor go through a period of rest, but at once undergoes further development, becoming transformed into a stalked fructification which produces a large number of spores. In accordance with their mode of origin these spores may be compared not merely with the zoospores developed from the zygospore of Pandorina, but also with the ascopores of the Ascomycetes, and even with the spores of the Muscineæ. The formation of this fructification out of the roundish plasmodium of Dictyostelium commences with the production in its centre by free cell-formation of a number of cells each surrounded by a cell-wall of cellulose, which unite into a parenchymatous tissue forming in the interior of the plasmodium a column or stalk standing erect on the substratum. As this column continues to grow in height, the rest of the protoplasm which surrounds it creeps up it, and collects at its summit into a round lump, the entire substance of which now breaks up into a number of spores. This example furnishes the simplest case of the course of development of a Myxomycete. In most other instances it is much more complicated, the development being more complete, and a reduction to this plan becoming constantly more difficult. But the first stage of the development is essentially the same in all Myxomycetes. Each spore gives birth to from one to eight

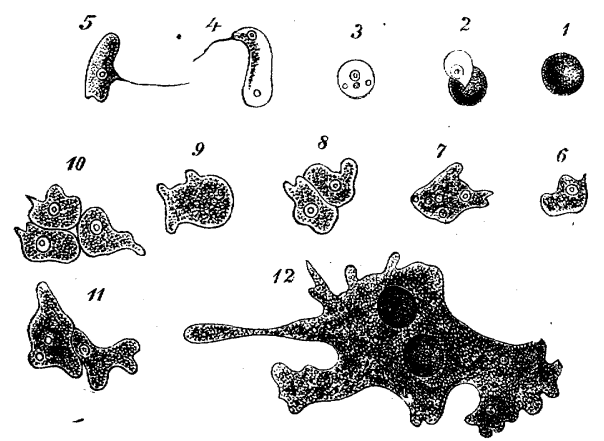

FIG. 173--Physarum album (after Cienskowski). 1 spore; 2 escape of its contents; 3 the contents when free; 4,5 the same in the form of a zoogonidium provided with a cilium; 6,7 after loss of the cilium; 9-Ir coalescence of the amœboid bodies; 12 a small plasmodium.

amoboid bodies, which grow and multiply by repeated division, subsequently coalescing with one another in large numbers and producing plasmodia. The plasmodia of other Myxomycetes, however, do not at once produce fructifications, but maintain for a longer period an independent life, creeping about in the moist interstices of their substratum; as, for example, the yellow plasmodia inside a tan-heap, which at length come to the surface, and then coalesce into the large bodies which are known as 'flowers of tan.' Other plasmodia creep about for a time on rotten wood or among decaying leaves, at length in the same way reaching the surface, and then usually producing simultaneously a number of fructifications. Fig. 172 $A$ may give an idea of the mode in which net-like structures are produced by these motions of the plasmodia. The substance of the plasmodium is thin and granular in the interior and bounded on the outside by a homogeneous pellicle; it is constantly changing its form; protuberances arise at various spots which move onwards with a flowing and creeping motion, ramify, and anastomose with one another, while the substance flows into them from behind, and in this manner enables the entire structure to creep gradually forwards. Immediately before the period when the fructification is produced, a tendency is manifested to creep up erect bodies, so that finally the fructification is frequently found on plants, stems, or leaves at a considerable distance from the original nutrient substratum. At this period the plasmodium collects at certain spots, and forms either 
a broad cake as in the 'flowers of tan,' or weak ascending outgrowths which gradually assume the form of the mature fructification, usually a stalked spherical or clubshaped body, or a spiral tube ${ }^{1}$, the growth of which is generally completed in a few hours. It has already been mentioned that the ripe fructification is usually surrounded by a firm wall, and that it often contains the so-called capillitium, in the interstices of which lie the numerous spores. Neither the wall of the fructification nor the capillitium is composed of cellulose, nor is the fruit-stalk, which is usually hollow; it must rather be supposed that the substance of the plasmodium, after it has already assumed the external form of the fructification, becomes differentiated into two distinct substances, one of which becomes hardened in various ways into pellicles, tubes, and solid threads, and thus forms the stalk, the wall of the fructification, and the capillitium, while the rest of the protoplasm, which has the capacity of further development, breaks up into small round portions which become invested with cell-walls and thus form the spores. The substance of the wall and of the capillitium corresponds, therefore, as Brefeld has pointed out, to the mass capable of swelling which fills up the space between the conidia in the conidiophore of the Mucorini. In the process of free cell-formation also by which the ascospores of the Ascomycetes are produced, there often remains within the ascus a considerable portion of its contents which is obviously not adapted to enter into the composition of a spore capable of germination.

In the differentiation of the protoplasm of the plasmodium into spores and into those portions (capillitium and wall of the fructification) which take no part in the further development, other portions of the contents which are useless for purposes of reproduction also become eliminated, and especially lime, which is often excreted in large quantities in the form of fine granules of calcium carbonate, and the yeilow substance which coats in loose flakes the fructification of the 'flowers of tan.'

It must be added in conclusion that under favourable vital conditions both the separate zoogonidia and the young or old plasmodia pass into a resting-state, the former simply becoming invested by a cell-wall, the latter becoming transformed into masses of cells ${ }^{2}$.

\section{B. Conjugation takes place between stationary cells.}

In the ZyGomyceTes ${ }^{3}$ the vegetative body, which, as in all Thallophytes destitute of chlorophyll (Fungi), is termed the mycelium, consists of a ramified hollow tubular cell (Fig. I74 B, m), in which septa only appear when it is fully mature and ready for sexual or non-sexual propagation. The ramifications of this mycelium all originate from a single germinating filament which is developed from a non-sexual reproductive cell (conidium), and may, in the course of a few days, cover with a

1 The term Athalium is given by Rostafinski to those large fructifications produced by the co-alescence of several simple ones, and which are therefore syncarps, as in the case of 'flowers of tan.'

${ }^{2}$ On the Chytridineæ, which are perhaps allied to the Myxomycetes, see Braun, Abhandl. der Berliner Akad. 1856, p. 22.-De Bary and Woronin, Berichte der naturf. Gesellsch. in Freiburg, 1863 , vol. III. Heft 2.-Woronin in Bot. Zeit. 1868, p. 8I. [Sorokin (Bot. Zeit. 1874) has discovered in Zygochytrium a process of conjugation resembling that of Mucorini; and in Tetrachytrium a conjugation of zoogonidia. Pfitzer (Monatsb. d. Acad. d. wiss., Berlin, I872) includes Chytridiacea with all the Coeloblasta destitute of chlorophyll in one group, the Phycomycetes. Sorokin suggests the name Siphomycetes for this, of which Amæebidium (Cienkowski, Bot. Zeit. 186I) is to be regarded as the simplest form.]

${ }^{3}$ Brefeld, Botanische Untersuchungen über Schimmelpilze, Heft I, 1872.-Van Tieghem, Recherches sur les Mucorinées, Ann. des Sc. Nat., $5^{\theta}$ ser., 1873 , vol. XVII, and in the French translation of this work, p. 336 et seq. [Quart. Journ. Micr. Soc. I87I, pp. 49-76.-Ann. des Sc. Nat., $6^{\circ}$ ser., vol. I, i 875 . p. I.] 
weft an area of several square centimetres. The mycelial filaments grow on organic substrata, such as fruits, bread, glue, or even on saccharine fluids or dung, absorbing their nutriment from them; many species even grow parasitically on others nearly allied, exhausting the contents of their cells through peculiar feeding organs, the Haustoria (see Fig. 175 b).

The non-sexual propagation of the- mycelium may continue through an endless number of generations, until the conditions are favourable for the formation of conjugating organs, i.e. for sexual reproduction. The conidia arise in two different ways:-In the family of Mucorini stout branches grow from the mycelium erect

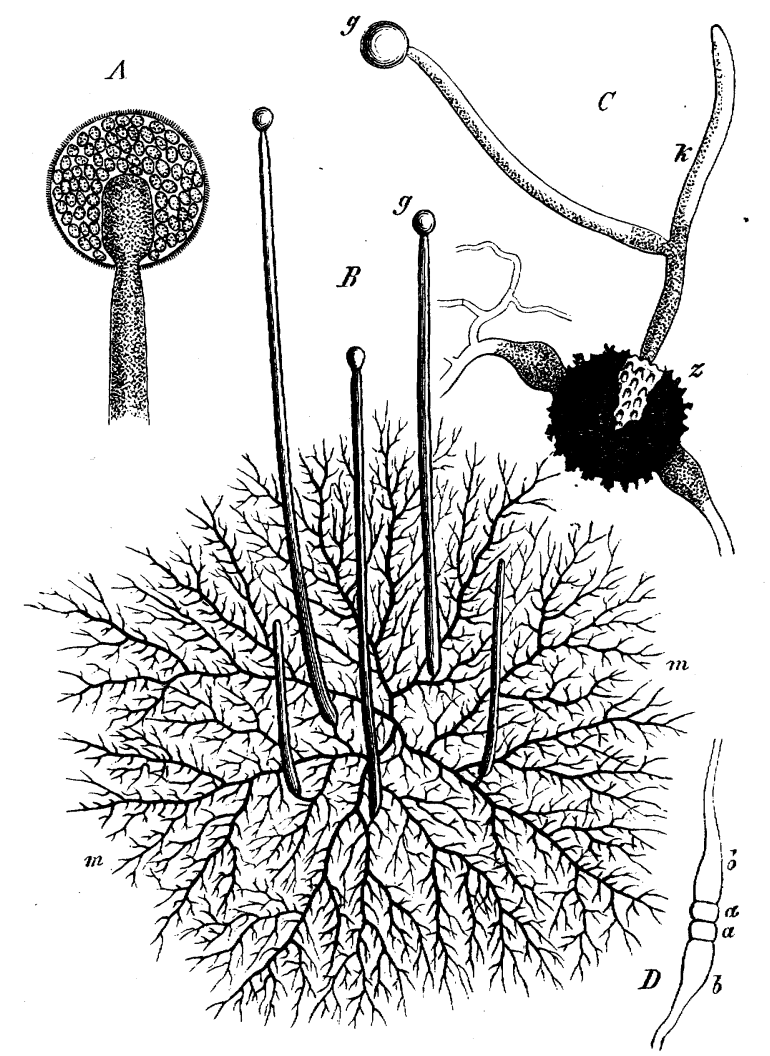

FIG. 174. $-B$ Mycelium three days old of Phycomyces nitens grown in a drop of gelatine with a decoction of plums; the finest ramifications are omitted; $g$ the conidiophore; $A$ a conidiophore of Mucor Mucedo in optical longitudinal section; $C$ a germiphore ; $A$ a conidiophore of Mucor Mucedo in optical longitudinal section; $C$ a germi-
nating zygospore of Mucor Mucedo $z$; the germinating filament $k$ is here putting out a lateral conidiophore $g$. $D$ Free conjugating branches $b b$, the extremities of which $a a$ have not yet coalesced, but are separated by a septum; the zygospore results from the coalescence of the cells $a$ a. ( $A, C, D$ after Brefeld; $B$ from nature.)

into the air, reaching a height of several centimetres, and at length swelling up at the summit into a sphere (Fig. $174 \mathrm{~B}, g$ ). Within this sphere are formed a number of round endogonidia, which become free by the rupture of the wall, germinate at once, and reproduce the mycelium. In a second family, the Piptocephalidx, the conidia are also produced on erect stalks, but these stalks branch copiously near their summit, and form numerous conidia (stylogonidia) by abstriction at the extremity, which produce a mycelium just as in the previous case. Besides these normal nonsexual reproductive organs, the mycelium also frequently produces gonidia of 
a different kind, the filaments breaking up by the formation of septa into short segments, which then become rounded off, and are able, under favourable conditions, to produce new mycelium. This explains the production of the so-called Mucor-yeast (from Mucor racemosus ${ }^{1}$ ), which bears a striking resemblance to true yeast (Saccbaromyces); the mycelial gonidia can multiply by a yeast-like budding when placed in an unsuitable nutrient fluid.

The mycelium has the power, under special circumstances, of reaching the morphological completion of its development by the formation of sexual reproductive organs. Thicker branchlets with a club-shaped extremity are produced on neighbouring filaments

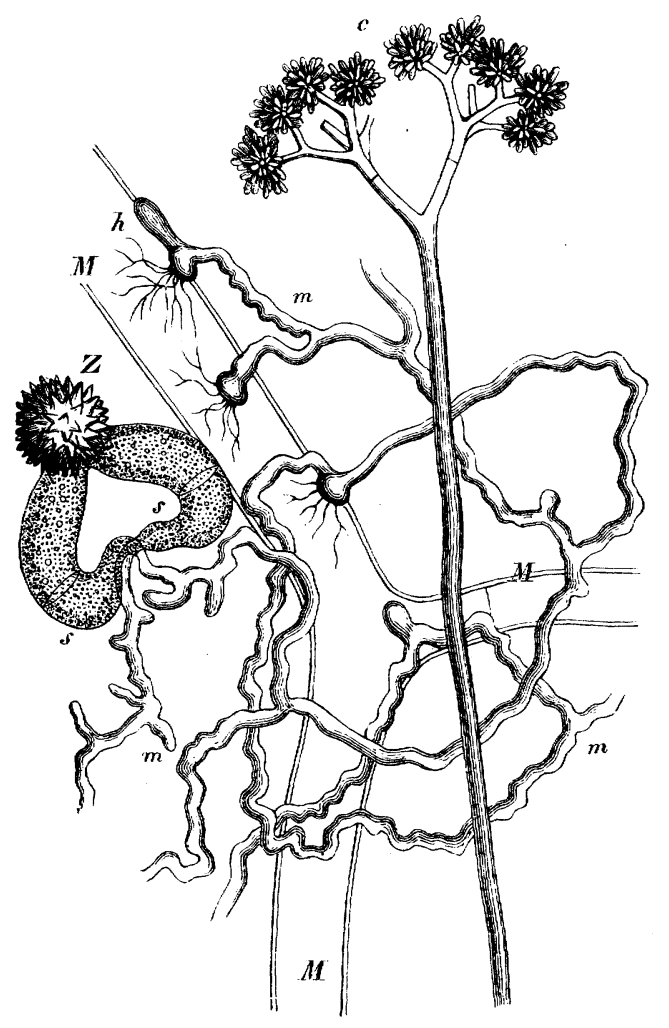

FIG. 175.-Piptocephalis Freseniana (after Brefeld). $M$ a piece of the mycelium of Mucor Mucedo from which the inycelium of Piptocephalis $m$ derives its nutriment; $h$ the haustoria of Piptocephalis which penetrate the derives its nutriment; $h$ the haustoria of Piptocephalis which penetrate the
mycelial filaments of Mucor; $c$ a conidiophore; ss the two conjugating branches of the mycelium which give rise to the zygospore $Z$.

of the mycelium; the apices of these branchlets come into contact, and coalesce by the disappearance of the cell-walls at the point where they touch (Fig. $174 \mathrm{D}$ and ${ }_{175} \mathrm{Z}$; see also 162 B) after a septum has been formed in each filament on each side of the point of conjugation. The protoplasm collects in the space which has been thus marked off; and a zygospore of comparatively large size is produced by the growth either of the entire space thus separated (Fig. I74 $C$ ) or of its central part only (Fig. $175 \mathrm{Z}$ ); the thick outer wall of the zygospore being usually of a dark colour and furnished with protuberances or spiny outgrowths. The zygospore ger- 
minates only after a long period of rest, and does not then give rise at once to a new mycelium of the ordinary kind; but the inner layer of the cell-wall bursts the outer layer and protrudes through it in the form of a tube (Fig. ${ }^{7} 74 \mathrm{C}$ ), which immediately developes into a conidiophore that breaks up into a number of conidia each of which developes into a new mycelium. This behaviour is evidently analogous to the germination of the zygospores of Pandorina, and to that of the oospores of EEdogonium and Cystopus hereafter to be described, which do not however produce conidiophores, but a number of zoospores which give birth to new individuals. The zygospore may therefore be considered as a sporocarp, forming, together with its conidiophores, a second generation, only that the carpospores are here precisely like the ordinary non-sexual conidia.

In our present state of knowledge the Zygomycetes may be divided into two families :-

I. The Mucorini, in which the conidia are formed, by free cell-formation, in the interior of spherical receptacles. In the genus Mucor the conidia are set free by the bursting of the fragile wall of the receptacle, while in Pilobolus this remains intact, but when ripe becomes detached at its base and, together with the conidia, is thrown to a distance by its elasticity. Mucor Mucedo is one of the commonest moulds, being found on fruits, bread, dung, and even in the interior of nuts and apples into which the mycelium penetrates. Mucor stolonifer covers in a short space of time large pieces of the same substrata, the mycelium putting out long stolon-like branches which attach themselves by their extremity, and produce conidiophores with black heads. The mycelium can even penetrate through the shell of fresh-laid eggs, and form conidiophores within them. Phycomyces nitens (Fig. ${ }^{7} 74 \mathrm{~B}$ ) is distinguished by its conidiophores, which are ten or fifteen centimetres in height and of a violet colour. Thamnidium bears an ordinary large conidiophore at the summit of each of the long stalks, and below these whorls of small branches with very small receptacles containing only a few conidia. Pilobolus almost always makes its appearance when fresh horse-dung is covered by a bell-glass ${ }^{1}$.

2. The Piptocephalidæ bear a number of stylogonidia on their conidiophores which are much branched towards the summit. The two genera described by Brefeld, Cbatocladium and Piptocephalis, are parasitic upon Mucor Mucedo, the latter being represented in Fig. 175 .

\section{CLASS III.}

\section{O O P O R E E.}

To this class belong all those Thallophytes, whether containing chlorophyll or not, which are reproduced sexually by means of oogonia. An Oogonium is a cell distinguished by its size and shape; the contents either form a naked primordial cell-the Germ-cell or Oosphere-by simple contraction and rounding off within the oogonium, which opens later, or divide into two or more portions, which similarly become oospheres. The fertilisation of these oospheres is effected by means of motile Antherozoids produced within

${ }^{1}$ [On Pilobolus crystallinus see Cohn, Nova Acta Acad. Nat. Curios. vol. XV. pt. I. p. 370.Klein in Pringsheim's Jahrb. fiir wiss. Bot. vol. VIII.] 
Antheridia, which penetrate into the oogonia and fertilise the oospheres; or a kind of conjugation takes place between the antheridium and oogonium, as Pringsheim has shown to be the case in the Saprolegnieæ. With the exception of the extremely simple Spharoplea, the antheridial cell is always smaller than the oogonium and different in form; the motile antherozoids, which are produced either by simple contraction of the contents, or more commonly by division into a great number of portions, are always much smaller than the oosphere, and generally many hundred or thousand times. This great difference in size is the essential difference between this mode of sexual union and conjugation, besides the fact that the immotile oosphere passively awaits fertilisation by the antherozoids which swim around it. After fertilisation the Oospore which results from the oosphere behaves precisely as a zygospore; it becomes invested by a firm cell-wall, and (except in Fucaceæ) must undergo a period of rest before germinating. Germination is in most cases indirect; i.e. the contents of the oospore do not at once develope into a new plant, but divide into a smaller or larger number of cells which escape in the form of naked zoospores, each of which grows into a new plant. In such cases therefore the oospore, as we have repeatedly seen in the Zygosporeæ, may be regarded as a very simple sporocarp or as a second generation. The oosphere is the analogue of the oosphere in the archegonium of Mosses; the ripe oospore, with its contents which break up into zoospores, is the very simple equivalent of the moss-capsule, as has been pointed out by Pringsheim, to whose researches we owe almost all that is here described. But cases occur, as in the Zygosporeæ, in which the oospore germinates directly, as in the Fucaceæ, and there is no period of rest.

The vegetative body of the Oosporeæ may consist of undifferentiated cells, as in Sphcroplea, in this respect resembling the Conjugatæ, the filiform thallus presenting no distinction of base and apex. In one large group (Cœloblastæ) the thallus consists, until the formation of fructification, of a single tubular cell, which often branches copiously, as in the Zygomycetes; in a further grade of development the thallus consists of branched and segmented filaments composed of cells of different kinds, and the plant, which is fixed to a substratum, manifests a wellmarked contrast between base and apex. Finally we find the Fucaceæ, in which the thallus is very massive, and forms an actual tissue in which differentiation may be recognised into epidermal layers and fundamental tissue.

There is no non-sexual multiplication by gonidia, either in the simplest form belonging to the class, Spharoplea, or in the most highly developed, the Fucaceæ. The thallus of the other intermediate forms, on the contrary, produces abundance of gonidia, by means of which propagation may take place through many generations, in the same manner as Marchantia is propagated by bulbils. The gonidia are produced either singly or in numbers as endogonidia in the interior of cells, escaping in the form of zoogonidia, or as stylogonidia by abstriction at the extremity of special branches; they are then immotile, as in the Peronosporex, though their contents may become transformed into motile zoogonidia.

In a systematic classification of the Oosporeæ, the forms which do not contain chlorophyll may be arranged as a special section of those that do, the genetic relationship being here unquestionable. 
A. SPHÆROPLEA annulina ${ }^{1}$ is at present the sole representative of the most simply organised of the Oosporex. It consists, when fully developed, of cylindrical filaments divided by septa into very long cells, the green protoplasm enclosing rows of large vacuoles, and thus forming girdle-like rings. When in the vegetative state the cells are all alike; it is only when the process of sexual reproduction is commencing that a difference makes its appearance, some of the cells forming nothing but antherozoids, the rest nothing but oospheres. The latter are produced in large numbers within each cell, the contents breaking up, after various previous changes, into several globular portions, each of which is characterised by a hyaline speck. The antherozoids are formed in extraordinarily large numbers by the breaking up of the entire contents of a cell which had previously assumed a yellowish brown colour. In both kinds of sexual cells a number of holes are formed by the absorption of portions of the wall; the antherozoids escape through these orifices, forcing themselves in quantities into the cells in which the oospheres lie. The antheridia and oogonia therefore resemble one another in external form, while the antherozoids and oospheres are very unlike; the latter are elongated and club-shaped, with two cilia at the pointed end. The oospore or fertilised oosphere clothes itself with a thick warty cell-wall; its contents become brick-red. Its further development commences after a resting stage; the red contents divide by successive bipartition into a large number of primordial cells which escape from the oospore, are provided with two cilia, swarm about, and germinate after a period of rest. The germinating cell, which is at first shortly fusiform, then grows in both directions into a capillary filament, so that the anterior and posterior ends are exactly alike, and there is no distinction between base and apex. After the filament has grown to a considerable size, transverse divisions take place and the filament is then composed of a number of equivalent cells.

\section{B.-Celoblaste.}

In this section are included for the present all those Oosporeæ in which the thallus consists of a single tubular cell, without a nucleus ${ }^{2}$, more or less branching, and usually fixed to one spot. It is only when reproduction is commencing that branches of special form are separated by septa. The Cœloblastæ are therefore not, accurately speaking, unicellular; it is only the vegetative body that is so. In all cases which have been accurately observed a non-sexual reproduction takes place by means either of stylogonidia or of motile endogonidia. The oogonia and antheridia are usually terminal cells of short lateral branches, and are very different in form; the former are more or less spherical, the latter tubular; the two being mostly placed close beside one another.

\section{FORMS CONTAINING CHLOROPHYLL.}

I. Vaucheria ${ }^{3}$ consists of a single elongated cell, branched in various ways, sometimes as much as 30 centimetres long, containing no nucleus, and developing on damp earth or in water. The fixed end is hyaline and branched in a wavy manner; the free part contains within the thin cell-wall a layer of protoplasm rich in chlorophyll-granules and drops of oil, and enclosing the large sap-cavity. This part of the thallus forms one or more main filaments which branch behind their growing point; only in $V$. tuberosa is

${ }^{1}$ Cohn, Ann. des Sci. Nat., $4^{\circ}$ ser., 1856 , p. 187.

${ }^{2}$ [According to Schmitz (Sitzber. d. niederrhein. Ges. in Bonn, 1879) a great number of nuclei are present in Vaucheria and its allies.]

3 Pringsheim, Ueber Befruchtung und Keimung der Algen, I855; Jahrbuch für wissen. Bot. Bd. II. p. 470.-Schenk, Würzburger Verhandl. Bd. VIII. p. 235.-Walz, Jahrbuch für wissen. Bot. Bd. V: p. 1 27.-Woronin, Bot. Zeit. 1869, nos. 9, 1o. [Stahl, Bot. Zeit. 1879.] 
the branching dichotomous; though commencing monopodially, the lateral branches often develope sympodially. At the commencement of the period of growth in the spring the plants arise from the oospores which have remained dormant through the winter, and are at first propagated through several generations in a purely non-sexual manner. This is effected either simply by the abstriction of the ends of branches, or by very large zoogonidia which escape from the interior of a cell, and are covered by very short cilia. Between these two forms the various species of Vaucberia show intermediate steps. In $V$. tuberosa, for example, branches swell up to a considerable size, become detached at the base, and put out at once one or more germinating tubes. In $V$. geminata the end of a branch swells up to an oval shape, its contents become separated by a septum, contract, and form a new cell-wall, and the gonidium thus formed either becomes free by the decomposition of the mother-cell-wall, or falls off along with it, germinating after some days. The gonidia of $V$. bamata are formed in the same way, but are thrown out with a jerk, remain at rest, and germinate during the next night. In other species, as $V$. sessilis, sericea, and piloboloides, the

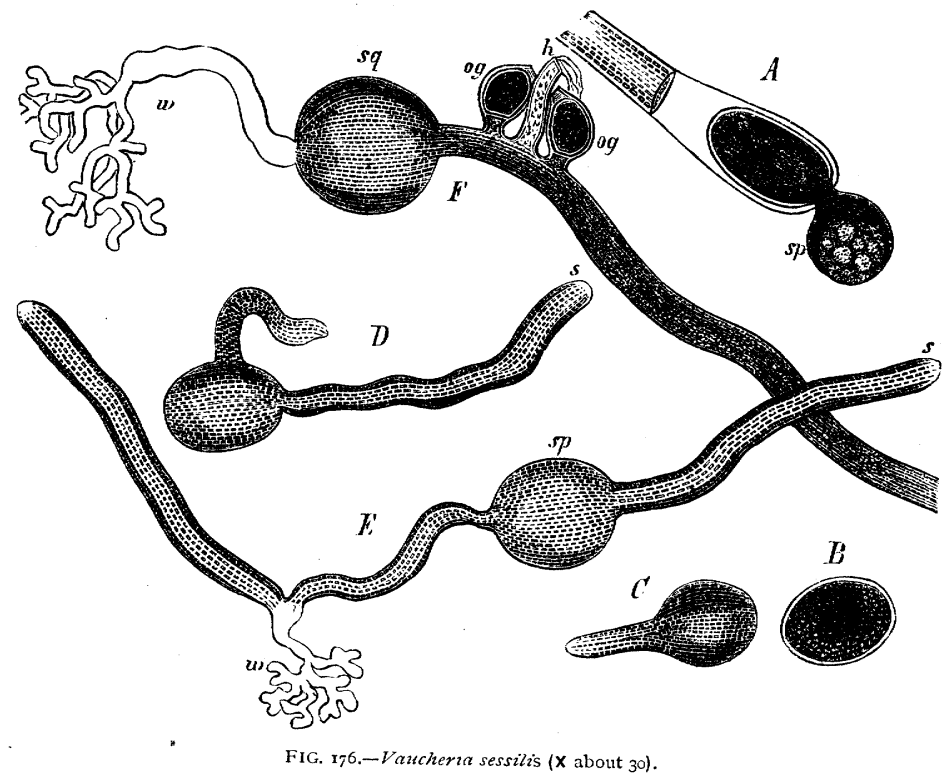

contents of a branch become separated and contract, and force themselves out as a zoogonidium through a crevice at the extremity. The motion of the zoogonidia lasts, in $V$. sericea, only for $\frac{1}{2}$ to $1 \frac{1}{2}$ minutes, but in other cases for hours. The rotation begins, in $V$. sessilis, as I have distinctly seen, during their escape; and if the opening of the mother-cell is too small, the zoogonidium splits into two pieces; each becomes rounded off and again constitutes an entire zoogonidium; the outer one swims away, while the inner one remains rotating within the mother-cell. The formation of the zoogonidia begins in the night, as is the case with most Algæ and Fungi; they escape in the morning, and their germination commences during the day or the next night. They put out on germination either only one or two tubes (Fig. I $76, C, D$ ), or form at the same time a root-like organ of attachment $(E, F, w)$. The oogonia and antheridia originate as lateral protuberances from a filament which contains chlorophyll (Fig. 177, $A$, $B$ ), sometimes even on the germinating tube of a zoogonidium. All the species of Vaucheria are monœcious, and the two kinds of sexual organs are mostly found very near together. The antheridia (Fig. 177, $b a$ ) are the terminal cells of slenderer branches, the contents 
of which contain but little chlorophyll but produce a large number of very small antherozoids (Fig. $177, D$ ); these escape from the antheridial cell which bursts at the apex. In several species the antheridia are curved like horns; in others they are straight $(V$. sericea) or curved sacs $(V$. pachyderma); in the $V$. synandra, discovered by Woronin, from two to seven small horns arise on the large ovoid terminal cell of a twocelled branch. The oogonia arise as thick protuberances (Fig. I $77, A, B$, og) filled with oil and chlorophyll. They swell up into an obliquely ovoid form, and finally the dense contents are separated by a septum $(F, o s p)$. The green and coarsely granular mass collects in the centre of the oogonium, while colourless protoplasm accumulates at its apex, at which spot it opens; at this moment the whole contents contract and form the oosphere. In some species a colourless drop of mucilage is expelled from the mouth. After the entrance of the antherozoids the oosphere clothes itself with a thick cell-wall; its contents become red or brown, and the oospore now commences its period of rest. The formation of the oogonia and antheridia begins in the evening,
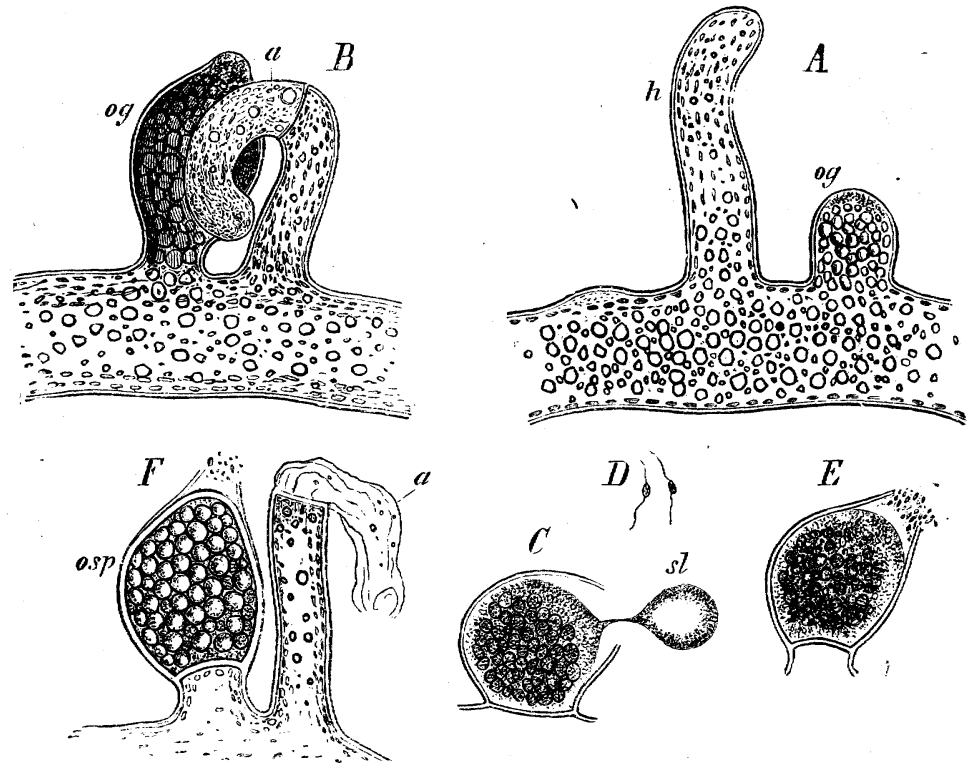

FIG. 177.-Vaucheria sessitis; $A, B$ origin of the antheridium $a$ on the branch $b$, and oogonium og. $C$ an open oogonium expelling a drop of mucilage $s l ; D$ antherozoids ; $E$ the antherozoids collected at the mouth of the oogonium; $F, a$ an empty antheridium; osp the oospore in the oogonium $(A, B, E, F$ from nature, $C, D$ after Pringsheim).

and is completed the next morning; fertilisation is accomplished between ro and 4 in the day ${ }^{1}$.

Closely allied with Vaucheria in the form of their thallus-which is usually a much-branched filament undivided by septa-are a series of other genera formerly comprised in the class SIPHONEE, the mode of sexual reproduction of which is however at present unknown, if indeed they have any. It cannot therefore yet be affirmed with certainty whether the Siphonex constitute a natural group. The mode in which the gonidia are formed is at least not opposed to this idea. The following forms may be especially mentioned :- In Botrydium ${ }^{2}$ the young plant is a spherical cell, from which

1 [Stahl has described a peculiar encysted form of Vaucheria geminata: this has been previously described by Kützing as a distinct plant under the name Gongrosira dichotoma. (Bot. Zeit., 1879.)]

${ }^{2}$ Braun, Verjüngung in der Natur, p. I36 [Ray Soc. Rostafinski and Woronin have detected (Bot. Zeit. 1877) the conjugation of zoogonidia in B. granulatum. The former considers the Botry. diacea allied to Pandorine and Hydrodictyece.] 
a hyaline prolongation is subsequently formed which branches like a root and penetrates the earth, while the upper part swells up into an ovoid vesicle, in which the protoplasm forms a parietal layer containing chlorophyll. From this arise, after growth is completed, a number of zoogonidia which are set free by the wall of the mothercell becoming gelatinous and deliquescing. This is evidently a more simple mode of growth than that of Vaucberia. A higher degree of branching is found in Bryopsis, which is also unicellular. This genus also forms on one side root-like organs of attachment, on the other upright much-branched stems (several centimetres in height) with unlimited growth at the apex; small branches with limited apical growth are formed on them in two rows or spirally, which clothe the stem like leaves, and after they have become shut off from it by septa, fall off; in them numerous zoogonidia are formed ${ }^{1}$. The branching of a single large cell is carried still further in the genus Caulerpa, which forms creeping stems growing at the apex with descending branched rhizoids and ascending leaf-like branches ${ }^{2}$. The growth of a unicellular thallus takes place in still another manner in Acetabularia. Here the plant, two or three centimetres high, has the form of a slender Hymenomycetous fungus, the stem of which developes a rhizoid below and bears a pileus above, consisting of a disc of closely crowded rays, which are themselves radial branches of the stem. The stem ends above in the form of a boss; at the base of the radial branches surrounding the boss stands an umbellate whorl of branched segmented hairs. In the rays of the pileus are formed the non-sexual zoogonidia ${ }^{3}$. Finally Udotea cyatbiformis must be mentioned here. This species forms a stalked leaf-like thallus, the stalk $\frac{1}{2}$ inch, and the thallus from $\frac{1}{2}$ inch to 2 inches long and broad, its thickness from $\frac{1}{1} \frac{1}{0} \sigma$ to $\frac{1}{20}$ line. When cut transversely it seems to consist of a cellular tissue, but in reality the thallus is composed of a great number of branched filaments, which, forming a cortical and medullary layer, are all ramifications of a $\sin _{3} l e$ cell ${ }^{4}$.

\section{FORMS NOT CONTAINING CHLOROPHYLL.}

2. The Saprolegnieæ ${ }^{5}$ are colourless parasites usually found attached to animal or vegetable organisms in water, especially dead insects, forming dense radiating tufts. The individual plants are long undivided filaments, penetrating deeply into the substratum by means of root-like branches, and branching more or less in the surrounding water, sometimes in an arborescent manner. The zoogonidia are formed in the branches after the contents have been separated by a septum; occasionally a number of such septa are formed, and they are then produced in each cell. The zoogonidia are formed simply by the simultaneous division of the contents into a very large number of portions (Fig. $178, A$ ); the cell opens at the apex and the gonidia are expelled; these at once swarm about in the water and become dispersed, or at first accumulate in a resting state in front of the opening; each gonidium then becomes immediately invested with a firm cell-wall, but after a short time it abandons it and then begins

1 Pringsheim, on Bryopsis, in the Monatsber. der Berlin. Akad. May I 871 .

2 Nägeli in Zeitschrift für wiss. Bot. I 844 . Bd. I. p. I 34 .

3 [Woronin, Ann. des Sc. Nat. $4^{\mathrm{e}}$ ser., vol. XVI. p. 200 . De Bary and Strasburger have detected (Bot. Zeit. 1877) the conjugation of zoogonidia.]

4 Nägeli, Die neueren Algensysteme, p. I77. [The remarkable fossil plant from Canada of Devonian age, Prototaxites Logani, was probably an enormous Siphonaceous Alga; see W. T. Thiselton Dyer, Journ. of Bot. I87 I, p. $25^{2}$; Carruthers, Monthly Micros. Journ. I872, p. 160.]

5 Pringsh€im in Jahrb. für wiss. Bot. vol. I. p. 285 [Ann. d. Sc. Nat. 1859, tom. XI. p. 349]; vol. II. p. 205 ; vol. IX. p. I9I.-De Bary, ditto, vol. II. p. I69.-Hildebrand, ditto, vol. VI. p. 249.Leitgeb, ditto, vol. VII. p. 257.-Cornu in Ann. de Sci. Nat, 5th ser., vol. XV. p. 328.-Schenk, Bot. Zeit. 1859 , p. 348.-Pfitzer in Monatsber. d. Berlin. Aliad. May I 872. - [De Bary und Woronin, Beit. zur Morphol. u. Physiol. d. Pilze, IV, I88 r.] 
swarming about (Fig. I78, B). It also sometimes occurs that the gonidia clothe themselves with a delicate cell-wall while still within the mother-cell, forming a kind of parenchyma, and then escape from the mother-cell through numerous holes in its wall. These various modes of formation of the gonidia, by means of which species and even genera have hitherto been distinguished, can, as Pringsheim has shown, take place simultaneously on the same plant, as in Saprolegnia and Acblya. In Saprolegnia, when the gonidia have escaped from the terminal cell of a branch; the septum bulges out and developes into a new gonidial receptacle which takes the place of the one that is now empty; in Acblya a lateral branch beneath the septum becomes the new gonidial receptacle. In the very simall and simple genus Pythium, which is parasitic,

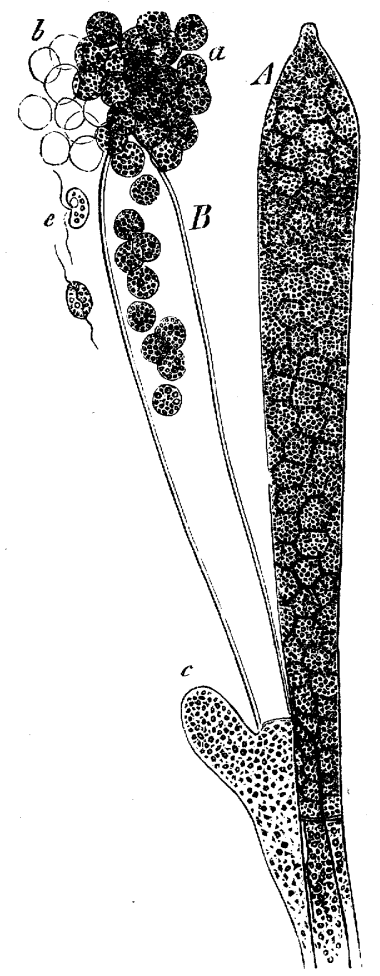

FIG. ${ }^{7} 78 .-T$ wo gonidial receptacles of Achlya; $A$ one still closed; $B$ with the zoogonidia escaping; a gonidium just escaped and still in a state of rest, $c$ aftercommencing to swarm, having abandoned their mencing to
cell-walls $b$.

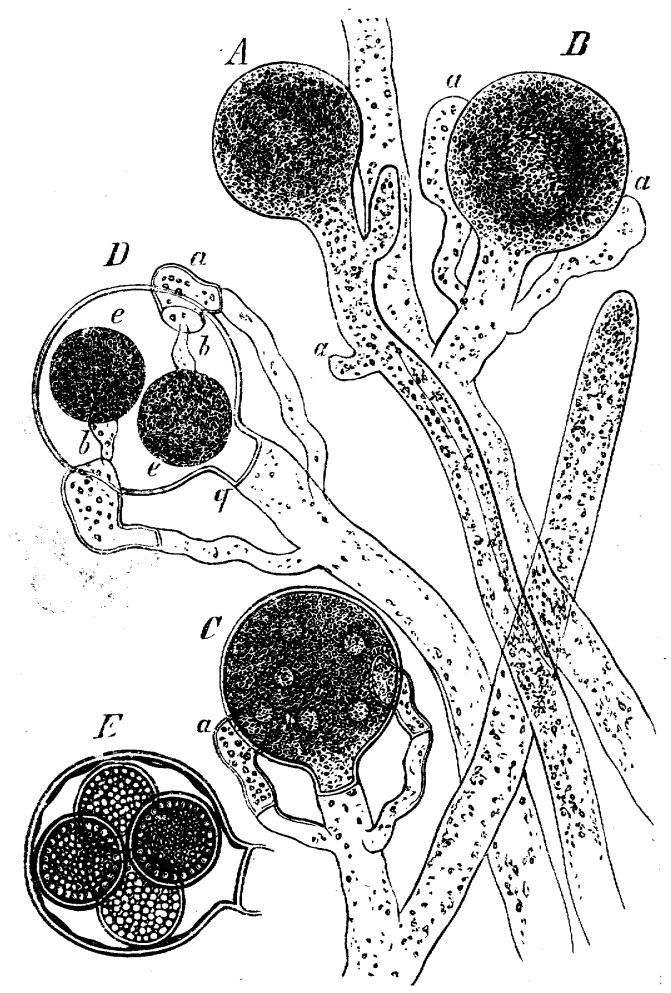

FIG. I79--Oogonia and antheridia of Achlya lignicola, growing on wood in water; the course of development is indicated by the letters $A-\dot{F}$ $a \cdot$ the antheridium, $b$ its tube penetrating into the cogonium $\left(\mathrm{X}_{550}\right)$.

filaments emerge which open at their apex and allow the protoplasm to escape in a ball which then breaks up into a number of zoogonidia. After coming to rest the zoogonidia of the Saprolegnieæ give rise to new plants, which, when they have access to a fresh substratum, such as a dead fly, produce in succession several generations of non-sexual gonidia-forming individuals.

It is only towards the close of the period of growth that sexual individuals make their appearance. The extremities of the filaments then swell up into a globular form (Fig. I 79), a septum is formed beneath the swelling, which constitutes the oogonium, and its protoplasmic contents either simply contract to form the oosphere, as in Pythium monospermum and Apbanomyces, or the contents divide into two or more 
oospheres, considerable contraction taking place. At the same time the antheridia are also formed, appearing as much slenderer lateral branches, beneath the oogonia but sometimes above their basal wall. These antheridial branches are usually much curved; their upper portion is separated by a septum, and the terminal cell thus formed; the antheridium usually applies itself firmly to the surface of the wall of the oogonium. The process of fertilisation itself, which had previously been only imperfectly known, presents, according to the most recent observations of Pringsheim, several remarkable peculiarities which throw considerable light on the genetic relationships of the Saprolegnieæ, and on the essential nature of the various modes in which it takes place. Even before the contents of the oogonium have broken up into oospheres, a larger or smaller number of spots of a lighter colour (Fig. 179, C) may be observed on the oogonium; at these spots Pringsheim states that the inner layer of the cell-wall protrudes in the form of a wart to form the conjugating organ by which fertilisation is subsequently effected: in some species of Saprolegnia and Acblya these warts remain covered by the outer layer of the cell-wall of the oogonium. As soon as an antheridial cell becomes closely applied to the oogonium, a protuberance of the inner layer of the cell-wall of the antheridium first of all penetrates into the outer layer of the cell-wall of the oogonium, and meets the wart already described belonging to the inner layer; this latter is then absorbed at the point of contact, and the protuberance of the antheridium grows into a narrow tube which penetrates deeply into the oogonium, its extremity burying itself in the midst of the oospheres. These processes are only partially represented in Fig. 179, which was drawn long before the discovery of the actual phenomena. According to Pringsheim the extremity of the fertilising tube opens in the interior of the oogonium; extremely minute bodies are repeatedly expelled in jerks and after long intervals, which are seen among the oospheres, and which are probably antherozoids or bodies with a similar function. But the substance expelled from a single fertilising tube may serve for the impregnation of several oosphores in an oogonium; and it is the more probable that this takes place since the number of these tubes which penetrate into an oogonium is usually different from that of the fertilised oospheres. With regard to the bright spots in the wall of the oogonium, through which, as we have seen, the fertilising tubes enter, it is evident that a process takes place here similar in its nature to conjugation, since not only does the antheridium grow into the oogonium, but the inner layer of the wall of the oogonium also, so to speak, grows towards the antheridium by the formation of the wart already described, in order to unite with it, although no actual coalescence of the contents takes place at that time, the wart being subsequently penetrated by the fertilising antheridial tube. But in some Saprolegnieæ the formation of this conjugating wart proceeds further; it penetrates through the outer layer of the wall of the oogonium, opens at the surface, and each wart thus becomes an orifice through which the antheridial tubes penetrate: sometimes, according to Pringsheim, the conjugating warts become raised above the spherical surface of the oogonium in the form of more or less elevated protuberances, or even grow into tolerably long tubes, the apices of which are occasionally met with in conjugation with an antheridial tube. These cases, although rare, manifest still more strikingly a certain resemblance to the conjugation of the Zygomycetes, and perhaps a still closer analogy to the process of fertilisation in some Ascomycetes, such as Peziza confuens; or a comparison might even be drawn between the trichogyne of Nemalion and the protuberances of the oogonium. The simiiarity of the process of fertilisation in the Saprolegnieæ to that already described in the case of Vaucheria is sufficiently clear; that spot in the oogonium of Vaucheria which subsequently opens and exudes a drop of mucilage may obviously be compared with the conjugating wart in those oogonia which contain only a single oosphere; only that in Vaucberia no fertilising tube is usually developed from the antheridium, since the antherozoids are larger, and remain motile for a considerable period, finding their own way into the open oogonium. Pringsheim has already pointed out the corresponding analogy with the Edogonieæ. 
Two other remarkable facts have still to be mentioned:-in the first place that determined by Pringsheim, that parthenogenesis is not an uncommon phenomenon in Saprolegnia and Acblya. Some or all of the oogonia on a plant may not be fertilised at all, the formation of antheridia on them being altogether suppressed; and yet the oospores are perfectly developed and are capable of germinating. We shall refer more in detail in Book III. Sect. 30 to this and other instances of parthenogenesis in the vegetable kingdom; here it need only be mentioned that the forms hitherto considered as diocious-no antheridial branches being found on the oogonia-are not distinct species, but only parthenogenetic forms of monœcious species. In contrast to the development of oospores without fertilisation is the occurrence of antheridia the tubes of which do not penetrate into oogonia, but open and expel the fertilising particles into the surrounding water. In these, as well as in those tubes which pierce the oogonia, Pringsheim observed that the expulsion does not take place all at once, but in repeated jerks and after considerable intervals.

The oospores or fertilised oospheres clothe themselves with a thick firm cell-wall and remain dormant in the oogonium for months. Their germination takes place subsequently in two different ways :- either the oospore puts out a germinating filament which at once developes into a branched plant in which zoogonidia are subsequently formed; or it puts out a short filament which opens at its apex and allows the whole of its contents to escape in the form of zoogonidia. Sometimes after the commencement of the formation of a filament the contents break up into gonidia, or the entire contents, surrounded by an inner cell-wall, escape from the outer envelope, and then germinate. In their mode of germination the Saprolegnieæ approach on one hand the Mucorini, on the other hand the Peronosporeæ.

3. The Peronospore $\boldsymbol{x}^{1}$ are all parasitic on the succulent parenchymatous tissue of living Phanerogams (Dicotyledons), the irregularly-branched mycelium spreading over large areas in the intercellular spaces, and putting out at a number of places appendages (Haustoria) which penetrate into the interior of the cells of the host, and enable it to absorb the cell-contents as food. As in the preceding families, the entire vegetative body or mycelium consists of a single tubular cell not divided by septa. Multiplication again takes place at the commencement of the period of growth exclusively in the non-sexual mode by the production of gonidia. These are abstricted and fall off from the end of branches, and are therefore stylogonidia, or, as they are usually termed, conidia. In the genus Peronospora, which comprises a large number of species, long slender branches of the mycelium emerge into the air through the stomata of the host for the purpose of the production of conidia, then branch in a somewhat arborescent manner, and form at the end of each branch a comparatively large ellipsoidal conidium. In Cystopus a large number of short club-shaped branches (Fig. 180, B) are formed densely crowded together on the mycelium beneath the epidermis of the host, and each of these produces at its extremity a series of conidia, until finally they burst the epidermis and escape in the form of a fine dust. The conidia germinate in different ways. In Peronospora there are some species, as $P$. infestans and nivea, in which the contents of the conidia, after falling off and reaching a drop of rain or dew, break up into a large number of swarming zoogonidia and escape (Fig. $180, C, D)$; in others, as $P$. pygmea, the whole of the protoplasm escapes out of the conidium and forms a roundish cell which at once puts out a germinating filament. In a third and fourth section of the-genus the conidium puts out at once a germinating filament which emerges either at a definite spot ( $P$. gangliformis) or indifferently at any spot ( $P$. parasitica, calotbeca, Alsinearum, \&c.). In Cystopus either all the conidia are alike, i.e. when they reach a drop of water they produce swarming zoogonidia

- 1 De Bary, Ann. des Sci. Nat., $4^{\mathrm{e}}$ sér., I860, vol. XIII ; I863, vol. XX. [On Phytophthora (Peronospora) infestans, see De Bary, Journ. of Bot. 1876, pp. 105-126, and 149-154: see also Bot. Ztg. I881.] 
(C. candidus), or the terminal conidium of each chain gives rise to a germinating filament, if it is capable of germinating, while the other cells of the conidial chain produce zoogonidia ( $C$. Portulaca). After the swarming is finished the zoogonidia become firmly attached to the cuticle of the host, invest themselves with a thin cell-wall, and, in the case of Peronospora infestans, put out a delicate germinating filament directly into an epidermal cell, piercing through its outer wall. After it has entered the cell (Fig. $180, H$ ) this filament absorbs the whole of the protoplasm of the zoogonidium, and, after piercing also the inner wall of the epidermal cell, reaches an intercellular space, where the mycelium developes. The zoogonidia of Cystopus are firmly attached near the stomata of their host, and push their germinating filaments into their orifices (Fig. I80, $G$ ),

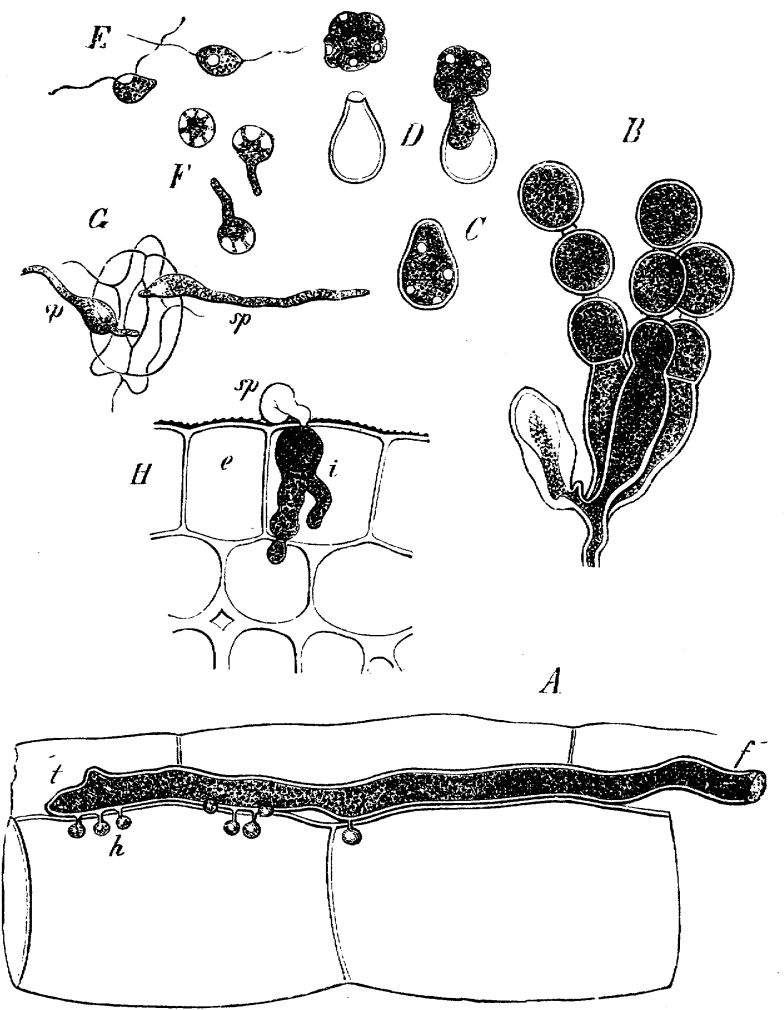

FIG. I80.-Cystopus candidus. $A$ branch of the mycelium growing at the apex $t$ with haustoria $h$ between the parenchymatous cells of Lepidium sativum; $B$ conidia-bearing branch of the mycelium; $C, D, E$ formation of zoogonidia from the conidia; $F$ zoogonidia germinating; $G$ a zoogonidium germinating on a stoma; $H$ zoogonidium of Peronospora infestans penetrating through the epidermis of a potato-stem (after De Bary, $\times 400$ ).

and thus find their way'at once into the intercellular spaces. When the mycelium has once obtained a footing in the parenchyma of the host, it continues to grow in it, and finally often spreads through the whole plant, putting out its conidia-bearing branches at various places in the stem, leaves, or inflorescence. In this manner the (unicellular) mycelium of $P$. infestans can even hibernate in the tubers of the potato, to undergo further development in the shoots in the following spring. The sexual organs of the Peronosporeæ are developed in the interior of the tissue of their host. Spherically dilated ends of branches of the mycelium shape themselves into oogonia (Fig. I $8 \mathrm{I}, A, 0 g$ ), in each of which an oosphere is formed out of a portion of the protoplasm $(B, o s)$. From another branch of the mycelium a branchlet grows towards the oogonium, 
Swells, and becomes closely attached to it; and the thicker part becoming separated by a septum (just as takes place with the oogonium itself), developes into an antheridium. As soon as the oosphere is formed, a fine branch of the antheridium $(B, a n)$ reaches it, penetrating the membrane of the oogonium. After fertilisation the oosphere becomes surrounded by a coat which thickens and forms an external rough dark-brown exospore and an inner endospore. These oospores remain dormant through the winter and then germinate; in the case of Peronospora Valerianella they form a mycelium on moist ground; those of Cystopus, however, produce zoospores; the endospore (i) forces itself like a bladder out of the ruptured exospore (Fig. I $8 \mathrm{I}, F$ ), and then bursting, the zoospores $(G)$ are set free, which behave in exactly the same manner as the zoogonidia produced from the conidia of this genus.

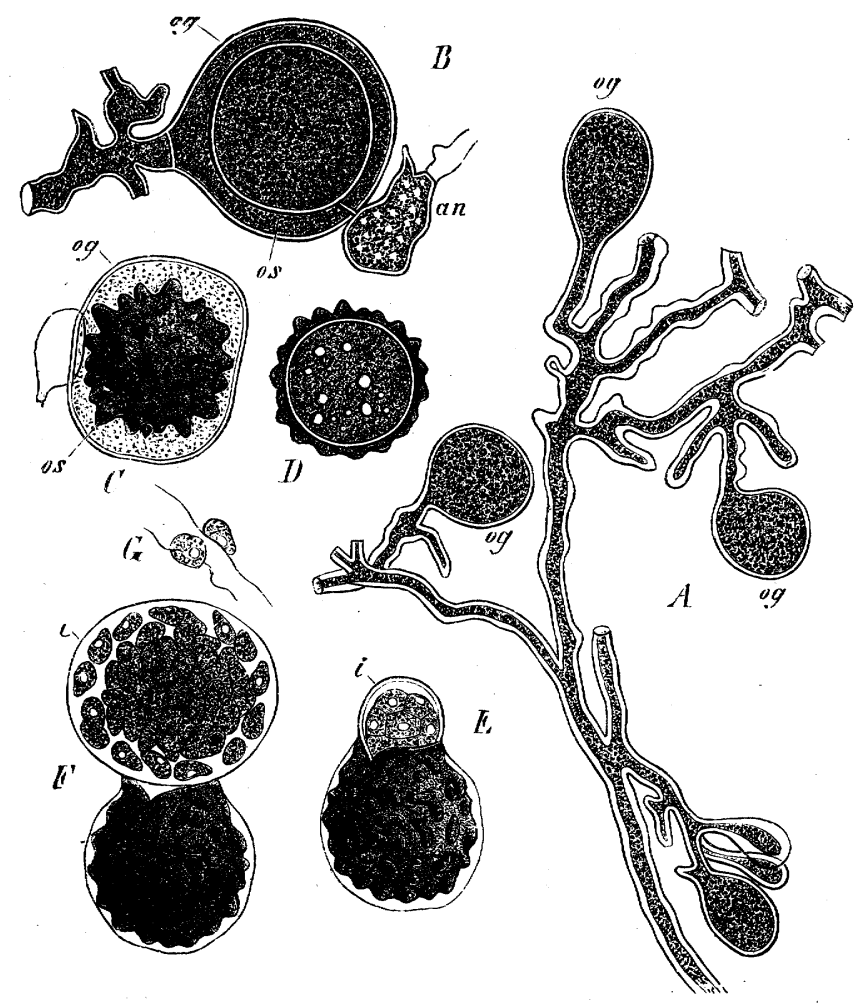

FIG. 18r.-Cystopus candidus. $A$ mycelium with young oogonia; $B$ oogonium og with oosphere os and antheridium $a n$;

$C$ ripe oogonium; $D$ ripe oospore; $E, F, G$ formation of zoospores from oospores; $i$ endospore (after De Bary, $\mathrm{X} 400$ ).

The genus Empusa, in which no sexual organs have as yet been discovered ', is probably related to the Saprolegnieæ and to the Peronosporeæ. Empusa musce is the parasite which proves fatal, more especially in the autumn, to house-flies. If these insects remain attached to the window-panes they become surrounded by a powdery substance which consists of extruded conidia. These conidia are capable of forming

1 [Brefeld, Untersuch. über die Entwickelung der Empusa Musca und E. radicans, I871. Nowakowski and Brefeld have both recently published observations on Empusa musca (Entomophthora) in the Bot. Zeit. for 1877 . While Nowakowski states that he has observed zygospores, Brefeld believes that the resting spores arise asexually. Nowakowski thinks the Entomophthoree allied with Piptocethalidece.] 
secondary conidia, and of projecting them to some distance. If one of these happens to strike a fly upon the white area of the under surface of its body, it sends a hypha through the skin, and there forms toruloid cells, which multiply by germination and are disseminated in the blood throughout the body of the fly. These finally grow into hyphæ which penetrate the skin, each forming a conidium, which is cast off with considerable force.

\section{C.-DOLVOCinea ${ }^{1}$.}

[This group includes two genera, Volvox and Eudorina, which resemble Pandorina in many respects, and were formerly included with it in one group. Like Pandorina, these plants are motile, and consist of a number of ciliated cells aggregated into a cœnobium. In Eudorina, which so much resembles Pandorina as to be sometimes mistaken for it, the number of cells is 16 or 32 ; in Volvox it is very great, amounting even to thousands. The cells are so arranged as to form the wall of a hollow sphere. The distinction between these plants and Pandorina is that in them certain cells of the cœnobium develope into antheridia and oogonia. In Eudorina four of the cells become antheridia, and the remainder oogonia: in Volvox a certain number only of the cells take on the function of reproductive organs. The antherozoids formed in the antheridia escape and make their way through the wall of an oogonium to the oosphere which it contains, and fertilise it. The oosphere then surrounds itself with a firm double cell-wall, and assumes a red colour: it becomes an oospore.

Non-sexual reproduction is effected by the repeated division in Volvox of certain cells (gonidia) of the cœnobium, and in Eudorina of all the cells. In the former, the young cœnobia which are thus formed escape into the cavity of the mother-cœnobium, where they remain until they are set free by its death and disintegration: in the latter, they are at once set free.

Sexual and non-sexual reproduction do not go on simultaneously in the same cœnobium of Volvox, and in one species (Volvox minor, Stein) the male and female organs are not produced in the same cœnobium, whereas this is the case in Volvox Globator, L.

The germination of the oospore has been observed by Kirchner in Volvox minor. The protoplasmic contents divide into two, and this is repeated until about 500 small cells have been produced; these are arranged like those of the adult cœnobium. The wall of the oospore gradually deliquesces, and the young cœnobium is set free.]

\section{D. - EDOGONIEE.}

The ordogonieæ ${ }^{2}$ include at present only the two genera $C$ Edogonium and Bulbocrete, the few species of which are common in fresh water, fixed by an organ of attachment at the lower end to solid bodies, mostly the submerged parts of other plants. The thallus consists of unbranched (Edogonium) or branched (Bulbocbxte) rows of cells, which multiply by intercalary growth, while the terminal cells elongate into hyaline bristles. The longitudinal growth of the cylindrical cells begins with the formation of an annular cushion of cellulose inside the cell, close beneath its upper septum; the cell-wall ruptures at this place circularly; the ring of cellulose then stretches, and a broad transverse zone is thus intercalated in the wall of the cell. The process is constantly repeated immediately beneath the older very short upper piece of the cell,

${ }^{1}$ [On Volvox, see Cohn, Beitr. z. Biol. d. Pflzn. I. 3, and Kirchner, ibid. II. I : on Eudorina see Carter, Ann. of Nat. Hist., ser. III. vols. 2 and 3.]

${ }^{2}$ Pringsheim, Morphologie der đEdogonieen, Jahrb. für wissen. Bot. vol. I. De Bary, Ueb. OEdogonium u. Bulbochate, I854-Duranyi, Jahrb. d. wiss. Bot. IX. [Ann. des Sci. Nat. 1856, vol. V. p. 25 I.-Carter, Ann. and Mag. Nat. Hist. I $85^{8}$, vol. I. pp. 29-39.] 
so that these pieces, forming small projections, give to the upper end of the cell the appearance of consisting of caps placed one over another, while the lower end of the cells appears to be enclosed in a long sheath (the lower old piece of cell-wall). This lower part of an elongated cell is always separated by a septum from the upper capbearing piece (see Fig. 17, p. 22). In Bulbocbrte the growth of all the shoots, even of the first which proceed from the spores, as far as it depends on cell-multiplication, is limited to the division of their basal cell; it follows therefore that the cells of each shoot must be considered to be the basal cells of the lateral branches which stand upon
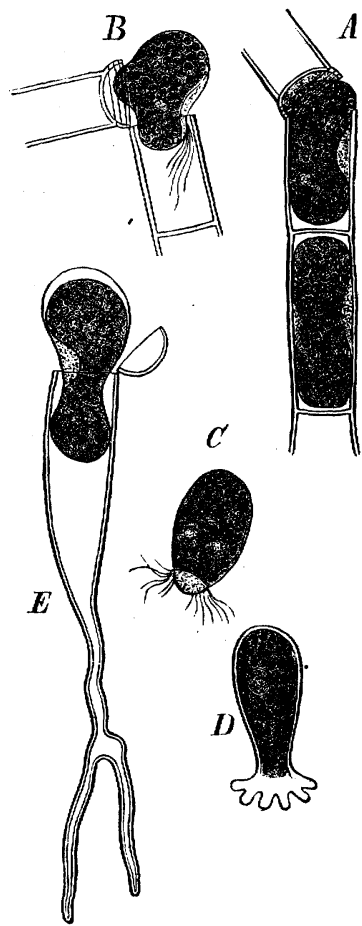

FIG. 182. - Development of the zoogonidia of Edogonium (after Pringsheim). $A, B$ their origin from an older filament; $C$ free zoogonidium in motion; $D$ commencement of its germination; $E$ a zoogonidium formed out of the entire contents of a germinating plant $\left(x_{35}\right)$.

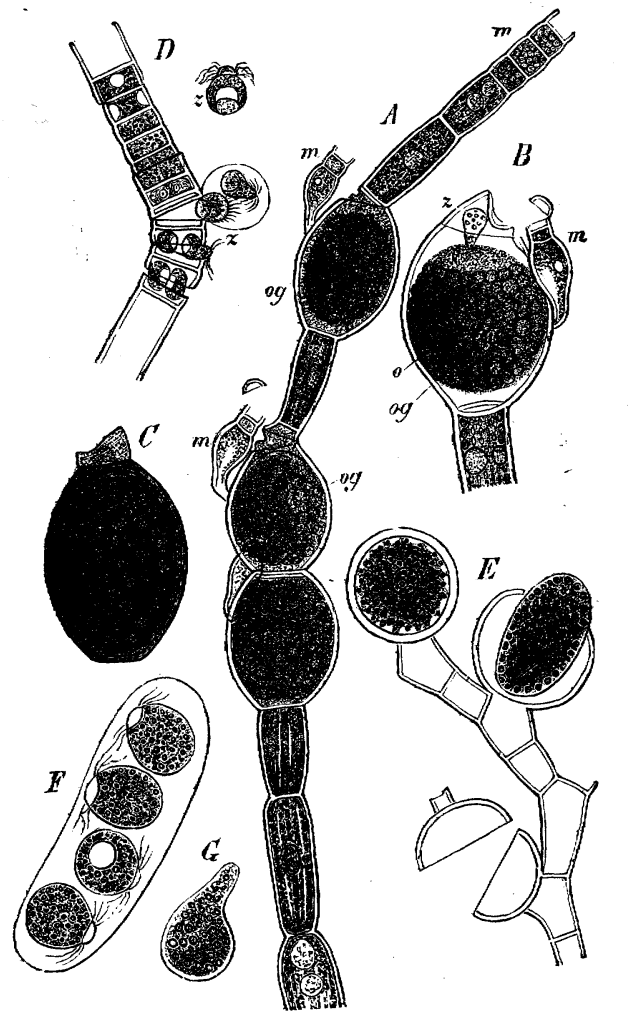

FIG. $183-A$ CEdogonium ciliatum ( $\times 250$ ) middle part of a sexual filament with an antheridium $(m)$ at the upper end, and two fertilised oogonia $(\circ g)$ and a dwarf male plant $m ; B$ oogonium at the moment of fertilisation; $o$ the oosphere, $z$ the antherozoid in the act of forcing its way in, $m$ dwarf male plant; $C$ ripe oospore; $D$ piece of the male filament of $O$. gemelliparum, plant; $C$ ripe oospore; $D$ piece of the male filament of $O$. gemelliparum,
$z$ antherozoids. $E$ branch of a hybernated plant of Bulbochate intermedia, $z$ antherozoids. $E$ branch of a hybernated plant of Bulbochate intermedia,
with one oogonium still containing a spore, another in the act of allowing it to escape; in the lower part an empty oogonium. F the four zoospores resulting from an oospore; $G$ zoospores from an oospore come to rest (after Pringsheim).

them. The cells contain chlorophyll-grains and a nucleus in a parietal layer of protoplasm. The zoogonidia, as well as the oogonia and antheridia, are formed from cells of the filaments, which only become enlarged and assume a more or less spherical form when they give rise to oogonia. From the oospores which have remained at rest for a considerable period several (usually four) zoospores are immediately formed, which produce asexual, i.e. zoogonidia-forming plants, from which again similar ones proceed, until the series of them is closed by a sexual generation (with formation of oospores); but the sexual plants produce zoogonidia as well. The sexual plants are either monœcious or diøcious; in many species the female plant produces peculiar zoogonidia (Androgonidia), 
out of which proceed very small male plants (dwarf males). Several generative cycles or only one may be completed in a vegetative period. The zoogonidium is formed in an ordinary cell of the filament (sometimes even in the first cell, Fig. $182, E$ ) by the contraction of its whole protoplasmic substance; it becomes free from the mothercell, the cell-wall splitting by a transverse slit into two very unequal halves (as in the division of the cells) (Fig. I82, $A, B, E$ ). It is at first surrounded by a hyaline membrane, which however it also breaks through. At its hyaline end-the-anterior end during the swarming-it is encircled by a circlet of numerous cilia. This end lies laterally in the mother-cell, and, after the movement ends, becomes the lower attached end which grows out into a rhizoid; the direction of growth of the new plant is thus at right angles to that of the mother-cell. The antherozoids are very similar in form to the zoogonidia, but much smaller (Fig. 183,D,z); their motion, due to a circlet of cilia, is also similar. The mother-cells of the antherozoids are cells of the filament, but shorter and not so rich in chlorophyll as the vegetative cells; they lie either singly or in groups (sometimes as many as twelve) above one another. In most species each antheridium-cell divides into two equal special mother-cells, each of which produces an antherozoid; they escape by the splitting of the mother-cell (as in the case of the zoogonidia) (Fig. 183,D). The androgonidia from which the dwarf male plants arise are produced from mother-cells similar to those which give birth to the antherozoids (without formation of special mother-cells). After swarming they fix themselves to a definite part of the female plant, on or near the oogonium, and after germination produce at once the antheridium-cells, and in them the antherozoids (Fig. I $83, A, B, m, m$ ). The oogonium is always developed from the upper daughter-cell of a vegetative cell of the filament which has just divided, and immediately after the division swells up into a spherical or ovoid form. In Bulbocbrte the oogonium is always the lowest cell of a fertile branch. This is not opposed to the law of growth above-mentioned, inasmuch as the mother-cell of a branch fulfils at the same time the function of its basal cell; the oogonium of Bulbocbrte is never the first cell of a branch, since this is always developed as a bristle. The oogonium becomes at first more completely filled with contents than the remaining cells; immediately before fertilisation the protoplasm contracts and forms, as in Vaucheria, the oosphere, in the interior of which the chlorophyll granules are densely crowded. The part of the oosphere which faces the opening of the oogonium consists simply of hyaline protoplasm. The opening of the oogonium is produced in a variety of ways. In some species of Edogonium and all of Bulbocbrte its wall has an oval hole in its side, out of which the colourless part of the oosphere protrudes in the form of papillæ, and takes up the antherozoids. In some species of Edogonium (Fig. I 83, $A, B$ ), on the other hand, the oogonium-cell splits, as when the zoogonidia are escaping; and the otherwise straight row of cells of the filament thus appears as if broken at this spot. In the lateral fissure appears some colourless mucilage, which the observer can actually see take the form of an open beak-like canal (Fig. I $83, B, z)$, through which the antherozoid enters. The antherozoid coalesces with the hyaline part of the protoplasm of the oosphere and disappears ${ }^{1}$. Immediately after fertilisation the oosphere surrounds itself with a membrane, which afterwards, like its contents, assumes a brown colour; but in Bulbochæte the contents of the oospore thus formed is of a beautiful red. The oospore remains enclosed in the membrane of the oogonium, which separates from the neighbouring cells of the filament and falls to the ground, where the oospore passes its period of rest. When it awakes to new activity, the oospore does not itself grow into a new plant; but in Bulbocbrte, where this process has been observed, its contents divide into four zoospores, which escape together with the inner skin of the oospore, and after this latter is dissolved, swim about. After becoming stationary each grows into a new plant.

1 In O. diplandrum, discovered by Juranyi, the large antherozoids creep in an amœboid manner over the oogonium until they reach the canal, which they slowly enter. 
The Confervaceæ, like the Edogoniex, consist of rows of cells or segmented filaments, which either remain unbranched, as in Cbxtomorpba, or become branched, as in Cladophora, Rbizoclonium, Stigeoclonium (Fig. 3), Draparnaldia, Cbxtophora ${ }^{1}$. With reference to their reproduction, it is only known that macro- and micro-zoogonidia are formed in the cells of the filaments (Cbrtomorpba, Cladophora), the sexual significance of which is still unknown ${ }^{2}$; and that in the other above-mentioned plants resting-spores are formed in certain cells of the filaments. Pringsheim suggests that they are probably equivalent to oospores, but that they are produced parthenogenetically.

\section{E. FUCOIDEE.}

The Fucaceæ comprise, in the narrow limitation proposed by Thuret $^{3}$, a few genera of large marine Algæ, the thallomes of which, often many feet long, have a
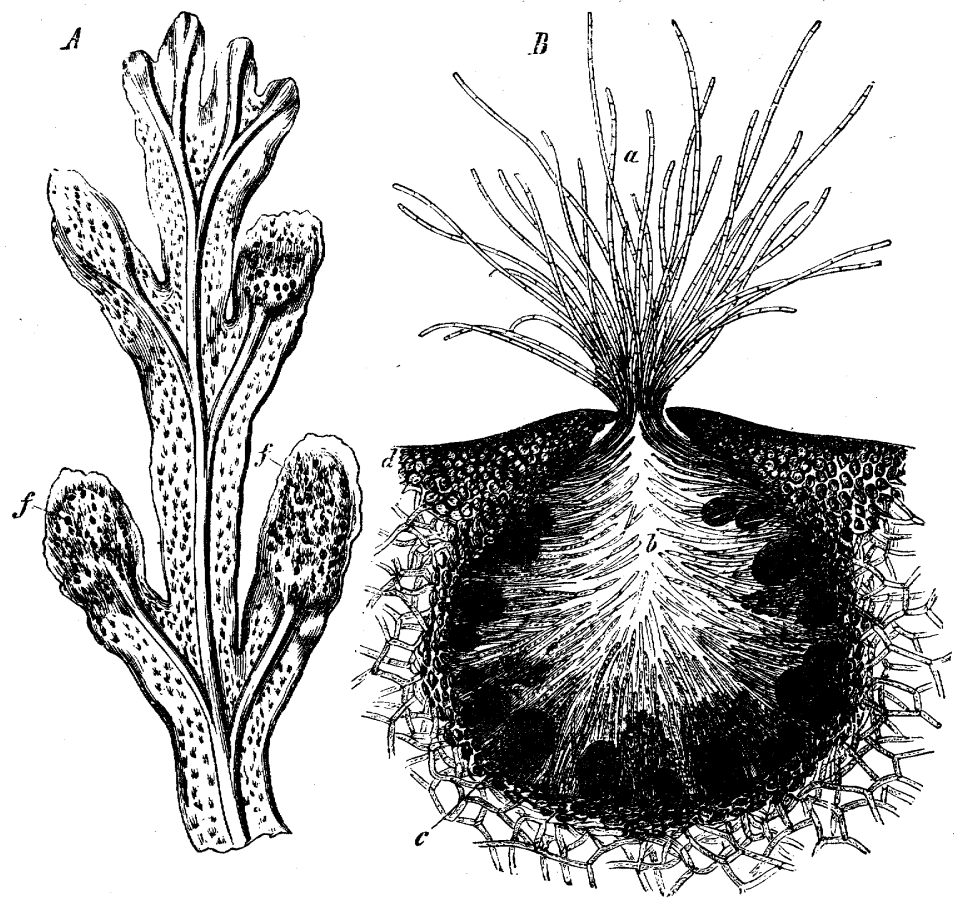

\footnotetext{
FIG. I84-Fucus platycarpus (after Thuret); $A$ end of one of the larger branches (natural size); $f f$ fertile branchlets : $B$ transverse section of a conceptacle; $d$ the surrounding epidermal tissue; $a$ the hairs projecting from the mouth; $b$ hairs in the interior; $c$ oogonia, $e$ antheridia (cf. Fig. 2, p. 3).
}

greenish-brown colour and a cartilaginous consistency. They are fixed to stones or other bodies by a branched attachment-disc. The thallomes branch dichotomously, and the further development is also frequently forked, but in other cases sympodial, as in Fig. 184. The ramifications, irrespectively of later displacements, all lie in one plane.

1 [The Ulvaceæ are probably allied to the Confervacex. In them the cells are arranged so as to form a delicate membrane.]

${ }^{2}$ [According to Areschoug (Nov. Act. reg. soc. sci. Upsal. ser. 3. vol. IX) the microzoogonidia of Cladophora conjugate in pairs.]

${ }^{3}$ G. Thuret, Aun. des Sci. Nat. II. 1854, p. I97. 
The tissue consists at the surface of small closely-crowded cells; in the interior it is laxer, and the elongated cells are often connected into articulated threads. The cell-walls often consist of two clearly distinct layers, an inner thin, firm, compact layer, and an outer gelatinous one, capable of swelling greatly in water, which fills up the interstices of the cells, and has the appearance of a more or less structureless 'intercellular substance ;' it is clearly the cause of the slimy character which the Fucaceæ assume after lying for some time in fresh-water. The granular cell-contents have been but little investigated; they appear to be mostly brown, but contain chlorophyll which is concealed by other colouring materials; from dead plants cold fresh-water extracts a buff-coloured substance $^{1}$. The tissue often becomes hollowed out internally into large cavities containing air which are forced outwards and serve as swimming bladders. The thallus has not, as far as I know, been further minutely examined ${ }^{2}$; the outer conformation especially has been but little investigated from a morphological point of view. (Cf. Nägeli, Neuere Algensysteme.)

The mode of sexual reproduction is far better known through the labours of Thuret and Pringsheim. The antheridia and oogonia are formed in spherical hollows (Conceptacles) which make their appearance in large numbers and densely crowded at the ends of the longer forked branches or of lateral shoots of peculiar form. These conceptacles are not formed in the interior of the tissue, but are depressions of the surface which become walled in by the surrounding tissue and so overgrown that at length only a narrow channel remains, opening outwards. The layer of cells which clothes the hollow is thus a continuation of the external epidermal layer of the thallus; and since the filaments which produce the antheridia and oogonia sprout from it, these latter are, morphologically, trichomes. Some species are monœcious, i.e. both kinds of sexual organs are developed in the same conceptacle, as in Fucus platycarpus (Fig. 184); others are diœcious, the conceptacles of one plant containing only oogonia, those of another plant only antheridia (e.g. Fucus vesiculosus, serratus, and nodosus, Himantbalia lorea). A number of hairs which grow in the conceptacles among the sexual organs are long, slender, articulated, but unbranched, and project in $F$. platycarpus out of the mouth of the receptacle in the form of tufts (Fig. $\mathrm{I}_{4}, B$ ). The antheridia are produced as lateral ramifications of branched hairs. Each antheridium consists of a thin-walled oval cell, the protoplasm of which splits up into numerous small antherozoids; these are pointed at one end, and each is furnished with two motile cilia; in the interior they contain a red spot. The formation of the oogonium begins with the papillose swelling of a parietal cell of the conceptacle ; the papilla is separated off by a septum, and divides, as it grows in length, into two cells, a lower, the pedicel-cell, and an upper, which forms the oogonium; this swells up into a spherical or ellipsoidal form and becomes filled with dark protoplasm. The protoplasm of the oogonium remains undivided in some genera (Pycnoplycus, Himantbalia, Cystoseira, Halidrys), and the whole contents of the oogonium thus form one oosphere; in others (Pelvetia) it divides it into two, four (Ozothallia vulgaris), or eight (Fucus). Fertilisation takes place outside the concep-

1 In a recent paper (Comptes Rendus de l'Acad. des Sci. Feb. 22, 1869) Millardet showed that from quickly-dried and pulverised Fucaceæ an olive-green extract is obtained by alcohol, which, shaken up with double its volume of benzine and then allowed to settle, produces an upper green layer of benzine containing the chlorophyll, while the lower alcoholic layer is yellow and contains phycoxanthine. Thin sections of the thallus, completely extracted with alcohol, contain also a reddish-brown substance which in fresh cells adheres to the chlorophyll-grains, and can be extracted by cold water, more easily when the dried Fucus has been previously pulverised. Millardet calls this reddish-brown substance phycophæine. (Compare further the interesting treatise of Rosanoff, Observations sur les fonctions et les propriétés des pigments de diverses Algues, in Mémoires de la Société des Sci. Nat. de Cherbourg, vol. XIII. I867; and Askenasy, Bot. Zeitg. no. 47, I869.) [See also Sorby, Proc. Roy. Soc. I873, vol. XXI. pp. 445, 454, 46I.]

${ }^{2}$ [See Rostafinski, Beit. z. Kenntniss der Tange, I876: also Bower, Quart. Journ. Mic. Sci. vol. 20 , new series.] 
tacles. The oospheres are expelled, surrounded by an inner membrane of the oogonium, and escape through the opening of the conceptacle; the antheridia at the same time become detached, and collect in numbers before the mouth of the conceptacle when the fertile branches are lying out of the water in moist air. When they again come into contact with the sea-water, the antheridia open and allow the antherozoids to escape, the oospheres at the same time escaping from the envelope which still surrounds them, and which is then seen to consist of two separated layers (Fig. 185, II). The antherozoids collect in numbers around the oospheres, become firmly attached to them, and when their number is sufficiently great, their movement becomes so energetic that they impart to the very large oosphere a rotatory motion which lasts for about half an hour. Whether the antherozoids force themselves into the oosphere Thuret leaves undecided; but analogy with the processes observed by Pringsheim in Vaucheria and Edogonium scarcely admits of a doubt that one or several of them mingle their substance with that of the naked ball of protoplasm. A short time after these processes are completed, the fertilised oosphere surrounds itself with a cell-wall, fixes itself to some body or other, and begins, without any period of rest, to germinate, and, lengthening at the same time,
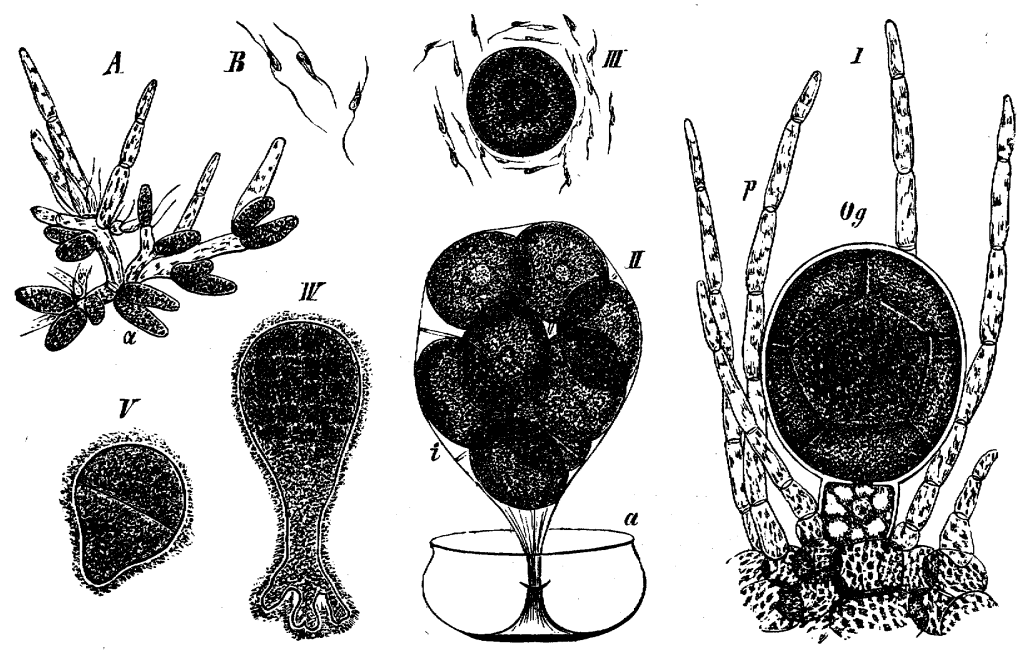

FIG. 185.-Fucus vesiculosus (after Thuret); $A$ a branched hair bearing antheridia; $B$ antherozoids; $I$ an oogonium, $O g$ after the contents have divided into eight portions (oospheres), surrounded by simple hairs $(p) ; I I$ commencement of the escape of the oosphere; the membrane $(\alpha)$ has burst; the inner membrane $i$ is ready to open (the two together constitute an inner layer of the cell-wall of the oogonium); $I I I$ oosphere surrounded by antherozoids; $I V$, $V$, germination of the oospore $(B \times 330$, all the rest $\times 160)$.

undergoes first of all a transverse division followed by numerous other divisions. The mass of tissue thus formed puts out from the part on which it rests a root-like hyaline organ of attachment, while the thick free end forms the growing apex (Fig. $185, I V$ ).

There are numerous marine Algæ, included in the group of Phæosporeæ, which resemble the Fucaceæ as well in the structure of their vegetative organs as in the presence of a colouring-matter mingled with their chlorophyll. To this group belong the often enormous Laminarieæ (Macrocystis, Laminaria, Lessonia, etc.), as also the smaller Ectocarpeæ, Sphacelarieæ, Chordarieæ, and Dictyoteæ.

The Phæosporeæ are reproduced non-sexually by zoogonidia, a mode of reproduction which does not occur among the Fucacex. In some, bodies which appear to be antheridia have been detected, but no oogonia ${ }^{1}$.

${ }^{1}$ [Goebel has observed (Bot. Zeitg. 1878) the conjugation of zoogonidia in two species of Ectocarpus: see also Berthold, in Mittheil. d. Zool. Stat. Neapel, II. I881.] 


\section{CLASS IV.}

\section{CARPOSPORE $A$.}

Under the name of Carposporeæ I include in a single class the Coleochæteæ, Characeæ, and Florideæ on the one hand, all of which contain chlorophyll, and on the other true Fungi, namely the Ascomycetes, Basidiomycetes, and Ficidiomycetes. The remarkable differences of their habit and mode of life offer no real obstacle to such an arrangement, any more than do the similar differences which exist between the Lemnaceæ and the Palms to their being both included in the class of Monocotyledons. In this case, as in that, it is the peculiarities which accompany the formation of the reproductive organs and become especially prominent in the product of fertilisation that indicate the existence of a relationship between plants which are at first sight so different.

All the plants belonging to this class are characterised by the formation of a spore-fruit (sporocarp) as the result of the fertilisation of the female reproductive organ, and differ herein from all those which have hitherto been considered. This spore-fruit consists, except in certain cases in which it is rudimentary, of two distinct parts, a fertile part, which is directly derived from the female organ and which produces at a later period one or more commonly numerous true spores, and an investing part, which encloses the spores. Occasionally this fruit attains a very considerable size, as, for instance, in the Truffle: in other forms it may remain comparatively small. In all cases, however, fertilisation not only causes a further development of the female cell, as in the Zygosporeæ and Oosporeæ, but certain processes of growth are initiated which may lead to comparatively insignificant results (as in Characeæ), but which are usually of an extensive character, so that a sporocarp is produced consisting of a considerable mass of tissue. In those cases in which the sporocarp remains relatively small, and in which it continues to derive its nourishment from the parent-plant until the spores are ripe (as in Characeæ, Florideæ, Coleochæteæ, and in some Fungi), it seems to be a mere appendage, like an apple on a tree: but in those cases in which it attains a considerable size, grows for a length of time and finally produces numerous spores, after that the parentplant has decayed, an alternation of generations becomes apparent. Such is the case among the true Fungi, especially in the Ascomycetes. In these, the fructification continues to grow for some time, and presents the appearance of being a perfectly independent plant. In fact, it is usually but erroneously regarded as being the whole Fungus, whereas it is only the product of a process of sexual reproduction which has taken place on the vegetative body, the mycelium. In such cases the process of the life-history of the plant is similar to that of a Fern; the insignificant mycelium corresponds to the prothallium, and the well-developed sporocarp of the Fungus to the spore-bearing Fern. If we consider from this point of view the various ways in which the fruit is formed among the Carposporex, we shall find that 
all possible intermediate stages exist between two extreme forms, the simplest being that form in which the sporocarp produces only a single spore or a single mothercell in which spores are formed, the most complex being that in which the sporocarp exists as an independent plant and produces countless spores.

The sporocarp is derived from a female organ which we will term the Carpogonium. It is only in the simplest case that the carpogonium is a single cell, and then it sometimes resembles very closely the oogonium of Class III, especially when, as in the Coleochæteæ, fertilisation is effected by means of motile antherozoids, or when the unicellular carpogonium is fertilised by a tube growing from the male organ (Sordaria, Podosphara), just as is the case in the Saprolegniex. In the majority of cases, however, the carpogonium is multicellular before fertilisation, and its cells contribute in different ways to its further development; some absorb the fertilising substance, whilst others give rise to that part of the fructification which produces the spores. This division of labour is very evident in the Ceramiex and other Florideæ, as also in many Ascomycetes (e.g. Ascobolus furfuraceus). It occurs both in unicellular and in multicellular carpogonia that a more or less elongated tubular projection arises from the carpogonium, the function of which is the absorption of the fertilising substance. This organ, which takes no part in the subsequent development of the fruit, is termed the Trichogyne, a name given by Thuret and Bornet to this organ in the Floridex. Like the style on the ovary of Phanerogams, the trichogyne of the Carposporeæ may be sometimes welldeveloped, and sometimes entirely absent. For instance, in Characeæ and in many Ascomycetes (Sordaria, Erysiphe) it is wanting; it is only imperfectly developed in Peziza confluens, and it is well-developed in Coleochæteæ and Florideæ. The male reproductive organ occurs in very various forms in the different groups of Carposporeæ, this variety being evidently dependent upon the varying form of the carpogonium and the habitat of the plant. In Coleochætex and Characeæ only is fertilisation effected by motile antherozoids; in Florideæ it is effected by cells which are conveyed passively, and in most Fungi by tubular outgrowths.

Should it be suggested as an argument against the existence of a relationship between the true Fungi and the green plants, which are included within this class, that the difference of habit between them is very great, it might be replied that the tissue of many Fungi presents striking resemblances to that of many Florideæ. The hyphal tissue of many gelatinous Fungi finds its analogue in the gelatinous tissue of many Florideæ. The rows of cells too, of which the mycelial filaments (hyphæ) of Fungi consist, differ only in habit from the branched rows of cells of which the thallus of many Coleochæteæ and of very many Florideæ consists.

It must be remembered that, in order to detect the relationships existing between different groups of plants, the simplest and not the highest forms are those which must be compared. If this be done in this case, it becomes evident that the simplest Florideæ are connected on the one hand with the Coleochæteæ and Characeæ, on the other with the simplest Ascomycetes. Each of these series of forms, however, becomes developed into higher forms in some particular direction, and so if the most perfect Ascomycetes be compared with the most highly-developed Florideæ and Coleochæteæ, oniy a very slight similarity between them will be detected. 


\section{FORMS CONTAINING CHLOROPHYLL.}

These are all submerged water-plants, the vegetative and reproductive organs of which have a well-marked tendency to clothe themselves with a peculiar cortex. This is especially remarkable in the genus Chara, and it will be described in detail hereafter when that genus is under consideration. It is also very evident in the Ceramiaceæ, and but rudimentary in the Coleochæteæ, where it is confined to the fruit. Side by side with forms possessing this cortex, there are others, very nearly related, which do not possess it.

In all the plants belonging to this group the fruit is small in proportion to the thallus which bears it, and the alternation of generations which finds its expression

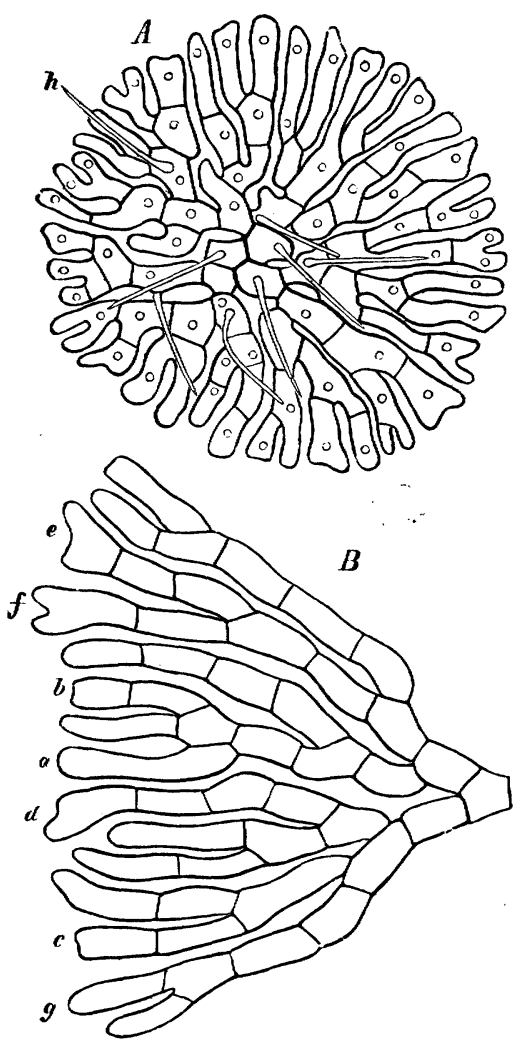

FIG. 186. $-A$ an asexual plant of Coleochate soluta $(\times 250)$; $B$ a piece of a similar disc; the letters $a \cdot g$ indicate the successive dichotomous branchings of the terminal cells fafter Pringsheim). in the formation of the fruit is therefore not very clearly marked.

\section{A. ThE COLEOCh \#TEx.}

The carpogonium is unicellular with a long trichogyne opening at its apex. Fertilisation is effected by antherozoids which are formed either in special small branches or in the cells of a filament which have undergone division. In the basal portion of the fertilised carpogonium there is a cell which grows considerably, and becomes invested by outgrowths derived from neighbouring cells. In the next period of vegetation it gives rise to numerous carpospores in the form of zoospores.

The Coleochætex ${ }^{\mathbf{l}}$ are small (about I-2 $\mathrm{mm}$.) fresh-water Algæ, of a bright green colour and constructed of branched rows of cells, attached in standing or slowly-running water to the submerged parts of other plants (e.g. Equisetum), and forming circular closelyattached or cushion-like discs. Their chlorophyll assumes the form of parietal plates or of large granules. The name of the genus Coleocbxte (sheath-hair) is due to the circumstance that certain cells of the thallus bear lateral colourless bristles fixed in narrow sheaths (Fig. I $86, A, b$ ). If the phenomena of growth of the different species are compared, two extreme cases are seen, connected by transitional forms. The one extreme is formed by $C$. divergens, which, as it developes from the spore, produces first of all creeping irregularly-branched articulated threads; from these spring ascending articulated branches which are also irregularly branched; the whole thallus does not assume any definite form. In C. pulvinata, on the contrary, the thallus forms a hemi-

1 Pringsheim in Jahrbuch für wissenschaftliche Botanik, vol. II. p. I. 
spherical cushion; the cellular filaments which are the result of germination branch somewhat irregularly in one plane, but form something like a disc; from them rise up ascending articulated branches, which again branch and form the cushion. In the following species no ascending branches are formed, but those which cling to their support form a more or less regular disc. In C. irregularis this takes place by irregular ramifications which lie in one plane gradually filling up all the interstices, till an almost uninterrupted layer of cells is obtained. In C. soluta (Fig. I86), on the other hand, a dichotomous ramification commences in the two first daughter-cells of the germinating spore, with corresponding cell-division of such a nature that even at a very early period a closed disc of radial forked branches is formed, which either lie loosely or are closely crowded side by side. While in the species already named the branches arise laterally from cells, but never from the terminal cell of a branch, in C. soluta we have the first instance of dichotomy as well as regular disc-shaped centrifugal growth, a condition which attains the highest development in C. scutata. In this species the cells which result from germination remain from the first united laterally and do not form isolated branches; the circular disc, when once formed, continues to grow by increase of its circumference, the marginal cells dividing by radial and tangential walls. This mode of growth may be explained in this way, that the first branches are united laterally and grow with equal rapidity in a radial direction, and then become divided by septa (in this case tangential); while the broadening of the terminal cell of each radial row corresponds with the succeeding radial division of a dichotomy. The law which prevails in the species previously mentioned, that only the terminal cell of a branch is divided by transverse septa, is exemplified in $C$. scutata by the marginal cells only of the disc being divided by tangential walls.

The Reproduction of the Coleochætex is brought about by asexual zoogonidia and by resting oospores produced sexually. The oospores do not at once produce new plants, but several zoospores; and the following alternation of generations takes place:-The zoospores, which arise in the early part of the year at the commencement of vegetation from a sporocarp of the previous year, produce only asexual plants, or, in other words, only such as can form zoogonidia. Only after a series of asexual generations varying in length does a sexual generation arise, which may be either monœcious or diœcious according to the species. Fertilisation produces one oospore in the carpogonia, which clothe themselves with a peculiar layer of cortical cells; and this oospore itself again developes into a parenchymatous reproductive body, from the cells of which the zoospores proceed in the next period of vegetation (Pringsheim). The zoogonidia (Fig. $187, D$ ) may arise in all the vegetative cells of the Coleochætex; in $C$. pulvinata especially from the terminal cells of the branches; they are always formed from the entire contents of the mother-cell, and escape through a round hole in its cell-wall.

The carpogonium is always the terminal cell of a branch, and hence in $C$. scutata the terminal-cell of a radial row (Nägeli). The peculiar mode of its development is subject, according to the growth of the plant, to some, though subordinate, modifications. One species, C. pulvinata (Fig. 187), may first of all be examined somewhat more closely. The terminal cell of a branch swells up and at the same time elongates into a narrow sac (Fig. $187, A, o g$, to the left), which then opens ( $\operatorname{og}^{\prime \prime}$, to the right) and exudes a colourless mucilage. The protoplasm of the swollen part, which contains chlorophyll, forms the oosphere in which a nucleus is visible. The antheridia are formed at the same time in adjoining cells, two or three protuberances $(A$, an $)$ growing out, which become separated by septa; each of the cells thus formed, which have somewhat the shape of a flask, is an antheridium; its entire contents form an antherozoid (z) of oval shape with two cilia which is endowed with motion like a zoogonidium; its entrance into the oogonium has not yet been observed. The effect of fertilisation is seen in that the contents of the carpogonium become surrounded with a proper membrane and form the oospore. This now grows considerably, and at the same time the formation of 
the cortical layer $(r)$ of the carpogonium commences; out of the cells that support it proceed branches $\left(A, \circ g^{\prime \prime}\right)$ which cling closely to it. These again form branches which also cling closely and divide transversely; the branchlets of other branches also ramify $(B)$; and only the neck of the carpogonium does not become covered with the cortical layer. All this happens between May and July; later, the contents of the remaining cells of the plant disappear, and the walls of the cortical layer of the carpogonium assume a deep dark-brown colour. The further development of the oospore within the carpogonium now covered with its cortical layer begins only in the next spring; a parenchymatous tissue is formed by successive bipartitions; the cortical layer splits and is

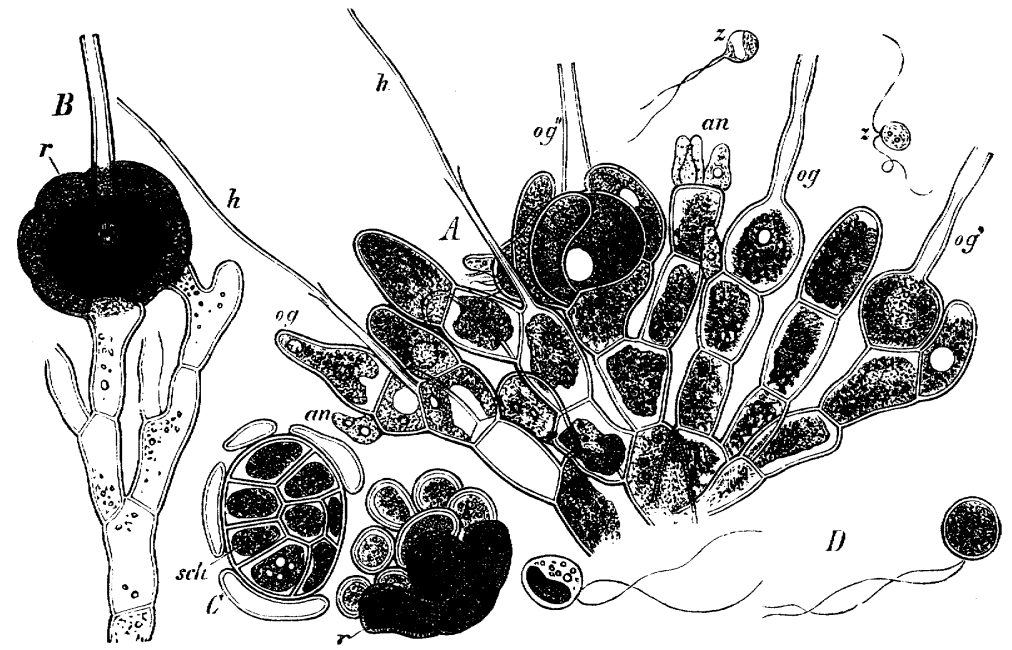

Fir 8 . $A$ part of fertile thallus of Coleochate pulvinata $\left(\mathbf{X}_{350}\right) ; B$ ripe carpogonium enclosed in its cortical layer $C$ germinating sporocarp, in the cells of which the zoospores are formed; $D$ zoospores $(B-D \times 28$, after Pringsheim).

thrown off $($ Fig. $187, C)$; and from each cell arises a zoospore, and from this again an asexual plant. C. scutata (the most abnormal species) deviates from these processes only so far that in it the carpogonia provided with their cortical layer lie on the surface of the disc, and the antheridia are the result of divisions of disc-cells into fours.

Pringsheim (loc. cit.) has already pointed out various relationships existing between the Coleochæteæ, the Florideæ, and the Characeæ.

\section{B. THE FLORIDEE.}

The carpogonium is either unicellular or composed of several cells, and it is provided with a permanently-closed trichogyne. If the carpogonium is multicellular, the trichogyne is borne by a lateral row of cells, which is termed the trichophore. Fertilisation is effected by non-motile rounded antherozoids which become attached to the trichogyne. As a consequence of fertilisation the basal portion of the carpogonium, which does not form the trichophore, forms a great number of spores by budding, each spore being the terminal cell of a short branch. The mass of spores is usually surrounded by an investment and thus a cystocarp is formed.

The Floride $\Re^{1}$ are a group of Algæ of extraordinarily variable form, belonging,

1 Nägeli und Cramer, Pflanzenphys. Unters. Zürich, Heft I. 1855; Heft IV. 1857.-Thuret, Ann. des Sci. Nat. 1855 , Recherches sur la fécondation, \&c.-Pringsheim, Ueber die Befruchtg. u. Keimung der Algen, Berlin I 855.-[Quart. Journ. Micr. Sc. I856, vol. IV. pp. 63, I 24.]-Nägeli, 
with few exceptions (Batrachospermum, Hildenbrandtia ${ }^{1}$ ), to the sea. In the normal condition they are of a red or violet colour; the green colour of their chlorophyll is concealed by a red pigment ${ }^{2}$, soluble in cold water.

The Thallus of the Floridex consists, in the simplest forms, of branched rows of cells, which elongate by apical growth and transverse division of their apical cell. An apparent formation of tissue occurs in many Ceramiacex (C. Cramer, Physiolog. u. system. Untersuch. über die Ceramiaceen, Zürich, I $86_{3}$ ) from the branch:s growing closely adpressed to their mother-axes, and thus surrounding them with a cortex, reminding one of the formation of the cortex in Cbara. In other Floridex the thallus is a flat expansion of cells, but often consisting of several layers; in some (as Hypoglossum and Delesseria) it assumes the contour of stalked leaves, even the venation being represented; in others (e.g. Spbxrococcus and Gelidium) it consists of filiform or narrow strap-shaped. masses of tissue, which ramify copiously (e.g. Plocamium, \&c.). In all these cases Nägeli asserts (Neuere Algensysteme, p. 248) that apical growth takes place from an apical cell (in Peissomelia possibly from several). In the simpler forms the segments of the apical cell are formed in one row by transverse divisions, in others in two or three rows by oblique walls. One group which comprises a large number of species, the Melobesiaceæ (Rosanoff, Mém. de la Soc. Imp. des Sci. Nat. de Cherbourg, vol. XII. I 866), forms disclike thallomes, which grow centrifugally at the circumference and are closely attached to the substance on which they grow, which generally consists of larger Algæ; they resemble Coleocbrte scutata in their size and mode of life, but their thallome generally consists of several layers, and the cell-wall is encrusted with lime.

The asexual organs of reproduction are gonidia: since four are usually formed in a mother-cell, they are termed Tetragonidia, but sometimes only one, or two, or eight are formed. They do not occur in the Nemaliex. When the thallus consists of rows of cells, the tetragonidia are produced in the apical cell of lateral branches; in the rest (with the exception of the Phyllophoracex, according to Nägeli) they lie imbedded in the tissue of the thallome, often in branches of peculiar shape, in great numbers.

The sexual organs, antheridia and carpogonia, are produced on other plants of the same species; the sexual plants are frequently diœcious.

Sitzungsb. der k. bayer. Akad. der Wissen.-Bornet and Thuret, Ann. des Sci. Nat. $5^{\text {th }}$ series, vol. VII. I 867.-Solms-Laubach, Bot. Zeitg. nos. 21, 22, 1867. [See also Agardh, Florideernes Morphologie, 1880; Kny, Ueb. Axillarknospen bei Florideen, 1873; Thuret, Études phycologiques, 1878; Bornet et Thuret, Notes Algologiques, 1876-80; Janczewski, Le développement des cystocarpes dans les Floridées, Cherbourg, 1876 ; Berthold. Zur Kennt. d. Bangiaceen (Mitth. d. Zool. Stat. z. Neapel, I880); Solms-Laubach, Corallineen (Fauna u. Flora d. Golfes von Neapel, I 881).]

1 [Also Lemaneaceæ, Sirodot, Ann. des Sci. Nat. 5th ser. I872, vol. XVI, and Bangia.]

2 Rosanoff extracted the red colouring matter by cold water, and examined it accurately. In transmitted light it is carmine-red, in reflected reddish-yellow ; the grains of chlorophyll also show this fluorescence, and when the red colouring matter (the phycoërythrine) has'escaped from them in consequence of injury to the cells they are green; the whole plant also remains green when the red colouring matter has been extracted by water or destroyed by heat. (Rosanoff in Compt. Rend. April 9, 1866.) Besides the chlorophyll-granules coloured red by phycoërythrine, Cohn found in Bornetia colourless crystalloids of an albuminous substance which are coloured a beautiful red by the colouring matter that escapes from the chlorophyll-granules when the cells are injured or killed. (Schultze's Arch. für mikr. Anat. III p. 24.) Cramer had previously observed crystalloids of this kind in Bornetia which had been preserved in a solution of sodium chloride, and had accurately described them; according to him they are partly hexagonal, partly octahedral (Rhodospermin). (Vierteljahrschr. der naturf. Ges. in Zürich, vol. VII.) Julius Klein (Flora, no. 1I, I87I) found colourless crystalloids in Griffithsia barbata and neapolitana, Gongoceras pellucidum, and Callithamnion seminudum; and states that the red crystalloids which are also found outside the cell-cavity only appear after treatment with sodium chloride, alcohol, or glycerine, since their colourless matrix takes up the diffusible red colouring matter of the Floridex. On Phycoërythrine see Askenasy, Bot. Zeitg. no. 30, 1867. - [Sorby, Monthly Mic. Journ. vol. VI. 1871, p. I 24. Van Tieghem has detected starch in the Florideæ, Compt. Rend. I 865.] 
The Antheridia are either single cells at the end of long articulated branches, when, as in Batrachospermum, each produces only one antherozoid, or the mother-cells of the antherozoids are congregated together in large numbers on a common axis as the terminal members of a very short branching-system (as in Ceramiacex). In Nitophyllum they densely cover certain portions of the surface of the thallus which consists of a single layer of cells; in the Melobesiaceæ they are produced in cavities which are formed by the overarching of the surrounding tissue. The roundish antherozoids have no cilia and do not swarm, but are moved along passively by the water; some of them are thus brought into contact with the trichogyne; they adhere to it, and, in consequence of the absorption of the cell-walls at the points of contact, their contents pass into it. The trichogyne remains otherwise permanently closed.

According to the structure of the Carpogonium, three types may be distinguished:

(r) In the Nemaliex, to which Batrachospermum belongs ${ }^{1}$, the entire female organ consists, as in the Coleochætex, of a single cell, which is prolonged upwards into a
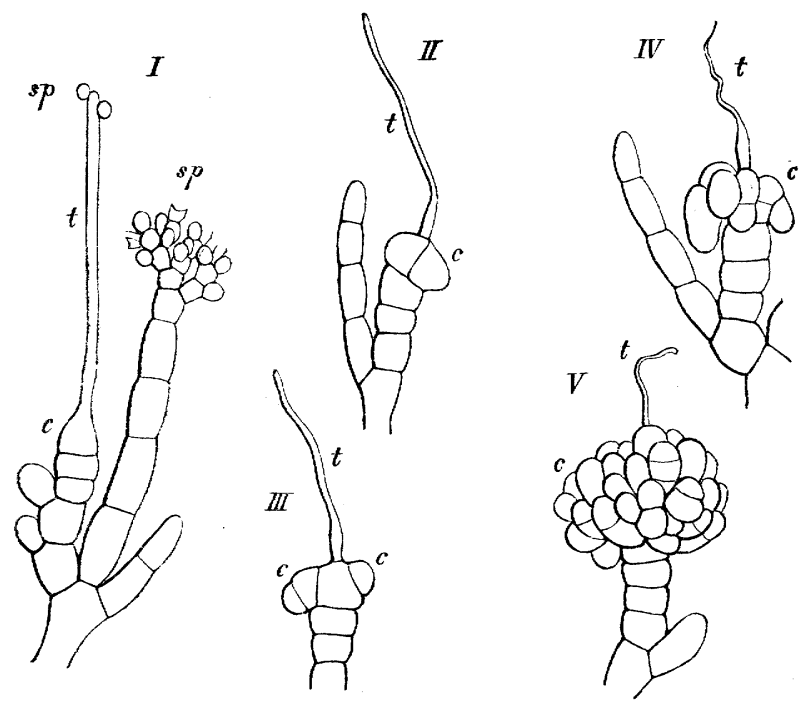

FIG. r88.-Nezualion multifidum; $I$ a branch with carpogonium $c$ and antherozoicis $s p ; I I$, $I I I$ commencement of the formation of the sporocarp; $I V, V$ development of the cluster of carpospores; $t$ the trichogyne; $c$ the carpogonium or sporocarp (after Thuret and Bornet).

trichogyne (Fig. $188, I, t)$. After fertilisation the basal portion of the carpogonium becomes multicellular in consequence of divisions having taken place (Fig. 188, II, c). The cells thus formed bulge outwards and give rise to a dense aggregation of short branches $(I V, V, c)$, the terminal segments of which are the carpospores. This simple sporocarp acquires in Batrachospermum a loose investment by the outgrowth of prolongations from the cells beneath the carpogonium.

(2) In the Ceramiex, Spermothamniex, Wrangeliex, \&c., the carpogonium is a multicellular structure before fertilisation, which has arisen from the terminal cell of a short branch. A lateral row of the cells bears the trichogyne, and is termed the trichophore (Fig. 189, $A, f$ ). This structure undergoes no further developement after the carpogonium has been fertilised. Certain other cells, however, lying in the neighbour-

1 As to Lemanea, which probably belongs to this group, see Sirodot, Ann. des Sci. Nat. $5^{\circ}$ série, vol. XVI. 1872. [Sirodot (Compt. Rend., I873 and, 1880) has found that the spores of Batrachospermum produce a Chantransia from which again the Batrachospermum is developed.] 
hood of the trichophore are stimulated by the fertilisation to fresh growth, and develope numerous closely-packed spores, each upon a short stalk (Fig. I89, B, g, b). In Lejolisia
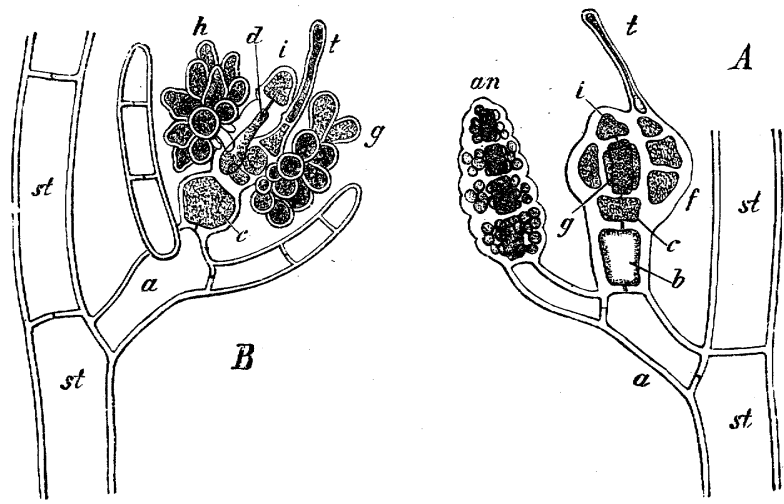

FIG. 189.-Herpothamnion hermaphroditum. $A$ a branch with a carpogonium $f$ and an antheridium $a n$. $B$ the mature cystocarp after fertilisation (after Nägeli).

(Fig. 190) the carpogonium is also multicellular, and it is from its central cell that the spores are developed, whilst the outer cells grow out into filaments forming a closed

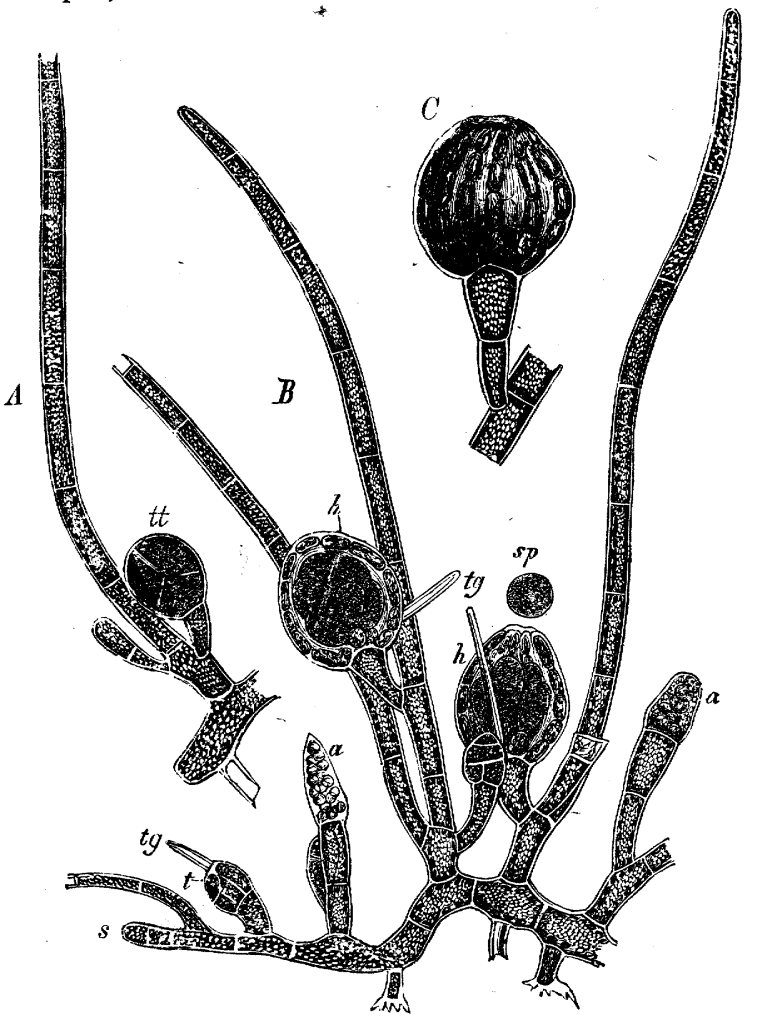

FIG. 190-Lejolisia mediterranea (after Bornet, $\mathrm{x}$ about I50). $A$ a small piece of a creeping filament with a root hair and n (he axial cell sithate row of cells being however not indicated; tg trichngyne by the side of the apex $t$ of the fertile branch; $h$ the envelope
of the cystocarp; sp a spore escaped from the cystocarp; $C$ an empty cystocarp, its envelope consisting of rows of cells. 
investment, which subsequently opens at the apex. The trichogyne and the trichophore can be observed lying externally to it (Fig. I $90, t g$ ). It is evident from these examples that neither the trichogyne nor even the cells of the trichophore undergo any further development as a consequence of fertilisation, but that it is in cells adjacent to them that the consequences of fertilisation are manifested, in their growth, branching, division, and final formation of spores. The formation of an investment is also a consequence of fertilisation. The fruits of Floridex are usually termed Cystocarps.

(3) The most complicated and most extraordinary process of fertilisation was found to occur by Thuret and Bornet in the genus Dudresnaya. Here the cystocarps are formed upon branches other than those which bear the trichophore. After that the long trichogyne, which is coiled at its base, has been fertilised, tubular branches spring from beneath it, which grow towards the true fertile carpogonial branches. Each of these latter has a spherical apical cell to which the outgrowth from the trichophore applies itself, and at the point of contact the cell-walls become absorbed. The apical cell of the carpogonial branch which has thus been fertilised becomes distended and filled with protoplasm; it becomes isolated by the formation of cell-walls, and then gives rise to the cystocarp. These tubular outgrowths convey the fertilising effect from a single trichogyne to numerous carpogonial branches, and thus one act of fertilisation suffices for the developement of several cystocarps on different branches ${ }^{1}$.

\section{The Charace ${ }^{2}$.}

The carpogonium consists of one relatively large cell and several smaller ones. The latter are known as 'Wendungszellen,' and probably represent a very rudimentary trichophore, the trichogyne of which is undeveloped. Fertilisation is effected by means of filiform antherozoids which are formed in very remarkable antheridia. The carpogonium is invested before fertilisation by five spirally-wound cells which arise from its stalk-cell. As a consequence of fertilisation the large cell of the carpogonium becomes a resting spore, producing, by its germination, a pro-embryo from which the sexual plant springs as a lateral shoot. No gonidia are formed.

The Characeæ are submerged aquatic plants, rooting in the ground and growing erect, attaining a height of from $\frac{1}{10}$ metre to a metre, and containing abundance of chlorophyll. They are very slender, forming stems and leaves only $\frac{1}{2}$ to $2 \mathrm{~mm}$. in thickness. With an alga-like habit, they possess a delicate structure, though sometimes attaining greater firmness from the deposition of lime on their surface. They live gregariously, mostly in crowded tufts, at the bottom of fresh-water ponds, ditches, and streams; they may grow in deep or in shallow, in stagnant or in quickly-flowing water; and are either annual or perennial.

In the greater number of species, which are distributed over all quarters of the globe, there prevails nevertheless so great a uniformity that they may all be arranged into two genera.

1 This mode of fertilisation has been detected by Thuret and Bornet in Polyides rotundus, also by Berthold in Halymenia Floresia and uivoidea, Nemastoma dichotoma and cervicornis, Grateloupia Consentinii, filicina, and dichotoma, and by Schmitz in the Squamariex: see Falkenberg, Die Algen, I88 r.]

${ }^{2}$ A. Braun, Ueber die Richtungsverhältnisse der Saftströme in den Zellen der Charen, in Monatsberichte dèr Berliner Akad. der Wiss. I $85_{2}^{2}$ and I 853.-Pringsheim, Ueber die nacktfüssigen Vorkeime der Charen, in Jahrb. f. wissen. Bot. 1864, vol. III.-Nägeli Die Rotationsströmung der Charen, in his Beitrảgen zur wissen. Bot I860, vol. II. p. 61.-Thuret, Sur les anthéridies des cryptogames, Ann. des Sci. Nat. I $8_{5}$ I, vol. XVI. p. I9.-Montagne, Multiplication des charagnes par division, ditto, I852, vol. XVIII. p. 65.-Göppert u. Cohn, Ueber die Rotation in Nitella flexilis, Bot. Zeitg. I 849.-De Bary, Ueber die Befruchtung der Charen, Monatsber. der Berliner Akad. May r87r. [For additional Bibliography, see Lindley, Vegetable Kingdom, 3rd edit. p. 28: also Journal of Botany, 1878 .] 
From the carpospore of the fruit of $\mathrm{Cbara}^{1}$ the sexual leaf-forming plant is not immediately developed, but a Pro-embryo precedes it, which attains only small dimensions and consists of a single row of cells with limited apical growth. The stem of the Leaf-bearing Sexual Plant springs from a cell which lies at some distance from the apex of the pro-embryo and grows in a direction nearly at right angles to that of its axis. The unlimited apical growth of the plant depends on an apical cell (Fig. 192, $C, t$ ) from which segments are cut off by transverse septa. Each segment immediately divides again by a transverse septum into two superposed cells, the lower one of which $(g)$ always grows without further division into a long internode (frequently 5 to $6 \mathrm{~cm}$. in length); the upper one scarcely lengthens, but is first divided in half by a vertical wall, and each half then divides by further successive septa so as to form a whorl of peripheral cells $(b)$. From the node thus constituted the leaves are developed, each from a peripheral cell, and the normal lateral branches, which always originate from the axil of the first or of the two first leaves of the whorl. The leaves of such a whorl, from 4 to ro in number, repeat in a modified manner the development of the stem, but their apical growth is limited: after the formation of a definite number of segments, the apical cell ceases to divide and grows into the terminal cell of the leaf which is usually pointed (Fig. $\left.192, A, b^{\prime \prime}\right)$. From these leaves lateral leaflets may arise in a similar manner to that in which the leaves themselves have been formed from the stem; and the leaflets may again in turn produce others of a higher order. The successive whorls of a stem alternate, and in such a manner that the oldest leaves of the whorl, in the axils of which the branches stand, are arranged on a spiral line winding round the stem. Each internode also usually undergoes a subsequent torsion in the same direction. The lateral branches, of which in Chara one is always developed in the axil of the oldest, in Nitella one in the axil of each of the two oldest leaves of the whorl, repeat the primary stem in all respects (Fig. 203). It has already been mentioned that the leaves undergo a segmentation similar to that of the stem; they also consist at first of very short internodes which are afterwards greatly elongated (Fig. 192, $B, \gamma$ ), and are separated by inconspicuous transverse plates or nodes. From these the leaflets arise in whorls the members of which are formed in succession, but they are directly superposed one above another, and do not alternate

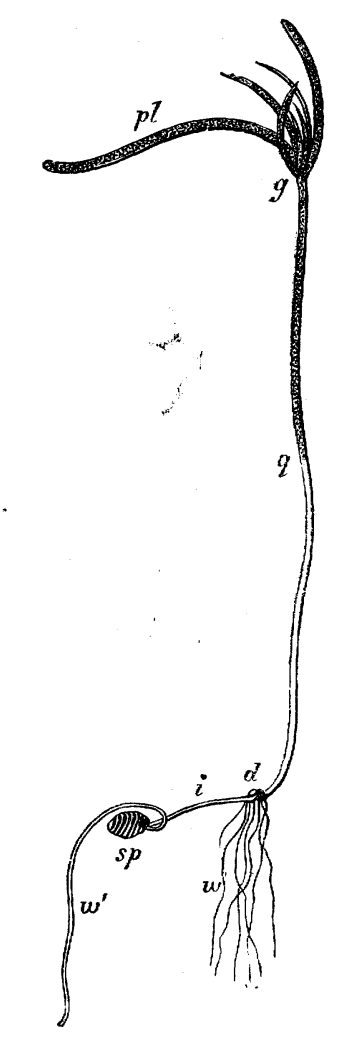

FIG. Igr.-Chara fragilis; sp germinating spore; id $q \not p l$ together form the proembryo ( $p l$ is segmented, which is not clearly indicated in the drawing); at $d$ are the rhizoids $w ; w^{\prime}$ the so-called primary root; $g$ the first leaves (not a whorl) of the second generation or leaf-bearing plant (after Pringsheim, $x$ about 4). like the whorls of primary leaves (Fig. 193, $C-E, \beta$ ). Each leaf begins with a node (the basal node), by which it is united with the stem-node, and so is each leaflet with its primary leaf. These basal nodes are the points of origin of the formation of the cortex which, in the genus Cbara, covers the internodes of the stem, but

1 This has not yet been observed in Nitella. [See De Bary, Zur Keimungsgeschichte der Charen, Bot. Zeitg. 1875. The carpospore is first divided by a wall at right angles to its long axis into a small upper and a large lower cell. The upper cell is then divided by a wall at right angles to the first into two equal cells : from one of these the pro-embryo is developed, from the other the 'primary root.'] 
is wanting in Nitella. From the basal node of each leaf one distinct cortical lobe runs downwards, and one upwards ${ }^{1}$ (Fig. 192, $r, r^{\prime}, r^{\prime \prime}$, and Fig. 194). In the middle of each internode therefore as many descending cortical lobes as there are

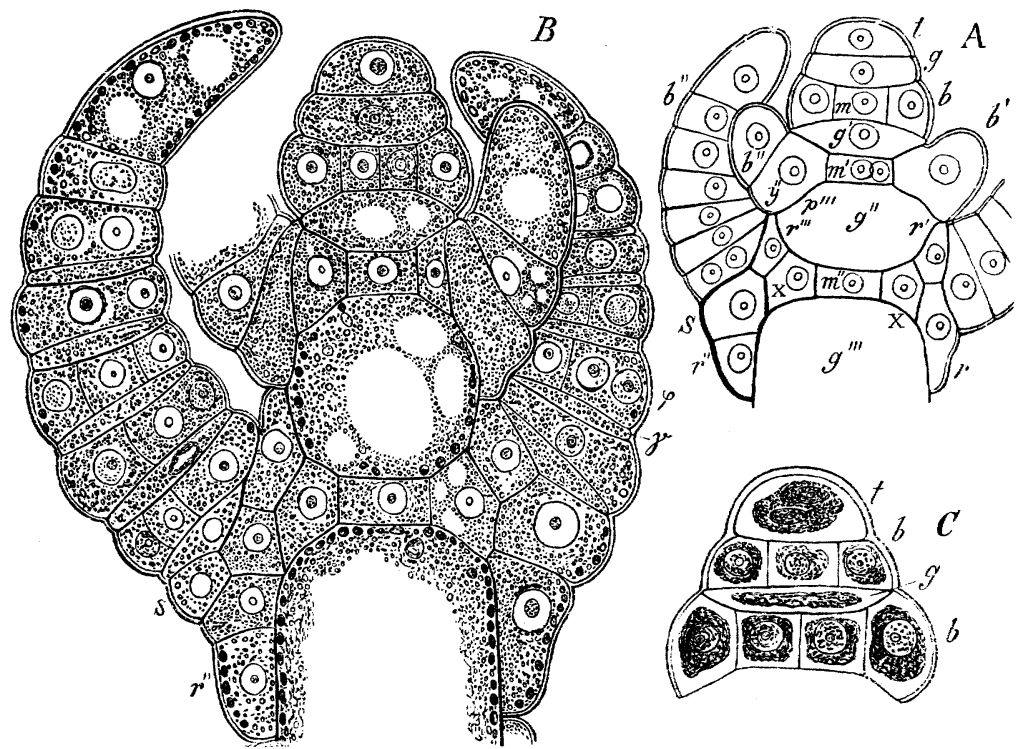

FIG Ig2 - Longitudinal section through a bud of Chara fragilis; in $A$ the contents of the cells have been left out; in $B$ the fine-grained substance is protoplasm, the larger granules are chlorophyll; the formation of vacuoles is shown; in $C$ the contents of the cells have been contracted by iodine solution ( $X 500)$.

leaves in the whorl meet with the cortical lobes that ascend from the whorl next below. The number of the latter is, however, smaller, because the leaf in the axil
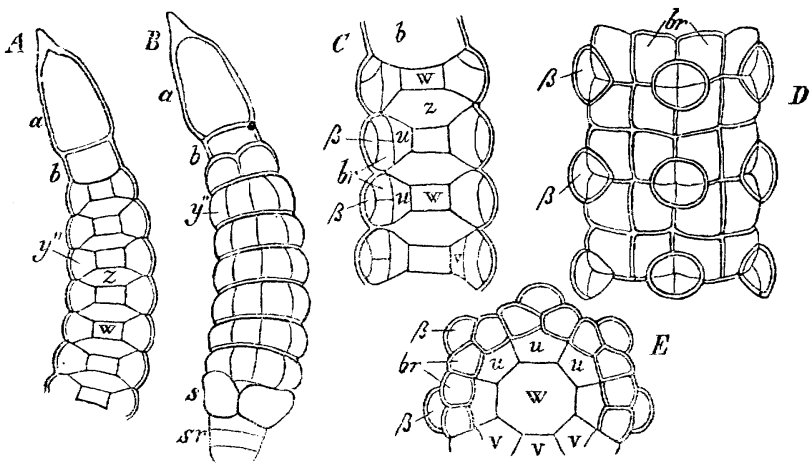

FIG. I93-Leaves of Chara fragilis; $a$ terminal cell, $b$ penultimate cell of a leaf; $z$ internodal cell ; $w$ cells of the leafnode; $y^{\prime \prime}$ mother-cell of a leaflet and of its basal node: from it arise $v$ and $u$ (the uniting cell), $b r$ the basal node which produces four simple cortical lobes and $\beta$ the leaflet. $A$ and $C$ in longitudinal section, $B$ an entire young leaf, external view, with the 'stipule' $s$ and its descending cortical lobe $s r ; D$ external view of the middle part of an older leaf, though still young; $E$ transverse section of a leaf-node, of the same age as $D$.

of which the lateral branch arises does not form an ascending lobe. The cortical lobes are in close contact laterally, and form a closed envelope round the inter-

1 The first internode of every branch and leaf becomes covered with a cortex derived only from the descending cortical lobes of the next node above. 
node, the ascending and descending lobes dove-tailing in a prosenchymatous manner. The formation of the cortex takes place so early that the elongating internode is covered by it from the first, the lobes keeping pace with its extension in length and thickness. Each lobe continues to grow, like the stem, by means of an apical cell, which becomes segmented by transverse septa; out of each of the segments cortical internodal and nodal cells are formed by repeated divisions. The latter divide, by successive septa, into an inner cell (Fig. 194, D,c), in contact with the internode of the stem, and three outer cells, the middle one $(f)$ of which commonly grows into the formr of a spine or knob, resembling a leaf. The outer lateral cells $(n n)$ of the cortical node, on the other hand, following the elongation of the internode itself, grow into longer tubes, so that each cortical lobe consists of three parallel rows of cells, the middle row however containing alternately short and long (internodal and nodal) cells. The cortex of the leaves is derived from the leaflets, and its formation is much simpler (Fig. 193, $C-E, b r$ ). From the basal nodes of Cbara other foliar structures also arise, both on the inner and outer side of the base of the leaf (Fig. I92, S), which Braun calls Stipules; they are always unicellular, and are sometimes very short, sometimes elongated.

The nodes are the parts from which all the lateral members of the Characex originate. The root-like structures or Rbizoids spring from the outer cells of the
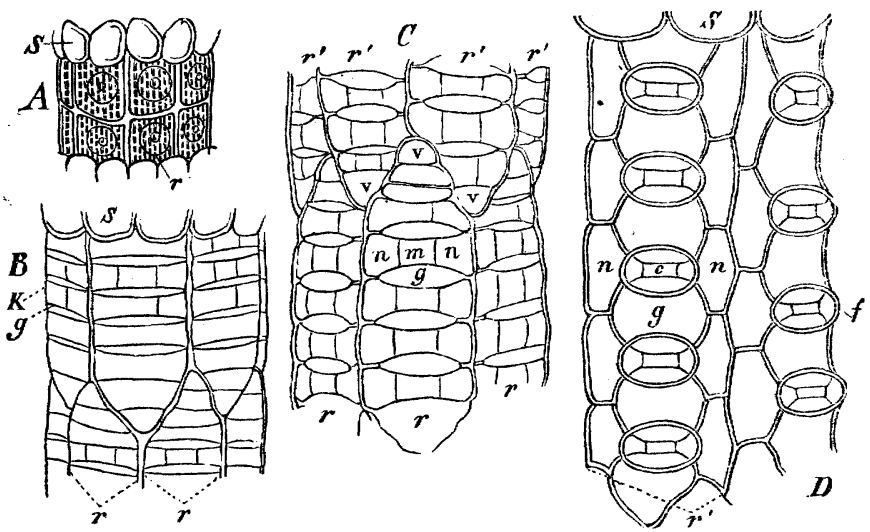

FIG. I94.-Development of the cortex of the stem of Chara fragilis; $A$ a very young internode of the stem with the cortical lobes $r$ still consisting of one cell ; $B-D$ its further development; $r r$ signifies in all the figures the cortical lobes that ascend from the lower, $r^{\prime} r^{\prime}$ those that descend from the upper leaves; $v v$ the apical cell of each cortical lobe; $g g$ its internodal cells; $n m n$ the commencement of the formation of the node; $D c$ the central cell of a cortical node; $S$ signifies in all the figures the unicellular 'stipules' which spring in pairs from the base of the leaves.

lower nodes of the primary shoot, and consist of long hyaline tubes growing obliquely downwards, and elongating only at their apex. They are formed by the outgrowth of flat cells at the circumference of the node, and are therefore attached to it by a broad base; but the bases of the stouter rhizoids themselves divide still further, giving rise, especially at the upper margin, to small flat cells from which slender rhizoids are developed. The rhizoids are segmented by only a few septa which lie far behind the growing apex, and have at first an oblique position. The two adjoining cells abut upon one another like two human feet placed sole to sole. The branching always proceeds only from the lower end of the upper cell (Fig. 195, B); a swelling is here formed which becomes cut off by a wall, and by further division produces several cells which grow into branches; these therefore stand on one side like a tuft. The tubular cells composing the rhizoids attain a length of from several millimetres to more than two centimetres, with a thickness of from $\frac{1}{40}$ to $\frac{1}{10} \mathrm{~mm}$.

The Vegeiative Reproduction of Characeæ always proceeds at the nodes, and has three modifications:-(I) Tuberous formations called Bulbils (starch-stars) which occur in Chara stelligera. They are isolated underground nodes with greatly abbreviated 
whorls of leaves of beautiful regularity; their cells are densely filled with starch and other formative materials; new plants are produced from shoots laterally developed. (2) The Branches ruith naked base of Pringsheim. These are formed on old nodes which have survived the winter or on cut nodes of Chara in the axils not only of the oldest but also of the younger leaves of a whorl, and are in fact only slightly different from the normal branches, the greatest difference being in the partial or entire absence of a cortex on the lower internode and on the first whorl of leaves. The cortical lobes which descend from the first node of the branch

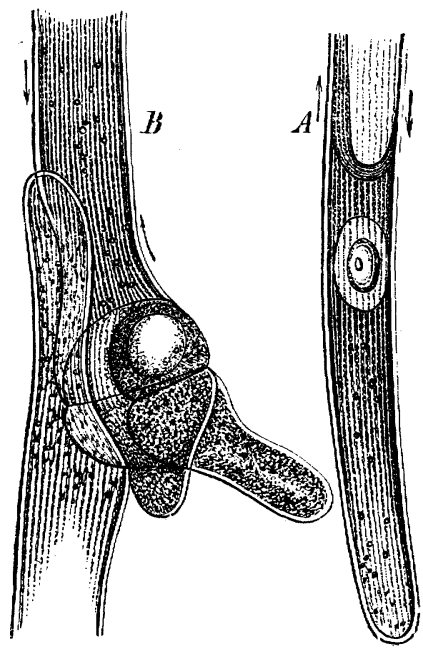

FIG. 195.-Rhizoids of Chara fragitis ; $A$ end in process of development ; $B$ a 'joint,' the lower part of the upper cell is branching (after Pring. sheim, $x$ 240). The arrows indicate the direction of the currents of protoplasm.

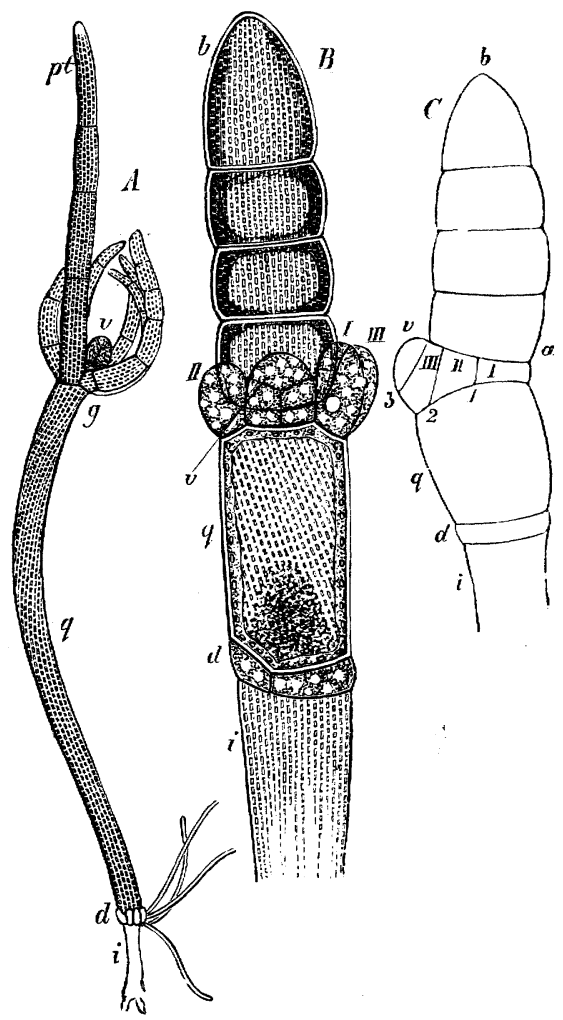

FIG. 196.-Charafragilis, $A$ an entire pro-embryonic branch; $i$ the lowermost colourless cell below the root-node; $d$ root-producing leafless node; $q$ the long cell proceeding from the middle cell of the bud-rudiment; $p t$ apex of the pro-embryo; $g$ the pseudo-whor of leaves, $v$ the bud of the leaf-bearing plant; $B$ upper part of a young pro-embryonic branch; $i, d, q$ as before, $b$ apex of the pro-embryo; $I, I I, I I I$ the young leaflets of the transitional node, $v$ the bud of the leafy stem ; $C$ still younger pro-embryonic branch $i, d, q, b$ as before; $I, I I, I I I$ the cells out of which the transitional nodes arise, $v$ apical cell of the stem-bud (after Pringsheim, $B \times$ izo).

often become detached from the internode and grow free, curling upwards, while the leaves of the lowermost whorl often do not form nodes. (3) The Pro-embryonic Branches. These spring, together with the last, from the nodes of the stem, but are essentially different from the branches, and have a similar structure to the proembryos which proceed from the spores. Like the last, they have only been observed in Chara fragilis (by Pringsheim). A cell of the node protrudes and grows into a tube, and its apex becomes separated by a septum. In this growing terminal cell 
further divisions take place, till the 'apex of the pro-embryo' which proceeds from it consists of a row of from three to six cells. Beneath the apex of the pro-embryo (Fig. 196, $C, a, b$ ) the tube swells, and the distended part becomes separated by a septum as a cell, which Pringsheim calls the 'bud-rudiment,' (Fig. 196, C, including the parts from $v$ to $d$ ). This cell is now divided by two oblique walls into three cells, the middle one of which $(q)$ lengthens into a tube (like an internode), while the upper and lower ones remain short. Out of the lower cell is afterwards formed a root-producing leafless node (Fig. $196, d$, and Fig. 191, $d$ ), while the upper one, which lies between the apex of the pro-embryo $a b$ and the elongated cell $q$ becomes the axis of the new generation. It becomes arched on one side outwards, and divides in succession into the cells $I, I I, I I I$, and $\tau$. Each of the cells $I, I I$, and $I I I$ becomes transformed by divisions into a disc of cells or transitional node, three of which thus stand over one another without intermediate internodes. Their lateral cells grow right and left, and form imperfect leaves of different lengths. The cell which lies outermost (Fig. I $96, C, v)$ now begins to undergo a series of divisions, corresponding to those of a normal leaf-bearing shoot. It is, in fact, the apical cell of the sexual leaf-bearing plant which arises from the proembryo. The displacement indicated in Fig. 196, $C$, subsequently causes the apex of the pro-embryo to be pushed to one side; and since this apex has the appearance of a simple leaf uncovered by cortex, the further development of the lateral leaves which spring from the cells $I, I I$, and $I I I$, brings about an appearance as if these different leaves together formed a whorl; and the bud of the lateral shoot thus comes to stand apparently in the centre of this pseudo-whorl (Fig. I96,

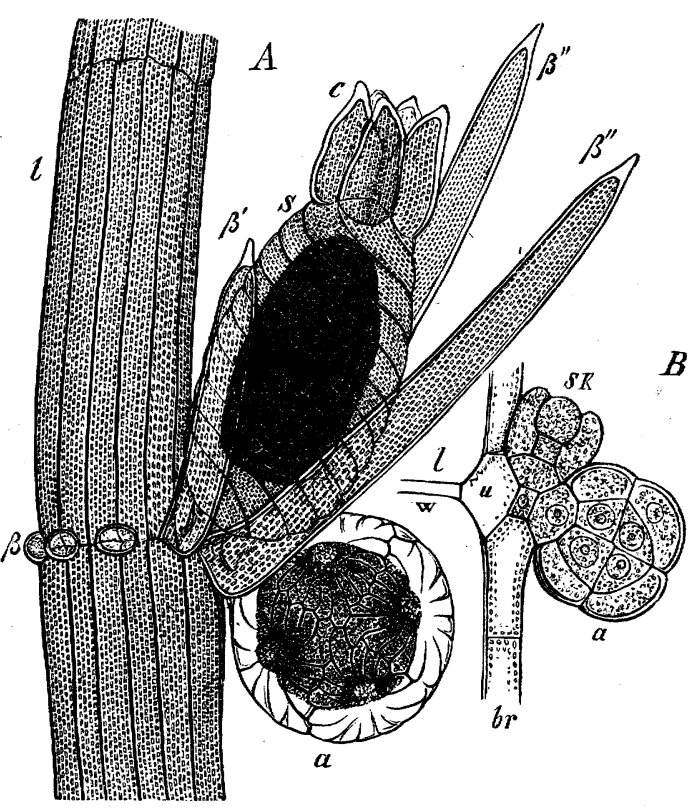

FIG. 197,-Chara fragilis. $A$ middle part of a leaf $b$ with an antheridium $\alpha$ and a carpogonium $S, c$ its crown; $\beta$ sterile lateral leaflet; $\beta^{\prime}$ large leaflets by the side of the carpogonium; $\beta^{\prime \prime}$ the bracteoles, spinging from the basal node of the carpogonium ( $\mathrm{X}$ about 50 ). $B$ a young antheridium $\alpha$ with a still younger carpogonium $S K ; v$ the nodal cell of the leaf, $u$ the uniting-cell between it and the basal node of the antheridium: $l$ cavity of the internode of the leaf; br cortical cells of the leaf $\left(x_{350}\right)(c f$. Fig. 203). A). If the structure which springs from the germinating spore is now compared with the pro-embryonic branch, the perfect homology cannot fail to be observed which Pringsheim pointed out in the parts that will be found indicated by the same letters in Figs. 191 and 196; but the pro-embryo of the spore has in addition a small node at the opening of the spore from which a rhizoid, sometimes called the primary root of Cbara, springs (Fig. 19I, $w^{\prime}$ ).

The Antheridia and Carpogonia are always borne by the leaves. An antheridium is in all cases the metamorphosed terminal segment of a leaf or of a leaflet, and the carpogonium, in the monœcious species, arises close beside the antheridium from the basal node of the same leaflet (Cbara) or from the last node of the leaf bearing a terminal antheridium (Nitella); hence in the monœcious Nitellæ the carpogonia are 
placed below, in the Charæ above or by the side of the antheridium. In the diœcious species of course no such relation of position can exist, but the morphological significance of these organs and the place of their origin are the same. We will now consider the structure of these organs when they are fully developed.

The Antheridia (Globules) are globular bodies $\frac{1}{2}$ to $1 \mathrm{~mm}$. in diameter, at first green, then red. The wall consists of eight flat cells, four of which, situated around the distal pole of the ball, are triangular, while the four situated around the base are quadrangular and become narrower below; each of these cells forms a segment of the shell of the ball, and are called Sbields. When unripe their inner cell-wall is covered with green chlorophyll-granules, which, in the ripe state, are of a red colour. Since
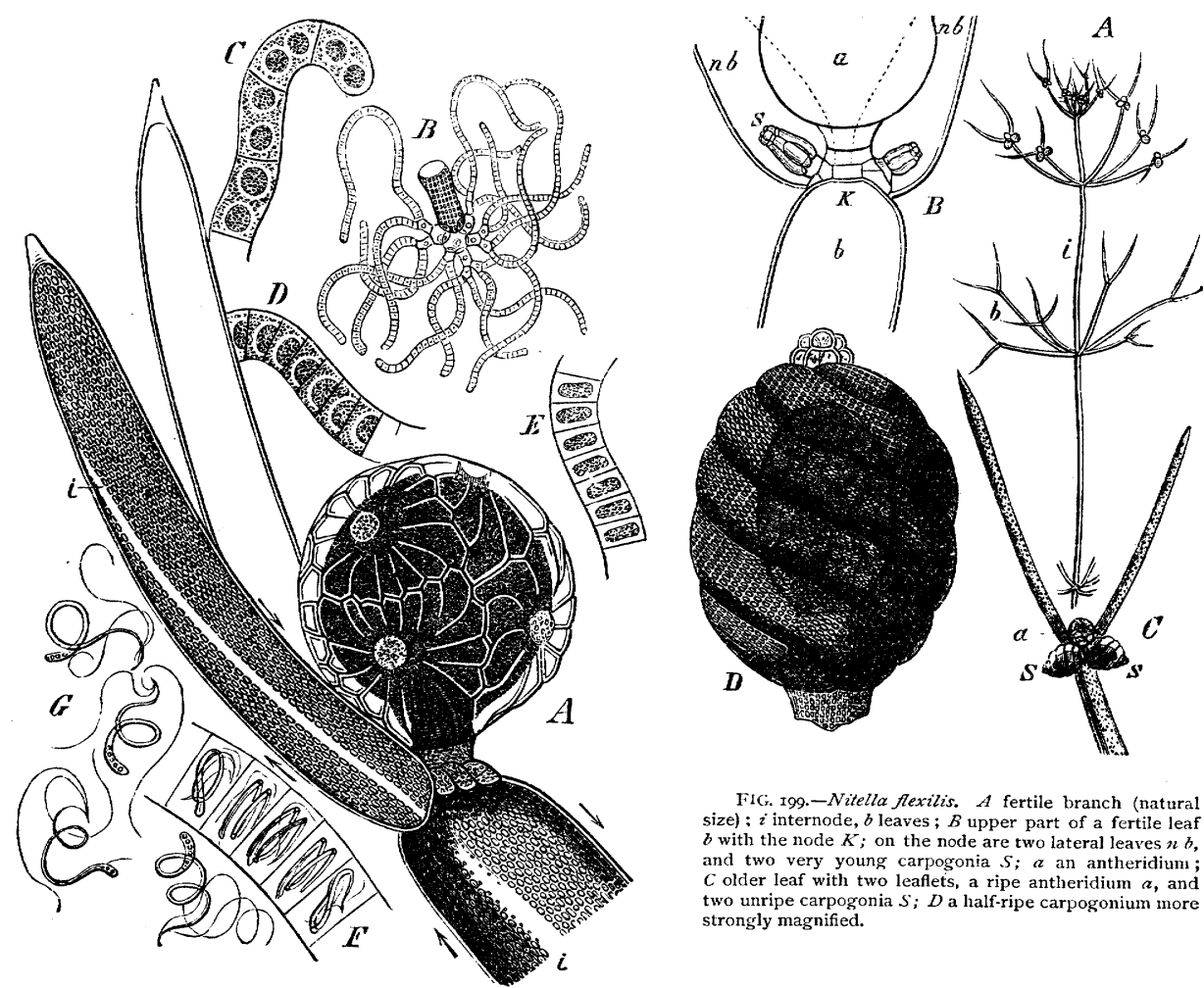

FIG. 199. - Nitella flexilis. $A$ fertile branch (natural size) : $i$ internode, $b$ leaves; $B$ upper part of a fertile leaf $b$ with the node $K$; on the node are two lateral leaves $n b$, and two very young carpogonia $S ; a$ an antheridium; $C$ older leaf with two leaflets, a ripe antheridium $a$, and two unripe carpogonia $S ; D$ a half-ripe carpogonium more strongly magnified.

FIG. ig8.- Nitella fexilis. A an almost ripe antheridium at the end of the primary leaf, by its side two lateral leallets, $i$ neutral lines; the arrows indicate the direction of the currents of protoplasm; $B$ a manubrium with its capitulum, secondary capitula, and the whip-shaped filaments, in which the antherozoids arise; $C$ end of one of the young filaments; $D$ middle part of an older one; $E$ of one still older: $F$ ripe antheridial filament with antherozoids $G(C-G \times 55$ ).

the outer wall is destitute of these granules, the outside of the ball appears clear and transparent (Fig. 197, $A$ ). From the lateral walls several folds of the cell-wall penetrate towards the middle of each shield, which gives them the appearance of being lobed in a radiate manner. From the middle of the inner face of each shield a cylindrical cell projects inwards, nearly to the centre of the hollow globule; this cell is called the Manubrium; at the central end of each of the eight manubria is a roundish hyaline cell, the Capitulum. The flask-shaped cell which supports the antheridium also penetrates into the interior between the four lower shields; and these twentyfive cells form the framework of the antheridium. Each capitulum bears usually six smaller cells (secontary capitula), and from each of these grow four long slender 
whip-shaped filaments, which, being coiled round and round, fill up the interior of the globule (Fig. 198, B). Each of these filaments (the number of which amounts to about 200) consists of a row of small disc-shaped cells (Fig. 198, D, E, F), numbering from roo to 200 . In each of these 20,000 to 40,000 cells is formed an antherozoid, a slender spiral thread, thickened behind, and bearing at its pointed end two long fine cilia (Fig. 198, G). When perfectly ripe, the eight shields fall apart, their spherical curvature becoming diminished; the antherozoids leave their mother-cells and move about in the water. This breaking up appears generally to happen in the morning, and the antherozoids are in motion for some hours, till evening.

The mature Carpogonium (Nucule), when ready for fertilisation, is a longer or shorter prolate spheroid; it is placed upon a short pedicel, visible externally only in Nitella, and consists of an axial row of cells, closely surrounded by five tubular cells which are coiled round it spirally. The whole must be considered as a metamorphosed branch. The lowest cell corresponds to the lower internode of a branch; it bears a short central nodal cell, around which the five enveloping tubes spring like a whorl of leaves. Above the nodal cell rises the peculiarly developed apical cell of the branch, very large as compared to the other parts, and ovoid. At its base, immediately above the nodal cell, an inconspicuous hyaline cell is separated at an early stage in Cbara; in Nitella a somewhat disc-shaped group of similar cells takes its place, which have been termed by Braun 'Wendungszellen,' and which I consider as forming a very rudimentary trichophore. The large apical cell of the carpogonium is filled with a number of drops of oil and grains of starch as well as with protoplasm; it contains pure hyaline protoplasm only in its apical region (the apical papilla). The enveloping tubes, which contain a quantity of chlorophyll, project above the apical papilla and bear the Crown, consisting in Cbara of five larger, in Nitella of five pairs of smaller cells, which have already been separated at an early stage from the enveloping tubes by septa. Above the apical papilla and beneath the crown, which forms a compact lid, the five enveloping tubes form the neck which encloses a narrow cavity, the apical cavity; above the papilla this cavity is of an obconical figure narrowing upwards, the five segments of the neck projecting and forming a kind of diaphragm, through the central very narrow opening of which the union with the upper roomy part of the apical cavity is effected. This is closed above by the crown; but, at the time of fertilisation, it opens externally by five clefts between the coronal cells; and through these clefts the antherozoids penetrate into the apical space filled with hyaline mucilage, to find their way into the apical papilla of the oosphere, where the cell-wall is apparently absent. After fertilisation the chlorophyll-granules of the envelope become reddish-yellow, the wall of the tubes which lie next the oosphere increases in thickness, becomes lignified, and assumes a black colour; and thus the oosphere, now transformed into a carpospore, becomes surrounded by a hard black shell with which it falls off, to germinate in the next autumn or spring.

With regard to the various processes of development, I will here describe only those of the antheridia and carpogonia.

Antheridia. The order of development of the cells has already been exhaustively described by A. Braun in the case of Nitella syncarpa and Chara Baueri; it agrees with that of Nitella flexilis and Chara fragilis. In Nitella the terminal cell of the leaf becomes the antheridium; the oldest leaf of a whorl first forms its antheridium, the others follow according to their age; the antheridia are recognisable even in the earliest state of the whorl of leaves. In Fig. $200, A$, is shown a longitudinal section through the apex of a branch, $t$ being its apical cell; its last-formed segment has already been divided by a septum into a nodal mother-cell $K$ and an internodal cell lying beneath it; beneath this lies the node with the last whorl of leaves; $b$ is its youngest leaf, $b K$ the basal node of the oldest leaf which already consists of the segments $I, I I, I I I ; a$ is the terminal cell of this leaf which is becoming transformed into the antheridium. While the antheridium is becoming developed, the leaf also undergoes still further changes which must be first 
considered. The segment $I I I$ becomes the first internode of the leaf, $I I$ becomes a node from which are developed the lateral leaflets $n b$ in $G$ and $D$. The cell $I$ divides into two

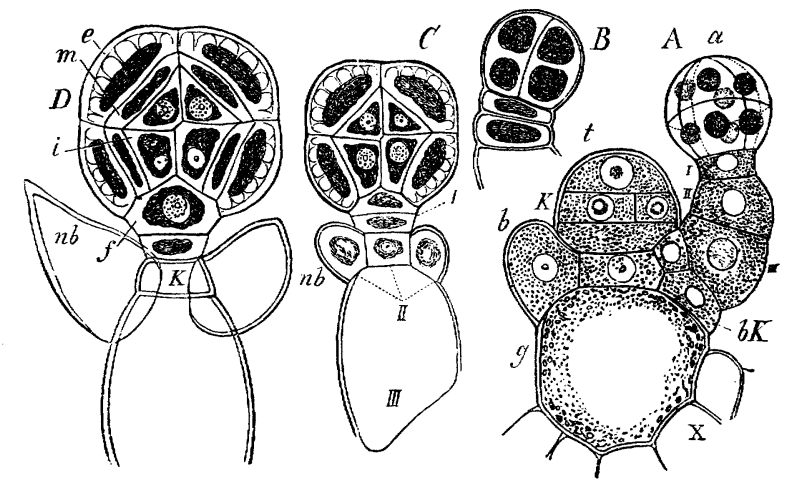

FIG. 200.-Development of the antheridia of Nitella flexilis. In $B, C$, and $D$ the protoplasm has been contracted by giycerine.

$(C, I)$, the lower of which remains short, while the upper grows into a flask-shaped cell (Fig. 200, $D, f$, and Fig. 20I, $f$ ).

The globular mother-cell of the antheridium (Fig. 200, $A, a$ ) first of all divides into two hemispheres by a vertical wall passing through the axis of the leaf; each of these is divided into two segments by a vertical wall at right angles to the first; in each of the four quadrants a third division takes place hori-

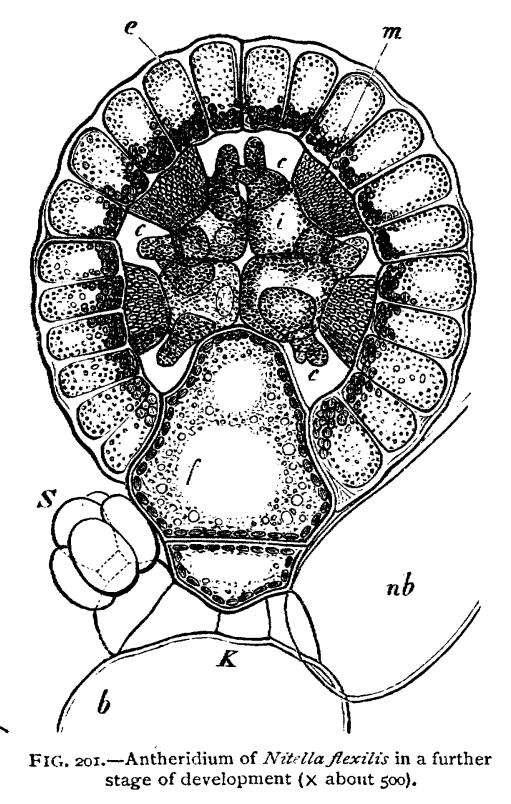
zontally and at right angles to the two last walls; and the antheridium now consists of four lower and four upper octants of a sphere. Contraction by glycerine clearly shows that each of these divisions of the protoplasmic body is completely effected before the appearance of the cellulose-wall (Fig. 200, $B$ ); the second division even takes place before the wall has arisen between the two first-formed halves; and the four quadrants may be made to contract without any wall being visible between them. In Fig. $200, B$, the third division has also taken place, the second vertical wall is already formed, and the two quadrants there visible are already divided; but no horizontal wall has yet appeared. In Fig. 200, $A, a$, are shown the eight octants in perspective together with their nuclei. Each octant now breaks up first of all into an outer and an inner cell (Fig. 200, $C$ ); the latter is again divided in all the eight octants $(D)$, so that each octant now consists of an inner, a middle, and an outer cell $(D, i, m, e)$. Up to this time the globe remains solid, and all the cells lie close to one another; but now commences an unequal growth, and with this the formation of intercellular spaces (Fig. 201). The eight outer cells (e) are the young shields, the side-walls of which show even at an earlier period the radial infolding already mentioned; they grow more strongly in a tangential direction than the inner 
cells, the outside of the globe increasing more rapidly than the inside: the middle cells $(m)$, which form the manubria, remain attached to the centres of the shields, but are separated from one another by the tangential growth of the shields; they grow slowly in the radial direction: the innermost cell $i$ of each octant is rounded off and becomes the capitulum.

The cell $f$ in Fig. 200, $D$, now also grows quickly, and forces itself between the four lower shields into the interior of the globe ; it becomes the flask-shaped cell, upon the apex of which rest the eight capitula. In Fig. 201 this condition of the antheridium is shown in longitudinal section; here the walls of the capitula bound the intercellular spaces which have now been formed and are filled with fluid; they put out branches $(c)$ which become septate, and again ramify; and these branches elongate by apical growth and also become septate. Their basal cells swell up into a roundish shape, and form the secondary capitula, upon which stand the whip-shaped filaments, consisting of the discoid cells which are the mother-cells of the antherozoids. (Compare Fig. 20r with Fig. $198, B$.)

The antheridia of Chara fragilis are produced by metamorphosis of those leaflets which form the innermost row on a leaf, and in fact, as is shown in Fig. 203, the development advances downwards on the primary leaf. The succession of cells and the mode of growth show no noteworthy differences from those of Nitella; the flask-shaped pedicel is here placed on a small cell wedged in between the cortical cells, which is the central cell of the basal node of the leaflet: Braun asserts that this cell is present also in sterile leaves, but I have not succeeded in finding it.

Antherozoids. The whip-shaped filaments in which the antherozoids arise do not grow merely at their apex, but have also an intercalary growth. This is shown by the elongated cells in the middle of young filaments, each with two nuclei, between which no division-wall has yet been formed (Fig. 198, C). The longer the filaments become, the more numerous are their divisions, until at length the individual cells have the appearance of rather narrow transverse discs. The further development of the contents of these mother-cells of the antherozoids progresses backwards from the end of the filament; the antherozoids are formed in basipetal order in each filament. At first the nucleus of each mother-cell lies in its centre, later it places itself in contact with one septum; the nucleus then disappears, and its substance becomes mixed with that of the protoplasm, which now forms a central discoid mass in the mother-cell, surrounded by a hyaline fluid (Fig. 198, E). From this is formed the antherozoid, and, when it is mature, there is no granular protoplasm left over in the cell ${ }^{1}$. The antherozoids begin to rotate even while within their cell, and escape out of it after the rupture of the antheridium; the filiform antherozoid has in Nitella 2 or 3 , in Chara 3 or 4 coils; the posterior thicker end contains a few glistening granules.

The Development of the Carpogonium has already been described in detail by A. Braun; I have also studied it in Nitella fexilis and Chara fragilis. In Nitella fexilis it springs from the node of the leaf beneath the antheridium (Fig. 199, $B$ and $C$ ); its origin is much later than that of the latter. Fig. 202, $A$, represents a very young carpogonium; the lowest cell of the pedicel $(b)$ bears the small nodal cell with the five rudiments of the enveloping tubes $(b)$, (two only are shown here in longitudinal section). Above the nodal cell lies the apical cell $(s)$ of the branch, for such is the nature of the carpogonium. $B$ represents a further stage of development, in which the first of the cells, designated by A. Braun the 'Wendungszellen,' has already made its appearance, and two septa have also appeared in the upper part of each enveloping tube; these upper short cells are raised up by the intercalary growth of the tubes, above the apical cell, and form the crown $K$ in $C$ and $D$. The lowest of the cells of the crown each forms a prolongation projecting inwards and downwards, as shown in Fig. $202 C$ and $D$, so that the whole carpogonium resembles a 'lobster-pot.' The spiral torsion of

1 Compare the opposite view of Schacht, Die Spermatozoiden im Pflanzenreich, 1864, p. 30. [The account given in the text is confirmed by Strasburger, Zellbildung u. Zelltheilung, I880.] 
the enveloping tubes does not begin till a later period; the coils become gradually flatter while the apical cell of the branch increases considerably in size and developes into the oosphere (Fig. 199). The development and fertilisation of the carpogonium of Chara has recently been described in detail by De Bary in the case of C. fotida. Here also it consists, from an early stage of its development, of an axial row of three cells, and five others consisting each of two cells which form an envelope round it. The lowermost cell of the axial row is the nodal cell, the second remains small and colourless, and corresponds to the first 'Wendungszelle' in Nitella. It becomes in this case also, as De Bary's drawings show, separated by a somewhat oblique septum at the base of the apical cell (now the third of the axial row). Originally almost hemispherical, the apical cell grows first of all into the form of a narrow cylinder, and then becomes ovoid; it is provided, until it attains its full size, with a thin very delicate cell-wall. Drops of fat and starch grains accumulate in its protoplasm: its apex however remains free from these, and forms a transparent finely granular terminal papilla, the receptive portion. The protoplasm of the apical cell of the carpogonium has therefore become transformed into an oosphere. The five enveloping tubes are from the first in close contact with the apical cell; after each has become divided by

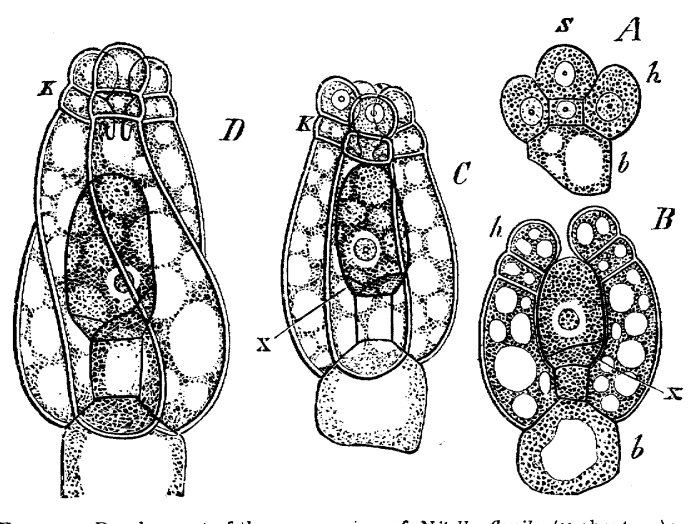

FIG. 202. Development of the carpogonium of Nitella flexilzs ( $\mathrm{x}$ about 300 ); $x$ 'Wendungszellen.'

a septum about half way up, the uppermost of the cells thus separated also become closely united with one another above the oosphere. This closing of the envelope takes place at least in Cbara fotida, before the 'Wendungszelle' has separated from the oosphere. The five upper cells of the envelope are at first as long as the five lower ones, and the septa which separate them lie about half way up the oosphere. As it now increases in size, the five lower ones become elongated into long tubes, which are at first straight but afterwards wind spirally round the oosphere. The five upper cells form the crown, which is elevated some distance above the apex of the oosphere. Between the crown and the apex of the oosphere the enveloping tubes grow inwards and increase in breadth, so that together they form, above the apical papilla of the oosphere, a thick diaphragm open only in the middle, by which a narrow space lying below the crown is separated from a still narrower one above the oosphere. The cells of the crown form a closed cover above the upper space; the upper and under space are united through the narrow opening in the diaphragm. De Bary found a similar structure in Nitella. As soon as the carpogonium attains its full size, the small space above the diaphragm enlarges, while the tubes between the diaphragm and the crown grow longer. This part of the envelope, which only attains its full size at a late period, De Bary calls the Neck. The tubes now separate laterally from one another, forming five clefts below the crown and above the diaphragm. Through these clefts the 
antherozoids force their way in great numbers into the apical space, which is filled with a hyaline mucilage. That one or more of them even find their way into the oosphere is rendered the more certain by the fact that about this time its papilla is protected by a very weak cell-wall or has none at all, as is shown by the small pressure required to expel its contents into the apical space.

A. Braun's account of the morphological value of the carpogonium of Chara is fully confirmed by our Fig. 203, $A$. It is necessary to explain, in the first place, that this is the lower part of a young fertile leaf of Chara fragilis, together with the contiguous piece of the stem and an axillary bud, represented in longitudinal section; $m$ is half of the nodal cell of the stem, $i$ its upper, $i^{\prime}$ its lower internode, $s r$ a descending, $y$ an ascending cortical lobe; $s r^{\prime}$ the cortical lobe of the lower internode which descends from the

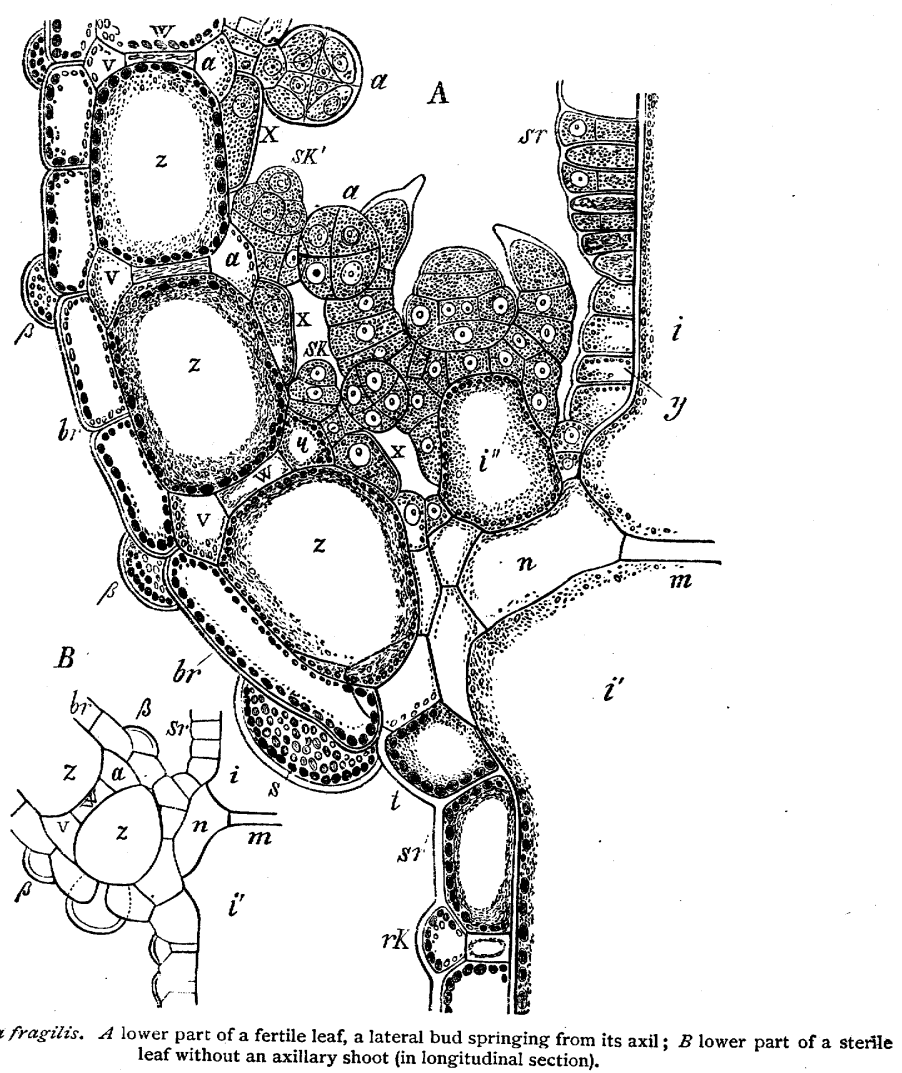

leaf, $r K$ a node of it; $i^{\prime \prime}$ the first internode of the axillary bud which rests upon the cell $n$ that unites the nodal cell $m$ with the basal node of the leaf. The leaf shows its three lower internodes, $z, z, z$, still rather short; they eventually attain from 6 to 8 times this length. Between them are the nodal cells $w, w ; v, v$ are the cells which unite the leaf-node with the basal node of the leaflets $\beta$ on the outer side of the leaf; $a$ the corresponding cells on the inner side of the leaf; $b r$ the cortical lobes of the leaf, two of which go upwards and two downwards from each leaflet $\beta$; the lowermost internode of the leaf is however covered only by descending lobes; by the side of one of them stands the stipule $s: x, x$ are the cortical lobes which descend on the inside of the internodes of the leaf, where the leaflets are transformed into antheridia, $a, a$; the ascending cortical lobes of the leaf are here absent, because one carpogonium always 
springs from the basal node of each leaflet. (Compare with this Fig. 197, $A$ and $B$.) In reference to the origin of the carpogonium, A. Braun says (l.c. p.69) that just as a branch springs from the basal node of a leaf, so does the carpogonium from that of a leaflet (in Chara fragilis from the basal node of an antheridium which stands in the place of a leaflet). As in the leaf which subtends a branch the ascending cortical lobes are wanting, so also in the leaflet which bears the carpogonium the cells forming the ascending portion of the cortex are also wanting. As it is the first leaf of the whorl on the stem that produces a branch in its axis, so it is also from the first (inner) leaflet of the whorl on the leaf that the carpogonium originates. The basal node of the antheridium in C. fragilis has, according to A. Braun, not four peripheral cells, as in sterile leaflets, but five; an upper odd one which is first formed, two lateral ones which follow, and two lower ones which are formed last of all. Of these five cells only the two lower ones are developed into cells which form the cortex (of the leaves), the upper one, wanting in the sterile basal nodes, is the mother-cell of the carpogonium; but the two lateral ones are developed into leaflets which stand laterally between the antheridium and carpogonium ( $c f$. Fig. $197, \beta^{\prime \prime}$ ); these latter Braun calls Bracteoles. The mother-cell of the carpogonium now grows out of the axil of the antheridium, and divides itself by a septum into an upper outer terminal cell and a segment which in its turn is broken up into two discs by a wall parallel to the previous one (Fig. 203, $A, S K$ ). The lower cell does not divide any further, it forms the concealed pedicel of the carpogonium, and corresponds to the first internode of a branch; but the upper one has the character of a nodal cell; it is divided by tangential walls into a zone of five outer and one inner cell $\left(S K^{\prime}\right)$; the former are the rudiments of the enveloping tubes, which are therefore morphologically leaves.

The Characex are distinguished by the size of their cells, and by the simple relations of the individual cells to the structure of the whole body. All the young cells contain nuclei, which at first always lie in the centre of the protoplasm that fills the whole cell; each bipartition of a cell is preceded by that of the nucleus and the formation of two new nuclei. As the cells grow, vacuoles form in the protoplasm which finally coalesce into a single large vacuole (the sap-cavity). The protoplasm, now clothing the wall as a thick layer, commences its rotatory motion which always follows the longest direction of the cell. Chlorophyll-granules are now formed: with the growth of the whole cell they also grow and multiply by repeated bipartition; they adhere to the inner side of the outermost thin stationary layer of protoplasm, and take no part in the rotation of the layers which lie further inwards; the nucleus, which is elongated and granular, also lies in this layer ${ }^{1}$. The rotating protoplasm becomes differentiated, as the cell grows, into a portion which is very watery and others which are denser, the former looks like hyaline cell-sap in which the latter float in the form of roundish larger or smaller lumps. Since these denser masses are passively swept along by the rotating clear protoplasm, as may be seen from their tumbling over one another, the appearance is presented as if the cell-sap caused the rotating motion. Together with the denser masses of protoplasm of less regular form, there are also a number of bodies of globular shape covered with delicate spines, consisting also of protoplasm. The current, as Nägeli has shown, is most rapid next the stationary parietal layer, and becomes gradually slower towards the interior; hence the spheres and globules which swim in the thin rotating protoplasm tumble over one another, because they become immersed at different spots in layers of different rapidity. Dependent on the direction of the current, the chlorophyll-granules are arranged in longitudinal rows on the stationary layer, and are deposited so thickly that they form a stratum; they are absent only at the neutral lines (Fig. I98, $A, i$ ). These neutral lines mark the position where the ascending

1 [In old internodal cells there are numerous nuclei which have been produced from the primary nucleus by division and 'fragmentation.' See Schmitz, Sitzber. d. niederrhein. Ges. in Bonn, 1879; Unters, ueb. die Zellkerne der Thallophyten. Strasburger, loc. cit. Johow, Bot. Zeitg. I 88I.] 
and descending portions, of the rotating protoplasm of a cell run side by side in opposite directions and neutralise each other, and where therefore there is no motion. The direction of the rotatory motion in each cell stands in a regular relation to that of all the other cells of the plant, and hence to its morphological structure, as has been shown by A. Braun.

\section{FORMS WITHOUT CHLOROPHYLL.}

To this second principal group of the class Carposporeæ belong all those plants, the fructifications of which have long been known as Fungi or Mushrooms, but which are now known under the names of Ascomycetes, Acidiomycetes, and Basidiomycetes. As a matter of fact a process of sexual reproduction has been actually observed as yet in only a few out of the very numerous genera of these plants, and these all belong to a single sub-division, namely that of the Ascomycetes. Among the Basidiomycetes mere traces of such a process have as yet been detected, and among the Acidiomycetes not even these have been observed. Nevertheless it is permissible to assume, until further information is obtained, not only that such a process actually takes place, but also that it agrees in its principal points with that observed in the Ascomycetes; at any rate such an assumption seems to be immediately suggested by the very similar course of development which obtains in these three groups ${ }^{1}$. We are, in fact, somewhat in the same position with respect to the sexual reproduction of these plants as were the botanists of the last century with respect to that of Phanerogams; they had observed the process of fertilisation in a few cases only, but they did not hesitate to assert, arguing from analogy based upon the similarity of the parts of the flowers and of the fruits developed therefrom, the sexuality of all Phanerogams.

Accepting the facts established by Tulasne, De Bary, and their followers with reference to the Ascomycetes, we may describe the life-history of one of these Fungi as follows. From the true spore (carpospore) there is developed a vegetative body, the mycelium, consisting of much-branched multicellular filaments, the hyphæ, which covers the surface or penetrates into the interior of the substratum upon which it grows ; it has often but a short term of existence, but it may occasionally continue to grow, as it appears, for years. In many cases this mycelium is capable of producing non-sexual reproductive cells which nearly always occur as conidia, and are usually developed upon special branches of the mycelium, the conidiophores, in large numbers by abstriction. These conidia, which correspond to the zoogonidia of Algæ and to the tetragonidia of the Florideæ, produce new mycelia on germination. Usually a mycelium reproduces itself thus asexually for many generations, and consequently many Fungi are only known in this stage of their life-history. In all cases, however, in which the life-history of a Fungus has been continuously traced, it has been observed that, under certain favourable conditions, the mycelium finally

1 [From the researches of Brefeld (Unters. ueb. Schimmelpilze, III, IV) it appears that there is no ground for assuming the existence of sexual reproduction in the Basidiomycetes. $\mathrm{He}$ is of opinion also that no sexual process takes place in the Ascomycetes, for, though they still possess sexual organs, these organs (except in Lichens) seem to have lost their function.] 
developes sexual organs, and that, as the result of fertilisation, a structure is produced which is quite different from the mycelium, a fructification, which, except in the simplest forms of these Fungi, consists of an aggregation of numerous hyphæ, and which presents great varieties of form. These fructifications are in many species small in proportion to the mycelium, and appear to be merely fruits developed upon it; in other cases, however, they continue to grow vigorously for some time, and attain a considerable size, obtaining their nourishment independently. Under these circumstances they appear to be independent plants, or, according to our modes of expression, to be alternate generations destined to produce true spores in usually very large numbers. The spores thus formed within a fructification (carpospores) are in these cases, as also among the Algæ, extremely different if not in their size at any rate in their form and other properties, from the conidia produced asexually on the mycelium. When the fructification is of considerable size it is commonly regarded as being the whole Fungus, just as a Horse-tail or a Fern is thought to be the entire plant, although the insignificant prothallium is an essential phase of the life-cycle of each of the latter. The mycelium, like the prothallium, is only the first stage of development, or, as it may be termed, the sexual generation (oophore), whilst the fructification corresponds to the fully-developed Horse-tail or Fern (sporophore). In those cases in which the fructification remains comparatively small and is nourished by the mycelium until maturity, a considerable similarity of habit becomes apparent between the Fungus and a Moss, for the sexually produced fructification of a Moss also derives its nourishment from the vegetative body of the first generation.

Like that of the Florideæ, the Characeæ, and the Coleochæteæ, the fructification of a Fungus consists of two essentially distinct parts, namely, of a sterile portion, - which is usually relatively large, and in some of the larger fructifications is by far the larger, and of a fertile portion in which, sooner or later, spores are formed. In the simpler forms the sterile tissue is merely an investing membrane which surrounds the spore-producing portion, but in larger and more complex fructifications, like those of Penicillium and Tuber, the sterile tissue is a compact mass into which the hyphæ which are to produce the spores penetrate, and within which they obtain nourishment and further ramify. A still higher degree of independence is attained by the sterile portion when the fertile hyphæ contained within it do not immediately give rise to spores but undergo a period of inactivity. Under these circumstances the fructification is, during the period of rest which may extend over weeks or months, simply a mass of tissue, which undergoes further development only when, under favourable circumstances, the contained fertile hyphæ produce spores. An inactive fructification of this nature is termed, at the suggestion of Brefeld who carefully studied these phenomena in Penicillium, a sclerotium.

When the formation of spores commences in the fructification, the fertile hyphæ may either grow towards the exterior and form the spores at the surface, when the fructification is said to be gymnocarpous, or they form the spores quite in the interior of the mass of sterile tissue, the outer layer of which then usually constitutes a firm cortex, the peridium, when the fructification is said to be angiocarpous. When numerous fertile hyphæ form a coherent layer on the surface of the fructification, such a layer is termed a hymenium; if, however, there is developed within the peridium 
of an angiocarpous fructification a peculiarly -formed mass of tissue in which very large numbers of spores are produced, such a mass is termed a nucleus or gleba.

The sexual organs, so far as they are known among the Ascomycetes, consist of a carpogonium as the female, and of a so-called pollinodium (antheridium) as the male. The two organs may differ but little in size and shape, and in Gymnoascus they are quite similar; more commonly, however, the carpogonium is larger and is multicellular, whereas the pollinodium is a thin, usually branched, tubular cell. In all cases the carpogonium differs from the pollinodium in that from it alone the fertile hyphæ take origin from which the spores are finally developed, the sterile tissue being derived from the hyphæ bearing the carpogonium or even from neighbouring cells.

Fertilisation is never affected by means of antherozoids ${ }^{1}$, but by the close application of the pollinodium in its whole length to the carpogonium, or merely of the apex of the pollinodium to the anterior portion of the carpogonium. Occasionally this portion of the carpogonium is prolonged into a narrow tube like the trichogyne of Nemalion. In the majority of the observed cases there is no direct exchange of protoplasmic substance between the pollinodium and the carpogonium in the process of fertilisation: the two organs remain closed and the fertilising matter passes from the pollinodium into the carpogonium apparently by diffusion. The fertile hyphæ of the fructification arise usually not from that part of the carpogonium which has been in direct contact with the pollinodium, but from nearer its base. Here again an analogy with the formation of the fructification of Florideæ presents itself.

Histologically considered, the mycelium and the fructification of these Fungi consist of hyphæ. The hyphæ are multicellular, usually much-branched, filaments which grow at their apices, and are generally very long and thin. In many cases, even in the larger fructifications, as for instance in the Mushrooms, it is easy to make out the individual hyphæ, but in other cases, although the tissue really consists of hyphæ, a so-called pseudo-parenchyma is formed by the close aggregation of their short thick segments. In angiocarpous Fungi (Tuberaceæ, Gasteromycetes) this pseudo-parenchyma is usually differentiated into well-defined concentric layers. Commonly, but not universally, the cell-walls of the pseudo-parenchyma are not coloured blue when treated either with iodine alone, or with iodine and sulphuric acid, but in certain cases this colouration is produced by the action of iodine alone (Asci of Lichens). Starch, as also chlorophyll, is absent from all Fungi, and this is the more remarkable since starch is found in Phanerogams which possess no chlorophyll.

Fungi grow exclusively upon organic substrata. Many grow in earth which is rich in humus or some other organic matter, others are parasitic upon and within animals and plants; this parasitism may present itself in the most varied manner, the most remarkable case being perhaps that which, as we shall hereafter see, occurs among Lichens. The mycelium usually buries itself in the nutrient substratum and can scarcely be separated from it, whereas the fructification comes to the surface.

As I do not propose to give an exact systematic account of the innumerable

1 [In Lichens the carpogonium is fertilised by non-motile antherozoids, termed spermatia, which resemble those of the Floridex.] 
species of Fungi, I will simply endeavour to illustrate the characteristics of the three principal groups above-mentioned by giving a complete account of such forms as have been accurately described by reliable observers, laying especial stress upon the more important features of their life-history.

\section{A. The Ascomycetes.}

In the fructification of the Fungi belonging to this group there arise from the ends of the fertile ascogenous hyphæ cells of a club-shaped or spherical form (asci), from the protoplasm of which numerous spores (ascospores) are developed by free-cellformation; usually a definite number of these spores, either four or eight, occurs in each ascus. The fructifications are either open (apothecia) or more or less completely closed (perithecia).

The spores always possess a firm cuticularised external membrane, the exospore, the surface of which usually presents asperities of different kinds: the inner membrane (endospore) of the spore forms, when the exospore has become ruptured in germination, the first hypha (or more than one) from which the mycelium takes its origin.

The mycelium produces in many instances conidiophores upon which the conidia are developed by abstriction. The conidia generally have a smooth surface and a very thin external membrane. In many genera they do not occur, although nearly-related genera possess them in abundance: for instance, they are absent in Tuber and present in Penicillium. In addition to the conidiophores there occur beside the fructification or even upon it, certain peculiar receptacles in which larger or smaller conidia (Stylogonidia in Pycnidia, Spermatia in Spermogonia) are developed, which, ever since the publication of the important mycological works of Tulasne, have been regarded as non-sexual reproductive cells of the Ascomycete upon which they exist. Since De Bary has shown that in many cases the pycnidia belong to other Fungi which are parasitic upon those in question, there has been some justification for the assumption that the so-called spermogonia also represent distinct genera of Fungi ; and this assumption gains in probability when it is considered that it deprives the doctrine of the pleomorphism of Fungi of its last remaining support.

(I) Gymnoascus ${ }^{1}$ is one of the simplest of the Ascomycetes. It is a small Fungus growing upon horse- or sheep-dung, the mycelium of which developes numerous sexual organs. Here the pollinodium and the carpogonium are completely similar before fertilisation, but after it has taken place the carpogonium divides so as to form a row of cells which grow out into short branched filaments bearing at their ends dense masses of asci each containing eight spores. As the investment is quite rudimentary the fertile portion of the fructification is in this case naked, and resembles, in this respect, that of the simplest Floridex. (Nemalion).

(2) Discomycetes ${ }^{2}$. In order to illustrate as fully as possible the formation of

1 Baranetzky, Bot. Zeitg. 1872, no. Io.

2 De Bary, Ueber die Fruchtentwickelung der Ascomyceten, Leipzig, I863, p. I I.-De Bary und Woronin, Beiträge zur Morphologie u. Physiologie der Pilze, and series, pp. I and 82, Frankfort I 866.-Tulasne, Annales des Sci. Nat. 5th series, vol. VI. p. 247. 1866.-Glinka-Janczewski, Bot. Zeitg. I87 I, no. I8. [Quart. Journ. Micr. Sci. I878, p. 438.-Brefeld, Unters. üb. Schimmelpilze IV.] 
a completely developed fructification, I select as an example Ascobolus furfuraceus, a Discomycete described by Janczewski. Fig. 204 represents a vertical section of the entire fructification of this Fungus, whilst still in connection with a portion of the mycelium, somewhat diagrammatically drawn for the sake - of clearness. The carpogonium $c$ and the pollinodium $l$ arise from branches of the mycelium. The former consists of a row of thick short cells, and is considerably curved; the delicate branches of the latter become closely applied to the anterior portion of the carpogonium. In consequence of fertilisation, one of the central cells of the carpogonium (which is designated the ascogonium) grows more vigorously than the others, assumes a somewhat spherical form, and developes by gemmation numerous filaments from which, at a later period, the asci are developed. In the meanwhile there has been formed from the hyphæ bearing the sexual organs a mass of filaments which completely invests the carpogonium, and which forms the large sterile portion of the fructification. Its hyphæ are so aggregated as to form a pseudo-parenchyma,

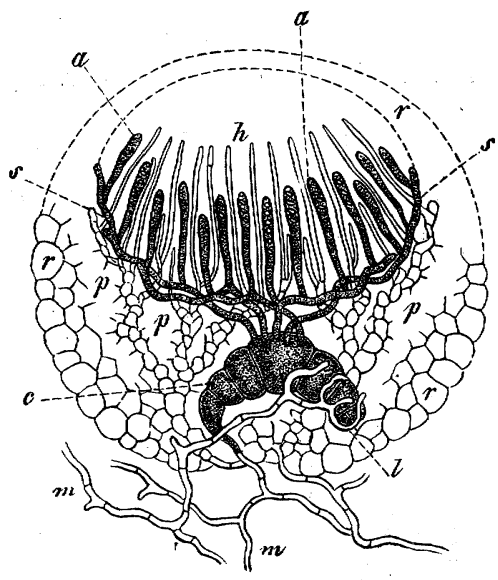

FIG. 204.-Diagrammatic section of the frustification of Ascobolus furfuraceus (after Janczewski); $m$ mycelium; $c$ carpogonium; $l$ pollinodium; $s$ ascogenous filaments; $a$ the asci; $r p$ the sterile tissue from which the paraphyses $h$ are developed.

$r$ in Fig. 204 being the cortical layer, and $p p$ the internal portion, in which the sterile hyphæ are diagrammatically indicated. The ascogenous filaments which have sprung from the ascogonium continue to grow forming a layer ss within the fructification, the subhymenial layer, and send upwards thick club-shaped branches, the asci, within which the spores are developed. In this way the hymenium $a a$ is formed, and it is completed by the upgrowth between the asci of parallel branches, the so-called paraphyses, from the sterile portion. Finally the cortex $r$ gives way at the apex, the hymenium comes to lie at the surface and expands in the manner represented in Fig. 205, in order that the spores may readily escape from the asci. In Peziza confluens, the species in which the sexual reproduction of the Ascomycetes was first discovered by De Bary in 1863 , the process is as follows, according to De Bary's and Tulasne's exhaustive researches:-The mycelium of $P$. confliens grows on the ground; branches arise at particular points of its hyphæ which are directed upwards and again branch abundantly; at the end of the branchlets the organs of conjugation or fertilisation are produced in large numbers close together, forming rosettes. The terminal cells of the stronger branchlets swell up into ovoid vesicles (Fig. 206, a), which put out a usually crooked prolongation $(f)$. From another cell of the same branch lying beneath this carpogonium grows a club-shaped branchlet, the pollinodium, the apex of which (i) unites with the prolongation just mentioned. After this has taken place, a number of fine hyphæ $(b)$ shoot out of the filament which bears these organs, and these surround the rosette of the organ of conjugation, enclosing it in a dense felt. This felt forms the body of the fructification; upon its upper side densely crowded hyphæ immediately rise up to form the hymenial layer; finally the fructification becomes an apothecium, which possesses somewhat the form represented in Fig. 205, and produces the ascospores in its asci. Woronin observed similar phenomena in P. granulosa and scutellata. In these species branches consisting of three or more cells arise from the mycelium; the terminal cell swells out into a globular or ovoid form, without, however, putting out a prolongation; from the cell lying beneath it arise two or more slender filaments which attach themselves closely to the former. The conjugating apparatus now becomes densely enveloped in numerous hyphæ which o:iginate 
beneath it ; from them is developed the wall of the apothecium. In Ascobolus pulcherrimus the carpogonium consists of a vermiform body, which Tulasne calls the Scolecite. It is a branch of the mycelium, consisting of a row of short cells which are much broader than those of the mycelium. The adjacent threads put out small branches, pollinodia, the terminal cells of which attach themselves firmly to the anterior part of the scolecite. It is subsequently covered over, together with this fertilising organ, by branched hyphæ

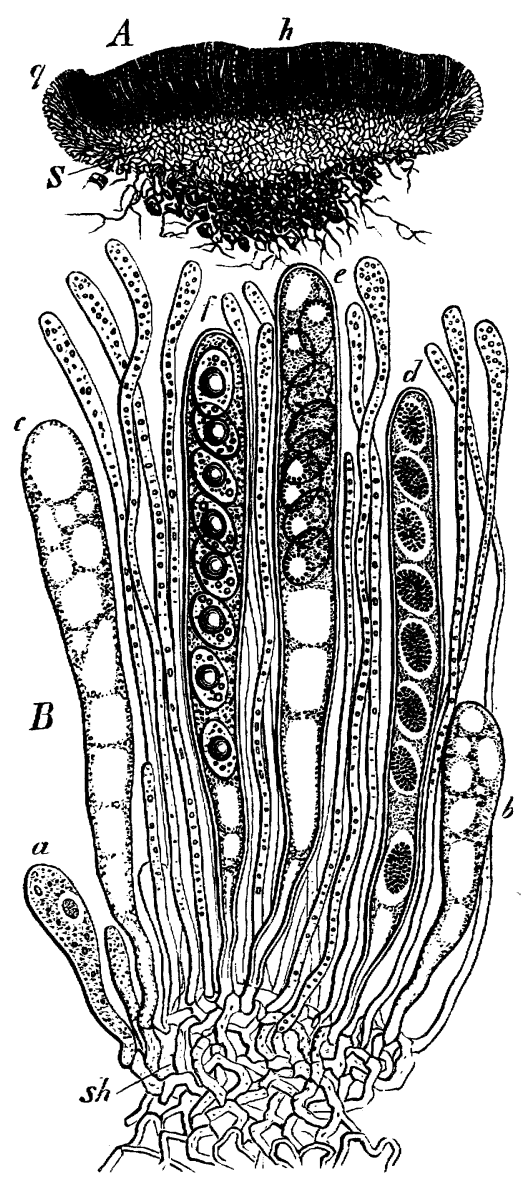

FIG. 205.-Peziza convexula; $A$ vertical section of the whole plant ( $X$ about 20 ); $h$ hymenium or layer in which lie the asci; $S$ the hyphal tissue surrounding the hymenium like a cup at its inargin $q$; at its base fine filaments proceed from the tissue, which penetrate into the soil; $B$ a small part of the hymenium ( $X$ about 500 ) ; sh subhymenial layer of densely interwoven hyphæ; $a-f$ asci, with intermediate slender para. physes, in which are red granules.

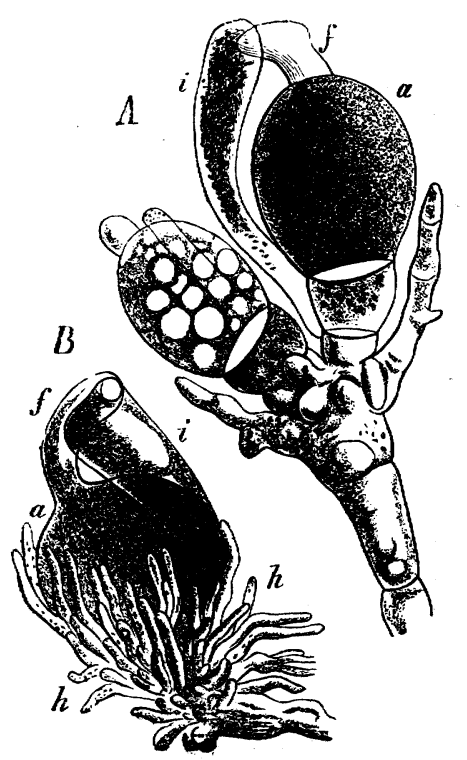

FIG. 206.-Conjugating apparatus of Peziza con. Auens (after Tulasne, very strongly magnified); in $B$ is shown the commencement of the formation of hyphæ $h$, in consequence of fertilisation, from which the apothecium is developed.

which spring from the neighbouring mycelium; and a ball is thus formed in the middle of which lies the scolecite; and this finally grows into the apothecium. In all these cases the origin of the ascogenous filaments from the carpogonium has not as yet been observed, but according to analogy there can be no doubt that such is the case.

In this group of the Discomycetes there are some individuals of which the mycelium. forms conidia, and the unripe fructification is in inactive sclerotium. Peziza Fuckeliana ${ }^{1}$

${ }^{1}$ [On Peziza Fuckeliana and Sclerotionum see Pirotta, Nuov. Giorn. Bot. Ital. I88I.] 
has been carefully investigated by De Bary with a view to the elucidation of these points. The mycelium of this Fungus is found in the autumn growing upon the wet dead leaves of the Vine. From it there arise erect segmented filaments, several millimeters in height, which ramify frequently towards their upper ends, numerous oval conidia being developed on each branch, which are capable of immediate germination and give rise to new mycelia. This stage of development of this Peziza was formerly regarded as representing a distinct Fungus, known as Botrytis cinerea. Later on the sclerotia are formed upon the mycelium, and although their origin from sexual organs has not been actually observed, yet after the observations of Brefeld upon Penicillium it cannot be doubted that such is the case. The sclerotia appear as callosities of various form, with a diameter of from $\frac{1}{2}$ to $I$ millimeter, in the tissue of the leaf which is infested with the Fungus, and persist after that this tissue has undergone decay. They consist of a dense felt of hyphæ with a black cortex. If they be placed upon moist earth soon after their formation, a great number of conidiophores are developed from them. If, however, the sclerotia have remained inactive for some months, they produce, when placed upon moist earth, small stalked cups, the flattened cavity of which bears a hymenium in which ascospores are formed (Fig. 205); this fructification is the Peziza Fuckeliana.

In addition to other genera which have small fructifications, this group includes also the Helvellacex, to which the genera Morcbella (Morel), Helvella, Spatbularia, and Geoglossum belong. In these the fructification is borne on a stalk, and is either hatshaped or club-shaped, and attains a considerable size. The hymenium covers the greater part of the surface of the fructification.

(3) The Erysiphem ${ }^{1}$ form spherical perithecia upon the surface of the substratum which they inhabit; but they remain so small that they can scarcely be seen with the naked eye, whereas the mycelium attains a considerable size. The investment of the fructification is a delicate hollow sphere consisting of pseudo-parenchyma, surrounding the few asci which spring from the carpogonium.

The very numerous species of the genus Erysipbe (Mildew) occur upon the surface of the leaves and green stems of Dicotyledons, and less frequently on those of Monocotyledons also ${ }^{2}$. The ramified mycelial filaments extend over the epidermis, crossing and re-crossing one another, and throw out haustoria at numerous points which penetrate into the cells of the epidermis. The mycelia are reproduced by means of conidia which are abstricted in rows at the upper end of the erect unbranched conidiophores. These reproductive organs, formerly termed Oidium, are the only ones at present known in many species, as, for instance, in Erysipbe (Oidium) Tuckeri, the Fungus which produces disease in grapes. In many other species, however, the sexually developed fruits may easily be found. Usually numerous filaments grow out from the cortical portion of these fruits, which either attach themselves, like the mycelial filaments, to the substratum, or remain quite free forming a delicate fringe. Both fruits and conidia may be developed upon the same mycelium.

The simplest mode of the formation of the fruit occurs in the sub-genus Sphrorotheca, Fig. 207. The carpogonia and pollinodia are developed together at the points at which the mycelial filaments cross one another, and are in contact from their first appearance. They are both small lateral branches; the one from which the carpogonium $c$ is to be formed assumes an ovoid shape, and is then shut off by a septum; the one which is to become the pollinodium $p$ curves over the apex of the carpogonium, and a septum is formed in its curved portion. After fertilisation, filaments spring from beneath the basal wall of the carpogonium, as also from the pollinodium (IV $b$ ), which closely invest the carpogonium and grow up and come together over its apex; they

1 Tulasne, Selecta fungorum carpologia, I. Paris 1860.-De Bary und Woronin, Beiträge zur Morphol. und Physiol. der Pilze, 3rd series. Frankfort 18 ;0.

${ }_{2}^{2}$ Perhaps Spharotheca pannosa (Rosarum) also penetrates into the tissues of its host. 
become multicellular in consequence of the formation of transverse septa within them, and, lying closely side by side, they give rise to a pseudo-parenchyma. From the internal surface of this investment, as it increases in size, short filaments are given off which fill up the space between it and the carpogonium which has not as yet undergone much change $(\mathrm{V} b)$. The still unicellular carpogonium now begins to grow vigorously; it is divided by a transverse septum into two cells, an upper and a lower, and may be regarded as a simple form of ascogenous filament, the apical cell of which is directly converted into an ascus $(\mathrm{V} a$ ). The apical cell, by its rapid growth, soon occupies the whole of the cavity of the fructification, and eight spores are produced by free-cell-formation in its protoplasm. Slight pressure upon the fruit causes the extrusion of the ascus (II $a$ ). In other Erysiphex, the perithecia of which contain several asci, as $E$. Umbelliferarum, communis, lamprocarpa, etc., the carpogonium is also originally unicellular, but it grows within the investment into a long, thick, curved filament, which is divided into segments by numerous septa. Many of these segments throw out lateral branches which bear the asci.
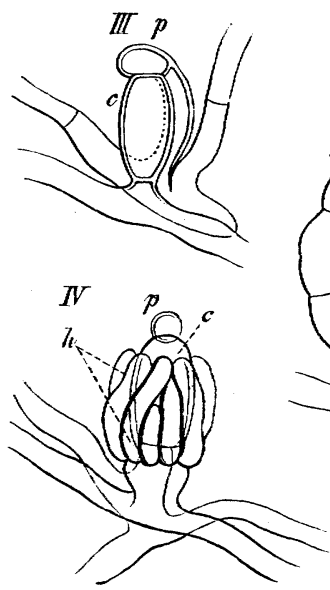

FIG. 207, $-I$ Conidiophore; $I I$ ripe perithecium of Erysiphe (Spharotheca) pannosa (after Tulasne); $I I I$ carpogonium and pollinodium; $I V$ the same after fertilisation; $V$ the young perithecium of Podosphara Castagnei (after De Bary) ; $c$ carpogonium ; $p$ pollinodium; $h$ wall of the perithecium; $a$ the single ascus.

These Erysipheæ which have numerous asci afford a transition to the Eurotieæ ${ }^{1}$ in which the carpogonium, even before fertilisation, elongates considerably and becomes spirally wound.

The life-history of Eurotium repens and that of Eurotium Aspergillus glaucus have been also recounted in detail by De Bary. Both species are found on the most various decaying or dead organic bodies, and are especially abundant on preserved fruit. The Fungus makes its appearance as a delicate flocculent white mycelium overspreading the surface, from which the upright conidiophores soon rise in large numbers. These swell in the upper part into a globular form, and on the upper half of the globe there arise a number of peg-shaped projections, densely crowded and arranged radially, the sterigmata, each of which produces gradually a long chain of greenish conidia; so that finally the head of the receptacle is covered by a thick layer of them. During this formation of conidia, the sexual organs appear on the same mycelium. The female organ, the carpogonium, is the corkscrew-like end of a branch of the mycelium (Fig. 208, $A, a s)$, the coils of which become gradually closer, until, when actually in contact, they form a hollow spiral $(B, C)$. During this process about as many septa are formed as there are turns of the helix (i.e. 5 or 6). From the lowest coil of the carpogonium two

' [Wilhelm, Die Pilzgattung Aspergillus, I877.] 
slender branches now shoot out at opposite points, and grow upwards on the outside of the helix; one of these developes more quickly, reaches the uppermost coil, and becomes closely attached to it by its apex $(B, p)$. This branch is the pollinodium. Conjugation takes place between its apex and that of the carpogonium, the cell-walls being absorbed at the point of contact, and the protoplasmic contents of the two cells commingle. Soon afterwards new filaments sprout out from the lower part of the pollinodium and of the carpogonium, which increase in number, cling closely to the spiral $(C)$, and finally entirely envelope it. From these filaments a layer of polygonal cells $(D)$ is formed by numerous transverse divisions, which envelopes the carpogonium. The cells of the enveloping layer grow inwards as papillæ which become septate $(E)$. While the enveloping layer is increasing in size, the cavity of the perithecium, which is thus enlarged, is filled up by the papillæ, and they finally insert themselves between the coils

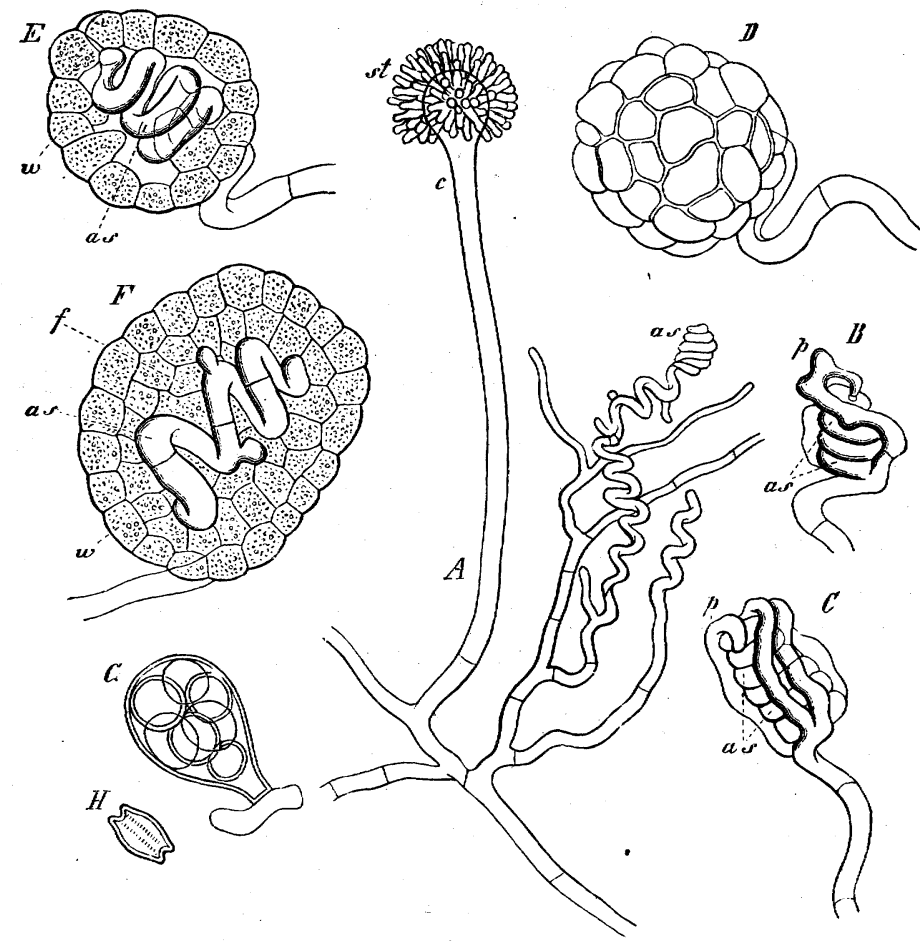

FIG. 208.-Development of Eurotium repens (after De Bary). $A$ small portion of a mycelium, with the conidia-bearing
hyphæ $c$ and young ascogonium (or carpogonium) as $: B$ the spiral ascogonium as with the pollinodiun $p ; C$ the same,
beginning to be surrounded by the threads out of which the wall of the perithecium is formed; $D$ a perithecium; $E, E$
section of young perithecia, $w$ parietal cells, $f$ pseudo-parenchyna, as ascogonium; $G$ an ascus; $H$ an ascospore.

of the carpogonium which have now become looser. These papillæ become divided by septa into numerous cells of similar diameter, so that at last the space between the enveloping layer and the coils of the carpogonium is filled by a pseudo-parenchyma $(F)$. During these processes a large number of septa arise in the carpogonium, and soon there shoot from its cells numerous commencements of branches, which penetrate on all sides between the cells of the pseudo-parenchyma, become septate, and ramify. Their last ramifications are the asci $(G)$, which therefore owe their origin to the fertilised carpogonium. These internal changes are accompanied by a considerable increase in size of the whole perithecium. During the development of the asci the pseudo-parenchyma becomes looser, its cells round themselves off, become capable of swelling, lose their fatty contents, and finally disappear; in the ripe perithecium 
it is replaced by the asci. The increase in size of the cells of the parietal layer keeps pace with that of the whole perithecium, and they become covered with a sulphuryellow coating which attains a considerable thickness and consists probably of a resinous or fatty substance. Finally these cells also collapse and dry up; the eight-spored asci also break up, till finally the perithecium consists only of the brittle yellow coating and of the mass of spores enclosed by it, which are set free by gentle pressure. In a similar manner to the perithecium, the mycelium also becomes covered by a coating, in this case of a chestnut colour, on which the perithecia are now individually visible to the naked eye as yellow granules. The ripe spores have the form of biconvex lenses $(H)$; when germinating the endospore which puts out the germinating filament swells up violently and splits the exospore into two halves. The mycelium which proceeds from the ascospores produces, like that which arises from the conidia, at first conidiophores and afterwards perithecia; but a proper alternation of sexual and non-sexual generations does not occur here.

(4) The Tuberaceæ (Truffles) form subterranean tuberous fructifications, which may be as large as a clenched fist. They are usually provided with a firm thick cortex of pseudo-parenchyma, and consist internally of a dense felt of hyphæ in which the ascogenous filaments ramify. The asci, within which the spores are produced, are imbedded in this tissue, and are arranged in groups or layers of various forms which, in a section, present the appearance of chambers or of a dark veining. Until recently the development of these fructifications had not been traced, and their morphological structure was but imperfectly understood. The discovery made by Brefeld that the commonest of all Moulds, Penicillium glaucum, is merely the conidiabearing mycelium of a small Truffle, has, however, thrown much light upon the morphology of this group of Fungi.

The mycelium of Penicillium glaucum grows upon nearly all organic substrata, even upon liquids, forming a dense felt. From it erect filaments at ise which form at their upper ends pencils of branches, and at the extremities of these long rows of greenish conidia are developed. These conidia are everywhere dispersed in the air, and it is for this reason that this Fungus is of such universal occurrence.

Like the Truffles, Penicillium only produces its fructification when deprived of air and of light, under circumstances in fact which are unfavourable to the development of the conidiophores. The fructifications are of a yellowish colour and attain the size of a small pin's head. On this account they were overlooked until Brefeld succeeded in producing them artificially. 'The mycelium ${ }^{1}$ must be cultivated upon a substratum which affords it abundant nourishment and enables it to attain, without any interruption, its most complete vegetative derelopment. This is reached as a rule in from seven to ten days after the spores have been sown. The access of the atmospheric oxygen must now be diminished by proper means, and the exhaustive formation of conidiophores will thereby be prevented. Since these conditions are not commonly fulfilled in nature, it is easy to understand why it is that only the asexual form of Penicillium has hitherto been known.

'The sexual organs of Penicillium agree in all essentials with those of Eurotium described by De Bary. They consist of a spirally-wound ascogonium (carpogonium), the female organ, and of a pollinodium, the male organ.

'After the fertilisation of the ascogonium, a process of development commences which differs very materially from anything of the kind as yet described among the Ascomycetes. In this case also the fertilised ascogonium becomes invested by filaments which arise, evidently in consequence of fertilisation, from beneath the ascogonium, but here the ascogonium itself at once begins to grow and its branches extend among the surrounding filaments. When the growing ascogonium is enclosed by eight or more (8-15) layers of filaments, no new layers are formed, but those which

${ }^{1}$ The following is taken literally from Brefeld's preliminary account in Flora, $18_{73}$, no. 2 I. 
already exist become further developed. This development consists in the division of the filaments into very numerous cells, which expand and thus form a coherent tissue. The further extension of the ascogenous filaments is first diminished and then arrested by the gradually increasing coherence of the investing cells, and they are seen, in a median section, as thick hyphæ running concentrically. After this formation of tissue has taken place an expansion of the cells, which is not uniform throughout, takes place, so that they attain six or eight times their previous size, and then their walls become much thickened. This thickening begins simultaneously at two points, internally, in the ascogenous hyphæ, and externally, in a zone which is separated from the periphery by a few layers of cells.

'The fructification, now free from the mycelium, is of the size and colour of a grain of coarse yellow sand. It is a sclerotium, consisting of from two to four peripheral layers of cells elongated tangentially, of a yellowish-brown colour, and internally of large cells arranged radially which become smaller towards the interior. Between these run the firm ascogenous hyphæ appearing like much-branched passages in the tissue.

'The sclerotia may be preserved in the dry state for as long as three months without losing their vitality. If they are placed upon moist blotting-paper, a further development of the ascogenous hyphæ takes place within six or seven weeks. They again acquire the appearance of living hyphæ, and become divided into numerous cells, each cell being capable of producing a branch which, at its first appearance, divides into a thick and a thin filament. The thick filaments are concerned in the development of the spores, whereas the thin filaments, which are but slightly branched and without septa, cause the absorption of the surrounding tissue and supply the thick ones with nutriment. The thick filaments form numerous closely-placed lateral branches immediately behind their apices, a septum being formed between each pair. These branches form a series of asci, each of which contains eight spores.

' The result of further development is that the whole internal sterile tissue is absorbed, the brown external layers alone remaining; the ripe asci together with the hyphæ bearing them and the nutrient filaments also disappear, so that finally, in six or eight months, the sclerotium, although it has not altered in external appearance, has become converted into a vesicle filled with a dense mass of countless spores of a bright yellow colour.

'When seen under favourable circumstances each ascospore gives rise to a mycelium quite similar to that developed from a conidium, which bears the characteristic conidiophores, each one of which can be genetically traced through the filaments of the mycelium to the individual spores.

'If, in consequence of desiccation, of a too advanced maturity, or for any other reason, the sclerotia lose their capacity for growth, that is, if the ascogenous filaments within them have lost their vitality, certain cells of their tissue may still be capable of germinating. The hyphæ springing from them come to the surface through fissures in the sclerotium, and proceed to form the ordinary conidiophores. In this process the physiological difference between the ascogenous filaments and the tissue surrounding them, which amounts to a perfect contrast, becomes more definitely manifest.'

The similarity of structure presented both by the ripe and the unripe fructifications of Penicillium with young and mature Truffles makes it at once evident that Penicillium belongs to the Tuberacex, and suggests that the formation of the fruit of the other members of this group takes place in the way so fully described with reference to Penicillium. Tulasne's ${ }^{1}$ figures, especially that of Elaphomyces Leveillei, represent the ascogenous filaments within the sterile tissue of the Truffles, and Brefeld observed them again in Tuber rufum. The well-known yellow bands of hyphæ correspond to the asco-

1 Tulasne, Fungi hypogæi, Paris 1862. [See also Reess, Parasitismus von Elaphomyces granulatus, Sitzber. d. phys. med. Soc. zu Erlangen, 1880.] 
genous filaments; they give rise to asci and at the same time absorb the surrounding sterile tissue.

The dark colour of the interior of Truffles is due to the numerous dark-coloured spores, and the marbled appearance with light and dark veins is the result of the distribution of the bands of sporiferous filaments in the colourless sterile tissue. The latter contains air between its hyphæ and therefore appears white when seen by reflected light. The spores of Truffles are formed in club-shaped or spherical asci of considerable size, by a process of free-cell formation. They are invested by an exospore which is covered with asperities, or has a rugose surface.

It is as yet unknown whether or not the mycelium of the Truffles, like that of Penicillium, lives exposed to the air and forms conidia at any period of its existence.

(5) The Pyrenomycetes ${ }^{1}$ usually produce their asci, which generally contain eight spores and are of an elongated club-shape, within small flask-shaped or roundish receptacles, which are termed perithecia. The wall of a free isolated perithecium (as in Spbxria, Sordaria, and others) consists of a firm pseudoparenchymatous tissue of a dark colour. The perithecium contains at first a delicate transparent tissue free from air, which is afterwards absorbed by the asci and paraphyses. These spring from a hymenial layer which clothes either the whole of the wall of the perithecium, or only its basal portion. The perithecia are either open from the first (as in Spharia.typhina, Sordaria), or they are originally closed and afterwards form an orifice clothed with hairs through which the spores can escape ( $X$ ylaria).

In a number of forms (Sphxrix simplices such as Pleospora, Sordaria) the free perithecia originate singly or in groups upon the inconspicuous filamentous mycelium which usually inhabits dead plants, but occurs also on living ones. It is certain from Woronin's observations upon Spbxria Lemannex and Sordaria that in these cases each perithecium is the result of a sexual act, and therefore represents an entire fruit. In other Pyrenomycetes, however, a so-called stroma is first formed from the mycelium. This is a cushion-shaped, mushroom-like, cup-shaped, or arborescent structure, consisting of a dense mass of apparently homogeneous tissue, in which numerous perithecia are developed. It remains uncertain whether, in such cases, the stroma is merely a peculiar form of the mycelium within which the sexual organs are subsequently developed and which bears a corresponding number of perithecia, or whether the entire stroma is the result of one act of fertilisation and is therefore to be regarded as a single fructification the asci of which are produced in numerous perithecia. Of these alternatives the latter is the more probable, for in Claviceps the stroma itself is derived from a scelerotium, which is doubtless the product of a sexual process.

The asexual reproductive cells or conidia are developed, among the Pyrenomycetes, not merely from the mycelium, but more especially from the stroma, and (as in Penicillium) even from the wall of the perithecium. They are formed on longer or shorter hyphal branches usually in considerable numbers, and occasionally larger and smaller conidia occur in the same species. It has already been pointed out that the receptacles known as spermogonia and pycnidia ${ }^{2}$, which also form larger and smaller conidia, are probably parasites, and do not form part of the cycle of life of the plant which they infest.

I select as an example for more detailed description the $\mathrm{F}$ ungus which produces the Ergot,-Claviceps purpurea ${ }^{3}$. Its development begins with the formation of a filamentous

1 Tulasne, Selecta fungorum carpologia, Paris $1860-65 .-$ Woronin und De Bary, Beiträge zur Morph. u. Physiol. der Pilze, Frankfurt I870.-Fuisting, Bot. Zeitg. I868, p. I 79. [Gilkinet, Rech. morph. sur les Pyrénomycetes, I, Sordariées, 1 874.]

${ }^{2}$ [Bauke, Beitr. z. Kennt. der Pycniden, Nov. Act. Leop-Carol. Akad. 1876, has shown that, in certain cases at least, the pycnidium with its stylogonidia is a definite part of the life-history of these Fungi.]

3 Tulasne, Annales des Sci. Nat. vol. XX. p. 5.-Kïhn, Mittheilungen des land'w. Inst. in Halle, yol. I. 1863 . 
mycelium, which attaches itself to the surface of the ovary of Grasses, especially of Rye, while still enclosed between the palex, covers it with a thick weft, and partially penetrates into its tissue, while the apex and often other parts of the ovary remain exempt from its attacks. The ovary becomes replaced by a soft white mycelial tissue which retains nearly its original form, the style being not unfrequently still borne on its summit. The surface of the tissue of the Fungus is marked by a number of deep furrows and forms a large number of conidia on basidia arranged radially, imbedded in a mucilaginous substance which exudes between the palex. In this condition the Fungus had been at one time considered a distinct genus, and described under the name of Sphacelia. The conidia can germinate at once and immediately again detach conidia, which, according to Kühn, again produce a sphacelia in other Grasses. The mycelium of the sphacelia forms, when the production of conidia has reached its height, a thick felt of firmer hyphr at the base of the ovary, which is at first still surrounded by the looser tissue of the sphacelia. This is the commencement of the sclerotium or Ergot; its surface soon assumes

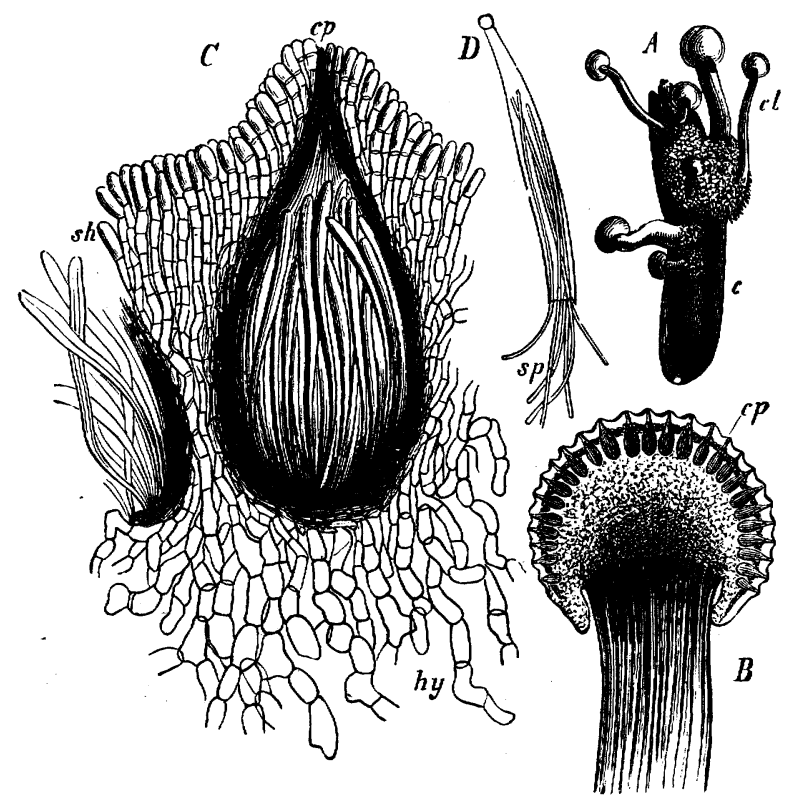

FIG. 209.-Ciaviceps purpurea. $A$ a sclerotium forming stromata $c l$ (Ergot); $B$ longitudinal section of upper part of a stroma, $c p$ the perithecia; $C$ a perithecium with the surrounding tissue (greatly magnified); $c p$ its orifice, $h y$ hyphæe of the pileus, sh epidermal layer of the pileus; $D$ an ascus ruptured and allowing the spores to escape (after Tulasne).

a dark-violet colour, and grows to a horn-shaped body, often as much as an inch in length. In the meantime the sphacelia ceases to grow, its tissue dies and is ruptured beneath by the sclerotium, and carried upwards on its summit, where it is placed like a cap and afterwards falls off. The hard ripe sclerotium now remains till the autumn, or usually till the next spring in a dormant state; the formation of the stroma begins when the sclerotium is lying on the damp ground. The stromata arise beneath the skin, a number of closely-packed branches being formed at definite points from the medullary hyphæ; the bundle breaks through the skin, and grows up into the stroma which consists of a long stalk and a globular head. In the latter a large number of flask-shaped perithecia (Fig. 209, $B$ and $C, c p$ ) appear, which do not possess a clearly-defined wall. Each perithecium is filled from the bottom by a number of asci, in each of which several slender filiform spores are produced. These spores swell up in damp situations, and put out germinating filaments at several points. When they reach the young flowers of 
Rye, or of other nearly allied grasses, Kühn states that the sphacelia arises from them, and the cycle of development is thus completed.

(6) Lichens'. From the researches of Schwendener ${ }^{2}$, there can no longer be any doubt that the Lichens are true Fungi belonging to the Ascomycetes (Discomycetes and Pyrenomycetes), but distinguished by a singular parasitism. Their hosts are Algæ, which grow normally in damp places but not actually in water, and belong, moreover, to very various groups (rarely Confervacex, frequently Chroococcaceæ and Nostocaceæ, more often Palmellaceæ, sometimes Chraolepidex). The Fungi themselves (Lichenforming Fungi) are not found in any other form than as parasites on Algæ; while the Algæ which are attacked by them, and which, when combined with the Fungus, are called Gonidia, are known in the free condition without the Fungus. When the species attacked by the Lichen-fungus is a filamentous Alga, and the development of the hyphal tissue is only moderate (as in Ephebe and Coenogonium), the true state of the case is at once clear; and as Lichens of this kind have become better known, the suspicion has frequently arisen that they are in fact only Algæ infested by Fungi. In the Collemacex also attention has frequently been drawn to the identity of the gonidia with the moniliform filaments of Nostocaceæ; but in this case the nourishing Alga usually undergoes considerable changes of habit, at least in its external contour, from the influence of the parasitic Fungus, like Euphorbia Cyparissias from its parasitic Ecidium. But the greater number of Lichen-fungi prefer as hosts the Chroococcacex and Palmellaceæ which grow as stains and incrustations on damp ground, the bark of trees, and stones. The separate cells and groups of cells of these Algæ become so involved by the tissue of the Fungus, that they are at last only interspersed here and there in the dense hyphal tissue, or appear in it as a special layer (the gonidial layer). The growth and multiplication of these Algæ, which thus become entirely enclosed by their parasites, is not hindered, but their development is disturbed in other ways. When, however, they are freed from their enclosing Fungus-tissue, their normal development proceeds, and in a few cases even the formation of zoogonidia takes place in them,

1 Tulasne, Mémoire pour servir à l'histoire organographique et physiologique des Lichens (Annales des Sci. Nat. 3rd series, vol. XVII).--Schwendener, Untersuchungen über den Flechtenthallus (in Nägeli's Beiträge zur wissensch. Botanik, 1860 and 1862 .-Ditto, Laub- u. Gallertflechten (Nägeli’s Beiträge zur wissensch. Botanik. I868).-Ditto, Flora, I872, nos. II-I 5.-Stahl, Beit. z. Entwickel.-Gesch. der Flechten, 1877. [Quart. Journ. Micr. Sc. 1873, p. 235, and 1878, pp. I $44,438$.

2 [The views of Schwendener have been corroborated by Bornet in an elaborate memoir published in the Ann. des Sci. Nat. I873, vol. XVII. He also put them to a synthetical test by sowing the spores of Parmelia parietina upon Protococcus. About the fifteenth day the hyphæ were well developed and ramified. Wherever they met isolated cells of Protococcus or groups of them, they attached themselves either directly or by means of a lateral branch. They did this to the Protococcus only, neglecting altogether the other bodies which were mixed with it. Similar results were obtained when the spores of Biatora muscorum were sown upon Protococcus. Spores of Parmelia sown separately ramified much less and developed no chlorophyll; Protococcus, on the other hand, during the same period remained unchanged and put out no hyphæ. Tulasne, however, sowed the spores of Lichens and believed that he twice detected the formation of gonidia upon the hyphæ (Ann. des Sci. Nat. 1852, XVII, pp. 96-98). De Bary indced described the green gonidium as originating by the expansion of a short lateral branch of the hypha into a globular cell, which is shut off by a septum and assumes a green colour; once formed, it increases independently by division, and a number of the gonidia eventually lie without stipites in the interstices of the Lichen-tissue (Morph. u. Phys. der Pilze, pp. 258, 263-265). Berkeley also believes that the gonidia originate from the hyphæ. having had 'a good opportunity of ascertaining their development from the threads of the mycelium in specimens developed within the vessels of pine wood' (Introd. to Crypt. Bot. p. 373). For a careful resumé of all the recent literature of the subject by Archer, see Quart. Journ. Micr. Sc. I873, p. 217. In this country Bentham has criticised Schwendener's view (Address to Lin. Soc. May 23, I873), and Thwaites and Berkeley have also expressed their dissent (Gard. Chron. I 873 , p. I 34I).] 
a fact first observed by Famintzin and Baranetzky, but incorrectly explained. It is to Schwendener's knowledge of the facts, the result of researches extending over many years, that the correct interpretation is due in these cases of the relationship borne by the Lichen-forming Fungus to the gonidia, i.e. to the Alga which it attacks'.

After these preliminary remarks the following description will be intelligible to the beginner. It is transferred, with but slight alterations, from the first edition of this book. We will consider first the Lichen as a whole, as it comes under observation, the nourishing Alga being distinguished as an elemental form of the thallus under the name Gonidia; and we will afterwards discuss the question of their algal nature more in detail.

The Thallus of Lichens is commonly developed in the form of incrustations which cover stones and the bark of trees, or penetrate between the lamellæ of the epidermis of woody plants, and then expose only the fructifications above the surface. These Crustaceous Lichens, as they are termed, have become so completely united in their growth to their substratum, at least on the under side, that they cannot be detached completely from it without injury to the thallus (Fig. $2 \mathrm{I} \circ, A, B, C$ ). The crustaceous Lichen-thallus

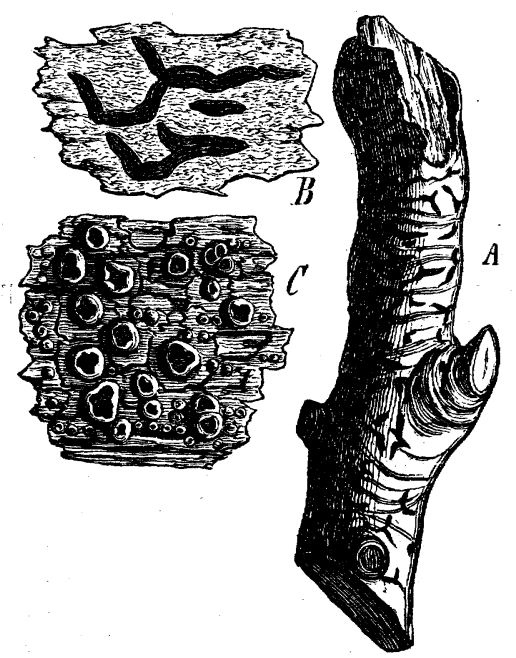

FIG. 210.- $A, B$ Graphis elegans, a crustaceous Lichen growing on the bark of the Holly; $A$ natural size, $B$ slightly magnified; C Pertusaria Wulfeni, another crustaceous Lichen (slightly magnified).

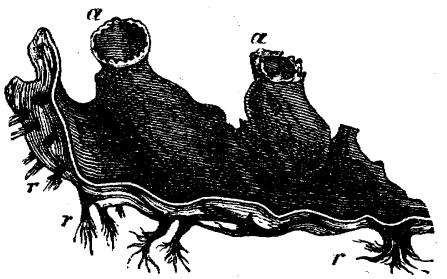

FIG. 2rr.-A piece of the foliaceous thallus of $P e l$. tigera horizontalis; $a$ the apothecia; $r$ the rhizines (natural size).

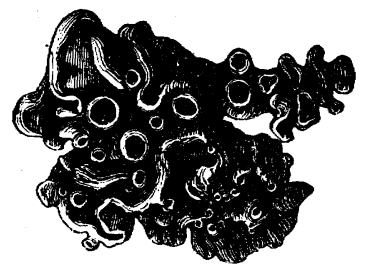

FIG. 2I2-Collema pulposum, a gelatinous Lichen (slightly magnified).

passes over, through various gradations, into that of the Foliaceous Lichens; the latter forms flake-like expansions often curled, which can be completely detached from the ground, stones, moss, bark, \&c. which support them, since they are attached to it only in places by a few organs of attachment, the Rbizines. The foliaceous thallus often attains considerable dimensions, in the large species of Peltigera and Sticta as much as a foot in diameter, and from $\frac{I}{2}$ to $I \mathrm{~mm}$. in thickness, and then generally assumes a circular form; at the growing margin it forms rounded indented lobes (Fig. 2 I 1 and Fig. $\left.21_{3}, B\right)$. A third form of the Lichen-thallus, also united with the previous one by transitional forms, is shown in the Fruticose Lichens, which are attached only at one spot and with a narrow base, and rise from it in the form of small much-branched shrubs. The branches of the thallus are either flat and ligulate, like the lobes of many foliaceous Lichens, or slender and cylindrical (Fig. 2 I 3,A). In Cladonia and Stereocaulon we have not so much a transition from the foliaceous to the fruticose thallus as a combination of the

1 A few additional historical notes will be found at the end of this section. 
two, a foliaceous expansion of small size being first formed, the cup-shaped or fruticosely branched thallus afterwards rising from this.

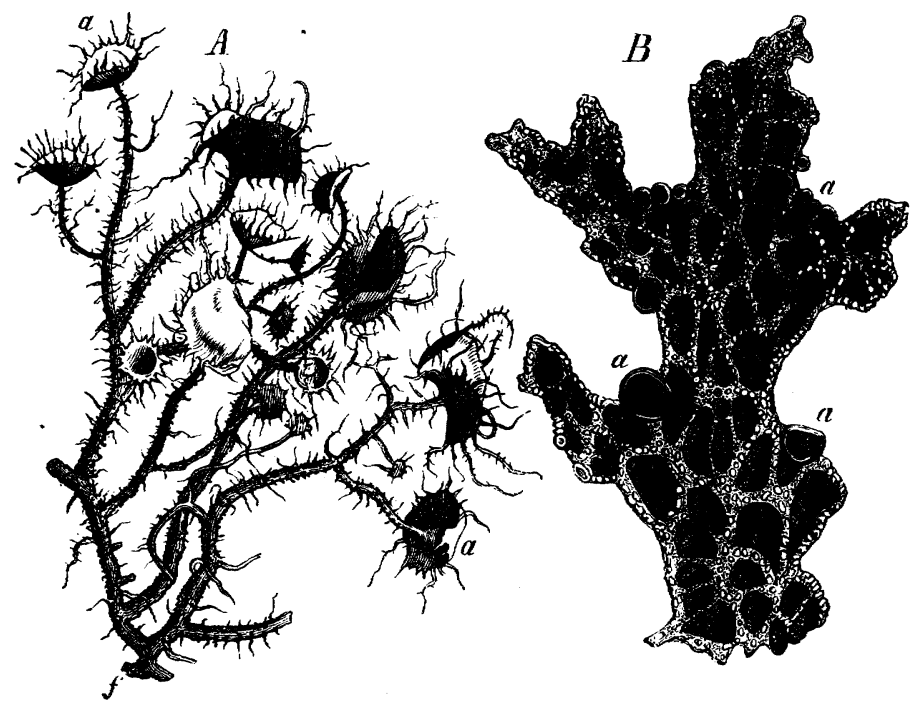

FIG. 213.-A Usnea barbata, a fruticose Lichen (natural size); B Sticta piulmonacea, a foliaceous Lichen (natural size) seen from beneath; $\alpha$ apothecia, $f$ the attaching disc of $A$, by which the Lichen becomes attached to the bark of a tree.

The thallus of Lichens can be diried, so as to be pulverised, without losing its vitality. When saturated with water it has generally a leathery consistence, is tough, elastic,

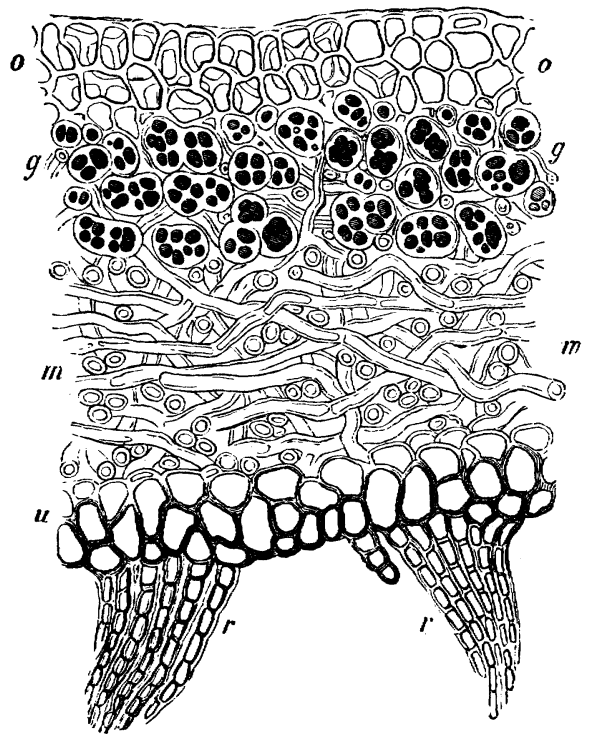

FIG. 2I4.-Transverse section through the foliaceous thallus of Sticta fuliginosa $(\times 500) ; o$ cortical or epidermal layer of the upper side; $u$ of the under side; $r r$ rhizines or attaching fibres, springing from the epidermal layer and therefore trichomes; $m$ the medullary layer, the hyphæ of which are seen cut, some transversely, some longitudinally. The upper and under cortical layers also consist of hyphæ, which however are much thicker, consist of shorter cells, and are united without interstices, forming a pseudo-parenchyma; $g$ the gonidia (their lightgreen masses of protoplasm are coloured dark); each gelatinous envelope encloses several gonidia produced by division. and flexible; but a large number of genera, which are remarkable also in other ways, are slimy and gelatinous in this condition. These Gelatinous Lichens, as they are termed, form cushion-like masses with an undulated surface, and in their growth are sometimes more like the fruticose, sometimes more like the foliaceous Lichens. A typical form is shown in Collema, Fig. 212.

The disposition of the gonidia and hyphæ in a thallus may be such that these two structures appear about equally mingled (as in Fig. 215), and the thalius is in this case called bomoiomerous; or the gonidia are crowded into one layer (as in Fig. 2I4), by which the hyphal tissue is at the same time separated according to circumstances into an outer and inner or an upper and under layer; the thallus-tissue is then stratified, and such Lichens are termed beteromerous (Figs. 214 and 217).

The mode of growth, branching, and external structure of the Lichen- 
thallus may either be determined by the ganidia, the hyphæ being concerned only in a secondary degree in its construction, or it may happen that the hyphæ determine the

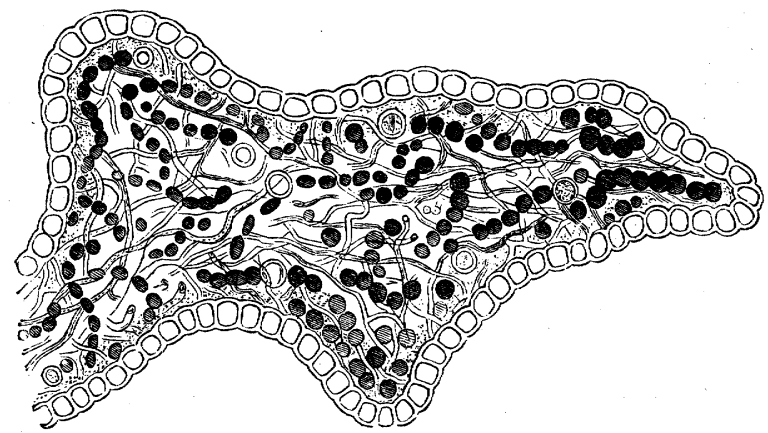

FIG. 215-Vertical section of the gelatinous thallus of Leptogizm scotinum ( $\mathrm{X} 500)$; an epidermal layer clothes the interior tissue, which consists mainly of amorphous and colourless jeily in which lie the coiled chains of gonidia; some of ti.e larger cells of the chains are colourless; between them run the fine hyphæ.

form and mode of growth, while the gonidia have only a secondary share in the formation of tissue. The former is the case in only a few Lichens; the latter is much
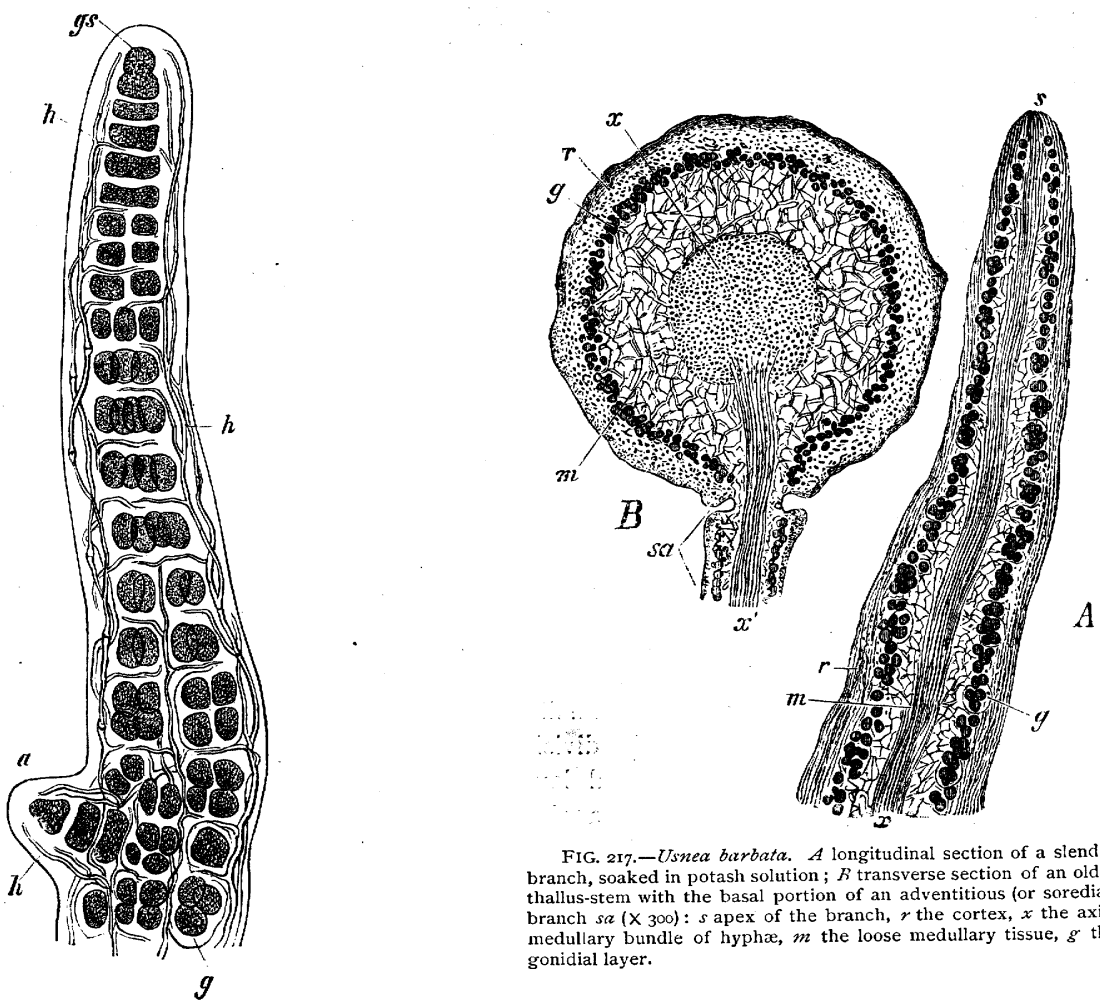

FIG. 217-Usnea barbata. A longitudinal section of a slender ( Fin. branch, soaked in potash solution , thallus-stem with the basal portion of an adventitious for soredial)
branch $s a(x, 00): s$ apex of the branch, $r$ the cortex, $x$ the axial medullary bundle of hyphæe, $m$ the loose medullary tissue, $g$ the gonidial layer.

FIG. 216.-A branch of the thallus of Ephebe pubescens ( $\times 550$ ).

the more common, and is that of the typical Lichens, especially of those that are heteromerous. In some homoiomerous gelatinous Lichens (as Fig. 215) it appears 
doubtful whether the change in the external form proceeds more from the gonidia or from the hyphæ. This relationship, which, although both morphologically and physiologically important, has not hitherto had sufficient attention paid to it by lichenologists, will be made sufficiently clear by an examination of Figs. 216 and 217 . In Fig. 2 I 6 is shown the longitudinal section of a branch of Ephebe pubescens; the large gonidia are left dark, and the very fine hyphæ are indicated at $b$. The branch increases at the apex by longitudinal growth and by transverse division of a gonidium ( $g s$ ), which is here the apical cell of the branch. The cells produced from the apical gonidium afterwards divide parallel to the longer axis of the branch; still later divisions are formed in different directions, and thus groups of gonidia arise at some considerable distance from the apex of the branch. The delicate hyphæ are represented in our figure as reaching to the apical gonidium; in other cases they come to a termination at a considerable distance beneath it. Even in this case it is only a few single hyphæ which follow the longitudinal growth of the branch; these grow within the gelatinous envelope which is evidently derived from the gonidia. At a considerable distance from the apex of the branch the hyphæ first put forth lateral branches which penetrate between the single or grouped gonidia, forcing their way through the deliquescent mass of their gelatinous cell-walls. Thus the whole form of the branch, its growth both in length and thickness, is determined by the gonidia; the hyphæ, from their small number and their fineness, produce scarcely any essential alteration either in the external form or the internal structure of the branch. This is clearly shown also in the origin of the lateral branches of the thallus. One of the exterior gonidia lengthens in a direction at right angles to the axis of the parent-branch, and becomes the apical cell of the lateral branch, producing at the same time new cells by transverse divisions, as is shown in Fig. $216, a$. Branches of the adjacent hyphæ turn in the same direction, and behave, in relation to the new apical cell, in the manner described above with respect to those of the primary branch.

In a manner similar to Epbebe pubescens, Usnea barbata, a fruticose Lichen, also forms a much-branched fruticose thallus. The branches of the thallus here also elongate by apical growth (cf. Fig. 217, $A$ ); but this is not brought about, as in Ephebe, by the gonidia, nor by a single apical cell. Each of the hyphæ at the end of the branch, which are nearly parallel and approximate at the apex, elongates by the apical growth of its terminal cell, and thus they produce in common the apical growth of the branch; this is followed further backwards by an intercalary growth, the result of the intercalary elongation of the hyphæ and of the formation of new hyphæ in different directions. The hyphæ lie so close together near the apex that they form a compact mass without interstices; it is only at some distance from it that the hyphal tissue is differentiated into a very dense cortex of fibres interwoven on all sides, an axial bundle of denselycrowded threads running in the direction of length, and a looser layer (the medullary layer) furnished with air-containing interstices. The point below the apex where this differentiation of the hyphal tissue begins is also that of the point of commencement of the gonidial layer, which consists of small roundish green cells, collected in small groups in consequence of multiplication by division; and these groups themselves form a layer between the medullary and cortical layers ( $c f$. Fig. 217, $B$, the transverse section). Below the growing apex of the branch of the thallus there are only single gonidia, by the division of which the cells of the gonidial layer are produced. It is evident therefore that in Usnea barbata the growth in length and thickness and the internal differentiation of the tissue depend entirely on the hyphæ, and that the gonidia behave like foreign bodies in the hyphal tissue; the formation of new branches proceeds also from the hyphæ and not from the gonidia. The branching may be dichotomous; and in this case the apical cells of the hyphæ converge towards two nearly adjacent points, and then continue to grow in corresponding directions, so that the two equal branches form an acute angle. Adventitious branches arise laterally below the apex of the thallus, the cortical fibres forming at a particular point a new apex and subsequently growing 
outwards. Gonidia are also to be found behind the new apex, while the base of the branch sends out medullary fibres and an axial bundle into the primary branch, so

t that the homologous forms of tissue of the two are continuous. The growth of Usnea may be compared, irrespectively of subordinate points, to that of the so-called stroma of the Xylarix; the formation of the gonidia is a subordinate element in the structure of the whole.

In some crustaceous Lichens the thallus possesses in general no defined contour, and no external differentiation takes place; the thallus appears as a somewhat irregular aggregation of masses of gonidia traversed by hyphæ. In other crustaceous Lichens (as Sporastatia Morio, Rbizocarpon subconcentricum, Aspicilia calcarea, \&c.) the thallus forms lobed discs which increase by centrifugal growth at the margin; the growing margin consists altogether of hyphal tissue, in which, further inwards, masses of gonidia appear at a few isolated spots and gradually spread; the cortical tissue is indented at the circumference of the spots where the gonidia are formed. Isolated scaly pieces of a true Lichen-thallus thus arise on a fibrous substratum called the bypotballus ${ }^{1}$.

The Formation of the Spores of Lichens takes place in receptacles termed Apothecia, when they are similar to those of the Discomycetes, or Peritbecia, when they are similar to those of some Pyrenomycetes. They are formed in the interior of the tissue of the thallus,

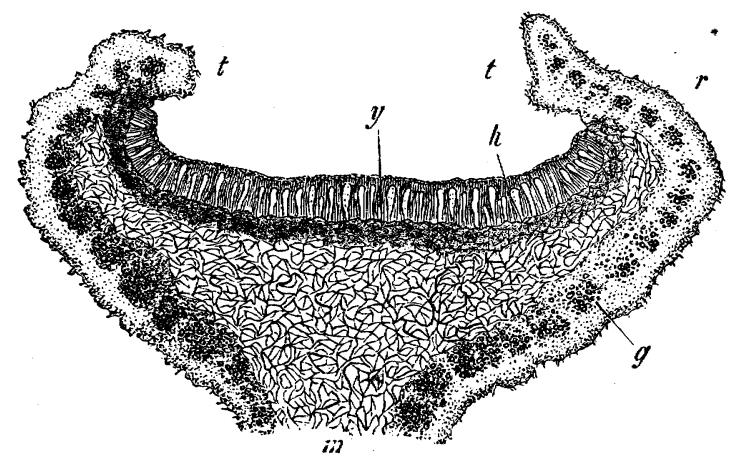

FIG. 218.-Vertical section of the apothecium of Anaptychia ciliaris (X about 50 ); $h$ the hymenium, $y$ sub-hymenial layer and excipulum; all the rest belongs to the thallus; $m$ its medullary layer, $r$ its cortex, $g$ its gonidia ; at $t t$ the thallus forms a cup-shaped rim round the apothecium.

and only appear above its surface at a later period, and then, in the one case they expand their hymenial layer to the air (Gymnocarpous Lichens), and in the other, they allow the spores to escape through an orifice (Angiocarpous Lichens). In all Lichens without exception the receptacle and all its essential parts take origin exclusively from the hyphal tissue; it is the Fungus alone that produces the receptacles; the nourishing Algæ, i.e. the gonidia, take no part whatever in it; or only in a secondary manner in so far as the thallus-tissue together with its gonidia grows like a wall round the apothecium and to a certain extent envelopes it (as shown in Fig. 218), or grows luxuriantly beneath the receptacle and raises it upon a kind of stalk above the surrounding thallus. The only exception to this endogenous origin of the receptacle occurs in Canogonium and similar forms, where it is impossible, because the hyphæ form only a very thin layer round the filamentous Alga which performs the part of gonidia ${ }^{2}$. These forms serve to show with especial clearness, as we know from Schwendener's researches, that the receptacle of Lichens belongs exclusively to the hyphal tissue.

The investigation of the development of the apothecium is attended with great difficulty, and more than one point is still obscure ${ }^{3}$. It originates, in heteromerous

1 See Schwendener, Flora, $186_{5}$, no. 26.

2 [See Archer, On Apothecia in some Algæ, Quart. Journ. Micr. Sci. 1875.]

3 What follows is taken from De Bary's account of his own researches, and from those of Schwendener and Fuisting. [See Stahl, loc. cit.] 
Lichens, beneath the cortical layer, in the lower part of the gonidial zone, or, in some crustaceous Lichens, in the deepest part of the thallus in immediate contact with the substratum; in homoiomerous gelatinous Lichens and in Ephebe it arises beneath the surface of the thallus. The commencement of the apothecium is, in heteromerous Lichens, a very small roundish ball of confused interwoven hyphæ, on the outer side of which a tuft of very delicate hyphæ-the first paraphyses-rises at a very early period. The most external hyphal investment of this ball, and therefore surrounding the tuft of paraphyses and opening above (outwards), is termed by lichenologists the Excipulum. The further growth of the rudiment of the apothecium is now occasioned by the increase in size of the excipulum by the formation of new fibres, while new paraphyses are intercalated among those already formed and outside the tuft, the extension of the apothecium being the immediate result of the fresh formation of these bodies. Growth is first completed in the centre of the apothecium; at the outside it continues longer, often even after the appearance of the apothecium above the surface of the thallus. The mother-cells of the spores, the asci, are formed, according to Schwendener and Fuisting, in a peculiar manner. 'Even in the young ball, and among the first rudiments of the paraphyses, thicker hyphæ are to be seen interwoven among the rest, rich in protoplasm, undivided by septa, and with numerous ramifications; the upright ends of the branches of these hyphæ which penetrate between the ends of the paraphyses develope into club-shaped asci; they may hence be termed ascogenous bypha. They are very readily distinguished from the paraphyses by their membrane being coloured blue by iodine after treatment with potash-solution, while that of the paraphyses remains colourless. They disappear at a very early period from the lower part of the rudiment of the apothecium, and remain only in one narrow layer which runs parallel to the upper surface of the apothecium, and extends below the lower ends of the ripe asci. In this layer they ramify in a centrifugal direction in proportion as the margin of the excipulum grows, and send out new asci among the new paraphyses. The first asci appear in the centre of the apothecium; and Schwendener states that no genetic connection exists between the ascogenous hyphæ and those from which the paraphyses are derived; the two form separate systems but interwoven into one another ${ }^{1}$. The layer in which the ascogenous hyphæ run is called the $S u b$ bymenial Layer; the hymenium itself consists of the paraphyses and the asci taken together. The term Hypotbecium is given to the mass of fibres which lies beneath the subbymenial layer, and is often strongly developed through subsequent growth; it consists of hyphæ the branches of which end in the hymenium as paraphyses, and of the remains of the primary ball; when mature, it can scarcely be distinguished from the excipulum. The growing apothecium bulges more and more, and finally breaks through the layer of thallus which overs it; the hymenium and the margin of the excipulum appear above the surface of the thallus, or the part of the thallus which surrounds the excipulum rises and grows with it forming a bowl-like rim. Among the medullary hyphæ which surround the apothecium a number of gonidia subsequently appear in many Lichens, so that a gonidial layer runs beneath the apothecium. In Peltigera and Solorina even the young apothecium is expanded flat, its paraphyses project vertically towards the surface of the thallus, and the layer of thallus which covers them is finally lifted like a thin veil. In Baomyces, Galycium, \&c. the basal portion of the hypothecium is developed into a long stalk which supports the apothecium.

1 From the newly-discovered processes in the formation of the reproductive organs of the Pyrenomycetes and Discomycetes, especially from the most recent statements of Janczewski on $A$ scobolus furfuraceus ( $f$. p. 309), it may be assumed that the tubular hyphæ of the sub-hymenial layer arise from a yet undiscovered ascogonium or scolecite; and that thus the apothecium of Lichens is the result of a sexual process in a similar manner to the perithecia of the Pyrenomycetes and the apothecia of Peziza and Ascobolus. [This has been discovered by Stahl. See the end of this section.] 
The perithecium of Angiocarpous Lichens is so similar in its mode of development and in its mature state to that of the Xylariæ, that there is no need to give an exact description of it.

The club-shaped asci of Lichens are similar in every essential point to those of the Pyrenomycetes and-Discomycetes; their wall is often very thick and capable of swelling; the spores (Fig. 219) arise simultaneously, as in those Fungi, by free-cell-formation, while a considerable portion of the protoplasm often remains unused in their production. The normal number of spores is eight, although sometimes only I-2 (in Umbilicaria and Megalospora), 2 or 3 or from 4 to 6 (in several Pertusarix); in Bactrospora, Acarospora, and Sarcogyne on the other hand their number amounts to some hundreds in one ascus.

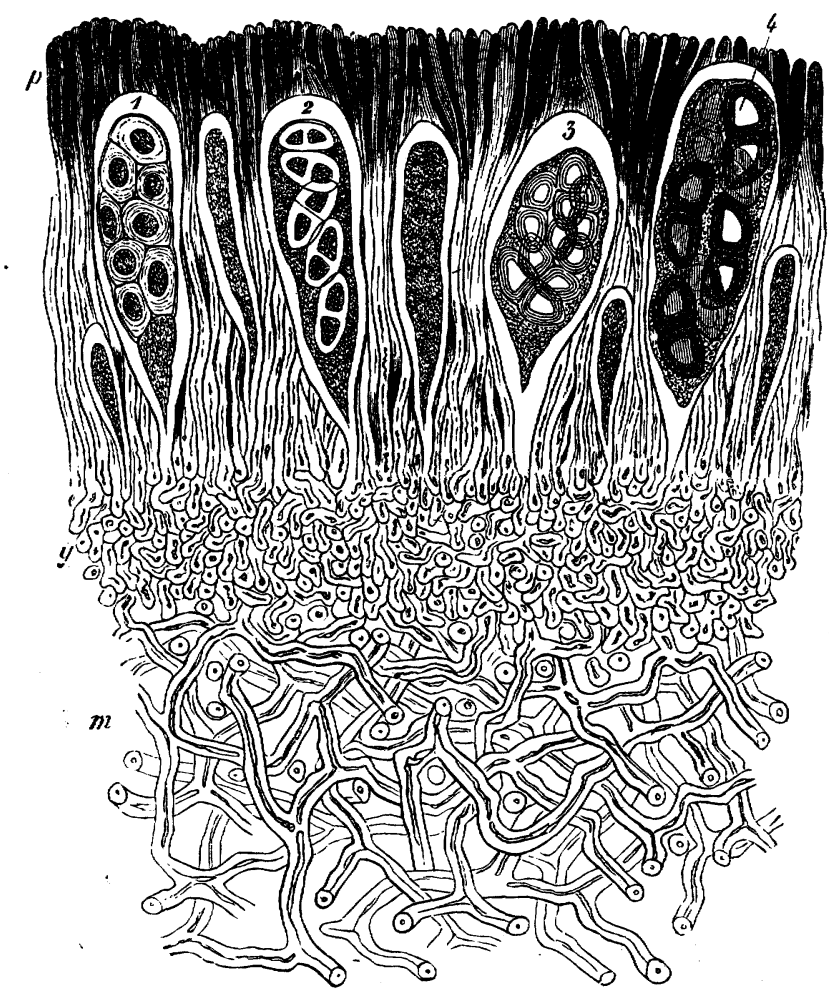

FIG. 219.-Vertical section of a small portion of the apothecium or Anaptychia ciliaris ( $\left.\times 55^{\circ}\right)$; $m$ the medullary layer of the thallus; $y$ the hypothecium, together with the sub-hymenial layer; $p$ the paraphyses of the hymenium, their upper ends of a brown colour; among them are the asci in various stages of development; in $x$ are the young spores not yet septate, in $2-4$ the spores more fully developed; the protoplasm in which the spores are imbedded is contracted by the drying up of the Lichen before the preparation was made.

The structure of the spores is very various, but in general similar to that of the Ascomycetes; very commonly they are septate and multicellular; the exospore is usually smooth and often variously coloured.

The spores are set at liberty by moisture penetrating the hymenium; they are suspended in the fluid which fills the ascus, and are expelled together with the fluid by the rupture of its apex. This expulsion is probably caused by the lateral pressure of the swollen paraphyses and the property of swelling possessed by the membrane of the ascus itself.

The germination of the spores of Lichens takes place by the endospore of each spore-cell putting out a filament which ramifies and extends over the damp substratum 
on which the spore is placed. The mode of germination of the very large spores of some genera, Megalospora, Ochrolecbia, and Pertusaria, differs from that of all the rest. They are simple, not septate, and densely filled with drops of oil (Fig. 220, $A, B$ ). Each spore puts out from different parts of its circumference a great number, even as many as a hundred, germinating filaments. The formation of each begins with the appearance in the endospore of a cavity widening from within outwards, which becomes surrounded by a very delicate membrane and grows outwards in the form of a filament (Fig. 220, $A, B$ ).

Besides the apothecia with ascospores capable of germination, Spermogonia are also generally present in Lichens, as in Ascomycetes; they generally occur on the same

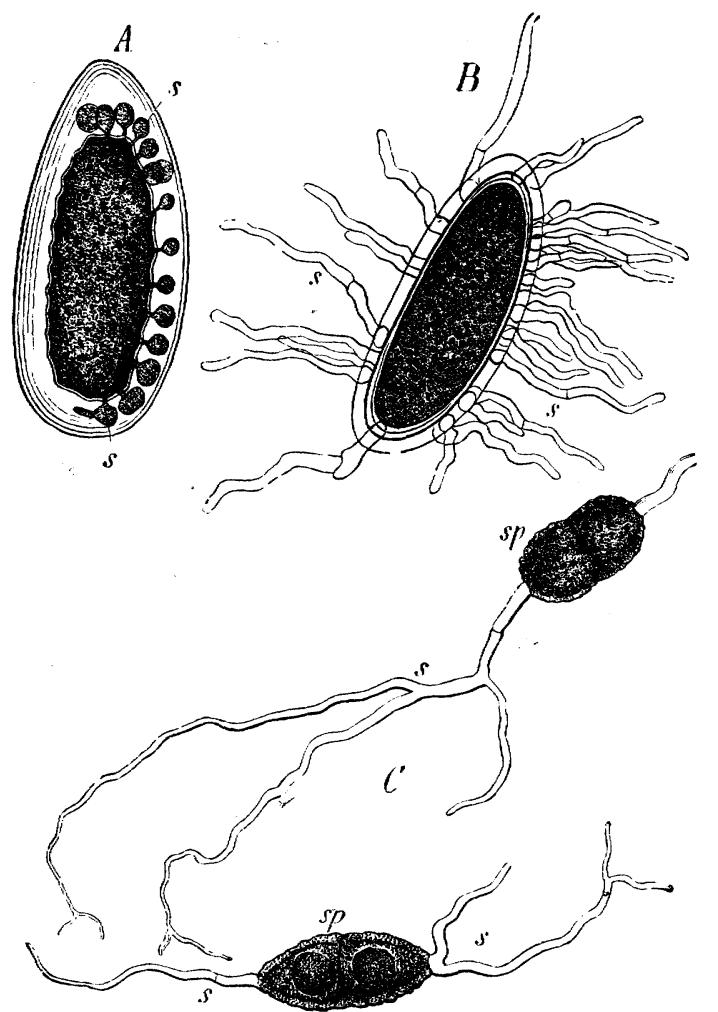

FIG. 220.-Lichen-spores germinating; $A$ longitudinal section of a spore of Pertusaria communis after lying 34 hours in glycerine, $s$ the rudiments of the germinating filaments; $B$ spore of Pertusaria leioplaca with a number of germinating filaments (after De Bary, $\times 390$ ); $C$ germinating septate spores of Solorina saccata (after Tulasne).

thallus as the apothecia. They are cavities in the thallus which are globular, flaskshaped, or sinuous, and densely clothed and almost filled with sterigmata; from these sterigmata the spermatia are detached in very large numbers, and escape through a fine orifice in the spermogonium. Sometimes also receptacles are found in which larger bodies, more like spores, are detached from the sterigmata ; receptacles of this kind are called Pycnidia, as in the Pyrenomycetes.

Besides the spores, most Lichens also possess organs termed Soredia, by which they are very extensively reproduced. They are single gonidial cells or groups of gonidia which, surrounded by a weft of hyphæ, are pushed out of the thallus, and are able, without any further process, to grow into a new Lichen-thallus. . The soredia are produced from the thallus in the non-gelatinous Lichens, as a fine powder, forming sometimes 
dense pulvinate masses (as in Usnea, Ramalina, Evernia, Physcia, Parmelia, Pertusaria, \&c.). In the heteromerous thallus the soredia appear in the gonidial layer; single gonidia, or sometimes several together becoming woven over by branches of hyphæ which cling
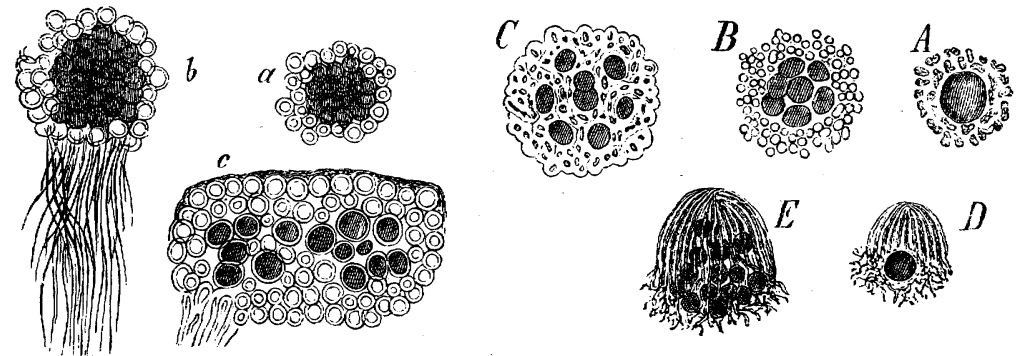

FIG, 22I $-A-D$ soredia of Uisuex barbata. $A$ a simple soredium, consisting of a gonidium covered with a web of hyphæ; $B$ a soredium, in which the gonidium has multiplied by division; $C$ a group of simple soredia, resulting from the penetration of the hyphæ between the gonidia; $D, E$ germinating soredia; the hyphæ are forming an apex of growth, and the gonidia are multiplying; $a-c$ soredia of Physcia parietina; $a$ with an envelope of pseudo-parenchyma; $b$ the envelope producing rhizines; $c$ a young thallus formed from a soredium (after Schwendener, $X_{500}$ ).

closely to them and form an envelope of fibres. The gonidia divide repeatedly, and each daughter-cell is again woven over. This process is often repeated, the soredia accumulate in great numbers in the gonidial layer, and finally rupture the cortex. After
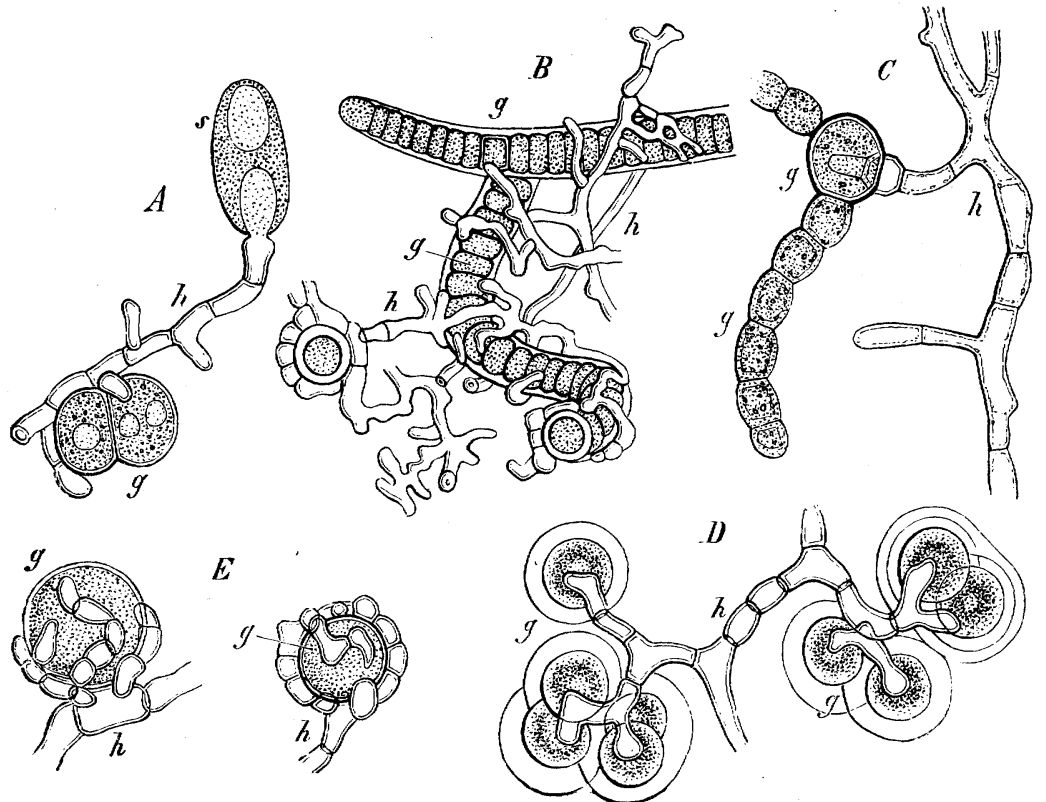

F1G. 222. -Examples of various Algæ which act as the gonidia of Lichens (after Bornet); $h$ in all cases is the hypha of the Fungus, and $g$ the gonidium. A, germinating spore $s$ of Physcia parietina, the hypha of which has attached itself to Protococcus viridis; $B$, a filament of Scytonema invested by the hyphæ of Stereocaulon ramulosum; $C$, taken from the thallus of the Lichen, Physma chalaganum; a branch of the hypha is penetrating into a cell of the Nostoc-filament (gonidium); $D$, taken from the thallus of Synalissa symphorea, the gonidia are the Alga Gloeocapsa; E, from the thallus of Cladonia furcata, the gonidium (Protococcus) is invested by hyphæ.

escaping in this manner, the soredia can still further multiply outside the thallus; but under favourable conditions either a single soredium or a mass of them grows out at once into a new thallus (Fig. $22 \mathrm{I}$ ). Schwendener states that in Usnea barbata this may occur. 
while the soredia are still included in the mother-thallus; soredial branches, as they are termed, are thus produced.

We may now turn to the consideration of the other elemental form out of which, in addition to the Fungus-hyphæ, the thallus of Lichens is constructed, the Gonidia. It has already been suggested that these are nothing but Algæ which are attacked and surrounded in their growth by Ascomycetes, and serve as hosts to them, the capability of assimilating inorganic materials being wanting on the part of their parasites.

Passing over the views of the older lichenologists, which will be found collated in the writings, cited below, of Baranetzky and of Schwendener, it may be pointed out here that De Bary (Handbuch der physiol. Bot. vol. ii. p. 29r) àrrived at the following alternative conclusions with respect to the gelatinous Lichens, such as Epbebe and similar forms; 'that either these Lichens are the completely developed fructifying states of plants the incompletely developed forms of which have hitherto been placed among Algæ, as Nostocaceæ and Chroococcaceæ, or the Nostocaceæ and Chroococcaceæ are typical Algæ which assume the form of Collemæ and Ephebæ \&c., in consequence of the penetration into them of certain parasitic Ascomycetes the mycelium of which extends throughout the growing thallus and often becomes attached to the cells filled with phycochrome (Plectospora, Omphalaria). In the latter case the plants in question might be termed Pseudolichens.' From the close of this quotation it appears that the writer does not apply the latter alternative to the heteromerous Lichens at any rate. Soon afterwards Famintzin and Baranetzky, and then the latter alone, published researches upon the further changes which the gonidia of Lichens undergo when they are set free by the decomposition of the hyphal tissue in water ${ }^{1}$. Baranetzky comes to the conclusion that 'the gonidia of the heteromerous chlorophyll-containing Lichens (Physcia, Evernia, Cladonia), as well as the heteromerous forms containing phycochrome (Feltigera) and of the gelatinous Lichens (Collema), are capable of carrying on an entirely independent life outside the lichen-thallus. When set free, the lichen-gonidia appear to extend their cycle of life; thus, for instance, the independently vegetating gonidia of Physcia, Evernia, and Cladonia produce zoogonidia.' He also found that all the cells of the spherical masses composed of the gonidia of Peltigera undergo a transformation so as to become extremely like the interstitial cells of a Nostoc, and he did not doubt that this was their permanent condition. 'Some, perhaps many, of the forms hitherto described as Algæ must be considered as independently vegetating lichen-gonidia, for the present at any rate; such are Cystococcus, Polycoccus and Nostoc.' The researches of Schwendener carried on, in part earlier, in part simultaneously and later, in the most careful manner, led to the opposite conclusion, that the gonidia are in fact Algæ which are more or less disturbed in their manner of life by the Fungus which is parasitic upon them. He first definitely stated and explained this view in his treatise 'Ueber die Algentypen der Flechtengonidien,' (Basel, 1869), as applying to all Lichens. In this memorable work, which assigned to the Lichens for the future their true systematic position among the Ascomycetes, he gives an account of those genera of Algæ which were to that time known as the hosts of lichen-fungi, that is, as playing the part of gonidia.

\section{Bluish-green Algæ. (Nostochineæ.)}

Name of group of Alga.

(I) Sirosiphonex

(2) Rivularieæ .

(3) Scytonemex

(4) Nostocaceæ . .

(5) Chroococcaceæ .
Lichen in aubich they occur as gonidia.

Ephebe, Spilonema, Polycbidium.

Thamnidium, Licbina, Racobienna.

Heppia, Porccyphus.

Collema, Lempbolemma, Leptogium, Pannaria, Peltigera. Omphalaria, Euchylium, Pbylliscium.

1 Mém. de l'Acad. Imp. des Sci. de St. Pétersbourg, 7th series, vol. XI. no. 9 and Mélanges biologiques tirés du Bulletin de l'Acad. Imp. de St. Pétersbourg, vol. VI. I 867.-[Ann. des Sci. Nat.

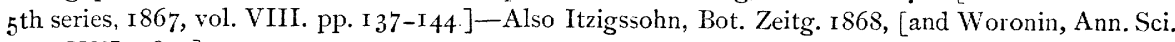
Nat. XVI, I $8 ; 2]$. 
II. Green Algæ.

(6) Confervacex (Cladophora) . . Conogonium, Cystocoleus.

(7) Chroolepideæ . . . . Graphideæ, Verrucarieæ, Roccella.

(8) Palmellacex . . . . Many fruticose and foliaceous Lichens.

Cystococcus bumicola . . Physcia, Cladonia, Evernia, Usnea, Bryopogon, and Anaptychia.

Pleurococcus . . . . Endocarpon and various crustaceous Lichens.

Protococcus . . . . Cladonia, Pbyscia.

Stichccoccus . . . . Spharomphale, Polyblastia.

(9) Coleochætex (Phyllactidium, Kütz.) Opegrapha filicina.

The inconceivable opposition offered to Schwendener's theory by lichenologists must surely be overcome by a recent publication of Bornet's ${ }^{1}$. After careful investigation of sixty genera of Lichens, he comes to the conclusion that each lichen-gonidium can be referred to some species of Algæ, and that the relations of the hyph $x$ to the gonidia are of such a kind as to exclude any possibility of assuming the existence of a genetic connection between them; that they find, in fact, their only satisfactory explanation in the theory of parasitism. Bornet shows that not only does the Alga which is the host of the Fungus become modified in consequence of the cohabitation, but that the Fungus itself often undergoes some change. He describes more accurately than had previously been done how the hyphæ of the Fungus attach themselves to the algal cells, and even penetrate into them, in order to absorb their contents, as the occurrence of the empty cell-walls of gonidia in the lichen-thallus suggests. He did not content himself with seeking in nature material which would show the Algæ being attacked by the lichen-fungus and the gradual formation of the thallus, but he sowed spores of Lichenfungi upon $A \lg ^{2}$ in order to be able to observe the manner in which the Fungus avails itself of the Alga. Of more especial interest is his proof of the fact that the same Alga may serve for very different F.ungi; for example, Clbroolepus umbrinum supports no less than thirteen genera belonging to five families of Lichens. Although many Lichen-fungi require to have particular Algæ as their hosts, a condition which occurs also in other cases of parasitism, it also happens that the same Lichen-fungus can avail itself of various forms of Algæ as its gonidia. The Alga which has been attacked by the Fungus and has become surrounded by its hyphæ is not always hindered in its growth, but in many cases is actually stimulated to more active vegetation. For further important details I must refer the reader to the work itself.

All reliable observations thus lead to the conclusion that a lichen-thallus is a mycelium which is nourished as a parasite by an Alga. The fructification of the Lichen, the apothecium or perithecium, belongs exclusively to the mycelium.

[The most conclusive evidence in favour of the truth of Schwendener's theory has been brought to light by the researches of $\mathrm{Stahl}^{3}$. In the first place, he succeeded, by cultivating the spores and hymenial gonidia of Endocarpon pusillum, in producing artificially a Lichen-thallus which bore perithecia and spermogonia. In the second place, he discovered that carpogonia are present in the thallus of certain Lichens which he investigated. The carpogonium of Collema microphyllum, for instance, is a hyphal filament which forms closely appressed coils at some distance below the surface of the thallus, and is then prolonged straight to the surface beyond which it projects. The carpogonium thus consists of two parts, the coiled portion, which Stahl terms the ascogonium, and the straight portion, which he calls the trichogyne. Spermatia, derived from

- Bornet, Recherches sur les Gonidies des Lichens, Ann. des Sci. Nat. t. XVII. I873.

${ }^{2}$ Compare also Reess, Monatsber. der Berl. Akad. I871 ; and Schwendener, Flora, I 872 ; also Treub, Bot. Zeitg. I873.

${ }^{3}$ [Beiträge zur Entwickelungs-geschichte der Flechten, I, II, I877: also de Bary, Die Erscheinung der Symbiose, I 879.] 
the spermogonia, are disseminated over the surface of the thallus by the agency of water, and are thus brought into contact with the projecting cell of each trichogyne, and to this they adhere. The contents of one at least of the spermatia pass into the trichogyne in consequence of the absorption of the cell-walls at the point of contact, and thus the carpogonium is fertilised. It will be seen that this mode of fertilisation is the same as that which has been described in the case of the Floridex. In consequence of fertilisation the cells of the ascogonium, as well as those of the adjacent hyphæ, are stimulated to growth, and the result is the formation of an apothecium; from the ascogonium are produced the asci, and from the adjacent hyphæ the paraphyses and the wall. It is clearly shown that the apothecium is derived solely from the fungal constituent of the Lichen. These results, besides throwing light upon the nature of Lichens, give a clue to the significance of the spermogonia and spermatia in other Ascomycetes and in the Ecidiomycetes.]

\section{B. The Acidiomycetes ${ }^{1}$.}

\section{(Uredinece.)}

If, in characterising this group, attention is confined, as in the case of the preceding groups, to those forms whose development is completely known, two extreme cases, as regard the conditions of reproduction and the alternation of generations, present themselves. In the simplest case the mycelium produces a fructification, the so-called Acidium, which consists, in its rnature condition, of a cup-shaped investment (peridium) and of a hymenium occupying its basal part; from the basidia of the hymenium spores are formed by abstriction. The spores thus produced (acidiospores) at once germinate, and each one developes a short filament consisting of but few segments, the growth of which soon ceases, and bearing upon short delicate branches smaller reproductive cells, the sporidia, which may be included under the term conidia in the sense in which that term has hitherto been used. The hypha bearing them is to be regarded as a promycelium. The sporidia, on germination, throw out hyphæ which penetrate into the epidermal cells of the host, and give rise to a mycelium which, in its turn, forms æecidium-fruits. In this case, which is found represented by Endophyllum Sempervivi, there occurs a simple alternation of generations, the alternating generations being the mycelium and the fructification (æcidium), with the slight variation that the æcidiospores give rise to the mycelium not directly but indirectly by means of the promycelium and its sporidia. The other extreme case is represented by Acidium Berberidis, Acidium Leguminosarum, and others. Here new mycelia are directly formed by the æcidiospores, without the intercalation of a promycelium. They do not, however, give rise to æcidium-fruits but develope conidia (the so-called Uredospores) upon basidia closely packed so as to form a kind of cushion, by means of which numerous generations of mycelia are produced during the period of vegetation. It is not until later that reproductive ceils of another kind, the Teleutospores, are produced in these generations to which the name Uredo has been given. These germinate in the following

1 Tulasne, Ann. des Sci. Nat. $3^{\text {rd }}$ ser. vol. VII; $4^{\text {th }}$ ser. vol. II. - De Bary, Ann. des Sci. Nat. 4th ser. vol. XX, and Monatsber. d. Berl. Acad. 1865.-Oersted, Bot. Zeit. 1865, p. 291.Reess, Die Rostpilzformen der deutschen Coniferen, Halle I869 (Abh. der naturf. Gesellsch. Bd. XI). -Oersted's System der Pilze, Lichenen, und Algen, translated into German by Grisebach and Reinke, Leipzig 1873, p. I9. [Schröter, Entwick. einig. Rostpilze, in Cohn's Beiträge, I, 18\%5.] 
spring and form promycelia, from the sporidia of which the mycelia which bear the æcidium-fruits are developed.

On comparing this second case with the first it becomes evident that here several generations of mycelia are intercalated between the formation of the æcidiospores and the formation of the promycelium. These generations give rise to peculiar reproductive cells, the uredospores and the teleutospores.

A sexual act has not as yet been observed even in these well-known forms of Acidiomycetes. If, however, we adhere to the rule that in the Thallophytes, as in Cryptogams generally, the most complex form of development is the result of a sexual act, and if we assume that an act of this kind does actually take place in this case, we cannot but regard the æcidium-fruit as the sexually-produced generation ${ }^{1}$. The æcidium-fruit will then correspond to the fructification of the Ascomycetes, the æcidiospores to the ascospores, and the uredospores, teleutospores, and sporidia to various forms of conidia. Should these probable assumptions be substantiated by future discoveries, it becomes at once evident that the nomenclature of the genera must be based not upon the forms bearing teleutospores, but upon those bearing æcidia, for instance the genus now known as Puccinia will have to be reconstituted

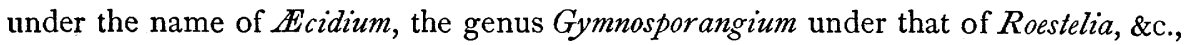
just as among the Ascomycetes not the conidia but the sporocarps afford the basis for their systematic arrangement.

That the uredospores and teleutospores are merely forms of conidia is demonstrated by the fact that they are present in some genera and species and absent in others, resembling in this respect the conidia of the Ascomycetes. They are both absent in Endophyllum, the uredospores are absent in Roestelia, and both are present in AEcidium Berberidis and in Acidium Leguminosarum.

The view which is here maintained is exclusively founded upon the well-known forms. There remains a much larger number of forms of which the life-history is only imperfectly traced. In a series of forms, for instance, the æcidium-fruits only are known (Acidium elatinum, Pini, abietinum ${ }^{2}$, \&c.), and it is still uncertain whether or not they reproduce themselves by the æcidiospores alone: in others only the teleutospores are known (Chrysomyxa, Puccinia Dianthi, compacta): in others again only the uredospores (Ccoma pinitorquum): uredospores and teleutospores, without æcidium-fruits, are known in Melampsora and Coleosporium. The last-named cases recall Penicillium and Eurotium in which, formerly, only the conidia were known and not the true fructifications, but they differ from them in that they possess two kinds of conidia. It appears that, like Penicillium and other Ascomycetes, certain Acidiomycetes can reproduce themselves for many generations solely by means of their conidia (uredospores and teleutospores) without attaining the completion of their development in the formation of a true fructification (æcidium).

The formation of the fructification of the Acidiomycetes, like that of many Ascomycetes, is accompanied by the development of peculiar receptacles, the

1 To this view I drew attention in the first edition of this book (1868). Oersted, loc. cit., and Brefeld also support it. [See also Stahl, Bot. Zeitg. I 874 .]

${ }^{2}$ [The life-history of $\mathbb{E} c$. abietinum has since been traced by De Bary (Bot. Zeitg. I879). The mycelium bearing uredo- and teleutospores infests the leaves of Rhododendron ferrugineum and hirsutum.] 
spermogonia, the significance of which in both cases is obscure. They make their appearance usually just before the æcidium-fruits and close beside them upon the leaves of the host. They extrude small conidia (spermatia) formed by abstriction, the significance of which in the life-history of the Acidiomycetes is completely unknown. It has been suggested that they are to be regarded as parasites, although the extraordinary constancy of their appearance certainly militates against this view.

The Æcidiomycetes exclusively inhabit living Phanerogams, mostly their stems and leaves, but also the living cortex of trees (Coniferæ). The extension of the mycelium in the intercellular passages of the host does not usually produce much injury, though in some cases the host becomes deformed, as for instance, the formation of 'witchesbrooms' in Firs by the growth of Acidium elatinum. Occasionally the mycelium is confined to certain circumscribed areas of its host (AEcidium Leguminosarum and others), but more commonly it extends throughout its tissues (Acidium Euphorbia cyparissix, Endophyllum Sempervivi). The fructifications, as also the conidia, are developed beneath the epidermis of the host, and penetrate through it to the surface only when they are mature.

Some of the best-known forms possessing conidia avail themselves of the same p'ant as a host throughout their whole life; for example, Acidium Leguminosarum and Tragopogonis : in others the various reproductive forms are developed upon different hosts, for example, the xcidium-fruits of Acidium Berberidis occur only on the leaves of Berberis vulgaris, whilst the uredospores and the teleutospores are formed only upon Grasses. Similarly the large æcidium-fruits of Roestelia cancellata occur only upon the leaves of Pomacex, the teleutospores only upon those of species of Juniperus. Such forms as these are said to be heterœcious (metœcious), to distinguish them from those above-mentioned which inhabit the same host throughout their whole life (autœcious).

The sporidia developed from the promycelium (whether this is derived from æcidiospores or from teleutospores) give off hyphæ which pierce the walls of the epidermal cells and penetrate into the interior of the host, whilst the hyphæ derived from the xcidiospores and from the uredospores grow upon the epidermis of the host until they reach a stoma through which they enter the intercellular passages. To this rule Puccinia Dianthi offers an exception, in that the promycelium derived from the teleutospores forms sporidia which send their hyphæ through the stomata.

Both the uredospores and the teleutospores protrude their hyphæ from certain definite portions of their surface at which the cuticularised external membrane (the exospore) is either wanting or is very thin. Each uredospore presents from three to six such areas lying in its equator, and each teleutospore has one in each cell. The teleutospores may be single, as in Uromyces, or in pairs, as in Puccinia, or three may be aggregated together, as in Triphragmium, or even four, as in Phragmidium. They germinate, usually after a considerable period of rest, in the spring, but occasionally immediately after their formation (Roestelia, Puccinia Diantbi).

In order to illustrate their life-history, I select as an example the Fungus the uredospores of which produce the 'rust' of Wheat, the Acidium Berberidis, hitherto known as Puccinia Graminis.

On the leaves of Berberis vulgaris are found in the spring yellowish swollen spots, where dense masses of mycelial filaments are interposed between the parenchyma-cells (Fig. 223, $A$ and $I$, the felted mycelium, lying between the cells, being indicated by dots). In these swollen spots are found two kinds of fructification, the Spermogonia, which are produced somewhat earlier, and the AEcidia. The spermogonia (Fig. 223, I, sp) are urn-shaped receptacles surrounded by a layer of mycelium as an envelope; hair-like threads which clothe the cavity protrude in the form of a brush from the opening of the spermogonium, penetrating the epidermis of the lcaf; the bottom of the spermo- 
gonium is covered with short mycelial branches, from the ends of which are detached numerous very small spore-like bodies, the Spermatia. It has already been stated that their significance in the development of the Fungus is unknown. The æcidium-fruits lie at first beneath the epidermis of the leaf, where they form a tuberous parenchymatous body - $(A)$, also surrounded by an envelope of fine mycelial filaments. When mature the xcidium breaks through the epidermis of the leaf and forms an open cup, the wall of which (the peridium, $p$ ) consists of a layer of hexagonal cells arranged in rows, which are produced at the bottom of the cup from basidium-like mycelial branches. The bottom of the cup is occupied by a hymenium, the hyphæ of which have their apices

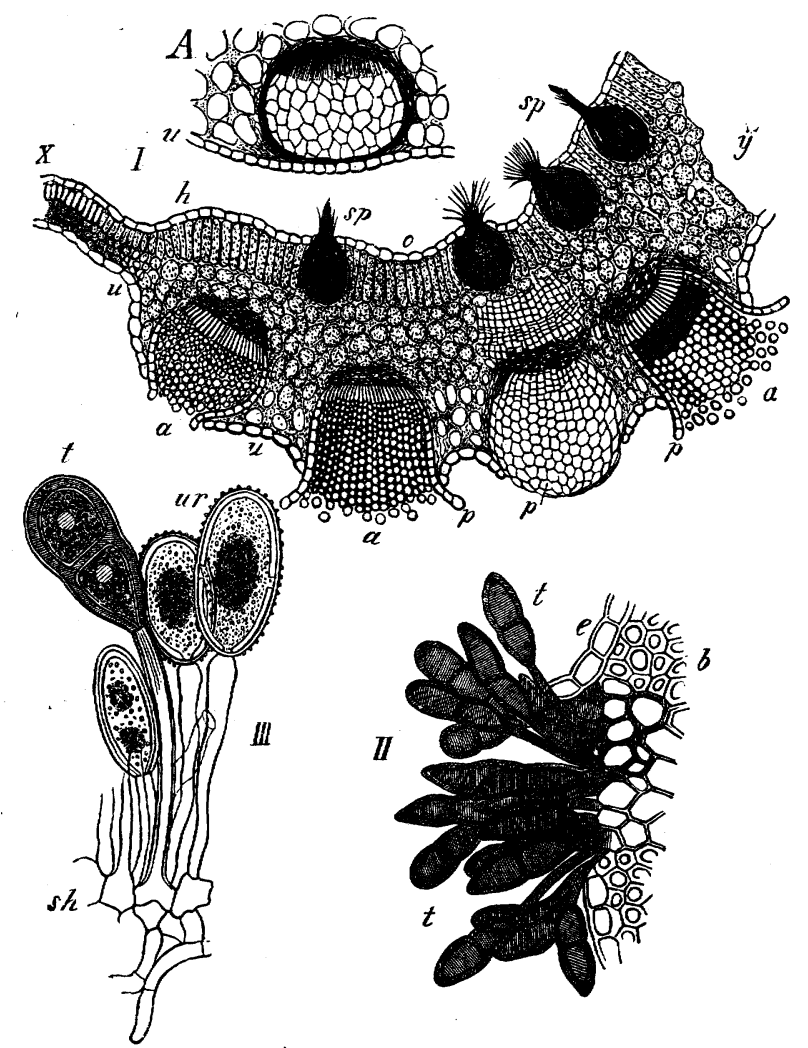

FIG. 223.-Puccinia Graminis. A part of a vertical section of a leaf of Berberis vulgaris with a young æecidium-fruit : $I$ section of leaf of Berberis with spermogonia $s p$ and æcidium-fruits $a ; p$ their peridium; at $x$ is the natural thickness of the leaf which is enormously thickened between $x$ and $y ; I I$ a mass of teleutospores on a leaf of Couch-grass; $e$ the ruptured epidermis; $b$ the hypodermal fibres; $t$ teleutospores; III part of a mass of uredospores $u r$ with one teleutospore $t$; $s h$ sub-hymenial hyphæ ( $A$ and $I$ from nature; $I I$ and $I I I$ after De Bary).

directed outwards and are continually detaching new conidia-like spores, which, originally of a polyhedral form in consequence of pressure from opposite sides, afterwards become rounded, and separate from one another at the opening of the cup $(I, a)$. The peridium itself has the appearance of a peripheral layer of similar spores; its cells however remain united, and, like the spores, contain red granules. The xcidiospores produced upon the leaves of Berberis only develope a mycelium when their germination takes place upon the surface of a leaf or stem of Grass (as Wheat or Rye). The germinating filaments then penetrate through the stomata, and the mycelium produced in the parenchyma of the Grass generates within 6 or Io days the uredospores $(I I I, u r)$, which are 
formed in cushion-like masses of mycelium upon densely-crowded branches (basidia) directed outwards immediately beneath the epidermis. They contain red granules and are perceptible with the naked eye as narrow long red projections upon the leaves and stems of Grasses. These uredospores are dispersed after the rupture of the epidermis, and germinate after some hours upon the surface of the Grasses (Fig. 224,D) : in these they form new mycelia which, in 6 or 10 days, bear uredospores again. While the Fungus is multiplying in this manner for several generations on Grasses during the summer in its uredo-form, the production of a new form of spores begins in the older uredo-fruits; the long two-celled teleutospores begin to be formed near the roundish uredospores (Fig. $223, I I I, t)$. The formation of uredospores in the uredo-

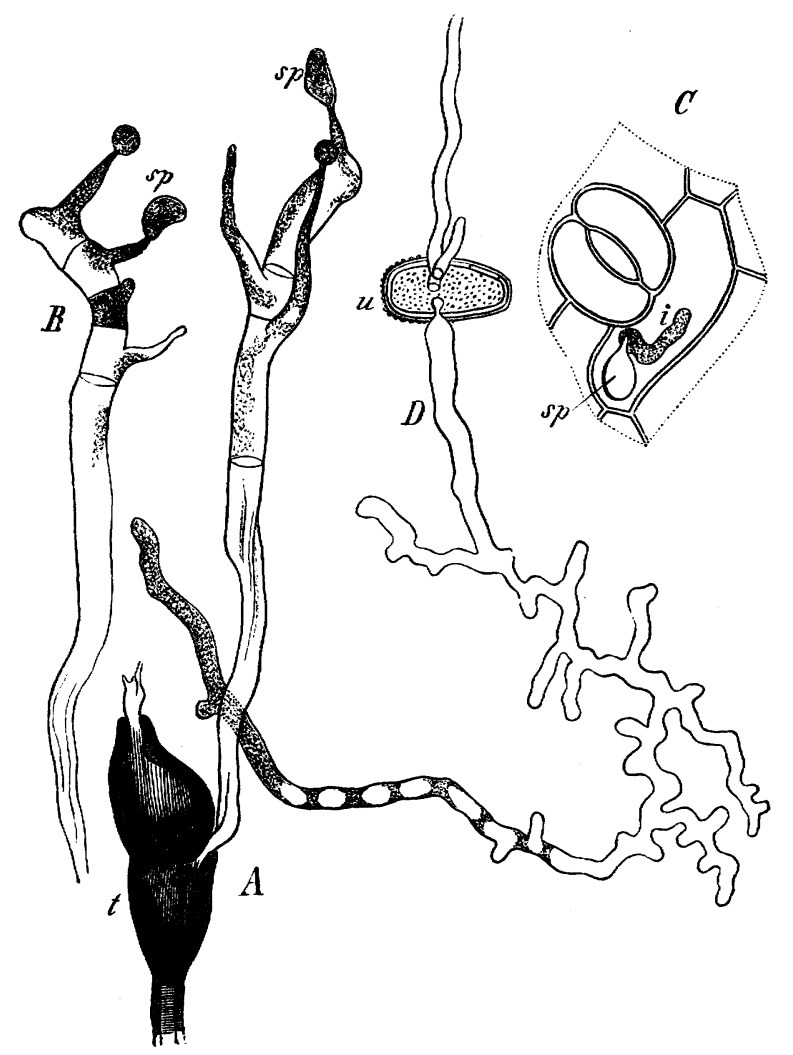

FIG. 224. - Puccinia Graminis. A germinating teleutospore $t$, the promycelium of which forms the sporidia sp: $B$ a promycelium (after Tulasne); $C$ a piece of the epidernis of the lower surface of the leaf of Berberis vulgaris with a germinating sporidium $s p ; i$ its germinating filament penetrating the epidermis; $D$ a germinating uredospore 14 hours after dissemination (after De Bary, l.c.).

fruits then entirely ceases, and teleutospores only are produced (Fig. $223, I I$ ), and with them the period of vegetation closes. The teleutospores persist on the grass-haulms through the winter, and do not germinate till the spring; they emit from their two cells short septate germinating filaments (Fig. 224, $A, B$ ), the promycelia, at the ends of which, on slender branches, the sporidia are produced. These sporidia develope a new mycelium only when they germinate on the surface of the leaves of the Barberry; their mode of germination differs from that of the other forms of spores, their germinating filaments penetrating, as in the Peronosporex, into and through the epidermis-cell (Fig. ${ }_{22}, C, s p$ and $i$ ), and thus reaching the parenchyma. They there form a mycelium 
which produces the swelling of the leaf constituting the first stage which we considered, a mycelium which bears spermogonia and æcidium-fruits.

The genus Roestelia possesses no uredospores. Its æcidium-fruits which make their appearance in July and August upon the leaves, petioles, and fruits of the Pomacer (Pyrus, Cydonia, Sorbus) resemble long-necked flasks and may become as much as eight millimetres long; they open either at their apices or laterally by means of slits. The chains of spores present a peculiarity which occurs also in other instances, that between any two spores there lies a sterile cell which subsequently decays. The teleutosporefruits belonging to Roestelia (formerly known as Gymnosporangium) appear upon species of Juniperus in the spring as spherical, conical, clavate, tongue-shaped, or palmate gelatinous masses of a yellow or brown colour. They consist of closely-placed basidia arising from the mycelium which extends beneath the epidermis of the leaves and in the cortex of the branches, and bearing the teleutospores. The teleutospores resemble those of Acidium Berberidis, and like them produce promycelia on germination, the sporidia of which reproduce Roestelia with æcidium-fruits upon the leaves of Pomaceæ.

Under the name of Hypodermieæ De Bary unites the Uredineæ with the Ustilaginex, which, however, do not seem to be very closely related to them. The Ustilaginem ${ }^{1}$ (Smuts) are parasitic in the tissues of Phanerogams, especially of Grasses, in which their mycelium ramifies without at first effecting any injury. It is only when the fructification, consisting of spherical dark-coloured conidia, is formed that the vegetable organ in which it occurs becomes deformed: this usually swells up into a vesicle, the whole of the internal tissue being absorbed and replaced by a black powder, the conidia of the Fungus. Maize seeds are in this way converted by the Ustilago Maidis into vesicles of the size of a nut, which, on bursting, liberate the powdery conidia; Oats attacked by the smut are entirely filled with the conidia of Tilletia caries. The germinating conidia produce a small promycelium which bears sporidia: the 'hyph $x$ developed from the sporidia penetrate into the sprouting grain and the mycelium continues to grow until it produces conidia in the ears.

\section{The Basidionycetes ${ }^{2}$.}

Although this division includes the largest and most beautiful of the Fungi, yet it is just here that our knowledge of their life-history is most imperfect. All that is certainly known is that the basidiospores developed upon the large fructifications consisting of masses of hyphæ, germinate, forming mycelia, and that at a later period these mycelia bear fructifications. A development of sexual organs, by means of which the formation of the fruit could take place, has not as yet been observed upon the mycelium; still, a consideration of our knowledge with regard to the Ascomycetes, more especially the Discomycetes, makes it at least probable that the spore-producing fructification is to be regarded as a true fruit which owes its origin to the as yet undiscovered sexual organs existing upon the mycelium ${ }^{3}$. However this may be, the whole process of development naturally divides itself in

${ }^{1}$ [Tulasne, Mémoires sur les Ustilaginées; Ann. Sci. Nat., sér. 3, VII, sér. 4, II.-De Bary, Unters. ueb. die Brandpilze, Berlin 1853.-Fischer von Waldheim, Aperçu systématique des Ustilaginées, Paris 1877.-De Bary, Protomyces microsporus und seine Verwandten, Bot. Zeitg. I874.]

${ }^{2}$ See De Bary, Morphol. u. Physiol. der Pilze, Flechten, und Myxomyceten, Leipzig I866.

${ }^{3}$ [From the researches of Brefeld (Basidiomycetes, 1877) it appears that these plants have no sexual reproduction. Their large fructifications are comparable to the asexual conidia-bearing fructifications of the Ascomycetes. The so-called 'basidiospores' are, like the 'uredospores' and 'teleutospores' of the Acidiomycetes, merely conidia.] 
this case also into two principal stages, the first being that of a filamentous mycelium derived from the spores, the second, that of a fructification of solid tissue producing numerous spores. No other organs of reproduction are known to recur with certainty in the Basidiomycetes (see De Bary, loc. cit.).

The external form and the internal structure of these fructifications vary to a very great extent, but the formation of the spores takes place upon one common plan. Certain branches of the fertile hyphæ become swollen so as to be clubshaped, and constitute the cells which bear the spores, the Basidia. Each basidium produces simultaneously two or more, usually four, basidiospores (or even eight). They are formed by the outgrowth of the wall of the basidium into delicate papillæ which become spherically or ovally dilated at their free ends : each of these dilatations becomes invested by a firm membrane, and is a spore which remains for a time upon the pedicle but at length falls off.

The basidia are developed simultaneously in large numbers and are usually closely arranged parallel to each other; in this way the hymenia are formed, which, among the Hymenomycetes, contain, as in the Discomycetes, sterile cells (paraphyses) among the fertile ones (basidia). If the hymenial layer clothes its free external surface, the fructification is said to be gymnocarpous; if, however, it lines cavities within its tissue, the fructification is said to be angiocarpous.

The majority of Basidiomycetes grow upon humus, or soil containing decaying vegetable matter; some develope their mycelium in old wood, as in the cortex of living tree-trunks; the smaller forms use fallen leaves and decaying branches as their substrata. More rarely they occur as true parasites upon living vegetable organs.

The following account will suffice to draw attention to some of the most widely differing forms which are of morphological importance.

(I) The simplest form of fructification is found in Exobasidium Vaccinii ${ }^{1}$, the mycelium of which is parasitic in the leaves and stems of Vaccinium Vitis idaa. On the surface of the organs which it has attacked, the mycelium directly bears a hymenium consisting of closely-packed basidia, each bearing four spores.

(2) The gelatinous Fungi, Tremellineæ, which grow upon dead wood or upon the trunks of old living trees, produce fructifications of a gelatinous consistency and of irregular form, usually occurring as thick rugose incrustations. The delicate hyphæ run in the gelatinous mass and form the hymenia at the surface. The formation of the spores takes place in a more complicated manner than in the other Basidiomycetes ${ }^{2}$.

(3) Among the Hymenomycetes the best known and most abundant species are those commonly known as Mushrooms. The structure which is usually called the Fungus is the fructification which springs from a mycelium vegetating in the ground, or on wood or some other substance. Usually, but not always, the cap (pileus) is stalked ; on its undersurface the hymenial layer lies upon projections of the substance of the pileus of various forms. In the genus Agaricus these projections consist of numerous lamellæ attached vertically and running radially from the summit of the stalk to the margin of the pileus; in Cyclomyces the lamellæ form concentric circles; in Polyporus and Dadalea they anastomose in a reticulate manner; in Boletus they form closely crowded vertical tubes; in Fistulina the tubes stand alone; in Hydnum the lower side of the pileus is covered with soft

1 Woronin, Bericht der naturf. Gesellsch. in Freiburg. vol. IV. 1867.

${ }^{2}$ See Tulasne, Ann. des Sci. Nat., 3rd series, vui. XIX. [Also, ib. sér. 5, XV, and Brefeld, loc. cit.] 
dependent spines like icicles, the surface of which bears the hymenium, \&c. In many cases the fructification is naked; in others the lower side of the pileus is covered with a membrane which is afterwards ruptured (velum partiale), or the pileus and stalk are both enveloped in such a membrane (velum universale); or finally, in a few species (Amanita) both are found. This formation of a velum is connected with the entire growth of the whole fructification; the naked pilei are essentially gymnocarpous, those covered by a velum indicate a transition to the angiocarpous fructifications of the Gasteromycetes. Agaricus variecolor is to a certain extent an intermediate form between those with naked pileus and those furnished with a universal velum. The fructification in this species arises as a slender cone on the mycelium (Fig. 225, I, a, b), consisting of parallel

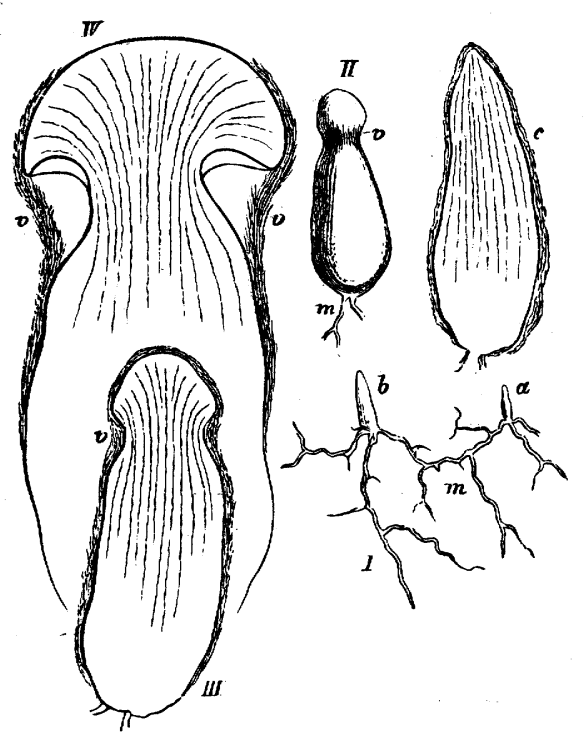

FIG. 225.-Agaricus variecolor. I mycelium $m$, with young fructifications $a b$ (natural size); $c$ longitudinal section of one of the latter (magnified); $I I$ an older fructification, with commencethe latter (magnified); $I I$ an older fructification, with commence-
ment of the formation of the pileus; $I I I$ the same in longitudinal section; $I V$ a more mature pileus, $v$ the velum. The lines in the sections indicate the course of the hyphæ.

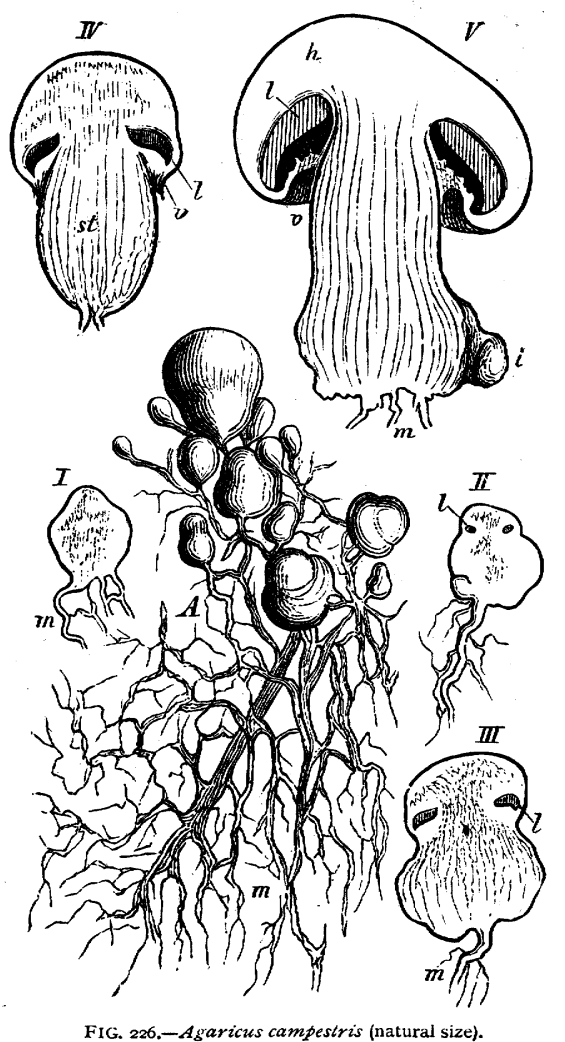

FIG. 226.-Agaricus campestris (natural size).

hyphæ growing at the apex $(I, c)$; an outer layer of hyphæ is present at an early stage surrounding the whole body as a loose envelope; afterwards the direction of growth alters, the branches of the hyphæ turn outwards beneath the apex (II, III) and thus form the pileus $(I V)$, the margin of which continues to grow centrifugally; the lamellæ are formed on its under-surface : as the distance of the margin of the pileus from the stalk increases, the loose peripheral layer of hyphæ becomes stretched $(I V, v)$, and forms a rudimentary universal velum. An example of the formation of a stalked pileus with a partial velum is afforded by the common mushroom (Agaricus campestris). Fig. 226 shows at $A$ a small piece of the greatly extended reticulately anastomosing mycelium $(m)$, from which spring a number of fructifications; these are at first solid pear-shaped bodies composed of young hyphæ all similar to one another. At an early stage the tissue of hyphæ gives way beneath the apex, leaving an annular air-cavity $(I I, l)$, the upper wall of which forms 
* the under-side of the pileus; and from this the radial hymenial lamellæ grow downwards $(I I I, l)$, filling up the air-cavity. The hyphæ run from the base of the whole fructification to the margin of the pileus, forming the outer wall of the air-cavity; the tissue lying in the centre elongates into the stalk $(I V, s t)$, while the distance from it of the margin of the pileus constantly increases; the hyphæ which lie beneath the air-cavity that contains the lamellæ become stretched in consequence, and separate from the stem from below upwards, forming a membrane $(V, v)$, running from the upper part of the stalk beneath the lamellæ to the margin of the pileus, into which their hyphæ are continued. When at length the pileus extends horizontally from the elongation of the tissues, the

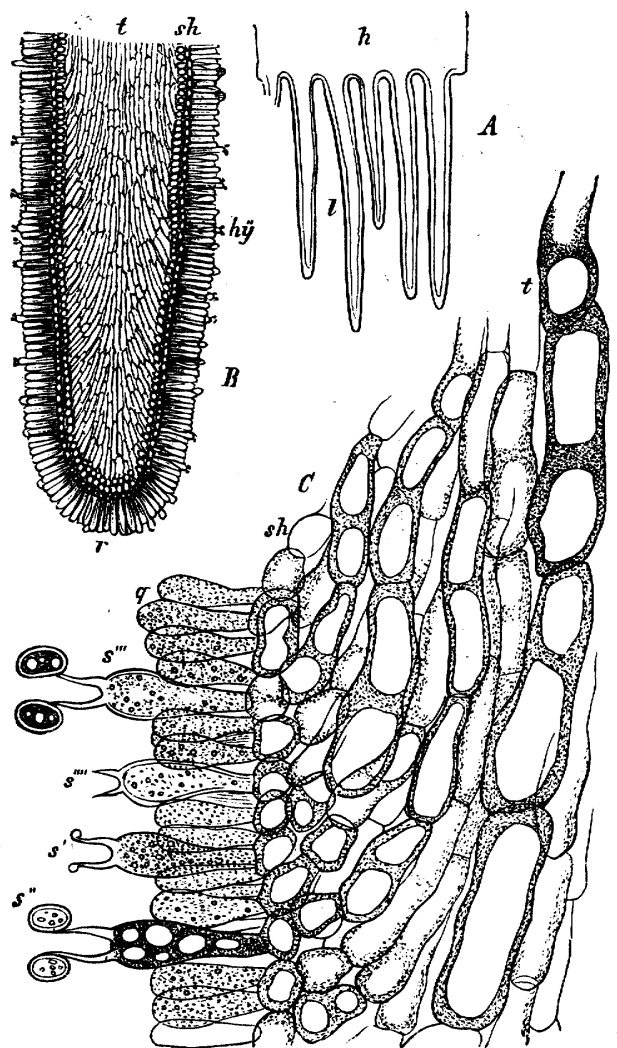

FIG. 227.-Agaricus campestris; structure of the hymenium; $A, B$ slightly magnified; $C$ a part of $B(\times 350)$. The protoplasm is indicated by fine dots. membrane (velum) becomes detached from its margin, and hangs from the stem like a ruffle (annulus). (Compare also Fig. 79, p. 96, Boletus favidus.)

The hymenium, as has already been mentioned, covers the surface of the lamelliform, peg-shaped, or tubular projections of the under-side of the pileus. A transverse section of the latter across the hymenium gives, in all three cases, nearly the same figure, as is seen in Fig. 227, drawn from Agaricus campestris. A shows a piece of the disc of the pileus cut transversely, $b$ the substance of the pileus, $l$ the lamellæ; in $B$ a piece of a lamella is more strongly magnified to show the course of the hyphæ. The substance of the lamella, called the Trama $(t)$, consists of rows of long cells, which diverge from the centre right and left to the outside, where the cells of the hyphæ are short and round, and form the sub-hymenial layer ( $s h$ in $B$ and $C$ ). From these short cells spring the club-shaped cells $(q)$, densely crowded and at right angles to the surface of the lamella, forming together the hymenial layer $(B, b y)$. Many of these remain sterile, and are called Paraphyses, others produce the spores and are the Basidia. Each basidium produces in this species only two, in other Hymenomycetes usually four spores. The basidium first of all puts out as many slender branches $\left(s^{\prime}\right)$ as

there are spores to be formed; each of these branches swells at the end, the swelling increases and becomes a spore $\left(s^{\prime \prime}, s^{\prime \prime \prime}\right)$, which falls from the stalk on which it was placed, leaving it behind $\left(s^{\prime \prime \prime}\right)$.

On the formation of the tissue of this group only one further remark need be made; that in the fructification of some Agaricinæ (e.g. Lactarius) some of the much-branched hyphæ are transformed into laticiferous vessels, from which large quantities of latex flow out when they are injured.

(4) The Gasteromycetes ${ }^{1}$ agree with the previous group in the mode of formation

1 [De Bary, Morphol, und Physiol. d. Pilze, I866.-Brefeld, Basidiomycetes, p. I 75.] 
of their spores (eight spores are often produced on a basidium); but their fructifications are always angiocarpous. The hymenia are formed in the interior of the fructification, which is at first usually spherical, or at any rate does not present externally any distinction of parts. The spores are disseminated by means of remarkable differentiations of the different layers, the growth of particular masses of tissue, or the simple bursting of the outer layer (the peridium). The nature of these processes, which are extremely various in their external appearance, may be understood from two examples. The first example, Crucibulum vulgare ${ }^{1}$, is selected from the beautiful Nidularieæ. The mycelium forms a small white crust of branched hyphæ, which extend over the surface of wood. In the middle of the crust the filaments are interwoven into a roundish body, the rudiment of the fructification; this grows by the intercalation of new branches of the hyphæ, and gradually assumes a cylindrical form. The outer hyphæ form at an early stage yellowish-brown branches, which are again branched and directed outwards, forming a dense covering of hair. While the spherical fructification is becoming changed
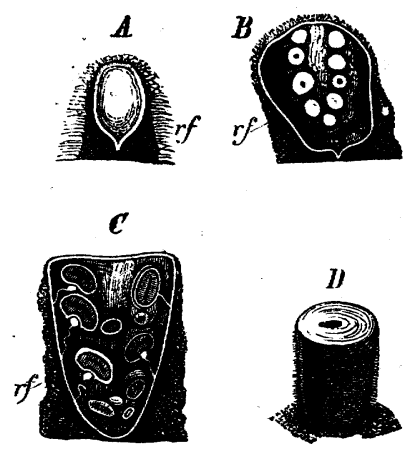

FIG. 228.-Crucibulum vulgare; $A, B, C$ in longitudinal section (slightly magnified); $D$ the entire plant nearly mature (natural size).

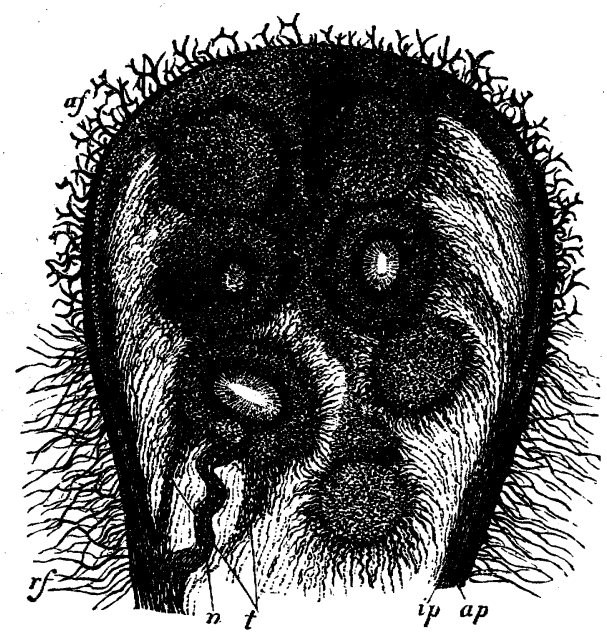

FIG. 229.-Crucibulum vulgare; longitudinal section through the upper part of a young fructification ( $X$ about the same as Fig. 228, B). The section is seen by transmitted light; the dark parts in the interior are those where air occurs between the hyphæ; at the light parts a transparent mucilaginous substance free from air has formed between the hyphæ. The light parts of this figure are dark in the previous ones.

into a cylinder, a large number of brown threads shoot out from it (Fig. 228, $C, r f$ ), which form a firmly-woven layer, the outer peridium, and on the outside of this a dense mass of radially projecting hairs. The walls of the hyphæ of this part assume a dark colour, but the inner tissue remains colourless (Fig. 228, $A$ ); its apex increases in breadth, the hairs separate from one another, and the outer peridium ceases to exist at the apex (Fig. 229, ap). In the meantime the differentiation of the tissue commences in the interior of the Fungus, which is at first formed of- densely-woven much-branched hyphæ, enclosing amongst them a considerable quantity of air which gives the whole a white appearance. Certain portions of the air-containing tissue become mucilaginous and freed from air; between the threads is formed in some places a hygroscopic transparent jelly, while in others none is produced. The conversion into mucilage begins first below the surface of the white medulla (Fig. 228, $A$ ), and its outer layer is thus transformed into an inner peridium which is a colourless sac projecting beyond the

1 Compare Sachs in Bot. Zeitg. 1855. [See also Tulasne, Annales des Sci. Nat. 1844, vol. I; Eidam, in Cohn's Beiträge, vol. 2, 1877.] 
dark outer peridium, and composed chiefly of branches of hyphæ running longitudinally upwards (Figs. 229 and $23^{\circ}, i p$ ). While this differentiation is proceeding from below upwards, small mucilaginous areolæ form at certain points in a deep layer of the white air-containing medulla, also proceeding from below upwards, like all the succeeding differentiations (Fig. 228, B, and Fig. 229). The formation of mucilage advances at the same time from the inner peridium inwards, and leaves round each of the mucilaginous areolæ a border of air-containing tissue (Fig. 229), which afterwards developes, by the dense interweaving of its branched hyphæ, into a firm envelope consisting of two layers, in which the mucilaginous areola lies. Each of these areolæ becomes a hymenial chamber. While the centre of the Fungus is becoming changed into mucilage,

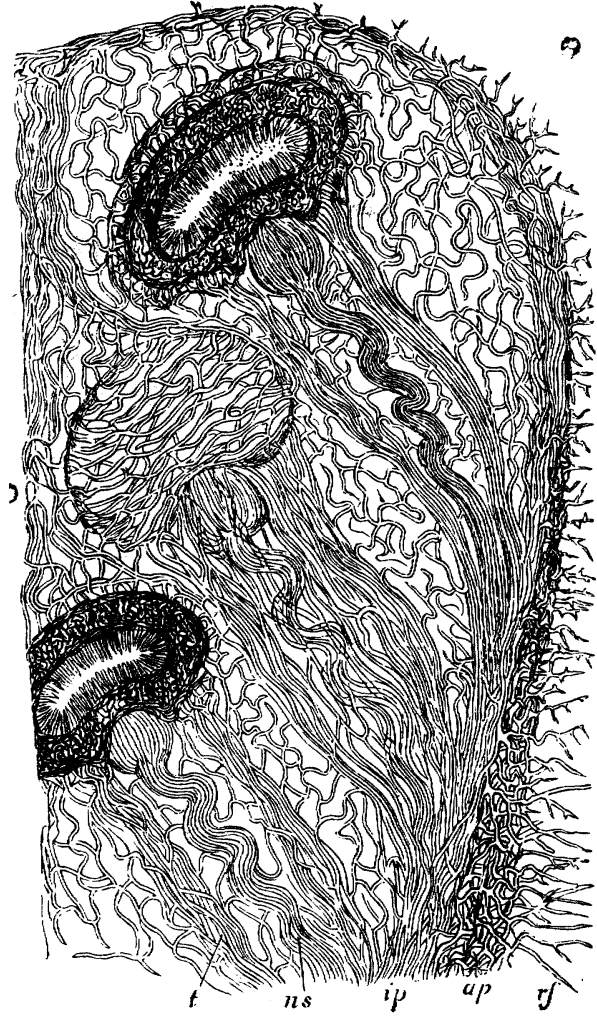

FIG, 230-Crucibulum vulvare; longitudinal section through the upper part of the right side of the mature fructification, showing the course of the filaments; for the sake of clearness the number of filaments has been reduced and their thickness increased.

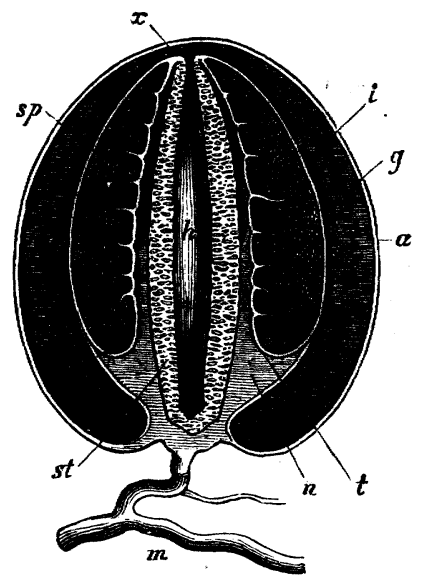

FIG. 23r.-Longitudinal section of a nearly ripe fructification of Phallus impudicus immediately before the elongation of the stalk ( $\frac{1}{2}$ the natural size); $a$ outer layer of the peridium; $g$ its gelatinous layer; $i$ inner peridium; st the stalk of the pileus $t$ not yet elongated, covered by the white honeycomb-like ridges; $s p$ the dark-green mass of spores (gleba); $h$ hollow cavity of the stalk, filled with watery jelly; $n$ the cup in which the base of the stalk remains after $n$ the cup in which the base of the stalk remains after its elongation; $x$ the place where the inner peridium
becomes detached by the elongation of the stem ; $m$ mycelial filament.

the chambers grow into lenticular bodies; a mucilaginous point has appeared at an early stage on the lower and outer part of each chamber, and forms its umbilicus. From it a denser bundle of threads runs downwards to the peridium, the umbilical bundle (Fig. 229, $n$, and Fig. $230, n s$ ); this is itself surrounded by a conical bag $(t)$ which surrounds the bundle like a loose sheath. This sheath eventually becomes mucilaginous; the bundle runs upwards into the mucilaginous depression of the umbilicus, where it is resolved into its threads which are now more loosely connected. The mucilaginous tissue in the interior of each chamber disappears, leaving a lenticular space similar in form to the chamber itself; and from the inner layers of the 
hyphæ of the chamber branches now arise which are directed inwards and form the hymenium. Each chamber is therefore clothed on its inner surface by a hymenial layer formed of paraphyses and basidia; each of the basidia produces four spores on short stalks. As the Fungus matures, the upper part of the peridium becomes stretched and flat, forming the Epiphragm; it afterwards ruptures and disappears, and the Fungus thus opens into a cup. The mucilage which surrounds the chambers dries up, and the chambers now lie free in the cup formed by the peridium, held by their umbilical bundles, which, when moistened, may be drawn out into long threads. If we imagine the chambers more numerous and more closely packed and with less dense walls, we obtain an explanation of the roundish cell-like loculi which occur in the fructification of other Gasteromycetes (as Octaviania, Scleroderma, \&c.).

Still more remarkable are the changes produced in the Phalloide ${ }^{1}$ by internal differentiation of the tissues; but of these only the most important points can be illustrated in the case of Pballus impudicus. Here also the young fructification formed on the underground perennial mycelium is at first a homogeneous convolution of hyphal filaments, in which differentiation begins and advances during growth. When the body has attained the size and form of a hen's or even a goose's egg, a longitudinal section gives the appearance represented in Fig. 23I. The tissue consists at this time of different portions which may be classified into four groups(I) The peridium, composed of an outer firm, thick, white membrane $(a)$, of an inner white, firm, but thin membrane (i), and of an intermediate thick layer of mucilaginous hyphæ $(g)$, (the gelatinous layer): (2) The spore-forming apparatus or Gleba ( $s p$ ), bounded on the outside by the inner peridium (i), on the inside by a firm thick layer $(t)$ from which walls project outwards united in a honeycomb manner dividing the gleba into a number of chambers. In these chambers the fertile branches of the hyphæ are found in great numbers, and on their basidia are formed four or more spores; so that, when ripe, the dark-green gleba appears to consist almost entirely of spores: (3) The stalk (st), formed of air-containing tissue hollowed into a large number of very narrow chambers; its axial portion is transformed into a deliquescent jelly, and the canal thus formed is open above in some individuals, in others it is closed by the inner peridium: (4) The $\operatorname{Cup}(n)$ forms a low broad column of firmer tissue, the outer part running upwards into the inner peridium, and sending up at the same time a layer which becomes softer between the stalk and the inner membrane of the gleba $(t)$; the base of the cup is continuous with the outer firm peridium. In this state the spores ripen; but for the purpose of their dissemination a great elongation of the stalk (st) takes place; the peridium is ruptured at the apex, the gleba becomes detached from the inner peridium, this latter splitting at $x$, and the membrane $t$ becoming detached below. The gleba is by this means raised up high above the peridium on the apex of the stalk, while the stalk attains the height of from 6 to 12 inches. This elongation is brought about by the expansion of its chambers, which give the mature stalk the appearance of a coarsely porous sponge; it increases in thickness in proportion to its increase in length. The spores now drop off the gleba in masses, the sporiferous hyphæ deliquescing into thick tenacious mucilage; till at last nothing remains of the gleba but the membrane $(t)$ with its honeycombed walls, which depends like a frill from the apex of the stalk, and is called the pileus. The peculiarities in the details of these processes exhibit the greatest variety in different species of the Phalloidex.

${ }^{1}$ [De Bary, Beitr. zur Morphol. u. Physiol. der Pilze, I. 1864.] 


\section{GROUP II.}

\section{U S C I N E $Æ$.}

The Liverworts (Hepaticx) and Mosses (Musci), which are comprised under the term Muscinex, are distinguished by a sharply-defined Alternation of Generations. From the germinating spore is developed either immediately a sexual generation rich in chlorophyll and self-supporting (as in most Hepaticæ), or a confervoid thallus is first formed (Protonema), out of which the sexual generation grows as a lateral shoot (as in some Hepatica and all Mosses). In the female sexual organ of this first generation there arises, as a new generation - the result of fertilisationa structure of an entirely different form, which is destined exclusively for the asexual production of spores. Without being organically united to the previous generation, this structure is nevertheless nourished by it, and appears, when observed externally, simply as its fruit, like the smaller fructifications of the Thallophytes. Since however it is an organism of an altogether peculiar kind, it may be desirable to give it a special name, which shall at once exclude any false analogy ${ }^{1}$; I propose therefore to call it the Sporogonium.

The Sexual Generation (Oophore) of Muscineæ which is produced directly from the spore or with the intervention of a protonema, is either a flat leafless thallus, as in many Hepaticæ, or a slender leafy stem, often much branched. In both cases, which are united by gradual transitional forms ${ }^{2}$, a number of root-hairs are usually formed, which fix the thallus or the stem to the substratum. In some cases this vegetative body scarcely attains a length of $\mathbf{I} \mathrm{mm}$., but in others as much as from to to $30 \mathrm{~cm}$. or even more, and ramifies copiously. In some of the smallest forms its term of life is limited to only a few weeks or months; in most it may be said to be unlimited, since the thallus or the leaf-bearing stem continually grows at its apex or by a process of renewal (Innovation), while the oldest parts die off behind. In this manner the branches become finally independent plants; and this, as well as the multiplication by gemmæ, stolons, detached buds, the transformation of hairs into protonema (in Mosses), \&c., serves not only to increase enormously the number of individuals formed by the asexual method, but is also the immediate cause of the social or cespitose mode of growth of these plants. Many Mosses in particular, even those which only rarely fructify, may in this manner form dense masses extending over considerable areas (as Sphagnum, Hypnum, Mnium, \&c.).

${ }^{1}$ It is incorrect, for instance, to regard the 'fruit' of a Moss as the morphological equivalent of the sporangium of a Rhizocarp or of the fruit of a Phanerogam.

${ }^{2}$ From the great similarity of the true leafless thallus of some Hepaticæ to the thalloid stems of others furnished with leaves on the under side, it will be convenient to use the term 'thalloid forms' for both; the term including both a true thallus (e.g. Anthoceros) and also a thalloid stem (as in Marchantia). 
The sexual organs are Antheridia and Archegonia. The mature antheridium is a body with a longer or shorter stalk, of a spherical, ellipsoidal, or club-shaped form, the outer layer of its cells forming a sac-like wall, while each of the small and very numerous crowded cells enclosed within it developes an antherozoid. The antherozoids are freed by the rupture of the wall of the antheridium at the apex; they are spirally coiled threads thicker at the posterior and tapering to a fine point at the anterior end, at which are placed two long fine cilia, the vibrations of which cause their motion. The female organs, which since the time of Bischoff have been called archegonia, are, when in a condition capable of being fertilised, flask-shaped - bodies bulging from a narrow base and prolonged into a long neck. The wall of the ventral portion encloses the central cell, the inferior and larger part of which forms the oosphere. Above this begins a row of cells which passes through the neck in an axial direction, and is continued as far as the cells which form the so-called 'Stigma.' The cells of this axial row become broken up before fertilisation, and transformed into mucilage which finally swells up and forces apart the four stigmatic cells. In this manner an open canal is formed, which leads down as far as the oosphere, and enables the antherozoids to enter it.

The great diversity in the origin of the sexual organs of Muscineæ is of great importance. In the thalloid Hepaticæ these organs arise behind the growing apex from the superficial cells of the thallus or of the prostrate thalloid stem, or on specially metamorphosed branches (as in the Marchantieæ); in the foliose Jungermannieæ and in the Mosses not only the antheridia but also the archegonia may be formed from the apical cell of the shoot or from segments of it; in this case they may take the place of leaves, or of lateral shoots, or even of hairs. Thus the antheridia appear as metamorphosed trichomes in the axils of the leaves of Radula, as metamorphosed shoots in Sphagnum, as apical structures and also as metamorphosed leaves in Fontinalis. In the same manner the first archegonium of the fertile shoots of Andrecea and Radula arises from the apical cell, the later ones from its last segments; and this is probably the case in Sphagnum.

The antheridia and archegonia are usually produced in great numbers in close proximity; in the thalloid forms of the Hepaticæ they are generally enveloped by later outgrowths of the thallus; in the foliose Jungermannieæ and in Mosses several archegonia are commonly surrounded by an investment formed of leaves which is termed the Perichatium; in Mosses the antheridia (with sometimes some archegonia) are usually borne in this manner also, while the antheridia of the Jungermannieæ and of Sphagnum stand alone. Very commonly, especially in the foliose kinds, Paraphyses, i.e. articulated threads or narrow leaf-like plates of cells, are developed by the side of the sexual organs. Besides the perichætium, there is also often in Hepaticæ (but not in Mosses) a so-called Perigynium, which grows as an annular wall at the base of the archegonia, and finally surrounds them as an open sac.

The Asexual Generation (Sporophore), the Sporogonium, arises in the archegonium from the fertilised oosphere (oospore). It first developes by repeated celldivisions into an ovoid embryo, growing at the end turned towards the neck of the archegonium, that is, the apex. Its final form is very different in different 
sections. In its lowest type (in Riccia) it is a globe, the outer cell-layer forming the wall, while all the inner cells become spores. In all other cases the sporogonium becomes differentiated externally into a stalk, which may be short or long and slender, termed the Seta, and which penetrates into the bottom of the archegonium and even into the underlying tissue, its base often becoming dilated, forming the Foot, and a Capsule (Urn or Theca) turned towards the neck of the archegonium, in which the spores arise. Together with the spores, long cells thickened by spiral bands, the Elaters, are also produced in most Hepaticæ. The internal differentiation of the spore-capsule is, in addition to this, very varied, and attains a very high degree of complexity, especially in the Mosses.

While the sporogonium is developing, the ventral portion of the archegonium also continues to grow; its cells rapidly increase in number, and it thus becomes broader, enclosing the young sporogonium, and, in this condition, is termed the Calyptra. Its behaviour supplies distinctive characters for the larger groups. In the lowest Hepaticæ (Riccia) the sporogonium remains always enclosed in the calyptra; in the higher Hepaticæ it protrudes only after the ripening of the spores, its seta elongating suddenly, and the capsule protruding from the ruptured calyptra for the purpose of disseminating the spores, the calyptra surrounding the base of the seta as a cup-like membranous structure. In the typical Mosses, on the other hand, the young sporogonium first assumes the form of a greatly elongated fusiform body, which, even before the development of the capsule, exerts a strong upward pressure upon the calyptra, which becomes ruptured at its base, and is raised up by the young sporogonium in various forms; the seta penetrates deep down into the tissue of the stem, by which it is surrounded as a sheath (Vaginula).

The spores of the Muscineæ arise in fours; the mother-cells-which had previously been united into a tissue with the surrounding cell-layers, but had become isolated even before the formation of the spores-show a rudimentary division into two previous to complete division into four. The number of the mother-cells and the place where they are produced in the sporogonium depends essentially on the internal differentiation of the latter. The ripe spores show a thin cuticle (the exospore) provided with small excrescences, which is ruptured on germination by the inner layer of the cell-wall (the endospore). Their contents consist, in addition to colourless protoplasm, of chlorophyll-granules, starch, and oil.

The Differentiation of the Tissues of Muscinea is very various, and more considerable than in the Algæ, but less so than in the Vascular Cryptogams. Fibrovascular bundles are not found; only in the stem and leaf-veins of the more perfect Mosses is an axial bundle of elongated cells differentiated, which may be considered as a slight indication of a fibro-vascular system. The Marchantieæ, on the other hand, show on the upper side of their thalloid stems, and the Mosses on their thecæ, a distinctly differentiated epidermis, which usually also forms stomata. The cell-walls of the Muscineæ are generally firm, often thick, tough, and elastic, and in this case frequently of a brown, bright red, or violet colour. The tendency towards the formation of jelly and mucilage, so general in the Thallophytes, is not found in the Muscineæ, with the exception of certain processes in the mother-cells of the spores. Various forms of thickening are not uncommon, especially in the 
spore-capsule, in the spiral bands of the elaters of Hepaticæ, and in the formation of the epidermis and peristome of the thecæ of Mosses.

Classification of Muscinea. The sexual generation is developed from the spore, generally after the previous formation of a protonema. It is the longest-lived of the two generations, and constitutes the self-supporting vegetative body of these plants, presenting either a flat dichotomously branched thallus, or a thalloid stem, or a filiform stalk furnished with from two to four rows of leaves. True fibro-vascular bundles are not developed. The archegonia and antheridia are, except in the simplest thalloid forms, stalked multicellular bodies, usually free, but sometimes buried in neighbouring masses of tissue from the subsequent growth of these latter. The central cell of the ventral part of the archegonium undergoes division, a ventral canal-cell being cut off, and the remainder of its protoplasm constitutes the oosphere by rejuvenescence. The antherozoids are spirally coiled threads with two cilia on the anterior pointed end.

The asexual generation or sporogonium arises from the fertilised oosphere within the actively growing ventral part of the archegonium, which becomes developed into the calyptra. The sporogonium is nourished by the sexual plant; it has therefore no independent existence, and appears externally as an appendage to it. It is usually a stalked capsule, in which usually a number of cells are developed into the mothercells of the spores; and from these the spores are formed by division into four after bipartition has commenced but has not been completed.

(I) Hepatica. The sexual generation arises either directly from the spore or with the intervention of a small inconsiderable protonema. It is developed as a flat dichotomously branched thallus or a thalloid stem, or finally as a filiform stalk furnished with two or three rows of leaves. This vegetative body is usually broadly expanded and clings closely to the ground or to some other substratum; even when the stems grow erect there is still an evident tendency towards the distinction of an upper (dorsal) and an under (ventral) surface. The mode of growth is hence always distinctly bilateral. The asexual generation or sporogonium remains surrounded by the calyptra until the spores are ripe; the calyptra is usually at length ruptured at the apex, and remains at the base of the sporogonium as an open sheath, while the free spore-capsule projects above its apex, to allow the escape of the spores. The mother-cells of the spores arise either from the whole of the cells except those of the single layer which forms the wall of the capsule, or the intermediate cells commonly become developed into elaters: a columella is present only in the Anthocerotex.

(2) Musci. The sexual generation is developed from the spore with the intervention of a protonema consisting of branched rows of cells and often vegetating for a considerable time independently, even when it has already produced leafy stems by lateral budding. The vegetative body is here always a cormophyte, a filiform stem furnished with leaves in two, three, or four rows, usually without any definitely indicated bilateral structure, and generally branched in a monopodial, never in a dichotomous manner. The asexual generation or sporogonium is only at first formed in the calyptra; afterwards this is usually ruptured below (at the vaginula), and raised up by the apex of the sporogonium, which it covers like a cap. The capsule, which is now first developed, produces the spores from an inner layer of tissue, while a large central mass of tissue remains sterile and forms the columella. The wall of the capsule is covered by a distinctly differentiated epidermis, the upper part of which usually becomes detached from the lower part (the Urn) in the form of a lid, in order to allow of the escape of . the spores. 
CLASS V.

\section{H E P A T I C $\AA^{1}$.}

(I) The Sexual Generation (Oophore) is developed, in some genera, directly from the germinating spore, its first divisions resulting in the formation of a cellular lamina or a mass of tissue which fixes itself by root-hairs and produces the thallus by growth at its apex, as in Anthoceros and Pellia. In other cases the body which results from the divisions of the spore first forms a narrow ribbon-like lamina of cells, the apical cell of which becomes subsequently the apical cell of a stem, and its segments form leaves, as in Jungermannia bicuspidata (according to Hofmeister). Or again, the bud of a leafy stem springs immediately from the spore (Frullania dilatata). In other cases, on the other hand, a protonema is formed; the endospore which grows out into the form of a tube produces a short articulated filament on which the rudiments of the thallus are formed as lateral shoots, in a manner similar to the leaf-buds of Mosses on the protonema (e.g. Aneura palmata, Marchantia). In Radula the spore produces first of all a flat plate of cells, from which the first bud of the leafy stem springs laterally (Hofmeister), a process which finds its analogue among Mosses.

The vegetative body of Hepaticæ is always formed in a distinctly bilateral manner; its free side, turned towards the light, is differently organised from that which faces and often clings closely to the substratum and is not exposed to light.

In the greater number of families and genera the vegetative body is a broad, flat or curled plate of tissue, varying in length from a few millimetres to several centimetres; and is either a true thallus without any formation of leaves, as in Anthoceros, Metzgeria, and Aneura, or lamelliform outgrowths arise on the under or shady side, which at the same time produces root-hairs; and these outgrowths may be looked on as leaves. For the sake of having a common expression for these forms extremely similar in habit, they may be comprised under the term Thalloid ${ }^{2}$,

${ }^{1}$ Mirbel, Ueber Marchantia, in the Mém. de l'Acad. des Sci. de l'Inst. de France, vol. XIII, I 835.-G. W. Bischoff, in Nova Acta Acad. Leopold. Carol. I835, vol. XVII. pt. 2.-C. M. Gottsche, ibid., vol. XX. pt. I.-Gottsche, Lindenberg u. Esenbeck, Synopsis Hepaticarum, Nürnberg, I 844.Hofmeister, Vergleich Untersuchungen, 1851 .- - O On the Germination, Development, and Fructification of the Higher Cryptogamia : Ray Society, 1862.]-Kny, Entwickelung der laubigen Lebermoose; Jahrb. für wiss. Bot. vol. IV. p. 66, and Entwickelung der Riccien, ibid., vol. V. p. 359.-Thuret, in Annal. des Sci. Nat. 18 I , vol. XVI (Antheridia). - Strasburger, Geschlechtsorgane u. Befruchtung bei Marchantia; Jahrb. für wiss. Bot. vol. VII. p. 409: [also Befruchtung und Zelltheilung, 1878]. - Leitgeb, Wachsthumsgeschichte der Radula complanata; Sitzungsber. der Wiener Acad. I87I, vol. LXIII.-Ibid., Bot. Zeitg. $187 \mathrm{I}$, no. 34 , and 1872 , no. 3.-A portion of what is said about the apical growth of Jungermanniex is derived from communications by letter from Leitgeb.-Janczewski, Bot. Zeitg. 1872.-[Leitgeb, Unters. ueb. Lebermoose, 1874-78. Goebel, Zur vergl. Anat. der Marchantieen, Arb. d. bot. Inst. in Würzburg, II. 3, I880.]

${ }^{2}$ [The term 'thalloid' is here, as on p. 342 , preferred to the one in more general use, 'frondose.'] 
in contrast to the Foliose Hepaticæ belonging to the family of Jungermannieæ, the vegetative body of which consists of a small slender filiform stem, bearing distinctly differentiated leaves (Jungermannia, Radula, Mastigobryum, Frullania, Lophocolea, \&c.). Between the thalloid and foliose forms of this family are some which present various stages of transition (as Fossombronia and Blasia).

The leaves of all Hepaticæ are simple plates of cells, in which even the mid-rib usual in the leaves of Mosses is always wanting.

In most of the thalloid forms the growing apical region of each shoot (Fig. $\mathbf{2 3}^{2}, s$ ) lies in an anterior depression, produced by the more rapid growth in length and breadth of the cells which are derived right and left from the segments of the apical cell. In the Anthoceroteæ, Riccieæ, and Marchanticæ, there is a group of apical cells, and the terminal branching is truly dichotomous. In the thalloid Jungermannieæ, there is a single apical cell; the terminal branches originate from the youngest segments of this cell, and, from their position in the

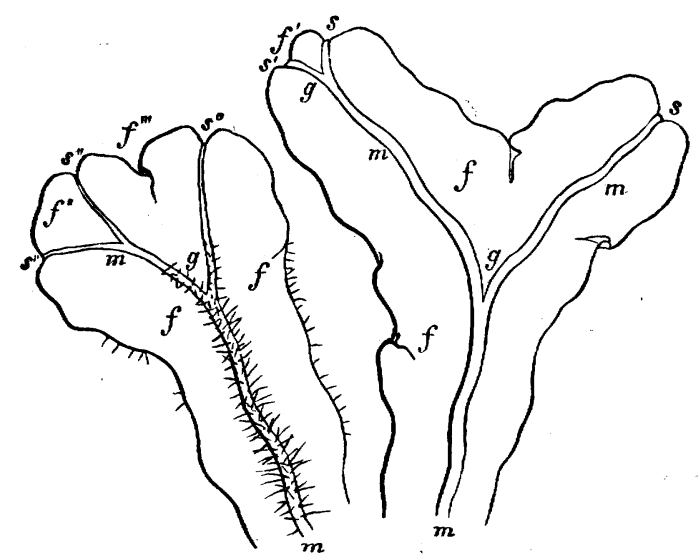

Fig. 232,-Metzgeria furcata; the right-hand figure seen from the upper, the left-hand figure from the under side; $m$ the mid-rib; $s, s^{\prime}, s^{\prime \prime}$ the apical region; $f, f$ wing-like expansion formed of a single layer of cells; $f^{\prime} f^{\prime \prime} f^{\prime \prime \prime}$ its mode of development after branching ( $\mathrm{X}$ about $\mathrm{Io}$ ).

depression and their active growth, push aside the apex of the primary shoot, and form with it a fork (false dichotomy). In the angle between the two bifurcations the permanent tissue increases more rapidly, and forms, so long as the two

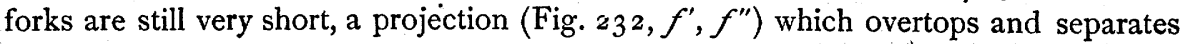
the apical regions, but which, when the forks are longer, is in turn overtaken by them, and now appears as an indented angle of the older fork $(f)$. The filiform stem of the foliose Jungermanniex, on the other hand, ends in a bud as a more or less prominent vegetative cone, with a strongly arched apical cell. In this case also the lateral branches spring from individual mother-cells, which, however, do not originate from the youngest segments of the apical cell, but lie, even at their first formation, some distance below the apex; the branching is therefore, at its commencement, distinctly monopodial.

We shall speak, under the separate sections, of the form of the apical cell, which forms two, three, or four rows of segments; as well as of the origin of the 
leaves and lateral shoots, since Leitgeb's researches show that great morphological differences occur in the different genera. For the same reason very little of a general character can be said, in addition to what has been mentioned above, on the habit and anatomical nature of the vegetative body, which must therefore be considered under the separate families.

The Asexual Propagation of Hepaticæ is often brought about by the dying off of the thallus or stem from behind, the branches thus losing their connexion and becoming independent. Adventitious branches, arising in the thalloid forms from cells of the older marginal parts, become detached in a similar manner. The propagation by gemmee is very common and characteristic; not unfrequently a number of cells of the margin of the leaf of foliose Jungermanniex (e.g. in Madotheca) simply detach themselves as gemmæ; in Blasia, on the other hand, as well as in Marchantia and Lunularia, peculiar cupules are formed on the upper side of the flat shoots exposed to the light, which are flask-shaped in Blasia, broadly cup-shaped

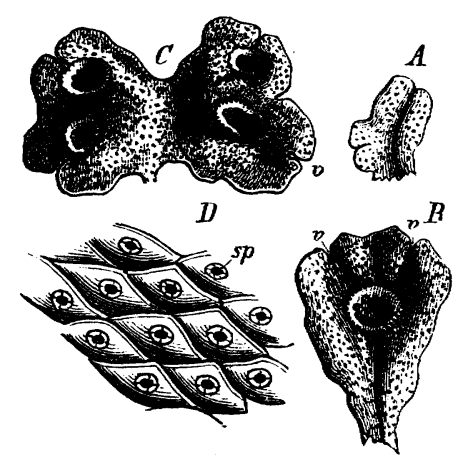

FIG. 233.-Marchantia polymorpha; $A, B$ young shoots; $C$ the two shoots which result from a gemma, with cupules; $v v$ the depressed apical region: $D$ a piece of the epidermis seen from above: $s p$ stomata on the rhomboid plates $(A-C \times$ slightly ; $D$ more on the rongly).
strom

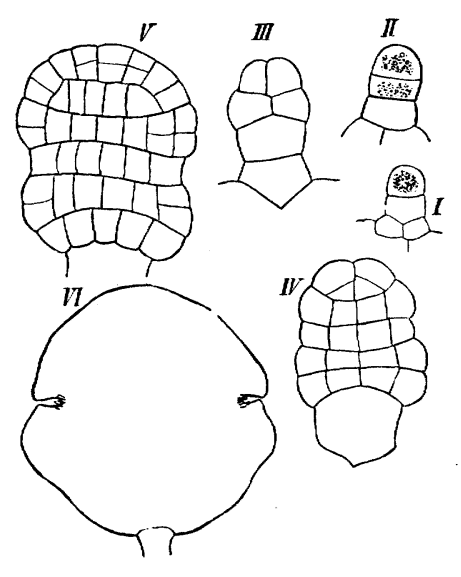

FIG. 234-Development of the gemmæ of Marchantia.

in Marchantia, crescent-shaped and deficient on one side in Lunularia. From the bottom of these cupules shoot out hair-like papillæ, the apical cells of which become transformed into a mass of considerable size constituting the gemma. (See Figs. 233, 234.) From the two depressions which lie right and left on the margin of the lenticular gemma (Fig. 234, $V I$ ) spring the first flat shoots (Fig. 233, $B, C$ ), when the gemmæ have fallen out of the cupule and lie exposed to light on damp ground.

The Sexual Organs are developed, in the thalloid forms, on the upper side exposed to light; in Anthoceros in the tissue of the thallus itself (endogenous); in the other thalloid forms from cells which project like papillæ and are of definite origin in reference to the segments of the apical cell. In the Marchantieæ branches of a very peculiar shape, which have a tendency to shoot upright from the flat stem, are formed, producing the antheridia on the upper, the archegonia on the under side; the male and female receptacles may be distributed either monœciously 
or diøeciously. There is a general tendency in the thalloid Hepaticæ for the sexual organs to be depressed into hollows by overarchings of the surrounding tissue, often opening externally by only a narrow mouth. An example of this is given in Fig. 235 .

In the foliose Jungermannieæ the origin of the antheridia and archegonia is very various, and they are also enveloped in different ways. Further reference will be made to this in describing the different families.

The antheridium consists, in the mature state, of a pedicel surmounted by a globular or ellipsoid body; in those which are imbedded in the tissue the former is usually short, in the free forms it is long, and composed of from one to four rows of cells. The body of the antheridium consists of a wall formed of a single layer of cells containing chlorophyll; the whole of the space enclosed by it is densely filled by the mothercells of the antherozoids; their escape is occasioned by the access of water and separation of the cells of the wall at the apex; sometimes, as in Fossombronia, these cells even fall away from one another. The small mother-cells of the antherozoids which escape in great numbers, separate in the water; the antherozoids become free, and have the appearance of slender threads curved spirally from one to three times, and provided at the anterior end with two long very fine cilia, by means of which they move in the water with a rotating motion. Usually they drag after them at the posterior end a small delicate vesicle, the origin of

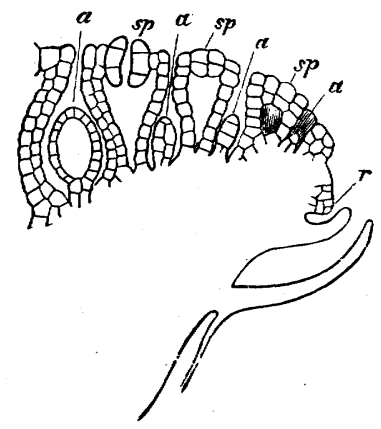

FIG. 235.-Anterior margin of the young antheridial disc of Marchantia polymor $p h a ; a$ the growing margin; $a, a, a$ the antheridia in different stages of development: $s p$ the stomata above the air-cavities between the antheridia (after Hofmeister, $\left.\mathrm{x}_{300}\right)$. which Strasburger traces to the central vacuole in the protoplasm of the mother-cell, in the periphery of which the antherozoid has been formed.

The succession of cell-divisions in the formation of the antheridia has been shown by the researches of recent observers to present great diversities in the different genera; they agree, however, in the antheridium always making its first appearance as a papilliform swelling of a cell from which it is separated by a septum. This papilla thus detached again divides into a lower and an upper cell, the former of which produces the pedicel, the latter the body of the antheridium (parietal layer and mother-cells of the antherozoids).

The succession of cell-divisions in the formation of the archegonia, from the observations of Janczewski ${ }^{1}$, Leitgeb, Kny, and Strasburger, appears to be essentially the same in the different families, even, mutatis mutandis, among the Anthocerotex. It is certain that the archegonium, like the antheridium, makes its first appearance as a simple papilla, which, in the case of the first archegonium of a receptacle of Radula, is itself the apical cell of the shoot. This papilla is shut off by a septum, and, in Riccia, is at once the mother-cell of the whole archegonium: in the other Hepaticæ it is divided by a second septum into two cells, the lower one of which

1 [Janczewski has made a series of comparative researches into the development of the archegonium of Muscineæ, Bot. Zeitg. I872, p. 869 et seq.] 
produces the pedicel, the upper one the archegonium itself. The lower cell undergoes numerous transverse and longitudinal divisions into several rows of cells. In the mother-cell of the archegonium there arise three longitudinal walls, by which three outer cells are formed; these, on their part, enclosing an internal cell which overtops them. The three outer cells are divided by radial longitudinal walls and thus form five or six investing cells; the median cell is divided by a transverse septum into an upper (stigmatic) and a lower cell. After the whole structure has increased somewhat in length, it comes to consist of two tiers in consequence of the

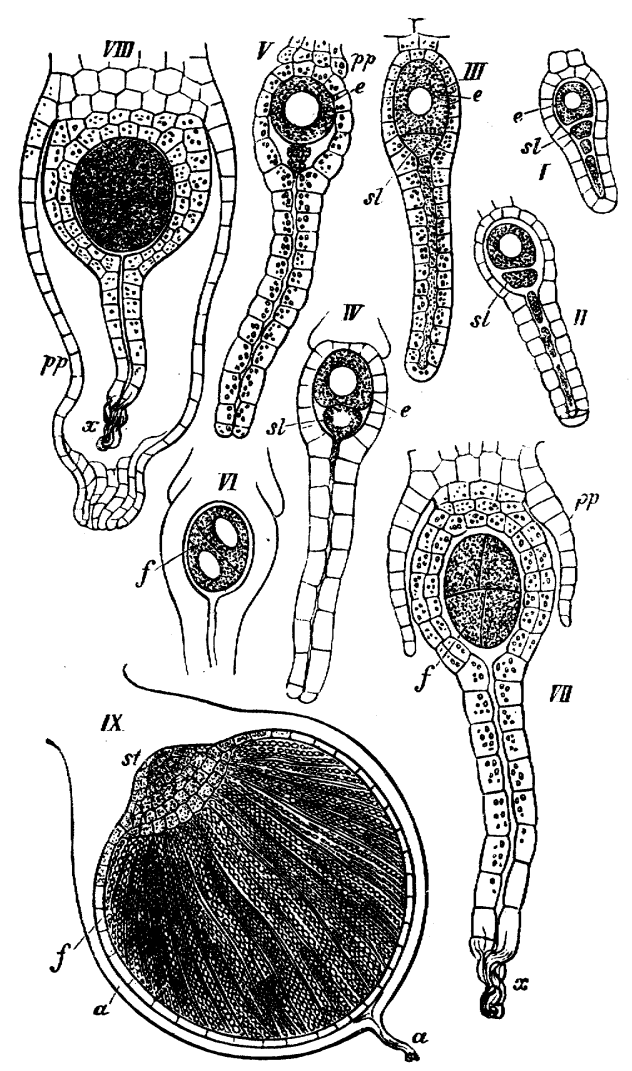

FIG. 236.-Later stages in the development of the archegonia and origin of the sporogonium of Marchantia polymorpha; $I, I I$, young archegonia; $I I I, I V$, after absorption of the axial row of cells of the neck; $V$ when ready for fertilisation; $V I . V I I I$ the cells of the mouth of the neck $x$ relaxed after fertilisation; the fertilised oosphere $f$ shows its first divisions. In these figures $I \cdot V s l$ is the ventral canal-cell which is last converted into mucilage, $e$ the unfertilised oosphere; $p p$ in $V \cdot V I I$ the perigynium in process of development; $I X$ the unripe sporogonium in the ventral portion of the archegonium which has developed into the calyptra; $a$ neck of the archegonium; $f$ wall of the sporogonium; st its stalk (foot); inside the sporogonium are the young elaters arranged in rays, among them the spores. (I-VIII $\times 300, I X$ about 30.$)$

division of each of the six investing cells, like the internal cell, by a transverse septum. The lower tier forms the ventral portion, the upper the neck of the archegonium. The internal cell of the ventral portion, the central cell, increases considerably in size and is divided by a transverse septum into a large inferior cell, the oosphere, and a small upper cell, the ventral canal-cell. Meanwhile the upper tier of cells, the neck of the archegonium, elongates; its axial cell dividing into four, eight, or sixteen long narrow cells, the canal-cells of the neck. Further transverse 
and longitudinal walls are formed in the external cells of the ventral portion, and a wall consisting of one or two layers of cells is produced; similarly, the wall of the neck, which consists of five or six longitudinal rows of cells, is formed by the transverse division of the peripheral cells of the upper tier. The primary stigmatic cell divides into the five or six stigmatic cells of the neck. The cell originally constituting the pedicel has also undergone both longitudinal and transverse divisions. Whilst the oosphere is being formed, the walls of the canal-cells of the neck and the transverse septum beneath the ventral canal-cell become converted into mucilage, the swelling up of which forces the protoplasm of the canal-cells out through the opened apex of the neck. (See Figs 236 and 256 .)

(2) The Asexual Generation, the Sporogonium, arises and is entirely formed within the growing ventral portion of the archegonium, which from this time is termed the Calyptra. The sporogonium does not anywhere unite in its growth with the surrounding tissue of the vegetative structure of the sexual generation, even when its seta penetrates into it.

The external form and internal structure of the sporogonium are very different in the different groups. In the Anthoceroteæ it is when mature an elongated twovalved pod projecting from the thallus. In the Ricciea it is a thin-walled ball entirely filled with spores, and, together with the calyptra, depressed in the thallus. In the Marchantiex it is a shortly-stalked ball enclosing elaters as well as spores, and, after it has broken through the calyptra, bursting irregularly or opening by a circular fissure and detaching an operculum. In the Jungermannieæ it also ripens within the calyptra, but breaks through it and appears as a ball borne upon a long slender stalk; the wall consists, as in the Marchantieæ and Riccieæ, when ripe, of a single layer of cells, but separates cross-wise into four lobes, to which the elaters remain attached. The elaters are, as in the Marchantiex, long fusiform cells, the delicate colourless outer layer of which is thickened within by from one to three brown spiral bands.

The sporogonium also originates in different ways. [The fertilised oosphere is always first divided into two cells by a wall (basal wall) which, in the lower forms, is inclined at an acute angle to the long axis of the archegonium, but in the Jungermanniex is at right angles to it. In Riccia the capsule is developed from the whole of the oospore: in the Marchantieæ and Anthoceroteæ the short seta of the sporogonium is developed from the lower or posterior (hypobasal cell) of these two cells, the capsule being developed only from the upper or anterior (epibasal) cell : in the Jungermannieæ the capsule and the seta are developed from the upper (epibasal) cell, the product of the development of the lower (hypobasal) cell being a small filamentous appendage upon the dilated base (foot) of the seta. This first division is followed by two others at right angles to it and to each other, so that the embryo now consists of eight cells (octants). The subsequent divisions take place more or less irregularly, but it appears that in the Jungermannieæ the four epibasal octants behave like apical cells, segments being continually cut off horizontally, but doubtless intercalary divisions also occur ${ }^{1}$.] When the young sporogonium has

1 [On the embryology of the Hepaticæ, see Hofmeister, loc. cit.; Kienitz-Gerloff, Bot. Zeitg. 1874-5; Leitgeb, Entw. d. Kapsel von Anthoceros, Sitzber. d. Wien. Akad, i876, and loc. cit.] 
in this manner attained its destined height, and partially even at an earlier period, a number of divisions of different kinds take place by which the structure is completed. The wall of the sporogonium becomes differentiated from the tissue from which the mother-cells of the spores are to arise; if elaters are formed they originate from the same tissue, the cells ceasing to divide transversely at an earlier period and remaining long, while the intermediate cells become rounded off and give rise to the mother-cells of the spores (Hofmeister).

The mode of division into four of the mother-cells of the spores also varies. Those of Anthoceros form at first two, and afterwards four, new nuclei which are arranged tetrahedrally, the protoplasm dividing before the nucleus; cellwalls are then formed, and thus the mother-cell breaks up into four spores ${ }^{1}$. In Pellia and Frullania, on the other hand, the division of the mother-cells commences by four protuberances arranged tetrahedrally, which at length are cut off by cellwalls; each contains a nucleus, and they form as many spores; in Pellia the spores immediately again divide several times, and thus give rise to a young plant.

The Hepaticæ are usualy divided into five families, viz.:-

$$
\begin{aligned}
& \text { I. Anthoceroteæ, } \\
& \text { 2. Riccieæ, } \\
& \text { 3. Monoclex, } \\
& \text { 4. Marchantieæ, } \\
& \text { 5. Jungermannieæ, }
\end{aligned}
$$

of which the first four include only thalloid forms, the fifth both thalloid and foliose genera.

I. Anthocerotem. Antboceros lavis and punctatus, which grow in summer on loamy ground, develope a perfectly leafless flat ribbon-like thallus, its irregularly developed ramifications forming a circular disc; the regularity of the dichotomous branching is disturbed by the adventitious shoots, which proceed from the margin of the thallus, and, in $A$. punctatus, also from the upper surface. The thallus consists of several layers of cells, and the apical cells of the branches which lie in the anterior depressions are divided by walls inclined alternately upwards and downwards (Fig. 237, C). In each of the cells of the thallus, the upper layer of which does not become differentiated into an epidermis, only one chlorophyll-granule is formed, surrounding the nucleus. On the under side of the thallus, Janczewski states that stomata are formed close behind the growing margin, through which filaments of Nostoc frequently penetrate, forming roundish balls in the tissue of the thallus (Fig. $237, B$ ), which were at one time considered to be endogenous gemmæ ${ }^{2}$. The antheridia and archegonia arise apparently without any definite arrangement in the interior of the upper side of the thallus. The formation of the antheridia commences by a circular group of cells of the outer layer separating from the subjacent tissue and thus producing a broad intercellular space, several of the lower bounding cells of which, after some vertical divisions, rise up in the form of papillæ, and form the antheridia (Fig. 237, B,an). It is only when the chlorophyllgranules in the walls of the antheridia have assumed a yellow colour and the antherozoids are mature that the roof of the cavity is ruptured, the antheridia opening at their apex and allowing the an:herozoids to escape. In the Riccieæ and Marchantieæ the archegonia, which are at first free, become gradually surrounded by masses of tissue, but in Anthoceros they are enclosed from the first. One of the superior

1 [On the development of the spores of Pellia and Anthoceros see Strasburger, Zellbiidung und Zelltheilung, 3 rd ed. p. I 56.$]$

${ }_{2}^{2}$ [See Waldner, Ueb. die Nostoc-Colonieen bei Blasia, Sitzber. d. Wien. Akad. 1878.] 
segmental cells, near to the growing-point of the thallus, divides into an outer and an inner cell; the outer (superior), which bulges slightly, is the mother-cell of the archegonium and is further divided (as is the case in other Liverworts) by three longitudinal walls vertical to the surface of the thallus, into one internal and three external cells. The latter produce the six primary investing cells which, at a later period, give rise to as many rows of cells which are enclosed on all sides by the tissue of the thallus. The internal cell is divided by a transverse septum into two cells, the lower of which becomes the central-cell, and by division gives rise to the oosphere and the overlying ventral canal-cell ; the upper undergoes transverse divisions in consequence of which a row of neck canal-cells is formed, as also the primary stigmatic cell which subsequently divides cross-wise to form the stigmatic cells. The variations from the mode of formation of the archegonium obtaining in the other Liverworts are thus seen to be slight, and scarcely justify the formation of the Anthocerotex into a distinct class. Fig. $237 C$ dates from a time at which the above-mentioned details were unknown, but it suffices nevertheless to give some idea of the important points in the process.

After fertilisation the oospore is divided in the manner described above. Whilst the developing sporogonium is gradually becoming a multicellular body dilated inferiorly
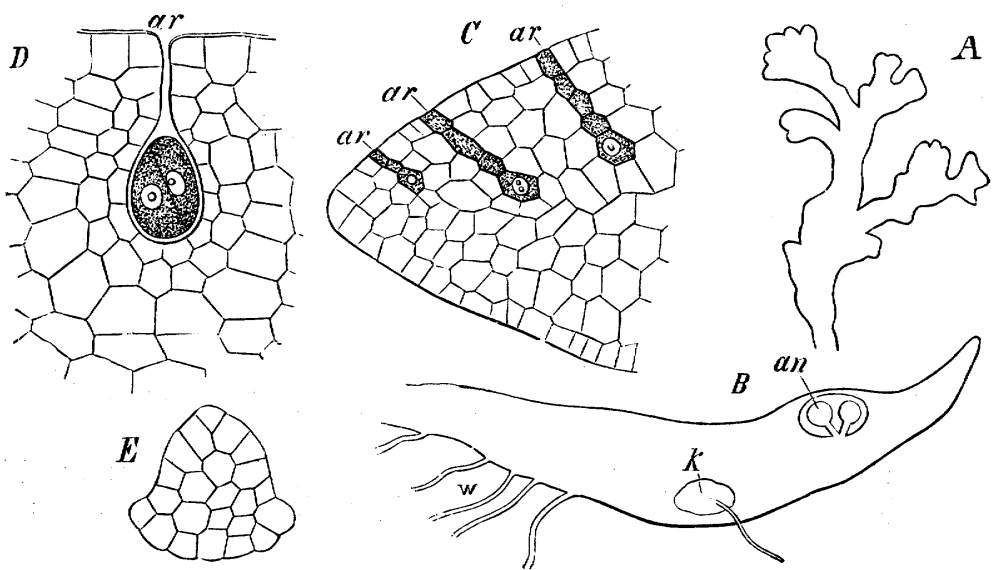

FIG. 237.-Anthoceros levis (after Hofmeister); $A$ a branched thallus; $B$ longitudinal section of a shoot (X 40); an antheridia beneath the layer of superficial cells; $C$ longitudinal section through the apical part of a shoot; $a r$ rudiments of archegonia $(X 500) ; D$ ar fertilised archegonium in the longitudinal section of a shoot, with an embryo consisting of two cells; $E$ multicellular embryo: $K$ in $B$ a colony of Nostoc settled in the tissue of the thallus.

(Fig. 237, E), the cells of the surrounding tissue of the thallus undergo numerous divisions and form an upwardly projecting involucre which is broken through at a later period by the elongating sporogonium. Differentiation now takes place in the homogeneous tissue of the sporogonium; a central cylinder of from twelve to sixteen rows of axially elongated cells is marked out, forming the columella, whilst the cells of the layer immediately adjacent to it undergo division by horizontal walls and give rise to the mother-cells of the spores and elaters; the external four or five layers of cells form the wall of the future 'pod.' Those cells of the layer investing the columella which are to form elaters undergo one or more vertical divisions. The elaters are here transversely directed rows of cells in which no spiral bands are formed. The mother-cells of the spores round themselves off, become gradually isolated, beginning from the apex of the sporogonium and proceeding basipetally, increase in size, and then divide into four, giving rise to the tetrahedral spores. The sporogonium elongates and becomes a pod-shaped structure of from fifteen to twenty millimetres in height, the brown wall of which, provided with an epidermis bearing stomata, splits from above downwards into two valves. 
2. The family Monoclem appears, according to the 'Synopsis Hepaticarum, to contain transitional forms between the Anthocerotex and the Jungermanniex. The long sporogonium has a longitudinal dehiscence and no columella; and the sexual generation is either thalloid or foliose.

3. The Ricciem form a flat dichotomously branched thalloid stem, floating in water or rooting in the ground, the apical cells of which, lying close to one another

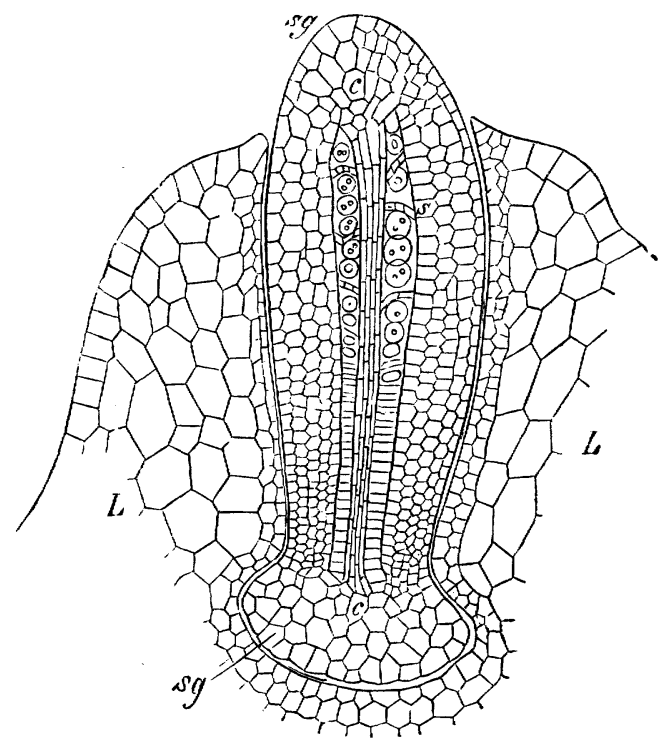

FIG. 238.-Anthoceros lavis; $s g$ the young sporogonium, the letters point to the foot; $L$ the involucre; $c-c$ the columella (after Hofmeister, $\mathrm{X}_{5} 5^{\mathrm{O}}$ ).

in the anterior depressions of the branches, are stated by Kny to become multiplied by vertical longitudinal partitions, and segmented by walls inclined upwards and down-
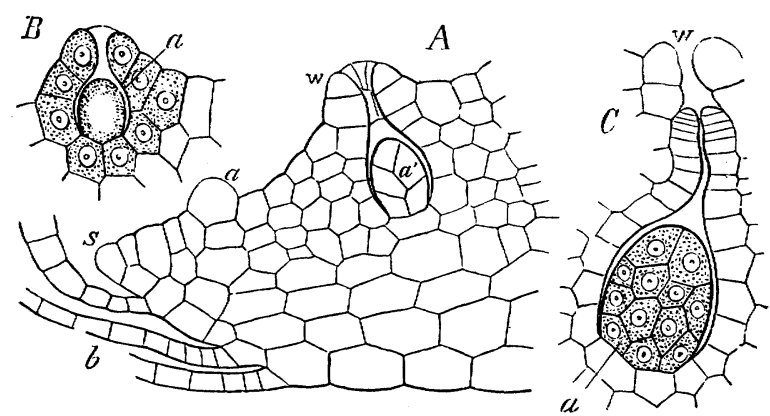

FIG. 239.-Riccia glazca; $A$ vertical longitudinal section through the apical region; $s$ apex, $b$ leaves, $a$ young antheridium, $a^{\prime}$ older antheridium already surrounded by involucral tissue $w ; B$ rudiment of an antheridium $a$ already overarched; $C$ young antheridium $a$ in longitudinal section (after Hofmeister, $\times 500$ ).

wards. On the upper side a distinct epidermis is differentiated, but without stomata, and beneath this lies the green tissue often provided with air-cavities, which is derived from the upper segments of the apical.cells; the under side is provided with a single longitudinal row of transverse lamellæ, which, resulting immediately from the lower segments of the apical cells, must be considered as leaves. Afterwards they split length- 
wise and form two rows; between them arise a number of root-hairs with conical thickenings projecting inwards.

The archegonia and antheridia are formed on the upper side from young epidermal cells which grow into papillæ, and are overarched, in consequence of their mode of development, by the surrounding tissue (Fig. 239). This involucre sometimes forms an elevated neck above the sessile antheridia. The archegonia project, at the time of fertilisation, above the epidermis; subsequently they are arched over, and develope from their fertilised oosphere the globular sporogonium with a wall consisting of a single layer of cells, and entirely filled with spores, without elaters. The spores are set free by the decay of the surrounding tissue.

4. The Marchantieø have all a thalloid stem extended flat upon the ground; it is ribbon-like, dichotomously branched, possesses a mid-rib, and is always composed of

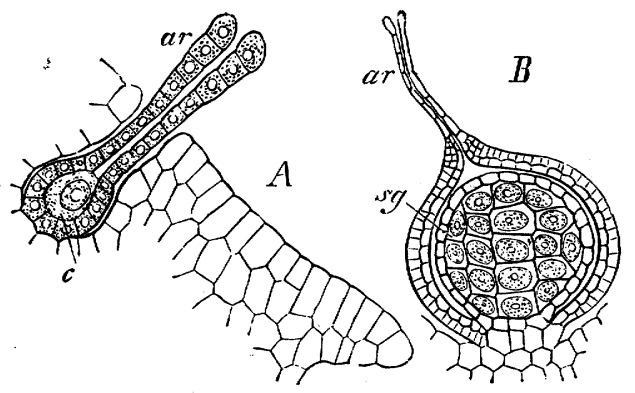

FrG. 240.-Riccia glauca; $A$ apical region in vertical longitudinal section ; $a r$ archegonium; $c$ oosphere $\left(\times 5_{600}\right) ; B$ the unripe sporogonium $s g$ surrounded by the calyptra, which still bears the neck of the archegonium $\operatorname{ar}(\times 300$, after Hofmeister).

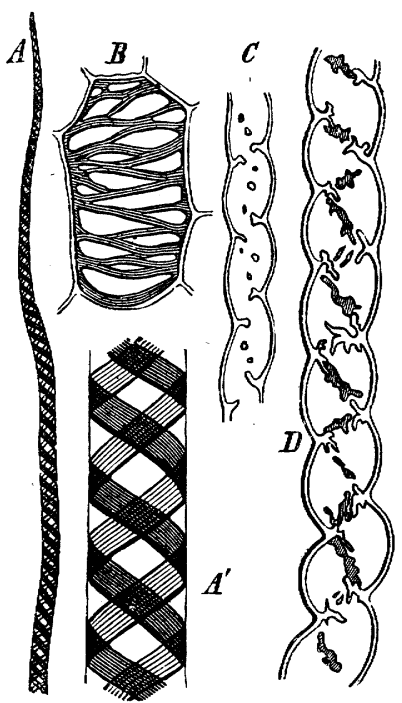

FIG. 240 bis.-Cell-forms of Marchantia polymorpha with thickenings; $A$ an elater (one-half) from the sporogonium, with two spiral bands; $A^{\prime}$ a portion more strongly magnified; $B$ a parenchyma-cell from the centre of the thallus, with thickenings projecting inwards in a lus, with thickenings projecting inwards in a
reticulate manner; $C$ a slender root-hair with thickenings projecting inwards, these are arranged on a spiral constriction of the cell-wall; at $D$ a thicker-root-hair, with thicker branched projections, and spiral arrangement still more evident.

several layers; the under side produces a number of hairs with conical thickenings projecting inwards placed upon a spiral constriction of the internal cavity (Fig. 240, bis, $C$ ), and also two rows of leaf-like lamellæ, like the Ricciex. The upper side is covered by a very distinctly differentiated epidermis, penetrated by large stomata ${ }^{1}$ of peculiar form. Each of these stands, in Marcbantia, Lunularia, \&c., in the centre of a rhombic plate; these plates are parts of the epidermis which overarch large air-cavities, from the bottom of which the cells containing chlorophyll spring in a conferva-like manner, while the rest of the tissue is destitute of chlorophyll and consists of long horizontal cells without interstices ( $c f$. Fig. $6_{5}$ ).

1 These stomata are formed (see Fig. 89) by the simple separation from one another of four or more epidermal cells which afterwards are divided by walls parallel to the surface of the thallus. (Leitgeb.) 
The sexual organs of the Marchantieæ are borne on monœcious or diœcious receptacles ${ }^{1}$. The antheridia, although springing from cells of the epidermis, are, as in Riccia, depressed in the upper side of the thalloid stem, and overarched by the surrounding tissue; they occur in larger or smaller numbers close together upon receptacles, which are discoid or shield-shaped sessile or stalked branches that have undergone a peculiar transformation. The archegonia are only in the Targioniex inserted at the apex of an ordinary shoot; in the other families they are produced on a metamorphosed branch, which rises like a stalk and developes in different ways at its summit; it bears the archegonia on its outer or lower side. With the variation in the form of the part which bears the archegonia is connected an equally varied mode of envelopment of the archegonia by involucres. Since it is impossible to describe these structures in a short space, we may take Marckantia polymorpha, the species most perfectly endowed in this respect, as an example. The explanation of the figures $24 \mathrm{I}-243$ will suffice to illustrate at least the most essential points.

The sporogonium of the Marchantiex, usually shortly stalked, contains elaters which radiate from the bottom towards the circumference ( $c f$. Fig. 236). It bursts either at
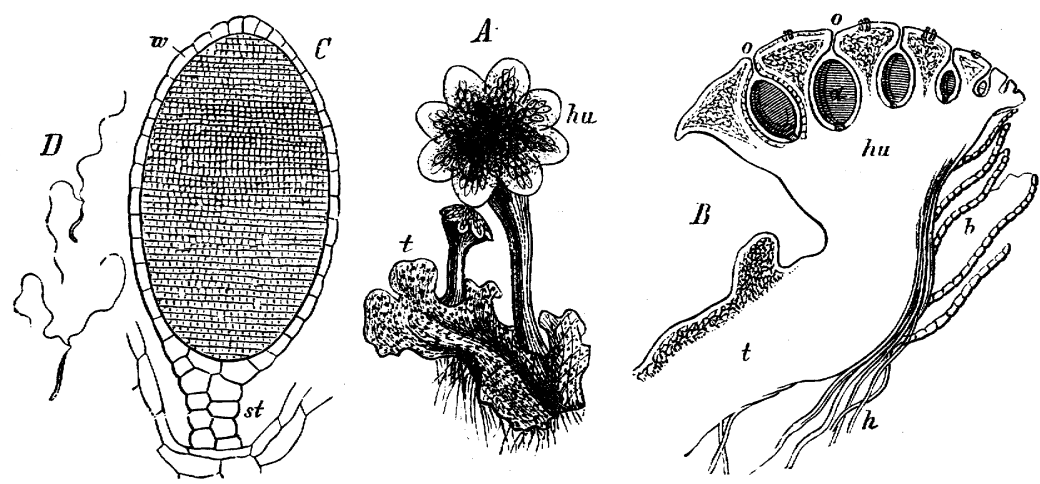

FIG. 241.-Marchantia polymorpha; $A$ a horizontal branch $t$ with two ascending branches which bear male receptacles $h u ; B$ vertical section through an incompletely developed male receptacle $h u$ and the part of the thalloid stem $a$ from which it springs; $b b$ leaves; $h$ root-hairs in a channel of the receptacle; $o o$ openings of the hollows in which the antheridia $a$ are placed; $C$ a nearly ripe antheridium; st its pedicel; $w$ the wall; $D$ two antherozoids (these last $x 800)$.

the apex with numerous teeth, or is four-lobed, or the upper part becomes detached as an operculum. The peculiar gemmæ and their cupules have a'ready been described.

5. The Jungermannieæ. In this family occur forms of which the vegetative body is a true flat leafless thallus, as Metzgeria and Aneura, as well as transitional forms whose flat thalloid stem forms leaves on the under surface (Diplolana), or whose stem, as in Blasia, elliptical in section in its early stage, becomes broad and leaf-like when older, and produces leaves on both surfaces. Closely allied to these is a genus ' with a less dilated stem, though still always greatly flattened on the upper side, and bearing leaves only above, (Fossombronia ?). The greater number of the genera, however, the foliose Jungermanniex, form a slender filiform stem, with numerous sessile leaves with broad insertions but distinctly differentiated; these leaves commonly occurring only in two rows situated on the upper side, as in Radula, some species of Jungermannia, Lejeunia, and Plagiochila. Typically, however, there are three rows of leaves, one being developed on the under or shaded side (hence termed Ampbigastria), the other two rows on the upper side (Frullania, Madotbeca, Mastigobryum). In the flagelliform branches the leaves remain very small, and are sometimes almost invisible.

1 [Leitgeb, Die Inflorescenzen der Marchantiaceen; Sitzber. d. Wien. Akad. 1880.] 
The bilateral structure is distinctly manifested not only by the thalloid forms, which mostly cling closely to the substratum, in that the sexual organs are formed only on the upper side or the one exposed to the light, and rhizoids and leaves on the under or shaded side; but in the foliose forms also this tendency is clearly shown, whether they cling closely to the substratum or rise from it obliquely. This bilateral structure is manifested not only in the different mode of the formation of the leaves on the two sides, and in the expansion of the ramifications in a single plane, but is also determined, both in the foliose and in the thalloid forms, by the growth of the apical region of the shoot. Even the youngest segments of the apical cell exhibit it, as is shown

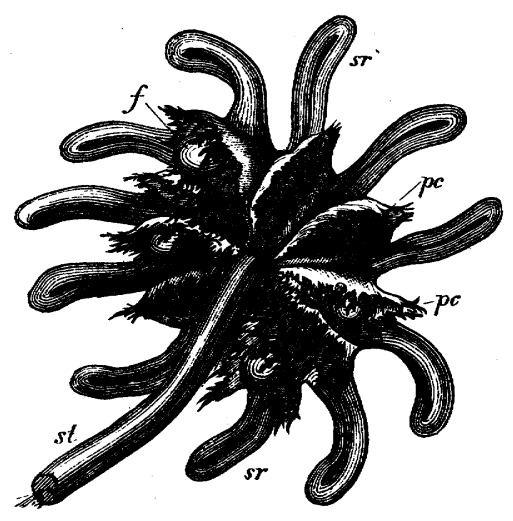

FIG, 242.-Female receptacle of Marchantia poly. morpha seen laterally from below; st stalk with two channels; $s r$ the radiate outgrowths of the disc; $p c$ the intermediate perichætium; $f$ sporogonia ( $x$ about 6 ).

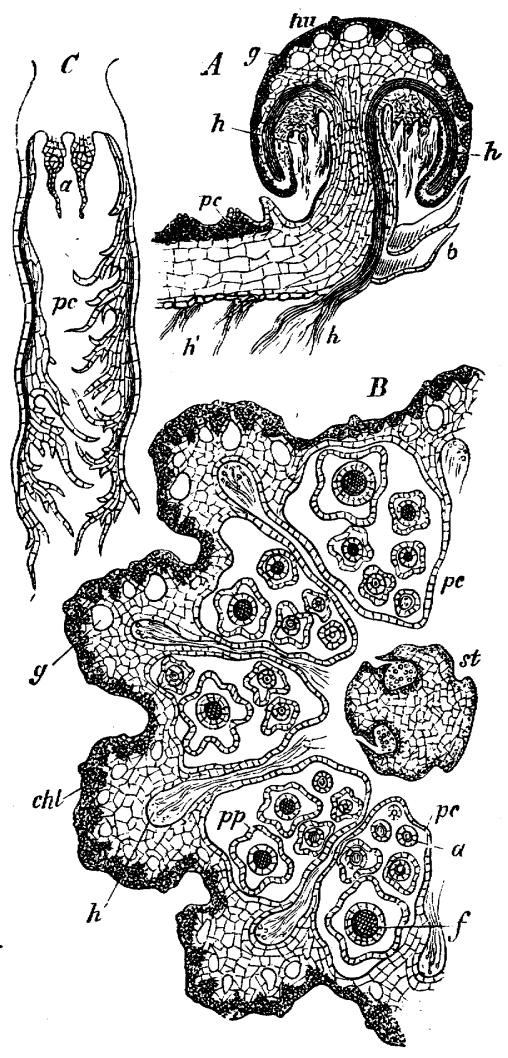

FIG. 243.-Marchantia polymorpha; $A$ vertical section through a female receptacle $h u$; $b b$ leaves; $h$ root-hairs in its channel; $g$ large cells between the air-cavities of the upper side; $B$ horizontal section of half an older receptacle and of its stalk st; $c h l$ the chlorophyll-bearing tissue of the disc, below which are large hyaline cells; $a$ unfertilised archegonia: $p p$ perigynia of fertilised archegonia ; $C$ vertical longitudinal section through the receptacle; $a$ two archegonia; $p c$ perichæetium ( $p c$ in Fig. 242).

in the different organisation of the upper and under sides, and in the similarity (though not symmetrical) of the right and left sides of the shoot.

Enough has already been said on the position of the apical region in an anterior depression in the thalloid forms, as well as on the termination of the filiform stem in the leaf-bud of the foliose genera. The form of the apical cell, and its segmentation in the thallus of Metzgeria, have been represented in detail in Fig. r ro; in Aneura and Fossombronia it is also two-sided. In Blasia, on the other hand, Leitgeb states that it is four-sided, and forms four rows of segments, a dorsal, a ventral, a right, and a left row. 'This may be most easily represented by supposing a wedge-shaped apical cell 
forming segments by walls inclined alternately upwards and downwards (towards the dorsal and ventral surfaces), as well as lateral segments from which the leaves proceed; a leaf is produced from the dorsal part of a lateral segment, a kind of leaf-tube from its central part, and a second leaf from its ventral part, though this last is more often absent' (Leitgeb, in lit.).

In the Jungermanniex with filiform stem and leaves arranged in two or three rows, the stem ends in a three-sided apical cell which forms three rows of segments in spiral succession; two rows being dorsal and lateral, while the third row forms the under or ventral side of the stem. The successive septa of each row of segments are parallel to one another, and the segments themselves are in straight rows, the rows being parallel to one another and to the axis of growth of the stem ${ }^{1}$. In the species with leaves arranged in two rows, a leaf springs from each of the dorso-lateral segments; .when the leaves are arranged in three rows each segment of the ventral side also produces a leaf, which is however smaller and of simpler structure and is also inserted transversely, while the insertion of the dorsal rows of leaves is oblique to the axis of the stem, so that the lines of insertion of each pair form an acute angle. Before a lateral segment has developed a papilla from which the leaf is formed, it divides by a longitudinal wall into an upper and a lower half facing dorsally and ventrally, each of which

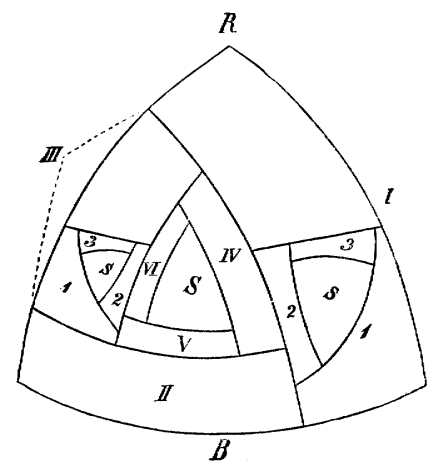

FIG. 244.-Diagram of the branching of those Jungermannieæ in which lateral shoots take the place of the ventral lobe of the dorsal leaves: $b$, ventral surface (after Leitgeb)

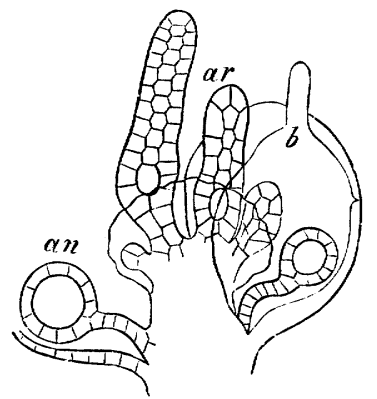

FIG. 245. - Inflorescence of Radula complanata; ar archegonium; an antheridium; $b$ leaf (after Hofmeister).

now forms a leaf-papilla. Hence it arises that the leaves of Jungermanniex are to a certain extent bisected or two-lobed; in the simpler leaves this is usually shown by a more or less deep incision of the anterior margin; but even when the leaves are quadripartite, as in Trichocolea, the primitive double origin can still be recognised. The lower lobe of the leaf is usually smaller, of peculiar form, and hollowed out.

The branching of the growing end of the shoot in the case of Metzgeria has already been represented in Fig. I 10 . According to Leitgeb it takes place in a similar manner also in the other thalloid forms with a two-sided apical cell, viz. in Aneura and Fossombronia. The very variable relation of the branching to the leaves discovered by Leitgeb ${ }^{2}$ is especially remarkable. In Metzgeria and Aneura no leaves, but only branches, are formed out of the segments; in Fossombronia the lateral shoot springs from the segment in place of a whole leaf; on the other hand, in the greater number of Jungermanniex with filiform leafy stem and three-sided apical cell, the lateral shoot springs from the segment in place of the lower or ventral lobe of the leaves of the dorsal side, so that in these cases the branch may be considered as a metamorphosed half-leaf. Fig. 244 will

1 Compare in reference to this what follows with respect to Mosses.

2 What follows is partially derived from Leitgeb's letters. [See his Untersuchungen, III.] 
serve to explain this remarkable process, where the apical view of a branching shoot is represented diagrammatically: $I, I I \ldots V I$ are the segments of the apical cell $S$ of the primary shoot; $I I, V$ being segments of the ventral, $I, I I I, I V, V I$ of the dorsal side. The two segments $I$ and $I I I$ are already divided by a longitudinal wall each into two halves respectively dorsal and ventral; and in the latter the apical cell $s$ of each lateral shoot has already been constituted by the formation of the walls $1,2,3$, while the dorsal half of each of these segments has developed into half a leaf. The other segments which do not form shoots develope normal two-lobed leaves. This is the process that occurs in Frullania, Madotbeca, Mastigobryum, Lepidozia, Trichocolea, and Jungermannia trichophylla. A third type of branching occurs finally in Radula and Lejeunia, where the formation of leaves is not disturbed by the branching, the branches springing from bebind the leaves at their base, and from the same segments.

Besides these modes of ramification of the segments of definite position, Leitgeb has recently discovered an endogenous formation of shoots, which are sometimes fertile branches, from the ventral segments provided with amphigastria, e.g. in Mastigobryum, Lepidozia, and Calypogeia; or they are formed without the production of a ventral row of leaves, as in Jungermannia bicuspidata and other Jungermanniex with leaves in two rows. In those especially which belong to the section Trichomanoidex the fertile branches are developed thus, and break out from the older parts of the stem as adventitious shoots; probably, however, their mother-cells always originate regularly in acropetal succession in the primary meristem of the vegetative cone, as in Mastigobryum and Lepidozia, but their development is deferred. Finally according to Leitgeb, the whole branching of many Jungermanniex appears to depend exclusively on the production of branches in this manner.

The reproductive organs are distributed monœciously or diœciously, and are formed, in the thalloid genera, on the dorsal side of the shoot ${ }^{1}$, in the foliose Jungermanniex at the end of primary shoots or of special small fertile branches, which commonly have the above-described adventitious origin on the ventral side. The antheridia are usually in the axils of the leaves, singly or in groups. The archegonia appear generally in large numbers at the summit of the shoot, either on those which bear antheridia below, or on special branches, which in the Geocalycex are hollowed out in such a manner that the archegonia are sunk in a deep pitcher-shaped hollow, an arrangement which may be compared, to a certain extent, with the structure of a fig. This occurs in an especially striking manner in Calypogeia. Where this peculiar enveloping of the archegonia does not occur, they are concealed by the nearest leaves (the perichrtium); and a perigynium is usually formed in addition, which grows round the archegonia as a special membranous envelope. The development of these organs has been accurately described by Leitgeb in the case of Radula complanata (Fig. 245). The primary and lateral shoots both bear, as a rule, both kinds of reproductive organs; such a shoot is always at first purely vegetative, but forms after a time antheridia, and finishes with the archegonia. Less often, however, it again recurs, after the production of antheridia, to a vegetative development. The antheridia of Radula are metamorphosed trichomes; they stand singly in the axils of the leaves, and are completely enclosed in the hollow formed by the very concave lower lobe of the leaf. They arise from the club-shaped protuberance of a cell belonging to the cortex of the stem and lying before the leaf at its base. The archegonia of Radula always stand at the end of the primary or of a lateral shoot, from three to ten together, surrounded by a perigynium, which is again enveloped by a perichætium of two leaves. The archegonia together with the perigynium are developed from the apical cell of the shoot and from its three youngest segments. The archegonia arise from the apical cell itself, and from the upper parts of its lateral

1 In Metzgeria furcata the antheridia and archegonia make their appearance diœciously on the concave dorsal surface of adventitious branches which arise from the ventral surface of the mid-rib and are so curved as to enclose the sexual organs. (Leitgeb.) 
segments; the lower parts together with the ventral segment are employed in the formation of the perigynium. The further development of the archegonia and antheridia has already been described.

In the species examined by Hofmeister the fertilised oosphere is first divided by a transverse septum, i.e. at right angles to the axis of the archegonium. Only the upper of the two cells, the one towards the neck of the archegonium, becomes further divided, and it gives rise to four apical cells arranged as octants of a sphere, as described above.

The basal portion of the growing archegonium becomes swollen out and penetrates down into the tissue of the stem, being nourished and firmly enclosed by it (the vaginula).

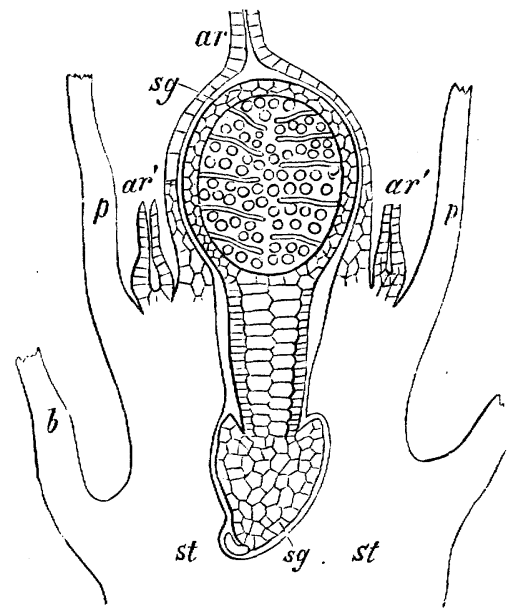

FIG. 246.- Fungermannia bicuspidata; longitudinal section of the unripe sporogonium $s g$, surrounded by the calyptra $a r ; a r^{\prime}$ archegonia which have remained unfer tilised ; $p$ base of the perigonium; st stem ; $b$ leaf : $s g$ points to the dilated base of the seta, which still bears the hypobasal cell (after Hofmeister). As soon as the young sporogonium consists of a number of cells, its wall becomes differentiated from the inner tissue which is to form the spores and elaters. In Frullania it is a single circular disc of cells lying transversely beneath the dome of the young sporogonium from which the vertical elaters, and by further divisions, the mother-cells of the spores arise, a process which reminds one of what occurs in Sphagnum. In most true Jungermanniex there is, on the other hand, a column of tissue consisting of vertical rows of cells (surrounded by the wall of the sporogonium consisting of two layers), out of which the elaters and spores are formed. The elaters lie, in this case, horizontally, and radiate from the ideal longitudinal axis to the wall of the sporogoninm (Fig. 246). In Pellia the inner fertile tissue forms, after the differentiation of the wall of the sporogonium, a hemisphere, from the cells of which arise the spores and the elaters radiating from below upwards, in a similar manner to what occurs in the Marchantieæ.

By a rapid extension of the hitherto short seta, the calyptra is ruptured at the apex, and the globular sporogonium with the already ripe spores is raised up on it. Whilst the spores are ripening, the inner layer of the wall of the sporogonium becomes absorbed; the single layer which still remains is ruptured at the apex, and splits into four (rarely more) longitudinal valves, which, flying asunder in the form of a star, carry with them at the same time the elaters, by which the spores are dispersed. The elaters, when mature, are long fusiform thin-walled cells, round the interior of which run from one to three brown spiral bands. 
The spore produces a conferva-like thallus, the Protonema, from which the leaf-bearing Moss arises by lateral branching with differentiation into stem and leaf. On this plant the sexual organs are formed; from the fertilised oosphere proceeds the sporogonium, in which the spores are formed from a small portion of the inner tissue.

The Protonema arises, in the typical Mosses, as a tubular bulging of the endospore, which elongates indefinitely by apical growth and becomes septate, the septa being oblique. The cells do not undergo any intercalary divisions, but form branches immediately behind the septa; these branches also become septate, and usually show a limited apical growth; they may, in turn, produce ramifications of a higher order. The part of the endospore which lies opposite the germinating filament may develope into a hyaline rhizoid, which penetrates into the ground. The cell-walls of the protonema-filaments are at first colourless, but as the primary axes lie upon the ground or even penetrate into it, their cell-walls assume a brown colour, while the cells above ground develope abundance of chlorophyll-granules ; and the protonema is hence nourished independently by assimilation; it not only attains a considerable size in some genera, covering a surface of from one to several square inches like turf with its densely matted filaments, but its term of life may be regarded as unlimited. In most Mosses it altogether disappears after it has produced the leafy stems as lateral buds; but where these latter remain very small and have only a short term of life, as in the Phascaceæ, Pottia. Physcomitrium, \&c., the protonema still remains vigorous after it has produced the leafy plants, and when the sporogonium has already been developed upon them. In such cases all three stages of the cycle of development are present simultaneously in genetic connexion. The Sphagnaceæ, Andreæaceæ, and Tetraphideæ differ from the typical

1 W. P. Schimper, Recherches anat. et pliysiol. sur les Mousses (Strassburg 1848). - LantziusBeninga, Beiträge zur Kentniss des Baues der ausgewachsenen Mooskapsèl, insbesondere des Peristoms (with beautiful illustrations) in Nova Acta Acad. Leopold. 1847.-Hofmeister, Vergleich. Untersuch. 1851. [On the Germination, Development, and Fructification of the Higher Cryptogamia, Ray Soc. 1862.]-Hofmeister, in Berichte der Kön. Sächs. Gesellsch. der Wissens. 1854.-Ditto, Entwickelung des Stengels der beblätterten Muscineen (Jahrb. für wissens. Bot. vol. III).--Unger, Ueber den anat. Bau des Moosstammes (Sitzungsber. der Kais. Akad. der Wissens. Vienna, vol. XLIII. p. 497).-Karl Müller, Deutschlands Moose (Halle 1853).-Lorentz, Moosstudien (Leipzig 1854).-Ditto, Grundlinien zu einer Vergleich. Anat. der Laubmoose (Jahrb. für wissen. Bot. vol. VI, and Flora 1867).-Leitgeb, Wachsthum des Stämmchens von Fontinalis antipyretica u. von Sphagnum; sowie Entwickelung der Antheridien derselben (in Sitzungsber. der Kais. Akad. der Wissens. Vienna 1868 and 1869).-Nägeli, Pflanzenphysiol. Untersuchungen, Heft I, p. I 5.-Julius Kühn, Entwickelungsgeschichte der Andreæaceen (Leipzig I 870 ). (Mittheilungen aus dem Gesammtgebiet der Botanik von Schenk u. Luerssen, vol. I).-Janczewski, Ueber Entwickelung der Archegonien, Bot. Zeitg. $187^{2}$. 
Mosses both in the structure of the sporogonia, and in the mode of formation of the protonema. The spores of the Sphagnaceæ produce, at least when they grow upon a firm substratum, a flatly expanded plate of tissue, which branches at the margin, and produces from its surface the leafy stems. In Andrecea, according to the investigations of Kühn, the contents of the spore divide, while still within the closed exospore, into four or more cells, and a tissue is thus formed similar to that produced in the spores of some Hepaticæ (as Radula and Frullania) ${ }^{1}$ : finally, from one to three peripheral cells grow into filaments which extend over the hard stony substratum. The branches of the protonema may now develope further in three different ways; longitu'inal as well as transverse divisions arise, and irregularly branched cellular ribbons are formed; or, divisions also taking place in addition parallel to the surface of these ribbons so that they come to be several layers thick, the protonema developed in this manner as a mass of tissue becomes

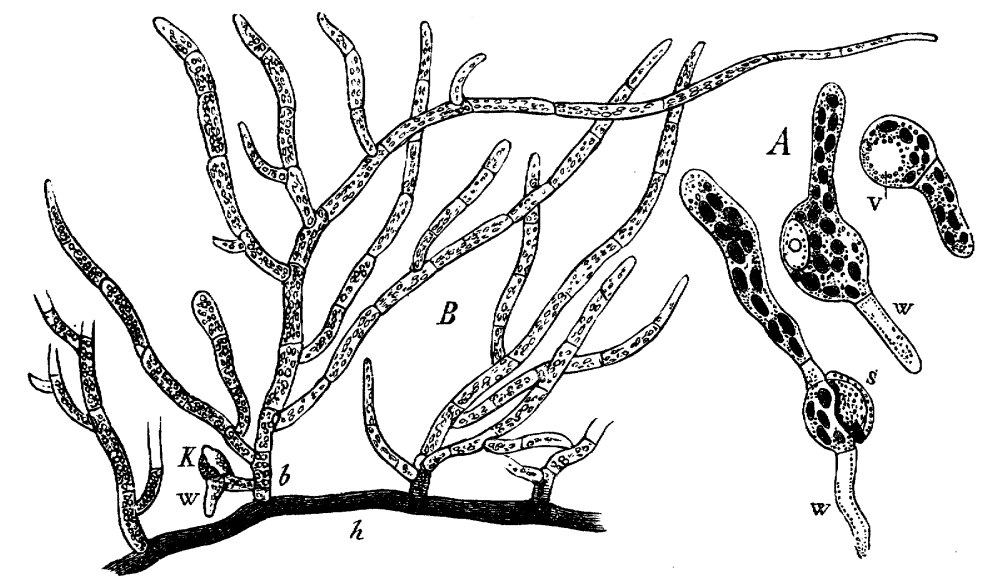

F16. 247.-Funariz hygrometrica; $A$ germinatung spores; $v$ vacuole; $v$ root-hair; $s$ exospore; $B$ part of a developed protonema, about three weeks after germination; $h$ a procumbent primary shoot with brown wall and oblique septa, out $B$ about arise the ascending branches with limited growth; $K$ rudiment of a leaf-bearing axis with root-hair $w\left(A \times 55^{\circ}\right.$;

erect and branches in an arborescent manner; finally, in the third form, the leaf-like branches of the protonema are plates of tissue of simple definite outline. Closely allied to this last form is the flat protonema of Tetraphis and Tetradontium, which, as will be further shown in a following illustration, arises at the end of longer and slenderer filaments ${ }^{2}$.

The buds which develope into the Moss-stems apparently never arise at the end of one of the principal protonemal filaments, but as lateral branches upon them. The idea suggested by me that the protonema, as also the equivalent rhizoids of the Bryineæ, represent a very rudimentary much elongated Moss-stem, just as the branches with naked base of Chara are merely simple forms of its stem, has been proved correct by the observations of Schuch (1870-1871) made

1 In true Mosses also (as Bartramia, Leucobryum, Mnium, and Hypnum) the first septum of the protonema is formed, according to Kiihn, even within the spore.

${ }^{2}$ Compare Berggren, Bot. Zeitg. I 871 . 
in the botanical laboratory at Würzburg, and concluded by Müller (1873). The principal filaments of the protonema and the large rhizoids have a very much elongated apical cell in which (and never in its segments) oblique septa are formed which are regularly inclined (spirally).in three or more directions, just in the same manner as the principal walls of the segments of the trilateral apical cell of the Moss-stem. These walls do not, however, intersect, as in the stem, since the segments (cells of the filament) are so long. Each segment is capable of forming a protuberance immediately behind its anterior wall, which is shut off by a wall corresponding to the 'foliar wall' formed in the stem-segments. After this protuberance has become elongated a wall is formed within it corresponding to the 'basal wall' formed in the segments of the stem. In this way the protuberance comes to consist of two cells; the one directed towards the growing apex of the filament corresponds to the mother-cell of the leaf, and the other, lying behind the preceding, developes a lateral branch, just as is the case in the stem ${ }^{1}$. Other unimportant divisions of these cells need not be mentioned here. Úsually

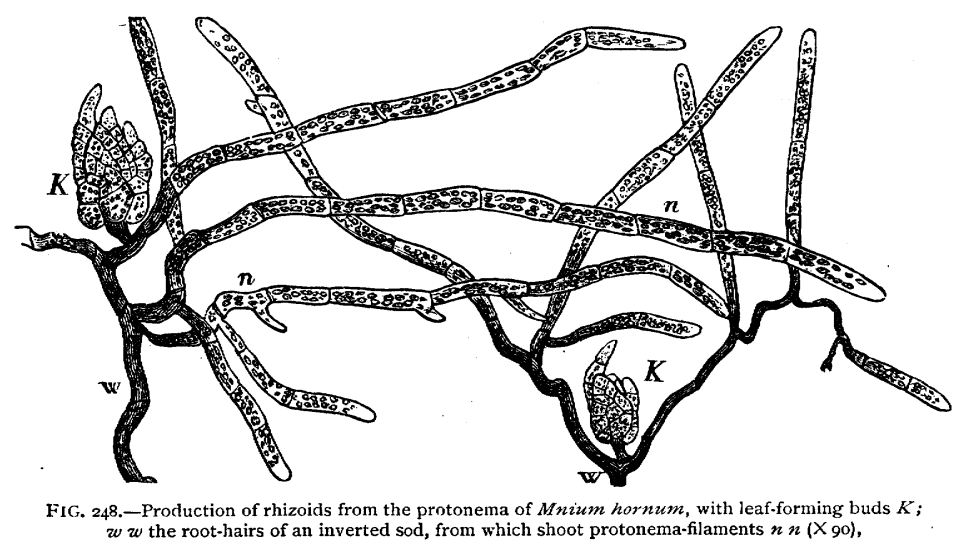

the anterior cell does actually give rise to a foliar organ, the posterior to a bud, but often one or both simply develope into rhizoids. The position of the walls of these cells is precisely similar to that of the corresponding cells of the stem; the protonema differs from the Moss-stem simply in the distance of one segment from the other, and in the suppression of those further divisions by which the tissue of the stem is produced from its segments. When a stem is developed from the protonema, it originates as a bud from the posterior of the two cells of the lateral protuberance. Its mode of origin is usually this, that the cell at first elongates into a filament by the formation of segments the primary walls of which do not intersect; after this segments are cut off by walls which do intersect, and from them foliar outgrowths, and later true leaves, are formed. From this it is evident that the formation of a Moss-stem from the protonema essentially depends upon the more rapid formation of segments one after another; a Mossstem is, so to speak, a protonemal filament with very short segments, forming 
mother-cells of leaves which at once grow out into expanded leaves instead of into filamentous structures. This interpretation is confirmed by all possible transitional forms, and a comparison of the ordinary characteristics of the stem and of the protonemal filament at once demonstrates its truth ${ }^{1}$. Whether or not the Andreæaceæ, Sphagneæ, \&c. resemble the Bryineæ in this feature, and the extent of such a resemblance, must be decided by future researches.

The apical cell of the stem is two-sided in Schistostega and Fissidens, and produces two straight rows of alternating segments; in the rest of the Mosses it is a three-sided pyramid, with the basal surface turned upwards (Fig. I i6). Each segment of the apical cell arches outwards and upwards as a broad papilla; this is cut off by a longitudinal wall (which Leitgeb calls a foliar wall), and developes, by further divisions, into a leaf, while the lower inner part of the segment produces, by further divisions, part of the inner tissue of the stem. Since each segment forms a leaf, the phyllotaxis is determined by the position of the consecutive segments. In Fissidens two straight rows of alternate leaves are thus formed; in Fontinalis three straight rows with the divergence $\frac{1}{3}$, the segments themselves lying here in three straight rows with the $\frac{1}{3}$ arrangement, because each newly formed primary wall is parallel to the last but three (both belonging to one segment). In Polytrichum, Sphagnum, Andrecea, \&c., on the other hand, each new primary wall encroaches on the ascending side with regard to the leaf-spiral; the primary walls of each segment are therefore not parallel; the segments themselves do not lie, even when first formed (without the assistance of any torsion of the stem), in three straight rows, but in three parallel spiral lines winding round the axis of the stem one above another; and the consecutive segments and their leaves diverge at an angle which, from what has been said, must be greater than $\frac{1}{3}$; the phyllotaxis is $\frac{2}{5}, \frac{3}{8}$, and so on ${ }^{2}$.

The primary meristem of the stem, situated beneath the punctum vegetationis, passes over into permanent tissue and usually becomes differentiated into an inner and a peripheral mass of tissue, which are not generally sharply defined; the cellwalls of the peripheral and especially of the outermost layers are usually strongly thickened and of a bright red or yellowish red colour; the cells of the inner fundamental tissue have broader cavities and thinner walls more slightly or not at all coloured. In some Moss-stems this differentiation goes no further than into an outer skin consisting of several layers and a thin-walled fundamental tissue $(e . g$. Gymnostomum rupestre, Leucobryum glaucum, Hedwigia ciliata, Barbula aloides, Hylocomium splendens, \&c., according to Lorentz); while in many other species a central bundle of very thin-walled and very narrow cells is formed in addition (Grimmia,

${ }^{1}$ I must content myself with the above brief account, for Herr Schuch has not yet published his observations which $I$ had the opportunity of following in all their details. Herr Müller is at present (1874) working at this subject, but is not yet in a position to publish his results. Figs. 247 and 248 were drawn at a time (1866) when the views expressed above were unformed. (See Arb. d. bot. Inst. in Würzburg, Heft IV. I874.)

${ }^{2}$ If the position of each fourth division of the apical cell is kept in view, it gives the impression as if the apical cell rotated slowly on its axis, producing, at the same time, leaf-forming segments. (Compare on this subject the work of Leitgeb mentioned above, Lorentz's work, Hofmeister's Morphologie, p. 194, and Müller, Bot. Zeitg. 1869, pl. VIII.) 
Funaria, Bartramia, Mnium, Bryum, and others) ${ }^{1}$. In Polytrichum, Atrichum, and Dawsonia alone do decided thickenings of the cell-walls take place in the central bundle in such a manner that each of several groups of originally thin-walled cells becomes surrounded by a thick wall and they together form the bundle. In Polytrichum commune there are found similar thinner extraaxial bundles. Sometimes bundles of thinwalled cells run from the base of the leaf-veins obliquely downwards through the tissue of the stem as far as the central bundle, which Lorentz regards as foliar bundles (e.g. in Splachnum luteum, Voitia nivalis, \&c.). If it is borne in mind that in some vascular plants fibro-vascular bundles of the most simple structure occur, and if the similarity of the cambiform cells of true fibro-vascular bundles to the tissue of the central and foliar bundles in Mosses is considered, these latter may with-

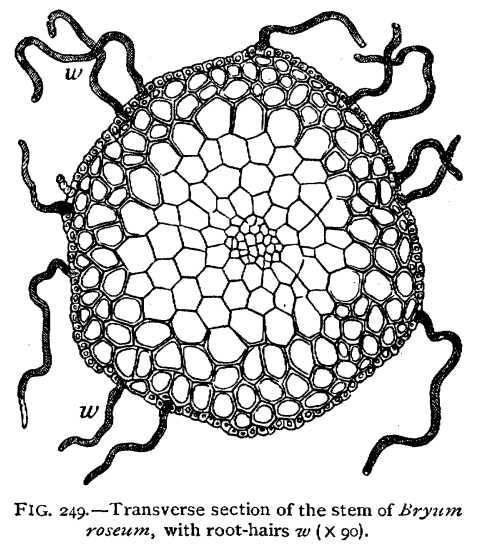

rosezem, with root-hairs $w(\times 90)$. out doubt be held to be fibro-vascular bundles of the simplest kind.

As has already been mentioned, the leaf originates from the broad papillose bulging of a segment of the apical cell of the stem : this is cut off by a wall. The lower (basal) part is concerned in the formation of the outer layers of tissue of the stem, whereas the apical part of the papilla constitutes the apical cell of the leaf; it forms two rows of segments by walls perpendicular to the surface of the leaf and inclined to the right and left. The number of the segments to be formed, in other words, the terminal growth of the leaf, is limited, and the formation of tissue from the cells thus formed advances downwards, ceasing finally at the base. The whole of the tissue of the leaf is sometimes (as in Fontinalis) a simple layer of cells; but very commonly a vein, i.e. a more or less broad bundle, is formed from the base towards the apex, dividing the unilamellar lamina into right and left halves, and consisting itself of several layers of cells. The vein is sometimes composed of uniform elongated cells, but more often various forms of tissue become differentiated in it, among which bundles of narrow thin-walled cells similar to the central bundle of the stem frequently occur, and these are sometimes continued to it through the external tissue of the stem as foliar bundles ( $c f$. Lorentz, l.c.). The shape of the leaves of Mosses varies from almost circular through broadly lanceolate forms to the acicular; they are always sessile and broad at their insertion; usually densely crowded; only on the stolons of some species, the pedicels of the cupules of the gemmæ of Aulacomnion and Tetraphis, as well as at the base of some leafy shoots, do they remain small and remote (cataphyllary leaves). In the neighbourhood of the reproductive organs they usually form dense rosettes or buds, and then not unfrequently assume special forms and colours. In Racopilum, Hypopterygium, and Cyathophorum, there are two kinds of leaves, a row of larger upon

${ }^{1}$ It is stated by Lorentz that the seta of the sporogonium is alw ays provided with a central bundle of this kind. 
one side, and a row of smaller leaves upon the other side of the stem. The leaves are not branched, but entire or toothed, rarely slit. In some kinds peculiar outgrowths are formed upon the inner or upper surface of the leaves; in Barbula aloides articulated capitate hairs. The lamina, which in other cases expands right and left from the median plane, is, in Fissidens, expanded in the median plane itself, proceeding from an almost sheathing base. The tissue of the leaf is, with the exception of the central vein, usually homogeneous and composed of cells containing chlorophyll, which sometimes project above the surface as mamillæ; in the Sphagnaceæ and Leucobryum the tissue is differentiated into cells containing air, and others which contain sap and chlorophyll, arranged in a definite manner.

The mode of branching of the stem of Mosses is apparently never dichotomous, but also probably never axillary, although connected with the leaves. Even when the branching is copious the number of lateral shoots is nevertheless usually much smaller than that of the leaves; in many cases the lateral branches are definitely limited in their growth, leading sometimes to the formation of definite ramified systems similar to pinnate leaves (Thuidium, Hylocomium). When the primary shoot produces reproductive organs at the summit, a lateral shoot situated beneath it not unfrequently displays a more vigorous growth, continuing the vegetative system; and by such innovations sympodia are formed. It sometimes happens that stolons, that is shoots either destitute of or furnished with very small leaves, creep on or beneath the surface of the ground, elevating themselves at a later period as erect leafy shoots. The mode of branching is very various, and is closely connected with the mode of life. The morphological origin of the lateral shoots has been carefully investigated by Leitgeb in the case of Fontinalis and Sphagnum, and admirably described. Since these two genera belong to very different sections, the results obtained in this case may be considered as of general application to the whole class. They agree in the fact that the mother-cell (which is at the same time the apical cell) of a branch originates beneath a leaf from the same segment as the leaf (Fig. I I6). In Fontinalis the branch arises beneath the median line of the leaf; but in Sphagnum beneath its cathodal half. In consequence of the further development of the mother-shoot, the lateral shoot in Sphagnum appears at a later period to stand by the side of the margin of an older leaf; and this is probably the explanation of the earlier statement of Mettenius that in Neckera complanata, Hypnum triqueirum, Racomitrium canescens, and others, the lateral shoots stand by the side of the margins of the leaves. When the shoot arises beneath the median line of a leaf, and the leaves are arranged in straight rows, the further growth of the stem may cause it to seem as if the shoot originated above the median line of an older leaf, in other words as if it were axillary. Leitgeb states that articulated hairs arise in the genera named in the axils of the leaves, or perhaps more correctly at the base of the upper surface of the leaves.

The dimensions attained by the leaf-bearing axes and axial systems of Mosses show a wide range. In the Phascaceæ, Buxbaumia, and others, the simple stem is scarcely $\mathbf{I} \mathrm{mm}$. in height; in the largest species of Hypnum and Polytrichum it is not unfrequently 2,3 , or more decimetres in length, and, if belonging to more than one axis, even longer, owing to the formation of innovations and sympodia (Sphagnum). The thickness of the stem is less variable; $\frac{1}{10} \mathrm{~mm}$. in the smallest, 
it scarcely exceeds $\mathrm{I} \mathrm{mm}$. in the thickest forms. Its dense tissue, coloured externally, is however very firm, often stiff, always very elastic, and capable of offering long resistance to decay.

The Root-hairs (Rhizoids) play an extremely important part in the economy of Mosses. It is only in the otherwise very abnormal section of the Sphagnacer that they are very sparsely and poorly developed; in most other forms they occur in large numbers at least at the base of the stem, often clothing it completely with a dense reddish-brown felt. Morphologically the rhizoids are not sharply distinguished from the protonema ${ }^{1}$; and it will be seen further on that they, like it, are capable of forming new leafy stems. They arise as tubular protuberances from the superficial cells of the stem, elongate by apical growth, and are segmented by oblique septa; at the growing end the wall is hyaline, and particles of earth become attached to it in the ground; subsequently these fall off; the wall becomes thicker and brown, as is also the case with the aërial root-hairs. The cells contain a considerable quantity of protoplasm and drops of oil (Fig. $250, B$ ). In many Mosses the root-hairs branch very copiously in the ground; they often form a dense inextricable felt; a felt of this kind may even arise above ground as a dense turf, and may serve as a soil for future generations. In Atrichum and other Polytrichaceæ, the stouter rhizoids coil round one another like the threads of a rope, the branches which proceed from them doing the same, and only the last and finest ramifications remain free.

The Vegetative Reproduction of Mosses is more copious and varied than is the case in any other section of the vegetable kingdom. It presents the peculiarity that the production of a new leaf-bearing stem is always preceded by the formation of a protonema, even when the propagation takes place by gemmæ. Exceptions are afforded only by the few cases in which leaf-buds become detached and commence immediately to grow.

In describing the different cases in detail, the first point that must be brought prominently forward is that both the protonema which proceeds from the spore itself and the leafy stems which spring from it are capable of reproduction of different kinds. The original protonema is so far an organ of reproduction that it may produce upon its branches a smaller or larger number of leafy stems in succession or simultaneously; sometimes the individual cells of the protonemabranch separate from one another after they have become rounded off into a spherical form, acquire thicker walls, and become for a time inactive (as in Funaria hygrometrica), forming, probably, at a later period again protonema-filaments. A secondary protonema may be formed from any root-hair when exposed to light in a moist atmosphere ( $c f$. Fig. 247 and Fig. $250, A, p$ ). In some species (Mnium, Bryum, Barbula, \&c.) it is sufficient to keep a turf of Moss damp for some days and turned downwards, in order to produce hundreds of new plants in this manner. Some apparently annual species, e.g. of Phascum, Funaria, and Pottia, persist perennially by means of their root-hairs; the plants disappear completely from the

1 The rhizoids appear to be distinguished from the protonema only by the absence of chlorophyll and by their tendency to grow downwards; the protonema developes certain branches as rhizoids; and the rhizoids may, on their part, develope single branches as a protonema growing upwards and containing chlorophyll; see p. $\mathbf{3}^{62}$. 
surface of the ground from the time that the spores become ripe till the next autumn, when the root-hairs again produce a new protonema, and upon this new stems arise.

Similar outgrowths from the roots occur also, according to Schimper, in the felted protonema of some species of Polytrichum ( $P$. nanum and aloides) on the slopes of hollow roads, and on that of Schistostega osmundacea in dark hollows. The root-hairs may also immediately produce leaf-buds, and behave, in this respect, exactly like the protonema. When the buds arise on underground ramifications of the root-hairs (Fig. $25^{\circ}, B$ ) they remain in a dormant state, as small microscopic

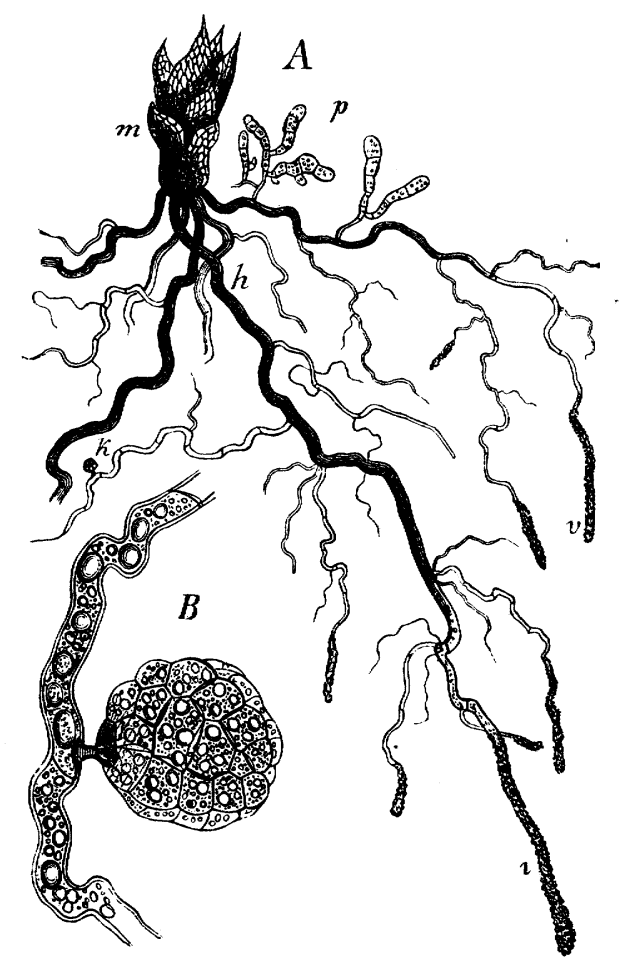

FIG. 250. $-A$ young plant of a Barbula $(m)$ with the root-hairs $h$, to the growing ends of which particles of earth have become attached; at $p$ a superficial root-hair is putting out branches containing chlorophyll, in other words a protonema. at $k$ a tuberous bud (bulbil) is growing from an underground branch of the root-hairs; $B$ this bud more strongly magnified $(A \times 20 ; B \times 300)$.

tuberous bodies (bulbils) filled with reserve food-material, until they chance to reach the surface of the ground, when they undergo further development (e.g. Barbula muralis, Grimmia pulvinata, Funaria hygrometrica, Trichostomum rigidum, Atrichum). The aërial root-hairs may, however, not only produce a protonema containing chlorophyll, but also leaf-buds without its intervention; and Schimper cites the remarkable fact that in Dicranum undulatum annual male plants are formed in this manner on the tufts of perennial female plants, and fertilise the latter.

Even the leaves of many Mosses produce a protonema, their cells simply growing, and the tubes thus formed becoming segmented. This occurs in Ortho- 
trichum Lyelli and obtusifolium; in O. phyllanthum tufts of club-shaped protonemal filaments with short cells arise at the apex of the leaves; and the same phenomenon occurs in Grimmia trichophylla, Syrrhopodon, and Calymperes. In Oncophorus glaucus a dense felt of interlacing protonema-filaments is formed at the summit of the plant where the reproductive organs are produced, which arrests its further growth, and hence produces at a later period new clumps of young plants. In Buxbaumia, especially $B$. aphylla, the marginal cells of the leaves form a protonema enveloping them as well as the stem with its filaments. Lastly, even

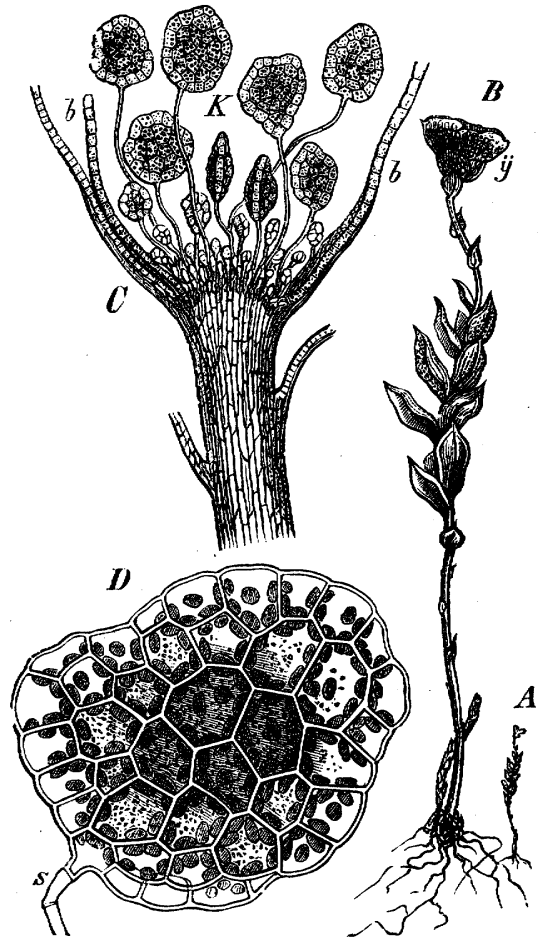

FIG. 25x.-Tetraphis pellucida; $A$ a plant producing gemmæ (natural size) ; $B$ the same, magnified; $y$ the cup in which the gemmae are collected; $C$ longitudinal section through the summit of the plant, $b$ the leaves of the cup, $K$ the gemmæ in various stages of development; the older ones are forced off their stalks by the later growth of the younger ones, and forced over the side of the cup; $D$ a mature gemma $(\times 500)$, consisting at the margin of one, in the centre of several layers of cells.

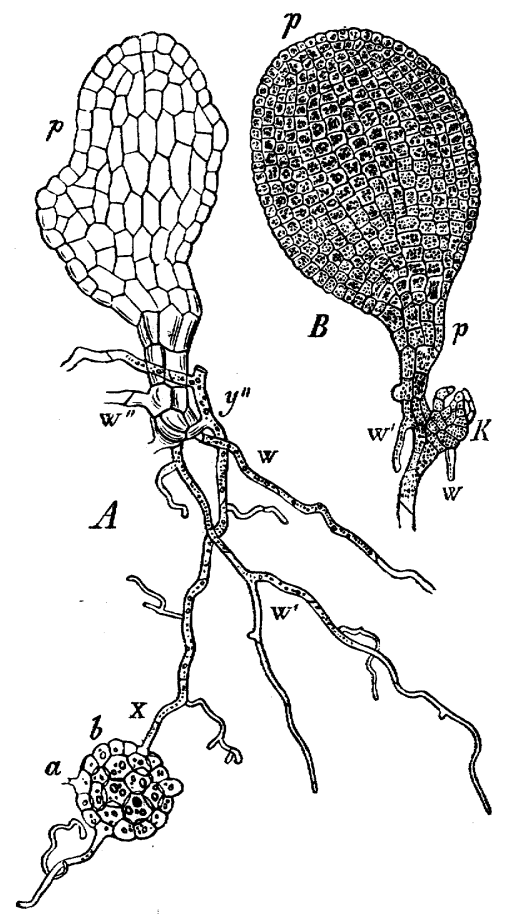

FIG. 252.-Tetraphis pellucida; $A, b$ a gemma, detached from its stalk at $\alpha$ the protonema-filament $x$ has been formed by the growth of a marginal cell of the gemma, and the flat by the growth of a marginal cell of the gemma, and the flat
structure $p$ as a lateral outgrowth from the protonema; this has also put out root-hairs $w, w^{\prime}, w^{\prime \prime}$ ( $\left.\times 100\right) ; B, p$ a flat protonema from the base of which a leaf-bud $K$ and root-hairs $w, w^{\prime}$ have sprung; the base of the protonema often puts out a number of new flat protonemata before a leaf-bud is formed.

detached leaves, if kept moist, may emit a protonema, as for instance those of Funaria hygrometrica ${ }^{1}$.

Gemma, which, like those of the Marchantieæ, are stalked fusiform or lenticular cellular bodies, occur in Aulacomnion androgynum at the summit of a leafless elongation of the leafy stem (Pseudopodium); in Tetraphis pellucida enveloped by an elegant cup composed of several leaves, out of which they subsequently fall. These

1 [It has been shown by Pringsheim (Jahrb. für wiss. Bot. XI) and by Stahl (Bot. Zeit. I876) that the sterile cells of the sporogonium and of the seta may give rise to protonema.] 
latter then put forth protonemal filaments, which produce first of all a flat membranous protonema; and upon this finally new leaf-buds arise (Figs. 25I, 252).

Finally the deciduous branch-buds of Bryum annotinum may also be considered as organs of reproduction; as also, according to Schimper, may the branches of Conomitrium julianum and Cinclidotus aquaticus, which likewise have the power of detaching themselves.

The Sexual Organs of Mosses usually occur in considerable numbers at the end of a leafy axis ${ }^{1}$, surrounded by enveloping leaves often of peculiar shape, and mixed with paraphyses, and the whole group of organs may, for the sake of

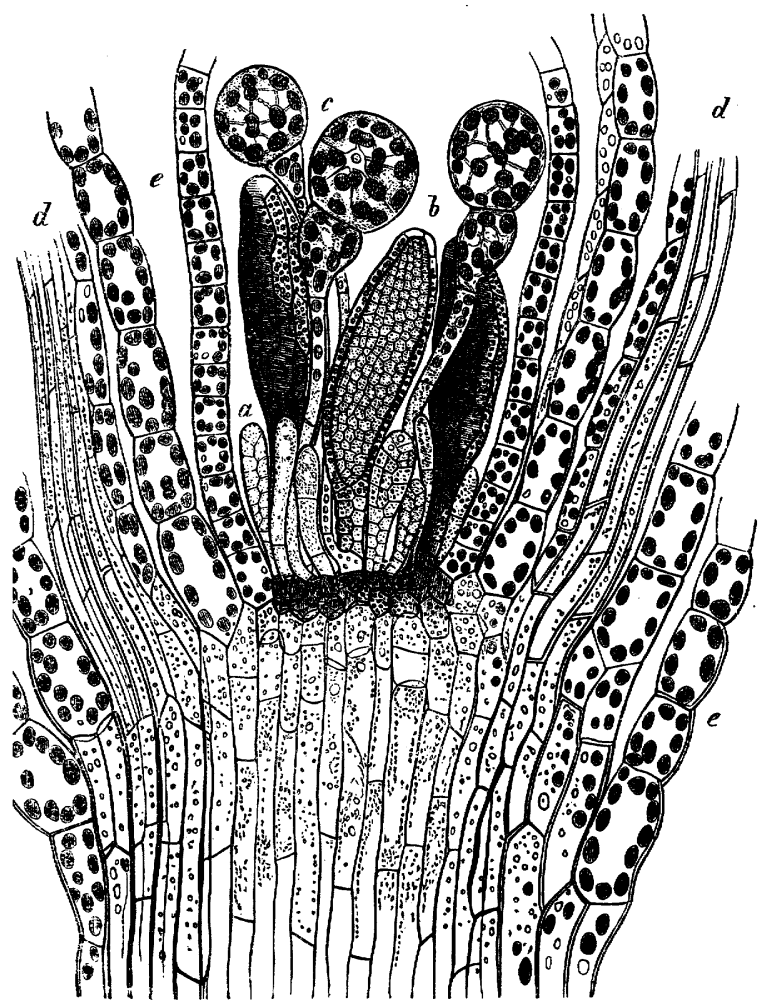

FIG. 253.-Longitudinal section of the summit of a very small male plant of Funaria hygrometrica; $a$ a young, $b$ a nearly ripe antheridium; $c$ paraphyses; $d$ leaves cut through the mid-rib; $e$ leaves cut through the lamina $(\times 300)$.

brevity, be called a 'Receptacle.' The receptacle of Mosses either terminates the growth of a primary axis (Acrocarpous Mosses), or the axis is indeterminate, and the receptacle is placed at the end of an axis of the second or third order (Pleurocarpous Mosses). Within a receptacle either both antheridia and archegonia are produced (bisexual receptacles), or it contains only one kind of sexual organ, and the receptacles may then be either monœcious or diœcious. Sometimes the male receptacles appear on smaller plants with a shorter duration of life (as Funaria hygrometrica, Dicranum undulatum, \&c.). In external appearance the bisexual are similar

1 The male branches of Sphagnum form an exception (vide infra). 
to the female receptacles, while the habit of the male receptacles is altogether different. In the former the archegonia and antheridia occur either close to one another at the summit of the stem in the centre of the envelope (Perichatium), either in two groups, or separated by peculiar enveloping leaves, and the antheridia stand in the axils of these arranged in a spiral, surrounding the central group of archegonia. The form of the perichætium is, in the female and bisexual receptacles, that of an elongated almost closed bud, formed by several turns of the leaf-spiral. Its leaves are similar to the foliage-leaves, and become smaller towards the interior, but grow all the more vigorously after fertilisation. The male perichætium consists of broader firmer leaves, and is of three different forms; usually it is bud-shaped, and resembles that of the female receptacle, but is shorter and thicker, its leaves often coloured red, and decreasing in size towards the outside; receptacles of this type are always lateral. Secondly, the male perichætia are sometimes shaped like capitula, and are, on the contrary, always terminal on a stouter shoot and globular; their leaves are broad, sheathing at the base, thinner and recurved at the upper part; they become smaller towards the interior, and leave the centre of the receptacle, with the antheridia, free; these receptacles are sometimes borne on a naked pedicel, a prolongation of the stem (Splachnum, Tayloria). Finally, the male perichætia are sometimes discoid and consist of leaves which are very different from the foliage-leaves; they are broader and shorter, expanded horizontally at the upper part, delicate and of a pale green, orange, or purple colour; they are always smaller the nearer the leaf-spiral approaches the centre; the antheridia stand in their axils (Mnium, Polytrichum, Pogonatum, Daresonia). The paraphyses stand between or by the side of the sexual organs; in the female receptacle they are always articulated filaments; in the male, filiform or spathulate, and consisting, in the upper part, of several rows of cells.

The Antheridia are, when mature, stalked sacs with a wall consisting of a single layer of cells containing chlorophyll-granules, which however, in the ripe state, assume a red or yellow colour. In the Sphagnaceæ and in Buxbaumia the antheridia are nearly spherical, but in all other Mosses of an elongated club shape. In the Sphagnacex they open in the same manner as in the Hepatica; in the other orders by a slit across the apex, through which the antherozoids still enclosed in their mother-cells are discharged as a thick mucilaginous jelly. The interstitial mucilage dissolves in water, and

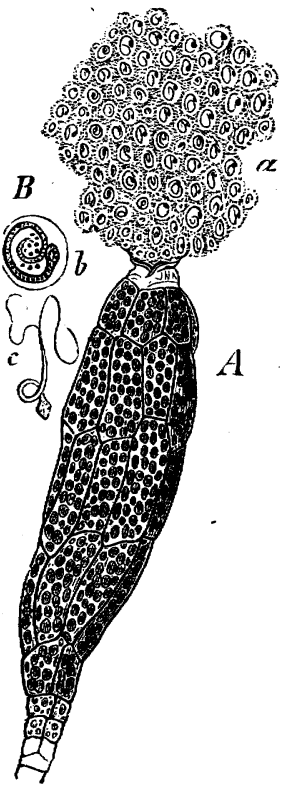

FIG. 254--Funaria hygrometrica; $A$ an antheridium bursting, $a$ the antherozoids $(x 350) ; B$ the antherozoids more strongly magnified, $b$ in the mother-cell; $c$ free antherozoid of Polytrichum $(\times 800)$. the antherozoids escape from their mother-cells and swim about free.

The careful investigations of Leitgeb show that the morphological significance of the antheridia is very various. In Sphagnum the mother-cell of the antheridium 
arises in exactly the place in which a branch would otherwise be formed, i. e. from the segment of the axis which lies beneath the cathodal half of the leaf; the antheridia may in this case be considered as metamorphosed branches. In Fontinalis, on the other hand, their morphological significance varies within the same receptacle; the one first formed is the immediate prolongation of the axis of the shoot, arising from its apical cell; the succeeding ones are developed from its last normal segments, and therefore resemble leaves in their origin and position; the last antheridia, finally, exhibit the morphological characters of trichomes, both in their variable number, their development as cells of the epidermis, and the want of definiteness in their place of origin. According to Kühn, Andreaa behaves in precisely the same way as Fontinalis. The mother-cell of the antheridium of Fontinalis is constituted as an apical cell forming two alternating rows of segments; in forming the oldest and terminal antheridium the apical cell changes from a triseriate to a biseriate segmentation. These segments are next divided by tangential walls in such a manner that the transverse section (which meets two segments) of the young organ shows four outer and two inner cells; the wall of the antheridium, one cell in thickness, arises from the former by further division; the small-celled tissue which produces the antherozoids from the latter. Andreca behaves also very similarly in these respects; the primary mother-cell of the antheridium appears as a papilla and is cut off by a septum; the lower cell produces a cushion-like support; the upper cell is again divided by a septum into a lower cell from the divisions of which the tissue of the stalk is formed, and an upper cell out of which the body of the antheridium arises; the formation of the latter takes place in the same manner as in Fontinalis. In Sphagnum the long stalk originates by transverse divisions of the growing papilla which produces the antheridium, the segments dividing again in a cruciform manner. The terminal cell then swells, and becomes divided by oblique walls of somewhat irregular position; a tissue is thus formed, which, at a subsequent period, consists also of a wall formed of a single layer of cells and an inner very small-celled tissue which produces the antherozoids.

The Archegonium consists when mature of a massive, moderately long base, which supports a roundish ovoid ventral portion; above this rises a long thin neck, generally twisted on its axis. The wall of the ventral portion, which consists, even before fertilisation, of a double layer of cells, passes up continuously into the wall of the neck consisting of a single layer of cells formed of from 4 to 6 rows (Fig. 256). Together they enclose an axial row of cells, the lowest of which, ovoid and lying in the ventral portion, produces the oosphere and the ventral canal-cell, the upper cells being the canal-cells of the neck. These and the ventral canal-cell become mucilaginous before fertilisation. This mucilage forces the four uppermost cells (stigmatic cells) of the neck apart, and thus opens the canal of the neck, allowing the antherozoids to penetrate to the oosphere. Fig. ${ }_{2} 5, B$, shows the row of canal-cells at the period when disorganisation is beginning, and when the stigmatic cells of the neck are still closed. In reference to the morphological significance of the archegonia, Leitgeb has already shown that at least the first archegonium of Sphagnum arises immediately from the apical cell of the female shoot; more recently Kühn found that in Andrecea the first is formed from the apical cell, the 
succeeding ones from its last segments, in the same manner as the antheridia of the same genus, and those of Radula and Fontinalis. According to preparations which Schuch obtained in the laboratory at Würzburg, the first archegonium arises also in typical Mosses from the apical cell of the shoot.

The order of succession of the cells in the development of the archegonium has been studied by Kühn in Andrecea, and by Janczewski in the Phascaceæ, Bryineæ, and in Sphagnum. As in the Liverworts so here also, the whole archegonium is derived from an outgrowth of a superficial cell of the punctum vegetationis. This is divided by a transverse wall ( $m m$, Fig. $255 A$ ) so as to form a lowèr cell (corresponding to the pedicel of the Liverworts) and an upper external cell, in
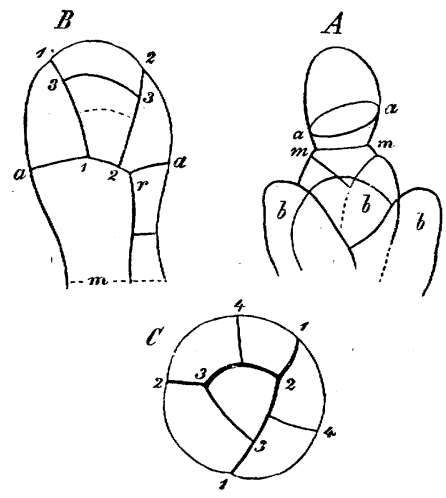

FIG. 255.-First stage of development of the archego nium of Andreace (after Kühn); $A$ terminal archegonium arising from the apical cell of the shoot; $b b$ the youngest leaves ; $B$ after the formation of the central cell and stigmatic cell; $C$ transverse section of the young ventral portion.

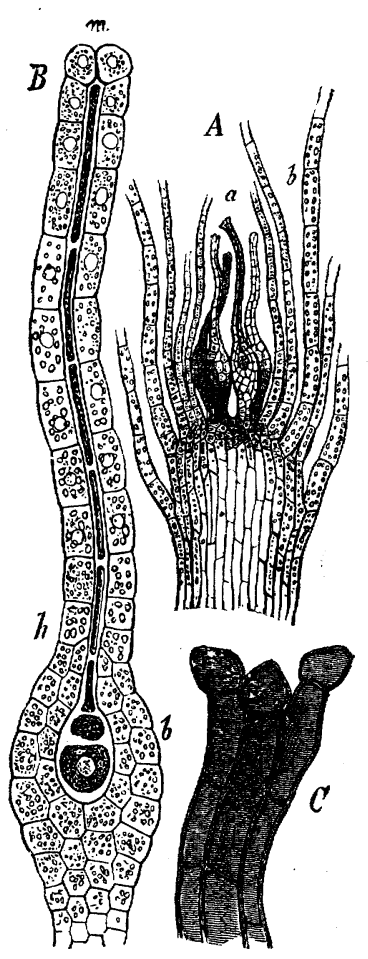

FIG. 256.-Funaria hygrometrica; $A$ longitudinal section of the summit of a weak female plant $(\times$ Ioo), $\alpha$ archegonia, $b$ leaves; $B$ an archegonium $(\times 550), b$ ventral portion with the oosphere, $h$ neck, $m$ mouth still closed; the canal-cells are beginning to be converted into mucilage (the preparation had lain three days in glycerine); $C$ the part near the mouth of the beginning to be converted into mucilage (the preparation

which, as in the corresponding cell of the antheridium, two oblique walls inclined in opposite directions appear. The two oblique ce!ls thus cut off give rise, at a later period, to the tissue of the lower part of the ventral portion of the archegonium, which is here more developed than in the Liverworts (Fig. $256, B$ ). The upper cell undergoes the same divisions as it does in the Liverworts; the mode of formation of the ventral wall and of the central cell is the same in this group as in that; but the formation of the neck is here quite different. Whereas in the Liverworts the first transverse division of the internal cell produces an upper cell which at once represents the 'stigma' of the archegonium, the cell thus formed 
in this group acts like an apical cell, giving rise by a series of longitudinal divisions to several tiers of cells, each tier consisting of three external cells and an internal canal-cell and in other respects resembling the single tier of neck-cells formed in the Liverworts. In this way a long and subsequently twisted neck is formed, consisting of six external rows of cells investing the central row of canal-cells. Below, the cells forming the neck become continuous with the wall of the ventral portion of the archegonium, which consists usually of two (four in Sphagnum) layers

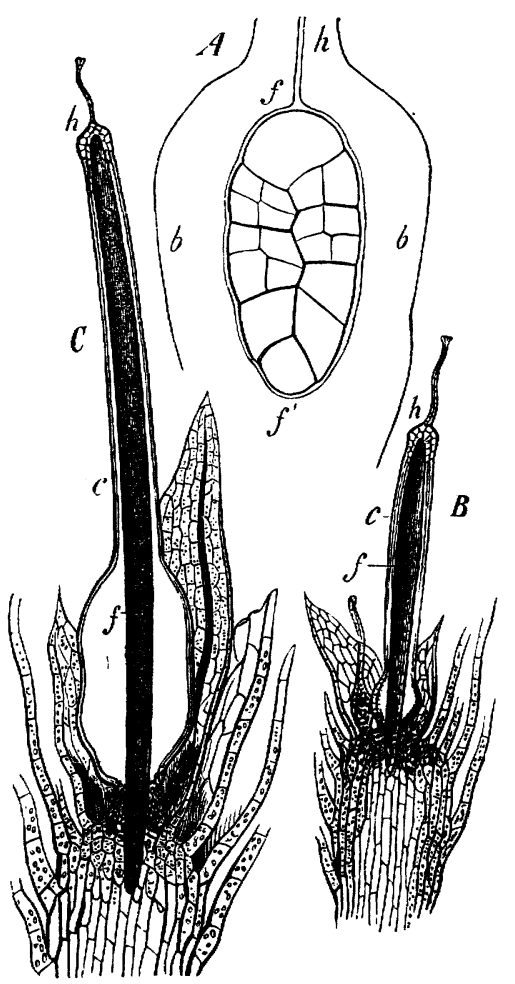

FIG. 257.-Funaria hygrometrica; $A$ origin of the sporogonium $f f^{\prime}$ in the ventral portion $b b$ of the archegonium
(longitudinal section $\times 500) ; B, C$ different further stages of development of the sporogonium $f$ and of the calyptra $c ; h$ neck of the archegonium ( $X$ about 40$)$. of cells. The central cell, which makes its appearance here earlier than in the Liverworts, becomes divided by a transverse wall into an upper cell, the ventral canal-cell, and a lower cell, the protoplasm of which contracts and forms the oosphere (Fig. 256, $B$ ). The conversion into mucilage of the canal-cells and the opening of the neck take place in the same manner as in the Liverworts.

The Sporogonium, which results from the fertilised oosphere, attains, in Sphagnum, almost perfect development within the actively growing ventral portion of the archegonium, which becomes transformed into the calyptra; but in all other Mosses the calyptra is torn away from the vaginula at its base, by the elongation of the sporogonium, usually long before the development of the spore-capsule, and (except in Archidium and its allies) is raised up as a cap. The neck of the archegonium, the walls of which assume a deep red-brown colour, still for some time crowns the apex of the calyptra. The sporogonium of all Mosses consists of a stalk (the Seta), and the spore-capsule (Theca or Urn); but the former is very short in Sphagnum, Andreaa, and Archidium, longer in most other genera, and with its base planted in the tissue of the stem, which, after fertilisation, grows luxuriantly beneath and around the archegonium, forming a sheathlike investment, the Vaginula. The unfertilised archegonia may frequently be seen on the exterior slope of the vaginula, since only one archegonium is usually fertilised in the same receptacle, or it is only in the one first fertilised that an embryo is developed. The capsule has in all Mosses a wall consisting of several layers of cells and a distinct epidermis which sometimes possesses stomata $^{1}$; the whole of the inner tissue is never used up in

1 The stomata upon the capsules of Mosses are peculiar, as Schimper has shown, in that the mother-cell of a stoma is not divided into two guard-cells, for the dividing wall does not extend 
the formation of spores, even when, as in Archidium, it is subsequently supplanted by them; a large part of the central tissue remains as the so-called Columella, and it is at the circumference of this that the mother-cells of the spores are formed. The structure of the mature capsule, and especially the contrivances for dispersing the spores, are, however, so different in the various principal sections of Mosses that it will be better to consider them more closely separately, and the more so because by this means we shall at the same time arrive at the distinctive characters of the larger natural systematic groups.

${ }^{1}$ In the mode of origin of the sporogonium there is, as might be expected, less variety. [The oospore is first of all clothed with a cell-wall, continues to grow considerably, and is then divided by a horizontal or slightly oblique wall (basal wall). The lower (hypobasal) of these two cells undergoes only one or two divisions, and, as in Jungermannieæ, contributes but little to the formation of the embryo. The upper (epibasal) cell gives rise to the capsule and the seta: a two-sided apical cell is formed in it, by means of two oblique divisions.] Hofmeister asserts that in Bryum argenteum the upper cell (that facing the neck of the archegonium) is divided once or twice by horizontal septa before the first oblique division, while in Phascum and Andrece a this oblique septum is formed immediately after the first horizontal one. The apical cell now forms two rows of segments by partition-walls inclined alternately, and these segments are next divided by radial vertical walls, followed by further numerous transverse divisions. By this process the young sporogonium is transformed into a multicellular body which is usually fusiform, the lower end not participating in the growth in length. A swelling of this lower end, such as usually occurs in Hepaticæ, takes place also in certain cases such as Sphagnum, Archidium, and Phascum. The apex of the sporogonium now becomes inactive, and beneath it the capsule is formed as a spherical, ovoid, cylindrical, or frequently unsymmetrical swelling which originates, in the typical Mosses, only after the elongation of the fusiform or cylindrical sporogonium, and after the raising. up of the calyptra. The internal differentiation of this mass of tissue, at first homogeneous, gives rise to the various tissues which compose the capsule of Mosses, and especially to the mother-cells of the spores which first of all become isolated and then divide so as to form four spores. The contents of the mother-cell begin to divide into

completely across it; it forms simply a trabecula between the outer and the inner wall, which splits into two lamella. Between these is the opening of the stoma.

1 [The embryology of Mosses is treated of in the following works. Hofmeister, On the higher Cryptogamia, Ray Soc. I862.--Schimper, Rech. anat. et physiol. sur les Mousses, 1848.-Kühn, Entwick. d. Andreæaceen, Bot. Mittheil. von Schenk und Luerssen, I874-Vouck, Entwick. d. Sporogoniums von Orthotrichum, Sitzber. d. Wien. Akad. 1876.-Leitgeb, Das Sporogon von Archidium, ibid. I879.-Kienitz-Gerloff, Bot. Zeitg. 1878 . It appears that the tissue of the sporogonium presents, at an early stage, a differentiation into an amphithecium, from which the wall of the capsule and of the spore-sac are derived, and a central endothecium, corresponding to the future columella and sporogenous layer. Leitgeb has however pointed out that in the Sphagnaceæ the sporogenous layer is probably derived from the amphithecium. It is of interest to note also that, as Goebel has pointed out, the spores of certain Vascular Cryptogams are developed, like those of Mosses, from a layer of cells (archesporium), (vide infra, p. 388). The development of the spores from a layer of cells occurs also in the developing sporogonia of some Liverworts: it is distinct in Anthoceros, less so in the Jungermannieæ, still less in the Marchantieæ, and does not take place in Riccia.] 
two, but this bipartition is usually not completed, the division into four taking place at once. The preparation for the formation of spores takes place simultaneously everywhere within the same capsule. The ripe spores are roundish or tetrahedral, surrounded by a thin finely granulated exospore, which is of a yellowish, brownish, or purple colour. Besides protoplasm, they contain chlorophyll and oil. In Archidium, where only sixteen are formed in each capsule, they are about $\frac{1}{5} \mathrm{~mm}$. in size, in the highly developed Dawesonia scarcely $\frac{1}{200} \mathrm{~mm}$. (Schimper). When kept dry the spores often retain their power of germination for a long time, but when moist they frequently germinate after a few days, those of Sphagnum after two or three months.

The time necessary for the formation of the sporogonium varies greatly in the different species, but is usually very long in comparison with the small size of the
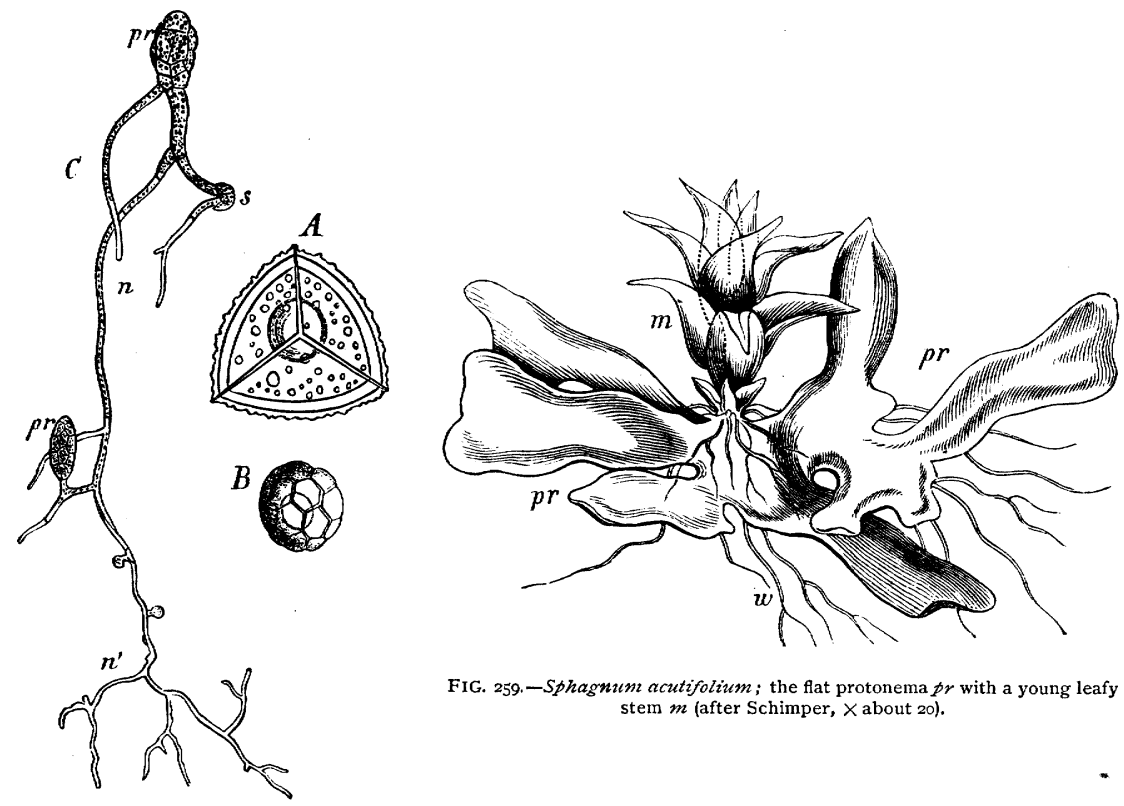

FIG. 259.-Sphagnum acutifolium; the flat protonema pr with a young leafy stem $m$ (after Schimper, $\times$ about 2o).

FIG. 258.-Sphagnum acutifolium; $A$ a large spore, seen from the apex ; $B$ a small spore; $C$ a protonema $n n^{\prime}$ resulting from the spore $s ; p r$ rudiments of young plants (after Schimper).

body concerned. The Pottieæ blossom in summer, and ripen their spores in the winter; the Funarieæ are perennially in blossom, and have constantly sporogonia in all stages of development, each occupying for its completion probably 2 to 3 months; Phascum cuspidatum developes in the autumn from its perennial underground protonema, and ripens its spores in a few weeks before the winter. The bog $H_{y}$ pna , on the other hand ( $H$. giganteum, cordifolium, cuspidatum, nitens, \&c.), blossom in August and September, and ripen their spores in July of the next year; they often require ten months for the development of their sporogonia. H. cupressiforme bears in autumn at the same time sexual organs and ripe spores, and hence requires one year. 
The same length of time is required by Philonotis, and by some species of Bryum and some of Polytrichum which blossom in May and June ${ }^{1}$.

Mosses may be distributed naturally into four parallel orders:-

$$
\begin{aligned}
& \text { I. Sphagnaceæ, } \\
& \text { 2. Andreæaceæ, } \\
& \text { 3. Phascaceæ, } \\
& \text { 4. Bryaceæ (True Mosses). }
\end{aligned}
$$

Of these the first includes a single genus, the second and third only a few; the fourth all the remaining extremely numerous genera. The first three groups recall, in many respects, the Hepaticæ; even the series of true Mosses commences with some genera which still resemble that class; the lowest forms of all the groups exhibit many resemblances which are wanting in the most highly developed. We have therefore four diverging series.

r. The Sphagnace ${ }^{2}$ include only the single genus Sphagnum. When the spores germinate in water, a branched protonema is developed, on which the leaf-buds immediately appear laterally (Fig. 258, C). On a solid substratum, on the other hand, the short protonema forms first of all a branching flat protonemal expansion (Fig. 259), on which (as in Tetrapbis) the leaf-buds appear. The leafy stems produce root-hairs only in the young state. The abundant protonema of true Mosses is entirely wanting. The stem, as it increases in strength, produces laterally, by the side of every fourth leaf, a branch, which, even at the very earliest period, is again much divided; tufts of branches arranged regularly thus arise which form a compact mass at the summit of the stem, but lower down are more distant from each other. The separate branches develope in different ways; one is produced each year beneath the summit after the ripening of the fruit, and developes in a similar manner to the primary stem, growing up along with the prolongation of the latter, so that each year a false dichotomy takes place on the stem. These innovations afterwards become separated by the slow decay of the plant advancing from below, and constitute independent plants. Some of the branches of

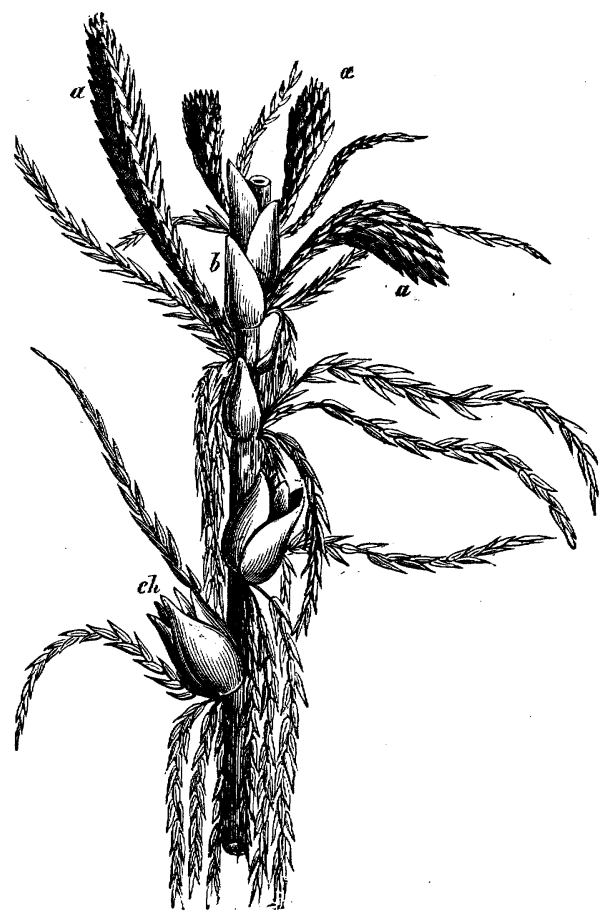

FIG. 260.-Sphagnum acutifolium; part of the stem below the apex; $a$ a the male branches, $b$ leaves of the primary stem; $c h$ perichætial branch with old still closed sporogonia (after Schimper, $\times 5^{-6)}$.

each tuft, however, turn downwards, become long, slender, and finely pointed, and are closely applied to the primary stem, forming a dense envelope around it; while other

1 Klinggräff, Bot. Zeitg. I860, p. 344 .

${ }_{2}$ W. P. Schimper, Versuch einer Entwickelungsgeschichte der Torfmoose. Stuttgart 1858 (with many beautiful plates). [Russow, Beitr. z. Kenntn. d. Torfmoose, I865.-Leitgeb, Wachsthum des Stammes und Entwickelung der Antheridien bei Sphagnum, Sitzber. d. Wien. Akad. I869.] 
branches of each tuft turn outwards and upwards. The leaves spring from the stem and the branches from a broad base, and are usually arranged with a divergence of $\frac{2}{5}$; they are tongue-shaped or apiculate, and, with the exception of the first on the young stem, are composed of two kinds of cells arranged regularly. The young leaf necessarily consists of homogenous tissue; but as the development progresses the cells of the veinless lamina become differentiated into large broad cells about the shape of a long lozenge, and into narrow tubular cells, running between the former, bounding them, and connected with one another into a network; they are, as it were, squeezed in among the larger ones. The larger cells lose the whole of their contents, and hence appear colourless; their walls show irregular narrow spiral bands with the turns some distance apart, as well as large dots, each of which has a thickened edge, while the part of the cell-wall which closes the dot is absorbed. Large, usually circular holes, are thus formed in the cell-wall of the colourless cells. The intermediate tubular narrow cells retain their contents,

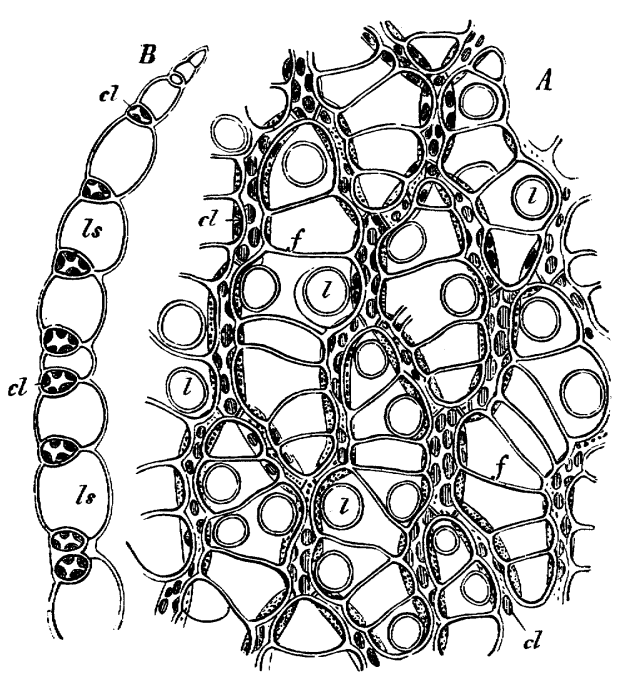

FIG. 26I.-Sphagnum acutifolizm; $A$ a portion of the surface of the leaf seen from above, $c l$ the tubular cells containing chlorophyll, $f$ the spiral bands, $l$ the holes in the large empty cells; $B$ transverse section of a leaf, $c l$ the cells that contain chlorophyll, $i s$ the large empty cells.

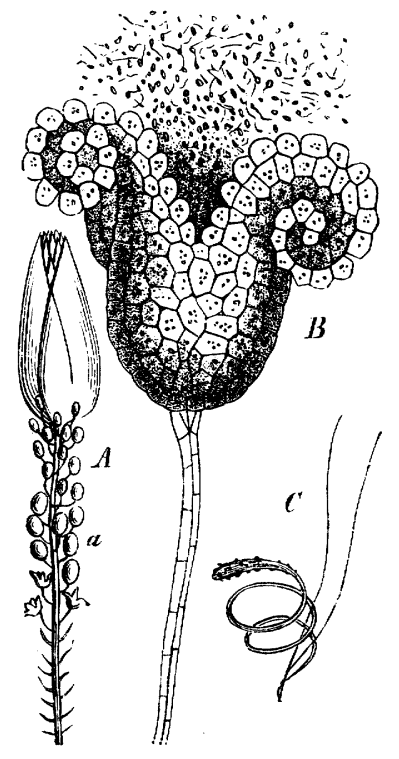

FIG. 262.-Sphagnum acutifolium; $A$ a male branch, with the leaves partially removed in order to show the antheridia $a ; B$ an open antheridium (very highly magnified); $C$ a free motile antherozoid (after Schimper).

form chlorophyll-granules, and thus constitute the functional tissue of the leaf, the entire area of which is, however, smaller than that of the colourless tissue (Fig. 26r). The stems consist of three layers of tissue, the innermost of which is an axial cylinder of thin-walled, colourless, elongated, parenchymatous cells; it is enveloped by a layer of thick-walled, dotted, firm (lignified?), prosenchymatous cells, with their walls coloured brown. The epidermal tissue of the stem, finally, consists of from $\mathrm{I}$ to 4 layers of very broad thin-walled empty cells, which, in $S$. cymbifolium, possess spiral thickenings and round holes similar to those of the leaves ( $c f$. Fig. 8r). These colourless cells, both those of the leaves and of the epidermal layer of the stem and of the branches, serve as a capillary apparatus for the plant, through which the water of the bogs in which it grows is raised up and carried to the upper parts; hence it results that the Sphagna, which always grow erect, are penetrated with water to their very summits like a sponge, even when their tufts stand high above the surface of the water. 
The archegonia and antheridia of Sphagnum arise on the fascicled branches, as long as they are still near the summit of the primary stem and belong to the terminal tuft. The time of bearing them is mostly in autumn and winter, but is not exclusively confined to these periods. The antheridia and archegonia are always distributed on different branches, sometimes even on different plants, and in this case the male and female plants form large distinct patches. When the primary stem does not continue to grow during the development of the sporogonia in consequence of dry weather, these are to be found on the branches of the terminal tuft at a later period; but when the supply of water is great and vigorous increase of length takes place, the fertile branches become separated from one another, and are subsequently found lower down on the stem; the sporogonia and older male branches are thus removed to a distance from the summit, although at the time of their development they stand near it. The branches which bear the antheridia are generally conspicuous externally by their imbricated leaves forming beautiful densely crowded orthostichies or spiral parastichies; the leaves are generally yellow, bright red, or especially dark green, and can hence be easily recognised (Fig. 260, $a$ a). The antheridia stand, on the mature shoot, by the side of the leaves; they are never terminal, and are found only in the middle part of the male branch, one standing beside each leaf; the male branch may therefore continue to grow at the summit, and become an ordinary flagellate branch. This position of the antheridia, and still more their roundish form and long pedicel, causes the Sphagnaceæ to resemble some Jungermanniex; the mode in which they open (Fig. 262) recalls the Hepaticx even more than the true Mosses. The archegonia arise at the blunt end of the female branch, the upper leaves of which form a bud-like envelope; but the young perichætial leaves are still contained within this at the time of fertilisation, although they afterwards become further developed. The archegonia are exactly like those of the rest of the Mosses; several of them are usually fertilised in one perichrtium, but only one perfects its sporogonium. The development of it occurs within the perichætium; the summit of the branch then begins to elongate; it grows out into a long naked receptacle, and elevates the sporogonium contained in its calyptra high above the perichætium. This so-called Pseudopodium must not, therefore, be confounded with the seta of other Mosses (see Andreæaceæ). At Fig. $2_{3}, B$, is shown in longitudinal section the nearly ripe sporogonium developed within the calyptra.

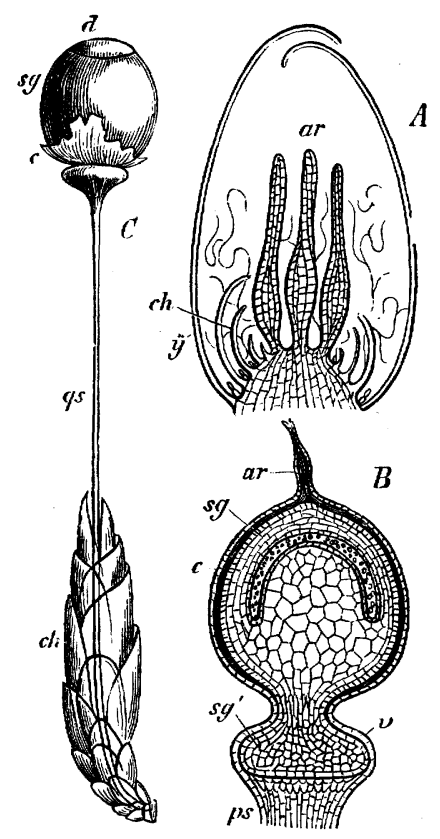

FIG. 263.- $A, B$, Sphagnum acutifolium; $A$ longitudina section of the female flower, ar archegonia, $c h$ perichætial leaves still young, $y$ the last perichætial leaves; $B$ longileaves still young, $y$ the last perichætial leaves; $B$ longi-
tudinal section of the sporogonium $s g$, the broad base of which $s g^{\prime}$ remains in the vaginula $v$, while the capsule is surrounded by the calyptra $c$, upon this is the neck of the archegonium ar, ps the pseudopodium; C Sphagnum squarrosum, ripe sporogonium $s g$ with its lid $d$ and ruptured calyptra $c ; q s$ the elongated pseudopodium growing from the perichætium $c h$ (after Schimper).

Its lower part forms a thick base imbedded

in the end of the pseudopodium which is transformed into the vaginula. The sporemother-cells are formed from a cap-shaped layer of cells beneath the apex of the spherical theca; the part of the inner tissue which is found beneath it forms a low nearly hemispherical column, which is in this case also termed the columella, although it is distinguished from the columella of true Mosses by not reaching to the apex of the theca. The mode of the formation of the spores from the mother-cells resembles that 
of true Mosses; but there occur, besides the ordinary (large) spores, also smaller spores in special smaller sporogonia, which owe their origin to a further division of the mothercells ( $c f$. Fig. $258, B$ ). The theca opens by the detachment as a lid of the upper segment of the wall of the spherical capsule, which is sometimes more strongly convex. The calyptra, which closely surrounds the growing sporogonium as a fine envelope, is ruptured irregularly.

2. The Andreæace ${ }^{1}$ are small cespitose Mosses which are very leafy and much branched; their very shortly stalked theca is elevated, as in Sphagnum, above the perichætium on a leafless pseudopodium. The long apiculate theca raises up the calyptra in the form of a pointed cap, as in the true Mosses, while the short seta remains buried in the vaginula. The body of the young sporogonium becomes differentiated into a parietal tissue consisting of several layers which surrounds the simple layer of the spore-mother-cells without any intermediate cavity, and a central mass of tissue, the columella: in the same manner as in the Sphagnacex, the layer of cells which produces the spores is bell-shaped and closed above, the columella terminating beneath

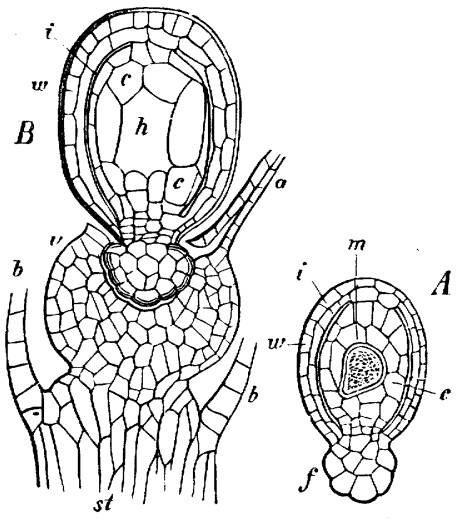

FIG. 264.-Archidium phascoides; $A$ longitudinal section of the young sporogonium, showing the mother-cell $m$ of the spores; $B$ longitudinal section through the young sporogonium with its calyptra and vaginula, $f$ foot of the sporogoniun, $w$ wall of the theca, $i$ intercellular space, $c$ columella, $h$ hollow out of which the spore-mother-cells have fallen, $v$ vaginula, st stem, $b$ leaves, $a$ neck of the archegonium. After Hofmeister $(X 200)$.

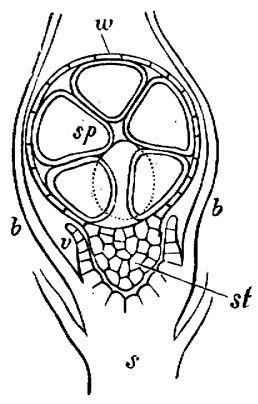

FIG. 265.-Archidium phascoides: longitudinal section through a nearly ripe sporogonium, $w$ its wall, $s p$ its spores, $v$ the vaginula, $b$ leaves of the stem, st base (foot) of the sporogonium. After Hofmeister $(\times 200)$.

it. The ripe theca does not open by an operculum, but by four longitudinal slits at the sides; four valves are thus formed united at the apex and at the base, which are closed in damp, but open in dry weather.

3. The Phascace $\boldsymbol{P}$ are small Mosses, the short stems remaining attached to the protonema until the spores are ripe; they may be considercd as the lowest form of the following group, to which the genus Phascum forms the transition. They are, however, all distinguished by their theca not opening by an operculum, but allowing the escape of the spores only by its decay. While in the genera Pbascum and Ephemerum ${ }^{2}$ the internal differentiation of the theca corresponds essentially to that of true Mosses, although more simple, the genus Arcbidium displays a more considerable deviation, and as an interesting transitional form may be examined a little more closely ${ }^{3}$. The

1 J. Kühn, Zur Entwickelungsgeschichte der Andreæaceen. Leipzig I870.

$2 \mathrm{~J}$. Müller, in Jahrbuch für. wiss. Bot. I867, vol. VI. p. 237 .

3 Hofmeister, in Bericht der königl. Sächsich. Gesellsch. der Wiss. 1854, April 22. [See also Leitgeb, Das Sporogon von Archidium, Sitzber. d. Wien. Akad. 1879. He shows that the mother- 
very short seta of the sporogonium swells, as in Spbagnum and Hepaticæ; the roundish theca ruptures the calyptra laterally, without raising it up as a cap. Archidium agrees with the true Mosses in the formation in the theca of an intercellular space running parallel to its lateral surface, which separates the wall from the inner mass of tissue. The latter appears as a column continuous at the base and apex with the wall of the theca. But while in the true Mosses a layer of cells parallel to this intercellular space produces the spore-mother-cells, it is here only a single cell lying eccentrically in the inner mass of tissue that becomes the primary mother-cell of all the spores (Fig. $26_{4}, A$ ). It grows considerably, and supplants the other cells, until it lies free in the hollow of the theca; it then divides into four cells, each of which produces four spores. The wall of the primary mother-cell remains entire, while the sixteen spores grow, and fill up the whole of the theca, the inner cell-layer of which is also absorbed (Fig. 265).

4. In the Bryacem or True Mosses the sporogonium is always stalked, and the seta is usually of considerable length. The seta is cylindrical, obtusely pointed

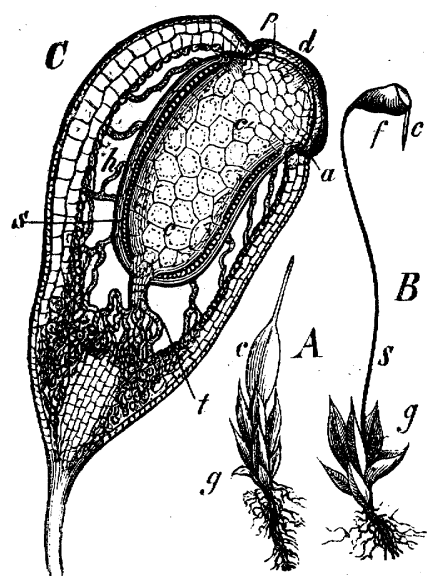

FIG. 266.-Funaria hygrometrica; $A$ a young leafy plant $g$ with the calyptra $c ; B$ a plant $g$ with the nearly ripe sporogonium, $s$ its seta, $f$ the theca, $c$ the calyptra; $C$ longitudinal section of the theca bisecting it symmetrically; $d$ operculum, $a$ annulus, $p$ peristome, $c c^{\prime}$ columella, $h$ air-cavity, $s$ the pri-, mary mother-cells of the spores.

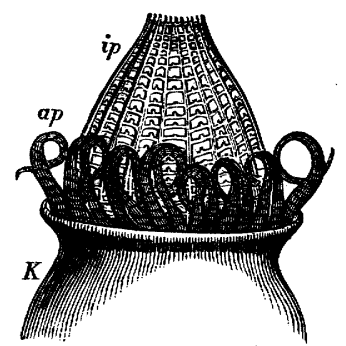

FIG. 267.-Mouth of the theca of Fontinalis antipyretica; ap outer peristome, $i$ inner peristome. After Schimper $(\times 50)$.

below, and firmly implanted in the vaginula; the theca always opens by the detachment of its upper part as a lid (Operculum); the operculum is either simply and smoothly detached from the lower part of the theca, or a layer of epidermal cells termed the Annulus is thrown off in consequence of the swelling of their inner walls, and the operculum in this way separated from the theca. Most commonly, after the operculum has fallen off, the margin of the theca appears furnished with appendages of very regular and elegant form arranged in one or two rows; the separate appendages are termed $\tau_{e e t h}$ or Cilia, the whole together the Peristome; if the peristome is wanting, the theca is said to be gymnostomous. The theca is at first a solid homogeneous mass of tissue; the differentiation of its interior begins with the formation of an annular intercellular space which separates off the wall of the theca consisting of several layers of cells; but the wall remains attached above and below to the colu-

cells of the spores are not derived from a single primary cell. A variable number of spore-mothercells $(I-7)$ are developed independently, each of which gives rise to four spores. They are arranged quite irregularly. 
mella. The intercellular space is traversed by rows of cells which stretch across from the wall of the theca to the inner mass of tissue; they resemble most nearly protonemal filaments, or those of Algæ, but have been formed by simple differentiation of the tissue of the theca. They contain chlorophyll-granules like the inner cell-layers of the wall. The outer layer of the wall of the theca is developed into a very characteristic epidermis strongly cuticularised externally. The third or fourth layer of cells of the inner mass of tissue, which is therefore separated from the annular air-cavity by two or three layers of cells (forming the spore-sac), produces the mother-cells of the spores. The cells of this layer are first of all distinguished by being densely filled with proto-
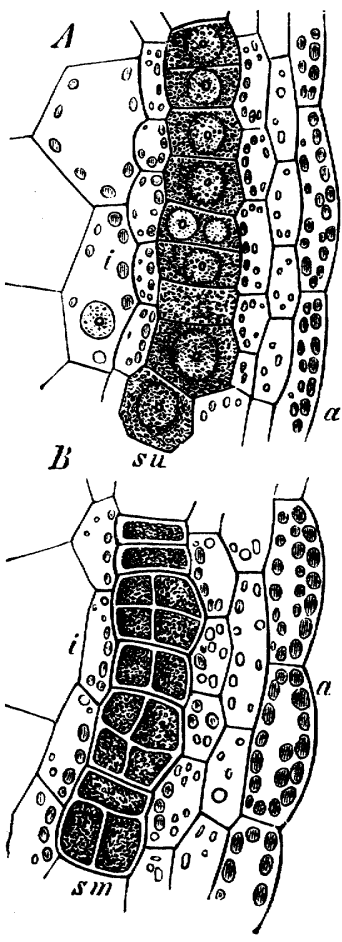

FIG. 268.-Funaria hygrometrica; transverse section through the sporesac; $A$, su the primary mother-cells $B, s m$ the spore-mother-cells not yet isolated; $a$ outer side, $i$ inner side. of the spore-sac $\left(\times 55^{\circ}\right)$.

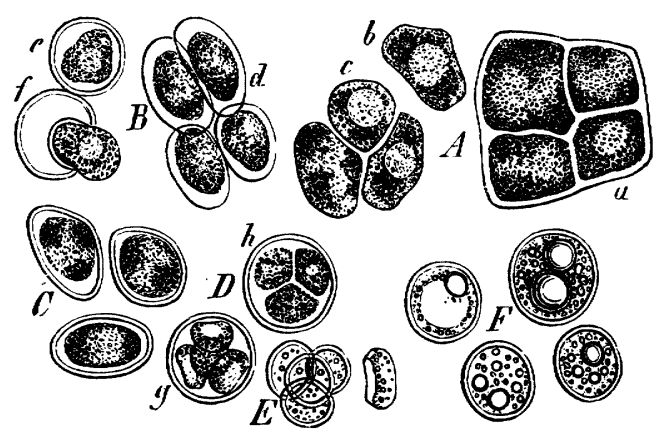

FIG. 269.-Development of the spores of Funaria hygrometrica observed in very dilute glycerine; $A$ mother-cells, at $a$ still united, at $b$ and $c$ the separation has cominenced; $B$ isolated mother-cells clothed with cell-walls; at $f$ expelling the protoplasmic contents; $C$ mother-cells with indication-of the commencement of protoplasmic contents ; $C$ mother-cells with indication - of the commencement of
the bipartition of the contents; $D$ the contents have divided into four masses of protoplasin, still surrounded by the primary cell-wall, but they themselves are naked; $E$ the spores enveloped by cell-walls; $F$ ripe spores $\left(\mathbf{X}_{550}\right)$.

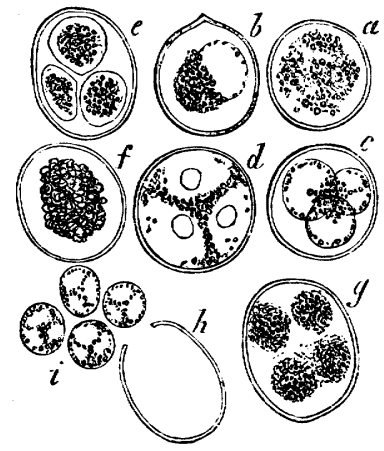

FIG. 270.-Various states of division of the mother-cells of the spores of Funarin hygrometrica, observed in water, the progress of development indicated by the
letters $a-i$.

plasm, in which lies a large central nucleus, and are attached without interstices to the surrounding tissue in a parenchymatous manner. From their division proceed the sporemother-cells, which become isolated by the deliquescence of the cell-walls, and then float in the fluid contained in the spore-sac, till they form the spores by repeated division. The Spore-sac is the term given to those layers of cells by which the large air-cavity is separated from the spore-mother-cells. It seems convenient to consider the layers which bound the spore-cavity on the axial side (Fig. 268, i) also as a part of the spore-sac; its cells contain on both sides starch-forming chlorophyll-granules. The inner large-celled tissue, which contains but little chlorophyll, and is thus surrounded 
on all sides by the spore-sac, is distinguished as the Columella. The spore-sac is ruptured by the casting off of the operculum, but the columella remains dried up, and in Polytricbum there remains also a layer of cells, the Epipbragm, attached to the points of the teeth of the peristome, and covering the opening of the theca.

We must now examine somewhat more closely the origin of the peristome. In those genera which, like Gymnostomum, do not form a peristome, the parenchyma which lies immediately beneath the operculum is homogeneous and thin-walled; when the theca is ripe, it contracts and dries up within the operculum, which is formed essentially only of the epidermis; or it remains attached to the columella and forms a thickening at its summit, which projects over the opening of the theca; or again it forms a kind of diaphragm, which closes the mouth of the theca after the casting off of the operculum (Hymenostomum). The transition to the genera provided with a true peristome is furnished by Tetraphis. In this genus the firm epidermis of the upper conical part of the theca falls off as the operculum, while the whole of the tissue immediately beneath the operculum, the two outer layers of which are thick-walled, splits across into four valves. These are also termed by systematists a peristome, although their origin and structure are widely different from that of the true peristome in other genera. For, except in the Polytrichacex, neither the teeth nor the cilia consist of cellular tissue, but only of thickened and hardened parts of the walls of a layer of cells, which is separated by some layers of thin-walled cells from the epidermis which forms the operculum; the latter layers, as well as the delicate parts of the former, become ruptured and disappear, while the thickened parts of the wall remain after the casting off of the operculum. This will be rendered clear by an example. Fig. $27 \mathrm{I}$ represents a part of the longitudinal section which bisects the theca of Funaria bygrometrica symmetrically, corresponding to the part in Fig. 266, $C$, designated $a$; $e e$ is the reddish brown epidermis strongly thickened on the outside; at the part where it bulges its cells are of a peculiar shape, forming the ring or annulus; se is the tissue lying between the epidermis of the theca and the air-cavity $b$; the large-celled tissue $p$ is the prolongation of the columella into the cavity of the operculum; at $S$ are seen the uppermost spore-mother-cells; directly above the air-cavity $b$ rises the layer of cells which forms the peristome; its walls $(a)$, which face outwards, are strongly thickened, and of a bright red colour; the thickening is continued also partially along the septa ; the longitudinal walls which lie on the axial side of the same layer of cells $(i)$ are also coloured, but less strongly thickened. In Fig. 272 is shown further a part of the transverse section through the basal part of the operculum; $r r$ are the epidermal cells placed immediately above the annulus, forming the lower edge of the operculum; $a$ and $i$ the thickened parts of the layer of cells concentric with the operculum, which form the peristome. A section near the apex of the operculum would show, instead of the broad thickening-masses $i, i^{\prime}, i^{\prime \prime}$, only the middle part of the inner wall, but more strongly thickened. If now it is supposed that when the theca is ripe the annulus and the operculum fall off, the cells $p$ and those which lie between $a$ and $e$ (Fig. 27I) disappear, and that the thin pieces of wall between $a, a^{\prime}, a^{\prime \prime}$, and between $i, i^{\prime}, i^{\prime \prime}$, in Fig. 272 , are also destroyed, then the thick red pieces of wall alone remain, forming sixteen pairs of tooth-like lobes pointed above, crowning the edge of the theca in two concentric circles. The outer row are termed Teeth, the inner row Cilia. The thickened cells at $t$, Fig. 27 I, unite the base of the teeth with the edge of the theca. According as the layer of cells which forms the peristome consists, in transverse section, of a larger or smaller number, and according as one or two thickened walls are formed within each one of these cells, the number of teeth and cilia varies; it is always however a multiple of four, generally 16 or 32 . In many cases the thickening at $i$ is wanting; the peristome is then simple, and formed only of the teeth of the outer row. The thickenings at $a$ are very commonly much stronger than is the case in Funaria, and the teeth therefore stouter. The thickened parts of the wall may also partially or entirely coalesce laterally with one another; and then the parts of the peristome either above or below 
form a membrane; in this case the teeth appear split from one another above, and the endostome (the inner peristome) is composed of a lattice-work of longitudinal or transverse ridges instead of cilia (Fig. 267). A great variety is met with here, which may

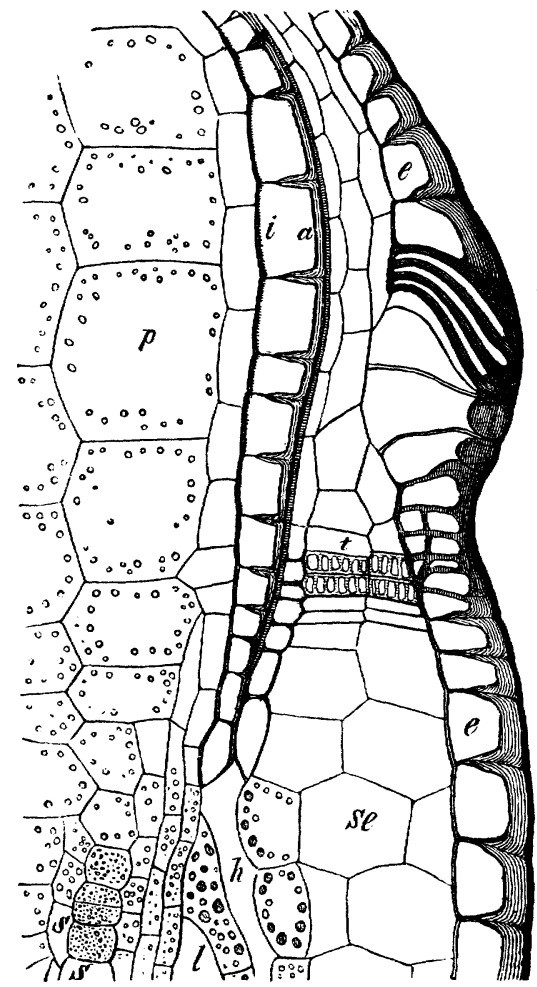

FIG. 27x.-Funaria hygrometrica; part of a longitudinal section of an unripe theca.

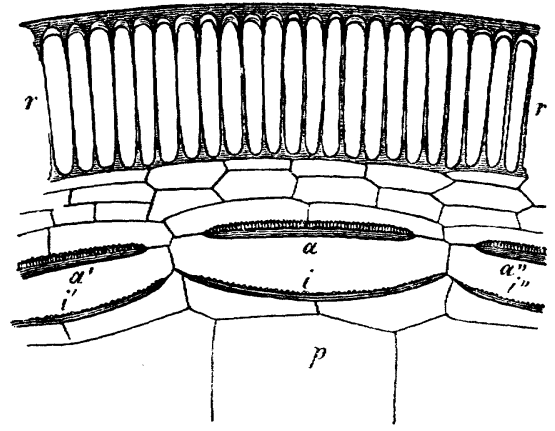

FIG. 272-Funaria hygrometrica; part of a transverse section through the operculum.

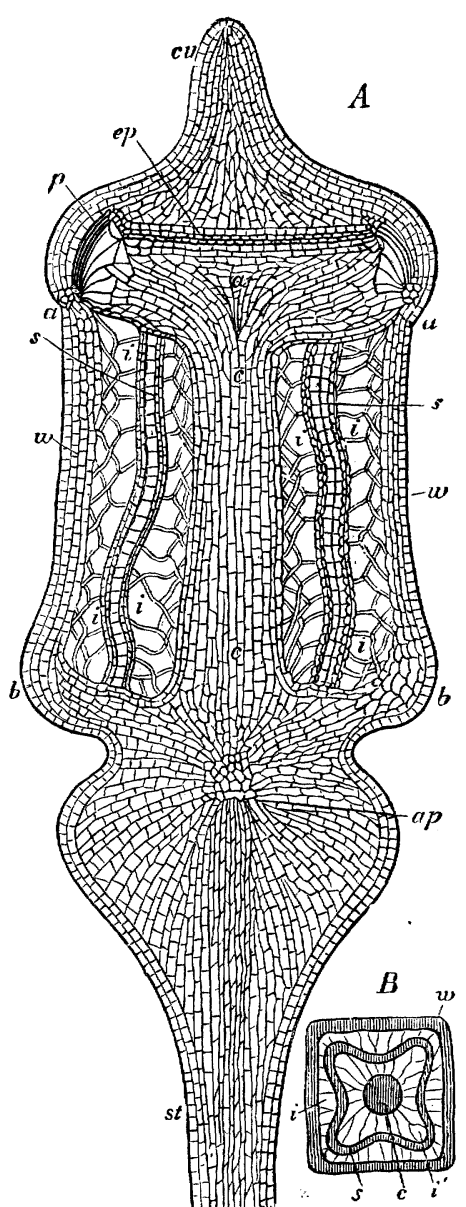

FIG. 273.- $A$ longitudinal section of the theca of Polytrichum piliferum (after Lantzius.Beninga, $\times I 5) ; B$ the transverse section $(X$ about 5$)$; $w$ wall of the theca, $c u$ operculum, $c c$ columella, $p$ peristome, ep epiphragm, $a$ a annulus, $i z$ the air-cavities penetrated by alga-like cellular filaments, $s$ spore-sac, containing the primary mothercells of the spores, st the seta, the upper part of which forms the apophysis $a p$.

easily be understood by the beginner when he has obtained a clear idea of the principle. The inner and outer sides of the teeth of the peristome are hygroscopic to a different 
degree; hence, as the amount of moisture in the air varies they bend inwards or outwards, or sometimes in a spiral whorl, as in Barbula.

The genus Polytrichum, to which the largest and most highly developed Mosses belong, differs from the other genera in several points in the structure of its theca. The teeth of the peristome are composed not simply of single pieces of membrane, but of bundles of thickened prosenchymatous cells; these bundles are horseshoe-shaped; the branches of two adjoining bundles directed upwards form together one of the 32-64 teeth. A layer of cells uniting the points of the teeth (Fig. 273, ep) remains, after the casting off of the operculum and the drying up of the adjoining cells, as an epiphragm stretched across the theca. The spore-sac is, in some species (e.g.P.piliferum), separated from the columella by an air-cavity, which is penetrated, like the outer air-cavity, by conferva-like rows of cells. In most species the seta is swollen beneath the theca, forming the Apophysis, a phenomenon which is repeated in a somewhat different manner in the genus Splachnum, where this part is sometimes expanded transversely as a flat disc.

\section{VASCULAR CRYPTOGAMS.}

UNDER this term are included in one group the Ferns, Equisetacex, Ophioglosseæ, Rhizocarpeæ, Lycopodiaceæ, Selaginelleæ, and Isoëteæ. As in the Muscineæ, the life-history of the plant is divided into two generations which are extremely different both morphologically and physiologically. From the spore proceeds first of all a sexual generation; from its fertilised archegonium is produced in the second place a new plant, which does not form sexual organs, but in their place a number of spores. In the Ferns, Equisetaceæ, and Lycopodiaceæ these spores are all alike; the Rhizocarpeæ, Selaginelleæ, and Isoëteæ, on the contrary, produce two kinds of spores, large and small, Macrospores and Microspores.

The Sexual Generation [Oophore] which is developed from the spore always preserves, in Vascular Cryptogams, the form of a thallus; it never attains, as in the more highly developed Mosses, to a differentiation into stem and leaf, but remains small and delicate, and closes its life with the commencement of the development of the second generation. It appears, therefore, externally as a mere precursor of further development, as a transitional structure between the germinating spore and the variously differentiated second generation. Hence the name Prothallium has been given to this first or sexual generation of Vascular Cryptogams.

In the Ferns and Equisetaceæ the prothallium resembles the thallus of the lowest Hepaticæ. The prothallia sometimes continue to grow for a considerable time; they contain a large amount of chlorophyll, and form numerous roothairs. After they have thus attained sufficient vigour by independent nourishment, they produce archegonia and antheridia, usually in considerable numbers. A tendency to become diœcious is sometimes manifested in these prothallia, although they proceed from similar spores; both kinds of sexual organs being, however, 
often produced on the same prothallium. In the Rhizocarpeæ, Selaginelleæ, and Isoëtex, on the other hand, the separation of the sexes is already prefigured by the two kinds of spores, the Macrospores being female, in so far as they develope a small prothallium, which produces exclusively archegonia, or sometimes only a single one ; the Microspores being male, inasmuch as they develope a still smaller prothallium bearing exclusively antheridia. The female prothallium of the Rhizocarpeæ is a small appendage of the macrospore, formed in its interior but afterwards developed externally although nourished by it; in Selaginelleæ and Isoëteæ the prothallium is developed in the spore itself, filling it up with a mass of tissue, the archegonia becoming exposed only by the splitting of the cell-wall of the spore. The male prothallium consists, in these groups of plants, of a single sterile or vegetative cell, and of a larger or smaller number of cells in which antherozoids are developed.

The Archegonia of Vascular Cryptogams, like those of the Muscineæ, are bodies, consisting of a ventral part which encloses the oosphere, and of a neck, usually short and composed of four longitudinal rows of cells. The two groups differ in the fact that in Vascular Cryptogams the tissue of the wall of the ventral part is formed from the prothallium itself; and the ventral part of the archegonium is therefore enclosed in the tissue of the sexual generation, the neck only projecting beyond it. The archegonium originates from a superficial cell of the prothallium which is divided by a tangential wall into two, an external and an internal. The former forms, by intersecting longitudinal and subsequent transverse divisions, the four rows of cells of the rather short neck; the latter grows outwards between the neck-cells into a projection which becomes separated, forming the neck-cell, and another segment is cut off from the large inferior cell (the central cell, Janczewski) to form the ventral canal-cell. Thus there arises from the original internal cell an axial row of three cells, the lowest of which is the oosphere. The two neck-cells become converted into mucilage as in the Muscineæ. The mucilage thus produced in the neck finally swells up considerably, forces apart the four apical cells of the neck, and is expelled; an open canal is thus formed, leading from without to the oosphere; the expelled mucilage appears to play an important part in the conduction of the 'swarming' antherozoids to the opening of the neck. Fertilisation is always effected by means of water, 'which determines the opening of the antheridia and archegonia, and serves as a vehicle for the antherozoids. The advance of these latter as far as the oosphere, and even their entrance into and coalescence with its protoplasm, has been directly observed in the different groups.

The Antherozoids ${ }^{1}$ are, like those of the Muscineæ, spirally coiled threads usually with a number of fine cilia on the anterior coils. In the cases hitherto observed they arise from the peripheral part of the protoplasm of their small mother-cells, a central vesicle of protoplasm, containing starch-grains, being left over, which, adhering to a posterior coil of the antherozoid, is often dragged along by it, but is detached before

1 [On the development of the antherozoids of the Vascular Cryptogams, see Strasburger, Zellbildung und Zelltheilung, 3 rd. ed. 1880 . The formation of the antherozoid is, in all cases, preceded by the disappearance of the nucleus of the mother-cell, its substance becoming diffused throughout the protoplasm.] 
its entry into the archegonium. The mother-cells of the antherozoids arise in the antheridia, which in Ferns and Equisetaceæ project free from the prothallium as roundish masses of tissue, but in the Ophioglosseæ and Lycopodium are imbedded in the prothallium. Among Rhizocarpeæ, Salvinia forms a very simple antheridium which projects from the microspore, while the Marsiliaceæ and Selaginelleæ produce their antherozoids within the microspore itself, after a few-celled mass of tissue has been formed in it which must be considered as a rudimentary prothallium.

The Asexual Generation [Sporophore] which produces spores, arises from the oospore or fertilised oosphere in the archegonium. In Ferns, Equisetaceæ, and Rhizocarpex, its earliest divisions, the rudiments of the first root, the first leaf, and the apex of the stem can be recognised, while at the same time a lateral outgrowth of its tissue, called the Foot, commences at the bottom of the ventral part of the archegonium, and draws from the prothallium the first nourishment for the young plant. The ventral part of the archegonium at first grows vigorously (except apparently in the Selaginellex), enveloping the embryo, until this latter finally protrudes free, leaving however, for some time, the foot still attached to it as a nutritive organ. This process offers an unquestionable analogy to the formation of the calyptra of the Muscineæ. While, however, the spore-producing generation of the Muscineæ remains a mere appendage of the sexual plant, appearing in a certain sense as its fruit, the corresponding generation of Vascular Cryptogams developes, on the contrary, into a conspicuous, highly organised, independent plant, which frees itself at a very early period from the prothallium, and obtains its own nourishment. It is this asexual generation which is called, in ordinary language, simply the Fern, Equisetum, \&c.; it always consists of a leafy stem, usually producing a number of true roots; roots may, however, occasionally be entirely absent, as in some Hymenophyllaceæ, and in Psilotum and Salvinia. In many cases, especially in Ferns, Equisetaceæ, and (especially the extinct) Lycopodiaceæ, the spore-producing generation attains great dimensions with an unlimited term of life; only a few species are (like Salvinia) annual, or very small, resembling Mosses in habit, as Azolla and some Selaginelleæ.

The Leaves are either simple, unsegmented, or variously branched (Filicineæ). There does not, however, occur so great a variety due to metamorphosis in the forms assumed by the leaves in the same plant as in Phanerogams.

The Roots usually arise in acropetal succession on the stem (or on the leafstalk in some Ferns), and branch monopodially or dichotomously; they always remain nearly uniform in size, the first root never attaining the dimensions of a tap-root, as in many Phanerogams. The lateral roots do not arise, as in Phanerogams, from the pericambium, but from the innermost cortical layer of the main root.

The Differentiation of the Systems of Tissue attains a high degree of perfection for the first time in this group of plants. The epidermis, fundamental tissue, and fibro-vascular bundles are always clearly distinct, and are composed of cells of various forms. The fibro-vascular bundles are closed; the phloëm usually surrounds the xylem of each separate bundle like a sheath.

The Branching of the Stem is very different in the different classes of Vascular Cryptogams; it is essentially monopodial, but it is often apparently dichotomous: 
axillary branching, in the sense in which the term is applied to Phanerogams, probably does not occur here.

The Development of the Sporangia ${ }^{1}$ is in most cases evidently a function of ordinary or of specially modified leaves; it is only when they arise singly upon the upper surface of leaves, as in Lycopodium, that they come to stand in the axils of the leaves: in Selaginella they arise from the stem, and in Psilotum and Tmesipteris from lateral branches. In the earliest stage of their development they are in the Filicineæ outgrowths of single epidermic cells: in the other Vascular Cryptogams they are developed from a group of cells. [The mother-cells of the spores are derived from one or more cells which lie at first immediately beneath the epidermis of the sporangium (hypodermal), and which Goebel terms the archesporium. The archesporium becomes invested by one or more layers of cells, derived either from the division of its own cells or of the cells forming part of the sporangium, to which the name of tapetum is given. As the sporangium ripens, the cells of the tapetum usually become absorbed, and the mother-cells of the spores become isolated from their combination into a tissue. From each mother-cell four spores are developed: this takes place in two ways; either the cell is divided into two by a cell-wall after the nucleus has divided, and this process is repeated in each of the two daughtercells, or the nucleus divides into two and each of these again into two before any cell-wall is formed. The latter is by far the more general mode. The distinction between macrospores and microspores in the Rhizocarpeæ and Selaginelleæ is manifested only after the division into four of the mother-cells which were previously alike.]

It is clear from what has now been said that the sporangium of Vascular Cryptogams is equivalent, from a physiological but not from a morphological point of view, to the sporogonium of Mosses. This latter forms by itself the whole of the asexual generation of Mosses; while the sporangium of Vascular Cryptogams

1 [On the Comparative Development of the Sporangia see Goebel, Bot. Zeitg. I880-8r. He classifies the Vascular Cryptogams according to the mode of development of their sporangia, as follows :-

Leptosporangiata. The sporangium is developed from a single epidermal cell. The archesporium is a single cell, and the tapetum is derived from it.

Filices; Rhizocarpeæ.

Eusporangiata. The sporangium is developed from several cells, which, except in the case of Isoëtes, are all superficial.

a. Archesporium unicellular; tapetum derived not from it, but from the tissue of the sporangium. Ophioglosseæ; Marattiaceæ; Equisetaceæ.

b. Archesporium unicellular; tapetum derived from it. Psilotum; Tmesipteris (?).

c. Archesporium unicellular; tapetum derived partly from it, and partly from the tissue of the sporangium.

Selaginella.

d. Archesporium multicellular (a row of cells); tapetum formed not from it but from the tissue of the sporangium. Lycopodium.

e. Archesporium multicellular (a layer of cells); tapetum derived from it. Isoëtes.

The Phanerogams also belong to the Eusporangiata, see infra.] 
is a relatively small outgrowth borne on either the leaf or the stem of the asexual generation which consists of stem, leaf, and root.

The proof that what is termed the Moss-fruit, i.e. the sporogonium, is, from its position in the alternation of generations, the equivalent of the entire leafy and rooting spore-producing plant of Vascular Cryptogams, was brought forward by Hofmeister as long ago as $185_{1}$ (Vergleichende Untersuchungen, p. $139^{1}$ ). In connection with the relationships pointed out by him between the Selaginellex and Isoëteæ on the one hand and the Coniferæ on the other, this discovery is one of the most fertile in results that has ever been made in the domain of morphology and classification. The researches of Pringsheim and Hanstein on the development of Rhizocarps, carried out with great acuteness and deep penetration, those of Nägeli and Leitgeb on the roots of Vascular Cryptogams, and of Cramer on the apical growth of the stem of Equisetaceæ and Lycopodiacex, to which numerous more recent observations may be added, have not only contributed to a more accurate knowledge of this group of plants, but have especially cleared up the fundamental morphological facts. Since the appearance of the first edition of this book, our knowledge of the alternation of generations has been enriched by Millardet's discovery of the male prothallium in Isoëteæ and Selaginelleæ; and the labours of Millardet, Strasburger, Kny, and especially of Janczewski, have resulted in a more complete acquaintance with the development of the sexual organs and of the process of fertilisation itself in its details.

Taxonomy. Our ideas as to the mutual relationships existing between the various divisions of Vascular Cryptogams are at present very variable, they are, in fact, in a state of transition. The division into "Isosporeæ and Heterosporeæ suggested by me in the first edition and retained in the third edition of this book seemed to be fully. justified as long as it could be assumed that in the Lycopodiex two forms of prothallium were developed as in the Selaginelleæ and Isoëteæ. This assumption has been proved untenable by Fankhauser's discovery of the monœcious prothallium of Lycopodium. Still a separation of the isosporous Lycopodiex on this account from the heterosporous Selaginelleæ and Isoëteæ would be unjustifiable. Besides, recent researches have shown that the Rhizocarpeæ are much more closely related to the true Ferns than to the heterosporous Selaginellex and Isoëtex. As a consequence, the division of Vascular Cryptogams into Isosporeæ and Heterosporeæ must be given up as being purely artificial, and we are led to assume that the differentiation of primarily similar spores into microspores and macrospores has taken place in two distinct groups of these plants; for the first time, in a developmental series which begins with the true Ferns, for the second, in a series which begins with the Lycopodieæ. This assumption is supported by the fact that the differentiation of two kinds of spores takes place in the Rhizocarpex in a manner very different from that in which it takes place in the Selaginellex and Isoëtex. Further, since I pointed out in the first edition (r 868) that the sporangia of some forms arise as multicellular bodies, whereas others are developed from single cells, younger botanists (especially Luerssen and Russow) have extended my observations and have laid stress upon the distinction of Trichosporangia from Phyllo(Caulo-) sporangia as a point of great systematic importance.

If, in accordance with the present state of our knowledge, I give up my former classification, I must also, on the same grounds, decline to accept the classifications suggested by Luerssen ${ }^{2}$ and by Russow ${ }^{3}$, for they are based on isolated characteristics,

1 [On the Germination, Development and Fructification of the Higher Cryptogamia, and on the Fructification of the Coniferæ, by W. Hofmeister; translated by F. Currey; Ray Soc. 1862, p. 434.]

${ }_{2}^{2}$ Luerssen, in den Mitth. aus dem Gesammtgebiet der Botanik von Schenk und Luerssen,

Bd. I. p. 107 .

3 Russow, Vergleichende Untersuchungen, St. Petersburg 1872. 
upon which too much importance is laid, and therefore run counter to natural affinities and cause forms which are widely different to be placed side by side.

The following new classification lays no claim to be regarded as definitely indicating for all time the relationships existing among Vascular Cryptogams, but I believe that it corresponds more closely than any previous arrangement with the present state of our knowledge on the one hand, and with those affinities which may be said to be self-evident on the other. It appears certain that the three Classes here formed, the Equisetineæ, the Filicineæ, and the Dichotomex, represent three distinct and separate types. Great difficulties are offered to the systematic sub-division of the Filicineæ, for certain groups of them, especially the Osmundaceæ, the Schizæaceæ and the Gleicheniaceæ, have not yet been thoroughly investigated from a morphological point of view.

\section{Systematic review of the Vascular Cryptogams.}

\section{L A S S VII.}

\section{EQUISETINEe.}

The spores are of one kind and give rise to prothallia which vegetate independently, and which are usually diœcious, the female prothallia being the larger, the male the smaller. The second generation consists of a copiously branched stem, with well-defined internodes, and bearing relatively small sheathing whorls of leaves. The branches arise in whorls and in strict acropetal order from the nodes of the stem. A root may arise below each branch; its ramification is monopodial. The sporangia are borne upon metamorphosed peltate leaves which form a terminal spike: they originate as multicellular protuberances (emergences), from five to ten in number upon each scale. The mother-cells of the spores, it appears, are derived from a unicellular archesporium. Both stem and root increase in length by means of a large apical cell which gives rise to three rows of segments. The fibro-vascular bundles of the stem are arranged in a circle; their xylem is rudimentary, resembling that of the bundles of Monocotyledons. The axial fibro-vascular cylinder of the root has no pericambium.
Family.
(I) Equisetaceæ.

\section{CLASS VIII.}

\section{FILICINE爪.}

The majority of these plants possess spores of one kind only, from which independent monœcious prothallia are developed: but the Rhizocarpeæ have macrospores and microspores forming rudimentary prothallia which never become free from the spore. The second generation is a stem bearing numerous well-developed leaves which are usually much branched. The stem either does not branch, or it does so but rarely, and it bears numerous roots. The sporangia are borne on ordinary leaves, or on leaves which have been specially modified: they are usually aggregated into small groups (Sori). The sporangia of the Ophioglosseæ and Marattiaceæ are from the first multicellular, and those of the Filices are developed from single superficial cells: the archesporium is unicellular. An apical cell is present in the stem and in the root: it forms, in the stem, two or three rows of segments, in the root usually three rows. The fibro-vascular bundles are generally very well developed, and the central xylem, which consists for the most part of scalariform tracheïdes, is usually surrounded by the phloëm.

Order I. Stipulatæ. The spores, so far as is at present known, are of one kind and give rise to independent monocious prothallia. The second generation is a simple, 
usually unbranched, erect or oblique stem of a somewhat tubercular form, bearing leaves arranged close together in a spiral manner. The leaves are relatively large, are usually much branched, and usually bear stipules at the base of their petioles. The sporangia are borne either upon the under surface of ordinary leaves, or in spicate or paniculate fructifications which are metamorphosed leaf-segments. They are derived from groups of cells which form projections upon the surface of the leaves. The mother-cells of the spores are found in large numbers. A single apical cell is present in the stem and root.
Families.
(1) Ophioglosseæ.
(2) Marattiaceæ.
(3) Osmundacex.
(4) Schizæaceæ.

Order II. Filices. The spores are of one kind and develope independent monœcious prothallia. The second generation is either an erect unbranched stem, or a stem which is more or less horizontal and bilaterally symmetrical and which branches occasionally. The leaves do not possess stipules, are circinate in their vernation, and bear upon their laminæ, which may or may not be more or less specially modified, very numerous sporangia usually arranged in sori covered by indusia. The sporangia arise from distinct epidermal cells, and usually sixteen spore-mother-cells are formed in each. The sporangia are opened by means of the so-called ring (annulus). Both stem and root possess a single apical cell. The ground-tissue tends to become converted into brown sclerenchyma, which especially supports the sheaths of the fibro-vascular bundles.
Families.
(I) Gleicheniaceæ (?), (Osmundaceæ, Schizæaceæ?).
(2) Hymenophyllacex.
(3) Cyatheacex.
(4) Polypodiaceæ.

Order III. Rhizocarpex. The sporangia are of two kinds, those of the one kind containing macrospores, those of the other microspores. Within the macrospore a small prothallium is formed which does not become separated from the spore; within the microspore the mother-cells of the antherozoids are formed from a very rudimentary prothallium. The second generation is a bilaterally symmetrical, horizontal, regularly branched stem, which bears leaves upon its dorsal surface in two or more rows, and roots upon its ventral surface. (Salvinia is rootless.) The sporangia arise from individual superficial cells of the placentæ and are enclosed in sporocarps which may be uni- or multilocular, and are formed by metamorphosed leaves or segments of leaves. The placenta of each loculus of the sporocarp bears a sorus of sporangia, and there are sixteen mother-cells of the spores in each sporangium. The microspores are formed in great numbers in each microsporangium $(4 \times 16)$, but only one macrospore in the macrosporangium comes to maturity. The stem grows by means of a two- or a three-sided apical cell; the root has always a three-sided apical cell.
Families.
(1) Salviniaceæ.

(2) Marsiliaceæ.

\section{L A S S IX.}

\section{Dichotomex.}

The prothallia are developed either from spores of one kind only and are then independent and monœcious, or they are developed from spores of two kinds (macroand microspores), and then remain within the spore until the period of fertilisation. 
The second generation is a simple or repeatedly branched stem, usually possessing roots and always bearing simple, unsegmented, comparatively small, but very numerous leaves, which are traversed by only a single fibro-vascular bundle. All the branchings of the stem and of the roots present the appearance of having originated dichotomously. The sporangia are borne singly upon the upper surface of the base of the leaves, or in their axils, or even in an extra-axillary position upon the stem. They originate as masses of cells derived in part (Isö̈tes) from internal tissues, covered by the epidermis which forms their walls. The archesporium is multicellular in some cases, unicellular in others.

Order I. Lycopodiaceæ. The prothallia, which are developed from spores of one kind only, are capable of independent growth and are monœcious. The roots branch dichotomously in alternate intersecting planes, and neither stem nor root possesses a single apical cell. The leaves have no ligula. The fibro-vascular cylinder of the stem consists of numerous xylem-bundles, each of which is surrounded by phloëm.

Families. (I) Lycopodiex.

(2) Psiloteæ.

(3) Phylloglosseæ.

Order II. Ligulatø. The spores are of two kinds. Each macrospore forms a rather large internal female prothallium, the archegonia of which only become exposed when the wall of the spore is ruptured. Within each microspore a rudimentary prothallium completely filling it is formed, certain cells of which give rise to the mothercells of the antherozoids. The second generation is of very different habit in the two families. The leaves are always provided with a ligula borne above their base, and below this lies the sporangium which contains either numerous microspores or four or more macrospores.

Families. (I) Selaginellex.

(2) Isoëtex.

CLASS VII.

EQUISETINEÆ1.

The Sexual Generation (Oophore). The spores of the Equisetaceæ, so soon as they have attained the ripe condition (they retain their power of germination only

${ }^{1}$ G. W. Bischoff, Die kryptogamischen Gewächse (Nürnberg I828).-W. Hofmeister, Vergl. Unters. (I $85 \mathbf{I}$ ). - Ditto, Ueber die Keimung der Equiseten (Abh. der königl. Sächs. Gesell, d. Wiss. I855, vol. IV. p. I68).-Ditto, Ueber Sporenentwickelung der Equiseten (Jahrb. für wiss. Bot. vol. III. p. 283).- [Germination, Development, and Fructification of the Higher Cryptogamia (Ray Society), pp. 267-306]. - Thuret (in Ann. des Sci. Nat. 1851, vol. XVI. p. 31).-Sanio, Ueber Epidermis und Spaltöffnungen des Equis. (Linnæa, vol. XXIX. Heít 4).-C. Cramer, Längenwachsthum und Gewebebildung bei $E$. arvense und sylvaticum (Pflanzenphys. Unters. von Nägeli und Cramer, 1855 , vol. III).-Duval-Jouve, Hist. Nat. des Equisetum (Paris I864).--H. Schacht, Die Spermatozoiden im Pflanzenreich (Braunschweig I 864).-Max Reess, Eritwickelungsgeschichte der Stammspitze von Equisetum (Jahrb. für wiss. Bot. 1867, vol. VI. p. 209).-Milde, Monographia Equisetorum, in Nova Acta Acad. Leop. Carolinæ, I 867, vol. XXXV.-Nägeli und Leitgeb, Entstehung und Wachsthum 
for a few days), show, when sown in water or on damp soil, the preparatory phases of germination after a few hours. In the course of some days the prothallium becomes developed into a multicellular plate, the further growth of which then proceeds very slowly. The spore, which contains a nucleus and chlorophyllgranules, increases in size as soon as germination commences, becomes pear-shaped, and divides into two cells, one of which is smaller with scarcely any except colourless contents, and soon developes into a long hyaline root-hair (Fig. 274,I,II, III, w), while the anterior and larger cell includes all the chlorophyll-granules of the spore which multiply by division. This cell produces by further divisions the primary plate of the prothallium, which increases by apical growth and soon branches $(I I I-V I)$. The process of multiplication of the cells is therefore apparently extremely irregular; even the very first divisions vary; sometimes the first wall in the primary apical cell which contains chlorophyll is but little inclined with respect to the longitudinal axis of the young plant (in $E$. Telmateia it sometimes coincides with it); in other cases, on the contrary, this cell developes into a longish tube, the apical part of which is cut off by a transverse septum (occasionally in $E$.arvense). The further growth is brought about by one or more apical cells dividing by transverse septa, and longitudinal walls are subsequently formed in the segments in an order very difficult to determine. Ramification takes place by the bulging out of lateral cells, which then continue their growth in a similar manner. The chlorophyll-granules in the cells also increase continuously by division. The young prothallia are, in $E$. Telmateia, usually narrow and ligulate, and consist of but a single layer of cells. The older prothallia are, both in this and in other species, branched in an irregularly lobed manner; one of

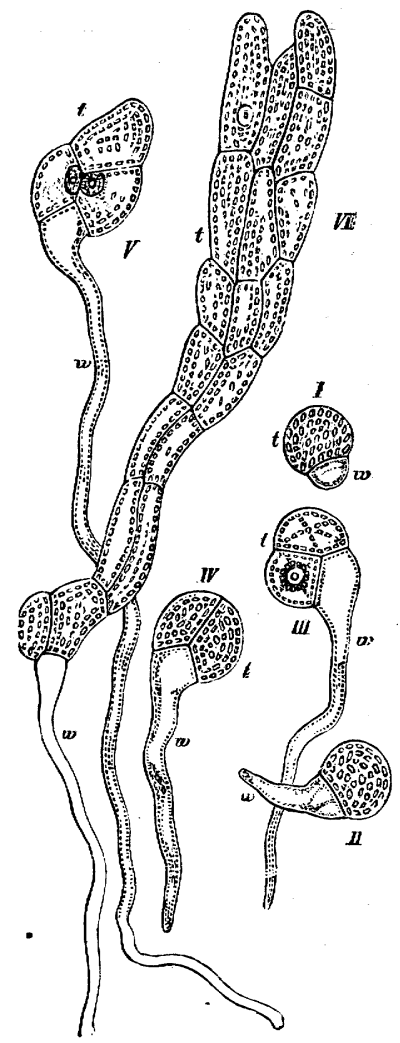

FIG. 274.-First stage of development of the prothalium of Equisetum Telmateia; $w$ the first root-hair; $t$ rudiment of the prothallium. The order of development follows the numbers $I-V I$ (X about 220). the lobes takes, sooner or later, the lead in growth, becomes thicker and fleshy, consisting of several layers of cells, and puts forth root-hairs from its under side.

The prothallia of the Equisetaceæ are, in general, diœcious. The male prothallia remain smaller, attaining a length of a few millimetres, and produce archegonia only in exceptional cases on shoots of later origin (Hofmeister). The female

der Wurzeln (Beitr. zur wissen. Bot. von Nägeli, Heft IV. München I867).-Pfitzer, Ueber die Schutzscheide (Jahrb. für wissen. Bot. vol. VI. p. 297).-Russow, Vergl. Unters. uber die Leitbündelkrypt. Petersburg I872, p. 41.-Janczewski, uiber die Archegonien, Bot. Zeit. I872, p 420. Van Tieghem, on the roots, in Ann. des sci. nat. sth series, vol. XIII.-[Sadebeck, Ueb. die Entwickelung der Prothallien der Schachtelhalme, Bot. Zeit. 1877.] 
prothallia are larger (as much as $\frac{1}{2}$ inch); Hofmeister compares them to the thallus of Anthoceros punctatus, Duval-Jouve to a curled endive-leaf. Duval-Jouve states that the antheridia appear about five weeks after germination, the archegonia much later. These statements refer especially to $E$. arvense, limosum, and palustre; according to the same writer, the prothallia of $E$. Telmateia and sylvaticum are broader and less branched; those of $E$. ramosissimum and variegatum slenderer and more elongated.

The Antheridia ${ }^{1}$ arise at the end or margin of the larger lobes of the male

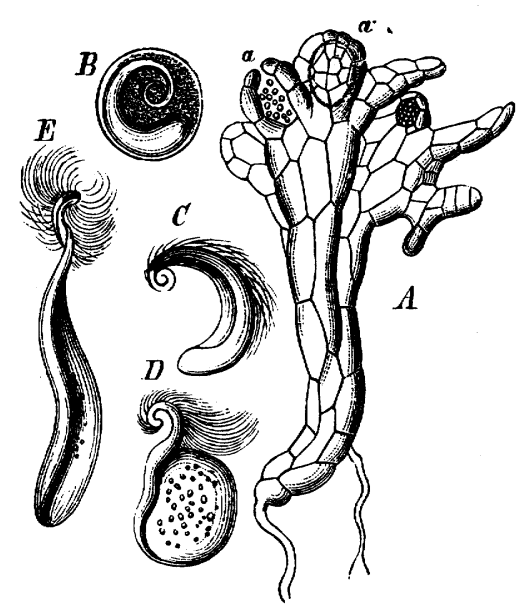

FIG. 275.-A male prothallium of Equisetum arvense with the first antheridia $a$ (after Hofmeister, $\times 200$ ) ; $B-E$ antherozoids of $E$. Telmateia (after Schacht).

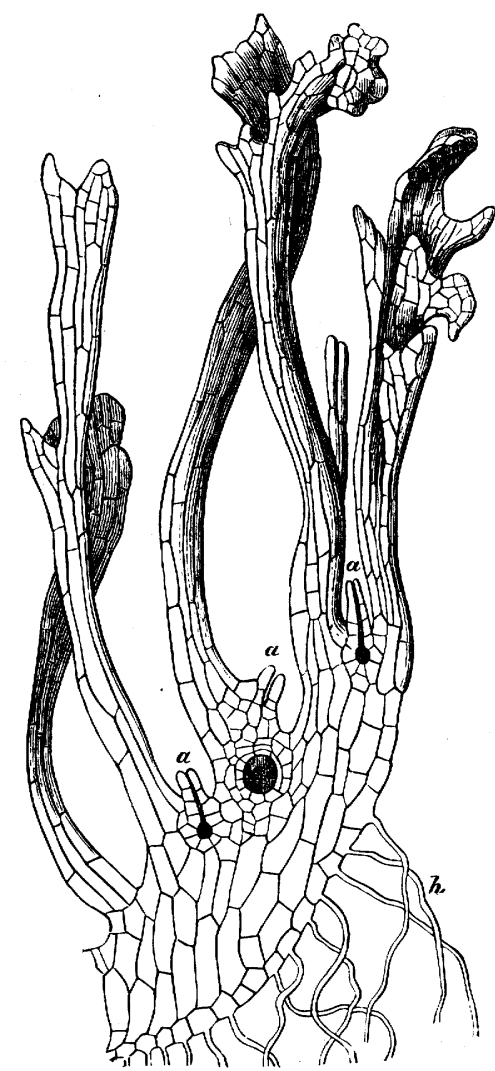

F1G. 276. - Lobe of a highly developed female prothallium of Equisetum arvense cut through vertically (after Hofmeister, $X$ about 60 ); $a$ a $a$ two abortive and one fertilised archegonium, $h$ root-hairs.

prothallium. The apical cells of the enveloping layer of the antheridium contain but little or no chlorophyll; they separate from one another on the addition of water (like those of Hepaticæ), to allow the escape of the antherozoids, which are still enclosed in vesicles and number from roo to $\mathbf{5} 5$. The hindermost and thickest of the two or three coils of the antherozoid, which is larger in this class than in all other Cryptogams, bears an appendage on the inner side which

${ }^{1}$ [Sadebeck, Ueb. die Antheridien-Entwickelung der Schachtelhalme Sitzber. d. Gesellsch. naturfor. Freunde zu Berlin, 1875.] 
Hofmeister terms an undulating Float, Schacht a thin-walled vesicle of protoplasm, and which contain granules of starch and sap (compare with Ferns and Isoëteæ).

The Archegonia are developed from single superficial cells of the anterior margin of the thick and fleshy lobes of the female prothallium. As the tissue of the prothallium beneath them continues its growth, the archegonia come, as in Pellia, to stand on its upper surface. The mother-cell of the archegonium, after it has become much curved, divides by a wall parallel to the surface of the prothallium. From the outer of the two daughter-cells is formed the neck, consisting, at a subsequent period, of four parallel rows each of three cells. Of these, the four upper cells become very long; the four middle ones remain shorter; the four lower ones scarcely elongate at all, and contribute by their multiplication, like the cells of the prothallium which surround the central cell, to the formation of the wall of the ventral part of the archegonium, which consists of one or two layers. The other daughter-cell, which is sunk in the tissue of the prothallium, elongates whilst the wall of the neck is being formed and projects between the four rows of cells constituting it. This projection is then cut off by a septum from the lower large portion of the cell. Of the two cells thus formed, the former is the single canal-cell of the neck, and the latter is the central cell of the archegonium. The central cell is divided again into two, the upper being the ventral canal-cell, the lower contracting and forming the oosphere. In these processes the archegonium of Equisetum resembles that of Ferns, the only difference being that in the former the canal-cell does not occupy the whole length of the neck (Janczewski). The four upper long cells of the neck curve radially outwards, when the canal of the neck is being formed, like a four-armed anchor. Immediately after fertilisation the canal of the neck closes, the oosphere, and which has now become the oospore, enlarges, and the cells of the wall of the ventral part-of the archegonium which surrounds it begin rapidly to multiply.

Development of the Asexual Generation (Sporophore) of Equisetum. The formation of the embryo from the oospore is the result of divisions, the first of which is inclined to the axis of the archegonium, and is followed, according to Hofmeister, in each of the two cells by a division-wall placed perpendicularly to the first. The embryo appears to be composed of four cells arranged like the quarters of a sphere. The same author states that the foot arises from the lower quarter, the rudiment of the first shoot from one of the lateral ones, turning upwards immediately afterwards and producing as the rudiment of the first leaf a projecting girdle, which then grows out into three teeth (Fig. $277 B$ ). The apical cell of the first root arises from an inner cell of the tissue ${ }^{1}$.

1 [On the Embryology of Equisetum see Sadebeck, Jahrb. f. wiss. Bot. XI. I 878, and also in Schenk's Handbuch, III. The embryology of Equisetum closely resembles that of Ferns (see infra, p. 426). The oospore divides by successive bipartitions into eight segments : of the four octants forming the upper (epibasal) half of the embryo, one gives rise to the stem, two give rise to the first leaf (cotyledon), and one gives rise to the second leaf: of the four octants forming the lower (hypobasal) half of the embryo, two give rise to the foot, one disappears, and the remaining one, which is diametrically opposite to the stem-octant, gives rise to the root.] 
The first leaf-bearing shoot grows upwards, and forms from ten to fifteen internodes with leaf-sheaths ending in three teeth. It soon produces at its base a new stronger shoot with four-toothed sheaths (as in $E$. arvense, pratense, and variegatum, according to Hofmeister), which in turn gives origin to new

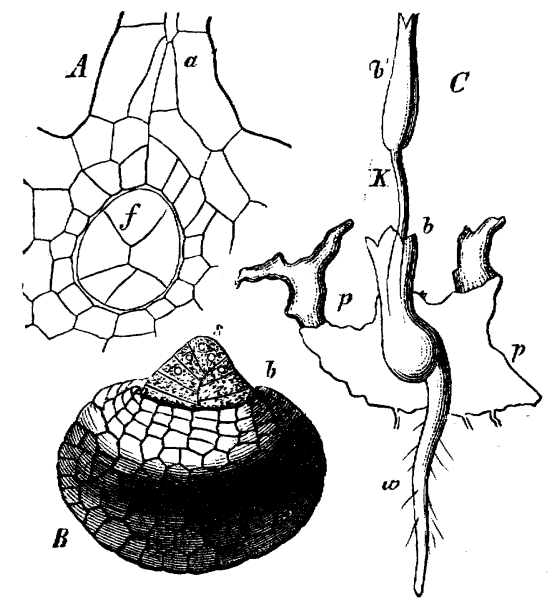

FIG. 277.-Development of the embryo of Equisetum ar. vense (after $\mathrm{H}$ ofmeister); $A$ archegonium cut through vertically with the embryo $f(\times 200) ; B$ embryo further developed and isolated, $b$ rudiment of a leaf, $s$ apex of the first shoot $(\times 200)$; $C$ vertical section of a lobe of a prothallium $p p$, with a young plant, $w$ its first root, $b b^{\prime}$ its leaf-sheaths ( $\times$ Io). generations of shoots, developing constantly thicker stems and sheaths with a larger number of teeth. Sometimes the third or one of the succeeding shoots penetrates downwards into the ground, forming the first perennial rhizome, which again produces from year to year new underground rhizomes and ascending leafy shoots.

In order to facilitate the understanding of the Mode of Growth of the Stem and Leaves, it is necessary to glance in the first place at their structure in the mature state. Every axis of an Equisetum consists of a series of joints (internodes) usually hollow and closed at their base by a thin septum. Each internode passes upwards into a leaf-sheath embracing the next internode, the sheath being split at its upper margin into three, four, or usually a larger number of teeth. From each tooth of the sheath a fibro-vascular bundle runs vertically downwards into the internode as far as the next node, parallel with the other bundles of the same internode; at the lower end each bundle splits into two short diverging limbs, by which it unites with the two neighbouring bundles of the next lower internode, where they descend into it from their sheath-teeth. The joints of the stem and their leaf-sheaths therefore alternate; and since in each joint the arrangement of bundles, leaf-teeth, projecting longitudinal ridges, and depressions or furrows, is exactly repeated in the transverse section, the different parts of a joint always correspond to the intervals between the homologous parts of the next upper and next lower joint. If the internode has projecting longitudinal ridges on its surface, one of these always runs downwards from the apex of each leaf-tooth parallel with the others as far as the base of the internode; between each pair of leaf-teeth commences a furrow or channel, which also continues as far as the base of the internode. The projecting ridges lie on the same radii as the fibro-vascular bundles, each of which contains an air-canal; the depressions or furrows lie on the same radii as the lacunæ of the cortical tissue (which are sometimes wanting), and alternate with the fibrovascular bundles. The branches and roots spring exclusively from within the base of the leaf-sheath; and as this forms a whorl, the branches and roots are also verticillate. A root may arise beneath the bud of each branch; both break through the leaf-sheath at its base. All the joints of the axis agree in these respects, however they may be modified as underground rhizomes, tubers, ascending stems, leafy branches, or sporangiferous axes. 
The end of the stem, enveloped by a large number of younger leaf-sheaths, terminates in a large apical cell, the upper wall of which is arched in a spherical manner, while the three infero-lateral walls are almost plane. The apical cell has therefore the form of an inverted triangular pyramid, the upturned basal surface of which is a nearly equilateral spherical triangle. The segments are cut off by walls which are parallel to the oblique sides of the apical cell, that is, to the youngest primary walls of the segment; the segments, disposed in a spiral $\frac{1}{3}$ arrangement, lie in three vertical rows. Each segment has the form of a triangular plate with triangular upper and under walls, rectangular lateral walls lying right and left, and an outer rectangular wall which is curved. Each segment is first divided-as was shown by Cramer and Reess and confirmed by myself-by a wall parallel to the upper and under surfaces into two equal plates lying one above

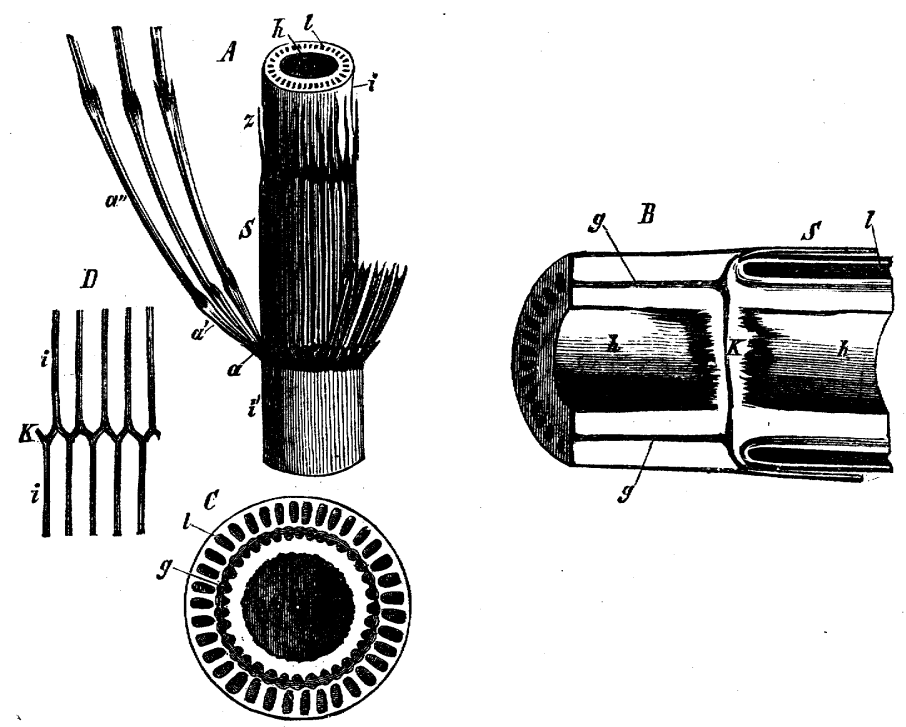

FIG. 278.-Equisetum Telmateia; $A$ piece of an upright stem (natural size), $i i^{\prime}$ internodes, $h$ its central cavity, $l$ lacunæ of the cortex, $S$ leaf-sheath, $z$ its apex, $a a^{\prime} a^{\prime \prime}$ the lower internodes of young shoots; $B$ longitudinal section of a rhizome ( $\times$ about 2 ), $k$ septum (diaphragm) between the cavities $h h, g$ fibro-vascular bundle, $l$ lacunæ of the cortex, $S$ leaf-sheath; $C$ transverse section of a rhizome ( $X$ about 2 ), $g$ and $l$ as before; $D$ union of the fibro-vascular bundle of an upper and lower internode $i i^{\prime \prime}, K$ the node.

another, and consequently each half the height of the undivided segment. Each half-segment is then again halved, in the most usual case, by a vertical nearly radial wall. The segment now consists of four cells, two of which lie one above the other and reach as far as the centre, but the other two do not because the vertical wall is not accurately radial but intersects one of the lateral walls of the segment (the anodal wall), (Fig. 279, E). Divisions now take place without any strict rule in the four cells of each segment parallel to the primary and the lateral walls; and tangential divisions also soon make their appearance, by which the segment is split up into inner and outer cells, in which further divisions afterwards take place. The former produce the pith, which is soon destroyed as far as the septum at the base of each internode by the expansion of the stem; the latter produce the leaves and the entire tissue of the hollow internodes. The segments are, as has 
been mentioned, disposed originally in a spiral with $\frac{1}{3}$ arrangement; and since each segment without exception (as in Mosses) produces a leaf or what corresponds to a part of a leaf-sheath, the leaves of Equisetum must also be arranged in a spiral. This does, in fact, sometimes occur when the growth is abnormal; but when the growth is normal, a small displacement takes place at a very early period, of such a nature that the three segments which form a cycle always come to be arranged into a disc transverse to the stem, their outer surfaces thus

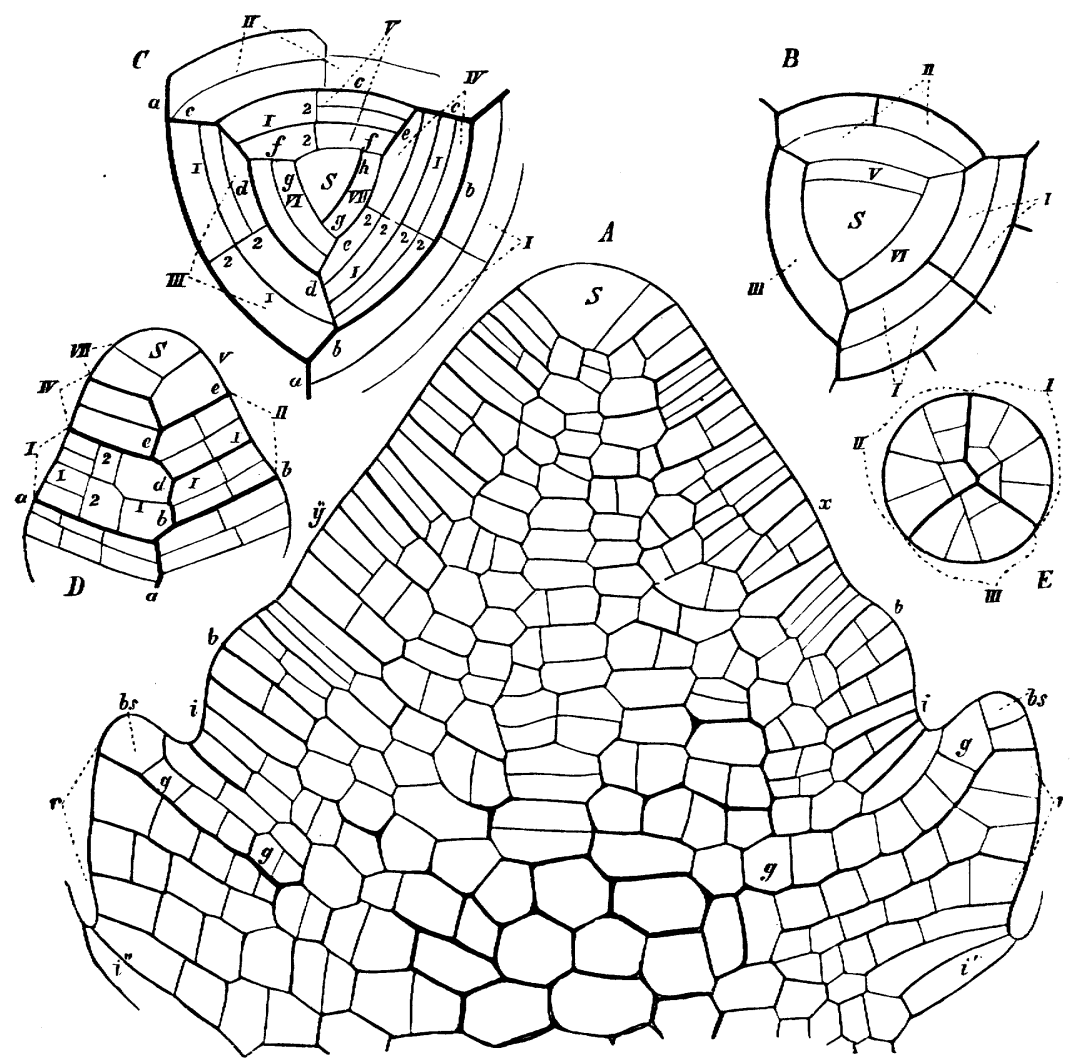

FIG. 279. $-A$ longitudinal section of the end of a stem in an underground bud of Equisetum Telmateia; $S$ apical cell, $x y$ first indication of the girdle from which the leaves are subsequently formed, $b \quad b$ a more advanced and distinctly marked foliar girdle, $b s$ the apical cells of a strongly projecting foliar girdle, $r r$ rudiment of the cortical tissue of the internodes, $g g$ rows of cells from which the leaf-tissue and its fibro-vascular bundle proceed, $i$ ithe lower layers of cells of the segment which take no part in the formation of leaves (from nature); $B$ horizontal projection of the apical view of the end of a stem of $E$. Telmateia ; $s$ apical cell, $I-V$ the successive segments, the older ones still further divided; $C$ horizontal projection of the apical view of $E$. arvense; $D$ optical longitudinal section of the end of a very slender stem; $E$ transverse section of the end of a stem after the formation of the vertical and first tangential walls. $(C, D, E$, after Cramer; the Roman numerals indicate the seginents, the Arabic numerals the walls formed in them in the order of their succession; the letters the primary walls of the segments.)

forming an annular zone or girdle. According to Reess, to whom this observation is due, the three segments of each cycle are formed in rapid succession, while a longer time elapses between the formation of the last segment of the preceding and that of the first of the succeeding cycle. Thus by the unequal growth of the segments in longitudinal direction each cycle of segments or turn of the spiral produces a whorl, which therefore, strictly speaking, is a pseudo-whorl, because resulting from subsequent displacement. Each whorl of segments now 
forms a leaf-sheath, and the corresponding internode or joint of the stem. The above-mentioned divisions take place in the three segments during their arrangement into a transverse disc, each segment becoming converted into a mass of cells consisting of from four to six layers. As soon as the transverse zone is formed, the formation of the leaves commences by the growth of the outer cells of the segments. They form an annular ridge; one of the upper transverse cell-layers of the whorl of segments projects outwardly, forms the apex (the circular apical line) of the ridge (Figs, 279, 280, $b s$ ), and those of its cells which lie most externally (the apical cells) divide by walls inclined alternately towards and from the axis. The circular apical line becomes more and more elevated, and thus the annular ridge becomes a sheath enveloping the end of the stem. This same layer, of which the outermost cells form the apical line of the annular ridge, produces in the interior of the sheath a meristem in which the fibro-vascular bundles of the leaf-sheaths arise. The lower transverse cell-layers of the whorl of segments grow only slightly outwards and upwards, become divided by vertical and afterwards rapidly by transverse walls, to produce the tissue of the internode, which passes gradually into that of the leaf. A vertical layer of this tissue forming a hollow cylinder (Fig. $280, v v$ ) is distinguished by numerous vertical divisions; it forms a ring of meristem (procambium, thickening-ring in Sanio's sense), in which the vertically descending fibro-vascular bundles of the internode are formed. These bundles form the prolongations of those of the leaf-teeth, which they

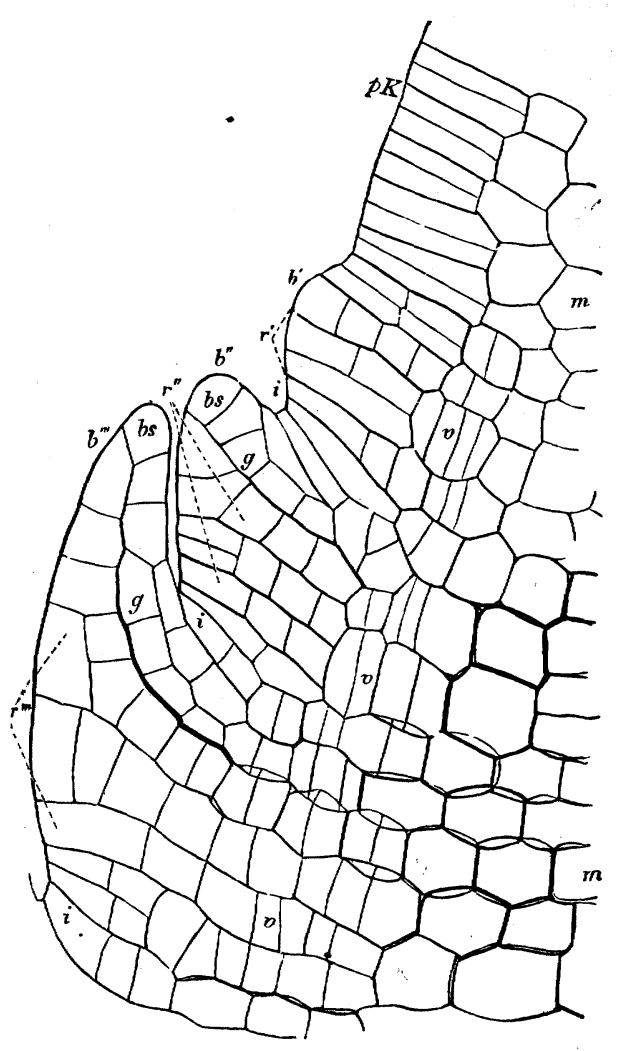

FIG. 280.-Left half of a radial longitudinal section beneath the apex of an underground bud of Equisetum Telmateia in September apex of an underground bud of Equisetum Telmateia in September;
$p K$ lower part of the vegetative cone, $b^{\prime} b^{\prime \prime} b^{\prime \prime \prime}$ leaves, $b s$ their apical $p K$ lower part of the vegetative cone, $b^{\prime} b^{\prime \prime} b^{\prime \prime \prime}$ leaves, $b s$ their apical
cells, $r^{\prime} r^{\prime \prime} r^{\prime \prime \prime}$ the cortical tissue of the corresponding internodes; $m m$ pith, $v v v$ thickening ring, $g g$ layer of cells from which the fibro-vascular bundles of the leaf-tooth arises.

meet, as shown in Fig. $28 \mathrm{r}, g g^{\prime}$, at an obtuse angle, and coalesce to form curved 'common' bundles. The layers of cells which lie outside the ring of meristem that gives rise to the bundles produce the cortex of the internode, in which airconducting canals soon arise. Even at an early period the first rudiments of the sheath-teeth appear as protuberances at regularly distributed points, each of them ending in one or two apical cells (Fig. 282) ${ }^{1}$.

1 On the original number and subsequent increase of the sheath-teeth, \&c., compare Hofmeister and Reess, $l$. $c$. 
The Branching of the Equisetaceæ depends exclusively on the formation of lateral buds. Each of these is formed from a superficial cell lying just above the youngest foliar girdle, and this takes place at points alternating with the sheath-teeth, long before the differentiation of the fibro-vascular bundles. [It was thought that these buds were of endogenous origin ${ }^{1}$, but the researches of Janczewski and of Famintzin have shown that this is not the case ${ }^{2}$.] Hofmeister was the first to show that each bud proceeds from a single cell of the tissue; and although $I$ have myself never seen it in a unicellular condition, I have found rudimentary branches composed of only two or four cells; and these showed that even the first three divisions of the mother-cell of the branch are inclined in three directions in such
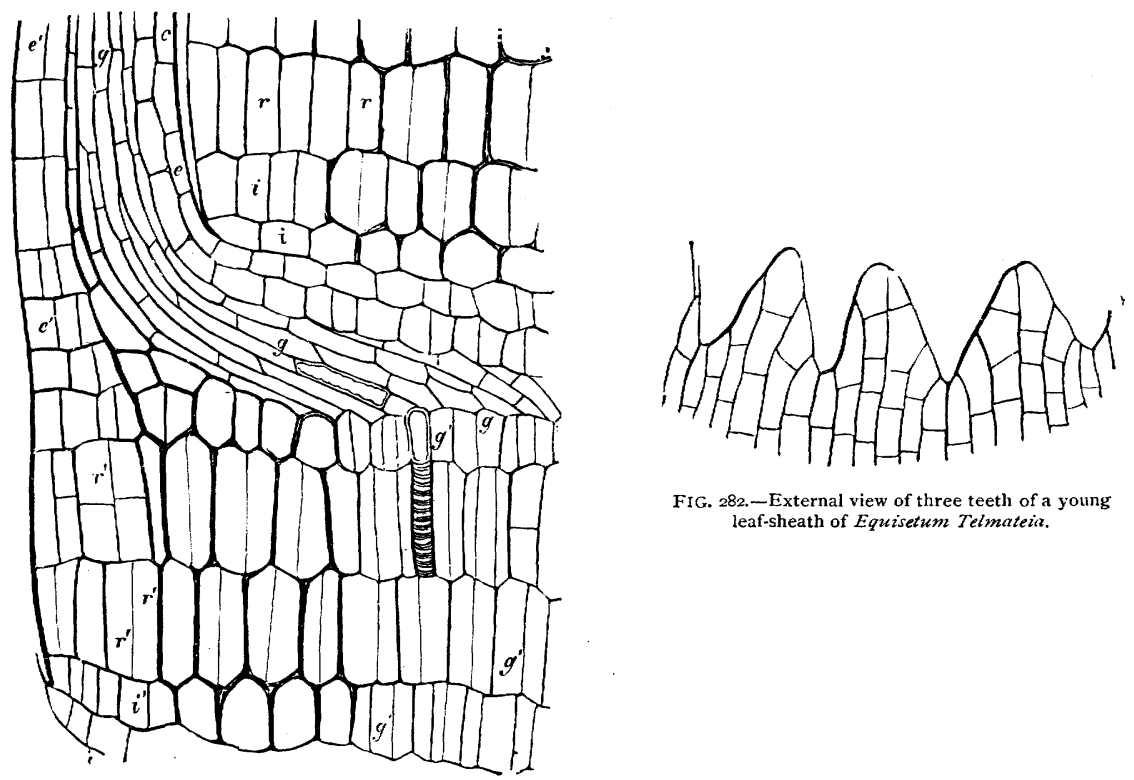

FIG. 282.- External view of three teeth of a young leaf-sheath of Equisetum Telmateia.

FIG. 28I.-The same as Fig. 280, but at a greater distance from the apex, showing a further advance of the differentiation of leaf-sheath and internode; $r r$ cortex of the upper, $r^{\prime} r^{\prime} r^{\prime}$ cortex of the lower internode, $e e$ the inner, $e^{\prime} e^{\prime}$ the outer epidermis of the leaf-sheath, $g g$ the foliar portion of the fibro-vascular bundle, $g^{\prime} g^{\prime} g^{\prime}$ its descending portion belonging to the internode; the first annular vessel is formed at their point of meeting.

a manner that a trilaterally pyramidal apical cell is produced; and the first three divisions thus form the first three segments. Lateral buds of the rhizome of $E$. Telmateia and $E$. arvense, late in the autumn or early in the spring, usually show in longitudinal section all the stages of development of the buds. After they have formed several foliar girdles and their apex is covered by a firm envelope of leaf-sheaths, they break through the base of the parent leaf-sheaths. They may also remain dormant for a long period, as is shown by the circumstance that buds break out when the underground nodes of ascending stems are exposed

1 See also the account of the Jungermannieæ, p. 359 .

${ }^{2}$ [Janczewski, Rech. sur le dév. des bourgeons dans les Prêles; Mém. Soc. de Sci. Nat. de Cherbourg, XX. 1876-77.-Famintzin, Bull. de l'Acad. Imp. d. St. Pétersbourg, XXII. 1876.] 
to the light. It may be assumed that there is always as large a number of buds in a rudimentary condition as there are sheath-teeth. On the erect leafy stems of $E$. Telmateia, $E$. arvense, and other species, they all attain complete development, and produce the numerous slender green leafy shoots of these species; in other species the development of the branches is more sparing; some, as $E$. hiemale, usually form no aërial lateral shoots at all except when the terminal bud of the stem is injured, and then the node next below produces a shoot. Branches do not usually make their appearance on rhizomes in the form of complete whorls, but in twos or threes, but on the other hand they are more vigorous; they become either new rhizomes or ascending stems. Since, in the cases first mentioned, the buds arise like the leaves in strict acropetal succession, it may be assumed that where the production of shoots is only induced at a later period by accidental circumstances, the buds have up to that time remained dormant in the interior.

The Roots arise in whorls, each immediately below a bud; but they may also often be suppressed, and may be developed, according to Duval-Jouve, even on aërial nodes, by humidity and darkness. Their development has been studied by Nägeli and Leitgeb (l.c.); in its earliest stages, which are represented diagrammatically in Fig. 284, it resembles essentially that of Ferns. The cortex is differentiated into an inner and an outer layer; the former forms air-conducting intercellular spaces, at first arranged, like the cells themselves, in radial and concentric rows, and afterwards combining by the rupture of the cells into a large air-cavity surrounding the central fibro-vascular cylinder. As the fibro-vascular cylinder of the root developes (seen in transverse section),

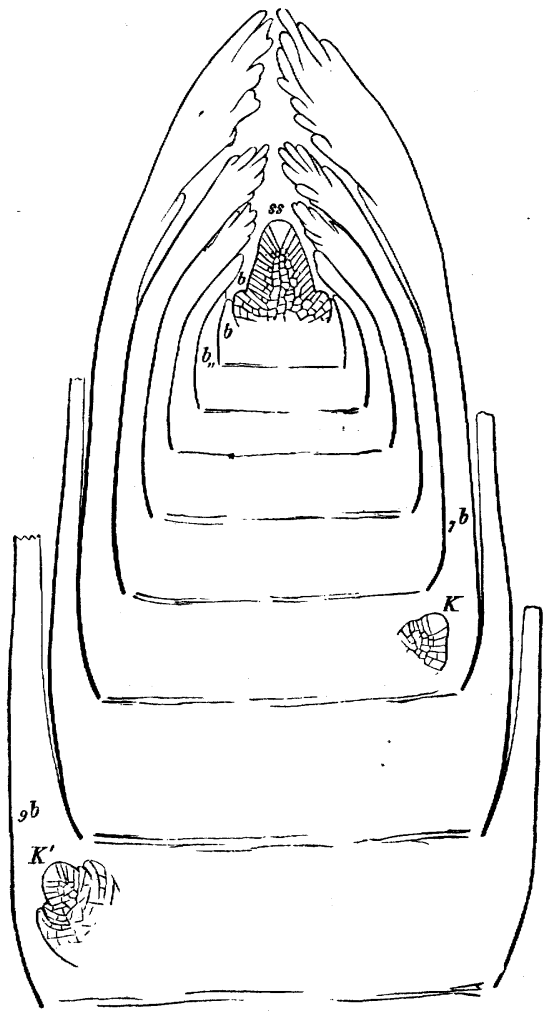

FIG. 283.-Longitudinal section through an underground bud of Equisetum arvense; ss apical cell of the stem, $b \rightarrow 9 b$ the leaves; $K K^{\gamma}$ two buds; the horizontal lines across the stem indicate the position of the diaphragms. each of the three primary cells which

alone of the six reach the centre is first of all divided by a tangential wall, so that the rudiment of the vascular bundle now consists of three inner and six outer cells. The six outer cells produce a cambial tissue in which the formation of vessels begins, commencing from two or three points of the circumference and advancing towards the interior. Last of all one of the three inner cells forms a broad central vessel; and phloëm is produced in the circumference of the vascular cylinder. In the other vascular Cryptogams the innermost layer of the cortical tissue forms the bundle-sheath (Pleromscheide, Schutzscheide), the radial walls of the cells 
having the characteristic folding, but in the Equisetaceæ it is the last layer but one of the cortical tissue which presents this appearance, whilst the innermost layer which directly abuts upon the axial cylinder seems to supply the place of the pericambium which does not exist in the roots of these plants. This innermost layer differs from the pericambium of the roots of other Vascular Cryptogams in that the lateral roots take origin from it, so that here also, as in all other Vascular Cryptogams, the lateral roots arise from the innermost layer of the cortical tissue. As a pericambium is wanting here, the commencing roots arise in immediate proximity to the external vessels of the axial cylinder. The cells, each one of which gives rise to a lateral root, are formed in strictly acropetal succession in the innermost cortical layer on the outer side of the primary xylem-vessels ${ }^{1}$.

The Sporangia of Equisetaceæ are outgrowths of peculiarly metamorphosed leaves which are generally formed in numerous whorls at the summit of ordinary
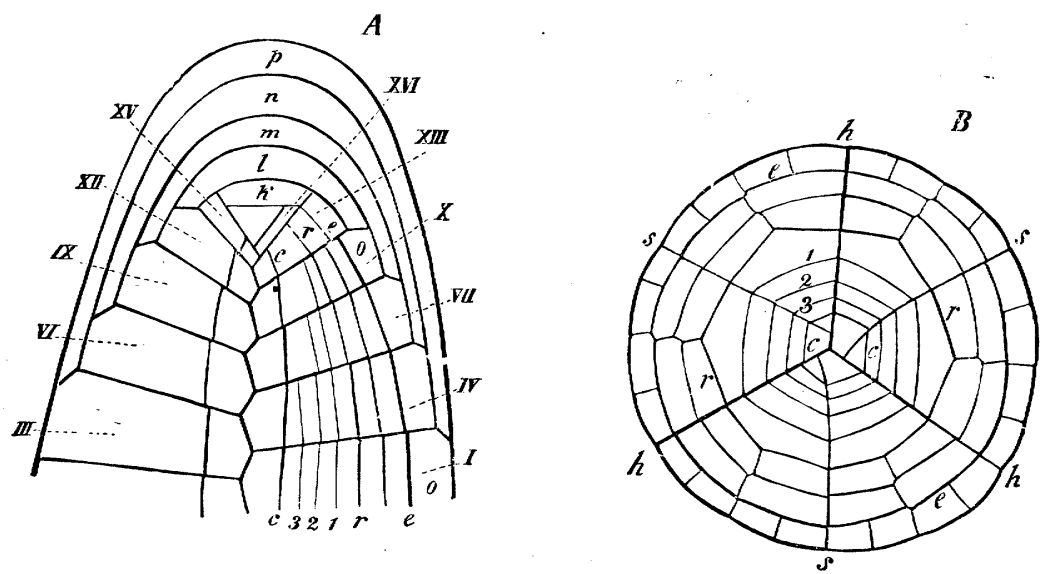

FIG. 284-Diagram of the succession of cell-divisions in the apex of the root of Equisetum hiemale (after Nägeli and Leitgeb), (this diagram will serve also in the main for Ferns and for Marsilia). $A$ longitudinal section ; $B$ transverse section at the lower end of $A$; $h h$ the primary walls, $s s s$ the sextant walls of the segments, indicated in $A$ by the figs. $I-X V I$, the lower end of $A$; $k m n$ the layers of the root-cap, all the further divisions is divided from the cortex of the root, $e$ the boundary-wall between which the rudimentary fibro-vascular cylinder (procambium) is divided from the cortex of the root, $e$ the boundary-wall between
the epidermis $o$ and the cortex (epidermal wall), $r r$ boundary-wall between the outer and inner cortex (cortical wall), $1,2,3$, the successive tangential walls by which the inner cortex is divided into several layers, the radial divisions being onitted.

shoots or of those specially destined for this purpose. Above the last sterile leafsheath of the fertile axis an imperfectly developed leaf-sheath is first of all produced (Fig. 285,a), a structure corresponding in some degree to the bracts of Phanerogams. The development of this structure is sometimes more, sometimes less leaf-like; foliar girdles are formed above it in acropetal succession beneath the growing end of the shoot, projecting however but slightly, as in the ordinary formation of leaves of Equisetum. A large number of protuberances project from each of these girdles, corresponding to the teeth of the ordinary leaf-sheaths; and thus several whorls of hemispherical projections are formed lying closely one over another, which, increasing more rapidly in size at their outer part, press against one another, and thus become hexagonal, the successive whorls alternating; while the basal (inner)

1 [See Van Tieghem, La Racine, Paris I87..] 
portion of each protuberance remains slender, and forms the pedicel of the hexagonal peltate scale. The outer surface of these scales is tangential to the axis of the spike; on its inner side, facing the axis, arise the sporangia, five or ten in number on each scale. In the early stages of development each single sporangium has the appearance of a small blunt multicellular wart; [an axial row of the cells grows more vigorously than the rest, and it is the terminal hypodermal cell of this row which constitutes the archesporium. The epidermal layer divides by walls parallel to the surface, so that four layers of cells invest the archesporium, of which the two outer form the wall of the sporangium, and the two inner the tapetum]. By repeated divisions the archesporial cell produces the spore-mother-cells which become isolated, while of the exterior cell-layers which at first envelope them only the outermost finally remains as the wall of the sporangium. The mother-cells of the spores, connected together in groups of fours or eights, float freely in a fluid which fills the sporangium and is interspersed with granules. The processes that take place in the mother-cells up to the time of the formation of the spores have already been described in detail in Chap. I (see Fig. Io, p. 14) ${ }^{1}$. It was there shown how the division into four of the mother-cells is preceded by an indication of a division into two, in a manner analogous to the corresponding process in Ferns. The ripe sporangium opens by a longitudinal slit on the side which faces the pedicel of the peltate scale. The very thin-walled cells of the wall have previously formed spiral thickening ridges on the dorsal, annular ones on the ventral side of the sporangium, arising, according to DuvalJouve, in the case of $E$. limosum, with extraordinary rapidity immediately before the dehiscence. The development of the spores of

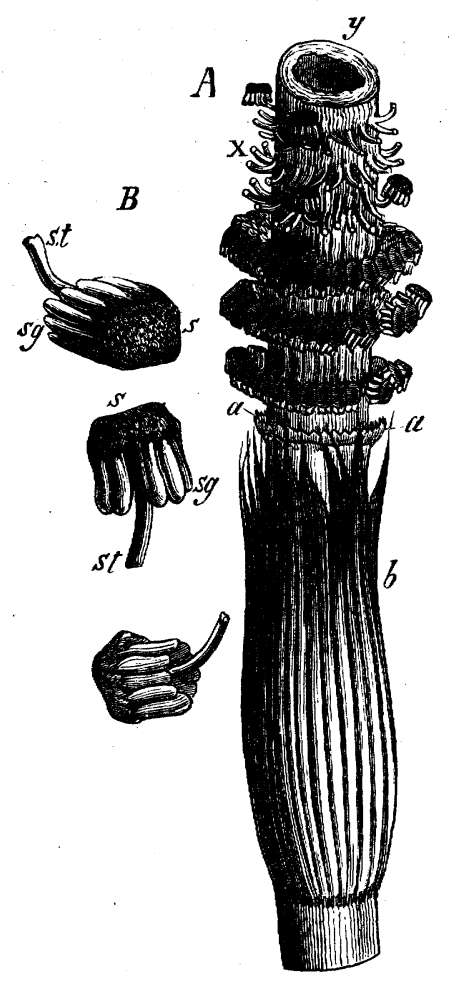

FIG. 285.-Equisetum Telmateia; $A$ upper part of a fertile stem with the lower half of the spike (natural size), $b$ leaf-sheath, $a$ the annular 'bract,' $x$ the pedi. cels of peltate scales which have been cut off, $y$ transverse section of the rachis of the spike; $B$ transverse section of the rachis of the spike; $B$
peltate scales in various positions (slightly magnipeltate scales in various positions (slightly magni-
fied); st the pedicel, $s$ the peltate scale, sg the sporangia.

Equisetum, after they have made their appearance as naked primordial cells by the division into four of their mother-cells, shows the peculiarity of a successive formation of distinct coats. Each spore forms first of all an outer non-cuticularised coat capable of swelling, which, splitting subsequently into two spiral bands, forms the so-called elaters, a second and third coat soon afterwards making their appearance within it. All three lie at first closely one upon another like successive layers of a single coat; but when the spore is placed in water, the outer one, even at this period, swells up strongly and becomes detached from the others (Fig. 286,

1 [On the development of the spores see Strasburger, Zellbildung und Zelltheilung, 3rd ed., p. I 55,] 
$B$ ). The three coats may be easily distinguished even in the quite fresh spore when placed in distilled water $(A)$, (in the case of $E$. limosum), the outer one (I) being colourless, the second (2) light blue, and the third (3) yellowish. As the development advances, the outer coat is separated like a loose investment from the body of the spore $(C, d, e)$, and at the same time its division into elaters is first indicated. The optical longitudinal section shows that the spiral thickeningbands of this coat are separated only by very narrow spaces of thin membrane $(D$, $E)$; these at length entirely disappear, and, when the surrounding air is dry, the thicker parts separate from one another as spiral bands, forming when unrolled a four-armed cross; they are united by their centre, and attached there to the second coat. It is probably this spot which may be recognised even in the unripe spore in the form of an umbilical thickening ( $n$ in $A$ and $B$ ). In the fully developed elaters an external very thin cuticularised layer may be distinguished. They are extremely hygroscopic; when the air is damp they are rolled round the spore, but when dry are again unrolled. When this alternation takes

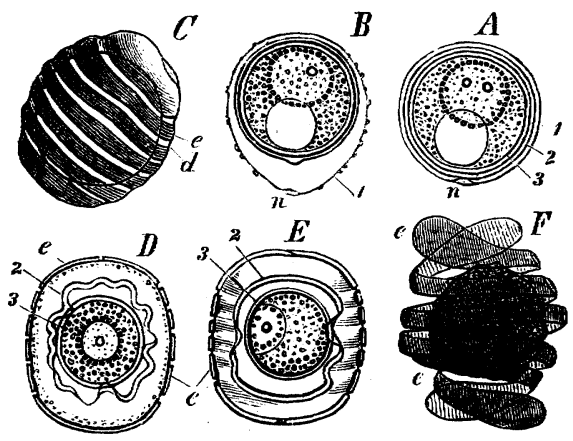

FIG. 286.-Development of the spores of Equisetum limosum $(\times 800) ; A$ unripe spore with three coats just placed in water; $B$ the same after two or three minutes in water, the outer coat having become separated, a large vacuole is seen by the side of the nucleus; $C$ commencement of the formation of the elaters on the outer coat $e(=1$ in Figs. $A$ and $B) ; D, E$ the same stage of development in optical section after lying twelve hours in glycerine, $e$ the outer coat; 2, 3, the inner coats separated from one another; $F$ the outer coat split into spiral elaters, coloured a beautiful blue by Schultz's solution.

place rapidly (as when lightly breathed on under the microscope), the spores are set in active motion by the bendings of the elaters. If spores, the outer coat of which has not yet become split up into elaters, but which already show the corresponding differentiations $(D, E)$, are allowed to lie for some time in glycerine, the spore contracts considerably, surrounded by its inner coat, while the second cuticularised coat raises itself from the former in folds. The inner coat is differentiated into a granular cuticularised exospore, and an endospore of cellulose.

Very little need be said about the Classification of Equisetacex, as all existing forms are so nearly related to one another that they may be included in a single genus, Equisetum. Even the Equisetacex of earlier geological periods, the Calamites, show, in the little that is still discernible of their organisation, the closest agreement with existing forms.

The Habit of the Equisetaceæ is, like their morphological structure, of a very characteristic kind. In all the plant is perennial by means of creeping underground rhizomes, from which ascending aërial shoots rise annually, mostly lasting only for one 
period of vegetation, less often for several years. The sporangiferous spikes appear either at the summit of these axes, which are at the same time the organs of assimilation, or on special fertile shoots which, when destitute of chlorophyll and unbranched, die after the dissemination of the spores ( $E$. arvense and $\mathcal{T}_{\text {elmateia }}$ ), or throw off the terminal spike and act as vegetative shoots (E. sylvaticum and pratense). The fertile axes are developed from the underground internodes of the erect vegetative axes; they remain during the summer, in which the latter are unfolded, in the budcondition beneath the ground, but during this period either develope their sporangiferous spikes so far that in the next spring nothing is necessary except elongation and the dissemination of the spores (E. arvense, pratense, Telmateia, \&c.), or the spikes attain their full development only in the spring after the elongation of the axes which bear them ( $E$. limosum). The habit of the aërial shoots is determined especially by the number and length of the verticillate usually very slender lateral branches; in some, as $E$. biemale, trachyodon, ramosissimum, and variegatum, they are generally entirely wanting; in others, as E. palustre and limosum, they are few ; in others again, as $E$. arvense, Telmateia, and sylvaticum, they are developed in large numbers. The height of the leafy stem is in our native species mostly from I to 3 feet; in E. Telmateia, where the ascending axis of the sterile shoots is colourless and destitute of chlorophyll, it attains a height of 4 or 5 feet and a thickness of about $\frac{1}{2}$ inch; while the green slender leafy branches are even in this case scarcely $\frac{1}{2}$ line thick. The tallest stems are produced by $E$. giganteum in South America, as much as 26 feet high, but only about the thickness of the thumb, and are kept in an upright position by neighbouring plants. The Calamites were as lofty, and as much as $\mathrm{r}$ foot thick. The rhizomes mostly creep at a depth of from 2 to 4 feet beneath the ground, and extend over areas ro to 50 feet in diameter; but are also found at a much greater depth. They prefer damp, gravelly, or loamy soil, their thickness varying from $I$ to 2 lines to as much as $\frac{1}{2}$ inch or more. The surface of the internodes of the rhizome is, in some species, as E. Telmateia and sylvaticum, covered with a felt of brown root-hairs, which also clothe the leaf-sheaths of the underground part of ascending stems, a peculiarity which reminds one of Ferns. In some species, as $E$. limosum and palustre, the surface is smooth and shining, while in others it is dull. The ridges and furrows of the aërial stems are usually but little developed on the underground stems; sometimes the rhizomes are twisted. The central canal of the internodes is sometimes wanting in the rhizomes; but the lacunæ of the fibro-vascular bundles (carinal canals) and those of the cortical parenchyma (vallecular canals) are always present; the air which the tissues require and which is not found in the usually very compact soil is carried by these canals from the surface to the underground organs. As in the case of the spikes, the formation of the branches of the leafy stems has already taken place entirely or at least to a great extent in the preceding year in the underground bud, so that in the spring the internodes of the ascending axis have only to extend and the slender lateral branches to unfold, as may be seen with especial ease in $E$. Telmateia. All the more important cell-formations and the processes of morphological differentiation thus take place underground; the aërial unfolding has for its main purpose only the dispersion of the spores and assimilation by the leafy shoots, by the exposure of the cortex, which contains chlorophyll, to light. The 'rapid growth of the upright stems in the spring is especially brought about by the simple elongation of the internodal cells already formed, although permanent intercalary growth of the internodes sometimes also takes place, and especially at their base within the sheaths. The tissues often remain there for a long time in the young state, and in $E$. biemale the shorter internodes grow out of their leaf-sheaths after passing through the winter, and they are then lighter in colour; the shorter they were before the winter, the more they elongate afterwards.

Special Organs for Vegetative Propagation, like those of Mosses, are not found in the Equisetacex any more than in Ferns; but every part of the rhizome, and the underground nodes of ascending stems, are adapted for the production of new stems. In 
some species some of the underground shoots swell up into ovoid ( $E$. arvense) or pearshaped (E. Telmateia) tubers about the size of a hazel-nut; Duval-Jouve states that these occur also in E. palustre, sylvaticum, and littorale, but in other species (E. pratense, limosum, ramosissimum, variegatum, and biemale) they have not yet been observed. The tubers are produced by the rapid increase in thickness of an internode at the end of which is situated the terminal bud; this may repeatedly form tuberous internodes so that the tubers become moniliform, or they may develope simply as a rhizome, or sometimes a central internode of a rhizome is developed in a tuberous manner. The parenchyma of these tubers is filled with starch and other food-materials; they may apparently long remain dormant and form new stems under favourable circumstances.

Among the Forms of Tissue of the Equisetace ${ }^{1}$ the epidermal system and the fundamental tissue are in particular developed in a great variety of ways. The fibro-vascular bundles, which in Ferns are so thick and so highly organised, especially in their xylemportion, appear to be less developed in the Equisetacex; they are slender, the lignification of the xylem-portion very slight (as in many water and marsh plants); the firmness of their structure is chiefly due to the epidermal system with its highly developed epidermis, and to the hypodermal fibres. What follows has special reference to the internodes; the leaf-sheaths are usually similarly constituted in their lower and central parts; at the teeth the tissue is simpler and somewhat different.

The Epidermal Cells are mostly elongated in the direction of the axis, and are arranged in longitudinal rows separated by transverse or slightly oblique walls; the boundary-walls of the adjoining cells are often undulating. The epidermis of the underground internodes is almost always destitute of stomata, and consists of cells with either thick or thin walls, usually brown, which, in some species, as E. Telmateia and arvense, develope into delicate root-hairs. The epidermis of the deciduous sporangiferous stems of the species just named is similar to that of the rhizome and without stomata; and the same is the case with the upright colourless sterile stem of $E$. Telmateia. On all the aërial internodes which contain chlorophyll, on the leaf-sheaths and on the outer surface of the peltate scales, the epidermis possesses numerous stomata which always lie in the channels, never on the ridges, and are arranged in longitudinal rows either single or lying close to one another. On the ridges the epidermal cells are long, in the channels between the stomata shorter. All the cells, even those of the stomata, have their outer walls strongly silicified, and exhibit very often on their outer surface protuberances of various forms, which are also and indeed peculiarly strongly silicified. These protuberances resemble fine granules, bosses, rosettes, rings, transverse bands, teeth, and spines; on the guard-cells they usually occur in the form of ridges, running at right angles to the orifice. The guard-cells are generally partially covered by the neighbouring epidermal cells. The mature stoma appears to be formed of two pairs of guard-cells lying one over another; Strasburger asserts that these four cells arise from one epidermal cell, and lie at first side by side at the same level. Only at a later period the two inner ones (the true guard-cells) become pressed inwards and overreached by the two outer ones which grow more rapidly. Bundles or layers of firm thick-walled cells (Hypodermal Tissue) are of common occurrence beneath the epidermis of rhizomes, of upright stems, and of their leafy shoots (with the exception of the deciduous sporangiferous stems). In the rhizomes they form a continuous stratum of brown-walled sclerenchyma consisting of several layers; in the aërial internodes they are colourless and are developed with especial prominence in the projecting ridges.

The Fundamental Tissue of the internodes consists in the main of a colourless thinwalled parenchyma occurring only in the rhizomes, the deciduous sporangiferous stems, and the colourless sterile axes of E. Telmateia. The green colouring of the other shoots

1 [For further details on this subject, see De Bary, Vergleichende Anatomie der Vegetationsorgane der Phanerogamen und Farne, 1877.] 
is caused by layers consisting of from $\mathrm{I}$ to 3 strata of parenchyma containing chlorophyll (the cells lying transversely). This green tissue lies especially beneath the furrows, corresponding to the stomata, and appears in a transverse section as ribbon-shaped masses concave outwardly; in the slender leafy branches, where the ridges sometimes cause the transverse section to have a stellate outline (e.g. E. arvense), the tissue containing chlorophyll is in excess. The vallecular canals, which correspond to the furrows, arise in the fundamental tissue by separation and partially by rupture of the cells; they may be absent from the slender leafy branches.

The Fibro-vascular Bundles are arranged, in a transverse section of the internode, as in Dicotyledons, in a circle, each corresponding to a ridge of the surface, between the cortical canals but somewhat nearer the centre. In the axis of the sporangiferous stems, where the diaphragms are wanting, they run in the same manner, and bend out singly into the pedicels of the peltate scales (as into the sheath-teeth). The bundles of a shoot are all parallel to one another; each bundle is the result of the coalescence of two portions; one of these belongs to the leaf-sheath and developes in the median line of one of its teeth from below upwards; the other portion developes in the internode itself from above downwards. At the angle where the two portions meet, the formation of tissue begins in both, and thence advances in opposite directions; the lower end of each bundle unites by two lateral commissures with the two next alternate bundles of the next lower internode (Fig. 278); the Equisetacex have therefore only 'common' bundles. In transverse sections these bundles resemble those of Monocotyledons, especially of Grasses; the first-formed annular, spiral, or reticulated vessels belonging to the inner side, together with the thin-walled cells which separate them, are subsequently destroyed, and a canal (carinal) remains in their place traversing the whole length of the fibro-vascular bundle on its inner side. Right and left of this lie on the outside a few not very broad vessels thickened reticulately; external to the canal lies the phloëm-part of the bundle, formed of a few wide sieve-tubes and narrow cambiform cells, and at the circumference of a few thick-walled narrow bast-like cells. A bundle-sheath, as it is termed, sometimes surrounds each bundle (E. limosum), but generally runs continuously outside the circle of all the bundles, as in most Phanerogams.

[Professor W. C. Williamson is led by a study of the internal organisation of Calamites and Calamodendra ${ }^{1}$ to the conclusion that in England at least we have but one group of these fossil plants. When young their vascular zone, separating a medullary from a cortical parenchyma, was scarcely more than a thin ring of longitudinal canals, each of which had a few vessels at its outer border. In this state the structure of the plant presented a close resemblance to that of a recent Equisetum. But as the plant grew in size, new vessels were added to the exterior of the preexisting bundles, so that each of the latter became the starting-point of a woody wedge which continued to grow peripherally until it assumed large dimensions. In some specimens these wedges measure fully two inches between the canal marking their medullary angle and their peripheral or cortical base. Each wedge is composed of vertical radiating laminæ of barred or reticulated vessels separated by cellular rays. The medullary portion became fistular, as in the recent Equisetacex, at an early age, and when the fistular cavities became filled with sand or mud, the very thin layer of medullary cells which remained did not prevent the sand from moulding itself against the inner angles of the vertical woody wedges, which thus produced the longitudinal grooves so characteristic of the casts commonly seen in collections. In such specimens most of the vegetable elements disappeared during fossilisation, and what remained, in the shape of a thin film of coal, moulded itself upon the medullary cast, and gave to the specimens the appearance of having had corresponding

1 [On Fossil Equisetaceæ, see Williamson, Mem. Lit. Phil. Soc. Manch. 3rd ser. vol. IV. pp. I55-183; Ditto, Trans. Roy. Soc. vol. CIXI. pp. 477-510: also vol. CLXIX. Part 2, 1878.Coemans, Journ. Bot. 1869 , pp. 337-340.-Dawson, Ann. Nat. Hist. $4^{\text {th }}$ ser. vol. IV. pp. 272-273.-

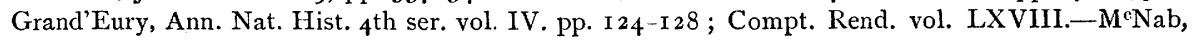
Journ. of Bot. 1873 , pp. $72-80$. 
grooves upon their outer bark surfaces. No single example of a specimen of which the internal organisation is preserved-and we now possess these in great numbers-sustains this latter conclusion. Wherever the true bark is preserved it exhibits an outline indicating a smooth surface, longitudinal internodal flutings and transverse nodal constrictions being alike absent.

In its young state the bark consisted of undifferentiated cellular tissue, and we now have evidence that a hypodermal layer of prosenchyma was developed in it which ultimately attained to an enormous thickness.

The woody werges extended vertically through each internode without interruption, but at the node each wedge split into two halves, each half coalescing with the contiguous half of the wedge nearest to it to form one of the wedges of the next internode. At each node in young stems numerous small cellulo-vascular bundles passed outwards through the vascular zone to supply some peripheral organs; probably verticillate leaves and branches. But most of the latter became abortive; the branches of the larger stems being few, and unsymmetrically disposed. Stems and branches were thickened simultaneously as in ordinary exogenous trees. Besides these diverticula, in most of the Calamites, immediately below each node, there passed outwards, through the cells of the large primary medullary rays, so conspicuous in all young shoots, and near the medullary surface of matured vascular axes, an exceedingly regular verticil of primary canals, with circular or oblong sections, from the central fistular cavity through the woody zone to the bark. One of these canals occupied the uppermost end of each of the large cellular rays which separated the vascular wedges of each internode. In the common fossilised casts these canals are indicated by a very regular verticil of small round or oblong impressions, which some writers have erroneously associated with roots, and others with vascular bundles going to leaves or branches. But they never contained any vascular tissues whatever. Of the leaves of Calamites we have but little knowledge, although some have identified them with those of Asterophyllites and Sphenophyllum.

Professor Williamson has only obtained one example of a fruit which he can with confidence identify with Calamites (On a new form of Calamitean Strobilus, Williamson, in Mem. Lit. Phil. Soc. Manch. 3rd ser. IV. p. 248). It is a strobilus the structure of the axis of which corresponds most closely with that of a young Calamitean shoot. At each node it has a curiously perforated transverse disk fringed with numerous peripheral bracts. From the upper surface of each disk there projects vertically upwards a ring of slender sporangiophores, around each of which were clustered three or four sporangia full of spores. These sporangia are so compactly compressed that a transverse section of this fruit presents the appearance of a compact mass of spores, amongst which the outlines of the sporangia are traceable with difficulty. Whilst he has failed to find any true stem in which the outer surface of the bark was fluted, that of the internodes of this fruit was undoubtedly so. The flutings of the fruit-bark do not, like those seen in the carbonaceous film covering the common casts, correspond in number and position with those caused by the woody wedges, since two vascular bundles are located in each projecting ridge of the axis of the former structure, instead of one as in the latter.

Mr. Carruthers believes the fruits figured by Mr. Binney, Professor Schimper, and himself, under the several names of Calamodendron commune, Calamostachys Binnoyana, and Volkmannia Binneyi (Journ. of Bot. 1867 , pp. 349-356), to belong to Calamites; and he further regards the spores as having been furnished with elaters similar to those of Equisetum. Professor Williamson is unable to agree with either of these conclusions. The numerous specimens which his cabinet now contains make it absolutely certain that the supposed elaters are merely fragments of the torn mother-cells of the spores, and it is his impression that these fruits, now known to be heterosporous, have closer affinities with the Lycopodiaceæ than with the Equisetaceæ; they certainly are not the strobili of Calamites.] 


\section{CLASS VIII.}

\section{F I L I C I N E $\nRightarrow$.}

THE plants included in the group of the Filicineæ are distinguished from the Equisetaceæ and from the Dichotomeæ by the various and complete development which their leaves attain. In proportion to the stem, the leaves are always of considerable size, and in their anatomical structure, as well as in their external form, they manifest a higher differentiation than do those of the two groups above mentioned. In these two groups the whole external form of the plant depends upon the formation and branching of the stem, and the most important physiological functions are performed by this organ; in the Filicineæ the stem is essentially an organ for bearing leaves and roots; its growth is slow, frequently its development is so imperfect that no internodes are formed, whereas the leaves are endowed with an active apical growth which continues for a considerable time and in some cases is unlimited. Further, the stem, in the Filicineæ, has but little tendency to branch; in whole families it remains simple, and not unfrequently the formation of buds is provided for by the leaves in which a strong tendency to branch is manifested, and which present, in consequence, the most varied forms of pinnate and palmate segmentation and of dichotomous branching. In the Equisetaceæ and Dichotomeæ it generally happens that the stem takes part in the formation of the fructification; in the Equisetaceæ it is always and in the Dichotomeæ it is usually an apical spike which is terminal upon the branch bearing it. In the Filicineæ this is never the case; the function of reproduction is discharged solely by the leaves, the stem taking no part whatever in it. The leaves bear very numerous sporangia (the number varying with the size of the leaf), whereas the peltate scales of the Equisetaceæ bear but a few, and the fertile leaves of the Dichotomeæ only one. The mode of development of the sporangia upon the leaves of the Filicineæ is not uniform; in the Stipulatæ each sporangium arises from a group of epidermal cells, in the Filices and Rhizocarpeæ from a single epidermal cell ${ }^{\mathbf{1}}$.

It appears, moreover, that the Rhizocarpeæ, which were formerly separated from the Ferns on account of the mode in which their fructification is developed, present (more especially the Salviniaceæ) a sufficient number of resemblances to the true Ferns to justify us in regarding them as a branch of the ancestral tree from which the Hymenophyllaceæ and Polypodiaceæ have sprung.

It is not easy to give a brief account of the relationships existing between the groups contained within this class, for a whole series of Ferns, the Osmundaceæ, Schizæaceæ, and Gleicheniaceæ have been as yet but imperfectly investigated from a morphological stand-point. Our present knowledge (1874) of these plants is very superficial, it suffices merely for the diagnoses of systematists. For those who can obtain the necessary material and who possess the requisite morphological

${ }^{1}$ [See Goebel, loc. cit.] 
education a field for work is here open. We must content ourselves now with briefly stating all that is actually known, and with merely mentioning the less known forms when occasion demands.

\section{Order I. Stipulat}

Under this name, which is based upon the peculiar formation of stipules common to the members of the two groups, I include the Ophioglosseæ and the Marattiaceæ, for the relationship existing between them is manifested in several important particulars. In both groups the prothallia are moncecious and capable of independent growth; but there is a tendency to diœcism in the Marattiaceæ. That the prothallia of the Ophioglosseæ are subterranean, whereas those of the Marattiaceæ are not so, is a difference of physiological and not of morphological importance. The stem of the second generation in both families is characterised by its very slight growth in length, the usual absence of internodes and of any branching, the whole of its surface being occupied by the insertions of the leaves, and by the development of roots acropetally immediately behind its apex. The absence or rudimentary development of bundle-sheaths and of brown schlerenchyma in the ground-tissue of the stem and of the leaves distinguishes these plants from the true Ferns. The Ophioglosseæ diverge most widely from the Ferns in that their sporangia are imbedded in the tissue of the leaf. The Marattiaceæ present an intermediate condition in that their sporangia are quite external to the leaf and are attached by a narrow base.

Although it is not improbable, it is still an open question whether or not the Osmundaceæ are nearly related to these two families. Their petioles bear at their bases lateral membranous wing-like appendages which may fairly be termed stipules, but which are certainly very different from those of the Marattiaceæ and Ophioglosseæ. Further, the stem of the Osmundaceæ, which is thickly covered with roots, is not erect like that of the other two groups, and it is uncertain whether the numerous lateral branches arise from it or from the petioles of the leaves. The fructification seems to indicate a relationship, for it recalls the paniculate fructification of the Botrychiæ, but the fertile segments of the leaves have no mesophyll. In this respect the Schizæaceæ resemble the Osmundaceæ, but in other features, more especially in the want of stipules, they differ from the Stipulatæ.

Although the connection of the Ophioglosseæ with the Marattiaceæ is tolerably evident, it may be advisable to give a separate account of each family.

Family I. Ophioglosseæ ${ }^{1}$. The Sexual Generation (Oophore). The prothallium is at present known only in Ophioglossum pedunculosum and Botrychium Lunaria. In both cases it is developed underground. It is destitute of chlorophyll, and forms a parenchymatous mass of tissue which, according to Mettenius, has at first, in the species first-named, the form of a small round tuber, out

1 Mettenius, Filices horti botanici Lipsiensis. Leipzig I 856, p. I 19.-Hofmeister, Abhandlungen der königl. Sächs. Gesellsch. der Wissens. I 857, p. 657 - - [On the Germination, Development and Fructification of the Higher Cryptogams, Ray Soc. 1862, pp. 307-31 7.]-Russow, Vergleich. Unters. St. Petersburg, I872, pp. I I 7 ff. - [Holle, Ueb. Bau und Entwickelung der Vegetationsorgane der Ophioglossen, Bot. Zeitg. 1875.$]$ 
of which is subsequently developed a cylindrical vermiform shoot, which grows erect underground, is rarely and slightly branched, and elongates by means of a single apical cell. When the apex appears above ground and becomes green, it forms lobes and ceases to grow. The tissue of this prothallium is differentiated into an axial bundle of elongated, and a cortex of shorter parenchymatous -cells, and the surface is clothed with root-hairs. With a transverse diameter of $\frac{1}{2}$ to $\mathbf{I} \frac{1}{2}$ lines, it attains a length of from 2 lines to 2 inches. The prothallium of Botrychium Lunaria is, according to Hofmeister, an ovoid mass of firm cellular tissue, the greatest diameter of which does not exceed $\frac{1}{2}$ line, and is often much less (Fig. $287, A$ ). It is light brown externally, yellowish white internally, and provided on all sides with sparse moderately long root-hairs. These prothallia are monœcious; each one produces a number of antheridia and archegonia, which are distributed with tolerable uniformity over the whole of its upper surface, with the exception, in $O$. pedunculosum, of the small primary tuber; in Botrychium it is the upper side which chiefly bears antheridia.

The Antheridia are cavities in the tissue of the prothallium covered externally by a few layers of cells, and in Ophioglossum only slightly projecting beyond the

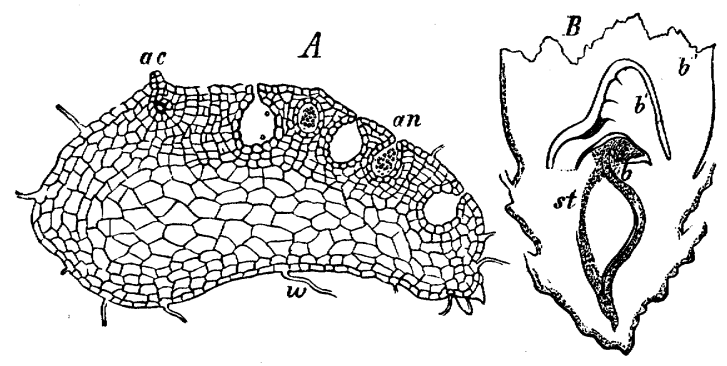

FIG. 287.-Botrychium Lunaria; $A$ longitudinal section of prothallium ( $\left.\times 50^{\circ}\right), a c$ an archegonium, att an antheri dium, $w$ root hairs; $B$ longitudinal section of the lower part of a young plant dug up in September $(\times 20) ; s t$ stem, $b b^{\prime} b^{\prime \prime}$ leaves (after Hofmeister).

surface. In this genus the mother-cells of the antherozoids originate by repeated divisions from one or two cells of the inner tissue (covered externally by one or two layers of cells); they form a mass of tissue of roundish form, and, as in Botrychium, give rise to the antherozoids, which are similar in form to those of the Polypodiaceæ, but larger; they escape through a narrow opening in the cover of the antheridium.

The Archegonia are apparently developed in a similar manner to those of other Vascular Cryptogams. Mettenius saw in Ophioglossum instances in which they consisted of two cells, a superficial cell and one lying below it; this latter, he considered, became the central cell, the former producing the neck of the archegonium by dividing into four cells arranged crosswise, which then produce, by further divisions, four vertical rows each consisting of two or more cells, and thus form the neck. The wall of the ventral part which surrounds the central cell is formed by divisions of the cells of the prothallium which surround it; the ventral part is therefore completely imbedded, and only the neck, which is usually very short, projects above the surface. Mettenius asserts that in Ophioglossum a 
prolongation of the oosphere (probably a canal-cell, as in Ferns and Rhizocarps), penetrates into the lower part of the neck.

The Asexual Generation (Sporophore). The first divisions of the oospore are not known; but the mode of formation of the embryo differs, as may be concluded from more advanced stages, from that of Ferns. Mettenius states that in Ophioglossum pedunculosum the end of the embryo which faces the apex of the prothallium developes into the first leaf, while the opposite end produces the first root. Unlike what occurs in Ferns, the concave upper side of the first leaf faces the neck of
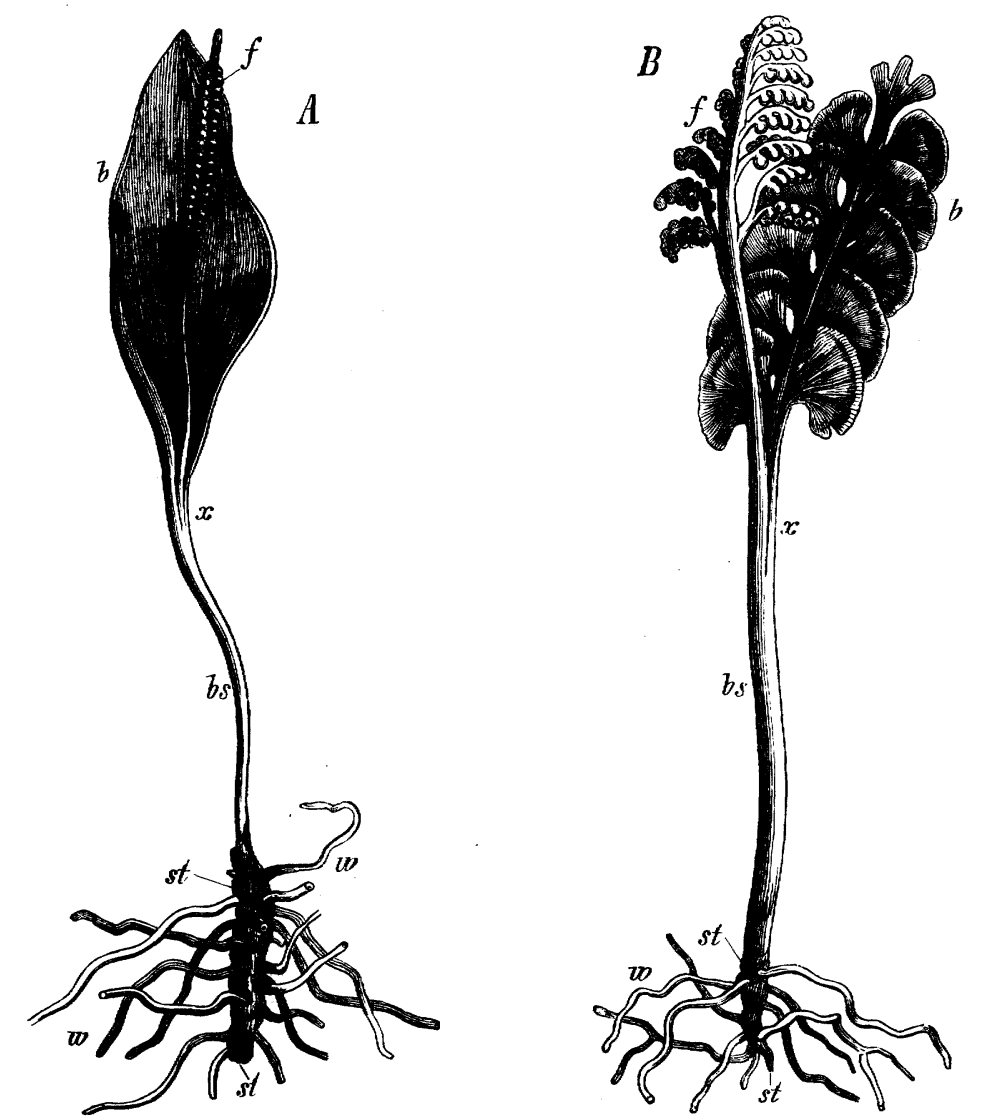

FIG. 288. - A Ophioglossum vulgatum; B Botrychium Lunaria (both natural size) ; w roots, st sten, bs leaf-stalk, $x$ point where the leaf branches, the sterile lamina $b$ separating from the fertile branch $f$.

the archegonium; the rudiment of the stem (which Mettenius terms the 'primary rudiment of the embryo') lies nevertheless on the side of the embryo which faces the base of the archegonium. Hofmeister, on the other hand, makes the following statement with regard to Botrychium :- 'The position of the embryo with respect to the prothallium differs widely from that which occurs in the Polypodiaceæ and Rhizocarpeæ; Botrychium approaches in this respect those Vascular Cryptogams the prothallium of which, like that of Ophioglossaceæ, is destitute of chlorophyll (Isoëtes, Selaginella). The punctum vegetationis of the embryo lies near the apical point of 
the central cell of the archegonium; the first roots arise beneath it, near the base of the archegonium' (l.c.p. 308).

The processes of growth of the mature plant have not yet been ascertained with as much certainty as in other Vascular Cryptogams. In Ophioglossum vulgatum and Botrychium Lunaria the erect stem, buried deep in the earth and growing very slowly in length, branches but rarely. Even the comparatively thick roots rarely branch, and it is not known whether the branching is then monopodial or dichotomous. [According to Holle, the roots have a trilaterally pyramidal apical cell. They do not branch in Ophioglossum, and in Botrychium their branches are probably produced laterally and not by dichotomy.] The flattened apex of the stem, surrounded by the insertions of the leaves, is buried deeply in the leaf-sheaths, and shows, in Ophioglossum vulgatum, according to Hofmeister, a threesided pyramidal apical cell as seen from above. The leaves have a sheathing base, and each is completely enclosed in the next older one, as shown in Fig. 289 in the case of Botrychium Lunaria. In Ophioglossum the relative positions of the parts at the end of the stem are still more complicated, from the fact that the rudimentary leaves, while completely enclosed one within another, produce stipular structures which grow together so completely that each leaf appears as if enclosed in a kind of chamber formed by the cohesion of the stipular parts of leaves of different ages, recalling a similar arrangement in Marattia. These cohesions however leave an opening at the apex of each chamber; the apex of the stem is therefore exposed to the air through a narrow canal (Hofmeister).

As soon as the plant has attained a certain age, each leaf bears a fructification, which forms a branch springing from the axial side of the leaf. In the genus Ophioglossum both the outer sterile and the fertile branch of the leaf are unbranched or only lobed; in the Brasilian $O$. palmatum the lamina is dichotomously lobed, and its margin bears on each side, as it joins the petiole, numerous fertile lobes or spikes of sporangia. In the genus Botry-

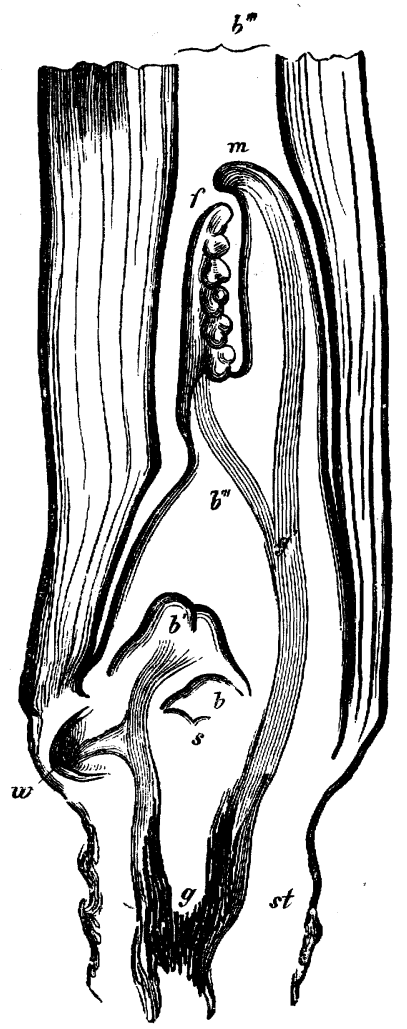

FIG. 289.- Longitudinal section through the lower part of a mature plant of Botrychium $L u$. naria. st stem, $g g^{\prime}$ fibro-vascular bundles, wa young root, $b$ apex of the stem, $b b^{\prime} b^{\prime \prime} b^{\prime \prime \prime}$ the four leaves already formed, $b^{\prime \prime \prime}$ the one unfolded during the present year: $b^{\prime}$ shows the first indication of the branching of the leaf; in $b^{\prime \prime}$ this has advanced further; $m$ is the median line of the sterile lamina, having already its lobes right and left which are not shown; $f$ the fertile lamina with the young branches, on which the sporangia will
the the young branches, on which
be produced ( $x$ about 10 ). chium both are branched and in parallel planes (Fig. 288, $A$ and $B$ ). The earlier hypothesis of a cohesion of the two leaf-stalks of a fertile and of a sterile leaf is at once negatived by the history of development (Fig. 289); the history of development rather indicates, as Hofmeister first showed, that the fructification originates on the inner side of the leaf. In the mature state the fertile leaf-branch either separates from the sterile (green) one at the base or at 
the middle of the lamina (O. pendulum), or the two branches of the leaf appear as if separated deep down to their origin (O. Bergianum), or, finally, the fertile branch springs from the middle of the leaf-stalk (Botrychium rutafolium and dissectum).

The Sporangia of the Ophioglossaceæ are so essentially different from those of Ferns and Rhizocarps that these plants cannot, for this reason, be arranged in either of these classes. They arise from several epidermal cells. The wall of the sporangium consists of several layers of cells, its outer limit being formed by the epidermis of the leaf itself. The mother-cells of the spores in Botrychium Lunaria

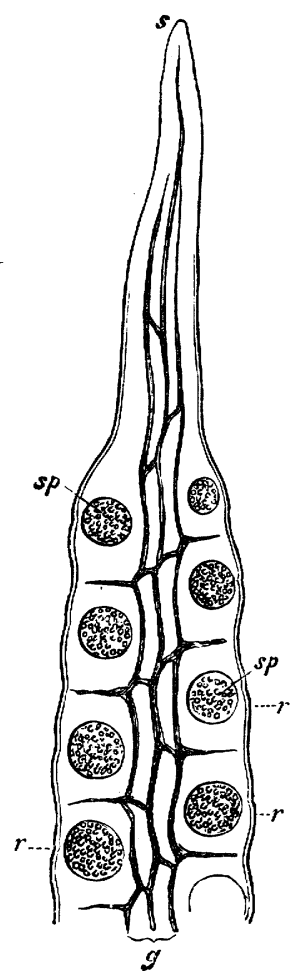

FIG. 290.-Longitudinal section through the upper part of a spike of Ophioglossum vulgatum; s its free apex, sp the sporanyial cavities, $r$ the part where they burst transversely; $g$ the fibrovascular bundles ( $X$ about 10 ). and probably also in Ophioglossum are derived, according to Goebel (loc. cit.), from a single central cell (archesporium) which is invested by peculiar cells (forming the tapetum) developed by the division of the surrounding cells of the sporangium. A longitudinal. section through the unripe so-called spike of $O$. vulgatum (Fig. 290) shows that the outer layer of the wall of the sporangium is a continuous prolongation of the epidermis provided with stomata and covering the whole of the fertile branch of the leaf. At the places where the lateral transverse line of dehiscence subsequently appears in each sporangium, these epidermal cells are elongated radially, and the whole layer exhibits an indentation at first scarcely perceptible. The spherical cavities which contain the masses of spores are imbedded in the tissue of the organ, and are therefore entirely surrounded by its parenchyma, there being several layers of it on the outer side where the transverse fissure subsequently arises. The middle part of the mesophyll is penetrated by fibro-vascular bundles which anastomose with one another into long meshes, and send out a bundle transversely between each pair of sporangial cavities. The course of development is the same in Botrychium, if the separate sporangiferous branches of the panicle are compared with the spike of Ophioglossum. The sporangia are similarly placed on them in two rows and alternate; only they project further because the tissue between each pair of sporangia is but slightly developed. Four spores are formed from each mother-cell. The mother-cell, after an indication of a division into two, divides into four segments, each surrounded by a delicate cell-wall. The protoplasm of each of these special mothercells becomes invested by a new wall, the true wall of the spore, and the primary walls become absorbed, so that the spores become free. In specimens of both genera preserved in spirit, the young spores, still connected together in fours, are found imbedded in a colourless, granular, coagulated mass of jelly, which in the living plant clearly corresponds to the fluid in which the spores of other Vascular Cryptogams float before they are ripe. The spores are tetrahedral; in Botrychium they are provided, even in a very early state, with knob-like projections on the cuticularised exospore. 
Among the Forms of Tissue of the Ophioglossacex, the prevailing one is parenchymatous fundamental tissue; there is no sclerenchyma. It consists, especially in the leafstalk, of long, almost cylindrical, thin-walled succulent cells with straight septa and large intercellular spaces; in the lamina the latter are, in $O$. vulgatum, very large, and the tissue spongy. In $O$. vulgatum and $B$. Lunaria, the epidermal tissue nowhere possesses special hypodermal layers; a well developed epidermis with numerous stomata on the upper and under side of the leaves immediately covers the outer layers of the fundamental tissue : at the periphery of the stem layers of cork are formed. The fibro-vascular bundles of $O$. vulgatum form, according to Hofmeister, a hollow cylindrical network in the stem, on which the leaves are arranged spirally, with a $\frac{2}{5}$ phyllotaxis; each of the meshes of this network corresponds to a leaf, and gives off to it the foliar bundles from its superior angle: the lower end of each foliar bundle terminates in a root. The leaf-stalk is penetrated by from 5 to 8 slender fibro-vascular bundles, which, in transverse section, are arranged in a circle, and between which the fundamental tissue forms wide lacunæ. Each of these bundles has on its axial side a strong fascicle of narrow reticulately thickened vessels, a broad fascicle of soft bast (phloëm) lying on their peripheral side. In the sterile lamina the slender bundles branch copiously and anastomose into a network; they run into the mesophyll which contains chlorophyll, without forming projecting veins. The slender stem of B. Lunaria has the same structure as that of Ophioglossum; its vascular bundles appear to be only the lower ends of the foliar bundles (Fig. 289), which are arranged in a circle in the stem and form a hollow fibro-vascular cylinder consisting of numerous xylem-bundles surrounded by a common investment of phloëm. In each leaf-stalk, which has a conical cavity below obliterated above, arise two broad ligulate bundles, which split above, below where the leaf divides into the fertile and sterile laminæ, into four narrower bundles. Each of these latter consists of a broad axial fascicle of tracheides thickened in a scalariform or reticulated manner, which is enveloped by a thick layer of phloëm. This layer shows an inner stratum of narrow cambiform cells, while the outside is formed of soft thick-walled bast-like prosenchyma (as in Pteris and other Ferns). In the lobes of the sterile lamina the bundles repeatedly split dichotomously, and run through the mesophyll without forming projecting veins.

The ground-tissue either forms no sheath round the fibro-vascular bundles of the leaves (Opbioglossum) or it forms a sheath consisting of collenchyma (Botrychium); the usual bundle-sheath of cells with sinuous walls appears to be wanting. According to Russow, the fibro-vascular cylinder formed in the stem of Botrycbium by the lower portions of the foliar bundles is surrounded by a sheath of this kind (Plerome-sheath). $\mathrm{He}$ also believes that the fibro-vascular bundles in the stem of Botrycbium undergo a slight subsequent growth in thickness. In the petiole of Opbioglossum I find, as Russow describes, that the thin fibro-vascular bundles have collateral phloëm and xylem and that the central xylem of Botrycbium is surrounded on all sides by phloëm.

[According to Russow, a formation of cork takes place at the surface of the rhizome of Ophioglosseæ; in this, so far as is known at present, they are unique among Vascular Cryptogams.]

Habit and Mode of Life. The number of leaves which appear each year is small, and constant in the species; thus $O$. vulgatum and $B$. Lunaria unfold only a single leaf annually, $B$. rutafolium two, a sterile and a fertile one; $O$. pedunculosum from 2 to 4 (Mettenius). The extremely slow development of the leaves is remarkable; in $B$. Lunaria each leaf requires four years, of which the three first are passed underground; in the second year the two branches (the sterile and fertile laminx) are formed, and further developed in the third; in the fourth year they for the first time rise above ground (Fig. 289), the process reminding one of the slow formation of the leaves of Pteris aquilina; the same occurs in $O$. vulgatum. In both genera the formation of the sporangia begins a full year before they ripen.

Vegetative Reproduction takes place in Opbioglossum by means of adventitious buds from the roots. $O$. pedunculosum is so far monocarpous that, after the production of fertile 
leaves, it as a rule dies down, but maintains a perennial existence by means of the rootbuds (Hofmeister). Most species are only, reckoning from the base of the stem to the apex of the leaf, 5 or 6 inches high; a few attain the height of a foot; B. lanuginosum of the East Indies is stated by Milde to be 3 feet high; the leaf is three or four times pinnate, and the stem contains from 10 to 17 fibro-vascular bundles.

Family 2. Marattiaceæ ${ }^{1}$. I. The Sexual Generation (Oophore). [The spores, which are of two forms, reniform and nearly spherical, germinate much in the same way as those of the Polypodiaceæ. In Angiopteris the first root-hair is developed at an early period, but in Marattia it does not make its appearance until some time after the commencement of germination, when the prothallium is already multicellular. The prothallium, like that of the true Ferns, is somewhat cordate and forms a flattened expansion upon the surface of the soil, but it is more fleshy and it is dark green in colour. Sometimes it grows by means of an apical cell, but this is not always formed. The antheridia are developed on either the under or the upper surface of the prothallium, from single superficial cells. The archegonia are developed in the same manner as those of the Polypodiaceæ, and more especially on the lower surface. The antheridia are not developed until some months after germination begins, and the archegonia still later.]

2. The Asexual Generation (Sporophore) when mature resembles a Fern in habit. The mode of its development is still unknown. It consists of a usually erect, short, thick, tubercular stem which bears large, closely-packed, spirally arranged leaves, with long petioles, the lamina being usually pinnatifid, but sometimes palmatifid. The resemblance to the true Ferns is rendered more striking by the circinate vernation of the leaves, and by their gradual unrolling from below upwards.

The Stem of Marattia, Angiopteris, and Dancea recalls on the whole the mode of growth of the stem of the Ophioglosseæ. It grows erect, but does not attain any considerable height. It is a tubercular mass, partially imbedded in the earth, and it is so completely covered with leaves that no portion of its surface is freely exposed. In some species it is small, but in the large Marattieæ and in Angiopteris evecta it may be from one to two feet high and broad in proportion. The stem of Kaulfussia assamica is a subterranean, creeping, bilateral rhizome, according to de Vriese, which bears leaves upon its upper and roots upon its under surface. It appears that the stem of the Marattiaceæ (except, according to Holle, in the case of Dancea trifoliata) never branches. The lower older portion of the stem is covered by the basal parts of the older petioles, bearing the stipules, from which the upper parts of the petioles, which at this point are provided with a large articular swelling, have become detached, leaving a smooth cicatrix encircled by the stipule (Fig. 29I, $n$ ). At the upper part of the stem, the still living leaves form a large rosette, in the centre of which lies a bud consisting of numerous young leaves of

${ }^{1}$ De Vriese et Harting, Monog. des Maratt. Leide et Düsseldorf. 1853.-Lürssen, Mittheilg. aus dem Gesammtgebiet der Bot., Bd. I. Heft. 3. 1872; id. Bot. Zeit. 1872, p. 768, and 1873 , p. 625.-Russow, Vergl. Unters. 1872, p. 105. Some information derived from drawings and letters communicated by Prof. Tschistiakoff has been embodied in the text. [Holle, Die Vegetationsorgane der Marattiaceen, Bot. Zeit. 1876. Jonkman, Entwickelung des Prothalliums der Marattiaceen, Bot. Zeit. 1878 .] 
different ages $(b, n b)$. The young leaves have a circinate vernation, and are completely invested by the stipules until the time when the petiole begins to elongate and the lamina to unroll itself. Each pair of stipules belonging to a petiole forms, as is shown in Fig. $29 \mathrm{r}, A$ and $B$, an anterior and a posterior chamber, which are separated by a longitudinal wall (commissure). In the posterior chamber lies the rolled-up leaf, to which the stipules actually belong, the two posterior wings of the stipules extending round it. The chamber formed by the anterior wings of the stipules encloses the group of young leaves. This is the arrangement in Angiopteris, and it appears from herbarium specimens to obtain also in Dancea, and from
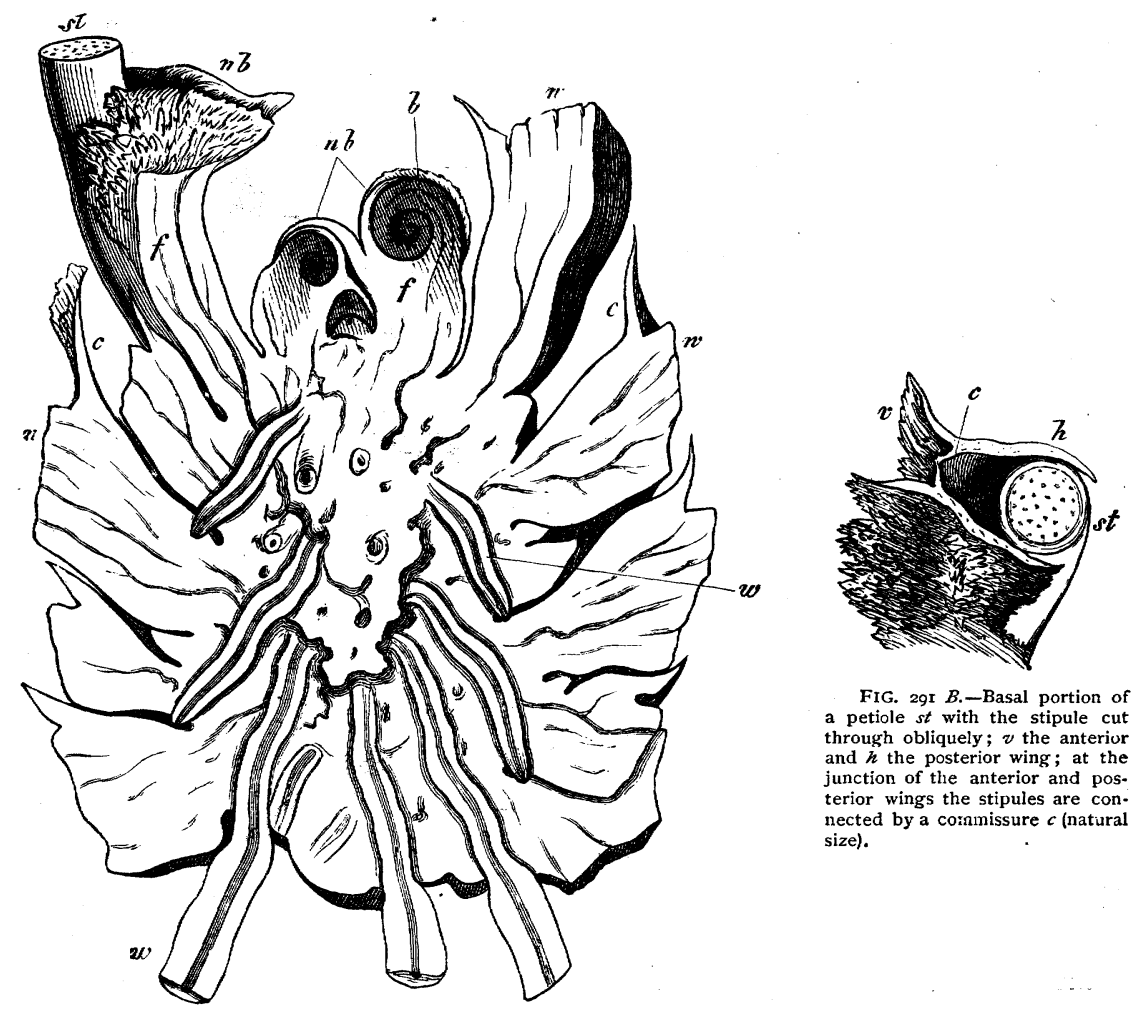

FIG. 29I B.-Basal portion of a petiole st with the stipule cut through obliquely; $v$ the anterior and $h$ the posterior wing; at the junction of the anterior and pos. terior wings the stipules are con. terior wings the stipules are con-
nected by a cotamissure $c$ (natural size).

FIG. 291 $A$.-Vertical section of the stem of a young Angiopteris evecta : above are the youngest leaves $(b)$ still completely surrounded by the stipules $n b$; st petiole of an unfolded leaf with its stipule $n b ; n$, in every case the cicatrix on the basal portion of the petiole $f f$, from which the upper portions have separated; $c c$ the comnissures of the stipules in vertical section; $w w$ roots (natural size).

drawings in Marattia. Harting's representation of the stipules is quite erroneous. These peculiar stipules remain fresh and succulent not only during the life of the leaves but also after they have fallen, and adventitious buds may originate from them.

The roots arise, as is shown in Fig. $29 \mathrm{I}, A$, in the tissue of the stem immediately below the growing-point. One arises apparently at the base of each young leaf. They grow obliquely through the succulent parenchyma of the stem and of the older basal portions of the leaves, and finally reach the surface at a 
lower level between them or through the cicatrix of a leaf. They are not so. numerous as those of most true Ferns, and they differ from them in their light colour, their more delicate structure and their greater thickness, peculiarities which they share with those of Ophioglosseæ. They ramify considerably in the soil, apparently in a monopodial manner.

The leaves which in the smaller species attain a height of from one to two feet, in the largest (Angiopteris) of from five to ten feet, have a long firm petiole, channelled on its inner surface, which bears the compound lamina which is either pinnate or bi-pinnate, or palmate as in Kaulfussia. The primary petiole is attached to the basal portion by means of an articular swelling, and the secondary petioles are connected with it, the leaflets with their rachis, in the same manner, just as is the case in the Leguminosæ.

The Marattiaceæ differ from the glabrous Ophioglosseæ in that they are hirsute, but not nearly so much so as the true Ferns.
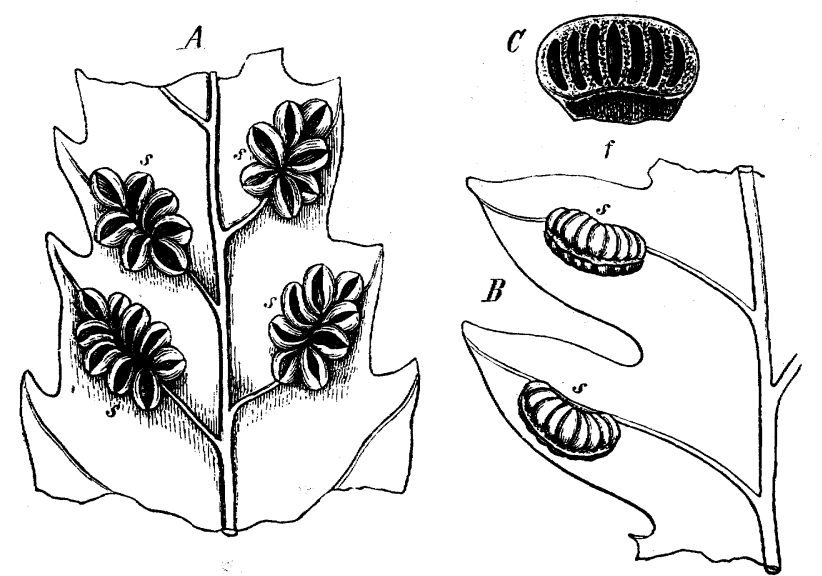

FIG. 292. $-A$ under surface of the upper part of a leaflet of Angiopteris caudata, with sori $s s$. $B$ some teeth of the margin of the leaf of Marattia $s p$. with sori $s$, . C half a sorus with opened sporangia (chambers).

The Sporangia of the Marattiaceæ are developed in considerable number on the underside of ordinary leaves which have undergone no further modification. Like those of the majority of true Ferns they are borne upon the veins of the leaves, and are usually arranged in two rows forming sori, which either cover the veins running from the midrib to the margin of the leaflet throughout their whole length (Dancea), or only for a short distance near the margin (Angiopteris, Marattia); in Kaulfussia they are placed upon the delicate anastomosing branches of the veins. The sorus is borne upon a cushion-like outgrowth of the tissue of the vein, the placenta. In Angiopteris alone are the individual sporangia free from each other; they are ovoid and sessile, and when mature they open by a longitudinal slit on their internal surface. If the sporangia of each row of the sorus be imagined to have become coherent, and the two rows to have become attached by their adjacent surfaces or to have completely coalesced, a structure results which actually occurs in Marattia (Fig. 292, $B, C$ ). This structure might well be regarded as a sporangium with numerous chambers arranged in two rows 
if the history of its development alone decided the question, and then it would offer no analogy to the same structure in Angiopteris. But analogy clearly indicates that in Marattia we have not to do with a multicellular sporangium but with a sorus, the individual sporangia of which have become united. Like those of Angiopteris, each of these sporangia opens by a longitudinal slit upon its inner surface. It is of but little importance for this interpretation that the apparently multicellular sporangium, which we regard as a coalesced sorus, is borne in Eupodium (Mar. Kaulfussii) on a stalk of considerable height, for the sorus in many of the true Ferns (Cyathea, Thyrsopteris) is also stalked. It can scarcely be doubted therefore that the multicellular fructification of Marattia is a coalesced sorus, and the same holds good also for Kaulfussia and Danca. In Kaulfussia the sporangia of a sorus (from eight to twenty in number) are arranged in a circle and are united to form a many-chambered ring. Each opens on its inner side by a longitudinal slit. This arrangement is even more striking in Danæa, where the united sporangia form two long rows covering the vein bearing them throughout its whole length, and where each chamber (sporangium) opens at its apex. The sorus is usually surrounded by flattened lobed hairs forming a kind of indusium, which, in Danca, appears like a kind of cup in which the sorus lies. Luerssen's argument that these outgrowths of the epidermis are not to be regarded as an indusium because they occur elsewhere upon the leaves and are therefore merely hairs, is not valid, for the indusium of the true Ferns is a hair-like outgrowth, and must be regarded as a trichome. As in the Ferns, so in the Marattiaceæ, the indusium does not occur in all species.

The development of the sori has been studied by Luerssen and by Goebel in Marattia, and by these observers and by Tschistiakoff in Angiopteris. In both cases the placenta arises as a cushion-like protuberance from the fertile vein of the epidermis and the subjacent tissue. In Angiopteris two separate rows of papillæ make their appearance upon the receptacle, each of which consists from the first of a group of cells derived from a group of the superficial cells of the placenta. Each papilla becomes one of the free sporangia of the sorus. In very young sporangia Tschistiakoff was able to detect an internal cell (archesporium) surrounded by two or three layers of cells which gave rise by repeated division to a group of spore-mother-cells. In Marattia two parallel swellings appear on the placenta, which soon become separated by a deep and narrow fold. In each of these swellings a row of cell-groups, the mother-cells of the spores, are differentiated, which have been formed by the division of the archesporium. Each of these groups corresponds to a sporangium, the walls of adjacent sporangia coalescing from the first. The inner surfaces of the two parallel swellings approach each other more and more closely as development proceeds, but they separate widely when the spores are ripe, so that the multilocular fructification splits longitudinally into two halves, and the loculi of each half open by vertical slits upon their inner surfaces.

The development of the spores, four from each mother-cell, differs but little from that of the Ophioglosseæ and the Ferns. It is important to note that in the Marattiaceæ the wall of the mature sporangium consists of several layers of cells, whereas in the Ferns it consists only of one. 
Histology. As a peculiarity of the epidermal tissue the very large, widely-open stomata of the leaves of Kaulfussia may be mentioned. They are developed in the usual way, but they soon become remarkable on account of the extraordinary size of the aperture and of the arrangement of the guard-cells in a narrow ring, surrounded by two or three rings of epidermal cells (Luerssen).

In the intercellular spaces of the parenchymatous ground-tissue of the leaves Luerssen found outgrowths from the walls of the surrounding cells. Where the spaces were small these outgrowths assumed the form of bosses or pegs, but where the spaces were large they were long thin filaments. They are quite solid and consist of cuticularised cell-membrane. The large intercellular spaces are quite filled with a felt-work of these filaments. Luerssen found this to be the case in Kaulfussia, Danca, Angiopteris, Marattia.

In the ground-tissue of the leaves bands and bundles of sclerenchyma are differentiated, but it is not so hard or so darkly-coloured as that of Ferns. In the articular swellings collenchyma is developed. Elongated cells containing tannin are to be found in all parts of the ground-tissue, and gum-ducts are scattered throughout the thin-walled parenchyma. Reference has been made on page 64 to the Sphærocrystals.

In the stem of Angiopteris, which I have investigated, there is no sclerenchyma.' It consists for the most part of large thin-walled parenchymatous cells, amongst which are scattered very numerous cells containing tannin, as well as gum-ducts. The contents of the latter cover a piece of the stem when placed in water with a thick layer of gelatinous mucilage.

The fibro-vascular bundles of the leaves and of the stem resemble those of the Ferns. The central xylem consisting of wide scalariform tracheides is surrounded by a layer of phloëm. In the leaf the bundles (of Angiopteris) are usually flattened, in the stem they have a circular outline. The usual bundle-sheath, consisting of a single layer of cells with a peculiar folding on their adjacent walls, which is especially constant in the Ferns, is absent in Marattia and Angiopteris from the fibro-vascular bundles both of the leaf and of the stem, but it is present in Danca. In the root it is present, and consists of large cells. Harting has described the roots which traverse the parenchyma of the stem (Fig. $29 \mathrm{I}$ $A, w)$ as fibro-vascular bundles, and has figured them on Plate VII. figs. 3 and 4 of his Monograph of the Marattiacex. He did not investigate the structure of the real fibrovascular bundles at all. It is necessary to draw attention to this mistake because Russow, relying upon Harting, describes the fibro-vascular bundles of the stem as possessing an external sheath (Scbutzscheide), and states that this structure occurs only in the roots which traverse the stem. It is difficult to imagine how Russow could have overlooked this obvious mistake of Harting's. It is by no means easy (in Angiopteris) to obtain a transverse section of one of the fibro-vascular bundles of the stem, for they are very irregularly curved and are everywhere covered with roots which traverse the network formed by the bundles. As I had only one stem at my disposal I was unable to satisfy myself as to the true form of the fibro-vascular system, but it appears that Harting's figure is not very true to nature. The numerous bundles which bend outwards into each leaf are formed by the division in the lower part of the petiole of the few bundles which spring from the fibro-vascular network of the stem (Fig. 29 I $A$ ).

According to Holle, the stem of Marattia grows by means of a four-sided apical cell. In the stouter roots, according to Harting and Russow, the place of the apical cell is taken by a layer of very large cells. In the slender roots of Marattia and Angiopteris Holle has found a four-sided apical cell. 


\section{Order II. Filices ${ }^{1}$.}

The Sexual Generation (Oophore) or Prothallium of Ferns is a thalloid body containing chlorophyll and obtaining its nourishment independently; its development presents striking resemblances to that of the simpler Hepaticæ, and to a certain extent even to the formation of the protonema of some Mosses. It produces simple tubular unarticulated root-hairs, and finally antheridia and archegonia. Its development and the duration of its life may embrace a considerable space of time, especially when the archegonia are not fertilised.

When the spores germinate, which usually does not take place till a considerable time after dissemination (but in Osmunda after only a few days), the cuticularised exospore, generally provided with ridges, bosses, spines, or granulations, splits along its edges; the endospore, which now protrudes and is not unfrequently already divided by septa, produces the prothallium, either immediately, as in Osmunda, or after the preliminary formation of a filamentous protonema, which presents in Hymenophyllaceæ certain resemblances to that of the Andreæaceæ and of Tetraphis among Mosses. The development of the prothallium has been more exactly investigated only in the Hymenophyllaceæ, the Polypodiaceæ, and also in Osmunda and Aneimia ${ }^{2}$; and the considerable differences which have thus been established necessitate separate descriptions.

In the Hymenophyllaceæ the contents of the spore are divided, even before germination, into three cells meeting in the centre; in some species of Trichomanes small cells are cut off at three points of the circumference, while a large central

1 H. von Mohl, Ueber den Bau des Stammes der Baumfarne (Verm. Schriften, p. Io8).-Hofmeister, Ueber Entwickelung und Bau der Vegetationsorgane der Farne (Abhandlungen der königl. Sächs. Gesells. der Wissen. 1857, vol. V).-Ditto, Ueher die Verzweigung der Farne (Jahrb. für wissen. Bot. vol. III. p. 278).-Mettenius, Filices Hort. Bot。 Lipsiensis (Leipzig 1856).-Ditto, Ueber die Hymenophyllaceen (Abhandlungen der königl. Sächs. Ges. der Wissen. I864, vol. VII).-Wigand, Botanische Untersuchungen (Braunschweig 1854). - [On the Germination, Development, and Fructification of the Higher Cryptogamia, \&c. Ray Society, 1862, pp. 1 28-266.]-Dippel; Ueber den Bau der Fibrovasalstränge, in the Berichte deutscher Naturforscher u. Aerzte in Giessen, $\mathbf{1}^{8} 6_{5}$, p. I42.-Reess, Entwickelung des Polypodiaceensporangiums (Jahrb. für wissen. Bot. I866, vol. V. p. 5).-Leszczyc-Suminski, Zur Entwickelungsgeschichte der Farnkräuter, I848.-Strasburger, Befruchtung der Farnkräuter (Jahrb. für wissen. Bot. 1869, vol. VII. p. 390).-Kny, Ueber Entwickelung des Prothalliums und der Geschlechtsorgane, in the Sitzungsberichte der Gesellschaft naturforschender Freunde in Berlin, Jan. 2 I and Nov. I 7, 1868.-Kny, Ueber Bau und Entwickelung des Farnantheridiums (Monatsberichte der kais. Akad. der Wissen. Berlin, May 1869).-Kny, Beiträge zur Entwickelungsgeschichte der Farnkräuter (Jahrb. für wissen. Bot. vol. VII. p. I).Russow, Verg1. Unters. Petersburg 1872.-Janczewski, Ueber die Archegonien. Bot. Zeit. 1872, p. 418.- - On the development of the prothallium, see also Bauke, Keimungsgeschiche der Schizæaceen, Jahrb. f. wiss. Bot. XI; Burck, Dévelop. du prothalle des Aneimia, Arch. Néerlandaises, X; Bauke, Entwick. d. Prothalliums bei den Cyatheaceen, Jahrb. f. wiss. Bot. X ; Goebel, Entwick. d. Prothalliums von Gymnogramme leptophylla, Bot. Zeit. 1877; Janczewski et Rostafinski, Le prothalle de l'Hymenophyllum Tunbridgense, Mém. soc. nat. d. sci. natur. de Cherbourg, XIX ; Prantl, Die Hymenophyllaceen, I 875.]

2 Although the Osmundaceæ, Schizæaceæ, and Gleicheniaceæ probably constitute a group apart from the Polypodiaceæ and Hymenophyllaceæ, I introduce here what little is known concerning them, for our knowledge is not sufficient to permit of any but an imperfect account of them being given. Where it is not expressly stated to be otherwise, the descriptions given above refer to the Polypodiaceæ and Cyatheaceæ. 
cell remains undivided. The cells develope into germinating filaments, bursting the exospore in three directions; these filaments then grow at their apex, and become segmented by septa; only one of them however generally attains a more decided development, the others soon assuming the form of hairs. In Hymenophyllum Tunbridgense this one frequently developes at once into a cellular plate; but in other species it forms a much-branched conferva-like protonema, on which flat prothallia 2 to 6 lines in length and $\frac{1}{2}$ to $1 \frac{1}{2}$ in breadth are formed as lateral shoots. Each cell of the filament may give rise to a branch which is given off behind the anterior septum, and is at once separated by another septum. . Some of these branches continue to grow indefinitely like the mother-shoot, others end in becoming hairs; a larger number are transformed into flat prothallia, but most develope into root-hairs. Here and there the rudiment of a filamentous branch becomes converted into an antheridium, or even into an archegonium. At the apex of the flat prothallia spherical cells arise in Trichomanes incisum on marginal flask-shaped cells : these must probably be considered as organs of propagation; but the marginal cells of the flat prothallia may develope into root-hairs and new protonemal filaments, and also into new flat shoots. The root-hairs are mostly short, with brown walls, and produce at their end lobed attaching-discs or branching tubes.

In the Polypodiaceæ and Schizæaceæ the endospore developes into a short articulated filamentous protonema, at the end of which, even at an early stage, a more or less considerable increase in breadth takes place; a plate of tissue is thus formed consisting at first of only one layer, which soon assumes a broadly cordate or even reniform shape, and has its growing apex situated in an anterior depression. Its apical cell forms two rows of segments right and left, by walls which are perpendicular to the surface, and from their further divisions the flat tissue is produced. The power of rejuvenescence of the apical cell is, however, limited; it ends in the formation of a septum by which a new apical cell is formed, which then divides by longitudinal walls, and thus forms a row of apical cells lying side by side which occupies the bottom of the depression of the prothallium-disc, in the same manner as in the thallus of Pellia. The root-hairs are all lateral structures, springing in large numbers from the under-side of the posterior part of the prothallium; among them are the antheridia, which in this case are only rarely marginal. The archegonia are also produced on the under-side, but on a cushion behind the anterior depression formed of several layers; in Ceratopteris several cushions are formed bearing archegonia.

Osmunda (examined minutely by Kny, and compared with the preceding, l.c.) is distinguished in the first place from the Polypodiaceæ and Schizæaceæ by the absence of the protonematous filament. The endospore undergoes divisions at the very commencement of germination, which form a plate of tissue of which a posterior cell is converted, as, in Equisetaceæ, into the first root-hair. The succeeding root-hairs arise from marginal cells and on the under-side of superficial cells of the prothallium, the apical growth of which follows a similar course to that of Polypodiaceæ. The mid-rib consisting of several layers is characteristic of Osmunda, penetrating the ribbon-like prothallium from the posterior end to the apex, and producing a large number of archegonia on both sides. The antheridia spring partly from the margin, partly from the lower surface with the exception of the mid-rib. 
Like many thalloid Hepaticæ, the prothallia of Ferns also produce adventitious shoots from single marginal cells ${ }^{1}$; this happens with especial profusion in Osmunda, where the adventitious shoots become detached, and play the part of vegetative organs of reproduction.

The prothallia show a tendency to be diœcious, which is manifested in the fact that all the spores from a sporangium sometimes produce prothallia bearing antheridia only (as in Osmunda regalis); while in other cases the archegonia appear later and in smaller numbers, and are fertilised by the antheridia of younger prothallia.

The Antheridia are, speaking morphologically, trichomes; they are produced in the same manner as the root-hairs, as outgrowths of the marginal or superficial cells of the prothallia; in the Hymenophyllaceæ they are also produced on the protonemal filaments. The projection is usually separated from the mother-cell by a septum, and swells up spherically at once or after the formation of a pedicel. In some cases the mother-cells of the antherozoids are formed at once in this globular cell; but it usually undergoes still further divisions ${ }^{2}$, in consequence of which the wall of the antheridium consists of a single layer of cells surrounding the central cell. Within the cells of this layer chlorophyll-granules are formed towards their inner wall, while the central cell of the antheridium divides further into the mother-cells of the antherozoids, which, however, are not numerous. The dehiscence of the ripe antheridium is the consequence of a rapid absorption of water in the parietal cells, which swell up violently and compress the contents of the central cell till the antheridium is ruptured at the apex. The antherozoid-cells thus escape, and out of each of them is set free an antherozoid coiled spirally three or four times. The finer anterior end of each antherozoid is provided with a number of cilia; the thicker posterior end often drags with it a vesicle furnished with colourless granules, which subsequently falls off and remains at rest, while the filament alone continues in motion. Strasburger states that this vesicle is formed from a central part of the contents of the mother-cell, the parietal protoplasm of which forms the filament and its cilia. The vesicle is hence properly not a part of the antherozoid; it is only attached to it, and swells up strongly in water by endosmose, as is shown in Fig. 293.

The Archegonium arises from a single superficial cell of the prothallium, which is at first only slightly arched and is divided by two walls parallel to the upper

1 [On the formation of gemmæ by Fern-prothallia, see Cramer, Ueb. geschlechtslose Vermehrung des Farn-prothallium, Basel r 88r.]

${ }_{2}$ These divisions take place in a very remarkable manner. In the hemispherical mother-cell of the antheridium of Aneimia hirta an arched wall arises, by which it is divided into an inner hemispherical cell, and an outer one which covers the former like a bell; the latter is then split up by a transverse annular wall into an upper lid-like and a lower hollow cylindrical cell. The same thing occurs in Ceratopteris; in other cases, as in Asplenium alatum, a funnel-shaped wall is formed in the hemispherical mother-cell of the antheridium, the wide end of the funnel being directed towards the upper surface of the mother-cell, and the upper part of the mother-cell is cut off by a transverse septum as a covering cell; two, or even three, funnel-shaped walls may be formed in succession, so that the parietal layer of the antheridium consists of two or three superposed cells forming its circumference and a covering cell (as in Fig. 293). The mode of formation of the antheridium-wall is quite different in Osmunda, where it consists below of two or three cells, upon which rest several of the upper cells which result from the division of the covering cell (Kny, l. c.).-[See also Strasburger, Theilungsvorgänge in den Antheridien der Farne; Zellbildung und Zelltheilung, zrd edition.] 
surface. The lowest of the three cells thus formed, which Janczewski calls the basal cell (Fig. 294, below $e$ ), subsequently divides in the same manner as the cells of the surrounding tissue, and thus contributes to the formation of the ventral wall of the archegonium which is completely embedded in the tissue of the prothallium. The most external of the three primary cells gives rise to the wall of the neck of the archegonium (Fig. 294, $A, h h$ ), by dividing crosswise into four cells from which the

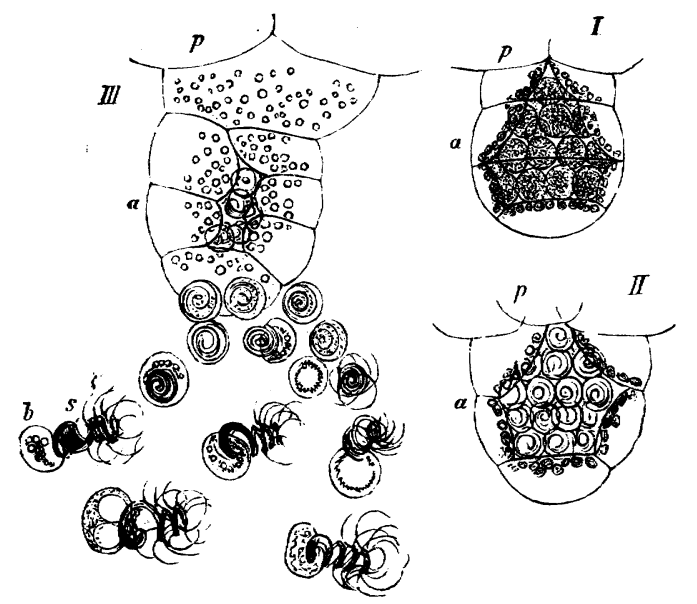

\footnotetext{
FIG. 293.-Antheridia of Adiantum Capillus-Veneris ( $\times 550$ ), in longitudinal optical section ; $I$ not yet ripe ; $I I$ the antherozoids alr ady mature; $I I I$ the antheridium burst, the parietal cells greatly swollen radially, the antherozoids mostly escaped; $p$ prothallium, $a$ antheridium, $s$ antherozoid, $b$ the vesicle containing starchgrains.
}

four rows of cells of which the neck of the archegonium consists are produced by oblique divisions. The anterior wall of the neck (that is, the wall which is directed towards the apex of the prothallium) grows more rapidly than the opposite wall and
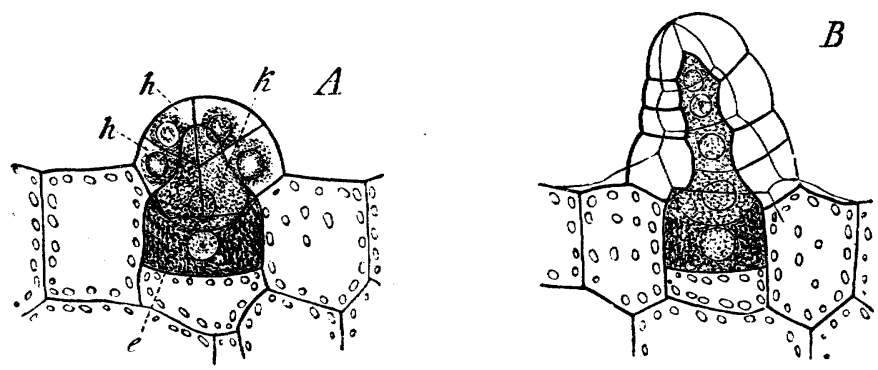

FIG. 294.-Young archegonia of Pteris serrulata (after Strasburger); $e$ the central cell, $h h$ the neck, $k$ the canal-cell.

becomes convex. Accordingly, the number of cells in the anterior row is larger than that of the posterior row, in the former it is usually six, in the latter, four.

From the middle one of the three primary cells the central cell and the canalcell of the neck are derived, that is, the axial row of cells of the archegonium. Whilst the wall of the neck is being formed, this middle cell becomes pointed above and penetrates between the cells of the neck (Fig. 294, $A$ ); the pointed portion is 
then cut off by a transverse septum and forms the single canal-cell of the neck, which elongates with the growth of the neck and fills its cavity. According to Strasburger a tendency to division, which however does not actually take place, is indicated (Eig. 294, $B$ ) by the appearance in the neck-cell of several nuclei, a view which is opposed by Janczewski. According to the latter observer the large centralcell divides into an upper small cell, the ventral canal-cell (Fig. 295, B, s), and into a lower much larger cell, the oosphere, which subsequently rounds itself off. The walls of the canal-cells swell up, become mucilaginous, and finally the watery mucilage together with the protoplasm of the canal-cells is forced out of the opened neck. The antherozoids are retained by this mucilage and collect in large numbers before the archegonium; a number force themselves into the canal of the neck, often finally stopping it up; a few reach the oosphere, force themselves into and disappear in it.

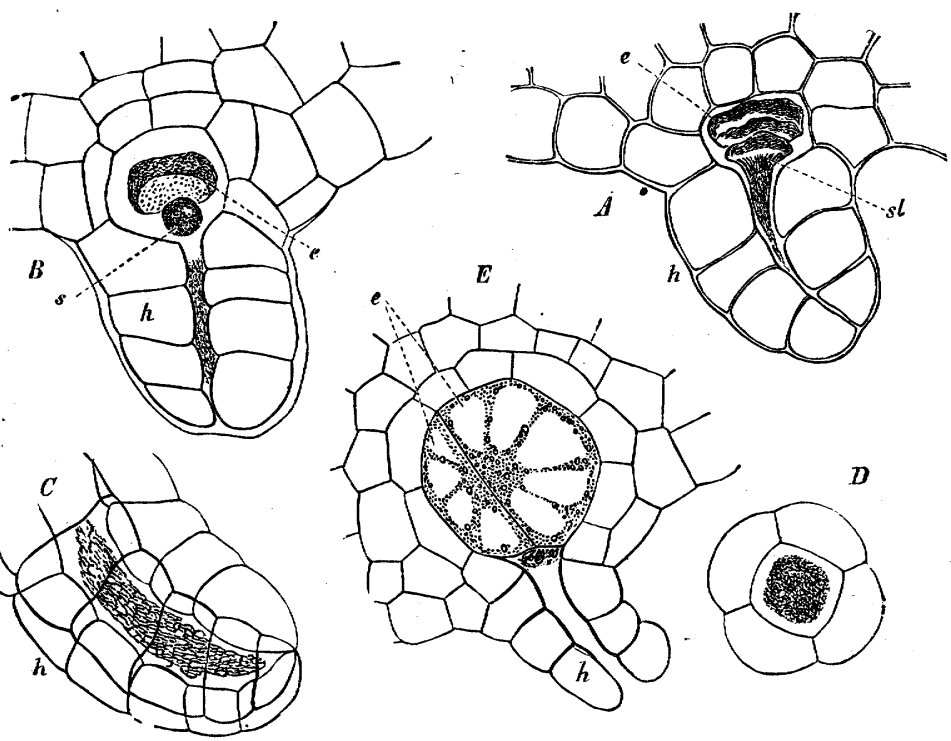

FIG. 295.-Archegonia of Adiantum Capillus.Veneris $(\times 800) ; A, B, C, E$ in longitudinal optical section; $D$ in transverse optical section; $A, B, C$ before, $E$ after fertilisation; $h$ neck of the archegonium, st mass of mucilage, $e$ oosphere; $E, e$ the two-celled embryo (observed after lying one day in glycerine).

The entrance takes place at a lighter spot of the oosphere facing the neck, which is termed the Receptive Spot ${ }^{1}$ (compare the oogonia of Algæ). After fertilisation the neck closes.

The Asexual Generation (Sporophore) or Fern (as it is popularly termed) is developed from the oospore or fertilised oosphere of the archegonium ${ }^{2}$. At first the surrounding tissue of the prothallium keeps pace with the increase of the embryo, so

1 Strasburger states that the act of fertilisation may be observed especially clearly in Ceratopteris; the forcible entrance of the antherozoids as far as the oosphere had previously been seen by Hofmeister.

${ }^{2}$ [From the researches of Farlow (Quart. Jour. Micr. Sci. 1874) and of De Bary (Bot. Zeit. I878) the sporophore is frequently developed from the oophore in some Ferns (Pteris cretica, Aspidium falcatum) without the intervention of sexual organs. The young Fern is produced as a bud from certain cells of the prothallium. De Bary terms this mode of development Apogamy.] 
that this latter remains for some time enclosed in a protuberance springing from the under surface, until the first leaf and root break through. The first processes of division of the oospore are, as Hofmeister has shown in the case of Pteris aquilina and Aspidium Filix-mas, not entirely alike in different Ferns ${ }^{1}$. It is certain, however, that the first division-wall (called the basal wall) of the oospore is transverse to the longitudinal axis of the prothallium, and inclined to it obliquely; as shown in Fig. 295, $E$, its inclination is the same as that of the neck of the archegonium. It is also certain that each of the two daughter-cells is at once divided again by a wall in the plane of the prothallium (called the transverse wall), so that the embryo now consists of four cells placed as quadrants of a sphere, and these are further divided by a wall parallel to the long axis of the prothallium (called the median wall). In Fig. 296 these first transverse divisions are indicated by thicker lines, the embryo being seen in longitudinal section. The explanation of the figure points out the interpretation which Hofmeister gives to the first four cells of Pteris aquilina, which the reader may compare with the corresponding development of Salvinia and Marsilia; but it must not be forgotten that the embryo of the Fern lies, so to speak, on its back. Although it is impossible in this place to go into a more

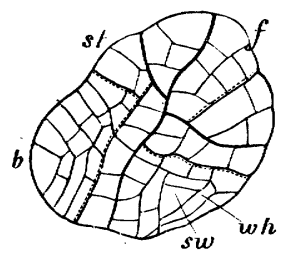

FIG. 296.-Vertical longitudinal section of the embryo of Pteris aquilina (after Hofmeister, Entwickelung und Bau der Vegetationsorgane der Farne, p. 607); the thicker lines are sections of the first two division-walls by which the embryo is divided into four cells (the continuous thick line represents the basal wall). The lower anterior cell forms the leaf $b$; from the upper anterior is derived the stem $s t$; from the lower posterior cell is produced the root, sw being its apical cell and wh its root-cap; the foot $f$ is formed from the upper and posterior of the first four cells.

minute description, it is still necessary at least to point out that a close resemblance exists between the embryo of Ferns and that of Rhizocarps.

[The embryo now consists of eight cells. Of the four octants which lie in front of the basal wall (the epibasal half of the embryo) the two upper (i.e. those nearest the neck of the archegonium) give rise the one to the growing point of the stem, the other to trichomes, from the two lower octants the first leaf (cotyledon) is developed. Of the four octants which lie behind the basal wall (forming the hypobasal half of the embryo), the upper two form the foot; and of the lower two, the one which is diametrically opposite to that which forms the stem gives rise to the root, and the other is gradually suppressed.]

1 [On the embryology of Ferns, see Kny, Keimung und Entwickel. von Ceratopteris, Bot. Zeit. 1874, and Die Entwickelung der Parkeriaceen, Nov. Act. Acad. Leop. Carol. 1875.-Vouk, Die Entwick. des Embryo bei Asplenium Shepherdi, Sitzber. d. Wien Akad. 1877.-Leitgeb, ibid. 1878, Zur Embryologie der Farne.-Kienitz-Gerloff, Entwickelung des Embryo bei Pteris serrulata, Bot. Zeitg. 1878.-A good summary is given by Sadebeck in Schenk's Handbuch, vol. I. Compare also the accounts given of the embryology of Equisetum, Marsilia, Salvinia, and Selaginella, as also of Muscineæ: further, Goebel, zur Embryologie der Archegoniaten, Arb. d. bot. Inst. in Würzburg, II. $3,1880$. 
The foot is an organ by which the embryo attaches itself to the tissue of the prothallium, in order to draw nourishment from it while the first roots and leaves are being formed. The first parts of the stem and the roots and leaves, which are now developed in succession from the embryo, are very small, and remain so; those which are formed later are gradually larger. The leaves become constantly more complex in form, and the structure of the stem more intricate as the new portions formed by its growth in length increase in diameter. The first parts of the stem, like the first leaf-stalks, contain each only one axial fibro-vascular bundle; the later ones a larger number when both stem and leaf-stalk have attained a considerable thickness. In this manner the Fern continues to gain strength, not by subsequent increase of size of the embryonic structures, but by each successive part attaining a more considerable size and development than the preceding ones; until at length a kind of stationary condition is arrived at in which the newly-formed organs are nearly similar to the preceding ones. The following observations refer especially to this mature condition of Ferns.

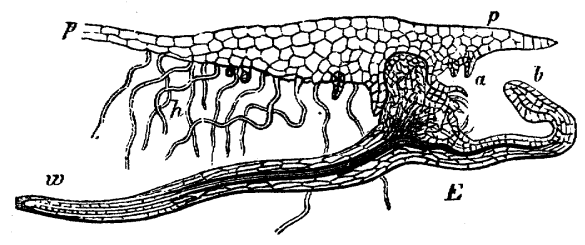

FIG. 297.-Adiantum Capillus.Veneris; vertical longitudinal section through the prothallium $p p$ and the young Fern $E$; $h$ root-hairs, $a$ archegonia of the prothallium, $b$ the first leaf, $w$ the first root of the young plant (X about ro).

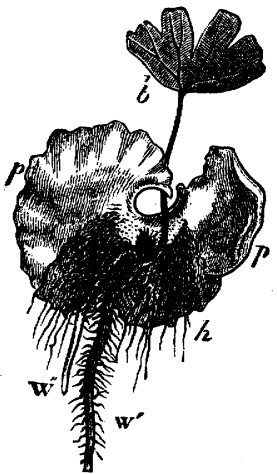

FIG. 298.-Adiantum Capillus-Veneris; the prothallium $p p$ seen from below with the young Fern attached to it; $b$ its first leaf; $w^{\prime} w^{\prime \prime}$ its first and second roots; $h$ root-hairs of the prothallium ( $X$ about 3o).

The mature Fern is, in some Hymenophyllaceæ, a small delicate plant, not much exceeding in dimensions the larger Muscineæ; in other sections the fully grown plants attain the size of considerable shrubs; some species, natives of the Tropics and of the Southern Hemisphere, assume even a palm-like habit, and are called Tree-ferns. The stem creeps on or beneath the ground (as in Polypodium and Pteris aquilina), or climbs up rocks and stems; in some it ascends obliquely (e.g. Aspidium Filix-mas); in Tree-ferns it rises up vertically in the form of a column. The roots are usually very numerous; in Tree-ferns the stem is often entirely covered by a dense mantle of them. They arise on the stem in acropetal succession; sometimes close to the growing apex of the stem (as in Pteris aquilina). When the internodes remain very short, and the stem is entirely covered with the bases of the leaves, the roots arise, as in Aspidium Filix-mas, from the leafstalks. In many Hymenophyllaceæ which have no true roots, branches of the stem assume a root-like structure. In creeping and climbing species the leaves are separated by distinct internodes which are sometimes very long; in thick, ascending, 
and vertical stems, the internodes are usually undeveloped, and the leaves so crowded that no free portion of the stem remains uncovered, or only a very inconsiderable one $^{1}$. The leaves of Ferns are usually characterised by a circinate vernation, and they only unroll in the last stage of their growth; the mid-rib and the lateral veins are curved from behind forwards. The forms of the leaves are among the most perfect in the whole vegetable kingdom; they manifest an enormous variety in their outline, the lamina being usually deeply lobed, branched, or pinnate. In comparison with the stem and the slender roots they are mostly very large, and sometimes attain extraordinary dimensions, even a length of from 6 to ro feet (as in Pteris aquilina and Cibotium). They are always stalked, and continue their growth at the apex for a long time; the leaf-stalks and the lower parts of the lamina are often completely unfolded while the apex is still growing (as in Nephrolepis). This apical growth is not unfrequently interrupted periodically (vide infra); in Lygodium

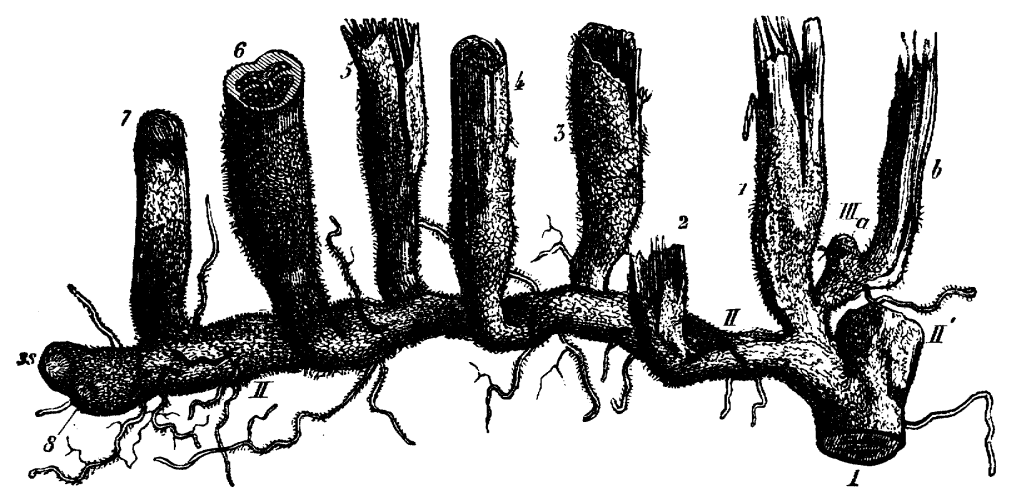

FIG. 299.-Pteris aquilina, a part of the underground stem with leaves and bases of the leaf-stalks (reduced about onehalf); $I$ older portion of the stem bearing the two bifurcations $I I$ and $I I^{\prime}$, ss the apex of the weaker branch $I I$; beside it the youngest leaf-rudiment $8 ; x-7$ the leaves of this branch, one being developed in each year; $1-5$ the leaves of earlier years, which have already died off at some distance from the stem; 6 the leaf of the present year with unfolded lamina, the stalk having been cut off; 7 the young leaf for next year; at the apex of the stalk is the lamina still very small and entirely clothed with hairs. The
culd leaf-stalk $I$ bears a bud $I I I a$, which has developed a leaf $b$ that has already died off. The more slender filaments are roots. All the parts shown in the figure are subterranean.

the leaf-stalk or the rachis resembles a twining stem with long-continued growth, the pinnæ presenting the appearance of leaves. The amount of metamorphosis of the leaves is, notwithstanding, very inconsiderable; on the same plant the same forms of leaves, mostly foliage-leaves, are constantly repeated; scale-like leaves occur on underground stolons (e.g. in Struthiopteris germanica), and in many cases the fertile leaves (those which bear sporangia) assume special forms. Such differences as occur in most Phanerogams are not found in the development of the leaves of one plant; Platycerium alcicorne must, however, be mentioned, as having the foliageleaves alternately developed as broad plates closely applied to the supporting surface and as long dichotomously branched ribbon-shaped erect leaves.

Among the various forms of trichomes of Ferns those termed Ramenta are

1 From the change of form and of size presented by the older cicatrices Brongniart concluded that the stems of the Tree-ferns continue to grow in length (and in thickness?) for some time after the leaves have fallen off. 
especially striking, from their great numbers and from being frequently flat and leaflike; the younger leaves are generally entirely covered and concealed by them.

After these preliminary particulars, we may now turn to a consideration of the mode of growth of the separate organs.

The growing end of the stem sometimes far outruns the point of attachment of the youngest leaves, and then appears naked, as in Polypodium vulgare, $\boldsymbol{P}$. sporodocarpum, and other creeping Ferns, as well as in Pteris aquilina, where, according to Hofmeister, it frequently attains in old plants a length of several inches without bearing leaves. Mettenius states that in many Hymenophyllaceæ leafless prolongations of the axis of this kind have been taken for roots. In other cases, on the contrary, especially in Ferns with an erect growth, the increase in length of the stem is much slower, its apex remaining enclosed in a leaf-bud. The stem generally ends in a flat apex; sometimes, as in Pteris, it is even imbedded in a funnel-shaped elevation of the older tissues (Fig. $3 \circ \mathrm{r}, E$ ). The apex of the stem is always occupied by a clearly distinguishable apical cell, which is either divided by walls alternately inclined, and then resembles, when viewed from above, the transverse section of a biconvex lens; or it is a three-sided pyramid, with a convex anterior surface and three oblique lateral surfaces, which intersect behind. The outlines of the segments, which are in the first case in two, in the second case in three rows, or arranged with more complicated divergences, soon disappear in consequence of numerous cell-divisions and of the displacement caused by the growth of the masses of tissue and leaf-stalks surrounding the apex. The apical cell, for instance, of Pteris aquilina, is wedge-shaped, the segments

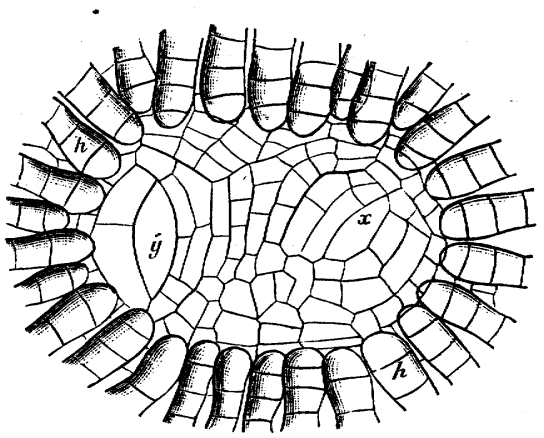

FIG. 300.-Apical view of the end of the stem of Pteris aqui lina; $y$ the apical cell of the stem; $x$ the apical cell of the youngest leaf; $h h$ hairs which cover the apical region surrounded by a cushion of tissue. on the horizontal stem forming a right and a left. row; the edges of the apical cell face upwards and downwards (Fig. 300). The same is also the case, according to Hofmeister, in Niphobolus chinensis and rupestris, Polypodium aureum and punctulatum, and Platycerium alcicorne. In Polypodium vulgare he states that it is sometimes wedge-shaped, sometimes pyramidal with three faces; the last-named form occurs also in Aspidium Filix-mas, \&c. As a rule it may for the present be assumed that creeping stems with a bilateral development have a wedge-shaped apical cell, upright or ascending stems with radiating rosettes of leaves one that is a three-sided pyramid.

The further relationships of the segments of the apical cell of the stem to the origin of the leaves and to the building up of the tissue of the stem itself are still but little known in detail. It cannot be doubted that each leaf results from a single segment only, and that this segment-cell is devoted from an early period to the formation of the leaf, but it appears doubtful whether the segments always form leaves, and if not what number of sterile segments intervenes between those from which a leaf is developed. 
The phyllotaxis of Ferns sometimes corresponds to the rectilinear arrangement of the segments of the apical cell. Thus the distichous arrangement of the leaves of Pteris aquilina, Niphobalus rupestris, and of some species of Polypodium, corresponds to the biseriate segmentation of the apical cell of the stem. But where the phyllotaxis is complicated and spiral, and the apical cell a three-sided pyramid, as occurs in Aspidium Filix-mas, the same processes may take place as in those Mosses which have their leaves arranged in many rows with a trilateral apical cell, such as Polytrichum ${ }^{1}$.

The Terminal Branching of the stem is considered by Hofmeister to be dichotomous in all Ferns ${ }^{2}$. The branches arise very near the end of the stem, and are, at least at first, equivalent to the primary stem, so that the branching is a bifurcation. That the branches are independent of the leaves is inferred by this writer from the fact that the ends of the stem of Pteris aquilina, which are leafless and often several inches long, regularly bifurcate. These branches are, in this and in many other cases, not axillary; and where, in other Ferns, they appear axillary, we must assume, with Hofmeister, that the bifurcation has taken place immediately in front of a youngest leaf, and that the limb of the fork which stands before the leaf developes to a smaller, while the other (the prolongation of the primary stem) does so to a greater extent. The branching at the end of the stem does not necessarily take place in the same plane as the insertion of the leaf immediately preceding; when it does, the branch stands laterally on the stem beside the leaf. To this class belongs, according to Mettenius's description, the extra-axillary branching of those Hymenophyllaceæ which have their leaves in two rows. That which distinguishes Ferns from Phanerogams with axillary branching, especially Angiosperms, is the rarity of terminal branching. While in the latter every leaf-axil, at least in the vegetative region, bears a bud, even the apparently axillary branches of creeping Ferns with long internodes occur mostly only at great distances, being wanting in a number of intermediate leaves. In those Ferns where the growth of the stem is slow and the apical region of considerable size, especially in erect species like Aspidium Filix-mas and the Tree-ferns, terminal branching of the stem is reduced to a minimum, or is entirely absent, or occurs only in abnormal cases.

The formation of new shoots from the bases of leaf-stalks must be distinguished from the normal terminal branching of the stem. These have nothing to do genetically with the stem, any more than the formation of adventitious shoots from the lamina of the leaves (vide infra).

The Development of the Leaf ${ }^{3}$ is exclusively basifugal and apical, the further growth being also basifugal. The leaf-stalk is first formed; at its apex the lamina begins subsequently to show itself; its lowest parts are formed first, its higher parts in basifugal succession. The extraordinary slowness of this growth is very re-

${ }^{1}$ See Hofmeister, Allgemeine Morphologie, p. 509; and Bot. Zeitg. 1870, p. 44I.

2 [According to Mettenius (Ueb. Seitenknospen bei Farnen, Abhdl. d. kgl. sächs. Ges. d. Wiss. V. I86I, this is not the case. Mettenius' view has been recently confirmed by Prantl, who has found that the branching in many Ferns, such as Cystopteris montana, Phegopteris vulgaris, Dryopteris calcarea, is effected by lateral buds (Flora, i 875 ). Ceratopteris appears to be the only Fern which does not produce them (Kny, loc. cit.)]

3 [See Sadebeck, Ueb. d. Entwick. d. Farnblattes; Verhandl. d. bot. Ver. d. prov. Brandenburg, 1874.$]$ 
markable, finding its parallel only among the Ophioglossaceæ. In old plants of Pteris aquilina the formation of the leaf commences fully two years before its unfolding:- at the commencement of the second year only the leaf-stalk is as yet in existence, about one inch high. Up to this period its growth has been effected by a single apical cell which is divided by oblique walls in alternating directions; it is now carried on by a number of marginal cells which divide. The pinnæ are derived from the segments of the single apical cell; the veins are formed by the repeated divisions of the marginal cells (Sadebeck). In the summer of the second year the lamina arises for the first time at the apex of this rod-like body, and may be found hidden in the form of a minute disc beneath the long hairs. It immediately bends downwards at its apex, and hangs down like an apron from the apex of the stalk (Fig. $301, B, C, D$ ). Its growth now proceeds underground, so that it does not begin to unfold till the third spring, when it is raised above ground by the elongation of the leaf-stalk. The whole of the leaves of a rosette of Aspidium

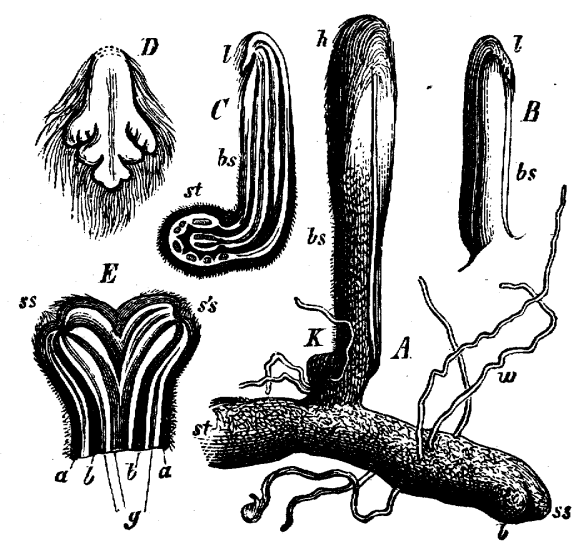

FIG. 3ot.-Pteris aquilina; $A$ the end of a stem st, the apex lying at ss; by its side at $b$ is the rudiment of a leaf, $\delta s$ the stalk of a leaf in the second year, at $h$ its lamina enveloped by hairs, $K$ a bud at the back of the leaf-stalk, $w$ roots; $B$ a young leaf in the second year, $b s$ its stalk, $l$ its small lamina with the hairs removed; $C$ longitudinal section of a sime st, $b s$ and $l$ as in $B ; D$ the lamina of a leaf in the of a similar leaf, connected with the transverse $(x$ about 5$)$; the first segments have begun to be formed; $E$ horizontal longitudinal section of a branching of the stem, $s s s^{\prime} s$ the two apices, $a$ a brown epidermal tissue, $b b$ brown sclerenchyma, $g$ fibro-vascular bundles. ( $A, B, C$ natural size.)

Filix-mas have been in course of formation two years before their unfolding; the leaf-stalk is in this case also formed in the first year, and the first formation of the lamina takes place on the oldest leaves of the young rosette.

The basifugal apical growth of the lamina of Fern-leaves is however most conspicuous when it continually advances for a considerable time without attaining a definite conclusion while the lower parts of the lamina have long been fully developed, as in Nephrolepis. The periodical interruption of the apical growth of the lamina already mentioned occurs in many species of Gleichenia and Mertensia, where the development of the leaves remains stationary above the first pair of pinnæ, and when the pinnation is compound this is often repeated in the several orders of branching, so that the apex, forming apparently a bud in the fork, either remains altogether undeveloped, or is developed in a succeeding period of vegetation, and then only incompletely. This intermittent development of the leaves may 
apparently extend over many years (see Braun, 'Rejuvenescence,' p. I23). According to Mettenius, the lamina of some Hymenophyllaceæ is capable of unlimited development, and is annually renewed. In Lygodium the primary branches of the lamina remain also in a bud-like condition at the end after the formation of each pair of pinnæ of the second order, while the rachis of the leaf grows without limit and resembles a twining stem.

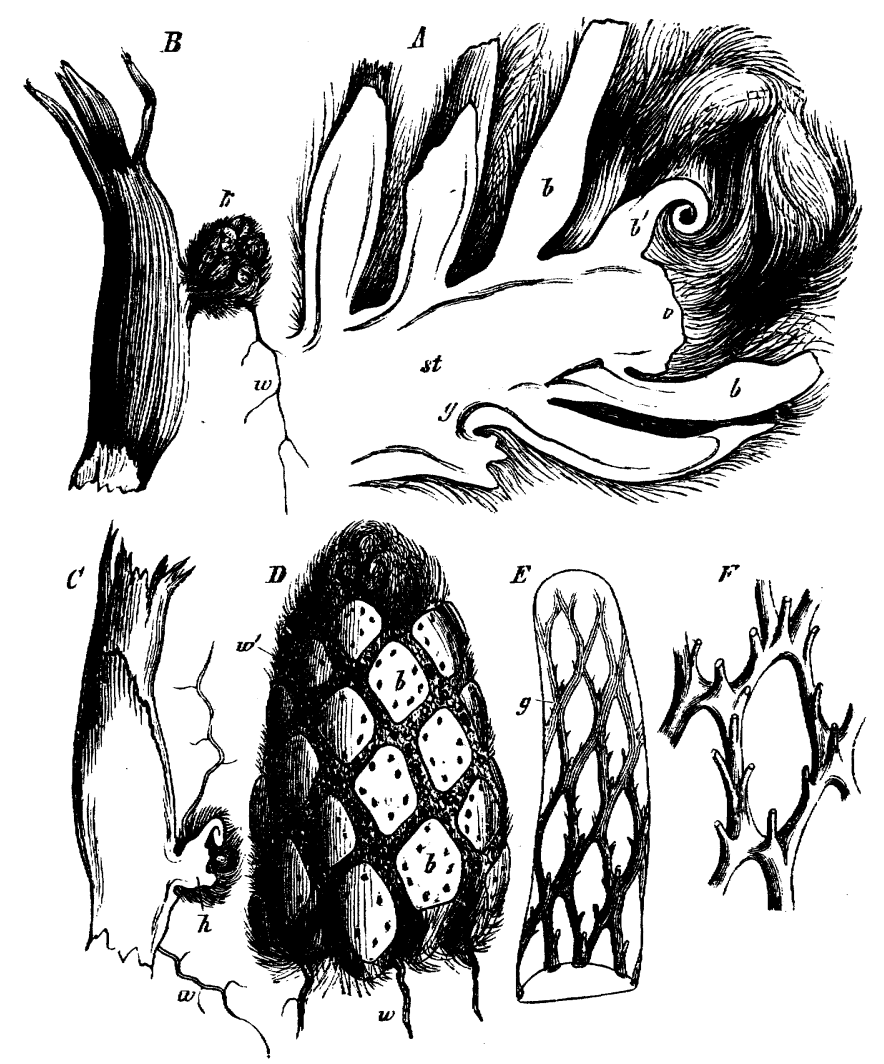

FIG. 302.-Aspidium Filix-mas; A longitudinal section through the end of a stem, $v$ the apical part of the stem st. $b b$ leaf-stalks, $b^{\prime}$ a young leaf still rolled uf, the rest enveloped in long hairs, $g$ fibro-vascular bundles; $B$ a leaf-stalk of the same plant broken off, bearing at $k$ a bud with several leaves, $w$ a root of this bud; $C$ a similar leaf-stalk cut through lengthways, bearing a root at $w$ and a bud at $h ; D$ end of a stem with the leaf-stalks cut off with the exception of the youngest leares of the terminal bud, in order to show the arrangement of the leaves, the spaces between the stalks $b b$ youngest leaves of the ter are filled with numerous roots which themselves all spring out of the stalks; $E$ end of a stem the cortex of which has
been peeled off in order to show the net-work of fibro-vascular bundles $g ; F$ a mesh of this net-work slightly magnified, showing the basal portions of the bundles which pass into the leaves.

The branching of the lamina of Fern-leaves is not unfrequently forked in the mature state, as in Platycerium, Schizcea, \&c. ; but Hofmeister refers also the pinnate forms to dichotomous branching at the commencement, which becomes sympodial with further development, a right and left limb of the bifurcation being alternately weaker in its growth, and forming the lateral pinnæ; while the branches, the growth of which is favoured, form the rachis of the leaf or of the branch of a leaf ${ }^{1}$.

1 It must be observed here also that Hofmeister applies the term 'dichotomy' in a much wider sense than is usually done. New examinations of a large number of species are greatly to be desired, 
The Formation of Adventitious Buds ${ }^{1}$ which do not result from the terminal branching of the stem, is, in Ferns, connected with the leaves. These buds make their appearance on the leaf-stalk or on the lamina itself. The shoots of Pteris aquilina which spring from the leaf-stalk (Fig. $30 \mathrm{r}$ ) stand at the back of the individual leaf-stalks near the base; in Aspidium Filix-mas (Fig. 302) they arise at a moderate height above the insertion, usually on one of the lateral edges of the leaf-stalk: In both cases Hofmeister states that they are formed on the young leaf-stalk even before the first appearance of its lamina, and before the differentiation of its tissue. A single superficial cell of the leaf-stalk is the mother-cell of the new shoot; and as the surrounding tissue of the leaf-stalk grows like a wall around them, they may, as in Pteris, be placed in a deep depression, where they sometimes remain dormant for a long period. Even when the leaf has long died away, the leaf-stalk still remains succulent up to the bud, and filled with food-materials; and in Aspidium Filix-mas vigorous stems are not unfrequently found with a number of leaves at their posterior end still attached to the leaf-stalk of an older stem. In some cases, as in Struthiopteris germanica, the buds produced on the leaf-stalks develope into long underground stolons furnished with scale-leaves, which become erect at the end and unfold a crown of foliage-leaves above ground. In Nephrolepis undulata they swell at the end into tubers. Adventitious buds spring from the lamina, especially in many species of Asplenium; in $A$. furcatum, e.g., often in large numbers from the middle of the upper surface of the pinnæ; in $A$. decussatum from the base of the pinnæ (or axillary on the mid-rib?). Ceratopteris thalictroides not unfrequently produces buds in the axils of all the divisions of the leaves, which, especially when the detached leaf is laid upon damp ground, develope rapidly, and grow into vigorous plants. According to Hofmeister, these buds also spring from superficial cells of the leaf. The long pendulous leaves of some Ferns touch the ground with their apices, root, and sometimes also put out new shoots from these points $(e . g$. Chrysodium flagelliferum, Woodwardia radicans, \&c.).

'The Roots. During its growth the stem is usually constantly forming new roots in acropetal succession, which, in the creeping species, become at once fixed to the substratum. [As a rule they are developed quite endogenously, but in Ceratopteris thalictroides, according to $\mathrm{Kny}$, they arise from cells immediately beneath the epidermis. According to Conwentz, a distinct relation can usually be observed between the number of the roots and that of the leaves.] In Pteris aquilina the new roots appear close behind the apex, and, both in this species and in Aspidium Filix-mas, they also proceed from the adventitious buds of the leaf-stalk while still very young. It has already been mentioned that, in the last-named species, when the mature stem is completely covered by leaf-stalks, all the roots spring from them and not from the stem, In Tree-ferns especially the lower part of the erect stem is entirely covered by slender roots, which grow downwards, forming an envelope several inches thick before they penetrate the soil, and thus give a

both in reference to the formation of leaves and to the terminal branching of the stem. [According to Sadebeck, the pinnæ are developed as lateral outgrowths, but the branching of the veins is the result of the dichotomy of the marginal cells; see supra.]

1 [On the development of these adventitious buds, see Heinricher, Sitzber. d. Wien. Akad. 18;8 and I $88 \mathrm{I}$. 
broad base to the stem although it is there really much more slender; but in the upper part there are also a great many roots. In small plants they are very slender; on large plants they attain a diameter of from $\mathbf{I}$ to $3 \mathrm{~mm}$; they are cylindrical, generally covered with a number of root-hairs which form a kind of felt, and are of a brown or black colour. The history of the growth of Fern-roots has been studied by Nägeli and Leitgeb'. 'The apical cell is a three-sided pyramid, with a convex equilateral base. The segments or layers of the root-cap, detached by convex septa parallel to the base, first divide into four cells placed crosswise, and the walls which effect this division alternate in successive layers by about $45^{\circ}$; each of the four cells of a layer then splits up into two external and one internal (central one), so that the layer is now formed of four internal cells arranged in a cross, and of eight external cells. Further divisions may then follow; the central cells of the layer grow more quickly in an axial direction, and may become divided by transverse septa, by which the layer is made to consist of two or more strata in the middle. The formation of a layer of the root-cap is generally followed by that of three root-segments before a further new layer is formed; these segments, corresponding to the faces of the threesided apical cell, lie in three straight longitudinal rows. Each of these triangular tabular segments includes a third of the circumference of the root, and is first divided by a radial longitudinal wall into two unequal portions. The transverse section of the root now shows six cells, three of which meet in the centre, while the other three do not reach quite so far. Each of these six cells is then divided by a tangential wall (parallel to the surface) into an inner and an outer cell; the inner ones form the fibro-vascular bundle, while the six outer cells form the rudiment of the cortex. If the root becomes thick, the six cortical cells divide by radial walls; if it remains slender, this division does not take place. The six or twelve cortical cells are now divided by a tangential longitudinal wall, and the fibro-vascular bundle is enclosed by two layers of cells, the outer of which forms the epidermis, the inner the fundamental tissue of the cortex (see Book I, p. I 44).

The roots of Ferns, like those of Equisetaceæ, branch monopodially, and the lateral roots arise on the outer side of the primordial fibro-vascular bundles in acropetal succession, usually in two rows, but occasionally in three or four. The cells from which the lateral roots spring belong to the most internal layer of the cortical parenchyma (bundle-sheath), and are opposite to the xylem-bundles: they are separated from the fibro-vascular bundles of the parent root by the pericambium. The rudiments of the roots make their appearance near to the growing-point before the vessels are formed. Adventitious lateral roots, arising behind those which already exist, do not occur. The three-sided pyramidal apical cell is formed in the mother-cell of a lateral root by three oblique septa, and then the first root-cap is produced from it. If two primordial fibro-vascular bundles are developed in the lateral root, they lie right and left with reference to the

${ }^{1}$ Sitzungsber. der bayr. Akad. der Wiss. Dec. 15, 1865. Compare with what follows the diagram of a root given under the Equisetacex, which serves in the main also for Ferns and Rhizocarps; also p. 144. [See also Conwentz, Beit. z. Kennt. des Stammskelets einheimischer Farne, Bot. Zeit. I 875 .] 
parent root. The cortex of the parent root is simply broken through, no root-sheath being formed.

The Trichomes of Ferns assume a great variety of forms. True root-hairs, simple unarticulated tubes, arise, not only on the roots themselves, but also on underground stems and on the bases of leaf-stalks (as in Pteris aquilina and Hymenophyllaceæ). On aërial creeping stems and on the leaf-stalks the numerous usually brownish or dark-brown flat multicellular hairs, the Palece or Ramenta, occur, soon becoming dry, often entirely enclosing the buds, and attaining a length of from $I$ to $6 \mathrm{~cm}$. (as in Polypodium, Cibotium, \&c.). Long strong bristles are sometimes found on the lamina (in Acrostichum crinitum), and very often fine, delicate, articulated hairs. They are formed from single superficial cells at the growing-point.

The Sporangia of Ferns are small rounded capsules, which are borne on long stalks in the Polypodiaceæ and Cyatheaceæ, but which are sessile in other Ferns. The wall of the sporangium, when mature, consists of but a single layer of cells. A

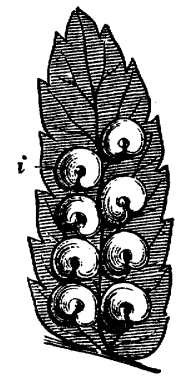

FIG. 303.-Under-side of a lacinia of a leaf of Aspidium Filix-mas, with eight indusia i with $\times 2)$. ring of cells belonging to the wall of the capsule and running across it transversely or obliquely or lengthwise is generally developed in a peculiar manner, and is then termed the Annulus. By its contraction when dried up the capsule bursts at right angles to the plane of the annulus. Sometimes, instead of the annulus, a terminal or lateral group of the cells of the wall of the capsule is developed in a similar manner.

The sporangia are generally combined into groups, each group being termed a Sorus; the sorus contains either a small definite number or a large indefinite number of sporangia, and among them also very commonly some slender articulated hairs, the Paraphyses. The whole sorus is very generally covered by an excrescence of the epidermis, the true Indusium ${ }^{1}$ : in other cases the indusium consists of an outgrowth of the tissue of the leaf itself, and is then composed of several layers, and even has stomata; or the covering of the sorus is simply the result of the margin of the leaf being recurved or rolled over it: in these cases the indusium is said to be false. In Lygodium each separate sporangium is covered by a pocket-shaped growth of the tissue of the leaf like a bract. Sori are nót usually formed upon all the leaves of the mature plant; sometimes groups of fertile and sterile leaves alternate in regular succession, as in Struthiopteris germanica. In some cases the sori are uniformly distributed over the whole of the lamina, in others they are connected with definite portions of it. The fertile leaves may be in other respects like the sterile ones, or they may be strikingly different from them; and this difference is not unfrequently occasioned by the partial or entire failure of development of the mesophyll between and near the fertile veins; the fertile leaf, or the fertile part of the leaf, then appears like a spike or panicle furnished with sporangia (e. g. Osmunda, Aneimia). The sporangia generally arise from the epidermis of the

${ }^{1}$ [On the development of the indusium, see Prantl, Die Hymenophyllaceen, 1875, and die Schizæaceen, I88I; Burck, Over de ontwikkelingsgeschidenis van het indusium der Varens, Haarlem i874.] 
veins of the leaf, and especially on the under side of the lamina ; but in the Acrostichaceæ they spring both from the veins and from the mesophyll; in Olfersia they cover both surfaces of the leaf at the sides of the mid-rib, or in Acrostichum only the under side. When, as is usually the case, the veins are the only parts that bear the sporangia, the fertile veins may be like the sterile ones, or they may undergo a variety of changes at the spots where they bear the sori ; they may be swollen into a cushion (forming a placenta), or they may project beyond the margin of the leaf, as in the Hymenophyllaceæ. The sorus may be seated on the end of a vein, which then frequently puts out two branches in the angle of which is placed the sorus, or it may be formed upon the vein and behind its end ; or the sorus may run for a considerable distance by the side of the vein. Sometimes the fertile veins run close to the margin of the leaf, in other cases close to the mid-rib of the lamina.

The Development of the Sporangium ${ }^{1}$ is accurately known only in the Polypodiaceæ; it arises there from a papillose outgrowth of one of the epidermal cells

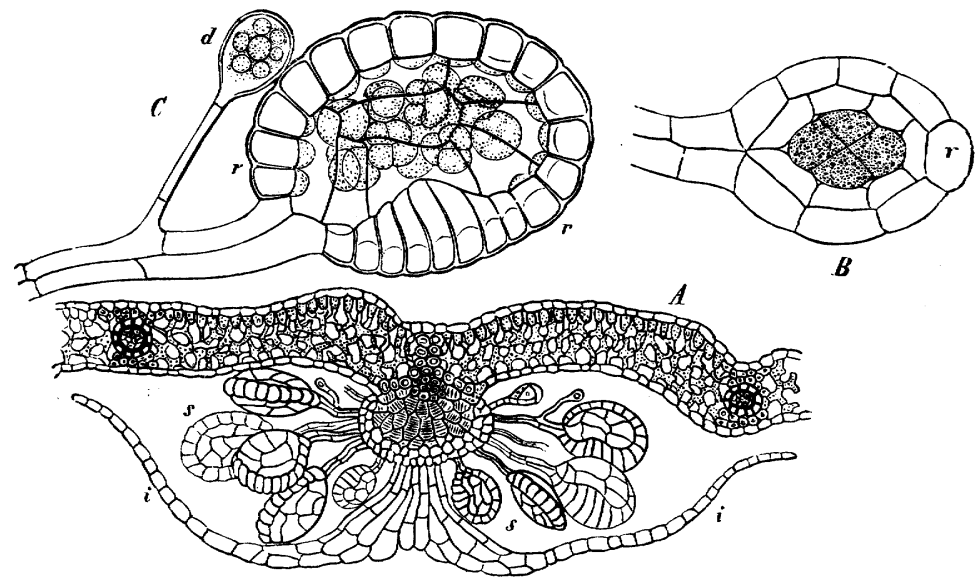

FIG. 304.-Aspidium Filix-mas. A transverse section of a leaf with a sorus consisting of the sporangia $s$ and the indusium $i$; right and left in the mesophyll of the leaf are two small fibro-vascular bundles, the sheath of which shows the dark brown thickenings on the walls that face inwards. $B$ a young sporangium, its annulus standing vertically to the plane of the paper, $r$ its apical cell; in the interior four cells are seen resulting from the division of the central cell $C$ lateral view of a nearly ripe sporangium, $r r$ its annulus, $d$ the stalked gland peculiar to this species; within the sporangium are seen the young spores already formed.

from which the sorus originates. Reess has shown that before the formation of the sporangium the epidermal cell concerned has been already divided cross-wise; the papilla is cut off by a septum, another septum arising, after further elongation, in the mother-cell of the sporangium thus formed; the lower cell forms the pedicel, the upper cell the capsule of the sporangium. The pedicel is usually transformed,

1 When the first sporangia are ripening, all stages of development of the younger ones may be found in the same sorus side by side. [The following are the more important works on the subject: Reess, Zur Entwickelungsgeschichte des Polypodiaceen-Sporangiums, Jahrb. f. wiss. Bot. V, and Bot. Zeit. 1867.-Tschistiakoff, Die Sporangien und Sporen der Polypodiaceen, Nuov. Giorn. bot. Ital. VI.-Russow, Vergl. Unters. Petersburg I 872.-Fischer von Waldheim, Ueb. die Entwickelung der Farnsporen, Jahrb. f. wiss. Bot. IV.-Kny, Ueb. Ceratopteris thalictroides, Bot. Zeit. 1874.-Prantl, Die Hymenophyllaceen, I 875, and Die Schizæaceen, I88 r.] 
by intercalary transverse divisions and longitudinal walls, into three rows of cells; the nearly hemispherical mother-cell of the capsule is next transformed, by four successive oblique divisions, into four plano-convex parietal cells and a tetrahedral inner cell (archesporium); in the former further divisions follow perpendicular to the surface, while the inner cell again forms four tabular segments which are parallel to the outer parietal cells and which constitute the tapetum. These inner parietal cells also divide perpendicularly to the surface of the capsule, and may form two layers. The cells of the outer parietal layer from which the annulus is to be formed are further divided by parallel walls perpendicular to the surface of the sporangium and to the median line of the annulus, until the prescribed number of cells of the annulus is reached; these cells then project above the surface of the capsule. While the tetrahedral central cell is now producing the mother-cells of the spores by successive bipartitions, the cells of the tapetum are absorbed, and the cavity of the sporangium is considerably enlarged by this means and by the superficial growth of the outer parietal layer; so that the mass of mothercells (according to Russow there are usually sixteen) floats entirely free in the fluid that fills the sporangium (Fig. $3 \circ 4$ ).

But little is known concerning the development of the sporangia in other families of Ferns. According to Russow and Prantl it takes place in Alsophila (Cyatheaceæ) in the same way as in the Polypodiacex, whilst
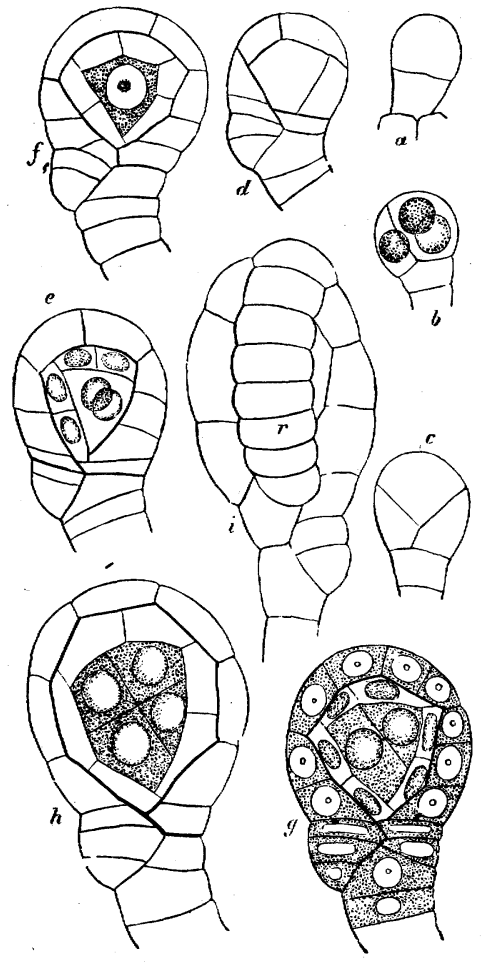

FIG. 305.-Development of the sporangium of Asplenium Trichomanes; the order of succession according to the letters $a-i$; in $i$ the annulus $r$ is shown; the other figures are seen in optical longitudinal section, and the annulus is perpendicular to the plane of the paper $(\times 550)$. in Aneimia and Mohria (Schizæacex), according to their figures and descriptions, each sporangium arises, before the differentiation of the epidermis, from a single cell. Probably the sporangia of the Osmundaceæ originate in the same way as those of the Schizæaceæ. In both families a much larger number of spore-mother-cells is formed in the sporangium than is the case in the Polypodiaceæ, and this fact also recalls the Ophioglosseæ and the Marattiaceæ.

Each spore-mother-cell is, in Aspidium Filix-mas (Fig. 306, I), provided with an evident nucleus; in consequence of its division, two new large clear nuclei arise $(I I I)$, between which an evident line of separation is sometimes to be seen. After the division of these nuclei, four new smaller nuclei appear $(I V)$, the mother-cell splitting up into four spore-cells $(V)$, the relative position of which varies (as is shown in Figs. VI, VII, and VIII). The spore now becomes clothed with its cell-wall, which is differentiated into an endospore consisting of cellulose 
and a cuticularised brown exospore furnished with ridges $(I X)$, and chlorophyll is formed within the spore.

In various other Polypodiaceæ, according to Russow, the course of the development of the spores is in so far different, that the mother-cell, as occurs also in the formation of the pollen in Phanerogams, divides into four thick-walled cells, the so-called special mother-cells; the protoplasm of each of these then forms around itself a permanent coat, and the walls of the mother-cell undergo absorption. Spores of the shape indicated in Fig. 306 are said to be bilateral in contradistinction to those which have been formed from a mother-cell in which the four nuclei were placed tetrahedrally, and which have therefore a rounded tetrahedral form. In the Hymenophyllaceæ, Osmundaceæ, and Cyatheaceæ the latter only occur, in the other families sometimes the one kind and sometimes the other.

The spores of many Polypodiaceæ are distinguished by the long period during which they retain their power of germination, and by the slowness of this process; those of Hymenophyllaceæ often begin to germinate while still in the sporangium.

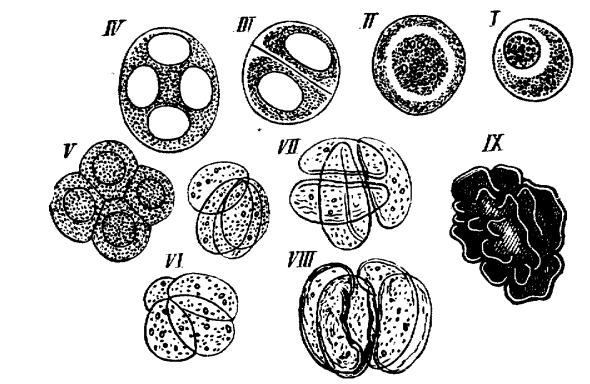

FIG. 306.-Development of the spores of Aspidizm Filix-mas $(\times 550)$.

(a) Histology ${ }^{1}$. With reference to the Epidermis, attention has been directed on p. 105 to the peculiar mode of development of the stomata in many cases. It may also be mentioned that the epidermal cells usually contain chlorophyll-granules.

The Fundamental Tissue of the stem and of the leaf-stalks consists, in some species (as Polypodium aureum and vulgare, and Aspidium Filix-mas), entirely of thin-walled parenchyma; in others (as Gleichenia, species of Pteris, and Tree-ferns), string-like, ribbon-shaped, or filiform portions of the fundamental tissue become differentiated, the cells of which undergo great thickening, and become brown-walled, hard, and prosenchymatous, forming sclerenchyma. In the stem of Pteris aquilina (Fig. $307, A$ ) two thick bands of sclerenchyma of this description $(p r)$ lie between the inner and outer fibro-vascular bundles, and fine threads of sclerenchyma appear on the transverse section of the colourless parenchyma as dark points. In other cases (as in Polypodium vacciniifolium and in Tree-ferns), dark layers of sclerenchyma, the nature of which was in these cases first correctly recognised by $\mathrm{H}$. von Mohl, form sheaths round the fibrovascular bundles, to which the erect stem more especially owes its firmness. The outer layer of the fundamental tissue of thicker stems and leaf-stalks lying beneath the epidermis is often dark brown and sclerenchymatous, forming a hard firm sheath, as again, for instance, in Pteris aquilina (Fig. $307, A, r$ ) and Tree-ferns. In order to facilitate, in spite of this firm coat, the communication of the outer air with the inner parenchyma

1 [For further details see de Bary, Vergleichende Anatomie der Phanerogamen und Farne, 1877.] 
which is rich in assimilated food-materials, it is, in Pteris aquilina, interrupted along two lateral lines, where the colourless parenchyma rises to the surface. In Tree-ferns, on the other hand, according to $\mathrm{H}$. von Mohl, depressed cavities appear on the enlarged base of the rachis of the leaf, where the sclerenchyma is replaced by a loose and pulverulent tissue.

It may be mentioned here in addition, as an isolated histological -peculiarity, that in Aspidium Filix-mas, according to Schacht, roundish stalked glands occur in the fundamental tissue of the stem, which I have also noticed in the green parenchyma of the leaves, and on the pedicels of the sporangia of the same Fern (Fig. 304, $C, d$ ).

The lamina of the leaf consists in Hymenophyllaceæ only of a single layer of cells, as in Mosses; in all other Ferns it is formed of several layers. Between the upper and under epidermis lies a spongy parenchyma containing chlorophyll, the mesophyll, penetrated by the fibro-vascular bundles which form the venation of the leaf. The course

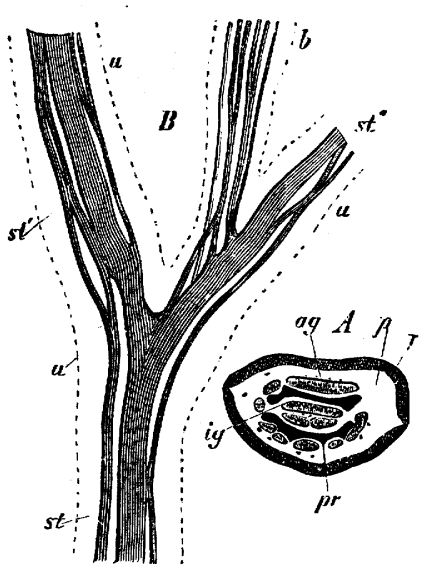

FIG. 307.-Pteris aquilina; $A$ transverse section of the stem, $r$ its brown sheath (the layer of sclerenchyma beneath the epidermis), $p$ the soft colourless parenchyma of the fundamental tissue; $i g$ inner fibro. vascular bundles; $\alpha g$ upper broad outer bundle; $B$ the separated upper fibro-vascular bundles of the stem st, and of its branches st' and st", b bundles of the leaf-stalk, $u u$ outline of the stem (natural size).

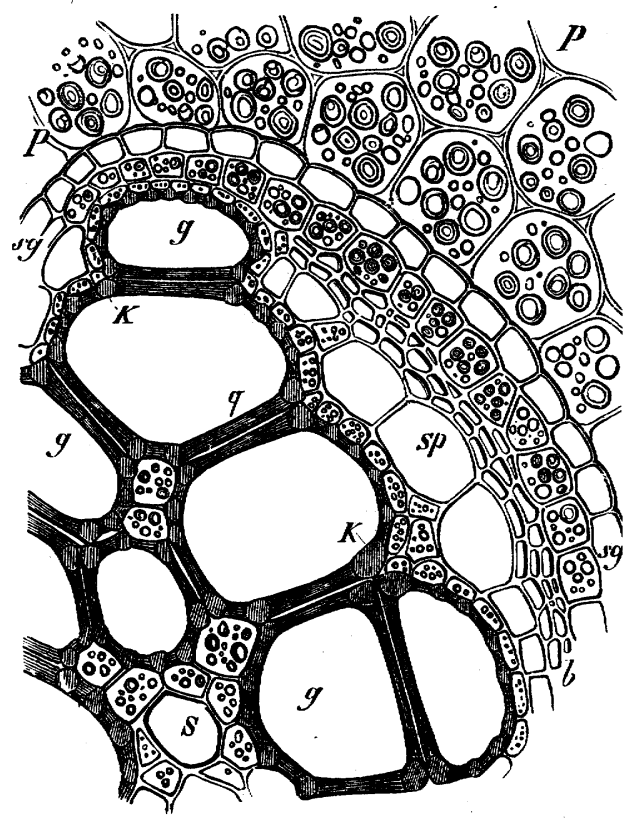

FIG. 309.-A quarter of the transverse section of a fibro-vascular bundle from the stem of Pteris aquilina, with the adjacent parenchyma $P$ containing starch, $s g$ the bundle-sheath, $b$ the layer of bast-fibres, $s p$ the large sieve-tubes, $g g$ the large vessels of the xylem thickened in a scalariform manner, $S$ a spiral vessel surrounded by cells containing starch $(\times 300)$.

of the veins is very various; sometimes they run branching dichotomously at acute angles, or spreading like a fan upwards and sideways, without anastomosing and without forming a mid-rib ; more often the undivided lamina, or a division of the lobed, incised, or pinnate leaf, is penetrated by a distinct median vein though but slightly projecting, from which spring more slender branches, which themselves again ramify dichotomously or apparently monopodially, and run to the margins. The finer veins frequently anastomose like those of the leaves of most Dicotyledons, and divide the surface into areolæ of characteristic appearance.

The Fibro-vascular Bundles of Ferns are closed; they consist of a mass of xylem, completely enveloped by a layer of phloëm. Besides a few narrow spiral vessels, lying at certain definite points in the transverse section, the xylem consists of vessels with bordered pits which usually resemble transverse clefts (scalariform vessels), their ends 
being mostly obliquely truncated, or fusiform and pointed. Between the vessels lie narrow thin-walled cells, which contain starch in winter. The phloëm, in addition to cells similar to those last named, contains wide sieve-tubes or latticed cells, and at the circumference narrow, bast-like, thick-walled fibres. The whole bundle is usually enclosed by a distinct sheath of narrower cells (vascular bundle sheath); the latter often, but not always, is invested by a layer of brown sclerenchymatous cells, the walls of which are very much thickened either, as in Platycerium, on that side which is next the bundle, or, as in Blecbnum brasiliense, on that side which is most distant from it. This layer is easily mistaken for the bundle-sheath itself. A single layer or several layers of cells may often be found at the periphery of the phloëm lying just inside the true bundle-sheath. Russow regards this structure as belonging, like the bundle-sheath, to the ground tissue, and he terms it the phloëm-sheath. Such a phloëm-sheath is represented in Fig. 308 as a layer of cells containing starch lying between $s g$ and $b$.

The fibro-vascular bundles are single and axial in very slender filiform stems, as in those of Hymenophyllaceæ, and in the young plants of larger species. When the stems of the latter become thicker with increase of growth, a network of anastomosing bundles is formed in place of the axial bundle, presenting, in typical cases, a wide-meshed hollow cylinder, by which the fundamental tissue of the stem is separated into an outer cortical layer and an inner medullary portion (Fig. 302, $A$ and $E$ ). Not unfrequently, however, isolated bundles also arise in addition; thus in Pteris aquilina two strong broad cauline bundles are formed within the medullary portion (Fig. 302, $\mathrm{A}$, ig), and in Tree-ferns a number of filiform bundles are scattered through it which enter into the leaf-stalk through the meshes of the primary bundle. The primary bundles which form the cylindrical network already mentioned are mostly ribbon-shaped, broad, and, in the case of Tree-ferns, commonly have their margins curved outwards, so that they with their thick, firm, brown sheaths of sclerenchyma occupy most of the circumference of the stem. From these margins spring the more slender filiform bundles which enter the leaf-stalk, and are more numerous in proportion to its thickness. These may also coalesce laterally into plates of different forms, or may run separately side by side. The leaf-stalk always corresponds to an opening of the meshes of the cylinder of the primary bundle. The thick bundles which run through the stem appear to be all cauline. Hofmeister found in Pteris aquilina ${ }^{1}$ that they exhibit the same distribution on the leafless elongated ends of the stem as on its leafy parts, a proof that the distribution does not depend on the leaves, as in Phanerogams. The end of the bundle may even be followed up to near the apical cell of the stem, in places where the nearest leaf-stalks have not yet begun to form bundles.

(b) Taxonomy. The Ferns may be classified as follows:-

Family 1. Osmundaceæ. In Osmunda the fructification is paniculate, the sporangia being borne on the laciniæ of leaves the mesophyll of which is not developed. In Todea the fertile leaves resemble the sterile ones. 'The shortly-stalked, unsymmetricallyrounded sporangia are furnished on one side of the apex with a group of peculiarlyformed cells, and they split open longitudinally on the other side. The stem, which is densely covered with roots, throws out lateral shoots resembling itself.

Family 2. Schizæaceæ ${ }^{2}$. Except in Mobria, where the sporangia lie on the under surface of the leaf near the margin which is incurved over them, the laciniæ bearing the sporangia are arranged in spikes or panicles. In Scljizcea and Lygodium the sporangia are arranged in two rows upon the under surface of a very much contracted lacinia, each sporangium of Lygodium being invested by a sac-like indusium. In Aneimia the two lowest branches of the lamina have no mesophyll, and form stalked panicles on the

1 I found a stem of Pteris aquilina in which the two internal cauline bundles had coalesced laterally so as to form a hollow cylinder enclosing one part of the parenchymatous ground-tissue as a medulla.

2 [Prantl, Die Schizæaceen, I 88I.] 
ultimate branches of which the sporangia are developed as if they were metamorphosed lacinix. The ovoid or pear-shaped sporangia are sessile, the apex of each being occupied by a cap-like zone of peculiarly-formed cells. The dehiscence is longitudinal. The stem (also in Lygodium) does not branch much, and is but feebly developed. A single fibro-vascular bundle traverses the petiole. The leaves of Lygodium resemble twining stems.

Family 3. Gleicheniaceæ. The sessile sporangia are borne upon the dorsal surface of ordinary leaves. They form sori of usually three or four sporangia, and no indusium is developed. The sporangium has a complete transverse annulus, and longitudinal dehiscence. The stem is a thin, creeping rhizome. The lamina of the leaf is remarkable for its innovation.

Family 4. Hymenophyllacem ${ }^{1}$. The sporangia have an oblique or transverse complete annulus; and therefore burst with a longitudinal slit; they are formed on a prolongation of the fertile vein (the Columella), projecting beyond the margin of the leaf, which is surrounded by a cup-shaped indusium. The mesophyll of the leaves usually consists of a single layer of cells, and is then necessarily destitute of stomata, which do however occur in Loxsoma on the leaf, which then consists of 'several layers. The stem is generally creeping and mostly very slender, and furnished with an axial fibro-vascular bundle. True roots are not present in all the species; where they are absent, the stem itself is clothed with root-hairs: a large number of species of Trichomanes are described by Mettenius as rootless, and in these cases branches of the stem assume a deceptive root-like appearance. The development of the axes precedes by a long space that of the leaves; several internodes have usually completely ended their growth while the leaves belonging to them are still very small; and these apparently (or actually?) leafless shoots often branch further to a great extent. The formation of the tissue of these families shows also many peculiarities, concerning which reference must be made to Mettenius (Hymenophyllacex, l.c.). The fertile end of the veins of the leaf projecting beyond its margin, the columella, elongates by intercalary growth, and the newly-formed sporangia are, in a corresponding manner, produced in basipetal succession. They are arranged in a spiral line on the columella. The almost sessile sporangia are biconvex, and are attached to the columella by one of their convex surfaces. The annulus projecting in the form of a cushion which separates the two convexities is usually oblique, and divides the circumference into two unequal portions. In Loxsoma the sporangia are pear-shaped and distinctly stalked. Paraphyses accur only in a few species of Hymenophyllum.

Family 5. Cyatheaceæ. The sporangia are shortly stalked and have a complete, oblique, eccentric annulus. They are borne upon a strongly-developed placenta forming a closely-packed sorus, which is either naked or invested by an indusium, which may be cup-shaped or completely encloses it. The genera Cibotium, Balantium, Alsopbila, Hemitelia, and Cyatbea include the so-called Tree-ferns, with a lofty, erect, unbranched stem, often thickly covered with roots, bearing at its apex a rosette of large usually compoundly pinnate leaves.

Family 6. Polypodiacem. The sporangia are borne in great numbers on the under surface of usually unmodified leaves. They have a vertical incomplete ring, and they dehisce transversely. The following subdivisions of this family, which contains the largest number of species of any, may be distinguished:-

(a) Acrostichex. The sori cover the surface and veins of the under side or of both sides, or are placed upon a thickened placenta which stands on the vein. There is no indusium. (Acrosticbum, Polybotrya.)

(b) Polypodiex. The sori are rounded or linear, and terminal or lateral on the veins. They are naked. The leaf-stalk is either articulated to the stem (Polypodium), or is not (Phegopteris.)

${ }^{1}$ [Prantl, Die Hymenophyllaceen, I875.] 
(c) Aspleniex. The sori are unilateral on the course of the veins, and are covered by a lateral indusium, or rarely without any; or they extend at their apex over the back of the veins, and are covered by an indusium springing from it ; or they occupy special anastomosing branches of the veins, and are unilateral and covered by an indusium free on the side of the vein. (Asplenium, Scolopendrium.)

(d) Aspidiex. The sori are dorsal on the veins, covered with an indusium, or terminal and without indusium. (Aspidium.)

(e) Davalliex. The sori are terminal on a vein or at a fork, and are furnished with an indusium; or are placed on an intramarginal anastomosing bend of the veins, and covered with a cup-shaped indusium, free at the outer margin. (Davallia, Nephrolepis.)

(f) Pteridex. The sori are continuous along the margin of the leaf, and are covered by a false indusium. (Pteris, Adiantum, Blecbnum.)

\section{Order III. Rhizocarpese ${ }^{1}$.}

The Sexual Generation (Oophore) of Rhizocarps is developed from spores of two different kinds; the smaller spores (microspores) produce antherozoids, and are therefore male; the larger spores (macrospores), which exceed the smaller kind several hundred times in size, produce a small prothallium, which never separates from them, and forms one or several archegonia; the macrospores may therefore be considered to be female.

The development of the antherozoids is preceded by the formation of a very rudimentary Male Prothallium. In the genus Salvinia the microspores lie imbedded in a mass of granular hardened mucilage (as they do also in Azolla, in which plant their germination is not known), which fills up the whole of the microsporangium; they do not escape, but the endospore of each of them grows out into a tube which pierces the mucilage and the wall of the sporangium and forms a septum at its curved end (Fig. $309, A$ and $B$ ). The terminal cell of the tube thus produced is again divided by an oblique wall, after which the protoplasm contracts in the two cells (which Pringsheim together calls the antheridium), and splits up by repeated bipartition into four roundish primordial cells, each of which forms an antherozoid. In addition a small portion of the contents remains inactive in each of the two cells. The antheridial cells burst by transverse slits to allow the escape of the antherozoids. The spirally-coiled antherozoid is still enclosed for a time in its

${ }^{1}$ G. W. Bischoff, Die Rhizocarpeen u. Lycopodiaceen (Nürnberg I828).-Hofmeister, Vergleich. Untersuch. I851, p. 103.- [On the Germination, Development, and Fructification of the Higher Cryptogams, Ray Soc. I862, pp. 318-335.]-Ditto, Ueber die Keimung der Salvinia natans (Abhand. der königl. Sächs. Gesellsch. der Wissensch. 1857, p. 665).-Pringsheim, Zur Morphologie der Salvinia natans (Jahrb. für wissensch. Bot. vol. 1II. I863).-J. Hanstein, Ueber eine neuholländische Marsilia (Monatsber. der Berliner Akad. 1862, Ann. des Sci. Nat. $4^{\text {th }}$ series, vol. XX, I863, pp. I49I66).-Ditto, Befruchtung u. Entwickelung der Gattung Marsilia (Jahrb. für wissensch. Bot. vol. IV, 1865).-Ditto, Pilulariæ globuliferæ generatio, cum Marsilia comparata (Bonn I 866). - Nägeli u. Leitgeb, Ueber Entstehung u. Wachsthum der Wurzeln bei den Gefässkryptogamen (Berichte der bayer. Akad. der Wissensch. I866, Dec. 15, and Nägeli's Beiträge zur wissensch. Bot. vol. IV. 1867). - Millardet, Le Prothallium male des Cryptogames vasculaires (Strasbourg 1869).-A. Braun, Ueber Marsilia u. Pilularia (Monatsber. der königl. Akad. der Wissensch. Berlin, Aug. I870).E. Russow, Histologie u. Entwickelung der Sporenfrucht von Marsilia (Dorpat I87I).-Strasburger, Ueber Azolla (Jena I873).-Juranyi, Über die Entwickelung der Sporangien und Sporen von Salvinia natans (Berlin 1873). - [Arcangeli, Sulla Pilularia globulifera e sulla Salvinia natans; Nuov. Giorn. Bot. Ital. 1876 .] 
mother-cell (Fig. 309, D). In Marsilia and Pilularia the antherozoids are produced in much the same manner; the protoplasmic contents of the microspore divide into three cells: when the exospore bursts, the endospore protrudes containing these three cells, of which one is sterile (rudimentary prothallium) and the other two produce antherozoids: within each of these two cells divisions take place so that a number of tetrahedrally-arranged primordial cells are formed which become surrounded with thin cell-walls, and are the mother-cells of the antherozoids. As in Ferns, we find also in Rhizocarps only a portion of the contents of the mother-cell applied to the formation of the antherozoid. According to Millardet,
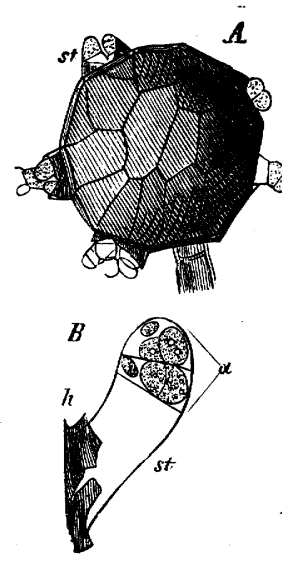

FIr. 309.-Salvinia natans; $A$ an entire microsporangium, the tubes of the microspores st breaking through ( $\mathrm{X}$ about 100 ) ; $B$ one of these tubes st protruding from the envelope of the microsporangium $h$ (X about 200), $a$ the envelope of the microsporangium $h$ ( $X$ about 200$), a$ the
antheridium still closed; $C$ tube with the empty antheantheridium still closed; $C$ tube with the empty
ridium ; $D$ antherozoids ( $\times 500)$ (after Pringsheim).
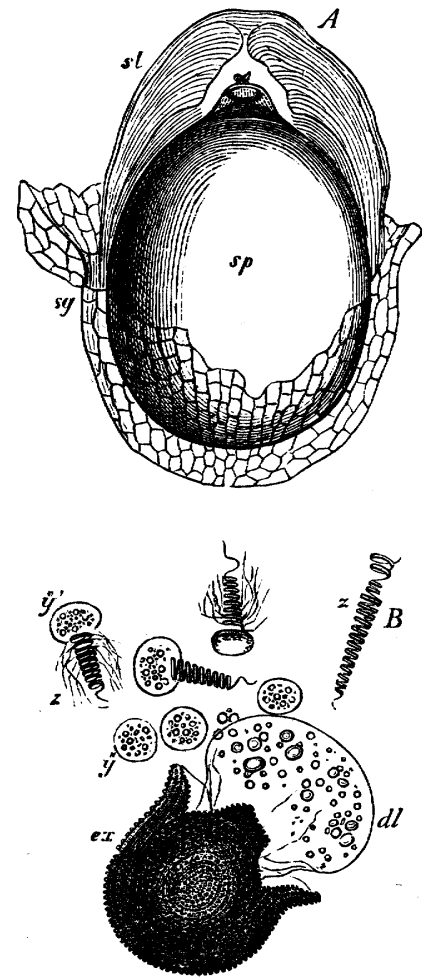

FIG. 310.-Marsilia Salvatrix; $A$ macrospore $s \not p$ with its epispore $s t$ and the apical papilla projecting into its funnel: in the papilla is a broad yellowish drop, $s g$ the ruptured wall of the macrosporangium ( $\times$ about 30 ). $B$ a microspore burst after the escape of the antherozoids; $e x$ the exospore, $d l$ the protruding endospore containing granules, $z, z$ the spiral antherozoids, $y y$ their vesicles containing starch-grains. The epispore of the microspore is no longer in existence $(\times 550)$. (The exospore does not show the regular arrangement of the protuberances, which is indicated erroneously in the figure.)

this portion assumes the form of a roundish turbid mass consisting of protoplasm and starch-granules, which, during the formation of the antherozoids, becomes gradually clearer, and, when the latter escape from the mother-cell, forms a vesicle consisting of the unused protoplasm and the starch-granules lying in it. In Pilularia, where the antherozoid is a thread coiled four or five times, this vesicle remains attached to the mother-cell. In Marsilia, on the contrary, it adheres to the posterior coils of the corkscrew-like antherozoid, which is coiled $\mathbf{2} 2$ or $\mathbf{1} 3$ times ; and is often carried about with it for a considerable time by its swarming motion, 
but finally becomes detached. When the antherozoids are formed in their mothercells, the exospore bursts at the apex, the endospore swells up as a hyaline bladder, which finally bursts and allows the escape of the antherozoids (Fig. 3 Io, $B$ ).

The Female Prothallium is formed within the apical papilla of the macrospore from a small part of its protoplasm, and only partially emerges at a later period from the spore-cavity, but remains united with the latter, closing it by its basal surface, for the purpose of using up the food-materials (starch-grains, fatty oil, and albuminous substances) which are stored up there. The separate stages in the first formation of the prothallium are still in many respects not clear; but it is certain that it arises from a collection of protoplasm in the cavity of the papilla; this protoplasm. immediately breaks up into several cells, which, according to Hanstein in Marsilia and Pilularia ${ }^{1}$ and according to Juranyi in Salvinia, become clothed only at a later period with cell-walls and thus form a tissue. The further

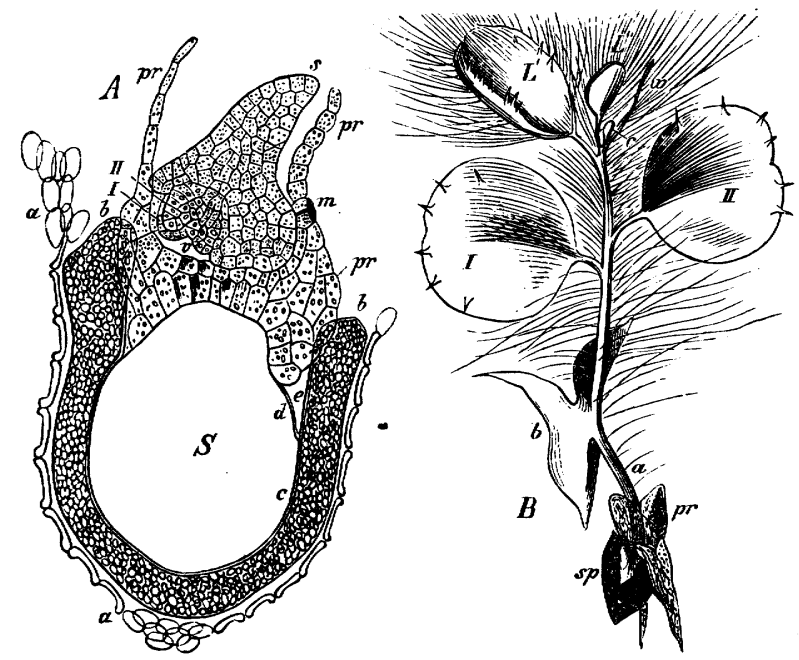

FIG. 3rr.-Salvinia natans (after Pringsheim). A longitudinal section through the macrospore, prothallium, and embryo in the median line of the prothallium ( $\times$ about $\gamma$ o), $a$ wall of the sporangium, $b$ epispore, $c$ proper wall of the spore, $e$ its prolongation, $d$ the diaphragm mentioned above which separates the prothallium from the spore-cavity, prthe prothallium already broken through by the embryo, $I, I I$ its two first leaves, $s$ the scutiform leaf; $B$ an older seedling with the spore $s p$ and prothallium $\operatorname{pr}(\times 20), a$ the caulicle, $b$ the scutiform leaf, $I, I I$ first and second single leaves, $L, L^{\prime}$ aërial leaves of the first whorl, $w$ submerged leaf of the first whorl.

processes seem to me, according to the statements of Pringsheim, Hanstein, and Hofmeister, compared with my own observations on Marsilia Salvatrix, to be briefly these:-the tissue of the prothallium is for a certain time completely enclosed in the apical papilla of the macrospore, covered above by the epidermal layers of the apex of the spore itself, and shut off from the spore-cavity below by a wall of cellulose which is stretched across like a diaphragm and is attached at the circumference to the endospore. By the further growth of the prothallium the epidermal layers of the papilla are ruptured above, the dorsal part of the prothallium projects into the funnel-shaped cavity which is left by the absence of the thick episporial layer of the macrospore; subsequently the diaphragm

1 Accordirg to Arcangeli (loc. cit.) the prothallium is formed in Pilularia by cell-division.] 
arches convexly, and the prothallium is thus pushed further outwards. This is the present state of our knowledge with respect to the position of the prothallium in the macrospore. (Compare the explanations of the figures further on.)

The prothallium of Salvinia natans attains a much more considerable size than that of the two other genera already mentioned; it contains abundance of chlorophyll, and forms a number (which may even be large) of archegonia in definite positions. After it has broken through the membrane of the papilla, it appears, seen from above, as three-sided between the three torn lobes of the epispore; one of these sides is anterior; the two posterior sides meet behind at an acute angle; a line from this angle to the centre of the anterior side runs above the elevated saddle-shaped back of the prothallium, and forms its median line. The anterior side projects above the back, and, where it meets the two posterior
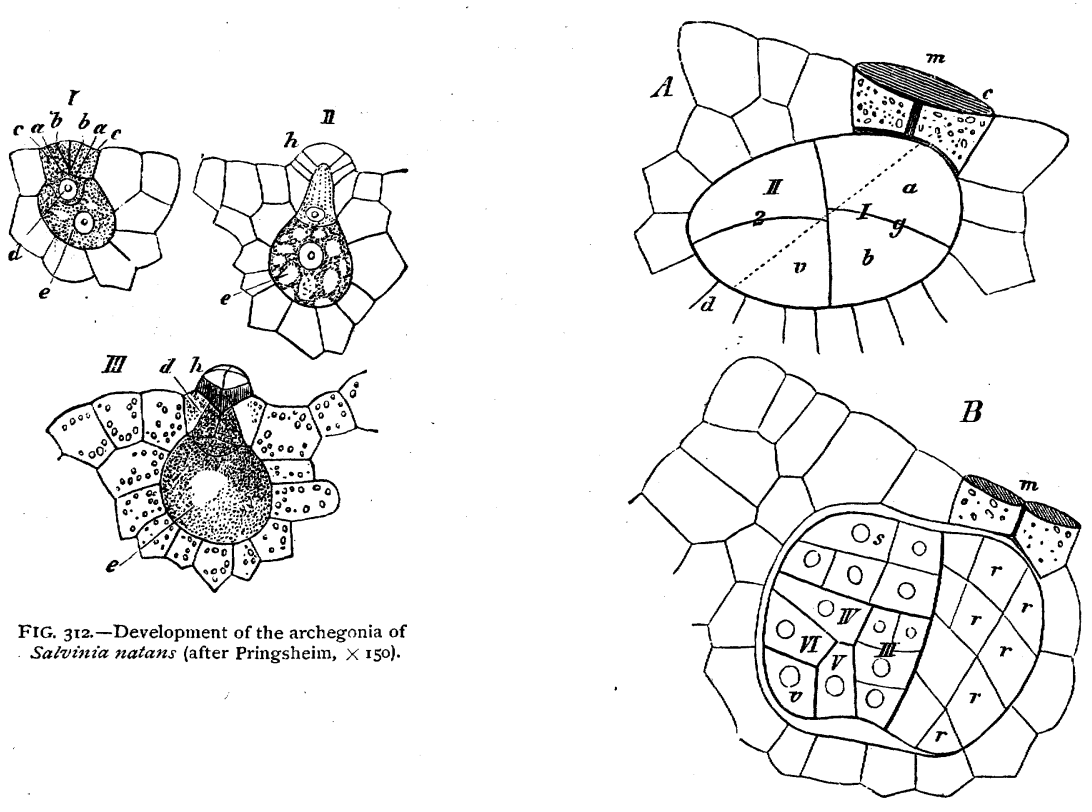

FIG. 312.-Development of the archegonia of Salvinia natans (after Pringsheim, $\times 150$ ).

FIG. 3r3.-Salvinia natans; median longitudinal section through the prothallium and young embryo; $A$ after the first three divisions of the oospore, $I$ and $I I$ are separated by the basal wall; $I$ the hypobasal segment divided by the wall $y$ into the cells $a$ and $b ; I I$ the epibasal segment,divided by the wall 2 (transverse wall); $c d$ axis of growth; $B$ embryo in a further stage of development, $r r r$ first stage of the foot, $s$ apical cell of the scutiform leaf, $I I I-V I$ the succeeding segments, $v$ apical cell of the stem; $m$ in $A$ and $B$ the closing cells of the archegonium (after Pringsheim).

sides, the two angles grow subsequently into long wing-like prolongations hanging down by the sides of the macrospore. The first archegonium makes its appearance on the median line of the elevated back immediately behind the growing anterior side of the prothallium; two other archegonia then invariably appear right and left of the first, so that they stand in a transverse row parallel to the anterior side. If one of these archegonia is fertilised there is an end of the growth of the prothallium; but if this does not happen, the prothallium continues to grow on its anterior side, and from $I$ to 3 new transverse rows of archegonia are produced, each of which contains from 3 to 7 . The long oosphere of each archegonium lies obliquely in the tissue of the prothallium; so that the outer (neck) 
end is directed backwards, its inner deeper end facing the anterior margin. At this latter point lies at a subsequent period the apical cell of the embryonal stem. Young archegonia have the apex of their central cell covered with four superficial cells arranged in the form of a cross ; in each of these latter a wall arises inclined from without inwards and downwards, followed in each inner cell by another similar partition (Fig. 3 $\mathbf{2} 2 I, a, b, c$ ). By the succeeding growth these cells are transformed into four rows, each consisting of three segments lying one above another, forming the neck $(I I, I I I)$, the lower of which are termed 'closing cells,' the upper pair the 'stigmatic cells' $(I I I, h)$. In the meantime a new cell arises at the apex of the central cell, which, with its conical point, forces itself between the closing cells $(I, d, I I I, d)$, and forms the canal-cell, first discovered by Pringsheim; according to Janczewski a small segment is cut off from the upper part of the central cell to form the ventral canal-cell, so that here, as in the other Vascular Cryptogams, two canal-cells are developed. The two canal-cells become transformed
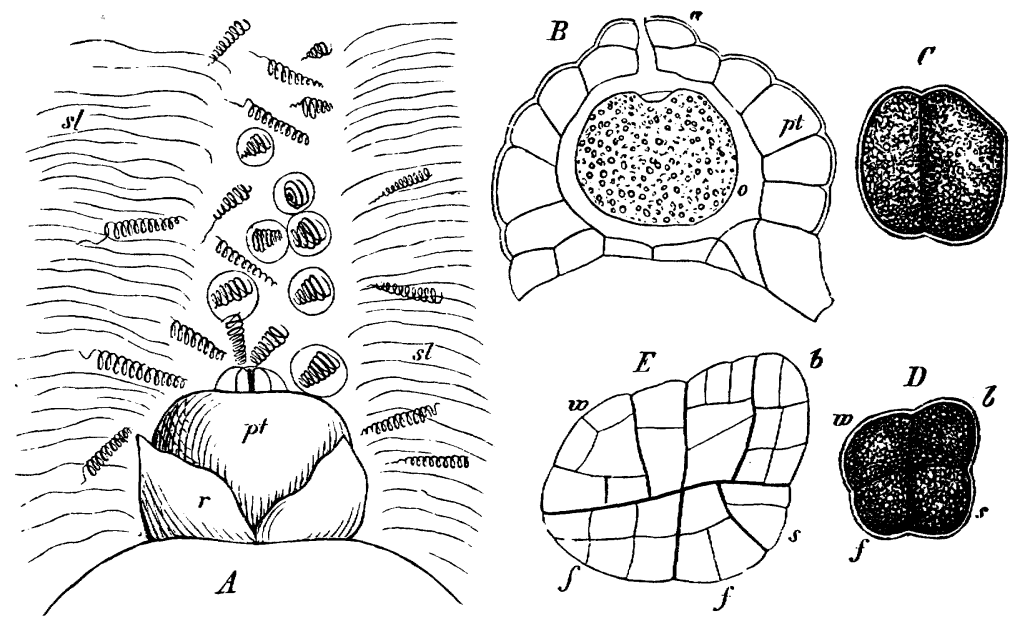

FIG. 3I4-Marsitia Salvatrix; $A$ pt the prothallium projecting through the ruptured membrane $r$ of the spore; $s l$ the mucilaginous epispore which forms the funnel, with a number of antherozoids ; $B$ vertical section of a prothallium $p t$ with an archegonium $a$ and oosphere $o ; C, D, E$ young embryos, $s$ apex of the stem, $b$ leaf, $w$ root, $f$ foot $(B-E$ after Hanstein).

into mucilage, which escapes from the canal laid open by the throwing off of the stigmatic cells. The large remainder of the central cell $(I, I I, I I I, e)$ becomes the oosphere. After fertilisation has been accomplished, the canal again closes by the lateral approximation of the 'closing cells.'

The prothallium of Marsilia and Pilularia projects as a hemispherical mass of tissue from the apical papilla of the macrospore, after it has ruptured the walls of the spore at that place (Fig. 3 i $4, A, B$ ), and remains buried at the bottom of the funnel formed by the epispore of the macrospore. Even at an early period, before the rupture, Hanstein asserts that the large central cell may be recognised in it, surrounded, in its entire circumference, at least at first, by a single layer of cells, so that the prothallium bears originally only a single archegonium. The central cell is here also covered by four cells arranged crosswise, which form at the same time the apex of the whole prothallium. By a similar process to that which occurs 
in Salvinia, they form the free neck-portion (which in Marsilia projects only slightly, in Pilularia very much) and the 'closing cells' of the archegonium. Above the central cell, the protoplasm of which contracts, a small canal-cell is visible, according to Hanstein, penetrating between the 'closing cells' and behaving as in Salvinia. Hanstein was unable to recognise any further cell-formation within the central cell, and he concluded that the whole of its protoplasmic body was converted into the oosphere; Janczewski, however, found here also the ventral canal-cell which occurs in other Vascular Cryptogams, as a small mass of protoplasm cut off from the central cell. After fertilisation the layer of tissue of the prothallium surrounding the central cell becomes double; a few chlorophyll granules arise in it, and the outer cells grow in Marsilia Salvatrix (Fig. $3^{\mathbf{I}} \mathbf{5}$ ) into long root-hairs, which are especially luxuriant when no fertilisation takes place. In the case of Marsilia Salvatrix the antherozoids collect in large numbers at the time of impregnation in the funnel above the prothallium, and force themselves into the neck of the archegonium.

Development of the Asexual Generation. The first processes of division by which, in Salvinia, the oospore is transformed, after fertilisation, into the embryo, have been most accurately described by Pringsheim. The first division is effected by a wall (basal wall) which separates the posterior (hypobasal) half of the oospore, above which is the mouth of the archegonium, from the anterior (epibasal) half, which is usually larger; this wall is nearly perpendicular to the median line of the prothallium. The two cells are next divided by walls (transverse) nearly at right angles to the previous one. If the angle enclosed by these two walls is bisected by a straight line (Fig. $\mathbf{3}^{\mathbf{I}} \hat{3}, A, c, d$ ), this line represents the axis of growth of the stem. This is followed by walls (median) at right angles to the two former, and thus the

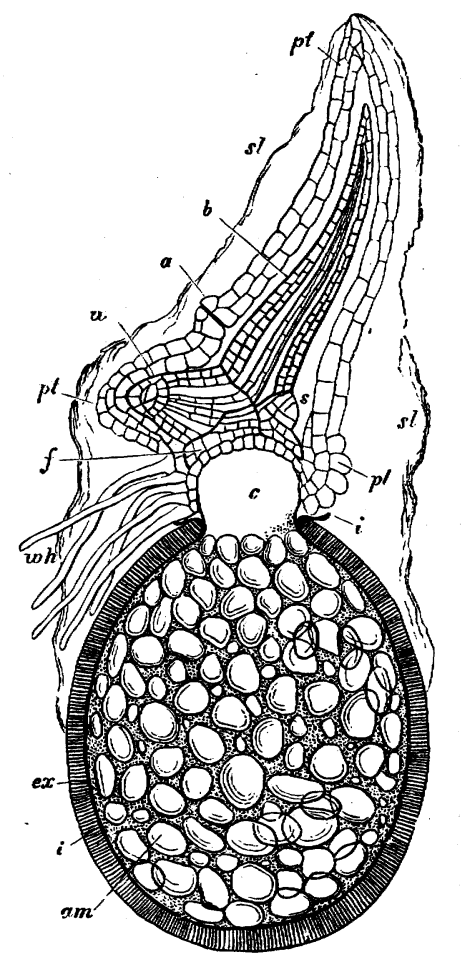

FIG. 315.-Longitudinal section through the spore prothallium and embryo of Marsilia Salvatrix ( $\times$ about 6o); am starch-grains of the spore, $i$ inner coat of the spore burst above into lobes, ex the coat of the spore burst above into lobes, ex the exospore consisting of prisms, $c$ the cavity beneath
the arched diaphragm on which is the basal layer of the arched diaphragm on which is the basal layer of
the prothallium. pt the prothallium, wh its root-hairs, $a$ the archegonium, $f$ the foot of the embryo, $w$ its root, $s$ the apex of its stem, $b$ its first leaf by which the prothallium becomes extended, $s l$ the mucilaginous epispore which at first forms the funnel above the papilla, and which still envelopes the prothallium fifty hours after the dissemination of the spores. embryo comes to consist of eight cells, octants of a sphere. Of the four epibasal octants, the two upper give rise to the first leaf (cotyledon), which, on account of its peculiar form, is known as the 'scutiform leaf:' one of the two lower gives rise to hairs, and the other to the apical cell of the stem which now lies in front and below $(A, v)$; in this latter walls are now formed inclined alternately upwards and downwards, and by this means the two rows of segments are formed out of which the structure of the stem of Salvinia is gradually developed. In Fig. 3 г $3 B$ are shown, at $I I I, I V, V$, and $V I$, these segment-cells 
undergoing still further division. The four hypobasal octants give rise to the foot. Thus no root is developed at this period, nor is one developed subsequently; Salvinia is absolutely rootless. In order to understand the subsequent processes of growth, Fig. 3II must be compared with Fig. 3 I 3 . The growing embryo bursts the prothallium; from $r r r$, in Fig. $3 \mathbf{r} 3 B$, arises the foot (caulicle) of the young plant (Fig. $3^{\text {I I }} a$ ); from $s$, Fig. $3 \mathbf{I} 3 B$, is formed the scutiform leaf (Fig. 3II $B, b$ ), by the growth of which the terminal bud of the stem becomes directed downwards (Fig. $3 \mathbf{I} \mathbf{I} A, v$ ). The epibasal part of the embryo faces the anterior side, its hypobasal part the posterior side of the prothallium; its axis of growth lies in the same plane with the median line of the latter.

The first divisions of the embryo of Marsilia Salvatrix agree in all essential points, according to Hanstein's observations and my own, with those of Salvinia; and Hanstein states that this is also the case with Pilularia; but in both these genera the rudiment of the first root is visible at an early period. The stem in these genera also creeps or floats in a horizontal direction from the first, as in Salvinia, and forms a number of roots in acropetal succession. Fig. $3 \mathbf{I} 4$ shows the first divisions of the embryo of Marsilia Salvatrix. The oospore is divided by a nearly vertical basal wall into an anterior (epibasal) larger and a posterior (hypobasal) smaller cell : these are divided, as in Salvinia and in Ferns, by a transverse and by a median wall, so that the embryo consists of eight cells. Of the four epibasal octants, one of the two upper ones becomes the apical cell of the stem, whilst the other gives rise to the second leaf (cotyledon); from the lower two the first leaf (cotyledon) is developed. Of the four hypobasal octants, the lower one, which is diametrically opposite to the apical cell of the stem, gives rise to the primary root, the other octant at the same level becoming suppressed: the two upper ones give rise to the foot (Fig. 3I4 $E, f$ ). The union between embryo and prothallium is brought about by the foot. The apical cell of the stem, Fig. $3 \mathbf{I} 4$ $E, s$, thus lies, after the formation of the first three walls, between the anterior margins of the first leaf and of the foot. In the stage represented in Fig. $3{ }^{1} 5$ this origin of the first leaf, first root, and foot, may still be recognised from the arrangement of the cells.

The further growth of the three genera, otherwise very different in their habit, to which we must add Azolla, although its development has not yet been investigated, agrees in maintaining the bilateral structure already manifested in the embryo in connection with the decidedly horizontal growth, although, as we shall see, the position of the apical cell and of its segments varies. In contrast with Muscineæ and Equisetaceæ, but in accordance with Ferns, a leaf is not produced in the Rhizocarpeæ from every segment of the stem; certain of the segments remain sterile, and these then go to the formation of internodes. The leaves grow, as in Ferns and Ophioglossaceæ, basifugally by means of an apical cell which forms two rows of alternating segments. Before the development has assumed a constant course, an increase of vigour of the young plant takes place, which is shown in the enlargement of the leaves and the greater perfection of their forms, as well as in a change of their relative positions. But in order to make this clear, it is necessary to observe separately Salvinia on the one hand, and the Marsiliaceæ (Marsilia and Pilularia) on the other. 
The embryo of Salvinia, as long as it is enclosed in the prothallium, forms, as we have seen, the segments of its apical cell alternately above and below; but when the apex of the stem is exposed in consequence of its elongation, a torsion takes place to the extent of about $90^{\circ}$, so that the two rows of alternate segments of the apical cell lie right and left, a peculiarity which has also been observed by Hofmeister in Pteris aquilina. The first leaf is the scutiform leaf mentioned above, which is placed medio-dorsaily; then follow a second and third aërial leaf standing singly, after which the definite verticillate arrangement of the leaves at length commences at the fourth node; each whorl thereafter consists of a submerged leaf springing on the ventral side (right or left), which at once branches, and forms a tuft of long filaments hanging down into the water; while two other leaves have quite flat laminæ and spring from the dorsal side, touching the water only with their under surface (Fig. 319). These three-leaved whorls alternate, and thus form two rows of ventral submerged, and four rows of dorsal aërial leaves. Their succession

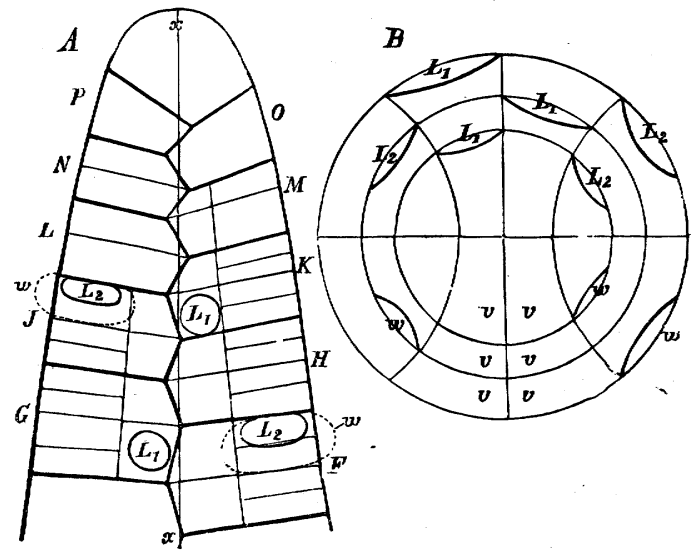

FIG. 315 a.-A the vegetative cone of the stem of Salvinia natans, represented diagrammatically and looked at from above; $x x$ projection of the plane which divides it vertically into a right and left half; the segments are indicated by stronger outlines, their divisions by weaker lines; the succession of the segments is denoted by the letters $F-P$; $B$ diagram of the stem with three whorls of leaves, its ventral side indicated by $v v ; w$ the first-formed submerged leaf; $L_{1}$ the aërial leaf formed next; $L_{2}$ the second aërial leaf of the same whorl formed last of all between the two first (after Pringsheim).

in age in the whorl, and the position of the whorls (antidromal among themselves), are indicated in Fig. $3 \mathbf{I} 5 a$. The node of the stem which produces a whorl of leaves is, as was shown by Pringsheim, formed of a transverse disc of the long vegetative cone, which in its length (or height) corresponds to a half-segment; while each internode corresponds to the whole height of a segment. Each nodal disc, as well as each internode, consists of cells of the right and left row of segments of different ages; in Fig. $\mathbf{3}^{\mathbf{I}} \mathbf{5} a$ an internode is formed of the segment $H$ on the right side, of the anterior half of the older segment, $G$, and of the posterior half of the younger segment, $J$, on the left side; the next internode is the product of the whole of the left segment, $L$, and of the two halves of $K$ and $M$ lying to the right; the intermediate nodal disc which forms the leaves $w, L_{1}, L_{2}$ consists, on the other hand, of the anterior half of the left older segment $J$ and of the posterior half of the right younger segment $K$; in the preceding and succeeding node the relationships 
are the same, right and left being transposed. In each whorl the submerged leaf is the oldest, the one further from it of the two aërial leaves the second; the nearer aërial leaf is the last formed. Each leaf arises from a cell of definite position, which becomes arched outwards (Fig. 3 I $6, B, L_{1}, L_{2}$ ), and, becoming the apical cell of the leaf, forms a row of segments on each side.

In the genus Azolla, which has been studied by Strasburger, the apical cell of the horizontal floating stem which curves upward near its growing end gives rise to a right and to a left row of segments, each of which is divided by a lateral longitudinal wall into a dorsal and a ventral half. Each of these halves is divided by a transverse wall into an acroscopic and a basiscopic portion, and each of these four cells is further divided into two by an oblique or a vertical longitudinal wall. The stem then consists (disregarding the subsequent divisions) of eight longitudinal rows of cells which have been. formed from two rows of segments. The two dorsal rows remain sterile and form neither leaves nor buds. The two rows of leaves lie right and left on the dorsal aspect, and from the neighbouring rows

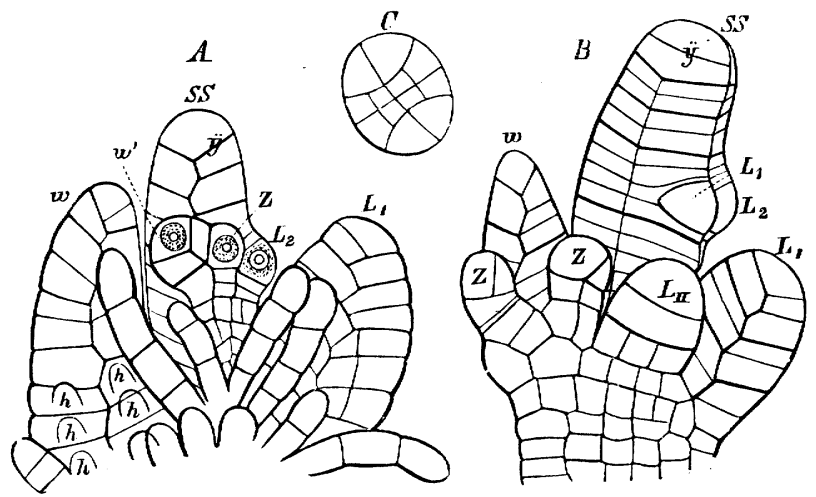

FIG. 316.-Apex of the horizontal floating stem of Salvinia (after Pringsheim); $A$ ventral side, $B$ left side, $C$ transverse section of the long vegetative cone, $S S$ apical cell of the stem, $y$ its last septum, $w$ submerged leaf, $Z$ its lateral teeth, $L L$ aërial leaves, $h h$ hairs.

of the ventral half, a little in front of or a little behind the leaves, arise the branches of the stem. Finally, the two inferior ventral rows bear the roots, each of which arises close to a bud and grows by means of a three-sided apical cell. If the leaves marked $L_{2}$ in Fig. $3{ }^{\text {I }} 5 a$ be regarded as the only ones present, the arrangement of the leaves in Azolla is approximately represented, with reference to which the buds and roots are placed in the manner above described. However, the arrangement of the leaves differs from this diagram in so far that, in Azolla, the leaves of the one row all arise from one cell of the acroscopic part of the segment, whereas those of the other all arise from one cell of the basiscopic part, in consequence of the position of the first leaf which always arises on the inner side of a branch and is directed towards the parent stem. Between any two leaves, which are placed alternately and in two rows, is an internode of the length of half a segment, one side of the internode being formed from the basiscopic, the other from the acroscopic half of a segment.

In Marsilia the apical cell of the embryo is so placed that dorsal and ventral 
segments in two rows are at first formed from it by walls inclined upwards and downwards; the dorsal median leaf also proceeds from the first dorsal segment. But a different arrangement is soon produced as the plant increases in strength; the apical cell of the stem forms segments arranged in three rows with a $\frac{1}{3}$ divergence, and in such a manner that one row of segments comes to lie below (ventrally), while the two other rows form the dorsal side of the stem. The ventral side of the stem forms roots in strictly acropetal succession, as in Azolla, the youngest being found near the apex of the stem. On the dorsal side of the stem the

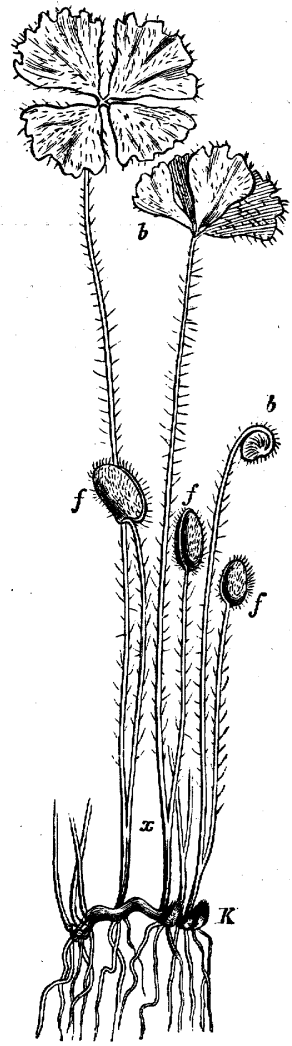

FIG. 317.-Anterior part of the stem of Marsilic Salvatrix with leaves (reduced one-half); $K$ terminal bud. $b b$ leaves, $f f$ sporocarps springing from the leaf. stalks at $x$.

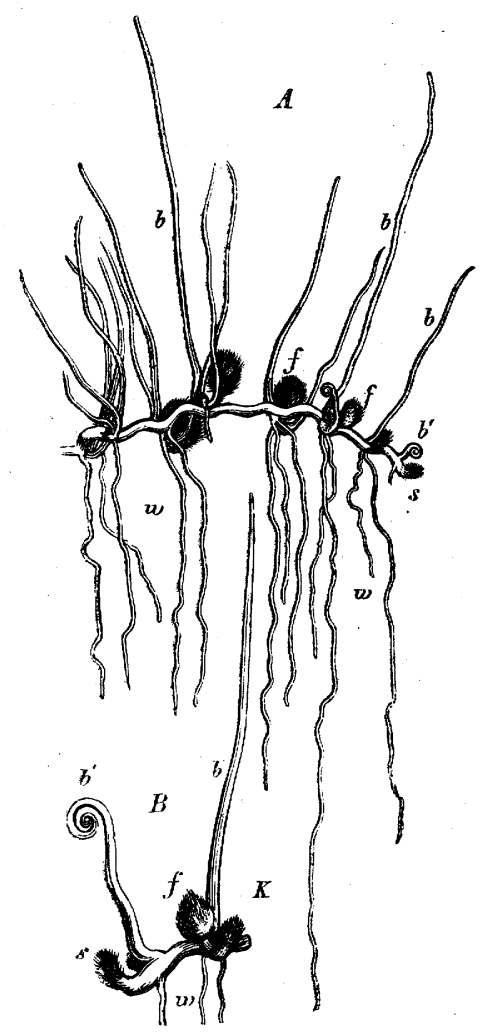

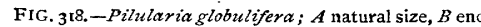
of a shoot magnified, $s$ terminal bud of the stem, $b b$ leaves, $w$ roots, $f$ sporocarps, $K$ lateral bud.

leaves arise in two alternating rows, some of the dorsal segments remaining at the same time sterile and serving for the formation of internodes. The first leaf of the young plant, lying in the median line and without a lamina, is followed, in the biseriate arrangement which now results, by a number of young leaves with a short stalk and a lamina at first entire but afterwards divided into two and four lobes; normal leaves, circinate in their vernation, are then for the first time formed with a long stalk and a quaternate lamina. In the processes which have now been described, Pilularia agrees, according to Hanstein's observations, with 
Marsilia, except that all the leaves remain destitute of a lamina (Fig. 318); they are long, conical, filiform, and at first rolled up spirally forwards.

The Branching of Rhizocarps is similar to that of Ferns. Pringsheim states that in Salvinia terminal branching never occurs ; new shoots arise, on the contrary, exclusively from the basal part of the submerged leaves, each leaf of this description forming a shoot on the side which faces the nearer aërial leaf; every branch produces at once a trimerous whorl of leaves. Strasburger, however, considers it possible that these branches arise, as in Azolla (see ante), from the stem itself close to the leaves. The branching of the Marsiliaceæ has been termed by Hanstein axillary, a designation with which, however, I am unable to agree. The lateral shoots have altogether the appearance of springing from the stem itself near to the leaves, but at a later period they appear to be lateral and not axillary to the leaves. As to their first origin, which has not yet been accurately ascertained, it appears to be most natural to refer to the relative positions of these organs in Azolla, where the lateral branches of the stem arise from cells lying anteriorly or posteriorly or even dorsally to the origins of the leaves.

The Growth of the roots of the Marsiliaceæ and their monopodial branching agree with that of Ferns and Equisetaceæ in all important points. It has already been mentioned that among the Salviniaceæ, Salvinia itself is quite destitute of roots, and that in Azolla the two ventral rows of cells of the stem give rise to roots by the side of the lateral buds. The apical cell of the stem of these plants forms segments in two rows, whereas that of the root is a three-sided pyramid like that of the Marsiliaceæ, Ferns, and Equisetaceæ. A root-sheath is formed, according to Strasburger, from the cells of the stem which lie over the endogenous mother-cell of the root of Azolla, which keeps pace with the growth of the root and invests it during the whole of its existence. It is still more remarkable that the root-cap of Azolla is formed from one single cell. From this cell two layers of cells are derived which grow with the growth of the root and completely invest it on all sides.

The Sporocarps. The formation of the fructification shows even more evidently than the structure of the vegetative organs, that the two families of the Rhizocarpeæ present considerable differences in certain particulars, which demand separate consideration, as well as an agreement in essential characteristics.

The Salviniacee, including the two genera Salvinia and Azolla, occupy with respect to their fructification an intermediate position between the Ferns and the Marsiliaceæ. Their sporangia are enclosed in unilocular capsules, two or more of which occur on the segments of the leaves (Fig. 319 A,B). In Salvinia these capsules are borne on the basal segments of the submerged leaves; in Azolla it is the external, downwardly directed segment of the deeply bisected leaf, and moreover of the first leaf of each branch, which bears them. The apex of the segment of the leaf which is to form the sporocarp grows out into a columella (placenta) upon which the sporangia are developed, and at the same time an annular wall arises round the base of the columella, and continues to grow until it closes over its apex, thus forming the wall of the sporocarp by which the sorus is completely enclosed. The sporocarp of the Salviniacex closely resembles the sorus of the Hymenophyllacex, but differs from it in that the indusium of the 
latter is cup-shaped and therefore open at the apex, whereas the wall of the former completely encloses the sorus, as does the indusium of Cyathea. The sporocarp of the Salviniaceæ is then a sorus, the term being used in the sense in which it is applied to the fructification of Ferns, but here the indusium is much more fully developed, consisting of two layers of cells, the walls of which, toward the upper part of the capsule, become lignified in Azolla.

Each sporocarp contains either microsporangia or macrosporangia, but both kinds of sporocarp occur in the same plant and even upon the same leaf, so

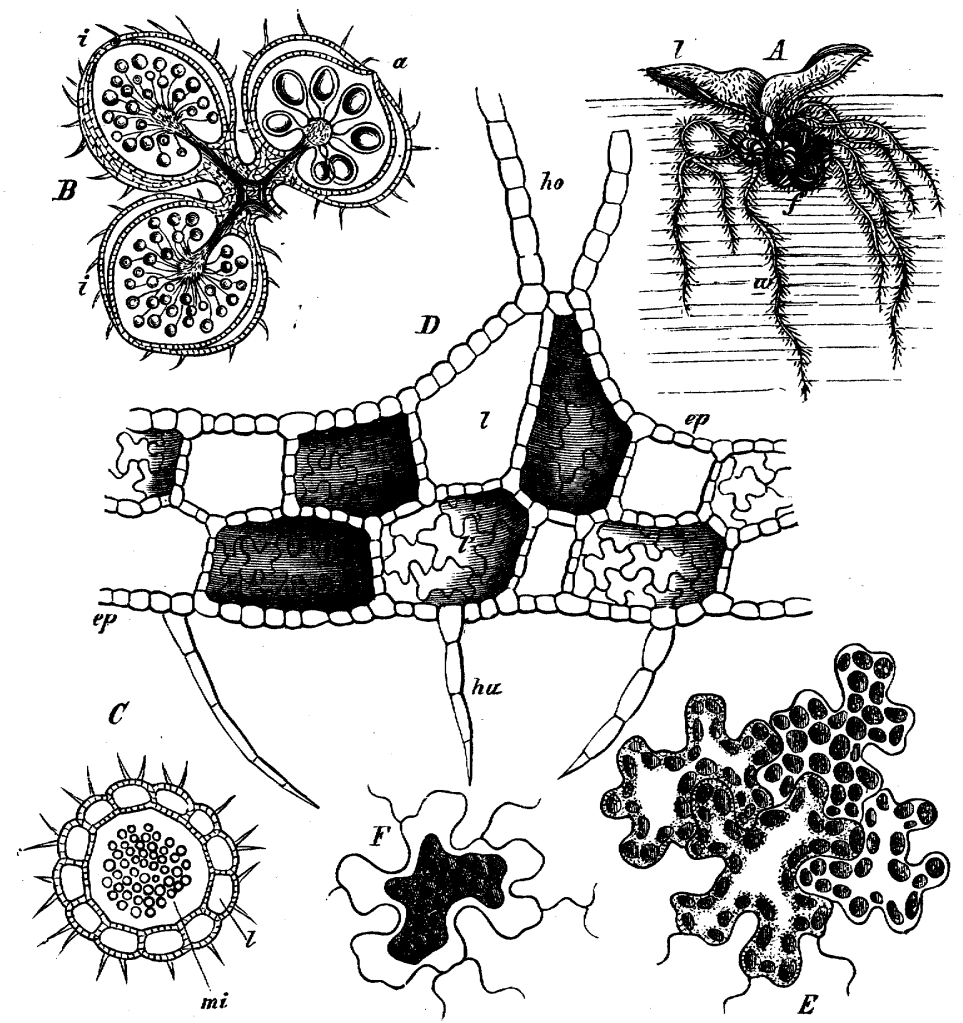

FIG. 319.-Salvinia natans; $A$ transverse section of a stem bearing a whorl of leaves, $l$ aërial leaves, $w$ submerged leaf with several teeth, $f$ sporocarps on it (natural size); $B$ longitudinal section through three fertile teeth of a submerged leaf, $a$ a sporocarp with macrosporangia, $i i$ two sporocarps with microsporangia; $C$ transverse section of a sporocarp with microsporangia $m i ; D$ transverse section of an aërial leaf, $h u$ hairs of the under side, $h o$ hairs of the upper side, ep epidernis, $l$ air-cavities, the dark ones show the vertical walls of the tissue in the background $(B-D \times 10) ; E$ cells of a lamella of tissue in the leaf; $F$ one of the cells after contraction of the contents in glycerine.

that the plant is monœcious. The microsporangia are contained in Azolla, as in Salvinia, in large numbers within the sporocarp; in Azolla only a single macrosporangium is formed in a sporocarp, whereas in Salvinia several macrosporangia are formed. All the spores derived from the mother-cells ( 16 ) in a microsporangium reach maturity, but only one of the $(4 \times 16)$ spores of a macrosporangium ever becomes ripe, so that in Azolla, where there is but one macrosporangium in a sporocarp, a single macrospore is enclosed by the wall of the 
sporocarp and by that of the sporangium (which subsequently disappears?). The sporangia are capsules borne on long stalks, the wall of which, when mature, consists of a single layer of cells. The macrosporangium is shortly stalked and probably arises from the apex of the columella.

The development of the sporangia in Salvinia has recently been investigated by Juranyi. The columella is covered with a layer of radially elongated cells, each of which grows out into a papilla and forms the rudiment of a sporangium. The papilla is divided by a transverse septum into a lower and an upper cell. The lower one undergoes repeated transverse divisions and forms the long, segmented stalk, which comes to consist of several rows of cells by means of longitudinal divisions in the case of the macrosporangia only; the upper one assumes a hemispherical form and gives rise to the body of the sporangium by means of divisions which are similar to those occurring in the Polypodiaceæ (see Fig. 305 ). A wall is formed consisting of a single layer of cells, within which is a single tetrahedral cell, the archesporium. From this, four flat segments are cut off which form a layer of cells containing much protoplasm surrounding the tetrahedral central cell. The central cell, by repeated division, gives rise to the sixteen mother-cells of the spores, and meanwhile the layer investing it has undergone division into two layers which together form the tapelum. Each of the sixteen mother-cells of the spores then divides into four tetrahedrally placed spore-cells. $U_{p}$ to this point the procasses are the same in both micro- and macrosporangia. In the microsporangia all the sixty-four cells develope; they become separate and are scattered irregularly in the cavity of the sporangium. The tapetum becomes disorganised and forms a frothy mucilage, which subsequently hardens, in which the spores are enclosed. In the macrosporangia only one of the sixty-four rudimentary spores continues to develope; it becomes enormously enlarged so that finally it nearly fills the cavity of the sporangium. A large nucleus lies at that end of the macrospore which is directed towards the apex of the sporangium. During the growth of the macrospore the tapetum, and subsequently all the other undeveloped spores, undergo disorganisation, becoming converted into a frothy plasma which is in contact with the outer coat of the macrospore, and is especially accumulated at its apex. It is this frothy plasma which becomes hardened and forms a thick investment for the mature macrospore, termed the Epispore (Fig. 3 I I, $A$ ), which splits even during its formation into three lobes over the apex of the spore, so as to permit of the subsequent outgrowth of the prothallium.

Strasburger had already demonstrated the existence of this hardened frothy mucilage in both kinds of sporangia of Azolla, but in this plant it assumes a far more conspicuous appearance. In the microsporangia the mucilage looks like a large-celled tissue, and forms from two to eight separate clumps (Massula), each of which encloses a number of microspores. In some species (A. filiculoides, Caroliniana) these massulæ have their surfaces covered with hair-like appendages, barbed at their free ends (Glochidia), by means of which they attach themselves, when they have escaped from the sporangia, to the floating macrospores. The macrosporangium of Azolla contains a single spheroidal macrospore, which does not nearly fill its cavity, the whole surface of which is completely covered with a thick rugose layer of this hardened frothy mucilage. It projects from the apex of the spore and runs 
out beyond it into a pencil of delicate filaments; three (or three times three) large masses of it are attached at this point. The frothy mucilage with its cavities full of air thus forms a float for the macrospore, and bears on its surface the upper part of the ruptured sporangium.

The sporocarps of the Marsiliaceæ are much more complex and more firmly. constructed than those of the preceding family. The sporocarp of Pilularia is a shortly-stalked spheroidal capsule, standing apparently by the side of a leaf towards its ventral aspect. Its morphological significance is not yet fully understood. The capsule has a very thick hard wall, consisting of several layers of cells, and contains, besides a considerable quantity of soft succulent parenchyma, hollow chambers which extend from base to apex. Pilularia globulifera (Fig. 320) has four such chambers, $P$. minuta two, and $P$. americana three. The external wall of each chamber bears a thickening along its median line of the inner surface throughout its whole length, and in this thickening runs a fibrovascular bundle. On this receptacle (placenta) the stalked sporangia are borne, forming a sorus which consists at its lower part mostly of macrosporangia, at its upper of microsporangia. Probably each chamber has, at an early stage of development, an aperture at its apex, but

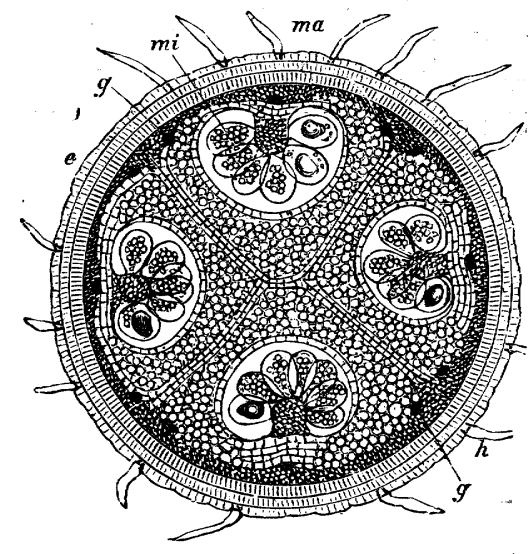

FIG. 320.-Transverse section of the sporocarp of Pilularia globulifera below the middle, where the macrosporangia and microsporangia $m a$ and $m i$ are intermingled; $g$ the fibrovascular bundles, $h$ hairs, $e$ epidermis of the outer surface. it is not clear here, any more than in Marsilia, how far a comparison of the delicate tissue which invests the sorus to an indusium is justifiable, a comparison which is made by many botanists.

The sporocarps of the various species of Marsilia are usually somewhat bean-shaped capsules with very hard walls, borne on long or short stalks, which arise either upon the ventral aspect of the petioles of ordinary foliage-leaves (Fig. $3^{\mathbf{I}} 7$ ) or at their bases. Their stalks may be simple, bearing but one sporocarp; or forked, bearing many sporocarps. From a petiole several usually arise together. The stalk is continued along the posterior edge of the sporocarp (Fig. $3^{2} 5$ ), giving off lateral branches right and left, which dichotomise and run towards the ventral edge. The ripe capsule is bilaterally symmetrical and contains two rows of chambers each of which extends from the ventral to the dorsal margin (Fig. $3^{21}, A, B$ ). In the very young fruit, according to Russow, each chamber communicates with the exterior by means of a narrow canal opening on the ventral aspect. A thickening (placenta) runs along the external wall of each chamber, bearing the macrosporangia on its central ridge, and the microsporangia on its flanks; thus each chamber. contains a sorus consisting of two kinds of sporangia. When the sporocarp bursts it becomes evident (Fig. 325) that the soft internal tissue forms, as in Pilularia, a completely closed investment for each sorus. When mature the microsporangia contain numerous $\left(4 \times \mathrm{r}^{\prime} 6\right)$ spores, the macrosporangia but a single one. 
The development of the sporangium begins with the outgrowth of one of the superficial cells of the placenta which bears the sorus. The subsequent divisions are the same as those above described with reference to Salvinia, so that here also the sporangium is soon elevated on a stalk and consists of a wall of a single layer of cells and of a tetrahedral central cell or archesporium (Fig. 322, I-III). From this a tapetum is cut off by four septa parallel to its sides, which, as in the Salviniaceæ and in the true Ferns, surrounds the cell from which the spores are subsequently derived (Fig. $3^{22}, I V, V$ ). The stalk of the sporangium of the Marsiliaceæ, in accordance with the foregoing description, consists at first of three rows of cells, but these rows are afterwards multiplied by longitudinal divisions. As the young capsule of the sporangium gradually increases in size, the cells of the wall undergo radial division and the cells of the tapetum both radial and tangential. The central cell by repeated bipartition forms the sixteen mother-cells of the spores,

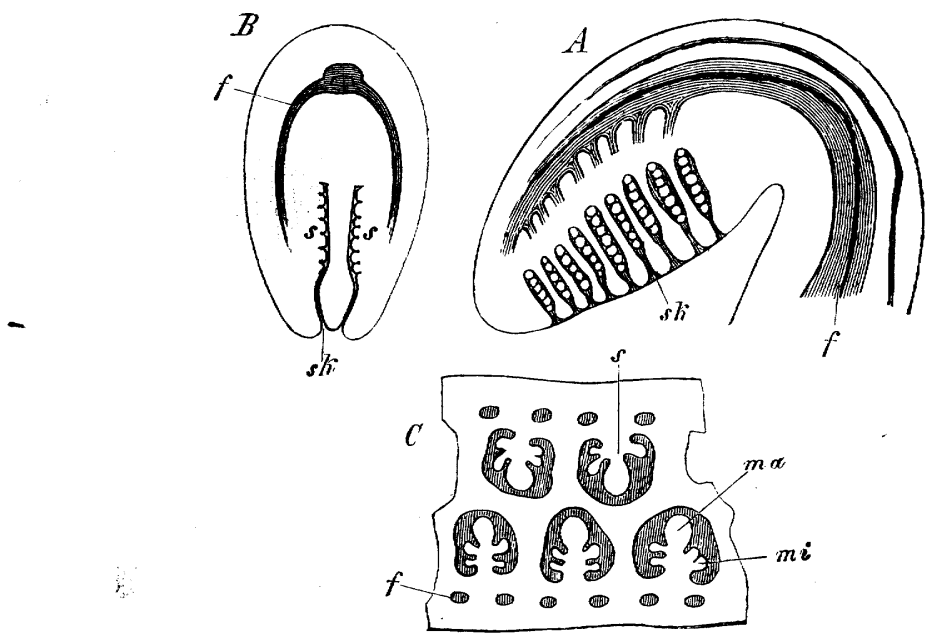

\footnotetext{
F1G. 32t.-A very young sporocarp of Marsilia elata (after Russow); $A$ a median longitudinal section; $B$ a transverse section; $C$ part of a longitudinal section at right angles to $A$. $f f$ fibro-vascular bundles, $s s$ sori, $s k$ canals of the sori, $m a$ macrosporangia, $m i$ microsporangia (compare Fig. 325 ).
}

each of which developes four tetrahedrally arranged spores in the usual way. During this process the cells of the tapetum gradually undergo disorganisation, and the cavity of the sporangium becomes filled with a granular plasma in which lie the mother-cells and the tetrads of spores and from which the remarkable epispore is subsequently formed. Up to this point the course of development in both kinds of sporangia is the same, but differences now become apparent. All the spores of the sixteen tetrads formed in the microsporangia reach maturity; each of the four spores within a mother-cell ${ }^{1}$ surrounds itself with a permanent coat, and then the wall of the mother-cell becomes absorbed. In the macrosporangia one of the young spores of each of the sixteen tetrads grows more vigorously than the other three; finally all the tetrads but one cease to develope, and the largest cell of this one-the future

1 Russow raises the objection, $l$. c. p. 62, that I make no mention of special mother-cells in describing the development of the spores. His mistake finds its correction in $\S 3$ of Book $I$. 
macrospore-increases very much in size whilst the other three gradually shrivel. Figures $3^{2} 3$ and $3^{24}$ illustrate the development of the macrospore of Pilularia globulifera, after drawings made by me in $\mathbf{1} 866$. They show the young macrospore in $I, I I, I I I$, still in connexion with its three sister-cells which are invested by the cell-wall of the mother-cell which has already become mucilaginous $(I)$. The four cells are attached to each other by means of rigid spine-like projections, that of the macrospore being the most strongly developed. At a later period the macrospore is
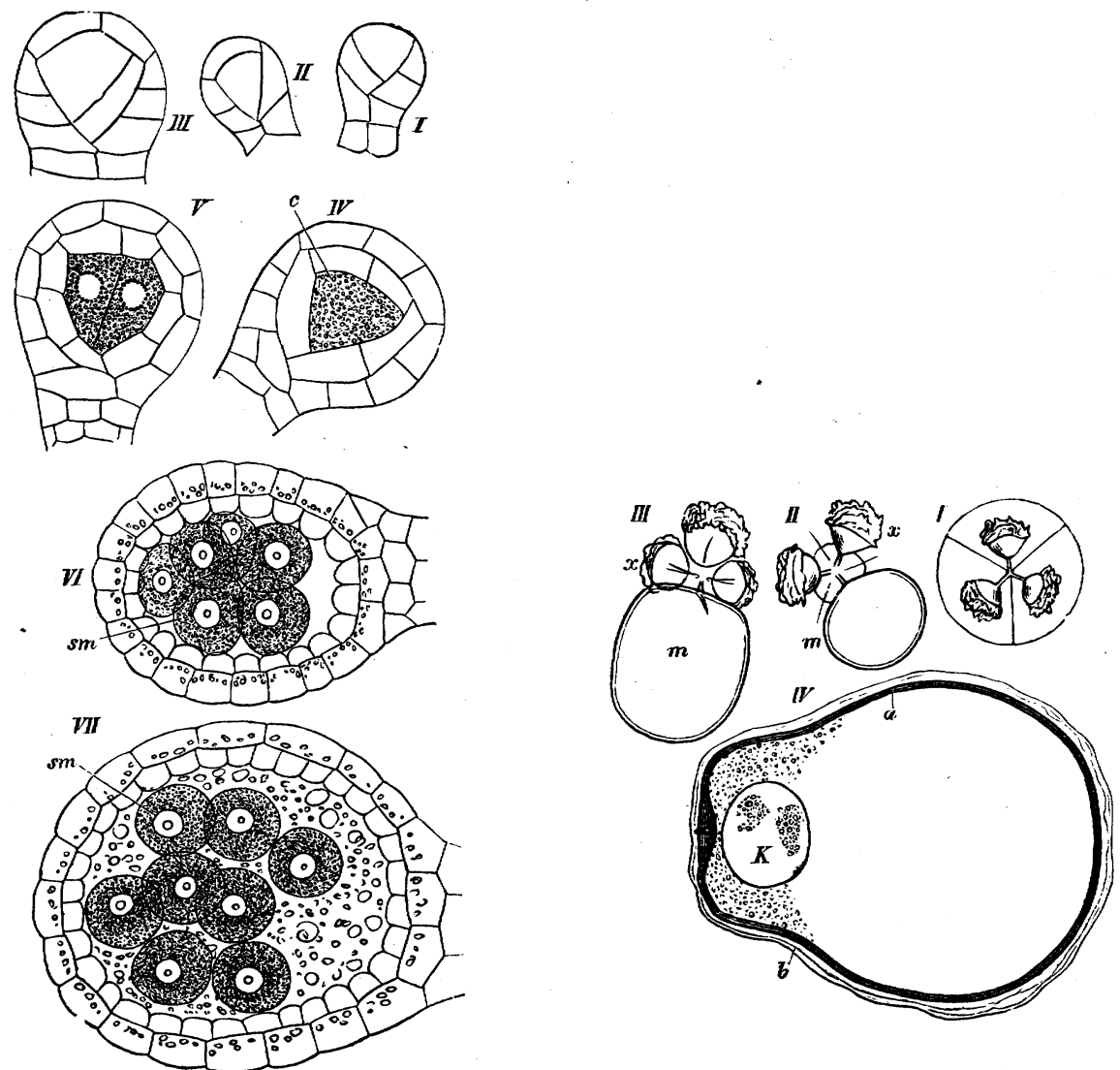

FIG. 322.-Development of the sporangium of Pilutaria globulifera, all the figures in optical longitudinal section; $I V c$ primary mother-cell of the spores invested by the tapetum; sm mother-cells of the spores $\left(\times 55^{\circ}\right)$.

FIG. 323-Development of the macrospore of Pilutaria globulifera; $x$ the abortive sister-cells, $m$ the macrospore, $K$ its nucleus, $a$ the inner, $b$ the outer coat.

of very considerable size; its aborted sister-cells are attached to it laterally (Fig. $3^{24}, x$ ), and its firm coat has become brown and is invested by a layer of mucilage (Fig. 323, $I V, b$ ) which often appears to be folded. It forms a papilla (Fig. $3^{24}, b^{\prime}$ ) at the apex which shrivels when the spore ripens. A layer of a soft substance, presenting a distinctly prismatic structure, makes its appearance (Fig. $3^{24}, C$ ) on the outside of the mucilaginous layer, and becomes in its turn invested by a third layer which is thicker than itself of a less distinctly organised structure. Both these layers leave the apex of the spore uncovered and form a funnel through which the 
antherozoids penetrate after germination (comp. Fig. 3 $3_{4}$ ). The macrospores of Marsitia possess a similar epispore, the development of which has been very fully but not very intelligibly described and figured by Russow. According to him the mucilaginous invęstment of the abortive sister-cells passes over to the macrospore and forms the first layer of its epispore; then a protoplasmic vesicle encloses the whole macrospore, and within this the thick prismatic layers of the epispore are developed. Russow's account confirms the view which I had already expressed that these investing layers are deposited from without, a view which is rendered even more probable by the facts known concerning these processes in the Salviniaceæ, but it makes the whole process somewhat obscure, and I have no material at present for a fresh study of the formation of the epispore in the Marsiliaceæ.

The ejection of the macro- and microspores from the very firm sporocarps is associated with some remarkable processes, for a knowledge of which we are indebted to Hanstein. The ripe fruits of Pilularia globulifera lie either in moist
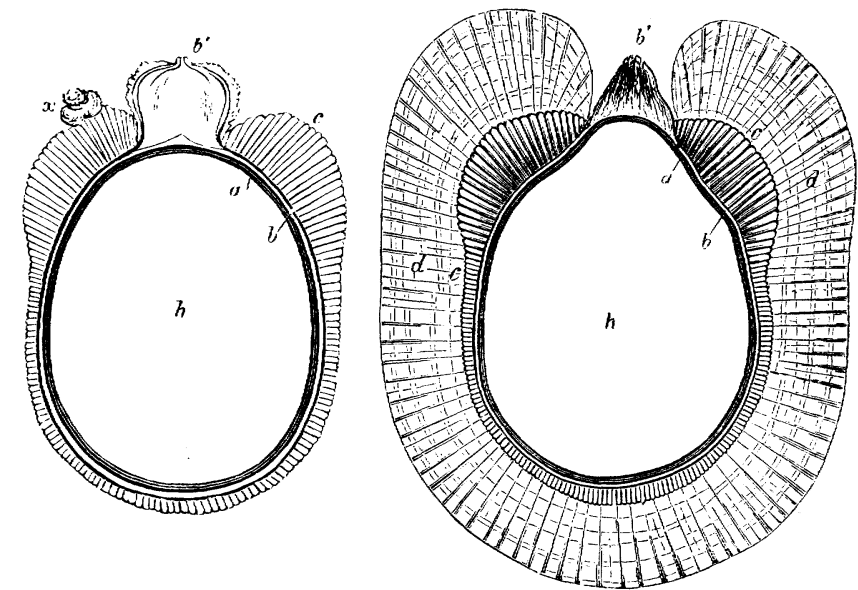

FIG. 324. -Further development of the macrospore of Pilularia globulifer $a ; h$ cavity of the spore, $a$ the first inner coat, $b$ the second, $c$ the third, $d$ the fourth coat; the second, third, and fourth together form the epispore $(\times 80)$.

earth or upon it; the wall splits at the apex into four valves, and a hyaline viscid mucilage, evidently derived from the tissue separating the chambers, escapes and forms a drop upon the surface of the soil which continues to increase in size for some days. In this mucilage the microspores and the macrospores are carried out, and they germinate in it. When fertilisation has taken place the drop of mucilage becomes fluid, and leaves the fertilised macrospores on the moist ground to which they are attached by the root-hairs of their prothallia until the first root of the embryo penetrates it. Fig. $3^{2} 5$ represents the most important of the corresponding processes in the case of Marsitia Salvatrix. If the sporocarp, which is as hard as a stone, be slightly injured along its ventral margin and be then placed in water, the water gains access to the interior and causes the tissue forming the walls of the chambers to swell-up, and the result is that the testa splits along its ventral side into two valves. It is shown in Fig. $325, B$, that a hyaline ring protrudes, which lay previously in the angle along the ventral edge, and carries with it the chambers 
containing the sori which are less capable of swelling-up. As the ring gradually expands the attachments of the chambers to the dorsal margin of the sporocarp become ruptured and they are drawn completely out of the testa. As a rule the attachment of the ring is broken through at one end, it then straightens itself and bears the chambers, which are still closed sacs, in two longitudinal rows, there being now a considerable interval between each pair of chambers, whereas they were closely packed together whilst still within the testa. These processes are completed in a few hours, both kinds of spores are set free, and, if the temperature be favourable, fertilisation takes place within from twelve to eighteen hours after placing the sporocarp in water.

(a) Histology ${ }^{1}$. The development of the tissues of the Rhizocarps agrees in all essential points with that of the true Ferns. The growth of the stem, of the root, and to a certain extent of the leaves, by means of an apical cell, is as evident here as in the Characeæ and Equisetaceæ, and has been thoroughly studied. The epidermis presents several peculiarities, more especially as to the stomata. . The fundamental tissue is remarkable, as is usually the case with water or marsh plants, for its large intercellular spaces. For information as to the occurrence of sclerenchyma in the leaves and in the testæ, the memoirs of Braun and of Russow must be consulted. The fibrovascular bundles, more especially those of the Marsiliacex, very closely resemble those of the true Ferns in their composition. There is a central xylem entirely surrounded by phloëm, and this again is invested by a single layer of cells with folded lateral walls forming the bundlesheath. A single bundle traverses stem, root, and leaf. In Marsilia, the bundle branches in the leaf, forming a dichotomous venation. A transverse section of the stem of the Marsiliaceæ shows the fibrovascular mass forming a ring surrounding a central mass of fundamental tissue. The fibrovascular mass consists evidently of several bundles which have coalesced; that this is so is indicated by the fact that the phloëm of its inner side is

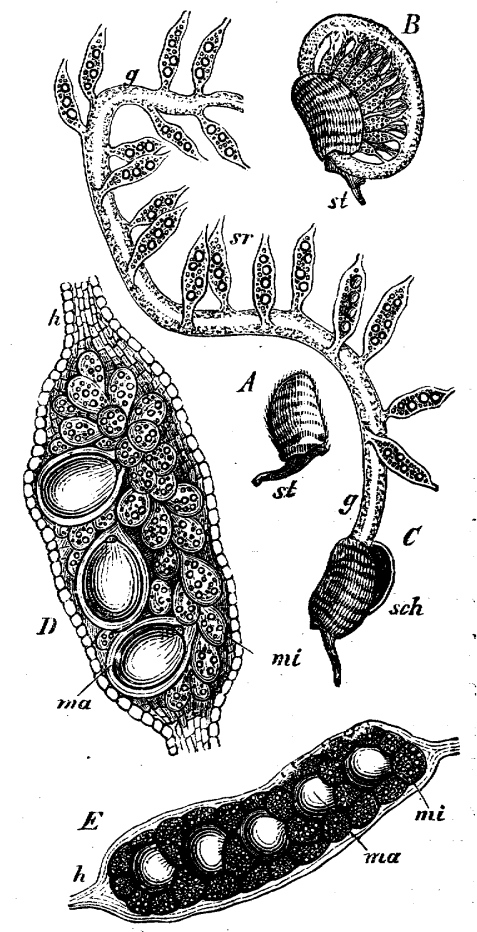

FIG. 325.-Marsilia Silvatrix; $A$ a sporocarp (natural size), st the upper part of its pedicel; $B$ a sporocarp which has burst in water and is protruding its gelatinous ring (after Hanstein); $C$ the gelatinous ring $g$ ruptured and extended, $s r$ compartments of the sporocarp; $s c h$ testa of the sporocarp; $D$ a compartment of an unripe sporocarp with its sporangia; $E$ one from a ripe sporocarp; $m i$ microsporangia, ma macrosporangia. bounded by a bundle-sheath just as the outer side is. In Pteris aquilina it often happens that the two wide cauline bundles of the stem unite laterally so as to form a tube enclosing a medulla.

(b) Classification. It is already evident from what has been already said, that the Rhizocarpeæ belong to two clearly-defined families, of which the one, the Salviniaceæ is closely allied to the true Ferns, whereas in the other, the Marsiliaceæ, the especial characteristics of the order find their most complete expression.

Family I. Salviniaceæ. Plants floating horizontally on the surface of water. The

${ }^{1}$ [For further details on this subject see De Bary, Vergleichende Anatomie der Vegetationsorgane, $1877 ;]$ 
apical cell of the stem forms two rows of segments, a right and a left. Sori either male or female: each sorus contained in a unilocular sporocarp. Spores invested by hardened frothy mucilage (Massulæ, Epispore). The prothallium derived from the macrospore is well developed and bears several archegonia. Azolla has roots, Salvinia has none.

Family 2. Marsiliacem. Plants creeping on moist earth, or floating to some extent in water. The three-sided apical cell of the stem forms two dorso-lateral rows, and one ventral row of segments. Each sorus includes both macro- and microsporangia, and two or more sori are contained in the multilocular sporocarp. The spores are invested by hardened mucilage-epispore-which presents a radially prismatic structure, and is to some extent capable of swelling-up. The prothallium of the macrospore bears a single archegonium. This family includes the genera Marsilia and Pilularia.

\section{CLASS IX.}

\section{Diснотоме ${ }^{2}$.}

Hitherto the Lycopodieæ, Psilotum, the Selaginellæ, the Isoëteæ, etc., have been included together in the group of the Lycopodiaceæ, and rightly so, for these genera exhibit not only in their habit, but also in their morphology, a degree of relationship which makes it impossible to separate any one of them from the others with the view of erecting it into a new class or of including it in one of the other two classes of Vascular Cryptogams. Recent investigations have, however, shown that this class includes two well-defined subdivisions which must be kept distinct. To one of these two orders belong the genus Lycopodium and its immediate allies, and it must therefore necessarily take the name of Lycopodiaceæ; consequently a new name has to be found for the whole class, and I select that of Dicнотоме e, because it brings into prominence one of the most obvious of the characteristics of these plants, that the branching presents the appearance of being the result of dichotomy, although, as a matter of fact, the branching of the stem is monopodial. It must be remembered, however, that these two modes of branching gradually pass one into the other. These plants are as remarkable among vascular plants for their evident tendency to branch dichotomously as are the Equisetaceæ for their whorls of leaves. That dichotomy is indeed a typical peculiarity of these plants is proved by the fact that their roots are the only ones at present known which branch dichotomously.

The dichotomous habit of the branching is by no means the only characteristic common to the members of this class, as a comparison with the Equisetaceæ and Filicineæ at once shows. They possess but little in common with the Equisetaceæ except perhaps the relatively slight development of the leaves, although that is brought about in this class in a very different way, for the similarity in the mode of development of the sporangia in the two classes does not merit consideration since, as we have seen in the Filicineæ, this is a characteristic which is critical only for the

1 [Inasmuch as dichotomous branching is not universal even among the Lycopodieæ, many authors prefer to designate this group of plants as Lycopodinæ.] 
smaller subdivisions. It is easier to find points of connection between this group and the Filicineæ, such, for instance, as the similarity in the composition of the fibrovascular bundles, and certain modifications of the fundamental tissue which occur in the two classes, but the external conformation is the most important point to be considered as an indication of relationship. In the Filicineæ the well-developed leaves form the principal morphological feature, whereas in the Dichotomeæ they are, though numerous, of very simple structure and of small size; in the one class the external conformation depends upon the stem, in the other upon the leaves. It is of great importance to note that each leaf, in the Dichotomeæ, produces a single sporangium, whereas each leaf, in the Equisetacex and Filicinex, bears several sporangia. In this group the sporangium arises typically near to the base of the leaf, and it is of but little consequence that in some cases it arises further back from the axil of the leaf or even from the stem; this is merely an example of an occurrence common among Phanerogams that organs of the same morphological value (in their case the branches) arise at one time at the base of the leaf, at another in its axil, or even from the stem above the axil. Although it has already been pointed out that the mode of development of the sporangia is insufficient to define the principal subdivisions of the Vascular Cryptogams, it must be remarked here that, in contrast to the various modes occurring in the Filicinex, the mode of development of the sporangia is constant, even when the other morphological characteristics are widely different; here the sporangium is always developed from a group of cells.

A short definition of the two orders-I. Lycopodiaceæ, II. Ligulatæ-is given at the close of the introduction to the Vascular Cryptogams.

\section{Order I. Lycopodiaces ${ }^{1}$.}

I. The Sexual generation (Oophore). The conditions which are necessary for the germination of the spores of Lycopodium, Psilotum, etc., are as yet unknown. In spite of the very numerous attempts made to cultivate them only one observer, De Bary, has succeeded in obtaining the early stages of the development of the prothallium of Lycopodium inundatum. The endospore protruded as a spheroidal vesicle from the exospore which had split into three valves: the vesicle was then divided by a transverse septum into an inner basal cell, which underwent no subsequent alteration, and an outer cell which grew like an apical cell and formed two rows of segments. Each segment was then subdivided by a tangential wall into an

1 Bischoff, Die cryptog. Gewächse. Nürnberg 1828.-Spring, Monographie de la famille des Lycopod. (Mém. de l'Acad. roy. Belge, $18_{42}$ et I849).-Cramer, über Lycop. Selago in Nägeli und 'Cramer, Pfl. phys. Unters. Heft 3, I855.-De Bary, uiber die Keimung der Lycop. in Ber. d. naturf. Ges. zu Freiburg-i-Br. and Ann. Sci. Nat. 1858. Heft IV.-Nägeli und Leitgeb, über die Wurzeln, in Nägeli's Beit. zur wiss. Bot. Heft 4, 1867.-Payer, Botanique cryptogamique. Paris 1868.Hegelmaier, Bot. Zeit. I872.-Russow, Vergl. Unters. Petersburg I 872.-Mettenius, über Phylloglossum, Bot. Zeit. 1867. - Juranyi, über Psilotum. Bot. Zeitg. 1871. - Fankhauser, Bot. Zeitg. 1873.Strasburger, Bot. Zeitg. 1873. [Arcangeli, Studii sul Lycopodium Selago, 1874.-Prantl, Bemerk. üb. d. Verwandtschaftsverhältnisse der Gefässkryptogamen; Verh. d. phys.-med. Ges. zu Würzburg, 1875. - Braun, Blattstellung und Verzweigung bei Lycopodium, Sitzber. d. bot. Ver. d. Prov. Brandenburg, 1874.--Hegelmaier, Zur Kenntniss einiger Lycopodinen, Bot. Zeitg. 1874.-Bruchmann, Ueb. Anlage $\mathrm{u}$. Wachsthum der Wurzeln von Lycopodium, Jenaische Zeitschrift, 1874.] 
inner and an outer cell, so that at this stage the young prothallium consisted of four short cells forming an axial row terminated above by the apical cell, below by the basal cell, and enclosed laterally by two rows of cells. It was impossible to trace the development any further. Fifteen years later (1872) Fankhauser found in Switzerland completely developed prothallia of Lycopodium annotinum among some Mosses, one of which was still connected with the young plant of the second generation (Fig. 326). These prothallia, which had grown in the absence of light, were irregularly lobed masses of cells of a pale yellow colour, furnished sparingly with small root-hairs. On the upper surface were numerous antheridia which are ovoid cavities in the tissue of the prothallium covered by a single layer of cells and filled with the very numerous mother-cells of the anthero-

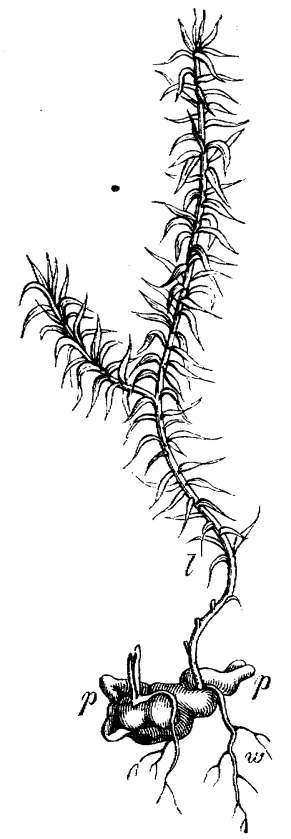

FIG. 326.-Lycopodium annotinum, after Fankhauser; $p$ the prothallium, $l$ the young plant $w$ its root (natural size). zoids. The form of the antherozoids themselves was not very clearly made out. These prothallia had no longer any archegonia, but they bore young plants: hence it appears that Lycopodium produces only one kind of spores, a conclusion which is quite in accordance with the results of direct observation, and that the prothallia are monocious, a peculiarity which at once sharply distinguishes the Lycopodiceæ from the Isoëteæ and Selaginelleæ, as does also the very considerable size attained by the prothallium and its complete independence of the spore. Probably these conditions are the same in those genera which possess but one kind of spores, Psilotum, Tmesipteris, Phylloglossum. The prothallia of Lycopodium evidently bear several archegonia, for Fankhauser found on them young plants in various stages of development. From the attachment of the young plant to the prothallium, it appears that the archegonia lie upon its upper surface in the grooves between the lobes.

2. The Asexual generation (Sporophore). From what has already been said it is evident that nothing is known concerning the development of the embryo. The young plants which Fankhauser found were attached to the prothallium by means of a small projection, about the size of a pin's head inserted into its tissue. This projection arises at the side of the base of the stem and of the first root; it evidently corresponds to the foot of Ferns.

The habit of the mature plants is very various in the different genera. Some species of Lycopodium have an erect stem and erect branches (L. Selago), in which case the roots arising from the lower part of the stem often grow downwards within it and issue in a tuft from its base (L. Phlegmaria, ulicifolium, \&c.). Very frequently the main stem and its largest branches creep upon the earth, sending out roots here and there into the soil, only certain branches, more especially those which bear the sporangia, being erect. In these forms there is a tendency towards bilateral organisation, which finds expression more particularly in the structure of the axial fibrovascular mass. In all species the stem is thickly covered with small, narrow, 
sometimes elongated leaves. The differences of habit are chiefly due to the greater or less development of one or other of the branches of the bifurcations. The sporangia are borne in L. Selago in the axils of ordinary foliage-leaves, but usually they are borne in the axils of leaves which are peculiar in shape and colour, and which form terminal spikes on special fertile branches which are frequently curiously modified.

In Psilotum the stem is thin, much branched, and developed throughout in a bifurcate manner, so that the plant presents the appearance of a straggling shrub. It possesses no true roots, but a number of underground branches discharge the functions of roots. The leaves are few, and are but small pointed scales even upon that part of the stem which is above ground. The sporangia are borne three or four together on small, short, lateral shoots of the branches, and do not form a definite fructification. Similar arrangements occur in Tmesipteris, but here the leaves are much larger. With these genera is associated Phylloglossum, a small Australian plant only a few centimetres high, which differs from them considerably. It consists of a stem arising from a small tuber, and bearing at its lower part a rosette of a few long leaves and one or more lateral roots; it is prolonged above this as a thin scape and terminates in a spike of small leaves bearing the sporangia. The plant is propagated by means of adventitious shoots consisting of a tuber with a rudimentary leafless bud: in this respect it resembles our native Ophrydeæ.

The development of the vegetative organs is only thus completely known with. reference to native Lycopodieæ; our knowledge of the other genera is very fragmentary. We are indebted for the most valuable information to the labours of Hegelmaier, of Leitgeb, and of Nägeli. According to Cramer, Pfeffer, and Hegelmaier, and this is corroborated by my own observations, the growing end of the stem of Lycopodium does not possess a single apical cell; this is true also of the leaf and of the root. The growing point of the stem consists of a small-celled primary. meristem which does not in all cases present a distinct differentiation of dermatogen and periblem, but there is a distinct axial plerome which extends to close below the apex. In $L$. Selago the apex is flat, but in L. complanatum, clavatum, annotinum, alpinum, \&c., it projects beyond the youngest leaves in the form of a dome. Just as in Phanerogams, the leaves and the rudimentary branches are not developed from single cells of the growing-point, but from groups of cells which include both the superficial and deep layers of the primary tissue.

The branching of the stem of Lycopodium may be generally described as being dichotomous, although, according to Hegelmaier, the first development of a branch begins in many cases at the side of the growing apex of the stem. In all cases, however, this takes place above the youngest leaves without any reference to their position, and herein it differs from the branching of Phanerogams. According to Cramer two new growing points of equal activity are formed side by side upon the flat apical surface of $L$. Selago, and they in turn develope dichotomously, and Hegelmaier found the same to be the case in the vegetative shoots of heterophyllous species (L. complanatum, chamacyparissus). In these cases then true dichotomy occurs. On the other hand, the rudiment of the branch appears as a lateral protuberance from the projecting growing point of the vegetative shoots of $L$. clavatum 
and annotinum and of the creeping shoots of L. inundatum; yet it arises in such a manner that the branching may be regarded as being closely allied to dichotomy. The bifurcation of the fertile branch of $L$. alpinum presents another form of dichotomy (false dichotomy), for here the growing-point is extended by the development of two new growing points, one on the right and the other on the left side of it; the central one then ceases its activity, whereas that of the two lateral ones commences, so that the apex of the parent shoot becomes indistinguishable. Those Lycopodieæ which have their leaves arranged in four rows, and which resemble the Selaginelleæ (L. complanatum, chamacyparissus), branch in one plane only, which coincides with that of the larger leaves; whereas those species which have their leaves arranged spirally or in whorls, branch in various intersecting planes. Hegelmaier recognised the bulbils which occur in several species (L. Selago, lucidum, reflexum) as being peculiarly modified branches. They arise on the shoot in the place of leaves. They fall off of themselves, and are provided with a few leaves and a rudimentary root.

Since the leaves are closely placed by the side of and above each other from the first, so as to cover the whole surface of the stem, there are no internodes (as also in Ophioglossum, Marattia, Aspidium, Isoëtes); and not only so, but the outer cortical layer of the stem is genetically connected with the tissue of the bases of the leaves. The leaves become separated the one from the other by subsequent intercalary growth, and in many cases the base of the leaf becomes sharply defined from the stem.

The first rudiments of the leaves of Lycopodieæ appear as multicellular lateral protuberances of considerable breadth upon the growing apex of the stem. They grow at first apically, but this soon terminates in the formation of a hair-like prolongation; all further growth is intercalary at the base of the leaf. The size and form of the leaves are very different in the various species, but they are always simple unbranched, and not stalked but sessile with a narrow base. Occasionally their surfaces, with the exception of the free apices, are closely applied to the stem (as is the case in Thuja), but more commonly the leaves are quite free. They are acicular, or at any rate of small width, and, as in all Dichotomeæ, a mid-rib only and no"lateral veins is present.

The phyllotaxis is sometimes verticillate, sometimes spiral, and both kinds may occur on the same plant. The whorls may consist of pairs of leaves which decussate, or of three, four, or more, and are arranged in creeping stems on zones the planes of which are oblique to the long axis of the stem. The number of leaves in a whorl varies even on the same shoot. According to Hegelmaier, the whorls are true ones; the leaves composing them are developed simultaneously and at the same level at the punctum vegetationis. Where the phyllotaxis is spiral it is so from the first, and the divergence undergoes no important modification. The small and very various divergences of the leaves are remarkable, as Braun pointed out; he found in $L$. clavatum the following divergences, $\frac{2}{9}, \frac{2}{11}, \frac{2}{13}, \frac{2}{15}, \frac{2}{17}$, as well as whorls consisting of from four to eight leaves; in L. annotinum they were $\frac{2}{7}, \frac{2}{9}$, and whorls of four or five leaves; in L. inundatum $\frac{2}{9}$ and whorls of five leaves (Bot. Zeit. $187^{2}$, p. $8 r_{5}$ ).

The much bifurcated, multiangular, thin stem of Psilotum grows by means of a three-sided apical cell, which, according to Nägeli and Leitgeb, forms (in the 
subterranean shoots) three spiral rows of segments, the segmental walls advancing in the anodal direction, as in many Mosses. The small widely separated leaves, which have no fibro-vascular bundles, are borne upon the angles of the stem and bear no apparent relation to its branches.

Psilotum triquetrum is a plant perfectly destitute of roots, forming however a number of underground shoots which serve the purpose of roots and are extremely similar to them. On the shoots of the rhizome which approach the surface of the ground may be detected with a lens minute leaves of a whitish colour and acicular shape ; the deeper root-like shoots have a blunter end, on which no trace of leaves can be detected, even with the lens. While the anatomical structure of the superficial shoots corresponds to that of the true stem of these plants, in these deeper shoots the vascular bundles are united into an axial group, as in true roots. The shoots which bear visible rudiments of leaves may turn upwards, become green and transformed into ordinary foliage-shoots, while the root-like shoots, which are more slender, may also turn upwards, become thicker, and assume the appearance of the ordinary superficial rhizome-shoots. In this point therefore they differ at once from true roots, but still more in the absence of a root-cap. They terminate in an apical cell, which forms oblique segments alternating in different directions. The most important point, however, is that these shoots really possess rudiments of leaves which consist of only a few cells and do not project above the surface, but remain concealed in the tissue. They are best recognised in longitudinal section, when they are seen to consist of an apical cell and from two to five cells with the characteristic arrangement of leaf-cells. Similar rudimentary leaves consisting of but few cells occur also on the ordinary rhizome-shoots, where, however, they do not undergo further development, especially when the end of the shoot appears above ground. The root-like shoots branch like the ordinary ones; a cell is cut off by an oblique wall from one of the youngest segments, and forms the apical cell of the new shoot.

The other genera all possess true roots. In the Lycopodieæ with creeping or climbing stems they arise singly and dichotomise in rectangularly intersecting planes in the soil. It has already been mentioned that in the Lycopodieæ with erect stems, such as in L. Selago, Phlegmaria, ulicifolium, the roots issue in a tuft from the base of the stem which is somewhat tubercular. These roots originate high up in the stem, as high as five centimetres and even above the first bifurcation according to Strasburger; they develope at the periphery of the axial fibro-vascular mass, but they are peculiar in that they grow down through the fundamental tissue of the stem and even dichotomise there. (Compare with Angiopteris, p. 41 7.)

The Sporangia, in the genus Lycopodium, occur singly on the bases of the leaves or in their axils. As in all Dichotomeæ, they are here larger than in the Ferns. They are borne on short broad stalks, and the capsule is somewhat reniform, its longer axis lying transversely to that of the leaf. They open by means of a slit running in this direction over the apex, two valves being formed which remained united at the base. The contained spheroidal or tetrahedral rather small spores are numerous; they all have the same shape and are provided with a sculptured exospore. After I had pointed out in the first edition of this book (1868) that the sporangia of the Lycopodieæ originate as multicellular protuberances of the tissue of the leaves, 
Hegelmaier and Russow have confirmed and extended the observation. They are developed from a small group of epidermal cells, as Goebel has shown, and, in consequence of repeated divisions, they soon appear as flat projections occupying the whole breadth of the base of the leaf, consisting of a group of internal cells covered by an external layer. Tangential divisions take place in the epidermal cells by means of which the wall becomes two-layered, and the upper or external portion of the tapetum is formed, the tapetum being completed by similar divisions taking place in

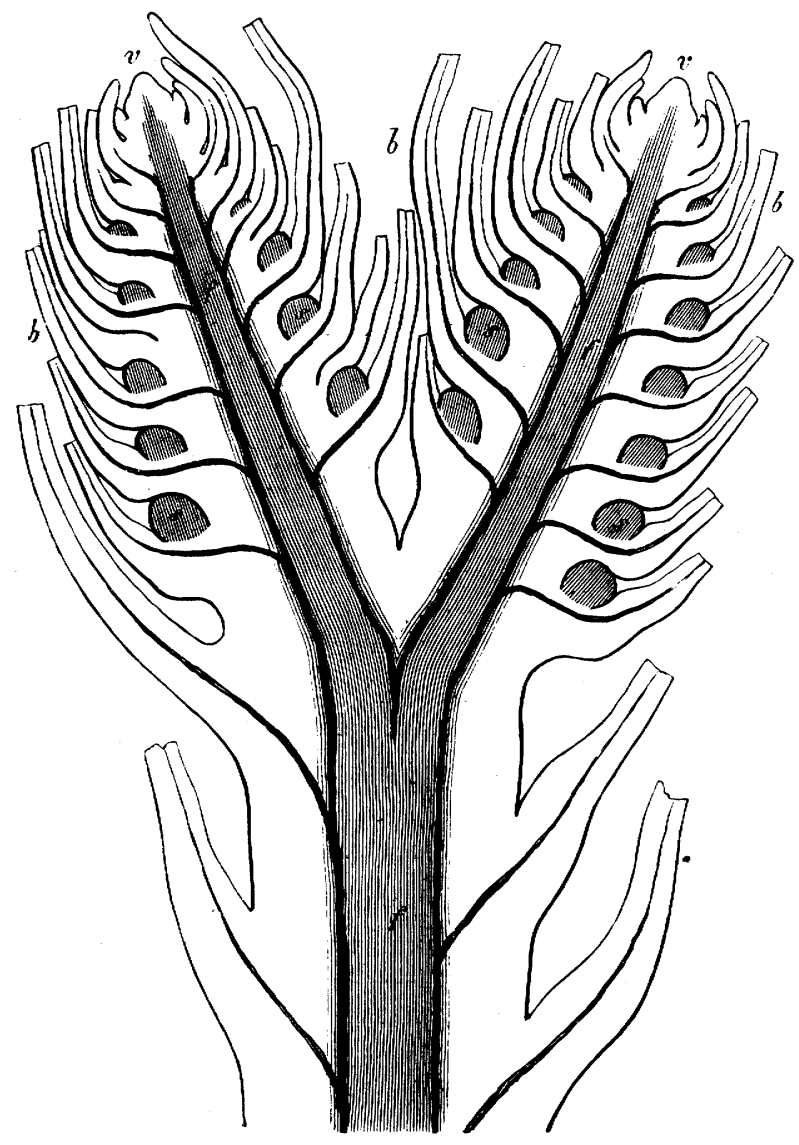

FIG. 327.-A dichotomously branched fertile shoot of Lycopodium Chamacyparissus, in longitudinal section, slightly magnified; $f f$ the axial fibrovascular mass, $b b$ leaves, $s s$ the young sporangia.

more deeply placed cells : the archesporium probably consists of a transverse row of cells; these undergo division and form a rounded mass of spore-mother-cells. These cells become isolated, their walls undergo considerable thickening, and, after an indicated division into two they form four chambers (the so-called 'special mothercells'), within each of which the contained protoplasm surrounds itself with a permanent spore-wall. It is not until the projections, spines, \&c., have been developed upon this wall that the walls of the chambers of the mother-cells are absorbed. 
The nature of the sporangia of Psilotum and of Tmesipteris is in many respects obscure. In Psilotam the short branches which bear the apparently trilocular sporangia ${ }^{1}$ arise as lateral papillæ on the growing point, and, according to Juranyi, are provided with a three-sided apical cell like the vegetative branches. A fibrovascular bundle runs from that of the parent shoot to the papilla, but does not extend beyond the half of its height. The two small leaves of this fertile branch, which were formerly regarded as being the segments of a single leaf, arise separately from the papilla and coalesce at a later period. Even at a tolerably advanced stage the papilla still consists of undifferentiated tissue which, as in the case of the anthers of Phanerogams, forms parietal layers and three groups of spore-mother-cells; in this way. three loculi are formed, which are separated by longitudinal walls and an axial mass of tissue, and which project considerably on the exterior. I regard these three loculi as so many sporangia which are formed in the apical part of the fertile shoot to which the axial fibro-vascular bundle extends. In Tmesipteris the sporangium, which is apparently divided into two loculi by a transverse septum, is borne upon a small lateral branch which bears a leaf to the right and to the left.

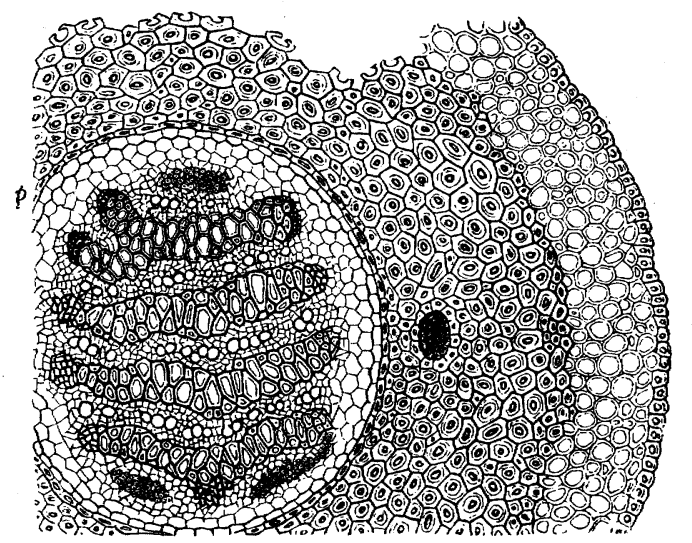

FIG. 328.-Transverse section of the stem of Lycopodium Chamacyparissus ( $\times \mathbf{I}_{5}$ ).

Histology ${ }^{2}$. The Epidermis of the leaves of L. annotinum, clavatum, and Selago, is provided with stomata on both surfaces; the stomata are often arranged in small groups. The leaves of the heterophyllous species which have them arranged in four rows, possess stomata on their inner surface; stomata occur on the outer surfaces of those portions of the leaves which adhere to the stem and which are directed towards the earth. The epidermis of the root is sometimes strongly cuticularised, as in L. clavatum.

The Fundamental Tissue of the stem consists of cells which are sometimes thin-walled throughout, as in L. inundatum, but usually the inner layers have thick walls, and are

1 [This has been cleared up by Goebel (Beitr. z. vergl. Entwick. der Sporangien, Bot. Zeit. I 88I). He finds that the 'trilocular sporangium' of Psilotum is really a group of three sporangia. each of which contains primarily a unicellular archesporium, from which a two-layered tapetum is subsequently derived, as also the mother-cells of the spores. The 'loculi' of the apparently bilocular sporangia of Tmesipteris are, like those of Psilotum, which they closely resemble in their development, really distinct sporangia.]

${ }^{2}$ [For further details see De Bary's Vergleichende Anatomie, 1877.$]$ 
prosenchymatous, or even sclerenchymatous, though they never become brown like those of the Ferns (Fig. 328).

The axial fibro-vascular cylinder is sharply defined from the ground tissue by a welldeveloped bundle-sheath consisting of from one to three layers of cells. In the leaves of the heterophyllous species air-cavities exist, and they are found also in the stem of $L$. inundatum. In this species Hegelmaier found gum-canals in the stem and leaves (one in the mid-rib), which are formed by the gradual separation of cells from each other. The bordering cells project into the canal like varicose hairs. In L. annotinum such canals occur only in the fertile branch.

The Fibro-vascular Bundles of the Lycopodiex are very characteristic. In the stem and in the root there is a large axial cylinder which has usually a circular outline. In this (Fig. 328) lie bands of xylem which are either isolated or united in various ways so as to form figures which would be symmetrically bisected by an axial longitudinal section. Transverse sections taken at different heights show the xylem arranged in different patterns, for the bands anastomose in their course. These bands of xylem consist, like those of Ferns, of tracheides which are pointed at the ends, which are wider in the centre than at the edges of the bundle, and which, when they are narrow, have round pits, when broad, the pits are fissure-like. Small spiral vessels (proto-xylem cells) are to be found at the edges of the bands of xylem. In creeping and oblique stems the concavity of the bands is always directed upwards. These bands are embedded in a mass of small-celled phloëm, in which rows of wider cells lying between the xylem bundles occur. Although, according to Hegelmaier, these cells possess no sieve-plates, they may be regarded as the representatives of the sieve-tubes.

The 'proto-phloëm cells' (bast-fibres) lie toward the exterior between the ends of the xylem bundles. The arrangement of the elements recalls that of the axial cylinder of roots. Within the bundle-sheath are several layers of rather large cells which invest the peripheral phloëm; to these Hegelmaier has given the name of 'phloëm-sheath,' and probably they truly correspond to the layer occurring in Ferns to which the same name has been given. I maintain the view which I formerly expressed that the axial cylinder of the stem of the Lycopodiex consists of several fibro-vascular bundles which have coalesced; for Hegelmaier's argument against it, that the bands of xylem are not isolated throughout their whole extent, is by no means conclusive, and moreover the resemblance between the axial cylinder of the stem and that of the root supports my view. Each leaf contains a single thin fibro-vascular bundle of very simple structure, which runs very obliquely from the base of the leaf through the cortex, to become connected lower down with the margin of one of the xylem bundles of the stem. The leaves of Psilotum contain no fibro-vascular bundles. According to Russow the xylem of the axial fibro-vascular cylinder of the stem forms an angular hollow cylinder at the projecting angles of which are groups of narrow spiral vessels.

The axial cylinder is cauline: it can be followed in the procambial condition to just beneath the apex of the stem. The rows of spiral cells are first formed within the xylem bands, with which the similar elements in the bundles of the leaves become connected (Fig. 327) long before the development of the tracheïdes.

\section{Order II. Ligulate ${ }^{1}$.}

I. The Sexual Generation (Oophore). Like the Rhizocarpeæ among the Filicineæ, the Ligulatæ, including the genera Selaginella and Isoëtes, are distinguished

${ }^{1}$ Hofmeister, Vergleich. Unters. 1851.-(Germination, Development, and Fructification of the Higher Cryptogamia, Ray Soc.) - Hofmeister, Entwick. der Isoëtes lacustris, in Abhand, d. Königl. Sächs. der Wiss. IV. I 855-Nägeli und Leitgeb, iiber Entstehung und Wachsthum der Wurzeln, in 
among the Dichotomeæ in that they possess spores of two kinds, the macrospores and the microspores. As in the Rhizocarpeæ so here the carrying back of sexual differentiation to the development of the spores is associated with this peculiarity, that the spores in germinating seem to attain their object, the formation of reproductive organs, as directly as possible, for the prothallium is not a plant capable of independent growth but a development of tissue within the spore. The mode in which this is brought about in the Ligulatæ differs in essential points from that obtaining among the Rhizocarpeæ.

The Microspores of Isoettes and Selaginella do not produce the mother-cells of the antherozoids immediately from their contents, as was formerly thought. To the treatise of Millardet mentioned in the foot-note we owe our knowledge of the fact, so important in connection with the relationship of the higher Cryptogams to the Gymnosperms, that at the period when the microspores are ripe, their contents are transformed into a mass of tissue consisting of but few cells. One of these cells
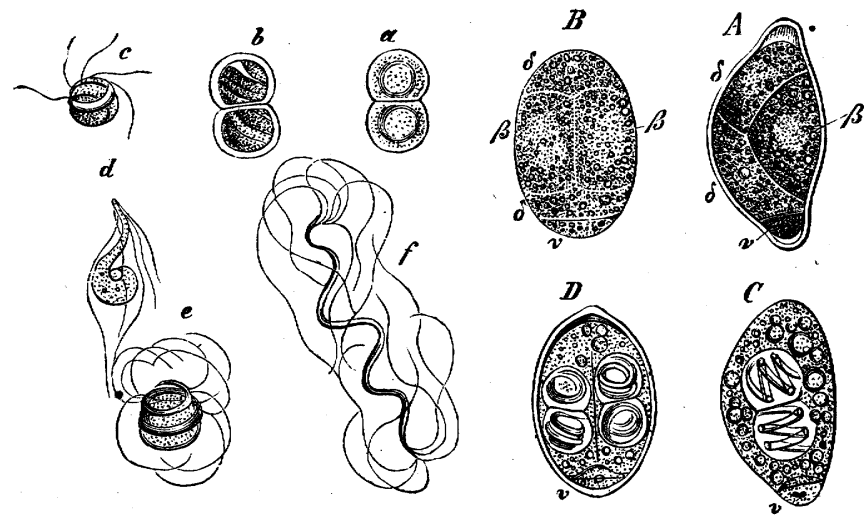

FIG. 329.-Germination of the microspores of /soëtes lacustris (after Millardet). $A$ and $C$ microspores seen on the right side, $B$ and $D$ on the ventral face; $A$ and $B$ show the formation of the antheridium, $\delta \delta$ its dorsal cells, $\beta B$ its ventral cells, $C$ and $D$ the formation of the antherozoids, $\beta$ and $\delta$ have disappeared : $v$ is the vegetative cell (prothallium of Millardet); $a-f$ development of the anthetozoids $\left(A-D\right.$ and $a-d \times 5^{80}, e$ and $\left.f \times 700\right)$.

remains sterile, and may be considered a rudimentary prothallium; while from the others originate the mother-cells of the antherozoids, and these may therefore be looked on as a rudimentary antheridium:

The microspore of Isoëtes lacustris breaks up, after hibernation, into a very small sterile cell and a large one comprising the whole of the rest of the contents (Fig. $329 A-C$ ). The former $(v)$, cut off by a firm wall of cellulose, does not undergo any further considerable changes; the latter, on the other hand, splits up into four primordial cells without cell-walls, of which the two ventral ones produce

Nägeli's Beitr. z. Wiss. Bot. IV. I867.-A. Braun, über Isoëtes, in Monatsber. d. Berl. Akad. I863.Milde, Filices Europæ et Atlantidis, Leipzig I 867.-Millardet, le prothallium male des crypt. vasc. Strasburg 1869.-Pfeffer, Entw. des Keims der Gattung Selaginella in Hanstein's Bot. Abhand. IV. 1871.-Janczewski, Bot. Zeitg. I872, p. 441.-Tschistiakoff, ïber Sporenentwickelung von Isoëtes, in Nuovo Giornale bot. Ital. I873.-Russow, Vergl. Unters. Petersburg I872.-[Braun, Ueb. Blattstellung und Verzweigung bei Selaginella, Sitzber. d. bot. Ver. d. Prov. Brandenburg, I874.-Hegelmaier, Zur Kennt. einiger Lycopodinen, Bot. Zeitg. 1874.-Treub, Recherches sur les organes de la végétation du S\&laginella Martensii, I877.] 
each two antherozoid-mother-cells, and therefore four in all. Pfeffer has confirmed the statements of Millardet that in Selaginella, long before the spores escape from the sporangium, a small sterile cell is first of all separated by a firm wall, while the other large cell breaks up into a number (6 to 8$)$ of primordial cells (Fig. 33 I $A-D$ ). He found, however, their arrangement different in Selaginella Martensii and caulescens from that which Millardet described in the case of $S$. Kraussiana, a variation which seems immaterial when compared with similar differences in the antheridium of Ferns. The essential difference between the results of the two observers consists in this :- that, according to Millardet, only two of the primordial cells produce the mother-cells of the antherozoids, which then, increasing in number, cause the absorption of the rest of the primordial cells, and fill up the spore; while Pfeffer found, in his species, that all the primordial cells underwent further division, and contributed to the formation of the antherozoids. As to the mode of development of the antherozoids they were both in accordance. In Isoëtes the antherozoids are long and slender, attenuated, and splitting up at both ends into a tuft of long slender cilia; in Selaginella they are shorter, thick behind, finely drawn out in front, and divided there into two long fine cilia. In the perfectly mature condition
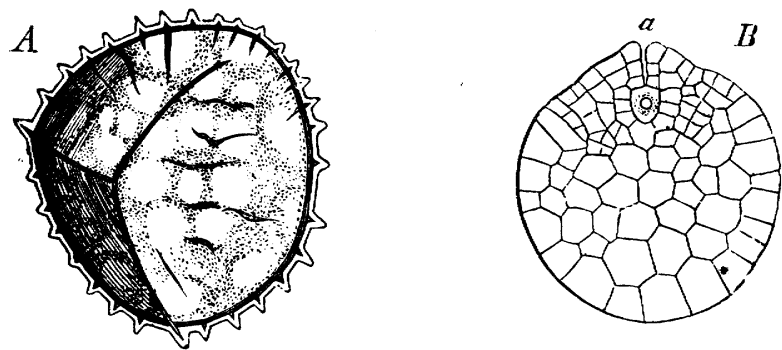

FIG. 330.-Isö̈tes lacustris (after Hofmeister); $A$ nacrospore, two weeks after its escape from the sporangium, rendered transparent by glycerine $(\times 60) ; B$ longitudinal section of the prothallium four weeks after the escape of the macrospore, $a$ archegonium $(\times 40)$.

the antherozoids are rolled up into an elongated helix or into a short spiral. The mode of their formation in the mother-cells is the same in both genera, and agrees in essential points with that of Ferns. A cell-nucleus is not present at the time when the antherozoid is first formed; the contents of the cell are perfectly homogeneous; the antherozoid originates from ‘ a shining scarcely granular mass of protoplasm which encloses a vacuole, the cilia at one end being formed first, and the spiral body becoming differentiated from before backwards by a kind of splitting of the protoplasm. The antherozoid is originally curved spirally round the central vacuole; this latter, surrounded by a fine membrane, not unfrequently remains attached to the posterior end of the antherozoid after it has escaped, and is carried along by it. The movement does not last longer than five minutes in the antherozoids of Isoëtes, in Selaginella from one-half to three-quarters of an hour. From the commencement of germination till the complete maturity of the antherozoids there is, in Isoëtes, an interval of about three weeks; the same period from the dissemination of the spores is necessary in Selaginella.

The Macrospores produce the female prothallium, which is an endogenous structure in a still higher degree even than is the case in the Rhizocarps. In this respect 
and in the mode of its development, it shows a still greater resemblance to the tissue that fills up the embryo-sac of Gymnosperms, and even of Angiosperms. In Isoëtes the cavity begins to be filled with cellular tissue a few weeks after the escape of the macrospores from the decaying macrosporangium; the cells of this tissue are all at first naked (without cell-wall); they appear to become enclosed in firm cell-walls only when the whole cavity of the endospore is filled with them (Fig. 330). In the meantime the endospore thickens, becomes differentiated into layers, and assumes a finely granular appearance, phenomena which, as Hofmeister insists, are exhibited in like manner in the embryo-sacs of Coniferæ. The spherical pro-

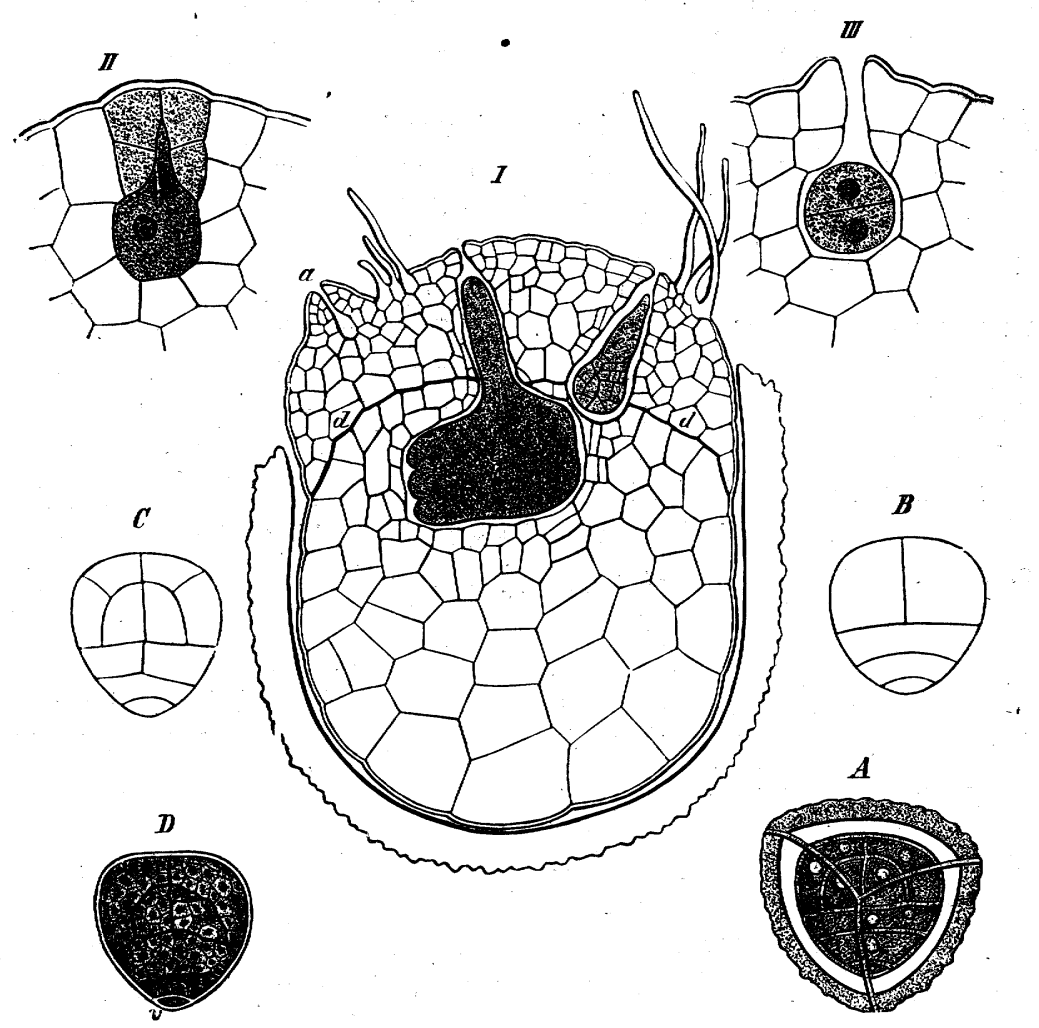

FIG. 33I-Germination of Selaginella (after Pfeffer); $I-I I I, S$. Martensii, $A-D, S$. caulescens; I longitudinal section FIG. 33r.-Germination of Selaginella (after 'endosperm,' $d$ the diaphragm, $e e^{\prime}$ ' two embryos in process of formation; of a macrospore filled with the prothallium and 'endosp with the oospore fertilised and divided once; $A$ a microspore $I I$ a young archegonium not yet open; $I I I$ an archegonium with the oospore fertilsed and the antherozoids in the perfect showing the primordial cells; $B C$ a
antheridium; $v$, vegetative cell.

thallium now swells up, the three convergent edges of the exospore burst lengthwise and thus form a three-rayed fissure, where the prothallium is covered only by the membranous endospore; this also peels off, and softens, finally exposing the corresponding part of the prothallium. At its apex appears the first archegonium; if this is not fertilised, several others are subsequently formed at its side. In Selaginella, even when the macrospores are still lying in the sporangium, the apical region is found to be clothed with a small-celled meniscus-shaped mass of tissue which is probably formed, during the ripening of the spores, by the division 
of an accumulation of protoplasm. This tissue afterwards produces the archegonia, and is therefore the true prothallium; but a few weeks after the dissemination free cell-formation begins beneath it in the spore-cavity, finally filling up the whole cavity, and forming a large-celled tissue, which Pfeffer, supported by considerations with which I also agree, compares to the endosperm of Angiosperms, and, following this analogy, calls by the same name. At the period of fertilisation and of the formation of the embryo, the macrospores of Selaginella contain, therefore, both a prothallium and an endosperm. The formation of the archegonia begins even before the rupture of the exospore, which occurs in this genus in the same manner as in Isoïles. The first archegonium originates at the apex of the prothallium; the others arise, whether the first is fertilised or not, in centrifugal succession on the exposed parts of the prothallium.

In both genera the archegonium originates by division of a superficial cell parallel to the surface; the upper of the two new cells divides into four cells placed crosswise, each of which splits by an oblique division into two, one lying over the other; in this way the neck is formed, consisting of four rows, each of two cells in Selaginella, and of four cells in Isoëtes. The lower of the first two cells sends out a narrow prolongation between the neck-cells, which becomes the canal-cell of the neck (Fig. 33 I II). The lower larger portion, the central-cell according to Janczewski, then has a small portion of its protoplasm cut off which corresponds to the ventral canal-cell of the other Archegoniata, the remainder constituting the oosphere. The two canal-cells become mucilaginous and are extended from the opened neck so as to permit the access of the antherozoids to the oosphere.

2. The Asexual Generation (Sporophore). The Development of the Embryo. The first division of the oospore (formation of the basal wall) differs from that of Ferns and Rhizocarps, taking place perpendicularly to the axis of the archegonium. According to Hofmeister, each of the two cells first formed is divided in Isoëtes in a plane at right angles to that of the first division, the relation of which to the first root, the first leaf, the stem, and the foot of the embryo, requires yet further elucidation ${ }^{1}$. The formation of the embryo of Selaginella has recently been investigated in detail by Pfeffer. From an elongation of the upper half (hypobasal) of the oospore is formed the Suspensor, a body which is wanting in all other Cryptogams, but universally present in Phanerogams, and through which Selaginella consequently approaches flowering plants. The suspensor seldom remains

1 [According to Bruchmann (Jenaische Zeitschrift, 1874) the upper (epibasal) cell grows rapidly towards the neck of the archegonium and produces the apex of the stem and the first leaf (cotyledon); at the lower part of the anterior surface of the cotyledon a cell grows out and gives rise to the 'ligula,' and it is the cells lying immediately at the base of this organ which constitute the growing point of the stem. The lower (hypobasal) of the two primary cells grows slowly downwards into the spore, forming the foot; from the superficial cells of that part which is diametrically opposite to the growing point of the stem the primary root is developed. From the more recent researches of Kienitz-Gerloff (Bot. Zeitg. I88I) it appears that the first divisions of the oospore resemble those in the Filicineæ (see supra, p. 426). The two anterior superior (epibasal) octants give rise to the cotyledon, the two posterior (hypobasal) superior octants to the root, and the four inferior to the foot. The ligula is developed from one of the cotyledonary octants; from the rootoctants a cotyledonary sheath is also developed. He does not agree with Bruchmann that the root has an exogenous origin.] 
a simple cell; a smaller or larger number of divisions usually takes place in its lower part (Fig. 332, $A-D$ ). The embryo itself originates from the lower (epibasal) half of the oospore. By the elongation of the suspensor and the compression and absorption of the surrounding cells, the embryo is forced into the endosperm, in which it now undergoes further development, as in Phanerogams. In the mothercell of the embryo (epibasal cell) two segments ${ }^{1}$ are in the meantime formed by the wall II (transverse wall); out of each proceeds an embryo-leaf (cotyledon), and a longitudinal half of the hypocotyledonary segment of the stem. The foot and root originate besides from the right segment, and in the left (in the figure) segment is formed the two-sided apical cell of the stem (Fig. 332, $A, B$ ). While the two segments are becoming transformed by a number of cell-divisions into masses of cells, of which an inner mass very soon separates itself as the procambium of the axial bundle and a peripheral mass as dermatogen and periblem, a swelling is produced laterally beneath the first leaf, forming the foot ${ }^{2}$; by its increase the
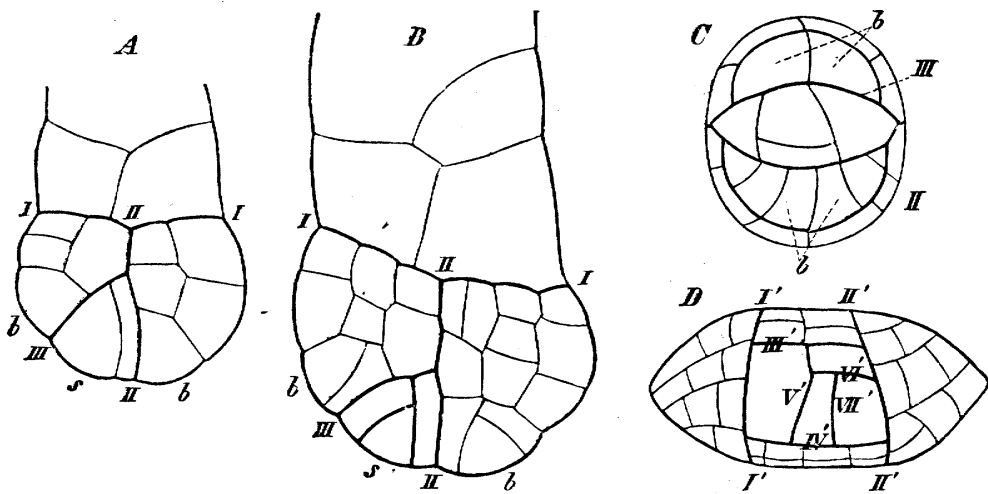

FIG. 332.-Development of the embryo of Selaginella Martensiz (after Pfeffer); $A, B$ lower part of the suspensor with the first much-divided segments of the embryo, and the apical cell $s$ of the future stem; $b b$ the first leaves; $C$ apical view of the same; $D$ the apex seen from above in the act of forming two new apical cells, right and left; $I, I I, I I I$ the primary walls of the primary apical cell; $I^{\prime}-V I I^{\prime}$ the longitudinal walls by which the two new apical cells are formed. (I basal wall ; $I I$ transverse wall.)

stem is forced over to the other side (that of the younger segment); so that the apex comes to lie horizontally, and afterwards is even directed upwards (Fig. 33 I $I$ ); and finally the bud, with its first leaves, the cotyledons, grows out upright from the apical part of the macrospore when the embryo begins to increase in length. The first root is formed a considerable time afterwards between the foot and the suspensor. It is lateral, and its apical cell is formed from an inner cell of the right segment; but the first layer of its root-cap originates from the splitting into two layers of the overlying dermatogen; the later layers of the root-cap arise from the apical cell of the root itself.

It has already been mentioned that in Pteris and Salvinia the position of the apical cell of the growing stem is placed at an angle of about $90^{\circ}$ with respect to

2 [There is reason to believe that four octants are formed from the epibasal cell as in the other Vascular Cryptogams.]

2 [On the propriety of regarding this organ as the morphological equivalent of the foot of the other Vascular Cryptogams, see Quart. Journ. Micr. Sci. 1878.] 
that of the embryo. Something of the same kind occurs in Selaginella; the apical cell, which lies between the rudiments of the first two leaves, is divided by walls in such a manner that a four-sided apical cell is formed (Fig. $33^{2} C, D$ ), the segments of which arise in decussate pairs. In the fifth or sixth segment a second four-sided apical cell is now formed by a curved wall with the convexity turned towards the primary apical cell, so that a longitudinal section through the two apical cells cuts at right angles the common median line of the first leaves, and that of the original two-sided apical cell. Each of the two four-sided apical cells now developes into a branch; but neither of the branches continues to grow in the direction of the hypocotyledonary axis; the branching therefore takes place immediately above the first leaves or cotyledons. The four-sided apical cells of the two rudimentary branches are soon transformed into two-sided apical cells each forming two rows of segments.

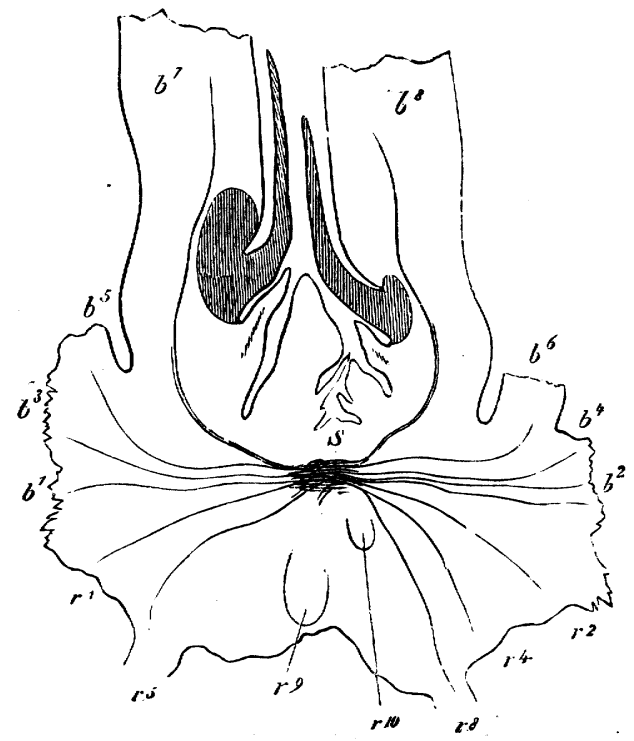

FIG. 333.-Longitudinal section of Isoëtes Lacustris at right angles to the furrows of the stem ten months old (after Hofmeister), $S$ stem, $b^{1}-b_{8}$ leaves, $r^{1-r 10}$ roots $\left(\times 3^{\circ}\right)$; the ligula of the two developed leaves is shaded.

The first formation of all the organs and the first branching always take place before the protrusion of the embryo from the spore.

External Differentiation. The Stem is distinguished in Isö̈tes, as has already been mentioned, by its extraordinarily small growth in length, with which is connected, in this as in other cases (Ophioglosseæ, Marattiaceæ, and many Ferns), an absence of branching; no internodes are formed, the leaves with broad bases of insertion constituting a thick rosette, without leaving between them any surface of the stem bare. The upper region of the stem, which is furnished with leaves, has the form of a shallow funnel, depressed in the centre or apex (Fig. 333). The long-continued increase in thickness, which distinguishes the stem of Isoëtes from that of all other Cryptogams ${ }^{1}$, is brought about by an internal layer of meristem, surrounding the

1 Compare what has been said already about Botrychium and what follows about Lepidodendron. 
central vascular body, and continually producing new layers of parenchyma on the outside. This takes place especially in two or three directions, so that two or three corresponding masses of tissue are formed, slowly dying off on the outside, between which lie as many deep furrows meeting on the under surface of the stem. From these a large number of roots are produced in rows in acropetal succession.

In the Selaginelleæ the stem remains slender, but lengthens rapidly, branching profusely, and forms distinct internodes. The end of the stem rises above the youngest leaves as a slender cone. In Selaginella a tendency prevails to sympodial scorpioid development of the branches which not unfrequently leads to the system of abundantly branched shoots developed bilaterally in one plane attaining a definite outline, and a corresponding resemblance to a compoundly pinnate leaf. In consequence of the small size of the leaves in this genus, the general habit is mainly dependent on the development of the systems of branches. The main shoots which result from the sympodial development of the branches may creep like rhizomes, may grow obliquely upwards, may climb, or may form the stems of arborescent and fruticose plants. In all cases the repeated branchings take place in one plane, for the bilateral symmetry which is so marked in the position of the branches and in the phyllotaxis already exists in the growing point.

The Leaves are always simple, unbranched, penetrated by only a single fibro vascular bundle, terminating in a simple point, and ending, in Selaginella, in a fine awn. The largest leaves occur in Isoëtes, where they attain a length of from 4 to $60 \mathrm{~cm}$. They are in this case divided into a basal part or sheath, and an upper part or lamina. The sheath does not entirely embrace the stem, but rises in a somewhat triangular form from a very broad insertion, and is acuminate; it is convex behind and concave in front, where there is a large depression, the $F_{\text {ovea, }}$

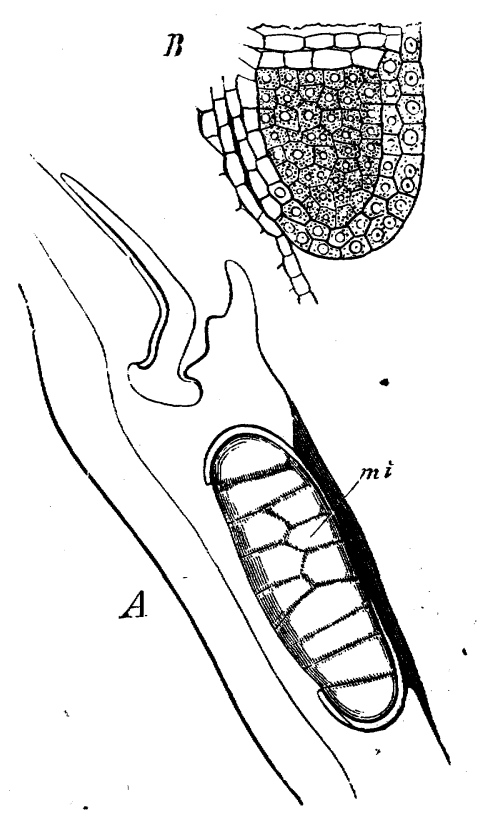

FIG. 334.- $A$ longitudinal section through the base of a leaf of Isoëtes lacustris with its microsporangium $m i$ still unripe; $B$ longitudinal section of the lower part of a young sporangium $(\times 3 \circ)$ (after Hofmeister). containing the sporangium; the margin of this depression rises in the form of a thin membranous outgrowth, which in many species lies above the sporangium and envelopes it, the Velum. Above the fovea and separated from it by the 'saddle,' lies a smaller depression, the Foveola, the lower margin of which forms a lip, the Labium, while from its bottom an apiculate membranous structure, the Ligule (or Lingula), with a cordate base, is prolonged beyond the foveola (Fig. 334, $A$ ). The lamina of the leaf, containing chlorophyll, into which the sheath passes above, is narrow and thick, almost cylindrical, but flattened in front, and penetrated by four wide air-canals, which are divided by septa. This form is exhibited by the fertile leaves of all the species of Isoëtes; a rosette of such leaves is produced annually; but between each pair of annual whorls is formed a whorl of imperfect leaves, which 
consist, in I. lacustris, of only a small lamina, but in the terrestrial species are destitute even of this, and are simply cataphyllary leaves (phyllades).

The leaves of Selaginella are never more than a few millimetres in length, and are usually cordate at the base with a narrow insertion, acuminate, and from lanceolate to ovate in form. In the greater number of species the sterile leaves are of two different sizes, the ventral leaves attached to the under or shaded side of the obliquely ascending stem are much larger than the dorsal leaves on the upper side exposed to the light (Fig. 335, A). Both kinds taken together form four longitudinal rows (vide infra). On its upper side and near the base each leaf bears a

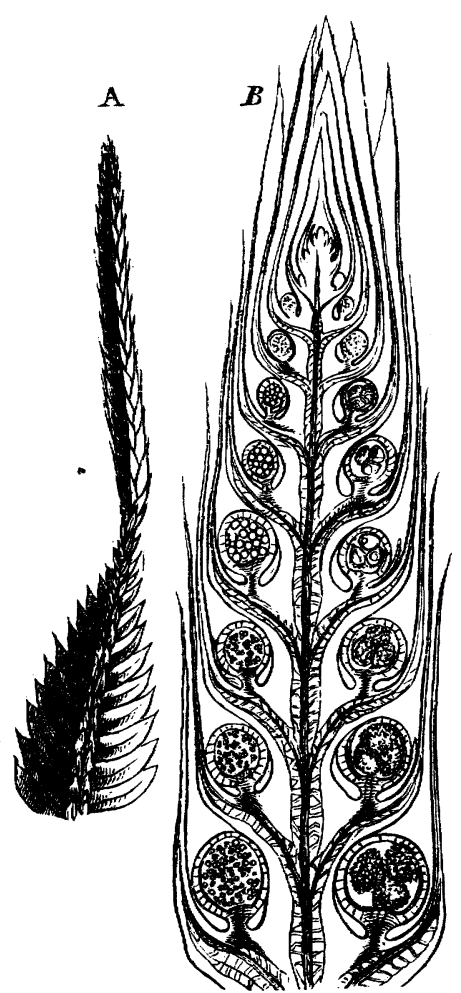

FIG. 335-Selaginella inaqualifolia; $A$ fertile branch (one-half natural size); $B$ apex in longitudinal section bearing microsporangia on the left, macrosporangia on the riglit (magnified). ligule; the point of attachment of the sporangium is below this on the fertile leaves. The fertile leaves form a quadrangular terminal spike, are uniform in size, and usually of somewhat different form from the sterile ones.

Phyllotaxis. In Isoëtes the rosettes are arranged spirally, with the divergences $\frac{3}{8}, \frac{5}{13}, \frac{8}{21}$, $\frac{13}{3}$, the fractions becoming more complicated the larger the number of leaves that are annually formed. In the species of Selaginella which have their leaves arranged in four rows, each dorsal and ventral leaf form together a pair, whose median plane, however, does not intersect that of the next pair at right angles but obliquely, an arrangement which is often clearly seen on old shoots of S. Kraussiana.

The Apical Growth of the stem takes place by means of an apical cell ${ }^{1}$. That of Isoëles lacustris is, according to Hofmeister, two-edged when the stem has two furrows; in the species with three furrows it is a three-sided pyramid. In young plants the leaves stand accordingly in the first case in two, in the second case in three rows; but later the phyllotaxis becomes more complicated and spiral, indicating perhaps that in the older stem the primary walls of the segments advance in the anodal direction, as is the case in those Mosses which have a threesided apical cell and a complicated phyllotaxis. In those species of Selaginella which have the leaves in four rows, the apical cell of the stem is, according to Pfeffer, two-

1 [Hegelmaier (Bot. Zeitg. 1874) was unable to find an apical cell in Isoëtes velata or in I. Durieui. He considers that, since in certain Selaginelleæ (S. arborescens, Pervillei, Lyallii; Russow, Vergl. Unters.) there is not a single apical cell, but a group of dividing cells, and in $S$. Wallichii (Strasburger, Bot. Zeitg. 1873) there are two apical cells, whereas in the other Selaginelleæ there is a single apical cell, it is possible that similar differences may exist in the genus Isoëtes; some species having a single apical cell, and others a group.] 
sided $^{1}$ (Fig. $33^{6}, A, B$ ). The two rows of segments here form an elevated vegetative cone, at the base of which the rudiments of the leaves first appear at the height of the fourth or fifth segment. The two edges of the apical cell are directed upwards and downwards (on the obliquely ascending shoot). The relationship of the leaves to the segments has not yet been entirely made out. The two leaves of each pair arise obliquely, one above, the other below, and alternately right and left where the pairs cross obliquely, by the outgrowth of zones of cells, each of which embraces about a fourth of the circumference of the stem. Divisions then take place in these cells which are directed obliquely upwards and downwards, and a row of apical cells is thus formed, by means of which the growth of the leaf is continued (Fig. 336, $A$ ). The branching of the shoot is effected by a second two-edged apical cell being formed from the youngest segment (Fig. 335, $C, D$ ), a wall being developed in it which is convex to the existing apical cell and which intersects the primary wall below. The two shoots which are thus formed grow right and left of the previous

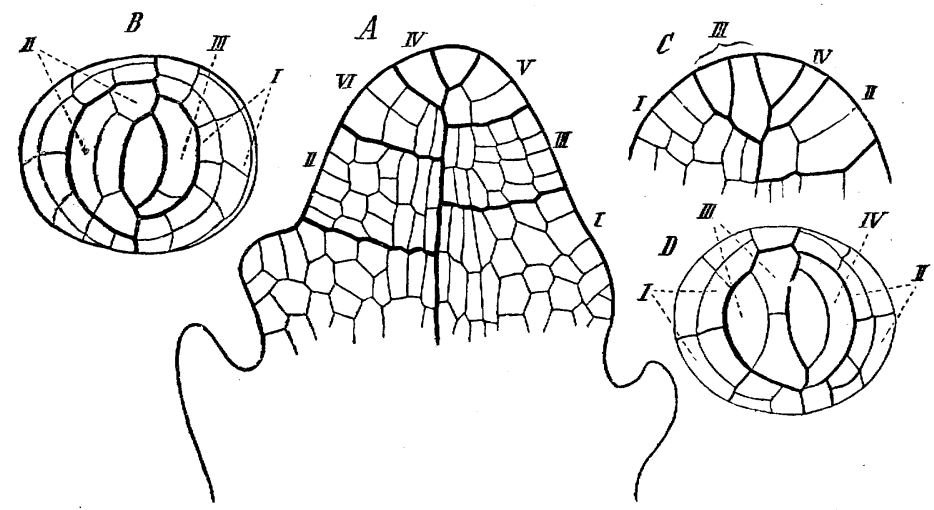

\footnotetext{
FrG. 336-Apex of the stem of Selaginella Martensii (after Pfeffer); $A$ longitudinal section of the end of the stem with the first rudiment of the leaves; $B$ apex of the stem seen from above; $C$ formation of an apical cell seen from the side $D$ the same seen from below. The primary walls of the segments are denoted by darker lines; the segments themselves are numbered with Roman figures.
}

direction of growth, and all the successive branchings take place in one and the same plane.

The Roots. All the species of Selaginella possess true roots; but in some, as S. Martensii and Kraussiana, they arise on a structure which Nägeli calls the Rhizophore, and which has no root-cap. In S. Kraussiana the rhizophores spring from the dorsal side of the stem, nearly at the base of the weaker of each pair of branches, curl themselves round it, and then grow downwards; it is only rarely in this species that two of these organs arise near one another. S. Martensii, on the other hand, forms at each branching two rhizophores, one on the dorsal and one on the ventral side (the plane which passes through them is perpendicular to the plane of branching), but usually only the ventral one undergoes further development, while the dorsal generally remains in the form of a small protuberance. The rhizophores

1 [Treub has shown (Recherches etc. sur le Selaginella Martensii) that the form of the apical cell is very variable; it is sometimes two-sided and sometimes three-sided in branches of the same plant.] 
arise very near the punctum vegetationis, probably at the same time as the branches; unlike the roots, they are exogenous structures, which, when young, possess a distinct apical cell. This is probably two-sided (it is four-sided in S. Martensii), but soon ceases to form new segments, the further growth being effected by intercalary division of the segments and elongation of the cells which proceed from them. After the cessation of the apical growth, the end of the still very short rhizophore swells up into a spherical form; its cell-walls become thicker, and in the interior of the swelling the first rudiments of the true roots originate, which however do not break through until the rhizophore has attained such a length by intercalary growth that its swollen end penetrates into the ground. The cells of this terminal part become disorganised and deliquesce into a homogeneous mucilage, through which the true roots penetrate into the ground. The rhizophores, as Pfeffer has shown (in S. Martensii, incequalifolia, and levigata), are often transformed into true leafy shoots, which at first show some deviations from the normal structure in their leaves, but afterwards continue to grow as normal shoots, and even produce sporangiferous spikes.

In Selaginella cuspidata, and some other species, there are no rhizophores, but roots spring immediately from the places nearest the ground where the stem branches, and, like the rhizophores of $S$. Martensii, they branch even before they reach the ground. These roots are also formed very early, near the punctum vegetationis, probably at the same time as the branches of the stem. The roots which spring immediately from the stem, as well as those which proceed from the rhizophores, branch in such a manner that the planes of the successive branchings cross one another at right angles. The branchings of the roots follow one another very quickly, and at the end of the mother-root are densely crowded; the apical cell is difficult to detect, but is probably, like those of the stem and of the rhizophore, two-sided (four-sided in S. Martensii). It soon ceases to form segments; the increase of length of each branch of the root takes place therefore almost exclusively by intercalary growth. Similar phenomena are observable in the roots which. proceed from the furrows of the stem of Isoëtes, and which branch (by true dichotomy) three or four times in planes at right angles to one another. Nägeli and Leitgeb failed to find in them any apical cell distinguished by its form or size, although they considered the existence of a two-edged apical cell probable ${ }^{\mathbf{1}}$. (See Fig. I $3^{8}$, after Hofmeister.) In Isoêtes the plane of the first dichotomy is parallel to the axis of the stem, in Selaginella (cuspidata and levigata) the plane of branching is at right angles to it.

The Sporangia of the Ligulatæ are of considerable size in proportion to the leaf, and are borne on short thick stalks. Each fertile leaf bears a single sporangium which always lies below the ligula either on the leaf itself (Isoëtes), or in its axil, or even on the stem (Selaginella).

The sporangia of Isoëtes are sessile in the fovea of the leaf-sheath, to which they are attached by a narrow base (Fig. 334, $A$ ). They are unquestionably products of the leaves; the outer leaves of the fertile rosettes produce only macro-

${ }^{1}$ [According to Bruchmann, there is not a single apical cell, but a meristem resembling that of some Phanerogams.] 
sporangia, the inner ones only microsporangia, the former containing a large number of macrospores. Both kinds of sporangia are imperfectly chambered by bands of tissue (trabeculce) which cross from the ventral to the dorsal side. The sporangia do not dehisce, but the spores escape by the decay of the wall.

In the Selaginellece the sporangia are shortly stalked roundish capsules. The macrosporangia contain usually four, less often two or eight macrospores. In the division of Articulatæ the lowermost sporangium only of a spike produces macrospores; in the other divisions there are several macrosporangia. The sporangia do not take origin, as Hofmeister's older accounts would seem to show, from single cells

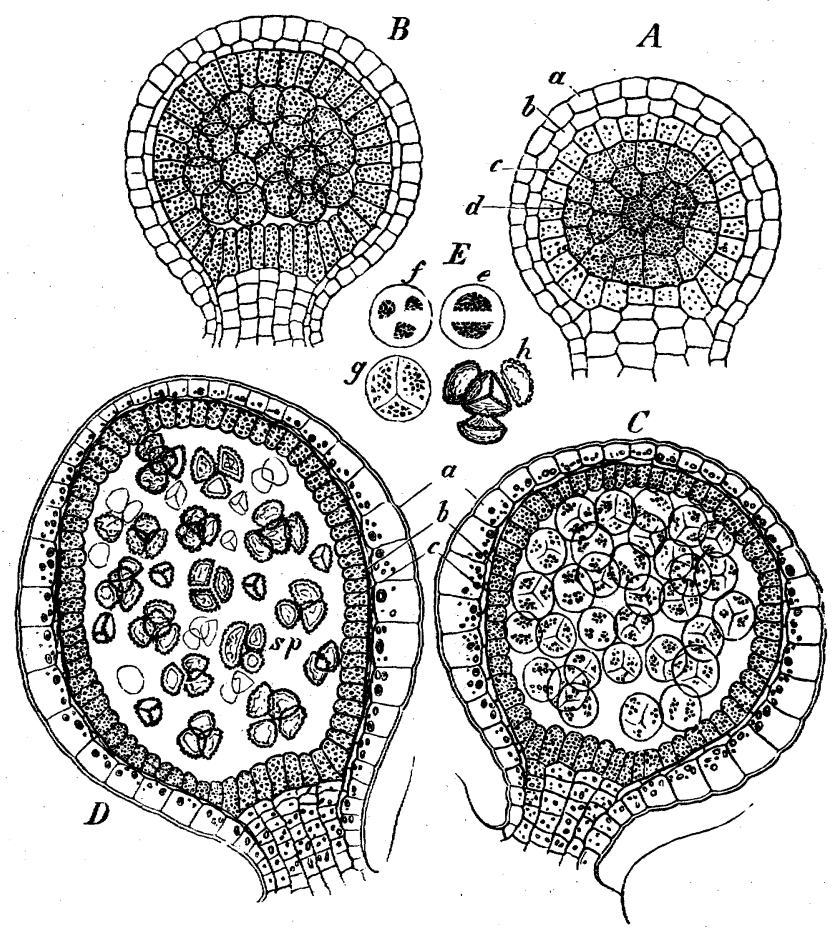

FIG, 337.-Development of the sporangia and spores of Selaginella inaqualifolia; the crder of succession is indicated by the letters $A-D ; A$ and $B$ serve for all the sporangia, $C$ and $D$ for the microsporangia only; $E$ division of the mother cells of the microspores, $h$ four nearly ripe spores; in $A, C$ and $D, a, b$ are the two layers of the wall of the sporangium, $c$ is the tapetum, $d$ the primary mother-cells of the spores $(A, B$ and $E \times 500 ; C$ and $D \times 200)$.

of the epidermis, but, as in Lycopodium, from a group of such cells (according to Goebel $^{1}$ ).

The sporangia arise on the growing-point of the stem immediately above the base of the corresponding leaves, but this by no means justifies us in regarding them as cauline organs as Russow does. Like those of Lycopodium, they at first appear as flattened protuberances which become more or less spheroidal at a later period and finally clavate. At a later period the sporangia appear to be inserted in the axil of the leaves or on their base. The fibro-vascular bundle of the leaf runs

1. [Beit. z. Vergl. Entwick. d. Sporangien; Bot. Zeitg. 1881.] 
beneath the sporangium without giving off a branch to it. By repeated divisions of the primary cells a mass of tissue is formed which is differentiated into an outer layer of cells, the wall of the sporangium, and an internal group of cells, one of which is the archesporium; the tapetum (Fig. 337, c) is formed toward the free surface of the sporangium, from cells which are cut off from the archesporium, and it is completed toward the base by cells which are cut off by tangential walls from the cells which surround the archesporium. The cells forming the wall also undergo division by walls parallel to the surface, and thus the wall of the sporangium comes to consist of two layers (Fig. 337, $a, b$ ). The mother-cells of the spores are produced by repeated divisions of the archesporial cells. These cells soon become isolated and round themselves off, and, in the case of the microsporangia, they all divide, after an indicated division into two, into four tetrahedrally-placed spores which retain their relative positions until they reach maturity (Fig. $337, E, g, h$ ). In the macrosporangia, on the other hand, one of the mother-cells grows more strongly than the rest; it divides

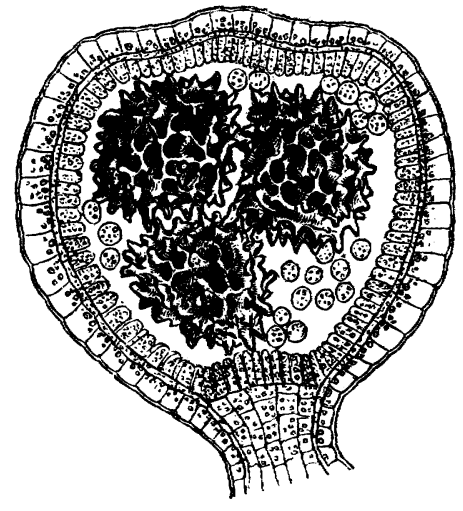

FIG. 338.-A nearly ripe macrosporangium of Selaginella inaqualifolia; the fourth spore which lies behind is not indicated ( $\times$ roo). and gives rise to the four macrospores, all the other mother-cells remaining undivided but continuing to exist (at least in Selaginella incequalifolia) for a considerable time. The macrospores are arranged, in consequence of the mode of division of the mother-cells, as the corners of a tetrahedron, an arrangement which persists until they are set free. Very commonly weakly macrospores are to be found in otherwise normal spikes of sporangia. The tapetum persists until the spores are ripe, whilst in the case of Ferns it is absorbed during the formation of the spores.

[In the case of Isoëtes, it has been shown by Tchistiakoff, by Hegelmaier, and by Goebel, that the sporangium arises from a group of cells at the base of the leaf, this group including cells belonging to the three superficial layers of their tissue. In consequence of cell-division and growth the sporangium soon appears as a swelling in the fovea. As in the Selaginellex, the fibro-vascular bundle of the leaf runs beneath the sporangium without giving off a branch to it. The most deeply-placed cells of the group form the short thick stalk of the sporangium; the superficial layer forms its wall; the intermediate layer constitutes the archesporium, from which the mother-cells of the spores as well as the trabeculæ are derived. In the microsporangium the archesporial cells elongate and are divided by walls parallel to the free surface of the sporangium, and thus rows of cells are formed. Of these rows some undergo no further change, and these form the trabeculæ. In the others either single cells or groups of cells increase in size and become divided by both transverse and longitudinal walls; these divisions produce a tapetal layer at an early stage which surrounds each group of spore-mother-cells. The differentiation of the macrosporangia proceeds in much the same manner, but here the sporogenous cells of the archesporium only undergo such divisions as are necessary for the 
formation of the tapetum, the remaining cells being spore-mother-cells. The tapetal cells then divide by transverse and longitudinal walls, so that the spore-mother-cells come to lie deeply within the tissue of the sporangium.

In the macrosporangium of Isöetes each spore-mother-cell divides to form four macrospores; its nucleus divides into two and each of these again into two, before any intervening cell-wall is formed. There is this peculiarity about the mode of the division, that the protoplasm, as in the delevopment of the spores of Anthoceros, begins to divide before the nucleus. The spore-mother-cells of the microsporangium divide in a different manner, the only other known instance of the kind occurring in the pollen-mother-cells of Monocotyledons ${ }^{1}$. In them the nucleus divides into two, and this is followed by the formation of a cellulose wall between the two cells: the nucleus of each of these then divides, and a wall is formed between the resulting cells. It is in this way that the four 'special' mother-cells of the microspores are produced.]

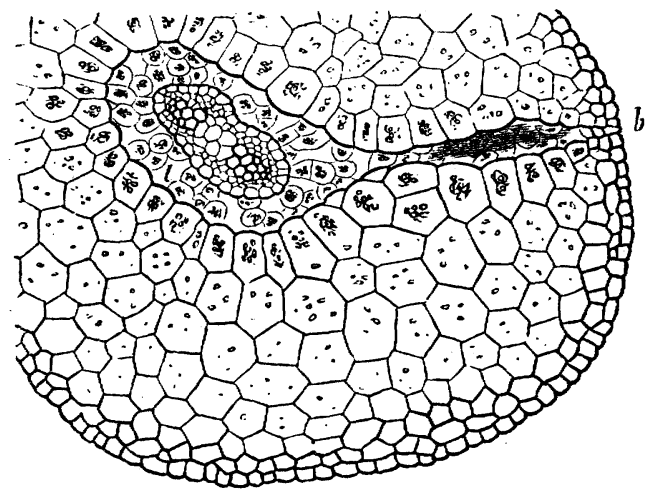

FIG. 339.-A transverse section of the stem of Selaginella denticulata, the central vessels of the bundle not yet lignified; $b$ air-cavity surrounding a bundle which is being given off to a leaf.

Histology ${ }^{2}$. In the Selaginellex, to which group the following remarks more especially apply, the epidermis of the stem consists of long prosenchymatous cells between which no stomata occur. The cells of the epidermis have often beautifully sinuous lateral walls, and, like those of the Ferns, they contain chlorophyll which occurs in these cells as well as in the cells of the fundamental tissue of the leaf in the form of large granules, only a few of which are to be found in each cell (Fig. 44). The leaves usually possess stomata on the under surface only, but they occur on both surfaces of the small leaves of $S$. pubescens. In several species (such as $S$. stenopbylla and Martensii) single epidermic cells occur with walls so thickened that the lumen is almost occluded. (Russow). In most of the species the epidermis of the upper differs from that of the under surface, in others (S. Galeotti, Kraussiana) the epidermis of the two surfaces is of the same nature.

The Fundamental Tissue of the stem consists, as in Lycopodium, of elongated cells with septa which are either oblique or transverse: these cells retain, however, their thin walls and large cavities, in contrast to what is usually the case in the Lycopodiex, the hypodermal layers only becoming thick-walled (Fig. 340). It appears that the cells of the fundamental tissue, and consequently those of the other tissues also, are capable

${ }^{1}$ [Strasburger, Zellbildung und Zelltheilung, 3rd ed., i880, p. 167.]

2 [For further details see De Bary, Vergleichende Anatomie, 1877.] 
of long-continued growth both in length and in circumference, which accounts for the intervals between the leaves of old stems and for considerable thickness of the stems themselves, a fact which is worthy of investigation not only with reference to these plants, but also to the Lycopodiex and many Ferns. It is a striking peculiarity of the Selaginellex that the ground-tissue (as also in the stem of Mosses) presents none of the usual small intercellular spaces, a result probably of the prosenchymatous arrangement of the cells. This is compensated for by the development of a large air-cavity, which everywhere surrounds each fibro-vascular bundle of the stem (Figs. 340 and $34 \mathrm{I}$ ). This cavity is traversed by transverse rows of cells forming trabeculæ attached to the bundle: if the cells are somewhat rounded, the bundle appears to be surrounded by a loose spongy parenchyma (Fig. 339), which is sharply defined from the firm compact ground-tissue. The ground-tissue of the leaf is a loose spongy parenchyma containing

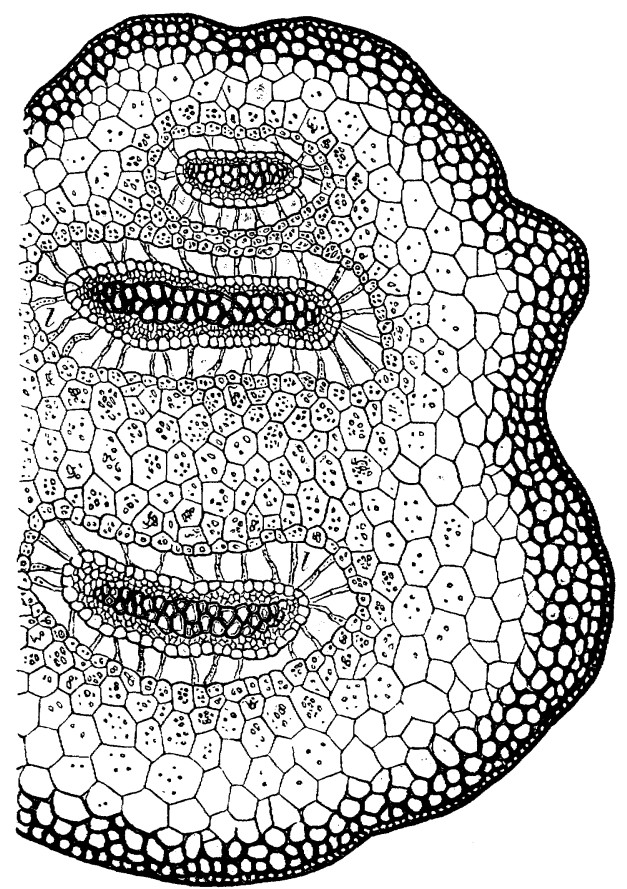

FIG. 340.-Tran sverse section of the stem of Selaginella inaqualifolia $\left(\times \mathrm{I}_{50}\right)$.

chlorophyll; in small species with thin leaves this tissue is developed only round the single fibro-vascular bundle traversing the leaf, so that at the margins the epidermis of the upper and that of the under surface come into contact.

The Fibro-vascular Bundles, one or more of which traverse the stem, are cauline, like those of the Lycopodieæ. They can be traced in the form of procambium beyond the youngest leaves up into the apex of the stem to close beneath the apical cell. The separate bundles coming from the leaves become united with the cauline bundles in these plants, as in the Lycopodiex, only at a later period. In their composition the fibro-vascular bundles resemble those of the true Ferns. They have usually an elongated elliptical form. The xylem is central, consisting for the most part of scalariform tracheides, and it is surrounded by the thin-walled phloëm (Figs. 339, 340). The very narrow spiral vessels (Fig. 34I) which are the primary elements of the xylem lie at the ends of the long axis of the bundle, and it is from these two points that the development 
and lignification of the wider tracheides proceed (Fig. 339). The layer of phloëm which invests the xylem is itself surrounded by two or three layers of parenchymatous cells, which Russow compares to the phloëm-sheath of the Ferns, but which must at any rate be regarded as forming a bundle-sheath belonging to the ground-tissue, investing the bundle within the above-mentioned air-cavity. A well-defined sheathing layer composed of cells with folded lateral walls is not to be found in the stem or in the leaves. In the latter, the fibro-vascular bundles are delicate and of simple composition: the xylem consists of spiral and reticulated tracheïdes, and it is invested by a scanty phloëm.

To this brief description must be added a few words with respect to Isoëtes. The short stem of the mature plant contains an axial woody body which can scarcely be termed a bundle, consisting of short tracheïdes loosely united, with spiral or reticulated

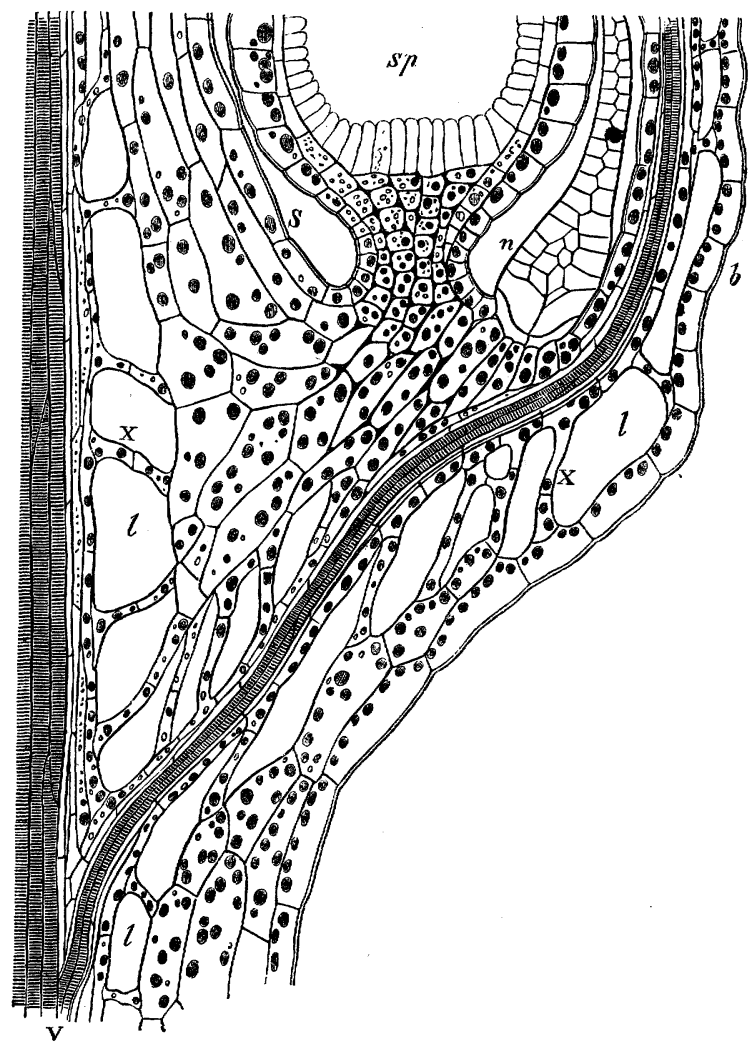

FIG. 341.-Selaginella inaqualifolia; longitudinal section through the right side of the axis of a spike $S$, the base of the leaf $b$, the ligule $n$, and the sporangium $s p ; V$ point where the cauline and foliar fibro-vascular bundles unite; $l$ air-condueting intercellular spaces; $X$ series of cells traversing the spaces.

thickening bands, and of delicate parenchymatous cells, which is invested by a layer of clear, shortly prismatic cells with large but delicate pits on their walls: these Russow considers to represent the phloëm. The bundles appear to be built upon the collateral type, the phloëm forming a continuous ring external to the xylem. The fibro-vascular bundles proceed, one into each of the very numerous leaves (Fig. 333) and into the roots. The stem of Iosëtes probably does not possess any cauline fibro-vascular bundle ${ }^{1}$

1 [Hegelmaier (Bot. Zeitg. 1874) and Bruchmann (Jenaische Zeitschrift, 1874) are of opinion that the apical portion of the fibro-vascular body is really a cauline bundle. De Bary, however (loc. cit.), does not accept this view.] 
at all; it would appear rather, from the position of the vessels, that the axial fibrovascular body consists only of the lower (inner) commencements of the foliar bundles, which are here densely crowded. In the same manner the basal disc-like woody body may consist only of the densely crowded commencements of the bundles of the roots. If this view is correct, the class of Dichotomex presents two extremes, one in Psilotum, where the foliar development is small, and where there are, according to Nägeli, no foliar bundles, but the elongated stem forms a fibro-vascular bundle belonging to it only; the other in Isoëtes, where the short stem possesses no cauline fibrovascular bundle, and only the strongly developed leaves have one each. The structure of the leaves of Isö̈tes varies according as the species grow submerged in water, in marshes, or on dry ground. In the first case they are long and conical, penetrated by four air-cavities divided by septa into channels, with a weak fibro-vascular bundle in the axis of the organ, and the epidermis destitute of stomata; in the second case they are similar, but provided with stomata and strands of hypodermal fibres; in the third case the epidermis is also provided with stomata, and the basal portions of the dead leaves (phyllopodes) for a firm black coat of mail round the stem. The groundtissue is not separated from the single fibro-vascular bundle traversing the leaf by a bundle-sheath; according to Russow it forms sclerenchyma under the epidermis which is usually colourless, in Isoëtes Hystrix, and dark brown sclerenchymatous strands which constitute most of the sheathing portion of the leaf.

Subsequent Continuous Growth in Thickness of the Stem. Outside the layer of clear tissue (phloëm) which surrounds the central woody mass of the stem of Isoëtes is a layer of meristematic cells by the activity of which the stem grows in thickness: it forms phloëm-cells internally, thus adding to the fibro-vascular mass, and cortical parenchymatous tissue on its outer side. The cortical tissue is formed much more rapidly than the fibro-vascular, and thus, in an old stem, the cortex is the preponderating tissue. This meristematic layer is evidently not analogous to the cambium of Dicotyledons and Conifers inasmuch as it forms fibro-vascular tissue on one side only, whereas in these groups of plants the cambium forms fibro-vascular tissues on both surfaces, xylem internally and phloëm externally. It is rather to be compared to the thickening-ring of Dracana and other arborescent Liliacex in which a continuous growth in thickness of the stem occurs. This view is supported by the fact that isolated bundles are occasionally formed by this meristematic layer in the stem of Isö̈tes. Thus Russow says that he found 'lying round the central woody mass of the stem of a robust specimen of Isoëtes lacustris, but separated from it by five or six layers of cells representing soft-bast, xylem bundles (consisting like the central xylem of shortly fusiform cells with irregular spiral thickenings) invested both on the outside and on the inside by tabular cells; between these, the bundles radiating from the central woody mass to the older dead leaves are disposed.' No connexion could be traced between these bundles and either leaves or roots.

A similar but much more considerable growth in thickness by means of a layer.of meristem surrounding the axial fibro-vascular bundle has been recently shown by Professor Williamson to have occurred in the extinct Lepidodendra which are so commonly present in the Coal Measures, and which are evidently closely allied to the Selaginellex. In these plants, however, if I rightly interpret Professor Williamson's account, it appears that a phellogen layer also existed at the periphery of the stem in correlation with the considerable growth of thickness resulting from the activity of the internal layer of meristem. These facts, taken in connexion with the probability of a growth in thickness of the stem of Botrychium, seem to indicate that this growth is generally wanting in the existing Vascular Cryptogams because they are less highly developed than their remote ancestors.

[Professor W. C. Williamson has contributed the following note on the Carboniferous Lycopodiaceæ :- - The large and varied group of the Lycopodiaceous plants of the Coal Measures exhibits so many modifications that it is difficult to give a brief statement of their characteristic features. But 
so far as the Lepidodendroid and Sigillarian forms are concerned, our British forms all exhibit one type of internal organisation. In the very young state each twig has a central bundle of scalariform vessels surrounded by a "bark," which usually exhibits an inner parenchymatous layer surrounded by a more prosenchymatous one, which is again invested by a second but more unequal parenchyma. This prosenchyma, as in Calamites, increases steadily in thickness as the growth of the stem advances, until it appears to constitute the chief tissue of the bark. Bundles of vessels given off by the central vascular axis proceed to each of the leaves. As the twig enlarges, the central axis almost invariably expands into a vascular cylinder, its interior becoming occupied by a cellular parenchyma of large size, and which now occupies the position and exhibits the appearance of a true medulla. The parenchyma of the leaves appears to be an extension of the outermost parenchyma of the bark. The above remarks appear to represent the common history of all the Lepidodendroid plants up to a certain stage of their growth. Beyond this stage their histories vary somewhat in the different groups. In some forms, e.g. those to which the Haloniæ belong, the branches attain considerable dimensions without undergoing any great change in their internal organisation; but in others a new development of vascular tissue invests the central cylinder at a period which seems to have varied in different species. This new growth takes place in successive layers, which are arranged in vertical laminæ disposed in radiating planes separated by tracts of muriform parenchyma; successive additions are made to the outer margins of the woody wedges previously formed through the agency of a pseudo-cambial layer of the innermost 'bark,' These exogenous growths continued until the woody zone attained to a great thickness in the larger trunks. These exogenous layers took no part in supplying the leaves with vessels. The foliar bundles invariably pass through them on their way from their source in the inner non-radiated vascular cylinder to the leaves. It being now admitted that Stigmaria was the general form of root of Lepidodendroid and Sigillarian types it is necessary to correlate its tissues with those of the aërial stem. It contains a "medulla" surrounded by a cylinder composed of radiating vascular laminæ separated by cellular rays, and enclosed in a thick "bark." Large vascular bundles are given off from the vascular wedges to supply the rootlets. Thus the structure of the root differs from that of the aërial stem in two ways. (1) The inner vascular cylinder of the latter, characterised by the non-radiating arrangement of its vessels, by the absence of "cellular rays," and by the numerous foliar bundles which it gives off to the leaves, is altogether wanting in the former. (2) On the other hand, the exogenous zone of the stem is prolonged into the roots, retaining all its more important features. These however are modified in two ways-Ist, in the absence of small passages for the transmission of foliar bundles of vessels; and, 2nd, in their replacement by much larger spaces having a lenticular section, and through which large vascular bundles, directly derived by enlarging from the exogenous laminæ themselves, pass outwards to the succulent rootlets. The rootlets of Stigmaria ficoides, which equally belong to Sigillaria and to Lepidodendron, have a very remarkable internal organisation, identical with that which is characteristic of the roots of recent Lycopods, a fact which affords additional confirmation of the close affinity of the Sigillarice and the Lepidodendra. That Lepidostrobi are the fruits of Lepidodendroid plants is certain. Equally so is it that many of the former produced microspores in the upper sporangia of each cone, and macrospores in those occupying its basal end. The incalculable myriads of these macrospores found in many coals render it probable that a very large number of the Lepidostrobi possessed both kinds of spores; indeed it is far from certain that any of them did otherwise. In the great majority of cases the sporangia of these fruits are shrivelled and empty, the spores having been shed; and this renders it impossible to say what their original character was $\left.{ }^{1}{ }^{\prime}\right]$

1 [For the literature of the Carboniferous Lycopodiaceæ see Brongniart, Archives du Mus. d'Hist. Nat. vol. I, and Journ. Bot. vol. VII. pp. 3-8.-King, Edin. New. Phil. Journ. vol. XXXVI. -Hooker, Mem. Geol. Surv. vol. II.-Carruthers, Monthly Mic. Journ. vol. I. pp. I77-181 and 225-227; Quart. Journ. Geol. Soc. vol. XXV. pp. 248-254.-Williamson, Phil. Trans. vol. CLXII. pp. I97-240, and Phil. Trans. vol. CLXXII. Part II, I881._-Thiselton Dyer, Quart. Journ. Mic..Sc. 1873 , pp. I52-156.] 
G R OUP IV.

\section{P H A N E R O G A MS.}

THE Alternation of Generations in Phanerogams is concealed in the formation of the Seed, which, at least in its earliest stage, consists of three parts:-(I) The Testa, which is a part of the mother-plant; (2) The Endosperm ${ }^{1}$; and (3) The Embryo, which is the product of the development of the fertilised oosphere.

In Vascular Cryptogams we have already seen the sexual generation which results directly from the spore, the prothallium, losing more and more of its character as an independent plant. In the Ferns, Equisetaceæ, and Ophioglossaceæ it grows independently of the spore, often for a considerable period; in the Rhizocarpeæ and Ligulatæ, where male and female spores are formed, it arises in the interior of the spore, the female prothallium still protruding, in the former group, out of the cavity of the macrospore, but remaining united with it; while in Isoëtes it fills up the interior of the macrospore as a mass of tissue which only bursts the cell-wall of the spore in order to render the archegonia accessible to the antherozoids. In the Cycadeæ and Coniferæ this metamorphosis is carried one step further; the prothallium ${ }^{2}$, which is now known as the Endosperm, remains during its whole existence enclosed in the macrospore or Embryo-sac; it produces before fertilisation archegonium-like structures, the 'Corpuscula', in which the oospheres arise. The processes which take place in the embryo-sac of Monocotyledons and Dicotyledons appear somewhat different, and bear a greater resemblance to what takes place in the macrospore of Selaginella. In this genus, besides the prothallium which produces the archegonia, there arises subsequently, by free cell-formation, another tissue which fills up the rest of the space of the macrospore; to this tissue the endosperm of Monocotyledons and Dicotyledons, which is formed by free cell-formation only after fertilisation, appears to correspond ${ }^{3}$. If, therefore, the embryo-sac is the

1 The only reason why the ripe seeds of many Dicotyledons do not contain any endosperm is because it has already been absorbed and supplanted by the rapidly growing embryo before the seeds become ripe; while in others this absorption happens only on germination after the ripening of the seeds, $i . e$. on the unfolding of the embryo; more rarely the formation of endosperm is from the first rudimentary.

2 The analogy of the endosperm with the prothallium of the higher Cryptogams was first shown by Hofmeister (Vergleich. Untersuch. $185 \mathrm{I}$ ), [Germination, Development, and Fructification of the Higher Cryptogamia, Ray Soc. 1862, p. 438].

3 Compare Pfeffer in Hanstein's Botanical Dissertations, Heft. IV. p. 24. The 'Antipodal Cells' in the embryo-sac of Angiosperms may probably be considered as a rudiment of the true prothallium. [According to Strasburger (Angiospermen und Gymnospermen, I879) not only the antipodal cells and the egg-apparatus, but also the endosperm of the embryo-sac of Angiosperms, represent the prothallium (endosperm) of the Gymnosperms and of the Vascular Cryptogams. This view leaves the 'endosperm' of Selaginella without any representative in other groups of plants. Goebel has however expressed the opinion (Bot. Zeitg. 1880) that the endosperm of Selaginella corresponds to the antipodal cells of Angiosperms.] 
representative of the macrospore, that part of the ovule in which the embryo-sac arises (the nucellus) must be considered the equivalent of the macrosporangium. But, as in the formation of the ovules of Monocotyledons and Dicotyledons, certain processes of development (the formation of the archegonia or 'corpuscula'), being no longer necessary, are suppressed, and the oosphere is immediately produced within the embryo-sac as the analogue of the macrospore, so also the production of the embryo-sac immediately from the tissue of the nucellus of the ovule is more direct. Its production is due to the increase in size of an inner cell of the nucellus which here represents the sporangium. But while even in the most highly developed Cryptogams the macrospore still becomes detached from the mother-plant, and the full development of the prothallium takes place only after the dissemination of the spores, so that the embryo always arises in structures distinct from those of the mother-plant, the embryo-sac (or macrospore) of all Phanerogams remains, on the contrary, enclosed in the ovule, the endosperm in the embryo-sac, and the embryo in the endosperm. In this manner arises that structure peculiar to Phanerogams, the Seed, the testa of which, the product of the envelopes of the ovule, closely invests both endosperm and embryo. The whole becomes separated from the mother-plant after the embryo has attained a certain very variable degree of development. Germination consists in the further development of the embryo at the expense of the endosperm.

If, on the other hand, the microspores of Selaginella and Isoëtes are compared with the pollen-grains of Phanerogams, a series of analogies is again seen which becomes intelligible on comparing the intermediate phenomena presented by Gymnosperms. Indications of the male prothallium and antheridium are indicated, as Millardet and Pfeffer have shown, by certain cell-divisions which may also be recognised in a simpler form in the pollen-grain of Gymnosperms and in a still simpler form in those of Angiosperms. Like the microspores, the pollen-grains contain the male fertilising substance, which, passing into the oosphere, causes it to develope the embryo; but a great difference is displayed in the mode in which the fertilising substance is conveyed. In Cryptogams the fertilising substance takes the form of antherozoids endowed with motion and adapted to force themselves, with the assistance of water, into the oosphere through the open neck of the archegonium. In Phanerogams, where the oosphere is enclosed in the embryo-sac and ovule, and in Angiosperms by the wall of the ovary in addition, such a conveyance of the fertilising substance would not serve the purpose intended; the pollen-grains are therefore themselves conveyed to the ovule by foreign agencies, such as the wind, mechanical contrivances in the flowers, and especially insects; and then germinating like spores, they emit their pollen-tubes, which, penetrating through the tissue of the ovule, finally reach the embryo-sac, and transmit the fertilising substance to the oosphere. The analogy of pollen-grains to spores becomes still more evident when we examine the mode of origin of both. The mass of tissue in which the pollen is formed, the pollen-sac, shows, not only in its morphological but also in its anatomical relationships, a striking resemblance to the sporangium of Vascular Cryptogams. As in the latter the spore-mother-cells are formed by the isolation of cells previously combined, so also are the mother-cells of the pollen; and as the former themselves usually produce the spores by division into four, after previous indication of a 
bipartition, the pollen-cells are produced from their mother-cells in a similar manner. Moreover, in the points here indicated Gymnosperms again appear as a connecting link between Cryptogams and Angiosperms; the pollen-sacs of Cycadeæ and of some Coniferæ closely resembling, in form and position, the sporangia of some Vascular Cryptogams.

The general result of these observations is that the Phanerogam, with its pollen-grains and its embryo-sacs, is equivalent to the spore-producing (asexual) generation (Sporophore) of the Vascular Cryptogams. But as in Vascular Cryptogams the sexual differentiation first makes its appearance (in Ferns and Equisetaceæ) in the prothallium only, and next (in Rhizocarpeæ and Ligulatæ) in the spores themselves, so, in Phanerogams, this process is carried back a step further, the sexual differentiation arises still earlier, being manifested not only in the formation of embryo-sac and pollen-grains, but also in the difference between ovule and pollen-sac, and between the leaves bearing them (carpels and stamens), and even earlier in the distinction between male and female flowers, and last of all in the diœcious condition of the plants themselves ${ }^{1}$. [The sexual generation (Oophore) is represented in the pollen-grains (microspores) by the formation of cells within them which correspond to a male prothallium, and in the embryo-sac (macrospore) by the formation of the egg-apparatus, antipodal cells, and endosperm, which together correspond to a female prothallium. A distinct alternation of generations can therefore be traced in the life-history of a Phanerogam.]

The fertilised oosphere of Phanerogams produces a Suspensor, growing towards the base of the embryo-sac and dividing, a structure which we have already met with in Selaginella, on the apex of which there is a mass of tissue at first almost globular, which is the embryo. The development of the embryo usually proceeds, even before the maturity of the seed, to such an extent that the first leaves, the primary axis, and the first root, can be clearly distinguished. It is only in parasites and saprophytes devoid of chlorophyll that the embryo usually remains rudimentary until the dissemination of the seeds without discernible external differentiation; while in those Phanerogams which contain chlorophyll the embryo not unfrequently attains a very considerable size and external differentiation (as in Pinus, Zea, Esculus, Quercus, Fagus, Phaseolus, \&c.) Independently of any curving of the embryo, the primary apex of its stem always lies originally pointing towards the bottom of the embryo-sac (the base, chalaza, of the ovule); the first root (primary root) coincides with a posterior prolongation of the primary stem; it faces the apex (micropylar end) of the embryo-sac, and is of distinctly endogenous origin, inasmuch as its first rudiment at the posterior end of the embryo is covered by the nearest cells of the suspensor.

The apical cell of the punctum vegetationis, which is easily recognised in many Algæ, in Characeæ, Muscineæ, Ferns, Equisetaceæ, and Rhizocarpeæ, as the primary mothercell of the tissue, has already, as we have seen, been replaced by a small-celled primary meristem in the Lycopodiaceæ. The apical growth of the axes, leaves, and roots of Phanerogams also can no longer be referred to the activity of a single apical cell from which the whole primary meristem has proceeded. Even in those cases

${ }^{1}$ Compare what is said on Dichogamy in Book III. 
where a single cell (not, however, of preponderating size) occupies the apex, and the arrangement of the superficial cells of the punctum vegetationis appears to point to it as the primary mother-cell, it is nevertheless by no means to be assumed that all the cells, and especially the internal mass of the primary meristem, have proceeded from it. The primary meristem of the punctum vegetationis consists of a large number of usually very small cells; more or less evidently disposed in concentric layers; an outer single layer, the dermatogen, may be recognised in Angiosperms as the immediate continuation of the epidermis of the older parts, and is continuous even over the apex of the punctum vegetationis. Beneath it lies a second meristematic tissue, [the periblem], consisting usually of a few layers of cells, which covers the apex and passes lower down into the cortex; this envelopes a third inner mass of tissue, [the plerome] terminating beneath the apex as a single cell ${ }^{1}$ (Hippuris, \&c.) or as a group of cells; and out of it proceeds either an axial fibro-vascular body. (in roots, and in the stems of water-plants), or the descending limb of the fibro-vascular bundles. In harmony with this the root-cap does not proceed, as in Cryptogams, from transverse divisions of an apical cell, but arises, on the contrary, in Gymnosperms from a luxuriant growth of the layers of periblem of the root and from their splitting away towards the apex, and in Angiosperms from a similar process in the dęrmatogen, or from a special meristematic layer the calyptrogen ${ }^{2}$. Even the first rudiments of lateral structures, leaves, shoots, and roots, cannot be traced back in Phanerogams to a single cell in the same sense as in Cryptogams. They are first observable as protuberances consisting of a few or a larger number of small cells; the protuberance which is to form a shoot or a leaf shows, even when it first begins to swell, an inner mass of tissue which is connected with the periblem of the generating vegetative cone, and is covered over by a continuation of the dermatogen.

The normal Mode of Branching at the growing end of the shoot, leaves, and roots, is, with few exceptions, monopodial: the generating axis continues to grow as such, and produces lateral members (shoots, lateral leaf-branchings, lateral roots) beneath its apex. Some cymose inflorescences appear however to be the result of dichotomous branching, and it is possible that in the Cycadeæ also the branching of the stem and leaves may be dichotomous. The monopodial branching of the axes is usually axillary; i.e. the new rudiments of shoots appear above the median plane of very young (but not necessarily the youngest) leaves, in the angle which they form with the shoot, or somewhat above it. In Gymnosperms every axil of a leaf does not usually produce a shoot; sometimes (in Cycadeæ), the branching of the stem, as in many Filicineæ, is reduced to a minimum. In Angiosperms, on the contrary, it is the rule that every axil of a foliage-leaf (i.e. one not belonging to the flower) produces a lateral shoot (sometimes even several side by side or one above another); but commonly the axillary buds, once formed, are inactive, or develope only at later periods of vegetation. In addition to the above-mentioned

1 As in so many other respects, here also Isoëtes shows an affinity to Phanerogams, as is evident from Nägeli. and Schwendener's researches on the apical growth of roots. (Compare Nägeli's Beiträgen, 1867, Heft. IV. p. I 36 .)

2 See Hanstein, Bot. Abhandl. Heft I, and Reinke, Göttinger Nachr. 187x, p. 533. [Janczewski, L'accroissement terminal des Racines, Mém. soc. nat. de Sci. de Cherbourg, I874, and Ann. d. Sci. nat. sér. 5, t. XX.] 
cases of apparent dichotomy, there are in Angiosperms only a few cases of actual or apparent extra-axillary branching, which will be mentioned when discussing the characteristic features of this class.

Phanerogams are distinguished from Cryptogams by an extraordinarily varied and complete metamorphosis of members bearing the same name; and this is connected with the almost infinite variety in the mode of life, and the more marked differentiation of the physiological functions of these plants; and the same is the case with the differentiation of tissues, which in Phanerogams greatly exceeds even that of Ferns. In these respects also Gymnosperms assume an intermediate position between Cryptogams and the rest of Phanerogams.

What has now been said will serve to explain on one hand the distinction between Vascular Cryptogams and Phanerogams, on the other hand the points in which they agree, and the affinity of the two groups in their main outlines. In order, however, to facilitate the comprehension by the student of the characteristics of the separate classes of Phanerogams which are now to be described, we must in the first place keep in view a few of their peculiarities, which have at present only been briefly touched upon, and attempt to settle the nomenclature, which has become to some extent obsolete and out of harmony with the most recent theories.

The Flower, in the broadest sense of the term, is composed of modified foliar organs and of an axis which bears them. [The most highly modified leaves of the flower are the stamens and the carpels: these so-called 'sexual organs' are really spore-bearing organs, comparable to the spore-producing leaves of the Vascular Cryptogams.] When the leaves which stand immediately beneath the sexual organs on the same axis differ from the rest of the leaves of the plant in their arrangement, form, colour, or structure, and are physiologically connected with fertilisation and its results, they are considered as belonging to the flower, and are termed collectively the Floral Leaves or Perianth. The separate flowers are distinguished from the Inforescence by including, together with their sexual organs and perianth, only one axis, while the inflorescence is an axial system with more than one flower ${ }^{1}$. Röper has termed the tout ensemble of the male sexual organs of a flower the Androcium, that of the female organs the Gynceceum. When a flower contains sexual organs of both kinds it is called bermaphrodite or bisexual; if it contains only male or only female sexual organs, and is therefore unisexual, it is termed diclinous; when flowers of both sexes occur on the same individual plant, the species is monæcious, when on different individuals it is diæcious. Usually the apical growth of the floral axis ceases as soon as the sexual organs make their appearance, and frequently even earlier; the apex of the floral axis is then concealed, and is often deeply depressed in the centre of the flower; but in abnormal cases (and normally in Cycas) the apical growth of the floral axis re-commences, again produces leaves, and sometimes even a new flower, and a Proliferous Flower is thus produced. The sexual organs and perianth of a flower are usually crowded (arranged in rosettes either spirally or in whorls); the part of the floral axis which bears them remains very short, no internodes being in general distinguishable in it; and it not unfrequently expands into the form of a club or disc, or becomes hollow, and this part of the floral axis is called the Torus or Receptacle. In Coniferæ and Cycadeæ (occasionally also in Angiosperms), it is however sometimes elongated to such an extent that the sexual organs appear loosely arranged along an axis in the form of a spike. Beneath the receptacle the axis is mostly

1 In some cases it is however difficult to distinguish between a flower and an inflorescence; as in some Coniferæ, and especially in Euphorbia. (On the latter, see Warming in Flora, 1870, no. 25 ; Schmitz, do. $187 \mathrm{I}$, nos. 27, 28; and Hieronymus, Bot. Zeitg. 1872, no. I 2.) [E. Warming, Er Koppen hos Vortemaelken en Blomst eller en Blomsterstand, Köbenhavn 1871.$]$ 
elongated and more slender, either entirely naked or bearing one or two small leaves or Bracteoles. This part of the axis is the Peduncle; if it is very short, the flower is said to be sessile. No shoots usually arise from the axils of the floral leaves, even when they are produced in all the other leaf-axils of the plant; there occur, however, abnormal cases (which are not very uncommon) of axillary branching or prolification even within the flower.

The male spores or Pollen-grains are equivalent to the microspores of the higher Cryptogams, and arise in receptacles corresponding to the sporangia in those plants, which may be termed in general Pollen-sacs. These are at first solid masses of tissue in which, as in the sporangia, an inner mass of cells becomes differentiated into the mother-cells of the pollen-grains (at first by more vigorous growth of the single cells), while the surrounding layers of tissue become developed into the wall of the pollen-sac. [The mother-cells of the pollen-grains are derived from one or more hypodermal cells constituting the archesporium, which is invested by a layer of peculiar cells, the tapetum : the tapetal cells are derived either entirely from the archesporium, or entirely from the tissue of the anther, or partly from the archesporium and partly from the tissue of the anther ${ }^{1}$.] It has already been mentioned that the mother-cells of the pollen become separated and detached from the tissue (though this rule is subject to exceptions), and then produce the pollen-cells by division into four after actual bipartition or at least an indication of it. A special description of these processes will be given under the heading of the separate classes; at present we must however premise a few facts relative to the morphological nature of the pollen-sac. Like the sporangia of most Vascular Cryptogams, the pollen-sacs of Phanerogams are usually products of the leaves, which however mostly undergo in this case a striking metamorphosis, remaining much smaller than all the other leaves. A leaf which bears pollen-sacs may be termed a Staminal Leaf or Stamen; the most recent researches have, however, shown cases in which the pollen-sacs arise on the elongated floral axis itself, as Magnus has illustrated in the case of Naias, Kaufmann in Casuarina, and Rohrbach in $T_{y p h a}$; in these cases it is still doubtful whether the pollen-sacs may not be the only surviving portions of otherwise completely abortive staminal leaves ${ }^{2}$. In the Cycadeæ the pollen-sacs grow singly or in groups on the under side of the relatively large stamens, often in large numbers, resembling in position the sporangia on Fern-leaves. In the Coniferæ the stamens have still more lost the appéarance of ordinary leaves; they remain small, and form several or only two relatively large pollen-sacs on the under side of the lamina which is still distinctly developed. In Angiosperms the stamen is usually reduced to a slender weak and often very long stalk called the Filament, bearing two pairs of pollensacs at its upper end or on both sides beneath the apex, which are included as a whole under the term Anther; the anther therefore usually consists of two longitudinal halves, united and at the same time separated by a part of the filament termed the Connective. The two pollen-sacs of each half of the anther are contiguous throughout their length, and frequently both halves of the anther are in close apposition. The separate pollensacs then appear as compartments of the anther, which is in this case quadrilocular, in contrast to those anthers (of rare occurrence) in which each half contains only a single pollen-sac, and which are therefore bilocular.

The female spore or Embryo-sac, the analogue of the macrospore, is usually derived from a bypodermal cell of the nucellus of the ovule, which must be regarded as the archesporium, the ovule itself corresponding to the macrosporangium of the heterosporous Vascular Cryptogams ${ }^{3}$. The nucellus is a small-celled mass of tissue of usually

1 [Warming, Unters. üb. Pollenbildende Phyllome und Caulome, in Hanstein's Bot. Abhandl. II. 1870: also Goebel, loc. cit.]

${ }^{2}$ [For instances of the production of pollen-grains in abnormal positions, even in ovaries or in the ovules themselves, see Masters, Vegetable Teratology, Ray Soc. London r869, pp. r82-188.]

3 [See Strasburger, Angiospermen und Gymnospermen, I879; and Goebel, loc cit.] 
ovoid form, and enclosed, with a few exceptions, in one or two envelopes, each of which consists of several layers of tissue. These envelopes or Integuments grow round the young nucellus from its base (the chalaza), and form at its apex-where they approach and often greatly overtop it-a canal-like entrance, the Micropyle or Foramen, through which the pollen-tube forces its way, in order to reach the apex of the embryosac. Very commonly the nucellus, enclosed in its integuments, is seated on a stalk, the Funiculus; but this is sometimes wanting, and the ovule is then said to be sessile; the point of attachment of the ovule to the funiculus is termed the bilum. The funiculus is, with a few exceptions (Orchidex), penetrated by an axial fibro-vascular bundle which usually ceases at the base of the nucellus. The external form of the ovule when in a state for fertilisation is very various. Independently of outgrowths of various kinds at the funiculus and the integuments, the direction of the nucellus (together with its coats), with respect to the funiculus, is of especial importance. The ovule is orthotropous when the nucellus lies in the same straight line as the hilum and chalaza, which coincide in position, and the apex of the nucellus is the apex of the entire ovule. Much more frequently the ovule is anatropous, i.e. the apex of the nucellus, and therefore the micropyle which projects beyond it, lies close to the hilum, the chalaza being at the opposite end, and the funiculus runs along the side of the nucellus, so that the ovule appears as if sharply curved at its base; the integuments (or at least the outer one) have united in growth with the ascending funiculus, which, so far as this union is complete, is termed the Raphe; the nucellus itself being in this case straight. Much less common is the campylotropous ovule, where the nucellus itself (together with its coats) is curved; its apical part, and therefore its micropyle, chalaza and hilum, lie close together at its base. These are, however, only the most striking forms, which are united by transitional states. The place from which the ovules spring is called the Placenta, and belongs to the axis of the flower, or more commonly to the carpels themselves. The placentæ often do not show any peculiar phenomena of growth; but more commonly they project like cushions, and may thus assume the appearance of special organs, finally becoming detached from the surrounding tissue. While after fertilisation, both the endosperm and the embryo are undergoing simultaneous development in the embryo-sac, the former most commonly increases considerably in size, and supplants the surrounding layers of tissue of the nucellus (sometimes even of the inner integument); and the tissue of the integument which is not displaced, or usually only certain definite layers of it, becomes then developed into the Testa. If a portion of the tissue of the nucellus, filled with food-materials, remains unchanged until the seed is ripe, it is distinguished as the Perisperm; its food-materials, although lying outside the embryo-sac, are consumed by the embryo during germination; and the perisperm may then act physiologically as the representative of the endosperm ${ }^{1}$. The seeds of Piperaceæ, Zingiberaceæ, and some Nymphæaceæ contain both endosperm and perisperm. Sometimes the ovule, during the period of its development into a seed, is enveloped from below by a new coating, which usually itself surrounds the tough testa as a soft mantle, and is termed the Aril. Of this nature is the red pulp which surrounds the hard-shelled seed of the Yew; and the origin is the same of the so-called 'mace' of the nutmeg, the seed of Myristica fragrans.

If we now turn our attention to the morphological nature of those structures from which the ovule immediately springs, we find a considerable variety. Only rarely does the orthotropous ovule appear as the prolongation or terminal structure of the floral axis itself, so that the nucellus forms directly the vegetative cone of the latter, as in Taxus and the Polygonacex. It is more usual for the ovule to grow laterally on the floral axis, thus corresponding in position to a leaf, as in Juniperus, Primulacex, and

1 [The endosperm and perisperm are generally both included in English text-books under the term 'albumen,' a term which should by all means be avoided, as conveying the idea of a definite chemical composition, whereas that of the endosperm varies greatly.] 
Compositæ. But the most common case is where the ovules spring from undoubted leaves-the carpels-and usually from their margin, like pinnæ from the leaf (this is very clear, e.g. in Cycas), more rarely from their upper (or inner) surface (as in Butomus, Akebia, Nymphaa, \&c.). If the ordinary morphological definitions are applied to these relationships, we should have in the first-named case ovules of an axial nature, or they would be metamorphosed caulomes ${ }^{1}$; where they spring laterally from the axis, they would have to be considered as metamorphosed entire leaves; and where they proceed laterally from the margins of carpellary leaves, as metamorphosed pinnæ. For those ovules which spring from the surface of carpels there is no clear analogy with any purely vegetative structures (i.e. with any that do not subserve the purpose of fertilisation); though in this case we may be reminded of the sporangia of Lycopodium. The ovules, finally, of some Cupressinex, which appear to have an axillary position on the carpeis, have not yet been sufficiently invèstigated with respect to their true relationships. In some cases the morphological interpretation is supported by malformations which not unfrequently occur. Cramer, to whom we are indebted for an admirable investigation of this question, has shown that the ovules of Primulaceæ and Compositæ, which arise laterally beneath the apex of the axis of the flower, become gradually transformed into entire leaves of the ordinary form; and that in the same manner the ovules of Delphinium, Melilotus, and Daucus, which spring laterally from the margins of the carpellary leaves, may become developed into ordinary parts of the lamina, as lacinix or leaflets. It appears on the other hand significant that nothing of the kind has yet been observed in those ovules which have been interpreted above as metamorphosed portions of the axis. The development not only of normal, but still more plainly that of abnormal ovules, shows further that a morphological distinction exists between the nucellus on the one hand and the funiculus together with the integuments on the other hand. In those anatropous ovules which may be regarded as metamorphosed leaves or parts of leaves, the nucellus makes its appearance as a new lateral structure inserted on the rudiment of the ovule, and when this latter becomes developed in a leaf-like manner it appears as an outgrowth of the surface of the leaf. This fact, the morphological importance of which was first insisted on by Cramer, is however not universal, as is especially shown in the development of the ovules of Orchidex, the nucellus of which unquestionably corresponds to the apex of the entire ovule, although it becomes anatropous by subsequent curvature; still less possible does it appear to consider the nucellus of the orthotropous ovule of Taxus and the Polygonaceæ as a lateral formation, since it is obviously an elongation of the apex of the floral axis (see Angiosperms) ${ }^{2}$.

The Carpellary Leaves are the foliar structures of the flower which stand in the closest genetic and functional relationship to the ovules. They either produce and bear the ovules, or are constructed so as to enclose them in a chamber, the Ovary, and to form the apparatus for the reception of the pollen, or Stigma. The varying morphological significance of the carpellary leaves is clearly seen by a comparison of the genera Cycas and Juniperus. In Cycas the carpels resemble the ordinary leaves of the plant, and the ovules are produced on their margins and remain entirely exposed; in Juniperus the ovules spring from the floral axis itself, corresponding, even in their position, to a whorl

1 Cramer, Bildungsabweichungen bei einigen wichtigeren Pflanzen-familien, u. die morphologische Bedeutung des Pflanzeneies (Zürich 1864), is inclined to consider all ovules as metamorphosed leaves or parts of leaves. To this view I have already expressed some hesitation in the first edition of this book; the description here given, which differs from the earlier one, is derived as much as possible from direct observation.

2 [In view of the very great variety of position in the development of the sporangia (including pollen-sacs and ovules) it will be on the whole simpler and more satisfactory, as Goebel has suggested, not to attempt to assign them to the categories of phyllome and caulome, but to regard them as organs having a morphological value of their own.] 
of leaves, but the preceding whorl of carpellary leaves swell up after fertilisation, and envelope the seeds in a pulpy mass, the berry-like fruit of these plants. In Primulaceæ the ovules spring from the elongated floral axis itself, and thus correspond in their position to entire leaves; they are however enclosed, even at the period of their formation, by an ovary, consisting of the carpels and an elongated style bearing the stigma. In most other Dicotyledons and Monocotyledons the ovules are seated on the revolute margins of the carpels which have grown together into an ovary, and which therefore in these cases both produce and enclose the ovules. But notwithstanding these very considerable morphological differences, the carpellary leaves are always alike physiologically in being excited by fertilisation to further development during the maturing of the seeds, and in taking a certain share in their future history.

Pollination and Fertilisation. By Pollination is meant the conveyance of the pollen from the anthers to the stigma of Angiosperms or to the nucellus of Gymnosperms. The pollen is detained there by a viscid substance, or often by hairs, and the emission is thus brought about of the pollen-tube which in Gymnosperms penetrates at once the tissue of the nucellus, but in Angiosperms grows downwards through the tissue of the stigma and the frequently very long style in order to reach the ovules; it then forces itself into the micropyle and advances as far as the embryo-sac. It is only when it reaches the embryo-sac (in Gymnosperms however it penetrates still more deeply) that fertilisation of the oosphere results. A considerable time, occasionally even months, often elapses between pollination and fertilisation; but commonly only a few days or hours.

Pollination is rarely effected by the wind alone, those plants in which this is the case are said to be anemophilous; in this case large quantities of pollen are produced in order to secure the result, as in many Coniferæ. In a few cases the pollen is thrown on to the stigma by the bursting of the anthers (e.g. in some Urticaceæ); but the means usually employed is that of insects, and the plants in which this is the case are said to be entomopbilous. For this purpose special and often very complicated contrivances are met with to allure insects and attract them to visit the flowers; and at the same time the object is accomplished of always conveying, where possible, the pollen to the stigma of a different flower to that which produced it (even when they are hermaphrodite). In reference to this object the parts of the flower also assume definite forms and positions, which will be followed out further in Book III. Here it need only be mentioned that insects are especially attracted to visit flowers by the nectar secreted in them; this usually sweet juice is generally produced deep down among the foliar structures of the flower, and the form of the parts is generally so contrived that the insect, while it is obtaining the nectar, must place its body in certain definite positions by which it at one time brushes the pollen out of the anthers, at another time attaches it to the stigma of another flower. The diversity in the forms of flowers depends especially on these relationships, a comparatively simple plan of structure underlying them all. The organs which secrete the nectar, the Nectaries, are therefore of extreme importance in the life-history of most Phanerogams; they are, nevertheless, usually very inconspicuous, and,--which is very significant with respect to the relationship of morphology with physiology,-notwithstanding their enormous physiological importance, they are attached to no definite part of the flower in a morphological sense; almost every part is able to perform the function of a nectary. This term therefore does not denote a morphological but a purely physiological idea. The nectary is usually only a small spot at the base of the carpels (as in Nicotiana), or of the stamens (as in Rheum), or of the petals (e.g. Fritillaria) which, without becoming more prominent, produces the nectar; but frequently it is in the form of glandular protuberances of the floral axis between the insertion of the stamens and petals (as in Cruciferæ and Fumariaceæ). A particular organ, e.g. a petal, is often transformed, for the purpose of secreting and storing up the nectar, into a hollow receptacle, forming a spur-like protuberance (e.g. Viola); or all the perianth-leaves become developed into 
hollow or pitcher-like nectaries (as in Helleborus), or they assume the most wonderful forms, like the petals of Aconitum ${ }^{1}$.

Even before fertilisation, pollination is usually followed by striking changes in the parts of the flower, particularly in the gynæceum, and especially when the parts concerned are delicate; thus the stigmas, style, and corolla wither, the ovary swells up (as in Gagea and Puscbkinia), and the like. The most striking result of pollination is shown in mány Orchidex, where the ovules are only formed as a consequence of this process.

[The process of Fertilisation is essentially this, that protoplasmic and nuclear substance passes from the pollen-tube into the oosphere; the protoplasmic substance coalesces with that of the oosphere, and the nuclear substance (male pronucleus) with that of the oosphere (female pronucleus) to form the definitive nucleus of the oospore.]

Those changes however which are excited by fertilisation are still more energetic and varied than those which are consequent on pollination; the oospore developes into the embryo; the endosperm-formed previously in Gymnosperms - is completed in Angiosperms only subsequently to fertilisation; the ovules grow along with the ovary, their layers of tissue are differentiated, become lignified, pulpy, dry, \&c. The increase in size of the ovary, which is frequently enormous (in Cucurbita, Cocus, \&c. several thousand times in volume), shows in a striking manner that the results of fertilisation extend to the rest of the plant, in so far as it affords the materials of nourishment. Striking changes in form, structure, and size take place after fertilisation, especially in the carpels; placentæ, and seeds; but very frequently similar changes result also in other parts. Thus, e.g., it is the receptacle that constitutes the fleshy swelling which is called the Strawberry, on the surface of which are seated the small true fruits; in the Mulberry it is the perianth of the flowers that swells up to form the succulent coating of the fruit; in Taxus it is a cup-shaped outgrowth of the axis beneath the ovule (the aril) that surrounds the naked seed with a red fleshy coating, \&c. Popular usage includes under the term Fruit all those parts which exhibit a striking change as the result of fertilisation, especially when they separate as a whole from the rest of the plant; in ordinary language the Strawberry, as well as the seed of the Yew surrounded by its aril, the Fig, and the Mulberry, are all fruits. Botanical terminology limits the idea of Fruit within narrower boundaries, which, however, are not yet sharply defined. In the most exact use of botanical terms, the whole of the gynæceum which ripens in consequence of fertilisation may be termed the Fruit. When the gynæceum consists of coherent carpels or of an inferior ovary, the flower produces a single entire fruit; if the carpels do not cohere, each forms a part of the fruit, or a fruitlet. This limitation of the term is often, however, inconvenient; and it would seem preferable to give it a definition which will vary in the different sections.

The point to be most clearly borne in mind by the student is that the fruit is not a new plant-structure. All the parts of the fruit which are morphologically determinable, originate and assume their morphological character before fertilisation; the result of fertilisation is merely a physiological change in the parts. The only new parts in a morphological sense are the embryo and the endosperm, which are produced in the ovule.

The Inflorescence. When a shoot which has previously formed a large number of foliage-leaves terminates in a flower, the flower is said to be terminal; if, on the other hand, a lateral shoot developes at once into a flower, with one or at most a few bracteoles beneath it, the flower is termed lateral. Sometimes the first primary axis which proceeds from the embryo terminates in a flower; but more often the axis continues to grow, or its growth comes to an end, without forming a flower, and it is only lateral shoots of the first, second, or a higher order that terminate in flowers. In the first case the

1 [On this subject see Müller, Befruchtung der Blumen durch Insekten, 1873; and Sir John Lubbock, British Wild Flowers in relation to Insects; also Book III. of this work.] 
plant may be termed, in reference to the formation of its flowers, uniaxial, in the other cases bi-, tri-axial, \&c. When a plant produces only terminal flowers, or when the lateral flowers spring from the axils of single large foliage-leaves, they are said to be solitary. When, on the other hand, the flowering branchlets are densely crowded, and the leaves within this region of ramification are smaller and of a different form and colour from the others, or are entirely absent, an Inflorescence arises, in the narrower sense of the term, usually sharply differentiated from the vegetative region of the plant, and not unfrequently assuming very peculiar forms which require a special terminology. This occurs however only rarely among Gymnosperms, the formation of multifloral inflorescences of peculiar form being characteristic of the more highly developed differentiation of Angiosperms; and it will therefore be convenient to defer a more detailed classification and definition of inflorescences until we are treating of that class.

With reference also to the Histology ${ }^{1}$, one point only need be mentioned here, in which Gymnosperms and Angiosperms agree. The Fibro-vascular Bundles of Phanerogams exhibit the characteristic peculiarity that every bundle which bends outwards to a leaf is only the upper limb of a bundle which runs downwards into the stem; in other words, we have here 'common' bundles, each of which has one limb that ascends and bends out into the leaf, and another which descends and runs down into the stem; the latter is called by Hanstein the 'leaf-trace.' In the most simple cases $(e . g$. in most Coniferæ) only one bundle bends out into each leaf; but when the insertion of the leaf is broad, or the leaf is large and strongly developed, a larger number of bundles pass from the stem into the leaf, in which they ramify when the lamina is broad; the leaftraces may consist therefore of one or more bundles. The bundles are usually thicker at the spot where they pass from the stem into the leaf than lower down in their course. Each bundle of this kind may pass downwards through only one internode or through several; in the latter case an internode with several leaves standing above it contains the lower parts of bundles which bend outwards above into leaves of different height and different age. The descending foliar bundle seldom has its lower extremity free; it is usually attached laterally to the middle or upper part of a lower (or older) bundle. This may take place by the bundle splitting below into two branches which anastomose with the lower bundles; or the thin ends of the descending bundles may intercalate themselves between the upper parts of older foliar bundles; or each bundle may bend right or left and become finally joined laterally to a lower bundle. In this manner the foliar bundles, originally isolated, are united laterally in the stem into a connected system; and this, when copiously developed, gives the impression of having arisen by branching, whereas it arises in fact from the coalescence of separate portions originally distinct.

Besides the descending limbs of the common bundles, others may however occur in the stem of Phanerogams; first of all net-works (as in Grasses) or girdle-like reticulations (as in Rubiacex or Sambucus) are frequently formed in the nodes of the stem by horizontal bundles. Furthermore, longitudinal bundles may become differentiated in the stem, which have nothing to do with the leaves; and the mode of formation of these 'cauline bundles' may vary greatly. They originate either at an early period in the primary meristem of the stem, immediately after the foliar bundles and in the pith (as in Begoniacex, Piperaceæ, and Cycadex), or only at a much later period in the outer layers of the stem when this has continued to increase in thickness, outside the foliar bundles (as in Menispermaceæ, Aloineæ, and Dracana).

The further development of the foliar bundles varies in Monocotyledons on the one hand and in Gymnosperms and Dicotyledons on the other. In the former they are closed; in the latter a layer of formative cambium remains, which, in stems that increase rapidly in thickness and become woody, usually prolongs itself across the medullary

1 [For further details see De Bary, Vergleichende Anatomie der Phanerogamen und Farne, 1877.] 
rays so as to form a perfect ring (the cambium-ring), and then produces regulariy new layers of phloëm on the outside and of xylem on the inside. In the primary roots and the stouter lateral roots of Gymnosperms and Dicotyledons an increase of thickness also takes place by the subsequent formation of a closed cambium-ring, which, like that of the stem, is not found in Cryptogams, and commonly leads to the formation of strong persistent root-systems, which are more often replaced physiologically in Monocotyledons by rhizomes, tubers, and bulbs. With the persistent increase in thickness is connected, finally, the active and extensive production of cork, a process foreign both to Cryptogams and to Monocotyledons. It will be more convenient, however, to defer the special discussion of these points also until we are treating of the characteristics of the separate classes.

\section{SYSTEMATIC REVIEW OF PHANEROGAMS.}

The distinguishing characteristic of Phanerogams, as contrasted with Cryptogams, lies in the formation of the Seed. This organ is developed from the ovule, which, in its essential part the nucellus, produces the Embryo-sac, and in this the Endosperm and the Oosphere. The latter is fertilised by the Pollen-tube, an outgrowth of the Pollengrain, and, after, produces the Embryo borne on a Suspensor. The phanerogamic plant which is differentiated into Stem, Leaves, Roots, and Hairs, corresponds to the spore-forming (asexual) (Sporophore) generation of Vascular Cryptogams; the Embryosac to the Macrospore; the Pollen-grain to the Microspore; the Endosperm is equivalent to the female Prothallium; and the Seed unites in itself, at least for a time, the two generations, the Prothallium (Endosperm), together with the young plant of the second generation, the Embryo.

Flowering Plants may be classified as follows:-

\section{Phanerogams without an Ovary.}

The ovules are not enclosed before fertilisation in a structure (the Ovary) resulting from a cohesion of carpellary leaves. The endosperm arises before fertilisation, and forms archegonia (i.e. 'corpuscula'), in which the oospheres originate. The contents of the pollen-grains are divided before the formation of the pollen-tube, corresponding to divisions taking place in the microspores of Selaginella.

I. Gymnosperms. The first leaves produced from the embryo are arranged in whorls of two or more.

A. Cycadea. Branching of the stem very rare, or entirely suppressed; leaves large, branched.

B. Conifere. Axillary branching copious, but not from all the leaf-axils; leaves small, not branched.

C. Gnetacece. Mode of growth very various; flowers similar in many respects to those of Angiosperms.

\section{Phanerogams with an Ovary.}

The ovules are produced in the interior of a structure (the Ovary) formed by the cohesion of carpellary leaves (often only of one carpel, the margins of which have become coherent), bearing at its summit the stigma upon which the pollen-grains germinate. The endosperm is formed after fertilisation at the same time as the embryo, both remaining rudimentary in some cases. A division of the contents of the pollen-grain is indicated. The branching is almost always axillary and from the axils of all the foliage-leaves. 
2. Monocotyledons. The first leaves produced from the embryo are alternate; endosperm usually large; embryo small.

3. Dicotyledons. The first leaves of the embryo form a whorl of two (or are opposite); endosperm very often rudimentary, often entirely absorbed by the embryo before the ripening of the seeds.

\section{CLASS $\mathrm{X}$.}

\section{G Y M N O S P E R M S}

This class embraces, in the orders Cycadeæ, Coniferæ, and Gnetaceæ, plants of strikingly different habit, but evidently closely allied in their morphological structure, in the peculiarities of the mode of formation of their tissue, and especially in their sexual reproduction. On these grounds they take up an intermediate position between Vascular Cryptogams and Angiosperms, while they approach Dicotyledons among the latter, especially in their anatomical structure.

The Pollen-grains suggest a homology with the microspores of Selaginella, their contents undergoing before pollination one or more divisions into cells which resemble a very rudimentary male prothallium. One of these cells (the largest) grows into the pollen-tube when the pollen-grain has reached the nucellus of the ovule. The pollen-sacs are always outgrowths from the under side of structures unquestionably foliar (staminal leaves), and bear a striking resemblance in many cases to the sporangia of some Vascular Cryptogams. They are produced either in larger or smaller numbers or in pairs on a staminal leaf, without cohering in their growth.

The Ovule, which is almost always orthotropous, and usually provided with only one integument, either appears to be the metamorphosed end of the floral axis itself, or it originates laterally beneath its apex (or is apparently axillary), or it grows from the upper surface or margins of the carpels. These never cohere so as to form a true ovary before fertilisation, although during the ripening of the seeds they often increase considerably in size, close together, and conceal the seeds, usually separating again when they are mature in order to allow them to fall out; the cases are, however, not rare in which the seeds remain quite naked from first to last. The embryo-sac is formed beneath the apex of the ovule, which consists of small-celled tissue and remains enclosed until fertilisation by a thick layer of the tissue of the nucellus. Sometimes the formation of several embryo-sacs commences in one nucellus, but only one of them attains its full development. The Endosperm arises by free cell-formation long before fertilisation in the embryo-sac, which is distinguished by its firm wall; but the cells soon become combined into a tissue and increase by division. Within this mass of tissue, corresponding to the endogenous prothallium of Selaginella, arise the Archegonia (or Corpuscula ${ }^{1}$ ) in larger or smaller numbers.

1 [The central cells of the archegonia of Gymnosperms were discovered by Robert Brown in I 834. He called them corpuscula or embryoniferous areolæ (Miscellaneous Botanical Works, 
Strasburger states that each of these bodies is formed from an endosperm-cell lying at the apex of the embryo-sac, which increases considerably in size and produces the neck and central cell of the archegonium by division. According to the same authority a small upper portion of the central cell beneath the neck is even separated as the canal-cell. There is little doubt that, as Strasburger asserts, the whole of the central cell is to be considered as the oosphere, although Hofmeister thought that a number of oospheres arose in it by free cell-formation ${ }^{1}$. After the pollen-tube has penetrated the tissue of the nucellus and forced itself as far as the archegonium (corpusculum), where its fertilising material is conveyed to the oosphere, the Suspensor is formed by division of a cell which is developed in the lower part of the oosphere. The cells of the suspensor are at first small, but the middle or upper ones elongate very much, and, pushing the lower ones before them, penetrate into a softened part of the endosperm. Sometimes the suspensors which are produced side by side separate; each bears at its apex a small-celled rudiment of an embryo. On this account, and also because several archegonia are often fertilised in one endosperm, the unripe seed contains several rudimentary embryos, of which, however, only one usually increases greatly in size, the others withering away.

During the development of the embryo, the endosperm becomes filled with nutrient materials and increases greatly in size; the embryo-sac which encloses it grows at the same time, and finally entirely absorbs the surrounding tissue of the nucellus; the integument, or an inner layer of it, becomes developed into a hard shell, while frequently (in naked seeds) its outer mass of tissue becomes fleshy and pulpy and gives the seed the appearance of a drupaceous fruit (e.g. Cycas, Salisburia). The effect of fertilisation not unfrequently extends also to the carpels or other parts of the flower, which grow considerably, forming fleshy or woody coatings to the seeds, or cushions beneath them.

The ripe Seed is always filled with the endosperm, in which the embryo lies and is distinctly differentiated into stem, leaves, and root. It fills up an axial cavity of the endosperm, is always straight, its radicle being turned towards the micropyle, its plumule towards the base of the seed. The first leaves which the embryonal stem produces stand in a whorl, consisting generally of two opposite, but not unfrequently of three, four, six, nine, or more members. At the period of germination the radicle first protrudes through the split testa; the bud which is formed between the Cotyledons or first leaves at the apex of the stem is forced out by their elongation, the cotyledons still remaining concealed in the seed, and remaining in it until its food-materials have been completely consumed by the embryo. Sometimes they remain concealed there as organs which have become useless; but in Coniferæ they are drawn out by the elongation of the embryonal stem and brought above the surface of the ground, where they unfold as the first foliage-leaves. The cotyledons of Coniferæ become green even within the seed in complete darkness, the

vol. I. pp. $5^{6} 7$ and 570 ). The structure of the neck of the archegonium was made out by Hofmeister, who applied to it the term rosette (On the Higher Cryptogamia, p. 4II). Archegonium and corpusculum do not seem exactly synonymous, since the latter, properly speaking, is only equivalent to the central cell of the former. Henfrey termed the central cells 'secondary embryo-sacs' (Elementary Course, and edition, p. 608).]

1 More will be said on this subject under Coniferæ. 
formation of chlorophyll taking place, as in Ferns, without the assistance of light. It is not known whether the same thing occurs also in the Cycadeæ and Gnetaceæ. The young plant, freed from the seed, consists of an erect stem, passing below insensibly into the vertically descending tap-root, from which numerous secondary roots soon proceed in acropetal order, usually forming finally a well-developed root-system. The embryonal stem grows vertically upwards, and is usually not only unlimited in its growth, but is much stouter than all the lateral shoots, even when these are formed in abundance, as is the case with Coniferæ. In the remarkable Gnetaceous Welzeritschia, however, the apical growth altogether ceases at a very early period, and even the production of new leafy shoots is suppressed, as is usually the case also in Cycadeæ.

An apical cell does not exist either at the ends of the shoots or at the apices of the roots of Gymnosperms. In this respect they resemble the other Phanerogams, but they differ from them in that the primary meristem of the Punctum vegetationis of the stem shows either no differentiation (Cycadeæ, Abietineæ), or only an indistinct differentiation, of Dermatogen (young epidermis) and Periblem (young cortex). At the apex of the root the well-defined axial fibro-vascular mass (Plerome) is covered by a continuation of the cortical tissue (Periblem). Layers of cells belonging to this tissue, which cover the apex, become thickened and split off, thus forming the rootcap. The root-cap therefore is not derived here, as in most Angiosperms, from the active growth and splitting of the young epidermis (Dermatogen), or from a proper meristematic layer (Calyptrogen).

The Flowers are usually developed on small lateral shoots, often of a high order of ramification; terminal flowers occur on the primary stem only in the Cycadex (and in them not exclusively). They are always diclinous; the plants themselves monœcious or diœcious. The male flower consists of a slender axis usually greatly elongated, on which the staminal leaves are arranged in large numbers usually spirally or in whorls. The female flowers are remarkably different in their external appearance, and usually very unlike those of Angiosperms. A kind of perianth of rather delicate leaves occurs only in Gnetaceæ; in Coniferæ and Cycadeæ it is wanting or is replaced by scales. But what makes the female flowers peculiarly strange, independently of the absence of an ovary, is the elongation of the floral axis, on which the foliar structures are placed not in concentric circles as in Angiosperms, but in a distinctly ascending spiral arrangement, or in alternating whorls when they are numerous. When only a few ovules are produced on a naked or small-leaved inforescence, as in Podocarpus and Salisburia, the last trace of resemblance in habit to the flowers of Angiosperms ceases. But to clearly understand the matter it is only necessary to retain distinctly in mind the definition of a flower, viz. an axis bearing members which are modified for the production of spores.

On the histology of the Gymnosperms see the remarks at the conclusion of the description of the whole class. 


\section{A. C Y C A D E $\mathbb{E}^{1}$.}

The Embryo, enclosed in the large endosperm, possesses two opposite unequal cotyledonary $^{2}$ leaves, which lie with their inner surfaces face to face, cohering towards their apices. The tendency of the subsequent foliage-leaves to branch is sometimes displayed even in these cotyledons, a rudimentary lamina being formed on the larger one, with an indication of pinnæ (as in Zamia, Fig. $342 B^{\prime}$ ). The seed germinates when laid in moist earth, but only after a considerable interval; the testa splits at the posterior end and allows the emission of the primary root, which at first grows vigorously downwards, but sometimes assumes afterwards a tuberous form or produces a system of rather thick fibrous roots. According to Fig. $342 C$ borrowed from Schacht, and a more recent statement by Reinke, the branching of the primary root is laterally monopodial; $\mathrm{Mi}$ quel, however, asserts the existence of bifurcations of the more slender roots in older plants of Cycas glauca and Encephalartos. According to Reinke and Strasburger, only those lateral roots which emerge from the ground branch dichotomously, probably as a pathological phenomenon. By the elongation of the coty-

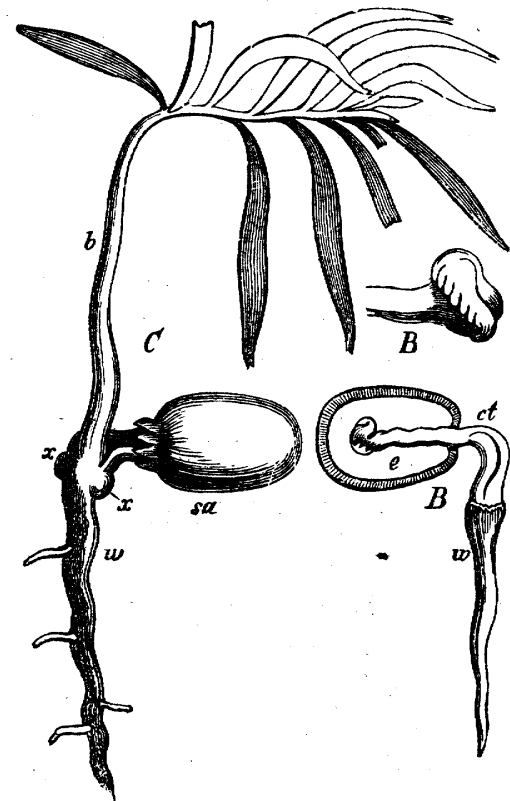

FIG. 342.-Germination of Zamia spiralis (after Schacht, reduced). $B$ commencement of germination, $c t$ the cotyledons coherent above their elongated base, one of them having at its apex (magnified at $B^{\prime}$ ) an indication of a pinnate lamina; $C$ seedling six months old; $s a$ seed, $w$ the primary root, $b$ the first pinnate leaf, $x x$ rudiments of the adventitious roots which afterwards grow upwards. ledons which remain in the endosperm

1 Miquel, Monographia Cycadearum, 1842. [Ditto, On the Sexual Organs of the Cycadaceæ; Journ. of Bot., March and April 1869].-Karsten, Organogr. Betracht. über Zamia muricata, Berlin I 857--Mohl, Bau des Cycadeen-stammes (Vermischt. Schrift. p. 195).-Mettenius, Beiträge zur Anatomie der Cycadeen (Abhandl. der königl. Sächs. Gesellsch. der Wissensch. vol. VII, I86r).[W. C. Williamson, Contributions towards the history of Zamia gigas, Trans. Linn. Soc. vol. XXVI, 1870.-Carruthers on Fossil Cycadean Stems from the Secondary Rocks of Britain, ibid.]-On the structure of the pollen see Schacht, Jahrb. für wissensch. Bot. vol. II. p. I42 et seq.-Kraus, Ueber den Bau der Cycadeenfiedern (Jahrb. für wissensch. Bot. vol. IV).--Reinke in Nachrichten der königl. Gesellsch. der Wissensch. in Göttingen, I871, p. 532.-De Bary, Bot. Zeitg. 187o, p. 574,-Juranyi, Bau u. Entwickelung des Pollens bei Ceratozamia (Jahrb. fuir. wissensch, Bot. vol. VIII. p. 382).Ueber Wachsthum und Verzweigung der Wurzeln, Reinke, Morphol. Abhand1. 1873.- [Warming, Undersogelser og Betragtninger over Cycaderne, and, Bidrag til Cycadeernes Naturhistorie, in the K. D. Vidensk. Selsk. Forhandl. 1877 and 1879 ; also Bot. Zeitg. 1878.]

2 Van Tieghem found in three seedlings, hybrids of Ceratozamia longifolia + with Cer. Mexicana t, only a single sheathing cotyledon, in another two very unequal cotyledons. Two embryos of Zamia spiralis had two unequal cotyledons, another had three, and another had only one. (See Van Tieghem in the French edition.) 
and absorb their nourishment from it, their basal parts and the intermediate plumule are pushed out of the seed. The portion of the axis which bears the cotyledons, as well as that which developes above them, remains very short, but a considerable lateral increase of size takes place beneath the apex, due to a large development of parenchymatous tissue. The stem thus acquires the form of a roundish tuber, which it retains even at a later period in some species; but in most it lengthens in the course of years into an erect tolerably stout column which sometimes attains a height of some metres. This slow increase in height, together with the considerable increase in thickness of the growing end, is correlated with the absence of a tendency to branch as in other similar cases (Isoëtes, Ophioglossum, Aspidium Filix-mas, \&c.). The stem of Cycadeæ usually remains perfectly simple, although old stems sometimes divide into branches of equal stoutness. But when several flowers are formed at the summit, this evidently depends on branching; and, as far as one is able to judge from drawings, it is probable that this branching is dichotomous. In old or sickly plants small bulbous or tuberous gemmæ are not unfrequently found at the base of the stem under or above ground, the morphological nature of which is still doubtful; in Miquel's opinion it is not impossible that they spring from old leaf-scales, and have therefore nothing to do with the branching of the stem.

The whole of the surface of the stem is furnished with leaves arranged spirally; no internodes can be distinguished. The leaves are of two kinds; dry, brown, hairy, sessile, leathery scales of comparatively small size, and large, stalked, pinnate or pinnatifid foliage-leaves. The scales and the foliage-leaves alternate periodically; a rosette of large foliage-leaves is produced annually or biennially, and among these the terminal bud of the stem is enveloped with scales, under protection of which the new whorl of foliage-leaves is slowly formed. This alternation begins at once on germination in Cycas and other genera, a number of scale-leaves following the leaf-like cotyledons, and enveloping the bud of the seedling; after these a pinnate though small foliage-leaf is then usually developed, which is again followed by scales. It is only as the strength of the plant increases after several years' growth that the foliage-leaves are produced in whorls constantly increasing in size, and forming, after the older ones have died off, the palm-like crown of leaves, the scales which stand above them enclosing at the same time the apical bud of the stem. In this bud the foliage-leaves are so far formed beforehand, that when they at length burst the bud they only have to unfold, this process then occupying only a very short time, while one or two years elapse before the unfolding of the next rosette of leaves. The leaves which proceed from the bud are in Cycas and other genera circinate like those of Ferns; in others the rachis of the leaf only is rolled up ; in others, finally, as Dion, the growth of the leaf is straight, its lateral leaflets being also straight before expansion ${ }^{1}$. The unfolding is, as in Ferns, basifugal, and, probably in consequence of this, there is also a permanent apical growth and a basifugal development of leaflets. The leaflets are usually simple, and generally stand alternately on the rachis, which is often $\mathbf{I}$ to 2 metres long. The mode in which

1 [This statement is not quite exact. In Zamia and Encephalartos the leaves are not circinate in vernation ; and even in Cycas it is only the leaflets and not the rachis that is so.] 
the lamina terminates above, points to a dichotomous branching of the leaf, the rachis of which may therefore be considered as a sympodium composed of the basal portions of the successive bifurcations, while the lateral leaflets represent the bifurcations of the lamina of the leaf, the growth of which is arrested and flattened. The whole leaf would therefore be a dichotomous cymose branch-system. Researches into the history of its development are however wanting, as in the case of the branching of the stem and root.

The Flowers of the Cycadeæ are always diœcious, and the plants are therefore either male or female. Both kinds of flowers appear at the summit of the stem, either singly, as in Cycas, as terminal flowers of the primary stem, or in pairs or larger numbers as in Zamia muricata and Macrozamia spiralis, where they may perhaps be regarded as metamorphosed bifurcations of the stem $^{1}$. The flower consists of a strong conical elongated axis, sometimes supported on a naked peduncle, but densely covered in other parts by a large number of staminal and carpellary leaves arranged spirally.

In Cycas the female flower is a rosette of foliage-leaves which have undergone but slight metamorphosis (Fig. 343), the apex of the stem developing again first of all scale-leaves, and then new whorls of foliage-leaves; the stem, therefore, grows through the female flower, thus furnishing an instance of prolification. The separate carpels are, indeed, much smaller than the ordinary foliage-leaves, but are essentially of the same structure; the lower pinnæ are replaced by ovules, which attain, even before fertilisation, the magnitude of a moderate-sized ripe plum, the fertilised seed acquiring the dimensions and the appearance of a moderate-sized ripe apple, and hanging quite naked on the carpel. Whether the male flower of Cycas also exhibits prolification I do not know, and it seems improbable; the very numerous staminal leaves are much smaller, 7 to $8 \mathrm{~cm}$. long, and undivided; they expand considerably from a narrow base and terminate in an apiculus. They are furnished on the under side with a number of densely-crowded pollen-sacs; the whole flower is from 30 to $40 \mathrm{~cm}$. long.

The male and female flowers of the remaining genera of Cycadeæ resemble fir-cones externally. The comparatively slender floral axis rises as a rachis on a short naked peduncle, and on this are seated the numerous staminal or carpellary leaves (Fig. 344). The axis terminates with a naked apex which undergoes no further development (Fig. $344 D$ ). The stamens are, indeed, but small in comparison to the foliage-leaves of the same plant, but are, nevertheless, the largest which occur anywhere among Phanerogams. In Macrozamia, as in Cycas, they are from 6 to $8 \mathrm{~cm}$. long, and as much as $3 \mathrm{~cm}$. broad; they spring, with rather a narrow base, from the floral axis, and expand into a kind of lamina, terminating in an apiculus (Macrozamia) or in two curved points (Ceratozamia), or the lower part of the stamen is thinner and stalk-like and bears a peltate expansion (Zamia). They are also distinguished from the stamens of most other flowering plants by their

1 The hypothesis that the male flower of Cycas Rumphii is one, the leaf-bud by which the stem is prolonged the other bifurcation of the dichotomising apex of the stem, is not supported by De Bary's recent researches. [According to Warming (loc. cit.) all the flowers are probably terminal; possibly the male flower (in Ceratozamia longifolia) is produced on a branch of a dichotomy of the stem : it is certainly not borne on a lateral branch.] 
persistence, becoming lignified and often very hard. The numerous pollen-sacs on the under side of the stamens are usually collected into small groups numbering from two to five, like the sori of Ferns, these again forming larger groups on the right and left side of the leaf. The pollen-sacs are globular or ellipsoidal, usually about $\mathbf{I} \mathrm{mm}$. in size, and are attached with a narrow base to the under side of the stamen; Karsten states that in Zamia spiralis they are even stalked. They dehisce longitudinally, and are in all respects much more like the sporangia

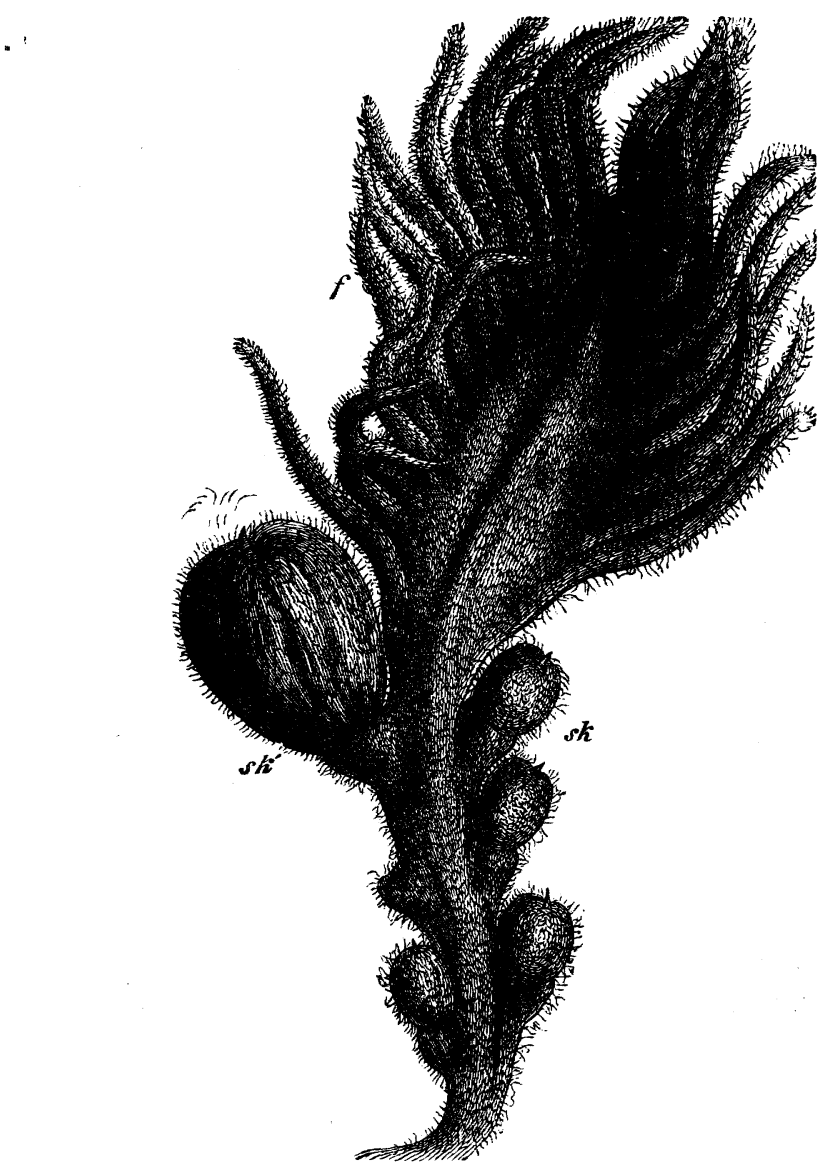

FIG. 343.-A carpel of $C y$ cas revoluta (reduced about $\frac{1}{2}$ ); $f$ finiz of the leaf-like carpel; sk ovules replacing the lower pinnæ; $s k^{\prime}$ an ovule further developed.

of Ferns than the pollen-sacs of other Phanerogams, from which they also differ in the firmness and hardness of their wall. The mode of development of the pollen-sacs and pollen-grains of Cycadeæ was till lately unknown; it has only quite recently been observed by Juranyi in Ceratozamia longifolia. The pollensacs are formed on the under side of the stamens in the form of small papillæ, probably consisting from the first of several cells over which the epidermis of the surface of the leaf is continuous. The inner tissue is next differentiated 
(as in the sporangia of Lycopodiaceæ, Equisetaceæ, and Ophioglossaceæ) into an outer layer of smaller cells enclosing a larger-celled tissue; the cells of the latter continue to grow and divide in all directions, and the mother-cells of the pollen are finally isolated, but densely crowded together, as in Dicotyledons. The mode of division of the mother-cells is nevertheless more like that of Monocotyledons in this respect, that they first of all divide into two daughter-cells, each of which again undergoes bipartition. The first division-wall is partially formed, as in Dicotyledons, by the slow growth of an annular ridge of cellulose, formed in the depres-
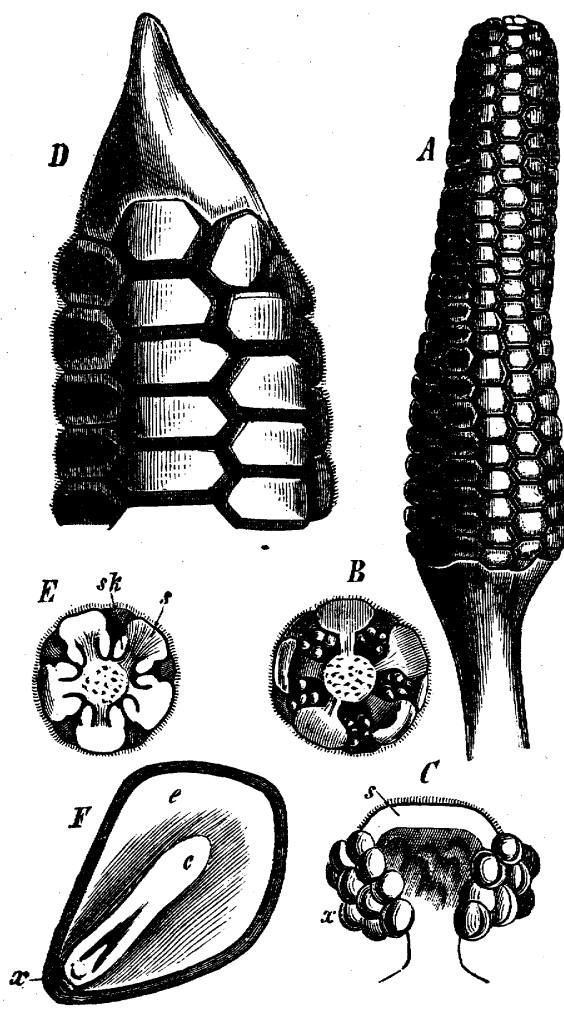

FIG. 344-Zamia muricata (after Karsten). $A$ a male flower (natural size); $B$ transverse section of one; $C$ one of its stamens with the pollen-sacs $x$ and the peltate expansion $s$ (seen from below); $D$ the upper part of a female flower (natural size); $E$ transverse section of one, $s$ the peltate scale bearing the ovules $s k ; F$ longitudinal section of a ripe seed; $e$ endosperm, $c$ cotyledons, $x$ the folded suspensor.

sion produced by the previous constriction of the protoplasm of the mother-cell; but in each of the two daughter-cells the second partition appears to be formed simultaneously, as in Monocotyledons. The four young pollen-cells are now freed by the rapid absorption of the cell-wall which surrounds and separates them. The pollen-grains, when free from their mother-cells, are unicellular and spherical; but, during their further growth, the contents, enclosed by an extine and intine, divide into two cells, a smaller and a larger one, each possessing a nucleus. The smaller of these two cells, lying on one side against the intine of the pollen-grain, becomes arched on the opposite side, and projects in the form of a papilla into the larger 
one. This smaller cell now again undergoes a transverse division parallel to the first, and this is sometimes followed by a second; a two- or three-celled body is thus formed, attached on one side to the intine, and projecting into the cavity of the larger cell, as in Abietineæ, which, moreover, further resembles Ceratozamia in the fact that, as in the Coniferæ, the large cell, formed by the first division of the - pollen-grain, developes into the pollen-tube, the mass of small cells remaining inactive in the pollen-grain. In Cycas Rumphii, Encephalartos, and Zamia, the pollen-grain also splits up, according to De Bary, into a larger and a smaller cell, the latter also in this case again dividing once, and the larger cell developing into the pollen-tube. The spot where the intine which developes into the pollen-tube breaks through the extine lies exactly opposite the mass of small cells (the secondary cells of the pollen-grain); the extine is in this place thinner, and in the dry pollen-grain deeply folded in, so that the transverse section of the dry pollen-grain is kidney-shaped. During the absorption of water which

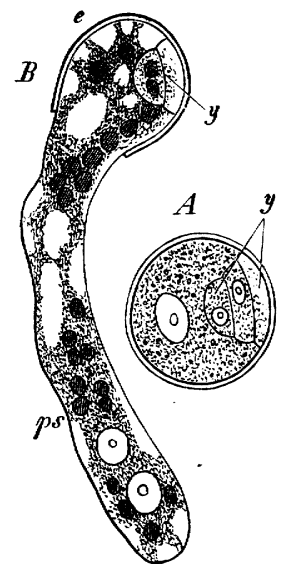

Fic. 345-Cera.ozamia longifolia (after Juranyi); $A$ pollengrain containing a group of three grain containing a group of three
(vegetative) cells $y ; B$ development of the pollen-tube from the large cell of the grain; $e$ the extine, $b$ s the pollen-tube covered by the intine. precedes the formation of the pollen-tube the pollen-grain again assumes a spherical form.

The carpellary leaves are arranged spirally or in apparent verticils, closely crowded on the axis of the female flower. Those of Cycas have already been described; in Zamia, Encephalartos, Macrozamia, and Ceratozamia, the carpels are much smaller, and each bears only two ovules, attached right and left to a peltate expansion which terminates a slender pedicel (Fig. 344). The ovule is always orthotropous, and consists of a large nucellus and a thick integument the inner layer of which (in contrast to that of other Phanerogams) is penetrated by a number of fibro-vascular bundles. The micropyle is a slender tube, formed by the prolongation of the contracted margin of the integument beyond the summit of the nucellus. According to De Bary's researches a second inner integument appears to exist in the case of Cycas revoluta. [In the nucellus a group of cells can be readily distinguished at an early stage, which Warming considers to be homologous with the mothercells of the spores in the sporangia of the Vascular Cryptogams; from one of these the embryo-sac is formed. The wall of the embryosac becomes thickened, and its cavity becomes filled with endosperm. From certain superficial cells of the endosperm the archegonia (corpuscula) are formed. The neck of the archegonium consists of two cells. From the large central cell a canal-cell is cut off, leaving the remainder as the oosphere. After fertilisation, each oospore gives rise to a single suspensor; the embryo is not developed at its apex until after the seed has been sown. The embryo of Ceratozamia has only one cotyledon; Cycas and Macrozamia have two; Van Tieghem has, however, found two cotyledons in some cases in Ceratozamia Mexicana, and three in Zamia spiralis.]

In consequence of the form and position of the carpels, the ovules are covered and concealed before and after fertilisation, except in Cycas; at the period of polli- 
nation, which is apparently brought about by insects, the carpels separate from one another, and the micropyle excretes a fluid to which the pollen-grains adhere. The outer layer of the testa is usually fleshy, the inner one hard, and the seed therefore resembles a plum, with its surface often brightly coloured.

\section{B. CONIFER Æ 1 .}

Germination. The endosperm surrounds the embryo in the form of a thickwalled sac open at the radicular end; the embryo lies straight in the central cavity of the endosperm; its axis is continuous behind with the rudiment of the primary root, and bears at its anterior end a whorl of two or more cotyledonary leaves, between which it terminates in a roundish apex (Fig. $346 I$ ). The Taxineæ and most Cupressineæ and Araucarieæ have two opposite cotyledons, although in some Cupressineæ there are from three to nine, and in some Araucarieæ whorls of four cotyledons; while among the Abietineæ there are rarely so few as two, more often four or even as many as fifteen. To refer this larger number of cotyledons to the division of two opposite ones, as Duchartre proposes, is entirely opposed to the other processes of leaf-formation in these plants, especially to the common occurrence of whorls consisting of several leaves on the growing axis of seedlings.

When placed in damp soil the endosperm swells up, bursts the testa at the radicular end of the embryo, which is then pushed out by the elongation of the axis, and grows into a strong descending tap-root, from which lateral roots proceed, succeeding one another rapidly in acropetal succession, and subsequently branching. This is the commencement of the root-system of Conifers, which is frequently strongly developed and persistent. After the emergence of the root, the cotyledons elongate in their turn, push out their bases from the seed and the end of the axis that lies between them, but they themselves remain in the endosperm until it has been absorbed. In Araucaria (sub-genus Colymbea) and in Salisburia the hypocotyledonary portion of the axis remains short, and the cotyledons remain

1 For the structure of the flowers, see R. Brown, On the Plurality and Development of the Embryos in the Seeds of Coniferæ: Misc. Bot. Works, London, 1866, vol. I. pp. 567-576.-H. von Mohl, Vermischt. Schrift. pp. 25 and 49.-Schacht, Lehrb. der Anat. u. Phys. vol. II. p. 433.-Eichler in Flora, 1863, p. 530, [and Nat. Hist.,Rev. 1864, pp. 270-290; Flora, 1873, and Trans. Bot. Soc. Edin. 1873, pp. 535-541.-Dickson, Trans. Bot. Soc. Edin. VI. p. 420; New Phil. Journ. 1861, pp. I98, 199.-J. D. Hooker, On the Ovary of Siphonodon in Trans. Linn. Soc. XXII. pp. I37, I38.-Caspary in Ann. des Sci. Nat. 4th series, vol. XIV. p. 200, and Flora, 1862, p. 377--Brongniart, Bull. Bot. Soc. France, XVIII. p. I4I.-Van Tieghem, Ann. des Sci. Nat. $5^{\text {th }}$ series, vol. X.] For the fertilisation, Hofmeister in Vergl. Unters. $185 \mathrm{I}$ [On the Germination, Development, and Fructification of the Higher Cryptogams, Ray Soc., pp. 400-433.]-Strasburger, Die Befruchtung der Coniferen, Jena 1869. For the pollen, Schacht in Jahr. f. wiss. Bot. vol. II. p. 142.-Strasburger, Ueber die Bestäubung der Gymnospermen, Jenaische Zeitschr. vol. VI. Also in addition: [Zuccarini, Morphology of the Coniferæ, Ray Soc. Rep. and Pap. on Bot. 1845.] - Pfitzer, Ueber den Embryo der Coniferen, Niederrhein. Ges. für Natur. u. Heilk. Aug. 7, 1871.-Reinke, Ueber das Spitzenwachsthum der Gymnosperm-Wurzeln, Göttinger Nachr. 187 I, p. 530.- [Strasburger, Die Coniferen u. die Gnetaceen; eine morphologische Studie, Jena 1872 .- Eichler, Sind die Coniferen gymnosperm oder nicht? Flora 1873, and Blüthendiagramme, I. 1875.-Strasburger, Die Angiospermen und die Gymnospermen, I879.] 
contained in the seed; in most Conifers, on the contrary, this portion becomes greatly elongated, making a sharp bend in an upward direction, pierces the soil, and draws the cotyledons with it. As soon as these are exposed to light, the hypocotyledonary portion straightens itself, the whorl of cotyledons expand, and, having become green while still underground, act as the first foliage-leaves of

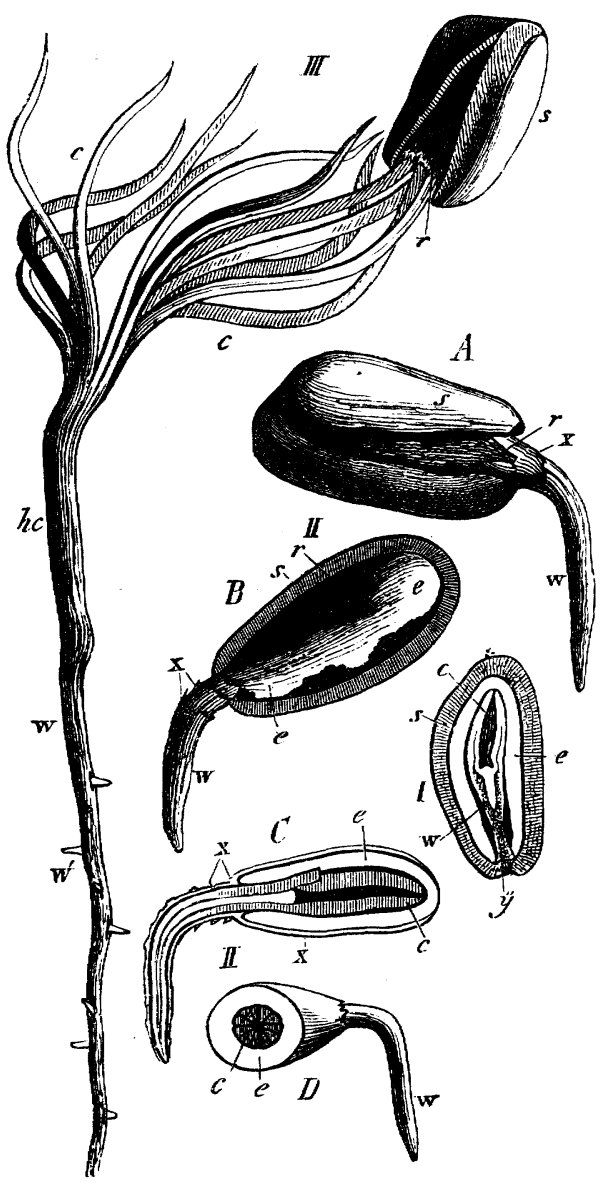

FIG. $346 .-$ Pintts Piken; $I$ longitudinal section through the middle of the seed, $y$ the micropylar end; $I I$ commencement of germination, emergence of the root; $I I I$ completion of germination, after the endosperm has been absorbed (the seed lay at too small a distance below the surface, and was therefore raised up by the cotyledons when the stem began to grow); $A$ shows the ruptured testa $s, B$ the endosperm $e$, one half of the testa having been removed, $C$ longitudinal section of the $A$ shows the primary root, $x$ the embryo-sac pushed out by it (ruptured in $B$ ), $h c$ hypocotyledonary portion of the axis, $w^{\prime}$ secondary roots, $r$ red mem. $x$ the embryo-sac pushed out
brane within the hard testa.

the seedling, the apex of its axis having in the meantime formed a bud with new leaves (Fig. 346).

Mode of Growth and External Differentiation. The terminal bud of the stem of the seedling grows more rapidly, though frequently interrupted, than the lateral shoots which arise subsequently. The primary stem is thus a direct prolongation of the axis of the embryo; it never ends in a flower, but grows indefinitely at the 
summit, becoming thickened to a corresponding extent by the activity of a cambiumring, and thus becomes a slender cone attaining a height of 100,200 , or even more feet ${ }^{1}$, and a diameter at the base of 2 or 3 or as much as 20 feet. On this highly-developed primary axis the lateral axes of the first order are produced; often periodically in rosettes at the apex (pseudo-whorls) or distributed irregularly and branching again in the same manner. Each primary axis usually grows more vigorously than its secondary axes; and hence the collective form of the system of branching, as long as the primary axis continues to grow vigorously, is that of a panicle of conical or pyramidal form. While in Cycadeæ the branching is almost entirely suppressed, the peculiar form and beauty of Conifers depends chiefly on the branching, the more so as the leaves are almost always small and inconspicuous, serving only, as far as the outward appearance of the plant is concerned, as a clothing to the system of branching. The branching is always axillary; but Conifers differ from Angiosperms in not producing buds in nearly all the leaf-axils; in Araucaria and some, species of Taxus, Abies, and other genera, it is chiefly or exclusively the youngest leaf-axils of a year's growth which produce branches, and these grow vigorously. In Juniperus communis, indeed, buds occur in most of the leaf-axils, but only a few develope. In Pinus sylvestris and its allies shoots are formed only in the axils of the cataphyllary leaves which are borne exclusively by the primary stem and the permanent woody branches, remaining however very short, and producing two, three, or more acicular foliage-leaves, from the axils of which no lateral shoots are produced. In Larix, Cedrus, and Salisburia, buds are formed in the axils of a considerable number-but not nearly all-of the foliage-leaves, a few growing rapidly, and serving for the development of the branch-system, while others remain very short, and form annually a new rosette of leaves without lateral buds. In Thuja and Cupressus also, which are distinguished by their copious branching, the number of small leaves is still very much larger than that of the axillary shoots. Many Conifers exhibit a very regular arrangement of those branches of different orders which arrive at their full development, the symmetry of the whole tree being at the same time increased by their difference in size. The branches of the first order on the upright primary stem are frequertly formed in a pseudo-whorl of several members at the conclusion of each period of vegetation, the same process being frequently repeated on the branches themselves (e.g. Pinus sylvestris, Araucaria brasiliensis, and especially Phyllocladus trichomanoides, and many others); more commonly a tendency to bilateral ramification appears on the horizontal branches of the first order (as in Abies pectinata); and not unfrequently besides these strong branches from which the framework of the tree is constructed, smaller ones are also formed between them (e.g. in Abies excelsa). In many cases the arrangement and growth of the branches are more irregular; the greatest deviation from this type being shown in the Cupressineæ, especially Cupressus, Thuja, and Libocedrus, in which the tendency to bilateral ramification ${ }^{2}$ is seen even on the primary stem, which is more perfectly developed on the lateral shoots. Branch-systems of three or

I [The trunk of Sequoia (Wellingtonia) gigantea of California attains the height of 400 feet.]

2 In many species also of Abies and Pinus there is an evident tendency to bilateral development in the horizontal lateral shoots, the spirally arranged leaves inclining over to the right and left, and thus forming two $\mathrm{c}$ )mb-like rows. 
four orders of shoots are developed in one plane in such a manner that a system of this kind assumes a definite contour and somewhat the appearance of a pinnate leaf. In Taxodium the foliage-leaves are formed in two rows on slender branches a few inches in length; in $T$. distichum these fall off in the autumn together with their leaves, thus presenting a still greater resemblance to pinnate leaves. Finally Phyllocladus produces on all its verticillate shoots only small colourless scale-like leaves, from the axils of which, but beneath the terminal bud, whorls of shoots spring with limited power of growth, developing their bilateral side-shoots in the form of flat-lobed foliage-leaves. These remarks, incomplete as they are, may suffice to turn the attention of the student to the phenomena of the branching, which are moreover easily observed.

The Leaves (with the exception of the floral leaves) are either all foliage-leaves containing chlorophyll, as in Araucaria, Juniperus, Thuja, \&c.; or all colourless or brownish scales, as in Phyllocladus, where the foliage-leaves are replaced by leaflike shoots; or, finally, scales and foliage-leaves are very frequently formed at the same time, and even on the same shoots (as in Abies), where the scales only serve the purpose of protecting the buds; or the two forms are distributed on different axes, as in the true Pines (Pinus), the permanent woody shoots of which produce only membranous scales, the axils of which develope sterile foliage-shoots which afterwards die off. The foliage-leaves of Conifers are mostly small, of simple structure, and scarcely ever compound; they are smallest and at the same time most numerous in the Cupressineæ, where they form a dense covering to the axes of the branchlets (as Thuja, Cupressus, \&c.); in most of the Abietineæ and in Taxus and Juniperus, they are larger, more sharply separated from the axis, narrow and comparatively thick, usually angular and prismatic (acicular); intermediate forms between these acicular leaves and the broad expanded leaves of Thuja occur in Araucaria excelsa, \&c. In Podocarpus and Dammara the leaves are flat and broader, and in Salisburia they are stalked and even two-lobed, with a deeply emarginate apex as if from dichotomous division. Not unfrequently, especially in the Cupressineæ, the foliage-leaves of the elongated primary axis are different in form from those higher up the same axis and from those on the lateral shoots; in Thuja, Juniperus virginiana, Cupressus, \&c., the former are acicular, patent, and of considerable size, the latter very small and closely appressed to the axis; the youthful foliage sometimes recurs on isolated branches of adult plants. The axis of the shoot within the bud is so densely covered with the bases of leaves that no free portion of the surface of the axis is visible between them. When the axis has attained a considerable length on the unfolding of the bud, the bases of the leaves generally grow at the same time in length and breadth, so that they entirely cover the surface of the enlarged shoot also, clothing it with a green cortex, in which the parts belonging to the separate leaves can be distinctly recognised. This is especially clear in Araucaria and many species of Pinus, but is very common also in other genera; in Thuja, Cupressus, Libocedrus, \&c., the axis of the shoot is also completely covered with these leaf-cushions; but the free parts of the leaves are very small and often project only as short points or projections. The phyllotaxis is spiral in the Abietineæ, Taxineæ, Araucarieæ, Podocarpus, \&c.; the Cupressineæ bear whorls which, above the cotyledons, contain generally from three to five leaves, but 
usually fewer at a greater height on the primary axis. The secondary axes usually begin at once with decussate pairs, which, in bilateral shoots, are alternately larger and smaller (as in Callitris and Libocedrus); in Juniperus and Frenela the whorls on the secondary axes also consist of from three to five leaves, and are alternate; the pairs of leaves of Dammara stand at an acute angle to one another. The foliageleaves of most Conifers are very persistent, and may live for several years, their leafcushions keeping pắce in growth for a long time with the increase in size of the axis; in Larix and Salisburia the leaves alone are deciduous in autumn, in Taxodium distichum the axes that bear them are also deciduous.

The Flowers of Coniferæ are always diclinous; either monocious, as in the Abietineæ and Thuja, or diœcious, as in Taxus, Salisburia, and Juniperus communis; the male are usually much more numerous than the female flowers. They are never terminal on the primary stem, differing in this respect from those of Cycadeæ; even the larger woody branches bear only rarely, as in Abies excelsa, terminal (in this case only female) flowers. Usually the flowers are produced at the apex of small foliage-shoots of the last order, or in the leaf-axils of the stronger foliage-shoots. In Thuja, for instance, male and female flowers appear at the end of small short green shoots of the bilateral system of branches; in Taxus and Juniperus, on the other hand, in the axils of foliage-leaves of larger shoots; in Abies pectinata they are found on the under side of shoots of a higher order at the summit of older trees, both kinds in the axils of foliage-leaves, the female flowers singly, the male in larger numbers. The flowers of Pinus sylvestris and allied species appear in the place of the undeveloped branches (tufts of leaves) which stand in the axils of the scales of growing woody shoots, the male flowers usually in groups forming an inflorescence the primary axis of which is the mother-shoot, the female flowers generally more scattered. In Salisburia the flowers appear exclusively on the short lateral branches which annually form new rosettes of leaves, and they are situated in the axils of the foliage-leaves or of the inner bud scales (Fig. 347, $A$ and $B$ ).

The part of the floral axis immediately beneath the organs of reproduction is densely covered with scales or foliage-leaves in the female plant of Taxus, Juniperus, \&c. (Figs. 348, 349); it is developed as a naked stalk in the Abietineæ, Salisburia, the male plant of Taxus, Podocarpus, \&c. (Fig. $347 A, B$ ). The flowers of Coniferæ resemble those of Cycadeæ in the peculiarity that the axis elongates even at the part that produces the organs of reproduction; if these are numerous, the whole flower presents the appearance of a long cone, resembling externally a catkin; and this term is indeed given to it in the superficial language of many systematists. In Angiosperms the flowering shoot usually undergoes a very peculiar development at its summit, the portion of the axis which bears the flower (the receptacle) remaining very short and broad, and the floral leaves and organs of reproduction being formed in positions which differ greatly from those of the foliage-leaves; in Coniferæ the distinction between a floral and a foliage-shoot is much less, and this is especially conspicuous in the arrangement of the leaves; if those of the foliage-branches are arranged spirally, so also are usually those of the flowers, as, e.g., in the Abietineæ; if, on the contrary, as in the Cupressineæ, they occur in alternating whorls, the staminal and carpellary leaves are arranged in the same 
way. In Juniperus communis even the ovules, here the representatives of whole leaves, are arranged in alternating whorls. But, occasionally, as in Taxus, greater differences are to be observed in the phyllotaxis of the flowering shoot as compared with that of the foliage-shoots.

The Male Flowers always consist of a distinctly elongated axis provided with staminal leaves, and ending above in a naked apex (Fig. $349 \mathrm{~A}$ ). The stamens are mostly more delicate and of a different colour from the foliage-leaves, and are usually divided into a slender pedicel and a peltate lamina bearing the pollen-sacs on its under side, as in Taxus, the Cupressinex, and Abietineæ (Fig. $348 B, 349 A, B$, $35 \circ A)$. The flat expansion at the end of the pedicel may, however, be entirely absent, as in Salisburia (Fig. $347 \mathrm{C}$ ), where it is reduced to a small knob on which the pollen-sacs hang. That the parts which bear the pollen-sacs in Coniferæ are beyond doubt metamorphosed leaves, is evident not only from their form, but still

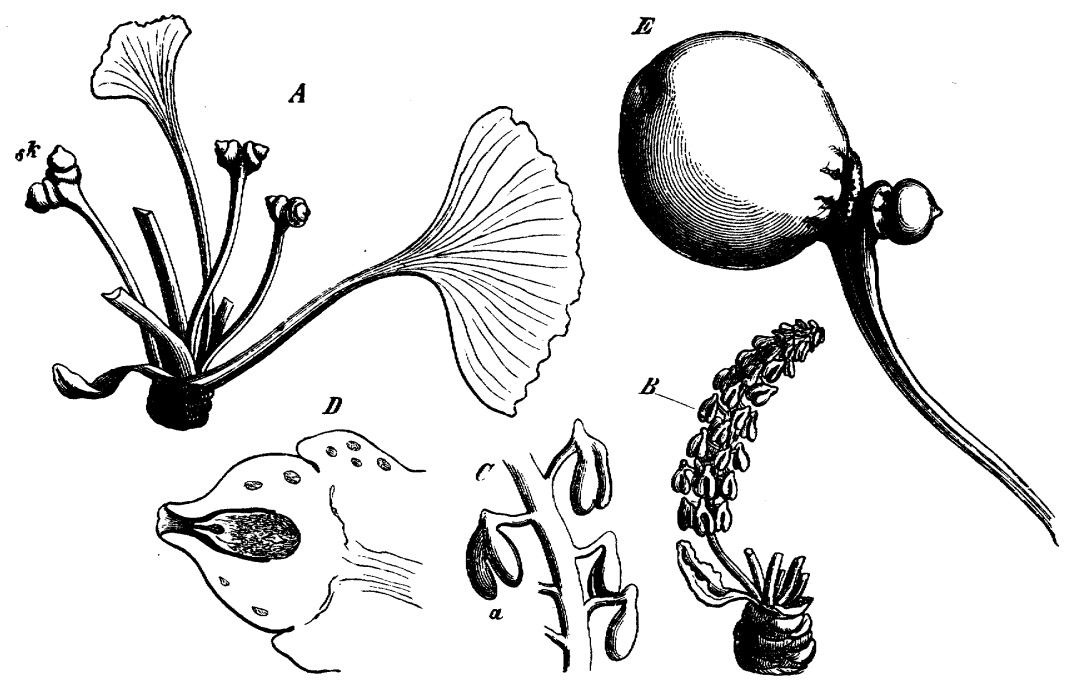

FIG. 347.-Salisburia adiantifolia (natural size). A a short secondary foliage-shoot with female flowers, on the naked axes of which are placed the ovules $s k ; B$ a male flower; $C$ part of one magnified, $a$ the pollen-sacs; $D$ longitudinal section of an ovule magnified; $E$ a ripe seed with an abortive one by its side on the foral axis.

more from their arrangement, which has already been spoken of. If the staminal leaves of the Cycadeæ show a resemblance in more than habit to the sporangiferous leaves of Ferns, those of Coniferæ may perhaps be compared to the peltate scales that bear the sporangia of Equisetaceæ; and not unfrequently, as in Taxus, Juniperus, \&c., the resemblance of the male flowers to the inforescence of Equisetum is as striking in external appearance as in the actual agreement between them from a morphological point of view. The pollen-sacs usually hang, with a narrow base, on the under side of their support, and do not cohere in their growth; their number is usually much smaller than in Cycadex, but much more variable than in Angiosperms; in the Yew the peltate part of the staminal leaf bears from three to eight, in the Juniper and most Cupressineæ three roundish pollen-sacs (Figs. 348, 349). Those of Pinus, Abies, and their allies lie in pairs parallel or placed obliquely to one another, right and left of the pedicel, which here resembles the connective of 
Angiosperms; in Araucaria and Dammara, on the other hand, the long sausageshaped pollen-sacs hang in larger numbers free beneath the peltate limb. The wall of the pollen-sacs is usually delicate, and finally dehisces longitudinally to allow the escape of the pollen-grains, which are produced in extraordinarily large numbers, since they have usually to be carried by the wind to the female organs of the same or of another tree. The pollen-grains which happen to fall on the opening of the micropyle of the ovules are retained by an exuding drop of fluid, which about this time fills the canal of the micropyle, but afterwards dries up,

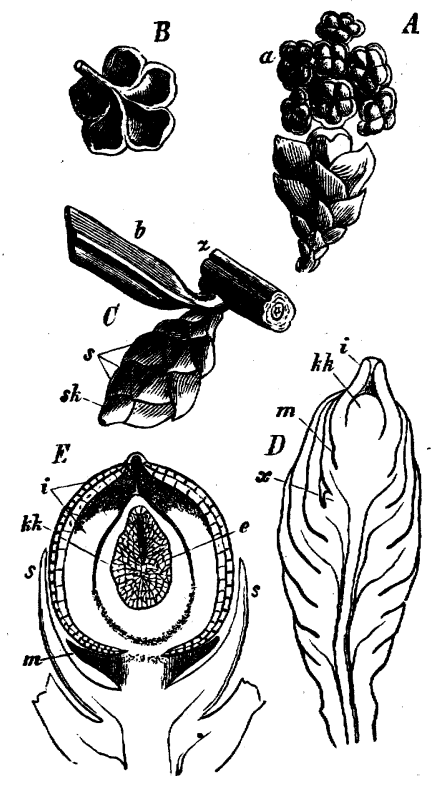

FIG. 348.-Taxus baccata; $A$ male flower (magnified), $a$ the pollen-sacs; $B$ a stamen seen from below with open pollen-sacs; $C$ piece of a foliage-shoot with leaf $b$, from the axil of which springs the female flower, $s$ its envelope of scales, $s k$ the terminal ovule; $D$ longitudinal section of the same (magnified), $i$ integument, $k k$ nucellus of the ovule, $x$ a rudimentary axillary ovule; $E$ longitudinal section through a more mature ovule before fertilisation, $i$ integument, $k k$ nucellus, $e$ endosperm, $m$ aril, $s$ upper scales of ment, $k k$ nucell
the envelope.
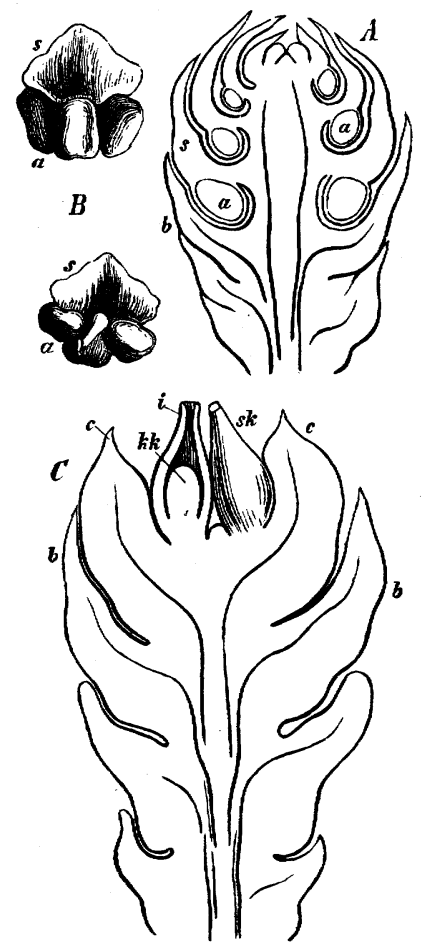

FIG. 349.-7uniperus communis; $A$ longitudinal section of a male flower, $B$ (upper figure) a stamen seen from the front and the outside, (lower figure) seen from from the front and the outside, (lower figure) seen from
the back of the axis; $C$ longitudinal section of a female flower; $a$ the pollen-sacs, $s$ the peltate lamina of the stamen, $b$ lower leaves of the floral axis, $c$ carpets, $s k$ ovules, $k k$ nucellus, $i$ the integument $(A$ and $C \times$ about 12).

and thus draws the captured pollen-grains to the nucellus, where they immediately emit their pollen-tubes into its spongy tissue. In the Cupressineæ, Taxineæ, and Podocarpeæ this contrivance is sufficient, since the micropyles project outwardly; in the Abietineæ, where they are more concealed among the scales and bracts, these themselves form, at the time of pollination, canals and channels for this purpose, through which the pollen-grains arrive at the micropyles filled with fluid (cf. Strasburger, l.c.). The large number and lightness of the pollen-grains enables them to be carried great distances by the wind; in the true Pines and the Podo- 
carpeæ their capacity for transport is increased by the vesicular hollow protrusions of the extine, as represented in Fig. $35 \mathbf{I}, I V, V, b l$. [The pollen-sacs (microsporangia) of the Coniferæ resemble the sporangia of the Vascular Cryptogams in the mode of their development. A section through the pollen-sac of one of the Cupressineæ, for example, shows that it resembles a sporangium of Lycopodium:

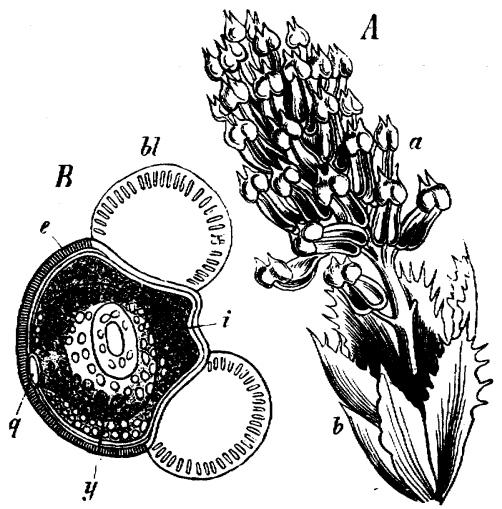

FIc. 350.-Abies pectinata; $A$ a male flower, $b$ the delicate bud-scales forming a perianth, $a$ the stamens; $B$ a pollen-grain (after Schacht), $e$ its extine, forming the two large vesicular protrusions $b l$.

in the centre is a group of sporogenous cells surrounded by a layer of flattened tabular cells, the tapetum, and externally is the wall of the sporangium. From' Goebel's ${ }^{1}$ researches it appears that the archesporium, in Biota orientalis at any rate, is a hypodermal cell, the terminal cell of one of the axial rows of cells of

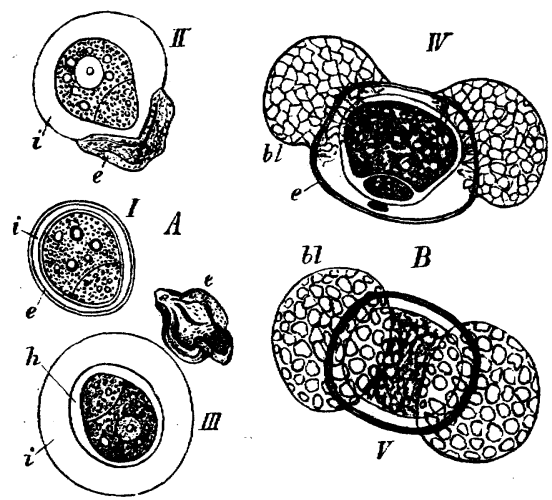

FIG. $35 \mathrm{r} .-A$ pollen-grain of Thuja orientalis before its escape from the pollen-sac, $I$ fresh, $I I, I I I$ after lying in water, the extine $e$ having been stripped off by the swelling of the intine $i ; B$ pollen-grain of Pinus finaster before its escape, $e$ the extine with its vesicular protuberances $b l$.

which the young pollen-sac consists. Possibly the archesporium consists in this case, às in Lycopodium, of a transverse row of such hypodermal cells. The cells of the tapetum are derived here, as in Selaginella, partly from the archesporium and partly from the tissue of the wall of the pollen-sac. In Pinus the pollen-sac

${ }^{1}$ [Goebel, Vergl. Entwick. d. Sporangien, Bot. Zeitg., 1881.] 
is developed from a group of superficial cells : these multiply by repeated division, so that a protuberance is formed consisting of a number of rows of cells invested by a parietal layer: the terminal cell of the lowest of these rows becomes the archesporium, and it is therefore, in this case also, a hypodermal cell. The cells of the tapetum are derived entirely from the parietal cells of the pollen-sac, just as is the case in the sporangium of Lycopodium. Goebel draws attention to the fact that in the majority of the Cupressinex the pollen-sacs are protected by an outgrowth of the staminal leaf which he considers to be analogous to the indusium of Ferns.]

A formation of cells, recalling the rudimentary development of a prothallium in the microspores of Selaginella and Isoëtes, takes place in the pollen-grains of the Coniferæ before their dissemination, in the same manner as in those of the Cycadeæ (compare Fig. 345). The process is a very simple one in Taxus Podocarpus, the Cupressineæ, Araucaria and in the true Pines. The contents of the pollen-grain are divided by a septum so as to form a large and a small cell, the latter undergoing no subsequent change (Fig. 35I). In the other Abietinea the septum becomes arched into the cavity of the larger cell, and a second septum is formed in the smaller eell, so that a two-celled body is formed which is situated, as in the Cycadex, at the posterior end of the grain. At this point the extine presents a split which was formerly regarded by Schacht as being the basal cell of the internal group, but which, according to Strasburger, arises in the same way as the vesicular expansions of the extine found in the grains of many species of Pinus. Strasburger has shown that in all cases it is the large cell of the pollen-grain which grows out into the pollen-tube, as is the case in Cycadex (Fig. 345). If we pursue the comparison with the highest Cryptogams which has been already initiated, we must regard the pollen-tube as representing the antheridium to some extent, and the internal group of cells, formed before the development of the pollen-tube, as corresponding to the vegetative cell in the microspore of the Ligulatæ, for, like it, it undergoes no subsequent change. A peculiarity which distinguishes the pollen-grain of Conifers from that of Angiosperms lies in the rupture and final stripping off of the extine by the swelling of the intine (Fig. 35I, I, II, III). Even in this apparently insignificant fact a resemblance is again seen to the microspores of Cryptogams, and especially to those of . Marsiliaceæ, in which the swelling endospore protrudes through the exospore.

The structure of the Female Flozeers is very different in the different sections of Coniferæ, and in some cases the homology of the separate parts is still doubtful. The position of the ovules, as far as can be judged from advanced stages of development, is, in particular, very variable, and with this is again connected the fact that different opinions may be entertained as to the part which should be called the carpel. The following description of these structures, a full discussion of which is not permitted by our limited space, is drawn immediately from the observation of advanced stages of development; it is possible, however, that the direct observations of the most rudimentary stage will cause an alteration in some points ${ }^{1}$.

The female flowers of Taxus spring from the axils of foliage-leaves belonging

3 Compare Strasburger and Eichler, loc. cit. 
to elongated woody shoots. They have the form of short branches covered with decussate scale-like bracts (Fig. 348,C,D); the axis of the shoot ends in an apparently terminal ovule, the nucellus of which has the appearance of being the vegetative cone of the axis, but axillary ovules also occur. In Salisburia the female flowers spring from the axils of foliage-leaves belonging to short lateral branches which annually produce new rosettes of leaves (Fig. $347 \mathrm{~A}$ ); the single flower consists of a stalk-like elongated axis which bears immediately beneath its apex two or more rarely three lateral ovules. Neither in this genus nor in Taxus are there any foliar structures close to the ovules which either from their position or from any other circumstance can be regarded as carpels. In the genus Podocarpus small flowering shoots are developed, springing in $P$. chinensis (according to Braun) from the axils of foliage-leaves, in $P$. chilina from the axils of very small scale-leaves at the end of elongated leafy shoots; they consist of an axial structure slender and stalk-like below, club-shaped above, and bearing three pairs of very small decussate scales. The floral axis terminates between the upper pair; the ovules, in this case anatropous, with their micropyle turned downwards and towards the floral axis, spring from the axils of this pair; one ovule however is usually abortive, and the flower becomes one-seeded. In Phyllocladus the lower lateral branchlets of the leaf-like flattened shoots are transformed into female flowers which are raised upon a pedicel and are swollen above into the form of a club, the large ovules standing (according to a drawing of Decaisne's ${ }^{1}$ ) in the axils of small leaves. In these two genera the small scales from the axils of which the ovules spring may be regarded as carpels, if it is thought necessary to assume the existence of these organs.

The ovules of Juniperus communis (Fig. 349,C) stand in whorls of threes beneath the naked extremity of the floral axis, the flower springing as a little shoot from the axil of a foliage-leaf, and its axis bearing whorls of three leaves. The ovules apparently alternate with the three leaves of the upper whorl, and hence must, from their position, be themselves considered as metamorphosed leaves; 'these leaves of the upper whorl swell after fertilisation, grow together and become fleshy, forming the pulp of the juniper-berry in which the ripe seeds are entirely enclosed; they may therefore be termed carpels. In the other Cupressineæ the flower consists of decussate whorls of two or three leaves, which grow considerably after fertilisation and attain a considerable size, enveloping the seed and forming a pericarp which may therefore correctly be said to be formed of carpels. In Sabina the pericarp is fleshy and berry-like, as in Juniperus; in the other genera, on the other hand (Thuja, Cupressus, Callitris and Taxodium), the carpels become woody and assume the form of stalked peltate scales, or of valves separating from one another longitudinally (Frenela); these are closely approximate during the development of the seed, but afterwards open to allow the ripe seeds to fall out. The erect ovules of Cupressineæ sometimes appear to stand in the axils of the carpels; but it is clear in other cases that they spring from the carpels themselves, either low down near their point of insertion

1 [See Le Maout and Decaisne's Descriptive and Analytical Botany, edited by Dr. Hooker, London 1873 , p. 747.] 
or at a greater height. In Sabina and Callitris quadrivalvis (Fig. 352) only two decussate pairs of carpels separate like a star at the time of flowering; in Sabina the ovules stand in pairs in the axils of the two lower carpels, right and left of their median line, some of them being frequently abortive; in Callitris quadrivalvis a pair occurs on each of the lower carpels and a pair higher up; but this position can only be explained by further investigation of the history of their development. In Thuja and Cupressus there are three or four decussate pairs of carpels, in Taxodium a still larger number; in Thuja and Taxodium two erect ovules are situated at the base of each of the central pairs of carpels, springing from the right and left of their median line; in Cupressus there are a considerable number at the base of each carpel. In Arceuthos drupacea and Frenela verrucosa the fruits (in the collection at Würzburg) consist of alternating whorls of three carpels, opening, in the last species, after the seeds become ripe, like a six-lobed capsule. Each carpel is swollen on its inner side into a thick placenta ascending from the base to the apex, and bearing numerous winged seeds which stand in transverse rows of threes; there are from four to six of these rows on each carpel, the whole inner side therefore bearing seeds nearly up to the apex.

So far as the relative positions of the parts of the flower can be explained
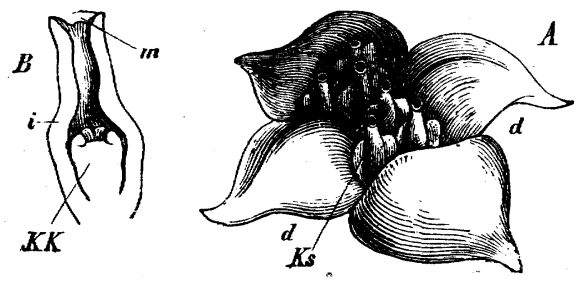

FIG. 352.-Callitris quadrivalvis; $A$ female flower (magnified); $d d$ two pairs of decussate leaves (carpels) in the axils of which are six ovules $(K s) ; B$ vertical longitudinal section of an ovule through its broader diameter; $K K$ the nucellus still without an embryo-sac; $i$ the tubular elongated integument with the micropyle $m$.

without going back to their earliest stage, a great diversity is thus shown in the two families of Taxineæ and Cupressineæ; the ovule is terminal in Taxus, lateral beneath the summit of the axis in Salisburia, carpellary leaves appearing to be entirely absent. In Podocarpus and Phyllocladus they are indicated indeed, as small scales, the ovules springing from their axils; but they are small and do not at any time constitute a pericarp. A structure of this kind, in the form of a berry or of a chambered woody fruit, is indeed formed after fertilisation in the Cupressinex, the carpels either becoming fleshy and growing together (as in Juniperus and Sabina), or becoming woody and closing in laterally by their peltate expansions (as in Cupressus, Thuja, and Callitris), or presenting the appearance of the lobes of a unilocular capsule (e.g. Frenela); but the carpels are in these cases at first entirely open. In Juniperus communis the ovules form a whorl alternating with the carpels; in the other genera they stand in pairs or in larger numbers at their base, or cover the whole of their inner side (as Frenela).

In the Abietineæ the well-known cones are the female flowers (or rather fruits). The cone is a metamorphosed shoot, its axis bearing a number of crowded woody scales arranged spirally, the ovules arising on them rarely singly, usually in pairs, occasionally in larger numbers. In the Abietineæ (Abies, Picea, Larix, Cedrus, and 
Pinus) the seminiferous scales (Fig. 353, $A, B, s$ ) appear to be axillary structures in the axils of bracts $(c)$ which spring from the axis of the cone; but the examination of very young cones of Abies pectinata shows that the seminiferous scale itself arises as a protuberance at the base of the bract $(c)$, and is therefore not axillary. While the bract afterwards grows very little or not at all, this protuberance increases greatly, and produces on its upper surface two ovules which are

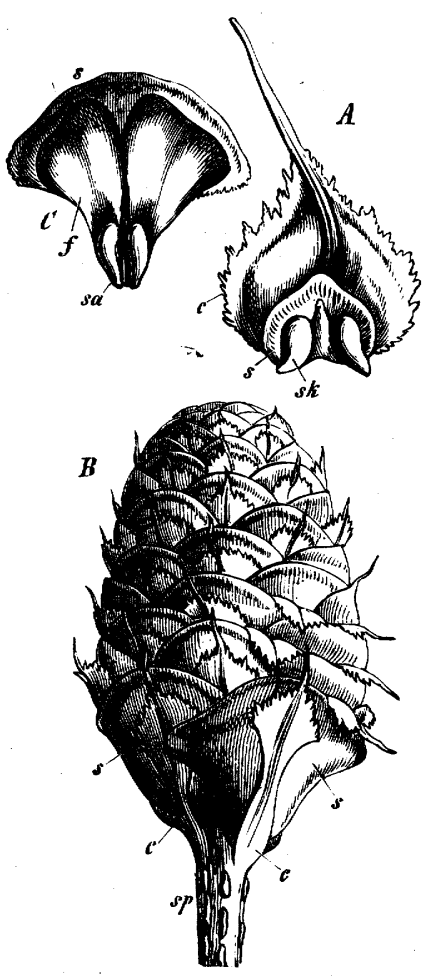

FIG. 353--Abies pectinata (after Schacht). A a leaf detached from the female floral axis seen from above, with the seminiferous scale $s$ bearing the ovules $s k$ (magnified); $B$ upper part of the female flower (or cone) in the mature state; sp floral axis or axis of the cone, $c$ its leaves, $s$ the largely developed seminiferous scales; $C$ a ripe seminiferous scale with the two seeds sa, $f$ the wing of the seed (reduced). attached to it by one side with the micropyle towards the axis of the cone. The seminiferous scale of these genera must therefore be considered as a greatly developed placenta growing out of a carpel (Fig. $353 A, B, c$ ) which is very small or even abortive ${ }^{3}$. According to this view the whole cone is a single flower with a number of small open carpels (hitherto considered as bracts), which are far outstripped in their growth by their seminiferous placentæ (the scales). In the other Abietinex also, the female flowers of which I have had no opportunity of examining, it may be concluded from the descriptions that the cone is a single flower with numerous seminiferous scales arranged spirally, not springing from the axils of leaves, but growing immediately out of the axis of the cone, and therefore themselves leaves and of a carpellary nature. Eichler (l.c.) says, in reference to Dammara, Cunninghamia, Arthrotaxis, and Sequoia:-'The scales of a cone are in these genera all of one kind; they consist simply of open carpels; and, in order not to introduce confusion into the definition of a flower, the whole of what is found on the axis, in other words the whole cone, must be considered a single flower; and this is also necessary in the case of the Araucariex, the Cupressineæ, and the male 'catkins' of all Coniferæ '.' In Araucaria each scale (or carpel) bears only a single ovule, which, according to Eichler, is so enveloped by it that the only

1 Braun, Caspary, and Eichler consider the seminiferous scale in Pinus and Larix as itself a flower; i.e. as a short axis which has coalesced with its two carpels and stands in the axil of the bract ( $c$ in our figure). In that case the cone of these genera, in contradistinction to that of the other Coniferæ and of Cycadeæ, would be an inflorescence ( $c f$. Caspary in Ann. des Sci. Nat. 4th series, vol. XIV, p. 200 , and Flora, 1862 , p. 377 ); but this view I have already contested more in detail in my first edition, p. 427. It is impossible to consider the seminiferous scale of Pinus and Abies itself as a single carpel. In opposition also to the most recent views of Mohl (Bot. Zeitg. I87 1, p. 22, and of Strasbuıger), I cannot bring myself to consider the seminiferous scale of the true Abietineæ as a coherent structure formed of two leaves of an undeveloped branch.

${ }^{2}$ Eichler thinks that an exception must be made in favour of Podocarpus and Cephalotaxus. 
opening left is that of the micropyle which faces the axis of the cone; in Cunninghamia there are three ovules, in Arthrotaxis from three to five, in Sequoia from five to seven, in Sciadopitys as many as seven or eight on one scale, and their micropyle here also faces the axis of the cone. In Dammara the scale bears, according to Endlicher ${ }^{1}$, only one ovule which, like those of Sequoia and Sciadopitys, are inserted near the apex and hangs down free ${ }^{2}$.

${ }^{1}$ [Van Tieghem has been led by studying the distribution of the bundles in the different parts of the female bud of Coniferæ to the opinion-different from that expressed by Sachs-that the female flower throughout this group of plants is in every case constructed after a single fundamental type which has undergone various secondary modifications. He has given in a note to his French translation of the present work the following abstract of the conclusions which are worked out in greater detail in his paper already cited in the Annales des Sciences Naturelles.

Neither the axis of the female bud nor its leaves or bracts of the first order ever bear ovules. It is always upon structures arising from the axils of these bracts that the ovules make their appearance. This establishes a fundamental distinction between Cycadeæ and Coniferæ. In the former group it is always the leaves of the female bud of the first order that produce the ovules directly. While therefore we may regard the female bud in Cycadeæ as well as the male as contributing a single flower, this does not hold good in the case of Coniferæ. We may if we please regard the male bud of Coniferæ as a single flower, but the female bud is an inflorescence. The structure which bears the ovule in Coniferæ is always a foliar organ - the first and only leaf of an axis which undergoes no further development. This leaf, which is more or less largely developed beyond the circumscription of the ovule or ovules which it bears, is an open carpel and in itself constitutes the whole female flower. It is always inverted, that is to say, it arises upon the suppressed axis which bears it with its ventral face opposite to and united with the ventral face of the primary bract. When the ovules do not terminate the carpel, it is upon its structurally dorsal - but in respect of position upper-face that they arise, just as it is upon its structurally dorsal - but in respect of position lower-face that the pollen-sacs arise upon the stamen.

This is the general type. It remains to consider the principal secondary modifications which are superinduced upon it in the different genera.

The axillary branch, which is reduced to its first leaf, is most frequently of the first generation in respect to the axis of the female bud; but it is also sometimes of the second (Taxus) and may even be of the third order (Torreya). The carpel itself is either entirely distinct from the parent bract (the Pineæ, Taxineæ) or the two leaves are united together by their ventral surfaces and are only separate towards their summit (Cupressineæ, Sequoiex, Araucariex). This difference merely depends upon a different localisation of the intercalary growth of the two leaves; it is a difference the same in kind as that which separates a dialypetalous corolla from a gamopetalous one. Whether free or united with the bract, the carpellary leaf bears its ovules sometimes towards its base (Cupressineæ), sometimes towards its middle (Pineæ), sometimes towards its summit (Araucarieæ); each represents a lobe, more or less developed, of the dorsal face of the carpel.

In the Taxinea the ovules terminate the carpellary leaf; they result in this case from the transformation of its whoie entire limb, whether each half of the limb forms an ovule (Salisburia, Cephalotaxus), or whether the entire limb has only produced a single one (Podocarpus, Phyllocladuis, Taxus, Torreya, \&c.). In this case it is evidently only the petiole of the ovuliferous leaf which represents the carpel; if the petiole is long (Salisburia) the carpel is obviously developed; but if it remains very short (Cephalotaxus, Podocarpus, Phyllocladus, Taxus, Torreya, \&c.) the carpel is almost absent-in otl.er words, the carpellary leaf is reduced to a sessile limb completely converted into a single ovule (Podocarpus, Taxus, \&c.) or into two ovules (Cephalotaxus). The number of the ovules which each carpellary leaf bears, as well as the number of carpellary leaves themselves, that is to say, of the female flowers which enter into the composition of the inflorescence, both vary, and may even be simultaneously reduced to unity, which is the ordinary case in Taxus.]

${ }^{2}$ [For a review of the literature of the question whether the ovules of Coniferæ are really naked or whether there is a true ovary, see Eichler, 'Sind die Coniferen gymnosperm oder nicht ?' in 'Flora' for 1873 , translated in Trans. Bot. Soc. Edin. 1873, pp. 535-541. Dr. Eichler here, in opposition to the contrary view of Strasburger, sums up the whole argument strongly in favour of the opinion that 
The Ovules (macrosporangia), as we have already seen, are in the Podocarpeæ anatropous and furnished with two integuments; in the rest of Coniferæ they are orthotropous and possess only one integument; in the Cupressineæ and Taxineæ they are erect, in the Abietineæ inverted, with the micropyle towards the base of the scale, to which the ovules are usually attached on one side. In these cases there is no funiculus, and the ovule consists only of the small-celled nucellus and one integument, which usually projects above it and forms a comparatively wide and long micropylar canal, through which the pollen-grains reach the apex of the nucellus, which is sometimes depressed (see Figs. $347,348,349,35^{2}$ ). Lateral outgrowths of the integument not unfrequently cause the ovule, and afterwards the seed, to appear winged on both sides, as in Callitris quadrivalvis (Fig. 352), Frenela, \&c. The wing-like appendage of the seed of Pinus and Abies, on the other hand, is the result of the detaching of a plate of tissue from the seminiferous scale, which remains attached to the ripe seed.

[The Development of the Ovule and of the Embryo-sac. The development of the ovule begins in Taxus (according to Strasburger, Angiospermen und Gymnospermen) with the division, parallel to the surface (periclinal), of a group of hypodermal cells at the punctum vegetationis of a lateral branch, and this is followed by similar divisions in the overlying epidermis. The rudiment of the ovule is surrounded by a projecting ring of tissue, also developed by the division of hypodermal cells, which is the first indication of the integument. The ovule elongates in consequence of growth and repeated cell-divisions, and consists internally of a number of longitudinal rows of cells, which have all been derived from the hypodermal layer, invested by several layers of cells which have been derived from the epidermal layer. The apical cells of these internal rows are distinguished by their size and by the granularity of their protoplasm; they constitute the archesporium: they are surrounded by tapetal cells, but it is not clear whether the tapetum is derived from the archesporium or from the surrounding cells of the nucellus. One, but sometimes more, of the archesporial cells, usually the central one, divides so as to form three cells lying one above the other, and it is the lowest of these which enlarges and becomes the embryo-sac. The development of the ovule in Ginkgo biloba and in Podocarpus chinensis follows essentially the same course, as does also that of Thuja occidentalis. It will be observed that in these plants several embryosacs are frequently indicated at first, but only one attains perfect development.

In the Abietineæ (Larix europaa, Pinus sylvestris and Pumilio) the multiplication of cells by the repeated division of the hypodermal and epidermal cells is much less considerable. The archesporium is unicellular; as it elongates a small cell is cut off from it towards the free surface of the ovule, which is a tapetal cell; the lower cell now divides into two, and of these two the upper divides again into two; the lowest of these three cells developes into the embryo-sac. Only one embryo-sac is ever indicated in the group.

A section of the young ovule of one of the Cupressineæ, before the embryosac is developed, presents, as Goebel has pointed out, a considerable similarity

the Conifers are really gymnospermous. In his most recent work on the subject (Die Angiospermen und die Gymnospermen) Strasburger accepts the view of the gymnospermous nature of the ovule.] 
to a section of a young sporangium of Lycopodium: the archesporium consists in both cases of a group of cells bounded by a tapetal layer. There is reason, inasmuch as their mode of development is the same, to regard these cells in the Cupressineæ as being, like the archesporial cells of Lycopodium, spore-mother-cells ; but whereas in Lycopodium each of these mother-cells produces four spores, in the Cupressineæ only one of them persists and it produces only one spore, the embryo-sac. The embryo-sac is then the equivalent of the macrospore of the heterosporous Vascular Cryptogams.

In the Abietineæ, the archesporium has become much reduced as compared with that of the Cupressineæ. The Cupressineæ resemble the higher Vascular Cryptogams in the mode of development of the ovule and embryo-sac, whereas the Abietineæ approach the Angiosperms.]

Development of the Endosperm (Prothallium). The first stage in the development of the endosperm is the division of the nucleus of the embryo-sac; each of the two nuclei divides again, and this process is repeated until a number of nuclei have been formed which lie in the peripheral protoplasm. Around each nucleus the protoplasm becomes marked off by an ectoplasmic layer, cell-walls are formed, and thus one or two layers of endosperm-cells are formed in the embryo-sac in contact with its wall. These cells grow in the radial direction, and divide in such a manner that the embryo-sac is filled with parenchymatous tissue. In those Coniferæ in which the seeds take two years to ripen, as Pinus sylvestris and Juniperus communis, the endosperm formed in the first summer is again absorbed in the spring, the protoplasm of the primary endosperm-cells is set free by the deliquescence of their cell-walls, and forms by division a qumber of new cells which, in May of the second year, again fill with parenchymatous tissue the embryo-sac now considerably increased in size.

The archegonia (corpuscula) are developed, according to Strasburger's observations, from superficial cells of the prothallium (endosperm) in the same manner as the archegonia of the highest Cryptogams. The mother-cell increases in size and is divided by a septum parallel to the surface of the investing embryo-sac; a large inner (lower) cell is thus formed, the central cell of the archegonium, and an upper small one, lying next the embryo-sac from which the neck of the archegonium is formed ${ }^{1}$. In Abies canadensis this neck remains simple and unicellular, and elongates considerably with the increase in size of the surrounding endosperm; but usually the original cell which constitutes the neck divides into several cells which either lie only in one plane (Figs. $354 A, d, 355 I, d$ ), the 'stigmatic cells,' or form several layers lying one over another (as in Abies excelsa and Pinus Pinaster). Seen from above the neck appears to form a four-celled, or, in Abies excelsa, even an eight-celled rosette. The homology of the 'corpusculum' with the archegonium of Vascular Cryptogams, already established by the earlier investigations of Hofmeister, is carried a step further by Strasburger, who discovered the formation also of a canal-cell. He considers that the part of the protoplasmic contents of the large central cell which lies immediately beneath the

1 Hofmeister (Vergleichende Untersuchungen, p. 129) gives a somewhat different account of the origin of the archegonium [Germination, \&c., p. $4^{\text {IO }}$ ]. 
neck is separated from the rest by division, and a small cell is thus formed shortly before fertilisation (i.e. before the access of the pollen-tube to the endosperm); this cell being clearly equivalent to the canal-cell so often mentioned in Vascular Cryptogams which is afterwards converted into mucilage ${ }^{1}$. In Abies canadensis and excelsa and Pinus Larix this canal-cell is, according to Strasburger, very evident; while in the Cupressineæ (Thuja, Juniperus, and Callitris) its demarcation from the rest of the contents of the central cell is only slight. As in those Vascular Crypto-

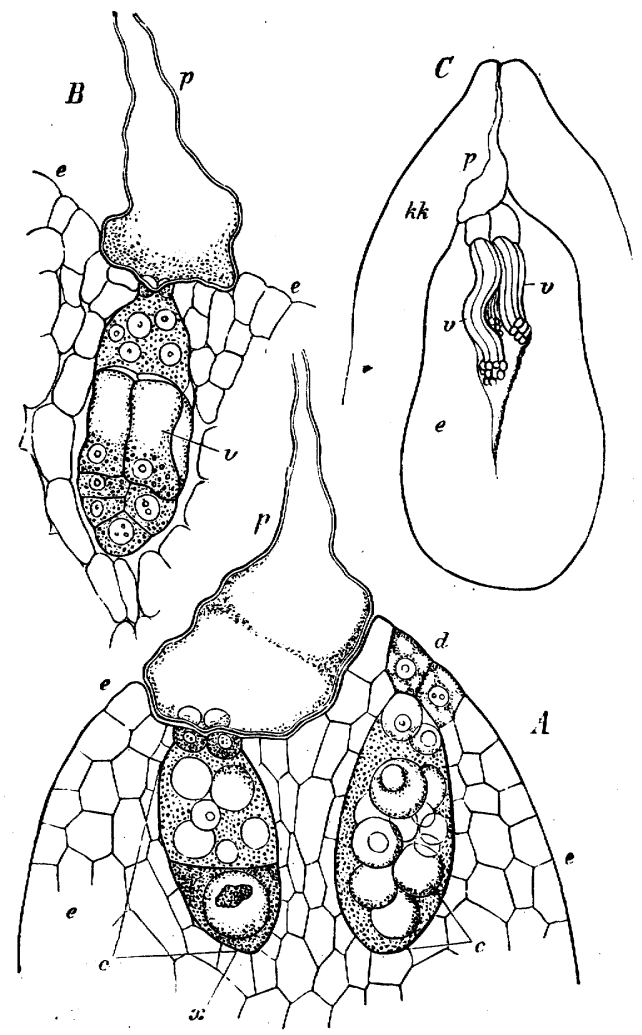

FIG. 354--Taxus canadensis (after Hofmeister). A longitudinal section through the upper end of the endosperm $e e$ and the lower end of the pollen-tube $p, c c$ the archegonia, $d$ their stigmatic cells, the left archegonium is fertilised, $x$ rudiment of the embryo (June 5$),(X 300), B$ part of the endosperm with an archegonium, the embryo of which $v$ is already further developed, $p$ the pollen-tube (June 10) ( $\times 200$ ); $C$ longitudinal section of a nucellus (June r5), $k k$ nucellus, $e$ e endosperm, $p$ pollen-tube, $v v$ embryos proceeding from two archegonia $(\times 50)$.

gams where the ventral part of the archegonium is plunged in the tissue of the prothallium, the neighbouring cells become transformed by further divisions into a parietal layer surrounding the oosphere, so the same thing takes place also in the endosperm of Coniferæ. In the Abietineæ each archegonium is separated from an adjacent one by at least one, often by a large number of layers of cells : those of the Cupressineæ, on the other hand (Fig. 355, $c p$ ), are in lateral contact. The archeindicated. 
gonia of Taxus are short; in those of the Abietineæ the oosphere is elongated; in the Cupressineæ it becomes angular from the pressure of the adjacent cells. The number of the archegonia which are formed in the endosperm beneath the apex of the embryo-sac is very various; Hofmeister and Strasburger state that in the Abietineæ it is from three to five, in the Cupressineæ from five to fifteen (according to Schacht it may even be thirty), in Taxus baccata from five to eight. The continuous growth of the surrounding endosperm causes the formation of funnel-shaped depressions above the archegonia, which in some Abietincæ are but

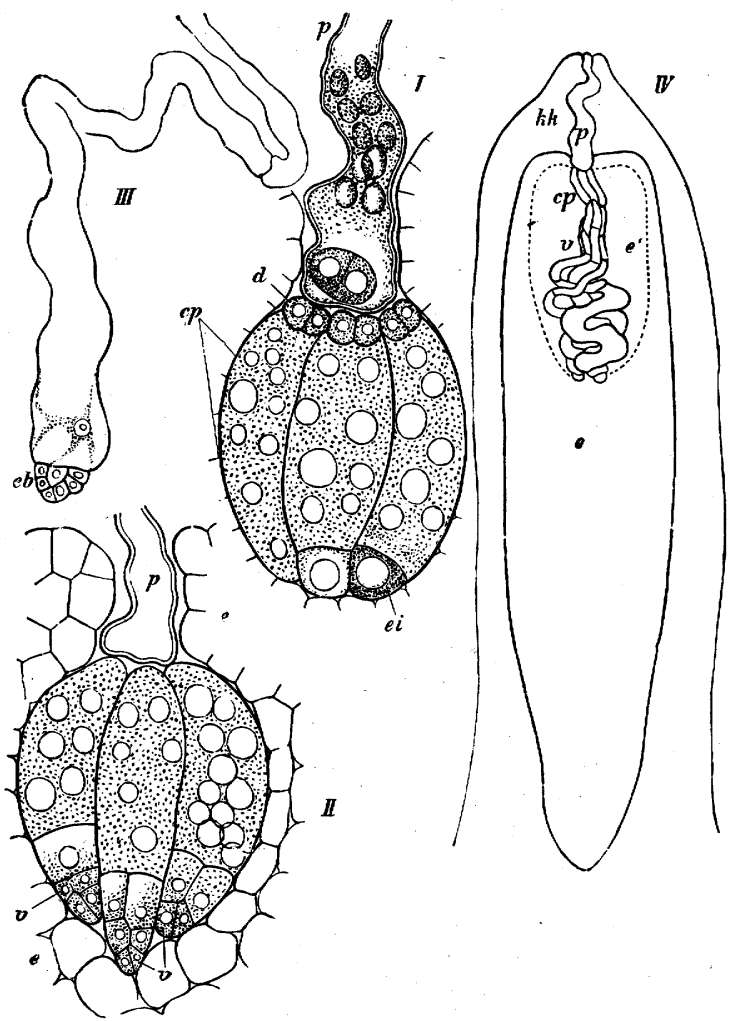

FIG. 355.-Funiperus communis (after Hofmeister). I three archegonia cp close beside one another, in two of them the rudimentary embryo $e i$ is seen at the lower end of the oosphere, $d$ neck-cells, $p$ pollen-tube $(\mathrm{July} 28)(\times 300)$. II a similar section, $e e$ the endosperm, $v v$ the embryos : III lower end of one of a suspensor with the rudiment of the embryo $e b$; $I V$ longitudinal section of the nucellus $k k, e$ the endosperm, $e^{\prime}$ portion of the endosperm that is broken up, $p$ pollentube, $c p$ the archegonia, $v$ the suspensors (beginning of August) ( $\times 80)$.

shallow, in Pinus Pinaster, P. Strobus, \&c., deep and narrow. In these species each of the funnel-shaped depressions leads down only to the neck of one archegonium; in the Cupressineæ (Callitris, Thuja, and Juniperus), where they lie closely crowded together, the cluster of them is walled round by the endosperm, and a funnel is formed common to them all, which still remains closed by the cell-wall of the embryo-sac.

Fertilisation. The pollination of the ovules takes place before the archegonia are formed in the endosperm; the pollen-grains, having reached the apex of the 
mucellus, put out their tubes at first only for a short distance into its tissue ; their growth is then for a time suspended. After the archegonia are completely developed, the pollen-tubes begin to grow again into the endosperm in order to reach them ${ }^{1}$. This interruption of their growth lasts, in those Coniferæ whose \&. seeds ripen in a single year, for only a few weeks or a month; when the seeds take two years to ripen, as in Juniperus sibirica and communis, and Pinus sylvestris and $P$. Strobus, it lasts until June of the next year. Whilst the pollen-tubes penetrate through the loose portion of the tissue of the nucellus, their width gradually increases at their lower end, their wall becoming at the same time thicker; until at length they meet the wall of the embryo-sac which has now become soft, break through it, penetrate into the funnel of the endosperm mentioned above, and attach themselves firmly to the cells of the neck of the archegonia. In the Abietineæ and Taxineæ each pollen-tube fertilises only one archegonium; and several tubes therefore penetrate into the funnel at the same time; in the Cupressineæ on the contrary one pollen-tube suffices for the fertilisation of the whole group of archegonia beneath the broad funnel of the endosperm. The tube entirely fills up the funnel and applies itself to the necks of the whole group of archegonia; short narrow protuberances from the wide pollen-tube now grow into the separate necks of the archegonia, forcing the neck-cells from one another and destroying them, and at length reaching the oosphere. The same process takes place in the Abietineæ and Taxineæ; the pollen-tube, after widening, becomes narrower and enters the neck of only one archegonium, and penetrates finally as far as the oosphere. A thin spot may be observed at the extremity of this protuberance of the thick-walled pollen-tube, which obviously facilitates the escape of the fertilising substance; and this is probably assisted by the pressure exerted by the tissue which lies above on the part of the pollen-tube outside the archegonium. Hofmeister states that a few free primordial cells (Fig. 355, $I$ ) are sometimes formed in the end of the pollen-tube, which he was inclined to consider as rudimentary indications of mother-cells of antherozoids (corresponding somewhat to those in Salvinia), and Strasburger has detected the existence of bodies of this kind in Juniperus and Pinus. [The process of fertilisation takes place, according to Strasburger ${ }^{2}$, as follows. In Juniperus virginiana he observed that when the apex of the pollen-tube reached the archegonia, the primordial cells in the end of the tube arrange themselves so that they lie over the archegonia. These cells now undergo absorption, the more anterior ones disappearing first. $\mathrm{He}$ frequently detected in the oosphere of Picea, at the time of fertilisation, two nuclei. One of these is the nucleus of the oosphere, and may be termed the 'female pronucleus;' the other appears to have passed into the oosphere from the pollen-tube, and is the 'male pronucleus' (spermakern). These two nuclei coalesce to form the definitive nucleus of the oospore. It seems that, in addition to the nuclear substance, a certain amount of protoplasm also passes into the oosphere from the pollen-tube, and fuses with the protoplasm of the oosphere. The pollen-tube of the Abietineæ contains a considerable number of starch-grains, and these too are

1 In Salisburia (Gingko biloba) fertilisation does not take place until October, when the seed is ripe and has already fallen off. The embryo is developed within the seed during the winter months. (See Strasburger, Die Coniferen und Gnetaceen, I872, p. 291.)

${ }^{2}$ [Ueber Befruchtung und Zelltheilung, $\left.18 ; 8.\right]$ 
conveyed, probably in a soluble form, into the oosphere. In the Cupressineæ, the pollen-tube contains no starch-grains, but they appear abundantly in the nucleus of the oospore. The pollen-tube remains completely closed during the whole process. The first manifest result of fertilisation in the oospore is the division of its nucleus $\mathbf{1}$. In Pinus and Picea the nucleus usually travels to the lower end of the oospore; it then divides into two, and each of these divides again into two, and thus four nuclei are formed which lie in one horizontal plane. Around each of them protoplasm becomes aggregated, and thus a formation of cells takes place. It is from these cells that the embryo or embryos are developed. Exactly the same takes place in Juniperus. In Ginkgo (Salisburia) the nucleus divides, and this is repeated until a large number of nuclei have been formed; a process of free cell-formation is then initiated round these nuclei ; the cells become separated by cellulose walls, multiply by division, and together constitute the embryo.]

The mode of origin of the embryo is very different in the various families, but in nearly all of them certain cells form a suspensor, and, by their great elongation, carry the rudimentary embryo, which lies at the apex of the suspensor, through the base of the archegonium out into the tissue of the endosperm where the embryo developes into a young plant ${ }^{2}$. In the Cupressineæ the cell from which the embryo is developed (Fig. 355) becomes divided so as to give rise to three superposed cells, of which, in Thuja occidentalis, only the upper two become divided into four by longitudinal walls, whilst in the lowest the rudiment of the embryo is formed by means of oblique septa : the embryo is then extruded from the archegonium by the elongation of the upper cells. In this case an oospore gives rise to a single embryo, growing at first by means of an apical cell from which two rows of segments are cut off but which it afterwards loses. In Juniperus, on the other hand, the lowermost of these three cells becomes divided into four by intersecting longitudinal walls, which are forced out by the elongation of the cells above them : these four cells become rounded off and they separate, each giving rise to a rudimentary embryo. In this case one oospore gives rise to four embryos, only one of which developes into a plant. The first development of the suspensor in the Abietineæ is somewhat different. The four cells lying side by side in one plane become divided by transverse septa so as to form three tiers; the cells of the second tier grow out into long coiled filaments, whereas those of the uppermost tier remain in the oospore as a rosette: the four cells of the lowest tier, which have been forced into the endosperm by the elongation of those above them, are repeatedly divided and thus contribute to the elongation of the suspensor; the four rows of cells then separate, each bearing a terminal cell from which the rudiment of the embryo is developed in such a manner that the possibility of the existence of an active apical cell is excluded ${ }^{3}$. Thus in the Abietineæ also four rudimentary embryos are developed from a single

1 [Strasburger, Zellbildung und Zelltheilung, $3^{\text {rd edition, p. 46.] }}$

2 [Strasburger has observed (Angiosfermen u. Gymnospermen, p. 149) that in Cephalotaxus Fortunei and in Araucaria brasiliana certain cells at the apex of the embryo are thrown off when it has made its way into the endosperma.]

${ }^{3}$ [Skrobiszewski has pointed out (Bull. de la Soc. Imp. des Natural. de Moscow, 1873), and his observations have been confirmed by Strasburger (Die Angiospermen und die Gymnospermen), that the embryo of Pinus Strobus, unlike that of the other Abietineæ, grows in length by means of a twosided apical cell.] 
oospore; but Picea vulgaris in this group resembles Juniperus in the preceding, in that only a single embryo is developed. In Taxus baccata the rudiment of the embryo consists of two or three tiers of cells, the upper of which elongate to form the suspensor: the lower tier consists of from four to six cells, one only of which gives rise to the embryo: the suspensors do not become isolated (Fig. 354). In Salisburia, according to Strasburger, no suspensor is formed: the cells which are formed in the oospore, after fertilisation, form a coherent mass of tissue, which increases by cell-division, and this constitutes the embryo.

It appears, therefore, that several embryos can be produced from one oospore; the number within a single endosperm being increased by the simultaneous fertilisation of severa larchegonia. Polyembryony, which is rare among Angiosperms (see infra), is thus the typical condition among Conifers and generally among Gymnosperms, but only in the very earliest stage; for usually only one of the rudiments developes into a vigorous embryo, such as has already been described. During its development the endosperm also continues to grow vigorously; its cells become filled with reserves of food-material (fat and albuminoids); the embryo-sac which surrounds it grows at the same time, and finally supplants the tissue of the nucellus, the tissue of the integument hardening at the same time into the testa. In Salisburiu, however, an outer strong layer of tissue forms the pulpy envelope which causes the seed to resemble a drupe. The elongated cells of the suspensor usually disappear during these processes, but according to Schacht are permanent in Larix.

During the period that the seeds are ripening, the carpels and the placentæ also continue to grow and to undergo changes in texture. In Taxus a red aril, which afterwards becomes pulpy, grows round the ripening seed (Fig. $348 \mathrm{~m}$ ); in Podocarpus the part of the floral axis that bears the scales and the seeds, and which was already considerably swollen, becomes fleshy; in Juniperus and Sabina the carpels themselves form the blue 'berry' which envelopes the seeds : in most other Cupressineæ the carpels grow, close up laterally and become woody; and the same occurs in those Abietineæ which are without bracts (in respect to Cunninghamia, vide supra); while in Pinus, Abies, Cedrus, and Larix, it is the placental scales which after fertilisation grow vigorously, outstripping in their growth the true carpels (bracts), become woody, and form the mature cone. In all these cases (except Podocarpus, Salisburia, and Taxus), the seed is closely and firmly enclosed during ripening by the carpels or placental scales; it ripens within the fruit, the parts of which do not again separate or become detached (as in Abies pectinata) in order to allow of the escape of the seeds until they are completely ripe.

So long as we are still in doubt as to the nature of the female flowers of various genera, the systematic arrangement of the Coniferæ can only be considered as provisional ; Endlicher (Synopsis Coniferarum, I 847) distinguishes the following families :-

Family r. Cupressinem. Leaves, including those of the flowers, opposite or verticillate (in Division $e$ single); flowers monœcious or diœcious; stamens terminating in a shield-like expansion bearing pollen-sacs in twos or threes or larger numbers; female flower consisting of alternate whorls of carpels, bearing at their base or on their inner surface two or a larger number of erect ovules (in Juniperus communis the ovules are alternate with the three carpels on the floral axis); embryo with two, rarely three or nine cotyledons. 
(a) Juniperinea. Fruit berry-like (Juniperus, Sabina).

(b) Actinostrobea. Carpels united into valves; afterwards separating as a four- or six-rayed star (Widdringtonia, Frenela, Actinostrobus, Callitris, Libocedrus).

(c) Thujopsidea. Carpels partially overlapping one another (Biota, Thuja, Thujopsis.)

(d) Cupressinea vera. Carpels peltate and polygonal in front (Cupressus, Cbamacyparis).

(e) Taxodinea. Carpels peltate or overlapping; leaves alternate (Taxodium Glyptostrobus, Cryptomeria).

Family 2. Abietineø. Leaves usually acicular and arranged spirally, singly, or in twos, threes, or rosettes on special short shoots ; flowers monœcious, rarely diøcious: stamens numerous, with two or more tong pollen-sacs; female flower consisting of a number of scale-like placentæ arranged spirally, which are either themselves carpels or are lignified outgrowths of small carpels; micropyle of the ovule turned towards the base of the placenta; embryo with from two to fifteen cotyledons.

(a) Abietinea vera. Seeds in pairs on a scale-like placenta which springs from a small open carpellary leaf (Pinus, Abies, Tsuga, Larix, Cedrus).

(b) Araucariea. Seed single on the carpel, and enveloped by it (Araucaria).

(c) Cunninghamiea. Seeds single or numerous on a carpel (Dammara, Cunningbamia, Artbrotaxis, Sequoia, Sciadopitys).

Family 3. Podocarpeø. Leaves acicular or broader, and arranged spirally ; flowers monœcious or diœcious; stamens short, with two roundish pollen-sacs; female flower consisting of an axis swollen above with small scale-leaves, from the axils of which (?) the ovules spring; embryo dicotyledonous.

Podocarpus (Dacrydium, Microcachrys).

Family 4. Taxinem. Leaves arranged spirally, acicular or often of considerable breadth; in Pbyllocladus there are no foliage-leaves, these being replaced by leaf-like branches; flowers always diœcious ; stamens of various forms, bearing two, three, four, or eight pendent pollen-sacs; female flowers always consisting of a naked axis or of one furnished with small leaves, bearing the erect ovules terminally or laterally; ripe seed enclosed in a fleshy aril or with the outer layer of the testa fleshy; embryo dicotyledonous.

Phyllocladus, Salisburia, Cephalotaxus, Torreya, Taxus.

\section{GNETACE $Æ 1$.}

This order includes three genera which differ strikingly in habit. The Ephedræ are shrubs with no foliage-leaves and with long, slender, cylindrical green-barked branches; at the joints of the stem are two opposite minute leaves which grow together into a bidentate-sheath, and from their axils the lateral branches spring. In Gnetum the leaves are also opposite on the jointed axes, but large and stalked, with a broad lanceolate lamina and feather-veined venation. Thirdly, Welwitschia mirabilis, so remarkable a plant in many other ways, possesses only two foliage-leaves of immense size. They are extended on the ground and become divided into strips as they become old; the stem remains short, rising only slightly

1. See Strasburger, Die Coniferen und Gnetaceen, Jena, I872, 
above the ground, and is broad above with a furrow across the top, while it is tuberous below, and passes into the tap-root ${ }^{1}$.

The Flowers of Gnetaceæ are unisexual, and are arranged in diœcious (Ephedra) or monœcious inflorescences; the inflorescence has a well-defined form, and in Ephedra and Gnetum springs from the axils of the opposite leaves. The male flower of these genera consists of a small bifid perianth, in the middle of which rises a staminal column, which in Gnetum is bifurcate above and bears two bilocular anthers, in Ephedra a larger number crowded into a head. The female flower of Gnetum (Eichler, in Flora 1863, p. $4_{63}$ ), like that of Ephedra, also possesses a perianth, flask-shaped in the former, obscurely trigonous in the latter genus; it envelopes a central ovule possessing in the case of Ephedra one integument, in that of Gnetum two, the inner of which is elongated like a style ${ }^{2}$. The more exact morphology of these flowers is still doubtful. In Gnetum the inflorescence, which springs from the axil of the foliage-leaves, consists of a jointed axis with verticillate leaves, in the axils of which the flowers, male and female, are agglomerated. The inflorescence of Welwitschia ${ }^{3}$ is a dichotomously branched cyme nearly a foot in

${ }^{1}$ For a full description of this remarkable plant see J. D. Hooker in Trans. Linn. Soc. vol. XXIV.

2 [According to Beccari (Della Organogenia dei fiori feminei del Gnetum Gnemon, Nuovo Giorn. bot. ital. IX, 1877) the female flowers are lateral axes, the nucellus being the end of the axis. Strasburger (Angiospermen und Gymnospermen) regards the two investments of the nucellus in Ephedra and the three in Gnetum as integuments.]

${ }^{3}$ [According to Professor W. R. M Mab, 'The cones of Welwitschia consist of numerous opposite and decussate bracts, with a sessile flower in the axil of each of the bracts. The perfect flowers in the male cone consist of two outer perianth leaves (calyx) placed right and left, two inner ones (corolla) placed anteriorly and posteriorly, six stamens united below, and two carpels anterior and posterior, the conical end of the axis projecting as a rudimentary axile ovule surrounded by the two carpels. The outer parts of the perianth are first developed appearing as two shoulders at the very base of the young floral branch. The flower next in age has the floral axis more elongated, the outer parts of the perianth larger, and a distinct swelling is visible above the outer parts. These swellings are anterior and posterior, and much larger than the outer parts. Above the inner parts of the perianth the axis is expanded, and contracts near the rounded apex. The expanded portions are superposed on the outer lateral parts of the perianth, and are the two primordial staminal cushions. These cushions are semilunar, and in the earlier stages show no trace of division into three. At this stage the parts of the perianth rapidly enlarge and cover in the central parts of the flower. A projection now forms anteriorly and posteriorly, the first indication of the two carpels. The next stage shows the two staminal cushions each forming three elevations, the central one larger than the two lateral ones. The six stamens are thus produced by the branching of two primordial stamens. In the next stage the carpels elongate and cover in the punctum vegetationis, ultimately developing the peculiar style and stigma-like process. The axis elongates slowly and forms a conical projection which is undoubtedly a rudimentary axile ovule, but it never shows any appearance of an embryo-sac.'

In the female flowers, which are produced in different cones from the male flowers, the development is very different. A very short stalk is developed in the female, which is wanting in the male; then two shoulders are developed exactly like the two outer parts of the perianth in the male flower, to which Dr. Hooker considtered them to be equivalent. Judging from the construction of the male flower, Professor $\mathrm{M}^{\mathrm{c}} \mathrm{Nab}$ was disposed to accept this view; but with hesitation, as he could not account for the stalk-like process. Strasburger however concludes that they are carpels, and in that $\mathrm{M}^{\mathrm{C}} \mathrm{Nab}$ quite concurs. Above the carpels the axis elongates slightly, and a ring is formed surrounding the punctum vegetationis. This ring is the ovular integument. Comparing the two flowers, it will be seen that in the male there are four series of parts, in the female the three outer series are wanting and only the carpels remain. But in the male flower the carpels are anterior and 
height, rising above the insertion of the two enormous leaves on the periphery of the broad apex of the stem. The branches of the inflorescence are terete and jointed, spring from the axils of the bracts, and bear upright longish cylindrical cones; these are furnished with from seventy to ninety broadly ovate scale-leaves standing closely one above another in four rows, a single flower being seated in each axil, male and female in different cones. The male flowers are pseudo-hermaphrodite, and possess a perianth consisting of two pairs of decussate leaves; the lower ones are entirely free, sickle-shaped and pointed, the upper ones broadly spathulate and coherent at their base into a compressed tube. Within this tube are six stamens, monadelphous at the base, with cylindrical filaments and terminal spherical bilocular anthers, which dehisce above the apex with a three-armed fissure ; the pollen-grains are simple (?) and elliptic. The centre of the flower encloses a single erect orthotropous sessile ovule with broad base, and with no other investment than a simple integument, which is drawn out into a style-like tube with a margin expanded in a discoid manner; the nucellus, however, has no embryo-sac, or is sterile. In the female flowers the perianth is tubular, greatly compressed, somewhat winged, and altogether undivided; there is no indication of any male organ; the ovule (in this case of course possessing an embryo-sac) is entirely enclosed in the perianth, and is similar in its external form to that of the male flower, but with this difference, that the elongated point of the integument is only simply slit, not expanded into the form of a plate. When ripe the cone is about two inches long and of a scarlet colour; the scales are persistent; the perianth enlarges considerably and becomes broadly winged; its cavity is narrowed above into a narrow canal, through which the apex of the integument passes. The seed is of the same form as the unfertilised ovule, and contains abundant endosperm, in the axis of which lies the dicotyledonous embryo ; the embryo is thick at the radicular end, and is there attached to the very long spirally-coiled suspensor. The formation of endosperm commences in the embryo-sac before fertilisation; archegonia without necks, consisting, that is, only of oospheres, are formed which grow out of the embryo-sac to the number of from twenty to sixty, and penetrate into the canal-like cavity of the nucellus, there they are fertilised by the pollen-tubes which have grown to meet them. [After fertilisation, the oospore elongates into a tube, a portion of which is cut off by a wall near its lower end. This cell at once divides into four, placed crosswise. These cells multiply by division, so that a group of cells is formed which, for the most part, constitute the embryo; the marginal cells of the group grow out into long tubes, the so-called ' embryonal tubes.' Although from two to eight archegonia are fertilised only one embryo is developed.

The development of the ovule and of the embryo-sac has been studied by Strasburger (Angiospermen und Gymnospermen) in Gnetum Gnemon. Groups of

posterior, while in the female they' are lateral. This is to be explained by the fact that the carpels are here the first leaves of a branch, and that it is very rare (except in grasses) that the first leaves of a shoot are anterior or posterior, and not lateral. The ovular integument of the female flower is wanting in the male. While therefore the male flower is complex, the female is remarkably simple.

For further details see Transactions of the Linnean Society, 1873, vol. XXVIII. pp. 507-51 2. On the general Morphology and Histology of Welwitschia see Bower, Quart. Journ. Micr. Sci. I88I.

1 [Strasburger (loc. cit.) now regards the 'perianth' as an outer integument.] 
epidermal cells, situated in the 'cupule' formed by the bracts, begin to divide, and then divisions take place in the hypodermal cells; by this means a number of protuberances are formed, usually six or eight, in a whorl, which are the rudimentary ovules. Around each of these the external integument begins to grow up: when the external integument covers about two-thirds of the nucellus, the middle integument begins to be formed, and this is immediately followed by the appearance of the innermost integument. The nucellus elongates above the insertion of the external integument, and in consequence the cells of which it consists can be distinctly seen to be arranged in longitudinal rows. The terminal cell of one or more of these rows; which is therefore hypodermal, elongates, and thus the archesporium is constituted. These cells now undergo divisions, cells being cut off from them towards the free surface of the nucellus; these cells thus cut off form part of the tapetum, the larger cells beneath them being the mother-cells of the embryo-sacs. Of these, one only gives rise to an embryo-sac, the others becoming obliterated. The formation of the endosperm begins with the division of the nucleus of the embryo-sac: a number of nuclei are formed by repeated division, and around these free cellformation takes place.

The development of the ovule of Ephedra is much the same as that above described in Gnetum. The endosperm of Ephedra produces from three to five archegonia, with an elongated oosphere, a distinct canal-cell, and a long neck consisting of rows of cells. After fertilisation the nucleus of the oospore divides, and the two new nuclei separate and travel towards the opposite ends of the cell. Here they undergo division, and this is repeated until usually eight nuclei have been formed. Around these a process of free cell-formation now takes place, and each of the cells thus formed becomes enclosed in a cellulose wall. Each of these cells, which are quite distinct from each other, now grows out into a tube which escapes from the archegonium and penetrates into the endosperm: a small portion of the tube is now cut off by a transverse septum near the apex. It is from this cell that the embryo is developed. It divides into two by a transverse wall parallel to the first, and these two cells grow and divide in various directions; sometimes a two-sided apical cell is formed by means of which the embryo grows.]

A small cell is formed in the pollen-grain of.Ephedra as in that of the Cupressineæ.

The Histology of Gymnosperms. From the abundant though still unsifted material I will only adduce a few particulars as a contribution to the special characteristics of this section.

The Fibro-vascular Bundles ${ }^{1}$ are similar in their structure to those of Dicotyledons. There is a system of bundles common to the stem and leaves; the portions which

${ }^{1}$ Mohl, Bau des Cycadeenstammes (Verm. Schr. p. I95).-Kraus, Bau der Cycadeen-Fiedern (Jahrb. f. wiss. Bot. vol. IV. p. 329). - Geyler, Ueber Gefässbundelverlauf bei Coniferen (ditto, vol. VI p. 68).-Thomas, Vergl. Anat. des Conifer-Blattes (ditto, vol. IV. p. 43).-Mohl, Ueber die grossen getüpfelten Röhren von Ephedra (Verm. Schr. p. 269).-J. D. Hooker, On Welwitschia (Trans. Linn. Soc. vol. XXIV). - Dippel, Histologie der Coniferen (Bot. Zeitg. I862 and I863).Rossmann, Bau des Holzes (Frankfurt-a-M. I863).-Mohl, Bot. Zeitg. I871.-[Bertrand, Anat. comp. des tiges et des feuilles chez les Gnetacées et les Conifères, Ann. Sci. Nat., sér. 5. XX. - Bower, On Welwitschia, Quart. Journ. Micr. Sci. I 88 I. - De Bary, Vergleichende Anatomie der Vegetationsorgane, I 877 .] 
descend into the stem forming a circle, where a closed cambium-ring is produced by the formation of interfascicular cambium. The ascending portion, which curves out into the leaf itself, assumes in Cycadeæ more or less the character of a closed bundle, while in the leaves of many Coniferæ it at least retains the appearance of an open bundle. [In most cases the growth in thickness of the stem is brought about by the division of the cells of the cambium-layer, but in Cycas and Encephalartos the activity of the cambium-layer is of limited duration. The subsequent growth in thickness of the stem in these two genera is effected by the production of successive new cambium-rings, which make their appearance just outside the bast of the older vascular bundles.] No exclusively cauline bundles are produced in the stem of Coniferæ or of Ephedra; but in some Cycadeæ accessory-bundles arise in the older stem which are independent of the common bundles and of those formed by the cambium-layers. Thus in the tissue of the pith of Encepbalartos slender isolated bundles occur; while in Cycas a system of thicker bundles is developed in the cortex which may form there in old age one or more apparent rings of wood. As far as we can judge from Hooker's description, bundles occur in the bark of Welavitschia which owe their origin to a layer of meristem enveloping the whole stem $^{1}$. The Coniferæ, as has been mentioned, possess only common bundles, the descending portions passing through a number of internodes, and then joining others lower down either unilaterally or on both sides by splitting into two arms and turning to both sides. The leaves of Coniferæ, when narrow, contain only one fibro-vascular bundle from the stem, which then usually splits into two halves running parallel to one another (Fig. 102); when the leaves are broader, two (Salisburia, Ephedra) or even three bundles occur; when the leaf forms a flat broad lamina, as in Salisburia and Dammara, the bundles ramify in it, but without forming a net-work; in Salisburia they repeatedly branch dichotomously. In Coniferæ these bundles seldom form prominent veins, but run through the middle of the tissues of the leaf. In the two gigantic leaves of Welwitschia there are a number of bundles, the parallel ramifications of which run into the iniddle layer of tissue. In the large pinnate leaves of Cycadex there are also several bundles which curve nearly horizontally within the cortical parenchyma, and split into a number of stout bundles in the leaf-stalk when it is thick; these bundles exhibit a beautiful arrangement when seen in transverse section (in Cycas revoluta, e.g. in the form of an inverted $\Omega$ ). They run parallel in the rachis of the pinnate leaf, and give off branches into the pinnæ, where they either run parallel in the middle layer of tissue (as in Dion) or dichotomise (e.g. Encephalartos); while in Cycas they form a mid-rib projecting beneath. The course of the bundles in - the leaf therefore shows a decided resemblance to that of many Ferns.

The substance of the wood of the stem is formed from the descending bundles, which are at first completely isolated, but soon coalesce into a closed ring by portions of cambium which cross the medullary rays. The primary wood or xylem, termed the Medullary Sheath, which consists of the xylem-portions of the descending limbs of the common bundles, contains, in all Gymnosperms, as in Dicotyledons, long narrow vessels with annular or spiral thickening-bands, while further outwards occur scalariform or reticulately thickened vessels. The secondary wood produced from the cambium-ring after the cessation of growth in length consists, in Cycadeæ and Coniferæ, of long tracheïdes grown one into another in a prosenchymatous manner (cf. p. 25) with a few large bordered pits, which are usually circular, at least when the wood is mature. Every possible stage of transition occurs between these tracheides and the spiral vessels of the medullary sheath. The secondary wood of Cycadeæ and Coniferæ is distinguished from that of Dicotyledons by the striking peculiarity that it is composed only of this prosenchymatous form of cells $^{2}$ ); and that the wide dotted vessels composed of short cells are wanting which penetrate the dense narrow-celled masses of the wood of Dicotyledons. In the younger stems of Cycadex the tracheides with broad bordered

1 [De Bary compares the structure of the stem of Welwitschia to that of Chenopodiaceæ and Amarantaceæ, etc. (see infra); that of Gnetum to that of Phytolacca, Polygaleæ, Dilleniaceæ, etc.]

${ }^{2}$ Wood-parenchyma is not formed, or only in small quantity. 
pits and hence with a more or less scalariform wall are very much like the long prosenchymatous vessels of Vascular Cryptogams; and this resemblance extends even to the tracheïdes of Coniferæ, so far as they are distinctly prosenchymatous, although the smaller number and round form of the bordered pits shows a more marked difference. The bordered pits of Coniferæ are usually developed only on the wall which faces the medullary rays, in one or two rows, but in Araucaria in larger numbers and densely crowded. In the structure of the secondary wood, as in that of their flowers and in their habit, Gnetaceæ approach Dicotyledons; in Ephedra broad vessels occur in it together with the usual tracheides in the inner part of the ring of wood, but their component cells are separated by oblique septa, and are therefore still prosenchymatous, and are penetrated by several roundish holes; their lateral walls show bordered pits like the tracheides, and furnish a striking evidence that the true vessels in the secondary wood of Dicotyledons are connected by intermediate forms with the vessels of Vascular Cryptogams formed from prosenchymatous cells. In the wood of Welwitschia tracheindes with doubly bordered pits are also present.

The medullary rays of the secondary wood of Coniferæ are very narrow, often only one cell in breadth; the cells are strongly lignified, and their lateral faces in contact with the adjoining tracheïdes are provided with closed bordered pits. In Cycadeæ the rays are broader, and their tissue bears a closer resemblance to the parenchyma of the pith and cortex; their number and width cause the whole substance of the wood to appear spongy, and its prosenchymatous cells are seen to be strongly curved in different directions in tangential sections. The phloëm-portion of the fibro-vascular bundles of Gymnosperms resembles that of Dicotyledons; it is mostly composed of true strongly-thickened bastfibres, cambiform cells, latticed cells, and parenchymatous cells; while in Coniferæ they are formed in alternate layers. Usually the soft bast predominates.

The Fundamental Tissue of the stem of Gymnosperms is separated by the ring of wood into pith and primary cortex. Both are very strongly developed in Cycadex, especially the pith, and consist of true parenchyma, while the woody portion is considerably smaller. In Welavitscbia the parenchymatous tissues appear also to predominate; but the greater part of their substance is the product of the activity of the meristem-layer of the stem already mentioned. A large number of so-called spicular cells occur dispersed in all the organs of this remarkable plant, they are fusiform or branched and greatly thickened; and a number of beautifully developed crystals are found imbedded close to one another in their cell-wall. Similar structures also occur in Coniferæ (p. 66).

The parenchymatous fundamental tissue of Coniferæ decreases greatly with the increase in age of the stem (and of the root). With the exception of the pith, which is here small, the stem consists exclusively of the products of the cambium-ring, since the primary cortex, and afterwards also the outer layers of the secondary cortex which always have a subsequent growth, are used up in the formation of cork. In the stem of Cycadeæ, the increase of which in thickness is inconsiderable, the formation of cork is also very smali ; in Welwitscbia it appears to be entirely wanting (?).

Intercellular Passages are widely distributed in Gymnosperms; their structure is that which has been explained generally at pp. 78 and 94. In Cycadeæ they are found in all the organs in large numbers, and contain gum, which exudes from incisions in thick viscid drops; in Coniferæ they contain oil of turpentine and resin. In this latter order they occur in the pith of the stem, in the whole substance of the wood, and in the primary and secondary cortex, as well as distributed through the leaves; always following the direction in length of the organs, like the gum-passages of Cycadex. In many Conifers with short leaves roundish resin-glands also occur in them (as in Callitris, Thuja, and Cupressus, according to Thomas); in Taxus the resin-canals are entirely wanting ${ }^{1}$.

1 [Van Tieghem (Ann. des. Sci. Nat. 1872) distinguishes the six following modifications of the distribution of the secretory organs in Coniferæ:- $r$. No canals in the root nor stem: Taxus. 2. No canals in the root; canals in the cortical parenchyma of the stem : Cryptomeria, Taxodium, Podo- 
The Leaves of Cycadeæ and Coniferæ are covered by a firm epidermis, usually strongly cuticularised, and furnished with numerous stomata, each with two guard-cells. In the Cycadeæ the guard-cells are more or less deeply depressed, and the stomata occur only on the under side of the lamina, and are either irregularly scattered, or arranged in rows between the veins (Kraus). In the leaves of Conifers the guard-cells are also, according to Hildebrand (Bot. Zeit. 1869, p. 149), always depressed in the epidermis; and the stoma has hence always a vestibule. In Coniferæ the stomata are developed either on both or only on one side of the leaf; when the leaf is broad, as in Dammara and Salisburia, they are irregularly scattered; when the leaves are acicular they mostly lie in longitudinal rows; and in the large leaves of Welavitscbia they are also arranged in rows. The firm texture of the leaves of Cycadeæ and Coniferæ is due to a hypodermal layer, often strongly developed, consisting of strongly-thickened, generally long, fibre-like cells lying parallel to the surface; in the leaf of Welavitscbia this hypoderma consists of spongy succulent tissue penetrated by bundles of fibres, which acquires its hardness from a mass of spicular cells. The chlorophyll-tissue of the leaves lies beneath this layer, and is developed on the upper side of the leaves of Cycadex and of the broader leaves of Coniferæ as the so-called Pallisade-tissue; i.e. its cells are elongated in a direction vertical to the surface of the leaf and are densely packed together. In Pinus, Larix, and Cedrus the cells which contain chlorophyll exhibit the infoldings of the cell-wall which have been already mentioned at p. 74. The middle layer of the tissue of the leaf, in which also the fibro-vascular bundles run, has usually a peculiar development in Gymnosperms ; in Cycadeæ and Podocarpeæ it consists of cells elongated in a direction transverse to the axis of the leaf and to the bundles, but parallel to the surface of the leaf, leaving large intercellular spaces (Transfusion-Tissue of Mohl). In the acicular leaves of the Abietineæ the fibro-vascular bundle, split into two, is enveloped by a colourless tissue, which is

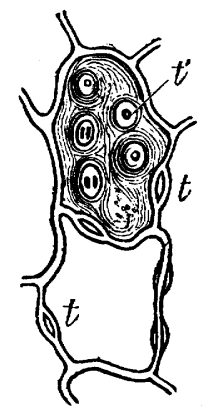

FIG. 356.-Pinus Pinaster; two cells of the colourless parenchyma surrounding the fibro-vascular bundle of the leaf; $t t$ the bordered-pits cut across, $t^{\prime}$ the same seen from the surface. sharply differentiated from the surrounding chlorophyll-tissue (Fig. 102). It is parenchymatous, and is distinguished by the large number of bordered pits which the walls of the cells bear (Fig. 356) ${ }^{1}$.

carpus, Dacrydium, Torreya, Tsuga, Cunninghamia. 3. No canals in the root; canals in the cortical parenchyma and in the pith of the stem: Salisburia. 4. A secretory canal in the root; canals in the cortical parenchyma of the stem: Cedrus, Abies, Pseudolarix. 5. Canals in the wood of the fibrovascular bundles of the root and stem; canals in the cortical parenchyma of the stem: Pinus, Larix, Picea, Pseudotsuga. 6. Canals in the liber of the fibro-vascular bundles of the root and of the stem; canals in the cortical parenchyma of the stem: Araucaria, Widdringtonia, Thuja, Cupressus, Biota. In Cycadeæ the canals are found disseminated through the cortical parenchyma of the stem; the pith of Cycas appears destitute of them. In their distribution they resemble therefore that which occurs in the second class of Coniferæ.]

1 For further details see Mohl, Bot. Zeit. 1871, Nos. 1, 2. 


\section{A N G I O S P E R M S¹.}

Monocotyledons and Dicotyledons are distinguished from Gymnosperms by the following characters:- their ovules are formed within a receptacle, the Ovary; the endosperm originates in the embryo-sac only after fertilisation,-characteristics, the importance of which has already been shown in the general introduction to Phanerogams. Concurrently with these distinctions there are however a number of other peculiarities in these plants taken as a whole which distinguish them from

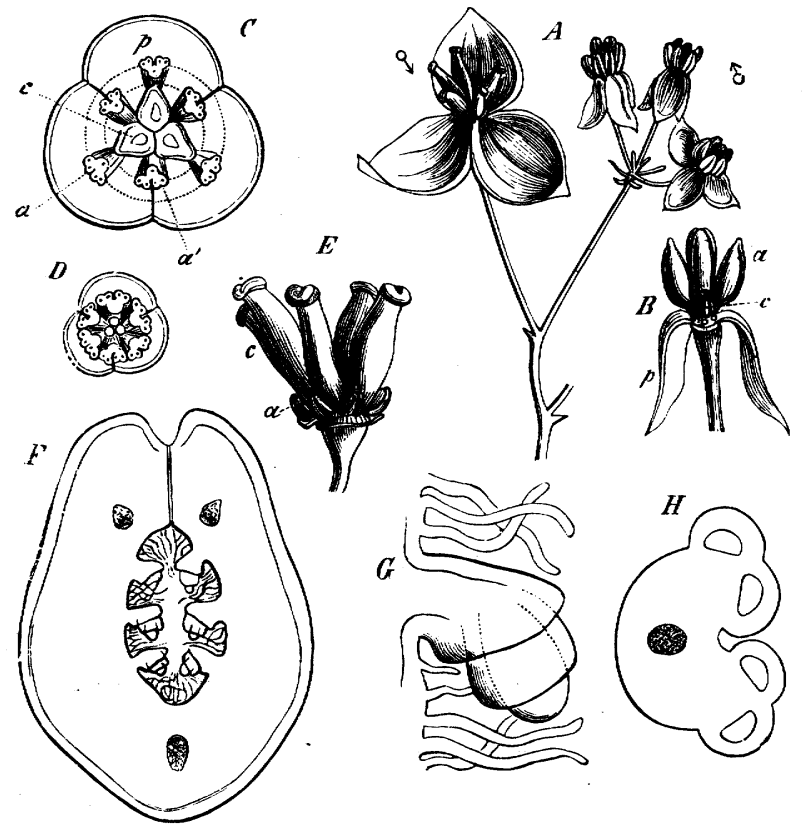

FIG. 357.-Akebia quinata; $A$ part of an inflorescence, $Q$ female, $\delta$ male flowers; $B$ a male flower cut through lengthwise, $c$ its sterile carpels; $C$ horizontal section of a female flower (magnified); $D$ horizontal section of a male flower; $E$ gynæceum of the female flower with the sterile stamens $a ; F$ an ovary cut through horizontally; $G$ an ovule; $H$ horizontal section of an anther ; $a$ (in $B$ and $C$ ) the outer, $a^{\prime}$ the inner stamens, $c$ (in $E$ ) the carpels ; $p$ (in $B$ and $C$ ) the perianth.

all other vascular plants; and this is especially the case with the structure of the flowers and the fruit, the normal morphological relations undergoing such peculiar combinations and changes that a more detailed description of them must precede the special description of the two classes which they include.

The Flower as a whole ${ }^{2}$. The flower of Angiosperms is rarely terminal, i.e. the primary stem, which is a prolongation of the axis of the embryo, rarely terminates

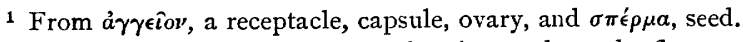

2 The most important and comprehensive work on the flowers of Angiosperms is Payer's Traité d'Organogénie de la Fleur (Paris 1857), with ${ }_{54} 4$ plates. Also Van Tieghem, Rech. sur la structure du pistil (Mém. des savants étrangers, XXI. I87I), and his notes in the French edition. [Eilcher, Bliithendiagramme, and Gray, Structural Botany.] 
in a flower, making the plant uniaxial. When this is the case a sympodial or cymose inflorescence is usually developed, new axes with terminal flowers arising beneath the first flower; but it is more common for only axes of the second, third, or a higher order to terminate in a flower, so that the plant may in this respect be termed bi-, tri-, or multi-axial.

While in Gymnosperms the flowers are typically unisexual or diclinous, hermaphroditism largely prevails among Angiosperms, although monœcious and diœcious species, genera, and families are not uncommon. The male flowers are sometimes essentially different in structure from the female flowers (as in Cupuliferæ and Cannabineæ), but in most cases the unisexuality arises merely from the partial or entire abortion either of the andræcium or the gynæceum, the flower being in other respects constructed on the same type (Fig. $357, A$ ); and in such cases it also frequently happens that hermaphrodite flowers are developed in addition to the male and female (polygamous species, as the Ash, Acer, Saponaria ocymoides, \&c.). But even in the greater number of cases where the male and female organs are completely developed in hermaphrodite flowers and functionally perfect, fertilisation

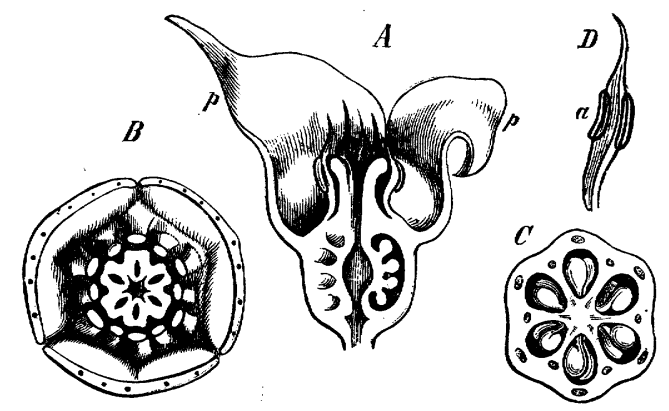

FIG. 358.-Asarum canadense; $A$ the flower cut through lengthwise, $p$ the perianth; $B$ horizontal section of the flower above the ovary; $C$ horizontal section of the sex-locular ovary; $D$ a stamen with its lateral anther-lobes $a$.

takes place by the conveyance of the pollen of one flower to the gynæceum of other flowers or even of other individuals of the same species, because either pollination within the same flower is impossible in consequence of special contrivances (such as dichogamy), or because the pollen is potent only in the fertilisation of ovules of another flower (as in Orchidex, Corydalis, \&c.): To these phenomena we shall recur more in detail in the Third Book, when speaking of the physiology of sexual reproduction.

While in Gymnosperms the floral axis is usually elongated to such an extent that the sexual organs, especially if numerous, are evidently arranged one above another in alternate whorls or in spirals,-in Angiosperms, on the contrary, the floral axis which bears the floral envelopes and sexual organs is so abbreviated that space can only be found for the various foliar structures by a corresponding expansion or increase in size of the receptacle or torus; this receptacle swells even before and during the formation of the floral leaves in a club-shaped manner, and is not unfrequently expanded flat like a plate or even hollowed out like a cup in such a manner that the apex of the axis is placed at the bottom of the hollow (p. 220 ), while 
the cup thus formed encloses the carpels (as in perigynous flowers), or even takes part in the formation of the ovary, which is then inferior (Fig. 358). The result of this abbreviation of the axis is that the separate parts do not usually stand one above another, but rather in concentric whorls, or in scarcely ascending spirals, for which reason the explanation of the relative positions expressed by a diagram in the sense explained on p. 188 appears the most obvious. This abbreviation of the axis is also obviously the immediate cause of the numerous cohesions and displacements which are nowhere met with so frequently as in the flowers of Angiosperms. The small development of the floral axis in length depends on the early cessation of its apical growth; the acropetal or centripetal order of succession of the floral leaves may therefore be disturbed ${ }^{1}$ by the production of intercalary zones of growth, although even in these cases the disturbance of the ordinary regularity remains inconsiderable. The acropetal order of succession is however even here in most cases strictly carried out, and the apical growth of the floral axis not unfrequently continues long enough

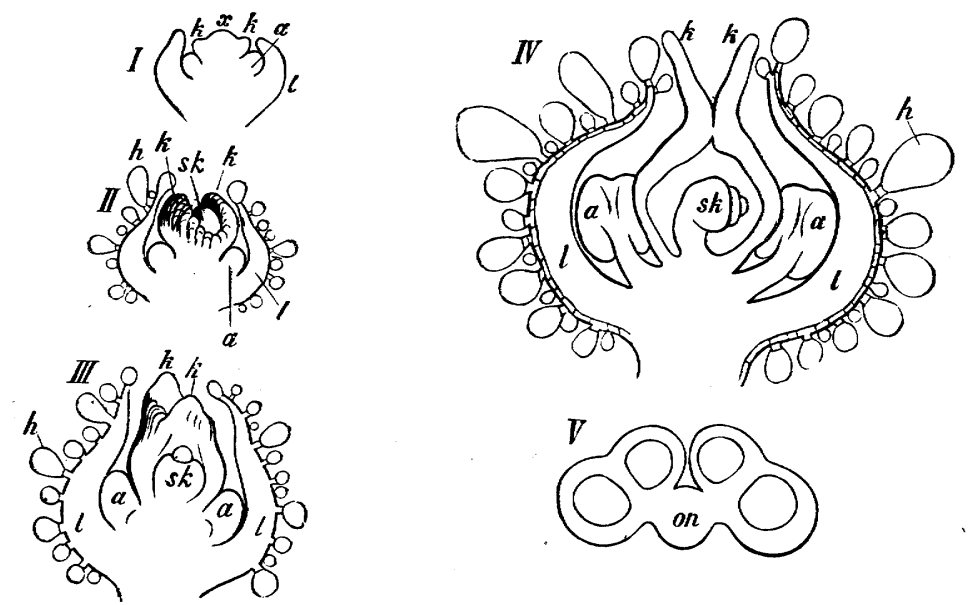

FIG. 359.-Chenopodium Quinoa; $I-I V$ development of the flower (in longitudinal section), $l$ the calyx furnished with glandular hairs $h, a$ anthers, $k, k$ carpels, $s k$ ovule, $x$ apex of the floral axis, $V$ horizontal section of an anther with four pollen-sacs on the connective on (strongly magnified).

to allow the foliar structures to arrange themselves in evident whorls placed one over another or in spirals (e.g. Magnolia, Ranunculaceæ, Nymphæaceæ). Occasionally also particular portions of the axis are greatly elongated within the flower, as the portion between calyx and corolla in Lychnis (Fig. 36I), in Passiflora that between corolla and stamens, in Labiatæ that between stamens and ovary.

The flower of Angiosperms, like that of Gymnosperms, is a metamorphosed shoot, a leaf-bearing axis ; but this section of the vegetable kingdom is especially characterised by the high degree of metamorphosis which the floral shoot has undergone, and by the very peculiar characteristics and the different arrangement of the foliar structures as contrasted with those of the purely vegetative shoots. As far as external appearance goes, the flower of Angiosperms is an altogether

1 The cases adduced by Hofmeister (Allgemeine Morphologie, § 10) of the absence of strict acropetal succession in the foliar structures all belong to this category. 
peculiar structure, sharply differentiated as a whole from the rest of the organism. This peculiar appearance is due not only to the special properties of its axis, but especially to the presence of the floral envelopes, and most of all to the circumstance that the foliar structures of the flower are arranged, with rare exceptions, in the form of whorls, even when the leaves of the vegetative shoots are alternate or distichous, or disposed in other similar arrangements. Each of the distinct appendicular organs of the flower, viz. the perianth, androcium, and gynæceum, is usually represented by several members arranged in concentric circles or in a spiral; so that one or more perianth-whorls are immediately succeeded within by one or more whorls of stamens, and these by the gynæceum in the centre of the flower. One or other of these whorls may however be absent, or each of the separate whorls may be represented by only a single member, as
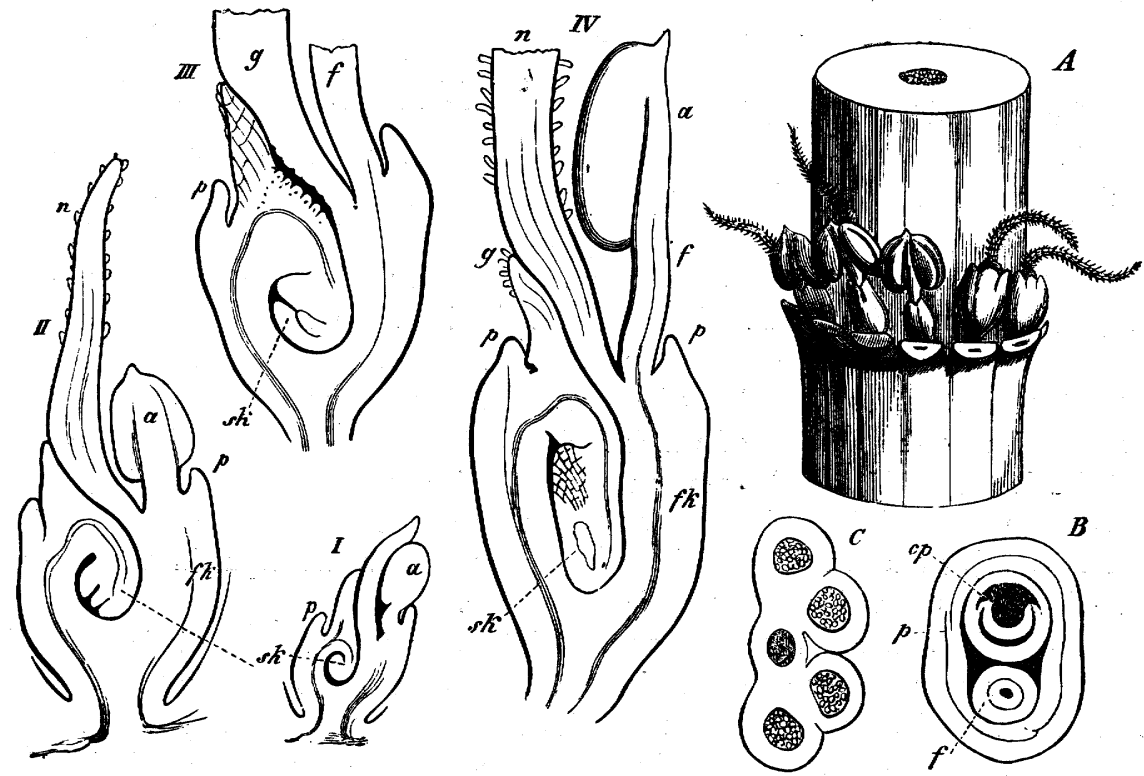

FIG. 360.- Hippuris vulgaris; $A$ piece of an erect stem, the flowers standing in the axils of the whorl of leaves (which have been cut off); $B$ horizontal section of a female fower above the ovary, $p$ perianth, $c p$ carpel; $C$ horizontal section of the anther ; $I-I V$ longitudinal section of flowers in various stages of development, $a$ anther, $f$ filament, $g$ style, $n$ stigma, $p$ perianth $f k$ the inferior ovary, $s k$ the pendulous and anatropous ovule.

in Hippuris (Fig. 360), where only one stamen and one carpel are contained within a scantily developed perianth. It is only rarely that the whole flower is reduced to a single sexual organ, as the female flowers of Piperacex, or the male and female flowers of some Aroidex; it is much more commonly the case that the flower is composed of successive whorls of members disposed from without inwards (or from below upwards), consisting of the same or multiples of the same number ${ }^{1}$, radiating from the centre on all sides like a rosette, an arrangement which is

1 [To this peculiarity of structure the term 'symmetrical' is generally applied in English textbooks; in the present work however this word is used in a very different sense, namely in reference to any structure (foliar or floral) which can be divided into two similar halves, or the parts of which are radially disposed around a central point; see p. 204.] 
frequently partially obscured at a subsequent period by bilateral development and abortion.

The Floral Envelope or Perianth is only rarely entirely wanting, as in the Piperacex and many Aroidex; more often it is simple, i.e. it consists of only one whorl of two, three, four, five, or rarely a larger number of leaves (as in Figs. $\left.357,35^{8}\right)$; in this case the perianth is frequently inconspicuous and composed of small green leaves, as in the Chenopodiaceæ and Urticaceæ, but is sometimes large, of delicate structure and brightly coloured (petaloid), as in Aristolochia, Mirabilis, \&c. But in both classes of Angiosperms (Monocotyledons and Dicotyledons) the perianth is usually composed of two alternating whorls consisting of the same number of leaves, two, three, four, five, or rarely more. In most Dicotyledons and many Monocotyledons the form and structure of these two whorls is very different ; the outer whorl or Calyx consisting of stouter, green, usually smaller leaves (Sepals), while the inner whorl or Corolla is more delicate, and is formed of white or bright-coloured, usually larger leaves (Petals). It is however more convenient, for the sake of brevity, as Payer has already suggested, to designate the inner whorl as corolla, the outer whorl as calyx, even in those cases where the structure of the two is the same ${ }^{1}$; and this is the more necessary since the contrast of structure referred to is frequently wanting, both whorls being either sepaloid, as in Juncaceæ, or both petaloid, as in Lilium; in Helleborus, Aconitum, and some other species, the outer whorl or calyx alone is petaloid, the inner whorl or corolla being transformed into nectaries. In some Dicotyledons the perianth does not consist of alternating whorls, but of a smaller or larger number of turns of spirally arranged leaves, the number of which is then usually large or indefinite; the outer or lower leaves of this spiral arrangement may in this case also be sepaloid, the inner ones alone petaloid (e.g. Opuntia), or they may all be petaloid (as in Epiphyllum and Trollius), or a gradual transition takes place from the sepaloid through the petaloid to the staminal structure (as in Nymphaa).

But besides the usual sepaloid and petaloid form and structure of the perianthleaves, there occur other considerable deviations from the ordinary foliar structure. Thus, for example, the (imperfect) perianth of Grasses consists of very small delicate colourless membranous scales (the Lodicules), that of some Cyperaceæ is replaced by hair-like bristles, the Seta ; in the place of the calyx of Compositæ a crown of hairs, the Pappus, surrounds the corolla ; and it has already been mentioned that the petals of Aconitum, Helleborus, \&c. are transformed into nectaries of a peculiar form.

Whether the perianth consist of one or two whorls, the leaves of the same whorl have very commonly the appearance of being coherent or of coalescing with one another, forming a cup, bell, tube, and so forth, the number of the coherent sepals or petals being determined by that of the marginal teeth. Coherent perianthwhorls are produced, after the formation of the distinct foliar structures at the circumference of the receptacle, by the common zone of insertion of these distinct structures being raised up by intercalary growth as an annular wall, and forming, as

1 The substantives calyx and corolla then designate the position of the whorl, the adjectives sepaloid and petaloid the nature of the part. 
it continues to develope, the part common to the whole whorl of floral leaves. The coherent tubular or campanulate part does not therefore consist of originally free portions which cohere subsequently by their edges, but from the very first it forms a whole which is intruded, so to speak, at the base of the perianth-leaves; the originally free leaves eventually forming the marginal teeth of the common basal portion. Applying the term Sepal to a calycine, Petal to a corolline leaf, a calyx consisting of coherent leaves is gamosepalous or synsepalous, a corolla consisting of coherent leaves gamopetalous or sympetalous; if the leaves of the perianthwhorl are not coherent, but free, this is expressed by the terms eleutherosepalous or aposepalous, and eleutheropetalous or apopetalous ${ }^{1}$. When there is only one perianth-whorl, and it is desired to state whether it consists of coherent or of free leaves, the terms gamophyllous or symphyllous and eleutherophyllous or apophyllous may be used. It sometimes happens moreover that two perianth-whorls coalesce into one, so that, for example, two alternating trimerous whorls have united into a six-toothed tube (as in Hyacinthus, Muscari, \&c.).

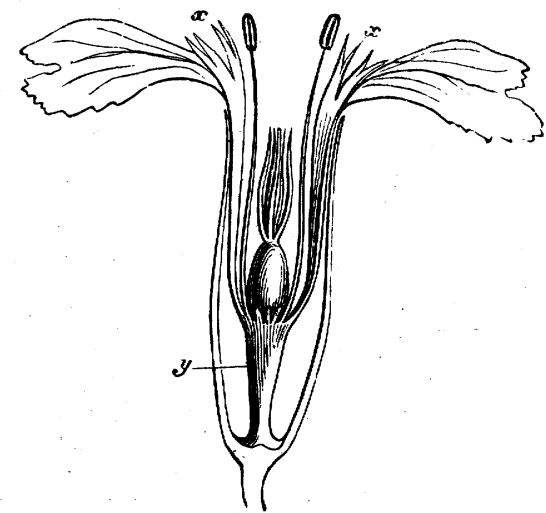

FIG. $36 \mathrm{r}$-Longitudinal section through the flower of Lychnis flos-Fovis; $y$ the elongated portion of the axis (anthophore) between calyx and corolla; $x$ ligule of the petals or corona.

If the leaves of the outer and inner whorls are free (not coherent), and if the distinction between calyx and corolla is clearly marked, then, in addition to the structural distinctions already named, other differences of form are also usually to be observed. The sepals have generally a broader base, are sessile, usually of very simple outline and pointed at the apex; the petals have mostly a narrower base, their upper portion is often very broad, and a distinction is not unfrequently apparent of claw (unguis) and blade (lamina), and the lamina is often divided or otherwise segmented. At the point where the lamina bends back from the unguis, ligular structures are often formed on the inner or upper side, which, when treating the flower as a whole, are comprised under the term Corona, as in Lychnis (Fig. 36ז), Saponaria, Nerium, Hydrophylleæ, \&c. When the corolla itself is

1 The terms 'polysepalous' and 'polypetalous' are objectionable, since these terms do not express the contrast correctly; still more so are 'monosepalous' and 'monopetalous,' as applied to the coherent whorls, because they have no reference to the true nature of the phenomenon. 
gamopetalous, the parts of the corona also coalesce, as in Narcissus, where it is very large.

The whole form of the perianth, especially when its structure is decidedly petaloid and its dimensions considerable, always stands in a definite relation to pollination by the aid of insects [or birds] : and large, brilliantly coloured, odoriferous flowers only occur where the fertilisation is brought about by this means. The purpose of these properties is to attract insects to visit the flowers; and the infinitely varied and often wonderful form of the perianth is especially adapted to compel certain positions of the body and certain movements on the part of insects of a definite size and species when searching for the nectar, by which the conveyance of pollen from flower to flower is unintentionally accomplished by them. We shall recur in detail to these physiological questions in the Third Book. The radial or bilateral symmetry of the perianth is usually associated with that of the other parts of the flower, and will therefore be discussed in connection with it.

Besides the perianth in the narrower sense which we have hitherto considered, there are often additional envelopes to the separate flowers. In the Malvacex and some other plants the true calyx appears to be surrounded by a second calyx (Epicalyx or Calyculus), the morphological significance of which, however, varies. In Malope trifida, for example, the three parts of the epicalyx represent a sub-floral bract with its two stipules; in Kitaibelia vitifolia, the six-parted epicalyx consists (according to Payer) of two such sub-floral leaves with their four stipules. Again, the epicalyx may be purely illusory from the production of stipular structures by the true sepals, as in Rosa and Potentilla. In Dianthus Caryophyllus and some other species a kind of epicalyx results from two decussate pairs of small bracts which are found immediately beneath the calyx; in the terminal flowers of Anemone a whorl of bracts stands at a short distance below the flower, which takes the form in the nearly allied Eranthis hyemalis of a kind of epicalyx ${ }^{1}$. The epicalyx of the small flowers of Dipsacacex is of special interest, each of them being surrounded, within the crowded inflorescence, by a membranous tube, which here forms the epicalyx. Sometimes, after the perianth and sexual organs have begun to be formed, an outgrowth of the flower-stalk, at first annular, is formed below the flower, growing up afterwards in the form of a cup or saucer, and bearing scaly or spiny protuberances. A structure of this kind is called a Cupule; and the cup in which the acorn of the various species of Oak is seated is of this nature ${ }^{2}$. In this case the cupule surrounds only one flower, in the Sweet-Chestnut and Beech on the other hand it encloses a small inflorescence. This spiny cupule afterwards splits from above, separating into lobes, to allow the escape of the fruit which has ripened within it. When an inflorescence is surrounded by a peculiarly developed whorl or rosette of leaves, as in Umbelliferæ and Compositæ, this is called an Involucre; when a single sheathing leaf envelopes an inflorescence springing from its axis, it is a Spathe. Both involucre and spathe may assume a petaloid structure, the former, for example, in Cornus florida, the latter in Aroidex.

1 [The garden Clematis known as 'Lucie Lemoine' possesses a well-marked seven-leaved involucre which has evidently originated from the growth of the axis above the outermost whorl of the multiplied petaloid sepals.]

${ }^{2}$ On the development of the acorn-cup see Hofmeister, Allgemeine Morphologie, p. 465. 
The Androcium is composed of the assemblage of the male sexual organs of a flower. Each separate organ is called a Stamen, and consists of the Anther and its stalk the Filament, which is usually filiform, but sometimes expanded like a leaf. The anther consists of two longitudinal halves (anther-lobes) placed on the upper part of the filament right and left of its median line; and the portion of the filament which bears the lobes of the anthers is distinguished as the Connective.

The lateral position of the stamens on the floral axis (the receptacle) is quite unmistakeable in all hermaphrodite and in most exclusively male flowers. Their lateral position, their exogenous origin from the primary meristem next the punctum vegetationis of the floral axis, their acropetal order of development, and the frequent monstrosities in which the stamens assume more or less the nature of petals, or even of foliage-leaves ${ }^{1}$, place it beyond doubt that they must be considered morphologically as foliar structures, and make it convenient to term them Staminal Leaves; the filament, together with the connective, being considered as the leaf, of which the two anther-lobes are appendages. From a morphological point of view it is therefore indifferent whether the filament greatly preponderates in size, or is inconsiderable as compared to that of the anther. Certain cases have, however, become known in which the anther appears itself to be a product of the floral axis, and the stalk, which corresponds to the filament, is the floral axis itself, but doubts suggest themselves as to the accuracy of these observations and as to the correctness of their interpretation. According to Magnus ${ }^{2}$, the vegetative cone of the male floral axis of Naias becomes transformed into a quadrilocular anther by the formation of pollen-mother-cells in four peripheral longitudinal strips of its tissue. Kaufmann had previously described a somewhat similar process in the case of the anther of Casuarina; and, according to Rohrbach ${ }^{3}$, the apex of the floral axis of Typha either itself developes into the anther, or it first of all branches and then forms an anther on each branch. Schenk asserts in a letter, that this latter statement is erroneous; according to his observations the stamens are developed like those of the Compositæ on the margin of the shallow depression at the apex of the parent axis. The question as to the nature of the organs bearing the anthers in the Euphorbieæ, whether they are modified branches (caulomes) or leaves, is discussed in a considerable literature which does not, however, lead to any decision ${ }^{4}$. Even if, as Warming states, the single anther of Cyclanthera is developed at the apex of the floral axis, this central organ is not necessarily a caulome any more than the axillary sporangia of many Lycopodiex. The true significance of such cases as these cannot be arrived at from a study of development alone, but comparisons must be instituted, as also in those cases in which complete abortion of certain parts of the flower occurs, with nearly related forms, that is, the 'phylogenetic method' must be followed. These remarks apply also to the above-mentioned peculiarities of the anther in Naias and Casuarina ${ }^{5}$.

1 [On 'phyllody' and 'petalody' of stamens see Masters, Vegetable Teratology, Ray Soc. 1869, pp. $253^{-25} 6$, and $285^{-296}$.]

2 Magnus, Bot. Zeitg. 1869, p. 77 r.

3 Rohrbach, in Sitzungsber. der Gesellsch. naturf. Freunde in Berlin, Nov. 16, 1869.

4 Warming, in Hanstein's Bot. Abhandl. Bd. II.

5 [See also Magnus, Beitr. z. Kennt. d. Gatt. Naias, Berlin, 1870.-Strasburger, Die Coniferen 
But, besides, the morphological homology of the separate parts of the ordinary stamens is not yet altogether determined, more precise investigations into the history of development being still wanting in this direction. Cassini and Röper consider the two anther-lobes as the swollen lateral halves of the lamina of the stamen; their loculi would therefore in that case be mere excavations in the tissue of the leaf; the pollen-mother-cells become differentiated inside the young tissue of the leaf, According to this view the furrow between the two pollen-sacs of an anther-lobe (see Fig. $357, H$ ) would correspond to the margin of the staminal leaf; but this cannot be the case ${ }^{1}$, at least not always, according to Mohl's observations. When the stamens become transformed into petals (by the so-called 'doubling' of the flower) as in the Rose, Poppy, Nigella damascena, \&c., it may be observed with certainty that the anterior and posterior loculi do not stand opposite one another, which would be the case if one belonged to the upper, the other to the under side of the staminal leaf; but that both are formed on the upper surface, the anterior loculus nearer the median line of the leaf, the posterior one nearer its margin. It is further observable that in such cases the two pollen-sacs of an anther-lobe do not always stand close to one another, but that they are frequently separated by a tolerably broad piece of the leaf, and that this intermediate piece contracts in the normal state into the partition-wall between the two pollen-sacs. The greater stress must be laid on these observations of Mohl, because in them the abnormal development only shows more plainly what can often enough be seen in a horizontal section of the anther and connective of normal stamens, viz. that the pollen-sacs of an anther-lobe evidently belong to one side of the stamen; it appears, however, that they must in some cases be referred to the under (Fig. 357, $C, H$ ), in others to the upper side (Fig. $3^{60}, C$ ). The origin of the pollen-mother-cells and the development of the wall of the separate pollen-sacs calls to mind so vividly in all essential features the corresponding phenomena in the sporangium of Lycopodiaceæ and even of Equisetaceæ, that it may be

und Gnetaceen, 1872.- Hieronymus, Zur Deutung sogen. axiler Antheren, Bot. Zeit. r872, ard Beitr. z. Kennt. d. Centrolepidaceen, Halle 1873.-Reuther, Beitr. z. Entwick. d. Blüthe, Bot. Zeitg. I876.-Engler, Beitr. z. Kennt. der Antherenbildung, Jahrb. f. wiss. Bot. X. I876.-Celakovsky, Teratologische Beiträge zur morphol. Deutung des Staubgefä:ses, Jahrb. f. wiss. Bot. XI. I878.

With reference to Typha, Magnus finds that the apparently axial stamen consists really of three coherent lateral stamens. In Naias, Casuarina and Cyclanthera, the stamen is undoubtedly axial. It would appear, therefore, that stamens are not always phyllomes. It is still possible, however, that they may be phyllomes in these cases. Celakovsky goes so far as to regard the stamen of Naias as a terminal leaf, a quite impossible morphological conception (Flora, 1874). The researches of Hieronymus tend to show that the statement made above on p. 491 may be near the truth, namely, that in these cases the pollen-sacs may be the surviving portions of otherwise abortive staminal leaves. In Brizula, one of the Centrolepidaceæ, he finds a single axial stamen; in Alepyrum and Centrolepis he finds that the stamen is developed from one longitudinal half of the growing-point, and that the other half, the persistent growing-point, is forced on one side by the growth of the stamen, so that the stamen lies in the same straight line as the long axis of the stem. He finds this to be the case alse in a Grass, Festuca pseudo-myurus; in the nearly-allied $F$. geniculata, which usually has three lateral stamens. it sometimes happens that only one stamen is present, and this is then developed in the manner described above. On the other hand, these facts may be used to prove that a stamen may be sometimes a caulome and sometimes a phyllome. It must be borne in mind that the ideas of caulome and phyllome are relative and not absolute.]

1 H. v. Mohl, Vermischte Schriften, p. $4^{2}$. 
assumed, until more exact observations bring something different to light, that each pollen-sac (i.e. each loculus with its wall) corresponds to a sporangium, and hence also to a single pollen-sac of Cycadeæ and Cupressineæ; and that therefore the anther usually consists of four pollen-sacs springing side by side from the anterior or posterior side of a staminal leaf, the sacs lying in pairs so close to one another right and left of the connective, that they coalesce more or less laterally to form one anther-lobe. But before we pass on to the consideration of the pollen-sacs and their contents, we must again recur to the discussion of the entire stamen and andrœcium.

The stalk of the anther (the filament with its connective) is either simple or segmented. The simple filament may be filiform (Fig. 359) or expanded into the form of a leaf (Fig. 358), sometimes even very broad, as in Asclepiadeæ and A pocynaceæ; or it may be broad below (Fig. $363, f$ ) or above; it generally terminates between the two anther-lobes, but is not unfrequently prolonged above them (Fig. $35^{8}, D$ ) as a projection, or in the form of a long appendage as in the Oleander. If the upper part of the stalk, the connective, is broad, the two anther-

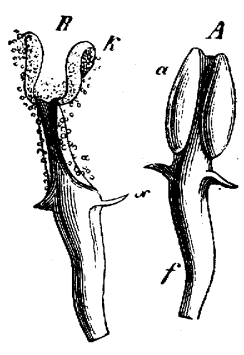

FIG. 362.-Stamen of Mahonia Aquifolium; $B$ with the anther open (by recurved valves).

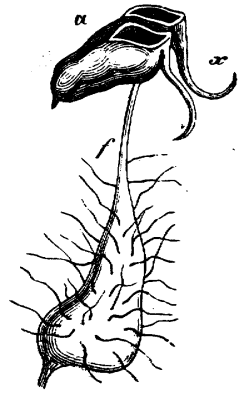

FIG. 363. -Stamen of Arbutus hybrida, anther open (by pores) ; $x$ appendage.

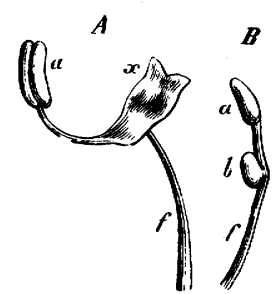

F1G. 364-Stamens of Centradenia rosea; $A$ a larger fertile one, $B$ a smaller sterile one of the same flower.

lobes are distinctly separated (Figs. $35^{8}, 3^{62}$ ); if it is narrow, they lie close to one another. The articulation of the stalk is very commonly the result of the connective being sharply separated from the filament by a deep constriction; the connection of the two is then maintained by so thin a piece that the anther, together with the connective which unites the anther-lobes, swings very lightly as a whole on the filament (versatile anther). The point of connection may be at the lower end, at the centre (Fig. $3^{6} 3$ ), or at the upper part of the connective; sometimes the detached connective attains a considerable size, and forms appendages beyond the anther (Fig. $3^{6} 4, A, x$ ), or it is developed between the two lobes like a cross-bar, so that the filament and connective form a $\mathrm{T}$, as in the Lime, and to a much greater extent in Salvia, where the transversely extended connective bears an anther-lobe on one arm only, while the other is sterile and is adapted for a different purpose. Whether the anther-lobes are parallel depends on the mode of their connection with the connective; if they are so, they are usually attached to the connective for their whole length; or in other cases they are separated above, or free below and coherent above, in which case they may become placed at such a distance from one 
another that the two lobes lie in one line above the apex of the filament, as in many Labiatæ. Not unfrequently the filament also has appendages; as, for example, the membranous expansions or appendages right and left below in Allium which resemble stipules, or a hood-shaped outgrowth behind as in Asclepiadeæ, or ligular structures in front as in Alyssum montanum, or conical prolongations beneath on one side as in Crambe, or on both as in Mahonia (Fig. $3^{62}, x$ ).

A phenomenon of great importance from a morphological point of view is the branching of stamens which occurs in many Dicotyledons, a peculiarity of structure which was erroneously confounded by the older botanists with their cohesion, although the two are fundamentally distinct. Sometimes the branching of stamens takes place, like that of foliage-leaves, bilaterally in one plane, right and left of the median line, so that the branched stamen has a pinnate appearance, as in Calothamnus (Fig. $365, s t$ ), where each division bears an anther. In other cases the branching takes place in a kind of polytomy, as in Ricinus (Fig. 366), where the

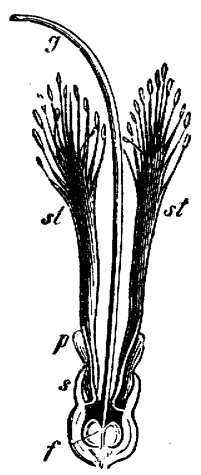

FIG. 365-Longitudinal section of the flower of Calothamnus; $f$ the ovary, $s$ calyx, $p$ petals, $g$ style, st branched stamens.

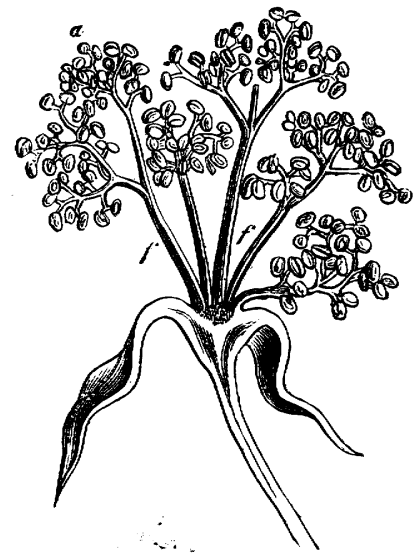

FIC. 366.-Part of a male flower of Ricinus communis cut through lengthways; $f f$ the basal portions of the compoundly-branched stamens; $a$ the anthers.

separate stamens arise in the form of simple protuberances from the receptacle, each one repeatedly producing new protuberances, which at length develope by intercalary growth into a compoundly and repeatedly branched filament; the ends of the branches all bearing anthers. In the Hypericineæ ${ }^{1}$, three or five large broad protuberances (Fig. $3^{67}, I I-V, a$ ) spring from the periphery of the floral axis after the formation of the corolla, on each of which smaller roundish knobs are produced in basipetal succession from its apex; these latter become the filaments, each of which eventually bears an anther, and are connected at their base with the primordial protuberance of which they are branches. A horizontal section through the flowerbud before the opening of the flower shows, especially in Hypericum calycinum, the numerous filaments which spring from one original protuberance densely crowded into one bundle. In this and many similar cases the common primordial basal

${ }^{1}$ [For further details see Molly, Unters. üb. die Bliuthenentwickelung der Hypericineen und Loasaceen, Diss. Inaug. Bonn, I875.] 
portion of the stamen remains very short, while the secondary filaments lengthen considerably and subsequently present the appearance of a tuft springing from the receptacle, the true nature of which can only be ascertained by the history of its development. If, on the contrary, the primordial basal portion lengthens, as in
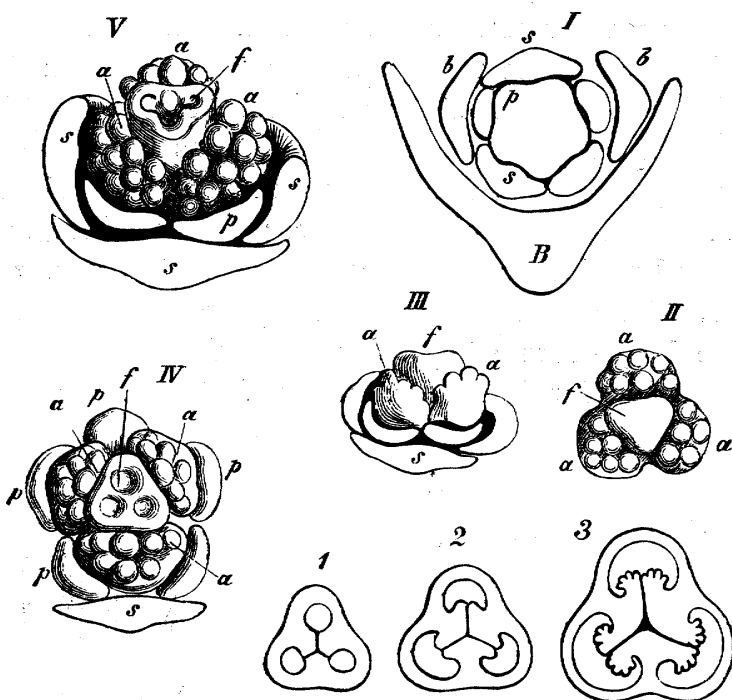

FIG. 367.-Development of the flower of Hypericum perforatum; $I$ young flower-bud in the axil of the bract $B$, with its two bracteoles $b b, s$ the sepals, $p$ first indication of the petals; $I I$ middle part of a somewhat older bud, $f$ rudiment of the ovary, $a, a, a$ the three stamens with the rudiments of their branches arising as protuberances; $I I I$ a flower-bud of nearly the same age as in $I I$, but seen from the side, $s$ a sepal, $a \alpha$ the stamens, $f$ the ovary; $I V$ and $V$ flower-buds in further stages of development, the letters indicating the same as in $I, I I$, and $I I I ; \mathrm{r}, 2,3$ ovary in various stages of development cut through horizontally.

Calothamnus, the whole stamen is easily recognised as branched even in the mature condition.

Of no less importance for understanding the entire plan of structure of a flower, and especially the relations of number and position which actually occur,
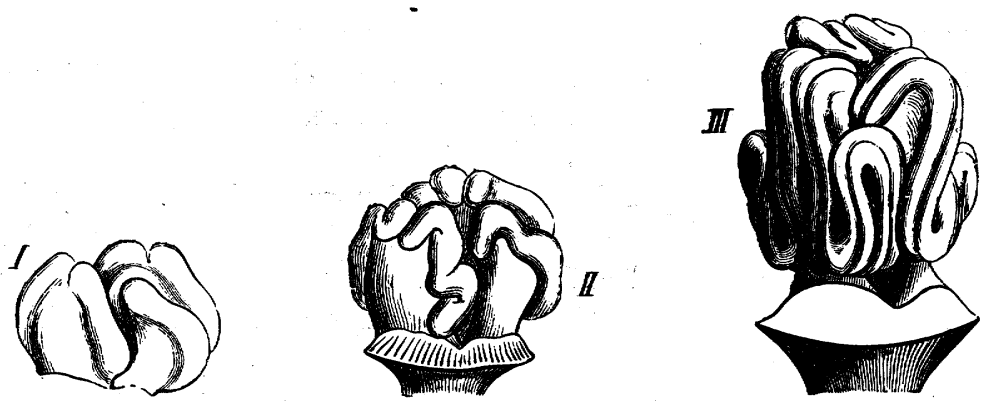

FIG. 368.-Development of the andrcecium of Cucurbita Pepo (after Payer); in all the figures the simple stamen is to the right, behind and to the left two double ones. The anthers grow vigorously in length and form vermiform coils,

is the cohesion of stamens which grow side by side in a whorl. In Cucurbita, for example, there are, in the earliest stage, five stamens, but at a later period only three are found, two of which are, however, broader than the third; these are each 
the result of the lateral coalescence of two stamens. In this case the filaments become combined into a central column, on which (as is shown in Fig. $368, I I I$ ) the pollen-sacs grow more rapidly in length than the filaments, forming vermiform coils.

The relationships are much more complicated and more difficult to understand when cohesion and branching of the stamens occur simultaneously, as in Malvacex. In Althea rosea, for instance, the filaments form a membranous closed tube which completely envelopes the gynæceum; springing from this tube are five vertical and parallel double rows of long filaments, each of which (Fig. ${ }_{369}, B$ ) again splits into two arms $(t)$, and each of these arms bears a single anther-lobe. The history of development and a comparison with allied forms shows that the tube is formed by the lateral coalescence of five stamens; but the coherent margins produce double rows of lateral ramifications, in other words, of filaments, which then again split into two arms. A horizontal section of the young staminal tube (Fig. $3^{69}, A$ ) shows plainly these double rows of split filaments ; the part $(v)$ which lies between two of these must be considered as the substance of a stamen, the margins of which
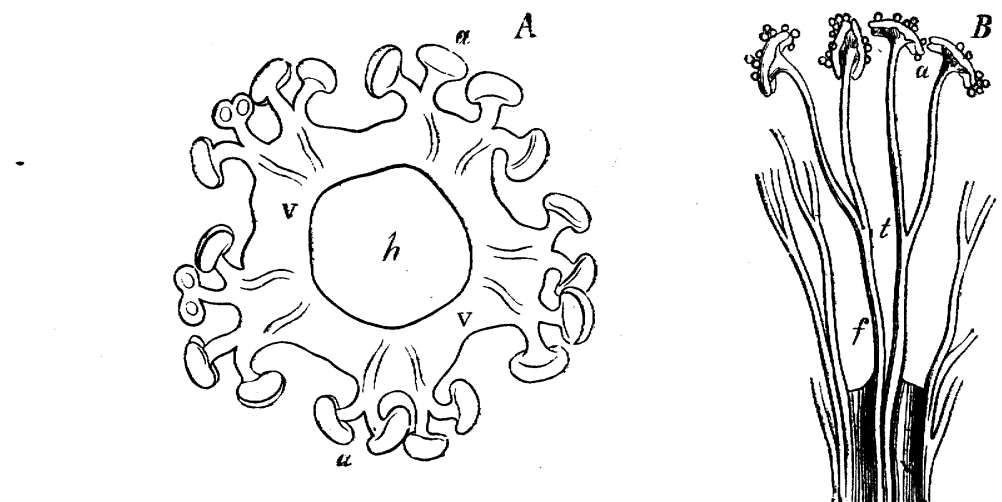

FIG. $3^{69}$ - Althea rosea; $A$ horizontal section through the young androcium ; $B$ a piece of the tube of a mature androcium with several stamens; $h$ cavity of the tube, $v$ substance of the tube, $a$ anthers, $t$ the spot where the filament divides, $f$ the spot where two filaments spring from the tube ( $A$ much more strongly magnified than $B$ ).

each bear right and left a simple row of filaments as laciniæ or branches ${ }^{1}$. In the Lime, where the five primordial stamens also branch at the margins, and form anthers on their brançhes, the stamens remain free, but in other respects the phenomena are altogether similar: (cf. Payer, l.c.)

The stamens not unfrequently suffer conspicuous displacements by the intercalary growth of the tissue of the receptacle in the region of their insertion; and such displacements are also ordinarily included under the term cohesion (or adhesion $)^{2}$. Thus the stamens often adhere to the calyx or corolla; and then, when

${ }^{1}$ The strangeness of this conception will disappear if the structure is recalled of a unilocular ovary with numerous carpels coherent at the margins, e.g. Viola, where the ovules arise in double rows on the lines of junction (the placentæ). What takes place in one case in the inside in reference to the ovules takes place in the other case on the outside in the formation of the filaments.

2 [It has come to be the usage in English works on descriptive botany to apply the term 'cohesion' to the apparent union of organs of the same kind, 'adhesion' to the apparent union of organs of a different kind.] 
mature, the filaments appear as if they sprang from the inside of the perianth; the earliest stages of development show, however, that the perianth-leaves and the stamens spring in succession and separately from the receptacle; it is not till a later period that intercalary growth begins at the part of the receptacle from which both spring; in this manner a lamella grows up which structurally forms the basal portion of the perianth-leaf, and which at the same time bears the stamen, so that the appearance is presented as if the stamen sprang from the centre of its inner surface. This is shown in Fig. 37o, $B$, where $p$ is a perianth-leaf and $a$ an anther sessile upon it; the two stand at first distinct on the young receptacle one over the other; the portion of leaf lying beneath $a$ and $p$ is not formed till a much later period by intercalary growth, and pushes up at the same time the true perianth-leaf $p$, and the stamen $a$. This kind of adhesion is especially frequent in those flowers whose petals have also become coherent laterally into a tube, such as Compositæ, Labiatæ, Valerianaceæ, \&c. On the other hand, the stamen may also become adherent in various ways to the gynæceum. In Sterculia Balanghas (Fig. 37I) this structure

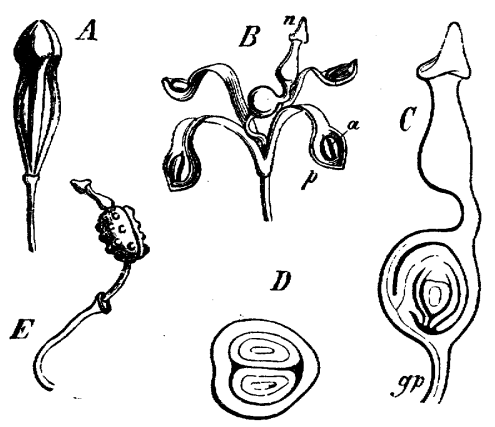

FIG. 370.-Flower of Manylesia glabrata; $A$ before opening; $B$ open ; $C$ the gynæceum, $g p$ the gynophore; $D$ horizontal section of the ovary; $E$ fruit ripening on its pedicel.

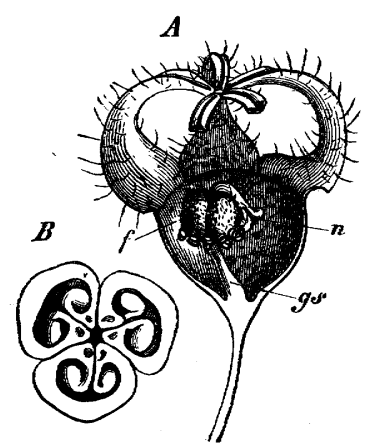

FIG. 37x--Flower of Sterculia Balanghas $A, g s$ the gynophore, $f$ ovary, $n$ stigma; $B$ horizontal section of the ovary.

is only apparent, depending simply on the small stamens, which are placed close beneath the ovary, becoming raised up together with it by the elongation of a part of the receptacle; from their small size they appear like a mere appendage of the large ovary; the part which bears both the organs, the Gynophore, is therefore in this case an internode of the floral axis. Much more complicated is the history of the formation of the true Gynostemium (column) which is formed above an inferior ovary, as in the Aristolochiaceæ, and especially in the Orchideæ, where these adhesions and displacements of the parts of the flower are also combined with abortion of certain members. Since these relationships will be explained in the sequel, the examination of Fig. 372 will suffice for the present, where the flower of Cypripedium is represented from the side $(A)$, from behind $(B)$, and from front $(C)$, after removal of the perianth $(p p)$. $f$ is the inferior ovary, gs the gynostemium, resulting from the adhesion of three stamens - two of which $(a a)$ are fertile, while the third $(s)$ forms a sterile staminode-with the carpel, the anterior part of which bears the stigma $(n)$. In this case the gynostemium consists entirely of coherent foliar structures, or of the basal portions of the staminal and carpellary leaves, both of 
which spring from the upper margin of the hollowed-out receptacle which constitutes the inferior ovary ${ }^{1}$.

The size and form of the stamens frequently varies within one and the same flower; thus, for instance, in the Cruciferæ there are two shorter and four longer (tetradynamous), in the Labiatæ two larger and two shorter (didynamous) stamens; in Centradenia, as was shown in Fig. ${ }^{6}{ }_{4}, A, B$, they are not only of different size, but are also differently segmented. A correct conception of the history of development and a comparison of the relationships of number and position in nearly allied plants enable one to apply the term stamen even to structures which have no anther and therefore want the ordinary physiological character of stamens. Thus, for example, in Geranium there are two whorls of fertile stamens, while in the nearly related genus Erodium those of one whorl are without anthers. Such sterile stamens or Staminodes generally undergo further metamorphosis, by which they become unlike the fertile ones and not unfrequently petaloid, as the innermost staminal leaves of Aquilegia; or assume very peculiar forms, as in Cypripedium (Fig. 372, s). In

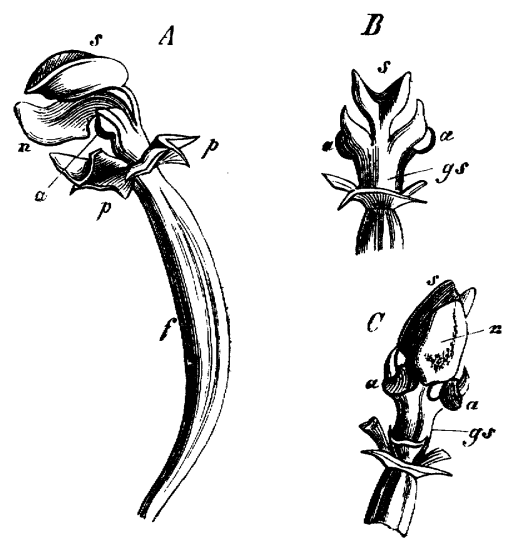

FIG. 372.-Flower of Cypripedium Calceolus after removal of the perianth.

some Gesneraceæ a glandular structure or nectary is found in place of the posterior stamen (compare the drawing of Columnea, Fig. 416). Metamorphoses of this kind may be considered as the first steps to a condition of abortion, the final stage of which is the production of a vacancy at the spot where the stamen should be, as in the Labiatæ, an order closely allied to the Gesneraceæ, where, in the place of this staminode there is no structure whatever; instead of the five stamens to which the plan of construction of the flower points, there are only four, even the rudiment of the fifth, the posterior one, being suppressed, as is seen in Fig. $373^{2}$. Phenomena of this kind altogether justify the hypothesis of abortion in those cases in which the absent organ does not disappear in the course of development, but never comes sequel.

1 Compare the account of the development and significance of the flowers of Orchideæ in the

${ }^{2}$ [Peyritsch however (Sitzungsb. der k. Akad. der Wissen. $z u$ Wien, $187^{2}$ ) infers, from the constant reversion to fours in the peloric flowers of Labiatæ, and from other considerations, that the original type of the flower is tetramerous.] 
into existence at all, if the hypothesis of the suppression of the part is confirmed by a comparison of the relationships of number and position in nearly-allied plants. The hypothesis of an abortion of this kind was, however, for the first time placed on a firm basis by the theory of descent.

The number of stamens in a flower is only rarely so few as one or two; like the perianth-leaves they are usually numerous, and they are then arranged in the form of rosettes, either spirally or in whorls. If the arrangement of the perianthleaves is spiral, that of the stamens is usually the same, and the number of the latter is then very commonly large and indefinite, as in Nymphea, Magnolia, Ranunculus, Helleborus, \&c.; but in this case they are sometimes also few in number and definite.

Much more often, however, the stamens are arranged in one or more whorls, those in one whorl being then usually equal in number and alternate with those in

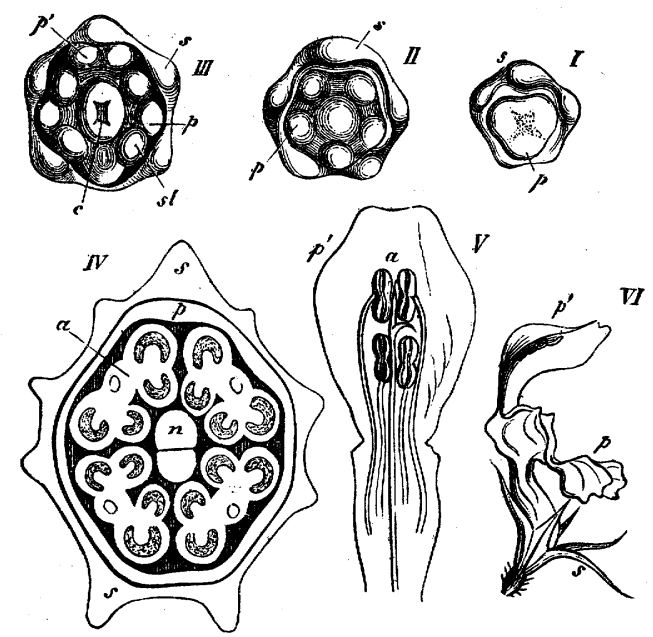

FIG. 373.-Various stages of developinent of the flower of Lamium album; I, II, III very young buds seen from above; in $I$ the rudiments of the sepals $s$ are formed, in $I I$ those of the petals $p$, in $I I I$ tinose of the stamens st and of the carpels $c ; I V$ horizontal section of an older bud, $s$ tube of the gamosepalous calyx, $p$ that of the gamopetalous corolla; $a$ anthers, $n$ stigmas; $V$ upper lip of the corolla with the epipetalous stamens;

$V I$ entire mature flower seen from the side.

the other whorls, and with the perianth-leaves [symmetrical flowers of English textbooks]. There are, however, numerous deviations from this rule [unsymmetrical flowers of English text-books] occasioned frequently by the abortion of particular members or of whole whorls, or by their multiplication, or by the superposition of consecutive whorls; and not unfrequently in the place of a single stamen two or even more will arise side by side (dédoublement). These phenomena, which are often difficult to make out, are nevertheless of great value in the determination of natural affinities, and will be still further examined in the sequel.

Development of the Pollen and of the Anther-wall ${ }^{1}$. The description given in this place will apply only to the ordinary cases in which the pollen is formed in

1 Nägeli, Zur Entwickelungsgeschichte des Pollens, Zurich, 1842. - Hofmeister, Neue Beiträge zur Kenntniss der Embryobildung der Phanerogamen, II. Monocotyledonen.-Warming, Unters. uib. pollenbildende Phyllome und Caulome, in Hanstein's Bot. Abhdl. Bd. II. I873.-[Goebel, Beit. z. Entwickgesch. d. Sporangien, Bot. Zeit. I881.] 
separate grains in the four loculi of the anther, and falls out of the anther after it has opened; some of the more important exceptions will be mentioned hereafter.

Immediately after the perianth-leaves, or their innermost whorl, first become visible on the receptacle as roundish protuberances, the rudiments of the stamens make their appearance in a similar manner, but usually obtain a considerable start in growth of the corolla, which not unfrequently remains for a considerable time in a very rudimentary condition. These bodies, which consist of homogeneous primary meristem, very soon show the outlines of the two anther-lobes united by the connective; the filament is still very short, subsequently it also grows slowly, and it is only just before the expansion of the flower that it elongates very rapidly by vigorous intercalary growth. When the four pollen-sacs make their appearance externally on the young anthers as longitudinal protuberances, a layer of cells becomes differentiated within them from the hitherto homogeneous tissue. The young anther consists at first of a small-celled proto-meristem in which a fibrovascular bundle becomes differentiated lying in the axis of the connective; the external peripheral layer forms the dermatogen or the young epidermis. According to Warming's comprehensive researches, it is usually only that layer which immediately underlies the epidermis (the most external layer of the periblem) which gives rise to the mother-cells of the pollen and to layers of the wall of the pollen-sac surrounding them. [These cells constitute the archesporium of Goebel. It will be seen that in the pollen-sac, as in the sporangia of the Vascular Cryptogams, the archesporium is derived from hypodermal cells.] Within each of the beforementioned four longitudinal protuberances, the layer of cells (archesporium) immediately underlying the epidermis splits into two, the inner of which gives rise to the mother-cells of the pollen. These cells soon become conspicuous on account of their large size, and, on a transverse section of the anther, they are seen to form a multicellular layer, concave internally, in each of the four protuberances. It does occur that the transverse section of one of these protuberances shows only a single mother-cell, so that here one row only of these cells is present (Compositæ, Malvaceæ). Less frequently the mother-cells are isolated (as in Mimosex). The mother-cells of the pollen divide but rarely until the formation of the tetrads begins; hence the number of mother-cells primarily formed is only slightly increased: but the simple row or layer of primary mother-cells may form several layers or a cylindrical mass of mother-cells as the result of divisions in all directions. That layer of cells which, as mentioned above, was formed by the longitudinal splitting of the layer (archesporium) from which the mother-cells are derived, lies between them and the epidermis. It divides, according to Warming, into usually three layers, in which radial, horizontal, and tangential divisions successively occur. The innermost of these three layers (Fig. 374, $A$ ep, Fig. 377, $B n$ ), which is completed by a corresponding layer on the inner side of the group of mother-cells, developes into a peculiar epithelium (the tapetum), investing the mother-cells of the pollen on all sides and lining the cavity of the pollen-sac; it corresponds to the tapetum in the sporangia of the Vascular Cryptogams, and, like it, it is finally absorbed in the process of the development of the pollen. The same fate overtakes the next outer layer of cells. The most external of the three above-mentioned layers, which lies immediately beneath the epidermis, 
forms the layer of fibrous cells which cause the dehiscence of the anther (Fig. $3^{82}$, $G \beta)$; to this we shall have to refer hereafter.

The mother-cells of the pollen are at first large and their. walls thin (Fig. 374, $A, s m)$; but these increase considerably in thickness, though generally not uniformly (Figs. $375,378, A$ ), the thickening matter being usually distinctly stratified. In many Monocotyledons the mother-cells now become completely separated, the pollen-sac becomes broader, and the cells float singly or in connected groups in a granular fluid which fills up its cavity, as is shown in Fig. 374, $B$, a phenomenon

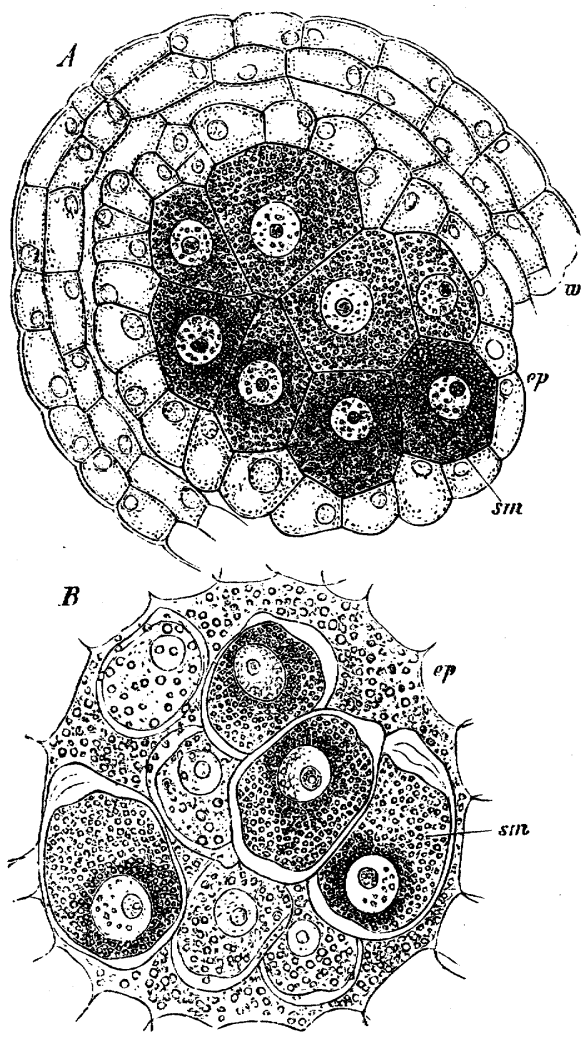

FIG. 374.-Funkia cordata; $A$ transverse section through a young pollen-sac before the isolation of the mother-cells $\mathrm{sm}$, $e p$ the tapetum which clothes the anther-lobe, $w$ wall of the pollen-sac; $B$ the anther-lobe after isolation of the mother-cells $s m$; ep the tapetum $(\times 500)$.

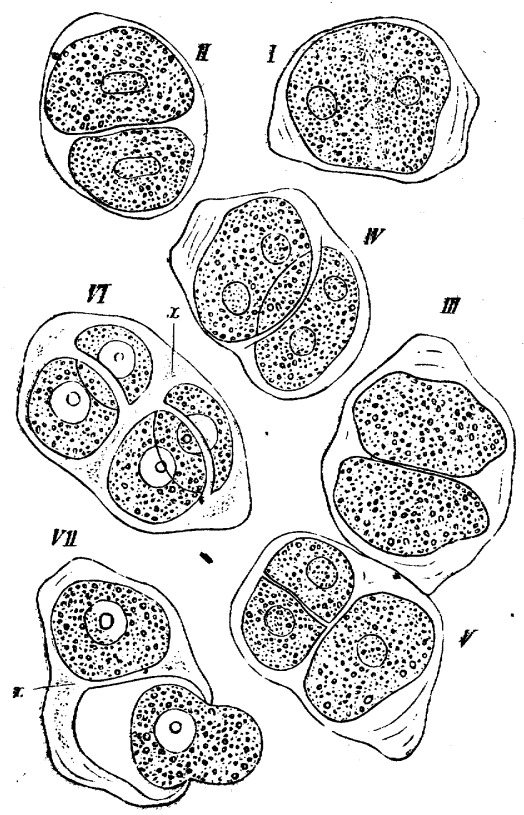

F1G. 375.-Mode of formation of the pollen of Funkia ovata $\left(\times 55^{\circ}\right)$. In $V I I$ the wall of the daughter-cell has absorbed water till it has burst; its protoplasm is forcing itself out through the fissure, and is lying before it rounded off into a spherical form.

which calls strongly to mind the formation of the spores of Vascular Cryptogams. In other cases, however, as for instance in many Dicotyledons (Tropaelum, Althea, $\& c$.$) , the very thick-walled mother-cells do not become isolated; they completely$ fill up the pollen-sac, but may become separated after the rupture of the antherwall in water. In most Monocotyledons the large central nucleus divides, and two fresh nuclei make their appearance; this is followed by the division of the protoplasm, and by the simultaneous formation of a cell-wall in the plane of division. The same process is repeated in each of the two cells, and thus the 
four 'special' mother-cells of the pollen are formed (Fig. 375). Thus the mode of development of the pollen-grains in Monocotyledons resembles that of the microspores of Isoëtes. It sometimes happens that only one of the two secondary cells divides, and then only three pollen-grains are formed, two of them being of normal size, the third considerably larger: or again, neither of the two cells may divide, or only imperfectly, and this leads to modifications of the size and shape
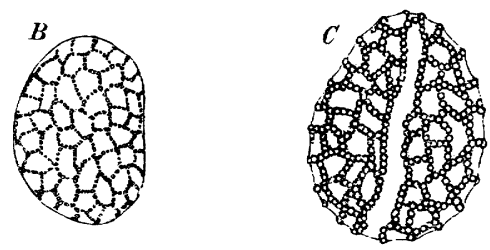

FIG. 376.-B a young pollen-cell of Funkia ovata; the thickenings which project outwardly are still small, in the older pollen-cell $C$ they are larger; they are arranged in lines connected into a net-work.

of the resulting pollen-grains. In other Monocotyledons (Asphodelus albus and luteus) the development of the pollen proceeds in the manner to be described below as being characteristic of Dicotyledons ${ }^{1}$. A second process is especially characteristic of Dicotyledons, in which, after the division of the nucleus of the mother-cell, the two secondary nuclei divide in planes at right angles to that of the first division and to each other; the four nuclei are thus arranged, as it were, in the corners of a tetrahedron. The protoplasm becomes then constricted into four
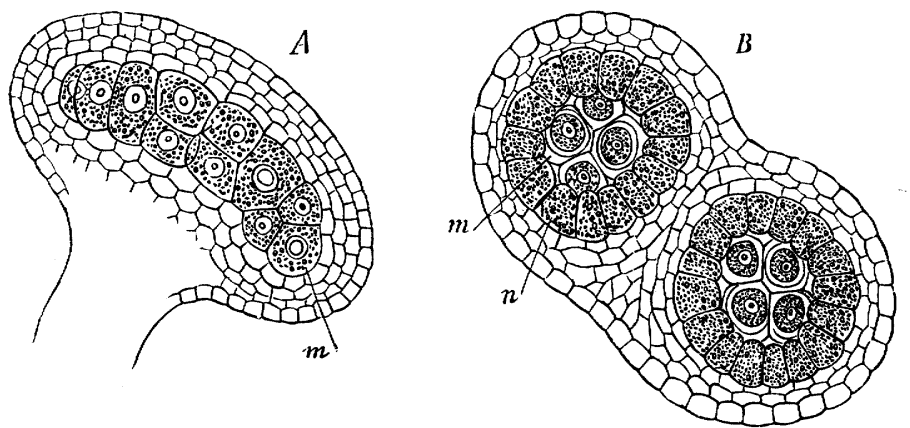

FIG. 377.- $A$ pollen-sac of Althaa rosea seen from the side, $m$ the archesporium; $B$ transverse section of an antherlobe showing the two pollen-sacs, $m$ the mother-cells of the pollen, in $A$ still united into a tissue, in $B$ already divided each into four pollen-cells, $n$ the tapetum of the pollen-sac. Each anther-lobe, consisting of two pollen-sacs, is here borne on a long branch of the filament.

lobes, each nucleus forming the centre of one of the lobes, by the ingrowth of the thick wall of the mother-cell ; a simultaneous formation of cell-walls now takes place in the planes of division, the walls being attached to the margin of the ingrowths of the wall of the mother-cell, and thus the four masses of protoplasm which have become rounded off during the division become four distinct cells (Fig. $378, A-E$ ). The mass of cellulose round each of the daughter-cells of the

1 [This account of the development of the pollen is taken from Strasburger (Zellbildung und Zelltheilung, 3rd edition, 1880.] 
tetrad now becomes differentiated into concentric systems of layers, and these are enveloped by layers which are common to the whole tetrad (Figs. $378 E, 379$ ). If the tetrads have lain for some time in water, the layers usually burst, and the protoplasmic contents of the young pollen-cells are forced out through the fissure, and become rounded off into a sphere (Figs. $375 V I I ; 378 F, G$ ). Soon after the conversion of the mother-cells of the pollen into a tetrad, each protoplasmic mass becomes clothed with a new cell-wall, at first very thin, which is continuous with the inner layers of the wall of the mother-cell, as is shown by its becoming detached from them when caused to contract by alcohol. This is the true cell-wall of the pollen, which now increases greatly in thickness, and becomes differentiated

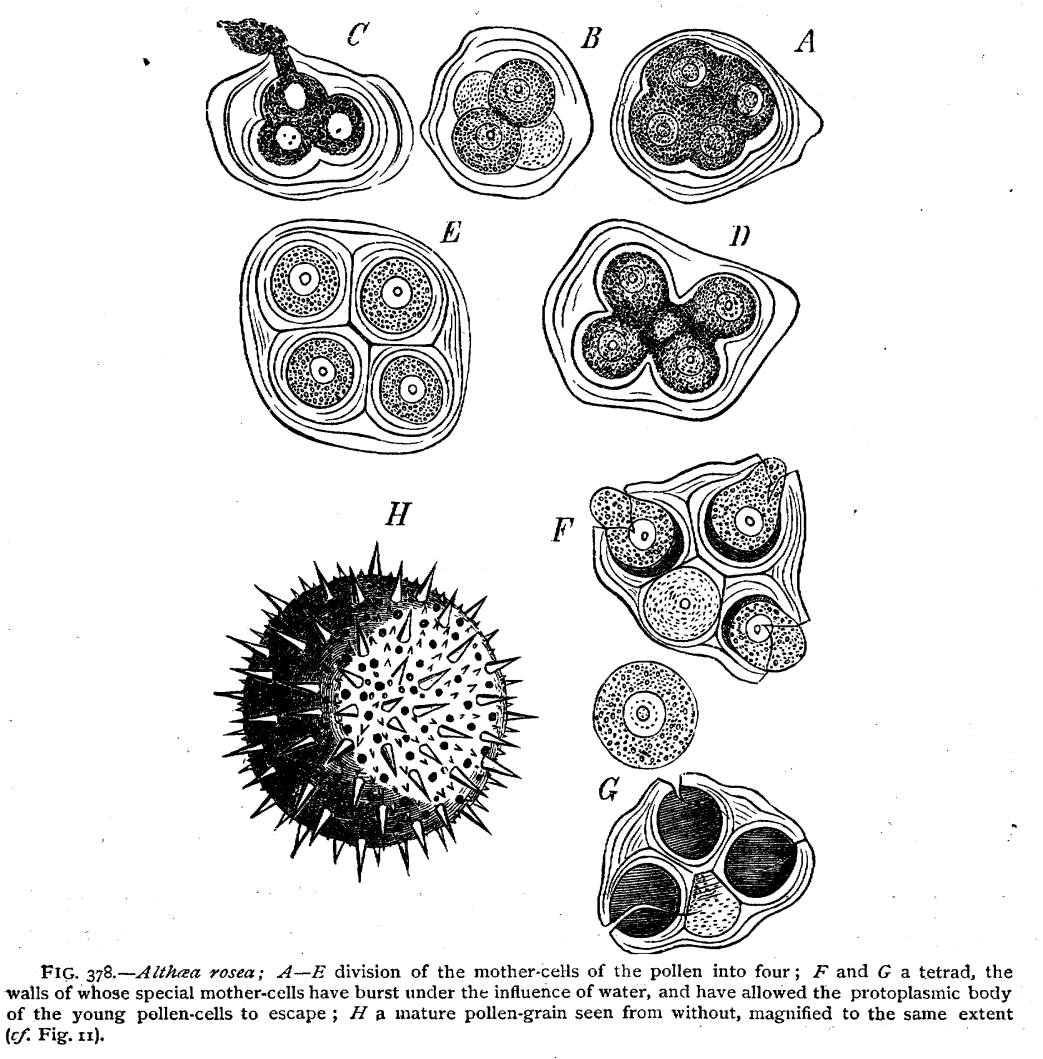

into an outer cuticularised layer and an inner one of pure cellulose, the Extine and the Intine. The former becomes covered on the outside with spines (Fig. 379, ph), warts (Fig. 376), ridges, combs, \&c.; while the latter frequently forms considerable thickenings which project inwards at particular spots (Fig. 379, v), and at a later period are employed to form the pollen-tube. During these processes the layers forming the envelope of the tetrad become slowly absorbed, their substance is converted into mucilage, and they at length entirely lose their form; their disorganisation may commence either on the inner (as in Fig. 375, VII, x) or outer side (Fig. $379, s g$ ) of the wall of the mother-cell. In consequence of this absorption 
the young pollen-cells become free, separate, and float in the granular fluid which fills up the cavity of the anther; and within this they now attain their definite development and size. The fluid being thus used up, the mature pollen-grains finally fill up the cavity of the anther in the form of a powdery mass.

[The ripe pollen-grain of Angiosperms has been found in many cases to contain two nuclei ${ }^{1}$. It appears that when the pollen-grains have become isolated from each other, the nucleus of the grain undergoes division into two, one larger, the other smaller. The smaller nucleus travels to the wall of the grain and becomes invested by protoplasm, thus constituting a primordial cell, which, in some cases, is cut off from the rest of the grain by a wall of cellulose : the larger nucleus remains as the nucleus of the larger cell of the pollen-grain. The smaller nucleus may divide once or twice, thus giving rise to a group of cells; the large nucleus does not divide: the form of the nuclei varies very much. These processes resemble those which have

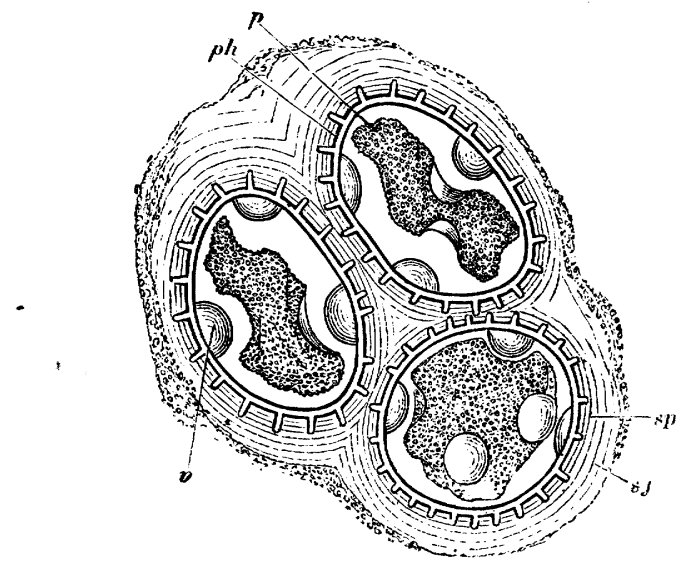

FIG. 379.-Mother-cell of the pollen of Cucurbita Pepo; $s g$ the outer common layers of the mother-cell in the act of being absorbed; $s p$ the so-called 'special mother-cells,' consisting of masses of layers of the mother-cell which surround the young pollen-cells; they also are afterwards absorbed; ph the wall of the pollen-cell; its spines grow outwards and penetrate the special mother-cell; $v$ hemispherical mass of cellulose on the inside of the pollen cell. wall, from which the pollen-tube is afterwards formed; $p$ the protoplasin contracted $(\times 550)$. (The preparation was obtained by making a section of an anther which had lain for some months in absolute alcohol.)

been described as taking place in the pollen-grains of the Gymnosperms: the small cell (or the cells derived from it) evidently corresponds to the 'vegetative' cells in the grains of Gymnosperms and in the microspores of the heterosporous Vascular Cryptogams.] The pollen-tube is formed from the large cell: it is developed as a protuberance of the intine, which perforates the extine at certain definite spots that have usually been prepared beforehand. The spots where this perforation takes place are often more than one, or even very numerous (Fig. $380 a, 3810$ ); yet, notwithstanding the possibility of the formation of this number of pollen-tubes from one grain, only one usually grows to an extent sufficient to effect impregnation. Independently of the structure of the extine itself which has already been mentioned, the external

1 [Hartig (in Karsten's Botan. Untersuch. III. I866) was the first to observe two nuclei in a pollen-grain: he found them in the grains of Tradescantia, Campanula, Enothera, Lilium, Clematis, Allium, etc. His observations have been extended by Strasburger (Ueber Befruchtung und Zelltheilung, 1878) and by Elfving (Jenaische Zeitschrift, I877, and Quart. Journ. Micr. Sci., XX. 1880.)] 
form and sculpture of the outer coat of pollen-grains depends chiefly on the number of the spots at which the perforation may take place, on the mode in which these are arranged, and on the circumstance whether the extine is at these spots merely thinner and the intine projects in the form of a wart (Fig. $3^{80}$ ), or whether roundish pieces of the extine become detached in the form of a lid, as in Cucurbitaceæ and Passiftora (Fig. 35, p. 33), or whether it splits into bands by spiral fissures, as in Thunbergia (Fig. $3^{6}$, p. 34), \&c. ${ }^{1}$ At the points of perforation the intine is generally thicker, often forming hemispherical protuberances which furnish the first material for the formation of the pollen-tube (Fig. $3^{81}, i$ ), or the extine only forms thinner longitudinal striæ which fold inwards when the pollen-grain becomes dry (as in Gladiolus, Yucca,
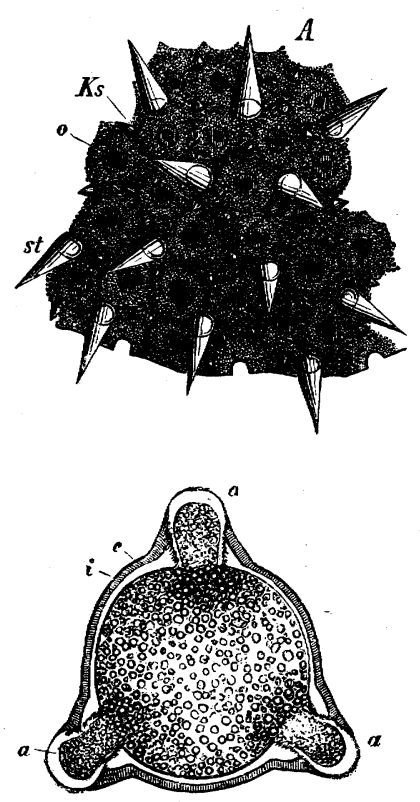

FIG. $3^{80}$.-Transverse section of a pollen-grain of Epilo. bium angustifolium: $a$ the points where the intine $i$ protrudes, the intine being there thicker and the extine $e$ thinner $(\times 500)$.

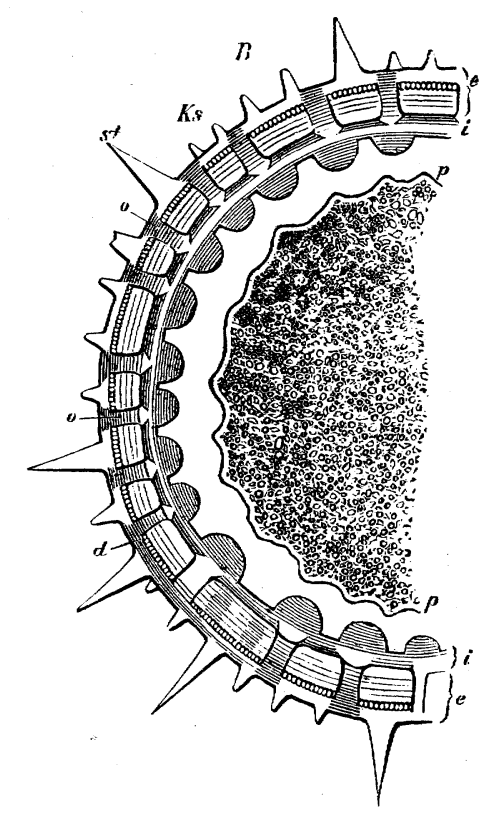

FIG. $3^{81}$ - - Pollen-grain of Althac rosea: $A$ a piece of the extine seen from without; $B$ the half of a very thin section through the middle of the pollen-grain, st large spines, $K s$ small spine of the extine, $O$ perforations through the extine $e$, $i$ the intine, $p$ the protoplasm of the pollen-grain contracted $(\times 800)$.

Helleborus, \&c.). Very commonly however the intine is uniformly and continuously thickened, as in Canna, Strelitzia, Musa, Persea, \&c. ; and in this case, according to Schacht, no definite spots are prepared beforehand where the perforation is to take place. The number of these peculiarly organised points of perforation is definite in each species, often in whole genera and families; there is only one in most Monocotyledons and a few Dicotyledons, two in Ficus, Justicia, \&c., three in the Onagrarieæ, Proteaceæ, Cupuliferæ, Geraniaceæ, Compositæ, and Boragineæ; four to six in Impatiens, Astrapaa, Alnus, and Carpinus, while the number is large in Convolvulaceæ,

${ }^{1}$ For more minute details see Schacht, Jahrb. für wissensch. Bot. II. p. ro9, and Luerssen, ibid. VII. p. 34.- [Fritzsche, Beiträge zur Kenntniss des Pollen, Berlin, I832.-Mohl, Beiträge zur Anatomie u. Physiologie der Gewächse, Ist Heft, Bern, 1834. - Edgeworth, Pollen, London, 1877.] 
Malvaceæ, Alsineæ, \&c. (see Schacht, l.c.). The extine is rarely smooth, more often marked on the outside by the sculpture to which reference has already been made. When it is very thick, layers of different structure and texture may frequently be detected, and differentiations sometimes occur in a radial direction, penetrating the thickness of the extine (Fig. $3^{8} \mathrm{I}$ ), and giving it in some cases the appearance of consisting of rod-shaped prismatic pieces or of honeycomb-like lamellæ, \&c., peculiarities of structure recalling those of the epispore of Marsiliacex. The contents of the ripe pollen-grain, the Fovilla ${ }^{1}$ of the older botanists, usually consists of a dense coarsegrained protoplasm in which grains of starch and drops of oil may be recognised. When the grain bursts in water, the fovilla escapes in masses connected by mucilage and often in long vermiform threads. The surface of the extine is commonly found coated with a yellow oil, or of some other colour, often in evident drops, which renders the pollen viscid and adapted to be carried by insects from flower to flower ; in only a comparatively few cases is it quite dry and powdery, as in Urticaceæ and many Grasses, where it is projected with violence from the anthers or simply falls out.

At the time when the pollen-grains are nearly mature, and the flower-bud is preparing to open, the wall of the pollen-sacs undergoes a further development ${ }^{2}$. The outer layer of cells or epidermis always remains smooth-walled (see Fig. 382, p. 558); the inner layers or endothecium are also smooth if the anther does not dehisce. If on the other hand it opens by recurved valves (Fig. $3^{62} k$ ), the cells of the inner layers of these valves only are provided with thickening-bands (or are fibrous) ; while, when the pollen-sacs dehisce longitudinally, the whole of their endothecium contains fibrous cells. There is usually only one such layer, sometimes several; in Agave americana as many as from eight to twelve. The thickening-bands of the fibrous cells which project inwards are usually wanting on their outer wall; on the side walls they are generally vertical to the surface of the pollen-sac; on the inner wall they run transversely and are united in a reticulate or stellate manner. Since the epidermal cells contract more strongly when the ripe anther-walls dry up than those of the endothecium which are provided with thickening-bands, they exert a force which has a tendency to make the anther-wall concave externally and to give way at its weakest point. The modes in which the anthers open are very various, and are always intimately connected with the other contrivances which are met with in the flower for the purpose of pollination with or without the agency of insects. Sometimes only a short fissure (pore) is formed at the apex of each anther-lobe, as in Solanum, Ericacea (Fig. ${ }^{6}{ }_{3}$ ), \&c., through which the pollen of both the contiguous pollen-sacs escapes; but more-commonly the wall gives way in the furrow between the two sacs (the suture) along its whole length, the tissue which separates them becoming at the same time more or less destroyed, and thus both pollen-sacs dehisce at the same time by the longitudinal fissure (Fig. 382). It is this phenomenon that has given rise to the erroneous description of these anthers as being bilocular; but if nomenclature is to have a scientific basis, they must be termed quadrilocular, in contrast to the

1 [On the constitution of the 'amyloid corpuscles' in the fovilla of pollen see Saccardo, Nuovo Giornale Botanico Italiano, 1872 , p. 241.]

${ }^{2}$ Compare H, v. Mohl, Vermischte Schriften, p. 62.-Purkyne, De cellulis antherarum fibrosis, Vratis. 1830 . 
really bilocular anthers of Asclepiadeæ and the octilocular ones of many Mimoseæ. Sometimes again the anther-lobes open at the apex by a pore which results simply from the destruction of a small portion of tissue at this spot (Hofmeister). In other respects we still want a detailed and comparative investigation of these processes, which are very various and of great physiological importance; only the additional remark need be made here, that it is very important from a systematic point of view whether the anthers open inwards towards the gynæceum (introrse), or outwards (extrorse), the difference depending on the position of the suture and hence on that of the pollen-sacs on the inner or outer side of the filament.

In several families of Monocotyledons and Dicotyledons more or less considerable deviations ${ }^{1}$ occur from the course of development of the pollen and from its final structure which has been here described. Naias and Zostera deviate only to this extent, that no thickening of the wall of the mother-cells takes place, and that the pollen-cells themselves are very thin-walled, acquiring in Zostera a very strange appearance from assuming, instead of the ordinary rounded form, that of long thin tubes lying parallel to one another in the anther. The deviations are more considerable in the formation of compound pollen-grains. The origin of these is either that only the four daughter-cells (pollen-cells) of one mother-cell remain more or less closely united, like the pollen-tetrads (four-fold grains) of some Orchideæ, Fourcroya, Typha, Anona, Rhododendron, \&c.; or the whole product of one primary mother-cell remains unseparated and forms a mass of pollen consisting of eight, twelve, sixteen, thirty-two, or sixty-four connected pollen-cells, as in many Mimoseæ and Acacieæ ${ }^{2}$. In these cases the cuticle or extine is more strongly developed on the outer surface of the daughter-cells lying at the circumference of the mass, and covers the whole as a continuous skin; while only thin ridges of the cuticle project from this skin inwards between the separate cells. In the various sections of Orchideæ every gradation occurs from the ordinary separate pollen-grains of Cypripedium, through the four-fold grains of Neottia, to the Ophrydeæ, where all the pollen-grains which are formed from each primary mother-cell remain united, and thus a number of pollen-masses lie in one pollen-sac ; and finally to the Pollinia of the Cerorchidex, where all the pollen-grains of a pollen-sac remain united into a cellular mass. In this case, as in the Asclepiadeæ with only bilocular anthers, where the grains of each pollen-sac are firmly united by a waxy substance, it is obvious that the pollen cannot be dispersed, nor can the pollen-masses fall out spontaneously from the anthers; but the flower is provided with very peculiar contrivances by means of which insects in search of honey extract from the pollen-sac the pollinia or the masses of pollen which are glued together, and again get rid of them on to the stigmas of other flowers of the same species (see Book III on Sexual Reproduction).

The Female Sexual Organs or Gynceeum ${ }^{3}$ (Pistil) of the flowers of Angiosperms

1 In reference to what follows compare Hofmeister, Neue Beiträge, pt. II. (Abhand der könig. Sächs. Gesellsch. VII); also Reichenbach, De pollinis Orchidearum genesi, Leipzig $18 \mathbf{5}^{2}$; and Rosanoff, Ueber den Pollen der Mimosen (Jabrb. für wissensch. Bot. VI. p. 44I).

${ }^{2}$ In many Mimoseæ the anther is, açcording to Rosanoff, octilocular, two pairs of small loculi being formed in each anther-lobe; the pollen-cells of each poilen-sac remain united into a mass.

3 Compare with this Payer's view (Organogénie de la fleur, p. 725 ), which differs in some essential points. 
consist of one or more closed chambers in which the ovules are formed; the lower, hollow, swollen part of each separate seed-chamber which encloses the ovules is called the Ovary; the place or the mass of tissue from which the ovules spring directly into the ovary is a Placenta. Above the ovary the seed-vessel narrows into one or more thin stalk-like structures or Styles, which bear the Stigmas; these are glandular swellings or expansions of various forms which retain the pollen that is carried to them, and by means of the moisture which is excreted from them induce the emission of the pollen-tubes.
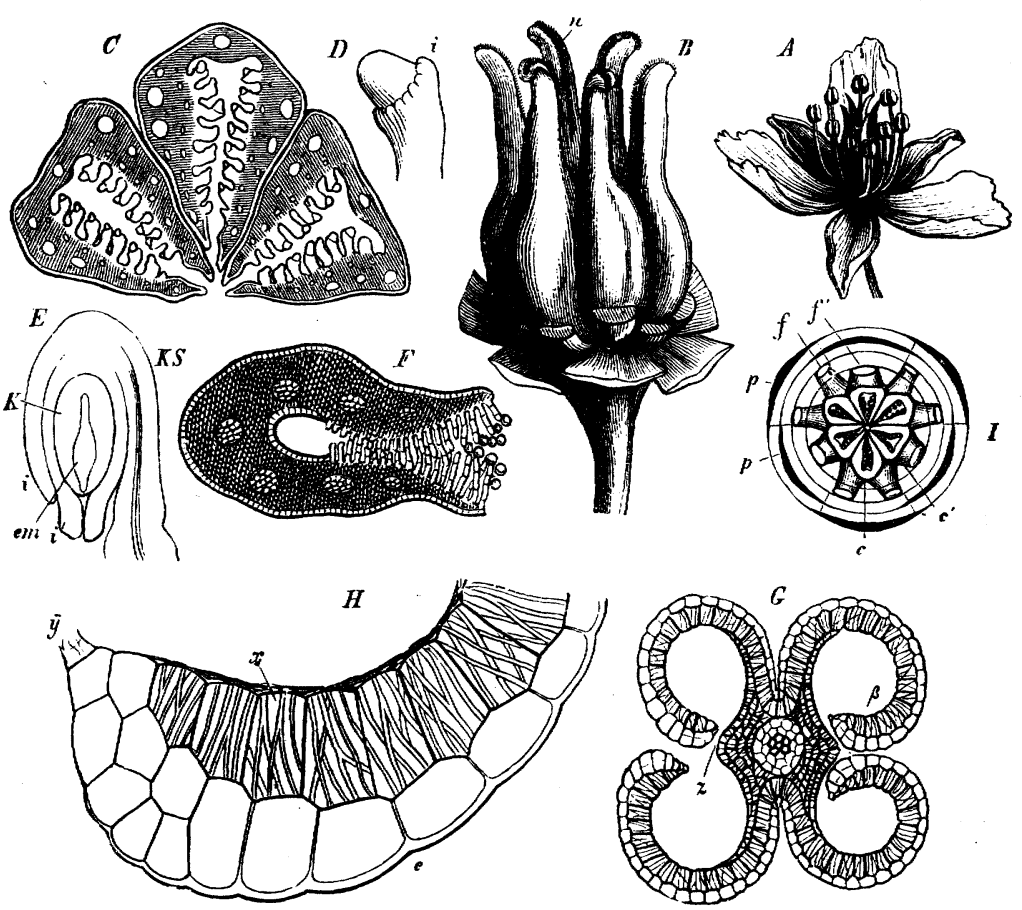

FIG. 382.-Butomus umbellatus: $A$ flower (natural size); $B$ the gynieceum (magnified), the perianth and stamens removed, $n$ the ovules ; $D$ a young ovule; $E$ an ovule immediately before fertilisation, $i i$ the integuments, $K$ the nucellus, $K S$ the raphe, em the ovules ; $D$ a young ovule; $E$ an ovule immediately before fertilisation, $i i$ the integuments, $K$ the nucellus, $K S$ the raphe, em the
embryo-sac; $F$ horizontal section through the stignatic portion of a carpel (strongly magnified), pollen-grains attached to the embryo-sac; $F$ horizontal section through the stignatic portion of a carpel (strongly magnified), pollen-grains attached to the
stignatic hairs; $G$ horizontal section of a quadrilocular anther, but the valves $z$ are so separated at $\beta$ that it then appears bilocular; $H$ part of an anther-lobe (corresponding to $\beta$ in $G$ ), $y$ the point where it has becone detached from the connective, $e$ the epidernis, $x$ the fibrous layer of cells (endothecium); $I$ diagram of the entire flower; the perianth $p p$ consists of two alternate whorl $f^{\prime}$ simple and thicker; the gynæceum also consists of two whorls of three carpels, an outer $c$ and an inner whorl $c^{\prime}$; there are therefore six alternate whorls of three, the members of the first staminal whorl being doubled.

The Gynæceum is always the final structure of the flower. When the floral axis has attained a sufficient length, the gynæceum is formed at its apex; if the axis is flat, disc-like, or expanded, it stands in the centre of the flower ; if it is hollowed out or cup-shaped, the gynæceum is placed at the bottom of the hollow, in the centre of which lies the apical point of the floral axis. In the diagram of the flower, Figs. $3^{82} I$, and $3^{84} B$, where each outer circle represents a lower transverse section, and each inner circle a higher one, the gynæceum necessarily appears always as the innermost central structure of the flower, the longitudinal displacements on the floral axis being neglected in the construction of the diagram. 
When the axial part of the flower, the Receplacle or Torus, is so elevated in the centre that the base of the gynæceum lies evidently above the stamens, or at least in the middle of the andrœcium, the perianth and the andrœcium, or even the whole flower, is said to be hypogynous (Fig. $3^{82}$ ). When, on the contrary, the receptacle is hollowed out like a cup or saucer, bearing the perianth and stamens on its annular margin, while the gynæceum springs from the bottom (Fig. $384, A$ ), the flower is said to be perigynous. It is obvious that intermediate forms are possible between extreme cases of hypogynous and perigynous flowers; and these are in fact common, especially among Rosifloræ. In both these forms of flower the gynæceum
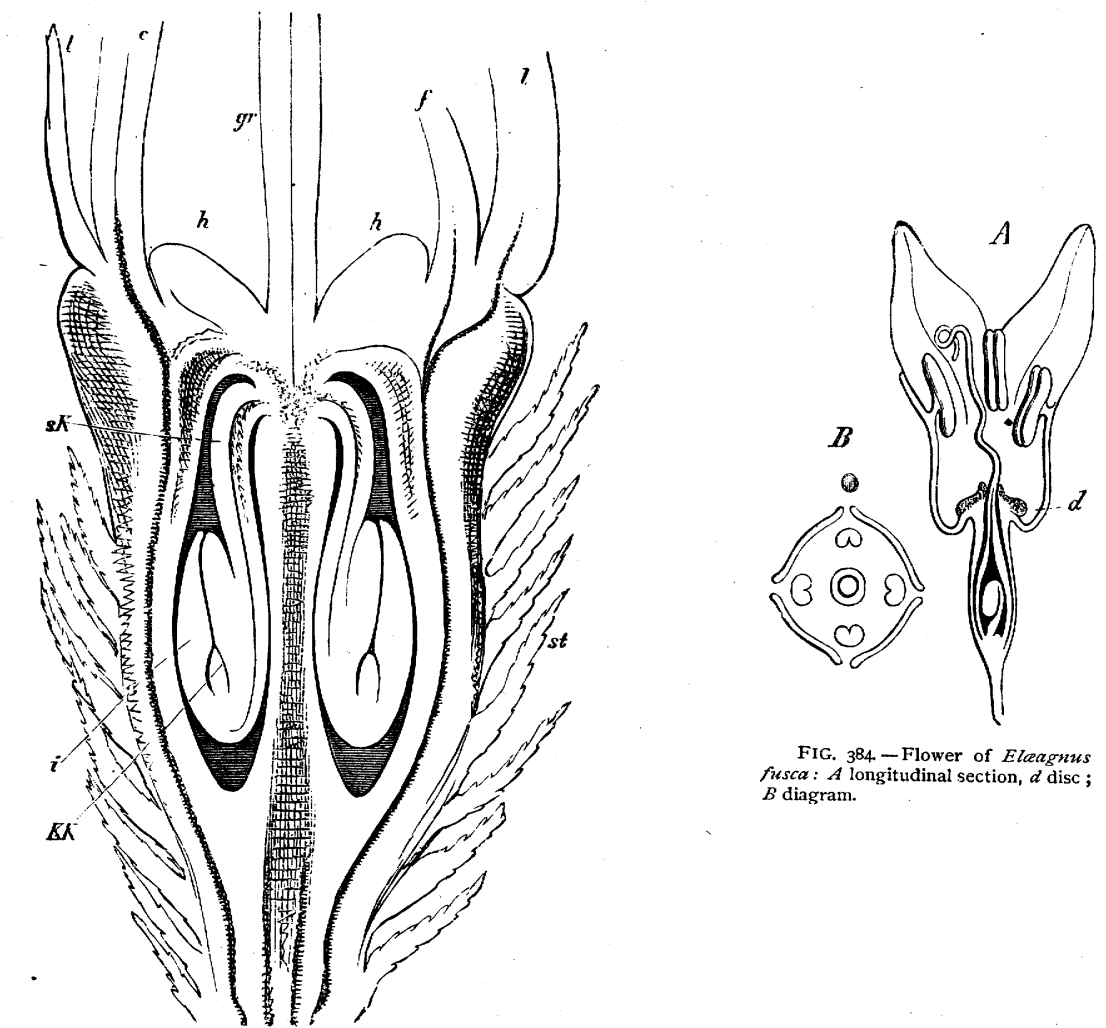

FIG. 384-Flower of Elaagnus fisca: $A$ longitudinal section, $d$ disc $B$ diagram.

FIG. 383 .-Longitudinal section through the inferior ovary of Eryngium campestre; $l$ sepals, $c$ petals, $f$ filament, $g r$ style, $h$ disc, $K K$ nucellus of the ovule, $i$ integument.

is free, the receptacle taking no part in the formation of the wall of the ovary, although this appears to be the case externally in some perigynous flowers, as Pyrus and Rosa. The flower finally is epigynous when it possesses an actually inferior ovary. This latter is distinguished from the ovary which is buried in the receptacle of perigynous flowers by its wall being formed of the receptacle itself hollowed out into the form of a cup or even of a long tube. The carpels, which in the case of the free superior ovary form its whole wall, spring in the inferior ovary (like the perianth and the andrœcium) from the margin of the hollow receptacle, and only close up the cavity above, where they are prolonged into the style and 
bear the stigmas (Fig. $3^{8} 3$ ). Intermediate forms are also not uncommon between the superior ovary of hypogynous and the inferior ovary of epigynous flowers; the ovary may, for example, be composed in its lower half of the receptacle, in its upper part of the coherent carpels; transitional forms of this kind are found especially among Saxifragaceæ. When the gynæceum of a flower consists of a single ovary only one fruit is formed, and the flower is said to be monocarpous (Figs. $3^{8} 3,3^{84}$ ), in contradistinction to the polycarpous flowers, the gynæceum of which consists of several isolated ovaries from which the same or a smaller number of fruits are developed (Fig. 382).

It will be easier to understand the different forms of the gynæceum if the more important ones are considered separately; and for this purpose the following ciassification may be made:-

I. Gynæceum Superior ; flower hypogynous or perigynous.

A. Ovules attached to the carpels.

a. Ovary monocarpellary;

(a) flower with one ovary,

$(\beta)$ flower with two or more ovaries.

b. Ovary polycarpellary;

$(\gamma)$ ovary unilocular,

$(\delta)$ ovary multilocular.

B. Ovules attached to the floral axis ;

$(\epsilon)$ ovule solitary, terminal,

$(\zeta)$ ovules one or more, lateral.

II. Gynæceum Inferior; flower epigynous.

C. Ovules attached to the carpels;

$(\eta)$ ovary unilocular,

$(\theta)$ ovary multilocular;

D. Ovules attached to the floral axis ;

(๖) ovule solitary, terminal,

(к) ovules one or more, lateral.

The Superior Gynceceum is constructed essentially of peculiar foliar organs, the carpellary leaves or carpels. These usually produce the ovules, which generally spring from the margins of the carpels, as in Fig. $3^{85}$, but frequently also from the whole inner surface, as in Fig. $357 F$, and Fig. $3^{82} C$. The ovary is monocarpellary (simple) when it consists of only a single carpel, the margins of which are coherent, so that the mid-rib runs along its back, and the ovules, when they are marginal, form a double row opposite to it. The inflexed margins of the carpellary leaf may swell up into thick placentæ (as in Fig. 386 ) and produce a larger number of rows of ovules. The number of ovules is, on the other hand, not unfrequently reduced to two (as in Amygdalus) ${ }^{1}$. In monocarpous flowers there is only one such carpellary leaf, as in Figs. $3^{84}, 3^{8} 5$; in polycarpous flowers there may be two, three,

1 With reference to the occurrence of a single ovule standing in the axil of the carpel (as in Ranunculus), see infra. 
or more, or even a very large number: if the number is two, three, or five, they usually stand in a whorl; if four, six, or ten, they are generally arranged in two alternating whorls (see Fig. $382, B, I$ ). When the number of monocarpellary ovaries in a flower is considerable, as in Ranunculaceæ, Magnolia, \&c., the part of the axis which bears them is commonly elongated (to a very considerable extent for example in Myosurus), and their arrangement is then spiral. The monocarpellary ovary is originally always unilocular, though it may subsequently become multilocular from the production of ridges by the luxuriant growth of the inside of the carpel, which divide the cavity longitudinally into compartments, as in Astragalus, or transversely, as in Cassia fistula. Ovaries of this kind may be distinguished as monocarpellary with spurious loculi, but ought not to be called polycarpellary.

A polycarpellary (compound) ovary is always the result of the union of all the carpels of a flower, the number being usually two, three, four, or five, arranged in one whorl, the floral axis terminating in the midst of them. If the separate

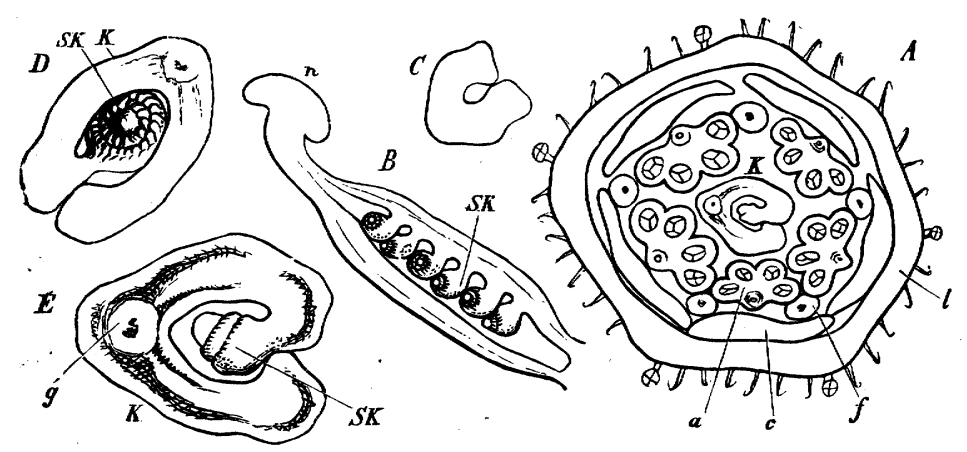

FIG. 385.-Phaseolus vulgaris; $A$ horizontal section through the flower-bud, $l$ calyx-tube, $c$ corolla, $f$ filaments of the outer, $a$ anthers of the inner staminal whorl, $K$ carpel; $B$ longitudinal section of the carpel, with the ovules $S K$ and stigma $n ; C, D, E$ horizontal sections of carpels of different ages, $S K$ the parietal (marginal) ovules, $g$ mid-rib of the carpel.

carpels remain open, and cohere in such a manner that the right margin of one unites with the left margin of another, the result is a unilocular polycarpellary ovary. The placentation is in this case parietal when the coherent margins project only slightly inwards, as in Reseda, Viola, \&c. But if the coherent margins of the carpels project further inwards, the cavity of the ovary becomes imperfectly multilocular, the chambers being connected with one another in the centre, as in Papaver, where the imperfect dissepiments are covered on both sides by a number of ovules. A bi- or multilocular polycarpellary ovary results when the margins of the carpels project inwardly so far that they meet or cohere either at or near the axis of the ovary, the elongation of the floral axis in the centre frequently contributing to this result. The mode of cohesion of the carpels in multilocular ovaries may vary greatly in other respects, according as it takes place along the whole length of their inflexed margins, or only below, while the upper parts resemble a whorl of monocarpellary ovaries (Figs. $3^{86-389}$ ). Since the margins of the carpels which meet in the centre become developed into the placentæ, the ovules make their appearance in the central angles of the loculi (axile placentation), as 
is seen in Fig. 388 ; but very commonly the margins of the carpels which turn in as far as the centre then split into two lamellæ which are bent back and swell out into placentæ in the middle of the loculi, as is shown in Fig. 387 . It is clear that in this case the two placentæ within each loculus correspond to the margins of the same carpel which forms the outer wall of the loculus.

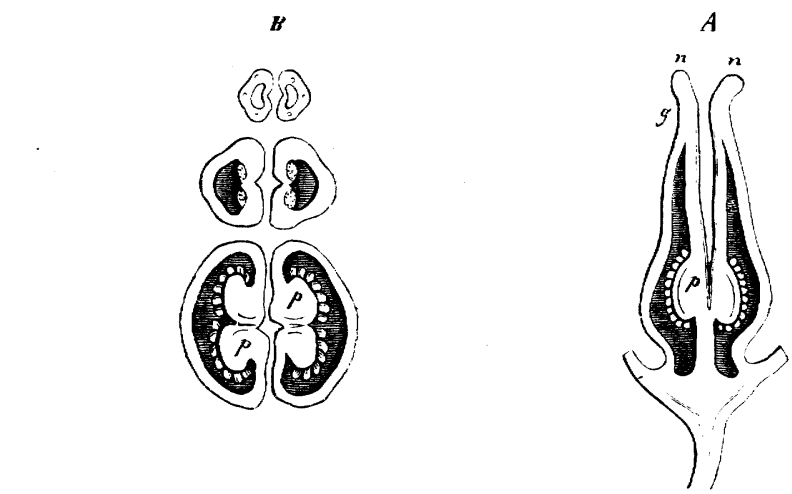

FIG. $3^{86 .-G y n a c e u m ~ o f ~ S a x i f r a g a ~ c o r d i f o l i a ; ~} A$ longitudinal section, $g$ style, $n$ stigma; $B$ horizontal section at different heights, $p$ placenta.

Spurious dissepiments may arise in polycarpellary as in monocarpellary ovaries; if the polycarpellary ovary consists of two loculi, it may thus become quadrilocular, or five original loculi may become divided into ten. The first case is universal in Labiatæ and Boragineæ. Fig. 390 shows that the ovary is formed of two coherent carpels, the margins of which $(I-I V)$ projecting inwards form a right and a left placenta $(p l)$; on each of these placentæ which correspond to the margins of the
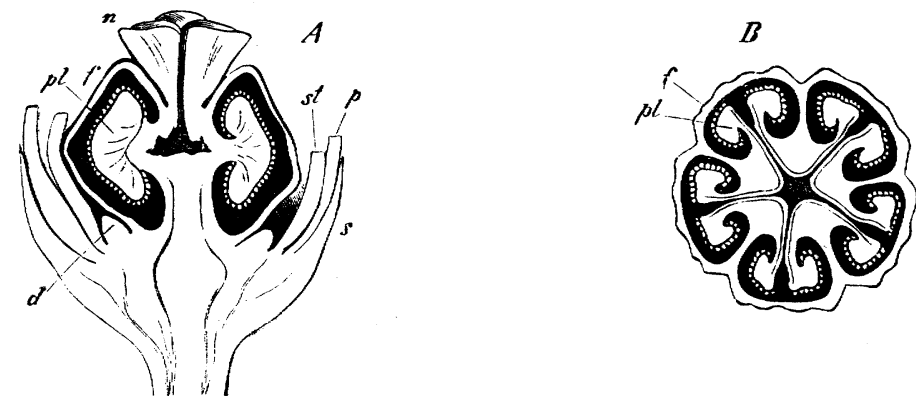

FIG. 387.-Gynæceum of Pyrula umbellata; $A$ longitudinal section, $s$ sepals, $\not$ petals, $s t$ filaments, $f$ ovary, $n$ stigma, $d$ nectar-glands; $B$ horizontal section through the ovary, $f$ the wall, $p l$ placentr.

carpels a posterior and an anterior ovule are produced, but an outgrowth from the mid-rib of the carpel $(I V, V I, x)$ inserts itself between the two ovules belonging to each loculus, dividing it into two one-seeded lobes. Since at a subsequent period the outer part of the wall of each of the four lobes bulges strongly outwards and upwards $(B)$, the separation of the bicarpellary ovary into four separate parts becomes still more distinct; and finally they completely separate as 
one-seeded lobes of the fruit; while in Boragineæ the separation is still more complete. The division of the five loculi of the ovary of Linum into ten by spurious dissepiments is not so perfect, the projections from the centres of the carpels not reaching the central axis of the ovary.

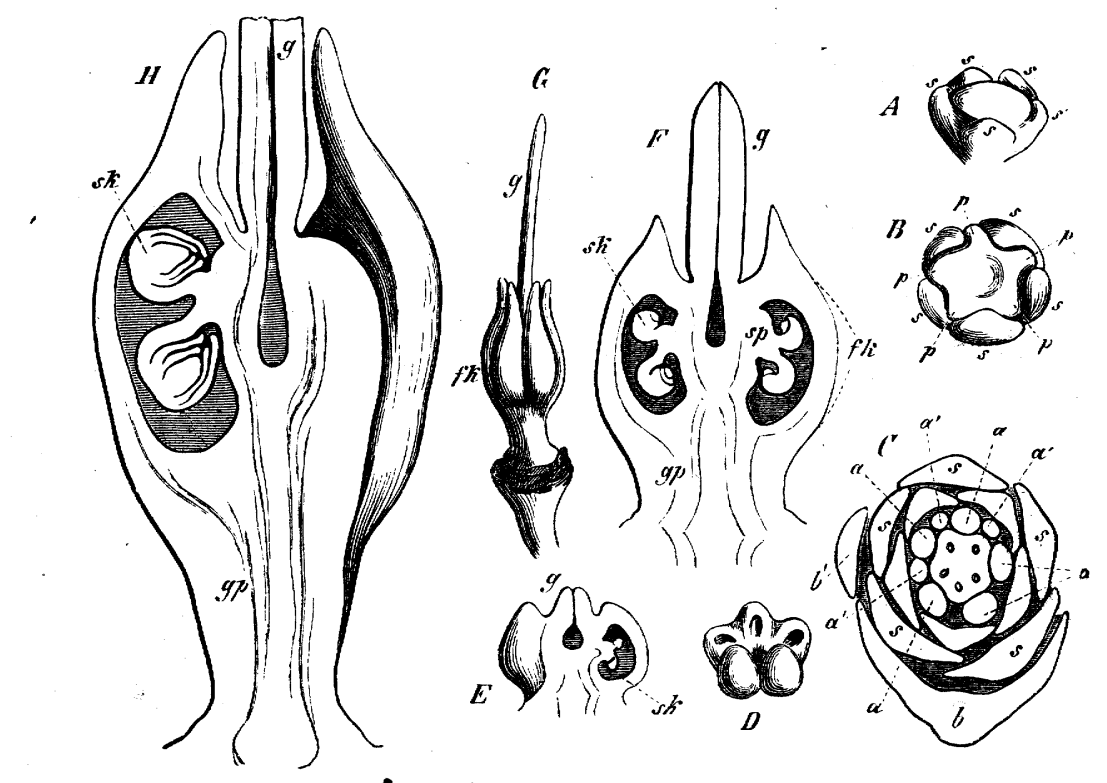

FIG. 388.-Dictamnus Fraxinella; $A$ young flower-bud, with rudiments of sepals $s ; B$ older flower-bud, with rudiments of petals $p$; $C$ still older state, with rudiments of the five stamens $a$, five more stamens $a^{\prime}$ arise between them, of which three are already visible; $b$ the bract, $b^{\prime}$ a bracteole; $D-H$ development of the ovary $f k$, sk ovules, $g \not p$ gynophore, $g$ style.

Before passing to the consideration of ovaries in which the ovules are borne by the floral axis (i.e. with axial placentation), it should be mentioned that there are cases in which the present state of our knowledge does not enable us to decide with

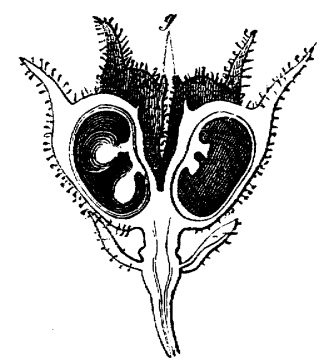

FIc. 389.-Ripe fruit of Lictamnits Fraxinella; the anterior carpel has been removed and the two lateral ones opened; $g$ gyrobasic style (natural size).

certainty whether the ovules arise from the axis or from the margins of the curpels which have become united to it; and these doubtful cases are possibly more numerous than is generally thought. Payer's observations on Cerastium and Malachium show that in Caryophylleæ the expanded apex of the floral axis becomes 
considerably elevated even before the formation of the carpels; the carpels are then seen in a whorl, and are attached by means of their coherent margins to the elevated axis; each forms what may be described as a pocket attached to the axis. As the axis becomes elongated, the margins of the carpels form radial dissepiments separating the pockets, which widen into loculi; and the carpels finally rise above the apex of the axis. In Cerastium and other genera the dissepiments also rise above it as free lamellæ which do not meet in the centre, so that the ovary is quinquelocular below, while in the upper part it remains unilocular. The ovules are produced in two parallel rows on the axial face of each loculus, this face being apparently formed from the axis itself. In some genera of Caryophylleæ it seems probable that the placentæ are axial, while in others they would appear rather to be carpellary.

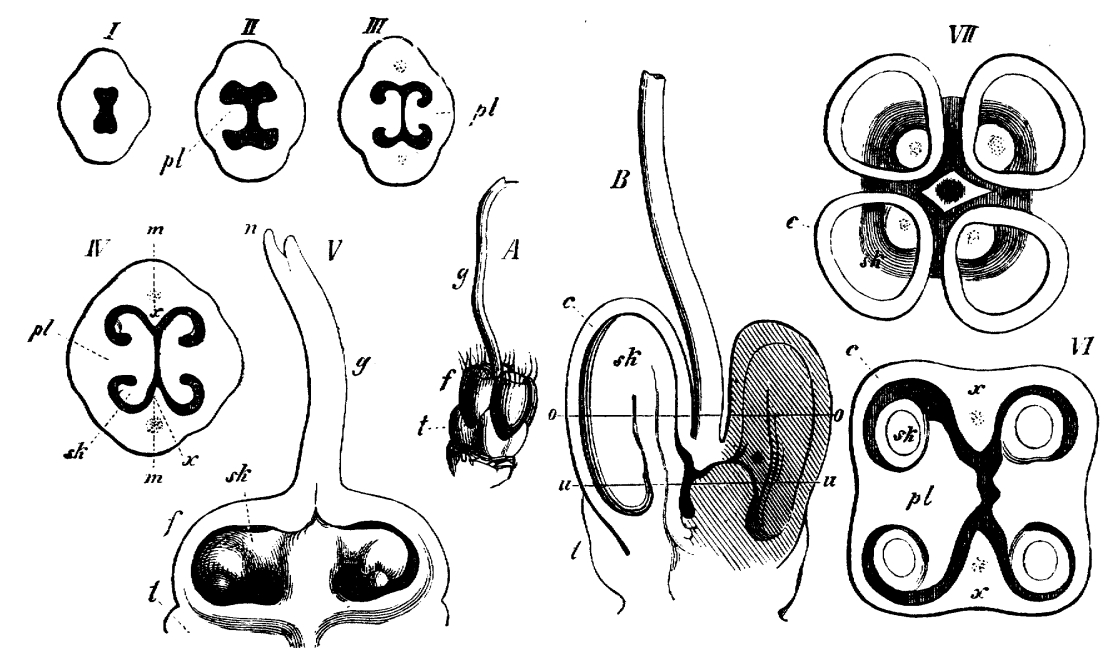

FIG. 390.-I-VII stages of development of the ovary of Phlomis pungens (a Labiate), $V$ in longitudinal, the rest in transverse section; $A$ a gynæceum seen from without ready for fertilisation; $B$ the same in longitudinal section, the lines $u x$, $0 o$ correspond to the transverse sections $V I$ and $V I I ; p l$ the placenta, $x$ the spurious dissepiment, $f$ loculi, $s k$ ovules, $c$ wall of the carpel, $t$ disc, $g$ style, $n$ stigma.

Among Superior Ovaries with axial Placentation, those of Typha, Naias, and Piperace ${ }^{1}$ require especial mention. In these cases the very simple female flower consists (with the exception of the perianth of $T y p h a$, which is represented by hairs) of nothing but a small lateral shoot transformed into an ovary with a central ovule ${ }^{2}$. The apex of the axis of this shoot itself developes into the terminal nucellus of the ovule, round which an annular zone grows up from below, overarches it, closes up above, and thus forms the wall of the ovary. In Typha only one style and stigma

1 Magnus, Zur Morphologie der Gattung Naias (Bot. Zeit. I 869, p. 772).--Rohrbach, Ueber Typha (in Sitzungsber. der Gesells. naturf. Freunde Berlin, Nov. 16, 1869). - Hanstein u. Schmitz, Ueber Entwickelung der Piperaceenblüthen (Bot. Zeit. 1870, p. $3^{8}$ ).

2 As in the case of the 'axial anthers,' so here also some uncertainty still exists. In a letter to me Schenk distinctly denies the axial nature of the ovule in Typha; he states that it is lateral, that it appears as a small protuberance on the wall of the ovary, a position which it retains until maturity. 
surmount the ovary, which may therefore be considered to be composed of a single carpel which rises up from the floral axis as an annular zone. In Piperaceæ however the stigma, which is sessile on the apex of the ovary, is often placed obliquely. or divided into several lobes; and this, like the two or four styles which surmount the ovary of Naias ${ }^{1}$, indicates that the ovary is not composed of one but of several carpels, which first make their appearance, like the leaf-sheaths of Equisetum, as an unbroken ring, which only at a later period becomes resolved at its upper margin into teeth. This hypothesis appears the more admissible since, in other Angiosperms where a comparison- with nearly allied forms justifies

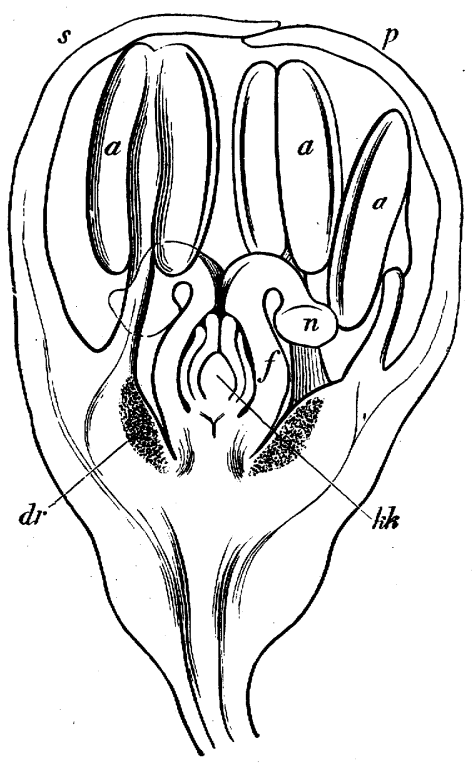

FIG. 39r.-Longitudinal section of the flower of Rheum undulatum; $s$ leaf of the onter, $p$ of the inner perianth-whorl; $a a a$ three of the nine anthers, $f$ ovary, $n$ stigma, $k k$ nucellus of the ovary, $d r$ glandular tissue at the base of the filaments forming the nectaries.

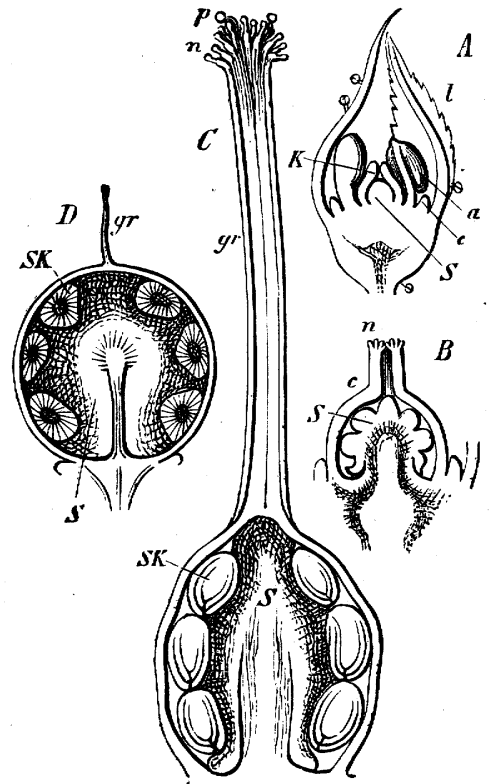

FIG. 392.-Anagallis arvensis; $A$ longitudinal section of a young flower-bud, $l$ sepals, $c$ corolla, $a$ anthers; $K$ carpel $S$ apex of the floral axis; $B$ the gynæceum further developed, the stigma $n$ being now formed, and the ovules on the central placenta $S$; $C$ the gynæceum ready for fertilisation, $p$ pollengrains on the stigma $n, g r$ style, $S$ central placenta, $S K$ ovules $D$ unripe fruit, the placenta $S$ has become fleshy and swollen so as to fill up the spaces between the ovules.

us in inferring a number of coherent carpels, these carpels originate as an undivided annular zone which developes into the ovary, style, and stigma; as, for instance, in Primulaceæ (Fig. 392) (free central placentation). In Polygonaceæ, on the other hand, where the ovary also forms eventually a closed cavity containing the central ovule (Fig. 39r), the cohesion of two or three carpels to form the ovary may not only be recognised from the corresponding number of the styles and stigmas; but separate carpels appear at first distinct on the floral axis, and only amalgamate in the course of their growth, their zone of insertion becoming elevated as a ring. Since the wall of the ovary does not in any of these cases form placentæ from

1 I am unable to understand why Magnus calls the wall of the ovary 'perianth.' 
the number and position of which the number and position of the carpels might otherwise be more easily determined, we are thrown back on the direct observation of the first stages of development and on the numbers of the styles and stigmas. Failing this, the solution of the question depends on morphological relationships which are still by no means made out with sufficient certainty, notwithstanding the numerous researches which have been made on the development of the flower.

Besides the number of the carpels which have coalesced to form the ovary, it is a question of interest whether in any particular case the ovules have been produced laterally on the floral axis or as its terminal structure. In the cases of Piperaceæ, Polygonaceæ, Naias, Typha, \&c., where only a single ovule springs from the base of the ovary, it is evident that this must be the terminal structure of the floral axis; and the investigations of Hanstein and Schmitz, Magnus, Rohrbach, and Payer, have proved in addition that not only the ovule as a whole, but the nucellus itself, must be considered as a terminal struciure ${ }^{1}$. It must not, however, be inferred from this that every ovule which springs from the base of the cavity of the ovary necessarily forms the apex of the floral axis; for it is conceivable that the axis itself may have ceased to grow, but has produced an ovule at the side of its apex, a case which we shall meet with further on in the inferior ovary of Compositæ. In a few cases the floral axis rises free within the spacious cavity of the ovary and produces ovules laterally, as occurs in Primulaceæ (Fig. 392) and Amaranthaceæ (in Celosia, according to Payer).

The Inferior Ovary of epigynous flowers results from the retardation or complete suppression of the apical growth of the young floral axis, its peripheral tissue rising as an annular zone, and producing on its free margin the perianth, stamens, and carpels (Figs. 393, 394). The hollow structure which is thus formed, and which is at first open above, is afterwards covered over by the carpellary walls which close in above it; the apex of the floral axis lies at the bottom of the elongated cup-shaped or tubular cavity. Notwithstanding this striking displacement of the axial parts, the structure of the inferior ovary resembles that of the free polycarpellary ovary in almost all respects; it may also be either unilocular or multilocular-if unilocular, the placentation may be basilar, lateral, or parietal. When the placentation is basilar, the ovule sometimes appears as if it were the terminal structure of the apex of the axis; as for instance the erect ovule of Juglandeæ. In Compositæ, on the other hand, the position of the single anatropous ovule is not terminal but lateral; the apex of the floral axis may often be clearly made out as a small elevation beside the funiculus, and in abnormal cases it undergoes further development into a leaf-bearing shoot ${ }^{2}$. In Samolus the apex of the axis rises within the unilocular inferior ovary as in the superior ovary of other Primulaceæ (Fig. 392), and bears a number of lateral ovules. If the placentæ of the unilocular inferior ovary are parietal, they form on the wall two, three, four, five or more ridges from above downwards or from below upwards, and bear two or a larger number of rows of ovules (as in Opuntia or Orchideæ). These placentæ, which project more or less

${ }^{1}$ [See infra, p. 574.]

${ }^{2}$ Cramer, Bildungsabweichungen und morphologische Bedeutung des Pflanzen-Eies (Zürich I 864). -Köhne, Die Blithenentwickelung der Compositen, Berlin 1869. - Buchenau, Bot. Zeit. I832, No. 18 et seq. 
into the interior, may be regarded as the prolongations of the margins of the carpels downwards on the inside of the ovary. A similar explanation may be given of the longitudinal dissepiments of the multilocular inferior ovary; the same differences occur in them as those which have already been described in the case of the superior ovary; for they may either meet in the middle and bear the ovules in the axile angles of the loculi (Fig. 358), or they may split into two lamellæ, bend back, and bear the ovules in the middle of the cavity of the loculus (as in Cucurbitaceæ). Usually two, three, or more carpels share in the formation of the upper part of the inferior ovary, their elongated margins being prolonged inwards and developing downwards into the parietal placentæ or the dissepiments of the multilocular ovary. In such cases the inferior ovary must be termed polycarpellary, like

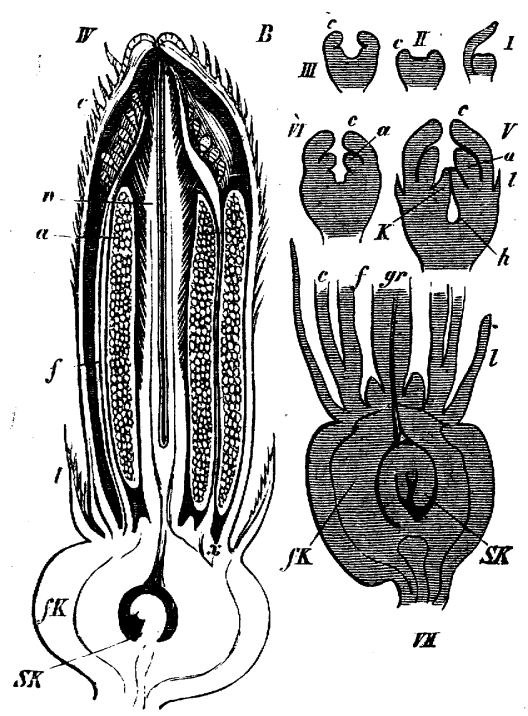

FIG. 393.-I-VII stages of development of the flower of Helianthus annuus; $l$ calyx, $c$ corolla, $f$ filaments, $a$ anthers, $x$ basal portion which afterwards developes into the lower part of the tube of the corolla which bears the epipetalous stamens, $f K$ the inferior ovary, $S K$ the ovule, $k$ carpel, gr style.
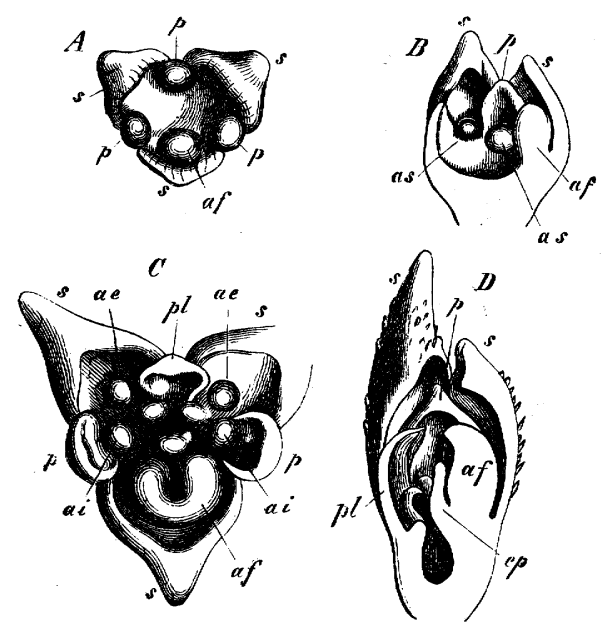

FIG. 394.- $-A-D$ stages of development of the flower of: Calanthe veratrifolia (after Payer); $A$ and $C$ seen from above, $B$ and $D$ in longitudinal section, $s$ sepals, $p$ petals, $p l$ the petal which developes into the labellum, $a f$ the single fertile anther, an and ai abortive anthers of the outer and inner whorl; in $B$ as are the sterile stamens, in $D$ ep one of the three carpels.

the superior ovary of similar structure. Examples of a monocarpellary inferior ovary appear to be very rare; Hippuris (Fig. $3^{60}$ ) affords one; its inferior ovary consists of a single carpel, and contains a solitary anatropous pendulous ovule:

The Style is a prolongation of the carpel above the ovary; in monocarpellary. ovaries there is therefore only one style (Figs. $3^{82}, 3^{84}$ ), which may however be branched; when the ovary is polycarpellary, the style consists of as many parts as there are carpellary leaves; these parts may be free for the whole distance above the ovary (Fig. 386 ), or coherent for a certain distance above it, separating only at a greater height; or, finally, they may cohere for their whole length (Figs. $388 \mathrm{G}$, 390 ). Although the style arises from the apex of the young carpel, it may subsequently stand on the axile side of the monocarpellary ovary, the carpel becoming considerably bulged outwards by the more rapid growth of the dorsal side of the 
ovary (as in Fragaria and Alchemilla). If this occurs with each of the carpels of a polycarpellary ovary, the ovary itself appears to be depressed in the middle, and the style rises from the depression (Figs. 387,388 ). In Labiatæ and Boragineæ this peculiarity is especially conspicuous, the four lobes of the bilocular ovary forming strong protuberances (Fig. 390, $A, B$ ), so that the style finally appears to spring from between four parts of the ovary which seem to have scarcely any connection with one another, and is hence termed a gynobasic style.

The style may be hollow, that is, it may be penetrated by a channel consisting of a narrow elongation of the cavity of the ovary, as in Butomus (Fig. 382, $B, F$ ), where it opens on the hairy surface of the stigma; or in Viola (Fig. 395), where the channel is broad, and opens above into the spherical cavity of the stigma; or in Agave and Fourcroya, where the style is hollow throughout its whole length

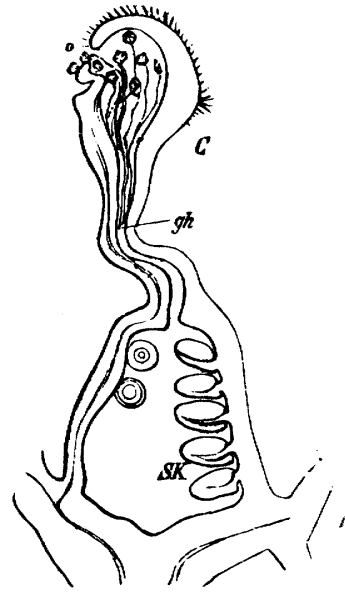

F1G. 395.-Longitudinal section through the gynæceum of Viola tricolor; $S K$ the anatropous ovules, $g h$ channel of the style. $o$ its opening: in the hollow of the stigma $o$ its opening: in the hollow of the stigma
which is filled with the stigmatic secretion are pollen-grains which are putting out their pollen-tubes. and open to the stigma, the simple channel dividing below into three tubes which run into the loculi of the ovary, a phenomenon which occurs also in other Liliaceæ $^{1}$. In other cases it is at first hollow, as in Anagallis (Fig. 392, $B$ ), but becomes afterwards filled up by the growth of the tissue. There is usually no channel to be detected in the style when the pistil is ready for fertilisation, or at least not in its upper part ; in the place of this its centre is occupied by a mass of loose tissue, the 'conducting tissue,' through which the pollen-tubes grow till they reach the cavity of the ovary. The external form of the style is usually cylindrical, filiform, or columnar, sometimes prismatic or ribbon-shaped; in the Irideæ it generally attains a considerable size; in Crocus it is very long, tripartite above, each division being deeply hollowed out like a cup; while the genus Iris is distinguished by its three free broad petaloid coloured styles. Sometimes the portion of the style which belongs to each carpel branches, as in Euphorbiaceæ, where a tripartite style, each arm of which bifurcates, corresponds to the three carpels. The style frequently remains very short, and then has the appearance of being a mere constriction between the ovary and stigma, as in Vitis.

The Stigma ${ }^{2}$, in the narrower sense of the term, is the part of the style which is destined for the reception of the pollen. When pollination takes place it is covered with a viscid secretion, and usually with delicate hairs or short papillæ, constituting a glandular structure which is sometimes merely a peculiarly developed portion of the surface of the style, sometimes a special organ of very variable appearance attached to it. The form of the stigma always has an intimate connection with the mode of conveyance of the pollen by insects or otherwise, and can be understood and explained only when these facts are taken into consideration. A few

\footnotetext{
1 Zuccarini, Nova Acta Ac. Leopold, XVI. pt. II. p. 665.

${ }^{2}$ [Behrens, Untersuch. ueb. d. anat. Bau des Griffels und der Narben, Göttingen 18 75 .]
} 
specially interesting cases will be described in Book III; it is sufficient now to mention that the surface of the stigma forms the exit of the open channel of the style when there is one; if this channel is closed or entirely absent, the stigma has the appearance of a superficial glandular structure upon or beneath the apex of the style or of its arms. If these arms are long and slender, and covered with long hairs, the stigma has the form of a pencil or tuft of hairs or feathers, as in Grasses; in Solanaceæ and Cruciferæ the moist surface of the stigma covers a knob-like indented thickening at the end of the style; in Papaver it forms a manyrayed star on the lobed style. Sometimes the stigmatic portion of the style is greatly swollen, as in the Asclepiadeæ, where the two monocarpellary and distinct ovaries cohere by the stigmas; the true stigmatic surface into which the pollen-tubes penetrate lies in this case concealed on the under side of the stigma ${ }^{1}$.

The Nectaries ${ }^{2}$. Wherever pollination is effected by insects, glandular organs are found in the flowers which secrete odoriferous and sapid (generally sweet) juices, or contain them within their delicate cellular tissue from which they are easily sucked out. These juices are included under the term Nectar, the organs - which produce them being the Nectaries. The position, form, and morphological significance of the nectaries are very various, and always stand in immediate relation to the special contrivances for the pollination of the flower by means of insects. The nectaries are often nothing but glandular portions of tissue on the foliar or axial parts of the flower; very often they project in the form of cushions of more delicate tissue, or take the form of stalked or sessile protuberances; or whole foliar structures of the perianth, of the andrœcium, or even of the gynæceum, are transformed into peculiar structures for the secretion and accumulation of the nectar. Since it is quite impossible to treat these organs morphologically in general terms, a few examples may serve to show the student where he will have to look for the nectaries in different flowers. In Fritillaria imperialis the nectaries are shallow excavations on the inner side of the perianth-leaves near their base, large clear drops of nectar exuding from them; in Elaeagnus fusca a glandular annular cushion on the gamophyllous perianth (Fig. $384 d$ ); in Rheum slight glandular protuberances at the base of the stamens (Fig. 391 dr); in Nicotiana an annular callosity at the base of the superior ovary; in the Umbelliferæ a fleshy cushion surrounding the bases of the styles united above the inferior ovary (Fig. $3^{8} 3 h$, p. 559); in Compositæ they are also at the base of the style (Fig. 393). In Citrus, Cobaa scandens, Labiatæ, and Ericaceæ, the nectary appears as a development of the floral axis or receptacle in the form of an annular zone beneath the ovary (Figs. $3^{87} d, 390 A, t$ ), \&c.; in Cruciferæ and Fagopyrum in the form of four or six roundish or club-shaped outgrowths or warts between the filaments, \&c. An abortive stamen is converted into a nectary in the Gesneraceæ; in Cucumis Melo (the Melon) the whole androcium is replaced in the female and the gynæceum in the male flowers by a similar organ. As a rule the nectaries occur deep down among the other parts of the flower; and when they secrete nectar, it collects at the

1 On the position of the lobes of the stigma in relation to the placentæ in different plants, see Robert Brown, Misc. Bot. Works, Ray Soc. I 867 , vol. I. pp. 553-563.

2 [Behrens, Die Nectarien der Blüthen, Flora, I879.-Bonnier, Les nectaires, Ann. d. sci. nat. sér. 6. t. VII.] 
bottom of the flowers, as in Nicoliana and Labiatæ. Frequently, however, special hollow receptacles are constructed for this purpose, as is especially the case with the bag-like appendages of the perianth-leaves (Fig. 396), usually called Spurs. In Viola only one of the perianth-leaves forms a hollow spur, into which the appendages of two stamens are prolonged and secrete the nectar. The cup-shaped stalked petals of Helleborus and the slipper-shaped petals of Nigella secrete at the bottom of their cavity the nectar which gathers there.

The Ovule (macrosporangium) of Angiosperms usually consists of a clearly developed, sometimes even very long stalk or Funiculus (as in Opuntia and Plumbagineæ) - which, however, is sometimes entirely wanting, as in Grasses-and one or two integuments which enclose the nucellus. [The general rule is that the ovules of the gamopetalous Dicotyledons have one integument, and that the ovules of the apetalous and polypetalous Dicotyledons as also those of the Monocotyledons have two integuments. Exceptions occur, however: thus, among gamopetalous Dicotyledons the Primulaceæ, Myrsineæ, Plumbaginaceæ, and Cucurbitaceæ have two integuments; and among apetalous and polypetalous Dicotyledons the following
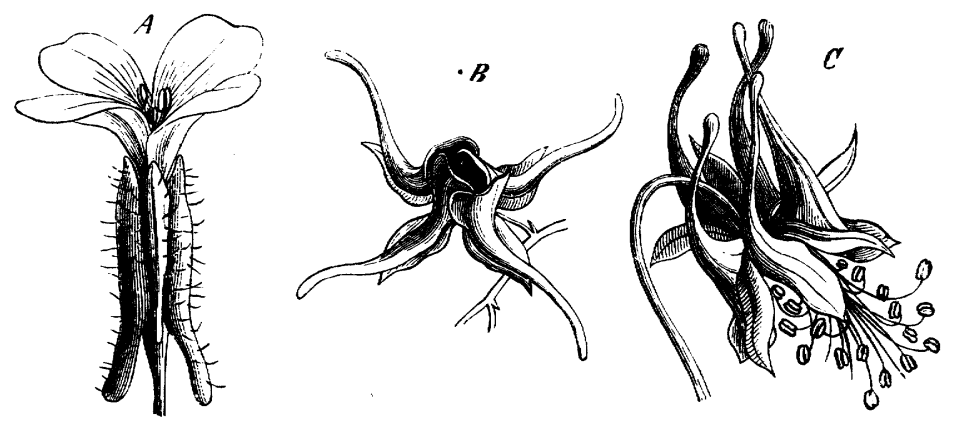

FIG. 396.-Flowers with spurred sepals $(A)$ and petals $(B . C) ; A$ Biscutella hispida, $B$ Epimedium grandiftorum, $C$ Aquilegin canarensis.

have only one integument, the Loaseæ, Pittosporeæ, Umbelliferæ, Callitriche, Empetrum, Hippuris, and Escallonia (Warming).] A third envelone, the Aril, is frequently formed subsequently (as in Myristica, Euonymus, Asphodelus lutea, Alö̈ subtuberculata, \&c. When the ovule is the terminal structure of the floral axis, and has a short funiculus, it is orthotropous, as in Piperaceæ and Polygonaceæ; the campylotropous form, i.e. where the nucellus together with its integuments is itself curved, is comparatively rare, but occurs in Grasses, Fluviales, Caryophylleæ, \&c. The usual form of the ovule of Angiosperms is the anatropous; the nucellus together with its integuments is inverted, so that the micropyle faces the point of origin of the funiculus from the placenta (hilum) (Figs. $3^{82}, E, 3^{8} 3$ ); in this case the funiculus runs up the side of the ovule, coalesces with it, and is termed the Raphe. The micropyle is frequently, especially in Monocotyledons, formed by the inner integument only of the nucellus; but sometimes, especially among Dicotyledons, the outer integument grows also above the opening of the inner one, and the channel of the micropyle is then formed at its outer part (the Exostome) by the outer, at its inner part (the Endostome) by the inner integument. 
When there are two or three integuments, the innermost (the Primine of Mirbel) is generally formed first, then the outer one (the Secundine), and finally, usually at a much later period, the Aril; the order of development is therefore basipetal in reference to the axis of the ovule. The transverse zone from which the single or the two true integuments spring is termed the Chalaza (more correctly the base of the ovule).

The integuments are usually only a few layers of cells in thickness, and have the appearance, especially when they enclose a large nucellus, of thin membranes

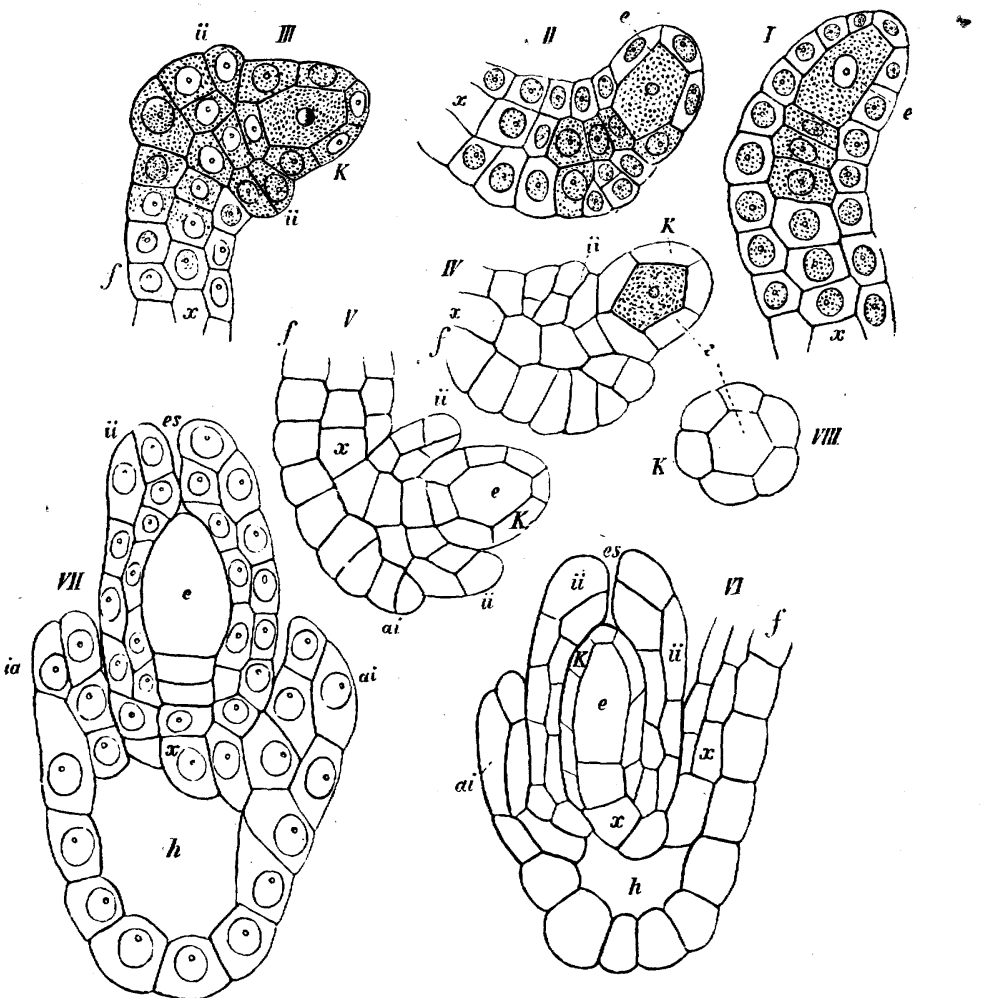

FIG. 397. $-I-V I I$ stages of development of the ovule of Orchis mititaris $\left(\times 55^{\circ}\right) ; I-V I$ seen frotn the side in longitudinal sectiou, $V I I$ from the front, the funiculus being behind, $V I I I$ a horizontal section of $I ; x x$ the axial row of cells, the upper one of which is the archesporium, $e, f$ the funiculus, $i i$ the inner, $a i$ the outer integument, $K$ the nucellus, es the micropyle, $h$ an intercellular space : in $V I I$ the embryo-sac $e$ has completely replaced the tissue of the nucellus.

(Fig. $382, E$ ). But when only one integument is developed, the nucellus usually remains very small, while the integument becomes thick and solid, extending far beyond the nucellus, and forming, before fertilisation, the principal mass of the ovule, as in Hippuris (Fig. 360), Umbelliferæ (Fig. 383), and Compositæ (Fig. 393).

There is still much doubt about the history of development of the separate parts of the ovule ${ }^{1}$; the following may be stated as certain or at least probable. In the formation of the erect orthotropous ovule the apex of the floral axis rises within the ovary as a roundish or conical ovoid protuberance which forms the

\footnotetext{
${ }^{1}\left[\right.$ For an account of the development of the ovule see infra, p. $\left.57^{6 .}\right]$
} 
nucellus; an annular wall grows up first, and finally envelopes the nucellus and extends beyond it as an integument. If a second outer integument is formed in addition, this arises in a similar manner, and grows up around the first (as in Piperaceæ, Polygonaceæ, \&c.). The anatropous ovule may be at first a straight or slightly curved projection of tissue (as in Fig. 397,I), but it immediately becomes evidently curved at the spot where the first or the single integument springs from it (Fig. 397, II, III, IV); the apical part enclosed by the integument then forms the nucellus, while the subjacent basal part becomes the funiculus. As the integuments arise, the curvature becomes gradually stronger, and the nucellus becomes inverted even before the outer integument has entirely developed. This latter is therefore not formed on the side next to the raphe, but clothes all the free part of the ovule, right and left of the raphe (Fig. 397, V,VI, VII). Cramer was the first to point out that anatropous ovules may originate in another way (and this is probably

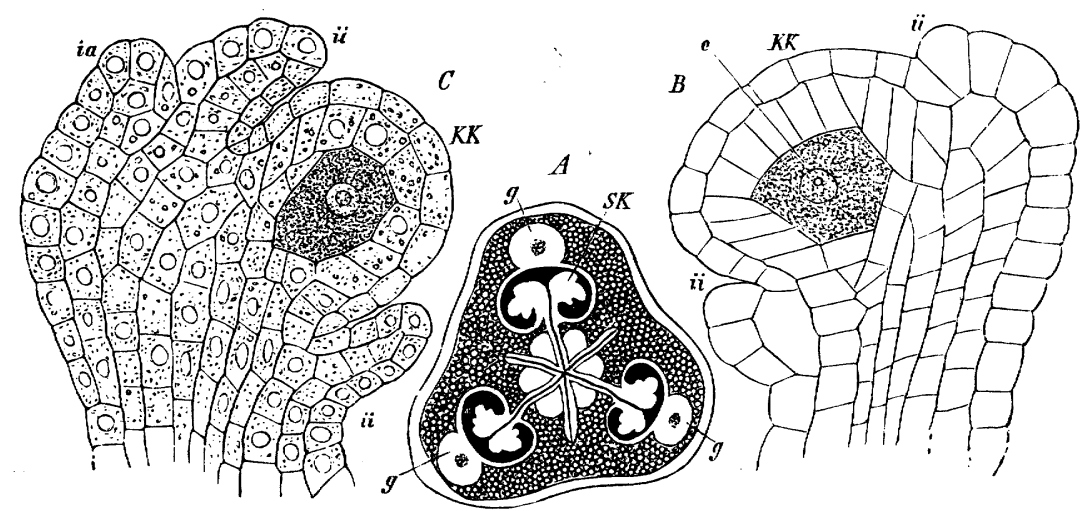

FIG. 398.-Funkia cordata; $A$ horizontal section of the young superior trilocular ovary, two ovules $S K^{\prime}$ are seen in each loculus, growing from the revolute margins of the carpels, $g$ a fibro-vascular bundle surrounded by light-coloured parenchyma ; $B$ and $C$ two successive states of the young ovule in longitudinal section, $K K$ tissue of the nucellus, $i i$ inner, $i a$ outer integument, $e$ embryo-sac ( $A$ slightly, $B, C$ very highly magnified).

the most common case), the ovule developing as a secondary lateral projection beneath the apex of the young conical funiculus, and curving backwards subsequently towards the base of the latter. This inversion takes place while the single or the inner integument is enveloping the nucellus from the summit of the funiculus; the second integument, if there be one, then similarly clothes the free part (see Fig. 398, B, C). Köhne ${ }^{1}$ has indeed thrown some doubt on the actual lateral origin of the nucellus, not only in Compositæ, but also in Solanum, Hedera, Fuchsia, Begonia, \&c. I have, however, had the opportunity of observing a number of different stages of development in this respect, and not only of convincing myself that the funiculus arises laterally with respect to the apex of the floral axis, but also that the nucellus, when first visible, stands laterally also below the apex of the funiculus. It is possible that the observation of peculiarly favourable cases will remove

1 Köhne, Ueber die Blïthenentwickelung bei den Compositen. Berlin I866. [Köhne's view is supported by Haenlein's observations (Beit. z. Entwickelungsgeschichte der Compositenblithe, Schenk's Mittheilungen, II. I8/5).] 
the last remaining doubt on this point ${ }^{1}$. Cramer has shown in a number of other instances that all stages of the metamorphosis of ovules occur when the flower is developed in a monstrous condition, leading also to the conclusion that the nucellus is a lateral development on the funiculus of the ovule. Malformations of Delphinium elatum, where the ovules spring from the margins of the carpels, show that the carpel is transformed into a flat open pinnate leaf, the lobes of which are the metamorphosed ovules. The nucellus here springs from the upper or inner side of the lobe of the leaf which represents the transformed funiculus together with the integument. In Melilotus, Primula chincnsis, and Umbelliferæ, Cramer found the same to be the case ${ }^{2}$. Relying on this and other facts, and on the hypothesis that the ovule is never a terminal structure of the floral axis, Cramer ${ }^{3}$ adopted the view that the ovule is either a metamorphosed leaf or part of a leaf (a tooth or outgrowth of the upper surface). The ovule of Primulaceæ and Compositæ he considered to be a whole leaf, and he supposed that closer observation would show the same to be the case in other flowers also, especially in those where the flower is said to possess a solitary 'reputed terminal ovule,' as Urtica (and Taxus), and perhaps also the Dipsacaceæ and others. The nucellus' would in this case be a new formation on the surface of the ovular leaf, the funiculus would correspond to the base of this leaf, and the integuments to its upper part, which is folded once or twice in the form of a cup or hood round the nucellus. On the other hand he would consider as only portions of the leaf (teeth or outgrowths of the upper surface) all those ovules which spring singly or in numbers from the margin or upper surface of carpellary leaves, as those of Cycadeæ, Abietineæ (?), Liliaceæ, Umbelliferæ, Ranunculaceæ, Resedaceæ, Cruciferæ, Leguminosæ, \&c. In these cases the nucellus would be a new formation on the surface of the lobe, the funiculus would correspond to its base, and the integuments to its upper part folded once or twice round the nucellus in the form of a cup. Only in those few plants in which the ovule has no integument would the naked nucellus or entire ovule correspond to this lobe of the carpellary leaf. In the first edition of this book I expressed my agreement with Cramer's view, but with a reservation with respect to Orchideæ, being especially influenced by the importance which I then attached to the morphological equivalency of the nucellus in all Phanerogams. Further reflection has, however, deprived this reason of its importance; and I am the more induced to ascribe different morphological significations to the ovules, according to their mode of origin and their position, because (as has been shown by Magnus, Rohrbach, Hanstein, and Schmitz ${ }^{4}$ ) in Piperaceæ, Typhaceæ, and Naiadeæ the ovule is actually the terminal structure of the floral axis, and in Naias this terminal ovule is also anatropous. In these statements I not only find the confirmation of my

1 Schenk writes, 'that which appears plausible enough in the Compositæ is certainly not the case in other families; the ovule is not a lateral branch of the primitive rudiment, but this itself developes into the ovule.'

2 Compare also H. von Mohl, Vermischte Schriften, pl. I. figs. 27-29.

3 Cramer, Bildungsabweichungen bei einigen wichtigeren Pflanzenfamilien und die morphologische Bedeutung des Pflanzeneies (Zürich I869; p. I 20), where the literature of this subject has been carefully treated.

4 These researches have been already quoted; [see also Eichler, Helosideen; Bot. Zeitg. I868, and Strasburger, Coniferen und Gnetaceen, 1872.] 
own observations on Chenopodiaceæ and Polygonaceæ, but they also warrant the assumption that the ovules previously described by Payer as terminal are really so. Since, however, it is not my object here to enter into a detailed proof of theoretical matters, it will be sufficient for the present to summarise the various phenomena.

With respect to position, the following classes may first of all be distinguished:-

A. Ovules produced on the Carpels and springing from the carpellary leaves ; and either

I. Marginal, from the reflexed margins of the carpels (Figs. $3^{8} 5,3^{86}$, $387,390)$; or,

2. Superficial, from the whole of the inner surface of the reflexed halves of carpellary leaves, always apparently with the exception of the mid-rib of the carpellary leaf (Fig. $357,3^{82}$ ).

3. Axillary or basal, arising from the base of the upper surface of the carpel or in the axil of the carpel (Ranunculus, Sedum, Zanichellia according to Warming ${ }^{1}$.

${ }^{1}$ See Warming, Rech. sur la ramification des Phanérogames, Kopenhagen $I^{8} 7^{2}$, p. 22. Tab. XI. fig. I-ro. Axillary ovules are no more to be regarded as buds (caulomes) than are the axillary sporangia of Lycopodium.

[The question of the morphological significance of the placenta and of the ovule is one which has been much discussed of late. With regard to the placenta, Schleiden, starting with the conception of the ovule as being a bud, considered the placenta to be necessarily an axial structure, inasmuch as only axial structures normally bear buds (Princip'es of Scientific Botany, 1849, pp. $382 \mathrm{ff}$.), a view which was adopted and developed more especially by French botanists (see Payer, Organogénie). According to a second view, the placenta is a portion of the carpel itself, usually of its margin, that is, that it is always borne by a leaf. This view has been revived of late years by Van Tieghem (Rech. sur la structure du pistil, I 871), by Celakovsky (Ueb. Placenten und Hemmungsbildungen der Carpelle, Sitzber. der k. böhm. Ges., Prag, I875; Vergl. Darstellung der Placenten, ibid. 1876), by Braun (Bemerk. ueb. Placentenbildung, Sitzber. d bot. Ver. d. prov. Brand. I 874), and Eichler (Blüthendiagramme, II. 1878) has now accepted it. In spite of all that has been written in support of these two theories, it cannot be admitted that either of them satisfactorily explains every possible case : if the former must evidently be forced when it is applied to a case of parietal placentation, this is equally the case with the latter when it is applied to free-central placentation, as in the Primulaceæ. Both these attempts at generalisation seem to be too arbitrary. In consequence a third view has been promulgated, more especially by Huisgen (Untersuch. ueb. die Entwickelung der Placenten, Bonn 1873) and formerly held by Eichler (Blïthendiagramme, I. 1875), that the nature of the placenta is not the same in all cases. In the Primulaceæ, for instance, it belongs to the floral axis. and, according to Huisgen, this is also the case in certain instances of axile placentation, as in the Solanaceæ. Lobeliaceæ, Ericaceæ, Malvaceæ, and Hypericaceæ, the placenta in these orders being a prolongation of the stem; it also belongs to the axis in such forms as the Piperacex, in so far as any placenta can be said to exist in them at all: in the Violaceæ and Leguminosæ and in Monocotyledons the placenta is a development of the carpels : finally, in Cruciferæ and Resedaceæ, and, according to Barcianu, in the Onagraceæ (Ueb. die Bliithenbildung der Onagraceen, Schenk's Mittheilungen, II. I875), the placenta is an independent organ, probably a phyllome, a view which was held by Treviranus (Physiologie, II. $183^{8}$ ).

Now with regard to the ovule. Schleiden, Braun, and most of the older botanists regarded the ovule as being a bud, but many regarded it as a leaf or part of a leaf (for the early history of the subject see Braun, Polyembryonie und Keimung von Calebogyne, 1860), a view which, as stated above in the text, has been more recently revived.by Cramer; this view has been further developed by Celakovsky (Ueb. die morphol. Bedeutung der Samenknospen, Flora, I874; Zur Discussion ueber das Eichen, Bot. Zeitg. 1875, and Vergrünungsgeschichte der Eichen von Alliaria officinalis, ibid. 1875, und von Trifolium repens, ibid. 1877), and so far modified that, according to him, the 
B. Ovules produced on the Axis and springing from the prolongation of the floral axis within the ovary, the carpels themselves being sterile; these may be either-

4. Lateral, when they stand beside or below the apex of the floral axis, which either rises as a columella and bears a number of ovules (as in Fig. 392), or is arrested in its development, so that the single ovule formed appears terminal (as in Fig. 393); or,

5. Terminal, when the apex of the floral axis itself becomes the nucellus (as in Fig. 391, and in Piperaceæ, Naias, Typha, \&c.).

To which of these classes the ovules belong in any given plant must be decided in each separate case; the position on the margin of the carpels is by far the most common among Angiosperms, both the superficial and the axial position belonging only to single families or genera. If these facts are compared with what occurs in Gymnosperms, the ovules of Cycadeæ must be classed with the marginal carpellary, those of many Cupressineæ with the superficial description; while those of Taxus are axial and terminal, and those of Salisburia lateral.

When the position of the ovules is given, so also is some information as to their morphological significance: the terminal ovules may be regarded as the terminal portion of the axis, the lateral as equivalents of whole leaves, the marginal as branches of leaves (laciniæ, pinnæ, or lobes); the superficial ovules may be included in the category of such foliar outgrowths as we have already found to occur in the form of

ovule is a metamorphosed segment of a carpellary leaf, a definition which Eichler has now accepted (Bliithendiagramme, II). A third view, the one stated in the text, is now held by many, that, as in the case of the placenta, the morphological value or 'dignity' of the ovule is not always the same.

Of these views, the one which ajpears to be the most true to nature is the one which allows the greatest latitude: but it is not always possible to refer an ovule to one of the categories, caulome and phyllome, for its position does not necessarily indicate its morphological significance. Thus, a lateral ovule, as in Compositæ and Primulaceæ, might be either a leaf or a bud; its probable leaf-nature in these cases depends entirely on teratological evidence, which is of very doubtful value, for an organ in a monstrous condition does not necessarily assume its primitive archetypical form. Again, an organ borne by a leaf does not necessarily represent some typical part of the leaf, witness the adventitious buds which are developed on leaves in many cases, and the ovules which cover the surface of the carpels in Nuphar luteum and Brasenia peltata (Strasburger, Angiospermen und Gymnospermen, p. 57).

The difficulties met with in endeavouring to regard the ovule as a caulome or a phyllome may be got over by regarding it as an 'emergence' (Strasburger, loc. cit.), borne sometimes on an axial, sometimes on a foliar member. This view, to be completely satisfactory, ought to be applicable also to pollen-sacs and to the sporangia of the Vascular Cryptogams. This cannot be quite accurately done, for an $\epsilon$ mergence (p. 162 ante) is described as being developed not only from the epidermal layer but also from the subjacent cells of the organ bearing it; and we know that the sporangia of the Vascular Cryptogams (except those of Isoëtes) and the pollen-sacs of Pinus are derived from one or more epidermal cells. Goebel (Bot. Zeitg. I 88I) has expressed the opinion that a sporangium (ovule, pollen-sac) is simply a sporangium, an organ sui generis as much as a stem or a leaf.

A few words may be added here with reference to the morphological significance of the integuments of the ovule. The view has been often expressed that the integuments of the ovule are homologous with the indusium of Ferns; this is opposed by Strasburger (Angiospermen und Gymnospermen) and by Goebel (Bot. Zeitg. 188I) on the ground that the integuments arise from the ovule itself, whereas the indusium is an outgrowth of the leaf bearing the sporangium. These organs are analogous, but not homologous.] 
sporangia in Lycopodium. These explanations are so far confirmed by the occurrence of malformations, that the lateral axial and the marginal carpellary ovules are often enough transformed into foliar structures of ordinary form, while this appears never to occur with terminal or superficial ovules.

These remarks have at present been confined to the ovule as a whole, although reference has already been made to the theory of Cramer on the various morphological relationships of the nucellus and of the other parts, the funiculus and the integuments. Malformations ${ }^{1}$, which in this respect are even more instructive than the normal development, led Cramer to the conclusion that when the ovule appears to be the equivalent of a lateral branch or of the whole of a leaf, the funiculus and the integuments together correspond to the foliar structure in each case; the nucellus arises from it as a lateral outgrowth, while the integuments correspond to the hoodshaped lamina of the leaf, growing over the nucellus.

The ovules are sometimes rudimentary; those of Balanophoreæ and Santalaceæ have no integument; the nucellus is naked, and in some species is itself composed of only a few cells. In Loranthaceæ the development does not even proceed so far as the formation of a distinctly differentiated ovule; the growth of the apex of the floral axis ceases so soon as the carpels begin to be formed; and the cohesion of these is such that it is scarcely possible to speak of a cavity of the ovary; the formation of the embryo-sac in the axial part of the tissue of the inferior ovary is the only indication that this spot corresponds to the ovule; and since more than one embryo-sac is formed, it still remains doubtful whether this mass of tissue must be regarded as the equivalent of one or of several ovules ${ }^{2}$.

The Development of the Ovule and of the Embryo-sac. . [The first indication of the development of the ovule is the division by a wall parallel to the surface (periclinal) of one or more cells lying immediately beneath the epidermis of the placenta ${ }^{4}$; in the case of ovules which have a simple structure when mature, as those

1 [See Masters, Vegetable Teratology, I869; and Peyıitsch, Zur Teratologie der Ovula, Festschrift d. k. k. Zool.-Bot. Ges. Wien, 1876.]

${ }^{2}$ Hofmeister, Neue Beiträge, I (Abhandl. der kon. sächs. Gesellsch. der Wissensch. VI). [The remarkable position of the ovule in Hydnora (Prosopanche) americana, immersed in the placental tissue, is comparable with that of the sporangium in Isoëtes (Fig. 334). It is not possible to say at present if the embryo-sac of Hydnora belongs, like that of the Loranthaceæ, to an ovule which arises as a prominence on the placenta and is subsequently overgrown by it, or if it is developed from a cell of the placental tissue, the ovule not becoming differentiated from the placenta. On Hydnora see De Bary, Abhandl. der naturf. Gesellsch. zu Halle, vol. X; Hooker, Journ. Linn. Soc. vol. XIV. p. I82.]

${ }^{3}$ Hofmeister, Neue Beiträge zur Kenntniss der Embryobildung der Phanerogamen, Abhandl. d. sächs. Ges. d. Wiss, VI, VII, I859.-[Strasburger, Die Coniferen und Gnetaceen, p. 409; id. Ueb. Befruchtung und Zelltheilung; id. Ueb. Zellbildung und Zelltheilung, 3rd ed.; id. Die Angiospermen und die Gymnospermen.-Warming, De l’ovule, Ann. d. Sci. Nat. 1878.-Vesque, Dév. du sac embryonnaire des Phanérogames, Ann. d. Sci. Nat. sér. 6, VI, I878; id. Neue Untersuchungen, Bot. Zeitg. I 879.-Treub et Mellink, Notice sur le dév. du sac embryonnaire, Arch. Néerlandaises, XV.Fischer, Zur Kenntniss der Embryosacentwickelung, Jenaisch. Zeitschr. XIV, I880.-Marshall Ward, Embryo-sac of Gymnadenia conopsea, Quart. Journ. Micr. Sci. XX, 1880; id. Journ. Linn. Soc. XVII, I880.]

${ }^{4}$ [In the fourth German edition, Prof. Sachs, relying upon the observations of Hofmeister, regards the ovules of Orchideæ as being trichomes, inasmuch as they are stated by Hofmeister to be developed from single epidermal cells of the placenta. Hofmeister's observations have been shown to be erroneous by Strasburger (Coniferen und Gnetaceen) and by Warming (loc. cit.). The ovules 
of Orchids, only a single cell is thus divided, in other cases several. By repeated divisions parallel to the first the cells are multiplied, and a.protuberance is formed consisting in Orchids (Fig. 397) of a single row of cells, in other cases of a number of rows, invested by the epidermis; this protuberance is the nucellus.

The general course of the development of the embryo-sac is as follows: the terminal cell of the row in simple ovules (Orchids, Monotropa), the terminal cell of the axial row in more complex ovules, a hypodermal cell therefore ${ }^{1}$, becomes distinguished by its size and by the granularity of its protoplasm. This cell elongates with the growth of the nucellus, and a segment is cut off from it towards its upper (micropylar) end by a transverse wall, and this may be followed by the cutting off of a second segment in a similar manner; only one such segment is cut off in Tritonia aurea, Anthericum ramosum, Triglochin palustre, Luzula pilosa, Tradescantia virginica, Chenopodium fretidum, Helianthemum Rhodax, etc., two in many Rosaceæ: when only one segment is cut off, it usually divides into two by a wall parallel to the long axis of the nucellus (anticlinal), and whether one or two segments have been primarily cut off, they may undergo division by transverse walls. The large remaining cell now usually is divided by a transverse wall into two of nearly equal size, and one or both of these may be divided in a similar manner. The walls which are formed in connexion with these divisions are remarkable for their thickness and their glistening appearance. The result of these divisions is the formation of a row of three or four cells lying in the long axis of the nucellus; it is usually the lowest cell of this axial row which enlarges and becomes the embryo-sac, causing by its growth the absorption of the others.

Before going into further detail it will be well to become acquainted with the terminology which is to be used in describing these phenomena, and this may be best done by comparing them with those which accompany the development of the sporangia in the Vascular Cryptogams. It has been already pointed out that the ovule corresponds to a sporangium, and we see, from the facts stated in the preceding paragraph, that in its first development it resembles the sporangia of Isoëtes in that it is derived not from the epidermis only but also from subjacent cells, and that, as in the sporangia of the majority of Vascular Cryptogams, there appears within it at an early stage a hypodermal cell which is readily distinguishable from the cells surrounding it : to this cell the term archesporium may be applied as well here as in speaking of the Vascular Cryptogams, Similarly we may call the cell or cells which are cut off from the archesporium toward its micropylar end tapetal cells, the tapetum being completed in these plants by cells of the nucellus, a condition which recalls that in Selaginella. The further divisions of the archesporium are comparable to those which take place in the sporangia of the Vascular Cryptogams and which result in the formation of the mother-cells of the spores: the axial row of cells is then a row of sporemother-cells, and, inasmuch as one of these developes into the embryo-sac, the

of Orchids are developed in the manner described above in the text. Warming also states that in many cases the first cell-divisions make their appearance in the layer next but one to the epidermis (Ribes, Viola, Ficaria, Geum, Lamium, Symphytum, Verbascum), or even in a deeper layer (Malva, Pisum).]

1 [In Carex pracox, according to Fischer, the archesporium is derived from a more deeply placed cell of the nucellus.] 
embryo-sac in the ovule of an Angiosperm is equivalent to one of the spore-mothercells in the sporangium of a Vascular Cryptogam.

Among the more important deviations from the above described mode of the development of the embryo-sac, the following may be mentioned. In Tulipa Gesneriana and in Lilium bulbiferum, according to Treub and Mellink, the archesporium undergoes no division, but simply enlarges and becomes the embryo-sac. In a number of cases described by Strasburger (Angiospermen und Gymnospermen) and Fischer (Myosurus minimus, Senecio vulgaris, Lamium maculatam, Delphinium tridactylon and villosum, Sisyrinchium iridifolium, Orchis pallens, Gymnadenia conopsea, Monotropa Hypopitys, and several Grasses) no tapetal cells are formed. In Alisma Plantago, Allium fistulosum, Chenopodium fotidum, and Sabulina longifolia, the archesporium only divides once, that is, it produces a row of only two cells, the lower of which becomes the embryo-sac.

It has been not unfrequently observed that the terminal cell of more than one of the rows of cells of which the nucellus primarily consists assumes the characters of an archesporial cell : in such cases the archesporium is multicellular, a condition which is the normal one in Isoètes among Vascular Cryptogams. This appears to be commonly the case among the Rosaceæ. In Fragaria vesca Strasburger found (and his observations have been confirmed by those of Fischer on Cydania japonica, Geum strictum, Sanguisorba pratensis, Rubus casius, and Agrimonia Eupatoria) several archesporial cells forming a hypodermal layer or row. Each of these cells behaves in the manner already described: one or two tapetal cells are cut off, and then the cell undergoes division so as to form a row consisting of three or four cells. The lowest cell of each of these rows now begins to develope into an embryo-sac, but the cell of the axial row developes more rapidly than the others, causing their absorption; it is this cell which constitutes the embryo-sac. In Rosa livida Strasburger has observed that the uppermost cells of the rows, which usually consist of four cells, but sometimes of five or even six, develope and enlarge, and the second cell of the row often does the same. In the process of development some of these commencing embryo-sacs become absorbed, as do also the tapetal cells and the layers of cells which have been formed at the apex of the nucellus by the repeated division of the epidermal layer; one of them generally extends to the integument and becomes the largest embryo-sac, but the mature ovule contains several embryo-sacs. Tulasne has pointed out ${ }^{1}$ that in the Cruciferæ (Cheiranthus Cheiri) several embryo-sacs are developed, but only one of them is persistent. These phenomena recall the fact that in Taxus, Ginkgo, Thuja and Gnetum, among Gymnosperms, several embryo-sacs are at first formed.] The multiplicity of embryo-sacs in the ovary of Viscum cannot be included under this head, for the absence of the differentiation of the ovule makes it uncertain whether the mass of tissue in the ovary, to which we have already alluded, is to be regarded as the equivalent of one or of several ovules.

[In a great number of cases the epidermis of the nucellus remains a single layer of cells, but not unfrequently two layers are formed at the apex of the nucellus by the periclinal division of the primary epidermis (dermatogen). In some cases several

1 [Études d'embryogénie végétale, Ann. d. Sci. Nat. XII, I 849 .] 
layers of cells are thus formed constituting a sort of cap at the apex of the nucellus : this is indicated in Hippuris, and it is well seen in Delphinium, Helianthemum, and in the Rosaceæ; to the last case reference has been made in the preceding paragraph. In Geum urbanum, Iris Pseudacorus, and Agrostemma the whole free surface of the nucellus is covered by a number of layers of cells which have been formed in this manner (Warming).

The integuments are developed as outgrowths of the nucellus. In those cases in which the ovule has only a single integument, the greater part of it is developed from the epidermis of the nucellus. When the ovule has two integuments, the inner one is developed principally from the epidermis, the outer principally from the subjacent cells ${ }^{1}$. Cell-division and growth take place in a zone encircling the nucellus, and thus the integument grows up, at first as a ring, but later as a continuous membrane investing it. Accordingly as this zone is narrow or broad, the integument will consist (in thickness) of one or more layers of cells. As a general rule the inner integument in those cases in which two are present is developed first; exceptions to it appear to occur, according to Warming, in Euphorbia, Cuphea, Mahernia glabrata.

In some cases three integuments appear to be present (Asphodelus luteus and creticus, Reseda lutea), but it seems probable that the external one is to be regarded as an arillus. On the other hand it appears, in some cases in which two integuments are developed, as if only one were present (Viola, Ficus, Convallaria, Orchis, Tropaolum, Delphinium); this is due to the very intimate connexion of the two integuments.]

The further behaviour of the embryo-sac of Angiosperms differs in many ways from that of Gymnosperms. In Gymnosperms it remains surrounded by a thick layer of the tissue of the nucellus till after fertilisation has taken place; it is comparatively small, and is surmounted by a strongly developed nuclear protuberance. In Angiosperms, on the other hand, the embryo-sac has grown considerably even before fertilisation; it usually supplants the surrounding tissue of the nucellus so far that it remains enveloped by only a thin layer of it, or is even in actual contact with the inner surface of the inner integument, as in Orchideæ (Fig. 397,VII). In such cases the tissue of the apex of the ovule often still remains entire (as in Aroidex), but frequently the apex of the embryo-sac bursts through it, and projects into the micropyle (as in Crocus and Labiatæ), or even grows out beyond it as a long tube (e.g. Santalum). The middle and lower part of the sac also frequently extends considerably; in many gamopetalous Dicotyledons it puts out vermiform appendages which penetrate into and destroy the tissue of the integument, as in Rhinanthus, Lathraa, and some Labiatæ. While this process of growth is proceeding, the protoplasm which at first fills up the whole sac becomes full of vacuoles; a large sapcavity arises surrounded by a parietal mass of protoplasm, which accumulates especially in the apical prominence and at the bottom of the embryo-sac, while

1 [According to Warming the integuments are developed in some cases from the dermatogen alone; as in Orchis, Primula chinensis, Centradenia floribunda, Lysimachia verticillata, Linnea perenne (Strasburger); and Begonia heracleifolia, among plants which have two integuments, and in Peperomia and Monotropa among those which have only one integument. This mode of development of the integuments is comparatively rare.] 
threads, in which currents are visible, radiate to the walls from the protoplasm which envelopes the nucleus.

[During the growth of the embryo-sac its nucleus divides, and the two new nuclei travel to the opposite ends of the sac, a large central vacuole being formed. Each of these nuclei divides into two, and each of these again into two, so that there are four nuclei at each end of the embryo-sac. One nucleus from each end now travels towards the centre of the sac, where they meet and coalesce to form the definitive nucleus of the embryo-sac; these two nuclei may be termed the polar nuclei. Round the three nuclei at the two ends of the sac a process of free cellformation now takes place, so that there are three cells at each end of the sac. The cells at its lower (chalazal) end soon become surrounded with cell-walls, and constitute the antipodal cells: the cells at its upper (micropylar) end remain naked, and constitute the egg-apparatus. Two of the cells of the egg-apparatus lie nearer the apex of the sac than the third; they are somewhat elongated superiorly, and the nucleus lies in this elongated portion, the rounded inferior portion containing a large vacuole: these cells have been termed by Strasburger the Synergida. In many cases the elongated superior ends of these cells presents a longitudinal striation (as in Gladiolus, Crocus, Santalum, Zea, Polygonum, etc.), first observed by Schacht ${ }^{1}$, and termed by him the Filiform Apparatus. The third cell, which lies at rather a lower level than the other two, is the oosphere: it is more or less rounded in form, and its nucleus lies towards its lower end. All three are usually attached to the wall of the embryo-sac. It is important to note that the nuclei of the synergidæ are sister-nuclei; the nucleus of the oosphere is the sister-nucleus of the polar nucleus which coalesces with the polar nucleus from the lower end to form the definitive nucleus of the embryo-sac.

The following are some of the principal deviations from the series of phenomena which have been described above. Sometimes, as in Ornithogalum nutans, only one of the synergidæ is present. It may be that this is due to the omission of one of the divisions of the nuclei at the apex of the embryo-sac; but, inasmuch as two are generally to be found in preparations of early stages and only one in preparations of later stages of development, it is possible that two are primarily formed but that one soon undergoes absorption. In Sinningia, according to Strasburger, only one synergida is present in some cases, and very rarely both are absent. In exceptional instances two oopheres have been found in the embryo-sac, in Sinningia by Strasburger, and in Gomphrena by Fischer. This peculiar abnormality appears to be the rule in Santalum album. Strasburger explains it by supposing either that one of the three nuclei of the egg-apparatus has undergone division, so that four cells are formed instead of three, or that the upper polar nucleus becomes the nucleus of the second oosphere; in the latter case, the definitive nucleus of the embryo-sac would be constituted by the lower polar nucleus alone. The antipodal cells are very imperfectly differentiated in the Orchids, and do not become clothed with cell-walls: it is this fact, doubtless, which caused Hofmeister to assert that the antipodal cells are frequently wanting in Orchids. In some cases,

1 Jahrb, für wiss. Bot. I and IV. [Schacht states that in Santalum album the two synergidæ are separated by a septum which is formed in the apical portion of the embryo-sac; this observation has been confirmed by Strasburger (Zelltheilung und Befruchtung).] 
such as Allionia and Delphinium, they are very large and well-developed. In the Gramineæ, Fischer has found that they divide and give rise to a considerable mass of cells, a fact which Strasburger has ascertained with regard to Ornithogalum, though here it is exceptional. In some instances the two polar nuclei meet, not in the centre, but towards the upper end of the embryo-sac; in this case the upper nucleus is stationary, and the lower nucleus has to travel nearly the whole length of the embryo-sac. This occurs, according to Fischer, in Elodea and in many Gramineæ, and apparently also in Allium fistulosum. The coalescence of the two polar nuclei usually takes place before fertilisation, but in Alisma and in Allium fistulosum it does not take place until the pollen-tube has reached the embryo-sac or even until fertikisation has been actually effected.

As regards the fate and the function of these various cells which are formed in the embryo-sac, the oosphere is the one which undergoes fertilisation and developes into the embryo, the others being transitory structures. The synergidæ appear to

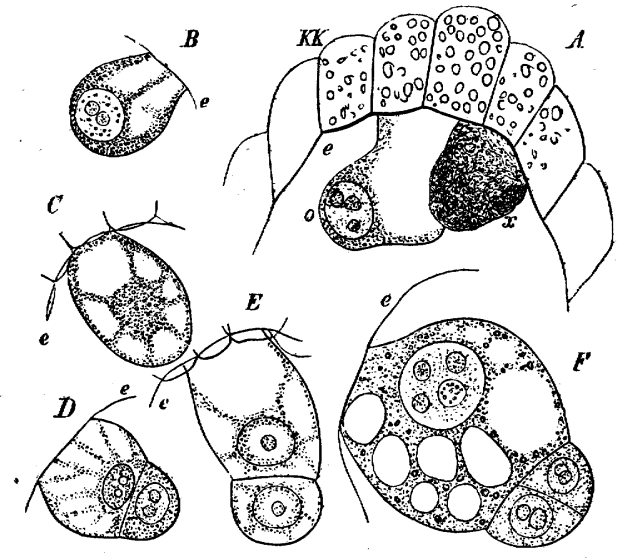

FIG. 399.-Funkia cordata; $A$ apex of the embryo-sac $e$, covered with a layer of cells belonging to the nucellus $K K, x$ one of the synergidæ, $\sigma$ the oosphere with its nucleus; $B, C$ oospore before, $D, E$ after the first division; $F$ the spherical suspensor with the two-celled rudimentary embryo $(\times 500)$.

cause the disintegration and in some cases at least the absorption of that part of the wall of the embryo-sac with which they are in contact, and besides this, they have a further function in the process of fertilisation to which reference is made below. In some cases (Crocus vernus, Torenia asiatica, Santalum album) their pointed ends become covered with a cap of a homogeneous substance which gives the reactions of cellulose: by this means they replace that part of the wall of the embryo-sac of which they have caused the absorption. After fertilisation the synergidæ undergo absorption. In many cases the antipodal cells soon undergo absorption, but in some they persist, and may be seen at the base of the endosperm in the fertilised ovule.

We will now proceed to discuss these phenomena from a morphological point of view. We have seen above that the cell which developes into the embryo-sac is the equivalent of one of the mother-cells of the spores in the sporangium of a Vascular Cryptogam, and to this we may add that it is equivalent to one of the mothercells of the pollen in the pollen-sac of the stamen. With this as the basis of their 
reasoning Warming and Vesque consider that the processes of cell-formation which go on in the embryo-sac correspond to the formation of spores or pollen-grains from their mother-cell, the four nuclei at each end of the embryo-sac representing a tetrad of spores. In order that this view may be perfectly consistent it is obviously necessary to assume that the embryo-sac is formed by the fusion of two cells equivalent to spore-mother-cells, inasmuch as two tetrads of nuclei are formed within it, and further, that the cells which are formed round these nuclei are each of them equivalent to a spore.

The view which is more generally held, and which is due to Strasburger, is that the cell which forms the embryo-sac is a spore-mother-cell which does not undergo any division into special spore-mother-cells, but which developes without dividing into a single spore, the embryo-sac. The processes of cell-formation which go on in the embryo-sac are not therefore to be compared to those which accompany the formation of spores or of pollen-grains from their mother-cell, but they are to be compared to those which accompany the germination of a spore or of a pollengrain. The six cells which are formed in the embryo-sac, three at the chalazal and three at the micropylar end, represent a rudimentary prothallium; they are comparable to the endosperm of Gymnosperms and to the prothallium in the macrospore of the heterosporous Vascular Cryptogams ${ }^{1}$. These cells, which we may term primary endosperm-cells, are, as we have seen, subsequently differentiated into the antipodal cells and the egg-apparatus; thus three of them are purely vegetative, whilst the other three are concerned in the sexual reproduction of the plant, one of them becoming the oosphere and the other two the synergidæ. In the Vascular Cryptogams we saw that the archegonium was developed from a single superficial cell of the prothallium; in the Gymnosperms we saw that the archegonium was developed from a single superficial cell of the endosperm, that it was a less complex organ than in the Vascular Cryptogams, and that it had undergone a reduction, a condition which we found most evident in Welwitschia in which the mature archegonium consists of only a single cell; in the Angiosperms this reduction is carried still further, the archegonium being represented only by the oosphere, which is one of the primary endosperm-cells. It was thought at one time that the Filiform Apparatus of Schacht represented the canal-cell of the archegonium which we have found to be present in the higher Cryptogams and in most Gymnosperms, but this cannot be the case inasmuch as this apparatus is, as stated above, simply the striated ends of the synergidæ; still less can the synergidæ be canal-cells, for they are the product of a nuclear division and cell-formation in which the oosphere is not directly concerned; moreover their function is entirely peculiar. The completion of the prothallium takes place when what we may term the secondary endosperm is formed; this is what is commonly termed the endosperm, and its formation in the embryo-sac does not commence until the fertilisation of the oosphere has been effected.]

Fertilisation ${ }^{2}$. The pollen-grains which germinate on the stigma send out

1 [Allusion has already been made (on p. 486) to Goebel's view that the antipodal cells of Angiosperms correspond to the ' endosperm' of Selaginella.]

${ }_{2}$ Besides the works of Hofmeister already quoted, see his historical account in Flora, 1857, p. 125, where the literature is collected. [Also Strasburger, Ueb. Befruchtung und Zelltheilung.- 
their tubes through the channel of the style where there is one, or more usually through the loose conducting tissue in its interior, down to the cavity of the ovary. Frequently both in erect basilar (Fig. 39I) and in pendulous anatropous ovules the micropyle lies so close to the base of the style that the descending pollen-tube can enter it at once: but more often the pollen-tubes have to undergo further growth after their entrance into the cavity of the ovary before they reach the micropyles of the ovules; and they are then guided in the right direction by various contrivances. We frequently find papillose projections of the placentæ or other parts of the wall of the ovary, to which the pollen-tubes attach themselves; in our species of Euphorbia a tuft of hairs conducts them from the base of the style to the neighbouring micropyle ; in the Plumbagineæ, the conducting tissue of the style forms a conical descending outgrowth, which conducts the pollen-tube into the micropyle; and so forth. [The conducting tissue is also secretory, and it appears that, as Amici originally suggested (and this view has been recently confirmed by Dalmer), the pollen-tube obtains the materials necessary for its growth from the secretion.]

[We have already seen that two cells (at least) are formed in the pollen-grain of Angiosperms, and that they are sometimes separated by a cellulose wall, but more commonly by an ectoplasmic layer (hautschicht) of protoplasm. The pollen-tube is formed from the larger of these two cells. It sometimes happens that the smaller (vegetative) cell of the pollen-grain is unaffected by the formation of the tube, but more commonly the layer separating the two cells is absorbed, and the two nuclei travel, together with protoplasm, into the growing tube, the nucleus of the larger cell frequently going first.]

Since every ovule requires one pollen tube for its fertilisation, the number of tubes which enter the ovary depends, speaking generally, on the number of the ovules contained in it; the number of pollen-tubes is however usually larger than that of the ovules; where these latter are very numerous, the number of pollen-tubes is therefore also very large, as in Orchideæ, where they may be detected in the ovary even by the naked eye as a shining white silky bundle.

The time that intervenes between pollination and the entrance of the pollentube into the micropyle depends not only on the length of the style, which is often very considerable (as in Zea and Crocus), but also on the specific characters of the plants. Thus, according to Hofmeister, while the pollen-tubes of Crocus vernus only require from twenty-four to seventy-two hours to penetrate the style which is from 5 to $10 \mathrm{~cm}$. in length, those of Arum maculatum take at least five days, although the distance they have to go over is scarcely more than 2 or $3 \mathrm{~mm}$., and those of Orchideæ require ten days or even several weeks or months, during which time the ovules first become developed in the ovary, or even are not formed till then.

The pollen-tube is usually very slender and thin-walled as long as it is increasing quickly in length; after entering the micropyle its wall generally thickens rapidly and often considerably, chiefly, as would seem, by swelling, so that its apical portion communicates with the rest of it by only a narrow channel, or is entirely cut off. Hofmeister compares it, in this condition, to a thermometer-tube (as e.g. in Lilium, I880.-Capus, Anat. du tissu conducteur, Ann. d. Sci. Nat. sér. 6. t. VII:] 
Cactus, and Malva); while sometimes the cavity of the tube becomes wider (as in Enothera and Cucurbitaceæ). Its contents consist of granular protoplasm, usually mixed with a number of starch-grains (the Fovilla).

Within the micropyle the pollen-tube either comes immediately into contact with the naked apex of the embryo-sac, or, as in Watsonia and Santalum, with the projecting striated ends of the synergidæ; but very commonly a portion of the tissue of the apex of the nucellus still remains through which it has to make its way to the embryo-sac. The wall of the embryo-sac is often weak at the apex, and is frequently inflexed by the advancing end of the pollen-tube, or even perforated.

[Strasburger has carefully studied ${ }^{1}$ the process of fertilisation in Torenia asiatica, Gloxinia hybrida, various Orchids, Monotropa, and Pyrola. He finds that when the pollen-tube first comes into contact with the synergidæ or with the embryo-sac, one or both of the nuclei can be distinguished in it near its apex. These very soon disappear, and its contents present a highly refractive, finely granular appearance. The appearance of one or both of the synergidæ now begins to change; its nucleus and its vacuole disappear, and its protoplasm becomes uniformly and highly granular, closely resembling in appearance the contents of the pollen-tube as described above; it loses its form, becoming irregular, and it is closely attached to the oosphere; portions of it may break off and fasten on to the oosphere here and there. The oosphere now becomes granular, and two nuclei can be detected in it; one of these is the nucleus of the oosphere (the female pronucleus), the substance of the other (male pronucleus) has doubtless been derived, through the synergidæ, from the pollen-tube. These two nuclei meet and coalesce, constituting the nucleus of the oospore; this nucleus is often seen to contain two nucleoli, the nucleoli of the male and female pronuclei, which coalesce somewhat later: the oosphere now surrounds itself with a cellulose wall, and with this its conversion into the oospore is complete.]

The further results of this process can usually be observed after a short time in the behaviour of the nucleus of the embryo-sac and of that of the oospore. It frequently however occurs that a considerable time elapses after the entrance of the pollen-tube before the commencement of the development which is induced by it; several days or even weeks in many woody plants, as Ulmus, Quercus, Fagus, Juglans, Citrus, AEsculus, Acer, Cornus, Robinia, \&c.; almost a year in the American Oaks, the seeds of which take two years to ripen; in Colchicum autumnale the pollen-tube enters the embryo-sac at the latest at the beginning of November, but it is not till May in the next year that the formation of the embryo begins. (Hofmeister.)

Even the advance of the pollen-tube through the conducting tissue of the style and into the cavity of the ovary often causes extensive changes in the flower; if the perianth is delicate it usually loses at this time its freshness, fades, and afterwards entirely falls off ; among Liliaceæ it is common for the ovary to commence growing actively even before the fertilisation of the ovules (Hofmeister); in Orchideæ not only is the active growth of the ovary, which often lasts for a considerable time, occasioned by pollination, but the ovules themselves are by it rendered capable of fertilisation; in some cases even their production is thus induced from the placenta which would otherwise remain sterile. (Hildebrand: see also Book III on the Sexual Process.)

${ }^{1}$ [Befruchtung und Zelltheilung, 1878, p. 52 .] 
Results of Fertilisation in the Embryo-sac; Formation of the Endosperm ${ }^{1}$ and Embryo. [The first result of fertilisation seen in the embryo-sac is the division of its nucleus. The action of the pollen-tube on the oosphere is apparent about the same time: the oospore increases in size, elongating in the direction of the long axis of the nucellus. The formation of the endosperm very commonly begins before the division of the oospore, at the latest during the formation of the suspensor. The two nuclei which are formed by the division of the nucleus of the embryo-sac divide again, and this process is repeated until a number of nuclei are formed, lying in the protoplasm which lines the wall of the embryo-sac, the number of nuclei becoming greater as the embryo-sac increases in size. When the embryo-sac has ceased to grow the protoplasm becomes aggregated around the nuclei, and the area of protoplasm surrounding each nucleus is marked out by an ectoplasmic layer, and in this layer a cellulose membrane is formed which is attached externally to the wall of the embryosac, and is free at its inner margin; thus the cells are separated from each other. The cells now increase in size, become vacuolated, and a cellulose wall is formed on their internal surfaces. These processes of cell-formation begin usually at the chalazal end of the embryo-sac and gradually extend to the micropylar.
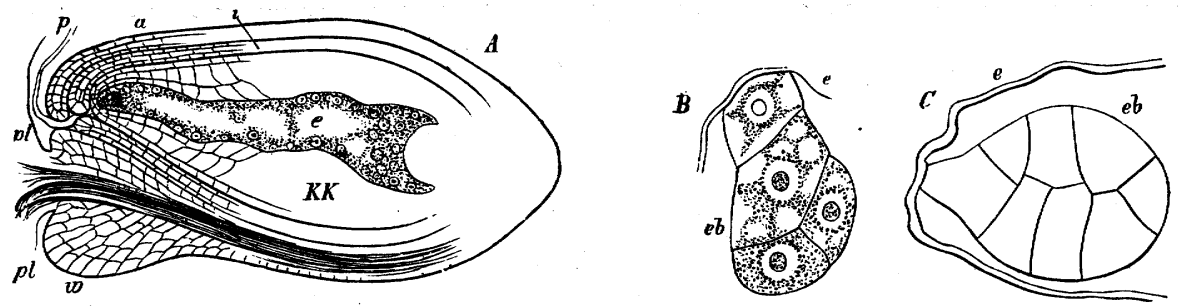

FIG. 400.-Viola tricolor; $A$ longitudinal section through the anatropous ovule after fertilisation, $p l$ the placenta, $w$ cushion on the raphe, $a$ outer, $i$ inner integument, $p$ the pollen-tube which has entered the micropyle, $e$ the embryo-sac containing the embryo (to the left) and a number of young endosperm cells; $B$ and $C$ the apices of two embryo-sacs $e$ with the embryo $e b$ attached to it; the suspensor in $B$ is two-celled.

In many cases the development of endosperm does not proceed beyond this point, for the growing oospore comes into contact with the parietal layer of cells and prevents any further cell-multiplication: in other cases the cells of the parietal layer multiply by division, and thus fill up the embryo-sac.] If the sac increases greatly in size, as, for instance, in Ricinus and in the large-seeded Papilionaceæ, the filling up with endosperm does not take place till later, and the centre of the sac is filled in the unripe seed with a clear vacuole-fluid. In the embryo-sac of the Cocoa-nut, which grows to an enormous size, this fluid-the cocoa-nut-milk-remains until the seed is fully ripe, the tissue of the endosperm forming a layer only some millimetres in thickness, which lines the inside of the testa. The very narrow elongated embryosacs of plants with small seeds, as Pistia and Arum, are filled up by a single longitudinal row of endosperm cells. In a large number of dicotyledonous plants (as Loranthaceæ, Orobancheæ, Labiatæ, Campanulaceæ, \&c.), with long narrow tubular

1 [Hofmeister, Neue Beiträge, Abhand. d. k. sächs. Ges. d. Wiss. VI.-Strasburger, Befruchtung und Zelltheilung; id. Zellbildung und Zelltheilung, 3rd ed.; id. Angiospermen und Gymnospermen. -Hegelmaier, Vergl. Unters. ueb. Entwick. dikotyledoner Keime, I878; id. Zur Embryogenie und Endospermentwickelung von Lupinus, Bot. Zeitg. 1880.-Darapsky, Der Embryosackkern und das Endosperm, Bot. Zeitg. I 879.] 
embryo-sacs, the space of the embryo-sac is first of all divided by two transverse septa, further transverse divisions succeeding in all or some of the cells thus formed, followed by longitudinal ones; the tissue of the endosperm is thus formed, and in this case often fills up only certain parts of the embryo-sac; or the sac is divided by a septum into two daughter-cells, the upper of which contains the rudimentary embryo, and produces endosperm in small quantities by free cell-formation (e.g. Nymphea, Nuphar, Ceratophyllum, Anthurium ${ }^{1}$ ). In a few families only the formation of endosperm is rudimentary, and limited to the temporary appearance of a few free cells or nuclei, as in Tropaolum, Trapa, Naiadeæ, Alismaceæ, Potamogetoneæ, Orchideæ; in Canna even this rudimentary production of endosperm appears to be suppressed.

During the first formation of the endosperm, the embryo-sac usually increases in size, and thus displaces the tissue of the nucellus which still to a certain extent surrounds it; only in a few cases is the nucellus still partially or entirely preserved; it becomes filled with food-materials, like the endosperm, and replaces this latter as a reservoir of reserve-materials for the embryo. In most of the Scitaminea (e.g. Canna), this tissue, the Perisperm, is very strongly developed, while the endosperm is altogether wanting; in the Piperaceæ and in many of the Nymphæaceæ there is a small endosperm in the ripe seed, lying in a hollow of the much larger perisperm.

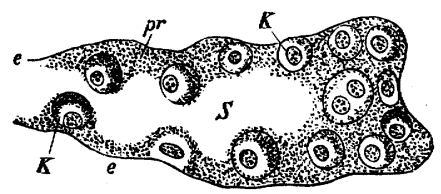

FIG. 401--Viola tricolor, posterior part of the cmbryo-sac, $e$ its cell-wall, $S$ the cavity of the cell, $K, K$ young endosperm-cells which have been produced in the protoplasm pr.

While the endosperm surrounded by the embryo-sac increases in size, the Testa is formed from the development of the integuments which accompanies that of the endosperm; but in Crinum capense and some other Amaryllidex the growing endosperm is stated by Hofmeister to burst the testa and even the wall of the ovary; its cells produce chlorophyll, and the tissue remains succulent and forms intercellular spaces (which does not occur in other cases). In Ricinus a similar growth takes place when the ripe seed germinates in moist earth, bursting the testa (according to von Mohl); and the endosperm, previously ovoid and from 8 to ro $\mathrm{mm}$. long, is transformed into a flat broad sac 20 to $25 \mathrm{~mm}$. in length, which surrounds the growing cotyledons until they have absorbed all the food materials from it.

In Monocotyledons and many Dicotyledons the embryo remains small and is either enveloped by the endosperm or lies by its side (as in Grasses); the cells of the endosperm, which are in close contact without intercellular spaces, become filled, until the seed is ripe, with a protoplasmic substance and fatty matter or starch or both, in which case they remain thin-walled; it then appears as the mealy (full of starch) or fatty portion of the ripe seed, the embryo being found by its side or within it; but it is often horny in consequence of a considerable thickening of its cell-walls which have the power of swelling (e.g. the Date and other Palms, Umbelliferæ, Coffea, \&c.) If this thickening has taken place to a very great extent;

1 For further details on this point see the account of the Dicotyledons. 
the endosperm may fill up the testa as a hard mass, forming, for instance, the 'vegetable ivory' in the Phytelephas. In these cases the thickened walls of the endosperm-cells, which are absorbed during germination together with their protoplasmic and fatty contents, serve for the first nourishment of the embryo. The ripe endosperm, when copiously developed, has usually the form of the entire ripe seed, being uniformly covered by its testa; its external form is therefore generally simple, often round; although considerable deviations from this frequently occur, especially among Dicotyledons. Thus, for instance, the substance known as 'the 'coffee-berry' consists, with the exception of the minute embryo which is concealed in it, entirely of the horny endosperm; but this, as a transverse section shows, is a plate folded inwards at its margins. The marbled (ruminated) endosperm which forms the nutmeg (the seed of Myristica fragrans) and the areca-nut (the seed of the Areca-palm) owes its appearance to the circumstance that an inner dark layer of the testa grows in the form of radiating lamellæ between narrow foldlike protuberances of the light-coloured endosperm. The ripe endosperm is either a perfectly solid mass of tissue, or it possesses an inner cavity, as in Strychnos $N u x$-vomica, where, like the seed itself, it is broad and flat. This is clearly the result of the endosperm which grows inwards from the periphery of the embryo-sac, leaving a free central space, which, as has already been mentioned, is very large and filled with fluid in the case of the cocoa-nut. In these cases the endosperm is therefore a hollow thick-walled sac, enclosing a roundish or flattened cavity.

In a large number of families of Dicotyledons, the first leaves of the embryo, the Cotylédons, grow, before the seeds are ripe, to so considerable a size that they displace the endosperm which was previously present, and finally fill up the whole space enclosed by the embryo-sac and the testa; while the axial part of the embryo, and the bud (plumule) that lies between the bases of the cotyledons, attain even in these cases only inconsiderable dimensions. In these thick fleshy or foliaceous cotyledons (which are then usually folded) the reserve of food-material accumulates, consisting of protoplasmic substance, of starch and fatty matter, which is in other cases stored up in the endosperm, and is made use of during the development of the seedling. This storing of the cotyledons with so large a quantity of food-materials appears to take place by its transference from the endosperm; and hence the difference between those seeds which in the ripe condition contain no endosperm ['exalbuminous'] and those which do contain it ['albuminous'] consists essentially only in the fact that the food-material of the endosperm has passed over in the former case before germination into the embryo; while in the latter case this only takes place during the process of germination. The presence or absence of the endosperm in ripe seeds is more or less constant within large groups of forms, and is therefore of value in classification. Of the better-known families, for example, the Compositæ, Cucurbitaceæ; Papilionaceæ, Cupuliferæ (the Oak and Beech), \&c. are destitute of endosperm. Sometimes also the embryo increases in size to such an extent that the endosperm appears as a thin skin surrounding it.

We must now recur to the oospore in order to follow the formation of the Embryo. The cell-wall of the oospore coalesces superiorly with the wall of the embryo-sac at its apical swelling, its free end being turned towards the base of the ovule; it then lengthens, and undergoes one or more transverse divisions. 
[A row of cells is thus formed, from the lowermost of which, usually a spheroidal cell which we may term the embryo-cell, the greater part of the embryo is in most cases formed; but in many cases (in many Papaveraceæ and Caryophyllaceæ, in Asclepias, Cuscuta, Nicotiana, Viola, and others, amongst Dicotyledons, and commonly among Monocotyledons) the two end cells of the row form the chief part of the embryo, Hanstein considered that the presence of two embryo-cells was characteristic of Monocotyledons, but Hegelmaier has found that a single embryocell occurs in this group (e.g. Ornithogalum natans), and that two embryo-cells frequently occur in Dicotyledons, as mentioned above. The upper cells of the row form the Suspensor. In some cases (Glaucium) the cells of the suspensor undergo longitudinal divisions, and it consists consequently of several rows of cells. Usually the lowest cell of the suspensor, the hypophysis, contributes to the formation of the embryo. In the Gramineæ the cells of the suspensor divide and form a multicellular appendage at the radicular end of the embryo (Keimanhang, Hanstein); when the primary root begins to elongate this mass of cells is split off and it forms a sort of sheath (coleorhiza) to the young root.] The suspensor usually remains short (Fig. 400); sometimes, as in Funkia, its basal cell swells up into a globular form (Fig. 399); in other cases (as, according to Hofmeister, in Loranthus) the oospore lengthens before division, and penetrates to the considerably enlarged base of the long tubular embryo-sac. In those Dicotyledons where the endosperm is formed only at certain lower parts of the embryo-sac by division, a similar elongation of the oospore is usual, although not to so great an extent (e.g. Pedicularis, Catalpa, Labiatæ). In the embryo-cell a longitudinal or only slightly oblique division-wall first of all makes its appearance, indicating the commencement of the formation of the embryo (see also Fig. 15, p. 18). As this is followed by rapidly repeated divisions, a spherical or ovoid mass of small-celled tissue is produced, from which the first foliar structures, the cotyledons, subsequently arise, while the rudiment of the first root may be observed in the differentiation of the tissue at the boundary-line of the suspensor and embryo. The first cells of the embryo are not unfrequently disposed as if they had resulted from oblique divisions of an apical cell in two or three directions (Fig. $400 \mathrm{C}$ ), a supposition which is completely supported by the oblique position of the first septum in the embryo-cell; in Rheum I also found the apex of young embryos to present an appearance which suggested the existence of a three-faced apical cell. According to Hanstein's new and prolonged researches, the process is, nevertheless, different; he asserts that the first longitudinal wall, even when it stands obliquely to the last transverse wall, is still in the median plane of the body of the embryo which is being formed, and is frequently at right angles to the last transverse wall, and therefore in the axis of growth of the suspensor ${ }^{1}$. The formation of this median longitudinal wall in

1 The description in the text is taken from Hanstein's preliminary publications (Monatsberichte der niederrhein. Gesellsch. für Natur- und Heilkunde, July 15 and August 2, 1869), as well as from more detailed communications in letters. Professor Hanstein has also had the kindness to allow me the sight of a number of drawings; and, with his permission, the figs. 402-405 are copied from them. I have also had the opportunity, in the summer of 1869 , of seeing preparations of Hanstein's similar to Fig. 403. Compare also Hanstein, Botanische Abhandlungen, Heft I, for a more detailed description of the development of the embryo in Monocotyledons and Dicotyledons. [See also Quart. Journ. Micr. Soc. 1873, p. 51. The following are some of the more important contributions 
the embryo-cell completely excludes the possibility of a bi- or pluri-seriate segmentation of the apical cell. We learn from Hanstein that the mode of formation of the embryo of Monocotyledons may be seen remarkably clearly in Alisma. In Fig. 402, $I I$, are shown, above the suspensor $v$, two other cells $a$ ( $a$ is formed by the division of the cell $v$ in $I$ ) and $c$ lying one over the other, the last of which is already divided by a longitudinal and a transverse wall into four. cells arranged like quadrants of a sphere. A comparison of the stages $I I-V$ shows that the further development advances first of all in a basipetal direction. A cell $w$ or $h$, the result of intercalary division, which arises at the end of the suspensor next to the embryo $a c$ already formed, is especially to be noted. It is from this that the root is subsequently developed. Hanstein calls it and the tissue which proceeds from it the hypophysis ${ }^{1}$. Before the body of the embryo undergoes any external
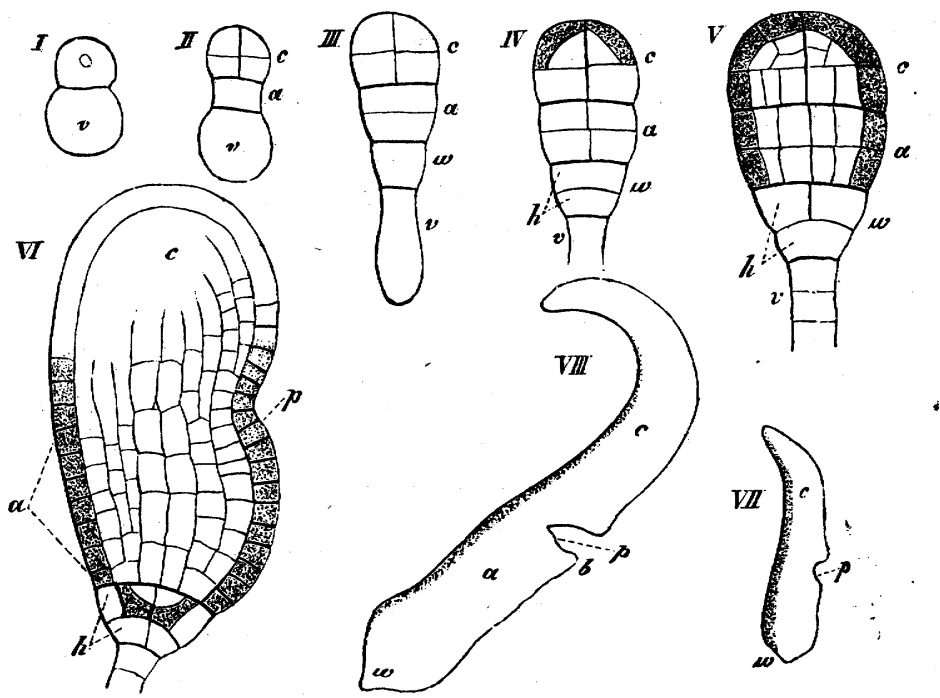

FIG $402,-$ Formation of the embryo of Monocotyledons (Alisma) (after drawings by Hanstein); $I-V I I I$ various stages of development; $v$ the stspensor, $h$ the hypophysis, $w$ the region in which the radicle is formed, $p$ the region in which the plumule is formed, $c$ cotyledon, $b$ first leaf ( $V I I$ and $V I I I$ much less magnified than the rest; the dermatogen is shaded). $I$ consists of the rudimentary suspensor $v$, and of the embryo.cell.

differentiation, its tissue differentiates into a single peripheral layer (shaded in the, drawing), and a tissue internal to this; the former is the primary epidermis or

to the recent literature of the subject: Fleischer, Beitr. zur Embryologie der Monokotylen und Dicotylen, Flora, 1874. - Hegelmaier, Zur Entwick. monokotyledoner Keime, Bot. Zeit. 1874; id. Vergl. Untersuch. üb. Entwick. dikotyledoner Keime, mit Berücksichtigung der pseudo-monokotylen, Stuttgart, 1878.-Solms-Laubach, Ueb. monocotyle Embryonen mit Scheitelbürtigem Vegetationspunkt, Bot. Zeitg. I878. -Treub, Sur l'embryogénie de quelques Orchidées, Amsterdam, I879.Westermaier, Die ersten Zelltheilungen im Embryo von Capsella Bursa pastoris, Flora, 1876.Famintzin, Embryologische Studien, Mém. de l'Acad. Imp. de St. Pétersbourg, sér. 7, t. XXVI, 1879.]

${ }_{1}^{1}$ [According to Famintzin, the cell $w$ or $h$ is derived, not from the suspensor, but from the division of the cell $a$ : this being the case, it is the cell $a$ which must be regarded as the hypophysis. If this is so, the hypophysis, in Alisma, contributes more largely to the formation of the embryo than in other instances. Possibly this explanation may be applied to all cases in which two embryo-cells are said to be present.] 
dermatogen, which continues to grow only in extent and divides only in a radial direction; the figures $I V-V I$ show that the dermatogen is marked off from the primary cells of the embryo by tangential divisions proceeding towards the base. The inner mass of tissue soon undergoes further differentiation; an axial string of tissue is produced by divisions, especially longitudinal, forming the plerome or tissue which subsequently produces the fibro-vascular bundles; the primary meristem lying between the plerome and the dermatogen, and which undergoes copious transverse divisions, is the periblem, i.e. the primary cortical tissue. At the same time that this differentiation of tissue is first indicated in the upper part $a c$ of the embryo, it begins also in the hypophysis $h$. The lower layer of the hypophysis takes no part in the formation of the dermatogen, while from its upper layer (in $V I$ ) is formed a prolongation of the dermatogen and of the periblem of the body of the embryo, from which, as will be explained further on, the root is developed as a posterior appendage of the embryo. Hanstein designates the apical part $c$ of the embryo the cotyledon, at the base of which $b$ the apex of the stem is afterwards formed laterally: the cotyledon is apparently developed from the cells $c$ in $I I$, the apex of the stem and its hypocotyledonary portion (hypocotyl) together with the upper part of the primary root from the cell $a$ in $I I$. But if this apical part is really the cotyledon, which seems to me to be not yet sufficiently established, the cotyledon cannot possibly be a foliar structure (phyllome), even if (as in Allium) it subsequently assumes altogether the appearance of a foliage-leaf.

The different stages in the development of the embryo from the oospore are much more clearly seen in Dicotyledons than in Monocotyledons, the Grasses in particular among the latter presenting difficulties. Hanstein has singled out Capsella Bursa-pastoris for detailed description. Fig. 403 shows first of all that the mass of the embryo is developed from the spherical apical cell of the suspensor $v$, and in what manner this takes place; here also a cell $h$ of the suspensor forms the hypophysis which contributes to the formation of the primary root (radicle). The spherical primary embryo-cell divides first by a longitudinal wall $\mathbf{I}-\mathbf{I}$ (in $I-I V$ ); [each of the two cells thus formed is then divided into two by a longitudinal wall at right angles to the first, so that the embryo now consists of four cells which are quadrants of a sphere;] this is followed in each of the quadrants by a transverse division 2-2, so that the body of the embryo now consists of eight cells (octants of a sphere), each of which next undergoes a tangential division, by which eight outer cells are formed as the rudiment of the dermatogen, and eight inner central cells $(I I)$. While the first only multiply by radial divisions, the inner mass of tissue grows in all directions, resulting at an early period in its differentiation into plerome (III, IV, $V$, shaded in the drawing) and periblem. The mass of tissue which is produced from the primary embryo-cell thus increases rapidly by the multiplication of its cells, and two large protuberances $(V, c c)$, the first leaves or cotyledons, soon make their appearance one on each side of the apex $(s)$; the apex of the stem exists for the present only as the end of the longitudinal axis of the embryo; an elevated mass of tissue, the vegetative cone of the stem, is not formed till later, deeply enclosed between the cotyledons. The posterior or basal end of the axis of the embryo after the differentiation of its primary meristem into dermatogen, periblem, and plerome $(I I, I I I, I V)$, is, so to speak, open, as long as this differentiation 
has not also taken place in the hypophysis $(h)$; but finally it takes place in it also and in such a way (as is shown in Fig. 403,V) that the upper of its two cells breaks up into two layers $\left(h^{\prime}\right)$, the outer of which becomes continuous with the dermatogen of the axis, while the inner layer forms a prolongation of the internal axial tissue. The lower cell of the hypophysis $(h)$ divides cross-wise ( $V b$, seen from below) and may be regarded as a transitional structure between suspensor and root (appendage of the root) or as the first layer of the root-cap. The root-cap is formed in this case simply by a luxuriant growth of the dermatogen. The dermatogen, which elsewhere remains simple, and passes over into permanent

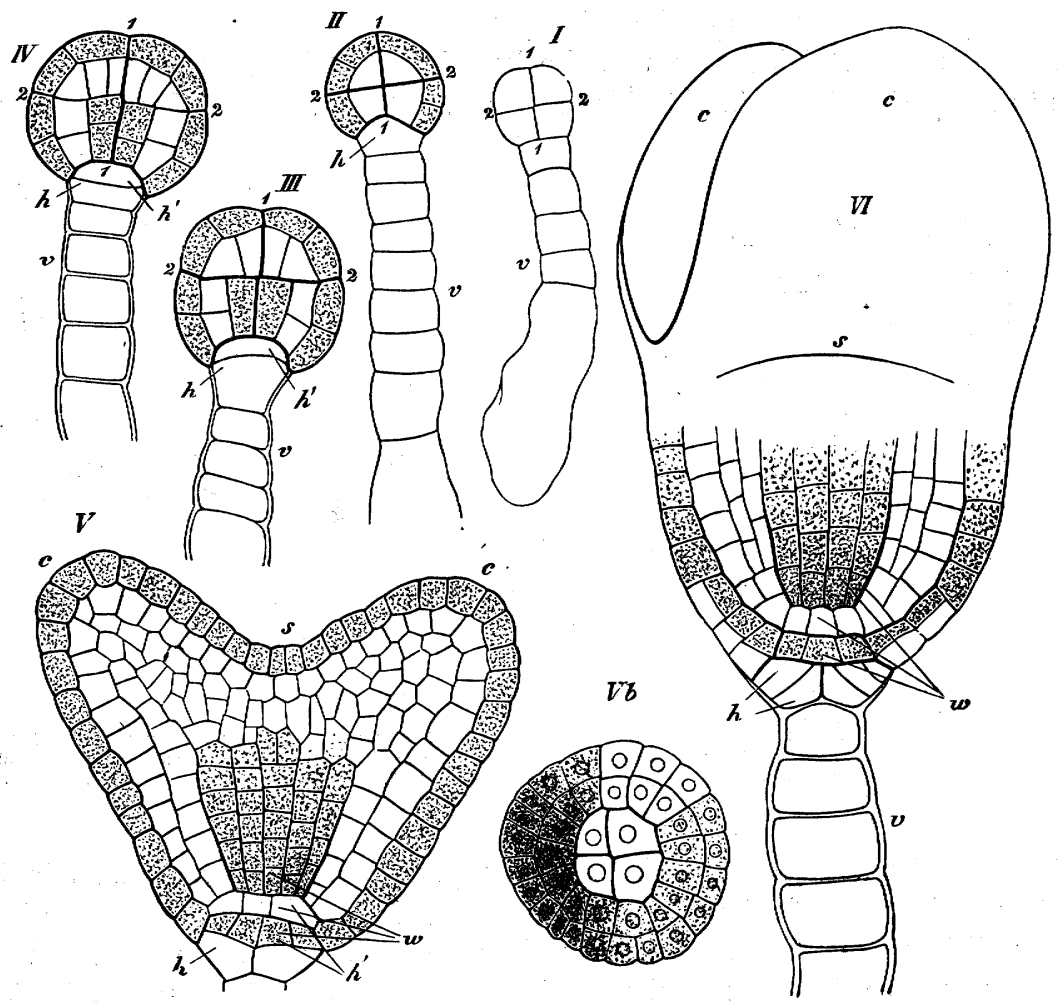

FIG. 403.-Development of the embryo of Capsella Bursa-pastoris (after Hanstein); I-VI various stages of development, $V b$ apex of the root seen from below; $x, I, 2,2$, the first divisions of the embryo-cell, $h h$ the hypophysis, $v$ the suspensor, $c$ the cotyledons, $s$ apex of the axis, $w$ root (the dermatogen and plerome are shaded).

tissue in forming the epidermis, increases in thickness, on the contrary, where it covers the punctum vegetationis of the root and undergoes repeated periclinal divisions (parallel to the surface). Of the two layers which are successively formed on each of these occasions, the outer becomes a layer of the root-cap (Fig. 404 wh, and Fig. 405, 2); the inner remains as dermatogen and again undergoes the same process. This dermatogen which covers the vegetative cone of the root behaves therefore like a layer of phellogen, with this difference, that the cells produced from cork-cambium become at once permanent cells, while those of the root-cap remain still capable of division; so that each layer split off as it 
were from the dermatogen forms a cap consisting of several layers of cells, its growth being most active in the centre, and diminishing towards the periphery. The splitting of the dermatogen into two lamellæ usually progresses from the centre towards the periphery of the apex of the root; in the secondary roots of Trapa, Hanstein and Reinke state that the reverse is the case ${ }^{1}$.

Lateral roots not unfrequently arise in the embryo even before the ripening of the seed, in addition to the primary root which we have hitherto alone considered; as, for instance, in many Grasses and some Dicotyledons (e.g. Impatiens, according to Hanstein and Reinke, Cucurbita from my own observations). In Trapa natans the primary root soon becomes abortive, lateral roots arising at an early period from the hypocotyledonary portion of the axis.

Hanstein and Reinke state that the lateral roots of Angiosperms have their origin in the pericambium, in Nägeli's sense of the term ${ }^{2}$. Their development was

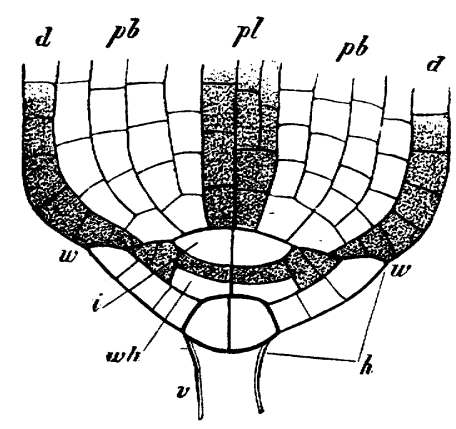

FIG. 404.- Diagrammatic representation of the formation of the primary root in Monocotyledons and its connection with the stem (after Hanstein); $v$ suspensor, $h$ hypophysis, $w w$ line of separation of the root and stem, wh layer of the root-cap, $d$ dermatogen, $p b$ periblem, $p l$ plerome.

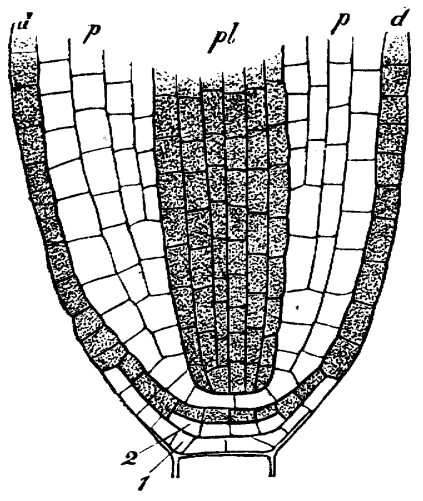

FiG. 405.-Diagrammatic representation of the same in the case of Dicotyledons (after Hanstein) 1,2, the first layers of the root-cap, $p$ periblem, $d$ derma togen, $p l$ plerome.

found in several plants to harmonise with this. In Trapa natans, for example, it is as follows :-A group of cells of the pericambium, which consists of only one layer, divides radially; the newly-formed cells elongate in the same direction, and then divide tangentially; the outer of the two layers produces the dermatogen, the inner the body of the root. The dermatogen, pushed outwards by the development of the body of the root, produces the root-cap in the way already mentioned; the tissue of the body of the root itself which is covered by it becomes differentiated into plerome and periblem. The same process takes place in Pistia, and probably also in Grasses. Hanstein and Reinke do not- find 'anywhere an apical cell which originates the growth, as in Cryptogams, but a group of cells which obey a common law of growth.'

The variation in the size of the embryo in the ripe seed of Angiosperms has

1 [See the account of the apical growths of roots and of the development of lateral roots which is given in the Appendix.]

${ }^{2}$ Compare what was said on Fig. 125, p. 167. 
already been mentioned when speaking of the endosperm. The external differentiation sometimes goes no further than the rudiment of the root (radicle) at the posterior end of the stem of the embryo, and the cotyledons (e.g. in Cucurbita, Helianthus, Allium Cepa, \&c.), between which lies the naked punctum vegetationis. But frequently this latter undergoes further growth before the seed is ripe, and produces additional foliar structures (as in Grasses, Phaseolus, Faba, Quercus, Amygdalus, \&c.), which are then included, in the ordinary nomenclature, under the term Plumule, but do not unfold until the germination of the seed. The systems of tissue are usually sufficiently clearly differentiated as such at the period of maturity of the seed; but the different forms of permanent tissue do not become developed till later, during germination. A striking exception to this advanced development of the young plant within the ripening seed is afforded by parasites and saprophytes destitute of chlorophyll, but especially by Orchideæ. In them the embryo remains until the seed is ripe as a small round body consisting sometimes of only a few cells, without any external differentiation into stem, leaves, and root; this takes place only after germination, and even then sometimes quite imperfectly.

Polyembryony and Parthenogenesis ${ }^{1}$. In a few cases polyembryony, that is the presence of more than one embryo in a single seed, has been found to occur in Angiosperms, but it is brought about in a way which is very different from that in which, as we have seen, it is caused in Gymnosperms. It was thought by Hofmeister that, in the cases which he investigated (Funkia corulea, Scabiosa, Citrus), a number of oospheres were formed in the parietal protoplasm of the embryosac, and that these were fertilised, but that of the large number of rudimentary embryos thus formed, which is very considerable especially in Citrus, only a few become fully developed. [This subject has been carefully investigated by Strasburger, and he has found that these embryos are not formed from oospheres, but are developed as outgrowths from the cells of the nucellus which bound the embryosac. In some cases (Funkia, Nothoscordum fragrans, Citrus) it appeared as if this adventitious development of embryos were dependent upon the fertilisation of the oosphere; a development of an embryo from the oospore, in addition to the adventitious development of embryos from the nucellus, was only observed in Citrus.

Coelebogyne ilicifolia has long been known as a plant with polyembryonic seeds, and it has been observed that these fertile seeds are produced without pollination of the female flower. It was concluded that this plant was an instance of parthenogenesis, that is, of the development of an embryo from an unfertilised oosphere. Strasburger has found that its numerous embryos are developed adventitiously in the manner described above: it is therefore not parthenogenetic.]

Development of the Seed and Fruit. While the endosperm and embryo are becoming perfectly formed in the embryo-sac, growth proceeds not only in the ovule but also in the wall of the ovary that encloses it. Since the testa is formed

1 [Hofmeister, Die Lehre von der Pflanzenzelle, i867, p. I 14.-Braun, Ueber Parthenogenesis bei Pflanzen, Berlin I857; id., Ueber Polyembryonie und Keimung von Calebogyne, Berlin 1860.Braun und Hanstein, Die Parthenogenesis der Coelebogyne ilicifolia, in Hanstein's Botanische Abhandlungen, III, I877.- Strasburger, Ueber Befruchtung und Zelltheilung, I878. See also the section on Parthenogenesis in Book III.] 
at the expense of the whole or part of the cellular layers of the ovular integuments ${ }^{1}$, and presents extreme diversities in its structure, the ovule, together with its contents which have resulted from fertilisation, becomes the Seed. The wall of the ovary, the placentæ, and the dissepiments, not only increase in dimensions, but undergo the most various changes of external form and still more of internal structure. Together with the seeds they constitute the Fruit. The transformed wall of the ovary now takes the name of Pericarp; if an outer epidermal layer is specially differentiated it is called the Epicarp, and the inner portion the Endocarp; while a third layer, the Mesocarp, frequently lies between these two. A number of typical kinds of fruit are distinguished according to the original form of the ovary and the structure of its tissue when ripe, the nomenclature of which will be given in the sequel. But sometimes the long series of deep-seated changes induced by fertilisation extends also to parts which do not belong to the ovary, and even to some which have never belonged to the flower. But as they are part of the fruit from a physiological point of view, and are usually associated with it as a whole, while sharply differentiated from the rest of the plant, a structure of this kind (such as the Fig, Strawberry, and Mulberry) may be termed a $P_{\text {seudocarp. }}$.

At a certain period either the fruit together with its seeds becomes detached from the rest of the plant, or the seeds alone separate from the dehiscent fruit; and this is the period of maturity. In many species the whole plant dies down when the fruit is ripe, and a plant of this description is termed monocarpic (bearing fruit only once). Monocarpic plants may be distinguished into those which fructify in the first period of vegetation (annual plants), those which do not till the second year (biennial plants), and finally not till after several or a large number of periods of vegetation (monocarpic perennial plants, as Agave americana). Most Angiosperms are however polycarpic; i.e. the vital power of the individual is not exhausted by the ripening of the fruit; the plant continues to grow and periodically fructifies afresh, or is polycarpic and perennial.

I. The Inflorescence ${ }^{2}$. It is comparatively rare for the flowers of Angiosperms to arise singly at the summit of the primary shoot or in the axils of the leaves; peculiarly developed branch-systems are much more commonly produced at the end of the primary shoot or in the axils of its foliage-leaves, which usually bear a considerable number of flowers and are distinguished by their collective form from the rest of the vegetative body; in polycarpic plants these may even be thrown off after the ripening of the fruit. Such a system of branching is termed an Inflorescence. The habit of the inflorescence does not depend merely on the number, form, and size of the flowers which it bears, but also on the length and thickness of the branches of different orders, as well as on the degree of development of the leaves from the axils of which the branches spring. These leaves are generally much simpler in form and smaller than the foliage-

1 [From the very extensive recent literature on the structure and development of the seed-coats the following may be cited: Lohde, Ueb. d. Entwickelungsgeschichte und den Bau einiger Samen. schalen, Schenk's Mittheilungen, II, I875.-Sempolowski, Beitr. z. Kennt. des Baues der Samenschale, Leipsig, 1874.-Hegelmaier, Ueb. Bau und Entwickelung einiger Cuticulargebilde, Jahrb. f. wiss. Bot. IX, 1874.-Chatin, Le dév. de l'ovule et de la graine, Ann. de Sci. Nat. sér. 5, t. XIX, I874.von Höhnel, Morphol. Unters. üb. die Samenschalen der Cucurbitaceen, Sitzber. d. Wien. Akad. LXXIII, 1876.-Haberlandt, Entwick. und Bau der Samenschale von Phaseolus, ibid. LXXV, 1877.]

2 [See also Eichler, Bluithendiagramme, I, and Asa Gray, Structural Botany, I880.] 
leaves; frequently coloured (i.e. not green) or altogether colourless. They are distinguished as Hypsophyllary Leaves or Bracts; and in this term are frequently included the small leaves which spring trom the pedicels and which often have no axillary shoots (Bracteoles). Leaves of this kind are sometimes entirely absent from the inflorescence or from certain parts of it; the ultimate floral axes or pedicels of the flowers are then not axillary, as in Aroidex, Cruciferæ, \&c.

A large number of different forms of inflorescence may arise by the combination in different ways of the determining characters already mentioned. Each form is constant in the same species, and is often characteristic of a whole genus or family; hence the form of the inflorescence often not only determines the habit of the plant, but is also of value to its systematic classification.

The most convenient basis for the classification of the forms of inflorescence is the mode of branching. This is less variable than the other features, and can be referred to a few types; it also affords distinctive characters for the principal groups, which might then be further sub-divided according to the length and thickness of the separate axes and other points.

With reference to the mode of branching, the first point to observe is that every inflorescence originates from the normal terminal branching of a growing axis; the mode of branching is always monopodial in Angiosperms with the exception of the cases mentioned under Division $\mathrm{r}_{4}$; i.e. the branches arise laterally beneath the apex of the growing mother-shoot. If the leaves on this shoot (the bracts) are conspicuously developed, the lateral axes arise in their axils; if they are inconspicuous or abortive, the lateral axes of the inflorescence are not indeet axillary, but their mode of branching and growth remain the same as if the bracts were present; and it is usual, in framing the divisions, not to lay great stress on this circumstance (see p. 176 ). But the presence of bracts is of great practical value, since it assists in the recognition of the true mode of branching even in the mature inflorescence, inasmuch as the axillary shont is always lateral. When the bracts are absent it is often difficult to distinguish a lateral from a primary axis, since the former often grows as vigorously as the latter, or even more so. In Section 24 of the chapter on General Morphology (p. I69 et seq.) the principles have been laid down according to which the various systems of branching may be generally classified; these will serve also in every respect for inflorescences, and form the basis of the characters of the larger groups in the following classification. Of the great number of separate forms of inflorescence only the more common ones, a nomenclature for which is already provided in systematic botany, will be enumerated ${ }^{1}$.

A. Racemose (monopodial), Centripetal, or Indefinite Inflorescences, in the widest sense of the terms, result from the primary axis or rachis of the branching system producing a larger or smaller number of lateral shoots in acropetal succession; the capacity for development of each lateral shoot being smaller, or at least not greater, than that of the portion of the primary axis which lies above it.

a. Spicate Inforescences arise when the lateral axes of the first order do not branch and are all floral axes; the rachis terminates with or without a flower.

(a) Spicate Inflorescences with elongated rachis:-

r. The Spike: Flowers sessile; rachis slender (as in some Grasses).

2. The Spadix: Flowers sessile; rachis thick and fleshy, usually enveloped in a large spathe; bracts generally undeveloped (Aroidex).

3. The Raceme: Flowers distinctly stalked (e.g. Cruciferæ, without bracts; Berberis, Menyanthes, Campanula, rachis terminating in a flower).

1 Compare the dissimilar descriptions in Ascherson's Flora of the Province Brandenburg, Berlin, I 864, and in Hofmeister's Allgemeine Morphologie, $\S 7$. 
( $\beta$ ) Spicate inflorescences with abbreviated rachis :-

4. The Capitulum : Rachis conical or tubular, or even hollowed out like a cup ; flowers sessile; bracts frequently absent (Compositæ, Dipsacaceæ).

5. The Simple Umbel: Flowers stalked and springing from a very short rachis (e.g. the Ivy).

b. Panicled Inflorescences arise when the lateral axes of the first order again branch and produce axes of the second and higher orders; every axis or only those of the last order may terminate in a flower; the capacity for development usually decreases from below upwards both on the lateral and on the primary axis.

(a) Panicled Inflorescences with elongated axes:-

6. The true Panicle: Axes and pedicels elongated (Crambe, Grape-vine).

7. The Compound Panicle made up of Spikes: The elongated lateral axes bear sessile flowers (Veratrum, Spirca Aruncus, the 'ears' of Wheat, Rye, \&c.).

( $\beta$ ) Panicled Inflorescences with abbreviated axes :-

8. Compact spike-like Panicle: The very short lateral axes are arranged on an elongated primary rachis (the 'ears' of Barley, Alopecurus, \&c.).

9. The Compound Umbel: The very short rachis bears a densely compact umbel of secondary (partial) umbels usually with long stalks ( $f f$. No. 5); if the compound umbel is surrounded by a whorl of leaves this is called the Involucre; a similar whorl surrounding the secondary umbel is an Involucel (secondary involucre); one or both may be absent; (most Umbelliferæ).

B. Cymose, Centrifugal, or Definite Inflorescences result from the primary axis branching beneath the first flower in such a manner that each lateral axis itself terminates in a flower, after producing one or more lateral axes of a second order which in their turn terminate in flowers and continue the system in this manner; the development of each lateral shoot is stronger than that of the primary axis beyond the point of origin (see Figs. 134-136, pp. 178-180).

a. Cymose Inflorescences witbout a Pseud-axis: Two or more lateral axes are developed beneath each flower, terminating in flowers; lateral axes of a higher order continuing the system in the same manner.

10. The Anthela: An indefinite number of lateral axes are produced on each axis, and overtopping the primary axis develope in such a manner that the entire inflorescence does not acquire any definite shape (e.g. Juncus lamprocarpus, tenuis, alpinus, and Gerardi, Luzula nemorosa, \&c. $\left.{ }^{1}\right)$. The anthela of these genera, as well as of Scirpus and $C y p e r u s$, exhibits a number of different transitional forms to the panicle and even to the spike, and on the other hand to the formation of cymose inflorescences with pseud-axes, e.g. in Juncus bufonius. The inflorescence of Spirca Ulmaria is included in this form by myself and others.

I I. The Cymose Umbel : A whorl of three or more equal axes springs from the primary one, secondary whorls of lateral axes being again produced from it, and the process being then again repeated (see Fig. 148). The whole system resembles a true umbel in habit;

${ }^{1}$ Compare the careful description by Buchenau in Jahrb. fuir wissensch. Bot. IV, p. 393 et seq. and Pl. 28-3o. 
very good examples are afforded by several species of Euphorbia, especially $E$. Latbyris and belioscopia. This form of cyme is not essentially distinct from the next, and in the highest orders of branching commonly passes into it; in Periploca graca, for example, even in the first ramification.

I 2. The Dichasium : Each primary axis terminating in a flower produces a pair of opposite or nearly opposite lateral axes, which in their turn produce pairs of the second order, and so on. The whole system appears as if composed of bifurcations, especially after the older flowers have fallen off; as in Euphorbia, many Sileneæ, some Labiatæ, \&c. The dichasium easily passes, in the first or a succeeding order of lateral axes, into a sympodial mode of development.

b. Cymose Inflorescences with a Pseud-axis (Sympodial Inforescences). Each axis which terminates in a flower bears only one lateral axis of the next order. The basal portions of the consecutive orders of axes may lie more or less in a straight line, and may become thicker than the flower-stalk (above the branching). A pseud-axis or sympodium may thus become either straight or curved first in one direction and then in another, the flowers appearing to be produced on it as lateral shoots (see Fig. $136, A, B, D, \mathrm{p}$. 180). If the sympodium is clearly developed, it resembles a spike or raceme, from which however it is easily distinguished when bracts are present by their being apparently opposite to the flowers (as in Heliantbemum); but displacement not unfrequently causes it to assume a different form (as in Sedum).

13. The Unilateral Helicoid Cyme (Bostryx) is a sympodial cyme in which the median plane of each of the successive axes which constitute the system is always situated on the same side, whether right or left, with respect to the preceding one (see Fig. I $36, D$ ); as for instance, in the primary branches of the inflorescence of Hemerocallis fulva and Aava, and in the partial inflorescences of Hypericum perforatum which are themselves arranged in a panicle. (Hofmeister.)

14. The Unilateral Scorpioid Cyme (Cicinnus) is one in which the successive axes arise alternately to the right and left of the preceding one (Fig. I $36 \mathrm{~A}$ ), as in Helianthemum, Drosera, Tradescantia, and Scilla bifolia. (Hofmeister.) The inflorescence of Echeveria belongs also to this kind of originally monopodial sympodium; the mature cyme has a pseud-axis on which the flowers are placed opposite the leaves. While the summit of each successive axis is converted into a flower, a lateral axis arises in the axil of the subtending leaf. This lateral axis developes further, forms a new leaf in a plane nearly at right angles to the last, and becomes transformed into a flower, while a lateral axis appears in the axil of its leaf which continues the development; the leaf which arises on this axis is in the same plane as the last but one. (Kraus.)

The inflorescences of the Boraginex and Solanacex differ both in their mode of development and in their external appearance from the plan described in $B b$. Kaufmann has already stated ${ }^{1}$ that the inflorescence of some Boraginex is the result of repeated

' Kaufmann, Bot. Zeitg. 1869, p. 886 [and Nouv. Mém. de la Soc. Imp. des Nat. de Moscow, XIII, p. 248]. [Kaufmann's observations have been confirmed by Warming (Ramification des Phanérogames, $187^{2}$ ), by Pedersen (Bot. Tidșkrift, 1873 ), and by Kraus (Bot. Zeitg. I871) in so far as bracteate scorpioid cymes are concerned. (See also Wydler, Zur Morph. d, dichotomen Blïthenstände, Jahrb. f. wiss. Bot. XI, 1878 ). Warming considers that dichotomy also occurs in naked scorpioid cymes, but Kraus states that these are monopodial, and Warming admits that lateral 
dichotomy of the apex of an axillary bud; and Kraus has also shown that the leafless inflorescence of Heliotropium and Myosotis is a monopodium, at all events when luxuriant. A thick and flattened vegetative cone developes two alternate rows of flowers on its upper side; on this side the longitudinal growth of the primary axis is at first stronger; and the younger part of the inflorescence is consequently rolled with its apex downwards in a circinate manner. An inflorescence which is formed in this manner, as will he seen from what has already been said, cannot properly be described as a scorpioid cyme, but corresponds rather to a raceme or spike which bears flowers only on one side of its rachis. The leafy scorpioid cymes of Ancbusa, Cerinthe, Borago, and Hyoscyamus are, on the contrary, the result of dichotomous branching; a leaf which stands on the primary axis ending in a flower bears in its axil a vegetative cone which is at first hemispherical; this becomes broader and dichotomises in a direction parallel to the surface of the leaf; one of the bifurcations becomes a flower, the other bears a new leaf at right angles to the last, and forms a dichotomy above it as before. The planes of dichotomy therefore cross one another at right angles; and this is the reason why the leaves always stand between the sympodial axis and the flower. Lateral displacements of the leaves begin at the second division and continue afterwards.

According to Kraus it is doubtful whether the sympodial inflorescences of Ompbalodes and Solanum nigrum are the result of dichotomous or of lateral branching. On the side of the primary axis which becomes a flower a leafless lateral axis arises which continues to branch, and the right and left lateral axes of which are alternately transformed into flowers. Kraus entertains a similar doubt respecting weak inflorescences of Myosotis and Heliotropium (vide supra).

It will be seen from what has now been said, that within an inflorescence which consists of several orders of axes there may be produced not only different forms of one section, but forms belonging to both sections ( $A$ and $B$ ), mixed inflorescences being thus formed. Thus, for example, a panicle may form dichasia in its last ramifications (as in some species of Silene); a dichasium may bear capitula (e.g. Silpbium), or even in its first branches or in those of a higher order may pass into a helicoid or scorpioid cyme (as in Caryophyllex, Malvaceæ, Solanaceæ, Linaceæ, Cynanchum, Gagea, Hemerocallis, \&c.). The mode of branching of the inflorescence is in most cases different from that of the vegetative stem. Not unfrequently it passes abruptly from one to the other, but often through intermediate modes of branching.

In the older systems of nomenclature a number of other terms are given to various forms of inflorescence, such as glomerulus, corymb, \&c.; but they all designate merely the habit or external form of the system, and must be referred, in a scientific description, to one or other of the above forms or to combinations of them.

2. With regard to the Change in the Mode of Branching accompanying the transition from the vegetative to the floral region of a shoot, Warming gives some very valuable information in his Recherches sur la ramification des Phanerogames (Kopenhagen I 872), from which it appears that the numerous cases of extra-axillary branching in inflorescences can be referred to axillary branching as the typical mode. He lays it down that the axillary branch with the corresponding leaf are to be regarded as a whole, one

branching usually takes place in weak inflorescences. Goebel (Arb. d. Bot. Inst. in Würzburg, II, I880) finds not only that Kraus' account of the development of the inflorescence of Myosotis and of Heliotropium is accurate, but that it applies also to that of Symphytum officinale, Anchusa, Cerinthe, Borago, Cynoglossum, Echium vulgare, Lithospermum arvense, and Caryolopha sempervirens; the development of the flowers is lateral also in Hyoscyamus niger, Klugia Notoniana, and in Helianthemum. Goebel's conclusions have been combated by Celakovsky (Flora, I880). It must be borne in mind that dichotomous and lateral branching are not absolutely distinct forms, but that they are connected by intermediate conditions, so that they gradually merge one into the other. (See also Henslow, On the Origin of the so-called Scorpioid Cyme, Trans. Linn. Soc., I 880 ; and the History of the Scorpioid Cyme, Journ. of Botany, I88I.)] 
part of which-the leaf,--or the other part-the branch,--may be developed earlier than the other or simultaneously with it, or more or less completely than it. It is evident in the vegetative region that the subtending leaf always arises first, and developes more actively, to begin with at any rate, than the corresponding branch which only becomes apparent when one or more young leaves have arisen above the leaf in question. (Figs. I29, I3 r.) In many inflorescences the development of the leaf precedes that of the axillary branch by a much shorter interval, as in the spikes and racemes of Amorpha, Salix, Rudbeckia, Lupinus, Veronica, Digitalis, Orcbis, Delpbinium. In the development of other inflorescences the axillary branches are formed immediately after their subtending leaves, so that no rudimentary leaf intervenes between the apex of the shoot and the youngest axillary branch (Plantago, Orcbis, Epipactis). Sometimes leaf and branch arise simultaneously, as in the Gramineæ, Cytisus, Trifolium, Orcbis, Plantago, Ribes. Or again, the axillary branch is formed first, before its subtending leaf, in which case the leaf attains only a slight development, its presence being merely indicated as in Sisymbrium, Brassica and other Cruciferæ, Umbelliferæ, Anthemis, Valeriana, Asclepiadex, Bryonia, Cucumis. Or the subtending leaf may not make its appearance at all, and no bracts are developed, as in many Cruciferæ (Fig. I 32), Compositæ, Gramineæ, Umbelliferæ, Papilionaceæ, Cucurbitaceæ, Asperifolieæ, Solaneæ, Hydrophyllex, Saxifragex, Potamogetonex. In all these inflorescences the youngest buds are nearer to the apex of the parent shoot than any foliar organs in so far as these have been developed, but the branching must not on this account be regarded as dichotomous. A dichotomy of the parent shoot only takes place when a vigorous branch is developed so near to the apex that a continuation of the direction of growth of the shoot is rendered impossible, its apez apparently dividing into two or more apices. According to Warming this is the case in Hydrocharis, Vallisneria, the Asclepiadex, the scorpioid cymes of the Solaneæ, Asperifolieæ, Hydrophylleæ, Cistaceæ, and many Cucurbitaceæ. This tendency to dichotomise shown by plants the vegetative parts of which branch in a lateral axiliary manner is doubtless connected with the suppression of the development of leaves in the inflorescences, and this is confirmed by the fact that the tendrils of Vitis and Cucurbita, on which the development of leaves is rudimentary, exhibit the same tendency.

The axillary branches of the vegetative region are usually so placed that they arise both from the basis of the leaf and from the tissue of the stem; but it sometimes happens that the branch is entirely transferred to the stem and becomes isolated from the leaf. In the floral region, on the other hand, it not unfrequently happens that the axillary branch (the inflorescence) arises solely from the leaf, as in Hippuris (Fig. I 19), Amorpha, Salix nigricans. If, however, the subtending leaf (bract) is developed later than the axillary branch (inflorescence), it may arise from it, so that the leaf has no direct connection with the parent shoot, but appears to be the first lowest leaf of the lateral branch; this is the case, according to Warming, in Antbemis, Sisymbrium, Umbelliferæ, and to a slight degree in Papilionacex, Orchidex, Valerianex, and others. These relations are usually evident at the earliest stage of development, but frequently the subtending leaf is found upon the axillary branch in the mature condition, as in Thesium ebracteatum, Samolus Valerandi, Boragineæ, Solaneæ, Crassulaceæ, Spiraa, Loranthaceæ, Ipomaa bona nox, Agave americana, Ruta, Paliurus, Tilia (in which this applies to the large bract of the inflorescence), and others.

3. Number and Relative Position of the Parts of the Flower ${ }^{1}$. Just as the forms of branching of the inflorescence are usually different from those of the vegetative stem, the arrangement of the leaves of Angiosperms is also usually different on the shoot which constitutes the flower from that on other parts of the same plant. The cessation of the apical growth of the receptacle, its great increase in breadth, or even hollowing out, before and during the time when the perianth and the sexual organs are being formed,

\footnotetext{
1 [See Eichler, Blüthendiagramme.]
} 
influences their order of succession and their divergence from one another. But since, notwithstanding the extraordinary variation of the other relations of form, the true position of the floral leaves varies but little - though it may often be difficult to determine - the knowledge of this position is often of great importance in the determination of the affinities of the species, and hence for purposes of classification. This is especially the case if we at the same time take into account the abortion of individual members which is here of so common occurrence, the multiplication of the parts which take place under certain circumstances, and their branching and cohesion.

In order to facilitate a description of these relationships, it is necessary to explain certain terms and methods of description.

In the first place it is important to denote the position of all the parts of a flower with respect to the mother-axis of the floral shoot. For this purpose the side of the flower which faces the mother-axis is termed the posterior, that which is most remote from it the anterior side. If a plane be imagined to divide the flower longitudinally from front to back, and to include the primary axis of the flower as well as that of the mother-shoot, this is the median plane of the flower, dividing it into a right and a left half. Floral leaves, as well as ovules and placentæ, which are bisected longitudinally by the median plane, are said to have a median position, either posterior or anterior. If another plane is now imagined at right angles to the first, and also including the axis of the flower, it may be termed the lateral plane; this plane divides the flower into a posterior and an anterior half, and parts which are longitudinally bisected by it are precisely lateral. The two planes which bisect the right angle between the median and the lateral planes may be called diagonal planes, and the parts which are bisected by them be said to have a diagonal position. Flowers usually have some of their floral organs placed exactly posteriorly or anteriorly, not so commonly exactly right and left or exactly diagonally; but usually other additional terms must be used, such as obliquely posterior or obliquely anterior.

If next the position of the parts of the flower with respect to one another be examined, their arrangement, as has already been mentioned, is either spiral or verticillate.

Flowers with a spiral arrangement of their parts are comparatively rare, and apparently occur only in certain orders of Dicotyledons (Ranunculaceæ, Nymphæaceæ, Magnoliacex, and Calycanthacex). Braun has termed such flowers acyclic, when the transition from one foliar series to another, as from calyx to corolla or from corolla to stamens, does not coincide with a definite number of turns of the spiral (as Nymphæaceæ and Helleborus odorus); bemicyclic when it does so coincide. This latter term may also be employed when some of the foliar structures are actually cyclic (verticillate), others spiral, as in Ranunculus, where the calyx and corolla form two alternating whorls, followed by the stamens and carpels arranged spirally. Parts which have a spiral arrangement sometimes occur in definite numbers, more often in larger indefinite numbers.

When on the other hand the parts of the flowers are arranged in whorls, the number of the whorls, as well as that of the members of each whorl, is constant in the same species, and within larger or smaller circles of affinity ${ }^{1}$. When the number of members

1 [The number of whorls in a flower may vary very widely, from one (Carex) to fifteen or sixteen (Aquilegia). In some cases the calyx, corolla, andrœcium, and gynæceum each consists of a single whorl, so that the flower has four whorls. More commonly, however, one or other of these series consists of more than one whorl. This is most frequently the case in the androecium, so much so in fact that it is customary to regard the typical flower as containing two whorls of stamens: in an isomerous flower, if the stamens are in a single whorl it is said to be isostemonous, if in two whorls diplostemonous, and so on. The calyx often consists of more than one whorl (Menispermaceæ, Berberidaceæ), and in most tetramerous flowers (Cruciferæ, Onagraceæ) it is composed of two decussating dimœrous whorls. More rarely the corolla consists of more than one whorl ; instances of this occur in the Fumariaceæ, Berberidaceæ, Papaveraceæ, Menispermaceæ. A gynæceum of two, 
of each whorl is the same, and those belonging to the different whorls are placed one over another so as to form orthostichies, I adopt Payer's expression of superposed (instead of the ordinary one of 'opposite'). When the stamens are superposed on the calyx or corolla, they are termed respectively antisepalous and antipetalous; if the members of a whorl fall between the median lines of those of the next whorl above or below, the whorls are alternate. When the number of members is the same in each.whorl, they are said to be isomerous, when this is not the case beteromerous; and Braun calls those flowers eucyclic in which the members of all the whorls are equal in number and alternate. It also happens however that members of the same kind arise subsequently between those of a whorl already formed; as, for instance, five later stamens between the five earlier ones in Dictamnus Fraxinella (Fig. 414), and probably in many eucyclic flowers with ten stamens. Members subsequently introduced in this manner into a whorl may be called interposed. (For further details, vide infra.)

The consideration of the number of the parts of the flower cannot be separated from that of their relative position. But before entering more minutely upon this subject, the construction of the Floral Diagram must be described.

The Floral Diagram is constructed differently according to the purpose it is intended to serve. Some treat it as a somewhat free drawing of an actual transverse section of the flower, and indicate on it not merely the number and position, but approximately the form, size, æstivation, cohesion, \&c. of its parts. This purpose is however clearly best attained by preparing as accurate drawings as possible of actual transverse sections of the flower-bud, which will then also contain much that would be superfluous for observations of a certain kind. But if it is merely required to represent the number and position of the parts of the flower in such a manner as to render as easy as possible the comparison in this respect of a number of flowers, it is best to disregard all other peculiarities, and to adopt one and the same plan for all diagrams, and that as simple as possible, so as to represent nothing but the variations in the relationships of number and position. This is the only purpose kept in view in the diagrams given in the remainder of this work, of which Figs. 406-408 may serve for the present as examples. They are constructed according to the rule already given on p. 188 ; the dot above the diagram always represents the position of the mother-axis of the flower; and the lower is therefore the anterior part. Although mere dots would be sufficient to indicate perfectly the number and position of the parts of the flower, different signs have nevertheless been

rarely more, whorls is found in many Butomaceæ and Alismaceæ; usually when the number of the carpels is great they are arranged spirally. When the members of a series (calyx, corolla, etc.) are in one whorl, the series is said to be monocyclic; if in more than one, di-, tricyclic, etc.; if in many, polycyclic.

In isostemonous flowers it frequently occurs that the stamens are antipetalous, as in Ampelideæ, Rhamnaceæ, Plumbaginaceæ, Primulaceæ. This is usually ascribed to the abortion of an exterior whorl of antisepalous stamens, an assumption which is based either on the presence of a whorl of staminodes in the place of the missing stamens, or on the presence of two whorls of stamens in allied Orders.

It not unfrequently happens in a diplostemonous flower that the stamens of the outer whorl are antipetalous (Limnanthes, Ruta, Dictamnus (Fig. 4I 4), Pyrola, Monotropa, Chrysosplenium, Epilobium, Enothera, Fuchsia, Geraniaceæ, Zygóphyllaceæ, Crassulaceæ, Ericaceæ, Rhodoraceæ, etc.): when this is the case the androcium is said to be obdiplostemonous; the carpels are superposed on the stamens of the outer whorl, and therefore also on the petals. The most satisfactory explanation of obdiplostemony is that given by Celakovsky (Flord, 1875), though, as we shall see below (note, p. 606), it cannot be applied in all cases : according to him the staminal whorls arise in regular acropetal succession, the antisepalous stamens being developed first, as in direct diplostemony; the antipetalous stamens are developed internally to the others but become gradually displaced outwards, so that they appear either to lie in the same whorl as the antisepalous stamens or externally to them. This is the real meaning of the 'interposition' mentioned above. (For further details on these points see Gray's Structural Botany, and Eichler's Blüthendiagramme.)] 
chosen for the various separate organs, in order to render the explanation more readily visible to the eye. The leaves of the perianth are represented by arcs of a circle, a kind of mid-rib being indicated on each of the outer whorl of these, or calyx, merely in order to distinguish them at a glance from the inner whorl. The sign chosen for the stamens resembles a transverse section of an anther, but without reference to the position of the pollen-sacs or of their mode of dehiscence whether inwardly or outwardly. When the stamens are branched, this is indicated by the signs being grouped, as in Fig. 408, where the five groups correspond to five branched staminal leaves. The gynæceum is treated as a simplified transverse section of the ovary, since it is thus most easily distinguished from the other parts; the marks within the loculi of the ovary indicate the ovules, which however are only represented in those cases where their actual position can be expressed by so simple a plan. The size, form, and cohesion of the separate parts are not taken into account at all. The construction of these diagrams is based partly on careful investigations of my own, but chiefly on the studies of Payer in the history of development (Organogénie de la fleur), as well as on the descriptions of other authors (Döll, Eichler ${ }^{1}$, and Braun).

I draw a distinction between empirical and theoretical diagrams. The empirical diagram only represents the relative number and position of the parts, just as a careful observation shows them in the flower; but if the diagram also indicates the places where members are suppressed-which can only be determined by the history of development and by comparison with allied species, especially if it points out relationships
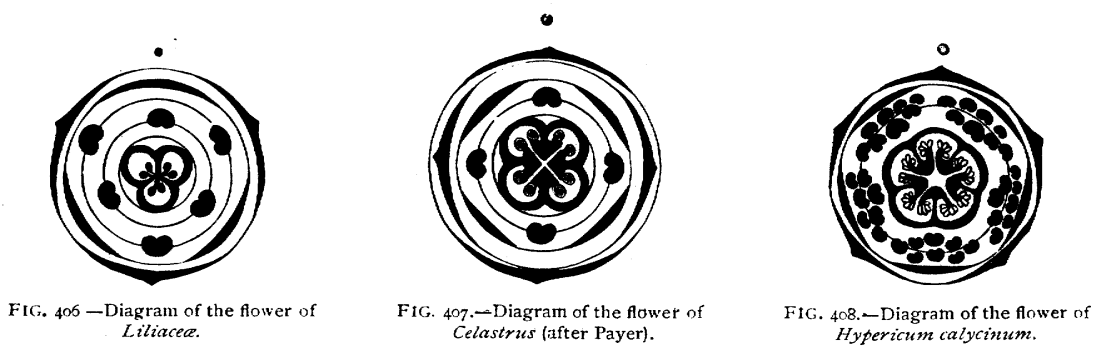

which are entirely the result of theoretical considerations-I call it a theoretical diagram. If the comparison of a number of diagrams shows that, although empirically different, they nevertheless yield the same theoretical diagram, this common theoretical diagram may be termed the type or typical diagram according to which they are all constructed. I consider the careful determination of such types an important problem, the solution of which may be extremely useful in the classification of Angiosperms. When the type has once been ascertained, the theoretical diagrams which correspond to it may be treated as derivative forms from which particular members have disappeared, or where they have been replaced by a number of members. From the stand-point of the theory of descent the type corresponds to a form still in existence or that has already disappeared, from which the species to which the derivative diagrams belong have arisen by degeneration (i.e. by abortion ${ }^{2}$ ) or by multiplication of the parts.

A few examples will explain this. The flower of Grasses, which is seated among the paleæ, may be deduced, as is shown in Fig. 409, on the theory of the abortion of certain

1 [Eichler, Blïthendiagramme, I, II, I875-8.]

2 The construction of the diagram itself shows that the theory of abortion is justified even where the earliest state of the flower-bud gives no indication of the absent member, if the number and position of the parts present point distinctly to such a hypothesis. If the idea of abortion in this sense is not admitted, neither can the increase in number of individual parts, or their replacement by several, be allowed. It is only the theory of descent that gives a rational explanation of either fact, and that a very clear one. 
parts from the typical flower represented in Fig. 406 , which is itself the typical diagram of Liliacex. $A$ is the diagram of Bambusa, which only deviates from the type in the absence of the outer perianth-whorl which is indicated by dots. But in most other Grasses $(B)$ the posterior leaf of the inner perianth-whorl (this whorl appearing generally only in the form of small colourless scales), the whole of the inner whorl of stamens, and the anterior carpel, are also wanting. In Nardus again $(C)$, the anterior carpel only is present (as far as the pistil is concerned); all the absent parts are represented by dots, and the diagram 'is therefore so far a theoretical one. If the dots are removed, we get the empirical diagram; the number and position of the carpels is here determined from those of the stigmas ${ }^{1}$.

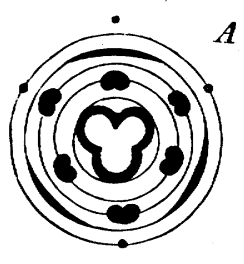

FIG. 409.-L iagram of the flower of a Grass; $A$

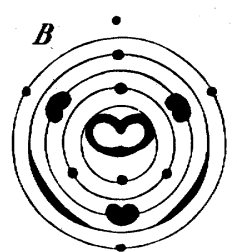

vol. I. pp. 105, 133).

The flowers of Orchideæ can also be derived, like those of Gramineæ, from the type represented in Fig. 406, the empirical diagram of Liliaceæ, although their external form is so remarkably different. While in Grasses the perianth is especially degenerated or even partially abortive, in Orchids both whorls are developed in a petaloid, and like the whole flower, in a zygomorphic or monosymmetrical manner. Of the andrœcium, which consists typically of two alternating whorls, each of three stamens, only a single stamen is completely developed in most Orchids (Fig. $4 \mathrm{IO}, A$ ), viz. the anterior one of the outer whorl, the others being abortive. Indications of these are however sometimes found in the young bud, as in Calanthe veratrifolia (according to Payer, cf. Fig. 394), where at
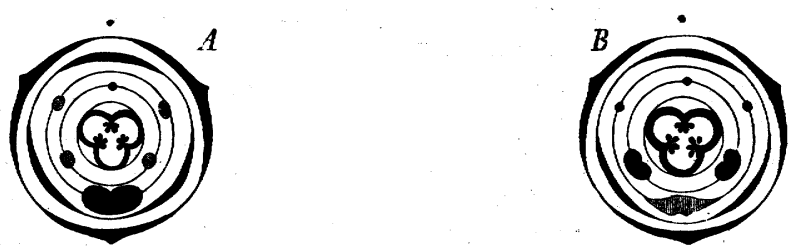

FIG. 410.-Diagram of the flower of Orchidex: $A$ the ordinary structure; $B$ that of Cypripedium (see Figs. 372 and 418 ) : the dots indicate stainens which are altogether abortive, the shaded figures rudimentary stamens which become abortive or transformed into staminodes.

least the two anterior ones of the inner whorl (but not the posterior one) appear as small elevations which soon disappear. In Cypripedium, on the contrary, a large staminodium (see Fig. 372) takes the place of the anterior stamen which is elsewhere fertile; while the two anterior and lateral anthers of the inner whorl are fully developed and fertile (Fig. 4 IO, B). In Ophrydex two small staminodes are found beside the gynostemium ( $c f$. Fig. $418, D$, st) in the place of the two fertile stamens of Cypripedium; while in Uropedium all three of the inner whorl are completely developed. (Döll.) The carpels which, by adhesion with the andrœcium form the gynostemium, are developed unequally, a difference which however is usually not discernible in inferior ovaries, and is therefore not indicated in the diagram. The student who desires to

1 Compare further, Döll, Beiträge, in the Jahresbericht des Mannheimer Vereins für Naturkunde, I870, where an actual pentacyclic trimerous flower of Strebtochate is described. 
investigate these relationships for himself must observe that the long inferior ovary of most Orchids undergoes a torsion (resupination) at the time of the opening of the flower, which causes the posterior side of the flower to assume an anterior position; but transverse sections even of advanced buds show clearly the true position of the parts of the flower in relation to their mother-axis.

The flowers of most Monocotyledons, like those of Orchids and Grasses, can be derived from a type which is actually seen in Liliacex, and which represents a flower consisting of five alternating whorls, each with three members, of which the two outer ones constitute the perianth, the two next the andrœcium, and the last the gynæceum; although the latter may sometimes be represented by two whorls. Occasionally instead of abortion an increase of number takes place in particular whorls, by the formation of one member instead of two (as in Butomus, Fig. 382 ).

Increase in the typical number of the members of a whorl may arise in different ways, as the following examples will show. According to the detailed researches of

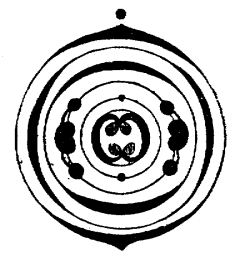

FIG. 4Ir.-Diagram of the flower of Fumariaceæ (after Eichler).

Eichler ${ }^{1}$, the flowers of Fumariaceæ may be referred to a type in which there are six decussate pairs of members (Fig. $4 \mathrm{II}$ ), viz.

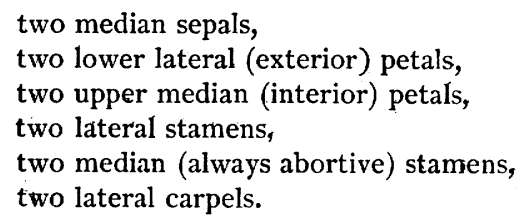

The two lateral stamens are however represented in some genera (as Dicentra and Corydalis) by two groups, each consisting of three stamens, an inner one with an entire quadrilocular anther, and two lateral stamens each with a bilocular anther, a structure which Eichler explains on the hypothesis that the lateral stamens are only stipular structures, and therefore branches from the base of the middle one. In Hypecoum Eichler assumes a cohesion of each pair of opposite stipular stamens so as to form an apparent whorl of four stamens.

Eichler also deduces the flowers of Cruciferæ and Cleomeæ (a section of Capparidex) from a type represented by Fig. $412 A$, which is also the empirical diagram for Cleome droserafolia, and for certain species of Lepidium, Senebiera, and Capsella. This typical flower consists of

two lower median sepals,

two upper lateral sepals,

four diagonal petals in one whorl,

two lower lateral stamens,

two upper median stamens,

two lateral carpels.

1 Eichler, Ueber den Blüthenbau der Fumariaceen, Cruciferen, und einiger Capparideen, in (Regensburg) Flora, 1865, nos. 28-35, and I869, p. I.-Peyritsch, Ueber Bildungsabweichungen der Cruciferenbliithen, Jahrb. für wiss. Bot. vol. VIII. p. I I 7 . 
Deviations from this type are produced by the formation of two or more stamens in place of each of the upper (inner) ones; in the Cruciferæ usually two (Fig. 4I3), in the Cleomex sometimes two, sometimes more (Fig. 4 I2 B). Such a replacement of one stamen by two or more is termed by Payer Dédoublement ${ }^{1}$, by Eichler and others Collateral Chorisis, and must apparently be considered as a branching of very early origin. This view is confirmed in this case by the fact that in the Crucifer Atelantbera the
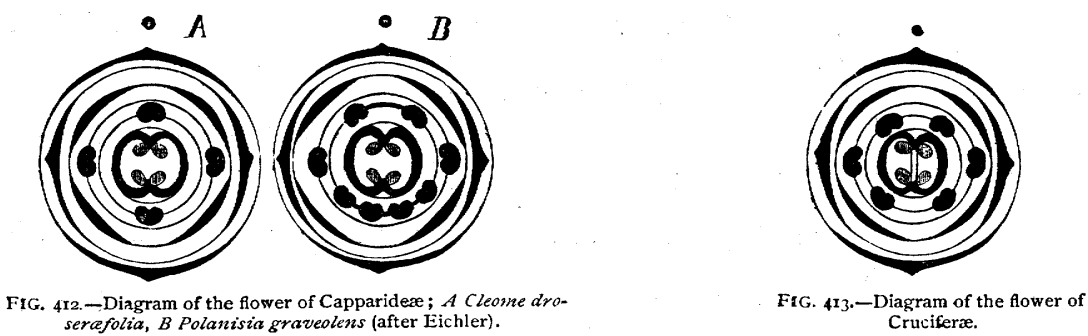

median stamens are only split and the two halves of each provided with half-anthers, while in Crambe each of the four inner stamens puts out a lateral sterile branch, which may be explained as the commencement of a further multiplication of the stamens such as actually occurs in the Crucifer Megacarpaea and in many Cleomex. Even if the way in which increase of the typical dimerous number of the inner whorl of stamens has been brought about be still obscure, it appears certain that the inconstancy of the number of the members of the staminal whorl proves that in Cruciferæ and Cleomex a deviation has arisen in this part of the flower from the typical dimerous number, while the other whorls have remained unchanged. The only deviation which occurs in the gynæceum of the Crucifers is in the genera Tetrapoma and Holargidium, where, besides the two lateral carpels, two median ones are also produced, thus forming a four-lobed ovary ${ }^{2}$.

An essentially different kind of increase in the typical number of the members of a flural whorl may be caused by the formation in the still very young bud of new members of the same kind between those already in existence and on the same zone

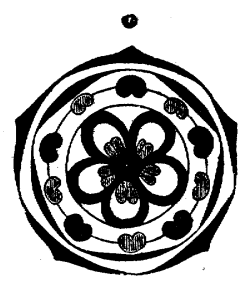

FIG. 4I4.-Diagram of the flower of Dictamnus Fraxinella (cf. Fig. $3^{8}$ ).

of the receptacle; i.e. by what we have already described as the Interposition of new members. This I found to occur, for example, in Dictamnus Fraxinella (Fig. 388), and is represented in the diagram, Fig. 4I4, by the stamens of later origin being shaded not so dark as those of earlier origin. It may, I think, be inferred from Payer's descriptions

1 The theory of an original dimerous symmetry in the flowers of Cruciferæ has been pushed still further by Meschaeff (Bull. Soc. Imp. Nat. Mosc.), who regards the four petals as also the result of a lateral dédoublement of a single pair (see Bentham, Ann. Address Linn. Soc. 1873).]

${ }^{2}$ [Holargidium is a section of Draba. According to Bentham and Hooker the four carpels of Tetrapoma are an abnormality not constant under cultivation. The same authors also mention the occasional occurrence of a similar abnormality in Brassica and Nasturtium.] 
and drawings that the same process occurs in the nearly related genus $R$ uta, and in the families Oxalidex, Zygophyllaceæ, and Geraniace included in the same circle of affinity; viz. that in these cases also five stamens are interposed between those already in existence $^{1}$. If the five interposed stamens are supposed to be removed, there remains in these families a regular pentamerous flower with four alternating whorls each consisting of five members, such as is found in the nearly related Linaceæ and Balsamineæ ${ }^{2}$.

Floral Formula. The diagram may, under certain circumstances, be substituted, at least partially, by a formula composed of letters and numbers. In a floral formula of this kind the relative positions of the parts cannot indeed always be represented with accuracy; but it has the advantage that it can be expressed by ordinary printer's type, and, what is perhaps of greater importance, is capable of a wider generalisation, since the numerical coefficients may be replaced by letters.

The construction and application of these formulæ will easily be made intelligible by a few examples ${ }^{3}$.

The formula $S_{3} P_{3} S t_{3}+_{3} C_{3}$ corresponds to the diagram of the Liliaceæ, Fig. 406 , and signifies that each of the two perianth-whorls - the outer whorl or sepals $S$, and the inner whorl or petals $P$-consists of three members, the andrœcium of two whorls each of three stamens $S t$, and the gynæceum of three carpels $C$. The diagram shows in addition that these trimerous whorls alternate without interruption; but since this is the usual case

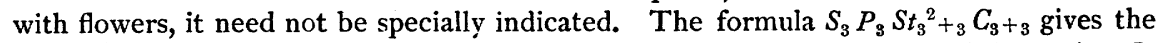
relative positions of the parts of the flower of Butomus umbellatus (Fig. 382). It is distinguished from the previous one by the gynæceum consisting of two whorls of three carpels each, and the androcium having the typical three stamens of the outer whorl ench replaced by two stamens, which is expressed by the symbol $3^{2}$. The formula $S_{0} P_{3} S t_{3}+{ }_{3} C_{3}$ corresponds to the diagram of the flower of Bambusa, Fig. $409 \mathrm{~A}$, and differs from that of Liliacex only in the suppression of the outer perianth-whorl, represented by $S_{0}$. The numerical relations of the parts of the flower of Orchidex, Fig. 4 I $0 A$, might be expressed by the formula $S_{3} P_{3} S t_{\mathrm{i}+}{ }_{0} C_{3}$, the symbol $S t_{i}+_{0}$ indicating that all the members of the inner staminal whorl are abortive, while on the other hand in the outer whorl the two posterior ones are suppressed, the anterior outer stamen being perfectly developed; the two dots over the number $\ddot{i}$ are meant to indicate that the absent members are the posterior ones; were the anterior ones deficient the dots would be placed beneath the number, as in the formula $S_{0} P_{\dot{2}} S t_{3}+{ }_{0} C_{2}$ which corresponds to the ordinary flower of Grasses represented by the diagram Fig. $409 B$. The formula $S_{2} P_{2} S t_{2}+{ }_{2} C_{2}$ expresses the whorls consisting of decussate pairs which form the flower of Maianthemum bifolium; the formula $S_{4} P_{4} S t_{4}+{ }_{4} C_{4}$ or $S_{5} P_{5} S t_{5}+{ }_{5} C_{5}$ the flowers of $P a r i s q u a d r i f o l i a$, in which all the whorls are either tetramerous or pentamerous. These

1 [These are all cases of obdiplostemony. In the case of Dictamnus and of Ruta this is to be explained by Celakovsky's theory of displacement. In the Oxalideæ and Geraniaceæ Frank has found (Jahrb. f. wiss. Bot. X), in opposition to Payer, that the antipetalous stamens are developed first. It is therefore difficult to give a satisfactory account of the obdiplostemony in these orders. Eichler regards it as due to constant deviation from the normal acropetal development of the whorls of the flower.]

2 Döll (Flora von Baden, vol. III. pp. II75, II77) and others suppose that a whorl has become abortive between the corolla and ovary in Rutaceæ and Oxalidex, a hypothesis which is not supported by the history of development, and which is superfluous on our hypothesis. To assume abortion merely because certain whorls do not alternate seems to me to be going too far. Besides, the ten stamens of Epacrideæ and Rhodoraceæ cannot belong to two but only to one whorl in which five are of earlier origin, and five have been interposed. (Compare Payer, Organogénie de la fleur, pl. II 8.)

${ }^{3}$ Grisebach (Grundriss der systematischen Botanik; Göttingen, 1854 ) has denoted the relative numbers of the parts of flowers in a different manner, placing the numbers of the members of a whorl simply one after another, and indicating cohesions by strokes. 
and most other formulæ for the flowers of Monocotyledons may now be combined into a general expression $S_{n} P_{n} S t_{n+n} C_{n(+n)}$, which signifies that the flowers belonging to this type are usually constructed of five alternating whorls each with the same number of members, two of which are developed in the form of perianth-whorls, two as staminal whorls, and generally only one as a carpellary whorl; the bracket $(+n)$ at the end of the formula indicating that a second carpellary whorl sometimes occurs in addition. The general number $n$ may, as the examples which have been adduced show, have the value $2,3,4$, or $5 ; 3$ is the most common. If a considerable increase of the number of members takes place in a whorl, and if this number, as is then usually the case, is variable, this is expressed by the symbol $\infty$; thus the formula for Alisma Plantago is $S_{3} P_{3} S_{3}+{ }_{3} C_{\infty}$.

As has already been mentioned, no further indication is given of the position of the whorls when they alternate; when a departure from this rule occurs, this can be more or less accurately expressed by special symbols. Thus, for example, the formula for the flower of Cruciferæ, Fig. ${ }_{4} \mathrm{I} 3$, might be represented by $S_{2+2} P_{\times_{4}} S t_{2}+_{2}{ }^{2} C_{2}\left(+_{2}\right)$, the symbol $P_{\times 4}$ signifying that the decussate pairs of sepals are followed by a corolla consisting of one whorl of four petals, which are however arranged diagonally to the sepals. In order to express the superposition of two consecutive whorls, a vertical stroke might be placed after the number of the first whorl; thus $S_{5} P_{5} \mid S t_{5}{ }^{\mathrm{v}} C_{5}$ might represent the formula for Hypericum calycinum (Fig. 408$), \mid S t_{5}{ }^{v}$ indicating that the androcium consists of five branched $\left(5^{\nabla}\right)$ stamens which are superposed on the petals. If, finally, it is desired to signify that members of a second whorl are interposed at the same level between those of one already in existence, the number of the new members may be placed simply beside those of the original whorl; thus the formula $S_{5} P_{5} S t_{5 \cdot 5} C_{5}$ would correspond to the diagram Fig. $4 \mathrm{I} 4$.

In the formulæ already given no cohesions of any kind have been indicated; they can however under certain circumstances easily be expressed by special symbols. Thus, in the formula for Convolvulus $S_{5} \overparen{P_{5}} S t_{5} \overparen{C_{2}}$, the sign $\widehat{P}_{5}$ indicates a gamopetalous corolla of five petals, $\widehat{C_{2}}$ a syncarpous ovary of two carpels. In the formula for the flowers of Papilionaceæ again $\widehat{S_{5}} P_{5} S t_{5} \overparen{t_{4}+1} C_{1}$, the expression $\widehat{S t_{5+4}+1}$ signifies that the five stamens of the outer and four of those of the inner whorl have united into a tube, while the posterior stamen of the inner whorl remains free ${ }^{1}$.

The mode of writing the formulæ must vary according to the object which one has in view; the greater the number of relationships it is intended to express, the more complicated will they become; and care must be taken that they do not lose their clearness by being overladen by too many signs.

The examples of formulæ which have hitherto been adduced all illustrate cyclic flowers; those parts of flowers which are arranged spirally may be denoted by the symbol - placed before them, and the angle of divergence may also be affixed to their number. Thus, for example, the relative numbers and positions of the parts of the flower of Aconitum, according to Braun's investigations, may be expressed by the formula $S-2 / 5{ }_{5} P-3 / 8{ }^{8} S t-8 / 21{ }_{\infty} C_{-3}$, which indicates that all the foliar structures of this flower are arranged spirally, and that the calyx consists of five sepals with the divergence $2 / 5$, the corolla of eight petals with the divergence $3 / 8$, and the andrœcium of an indefinite number of stamens with the divergence ${ }^{8} / 21$. It would however be sufficient in this case, since the spiral arrangement runs through the whole flower, to place the symbol only once before the whole formula, thus $-S_{2 / 5}{ }_{5} P_{3} / 8{ }_{8} S t_{8} /_{21} C_{3}$.

In flowers with a cyclic arrangement of their parts a statement of the angle of divergence is generally unnecessary, since the members of each whorl usually arise simultaneously, and are arranged so as to divide the circle into equal parts. When they do not arise simultaneously but successively in the circle with a definite angle

I Sce also Rohrbach, Bot. Zeitg. 1870, pp. 816 et seq. 
of divergence, as in most trimerous or pentamerous calyces, this can be indicated by placing the angle of divergence after the number of the members; thus the formula for Linaceæ would be $S_{5}^{2} / 5 P_{5} S t_{5} C_{5}$. If, on the other hand, the members of a whorl are formed in succession from front to back, this may be shown by an arrow pointing upwards $\uparrow$, as in the formula for Papilionaceæ $S_{5} \uparrow P_{5} \uparrow S t_{5} \uparrow+_{5} \uparrow C_{1}$. If they are formed in succession from back to front, the arrow may be made to point downwards $\downarrow$, as in the formula for Reseda $S_{n \downarrow} P_{n \downarrow} S_{t_{p} !+q \downarrow} C_{r}$, where the number of the parts is expressed by letters instead of figures in consequence of its variability ${ }^{1}$.

4. Order of Development of the Parts of the Flower. The foliar structures arise on the axis of the floral shoot, as on other axes, in acropetal order below the growing apex. It is however not uncommon in the formation of flowers for the apical growth of the axis to cease altogether or to become extremely slow, while the receptacle continues to increase in breadth, and to develope transverse zones of intercalary growth. When this is the case the acropetal order of development is disturbed, and new whorls may become interposed between those already in existence. But even within the same floral whorl the individual members may be formed in a very different order of succession, according as the zone of the receptacle which bears the floral leaves is developed in a uniform manner all round (as in polysymmetrical flowers) or more rapidly on the anterior or the posterior side (which is especially the case in monosymmetrical or zygomorphic flowers).

In flowers with a spiral arrangement of their parts ${ }^{2}$, disturbances of the acropetal order of development are of less importance the more numerous the parts with a spiral arrangement, and the longer the apical growth of the floral axis continues. Those members which have a spiral arrangement arise one after the other in ascending order; the angle of divergence may either be constant or may change. Thus, according to Payer, in Ranunculaceæ and Magnoliaceæ the perianth-leaves and stamens arise in a continuous spiral, but each turn of the spiral consists of a larger number of stamens than of perianth-leaves; thus, e.g., in Helleborus odorus, where all the organs of the flower are arranged spirally, the corolline turn includes only thirteen petals, while each turn of stamens numbers twenty-one. According to Braun the turns of the calyx of Delphinium Consolida have a $2 / 5$ arrangement ${ }^{3}$; the divergence then undergoes a small change, but without materially deviating from ${ }^{2} / 5$; the first turn with this altered arrangement is the corolla; the three following ones are the stamens, and the spiral terminates with a single carpel. In the section Garidella of Nigella the first of the turns with $\mathrm{a}^{2} / 5$ angle of divergence is the calyx and the second the corolla; then follows a slight change in the angle to $3 / 8$, the stamens forming one or two turns with this arrangement; and the spiral closes with three or four carpels. In the section Delpbinellum of Delpbinium the calyx constitutes a turn with $2 / 5$, the corolla one with $3 / 8$ angle of divergence; then follow two or three turns of stamens with the angle very near $3 / 8$, the spiral closing with three carpels. In the section Staphisagria of the same genus, and in Aconitum, the calyx forms a turn with $2 / 5$, the corolla one with $3 / 8$ angle; the stamens stand in one or two turns with the divergence $8 / 21$ or $13 /{ }_{34}$; concluding with three, five, or rarely a larger number of carpels. It must be noted in reference to these arrangements that the members of successive turns stand in orthostichies when the angle of divergence remains constant; but that the orthostichies pass into oblique rows when the divergence undergoes a small change.

The first thing to observe in cyclic flowers (i.e. those in which the parts are arranged in whorls) is the order of formation of the whorls with respect to one another, and then

1 See Payer, Organogénie de la fleur; also the next paragraph.

2 Compare Payer, Organogénie de la fleur, pp. 707 et seq.; and Braun, Jahrb. fiur wissensch. Bot. I, Ueber den Blüthenbau der Gattung Delfhinium.

${ }^{3}$ Compare with this what is said below respecting sepals and petals which are formed with the angle of divergence $1 / 3$ and $1 / 5$. 
the order in which the members of each whorl are themselves formed; although the two are in fact closely connected. A disturbance of the acropetal order of succession in the formation of the whorls occurs when the carpels have begun to be formed before all the stamens which stand below them have been produced, as in Rubus, Potentilla, and Rosa', or when the calyx is not formed until after the androcium (as in Hypericum calycinum according to Hofmeister), or when the calyx is not observable until after the corolla has become considerably developed or even after the formation of the stamens and carpels, as in Compositæ, Dipsacaceæ, Valerianaceæ, and Rubiaceæ.

One of the most remarkable deviations from the general rule of the order of development of the floral whorls occurs in Primulaceæ, where five protuberances (primordia) appear on the receptacle above the calyx, each of which grows up into a stamen, while on the posterior or lower side of the base of each primordial stamen a lobe of the corolla subsequently appears ${ }^{2}$. Pfeffer, who has observed this order of development (Jahrb. für wissensch. Bot. vol. VII. p. 194), considers that the same probably also happens in the pentandrous Hypericineæ and in Plumbagineæ; he therefore explains the corollalobes as posterior outgrowths of the stamens (a posterior ligular structure), such as, for instance, occur on the stamens of Asclepiadeæ in the form of hood-shaped nectaries, where a true corolla is also present. The flowers of Primulacex would therefore be strictly apetalous in the morphological sense of the word, since their corolla is not a true floral whorl, but only an outgrowth of the staminal whorl. In other families of Dicotyledons, on the other hand, superposed corollas and androcia arise separately and in acropetal order; as, for instance, in Ampelideæ, probably also in Rhamnaceæ, Santalaceæ, and Chenopodiacex.

The individual members of a floral whorl may arise in succession from front to back or the reverse, especially when the flowers themselves are subsequently developed zygomorphically. Thus, for instance, in Papilionaceæ the anterior median sepal is formed first, then simultaneously one to the right and one to the left, and finally the two posterior ones; but before these last arise the two anterior petals appear, followed by the two lateral and finally the posterior one; and the androcium, consisting of two alternating whorls of five stamens each, is formed in the same manner from front to back $^{3}$. In the Resedacex, on the contrary (Reseda and Astrocarpus), Payer states that the petals, stamens, and carpels are developed from behind forwards on both sides $(c f$. Fig. I 45, p. I 87 ).

When the calyx consists of pairs of sepals, those of each pair are formed, as Payer has shown, simultaneously; but if the calyx consists of three or five sepals, they are usually formed one after another, and with the angle of divergence in the one case $1 / 3$, in the other $2 / 5$; but the succeeding whorls, the petals, stamens and carpels, usually arise as simultaneous whorls, with the exceptions already named and others still to be spoken of.

It is well to draw attention here to the circumstance that it does not follow from the order of succession advancing from one point, with a definite angle of divergence, say $1 / 3$ or $2 / 5$, that the arrangement is a spiral one ${ }^{4}$; it may just as well in such cases be a whorl. The nature of the arrangement depends on the circumstance whether the foliar structures in question are formed at the same height or not, i.e. at an equal distance from the centre of the flower; if this is the case, we have a whorl; but if the members

1 Compare Hofmeister, Allgemeine Morphologie, pp. $4^{6} 3$ et seq., where Payer's observations on this point will also be found.

${ }_{2}^{2}$ [According to Frank (Ueb. d. Entwick. einig. Blïthen, mit bes. Berïcksichtigung der Theorie der Interponirung, Jahrb. f. wiss. Bot. X. 1876 ), the stamens and petals of the Primulaceæ arise independently, but they fuse with the stamens during their development, and subsequently become free again.]

3 On the nearly related Cæsalpineæ see Rohrbach, Bot. Zeitg. I870, p. 826.

${ }^{4}$ Compare the successive true whorls of Chara and Salvinia, pp. 187, 191, 293, 449. 
arise in acropetal order at different heights, i.e. approaching the centre of the flower with each step in the divergence, the arrangement is a spiral one. The last appears to be actually the case in many calyces; but it is doubtful whether it ever occurs where the angle of divergence of the sepals is $1 / 3$ or $2 / 5$.

We must now refer again to the cases already mentioned, where new members of a whorl are formed between those already in existence and at the same height ${ }^{1}$. In the Oxalidex, Geraniaceæ, Rutaceæ, and Zygophyllaceæ, an entire whorl of five stamens is thus interposed between those already present; according to Payer, in Peganum Harmala, a whorl of ten stamens is even formed in this manner, arising, not in pairs between the first five, but lower down at the bases of the petals; whether the later formed stamens arise on the same level with the first, or lower down, is obviously regulated according to the space afforded by the changes of form of the growing receptacle. A still further departure from the ordinary process occurs in the Acerineæ, Hippocastaneæ, and Sapindacex, where Payer asserts that a whorl of five stamens is first of all formed alternating with the corolla, in which an imperfect whorl of two or four stamens is subsequently interposed at the same height, as is shown by his illustrations. In Tropaolum, on the

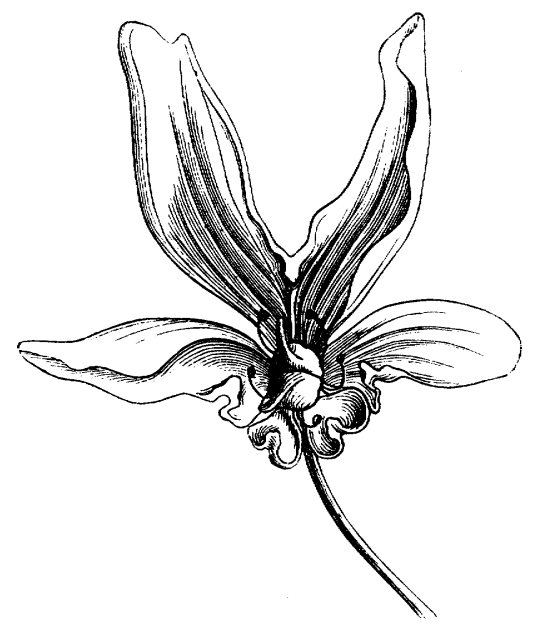

FIG. 4r5.-Flower of Heracleum pubescens with zygomorphic corolla.

other hand, according to Payer and Rohrbach ${ }^{2}$, three stamens first of all appear after the formation of the petals, and then between them five others, the distance of which from the centre of the flower is however rather greater than that of the three earlier ones.

5. Symmetry of the Flower. If the observations which will be found on pp. I 87 et seq. under the head of General Morphology are now applied to the floral shoot, it is seen that true symmetry and distinctly bilateral structure occur here far more commonly

${ }^{1}$ Compare also on this point Pfeffer, Jahrb. für wiss. Bot. vol. vIII. p. 205.

2 Rohrbach (Bot. Zeitg. 1869, Nos. 50, 5r) however gives a different explanation to these observations from that mentioned here. The equal or greater clistance at which the later stamens arise from the centre of the flower is a distinct proof that one cannot in this case suppose that the parts are produced in a spiral arrangement advancing from without inwards. [See note on p. 6or. Assuming the correctness of Payer's observations, these are instances of incomplete obdiplostemony. According to Buchenau (Morph. Bemerk. üb. einige Acerineen, Bot. Zeitg. I861), all the stamens are developed simultaneously in the Acerinex.] 
than on the vegetative shoots. In contrast to the lax mode of expression used by many botanists, I understand by Symmetrical Structures those which may be divided into two halves, each of which is an exact reflex image of the other. If a flower can be divided in this manner by only one plane, I call it simply symmetrical or monosymmetrical; if, on the contrary, it can be symmetrically divided by two or more planes, it is, as the case may be, doubly or polysymmetrical. The happy expression zygomorpbic already used by Braun may be applied equally to monosymmetrical flowers and to those polysymmetrical ones in which the median section produces halves of quite a different form from those caused by lateral section (e.g. Dicentra). I apply the term regular to a poly-

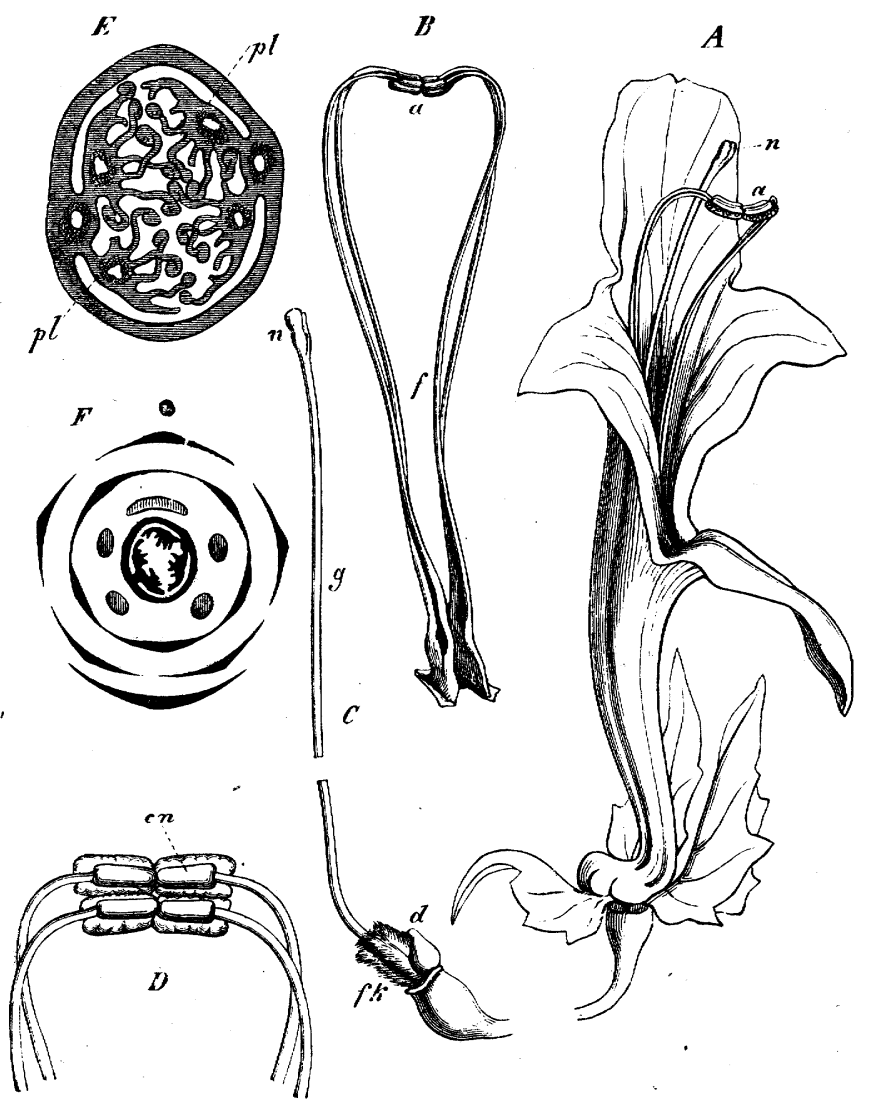

FIG. 416.-Zygomorphic flower of Columnea Schiedeana: $A$ entire flower after reinoval of two sepals; $B$ androcium ; $C$ gynæceum; $D$ the coherent anthers magnified and seen from behind; $E$ horizontal section of the ovary; $F$ diagram; $a$ anthers, $n$ stigma, $g$ style, $f k$ ovary, $d$ the staminode developed into a nectary, $p l$ the lateral oblique placentæ.

symmetrical flower only when the symmetrical halves produced by any one section are exactly like or very similar to those produced by any other section; or-which comes to the same thing - when two, three, or more longitudinal sections divide a flower into four, six, or more equal or similar portions.

In exactly defining the symmetrical relations of a flower, the relative positions of the parts, as represented by the diagram, must first of all be distinguished from the entire form of the flower, such as is realised in the development of the organs.

If attention is paid first of all only to the relative positions of the parts, it is clear that they can never be distributed symmetrically in flowers with a truly spiral structure; 
while in hemicyclic flowers those members at least which are arranged in whorls may possibly be distributed symmetrically. If, on the contrary, the parts are all arranged in whorls, they are usually distributed monosymmetrically or polysymmetrically on the receptacle. Thus, for example, the diagram Fig. 406 can be divided symmetrically and regularly by three planes, Fig. 407 by four, and Fig. 408 by five planes. The diagrams Fig. $409 B$ and $C$, as well as Fig. 4 IO, can, on the contrary, be symmetrically halved by only one plane, which is at the same time the median plane. The diagram Fig. 4I I can be divided by the median plane into two symmetrical halves which are unlike those produced by the lateral section; this diagram is, like those in Figs. $409 B, C$ and $4 \mathrm{r} 0$, zygomorphic, but is doubly while these are only singly symmetrical ${ }^{1}$.

The symmetry of mature unfolded flowers is indeed usually connected genetically with the relations of symmetry of the diagram (which represents only the position and number of the parts); as will be made clear by a comparison of Figs. $4 \mathrm{I} 6$ and $4 \mathrm{I} 8$ with Fig. 4 ro $A$. But inasmuch as the entire form of the mature flower is essentially deter-

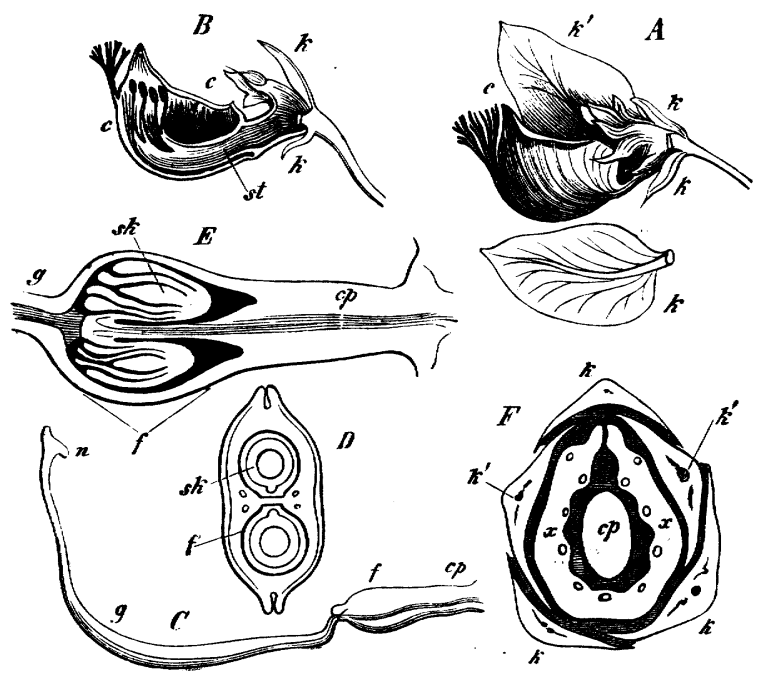

FIG. 417.-Zygomorphic flower of Polygala grandiflora: $A$ entire flower seen from the side after removal of one sepal $k ; B$ flower divided symmetrically without the gynæceum; $C$ the gynæceum magnified; $D$ horizontal section of the ovary; $E$ median longitudinal section of the ovary; $F$ horizontal section of the flower; $k$ calyx, c corolla, $s t$ staminal tube, $c p$ gynophore, $f$ ovary, $g$ style, $n$ stigma, sk ovules, $x x$ the tube formed by the adhesion of the petals and stamens.

mined by the shape, size, torsion, and curvature of the separate parts, these circumstances also exert a preponderating influence on the relations of symmetry of the open flower, and to such a degree that even flowers which have their parts arranged spirally may become monosymmetrically zygomorphic in reference to their entire form, as is the case to a high degree, for example, in Aconitum and Delpbinium. It must however be observed that the zygomorphism of the flower is here brought about principally or entirely by the calyx and corolla, the spiral arrangement of which may perhaps still be doubtful, but which always occupy so narrow a zone on the receptacle that their position may be considered practically to be verticillate. If, on the other hand, the floral axis is sufficiently elongated to show that the arrangement is a distinctly ascending spiral one, as in the perianth and andrœcium of Nymphaea and the andrœcium and gynæceum of Magnolia, the subsequent development of the organs appears also not to show any zygomorphic nor indeed generally any kind of actually symmetrical arrangement.

1 The beginner may make these relations more evident to himself by placing a small mirror with a smooth edge vertically upon the paper so as to bisect the diagram. 
The zygomorphic and monosymmetrical form occurs, on the contrary, very commonly in those flowers the parts of which are arranged in whorls. A very distinctly zygomorphic arrangement is not unfrequently united with a partial or entire abortion of particular members, as, e.g., in Columnea, Fig. 416, and other genera of Gesneracex, where the posterior stamen is transformed into a small nectary; while in Labiatæ it is entirely wanting. This abortion is carried still further in Orchideæ, where, of the six typical stamens, only the median anterior one of the outer whorl or the two lateral anterior ones of the inner whorl are developed (see Fig. 4Io). The ultimate monosymmetrical arrangement is sometimes to a certain extent indicated by the order of their formation, even in the rudimentary condition of the parts of the flower, when their origin is not simultaneous in the whorl, and does not progress with a definite angle of divergence, but is so arranged that the development commences with one anterior or one posterior member, and then advances simultaneously right and left from the median line towards the opposite side of the whorl. Examples have already been given of this arrangement in Papilionacex in the one case and Resedace in the other.

In the zygomorphic flowers of $\mathrm{Fu}$ mariacex, the diagram (Fig. $4 \mathrm{II}$ ) is, as we have already pointed out, symmetrically divisible in different ways by two planes. The anterior and posterior halves, symmetrically similar to one another, are unlike the right and left halves, which again are symmetrically alike. This is the arrangement of the parts in the mature flower of Dicentra; in Fumaria and Corydalis the right side is developed differently from the left, one producing a spur, the other not; while the anterior and posterior sides remain symmetrical. In this case therefore the plane of symmetry coincides
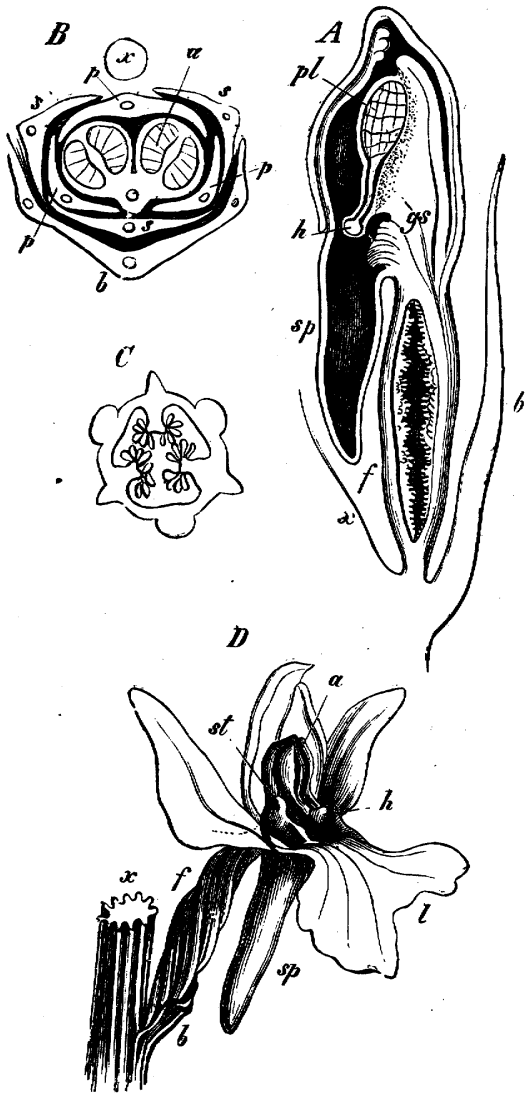

FIG. 418.-Zygomorphic flower of Orchis maculata: $A$ bud divided symmetrically through the middle: $B$ transverse section of the bud; $C$ horizontal section of the ovary; $D$ entire mature flower, one of the lateral perianth-leaves having been removed; $x$ mother-axis of the flower; $b$ bract, $s$ outer, $p$ inner perianthleaves-the posterior one $l$ becomes the labellum $a$ the single stamen, st staminodes, $g s$ gynosten. disc (retinaculum), sp spur the labcid disc (retinaculum), $s p$ spur of the labellum, $f$ the inferior ovary, twisted in $D$ (resupinate) (compare the diagram Fig. 4ro). with a lateral section. In the zygomorphic flowers of some Solanaceæ the plane of symmetry and the median plane intersect at an acute angle. But by far the greater number of zygomorphic monosymmetrical flowers are so constructed that the median plane coincides with a longitudinal section which divides the flower symmetrically; as for instance in Labiatæ, Papilionaceæ, Orchideæ, Scitamineæ, Lobeliaceæ, Compositæ, Delpbinium, and Aconitum ${ }^{1}$. The zygomorphic development is especially prevalent in the lateral flowers of spicate, racemose,

1 In observations of this kind attention must be paid to torsions, such as occur in the ovary of Orchideæ, the flower-stalk of Fumariaceæ, the Laburnum, \&c. 
or paniculate inflorescences; but is found also in those that are cymose and that have all the flowers terminal (Labiatæ and Ecbium). It seems as though the vigorous development of the principal rachis of the entire inflorescence-whether the final ramifications are cymose or not-often determines a zygomorphic development of flowers, as is shown in Labiatæ, Scitamineæ, and Esculus. The formation of a vigorous pseud-axis appears to exercise a similar influence in the case of sympodial inflorescences (as in Ecbium).

6. The Fruit of Angiosperms is the mature ovary which contains the ripe seeds and has undergone physiological changes as the result of fertilisation. The style and stigmas are frequently deciduous (as in Cucurbita, Grasses, \&c.). Some of the ovules not unfrequently disappear, and the number of seeds is thus less than that of the ovules. When all the ovules of one or more loculi of a multilocular ovary disappear in the process of ripening, only the fertile loculus continues to grow; the others become partially or entirely suppressed, and can be recognised only with difficulty or not at all. A multilocular ovary may thus produce a unilocular, and often a one-seeded fruit. Thus from the trilocular ovary of the Oak, each loculus of which contains two ovules, results a unilocular one-seeded fruit, the acorn. A less complete disappearance of two or four loculi together with their ovules occurs in the tri- or quinqui-locular ovary of the Lime, the fruit usually containing only one seed.

Parts of the flower again which do not belong to the gynæceum, or even not to the flower, undergo changes resulting from fertilisation. The entire structure which is thus formed may be termed a Pseudocarp, and may be composed of a single fruit or of a number of true fruits together with the surrounding parts which have undergone peculiar development. Thus, for example, the strawberry is a pseudocarp, the axial part (or receptacle) of the flower swelling out and becoming fleshy, and bearing on its surface the true small fruits. In the 'hip' of the Rose the hollow urn-shaped flowerstalk (asain the receptacle) encloses the separate ripe fruits in the form of a red or yellow succulent envelope. The apple is also in the same sense a pseudocarp; and the mulberry results from a whole spike of flowers, the perianth-leaves of each separate flower swelling and becoming fleshy and enclosing the sinall dry fruit. In the fig the hollowed-out stalk of the whole inflorescence forms the pseudocarp, bearing the fruits inside.

Starting from the definition that a fruit is always the product of a single ripe ovary, it follows that several fruits may arise from one flower, whenever, namely, there is more than one monocarpellary ovary in the flower; in other words, when the flower is polycarpellary and apocarpous; each carpel therefore produces a simple fruit. The simple fruits, taken together, may be termed an aggregate fruit, but it would be much better to apply to it the term Syncarp. Thus, for example, the small fruits resulting from the flower of Ranunculus or Clematis or the larger ones from the flower of Paonia or Helleborus, form together a syncarp. Of a similar character is the blackberry, consisting of a number of drupe-like fruits, the product of a single flower. The fleshy receptacle of the Rose-hip again encloses a syncarp, but the separate fruits constituting it are in this case dry and not fleshy. The syncarp must not be confounded with the pseudocarp resulting from an entire inflorescence, as in the cases of the mulberry and fig already named, or the pine-apple, or Benthamia fragifera.

The single multilocular ovary of a flower may undergo transformation so as to produce two or more parts, each containing seeds, and appearing like simple fruits, and hence termed Mericarps, while the whole fruit is called a Scbizocarp. This separation may take place at a very early period in the process of the formation of the fruit; as in Tropcolum, where each loculus, enclosing a single seed, becomes rounded and at length entirely separated from the others as a closed mericarp; and in Boragineæ and Labiatæ, where each of the two carpels produces two one-seeded chambers, all four becoming at length completely separated, and surrounding the style as distinct mericarps (here called Carceruli); or the separation only takes place by the splitting and rupture 
of certain plates of tissue in the fully ripe fruit (as in Umbelliferæ and Acer), then termed a Cremocarp, where the fruit breaks up into two one-seeded halves or mericarps by the splitting of the dissepiment or 'carpophore' along its length. The quinquilocular fruit of Geranium splits up in the same manner into five one-seeded mericarps.

True single fruits are in general unilocular or multilocular, according as the ovary was divided or not. But the unilocular ovary may produce a multilocular fruit by spurious dissepiments, i.e. such as cannot be considered as the reflexed margins of the carpeis; and the loculi of such a fruit may lie either one above another or side by side. The compartments, for example, of the legume (lomentum) of some Papilionaceæ and of Cassia fistula lie one over another, while the two spurious loculi of the legume of Astragalus lie side by side. A multilocular ovary may, vice versâ, produce a unilocular fruit by the suppression of one or more loculi, as in the Oak and Lime. A classification or fruits into monocarpellary and polycarpellary cannot therefore be carried out as it can be in ovaries; the terms having now a different application.

The wall of the ovary becomes the wall of the fruit or Pericarp. If sufficiently thick, it can generally be divided into two or three layers, the tissue of which is developed differently; the outer one, often nothing but the epidermis, is then called the Epicarp, and the inner one the Endocarp. If another one lies between these, it is called the Mesocarp, or when it possesses a fleshy character, the Sarcocarp.

Using the nomenclature which has now been described, we may classify all true fruits into two principal sections, and each of these again into subdivisions, according to whether the pericarp consists, when the fruit is ripe, of succulent fleshy layers or not, and whether the fruit dehisces in order to allow the escape of the seeds which become detached from the placentæ, or not; viz.

A. DRY FRUits. Pericarp woody or tough and leathery, the cell-sap having disappeared from its cells.

a. Dry Indehiscent Fruits. The pericarp does not split open, but encloses the seed till germination; the testa is thin and membranous, and but little developed.

(a) One-seeded dry indehiscent fruits.

r. The Nut or Glans: the dry pericarp is thick and hard, and consists of lignified sclerenchymatous tissue; $\bullet . g$. the Hazel-nut.

2. The Caryopsis or Acbenium: the dry pericarp is thin, tough, and leathery, in close contact with the seed, and separable or not from the testa; as the fruit of Compositæ, Grasses, the Sweet-Chestnut.

( $\beta$ ) Bi- or multilocular dry indehiscent fruits.

3. These are mostly Scbixocarps splitting up into Mericarps, each of which resembles a nut or achenium; e.g. Umbelliferæ, Geraniaceæ. When the mericarp is winged, as in Acer, it is called a Samara.

b. Dry Dehiscent Fruits or Capsules in the more general sense. When the fruit is perfectly ripe, the pericarp bursts or splits to allow the escape of the seeds, which are themselves clothed with a strongly developed usually hard or tough testa. They generally contain more than one seed.

(a) Capsules with longitudinal dehiscence :-

4. The Follicle, consists of a single carpel which splits along the ventral suture or coherent margins of the carpels which bear the seeds; as in Pconia, Aquilegia, and Illicium anisatum; in Asclepias the thick placenta also becomes detached.

5. The Legume consists also of a single carpel, which however splits not only along the ventral but also along the dorsal suture, and thus separates into two halves; Pbaseolus, Pisum. 
6. The Siliqua consists of two carpels which form a bilocular fruit with a longitudinal (spurious) dissepiment; the two halves of the pericarp separate from the dissepiment and the placentæ which remain behind (replum); Cruciferæ.

7. The Ciapsule (in the narrower sense of the term) results from a polycarpellary unilocular or multilocular ovary, and splits longitudinally into two or more lobes and valves, which separate from one another only partially from the apex downwards (as in Cerastium), or entirely to the base. If, in a multilocular fruit, the fissures cause the dissepiments themselves to split, the dehiscence is septicidal (as in Colcbicum); if, on the contrary, the fissure is in the middle between each pair of dissepiments (i.e. along the dorsal suture of the carpels), the dehiscence is loculicidal (as in Tulipa and Hibiscus); if again a part or the whole of each dissepiment remains attached to a central column (which in the latter case is winged), from which the valves become detached, the dehiscence is septifragal (as in Datura) ${ }^{1}$. If the capsule results from a unilocular polycarpellary ovary, the separation of the valves may take place either at the sutures corresponding to the septicidal dehiscence (as in Gentiana), or in the middle between them, corresponding to the loculicidal dehiscence (as in Viola).

( $\beta$ ) Capsules with transverse dehiscence :-

8. The Pyxidium opens by the separation of an upper part of the pericarp which falls off like a lid, while the lower part remains attached to the flower-stalk in the form of an urn (e.g. Plantago, Hyoscyamus, Anagallis).

( $\gamma$ ) Capsules opening by pores:-

9. The term Pure-capsule might be given to those in which openings of small size result from small valves becoming detached at certain points of the pericarp; the small seeds being shaken out by the wind through these openings (e.g. Papaver, Antirrbinum).

B. SUCCUlENT FRUits. The tissue of the pericarp or certain layers of it remain succulent until the fruit is ripe, or assume a fleshy pulpy texture.

c. Succulent Indehiscent Fruits. The succulent pericarp does not burst, and the seeds therefore do not escape.

Io. The Drupe or Stone-fruit. A mesocarp of fleshy texture and usually considerable thickness lies within a thin epicarp; the endocarp forms a thick hard layer (the stone, called also the putamen) which usually encloses only one seed with a membranous testa (the Plum, Cherry, Peach, \&c.).

II. The Berry. The rest of the tissue of the pericarp is developed in the form of a succulent pulp within a more or less tough or hard epicarp, the seeds being imbedded in the pulp and surrounded by a firm or even hard testa. The berry is distinguished in general from the drupe by the absence of a hard endocarp, and usually contains more than one seed (as the Currant, Gourd, Pomegranate, Potatoberry), but sometimes only one (as the Date). Closely resembling the berry is the fruit of the various species of Citrus, sometimes called Hesperidium, the pericarp of which consists of a leathery

1 [Septifragal dehiscence may take place either septicidally (as in Rhododendron, Kalmia) or loculicidally (Datura).] 
outer layer and a pithy inner layer; at a very early period multicellular protuberances are developed from the innermost layer of tissue of the wall of the multilocular ovary, which gradually fill up the cavity of the loculi of the fruit with isolated but closely crowded succulent lobes of tissue, and form in this case the pulp.

d. Succulent Dehiscent Fruits. The succulent but not fleshy pericarp.splits and allows the escape of the seeds which have usually a strongly developed testa.

12. The term Succulent Capsule might be given to those fruits the succulent pericarp of which opens by dividing into lobes, and allows the seeds to escape (as in the Horse-Chestnut and Balsam).

13. The fruit of the Walnut corresponds again to the drupe; the outer succulent layer bursts, a stony endocarp surrounding the thinskinned seed. It might be called a Debiscent Drupe.

14. The fruit of Nuphar bears more resemblance to a berry, but differs in the bursting of the outer firm layer of the pericarp; it may be termed a Debiscent Berry; in N. advena this exposes an inner coating of each loculus of the fruit, which floats for some time on the water like a bag filled with seeds.

The enumeration here given includes only the more common forms of fruits; there are a number of others which cannot be placed exactly in any of the above categories, but to which no special name has been given ${ }^{1}$.

The Ripe Seed depends, as respects its external nature, on the development of the pericarp. The testa is in general thicker, firmer, and harder in proportion to the softness of the pericarp, especially when this latter bursts to allow the dispersion of the seeds. When, on the contrary, the pericarp is tough or woody, and encloses the seeds until they germinate, as in the caryopsis, nut, drupe, and schizocarp, the testa remains thin and soft, as also when the endosperm is strongly developed and very hard and encloses a small embryo, as in the Date and Phytelephas. The testa of the seeds of dehiscent fruits is usually covered by a distinctly differentiated epidermis; and it depends on the configuration of this epidermis whether the seed has a smooth appearance (as in the Pea and Bean), or displays a variety of sculpturing, such as pits, warts, bands, and so forth (as in Hyoscyamus, Datura, Papaver, Nigella, \&c.). The epidermal cells of the seed not unfrequently grow into hairs; cotton consists, for example, of the long woolly hairs which clothe the seed of Gossypium; in some cases only a pencil-like tuft of long hairs is developed, as in Asclepias syriaca. The epidermal cells of some seeds, as the Flax, Quince, Plantago Psyllium, arenaria, and Cynops, contain layers of cellulose which have become converted into mucilage, swell up strongly with water, become separated, and envelope the seeds when moist in a layer of mucilage. Pericarps which are indehiscent and which contain small seeds not unfrequently assume a character closely resembling that of the testa of the seeds of dehiscent fruits; and this is especially the case with the achenium and caryopsis, which are hence popularly called seeds. The corona of hairs which serves as an apparatus for the dissemination of many seeds through the air is frequently developed in the caryopsis as an appendage of the pericarp (as the pappus of Compositæ, which properly replaces the superior calyx). The wings answering the same purpose which are formed during the development of the testa of some seeds in dehiscent fruits (seen in an especially beautiful manner in Bignonia) recur again on the pericarp of indehiscent fruits (as in Acer). The mucilaginous epidermis spoken of above of the seeds of dehiscent fruits recurs in the epidermis of the carcerulus of Salvia and other Labiatæ, \&c. These and a number of other facts show that all that is essentially required in the development both of the

1 [For other recent attempts to classify fruits, see Dickson, Brit. Assoc. Rep. 187r, also Nature, vol. IV. p. 347, and Journ. of Bot: I87I, p. 3Io; McNab, Nature, vol. IV. p. 475 ; Masters, Nature, vol. V. p. 6 ; and Gray, Structural Botany.] 
pericarp and the testa is to furnish means for the dissemination of the seeds in various ways; structures which are morphologically very different thus attaining the same physiological development, while those which are morphologically similar attain the most various physiological development. A detailed enumeration is therefore more in the province of physiology and biology than in that of morphology and classification. (See Book III.)

To complete the subject of nomenclature, it only remains to remark that the part of the seed where it has become detached from the funiculus-usually easily distinguished after falling out-is termed the Hilum or umbilicus. The micropyle is often also to be recognised, lying, in anatropous and campylotropous seeds, close beside the hilum (as in Faba, Pbaseolus, and Corydalis), generally as a wart pitted in the middle. When outgrowths occur on the seed, either along the raphe, as in Chelidonium majus, Asarum, Viola, \&c., or as a cushion covering the micropyle as in Euphorbia, they are variously called Crest, Stropbiole, or Caruncle. The Aril which envelopes the base of the ripe seed or the entire seed as a fleshy succulent mantle and is easily removed from the true firm testa has already been described in detail.

\section{CLASS XI.}

\section{O N O C T Y LE D O N S.}

The Seed of Monocotyledons usually contains a strongly developed endosperm and a comparatively small embryo; and this is exhibited in an especially striking manner in large seeds, such as those of Cocos, Phonix, Phytelephas, Crinum, \&c. In the Naiadeæ, Juncagineæ, Alismaceæ, and Orchideæ, the endosperm is wanting from the first; and in the Scitamineæ, where it is usually wanting, it is replaced by a copious perisperm.

The Embryo is usually cylindrical, fusiform, and sometimes considerably elongated, and is then also curved spirally (e.g. in Potamogeton and Zanichellia); its form is not unfrequently that of an erect or inverted cone, in consequence of a considerable thickening of the upper end of the cotyledon. The axis of the embryo is generally very short and small in comparison to the cotyledon; in the Helobiæ on the contrary the axial portion of the embryo forms the greater part of it. At the posterior end of the axis is the rudiment of the primary root, in addition to which two or more lateral roots also originate in Grasses, which, like the primary one, are surrounded by a root-sheath (Fig. 124, see also p. 588). The embryo of Grasses is also distinguished by the presence of the Scutellum, an outgrowth of the axis beneath the cotyledon, which envelopes the whole of the embryo like a mantle, and forms a thick peltate plate on the posterior side where it is in contact with the endosperm ${ }^{1}$. In the Orchideæ, Apostasiaceæ, and Bur-

${ }^{1}$ [Van Tieghem (Ann. des Sci. Nat. $5^{\text {th }}$ series, vol. XV, 1872) gives a useful summary of the various views which have been held with respect to the homology of the parts of the embryo of Grasses. He regards the scutellum as the cotyledon, and what Sachs considers the cotyledon as only its strongly developed ligule. According to Hegelmaier (Bot. Zeitg. 1874) the cotyledon of Grasses consists of two parts; the one forms the scutellum, the other forms a sheath round the plumule.] 
manniaceæ, the parts of the embryo of the ripe seed are not differentiated; it consists of a round mass of tissue on which the plumule is developed only during germination.

Germination $^{1}$ either begins at once with the lengthening of the roots-their

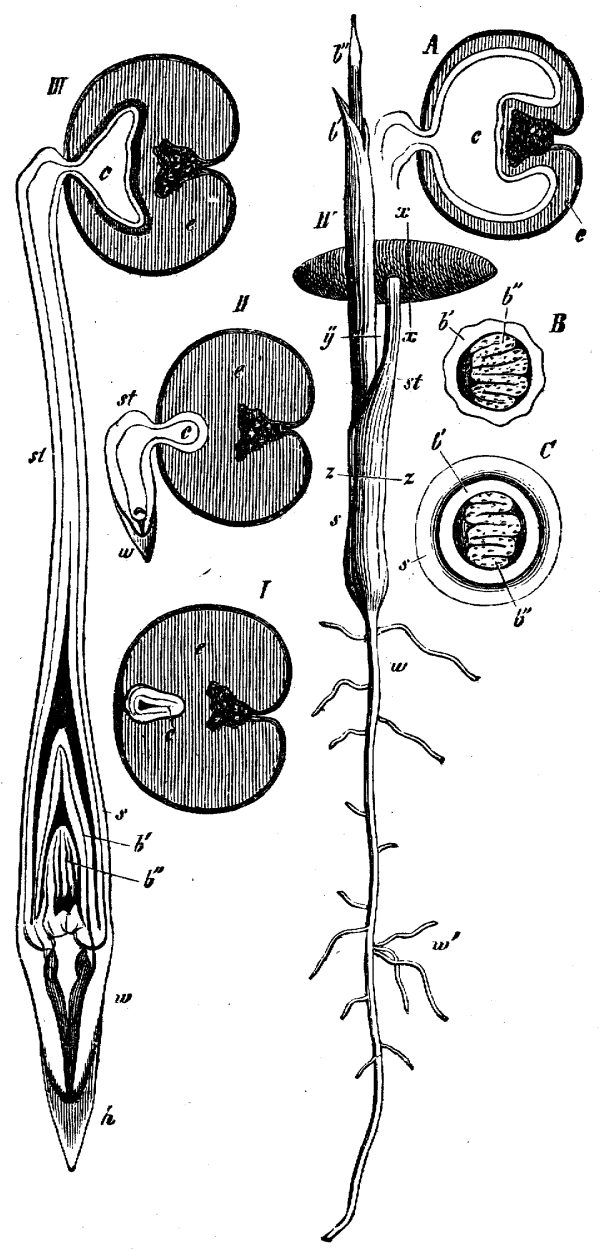

FIG, 4I.-Germination of Phenix dactylifera: $I$ transverse section of the dormant seed; $I I I-I V$ different stages of germination $(I V$ the natural size); $A$ transverse section of the seed at $x x$ in $I V ; B$ transverse section at $x y, C$ at $z z$; $e$ the horny endosperm, $s$ the sheath of the cotyledon, st its stalk, $c$ its apex developed into an organ of absorption which gradually consumes the endosperm and at length occupies its place, $w$ the primary root, $w^{\prime}$ secondary roots, $b^{\prime} b^{\prime \prime}$ the leaves which succeed the cotyledon, $b^{\prime \prime}$ becomes the first foliage-leaf: in $B$ and $C$ its folded lamina is seen cut across.

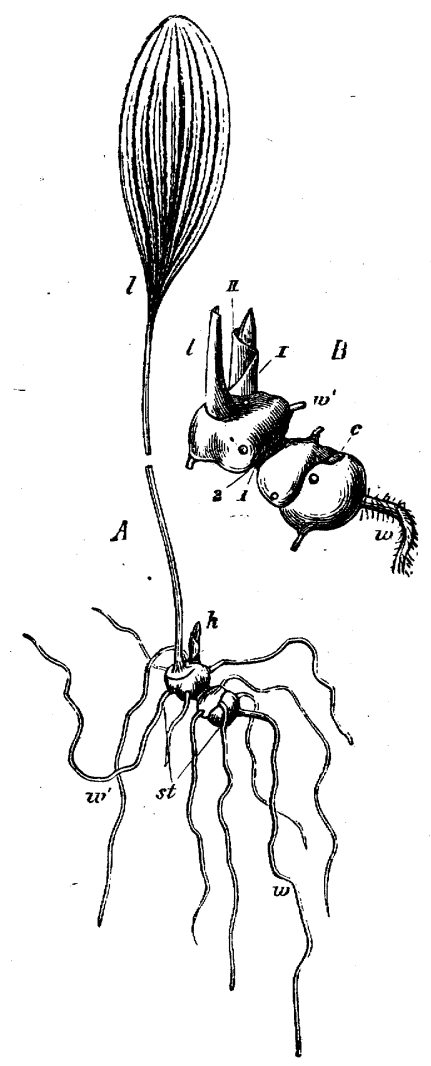

FIG. 420.-Plant of Polygonatum multiflorum in its second year; $B$ its stem magnified, $w$ the unbranched primary root, $w^{\prime}$ lateral roots springunbranched primary root, wr lateral roots sping ing from the stem $s t, l$ foliage-leaf of the second year, $k$ bud, $c$ the scar where the cotyledon was attached, $I$ and 2 scars of the first sheath-leaves which precede the foliage-leaf $l . I, I I$ the succeeding sheath or cataphyllary leaves of the bud in $B$. (Cf. Fig. 143.)

protrusion causing in Grasses the rupture of the root-sheath which envelopes them, and which remains attached to the axis of the embryo as the coleorhiza (Fig. 123); -or, as is more commonly the case, the lower part of the cotyledon lengthens,

1 See Sachs, Bot. Zeitg. 1862 and 1863. 
and pushes the end of the root, together with the plumule which is enveloped by the sheath of the cotyledon, out of the seed (Fig. 4I9), while its upper part remains in the endosperm as an organ of absorption, until the endosperm is consumed. In Grasses, however, the whole of the plumule projects from the seed, the scutellum only remaining behind in it, in order to convey to the embryo the food-material contained in the endosperm.

The growth of the primary root of Monocotyledons soon ceases even when it is very strongly developed during germination, as in Palms, Liliaceæ, Zea, \&c.; lateral roots are produced in its place, springing from the axis, which are stronger the higher up they are produced on it. No such permanent root-system is developed from the primary root of Monocotyledons as is found in Gymnosperms and in many Dicotyledons; sometimes no roots at all are produced, as in some Orchidaceous saprophytes destitute of chlorophyll (as Epipogium and Corallorhiza), which never possess any roots.

The plumule of the embryo is usually completely enclosed in a single sheath-like structure, the first leaf or cotyledon, which developes either into a sheath-like cataphyllary leaf or at once into the first green foliage-leaf of the young plant (as in Allium). Within the cotyledon there is generally a second and sometimes (in Grasses) a third and fourth leaf, which protrude on germination out of the sheath of the cotyledon, increasing by intercalary growth at their base ; these and the leaves which are formed subsequently are larger the later they are formed on the growing axis. The axis usually remains very short during germination without forming any distinct internodes (Allium, Palms, \&c.), or it lengthens more rapidly and becomes segmented into evident internodes ( $Z e a$ and other Grasses).

The increase in strength of the plant may take place by the powerful growth of the axis of the embryo itself, so that this at length forms the primary stem of the mature plant bearing the organs of reproduction, as for instance in most Palms, Aloes, $Z e a, \& c$. If the axis of the embryo remains short while it increases in strength, it may grow considerably in thickness and form a tuber (Fig. 420), or, if the bases of the leaves become thick (as in Allium Cepa), a bulb. If the axis of the embryo itself developes into the primary stem, whether into an upright one or a creeping rhizome, it first of all takes the form of an inverted cone, which is elongated or abbreviated according to the length of the internodes. This peculiarity, which belongs to most Monocotyledons in common with Ferns, depends on the absence of any subsequent increase in thickness; the portions of the stem first formed retain their size, while each successive portion is larger ; the diameter of the stem is therefore so much larger the nearer it is taken to the apex. As long as this growth proceeds, the stem continues to grow stronger; but sooner or later there comes a time when every portion of the stem acquires the same thickness as the previous one; the stem then becomes cylindrical, or, if it is compressed like some rhizomes, still with a uniform breadth. The lateral shoots exhibit the same peculiarity when they spring low down from the primary stem (as in Alö̈, \&c.). But the primary shoot which springs from the embryo not unfrequently disappears after producing lateral shoots which grow more vigorously than it, and these again transfer the further growth to new shoots, which now 
produce from generation to generation thicker axes, larger leaves, and stouter roots, until at length a condition again results in which each successive generation of shoots produces others of equal strength. If the portions of the axes of the shoots beneath the points where the shoots of the next order arise are persistent, sympodia arise (as represented in Fig: 143); but frequently each shoot entirely disappears after producing one of the next order, as for instance in our native tuberous Orchids (Fig. $15^{8}$ ), or in the Crown-Imperial (Fig. 42r), or Autumn Crocus (Fig. 422) ${ }^{1}$.

The normal Mode of Branching of Monocotyledons is always monopodial and usually axillary ${ }^{2}$; a bud is generally formed in the axil of each leaf, but often does not unfold, so that the number of branches visible is often less than that of the leaves (as in Agave, Aloë, Draccena, Palms, many Grasses, \&c.). But sometimes several buds are formed in the axil of a leaf, and if the insertion of the leaf is broad these are placed side by side, as occurs in many bulbs (Fig. I30).

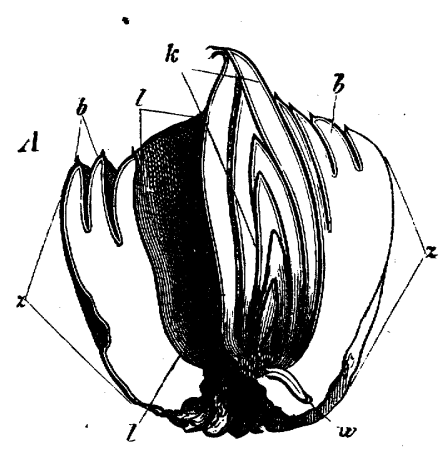

FIG. 421, - Bulb of Fritillaria imperialis in November: $A$ longitudinal section of the whole bulb reduced, $z z$ the FIG. 42 . - B decayed flower-stem : next year's bud is formed in the axil of the innermost scale; its first leaves will form the new bulb, while its axis will develope into the flower-stem; the root $w$ springs from the axis of this bud. $B$ longitudinal section of the apical region of next year's bud, $s$ apex of the stem, $b b^{\prime} b^{\prime \prime}$ youngest leaves.

In Musa a number of flowers even stand side by side in the axil of a bract, and in Musa Ensete two rows one over the other. In the Spadicifloræ the bracts are often absent ${ }^{3}$, and the ebracteate flowers stand on the rachis of the inflorescence, but are distinctly lateral in their origin. This is also the explanation of the branching of Lemna, which does not in general form any foliage-leaves, but the vegetative body of the plant consists of disc-like or swollen portions of the axis containing chlorophyll which branch laterally out of one another, and are connected together only by slender stalks, or soon separate. The plane of ramification coincides with the surface of the water on which they float; each shoot

1 Further details of the great variety of modifications of these processes of growth will be found in Irmisch, Knollen und Zwiebelgewächse (Berlin 1850), and Biologie und Morphologie der Orchideen (Leipzig 1853).

2 According to Magnus (Bot. Zeitg. 1869, p. 770) the flower of Naias occupies exactly the place of the first leaf of a branch; but it appears from p. 771 as though the flower and the shoot that bears it were the bifurcations of a dichotomy.

${ }^{3}$ Compare under Dicotyledons, p. $63^{8}$. 
produces only one or a pair of opposite lateral shoots, and the branching is therefore distinctly cymose, sympodial, or, as in Lemna trisulca, dichasial.

Besides the formation of shoots by the branching of the axis, adventitious shoots also sometimes occur on leaves which perform the function of gemmæ; as for instance on the margins of the leaves of Hyacinthus Pouzolsii and some Orchids (Döll, Flora, p. 348) ${ }^{1}$. The large gemmæ which appear very regularly at the point of junction of the leaf-stalk and lamina, and at the base of the lamina of Atherurus ternatus, are especially striking. The small bulbs on the stem of

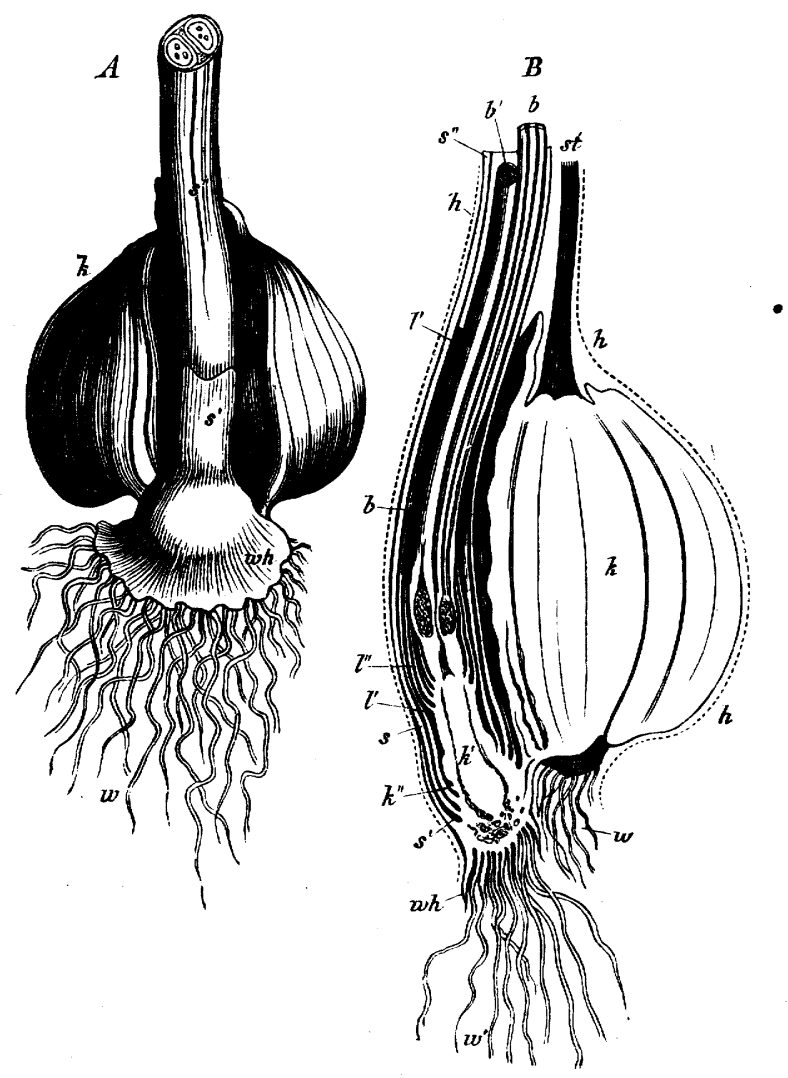

FIG. 422. -The underground part of a flowering plant of Colchicum autumnale: $A$ seen in front and from without, $k$ the corm, $s^{\prime}, s^{\prime \prime}$ cataphyllary leaves embracing the flower-stalk, wh its base from which proceed the roots $w ; B$ longitudinal section, $h h$ a brown skin which envelopes all the underground parts of the plant, st the flower- and leaf-stalk of the previous year which has died down, its swollen basal portion $k$ only remaining as a reservoir of food-materials for the new plant now in flower. The new plant is a lateral shoot from the base of the corm $k$, consisting of the axis from the base of which proceed the roots $z^{\prime}$, and the middle part of which $\left(k^{\prime}\right)$ swells up in the next year into a corm, the old corm $k$ disappearing; the axis bears the sheath-leaves $s, s^{\prime}, s^{\prime \prime}$ and the foliage-leaves $\gamma^{\prime \prime}, l^{\prime \prime}$; the flowers $b b^{\prime}$ are placed in the axils of the uppermost foliage-leaves, the axis itself terminating amongst the flowers. The foliage leaves are still small at the time of flowering; in the next spring they emerge from the ground together with the fruits; the portion of the axis $k$ then swells up into the new corm, on which the axillary bud $k^{\prime \prime}$ developes into the new flowering plant, while the sheath of the lowermost foliage-leaf is changed into the brown enveloping skin.

1 [On the buds developed on the leaves of Malaxis which exhibit a striking resemblance to the ovules of Orchideæ, see Dickie, Journ. Linn. Soc. vol. xiv. pp. I and I80. Dr. Dickie considers the structure of these buds to favour the theory that the ovule is homologous to a bud, the nucellus-like body of the bud corresponding to an axis. See also Henslow on Malaxis, Mag. Nat. Hist. vol I. I 829 , pp. 44I, 442.] 
Lilium bulbiferum are, on the other hand, normal axillary shoots, and probably the same is the case with those on the infloresence of some species of Allium. Adventitious buds are stated by Hofmeister to occur on the roots of Epipactis microphylla.

The Leaves of Monocotyledons are seldom verticillate, though this occurs in the foliage-leaves of Elodea and the bracts of Alisma; they are very commonly

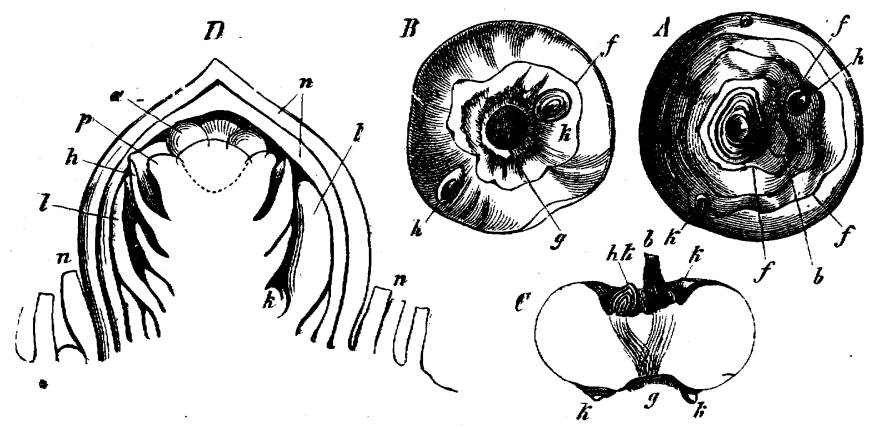

FIG. 423-Crocus vernus: $A$ the bulbous stem seen from above, $B$ seen from below, $C$ from the side and cut through lengthwise; $f f f$ the circular line of scars of the cataphyllary leaves, $k k$ the corms which grow in their axils; $b$ the base of the decayed flower- and leaf-stem, by its side ( $h k$ in $C$ ) next year's bud, from which a new corm and flower-stem will be produced; $D$ longitudinal section through this bud, $n n$ its cataphyllary leaves, $l l$ foliage-leaves, $h$ bract, $p$ perianth, $a$ anthers, $\dot{k}$ a bud in the axil of a foliage-leaf.

arranged alternately in two rows, as in Graminex, Iridex, Phormium, Clivia, Typha, \&c. This arrangement either prevails over the whole shoot together with its secondary shoots, or occurs only at first, and then passes into spiral arrangements, which very commonly lead to the formation of rosettes radiating on all sides, as in Aloë (see Fig. 152, p. 193), Agave, Palms, \&c. The arrangement with the angle
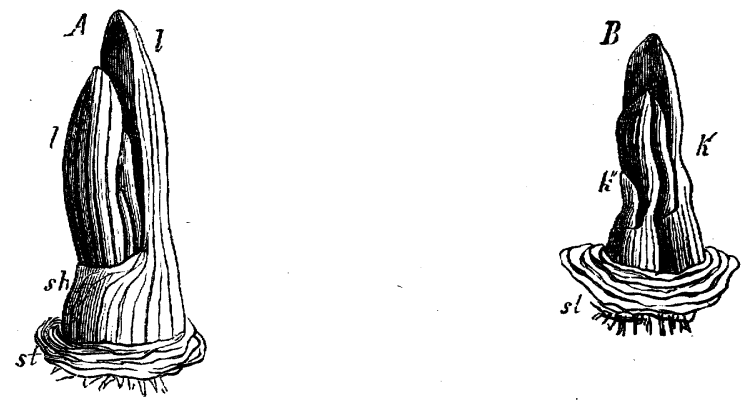

FIG. 424.-Bud in the inside of a bulb of Allinm Cepa, the scales having been removed; st the short flat base of the
tem on which the bulb-scales are inserted; $l$ in $A$ lamina, sh the sheath of the foliage-leaves still short; in $B$ the outer stem on which the bulb-scales are inserted; $l$ in $A$ lamina, $s h$ the sheath of the foliage-leaves still short; in $B$
leaves have been removed, and an axillary bud $k^{\prime \prime}$ has made its appearance in addition to the terminal bud $k^{\prime}$.

of divergence $1 / 3$ is much rarer, but occurs in some species of Alö̈, Carex, Pandanus, \&c. Spiral arrangements with a smaller divergence than $1 / 3$ also occur sometimes; as e.g. in Musa (in M. rubra the angle is, according to Braun, $3 / 7$ in the foliage-leaves, $4 / 11$ in the bracts), and Costus (where the angle of the foliageleaves is from $1 / 4$ to $1 / 5$ ), \&c. The axillary shoots of Monocotyledons mostly begin with a leaf in close contact with the primary axis and with its back turned towards 
it, and usually bicarinate. Of this character must be considered, for instance, the upper pale of the flower of Grasses, which is itself an axillary shoot of the lower pale. When the phyllotaxis of successive orders of shoots is alternate in two rows, the result of this arrangement is that a whole system of shoots is bilateral, or may be divided by a plane which bisects the leaves (as in Potamogeton, Typha, \&c.).

The mode of insertion of the cataphyllary and foliage-leaves, and very often that of the hypsophyllary leaves (as for instance that of the spathe which is of common occurrence), is entirely or at least generally amplexicaul, and the lower part of the leaf is in consequence sheathing; and this is evidently connected with the want of stipules, which are so frequent

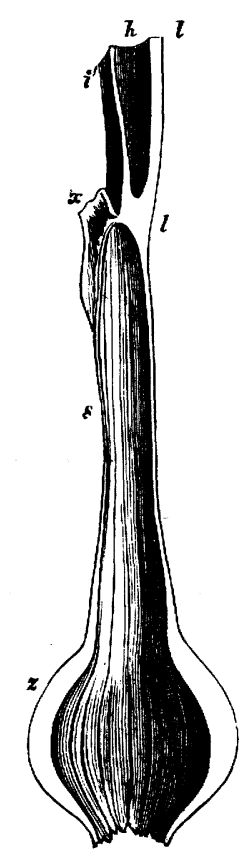

FIG. 425.-A leaf of Allium Cepa divided lengthwise; $z$ the thickened base of the sheath, which persists as a bulb-scale after the upper part of the lea has died down, $s$ the membranous part of the sheath, $l$ the hollow lamina, $h$ cavity of the lamina, $z^{y}$
inner side of the lamina, $x$ ligule. among Dicotyledons. The cataphyllary and many of the hypsophyllary leaves are usually reduced to this sheathing part, which generally passes immediately into the green lamina in the case of the foliage-leaves; but in Scitamineæ, Palmaceæ, Aroideæ, and some others, a long and comparatively slender stalk developes between the sheath and the lamina. When the leaf-stalk is absent, and the lamina sharply marked off from the sheath, a Ligule is not unfrequently present at the point where the two meet, as in Grasses and Allium (Fig. 425).

The lamina is generally entire and of a very simple form, commonly long and narrow (ligulate), rarely roundish and disc-shaped (e.g. Hydrocharis), or cordate or sagittate (as in Sagittaria and some Aroidex). Branching of the lamina is a rather rare exception among. Monocotyledons; and then takes the form either of lobes from a broad common base or less often of deep divisions, as in some Aroideæ (e.g. Amorphophallus, Fig. 141, Atherurus and Sauromatum). The division of the compound and pinnate leaves of Palms is not due to a branching occurring at an early stage, but to a splitting which takes place on unfolding, and is caused by the drying up of certain strips of tissue within the lamina, which is at first sharply folded up. The formation of the tendrils of Smilax appears, on the other hand, to depend on actual branching of the leaf-stalk.

The Venation of the foliage-leaves differs from that of most Dicctyledons, in that the weaker veins do not generally project on the under side of the leaf, but run through the mesophyll; in the smaller leaves there is even no projecting mid-rib. The mid-rib is, on the other hand, strongly developed in the large stalked leaves of the Spadicifloræ and Scitamineæ, and is permeated by a number of fibro-vascular bundles. When the leaf is ligulate and its insertion broad, the fibro-vascular bundles run nearly parallel to one another; in broader leaves without a conspicuous mid-rib they describe curves from the median line to the margins (as in Convallaria). But when a strong mid-rib occurs in a broad lamina, as in Musa \&c., the fibro-vascular bundles which run through it give off laterally smaller thin bundles which run parallel to one another 
in large numbers to the margin of the leaf. These parallel transverse nerves are sometimes united into a lattice-like network by short straight anastomosings (as in Alisma, Costus, and Ouvirandra, the mesophyll being absent within the meshes of the latter). It is only rarely (as in some Aroideæ) that projecting lateral veins are given off from the mid-rib, a finer reticulated venation springing from them.

The Flower of Monocotyledons usually consists of five alternating isomerous whorls; viz. an outer and an inner perianth-whorl, an outer and an inner whorl of stamens, and a carpellary whorl, which is succeeded by a second carpellary whorl

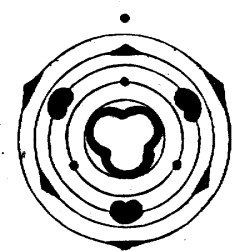

FIG. 426.-Diagram of Scirpus (Cyperaceæ).

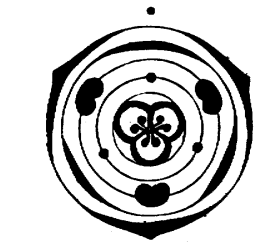

FIG. 427.-Diagram of Irideæ.

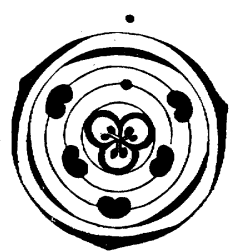

FIG. 428,-Diagram of Musaceæ.

only in Alismaceæ and Juncagineæ. The most common typical flora formula is therefore $S_{n} P_{n} S t_{n+n} C_{n(+n)}$. It is only in the Hydrocharideæ and a few other isolated cases that the number of whorls of stamens is larger. Where in other cases, as Butomus, an increase of the typical number of stamens occurs, this takes place by dédoublement without any increase of the number of whorls (Fig. 43 I A).

The number of members in each whorl is two $\left(S_{2} P_{2} S t_{2+2} C_{2}\right)$, in only a very few cases scattered through the most different families (e.g. in Maianthemum and some Enantioblastæ; it is sometimes four or five (occasionally in Paris quadrifolia and

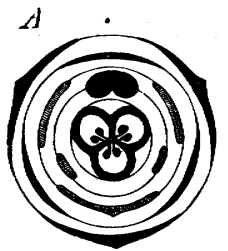

FrG. 429.-Diagram of Zingiberaceæ; A Hedychium (after Le Maout and Decaisne), $B$ Alpinia (after Payer).

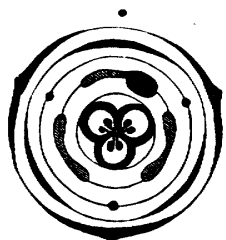

FIG. 430.-Diagram of Canna (Musacex), after Payer.

in some Orontiaceæ); but the usual number of members in each whorl is three, and the typical formula therefore $S_{3} P_{3} S t_{3+3} C_{3(+3)}$. In the large section of Liliifloræ, in some Spadicifloræ, and in many Enantioblastæ, Juncagineæ, and Alismace ${ }^{1}$, this typical floral formula is at once obtained empirically; in most others particular members or whorls are wanting, but the abortion of these is generally at once evident from the position of those that are present. In the Scitamineæ with only one or even with only half an anther (Fig. $429,43^{\circ}$ ) the rest of the members of the andrœcium are present or only partially deficient, but are trans-

1 The dimerous flower of Potamogeton $\left(S_{2} P_{2} S_{t_{2}}+{ }_{2} C_{4}\right)$ (see Hegelmaier, Bot. Zeitg. 187o, p. 287) differs from the typical formula only to this extent, that the four carpels arise simultaneously, and are placed diagonally to the preceding pairs. 
formed into petaloid staminodes. It has already been pointed out how the flowers of Gramineæ and Orchideæ can be traced back to the trimerous pentacyclic type ; the theoretical diagrams here given (Figs. 426-433) will answer the same purpose for some of the other more important families.

If the pentacyclic flower with the formula $S_{n} P_{n} S t_{n+n} C_{n(+n)}$ is considered as the typical one for Monocotyledons, it will be seen that the great majority of families the number of whose parts deviates from this type do this only by the suppression of single members or of whole whorls, the typical position of those that still remain with respect to one another not being disturbed. The

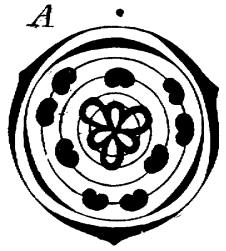

FLG, 43r.-Diagram of Alismaceæ; A Butomus, $B$ Alisma.

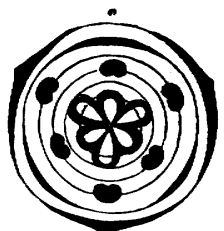

FIG. 432.-Diagram of Triglochin (Juncagineæ).

variety in the forms of flowers in this class is therefore brought about almost entirely by abortion ${ }^{1}$; and it is not uncommon for abortion to be carried to such an extent in Monocotyledons that nothing is left at last of the whole flower but a single naked ovary or a single stamen, as happens frequently in Aroideæ. In these cases a similar explanation of the relationships of the parts of the flower is rendered possible and even evident by the occurrence of flowers with the actual typical structure, and by a complete series of transitions caused by partial abortion. It is especially in small closely crowded flowers, as those of Spadicifloræ, Glumifloræ, \&c., that so great a reduction of the typical number of members is observed;

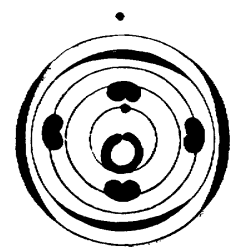

FyG. 433.-Diagram of Gymnostachys (Aroidex), after Payer.

while in larger and more isolated flowers the number of members in each whorl is usually complete or even excessive (as Butomus and Hydrocharis), and deviations usually result from petals (or petaloid staminodes) being formed in the place of fertile stamens (e.g. Scitamineæ). With reference to the abortion which is often carried to so great an extent in small flowers, it may in certain cases even be doubtful whether in an assemblage of stamens and carpels we have a single flower or an inflorescence consisting of several flowers reduced to a very simple state by abortion, as for example in Lemna.

${ }^{1}$ Compare what was said on Abortion at p. 222 and in the Introduction to Angiosperms. 
When both the perianth-whorls are well developed, they are usually similar in structure; in large flowers they are generally delicate and petaloid and either brightly coloured or not (Liliaceæ, Orchideæ, \&c.); in small flowers on the contrary they are firm, dry, and membranous, as in Juncaceæ, Eriocauloneæ, \&c. Sometimes however the outer perianth-whorl is green and sepaloid, the inner whorl larger, delicate, and petaloid (Canna, Alisma, Tradescantia); in the very small and closely crowded flowers of the Glumifloræ, the perianth-leaves, when present, take the form of hairs (the setæ of Cyperaceæ) (Fig. 426), or of small membranous scales (the lodicules of Grasses).

The Stamens generally consist of a filiform filament and a quadrilocular anther; though variations frequently occur, especially in the form of the filament and connective. Among the most striking deviations from the ordinary type are the petaloid staminodes of Cannaceæ and Zingiberaceæ. It has already been pointed out (pp. 49I, 54 I), that the foliar nature of the stamens is subject to an exception in the Naiadeæ (at least in Naias) according to the researches of Magnus. The stamens of Monocotyledons scarcely ever branch, as is often the case in Dicotyledons; and this corresponds to the customary absence of branching in the other foliar structures also. If the diagram of the flower of Canna (Fig. 430), drawn according to Payer's description, is correct ${ }^{1}$, the petaloid staminodes are branched.

The Gynceceum has usually a trilocular ovary; less often it is tricarpellary but unilocular; in both cases it may be either superior or inferior, but the latter occurs only in plants with large flowers (Hydrocharis, Irideæ, Amaryllideæ, Scitamineæ, Orchideæ, \&c.). The formation of three or more monocarpellary ovaries is limited to the alliance of the Juncagineæ and Alismaceæ, in which the ordinary number of members and of whorls of the gynæceum is also exceeded, reminding one of the Polycarpæ among Dicotyledons.

Adhesion and displacement are not so common in the flower of Monocotyledons, and usually not so complicated as among Dicotyledons; among the most striking phenomena of this nature are the formation of the gynostemium of Orchids ; the cohesion of the six similar perianth-leaves into a tube in Hyacinthus, Convallaria, Colchicum, \&c.; and the epipetalous and episepalous position of the stamens in the same plants and in some others. Adhesion of the stamens to the calyx or corolla occurs much less constantly in particular families among Monocotyledons than among Dicotyledons.

Terminal flowers to a leafy primary shoot occur very rarely among Monocotyledons (e.g. in Tulipa); terminal inflorescences are more common. The flower acquires a tendency to zygomorphism, especially as it increases in size; but this is often only feebly indicated, and attains its highest development in Scitamineæ and Orchideæ.

The Ovules of Monocotyledons usually spring from the margins of the carpels, rarely from their inner surface (as in Butomus); the single orthotropous ovules of Naias (according to Magnus) and Typha (Rohrbach) arise by the transformation of

1 According to Eichler's masterly description of the flower of Canna (Bot. Zeitg. I873) the relations of the andrœcium are not quite those expressed in the diagram Fig. 430. Eichler gives the formula as being $S_{3} P_{3} S t_{(0)+\left(0.1^{2}, \mathrm{x}\right)} C_{3}$. 
the end of the floral axis itself (see pp. 492, 57 I); in Lemna and in some Aroidex one or more ovules stand at the bottom of the cavity of the unilocular ovary. The prevailing form of the ovule is anatropous; but in Scitamineæ, Gramineæ, and some other orders, campylotropous ovules occur; in the Enantioblastæ and a few Aroideæ they are orthotropous, either erect or pendulous. 'The nucellus is almost without exception enclosed in two envelopes (Crinum however forms an exception).

The Embryo-sac ${ }^{1}$ generally remains surrounded by one layer of the tissue of the nucellus till the time of fertilisation; the apex is sometimes destroyed so that the embryo-sac projects (as in Hemerocallis, Crocus, Gladiolus, \&c.); but, on the other hand, the apex not unfrequently remains as a cap of tissue covering the top of the embryo-sac (as in some Aroideæ and Liliaceæ). In Orchideæ the growing embryo-sac completely destroys the layer of tissue that envelopes it together with the apex of the nucellus; and this happens after fertilisation in all the other Monocotyledons that possess an endosperm, and in this case the embryo-sac sometimes advances even to the inner integument and destroys it (Allium odorans, Ophrydeæ).

In the greater number of Monocotyledons a copious development of endosperm-cells in the parietal protoplasm follows quickly after fertilisation. They soon unite into a layer of tissue and divide tangentially, until at length the embryosac is filled with radial rows of cells the result of division. Narrow embryo-sacs are filled up by the growth of the first endosperm-cells; but sometimes the cells formed by free cell-formation in the parietal layer of protoplasm constitute at first a loose mass which fills up the embryo-sac and only closes up into a tissue at a later period (e.g. Leucojum, Gagea). The narrow embryo-sac of Pistia is filled up by a row of broad disc-shaped cells which lie in it like transverse compartments and are perhaps the result of division of the sac itself. In some Aroideæ only a part of the embryo-sac is filled with endosperm, the rest remaining empty.

The endosperm still continues to grow after it has filled up the embryo-sac, the seed which it fills increasing also in size. It has already been mentioned how considerable this growth is in Crinum (p. $5^{86}$ ).

In all those Monocotyledons which form an endosperm (albuminous), it becomes closed up into a continuous tissue enveloping the embryo before this has completed its growth. By the growth of the embryo a part of the endosperm which surrounds it is again forced aside; and on this displacement depends the lateral position of the embryo in Grasses by the side of the endosperm, and the absence of this latter in some Aroideæ. But in all the other Monocotyledons which have no endosperm (exalbuminous), Naiadeæ, Potamogetoneæ, Juncagineæ, Alismaceæ, Cannaceæ, and Orchideæ, its formation is altogether suppressed, or transitory preparations for it only take place.

On the first origin of the embryo reference must be made to what was said in the Introduction to Angiosperms (p. 589); there are many points which are still doubtful in the formation of the plumule, scutellum (in Grasses), and root, from the original small-celled mass of tissue of the embryo.

${ }^{\mathfrak{}}$ See Hofmeister, Neue Beiträge (Abhandl. der königl. Sächs. Gesellsch. der Wissensch. vol. VII); also supra, p. 576 . 
With respect to their Histology $y^{1}$, Monocotyledons differ from Dicotyledons and Gymnosperms chiefly in the course of the fibro-vascular bundles in the stem, and in the want of a true cambium-layer. A number of common bundles (i.e. those common to the stem and leaves) enter the stem side by side from the broad insertions of the leaves, pass obliquely downwards into it, and then again bend outwards as they descend, approaching gradually the surface of the stem. The common bundle is usually thickest and most perfectly developed at the curved portion which lies deepest in the stem, while the limb which bends upwards into the leaf becomes thinner and simpler upwards, and the descending limb of the bundle behaves similarly downwards. Hence a transverse section of the stem which cuts through the different descending limbs at different heights in their course shows bundles of different structure and of various sizes. A radial longitudinal section through the bud or through mature stems with short internodes (as Palm-stems, thick rhizomes, bulbs, \&c.) shows how the bundles which descend from different leaves, the curves of which lie at different heights, cross one another radially, some of them bending inwards where others are already turning outwards. In elongated internodes, as for instance those of the stalks of Grasses and of some Palm-stems (like Calamus), the long scapes of Allium, \&c., the bundles run nearly parallel to one another and to the surface; the curves and intersections of the bundles may be easily distinguished at the apex of such stems, and localise themselves in the transverse plates or nodes which do not elongate between each pair of internodes. The nodes are not unfrequently traversed by a network of horizontal bundles; this is very conspicuous in the Maize.

The course of the fibro-vascular bundles which has now been described renders impossible the separation of the fundamental tissue of the stem into pith and cortex in the sense in which this occurs in Conifers and Dicotyledons. The parenchymatous fundamental tissue fills up homogeneously the spaces between the bundles which are generally numerous; but a separation takes place not unfrequently into an outer peripheral layer and an inner region, a layer of tissue being formed between the two the cells of which are thickened and lignified in a peculiar way (as for instance in most thickish rhizomes, in the hollow scape of Allium, \&c.).

In consequence of their not being parallel, and of their scattered distribution in the transverse section of the stem, the descending bundles of Monocotyledons have not the power of coalescing into a closed sheath by connecting bands of cambium (interfascicular cambium), as is the case in other Phanerogams. In correlation with this the layer of cambium between the phloëm and xylem is also absent; the fibro-vascular bundles are closed. When a portion of the stem ceases to grow in length, the whole of the tissue of the bundles becomes transformed into permanent tissue (see e.g. Fig. 92, p. Iro); and there is in consequence usually no subsequent increase in thickness; each portion of the stem, when once formed, maintains the thickness which it had already attained within the bud near the apex of the stem. But in Dracana, Alö, and $r_{u c c a}$, a renewed increase of thickness begins afterwards at a considerable distance from the apex of the stem, which may even continue for centuries and may cause a considerable though slow increase in its circumference. But this subsequent growth in thickness takes place in a way quite different from that which occurs in Gymnosperms and Dicotyledons;-a layer of the fundamental tissue parallel to the surface of the stem becomes transformed into meristem which continually produces new closed fibro-vascular bundles, and between them parenchymatous fundamental tissue (Fig. 104). A more or less evidently stratified network of slender anastomosing bundles is thus formed, the position and connection of which is easily recognised on stems which have been exposed to the weather, and in which the parenchyma which fills up the interstices has

1 Von Mohl, Bau des Palmenstammes, in his Vermischte Schriften, p. r29. - Nägeli, Beiträge zur wissensch. Bot. Heft. I.-Millardet, Mémoires de la Soc. Imp. des Sci. Nat. de Cherbourg, vol. XI, I865-[De Bary, Vergleichende Anatomie der Vegetationsorgane, I877.] 
decayed. This network of closely-placed closed fibro-vascular bundles now forms a kind of secondary wood which surrounds like a hollow cylinder the space in which the original fibro-vascular bundles of the stem run isolated and loose in the form of long threads. This thickening ring of the arborescent Monocotyledons resembles the secondary woody mass of Conifers and Dicotyledons in the fact that it belongs altogether to the stem and has no genetic connection with the leaves, differing in this from the original common bundles. An exception to the ordinary structure of Monocotyledons occurs in submerged water-plants (Hydrilla and Potamogeton), in which, according to Sanio (Bot. Zeitg. 1864, p. 223 , and 1865 , p. I 84 ), an axial cauline bundle in the stem lengthens continuously, while the foliar bundles do not unite with it till a later period, a peculiarity which recurs in some dicotyledonous water-plants, and reminds one of the corresponding processes in Selaginella.

The Systematic Classification ${ }^{1}$ of the sub-sections of Monocotyledons here adopted is that of A. Braun (in Ascherson's Flora of the province Brandenburg, Berlin 1864); but with the variation that the order Helobix there given is broken up into a series of orders. In the short diagnoses of the orders only a few of the characters are specified which are most important from a systematic point of view; the figures placed within brackets refer to those attached to the families belonging to the order in which the characters named are present or absent. A complete account might have been given of the characters of the separate families of Monocotyledons; but since a similar treatment of the class of Dicotyledons would have far exceeded our limits, the mere enumeration of the families must, for the sake of uniformity, suffice.

\section{SERIES 1.-HELOBIX.}

Water-plants; seed with little or no endosperm, but a strongly developed hypocotyledonary axis to the embryo. The number of parts of the flower usually varies from the ordinary type of Monocotyledons.

Order 1. Centrospermm (so named from the central position of the seed in (I) and in Naias). Flowers imperfect, very simple, usually without a perianth; in (r) consisting of two stamens and a unilocular ovary (containing from $\mathrm{I}$ to 6 basilar ovules) surrounded by a sheath (perianth or spathe); ovary in (2) unilocular, usually one-seeded; seed with but little endosperm. The Lemnacex consist of small branched leafless floating vegetating bodies, generally with true pendent roots; the Naiadex are slender branched long-leaved submerged plants; this family is not definable systematically, and should be split up into several. (The Lemnacex should perhaps be united to the Aroidex.)

Families: r. Lemnaceæ.

2. Naiadeæ.

${ }^{1}$ [The systematic classification adopted in this book is not one which the reader will find followed in any standard English work, either as respects Monocotyledons or Dic tyledons. The work now generally adopted as containing the most satisfactory system of distribution of the vegetable kingdom into classes, orders, and genera, is Bentham and Hooker's Genera Plantarum (London, 1862 1873), which is however at present only completed so far as to include the Gamopetalæ with inferior ovary. In Dr. Hooker's edition of Le Maout and Decaisne's Traité Générale de Botanique (London, 1873) will be found the outlines of this classification completed as far as relates to the classes and orders. De Cando!le's Prodromus Systematis Naturalis Vegetabilium in I 7 vols. (Paris 1818-1873) contains a description of every known species of Dicotyledons; Walpers' 'Repertorium' and 'Annales' serving as supplements to the earlier volumes, which are far less complete than the later ones. For an admirable epitome and illustrations of the character of each of the natural orders see also Oliver, Illustrations of the Principal Natural Orders of the Vegetable Kingdom; London, 1874.$]$ 
Order 2. Polycarpæ. Flowers pentacyclic or hexacyclic $(2,3)$; whorls in (I) dimerous and decussate, with four monocarpellary ovaries placed diagonally; in $(2,3)$ trimerous, or with a larger number of stamens and carpels (see p. 626); the gynæceum consists of three or more monocarpellary ovaries, which are one- or more-seeded; endosperm absent. Perennial floating water- or upright bog-plants, with large lattice-veined or long narrow (2) leaves.

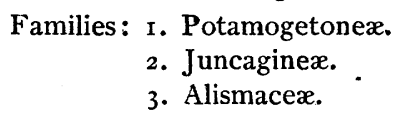

Order 3. Hydrocharideæ. Flowers diœcious or polygamous, with trimerous whorls, and perianth consisting of both calyx and corolla; male flowers of from one to four whorls of fertile stamens and within these several whorls of staminodes; female flowers with an inferior tripartite or six-chambered (3) many-seeded ovary; endosperm absent. Perennial submerged or floating water-plants with spiral or verticillate $(\mathrm{r})$ leaves.

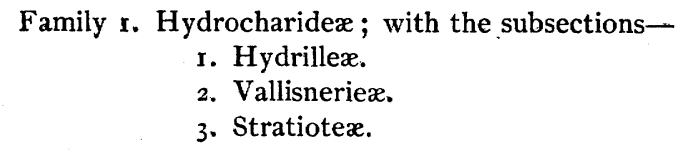

\section{SERIES II.-MICRANTHE.}

Land- or bog-plants; the individual flowers usually very small and inconspicuous, but collected in large numbers in the inflorescence, and almost always referable to the dimerous or trimerous pentacyclic type.

Order 4. Spadiciflorø. Inflorescence a spadix or panicle with thick branches (4), generally enveloped in a large sometimes petaloid (I) spathe; bracts small or altogether absent; perianth never petaloid, usually inconspicuous or altogether abortive $(\mathrm{I}-3)$; sexual organs generally diclinous by abortion; fruit always superior and often very large $(2,4)$; the seed mostly large or of an immense size and with a very large endosperm; embryo small, straight. Mostly large strong plants with the stem strongly developed, chiefly above ground, and a great number of large foliage-leaves; in $(\mathbf{r}, 3,4)$ they have a broad branched or apparently pinnate or compound lamina, a leaf-stalk and sheath, in (z) they are sessile, very long and narrow.

Families: 1. Aroideæ.

2. Pandanacex.

3. Cyclantheæ.

4. Palmaceæ.

Order 5. Glumiflorø. Inflorescence spicate or panicled, without a spathe; flowers very small and inconspicuous, usually concealed among thickly-placed dry hypsophyllary leaves (glumes or pales) $(2,3)$; perianth absent, or replaced by hairlike structures or scales; fruit superior, small, one-seeded, dry and indehiscent (a caryopsis); embryo in (1) long and in the axis of the endosperm, in (2) by its side and very small, in (3) also by the side of the endosperm, but considerably developed and provided with a scutellum. Plants with persistent underground elongated rhizomes, and upright foliage-leaves in two or three (2) rows; (I) should perhaps rather be included in the fourth order.

Families: I. Typhaceæ.

2. Cyperaceæ.

3. Gramineæ. 
Order 6. Fnantioblastæ. Flowers in crowded (4) cymose inflorescences, inconspicuous $(\mathrm{I}, 2)$, or conspicuous $(3,4)$, pentacyclic, and usually trimerous (in $(\mathrm{I}, 2)$ often dimerous); perianth-whorls glumaceous in $(\mathrm{I}, 2)$, developed into calyx and corolla in $(3,4)$; fruit a superior bi- or trilocular capsule with loculicidal dehiscence; ovule orthotropous, and the embryo ( $\beta \lambda \dot{\alpha} \sigma \tau \eta)$ therefore opposite ('́) $\nu a \nu-$ rios) the base of the seed (hilum). Plants with grass-like (I-3) or succulent habit (4).

Families : r. Restiacex.

2. Eriocaulonex.

3. Xyridex.

4. Commelynaceæ.

\section{SERIES III.-COROLLIFLORE.}

Both the perianth-whorls conspicuous, usually large and petaloid; the two staminal whorls completely developed or partially wanting by abortion, and then replaced by staminodes; one carpellary whorl; the five whorls, with few exceptions, trimerous.

Order 7. Liliiflorø. Inflorescence very various, racemose or cymose; the large flowers sometimes single. Flowers pentacyclic and trimerous, except a few cases where they are dimerous, tetramerous, or even pentamerous; in (3) the inner staminal whorl is wanting; perianth-whorls similar, in (I) inconspicuous and membranous, but usually petaloid $(2,3,5-8)$ and often large; sometimes all the six leaves are coherent into a tube ( 6 and elsewhere), often with epipetalous and episepalous stamens; ovary superior in $(\mathrm{I}, 2)$, inferior in the other families, usually forming a trilocular capsule or berry; embryo surrounded by endosperm. Plants of very various habit; with strong woody stems increasing in thickness in Aloë, rucca, and Dracana (2); more often with underground rhizomes, corms, or bulbs, from which spring leafy annual shoots; leaves mostly long and narrow, in (4) with a broad lamina and slender stalk.

Families: r. Juncacex.

2. Liliacex.

3. Irideæ.

4. Dioscoreæ.

5. Taccaceæ.

6. Hæmodoraceæ.

7. Pontederiaceæ.

Order 8. Ananasinem. Flowers consisting of the typical five trimerous whorls; outer perianth-whorl developed into calyx, inner one into corolla; ovary trilocular and many-seeded, superior or inferior; embryo by the side of the endosperm; leaves long, often very narrow.

Family: r. Bromeliaceæ.

Order 9. Scitamineæ. Floral whorls trimerous and zygomorphic; both perianth-whorls or only the inner one $(2,3)$ petaloid; of the stamens the posterior one of the inner whorl is abortive in $(1)$, this alone being fertile in $(2,3)$ (in 3 with only half an anther), while the rest are changed into petaloid staminodes

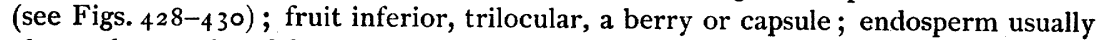
absent, but replaced by a copious perisperm. Usually handsome, often very large (I) leafy shrubby plants springing from a persistent rhizome, with large leaves, generally divided into a broad lamina, leaf-stalk, and sheath.

\footnotetext{
Families: ז. Musacex.

2. Zingiberaceæ.

3. Cannaceæ.
} 
Order 10. Gynandræ. The entire flower zygomorphic in origin and development; by the torsion of the long inferior ovary ( $r$ ) the anterior side of the mature flower usually becomes posterior; both of the trimerous perianth-whorls petaloid, the posterior leaf of the inner one (the labellum) generally provided with a spur; of the six typical stamens of the two whorls only the anterior ones are eventually developed, and in (I) (with the exception of Cypripedium) the anterior one of the outer whorl is alone fertile and has large anthers, the two anterior ones of the inner whorl forming small staminodes; but in Cypripedium it is these latter that are fertile, the anterior one of the outer whorl forming a large staminode; in (2) the same occurs, or the three anterior ones are fertile; filaments of the fertile and sterile stamens coherent with the three styles into a gynostemium ; pollen in single grains, tetrads, masses, or pollinia ; ovary inferior and unilocular with parietal placentation ( $\mathrm{r}$ ) or trilocular with axile placentation (2); ovules anatropous; seeds very numerous, very small, without endosperm, and with the embryo undifferentiated. Small herbs or larger shrubby plants; the tropical Orchidex often epiphytal and furnished with peculiar aërial roots; our native species perennial with underground rhizomes or tubers; some Orchidex are saprophytes destitute of chlorophyll, and a few have even no roots (Epipogium, Corallorbiza).

Families: I. Orchidex.

2. Apostasiaceæ.

The Burmanniaceæ with cymose inflorescence, three or six fertile epipetalous stamens, free tripartite style, and uni- or tri-locular inferior ovary, are allied to the Gynandræ by their small seeds without endosperm and their undifferentiated embryo; and in this order, which consists for the most part of small plants, there are some saprophytes destitute of chlorophyll.

\section{CLASS XII.}

\section{DICOTYLEDONS.}

The ripe Seed of Dicotyledons contains either a large endosperm and a small embryo (as in Euphorbiaceæ, Coffea, Myristica, Umbelliferæ, Ampelideæ, Polygonaceæ, Cæsalpineæ, \&c.); or the embryo is comparatively large, and the endosperm occupies but a small space (e.g. Plumbagineæ, Labiatæ, Asclepiadeæ, \&c.); or, thirdly, the endosperm is entirely wanting, and the embryo fills up the whole of the space enclosed by the testa, and thus, when ripe, often attains a very considerable size (e.g. Assulus, Juglans, Cucurbita, Tropaolum, Cupuliferæ, Leguminosæ, \&c.); though in small seeds it still remains of moderate dimensions (as in Cruciferæ, Compositæ, Rosifloræ, \&c,). The absence of endosperm generally results from its absorption by the rapid growth of the embryo before the ripening of the seed; only in a very few cases is it rudimentary from the first (Tropcoolum, Trapa). In most of the Nymphæaceæ and in the Piperaceæ the embryo and the endosperm which surrounds it both remain small, the rest of the space within the testa being occupied by perisperm. 
The Embryo generally attains but very small dimensions in the small-seeded parasites and saprophytes destitute of chlorophyll, and remains without differentiation until the time of ripening of the seed; in Monotropa it never consists of more than two cells, and even in Pyrola secunda, which possesses chlorophyll, only of from eight to sixteen (Hofmeister). The ripe seeds of Orobanche, Balanophora, Rafflesiacex, \&c. contain a very small undifferentiated embryo in the form of a roundish mass of tissue; the embryo of Cuscuta is of moderate size and length, but the formation of leaves and roots on the filiform stem ${ }^{1}$ is suppressed. The Mistletoe (Loranthaceæ), on the other hand, parasitic but containing chlorophyll, produces an embryo which is not only large but well-developed.

If the embryo of the ripe seed is differentiated, as is generally the case, it consists of an axis and two opposite primary leaves (cotyledons) between which the axis terminates as a naked vegetative cone (Cucurbila), or bears a bud which sometimes consists of several leaves (Vicia Faba, Fig. 436, Phaseolus, Quercus, \&c.). Instead of the two opposite cotyledons, a whorl of three is not unfrequently formed in those plants which normally possess only two ${ }^{2}$ (Phaseolus, Amygdalus, Quercus, \&c.). The opposite cotyledons are usually alike in form and vigour; in Trapa however one remains much smaller than the other; and cases even occur in which

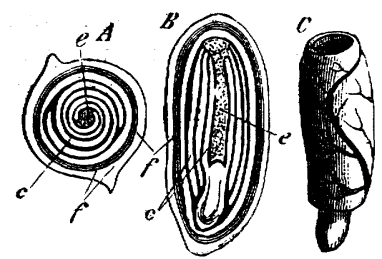

FIG. 434.-Chimonanthus fragrans: $A$ horizontal section of the nearly ripe fruit; $B$ longitudinal section of the same, $f$ the thin pericarp, $e$ remains of the endosperm, $c$ cotyledons; $C$ the embryo removed from the seed, showing the cotyledons rolled round one another, the radicular end below.

only one has been formed, as in Ranunculus Ficaria ${ }^{3}$, where it remains below in the form of a sheath, and in Bulbocapnos, a section of Corydalis*. The two cotyledons generally form by far the larger part of the ripe embryo, so that the axis has the appearance only of a small fusiform appendage between them; and this structure is especially striking when the embryo attains a very considerable absolute size in those seeds which possess no endosperm, and the cotyledons swell up into two thick fleshy bodies (as in Assulus, Castanea, Quercus, Fig. 438, Amygdalus, Vicia Faba, Phaseolus, the Brazil-nut, \&c.); but more often the cotyledons remain thin like shortly stalked foliage-leaves of simple form (as in Cruciferæ, Euphorbiaceæ, and Tilia, the last with a three- to five-lobed lamina). Most often they

1 According to Uloth (Flora, I86o, p. 265) the root-cap is also absent. On parasites see especially Solms-Laubach in Jahrb. für wissensch. Bot. vol. VI. pp. 599 et seq. [Uloth's statement is confirmed by Koch (Ueb. die Entwick. der Cuscuteen, Hanstein's Bot. Abhdl. II. 1874).]

2 Numerous additional instances are given in the Bot. Zeitg. 1869, p. 875. [Masters, Vegetable Teratology, Ray Soc. 1869, p. 370.]

${ }^{3}$ Irmisch, Beiträge zur vergleichenden Morphologie der Pflanzen, Halle I854, p. I 2.

4 [To these instances of what is termed a 'pseudo-monocotyledonous' development may be added Carum Bulbocastanum (see Hegelmaier, Entwick. dicot. Keime, 1878).] 
lie with their inner faces flat against one another (Figs. 435, 436); but are not unfrequently folded or wrinkled and curved backwards and forwards (as in Theobroma with thick, Acer and Convolvulaceæ, \&c. with thin cotyledons); less often they are rolled spirally round one another (Fig. 434).

The axis of the embryo beneath the cotyledons is generally elongated and fusiform, and when of this shape is described in works on descriptive botany as the Radicle. This fusiform body consists however in its upper and usually larger part of the hypocotyledonary portion of the stem, and only the lower posterior terminal piece, which is often very short, is the rudiment of the primary root (Fig. 437). The rudiments of the secondary roots can sometimes be distinguished in the tissue of the primary root (in Cucurbita, and according to Reinke in Impatiens).

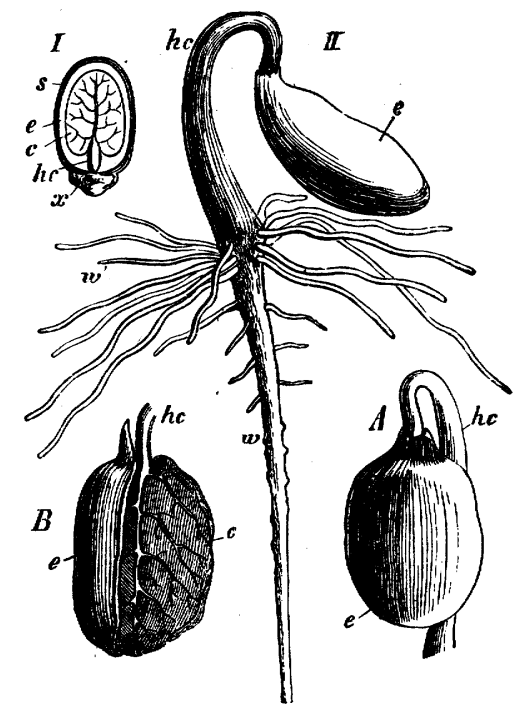

FIG: 435-Ricintus communts; $I$ longitudinal section of the ripe seed; $I I$ germinating seed with the cotyledons still in the endosperm (shown more distinctly in $A$ and $R$ ), $s$ testa, $e$ endosperm, $c$ cotyledon, $k c$ hypocotyledonary portion of the stem, $w$ primary root, $w w^{\prime}$ secondary root, $x$ the caruncle, an appendage of the seed characteristic of Euphorbiaceæ.

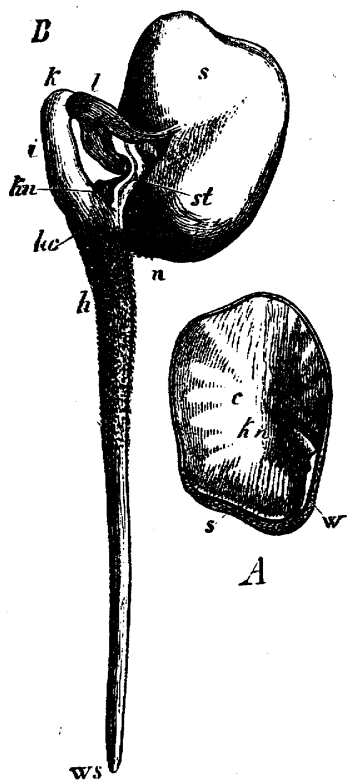

Fig. 436.-Vicia Faba: $A$ seed with one of the cotylednns removed, $c$ the remaining cotyledon, $w$ radicle, $k n$ plumule, $s$ testa ; $B$ germinating seed, $s$ testa, $l$ a portion of the testa torn away, $n$ hilum, st petiole of one of the cotyledons, $k$ curved portion of the axis above the the cotyledons, $k$ curved portion of the axis above the cotyledons, $h c$ the very short hypocotyledonary portion of
the axis, $h$ the primary root, ws its apex, $k n$ bud in the axil of one of the cotyledons.

Germination generally takes place-after the testa, or in dry indehiscent fruits the pericarp, has burst from the swelling of the endosperm or of the cotyledons themselves-by the elongation of the hypocotyledonary portion of the axis to such an extent as to push the radicle out of the seed, the root then beginning to grow rapidly and generally attaining a considerable length and forming secondary roots in acropetal succession, while the cotyledons and plumule still remain in the seed (Figs. 435, 436, 437). Thick fleshy cotyledons usually remain in the seed during germination, finally perishing after their food-material has been consumed (as in Phaseolus multiflorus, Vicia Faba, Fig. 436, Quercus, Fig. 438). In this case the petioles of the cotyledons lengthen so much that the plumule which is concealed 
between them is pushed out (Fig. 438), and now grows upright so that the seed and cotyledons together have the appearance of being a lateral appendage of the axis of the embryo. But usually the cotyledons are destined for further development, especially when they are thin, and form the first foliage-leaves of the plant. In order to liberate them and the plumule which lies between them from the seed, the hypocotyledonary portion of the axis increases considerably in length, making

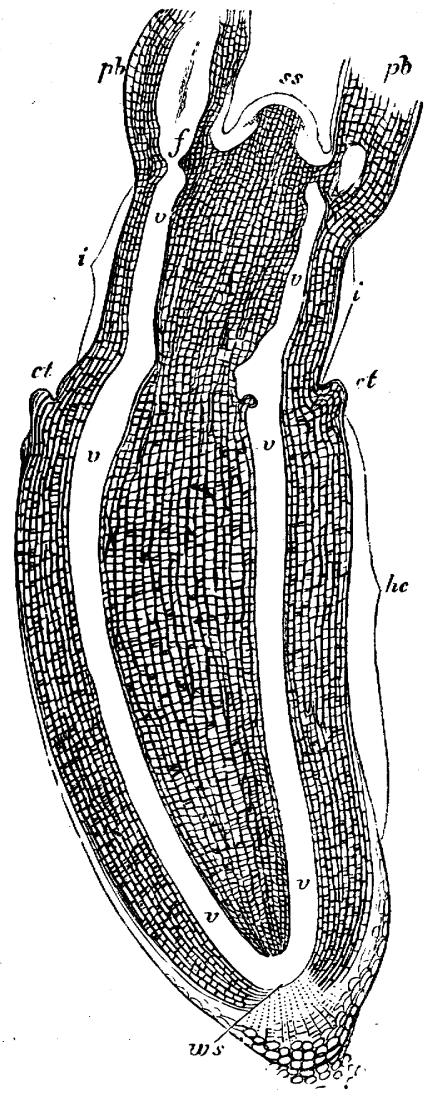

F1G. 437.-Longitudinal section of the axis of the embryo in the ripe seed of Phaseolus multiflorus, parallel to the cotyledons ( $\times$ about 30 ) ; ss apex of the stem, zus of the root. $c t$ cushion at the insertion of the cotyledons, $i$ the first internode, $p b$ the petioles of the first foliage leaves, $v, v, f$ the procambium of the fibro-vascular bundles.

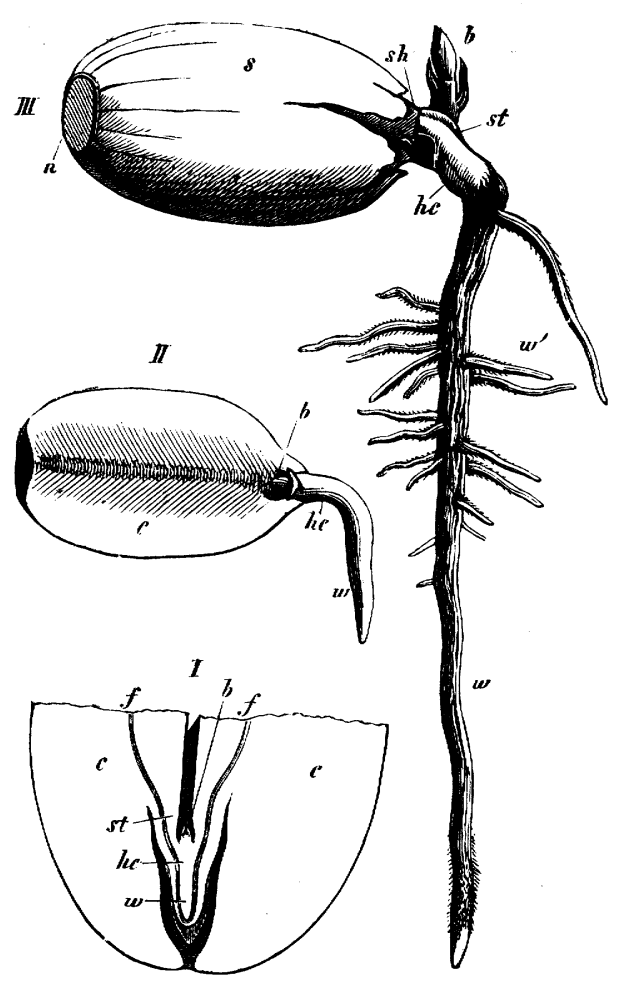

FIG. 438.-Quercus Robur; $I$ longitudinal section of the embryo (mag. nified) after removal of the anterior half of both cotyledons $c, c$, the hypocotyledonary portion of the axis $h c$, the primary root $z$, and $c$; the $b$ are concealed between the lower portion of the thick cotyl plumule petiole of the cotyledons; $I I$ seed at the time when germination is ; $s t$ petiole of the cotylons mencing (naturalsize), the pericarp and one cotyledon have been removed, the hypocotyledonary portion of the axis and the radicle have elongated $I I I$ further stage of germination, the plumule having emerged from the testa $s h$ and pericarp $s$ by the elongation of the petiole of the cotyledons $s t, w$ primary root, $w^{\prime}$ secondary roots.

first of all a curve which is convex on the upper side (Fig. 435), because the cotyledons still remain in the seed while the lower end of the stem is attached by the root to the ground. Ultimately, by a final lengthening of the hypocotyledonary portion, the upper part of the axis together with the cotyledons is drawn out of the seed in a pendent position. The axis now straightens as it continues to grow, and the cotyledons expand in the air, the plumule developing more completely and 
pushing up between them. The cotyledons which thus become exposed to the light usually. increase rapidly in size, and constitute the first green leaves of the plant, which are of simple form (e.g. Cruciferæ, Acer, Cucurbita, Convolvulaceæ, Euphorbiaceæ, \&c.). If the seed contains an endosperm, the cotyledons do not emerge till after it has been absorbed (Fig. 435). Many transitional forms occur between the different modes of germination now described; peculiar phenomena sometimes appearing which are caused by special vital conditions. In Trapa, for example, the primary root is from the first rudimentary, and remains altogether undeve. loped; the hypocotyledonary portion of the stem lengthens considerably, curves upwards, and protrudes a great number of lateral roots in rows which fix the plant into the ground ${ }^{1}$.

The further development of the young plant may take place by the rapid enlargement of the primary axis of the embryo. While the axis is growing, generally in an upright direction, the shoot which developes from the plumule becomes the primary stem of the plant, lengthening at the summit, and usually producing weaker lateral shoots (e.g. Helianthus, Vicia, Populus, Impatiens, \&c.). When the main stem is perennial, it sooner or later ceases to develope further at the apex, or the lateral shoots nearest to the apex become equally strong. An arborescent head is thus formed, the main stem or trunk becoming denuded by the dying off of the lower branches, or the main stem continues to grow erect as a sympodium (as in Ricinus, the Lime, \&c.); or lateral shoots are formed at an early period at the base of the primary stem which grow as strongly, and thus give rise to a shrubby plant. When the axis of the embryo grows vigorously, the primary root generally also grows vigorously in a downward direction ${ }^{2}$; and a Tap-root is thus formed, from which, as long

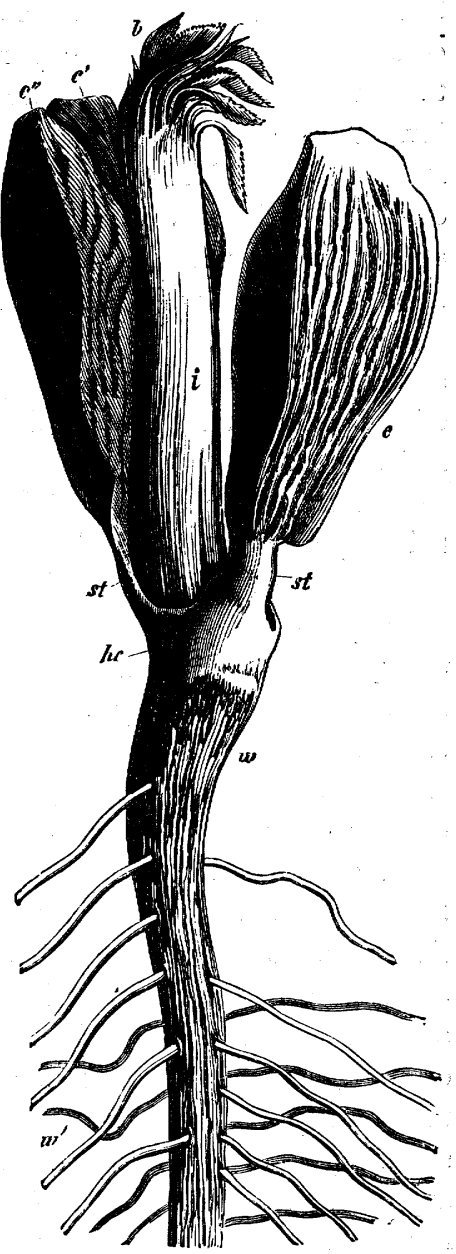

FIG. 439.-Almond-seed germinating, one of the cotyledons $c^{\prime} c^{\prime \prime}$ being split; the letters as in Fig. $43^{8}$, $i$ the first internode strongly developed. as it increases in length, the lateral roots spring in great numbers in acropetal succession. When the growth in length of the tap-

1 [See De Candolle, Organographie Végétale, P1. 55.]

${ }^{2}$ One of the most remarkable exceptions is afforded by the genus Cuscuta, which has no primary root, the posterior end of the axis penetrating into the ground on germination, but soon dying off when the upper filiform portion of the stem has embraced the plant on which it becomes parasitic, and has fixed itself on to it by its short suckers; the plant afterwards grows vigorously and branches. 
root ceases, adventitious roots become intercalated among the lateral roots already formed, and like them, grow vigorously, and may themselves produce lateral roots of higher orders. A strong root-system is thus produced with the primary root of the embryo for its centre, which endures as long as the stem itself. By the subsequent increase in thickness the primary stem (as well as its branches) assumes the form of a slender upright cone, the base of which rests on the base of the inverted cone formed by the primary root which has also increased in thickness. While these processes, which are here described in their main outlines, take place almost invariably among Conifers, a number of deviations occur, on the other hand, among Dicotyledons similar to those which have been spoken of under the head of Monocotyledons. The primary axis may die soon after germination or at the end of the first period of vegetation, the primary root often perishing as well, while the axillary shoots of the cotyledons or of subsequent leaves continue the life of the individual. Thus, for example, in the Dahlia, a strong adventitious root is given out laterally from the hypocotyledonary portion of the axis at the close of the first period of vegetation of the young plant, and swells into a tuber; the primary root-system and the portion of the axis above the cotyledons disappear, and there remain only for the continuance of the life of the plant the new tuberous root, the hypocotyledonary portion of the axis, and the axillary buds of the cotyledons. The process is still more striking in Ranunculus Ficaria, where, after the development of the primary root, a tuberous lateral root is produced below the primary axis of the embryo, sheathed by a coleorhiza, and maintains its existence together with the axis, while the primary root and the first leaves perish. Among the numerous cases belonging to this category may be mentioned also Physalis Alkekengi, Mentha arvensis, Bryonia alba, Poljgonum amphibium, and Lysimachia vulgaris ${ }^{1}$. The production of bulbs also occurs among Dicotyledons (as in species of Oxalis), though not so commonly as among Monocotyledons; of more common occurrence are tubers or swellings of underground branches, stolons, or rhizomes of greater or less thickness. The greater number of Dicotyledons have perennial underground roots or stems which send up periodically leafy and flowering shoots that die at the end of each period of vegetation. In all such cases, where the primary root-system of the seedling perishes, new roots are repeatedly developed from the stem; and the power possessed by most Dicotyledons of producing adventitious roots from the stem, especially when kept moist and dark, enables them to be reproduced to almost any extent from branches and portions of branches. Some species climb, like the Ivy, by roots put out regularly from the weak stem which requires a support; others send out runners to a distance, on which the bud forms a new plant, as in the Strawberry, the stem which is thus formed putting out roots. The order of succession of new roots from the stem is in general acropetal, but they do not usually make their appearance except at a considerable distance behind the growing bud; many Cactaceæ however not unfrequently produce them close below it.

The Mode of Branching. The normal monopodial branching is axillary; the lateral shoots are produced in the angle which the median line of the leaf forms

1 The above is taken from Irmisch's detailed descriptions in his Beiträge zur vergleichenden Morphologie der Pflanzen, Halle, 1854,1856 ; Bot. Zeitg. 1861 ; and elsewhere. 
with the internode. On a vegetative shoot at least one lateral shoot is produced in the axil of each leaf, although only a few of the axillary buds unfold. Sometimes other axillary buds are produced in rows above the original one; as, for instance, above the axils of the foliage-leaves in Aristolochia Sipho, Gleditschia, Lonicera, \&c. ${ }^{1}$, above the axils of the cotyledons in Juglans regia, and that of the larger cotyledon in Trapa. In woody plants the axillary buds destined to live through the winter are not unfrequently so completely surrounded by the base of the leaf-stalk that they are not visible until the leaf has fallen off, as in Rhus typhinum, Virgilia lutea, Platanus, \&c., and are then called Intrapetiolar Buds. Besides the ordinary axillary branching, some cases are known among Dicotyledons of lateral and monopodial but extra-axillary branching. To this description belong the tendrils of Vitis and Ampelopsis which are produced (according to Nägeli and Schwendener) beneath the punctum vegetationis of the mother-shoot, opposite to the youngest leaf and somewhat later than it. In Asclepias syriaca and some other plants a lateral vegetative branch stands beneath the terminal inflorescence between the insertions of the foliage-leaves, which themselves also produce shoots in their axils. According to Pringsheim ${ }^{2}$ lateral shoots arise on the concave side of the long spirally-curved vegetative cone of Utricularia vulgaris which he considers to be extra-axillary branches, while normal shoots are formed in the axils of the leaves which stand in two rows on the convex side of the shoot or by their side. It appears to me however certain that these extra-axillary structures on the concave side of the mother-shoot are leaves of peculiar form ${ }^{3}$, since inflorescences are produced in their axils.

The suppression of the bracts of the inflorescence, which is not uncommon, cannot be placed in the same category as the cases just mentioned of extra-axillary branching, where large leaves in the axils of which buds are also formed exist near the extra-axillary lateral branches. Here, on the contrary, as for instance in Cruciferæ and the capitulum of many Compositæ, the formation of leaves on the branching axis of the inflorescence is itself entirely suppressed; there are no leaves in the axils of which the branches could stand. The branches are however produced as if the leaves were actually there. With reference to the changes in the mode of branching met with in passing from the vegetative to the floral region and to the frequent transference of the bract on to the branch axillary to it, the remárks on p. 598 may be consulted.

Adventitious buds are rare in Dicotyledons, as they are in Phanerogams generally. Those which are commonly formed with an exogenous origin in the indentations of the margins of the leaves of Bryophyllum calycinum are well known, and serve to propagate the plant. They sometimes occur (according to Peterhausen ${ }^{4}$ ) in Begonia coriacea in the form of small bulbs on the peltate surface of

\footnotetext{
1 See Guillard, Bull. Soc. Bot. de France, vol. IV, I857, p. 239 (quoted by Duchartre, Eléments de Botanique, p. 408).

2 Zur Morphologie der Utricularien, Monatsber. der königl. Akab. der Wissench. Feb. I869.

3 This of course depends on what is considered leaf and what shoot; this is not however a matter of simple observation, but rather of conventional conceptions convenient for a special purpose.

4 Beiträge zur Entwickelung der Brutknospen (Hameln 1869), where various examples are also given of axillary buds of Dicotyledons which form deciduous gemmæ; as in Polygonum viviparum,
} 
the leaf where the principal veins radiate ${ }^{1}$. On the adventitious buds on the leaves of Utricularia, Pringsheim's treatise already quoted may be consulted. Adventitious buds more often spring from roots, e.g. in Anemone japonica, Linaria vulgaris, Cirsium arvense, and Populus tremula, according to Irmisch ${ }^{2}$. The shoots which spring from the bark of the older stems of trees must not at once be set down as the development of adventitious buds; since the numerous dormant buds of woody plants may long remain buried and yet retain their vitality.

The Leaves of Dicotyledons exhibit a greater variety both in their position and their form than those of all other classes of plants put together. The ordinary phyllotaxis of seedlings begins with a whorl of two cotyledons, and continues either in decussate pairs or passes into a distichous arrangement or into whorls consisting of larger numbers or spiral arrangements with the most various angles of divergence. More simple arrangements, especially that of decussate pairs, are generally constant in whole families, the more complicated arrangements usually less constant. Axillary branches usually begin with a pair of leaves which are either opposite or alternate, and stand right and left of the median line of the mother-leaf.

It is quite impossible to give in a short space even a general account of the forms of leaves, even apart from cataphyllary leaves (scales on underground stems and those which envelope persistent buds), hypsophyllary leaves or bracts, and floral leaves; only a few of those forms of foliage-leaves can be mentioned here which are peculiar to or characteristic of Dicotyledons. The foliage leaves are usually divided into a slender leaf-stalk (petiole) and a flat blade (lamina); the lamina is very commonly branched, i.e. lobed, pinnate, compound, or incised; and even where it forms a single plate (simple leaf) the tendency to branching is generally indicated by indentations, teeth, or incisions in the margin. The branching of the lamina has usually a distinctly monopodial origin, but its development may continue in a cymose manner, a helicoid succession of lateral lobes being formed on each side right and left of the centre of the leaf (as in Rubus, Helleborus, \&c., see Fig. 14I). The sheathing amplexicaul base is not common in Dicotyledons (but occurs in Umbelliferæ); and the occurrence of Stipules in its place is more common. The cohesion of opposite leaves into a single plate pierced by the stem is not uncommon ('perfoliate' leaves, as in Lamium amplexicaule, Dipsacus Fullonum, Lonicera Caprifolium, species of Silphium, Eucalyptus, \&c.); as well as the downward prolongation of the lamina of the leaves ('decurrent leaves'), which distinguishes the 'winged' stem of Verbascum, Onopordon, \&c. The not uncommon 'peltate' leaf also scarcely occurs in so marked a manner in any other class (Tropcolum, Vicloria regia, $\& c$.). The power of Dicotyledons to develope from their foliage-leaves organs of the most diverse functions adapted to the most various conditions of life is seen in a very striking manner in the common occurrence of leaf-tendrils and leaf-spines, and still more in the formation of the ascidia or 'pitchers' of Nepenthes, Cephalotus, Sarracenia, \&c.

Saxifraga granulata, Dentaria bulbifera, Ranunculus Ficaria, \&c. [Berge, Ueb. Bryophyllum calycinum, Zürich 1877 .]

1 The common method of propagating Begonias is by cutting or tearing the leaf, which, if then placed on moist soil, produces buds on the edges.]

${ }^{2}$ [Irmisch, Bot. Gaz. III. pp. I 46 and 160.$]$ 
The Venation of the foliage-leaves (with the exception of the thick leaves of succulent plants) is distinguished by the numerous veins which project on the under side, and by their curvilinear anastomoses by means of fibro-vascular bundles running through the mesophyll itself. The mid-rib, which usually divides the leaf into two symmetrical but sometimes into very unsymmetrical halves, gives off lateral veins right and left; one, two, or three strong nerves, similar to the mid-rib, often springing in addition from the base of the lamina right and left of the median line. The whole system of the projecting veins of a foliage-leaf behaves like a monopodial branch-system developed in one plane, the interstices being filled up by the green mesophyll in which lie the anastomoses combined into a small-meshed network. Within the meshes still finer bundles are usually formed which end blindly in the mesophyll. In membranous cataphyllary and hypsophyllary leaves and the perianth-leaves of the flowers the projecting veins do not usually occur; the venation is more simple and more like that of Monocotyledons ${ }^{1}$.

The Flower ${ }^{2}$. In the great majority of Dicotyledons the parts of the flower are arranged in whorls, or the flowers are cyclic; only in a comparatively small number of families (Ranunculaceæ, Magnoliaceæ, Calycanthaceæ, Nymphæaceæ, and Nelumbiaceæ) are all or some of them arranged spirally (acyclic or hemicyclic ${ }^{3}$ ).

In Cyclic Flowers the whorls are usually pentamerous, less often tetramerous, both numbers occurring in nearly-related plants. Dimerous or trimerous or combinations of dimerous and tetramerous whorls are much less common than pentamerous, and are usually characteristic of smaller groups in the natural system.

When the floral whorls are tetramerous or pentamerous, they are generally four in number, and are developed as Calyx, Corolla, Andrœcium and Gynæceum. In dimerous or trimerous flowers the number of the whorls is much more variable, and then it is not uncommon for each series of organs to be made up of two or three whorls; while in the previous case the multiplication of the whorls is almost entirely confined to the andrœcium.

The corolla is frequently absent, and the flowers are then said to be apetalous. When the calyx and corolla are both present the number of their parts (sepals and petals) is almost always the same (Papaver is an exception); but this is not the case with the number of the whorls. In Cruciferæ, for example, the calyx consists of two decussate whorls of two sepals each, the corolla of one whorl of four petals. When the perianth and andrœcium are both present (whether the former consist of calyx only or of both calyx and corolla), the number of their parts is usually the same, that is, the flower is isostemonous, but the stamens are often more, rarely fewer in number than the parts of the perianth, and the flower is then anisostemonous. When the

1 [The structure of the leaf compared with that of the stem has been worked out by Casimir De Candolle, Archives des Sciences, I868; the 'Student' for the same year contains an abridged translation of his paper.]

${ }^{2}$ The floral diagrams given here are drawn partly from my own investigations, but chiefly from the researches of Payer into the history of development, assisted by Döll's Flora of Baden. The figures placed beneath the diagrams are intended to indicate the number and cohesion of the carpels as well as the placentation in those plants the diagram of which is otherwise the same. [See also Eichler, Blïthendiagramme; Gray, Structural Botany.]

3 Compare pp. 600 and 608. 
flower is tetramerous or pentamerous the number of carpels is usually less; when the flower is dimerous or trimerous, or when the parts are arranged spirally, the number of carpels is not unfrequently larger.

It will be seen from this brief outline that the relations of number and position in the parts of the flowers of Dicotyledons are very various, and cannot be referred, as is the case with Monocotyledons with but few exceptions, to a single type. Even the establishment of different types for the larger groups is attended with great uncertainty, since the knowledge of development necessary in order to refer particular forms of flowers to general formulæ is often wanting. The too universal application of the spiral theory of phyllotaxis in the case of cyclic flowers has often increased the difficulty, and has even occasioned doubts which would not have arisen without the theory.

For the great majority of Dicotyledons the floral formula may be given $S_{n} P_{n} S t_{n(+n+\ldots)} C_{n(-m)}$. This formula holds good for most pentamerous flowers and for those which are truly tetramerous (or octamerous as Michauxia); so that $n$ is in these cases 5 or 4 (or 8 as the case may be). In the andrœcium an indefinite number of (alternating) whorls $S t_{n(+n+\ldots)}$ must be assumed in order to include
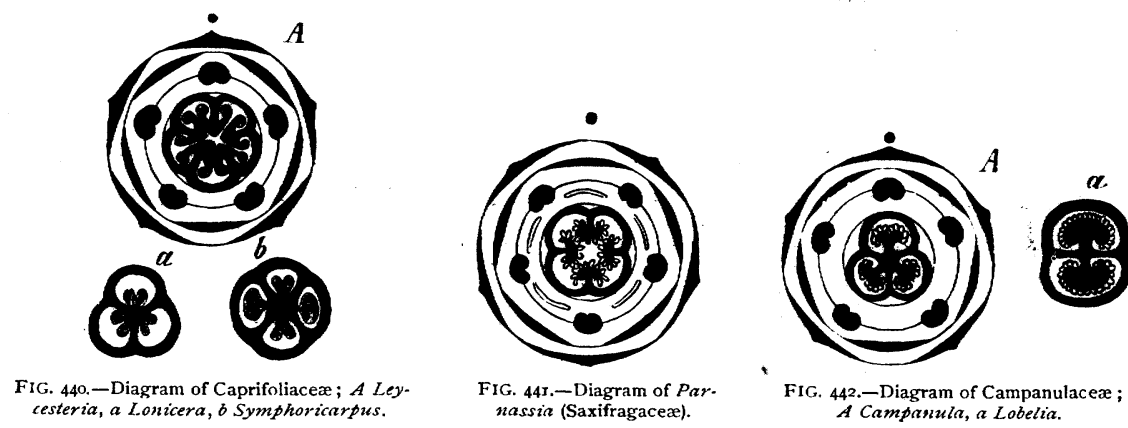

the large number of flowers in which the andrœcium consists of more than one whorl (as e.g. Fig. 45I). The mode of expressing the gynæceum $C_{n(-m)}$ is intended to show that very commonly the number of carpels is fewer than 5 or 4 (or 8 as the case may be); $m$ may be of any value from o to $n$. In the majority of gamopetalous orders and elsewhere there are very commonly only two carpels; and in this case they stand in a median line posterior and anterior; but on- the hypothesis that the typical gynæceum consists of five alternating carpels and has been reduced to two by abortion, one must stand in the median position in front, the other obliquely behind. A similar difficulty is also presented when the gynæceum consists of three or of only one carpel. It would carry us too far to detail the reasons which nevertheless determine me to retain the formula above given for the gynæceum of flowers of this description; it need only be mentioned that species or genera with the typical five carpels occur in the most diverse families and orders where a smaller number is the normal one.

The diagrams Figs. 440-450 represent a selection of cases which can be reduced (if no further reference is made to the consideration mentioned above) to the general formula which here assumes the simpler expression $S_{n} P_{n} S t_{n} C_{n(-m)}$. 
A comparison with nearly-allied forms leaves little room for doubt that the vacant spaces indicated by dots in the three outer whorls correspond to abortive members in the sense already frequently indicated, even when the absence of these members is so complete that the earliest stages of development of the flower give no indi-

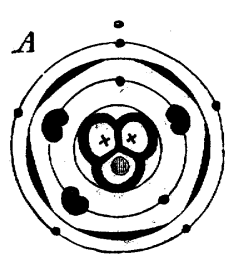

IIIG. 443.-Diagram of Valerianacex; $A$ Voleriana, $B$ Centranthus.

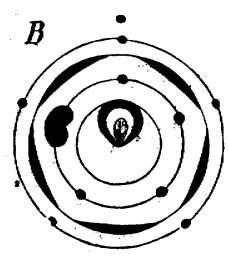

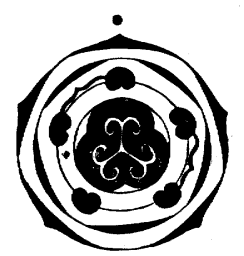

FIG. 444-Diagram of $\mathrm{Cu}$ curbitaceæ.

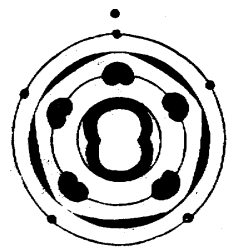

FIG. 445.-Diagram of Com. positæ.

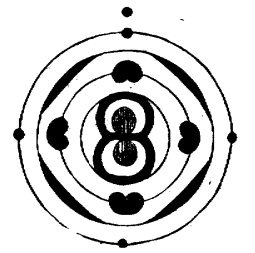

FIG. 446.-Diagram of some Rubiac æ.

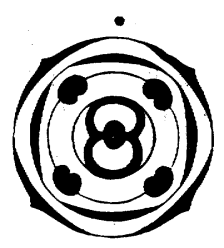

FIG. 447.-Diagram of Plantagineæ

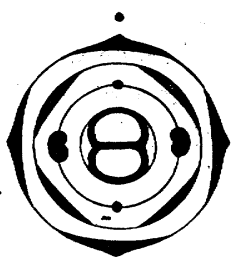

FIG. 448.-Diagram of Oleacex.

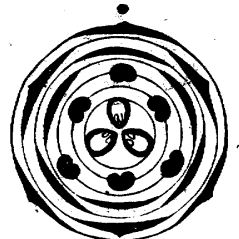

FIG. 449.-Diagram of Menispermaceæ.

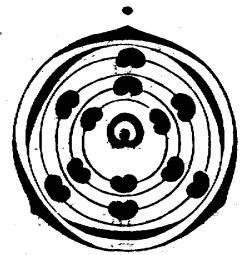

FIG. 450.-Diagram of Cinnamomum (Lauraceæ).

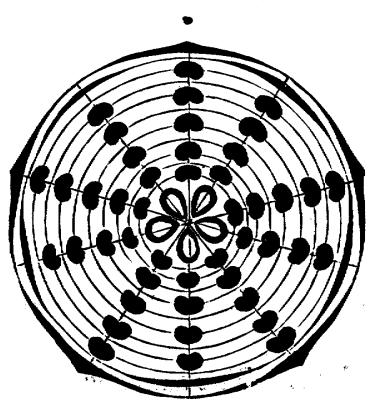

FIG. 451.-Diagram of Aquilegia (Ranunculaceæ).

cation of them. The same is the case also when the number of carpels is less than the typical one. Other cases however occur, as in the case of Rhus (Fig. 452), where certain members, in this case two out of the three carpels, disappear in the course of development. Crozophora tinctoria (Fig. 453) is especially instructive in 
regard to the relationships here suggested, the flowers becoming diclinous from the stamens in the female flowers developing as sterile staminodes, which may be considered as the first step towards abortion, while in the male flowers the three carpels are replaced by as many fertile stamens (Payer).

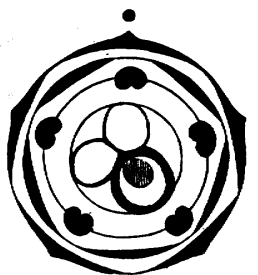

FIG. 452.-Diagram of $R$ hus (Anacardiaceæ).

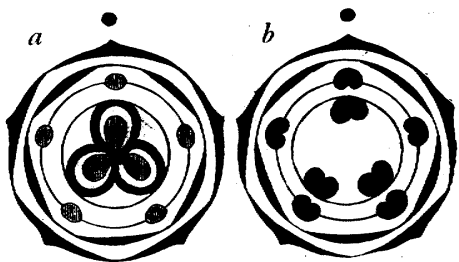

FIG. 453-Diagram of Crozophora (Euphorbiaceæ), $a$ female, $b$ inale flower.

Reference was made in the Introduction to Angiosperms (pp. 601, 6ro) to the interposition of a whorl of stamens between the members of a previously formed staminal whorl; and it was mentioned that the interposed whorl has sometimes not

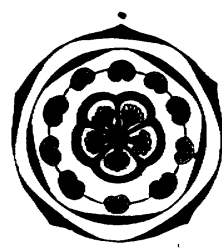

FIG. 454.-Diagram of pentamerous Ericaceæ and Epacrideæ.

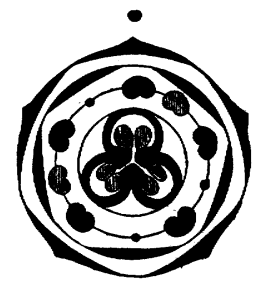

FIG. 455-Diagram of .Esculus (Hippocastaneæ)

the full number of members. These phenomena occur in various large groups of Dicotyledons ${ }^{1}$. In Fig. 454 the five stamens of the decandrous flower of the group of Bicornes which are interposed as a whorl of full number within the first whorl

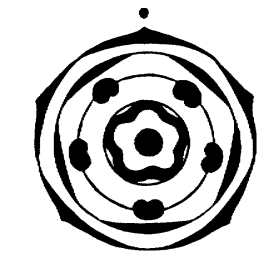

FIG. 456.-Diagrain of Primulaceæ.

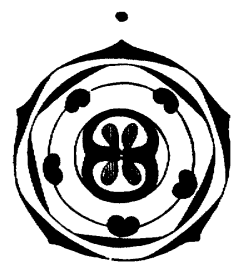

FIG. 457.-Diagram of Vitis (Ampelideæ).

are indicated by the lighter colour. The same is the case with the larger number of Gruinales, among which however the Balsaminex possess only the typical five stamens; the Lineæ and the genus Erodium have five additional rudimentary stamens interposed between them; while in Peganum Harmala and Monsonia the

1 Payer's figures show that the interposed whorl, although of later origin, is sometimes exterior to the typical whorl. The main point is that the position and number of the other parts of the flower are exactly as if there were no interposed whorl. 
number of stamens in the interposed and outer whorl is doubled. The order : Esculineæ is of special interest in this connection, since in some of its families (Acerineæ and Hippocastaneæ, Fig. 455) the interposed staminal whorl remains incomplete, so that the total number of stamens is not a multiple of the typical fundamental number (five). Among pentamerous flowers Lythrarieæ, Crassulaceæ, and Papilionaceæ may be mentioned in addition, and among tetramerous ones Enothereæ, in which a complete staminal whorl is interposed.

One of the most remarkable deviations from the ordinary structure takes the form in not a few families of Dicotyledons of the simple staminal whorl being superposed on the corolline whorl, as shown in Figs. 456, 457, and as occurs also in the Rhamnaceæ, Celastrineæ, the pentandrous Hypericineæ, and Tilia. Pfeffer ${ }^{1}$ has shown that the two superposed whorls of Ampelideæ arise independently of one another and in acropetal order, while on the other hand in Primulaceæ they first appear in the form of five projections each of which forms a stamen, and from each of which a petal subsequently grows outwards. In these cases we have no sufficient ground for the hypothesis that an alternating whorl has been suppressed between the two superposed ones; although in other cases this supposition is justified, or at least is very probable. Thus in the order Caryophyllineæ, families, genera and
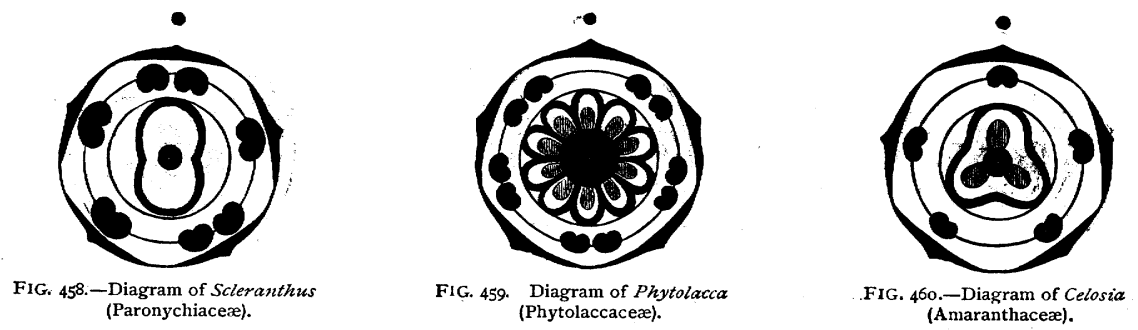

speeies occur in which the corolla is absent and the stamens are superposed on the sepals; and since in the same natural group species also occur with a corolla, it may be assumed that where the corolla is absent this is the result of abortion. The diagram of these plants (Figs. $45^{8}, 459$ ) is complicated still further by the tendency which they exhibit to a dédoublement of the stamens and even of the carpels.

When a flower has more stamens than sepals or petals, this may be the result; as has already been mentioned, on the one hand of an increase in the number of staminal whorls (as in Fig. 45r), or on the other hand, of the interposition of a perfect or imperfect whorl among the typical ones, or of dédoublement of the stamens (as in Fig. 458). These cases must be clearly distinguished from those in which a larger number of stamens results from the branching of the original ones; a phenomenon which is found in different sections of Dicotyledons, and is some times constant in whole families (see p. 544). Thus, for instance, in Dilleniaceæ (Fig. 46r), Aurantiaceæ (Fig. 462), and Tiliaceæ (Fig. 463), each symbol which indicates a group of anthers corresponds to a single original stamen. In this case the number of original stamens is the same as that of the petals and sepals;

1 Pfeffer, Bot. Zeitg. 1870, p. I43; and Jahrb. fiur wissensch. Bot.. vol. VIII. p. 194: (see supra, p. 609). 
but sometimes it is less (as in Hypericum perforatum with three staminal bundles in the pentamerous flower); so that an increase in the number of stamens is united with a decrease of the typical number of staminal leaves.

The branching of carpels is much less common than that of stamens. It occurs very clearly in Malvaceæ, where the typical number of carpels is five, and they are often developed as such (as in Hibiscus). In some genera however (as Malva, Malope, and Althae ) five original rudiments of carpels first of all make their appearance in the form of a low cushion. Each of these forms very early a larger number of outgrowths lying side by side, and each of these produces a style and a one-seeded compartment of the peculiarly-shaped gynæceum ${ }^{1}$.

This short sketch will be sufficient to show what variations are possible in the numbers and positions of the parts that may be included under the expression $S_{n} P_{n} S t_{n(+n+\cdots)} C_{n(-m)}$, which, as has already been said, is especially characteristic of flowers with pentamerous or truly tetramerous whorls. True tetramerous flowers are allied not only to those that are octamerous (like Michauxia), but also to those with dimerous whorls, among which Enothereæ may be especially mentioned. Of genera belonging to this family, Epilobium, for example, is constructed on the formula $S_{2+2} P_{\times 4} S t_{4.4} C_{4}$, Circea on that of $S_{2} P_{2} S t_{2} C_{2}$; and Trapa, with

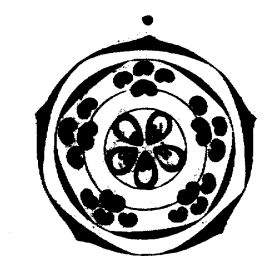

FIG. 46r.-Diagram of Candollea (DHlleniaceæ).

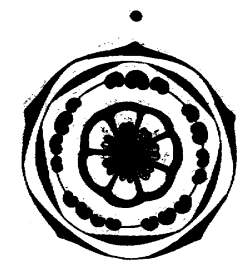

F1G. 462.-Diagram of Citrus (Aurantiaceæ).

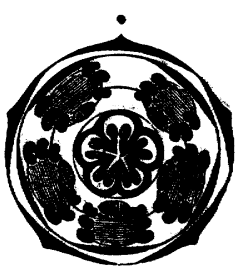

FIG. 463.-Diagram of Tiliacex

the formula $S_{2+2} P_{x_{4}} S t_{4} C_{2}$, must also be included here. Although in Epilobium and Trapa the calyx really consists of two whorls, this pseudo-whorl formed of two decussate pairs is followed by the other whorls exactly as if it were a true tetramerous whorl. But other dimerous and tetramerous flowers exhibit a more considerable deviation from the type, inasmuch as the two dimerous perianth-whorls which develope as if they were a tetramerous calyx or corolla are followed by a staminal whorl which is superposed on the pseudo-whorl consisting of two decussate pairs, as in Urtica and other genera of the order, and in Proteaceæ with the formula $S_{2+2} S t_{4} C_{1}$ (Fig. 370).

Among the dimerous and trimerous flowers of the orders Polycarpæ and Crucifloræ, where they are the most perfectly developed, a tendency prevails for more than one whorl to go to the formation of the calyx, the corolla, the andrœcium, and even the gynæceum, a tendency which may be expressed by the formula $S_{p(+p+\cdots)} P_{p(+p+\cdots)} S t_{p(+p+\cdots)} C_{p(+p+\cdots)}$; for example,

Fumariaceæ, $S_{2} P_{2+2} S t_{2+} \ldots C_{2}$.

Berberideæ,

Epimedium, $S_{2+2} P_{2+2} S t_{2+2} C_{1}$,

1 See Payer, Organogénie de la fleur, Pl. 6-8. 


\section{Berberis, $S_{3+3} P_{3+3} S_{3+3} C_{1}$, Podophyllum, $S_{3} P_{3+3}{ }^{2} S t_{3+3}^{3} C_{1}$. Cruciferæ, $S_{2+2} P_{\times 4} S t_{2+2}{ }^{2} C_{2(+2)}$.}

A large number of examples of this general formula are afforded by the family Menispermaceæ, in which the whorls are sometimes dimerous, sometimes trimerous, while sometimes whorls of each description occur in one flower; and where almost every one of the organs may disappear by abortion ${ }^{1}$.

In addition to the trimerous flowers already mentioned, there are also some which come under the first-mentioned general formula $S_{n} P_{n} S t_{n(+n)} C_{n(-m)}$; as, for example, Rheum with the formula $S_{3} P_{3} S t_{3}^{2}{ }_{+3} C_{3}$. Other trimerous flowers again appear to belong to a third type, as Asarum with the formula $S_{3} S t_{3+6} C_{6}$.

When the number of staminal whorls is considerably increased, it not unfrequently happens that the number of stamens in each whorl also undergoes change, and complicated alternations arise. Flowers the structure of which is otherwise altogether different resemble one another in this respect, as is shown by the Papaveracea on the one hand (Fig. ${ }_{464}$ ), and by the Cistineæ and some Rosaceæ on the other hand.
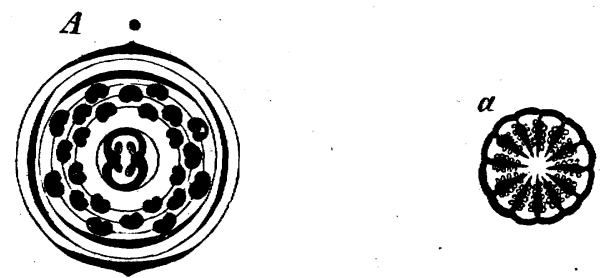

FIG. 464.-Diagram of Papaveraceæ; A Chelidonium, a Papaver.

The reduction of the flower to a simpler condition is often carried so far in many Dicotyledons (as in Monocotyledons) that each individual flower consists only either of an ovary with one or several stamens, or, when the arrangement is diclinous, even only of a single ovary or of a single or several stamens; the perianth being either entirely absent (as in Salix and Piperacex) or reduced to a cup-like structure (Populus, the female flower of Cannabineæ, \&c.) or to hair-like scales among the sexual organs which represent the flower (e.g. Platanus). Flowers of this kind are generally very small and densely crowded in large numbers in the inflorescence (such as capitula, spikes, or catkins). In some cases it may even be doubtful whether we have an inflorescence or a single flower, as in the genus Euphorbia ${ }^{2}$.

The development of the separate parts and the entire form of the flower in the mature state is so various that it is scarcely possible to state any general

' Eichler, Ueber die Menispermaceen, Denkschrift der k. bayer. Ges., Regensburg 1864.Payer, Organogénie de la fleur, Pl. 45-49.-Eichler, Flora, 1865, Nos. 2-8 et seq.

${ }^{2}$ See Payer, l.c. p. 529 ; [also foot-note to p. 490 . It is now generally admitted that the Cyathium of Euphorbia is an inflorescence, a view which was first enunciated by Robert Brown in opposition to Linnæus, who regarded it as a single flower. It appears that the stamens are here axial (Warming, Ueb. pollenbildende Caulome und Phyllome, in Hanstein's Bot. Abhdl. 1I, 1873)]. 
facts concerning them. The perigynous structure of the flower is peculiar to Dicotyledons, as is also the occurrence of hollowed axes of the inflorescence, like the fig and similar structures, and the cupule, which occur in some families, and are dependent on similar processes of growth.

The Ovules exhibit, in the different divisions of Dicotyledons, all those varieties of structure which have already been mentioned in the introduction. Very commonly, especially among the Gamopetalæ, the nucellus is covered by only one integument, which is then often very thick before fertilisation. But on the other hand the third integument or aril is much more common than among Monocotyledons. When there are two integuments, the outer one-differing again in this respect from most Monocotyledons - takes part in the formation of the micropyle, enveloping the exostome or entrance to it. In some parasites the ovules are rudimentary, and in many Balanophoraceæ are reduced to a naked few-celled nucellus; while in Loranthaceæ they are coherent with the tissue of the floral axis in the inferior ovary.

The behaviour of the Embryo-sac ${ }^{1}$ before and after fertilisation is similar in most Dicotyledons to that which occurs in Monocotyledons. The endosperm usually originates by free cell-formation, and is transformed by repeated divisions of the first cells which are formed in this manner into a more or less dense tissue, which fills up the embryo-sac either before or after the formation of the multicellular rudiment of the embryo. But in a very considerable number of families belonging to altogether different groups the embryo-sac exhibits on the one hand striking phenomena of growth, elongating considerably before impregnation into a long tube, and emitting after impregnation one or more vermiform protrusions which penetrate into and destroy the tissue of the nucellus and of the integuments, or even protrude altogether out of the ovule (as in Pedicularis, Lathrea, and Thesium). On the other hand, in those plants in which the endosperm originates by cell-division we learn from Hofmeister that the following variations occur:- 'The whole of the cavity of the embryo-sac behaves like the first cell of the endosperm in Asarineæ, Aristolochiaceæ, Balanophoraceæ, Pyroleæ, and Monotropeæ; the first division of the sac is the result of a partition-wall which divides it into two nearly equal halves, each of which encloses a cell-nucleus and again divides at least once into daughter-cells. In other cases the first cell of the endosperm includes the upper end of the embryo-sac; the embryo-sac, immediately after fertilisation, appears to be divided by a transverse septum into two halves, the upper one of which developes into the endosperm by a series of bipartitions; while no such bipartition of the lower one occurs in Viscum, Thesium, Lathrea, Rhinanthus, Mazus, Melampyrum, or Globularia. The first cell of the endosperm fills up the middle part of the embryo-sac in Veronica, Nemophila, Pedicularis, Plantago, Campanula, Loasa, and Labiatæ; its lower end in Loranthus, Acanthus, Catalpa, Hebenstreitia, Verbena, and Vaccinium.' In Nymphaa, Nuphar, and Ceratophyllum, the upper end of the embryo-sac is cut off from the rest of the space by a septum soon after fertilisation, and the further development of

1 Hofmeister, Jahrb. fuir wiss. Bot. vol. I. p. I85; and Abhandl. der kön. Sachs. Ges. der Wiss. vol. VI. p. 53, , see supra, p. 579). 
the daughter-cells or endosperm takes place only in the upper part which also encloses the oospore. This mode. of formation of the endosperm differs however from that which occurs in the plants mentioned above, in taking place in the upper half of the embryo-sac by free cell-formation.

In the very large majority of true parasites (except Cuscuta) and saprophytes, the endosperm is formed by cell-division; in Cuscuta however by free cell-formation. Hofmeister states that only slight indications of the formation of endosperm are to be found in Tropaolum and Trapa.

The mode of formation of the Embryo of Dicotyledons, as it has now been elucidated by Hanstein's recent researches, has already been explained in the introduction to Angiosperms (see Fig. 403). It need now only be stated in addition that in parasites destitute of chlorophyll and in some saprophytes the seeds become ripe before the embryo has emerged from the condition of a roundish mass of tissue still without external differentiation of parts $(e . g$. in Monotropa, Pyrola, Orobanche, Balanophoraceæ, and Rafflesiaceæ).

With reference to the Histolog $y^{1}$, I will confine my remarks here to a description of the behaviour of the fibro-vascular bundles and of the mode in which the stem increases in thickness.

With the exception of a few water-plants of simple structure, in which a purely cauline fibro-vascular cylinder runs through the stem and increases in length at its summit, the foliar bundles originating from it later (in Hippuris, Aldrovanda, Ceratophyllum, and to a certain extent also Trapa, according to Sanio), it is the general rule that 'common' bundles are first formed, the ascending branches of which enter the stronger foliage-leaves generally in large numbers, and then pursue their course as isolated bundles in the leaf-stalk and mid-rib, giving off the secondary bundles which constitute the venation of the lamina ${ }^{2}$. The branches which descend into the stem mostly run downwards through several internodes, become first interposed between the upper parts of the older bundles, and sometimes (Fig. 465) first split and then coalesce laterally with the older bundles lower down. Sometimes (as in Iberis) every bundle is twisted in the stem and in the same direction, so that the bundles which have coalesced sympodially, belonging to leaves of different heights on the stem, ascend spirally within the cortex. But most commonly they run parallel to the axis of the stem, until they anastomose with older bundles lower down. The bundles do not bend deeply into the inner tissue of the stem, but turn downwards and run parâllel to one another at the same distance below the surface, so that they lie in one layer, which presents the appearance of a ring on transverse section separating the fundamental tissue into pith and primary cortex. The portions of the fundamental tissue which lie between the fibro-vascular bundles connect the pith with the primary cortex, and form the primary Medullary Rays. If there is no subsequent increase in thickness no further change takes

1 Hanstein, Jahrb. für wiss. Bot. vol. I. p. 233 et seq.; and for the girdle-shaped combinations of vascular bundles, Abh. der Berl. Akad. I857, 8.-Nägeli, Beiträge zur wiss. Bot. Leipzig, Heft I, $185_{5}$; and Dickenwachsthum und Anordnung der Gefässstränge bei den Sapindaceen, München I $86_{4}$. -Sanio, Bot. Zeitg. I 864, p. I93 st seq., and 1865, p. I65 et seq.-Eichler, Denkschrift der kön. bayer. Bot. Gesells. vol. V. Heft I. p. 20, Regensburg 1864.-[De Bary, Vergleichende Anatomie der Vegetationsorgane der Phanerogamen und Farne, 1877.]

2 When several fibro-vascular bundles enter a leaf-stalk, they are generally widely separated by the fundamental tissue; but sometimes, as in the Fig, the bundles are arranged in a circle on transverse section, and form a closed hollow cylinder which divides the fundamental tissue of the leaf-stalk into pith and cortex. Isolated fibro-vascular bundles also run into the pith of the leaf-stalk in the Fig, as occurs also in the stems of some Dicotyledons. 
place. But usually, even in annual stems (as Heliantbus and Brassica) and invariably in woody stems and branches, several years old, the subsequent increase in thickness begins after the elongation of the internodes. A layer of cambium is formed in each bundle between the phloëm, which is external, and the xylem which is turned towards the axis of the stem; the cambium layers of the bundles, which are at first separated by the medullary rays and lie side by side in a ring, unite into a closed mantle of cambium, an interfascicular cambium being formed by divisions in the intermediate cells of the medullary rays, which bridge over the spaces between the layers of the cambium of the separate fibro-vascular bundles (see Fig. 93). The Cambium-ring thus formed produces on the outside layers of phloëm, on the inside layers of xylem, while it is at the same time itself constantly increasing in circumference. All the tissue formed in the fibro-vascular bundles from the cambium-ring on the outside may be termed Secondary Bast, all the xylem formed on the inside Secondary $W o o d$, in opposition to the Primary Bast and the Primary Wood, which consist of the isolated bundles of phloëm and xylem of the foliar bundles which were already in existence before the formation of the cambium-ring. While the wood which is produced from this cambium-ring forms a hollow cylinder, the primary woody bundles project from the inside of the ring into the pith as ridges, and often cause it to present on transverse section the appearance of

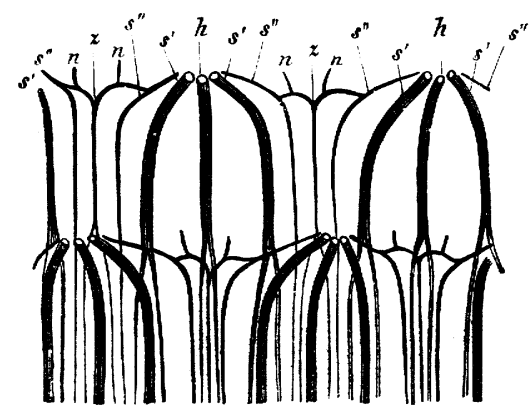

Fit. 465.-The course of the bundles in two internodes of Sambuctes Ebulus: they lie in a cylinder which is here flattened out; each internode bears two opposite leaves, and each leaf receives from the stem a middle bundle $h h$ and two strong lateral bundles $s^{\prime} s^{\prime}$; the descending limbs of the bundles split and interpose between the lower bundles; there are in addition weaker bundles $s^{\prime \prime} s^{\prime \prime}$ united by horizontal branches, from which bundles $n n$ ascend into the stipules. (After Hanstein.)

a star. The whole of these primary xylem-bundles are included in the term Medullary Sheath; and in the same sense one may adopt Nägeli's term of Cortical Sheath to express the whole of the primary bast-bundles at the periphery of the bundles. The growth in length of the medullary and cortical sheaths accompanies that of the internodes which takes place before the formation of the cambium-ring, and they therefore generally consist of very long elements; - the medullary sheath of very long annular, spiral, and reticulated vessels intermixed with long woody fibres; the cortical sheath containing bundles of long bast-fibres which become widely separated from one another by the increase in circumference of the stem, and which are often strongly thickened but long and flexible; in addition to these, long cambiform cells and elongated bast-vessels (latticed and sieve-tubes) occur in it. The structural elements of the secondary cortex are, like those of the secondary wood, shorter; in the secondary wood there are no annular or spiral vessels, these being altogether replaced by shorter and broader vessels with bordered pits, surrounded by wood-fibres intermixed with woody parenchyma (see p. I 16). The secondary cortex forms either a number of layers of thick-walled as well as thin-walled bast-fibres, and partially parenchymatous masses of phloëm, or these last only, or the most various combinations of both. Finally the primary cortex and the epidermis are both generally supplanted by the formation of periderm and bark; although they may sometimes undergo a considerable growth in thickness by increasing in diameter at the same time 
that longitudinal divisions are formed (as in Viscum, Helianthus annuus, \&c.). The masses of xylem and phloëm formed by the activity of the cambium-ring are penetrated lengthwise in radial direction by secondary medullary rays consisting of horizontal cells which in the wood are not always lignified, and in the secondary cortex are generally soft and parenchymatous. In the one case they are called xylemrays, in the other phloëm-rays, and always have the power of taking up assimilated food-materials. In proportion as the cambium-ring increases in size, the number of these rays increases; and the later layers of wood are always traversed by a larger number of rays. They are one or more layers of cells in thickness, and form thin vertical plates wedge-shaped at their upper and lower edges, which have the appearance in a longitudinal section of ribbon-like structures (the 'silver-grain'). In a tangential section the fibro-vascular bundles which run through the length of the stem are seen to form a network of elongated meshes, through which the rays pass (especially clearly seen in decaying cabbage-stumps). The medullary rays, like the fibro-vascular bundles, are added to by means of the cambiums-ring outwards and inwards; and as the ring increases in thickness, it produces new rays between the old ones.

[The following tabular account of the structure of the secondary wood (xylem) in Dicotyledons is taken from De Bary (Vergleichende Anatomie) :-

r. Wood consisting only of tracheïdes with bordered pits :-

Winterex (Drimys Winteri, Tasmannia aromatica; also Trocbodendron aralioides): (Conifers ${ }^{1}$ ).

2. Wood consisting of vessels, tracheïdes, parenchyma, and intermediate cells (ersatzfasern $\left.{ }^{2}\right):-$

a. With no intermediate cells; Ilex aquifolium, Staphylea pinnata, Rosa canina, Cratagus monogyna, Pyrus communis, Spirca opulifolia, Ćamellia, \&c.

b. With no parenchyma; Porlieria.

c. With both parenchyma and intermediate cells; Jasminum revolutum, Kerria, Potentilla fruticosa, Casuarina equisetifolia and torulosa, Aristolocbia Sipho, \&c.

3. Wood consisting of vessels, tracheïdes, fibres, parenchyma, and intermediate cells:-

a. With no intermediate cells; fibres unseptate; e.g. Sambricus nigra and racemosa, Acer platanoides, pseudoplatanus, and campestris.

$b$. With both parenchyma and intermediate cells; fibres unseptate; Berberis vulgaris, Mabonia; (Ephedra).

c. With no intermediate cells; fibres septate and unseptate; Punica, Euonymus latifolius and europaus, Celastrus scandens, Vitis vinifera, Fucbsia globosa, Centradenia grandifolia, Hedera Helix, \&c.

d. With all four kinds of cells; Müblenbeckia complexa, Ficus (?).

4. Wood consisting of vessels, tracheïdes, fibres, parenchyma, and intermediate cells. This is the most common, and may be taken as the typical structure:-

a. With no intermediate cells; Sparmannia africana, Calycantbus, Rbamnus cathartica, Ribes rubrum, Quercus, Custanea, Carpinus sp., Amygdaleæ, Melaleuca, Callistemon sp., \&c.

1 [According to Sanio, Taxus baccata has wood of this composition, but Hartig and Kraus state that some parenchyma is also present; this parenchyma is better developed in the other Conifers.]

${ }^{2}$ [See supra, p. I I9. These cells were first distinguished by Sanio, who termed them 'ersatzfasern' (replacing-fibres), because they frequently take the place of the wood-parenchyma. The term 'intermediate' used above refers to the fact that they are intermediate in form between prosenchymatous and parenchymaious cells.] 
b. With no parenchyma; Caragana arborescens.

c. With both kinds of cells; most foliage-trees and shrubs, e.g. Salix, Populus sp., Liriodendron, Magnolia acuminata, Alnus glutinosa, Betula alba, Juglans regia, Nerium, Tilia, Hakea suaveolens, Ailantbus, Robinia, Gleditscbia sp., Ulex europaus, \&c.

5. Wood consisting of vessels, fibres, parenchyma, and intermediate cells:-

a. With no parenchyma; Viscum album.

$b$. With no intermediate cells; Avicennia.

c. With both kinds of cells; Fraxinus excelsior, Ornus, Citrus medica, Platanus, \&c.

6. Wood consisting of vessels, fibres, and parenchyma:-

Cheiranthus Cheiri, Begonia. Also many Crassulaceæ and Caryophyllaceæ.

7. Wood consisting of vessels, fibres, parenchyma, and true woody fibres ${ }^{1}$ (?) :-

Coleus Macraei, Eugenia australis, Hydrangea bortensis.

8. Wood consisting of vessels, tracheïdes, woody fibres, septate fibres, parenchyma, and intermediate cells:-

Ceratonia siliqua, Bignonia capreolata; it is however still doubtful if true woody fibres are present.]

When the increase in thickness of a stem ceases periodically and is renewed with each new period of vegetation, as in our woody plants, a layer of wood is formed during each period of growth (and usually also a secondary cortical layer), which is sharply marked off from those of the preceding and of the following year, and is called an Annual Ring of the wood. These annual rings are usually distinctly visible to the naked eye, because the mass of wood formed in the early part of each period of vegetation has usually a different appearance from that formed in the autumn, the latter being denser, the former less dense and generally with a greater number of vessels. The wood formed in the spring consists also of wider cells than that produced in the autumn, and the radial diameter of the cells is usually greater. The cells formed in the autumn appear compressed radially and broad in the tangential direction; their cavities are smaller, and hence, other things being equal, the thickness of their wall is greater. A given quantity of wood produced in the autumn is therefore denser than a like volume formed in the spring ${ }^{2}$. While Dicotyledons differ so widely from Mono-

1 [The distinction which is drawn between 'fibres' and 'true woody fibres' is in their contents : the former nearly always contain starch, in some cases (Spircea salicifolia, young wood of Vitis vinifera and Centradenia grandifolia) chlorophyll, and in others (Syringa vulgaris) tannin; the latter contain air and water, and sometimes a mere residue of protoplasm. These two kinds of fibres usually occupy the same relative position in a fibro-vascular bundle, and it is doubtful if they are ever both present in the same bundle: the 'fibres' gradually become converted into 'woody fibres.']

2 The cause of this difference is not yet known; but I suppose that it depends simply on the difference in pressure to which the cambium and the wood are subjected from the surrounding cortex. This pressure is less in the spring, and constantly increases till the autumn. I have no direct measurements of this, but conclude it from the fact that the longitudinal fissures in the bark become wider in February and March, as may be clearly seen in the Oak, Maple, Poplar, Walnut, \&c. I cannot here explain the cause of this; but in any case the bark, the longitudinal fissures of which have become wider in winter, must exert less pressure on the cambium in the spring, and the cells of the wood must therefore be able to extend more easily in a radial direction. The pressure which the bark exerts on the cambium must continually increase by the thickening of the ring of wood internally and the drying up of the bark in summer externally, and must affect the radial growth of the young cells of the autumnal wood. Further investigations which I am proposing to make will determine whether my theory is correct.'-This hypothesis, which I brought forward in the first edition, has recently been fully confirmed by the researches of $H$. de Vries. (See Flora, $187^{2}$, no. I6, and sect. $\mathrm{I}_{5}$ of Book III of this work.) 
cotyledons in this mode of increase of their stems in thickness, they agree almost entirely in this respect with Gymnosperms, except that in these latter there are no pitted vessels in the secondary wood. In this respect however, according to von Mohl, Ephedra indicates a transition to Dicotyledons. The organisation of Dicotyledons shows also in some sense a higher stage of development in the greater varieties of the forms of cells of which the xylem and phloëm are composed.

A remarkable deviation from these normal processes is exhibited by the Sapindacex. In some plants of this order the stem has the ordinary structure; but in others a transverse section shows several smaller woody cylinders of various sizes outside the usual one and lying in the secondary cortex. Each of these increases in thickness, like the normal ones, by a cambium-layer which surrounds it. Nägeli supposes the cause of this structure to be that the primary fibro-vascular bundles of the stem do not lie in a circle on the transverse section, but in groups more towards the outside or inside. When the connecting bands of cambium are formed in the fundamental tissue, the isolated bundles become united on the transvérse section, according to their grouping, into one (as in Paullinia) or several (e.g. Serjania) closed rings.

The cause of a large number of deviations of different kinds from the normal structure of the stem in Dicotyledons which occur in various families, is the formation of other cauline bundles of later origin in the stem besides the common bundles, either within the primary pith or outside the ring in which the common bundles lie. We owe to Nägeli a more exact knowledge of these cases, and more especially to the very exhaustive labours of Sanio, which form for the most part the basis, in addition to my own observations, of the following short sketch, without going in detail into special cases $^{1}$. I must refrain, in particular, from giving a detailed account of the behaviour of Sanio's thickening-ring or of Nägeli's meristem-ring, as this would involve considerable prolixity.

These phenomena may be classified into two groups, according as the secondary (cauline) bundles originate within or without the circle of the primary (common) bundles. Sanio calls the former the endogenous, the latter the exogenous mode of origin.

First Group. The secondary bundles are formed outside the primary bundles (exogenous).

a. The primary (common) bundles lie near the axis of the stem, and remain more or less isolated, while the secondary (cauline) bundles are formed by a closed cambium-ring external to the primary bundles, which continues to grow on the outside (originally a 'thickening-ring' in Sanio's sense). Examples are furnished by Mirabilis, Amarantbus, Atriplex, Ghenopodium album, and probably by all the Nyctaginex and Mesembryanthemacex.

$b$. The primary (common) bundles lie in a ring on the transverse section and continue their growth by means of a closed cambium-ring, which however soon disappears. A new cambium-ring is then formed outside the one which has disappeared, and another one again outside this one when it has in turn disappeared. Several circles of fibrovascular bundles are thus formed, continually increasing in number. In many Menispermacex (e.g. Cocculus), the new outer circle of vascular bundles together with its cambium-ring is developed from a ring of meristem which lies in the primary cortex and therefore outside the primary bast, - a phenomenon which is repeated in the cortex as its growth proceeds (Nägeli). In Phytolacca, on the other hand, and, according to Eichler, also in Dilleniaceæ, Wistaria, Baubinia, Polygaleæ (Securidaca and Comesperma), Cissus, and Phytocrene, the successive circles of bundles originate in the secondary bast.

Second Group. The secondary bundles arise early after the primary bundles further inwards or nearer the axis of the stem (endogenous).

1 [Oliver has collected the bibliography of the structure of the stem of Dicotyledons in the Nat. Hist. Rev. 1862 , pp. $298-329$, and_I 863 , pp. $25^{1-2} 5^{8}$.] 
a. Both the primary and the secondary bundles remain isolated; they are not united by a closed cambium-ring, but anastomose with one another, as in Cucurbita, Nymphæaceæ, and Papaver. In the Cucurbitaceæ and Piperaceæ the more internal bundles are arranged in a ring, but in the Nymphæaceæ they are arranged irregularly, so that the transverse section of the stem bears a greater or less resemblance to that of a Monocotyledon.

$b$. The primary bundles lie in a ring on the transverse section, and are united by a cambium-ring; the secondary bundles arise at an early period in the pith and remain isolated and scattered on the transverse section; they anastomose with one another and with the primary bundles in the nodes of the stem. Examples are furnished, according to Sanio, by Begoniacex, Aralia, and some Umbelliferæ.

The Cell-forms of the phloëm and xylem of Dicotyledons have already been described in general terms (see p. I 6 et seq.). Only two peculiar phenomena need be mentioned here. In Cucurbitacex, some Solanacex, and Nerium (and in a certain sense also in Tecoma radicans ${ }^{1}$ ), a phloëm-tissue is found not only on the outside but also on the inside of the fibro-vascular bundles, which is developed with especial strength in Cucurbitacex. The isolated fibro-vascular bundles in the pith which are enclosed by the ring of wood sometimes show an abnormal arrangement of their phloëm and xylem. Thus, according to Sanio, Aralia racemosa has, within the normal circle which grows by means of a cambium-layer, an endogenous circle of closed fibro-vascular bundles in which the xylem is peripheral and the phloëm central as regards the stem. The isolated bundles in the pith of Phytolacca dioica on the other hand consist, according to Nägeli, on a transverse section, of a hollow woody cylinder which surrounds the phloëm on all sides and is penetrated by xylem-rays. The isolated fibro-vascular bundles of the pith in the rachis of the inflorescence of Ricinus communis also consist of a thin axial bundle of phloëm (?), surrounded by a sheath of cells (xylem ?) arranged in rays.

A layer of collenchyma is very common in Dicotyledons beneath the epidermis of the internodes and leaf-stalk.

The Classification of Dicotyledons ${ }^{2}$ has now been carried out so completely that the smaller groups which are called Families ${ }^{3}$, and which usually comprise genera very nearly related to one another, have been united into larger groups or orders; so that at present only a few families remain unplaced. The greater number of the orders can also be again arranged into larger groups which are clearly connected by actual relationship. Systematists have not however up to the present time agreed as to how many of these cycles of affinity should be established, so as to make the primary division of the whole class of Dicotyledons in accord with the requirements of scientific classification. The grouping of all Dicotyledons into three sections, Apetalæ, Gamopetalæ, and Eleutheropetalæ, proposed by De Candolle and Endlicher ${ }^{4}$, is now abandoned by most, although still much in use for practical purposes. A. Braun ${ }^{5}$ placed among the Eleutheropetalæ the greater number of plants previously classed among Apetalæ; and Hanstein ${ }^{6}$ has now distributed among them the remainder, so that the whole class consists of only two sub-classes, Gamopetalæ and Eleutheropetalæ. This classifica-

1 [A cambium-ring is formed internally to the primary bundles in the stem of this plant; see Nägeli, Beiträge, I: also Sanio, Bot. Zeitg. 1864, p. 228.]

${ }^{2}$ [See note to p. 630 .]

3 Le Maout and Decaisne's Traité général de Botanique, descriptive et analytique, is strongly to be recommended for a study of the diagnosis of the families [translated by Mrs. Hooker; London I873].

${ }_{4}^{4}$ Endlicher, Genera plantarum secundum ordines naturales disposita, Vindobonæ, 1836-1840; and Enchiridion botanicum, Lipsiæ-Viennæ, $\mathbf{1} 84 \mathbf{I}$.

${ }^{5}$ A. Braun, Uebersicht des natürlichen Systems, in Ascherson's Flora der Provinz Brandenburg, 1864 .

- Hanstein, Uebersicht des natiirlichen Pflanzensystems, Bonn 1867. In the first edition of this 
tion however assigns far too great an importance to this particular point of structure, considering that on the one hand flowers occur among the Eleutheropetalæ which differ greatly from one another not only in this but also in every other respect; while on the other hand the most intimate relationship exists between particular sections of Eleutheropetalæ and of Gamopetalæ. I therefore think it convenient, while retaining the largest sub-divisions of the class, to employ also other characters in the classification; and to make use of the character drawn from the cohesion or non-cohesion of the petals in the subdivision of the largest group, that provided with two perianthwhorls. In the following classification Dicotyledons are split up into five divisions of equal systematic and morphological value, which should rather be arranged parallel to one another than in a single linear series. This classification has also, I think, a practical advantage; since the extraordinarily large number of families and orders can be more easily kept in the memory when they are at once arranged in several comprehensive groups of equal value.

\section{DICOTYLEDONS.}

I. Julifloræ :
A. Piperineæ,
B. Urticineæ,
C. Amentiferæ.

II. Monochlamydem :
A. Serpentarieæ,
B. Rhizantheæ.

III. Aphanocyclø:
A. Hydropeltidinex,
B. Polycarpæ,
C. Crucifloræ.

IV. Tetracyclø :

(a) Gamopetale:
A. Anisocarpæ,
B. Isocarpæ.

(ß) Eleutheropetala :

C. Eucyclæ,

D. Centrospermæ,

E. Discophoræ.

V. Perigynæ:
A. Calycifloræ,
B. Corollifloræ.

The sections designated by capital letters correspond partly to single ordèrs, partly to whole series of orders in the system referred to above.

\section{JULIFLORE.}

Flowers very small or inconspicuous, crowded in dense inflorescences-spikes, capitula, or less often paanicles-which are often of very peculiar form; naked or with a simple sepaloid perianth, and usually diclinous; the male and female flowers often different. Leaves simple.

A. Piperinea. Flowers very small, in dense spikes subtended by bracts, without

book I followed this work with but little deviation.' Compare also Grisebach, Grundriss der systematischen Botanik. 
a perianth. The small embryo lies, surrounded by the endosperm, in a hollow of the copious perisperm. Herbs or shrubs, often with verticillate leaves.

$$
\begin{aligned}
\text { Families: } & \text { I. Piperaceæ, } \\
& \text { 2. Saurureæ, } \\
& \text { 3. Chlorantheæ. }
\end{aligned}
$$

B. Urticinea. Perianth simple, sepaloid, three- to five-partite, sometimes absent; stamens superposed on the segments of the perianth; flowers hermaphrodite or diclinous, and then the male and female flowers different (3), usually in densely crowded inflorescences, the female flowers in spikes, umbels, capitula (2) or sometimes panicles (3), not unfrequently developing into peculiar pseudocarps (as the Mulberry, Fig, Bread-fruit, and Dorstenia). Fruit usually unilocular, rarely bilocular; ovules one or rarely two in each loculus; seed usually with endosperm. Large shrubs or trees ${ }^{1}$; leaves stalked, usually stipulate.

$$
\begin{aligned}
& \text { Families: } \text { I. Urticaceæ, } \\
& \text { Urticeæ, } \\
& \text { Moreæ, } \\
& \text { Artocarpeæ, } \\
& \text { 2. Platanaceæ, } \\
& \text { 3. Cannabineæ, } \\
& \text { 4. Ulmaceæ (including Celtideæ). }
\end{aligned}
$$

C. Amentifera. Flowers diclinous, epigynous, in compact panicles (false spikes); the female few-flowered inflorescence in (2) surrounded by a cupule. Fruit dry, indehiscent, one-seeded; seed without endosperm. Trees with deciduous stipules.

$$
\begin{aligned}
\text { Families: } & \text { I. Betulaceæ, } \\
& \text { 2. Cupuliferæ. }
\end{aligned}
$$

\section{Monochlamyde正.}

Flowers large and conspicuous and consisting of a simple more or less petaloid, usually gamophyllous perianth, one or more staminal whorls, and a polycarpellary ovary; carpels equal in number to or double that of the segments of the perianth. The number of members of the whorls is derived from the typical numbers two, three, four, or five, and generally increases in the inner whorls. Ovary generally inferior and surmounted by a short thick columnar style, to which in the hermaphrodite flowers the stamens are usually partially or entirely adherent. Flowers often diclinous. Seeds numerous.

A. Serpentariea. Creeping or climbing plants with slender stems and large simple leaves; floral whorls dimerous and tetramerous ( $\mathrm{I}$ ) or trimerous and hexamerous; perianth-leaves free (I) or coherent into a tube; ovary of four or six loculi ; embryo small but differentiated.

$$
\begin{aligned}
\text { Families: } & \text { I. Nepentheæ, } \\
& \text { 2. Aristolochiaceæ, } \\
& \text { 3. Asarineæ. }
\end{aligned}
$$

B. Rbizanthea. Root-parasites without chlorophyll or foliage-leaves, generally with stunted vegetative organs and very large solitary flowers or small flowers in a dense inflorescence (I); whorls dimerous to octamerous ( $\mathrm{I}$ ), trimerous (2), or pentamerous and decamerous (3); ovary with one or eight (I) loculi ; the placentæ and anthers of very peculiar form; a very great number of small seeds with rudimentary embryo.

Families: r. Cytineæ,

2. Hydnoreæ,

3. Rafflesiaceæ.

1 [The Urticer include a number of herbaceous genera.] 


\section{APHANOCYCLE.}

Flowers hemicyclic or cyclic, or the parts arranged spirally; the members of each whorl usually free, not coherent with one another, or only in the gynæceum; perianth generally distinctly differentiated into calyx and corolla; the numbers of the parts in the four whorls very variable; stamens usually more in number than perianth-leaves; carpels forming generally one, several, or a large number of monocarpellary ovaries; the ovary is usually superior and, when polycarpellary, is uni- or multi-locular. Ovules springing occasionally in all the groups from the inner surface of the carpels.

A. Hydropeltidinea. Water-plants with solitary lateral and usually large flowers, the perianth-leaves and stamens variable in number and arranged spirally; ovaries several and monocarpellary $(1,2)$, or one only polycarpellary and multilocular; embryo small, usually surrounded by a small endosperm in a hollow of the perisperm.

$$
\begin{aligned}
& \text { Families: } \text { 1. Nelumbiaceæ, } \\
& \text { 2. Cabombex, } \\
& \text { 3. Nymphæaceæ. }
\end{aligned}
$$

B. Polycarpa. Parts of the flowers arranged spirally or in whorls, when in whorls usually dimerous or trimerous, each series generally consisting of more than one whorl, rarely in four pentamerous whorls (2); gynæceum consisting of one, several, or a larger number of monocarpellary ovaries (trimerous and unilocular in 8), which are one- or many-seeded; embryo small; endosperm none (8), abundant, or very large (9).

$$
\begin{aligned}
& \text { Families: } x \text {. Ranunculaceæ, } \\
& \text { 2. Dilleniaceæ, } \\
& \text { 3. Schizandreæ, } \\
& \text { 4. Anonaceæ, } \\
& \text { 5. Magnoliaceæ, } \\
& \text { 6. Berberideæ, } \\
& \text { 7. Menispermaceæ, } \\
& \text { 8. Laurineæ, } \\
& \text { 9. Myristicaceæ. }
\end{aligned}
$$

C. Cruciflora. Perianth-whorls dimerous; in (3) and (4) corolla of four petals placed diagonally; staminal whorls two or more, each consisting of two stamens or divisible by two; ovary polycarpellary, unilocular, or (spuriously) bilocular, or multilocular; seed with $(\mathrm{I}, 2)$ or without endosperm.

$$
\begin{aligned}
& \text { Families: } \text { 1. Papaveraceæ, } \\
& \text { 2. Fumariaceæ, } \\
& \text { 3. Cruciferæ, } \\
& \text { 4. Capparideæ. }
\end{aligned}
$$

\section{Tetracycle.}

Parts of the flower always arranged strictly in whorls; the typical number of whorls is four, the calyx, corolla, andrœcium, and gynæceum each consisting of a single whorl; whorls generally pentamerous, rarely tetramerous (very rarely dimerous or octamerous); any one of the whorls may be entirely wanting, or individual members may be abortive; this occurs most often with the stamens and carpels. Increase in number of the stamens usually takes place by the interposition of one perfect or imperfect whorl between the members of the typical whorl or a little outside it, or by doubling of the members, or by branching of the original staminal leaves; increase in number of the staminal whorls themselves is rare. All the whorls usually alternate, but the stamens are not unfrequently superposed on the petals. A tendency prevails in all the sections to a diminution of the number of carpels below that of the members of the perianth-whorls; very commonly 
there are only two, one anterior and one posterior. Ovary almost always single and polycarpellary, inferior or superior, unilocular or multilocular.

\section{Gamopetalø or Sympetalø.}

The petals united at the base into a tube or cup; corolla never wanting.

A. Anisocarpa. The number of whorls or of members of the whorls is never larger than the typical number; calyx or some of the stamens sometimes abortive; carpels usually only two, one anterior and one posterior, or three and united into a single ovary ${ }^{1}$.

\section{a. Hypogynæ.}

Order 1. Tubifloræ.

Families: I. Convolvulaceæ (including Cuscuteæ),

2. Polemoniacex,

3. Hydrophyllacex,

4. Boraginex,

5. Solanacex.

Order 2. Labiatifloræ.

Families : I. Scrophulariacex,

2. Bignoniaceæ,

3. Acanthaceæ,

4. Gesneraceæ,

5. Orobancheæ,

6. Ramondiex,

7. Selaginex,

8. Globulariaceæ,

9. Plantagineæ,

10. Verbenaceæ,

II. Labiatæ.

Order 3. Diandræ.

Families: I. Oleaceæ,

2. Jasminiacex.

Order 4. Contortæ.

Families: 1. Gentianaceæ,

2. Loganiaceæ,

3. Strychnaceæ,

4. Apocynaceæ,

5. Asclepiadeæ.

b. Epigynæ.

Order 5. Aggregatæ.

Families : r. Rubiaceæ,

2. Caprifoliacex,

3. Valerianaceæ,

4. Dipsacacex.

Order 6. Synandræ.

Families: 1. Cucurbitaceæ,

2. Campanulaceæ,

3. Lobeliaceæ,

1 The orders are arranged mainly after Braun and Hanstein. 
4. Goodeniaceæ,

5. Stylidiex,

6. Calycereæ,

7. Compositæ.

B. Isocarpa. Carpels equal in number to the sepals and petals, usually five, rarely four, and coherent into a generally superior ovary (except Order I, Family I, where there are only two median carpels); diminution of the number of stamens does not occur (except in Order I, Family I); in Orders 2 and 3 , on the other hand, a perfect staminal whorl is usually interposed; in Order I the stamens are superposed on the petals, and a number of seeds spring from an elevated axial placenta in the unilocular ovary; in Orders 2 and 3 the ovary is multilocular and many-seeded.

\section{Order 1. Primulineø.}

Families: I. Lentibulariacex,

2. Plumbagineæ,

3. Primulacex,

4. Myrsinaceæ.

Order 2. Diospyrineæ.

Families: I. Sapotaceæ,

2. Ebenaceæ (including Styracaceæ).

Order 3. Bicornes.

Families: 1. Epacrideæ,

2. Pyrolacex,

3. Monotropex,

4. Rhodoracex,

5. Ericacex,

6. Vaccinieæ.

\section{Eleutheropetalæ or Dialypetalø.}

Petals free, sometimes wanting.

C. Eucycla. Corolla very rarely wanting; stamens very commonly twice or three times as many as petals by the interposition of a perfect or even double (Orders 6,7 ) whorl, or by the interposition of an imperfect whorl differing in number from the corolla (Order 5); in the isostemonous flowers the stamens are sometimes superposed on the petals (Order 4 ), or the original stamens branch (especially in Orders 2,3 , and 8 ); the number of carpels often the same as that of the sepals and petals (Orders 7, 8), but commonly less-two, three, or four; ovary unilocular with parietal placentæ in Order $\mathrm{I}$, in the others multilocular; seed generally without endosperm.

Order 1. Parietales.

Families: x. Resedaceæ,

2. Violacex,

3. Frankeniaceæ,

4. Loasacex,

5. Turneraceæ,

6. Papayaceæ,

7. Passifloracex,

8. Bixaceæ,

9. Samydaceæ,

ı. Cistinex. 
Order 2. Guttiferæ.

Families: I. Salicineæ,

2. Tamariscineæ,

3. Reaumuriaceæ,

4. Hypericineæ,

5. Clusiaceæ,

6. Marcgraviaceæ,

7. Ternstrœmiaceæ,

8. Chlænaceæ,

9. Dipterocarpeæ.

Order 3. Hesperidæ.

Families: r. Aurantiaceæ,

2. Meliaceæ (including Cedreleæ),

3. Humiriacex,

4. Erythroxylaceæ.

Order 4. Frangulinæ.

Families: r. Ampelideæ,

2. Rhamnacex,

3. Celastrinex,

4. Staphyleacex,

5. Aquifoliaceæ,

6. Hippocrateaceæ,

7. Pittosporeæ.

Order 5. Assculinæ.

Families: I. Malpighiaceæ,

2. Sapindaceæ,

a. Acerineæ,

b. Sapindeæ,

c. Hippocastaneæ,

3. Tropæolaceæ,

4. Polygalaceæ.

Order 6. Terebinthinæ.

Families: I. Terebinthacex,

a. Anacardiaceæ,

b. Burseraceæ,

c. Amyridex,

2. Rutacex,

a. Rutex,

b. Dissmeæ,

c. Xanthoxyleæ,

d. Simarubeæ,

3. Ochnaceæ.

Order 7. Gruinales.

Families: 1. Balsaminex,

2. Limnanthaceæ,

3. Linaceæ,

4. Oxalidex,

5. Geraniacex,

6. Zygophyllaceæ. 
Order 8. Columniferæ.

Families: I. Sterculiaceæ,

2. Byttneriace

3. Tiliaceæ,

4. Malvaceæ.

Order 9. Tricocc ${ }^{1}$.

Families: I. Euphorbiaceæ,

a. Euphorbiex,

b. Acalyphex;

2. Phyllanthaceæ,

a. Phyllantheæ,

b. Buxineæ.

D. Centrosperma. Corolla usually wanting (except in Fam. 6); stamens fewer or more often more numerous than the sepals, in the last case generally double as many (4 or 6); ovary usually superior and unilocular, with one or more basal often campylotropous ovules, less often multilocular with central placentation.

Order 1. Caryophyllineæ.

Families: r. Nyctaginex,

2. Chenopodiaceæ,

3. Amaranthaceæ,

4. Phytolaccaceæ,

5. Portulacaceæ,

6. Caryophyllex:

a. Paronychiex,

b. Scleranthex,

c. Alsinex,

d. Sileneæ.

E. Discophora. Ovary inferior (Order $\mathbf{I}$ ) or half inferior or even superior, and then (Order 2, Family 5) carpels distinct; carpels as many as or fewer than sepals and petals (often two); when the ovary is inferior or half inferior a nectariferous disc usually occurs between the styles and the stamens; stamens equal in number to sepals and petals (Order $\mathrm{I}$ ) or twice as many, or even a still larger number; calyx-limb usually obsolete in Order I; seed generally with copious endosperm.

\section{Order 1. Umbelliflorø.}

Families: 1. Umbelliferæ,

2. Araliaceæ,

3. Cornaceæ.

Order 2. Saxifragine ${ }^{2}$.

Families: I. Saxifragaceæ (including Hydrangex,

Escallonieæ, and Cunoniaceæ),

2. Grossulariaceæ (?),

3. Philadelpheæ (?),

4. Francoaceæ (?),

5. Crassulaceæ (?).

V. PERIGYNe.

Flower displaying a tendency towards the perigynous structure. An annular body is elevated from the floral axis bearing the perianth and the stamens, and enveloping the gynæceum as a cup-, saucer-, or urn-like receptacle; or it becomes adherent in its

1 The position of this order is doubtful.

2 The position of the families marked (?) is doubtful. 
growth to the carpels (B, Order 2, Family 2). In a few families which are placed here provisionally (B, Order 3 , Families $4^{-6}$ ) the ovary is truly inferior.

A. Calycifora. Perianth simple, either sepaloid or petaloid and usually tetramerous; the tubular receptacle is generally of the same nature, and in Family 3 is even quadripartite, corresponding to the four perianth-leaves and to the four stamens superposed on them (see Fig. 370); stamens fewer than, as many as, or twice as many as the perianth-leaves; ovary monocarpellary, rarely bilocular, with one or a few seeds; seed with little or no endosperm.

\section{Order 1. Thymelæineœ.}

Families: I. Thymelæaceæ,

$$
\begin{aligned}
& \text { 2. Elæagnaceæ, } \\
& \text { 3. Proteaceæ. }
\end{aligned}
$$

B. Corolliffora. Calyx, corolla, and androcium placed on a flat (Order 1 ) or cup-shaped receptacle, or on one hollowed out into a deep urn-shape (Order 2 and in part 3), which is often (Order 2) thick and succulent (as in the Apple, Rose-hip, \&c.) ; sepals distinct or coherent (Order I); petals always distinct (corolla dialypetalous); the two perianth-whorls usually pentamerous, sometimes tetramerous; stamens as many as or twice as many as (Order 1 ) sepals and petals, or a much larger number (Order 2), in Order 3, Family 3, commonly branched; gynæceum composed of one (Order I, and in part 2) or several or a large number of monocarpellary ovaries; or (in Order 3 ) ovary polycarpellary, and sometimes inferior (Families 4-6).

\section{Order 1. Leguminosæ.}

Families: I. Mimosex,

2. Swartzieæ,

3. Cæsalpineæ,

4. Papilionacex.

Order 2. Rosifloræ.

Families: I. Calycanthaceæ,

2. Pomex,

3. Rosacex,

4. Sanguisorbex,

5. Dryadeæ,

6. Spiræeæ,

7. Amygdaleæ,

8. Chrysobalaneæ.

Order 3. Myrtiflorø.

Families: ז. Lythrarieæ,

2. Melastomaceæ,

3. Myrtaceæ,

4. Combretaceæ,

5. Enothere $\mathfrak{x}$,

6. Haloragideæ.

\begin{tabular}{|c|c|c|}
\hline Santalaceæ. & Callitrichaceæ. & Begoniaceæ. \\
\hline Loranthaceæ. & Ceratophyllacex. & $\begin{array}{l}\text { Mesembryanthemeæ. } \\
\text { Tetragonieæ. }\end{array}$ \\
\hline Podostemoneæ. & Empetraceæ. & Cactaceæ. \\
\hline
\end{tabular}

Families of unknown or very doubtful affinity.

1 The position of these families here is very doubtful. 


\title{
B O O K III.
}

\section{P H Y S I O L O GY.}

\author{
CHAPTER I.
}

\section{MOLECULAR FORCES IN THE PLANT.}

\section{SECT. 1. - The Condition of Aggregation of organised structures 1 .} Cell-walls, starch-grains, and protoplasmic structures consist, in their natural condition, at every point that can be seen even under the microscope, of a combination of solid material with water. If these organised structures are placed in a substance capable of removing water, a part of their aqueous contents is withdrawn; while, on the other hand, if they are in contact with aqueous solutions possessing certain chemical properties and of a proper temperature, they absorb more water. The volume alters with the change in the proportion of water; loss of water causes diminution, absorption of water a corresponding augmentation of volume. Since the absorption of water occasions a considerable elevation of temperature (air-dry starch rises $2^{\circ}$ or $3^{\circ} \mathrm{C}$. when mixed with water of the same temperature), it must be supposed that the water becomes denser as it is absorbed ${ }^{2}$. Within certain limits these variations in the proportion of water may occur without occasioning any permanent change in the intimate structure; but if, with a higher temperature and in the presence of chemical reagents, the proportion falls below a certain minimum or exceeds a certain maximum, permanent changes of the intimate structure take place which can no longer be reversed; and the internal organisation of the body becomes partially or entirely destroyed.

1 See Sachs, Handbuch der Experimental-Physiologie, p. 398 et seq.-Nägeli u. Schwendener, Das Mikroscop, p. 422 et seq.; compare also Book I of this work, p. 28 et seq.-Cramer, Naturforsch. Gesells. in Zürich, Nov. 8, 1869 .

${ }^{2}$ Jungk, in Pogg. Ann. 1865, vol. I25. p. 292 et seq. 
These facts, in connection with a number of other phenomena, first led Nägeli to the hypothesis that organised bodies consist of isolated particles or Micelle ${ }^{1}$ between which the water penetrates, and which are solid and relatively unchangeable, and invisible even with the most powerful microscopes. Every micella of a saturated organised body is, on this hypothesis, surrounded by layers of water by which the adjacent micellæ are completely separated from one another. These micellæ may be supposed to be of various sizes, and it is evident a priori that, if the thickness of the aqueous envelope is the same, larger micellæ will form a denser, smaller micellæ a less dense substance; and it may therefore be concluded conversely that the layers and lamellæ of organised bodies of different densities, especially those of the cell-wall and of starch-grains, are composed of micellæ of different sizes; and the difference in the proportion of water in such cases leads to the hypothesis that the densest substance consists of micellæ which are several thousand times larger than those of the more watery substance. As the micellæ increase in size, the density of the whole substance is moreover increased by the smaller distance that intervenes between them, so that larger micellæ are separated from one another by thinner layers of water. The changes in volume of organised bodies due to the removal of water or its absorption depend, according to this view, on the fact that when swelling takes place the micellæ are forced further apart by the water which penetrates between them; while, on the other hand, when water is removed they approach one another in proportion as the water is withdrawn from their interstices.

The forces which are concerned in these processes in the interior of an organised body may be divided into three kinds:-(I) the Cohesion within each separate micella impermeable to water, which is itself an aggregate of molecules and atoms; (2) the Attraction of the adjacent micellæ for one another, in consequence of which they tend mutually to approach; and (3) the Attraction of the surfaces of the micella for the absorbed water, which counteracts the mutual attraction of the neighbouring micellæ.

In starch-grains, cell-walls, and to a certain extent in crystalloids ${ }^{2}$, the absorbed water is not deposited uniformly in all directions; the micellæ are, on the contrary,

1 [The term Micella was applied by Nägeli to the aggregates of molecules of which organised bodies consist in the second edition of his work on the Microscope (1877). Pfeffer, in his Osmotische Untersuchungen, published in the same year, applies the general term tagma to all aggregates of molecules, and the term syn'agma to bodies which are built up of tagmata. In his Theorie der Gährung (1879), Nägeli gives the following definitions of the terms which he suggests for describing the constitution of matter:-Atom, the ultimate particle of a chemical element: Molecule, an aggregate of atoms, and hence the ultimate particle of a chemical compound: Pleon, an aggregate of molecules, which, like the molecule, can be neither increased nor diminished without a change in its chemical nature; examples of this are afforded by compound salts (e.g. alum), and by salts which contain water of crystallisation (hydropleon): Micella, like the pleon, an aggregate of molecules, but differing from the pleon in that it consists of a much larger number of molecules, and in that increase or decrease of size does not affect its chemical constitution; in this latter respect it behaves like a crystal. The micellæ may combine to form a Micellar Aggregate (Micellverband), and this may be so large that it is readily visible; the crystalloids are examples of large micellar aggregates. It will be noted that the Atom, Molecule, and Pleon are chemical ideas, whereas the Micella and the Micellar Aggregate are purely physical.]

2 [See Book I. p. 49, on Crystalloids.] 
forced further from one another in certain directions, as is clearly seen upon the change of form of the whole, from the formation of fissures, \&c. One of the most remarkable effects of the tensions thus caused in the interior of the body is the fact - that when swelling takes place particular dimıensions may even decrease; thus, for example, the layers of stratification of bast-fibres become very considerably shorter when they swell up under the influence of dilute sulphuric acid, the coils of the spiral striation becoming closer and larger in circumference. Crystalloids change their angles several degrees when they swell. These phenomena are explicable only on the supposition that the micellar forces in the interior of organised substances vary in intensity in different directions ; and this again is conceivable only on the hypothesis that the form of the micellæ is not spherical. Nägeli and Schwendener obtained a deeper insight into these laws by a very careful observation of the phenomena produced by polarised light in cell-walls, starch-grains, and crystalloids ${ }^{1}$. They inferred from these facts a crystalline structure of the individual micellæ, and that the crystals are doubly refractive and have two optical axes which are so arranged, at least in the greater number, that one axis of elasticity within each micella of starch-grains and cell-walls is placed radially, but the two other axes of elasticity tangentially. In crystalloids the micellæ are probably arranged as in true crystals, but separated also by layers of water parallel to the faces or lines of cleavage.

The behaviour of chlorophyll-granules and of colourless protoplasm towards polarised light, as well as under the addition and removal of water, is at present but little known; and a more definite idea of the form of their micellæ is therefore not yet possible.

The solid micellæ of one and the same organised body which are separated by aqueous envelopes always vary in their chemical nature; so that at every visible point micellæ which possess chemically different properties lie by the side of and among one another separated by layers of water. In starch-grains, cell-walls, and crystalloids this fact is inferred from the circumstance that certain substances are extracted by the application of certain solvents, while other substances remain behind, constituting what is called the skeleton. This skeleton is of course less dense than the original substance; and it is evident that the extraction has taken place at all visible points, without the external form or internal structure having undergone any essential change. Thus, for example, a skeleton of cellulose remains behind when the lignin has been extracted from wood-fibres by maceration in nitric acid and potassic chlorate; and again, a skeleton of silica remains behind with all the optical properties of the cell-wall when the organic substance has been burnt away. In the same manner a grain of starch leaves behind a skeleton containing very little solid material when the granulose has been extracted by saliva or some other reagent. From crystalloids also a skeleton in this sense of the term containing very little solid matter can be obtained by the solution of a part of their substance. The properties of these skeletons show that the micellæ which remain behind after solution of the rest still occupy essentially the same position and are endowed with the same forces

1 Hofmeister (Handbuch der phys. Bot. vol. I. p. 348) has arrived at altogether different conclusions, with which I cannot agree. 
as before; it is therefore probable that the extracted substance lay previously between these micellæ without being contained in them. This view is also more or less probable in the case of chlorophyll-granules and protoplasm; in the former the fundamental protoplasmic substance remains behind as a very solid skeleton when the green colouring matter is extracted by ether, alcohol, oil, \&c. Very different substances are certainly combined in the protoplasm; and when a naked primordial cell secretes a cell-wall, it may be assumed that the micellæ which form the cell-wall were previously distributed between those of the protoplasm, and only change their position and their chemical nature when they are secreted in the formation of the cell-wall $^{1}$; the protoplasm which remains behind retaining essentially its original properties. The same is the case when grains of starch or chlorophyll-granules are formed in the protoplasm. A fundamental substance is clearly present in the protoplasm which always retains the essential properties of protoplasm; but various other substances penetrate between its micellæ which are afterwards excreted. This is especially observable in the formation of zygospores and swarmspores.

The nutrition and growth of organised structures takes place, as has already been shown in Book I, by intussusception; the nutrient solution penetrates between the micellæ already in existence, and either occasions by apposition an enlargement of the individual micellæ, or new micellæ of small size are produced in the spaces filled with water, which then increase by the apposition of new matter, or the increase takes place in both ways at different points. The increase in surface of the cell-wall, starch-grain, \&c. is therefore brought about by the micellæ being forced apart from within. Connected with the growth of the micellæ already in existence and with the formation of new ones is a continual disturbance of the osmotic equilibrium between the surrounding fluid (the cell-sap in the widest sense of the term, see p. 62) and that within the body, which has the effect of constantly drawing fresh particles from the surrounding fluid to the interior of the body which is undergoing augmentation.

Chemical processes in the interior of the growing body are also always connected with these processes of growth. The nutrient fluid which penetrates from without contains in fact the material for the formation of micellæ of a definite chemical nature; but this material is chemically different from the micellæ which it produces. Thus starch-grains are nourished by a fluid which clearly does not contain any starch in solution; and again the cell-wall grows by the absorption of substances out of the protoplasm which are not dissolved cellulose. The colouring matter of the chlorophyll arises in the interior of the chlorophyll-granule; and the substances by which the protoplasm is nourished by intussusception are clearly only produced in the interior of the protoplasm, as is shown in particular by naked plasmodia and by unicellular Algæ and Fungi. Growth by intussusception is therefore connected not only with a continual disturbance of the molecular equilibrium, but also with chemical processes in the interior of the growing structure. Chemical compounds of the most various kinds meet between the micellæ of an

${ }^{1}$ [According to Schmitz (Sitzber. d. Niederrhein. Ges. f. Natur. und Heilkunde, Bonn, I880), the cell-wall is formed, at least in the cases which he observed, by the actual conversion of a peripheral layer of protoplasn into cellulose.] 
organised body, so that they act upon and decompose one another. It is certain that all growth continues only so long as the growing parts of the cell are exposed to atmospheric air; the oxygen of the air has an oxidising effect on the chemical compounds contained in the organised structure; with every act of growth carbon dioxide is produced and evolved. The equilibrium of the chemical forces is also continually disturbed by the necessary production of heat; and this may also be accompanied by electrical action. The movements of the atoms and molecules within a growing organised body represent a definite amount of work, and the equivalent forces are set free by chemical changes. The essence of organisation and of life lies in this:- that organised structures are capable of a constant internal change; and that, as long as they are in contact with water and with oxygenated air, only a portion of their forces remains in equilibrium even in their interior, and determines the form or framework of the whole; while new forces are constantly being set free by chemical changes between and in the molecules, which forces in their turn occasion further changes. This depends essentially on the peculiarity of micellar structure, which permits dissolved and gaseous (absorbed) substances to penetrate from without into every point of the interior, and to be again conveyed outwards.

This internal instability attains its highest degree in chlorophyll-granules and protoplasm. In the former chemical processes take place with great energy and activity under the influence of light, such as the formation of the green colouring matter and of starch; and when deprived of light other chemical changes at once ensue, which terminate only with the complete destruction of the entire chlorophyll-granule. The remarkable properties of protoplasm, which we have already examined from different sides in discussing the structure of the cell, attain their climax in its spontaneous automatic power of motion, and in its capacity of assuming different forms and changing both its shape and its internal state, and therefore of bringing into action internal forces, even when corresponding impulses from without cannot be observed. It is impossible to enter here in detail into the explanation of these remarkable facts; but they will be understood, at least generally and to a certain extent, if it is borne in mind that neither the chemical nor the molecular forces are ever in equilibrium in the protoplasm; that the most various elementary substances are present in it in the most various combinations; that fresh impulses to the disturbance of the internal equilibrium are constantly being. given by the chemical action of the oxygen of the air ; and that energy is continually being set free at the expense of the substance of the protoplasm itself, which must lead to the most complex actions in a substance of so complicated a structure. Every impulse from without, even when imperceptible, must call forth a complicated play of internal movements, of which we are able to perceive only the ultimate effect in an external change of form.

The destruction of the micellar structure of organised bodies may take place in many different ways, and affords an insight into many physiological processes.

The most important forces by which the micellar condition of organic substances is permanently altered are changes in temperature, chemical reagents, and substances which have a powerful attraction for water. But these agencies do not in general cause destruction until they have exceeded a definite degree of intensity; while different changes of temperature and different states of concentration of the reagents 
not unfrequently give rise to phenomena differing not only in degree but even in kind. The effect of most external influences depends moreover to a great extent on the chemical nature of the substance which forms the material and micellar framework of an organised body. Cell-walls ${ }^{1}$ and starch-grains for instance differ from crystalloids, chlorophyll-granules, and protoplasm, since the former consist mainly of carbo-hydrates insoluble in water, the latter chiefly of albuminoids.

The following are some of the more obvious phenomena selected from the great mass of existing observations, which are, however, still incomplete.

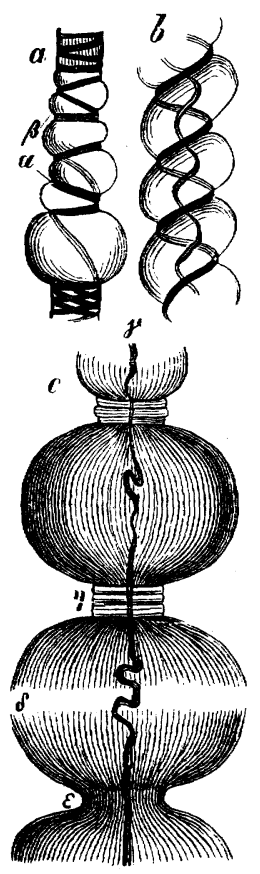

FIG. 466. -Trichoblasts from a leaf of Hoya carnosa (see Fig. 3o, p. 29); $a$ and $b$ after the commencement of the action of iodine and dilute sulphuric acid; $c$, when the swelling in dilute sulphuric aeid has proceeded further. $\alpha$ and $\beta$ in $\alpha$ are the outermost layer not capable of swelling, and coloured dark-blue, which breaks up somewhat irregularly in these cases, but in $c$ more regularly, into a spiral band, while the inner layers swell between them, and are coloured light-blue by iodine; $\gamma$ in $c$ is the cavity of the cell; $\epsilon$ and $\eta$ are constrictions at points where the outer layer is especially firm; at $\delta$ the greatly swollen substance is beginning to become disor. ganised $(\times 800)$.

(b) Acids (especially sulphuric acid) when greatly diluted cause starch-grains and cellulose at the ordinary temperature to swell up much more violently than pure water, without however destroying their organisation; and the previous condition returns when the acid is washed out. If, on the other hand, the acids are more highly concentrated,

1 The cell-wall I suppose here and in the sequel to be neither cuticularised, lignified, nor converted into mucilage.

${ }^{2}$ See Sachs, Handbuch der Experimental-Physiologie, pp. 63 et seq. 
a violent swelling takes place in cellulose and starch-grains, and they pass into a pasty state. Protoplasmic substances, on the contrary, coagulate, as they do under the influence of higher temperatures. Concentrated sulphuric acid finally completely destroys the micellar structure of both with a smaller or larger amount of chemical change, and they deliquesce.

(c) Solution of Potash acts on starch-grains like sulphuric acid, especially in causing them to swell up. Its action on protoplasmic substances is on the other hand very different from that of acids; if the solution is dilute they swell up strongly or deliquesce, and this is especially the case with protoplasm and the nucleus of very young cells (the nuclei of older cells often resist the action strongly). But in a highly concentrated solution of potash protoplasmic structures often retain their form and apparently their structure; they neither coagulate nor deliquesce. The fundamental destruction of their micellar structure which has nevertheless taken place is evident from the fact that they immediately deliquesce if water is added copiously.

(d) Mechanical Infuences. Organised structures bear without injury mechanical forces such as pressure, impact, or slight traction; they are either sufficiently elastic, like starchgrains and cell-walls, again to bring into equilibrium the changes which are thus caused in their internal tension and external form; or they are inelastic like protoplasm and chlorophyll-granules, and can then equalise small passive changes of form in another way. But stronger forces cause disruptions of the micellæ which cannot be again effaced. The micellar structure of the separated portions may however still be perfectly retained, as is shown by fragments of starch-grains and cell-walls. This is still more evident in motile protoplasm, where the separated portions of the previously continuous substance behave like so many individuals, and have the power of independent motion; as, for example, separated portions of plasmodia, the detached halves of the rotating protoplasm in the root-hairs of Hydrocharis when contracted by a solution of sugar, \&c. In the same manner two or more separated portions of protoplasm may unite into a whole, as in the formation of large plasmodia and of zygospores, the fertilisation of oogonia, \&c. The only purely mechanical mode in which complete destruction of an organic structure can be accomplished is by crushing; i.e. by complete disseverance of its micellæ and their subsequent promiscuous intermixture. In this case a chemical change usually directly follows the mechanical destruction of the micellar structure of the protoplasmic substance. In some cell-walls the mere interruption of continuity by a cut causes striking changes in the adjoining and the more distant parts; thus, according to Nägeli, cell-walls of Scbizomeris that have been cut through become shorter and thicker to a remarkable extent.

(e) Changes in the micellar structure of organised structures caused by injurious influences determining their death are often accompanied by striking changes in their relations to diffusion. With respect to starch and cellulose but little is known in this respect ; but the phenomena connected with protoplasm, including the nucleus, are very remarkable ${ }^{1}$. Normal living protoplasm does not, for example, absorb any colouring material from the surrounding solution; but as soon as it has been killed by heat or by a chemical reagent, the dissolved colouring material not merely penetrates into it, but accumulates in it to such an extent that the dead protoplasm appears of a much deeper colour than the surrounding solution of the colouring substance. Starch and cellulose, on the contrary, even in a fresh unchanged condition, absorb from a solution of iodine a comparatively much larger quantity of iodine than of the solvent, and become of a much deeper colour than the surrounding solution; the colour is also different, usually blue, while the surrounding solution is yellowish brown. The protoplasm which fills the cells and has been killed in any manner, by frost, heat, or chemical agents, is more

1 Nägeli, Pflanzenphysiologische Untersuchungen, vol. I. p. 3 et seq.-Hugo de Vries, Sur la perméabilité du protoplasm des betteraves, Arch. Neérland. vol. VI, i871 : [also id., Unters. ueb. die Mechanischen Ursachen der Zellstreckung, 1877.-Pfeffer, Osmotische Untersuchungen.] 
permeable (whether cellulose is so also is not known); it allows the cell-sap, which in living and growing cells is always subject to high pressure, to filter out as if it had become porous. This is well seen when coloured cells or tissue are frozen or heated above $50^{\circ} \mathrm{C}$.; they then allow their coloured contents to diffuse out, which they do not do when living.

( $f$ ) The true nature of the change which the micellar structure of moist organised bodies undergoes by heating above $50^{\circ}$ or $60^{\circ} \mathrm{C}$., or when they are made to swell up strongly by treatment with acids or alkalies, is considered by Nägeli to lie in the destruction of the crystalline micellæ. In the case of starch-grains and cell-walls this view is supported by a few facts which have hitherto not been explained in any other manner. The increase of the power of absorbing water under such conditions is then explained on the hypothesis that the number of particles which attract water is increased and their size diminished by the destruction of the micellæ; and this must necessarily be connected with an increase in the proportion of water and a corresponding increase in volume. It is especially noteworthy that the denser layers of starch-grains and cellwalls become under these circumstances homogeneous with the least dense and most watery layers. But since the denser layers probably consist of large, the less dense layers of small micellæ, the explanation may lie in the fact that the large micellæ of the dense substance are broken up into a number of small micellæ, and thus become similar to those of the less dense substance. The same explanation may be given of the fact that when the organised structure is changed by undergoing strong swelling, the optical properties of starch and cellulose also undergo change; their previous action on polarised light disappearing altogether. This is also explained if we suppose that under the action of these agents the micellæ which produce the optical effect lose their form, and that their fragments are irregularly intermixed.

How far these views can be applied also to protoplasmic structures and their coagulation remains at present uncertain.

(g) The disorganisation of the micellar structure of organised bodies may take place gradually; and when it has exceeded a certain limit, a new substance is produced from the originally organised material, the molecular condition of which has, since the time of Graham, been termed colloidal. From the similarity which, according to Nägeli and Schwendener, exists between organised and crystalline bodies, it is not surprising that there are also mineral substances which, like silica, are usually crystalline, but become under certain circumstances colloidal ${ }^{1}$. Organised bodies absorb water and other fluids, increasing at the same time in volume up to a certain maximum at which they are saturated; crystalline bodies dissolve in a definite minimum of water and produce a saturated solution which can be diluted ad libitum. Colloidal bodies show in this respect intermediate properties; they can be mixed with water in all proportions without any minimum or maximum. Solvents cause in organised and crystalline bodies a sudden passage from the solid to the fluid condition. Colloidal bodies pass from the solid to the fluid condition, when they are soluble, through all stages of softening; in a certain state when they contain but little water they are hard, then tenacious, then viscous and scarcely fluid, finally when mixed with abundance of water perfectly fluid. Even in the fluid state they may be mucilaginous, adhering strongly to organised, less strongly to crystalline substances; and even when greatly diluted they diffuse very slowly, and some of them appear unable to penetrate organic membranes such as cell-walls. On drying they afford a homogeneous substance which differs greatly in its capacity for swelling and in its optical properties from crystals and from organised bodies. In contradistinction to these latter, colloidal bodies may be considered amorphous internally as well as externally. Colloidal bodies occur abundantly in plants as products of the decomposition of organised bodies, and under certain circumstances they supply material for the production of new organised bodies. Thus gum-bassorin and perhaps also gum-arabic, as well as the

${ }^{1}$ See, among other authorities, Graham, Phil. Trans. I862; Journ. Chem. Soc. 1862. 
mucilage of quince and linseed, result from the decomposition of cell-walls; perhaps also the formation of the substance of the cuticle must be included in this category. Viscin is the product of decomposed cellulose; the origin of colloidal pectin and caoutchouc is still unknown; but none of these substances are of any further use to the plant.

(b) Traube's Artificial Cells ${ }^{1}$. Among the most important of the phenomena belonging to the growth of the plant are those connected with the cell-wall; and everything which contributes to a more exact knowledge of its development must always be welcome. The researches of Traube, of which an abstract is here given, are of great interest from this point of view; even though it may not always be possible to transfer all the properties of his artificial cells to the real plant.

Starting from Graham's observation that dissolved colloids cannot diffuse through colloidal membranes, and from the empirical fact that precipitates of colloidal substances are usually themselves colloidal, Traube found that a drop of a colloid $A$ placed in a solution of a colloid $B$ must become surrounded by a pellicle. If $A$ is also more concentrated (or rather if its attraction for water is greater) the cell must become turgid, i.e. the precipitated pellicle must become stretched by the additional water that is absorbed; and the micellx of the pellicle thus become separated to such an extent that a fresh precipitate takes place between them which occasions increase in the superficies of the pellicle. For a more exact study Traube chiefly employed cells the pellicle of which consisted of a precipitate of gelatine tannate. For this purpose the tendency of the gelatine to coagulate was destroyed by boiling for thirty-six hours. A large drop of this so-called $\beta$ gelatine of the consistency of syrup was taken up by a glass rod, allowed to dry for some hours in the air, and then plunged into a flask half filled with a solution of tannic acid, into the cork of which the rod was fixed. The portion of gelatine which undergoes solution on the outside of the drop immediately forms a completely closed pellicle with the surrounding solution of tannin; and the water which penetrates through it constantly dissolves the gelatine within. In a dilute solution of tannin of 0.8 to ${ }^{\circ} 8$ p.c. a tense pellicle which is not iridescent and is therefore thick is formed; in a concentrated solution of from 3.5 to 6 p.c. (in which therefore there is a smaller difference between the concentration of the two fluids) a thin flaccid iridescent pellicle is formed ${ }^{2}$. Traube found that the cells which are at first thick-walled go through various stages of development; they remain spherical so long as the nucleus of gelatine is not completely dissolved; a turbidity then sets in from above downwards owing to the solution of a part of the pellicle in the solution of gelatine which is more dilute in its upper part; the pellicle at the same time begins to collapse and to become iridescent; and finally the contents become clear and tension is again set up. After the lapse of some weeks the cell still allows gelatine to escape when torn. The greater the difference in the concentration of the two fluids, the firmer and more tense is the pellicle; i.e. the greater the intensity of the endosmotic attraction the greater is the number of layers of atoms which coagulate so as to produce the pellicle, and therefore the thicker it is.

With reference to the properties of the pellicle, Traube shows that all pellicles hitherto employed in experiments on diffusion have perforations ${ }^{3}$, while the precipitated

1 Traube, Experimente zur Theorie der Zellbildung u. Endosmose, in Arch. für Anat., Phys., u. wissensch. Medicin, von Reichert u. Du Bois, 1867 , p. 87 et seq.: [also Pfeffer, Osmot. Unters.]

${ }^{2}$ Only pellicles of gelatine behave in this way; all others are iridescent when tense.

${ }^{3}$ It is easy to convince oneself of the presence of actual perforations in pig-bladder, ox-bladder, the pericardium, amnion, collodion-membrane, or parchment, with which experiments on diffusion have hitherto usually been made, by stretching them over a wide glass tube, pouring in a column of water from 20 to $40 \mathrm{~cm}$. high, and repeatedly drying the free surface of the membrane with filtering paper. Water is then almost always seen to ooze out at particular spots; a piece of membrane 2 or $3 \mathrm{~cm}$. square is seldom water-tight. The perforations are still more evident if the tube is filled with a concentrated solution of common salt and the membrane dipped in water. Instead of a 
pellicles have only micellar interstices; and indeed these latter are, according to him, smaller than the molecules of the precipitate of which the pellicle is composed. But in spite of the greater density, the endosmose is quicker than with all other membranes, because they are thinner. The pellicle becomes firmer (stiffer?) when lead acetate or copper sulphate is added to the $\beta$ gelatine. As soon as the micellæ of the stretched pellicle have become so far separated by the pressure of the cell-contents which have increased in quantity by the action of endosmose that their interstices allow the passage of the two substances from which the pellicle is formed, these substances must obviously again at once mutually react upon one another at those points, and must cause the production of new micellæ of pellicle, which are deposited between those already in existence. Growth therefore takes place by intussusception, and is caused by the stretching of the pellicle, which stretching is on its part occasioned by endosmose. That the growth takes place not only by stretching but also by deposition Traube proved by replacing the tannic acid by water. As soon as this was done (i.e. as soon as the formation of new molecules of the precipitate in the pellicle was prevented, the endosmose still continuing) the growth ceased.

As long as the concentration of the contents of the artificial cell is everywhere the same, the pellicle remains everywhere equally thick, and the cell retains its spherical form. But when the contents become diluted, a denser solution is formed in the lower part of the cell, a more watery solution in the upper part. The pellicle becomes in consequence thinner above, because the difference of concentration is smaller there, and therefore more extensible; hence the pellicle becomes more strongly stretched above and increases more rapidly in superficies, and protuberances directed outwards are not unfrequently formed. This may be expressed shortly by saying that endosmose takes place principally in the lower part of the cell, growth in the upper part. The difference however in the concentration in the interior of the cell which causes this is the consequence of the water which penetrates by endosmose not mixing at once uniformly with all parts of the interior solution, so that layers of different specifis gravity lie one over another.

Further experiments showed that growing pellicle-precipitates having the form of cell-walls are produced also by mixing colloids with crystalloids $^{1} ;$ e.g. tannic acid with copper and lead acetates, gelatinous silica with the same substances or with copper chloride, or finally crystalloids with one another, as potassium ferro-cyanide with copper acetate or chloride. Traube came to the conclusion that every precipitate the interstices of which are smaller than the molecules of its components must assume the form of a pellicle when the solutions of its components come into contact with one another. Since the pellicle-precipitates, as has already been mentioned, contain micellar interstices but no perforations, they are peculiarly well adapted for the study of endosmotic processes. They behave in this respect very differently from other membranes, being themselves often perfectly impermeable to the most diffusible substances, but allowing other chemical compounds to pass through them; and every kind of pellicle has in this respect its own peculiarities. Independently of the fact that every pellicle-precipitate is impermeable to the fluids from which it is itself produced, the $\beta$ gelatine tannate is, moreover, impermeable for example also to potassium ferro-cyanide, but permeable to ammonium chloride, barium nitrate, or water. The pellicle of copper ferro-cyanide which is formed round a drop of copper chloride in potassium ferro-cyanide is impermeable to barium chloride, calcium chloride, potassium sulphate, ammonium sulphate,

diffusion-current equal over the whole surface of the membrane, separate threads of the solution of salt are seen to sink down into the water. These experiments show how little dependence is to be placed on the researches hitherto made on diffusion with membranes.

'[The term 'crystalloid' is here used in the sense in which it was first employed by Graham, to indicate those substances - as opposed to 'colloids'-which may be susceptible of crystallisation, and which are endowed with the power of diffusion through a porous septum.] 
or barium nitrate, but permeable to potassium chloride or water. Traube considers that in the permeability of the pellicle-precipitates we have a means of determining the relative size of the molecules of different solutions, since only those molecules can pass through the pellicle which are smaller than its micellar interstices and therefore smaller than the molecules of the solutions which produce it.

If a small quantity of ammonium sulphate is added to a solution of $\beta$ gelatine, and a small quantity of barium chloride to one of tannic acid, and the two mixtures thus obtained are themselves mixed, a pellicle is formed of gelatine tannate, and in it a precipitate of barium sulphate which diminishes the size of the interstices; the two solutions which cause the deposit can no longer diffuse; but the incrusted pellicle is still permeable to the smaller molecules of ammonium chloride and water.

Traube maintains that there is no such thing as an endosmotic equivalent in the sense of the older theory. Endosmose is independent of any interchange, since it results entirely from the attraction of the soluble substance for the solvent; and this attraction is invariable at any given temperature and may be termed Endosmotic Force. The endosmotic force of grape-sugar, for instance, is very great, that of gelatinous substances very small.

To these researches, which are of extreme importance in reference to vegetable physiology, and of which we shall make much use in the sequel, though with a cautious selection, Traube has added observations on the growth of the pellicle-precipitates of copper ferro-cyanide, the main results of which however I have been unable to confirm after a number of experiments.

If a drop of a very concentrated solution of copper chloride is dropped into a dilute solution of potassium ferrocyanide, it immediately becomes coated with a thin brownish pellicle of copper ferrocyanide which exhibits peculiar phenomena. It is more convenient to place small pieces of copper chloride in the ferrocyanide solution, where a green drop is immediately formed at the expense of the water of the solution, producing the pellicle on its surface, and still enclosing the solid copper chloride which dissolves gradually from the permeation of the water. These cells manifest active growth and a variety of differences not easy to explain and dependent on secondary circumstances. Some have very thin pellicles, are roundish, and exhibit a slight tendency to grow upwards; they usually form a number of small wart-like outgrowths and attain very considerable dimensions (from $\mathrm{I}$ to $2 \mathrm{~cm}$. in diameter); they appear to be formed chiefly by the solution of large pieces of the copper chloride. Others have thick reddish brown pellicles, grow quickly upwards in the form of irregular cylinders, rarely branch, and attain a diameter of from 2 to $4 \mathrm{~mm}$. and often a height of several centimetres. Combinations of the two forms also occur which sometimes form a kind of horizontal tuberous rhizome-like structure from which long stalk-like outgrowths arise upwards, and root-like protuberances downwards.

It is impossible, in the space at our disposal here, to give a detailed description of these phenomena; one only may be specially mentioned:-that these pellicles of copper ferrocyanide do not grow, as Traube supposes, by intussusception, but in quite a different way (by eruption). When a brown pellicle has been formed round the green drops, water penetrates quickly from without through it to the copper chloride; the pellicle becomes rapidly stretched, and, as may be clearly seen, at length ruptured, The green solution immediately escapes through the fissure, but becomes at once coated with a pellicular precipitate which appears either as an intercalated piece of the previous one, or as an excrescence or branch of it, a process which is repeated as long as any copper chloride remains inside the cell. We cannot therefore in this case conclude that deposition of fresh micellæ of the pellicle takes place between those already in existence. These cells cannot, so to speak, be injured; if they are pricked, then at the moment when the point which pricks them is withdrawn an outgrowth follows immediately, which is easily to be explained from what has been said. In consequence of the rapid flowing in of water through the perforation, the dissolved or the 
still solid copper chloride has no time to form a homogeneous solution; a stratification arises which begins in the lower part of the cell with a very concentrated solution, and passes in the upper part into almost pure water when the cell has already grown to some height. Since the dilute upper fluid is lighter than the surrounding solution, it exerts an upward pressure upon the membrane-just as a cork held down under water attempts to rise-till it is ruptured below or at the apex (in the second form of cell). But the lighter fluid, when on the point of ascending, becomes at once surrounded by a pellicle which remains attached to the walls of the fissure of the old one; and thus apical growth takes place in cells of this description in the form of eruptions, just like the formation of branches and excrescences in the round ones. If the fluid in the upper part of the cell is pure water, large pieces of the pellicle break off and rise up into the surrounding solution like air-balloons open below. If the copper chloride is entirely consumed in the formation of the pellicle, the opening caused by the tearing off of the upper cap does not close, or the whole cell ascends like an airballoon. If rapidly growing cells of the second form are placed in a horizontal position, an outgrowth takes place at the extreme apex as the least solid point, which is directed vertically upwards, and then grows in this direction like the former apex of the cell. This process, even though it calls to mind distantly the bending upwards of growing stems which are placed horizontally (geotropism), bears in fact not the least actual resemblance to this phenomenon, as will be shown in Chap. IV; and this is at once evident if it is remembered that in these cells there is no such thing as growth by intussusception.

SECT. 2. Movement of Water in Plants ${ }^{1}$. The growth of the cells of plants is always connected with the absorption of water, not only as regards the increase of size of the vacuole, but the growth of the cell-wall and of other organised structures is also accompanied by the intercalation of particles of water between the solid micellæ. Water must therefore be conducted to the growing cells and tissues; and when the organs which absorb the water lie at a distance from those which require it for their growth, the movement which results is necessarily considerable. Water is in the same manner required by the organs of assimilation, since it furnishes the hydrogen required for organic compounds. The reservoirs of food-material in which the assimilated compounds are for a time accumulated also require water for the purpose of again dissolving these substances, in order that they may be carried as formative materials to the leaves and the growing apices of roots and stems. All these movements of water, which are necessarily connected with nutrition and growth, proceed slowly like growth itself; their direction is in general determined by the relative positions of the organs which absorb the water from without and of those which make use of it.

In plants which grow under water or beneath the ground where no loss of water takes place or only to a very considerable extent, there is no need for these processes. The case is nearly the same also with some land-plants which are almost completely protected by a peculiar organisation from loss of water by evaporation (transpiration) when it has once been absorbed, as the Cactus-like Euphorbias, Stapelias, \&c., which are by this means enabled to live in the most arid localities. But the great majority of plants have foliage with a very large

${ }^{1}$ See Sachs, Handbuch der Experimental-Physiologie, the section on the movement of water, p. 196, where the literature up to $186_{5}$ is mentioned; the most important of the more recent publications are quoted in the sequel. 
superficial development; when the leaves are also delicate, as in most plants with a rapid growth, a very considerable portion of the water of their cell-sap is removed by transpiration within a short time, so that in the course of a single period of vegetation the quantity of water which has been withdrawn by transpiration may exceed many times the weight and volume of the plant itself. It is easy to understand that this is possible only when the loss is compensated by the absorption of corresponding quantities of water through the roots, and that the water withdrawn from the leaves is replaced in this way. As long as the tissue of plants in which transpiration takes place remains turgid, the supply must nearly equal the loss; so long therefore as transpiration proceeds continuously from the leaves or other surfaces, a constant current of water exists from the roots to the leaves. When transpiration ceases, as in very moist air, or when the leaves are wetted by dew or rain or after the falling of the leaves, the current of water also ceases as soon as the tissues which have become. somewhat flaccid are again turgescent. Since transpiration is accelerated by a high temperature of the air, by its dryness, and above all by sunshine, and as these conditions are constantly changing, the rapidity of the current of water is also subject to continual change.

The current of water occasioned by transpiration has, as will be seen, no immediate connection with the processes of growth and nutrition; the HorseChestnut and other trees and shrubs which put out in spring only a definite number of leaves, and during the summer do not any further increase their foliage, transpire the most rapidly during this time; and at this time also the current of water is most considerable in them. In winter both growth and transpiration, and with the latter the amount of water also in the tissues, remain stationary; when the buds are put out, the water is first of all only set in motion to the extent required by the increase of the growing organs; but as the development of the organs increases their surface, the amount of evaporation again rises, and the current begins afresh.

While the movement of water required for purposes of growth and nutrition must take place in the most different forms of tissue-in the parenchyma and even in the primary meristem of buds and of the apices of roots-it is nevertheless certain that the current of water caused by transpiration passes exclusively through the woody portion of the fibro-vascular bundles; all the rest of the tissue may be destroyed at any place without the current of water ceasing, if only the wood remains entire. In Conifers and Dicotyledons which have a compact wood, one main current passes through the root and stem, dividing in the branches and leaves into constantly narrower channels; while in Ferns and Monocotyledons the current of water passes, even in the primary stem, through isolated narrower channels corresponding to the course of the isolated woody bundles. That the lignified elements of the xylem of the fibro-vascular bundles determine the channel of the current, is seen not only from direct observation, but also from the fact that the formation of wood is the more considerable, the greater the evaporation and the stronger the current of water in a plant. In submerged and underground parts of plants from which no transpiration takes place the xylem remains entirely or nearly unlignified; in Dicotyledons and Conifers, where the transpiring surface increases with age, the channel taken by the current is also annually widened by the increase of the wood. The crown of leaves of Palm-trees remains after a 
certain time of nearly the same size, and the stem and the channels of the current (woody bundles) which traverse it consequently retain their diameter unchanged.

The movements of water caused by growth as well as those induced by evaporation have this in common, that their direction is towards the places where they are required. If growth or transpiration begins at a certain time at a definite spot, the nearest portions of the tissue give up their water first of all, then the more distant ones, the organs at the greatest distance, generally the roots, absorbing water from without. The movement therefore propagates itself continually further and further from the point to which

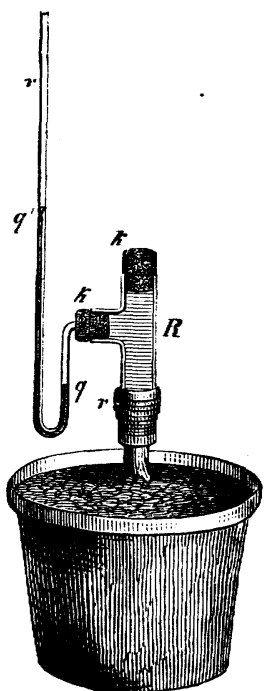

FIG. 467--Apparatus for observing the force with which water escapes under rootpressure from the transverse section of a stem $r$. The glass tube $R$ is first of all firmly fastened to the stem, and the tube $r$ then fixed into it by the cork $k, R$ is com. then fixed into it by the cork $k . R$ is com-
pletely filled with water, the upper cork $k$ then fixed in it, and mercury poured into the tube $r$ so as to stand from the first higher at $q^{\prime}$ than at $q$, the level $q^{\prime}$ rising above $q$ according to the intensity of the root-pressure. The apparatus is much more convenient to handle than that hither. to in use.

(Fig. 467 ) it shows that even in smaller plants with but little wood (as Tobacco, Maize, the Stinging Nettle, \&c.) the water which exudes stands at a pressure which holds in equilibrium a column of mercury several centimetres in height; while in some woody plants, as for instance the Vine, this pressure may amount to $76 \mathrm{~cm}$. (or one atmospheric pressure).

In many plants of small height this root-pressure is observable from the fact that water exudes at particular points of the leaves in the form of drops, provided that the internal supply of water is nowhere diminished by powerful transpiration, and the pressure thus removed. Thus drops of water appear abundantly and repeatedly on the margins and apices of the leaves of many Grasses (especially 
striking in the Maize), Aroidex, Alchemilla ${ }^{1}$, \&c., when transpiration is diminished by the absence of light and the cooling of the air, and the activity of the roots increased by warm damp earth. Even in unicellular plants, or those which consist only of rows of cells, as the Mucorini (e.g. Pilobolus crystallinus), Penicillium glaucum, and the large Fungi (as Merulius lacrymans), the water is forced out in drops from the upper part, it having been absorbed by the lower parts which perform the function of roots and press it upwards.

Fluid however not unfrequently appears in drops in places where there can be no pressure directed upwards from the root. Thus the nectaries of flowers, as those of Fritillaria imperialis, and the glands in the pitchers of $N_{e p e n t h e s}{ }^{2}$, \&c., exude drops of liquid even when the, stem is cut off from the root and merely placed in water. In this case the forces which cause the pressure must arise in the upper masses of tissue, perhaps even in the organ itself, for the water is conveyed to the cut stem not by pressure but by suction.

The phenomenon known as the 'bleeding' of wood cut in the winter must not be confounded with this. This bleeding occurs when the cut branch or piece of stem, previously cold and saturated with water, is rapidly warmed; the air. which is enclosed with the water in the cells and vessels of the wood expands, and forces the water out where it can find an opening. If the piece of wood is again cooled, the air contracts, and the water in contact with the section is again sucked in. It is evident that these expansions and contractions of air in the wood must also take place when the woody substance of the tree is uninjured; and hence currents are set up from the parts which are becoming warmer to those which are becoming cooler, and tensions are brought about. All this however happens only so long as air as well as water is found in the cavities of the wood, as is the case in the winter and spring before the leaves unfold and transpiration begins.

Although the movements of water in plants have been copiously investigated and discussed for nearly 200 years, it is nevertheless still impossible to give a satisfactory and deductive account of the mode of operation of these movements in detail ${ }^{3}$. This

1 According to Duchartre, De la Rue, and Rosanoff, the exudation usually takes place through stomata, which are either developed in a peculiar manner, or are very large (water-pores), or possess the ordinary form. De Bary remarks in connection with this:- 'If water is forced into the wood of a branch of a plant adapted to the purpose, e.g. Fuchsia globosa, by the moderate pressure of a column of mercury, drops of water at once exude from the large stomata' (Bot. Zeitg. I869, No. 52, p. 882). [This subject has been further investigated by Moll (Med. d. k. Akad. v. Wet. XV. 2, 1880: see also Nature, vol. XXII, I880.]

${ }^{2}$ [The liquid contained in the pitcher-like organs of Sarracenia, Nepenthes, Cephalotus, \&c. is not pure water. Dr. Völcker (Ann. and Mag. of Nat. Hist. vol. IV. p. I 28, and Phil. Mag. vol. $\mathrm{XXXV}$. p. 192) states that it is generally clear and colourless, rarely yellowish, and reddens litmus. The proportion of residue left on evaporation varies from $0^{\circ} 27$ to $0^{\circ} .92 \mathrm{p}$.c. This residue consists of 38.61 p. c. organic matter, chiefly malic acid with a little citric acid, 50.02 p. c. potassium chloride, 6.36 p. c. soda, 2.59 p. c. lime, and 2.59 p. c. magnesia. Dr. Buckton (Nature, vol. III. p. 34) found that the liquid contained in the pitcher-like labellum of Coryanthes consists of $98.5 \mathrm{I}$ p. c. water and volatile oils, and $\mathrm{r}_{4} 49 \mathrm{p}$. c. non-volatile residue. It is clear and somewhat glutinous in consistence, with a high refractive power, and a sp. gr. $\mathbf{I} \cdot 062$; neutral to test-paper; on evaporation it becomes milky, finally yielding a transparent gum insoluble in alcohol. See paragraph $(e)$ in the appendix to this section.]

${ }^{3}$ Although Dr. Muiller, in the second part of his 'Botanische Untersuchungen' (Heidelberg I872), assumes that he has actually accomplished this, those only will believe it who are entirely ignorant of vegetable physiology. 
much appears certain, that the ultimate force concerned is diffusion (in the broadest sense of the term). But since in the living plant this force acts under conditions widely different from those in operation in artificial apparatus, we are compelled on all essential points to draw our conclusions as to the internal processes from the careful study of the phenomena in the plants themselves. Our space will however only permit us to refer to these in general terms. The main result of the investigations hitherto made is to maintain the distinction between the different causes of motion in the fluids of the plant to which we have already alluded, until a more thorough knowledge justifies some other interpretation. What follows is less for the purpose of explaining the phenomena than of illustrating by examples what has already been said.

(a) The slow movement of water caused merely by Growth and Assimilation is seen in its simplest form in unicellular Fungi and Algæ and in those in which the cells are arranged in rows and plates, and in germinating spores and pollen-grains; since in these cases the growing and assimilating cells absorb the water which they require immediately from their moist environment. That this is caused by the imbibing power of the cell-wall and of the protoplasm as well as by endosmose (i.e. the attraction of the dissolved substances within the cell for water), is certain, although we have not yet sufficient knowledge of the exact mode in which these processes go on. On the other hand, in plants which consist of masses of tissue the young growing parts withdraw the water of vegetation from the older mature parts, and these latter become in consequence empty if they receive no fresh supply from without. This is seen clearly when tubers, bulbs, trunks of trees which have been cut down, \&c., put out buds in ordinary moderately dry air, and thus gradually lose the water they have contained ${ }^{1}$.

(b) Transpiration ${ }^{2}-i . e$. the evaporation of water from cells and masses of tissue-is produced and modified by external and internal conditions and causes. Among external causes those must first be noted which produce evaporation from moist surfaces, such as the relative temperature and dryness of the air and that of the transpiring tissue itself. Evaporation will generally increase as the temperature of the surrounding air rises and its degree of saturation consequently decreases; and this must for our purpose be considered the most direct measure of the greater or less tendency to evaporation. It must not however be expected that the amount of evaporation from plants is simply in proportion to any one of these conditions. It is still doubtful whether light, i.e. radiation as such, independently of the elevation of temperature caused by it, influences transpiration ${ }^{3}$. The stomata of most plants open more widely in light than in the dark ${ }^{4}$; that is, the openings which allow of the escape of the aqueous vapour formed in the interior of the tissue become larger, and this must have the effect of promoting further evaporation within. It is not yet decided whether light acts on the stomata as such, or by means of the heat which accompanies it, or the chemical changes which it causes.

Among the conditions connected with the organisation of the plant itself which determine the amount of transpiration must be noticed the nature of the cortical tissue, the size and number of the intercellular spaces, and the character of the substances dissolved in the cell-sap. When the cortical tissue is a continuous and thick layer of periderm as in many woody branches, potato-tubers, \&c., or a thick layer of bark as in older trunks of trees, the evaporation of water from the succulent tissues which lie beneath is rendered

1 For further details see Nägeli, Berichte der kön. bayer. Akad. I86ı; Botanische Mittheilungen, vol. I. p. $4^{\circ}$.

${ }^{2}$ Sachs, Experimental-Physiologie, p. 22 1.-Müller, Jahrb. für wiss. Bot. vol. VII, I868.Baranetzky, Bot. Zeitg., 1872, Nos. 5-7.- [See also Vesque, Ann. Sci. Nat. 1877, and Burgerstein, Ueb. den Einfluss aeusserer Bedingungen auf die Transpiration, Wien, 1876.]

3 Dehérain's researches (Ann. des Sci. Nat. 1869, pl. XII. p. I) do not decide the question.

4 Von Mohl, Bot. Zeitg. I $83^{6,}$ p. 697 . 
difficult in the extreme ${ }^{1}$. The cuticularised outer wall of the epidermis of young leaves and internodes is less efficacious in this respect; if it is very thin as in many quickly-growing- leaves, especially those of water-plants, or altogether imperceptible as in roots, these parts dry up very quickly in ordinary air. In contradistinction to this the evaporation is very small from hard evergreen leaves, Cactus-stems, \&c., which are covered by a thick cuticular coating. It may be assumed that in plants provided with a thick cuticle transpiration takes place principally through the stomata, and is therefore dependent on their smaller or larger number and size. The evaporation does not in this case proceed from the surface of the organ (or only to an imperceptible extent) but in its interior, viz. at the places where the cells of the parenchyma bound the intercellular spaces. These spaces may be supposed to be always at least nearly saturated with aqueous vapour; but the vapour will escape through the stomata with every increase of its tension or decrease of the tension of the vapour without, and will thus give rise to the production of more vapour in the inside. The production of vapour in the intercellular spaces is moreover the more abundant the larger they are themselves, or in other words the larger the superficies of cell-wall which bounds them. This circumstance, and the much larger number of stomata on the under side of the leaves, are clearly the reason why evaporation is generally so much more copious from it than from the upper side. Since water containing any substance in solution evaporates more slowly than pure water, and the more slowly the more concentrated and denser the solution, this must also be considered among the conditions which limit the transpiration of water from the sap of plants. It must not however be forgotten that evaporation takes place only on the external surfaces of the cell-walls of tissues, which on their part remove the water by imbibition from the cell-sap.

The conditions now named which regulate transpiration are combined in the most various ways, and not only cause different plants to show different amounts of transpiration, but also the amount to be very different in the same plant at different times. A definite statement cannot however be made of the total amount of transpiration, i.e. of the quantity of water required by a plant during its period of vegetation, although certain very variable limits can always be assigned to each species in this respect. Two plants of the same species may, as any one may see, thrive equally well if one grows in damp soil and dry air, the other in dry soil and damp air, the former thus using up a large, the latter a small amount of water. In general the conditions of transpiration which have been mentioned exhibit periodic variations related to the meteorological distinction of day and night; the temperature, the moisture of the air, and light, are usually favourable to evaporation by day, unfavourable by night; but under certain circumstances this condition may even be reversed.

(c) Currents of Water in the Wood. Superficial cells or those which bound intercellular spaces and lose water directly by evaporation would very soon collapse and dry up if they were not able again to replace that which they have lost. This can only take place by the flow of water from the adjoining cellular tissue from which no evaporation occurs; but when this tissue is placed in the same condition as the former, it must also compensate its loss from more distant layers of tissue, and these again from those which are connected with the conducting organs or woody bundles which convey the water from the roots. The question here presents itself whether this movement of water within the succulent tissue (especially in the parenchyma of the leaves) is caused by endosmose from cell to cell, or whether it does not occur at least principally along the cell-walls, these latter forming the channels of communication between the woody bundles and the surfaces where the evaporation takes place, the contents of the cells being only incidentally concerned in the process.

The chief evidence of the fact that the rapid currents of water in the roots, stem, and branches caused by transpiration take place only in the wood, i.e. in the lignified

${ }^{1}$ [It may be effected in the summer by means of the lenticels; see ante, p. 108.] 
xylem, has already been stated. It can be demonstrated in a more conspicuous manner by placing a cut stem or branch with its cut surface in a coloured solution ${ }^{1}$ while the leaves are transpiring. If the stem or branch is cut through at various heights after a few hours, or according to circumstances after a longer period, the colouring of the wood will show how high the solution has been sucked up in it, and will be seen only in the woody bundles and not in the cortex or pith. If branches with pure white flowers are employed in this experiment, such as a white-flowered Iris or Deutzia, and if they are placed in a dark aqueous solution of aniline, the white petals are found, after from ten to fifteen hours, to be permeated by dark blue veins corresponding to the fine woody bundles of the venation. This beautiful appearance however soon vanishes, the poisonous colouring material subsequently killing the adjoining layers of parenchyma, and colouring the spaces between the veins blue by diffusion, and the corolla consequently becomes flaccid ${ }^{2}$.

The difference in the amount of transpiration under different external conditions must also correspond to a difference in the rapidity of the current of the water in the wood. In rainy weather, when there is no evaporation or but very little from the leaves, the movement of the water in the stem will be very slow; but when the transpiration increases with sunshine and wind, the current of water in the woody bundles is also accelerated. Under the hypothesis that the water moves only in the woody substance of the walls of the wood-cells themselves and not in their cavities, I have calculated the rapidity of the ascending current of water in a branch of the Silver Poplar in which there was strong transpiration, and obtained a rate of $23 \mathrm{~cm}$. per hour. $\mathrm{M}^{\mathrm{c}} \mathrm{Nab}$ placed branches of Prunus Laurocerasus ${ }^{3}$ from which transpiration was taking

${ }^{1}$ I must take this opportunity of making the remark that I still entertain, and in a high degree, the doubt previously expressed, whether it is not a purely pathological phenomenon that is produced in this manner.

2 [This is a method of experimentation which has been practised by numerous observers since the commencement of the last century, when it was apparently first tried by Magnol. Sarrabat (otherwise Delabaisse) coloured the veins of the flowers of the tuberose (Polyanthes tuberosa) and Snapdragon (Antirrhinum majus) by watering the plants with the juice of the berries of Phytolacca. (Dissert. sur la circul. de la Sève, Bordeaux, I733.)

Van Tieghem (in the French edition of this work, p. 791) quotes Reichel as having plunged the roots of a flowering plant of Datura Stramonium into a decoction of the wood of Fernambouc; the liquid followed the course of the vessels, and after eight days veined the corolla with red, and made its appearance also in the stamens, the walls of the fruit, and even in the style. (De vasis plantarum spiralibus, Leipzig, I 748.) For other old authorities see De Candolle, Phys. Veg. i. 82.

De Saussure found that the stem of a bean became coloured by a decoction of Brazil-wood; and this was one of the facts upon which he based the conclusion that organic matters were capable of being taken up by the roots of plants (Ann. des Chem. u. Phys. xlii. p. 275). Biot noticed that the red colouring matter of Phytolacca was absorbed by white hyacinths when poured upon the soil in which they were grown; after two or three days, however, the red colour disappeared from the flowers. (Comptes Rendus, 1837, i. I 2.) Unger also made the same experiment (Botanical Letters, p. $\left.3^{8}\right)$. Hallier immersed the ends of cuttings of plants in solution of indigo or black cherry juice. (Phytopathologie, 1868, p. 67). Persoz states (Introd. à l'étude de la Chimic moléculaire, p. 553) that plants of Impatiens parviflora, the roots of which are immersed in a solution of sulphindigotic acid, absorb that fluid in a reduced or colourless state due to the action of the roots upon it; in the petals it again undergoes oxidation and becomes blue. The experiments of Herbert Spencer (Principles of Biology, i. p. 538) may also be referred to.]

${ }^{3} \mathrm{M}^{\mathrm{c} N a b}$, Transactions of the Botanical Society of Edinburgh, I87I. [Dr. Pfitzer has suggested that the result may be arrived at by the much simpler mode of allowing the plant grown in a pot to become so flaccid from want of water that the leaves droop perceptibly, and then, after supplying the root with water, to observe the length of time that elapses before the leaves at various heights from the ground recover their normal position. Pfitzer found by this means a much more rapid rate of ascent indicated than that stated by $\mathrm{M}^{\mathrm{c} N a b}$; and believes that there is a serious source of error in MNab's experiments, from the saline solution not rising so fast as pure water: (also Trans. Roy. 
place in a solution of lithium citrate, and then examined the ashes of successive internodes by the spectroscope. The solution was found to rise from $4^{2}$ to ${ }_{4} 6 \mathrm{~cm}$. in one hour. But neither method of calculation is exact or probably of much value.

The current of water in the wood which replaces the loss occasioned in the leaves by transpiration is not caused by osmose, since at the time when the transpiration is strongest and therefore the current in the wood quickest, the cavities of the conducting wood-cells do not contain sap but air, or at the most are only partially filled with fluid. If the rising of the water took place by endosmose from cell to cell, the cells would necessarily possess closed cell-walls and be full of sap, the concentration of which would constantly increase from below upwards in the wood. But the conducting cells are at this time not closed, but partially or altogether (as in Coniferæ) connected with one another by open bordered pits ${ }^{\text {}}$. In the spring, before strong transpiration sets in, and therefore at a time when the water in the wood is comparatively at rest, the wood-cells also, it is true, contain sap, flowing in quantities out of their communicating cell-cavities when holes are bored in the trunks (as in the Birch, Maple, \&c.). But this sap does not, as is proved by analysis ${ }^{2}$, show a concentration increasing from below upwards. The fact ${ }^{3}$ also that water rises in cut leafy stems placed with their upper end in water though planted and rooted, and flows therefore in a direction opposite to the ordinary one in the stem, shows that endosmose depending on a definite distribution of the concentration of the sap cannot be the cause of the current of water. Since vessels and wood-cells communicating with one another through their open pores form narrow cavities which sometimes become wider as they proceed, sometimes narrower, the woody substance may be represented by a bundle of narrow glass tubes alternately bulging and contracting, in which the water which fills them rises by capillary attraction. But how little efficacious a contrivance of this kind would be is seen at once from the width of the capillary tubes, which is much too great to raise water to a height of roo feet or more. It must also be pointed out that in the summer, when the current of water is strongest, it is principally air and not fluid that is conveyed through the cavities of the cells.

Since it is evident from what has been said that the movement of the water takes place in the woody substance and not in the cell-cavities filled with water, there remain only two hypotheses; viz. (I) that the movement takes place in the water contained

Irish Acad. vol. XXV, 1874 ). Sachs has found that salts of lithium do travel along cell-walls as fast as the water in which they are dissolved. By supplying the roots of plants with a solution of a salt of lithium, he has obtained the following rates at which it travelled in the root and stem :-

\begin{tabular}{|c|c|c|c|c|}
\hline Plants with roots in water. & & & & Rate per hour. \\
\hline Salix fragilis & . & • & • & $85^{\circ} \circ \mathrm{cm}$ \\
\hline Zea Mais & • & - & . & $3^{6 \circ} \circ$, \\
\hline Plants with roots in earth. & & & & \\
\hline Nicotiana Tabacum & . & - & . & I 18.0, \\
\hline Albizzia lophantha & - & - & . & $154^{\circ} \circ$, \\
\hline Musa Sapientum & & • & • & $99^{\circ} 7 "$ \\
\hline Helianthus annuus . & . & . & . & 630, \\
\hline Vitis vinifera & - & • & • & 98.0, \\
\hline
\end{tabular}

In all these cases the plants were under such conditions as to promote transpiration to the utmost. (Sachs, Beitr. z. Kennt. d. aufsteigenden Saftstroms in transpirirenden Pflanzen, Arb. d. bot. Inst. in Würzburg, II. I, 1878 .]

${ }^{1}$ [Sachs has found that in Abies pectinata the bordered pits of the spring-wood are closed (Porosität des Holzes).]

${ }^{2}$ The older statements of Unger are referred to in my 'Experimental-Physiologie ;' others will be found in Schröder, Jahrb. für wiss. Bot. vol. VII. p. 266 et seq.

3 The conduction is however by no means so considerable in the reversed as in the ordinary direction, as Baranetzky found in the laboratory at Wuirzburg; but this may be connected with other peculiarities of the organisation. 
in the lignified cell-walls (or in other words imbibed by them); and (2) that it is caused by a very thin stratum of water which overspreads the inner surface of the wood-cells and vessels ${ }^{1}$. In both cases it must be assumed that the transpiration in the tissue of the leaves causes the upper parts of the wood to contain less water, and therefore to draw up the water from the parts which lie lower. The woody bundles of the roots are surrounded by succulent parenchyma, from which they remove the water; and these again absorb it from the soil by endosmose. It may however be imagined that this movement in the substance of the cell-walls (the contents not participating in it) extends as far as the surface of the parenchyma of the root, where the water contained in the soil is absorbed. The question whether the attraction of the cell-walls for water,-putting aside the question whether it moves in their substance or only on their surface,-is sufficiently powerful to sustain the weight of a column of water of the height of 100 or even 300 feet or more attained by some trees, may be answered without hesitation in the affirmative, since we have to do here with molecular forces in relation to which the action of gravity altogether disappears. But it is another question whether the rapidity of the molecular movements of water of this nature is sufficient to cover the requirements of the foliage of a tree which amounts on a hot day to hundreds of pounds ${ }^{2}$.

The hypothesis finally that the water necessary to supply the loss by transpiration is forced up into the stem as far as the leaves by root-pressure must be abandoned, since this could only operate in the cavities of the wood; and these are always empty in energetically transpiring plants. In the case of tall trees the pressure would also not be sufficient; and if I at one time assumed that this might be a cooperative cause at least in shrubs and annual plants, I must retract this after my observations made in the year 1870 ; since these show that the root-stock of such plants as the Sun-flower, Gourd, \&c., is even subject to a negative pressure when they are transpiring strongly; i.e. does not press water up, but greedily sucks it in at a cut surface above the ground (vide infra) ${ }^{3}$.

The insufficiency of all attempts hitherto made to explain the transpiration-current in the wood is especially noticeable from the fact that it is only under certain internal conditions which cannot be more accurately ascertained that wood is capable of conducting water with the force and rapidity required by the transpiration from the leaves. Woody but air-dry branches with a lower cut surface placed in water are never able.to raise up as much water as is necessary to compensate the evaporation even from an upper cut surface; while the same branch in a fresh state conducts water fast enough to replace the much greater amount of transpiration from the numerous leaves. A change is thus caused in wood simply by drying up which deprives it of the power of conducting water rapidly. The natural alteration which takes place in wood, by which it is transformed as it increases in age into 'duramen'the cell-walls becoming harder and of a deeper colour-also deprives it of this power. If a tree is deprived not only of the bark but also of the 'alburnum' (the light-coloured younger wood on the outside), in an annular zone, the foliage of the tree, according to the statement of different writers, dries up, because the water is not conducted sufficiently rapidly through the duramen.

1 This hypothesis follows from the discoveries of Quincke on capillarity, and has been communicated to me by him. [In consequence of subsequent researches, Sachs is now of opinion that the transpiration-water travels only in the cell-walls of the wood (Ueb. die Porosität des Holzes, Arb. d. bot. Inst. in Würzburg, II. 2, I879).]

${ }^{2}$ See Nägeli u. Schwendener, Das Mikroskop, p. 365 et seq.

3 [This negative pressure is due to the fact that, in consequence of active transpiration, the air which is contained in the vessels of the wood is more rarified, i.e. is at a lower pressure, than that of the atmosphere. Consequently, when the stem of an actively transpiring plant is cut through under water or mercury, the liquid is violently injected into the cavities of the vessels by the atmospheric pressure. (See von Höhnel, in Mittheil. d. k. k. Landwirth ch. Laborat. in Wien, 1877.)] 
Among the most remarkable of the phenomena related to this is the fact that the younger terminal portions of the stems of large-leaved plants partially lose the power of conducting water when cut off in air. If the cut leafy end of the stem of Heliantbus annuus, H. tuberosus, Aristolocbia Sipho, \&c. be placed with the cut section in water, the suction is not sufficient to compensate the evaporation from the leaves, which therefore wither after a shorter or longer time ${ }^{1}$. As I have already shown in the second edition of this book, the withered shoot may in a short time be revived by forcing in water by means of the contrivance represented in Fig. 468. I did not discover till afterwards that the shoot remains turgid even when the pressure is reduced to zero, and even when the mercury is raised up by the suction of the shoot in the same arm of the tube $(q)$, when therefore a force acts on the section of the shoot in the opposite direction. This shows that the forcing in of water is only necessary at first, but that the revived shoot has itself sufficient power of suction to raise up a column of mercury several centimetres in height, and thus to replace the loss by transpiration from the leaves. Thus much was known about the phenomenon of the withering of cut shoots placed in

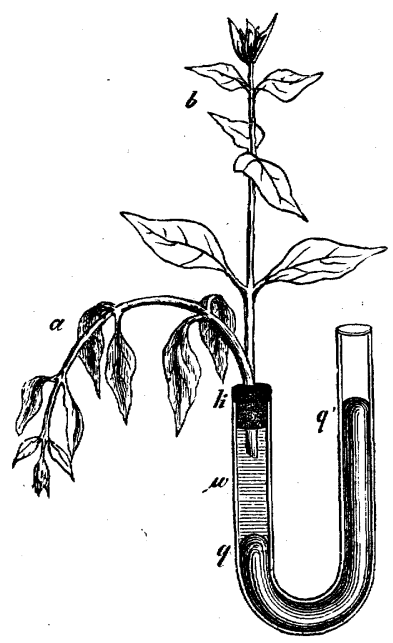

FIG. 468,-Apparatus for showing the revival of withered shoots by forcing water into them. The U-shaped glass tube is first filled with water, and the perforated stopper of caoutchouc $k$ in which the stalk of the plant is inserted is then fixed in. When the shoot is withered, as represented by $a$, mercury is poured into the other arm of the tube, so as to stand at $q^{\prime}$ some 8 or $10 \mathrm{~cm}$. above $q$, and the shoot then revives, as represented by $b$, even when the level $q$ becomes subsequently higher than $q^{\prime}$.

water, when Dr. Hugo de Vries ${ }^{2}$ took up the further investigation of it in the laboratory of the Würzburg Institute. The results obtained by him I will now quote :-

'If rapidly-growing shoots of large-leaved plants are cut off at their lower part which has become completely lignified, and are placed with the cut surface in water, they remain for some time perfectly fresh. But if they are cut through at the younger parts of their stem and are then placed in water, they soon begin to wither, and the more rapidly and completely the younger and less lignified the part where the section is made. This withering can be easily prevented by making the section under water, and taking care that the cut surface does not come into contact with the air, the conduction

1 [Von Höhnel has shown (Bot. Zeitg. 1879) that the rapid loss of conductivity for water shown by branches which have been cut off and placed in water is due to the fact that the contents of the injured cells escape and form a layer on the cut surface; this becomes infested with Bacteria, and these form a membrane (zooglœa) over the surface which prevents the absorption of water.]

${ }^{2}$ [Arb. d. bot. Inst. in Würzburg, I. 3, 1873; Ueb. das Welken abgeschnittener Sprosse.] 
of water through the stem thus suffering no interruption. If care is taken that while the section is being made in the air the leaves and upper parts of the stem lose only a very small quantity of water by transpiration, withering does not begin till later and increases only slowly after the cut surface is placed in water and the leaves again transpire.'

It results from these experiments that the cause of withering is the interruption in the conduction of water from below; and this interruption produces withering not only from the conduction of the water ceasing for a short time, but chiefly also from the power of conducting water in the stem being diminished by the loss of water above the cut surface, which loss cannot be restored simply by placing the cut surface in contact with water.

If the cut surface does not remain too long in contact with the air, the diminution of the capacity for conduction takes place in only a short piece of the stem above the cut. When placing in water ends of shoots which have begun to wither after being cut off, it is only necessary to remove by a new cut a sufficiently long piece above the first cut, but this time beneath the water, for the shoot to revive. In the case of shoots 20 centimetres or more in length which at this distance from the apex are not lignified, the removal of a piece $6 \mathrm{~cm}$. long is usually sufficient to revive the withered shoot (e.g. in Heliantbus tuberosus, Sambucus nigra, Xantbium ecbinatum, \&c.). This experiment proves beyond question that the change, whatever its nature may be, takes place only in this relatively short piece above the cut. That it consists in a diminution of the power of conducting water is shown by the following experiment:-When a sufficient number of the lowest and largest leaves have been removed from a stem of Heliantbus tuberosus cut off in the air and placed in water, and which has begun to wither, the leaves that are left and the terminal bud will after some time begin to revive even without again cutting the stem. The water which is required for the transpiration of a great number of leaves can therefore no longer be conducted through the stem after it has been cut off in air, although that which is wanted for the transpiration of a few leaves can be.

The cause of this phenomenon is therefore a diminution in the power of conducting water in a short piece above the cut surface of the stem. This is evidently occasioned by the loss of water from the cells caused by the suction of the higher parts not being compensated by absorption from below. All circumstances which favour this loss of water increase also the loss of power of conducting it, and cause the shoot which is placed in water to wither more rapidly and completely. It must therefore be assumed that the conducting power of the cells depends on the quantity of water they contain. The probability of this hypothesis is increased by the fact that by artificially increasing the amount of water in the cells of this piece its conducting capacity can also be increased, as is proved by forcing in water from below. If the modified portion is dipped in water of from $35^{\circ}$ to $40^{\circ} \mathrm{C}$., the withered shoots soon revive, and if then placed in water of $20^{\circ} \mathrm{C}$., remain fresh for days (as in the case of the Elder), or at least wither more slowly (e.g. the Artichoke).

(d) Water retained in the wood by Capillary Attraction. If the capillarity of the cavities in the wood must be considered as without any immediate action on the currents of water, this force must nevertheless be taken into account with respect to other processes connected indirectly with the movement of water in the plant. In winter and after long-continued rain in summer a large quantity of water is found in the cavities of the wood together with bubbles of air which occupy the wider spaces. It is not known how this water has reached the higher parts of the trees, though it is possibly by the formation of dew as the temperature varies; it is however to a great extent retained by capillarity. A part of the water flows out in many cases through holes bored in the stem if they are not placed too high, as in the Birch, Maple, Vine, \&c. It may be supposed that the water which flows out has been forced up by the root-pressure which must also be taken into account; though how far up this pressure extends is not yet 
ascertained. The water which is present in the cavities when there is feeble transpiration and which will not flow out of them is clearly retained by capillarity, assisted by the air in the cell-cavities; for Montgolfier and Jamin have shown that in capillary spaces which contain water and air, the water is not easily set in motion. This explains also the phenomenon already mentioned, that water escapes when pieces of wood which have been cut off in cold weather are warmed, because the air expands and forces out the water. Subsequent cooling causes on the contrary water to be sucked in at the cut surface, because the air contracts, and the pressure of the external air forces in water from without.

(e) The ascent of water from the root into the stem ${ }^{1}$. The most important features of this phenomenon have already been briefly mentioned. It is to be observed in the open air in plants of the most different kind, if they possess vigorous root-systems and well-developed wood; as, for instance, in the Birch, Maple, and Vine, and among annual plants, in the Sunflower, Dablia, Ricinus, Tobacco, Gourd, Maize, Stinging Nettle, \&c. In order to study the phenomenon accurately, it is best to grow the plants for some time previously in large flower-pots until they have developed a strong root-system. Landplants such as Maize grown in water and artificially fed by nutrient substances are also well adapted for the investigation. If the stem of such a plant is cut across smoothly 5 or $6 \mathrm{~cm}$. from the ground, and a glass tube fixed to the stump by means of an india-rubber tube, the result will be seen as follows. If the plant was in a condition to transpire freely before it was cut, the cut surface of the root-stump remains at first quite dry, and if water is poured into the glass tube it is at once sucked up ${ }^{2}$. The woody substance of the root-stump has evidently been exhausted by transpiration before the operation, and contains but very little water; not only are its cavities empty, but even the cell-walls of the wood may not be saturated. After a shorter or longer time however the exudation of water at the cut surface begins-rising higher and higher in the tube-and continues from six to ten days if the plant is properly treated, becoming during the earlier part of the time continually more copious, attaining a maximum, and finally diminishing until it ceases with the death of the root-stock. If the cut section is repeatedly dried with blotting paper during the time that the water is flowing, it is clearly seen that the water exudes from the woody tissue-in Monocotyledons from the xylem of the separate bundles-and that it comes principally from the openings of the larger vessels. That the water which flows out had previously been absorbed by the roots out of the ground, and not merely from the store in the rootstock, is at once evident from the fact that the quantity which exudes at the cut section is after a few days greater in volume than the whole of the stock. Under the conditions here described, the water which flows out contains only traces of organic substances in solution; but the presence of mineral constituents can be easily proved, especially lime, sulphuric acid, phosphoric acid, and chlorine, which the plant has absorbed out of the ground. The water which flows in the spring from holes bored in trees such as the Birch and Maple, contains however considerable quantities of sugar and albuminous substances; since the longer stagnation in the cavities of the wood gives it the opportunity of absorbing these substances out of the closed living cells of the wood and out of the surrounding parenchyma, a result which cannot be expected, or only in a smaller degree, in the case of the rapid flow from the smaller root-stocks of quickly-growing plants.

In order to determine the quantity of the outflow, a narrow burette may be used instead of the tube, in which the amount can be read off hourly in cubic centimetres when the outflow is at all considerable. The root-pressure which acts upon the cut surface is however then considerably altered. In order to avoid this, a tube of the

1 See in particular Hofmeister, On the tension and the quantity and rapidity of the flow of the juices of living plants; Flora, 1862, p. 97.

2 This fact is sufficient to prove that the root-pressure has no share in the ascent of the water at the time when transpiration is active. 
form shown in Fig. ${ }_{467} R$ is fixed to the stump, and to it is attached a narrow tube instead of the manometer; the free end of this tube is bent downwards into a graduated burette. If the tubes are from the first filled with water, as much runs into the burette as flows out from the cut section, and the pressure therefore remains constant. From this experiment it has been ascertained that the flow of water varies from day to day, from one time of the day to another, and even from hour to hour; but the causes of these variations in the outflow, which must depend on the activity of the roots, are not yet known; it would even seem as if a periodicity were established independently of the temperature and of the moisture of the ground ${ }^{1}$.

The measurement of the highest pressure at which the outflow can still take place at the cut surface can be effected by the apparatus figured in Fig. 467, where it is expressed by the difference of level of the mercury in the two arms of the tube, or by $q-q^{\prime}$. This will however only afford a measurement of the pressure which the outflowing water may be able to overcome at the cut surface; but it has obviously had also to overcome other resistances of unknown magnitude in the interior of the root-stock. With respect to this point I was interested in ascertaining how great is the difference in the outflow if one of two equal root-stocks has no pressure to overcome at the cut surface, the other a considerable but constant pressure. If, in Fig. $469, a$ indicates the cut stem of a Sunflower or similar plant grown in a pot, $c, d, e$ the tube which is attached to it by the india-rubber tube $b$, and $f$ a glass tube bent downwards, which (not as in the figure) reaches beyond the rim of the pot and terminates in a burette, while the opening of $f$ lies exactly on the level of the cut surface of the stem; then, when the tube $c, d, e, f$ has been filled with water, we have an apparatus for observing the outflow when the pressure at the cut surface is at zero. A second root-stock from a plant of exactly the same age and vigour and grown in a pot of the same size is provided with the apparatus figured in Fig. 469, where the tube $f$ through which the outflow takes place reaches the vessel $b$ through the cork $g$. This vessel contains water in its upper, mercury in its lower part. A tube $k$ rises from the cork $i$ to a certain height and is bent round at the free end $o$ where it dips into a graduated tube. If the apparatus is so contrived that, for example, the opening for the outflow $o$ stands about $15 \mathrm{~cm}$. above the level $n$, then the column of mercury on exercises a pressure of $15 \mathrm{cms}$. on the water $b$, and through it on the cut surface at $b$. When the water begins to flow out from the cut surface at $b$, the quantity of water in $b$ will be increased, and an equal volume of mercury will flow out at $o$. The mercury collects in the burette, and its level enables the quantity of water which has flowed from the cut surface to be read off from hour to hour, and to be compared in the other apparatus where there is no pressure. After a long period of observation, the level $n$ falls sensibly and the pressure on augments a little. But it is easy to bring it again to the original amount if a fresh quantity of mercury is poured in every twelve hours.

1 Very detailed observations on this point were made by Baranetzky in the Würzburg laboratory, in the summer of 1872 . For this purpose he availed himself of the autographic auxanometer described hereafter. The sap flowed from the root-stock into one limb of a long narrow U-shaped tube, in the other limb of which was a float bearing an index which marked the changes of level upon the smoked paper of the rotating cylinder. This method gives very accurate results in so far as the quantity of the sap is concerned. Considerable variations of temperature ( $10^{\circ}$ in 24 hours) affect the flow in such a way that every rise increases, every fall diminishes it. If the variations of temperature are small, a daily periodicity which is independent of such variations can be detected, a maximum and a minimum being attained daily. According to Baranetzky, the time of their occurrence depends upon the periodical exposure of the plant to light during the time preceding the experiment. I am unable to assure myself of the correctness of this conclusion from Baranetzky's experiments. I do not deny the possibility of it, but I reserve my opinion until further experiments have been made. (See Baranetzky, Bot. Zeitg. 1873 , No. 5, and Die Periodicität des Blutens, Abhandl. d. naturfor. Ges. zu Halle, XIII, I873.) 
I observed in this manner in the summer of 1870 for five days two equally strong root-stocks of the Sunflower ${ }^{1}$; and the result was that the difference of the outflow was but small, although the amount of pressure in one case was zero, in the other case $17 \mathrm{~cm}$. of mercury. In the first thirty-three hours the outflow where there was no pressure at the cut surface amounted to 26.45 cubic $\mathrm{cm}$.; when the pressure was $17 \mathrm{~cm}$. of mercury it was $20^{\circ} 9$ cubic $\mathrm{cm}$. A sudden change in the pressure of the mercury of $\mathrm{I}$ or $2 \mathrm{~cm}$. also caused no considerable alteration in the rapidity of the outflow.

Our object now is to form some idea as to the cause of this powerful ascent of water in the wood of the root-stock, and to explain how it happens that the water absorbed at the surfaces of the roots not only passes into the cavities of the wood, but is pressed upwards with so great a force as to be able to overcome a considerable resistance at the

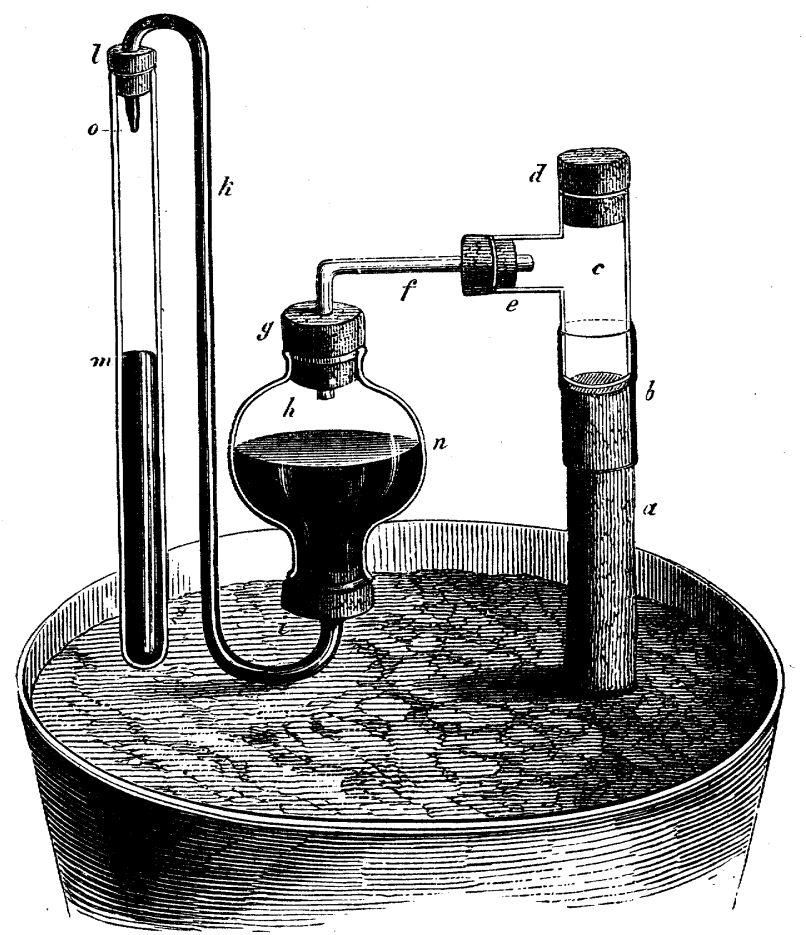

FIG. 469.-Apparatus for measuring the outflow under a considerable and constant pressure. The cork $l$ has a lateral incision in order to allow of the escape of the air when the mercury is dropped in.

cut surface; for it is obvious that the water which flows out above must have been absorbed below at the surfaces of the roots. This absorption can only be induced by the endosmotic action of the parenchymatous cells of the cortex of the root. If we suppose that this endosmotic force is very considerable, these cells will swell greatly; and as much water will filter through the cell-walls into the cavities of the wood as is absorbed from without by endosmose. The parenchymatous cells which are gorged by endosmose drive into the vessels the water which presses into them in consequence of the endosmose, with such force that in flowing out above from the vessels it is still able to overcome a considerable pressure. It follows from this explanation that the pressure which acts at the cut surface must, in accordance with the laws of hydrostatics, be

1 I cannot here describe the whole series of minute observations. 
exerted also against the inside of the vessels which receive the water from the turgid parenchymatous cells. But the water which enters them has also to overcome the resistance to filtration exercised by the cell-walls. The endosmose of the cortical cells of the root must overcome these resistances. Although we do not know the magnitude of the endosmotic force, yet we have ground for supposing that it is much greater than that given by Dutrochet's experiments on animal membranes; and this explanation would therefore be very probable. But a difficulty occurs in answering the question why the turgescent cortical cells of the root expel their water only inwards into the woody tissues and not also through their outer walls. We may however here be helped by the supposition that the micellar structure of the cell-walls is different on the outer and inner sides of the cells, and that those facing the exterior of the root are best adapted to allow endosmose, while those facing the interior of the root are best adapted for permitting filtration under high endosmotic pressure. It must however be observed that this supposition is at present only a hypothesis for the purpose of explaining to a certain extent the processes which take place in the root. The exudation of drops of water from the upper cell of the Fungus Pilobolus crystallinus, from the root-hairs of a Marchantia grown in damp air, \&c., shows moreover that cells distended by endosmotic tension can in fact exude water at certain spots. It is difficult to give any other explanation of the exudation of nectar in flowers; the excreting cells must evidently absorb the water or the sap with great force on one side, and then exude it on the other side. That in this case pressure from the root does not directly cooperate is shown by the fact that this exudation of nectar, which is often very copious, as in the flowers of Fritillaria imperialis and in the pitchers of Nepentbes ${ }^{1}$, takes place even when cut flowers or pitchers are simply placed in water. In this respect these exudations of fluid differ from the exudation of drops on the leaves of many plants, which only takes place when they are still in connection with the root, and which is clearly caused by the forcing power of the root (as in Aroidex, \&c.). It also happens however sometimes that drops of water are exuded from cut surfaces of the tissue, while another cut surface of the organ absorbs water. This I found, for instance, to be the case with pieces of the young stems of different Grasses, cut off from 6 to $10 \mathrm{~cm}$. in length, which were placed with the lower end in damp soil ; the free upper end then repeatedly and continuously exuded drops of water in darkness and in an atmosphere saturated with moisture. Here the parenchymatous cells of the lower cut surface clearly acted as the cortical cells of the root, absorbed by endosmotic action, and probably pressed the water thus absorbed into the vessels, from which it then escaped to the upper cut surface.

$(f)$ The combined action of transpiration, conduction, and absorption of water by the roots takes place under ordinary and favourable conditions in such a manner that nearly as much water is absorbed through the roots and conducted upwards through the wood as is transpired from the leaves. As long as this equilibrium lasts, the plant is turgid and tense in all its parts ; and conversely, it may be concluded from the unaltered turgidity and tenseness of the leaves and internodes that the conduction of water is sufficient to compensate the transpiration by the leaves. Hence, under these conditions, the quantity of water transpired may be taken as the measure of the absorption of the root (or of a cut surface), and conversely the absorption observed as the measure of the

1 Compare Wunschmann's dissertation, 'Ueber die Gattung Nepenthes' (Berlin, 1872 ), where my Handbook only, and not the above taken from the third edition of this work, is quoted. It is questionable if there is any ground for distinguishing between 'excretions' and the sap which escapes from a root-stock in so far as the mechanism of the excretion is concerned, as the older botanists and Wunschmann do. The facts above mentioned render it improbable. They tend to show, on the contrary, that in other vegetable organs, as well as in roots, hydrostatic pressures may be set up which tend to force the fluids out of the tissues. It is a matter of merely secondary importance that 'excretions,' such as nectar and the fluid contained in the pitchers of Nepenthes, are of higher concentration than the sap which escapes from a root-stock. 
transpiration from the leaves. Since however the tissues can be more or less turgid without its being immediately perceptible, transpiration and absorption are not usually exactly equal. But for most observations the small occasional difference may be neglected so long as no actually perceptible amount of flaccidity, i.e. of withering, caused by the collapse of the cells, takes place when the transpiration is stronger and the absorption weaker; or so long as, in the opposite case, no exudation of drops of water results on the leaves of rooted plants. It is only when longer observations are made on growing plants that the comparatively small quantities of water have to be taken into account which are needed for the increase in size of growing organs.

Without going more minutely into the various cases which present themselves ${ }^{1}$, it need only be pointed out in addition that withering is the consequence of the quantity of water transpired being greater than that absorbed through the roots or through a cut surface of the stem. This only occurs in general when the amount of transpiration is very considerable, or when the ground is very dry, or when in cut shoots the power of the stem to conduct water has ceased. The exudation of drops of water already mentioned is, on the other hand, the consequence of a smaller quantity of water evaporating from the leaves than is absorbed by the roots and forced up into the upper organs. If a branch of a Potato-plant, a leaf of an Aroid, a cut stem of Maize, or the like, is fixed in the cork $k$ in Fig. 468 , and if, when the transpiration is weak a pressure of mercury of 10 or $12 \mathrm{~cm}$. is allowed to act for some time, drops of water appear at the same spots on the apices or margins of the leaves, where they would appear in plants with roots in the evening or night or in damp weather. In the same manner the exudation of drops from plants with roots can be produced or increased by warming the ground and covering the leaves with a bell-glass in order to hinder evaporation ${ }^{2}$.

The pressure due to the root which is so conspicuous in stems when cut across and when the amount of evaporation is very small, can scarcely be of any considerable use in promoting the current of water in the wood caused by strong transpiration. The fact already mentioned that strongly transpiring plants suck up water at the cut surface of their stems immediately after the upper part has been cut off, shows that the propelling force of the root does not act sufficiently quickly to protect even the vessels of the root-stock of strongly transpiring plants from complete exhaustion; that is, although the force which drives the water into the root-stock is great, as we have seen, it acts too slowly to be taken into account when the transpiration is rapid.

The same conclusion is reached if the quantity of water which exudes in the same time from the cut stem of a plant above the root is compared with that which is absorbed at the lower cut surface by the upper part of the same plant. The absorption of the upper part is always much more considerable in amount than the outflow from the root-stock, even when the withering of the upper part indicates that the capacity of its wood for conduction has diminished, and that it absorbs less than it would absorb in the normal condition. Thus, for example, the water absorbed by the cut leafy top of a Tobacco-plant amounted in five days to 200 cubic $\mathrm{cm}$., while the root-stock exuded only 15.7 cubic cm. In the same manner in Cucurbita Pepo (when much withered) the amount absorbed was $\mathrm{I}_{4}$ cubic $\mathrm{cm}$., the exudation from the root-stock only $\mathrm{Ir}^{\circ} 4$ cubic $\mathrm{cm}$. The withered upper part of a Sunflower absorbed in a few days 95 cubic $\mathrm{cm}$., while the root-stock exuded only $5^{2^{\circ}}$ cubic $\mathrm{cm}$. The result is also the same when the relative amounts which extend over a shorter time are compared ${ }^{3}$.

1 See Rauwenhoff, Phytophysiologische Bijdraden in Verslagen en Mededeelingen der kon. Akad. van Wetens., Afdeeling Natuurkunde, $2^{\text {do }}$ Reeks, Deel III, 1868, where however the indispensable thermometric observations are wanting.

2 The exudation of drops on the margins of the leaves of plants, the roots of which are surrounded by damp warm earth, their foliage rising into moist air, is a very common phenomenon, as I know from the experience of many years.

3 For a more complete account see Arb. d. Bot. Inst. in Würzburg, I. 3, 1873 . 
It follows from these facts that, with the exception of times when the amount of transpiration is small or when drops of water exude from the leaves, no root-pressure at all exists when the plant is uninjured; and that this pressure can only be detected when transpiration and absorption have ceased or when they are very small. The exhaustion of the root-stock of a strongly transpiring plant (as after it has been cut off) proves rather that a plant with roots behaves in exactly the same way as a cut shoot. Just as the latter absorbs water from a receiver, so the wood of the root-stock which has lost water in consequence of transpiration above absorbs water from the cortical cells of the root which obtain it by endosmose. From all this it still remains in doubt whether in such cases the contents of the cortical cells of the root must not be left altogether out of consideration, since it is possible that the conduction of water by the cell-walls alone, reaches as far as the surface of the roots.

(g) The parts of land-plants which are covered with a cuticle and in which transpiration takes place appear to have no power of absorbing in any considerable quantity the water by which they are moistened, such as the rain and dew which is deposited on the leaves. As long as the tissues and leaves of uninjured plants with roots become turgid and are supplied with water from below, any considerable absorption through the surfaces of the leaves themselves, even when they are thoroughly wetted ${ }^{1}$, is not to be expected, since it is not easy to see where the water can go in cells that are already gorged $^{2}$. But even when a rooted plant has withered, it is still doubtful whether the revival which takes place when its leaves are wetted depends on the absorption of water by the leaves, since it is not impossible for an upward pressure to take place subsequently. Greatly withered shoots do not become turgid when placed in water, or do so only very slowly unless the cut surface is immersed, and even in this case there is doubt as to the absorption of water through the surfaces of the leaves.

In harmony with this Duchartre found also ${ }^{3}$ that rooted plants (Hortensia, Heliantbus annuus), which withered in the evening in consequence of the dryness of the earth in the pot, did not recover or become turgid if copiously moistened by dew during a whole night, the pots in which the roots spread being provided with a closed cover. Epidendral Orchids, Tillandsias, \&c. behave in the same way in this respect; they also absorb neither water nor aqueous vapour through their leaves, nor even in any considerable quantity through the roots. The water which they require for their transpiration and growth must be conveyed to them in the form of rain or dew which moistens the root-envelope (velamen) or wounded surfaces ${ }^{4}$.

When land-plants wither on a hot day and revive again in the evening, this is the result of diminished transpiration with the decrease of heat and increase of the moisture in the air in the evening, the activity of the roots continuing-not of any absorption of aqueous vapour or dew through the leaves. Rain again revives withered plants not by penetrating the leaves, but by moistening them and thus hindering further transpiration, and conveying water to the roots, which they then conduct to the leaves.

A simple experiment will afford much instruction to the student in these matters. The pot in which a leafy plant is growing is enclosed in a glass or metal vessel provided

1 On this subject see my Experimental-Physiologie, p. I 59.

2 Duchartre has neglected this obvious reflection in his researches (Bulletin de la Soc. Bot. de France, Feb. 24, 1860); in other respects also these experiments are very defective.

3 Duchartre, l.c. 1857 , pp. 940-946.

4 Duchartre, Expériences sur la végétation des plantes epiphytes (Soc. Imp. et centrale d'horticulture, Jan. 1856 , p. 67 ; and Comptes Rendus, I868, vol. I.XVII. p. 775). [This subject has been recently investigated by Detmer (Theorie des Wurzeldrucks, 1877), by Boussingault (Les fonctions physiques des feuilles, Agronomie, VI, I878), and by Henslow (On the Absorption of Rain and Dew by the green parts of plants, Journ. Linn. Soc. XVII, r879). It appears that leaves, like all other parts of plants, will absorb water if they are immersed in it long enough, but there is no evidence that the absorption of water, either as vapour or as liquid, is in any sense one of their functions.] 
above with a lid in two portions, and surrounding the stem so as completely to cover the earth in the pot. If the soil is dry the plant withers. If a bell-glass is placed over it the plant revives, and again withers if it is removed. This shows that the withering is the result of increased, the revival the result of diminished evaporation from the leaves when the roots convey but very little water to the plant. If cut shoots are allowed to wither and are then suspended in air nearly saturated with aqueous vapour, the leaves and younger internodes again revive, although the whole shoot continues to lose weight from evaporation. This phenomenon results from the water passing from the older parts of the stem to the younger withered parts, as must be concluded from Prillieux's experiments ${ }^{1}$.

Sect. 3.-Movements of Gases in Plants'. All growing cells of a plant, or all that are otherwise in a condition of vital activity, are continually absorbing atmospheric oxygen and giving back in its place a nearly equal volume of carbon dioxide. The cells which contain chlorophyll have in addition the property, under the influence of sunlight, of absorbing carbon dioxide from without, exhaling at the same time a nearly equal volume of oxygen mixed with some nitrogen. In proportion to the activity of the chemical processes which take place within the cells, the movements of gases occasioned by them vary greatly in rapidity. The formation of carbon dioxide at the expense of the atmospheric oxygen takes place continuously and in all the cells; but the quantities concerned are small in proportion to the large amount of carbon dioxide which is decomposed in the green tissues, and in exchange for which equal volumes of oxygen are exhaled. Some idea of the activity of this last-named process is obtained by reflecting that about one-half the (dry) weight of the plants consists of carbon which has been obtained by the decomposition of atmospheric carbon dioxide in tissues containing chlorophyll under the influence of light.

Oxygen and nitrogen are permanent gases, as also is carbon dioxide within the limits of the temperature of vegetation, and indeed far below it. Aqueous vapour, on the contrary, is only produced from water within these limits, and under certain conditions even returns to the liquid state. In other respects aqueous vapour behaves just like oxygen and nitrogen in reference to the processes to be considered here.

When the gases with which we have to do are traversing closed cell-walls, diffusing themselves through the cell-sap, or permeating or escaping from the protoplasm, chlorophyll-granules, \&c:, their motion is a molecular one of diffusion. When they fill in their elastic condition the intercellular spaces, vessels, cells destitute of sap, or the large air-cavities among the tissues, it is a movement of the whole mass depending exclusively on expansive force. The movements of diffusion tend to bring about conditions of equilibrium which depend on the coëfficient of absorption of the gas by a particular cell-fluid, on the composition of the cell-wall, \&c. on temperature, and on the pressure of the air. But these conditions are continually varying; and the equilibrium which is aimed at is being still more continually disturbed by chemical changes on which depend the metamorphosis

1 Prillieux, Comptes Rendus, 1870 , vol. II. p. 80.

${ }^{2}$ Sachs, Handbuch der Experimental-Physiologie, p. 243-Müller, Jahrb. für wiss. Bot. vol. VII. p. I 45 . 
of substances in the plant, assimilation, and growth; so that a state of rest can very seldom occur. The ordinary condition of the gases which are diffused through the cells of plants is that of movement.

But even the masses of gas found in the cavities of plants are not generally at rest. By the setting free or absorption of carbon dioxide or oxygen in the cells, the equilibrium is disturbed also in the neighbouring cavities; and changes in the pressure of the air or in temperature also exert an influence. The flexions again of the stem and leaf-stalk produced by the wind cause compressions and dilatations of the gases which fill the cavities, and these again give rise to currents of gas in the interior. The rapidity of the movement in the cavities varies greatly in proportion to their size ; within the very narrow intercellular spaces of ordinary parenchyma the motion is slow and inconsiderable even under considerable pressure, as contrasted with the rapid currents which are possible in the large intercellular spaces of most foliage-leaves and similar organs, or in the wide air-canals of hollow stems, or in the lacunæ of the tissue of water-plants.

In attempting to collect the most common phenomena into a more definite arrangement from this general point of view, the following appear to be the more important points.

(a) Unicellular plants, as well as those which consist merely of filaments or plates of cells such as occur in Algæ, Fungi, and Mosses, are in immediate contact with the air or with the surrounding water which contains gas in solution. The only essential condition here is that the gases shall be able to enter and escape from the cells by diffusion. If, for example, a cell of this kind containing chlorophyll is placed in sunlight, the carbon dioxide absorbed by it is decomposed; a fresh supply of the gas is therefore continually penetrating into it from without, because it is prevented from saturating the cell-sap; oxygen, on the contrary, is being constantly disengaged, the cell-sap receives more than it can contain, and gives off the excess by outward diffusion. Under these conditions therefore two molecular currents are set up in opposite directions which permeate the cell-wall, the protoplasm, and the cell-sap; and since carbonised products are formed in the cell at the expense of the decomposed carbon dioxide, this decomposition is the simultaneous cause of fresh quantities of the gas perpetually diffusing into the cell. The quicker the decomposition of the carbon dioxide, the more quickly is it replaced. The conditions are similar in cells containing chlorophyll when in darkness and in cells destitute of chlorophyll, though the chemical process is different; they absorb oxygen and produce carbon dioxide; only the process is much slower and less active. The cell acts as a centre of attraction for the gas which is decomposed in it, and as a centre of repulsion for the gas which is produced in it. This rule holds good also for the individual cells of a tissue, only that in this case the processes are more complicated, inasmuch as the diffusion currents of the gases do not take place between the cells and an unlimited external volume of gas, but between cells and cells on the one hand, between cells and internal air-cavities of limited size on the other hand.

(b) Among plants consisting of complicated aggregates of cells, submerged Waterplants are of peculiar interest, because their intercellular spaces do not open outwardly through numerous stomata, but communicate with large cavities which are formed in the interior of the tissues by the disjunction of cells or by the rupture of their walls. The underground stems of Equisetum and of many bog-plants show a similar structure. Uninjured plants of this kind are closed and air-tight outwardly; the gases which collect in the cavities can originate only from the surrounding tissues, which absorb oxygen, nitrogen, and carbon dioxide by diffusion from the surrounding water. These gases cannot simply diffuse through the surrounding tissues, but they undergo change within them, and when once collected in the spaces they are still further influenced by 
the chemical processes that go on in the surrounding tissues. A submerged water-plant, for example, which contains chlorophyll, absorbs carbon dioxide from without under the influence of sunlight; and at least a portion of the disengaged oxygen collects in the cavities. When it becomes dark this process ceases; the collected oxygen is now absorbed by the fluids of the tissue and gradually transformed into carbon dioxide, which can again diffuse back into the cavities, but partially also through the layers of tissue into the surrounding water. This, as well as the different coëfficients of diffusion of the gases, causes the air contained in the cavities to have an altogether different composition from that in solution in the surrounding water, and this composition to be subject to continual change. But it is not only the chemical composition of the gas in the cavities that is altered in this way; the pressure is also subject to variation. When the oxygen which is liberated from the green tissues collects rapidly in the cavities under the influence of bright light, the gas is then subject to high pressure, and escapes with force, injuring the surrounding layers of tissue.' The greater rapidity of diffusion of carbon dioxide, and its slower production in the tissue in darkness, do not, on the other hand, allow an increase of tension of the gas to arise easily in the cavities of the plant when kept in the dark.

The nitrogen of the atmosphere takes a more subordinate and secondary part in all these processes. It is indeed never absent from the air contained in the cavities, but is generally present in large quantities in it, together with oxygen and carbon dioxide. It is not however subject to such rapid and considerable variations, being neither used up nor disengaged in the changes connected with the assimilation of food in the tissues.

(c) Land-plants differ from water-plants in that their internal cavities, when present ${ }^{1}$, communicate directly with the atmosphere through the stomata. The anatomical conditions show at once that these organs are only the channels of exit from the intercellular spaces which are in connection with one another through the whole plant; and we know from experiment that these are in their turn in complete connection here and there with the cavities of the vessels and with the wood-cells ${ }^{2}$. The large air-cavities which are abundant even in land-plants (in hollow stems, leaves, fruits, \&c.), the woody tubes (or vessels) and wood-cells, and the usually extremely narrow capillary intercellular spaces of the parenchyma, form therefore a system of cavities full of air and in communication with one another, which are all closed below at the root, but which open outwardly above in the leaves, internodes, \&c., through numberless extremely narrow capillary openings.

What was said in paragraph $b$ on the changes which take place in the air contained in the cavities of water-plants, applies in general also to that of land-plants; but the equalising of the difference in the pressure at the various parts of a large plant is facilitated by the occurrence of vessels, that of the difference between the internal and external air by the stomata. This equalisation however proceeds in general extremely slowly, because the stomata, in consequence of their small diameter, can allow only small volumes of gas to pass through them in a short time. Notwithstanding their uninterrupted connection, there may therefore be considerable differences of pressure and great variations in the composition of the internal and external gas, as in water-plants. It must also not be forgotten that those layers of tissue in which a rapid interchange of gases is proceeding are covered with an epidermis containing a greater number of

1 Large Fungi and Algæ have indeed no stomata; but their internal air (among the hyphæ) is certainly in communication at least in places with the surrounding air by cavities among the superficial hyphæ. The stems of Mosses possess neither internal cavities nor stomata, while their sporecapsules possess both.

2 [The fact to which allusion has been made on p. 682, that namely the air in the vessels of an actively transpiring plant is at a lower pressure than that of the atmosphere, proves that the cavities of the vessels do not communicate with the intercellular spaces. If they did so communicate, such a difference of pressure could not possibly arise.] 
stomata than those which require a less active interchange in consequence of slower growth and assimilation. In addition to this, organs with a thin cuticle are better adapted to bring about interchange of gas by diffusion than those whose epidermis is provided with a thicker cuticle which hinders the diffusion-current. This is clearly the reason why roots require no stomata, since, in consequence of their slow increase in size and their thin-walled slightly cuticularised epidermis, they can accomplish the interchange of oxygen and carbon dioxide by diffusion alone; while the leaves, in consequence of their thick cuticle, require a large number of stomata in order rapidly to interchange large volumes of carbon dioxide with as large volumes of oxygen in sunshine. Even flowers and rapidly growing parasites which contain no chlorophyll possess stomata, though in smaller numbers, because they absorb a quantity of oxygen and exhale carbon dioxide. When the epidermis is replaced in the older parts of stems and roots by corkperiderm, the parts are not only externally impervious to air (with the exception of occasional fissures) in the ordinary sense, but even the interchange of gas by external diffusion practically ceases. But this case occurs only in those parts of plants where the fibro-vascular bundles form air-conducting vessels and usually also air-conducting woodcells, by means of which an interchange of gas is brought about internally with that contained in the parenchyma enveloped by the cork. This is especially the case with woody Dicotyledons and Conifers.

These considerations apply also to a great extent to aqueous vapour. The evaporation of the water of vegetation, resulting, as we have seen in the previous paragraph, in the production of currents in the plant, is almost entirely prevented by cork-periderm and bark, and at least very much hindered by cuticularised epidermal cells. Since the parts of plants exposed to the air are covered with one or other of these epidermal structures, evaporation can in general only take place to a subsidiary extent from their surface; the greater part of the aqueous vapour which these parts of the plant lose is evidently given off from the moist cell-walls in the interior of the tissue where they adjoin intercellular spaces and larger air-cavities. If these spaces are saturated with aqueous vapour, evaporation ceases; but if the external air is comparatively dry the vapour escapes through the stomata, and evaporation into the intercellular spaces recommences. If the transpiring tissue is heated, as by sunshine, the formation of vapour proceeds more rapidly in the interior, and the greater tension of the vapour causes its more rapid passage through the intercellular spaces and stomata.

The surfaces of the organs of plants which are constantly in contact with water cannot exhale aqueous vapour through such fine openings as the stomata under the existing conditions of temperature ; stomata are therefore wanting in submerged plants, or occur only occasionally. The leaves, for instance, of Water-Lilies, which float on the water, are especially instructive in this respect; on the side in contact with the water they have no stomata or very few, on the upper side exposed to the air a large number. This is the more striking since leaves entirely exposed to the air have generally a larger number of stomata on the under than on the upper side, where they are sometimes entirely wanting. 


\section{CHAPTER II.}

\section{CHEMICAL PROCESSES IN THE PLANT.}

SECT. 4.-The Elementary Constituents of the Food of Plants ${ }^{1}$. If we dry at a temperature of $100^{\circ}$ or $110^{\circ} \mathrm{C}$. any fresh vegetable structure so as to expel all the water which it contains, a friable residue will be left which no longer loses weight. In ripe seeds this usually amounts to about $\frac{8}{9}$ of the weight; in seedlings after the supply of reserve-material has been consumed, to generally less than $\frac{1}{10}$, increasing in subsequent stages of vegetation to $\frac{1}{5}$ or $\frac{1}{3}$; in submerged water-plants and Fungi it often amounts to less than $\frac{1}{10}$, and sometimes even to only $\frac{1}{20}$. These proportions, which are only roughly estimated, vary within wide limits according to the nature and age of the plant and of the particular organ.

If the dried residue of the plant is further exposed to a red heat in the presence of oxygen, by far the greater part of it is consumed and disappears in the form of products of combustion, chiefly carbon dioxide and aqueous vapour. The residue which now remains behind, usually a fine white powder, is the $A s h$, constituting generally only a small percentage of the dried substance, a proportion which is again subject to great variations with the specific nature of the plant and the kind and age of the particular organ.

Chemical analysis of the combustible.part of the dried substance shows that it consists in all plants of Carbon, Hydrogen, Oxygen, Nitrogen, and Sulphur; the latter remains behind after combustion in the form of sulphuric acid in combination with the bases of the ash.

In the ash are invariably found in addition Potassium, Calcium, Magnesium, Iron, and Phosphorus, and generally Sodium (Lithium?), Manganese, Silicon, and Chlorine; in marine plants also Iodine and Bromine. With these constituents there are sometimes associated, in rare cases and under special circumstances, very small quantities of Aluminium, Copper, Zinc, Cobalt, Nickel, Strontium, and Barium. The presence of very small quantities of Fluorine in plants is also inferred from the presence of calcium fluoride in the bones of animals which obtain the whole of their food directly or indirectly from plants.

${ }^{1}$ For a preliminary acquaintance with the very copious literature, my Handbuch der Experimental-Physiologie (Sects. 5 and 6) will be sufficient. A study of Th. de Saussure's Recherches chimiques sur la végétation, Paris 1804 , is also indispensable to any one who wishes to form an independent judgment for himself. A detailed description of the theory of nutrition is contained in, among other works, Mayer's Lehrbruch der Agriculturchemie, 1876. A variety of fundamental researches will also be found in Boussingault's Agronomie et Physiologie végétale. E. Wolff's Aschenanalyse für landwirthschaftliche Prod. \&c., Berlin $187 \mathrm{I}$, is also very valuable; as well as his Vegetationsversuche in wässerigen Lösungen ihrer Nährstoffe (Hohenheimer Jubiläumsschrift, 1862). 
It is self-evident that we have only to consider as elementary food-substances those which are indispensably necessary for the process of nutrition; while those the presence of which in the plant is proved by analysis, but which may also be absent without its nutrition being impaired; may be considered as accidental admixtures.

Of the first importance among the indispensable food-materials are the elements of the combustible substance which are present in all plants without exception, viz. Carbon, Hydrogen, Oxygen, Nitrogen, and Sulphur; because they are included in the chemical formula of cellulose or of the albuminoids which constitute protoplasm, and because therefore without these substances the plant-cell itself could not exist. It may be inferred also from the invariable presence of Potassium, Calcium, Magnesium, Iron, and Phosphorus in plants, that they are indispensable constituents of their food, and still more from the fact established by actual experiment, that the nutrition and growth of all plants hitherto examined for this purpose is impossible or abnormal if any one of these elements is wanting. In the case of Sodium, Manganese, and Silicon this has not yet been proved; it would appear rather that they may be dispensed with in the chemical process of nutrition. That Chlorine is necessary for the perfect nutrition of Polygonum Fagopyrum has been shown by Nobbe ${ }^{1}$. Whether Iodine and Bromine play the part of true food-materials in the marine plants in which they are found has not yet been ascertained; and these two elements may, from the mode of their occurrence, be for the present neglected as unimportant in this respect.

In the more general considerations as to the nutrition of plants we have therefore chiefly to do with the following elements :-

Carbon, Hydrogen, Oxygen, Nitrogen, Sulphur ;

Potassium, Calcium, Magnesium, Iron;

Phosphorus, Chlorine;

to which are to be added, under certain circumstances, Sodium and Silicon.

The physiological importance of these elementary substances is however very different. Those placed in the first line compose, as already mentioned, the greater part of the substance of the plant; they mainly form the organised and organisable part of the plant and of every individual cell ; their importance therefore lies in the fact that they furnish the chief materials for the construction of the plant. The constituents of the ash, on the other hand, are of less importance in this respect, if only in consequence of their much smaller quantity; they appear to promote chemical decompositions and combinations in plants, in consequence of which the far more abundant combustible principles are constructed out of the first-named five elements.

Carbon is a necessary constituent of every organic compound in varying proportion; usually about one-half the weight of the entire dried substance of the plant consists of this element. If the large quantity of vegetable matter which is annually produced is taken into account, the fact becomes the more remarkable that this enormous quantity of carbon is derived from the carbon dioxide of the atmosphere of which it forms on the average only about 0.04 per cent. It is only the cells which contain chlorophyll-and these only under the influence of sunlight-that have the power of

${ }^{1}$ Landwirthschaftliche Versuchsstationen, vol. VII, 1865. 
decomposing the carbon dioxide taken up by them, and at the same time of setting free an equal volume of oxygen, in order to produce organic compounds out of the elements of carbon dioxide and water, or in other words to assimilate. It is very probable that under these circumstances carbon dioxide loses only one-half its oxygen, while the other half of the oxygen which is exhaled is derived from the decomposition of water.

The fact is unquestionable-partly established by direct researches on vegetation, partly inferred from the circumstances under which many plants live in a natural condition - that most plants which contain chlorophyll (e.g. our cereal crops, Beans, Tobacco, Sunflower, many saxicolous Lichens, Algæ, and other water plants) obtain the entire quantity of their carbon by the decomposition of atmospheric carbon dioxide ${ }^{1}$, and require for their nutrition no other compound of carbon from without. But there are also plants which possess no chlorophyll and in which therefore the means of decomposing carbon dioxide is wanting; these must absorb the carbon necessary for their constitution in the form of other compounds. But since plants destitute of chlorophyll are either parasites or saprophytes, they absorb their carbon in the form of organic compounds which have been produced by other plants that contain chlorophyll. Parasites draw these products of assimilation directly from their hosts, while saprophytes (as Neottia Nidus-avis, Epipogium Gmelini, Corallorhiza innata, Monotropa, many Fungi, \&c.) make use for the same purpose of the materials of other plants which are already in a state of decomposition. Even the food of Fungi which are parasitic in and on animals is derived from the products of assimilation of plants containing chlorophyll, inasmuch as the whole animal kingdom is dependent on them for its nutrition. The compound of carbon originally present on the earth is the dioxide, and the only abundantly active cause of its decomposition and of the combination of carbon with the elements of water is the cell containing chlorophyll. Hence all compounds of carbon of this kind, whether found in plants or in animals or in the products of their decomposition, are derived directly or indirectly from the organs of plants which contain chlorophyll.

Hydrogen is present, equally with carbon, in every organic compound; in consequence however of the smallness of its combining equivalent, it falls far. below it as a percentage constituent of the weight of the dried substance of plants. As has already been mentioned, the hydrogen of the plant is probably derived from the decomposition of water in cells containing chlorophyll in the presence of sunlight. It probably enters into combination with the carbonic oxide (CO) simultaneously presented to it by the reduction of the carbon dioxide ${ }^{2}$.

1 [From the researches of Moll it appears that the roots take no part in supplying the plant with carbonic dioxide (Moll, Die Herkunft des Kohlenstoffs der Pflanzen, Arb. d. bot. Inst. in Würzburg, II. I, 1878).]

2 [The abstract of Adolph Baeyer's paper on the Chemistry of Vegetable Life in Journ. Chem. Soc. $187 \mathrm{I}$, pp. $33 \mathrm{I}-34 \mathrm{I}$, should be consulted. It is shown to be probable that chlorophyll fixes carbon oxide just as hæmoglobin does. When sunlight falls upon chlorophyll which is surrounded by carbon dioxide, that compound seems to suffer the same dissociation as at high temperatures, oxygen is liberated and carbon oxide remains combined with the chlorophyll. The simplest reduction of carbonic oxide is to formic aldehyde; it need only take up hydrogen, $\mathrm{CO}+\mathrm{H}_{2}=\mathrm{COH}_{2}$, and under the influence of the cell-contents, just as by the action of alkalies (which Butlerow has shown to be the case), the aldehyde is transformed into sugar.] 
Only a very small portion of the hydrogen contained in the nitrogenous vegetable substances can be supplied to the plant in the form of ammonia.

Oxygen is always present in organic compounds in smaller quantities than would be sufficient to oxidise the hydrogen and carbon present in them into water and carbon dioxide, because organic compounds are produced from carbon dioxide and water with the elimination of a part of their oxygen. The proportion of oxygen in vegetable substances is moreover very variable; and some even contain none at all of this element. But the total quantity of oxygen forms, next to carbon, the largest proportion of the weight of the dried substance. Oxygen is introduced into the plant in the form of water, carbon dioxide, and oxy-salts, in larger quantities than any other element; while extraordinarily large quantities of oxygen are set free into the air by the process of assimilation in the green organs. All the other organs of the plant also absorb atmospheric oxygen, and thus slowly reproduce carbon dioxide and water at the expense of the assimilated substances. Together with the process of deoxidation which is very active in the cells containing chlorophyll, another process of oxidation is proceeding comparable to that of the respiration of animals, but not generally very active, by which a part of the assimilated substance is again decomposed.

Nitrogen, an essential constituent of the albuminoids which form protoplasm, of vegetable alkaloids, and of asparagine, always forms only a small fraction of the weight of the dried substance of plants, - often less than $\mathbf{r}$, seldom more than 3 p. c. The nitrogen contained in the chemical compounds just mentioned is obtained from compounds of ammonia and nitric acid ${ }^{1}$; parasites and saprophytes perhaps also absorb organic nitrogen-compounds from without. It is on the other hand certain from a great number of experiments on vegetation, especially those of Boussingault, that plants have no power of using the free nitrogen of the atmosphere for the production of their nitrogenous compounds ${ }^{2}$. If plants are artificially supplied with all other food-materials, but it is rendered impossible for them to absorb ammonia or compounds of nitric acid as their source of nitrogen, no increase takes place of the albuminoids or of the nitrogenous substances generally, although the nitrogen of the atmosphere is at the command of the plant in such great quantities, filling up the intercellular spaces and diffusing through the fluids of the tissue ${ }^{\text {? }}$.

Sulphur, a constituent of albuminoids, of allyl, and of the essential oil of mustard, is taken up in the form of soluble salts of sulphuric acid, and chiefly

1 [Although plants containing chlorophyll usually absorb their nitrogen in the form of ammonia or nitrates, they can nevertheless absorb it also in the form of organic compounds such as urea, leucin, tyrosin, creatin, hippuric acid, and similar bodies; they cannot absorb it in the form of alkaloids.]

${ }^{2}$ [The important researches of Lawes, Gilbert, and Pugh on the sources of the nitrogen of vegetation (Phil. Trans. I86I, pt. 2, and Journ. Chem. Soc. 1863, p. I00) should be carefully studied on this point.]

${ }^{3}$ [Adolf Mayer has recently carried out a number of experiments to determine whether the aërial parts of plants have the power of absorbing ammonia or not. Preventing access of ammonia through the roots, he subjected the leaves to the influence of ammonium carbonate both in the gaseous and dissolved state, and found that it was absorbed in appreciable quantities, although the plants did not appear to thrive when access of ammonia through the roots was entirely prevented. Similar results have also been obtained by T. Schlosing (see Comptes Rendus, vol. LXXVIII. p. 1700).] 
(or perhaps always) of calcium sulphate. This salt is probably, as Holzner first pointed out ${ }^{1}$, decomposed by the oxalic acid which is formed in the plant itself, and the insoluble calcium oxalate is thus formed, while the sulphuric acid parts with its sulphur to form the organic compounds which have been mentioned.

Iron $^{2}$ (often accompanied by very variable quantities of Manganese) is indispensable for the production of the green colouring substance of chlorophyll, as is shown by experiments on vegetation; and since the green organs which contain chlorophyll form organic substances out of water and carbon dioxide, the importance of this element for the life of the plant is very evident, although extraordinarily small quantities of it are sufficient for this purpose. It may be taken up by the plant in the form of the chloride or sulphate or of some other compound. If larger quantities of solutions of iron become distributed through the tissues, the cells quickly die. Although small quantities of iron are essential for producing the green colour of chlorophyll, it is nevertheless uncertain whether the green colouring substance itself contains iron as an integral constituent of its chemical formula ${ }^{3}$.

Potassium is as essential for the assimilating activity of chlorophyll as iron for its production. Nobbe ${ }^{4}$ has recently shown that if food-materials otherwise complete but possessing no potassium are supplied to plants (as Buckwheat), they behave as if they were absorbing only pure water instead of the solution of foodmaterial. They do not assimilate and show no increase in weight, because no starch can be formed in the grains of chlorophyll without the assistance of potassium. The chloride is the most efficacious form in which potassium can be offered to Buckwheat; the nitrate comes next to it. If the potassium is offered only in the form of sulphate or phosphate, a very evident sickliness is apparent sooner or later, which results from the starch which is formed in the grains of chlorophyll not passing into the growing organs and thus becoming available for purposes of vegetation. Sodium and Lithium cannot replace potassium physiologically, because the former is simply useless to the plant, while the presence of the latter in the cell-sap is injurious to the tissues.

Phosphorus, Chlorine, Sodium, Calcium, and Magnesium have, as far as is yet known, no definite relation to special physiological purposes. The constant occurrence however of compounds of phosphoric acid in company with albuminoids, as well as of potassium salts in organs containing starch and sugar, points towards definite relations which they may possess to those chemical processes that immediately precede the processes of construction in plants. A large part of the calcium taken up by plants is, as has been mentioned, precipitated by oxalic acid, and remains inactive. The importance of calcium must therefore be sought partly in its serving as a vehicle for sulphuric and phosphoric acid in the absorption of

${ }^{1}$ Holzner, Ueber die Bedeutung des oxalsauren Kalkes, Flora, 1867.-Hilgers, Jahrb. für wiss. Bot. vol. VI. p. I.

${ }^{2}$ For special proof of the importance of iron see my Handbuch der Experimental-Physiologie, p. $\mathbf{I 4 2}^{2}$.

$s$ [On the chemical composition of chlorophyll, see infra.]

4 Nobbe, Schröder, and Erdmann, Ueber die Organische Leistung des Kaliums in der Pflanže; Chemnitz, I87r. 
food-material, and partly in its fixing the oxalic acid which is poisonous to the plant, and rendering it harmless. The elements just named are taken up by the plant when they are offered to it in the form of phosphates, sulphates, nitrates, or chlorides.

Silicon finally is taken up by a very large number of plants in the form of a very dilute aqueous solution of silicic acid; by some in larger quantities than all the other constituents of the ash. By far the larger part of the silicic acid passes into the insoluble state within the cell-walls, and remains behind after the destruction of its organic substance together with calcium (magnesium and potassium?) as a skeleton possessing the structure of the cell-wall. In land-plants it accumulates chiefly, though not exclusively, in the tissues exposed to evaporation, and especially in the cuticularised walls of the epidermis. In Diatoms, the cell-wall of which is very strongly silicified, this arrangement of course does not exist. Since it is possible to cause, by artificial feeding, plants which usually contain abundance of silica (like Maize) to grow almost entirely without it, and without any obvious departure from their normal structure, silicic acid appears to be of very subordinate importance for the chemical and organic processes; and its deposition in the cell-walls does not take place to any great extent until they are already fully developed.

The compounds serving as food-material must be subject within the tissues to progressive changes of position in addition to and in consequence of their chemical transformations. The equilibrium of diffusion is disturbed by the decomposition of a salt; immediately round the spot where this takes place the fluid of the tissue contains fewer molecules of the compound; and the more distant molecules of the same salt in a state of solution move therefore towards the spot where they are wanted. Every cell therefore which decomposes any particular salt acts as a centre of attraction upon the fluids of the tissue surrounding it, and the salt in question is drawn towards this centre. But this process does not affect any other salt dissolved in the same fluid. If, for example, calcium sulphate is decomposed in a cell and crystals of calcium oxalate formed, this itself supplies a cause for the more distant molecules of sulphate to be drawn towards that cell; but it affords no reason for the molecules of potassium nitrate which are also present to move in the same direction. Every substance dissolved in the cell-sap is set in motion only in so far as the equilibrium of diffusion and the uniform distribution of its own molecules is disturbed. It follows therefore clearly that there can be in general no such thing as a continuous uniform motion of a so-called 'nutritive sap.' It is only when a number of compounds which supply food-material are taken up at one spot such as the root, and are decomposed at another spot such as the buds or green leaves, that the direction of movement is nearly the same for all; but even in this case the rapidity with which the molecules of each particular salt move will vary, because this depends on the rapidity of consumption at the point towards which the movement is directed, and on the special rate of diffusion of each compound. It is only when some external pressure drives the whole of the cell-sap in one direction that the motion of different substances is uniform, provided that the fluid moves in open channels such as the laticiferous vessels or sieve-tubes; but if the pressure causes filtration through closed cell-walls, then the molecules of different salts are urged forward with a different speed, because the rapidity of filtration of different solutions varies with their composition and degree of concentration.

The same principles hold good also for the absorption of combinations of foodmaterial from without into the absorbing organ. It has already been shown in the previous paragraph how the decomposition of carbon dioxide in a cell containing chloro- 
phyll induces new quantities of the dioxide at once to enter this cell, whether the gas be at the time dissolved in water or present in the atmosphere. If no carbon dioxide were decomposed in the cell, its contents would become saturated with the gas in proportion to the pressure and the temperature, and every cause for further motion would be removed. But the decomposition is constantly providing more space for the entrance of fresh molecules of carbon dioxide; and this gas, although present in such small quantities in the atmosphere, collects here and supplies the material for the production of compact masses of carbon-compounds.

A water-plant acts in the same manner on the salts dissolved in the surrounding water. The external water and the internal cell-sap are in continuous connection through the fluid saturating the cell-walls. If the chemical processes within the plant are supposed to be at rest, an equilibrium of diffusion will tend to become established between the external and internal fluid according to the prevailing conditions. But the chemical processes in the interior are continually disturbing this equilibrium, and molecules of the salts which are being decomposed are continually streaming from without to the places in the interior where they are to be used. If molecules of calcium phosphate are even very sparingly distributed through the surrounding water, a dense accumulation will gradually arise in the plant, not of calcium phosphate, but of some other compounds of phosphoric acid and of calcium, because the molecular equilibrium is being continually disturbed by the separation of the phosphoric acid from the calcium, that is, by the chemical change. If the calcium phosphate remained as such within the plant, the movement would cease so soon as the equilibrium of diffusion was established. It will be at once clear from a consideration of these facts that the accumulation of certain substances in the interior of plants depends in the first place on whether the compound of them which is present in the surrounding water is decomposed in the plant; that moreover the constituents of the different compounds must accumulate in the plant in different quantities according to the extent to which these compounds are decomposed; and that finally the relative quantities of the substances in question within the plant need not bear any resemblance to those of the substances present in the surrounding water. Substances which are present in the water in the form of extremely dilute solutions occur in the plant in great quantities; while others which are abundant in the water are much less so in the plant. Thus, for instance, marine plants take up a much larger quantity of potassium and a smaller quantity of sodium than corresponds to the composition of sea-water; again, species of Fucus collect considerable quantities of iodine which is present in sea-water only in extremely small quantities. Since moreover different plants decompose the same compounds with different degrees of rapidity, it is obvious that different plants which draw their food-materials from the same water must exhibit an entirely different composition of their ash.

The processes are more complicated when a land-plant has to take up the saline compounds of its food-material from the soil which contains but little water. By far the greater number of land-plants thrive in soil which usually contains a quantity of water much below its full capacity of absorption, its pores being almost entirely filled with air. The small quantity of water present adheres completely to the minute particles of soil, and for this reason does not flow away; and this adherent water covers the surface of the particles of earth in the form of a fine stratum. The roots can only absorb this water when they are in the closest contact with the particles of soil; hence plants freshly planted wither even in moderately moist ground until a sufficiently large number of particles of earth become attached by means of new roothairs to the newly-formed rootlets. At these points of intimate connection between the root-hairs and the soil the adhering water of the latter is directly continuous with the cell-sap of the root by means of the water saturating the cell-walls of the roothairs. In this manner it is possible for the root to absorb the water of the soil; as this water enters at the points of contact, the equilibrium of the strata of water that 
cover contiguous particles of earth is disturbed, and the water of the soil retained by capillary attraction is set in motion towards these points of contact. This process spreads centrifugally from every root, and thus gradually makes the most distant parts of the soil subserve the nutrition of the plant. If salts, such as calcium sulphate, are present in solution in the enveloping strata of water, these salts follow the movements of the water, and finally enter at the points of contact with the roothairs.

But a large portion of the food-material, especially compounds of ammonia, potassium, and phosphoric acid, occur in the ground in a fixed condition, or, as it is generally termed, absorbed; they cannot be extracted from the soil even by very large quantities of water; the roots nevertheless take them up out of it with ease. It may be supposed in these cases that these food-materials occur as an extremely fine coating over the particles of soil, and can therefore only be taken up at the points of contact of the root-hairs with these particles; and they are there rendered soluble by the carbon dioxide exhaled by the roots. This action of the root is limited to the points of contact; only those particles of substance which come directly into contact with the root-hairs are dissolved and absorbed. But since the number and length of the roots is very considerable in all growing land-plants, and since also they are continually lengthening and forming new root-hairs, the root-system comes gradually into contact with innumerable particles of earth, and can thus take up the necessary quantity of the substance in question. This power of the roots of taking up, by means of the acid sap which permeates the walls of even their superficial cells, substances which are insoluble in pure water, presents itself in an extremely evident manner, as I was the first to show, when polished plates of marble, dolomite, or osteolite (calcium phosphate) are covered with sand to the depth of a few inches, and seeds are then sown in the sand. The roots which strike downwards soon meet the polished surface of the mineral and grow upon and in close contact with it. After a few days an impression of the root-system is found corroded in rough lines on the smooth surface; every root has dissolved at the points of contact a small portion of the mineral by means of the acid water which permeates its outer cell-walls ${ }^{1}$.

In taking up those constituents of the soil which are insoluble in pure water, the solution is therefore first of all accomplished by the plant itself; and it is at the point where solution takes place at the surface of the root that absorption inwards is also effected by endosmose. But in spite of this complication the same principles hold good for the absorption of material from the soil as have been explained in the case of absorption from a solution. Here also it is the consumption, the decomposition of the compounds in the plant, that regulates the absorption of the material. The quantitative composition of the ash has therefore no resemblance to that of the soil; and the ash of plants of different kinds growing side by side and deriving their nutriment from the same soil may be altogether different ${ }^{2}$. But the composition of the soil is important to the plant in a secondary degree; since plants of the same kind, if they grow for example on a soil rich in lime, will take up a greater quantity of lime than if the soil contained but little of it. This is obviously not in contradiction to the principle laid down, but only shows that the decomposition of a salt in the plant will take place more largely the more easily it is enabled to take it up.

1 For a more detailed account see the Handbook of Experimental Physiology, 1865, p. 189 .

${ }_{2}$ [Messrs. Lawes and Gilbert's long series of experiments on this subject are of especial value. (See Journ. Roy. Agric. Soc. vol. VIII. p. 496 et seq., I847 ; Journ. Chem. Soc. vol. X. p. I, I857; Report Brit. Assoc. 1861 and 1867.) Their latest publication, 'Report of Experiments on the growth of Barley for twenty years in succession on the same land' (Journ. Roy. Agric. Soc., second series, vol. IX) contains much information as to the power possessed by plants of extracting different substances from the soil.] 
SECT. 5-Assimilation and Metastasis (Stoffwechsel) ${ }^{1}$. The food-materials absorbed by the plant are, with a few exceptions, compounds of oxygen containing the highest possible proportion of that element. The assimilated substances, on the contrary, which form the greater part of the dried substance contain but little oxygen, some even none at all. It follows from this that assimilation must be a process of deoxidation. The transformation of food-materials containing a large proportion into the substance of plants containing but little oxygen must necessarily be accompanied by elimination of that element; and since we already know that this takes place only in cells containing chlorophyll and under the influence of sunlight, we have at once the locality, the conditions, and the time of the assimilation thus determined. No organs which are destitute of chlorophyll can assimilate; and in the dark or when the amount of light is small, even those assimilating organs which contain chlorophyll lose the power of producing organic substances out of water and carbon dioxide with the assistance of other foodmaterials, - a process to which we shall henceforward exclusively apply the term Assimilation.

The products of assimilation of the cells containing chlorophyll may undergo various kinds of chemical metamorphosis either in these cells themselves or after passing into other organs; and the aggregate of these processes may be distinguished from assimilation as Metastasis. It is important to bear clearly in mind the difference between these two processes, both in respect to their external conditions and to their results, the following being the chief points:-(I) Assimilation takes place only in those organs that contain chlorophyll; metastasis in all alike. (2) Assimilation occurs only under the influence of light; metastasis equally well in the dark. (3) Assimilation is necessarily accompanied by the elimination of a large quantity of oxygen; metastasis is usually connected with the absorption of small quantities of oxygen and the exhalation of small quantities of carbon dioxide. (4) Assimilation increases the dry weight of a plant; metastasis only alters the nature of the assimilated materials, and these usually suffer a diminution of their mass, the destruction of a part of the assimilated organic compounds being necessarily associated with the inhalation of oxygen and exhalation of carbon dioxide necessary for metastasis. (5) The increase in weight of a plant which contains chlorophyll depends on the accession of assimilated substance in the organs that contain the chlorophyll being greater during the time that they are exposed to light than the loss in the dry weight connected with the exhalation of carbon dioxide accompanying metastasis in all the organs and at all times of vegetation. (6) Organs containing no chlorophyll and plants entirely destitute of it (parasites and saprophytes) do not assimilate but absorb substances already assimilated; no process takes place in them except metastasis; and since this is associated with inhalation of oxygen and exhalation of carbon dioxide, they decrease the entire store of assimilated substances.

Growth, i.e. the formation and enlargement of cells, always takes place at the expense of substances already assimilated; and these therefore must be subject to continual chemical change.

${ }^{1}$ See Sachs, Handbuch der Experimental-Physiologie, the section on the Transformation of Food-material. 
Growth is only possible as a result of assimilation; but the two processes do not usually concur either in time or locality. The assimilated substances may remain in the plant for a longer or shorter time without becoming employed in the growth of cell-walls or in the production of protoplasmic substances (protoplasm or chlorophyll-granules); and in this case they are termed Reservematerials. Every cell, tissue, or organ in which assimilated substances are stored up for subsequent use is called a Reservoir of Reserve-material. The assimilating cell may itself serve as a reservoir for reserve-material (as unicellular Algæ or the leaves of evergreen plants); but usually a physiological division of labour is effected in the plant of such a nature as to transfer the products of assimilation from the organs that contain chlorophyll to other organs or masses of tissue which serve as reservoirs of the reserve-material and give it up to the parts destined for the formation of new organs (buds, the rudiments of roots, or cambium). In Mosses, Vascular Cryptogams, and woody Phanerogams, the tissue of the stem is usually also the reservoir for this purpose; in perennial herbs and shrubs it is more often the persistent bulbs, tubers, and rhizomes that perform this function. The spores of Cryptogams which have the power of germination always contain a small quantity of reserve-material, at the expense of which the first processes of germination take place; in Rhizocarpeæ and Ligulatæ the whole of the prothallium and embryo is produced in this manner. The seeds of Phanerogams remove much greater quantities of reserve-material from the mother-plant, which are accumulated either in the endosperm or in the cotyledons; the greater the quantity of this reserve-material the more numerous and the larger are the stems, roots, and leaves which the seedling can produce before it begins to assimilate. The minute seedlings, for instance, of Nicotiana and Campanula may be contrasted with the strong ones of the Bean, Almond, Oak, \&c. Since no assimilation takes place in the dark, it is only necessary to allow seeds, tubers, bulbs, rhizomes, \&c. to germinate and develope in the dark in order to form an idea of the number and size of the organs which can be formed from the reserve-material.

Since the organs of assimilation which contain chlorophyll are usually at a distance from the reservoirs of reserve-material and from the growing buds and roots, the products of assimilation have to be conveyed to the localities where they are required and where they are temporarily deposited. Growth and the deposition of reserve-material are therefore necessarily associated with corresponding movements of the products of assimilation and of those undergoing metastasis.

All these statements may be proved without any more accurate knowledge of the substances themselves which are produced by assimilation in the cells that contain chlorophyll and which undergo metastasis. But before entering on this question, we may first of all discuss the other:-whether all the products of metastasis are immediately applicable to the building up of new organs; and if not; what substances furnish the material for the production of cell-walls, protoplasm, and chlorophyll-granules. Among the extraordinarily large number of the products of metastasis which are proved by chemical analysis to exist in various plants, there is a comparatively small number of substances the behaviour of which in the growth of the organs and whose universal distribution through the vegetable 
kingdom clearly show that they furnish the material for the growth of cell-walls and of other organised structures. These substances may be termed, without reference to their chemical nature, Formative Materials. Starch, the different kinds of sugar, inulin, and the fats must be considered the formative materials of the cell-wall ; the albuminoids the formative materials of protoplasm and of the chlorophyll-granules.

Among the remaining products of metastasis are some which stand in genetic relation to the production of sugar; the glucosides, to which also belong certain tannin-substances. Asparagin is formed at the expense of the albuminoids contained in the reservoirs of reserve-materials, and is afterwards again used in the formation of albuminoids in the young organs.

All those organic compounds may be termed Degradation-Products which are produced by subsequent change in the substance of the organised structures of plants, and which have no further use in the building up of new cell-walls or protoplasmic structures. Thus bassorin is a degradation-product of cell-walls, as also is the mucilage of quince and linseed; the substances which cause lignification, suberisation, or cuticularisation are also probably the result of a partial degradation of the cellulose of the cell-walls. A residue of the protoplasm of older parenchymatous cells often remains until they entirely die away, and may also be considered a degradation-product. In the same manner a small residue of the chlorophyllgranules of leaves which die in the autumn remains over in the form of minute yellow granules which have no further use. The red and yellow granules also which cause the colour of ripe fruits and of the antheridia of Characex and Mosses result from the degradation of chlorophyll-granules, and have no further physiologicochemical use.

Those substances may be termed Secondary Products of Metastasis which are formed during this process, but have no further use in the building up of new cells, remaining inactive at the place where they are produced. Thus in the germination of many seeds (the Date, Ricinus, Phaseolus, Faba, \&c.) tannin-like compounds are formed in particular cells, and in many cases red colouring substances which, without undergoing any perceptible change, remain in these cells, while the rest of the substances of the seedling go through the most various chemical transformations and changes of place in the course of its growth. The same is the case with the essential oils in the glands of leaves, of caoutchouc in the laticiferous vessels, of resin and resin-forming substances in the resin-passages, and of the gummy compounds contained in the gum-passages of many plants. In this category may also be included the greater number of vegetable acids and many alkaloids. No interpretation has yet been given of the function of these substances in the internal economy of the plant; in the case of calcium oxalate Holzner's theory has already been mentioned that it is formed as a secondary product when the sulphuric acid combined with the calcium is replaced by oxalic acid; and that the free sulphuric acid then undergoes various further decompositions, while the base of the salt remains unused and inactive in combination with the oxalic acid produced as a secondary product, as calcium oxalate in the crystalline form. Among colouring substances no relation to the chemical processes which proceed in the plant has been traced except in the case of the green colouring substance of chloro- 
phyll; it is only in the presence of this substance that elimination of oxygen, and therefore assimilation, can take place. In the case of a long series of other substances, many colouring matters, acids, alkaloids, wax, tannin, pectinaceous substances, \&c., no relation to the other processes of metastasis is known, nor any physiological signification which they possess in the life of the plant.

In some cases substances which have ceased to take part in the processes of growth and of metastasis are nevertheless important or even indispensable for other purposes of vegetation. Of this class are the saccharine juices secreted by nectaries, which are of service to the plant only so far as they attract insects which thus bring about the conveyance of the pollen to the stigma. For a similar purpose a portion of the tissue of the anthers of Orchids is transformed into a viscid glutinous substance by which the pollinia become attached to the proboscis of insects. Thus again the sapid and nutritious substances which constitute the pericarps of some fruits are of no direct use for the growth of the seeds, but cause their dissemination by animals which feed on the fruits and thus disperse the seeds.

We must now again turn, after this preliminary explanation of the various parts played by the products of metastasis in the life of the plant, to the most important group of organic compounds, those which have been distinguished above as formative materials.

The determination whether any chemical compound belongs to the class of formative materials of the cell-wall and protoplasmic substances depends on its behaviour during growth, on its chemical composition, on its appearance and disappearance in growing cells and tissues, and on its chemical relations to other substances, especially to cellulose and to protoplasmic substances. Spores, seeds, bulbs, tubers, rhizomes, the persistent parts of woody plants, and other reservoirs of reserve-material, always contain chemical compounds belonging to two different groups. On the one hand nitrogenous substances are always present in the form of albuminoids (often several different ones as in the grains of cereals) which scarcely differ chemically from protoplasm, and when contained in the succulent reservoirs of reserve-materials preserve even the form of protoplasm. From this similarity, and still more when the migration and other relations of these substances are kept in view, the conclusion must be drawn that we have in them the material for the formation of protoplasm in the newly-formed organs. On the other hand all these reservoirs of reserve-material contain one or more nonnitrogenous substances belonging to the series of carbo-hydrates and oils. In seeds and spores there is generally a great deal of oily matter and little or no starch ; but many seeds contain on the other hand a great deal of starch with but little oily matter. In tubers, many bulbs, rhizomes, and stems, there is usually much starch stored up with but little oily matter; while in some tubers (as the Dahlia, Artichoke, \&c.), the starch is replaced by inulin; in the bulbs of Allium Cepa by a substance resembling grape-sugar; in the root of the Beet by crystallisable cane-sugar. Small admixtures of oily matter appear to be never absent, and $\ln$ some cases, especially in many seeds, this alone is present without any carbo-hydrate (as the Almond, Gourd, Castor-oil plant, \&c.).

Together with albuminoids, carbo-hydrates, and oils, a variety of other compounds may also occur in the reservoirs of reserve-material; but the limitation of 
substances of this kind to particular species of plants shows that they are not of the same significance as the former. They may be of great importance for the growth of the species; but more accurate knowledge is still wanted in all cases.

Since seeds, tubers, and other parts of plants that are filled with reserve-material can be made to unfold buds, to put out roots, and even to form flowers and the rudiments of fruits by supplying them with pure water and oxygenated air when the conditions for assimilation (chlorophyll and sunlight) are absent, it follows that the substances stored up in these reservoirs furnish the material for the growth of the new leaves, roots, and flowers. The reservoirs are therefore emptied in proportion as the growth of the new organs progresses; and when finally they become completely empty, all further growth ceases, if sunlight and chlorophyll do not cooperate to produce new formative material by assimilation. It is moreover easy to follow the reserve-materials by means of micro-chemical reactions in their course from the reservoirs through the conducting tissues to the growing organs, and to recognise their relation to the growth of particular tissues. A close study leads first of all to the conclusion that the albuminoids contained in the reservoirs of reserve-material reappear as such in the protoplasm of the newly-formed organs, having, independently of temporary qualitative changes; only altered their position. On the other hand it shows that the oily matter and the carbo-hydrates which had accumulated in the reservoirs finally entirely disappear as such or leave only a small residue (oil); while in their place a mass of new cell-walls is formed which were not in existence before; and the material for the construction of these can only have been derived, under the given conditions, from the carbo-hydrates, or, when these are absent, from the oily matter which has now disappeared. If we thus come to the conclusion that starch, sugar, inulin, and oil are the substances from which are formed the cell-walls of plants, at all events in so far as they are nourished from a reservoir of reservematerial, it by no means follows from this that the whole of the store is used up entirely in the production of cellulose; on the contrary a variety of other substances are formed during growth, such as vegetable acids, tannin, colouring-matters, \&c., which are probably also derived from the same non-nitrogenous reserve-materials. A part of the non-nitrogenous substance is also entirely destroyed and converted into carbon dioxide and water, a process which may cause a loss of 40 or even 50 per cent. of the weight of the organic substance of those seeds which germinate in the dark.

If the reserve-materials stored up in different seeds, tubers, bulbs, \&c. are compared, it is seen that starch, the various kinds of sugar, inulin, and oil, are of the same physiological value with regard to their most important purpose, viz. the formation of new organs; inasmuch as these substances can replace one another. Thus the cell-walls of the embryo of Allium Cepa are formed at the expense of the oily matter of the endosperm; but the cell-walls of the leaves and roots which grow from the bulbs evidently obtain their formative material from the glucose-like substance which fills the bulb-scales in a state of solution. In the Beet canesugar is stored up for the same purpose, inulin in the tubers of the Dahlia, and starch in the tubers of the Potato, the bulbs of the Tulip, \&c.; and these are subsequently consumed. But in most seeds all these carbo-hydrates are replaced 
by oily matter; and it cannot be doubted that this furnishes the material for the growth of the cell-walls when the new organs are being formed.

To the series of these substances of the same physiological value belongs finally cellulose itself, which may also be deposited in considerable quantities as a reserve-material, as in the endosperm of the Date, the greater part of the hard kernel of which consists of cellulose in the form of the pitted thickening masses of the cell-walls. These are dissolved during germination, and the products of their solution conveyed to the growing parts of the embryo, where they finally supply the material for the growth of the new cell-walls.

If on the other hand the substances which occur in dormant seeds, bulbs, tubers, and other reservoirs of reserve-material, are compared with those which are found in the conducting tissues and growing organs of seedlings and young rootswhich we already know must necessarily be produced from the former, because there is no other material which can produce them-it is seen that these reserve-materials must undergo repeated Metamorphosis while they are being conveyed to the growing organs and are being consumed in the process of growth, and before the permanent form of cellulose has been attained. Thus sugar and starch are found temporarily in all oily seeds during germination, and are often accumulated in great quantities, disappearing when germination is completed. In proportion as they are formed the amount of the original oil decreases; and in proportion as they again disappear the quantity of cellulose in the cell-walls increases. In other cases starch is conveyed from reservoirs of reserve-material to the growing organs, sugar being at the same time formed ${ }^{1}$; and fine-grained starch is again temporarily formed in the growing tissues themselves, disappearing once more with the growth of the cell-walls. This temporary formation of starch in the growing tissues themselves is an extremely common phenomenon, whether the reservoirs of reserve-material were filled with oily matter, inulin, sugar, starch, or cellulose. This transitory starch appears in the cells of the parenchyma and epidermis of young organs (only rarely in those of the fibro-vascular bundles) after they have become differentiated from the primary meristem; and disappears when the final elongation of the organs is completed, generally becoming transformed into sugar (glucose), which in its turn speedily disappears.

Transitory metamorphoses also take place when the albuminoids stored up in the reservoirs of reserve-materials are being transported and consumed ${ }^{2}$; although these metamorphoses cannot be followed by micro-chemical observations, as in the

${ }^{1}$ [The conversion of starch into sugar is effected by means of unorganised ferments; some of these have long been known, such as Emulsin (in Almonds), Diastase (in Barley), Myrosin (in Black Mustard seeds). More recently they have been detected in various plants and parts of plants by Kossmann (Journ. Pharm. Chem. (4) 22) and by Krauch (Landwirthsch. Versuchsstat. 23). Von Gorup-Besanez has found ferments of this kind in the germinating seeds of Vetches, Hemp and Flax, which have also a peptic action (see infra):]

${ }^{2}$ [The first stage in the metamorphosis of the reserve-proteids is, doubtless, their conversion into peptones, into proteid substances, that is, which are readily soluble in water and which diffuse rapidly. This is effected by the action of unorganised ferments. These bodies have been found in germinating seeds (v. Gorup-Besanez and Will) and in the secretion of 'carnivorous' plants such as Nepenthes, Drosera, and Darlingtonia. Recently a very active peptic ferment has been found in the green fruits of Carica Papaya (Würtz and Bouchet, Le Papaï, Comptes rendus, tom. 89, 9o, and 9I).

A good résumé of our knowledge on the action and distribution of unorganised ferments in plants is given in the second edition (I882) of Husemann's Pflanzenstoffe, I. p. 237.] 
case of the oils and carbo-hydrates. Thus a portion of the casein in the cotyledons of Leguminosæ passes over into albumin during germination; the insoluble proteids in the endosperm of Wheat are dissolved and carried up into the seedling plant. The albuminoids contained in seeds appear to be subject during germination to still more complete decompositions. The asparagin which occurs temporarily in parts of the embryo can only be formed by partial decomposition of the albuminoids ${ }^{1}$. It appears however that these products of the decomposition of the albuminoids under the influence of the energetic oxidation which takes place in the germinating seed are used in the formation of albuminoids in the growing parts of the embryo.

The preceding remarks refer to the processes of growth which are associated with the consumption of the substances stored up in the reservoirs of reservematerial. If those plants are now examined in a similar manner whose reserve foodmaterial has been consumed, whose green leaves have begun to assimilate under the influence of light, and which are forming the substances necessary for the growth of their buds, roots, \&c., the same substances are found similarly distributed through the conducting tissues of the internodes and the petioles and veins of the leaves as far as the buds and apices of the roots, and subject to the same metamorphoses as in the seedlings. It follows that the assimilating organs which contain chlorophyll perform the same function for the growing parts of the mature plant that the reservoirs of reserve-material do for the seedling; but with this difference, that the former produce the formative materials afresh, while in the latter they are not formed but only stored up.

The organic compounds originally formed in the cells containing chlorophyll by the decomposition of carbon dioxide and water under the influence of light are generally carbo-hydrates. The most common of these is starch; sugar occurs less often; oily matter perhaps occasionally. It has been shown (p. 46) that the starch which so commonly occurs in the chlorophyll-granules of plants that vegetate under normal conditions can only be produced when the plant is subject to the wellknown conditions of assimilation, i.e. when it decomposes carbon dioxide and water under the influence of sunlight. Seedlings which have completely exhausted their supply of reserve-materials by growth in the dark, and are afterwards exposed to the action of light, do not till then develope their chlorophyll. 'The first grains of starch which are found a little later in the plant are those enclosed in the chlorophyllgranules, and are at first small, but gradually grow larger. It is only afterwards that starch is found also in the conducting tissues of the internodes and leaf-stalks up to the buds, which then begin to grow anew. It has been shown further that this starch which is formed in the chlorophyll-granules disappears in the dark; i.e. becomes dissolved and transferred to the conducting tissues. In Allium Cepa the chlorophyll forms no starch; but a substance similar to grape-sugar is found in large quantities in the green leaves, and is distributed through all the tissues of the plant: it is still uncertain whether or not mannite is formed in a similar manner in the leaves of the Olive. Where drops of oil are found in the chlorophyll, they appear

1 According to Hosæus, ammonia is also formed during germination; and Borscow maintains that ammonia is set free during the vegetation of Fungi (Mélanges biol. tirés du Bullet. de l'Acad. imp. des Sci. Nat. Pétersbourg, vol. VII, I868). This is however denied by Wolf and Zimmermann (Bot. Zeitg. 1871, nos. 18, 19). For a further account of Asparagin see the appendix to this section. 
to be formed at the expense of the starch which has been produced there ; this conclusion being derived especially from the observation of what takes place in Spirogyra and Cereus ${ }^{\mathbf{1}}$.

The result of tracing by micro-chemical observation the products of assimilation in the conducting tissues leads once more to the conclusion that the starch which is formed in the cells containing chlorophyll is subject to a variety of chemical metamorphoses before it reaches the growing tissues and the reservoirs of reserve-material. Even during the period of vegetation the substances which are conducted to the young parenchyma of growing parts, as soon as this has been differentiated from the primary tissue, give rise to the formation of fine-grained starch which accumulates there temporarily, and disappears with the final and rapid increase in size of the cells. Starch and other substances are then produced afresh by assimilation in the fully developed leaves; and starch and the products of its transformation again appear in the conducting tissues, not to be consumed there, but only to be conducted to the still younger parts. The metamorphoses of the formative materials which are conveyed from the assimilating organs to the reservoirs of reserve-material, generally show a reversed order of succession to that which takes place during germination; the starch produced in the leaves is transformed in the leaf-stalks of growing Beet into glucose, from which crystallisable cane-sugar is formed in the swollen tuberous roots; in the Artichoke the starch is converted into inulin which is conducted through the stem to the underground tubers; in the Potato, the mature leaves of which form starch, a substance similar to glucose is chiefly found in the conducting tissues, which is conveyed to the growing tubers, and there evidently forms the material from which the large masses of starch are formed. In ripening fruits and seeds a large quantity of glucose is generally found which disappears from the seeds when they become ripe, starch being formed in these reservoirs of reserve-material; in Ricinus the oil of the endosperm is evidently formed at the expense of the saccharine substance which is conveyed to the seed; in the embryo of the same plant, as well as in that of Crucifers, fine-grained starch is formed temporarily, which disappears when the seeds are ripe, and is replaced by oily matter.

Whether the albuminoids also are first formed in the assimilating cells which contain chlorophyll and whether they can be formed only in them is still an undecided point. It is certain that they are formed in the chlorophyll-containing cells of Algæ; but it cannot be concluded from this that they can only be produced in the corresponding cells of plants with differentiated tissues; at all events experiments on the artificial production of the yeast-fungus show that it is able to form out of sugar and an ammonium-salt (with the assistance of the constituents of the ash) not only cellulose but also albuminoids, as may be inferred from the increase

1 [Briosi. states (Bot. Zeitg. 1873) that starch-grains are never found in the chlorophyll-granules of Musa and Strelitzia, but that drops of oil are present instead. His observations have been shown to be erroneous by von Holle and Godlewski (Flora, I877). Pringsheim (Ueb. Lichtwirkung und Chlorophyllfunction, Jahrb. f. wiss. Bot. XII, I880) enumerates a number of plants in which oil is present, and not starch, in the chlorophyll-granules; Vaucheria sessilis, Selaginella, Cycas, Stratiotes aloides, Lilium Martagon, Olea europaa, Begonia. He is of opinion that the first product of assimilation is a waxy substance to which he gives the name of Hypochlorin.] 
of the protoplasm in the rapidly multiplying cells. If the colourless cells of yeast are able to do this, it may be inferred, until the contrary is proved, that those cells of other plants which do not contain chlorophyll can also produce albuminoids, if only a carbo-hydrate or oil (or both) is conveyed to them from the leaves, and an ammonium-salt or a nitrate from the roots. That the formation of albuminoids probably takes place in this way within the conducting tissues of internodes and petioles may be concluded from the deposition of calcium oxalate in these tissues; since in the formation of this salt sulphuric acid becomes separated from the calcium, and its sulphur enters into the chemical formula of albuminoids ${ }^{1}$.

When the cells of the leaves become emptied of their contents at the close of the period of vegetation, and the deciduous parts die off, not only the last portion of starch which was formed in the latter, but also the material of the chlorophyllgranules, is itself absorbed and conveyed through the leaf-stalks to the reservoirs of reserve-material; all the serviceable substances contained in the leaves become incorporated in the permanent organs. The leaves change colour; a small quantity of very small shining yellow granules usually remain behind in the cells of the mesophyll as a residue of the absorbed chlorophyll-granules; and the leaves which are emptied in the autumn are therefore yellow. If they are red this is in consequence of a red sap which fills the cells in addition to the chlorophyll-granules ${ }^{2}$. Enormous quantities of crystals of calcium oxalate often remain behind in the deciduous leaves; the constituents of the ash which are serviceable to the plant, especially phosphoric acid and potash, are conveyed with the starch and the protoplasmic substances to the persisting parts; so that the falling leaves thus consist only of a skeleton of cell-walls and of the subsidiary products of metastasis which are of no value to the plant.

The direction of the Transport of the assimilated substances in the plant is determined by the fact that it must take place from the assimilating organs to the growing parts and to the reservoirs of reserve-material; while at the commencement of every new period of vegetation its direction must be from these reservoirs to the growing organs; and since new organs are usually formed above as well as below these reservoirs and the assimilating leaves, it is obvious that the movements of the assimilated substances must take place at the same time in opposite directions:

The Conducting Tissue for the transport of the formative materials consists, in plants with differentiated systems of tissue, of the parenchyma and the thinwalled cells of the phloëm of the fibro-vascular bundles. By. the parenchyma of the fundamental tissue, which always has an acid reaction, are conveyed the carbo-hydrates and oils; by the soft bast, the albuminoids which have an alkaline reaction. Small starch-grains often occur, as Briosi has recently shown, in the protoplasm of the sieve-tubes; I had already pointed out that this accompanied the absorption from the leaves in the autumn as well as very rapid growth ${ }^{3}$. Where

${ }^{1}$ See Sachs, Handbuch der Experimental-Physiologie, p. 345 .

${ }^{2}$ [On the colouring matter of the leaves in autumn, see Sorby, Quart. Journ. of Science, $187 \mathbf{r}$, p. 64 ; and 1873, p. 215.$]$

${ }^{3}$ Briosi, Bot. Zeitg. 1873 . It is by no means certain that the occurrence of small quantities of starch in the sieve-tubes demonstrated by Briosi, and the possibility of their passage through the 
there are laticiferous vessels, they furnish an open communication between all the organs of the plant; they contain albuminoids, carbo-hydrates, and oils, as well as the secondary products of metastasis, as caoutchouc and poisonous substances, the occurrence of which does not affect the significance here attributed to the laticiferous vessels; the occurrence of products of decomposition in the blood does not prevent us from regarding the blood-vessels as organs which serve to transport nutritious substances ${ }^{1}$.

The mode of motion of the assimilated substances is usually molecular; i.e. it is a movement of diffusion, especially where the transport takes place through closed cells. The pressure caused by the tension and turgescence of the tissues has in addition a tendency to propel the fluids in the direction of least resistance, which is also that in which they are consumed. In the system of communicating sieve-tubes and laticiferous vessels the movement of the substances is necessarily one of the entire mass, caused by inequalities of pressure, and by the distortions and curvatures which the wind produces.

As far as concerns the movements of diffusion, it is a general rule that every cell which decomposes any substance, renders it insoluble, or uses it for its growth, acts upon the dissolved molecules of this substance in the neighbourhood as a centre of attraction; the molecules stream to the parts where they are wanted because the molecular equilibrium of the solution is disturbed by its consumption. On the other hand every cell which produces a new soluble compound acts on the dissolved molecules as a centre of repulsion, because the continually increasing concentration occasions at the point of production a streaming of the molecules away from it towards the point of less concentration, the concentration continually decreasing towards the points where the substances are consumed. When the movement of diffusion is caused by the production and consumption of definite compounds of this nature, the proximate cause of the molecular movement of the dissolved substances must be the chemical processes involved in their metamorphoses. These metamorphoses take place, as we have seen, not only at the points where the substances are consumed in the process of growth, but also in the conducting tissues; and this production of transitory compounds must therefore favour movement towards the points of deposition and of growth. The formation of insoluble starch is in this sense a fact of peculiar importance. If for instance the starch produced in the leaves of the Potato is required to be transported to the tubers, it must necessarily be conveyed in a soluble form, and we find such a substance in the conducting tissues of the stem, namely, glucose. But if this glucose had to undergo no further change in the tubers, a solution of glucose of constantly increasing concentration would be uniformly distributed through the conducting

pores of the sieves, warrants the view that the sieve-tubes are conducting organs for starch in the same sense that they are for albuminoids. These small quantities of starch may pass into the sievetubes from the neighbouring parenchyma, to be used there, in young organs, as plastic material, or, in older organs, to take part in the formation of albuminoids. It may be that these substances are formed in the sieve-tubes out of carbo-hydrates and nitrogenous compounds, calcic sulphate being decomposed and a formation of crystals of calcic oxalate taking place in the cells surrounding the phloëm.

1 Sce also Faivre, Sur le latex du murier blanc; Ann. d. Sci. Nat. sér. V. t. Io. 
tissues and the tubers; and the accumulation of the whole of the reserve-material in the tubers would be impossible. The glucose is used up in the tubers in the formation of starch-grains; and a fresh quantity therefore continually streams in that direction; the whole mass of the material produced in the leaves is therefore gradually transferred to these reservoirs of reserve-material. The starch is first transformed into glucose, and then back into starch; and it is in this chemical process that the vehicle for the movement consists. Starch is even produced temporarily in the conducting parenchyma, but of course cannot be transported as such from cell to cell; its movement being effected by the solution of grains in one cell, - the product of solution diffusing into the adjoining cell, and being there employed in the formation of starch-grains which are then again dissolved, and so on. When again cane-sugar is formed in the tuberous roots of the Beet, the movement towards the root of the glucose which is produced from the starch assimilated in the chlorophyll is brought about in this way,-every particle of glucose undergoes chemical transformation when it reaches the root, and the molecular equilibrium of the solution of glucose is thus disturbed; the root acting as a centre of attraction on the glucose in the leaf-stalks. But the continual formation of the solution of glucose in the leaves at the expense of the starch causes in them an increase of concentration and a streaming of molecules towards the root, where the concentration of the solution of glucose is continually decreasing, while that of the solution of cane-sugar increases. The same is evidently the interpretation of the formation of inulin in the tuberous roots of the Dahlia and the tubers of the Artichoke, and of that of oil in ripening seeds at the expense of the sugar which is conveyed to them.

I infer the co-operation in the movement towards the parts where the substances are chemically altered, of the pressure exercised on the cell-sap by the tension of the tissues, even where we have to do with closed cells, from the fact that considerable quantities of the cell-sap appear on the surface of a transverse section of succulent organs, both from the parenchyma and from the cambiform cells, and this is clearly forced up by internal pressure. Since the tension and turgescence of the tissue are always less in the buds and apices of the roots than in the older parts, there must always be a tendency for the filtration of the sap towards the latter, which must act in the same way as diffusion.

That the contents of the perforated sieve-tubes and laticiferous vessels are also subject to considerable pressure from the surrounding tissue is shown by the extent to which these fluids flow out when the organ is cut through. The fluid which is subject to pressure will have a tendency to escape from these tubes to parts of the plant where the lateral pressure is less, which is the case in the buds and apices of the roots. The flexions and distortions occasioned in the organ by the wind will at the same time cause the fluid contents of the sievetubes and laticiferous vessels to be pressed away from the older bent parts towards the buds where the tension is less.

The statements here compressed into a very brief space rest on a series of detailed micro-chemical and experimental researches which $I$ have described in the Botanische Zeitung, 1859 and 1862-1865; Pringsheim's Jahrbücher für wissenschaftliche Botanik, vol. III. p. I83 et seq.; Flora, I862, pp. 129 and 289, and 1863, pp. 33 and 193 ; and have 
presented in a connected form in the section on the Transformation of Food-materials in my Handbook of Experimental Physiology ${ }^{1}$. The reader will there find the reasons for the views here given; and a few examples will now be sufficient to render somewhat clearer the general statements with regard to metastasis and the migration of the assimilated substances. In the outset it must be stated that by grape-sugar or simply sugar I understand a substance soluble in the cell-sap, easily reducing copper oxide, and readily soluble in strong alcohol, although it may not always exactly correspond to the grape-sugar of chemists, a point which is of but little importance for our present purpose.

I. The parenchyma of the bulb-scales of the Tulip-i.e. the four or five thick colourless leaves which serve as reservoirs of reserve material-contains, as long as the plant is dormant, in addition to considerable quantities of albuminous substances, a very ${ }^{\circ}$ large quantity of coarse-grained starch. The presence of sugar cannot be determined at this time by micro-chemical processes. As soon as the bud of the leaf- and flowerstem which is concealed within the bulb, but which had already been formed with all the parts of the flower during the previous summer, begins to elongate in February, and roots make their appearance from the base of the bulb, small quantities of sugar are found with the starch in the parenchyma of the bulb-scales. The whole of the parenchyma and of the epidermis of the leafy stem, of the young foliage-leaves, of the perianth, of the stamens, and of the carpels, becomes filled with fine-grained starch, the substance of which has already been derived from the bulb-scales, where the starchgrains have become transformed into sugar, which diffuses into the growing organs, and there, as far as it is not directly consumed, again supplies material for the formation of starch-grains.

Together with its consumption in the growth, at first slow, of the cell-walls, this temporary re-formation of starch at the expense of that contained in the bulb-scales continues at first in the young internodes, leaves, and flowers. The cells enlarge and become continually more filled up with fine-grained starch till the time when the bud comes above ground (Fig. 470). Then follows the rapid extension of the stem; the leaves expand, and the flower unfolds. With the considerable and rapid increase in size of the cells accompanying this unfolding, the fine-grained starch disappears in all these parts, sugar being temporarily produced which furnishes the material for the growth of the cell-wall. When all the parts above ground are fully unfolded, the cells, although much larger, are now devoid of starch. The corresponding loss which the bulb-scales have experienced up to this time is clearly seen from the decrease of their starch-grains; they may be found in all stages of absorption. The turgescence of the bulb-scales at the same time decreases, and they become wrinkled; but the formation of sugar in them still continues at the expense of the starch, even when the parts above ground have already done growing. The starch stored up in the bulb-scales finds in fact still another use; while the flower-stalk is extending, the bud in the axil of the uppermost bulb-scale begins to develope rapidly (it had already been formed in the previous summer); its cataphyllary leaves swell and become filled with starch; and the residue of the starch not consumed in the growth of the flower-stalk is transported from the scales of the mother-bulb through its base into the young bulb (Fig. 470, 2). These scales become gradually entirely emptied of starch, and while the green foliageleaves exposed to light are assimilating and contributing their share to the growth of the new bulb, they finally wither and dry up from the simultaneous loss of water and of assimilated matters. The scales of the mother-bulb form thin brown membranes

1 The researches of Schröder (Jahrb. für wiss. Bot. vol. VII. p. 26I), Sorauer, Siewert, Rœstell, \&cc. (collected in Hoffmann and Peters' Annual Report on the Progress of Agricultural Chemistry for I 868 and 1869 , Berlin $187 \mathrm{I}$ ) contain fresh confirmations of the account here given. [See also De Vries, On the Germination and Growth of Seeds, Tubers, and Roots of cultivated Plants (Clover, Potato, Beetroot) in Landwirthsch. Jahrbiicher, vols. 6-8, 1877-79.] 
which serve to protect the new bulb; the inflorescence subsequently dies down. The reserve-materials which accumulate in the daughter-bulbs are partly derived from those of the mother-bulb; but are completed by the products of assimilation of the green leaves of the flower-stalk. When the flower-stalk has also died down, nothing remains of the whole plant but the bud which has developed into a new bulb. For a time it does not put out any new organs, but is apparently dormant; but in the interior the end of the stem continues to grow slowly, and produces new rudiments of leaves and the flower-bud for the next year; when the process now described is repeated.

So far we have only pointed out the relation of the starch and of the sugar produced from it to the growth of the plant; there are formed however along with it, and probably likewise at the expense of these carbo-hydrates, other substances, such as the colouring matter of flowers, the oil in the pollen-grains, \&c. The albuminoids at first contained in the bulbscales become transported to a distance from them, and furnish the material for the formation of the protoplasm in the young cells of the growing flower-stalk; a large part is evidently employed in producing the chlorophyllgranules in the foliage-leaves as they become green. Their function is now to produce at least as much formative material by assimilation as is required to build up the transitory inflorescence and to supply the bulb.

2. The ripe seed of Ricinus communis contains a very small embryo in the middle of a very large endosperm; neither contains starch, sugar, nor any other carbo-hydrate, if we exclude the very small amount in weight of the cellulose of the thin cell-walls. The reserve food-material consists of a great quantity of oil (as much as 6o per cent.) and albuminoids, the admixture and composition of which have already been described on p. 53 . The very small quantity of these substances contained in the embryo would only suffice for the first and very inconsiderable development of the seedling; its enormous increase in size during germination must therefore be attributed almost entirely to the substances deposited in the endosperm. The endosperm of Ricinus enlarges very considerably, as Mohl first showed, during germina-

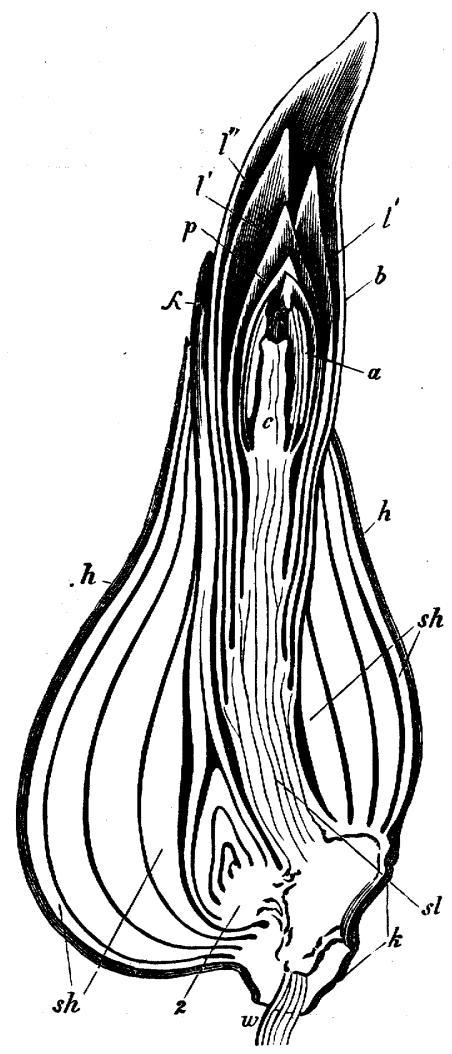

FIG. 470.-Longitudinal section through a germinating bulb of Tulipa pracox: $h$ the brown enveloping membrane $k$ the flattened stem which forms the base of the bulb and bears the bulb-scales $s h$; $s i$ the elongated part of the stem which bears the foliage-leaves $l^{\prime} l^{\prime}$, and terminates in the flower; $c$ the ovary, $p$ perianth, $a$ anthers; 2 a lateral bulb in the axil of the youngest bud-scale, which developes into the bud of next year's bulb; $w$ the roots which spring from the fibro-vascular bundles of the base of the bulb. tion, and the material required for its growth must therefore be diverted from the embryo. The two thin broad cotyledons remain in the endosperm, with their surfaces in contact with one another, long after the root and the hypocotyledonary part of the stem have emerged from the seed; they are in contact by their backs with the tissue of the endosperm which surrounds them on all sides, and absorb the reserve-materials from it, while they keep pace slowly with its enlargement. When the parts of the seedling have increased very considerably and the root has developed a number of lateral roots, the hypocotyledonary portion of the stem elongates so that the cotyledons are drawn out of the 
endosperm which is then completely emptied and reduced to a thin membranous sac. They now rise above the ground, become expanded to the light where they continue to grow rapidly and become green, and serve from this period as the first assimilating organs.

In this case, as in the germination of all oily seeds, sugar and starch are produced here in the parenchyma of every growing part, disappearing from them only when the growth of the masses of tissue concerned has been completed. Since the endosperm grows also independently, starch and sugar are, in accordance with the general rule, temporarily produced in it. The cotyledons apparently absorb the oil as such out of the endosperm, whence it is distributed into the parenchyma of the hypocotyledonary portion of the stem and of the root, serving in the growing tissues as material for the formation of starch and sugar, which on their part are only precursors in the production of cellulose. In these processes of growth tannin is also formed which is of no further use, but remains in isolated cells, where it collects apparently unchanged

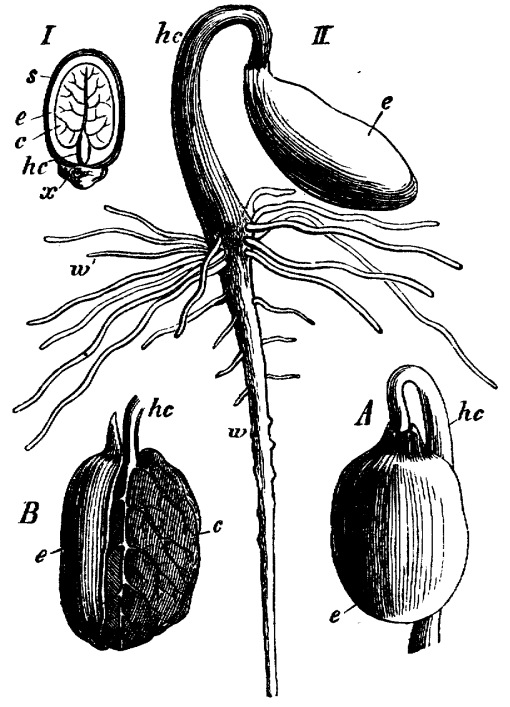

FIG. 47r.-Ricinus communis; I longitudinal section of the ripe seed; $I I$ germinating seed with the cotyledons stilt in the endosperm (shown more distinctly in $A$ and $B$ ), $s$ testa, $e$ endosperm, $c$ cotyledor, hc hypocotyledonary portion of the stem, $w$ primary root, $w^{\prime}$ secondary roots, $x$ the caruncle.

until germination is completed. It can scarcely be doubted that the material for the formation of this tannin is also derived from the oil of the endosperm, although perhaps only after a series of metamorphoses. The absorption of oxygen, which is an essential accompaniment of every process of growth and especially of germination, has in this case, as in that of all oily seeds, an additional significance, inasmuch as the formation of carbo-hydrates at the expense of the oil involves the appropriation of oxygen.

Since the metamorphoses of material proceed pari passu with the growth of the separate parts, the distribution of the products of metastasis through the tissues is continually changing, and can only be understood by a consideration of all the surrounding circumstances. The micro-chemical investigation of seedlings in the state represented in Fig. 47 I II, gives, for instance, the following result:-in the endosperm is found a great deal of oil and a little starch, with sugar at the outside; the epidermis and parenchyma of the slowly growing cotyledons are filled with drops of oil; a large number of the epidermal cells contain tannin; starch-granules are found 
only in the parenchyma of the leaf-veins; the parenchyma of the hypocotyledonary portion of the stem, which is at present growing the most rapidly, contains only comparatively little oil but much starch and sugar; and a number of the cells of the epidermis and parenchyma are filled with tannin. The primary root has first of all completed its growth in length and thickness (after germination it begins afresh); in its lower part it contains neither starch nor sugar (the former is present in the rootcap); in its upper part from which the lateral roots spring and in the lateral roots themselves sugar is still present, which is conveyed into the growing apices of the latter. When the hypocotyledonary portion of the stem has subsequently become vertical and has temporarily ceased to grow, the oil, starch, and sugar have almost entirely disappeared from it, and in their place the cell-walls have become thick, and the vessels and first cells of the wood and bast are already thickened. After the stem of the young plant has become upright, the cotyledons expand and grow rapidly, and the remainder of the oil which they had taken up from the endosperm now also disappears from them together with the starch and sugar. The seedling has now entered on a state in which the non-nitrogenous reserve-materials are consumed; a framework of large and solid cell-walls is produced in their place; and a quantity of tannin remains behind in some of the cells as a secondary product, as well as various other substances not present in the seed.

The albuminoids which form so peculiar and intimate a mixture with the oil in the ripe seed, and which are partially contained in the aleurone-grains of the endosperm in the form of crystalloids, are, during the processes which have been described, transferred to the embryo, where they produce the protoplasm. During the whole of the period of germination the cells of the fibro-vascular bundles are found to be densely filled with albuminous substances, subsequently only those of the phloëm; these substances are evidently in motion toward the apices of the roots where new cells are continually being formed. Every young rudiment of a lateral root behaves to reagents as an accumulation of albuminous substance on the side of the fibro-vascular bundles of the primary root. But a very considerable portion of this material remains in the upper part of the stem of the seedling where new leaves are formed, and a still larger portion in the cotyledons themselves, where it furnishes the material for the formation of the numerous chlorophyll-granules.

After the consumption of the reserve-material at the end of the period of germination, the cells - with the exception of the youngest parts of the buds and the apices of the roots-are destitute of any formative material; although it has grown to a large size and contains a great quantity of water, the dried weight of the plant is very small and even less than that of the seed, because a portion of the substance has been destroyed in the process of respiration. But active organs have been formed from the earlier inactive store of material; the roots absorb water and dissolved foodmaterial; the green cotyledons begin to assimilate; they produce starch in their chlorophyll; and the same substance is subsequently found also in the parenchyma of the petioles and in the stem as far as the bud, the young leaves of which grow from the products of the assimilation of the chlorophyll. At first the unfolding of new leaves and the increase in length and thickness of the stem and roots are very slow; but the capacity for work possessed by the plant increases with every freshly developed leaf and every new absorbing root; on each successive day it can produce a larger quantity of formative material than on any preceding one, and thus the rate of growth also increases.

If a Castor-oil plant is examined at the time when vegetation is most active, when the green leaves supply the material for metastasis in all the organs, starch is found in their chlorophyll-granules and distributes itself from them through the parenchyma of the veins and petioles downwards into the stem as far as the root, and upwards to the young leaves which are not yet in a condition to assimilate. The excess which is not immediately required for the purposes of growth becomes deposited in the pith 
and medullary rays, where (this is not the case in the chlorophyll) it is always accompanied by sugar; and it is evidently this latter substance which brings about the diffusion from cell to cell, and at the same time furnishes the material for the formation of new starch-grains. The sugar is the migratory product which takes part in the diffusion; the starch-grains are the temporarily stationary product.

The distribution of starch and sugar shows moreover that they move from the primary stem through the rachis of the inflorescence and the pedicels into the parenchymatous tissues, and penetrate into the young tissue of the flower, the growing fruit, and the ovules, there to be employed in the production of cellulose. The distributed starch collects more abundantly, especially in the immediate neighbourhood of those layers of cells which afterwards form the hard endocarp and the solid testa of the seed, in consequence of its being required here in greater quantity, disappearing also from them after the complete development of these layers of tissue.

The sugar and starch are conveyed through the funiculus to the ovules; they are distributed through the integuments and the parts surrounding the nucellus; and a large quantity. of sugar enters the growing endosperm, which supplies the material for the formation of the oil which gradually accumulates, while fresh supplies of sugar are constantly entering from without. In the growing embryo the cells are filled at a certain period with fine-grained starch, which then entirely disappears and is replaced by oil. All this indicates that the oil of the ripe seed of Ricinus is produced from the starch and sugar which were transported to it from the assimilating organs during the period of ripening; and even the hard woody pericarp and the testa obtain their formative material from those substances. The albuminoids which collect also in the young leaves and from which the chlorophyll-granules are formed, as well as that portion of these substances which accumulates in the seed as reserve food-material, are transported from the stem by the sieve-tubes and the cambiform cells of the fibrovascular bundles.

3. In the Leguminosæ ${ }^{1}$ a very important part in the transport of the reserve proteid substances is played by Asparagin. To demonstrate this, moderately thin sections are placed in alcohol, and the saturation assisted by shaking. This mode is however applicable only when the asparagin is abundant; when it is present in small quantities it can still be demonstrated by placing a thin cover-glass on the sections, and running in underneath a little absolute alcohol. In this case the asparagin crystallises out round the section; while in the former case it is precipitated in the cells in the form of crystals. These can easily be recognised; they are comparatively large, and cannot be mistaken for other crystals which are formed in all plants on treatment with alcohol, even where no asparagin is present, since these-which belong to various salts, among others to nitrates-always remain very small and have an entirely different appearance.

Lupinus luteus is a good object for examination, and possesses the great advantage that we have in its case an analytical investigation of Beyer's ${ }^{2}$ in which the organic constituents and especially the asparagin have been quantitatively determined in the root, hypocotyledonary portion of the stem, and cotyledons, at two stages of germination, the last shortly before the cotyledons have thrown off the testa.

The migration of the non-nitrogenous reserve-materials takes place in the usual manner. Starch is first of all formed in the hypocotyledonary portion and root, then disappears and remains only in the endoderm, the rest being transformed into sugar.

1 What follows is taken from a letter from Dr. Pfeffer. (Compare Book I. Sect. 8. p. 5I ; also Jahrb. für wiss. Bot. VIII. p. 429 et seq.)

[It must not be thought, however, that this substance is confined to this group of plants, for it is very widely distributed, nor is it the only substance of this kind, for leucine and tyrosine have also been detected in germinating seeds.]

2 Landwirthschaftliche Versuchsstationen, vol. IX. 
Asparagin is first found in the hypocotyledonary portion and root when they are about Io $\mathrm{mm}$. long, but then rapidly increases in quantity while these parts elongate; and it is now found also in the petiole of the cotyledons, and in the cotyledons themselves before they have become green and thrown off their testa, especially in their lower part. The conditions remain the same during the whole of the time that the reserve albuminous substances are being consumed. Asparagin is now found in large quantities in the petiole of the cotyledons, almost to the extent of a saturated solution ( 1 part dissolves in 58 parts of water at $13^{\circ} \mathrm{C}$.), as well as in the hypocotyledonary portion and in the stem as soon as it begins to grow. The asparagin extends in the root and stem towards the puncta vegetationis almost exactly as far as the sugar, becoming finally, like the latter, less abundant. Beneath the cotyledons it is wanting in the pith, while in the stem it is as abundant there as in the cortical tissue; it is never found in the vascular bundles. The asparagin also extends into the petiole of young leaves as far as the base of the unfolding pinnæ, as well as into the lateral roots. As long as asparagin is formed out of the albuminous substances in the cotyledons, it may also be found in the plant distributed as has been described; but when the cotyledons have been entirely emptied, the asparagin also disappears; but this does not happen in the case of Lupinus luteus until several leaves have completely unfolded.

The process is quite analogous in Tetragonobolus purpureus and Medicago tuberculata; in Vicia sativa and Pisum sativum the presence of asparagin in the cotyledons themselves cannot be proved with certainty, but is found at their base and usually also in their petiole, although these plants produce decidedly less of it than Lupinus luteus. Since moreover chemical analysis has established the production of great quantities of asparagin on germination in the case of a large number of other species of the order, we may regard this substance as the form of transport for the albuminous substances characteristic of all Papilionaceæ. Albuminous substances are moreover found in these plants also in the thin-walled elongated cells of the vascular bundles; and it is quite possible that they are at the same time also transported by these structures. It is evident that the source of the asparagin must be the albuminous substances, because the absolute amount of nitrogen remains the same during germination; and the nitrogen of seeds is all or nearly all contained in their albuminous ingredients.

The following numbers show the percentage composition of asparagin, and the composition of an amount of legumin, containing an equivalent quantity of nitrogen.

\begin{tabular}{|c|c|c|}
\hline Asparagin. & Legumin. & Difference. \\
\hline $\mathrm{C}=3^{6 \cdot} \cdot 4$ & $\mathrm{C}=64^{\circ} 9$ & $+28^{\circ} 5$ \\
\hline$H=6 \cdot I$ & $\mathrm{H}=8.8$ & +2.7 \\
\hline $\mathrm{N}=2 \mathrm{I}^{\prime} 2$ & $N=2 I^{\prime} 2$ & $0^{\circ} \circ$ \\
\hline $\mathrm{O}=36^{\circ} 4$ & $\mathrm{O}=30.6$ & -5.8 \\
\hline
\end{tabular}

Asparagin contains less Carbon and Hydrogen but more Oxygen than Legumin and other proteids. Consequently if the whole of the Nitrogen of Legumin is used in the formation of asparagin, a considerable quantity of Carbon and Hydrogen must be given off and a certain amount of Oxygen absorbed. Exactly the opposite will take place upon the conversion of asparagin into proteid. Pfeffer points out (Monatsber. d. Berl. Akad., 1873) that this regeneration of proteid from the asparagin formed during germination depends in so far upon the action of light as this is necessary for the decomposition of carbonic acid in cells containing chlorophyll. Asparagin remains in a plant exposed to light if carbonic acid be not supplied to it.

In Tropaolum majus asparagin occurs in the earliest stages of germination only; it disappears at a later period whether germination is taking place in light or in darkness. In this case the asparagin is converted into proteid before the non-nitrogenous reservematerials' are exhausted; hence the regeneration can take place without assimilation. The formation of asparagin during germination takes place in light or in darkness: it is 
only the regeneration of the proteids which is dependent, as in the Papilionacex, in the manner above mentioned upon light, that is, upon assimilation ${ }^{1}$.

The existence of asparagin has also been proved in the leaves and stems of some plants (see Husemann, Pflanzenstoffe); and its presence in the underground perennial parts of Stigmaphyllon jatropbafolium almost gives the impression of its being there also a reserve-material.

The absorption of assimilated substances into the plant from without takes place in seedlings, the reserve-materials of which are contained in the endosperm, in parasites ${ }^{2}$, and in saprophytes which contain no chlorophyll. Seedlings, which have been most studied in this respect, show how the reserve-materials of the endosperm may pass into the absorbing organs (in this case almost always foliar structures) without there being any actual continuity of the absorbing organ with the endosperm; they only lie in close apposition, and can be separated without any injury $^{3}$ (as in Ricinus, Fig. 47 I). It cannot be doubted that the metamorphoses which take place in the nutrient endosperm are brought about by the absorbing organ, that is by the embryo itself; the behaviour of the endosperm of the germinating Date, which is absorbed by the delicate tissue of the absorbing organ belonging to the cotyledon, shows clearly that the hard thickening-layers of the cell-walls of the endosperm are first of all transformed into sugar under the influence of this organ, and then absorbed. A substance evidently passes out of the absorbing organ into the endosperm which causes this metamorphosis of the cellulose. The oil and albuminoids of the endosperm are at the same time taken up into the embryo, where all the conducting parts of the parenchyma are filled with sugar and starch as long as the endosperm is not entirely absorbed. In the same manner also in Grasses substances possibly pass out of the embryo into the endosperm, and there bring about the chemical metamorphosis and solution of the starch and albuminoids before they are absorbed by the scutellum which is applied to the surface of the endosperm. It is possible however that in this case there may be some means in the endosperm itself of bringing about the solution of the starch and gluten in the presence of water independently of any chemical action of the embryo.

The absorbing roots of parasites penetrate into the tissue of the host, and often grow into it in the most intimate manner. It is certain that the exciting cause of the transport of the products of assimilation from the host to the parasite resides in the latter; the parasite acts on the conducting masses of tissue of the host like a growing bud of the host itself; the food-materials penetrate into it because it consumes and changes them.

The influence exerted by the absorbing organ of the embryo on the substances

1 [See Schulze, Ueb. Zersetzung und Neubildung von Eiweissstoffen in Lupinenkeimlingen, Landw. Jahrb. VII, r878.]

2 Parasites which contain chlorophyll, like the Loranthaceæ, can themselves assimilate, and only require therefore to draw water and mineral substances from their host (see Pitra in Bot. Zeitg. I86I, p. 3). Those parasites which are apparently destitute of chlorophyll (like Orobanche), and saprophytes (as Neottia), contain, according to Weisner (Bot. Zeitg. 187 , p. 37), traces of chlorophyll, which however can hardly be taken into account in assimilation.

${ }^{3}$ For further details see the accounts given by me in Bot. Zeitg. 1862 and 1863 , of the germination of different plants. 
in the endosperm, dissolving and chemically changing them, points to the way in which the absorption of food-material is effected by saprophytes which possess no chlorophyll, their absorbing organs probably first causing the solution and chemical transformation of the decaying organic constituents of the humus. The decaying foliage in which Monotropa, Epipogium ${ }^{1}$, and Corallorhiza grow, does not give up to water the serviceable materials which are still present in it, any more than the cellulose of the endosperm of the Date, or the starch of the endosperm of Grasses, or the oil of the seed of Ricinus, can be extracted by water; but these saprophytes nevertheless obtain their nutriment from them. The fact that the roots of plants of this kind are so few in number and so diminutive in length, as in Neottia, or are entirely wanting, as in Epipogium and Corallorhiza, is very remarkable in connection with this. These plants are concealed in the nutrient substratum till the time of flowering, and may act upon it by their whole surface; and it is important to note that the absorbing surface of seedlings is very small in proportion to the great amount of work done, as is also the case with the absorbing roots of Cuscuta, Orobanche, \&c.

Sect. 6. - The Respiration of Plants ${ }^{2}$ consists, as in animals, in the continual absorption of atmospheric oxygen into the tissues, where it causes oxidation of the assimilated substances and other chemical changes resulting from this. The formation and exhalation of carbon dioxide-the carbon resulting from the decomposition of organic compounds-may always be directly observed; the production of water at the expense of the organic substance in consequence of the process of respiration is inferred from a comparison of the analysis of germinating seeds with the composition of those which have not yet germinated. Experiments on vegetation show that growth and the metastasis in the tissues necessarily connected with it only take place so long as oxygen can penetrate from without into the plant. In an atmosphere devoid of oxygen no growth takes place; and if the plant remains for any time in such an atmosphere it finally perishes. The more energetic the growth and the chemical changes in the tissues, the larger is the quantity of oxygen absorbed and of carbon dioxide exhaled; hence it is especially in quickly germinating seeds and in unfolding leaf- and flower-buds that energetic respiration has been observed; such organs consume in a short time many times their own volume of oxygen in the pro. duction of carbon dioxide. But in all the other organs also-in every individual cell-respiration is constantly going on ; and it is not merely the chemical changes connected with growth that are dependent on the presence of free oxygen in the tissues; the movements of the protoplasm also cease if the surrounding air is deprived of this gas; and the power of motion possessed by periodically motile and irritable organs is lost if oxygen is withheld from them;

1 See Reinke, Flora, 1873 , No. I0-r4.

2 The special references for what is said on this subject will be found in my work on Experimental Physiology, sect. 9, On the action of atmospheric oxygen. Of more recent works may be mentioned especially, Borscow, On the behaviour of plants in nitrogen (Mélanges biologiques tirés du Bulletin de l'Acad. Imp. des Sci. Nat. de St. Pétersbourg, vol. VI, 1867); also Wiesner, Sitzungsber. der Wiener Akad. vol. LXVIII, 1871 ; Bert, Comptes Rendus, 16 Juin, 1873 . [See also Wortmann, Arb. d. bot. Inst. in Würzburg, Bk. II, I88o; Pfeffer, Das Wesen und die Bedeutung der Athmung in der Pfianze, Landwirth. Jahrb. VII, 1878.] 
but if this happens only for a short time the motility returns when the oxygen is again restored.

The respiration of plants is, like that of animals, associated with a loss of assimilated substance, this loss being always a great deal smaller in assimilating plants than the gain of substance by the activity under the influence of light of the cells which contain chlorophyll; but when, as in the germination of seeds, an energetic growth is combined with powerful respiration, no new products of assimilation replacing the loss, the loss in weight of the growing plant may be very considerable. Seeds which germinate in the dark may in this way lose almost one-half of their dry weight, and it would seem that this loss is occasioned exclusively by the decomposition of the non-nitrogenous reserve material ${ }^{1}$ and its combustion into carbon dioxide and water. If the non-nitrogenous reserve-material consists of oil, i.e. of a substance containing very little oxygen, a portion of the inhaled oxygen remains in the germinating plant, carbo-hydrates containing a large quantity of oxygen such as starch and sugar being formed at the expense of the oil.

The loss of assimilated substance caused by respiration would appear purposeless if we had only to do with the accumulation of assimilated products; but these are themselves produced only for the purposes of growth and of all the changes connected with life; the whole life of the plant consists in complicated movements of molecules and atoms; and the forces necessary for these movements are set free by respiration. The oxygen, while decomposing part of the assimilated substance, sets up important chemical changes in the remaining portion, which on their part give rise to diffusion-currents, and these bring into contact substances which again act chemically on one another, and so on. The dependence on respiration of the movements in protoplasm and motile leaves is very evident, since, as has been mentioned, they lose their motility when oxygen is withheld from them. These considerations lead to the conclusion that the respiration of plants has the same essential significance as that of animals; the chemical equilibrium of the substances is being continually disturbed by it, and the internal movements maintained which make up the life of the plant. Respiration is, it is true, a source of loss of substance; but it is also in addition the perpetual source from which flow the forces necessary to the internal movements ${ }^{2}$.

1 [According to Borodin (Ueb. die physiol. Bedeutung des Asparagins im Pflanzenreiche, Bot. Zeitg. 1878$)$ this is not the case. In the process of respiration the nitrogenous substances constituting the protoplasm become oxidised, and of this oxidation asparagin is one of the products. The nonnitrogenous materials are used up in supplying plastic material to the protoplasm.

Asparagin is regarded, from this point of view, as a nitrogenous waste-product (metabolite), and it therefore corresponds physiologically to the urea formed in the animal body, a comparison which was long ago suggested by Boussingault.]

${ }^{2}$ [M. Corenwinder, from a series of observations on the Maple and Lilac, has confirmed the view to a certain extent held by Mohl, that the process of respiration is always going on in a plant even when concealed by the greater activity of the decomposition of the carbon dioxide by the parts containing chlorophyll. He distinguishes two periods in the vegetative season of the plant:the first period, when nitrogenous constituents predominate, is that during which respiration is most active ; the second, when the proportion of carbonaceous substance is relatively larger, is the period when respiration is comparatively feeble, the carbon dioxide evolved being again almost entirely taken up by the chlorophyll, decomposed, and the carbon fixed in the process of assimilation. He 
The combination to form carbon dioxide of the inhaled oxygen with a portion of the carbon of the assimilated substance is, like all combustion, accompanied by the production of a corresponding amount of heat; but this only rarely leads to a sensible increase of temperature of the masses of tissue, because respiration, and in consequence the production of heat, is not in general very copious, while the circumstances are very favourable to the loss of heat by the plant. In this respect also plants may be compared to cold-blooded animals. When an amount of heat is set free in the cells by the process of respiration, it first of all distributes itself over the large mass of water which permeates the cells and the adjoining tissue. In the case of a water-plant the least excess of temperature is at once equalised by the surrounding water; while in the case of a land-plant evaporation has a powerful cooling effect on the aërial parts, quite independently of the action of the radiation of heat which is favoured by the large superficial development of most plants, and especially by their hairiness. With these causes of a rapid loss of heat, it is not surprising that the parts of a plant which are expanded in the air are even colder than it, although their respiration is continually producing small quantities of heat. But if the causes of the loss of heat are removed, it is possible to observe with the thermometer the increase of temperature caused by respiration. This can be done by accumulating rapidly germinating seeds, as is shown in the considerable elevation of temperature of grains of Barley in the manufacture of malt ; and this elevation can also be proved in the case of other germinating seeds, or growing bulbs and tubers. The proof is more difficult in plants with green leaves.

In some flowers and inflorescences the production of carbon dioxide which accompanies the inhalation of oxygen is very energetic, the radiation of the heat produced being at the same time diminished by the small superficial extent of the organ and by protecting envelopes; and in such cases a very considerable elevation of temperature of the masses of tissue has been observed. The best illustration of this is the spadix of Aroider at the time of fertilisation, where (especially in warm air) an excess of temperature of from $4^{\circ}$ to $5^{\circ}$ or even of $10^{\circ} \mathrm{C}$. or more has been detected. Less considerable elevations of temperature have also been observed in the separate flowers of Cucurbita, Bignonia radicans, Victoria regia, \&c.

In the few cases in which up to the present time the development of light or Phosphorescence has been observed in living plants, this phenomenon is also dependent on the respiration of oxygen. In Agaricus olearius (of Provence) this has been definitely proved by Fabre. This Fungus emits light only so long as it is alive, and ceases to do so at once when it is deprived of oxygen; the respiration is in this case also very copious. Besides this Fungus, Agaricus igneus (of Amboyna), A. noctilucens (of Manilla), A. Gardneri (of Brazil), and the Rhizomorphs are known to emit light spontaneously; the statements with respect to the light emitted from various flowers are of extremely doubtful value ${ }^{1}$.

found that the proportion of nitrogenous matter in leaves gradually diminishes, while that of carbonaceous matter increases, between autumn and spring. (See Revue scientifique, Aug. 1, 1874.)]

1 [For a collection of recorded instances of phosphorescence in plants sec Hardwicke's Science Gossip, 1871, p. 121.] See my Experimental Physiology, and Schmitz (Linnæa, 1843, p. 523) and Bischoff (Flora, I 82., II. 426) on the phosphorescence of Rhizomorphs. 
The apparatus described in my Handbook of Experimental Physiology, p. 27i, may be easily employed, with the necessary modifications, for the observation of the production of carbon dioxide and the elevation of temperature of germinating seeds. The following experiment is also adapted for the demonstration of these points in a lecture. One-third of a glass cylinder of 2 litres capacity is filled with soaked peas or some other seeds or with flowers in the act of unfolding (e.g. small flower-heads of Compositæ, as Matricaria or Pyrethrum), and closed with a well-fitting glass stopper. If the vessel is opened carefully after several hours, the air contained will be found to extinguish a burning taper let down into it, as if it had been filled with carbon dioxide.

In order to observe the development of heat also in small quantities of seeds and even in single flowers of larger size, I use various forms of the apparatus represented in Fig. 472. The flask $f$ contains a strong solution of potash or soda $l$ which absorbs the carbon dioxide set free from the plants. In the opening of the flask is placed a funnel $r$, containing a small filter-paper perforated with

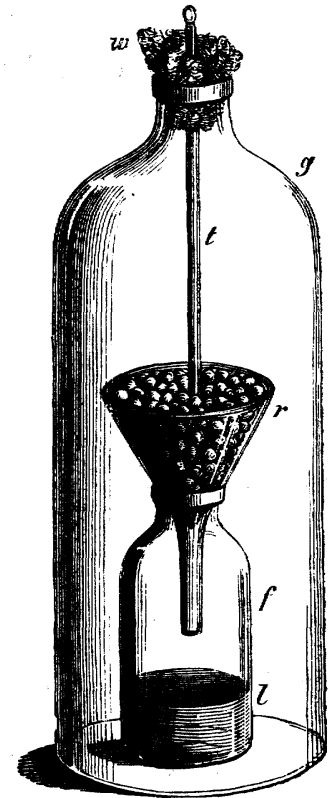

FIG. 472.-A pparatus for observing the rise of temperature in flowers and germinating seeds. a needle. The funnel is filled with soaked seeds or with cut flower-buds in the act of opening; and a bell-glass $g$ is now placed over it, through the tube of which a thermometer graduated to tenths of degrees is let in so that the bulb is surrounded on all sides by the plants. A loose plug of cotton-wool $z v$ closes the tube. In order to compare the temperature, a similar apparatus is placed close beside, in which the seeds or flowers as the case may be are or are not replaced by pieces of moist paper or green leaves. It is convenient to place both apparatuses in a large glass case in order still more completely to shield them from slow changes of temperature in the air of the room. As the isolation is not complete, the access of fresh oxygenated air to the plants is not hindered, and the continuance of respiration is therefore not prevented; the arrangement is on the other hand sufficient to reduce to a minimum the loss of heat by radiation and evaporation. The thermometers of both apparatuses, previously compared, must be frequently read off in order to detect the variations of temperature. If the bulbs are small enough, the elevation of temperature in the funnel may

be observed even with single flowers. In order to reduce still further the amount of evaporation and radiation, it is convenient, before the bell-glass $g$ is placed over, to cover the funnel with a perforated glass plate, the thermometer being inserted through its perforation.

It is possible under favourable circumstances to observe by means of this contrivance a rise of temperature of $\mathrm{I}_{5} 5^{\circ} \mathrm{C}$. with 100 or 200 peas, while the roots are developing; the anthers of a flower of the Gourd caused a rise of about $0.8^{\circ} \mathrm{C}$. in a tolerably large thermometer with the bulb of which they were in contact on only one side. A single capitulum of Onopordon Acantbium produced an elevation of $0.72^{\circ} \mathrm{C} . ;$ the stamens of a single flower of Nymphaa stellata one of about $0.6^{\circ} \mathrm{C}$. The temperature of a number of flower-buds of Anthemis cbrysoleuca heaped round the thermometer rose as they unfolded about $\mathrm{r} \cdot 6^{\circ} \mathrm{C}$.

It will be readily understood that flowers must not be used for these experiments as 
soon as they have been gathered; but that it is necessary to wait for some hours till they have acquired the temperature of the room. (Further details will be given elsewhere.) $\mathrm{M}^{\mathrm{c}} \mathrm{Nab}$ found that a large specimen of Lycoperdon giganteum produced a rise of temperature of $1 \cdot 2^{\circ}$ (F. or C.?). Bot. Zeitg. 1873, p. 560 .

C H A P T ER I I I.

\section{GENERAL CONDITIONS OF PLANT-LIFE.}

SECT. 7. The Influence of Temperature on Vegetation ${ }^{1}$ can only be investigated scientifically by observing the influence of definite and different degrees of temperature on the separate vital phenomena of plants, i.e. on the various processes of assimilation and metastasis, of diffusion, of growth, of the variations in the turgidity of the cells and tension of the tissues, of the movements of protoplasm and irritable organs and of those endowed with periodic motion, \&c.

The determination of the facts which have here to be investigated depends on an accurate determination of 'the temperature of the plant in any given case, or rather on that of the part of the plant in question on which the experiment is to be made. This is often attended with great difficulties, and is sometimes almost impossible, Independently of the changes of temperature, usually inconsiderable, caused by respiration in the interior of the plant, the temperature of each cell depends on its position in the mass of tissue and on the variations of the surrounding temperature. A constant interchange of heat is going on between the plant and its surrounding medium by conduction and radiation which essentially determines the temperature of any part of a plant at any particular time.

In reference to the conduction of heat, it must be mentioned in the first place that all parts of plants are bad conductors; the differences of temperature between them and the air, earth, or water that is in contact with them become only very slowly adjusted in this way. The conductivity for heat is probably also always different in different directions; that in the longitudinal direction in dry wood bears the proportion to that in the transverse direction of e.g. $\mathbf{I} \cdot 25: \mathbf{I}$ in the Acacia, Box, and Cypress, of $\mathrm{x} \cdot 8: \mathrm{I}$ in the Lime, Alder, and Pine.

The radiation of heat is on the other hand a very frequent and rapid cause of changes of temperature in most parts of plants; the chief effect of these changes being to bring about differences between the temperature of the surrounding medium and that of the plant, especially when the parts of the plant are of small size but have a large hairy surface, as is the case with many leaves and internodes. It must be noted in this connection that the radiating power of a body is equal to its

\footnotetext{
1 For more detailed proofs see my Handbook of Experimental Physiology, p. $4^{8}$ et seq.
} 
absorptive power; and that radiation depends not merely on the temperature, but also on the diathermancy of the surrounding medium.

In the aërial parts of plants, transpiration is an energetic additional cause of loss of temperature; inasmuch as water in the act of evaporation withdraws from the plant the amount of heat necessary for its vaporisation, and hence makes it colder.

In investigations of the influence of temperature on the various processes of vegetation, the conditions noticed above must always be carefully considered. It may be assumed in general that the result of their united action is that small waterplants and the underground parts of plants have usually nearly the same temperature as that of the surrounding medium when this temperature is not subject to too great variations; but that on the other hand leaves and slender stems exposed to air are generally colder than the air; while the thick stems of woody plants are sometimes warmer, sometimes colder, in consequence of their slow conducting power. How greatly the temperature of parts of plants of considerable superficial extent may be depressed by radiation below that of the air is shown by the fact that a thermometer placed on the grass and exposed to radiation indicates on clear nights a temperature several degrees lower than one placed in the air. If the latter is only a few degrees above the freezing-point, the temperature of the leaves of plants may in this manner fall below zero and they will suffer the effects of frost. The formation of dew on summer nights, and of the hoar-frost which is deposited in such large quantities on plants especially in the late autumn, are striking proofs of the effect of radiation in lowering their temperature. The relation of the temperature of plants to that of their surrounding medium is however very complicated when we have to do with solid bodies like trunks of trees, because the different powers of conduction in the longitudinal and transverse directions of the wood, and other causes, then cooperate -with the action of radiation and of absorption of heat through the bark. In general, as has been shown by Krutsch's beautiful experiments, the trunk is cooler during the day than the surrounding air, but warmer in the evening and night ${ }^{\text {. }}$.

With respect to the changes of volume in masses of tissue and in individual cells as the temperature varies, nothing is known with certainty except as regards dry wood. The numbers given by Caspary as the coëfficients of the expansion of wood caused by heat depend on untrustworthy observations and on a complete misunderstanding of the phenomena which take place in the objects observed ${ }^{2}$. When leaf-stalks and the branches of trees become curved at temperatures far below the freezing-point, this is obviously not altogether, if at all, caused by the different layers of tissue having different coëfficients of heat-expansion; but is mainly a consequence of the fact that the water of vegetation freezes, while the cellwalls lose water and in consequence contract more or less according to their state

1 [According to Becquerel, trees warm surrounding layers of air during the day and a good part of the night; they begin to cool them as soon as they have attained the same temperature. The maximum temperature is reached by the air two or three hours after midday; in the tree it is reached after sunset, in summer towards 9 p. m. See Mémoire sur les forêts et leur influence climatérique: Mém. de l'Inst. vol. XXXV. pp. 460-470.]

2 Proceedings of the International Horticultural Exhibition and Botanical Congress held in London, 1866, p. I16. 
of imbibition and of lignification. The phenomenon depends therefore in the first instance on a change in the state of imbibition and turgidity produced by different temperatures. Villari has carefully measured ${ }^{1}$ the coëfficients of heat-expansion for different dry woods. Like the expansion caused by the absorption of water, that caused by heat is much less in the direction of the fibres than in the radial direction across the fibres; but with the difference that the coëfficients of expansion for absorption are reckoned by hundredths in the radial and thousandths in the longitudinal direction, those for heat by hundred-thousandths and millionths; so that the alterations of the dimensions of dry wood in the two directions caused by changes of temperature are about rooo times smaller than those caused by the absorption of water. The following table is from Villari for temperatures between $2^{\circ}$ and $34^{\circ} \mathrm{C}$ : :-

\begin{tabular}{|c|c|c|c|}
\hline \multicolumn{3}{|r|}{ EXPANSION FOR $\mathbf{I}^{\circ}$} & Proportion. \\
\hline Box-wood. & $0.00006 \mathrm{r}_{4}$ & 0.00000257 & $25: 1$ \\
\hline Fir & $0.00005^{84}$ & $0.0000037 \mathrm{r}$ & I6: I \\
\hline Oak & 0.0000544 & $0.0000049^{2}$ & I $2:$ I \\
\hline Poplar . & $0.00003^{6} 5$ & $0.000003^{8} 5$ & $9: \mathbf{r}$ \\
\hline Maple & 0.0000484 & $0.0000063^{8}$ & $8: \mathrm{I}$ \\
\hline Pine. & 0.0000341 & $0.000005 \mathrm{I} \mathrm{I}$ & $6: \mathbf{I}$ \\
\hline
\end{tabular}

Since these numbers only hold good for dry wood, while wood as a constituent of the living plant can be observed only in the moist state, they cannot be applied directly to the explanation of the physiological phenomena due to changes of temperature; but they are nevertheless of great interest, since they give us an insight into the molecular structure of wood, especially as to its elasticity in different directions.

Something more is known as to the influence of different degrees of temperature on the vital phenomena of plants. On this subject the important fact must first be noted that the exercise of every function is restricted to certain definite limits of temperature within which alone it can take place; i.e. all functions are brought into play only when the temperature of the plant, or of the particular part of the plant, rises to a certain height above the freezing-point of the sap, and cease when a definite maximum of temperature is attained, which can apparently never be permanently higher than $50^{\circ} \mathrm{C}^{2}$ Hence the life of the plant, i,e. the course of its vital processes, appears to be confined in general within the limits zero and $50^{\circ} \mathrm{C}$. It must however be noted that the same functions may have very different limits between $0^{\circ}$ and $50^{\circ} \mathrm{C}$. in different plants; as is also the case with different functions in the same plant. A few examples will serve to explain this.

Since the cell-fluids, consisting of aqueous solutions often in a state of high concentration, do not usually freeze at zero, it is always possible for certain processes of growth to take place when the temperature of the surrounding air is as low as this, although this fact has not yet been sufficiently established. Uloth (Flora,

\footnotetext{
1 Poggendorff's Annalen, I868, vol. I33. p. $4^{\text {I } 2 .}$

${ }^{2}$ Sachs, Ueber die obere Temperaturgrenze der Vegetation, Flora, I864, p. 5; Krasan, Beitr. z. Kenntniss des Wachsthums, Sitzber. d. Wien. Akad. 1873.
} 
187 r, no. I2) observed the remarkable fact that seeds of Acer platanoides and of Wheat which had fallen between pieces of ice in an ice-house germinated there and pushed a number of roots several inches deep into the fissureless pieces of ice. From this observation he concluded that these seeds had the power of germinating at or even below the freezing-point of water; and that the penetration of the roots into the ice is caused by the development of warmth in the seed and by the pressure of the growing roots. It seems to me however that another explanation is possible. The ice was evidently surrounded by warmer substances, such as the walls of the house, which emitted to it rays of heat. Now it is a well-known fact that rays of heat, when they strike upon bubbles of air or bodies firmly frozen into a piece of ice, warm them and melt the surrounding ice. In this way not only the seeds but also their roots were warmed by the radiation of heat which passed through the ice, and thus the particles of ice in contact with them were melted. This experiment gives us therefore no certain knowledge of the actual temperature of the germinating seeds. The statements of different observers as to the highest temperature of the water in which some of the lower Algæ grow vary greatly; and Regel's assertion is perhaps the most probable that water must be below $40^{\circ} \mathrm{C}$. for plants to grow in it. I have convinced myself that a considerable number of plants are killed by an immersion for only ten minutes in water of $45^{\circ}$ or $46^{\circ} \mathrm{C}$., while flowering plants endure for a longer period an air-temperature of $48^{\circ}$ or $49^{\circ} \mathrm{C}$.; but at $5 \mathrm{r}^{\circ} \mathrm{C}$. lose their vitality after from ten to thirty minutes (any possible injury by drying up being of course prevented) ${ }^{1}$. As to the high temperatures which the spores of Fungi can endure without losing their power of germination, very different statements, some of them altogether incredible, have been made, according to which temperatures of more than $100^{\circ}$, even as high as $200^{\circ} \mathrm{C}$., would seem not to be injurious. Of ninety-four experiments which were made by Tarnowsky with all possible precautions ${ }^{2}$, the result was that the spores of Penicillium glaucum and Rhizopus nigricans exposed for from one to two hours to air of a temperature between $70^{\circ}$ and $80^{\circ} \mathrm{C}$. germinated only very rarely, while a temperature of $82^{\circ}$ or $84^{\circ} \mathrm{C}$. altogether killed them. Spores heated in their proper nutrient fluids entirely lose their power of germination at $54^{\circ}$ or $55^{\circ} \mathrm{C} .{ }^{3}$

The growth of parts of the embryo at the expense of the reserve-materials begins, as my experiments show ${ }^{4}$, in the case of Wheat and Barley even below $5^{\circ} \mathrm{C}$.;

${ }^{1} \mathrm{H}$. de Vries, Matériaux pour la connaissance de l'influence de la temperature, in Archives Néerlandaises, vol. V, 1870 , arrived at the same results from a number of experiments on Cryptogamia and flowering water and land-plants. According to Schmitz (Linnæa, 1843) Spharia carpophila is killed in ten minutes by water of $35^{\circ}-38^{\circ} \mathrm{R}$. $\left(43.5^{\circ}-47.5^{\circ} \mathrm{C}\right.$. $)$.

${ }^{2}$ One of the most important of these precautions is to prevent with certainty the entrance of spores after the temperature has been raised in the apparatus to the required point.

According to Wiesner, (Sitzber. d. Wien. Akad. 1873) spores of Penicillium glaucum sown on lemon pulp will not germinate below $\mathrm{r}^{\circ}{ }^{\circ} \mathrm{C}$. or above $43^{\circ} \mathrm{C}$. Any further development is confined to narrower limits. The most favourable temperature is from $22^{\circ}$ to $26^{\circ} \mathrm{C}$.

${ }^{3}$ For further details see pt. III of the Proceedings of the Botanical Institute of Wuirzburg.

4 Sachs, Abhängigkeit der Keimung von der Temperatur, Jahrb. für wissensch. Bot. vol. II. p. 338, 1860.-A. De Candolle in Bibliothèque universelle de Genève, I865, vol. XXIV. p. 243 et seq.Köppen, Wärme und Pflanzenwachsthum, eine Dissertation, Moscow 1870.-According to Kerner (Nat. wiss. Verein Janobrock, 1872 ) most plants, especially alpine plants, can germinate below $2^{\circ} \mathrm{C}$. See also further under chap. IV. 
in Phaseolus multiftorus and Zea Mais at $9.4^{\circ} \mathrm{C}$.; in Cucurbita Pepo at $13.7^{\circ} \mathrm{C}$ : But when the reserve-materials of the seed have been consumed, a higher temperature is apparently always necessary to enable growth to proceed at the expense of freshly assimilated material. The highest temperatures at which my observations indicate that germination can take place were about $42^{\circ} \mathrm{C}$. in the case of Phaseolus mulliflorus, Zea Mais, and Cucurbita Pepo; in Wheat, Barley, and Peas, about $37^{\circ}$ or $38^{\circ} \mathrm{C}$.

The lowest temperature at which the chlorophyll-granules turn green was determined for Phaseolus multiflorus and Zea Mais at above $6^{\circ}$, and probably below $15^{\circ} \mathrm{C}$.; for Brassica Napus above $6^{\circ} \mathrm{C}$.; for Pinus pinea between $7^{\circ}$ and $\mathbf{I I}^{\circ} \mathrm{C}$. The highest temperature at which leaves already formed and still yellow turn green was for the first-named plants above $33^{\circ}$; for Allium Cepa above $36^{\circ} \mathrm{C}$.

The exhalation of oxygen and the corresponding assimilation begin, according to Cloëz and Gratiolet, in the case of Potamogeton between $10^{\circ}$ and $15^{\circ} \mathrm{C}$; ; in Vallisneria above $6^{\circ} \mathrm{C}$. In many Mosses, Algæ, and Lichens, assimilation may possibly take place at still lower temperatures; according to Boussingault (Compt. Rend. vol. 68. p. 4 IO), carbon dioxide is decomposed by the leaves of the Larch at $0.5^{\circ}$ to $2.5^{\circ} \mathrm{C}$., and by those of Meadow-grasses at $\mathrm{I} \cdot 5^{\circ}$ to $3.5^{\circ} \mathrm{C}$. Heinrich found the minimum temperature at which bubbles of gas were given off by Hottonia palustris to be $2 \cdot 7^{\circ} \mathrm{C}$. The upper limit of temperature for this function has not been ascertained, except for Hottonia palustris, in which case Heinrich found it to be $50^{\circ}-56^{\circ} \mathrm{C}$.

The irritability and periodical movement of the leaves of .Mimosa do not begin till the temperature of the surrounding air exceeds $15^{\circ} \mathrm{C}$; ; the periodical movements of the lateral leaflets of the leaf of Desmodium gyrans only at temperatures above $22^{\circ} \mathrm{C}$. The upper limit of temperature for the sensitiveness of the leaves of Mimosa depends on the continuance of the warmth; in air of $40^{\circ} \mathrm{C}$. they become rigid within an hour; at $45^{\circ} \mathrm{C}$. within half an hour; at $48^{\circ}$ to $50^{\circ}$ within a few minutes, but may again become sensitive when the temperature falls. A temperature of $52^{\circ} \mathrm{C}$. causes permanent loss of the power of motion and death.

The lower limit of temperature for the motility of the protoplasm in Nitella syncarpa is stated by Nägeli to be zero; for the hairs of Cucurbita my observations place it at a temperature of $10^{\circ}$ or $1 \mathrm{I}^{\circ} \mathrm{C}$. The upper limit is $37^{\circ} \mathrm{C}$. in the case of Nitella syncarpa according to Nägeli ; in the hairs of Cucurbita, when immersed in water of $46^{\circ}$ or $47^{\circ} \mathrm{C}$., the current is arrested within two minutes; in the air exposure to a temperature of $49^{\circ}$ or $50^{\circ} \mathrm{C}$. for ten minutes does not stop the current. The current in the hairs on the filaments of Tradescantia ceases within three minutes in air at $49^{\circ} \mathrm{C}$., beginning again when the temperature is reduced.

The absorption of water through the roots is also confined to certain limits of temperature. Thus I found that the roots of the Tobacco-plant and Gourd no longer absorb sufficient water to replace a small loss by evaporation in a moist soil of from $3^{\circ}$ to $5^{\circ} \mathrm{C}$; the heating of the soil to from $12^{\circ}$ to $18^{\circ} \mathrm{C}$. suffices to raise their activity to the needful extent. The roots of the Turnip and Cabbage on the contrary absorb a sufficient quantity of water from soil reduced nearly to the freezingpoint to replace a moderate loss by transpiration.

A second result of the observations hitherto made may be stated as follows:The functions of a plant are assisted and accelerated in their intensity when the 
temperature rises above the lower limit for that function; on reaching a definite higher degree a maximum of intensity is attained; the activity then decreases with a further increase of temperature, until it entirely ceases at the upper limit. There is therefore no proportionality between a rise in the temperature and in the intensity of the function. Thus, according to my observations, the rate of growth of the roots of a seedling of Zea Mais attains its maximum at $27 \cdot 2^{\circ} \mathrm{C}$, of the Pea, Wheat, and Barley at $22.8^{\circ} \mathrm{C}$; while an increase of the temperature of the soil beyond these points causes in each case a decrease in the rapidity of growth ${ }^{1}$.

The irritability of the leaves of Mimosa is rather sluggish between $16^{\circ}$ and I $8^{\circ} \mathrm{C}$, and appears to reach its maximum at $30^{\circ} \mathrm{C}$. The periodically motile lateral leaflets of the leaf of Desmodium gyrans oscillate, according to $\mathrm{Kabsch}$, in from eighty-five to ninety seconds at $35^{\circ} \mathrm{C}$, , in from 180 to 190 seconds between $28^{\circ}$ and $30^{\circ} \mathrm{C}$; at lower temperatures the oscillations are imperfect, and at $23^{\circ}$ or $24^{\circ} \mathrm{C}$. they become almost imperceptible.

The rapidity of the movement of the protoplasm in Nitella syncarpa attains its maximum, according to Nägeli, at $37^{\circ} \mathrm{C}$; ; at a higher temperature the movement ceases. In the hairs of Cucurbita, Solanum Ly'copersicum, and Tradescantia, as well as in the leaves of Vallisneria, I found the motion of the protoplasm slow between $12^{\circ}$ and $16^{\circ} \mathrm{C}$., very rapid between $30^{\circ}$ and $40^{\circ}$, slower again between $40^{\circ}$ and $50^{\circ} \mathrm{C}$.

Very great and rapid variations of temperature between zero and $50^{\circ} \mathrm{C}$. have been shown by experiments made by De Vries on a number of different growing plants not to be attended with danger to life, inasmuch as no injury could be detected either at the time or afterwards. It does not however follow from this that considerable changes of temperature are without effect. It would appear rather that when a plant is generally exposed to a favourable temperature, its functions are carried on the more energetically the more constant this favourable temperature remains. This is shown by ordinary experience in horticulture, and still more by the experiments of Hofmeister (Pflanzenzelle, p. 53) and De Vries (l.c.) on the movement of protoplasm, and of Köppen (l.c.) on the growth of roots. The influence of sudden variations of temperature in producing an injurious effect on the plant is however very complicated, and has not yet been thoroughly investigated. I have shown that any rapid increase or decrease of temperature is accompanied by an increase or decrease of the rapidity of growth; although, according to Köppen, the increase of growth during a long period is less when the temperature is variable than when it is constant, the mean temperature being the same in both cases, a conclusion which the more recent experiments of Pedersen ${ }^{2}$ render questionable.

If the upper and lower limits mentioned above are exceeded, the functions of the plant may, according to circumstances, simply come to rest, again to become active on the return of a favourable temperature, or permanent changes are brought about, resulting in injury and finally in the destruction of the cells.

1 Further details on this subject will be found in my treatise already named, and in De Vries and Köppen (l.c.). Compare also what is said in chap. IV, on the influence of temperature on the rapidity of growth.

${ }^{2}$ [Haben Temperaturschwankungen als solche einen ungünstigen Einfluss auf das Wachsthum? Arb. d. bot. Inst. in Würzburg, I. 4, 1874 .] 
Cells killed by too high a temperature or by freezing show in general the same changes as if they had been killed by poison, electricity, \&c.; the protoplasm becomes stationary, turgidity ceases because the resistance of the cell-walls together with that of the protoplasm diminishes, and allows the sap to filter out; the tissues become flaccid; secondary chemical changes of the sap produce the same dark colour as in expressed juices; and rapid evaporation soon causes a complete drying up of the dead tissue.

The injury resulting from too high or too low a temperature may, under certain circumstances, be indirect and slow in its manifestation; this will be the case. when a particular function is too highly excited or too much depressed, and thus the harmonious co-operation of the various vital processes is disturbed. Thus growth may be so excited by too high a temperature that assimilation, especially when the light is deficient, is not sufficient to supply the necessary formative material; and the transpiration of the leaves may in addition be so much increased that the activity of the roots is insufficient to replace the loss. On the other hand, too low a ground temperature may so depress the activity of the roots that even small losses by transpiration from the leaves can no longer be replaced. We shall refer in the sequel to the injuries caused immediately to the cells by too high a temperature and by the freezing and thawing of the tissues.

I. The destruction of the life of cells by too high a temperature depends, like freezing, on their containing water. While succulent tissues are killed below or at $50^{\circ} \mathrm{C}$., air-dry seeds of Pisum sativum can resist a temperature of over $70^{\circ} \mathrm{C}$. for an hour without losing their power of germination; of grains of Wheat and Maize heated to $65^{\circ}$ for an hour, 25 p.c. germinated in one case. Peas soaked in water for an hour and exposed to a temperature of $54^{\circ}$ or $55^{\circ} \mathrm{C}$. were all killed; Rye, Barley, Wheat, and Maize at $53^{\circ}$ or $54^{\circ} \mathrm{C}$. Spores of Fungi showed similar phenomena, as is seen from Tarnowsky's experiments. The cause of death appears to be the coagulation of the albuminoids of which the protoplasm is composed, and this again depends on their containing water and on other circumstances, since these render a different temperature necessary for coagulation in different cases. The disorganisation of the cell-wall is perceptible only at higher temperatures; and that of starch, which only takes place between $55^{\circ}$ and $60^{\circ} \mathrm{C}$., need not be taken into consideration here, since cells which contain no starch are also killed by a rise of temperature above $50^{\circ} \mathrm{C} .{ }^{1}$

2. Freezing, or the destruction of cells by the solidifying of the water contained in them into ice and by the subsequent thawing of the latter, depends also mainly on the quantity of water in the cells. Air-dry seeds appear to be able to withstand any degree of cold without injury to their power of germination; the winter-buds of woody plants the cells of which contain a great quantity of assimilated substances but only a small quantity of water, stand the cold of winter and frequent rapid thawing; while the young leaves at the time of their unfolding in the spring succumb to a slight nightfrost. An at least equally important condition lies however in the specific organisation of the plant; varieties of the same species frequently differing in their power of resistance to cold and thawing. Some plants, like Mosses, Hepaticæ, Lichens, some Fungi of a leathery texture, the Mistletoe, \&c., appear in particular never to freeze; Pfitzer states that the Naviculex freeze between $-10^{\circ}$ and $-20^{\circ} \mathrm{C}$. and continue to live after thawing; while many flowering plants from a southern climate are killed by rapid changes of temperature near the freezing-point ${ }^{2}$. Schmitz (Linnæa, 1843 ) observed that an Agaricus fascicularis which had been frozen stiff grew after thawing.

1 The statements of Wiesner (Sitzungsber. der Wien. Akad. I87i, Oct., vol. LXIV. pp. I4, I5) I am unable to understand. A variety of recent statements as to the high temperatures which the spores of Fungi are said to be able to resist without losing their power of germination are so incredible and require such critical sifting that $I$ pass them by altogether.

${ }^{2}$ On the minimum of temperature which vegetation can in general bear see Göppert, Bot. Zeitg., $187 \mathrm{I}$, nos. 4 and 5 : [also Bot, Zeitg. 1875.] 
Whether the tissue of a plant can be killed simply by the solidifying of the water contained in its cells into crystals of ice is uncertain; while on the other hand it is unquestionable that in a great number of plants death is caused only by the mode in which the thawing takes place. The same tissue which retains its vitality if thawed slowly after the freezing of the water of its cell-sap, becomes disorganised if thawed rapidly after exposure to the same degree of cold. Death is therefore caused in these plants not by the freezing but by the thawing .

When ice is formed in the tissues of a plant, two points must be taken into consideration. The water, when about to freeze, is on the one hand contained in a mixed solution, the cell-sap; on the other hand it is retained by the force of cohesion as water of imbibition in the mice!lar interstices of the cell-wall and of the protoplasm. Now it is an established fact in physics that a solution when freezing separates into pure water which solidifies into ice and a concentrated solution with a lower freezing-point ${ }^{2}$. When therefore a portion of the cell-sap-water freezes, the remainder of the cell-sap becomes more concentrated; and chemical changes may possibly be induced, as Rüdorff has shown, by new combinations actually arising in a freezing solution. How far this circumstance must be considered in the destruction of cells by freezing and thawing is not yet decided.

What takes place in the freezing of a saturated organised body capable of swelling up is somewhat similar to that which occurs in a freezing solution. In this case also, when the temperature falls to a certain point, only a portion of the water freezes; the rest remains as water of imbibition between the micellæ of the body, which contracts, while the freezing portion of the water of imbibition separates to form ice-crystals. This phenomenon happens in a striking manner in starch-paste; a homogeneous mass before freezing, it has the appearance after thawing of a spongy coarsely porous structure, the water running off clear from its large cavities. The behaviour of coagulated albumen on thawing is exactly the same. In these cases a permanent change has clearly been brought about by the freezing of a portion of the imbibed water; the molecules of the substance which group themselves into a network containing but little water when ice is formed in paste or coagulated albumen, on thawing no longer combine with the portions of the water which separated from them on freezing into a homogeneous whole; the thawed paste is in fact no longer paste.

When living succulent tissue freezes, a portion only of the water separates and freezes as pure water, the rest remaining as water of imbibition in the protoplasm and the cell-walls, at least as long as the temperature does not sink very low. In leaves and succulent stems frozen at a temperature between $-5^{\circ}$ and $-10^{\circ} \mathrm{C}$. it is easily seen that only a portion of the water is present in the form of crystals of ice; another portion permeates the cell-walls which are not rigid but still flexible. If the congelation takes place slowly, the water assumes on the surface of the succulent tissue the form of a coating of ice consisting of densely crowded small crystals. These crystals stand at right angles to the surface of the tissue, and increase by growth at their base. A very large portion of the water of a tissue may in this way take the form of a coating of ice, while the tissue, becoming less watery, contracts in proportion ${ }^{3}$, and loses its

1 The correctness of this statement is supported by a careful series of observations which I communicated to the königl. sächs. Gesellsch. der Wissensch. I960, On the formation of crystals, \&c., and which will be found also in the Landwirthschaftliche Versuchsstationen, I86o, Heft. V. p. 167 , and in my Handbook of Experimental Physiology. I do not find that Göppert's objections (Bot. Zeitg., r87I, no. 24) affect my results; to his experiment on Calanthe veratrifolia quite a different explanation can be given from that suggested by him.

${ }^{2}$ Rüdorff, Pogg. Ann. I861, vol. CXIV. p. 63 ; and i 862 , vol. CXVI. p. 55 .

3 When this contraction operates unequally on different sides of a leaf or branch, it is easy to see that curvatures must result which are indeed actually frequently observed. The splitting of the trunks of trees in consequence of frost is probably only the result of changes of this nature. 
turgidity. This phenomenon is seen with remarkable clearness in the large leaf-stalks of Cynara Scolymus when they freeze slowly. The succulent parenchyma separates from the epidermis, which surrounds the former like a loose sack; the parenchyma itself splits apart in the interior so that each fibro-vascular bundle is enclosed in an envelope of parenchyma. Fig. 473 shows how the coatings of ice project from the masses of parenchyma. From pieces of the leaf-stalk which weighed 396 grammes I have collected $99 \mathrm{gr}$. of ice, which, when evaporated to dryness after thawing, left only slight traces (about $0^{\circ}$ I p. c.) of solid substance. I have often observed similar phenomena in other plants; the formation of ice is however not so regular as here. In the cavities of the ruptured tissue (as in the succulent stems of the Cabbage) small irregular flakes of ice are formed; sometimes the ice splits the epidermis and projects in the form of combs above the surface of succulent stems (Caspary). I have already shown elsewhere ${ }^{1}$ that when sections of succulent parts of plants (such as the Beet) are protected from evaporation and allowed to freeze slowly, continuous coatings of ice are produced on the surfaces of the section, consisting of prisms growing at the base. The formation and growth of these ice-crystals may be explained in this way. The temperature of the tissue falls to a certain point, thereby causing the freezing of an extremely thin stratum of water which overspreads the outside of the uninjured cell-walls. A new very thin stratum of water then immediately passes out of the cell-wall to its surface and also freezes,

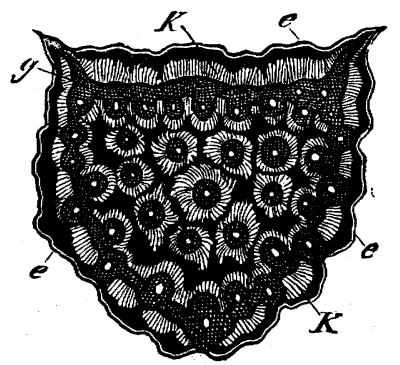

FIG. 473-Transverse section of a slowly frozen leaf-stalk of Cynara Scolymus; $e$ the detached epidermis; $g$ the parenchyma in which lie the transverse sections of the fibro-vascular bundles (left white). It forms a tough but pliant mass, which is ruptured during the process of freezing; a peripheral layer has become separated from the inner parts which surround the bundles; the surface of each portion of the parenchyma is covered with a crust of ice $K K$ con. sisting of densely crowded prisms (the cavities of the ruptured tissue are left black in the figure).

thickening the stratum of ice already formed; and thus it goes on. The cell-wall is constantly absorbing cell-sap-water from within, and at the same time allows the outermost molecular stratum of its water of imbibition to freeze. The first thin layers of ice on the exterior of the uninjured cells form polygonal plates in contact with one another; each plate becomes a prism by growth on its lower side; and the closely crowded prisms form a coating of ice which easily crumbles. These processes cause tbe cell-sap to become a more and more concentrated solution, while the cell-wall and the protoplasm contain a gradually diminishing quantity of water. It can now be to a certain extent understood why a rapid thawing kills the cells, while a slow thawing does not; for if the thawing take place slowly, the ice-crystals melt at their base where they touch the cell; the water as it becomes fluid is at once absorbed into the cell; and the original conditions of the cell-sap, cell-wall, and protoplasm may be re-established, if they have not been permanently impaired during the freezing. If on the contrary the coating of ice

1 Sachs, Formation of Crystals in the Freezing, and change of the Cell-walls in the Thawing of Succulent Parts of Plants (Bericht der kön. sächs. Ges. der Wiss. r 860). I have already mentioned in the first edition of this work the formation of crystals in the interior of frozen plants described above, and applied it to the explanation of freezing. Prilieux (Ann. des Sci. Nat. vol. XII. p. I28) afterwards, in 1869 , also described similar phenomena in a variety of plants. 
melt off very quickly, a portion of the water runs into the interstices of the tissue before it can be absorbed; the original normal degree of concentration of the cell-sap and degree of imbibition of the cell-wall and protoplasm cannot be re-established in the cells; and this, depending upon the chemical nature of the substances dissolved in the cell-sap and upon the conditions of the micellar structure of the protoplasm and of the cell-wall, may be fatal. It is evident, on the view here taken, that the danger of freezing increases with the amount of water in the tissue; for the less watery the tissue the more concentrated is the cell-sap and the larger is the proportion of water retained by the force of imbibition; only a small portion of the water can therefore form ice-crystals, and when they thaw the injurious effects are not so great.

We can now also understand why some plants are killed by being thawed too quickly when they have been frozen by very severe cold, while freezing by a moderate amount of cold is not injurious to them; for the lower the temperature falls the larger is the proportion of the cell-sap and water of imbibition that is converted into ice; the disturbance of the degree of concentration of the sap and of the imbibition of the cellwall is always greater with the increase of the cold; and therefore the restoration of the normal condition on thawing more difficult. That the splitting asunder of whole masses of tissue during freezing such as has been described has but little effect on the continuance of the life of the organ after thawing, is shown by the fact that even the leaf-stalks of the Artichoke, the frozen state of which is represented in Fig. 473, remain uninjured till the following summer if thawed slowly. These internal rupturings have as little to do with the sudden destruction of the life of the cells from cold as the splitting of the trunks of trees caused by frost, which, when the temperature falls very low, is produced by the contraction of the bark and outer layers of wood, the crevices again closing when the temperature rises.

The idea that growing plants, especially those which require a high temperature for their growth, can be directly killed by the cooling of their tissues for a short time nearly to the freezing-point is shown by $\mathrm{H}$. de Vries' experiments $(l . c$. $)$ to be fallacious. The older observations of Bierkander and Hardy that some plants of this description (e.g. Cucurbitacex, Impatiens, the Potato, Bixa Orellana, Crescentia Cujete, \&c.) freeze when exposed to the air at low temperatures above the freezing-point, may nevertheless be explained if it is recollected that the temperature of their tissues may fall below the freezing-point from radiation, even when that of the air is $2^{\circ}$ or $3^{\circ}$ or even $5^{\circ} \mathrm{C}$. above it. But there is another way in which low temperatures above zero are injurious to plants from southern climates, viz. when the soil about the roots remains for a considerable time at this low temperature while the leaves continue to transpire. In this case the absorption of water through the roots becomes so slow that they are no longer able to replace the loss caused by evaporation from the leaves, which in consequence wither, and at length altogether dry up. It is then sufficient to warm the soil about the roots, in order to revive the withered leaves; as I found in the case of plants of Nicotiana, Cucurbita, and Pbaseolus grown in pots. In England the branches of a Vine which were made to grow into a hothouse, while the roots stood in the ground outside, withered in winter, evidently only from the low temperature of the ground; for when this was watered with warm water, the branches in the hothouse recovered.

3. Among the changes caused in plants by long-continued depression of temperature, one of the most striking is the change in colour of leaves which persist through the winter, originally observed by $\mathrm{Mohl}^{2}$, and recently more minutely studied by Kraus ${ }^{3}$.

${ }^{1}$ Sachs, in Landwirthschaftliche Versuchsstationen, 1865 , Heft V. p. 195.

2 Mohl, Vermischte Schriften; Tübingen, 1845 , p. 375.

3 Kraus, Observations on the winter colouring of evergreen plants; in the Sitzungsber. der phys-med. Societät zu Erlangen, Dec. 19, I87I, and March 11, I872; also Bot. Zeitg.. 1874. [Batalin has shown (Bot. Zeitg., I874), and his observations have been confirmed by Askenasy (Bot. Zeitg, 1875), that this change of colour is due rather to the influence of light than to that of cold.] 
This change is of two kinds; the leaves either merely lose their colour and become brownish, yellowish, or rusty brown, as in Taxus, Abies, Pinus, Juniperus, and Buxus; or turn a decided red on the upper surface, as in Sedum, Sempervivum, Ledum, Mabonia, Vaccinium, \&c. The loss of colour of the first group depends, according to Kraus, on a change in the chlorophyll-granules, which lose their form and definition, a cloudy mass of protoplasm of a reddish brown or brownish yellow colour being formed, while the nucleus of the cell remains colourless. These changes are usually more complete in the 'pallisade cells' on the upper side than in the parenchyma which lies deeper. A spectroscopic examination shows that of the two pigments, a mixture of which forms, according to Kraus, the colouring substance of chlorophyll, the golden-yellow one remains unchanged, while the spectrum of the bluish-green substance undergoes a slight change.

The winter-leaves of the second group, which are coloured red or purplish-brown on the upper side, owe this colour to a rounded hyaline strongly refractive mass lying in the upper part of the pallisade-cells, which appears of a beautiful carmine-red where the leaves are red, but elsewhere of a pale-yellow, and consists mainly of tannin. The chlorophyll-granules, intact and of a beautiful green, are all crowded together in the inner end of these cells. In the spongy parenchyma of the mesophyll a colourless or red mass of tannin occurs in the centre of each cell, while the chlorophyll-granules, also intact, are collected in roundish or irregular lumps, sometimes in one place, sometimes in several, but always on the sides towards the adjoining cells. In these cases the colouring matter of the chlorophyll is unchanged with regard to either of its constituent pigments. The red-colouring matter is soluble in water, and cannot be distinguished by spectrumanalysis from the red colouring substances of flowers.

In all leaves which persist through the winter, and in the green parts of bark, Kraus found that the chlorophyll-granules had removed from the walls to the interior of the cell, and had collected there in lumps (see Sect. 8). When the weather has become sufficiently warm in the spring, the normal condition is restored; the red colouring substance disappears, and the chlorophyll-granules again take up their normal position on the cell-walls. Kraus shows that the winter change of the leaves depends on the fall of the temperature, since it is restored to the normal state by a simple rise in the temperature, whether in the dark or the light. By taking cut branches of Box into a warm room when the cold was severe and placing them in water, he found that the protoplasm of the cells, which had become homogeneous after one or two days, collected on the walls, and then divided into grains (as in the formation of chlorophyll-granules in the dark); the red colouring matter being changed first to a yellowish-green and finally to pure green. After the lapse of three, five, or at most eight days, the walls of the cells became lined with bright green sharply-defined chlorophyll-granules. In Thuja the process required two to three weeks (with me however only a few days). The restoration is therefore rather a slow process; while, according to Kraus, a single frosty night suffices to bring about the change in the form and colour of the chlorophyll-granules in the case of Buxus, Sabina, and Tbuja. That light has no share in the restoration of the normal condition of the chlorophyll is shown by the fact that it takes place also in branches which are kept in a dark room. On the other hand, the fact that the parts protected by being covered by other leaves show no change of colour would seem to indicate that the whole phenomenon has less to do with the low temperature of the air than with the cooling produced by radiation.

4. Convenient contrivances for observing the action of particular higher or lower temperatures on plants or parts of plants of considerable size are easily arranged ${ }^{1}$. It is more difficult to expose microscopic objects to a particular higher or lower temperature in such a manner that it can easily and certainly be observed, and that the temperature of the object is also that indicated by the thermometer, or nearly so.

${ }^{1}$ See Sachs, Handb. der Exp.-Phys. pp. 6.4, 66. 
All these requirements are fulfilled by the very cheap heating apparatus for the microscope represented in Fig. 474. Since I have not only made great use myself of this apparatus for three years, but have also recommended it to others, a description is the more in place here as it is well adapted for demonstrations in lecture-rooms.

The size of the heating apparatus must vary with that of the microscope; mine is constructed for one of Hartnack's ordinary instruments. The box is nearly cubical, and has double walls of sheet-zinc at the bottom and sides, enclosing a space $25 \mathrm{~mm}$. thick, which is filled with water through the hole $l$. It is quite open above; but in the front

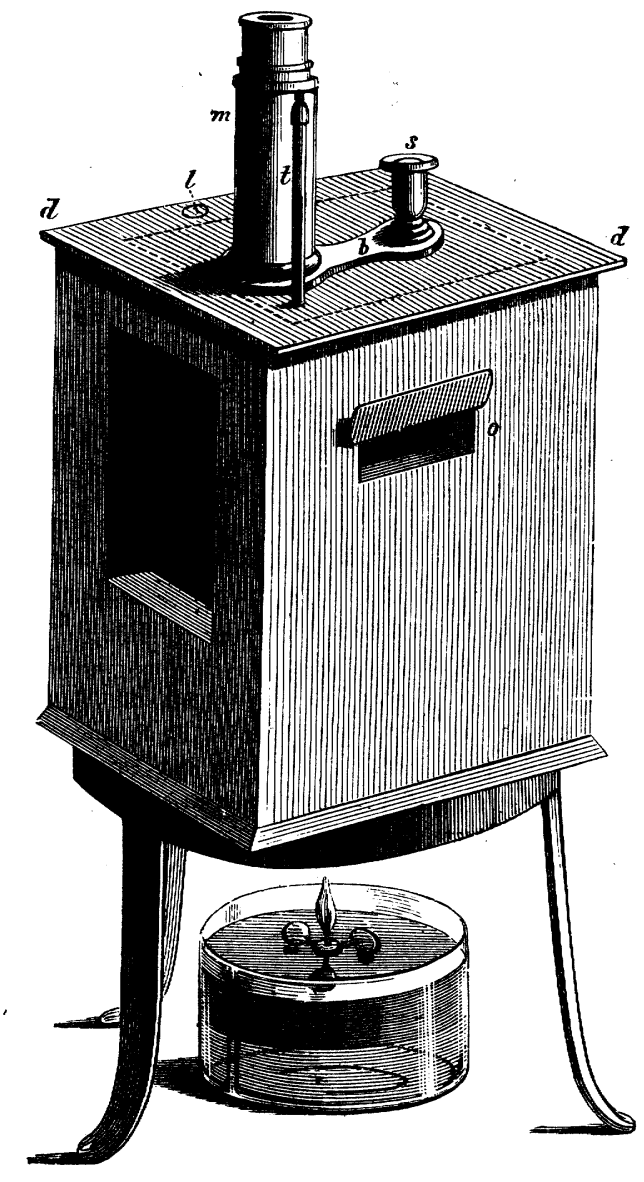

FIG. 474-Heating apparatus for the microscope.

side-wall is an opening $f$, which is closed by a glass plate well fitted but not otherwise fixed. This window is sufficiently large, and is so placed that it allows enough light to fall on the mirror of the microscope which stands in the box. The height of the box is so arranged that the upper rim of the double wall is on a level with the arm $b$ of the microscope. The opening of the box is closed by a thick cardboard cover $d d$, in which an opening is cut exactly to fit the $\operatorname{arm} b$. By the side of the tube of the microscope a round hole is cut in the cover through which a closely fitted small thermometer $t$ is passed, so that its bulb hangs near the object. The box is painted on the inside with black varnish, and a piece of cardboard moistened with water lies beneath the foot 
of the microscope in order to prevent its moving and to keep the air within moist. The focus is easily adjusted to the object by means of the fine adjustment $s$ which projects above the cover; two openings in the side, one of which is shown at $o$, enable the slide bearing the object to be moved, when necessary, by a pair of forceps. It is still more convenient to fix the slide on a wire which goes through a cork fitted to the opening 0.

If observations are required at a high temperature, the water in the box is heated by a spirit-lamp placed underneath. When the temperature has reached nearly the desired point, the spirit lamp is replaced by an oil-lamp with a floating light; the temperature will after a time become constant. In order to obtain higher or lower constant temperatures, one, two or three floating night-lights are placed in the lamp. If care is taken that the combustion be uniform, the temperature in the box remains for several hours so constant that it will vary only about $I^{\circ} \mathrm{C}$. This constancy of temperature ensures that the temperature of the object itself is that indicated by the thermometer.

It is easy by means of this heating apparatus to observe and demonstrate the influence of temperature on protoplasm-currents. To take observations at low temperatures it is sufficient to enlarge the hole $l$, in order from time to time to place pieces of ice in the cold water ${ }^{1}$.

Sect. 8.-Action of Light on Vegetation". A. General. The entire life of the plant depends on the action of light on the cells that contain chlorophyll, this being the essential condition under which new organic compounds are formed out of the elements of carbon dioxide and water. The amount of oxygen evolved in this process is nearly the same as that required for the combustion of the substance of the plant; and the amount of work equivalent to the heat produced by this combustion gives a measure of the amount of work performed by light in the chlorophyll-containing cells of the plant.

After a certain quantity of assimilated substance has been produced under the influence of light, a long series of vegetative processes may be carried on at its expense without any further direct action of light. The growth of new organs and the metastasis connected with it kept up in the organs by means of respiration is entirely or to a certain extent independent of light, and can even be carried on in absolute darkness. This is the case in the germination of seeds, bulbs, and tubers, the development of buds from woody branches and underground rhizomes, \&c. Even leafy plants which have accumulated a sufficient quantity of reserve-material in the light put out shoots and even flowers and fruits when placed in the dark.

As the parts of chlorophyll-containing plants which are underground or otherwise excluded from light are nourished by the products of assimilation produced in the light, so also parasites and saprophytes destitute of chlorophyll live, as has already been explained, on the work performed by plants that contain chlorophyll, and are therefore dependent indirectly on light, even though the whole of their development may be completed in darkness, as in the Truffle; in other instances they only emerge to unfold in the air the flowers already formed underground, and to

${ }^{1}$ [For further arrangements for maintaining a constant temperature under the microscope, see Stricker and Burdon-Sanderson, Quart. Journ. Micr. Sci. 1870; Schafer, ibid. 1874.]

${ }^{2}$ A. P. De Candolle, Physiologie végétale, 1832.-Sachs, Ueber den Einfluss des Tages-lichtes auf Neubildung u. Entfaltung verschiedener Pflanzenorgane; Bot. Zeitg. I863, Supplement.-Sachs, Wirkung des Lichtes auf die Blüthenbildung u. Vermittlung der Laubblätter; Bot. Zeitg. 1865, p. I17.-Sachs, Handb. der Exp.-Phys. 1865, p. I. 
disseminate their seeds, as is the case with Limodorum abortivum, Epipogium, Corallorhiza, Monotropa, Lathrea, Orobanche, \&c. Even many plants which do contain chlorophyll and which live on inorganic food complete their growth and the processes connected with it in complete darkness, only putting forth their green leaves at certain times for the purpose of again accumulating beneath the ground fresh formative material. This is the case with the Autumn Crocus, Tulip, Crown Imperial, terrestrial Orchids, and many others, and especially with plants which form bulbs, tubers, and rhizomes. If the growing end of a stem of a green-leaved plant $(e . g$. Cucurbita, Tropaolum, Ipomea, or Hedera) is secluded from all light while the green leaves remain exposed to it, the buds develope in the dark; leaves and flowers are produced, which latter attain their full size and beauty of colour, are capable of fertilisation, and produce fruits and even fertile seeds at the expense of the substance assimilated in the light in the green leaves and carried to them by the stem.

These and a number of other facts show that growth, i.e. the processes by which the form of the plant is attained, and metastasis are not necessarily dependent, or only to a subordinate extent, on the influence of light, if only the necessary qu untity of assimilated material has previously been accumulated.

This is a general statement of the case. If however the various separate processes of vegetation are observed-the behaviour of protoplasm, the formation, arrangement, activity, and destruction of chlorophyll, the growth of the younger and older parts, the movements resulting from the tension of the tissues, \&c.a long series of very varied facts presents itself which require detailed consideration, because the rays of different refrangibility which are mingled in white daylight affect vegetation in a manner altogether different; certain functions are induced only by the highly refrangible rays, others only or chiefly by those of lower refrangibility. These effects moreover vary not only with the temperature but also with the intensity of the particular rays. Finally it must be observed that light affects plants only when its rays penetrate into their organs; this however modifies their intensity and to a certain extent also their refrangibility. In every investigation of the action of light these points must therefore be kept in view. The following summarises what is at present known as to the general facts.

(I) Action of rays of different refrangibility. The rays of different refrangibility commingled in white sunlight which appear as variously coloured bands in the spectrum vary in their physiological action on the processes of vegetation. Chemical changes, so far as they are in the main dependent on light, are produced chiefly or solely by rays of medium or low refrangibility (viz. the red, orange, yellow, or green). This is the case for instance with the production of the green colour of chlorophyll, the decomposition of carbon dioxide, and the formation in chlorophyll of starch or sugar.

On the other hand the rays of high refrangibility (the blue or violet, as well as the invisible ultra-violet rays) are the principal or the only ones which produce mechanical changes so far as these are dependent on light. It is these rays which influence the rapidity of growth, alter the movements of the protoplasm, compel swarm-spores to adopt a definite direction in their motion, and change the tension of the tissues of the motile organs of many leaves and hence affect their position. 
These two laws, the result of careful observation, are only in apparent contradiction to the division of the rays of light which is current in chemistry and physics into those called chemically active, including the highly refrangible blue, violet, and ultra-violet, and the chemically inactive, or at least less active, including the less refrangible red, orange, and yellow, and partly also the green rays. This division has long been familiar; silver-salts, nitrogen chloride, and other inorganic compounds, are powerfully acted on by the former, scarcely at all by the latter. But when it was shown that the organico-chemical processes in plants were caused mainly or solely by the latter kind of rays, it was seen that this classification into chemical and non-chemical rays resulted from an imperfect induction, and that the correct statement of the fact is rather that there are chemical processes (generally dependent on light) which are related to rays of particular refrangibility. As far as concerns the mechanical effect on the plant of the highly refrangible rays, it is at present uncertain whether they are not ultimately due to chemical changes. In any case the action is visible to the observer only in the form of mechanical effect (movements, tensions, \&c.); and this is in harmony with the classification given above.

If sunlight is made to pass through sufficiently thick strata of solutions of potassium bi-chromate and-ammoniacal copper oxide ${ }^{1}$, the first only permits the passage of light consisting of the less refrangible half of the spectrum (red, orange, yellow, and some green), while the blue solution allows, in addition to some green, only the blue, violet, and ultra-violet rays to pass through. The sunlight is therefore in each case halved by absorption in such a way that the spectrum beneath the orange solution extends from the red to the green, that beneath the blue solution from the green to the ultra-violet. If the light after passing through one or other of these fluids is directed on plants capable of decomposing carbon dioxide and of curving heliotropically, and pieces of very sensitive photographic paper are at the same time exposed by their sidè, it is seen that the less refrangible rays of light (transmitted through the potassium bichromate) effect the decomposition of carbon dioxide and the colouration and decolouration of the chlorophyll almost as energetically as white daylight, while they produce only a very slight effect on the photographic paper. The growth of seedlings, on the other hand, proceeds in this light exactly as in the dark, although the leaves turn green. Conversely the light which has passed through the ammoniacal copper oxide has very little effect in decomposing carbon dioxide, although the action on photographic paper is very vigorous. The growth of seedlings is on the other hand the same as in white light; and the mechanical process of heliotropic curvature is very manifest. A number of more recent observations have confirmed and extended the results previously obtained ${ }^{2}$.

(2) Variation in the action of light on plants in proportion to its intensity ${ }^{3}$. That

${ }^{1}$ Sachs, Bot. Zeitg. 1864, p. 253 et seq., where the labours of previous observers are referred to in detail.

2 I have replied, in the second part of the 'Arbeiten des botan. Inst. in Würzburg,' 1872, to the objections urged by Prillieux to this statement, which rest on an entire confusion of the ideas Intensity of Light (objective), Brightness (subjective), Refrangibility (an objective), and Colour (a subjective) property of light.

3 With respect to the distinction which must here be borne in mind between the objective 
the action of light on plants varies with its intensity, as that of temperature with its elevation, does not admit of a doubt, and is obvious in all physiological observations. There can scarcely be said, however, to be any exact investigations on this point; and the great obstacle to their accomplishment is that we have at present no method of measuring the intensity of rays of light of any particular refrangibility in terms of a fixed unit which can be applied to plants. As far as concerns the highly refrangible rays, i.e. those which have the greatest mechanical effect, we are compelled to adopt the photo-chemical method of Bunsen and Roscoe ${ }^{1}$, which however gives no information respecting the different intensity of the red, orange, and yellow light, and can only be applied with great difficulty to experiments on vegetation. In the photometry of the less refrangible rays, on the contrary, we must always have recourse, according to the ordinary method, to the sensitiveness of the eye, i.e. to brightness, which cannot be considered in itself to be an actual objective measure of the intensity of the light, though it may be assumed under certain circumstances that increase or diminution of subjective brightness corresponds to increase or diminution of objective intensity. In describing the relation between the intensity of light and vegetation, we have therefore at present, with a few exceptions, to employ the ordinary expressions dark, dull, bright, dazzlingly bright, \&c., and to assume that they correspond to certain objective intensities. There is one case in which this relation between the subjective sensitiveness of the eye and the action upon vegetation of the light which causes it can be very strikingly proved; Pfeffer has shown that the curve of the subjective sensitiveness of the eye for the colours of the solar spectrum coincides exactly with the curve expressing the power of different regions of the spectrum in decomposing carbon dioxide ${ }^{2}$. This coincidence must however at present be considered purely accidental ${ }^{3}$, and cannot be extended to other phenomena. If the sunlight or diffused daylight which reaches the observer were always of the same intensity, it would be easy to regulate artificially, according to definite gradations, the intensity of the light that acts on the plant. But since the light of incandescent bodies (such as the Drummond's light ${ }^{4}$ ) contains the same rays as sunlight and acts similarly on the functions of plants, constant sources of light of a definite intensity can in this way be arranged, which will admit of gradual adjustment, in order to study the influence on vegetation of light of different intensities.

If we now turn to the observations on record, those of Wolkoff are the only ones in which actual measurements have been made. With the assistance of the photometric method contrived by Bunsen and Roscoe ${ }^{5}$, he showed first of all that changes in the intensity of the highly refrangible light do not stand in any appreciable relation to the exhalation of gas by water-plants. This is an

intensity of light and its brightness to the eye, see the paper quoted above and the literature there referred to.

1 See the admirable paper by Wolkoff in the Jahrb. für wiss. Bot. vol. V. p. I.

2 Pfeffer in Sitzungsber. der Ges. zur Beförderung der ges. Naturwiss. für Marburg, 1872, May 16.

3 See note on p. 747 .

4 See Hervé Mangon, Comp. rend. I861, p. 243.-Prillieux, ibid. I869, p. 408.

5 Bunsen and Roscoe, Pogg. Ann. vol. 108. 
additional proof that these rays play only an extremely small part in this process, so small indeed that in the experiments the actual effect might be concealed by other causes (see p. 744). He next used as the source of light a dull glass plate illuminated by daylight, at different distances from which he exposed the plants (Ceratophyllum, Potamogeton, Ranunculus fuitans) in a dark room; and he ascertained that the exhalation of gas was, within certain limits, nearly proportional to the intensity of the light ${ }^{1}$. There is probably however some particular intensity of the efficient rays at which a maximum of gas is exhaled, and above which the "apidity of the process again decreases and the plant suffers injury; but whether this maximum intensity of light is attained or exceeded by the sunlight as it falls on the surface of the earth cannot at present be determined. In reference to the smallest degree of intensity, of light at which exhalation of gas can still take place, we have only the statement of Boussingault that a leaf of Oleander ceased to exhale oxygen after sunset ${ }^{2}$.

The green colour of the chlorophyll of Monocotyledons and Dicotyledons is not produced in the dark, as may be seen by enclosing plants in closely shutting boxes of wood or metal, or in a dark cellar. The colouration begins however when the amount of light is barely sufficient to read a book by; and when it increases to the ordinary brightness of a sunny summer day, the rapidity of the change increases, and the colour becomes a deeper green than that produced when plants are placed for a longer time in places not so strongly illuminated. Famintzin nevertheless showed ${ }^{3}$, in the case of Lepidium satizum and Zea Mais, that bleached seedlings become green more slowly in direct sunlight than in diffused daylight.

The small intensity of light which suffices for the formation of chlorophyll is not sufficient for assimilation or for the formation of starch in the chlorophyllgranules. Plants (such as Dahlia, Faba, Phaseolus, Cucurbita, \&c.) which rapidly become green in the normal condition of full daylight, as well as in the diffused light of the back of a room, still form no starch in their chlorophyll-granules. They do however produce starch when placed in a window where, at the most, they enjoy but half the direct sunlight and diffused daylight; but, in harmony with this, the assimilation of these plants is much less active in the window than in full daylight in the open air ${ }^{4}$. The following experiment gives a somewhat more precise result. Four plants of Tropaolum majus grown from seed in the back of a room, all gave, when dried at $110^{\circ} \mathrm{C}$, a smaller weight than the seed; they had not assimilated, and died after consuming the reserve-material, although in the shade of the room they all produced green leaves. Four other plants of the same species which germinated at the same time grew for three months, exposed for only seven hours each day to the diffused light of a west window in the forenoon; they formed nearly 5 grammes of dry substance. Four other plants which were exposed in a west window from I p.m. till the following morning, and therefore to the afternoon sunshine, produced also only 5 grammes; while four other plants which stood in the

1 See also Pfeffer, Arbeiten des botan. Inst. in Würzburg, Heft I. p. 4 I.

${ }^{2}$ Comp. rend. vol. 68. p. 4 IO.

${ }^{3}$ Famintzin, Mélanges biologiques; Pétersbourg, vol. VI. p. 94, 1866.

${ }^{4}$ Sachs, Bot. Zeitg. 1862, No 47 ; and 1864 , p. 289 et seq. 
window during the same time day and night produced nearly twenty grammes of dry substance ${ }^{1}$. It is a necessary conclusion from the increase in weight of these plants, that in the diffused daylight of the window of a room carbon dioxide is decomposed by the cells which contain chlorophyll, and that this does not take place with great activity. The same conclusion is drawn from the observation that Vallisneria spiralis and Elodea canadensis give off bubbles of gas when the light falls on them for only a rather short time from the northern sky on a clear day, although the exhalation is much more rapid in direct sunlight. In the case of most plants which grow in full daylight, especially our cultivated plants, the increase of weight by assimilation is greatly diminished when they are grown in a window. Within a room itself they usually become exhausted by their own growth in consequence of the defective assimilation, which is not sufficient to replace the material consumed in growth and in respiration; and the plant ultimately dies. Many Mosses on the other hand, and wood-plants of various kinds which grow in the deep shade (as the Wood-Sorrel), are killed by constant exposure to broad daylight; but whether in these cases it is the intensity of the light or the transpiration that is too great, and which of the two is the direct cause of injury, is unknown. Stems which attain an enormous length in complete darkness remain perceptibly shorter in the shade of a room; in a window their growth is still less, and least of all in the open air in full daylight. The reverse is the case with the leaves of Dicotyledons and Ferns; in the dark they are often very small; in deep shade they are considerably larger, and still more so in a light window; in this position they even appear in many plants (Phaseolus, Begonia, \&c.) to attain their maximum of superficial development, remaining smaller in the open air ${ }^{2}$.

(3) Penetration of the rays of light into the plant. In order to determine the dependence on light of certain phenomena of vegetation, it is of special interest to know the depth to which rays of a given refrangibility can penetrate any tissue of a plant, and the intensity with which the different elements of daylight act on particular internal layers. With the exception of the underground parts of plants, stems enveloped in bark, young organs enclosed in leaf-buds, and the like, which are in complete darkness, the assimilating and growing organs are penetrated by light. The deeper the light penetrates, the more does it lose in intensity by absorption, reflexion, and dispersion. This loss however affects the different elements of white light in very different degrees, as was shown by my investigations made in $1859^{3}$, at present the only ones on this subject. The rays of greatest refrangibility are in general almost entirely absorbed by the superficial layers of tissue, while the red light penetrates most deeply. Of successive layers of an

1 Sachs, Exp.-Phys. p. 21. It must however be observed that the shorter the duration of the light in these cases, the longer was the time of their exposure to the dark in which they again lost a portion of the assimilated substance by respiration.

2 The statement made by Famintzin (Mél. biol. vol. VI. p. 73, 1866) that the motile Algæ, Chlamydomonas pulvisculus, Euglena viridis, and Oscillatoria insignis turn both from direct sunlight and deep shade to a light of medium intensity, is contradicted by Schmidt (quoted infra), who found that they always turn to light of greater intensity, and even to direct sunlight. The method of observation of both authors was however very imperfect. [See also p. 752.]

3 Sachs, Ueber die Durchleuchtung der Pflanzentheile; Sitzungsber. der Wien. Akad. I860, vol. 43 ; and Handb. der Exp. Phys. p. 6 . 
apple, gourd, succulent stems, \&c. only the outermost receives the light that falls on it unchanged (independently of the reflexion from the surface); each deeper layer is penetrated by light less intense than the preceding one, and of a different composition. This change in the light which penetrates the tissue is principally caused by colouring materials, especially chlorophyll, which have an absorptive power for particular groups of rays, allowing others to pass through, and producing in addition rays by fluorescence. which were not contained in the incident light. But the relations of these changes of light in the tissues to the changes which the light causes are not yet accurately known; not even in reference to chlorophyll, to which we shall again recur. What we have now said is intended only to draw the attention of the student to the subject; more exact investigations must be made in working out the different questions which arise.

B. Special. (1) Chemical Action of Light on Plants. (a) Formation of Chlorophyll ${ }^{1}$. In the formation of the chlorophyll-granules the protoplasm becomes differentiated into a colourless continuous part which forms the proper motile protoplasnic body of the cell, and into smaller distinct green portions which $r \in$ main imbedded in the former, the chlorophyll-granules. This process, as far as concerns the differentiation, is independent of light, at least in flowering plants, where the chlorophyll-granules are formed in the cells of the leaves even in the dark. The chemical process, on the contrary, by whtch the green colour is produced has a complicated dependence on light. If, for instance, the temperature is sufficiently high, the green colouring substance is formed in the cotyledons of Conifers and in the leaves of Ferns in complete darkness as well as under the influence of light ${ }^{2}$. In Monocotyledons and Dicotyledons, on the contrary, the chlorophyll-granules which are formed in the dark remain yellow ${ }^{3}$, until they are exposed to light even of small intensity, when they become green if only the temperature is sufficiently high; and the nearer, as $I$ have shown, the temperature approaches a definite maximum ( 25 to $30^{\circ} \mathrm{C}$.) the quicker does the chlorophyll of Angiosperms become green in the light. Provided therefore that the temperature is favourable, the chlorophyll in the cotyledons of Conifers and the leaves of Ferns does not require light in order to assume its green colour ; while that in Angiosperms does require it; and in both cases the change does not take place at a low temperature (see p. 729). It may be added here that the subterranean protonema of Mosses contains chlorophyll, though but in small quantity.

It may be concluded from such observations as have been made that all the visible parts of the solar spectrum have the power of turning the etiolated chlorophyll-

${ }^{1}$ Sachs, Bot. Zeitg. 1862, p. 365, and Exp.-Phys. pp. Io and 318.-Sachs, Flora, 1862, p. 213 , and 1864 , no. 32.-Mohl, Bot. Zeitg. I 861, p. 238.-Böhm, Sitzungsber. der Wiener Akad. vol. II. Compare also Book I. sect. 6 of this work.

${ }^{2}$ P. Schmidt (Ueber einige Wirkungen des Lichts auf Pflanzen; Dissertation, Breslau 1870 , p. 22) believes that these facts can be at least partially combated; but his experiments only prove that the chlorophyll which is formed in the dark is again destroyed by long exposure to dark at a high temperature $\left(33^{\circ} 7^{\circ} \mathrm{C}\right.$.), as is also the case with other plants.

3 [Elfving has found (Arb. d. bot. Inst. in Würzburg, II. 3, I880) that exposure to light at a temperature which is not sufficiently high to produce chlorophyll leads to an increased formation of the yellow colouring matter (etiolin) in etiolated seedlings.

There is reason to believe that chlorophyll is derived from etiolin (see Wiesner, Entstehung des Chlorophylls, Wien, 1877).] 
granules of Angiosperms green; but that the yellow rays and those nearest to them on each side are the most powerful; and that this is also the case with the exhalation of oxygen from cells containing chlorophyll ${ }^{1}$.

(b) The Decomposition of carbon dioxide in cells containing chlorophyll, on which depends the assimilation of plants, and which is perceptible externally by the exhalation of a volume of oxygen nearly equal to that of the carbon dioxide absorbed, is brought about at a favourable temperature (see p. 729) by rays of light. In submerged water-plants the gas (always mixed with a larger or smaller quantity of nitrogen) escapes in the form of bubbles from wounds, especially transverse cuts of the stem; and it has been shown by Pfeffer and myself that when their size is constant the rapidity of these bubbles, i.e. the number of them formed in a unit of time, may even be used to give an exact measurement. In observations on landplants it is on the other hand necessary to expose the leaves to light together with air containing carbon dioxide in glass vessels of a suitable size and form, and to measure the quantity of gas by a eudiometer.

The smallest intensity of light necessary for the evolution of oxygen is judged by the subjective measure of its brightness to our eye-rather considerable (see p. 742). This evolution is always taking place with considerable energy in diffused daylight, even when the rays reach the plant only from a small portion of the sky; but it is much stronger in direct sunlight.

The specific effect on the evolution of oxygen of the variously refrangible elements of sunlight, in other words of the different coloured bands of the solar spectrum, has been carefully investigated by Draper and very recently again by Pfeffer ${ }^{2}$. The observations were made partly with the solar spectrum, partly with solutions of different colours which transmitted light of a particular refrangibility. The amount of gas exhaled was measured partly by the eudiometer, partly by the number of bubbles. Pfeffer points out 'that each portion of the spectrum exercises a specific quantitative influence on the power of assimilation; and that this remains unchanged whether the particular rays act separately on the parts of plants that contain chlorophyll, or combined with some or with all the other rays of the spectrum.'

The following additional result was also obtained from Draper's and Pfeffer's observations, and from mine already quoted:- ' Only those rays of the spectrum which are visible to our eye have the power of decomposing carbon dioxide; and indeed those which appear brightest to the eye, the yellow rays, are alone as

${ }^{1}$ See in particular Guillemin, Ann. des Sci. Nat. 1857, vol. VII. p. I60. [According to Wiesner (Unters. ueb. d. Beziehungen des Lichtes zum Chlorophyll, Sitzber. d. Wien. Akad., vol. 69, 1874 ; also Bot. Zeitg. 1874), etiolated plants become green much more rapidly in blue than in yellow (intense) light. He attributes this to the more active decomposition of the chlorophyll in the yellow light. This view is supported by the observation of Guillemin (Ann. d. Sci. Nat., 1854) and of Famintzin (Mélanges biologiques, Acad. Imp. de St. Pétersbourg, vol. 6, I866) that the leaves of etiolated plants become green more rapidly in diffuse daylight than in sunshine.]

${ }^{2}$ Draper, Annales de chimie et de physique, 1844, p. 214 et seq.-Pfeffer, Arbeiten des Botanischen Instituts in Würzburg, Heft I. p. ${ }^{8}$, where reference is also made to the whole of the rest of the literature.-Pfeffer, Sitzungsber. der Gesellsch, zur Beförderung der gesammt. Naturwiss. zu Marburg, 1872, May I6; and Bot. Zeitg. I872, no. 23 et seq., where the paper by Müller, Botanische Untersuchungen, Heft I, Heidelberg I $87 \mathbf{I}$, is also discussed. [For an account of Draper's researches into the relations existing between plants and light, see his Scientific Memoirs, London, 1878.] 
efficacious in this process as all the others put together. The most refrangible rays of the visible spectrum which act most energetically on silver chloride, \&c., play a very subordinate part in the process of assimilation.'

Draper placed glass tubes filled with water saturated with carbon dioxide in which he had placed green parts of plants, in the different coloured portions of a solar spectrum. Seven of these tubes were exposed simultaneously in the same spectrum. The following table gives the result of two experiments of this kind :-

\begin{tabular}{|c|c|c|c|c|}
\hline \multirow{2}{*}{\multicolumn{2}{|c|}{ Part of the Spectrum. }} & & \multicolumn{2}{|c|}{ Gas evolved. } \\
\hline & & & Experiment I. & Experiment II. \\
\hline Dark-red & . & . & 0.33 & 0.0 \\
\hline Red-orange & - & . & 20.00 & 2475 \\
\hline Yellow-green & . & . & $3^{6 \cdot 00}$ & 43.75 \\
\hline Green-blue & . & . & 0.10 & $4 \cdot 10$ \\
\hline Blue & . & . & 0.0 & I'00 \\
\hline Indigo . & . & . & 0.0 & 0.0 \\
\hline Violet . & - & . & 0.0 & 0.0 \\
\hline
\end{tabular}

Pfeffer experimented chiefly on leaves of the Cherry-Laurel and Oleander, which were placed in air containing carbon dioxide (shut off by mercury) in suitable glass vessels, and received the sunlight through coloured solutions (tested by the spectroscope). The following was the result of sixty-four experiments:-If the amount of gas evolved in light which has passed through a stratum of water of standard thickness is represented by 100 , the numbers here given are the corresponding quantities of carbon dioxide decomposed in light which has passed through equal thicknesses of the solutions named.

$$
\text { Solution. Colour of light. } \quad \begin{gathered}
\text { Amount of carbon dioxide } \\
\text { decomposed. }
\end{gathered}
$$

Red, orange, yellow, green

Potassium bichromate

Ammoniacal copper oxide

Orcin

Aniline-violet

Aniline-red

Chlorophyll

Iodine solution
Green, blue, violet

Red, orange-green, blue, violet

Red, orange-blue, violet

Red, orange

Red-orange, yellow, green

Quite dark
$88 \cdot 6$

$7 \cdot 6$

$53^{\circ} 9$

$38 \cdot 9$

$3^{2 \cdot 1}$

$15 \cdot 9$

(14.1 carbon dioxide produced).

From a comparișon of these numbers Pfeffer deduced the following values for the decomposing power of the different regions of the spectrum, the action of white light being again placed at roo:-

$$
\begin{aligned}
& \text { For Red-orange . } \quad \text {. } \quad \cdot \mathbf{3}^{2 \cdot 1} \\
& \text { Yellow . . . . } 46 \cdot 1 \\
& \text { Green . . . . . } \mathbf{I}_{5} \mathrm{O} \\
& \text { Blue-violet . • • . } 7.6 \\
& 100.8
\end{aligned}
$$

and from these is deduced the first statement of Pfeffer given above. 
If these values are erected as ordinates upon the solar spectrum, taking its corresponding parts as abscissæ, the result, as shown in Fig. 475, is that the curve of the different powers of light for causing evolution of gas corresponds in the main with the curve of subjective brightness of the same regions of the spectrum; but does not coincide with the curve of heating power.

Pfeffer's experiments had shown that the method first employed by me for determining the intensity of the action of light on water-plants, viz. counting the number of the bubbles of gas given off in a unit of time, gave nearly the same results as actual measurement of the gas, the result being in fact somewhat too great, and inexact in inverse proportion to the amount of gas given off. He then applied this method to determine the amount of oxygen given off from a small water-plant (Elodea canadensis) when exposed to a portion $\mathbf{1} 3 \mathrm{~mm}$. in breadth of a very intense solar spectrum $23 \mathrm{~cm}$. long. In this experiment he had the advantage

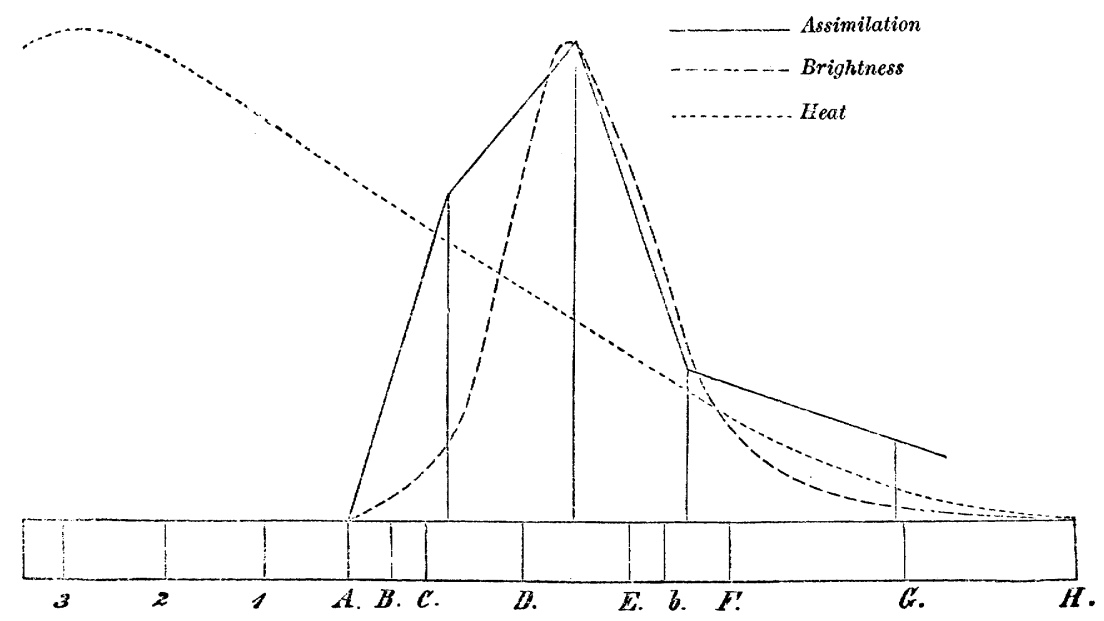

FIG. 475.-Graphic representation of the efficacy of rays of different refrangibility in causing the evolution of oxygen, com. pared with their brightness and heating power. The solar spectrum $A-H$ serves as a base, on which lines to represent the three different effects are erected as ordinates; the three curves are thus obtained which represent assimilation, brightness, and heat.

of being able to determine the amount of gas given off by the same plant in all the regions of the spectrum in successive very short spaces of time, and of thus avoiding various errors of observation which inevitably accompany eudiometric observations, or at least are very difficult to get rid of. A number of observations conducted in this manner gave the following result as the mean capacities for decomposition possessed by the different regions of the solar spectrum, yellow being placed at 100:-

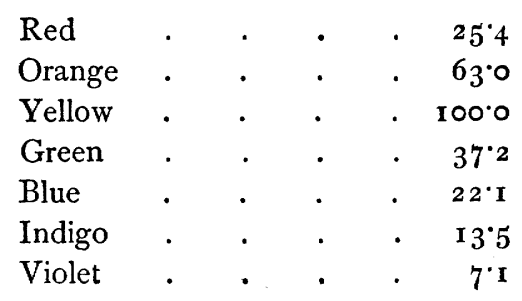


If allowance is made for the small error mentioned above incident to the method of counting the number of bubbles, we find that the curve of capacity for exhaling oxygen agrees still more exactly with the curve of brightness than is represented in Fig. 475, which was drawn from only a few data obtained with difficulty.

Since a comparison of the curve of brightness with that of the evolution of oxygen, otherwise convenient, has turned the attention of observers in a wrong path, and has led to many erroneous theories, it will be convenient to state the only relation between the two with which we have to do here, in precise terms:- The evolution of oxygen caused by chlorophyll is a function of the length of the waves of light; only those wave-lengths which are not greater than $0 \cdot 0006866 \mathrm{~mm}$. and not less than $0 \cdot 0003968 \mathrm{~mm}$. being able to produce this effect. Starting from the two extremes, the capacity of light for causing evolution of oxygen rises till it reaches its maximum at a wave-length of $0.0005889 \mathrm{~mm}$. Or, starting with the medium wave-lengths of the coloured region of the spectrum measured in hundred-thousandths of millimetres, the evolution of oxygen is effected by waves of light of a minimum length of 39 ; it increases with the increase of wavelength until the latter reaches about 59 ; it then diminishes if the wave-length continues to increase until it entirely ceases when the wave-length is 68 . It will be at once seen that we have here a similar phenomenon to that of the relation of vegetation to temperature; for we found (see p. 729) that this function also rises with the rise of temperature, attains a maximum at a definite temperature, and again decreases as the temperature rises still higher ${ }^{1}$.

Godlewski ${ }^{2}$ obtained the following results by a long series of eudiometric experiments as to the influence of the percentage of carbonic acid in the air upon the extent of the decomposition of this gas and upon the corresponding evolution of oxygen. An increase of the amount of carbonic acid present in the air, up to a certain limit (optimum), increases the evolution of oxygen, an increase beyond this limit diminishes it. The limit is different for different plants ; for Glyceria spectabilis on bright days it was from 8-10\%; for Typha latifolia from $5-7 \%$; for the Oleander probably rather lower. The increase of the evolution of oxygen consequent upon an increased amount of carbonic acid being in the air is much greater than the diminution produced when the optimum is exceeded by an equal amount. The greater the intensity of light, the more is the evolution of oxygen promoted by an increase of the carbonic acid up to the optimum, and the less is it diminished by excess. It follows that the influence of light upon the evolution of oxygen is the greater the more carbonic acid is contained in the air.

(c) Formation of Starch in Chlorophyll-granules ${ }^{3}$. The yellow chlorophyll (etiolin)granules formed in the dark are small; after turning green on exposure to light they become considerably larger, corresponding to the increase in size of the cells in which

1 The same law of dependence is also evidently applicable to the sensitiveness of the eye to brightness; and this is the cause of the curve of the brightness of light running nearly parallel to that of the evolution of oxygen.

${ }^{2}$ Godlewski, Arb. d. Bot. Instituts in Würzburg, Heft 3, 1873 .

${ }^{3}$ Sachs, Ueber die Auflösung und Wiederbildung des Amylums in den Chlorophyll-körnern bei wechselnder Beleuchtung: Bot. Zeitg. I864, p. 289 . 
they are contained. It is only after they have assumed their green colour and under the continued action of more intense light, in other words under conditions favourable to assimilation, that the formation commences of the starch which is enclosed within the chlorophyll-granules (see p. 46). When cells whose chlorophyll has produced starch after exposure to light are placed in the dark, the starch is absorbed and disappears completely from the chlorophyll-granules, and does so the quicker the higher the temperature. If light is again allowed access, starch is again formed in the same chlorophyll-granules; and the formation of starch is therefore a function of chlorophyll-granules exposed to light, its absorption a function of chlorophyll-granules not exposed to light. If complete or partial darkness is continued for a length of time, the chlorophyll-granule is usually itself destroyed; it first loses its form, is then absorbed, and finally disappears from the cells together with the colourless protoplasm; in the case of leaves of rapidly growing Angiosperms this takes place after a few days when the temperature is high. Cactusstems with slow growth and the shoots of Selaginella on the contrary remain green for months in the dark.

The absorption and re-formation of starch in the chlorophyll-granules-a process which I was the first to demonstrate in the leaves of Phanerogams - can be seen more readily in Algæ of simple structure like Spirogyra, which may therefore serve for purposes of investigation. I had already shown that the formation of starch in chlorophyll-granules depends on conditions which favour assimilation, and that the principal feature of this process, the evolution of oxygen, proceeds vigorously in light transmitted through potassium bichromate, and consists therefore of red, orange, yellow, and to a certain extent green. rays ; while the more strongly refrangible half of the spectrum, consisting of green, blue, violet, and ultra-violet rays, obtained by passing the light through ammoniacal copper oxide, has only a very slight effect. The conclusion at once followed from this, that the formation of starch must take place in the set of rays first named to the same extent that it does in full sunlight, but only to a very small extent in the latter set. This was confirmed by Famintzin's experiments ${ }^{1}$, in which he found that in Spirogyra the formation of starch in the chlorophyll-granules took place only in the mixed yellow light (that had passed through potassium bichromate), and not in the mixed blue light (that had passed through ammoniacal copper oxide) in which the starch already formed even disappears. Since however a small exhalation of oxygen takes place even in the mixed blue light, it must be supposed that a small production of starch occurs in it. Kraus's experiments ${ }^{2}$ with Spirogyra, Funaria, and Elodea, confirm this. He also found that in plants of Spirogyra which had lost their starch from exposure to dark, the formation of this substance in the chlorophyll-granules recommenced in five minutes in direct sunlight, in two hours in diffused daylight. In Funaria the formation of starch recommenced in the same manner within two hours in direct sunlight, within six hours in diffused daylight; and similar results were obtained with leaves of Elodea, Lepidium, and Betula ${ }^{3}$.

${ }^{1}$ Famintzin, Action of Light on Spirogyra; Mélanges biologiques, Petersburg 1865, Dec.; and I867, p. 277 .

2 Kraus, Jahrb. für wissensch. Bot. vol. VII. p. 5 I I .

${ }^{3}$ [From the observations of Weber (Ueb. specifische Assimilationsenergie, Arb. d. bot. Inst. in 
In accordance with the theory propounded by me that the starch formed in the chlorophyll-granules under the influence of light is the first product of assimilation produced by the decomposition of carbon dioxide, Godlewski has found (Flora, I873, p. 383 ), as the result of experiments as simple as ingenious, that in an atmosphere devoid of carbon dioxide no starch is produced in the chlorophyll-granules even in the light; that the starch contained in them disappears when the carbon dioxide is removed from the surrounding atmosphere, not only in the dark, but even in bright light. It may be inferred from this that the starch which is at any time found in the chlorophyll-granules is only the excess of the whole product of assimilation which has not yet been taken up. Of especial importance is his observation, which agrees with his eudiometrical experiments, that an increase in the proportion of carbon dioxide in the atmosphere to $8 \mathrm{p}$. c. in a bright light increases the rapidity of the formation of starch four or five fold, while in a diffused light the action is much less. A very large quantity of carbon dioxide in the atmosphere, on the contrary, retards the formation of starch in inverse proportion to the intensity of the light. Godlewski's experiments, made on the cotyledons of seedlings of Raphanus sativus, are opposed to the statement of Böhm (Sitzungsber. der Wien. Akad. March 6, I873), that the starch contained in the chlorophyll-granules is not a product of assimilation, a view which has already been sufficiently refuted by my earlier investigations.

(2) Mechanical Action of Light on Plants, (d) The influence of light on the movemeut of protoplasm varies according to the nature of the motion. Those movements which are the cause of the formation of new cells are not in general directly dependent on light (see p. 752); since they take place, in the great majority of cases, in partial or complete darkness. The 'streaming' motion of the protoplasm in older cells, or rotation and circulation, also goes on in continuous darkness as well as in alternate daylight and night; and even in the hairs of etiolated shoots which are developed in darkness. ${ }^{1}$. It has not been ascertained whether in these cases the rapidity and direction of the movement, the mode of distribution of the currents, and the accumulation of the protoplasm at particular spots, are influenced by the direction of the rays of light. An influence of this kind is apparently exercised by light on the plasmodia of Ethalium ${ }^{2}$. As long as the plasmodia are still in motion and not ripe for the production of spores, they appear on the surface of the tan when it is dark; but in the light, as in a sunny window, they again conceal themselves in the dark parts of the tan,- - a process which the

Würzburg, II. 2, 1879) it appears that equal areas of the leaf-surface of different plants produce different amounts of organic substance in a given time, the conditions being the same. He obtained the following numerical proportion for the energy of assimilation :-

\begin{tabular}{|c|c|c|c|c|c|}
\hline Tropaolum majus & • & . & • & • & 4.466. \\
\hline Phaseolus multiflorus & - & - & $\cdot$ & • & 3.215. \\
\hline Ricinus communis . & - & - & $\cdot$ & • & $5.29^{2}$. \\
\hline Helianthus annuus . & . & - & • & - & $5 \cdot 559]$. \\
\hline
\end{tabular}

1 Sachs, Bot. Zeitg., I863, Supplement.

2 [This subject has been investigated by Baranetzky (Mém. d. 1. soc. nat. d. sci. nat. de Cherbourg, XIX, I876): he found that the plasmodia, whilst still young, always avoided light. Schleicher has found on the contrary (Strasburger, Wirkung des Lichtes und der Wärme auf Schwärmsporen, Jen. Zeitschr. XII, I878) that the young plasmodia seek the light when its intensity is small: older plasmodia seek the light even when it is very intense.] 
- plant may be made to repeat two or three times in a day. It is not till the plasmodium has collected into a thick firm mass, and is preparing for the production of spores, that it comes to the surface in places exposed to light, but apparently only in the night or early morning.

The protoplasm which envelopes the chlorophyll-granules in the green leaves of Mosses and Phanerogams and in the prothallia of Ferns is induced, by the varying intensity of the light, to accumulate to a greater or less degree at different parts of the cell-walls, carrying the chlorophyll-granules along with it, and thus altering their distribution in the cell. It is still uncertain whether in this case the light affects the protoplasm only, the chlorophyll-granules being carried passively along with it; or whether the influence of the light is not first of all on the latter, which then give the impulse to the protoplasm. In either case it appears certain that the chlorophyll-granules do not of themselves possess any power of free motion, but are carried about by the motile protoplasm. Famintzin and Borodin ${ }^{1}$ found that under the influence of continued partial darkness the chlorophyll-granules in various Mosses and in the prothallia of Ferns collect on the side-walls of the cells (those at right angles to the surface of the organ); and that when these parts are exposed to light they leave them and distribute themselves over the parts of the cell-walls which are parallel to the surface of the organ. Prillieux ${ }^{2}$ and Schmidt have confirmed these statements. The view which I adopted long ago (see the first and second editions of this work), that these changes of position in the chlorophyllgranules are caused by the protoplasm, is confirmed by Frank's recent researches ${ }^{3}$. He shows that when the light falls only from one side, the protoplasm and the chlorophyll-granules collect mostly on those parts of the cell-walls on which the strongest rays fall, if the cells are sufficiently large to allow the light to be so arranged and these changes to take place in the position of their contents (as in the prothallia of Ferns and leaves of Sagittaria). Frank brought under a general point of view the changes in position of the chlorophyll-granules described by Famintzin and Borodin; he shows that the protoplasm in these cells is capable, according to circumstances, of adopting two different modes of distribution. In one mode, which he calls Epistrophe, the protoplasm and chlorophyll-granules collect on the free cell-walls, i.e. those which do not immediately adjoin other cells; for instance, next the surface in the superficial cells of organs consisting of several layers (the leaves of Sagittaria, Vallisneria, and Elodea); on the upper and under walls in organs consisting of only one layer of cells (leaves of Mosses, prothallia of Ferns); and in internal cells on the parts that bound the intercellular spaces. This is the position assumed in the normal conditions of vegetation and the mature state of the cells, but before they become too old. The second mode, or Apostrophe, takes place under unfavourable external conditions; as for instance in small fragments of tissue, when respiration is defective, turgidity diminished, the temperature too low, the cells too old, or-what is of most interest here-when light is cut off for a considerable time. Under these circumstances the protoplasm

1 Bohm, Sitzungsber. der Wien. Akad. 1857, p. 510.-Famintzin, Jahrb. für wissensch. Bot. vol. IV. p. 49.-Borodin, Mélanges biologiques; Petersburg, vol. VI, r867.

2 Prillieux, Compt. rend. I870, vol. LXX. p. 60.-Schmidt, l.c.

${ }^{3}$ Frank, Bot. Zeitg. 1872, Nos. 14, I5; and Jahrb. fïr wissensch. Bot. vol. VIII p. $216 \mathrm{et} \mathrm{seq.}$ 
and chlorophyll-granules collect chiefly on the walls that are not free, i.e. on those adjacent to other cells. The occurrence of apostrophe under direct sunlight which Borodin asserts ${ }^{1}$ (in various Phanerogams, as Lemna, Callitriche, and Stellaria), is denied by Frank, who maintains that what takes place in these cases is rather a collection of the protoplasm at the spots where the light is strongest, which may happen to be at the sides ${ }^{2}$.

It is evidently these aggregations of chlorophyll-granules on the side-walls of the cells caused by sunlight which were observed by Borodin that produced the phenomenon pointed out by Marquard and more exactly described by myself ${ }^{3}$, viz. that green leaves (e.g. those of Zea, Pelargonium, Oxalis, Nicotiana, \&c.) when exposed to sunlight assumed a bright green colour in a shorter time than in diffused light or in deep shadow. This can be made very evident by shading particular parts by pressing closely on them a strip of lead or tinfoil; if this strip is removed after five or ten minutes, the parts that were shaded show a dull green, those exposed to the sun a bright green colour. It is obvious that the tissue will appear to the eye a deeper green in proportion as the green granules are distributed uniformly over the surfaces facing the eye, a less deep green in proportion as they collect on the side-walls. Borodin's observations directiy confirm this hypothesis. This alteration in the grouping of the chlorophyll-granules which accompanies a change in the intensity of the light is caused only by the highly refrangible rays; the less refrangible rays (the bright and red ones) have the same effect as darkness ${ }^{4}$. It results therefore, as I showed in 1859, that if a strip of blue glass is laid on a leaf exposed to sunshine, it will produce no change of colour, while one will be caused by a strip of red glass.

Since these movements of the chlorophyll-granules are produced by the colourless protoplasm in which they are imbedded, it might be expected that the protoplasm of hairs which contain no chlorophyll or only a small quantity would be similarly influenced by the colour and intensity of the light. But the statements of Borscow and Luerssen ${ }^{5}$ which might be interpreted in this direction at least to some extent have not been confirmed by the observations of Reinke ${ }^{6}$.

The swarming of zoogonidia is also connected with protoplasmic movements. Their motile organs, the cilia, are supposed to be slender threads of protoplasm, by the vibration of which both the rotatory and the advancing movement of the zoogonidia is caused. The axis of rotation becomes subsequently the axis of growth; the anterior end in the advancing motion (where the zoogonidium is usually narrower,

1 Borodin, Mélanges biol., Petersburg I869, vol. VII. p. 50.

2 [From Stahl's investigations it appears that apostrophe is produced by direct sunlight (Bot. Zeitg. 1880). He finds that exposure to diffuse daylight produces epistrophe, that is, the position of the chlorophyll-granules in which the greatest area of their surface is exposed to the incident rays, whereas sunlight produces apostrophe, that is, the position in which the least possible area of their surface is exposed to the incident rays. In the one case they present their flat surfaces, in the other their edges to the incident rays.]

${ }^{3}$ Sachs, Berichte der math.-physik. Klasse der k. sächs. Ges. der Wiss. 1859.

4 Borodin, l.c.; Frank, Bot. Zeitg. 1871, p. 238.

5 Borscow, Mélanges biol., Petersburg 1867, vol. VI. p. 31 2.-Luerssen, Ueber den Einfluss des rothen $u$. blauen Lichts u. s. w., Dissertation, Bremen, 1868 .

- Reinke, Bot. Zeitg. I 87 I, Nos. 46, 47 . 
hyaline, and provided with cilia) becomes the base of the germinating plant when the zoogonidium has come to rest. These movements of zoogonidia and the very similar ones of the Pandorineæ are affected by light to this extent, that when the light comes from one side they either tend towards or away from the source of light, this depending apparently partly on the species and partly on the age of the individual. Cohn states that here also the less refrangible rays have the same effect as darkness, while the direction of the motion is determined by the blue and the more highly refrangible rays ${ }^{1}$.

(e) Cell-Division and Growth ${ }^{2}$. The first formation and early growth of the new organs in the higher plants consisting of masses of tissue is accompanied by a great number of cell-divisions, which usually take place in complete darkness; as for example, in the roots of land- and marsh-plants, the buds on underground rhizomes, and leaves and flowers which are produced within the dense envelopes of the bud. Cell-formation of the same kind may however take place under the influence of light which may even be intense, as is shown by the growth of the roots of land-plants in water exposed to light, or that of the aërial roots of Aroideæ (which are highly transparent at their cell-forming apex). The formation of stomata and hairs which is the result of cell-division may take place either in the light or in complete darkness within the bud, without any essential difference being observable in the two cases. In the same manner the cambium of the trunks of trees is covered by completely opaque envelopes, such as bark; while that of many annual stems (as Impatiens) is exposed to the light which penetrates the thin succulent cortex. Similar phenomena are presented in the formation and ripening of ovules within transparent or completely opaque ovaries. They are most obvious when shoots or even flowers which under ordinary circumstances are developed in the light are made to grow in complete darkness from bulbs, tubers, or seeds. The small variations from the normal condition which occur in such cases do not affect the early development of the organs; but their later growth which does not depend on cell-division is necessarily interfered with, as well as the development of chlorophyll. An obvious and necessary condition of these processes of growth, whether in the dark or the light, is the presence of a supply of assimilated reserve-materials, at the expense of which the formation of new cells can take place. In the case of the buds of the higher plants their reservoirs of reserve-

1 Cohn, Schles. Ges. für vaterl. Cultur, Oct. 19, 1865. The facts have however recently been questioned by Schmidt. [See Sachs, Ueb. Emulsionsfiguren, Flora, I876; Strasburger, Wirkung des Lichts und der Wärme auf Schwärmsporen, Jen. Zeitschr. XII, 1878; Stahl, Ueb. den Einfluss des Lichts auf die Bewegung der Schwärmsporen, Bot. Zeitg. 1878, and Verh. d. phys.-med. Gesellsch. in Würzburg, I879. It appears that the zoogonidia place themselves so that their long axes coincide with the direction of the incident rays. They move either towards the source of light or away from it, the direction of their movement being dependent upon a number of conditions, such as the intensity of the light, the relative temperature of different portions of the water in which the zoogonidia are, the age of the zoogonidia, and the amount of oxygen in the water. Zoogonidia which exhibit these phenomena are said, by Strasburger, to be phototactic. Some zoogonidia (such as those of Saprolegnia) do not appear to be affected by light.]

${ }^{2}$ Sachs, Ueber den Einfluss des Tageslichtes auf Neubildung u. Entfaltung verschiedener Pflanzen-organe, Bot. Zeitg. 1863 , Supplement. If I here consider cell-division and growth as essentially mechanical processes, this does not imply that chemical changes do not also accompany every process of growth. 
material are the bulbs, tubers, rhizomes, parts of the stem, cotyledons, and endosperm; "after the complete exhaustion of these growth ceases in the dark but continues in the light, because the assimilating organs can then produce new material. This relation of growth which is connected with cell-division to assimilation is especially clear in Algæ of simple structure (as Spirogyra, Vaucheria, Hydrodictyon, Ulothrix, \&c.), which assimilate in the day-time under the influence of light, while cell-division proceeds exclusively or at least chiefly at night. The swarm-spores are also formed in the night, but swarm only with access of daylight. In some Fungi also, as Pilobotus crystallinus, the splitting up of the protoplasm in the sporangium into a number of spores takes place only in the night, the spores being thrown out on access of light. While therefore in the larger and more highly organised plants assimilation and the construction of new cells out of the assimilated substances is carried on in different parts but at the same time, in small transparent plants in which the parts where these functions are effected are not surrounded by opaque envelopes they take place at different times. We have here a case of division of physiological labour which shows us that the cells which have to do with chemical work (assimilation) cannot at the same time perform the mechanical labour of cell-division; the two kinds of labour are distributed in the higher plants in space; in very simple plants in time. Provided there is a supply of assimilated reserve-material, cell-division can therefore take place either in the light or the dark. Whether there are special cases in which light promotes or hinders cell-division is not known with certainty. We might suppose we have such a case when Fern-spores and the gemmæ of Marchantia ${ }^{1}$ germinate in the light but not in the dark; but Borodin has shown that the less refrangible rays are alone active in this process of growth, mixed blue light (passed through ammoniacal copper oxide) acting like complete darkness. But since the less refrangible rays, as we have seen, have exactly the same effect on growth as the absence of light, but on the other hand are the efficient agent in assimilation, it may be supposed that these spores and gemmæ do not contain certain substances necessary for germination which must therefore be produced by assimilation. On the other hand it has not yet been explained on what depends the formation in long-continued darkness from many stems (as those of Cactus, Tropcolum, Hedera, \&c.) of roots which are not produced under the ordinary amount of light. Whether the degree of humidity is an element in this is uncertain but not improbable.

When the young organs emerge from the bud-condition, an active growth commences which is chiefly occasioned by the absorption of water into the cells and by a corresponding superficial extension of the cell-walls, cell-division still taking place only occasionally or not at all. This process of expansion takes place, in the case of aërial stems and foliar structures, in the daylight which penetrates deep into the transparent succulent tissues. In order to estimate the amount of its influence on these processes, it is best to grow seedlings or shoots of the same species of plant in continuous complete darkness, and others under an alternation of day and night, especially in the height of summer. Independently

1 Borodin, Mélanges biol., Petersburg 1867 , vol. VI; Pfeffer, Arbeiten des bot. Inst. in Wïrzburg, vol. I, I 87 I, p. 8 o. 
of the fact that the chlorophyll (with the exceptions already named) does not assume its green colour in the dark but remains yellow, differences of form which are often very striking are exhibited by plants grown in the dark, and constitute the bleached or etiolated condition. The internodes of etiolated plants are in general much longer than those of plants of normal growth; and the long narrow leaves of Monocotyledons are subject to the same change. On the other hand the leaves of Dicotyledons and Ferns usually (but not always) remain very small and do not completely outgrow their bud-condition, or exhibit peculiar abnormalities in their expansion. These peculiarities will be explained more in detail in Chap. IV. It is not necessary however to contrast etiolated plants with those of the normal green colour, in order to establish the influence of light on their growth. If plants of the same species are compared when grown in more or less deep shade with others grown in full daylight, these differences are still very conspicuous, varying according to the intensity of the light. Different species are however affected to a different extent by etiolation; the internodes of climbing plants, which are very long even under normal conditions, become much longer still in the dark; and some leaves of Dicotyledons, as for instance those of the Beet, become tolerably large under the same circumstances, while on the other hand the abnormally elongated internodes of etiolated potato-plants put out leaves of only a very small size $^{1}$. It is remarkable that etiolation, as I have already shown ${ }^{2}$, does not extend to the flowers ${ }^{3}$. As long as sufficient quantities of assimilated material have been previously accumulated, or are produced by green leaves exposed to the light, flowers are developed even in continuous deep darkness which are of normal size, form, and colour, with perfect pollen and fertile ovules, ripening their fruits and producing seeds capable of germination. The calyx however, which is ordinarily green, remains yellow or colourless. In order to observe this it is only necessary to allow tulip-bulbs, the rhizomes of Iris, or the like planted in a pot, to put up shoots in complete darkness, when perfectly normal flowers are obtained with completely etiolated leaves. Or a growing bud on a stem of Cucurbita, Tropaolum, Ipomaa, \&c., with several leaves, is made to pass through a small hole into a dark box, the leaves which remain outside being exposed to as strong light as possible. The bud developes in the dark a long colourless shoot with small yellow leaves and a number of flowers, which, except in the colour of the calyx, are in every respect normal ${ }^{4}$. The extremely singular appearance of these abnormal shoots with normal flowers shows in a striking manner the difference in the influence of light on the growth of different organs of the same plant.

1 [For a discussion of Etiolation, see Godlewski, Zur Kenntniss der Ursachen der Fornändeı ung etiolirter Pflanzen, Bot. Zeitg. I879, where the literature of the subject is quoted.]

2 Sachs, in Bot. Zeitg. I $86_{3}$, Supplement; and I£65, p. I I 7 .

3 [An exception to this rule is afforded by the coloured kinds of lilac which are forced during the months of February and March by the market-gardeners of Paris, at a temperature of from $33^{\circ}$ to $35^{\circ} \mathrm{C}$., and in almost complete darkness. The flowers expanded under these conditions are completely white. See Duchartre, Journ. de la Soc. Imp. et cent. d'hort. de France, 1860, pp. 272-28o.

* Sometimes however abnormal flowers appear in the dark as well as the normal ones. See Sachs, Exp.-Phys. p. 35 . 
The retarding effect of light on the growth of the shoot is evident even in a short time; and, as I have already briefly shown ${ }^{1}$, a periodical oscillation in the rapidity of growth is caused by the alternation of day and night (when the temperature is nearly constant). This variation is shown by the growing internode exhibiting a maximum of hourly growth towards sunrise, decreasing gradually from the advent of daylight till mid-day or afternoon, when it reaches its minimum, and increasing from this time till morning, when it again attains its maximum.

Prantl ${ }^{2}$ has shown that a similar periodicity exists in the growth of leaves when day and night alternate norinally. The fact that the leaves of the same plants (Cucurbita, Ferdinanda, Nicotiana), when they become etiolated by remaining in continuous darkness, are much smaller, is in apparent contradiction to this. But in such a case we have not to do with leaves which are heallhy and which are periodically assimilating, but with sickly leaves which contain no chlorophyll. These small yellow leaves, developed in darkness, are not exposed to the favourable influence of light upon which assimilation and its effect upon growth depend in normal leaves.

One of the best-known phenomena occasioned in plants by light is the fact that growing stems and leaf-stalks, when the amount of light which they receive is very different on different sides, bend or become concave towards the side exposed to the most intense light. This curvature is caused by the slower growth in length of the illuminated than of the shaded side; and parts of plants which show this behaviour to light are called heliotropic ${ }^{3}$. From the fact of heliotropis curvature towards the side which receives the most light, it is obvious that the plant would grow more quickly if shaded on all sides than if the light were more intense. The observation that leaves, some roots, Fungi, filamentous Algæ (like Vaucheria), \&c., curve heliotropically, indicates that their growth is retarded by light. That the chlorophyll has no share in causing this heliotropism is shown by the fact that organs which contain none, like some roots, or Fungi, as the perithecia of Sordaria fimiseda (according to Woronin), the stipes of the pileus of Claviceps (according to Duchartre ${ }^{4}$ ), and colourless etiolated stems, bend towards a stronger light. Since most heliotropic parts of plants are highly transparent, the light which falls on one side must penetrate more or less to the other side, on which also some light falls; it follows therefore that even inconsiderable differences in the intensity of the light which falls on the two sides must cause heliotropic curvature; i.e. difference in the rate of growth ${ }^{5}$. If plants which show heliotropic properties are grown in a box which receives light from one side that has passed in one case through a solution of potassium bichromate, in another case through

1 Sachs i Heft II of the Arbeiten des Bot. Inst. in Würzburg. 1872.

${ }^{2}$ Compare infra, Chap. IV. Sect. 20 ; also Arb. d. bot. Inst. Würzburg, Heft III.

${ }^{3}$ Further details on heliotropism will be given in Chap. IV.

4 Duchartre, Compt. rend. 1870; vol. LXX. p. 779.

5 It must however be noted that in the case of parts containing chlorophyll the light in penetrating the tissues loses its more refrangible rays which are the only ones that produce the effect; as has been already shown, only the less refrangible rays pass through the superficial layers (see p. 742). 
one of ammoniacal copper oxide, the internodes of the first remain quite straight and lengthen considerably as if they were in the dark, while those exposed to the mixed blue light grow less and at the same time bend strongly towards the light. It follows from this that only rays of high refrangibility, the blue, violet, and ultra-violet, cause the curvature by retarding growth ${ }^{1}$.

In addition to the large number of the parts of plants which, when illuminated unequally, bend so as to make the more strongly illuminated side concave, there are a much smaller number which bend in the opposite direction, i.e. become concave on the shaded side. In order to distinguish between them the former are termed positively, the latter negatively heliotropic ${ }^{2}$.

Both positive and negative heliotropism occur not only in organs containing chlorophyll, but also in those that are colourless; among the former in the green tendrils of Vitis and Ampelopsis ${ }^{3}$; among the latter in the colourless root-hairs of Marchantia ${ }^{4}$, the aërial roots of Aroideæ, Orchideæ, and Chlorophytum Gayanum, and the rootlets of some Dicotyledons, as Brassica Napus and Sinapis alba ${ }^{5}$. From the statement that positive heliotropism depends on a retardation of the growth of the organ exposed to the stronger light, it might be inferred that negative heliotropism is occasioned conversely by a more vigorous growth of the side exposed to the stronger light. This conclusion would be confirmed by a superficial examination of the phenomena; but if the attendant circumstances are observed more closely, some considerations arise which I shall examine in detail in Chap. IV. It need only be mentioned here that according to a theory started by Wolkoff, two different explanations are possible:-Very transparent organs, like the apices of the roots of Aroideæ and of Chlorophytum, refract the light which falls upon them in such a manner that the shaded side of the organ may actually be more strongly illuminated than the other; and its negative heliotropism is then only a special case of positive heliotropism. But in other cases, as in the Ivy and Tropcolum majus, the internodes are positively heliotropic when young, but negatively when old before growth ceases; and Wolkoff supposes that the curvature which is in these cases convex on the illuminated side is caused by the more vigorous assimilation and consequent longer duration of growth. It depends therefore upon nutrition which only affects the mechanism of growth in a secondary degree.

( $f$ ) Action of Light on the tension of the tissue of the contractile organs of leaves

1 See Sachs, Bot. Zeitg. $186_{5}$, On the action of coloured light on plants, where the literature is also quoted. I consider experiments with absorbent fluids more decisive than those with the spectrum; in this latter Guillemin states that not only do all the rays act heliotropically, but that there is even a lateral curvature towards the blue end of the spectrum. When the light is sufficiently strong the spectrum is certainly never free from diffused white light, which will cause heliotropism even when its intensity is very small. [Wiesner has found (Heliotropische Erscheinungen, noticed in detail in Chap. IV) that although the yellow rays do not give rise to heliotropic curvatures, they exercise, nevertheless, a retarding influence on growth.]

2 [Darwin (Movements of Plants) uses the terms 'heliotropic' and 'apheliotropic' instead of 'positively' and 'negatively heliotropic.' He considers that the heliotropic movements are modified forms of circumnutation (see infra).]

${ }^{3}$ Knight, Phil. Trans. I81 2, Pt. I. p. 3 I4.

4 Pfeffer, Arbeiten des bot. Inst. in Würzburg, 187 I, Heft I. Div. 2.

5 For the literature on this subject see Sachs, Exp.-Phys. p. $4^{I}$. 
endowed with motion ${ }^{1}$. The leaf-blades of Leguminosæ, Oxalideæ, Marantaceæ, Marsiliaceæ, \&c. are borne on modified petioles which serve as contractile organs, bending upwards or downwards under various external and internal influences, and thus giving a variety of positions to the leaf-blades. If these plants are placed in permanent darkness, the curvatures due to internal changes alternate upwards and downwards. Light exercises an immediate influence on these periodically contractile organs; any increase of its intensity tends to give the blade an expanded position, such as it occupies in the day-time; any diminution tends to cause it to assume a closed position upwards or downwards such as it has in the night. This effect, which I formerly termed 'the paratonic action of light,' is not the cause of the periodic movements; but rather counteracts the periodicity caused by the internal forces. In most leaves endowed with periodic movements the paratonic influence of light is so strong that it neutralises them, and induces in their place a periodicity dependent on the alternation of day and night. In the lateral leaflets of the leaves of Desmodium gyrans on the contrary the internal causes of the rapid periodic oscillations are so powerful as to overcome the paratonic action of light; and these leaflets move upwards and downwards when the temperature is high even in spite of changes in the amount of light. My earlier researches ${ }^{2}$ show that it is only the more refrangible rays that produce a paratonic effect, while red rays act like darkness.

The influence of light on the position of the contractile organs is not however only of this direct character; the motile condition is also indirectly dependent on it. Both the periodic and paratonic movement, as well as that (Mimosa) due to mechanical irritation-in fact, the power of movement-is lost when the leaves have remained in the dark for a considerable time, such as a whole day; in other words, they become rigid by long exposure to darkness. From this rigid condition they do not immediately recover when again exposed to light; the exposure to light must continue for a considerable time, some hours or even days, before the motile condition which I have termed 'Phototonus' is restored. It is only in this condition that the leaves are motile and sensitive to changes in the intensity of the light or to mechanical irritation. The paratonic curvatures of fully developed contractile organs caused by the action of light are distinguished from the heliotropic curvings of growing organs by the fact that, firstly, they are connected with phototonus, while the latter are not; and secondly, that they always take place in a plane determined by the bilateral structure, while the plane of heliotropic curvature depends only on the direction of the rays of light.

[The Chemistry of Chlorophyll. Gautier (Comptes Rendus, 1879, and Bot. Zeitg. 1880) and Hoppe-Seyler (Ber. deut. chem. Ges. 1879, and Bot. Zeitg. 1879) have succeeded in obtaining green crystals on the evaporation of an alcoholic solution of chlorophyll. Gautier considers these crystals to consist of chlorophyll, but Hoppe-Seyler is of opinion that they are a modification of chlorophyll, to which he gives the name of chlorophyllan. The following are the analyses:-

1 See Sachs, Ueber vorïbergehende Starrezustände, \&c., Flora, 1863.-Further details will be given in Chap. IV. [Also Darwin, Movements of Plants, I 880.]

${ }^{2}$ Sachs, Ueber die Bewegungsorgane von Phaseolus und Oxalis, Bot. Zeit. 1857, p. 8 I I $\boldsymbol{e}_{t}^{t} \mathrm{~s} \epsilon q$. 


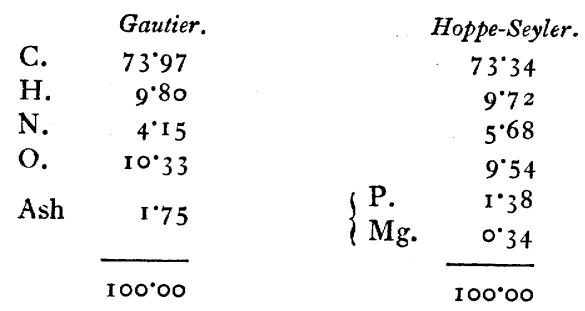

From this percentage Gautier deduces the formula $\mathrm{C}_{19} \mathrm{H}_{22} \mathrm{~N}_{2} \mathrm{O}_{3}$, and he points out its relation to that of Bilirubin $\left(\mathrm{C}_{16} \mathrm{H}_{18} \mathrm{~N}_{2} \mathrm{O}_{3}\right)$. It is of interest to note that iron was not found in either case. The spectrum of Hoppe-Seyler's chlorophyllan is the same as that of chlorophyll.]

Optical Properties of Chlorophyll. If parts of plants that contain chlorophyll are repeatedly boiled in water and then quickly dried at a temperature not too high and pulverised, a substance is obtained which is easily examined and can be preserved for a long time unchanged. From this powder the green colouring matter can be extracted by alcohol, ether, or oil. The green solution is speedily changed by the action of light in proportion to its intensity, the less refrangible rays of the spectrum acting most actively and rapidly. It then assumes a dirty brownish yellow-green colour, the green colouring matter having become modified or lost its colour.

If sunlight that has passed through a stratum of the pure green solution not too thick or too dark is decomposed by a prism, an extremely characteristic spectrum is obtained in which rays of very various refrangibility appear to have been more strongly absorbed the darker the solution or the thicker the stratum. This chlorophyll-spectrum has been the subject of much research; the most recent and comprehensive being that of Kraus, from whose description I borrow the following ${ }^{1}$ :-

The spectrum of an unchanged alcoholic solution of chlorophyll shows seven absorption-bands, four of which are narrow (Fig. $476 \mathrm{~A}, I, I I, I I I, I V)$, and are situated in the less refrangible half; while three $(V, V I, V I I)$ are broad and are situated in the more refrangible half. The latter, distinguishable as distinct bands only in very dilute solutions, coalesce, even in the solutions of medium concentration which are ordinarily examined, into a single continuous absorption-band occupying the whole of the more refrangible half of the spectrum.

The bands $I, I I, I I I$, and $I V$ are situated in the red, orange, yellow, and yellow-green. The deep black band $I$, sharply defined on both sides, lies between Fraunhofer's lines $B$ and $C$; the three others, shaded off on both sides, diminish in strangth in the order of their numbers. Between these bands the illumination is dim, and progressively in the order of the numbers; i.e. is less dim between $I I$ and $I I I$ than between $I$ and $I I$, \&c. To the left of $I$ the light is undiminished.

The bands $V, V I$, and $V I I$ in the more refrangible half of the spectrum are shaded on both sides; $V$ is situated to the right of Fraunhofer's line $F ; V I$, which is dark in the middle, to the left of and on the line $G$; VII may be regarded as the total absorption of the violet end. This spectrum has been found in all observations made on the most different plants; Mono- and Dicotyledons, Ferns, Mosses, and Algæ.

The Spectrum of living leaves agrees with that of the solution in its main characteristics $^{2}$. The bands $I-V$ are, according to Kraus, easily made out in all ordinary leaves

' Kraus, Sitzungsb. der phys.-med. Soc. in Erlangen, June 7 and July ro, I87r. See also Askenasy, Bot. Zeit. I867, p. 225 ; Gerland und Rauwenhoff, Archives néerlandaises, vol. VI, I87 I ; and Gerland, Pogg. Ann. I87 I, p. $5^{8} 5$. [Kraus, Zur Kenntniss der Chlorophyllíarbstoffe u. ihrer Verwandten; Stuttgart, $\mathbf{1} 8 \mathbf{7}_{\mathbf{2}}$. For reference to Mr. Sorby's papers see Sect. 8 a.]

${ }^{2}$ For further evidence of this very remarkable fact see Gerland und Rauwenhoff, l.c., p. 604. \begin{tabular}{l} 
It is not easy to understand how certain physicists can maintain the contrary. [Pringsheim points \\
\hline
\end{tabular} 
of Dicotyledons, Monocotyledons, and Ferns. But this spectrum differs constantly from that of the solution in all its bands being always nearer the red end; a point which was determined by Kraus by the use of Browning's micro-spectroscopic apparatus. This difference in position of the absorption-bands of the spectrum is, as he shows, an illustration of the universal rule that the absorption-bands approach nearer to the red end in proportion to the specific gravity of the solvent of the colouring substance. It follows from this that the green colouring matter is distributed in such a manner in the colourless matrix of the chlorophyll-granules that it must be considered in a state of solution. In no case can the colouring matter of chlorophyll in living cells be in a solid state, or equivalent to the residue left behind when the solution is evaporated.

If an alcoholic solution of chlorophyll is agitated with any quantity of benzol (say double its volume) two very sharply separated strata are formed after the fluid comes to rest, a lower alcoholic stratum of a pure yellow colour, and an upper blue-green stratum of benzol. Kraus considers this process to be a dialytic one; there are, ac-

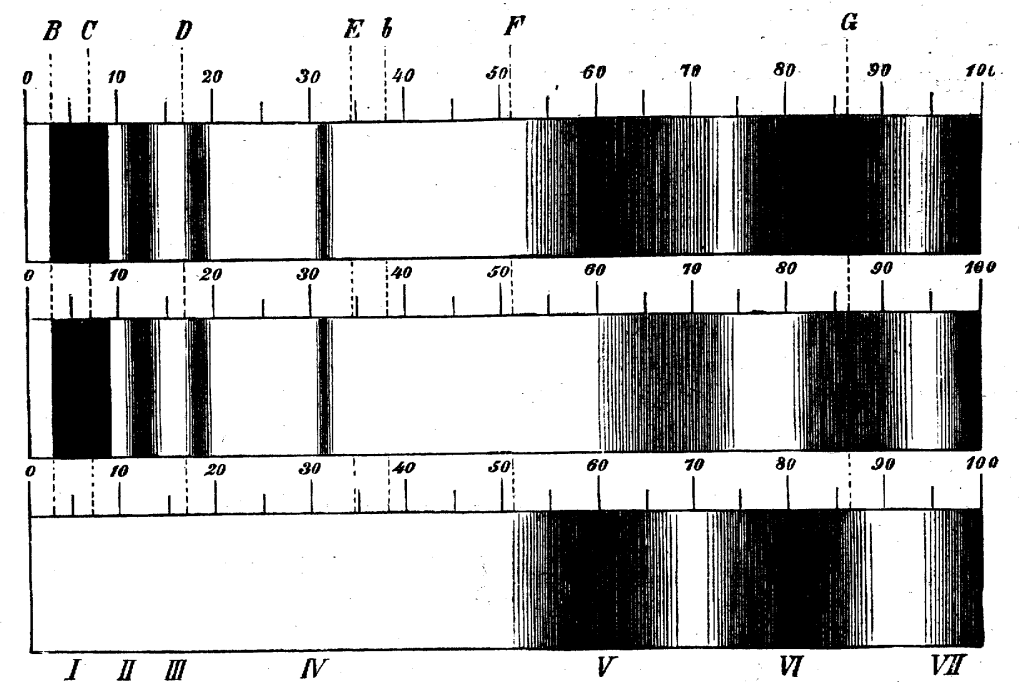

FIG. 476.-Absorption-spectra of the colouring matter of chlorophyll (after Kraus). $A$ the spectrum of the alcoholic extract of green leaves; $B$ that of the blue-green constituent soluble in benzol; $C$ that of the yellow constituent. The absorption-bands of $A$ and $B$ are indicated in the less refran yible (left-hand) portion as they would be produced by a more concentrated, in the more refrangible (right-hand) portion of the spectrum as they would be produced by a less concentrated solution; the letters $B-G$ indicate the well-known Fraunhofer lines of the spectrum; the figs. $I-V I I$ Kraus's absorption-bands in succession from the red to the violet end; the spectra are divided into twenty equal parts

cording to him, two colouring substances in the ordinary chlorophyll-solution, a bluegreen and a yellow one, soluble in very different degrees in alcohol and benzol.

Kraus therefore holds the spectrum of chlorophyll to be a combination-spectrum, i.e. that it arises from the superposition of the two spectra of the blue-green and the yellow colouring-matter. The blue-green substance gives the four narrow absorption-bands in the less refrangible half of the spectrum (Fig. $476, B$ ), and part of the band $V I$ which is situated at $G$ in the more refrangible half. The band $V$ (Fig. $476 C$ ) results from the yellow colouring matter which has absorption-bands in the more refrangible half of the spectrum. The band $V I$ of the chlorophyll-spectrum is the result of partial superposition of corresponding bands in the spectra of the yellow and the blue-green substances, which however do not perfectly coincide. Both colouring substances alike produce the absorption-band $V I I$ at the violet end.

According to Kraus' statements it is possible to decompose chlorophyll into two

out (Ueb. Lichtwirkung und Chlorophyllfunction, Jahrb. f. wiss. Bot. XII, I88I) that the chlorophyll-, spectrum is the same in leaves which are assimilating as in those which are not.] 
distinct colouring matters by a simple dialytic process. Conrad has shown, however (Flora, 1872, p. 396), that if a solution of chlorophyll in absolute alcohol be treated with benzol, a separation of the green and the yellow never occurs. This only takes place when dilute alcohol, of a strength less than 65 per cent., is used. Conrad points out forcibly that Kraus used dilute alcohol, which may be at once inferred from the fact that he extracted the boiled leaves with alcohol without having previously dried them. According to Conrad, it is very doubtful if this decomposition of the chlorophyll is simply a dialytic phenomenon. More probably a decomposition had previously been effected by the water, a suggestion which is supported by the fact that solutions of chlorophyll in dilute and in absolute alcohol when evaporated give in the former case, but not in the latter, a residue containing a yellow colouring-matter soluble in water ${ }^{1}$.

The yellow colouring matter is soluble in alcohol, ether, and chloroform, but not in water. On addition of hydrochloric or sulphuric acid (as Micheli had already shown) it becomes first emerald-green, then verdigris-green, and finally indigo-blue; the spectrum of the yellow substance which has in this manner become green shows altogether different absorption-phenomena to those of chlorophyll. The spectrum of the yellow ingredient of chlorophyll is identical (Kraus) with that of most yellow flowers (as Ranunculus, Mimulus, Gentiana lutea, Brassica, Taraxacum, Matricaria, \&c.), and agrees with it also in the reactions just named, as also does that of the yellow colouring substance of fruits and seeds (Euonymus, Solanum Pseudocapsicum, \&c.). This yellow substance is, like chlorophyll, combined with protoplasm. The substance present in the cells in the liquid form, as for instance in the flowers of the Dahlia, is different; it is soluble in water, and does not give a spectrum consisting of bands, but a continuous absorption of the blue and the violet. The colouring substance of some orange flowers, e. g. Eschscholtzia, also soluble in alcohol, is again different, possessing a fourth band in the blue-green to the left of the three bands of the ordinary yellow substance. The colouring matters of bright-coloured lower organisms which are soluble in alcohol are not identical with either of the two which constitute chlorophyll, but are related to them.

According to Kraus, the yellow substance of etiolated leaves also exactly resembles the yellow constituent of chlorophyll.

The Fluorescence of the colouring-matter of chlorophyll is seen from the fact that a sufficiently dark concentrated solution appears dark-red by reflected but green by transmitted light. The fluorescence is much more decided if the pencil of converging rays of the sun is made to fall on the green fluid through a condensing lens. If the solar spectrum is thrown upon the surface of a solution of chlorophyll ${ }^{2}$, it may be ascertained which rays of the sunlight cause the fluorescence; the red begins a little to the left of the line $\dot{B}$ of the solar spectrum, and stretches, although varying in intensity, over the violet end. On the dark-red ground are seen seven intensely red bands, each corresponding exactly both in position and in strength to an absorption-band in the spectrum of chlorophyll. If the fluorescence caused by the solution of chlorophyll is itself observed through a prism, it is seen to consist only of red rays, the refrangibility of which coincides with the strongest absorption-band of chlorophyll between $B$ and $C$. Every ray produces by fluorescence only such as correspond in their refrangibility to the absorption-band $I$. Whether the chlorophyll contained in living cells is subject to the same fluorescence is not certain, from the imperfect

1 [Pringsheim (Ueb. d. Asorptionsspectra der Chlorophyllfarbstoffe, Monatsber. d. k. Akad. d. Wiss. zu Berlin, 1874) confirms Conrad's observations. He shows that the separation into two layers depends upon the fact that benzol will not mix with weak alcohol, but will do so with strong. $\mathrm{He}$ also points out that under any circumstances some of the benzol is retained in solution in the alcohol, and further that benzol will dissolve more chlorophyll than alcohol and will therefore become more deeply coloured. He maintains that the yellow colour of the alcohol is due to the presence of some chlorophyll in it, as shown by the spectroscope, though some yellow colouringmatter (etiolin or xanthophyll) is also present.]

2 Hajes.bach, Pogg. Ann. vol. 14I. p. 245; Lommel, $i b$. vol. 143. p. 572. 
observations at present made; but it is probable, from the absorption-phenomena and their connection with fluorescence.

The question whether the absorption-bands of the spectrum of the colouringmatter of chlorophyll have any causal connection with the function of the chlorophyllgranules in decomposing carbon dioxide has recently been answered by Lommel in the affirmative, on purely theoretical grounds, in support of which he brings forward the following statements ${ }^{1}$ :-

'The most efficacious rays in promoting assimilation in plants are those which are most strongly absorbed by chlorophyll, and which at the same time possess a high mechanical intensity (heat-action); these are the red rays between $B$ and $C .^{\prime}$ But a glance at the carefully prepared tables given at pp. $745^{-6}$, shows that this theoretical reasoning is incorrect. If Lommel's hypothesis were correct, the evolution of oxygen would be seen, on observing the solar spectrum, to attain its maximum between $B$ and $C^{2}$, which however, as Pfeffer has shown, is by no means the case. The second of Lommel's statements is:- "The yellow rays can produce only a small effect notwithstanding their considerable mechanical intensity, because they are absorbed only to a small extent; and the same is the case with the orange and green rays.' This statement is again entirely opposed to observation; for it is these very rays that are the most efficacious in promoting evolution of oxygen. Lommel says indeed (l.c. p. 584) that 'this inference is incorrect;' it is however no inference, but the result of actual observation. That the light which has passed through a solution of chlorophyll causes only an inconsiderable evolution of oxygen is easily explained when it is recollected that even the yellow is considerably weakened in the spectrum of chlorophyll. But according to Lommel's theory there ought to be no evolution of oxygen at all when light has passed through a solution of this kind if it shows the absorption-bands very dark, since those rays which according to him are alone efficacious are wanting.

There is however no need for this direct contradiction; for a correct estimate of known facts leads to the conclusion that it cannot be those rays which are absorbed by the colouring matter of chlorophyll that cause the evolution of oxygen; for the rays absorbed in such a solution are the same as those absorbed in a green leaf. In the former there is however no evolution of oxygen (and apparently also no oxidation); and there is nothing to justify the supposition that the same rays which are absorbed by chlorophyll in solution without causing evolution of oxygen should cause it in the living leaf. It must certainly be right to suppose, as a necessary result of the principle of the conservation of energy ${ }^{3}$, that the rays which are efficacious in causing evolution of oxygen must be absorbed, inasmuch as they perform chemical work; but

1 Lommel, Pogg. Ann. vol. 143. p. $5^{81}$ et seq.

2 Muiller (Botan. Beobachtungen, Heidelberg 1871, Heft I) has adduced a great array of figures in support of this conclusion. But any one who knows how such observations shouk be made knows also what value is to be attached to these. See also Pfeffer, Bot. Zeit. 1872, No. 23 et seq.

${ }^{3}$ See also what I said on this subject long ago in my Experimental Physiology, p. 287. [Pringsheim has made a series of researches on Chlorophyll (Monatsber. d. k. Akad. zu Berlin, I874-188I; Jahrb. f. wiss. Bot. XII, I88I ; see also Nature, vols. XXI, XXIII, and Quart. Journ. Micr. Sci. I882), and has come to the following conclusions with respect to its function: (I) that the rays absorbed by it are not those which promote assimilation; (2) that, on the contrary, these rays promote the respiration of the protoplasm; (3) that it is the protoplasm of the chlorophyllgranule, and not the chlorophyll, which decomposes the absorbed carbonic acid and forms organic compounds; (4) that the energy for this purpose is derived -from light, but it has not yet been ascertained. which rays are absorbed by the protoplasm; (5) that the function of the chlorophyll in the process is protective, that is, that it absorbs the rays which would promote respiration in the chlorophyll-granule and this renders it possible for the synthetical processes to take place within it.] 
observation shows that it is not the rays absorbed by the green colouring matter that perform this work either in the solution or in the living plant ${ }^{1}$.

The Relation of Cell-division to Light has, as I have already explained, been completely misunderstood by Famintzin. In my paper ' On the influence of daylight on the formation and unfolding of various organs of plants' (Bot. Zeit. 1863, Supplement) I described in detail a long series of phenomena which show that the fresh formation of parts connected with cell-division is in general independent of light as long as there is a supply of reserve food-material to support growth. The main results were again collected in my 'Handbook of Experimental Physiology,' p. 31, referring also to that paper. Notwithstanding this, Famintzin ${ }^{2}$ commences his paper quoted above (three years later than one, and five than the other of my works) with the words: 'The action of light on cell-division has not yet been carefully examined by any one. All that I-have been able to find on this subject is limited to a remark of A. Braun's on Spirogyra and a statement of Sachs relating to cell-division in general.' He then quotes a passage from Braun cited also by me, and continues:- 'Basing his remarks on these statements, Sachs expresses himself as follows,' and then quotes some passages from my Handbook, p. 3i, no reference being made to the earlier paper or its conclusions. He then maintains that his own observations lead to entirely different results; but it is easy to show that they rather lead to the same as mine. At the end of his memoir (p. 28) he says:- 'The cell-division of Spirogyra is not prevented by light, as has hitherto been supposed, but on the contrary is promoted by it' (which is incorrect). According to Famintzin's observations, this acceleration of cell-division by light depends on the fact that light induces the assimilation of foodmaterial; which is obviously a different question from that argued by me and opposed by him; since, presupposing the presence of a supply of food-material, I only argued the question whether light exerts any influence on the physical fact of cell-division.

'The cell-division of Spirogyra,' continues Famintzin, 'has been proved to be dependent on light to the same extent as the formation of starch; but the relationship in the former case differs from that in the latter in the following respect:- the formation of starch is induced by a very brief exposure to light (about half an hour) and requires that its action be direct; starch is formed only under the influence of light; in its absence the formation at once ceases. Cell-division, on the other hand, is induced only after light has acted for some hours; it then commences in the cells whether these are exposed to light for a longer time or are removed into the dark.' This shows therefore that when food-materials are formed cell-division takes place in the light as in the dark; a fact which I had proved five years before by a greater number of observations.

Better in more than one respect is Batalin's treatise 'On the action of light on the development of leaves' (187I) ${ }^{3}$. Starting from the facts discovered by himself and by Kraus that cells have the same size in small etiolated leaves as in large letves of the same species grown in light, he concludes with justice that the number of cells is larger in the normal than in the etiolated leaf, and that the size of leaves is proportional to the number of cells in them. But from this he draws the following erroneous conclusion:- 'The leaf grows so long as it produces new cells; and the growth of the leaf does not depend on the increase in size of the cells.' It should rather be,- The growth of the leaf depends firstly and directly solely on the increase in size of the cells, and is proportional to this; but the cells, when they have grown larger, divide so that they are actually of about the same size in the small etiolated as in the large green leaf.' He continues:- 'Leaves do not grow in the dark because

1 Gerland (l.c. p. 609) has also arrived at a similar conclusion.

2 Famintzin, Mélanges phys. et chim., Pétersbourg 1868, vol. VII, On the action of light on the cell-division of Spirogyra.

3 Batalin, Bot. Zeit. 187 1, p. 670. 
their cells cannot divide without the assistance of light;' while the exact converse is the fact,- - they do not divide because they do not grow. This error prevails throughout. the whole treatise, which in other respects contains a number of instructive observations. Moreover, Prantl's measurements show that even in small etiolated leaves (Pbaseolus) numerous cell-divisions take place ${ }^{1}$.

It must be observed in addition that the very small growth of leaves in the dark is not a universal phenomenon even amongst Dicotyledons. The leaves produced from the tuberous roots of the Dahila and Beet grown in the dark, and even those of Pbaseolus, attain very considerable dimensions, and sometimes, especially when the temperature is high, almost the size of those developed in the light ${ }^{2}$.

Contrivances for observing plants in light of different colours (or of different refrangibility). In order to allow light of different degrees of refrangibility to act upon plants, three methods may be adopted:-(I) The use of the spectrum; (2) The removal of particular rays by absorbent media (glass or fluids); and (3) Coloured flames.

(I) If a ray of light is decomposed by passing it through a prism, it is possible to expose small plants or parts of plants to the action of narrow zones of the spectrum; and hence to allow light of approximately equal refrangibility to act upon them. Draper, Gardner ${ }^{3}$, Guillemin, and Pfeffer have worked in this manner. In using the spectrum it must however be observed that the intensity of the light in its different parts is less than that of the light that passes through the slit in proportion to the breadth of each part. If the spectrum at the distance from the prism where the observation is made is, for instance, $200 \mathrm{~mm}$. long, but the slit only I $\mathrm{mm}$. broad, the mean intensity of light of the whole spectrum is only $1 / 200$ of that which passes through the slit, even if no light is otherwise lost, which is seldom the case. Only a small luminous intensity must therefore be expected in the spectrum. In order to obviate this difficulty, it is necessary that very intense light pass through the slit, which may be effected by the use of condensing lenses. If, as is usually the case, sunlight is employed, the ray to be decomposed must be kept in a fixed position by a heliostat, or at least by a moveable mirror.

(2) Absorbent media. The defects which have been mentioned in observations with the spectrum, as well as the considerable cost of a heliostat, are avoided when coloured light is obtained by means of absorbent media. For this purpose discs of coloured glass or strata of fluids enclosed between colourless glass plates may be used. These last possess the advantage that almost any required amount of space may be illuminated by the light in question, and that the transmitted light only loses so much in intensity as is due to the small amount of absorption of the transmitted rays by the coloured medium. It is a mistake, though a very common one, to think that observations made with coloured screens are less exact than those made with the spectrum; in general it is just the reverse; and which method should have the preference must be decided in each particular case.

The use of absorbent media is always subject to the disadvantage that they do not generally transmit light of a single colour, but several different kinds of rays. This disadvantage is especially the case with coloured glass plates; and, with the exception of the deep red ruby and the very dark blue cobalt glass, there are scarcely any kinds which answer our purpose. It is more practicable- to obtain coloured fluids of the desired quality, although here also the number that can be used is small. The two which have been already mentioned are particularly useful, viz. a saturated solution of potassium bichromate, and a dark solution of ammoniacal copper oxide; by means of these, with the right concentration and thickness of the stratum, experiments

${ }^{1}$ Arb. des bot. Instit. Würzburg, I873, Heft III. p. 384 .

${ }^{2}$ See infra, Sect. 20.

${ }^{3}$ Gardner, Froriep's Notizen, 1844, vol. 30. No. I I.-Guillemin, Ann. des Sci. Nat. 1857, vol. VII. p. 160 . 
can be contrived so as to split white daylight exactly into two halves, the first solution transmitting the less refrangible rays from the red to the green, the blue solution all the more refrangible rays from the green to the ultra-violet. Those fluids also are of great use which transmit the whole spectrum with the exception of a few groups of rays as sharply limited as possible. If certain phenomena occur when plants are exposed to light transmitted through these solutions, it is certain that they are not caused by rays of that particular refrangibility which are absent, and vice versâ. It is obvious that absorbent media are of use in experiments only when the spectrum of the light that passes through them is accurately known. Glass plates are employed as windows in dark boxes closed on all sides in which plants are placed; coloured fluids can also be employed for the same purpose by placing them in glass vessels with parallel sides and using these as a window. When it is not necessary to allow light to fall in parallel rays upon the plant, the most convenient use of coloured fluids is to fill with them the space between the two walls of a double glass bell which is then placed like an ordinary bell-glass over the plants to be observed ${ }^{1}$.

For microscopic observations in coloured light I employ boxes like that represented in Fig. 474; only that instead of the colourless plate of glass, a double window is used, the space between the two panes being filled with coloured fluids.

(3) Coloured Flames-i.e. the light of bodies in a finely divided state heated to incandescence in a flame which is itself non-luminous-have not hitherto been employed for accurate observations on plants. I know only of one statement by Wolkoff:; that etiolated seedlings of Lepidium sativum became green when placed for seven or eight hours at eight inches distance from a non-luminous gas-flame in which sodium carbonate had volatilised and become incandescent. This light, as is well known, consists only of rays which correspond to Fraunhofer's line $D$. The red light of the flame of lithium or the blue light of that of indium \&c. may be employed in the same manner as this yellow flame, if sufficient intensity and the necessary permanence can be attained with these flames.

[The foregoing account would be incomplete without some statement of the results attained on this subject by Mr. H. C. Sorby. The following is a brief abstract, supplied by him, of investigations which will be found reported in detail in his published papers $^{3}$ :-

Vegetable colouring-matters may be divided into two principal classes, fundamental and accidental. The fundamental are those which are essential to the healthy growth of the plant ; and by carefully studying the position of the absorption-bands in living leaves these substances are often found in a free and solid state, even when they are soluble in water, or could easily combine with the closely associated oils or wax. When set free by boiling in water or by decomposition, they dissolve according to their properties in this respect in water, or combine with oil or wax if these be present. The petals and other portions of the organs of reproduction often contain some of the fundamental colouring-matters of the leaves, but frequently others are developed.

Accidental colouring-matters are those which may be present or absent without apparently interfering with the healthy growth of the individual plant, and are often so conspicuous as to make mere colour of very little importance if it depend upon them, and not on the difference in the kind or relative proportion of the fundamental colouring-

${ }^{1}$ Such double-walled bell-jars can be obtained from Warmbrunn and Quilitz, Berlin.

2 Wolkoff, Jahrb. fïr wiss. Bot. I866, vol. V. p. 11.

${ }^{3}$ [Proceedings of the Royal Society, vol. XV. 1867, p. 433.-Quarterly Journal of Microscopical Science, vol. IX. 1869, p. 358; vol. XI. 1871, p. 21 5.-Monthly Microscopical Journal, vol. III. 1870, p. 229; vol. VI. 187I, p. 124.--Proceedings of the Royal Society, vol. XXI. 1873, p. 442, Researches on the spectrum-analysis of the green colouring-matter of plants are given by Chautard in Ann. de Chim. et de Physique, Sept. 1874.] 
matters. These non-essential substances are far more common in the petals than in the leaves, and if of any use to the plant, are only indirectly advantageous, as, for instance, in attracting insects. It is doubtful to which of these two divisions certain substances should be referred, and perhaps some may not be essential for the healthy performance of vital functions, but merely necessary products; and some may be essential to one plant and not to others.

It has been found convenient to arrange the colouring-matters of plants in the following groups, which are as it were of generic value, and include several different species.

Cblorophyll group.-The green substance described as chlorophyll by many writers must often have contained two perfectly distinct green substances, and the product of the action of acids on one of them, mixed with one, and in some cases with three, different species of xanthophyll, and one or two of lichnoxanthine. These two green substances are blue cblorophyll and yellow chlorophyll ${ }^{1}$. Blue chlorophyll dissolved in alcohol is of a splendid blue-green colour, the whole of the green part of the spectrum and a considerable part of the contiguous blue being readily transmitted. rellow chlorophyll absorbs the whole of the blue and the blue end of the green, so that the general colour is a bright yellow-green. Chlorofucine is of a clear yellow-green colour.

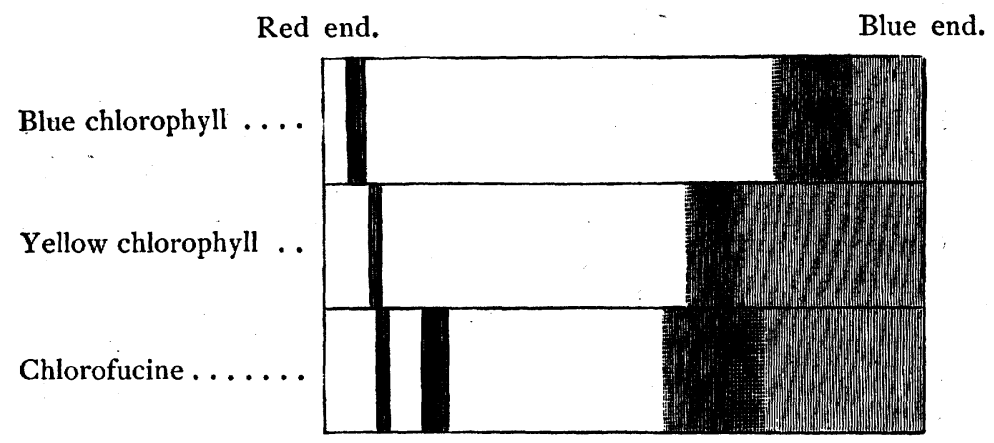

FIG. 476 b. - Spectra of the chlorophyll group compared.

It has many properties in common with the above-named two kinds of chlorophyll, being, like both of them, highly fluorescent and easily decomposed into another modification by acids. All three are insoluble in water and soluble in absolute alcohol, but not always in carbon bisulphide.

The difference between their spectra will be better understood by means of the figure, $476 b$, which represents the absorption-bands as seen in solutions diluted so as to show those at the blue end, and only the darkest and most characteristic of those in the red.

Xantbopbyll group.-This group includes a number of yellow or orange-coloured substances, insoluble in water but soluble in carbon bisulphide, giving spectra with two more or less well-marked absorption-bands in different positions, according to the particular species. They are not fluorescent, and when dissolved in absolute alcohol, after addition of a little hydrochloric acid, they all gradually become colourless, but two of them are first changed into a blue substance. Nearly all green leaves contain three perfectly distinct fundamental species, which Mr. Sorby has named orange xantbophyll, xantbophyll, and yellow xanthophyll. The spectrum given in Fig. 476, copied from Kraus,

1 [The spectrum given by Kraus (Fig. $476 \mathrm{~B}$ ) is due to a mixture of these with some of the products of the action of acids. See Pringsheim, Ueb. natiirliche Chlorophyllmodificationen, Monatsber. d. k. Akad. d. wiss. zu Berlin, I876.] 
must have been due to a mixture of the latter two. Olive Algæ contain another fundamental species, fucoxantbine. In many Fungi, and in the petals of flowers, occur other more orange-coloured species, of which that in Peziza aurantiaca is a good example. Sorby adopted the name proposed by $\mathrm{Kraus}^{1}$ for a still more red orangecoloured species; but what Kraus describes as phycoxantbine must have been a mixture of this substance with fucoxanthine and lichnoxanthine. The difference between the spectra of some of the above-named species will be better understood by means of the following figure $\left(4{ }^{6} 6 c\right)$, which represents those of the solutions in carbon bisulphide.

When these various substances are dissolved in benzol, their absorption-bands are all equally raised towards the blue end, so that we appear to have a remarkable series of very closely related substances.

Licbnoxantbine group. - T he colouring-matters belonging to this division are insoluble in water, soluble in absolute alcohol, and sometimes also in carbon bisulphide. They all give spectra without bands, and absorb more or less from the blue end. Some are yeilow, and others so red that they may be called licbnoerytbrines. Lichnoxanthine

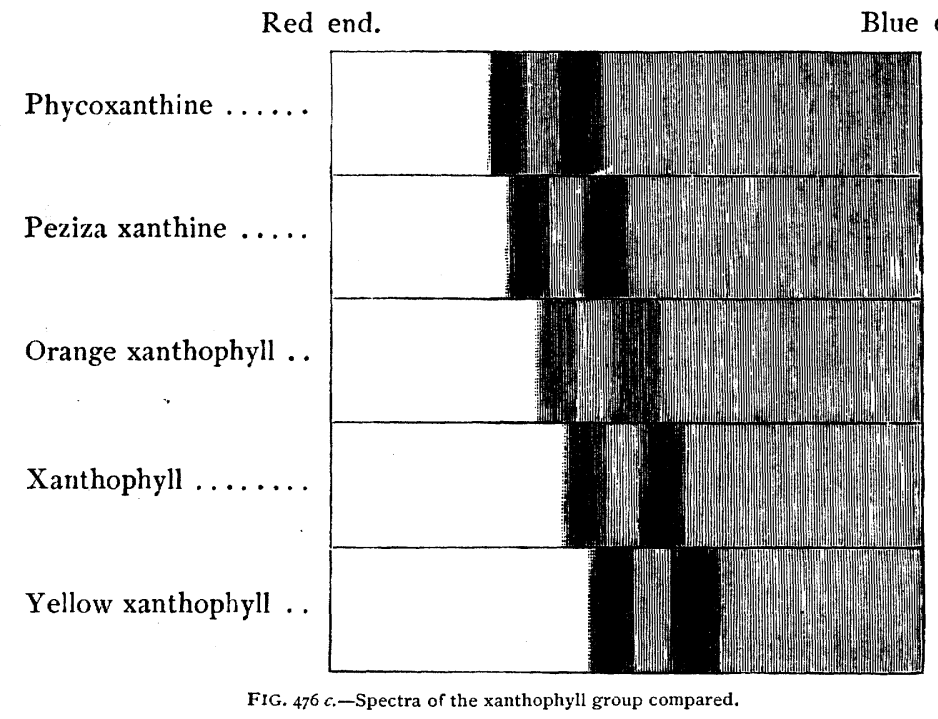

occurs in both the highest and lowest classes of plants, but the whole group is more especially developed in Lichens and Fungi. It is not yet possible to say what part they play in the economy of plants, and in some cases they are probably only products of the oxidisation of chlorophyll and resins, from which they may be prepared artificially.

We now come to a number of different groups, soluble in water but insoluble in carbon bisulphide.

Pbycocyan and Pbycoerytbrine grcups.-There are at least five distinct colouringmatters included in these two groups, which differ from one another in many well-marked particulars. The phycocyans are highly fluorescent, but the phycoerythrines little if at all. They give remarkable spectra with one main absorption-band. Some are connected with albuminous substances in much the same manner as the hæmoglobin of blood, being like it decomposed at exactly the same temperature as that at which albumen coagulates, whilst the others appear to be associated with some different but related substance. They are especially characteristic of red Algæ, but also occur in a few Lichens.

${ }^{*}$ Chlorophyllfarbstoffe, p. I09. 
Erythrophyll group.-The colouring-matters belonging to this group are very numerous, and their production often depends upon obscure and accidental causes, easily modified by slight variations in the internal or external conditions. They may be divided into three well-marked sub-groups, according as they are changed by the action of sodium sulphite. They are soluble in water, and are usually, if not always, dissolved in the juices of the plant, and disseminated in cells of various kinds. A greater number of different species occur in the petals than in the leaves. They are usually indicative of low constructive energy, but yet are not merely products of chemical decomposition.

Chrysotannin group.-Much remains to be learned with respect to these more or less pale yellow or even colourless substances, and the part they play in plant-life. The most striking fact connected with them is that when oxidised they give rise to the various brown substances which are the cause of many of the characteristic tints of autumnal foliage. These changes are mainly, if not entirely, due to chemical action, and can easily be imitated artificially.

Exposure to a greater or less degree of light may produce a great quantitative or even qualitative difference in the colouring matters. Rudimentary petals and rudimentary leaves correspond closely, but subsequently development takes place in two different directions; and very often when the petals of the more highly developed varieties are only partially grown, the constituent colouring-matters are both qualitatively and quantitatively the same as those in some other variety, as though this were due simply to a natural arrest of development. By growing almost in the dark flowers coloured by more or less of the orange species of the xanthophyll group, the-petals are obtained of the full size, but only yellow and corresponding exactly to the normally yellow variety; and there is this remarkable peculiarity, that the relative proportion between the different colouring-matters approximates more or less closely to what is obtained by exposing to light a solution of those found in the normal petals; that is to say, absence of light tends to prevent the formation in the petals of those more orange-coloured substances which are the most readily decomposed by exposure to light when they are dissolved out from the petals.] ${ }^{1}$

${ }^{1}$ [The occasional occurrence of 'chlorophylloid green colouring-matters' in the tissues of animals is a matter of considerable significance. Mr. E. R. Lankester has obligingly drawn up the following list of such cases. Those marked with an asterisk have been observed by hirn with the spectroscope for the first time:-Infusoria; Stentor Mulleri and others. Foraminifera. Radiolaria ; Rhaphidiophrys viridis, Heterophrys myriapoda (Quart. Journ. Micr. Sc. 1869). Cœeltnterata; * Spongilla fluviatilis (Journ. Anat. and Phys. 1869), * Hydra viridis, Anthea cereus var. smaragdina (chlorofucine). Vermes; Mesostomum viride (Planariæ), *Bonellia viridis (in the skin), *Chatopterus Valenciennesii (in the walls of the alimentary canal). Crustacea; *Idotea viridis (Isopoda). The chlorophylloid substance is not present in the same physical or chemical condition in all these cases. In Rhaphidiophrys, Heterophrys, Spongilla, and Hydra, it is localised in granules imbedded in the protoplasm; this is also the case in Bonellia, but the granules are finer. In Idotea it is not in granules but diffused in the chitino-calcareous integument. In all cases the chlorophylloid substance agrees in having a strong absorption-band in the red-a little to the right or left; and, except in Idotea, in being soluble in alcohol; and in having strong red fluorescence and in finally losing its colour when dissolved. In Bonellia, Chatopterus, and Spongilla, the absorption-spectrum presents differences in other respects in each case, and the green tint is itself different-being black olivegreen in Chatopterus, bluer but equally dark in Bonellia, and apple-green in Spongilla and Idotea. In Spongilla the green colour is not developed if the animal grows in the dark. But like etiolated vegetable tissues, Spongilla, when immersed in strong sulphuric acid, gradually developes a strong leaf-green colour, fully as intense as that of the naturally green specimens (Quart. Journ. Micr. Sc. 1874, p. 400). Bonellia, on the other hand, always lives in a dark hole excavated by it in calcareous rock, and Chatopterus lives in a thick opaque tube. Geddes has found that a green Planarian (Con-

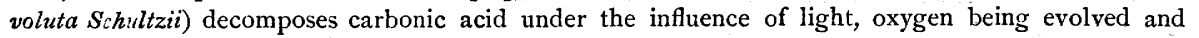
starch formed in the cells.] 
SECT. 9.-Flectricity ${ }^{1}$. The chemical processes within the cells of a plant, the molecular movements connected with the growth of the cell-wall and protoplasm, and the internal changes on which the activity of the protoplasm depends-whether exhibited in the formation of new cells or in movements of rotation-are probably connected with disturbances of the electrical equilibrium, although no actual empirical proof of this has yet been obtained. The fluids with different chemical properties in adjoining cells, the diffusion of salts and of assimilated compounds from cell to cell, and their decomposition, must also bring electromotive forces into play; but even this has not yet been observed directly. Even the electrical currents which must no doubt be set up by the evolution of oxygen from cells containing chlorophyll, by the formation of carbon dioxide in growing organs (as in seedlings), and by the transpiration of land-plants - although investigated by a few physicists-has not yet been actually established or accurately determined. According to Buff's careful observations, which have been confirmed by Jürgensen and Heidenhain, the internal tissue of land-plants is always electro-negative to its strongly cuticularised surface; the surface of roots, saturated with sap (like a transverse section of the tissue), is also electro-negative to the surface of the stems and leaves. If a plant or a cut part of a plant is placed, with the necessary precautions, in the circuit of a very sensitive galvanometer, a current passes from the external surface to the cut surface or to the surface of the root; this is in consequence of the contact of the cell-sap of the surface of the root or of a cut surface with the pure water employed to complete the circuit. The alkaline fluids of the thin-walled phloëm of the fibro-vascular bundles are surrounded by the acid fluids of the parenchyma, and diffusion-currents doubtless exist between them. These must certainly produce electromotive effects, but hitherto no investigations have been made on this subject $^{2}$.

The leaves and branches of plants present a large surface to the air; and the tissue of the whole plant is permeated with electrolytic fluids. These conditions appear to adapt plants to be the medium for equalising electrical differences between the earth and air by means of currents traversing the plant. Since the electrical tension of the air is generally different from that of the earth, and the relationship of the two is constantly varying with changes of weather, it may be assumed that in all probability constant electrical interchanges are going on through the agency of plants $^{3}$. Whether these have a favourable effect on the processes of

1 Villari, Pogg. Ann. I 868, vol. I 33. p. 425.-Jürgensen, Studien des phys. Inst. zu Breslau, 1861 ; Heft I. p. $3^{8}$ et seq.-Heidenhain, ditto 1863, Heft 2. p. 65.-Bruicke, Sitzungsb. der Wien. Akad. I 862. vol. 46. p. I.-Max Schultze. Das Protoplasma der Rhizopoden; Leipzig 1863, p. 44.-Kühne, Untersuchungen uiber das Protoplasma, I864, p. 96.-Cohn, Jahresber. der schles. Ges. für vaterlandische Cultur, I86I ; Heft I. p. 24.-Kabsch, Bot. Zeit. 1861, p. 358.-Riess, Pogg. Ann. vol. 69. p. 288.-Buff, Ann. der Chem u. Pharm. 1854, vol. 89. p. 80 et seq.-[J. Ranke, Untersuchungen über Pflanzenelektricität, Akad. der Wissen. München, Math.-Phys. Klasse, July 6, I872.-Kunkel, ueb. elektromotorische Wirkungen an unverletzten Pflanzentheilen, and Ueb. einige Eigenthümlichkeiten des electrischen Leitungsvermögens lebender Pflanzentheile, Arb. d. bot. Inst. in Würzburg, II. I, 2, I878-9.

2 Sachs, Ueber saure, alkalinische, und neutrale Reaction der Säfte lebender Pflanzen; Bot. Zeit. I862, No. 33 .

${ }^{3}$ [Becquerel thought that the evaporation from leaves forms an upward current of vapour which 
vegetation has at present, like the whole subject, not been investigated scientifically. The destructive discharges of atmospheric electricity which are effected through trees by means of flashes of lightning ${ }^{1}$, at least show that smaller differences of electrical equilibrium between the air and the earth may also be equalised by means of plants ${ }^{2}$.

The researches on the action of the electric stimulus on the movements of protoplasm and of leaves the motion of which is caused by tension of the tissues have not at present led to any important result from a physiological point of view, although distinguished observers have paid attention to this subject. It can only be said in a general way that very weak constant currents or induction-shocks (for a short time) produce no perceptible effect; that sufficiently strong electromotive force produces effects on the protoplasm and in the contractile tissues similar to those produced by a high temperature and by mechanical means; and that finally, when the strength of the current is still further increased, the protoplasm is killed and the motility of the leaves permanently destroyed, but sometimes in the latter case without causing death.

Jürgensen allowed the current from a battery of small Grove's elements, the force of which was regulated by a rheochord, to act under the microscope on the tissue of a leaf of Vallisneria spiralis. A constant current from one element produced no perceptible action; two or four elements caused a retardation of the protoplasmic movement, and when continued for a longer time completely stopped it. When the current was interrupted, the movement, if it had only been retarded, was restored to its original rapidity after the lapse of a short time; if it had entirely ceased, it was not recommenced even if the current was at once stopped. When the movement is thus arrested,

acted as a conductor to electricity. In this way, by destroying the necessary electrical conditions, he thought forests tended to dissipate hail-clouds. Mém. de l'Inst. vol. XXXV. pp. 806, 807.]

${ }^{1}$ [The disruptive effect of lightning upon trees is probably due to the sudden conversion of moisture into steam. See Osborne Reynolds, Proc. Phil. Soc. Manch. 1874, p. I5.]

${ }^{2}$ [Edwin Smith (Chemical News, Dec. I 7, I869) has detected constant currents of electricity passing in certain directions in plants, as follows :-In a cut piece of leaf-stalk (Rhubarb) from the end nearest the root to the end nearest the blade of the leaf; from the outer side of the leaf-stalk nearest the cuticle to the inner axis; from the lower end of the flower-stalk (Pæony) to the bract or petal; from the upper to the under surface of the leaf; in the stem (Hawthorn) from the cambium to the outer cuticle; in the root (several plants) from the outside to the axis, and from the root-stock towards the apex; in the hollow stems of monocotyledonous plants (Grass) from the inner to the outer surface; in the Potato from the centre to the outside; but in the Lemon, Pear, Gooseberry, and Turnip from the outside to the centre; in a living plant (Tropaolum) from the plant itself to the soil. Kunkel (loc. cit.) obtained similar results with the leaves and stems of a number of plants. He found that the direction of the current depended upon the relative moistness of the points in contact with the electrodes, any point being always positive with respect to another which is relatively dry. The currents which are observed appear therefore to be due to the travelling of water in the tissues. Phenomena of the same kind have been observed by Quincke in diaphragms.

Dr. Burdon-Sanderson has made a remarkable series of observations on the electric currents in Dioncea muscipula (see Report of British Association for 1873 ; also Nature, vois. VIII, X, and XV, and Proc. Roy. Soc. vol. XXI. p. 495). By the aid of Thomson's galvanometer he has shown that these currents are subject, in all respects in which they have been as yet investigated, to the same laws as those of animal muscle and nerve. Further, The Electrical Disturbance which accompanies the Excitation of the Stigma of Mimulus luteus, Nature, XVI, 1879. See also Munk, Die elektrischen und Bewegungs-Erscheinungen am Blatte der Dionaa muscipula, Arch. f. Anat. u. Physiol. Du BoisReymond, 1876 .] 
the chlorophyll-granules which are carried along by the very watery protoplasm accumulate at various spots. A current from thirty elements causes permanent cessation of the movement even if the connection is only momentary. Induced currents act like constant ones; but the number of induction-shocks which pass through the cells in a unit of time appears to have no considerable influence on the action.

The changes of form of protoplasm under the influence of a sufficiently strong electric current are, according to the observations of Heidenhain, Brücke, Max Schultze, and Kühne, similar to those caused by a high temperature near the extreme limit or beyond it. From those of Kühne it appears to result that protoplasm is a very bad conductor of electricity, and that the excitement caused by a current at particular spots in the protoplasm is not easily transferred to other spots.

Cohn, Kabsch, and others, state that weak induction-currents act on the sensitive parts of the leaves of Mimosa, the stamens of Berberis, Mabonia, and Centaurea Scabiosa, and the gynostemium of Stylidium graminifolium like concussion or contact, the parts moving as if under the influence of these agencies. According to Kabsch, stronger induction-currents, which permeate the whole plant, destroy the sensitiveness of the gynostemium of Stylidium even for mechanical excitation; but after half an hour the sensitiveness again returns. The statement of Kabsch is noteworthy that the movement of the leaflets of Desmodium gyrans are permanently prevented by stronger induction-currents, which however do not kill them.

\section{Sect. IO. - Action of Gravitation on the Processes of Vegetation .} Since the attraction of the earth acts uninterruptedly on all parts of the plant, the entire vegetable organisation must be so contrived that the weight of the separate parts of the plant is serviceable, or at least not injurious, to the various purposes of the life of the plant.

In observing these relationships the first thing is to distinguish between those contrivances which have for their object to bring the weight of the parts of the plant into harmony with the purposes of its life-gravitation itself not taking any direct recognisable part in the attainment of these objects-and those phenomena of vegetation on the other hand which are brought into existence by the direct influence of gravitation on the mechanism of growth.

To the first of these groups belongs the fact that the branches and foliage of upright stems are distributed nearly equally on all sides, and that in larger plants the firmness and elasticity of the masses of tissue in the stem is promoted by the formation of wood, or is brought about by other means, as for instance in the trunk of Musa. But since it is very common in the organic world for the same purpose to be attained by very different means, slender delicate stems with but little wood can protect themselves from sinking down and can expose their foliage to the light by twining round firm supports, or by climbing with the help of tendrils, hooks, spines, \&c. The same purpose is evidently served by the various floating contrivances of water-plants and those of fruits and seeds; in all these cases the structure is obviously adapted to make the weight of the part of the plant serviceable or at least not injurious to its life; although it cannot be maintained that gravitation takes any part in the formation of wood, in the sensitiveness of tendrils, or in the production of a floating apparatus. The only explanation of these arrangements lies in

1 These statements are intended in the first place to draw the attention of students to the processes of vegetation which are especially influenced by gravitation. Its action on the mechanism of growth will be fully described in Chap. IV, where also the literature is quoted. 
Darwin's Theory of Descent ; viz. that, under the influence of long-continued natural selection, only those structures are finally able to maintain their existence which, while sufficient for the other requirements of life, are so arranged that the weight of the part is not injurious or is even useful. It must not be inferred from this, nor does observation render it probable, that gravitation takes any direct part in these phenomena.

Gravitation however exerts a direct influence on the growth of young parts of plants as soon as the longitudinal axis of the growing organ is inclined obliquely to the perpendicular and therefore to the action of gravitation. In this case the growth in length of the oblique organ is different on the upper and under sides, and the more so the more nearly horizontal the axis of growth. According to the nature of the organ and its purpose in the economy of the plant, either the upper side grows more strongly than the under side, or the reverse. A curvature concave either downwards or upwards is thus caused by the influence of gravitation and growth, and this curvature increases until the free-growing end is directed

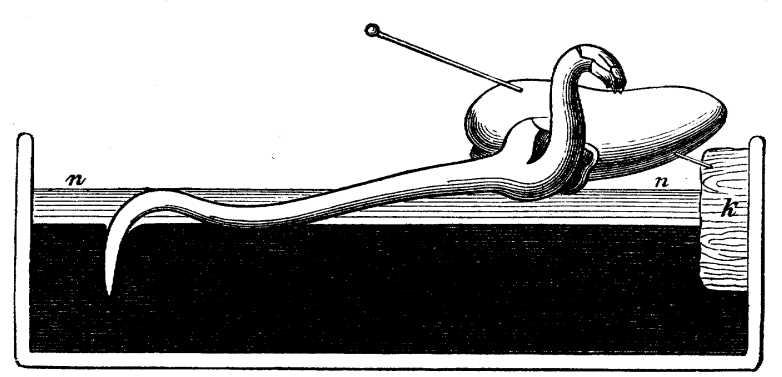

\begin{abstract}
FIG. 477-A seedling of Vicia Fixba, the root and stem of which were straight, which was so fixed that the apex of the FIG. 477.-A seedling of Vicia Faba, the root and stem of which were straight, which was so fixed that the apex of the
root lay nearly horizontally upon the surface of the mercury (black in the figure), by means of a pin to the cork $k$; $n n$ is the layer of water on the mercury. The figure shows the seedling 24 hours after its fixture; the root has bent sharply downwards at its growing portion, so that its apex penetrates the mercury; the resistance experienced by it in so doing is shown in the form of the part of the root lying just behind the downward curve. The stem has curved upward from its base. The position of the bud is independent of the action of gravitation; it is a phenomenon of nutation.
\end{abstract}

vertically either downwards or upwards; the former, for example, in primary roots, the latter in many primary stems. In lateral branches, leaves, and secondary roots, similar phenomena occur, though not so markedly. Internal processes of vegetation, the weight of the upper parts, or the influence of light, act in opposition to that of gravitation, so that conditions of equilibrium arise which cause the organs to stand horizontally or obliquely to the perpendicular.

Thus the vertical direction of primary roots and stems, and the oblique direction of their lateral branches, are determined solely by gravitation, or at any rate to some extent, so long as these parts are still growing; when they subsequently become lignified or cease to grow, they maintain the position once acquired. If therefore a growing plant rooting in the ground (inside a pot) is placed horizontally, the mature parts remain in this position; but the apex of the primary root turns downwards, and the growing internodes of the end of the stem turn upwards, the leaves, branches, and secondary roots also bend until they make about the same angle with the horizon that they did before the change in their position. The parts 
which were actually growing when the change was made are shown by the curvatures caused by the influence of gravitation.

Although we must defer till the fourth chapter the consideration of the internal changes which accompany these curvatures, the proofs that they are really caused by gravitation may be presented in the two following forms :-

(1) Individuals of the same species have everywhere on the earth's surface the same position with respect to the horizon, and therefore also with respect to the earth's radius. Upright stems therefore, such as those of Pines, grow in South America in totally different directions from what they do with us; if their axes of growth were elongated downwards, they would intersect in the centre of the earth, and coincide with its radii. It follows therefore that their direction of growth must be determined by a force which stands in a perfectly definite relation to the position of the earth's centre of gravity. But there is only one such force, viz. gravitation or the attraction of the mass of the earth. The same argument holds for horizontal or oblique branches, leaves, and roots, since these form a constant angle with the primary stem.

(2) Gravitation differs from other forces in acting independently of the chemical or other properties of the body, being regulated only by its mass; but the same property is also possessed by centrifugal force. If, as Knight ${ }^{1}$ first showed, a growing seedling is made to rotate with a rapidity sufficient to bring centrifugal force into play, this force acts on the different parts like gravitation; i.e. the parts which would otherwise be influenced by gravitation (as the primary root) now follow the direction of the centrifugal force and grow outwards from the centre of rotation, while the stem, which would otherwise grow upwards contrary to the direction of gravitation, now assumes a direction towards the centre of rotation, i. e. in a direction opposite to that of the acting force. This law is strikingly illustrated when seedlings, the roots and stems of which had previously grown in one straight line, are fixed upon a rotating disc (protected from evaporation by a bell-glass) in such a manner that the axis of growth has a tangential direction. The mature parts maintain this direction during the rotation, while those which are still growing bend so that the apices of the roots point outwards and the apices of the stem inwards (towards the centre of rotation). If the rotation takes place in a horizontal plane, gravitation acts, in addition to centrifugal force, on the growing parts, and the direction of the stem and root becomes oblique. But when the rotation is very rapid, it is possible to increase the centrifugal force to such an extent that the axis of growth remains nearly horizontal. If, on the contrary, the seedlings are fixed to a disc rotating in a vertical plane, each side of the growing part is in turn directed for a short time upwards, downwards, to the right, and to the left. The action of gravitation therefore affects all sides equally; $i$.e. the growth of the organ is practically independent of gravitation. Centrifugal force is therefore the only force that acts on the growing parts; and the root takes an outward radial direction even when the disc is not rapidly turned, the stem an inward radial direction. If however the disc is made to turn very slowly in a vertical plane (round a horizontal axis), so that

1 Knight, Phil. Trans. I806, part I. p. 99. 
there is in fact no centrifugal force (as by intermittent turns, one revolution in ten to twenty minutes with a radius of from 5 to $10 \mathrm{~cm}$.), I have shown ${ }^{1}$ that the organs then grow neither in the direction of gravitation nor in that of the centrifugal force, but just in those directions in which they had happened to be placed when fixed in the vessel. Under such conditions parts which normally grow straight often curve in a plane quite independently of external forces, and this can only be due to internal causes of growth which are distributed unequally round the axis of growth. Thus, for example, primary roots and stems of germinating seeds (Faba, Pisum, Fagopyrum, Brassica) will not lie in a straight line, but their respective axes of growth will intersect at any angle up to a right angle, the anterior side of the base of the stem growing more rapidly than the posterior side, and thus causing a curvature. It is clear that the direction of the secondary roots which spring from the primary root, as well as that of the leaves on the stem, is also, under these conditions, affected only by internal causes of growth. It is only in this way that we can explain the directions and forms assumed by parts of plants when uninfluenced by gravitation, centrifugal force, or heliotropic curvatures, which could not occur in these experiments.

CHAPTER IV.

\section{THE MECHANICS OF GROWTH.}

SECT. $\mathrm{t}$ I. Definition. The growth of crystals consists in an increase of their volume by the apposition of homogeneous particles in definite directions. In plants the process which we call growth is much more complicated; and the term is employed in different senses, according as we are speaking of the growth of a grain of starch or of a chlorophyll-granule, of part of a cell-wall, of a whole cell, or of a multicellular organ. The common point in all these processes is that they depend at last on the intercalation of new micellæ between those already in existence, in other words on intussusception, as has already been explained in the first section of Book III. But even in structures so simple as grains of starch or parts of cell-walls, we are met with insurmountable difficulties when we attempt to explain the mechanical process of growth in all its details; and the present state of our knowledge by no means enables us to propound a connected theory of the growth of the entire cell or of a multicellular organ. We are in fact at present able only to follow empirically the processes of growth in detail, their causes and results. After this we may attempt to form definite ideas of the separate

1. Würzburger Med.-Phys. Gesellschaft, March 16, I872: [also, Ueb. Ausschliessung der geotropischen und heliotropis hen Krümmungen während des Wachsens, Arb. d. bot. Inst. in Würzburg, II. 2, 1879. Sachs calls the apparatus used for this purpose a Clinostat.] 
processes, taking for granted at the outset the purely formal phenomena of morphology, and regarding as the ultimate object of the enquiry the obtaining an insight into the mechanism of growth. If the solution of this difficult problem must be deferred to a distant future, it at any rate lies within the scope of this work to collect together the ascertained phenomena. But even here we meet with the difficulty that no one has as yet undertaken to linit the term Growth to a definitely circumscribed idea. The term is however always employed in the case of plants and animals to designate permanent changes in form or volume or both, brought about by internal causes, themselves the result of organisation, and in their turn excited and maintained by definite external causes, as heat, light, gravitation, the supply of food-materials, water, \&c. Changes in the form or volume of parts of plants that remain quite passive to external forces, and in which changes no organising process cooperates, ought not to be included in the term Growth. Thus, for example, there is no growth when the form or length of an internode or root is altered by simple stretching, pressure, twisting, or bending (it may be by the hands). It is quite possible however that under certain circumstances internal changes might be brought about by external influences to which the part of the plant is at first altogether passive, but which, combined with organising processes, cause true growth or changes of growth. By organising processes I understand those internal changes which fulfil the two following conditions:-firstly, they are caused by the specific organisation of the part of the plant, which is of such a nature that any external influence can only effect changes in accordance with it; secondly, they result in a permanent change of the organised part which is not at once reversed by opposite external influences. If, for example, the elevation of the temperature above the inferior limit (see Sect. 7) has caused an increase in volume of the embryonic structures already saturated with water, the parts will not contract to their previous volume when the temperature again falls below this point, but will retain the increase acquired during the higher temperature; in other words the process is not reversed, it only ceases. Microscopic as well as other kinds of examination also show that the internal organisation has undergone permanent change varying with the specific properties of the plant. If on the contrary a stem is allowed to wither from want of water, it becomes shorter and ceases to grow; when it again absorbs water it becomes longer and thicker and begins to grow. The contraction on withering and the lengthening on the absorption of water are mere physical phenomena; but the lengthening and thickening of a part resulting from continued turgescence may actually depend upon growth, the organisation of the plant being altered permanently and to an amount varying with the species by the operation of the turgescence. It is again the result of permanent and specific change of organisation when a tendril, in consequence of the light pressure of the body to which it clings, lengthens less on the side in contact, more on the opposite exposed side; the curvature thus caused does not disappear if the pressure has lasted long enough; the whole phenomenon is therefore one of growth. When, on the contrary, the motile organ of a Mimosa-leaf bends downwards in consequence of irritation, and afterwards again bends upwards, this is, it is true, caused by the peculiar organisation of the plant; but the movement induces no change in the organisation itself, and its effects are not permanent, the leaf soon returning to 
its original condition. The sensitiveness of the leaves of Mimosa does not therefore depend on a change of growth caused by the irritation; while the power of tendrils to curl round supports depends, it is true, on sensitiveness, but of such a character as to cause a change in the processes of growth.

If increase in volume is included in the idea of growth, as is the case in ordinary language, the rigorously scientific use of the word would require special care ; for if we simply say that a plant or a part of a plant of considerable size grows, this may be accompanied actually by a decrease of the whole volume. Thus, for example, when bulbs sprout or seeds germinate in the air, the whole does not grow, but only the younger parts develope at the expense of the older, which in addition give off aqueous vapour and carbon dioxide. It is therefore necessary to distinguish accurately the growing parts from those connected with them which do not grow:

There are however changes of form in the parts of plants which are not associated with increase, and which may even be attended with decrease in volume, but which nevertheless depend on a permanent and irreversible change of organisation. Thus, for instance, the pith, after removal from the internodes, increases in length for days even while it loses water by evaporation in air that is not saturated. It would scarcely seem convenient to exclude these and similar phenomena from the idea of growth; and it is therefore necessary to distinguish between growth with and growth without increase in volume; in the latter case growth consists in a mere change of form which again depends on an alteration of position of the smallest particles. Every case of increase in volume of a grain of starch or of a cell must not be regarded as growth, inasmuch as it may be caused by absorption, and may be reversed by loss of water; nor is it necessary that growth in a single cell should be associated with increase in volume, since particular parts of the cell may furnish material for the increase of other parts. In this case the cell considered as a whole only changes its form; and if this change is caused by internal organising forces, it must be considered as a kind of growth. Those changes in the form and volume of cells must, on the other hand, be excluded from the idea of growth which occur only occasionally and admit of being completely reversed, as is the case with the contractile organs of sensitive and periodically motile leaves.

An error which is constantly made by those who are unacquainted with physiology is to confuse the ideas Growth and Nutrition, or to consider them identical. It is no doubt true that all growth must be associated with the conveyance of food-materials to the growing parts; but these food-materials are usually withdrawn from older parts where they were previously inactive; the whole organism, consisting of both growing and non-growing parts (for instance a bulb suspended and putting out leaves in the air), is not nourished as such from without. The growth of certain parts is therefore no indication of nutrition of the whole. Still less necessary is the connection between growth and nutrition from without; the special organs of nutrition, the green leaves, do not grow after they are mature, although they carry on the process of nutrition. The two processes may coincide both in place and time, i.e. in the same cell; but may also be separated in both space and time; and this is indeed usually the case, as has been sufficiently shown in Sect. 5 .

Sect. I 2. Various Causes of Growth. Growth, like all vital processes, takes place only when certain favourable external conditions coexist. These are the presence 
of assimilated food-material, water, oxygen, and a sufficiently high temperature. Under these conditions individual cells or masses of tissue may grow, provided that their organisation permits it. But independently of these conditions there are others, as we have seen in the last chapter, which, without absolutely causing or arresting growth, nevertheless influence it; as light, gravitation, and pressure. The firstnamed may be called the necessary, the last the secondary conditions of growth. In all growth all the necessary conditions must concur while the secondary conditions intervene only in certain cases, and exert their modifying influence very differently on the corresponding parts of different plants.

The conditions spoken of as Necessary and Secondary depend upon the environment of the plant, and act upon it from without. They may therefore be described as External Conditions or causes of growth, in contradistinction to the Internal Conditions dependent on the organisation of the plant. The existence of the latter conditions is most strikingly manifested in the fact that all parts of plants are able to grow only during a certain time; when this time-the period of youth and development - is past, they no longer grow, even when all the favourable conditions concur. This shows that the internal organisation undergoes changes, which at length render the continuance of growth impossible. But even in organs which are still growing a certain independence of external circumstances may be perceived; an Oak-leaf invariably grows differently from an Elm-leaf, an Oak-fruit from an Oak-root. The differences of these processes of growth is at once manifest in the difference of form and of the other properties of the organ; and no combination of external circumstances has the power of giving to a root, by change in its growth, the form of a leaf or to an Oak-leaf the structure of an Elm-leaf. There are also certain internal conditions of growth which do not decide, like the age of an organ and the necessary external conditions, whether growth shall take place, or at what rate; but determine how it shall proceed, and what specific and determinate organisation shall be attained by it. This latter circumstance depends only on the parent plants, or in other words on the species or variety to which it belongs. Descent determines the specific character of the growth; all the other conditions determine only whether growth shall take place at all, and with what rapidity and energy. The innate internal conditions that regulate the nature of the growth of the plant, when once present cannot again be destroyed or reversed; while the external conditions may be at one time brought into action, at another time set aside. The internal and external conditions of growth may therefore be distinguished as the historical and the physical; but those properties of a plant which have been obtained historically are generally termed hereditary. This expression is not open to objection unless heredity be considered, as has recently been done by many, as a kind of natural force requiring no further analysis. For in distinguishing hereditary conditions of growth-i.e. those that have been acquired historicallyfrom physical ones, it is not meant that the former do not also owe their existence to physical causes, but only that besides the accidental concurrence of physical conditions, it is also necessary to take into account certain characters which the plant has acquired when in the embryoric condition (in the broadest sense of the term) in the form of definite specialities of organisation through the influence of its parents. 
These suggestions must suffice here. The extremely difficult question which has been raised may be illustrated by far-fetched and elaborate explanations, but cannot be satisfactorily answered.

The external or physical causes of growth are the only ones that can be submitted to direct experimental investigation; the internal hereditary causes must be considered simply as something that exists and that is in the main unalterable; for if it were possible to change some of the mechanical and chemical properties of a tissue by means of external influences, this could not affect the true kernel of the hereditary characteristics; and again conversely, changes in these hereditary peculiarities, or variations, are never brought about by direct external influences, but only by unknown internal changes. Since therefore the specific peculiarities in the organisation of a plant are something in its nature that is entirely unknown, any investigation of the processes of growth must rest satisfied with showing the mode in which they are always associated with constant internal conditions, and what visible changes are produced in the processes of growth by physical influences. We cannot therefore be astonished if in the action of known external causeslight, gravitation, \&c. - on plants, effects are produced which appear altogether strange to one accustomed to examine purely physical processes ; but this astonishment disappears when it is borne in mind that the specific organisation of a plant itself represents a complexity of causes which we cannot analyse, and therefore are unable to estimate. It is in the constant recognition of this unknown factor-which causes physiological effects to turn out so entirely different from purely physical ones-that the difference between physiology and physics consists. The most striking mode however in which the aggregate of conditions of growth manifests itself in the inherited organisation, is when the same external causes produce entirely opposite effects on plants belonging to different species and even on different parts of the same plant.

To understand correctly the phenomena of vegetation, it is also necessary to distinguish between the direct and indirect action of external conditions on growth. For since growth is always dependent primarily on the presence of assimilated food-materials, light, temperature, or other external conditions may indirectly influence growth by affecting the formation and transport of the food-materials. But it is also possible and even probable that the mechanical process of intussusception itself on which growth is directly dependent may be modified by those and other causes the influence of which on growth is therefore in that case a direct one. The growth of one part may also be indirectly promoted or retarded by the growth or the removal of another part.

The unknown factor which exists in the inherited properties of organisms is by no means without analogy in inorganic nature. Chemists and physicists have also to assume peculiar properties of elementary substances. The aggregate of properties by which a particle of iron is absolutely distinguished from a particle of oxygen is as unknown and much more invariable than the aggregate of physiological causes which distinguish the inherited properties of an Oak from those of a Pine.

So far as the definition given above of historical properties concerns the inherited specific peculiarities of plants, the expression is not a metaphor from the point of view of the Theory of Descent, but must be taken in its literal signification. The specific properties which determine qualitatively the growth of each organ have 
sprung up successively in the course of time, i.e. in a series of generations. The chief evidence in favour of this view will be given in the last chapter of this work. It need only be mentioned now that this theory of the genesis of specific properties indicates the only possibility of arriving at an understanding of them in accordance with the laws of causality. At the present time this is possible only in the most general outline.

The use here made of the terms 'historical' and 'physical' may also be illustrated from another subject in the following manner. The nature of the geological formations of which the crust of the earth consists can be understood only from a historical point of view, because it is only at particular spots and at particular times that the conditions have concurred which produced, for example, the Chalk or the Old Red Sandstone. The formation of these rocks was dependent on chemical and physical processes, which must however have been preceded by other physical changes in the crust of the earth, in order that these rocks should be formed exactly at particular spots and particular periods. A crystal of sodium chloride can, on the contrary, be produced at any time, for the necessary conditions may be artificially brought together. Pseudomorphism of crystals can again be explained only from a historical point of view, although it is certain that the chemical and physical properties of the substances are alone concerned in the process.

We see therefore-and this is the object of these remarks-that the historical explanation of a natural phenomenon does not exclude its explanation from a physical point of view, but on the contrary includes it where we have to do with natural phenomena; and this principle is equally applicable to those properties of vegetable species which have been acquired hereditarily or historically, even when the application is practically much more difficult than in the case of inorganic nature.

SEct. I3. General Properties of the Growing Parts of Plants ${ }^{1}$. From the consideration of this subject the true crystals which are found in cells may be entirely excluded, since they do not differ in their general properties from those which occur elsewhere. The organised elementary structures on the contrary, the protoplasm, the nucleus, chlorophyll-granules, starch-grains, and the cell-walls, exhibit properties which distinguish them from all unorganised bodies.

These organised bodies are, in the first place, all capable of swelling; i.e. they have the power of absorbing water or aqueous solutions between their solid particles with such force that the particles are forced apart; the whole structure increases in size, and can thus exercise considerable pressure on the surrounding parts. If water is by any means withdrawn from the body which has thus swollen up, its particles again approach one another, and with such force that considerable strains may be exerted on the adjoining parts connected with it; as, for example, is shown in the bursting of dry capsules. The swelling and desiccation of organised parts may therefore cause change of form in the surrounding parts, i.e. in other organised parts. This power of swelling is of still greater importance, since it is this process that renders possible the interchange of sap between the individual cells as well as between whole masses of tissue. In order that growth by intussusception may take place, the dissolved food-materials must be able to enter by imbibition between the particles of the growing structure, and the chemical processes must take place there which construct from the dissolved food-materials solid particles to be intercalated between those already in existence, and in consequence of which the organic mass alters its volume and form (see Book III. Sect. I).

\footnotetext{
1 See Nägeli u. Schwendener, Das Mikroskop, p. 540 et seq.
} 
A second general property of the organised parts of plants is that they change their form when the external conditions remain perfectly unaltered, internal changes being the only efficient cause. Almost every process of growth is associated with change of form. These facts may be more briefly described by ascribing to organised structures endowed with the power of growth internal forces or plastic tendencies, if it is clearly understood that the term is only used to express a still unresolved aggregate of causes. As a result of these internal forces, organised structures have the power of overcoming resistance. Thus, for example, plasmodia which are constantly altering their form are able, notwithstanding their gelatinous and very soft nature, to overcome their own weight, and to creep up solid bodies. In the same manner the growth of the wood takes place with such force as to overcome the very considerable pressure of the surrounding bark.

But although the internal causes of these plastic tendencies are able to overcome certain obstacles, it is on the other hand certain that growth is also influenced by external forces, such as pressure, traction, stretching, bending, \&c., which are able to alter the form of solid bodies. The observations which have been made on this subject will be collected in the following sections; but it is in the first place necessary to define certain terms which will frequently be employed.

Like unorganised solid bodies, those which are organised oppose a greater or less resistance to the external forces which tend to alter their form; and are hence divided into hard and soft bodies. A hard body is one which offers considerable resistance, like many lignified or silicified cell-walls; a soft body is one which offers very little resistance, like protoplasm, chlorophyll-granules, or swollen cell-walls which have ceased growing, as gum-tragacanth. Structures which become disintegrated under pressure and traction rather than undergo any considerable change of form are brittle, like grains of starch or crystalloids of aleurone. If, on the contrary, they are capable of undergoing considerable changes of form, whether this take place by pressure or traction, they are extensible. It is clear that flexibility depends to a certain extent on extensibility, since the side of the bent part which becomes concave is compressed, the convex side stretched. All these properties are relative, and the same body may exhibit different phenomena according to the nature of the external forces which act upon it. Thus, for example, under a sudden blow the apex of a root behaves like a brittle body, and breaks easily, while it is flexible if slowly bent.

If the form of an extensible body has been changed by pressure, traction, or bending, and if, when then left to itself, it retains the form to which it has been forced, it is called inelastic; if, on the other hand, it resumes its original form, it is elastic. If the changes of form produced by external causes are small, they are usually completely reversed when the body is left to itself, and within these limits the body is perfectly elastic; but if the change of form exceeds certain limits dependent on the nature of the body and the length of time during which the force has been acting, it does not again assume exactly its previous form. The greatest amount of change which yet permits a complete restoration of the original form determines the Limit of Elasticity of the body; when this is exceeded, the stretched substance partially retains the form which it has been made to assume, and the less complete the return to its primitive shape the more imperfect is its elasticity. It 
would appear as if all bodies were imperfectly elastic to any long-continued stretching or alteration of form, and as if there were no limit of elasticity in the case of very long-continued but weak external influence. In all these points organised bodies, especially the growing parts of plants, exhibit the same phenomena as unorganised bodies. It must however be remembered that the terms explained above have reference only to effects visible externally; the internal changes which bring about the same external effect may be very different in different bodies. Rigidity, i.e. resistance to bending, depends, for example, evidently on very different internal conditions in the case of a woody cylinder and of a succulent stem or root consisting mainly of parenchyma. This is at once experimentally proved by the woody cylinder becoming less flexible and even brittle from loss of water, while the flexibility of succulent parenchyma is thereby increased. This is readily understood on recollecting that the flexibility of the woody cylinder depends on that of the walls of the wood-cells, which are not closed cavities, and therefore cannot become turgid, while the flexibility of parenchymatous tissue depends on the change of form of the closed turgescent cells, the extensibility and elasticity of the cell-walls taking only a subordinate part. Changes of form take place however more easily the less the turgidity of the cells; a parenchymatous tissue may be compared to an aggregation of bladders each of which is full of water; if they are all turgid with water, each bladder is tense and rigid, as also is the whole; if, on the contrary, they contain only enough water to fill without distending them, each separate bladder is flaccid, as also is the whole, which can therefore be bent in any direction. A mass of parenchyma may therefore be stiff and rigid even if its cell-walls are thin and very flexible, if only they are firm enough not to give way from the pressure of the water which stretches them, or to allow it to filter through. The flexibility and elasticity of the moist cell-wall cannot however be compared directly with these properties in a perfectly dry cell-wall or a strip of metal, as Nägeli and Schwendener (l.c. p. 399) have already shown. 'If we consider first of all,' they say, 'a fragment of moist cell-wall, say a lamella of the thallus of Caulerpa, a bast-fibre thickened so that the cell-cavity has disappeared, a spiral vessel, and so forth, it is proved by their behaviour to polarised light that stretchings, bendings, and other similar forces do not perceptibly change the arrangement of the atoms in the crystalline micellæ, but that only the distance of the micellæ themselves from one another is increased or diminished. On the other hand it is known that water is retained in the moist cell-walls with great force; and microscopic examination has shown that it cannot be forced out by bending or by compression of the part. No other hypothesis is therefore possible, except that the amount of water in a tense cell-wall is the same as in one in a neutral condition. The particles of water are therefore merely displaced by external forces, but are not forced out; they move, for example, with the bending of the part from the concave to the convex side, but afterwards fill up as completely as before the micellar interstices of the substance; and, since the sum of their tensions is but slightly altered, also occupy nearly the same space. If the same reasoning is applied to tissues without intercellular spaces and filled with sap, it is perfectly obvious that the cell-walls are not susceptible of change of volume any more than in the previous case. The same is the case also with the fluid contained in the cells. The only question now remaining is 
whether the changes of tension which are caused by external forces modify the permeability of the cell-walls at least in places. If this were the case, then when a tissue is compressed-since the hydrostatic pressure (turgidity) is in no case decreased by it, but the resistance of the cell-wall weakened ${ }^{1}$ - a part of the cellfluid must obviously be forced out, until the hydrostatic pressure has again reached an equilibrium with the diminished resistance of the cell-walls. In the same manner the effect of traction on a tissue must be to cause an influx of water into it, or, if this is prevented, the formation of an empty space ${ }^{2}$. If, on the other hand, the changes of tension which occur in plants have no perceptible influence on permeability, the tissues simply possess the properties of moist cellwalls; in any condition of tension ${ }^{3}$ they always occupy the same space ${ }^{4}$.'

In order to understand many of the phenomena now to be described, it is necessary to have a clear conception of the changes which a cell filled with sap undergoes in reference to its turgidity when it is compressed or stretched or simply bent by external forces. By Turgidity we understand the hydrostatic pressure which the water absorbed by endosmose exercises equally on all sides on thecell-wall, and which reacts on the contents in consequence of the elasticity of the cell-wall; so that in a turgid cell, while the cell-wall is stretched, the contents are compressed. A clear conception of this state of mutual tension of the cellwall and cell-contents may be obtained by closing a short wide glass tube at one end with a firm fresh bladder free from holes, pouring in a concentrated solution of sugar or gum, and finally closing also the other end with a thick bladder. This artificial cell, placed in water, absorbs it by endosmose with great force; the pieces of bladder which were previously stiff and tense arch into a hemispherical form and offer great resistance to pressure. If a hole is punctured by a fine needle in the bladder, a jet of fluid several feet in height springs from it. The force which drives out the fluid with such violence is the elasticity of the stretched bladder; but the cause which brings this elasticity into play is the endosmotic attraction for water of the fluid contained in the cell.

If we suppose in the case of a vegetable cell enclosed on all sides a degree of turgidity sufficient to stretch the cell-wall perceptibly, but leaving it still capable of further tension without bursting, and if this cell-wall is supposed to be extensible and elastic-as is especially the nature of growing and non-lignified cell-walls-the question presents itself :-What changes does the turgidity of the cell undergo when it is stretched or compressed by external forces or otherwise altered in form? This question can be sufficiently answered for our purpose by the simple contrivance represented in Fig. $478 . K$ is a wide and thick india-rubber tube to which the glass tube $S$, closed at $g$, acts as a stopper. After filling $K$ with water, the glass-tube $R$, open below at $o$, is fixed in and firmly fastened, the level of the water standing

1 These words are not clearly intelligible. Turgidity or the tension of the cell-wall is always increased, as we shall see directly, by pressure from without on a turgid cell; its resistance to filtration may in this manner be at length entirely overcome.

${ }^{2}$ Of course only when the cell-wall does not become folded.

${ }^{3}$ By tension is here clearly meant bending, stretching, or pressure from external forces.

4 The discussion given on p. 354 of the work quoted with respect to the alteration of the micellar structure of cell-walls by violent mechanical and chemical forces is of no importance for our present purpose. 
somewhere about $n$ in the thin drawn-out upper end of the tube. In order to give to the india-rubber tube, which here represents the cell-wall, a sufficient tension from the outset, it is convenient to make the thin end of the tube $R$ from 20 to $30 \mathrm{~cm}$. long, and to raise the level $n$ in proportion. The wide part of $R$ is fixed in a holder, so that the cell hangs down. A condition of equilibrium is thus established between the elasticity of the india-rubber tube and the hydrostatic pressure which can be compared with the turgidity of the vegetable cell; and in this condition the water-level stands at $n$. If the tube $S$ is now pulled downwards, the elastic tube is lengthened and at the same time made narrower, but the amount of space enclosed

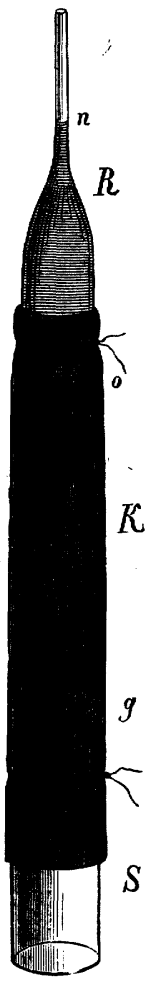

FIG. 478.-Apparatus for illustrating the change in the turgidity of cells caused by external distension or compression. by it is increased, as may be seen by the falling of the water-level $n$ in the narrow glass tube. If on the other hand the glass tube $S$ is pushed up and the india-rubber tube thus compressed without any bending or creasing taking place in $K$, the space enclosed by the tube $K$ is diminished, as is shown by the rising of the water-level $n$. The same thing takes place when the tube $K$ is bent in any way, or when it is compressed on any side ${ }^{1}$.

It is evident that if the upper glass tube $R$ were closed at $n$ so as to prevent a rise or fall of the water-level, any change which previously caused a rise of the level would now occasion an increase of the hydrostatic pressure, and vice versá. It may therefore be stated that in a closed and turgid cell any pressure acting from without or any curvature increases the turgidity, while any stretching of the cell diminishes it. If we imagine a straight succulent stem or a growing root to be bent, the cells on the convex side will be stretched, those on the concave side compressed, and the turgidity will be diminished in proportion in the former and increased in the latter. This result is very clearly confirmed if a very succulent rapidly growing internode of the Grape-Vine is slowly but firmly bent till it describes about a semicircle. It will be observed that during the bending a number of small drops of water escape in rows from the epidermis on the concave compressed and shortened side. It is indifferent whether they escape through fissures or are forced out through the cellwalls; in either case they show that the cells display a higher degree of turgidity on the concave compressed side than when the internode was straight.

In the present state of our knowledge, if we would keep clear of uncertain speculations, the considerations now given must be considered as by no means complete; but they are sufficient to draw the attention to conditions which must be taken into account as existing in the interior of the growing parts of plants when they are subject to pressure, traction, bending, and so forth, from external forces.

1 See also Pfeffer, Physiol. Unters. 1873, p. I 21. 
..

But if these internal changes are for the time left out of account, the purely external effect of the forces already mentioned is deserving of greater attention than it has hitherto received ${ }^{1}$. It would be of essential service, for instance, to ascertain at what point a growing internode, root, leaf, \&c. possesses the greatest extensibility, flexibility, and elasticity, and whether this point coincides or not with that of the most vigorous growth, and how perfect is the elasticity of the part; and so forth. We shall see that even somewhat crude observations in this direction afford results which enable us to remove old errors and avoid new ones.

Compared with the extensibility of mature internodes and parts of internodes, that of rapidly growing parts is very considerable, but their elasticity, on the contrary, is very imperfect. But the greater the development of the wood of a growing part, the greater is its elasticity and the less its extensibility. In young non-lignified roots, on the contrary, the resistance to bending is greater in the youngest than in the older parts, especially those whose growth in length has long been completed. The extremities of roots, very young leaves, and the ends of stems still enclosed in the bud, are generally brittle under a blow or pressure, but pliable and plastic to long-continued action of this kind, a condition that gives place during growth to an increasing resistance to sudden blows, which is in the first place due to increase of extensibility, afterwards to increase of elasticity.

In rapidly growing stems, leaves, and roots, the limit of elasticity is easily overstepped even by momentary flexion; and they always retain afterwards a slight though distinct curvature. It is often even possible, especially with roots and slender internodes, to give them any desired form by repeated bending with the fingers in different directions, like a thread of wax or a red-hot iron wire, without the power of growth being at all injured by the process. This effect is attained with greater certainty by exerting on the growing structure a flexion which is prolonged although small in amount. Thus the pedicels of many flowers are bent downwards by their weight, and retain this curvature even when the weight is removed, until a new condition of growth imparts greater elasticity and firmness to the tissues: under the influence of gravitation they then grow more rapidly on the lower side, become upright, and raise up the still greater weight of the fruit; as is strikingly seen in Fritillaria imperialis, Anemone pratensis, and many other plants with pendent flowers and erect fruits. In other cases again the curvature, which was at first due merely to external causes, becomes permanent and fixed in the tissue itself by the processes of growth, as in the fruit-stalks of Solanum Dulcamara.

One of the most striking phenomena of this class is that a lateral blow below a growing internode causes it to assume a curvature in the direction assumed by the internode at the moment of impact. The same thing occurs when the upper part of a shoot is taken in the hand and a curvature imparted to it similar to that caused by the blow. The upper part acquires in consequence a pendent position, which may however be again neutralised by subsequent growth.

There has been as yet no exact or detailed investigation of the elasticity of growing shoots, roots, and leaves; and the enquiry is, as I have convinced myself, attended with considerable difficulty. Observations sufficient to enable us to study

${ }^{1}$ See A. P. De Candolle, Physio'ogie Végétale, vol. I. p. I I. 
some of the phenomena of vegetation to be described in this chapter can however be made with the simplest methods and apparatus.

(a) Extensibility of growing Internodes. The upper and lower end of an internode - of a freshly cut fragment of a stem were marked with Indian ink. The shoot was held above and below the marks, laid on a micrometer graduated to millimetres, and stretched as strongly as possible without breaking ${ }^{1}$. The result is shown in the annexed table:-

\begin{tabular}{|c|c|c|c|}
\hline Name. & $\begin{array}{l}\text { Original length } \\
\text { of internode. }\end{array}$ & $\begin{array}{l}\text { Amount of temporary } \\
\text { elongation. }\end{array}$ & $\begin{array}{l}\text { Amount of perma- } \\
\text { nent elongation. }\end{array}$ \\
\hline 1. Cimicifuga racemosa & $296 \mathrm{~mm}$. & 6.8 p.c. & 3.5 p.c. \\
\hline 2. Sambucus nigra & 26 & $18 \cdot 0$ & $5 \%$ \\
\hline The next older internode & 65 & $3^{\cdot} \mathrm{I}$ & $I \cdot I$ \\
\hline A still older internode & 115 & $\cdot 8$ & $\cdot 0$ \\
\hline 3. Aristolocbia Sipbo & 102.5 & $4^{\circ} 4$ & I.O \\
\hline The next older internode & 242 & $2 \cdot 2$ & $\cdot 4$ \\
\hline 4. Aristolocbia Sipbo & $33^{\circ} 5$ & $10^{\circ} 4$ & $1 \cdot 5$ \\
\hline The next older internode & $252^{\circ} 5$ & $1 \cdot 8$ & 4 \\
\hline 5. Aristolocbia Sipbo & $7 I^{\circ} 5$ & $6 \cdot 3$ & $3 \cdot 5$ \\
\hline The next older internode & 226 & $2 \cdot 6$ & $\cdot 8$ \\
\hline
\end{tabular}

Imperfect as was the method of observation, these figures nevertheless show ( $\mathrm{r}$ ) that growing internodes are highly extensible, (2) that extensibility decreases with age, (3) that elasticity increases with age.

(b) Elasticity to flexion of growing Internodes. Internodes of fresh turgescent shoots were cut off, and bent on a card on which concentric circles were drawn; the axis of the internode was made to coincide as nearly as possible with one of the circles; the radius of this circle is recorded in the following table as the radius of curvature. The internode was then left to itself, and its permanent curvature determined in the same manner. The branch was then bent on the other side, and so on, as shown by the table. The internode was finally laid with its concave side on the measuring rod and pressed straight on to it.

\begin{tabular}{|c|c|c|c|c|}
\hline Name. & $\begin{array}{l}\text { Length of the } \\
\text { internode. }\end{array}$ & $\begin{array}{l}\text { Radius of } \\
\text { curvature } \\
\text { when bent. }\end{array}$ & $\begin{array}{l}\text { Radius of cur- } \\
\text { vature when } \\
\text { left to itself. }\end{array}$ & $\begin{array}{l}\text { Thickness of } \\
\text { the middle part } \\
\text { of the internode. }\end{array}$ \\
\hline
\end{tabular}

Valeriana officinalis; stalk of young inflorescence.

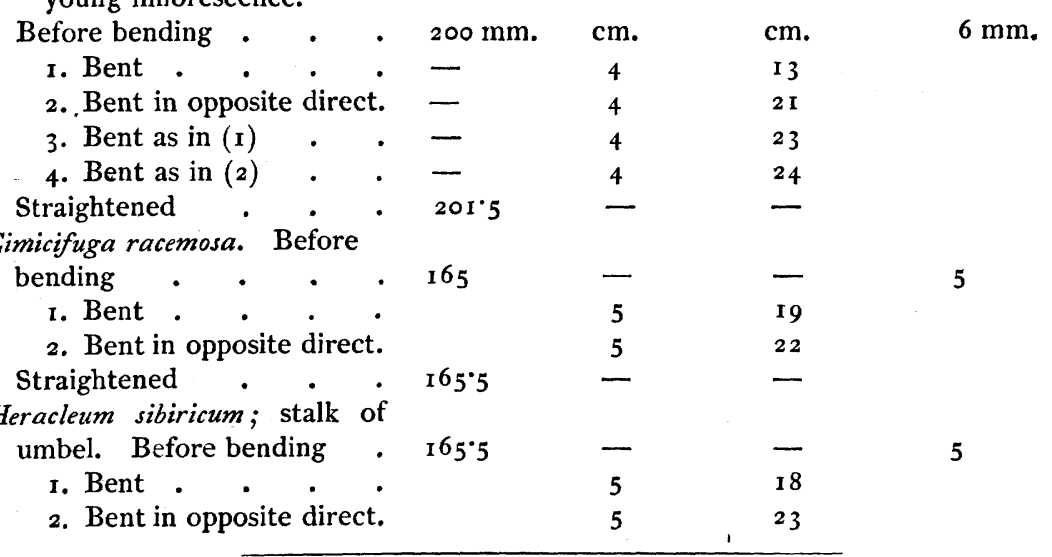

1 This somewhat primitive method of stretching, which of course does not furnish an exact. measure of the extensibility of different internodes, was employed because stretching by means of weights necessitates fastening the shoot, which is attended with great inconveniences. 


$$
\text { Name. }
$$

3. Bent as in ( $\mathrm{r}$ )

4. Bent as in (2)

Straightened

$V$ itis vinifera; young internode.

Before bending . . .

I. Bent • . . .

2. Bent in opposite direct.

3. Bent as in (I) . .

4. Bent as in (2) . .

Straightened . . .

Vitis vinifera; older internode.

Before bending . . .

I. Bent . . . .

2. Bent in opposite direct.

3. Bent as in ( $I)$. .

4. Bent as in (2) . .

Straightened . . .

$\begin{array}{ccc}\begin{array}{c}\text { Length of the } \\ \text { internode. }\end{array} & \begin{array}{c}\text { Radius of } \\ \text { curvature } \\ \text { when bent. }\end{array} & \begin{array}{c}\text { Radius of cur. } \\ \text { vature when } \\ \text { left to itself. }\end{array} \\ \text { I67.0 } & 5 & 25 \\ & 5 & 22 \\ \end{array}$

$47^{\circ} 5$

\begin{tabular}{ll}
- & - \\
2 & 4 \\
2 & 6 \\
2 & 6 \\
2 & 9 \\
\hline
\end{tabular}

Thickness of

the middle part

of the internode.

These examples, selected from a long series of observations, show:-( $\mathrm{r})$ that growing internodes are very flexible, (2) that after bending they do not altogether recover their straightness, or that the elasticity of curvature is imperfect; (3) that repeated bendings constantly in opposite directions leave progressively smaller curvatures $^{1}$; (4) that one vigorous bending, and to a still greater extent repeated ones in opposite directions, leave the internode flaccid, or deprive it of its rigidity (of which no special account is taken in the table); and (5) in the case of the three first examples, that an internode bent first in one and then in another direction lengthens slightly, while in the case of the two last there was no lengthening, but in one even a perceptible contraction.

(c) Change of length of the concave and convex sides of a bent internode. Here again, as in paragraph $b$, the bending was done by the hands, and measured by the radius of curvature on a card on which concentric circles were drawn. The original length, as well as those of the sides which remain concave and convex after the object is left to itself, were measured by means of a carefully applied strip of card divided into millimetres. In order to get a great difference between the concave and convex sides, very thick internodes were selected, and their thickness measured in the middle.

\begin{tabular}{|c|c|c|c|c|c|}
\hline Name. & $\begin{array}{l}\text { Length of } \\
\text { internode. }\end{array}$ & $\begin{array}{l}\text { Radius of } \\
\text { curvature } \\
\text { when bent. }\end{array}$ & $\begin{array}{l}\text { Radius of } \\
\text { curvature } \\
\text { when left } \\
\text { to itself. }\end{array}$ & $\begin{array}{l}\text { Contraction } \\
\text { of the } \\
\text { concave side. }\end{array}$ & $\begin{array}{l}\text { Lengthening } \\
\text { of the } \\
\text { convex side. }\end{array}$ \\
\hline
\end{tabular}

\section{Silpbium perfoliatum}

$13^{\circ} \mathbf{m m}$. thick.

Before bending . $185 \mathrm{~mm}$.

Bent in opposite direct. .

Straightened . . $\quad 185$

Ligularia macrophylla

$7.5 \mathrm{~mm}$. thick.

Before bending . $\quad 199$

Bent . . . . .

Bent again. .5

Bent in opposite direct. .

I $4 \mathrm{~cm}$. $26 \mathrm{~cm}$. I mm. $2 \mathrm{~mm}$.

I $40 \quad$ I $\quad I^{\circ} 5$

1 The curvature is less the greater the radius of curvature. 
These observations show, as was to be expected, that the permanent curvature of an internode is connected with a permanent contraction of the concave and lengthening of the convex side.

(d) As to the Distribution of Extensibility in growing shoots, the observations of de Vries $^{1}$ lead to this result, that in growing strongly turgescent shoots a maximum of extensibility and of flexibility exists immediately below the terminal bud. From this point they diminish as the distance from it increases, and therefore also with the age of the parts. This statement holds good independently of the age of the growing shoots.

(e) Sudden curvature of growing shoots from a blow or concussion. If upright growing shoots $^{2}$ are suddenly and violently struck below at a point where growth has ceased, the curvature thus caused advances upwards in the form of a wave, so that immediately after the blow has been given to the lower part the apex of the shoot is strongly bent, the concavity of the curvature lying on the side from which the blow was received. The elasticity of the bent part causes the apex to spring back immediately; but when, as we have seen, the elasticity is very imperfect, the shoot retains a part of its curvature. As soon as the shoot has come to rest after some oscillations, it may be observed that below the apex, where the shoot is most flexible to an ordinary passive curvature, a permanent curvature is established, the apex bending over, and always on that side fro $n$ which the blow was received. In many cases this phenomenon is produced by a single blow from a stick, as e.g. in Fagopyrum, Lytbrum, and Senecio, flower-stalks of Digitalis, Cimicifuga, Aconitum, \&c.; in more rigid stems which are less flexible and more elastic at the corresponding part, the bending over of the apex does not take place till after three or four or even from twenty to fifty blows have been given to the lower woody part; the amount of curvature also varies in different plants. If shoots are cut off low down so that a woody piece which has ceased to grow can be taken in the hand, and the shoot made to oscillate rapidly backwards and forwards, it assumes, when it comes to rest, a distinct curvature below the apex in the region of greatest flexibility. The plane of curvature coincides with that in which the oscillations take place, and the apex may bend to either side; but the permanent curvature will always be concave on the side on which the oscillations were strongest. If finally a rooting shoot or one firmly held in the hand receives repeated lateral blows at its summit, that is, above the most flexible part, a permanent curvature is produced in this region, but it is in this case convex to the side from which the blows came.

In all the cases which I have described the position of the permanent curvature is the same as that of the strongest curvature, even if acquired only momentarily by the shoot. The appearance is precisely the same as if the shoot were taken in the hand and then strongly bent once, or as if it were repeatedly bent backwards and forwards, but more strongly in one direction. Mere concussions which produce no strong flexion of the shoot cause no permanent curvature; if shoots are enclosed in glass tubes and violent impulses repeatedly imparted to them by jerking the tubes upwards or swinging them from side to side no change is visible when the shoots are removed from the tube.

If the part of a shoot susceptible of curvature is marked with ink in equidistant divisions, and is then made to oscillate by blows below this part, the convex side of the permanent curvature is found to have become longer, the concave side

1 Ueber die Dehnbarkeit wachsender Sprosse, Arb. des Bot. Inst. in Würzburg, Bd. I, I874.

2 The phenomenon here described was first observed and studied by Hofmeister (Jahrb. für wissensch. Bot. vol. Il, I860); and a few important corrections of his description were given by Prillieux (Ann. des Sci. Nat. sér. 5, vol. IX). The statements here made, which confirm the previous observations in all essential points, while differing from them in a few others, are entirely based on my own observations. 
shorter, precisely in accordance with the phenomena described in paragraphs $b$ and $c^{1}$. For the measurements in the following table as thick shoots as possible were used, since they give considerable differences in length between the convex and concave sides even when the curvature is slight. The measurements were made with strips of card graduated in millimetres, and which I applied closely to, the concave and convex sides.

\begin{tabular}{|c|c|c|c|c|}
\hline Name. & $\begin{array}{l}\text { Original } \\
\text { length. }\end{array}$ & $\begin{array}{c}\text { Approximate } \\
\text { radius of } \\
\text { curvature. }\end{array}$ & $\begin{array}{l}\text { Lengthening } \\
\text { of the } \\
\text { cunvex side. }\end{array}$ & $\begin{array}{l}\text { Contraction } \\
\text { of the } \\
\text { concave side. }\end{array}$ \\
\hline Silpbium perfoliatum & - $\quad 152 \mathrm{~mm}$. & r $8 \mathrm{~cm}$. & $3 * 4$ p.c. & o:o p.c. \\
\hline do. . & - 120 & - & $1 \cdot 7$ & $\cdot 6$ \\
\hline Macleya cordata & $87 \cdot 5$ & 7 & $2 \cdot 3$ & $x \cdot 7$ \\
\hline do. & . $\mathrm{IO}_{4}$ & 24 & $\cdot 5$ & $1 \cdot 5$ \\
\hline Polygonum Fagopyrum & 63 & 8 & $2 \cdot 1$ & $x \cdot 6$ \\
\hline Heliantbus tuberosus & 98 & - & $2 \cdot 0$ & $x^{\prime} \cdot 4$ \\
\hline Valeriana exaltata & . $\mathbf{I}_{50}$ & 32 & $\cdot 8$ & $\cdot 7$ \\
\hline do. . & - IIO & - & $\cdot 7$ & $2 \cdot \mathbf{I}$ \\
\hline Vitis vinifera & . 149 & 6-10 & $1 \cdot 3$ & $2 \cdot 0$ \\
\hline
\end{tabular}

The permanent curvature which remains after violent oscillations of a shoot, or the Curvature of Concussion, is the result of a lengthening of the convex and a simultaneous shortening of the concave side. A proof is thus afforded that the whole phenomenon is dependent on the very imperfect elasticity and the great flexibility of the region that is capable of flexion ${ }^{2}$. A shoot bent in this way shows the same changes as one that is simply bent between the hands. This result would not be at all altered were it found, in harmony with what was said in paragraph $b$, that the concave side was also sometimes slightly lengthened, since it is stretched by the recoil of the oscillations; and this elongation is not always entirely neutralised. Prillieux has compared this curvature to that of a lead-wire fixed to an elastic support, when the support was struck; he was unable however to see the reason why the older and younger parts of the shoot did not exhibit the phenomenon. In the older parts this depends on their more perfect elasticity, in the younger on their smaller flexibility, and on the circumstance that they are not strongly bent, but are only thrown backwards and forwards by the oscillations of the lower and more flexible parts.

The subsequent neutralisation of the curvature by growth must depend first of all on the increase of turgidity in the concave and its diminution in the convex side, and on the growth being consequently promoted in the former. This may be assisted also by the secondary effect of elasticity, in consequence of which the stretched epidermis of the convex side shortens, while the compressed tissues of the concave side expand.

Secr. I 4.-Causes of the condition of Tension in Plants. The elasticity of the organised parts of plants results in tension chiefly from the operation of three causes; viz. (I) the turgidity, in other words the hydrostatic pressure of the contents of the cell on the cell-wall; (2) the swelling and contraction of the cell-walls when

1 According to Hofmeister all the sides of the shoot become longer. He calculated the length of the curve which he took for an arc of a circle; and Prillieux measured only the concave side, which he found to be always shorter; the contraction of the whole shoot, i.e. of its neutral axis, cannot however be inferred from that of the concave side. The thickening which, according to Hofmeister, should take place, if the shoot becomes longer on all sides, I consider cannot be demonstrated, in consequence of the extremely small change in diameter which takes place in such cases.

2 Compare the different description given by Hofmeister in his paper On the Bending of the Succulent Parts of Plants, in the Berichte der kön. sächs. Ges. der Wiss., 1859. 
they imbibe or lose water; and (3) the changes in volume and form caused by. the growth of the cells. .

I. Turgidity. The force by which water is drawn by endosmotic attraction ${ }^{1}$ to the cell from the parts that surround it is not merely sufficient to fill the space enclosed by the cell-wall, but also to enlarge it, the increasing amount of sap distending the cell-wall until its elasticity is brought into equilibrium with the endosmotic absorption. In this condition the cell-wall is stretched to its full capacity, or the cell is turgid. If the cell loses a portion of its water by transpiration or by neighbouring cells withdrawing it, the tension of the cell-wall is decreased and the volume of the cell diminished. The hydrostatic pressure produced by the endosmotic action of the cell-wall acts from within and is the same at all points within the small cell-cavity; but this does not prevent different points of the cell-wall stretching and contracting in different degrees as the turgidity increases, in consequence of local variations in extensibility. Hence not only may the volume but also the form of the cell be changed by turgidity. The greater the tension between the cellwall and its contents, in other words the greater its turgidity, the greater is the resistance offered by the cell to external forces which tend to alter its form by pressure, but the more readily does it burst in consequence. If the cell loses so much water that the space enclosed by the flaccid cell-wall is no longer filled, it may become folded inwards by the external pressure of the air or of the surrounding water, and in this case the cell is said to collapse ; if the cell-wall is thick, firm, and inflexible, a tension of an opposite character to turgidity takes place in the cell. Since turgidity is nothing but the mutual tension of the cell-wall and contents, or a state of equilibrium between endosmotic absorption and the elasticity of the cell-wall, it is evident that only closed cells, i.e. such as have no orifices, can be turgid. The micellar interstices through which the water set in motion by endosmose forces its way into the cells are essentially different from pores; the former are so small that their diameter is completely under the control of molecular forces, while even the smallest pore withdraws at least the middle portion of its space from the influence of the molecular action of the substance that bounds it. Microscopic openings, like the pores of bordered pits, are orifices of this latter kind, and are excessively large compared with the micellar interstices through which endosmose acts. Cells with pits penetrating the cell-wall cannot therefore be turgid, because any tension however small between cell-wall and contents is at once neutralised by the superfluous sap becoming pressed out through the orifices. It is indeed possible for water to be forced out in this way even through closed cell-walls, but only when the turgidity is very great, and the hydrostatic pressure of the cell-sap on the perfectly tense cell-wall is sufficient to force out the water through the micellar interstices $^{2}$. The resistance offered by the cell-wall to this may be called resistance to

1 [It appears probable that the organic acids which are present in the cell-sap of all cells which are or can be turgid are the substances which induce endosmosis (see de Vries, Ueb. die Bedeutung der Pflanzensäuren für den Turgor der Zellen, Bot. Zeitg. 1879). De Vries is of opinion (Bot. Zeitg. 1879, Ueb. die inneren Vorgänge bei den Wachsthumskrümmungen mehrzelliger Organe) that growth in length depends upon the continuous production of actively osmotic substances in the cell-sap of the growing cells.]

2 That the water which filters through under such circumstances actually passes through 
filtration. It is very different in amount in cells of different kinds, and on it the degree of turgidity depends, when the intensity of the endosmotic force of the sap and the elasticity of the cell-wall are constant.

What follows with respect to the turgidity of the individual cell is equally true in general of masses of tissue; only that a much greater variety of phenomena may arise in this case according to circumstances. If, for example, a number of similar layers of tissue are united into a system, a curvature of the system may take place. when one layer loses water by evaporation and thus becomes shorter, or when it absorbs more water than another layer and thus becomes longer. For instance, the primary roots of seedlings which have become partially flaccid by evaporation and perceptibly shorter, quickly bend upwards concavely if placed with one side on water; if placed entirely in water they become straight and longer. Curvatures arise in the same manner when layers of different tissues, united with one another, are subjected to variations of turgidity. Stems of the Dandelion for instance split lengthwise and placed in water roll up in a spiral manner, the outside being concave, because the medullary parenchyma absorbs much more water, and consequently, from the extensibility of its cell-walls, expands more than the epidermis or the cortex, which absorb water more slowly, and whose cell-walls are besides not so extensible ${ }^{1}$.

As a single cell, with increasing turgidity, opposes greater resistance to forces which tend to change its form, so also a mass of tissue becomes more rigid when all its cells are more strongly turgid, and vice versâ. If, for example, a cylinder of pith is cut out from a growing internode, it is flaccid and flexible; but if it is placed for a quarter or half an hour in water, it not only becomes considerably longer, but also very rigid and even brittle in consequence of all its cells becoming rapidly filled with water. This effect is still more visible when the pith is surrounded by other less extensible tissues, as in an uninjured internode. If this internode has become flaccid from transpiration, and it is placed in water, the pith very soon begins to become turgid and to expand; but since it is surrounded by other tissues of different properties, it must stretch them in order to lengthen itself; this is only possible however until the elasticity of these layers is in equilibrium with the tendency of the pith to expand. In this case the elongation of the whole caused by the turgidity of the pith is much less than that of the pith alone would be; but on the other hand there is now a violent tension between the pith and the surrounding tissues, in consequence of which the whole internode appears very rigid or but slightly flexible. The whole internode may be compared to a cell the contents of which are represented by the pith, its cell-wall by the surrounding tissues. If the pith loses water the whole becomes smaller, the passively stretched tissues contracting elastically; and since the tension is thus decreased, the whole becomes more flaccid; the reverse when the change is in the opposite direction.

micellar interstices is clear from the fact that the amount of soluble substances contained in the water is altered by the filtration.

1 [De Vries has shown that the turgidity of cells may be diminished by placing them in solutions of neutral salts $\left(\mathrm{KNO}_{3}, \mathrm{Na} \mathrm{Cl}\right)$ of $4-6$ per cent.; water is withdrawn from the cells, and they consequently become smaller; if they are then placed in distilled water they regain their original size (Ueb. d. mechanischen Ursachen der Zellstreckung, I877).] 
2. Imbibition is the term given, as we have already seen, to the capacity ${ }^{1}$ of organised structures to absorb water between their micellæ with such force that they are thereby driven apart, their cohesion being partially or entirely overcome, and the whole thus increasing in volume. Loss of water, on the other hand, as by evaporation, causes approximation of the micellæ and a corresponding decrease in volume of the whole. Both distension and contraction take place with such force as to overcome external resistances of considerable magnitude. While in closed and thin-walled cells the changes in form and volume are chiefly caused by turgidity, in very thick-walled cells on the contrary with a small cavity (as many bast-fibres and collenchymatous cells) they are brought about mainly by imbibition and desiccation of the cell-wall, and especially when it is to a high degree capable of swelling, in other words is in a state to absorb or give off large quantities of water. In cells with open pores, where there can be no hydrostatic pressure or turgidity, as in wood-cells and vessels with bordered pits, imbibition and the desiccation of the perforated cell-wall are the only means of changing the size and form of the cell.

If, as is usually the case with thick cell-walls, the different concentric layers of cellulose have different degrees of capacity for imbibition and swelling (see Book I. Sect. 4), tensions are caused between these layers by the absorption or loss of water, which may even end in the layers becoming detached from one another; as, for example, occurs in transverse sections of thick-walled bast-cells and in starch-grains. But it is not only the quantity of water absorbed and given off that varies in the different layers of a cell-wall, but also the direction in which the water is principally absorbed or allowed to escape between the micellæ. Tensions are thus caused which may lead to the production of torsions and oblique fissures, to the rolling or unrolling of spiral bands of the cell-wall, and to a change in the obliquity of the spirals ${ }^{2}$.

All these changes, which are necessarily associated with the tensions of layers that have become convex and concave, take place also in masses of tissue and organs the cells of which have lost their contents and consequently their turgidity, while their cell-walls have become capable of imbibition, or, as it is generally termed, hygroscopic. The layers of cell-walls and the thin-walled masses of tissue which in the living state contain most water, contract most strongly after death and from desiccation; with change of form they become concave, or are ruptured by the contraction of the intermediate lignified tissue. Without entering at present into a detailed consideration of these extremely various phenomena, which, though often of extreme importance in the life of the plant, do not influence growth, it need only be mentioned that on them depend the bursting of most sporangia, anthers, and capsular fruits, the remarkable movements of the awns of various species of Avena and Erodium, as well as those of the Rose of Jericho (Anastatica hierochuntica) and of the so-called asthygrometer ${ }^{3}$. Of direct importance on the other hand, as respects the mechanical laws of growth, are the changes in volume of the

1 See Nägeli u. Schwendener, Das Mikroskop, p. 427 et seq. (1877).

2 Compare Cramer, in Nägeli u. Cramer's Pflanzen-physiologische Untersuchungen, 1855, Heft 3. p. 28 et seq.; and Sachs, Experimental-Physiologie, p. 429.

${ }^{3}$ Compare Cramer's statements in Wolff's treatise, Die sogenannte Asthygrometer; Zürich, 1867 . 
wood and bark of trees which accompany the variation in the quantity of water they contain, and the very powerful tension between them thus caused in woody plants, to which I shall again recur in detail. The attention of the student need now only be called to one point, viz. that when wood distends on imbibition or contracts on desiccation, this is caused entirely by the alteration in form and volume of the cell-walls, since turgidity cannot take place in wood as it does in a tissue consisting of closed cells. The distension and contraction of wood when it absorbs or loses water are very different in different directions, strongest in the tangential, weaker in the radial, weakest of all in the longitudinal direction ${ }^{1}$. This is the cause, for instance, of the longitudinal splits in woody stems when they become dry, which close again when water is absorbed; and the changes of dimension due to these phenomena take place with extraordinary force.

3. Growth itself must cause states of tension in the layers of a cell-wall or of the tissue of which an organ is composed, if the layers, although firmly united to one another, grow unequally. It is however much more difficult to understand the modifications of tension due to growth than those due to turgidity and imbibition, as the former cannot be altered artificially without a material change being caused also in the latter. Since the growth of every organised structure, such as a cell-wall, can only proceed so long as it is permeated with water, and since moreover the growth of the entire cell requires it to be in a turgid condition, and this condition itself has an influence on growth, it is extremely difficult to decide how far each of these phenomena is the cause of the other. If by growth we understand, according to the definition already given, only permanent and irreversible changes of organisation, affecting in the first place the micellar structure of the organism, it may be assumed, in accordance with the present state of our knowledge, that growth is always preceded by imbibition and turgidity, and that it is the

1 The measurements of Laves given below illustrate these relative changes of dimension. (See Sachs, Experimental-Physiologie, p. 43I.)

\begin{tabular}{|c|c|c|c|}
\hline & $\begin{array}{l}\text { In the direction of } \\
\text { the axis. }\end{array}$ & $\begin{array}{l}\text { In the direction of } \\
\text { the radius. }\end{array}$ & $\begin{array}{l}\text { In the direction of the } \\
\text { circumference. }\end{array}$ \\
\hline Maple & 0.072 & $3 \cdot 35$ & 6.59 \\
\hline Birch & 0.222 & $3 \cdot 86$ & 6.59 \\
\hline Oak & 0.400 & 3.90 & $7 \cdot 55$ \\
\hline Fir & 0.076 & $2 \cdot 41$ & $6 \cdot 18$ \\
\hline
\end{tabular}

The change in volume of wood was investigated by Weisbach (l.c. p. 432).

\begin{tabular}{|c|c|c|}
\hline & $\begin{array}{l}\text { Water absorbed by roo parts } \\
\text { by weight of dry wood. }\end{array}$ & $\begin{array}{l}\text { Distension of roo parts by } \\
\text { volume of dry wood. }\end{array}$ \\
\hline Maple & 87 parts & $9 \cdot 4$ \\
\hline do. & 87 & $7 \cdot 1$ \\
\hline Birch & 97 & $\begin{array}{l}7.0 \\
8.8\end{array}$ \\
\hline do. & 91 & $\begin{array}{l}8.8 \\
7.2\end{array}$ \\
\hline $\begin{array}{l}\text { dok } \\
\text { do. }\end{array}$ & $\begin{array}{l}00 \\
91\end{array}$ & $\begin{array}{l}7 \cdot 2 \\
7 \cdot 8\end{array}$ \\
\hline Fir & 94 & $5 \cdot 7$ \\
\hline do. & 130 & $5 \cdot 1$ \\
\hline
\end{tabular}

In comparing the change in volume with the amount of water absorbed, it must be borne in mind that the numbers in which the latter is expressed do not give merely the amount of water imbibed by the cell-walls, which alone causes the distension, but also that retained in the cavities by capillary attraction. It may therefore happen that there appears a smaller increase in volume when a larger quantity of water is absorbed. 
modification of the micellar forces caused by these conditions which render possible the intercalation of new solid particles among those already in existence. If, for example, a cell-wall is stretched by turgidity, the distance of its micellæ increased, and possibly a different arrangement of them brought about, this state may be reversed on the cessation of the turgidity, by the elasticity of the cell-wall. But if, during the condition of tension, growth takes place by the intercalation of new solid micellæ, the tension of the cell-wall is altered and in general diminished. If now the turgidity ceases as before, a new condition of equilibrium occurs in the cellwall; a permanent change has been effected by growth, which was rendered possible by hydrostatic pressure and imbibition.

The share taken by growth in the tension of the tissues amounts to this: new solid micellæ are intercalated, and the tension due to imbibition and turgidity is thereby partially neutralised. This is however only momentary; for after the intercalation of new micellæ the turgidity again increases, the degree of imbibition is modified, new tensions are again caused, which on their part are partially neutralised by the intercalation of fresh solid micellæ. It is probably near the truth to suppose that the limit of the elasticity of the growing cell-walls is constantly nearly reached by turgidity and imbibition as well as by the secondary tensions produced by them, and that on the other hand the tension is constantly being diminished by the intercalation of new micellæ. Growth may therefore be described as a constant overstepping of the limit of elasticity of the growing cell-wall which is constantly neutralised by the intercalation of additional solid micellæ.

It will of course be understood that in the brief description now given we do not mean to state a theory of growth, but only to indicate in general terms the mechanical effect exercised by growth on the tension of tissues, and conversely. It would be easy to deduce the explanation in particular cases. If, for example, a cell-wall is imagined distended by turgescence or by traction exerted by the surrounding tissue, the intercalation of solid particles in the layers of cellulose already present may take place to a greater or less extent, causing a differentiation in their extensibility, elasticity, and power of imbibition, and thus leading to mutual tensions of the layers, as may be seen almost invariably in thin transverse sections of the cells of plants, and especially in the outer walls of those of the epidermis. But these differences in the mode of intercalation in the different layers of the same passively distended cell-wall may depend on a variety of circumstances; as, for instance, on the degree of proximity of the layers to the protoplasm, on whether they are in contact externally with the air, \&c. But growth by intercalation may also vary according to the nature of the tissue of which the cell forms a part, or the chemical properties of the cell-contents, and according as the cells are passively distended or compressed by other cells. All these considerations are however merely hypothetical, and simply indicate the nature of the relations between growth by intercalation and the tensions caused directly by imbibition and turgidity. It may in any case be regarded as certain that intercalation is only possible as the result of imbibition and turgidity; but that these properties, as well as extensibility and elasticity, must, or at least may be, in their turn modified by it. The volume of the growing part increases; and since this takes place in different degrees in different layers of the same cell-wall, and in different layers of the tissue of the same organ, tensions varying in degree must be produced between these different layers.

It may not be superfluous to add some explanatory observations relative to what we understand by Tension. 
Corresponding to every tension is an opposite tension. If a tissue which has a tendency to become distended is prevented from doing so by its connection with surrounding tissues, both are in a state of tension, the one negative, the other positive. The tissues which are passively distended may be said to be in a state of negative tension, those which are compressed or hindered in their distension to be in a state of positive tension. In a turgid cell, the cell-wall is therefore in. a state of negative, the contents in a state of positive tension.

As long as there is no movement or change of form, the two opposing tensions must be equal; i.e. the work which the part in a state of positive tension would perform is equal to the work which would be performed by means of its elasticity by the part in a state of negative tension if the two were disconnected; or the elastic forces set in action must perform the same amount of work in two layers with opposite tensions and in equilibrium with one another. If, for example, a steel cylinder $1000 \mathrm{~mm}$. long is supposed to be placed in an india-rubber tube $500 \mathrm{~mm}$. long and closed below, and if the tube is stretched so that it can be fastened above the upper end of the steel cylinder, we have a system in a state of tension, the india-rubber negative, the steel positive; and since the system is at rest, the opposing tensions must be equal; i.e. all the particles of the india-rubber tend to contract with the same force as that with which those of the steel, which are now compressed, tend to separate from one another.

This example shows at the same time that the amount or intensity of the tension can by no means be measured by, the changes in dimension which the layers experience at the moment when they are set free from it. Let us, for example, suppose, in our system of steel and india-rubber, that the steel cylinder is shortened $0.1 \mathrm{~mm}$. out of 1000 by the india-rubber, while the india-rubber tube must be stretched $500 \mathrm{~mm}$. out of 1000 in order to produce an equilibrium. If the tube is now opened above, it at once contracts $500 \mathrm{~mm}$. (supposing it to be perfectly elastic), while the steel cylinder elongates only $0^{\circ} \mathrm{I} \mathrm{mm}$. ; the change of dimension is therefore 5000 times greater in the case of the india-rubber than in that of the steel, although the actual tension of the two was the same. But the alteration of dimension indicates only the amount of stretching to which the india-rubber, and of compression to which the steel was subjected. If therefore the layers of the tissue of an internode are separated from one another, the alterations of dimension which then ensue depend on the extensibility and compressibility of the layers as well as on the amount of tension. There is only one case in which the amount of tension can be inferred from the changes in dimension of the tissues when freed from a state of tension, viz. when their extensibility and compressibility are the same, and when perfect elasticity also exists in both. But the case is quite different with growing internodes; the extensibility of the tissues when in a state of tension is constantly changing in consequence of growth. In a young internode the epidermis and wood are very extensible; if they are separated from the pith this latter only lengthens slightly, because it was only slightly compressed, but the epidermis and the wood contract very considerably because they are very extensible and were stretched by the pith. On the other hand the alterations of dimension in layers of an older though not mature internode will be the reverse. The pith, when freed from the tension, elongates considerably, but the wood contracts only slightly, because its extensibility is now but small and it was but slightly stretched by the pith; the pith on the contrary being very compressible, was prevented from lengthening by the resistance of the wood. The intensity of the tension cannot by any means be determined in either case from the changes of dimension; these only show that there are tensions, and indicate also what parts are extensible and compressible, and which are in a state of positive and negative tension $^{1}$. It may be laid down as a rule that when the separation of two tissues causes

1 In his treatise On the Tension of the Tissue of the Stem and its Results (Bot. Zeitg. I867, No. I09) Kraus has employed the differences of length between the entire internode and its isolated 
one of them to contract or expand, while the length of the other apparently does not change, both layers were nevertheless in a state of tension, only the one which remained unchanged in length was but slightly extensible or compressible, while the other possessed these properties in a higher degree. When, on the other hand, an internode consists of very extensible cortex and very compressible pith, both will alter very considerably in length when separated; and yet the tension is not necessarily as great as in another internode where the cortex is less extensible and the pith less compressible, and where both undergo smaller alterations of length when separated. Similarly in our system of steel and india-rubber, if the steel is supposed to be replaced by a cylinder of india-rubber, this cylinder would be very strongly compressed by the tube of india-rubber which in its turn would be stretched by it; and when the system was broken up a smaller contraction would take place of the tube but a much greater elongation of the cylinder than in the case of the steel, even if the tension put into action had been the same in amount as in the system of steel and india-rubber.

\section{Secr. I5.-Phenomena due to the Tension of Tissues in the growing} parts of Plants ${ }^{1}$. A. Tension of different layers of a cell-wall. By cutting as large pieces as possible out of the walls of living cells and placing them in water, it is possible to demonstrate the existence of tensions in them; it is found that if the cell-wall consists of layers of which the outer ones have a less and the inner ones a greater capacity of imbibition, the piece of cell-wall will bend so that the outer side becomes concave, the inner side convex. If the greater part of the water of imbibition is withdrawn from the piece of cell-wall by placing it in a solution of sugar or in alcohol or thick glycerine, the bending diminishes or even changes into the opposite direction, the inner side becoming concave; this direction being again reversed by again placing the object in water. Narrow strips which may be cut at right angles to the surface out of pollen-grains of Cucurbita or Althaa or the cells of the internodes of Nitella are well adapted for this experiment.

The concave curvature outwards evidently depends on the inner layers of the

layers of tissue as a general measure of the intensity of the tension; but this, it will be seen from what has here been said, is inaccurate. If, for example, the wood and pith of an old internode are isolated, the contraction of the former is scarcely perceptible, while the latter elongates considerably; the pith of the internode was therefore, according to this method, in a state of great tension, while the wood was not; although the degree of tension of the two was really the same, differing only in sign (positive and negative). On p. II2 (l.c.), Kraus gives a correct account of the behaviour of the layers of tissue of growing interrodes.

1 The phenomena here described were first observed, although somewhat superficially, by Dutrochet (Mém. pour servir à l'hist. des végét. et des anim. 1837, vol. II). Hofmeister, in his treatise On the Bending of Succulent Parts of Plants (Berichte der kön. sächs. Gesells. der Wissensch. 1859), made some important corrections of the theory. On the Direction of the Parts of Plants caused by Gravitation, see ibid. 1860; on the Mechanics of the Movements due to the Stimulation of Parts of Plants, Flora, 1862, No 32 et seq. A connected account of the phenomena was given in my Experimental-Physiologie, p. $46_{5}$ et seq. Very minute investigations were published by Kraus in Bot. Zeitg. I867, No. I4 et seq., where the transverse tension of wood caused by the increase of its diameter was also for the first time described. Nägeli and Schwendener also contributed to the development of the theory in their 'Mikroskop,' p. 396 et seq. Still these phenomena require a much more exhaustive examination than has yet been given them; the account here given will only serve to introduce the student to facts which are easy of observation. In explaining the processes in the interior I differ greatly from the views of Hofmeister (Lehre von der Pflanzenzelle, p. $27^{2}$ et seq.). The difference in our views is so complete that it would be useless to point out particular points of difference. 
cell-wall absorbing more water in the direction parallel to their surface than the outer layers, and thus stretching more and becoming the convex side of the system. When water is withdrawn the opposite result must ensue. Let us suppose the cell to be closed and entire and not at all or scarcely turgid, i.e. with no hydrostatic pressure between cell-wall and cell-contents : the inner face of the cell-wall will then be in contact with the cell-sap, and will absorb more water than the outside; a tension will therefore be produced, the inner layers of the cell-wall having a tendency to stretch, and being partially prevented from so doing by the outer layers. This tension of its layers will impart to the cell-wall a certain stiffness and rigidity which is quite unconnected with turgidity. But since in the normal state, and especially when they are growing, cells are always turgid, the whole system of tissues will be distended independently of this.

If narrow strips are cut out of large succulent cells, or very thin slices of tissue are made so as not to contain any perfect cells, a concave outward curvature is obtained at the moment of making the section. This is at once explained by recollecting that the outer layer, especially when cuticularised, was in a state of passive tension before the section was made; while the inner layer, which was in an absorbent condition, was swelled up from contact with the cell-sap. At the moment of division this inner layer retains its water of imbibition; but the outer layer, which was in a state of greater tension, obeys its elasticity, and in consequence of its contraction becomes the concave, the inner the convex surface of the section. It is clear however that these phenomena must also occur when water is removed or absorbed. It is only in this way that it seems to me possible for the cell-walls to take any part in the tension of the tissues, a part which however must always be subordinate in the closed living cell to the influence of turgidity, since this stretches both the inner and outer layers, and every change in the degree of turgidity must cause contraction or distension of the entire cell-wall.

It is a question not without importance in what relation the imbibition and swelling of the cell-wall stand to the turgidity of the whole cell. If we imagine a single turgid cell, and suppose that from any cause the cell-wall (whether the layers are in a state of tension or not) is able to absorb more water from its contents than it had before, the question arises whether the turgidity is thus increased or diminished. By the increased amount of water absorbed from the contents by the cell-wall, they must be diminished, as also must the hydrostatic pressure on the cell-wall, and the more so when the size of the cell is increased by the imbibition. But since the cell-wall may also increase in thickness, the pressure on the contents may be supposed to increase from this cause. If however we take the simplest and least favourable case, viz. that the size of the cell remains unaltered but the thickness of the wall increases, and therefore that it distends inwardly, this will nevertheless not cause any increased pressure between cell-wall and contents, because the water which was the sole cause of the thickening of the cell-wall and diminution of the cell-cavity was withdrawn from the cavity. The swelling of the cell-wall can at the most diminish the size of the cell-cavity ${ }^{1}$ by the volume occupied by the water with-

1 When an amount of water $v$ penetrates into an organised body, and increases its volume, the increase of volume can never be greater than $v$, but at the most as large. 'The evolution of heat 
drawn from it. No increase of turgidity can therefore take place in this case, and still less when the cell also increases in size. The same argument of course applies also to a multicellular mass of tissue. But the case is different when the water withdrawn from the cell-contents by the cell-wall is replaced by means of endosmose, and the turgidity thus again increased ; in this case in proportion as water is absorbed by the cell-wall the turgidity and volume of the whole cell must also increase.

B. Mutual Tension of the layers of tissue of an organ. ( $\mathrm{r}$ ) Tension in the direction of length; i.e. parallel to the axis of growth of the organ. In the internodes of upright stems some idea may be obtained, if not of the intensity of the tension, at least of its kind (whether negative or positive), and of its variation in the different layers of tissue, by measuring the length of the internodes, and then separating the layers of tissue by a sharp knife, and comparing their length with that of the entire internode. It is obvious that the length of the entire internode is the result of the mutual tensions of its layers, some being, in this experiment, shorter and some longer than the entire internode; and it results from what has already been said about opposite tensions that if any particular layers have not changed in length after being separated, this does not prove that they were not distended or compressed when forming a part of the system, but only that they opposed a strong resistance to the tension then in existence, which resistance rendered the alteration of their length imperceptibly small. But the opposite is also possible; viz. that a layer of tissue when separated will show no perceptible contraction because it was so extremely extensible and inelastic that it yielded with extremely little resistance to the traction of the layers which were in a state of positive tension, the limit of its elasticity being continually overstepped.

If this method is applied to rapidly growing internodes, it is generally found that isolated strips of the epidermis, of the cortex, or of the wood (xylem), are shorter than the entire internode, while the isolated pith is considerably longer; the former therefore were in a state of negative, the latter was in one of positive tension. All the isolated layers are flaccid, while the entire internode was rigid from the mutual tension.

If a median longitudinal lamella bounded by two strips of epidermis is cut out of a growing internode with its xylem still unlignified, and if its tissues are then isolated so as to lie side by side, then, indicating the epidermis by $E$, the cortical layer by $C$, the xylem by $X$, the pith by $P$, the respective lengths after isolation may be stated as follows:-

$$
\mathrm{E}<\mathrm{C}<\mathrm{X}<\mathrm{P}>\mathrm{X}>\mathrm{C}>\mathrm{E} \text {. }
$$

It is at once evident from this that every layer was before the separation in a state of negative tension towards the next one inside, of positive tension towards the next one outside. The epidermis alon'e was in a state of passive tension; the pith alone was passively compressed, or rather prevented from extending.

The extensibility and elasticity of tissues are altered during the growth of an internode, as may be seen by comparing internodes of various ages; the extensibility of the wood decreases rapidly, that of the epidermis and cortex more slowly,

during imbibition indicates that a decrease of volume is taking place, and therefore that although $v$ is the amount of water absorbed by imbibition, the increase of volume is only $v-d$. 
as may be inferred from the decreasing rapidity with which these tissues contract on their isolation, and from the thickening of the cell-walls ${ }^{1}$. The pith from internodes of different ages shows on isolation at first an increasing, afterwards a decreasing amount of elongation. If the tendency of the pith to expand remained the same at all ages, it would, when isolated, elongate more in older than in younger internodes, in consequence of the increasing resistance of the tissues which are in a state of passive tension; but when the growth in length has ceased, or soon after, the pith loses its tendency to expand, as may be concluded from the fact that on isolation from such internodes it elongates less, and finally not at all $^{2}$, although the resistance of the wood has greatly increased; were the pith now as elastic as before, it would expand more rapidly when freed from the very great resistance of the wood.

The following table will now be understood; the length of the entire internode being always placed at 100 , and the amount of contraction indicated by negative, of expansion by positive percentages.

Number of the internode, Change of length of the isolated tissue counting from the youngest. in percentage of the entire internode.

\begin{tabular}{|c|c|c|c|c|}
\hline \multirow{3}{*}{ Nicotiana Tabacum } & & Cortex. & Xylem. & Pith. \\
\hline & $\mathrm{I}-\mathrm{IV}$ & $-5^{\circ} 9$ & $-I \cdot 5$ & $+2 \cdot 9$ \\
\hline & $\mathrm{V}-\mathrm{VII}$ & $-3^{\cdot 1}$ & $-I \cdot I$ & +35 \\
\hline \multirow{6}{*}{ do. } & . VIII-IX & -3.5 & $-I \cdot 5$ & +0.9 \\
\hline & $\mathrm{X}-\mathrm{XI}$ & -0.5 & -0.5 & +2.4 \\
\hline & $\mathrm{I}-\mathrm{II}$ & $-2 \cdot 2$ & & $+2 \cdot 3$ \\
\hline & III- IV & $-I \cdot 2$ & & $+4 \cdot 2$ \\
\hline & $\mathrm{V}-\mathrm{VII}$ & $-r \cdot 0$ & & $+2 \cdot 8$ \\
\hline & VIII-IX & $-I \cdot 8$ & & $+2 \cdot 7$ \\
\hline \multirow[t]{3}{*}{ Sambucus nigra } & I & $-2 \cdot 6$ & $-2 \cdot 6$ & $+4^{\circ} 0$ \\
\hline & II & $-2 \cdot 0$ & $-2 \cdot 8$ & +55 \\
\hline & III & $-1 \cdot 5$ & -0.0 & $+1 \cdot 5$ \\
\hline \multirow[t]{3}{*}{ do. } & I & -0.6 & & +37 \\
\hline & II & $-1 \cdot 6$ & & $+5^{\cdot 1}$ \\
\hline & III & -0.0 & & +0.9 \\
\hline \multirow[t]{3}{*}{ do. } & I & $-1 \cdot 3$ & & +6.5 \\
\hline & II & $-I \cdot 5$ & & $+10 \cdot 1$ \\
\hline & III & -0.6 & & $+2 \cdot 3$ \\
\hline
\end{tabular}

These numbers, taken from my Handbook of Experimental Physiology, may be supplemented by some others, calculated from the statements of Kraus ${ }^{3}$ ( $l . c$. Table I).

${ }^{1}$ The decrease in the extensibility of the epidermis was determined by Kraus (l.c., tables, p. 9), by attaching weights to strips of epidermis.

2 The relation between the tension of tissues and the state of growth of the internode (i.e. the phase of its greatest period of growth) requires fresh and detailed investigation. Kraus's Table III (Bot. Zeitg. 1867) shows that the greatest difference of length between cortex and pith does not always occur at the time of the greatest growth; and that even after growth has ceased, tensions may still continue. It must however be remarked that the method by which these numbers have been obtained is liable to considerable suspicion.

${ }^{3}$ Kraus has only given the absolute numbers; but a correct notion can be obtained only by comparing them with the length of the internode. 


\begin{tabular}{|c|c|c|c|c|c|}
\hline $\begin{array}{r}\mathrm{N} \\
\text { nod }\end{array}$ & $\begin{array}{l}\text { mber of the int } \\
\text { counting from } \\
\text { youngest. }\end{array}$ & $\begin{array}{l}\text { Char } \\
\text { in } p\end{array}$ & $\begin{array}{l}\text { of length of } \\
\text { entage of the }\end{array}$ & $\begin{array}{l}\text { e isolated ti } \\
\text { ntire interno }\end{array}$ & \\
\hline & & Epidermis. & Cortex. & Xylem. & Pith. \\
\hline Nicotiana Tabacum & III- IV & $-2 \cdot 9$ & & $-I \cdot 4$ & +3.5 \\
\hline & $\mathrm{V}-\mathrm{VI}$ & $-2 \cdot 9$ & $-I \cdot 3$ & -0.8 & $+2 \cdot 7$ \\
\hline & VII- IX & $-2 \cdot 7$ & $-2 \cdot I$ & -0.0 & +34 \\
\hline & $\mathrm{X}-\mathrm{XII}$ & $-\mathrm{I} \cdot 4$ & -0.5 & -0.0 & +34 \\
\hline & $X I I I-X V$ & $-I \cdot 05$ & -0.0 & $-0.8(?)$ & $+4 \circ$ \\
\hline Vitis vinifera & I & & $-3 \cdot 1$ & $-1 \cdot 6$ & $+6 \cdot 0$ \\
\hline & II & & $-I \cdot 7$ & -0.0 & $+8 \cdot 7$ \\
\hline & III & & $-2.5(?)$ & $-1 \cdot 0(?)$ & $+7 \cdot \mathbf{I}$ \\
\hline & IV & & -0.0 & -0.0 & +6.0 \\
\hline & $\mathrm{V}$ & & -0.0 & -0.0 & +27 \\
\hline Sambucus nigra & I & & $3^{\cdot I}$ & -0.0 & +0.0 \\
\hline & II & & $-1 \cdot 5$ & $-I \cdot 0$ & $+6 \cdot 4$ \\
\hline & III & & $-\mathrm{I} \cdot 6$ & & $+6 \cdot 5$ \\
\hline & IV & & $-I \cdot 6$ & $+0.3(?)$ & $+6 \cdot 1$ \\
\hline & $\mathrm{V}$ & & -0.2 & $+0.2(?)$ & +0.7 \\
\hline & VI & & -0.5 & -0.5 & +0.1 \\
\hline Helianthus tuberosus & $\mathrm{I}-\mathrm{IV}$ & $-4 \cdot 3$ & -1 & & +6.8 \\
\hline & $\mathrm{V}-\mathrm{VI}$ & $-1 \cdot 7$ & $-c$ & & $+6: 6$ \\
\hline & $\mathrm{VI}-\mathrm{VII}$ & -0.9 & $-c$ & & +44 \\
\hline & VIII & -0.5 & $-c$ & & $+3 \cdot 2$ \\
\hline & $\mathrm{IX}-\mathrm{XI}$ & -0.0 & $+c$ & & $+2 \cdot 0$ \\
\hline
\end{tabular}

It is easy to establish the existence of similar contractions of the outer tissues and elongations of the parenchyma in the case of growing leaf-stalks, as those of Beta, Rheum, Philodendron, \&c.

If a growing internode or a leaf-stalk is split by two longitudinal sections at right angles to one another, the parts will bend concavely outwards, evidently in consequence of the lengthening of the pith and contraction of the outer tissue. This phenomenon is seen most clearly if a thin longitudinal slice is taken from the middle of the internode, laid flat, and the pith then halved lengthwise; as the knife advances the two halves will bend concavely outwards. If, instead of cutting the section in two, thin strips of tissue are cut proceeding from without inwards, first one including the epidermis, next one including the cortical tissue, and finally one including the wood, they will all bend concavely outwards, because the adjacent layers are all in a state of negative tension on the outside, of positive tension on the inside, and when separated, the outer side always becomes shorter, the inner side longer.

That this bending is caused by simultaneous contraction of the outside and lengthening of the inside is at once clear from the measurements already given, but may also be observed directly, as will be seen from the following table. Longitudinal slices of considerable thickness were cut from the middle of growing internodes, laid flat, and the pith then halved by a longitudinal cut; the radius of the curvature which each half at once assumed was determined, and the length of the convex inner and the concave outer side measured by means of a strip of card graduated in millimetres. 


\begin{tabular}{|c|c|c|c|c|}
\hline eng & $\begin{array}{l}\text { Radius of } \\
\text { curvature of } \\
\text { the segment. }\end{array}$ & $\begin{array}{l}\text { Shorten- } \\
\text { ing of the } \\
\text { concave }\end{array}$ & $\begin{array}{l}\text { Lengthen- } \\
\text { ing of the } \\
\text { convex } \\
\text { inner (pith) }\end{array}$ & $\begin{array}{l}\text { Semidiameter } \\
\text { of the } \\
\text { internode. }\end{array}$ \\
\hline
\end{tabular}

Silphium perfoliatum.

\begin{tabular}{|c|c|c|c|c|c|}
\hline Left half & $69^{\circ} 5 \mathrm{~mm}$. & $4 \mathrm{~cm}$. & 2.8 p.c. & 9'3 p.c. & $3 \mathrm{~mm}$ \\
\hline Right half & $69^{\circ} 5$ & 4 & $2 \cdot 4$ & $9 \cdot 3$ & 3 \\
\hline hium perfolic & & & & & \\
\hline Left half & 190 & $3-4$ & $2 \cdot 8$ & $9^{\circ} 5$ & 3.5 \\
\hline $\begin{array}{l}\text { Right half } \\
\text { cleya cordata. }\end{array}$ & I go & $3-4$ & $2 \cdot 6$ & 10.8 & 4.5 \\
\hline Hollow & $134^{\circ} 5$ & $5-6$ & 0.74 & $7 \cdot 1$ & $3 \cdot 3$ \\
\hline
\end{tabular}

As we have already seen from the measurements of the layers when entirely isolated, it was also evident from the curvature of the two halves of the longitudinal slice that the contraction of the epidermis is less than the elongation of the pith. Since a slice is somewhat longer than the entire internode, if the length of the slice were taken as $=100$, the proportionate contraction of the outside would be greater, the lengthening of the inside less.

A rapid rate of growth, united with a certain amount of physical differentiation of the different layers of tissue, such as occurs in erect leafy shoots, stout leaf-stalks, and tendrils, appears generally to be favourable to the production of the tensions in tissues of which we have been speaking, as they are not found in stems of very slow growth, like stout rhizomes, the thick stolons of Yucca and Draccena, \&c. That the existence of tension has more to do with a physical differentiation in the elasticity and extensibility of the layers than with a morphological one, is shown by the fact that very considerable tensions are found even between the outer and inner layers of the hyphal tissue of the stipes of the larger Hymenomycetous Fungi, which are morphologically similar. Within the growing apical region of roots, on the contrary, where we have a combination of two layers of tissue sharply differentiated morphologically, viz. an axial fibro-vascular cylinder surrounded by a parenchymatous cortex, we do not find any considerable tension when the part is split by two longitudinal cuts at right angles to one another, or when the layers are completely isolated. But since it is easy to prove that the cortex of the root grows more rapidly and for a longer time than the axial cylinder ${ }^{1}$, it may be assumed that in an uninjured growing root there is nevertheless a small tension between them, positive in the case of the cortex, negative in that of the axial bundle; but it is only rarely that this tension becomes strong enough to be perceptible by the parts bending inwards when cut lengthwise; probably because the axial cylinder, which still consists of procambial tissue, is so extensible that it yields almost without resistance to the traction of the cortex. The case is different in the older parts of the root behind the growing end (which does not exceed ro $\mathrm{mm}$. in length). If this portion is split, the parts generally gape concavely outwards, although much less so than the growing part of erect stems. The curvature is however considerable in the aërial roots

1 The halves of roots split lengthwise continue to grow for days, and bend concavely on the cut surface. 
of Aroidex, where the opposite curvature which takes place at the apex is also sometimes well-marked.

The description now given of the states of tension in the case of stems is also applicable to all expanded internodes and leaf-stalks. Within the bud itself, and especially at the punctum vegetationis, there appears to be no tension of the tissues, or only one as slight as in the apices of roots. It is only when the epidermis is becoming cuticularised and the walls of the bast-cells are beginning to thicken that the tensions become perceptible.

The individual parts of fully mature organs, especially leaves, not unfrequently retain the tensions acquired during growth, which are in such cases often particularly strong. This is the case, for instance, in the contractile organs of the sensitive and periodically motile leaves of Papilionaceæ, Mimoseæ, Oxalideæ, \&c., to which we shall recur. While in these cases the true leaf-stalks and the internodes from which they spring have long become rigid, and no longer show any considerable tension of the tissues, an extraordinary elongation of the parenchymatous cortex occurs in the contractile organs, if they are separated from the solid axial fibro-vascular bundles; and considerable flexion results when these organs are split lengthwise. The opposite to this occurs in the nodes of the stems of Grasses, i.e. in the annular thickenings at the base of the leaf-sheaths ; no perceptible tension is observable in these. If a median longitudinal section is made and divided into its inner and outer layers, they exhibit none of the curvatures which are so striking in portions of young internodes. This flaccidity of the tissue, or at least the insignificance of the tension, must depend on the concurrence of two causes; on the one hand on the cessation of the growth of the parenchyma in the node (although it remains in a state capable of growing, and under certain circumstances begins to grow again), and on the other hand on the extensibility of the fibro-vascular bundles which do not become lignified within the node, or not till a late period when the cells of the same bundles, where they lie in the leaf-sheath and the internode, have long become lignified and rigid. While, therefore, the parenchyma of the node continues to grow, it stretches the unresisting fibro-vascular bundles, and when its growth ceases no perceptible tension remains. In the contractile organs of sensitive and periodically motile leaves, on the contrary, the axial fibro-vascular bundle becomes elastic and resistant before the growth of the surrounding parenchyma has ceased; and when this is the case a tension remains which is further increased by the extraordinary capacity of the parenchyma for becoming turgid.

If we now attempt to give an account of the causes which render the tension at first (when in the bud) imperceptible in the internodes of erect rapidly-growing stems, and make it subsequently increase and finally altogether disappear when the internodes are fully mature, we find that we must content ourselves with probable conjectures rather than with fully demonstrated propositions.

The origin of tension between the layers must in any case be referred mainly to differences in the growth of the cell-walls of such a nature that the intercalation of fresh material takes place less rapidly in those of one layer than in those of another; and it is especially manifest in those cases in which the cell-walls subsequently undergo thickening. From the first of these causes the layers which lengthen more slowly are placed in a state of passive tension by those that grow more rapidly; while the second 
cause diminishes their extensibility to an increasing extent, especially when, as in the xylem of the fibro-vascular bundles, the cell-walls become lignified, which renders them capable of resisting extension. The more quickly, on the other hand, the thin cell-walls in the pith and parenchyma generally increase in size (especially in length) by superficial growth, the stronger becomes the tension of the passively stretched layers of tissue. To this must be added the peculiar power of the medullary cells to absorb water from the older parts with great force and rapidity, and thus to maintain themselves in a state of the highest turgidity. This distends the pith independently of the superficial growth of its cell-walls, and besides influencing the more slowly growing layers of tissue, also contributes to increase the superficial growth of the cell-walls of the pith. If the woody bundles then become lignified as the tissues become more developed internally, and the resistance of the epidermis, which is constantly becoming more cuticularised, becomes too great, these tissues oppose an insuperable resistance to the further distension of the pith by growth and turgidity, and no further elongation of the internode is possible. The tendency of the pith to expand ceases; its cells lose their turg:dity, they give off their water to adjacent tissues, and become filled with air.

According to this view, which has been fully established in the main, the actual motive power of growth in internodes emerging from the bud-condition is the pith, and the thin-walled parenchyma generally. It is only the force thus exercised that causes the other tissues to increase in length as long as they are sufficiently extensible. The extraordinary absorbent power possessed by the pith enables it when growing to withdraw the water from the surrounding layers of tissue, and thus prevents the cells from becoming more strongly turgid, neutralising by this means one of the causes of the superficial growth of the cell-walls. It must also be remembered, as has already been shown (Fig. 478), that the turgidity of the cells of the stretched layers is even diminished, while that of the compressed cells (in the pith) is increased by the tension; and we consequently have here another cause of differences in the superficial growth of the cell-walls. Finally, it must be borne in mind that the internodes, at least of land-plants, are exposed to transpiration as soon as they emerge from the bud; but this cause of diminished turgidity will affect chiefly the epidermal cells and the subjacent layers, least of all the pith.

The great importance which is here attached to turgidity as a cause of growth is justified by the fact that the growth of the internodes is at once stopped by its decrease, i.e. by the withering of the shoot; while it is promoted by its increase, i.e. the growth of the shoot in water or damp air.

The first and most efficient cause of the tension of tissues in a growing internode is therefore the different capacity for turgidity of the different tissues; this depending partly on the nature of their fluids, partly on the structure of their cellwalls, and partly on their relative position in the internode. A more secondary place must be assigned to the swelling of the cell-walls caused by imbibition ; since it may be assumed that even when the turgidity of the cell is slight, the cell-wall still obtains sufficient water to satisfy its capacity for imbibition. If it were directly dependent on this, all the layers of tissue would grow equally rapidly, even when the turgidity was small, or had entirely disappeared. I rather hold the state of the case to be that 
when the cell-wall is passively distended by turgidity or by the tension of the surrounding layers of tissue, it is only enabled to deposit fresh substance in the direction of its surface when perfectly saturated; this does not however imply that other causes do not cooperate in promoting the intercalation.

The importance of turgidity as a cause of growth may be very strikingly illustrated in the case of isolated cylinders of pith, as we shall show presently.

When, in consequence of their separation, the tissues which were in a state of passive tension become suddenly shorter, and the pith which was in a state of positive tension suddenly longer, this process must be connected with a corresponding change in the form of the cells ${ }^{1}$; the cells which contract must at the same time become wider in diameter, while those of the pith which lengthen must on the contrary become narrower. It is impossible however to measure directly these changes of diameter, which are so small that ordinary methods are inapplicable.

It is, however, a necessary consequence of what has been said that the passive lengthening of the epidermal cells, \&c. in a growing internode makes them narrower; the young epidermis must therefore be too narrow, besides being too short for the inner masses of tissue. Similarly the pith, being prevented from elongating in the growing internode by the surrounding layers, must in consequence have a tendency to enlarge transversely; besides being too long for the elongated tissues, it will also be too thick for them, and must have a tendency to force them apart. It follows therefore from the longitudinal tension which has been observed in the layers of tissue of a growing organ, that a transverse tension must also exist in it of such a nature that the outer layers are in a state of passive tension, while the medullary cells which are prevented from lengthening have a tendency to dilate transversely.

If thick transverse sections ${ }^{2}$ from growing stems are cut radially, they gape open, evidently because the epidermis contracts in the peripheral direction, having been previously of too small circumference for the inner tissue, in other words, in a state of passive tension. The tendency of the medullary cells which are prevented from lengthening to become broader transversely does not appear, on the other hand, to be always hindered by the surrounding wood and cortical tissue, but often to be even promoted by them; so that these layers of tissue which surround the pith grow more rapidly in the peripheral direction than does the pith itself, and therefore exercise a radial traction upon it. A striking proof of this phenomenon is afforded by the frequent formation of cavities in stems and leaf-stalks at the time and place where the growth in length is most rapid. The increase in thickness of the pith is not sufficient to fill up the space which is enclosed by the surrounding tissues, and which increases in size; its cells separate in the longitudinal direction, and the woody cylinder remains clothed on the inside by a layer of pith, the longitudinal tension of which still continues. The existence of an outward traction upon

1 Any considerable change in the volume of the medullary cells when isolated must not indeed be expected, when it is recollected that neither the water contained in the cells nor the cell-walls permeated with water alter their volume under the forces exerted in this case. An alteration in the volume of the entire pith could at most arise from a change in the size of the intercellular spaces in consequence of the change in form of the cells.

2 Sachs, Experimental-Physiologie, p. 47 I. 
the pith can also be demonstrated in the case of internodes with solid cylinders of pith which are growing and at the same time increasing rapidly in diameter (e.g. Nicotiana, Silphium perfoliatum), by dividing a fresh transverse section (laid on glass) through the centre. The two cut surfaces of the pith now become curved outwardly and separate from one another, while the cortical parts of the segment still touch. This is an indication of the outward traction of the pith, and of the tendency of the cortical envelope to dilate peripherally.

These statements rest however at present on but a small number of observations, and better results may be expected from their repetition. It may nevertheless be assumed that in young internodes, before the fibro-vascular system has begun to become lignified, the pith exerts an outward pressure. This is accompanied later, when the tangential growth of the wood and cortex is more rapid, by an outward traction, which at length becomes so strong as to exceed the tendency of the pith to dilate transversely. The pith is therefore now actually in a state of passive tension transversely (and at the same time compressed longitudinally), until at length the cells in the centre of the pith become detached from one another, and a hollow is formed, if the whole does not lose its sap and become dried up, as for example in the Elder. Kraus observed ${ }^{1}$ that the medullary cells of an internode are longer when it is growing than when mature; but this is to be attributed, in accordance with what has been said, to the loss by the cells of the pith of their power of elongating when isolated. In the internode they are certainly not at first longer, and are afterwards actually shorter; the difference is only observable on isolation, and indicates that these cells at length lose the property of changing their form when isolated, or in other words become rigid.

The views here brought forward respecting the tension of the tissues of growing internodes and leaf-stalks are, I think, supported by the fact that the sudden and very considerable lengthening of the pith at the moment of its separation from the surrounding layers of tissue is followed by a slow lengthening which lasts for some days, while, on the contrary, the cortex and epidermis, which are in a state of passive tension, scarcely experience afterwards any perceptible contraction (but, according to Kraus, do not become longer even when placed in water). This subsequent lengthening of the isolated pith takes place with extreme force when it absorbs water, as Kraus has already shown; but the lengthening also continues in dry air when the pith even loses small quantities of its water, a point which had been previously overlooked.

The isolated cylinder of pith of a growing internode is very flaccid, flexible, and extensible; but if placed in water it soon becomes tense, rigid, and elastic, longer and apparently also thicker. The lengthening may amount in a few hours to as much as $4 \circ$ p.c., or even more. These phenomena are explained if we suppose the contents of the medullary cells to be very strongly endosmotic ${ }^{2}$, by which they. become in a high degree turgid, and thus not only increase considerably in size,

1 Bot. Zeitg. I867, p. II 2 .

2 Notwithstanding this powerful endosmose, the amount of solid substance dissolved in the cell-sap of the parenchyma is very small, as is shown by the fact that in cylinders of pith of this kind I found the dry weight only from 2 to 5 p. c., a considerable portion of which belonged to the cell-walls and protoplasm. 
but also become more rigid. The considerable increase in size presupposes, however, from the rapidity with which it takes place, great extensibility in the cellwalls. Isolated prisms of pith exposed to the air become shorter even than the length they possessed in the internode ${ }^{1}$; the cell-walls which were previously in a state of tension evidently contract elastically, as the turgidity diminishes from loss of water.

But if care is taken that isolated cylinders of pith do not absorb any water, while at the same time they can only lose a very small quantity of it, by enclosing them in a glass tube containing about $\mathbf{I}$ litre of dry air, they nevertheless continue to lengthen perceptibly for some days, although not so considerably as when they absorb water; and this lengthening affects chiefly the older parts, while the youngest parts sometimes contract. The whole cylinder becomes dry and rigid on the surface. Out of a large number of observations the following may be chosen to elucidate this point.

A prism of pith from a part of a shoot of Senecio umbrosus $235.5 \mathrm{~mm}$. long, lengthened about 57 p.c. on isolation, and weighed 5.3 grammes. It was divided into three parts by marks of indian ink; their lengths being:-i. (the oldest) $100 \mathrm{~mm}$., ii. $100 \mathrm{~mm}$., iii. (the youngest piece) $49 \mathrm{~mm}$. The prism of pith was now fixed in a dry glass tube, which was then corked at both ends. After fourteen hours the parts had lengthened as follows:- - part i. about $4.5 \mathrm{~mm}$., part ii. about $6.5 \mathrm{~mm}$., part iii. about $2 \mathrm{~mm}$. or 4.1 p. c., while the pith had lost O'I $5 \mathrm{grm}$. of water. After remaining for twenty-six hours more in the glass tube the following further changes had taken place; part i. had again lengthened about $2^{\circ} 5 \mathrm{~mm}$., part ii. about $0.5 \mathrm{~mm}$., while the length of part iii. had diminished about $0.5 \mathrm{~mm}$. No further loss of water had taken place, because the glass tube had become covered with moisture. The pith was now placed in water, and after six hours the following increase of length had taken place:-in part i. about $18 \mathrm{~mm}$. or 16.8 p. c., in part ii. about $23 \mathrm{~mm}$. or $2 \mathrm{r} \cdot 6$ p.c., in part iii. about $\mathrm{I} \mathrm{Imm}$. or $2 \mathrm{I}^{\circ} 6 \mathrm{p}$.c. (as compared with the length before placing in water). The pith had also become considerably thicker, having absorbed 6 grammes of water. The estimation of the dry weight showed that the pith contained only $0.22 \mathrm{grm}$. of solid substance; this was combined, when the pith was isolated, with $5^{\circ} 08 \mathrm{grm}$. of water; it subsequently lost $0^{\circ} .5 \mathrm{grm}$., but by the end of the experiment had again absorbed $6 \mathrm{grm}$. At first therefore the pith contained 4.23 p.c., at last only $\mathrm{r} \cdot 97$ p.c. of solid substance. Experiments of this kind show that the pith of the youngest internodes loses its water most easily by evaporation, as is shown by its decrease in length. Kraus was led by other experiments to the same conclusions; and he also showed-not in contradiction, as he thought, but in harmony with these results (l.c. p. 123)-that the older pith of growing internodes attracts water more powerfully and expands more than the younger.

If the question is now asked how the lengthening of the pith can take place in spite of the loss of water (though this may be small), it must first of all be noted that its surface becomes remarkably dry under the circumstances described. It is scarcely possible to attribute this significant desiccation of the surface to the small loss of

1 Kraus, l.c., Tables, p. 29. 
water indicated by the weight; it is probably rather caused by the inner cells of the pith withdrawing water from the outer cells, and thus lengthening; but the outer cells would become shorter if they were not stretched by the inner ones. That this is actually the case is shown by the rigidity of the pith under these circumstances, caused by the tension that subsists between the dry outer layer and the moister inner mass. If the prism of pith is divided lengthwise, the parts curve outwards ; and sometimes the outer surface becomes even strongly concave. If the inner cells of the pith are able to withdraw water from the outer ones, it may be inferred that the outer cells are also able to withdraw it from the surrounding wood and especially from the peripheral tissues, preventing these from becoming strongly turgid; their growth being thus retarded in favour of that of the pith, by which they are now placed in a state of passive tension. It is noteworthy that the medullary cells which contain a minimum quantity of dissolved substances nevertheless absorb water sufficiently powerfully to abstract it from the surrounding tissues which must evidently contain a much greater quantity of dissolved substances.

It is now clear from the observations which have been described, why portions of shoots cut lengthwise in half or in four and placed in water curve outward to such a remarkable extent; and why a curvature, which may be small but continues to increase for some time, takes place when such pieces are placed in a closed glass tube in dry air.

(2) Transverse tension caused by subsequent growth in thickness of the wood ${ }^{1}$. It has already been shown that transverse tensions also arise during growth which are caused by the longitudinal tension; a more exact knowledge of these is still a desideratum. With the commencement of the increase in thickness of the stem by means of the cambium-ring, a new cause of tension arises, acting in both a radial and peripheral direction; and this transverse tension generally continues as long as the cambium-ring remains active. The layers of tissue formed from the cambiumring have at first a tendency to expand in the tangential direction to an extent greater than the space enclosed by the epidermis and the primary cortex permits. These outer tissues therefore become stretched in the peripheral direction; and, since they are elastic and have a tendency to contract, they exert a pressure in the radial direction on the cambium and the tissue formed from it. It happens however also that the rings of wood produced on the inside of the cambium grow more strongly in the tangential direction than the phloëm produced on the outside, which is therefore passively stretched. A tension is hence set up in the transverse diameter of the stem during its increase in thickness of such a kind that each layer is stretched peripherally on its outside and compressed radially on its inside; in other words, is in a state of negative tension on its outside, of positive tension on its inside. If the separate layers of a transverse segment-epidermis, primary cortex, phloëm, and xylem-are separated, and their peripheral length compared, we get the following expression for the transverse tension:-

$$
\mathrm{E}<\mathrm{C}<\mathrm{Ph}<\mathrm{X} \text {. }
$$

As the increase in thickness proceeds the transverse tension increases, as is shown

1 [See Detlefsen, Ueb. Dickenwachsthum cylindrischer Organe, Arb. d. bot. Inst. in Würzburg, II. $1,1878$. 
by Kraus's very complete experiments; $i . e$. if the rings of tissue in a transverse section of the stem or in a woody branch are separated from one another, by dividing it longitudinally and then separating the rings, they contract the more the nearer they lie to the circumference, and the contraction is the more considerable, compared with the original circumference of the whole, the older the internode from which the section is taken. The traction upon the cells of the epidermis and of the primary cortex caused by the transverse tension is easily observed by the microscope in the transverse section, if young internodes of plants which increase rapidly in thickness, as Helianthus, Ricinus, or Ribes, are compared with those which have already been forming wood for some weeks or months. The form of the cells shows that they have been stretched in the peripheral direction (see Fig. 56), and have in consequence grown rapidly in that direction; the cells which have been thus altered in form are divided by radial septa. But at length the epidermis and primary cortex are no longer able to obey the peripheral traction; longitudinal fissures occur in the cortical tissue, generally after the commencement of the forma. tion of cork. When the periderm and cork have been formed on the older parts of stems, these secondary epidermal tissues undergo a continuous strain in the peripheral direction, and exert in turn a radial pressure on the living phloëm, cambium, and xylem. The first result of this pressure exerted by the growing inner tissues is the splitting of the layers of bark, especially longitudinally. The form of the fissures depends, however, on the course of the bundles of bast which take part in the formation of the bark, and on other relations of the tissues to one another. If a stem does not in its growth take the form of a cylinder or slender cone but of a spherical tuber, as in Beaucarnea and Testudinaria, the layers of periderm split apart in the form of tolerably regular polygons which cover the spherical surface of the stem like shields. These examples show at the same time that in those Monocotyledons also which grow in thickness tensions are produced by the subsequent increase of the stem in thickness similar to those caused by the activity of a true cambium-ring; for in this case it is replaced by a thickening-ring, in which new layers of fibro-vascular bundles and intermediate parenchyma are constantly being produced. (See Fig. 104.)

It is evident that before the bark splits or fissures already in existence become wider and penetrate inwards, the transverse tension must attain a certain intensity, which, from the great firmness of the bark, cannot be inconsiderable. At the moment when the splitting takes place at least a portion of the tension must, however, be destroyed. This is clearly the reason why the transverse tension attains its maximum (measured in the way described above), as Kraus has pointed out, above the part of the stem where the scaling-off of the bark begins. But even in annual stems which increase rapidly in thickness, as Helianthus, Dahlia, \&c., the transverse tension does not progressively increase from the apex to the root, but attains its maximum at an intermediate height, below which it diminishes. An explanation of this phenomenon is afforded by the fact that the limit of the elasticity of the bark is gradually exceeded by the long-continued pressure to which it is subject from within, and that the cell-walls which are strained grow at the same time by intussusception, and thus a portion of their tension becomes neutralised.

While we may consider the turgidity of the pith and its.enormous endosmotic 
power as the principal cause of the longitudinal tension of growing internodes and leaf-stalks before they become lignified, it is on the other hand probable that the imbibition and swelling of the cell-walls are the chief cause of the transverse tension. The wood, where the transverse tension chiefly originates, is, when mature, scarcely adapted for any distension by turgidity; while at all events in cells or vessels with bordered pits it is altogether impossible. Closed wood-cells, when turgidity is possible in them, cannot however expand greatly; since their own wall and the woody substance which surround them are far too inextensible to stretch to any considerable extent under the influence of hydrostatic pressure. It has, on the other hand, been already shown (Sect. 13) what considerable alterations of dimension the wood experiences especially in the peripheral and radial direction simply by imbibition ${ }^{1}$. Every layer of wood freshly formed on the inside of the cambium-ring has a tendency to grow wider in the peripheral direction, as long as the supply of water is sufficient to cause a decided swelling of the cell-walls. The cambial tissue is by this means stretched tangentially, and the enlargement of its cells thus caused is increased by turgidity; and from the thinness of their walls it may be assumed that it is their turgidity that protects them from becoming destroyed by compression between the wood and the bark. The elements of the secondary phloëm-the bastcells and the phloëm-parenchyma - can scarcely experience any great change of dimensions owing to the swelling of their cell-walls; the former are indeed thickwalled, but they are not so arranged as to form a layer which increases in size from this cause. The cells of the latter have such thin walls that their swelling produces but little expansion, and experience teaches that they do not increase much in size in consequence of turgidity. Finally, the periderm and the bark dry up and contract, if not to any great extent, yet with considerable force.

The experience of every year shows that the fissures in the bark-especially of thick trunks at the end of winter in February and March-become deeper and wider, evidently in consequence of the great swelling of the wood, which at this time contains the greatest quantity of water; while the bark had time to dry up and contract during the dry weather in winter. If the fissures increase in width by the strong tension thus produced-which can be easily seen when fresh-the damp weather in spring causes the bark to swell; the tension between it and the wood becomes much less, and the production of wood now begins afresh in the cambium. While the wood is becoming thicker during the summer, the bark dries up and shrinks, and the tension between the outside and inside again increases, to cease once more in the following spring. Not only does an annual period of transverse tension thus arise, but this is also the cause, as we shall see presently, of the difference between the spring and autumn layers of wood.

The statements made in this section may be briefly summed up as follows:-The tissues, at first homogeneous, become first of all differentiated in such a manner that chemico-physical differences are set up, in consequence of which certain layers, especially the pith, absorb the water in the tissues more strongly than the others, and consequently grow more rapidly; and the layers which are less turgid and grow more slowly are exposed to a passive traction which promotes their growth. After growth

1 [Nevertheless the amount of water of imbibition which a single lignified cell-wall can take up is small. (See Sachs, Ueb. d. Porosität des Holzes, Arb. d. bot. Inst. in Würzburg, II. 2, I\& 79.)] 
in length has ceased it is principally the stronger imbibition and swelling of the wood that presses the surrounding layers of tissue outwards and promotes their peripheral growth.

The intensity of the longitudinal and transverse tensions consequently depends mainly on the addition of water to the turgescent pith and the swelling wood; any decrease of the turgidity of the pith must cause it to contract, and hence the whole shoot to become shorter and flaccid. This is in complete accord with observation, since withered shoots, i.e. such as have lost water by transpiration, have not only become shorter but also flaccid. Any diminution of the amount of water absorbed by the wood must in the same manner diminish the transverse tension and the diameter of the shoot. A small loss of water in the peripheral tissue when in a state of passive tension does not on the other hand usually cause directly any considerable increase in its tendency to contract; since the increase in its size due to turgidity and imbibition are generally much less considerable than in the pith and wood.

If now there are circumstances which cause a daily periodic change in the quantity of water contained in the tissues, the result will be also a periodic increase and decrease in the intensity of the longitudinal and transverse tensions. Such a daily periodicity of the tension has been actually discovered by Kraus (l.c. p. I22), who has observed that the longitudinal tension estimated by the difference in length of the pith and the bark, as well as the transverse tension estimated by the contraction of the bark when detached from woody stems, decrease, under the normal conditions of life, from early morning till midday or early in the afternoon, when they reach their minimum, and then again increase, attaining their maximum early the next morning. Millardet determined this periodicity in quite a different way; and since the objects on which he experimented permitted an exact measurement, he detected in addition an increase, usually small, of the tension in the afternoon. Notwithstanding the statements of Kraus-which are partly opposed to this conclusion, but on the whole confirm it-I am inclined to attribute this periodicity chiefly or altogether to the variation in the amount of water contained in the tissues of the plant at.different periods of the day. When transpiration is greatly diminished during the night, the quantity of water in the plant must increase, and with this the tension; and conversely the increase of transpiration during the early part of the day must diminish the tension. Space does not permit me to give in detail the opposing statements of other observers; but this will be done in part further on. Here I need only point out that the periodicity, especially of the longitudinal tension, may possibly be also directly dependent on light, independently of the heat which accompanies the light and of the increase of transpiration caused by it (although this cannot be proved by Kraus's experiments, l.c. p. 125). As far as concerns a daily periodicity independent of temperature, light, and the amount of water contained in the tissues, I could only admit it when any other explanation of the phenomena was shown to be impossible. At present this is not the case. From the intimate dependence and correlation of growth and tension, from the fact discovered by $\mathrm{me}^{1}$ that the daily periodicity of growth in length coincides in every particular with the daily periodicity of tension observed by Millardet and Kraus, and that it is caused simply by changes in temperature and light, I consider it very probable that the daily periodicity of tension is also dependent on these agencies. On the one hand they influence growth and through it the tension, while on the other hand they affect the amount of water contained in the tissues by modifying transpiration and its conduction from the roots. Like all other periodic phenomena of vegetable life, that of tension requires a very careful investigation of its external causes before we resort to the last expedient of assuming internal periodic changes, of which no explanation can be given in the present state of our knowledge ${ }^{2}$.

1 Arbeiten des Bot. Inst. in Würzburg, I872, I, Heft 2. p. I68.

2 [A daily periodicity of thickness in the trunks of trees has been detected by Kaiser (Ueb. die 
SEcT. 16.-Modification of Growth caused by Pressure and Traction. Cells or whole masses of tissue may be subjected to pressure and traction in very different ways. On the one hand these forces may result, in a perfectly normal manner, from the tension of the tissues; on the other hand, external and more accidental circumstances may cause single cells or masses of tissue to be compressed or stretched in particular places by solid bodies, or tissues to become accidentally freed from the pressure and traction to which they are normally subject. The numerous phenomena which indicate or prove that growth is altered in this way have however at present been exactly investigated from this point of view in only a few cases. The following will therefore only serve to draw attention to a subject further discoveries in which will doubtless contribute largely to the establishment of a mechanical theory of growth.

I. Every cell-wall is subject to Pressure from within, by which it is distended, so long as the cell is turgid. But since the daily experience of microscopists teaches us that all growing cells are turgid; and that no cell which is unable to become turgid in consequence of openings in its cell-walls has any power of growth; and that withered internodes, leaves, and roots do not grow, while these organs grow more rapidly the more strongly turgid they are, it may be inferred that turgidity is an essential condition of the growth of the cell-wall ${ }^{1}$. This appears to a certain extent intelligible if Nägeli's theory of growth and Traube's experiments on artificial cells described in Sect. I of Book III are accepted. It may then be assumed that the interstices between the solid particles of the cell-wall which are occupied by water increase slightly in consequence of the distension of the cell-wall caused by the hydrostatic pressure of the sap; and that space is thus obtained for the intercalation of fresh particles of solid substance; the distension caused by turgidity then begins afresh and produces the same effect.

The distension which takes place at any particular spot of the cell-wall and the consequent intercalation of fresh solid substance depend however chiefly on the internal properties of the cell-wall itself. Not only do different parts of the cellwall differ in their extensibility, but they may even vary at the same spot in this respect in the longitudinal and in the tangential or the oblique direction, as may be seen from the swelling of the cell-wall. But that there is actually such a general difference in the extensibility in different directions is at once shown by the fact that growing cells assume the most various forms,-cylindrical, stellate, \&c.; while, if the extensibility of the cell-wall were the same in all directions, the cells must all become spherical as the result of turgidity, or polyhedral under that of mutual pressure. This little is nearly all that we know at present with reference to extensibility, turgidity, and growth by intussusception. It must be borne in mind that

tägliche Periodicität der Dickendimensionen der Baumstämme, Diss. Inaug., Halle r879). See also Kraus, Ueb. Wasservertheilung in der Pflanze, III, Die tägliche Schwellungsperiode der Pflanzen, Halle i 88r.]

1 [De Vries has shown (Mechan. Ursachen d. Zellstreckung) that when a growing internode is placed in a 5-1o per cent. solution of a neutral salt, its cells lose their turgidity, and that growth ceases; when the salt solution is washed out with distilled water, the cells regain their turgidity and growth is resumed. Sorauer (Bot. Zeitg. 1878) has pointed out that individuals grown in dry air are much smaller than others of the same species grown in moist air. These facts, and many others which might be mentioned, show the dependence of growth upon turgidity.] 
the rapidity of the growth of cells is in proportion to the thinness and therefore the extensibility of their walls. The growth in thickness of the cell-wall usually begins when the increase of the cell in volume begins to diminish or has altogether ceased.

If then the distension of the cell-wall caused by turgidity is the origin of its superficial growth, something similar must also occur when the cell-wall is stretched in some other way by external forces, the turgidity being less. This is the case with the epidermis and cortex of shoots as a result of the tension of the tissues. Since in long internodes and leaves these cells usually grow principally in the longitudinal direction, while in broad leaf-blades they assume the form of polygonal plates, this may be referred in the first case partly to the traction to which they are subject being chiefly in the longitudinal direction, in the second case to its being in all directions parallel with the surface ${ }^{1}$. It has already been stated that the cells of the primary cortex of shoots which are increasing rapidly in thickness are not merely stretched but also grow rapidly in the tangential direction ${ }^{2}$.

2. Pressure from without on the cell-wall which is distended by turgidity occurs in a very simple form when the apices of growing cells come into contact with solid bodies; as the root-hairs of land-plants with the particles of the soil ${ }^{3}$. The very thin and extensible cell-walls are in close contact with the irregular surface of the particles, just as when an elastic bladder filled with water is pressed externally by an angular body, only that they retain, after the pressure is removed, the form which has thus been given them, evidently in consequence of the intercalation of fresh particles of solid matter which perpetuates the form at first acquired only by distension. The reverse takes place when the external pressure on the cell-wall is removed. A very simple instance of this is afforded by the formation of the socalled 'Tüllen' in vessels ${ }^{4}$. They are formed where the thin non-lignified wall of a cell of the wood-parenchyma, still capable of growth, adjoins the pits of a vessel. The portion of wall which is stretched over the opening is forced through it by the pressure of the sap of the cell and swells out in the form of a papilla into the cavity of the vessel. As long as the vessel contained sap and was in a turgid state, its turgidity was in equilibrium with that of the adjoining cell; but as soon as the cell-sap of the vessel was absorbed, the portion of cell-wall which covers the bordered pit was subject to pressure on one side only, and was therefore forced in the opposite direction. These phenomena can be produced artificially by the removal of the pressure to which the cells are subject from the adjacent tissues; thus, for example, the cambium swells up on the cut surface of woody branches when placed in moist sand or air, in the form of a cushion between the bark and the wood. This 'Callus,' as it is termed, results from the growth of the uninjured cambial and adjoining cortical cells next the cut, where their growth was previously

1 For further details on the possible influence of tension on the formation of stomata, see Pfitzer, Jahrb. für wiss. Bot. vol. VII. p. 542.

${ }^{2}$ On the connection of the radial and peripheral arrangement of rows of cells in a transverse segment with the increase in diameter, see the lucid description of Nägeli in his Dickenwachsthum des Stengels bei den Sapindaceen, Munich I864, p. I3 et seq.: [also Detlefsen (loc. cit.).]

${ }^{3}$ Sachs, Experimental-Physiologie, p. 185.

${ }^{4}$ See Book I. p. 26 [and references in foot-note. These growths are frequently termed 'tyloses.'] 
prevented by the cells which have now been removed. When once projecting beyond the cut, they grow more rapidly than before in a lateral direction in consequence of the turgidity, and become divided by transverse and longitudinal walls.

The further development of such a callus where branches have been cut off leads to the well-known overgrowth on the stumps. In internodes of seedlings of Phaseolus which had accidentally become hollow, I found the medullary cells which surrounded the cavity to have grown into it in the form of spherical or club-shaped papillæ; divisions ensued, and nuclei were formed in the cells thus produced. The medullary cells which exhibited this active growth on the free surfaces of their walls would have retained their polyhedral form had the pith remained solid, because every surface of the cell-wall would have been exposed to the pressure of the two adjoining cells; but in consequence of the formation of the hollow, the pressure was removed on one side, and the turgidity, being no longer neutralised, caused the cellwall to swell out, and induced in it an active superficial growth ${ }^{1}$. These phenomena and others of the same kind show that it is often sufficient merely to remove the pressure to which tissues or individual cells are subject in order to bring about an active growth of the free surfaces of their cell-walls. The first cause at least of the new growth is the distension of the free surfaces of the cell-walls in consequence of the turgidity of their cells which was previously neutralised by that of the adjoining cells. But that a very small pressure from without is sufficient to prevent the growth of softer tissues at the points of contact is seen in the case of many large Fungi which develope among the vegetable mould of woods, and enclose in the margin of their pileus light loosely lying leaves, pieces of stick, and the like. The small pressure from without clearly prevents in these cases the superficial growth of the walls of the cells with which these bodies are in contact, while the adjoining cells extend laterally and enclose them.

But the most remarkable illustration of this law is seen in the effect produced by a slight pressure on the growth of tendrils, the longitudinal growth of the cells being thus greatly hindered and sometimes even stopped, while the cells of the opposite free side elongate rapidly, as is seen even at the first glance without measurement by making a longitudinal section of a tendril curling round a slender support. In what way the slight pressure which acts in a radial direction, and is generally combined with friction, exerts an influence on the longitudinal growth is however entirely unknown. Very similar phenomena are exhibited by the primary and secondary roots of seedlings (as $Z e a, F a b a$, and Pisum). If they are allowed to grow in a damp locality, and the growing parts are made to press on one side some solid body as a pin or another root, the root bends like a tendril round the body with which it is in contact, this side growing more slowly than the opposite one. It is evidently in consequence of a similar influence of pressure on growth that the aërial roots of Aroideæ and Orchideæ become closely attached to solid bodies, following exactly their inequalities. But even unicellular tubes, such as the hyphæ of Fungi and pollen-tubes (Fig. 479), are induced by contact with a solid body to grow closely applied to it. In this simplest case, where the hydrostatic pressure is uniform over the cell and distends the cell-wall, it does not admit of a doubt that the pressure

\footnotetext{
${ }^{1}$ Prantl succeeded in artificially inducing similar phenomena in the tubers of Dahlia.
} 
from without impedes the growth of the cell, independently of turgidity, while the growth proceeds unhindered on the side which is not in contact.

But the mechanical processes by which pressure on an organ in the radial direction impedes its growth on that side are unknown. The solution of the question must depend in the first place on whether the pressure acts on the cell-wall directly or in some way or other through the protoplasm ${ }^{1}$.

But in contrast to the phenomena which have now been described, external pressure also sometimes causes growth at places where otherwise there would be none. Thus Pfeffer has shown ${ }^{2}$ that certain hyaline superficial cells on both of the flat sides of the gemmæ of Marchantia possess the power of growing out into tubular root-hairs when they remain in contact for some time with a moist solid body; while contact with water produces no effect of the kind. These cells usually develope into root-hairs only when their outer surface is directed downwards, while those on the upper side, not being in contact with a solid body, do not grow out. This, as we shall see presently, is an effect of gravitation, which is however overcome by the action of the slight continuous contact, since this causes the cells on the upper side of the gemmæ also to grow out into root-hairs. The 'haustoria'

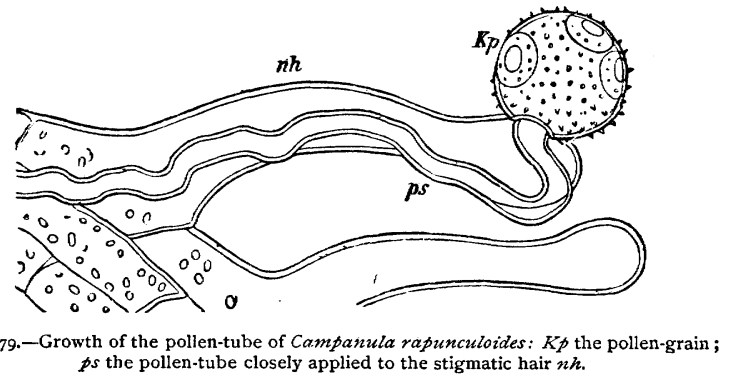

of Cuscuta and Cassytha and the adhesive discs on the tendrils of the Virginian Creeper are only formed, as was shown by v. Mohl, on the continuous contact of the surfaces of the tissue with a solid body; and this has been confirmed by recent experiments of Pfeffer's $\left(l . c . \text { p. } 9^{6}\right)^{3}$. In these cases a growth combined with celldivision and differentiation of tissue is caused by contact or slight pressure on a part of the organ, and would not take place without this pressure. The haustoria and adhesive discs thus formed are altogether indispensable for the life of the plant; for Cuscuta is nourished exclusively by the haustoria which penetrate into the tissue of the host; and it is by the formation of adhesive discs on the tendrils that the Virginian Creeper is enabled to climb up walls. If the tendrils do not meet with any solid body to which they can attach themselves by means of these discs,

1 If the relation between protoplasm and the growth of the cell-wall were better known, stress might be laid on the fact that even a very slight pressure on the cell-wall disturbs the movement of the protoplasm, and may even cause it to become detached from the cell-wall (see Hofmeister, Lehre von der Pflanzenzelle, p. 5I).

2 Arbeiten des Bot. Inst. in Würzburg, Heft I. p. 22.

3 [See also Darwin, On the Movements and Habits of Climbing Plants, London 1875, p. 84 et $s e q$. 
they dry up and fall off, while those which have formed discs increase in thickness and become woody.

r. The retarding effect on growth of an external pressure on the cells is very evident in the formation of the annual rings in wood. In the earlier editions of this work I called attention to the fact that the larger radial diameter of the wood-cells in the portion of the rings formed in the spring, and their smaller radial diameter in the portion formed in the autumn, might possibly depend on a difference in the pressure from the surrounding bark to which the cambium and the wood are subject, this pressure being less, as we have shown, in the spring, and constantly increasing during the summer. This hypothesis has been fully confirmed by $\mathrm{H}$. de Vries's investigations ${ }^{1}$. In branches two or three years old he increased the pressure of the bark in the spring by firmly winding string round them at particular places. The experiment showed in all cases, firstly, that the absolute thickness of the annual ring was less beneath the ligature than the mean thickness of the same annual ring at some distance above or below that spot. In several instances the difference was so considerable that the spot where the experiment was made appeared of considerably less diameter even to the naked eye, and this effect was increased by the formation of cushions of wood immediately above and below the ligature. Secondly, the absolute thickness of the 'autumnal layer' of wood (up to the middle of August, when the increase in diameter of the tree on which the observations were made ceased) was always greater, and generally considerably so, at the spot where the experiment was made, than the normal thickness. In the trees examined (Acer Pseudo-platanus, Salix cinerea, Populus alba, Pavia) the autumnal wood formed at this spot consisted of fibres flattened radially, between which were a smaller number of vessels than in the normal wood; its composition was therefore the same as that of the normal 'autumnal wood.' The normal autumnal wood of Ailantbus glandulosa consists almost entirely of wood-parenchyma-cells flattened radially; while the autumnal wood formed beneath a ligature made in May consisted of a thick layer of flattened fibres, between which a few vessels could be seen. These results show that when the pressure is increased, the formation of the autumnal wood begins at a time when, under normal pressure, a large-celled woody tissue is still being formed.

A diminution of pressure is obtained by making radial longitudinal incisions into the bast-tissue. The strips of bast contract somewhat tangentially, since their tension ceases. Near the incisions the pressure of the bast upon the wood is entirely removed; but in the middle between two adjacent incisions a considerable pressure always remains. The fresh portions of tissue which are formed next to the wounds differ to the greatest extent in their composition from the ordinary structure of the wood. A layer of wood of the ordinary structure is formed, on the other hand, in the portions of the cambium at the greatest distance from the incisions, and afterwards also on the outside of the abnormal portions of tissue. But it is only the tissue consisting of wood formed under artificially diminished pressure that we have at present to consider. The incisions were mostly 2 to $3 \mathrm{~cm}$. long, and were made in the periphery of two- to three-year-old branches at distances of from 4 to $6 \mathrm{~cm}$. in the middle of June and the middle of July, and therefore after the formation of the normal autumnal wood had already begun. The effect of the decrease of pressure was first of all shown, after the branches had been cut off in the middle of August, by a considerably greater increase in thickness at the spots than above or below them. On the transverse sections the thickness of the annual ring was greatest near the incision and decreased gradually from there to the middle points between two incisions. The layer of wood formed after the commencement of the experiment was often more than twice as thick at the former as at the latter spots. For a more exact investigation only those pieces were used in which a layer of distinctly flattened fibres of autumnal wood had been formed before

1 H. de Vries, Flora, I872, No. 16. 
the incision was made. But in all cases (the trees already named) the wood outside this layer of autumnal wood-and therefore all that was formed after the decrease of pressure-consisted of fibres which were not at all flattened radially, but had the same diameter, or even one somewhat greater, than those in the middle of the normal annual ring; it contained also as many vessels, or even more, than the normal wood. At the time therefore when autumnal wood is being formed in the normal parts of the branches, a woody tissue is produced, if the pressure is artificially diminished, agreeing in its structure with the ordinary wood formed in the middle part of the annual ring. For the normal production of autumnal wood it seems therefore necessary for the bark and the bast to exercise a considerably greater pressure on the cambium and the young wood.

These results explain the older experiments of Knight in I8or. He fastened young apple-trees with a stem of about one inch diameter so that the lower part, about three feet long, was immoveable, while the upper part with the foliage could bend under the pressure of the wind. During the period of vegetation the upper moveable part of the stem increased considerably in diameter, the lower fixed part only slightly. This is easily explained if we bear in mind that the swaying of the upper parts of the stem in different directions by the wind must always stretch the bark on the convex side, and therefore eventually relax it; it must thus become looser, and therefore the pressure of the bark at these points is always somewhat less than at the lower and immoveable parts of the tree. This explanation is completely confirmed by the fact that in one of the trees which could be swayed by the wind only in a northerly and southerly direction, the diameter of the stem increased so much in this direction as to bear the proportion of $I_{3}$ to $I_{I}$ as compared with the diameter in the easterly and westerly direction. It is obvious that this explanation is much more probable than that given by Knight himself, who thought the movement of the sap in the wood was promoted by the swaying of the stem caused by the wind.

The great assistance to the increase in diameter of trees afforded by the diminution of the pressure of the bark on the cambium has been long employed in horticulture. The bark of young trees is split from above downwards in summer; cushions of wood are formed at the edges of the incisions, which soon close up the wounds. The use of this process is that from the more rapid increase of the wood in thickness, the conduction of water to the leaves becomes more copious and the loss by transpiration is more easily replaced. The development of the buds and hence the formation of the organs of assimilation will be promoted by the increase of turgidity in the young branches.

2. If the roots of a plant which is transpiring through its leaves can obtain but a small quantity of water from the soil, the turgidity of the tissues in the growing organs will be less than it would be if the roots were surrounded by more moist earth. The immediate result of this will be, that the cells, and therefore also the organs which consist of them (leaves, internodes), will grow more rapidly when the supply of water is increased: in this way the assimilating leaf-surface will be increased, and, as a secondary result, there will be increased assimilation. This, in conjunction with an adequate supply of water, will produce a more vigorous growth of the whole plant, which will thus attain a more considerable size and a greater dry-weight than a plant which cannot obtain a sufficient supply of water. These far-reaching effects of the turgidity produced by an abundant supply of water are very prominent in a series of experiments made by Sorauer (Bot. Zeitg. 1873) upon Barley-plants. These plants were grown so that all the conditions, except the amount of water in the soil, were the same in each case. In one case the amount of water present in the soil was ro per cent., in others $20,40,60$ per cent. of the amount requisite for complete saturation. It appeared the more moist the soil the more fully were the leaves developed, that is, the greater the turgidity of the tissues. With the increase of breadth of the leaves, the number of the fibro-vascular bundles in them increased; and not only was the 
number of the vessels increased, but also that of the cells, so that the more vigorous growth of the cells had induced more numerous divisions. The dry-weight also increased with the moisture of the soil: but if the latter exceeded 60 per cent. of the quantity of water necessary for complete saturation, the dry-weight began to diminish. It appears that in this particular also, as in the case of heat and light, there is an optimum, below which every increase is favourable but above which it is injurious.

SEcr. i 7. Course of the Growth in Length under Constant External Conditions $^{1}$. It has already been explained in the morphological portion of this work that the organs of a plant do not grow simultaneously and uniformly at all points; but that roots and stems always increase slowly in size at the apex, as leaves also do at least at first. The growing-cells multiply by cell-divisions which take place regularly, but do not as a whole exceed a certain size, which is always small. Below this punctum vegetationis, consisting of primary meristem, not only does the differentiation of the homogeneous tissue into layers of different kinds begin, but also a more rapid increase in size of the cells, which do not now divide so often as before. In the parts of the organ which lie further from the punctum vegetationis cell-division ceases altogether (but at different periods in the different layers of tissue), while the growth of the cells still actively continues, until at length, when they have attained their ultimate form and size, the growth of the whole ceases. The cells are then several hundred or even thousand times larger than at the time of their formation beneath the punctum vegetationis. When the growth of stems, leaves, and roots has reached a sufficiently advanced stage of development, we are able therefore to divide their tissue into three regions:-(I) the punctum vegetationis, where new cells are chiefly formed, and increase in size is slow; (2) the portion where the main part of the increase in size takes place, but where there is no longer any cell-division or only to a subordinate extent; this is the elongating portion of the organ; and (3) the portions which no longer grow, at least in length, i.e. the mature portions of the organ. When growth entirely ceases at the punctum vegetationis, as is usually the case with leaves, all the cells continue to enlarge until the whole is mature. If the stem produces a number of closely crowded leaves, as it usually does, at its growing end, the whole of the region in which the chief part of the cell-division takes place is clothed with young leaves, which also themselves consist of cells undergoing division. But as soon as the leaves enter the second stage of development and begin to lengthen, they incline outwards; and when the stem is growing rapidly in length and forming evident internodes (which is by no means always the case) the lengthening begins at those points where it bears the leaves, which also begin to lengthen at the same time; the older mature leaves are generally found on mature internodes. If the internodes are clearly marked off from one another, as is especially the case when the leaves are verticillate or sheathing at their base, each internode forms a more or less individualised whole

1 Ohlert, Längenwachsthum der Wurzel, Linnæa I837, vol. XI. p. 615.-Münter, Bot. Zeitg. 1843, p. I25, and Linnæa, I84I, vol. XV. p. 209.-Griesebach in Wiegmann's Archiv. 1843, p. 267.Sachs, Jahrb. für wissensch. Bot. 1860, vol. II. p. 339.-Müller, Bot. Zeitg. I 869, No. 24.-Sachs, Arbeit. des Bot. Inst. in Würzburg, I872, Heft II. p. I02 ; ditto, Heft III, 1873, and Flora I873, No. 21.-Askenasy, Flora 1873, No I5, [and Verhandl. d. nat.-med. Vereins zu Heidelberg, N. F., Bd. II, 1878 , Ueb. eine neue Methode um die Vertheilung der Wachsthumsintensität zu bestimmen; Strehl, Unters. ueb. das Längenwachsthum der Wurzel, Diss. Inaug., Leipzig 1874.] 
as soon as it emerges from the bud, and different stages of growth may be distinguished in it, advancing from below upwards. This may take place in two different ways, according as the uppermost or lowermost part of an internode remains in an undeveloped condition, the other end being completely mature. This zone which continues for some time in an undeveloped state-cell-division taking place actively in it-is commonly found at the upper end of the internode (as in Phaseolus), and less frequently at the lower end, and this usually when it is enveloped by closely appressed leaf-sheaths or when it is in a bulb, as e.g. in Equisetacex (especially E. hyemale), Umbelliferæ, the bulbous Liliaceæ, the haulms of Grasses, \&c. If the internodes are not sharply defined, as in stems with small leaves and the floral axes of Dicotyledons, the various states of growth which have been described pass insensibly into one another on the stem; and this is always the case with roots. If leaves when once expanded continue to grow for some time, the process is the same as in stems or branches; while the lower portion of the leaf-stalk is fully mature, the upper parts present successively younger or less developed states. The formation of cells finally ceases at the apex and all the parts then become fully mature. This is strikingly the case in Ferns, less so in the pinnate leaves of Papilionaceæ or the incised leaves of Araliacex. But very often the activity of the punctum vegetationis of the leaves lasts for only a short time and its tissue completes its growth while cell-divisions still continue at the base of the leaf, and all the transitional states of growth are to be found between the base and the apex. This occurs, for instance, in the long leaves which grow from the bulbs of Liliaceæ and allied Monocotyledons. When a cell-producing zone of this kind occurs at the base of an internode or of a leaf, with more mature tissue lying above it, the whole organ behaves as if this zone were a punctum vegetationis; the states of growth succeeding one another in the reverse order. Such a zone, intercalated between mature portions of tissue, may be called an Intercalary Vegelative Zone. The growth of the internode or leaf may be termed basipetal, in contrast to the acropetal development where the punctum vegetationis lies at the apex of the internode or leaf.

According as the conditions of growth-temperature, the supply of water, and illumination-are favourable, these phenomena proceed more or less rapidly and uniformly. Every young cell formed at the punctum vegetationis grows and matures more rapidly the more favourable these conditions are. But if the organs are observed under the most constant possible conditions as they emerge from the bud, it is seen that their growth, both in length and thickness, dependent on the gradual development of the cells, does not advance by any means uniformly. The growing portion of a root, internode, or leaf does not lengthen to an equal amount in equal consecutive intervals of time; and the same is the case with stems consisting of a number of internodes, and with each zone, however small, of a growing organ. It is seen in fact that the growth of each part begins at first slowly, becomes gradually more rapid, and finally attains a maximum of rapidity, after which the growth becomes again slower, and finally ceases when the organ is fully mature.

If successive equal intervals of time are represented by $\mathrm{T}_{1}, \mathrm{~T}_{2} \ldots \mathrm{T}_{n}$, and the increments during these intervals by $\mathrm{I}_{1}, \mathrm{I}_{2}, \ldots \mathrm{I}_{n}$, then it may be stated as a general rule that- 


$$
\begin{aligned}
& \text { for } \mathrm{T}_{1} \mathrm{~T}_{2} \mathrm{~T}_{3} \mathrm{~T}_{4} \mathrm{~T}_{5} \mathrm{~T}_{6} \mathrm{~T}_{7} \\
& \text { we shall have } \mathrm{I}_{1}<\mathrm{I}_{2}<\mathrm{I}_{3}<\mathrm{I}_{4}>\mathrm{I}_{5}>\mathrm{I}_{6}>\text { zero. }
\end{aligned}
$$

This rule holds good for the separate zones of roots, internodes, and leaves, as well as for the entire organs from their first formation to the time of their full maturity. This course of growth I have termed The Grand Period ${ }^{1}$, or Grand Curve of Growth; since it is at once evident that if the values $I_{1}, I_{2} \ldots . I_{n}$ are drawn as ordinates with the intervals of time as abscissæ, a curve will be obtained which, starting from the axis of abscissæ, reaches a maximum of elevation, and returns again to the axis. The following examples will render this more clear.

Köppen ${ }^{2}$ found the following increase of length attained in periods of twentyfour hours with a nearly uniform mean temperature :-

\begin{tabular}{|c|c|c|}
\hline & Increase in length. & Mean temperature. \\
\hline First three days: per diem & Io $\mathrm{mm}$. & $17 \cdot 2^{\circ} \mathrm{C}$ \\
\hline Fourth day & 18 & $16 \cdot 6$ \\
\hline Fifth day & 44 & $17 \cdot 1$ \\
\hline Sixth day & $3^{2 \cdot 6}$ & r6.9 \\
\hline Seventh day & $27 \cdot 9$ & $17 \cdot 1$ \\
\hline Eighth day & 28 & $16 \cdot 4$ \\
\hline
\end{tabular}

\section{Roots $^{3}$ of Lupinus albus.}

In an internode of the flowering stem of Fritillaria imperialis I found the following increase in length in each period of twenty-four hours ${ }^{4}$ :-

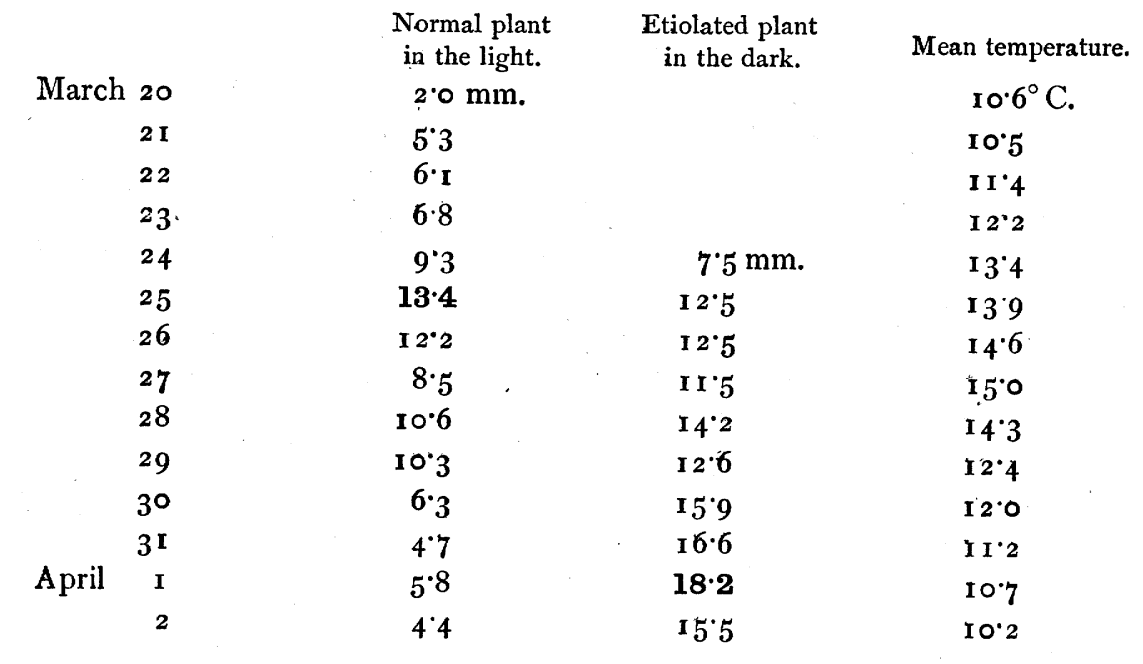

1 'Grand period,' in contrast to the small periodic oscillations of growth which, if represented graphically, would appear as smaller elevations and depressions on the grand curve.

${ }^{2}$ Köppen, l.c. p. $4^{8}$. I have calculated the daily growth from the lengths given in his tables.

3 That is, the root together with the hypocotyledonary portion of the stem.

4 A few irregularities in the course of the growth are explained by the temporary acceleration of the growth from the watering of the ground. Compare the curve in pl. I of the Arbeiten des bot. Inst. in Würzburg, vol. I, Heft II. p. I 29. 


\begin{tabular}{|c|c|c|c|}
\hline & Normal plant & Etiolated plant & Mean temperature. \\
\hline April 3 & $3 \cdot 8 \mathrm{~mm}$. & $14^{\circ} \circ \mathrm{mm}$ & $9 \cdot 4^{\circ} \mathrm{C}$. \\
\hline 4 & $2 \cdot 0$ & $13 \cdot 8$ & $10 \cdot 6$ \\
\hline 5 & $I \cdot 2$ & II'9 & 10.7 \\
\hline 6 & 0.7 & $8 \cdot 8$ & II'O \\
\hline 7 & 0.0 & 4.4 & $11 \cdot 0$ \\
\hline 8 & & $2 \cdot I$ & $11 \cdot 2$ \\
\hline 9 & & 0.6 & $11 \cdot 5$ \\
\hline Io & & 0.0 & 12.5 \\
\hline
\end{tabular}

An internode of Humulus Lupulus gave--

$\begin{array}{rcc} & \begin{array}{c}\text { Increase in length } \\ \text { in } 24 \text { hours. }\end{array} & \begin{array}{c}\text { Mean } \\ \text { temperature. }\end{array} \\ \text { April } 22 & 19^{\circ} 0 \mathrm{~mm} . & 14^{\circ} 9^{\circ} \mathrm{C} . \\ 23 & 25 \cdot 0 & 14.5 \\ 24 & 26.0 & 14.3 \\ 25 & 17 \cdot 2 & 13.9 \\ 26 & 4.8 & 14.1\end{array}$

Harting found that a Hop-stem, consisting of a number of internodes, which was 492 millimetres long on May $15^{\text {th }}$ had attained by the end of August a length of $7 \cdot 263$ metres, this growth being distributed as follows over the different months :-

$\begin{array}{ll}0.492 & \text { metres in April. } \\ 2 \cdot 23^{\circ} & \text { May. } \\ 2 \cdot 722 & \text { June. } \\ 1 \cdot 767 & \text { July. } \\ 0.05^{2} & \text { August. }\end{array}$

These observations and a number of others show that the grand period of growth manifests itself even when the course of the changes of temperature acts in opposition to it; i.e. when the temperature rises while the rapidity of growth decreases owing to internal causes, and vice versá. The course of growth may no doubt be so modified by great changes of temperature that the curve of the grand period can no longer be recognised in the measurements.

In order to determine the grand period of growth in a piece of a growing root, internode, or leaf-stalk, it is sufficient to mark a zone of the organ at the part where elongation is beginning by two lines of indian ink, and to measure the daily (or half daily) growth of this piece until it ceases.

By applying this method to the primary root of Vicia Faba, the temperature varying each day between $18^{\circ}$ and $21{ }^{\circ} 5^{\circ} \mathrm{C}$., I found the following increase to take place in each period of twenty-four hours in a piece originally $1 \mathrm{~mm}$. long situated immediately above the punctum vegetationis :-

$\begin{array}{lr}\text { Ist day } & 1.8 \mathrm{~mm} . \\ \text { 2nd } & 3.7 \\ \text { 3rd } & 17.5 \\ 4^{\text {th }} & 16.5\end{array}$




$\begin{array}{lc}5^{\text {th }} \text { day } & 17.0 \mathrm{~mm} . \\ 6 \text { th } & 14.5 \\ 7^{\text {th }} & 7 \% \\ 8 \text { th } & 0.0\end{array}$

In the same way I found that a piece at first $3.5 \mathrm{~mm}$. long of the first internode of Phaseolus multiflorus beneath the first pair of foliage-leaves, with a daily variation of temperature between $12.75^{\circ}$ and $13.75^{\circ} \mathrm{C}$., showed the following increase :-

$\begin{array}{lc}\text { Ist day } & \text { I.2 } \mathrm{mm} \text {. } \\ 2^{\text {nd }} & \mathrm{I} \cdot 5 \\ 3^{\text {rd }} & 2 \cdot 5 \\ 4^{\text {th }} & 5 \cdot 5 \\ 5^{\text {th }} & 7 \cdot 0 \\ 6 \text { th } & 9 \cdot 0 \\ 7^{\text {th }} & 14 \cdot 0 \\ 8 \text { th } & 10 \cdot 0 \\ 9^{\text {th }} & 7 \cdot 0 \\ \text { 10th } & 2 \cdot 0\end{array}$

Since every organ that is growing in length consists of zones of different ages; which are produced in succession from the primary meristem of the punctum vegetationis (or of an intercalary vegetative zone), the successive zones of an internode or a root indicated by ink-marks must show different increments of growth in equal times. While the zone nearest the punctum vegetationis is beginning to grow, the next one has already entered on a later phase of its grand period, while one at a greater distance would have attained the maximum of its rapidity of growth, and a still further one would have ceased to grow. In other words, a number of, zones below the cell-producing punctum vegelationis are in the ascending phase; while those lying further backwards are in the descending phase of their grand period; or again, each zone is in a later phase of its period of growth the greater its. distance from the punctum vegetationis. If the successive zones of a growing organ are indicated by the figures I, II, III, \&c., and the increments of growth observed; at the same time in each of them by $\mathrm{I}_{1}, \mathrm{I}_{2}, \mathrm{I}_{3}$, \&c.; then we have the following relationship :-

$$
\begin{aligned}
& \text { I II III IV V VI VII VIII } \\
& I_{1}<I_{2}<I_{3}<I_{4}>I_{5}>I_{6}>I_{7}>\text { zero. }
\end{aligned}
$$

There is therefore in the organ a region of maximum rapidity of growth. Thus, for example, I found in the first internode of Phaseolus multiflorus, which was divided into twelve zones, each $3.5 \mathrm{~mm}$. long, in the first forty hours :-

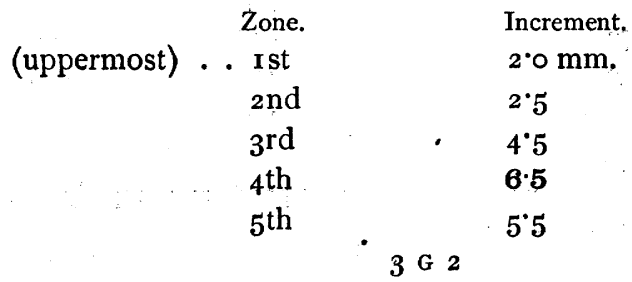




$\begin{aligned} \text { Zone. } & \text { Increment. } \\ 6 \text { th } & 3 \cdot 0 \mathrm{~mm} . \\ 7 \text { th } & 1 \cdot 8 \\ 8 \text { th } & 1 \cdot 0 \\ 9 \text { th } & 1 \cdot 0 \\ \text { I oth } & 0.5 \\ \text { I Ith } & 0.5 \\ \text { (lowest) . . I 2th } & 0.5\end{aligned}$

The maximum rapidity of growth lay therefore in the fourth zone, which was originally situated at a distance of about $10.5 \mathrm{~mm}$. from the upper end of the internode.

As it is usual for several contiguous internodes of stems to be growing at the same time, and the maximum rapidity of growth occurs, according to circumstances, in the second, third, fourth, or fifth internode beneath the bud, the region of most rapid growth is at a considerable distance from the apex of the stem, and especially when the internodes attain a considerable length and several are growing at the same time. In roots, on the other hand, the maximum rapidity of growth occurs much nearer the punctum vegelationis, usually at a distance of only a few millimetres; and the portion of the root beneath its apex in which the chief part of the growth takes place is consequently only a few millimetres long, while in stems with long internodes it is often many centimetres in length. If therefore a root and a stem with long internodes are divided into zones of equal lengths, e.g. I mm., commencing from the punctum vegetationis, the law of growth, as expressed by the general formula given above, is the same in both cases, but with this difference, that in the stem the number of zones that are increasing in length at the same time is much greater than in the root, in consequence of the fact that in the last case each zone completes its period of growth more quickly ${ }^{1}$; its curve is shorter and more abrupt.

Thus, for example, in a primary root of Vicia Faba which grew in damp air and which was divided, starting from the punctum vegetationis, into zones each I $\mathrm{mm}$. in length, I found the following increments of growth in the first twenty-four hours at a temperature of $205^{\circ} \mathrm{C} .:-$

$\begin{array}{cl}\text { Zone. } & \text { Increment. } \\ \text { Ioth } & 0 \cdot 1 \mathrm{~mm} . \\ 9^{\text {th }} & 0 \cdot 2 \\ 8 \text { th } & 0 \cdot 3 \\ 7^{\text {th }} & 0 \cdot 5 \\ 6 \text { th } & 1 \cdot 3 \\ 5^{\text {th }} & 1 \cdot 6 \\ 4^{\text {th }} & 3 \cdot 5 \\ 3^{\text {rd }} & \mathbf{8} \cdot \mathbf{2} \\ 2^{\text {nd }} & 5 \cdot 8 \\ \text { apex } & 1 \cdot 5\end{array}$

1 It by no means however follows from this that the root grows more rapidly, i.e. attains in the same time a greater length than the stem. 
In this case, therefore, the third zone, where the maximum increase of growth took place, was at first at a distance of only $2 \mathrm{~mm}$. from the apex.

It is clear that if an organ is divided into zones of small length, each zone will in general contain a larger number of cells the nearer it is to the punctum vegetationis, since the cells are longer the further they are from the apex. But from the point where growth ceases the number of cells in the successive zones of an organ of uniform structure will be the same. If therefore the zones are again designated by the numbers I, II, III, \&c., the number of cells in them by $N_{1}, N_{2}$, $\mathrm{N}_{3} \ldots \mathrm{N}^{\mathrm{n}}$, then we have :-

$$
\begin{array}{ll}
\text { I } & \text { II } \\
N_{1}>N_{2}>N_{3}>N_{4}>N_{5}>N_{6}>N_{7}=N_{8} .
\end{array}
$$

But the difference in the number of cells in the zones is very far from being the cause of the difference in the rapidity of growth that prevails in them; as is seen at once if it is recollected that the number continually decreases from the apex throughout the growing region, while the rapidity of growth first increases and then decreases. This may be expressed by the following formula:-

$$
\begin{aligned}
& \text { I II III IV } \mathrm{V} \text { VI VII VIII } \\
& \mathrm{N}_{1}>\mathrm{N}_{2}>\mathrm{N}_{3}>\mathrm{N}_{4}>\mathrm{N}_{5}>\mathrm{N}_{6}>\mathrm{N}_{7}=\mathrm{N}_{8} \text {. } \\
& \mathrm{I}_{1}<\mathrm{I}_{2}<\mathrm{I}_{3}<\mathrm{I}_{4}<\mathrm{I}_{5}>\mathrm{I}_{6}>\mathrm{I}_{7}>\text { zero. }
\end{aligned}
$$

If it were possible to divide in the same manner a filament of Vaucheria, a root-hair of Marchantia, or a similar unicellular organ, into small zones, it can -scarcely be doubted (as we may conclude from other circumstances dependent on growth) that we should find the same law to regulate the distribution of the rate of growth in individual cells endowed with a power of apical growth. Since the same law applies to roots and stems-whether zones 1 or 2 millimetres or, in the case of stems, $\mathbf{I}$ or 2 centimetres in length are observed-it is to be expected that this formula would hold good also if zones of only a tenth or hundredth, or even thousandth of a millimetre could be marked out and measured. In other words, we should find that the law of the grand period holds good for each single minute area of the surface of the wall of a young cell.

If the power of any particular zone to attain a definite length is called its Energy of Growth, then a zone which up to the time when its growth ceases reaches a length of ro mm. would have a smaller energy than one which continues to grow until it has reached a length of $100 \mathrm{~mm}$. Thus, for example, the successive internodes of most stems each of which was at one period $1 \mathrm{~mm}$. long, differ very greatly in length when mature; the internodes first formed are short, the next longer, and finally we have one the longest of all, followed again towards the apex by shorter ones. If we designate the energy of growth of the internodes I, II, III, \&c., by $\mathrm{E}_{1}, \mathrm{E}_{2}, \mathrm{E}_{3}$, \&c., we get the series-

$$
\begin{aligned}
& \text { I II III IV IV V VI VII VIII } \\
& \mathrm{E}_{1}<\mathrm{E}_{2}<\mathrm{E}_{3}<\mathrm{E}_{4}>\mathrm{E}_{5}>\mathrm{E}_{6}>\mathrm{E}_{7}>\mathrm{E}_{8} .
\end{aligned}
$$

With this increase and decrease in the energy of growth of the various internodes of a stem is usually associated a similar relationship between the size of their 
leaves, the lower ones forming smaller, the upper ones larger leaves, and then a largest of all (or whorl of largest leaves), usually followed again by smaller ones ${ }^{1}$. The secondary roots also which spring from the same primary root show similar relationships, the first attaining a smaller length than those that follow, and these being again followed by a graduated succession of shorter ones. The same is the case also with the lateral branches of the stems of annual plants, as well as of trees, especially when the order of development is distinctly monopodial.

It seems probable that an investigation of the zones of a root, stem, or leaf would also show that the energy of growth of successive zones first increases, then reaches a maximum, and finally decreases. The cells in the zone in which the maximum energy of growth prevails would also be the largest, while their number would be least. This hypothesis is in harmony with Sanio's measurements ${ }^{2}$ of the wood-cells of Pinus sylvestris; for he found that the final constant size of the woodcells of the stem varies, increasing gradually from below upwards, till it attains a maximum at a definite height, and then again decreases towards the apex. The same is the case with the branches.

If it be permissible to ascribe a special energy of growth to each separate zone of an organ, it becomes possible to understand how it is that, as is actually the case, every zone has its separate period of growth, and that a grand period for the whole organ itself may be determined. The maxima of rapidity of growth attained in the successive zones first rise and then fall; the duration of growth also of the zones probably at first increases and afterwards diminishes. Consequently the measurements of the whole organ represent the sum at first of only few and small partial increments, later of more numerous and larger ones, until finally the sum of the partial increments diminishes, because the number of zones growing at any one time and the energy of their growth alike diminish. Further investigation will show whether this hypothesis, which is at least an approximate one, is correct.

If the increments of length of an internode, stem, or leaf, in short intervals of time such as half-an-hour or an hour, are compared, it is usually found that they do not increase and then decrease regularly, but irregularly, the growth being sometimes greater, sometimes smaller. If the grand curve of growth is constructed directly from them, it does not assume the form of a continuous curve, but shows a number of small zigzags, which however disappear, if, for example, the interval is extended from one to three hours or more. These phenomena I call irregular variations of growth ${ }^{3}$. They appear to result from the plant being subjected to continual small variations of temperature, air, light, and moisture of the soil, which alter the turgidity, and therefore the extensibility and elasticity of the growing cells. I come

1 This phenomenon has not at present been sufficiently investigated. "In many stems, especially creeping ones, when the leaves have reached a certain size, this size remains constant in a long series of leaves before any decrease occurs.

${ }^{2}$ Jahrb. für wissensch. Bot. 1872 , vol. VII. p. 402. By a 'constant' size of the wood-cells I understand that which they possess in the later annual rings; in the inner annual rings they gradually increase, until in the following ones they attain a constant size.

${ }^{3}$ For further details see Reinke, Verhandl. des bot. Vereins fuir die Provinz Brandenburg, Jahrg. VII ; and Sachs, Arbeit. des bot. Inst. in Würzburg, Heft II. p. I०3. [See also Drude, Die stossweisen Wachsthumsaenderungen in der Blattentwickelung von Victoria regia, Halle $\mathrm{I} 88 \mathrm{I}$.] 
to this conclusion from observing that irregular variations of growth become less the more the plant is protected from variations in the surrounding conditions. Partial irregular neutralisations of the tension of the tissues may also cooperate to produce this result.

SECT. 18.-Periodicity of Growth in length caused by the alternation of day and night. The alternation of day and night implies varying combinations of the conditions of plant-life, especially of those that affect growth. Day and night are distinguished not only by the presence and absence of sunshine, but also by a consequent higher and lower temperature, which again causes variations in the moisture of the air. Independently of special meteorological phenomena, the temperature falls daily with the diminishing elevation of the sun till sunrise the next day, that of the air rapidly, that of the ground more slowly; at sunset the fall is sudden, as is the rise at sunrise. In general the atmosphere approaches a state of saturation as the temperature falls, i.e. the hygrometric difference decreases, as it increases with the rising temperature. But these general daily alternations act in a variety of ways, and even in opposite directions on the growth of plants; the increasing intensity of the light after sunrise retards growth, while the increasing temperature promotes it, as long as the other conditions remain the same; but the increase of the hygrometric difference caused by the increasing temperature of the air occasions also an increase of transpiration, which effects a diminution of the turgidity of the tissues, and this again retards growth.

It is important to ascertain which of these variable causes exercises the greatest influence on growth; and it will depend on this whether the growth of the plant is most rapid by day or by night. On a cloudy but warm and damp day the weak light has only a slightly retarding effect, but the temperature and the great amount of moisture greatly promote growth; under these circumstances the growth may be greater than in the succeeding night (equal periods of time being compared), when the total absence of light promotes growth, but the lower temperature is less favourable to it. But the proportion may be reversed; the plant may grow more slowly by day than by night when the difference in the temperature and moisture of the air during each is but small and very bright days intervene between dark nights, the intense light retarding growth by day more than the depression of the temperature by night.

The greatest variety of combinations may be imagined in this respect; and from the extreme changeableness of the weather the plant will, according to circumstances, sometimes grow more quickly by day, sometimes by night, without exhibiting any exactly recurrent periodicity. The numerous observations which have been made in this direction do not therefore point to any general law ${ }^{1}$. It has however been ascertained that, especially when long periods of time such as entire days are

1 These will be found described by me in detail in the Arbeiten des bot. Inst. in Wuirzburg, I872, p. I 7o. [Baranetzky (Die tägliche Periodicität im Langenwachsthum der Stengel, Mém. de l'Acad. imp. de St. Pétersbourg, XXVII, I879) finds that there is a daily periodicity of the growth of stems which is independent of the direct influence of any external conditions: (see also Bot. Zeitg. I877).] 
compared, all the other conditions of growth are outweighed by the effects of the variations of temperature, so that in general the rapiclity of growth increases with a rising and decreases with a falling temperature. The result of a number of measurements made by Rauwenhoff during several months in the most various weather was that the mean growth was greater in twelve hours of the day than in twelve hours of the night; viz.

$\begin{array}{lll} & \text { By day. } & \text { By night. } \\ \text { in Bryonia } & 59^{\circ} \circ \mathrm{p} . \mathrm{c} . & 4 \mathrm{I}^{\circ} \circ \mathrm{p} . \mathrm{c} . \\ \text { Wistaria } & 57^{\circ} 8 & 42^{\circ} \cdot 2 \\ \text { Vitis } & 55^{\circ} \mathrm{I} & 44^{\circ} 9 \\ \text { Cucurbita } & 5^{\circ} \cdot 7 & 43^{\circ} 3 \\ \text { do. } & 57: 2 & 42^{\circ} \cdot 8 \\ \text { Dasylirion } & 55^{\circ} 3 & 44^{\circ} 7\end{array}$

A similar tabular statement shows that the favourable influence of a higher temperature by day outweighs the retarding influence of daylight. Rauwenhoff's measurements show accordingly that the mean growth during six hours of the forenoon is less than that during six hours of the afternoon; since, while the average amount of light is the same, the temperature is higher in the afternoon than in the forenoon. If the afternoon growth is placed at roo, then the morning growth is -

$\begin{array}{ll}\text { in Bryonia } & 86 \\ \text { Wistaria } & 7 \text { I } \\ \text { Vitis } & 67 \\ \text { Cucurbita } & 79 \\ \text { do. } & 8 \text { I }\end{array}$

If however we calculate from Rauwenhoff's measurements the daily and nightly and the morning and afternoon values for shorter periods in which the changes of the weather do not neutralise one another, it will be found that the growth by night sometimes exceeds that by day, and that the afternoon is not always more favourable than the morning.

It is clear from what has been said that it is impossible to determine from observations in the open air, where the variations of temperature, light, and moisture are very great and are combined in a great variety of ways, in what manner each separate condition of growth affects the plant, and whether the alternation of day and night causes a similar alternation of growth, or whether there exist in the plant itself causes of daily periodicity independently of external changes. In order to decide this question, it is necessary first of all to make the observations independent of the accidents of weather, which is only possible by carrying them on in well-closed rooms where the temperature can be kept constant or made to vary, and where the amount of light can be increased or decreased, and the moisture regulated in the air and in the soil of the flower-pot. Under these circumstances it is possible to study the action of an increasing or decreasing amount of light upon a plant exposed to constant conditions of humidity and temperature, and therefore exhibiting a constant degree of turgidity; it is sufficient to measure and compare the increments of growth during short periods of time. 
A long series of observations of this kind on internodes has given me the following results ${ }^{1}$ :-

(I) The more exactly a constant temperature is maintained, darkness being constant and the amount of moisture being also constant, the more uniform is the course of growth at different periods of the day. There does not appear to be any daily periodicity of growth independent of external influences. The irregular variations of growth mentioned above were however observed.

(2) If great variations of temperature are allowed to act on a plant growing in darkness and with a constant amount of moisture, to such an extent that the temperature of the air round the plant alters some degrees C. from hour to hour, the rate of growth of the internodes rises and falls with the rising and falling temperature. If the hourly increments are taken as ordinates, and the intervals of time as absçissæ, the curve of growth follows all the elevations and depressions of the curve of temperature, without however any actual proportion being observable between the growth and the temperature; the curves do not run parallel but are only of the same description.

(3) If care is taken that during the period of observation the temperature undergoes only slight and gradual changes, while (the moisture being sufficiently uniform) the amount of light changes in the ordinary manner, increasing from morning till midday and decreasing from midday till evening to complete darkness at night, it will be found that the increments of growth are always greater from evening till sunrise, diminishing suddenly after sunrise, and then more slowly till evening. The alternation of day and night causes therefore under these circumstances a periodical rising and falling of the curve of growth of such a nature that a maximum occurs in the morning at sunrise and a minimum before sunset. A second rising of the curve of growth usually takes place also in the afternoon; but this, as I have shown, is a consequence of the higher temperature in the afternoon which overcomes the influence of light. The retarding influence of light is therefore strong enough to overbalance the favourable influence of the slight elevation of temperature in the forenoon, but not sufficient to overcome that of the stronger elevation of temperature in the afternoon.

The fact is of great interest that when a plant has been exposed to light during the day, its curve of growth after sunset, or if placed in the dark in the evening, does not immediately rise abruptly; i.e. that the most rapid growth which is independent of light is not at once attained when it is suddenly placed in the dark; but that-as is shown by the curve rising slowly till morning-the growth which has been retarded during the day only becomes gradually more rapid in the course of some hours, until the light to which the plant is again exposed in the morning causes a fresh retardation of growth, which again increases from hour to hour till the slowest rate is attained in the evening, if the temperature remains constant. In other words, the two internal conditions of the plant which correspond to darkness on the one hand and to daylight on the other hand pass over only gradually into one another. Light

1 Sachs, Arbeit. des bot. Inst. Würzburg, I872, vol. I, p. 168 et seq. The plants observed were chiefly Fritillaria imperialis, Humulns Lupulus, Dahlia variabilis, Polemoniam reptans, and Richardia athiopica. 
requires a considerable time in order to overcome the nocturnal, darkness a considerable time to overcome the diurnal condition of growth of the plant. If this were not the case, the curve of growth would at once rise abruptly in the evening when the room is suddenly darkened, would then continue at the same elevation till morning, would fall abruptly when light is again let in, and would then continue at the same height till the evening. But this does not correspond to the observed phenomena.

In order to study more closely the changes of growth occasioned by internal causes, or the dependence of these changes on external conditions, it is necessary to measure

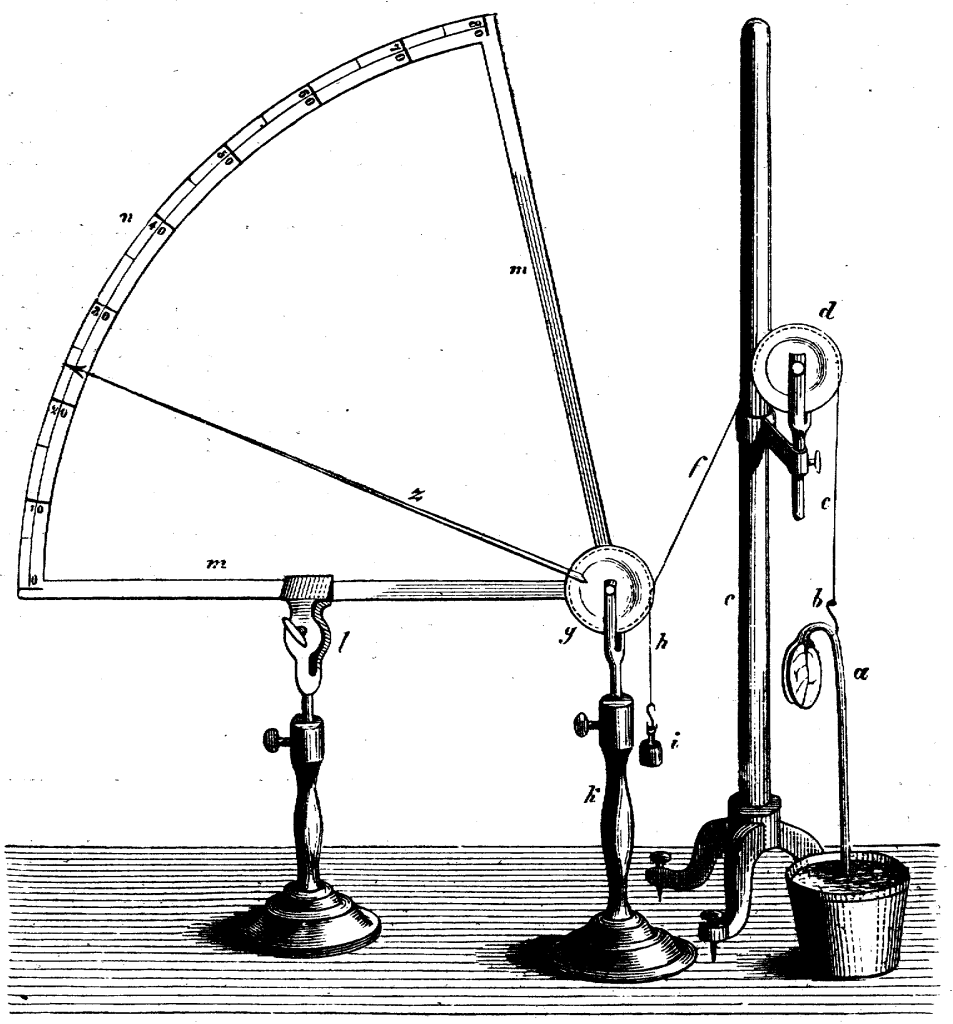

FIG. 480.-Arc-indicator, or apparatus for measuring the growth in length of a plant during short periods of time.

the increments in short spaces of time such as an hour or two or three hours. In the case of internodes or leaves of large plants which are growing very rapidly, as the flowerstems of Agave or the leaves of Musacex, this can be done with a certain degree of exactness by simple measurement with a measuring-rod. But for the purpose of more exact observations it is more convenient to make use of smaller plants which do not grow so rapidly, the growth during an hour not amounting to more than a millimetre, or even less. In such cases a simple measuring-rod is not sufficiently exact; and I have employed in its place three different methods. In each of them a thin but strong thread of silk is fixed to the upper end of the stem or internode of the plant growing in a 
pot, the thread passing vertically over an easily moveable pulley and moving an index fixed to the free end of the thread or to the pulley.

x. The Thread-indicator is a simple contrivance in which the free end of the thread

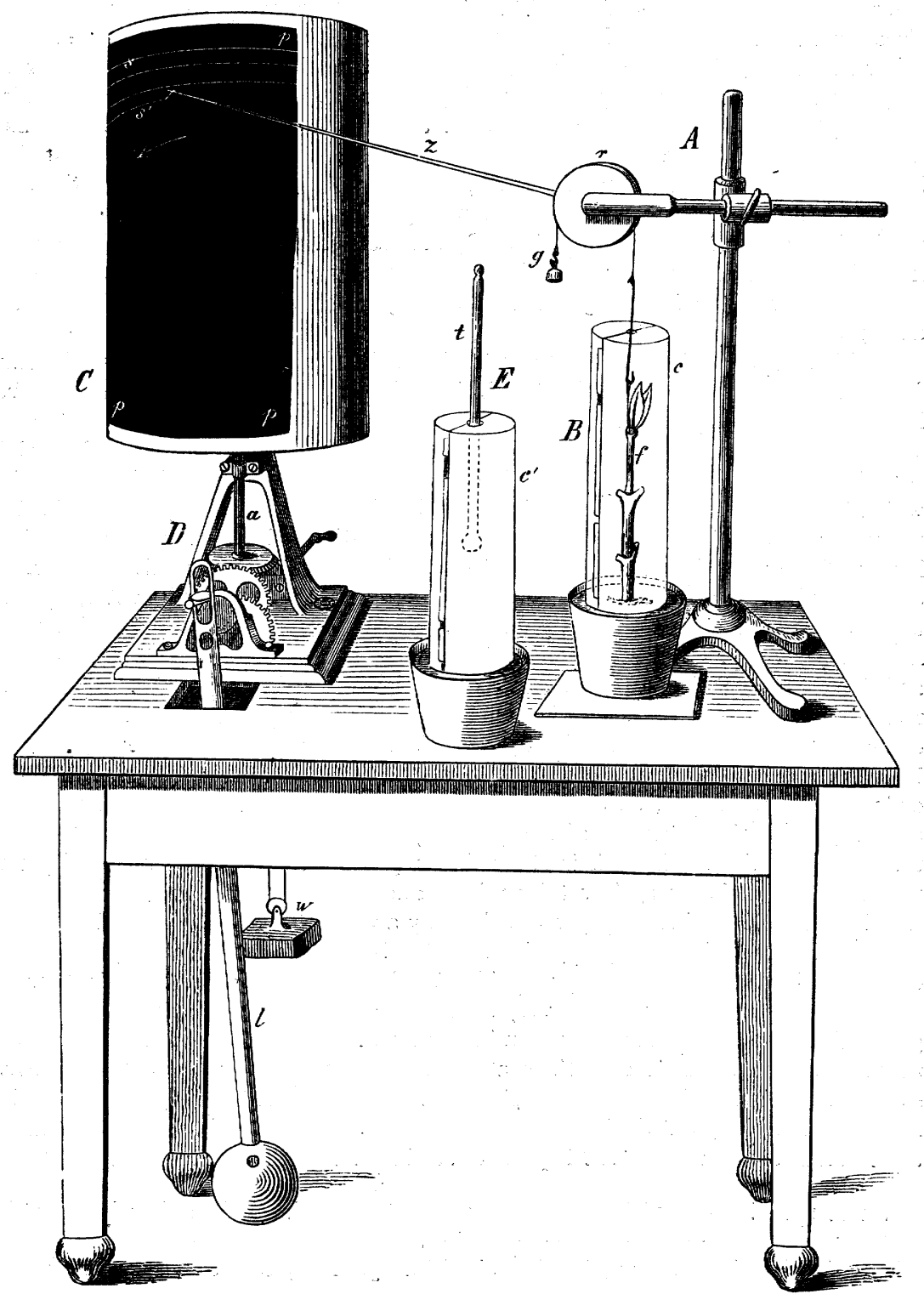

FIG. 48r.-Autographic Auxanometer, for recording the growth in length during short periods of an internode of a growing plant $f ; s s^{\prime}$ the lines scored by the index $z$ on the blackened paper $p$ fixed to the cylinder $C$ which is made to rotate eccentrically by means of the clock-work $D$.

which hangs down from the pulley and is kept tight by a weight of a few grammes carries a horizontal needle which moves freely over a graduated scale as the end of the thread which is fixed to the plant rises with its growth. 
2. In the Arc-indicator the thread $c f$ (Fig. 480) fixed to the plant $a$ is carried over the pulley $d$ and fixed to a pin which is attached to a second pulley $g$. An index $z$ made of a straight and firm straw is fastened to this second pulley in the radial direction, its free end pointing to a graduated scale on the arc of a circle $m n$. The equilibrium of the index is secured by the small weight $i$ which tends to turn the pulley in the opposite direction with a force which keeps the thread $c f$ in a state of tension. As the internode below the hook $b$ lengthens, the weight $i$ sinks, and a piece of the thread $c f$ of equal length is rolled off the pulley $g$, thus raising the index on the arc. If the index is, for example, ten times as long as the radius of the pulley, the portion of the arc which it will pass over represents ten times the increase in length of the internode. But since it is not usually required to know the absolute amount of the increase but only the relative amount in different times, it is sufficient merely to read off and compare the movements of the index on the graduated scale. By this instrument we are able to measure very small increments of growth; but, like the first process, it has the disadvantage that the observer must watch it during the whole time, which renders the investigation very difficult, especially at night.

3. The Autograpbic Auxanometer gets rid of this difficulty. It consists of a simpler form of the instrument already described. The thread $f$ fastened to the plant sets directly in motion the pulley which carries the index $z$, being fixed to it by a pin at $r$. The tension of the thread caused by the index itself is still further increased by the weight $g$. By this contrivance the point of the index falls as the stem grows below the point to which the thread is fastened. By means of the clock-work $D$ the cylinder $C$ fixed upon the vertical axis $a$ is made to rotate slowly, the rotation being arranged by adjusting the length of the pendulum $l$ so that a revolution is completed in exactly an hour. The cylinder is however fixed eccentrically on the axis $a$, so that during the rotation one side describes a larger circle than the other side. On the former side is fastened a piece of smoked paper $p p$. When the index is properly adjusted, its point touches the paper and describes on it a white line $s s^{\prime}$ during the rotation of the cylinder. But after the rotation has continued for some time the index is no longer in contact with the paper owing to the eccentricity of the cylinder, but becomes so again afterwards when it inscribes another line lower down. The distances between the lines described on the cylinder evidently depend on the rapidity of growth of the plant ${ }^{1}$. When, in consequence of this growth, the index has, after say twenty-four hours, reached the lower margin of the paper $p p$, the clock-work is stopped, the paper removed and replaced by a fresh piece, the index being again set by raising the pulley, and the observation repeated. The lines on the blackened paper are fixed by a varnish of collodion and dried, and the distances between them are proportional to the hourly growths of the internode. It is clear that the apparatus not only magnifies the increments, but also records them in the absence of the observer, which is very convenient, especially for observing the nocturnal growth. It is however necessary even in this case for the observer to note the temperature and the hygrometric conditions, at least between morning and evening. Fig. $48 \mathrm{I}$ shows in addition a tin vessel $B$, consisting of two halves united by a hinge, which may be used for shutting out the light from the plant, even after the thread has been attached to it. At $E$ the thermometer $t$ is placed in a similar vessel near the plant.

Sect. 19.-Effect of Temperature on Growth ${ }^{2}$. It has already been shown

1 See Arbeiten des Würzburg. bot. Inst., Heft II. [Wiesner, Ueb. eine neue Construction des selbstregistrirenden Auxanometers, Flora 1876.]

${ }^{2}$ F. Burkhardt in Verhandl. der naturf. Ges. in Basel, $185^{8}$, vol. II. I, p. $67 .-$ Sachs, Jahrb. für wissensch. Bot. 1860, Heft 1I. p. 338.-Alph. De Candolle in Biblioth. univ. et rev. Suisse, Nov. 1866. -H. de Vries, in Archiv. néerlandaises, I87o, vol. V.-Köppen, Wärme und Pflanzen-Wachsthum, Dissertation, Moskow 1870. [See also Haberlandt, Landw. Versuchsstationen, XVII, 1874 ; Just, Cohn's Beiträge, II, I877; Uloth, Flora, $187 \mathrm{I}$ and 1875 ; von Höhnel, in Haberlandt's W'iss. prakt. Unters. II, I $877 \cdot]$ 
in Sect. 7 that the life of a plant generally and its growth in particular is carried on only within certain limits of temperature (in general between zero and $50^{\circ} \mathrm{C}$.), and that each function has apparently in every plant its inferior and superior limits; so that, for example, the lowest temperature at which a plant of Wheat can grow is different from the lowest at which a Gourd can grow, \&c. It has also been shown that growth, like other phenomena, is more active the higher the (constant) temperature above the inferior limit, but that there is a certain temperature at which growth reaches its maximum activity, and above which any further rise of temperature causes a diminution of its rapidity. There is not, in the mathematical sense of the term, any proportion between the rapidity of growth and the height of the temperature, and the more accurately the relation between the two has been investigated, the more difficult is it to express this relation by any mathematical formula. It cannot, on the other hand, be doubted that it is of the utmost importance for any future theory of the mechanical laws of growth to ascertain the extent to which growth depends on temperature, at least in a few particular cases.

The difficulties of investigations of this kind are however much greater than is generally thought; and the results obtained hitherto, valuable as they are, go no further than what is stated above, and give us no deeper insight into the way in which that particular mode of motion of the molecules which we call heat is connected with that mode of motion which causes growth.

Restricting ourselves to the results at present obtained, it will be seen that they have a great practical value in addition to their theoretical significance. A knowledge of the cardinal points of temperature, viz. its superior and inferior limits and the particular temperature at which the maximum of action takes place, is indispensable to investigations of various kinds, in order to get at a correct interpretation of the phenomena. On this account a few of the more trustworthy observations may be given here.

In order to determine the cardinal points of temperature to which allusion has been made, observations are of value only when conducted at nearly constant temperatures; the means deduced from very variable temperatures may, as I have shown, lead to very erroneous conclusions. It is however by no means easy to maintain a sufficiently constant temperature for a whole day even by artificial heating or cooling. Special difficulty is met with in the determination of the inferior limit or specific zero, since the observation must extend over a considerable time-in the case of germination, several weeks-to be certain that growth does not take place. It would be possible, by means of the apparatus already described, to determine in the course of a few hours whether growth still takes place in an internode at a very high or at a very low temperature, and at what temperature it is the most rapid, if it were not extremely difficult to regulate the temperature of the plant in the apparatus with sufficient exactness. The auxanometer will however be very, useful even in this case. The observations on this point hitherto made, at least those which have any physiological value, have been on germinating seeds, as the temperature and moisture of the soil in which they grow can be more easily regulated than of the air in the case of internodes. Special facilities are offered by the roots of seedlings, as they do not emerge from the soil, and are more easily measured, from their simpler and more regular form. The following figures refer only to the roots of seedlings, the hypocotyledonary portion of the stem being also, in the case of Dicotyledons, included in the root. That exactly the same figures are not always obtained by different observers is the result of differences in the mode of observation, the amount of water, the nature of the soil, the inaccuracy of thermometers, \&c. 
The first point to determine is, whether germination--i.e. the growth of the embryo at the expense of the reserve materials in the seed-takes place only at certain temperatures, and at what temperature it takes place most quickly. Observations of my own gave the following results :--

Triticum vulgare
Hordeum vulgare
Cucurbita Pepo
Phaseolus multiflorus
Zea Mais

$\begin{array}{cc}\text { Inferior limit. } & \text { Most rapid growth. } \\ 5^{\circ} \mathrm{C} . & 287^{\circ} \mathrm{C} . \\ 5 & 28{ }^{\circ} 7 \\ 13^{\circ} 7 & 33^{\circ} 7 \\ 9^{\circ} 5 & 33^{\circ} 7 \\ 9^{\circ} 5 & 33^{\circ} 7\end{array}$

Superior limit.
$42^{\circ} 5^{\circ} \mathrm{C}$.
$37^{\circ} 7$
$46 \cdot 2$
$46 \cdot 2$
$46^{\circ} \cdot 2$

This table shows, if the ascertained temperatures are correct, that grains of Wheat cannot germinate below $5^{\circ} \mathrm{C}$., or seeds of the Gourd below $13^{\circ} 7^{\circ}$, \&c., however long they may lie in moist earth; and that they no longer germinate, but quickly perish at temperatures above those named in the third column; while at the temperatures named in the second column germination takes place in a shorter time than at either higher or lower temperatures. It may however be taken for granted, from the great difficulty of obtaining these numbers, that the result of further observations will not be identical, though probably approximate. It is clear that many series of experiments will be necessary in order to determine each of the cardinal points. The following figures, obtained by Köppen, agree moderately well with mine, as far as they relate to the same plants :-

$\begin{array}{lcc} & \text { Inferior limit. } & \text { Most rapid growth. } \\ \text { Triticum vulgare } & 75^{\circ} \mathrm{C} . & 29^{\circ} 7^{\circ} \mathrm{C} \text {. } \\ \text { Zea Mais } & 9^{\circ} 6 & 3^{\circ} 4 \\ \text { Lupinus albus } & 7.5 & 28.0 \\ \text { Pisum sativum } & 6.7 & 26.6\end{array}$

The following figures were obtained by $\mathrm{H}$. de Vries:-

\begin{tabular}{|c|c|c|}
\hline Phaseolus vulgaris & $\begin{array}{c}\text { Most rapid growth. } \\
315^{\circ} \mathrm{G} \text {. }\end{array}$ & $\begin{array}{l}\text { Superior limit. } \\
\text { above } 4^{2} 5\end{array}$ \\
\hline Heliantbus annuus & $3 I \cdot 5$ & below $42^{\circ} 5$ \\
\hline Brassica Napus & $31 \cdot 5$ & $42 \cdot 5$ \\
\hline Cannabis sativa & $31^{\circ} 5$ & above $42^{\circ} 5$ \\
\hline Cucumis Melo & $37 \cdot 5$ & \\
\hline Sinapis alba & $27^{\circ} 4$ & above $37^{\circ} 2$ \\
\hline Lepidium sativum & $27^{\circ} 4$ & below $37^{\circ} 2$ \\
\hline Linum usitatissimus & $27^{\circ} 4$ & above $37^{\circ} 2$ \\
\hline
\end{tabular}

The following results ${ }^{1}$, obtained by Alphonse de Candolle, are moderately trustworthy as far as relates to the inferior limit, but hardly so much so with respect to the superior limit and the temperature of most rapid growth, as may be concluded from various statements made by the observer.

\begin{tabular}{|c|c|c|c|}
\hline Sinapis alba & $\begin{array}{l}\text { Inferior limit. } \\
0^{\circ} 0^{\circ} \mathrm{C} \text {. }\end{array}$ & $\begin{array}{r}\text { Most rapid growth. } \\
\qquad 2 \mathbf{I}^{\circ} \mathrm{C} .\end{array}$ & $\begin{array}{l}\text { Superior limit. } \\
\qquad 28^{\circ} \mathrm{C} .\end{array}$ \\
\hline Lepidium sativum & $I \cdot 8$ & $2 \mathrm{I}$ & 28 \\
\hline Linum usitatissimum & $r \cdot 8$ & $2 \mathrm{I}$ & 28 \\
\hline Collomia coccinea & $5^{\circ} 0$ & 17 & about 28 \\
\hline Nigella sativa & 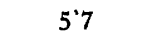 & above 2 I (?) & $\Rightarrow \quad 28$ \\
\hline 1beris amara & $5 \cdot 7$ & & \\
\hline Trifolium repens & $5 \cdot 7$ & $21-25$ & below 28 \\
\hline Zea Mais & $9^{\circ} \circ$ & $21-28$ & about $35^{2}$ \\
\hline Sesamum orientale & $13^{\circ} 0$ & $25-28$ & below 45 \\
\hline
\end{tabular}

1 I take the figures from the table of curves in De Candolle's treatise, with the assistance of the text.

${ }^{2}$ De Candolle remarks that the seeds of Maize, Melon, and Sesamum become brown, the first 
When De Candolle's inferior limits are below $5^{\circ} \mathrm{C}$., they are most probably correct ; his superior limits and temperatures of most rapid growth are, on the other hand, for the most part certainly too low.

More accurate information is afforded by the figures which give the lengths attained by roots in equal periods of time at different temperatures, and express therefore the rate of the growth of the roots of seedlings at different constant temperatures. These numbers increase from the inferior limit to the temperature of most rapid growth, and fall again from it to the superior limit.

In Zea Mais, for example, I found-

in $2 \times 48$ hours
48
48
48
48
48

$\begin{array}{lc}\text { Temperature. } & \text { Length attained by the root. } \\ 177^{\circ} \mathbf{1}^{\circ} \mathrm{C} . & 2{ }^{\circ} \mathrm{mm} . \\ 26.2 & 24^{\circ} 5 \\ 33^{\circ} 2 & 39^{\circ} \\ 34^{\circ} \circ & 55 \cdot 0 \\ 3^{\circ} \cdot 2 & 25^{\circ} \cdot 2 \\ 4^{\circ} 5 & 5{ }^{\circ} 5\end{array}$

Köppen obtained the following growth in length of roots in periods of forty-eight hours :-

\begin{tabular}{|c|c|c|c|}
\hline $\begin{array}{l}\text { Temperature. } \\
14^{\circ} 1^{\circ} \mathrm{C} \text {. } \\
18^{\circ} 0^{\circ}\end{array}$ & $\begin{array}{l}\text { Lupinus albus. } \\
9^{\circ} \mathbf{I} \mathrm{mm} . \\
\mathbf{1} \mathbf{I}^{\circ} 6\end{array}$ & $\begin{array}{l}\text { Pisum sativum. } \\
5^{\circ} 0^{\circ} \mathrm{mm} \text {. } \\
8.3\end{array}$ & $\begin{array}{l}\text { Zea Mais. } \\
\mathrm{I} \cdot \mathrm{I} \mathrm{mm}\end{array}$ \\
\hline $\begin{array}{l}23.5 \\
26.6\end{array}$ & $\begin{array}{l}3 \mathrm{I}^{\circ} 0 \\
54 \cdot 1\end{array}$ & $\begin{array}{l}30 \cdot 0 \\
53 \cdot 9\end{array}$ & $\begin{array}{l}10.8 \\
29.6\end{array}$ \\
\hline $28 \cdot 5$ & $50^{\circ} \mathrm{I}$ & $40^{\circ} 4$ & 26.5 \\
\hline $30^{\circ} 2$ & $43^{\circ} 8$ & $38 \cdot 5$ & $64^{\circ} \cdot 6$ \\
\hline $33^{\circ} 5$ & $14^{2}$ & $23^{\circ} \circ$ & 69.5 \\
\hline $3^{6 \cdot 5}$ & 12.6 & $8 \cdot 7$. & $20^{\circ} 7$ \\
\hline
\end{tabular}

The following are De Vries' results, also in periods of forty-eight hours:-

\begin{tabular}{|c|c|c|c|c|}
\hline $\begin{array}{l}\text { Temperature. } \\
15^{\circ} \mathrm{I}^{\circ} \mathrm{C} \text {. } \\
2 \mathrm{I}^{\circ} 6\end{array}$ & Cucumis Melo. & $\begin{array}{l}\text { Sinapis alba. } \\
\quad 3^{\circ} 8 \mathrm{~mm} \text {. }\end{array}$ & $\begin{array}{l}\text { Lepidium sativum. } \\
5^{\circ} 9 \mathrm{~mm} \text {. } \\
38^{\circ} 9\end{array}$ & $\begin{array}{l}\text { Lirum usitatissimum } \\
\mathrm{I}^{\circ} \mathrm{I} \mathrm{mm} . \\
20^{\circ} 5\end{array}$ \\
\hline $27^{\circ} 4$ & $18.2 \mathrm{~mm}$ & $52 \cdot 0$ & $71 \cdot 9$ & $44 \cdot 8$ \\
\hline $30 \cdot 6$ & $27^{\circ} \mathrm{I}$ & $44^{\circ} \mathrm{I}$ & $44^{\circ} 6$ & $39^{\circ} 9$ \\
\hline $33^{\circ} 9$ & $38 \cdot 6$ & 30.2 & $26 \cdot 9$ & $28 \cdot I$ \\
\hline $37^{\circ} 2$ & $70 \cdot 3$ & $100^{\circ}$ & 0.0 & $9^{\circ} 2$ \\
\hline
\end{tabular}

The assertion made by Köppen, in support of which he brings forward an array of figures, that similar parts of plants grow at different rates at the same mean temperature, whether the mean temperature is constant or whether it varies above and below the mean, and further that the rapidity of growth is diminished by the variations of the temperature even when the variations take place below the optimum, was inserted in the third edition of this book. This assertion, however, has not been confirmed by the careful observations made in different ways by Pedersen in the laboratory at Würzburg ${ }^{1}$. We shall see in Sect. 26 that variations of temperature act as stimuli which affect the rapidity of growth of many foliage and floral leaves in a remarkable manner. A thorough investigation of the subject from this point of view is much to be desired.

as if burnt at $40^{\circ \circ} \mathrm{C}$, a phenomenon which has not been noticed by others. These 'burnt' seeds however germinated afterwards at a lower temperature.

1 Haben Temperaturschwankungen als solche einen ungiinstigen Einfluss auf dass Wachsthum? Arb. d. bot. Inst. in Würzburg, Bd. I, I 874 . 
Sect. 20.-Action of Light on Growth in Length ".-Heliotropism ${ }^{2}$. Since we shall now pay exclusive attention to the questions whether and in what way light promotes or retards quantitatively the superficial growth of the cell-wall, we may for the time leave entirely out of consideration those cases where it changes

1 A. P. De Candolle, Physiologie végétale, Paris I832, vol. III. p. Io79.-Sachs, Bot. Zeitg. 1863, Supplement, and 1865, p. I 1 7.-Ditto, Experimental-Physiologie, Sect I 5.-Hofmeister, Lehre von der Pflanzenzelle, Sect. 36.-Kraus, Jahrb. für wissensch. Bot. vol. VII. p. 209 et seq.-Batalin, Bot. Zeitg. I87 1 , No. 4 o.

${ }^{2}$ [H. Müller (Thurgau), Ueb. Heliotropismus, Flora, I 876; Wiesner, Die Heliotropischen Erscheinungen, Denkschr. d. k. k. Akad. in Wien, I878, I880; Darwin, The Movements of Plants, I880.

A brief account of Wiesner's conclusions may be found useful; they are as follows :-

\section{Influence of the Intensity of Light.}

I. The maximum of heliotropic effect is produced by a certain intensity of light: increase or decrease of intensity diminishes the heliotropic effect until it is no longer produced. The optimum intensity varies in different plants.

2. The upper limit of intensity is either greater or less than that degree of intensity at which the parts of plants in question can grow at all: this depends upon the relative sensitiveness of the plants.

3. Hence it appears that sunlight may absolutely arrest growth: young stems are protected by their strong negative geotropism from the action of sunlight.

4. The degree of intensity at which heliotropism ceases corresponds, doubtless, to the intensity at which the plant no longer reacts by growth; an intensity which affects the plant no more than complete darkness.

\section{Influence of Refrangibility.}

I. Not only do the rays of high refrangibility possess heliotropic power, but those also of lower refrangibility: it is possessed by all rays from the ultra-red to the ultra-violet except the yellow rays.

2. The most marked effects are produced by the rays at the junction of the violet and ultraviolet : from these to the green the heliotropic effect gradually diminishes; in the yellow it is zero: it recommences in the orange and gradually increases until it attains a second maximum (small) in the ultra-red.

If the parts are not very sensitive, the effect is diminished in each of the colours in proportion to their heliotropic power, so that the orange, red, green, ultra-red, blue, etc. become inert in succession.

3. The heliotropic effect is not proportional to the mechanical intensity (thermic power) of the rays.

4. Negatively heliotropic organs exhibit the same phenomena.

III. Concomitant action of Heliotropism and Geotropism.

In strongly heliotropic organs, geotropism does not interfere with the exhibition of heliotropism provided that the light is intense.

\section{Presence of Oxygen.}

No heliotropic phenomena occur in the absence of oxygen.

\section{Photomechanical Induction.}

I. Both heliotropism and geotropism are exhibited after the removal of the organ under experiment from the action of light or of gravity respectively: this effect is an induced effect.

2. Successive exposures to the action of light or of gravity produce their effects distinctly; there is no summation.

\section{Relation of Heliotropism to Turgidity.}

In many cases positive heliotropic curvature does not take place in the zone of most rapid growth, where the turgidity is greatest, but in a zone below it, where the turgidity is less.

Etiolated organs become more sensitive to the heliotropic action of light after they have been exposed on all sides to diffuse light, probably because the turgidity of the growing cells is thereby diminished.] 
or may possibly change qualitatively the physiological and morphological naturé of the newly-formed organs.

The dependence of growth on light has already been spoken of in general terms in Sect. 8; and it was there especially insisted on that, in order to avoid serious misconceptions, this must be distinctly separated from the question of the part taken by light in assimilation. Here also we are concerned only with the processes of growth itself, since we always start from the point at which the cells or organs concerned have already obtained a sufficient quantity, or even excess, of formative materials.

It has been already stated that the various parts of the flower grow as readily in permanent darkness as in light. Most internodes, on the contrary, as has been explained in Sect. 18, grow more slowly when exposed to light on all sides, and remain shorter than when growing in the dark; when the light reaches them from one side only, they curve concavely towards the source of light. Other organs however, as root-hairs, tendrils, and some internodes, become longer on the side exposed to light than on that left in the dark. We have seen also that the leaves of Ferns and Dicotyledons soon cease growing in the dark and remain small. These observations show clearly enough that different cells and organs are differently affected by light as respects their growth. Since the light itself remains the same and there is a supply of formative materials, any explanation of these differences must aim at showing how it is that the inherited organisation of the plant in each case is affected just in one particular way and no otherwise by the oscillations of the ether. It is however at present quite impossible to give such an explanation ${ }^{1}$, since far too little is yet known of the phenomena themselves; the ascertained facts cannot yet even be reduced to a general law, especially in consequence of the obscurity which involves the behaviour of leaves (see infra) and of negatively heliotropic organs under the action of light. If these difficulties, which were referred to in Sect. 8, were solved, the organs of plants might be divided in respect of their behaviour towards light into three kinds:-(r) those the growth of whose cells is in general independent of light; as petals, stamens, fruits, and seeds; (2) those whose growth is retarded by light; the positively heliotropic organs which become abnormally elongated by absence of light; and (3) those whose growth is promoted by light. To this last category would belong negatively heliotropic organs if we could be certain that negatively heliotropic organs grew more slowly in darkness than in light. The observations of Schmitz ${ }^{2}$ on Rhizomorphs show, however, that this is not usually the case, for, although they are negatively heliotropic, they grow, like positively heliotropic organs, more quickly in darkness than in light.

The question in what manner light affects the mechanism of the growth of the cell-wall can therefore, in the present state of our knowledge, have a definite

${ }^{1}$ If Müller, in the second part of his Botanische Untersuchungen (Heidelberg 1872 ), gives the impression of having achieved this with but little difficulty, this only shows how far he is from a true method of investigation.

${ }^{2}$ Schmitz, Linnæa, 1843 , p. 513. [Similar results have been obtained by Müller (Thurgau) with the negatively heliotropic roots of Chlorophytum and of Monstera Lennei (Ueb. Heliotropismus, Flora, 1876), and by F. Darwin with those of Sinxpis alba (Ueb. das Wachsthum negativ heliotropischer Wurzeln, Arb. d. bot. Inst. in Wiirzburg, II, 1880 ).] 
meaning only in reference to positively heliotropic organs; inasmuch as it is in these cases certain that the growth of the cell-wall in the direction of the axis of growth of the organ is retarded and limited by light. But even in this case the question cannot at present be answered, since several others must first be solved. It must first of all be decided whether light acts in this manner on the cell-wall only when its plane of incidence is inclined to the axis of growth. A similar problem, as we shall see, is presented in the action of gravitation on growth. The various phenomena of positive heliotropism allow in fact of the supposition that rays of light which penetrate the cell-wall in a direction parallel to the axis of growth of the organ do not hinder growth, while they do so more strongly the more nearly vertical they are to it, whether the organ be multicellular or a simple filament. Light therefore acts more intensely the more nearly the transverse vibrations of the ether are parallel to the surface of the cell-wall. But the solution of these questions would by no means explain the action of light on the growth of the cell-wall; in the first place we must know whether light acts directly on the cell-wall, or if the effect is produced by means of the protoplasm, or by chemical changes in the cell-sap. But since we know that the cell-wall only grows so long as it is in contact on the inside with living protoplasm, and that the protoplasm itself is set in motion by light, in consequence of which it accumulates at particular parts of the cell-wall (see Sect. 8); and since this, like the growth of the cell-wall, is caused by the highly refrangible rays - the hypothesis must not at once be set aside. The question may moreover be asked whether light does not influence the growth of the cell-wall by means of chemical effects which it brings about in the cell-sap or the protoplasm, which however cannot be referred to assimilation, since they take place even in cells destitute of chlorophyll, as for instance in the positively heliotropic neck of the perithecium of Sordaria fimiseda, the stipes of Claviceps, and in many roots of seedlings; and since the leaves of Dicotyledons exhibit relations to light (vide infra) which indicate a chemical action on assimilated substances, but not on the process of assimilation itself.

So long as we take into account multicellular organs alone and merely contrast green and etiolated plants, great weight might be allowed to the hypothesis of a change in the turgidity caused by light (brought about by some chemical alteration in the cell-sap and the consequent change in diosmose ${ }^{1}$ ). But the fact that even unicellular tubes like those of Vauckeria and the internodal cells of Nitella are positively heliotropic, forbids this hypothesis, since in these cases the side exposed to light grows more slowly than the other, although all the parts of the cell-wall are subject to the same hydrostatic pressure from the sap.

The examples already given of positive heliotropism in submerged unicellular filaments, as well as the heliotropic curvings of multicellular internodes under water, show at once that they have nothing to do with a more rapid transpiration induced by light or its results.

The suggestion would appear on the contrary to be worth more attention that the reason why light retards the superficial growth of positively heliotropic cells is because it first of all promotes increase of thickness, and therefore diminishes the

${ }^{1}$ See Dutrochet, Mémoires pour servir, etc., Paris I837, vol. II. p. 60 et seq. [This question is discussed in the following small print.] 
extensibility of the cell-wall under the influence of the pressure of the sap on the side exposed to the strongest light. This hypothesis would be confirmed by Kraus's observations, according to which the cuticularisation of the epidermis as well as the thickening of the walls of the cortical and bast-cells is in fact imperfect in etiolated internodes, and the extensibility of these cell-walls consequently increased by the want of light. This explanation would apply not only in the case of the shaded side of a multicellular internode which curves towards the light, but also in that of a Vaucheria-tube or internode of Nitella; since it may be supposed that the wall is in the first place more strongly thickened on the side exposed to light and hence becomes less extensible, and therefore yields less to the pressure of the sap, and, in consequence, grows more slowly. We have at present no observations on heliotropic unicellular filaments.

If it be proved, as the recent researches of Wolkoff give ground for believing, that the negative heliotropism of organs which contain chlorophyll depends as little as that of roots on the stronger power of assimilation possessed by the side exposed to the source of light, it must be assumed that all the actions which have been mentioned as possible in one direction may take place also in an opposite direction; and this will show the great difficulty of the investigation.

A complete account of the mode in which growth depends on light is scarcely possible at present; what has now been said will call the attention of the reader to the most important questions involved in the investigation. It may be desirable however to collect some of the more important facts at present known, and to add some critical remarks.

(a) Organs whose growth is retarded by light. To take first the case of those internodes (including, according to Hofmeister, the unicellular ones of Nitella) which, when the light is unequal on the two sides, curve so that the side facing the source of light is concave while the other side is convex, or in other words are positively heliotropic. These exhibit a periodicity in their longitudinal growth corresponding to the alternation of day and night, when the temperature is sufficiently constant. The growth is more rapid from evening to morning, and less so from morning to evening. Both these facts are consistent with the phenomenon that the same internodes grow longer, and often considerably so, in permanent darkness than they would under normal conditions. These three results lead naturally to the conclusion that it is the direct action of light (and only in fact of its more refrangible rays, see Sect. 8) which retards the growth of these internodes. In the case also of positively heliotropic roots (as those of Ziea Mais, Lemna, Cucurbita, Pistia, \&c.), it may be supposed that if exposed to daylight they would exhibit the same alternation as internodes; but this is not yet fully established. Wolkoff has, on the other hand, already shown in the case of some roots, grown in water behind a transparent glass plate, that they grow more quickly in permanent darkness than under the alternation of day and night. Twelve primary roots of seedlings of Pisum sativum gave, for example, the following results :-

\begin{tabular}{lll} 
Day. & \multicolumn{2}{c}{ Successive increments. } \\
Ist & In the dark. & In difuse light. \\
I6 $\mathrm{mm}$. \\
2nd & $195 \mathrm{~mm}$. & 153 \\
3rd & 239 & 210 \\
$4^{\text {th }}$ & 250 & 113 \\
$5^{\text {th }}$ & 126 & 78 \\
the 5 days & 113 & $715 \mathrm{~mm}$.
\end{tabular}


The increments of growth of primary roots of seedlings of Vicia Faba were as follows :-

$\begin{array}{cccc}\text { In } 5 \text { roots } & \text { In the dark. } & \text { In diffuse light. } \\ \text { I I } & 309 & \text { as } & 272 \\ 9 & & & 612 \\ 6 \text { I } 2 & & 416\end{array}$

'In these cases a tendency of the roots was observed, though not a very decided one, to positive heliotropic curvature. The difference in the rapidity of growth would no doubt have been greater if the increments in the same time had been compared during the day only.

The long narrow leaves of many Monocotyledons exhibit the same phenomena as internodes and roots, becoming considerably longer in permanent darkness than under normal conditions, and showing positive heliotropic curvature when the light from the two sides is unequal. The plane of curvature may coincide with the plane of the leaf, so that one margin may be considerably longer than the other, and the whole leaf therefore unsymmetrical. I have observed this very evidently in a plant of Fritillaria imperialis grown in a window; those leaves only which sprang exactly from the side of the stem exposed to light being symmetrical like those growing in the open air. We have at present no observations on the daily periodicity in these leaves caused by light.

Observation of the broad netted-veined leaves of Dicotyledons is much more difficult. From the fact that in the dark they remain smaller, and often very much so, than under normal conditions, it might be concluded that their superficial growth presents exactly opposite phenomena to those of internodes and the long leaves of Monocotyledons. But Batalin has shown that it is sufficient to expose etiolated plants now and then to light-the time not being long enough for them to become green-for their growth in the dark to be afterwards considerably promoted. This leads to the supposition that light causes in etiolated leaves a chemical change which is not connected with assimilation, by which they are enabled to grow further in the dark. In any case this phenomenon shows that there is no real contradiction between the growth of these leaves and that of internodes, and that the reason why they become larger under the normal conditions of light than in permanent darkness is not because light has a directly favourable influence on the growth of the cells of these leaves. The recent experiments of Prantl $^{1}$ rather favour the hypothesis that green-and therefore healthy and normal-leaves exhibit the same diurnal periodicity of growth as positively heliotropic internodes. He succeeded, by a number of measurements both in breadth and length of the leaves of Cucurbita Pepo and Nicotiana Tabacum, taken at intervals of three hours, in constructing curves of growth, which, in spite of adverse fluctuations of temperature, rose from evening to morning, attained a maximum after sunrise, and then fell during the day till evening; exactly what I showed to be the case with positively heliotropic internodes. If this general law is established, it results that the broad nettedveined leaves of Dicotyledons grow more quickly in the dark than in the light, and are therefore hindered in their growth by light. But when such leaves remain nevertheless smaller in permanent darkness because they cease growing earlier, this must be interpreted as an unhealthy condition depending on the suspension of certain processes of metastasis which must precede growth and which are induced by light. In conformity with this hypothesis we must suppose that in leaves which unfold under the alternate influence of day and night, growth is directly hindered by light; but that at the same

1 Arbeit. des bot. Inst. in Würzburg, Vol. I, p 382. [See also Stebler, Ueb. Blattwachsthum, Jahrb. f. wiss. Bot. XI, I877. From his observations Stebler draws the erroneous conclusion that light promotes the growth of leaves (of Monocotyledons). For a criticism and further observations see Vines, The Influence of Light upon the Growth of Leaves, Arb. d. bot. Inst. in Wiirzburg, II, I $8 ; 8$. 
time certain chemical changes take place which in general make growth possible, and enable it to continue in the succeeding darkness, if it does not last too long. That this has nothing to do with assimilation is shown by Batalin's experiments with leaves destitute of chlorophyll.

If we now enquire what are the mechanical changes which light causes in the organs we have been considering, and by which their growth is retarded, it is to be regretted that no attempt has yet been made to study them in unicellular organs which exhibit positive heliotropism, as Vaucheria-tubes and internodes of Nitella, since they present the most simple case from a mechanical point of view. In the case of the internodes of Phanerogams which consist of tense layers of tissue, Kraus found in the etiolated state a smaller tension between the medullary and cortical layers, and that the cellwalls of the layers of tissue placed in a state of passive tension by the pith were less thickened, lignified, and cuticularised. It follows that these last are more extensible than in the normal internode, and therefore offer less resistance to the tendency of the pith to elongate. If we suppose that in unicellular tubes light also increases the cuticularisation and thickening of the cell-wall, the wall will offer greater resistance to the pressure of the cell-sap, will become less stretched, and will therefore grow more slowly ${ }^{1}$.

But little can be inferred as to the mechanical influence of light on growth from the changes in the tension of the tissues on the convex and concave sides of internodes with positive heliotropic curvature. If such an internode is split lengthwise so that the side exposed to light is separated from the other side, the former becomes more concave, while the latter becomes less convex or even somewhat concave towards the shaded side. In other words, the tension between the outer and inner layers is greater on the concave side exposed to light than on the convex shaded side. But the same phenomenon occurs also in internodes with an upward geotropic curvature, and with negatively heliotropic internodes, as well as with twining tendrils; and could not in fact be otherwise.

(b) Of Negatively beliotropic organs ${ }^{2}$ only a comparatively small number are at present known. Among those which contain chlorophyll may be named the hypocotyledonary portion of the stem of the seedling of the Mistletoe, the older nearly mature internodes of the Ivy and Tropeolum majus, and the basal portions of the tendrils of the Vine, Virginian Creeper, and Bignonia capreolata. I pass over at present the doubtful negative

${ }^{1}$ [With reference to the action of light upon growth, it is now universally admitted that the effects either of retardation or of curvature which it produces are the expression of modification of the turgidity of the growing cells. Three suggestions have been made as to the way in which this modification is brought about; (I) by a change in the elasticity of the cell-wall, (2) by a change in the osmotic properties of the cell-sap, (3) by a change in the permeability of the protoplasm. Some evidence in favour of the first of these is given above in the text, and it is further supported by Pfeffer (Physiologie, II. 145, I88I) and by Wiesner (Heliotropische Erscheinungen, II. p. 5). De Vries states (innere Vorgänge bei den Wachsthumskrümmungen) that 'external and internal causes produce curvatures in growing multicellular organs because they promote the formation, in the cells of one side of the organ, of substances which are osmotically active.' For the arguments in favour of the third suggestion see Vines, The Influence of Light on the Growth of Unicellular Organs, Arb. d. bot. Inst. in Würzburg, II, 1878 .

It is impossible to enter here upon a detailed criticism of these various views. The following remarks must suffice. With reference to No. I, it is difficult to understand how it can be satisfactorily applied to explain the action of light upon unicellular organs, seeing that the effects are so rapidly produced, nor is it clear how both positive and negative heliotropism can be explained by means of it. No. 2 clearly cannot account for positive and negative heliotropism in the case of unicellular organs, and its application to the case of multicellular organs is not obvious. As to No. 3, we know at least that light does act upon the protoplasm of zoogonidia, chlorophyll-granules, etc., and there seems to be no reason why it should not act directly upon that of growing cells.]

${ }^{2}$ Knight, Phil. Trans. 1812, p. 314.-Dutrochet, Mémoires, \&c., vol. II. p. 6 et seq.-Durand and Payer's statements.-Compare Sachs, Exper.-Phys., p. 4r. 
heliotropism, as I think, of the thallus of Marchantia and the prothallia of Ferns, as well as of other decidedly bilateral organs. Among organs which are not green must be especially mentioned the negatively heliotropic aërial roots of Aroidex and epidendral Orchids ${ }^{1}$; but, beyond all others, the roots of Cblorophytum guayanum, which are extremely sensitive to light coming from one side. Negative heliotropism has, in addition, been stated to occur in the roots of seedlings of Cichoriacex, Cruciferæ, \&c., and has recently been certainly determined by Wolkoff in the case of Brassica Napus and Sinapis alba. Among unicellular organs destitute of chlorophyll the only ones known at present with certainty to be negatively heliotropic are the root-hairs of Marcbantia (Pfeffer).

The observation that a number of organs destitute of chlorophyll and endowed with negative heliotropism, and in particular the highly sensitive roots of Cblorophytum, are very transparent, led Wolkoff to the hypothesis that the rays of light may be refracted by their cylindrico-conical shape, so as to produce a more intense illumination of the tissue on the side removed from the source of light than on that exposed to it; and that therefore the concave curvature on the former side is in fact a form of positive heliotropism. The apices of roots, when separated by a transverse section, if illuminated from one side and viewed from above, exhibit exactly the optical conditions which are assumed by this hypothesis. It must however not be forgotten that the apices of roots which are by no means negatively but at an earlier period even positively heliotropic, like those of Vicia Faba, manifest the same phenomenon, though perhaps to a lesser degree. Whether, on the other hand, it is possible to suppose a similar refraction of light in the case of the very thin-walled negatively heliotropic root-hairs of Marcbantia, is still in doubt. Further researches must show whether Wolkoff's suggestion is tenable or not. The Rhizomorphs would probably afford good material for observations on this subject, since, according to the researches of Schmitz, they are distinctly negatively heliotropic, and yet they grow more slowly in light than in darkness.

In the cases of the older internodes of the Ivy, which are only very slightly transparent, the older and lower parts of tendrils, \&c., the existence of an active focal line on the shaded side cannot be admitted, because this would evidently imply that it included more intense blue and violet light than, from the fact that the tissue which is penetrated by the light contains chlorophyll, it is probable it does. The negatively heliotropic curvature takes place however, at least in the Ivy as well as in the roots of Chlorophytum, only in highly refrangible light (after passing through an ammoniacal solution of copper oxide), not in yellow light (which has passed through potassium bichromate). If, as Wolkoff at one time supposed, the more vigorous nourishment, i.e. accumulation of assimilated substances, were the cause of the more rapid growth on the side exposed to light in this class of negatively heliotropic organs, they ought to curve much more strongly in the less refrangible (red, orange, or yellow) than in the more refrangible rays. This hypothesis would moreover fail to explain why the same internodes which when young showed decided positive heliotropism, at a later period when their growth has almost ceased manifest the opposite behaviour towards light.

The experiments which Wolkoff is now ( 1873 ) carrying on in the botanical laboratory at Würzburg, and which are not yet completed, lead at present to the conclusion that there are two kinds of negatively heliotropic organs. In one kind are included roots, in which the negatively heliotropic curvature takes place near the apex at the spot where growth is most rapid; to the other kind belong internodes where the negatively heliotropic curvature takes place only at the older parts whose growth is completed, while the young quickly-growing parts manifest positive heliotropism. In these latter cases the additional peculiarity occurs that the older parts, after being exposed to light on one side, will continue for some time to curve in the dark so that the side previously exposed to light becomes still more convex. This is a property which appears to be wanting in organs of the first kind as well as in those that are positively heliotropic.

1 According to a great number of observations of my own and statements of others. 
It is evident that we are here confronted with an unsolved problem; and when all the facts have been taken into consideration, the theory that there are two kinds of cells, the growth of one of which (positively heliotropic) is retarded by light, whilst that of the other kind (negatively heliotropic) is promoted by it, may be the simplest and most in accordance with facts. This difference is the less remarkable since in the behaviour of growing cells with respect to gravitation we find a precisely similar difference, but much more strongly marked ${ }^{1}$.

SECr. 21.-Action of Gravitation on Growth in Length :-Geotropism ${ }^{2}$. It has already been shown in Sect. Io that, when the access of light is equal on all sides or when heliotropism is prevented by the exclusion of light, gravitation is the cause of certain organs turning downwards, others upwards, and others again in a direction oblique to the horizon. At present we shall speak only of those which take a direction directly upwards or downwards, since other causes co-operate to bring about an oblique growth.

Just as organs, according to their internal nature, grow either more rapidly or less rapidly on the side which faces the source of light than on the other side, so also gravitation effects, in accordance with the nature of the organs, either an acceleration or a retardation of growth on the side which faces the earth. Those organs which are thus retarded in their growth are called positively geotropic, those which are accelerated negatively geotropic organs. Positively geotropic organs consequently become concave on the under side, and direct their growing apex downwards if their axis of growth is brought into a horizontal or oblique direction; negatively geotropic organs, on the contrary, become convex on the under side under similar conditions, and elevate their growing apex until it stands erect.

It has not yet been ascertained whether positively geotropic organs would manifest a different rapidity of growth if entirely withdrawn from the influence of gravitation (like positively heliotropic organs when withdrawn from the influence of light) from that displayed when gravitation acts in a direction parallel to the axis of growth $^{3}$. It would seem however as if gravitation only affected the rapidity of

1 Schmitz, Linnæa, I 843 , p. $5^{\mathrm{I}} 3$ et seq. If, as can scarcely be doubted, Schmitz's statements with regard to Rhizomorphs are confirmed, it results that no certain inference can be drawn as to the positive heliotropism of an organ from the fact that its growth is more rapid in the dark. We could scarcely have a better proof of the necessity for a fresh and more accurate investigation of all the phenomena of heliotropism. [Schmitz's observations have been confirmed, in other cases, by Muiller and F. Darwin (see ante).]

${ }^{2}$ Knight, Phil. Trans. I806, vol. I. pp. 99-108.-Johnson, Edinburgh Phil. Journ. 1828, p. 312. -Dutrochet, Ann. des Sci. Nat. 1833, p. 413.-Wigand, Botan. Untersuch. Braunschweig 1854, p. 133.-Hofmeister, Jahrb. für wissensch. Bot. vol. III. p. 77.-Dit1o, Bot. Zeitg. 1868, Nos, 16, 17, and 1869, Nos. 3-6.-Frank, Beiträge zur Pflanzen-Phys. Leipzig r 868, p. I.-Müller, Bot. Zeitg. I869 and I87I.-Spescheneff, Bot. Zeitg. I870, p. 65.-Ciesielski, Untersuch. über die Abwärtskrümmung der Würzeln, Breslau I871.-Sachs, Arbeit. des bot. Inst. in Würzburg I872, Heft 2. Abh. 4 and 5.-Ditto, Exper.-Phys., p. 505.-Ditto, Flora, 1873, No. 21. [Darwin (Movements of Plants) terms what are here termed positive and negative geotropism, 'geotropism' and 'apogeotropism' respectively. He considers that both positive and negative geotropism are modified forms of circumnutation (see infra).]

3 [Elfving has found (Beit. z. Kennt. d. physiol. Einwirkung der Schwerkraft, Helsingfors I880) that when the sporangiophores of Phycomyces, which are negatively geotropic, are grown in an inverted position, their growth is not so rapid as it is under ordinary conditions; that is, that they grow less rapidly in the direction of the action of gravity than in the opposite direction.] 
growth when its direction cuts that of the axis of growth at an angle, and the more so the nearer the angle' approaches a right angle.

The positive or negative character of geotropism depends as little as that of heliotropism on the morphological nature of the organ. Not only, for example, are all the primary roots of the seedlings of Phanerogams positively geotropic, and most secondary roots which spring from underground stems, as tubers, bulbs, or rhizomes; but also many leafy lateral shoots, especially those which are destined to produce rhizomes or to form new bulbs (e.g. Tulipa, Physalis, Polygonum, \&c.), and even foliar structures, like the cotyledonary sheaths of Allium, Phonix, and many other Monocotyledons. Among positively geotropic organs must also be included the lamellæ and tubes of the hymenium of Hymenomycetous Fungi. All axes which grow upright (and are not bilateral), petioles, and the stipites of many Hymenomycetous Fungi, exhibit, on the other hand, decidedly negative geotropism.

The geotropism, like the heliotropism, of different organs varies in all degrees. It is, for example, manifested very strongly in the primary roots and upright primary stems of seedlings; much less strongly in the secondary roots and in lateral branches of erect stems, \&c. It appears to be the general rule that when lateral shoots of the same kind spring from a vertical and therefore decidedly geotropic organ, the branches of the first order are less geotropic, and the further ramifications still less so the higher the order to which they belong; the exceptions to this rule may be caused by special circumstances. This gradation is very obvious in roots. From the primary root or a strong root springing from the stem with well-marked positive geotropism, proceed secondary roots of the first order which exhibit the phenomenon much less decidedly; and from these again secondary roots of the second order which apparently are not at all geotropic, and therefore grow in all directions as they may chance to originate. Geotropism, like heliotropism, does not depend on the organ containing or not containing chlorophyll, nor on whether it consists of masses of tissue or of a simple row of cells or of a single cell. To this last category belong, for example, the positively geotropic radical hyphæ of the $\mathrm{Mu}$ corini and the negatively geotropic sporangiophores of the same family and of numerous other Mould-fungi. In the same manner the rhizoids of Chara display positive, the stems negative geotropism, both consisting of unicellular segments, the former destitute of chlorophyll, the latter green. Whether and how strongly an organ is positively or negatively heliotropic or geotropic depends altogether on its importance in the economy of the plant, and hence on its physiological functions.

From the remarkable fact that there are organs endowed with positive and negative heliotropism and geotropism, and from many similarities exhibited by the two phenomena, the question presents itself whether all positively heliotropic organs must not possess one description of geotropism either positive or negative, or vice versa ; in other words, whether the two properties do not stand in some definite relation to one another. This does not however appear to be the case. Of primary roots, all of which are positively geotropic, some display positive, others negative heliotropism; and again, the aërial roots of Chlorophytum, Aroideæ, and Orchideæ display very distinct negative heliotropism, but are scarcely at all geotropic. According to Schmitz the same is the case with the Rhizomorphs. There appears therefore to be no necessary connection between the two phenomena. 
It is clear that organs which are both heliotropic and geotropic, and on which, since they lie obliquely to the horizon, the light falls from above or from below, are subject to changes in their growth dependent both on light and on gravitation. Thus, for example, the bending upwards of a branch placed horizontally on which the light falls from above may be caused at the same time by positive heliotropism and by negative geotropism. An erect stem, on the other hand, which turns heliotropically towards a source of light at the side and thus makes a curvature which is concave below, will have a tendency to become erect in consequence of its negative geotropism, and would do so if the light falling on it were removed. Stems therefore which in the evening were bent by positive heliotropism, will stand upright in the morning. These considerations are evidently of the first importance in making observations on the two phenomena.

We have already seen that no clear idea has yet been obtained of the mode in which light acts in influencing the growth of heliotropic organs. As little are we at present in a condition to affirm how the acceleration or retardation of the growth of the cell-walls results from the action of gravitation ${ }^{1}$. The hypotheses and considerations there stated may be repeated here mutatis mutandis. Particular stress must be laid on the fact that movements are induced in protoplasm by the action of gravitation just as by the action of light. Thus Rosanoff showed ${ }^{2}$ that the plasmodia of $A$ thalium septicum are negatively geotropic, creeping, under the influence of gravitation, over steep moist walls, and turning, under the action of centrifugal force, towards the centre of "rotation; they take therefore those directions which would be least expected from their apparently fluid condition. The question suggests itself whether there is not also protoplasm which behaves in this respect in an exactly opposite manner; and from the dependence of the growth of the cell-wall on the activity and probably also on the disposition of the protoplasm in the cell, the hypothesis must not be altogether set aside that all geotropic phenomena are in the first place caused by the protoplasm taking up definite positions in the cells under the influence of gravitation, and thus accelerating or retarding the growth of the cell-walls on the under sides. Since nothing is known on this subject, we must direct our attention solely to the growth of the cell-walls, leaving it undecided whether the effect of gravitation be direct or indirect.

In order to state clearly the problem how gravitation acts on the growth of the cell-wall ${ }^{3}$, we may consider as the simplest example a unicellular filament, such as we find in Vaucheria, the posterior end of which developes as a positively geotropic root, the anterior end as a negatively geotropic stem. Fig. $482 \mathrm{~A}$ may represent this, assuming that the whole filament grew at first in a vertical direction either upwards or downwards, but was then placed in a horizontal position, as shown by the light outlines $S$ and $W$. After some time the radical end would show a downward curvature,

[See note on p. 837.]

${ }^{2}$ Rosanoff, De l'influence d'attraction terrestre sur la direction des plasmodia des Myxomycètes (Mémoires de la Société impériale des sciences de Cherbourg, vol. XIV). [According to Strasburger (Wirk. des Lichts und der Wärme auf Schwärmsporen, Jenaisch. Zeitschr., XII, I878) this apparent negative geotropism of the plasmodia is due simply to the fact that they tended to travel against the direction of the stream of water by which they were kept moist during the experiments.]

${ }^{3}$ Duchartre's assertions on geotropism in his Observations sur le rétournement des Champignons (Compt. rend. I870, vol. LXX. p. 78I) show that he has not clearly comprehended the question. 
like $W^{\prime}$, the part $S$ on the contrary the upward curvature, as $S^{\prime}$. It is self-evident that each of these curvatures can only result from the growth, equal on all sides when the organ is erect, having now become unequal on the upper and under sides, the convex growing in both cases more quickly than the concave side.

If we now apply the results of my experiments on internodes and nodes of Grasses which curve upwards to the simple tube, the growth is found to be more rapid on the convex under side, less rapid on the upper side of the upwardly curved part, than when it grew erect. It may be assumed, from Ciesielski's measurements of roots, that when the filament curves downwards the growth has been more rapid on the convex upper side, less rapid on the concave under side, than if the curved part had grown onwards in a vertical direction. In other words, when the filament is placed in a horizontal position the growth is accelerated on the upper side of the positively geotropic part and on the under side of the negatively geotropic part, but always retarded on the opposite sides.

If therefore we assume that in Fig. $48 z B$ the two side walls of a transverse disc of the part $S$ of the filament when in an upright position had lengthened in a definite time to the equal lengths $o o$ and $u u$, it would have remained straight; but if the

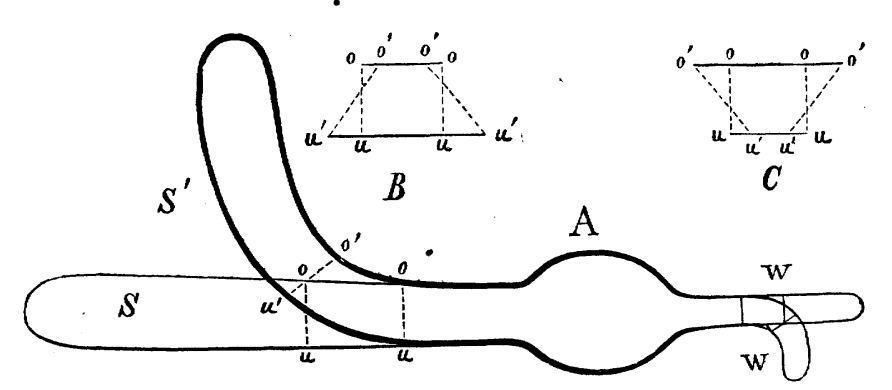

FIG. 482.-Diagram for illustrating geotropic upward and downward curvature.

tube had been placed horizontally during this time, the lower side would have attained the greater length $u^{\prime} u^{\prime}$, the upper side the shorter length $o^{\prime} o^{\prime}$, and the piece must in consequence become curved. Exactly the opposite would be observed, as shown in Fig. $482 C$, if the growing piece belonged to the part $W$ of the filament.

If now the unicellular filament $A$ were supposed divided by transverse and longitudinal divisions into a tissue consisting of a number of layers of cells; or if, what amounts to the same thing, a stem of a seedling were supposed to be substituted for the part $S$ of the filament, and a root for the part $W$, the same phenomena would occur, as experiments have shown, in every cell of the growing part, as those previously observed in the filament. In the part $S$ every cell would grow more rapidly on the under side, less rapidly on the upper side than if the part were upright, the reverse in the part $W$. We should find that in $S$ both the upper and under sides of any cell (i.e. upper and under in relation to the radius of the earth) are longer than those of the cells situated above it, the reverse in $W$; in other words, that every individual cell of a part which shows geotropic curvature behaves in the same way as if the part previously straight were held firmly by the two ends and then bent. This will be made clearer to the student if in the portion of the curved part included in 
$A$ lines are drawn parallel both to the straight and the curved outlines, and the septa of the cells are then indicated in the straight piece simply by parallel lines crossing the first at right angles, in the curved part by lines corresponding to the radii of curvature. The cells exposed by longitudinal sections through nodes of Grasses and roots endowed with geotropic curvature exhibit this phenomenon, although with many irregularities.

Now that the facts connected with the geotropism of the cell-wall have thus been made clear, we may proceed to the question, why or by what effect of gravitation these differences are occasioned in the growth on the upper and under sides of every cell of a geotropic organ when placed in a horizontal position. We have at present however no answer to this question, any more than in the case of heliotropism, the same diagram availing, mutatis mutandis, for the two phenomena.

The view brought forward by Hofmeister, and for some time adopted by me, that positive geotropism occurs only in those organs and in those parts of organs in which there is no tension in the tissues, while the organs in which there is strong tension are negatively geotropic, rested on imperfect induction. On the one hand, : the parts of the roots of seedlings which curve downwards (as I have shown elsewhere) are not entirely without tension between the cortex and the axial fibrovascular cylinder; while, on the other hand, in the nodes of Grasses, although they display a high degree of negative geotropism, there is no or very little such tension. Even in the negatively geotropic contractile organs of the petioles of Phaseolus ${ }^{1}$ the tension between the cortex and the axial bundle is of a similar character to that which occurs in positively geotropic roots, but extremely intense. If therefore the tension of tissues and the alteration effected in it by the influence of gravitation cannot be considered as the cause of the upward curvature, it may still be admitted that it is useful to upright organs by increasing their rigidity and elasticity, thus making them more readily assume the erect position; while this would be quite unnecessary in those that grow downwards.

A good illustration of the part played by rigidity and elasticity in producing the erect position of negatively geotropic organs is afforded by the pendent pedicels of many flowers and flower-buds, in which the tendency to curve upwards is altogether obscured, the weight of the flower being sufficient to bend the pedicel downwards. If in such cases the flower-buds are cut off, the pedicel becomes erect ${ }^{2}$ from the stronger growth of the under side, as e.g. in Clematis integrifolia, Papaver pilosum and dubium, Geum rivale, and Anemone pratensis. The tension in the tissue of such pedicels is not sufficient to give them the rigidity needful to overcome the weight of the flower by their geotropic curvature upwards; this weight, on the contrary, overcomes the tendency of the pedicel to curve convexly on the lower side, which tendency comes into play when the weight is removed. The same is the case in very long but not very rigid shoots, as those of the Weeping Willow, Weeping Ash, \&c.

If a number of organs grow in a horizontal or oblique direction without curving either upwards or downwards, this may result from their not being geotropic ${ }^{3}$ and

1 Sachs, Experimental-Physiologie, p. 105.

2 See De Vries, in Arbeiten des Bot. Inst. Würzburg, Heft II. p. 229.

3 [Elfving (Ueb. einige horizontal-wachsende Rhizome, Arb. d. bot. Inst. in Würzburg, II. 3, I880) has found that the rhizomes of certain plants (Heliocharis palustris, Sparganium ramosum, Scirpus 
growing straight forward in the direction of their first origin, just as rootlets of a. high order grow downwards from the under side of their parent root, upwards from the upper side, horizontally from the vertical sides, or continue to grow straight and oblique according to the direction of the primary root. To this must be referred, among other phenomena, the striking one described by me that plants which grow in uniformly moist soil emit a large number of fine roots out of it with their apices pointing upwards ; these are rootlets of the first or second order which spring from the upper side of horizontal or oblique parent roots and grow straight upwards without being geotropic. If the air is able to enter the ground freely, its surface is often dry, and the fine roots which are directed upwards die off, as I have ascertained by growing plants in glass vessels filled with earth.

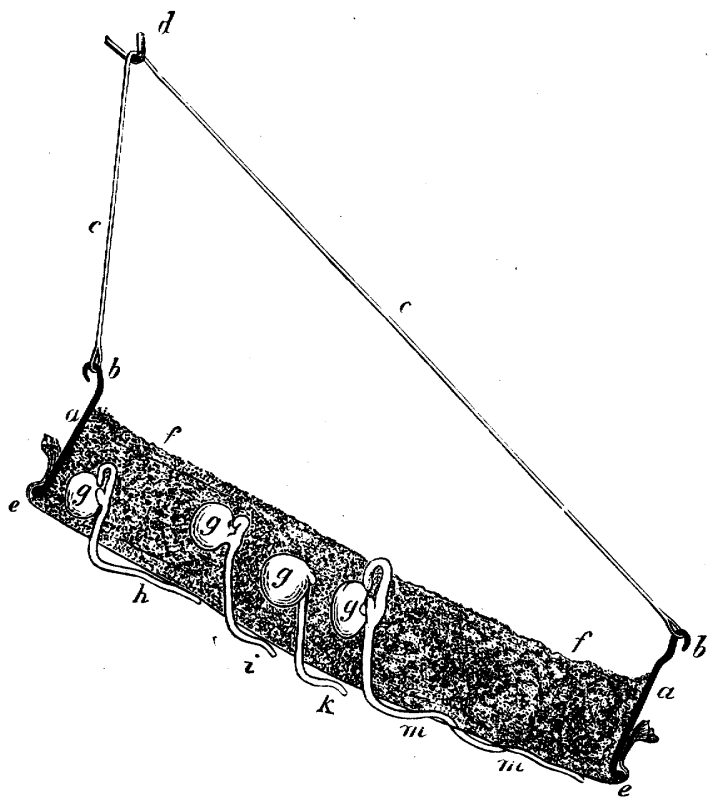

FIG. $483 .-$ A pparatus to illustrate the mode in which the geotropism of the roots $h i k \mathrm{~m}$ of seedlings $g \mathrm{~g} g$ is overcome when they come into relation with a moist surface (hydrotropism).

But even geotropic organs may grow obliquely or horizontally when other causes oppose or counterbalance their geotropism. One of the most common of these causes is the bilateral organisation which makes an organ grow more strongly. on one side from internal causes. Since I shall recur to this subject in the next section, only a single example need be given here. In the case of seedlings, rootlets of the first order not unfrequently appear above the surface of the soil obliquely when it is uniformly moist; and I have convinced myself that this is the result in cases which have been observed (e.g. Vicia Faba) of a stronger growth of their lower side altogether independent of geotropism, in consequence of which they always

maritimus), which are not bilaterally organised, tend to maintain a horizontal direction of growth; they are therefore not geotropic.] 
grow in a flat curve concave upwards. But external causes may also act in opposition to geotropism even when this is very strongly developed. Thus Knight and Johnson have shown, as I have recently described more in detail, that primary roots with strong positive geotropism, as well as secondary rootlets, when growing in moderately damp air, deviate from their vertical or oblique direction when there is a moist surface near them ${ }^{1}$. Under these circumstances a curvature concave to the moist surface takes place at the region below the apex where there would otherwise be a downward curvature, the apex being by this means conducted towards the moist surface so that it may penetrate into the moister soil or grow in contact with it. The apparatus represented in Fig. 483 is well adapted to exhibit this phenomenon. It consists of a zinc frame $a a$ covered below with wide-meshed network, thus forming a sieve hanging obliquely and filled with moist sawdust $f f$. The seeds $g g g$ germinate in the sawdust, their roots penetrating at first vertically downwards into it. When the apex of a root escapes through the network into air, which is not too dry, it turns towards the moister surface $h-m$, its geotropism being thus evidently overcome.

The foregoing account is intended to give the reader a general idea of the various debatable points which are especially to be remembered in the study of Geotropism and to which frequent reference is made in the literature of the subject. Until recently - there were no complete observations or measurements of the growth which necessarily accompanies geotropic curvature, or as to the true form of the curvature and its relation to time and other conditions, which might give some clue as to the nature of the internal changes which effect externally the upward or downward curvature. I have endeavoured to supply these in the papers mentioned at the beginning of this section. The observations were made upon organs of which the geotropism was wellmarked, such as erect growing stems, the nodes of grass-haulms, and downward-growing tap-roots.

I. The upward curvature of stems which normally grow erect ${ }^{2}$. My observations were made for the most part on the thick, firm, long internodes of scapes which attain a considerable height in a short time, the smooth surface of which can be marked with Indian ink and allows of accurate measurement of the portions thus indicated. The measurements of straight shoots as well as of the convex and concave sides of curved ones were made by means of flexible measures of stout paper upon which the scale was printed.

In order to be in a position to form an opinion as to the phenomena connected with the upward curvature of stems or internodes placed horizontally, the distribution of growth in these organs must first be understood. A general account of this was given in Sect. 17. At first the whole internode, or a shoot consisting of several internodes, is undergoing elongation. At a later period growth ceases at the base of the shoot, and only a certain number of internodes lying below the terminal bud (this bud is not taken into consideration here) constitute the region of the shoot which is growing and which is capable of making a geotropic curvature. In the case of single internodes, the region in which growth is to continue may lie near either to the base or to the apex; apical growth is the usual, basal growth the more uncommon case. It is remarkable that similar internodes of closely allied plants behave differently in this respect: thus in the scapes of Allium atropurpureum there is apical growth, whereas in that of

' [This exhibition of sensitiveness to moisture has been termed 'Hydrotropism.' (See Darwin, Movements of Plants, p. 180$)$.]

${ }^{2}$ Sachs, Flora, 1873 , No. 2 I. 
Allium Porrum and of Allium Cepa the growth is basal. The position and the form of the geotropic curvature is therefore different in the two cases.

The length of the growing region, when fully developed parts already exist, is greatest at a certain time, after which it diminishes as the stem gradually approaches its complete development, and finally disappears. The following measurements were made whilst growth was still active and the growing region of considerable extent. The length of the growing region behind the bud was in

Fritillaria imperialis
Allium Porrum
Allium Cepa
Allium atropurpureum
Cephalaria procera
Polygonum Sieboldi
Asparagus asper
Valeriana Pbu
Dipsacus Fullonum

\begin{tabular}{|c|c|c|c|}
\hline \multirow{9}{*}{ about } & \multicolumn{2}{|c|}{$7-9 \mathrm{ctm}}$. & \multirow{3}{*}{$\begin{array}{c}\text { in one internode } \\
\text { of the scape. }\end{array}$} \\
\hline & 40 & " & \\
\hline & 30 & , & \\
\hline & 50 & $"$ & \\
\hline & 35 & $"$ & ( 3 internodes). \\
\hline & 15 & $"$ & (4-5 internodes). \\
\hline & 20 & $"$ & (numerous internodes). \\
\hline & 25 & $"$ & (4 internodes). \\
\hline & 40 & ", & (3-4 internodes). \\
\hline
\end{tabular}

The measurement of portions of equal length (of one or of five ctm.) shows that the growth of each such portion is greater the more distant the portion measured is from the terminal bud, or, in the case of basal growth, from the base. At a certain distance from the apex (or the base) the maximum occurs; beyond this distance the growth of each portion diminishes until it altogether ceases at the limit of the growing region. The form of the geotropic curvature and its modifications essentially depend upon these conditions. In these particulars stems which consist of numerous internodes but without well-defined nodes (Asparagus) resemble long single internodes, such as the scapes of the various species of Allium. If however the stem is distinctly articulate, a curve of fractional growth may be obtained from each internode, which rises as we pass from its lower end until a maximum is reached, and then sinks as we pass towards its upper end. The node itself ceases to grow in length at an early period. As the result of this, the geotropic curvature of the whole stem is interrupted at the nodes, and the quickly-growing central portions of the internodes describe sharper curves. With the exception of this peculiarity, an articulate stem behaves generally in the manner above described with reference to a long scape consisting of a single internode. Finally, it is to be noted that each transverse zone of a growing stem grows at first slowly, then more rapidly until a maximum rapidity is attained, and then more slowly until growth ceases altogether. This also determines the form of the geotropic curvature.

Those portions of a stem which have ceased to grow and which are incapable of renewed growth in consequence of a change of position (see what is said below about the nodes of the haulms of Grasses) will not assume an erect position when placed horizontally or obliquely. Only those portions of a stem laid horizontally or obliquely take part in the assumption of an erect position which are growing (as in the downward curvature of the root), and this in proportion to the phase of their growth, their thickness, rigidity, etc. This curvature is a consequence of a modification of the growth in length of the stem produced by its abnormal position, of such a nature that the growth of the under side is more rapid and that of the upper side less rapid than that of the stem in the erect position. In quickly-growing parts the upper side evidently increases in length when the geotropic curvature is taking place, but in older more slowly growing parts the length of the upper side does not increase, and it may even become a little shorter if the curvature is very sharp, whilst the lower side elongates considerably. These statements can be easily verified by direct measurement of thick firm stems before and after the curvature has taken place. It is obvious that the convex side of a curved stem must be longer than the concave, but the question as to whether or not the growth of the concave side is slower and that of the convex 
side more rapid than is the case when both these parts are in the normal erect position, is answered in the affirmative not only by the direct measurement just mentioned, but also by some previous results which I obtained in another way, namely, by taking a number of similar shoots, splitting up some of them at once into strips of tissue which were measured, and by splitting up and measuring the others in the same way after continued growth, some in a vertical, some in a horizontal direction, finally comparing these measurements with each other ${ }^{1}$. It became apparent in all cases that of any two similar strips of tissue, the one belonging to the lower convex side had grown more rapidly, the one belonging to the upper concave side less rapidly than the corresponding strips of tissue in an erect shoot within the same time. As a consequence of this, the difference in length between the cortex and the pith belonging to the upper (concave) half of the upwardly curved shoot is increased, and that between the cortex and pith of the lower (convex) half is diminished, so that the upward curvature causes an increase of the tension between the tissues of the upper half and a diminution of the tension between those of the lower half. This may be illustrated by the following example. Twelve pieces of stem of Sida napaa cut off above and below, the leaves having been removed, each consisting of six or seven internodes and $300 \mathrm{~mm}$. in length, were taken: of these, four were at once split up into strips of tissue, four were laid horizontally in damp sand in a box, and four were placed nearly erect upon moist sand in a cylinder. The two following tables give the mean measurements of the strips of tissue belonging to four pieces of stem:-

\section{Lengths of the strips of tissue in Millimetres.}

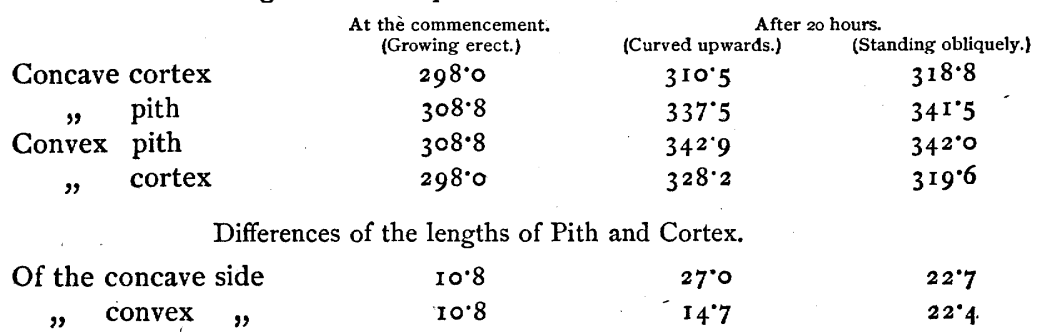

Increments of length in 20 hours.

\begin{tabular}{|c|c|c|c|}
\hline \multicolumn{2}{|c|}{ Of the concave cortex } & $\begin{array}{l}\text { Curved upwards. } \\
\quad 12^{\circ} 5\end{array}$ & $\begin{array}{l}\text { Standing obliquely. } \\
20^{\circ} 8\end{array}$ \\
\hline & pith & $28 \cdot 7$ & $32 \cdot 7$ \\
\hline$"$ & convex pith & $34^{\circ} \mathrm{I}$ & $33^{\circ} 2$ \\
\hline " & cortex & $30 \cdot 2$ & $2 \mathrm{I} \cdot 6$ \\
\hline
\end{tabular}

If a shoot which has lain for some time ( $1 / 2-2$ hours) in a horizontal position and has begun to show the first traces of an upward curvature be placed vertically, or be moved so that the plane of curvature becomes horizontal, the commencing curvature increases; hence it appears that the action of gravitation has a persistent effect, which may continue as long as three hours, and may produce considerable curvature. In the second of the two cases the curvature lies in a horizontal plane, and simultaneously with its increase an elevation of the free apex occurs in consequence of the geotropism induced by the new position. The persistent effect manifests itself even when the shoot is strongly curved upwards.

My observations afford the following information as to the form of the curvature of a shoot which is assuming the erect position under the action of gravity.

On experimental as well as on theoretical grounds it appears that the curvature (with a few exceptions) is not and cannot be a segment of a circle: this is only the

${ }^{1}$ Sachs, Arb. d. bot. Inst. Würzburg, I872, Heft II. p. 194. 
case where the curvature is greatest, that is where its radius is smallest; above and below this the curvature is less, and therefore the radii are larger.

It appears also that from the commencement to the termination of the process the form of the curvature is always altering, the maximum of curvature being attained by parts which were at first not curved at all or only slightly so, and parts which were previously strongly curved becoming straight.

The following paragraphs serve to explain the foregoing. We assume, for the sake of simplicity (excluding other possible cases), that the horizontally-placed shoot is rooted, or that its base which has ceased to grow (and which can absorb water) is fixed, whilst its apex can move freely. To make it more intelligible, let us consider the whole growing region, the region, that is, which takes part in the upward curvature, as divided into three parts, an apical, a middle, and a basal portion, which we may assume to be of equal lengths.

Since the form of the curvature of the whole curved portion is determined by the degree of curvature of each transverse zone, it is essential to know upon what conditions the curvature of each zone depends. The following are the determining conditions :-

I. The rate of growth.

2. The thickness.

3. The deviation from the vertical.

4. The time during which any zone lies in any given direction inclined to the vertical.

5. The persistent effect.

6. The rigidity and elasticity.

The curvature is greater, ceteris paribus, in any given short period of time, the more rapid the rate of growth in length, and the more nearly the deviation from the vertical approaches the horizontal; on the other hand, geotropism is slower the thicker the curving region is. Further, the curvature increases, that is the radius of curvature becomes smaller, the longer the time during which the curving region is inclined at an angle to the vertical. Moreover each transverse zone tends, according to what was said above, to curve more strongly than is due to its inclination to the vertical and to the length of time during which it is in that position; that is, each transverse zone which has been exposed for a certain time to the action of geotropism undergoes in consequence of its persistent effect a subsequent curvature, which is in excess of that produced by the other conditions. Finally; as regards rigidity and elasticity, it is clear that each transverse zone of a shoot lying horizontally must, by reason of the flexibility of the shoot, tend to bend downwards, that is, in opposition to the geotropic curvature, and this tendency will be greater the greater the weight which the shoot has to bear at its growing end and the more distant the section is from that end. It must be further borne in mind that the flexibility alters with age and that it diminishes as the thickness increases.

If the growing region of a horizontally-placed internode or stem were of the same thickness throughout, and if the rate of growth of all transverse zones were uniform and the flexibility so slight that it might be neglected (as is the case in short, thick stems), the curvature, at its first appearance, would have the form of a segment of a circle of large radius. Of these conditions, however, one at least, viz. the uniform rate of growth of all transverse zones, is never fulfilled, and since the region of most rapid growth is also that of greatest curvature, it is impossible that the curvature should be, even at the commencement, a segment of a circle.

Taking now the usual case, in which we have a shoot growing at its apex, of a conical form, growth being more active near the apex than near the base, the curvature resulting from a horizontal position will first be manifested by the apical portion, for its growth is the most rapid, it is the thinnest portion, and it has the least weight to raise; at a later period a less sharp curvature of the middle portion will be observed, and 
still Iater a very gentle curvature of the basal portion of the growing region, for the rate of growth diminishes from the apex towards the base whereas the thickness increases, and the further from the apex any portion is the greater the weight which it has to raise when curving. In consequence of the continued action of gravitation, or in consequence of its persistent effect, the curvature rapidly increases, but more rapidly in the apical than in the other portions.

As the result of this, the apical portion, and then the middle portion, becomes more and more nearly erect, and the inclination to the vertical of the shoot is less the nearer these portions are to the apex. For instance, a line drawn tangentially to the apical portion will very nearly coincide with the vertical, whereas a tangent to the centre of the middle portion will be inclined to it at an angle of about $45^{\circ}$, and a tangent to the centre of the basal portion deviates not more than perhaps $5-10^{\circ}$ from the horizontal. Consequently the apical portion will not be affected any longer by the action of gravitation, whereas the middle portion will continue to curve considerably, for its growth is still tolerably rapid and it is in a position which is favourable for curvature: the basal portion grows but slowly, but its position is very favourable for curvature. In consequence of the continuing curvature of the middle and basal portions the now erect apical portion becomes bent over out of the vertical, and this is increased by the persistent effect of the action of gravitation. Thin very rapidly-growing stems acquire this form of curvature in from 3 to 5 hours, thicker ones in from 12 to 15 hours, and very thick ones in from 24 to 30 hours.

After this condition has been attained a remarkable change of the form of the curvature begins. Whilst the apical portion which is erect or has curved even beyond the vertical is straightening itself in consequence of the more rapid growth of its concave side, the basal portion continues to curve slowly upward by reason of its still nearly horizontal position. In consequence of this the middle portion is passively elevated, in addition to its own active curvature, so that it comes to assume, like the apical portion, a position which is unfavourable to its geotropism, and like it, it begins to straighten itself (at least in its anterior part). Finally, the whole anterior part (including the apical and middle portions) stands erect, whilst the mature portion, lying behind the basal portion, is horizontal, the two being connected by the sharply-curved basal portion of the growing region.

It appears, therefore, that the greatest curvature occurs first in the thin quicklygrowing apical portion, then in the thicker middle portion which grows less rapidly, and finally in the still thicker slowly-growing basal portion.

If, on the other hand, we consider a scape of Allium Cepa or of Allium Porrum in which the growth is basal, the first effect of being placed in a horizontal position is that the greatest curvature is exhibited by that part of the basal region which is growing most rapidly, the mature apical portion remaining straight and being passively elevated. The curvature of the basal portion takes place but slowly, for it is very thick and it has to support the overhanging weight of the anterior portion. In this case also the apical portion may be elevated beyond the vertical, since the transverse sections of the basal portion which lie behind the region of greatest curvature continue to curve slowly and the position of the whole of the scape which lies in front of them is passively altered.

If a conical shoot with apical growth, the growth being more active toward the apex, be placed in such a position that the apex is directed downwards in a direction which deviates but little from the vertical, all the parts are at first in a position which is very unfavourable for geotropism, since gravitation acts upon the shoot at a very acute angle. The time which will elapse before the first appearance of curvature must therefore be greater than when the shoot is lying horizontally. It must be borne in mind that, as the curvature proceeds, the parts which are affected by it come to occupy a more favourable position for geotropism, for they approach the horizontal more and more closely; the action of gravitation will therefore increase as the curvature increases. The apical portion comes, at length, to occupy a horizontal position; it commences to 
elevate itself, and in consequence of the persistent effect of gravitation and of the curvature of the middle and basal portions, it may pass beyond the vertical; finally it stands erect. The middle portion remains, sharply curved; the basal portion is but slightly curved, for its growth ceases before it is possible for it, in consequence of its unfavourable position, to undergo any great curvature.

Growth and Curvature without Absorption of Water. If shoots consisting of a growing part and of a part which has ceased to grow be cut off and placed erect (the apex being uppermost) in a dry glass cylinder which is then closed in order to prevent excessive evaporation, they continue to grow for a considerable time without any absorption of water, and at the same time they lose a portion of their water by evaporation into the closed space ${ }^{1}$. It might be assumed that the water requisite for the elongation of the growing portion was derived from the part which had ceased to grow. If, however, the growing region alone be cut off and the terminal bud removed, and then marks be made on the shoot, it becomes evident that all the segments of the shoot grow without absorbing any water. The elongation is certainly less than usual, but it is distinct.

If shoots consisting of a part which is growing and of a part which has ceased to grow be cut off and placed horizontally in a closed space and protected from excessive evaporation, a curvature occurs in the growing region which may result in the elevation of the apex into an erect position. In this case the water which is necessary for the more rapid growth of the under side of the shoot might be absorbed from the posterior fully-developed parts. If, however, only the growing region of the shoot be cut off, or a single internode, the upward curvature will still take place, and, in this case, throughout the whole piece. Accompanying this process we have (I) a loss of weight due to the evaporation of water into the unsaturated atmosphere, (2) an elongation of the convex lower surface, corresponding to the upward curvature, and (3) no elongation, or a very slight one, but more generally a contraction, of the upper concave side.

Curvature of split shoots. If the growing region of a shoot is split into two symmetrical halves which remain connected posteriorly by a portion of the shoot which has completed its growth, they will curve concavely outwards in consequence of the tension of their tissues. If, whilst thus curved, the epidermis of the two concave surfaces and the two convex cut-surfaces of the pith be measured, and if then the shoot be placed in such a way that the epidermis of one surface is directed downwards and that of the other upwards, each half will exhibit geotropism. The growth of the pith of the upper half will be accelerated, whereas that of the cortex of the same half will be retarded or the cortex may even become shorter; in the lower half, the growth of the pith will be retarded and that of the cortex accelerated. The following were the increments of growth in 24 hours observed in Sylpbium connatum :-

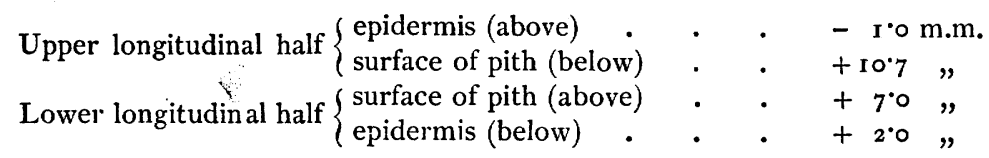

The same takes place, only in a more marked manner, in the case of the haulms of Grasses, which are more adapted for observations of this kind, for, when they are split, the two halves do not curve outwards.

If a longitudinal slice be taken from the middle of the stem of some Dicotyledon which is not hollow but which has a thick pith (e.g. Senecio Doria), by paring away the wood symmetrically on each side, it is possible to place it horizontally in two ways, (a) that in which the cut surfaces are vertical, and $(b)$ that in which the cut surfaces are

1 It is to be remembered that many shoots, such as those of Fritillaria imperialis, are much disturbed in their growth if the apex be cut off; and they hardly grow at all if they are cut off at the base. As a consequence the curvature of such shoots is very slight or entirely absent. 
horizontal. In the position (a) the vertical arrangement of the tissues will be as follows :-

$$
\begin{aligned}
& \text { Cortex, } \\
& \text { Pith, } \\
& \text { Cortex : }
\end{aligned}
$$

in this position the slice will always curve upwards. In the position $(b)$ the different tissues lie side by side in a horizontal plane, thus,

$$
\text { Cortex, Pith, Cortex, }
$$

and nearly the whole of both the upper and under surfaces will be occupied by the section of the pith. In this position it often happens that no geotropic curvature is exhibited, but accurate observation is rendered difficult by the unfavourable conditions.

If a prism of pith be cut out of the growing region of a solid shoot, without any other tissues being attached to it, and if this be placed for five or ten minutes in water so that it becomes rigid and turgid, and be then laid horizontally in moist air or in water, one end being fixed and the other free, no upward curvature takes place.

2. Uprward Curvature of Grass-baulms. In the case of the stems considered in the preceding paragraphs, the whole region of growth, which is of considerable length, is geotropic; hence the curvature is gradual and therefore also of considerable length, and every portion of the stem which has completed its growth in the erect position has become incapable of curvature. In the haulms of Grasses, on the other hand, the capacity for curvature is concentrated at the nodes, the long internodes remaining straight. Hence, a haulm possessing several nodes, if laid horizontally, will exhibit after a short time a number of sudden angular curvatures at the nodes, between which lie the straight internodes. If the oldest internode be fixed in a horizontal position, the third or fourth internode will have assumed an erect position in from one to three days. It is on this that the upgrowth of 'layered' Wheat depends.

It is an especial peculiarity of the haulms of Grasses that they retain for a considerable time the property of becoming erect, that is, that their under surfaces will grow rapidly if they lie horizontally, after that they have ceased to grow while in the normal vertical position. An abnormal position does not only affect the growth of the nodes of a haulm, but even causes it to recommence after it has already ceased; in this particular the motile organs of periodically motile leaves, e.g. those of Pbaseolus, resemble the Grass-haulm.

These nodes which are thus capable of curvature are, as is well known, the basal portions of the leaf-sheaths, which surround the base of the internode as a more or less well-developed annular swelling of considerable thickness but of delicate succulent structure. My observations were made upon Triticum, Dactylis, Glyceria spectabilis, Andropogon niger and Zea Mais, in which plants the nodes are sufficiently large to admit of tolerably accurate measurements. Portions of the haulms were cut off in such a way that there was a node in the middle of each, connected with an internode above and below; the lower cut end was fixed laterally into moist sand in such a way that the whole piece was horizontal: it was then put in a closed metal box, the atmosphere of which was damp. The free internode was found to become erect after two, three, or four days, according to the thickness of the node: sometimes it became vertical, but more commonly oblique. It was easy to see that the lower surface had elongated considerably. If the piece of haulm be now turned in such a way that the convex surface of the node is uppermost, the concave side begins to grow very vigorously; the node becomes straight, and appears uniformly elongated on all sides.

The convex lower surface of a sharply bent node appears smooth, transparent and glistening, whereas the concave upper surface is dark, opaque, and rough in consequence of transverse folds formed by its epidermis and parenchyma. A deep indentation may often be observed in addition, so that it appears as if the node had 
been artificially bent until it was dislocated. These changes are produced by a considerable shortening of the upper surface which accompanies the very vigorous growth of the lower surface. That this is the case is shown by the following measurements made on the nodes of Maize, the thickness of which in the plane of curvature was from $10-12 \mathrm{~mm}$.

Length of the Node.

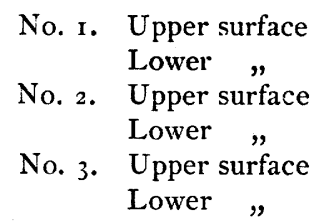

Before curvature.
$4^{\circ} 3 \mathrm{~mm}$.
$4^{\circ} \mathrm{I} "$
$4^{\circ} \circ "$
$5^{\circ} \circ "$
$5^{\circ} \circ "$
$5^{\circ} \circ "$

After curvature ${ }^{\text {. }}$
$2^{\circ} 5 \mathrm{~mm}$.
$9^{\circ} \circ "$
$3^{\circ} 0 "$
$11^{\circ} 0 "$
$4^{\circ} 5 "$
I $2^{\circ} 5 "$

Median longitudinal sections through the curved nodes showed that the cells of the epidermis and of the subjacent tissue of the underside had undergone a corresponding elongation, but that they had not undergone division, whilst those of the upper surface had not grown and had become so compressed by the curvature that the above-mentioned folds of the tissue had been produced.

3. The downward curvature of the tap-roots of seedlings ${ }^{2}$ was studied especially in a large-seeded variety of Vicia Faba, in Peas, Acorns, and Horse-Chestnuts. They were placed horizontally either in moist air, or in water, or in damp earth. In the last case, the seedlings were in a box having oblique walls of glass or of talc, which permitted the observation of the roots during their growth and curvature.

The statement that it is only those portions which are still growing that are capable of curvature holds good also for roots. I showed, in opposition to earlier views, that it is not one part only of the growing region but the whole of it, as in the case of stems, which exhibits geotropism (Fig. 484). Since the whole growing region (as was shown in Section 17 ) is only from 8 to $10 \mathrm{~mm}$. long, and in many roots even shorter, and since the curvature can only be considerable in the middle zones, the curvature appears, especially after a considerable time, sudden and sharp with a very small radius, a condition which is of considerable mechanical advantage to the penetration of the roots into firm soil. Since the considerations which were stated above with reference to stems may be generally applied with propriety to the curvature of roots, the form of the curvature appears to be in this case, as in the former, only at first that of the segment of a circle of large radius; but this is merely apparent, for, since the apex of the root is directed downwards in consequence of the curvature, the younger transverse zones are brought into a position which is unfavourable to geotropism, whilst the oldest soon cease to grow and can therefore curve no further. It is the zones which are in the middle phase of growth which undergo the greatest curvature, for these not only grow rapidly and for a considerable time, but they have also this advantage, that they do not at once come to occupy, in consequence of their curvature, a position which is unfavourable to geotropism, as is the case with the youngest zones. A more detailed account of the conditions which determine the form of the curvature of roots which are either horizontal, oblique, or erect, will be found in my paper which is here quoted.

The measurement of the growth of the upper and under sides of roots is much more difficult than in the case of stems and of the nodes of Grasses. I found that the growth of the upper side was as vigorous, or even more so, as it was when the root retained its normal position and form. The lower surface, however, is considerably hindered in its growth, and it appears from Ciesielski's statements, that

1 The curvature took place in six days.

2 Sachs, Arb. d. bot. Inst. Würzburg, I 873 , Heft III. 
folds are found upon the concave under surface in consequence of compression, just as in the nodes of Grasses. When the root is curving geotropically, all the cells within the curving portion usually grow, but their growth is slower the nearer they are to the lower surface which is becoming concave. In passing from the convex surface, where the cells are fully developed and contain much sap, to the concave surface, where the cells present the appearance of young undeveloped cells containing much protoplasm, all intermediate forms may be met with. Since, therefore, the development of the cells of the under side is very considerably impeded, it is possible for those of the upper side to undergo a more or less excessive elongation. Some observations, as yet incomplete, seem to indicate that the retardation of the growth in length of the under side is accompanied by a more vigorous growth of the cells in a radial direction, and the acceleration of that of the upper surface by a less vigorous radial growth.

If thick primary roots be split and be treated in the manner described with reference to stems, the same phenomena (though in exactly the opposite direction) are generally produced: this shows that geotropism is not merely a property of the root as a whole, but also of each of its constituent parts. These observations are, however, very difficult to carry out. A persistent effect of the commencing geotropic action, which was so well-marked in the case of stems, is stated to occur in roots also by Ciesielski and Frank. I have not yet succeeded in detecting it, but I will not reject the fact, for other methods will perhaps afford more satisfactory results.

4. The chief result of the observations which $I$ have made hitherto is, I believe, this, that the phenomena of upward geotropic curvature are essentially the same, though taking place in the opposite direction, as those of downward geotropic curvature, and that therefore the mechanical explanation of the one will include that of the other. This necessarily implies the incorrectness of the older explanations offered by Knight and by Hofmeister.

Knight, the discoverer of the fact that it is gravitation which induces geotropic curvature, believed the upward curvature of the stem to be due to an accumulation of nutrient materials towards the lower surface, which would induce more vigorous growth. Hofmeister, who regarded the state of tension of the tissues to be the most important factor in producing the curvatures of parts of plants, considered that the action of gravitation in producing an upward curvature was to increase the extensibility of the passively stretched tissues of the lower side. I pointed out that the growth of the under surface of an organ capable of curving upwards

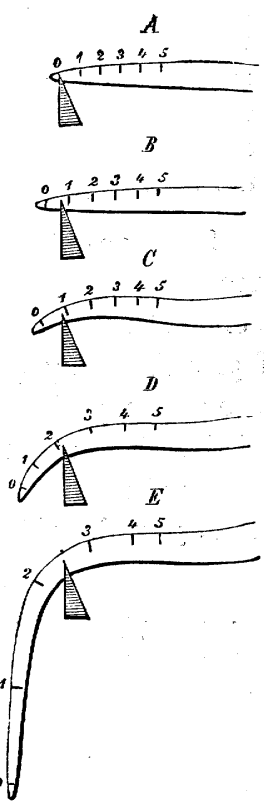

FIG. 484.-The growing and curving end of the primary root of Vicia Faba placed horizontally in loose earth behind a thin lamina, of talc. The root was marked out into five portions, each two millimetres in length, beginning from the punctum vegetationis. A tri angular index of paper was fixed to the talc so as to correspond to the mark $o$ on the root; by means of this the change of position of of this the change of position of the marks could be detected. $A$ at the commencement of the experiment, $B$ after one hour, $C$ at
the end of two hours, $D$ after seven hours, $E$ after twenty-three hours. was accelerated, and that of the upper surface retarded: I did not at the time express an opinion as to whether these modifications of growth were due to an altered distribution of plastic material or to a change in the extensibility of the passive layers of tissue.

Knight explained the downward curvature of primary roots in a somewhat obscure manner by referring it to the softness and flexibility of the growing apex, a view which was adopted by Hofmeister in a more precise and logically complete form, and one which I for a time accepted. It was assumed that the tissue of the growing root was comparable to soft dough, and that the unsupported end tended to curve downwards under the influence of its own weight. I considered that the weight of 
the free apex exercised a traction upon the growing cell-walls of the curving portion of the upper surface, in consequence of which the growth, the intussusception of this side, was accelerated, whilst exactly the opposite took place on the other side, and I believe that Hofmeister held the same opinion. Frank did not make a new suggestion when he pointed out that the downward curvature of the apex of the root was a phenomenon of growth, that is, of the more rapid growth of the upper surface: we held that view already. The point was to determine why the growth of the upper surface of the apex of a root when placed in a horizontal position is more vigorous than that of the under surface. Frank was right in asserting that the theory of Knight and Hofmeister was untenable, for, as Johnson had already shown, the apex of a root will curve downwards when its weight is counterbalanced and even when the counterbalancing weight is greater, and also because when the apex of a root is placed upon a firm horizontal support or upon the surface of mercury ${ }^{1}$ it exhibits the same phenomena of growth which effect the downward curvature. The explanation of Frank and the more recent ones of Müller are inadequate to answer the crucial questions.

SECT. 22.-Unequal Growth ${ }^{2}$. Our observations have hitherto had reference almost exclusively to the growth of multilateral or polysymmetrical organs, such as erect stems and descending roots. Organs of this kind offer the simplest example of growth taking place equally on all sides. But they form only a small minority, since not only a large number of primary stems like those of Hepaticæ, Rhizocarpeæ, and Selaginelleæ, but also by far the greater number of lateral branches of erect stems, and all leaves, display a decidedly bilateral organisation, i.e. two sides of their axis of growth exhibit different properties. With this bilateral organisation is also

1 See Fig. 477, and Arb. d. bot. Inst. Würzburg, Heft III. p. 448 et seq.

2 A. B. Frank, Die natürliche wagerechte Richtung von Pflanzentheilen (Leipzig, 1870). The views propounded in Frank's treatise are opposed by H. de Vries in the second Heft of the Proceedings of the Würzburg Bot. Inst. $187 \mathrm{r}$, p. 223 et seq.-See also Hofmeister, Allgemeine Morphologie der Gewächse, Leipzig I 868, Sect. 23, 24. [It has been noticed by many observers that the position of members of plants, such as leaves and the thallus of Liverworts, which have a distinctly bilateral structure (that is, which are dorsiventral), is at right angles to the direction of incidence of the rays of light and to the action of gravity. Frank considers that this is due to certain properties with which these members are endowed and by virtue of which their reaction to these forces is different from that of polysymmetrical members, such as stems and roots, which tend to place themselves in the line of incidence of the rays of light and of the action of gravity. This peculiar form of heliotropism and of geotropi $-m$ he terms transverse. In the note on p. 843 it is stated that Elfving has found instances of what appears to be transverse geotropism in certain rhizomes, which do not however possess a bilateral structure. Frank's views are adopted by Darwin (Movements of Plants); he uses the terms diaheliotropism and diageotropism.

De Vries concludes that the position of these dorsiventral members is the expression of the resultant action of several forces, such as negative heliotropism, negative geotropism, hyponasty, epinasty, and does not admit that these members react differently to polysymmetrical members when exposed to the action of light or of gravity. Sachs (Ueb. orthotrope und plagiotrope Pflanzentheile, Arb. d. bot. Inst. in Würzburg, II. 2, I879) comes to much the same conclusions. He divides all parts of plants into two classes, according to the position which they assume under the influence of ordinary external conditions. He terms those parts orthotropic which assume a vertical position and those parts plagiotropic which assume a position inclined to the vertical. All sides of the former react similarly to light and to gravity, whereas in the latter one longitudinal half reacts differently to the other longitudinal half. In the former the polysymmetrical structure is correlated with a polysymmetrical organisation; in the latter the bilateral structure is correlated with a bilateral organisation. In some cases, however, members with polysymmetrical structure are bilaterally organised, as is shown by the fact that they are plagiotropic.] 
usually connected a difference in the growth of the two dissimilar sides, which causes curvatures and hence changes in the position of the apex. The two dissimilar sides of bilateral organs must also be acted on differently by external agencies which affect growth, such as light, gravitation, and pressure. We do not attempt here to solve the question of the causes which produce the bilateral structure in any particular case; it need only be shown incidentally that this structure of lateral organs (as we have already seen in Book I. Sect. 27) is probably always brought about by internal. causes, and is independent of the action of external circumstances. This is in general evident from the fact that the median plane of bilateral appendicular organs has always a perfectly definite geometrical relation to the axial structure which bears them, and that moreover in the dark and under the influence of slow rotation round a horizontal axis, which eliminates the effect of gravitation, the bilateral structure and relation to the axis remain unchanged.

But before we proceed to the consideration of the growth of bilateral organs, it must be premised that even in multilateral erect stems and vertically descending roots growth does not always proceed equally and with equal rapidity on all sides of the longitudinal axis; it is much more common for first one side and then another of the organ to grow more rapidly than the rest, curvatures being thus caused the convexity of which always indicates the side that is at the time growing most rapidly. If another side then. grows more rapidly, it becomes convex, and the curvature changes its direction. Curvatures of this kind caused by the unequal growth of different sides of an organ may be called Nutations, and in so far as they are produced entirely by internal causes they may be said to be spontaneous. They occur most commonly and evidently when growth is very rapid, and consequently in organs of considerable length, and are produced under the influence of a high temperature either in darkness or when the amount of light is very small.

When two opposite sides of an organ grow alternately more and less rapidly, curvatures are caused first on one side and then on the other; it will, for example, bend first to the left, then become erect, and then bend to the right side; as occurs, e.g. in the long flower-scapes of Allium Porrum, which finally take an erect position when their growth is ended. It is much more common for the apices of erect stems above the curved growing part to move round in a circle or ellipse, the region of most active growth moving gradually, as it were, round the axis : it lies, for instance, at one time towards the north, then towards the west, south, and east in succession until it comes again to lie towards the north. This kind of nutation may be termed a Revolving Nutation ${ }^{1}$. Since the apex of the stem is constantly rising higher during the nutation owing to the elongation of the part below it, its revolving motion does not take place in a plane, but describes an ascending spiral line. This form of nutation occurs in many flower-stalks before the unfolding of the flowers, as in those

1 [Darwin is of opinion (Movements of Plants) that all nutation is revolving nutation, or, as he terms it, circumnutation. He regards all the movements connected with growth (heliotropism, geotropism, hyponasty, epinasty) as well as those of mature parts (spontaneous or induced movements) as being modified forms of circumnutation.

The unequal growth is of course the expression of an unequal turgidity of different parts of the growing organ (see de Vries, Ueb. die inneren Vorgänge bei den Wachsthumskrümmungen mehrzelliger Organe, Bot. Zeitg. 1879.] 
of Brassica Napus, where the movement ceases when growth is completed, and the stem finally becomes erect. It is very general in climbing stems and in almost all erect stems that bear tendrils; but bilateral tendrils also revolve at the time when they are about to take hold of a support ${ }^{1}$.

In bilateral appendicular organs nutation does not usually take the form of a revolving motion, or only to a subordinate extent, as in tendrils. The outer or dorsal side more often grows more rapidly so that the organ is curved concavely to the primary axis, and the inner side afterwards begins to grow more quickly, so that the organ finally becomes straight, or even concave on the dorsal side. This is the case in all strongly developed foliage-leaves, very strikingly in those of Ferns, which are at first rolled up towards the axis, and then unroll, often bending over backwards, becoming finally straight. The same phenomenon occurs in the tendrils of Cucurbitacee, which are also at first rolled up inwards, then become straight, and are finally rolled up outwards. Other tendrils are at first straight or only slightly

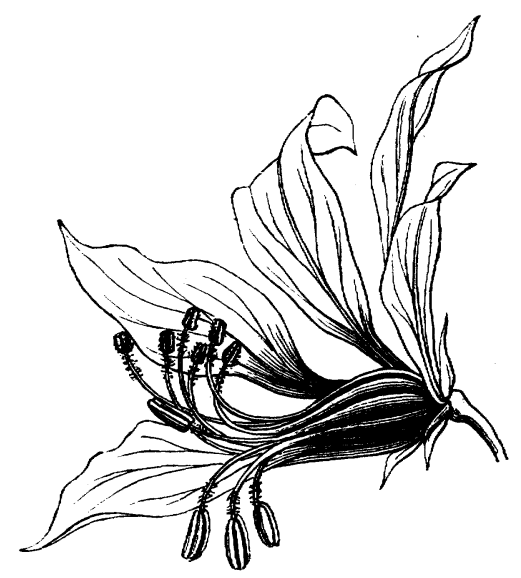

FIG. 485,-Nutation of the filaments of Dictamnis Fraxinella; the filaments of the stamens whose anthers have not yet opened are bent downwards; those with anthers already burst are bent upwards.

concave inwards, like leaves in vernation, but are afterwards rolled backwards. Movements of nutation are very common and easily observed in stamens with long filaments, as Tropcoolum majus, Dictamnus Fraxinella (Fig. 485), Parnassia palustris ${ }^{2}$, \&c., and in long styles like those of Nigella sativa, \&c. They occur at the time of the maturity of the sexual organs, and serve to place the stigmas and anthers in the positions adapted for the conveyance of pollen by insects from one flower to another ${ }^{3}$. Most lateral shoots behave in the same manner as ordinary leaves, growing at first only quickly enough on the outer side to become appressed to the primary axis in vernation, afterwards more rapidly on the inner side, by which they become straight and diverge at a greater or smaller angle from the primary shoot.

${ }^{1}$ See Sect. ${ }^{25}$, On the Twining of Tendrils.

2 [On the stamens of Parnassia, where there is not properly any movement of nutation, see Gris, Comp. rend. Nov. 2, I868; and A. W. Bennett, Journ. Linn. Soc. vol. XI. p. 24, 1869.]

${ }^{3}$ Vide infra under Fertilisation, Chap. VI. 
These movements of nutation of bilateral appendicular organs take place mostly. in one plane which coincides with the median plane of the organ. As long as the organ grows most rapidly on the dorsal side, it may be termed, after de Vries, hyponastic; afterwards, when it grows most rapidly on the inner or upper side, epinastic. Since in the later stages of development of an organ growth ceases at certain places-while at different distances from these places it presents different stages of growth, until it finally ceases everywhere-it is clear that in the same organ, together with areas where growth is completed and nutation no longer takes place, others occur with hyponastic and others again with epinastic growth, until at length nutation and growth alike cease altogether, as in Fern-leaves.

Seedlings of Dicotyledons afford a remarkable illustration of bilateral structures which nutate in one plane.; although their stem and primary root become afterwards multilateral and grow vertically upwards and downwards. The stem terminates in a pendent or nodding bud; and the curvature, which is generally very great, exhibits itself also in germination when it takes place out of the ground in a vessel that rotates slowly round a horizontal axis; it is a true curvature of nutation independent of light and gravitation. But the older portions of the stem become straight as they develope from the curved portion; and in proportion as the stem increases in length, the straight part which bears the nodding bud also lengthens. When germination takes place in a feeble light, or better in a slowly rotating vessel, a more rapid growth occurs of the side of the older portion of the stem which was at first concave, causing it to become convex; and hence the older and younger parts of the stem form together a letter S, as in Phaseolus, Vicia Faba, Polygonum Fagopyrum, Cruciferæ, \&c. But the primary roots of dicotyledonous seedlings also manifest a tendency to a bilateral organisation; since, when they develope under slow rotation round a horizontal axis, they seldom continue to grow straight, but curve concavely either in front or behind, sometimes even becoming rolled up. These and other instances of nutation are not clearly seen when the development takes place under normal conditions, because the growth of the stem of the seedling is retarded by light, and the curvature both of stem and root prevented by geotropism.

A knowledge of the different capacity for growth possessed by the anterior and posterior sides of bilateral organs lies at the root of an understanding of the fact that leaves, lateral shoots, and many secondary roots, although they are heliotropic and geotropic, yet assume definite positions with respect to the horizon, but without growing vertically upwards or downwards. When multilateral (orthotropic) primary stems and roots grow vertically, the essential cause is their growth being uniform on all sides of the axis of growth; the different sides of the organ are. in equilibrium with one another. Every deviation from the vertical position, to the right, left, front, or back, is counterbalanced by geotropism; the growing part curves until the free apex stands erect, in which position the action of gravitation is again equal on all sides. In the same manner light acts equally strongly on all sides of such organs. If therefore one side is exposed to stronger light, a heliotropic curvature takes place which finally brings the free part into a position in which all sides receive equally strong light on all sides, and therefore grow uniformly without any further curvature. The case is different with bilateral organs the anterior and posterior sides of which possess independently different capacities for growth (plagiotropic), and which there- 
fore exhibit a tendency for the more rapidly growing side to become convex. If the growth is very strongly hyponastic or epinastic, the curvature thus caused may take place in spite of the opposing action of light and gravitation, supposing the organs to be actually heliotropic or geotropic. Organs which grow horizontally or obliquely to the horizon must not be assumed to be on that account wanting in heliotropism or geotropism; still less is it necessary to suppose in these cases any special or altogether abnormal relations to light and gravitation ${ }^{1}$. It is sufficient, as de Vries has clearly shown, to suppose that light and gravitation act in the ordinary way on the growth of bilateral organs, in order to explain their directions of growth, if only it is borne in mind that their heliotropism and geotropism cooperate with their hyponastic and epinastic properties, and thus bring about positions of the organs which must be considered as the resultants of these different forces. The weight of the overhanging part must however also be taken into account, its tendency being always to change the lateral direction of the organ into a more horizontal or even pendulous one; and this must occur more decidedly the less the elasticity of the organ. When large leaves assume oblique or horizontal positions, it is because their epinasty tends to make them concave downwards as they unfold, while their positive heliotropism tends to make them concave upwards. The result is consequently a more or less flat expansion of the leaf, the position of which depends on the relation of the weight of the lamina to the flexibility of the petiole and mid-rib. The same phenomena are observable in horizontal or oblique lateral shoots, in which however the hyponasty of the axis often counterbalances the greater mass of the pendent parts (as in Prunus Avium, Ulmus campestris, Corylus Avellana, Picea nigra, \&c.). As soon as the position resulting from these forces is attained, it becomes permanent, from the mature parts becoming lignified, rigid, and hard, and thus in a condition to maintain the weight of the pendent parts.

If leaves which are unfolding or still growing have their under side turned upwards or towards the light, very strong curvatures take place, generally combined with torsions, by which the lamina finally resumes more or less completely its normal position; and the impression is given as if the under side were more sensitive to the influence of light, and the upper side to that of gravitation than the reverse. But this hypothesis is superfluous if it is borne in mind that in this case epinasty works concurrently with heliotropism and geotropism, and hence much stronger curvatures must take place than in the normal position where the former acts in opposition to the two latter forces.

The results here described are derived from the experiments of de Vries, which have been already quoted. For the following I am also indebted to him.

(a) Leaves. If a strongly developed mid-rib is separated from a leaf in active growth, it curls up concavely on the under side, showing that a tension exists between it and the lamina. De Vries found this to be the case in nearly two hundred species, with only a few exceptions. This curvature does not take place equally strongly at all ages; in leaves which have but just emerged from the bud it does not occur at all; it increases with age, and attains its maximum when the leaf is nearly fully grown, then decreases, and altogether disappears when the leaf has reached full maturity. This tendency to curve is at first apparent along the whole length of the mid-rib; it disappears first of all at the base, the part capable of curvature becoming constantly

${ }^{1}$ [See note 2 on p. $85+$ with reference to diaheliotropism and diageotropism.] 
smaller and nearer to the apex. If mid-ribs of leaves are separated in this last stage of growth and fixed upright in a damp and dark place (e.g. in wet sand in a spacious closed zinc box), they will continue to grow for some time; and since growth is more vigorous on the inner (anterior or upper) side, they will curve concavely on the posterior (or under) side, the curvature being however partially counteracted by geotropism. If separated mid-ribs of leaves are fixed horizontally in wet sand, so that the median plane lies horizontal, the epinastic curvature will take place without hindrance in a horizontal direction; but a geotropic curvature will at the same time ensue in a vertical plane, so that the two kinds combine to produce an obliquely ascending position. If, on the other hand, two similar mid-ribs are separated and fixed horizontally in wet sand, with the posterior side in one case below, in the other case above, geotropism will act in the former in opposition to epinasty, while in the latter the two will cooperate; and the consequence will be that in the former case the epinastic curvature will be more or less neutralised, while in the latter a strong curvature will take place upwards, the two forces acting in unison.

Phenomena of the same kind are produced by a combination of epinasty with heliotropism, if the separated mid-rib is fixed vertically in wet sand in a closed vessel into which light is admitted from one side,through a glass plate. Heliotropism is generally but not always exhibited, and is then always positive; but in all the cases hitherto observed is too weak to overcome epinasty. It will be seen from what we have said that all these movements of the mid-rib will be much less considerable when it is still in connexion with the lamina. Petioles show in general the same phenomena as midribs, but their motions which result from heliotropism, geotropism, and epinasty are unimpeded.

(b) Bilateral secondary shoots, such as branches of an inflorescence, horizontal or erect leafy branches, and stolons, were experimented on in a similar manner. It was thus proved that the branches of the inflorescence of Isatis tinctoria, Archangelica oficinalis, Crambe cordifolia, and all others that have been observed, the horizontal branches of Pyrus Malus, Asperugo procumbens, \&c., as well as the runners of Fragaria, Potentilla reptans, Ajuga reptans, \&c., are epinastic. When fixed horizontally in wet sand, they all curl upwards, whether the side that normally faces downwards (the posterior side) be placed below or above, but in the latter case more strongly, because geotropism and epinasty then cooperate. In some species (as Tilia and Pbiladelpbus) a branch, when stripped of leaves and placed in its normal position, did not curl upwards, while one placed in a reverse position did so, proving that there was in these cases an equilibrium between geotropism and epinasty. The horizontal branches of Prunus Avium, Ulmus campestris, Corylus Avellana, and some other plants were found on the other hand to be hyponastic; when laid horizontally in their natural position they curved upwards, but downwards if reversed, because their hyponasty was stronger than their geotropism.

Similar experiments to those made on petioles with respect to heliotropism showed in many cases the absence of this phenomenon, especially in the case of stolons; and that in other cases it was always positive, but too feeble to overcome the influence of their epinasty. In the case of branches, especially such as are long and slender, more account must be taken of weight in modifying the direction of growth than in that of leaves. The removal of the leaves $(e . g$. in Corylus) is in this case followed by a sudden curving upward, the result of elasticity; but this is subsequently intensified by geotropism and in many cases (as in Abies) also by hyponasty.

It may be left to the ingenuity of the student to ascertain the conditions determining the direction of an organ in any particular case, from the points of view stated above.

Sect. 23.-Torsion ${ }^{1}$. Organs of any considerable length very commonly ex-

${ }^{1} \mathrm{H}$. de Vries in the second Heft of the Proceedings of the Würzburg Botanic Institute, $187 \mathrm{r}$, p. 272.-Wichura in Flora, 1852, No. 3, and Jahrbuch für wissensch. Bot. vol. II, 1860.-Braun in Bot. Zeitg. 1870, p. 158. 
hibit torsions about their axis of growth; the striations on the surface of the organ are not parallel to its axis of growth, but run round it in the form of more or less oblique spiral lines, as if the organ were fastened at one end, and then twisted at the other. Torsions of this kind occur in the unicellular internodes of Nitella; they are common in the elongated multicellular internodes of the erect stems of Dicotyledons, universal in climbing internodes; the setæ of Mosses are generally very strongly twisted. Even in flat leaves, as Wichura has shown, torsions of the lamina occur very commonly; they behave like strips of paper fastened at one end and twisted by the other round their median line. These torsions are particularly conspicuous in the leaves of many Grasses, of Allium ursinum, species of Alstromeria, $\&$ c., causing the under side of the lamina to lie uppermost towards the apex ${ }^{1}$.

Since the striæ on a twisted organ run spirally round the axis, they must exceed the axis in length; if therefore the torsion is the result of growth, the growth of the outer layers of cylindrical, conical, or prismatic organs (internodes, roots, \&c.) must be more rapid or must last longer than that of the inner layers; and in twisted leaves there must be the same difference as respects the growth of the mid-rib in comparison to that of the margins. The fact that at the time of most rapid growth the inner layers generally grow more rapidly than the outer ones (Sect. I3), thus preventing the possibility of torsion, the additional fact that torsion does not generally take place until growth is ceasing, and lastly, the circumstance that etiolated internodes, which in a normal state do not exhibit torsion, usually manifest this phenomenon at the close of their growth, lead to the conclusion that torsion is the result of growth continuing in the outer layers after it has ceased or begun to cease in the inner layers. In twisted leaves, especially those of Alstromeria, the torsion however begins earlier. If the growth of the outer layers, besides being greater, were also exactly parallel to the axis, and if the resistance to the strain thus caused of the outer against the inner layers were exactly in the direction of the axis, there would be no torsion, but only a longitudinal tension between them, which would be directly opposed to the tension of the layers already described. It is however evident that this would be possible only if all the parts were arranged with mathematical precision; but that any irregularity, however small, must give a lateral direction to the strain in the outer layers, and thus cause a torsion ${ }^{2}$.

Torsions are also very often the result of an increase in diameter or are made more evident as the formation of wood advances, as is often seen in the bark of old stems of Dicotyledons and Conifers, and more clearly in the oblique course of the fibro-vascular bundles. It may be concluded with probability that the phenomenon is the result of the small but powerful increase in length of the young wood-cells; if these did not increase at all in length no torsion would take place.

1 [Similar torsions occur in petals as Cyclamen, fruits as Ailanthus malabarica, and not unfrequently in pedicels or inferior ovaries as Orchidex, causing the anterior part of the flower to become apparently posterior, and vice versa.]

2 This can easily be made clear to the student in the following way. If an india-rubber tube is strongly stretched, and another tube only a little wider is drawn over it, and the first is then released, it contracts and is then too short for the outer tube. If the two tubes were perfectly uniform in structure in the longitudinal and transverse directions, the only result would be a longitudinal tension; but torsion takes place also because a transverse is combined with the longitudinal tension. 
The examples of torsion we have been considering so far are produced solely by internal causes; the direction in which the striæ run round the axis is usually constant in the same species; but other instances of torsion frequently occur which result from external and accidental circumstances. It is evident that when any weight is attached to the side of an organ growing in a horizontal or oblique direction, such as an internode, leaf, or tendril, the tendency will be to produce a twisting of the organ round its axis. If the organ which is twisted in this manner is very elastic, the torsion will disappear when the weight is removed; but if it is only very imperfectly elastic, the torsion will remain permanently, as in a twisted thread of wax; and this will be the case if the organ is in a growing state. This does in fact occur in growing internodes, petioles, the mid-ribs of leaves, \&c. If an organ of this kind is fixed horizontally in wet sand, after a pin slightly weighted on one side, as by a drop of sealing-wax, has been passed horizontally through its summit, the small twisting force is sufficient, as de Vries has shown, to cause a permanent torsion in the growing part. The same result will of course ensue if a leaf or branch instead of a pin is attached to the side of the organ. Branches which grow horizontally and bear decussate pairs of leaves usually exhibit alternate torsions of their internodes to the right and left, so that the leaves all stand in two rows along the branch instead of in four. De Vries has shown that this is occasioned by the unequal twisting force of the leaves of each pair. If the young leaves are cut away no torsion results; if only one of each pair is removed, the torsion is determined by the weight of the remaining leaf.

Torsions of this kind also occur frequently when leafy shoots rise in consequence of geotropism from a horizontal position, and are caused by the unequal distribution of the weight of the leaves, and by their various geotropic and heliotropic curvatures twisting the stem as it becomes erect. Very clear instances are furnished by long petioles as those of Cucurbita, when the branch from which they spring is fixed in a reverse position. The effect of geotropism alone or combined with heliotropism would be simply to cause the petiole to curl upwards in a vertical plane; but the weight of the lamina is scarcely ever equally distributed on the two sides of the plane of curvature; one side is more heavily weighted, and causes the plane of curvature of the petiole to bend obliquely to that side, and other parts of the petiole to be thus exposed to the influence of gravitation and heliotropism. Complicated curvatures and torsions of the petiole and of the lamina itself are caused in this way, the final result being again to reverse the lamina, so as to bring its proper upper side uppermost and expose it to the light as much as possible.

It will be seen therefore that a distinction must be drawn between two kinds of torsion; firstly, that of erect organs; and secondly, that of organs which grow in a horizontal or oblique position. In the former case the torsion results from internal conditions of growth, and especially from the outer layers growing more rapidly than the inner ones; the arrangement of the internal parts-in the internodes of higher plants probably the course of the fibro-vascular bundles-determines the direction of the torsion.

Torsions of the second kind are caused in quite a different way. The outer layers of the growing organ are in a state of passive tension, and there is no internal tendency to torsion; but the weight of the parts attached to it causes a torsion of the growing organ, which is rendered permanent by growth and by the very imperfect elasticity of the organ. 
SECT. 24.-The Twining of Climbing Plants'. The stems of climbing plants, composed of long internodes, have the power of twining spirally round upright slender supports; and the long petioles of the Fern Lygodium possess the same property. This twining is a consequence of unequal growth, of a revolving nutation. It is not caused, as Mohl held, by an irritation exercised by the support on the growing internodes, and is therefore essentially distinct from the twining of tendrils round supports, which depends on the irritation caused by constant and permanent pressure $^{2}$.

Only a few plants twine to the right (i.e. from right to left as one looks at the support round which the plant twines), following the course of the sun or of the hands of a watch; among these are the Hop, Tamus elephantipes, Polygonum scandens, and the Honeysuckle; the greater number twine to the left, as Aristolochia Sipho, Thunbergia fragrans, Jasminium gracile, Convolvulus Sepium, Ipomaa purpurca, Asclepias carnosa, Menispermum canadense, Phaseolus, \&c.

The first internodes of twining stems, whether they are primary stems as in Phaseolus, lateral shoots from rhizomes as in Convolvulus, or from aërial organs as in Aristolochia, do not twine but grow erect without any support. The succeeding internodes of the same shoot twine; they first of all elongate considerably, while their leaves grow only slowly. The long young internodes incline to one side in consequence of their weight, and in this position revolving nutation begins; the overhanging part curves and executes a movement which causes the terminal bud to describe a circle or ellipse. This circular motion is caused entirely by the curving of nutation. If a black line is painted along the convex side of an internode of a plant that twines to the right, like the Hop while the bud is pointing to the south, then, when the bud points to the north it will be found on the concave side; when to the west or east, on the lateral surface between the convex and concave sides. Usually two or three of the younger internodes are in a state of revolving nutation at the same time; and, since they are in different stages of growth, the curvature of the older internode does not generally coincide with that of the younger one; the whole does not therefore form a simple arc, but often an elongated letter $\mathrm{S}$, with the different parts lying in different planes. As new internodes develope from the bud, they begin to revolve, while the third or fourth internode ceases to do so, becomes erect, and manifests another form of movement, becoming twisted, until its growth ceases $^{3}$.

1 [L. Palm, Ueber das Winden der Pflanzen: Preisschrift, Stuttgart 1827.-Mohl, Ueber den Bau und das Winden der Ranken und Schlingpflanzen, Tübingen I827.-Dutrochet, Comptes rendus, I84., vol. XIX, and Ann. des Sci. Nat. 3rd ser. vol. II.-Darwin, On the Movements and Habits of Climbing Plants, London 1875).

"Darwin has already attempted to show that Mohl's view of the irritability of climbing internodes is untenable, without however bringing forward any convincing proof. But this proof has been afforded by $\mathrm{H}$. de Vries in a series of investigations carried on in the Würzburg laboratory, published in the third part of vol. I of the Proceedings of the Würzburg Bot. Inst. (1873). The description here given of the mechanical principles is based principally on his results.

[See also Darwin, Movements and Habits of Climbing Plants, 1875, and Movements of Plants, 1880.]

3 Torsion is therefore not the cause of the revolution of the apex of the shoot, as is seen at once from the fact that the number of revolutions of torsion in the same time is different from that of the revolutions of nutation. 
The direction of revolving nutation and of torsion is, in all climbing plants, the same as that in which they twine round their support ${ }^{1}$. If a point in the terminal region exhibiting nutation is prevented from moving by some external cause, as by being fixed, the revolving movement of the free part will continue for some time, but the free part will then grow in a spiral ascending in the direction of nutation. The revolving movement of nutation thus combines with the induced torsion of the lower parts which are already coiled spirally; but this torsion is opposed in its direction to the revolving nutation, and therefore also to the torsion previously mentioned which exists in the lower portion of the free part. This latter torsion is probably occasioned by the weight of the free overhanging apex of the shoot; at all events it causes the concave side of the part in a state of revolving nutation to face from that time the axis of the spiral which has been formed.

The most common case in which revolving nutation is hindered in this way is when the apex of a shoot comes, in consequence of this motion, into contact with an erect support. If the support is not too thick, it forms the axis of the spiral curvatures which the climbing stem makes round it; when the support is very slender, the stem winds in such large coils that they do not touch the support at all, or only accidentally at a few places.

But revolving nutation can also be artificially interfered with in various other ways; as, for example, by placing a support on the posterior side of the shoot as respects its revolution, and fastening it by means of gum to the apex of the shoot, which would otherwise become detached from it. The first spiral coil is in this case formed in precisely the same manner as if the support were in its normal position, but the support stands outside the coil which does not therefore embrace it. Spiral coils of this kind, not embracing any support, are frequently produced when the stem rises above its support.

The youngest coils of a twining stem are not usually in contact with its support; they are wide and flat; while the older coils are in close contact with it, and are narrower and more oblique. This shows that the close clinging of climbing stems to their support is a subsequent result, the coils being at first looser and wider, and becoming afterwards narrower and more oblique. This fact, which is of great importance in the interpretation of the phenomena of climbing plants, was placed beyond doubt by de Vries, who caused the summits of climbing plants to coil in this manner without having any support in the middle. In this case also the coils were at first wider and flatter, and became narrower and more oblique with increasing age, until at length the piece became quite erect, a revolution of torsion being all that represented each spiral revolution. It is not improbable that geotropism is the cause of the coils-at first flat and sometimes almost horizontal-becoming afterwards more oblique. It is clear that the stronger the force with which the coils become narrower and more oblique, the more closely must they cling to their support. If there is a support in the axis of the coils, the younger parts of the summit will be constantly prevented by it from performing their normal revolution of nutation, and the apex will therefore continue to grow in a spiral, and will climb continually further up the support, the older coils always becoming more oblique

1 What follows is from de Vries. 
and clinging to the support. If the support is removed soon after a few loose coils have been formed round it, the shoot will retain its spiral form for a time, but will then straighten itself and recommence the revolution at its apex.

A revolution of torsion of the twining internodes must, on purely mechanical grounds, accompany every revolution of twining; but torsions of the parts which have already coiled also occur, especially with round rough irregular supports; their direction is sometimes to the right, sometimes to the left.

During the course of the twining the leaves must sometimes stand on the outside, sometimes on the inside of the coils ${ }^{1}$; in the latter case the leaf-stalk will be pressed against the support on which it slips laterally under the pressure of the contracting coil, dragging the internode sideways with it, and thus causing a local torsion.

What has now been said includes almost all that we at present know on the mechanism of the twining of climbing stems. A few remarks, borrowed from Darwin, may be added.

The revolution of the free overhanging apex is often strikingly uniform in the same plant under the same external conditions (as e.g. in the Hop, Micania, Pbaseolus, \&c.).

The following table of Darwin's gives some idea of the time required, under favourable conditions, for a revolution:-

\begin{tabular}{|c|c|c|c|c|}
\hline$y p h$ & & lour & 17 & \\
\hline Akebic & I & » & 30 & \\
\hline Conv & $\mathrm{I}$ & " & $4^{2}$ & \\
\hline Pbaseolus & I & $"$ & 55 & \\
\hline Adbatoda & & & & \\
\hline
\end{tabular}

The direction of the twining is usually constant in the same species; but it does sometimes happen, as in Solanum Dulcamara and Loasa aurantiaca, that different individuals twine in opposite directions. Darwin found, in these two species and in Scyphanthus elegans and Hibbertia dentata, that the same stem will sometimes twine first in one. and then in the other direction.

The positive heliotropism of twining internodes is generally feeble; a powerful heliotropism would obviously be only a hindrance to the twining and especially to the revolution, by which an effort, so to speak, is made to reach the support. Heliotropism is however shown by the fact that when the light falls from one side only, revolution takes place more quickly towards the source of light than away from it; as e.g. in Ipomaa jucunda, Lonicera brachypoda, Phaseolus, and Humulus.

It may be concluded from what has been said on the mechanism of twining that there is for every species a certain maximum of thickness of the support at which the twining is possible. The support must not be much thicker than the diameter of the coils which the shoot can make without a support; if the support is too thick, the apex of the shoot attempts to make coils by its side, and these eventually become effaced. Darwin (l.c. p. 22) acknowledges his ignorance of the cause why the climbing plant cannot twine round supports which are too thick; de Vries's experiments however seem to give a sufficient explanation.

The movements of twining internodes are more energetic the more favourable the external conditions of growth, and the more rapid the growth itself; they are therefore vigorous when food is abundant, temperature high, and the plants contain abundance of

${ }^{1}$ I may take this opportunity of remarking that, according to Dutrochet, the genetic spiral of the phyllotaxis takes the same direction in climbing plants which have their leaves arranged spirally as the twining; and therefore also the same as the spontaneous torsion and the revolving nutation of the same plants. 
sap. The direct action of light is not necessary for twining, since even etiolated plants (as Ipomea purpurea and Phaseolus multiforus) cling closely to their support in the dark. The assertion of Duchartre that Dioscorea Batatas does not twine in the dark reduces itself, according to de Vries's more recent observations, to the fact that while normal green shoots continue to climb in the dark, they cease rotating and twining when they become etiolated.

SECT. 25.-The Twining of Tendrils ${ }^{1}$. Under the term tendril may be comprised all filiform or at least slender long and narrow parts of plants which possess the property of curving round slender solid supports with which they come in contact during their growth, clinging to them in consequence, and thus at length fixing the plant to them. Tendrils are therefore at once distinguished from climbing internodes by their irritability to contact or pressure.

Organs of the most various morphological description may assume this physiological property. Sometimes tendrils are metamorphosed branches, as in Vitis, Ampelopsis, Passiflora, and Cardiospermum Halicacabum, where they may be considered more accurately as metamorphosed flower-stalks or inflorescences. In

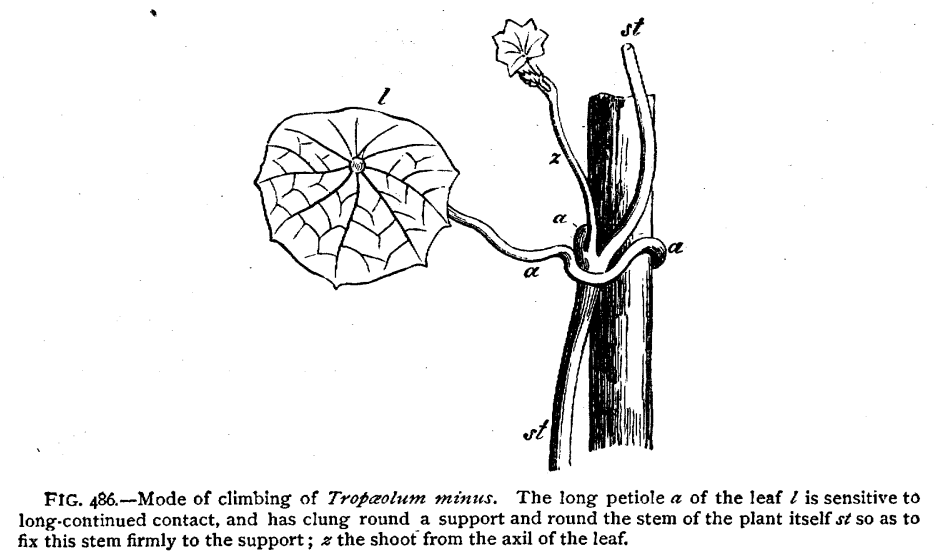

Cuscuta the whole stem may be regarded as a tendril rather than as a climbing stem. In other cases, as in Clematis, Tropaolum (Fig. 486), Maurandia, Lophospermum, Solanum jasminoides, \&c., the petioles may serve as tendrils. In Fumaria officinalis and Corydalis claviculata the whole of the finely-divided leaf is sensitive to contact, and its separate parts have the power of twining round slender bodies. In Gloriosa Plantii and Flagellaria indica the mid-rib protruding beyond the leaf serves as a tendril. In many Bignoniaceæ, in Cobca scandens, in Pisum, $\& c$. the anterior (upper) part of the pinnate leaf is transformed into slender filiform tendrils inclined forwards, while the basal part of the leaf is rigid and divided into leaflets; sometimes, as in Lathyrus Aphaca, the whole of the leaf is replaced by a filiform tendril. The morphological character of the tendrils of Cucurbitaceæ is still doubtful, though they must probably be regarded as metamorphosed branches.

I See the literature quoted in the preceding section, and de Vries, Arb. d. bot. Inst. in Würzburg, I. 
The distinguishing properties of tendrils are more perfectly developed the more exclusively they serve as organs of attachment for the sole purpose of climbing, the less therefore they partake of the normal character of leaves or parts of the stem; in other words, the more perfectly the metamorphosis is carried out. To this category belong especially the simple or branched filiform tendrils of the Cucurbitaceæ, Ampelideæ, and Passifloreæ. A typically developed tendril of this kind is represented in the mature state in Fig. 487 , after it has seized hold of a support by its apex and then coiled up. What is said here refers especially to true tendrils of this description.

The characteristic properties of tendrils are developed when they have completely emerged from the bud-condition, and have attained about three-fourths of their ultimate size. In this state they are stretched straight; the apex of the shoot which bears them usually revolves, the tendril itself exhibiting the same phenomenon, curving along its whole length (with the exception usually of the oblique basal portion and the hooked apex) in such a manner that the upper side, the right side, the under side, and the left side become in turn convex. No torsion takes place. During this revolution the tendril is rapidly growing in length and is sensitive to contact; i.e. any contact of greater or less intensity on the sensitive side causes a concave curvature first of all at the point of contact, from which the curvature extends upwards and downwards. If the contact is only temporary, the tendril again straightens itself. The degree of sensitiveness ${ }^{1}$ is very different in different species; in Passiflora gracilis a pressure of $\mathrm{I}$ milligram is sufficient to cause curvature in a very short time $(25 \mathrm{sec})$; in other species a pressure of 3 or 4 milligrams is required and the curvature does not take place so soon (30 sec. in Sicyos); the tendrils of other species curve, when slightly rubbed, in a few minutes; in the case of Dicentra thalictrifolia in half an hour; in Smilax only after more than an hour; in Ampelopsis still more slowly.

The curvature on the side which has been touched increases for some time, then remains stationary, and finally (often after some hours) the tendril again straightens itself, in which state it is once more sensitive. A tendril the apex of which is slightly curved is sensitive only on the concave under surface; others, as those of Cobcea and Cissus discolor, are sensitive on all sides; in Mutisia and Clematis the under and lateral surfaces are sensitive, but not the upper surface.

While the revolving nutation and sensitiveness last the tendril attains its full size in a few days; the revolving motion then ceases, and with it the sensitiveness; and further changes then follow, differing in different species. In some the tendrils remain straight after they have completely developed and become motionless; in others they become abortive and fall off, as e.g. Bignonia, Vitis, and Ampelopsis. It is more common for the tendrils to roll up from the apex slowly to the base, when growth has ceased with the concave side undermost, so that they at length form a spiral (as in Cardiospermum and Mutisia) or more often a helix narrowing conically upwards (as in Cucurbitaceæ, Passifloreæ, \&c.), in which state they then dry up and become woody.

1 This and what follows is from Darwin, Movements and Habits of Climbing Plants (1875), p. I 7 r et seq. 
These processes must however be considered as abnormal, the tendrils having failed of performing their purpose of coming into contact, by means of their revolving nutation, with a support during the period that they are sensitive and still in a growing state. If this contact takes place on the sensitive side, a curvature arises at the spot, and the tendril clings to the support; fresh sensitive spots are thus constantly brought into contact with it, and the free apex twines firmly round the support in a larger or smaller number of coils (Fig. 487). The nearer the spot where contact first takes place to the base of the tendril the larger are the number of revolutions round the support, and the stronger the attachment; though even a small number of coils is sufficient to attach it with considerable force. The portion of the tendril between its base and the point of attachment is obviously unable to twine round the support like the free apex; and therefore the irritation caused by the contact extending to the portion that is not in contact produces a different form of curvature, consisting in a rolling up of this portion into the form of a corkscrew, as shown in Fig. $487 u, w, w$. This coiling is similar to that already mentioned as taking place of its own accord in many tendrils which do not take hold of a support, especially in the circumstance that the under or dorsal side of the tendril is always the concave one; but it differs from a spontaneous coiling in being always the result of irritation, occurring invariably when tendrils take hold of a support, and also in taking place some time (half a day to a day) after the attachment, at a time when the tendril is still perfectly sensitive and growing rapidly in length; while the spontaneous coiling occurs only with the cessation of growth and of irritability. The coiling which is the result of the irritation caused by contact also takes place much more rapidly than that which is spontaneous; both can be readily observed by noticing older tendrils which are still straight and have

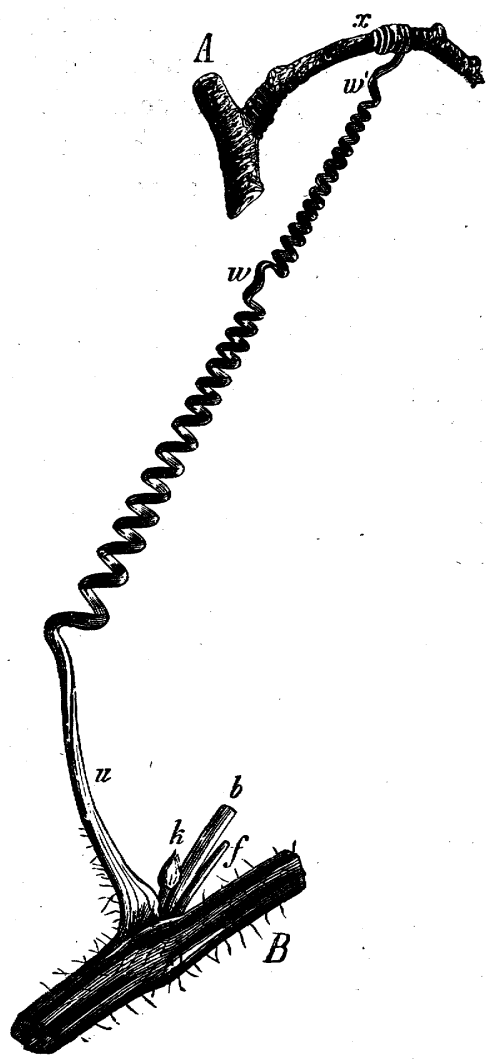

FIG. 487.-Coiling of a tendril of Bryonia dioica. B a portion of the branch from which the tendril springs by the side of the petiole $b$ and the axillary bud $k$; the lower part of the tendril $u$ is straight; the upper part $x$ has coiled round a twig $A$; the long intermediate part between the rigid basal portion $u$ and the point of attachment $x$ has coiled spirally, and thus raised the branch $B ; w z w^{\prime}$ the two spots where the direction of the coil is reversed. not attached themselves, and younger ones on the same shoot that are attached and already coiled up. The coiling of tendrils attached to supports is therefore a result of stimulation in the same sense as the twining of the free portion round a support; and it is only the physical impossibility of also twining round the support that forces the portion of the tendril between its base and the support to coil up like a corkscrew. The coiling of this intermediate portion, like the curvature of a longer piece of a tendril in consequence of the contact of a single point, is a proof that the local 
irritation is communicated along the tendril. The whole consequence of irritation does not however end with these phenomena ; for tendrils that are fixed to a support also increase subsequently in thickness, sometimes very considerably, like the petioles of Solanum jasminoides; they become woody, and have a longer term of life than those which have coiled spontaneously, or generally than those that have not attached themselves.

There is still another point in which attached tendrils differ from those that have coiled spontaneously. In the latter all the coils of the spiral run in one direction; those of a tendril attached to a support have, on the contrary, points (Fig. 487 , $\left.w, w^{\prime}\right)$ at which the direction changes; between any two of these points is a number of coils in the same direction, those beyond them being in the opposite direction; in long tendrils with close coils there are often as many as five or six of these points. Darwin has already shown that this is no special property of tendrils, and still less a specific result of irritation, but is rather a physical necessity; for if a body which coils up is fixed at both ends so that no twisting can take place at either end, the coils must necessarily be produced in opposite directions in order that the torsions which are unavoidably produced may counterbalance one another. This behaviour of fixed tendrils can be imitated by cementing a narrow stretched strip of indiarubber firmly along another strip which is not stretched, and then releasing the former; it contracts and forms the inside of a spiral, the outer side of which is formed by the strip that is not stretched. If the double strip is held at each end and first stretched out straight and then relaxed, coils will be produced, some to the right, others to the left, as in a tendril. If one end is now let go, the strip will twist itself anew into a spiral.

Since all the movements of tendrils that have been described are the result of growth, they take place only when the external conditions of growth are favourable, and the more energetically the more favourable they are; this is the case when food is abundant, temperature high, and the plant contains abundance of sap, the result of a copious supply of water combined with small loss by transpiration. Under these conditions tendrils can, as I have shown, carry on their nutation and sensitive movements even in the dark, and can twine and coil round supports. An instance is afforded by plants of Cucurbitu Pepo, the upper parts of which are grown in a dark vessel, and which are nourished by green leaves exposed to light.

As regards the mechanism of the curvatures caused by contact, as well as the coiling of free tendrils, it cannot be doubted that we have here to do with processes of growth and of its alteration by transverse pressure on the side which is growing less rapidly. The tendrils are only. sensitive to contact or pressure so long as they are in a growing state. A curvature due to irritation may be effaced during growth, in the same manner as the curvature of growing shoots caused by concussion; but if the irritation from the support lasts for a longer time and a coiling takes place, the difference in length between the convex and concave surfaces becomes permanent. The cells of the convex are longer than those of the concave surface (as in roots which have curved downwards or nodes of Grasses which have curved upwards); in thick tendrils which coil round slender supports the difference in length is so great that it strikes the eye at once without measuring. De Vries's recent experiments on tendrils that have not yet coiled, which he marked with trans- 
verse streaks and measured after they had coiled, show that the growth of the convex surface is more considerable, that of the concave surface less so than in the portions of the same tendril that have remained straight above and below the curved

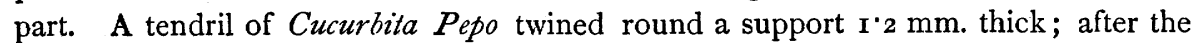
curvature was complete, the increment of the curved part for each millimetre of original length was $\mathrm{r}_{4} \mathrm{~mm}$. on the convex surface, while on the concave surface it was only $\circ .1 \mathrm{~mm}$.; the mean increment on both surfaces in the portion that remained straight amounted to $0^{\circ} \mathbf{2 m m}$. If the growth which takes place in the entire tendril at the time of contact with a support is small, a considerable acceleration occurs on the convex surface, but in general there is no elongation on the concave surface, or there may even be a contraction; in the case of a tendril of Cucurbila this contraction amounted to nearly one-third of the original length.

Similar alterations in the length of the convex and concave surfaces are observable in the spontaneous coiling of free, as well as in the coiled portion of attached tendrils between the base and the point of attachment; and since in these cases the amount of growth which takes place in the entire tendril is usually small a short time previously, the contraction of the concave surface is, according to de Vries, a very common phenomenon.

The conclusion to be derived from these phenomena and from others not described here is that the growth of the surface not in contact is first of all increased by the pressure of the support; the support presses the surface that is in contact, and the pressure which the concave surface undergoes arrests its growth, or even causes a contraction in it. It seems probable that a relaxation of the parenchyma of the surface in contact (by giving off water to the parenchyma of the upper surface) and a consequent elastic contraction of its cell-walls contribute to this result; at least this seems the only explanation of the contraction of the surface in contact in the case of tendrils the growth of which has already become slow. We have however as yet no knowledge of the mode in which the slight pressure of a light thread or that of the revolving tendril on a support causes this alteration of growth not only at the point of contact, but along the entire tendril.

The only cause of the spontaneous coiling of tendrils when not fixed to a support is that the upper surface continues to lengthen for a considerable time after the growth of the under surface has ceased. The cells of the growing upper surface probably withdraw from those of the under surface a portion of their water (as the inner layers of the pith from the outer layers, see p. 805), which causes the latter to become shorter, and the former to become longer.

Without entering further into the numerous questions of a purely mechanical character connected with the curving of tendrils, it may at least be explained why thick tendrils are unable to twine round very slender supports. If two tendrils are compared one of which twines round a slender, the other round a thicker support, it will be seen that in the former the proportional difference in length of the outer and inner sides must be greater than in the latter. If a thick and a slender tendril twining round supports of equal thickness are compared, the proportionate difference in length of the outer and inner surfaces will be greater in the former than the latter case; and if the support is supposed to decrease constantly in thickness, the difference will increase more rapidly in the case of the thick than in that of the slender tendril, and 
the question arises whether the difference in growth of the two surfaces of the tendril can reach to any given amount or not. The difference in length between the two surfaces caused by unequal growth has, in fact, a limit, as is shown by experiment. The slender tendrils of Passiflora gracilis twine firmly round threads of silk; the thick tendrils of the Vine on the other hand twine only round supports which are at least from 2 to $3 \mathrm{~mm}$. thick. The most strongly curved tendril of a Vine which I could find had twined firmly round a support $3.5 \mathrm{~mm}$. thick, and in a nearly circular coil ; the mean thickness of the tendril at this spot was $3 \mathrm{~mm}$. The concave surface of a coil was nearly II mm., the convex outer surface nearly $29 \mathrm{~mm}$. long, the proportionate length of the two surfaces therefore nearly as $1: 2 \cdot 6$. If this tendril $3 \mathrm{~mm}$. thick were forced to twine round a support only $0.5 \mathrm{~mm}$. in thickness, an almost circular coil would have on the concave surface a length of $\mathbf{r} \cdot 6 \mathrm{~mm}$., on the convex surface a length of $20^{\circ} 4 \mathrm{~mm}$; the relative length of the two surfaces would therefore be as $\mathbf{I}: \mathbf{r} 3$; and it does not seem possible for growth to cause so great a difference in length between the two surfaces of a tendril. If, on the other hand, the problem were to cause a tendril $0.5 \mathrm{~mm}$. thick to twine firmly round a support of the same thickness in nearly circular coils, it would only be necessary that the inside of a coil should be $\mathrm{I} .6 \mathrm{~mm}$., the outside $4^{\circ} 7 \mathrm{~mm}$. long, or that the proportion between the two surfaces should be as $\mathbf{I}: 3$.

In order for a tendril to attach itself firmly to a support, it is not sufficient that its coils should merely be in contact with it; they must be firmly appressed to it. That this is actually the case is seen when a tendril is made to twine round a smooth support, and the support is then withdrawn; when, as de Vries has shown, the coils become at once narrower and increase in number. This fact shows also that a tendril which is irritated by contact with a support endeavours to form coils the radius of whose curvature is less than that of the support, provided the support is not too slender nor the tendril too thick.

The cases are very instructive, in reference to the pressure which the coils of tendrils exercise on their supports, where leaves are embraced by strong tendrils, and are folded and compressed by them.

What has now been said is merely intended to draw attention to the more important mechanical principles which must be taken into account in the twining of tendrils. The biology of climbing plants and of those furnished with tendrils, so fertile in extraordinary adaptations, cannot be gone into in detail. On this subject the reader will find in Darwin's treatise quoted above a mass of beautiful observations most admirably described.

Since the physiological function of tendrils is to take hold of supports (generally other plants) in order to allow the slender-stemmed plant which is furnished with them to climb up, the point of greatest importance is for the tendril to be brought into contact with a support. This is usually effected with extraordinary perfection by the revolving nutation not only of the tendril itself but also of the apex of the shoot that bears it at the time when it is sensitive, thus causing every object anywhere within reach of the tendril which could be used as a support to be brought almost inevitably into contact with it. The apex of the shoot which bears the tendril usually describes an ascending elliptic helix, the revolution being completed in from one to five hours. As in the case of twining stems, a strong positive heliotropism would be injurious, as it would often carry the tendril away from the supports. Some tendrils appear in fact to be not heliotropic (those of Pisum according to Darwin), in others a weak positive helio- 
tropism is shown by the fact that the revolving nutation takes place more quickly towards the light than away from it. Some tendrils, strikingly those of the Virginian Creeper and Bignonia capreolata, have the remarkable power of developing broad discs at the end of their branches when they remain in contact for some time with hard bodies, which attach themselves like cupping glasses to rough surfaces, and enable the plant to climb up vertical walls when it finds no slender support round which it can coil. In this case it is obviously necessary that the tendril should turn towards the wall which serves as its support in order to become attached to it, and this is effected by negative heliotropism, which causes the tendril to approach the wall shaded by foliage, where it now performs its revolving movements of nutation-one might almost say its groping movements-creeps along the surface, finds out the crevices and depressions, and developes its adhesive discs.

SECT. 26. - Movements of growing Leaves and Floral Organs produced by variations of Light and of Temperature ${ }^{1}$. In the foregoing paragraphs we have become acquainted with the movements of curvature of growing organs which take place when the external conditions are constant, movements which are produced by the more rapid growth of the one or of the other side of the organ under the action of purely internal causes. These movements were termed 'spontaneous nutations.' Amongst the organs endowed with spontaneous nutation we found that tendrils are peculiar in being sensitive to contact on one side and in that the slight pressure of the support induces a more rapid growth of the free surface, and a much less rapid growth of the surface with which it is in contact. Many growing foliageleaves and floral organs, possessing, like tendrils, a bilateral organisation, are stimulated to curve by variations of temperature or of the intensity of light, the growth of one side or the other being either accelerated or retarded.

It is not all growing leaves and flowers that are sensitive to these meteorological influences; among plants which are very closely allied some do and some do not possess this property. Pfeffer mentions, as examples of sensitive growing leaves, in addition to the very sensitive leaves of Impatiens nolitangere, those of Chenopodiex, Atripliceæ, Solaneæ, Mimulus tigrinus, Mirabilis Jalapa, of species of Silene and Alsine, and of many Compositæ; to this list Batalin adds Malva rotundifolia, Enothera sp., Portulacca oleracea, Linum grandiflorum, Stellaria media, Gnaphalium uliginosum, various species of Polygonum, Senecio vulgaris, Sida Napaa, Rumea'x Hydrolapathum, Ipomcea purpurea, and Brassica oleracea, and doubtless further investigation will increase the number. The movements of these leaves are not effected by means of special organs, but it is the petiole or the lower part of the lamina of the leaf which curves, under the meteoric influences, upwards or downwards accordingly as the growth in length of the upper or of the lower surface has been accelerated by them. The mode of antagonism of the two sides of the bilateral organ is different in different species: in some the leaves are raised at night, as in Chenopodium, Brassica, Polygonum aviculare, Stellaria, Linum, in others the leaves fall, as in

1 Pfeffer, Physiologische Untersuchungen, Leipzig 1873, and Sitzungsber. der Ges. zur Beförd. der ges. Naturwiss. zu Marburg, i 873 --Batalin, Flora, 1873 .

[Pfeffer, Die Periodischen Bewegungen der Blattorgane, I 875.-Darwin, Movements of Plants. -Wiesner, Die heliotropische Erscheinungen, II.]

In these works full references are given to the older literature of the subject. 
various species of Impatiens, in Polygonum Convolvulus, and in Sida Naprea. The amplitude of these curvatures which follow the alternation of day and night appears to increase and diminish with the increase and the diminution of the rapidity of growth. 'In young leaves,' says Batalin, 'the curvatures are the greatest, and in Chenopodium and Stellaria, for example, they go so far that at night the young leaves close to form very large buds: the older leaves curve but little, the oldest not at all.'

Among floral organs it is more especially the movements of the petals and of the tubes of gamopetalous flowers which have attracted attention; no movements which could be included in this category are known to occur in stamens or styles. The movement consists in the curvature of the petal or of the segment of a corolla outwards at certain times of the day and inwards at others, in such a way that in the ordinary course the flower opens and shuts once in a day. The opening occurs usually in the morning or during the day when the intensity of light and the temperature are increasing, the closing towards evening when light and heat are diminishing, although the contrary is sometimes the case. In the Compositæ the movements of the individual flowers effect not so much their own opening and closing as that of the whole capitulum.

Among the very numerous instances of motile flowers the following have been more particularly investigated: Crocus, Tulipa, Colchicum, Ornithogalum, Anemone, Ranunculus, Nymphaa, Malope, many Compositæ, especially Taraxacum, Leontodon, Scorzonera, Hieracium, Calendula, Venidium, Bellis, \&c.

As in the case of leaves so here there are no special motile organs, but certain parts of the corolla continue to grow for a considerable time and are stimulated by meteoric influences to a more rapid growth either of their upper or their under surface (or internal and external), which effects the opening or closing of the flower. The region of curvature usually lies in the basal half of petals, but in Oxalis rosea in the upper half. Among the Compositæ with ligulate corollas there are some in which the motile zone lies immediately above the tube at the base of the ligulate corolla (Venidium, Bellis, Calendula), whilst in others, such as Taraxacum, Leontodon, \&c., the tube itself undergoes curvature; in both cases the centre of the capitulum is the centre of the movements which take place inwards or outwards along radii proceeding from it.

All the movements which are now under consideration agree in this respect, that the plane of curvature of the organ (leaf, petal, tube) coincides with its median plane, which is also the plane of symmetry, since it is the two sides of the organ which are different, that is the anterior and the posterior, which produce the curvatures in consequence of the different way in which they react to the influence exercised upon them by variations of light and of temperature.

The following paragraphs give an account of the mechanism of the movements produced by variations of light and of temperature, taken from the exhaustive researches of $\mathrm{Pfeffer}$ with some additions from Batalin.

I. The movements which are now under consideration differ from the periodical movements and the movements due to stimulation which are manifested by leaves possessing motile organs, in that they only take place so long as growth continues, and that they cease with it.

2. They are effected in consequence of a more vigorous growth of the internal 
(upper) surface of the organ due to an increase, within certain limits, of the temperature or of the intensity of light; when the temperature and the intensity of light are diminishing the growth of the external surface is greater than that of the internal. In the former case the curvature is convex inwards (opening), in the latter it is convex outwards (closing ${ }^{1}$ ). This is of course only the case when the diurnal condition of the organ is the open one; when the contrary is the case the meteoric influences affect the internal and external surfaces in just the opposite way.

3. The curvature of the growing organs which are sensitive to meteoric influences is not effected like that of fully-developed motile organs by an alternating expansion and contraction of the tissue, but by an alternating more vigorous growth of one side and then of the other, so that the organ, whilst making these movements, continues to increase in length. This by no means excludes the possibility of a slight shortening of the concave side occurring temporarily just as in the case of growing tendrils and of geotropically curved stems and nodes of Grasses.

4. Many of the organs now under consideration are especially sensitive to changes of temperature, others to variations in the intersity of light. Many are affected by very slight variations, others are less sensitive, and thus form a connection with those leaves and flowers which exhibit no such movements. In many cases each variation of temperature or of light has an immediate effect, in other cases the effect is produced only after the lapse of a considerable time since the last movement.

5. From the various differences mentioned in the preceding paragraph it becomes evident why certain flowers (and leaves) open very early in the morning, and others only later in the day; and why it is that some are affected by every change of weather, whereas others complete their daily period with great exactitude.

If we ask, finally, what the biological meaning of these phenomena may be in the economy of the plant, it is not easy at present to give any satisfactory answer in so far as leaves are concerned ${ }^{2}$. 'The opening and closing of flowers, however, has an obvious connection with the process of pollination ${ }^{3}$; the flowers which are open by day are visited by the winged insects which effect pollination, and their closure in the evening, or during cold damp weather during the day, serves as a protection to the pollen in the anthers. Like many similar useful adaptations, these can be readily explained on the Darwinian theory, since they depend upon the further development of properties which belong also to allied plants but are less developed in them, and are not accompanied in them by corresponding collateral arrangements.

1 In order to be able to apply the expressions 'opening' and 'shutting' to foliage-leaves these organs may be regarded as standing on a short axis or even as being in the bud.

${ }^{2}$ [Darwin includes these movements of leaves, as well as those described in the next chapter, under the head of nyctitropic or sleep-movements. The object of the closing up of the leaves at night is, he believes, to diminish the radiation from them and thus to prevent injury due to an excessive fall of their temperature. In addition to the nightly sleep there is a diurnal sleep (Paraheliotropism of Darwin), in which the leaves present their margins to the incident light; the object of this is to protect their chlorophyll from the action of too intense light. (See Darwin, Movements of Plants, p. 445.-Wiesner, Die natürlichen Einrichtungen zum Schutze des Chlorophylls, Wien I876.-Stahl, Ueb. sogennante Compasspflanzen, Jena 188 r).]

${ }^{3}$ [On the protection of the pollen from the influence of the weather, see Kerner, Die Schutzmittel des Pollens gegen die Nachtheile vorzeitiger Dislocation und Befeuchtung, Innsbrïck 18 73 .] 
(a) The most important result of Pfeffer's researches is doubtless the establishment of the fact that these movements depend not upon alternate expansion and contraction of the tissue, as was formerly thought, but upon modifications of growth. We must therefore distinguish between these movements and those of special organs which are no longer growing, and we may classify the former along with heliotropic and geotropic curvatures and with the movements of tendrils. It must not, however, be forgotten that all those external and internal conditions which increase or diminish the turgescence of the tissues must also accelerate or retard growth; hence it follows that the same causes which modify the state of tension of a fully-developed organ may also modify the growth of organs which are still growing. If this takes place to a differing extent in the two sides of a bilateral organ, movements produced by growth will be exhibited. It might be suggested that all the movements of curvature treated of in this and in the following chapter should be considered together. I quite agree with this suggestion as far as it goes, but an account contained in a text-book must possess clearness and precision, above all things, in the arrangement of the matter in hand. In the present incomplete state of our knowledge of the movements of curvature, these objects will be best attained if those movements which are results of growth be sharply separated from those which are independent of growth.

(b) As regards Leaves the following may be appended to the account previously given. In order to demonstrate that each upward and downward movement of the leaves of Chenopodium album is accompanied by an increase in length, Batalin fixed a straw seven or eight centimetres long as an indicator to the base of the lamina of a leaf attached to a stem which had ceased to grow; the indicator projected laterally from the leaf and its movements were recorded by a tracing made by its free end upon a surface of sooted paper. It became apparent that the curves described by the point of the indicator corresponding to each upward and downward movement did not coincide, but formed a zigzag line tending away from the stem.

According to Pfeffer, the leaves of Impatiens, Chenopodium, Nicotiana, and Wigandia exhibit, when in continuous darkness, a movement resembling that of the ordinary daily period, but this periodic movement does not continue for any length of time. The circumstance that the movement takes place, under these conditions, with the same intervals of time as in the ordinary period when the plant is exposed to the alternation of day and night, opposes the assumption that the movement is due entirely to internal causes, that is, that there is any 'independent periodicity.' On the contrary, Pfeffer is inclined to assume that we have in this an instance of persistent effect whereby those movements are produced in total darkness which had been previously brought about by the daily alternation of light and darkness. These movements, produced by persistence of effect, are accompanied by the growth of a particular side at a particular hour. It is not certain if, in addition to this, spontaneous nutations take place at shorter intervals of time.

From Pfeffer's manuscript I take the following. When motile leaves are placed in the dark an acceleration of the growth of both sides is the result, just as greater turgescence and tension of the tissues is produced in the motile organs of leaves which have ceased to grow (Mimosa, Papilionaceæ).

A slight shortening of the side which is becoming concave may occur, as in the case of the geotropic curvature of the nodes of Grasses and of the curvature of stimulated tendrils.

The property in virtue of which the leaves respond by an acceleration of growth to exposure to darkness is gained by previous exposure to light, but quite independently of assimilation. Leaves of Impatiens nolitangere make a distinct movement when replaced in darkness after an exposure of five minutes to light, and after an exposure of ten minutes the movement is well-marked. Growth is accelerated when the plant is replaced in darkness and takes place with greater rapidity than if the plant had remained continuously in the dark. The leaves of other plants (Siegesbeckia, Cbenopodium) require to 
- be exposed to light for a longer time in order that a movement may take place when they are again in darkness. Each movement produced by darkness is followed by a kind of rebound; thus the downward curvature of the leaf of Impatiens is followed by a rise. The rise takes place more quickly in the light.

The rigidity of the leaves of Impatiens is not materially affected by their assuming the nocturnal position.

A rise of temperature at 3 a.m. distinctly accelerated the assumption of the diurnal position by the leaves of Impatiens, but it seemed to have little or no effect upon other meteorically sensitive leaves (Chenopodium).

(c) Flowers (a). Pfeffer made measurements to ascertain the amount of growth during curvature upon flowers which perform movements at all times in consequence of slight changes of temperature (see infra). He found the Crocus (vernus and luteus) to be especially adapted for this purpose. In this flower the zone of curvature of the six segments of the perianth lies above their separation from the tube, and occupies from one-fourth to one-sixth of each segment of the perianth. A portion of this zone about $3 \mathrm{~mm}$. in length is especially capable of curvature, and upwards and downwards from this the capacity gradually diminishes. Black marks were made upon the most motile portions to serve as a means of measurement. The measurements were made with a magnifying power of eighty diameters. One division of the micrometer eye-piece was equivalent to $0.0076 \mathrm{~mm}$. The following table may serve as an illustration:-

Crocus vernus.

Lengths of the marked portion.

On the external surface.

Closed.

214 divisions.

217

187,

$209^{\circ} 5$,

$196 \%$

On the internal surface.
Open.

214 divisions.

$218 \%$

188 "

210,

196.

220

185,

210.5,

$2 \mathbf{I} 2$

196.5

In the closed position, the portion measured was nearly straight; in the open position, it was concave outwards, the curvature having a radius of from I $_{5}$ to $30 \mathrm{~mm}$.

These and other measurements made upon Tulipa, Oxalis, Taraxacum, Leontodon, and Venidium show that the side towards which the movement takes place undergoes no perceptible elongation, whilst the other side increases considerably in length.

It is possible, in the case of Crocus, to remove the epidermis of one side and to determine that the same movements are effected as before in consequence of variations of temperature. This took place even when the epidermis of both sides was removed. The negative tension and the elasticity of the epidermis therefore play but an unimportant part in producing the movement.

The growth of the motile zone is by no means at a standstill when the flowers are not performing any movements; on the contrary, it is continuing slowly and uniformly in the two antagonistic halves, and may be retarded or accelerated in either half at any time by a variation of the temperature or of the intensity of light: a movement (curvature) will of course be the result.

( $\beta$ ) The effect of Variations of Temperature may become apparent at any time in many flowers, such as those of Crocus and of Tulipa. If they be placed first in a cold 
and then in a warm atmosphere, it is easy to observe that an increase of temperature causes a movement of opening, and a decrease a movement of closing. Pfeffer succeeded in making a Crocus flower open and close eight times in one day. The opening is more complete if the flower has remained closed for a considerable time, and vice verisa. Particularly sensitive Crocus flowers will open or close in as few as eight minutes in consequence of a variation of temperature amounting to $5^{\circ} \mathrm{C}$. : a variation of from $12^{\circ}$ to $22^{\circ} \mathrm{C}$. will cause it in three minutes. Immersion in warm or cold water has the same effect. It is possible to ascertain, by appropriate arrangements, that Crocus flowers are sensitive to a variation of $0.5^{\circ} \mathrm{C}$. Flowers of Tulips are not so sensitive, but they will react to a variation of $2^{\circ} \mathrm{C}$. A reversed variation of temperature is not immediately followed by a reversal of the movement ; this continues for some time before it exhibits the effect of the more recent stimulus. The lower limit at which variations of temperature will still induce movements lies, for the Crocus, above $8^{\circ} \mathrm{C}$., according to Pfeffer. The flowers of Lecntodon bastilis, Hieracium vulgatum, Scorzonera bispanica, and of Oxalis rosea, open between $8^{\circ}$ and $10^{\circ} \mathrm{C}$., whilst between $1^{\circ}$ and $3^{\circ} \mathrm{C}$. they remain closed in the light.

When a certain maximum of temperature is exceeded, a partial closure of the flowers of Crocus and of Tulipa begins, although previously the opening had become more complete with every rise of temperature. These phenomena are rendered intelligible by Sect. 19.

Pfeffer mentions, after Crocus and Tulipa, the flowers of Adonis vernalis, Ornithogalum umbellatum, and Colcbicum autumnale, as being very sensitive to variations of temperature; and in a less degree those of Ficaria ranunculoides, Anemone nemorosa, and Malope trifida, all of which perform movements at any time of the day in consequence of variations of temperature, the more energetically the longer the period since the last movement. This is very evident in Nympbaa alba, Oxalis rosea and valdiviana, Mesembryantbemum tricolorum and ecbinatum, and in all motile flowers of Cumpositæ. When these have closed in the evening a rise of temperature from $10^{\circ}$ to $28^{\circ} \mathrm{C}$. produces scarcely any opening; in the morning, on the contrary, a rise of temperature causes them to open even in the dark.

( $\gamma$ ) The action of Light. Sudden obscurity suffices to cause the closure of open flowers. Evident closing was observed in Calendula officinalis, Leontodon bastilis, and in Venidium calendulaceum, when the flowers, which had fully opened in diffuse daylight, were placed in darkness from eleven to twelve o'clock in the morning; the temperature varied between $19^{\circ}$ and $20^{\circ} \mathrm{C}$. In the afternoon, after longer exposure to light, the closing consequent upon sudden obscurity is more marked. It appears also in the case of Compositæ and of Oxalis, that a sudden increase of the intensity of light causes a more vigorous opening if the flowers have been previously kept in darkness for a considerable time. The motile organ reacts the more vigorously to variations in the intensity of light, as also to variations of temperature, the longer the time since the occurrence of the last movement due to a stimulus acting in the opposite direction.

According to Pfeffer, it is only darkness which accelerates growth; but a considerable time must elapse before the acceleration is perceptible in one (the inner) of the two antagonistic masses of tissue. At the same time this side becomes compressed by the other.

It appears from Pfeffer's manuscript that as in the case of leaves so in flowers which are motile and which are sensitive to variations in the intensity of light, a persistent effect may be observed of such a kind that the daily periodicity of movement brought about by the alternation of day and night will continue to be manifested for some time in continued darkness. An instance of this is afforded by Tolpis barbata. The ligulate peripheral flowers of Bellis perennis curve outwards between seven and eight o'clock in the morning in August (at a window facing south), and close between five and six o'clock in the evening. In obscurity, the opening begins one or two hours later, and the closure at night is incomplete. The flowers of Taraxacum officinale, Leontodon bastilis, and of 
Barkbausia rubra, placed in the dark towards evening and allowed to remain there, only half-opened on the following day, and remained partially open during the night. On the second day the movements were much feebler. These movements could not be attributed to variations of temperature.

( $\delta$ ) The differing sensibility of leaves to variations of temperature on the one hand, and to variations in the intensity of light on the other, is evident from Pfeffer's statements. The flowers of Crocus and of Tulipa which are so very sensitive to variations of temperature, close in consequence of sudden obscurity, and open when exposed to light, with an energy which is sufficient to overcome the counteracting effect of a temperaturestimulus. Considerable variations of temperature may however reverse the opening or closing effected by light or darkness. In Oxalis, Nymphaa alba, Taraxacum, and Leontodon bastilis, however, the closing in the evening cannot be prevented by a rise of temperature, and a fall of temperature does not arrest the opening in the morning. But if these flowers be kept in darkness during the day-time, they may be made to open in the evening by a rise of temperature. It is necessary, in order that this opening may take place, that a considerable time shall have elapsed since the occurrence of the stimulating effect of darkness.

The conditions which are here stated explain why it is that many flowers growing in the open manifest an exact daily periodicity, whereas others open and close at all periods of the day in consequence of sudden changes of the weather.

It must be mentioned, in conclusion, that many flowers close in consequence of an excessive intensity of light, as they do when the heat is too great. Thus Oxalis valdiviana, Calendula, Venidium, and others close when direct sunlight falls upon them. However, it is still uncertain whether the light or the heat is the active cause.

$(\epsilon)$ It is scarcely possible at present to formulate a brief general explanation of the phenomena exhibited by leaves and flowers which are sensitive to the action of meteoric influences, for we do not fully understand the internal changes effected by these stimuli nor the mutual relations of the two antagonistic halves of the organ. It is important to discover whether a variation of temperature or of light acts in each case upon both the antagonistic halves in contrary ways, or if one side only is sensitive undergoing changes which affect the other side indirectly, as occurs in the case of the sensitive motile organs of Mimosex and probably also in the case of twining tendrils.

As a general result, it is to be remembered that each variation of temperature and of light acts the more energetically as a stimulus the longer (within certain limits) the time which has elapsed since the action of the last stimulus in the contrary direction. The matter may be put thus: a difference, which disappears at a later period, is produced between the upper and the lower side of a growing organ by each stimulus due to temperature or to light; the more nearly this difference has disappeared the more easy it is for a fresh stimulus to produce an effect, that is to produce again a difference between the upper and the lower surface, or, in other words, to cause a movement or a curvature. 


\section{PERIODIC MOVEMENTS OF THE MATURE PARTS OF PLANTS AND MOVEMENTS DEPENDENT ON IRRITATION.}

SECT. 27.-Introduction ${ }^{1}$. The greater number of the movements which are brought into play during growth-as the curvatures caused by heliotropism or geotropism or by the pressure of supports on tendrils and climbing plants-produce new permanent conditions, since it is growth that is modified. It is only when the action has been a very transitory one that heliotropic or geotropic curvature or that of tendrils due to irritability can again be effaced by further growth. During these processes the organ is advancing towards maturity; the changes which have not been effaced are therefore, as it were, stereotyped.

The case is quite different with the changes now to be described. They take place in organs whose growth is completed, but whose structure allows the tissues to assume different conditions which alternate under the influence of external or internal causes.

In those movements which occur during growth the tension of the tissue is concerned only so far as any change in it reacts on growth and modifies it. Periodic movements and those due to irritation, on the contrary, depend entirely on changes in the tension of the tissues, which, in this case, are fully developed only when the organ has attained maturity. These alterations of the tension of the tissues do not however induce new permanent conditions, but can be effaced; every change is again reversed by internal forces, and the previous condition restored so long as there has been no structural injury.

Various objections might be raised to the distinction drawn between the movements exhibited by growing organs and those performed by organs which have ceased to grow. It might be argued that motile organs begin to be irritable and to perform movements whilst they are still growing. In reply to this it may be urged that the motility persists after growth has ceased, and that it is only then that it is fully developed, whereas the motility of the organs considered in the previous chapter ceases with growth. A possible objection is that the motile organs under consideration have not ceased to grow, as a matter of fact, at the time when they are especially irritable and in periodic movement, for they are capable, when in this

${ }^{1}$ [Pfeffer, Physiologische Untersuchungen, 1873 ; id., Die periodischen Bewegungen der Blattorgane, 1875 .-Darwin, Movements of Plants, 1880 .] 
condition, of curving geotropically and heliotropically. I observed both these sets of phenomena, for example, in the motile organs of Phaseolus, and in the irritable filaments of the Cynareæ. These heliotropic and geotropic curvatures are necessarily accompanied by growth. To meet this it may be pointed out, (I) that the periodic movements and the movements due to irritation which we are now considering are not dependent upon growth but upon an alternate contraction and dilatation of the cells; (2) that there are other organs which are also capable of renewed growth under abnormal conditions after their growth under normal conditions has ceased. We found this to occur in the nodes of Grasses. When these have ceased to grow in the erect position, they exhibit sharp geotropic curvatures due to a vigorous growth of the lower surface when they are placed horizontally. The periodically motile and irritable organs evidently possess the same property. They are not only irritable and periodically motile after their growth under normal conditions has ceased, but they are capable of renewed growth under abnormal conditions. The conditions are abnormal when light falls upon these organs from one side only, or when they are placed in an unusual position which is more or less nearly horizontal; then heliotropism and geotropism are brought into play.

Now that sufficient stress has been laid upon the distinction between organs which are motile while growing and organs which are motile after they have ceased to grow, the points of resemblance of the two may be considered. The first of these is the fact that for each kind of movement which is exhibited by the special motile organs, a corresponding kind may be observed in growing organs. Thus, the spontaneous periodic movements of the former correspond to the spontaneous nutations of growing stems, leaves, tendrils, and flowers. The variations of temperature and of the intensity of light which act as stimuli upon the former, by increasing or diminishing the turgescence of the tissues, also affect many growing leaves and cause flowers to open or shut, by accelerating the growth of one side or the other. The motile organs of Mimoseæ, Oxalideæ, and Cynareæ, which are sensitive to contact, correspond to the growing tendrils and roots which are sensitive to pressure.

This comparison tends to show that the causes which induce contraction and dilatation of the cells of motile organs by modifying their turgidity, may also retard or accelerate the growth of growing organs. The deeply-seated connection between these phenomena will become evident if what was said in Sect. 44 as to the causes of growth be compared with the following account of the causes of the periodic movements and of those due to stimulation. According to my theory, the hydrostatic pressure which the cell-sap exercises, in consequence of its increase in quantity by endosmosis, upon the extensible cell-wall, is an essential condition of the growth of the cell; fresh solid matter is deposited between the micellæ of the stretched cell-wall, it therefore grows, and thus growth is a perpetual over-stepping of the limit of elasticity of the stretched cell-wall. Everything which increases the turgescence of the cell promotes its growth, everything which diminishes the turgescence is prejudicial to its growth. If these effects are produced in different degrees upon the two sides of a growing organ, corresponding curvatures will be produced. If these effects are produced in a mass of tissue the cell-walls of which have ceased to grow but are very extensible and very perfectly elastic, an increase of 
turgidity will cause an expansion, a diminution of turgidity will cause a contraction of the mass of cells, and these changes in volume will be accompanied by corresponding curvatures of the organ. The conditions which modify the turgescence of growing cells may be identical with those which modify the turgidity of cells which have completed their growth; in the former case every variation of turgescence involves an alteration of the volume of the cell which is made permanent by growth, in the latter case the alteration of volume is only temporary, and can be effaced by a variation of the turgidity in the opposite direction. From these considerations it becomes apparent that the study of the phenomena of movement will contribute to the development of a mechanical theory of growth, and vice versa.

SECT. 28. Review of the phenomena connected with periodically motile and irritable parts of plants. It is remarkable that all organs at present known as coming under this category are, in a morphological sense, foliar structures, as green foliage-leaves, petals, stamens, or occasionally parts of the carpels (styles or stigmas). It is the more striking that no axial structures or parts of stems are contractile in this sense, because the contractile parts of leaves are usually cylindrical, or at least are not expanded flat, and therefore possess the ordinary form of an axis. There is this further agreement in the anatomical structure of all parts which exhibit these phenomena; - that a very succulent mass of parenchyma envelopes an axial fibro-vascular bundle or a few bundles running parallel to one another; the elements composing these bundles being only slightly or not at all lignified, and therefore remaining extensible and flexible, a fact of importance in reference to the possibility of the movement, which consists of flexions upwards and downwards, generally in the median plane of the organ, the fibro-vascular bundle thus forming the neutral axis of the curvature ${ }^{1}$. The mass of parenchyma which envelopes the fibro-vascular bundle often has the form of a pulvinus, and does not contain in its outer layers any air-conducting intercellular spaces, or only very small ones, while in the inner layers they are larger, especially in the immediate vicinity of the bundle; these being, according to Morren, Unger, and Pfeffer, wanting only in the irritable stamens of Berberis and Mahonia. The tension of these layers of tissue which is generally very considerable, is caused by the stronger turgidity of the parenchymatous cells on the one hand and the elasticity of the axial bundle and epidermis on the other hand. As far as observations go at present, especially those made on the larger contractile organs, the tendency to extension is greatest in the middle layers of the parenchyma between the epidermis and the axial bundle, but the elastic resistance of the epidermis is less than that of the bundle.

If we now consider the nature of the movements in reference to the causes which directly operate to produce them, we may, in the present state of our knowledge, distinguish between three different kinds, viz.

(I) Those periodic movements which are produced entirely by internal causes, without the cooperation of any considerable external impulse of any kind. Such movements may be termed automatic or spontaneous.

(2) Spontaneously motile foliage-leaves are also sensitive to the influence of light, in such a way that within certain limits any increase in the intensity of the light

1 This is also true for Dionaa if the motile parts and not the whole leaf be considered. 
causes such a curvature of the contractile organs as to place the leaves in an expanded and completely unfolded position; while any decrease in the intensity of the light produces the opposite curvature, causing the leaves to fold up. The expanded position is called that of waking or the diurnal position, the opposite one that of sleep or the nocturnal position. In consequence of this sensitiveness to fluctuations in the light, these organs make periodic movements depending on the alternation of day and night, which, being induced by external causes, must be clearly distinguished from the automatic or those brought about by internal causes; and the more so because both kinds usually occur in the same organ, and are combined in various ways ${ }^{1}$. In their sensitiveness to variations in the intensity of light these fully-developed organs resemble the growing organs referred to in Sect. 26 . It has not yet been determined if any such parallelism exists with reference to variations of temperature.

(3) In a smaller number of instances periodically motile foliage-leaves, as well as some reproductive organs which do not exhibit periodical movements, are irritable to touch or concussion. If a particular spot of the organ is only lightly touched or subjected to a slight rubbing from a solid body, the side which is touched becomes concave or contracts. The same effect is produced if a stronger impulse acts on any other part of the irritable organ, which then excites the irritable part. If the motile part has curved in consequence of the mechanical irritation, it afterwards resumes its previous position, and is then again irritable.

The biological significance of these various forms of movement in the economy of the plant is known only in a few instances, as in the case of irritable stamens, where the insects that visit the flowers cause the irritation and consequent alteration in the position of the stamens, these movements being serviceable for the conveyance of the pollen either to the stigma of the same flower (as in Berberis ${ }^{2}$ ) or to those of other flowers (as in Cynaraceæ). We have no knowledge, on the other hand, of any purpose in the economy of the plant served by the periodic and irritable movements of foliage-leaves ${ }^{3}$.

(I) The spontaneous periodic movement is seen most conspicuously in the few cases in which the period extends only over a few minutes, and the oscillation of the organ takes place by day and night under a sufficiently high temperature, as in the small lateral leaflets of the trifoliolate leaf of Desmodium gyrans (the Indian 'Telegraph-Plant'), and the labellum of the flowers of Megaclinium falcatum (an African Orchid). The lateral leaflets of Desmodium gyrans ${ }^{4}$ are attached to the common petiole by slender petiolules 4 to $5 \mathrm{~mm}$. in length, the petiolules being the organs by the movements of which the leaflets are carried round, their apices describing nearly a circle. One revolution takes, when the temperature is above $22^{\circ} \mathrm{C}$., from 2 to 5 minutes; the motion is often irregular,

1 This distinction, partly founded on facts that have long been known, is very necessary for a clear insight into the phenomena, and was first brought forward by me in the treatise on the various immobile conditions of the periodically motile and irritable parts of plants ('Flora,' r863).

${ }^{2}$ [H. Muiller (Befruchtung der Blumen durch Insekten, Leipzig 1873) has shown that the irritability of the stamens of Berberis is a contrivance for cross-fertilisation rather than for selffertilisation.]

3 [See note 2 on page 873.$]$

4 For further illustrations see Meyen, Neues System der Pflanzen-Physiologie, I839, vol. III. p. 553. [The first account of Desmodium gyrans, based on Lady Morison's observations, is by Broussonet, Mém. Acad. de Paris, 1784, p. 616.] 
sometimes interrupted, and then recommencing suddenly in jerks. The labellum of Megaclinium falcatum ${ }^{1}$ narrows below into a claw traversed by three slender fibrovascular bundles, the curving of this portion imparting to the labellum a swinging motion up and down. In a much larger number of other foliage-leaves endowed with periodic motion the spontaneous periodicity is almost entirely concealed by the contractile parts being also very sensitive to light, so that a cursory observation detects only the daily period, or the different positions by day and night. If however these plants, or even cut branches placed in water, remain for some days in the dark or in artificial light of unvarying intensity, it is seen that the periodic movements do not cease, but continue when the temperature also is constant, i.e. independently of any irritation resulting from change of temperature. Under these circumstances the leaves are in a constant slow motion, indicated by the varying positions at short intervals (as e.g. in Mimosa, Acacia lophantha, Trifolium incarnatum and pratense, Pbaseolus, various species of Oxalis, as $O$. Acetosella, \&c. ${ }^{2}$ ). After a certain time these movements cease. The behaviour of the lateral leaflets of Desmodium gyrans and of the labellum of Megaclinium falcatum on the one hand, and that of leaves which assume different positions by day and by night on the other hand, offer a contrast in the following respect; in the former the internal periodic causes of the movement are stronger than the irritation of the light to which they may happen to be exposed, while in the latter these internal causes are outweigh ed by the irritation caused by the varying amount of light under ordinary conditions, so that only the daily periodicity induced by the alternation of day and night is apparent. To this last category belong the movements of the compound leaves of Leguminosæ, of many species of Oxalis, and of Marsilia. In the Leguminosæ the common petiole is often attached to the stem by a larger contractile organ or 'pulvinus;' and in all the cases just named the petiolule of each leaflet possesses a similar organ. If, as in the bipinnate leaves of Mimosa, there are secondary common petioles, these are also attached to the primary petiole by contractile organs. These organs always consist of an axial fibrovascular bundle surrounded by a thick layer of turgid parenchyma. The other parts of the leaves, the petiole as well as the lamina, are not spontaneously contractile, but the alterations in their position are caused by the curvatures of the organs at their base. The movement is either a curving upwards and downwards, as in Phaseolus, Trifolium, Oxalis, and the common petioles of Mimosa, or is directed from behind and below in a forward and upward direction, as in the leaflets of Mimosa.

(2) Sensitiveness to variations in the intensity of light is exhibited with peculiar distinctness in the leaves of Leguminosæ and Oxalidex and of Marsilia, and the consequent movements are effected by the organs which also produce the spontaneous periodic movements ${ }^{3}$. These organs occur also in the leaves of many other plants, as Cannacex and Marattiacex, but their irritability has not as yet been investigated.

In the diurnal position produced by increasing intensity of light the leaves generally have their surfaces completely unfolded and expanded flat; in the nocturnal position they are on the contrary folded up in different ways, being turned upwards, downwards, or sideways. The leaflets of Lotus, Trifolium, Vicia, and Latbyrus are, for example, folded upwards at night, those of Lupinus, Robinia, Glycyrrbiza, Glycine, Phaseolus, and Oxalis downwards; the common petiole of Mimosa turns downwards at night, that of Phaseolus becomes erect; the leaflets of Mimosa and Tamarindus indica ${ }^{4}$ turn laterally forwards and upwards in the dark, those of Tepbrosia caribaa backwards. When the petiole and other parts of the same leaf are contractile, the curvatures of the various motile parts may differ; thus, for example, the petiole of Pbaseolus turns upwards in the evening, while the leaflets turn downwards; the petiole of Mimosa on the other hand

1 C. Morren, Ann. des sci. nat. 1843 , 2nd series, vol. XIX. p. 91.

2 For further proof see Sachs, Flora, $186_{3}$, p. 468, where the literature of the subject is quoted.

3 [See Somnus Plantarum, P. Bremer, Linn. Amœn. Acad. iv. p. 333.]

4 See Meyen, Neues System der Pflanzen-Physiologie, vol. III. p. 476 . 
turns downwards while the leaflets turn forwards and upwards, till they partially cover one another in an imbricate manner. The folding-up of a leaf in the diurnal position when suddenly placed in darkness is the more energetic the longer the period of illumination has been, and inversely. If a plant which has been exposed to alternations of day and night be kept in darkness for a considerable time, the daily periodicity may continue for a time, according to Pfeffer, as a persistent effect, and then the more rapid spontaneous movements make their appearance. In cases in which they take place with considerable force (Oxalis, Trifolium) this persistent effect is not exhibited. In general the sensitiveness to the action of light is very different in different species.

(3) Many leaves endowed with periodic motility or sensitiveness to light are also irritable to contact and concussion ${ }^{1}$, as those of Oxalis Acetosella, stricta, corniculata, purpurea, carnosa, and Deppei ${ }^{2}$, Robinia pseud-Acacia ${ }^{3}$, various species of Mimosa, as sensitiva, prostrata, casta, viva, asperata, quadrivalvis, dormiens, pernambucina, pigra, bumilis, and pellita, Ascbinomene sensitiva, indica, and pumila, Smitbia sensitiva, Desmantbus stolonifer, triquietrus, and lacustris. In the greater number of these plants a rather violent or often repeated concussion is requisite to set the parts in motion, which then always assume the position of sleep; in other words, mechanical stimulation acts in the same way as a diminution of light. The sensitiveness is greater in Oxalis (Biophytum) sensitiva and Mimosa pudica, where a very slight concussion or simple contact on the contractile organ suffices to cause immediate and considerable motion, which is due, when the plant is highly irritable, to conduction of the stimulus to the parts not touched. Although the position assumed in consequence of contact or concussion resembles the nocturnal position, yet it differs from it in its conditions; the former is accompanied by flaccidity of the contractile organ, the latter by increased turgidity.

Among irritable stamens may be enumerated the various species of Berberis ${ }^{4}$ (e.g. vulgaris, emarginata, cretica, and cristata), and of the sub-genus Mabonia. In contact with the corolla when at rest, they curve concavely inwards when the base of the inner side of the filament is lightly touched, so that the anther comes into contact with the stigma.

There is a greater diversity in the phenomena produced by a slight blow or friction on any part of the filaments of various Cynaraceæ (as Centaurea, Onopordon, Cnicus, Carduus, and Cynara) and Cichoriaceæ (as Cichorium and Hieracium). The filaments which spring from the tube of the corolla bear the five firmly attached (not coherent) anthers, which together form a tube through which the style grows up while the pollen is escaping. At this time the filaments are irritable; when at rest they are curved concavely outwards as far as the width of the corolla-tube will permit; on contact or concussion they contract, become straight, and hence come into close contact along their whole length with the style which they enclose, lengthening again after some minutes and resuming their curved form. Since each separate filament is independently irritable, touching a single filament or a blow on one side only of the capitulum will irritate, according to circumstances, only one, two, or three of the filaments, and by the contraction of one side the whole of the reproductive organs will be bent to one side. By the displacement connected with this or the pressure of the other filaments on the corolla they are also irritated, and thus arises an irregular oscillating or twisting motion of the reproductive organs of the flower. If the whole capitulum is shaken, or if the hand is passed over the surface of the flower, or the flower is blown into, a 'creeping' motion ensues of all the flowers in the capitulum. This phenomenon occurs only while the style is growing through the anther-tube and the pollen is being emptied into the tube; the motion of the filaments effected by insects causes the anther-tube to be drawn

1 [Movements may also be caused by chemical stimuli; see Darwin, Insectivorous Plants, 1875.]

2 From Unger, Anatomie und Physiologie der Pflanzen, 1853, p. 417.

3 Mohl, Flora, 1832, vol. II. No. 32, and his Vermischte Schriften.

${ }^{4}$ Goeppert, Linnæa, 1828, vol. III. p. 234 et seq. 
downwards and a portion of the pollen thus to escape above it, which is then carried away by insects to other flowers and capitula where the stigmas are already unfolded ${ }^{2}$.

Among irritable female reproductive organs are the lobes of the stigmas of Mimulus, Martynia, Goldfussia anisophylla, \&c., which close when their inner side is touched, evidently in order to retain the pollen brought to them by insects. More striking are the movements which follow a light touch on the gynostemium of Stylidium, a genus almost peculiar to Australia (e.g.S. adnatum and graminifolium). The cylindrical gynostemium which bears the stigma and close beside it two anthers is, when at rest, turned sharply downwards; irritation causes a sudden elevation and even reversal to the other side of the flower.

A more detailed description of these and other contractile organs will be found in Morren's treatise named below ${ }^{2}$.

(4) Mobile and immobile condition of the motile parts of plants ${ }^{3}$. The parts of plants endowed with periodic motion and irritability may present alternately two different conditions according to the external influences to which the plants are subjected. Their properties may be suspended for a shorter or longer time, and may give place to a condition of immobility which again disappears if the external influences are favourable, provided the organ is not in the meantime killed. This immobile condition differs from that caused by death in the fact that it is transitory, and that the internal changes which cause it are reparable.

The following particulars are taken from the detailed illustrations in my work already quoted.

(I) Transitory rigidity from cold occurs in the leaves of Mimosa pudica when the influences are otherwise favourable if the temperature of the surrounding air remains for some hours below $15^{\circ} \mathrm{C}$.; the lower the temperature falls below this point, the more quickly does the rigidity set in; the irritability to touch and concussion disappears first, then that to the action of light, and finally also the spontaneous periodic movement. The lateral leaflets of Desmodium gyrans are, according to Kabsch, immotile when the temperature of the air is below $22^{\circ} \mathrm{C}$.

(2) Transitory rigidity from beat occurs in Mimosa within an hour in damp air at $40^{\circ} \mathrm{C}$., within half an hour in air at $45^{\circ} \mathrm{C}$., in a few minutes in air at $49^{\circ}$ to $50^{\circ} \mathrm{C}$.; the sensitiveness returns after exposure for some hours to air at a favourable temperature. In water the rigidity from cold of Mimosa sets in at a higher temperature, viz. in a quarter of an hour between $16^{\circ}$ and $17^{\circ} \mathrm{C}$., and the rigidity from heat at a lower temperature than in air, viz. in a quarter of an hour between $36^{\circ}$ and $40^{\circ} \mathrm{C} .{ }^{4}$ During the rigidity from heat, whether in air or water, the leaflets are closed, as after irritation, but the petiole is erect, while when irritated it is directed downwards.

1 These phenomena were discovered as long ago as $176_{4}$ by Count Battista dal Covolo, and are well described by Kölreuter in his preliminary Nachrichten von einigen das Geschlecht der Pflanzen betreffenden Versuchen; 3rd Appendix, I 766, pp. I 25, I 26.

2 C. Morren, On Stylidium, Mém. de l'Acad. roy. des sci. de Bruxelles, 1836; on Goldfussia, ditto, 1839; on Sparmannia africana, ditto, 1841; on Megaclinium, ditto, 1862. Also on Oxalis, Bull. de l'Acad. roy. des sci. de Bruxelles, vol. II. No. 7 ; on Cereus, ditto, vols. V and VI. [On the irritability of the stamens of Ruta, see Carlet, Comp. rend., August 25, 1873, and May 18, 1874; Heckel in Comp. rend., July 6, I874. On Sparmannia, Cistus, and Helianthemum, see Heckel, in Comp. rend., March 23 and April 6 and 20, 1874.]

3 Sachs, Die vorübergehende Starrezustände periodisch beweglicher und reizbarer PflanzenOrgane, Flora, I863, No. 29 et seq.-Dutrochet, Mém. pour servir, vol. I. p. 562.-Kabsch, Bot. Zeit. I 862 , p. 342 et seq.

4 A plant of Mimosa immersed in water of from 19 to $21 \cdot 5^{\circ} \mathrm{C}$. remains sensitive to impact and light for eighteen hours or more. Bert's statement (Recherches sur le mouvement de la sensitive, Paris 1867, p. 20) that Mimosa remains irritable up to $5^{6}$ or even $60^{\circ} \mathrm{C}$. is not suffciently confirmed, and is opposed to all that we know about the superior limits of temperature for vegetation. 
(3) Transitory rigidity from darkness. If plants whose leaves are periodically motile and irritable to light and concussion, as Mimosa, Acacia, Trifolium, Phaseolus, and Oxalis, are placed in the dark, the spontaneous periodic movements take place without the changes in position caused by the action of light, and therefore all the more clearly, and the irritability to touch is also not at first injured. But this motile condition disappears completely when the darkness lasts for one day or more. If a plant rendered rigid by exposure to darkness is again placed in the light, the motile condition is not restored for some hours or even for some days.

Perfect darkness is however by no means necessary in order to produce rigidity. It may be brought about by placing a plant that is very dependent on light, like Mimosa, for some days in a deficient light, as in an ordinary dwelling-room, at some distance from the window.

In contrast to the rigidity caused by darkness, I have applied the term Pbototonus to the normal motile condition resulting from the alternation of day and night. A plant in this condition, if placed in the dark, will, as we have seen, remain for some time (hours or even days) in a state of phototonus, which then disappears gradually; the plant is therefore, under normal conditions, in a state of phototonus even during the night. In the same manner a plant which has become rigid in continued darkness retains its rigidity for some time (hours or even days) after being exposed to light. The two conditions therefore pass over into one another only slowly.

In the case also of rigidity caused by darkness, the irritability of Mimosa to concussion disappears first, and then the spontaneous periodic motion. In the same manner a plant which has thus become rigid reassumes first of all its periodic movement, then its irritability.

The position of the various parts of the leaves of Mimosa when in a state of rigidity caused by darkness is different from that caused by darkness in phototonic plants, and also different from that produced by rigidity due to heat. In the first case the leaves remain quite expanded, the petiolules directed downwards, the common petiole almost horizontal.

Changes in the intensity of the light produce the same effect as irritants, but only on healthy phototonic plants; leaves which have become rigid from exposure to darkness show no irritability to variations in its intensity until they have again become phototonic from long-continued exposure to light. A plant of Acacia lophantba, left for five days in the dark, was found to have lost during the last forty-eight hours every trace of its spontaneous movements. It was then placed in a window, where within two hours it directed its leaflets strongly downwards, the sky being cloudy, and other small changes of position took place in the petiolules. In this condition the plant was still rigid; when it was then placed about noon in the dark with another phototonic plant of the same species, the position of its leaves did not change, the leaflets remained expanded, while the other plant within an hour closed its leaflets and assumed the most complete nocturnal position. Both plants were then once more placed in the window, when the first again retained the position of its leaves unchanged, while the normal phototonic plant expanded its closed leaflets in an hour, the sky being still cloudy. By the evening the lowest six leaves still remained rigid and expanded, but the upper eight or nine leaves closed; the next morning all the leaves again expanded into their normal diurnal position ${ }^{1}$.

Trifolium incarnatum exhibited similar phenomena, with only immaterial differences.

1 [Bert (Bull. de la Soc. bot. de France, vol. XVII, 1871, p. 107) found that the irritability of the leaves of Mimosa was destroyed by placing them under bell-glasses of green glass almost to the same extent as if placed in the dark; the plants being entirely killed in twelve days under blackened, in sixteen days under green glass; plants placed in the same manner beneath white, red, yellow, violet, and blue glasses being still perfectly healthy and sensitive, though varying in the rapidity of their growth.] 
It is worth noting that in the plants observed by me the positions of the leaves induced by the rigidity caused by darkness resemble the diurnal more than the nocturnal position of phototonic plants. Rigidity produced by darkness is apparently only exhibited by organs containing chlorophyll, for, according to a communication from Pfeffer, the stamens of Cynaracex, though developed in the dark, are irritable. This, as well as the fact that individual organs of a plant may be made rigid by darkness ${ }^{1}$, shows that this condition is not due to an excessive accumulation of carbonic acid in the tissues.

(4) Transitory rigidity from drought I have observed only in Mimosa pudica. If the earth in the pot in which a plant is growing is left unwatered for a considerable time, the irritability of leaves perceptibly diminishes with the increasing dryness, and an almost complete rigidity ensues, causing the common petiole to assume a horizontal position, and the leaflets to expand. Leaves which have lost their irritability are not withered nor flaccid; but the watering of the soil causes a return of the irritability within two or three hours.

(5) Transitory rigidity resulting from chemical infuences. In this category I include especially the condition termed by Dutrochet ${ }^{2}$ Asphyxia, which occurs in Mimosa when placed in the receiver of an air-pump. While the air is being pumped out, the leaves fold up, no doubt in consequence of the concussion; but the leaflets then expand, the petiole becomes erect, and while the leaves assume the same position as after prolonged withdrawal of light, they become rigid and possess neither periodic motility nor irritability to concussion. When brought into the air the plant again becomes motile. It can scarcely be doubted that the effect of the vacuum is essentially a result of the removal of the atmospheric oxygen, and therefore causes rigidity by suspending the respiration.

$\mathrm{Kabsch}^{3}$ confirmed these statements, and showed that the stamens of Berberis, $M a-$ bonia, and Helianthemum also lose their irritability in vacuo, regaining it in the air.

The cessation of the irritability of the stamens of these plants which Kabsch states to take place when they are placed in nitrogen or hydrogen gas may also be ascribed to a simple suspension of respiration, the irritability returning on access of air. The destruction of the irritability which takes place, on the authority of the same observer, in the stamens of Berberis in pure carbon dioxide or in air containing more than $4 \circ \mathrm{p} . \mathrm{c}$. of this gas must, on the contrary, be considered a positively injurious chemical action of the nature of poisoning. If they remain from three to four hours in carbon dioxide, the irritability returns only after some hours on replacing them in air. Carbonic oxide mixed with air in the proportion of from 20 to $25 \mathrm{p}$. c. destroys irritability, while nitrous oxide produces no effect. The stamens, on the other hand, bend towards the pistil in nitric oxide, and lose their irritability in $\mathbf{1} \frac{1}{2}$ or 2 minutes. Ammoniacal gas appears to cause transitory rigidity after a few minutes".

Kabsch states that rigidity ensues after from $I \frac{1}{2}$ to 2 hours in pure oxygen, the stamens again recovering in the air.

The vapours of chloroform or of ether destroy the irritability (also for variations of light?) of motile organs, without however causing death unless the action be too prolonged. If entire plants of Mimosex or branches which have been cut off be

1 Pfeffer, Physiol. Unters., Leipzig I873, p. 66.

2 Dutrochet, Mém. pour servir, vol. I. p. $5^{62}$.

${ }^{3}$ Kabsch, Bot. Zeit. I862, p. 342 .

${ }^{4}$ [J. B. Schnetzler (Bull. de la Société vaudoise des Sciences naturelles, I869) points out that the substances which destroy the contractility of animal 'sarcode' also destroy the irritability of the stamens of Berberis and the leaves of Mimosa. Curare has no prejudicial effect in either case; while nicotine, alcohol, and mineral acids destroy both. In the Comptes rendus for April $23^{\text {rd, }}$ I 870 , is a record of a series of experiments on the effect of chloroform on the irritability of the stamens of Mahonia.] 

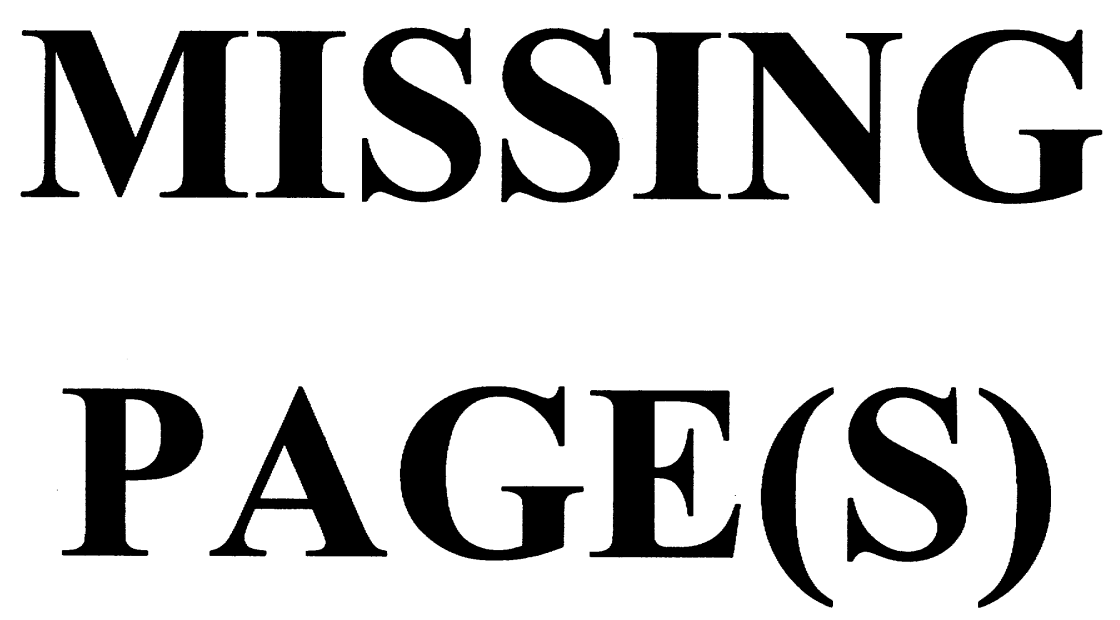

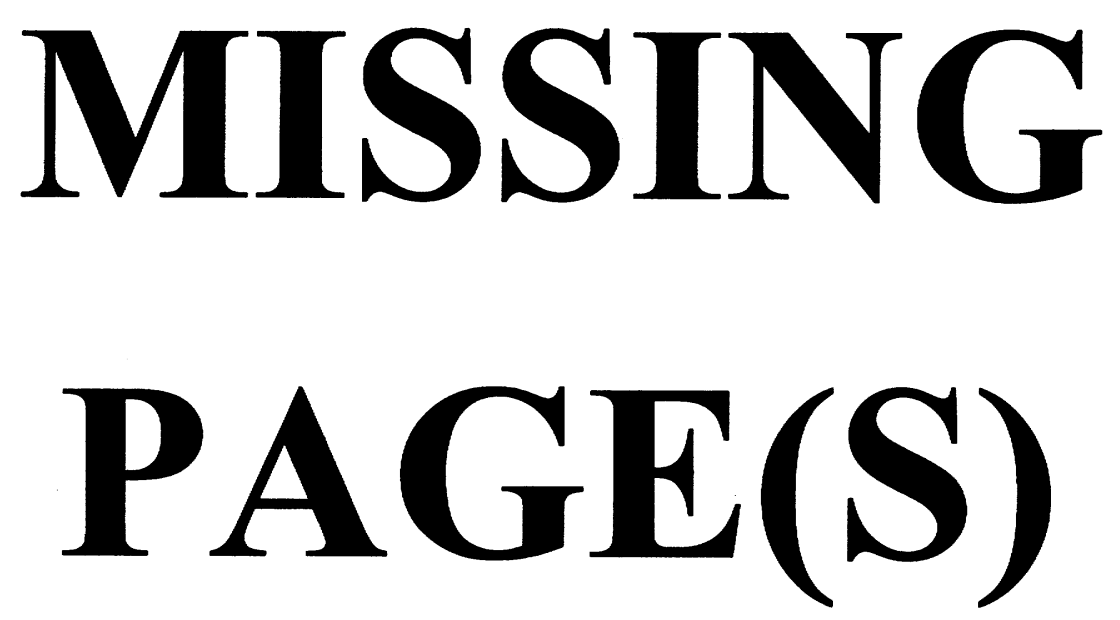

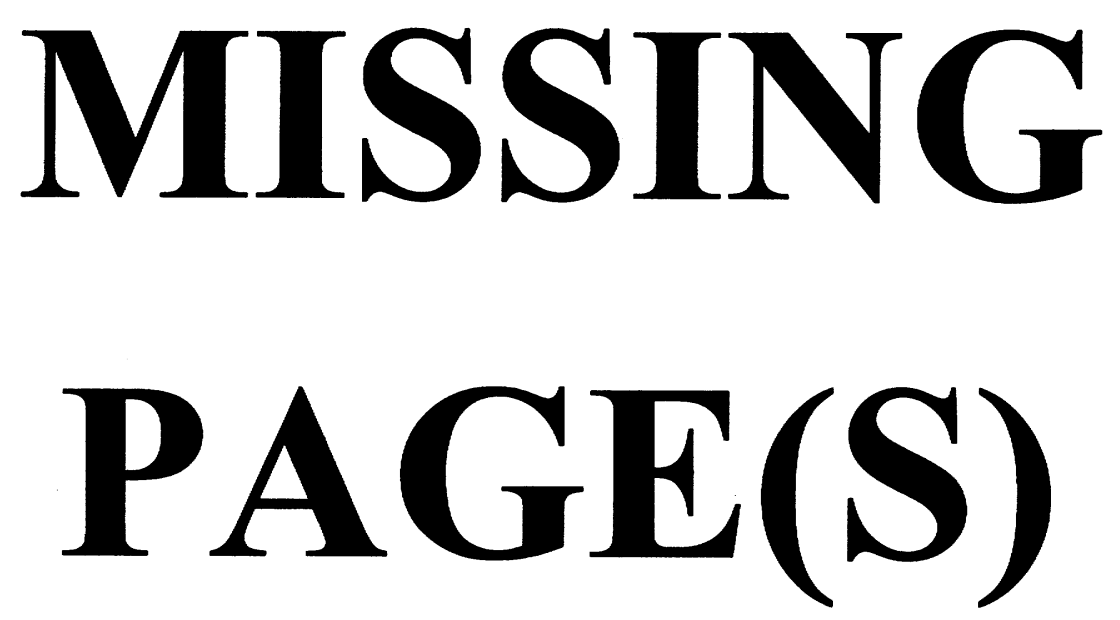

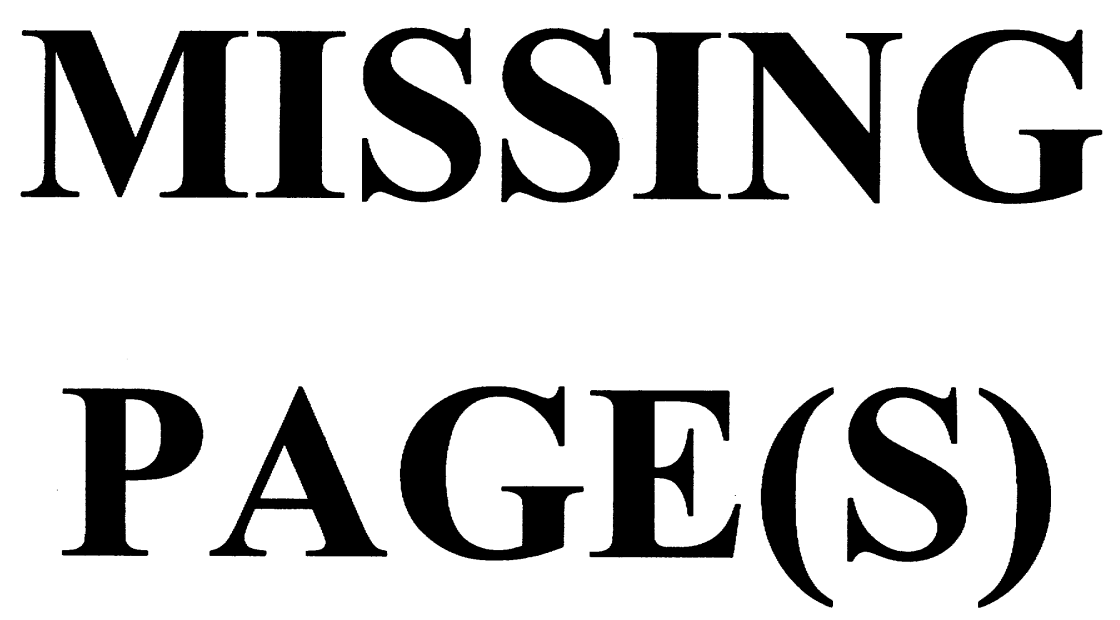
parenchyma has been removed, water may sometimes be seen to escape also from the horizontal cut surface of the parenchyma. It is therefore certain that during the movement produced by stimulation water escapes from the lower parenchyma; it gives off a small portion of it to the upper parenchyma (as is shown by the measurements that have been quoted), a larger portion flows off at the sides through the intercellular spaces, and a smaller portion apparently enters the central fibro-vascular bundle. The whole amount of water that escapes from the lower parenchyma is so small that it is no doubt at once absorbed by these parts at the moment of irritation.

Since water escapes from the parenchymatous cells of the under side when stimulated, and passes into the intercellular spaces, the air must be at least partially expelled from the latter; and this is evidently the cause of the darker colour of the irritated parts already observed by Lindsay. Pfeffer fixed a petiole in the normal condition so that the contractile organ could not bend when irritated; when he touched a point of the irritable side he saw the darker colour spread instantaneously from the point of contact. No other explanation of this phenomenon is possible than that the air is expelled from the intercellular spaces and replaced by water, which would cause a smaller amount of light to be reflected from the interior. The expelled air will collect, in consequence of the laws of capillarity, in the larger intercellular spaces round the central fibro-vascular bundle, from which it will easily reach the petiole.

In the diurnal position of the organ slight transverse folds are seen to run along both sides which after stimulation become more shallow on the upper but deeper on the under side, showing that the consequent curvature causes a slight passive compression of the under side. This side first of all contracts in consequence of its loss of water and of the elasticity of its cell-walls, and then becomes still further compressed by the downward curvature of the upper side.

How it comes about that a slight touch or concussion should cause an escape of water from the strongly turgid cells of the lower side, followed by an energetic reabsorption, cannot for the present be explained. Pfeffer's observations on the stamens of Cynareæ seem to warrant the assumption that the protoplasm of the irritable cells undergoes a change, in consequence of a touch or of concussion, of such a nature that it becomes more permeable to water, and that the water which has passed through the protoplasmic layer simply filters through the cell-wall, which then contracts in virtue of its elasticity.

The propagation of the stimulus in Mimosa, to which frequent reference has been made above, has been shown by Pfeffer (Jahrb. f. wiss. Bot. IX. p. 308), in confirmation of Dutrochet's results and of my own, to be effected by the fibro-vascular bundles. Since each movement of a leaf produced by stimulation is accompanied by an escape of water from its parenchyma, the water of the axial bundle and of the bundles connected with it is set in motion. If, when an incision is made into the wood of a stem, a drop of water exudes, a movement of the water in the fibro-vascular system is set up, which affects also that of the axial bundle of the contractile organ and of the irritable parenchyma.

In the contractile organs of the leaflets of Oxalis Acetosella ${ }^{2}$, where the anatomical and mechanical contrivances are similar to those of Mimosa, this compression is much stronger, and the under side contracts when the organ is irritated. Pfeffer states that a decrease in volume also takes place, and since a very considerable elongation of the upper parenchyma is required for the movements, there must be a more considerable transference of water from the under side. The organs of Oxalis differ from those of Mimosa in remaining irritable when the intercellular spaces are injected with water; but when in this state they become flaccid on irritation; it is probable

${ }^{1}$ [For a discussion of this subject see Vines, The Influence of Light on the Growth of Unicellular Organs, Arb. d. bot. Inst. in Würzburg, II. 1, 1878.]

${ }^{2}$ See Sachs, Bot. Zeit. I 857 , pl. XIII. 
therefore that a portion of the water passes from the contractile organ into the tissue of the petiole and lamina. The depression of the leaves of $O$. Acetosella and stricta when sunlight falls suddenly upon them is, like the irritable movements, attended with flaccidity, and according to Pfeffer, is of the same nature.

(b) The external features of the phenomena of irritability exhibited by the stamens of Cynaraceæ ${ }^{1}$ in the normal condition have already been described. For a close examination of them it is necessary to remove single flowers from the capitulum, and to cut away the corolla from below as far as the point of insertion of the filaments, or to cut across the corolla-tube, stamens, and style above the insertion of the filaments, and to fix the reproductive organs which are thus isolated by means of a pin in damp air. When the filaments have recovered from the irritation caused by this operation, they are convex outwards. The filaments are flat and strap-shaped; they consist of three or four layers of long cylindrical parenchymatous cells, separated by thin straight walls, and surrounded by a layer of epidermal cells of similar form, strongly cuticularised and growing out in many places to hairs, each of which is cut off by a longitudinal wall. Intercellular spaces of considerable size lie between the parenchymatous cells; through the middle of the parenchyma passes a delicate fibro-vascular bundle, which, like the epidermis, is strongly stretched by the turgid parenchyma.

If the flower has been dissected according to the plan first described, and one of the filaments, curved convexly outwards and fixed below to the corolla, above to the anthertube, is touched, it becomes straight and therefore shorter and in contact along its whole length with the style. If all the filaments are touched, it is seen that they have considerably decreased in length so as to draw down the anther-tube. After a few minutes they resume their original length and curvature, and are then again irritable. If the corolla has been dissected according to the second mode, where the filaments are cut away and can move freely below, it is easy to see that every time they are touched a curvature immediately ensues; if the outer side is touched, it becomes at first concave, then convex; if the inner side is touched, it becomes concave, and sometimes afterwards convex. The contraction of the stimulated filament begins at the moment of contact, after some time reaches its maximum, and the organ then at once begins again to lengthen, at first quickly, then more slowly.

With regard to the mechanism of these movements, we are in possession of Pfeffer's most acute observations made for the most part upon the filaments of Cynara Scolymus and Centaurea jacea. The following is a summary of his most important results.

The filaments are from 4 to $6 \mathrm{~mm}$. long in these species: the tangential diameter of those of Cynara is $0^{\circ} 42 \mathrm{~mm}$., the radial $0^{\circ} 2 \mathrm{~mm}$.; in Centaurea the measurements are 0.24 and $0^{\circ} \mathrm{I} 4 \mathrm{~mm}$. The axial fibro-vascular bundle is thin and delicate. The irritable parenchymatous cells are in Cynara two or three times, in Centaurea from four to six times as long as they are broad, and their transverse walls are at right angles to the long axis. All the walls of the cells, even of those forming the bundle, are thin: only the external walls of the cells of the epidermis are somewhat thickened. The abundant cell-sap of the parenchymatous cells is surrounded by a parietal layer of protoplasm of moderate thickness, in which lies a nucleus. The protoplasm exhibits rotation. Some tannin and a considerable quantity of glucose is dissolved in the cell-sap.

The filaments are irritable throughout their whole length, that is, they will contract at any point if touched. Pfeffer succeeded by especial contrivances to magnify the contractions one or two hundred times. The contraction may amount to from eight to twenty-two per cent. of the length of the filament when at rest. It is accompanied by a thickening of the filament, which is, however, too slight to suggest that the contraction produces merely a change of form; it rather indicates a considerable

1 Cohn, Contractile Gewebe im Pflanzenreich, Breslau I 86I ; ditto, Zeitschrift für wiss. Zoologie, vol. XII. Heft 3.-Kabsch, Bot. Zeit. 1861, No. 4.-Unger, Bot. Zeit. 1862, No. I5, and 1863, No. 46.-Pfeffer, Physiologische Untersuchungen, p. 80. 
decrease in bulk. This decrease is due to an escape of water from the cells into the intercellular spaces, from which it flows, as Pfeffer directly observed, when a filament is cut across, just as is the case with the organs of Mimoseæ. If the intercellular spaces be injected with water, the filaments remain irritable and on stimulation the escape of fluid from the cut surface is more evident.

The filaments are very extensible and at the same time very perfectly elastic. They may be stretched to twice their usual length, and, on being released, they return to their original dimensions.

When the filament is irritable, the axial bundle and the epidermis are stretched by the turgid parenchyma, and even after stimulation a tension of the same kind but less considerable still exists.

The possible assumption that the movement is due to an increase of the elasticity of the cell-walls under the influence of a stimulus, a contraction of all the cells and an escape of water from them being the result, is shown by Pfeffer to be incorrect, for the elasticity of the stimulated and of the unstimulated filament is the same.

The assumption that the permeability ' of the cell-walls is suddenly increased by a stimulus and that thus the escape of water is rendered possible, is also shown to be very improbable. Pfeffer points out that the water filters through under high pressure, and proves that the ordinary permeability of organic membranes is quite sufficiently great to permit of the passage of the small quantity of water which escapes from stimulated cells. It is therefore unnecessary to assume a sudden change in the properties of the cell-wall which increases its permeability.

Pfeffer overthrows Hofmeister's theory that the escaping water comes not from the vacuole of the cell but from the cell-wall itself, by the fact that the lateral walls of the parenchymatous cells become thicker on contraction, and he might have added that on this theory a contraction of empty cells but not of full tense cells was possible.

After having shown-though without absolute proof-how improbable it is that the cell-wall undergoes a sudden change in consequence of stimulation, Pfeffer goes on to point out how probable it is that some change is produced in the protoplasm which lines the cell-wall as a closed sac. For a complete discussion I refer the reader to his exhaustive treatment of the subject; I will only append the following account for the sake of clearness. It is evident that if permeability of the tense cell-wall remain unaltered, the escape of water from the cell may depend upon the permeability of the layer of protoplasm which lines the cell-wall. If it is not permeable, it becomes more closely pressed to the cell-wall by the increased hydrostatic pressure effected by endosmosis ; if now any force affects the protoplasm in such a way that the protoplasm becomes permeable to water, an escape of fluid will take place not only through the protoplasmic layer, but also through the cell-wall which has already been shown to be sufficiently permeable. It bas now to be shown that the occurrence of such a change in the protoplasm is possible, and to be explained why it is that this suddenly increased permeability of the protoplasm ceases after the movement, a fact which is essential to the restoration of the irritability. On these points I would refer the reader to the explanations given in Pfeffer's work; I would only add that such changes in the permeability of protoplasm as are here assumed are already known to occur. When the protoplasm of a cell of Spirogyra contracts before conjugation, it must necessarily become more permeable, for most of the water escapes from it; this escape does not take place when the cell is turgid and actively growing. If the cell-wall of the conjugating cell were very tense and if it were at the same time very elastic, it would contract simultaneously with the protoplasm, and would permit of the escape of the water through it. As a matter of fact the cell-wall of Spirogyra is not very tense, and it is rigid, so that it does not materially alter its form when the protoplasm contracts; the water which escapes through the protoplasm therefore occupies the space between it and the cell-wall. It may be objected that this contraction preparatory to the conjugation of the cell of Spirogyra is not the result of the action of an external stimulus; this is quite true, but 
it serves to prove that an alteration of the permeability of protoplasm is possible. Moreover it is known that the protoplasm of many cells contracts when they are subjected to pressure from without, and this is only possible if an escape of water takes place. If, under these circumstances, the cell-wall remains fixed, it is because it was but slightly stretched and comparatively inextensible before. If the wall of a cell which has been so treated were to contract as considerably as the protoplasm, we should have a result similar to that which was assumed above with reference to a stimulus. These considerations suggest that the irritability, in its narrowest sense, is a property of the protoplasm only, and that it is essential to the existence of irritable organs that the irritable protoplasm should be surrounded by cell-walls which are tense in consequence of the turgidity of the cells and which can follow the contractions of the protoplasm in virtue of their perfect elasticity.

(c) The irritable stamens of Berberis ${ }^{1}$ differ considerably in the mechanism of their movements from those of Cynarex, especially in that they are irritable not upon the outer side but upon the inner side only; further, the irritable parenchyma includes no intercellular spaces, but between the thin-walled cells there is a quantity of 'intercellular substance' which is capable of swelling. If the inner side of the filament be touched, it curves throughout its whole length. Pfeffer was able to observe in this case also that when the filament is cut across, stimulation causes the escape of a drop of water from the cut surface.

(d) Too little is at present known of the mechanism of the irritable gynostemium of Stylidium, of the leaves of Dionca muscipula, of the glands upon the leaves of Drosera, of the segments of the stigma of Mimulus, \&c., to admit of brief yet satisfactory treatment ${ }^{2}$.

SECT. 30.-Mechanism of the Movements produced by Variations of Temperature and of Light ${ }^{3}$. If plants with motile leaves, like Papilionaceæ and Oxalideæ, after having remained in the light, are suddenly placed in the dark, the leaves after some time take up their nocturnal position, closing upwards or downwards according to the species (Sect. 28). If light is now let in upon the plant in the state of sleep, the leaves again open and assume their diurnal position. Placing them in the shade has the same effect as complete darkness, but not so strongly.

These facts show that fluctuations in the intensity of the light cause curvatures of the motile parts of plants. If these parts are also irritable to concussion, as in Mimosa and Oxalis acetosella, darkness causes a similar position of the leaves to concussion. But the internal conditions are, as has been mentioned, very different in the two cases; for the folding up caused by darkness is associated with an increase

1 Unger, Anat. und Physiol. der Pflanze, 1855, p. 419.-Kabsch, Bot. Zeit., I86I, p. 26.-Pfeffer, Physiol. Unters., p. 157 .

${ }^{2}$ See also Unger, Anat. u. Phys., 1855, p. 419.-Suringar (on Drosera), Vereenigung voor de Flora van Nederland eng. den I 5 Juli, I 853.-Nitschke (on Drosera), Bot. Zeit. 1 860. - Schnetzler (on Berberis), in Bulletin de la Société vaudoise des Sci. Nat. X, I869.-Kabsch (on Berberis, Mimulus, \&c.), Bot. Zeit. 186r.-Kabsch (on Stylidium), Bot. Zeit. 1861.-A. W. Bennett, The Movements of the Glands of Drosera, Quart. Journ. Micr. Sci. I873.

[See also Kurz, Anatomie des Blattes der Dionca muscipula; Du Bois Reymond's Archiv, 1876 : also Munk, Die elektrischen und Bewegungs-Erscheinungen am Blatte der Dioncea Muscipula, ibid.; further, Darwin, Insectivorous Plants, 1875.]

${ }^{3}$ Dutrochet, Mém. pour servir, vol. I. p. 509.-Meyen, Neues Syst. der Pflanz.-Phys. vol. III. p. 487.-Sachs, Bot. Zeit. 1857 , Nos. 46, 47.-Bert, Recherches sur les mouvements de la sensitive, Paris 1867--Millardet, Nouvelles recherches sur la périodicité de la sensitive, Marburg 1869.Pfeffer, Physiol. Unters., Leipzig 1873. 
in the rigidity of the part, and therefore with an increase in its turgidity; while in that caused by irritation there is a decrease in all these, as Brücke was the first to show in the case of Mimosa. In the leaves of Phaseolus which are not irritable to concussion Pfeffer also found an increase of rigidity in the nocturnal position. - Conversely the diurnal position caused by the action of light or an increase in its intensity is the result of a diminution of the rigidity or turgidity. The effect produced upon the turgidity of motile organs by variations in the intensity of light causes upward and downward curvatures, since the variation of the turgidity of the one side of the organ is more considerable and occurs more rapidly than that of the other. A rise of temperature, on the contrary, which affects the motile part directly, is, according to Pfeffer, associated, in Oxalis, and in a less degree in Phaseolus, with increase of rigidity, and therefore also of turgidity, and causes a movement towards the nocturnal position, and hence a stronger turgidity of the upper side. When, on the other hand, an increase in the intensity of the light and a rise of temperature act on a contractile organ at the same time, its curvature is a resultant of the two changes; according as the one or the other preponderates, the leaf approaches more nearly the diurnal or the nocturnal position. Beyond this we have less certain knowledge as to the action of variations of temperature, than we have with reference to light.

Pfeffer, who has been especially engaged for a considerable time in the study of the mechanism of the movements produced by stimulation, has supplied me with the following:- The tendency to expand is increased by darkness equally in both the antagonistic halves, and in the tissues of the organ generally: light has the contrary effect, and the one half always reacts more powerfully than the other. The expansive force increases more rapidly in the half which is becoming convex, but it may become more considerable in the other half: it is for this reason that every movement produced by removal into darkness is followed after a time by a movement in the opposite direction, tending to the resumption of the position of equilibrium. It is quite certain that darkness not merely produces a closure but that it has a persistent effect, just as the movement imparted to a pendulum persists for a time whilst the amplitude of the oscillations is rapidly diminishing.'

Bert ${ }^{1}$ showed that if a Mimosa be continuously exposed to light for five days, the amplitude of its periodic movements diminished considerably, whereas the irritability increased. Pfeffer also found that continuous illumination for a period of one or more days arrested the daily periodic movements of Acacia lophantha. If a plant so treated be placed in the dark, closure takes place and then opening, and in continuous darkness for one or more days opening and closing repeatedly alternate. When the plant had been exposed to a strong light before being placed in the dark, the interval between the movements of opening and closing was about 24 hours. This is also the case if the plant be placed in the dark in the morning, so that the first closure takes place during the day. Pfeffer regards these phenomena (like those of motile flowers and of growing leaves) to be due to the persistent effect of the previous alternation of day and night; they are not to be confounded with the spontaneous periodic movements of these plants, for these latter continue when the former have disappeared, and the interval at which they occur is shorter.

SECT. 31.-Mechanism of spontaneous periodic Movements. The existence of spontaneous periodic movements which are not directly produced by the

1 Bert, Mém, de l'Acad, d. Sci. Phys, et Nat. de Bordeaux, I866, Bd. VIII. 
action of external influences has been already mentioned in Sect. 28, the movement of the lateral leaflets of Hedysarum gyrans being cited as a striking example. The rapid upward and downward movement of these leaflets take place, if the temperature be sufficiently high, both in continuous illumination and in continued darkness. The spontaneous movements of the leaflets of Oxalis acetosella and of Trifolium pratense are also very evident; the leaflets move in continued darkness and at a constant temperature through an angle of from $30^{\circ}$ to $90^{\circ}$ in from $\mathrm{I}$ to 4 hours.

It was pointed out in the foregoing paragraph that these spontaneous movements are independent of the persistent effect of the stimulating influence of light. Pfeffer clearly made this out in the case of the large terminal leaflet of Hedysarum (Desmodium) gyrans, which makes upward and downward movements of small amplitude in short periods of time. Whilst the daily periodicity (Sect. $3^{\circ}$ ) is manifested only for a short time in prolonged darkness, the spontaneous movements continue. In Trifolium and Oxalis the persistence of the daily periodicity is very slight, whereas the spontaneous movements are very evident in prolonged darkness.

It can scarcely be doubted, after what has been already said with reference to the various movements of growing and of mature organs, that the spontaneous periodic movements are effected by variations of turgidity, that is by the absorption and the escape of water. Since they are not accompanied, as Pfeffer has observed, by any alteration of the rigidity of the organ, it is probable that one half of the tissue gains in expansive force what the other loses and at the same moment; this almost amounts to saying that first one and then the other half of the tissue absorbs water from the other half.

Batalin (Flora, 1873) asserts that each periodic movement in Mimosa and Phaseolus is accompanied by a slight increase in length. Simple calculation suffices, however, to show that the periodic movements of these organs cannot be due to periodic growth of the upper and under surfaces.

The question why it is that the two sides of the organ are alternately more or less strongly turgid cannot be answered at present any more than the question why it is that first one side and then the other grows more rapidly in growing leaves, stems, tendrils, \&c. which exhibit nutation.

\section{CHAPTER VI.}

\section{THE PHENOMENA OF SEXUAL REPRODUCTION.}

Secr. $3^{2}$. Sexuality consists essentially in the formation, in the course of development of the plant, of reproductive cells of two different kinds, which have no independent power of further development, but which, by their coalescence, give rise to a product which possesses that power.

1 The facts upon which the considerations contained in this section are based are fully detailed in Book II, with references to the literature. 
It is only in a comparatively small number of cases, and in plants of very simple structure, like the Desmidiex, Mesocarpeæ, and Pandorinex, that the two cells which coalesce are alike in their mode of production, size, form, and behaviour when coalescing; and even in these cases they probably differ internally, since it is difficult to explain on any other hypothesis the necessity for their union into a pro:duct capable of development (the Zygospore). In some other Conjugatæ, as Spirogyra, this internal differentiation is exhibited at least to the extent that the contents of one of the conjugating cells pass over into the other, the contents of which remain stationary. But usually, as in most Algæ (Vaucheria, EEdogonium, Coleochate, Fucus, \&c.), and in all Characeæ, Muscineæ, and Vascular Cryptogams, a great variety of differences are manifested between the sexual cells as to size, form, motility, mode of production, and the share they take in the formation of the product of the union. This differentiation presents, especially in the Algæ, a most complete series of gradations between the conjugation of similar cells and the fertilisation of oospheres by antherozoids, any boundary line between these two processes being unnatural and artificial. The difference also between the sexual cells is developed only gradually and step by step, like the external and internal differentiation of plants ; and it is this that renders it probable that in the lowest forms of the vegetable kingdom, as in the Nostocacex, no process at all of this kind exists, or that at all events there are plants of extremely simple structure in which no such process occurs.

Wherever there is an evident external difference between the two sexual cells, one behaves actively in the union, and loses in the process its individual existence, the other behaves passively, absorbing into itself the substance of the active one, and furnishing by far the larger proportion of the first materials for the formation of the immediate product of the union. The former is termed the male cell or antherozoid, the latter the female cell or oosphere.

These most essential features of the sexual process may also be recognised in the fertilisation of the Ascomycetes and Floridex, although the external appearance of the female organ, the carpogonium, on the one hand and of the male organ (in certain Ascomycetes at least) on the other hand, are strikingly different from those which occur in any other class of plants.

The usual condition of the female cell during the sexual process (except in the Ascomycetes and Floridex) is that of a naked primordial cell (oosphere), formed either by simple contraction of the protoplasm of a cell previously enclosed within a cell-wall (as in the oogonium of Vaucheria, Edogonium, and Coleochate, and in the archegonium of Muscinex, Vascular Cryptogams, and Gymnosperms) or by the division of the protoplasm of a mother-cell combined with contraction and rounding off of the daughter-cells: (as in Saprolegnia and Fucaceæ), or by free cell-formation (as in the embryo-sac of Angiosperms). In all these cases the oosphere is spherical or ellipsoidal, except that in the Angiosperms it is sometimes elongated ; in general its form is the simplest that the vegetable cell can assume. The rounding off is not connected with any internal differentiation; at least where any internal differentiation is exhibited (as in the formation of chlorophyll and the granular contents in $E d o-$ gonium and other $\mathrm{Alg}$ ), the phenomenon is a secondary one in the process of fertilisation. The oosphere is never actively motile, even when, as in the Fucacex, 
after it is extruded it is set in rotation by the attached antherozoids; it usually remains enclosed in the mother-cell that produces it (the oogonium of Algæ and Fungi, the archegonium of Muscineæ, Vascular Cryptogams, and Gymnosperms, and the embryo-sac of Angiosperms), where it awaits fertilisation. While the male cell loses during the union its character as an individual cell, the oosphere is rendered capable of a more complete individual existence, which is first indicated by the invariable formation of a wall of cellulose, even when the oosphere results simply from the contraction of the protoplasm of an oogonium and still remains enclosed in its cell-wall, as in $\mathscr{E}$ dogonium and Vaucheria. In this respect the zygospore of Conjugatæ and Mucorini behaves also like a fertilised oosphere or oospore.

The male cell is more variable in its form and in its behaviour in the process of fertilisation. It always moves to the oosphere which remains at rest; in the Florideæ it is carried passively by the water; in the Fucaceæ, in Vaucheria, Edogonium, and other Algæ, in all Characeæ, Muscineæ, and Vascular Cryptogams, it swims actively. In other cases the male organ becomes attached in its growth to the female organ, as in the antheridial branches (pollinodia) of some Saprolegnieæ and of some Ascomycetes, and the pollen-tube of Phanerogams. The great variety of form of the male cell becomes especially conspicuous if we compare the roundish swarm-spore-like antherozoids of $E$ dogonium and Coleochate with the filiform antherozoids of Characeæ, Muscineæ, and Vascular Cryptogams, and with the rounded non-motile antherozoids of the Florideæ which (like the spermatia of Lichens) possess a cell-wall. The form is in each case evidently adapted to produce the right kind of motion in order to convey the fertilising substance to the female organ in a manner in harmony with its structure; while in the fertilisation of the latter the quality of the substance only is concerned.

According to the present state of our knowledge it may be assumed that fertilisation essentially consists in a union of protoplasm and nuclear substance derived from the male organ with protoplasm and nuclear substance of the female organ. In conjugation this union is brought about by the coalescence of the two conjugating cells. In the fertilisation of Edogonium and Vaucheria, the entrance of the antherozoid into the protoplasm of the oosphere and its absorption in it has been observed by Pringsheim. The antherozoids of Muscineæ and Ferns were observed by Hofmeister, and those of Marsilia by Hanstein, to enter the archegonium, those of Ferns by Strasburger to penetrate to the oosphere itself. It must therefore be inferred from analogy that in Phanerogams a union ${ }^{1}$ takes place of some substance contained in the pollen-tube with the oosphere; and in certain Ascomycetes of the contents of the pollinodium with those of the ascogonium. It would be impossible otherwise to explain how in these cases the mere contact of the often thick-walled pollen-tube with the embryo-sac, or of the pollinodium with the ascogonium, can effect fertilisation, while in the former cases such a complete coalescence of the male and female cells is necessary for this purpose.

${ }^{1}$ [See pp. 524 and 584.$]$ 
The product resulting from the sexual process is usually a new individual which has no longer any organic connexion with the mother-plant and is not united with it in growth. This is the case in the Muscineæ, where the sporogonium, and in Phanerogams, where the embryo, is nourished by the mother-plant, but there is no actual continuity of tissue between it and the latter. The case is quite different in the Ascomycetes (e.g. Lichens, Eurotium, and Erysiphe) and Floridex, in which the female organ itself or certain cells connected with it are stimulated by fertilisation to produce new shoots from which results a fructification containing spores; and it is only after the completion of this complicated vegetative process brought about by the sexual union that the spores are set free, and produce new individuals independent of the mother-plant.

The reproductive cells of the same plant do not differ merely externally; the inability of either to originate by itself a new course of development, while the two together produce an organism capable of germinating, shows that the properties of the two are complementary to one another. The sexual differentiation, or difference between the male and female cells, which is neutralised by the act of fertilisation, has been preparing for a longer or shorter time; the product which is the result of fertilisation owes its formation to the neutralising of the sexual difference. In the Conjugatæ and other families where the sexual difference is extremely small or even imperceptible, the preceding processes of development are also alike; the mothercells of the two kinds of reproductive cells even to the earliest stage of development do not differ externally. But where the sexual difference is greater, it is foreshadowed in the preceding processes of development. Thus the mother-cell of the antherozoids of $\mathscr{E}$ dogonium differs in form from that of the oosphere; and this is especially seen in the development of the OEdogonieæ with 'dwarf males.' In Vaucheria the branches which subsequently become antheridia differ at an early. stage from those which form the oogonia. The sexual differentiation of the Characeæ is inaugurated long beforehand in the great difference in the development of the antheridia and carpogonia, the position of the two organs on the leaf being also different. In the Muscineæ and Vascular Cryptogams again preparation is made for the production of the antherozoids and oospheres in different ways by the formation of antheridia and archegonia. But this preparation is not confined to the difference between the organs which immediately produce the reproductive cells; in many classes of plants it even goes back so far that the entire plant developes as a male or as a female plant, producing only male or only female reproductive organs. This occurs in some Algæ, Characeæ, Muscineæ, and in the prothallia of some Vascular Cryptogams.

The fact is very remarkable that this preparation may be carried back in the development of the individual even beyond the limit marked by the alternation of generations. In the Algæ, Characeæ, Muscineæ, Ferns, and Equisetaceæ, the nature of the alternation of generations is such that the sexual differentiation is developed in one of the generations, while it is neutralised in the succeeding generation. In these cases therefore we have a sexual and an asexual generation in the course of the development of the same individual; the asexual generation is the product of the neutralising of the sexual differentiation of the sexual generation. The two generations, especially in Muscineæ and Vascular Cryptogams, differ essentially from 
a morphological point of view ; they follow altogether different laws of development ; one of their limits always occurs in the fertilised oosphere. The prothallium developed from the asexual spore of Ferns and Equisetaceæ is, for example, morphologically a thallus without leaves or roots, while its physiological significance is determined by the production of antheridia and archegonia. From the fertilised oosphere on the other hand is produced the Fern or Horsetail, characterised morphologically by the differentiation of stem, root, and leaf; but sexually this differentiated plant is neuter, producing neither male nor female cells, but only asexual spores. If the process of development of Rhizocarpeæ and Selaginelleæ is compared with these phenomena, it will be seen that in these classes the two generations, the prothallium and the spore-forming leafy plant, stand essentially in the same relation to one another as in Ferns and Equisetaceæ, only that the sexual differentiation goes back to the spore itself; the spores are of two kinds, large female spores which produce the small female prothallium, and small male spores which produce a still smaller prothallium and antherozoids. The preparation for this sexual difference is manifested even in the asexual generation, by the sporangia producing only female or only male spores according to their position. In Salvinia the preparation goes back still further, each entire capsule producing only female or only male sporangia. It has already been pointed out how in Phanerogams the embryo-sac corresponds to the large, the pollen-grain to the small spore of heterosporous Vascular Cryptogams, and the endosperm to the prothallium. The endosperm of Phanerogams no longer appears as an independent structure, but only as a constituent part of the preceding generation; in Angiosperms it is often from the first rudimentary and sometimes entirely absent, and the female sexual cell, the oosphere, is then the immediate product of the embryo-sac which corresponds to the large spore. The true sexual generation therefore becomes less and less important; as such it becomes devoid of significance, while the sexual differentiation is carried back to the spore-forming generation, in which it determines the formation of the two kinds of reproductive organs, $i . e$. the pollen-sacs and ovules; the flower may be exclusively male or female (monœcious diclinous), and, where the plant is dicecious, the sexual differentiation affects the entire individual, which is either male or female. In all Cryptogams, on the other hand, diœcism is only displayed in one (the sexual) generation in the course of development of the individual.

The process of development brought about by fertilisation or the union of the reproductive cells is usually not confined to the resulting embryo, but shows itself also in a variety of changes in the mother-plant itself. In Coleochate the oospore becomes invested with a cortical layer; in Characeæ the enveloping tubes of the carpogonium grow after fertilisation, their coils increase in number, and their membranes become lignified on the inside; in the Hepaticæ a variety of envelopes arise from the mother-plant; in the Mosses the vaginule and in all Muscineæ the calyptra becomes developed; the tissue of the prothallium which surrounds the growing embryo of Ferns grows at first rapidly along with it; in Phanerogams the entire development of the seed and fruit depends on the changes caused in the motherplant by the fertilisation of the oosphere. The two most remarkable cases occur in Florideæ and Ascomycetes on the one hand, and in Orchideæ on the other hand. In the former fertilisation does not in general directly cause the formation of an 
embryo, but brings about processes of growth in the mother-plant, in consequence of which the cystocarp is produced in Florideæ and the spore-fruit in Ascomycetes. In the Orchideæ the action of the pollen-tube is visible on the mother-plant even before fertilisation; Hildebrand has shown (Bot. Zeit. 1863 , p. 34 I) that in all Orchids which he examined the ovules were not in a condition to be fertilised at the time of pollination; and in some (as Dendrobium nobile) they have not even begun to be formed; it is only during the growth of the pollen-tubes through the tissue of the stigma and style that the ovules become so far developed that fertilisation can at length be effected. In the Orchideæ the formation of the female cell is therefore a result of pollination; it is determined by the action of the male pollen-tube on the tissue of the mother-plant ${ }^{1}$.

When the embryo is being developed within the mother-plant, as in the Muscineæ and Vascular Cryptogams, it obtains its food-material from the plant; and this is connected in the Vascular Cryptogams with complete exhaustion and the dying off of the prothallium. In Phanerogams not only does the embryo usually acquire a considerable development, even within the fruit, but a great quantity of the products of assimilation is also withdrawn from the plant by the accumulation of reserve-material in the seed and by the development of the fruit; in many cases the plant itself is also completely exhausted, all its disposable formative substances are given up to the seed and the fruit, and it dies off (monocarpous plants). It is clear that all these changes and the various movements of materials in the mother-plant connected with them are results of fertilisation, results : of immense importance caused by the union of microscopic cells, imponderable by the best balance.

(a) A careful consideration of the phenomena occurring among the Thallophytes would probably lead to the formation of a tolerably clear idea of the mode of the Development of Sexuality in the Vegetable Kingdom. The space at our disposal will only suffice for a few general remarks.

The labours of Pringsheim ${ }^{2}$, which open up the way for a complete theory of sexuality in the future, seem to indicate that the conjugation of the motile cells of Pandorina, Ulothrix, \&c. is one of the primitive phases of a sexual act. If this be so, then it follows that a sexual coalescence of cells first made its appearance when the Thallophytes had already attained a considerable degree of morphological and of physiological development. The same result is reached by a consideration of the fact that sexuality is apparently absent in the Hydrodictyex ${ }^{3}$ and in the immediate allies of Ulotbrix, and that comparatively highly-developed forms, such as the Rivulariex, exist among the Protophyta in which no trace of sexuality can be discovered.

If it be also remembered that the simplest forms of sexuality, conjugation and the formation of zygospores, occur in very different groups of the Thallophytes, and that the mode of conjugation varies with the form and habit of the plants and that it may be very different in different groups, the thought is at once suggested that the sexual coalescence of cells may have commenced at different times and quite independently in

1 [For a summary of the instances in which pollen appears to have influenced the fruit of the mother-plant, see C. J. Maximowicz, Journ. Roy. Hort. Soc., new series, vol. III. p. 16I ; and Darwin, Animals and Plants under Domestication, vol. I. p. 397.]

2 Monatsber. d. k. Akad. der Wiss. in Berlin, 1869.

3 [Conjugation has since been observed in Hydrodictyon (see page 251 ).] 
different groups of Thallophytes. The variety of the modes in which the auxospores are formed among the Diatomeæ indicates that conjugation has become developed here from the first quite independently of the connection of this group with the Conjugatæ; and in the latter group so many modes of the formation of zygospores occur that possibly the development of sexuality began in several different species included within it. The mode in which conjugation takes place among the Zygomycetes seems to indicate that the sexual act originated independently, and it is still difficult to trace a historical connection between the fertilisation of the Ascomycetes and of the Florideæ and that of any other group of Thallophytes. It is more feasible, as Pringsheim has already suggested, to regard the fertilisation of the Edogonieæ and of the Vaucheriaceæ as being a further development of the conjugation of two motile cells. If, as appears to be the case, a sexual act originated at different times in different Thallophytes, a series of further developments should be found to correspond to each distinct origin, but at present it is impossible to say from which of these origins the formation of the archegonia of Mosses and of Vascular Cryptogams and that of the reproductive organs of Phanerogams has been evolved.

The question arises with reference to the simplest phases of sexuality, as to whether conjugation was effected primarily in consequence of a sexual differentiation of the cells, or whether conjugation preceded any sexual differentiation and that this only made its appearance as a secondary phenomenon when plant-forms had become more highly developed ${ }^{1}$. The former of these two alternatives is supported by those phenomena which indicate a mutual action at a distance of the conjugating cells and which have been frequently mentioned by observers as a sort of mutual search. The latter is borne out by the consideration that the conjugating processes are developed from those points only of the mycelium, in Zygomycetes and in many Ascomycetes, at which they are in contact. It is known that similar, but quite infertile, connections occur between mycelial filaments, and there are good grounds for believing (Sect. 16) that these phenomena of growth are induced by pressure; and therefore it may be reasonably inferred that the growth of the conjugating filaments and the formation of zygospores is a further development of the sterile coalescence of mycelial filaments (in the form of the letter $\mathrm{H}$ ) which is simply the result of pressure. If it be admitted that the sexual coalescence of cells originated at different times and in different plant-forms, it may also be admitted that in one case a sexual differentiation first took place which rendered a coalescence necessary, while in other cases the processes of growth initiated by pressure resulted in the developing of conjugating organs which were sexually differentiated.

(b) Parthenogenesis ${ }^{2}$ is the term used to express the fact that plants which possess normal male organs of fertilisation "and in which embryos are developed by the fertilisation of the oospheres may occasionally develope embryos from female cells which have not been fertilised, but which are nevertheless capable of complete development. This phenomenon, which is of frequent occurrence in the Animal Kingdom, especially among Insects, has been satisfactorily observed in only a few cases among plants. The doubts as to the parthenogenesis of the Coelebogyne ilicifolia which is cultivated in Europe still exists. It appears, however, that Chara crinita is represented in certain places by the female form only, and that nevertheless it bears an enormous number of spores which are capable of germination. The most satisfactory cases of parthenogenesis are those of Saprolegnia ferax and Acblya polyandra ${ }^{3}$. Pringsheim has shown that their oospheres are usually fertilised, but that frequently they germinate and develope

1 [From the fact that, as in Ulothrix for instance, the microzoogonidia come to rest and germinate as well without as with previous conjugation, it appears probable that the latter is the correct view.]

2 Braun, Die Parthenogenesis bei Pflanzen, in den Abhandl. der Berl. Akad. I856.-Pringsheim, Jahrb. f. Wiss. Bot. IX. [See page 593.]

3 [From de Bary's researches it appears that parthenogenesis is the rule in the Saprolegniæ.] 
new plants without fertilisation and without any difference in the mode of their germination from that of true oospores, with this single exception, that the quiescent period of the parthenogenetic cells is shorter.

Pringsheim's account of the development of the parthenogenetic forms of these plants which is appended here is of great interest:- 'The successive generations both of Saprolegnia ferax and of Acblya polyandra produced by cultivation become smaller, and the number of male filaments diminishes in each succeeding generation until they finally cease to be formed, and thus the monœcious forms become replaced by purely female ones.' These observations show that as the result of continued cultivation combined with the action of certain unfavourable conditions which accompany every attempt at cultivation, the formation of male sexual organs at length ceases. Possibly it is in consequence of the action of similar adverse conditions upon its internal constitution that Chara crinita ceases to form antheridia after having grown for a considerable time in certain waters. Possibly also these internal disturbances may affect the nature of the female cells, though they are developed in the usual external form, so that they are not sexually differentiated or only imperfectly so. In this case the effect would be one of the obliteration of the existing sexual differentiation, or in other words, a case of retrograde metamorphosis, and this is quite as conceivable as the first origin and the subsequent development of sexuality. In future investigations of the subject attention ought to be paid to the question whether the oospheres developed upon plants of Saprolegnia ferax and of Acblya polyandra which bear antheridia also are capable of parthenogenetic development, or whether this property belongs only to oospheres developed by plants destitute of antheridia ${ }^{1}$. However difficult it may be to answer this question by experiment, it must be done before it can be possible to decide whether or not the development of the male organs deprives the oospheres of their power of independent development, so that in proportion as the development of the male element diminishes the parthenogenetic property increases. Since we may assume that the essential object of fertilisation is to give to the oosphere something which it lacks but which is necessary for its further development, a parthenogenetic oosphere must possess, independently of fertilisation, that which it requires for its further development, that is, it is not sexually differentiated, and this probably because the differentiation of the male element has been suppressed.

(c) The Effects of Sexual Coalescence. Since nearly all plants, and more especially the majority of Thallophytes, are capable of reproducing themselves asexually, and since this is the usual mode of reproduction in many species, it may well be asked what the significance of sexual reproduction really is. If sexuality is merely concerned in the development of new individuals, it is difficult to understand why the asexual reproduction should not suffice. This question is of especial interest with reference to those lower forms of Thallophytes which reproduce themselves through many generations by asexual cells which may be either motile or non-motile : with reference to many Phanerogams, for instance, the Conifers, it seems as if without sexuality, which induces the formation of the seed, no reproduction would be possible.

The significance of sexuality is seen in quite another light when those plants are considered which exhibit a distinct alternation of generations, such as the Ferns, Equisetacex, the Mosses, and others. In Sect. 29 of Book I, I endeavoured to show that the alternation of generations, wherever it occurs in the Vegetable Kingdom, is produced by sexuality, and that without it no such alternation is possible. In all cases of well-marked alternation of generations an organism which finally bears sexual organs is developed from a spore which has been produced asexually; fertilisation initiates a new process of development which closes with the development of the spore. Before fertilisation, there is merely the organism developed from the spore, the first or sexual generation: after fertilisation the second or asexual generation is developed

1. [According to de Bary's recent investigations, the former of these two answers to the question is the correct one.] 
which bears spores. If we compare the histological and the morphological development of the two generations, it becomes strikingly evident, among the Vascular Cryptogams at least, that the generation which has been developed as the result of fertilisation is much more highly organised than the generation (prothallium) which has been developed from the spore. In the Mosses it might appear that the contrary is the case, for in these plants the sexual generation is the one which grows independently and which is differentiated into leaf and stem; however, the histological differentiation of the sporogonium is far more perfect than that of the moss-plant, so that it is true for Mosses also that the product of fertilisation is the more highly organised of the two generations.

All these cases of evident alternation of generations lead to the conclusion that a process of development of a more complex kind is initiated by the sexual act. To a certain extent this is true also of the Zygomycetes, the zygospore being a more highly organised cell than any of those of the mycelium, and it can scarcely be doubted with reference to the Coleochætex, the Characeæ, and the Florideæ that the sexuallyformed spore-fruit is histologically the most complex product of these plants. Although the same cannot be said with reference to the Oosporex and to the Conjugatæ, still this by no means affects the significance of the sexual act for other plants. A complete discussion of the facts would, on the contrary, probably show that the higher development of the Pandorineæ, of the Conjugatæ, and of the Diatomaceæ when compared with the Protophyta has been probably promoted by the evolution of sexuality, even though th:is is not expressed by a well-marked alternation of generations. The Phanerogams afford a similar, though exactly opposite case: in them the alternation of generations is exhibited in only a rudimentary form, for, in the course of the development of these plants from some primitive type allied to the Vascular Cryptogams the sexual generation (prothallium) has been reduced to its simplest expression. Whereas in the Oosporex the sexual generation is the predominating one and the product of fertilisation is but imperfectly developed, in Phanerogams it is the generation produced in consequence of a sexual act which comes to be completely developed, and it is the sexual generation (Prothallium, Endosperm) which is rudimentary. In the latter case we have the end, in the former the beginning of phytogenetic series; in the latter the alternation of generations is disappearing, in the former it is in the first stage of its evolution. If therefore we desire to understand the significance of sexuality in the history of the development of a single plant or in that of the whole Vegetable Kingdom, we must fix our attention upon those groups in which an alternation of generation is evident: in such cases (Vascular Cryptogams, Muscinex) the effect of sexuality is obvious. We may then conclude that the coalescence of the male with the female cell causes the development of an organism which is more highly differentiated both histologically and morphologically.

SECT. 33. Influence of the origin of the reproductive cells on the product of fertilisation. The male and female cells or the organs that produce them are formed at a greater or lesser distance from one another on the same plant, or on different individuals of the same species. The male and female cells of the same species may thus be more or less nearly related to one another as having been immediately or more remotely derived from the same parent-cell. The question arises what influence this genetic relationship of the male and female cells exercises on the product of fertilisation. At present we are unable to lay down any general law in this respect; but the overwhelming weight of evidence points to the law that the sexual union of nearly related cells is detrimental to the preservation of the plant, and in general the more so the further the morphological and sexual differentiation of the species has advanced. Only in a few plants of low organisation does a fertile union take place between sister-cells, as in Rhynchonema among Conjugatæ. But in most Algæ and Fungi (as Spirogyra, Edogonium, 
Fucus platycarpus, \&c.) the reproductive cells of the same plant are not so closely related, and especially where fertilisation is caused by actively or passively motile antherozoids, there being at least a possibility of their meeting with oospheres of more remote origin. Even in Vaucheria, where the antheridium is the sister-cell of the oogonium, the curving of the former, and the direction in which the antherozoids escape, indicates that fertilisation does not usually take place between the contiguous organs, but between those more remote or even between those produced by different individuals. The tendency for fertilisation to occur only between reproductive cells of as remote relationship as possible within the same species is manifested in a great variety of contrivances, the simplest being that on each individual of the sexual generation only male or only female organs are produced. Thus between the two uniting reproductive cells there lies the entire course of development of the two plants when the plants are derived from the same mother-plant, and a still longer course of development when they are derived from different mother-plants. This distribution of the sexes, which is generally termed Diceism, occurs in all classes and orders of the vegetable kingdom, showing that it is a useful contrivance for the maintenance of different species. Thus we find this phenomenon in many Algæ, as in most Fucaceæ, in some Saprolegnieæ and Characeæ (Nitella syncarpa, \&c.), in many Muscineæ, in the prothallium of many Ferns (Osmunda regalis) and of most Equisetaceæ, and in many Gymnosperms and Angiosperms.

If the plant which produces both kinds of sexual organs is large or at least highly differentiated, distance in the relationship of the two kinds of reproductive cells is still attained by the male and female organs being produced on different branches; and this phenomenon, which is in general termed Monoecism, is also common in the vegetable kingdom, as in some Algæ, many Muscineæ, and a very large number of Gymnosperms and Angiosperms '

But another condition which, according to the law just stated, should apparently be very unfavourable, is also of very common occurrence in the vegetable kingdom, namely, that the reproductive organs are in close contiguity, and the sexual cells are therefore of near even if not always of the closest affinity. Thus, for example, the same cellular filament of $O E$ dogonium produces both male and female cells, the same Vaucheria-filament antheridia and oogonia in close proximity, the same conceptacle of Fucus, platycarpus produces both oogonia and antheridia; the carpogonia of most Characeæ are produced close beside the antheridium on the same leaf; the archegonia and antheridia of 'some Mosses (species of Bryum) are collected together in hermaphrodite receptacles, the prothallia of many Ferns produce both kinds of reproductive organs side by side; in the flowers of Angiosperms hermaphroditism is the typical and most common arrangement. But in all these cases where the aim is apparently to favour the union of sexual cells nearly related to one another, there are at the same time contrivances which hinder the male cells from reaching the contiguous female cells; or at least to render it possible that this should not always happen. This fact was first recognised by Kölreuter ( $I 761$ ) and Karl Conrad Sprengel (I793), and has been further illustrated recently by Darwin,

1 The arrangement of the reproductive organs termed Polygamy is also a contrivance intended to hinder perpetual self-fertilisation of a flower or of an individual. 
Hildebrand, and others ${ }^{1}$. In spite of the hermaphrodite flowers of Phanerogams and the similar sexual arrangements of Cryptogams, it appears very certain that the union of nearly related sexual cells must be unfavourable to the perpetuation of most plants, since such various and often astonishing means are provided in order to prevent self-fertilisation when the sexual organs are contiguous.

One of the simplest and commonest means for ensuring cross-fertilisation is Dichogamy, i.e. the arrangement by which the two kinds of reproductive organs, when they are contiguous, are mature at different times, so that the sexual cells which are in close contiguity and are therefore nearly related are not capable of performing their respective functions simultaneously. The male cell must in these cases unite with a female cell in a different group of sexual organs. This is in fact usually the case with the hermaphrodite flowers of Angiosperms, as also with most prothallia of Ferns and the monœecious Characex, in which the carpogonium is situated close to the antheridium but becomes mature only at a later period (this is very strikingly the case in Nitella flexilis). Insects are the main agents in the conveyance of the pollen to the stigma of other flowers of dichogamous Phanerogams, for which purpose the parts of the flower possess special adaptations which will be described presently. In the dichogamous species of Nitella and prothallia of Ferns the motility of the antherozoids is sufficient to enable them to reach the archegonia of neighbouring prothallia, or the carpogonia on other leaves of the same plant, or even on other plants of the same species. Whether the Algæ named above and some Muscineæ are dichogamous is doubtful ; but the motility of the antherozoids renders it possible for them to reach the oospheres of other plants or those on other branches of the same plant.

Among Angiosperms, in addition to the common occurrence of dichogamy, there are also other contrivances of a very different nature which have the sole purpose of transferring the pollen of hermaphrodite flowers, by the help of insects, to the stigma of another flower of the same or of a different plant. In most Orchideæ, Asclepiadeæ, Viola, \&c., the reproductive organs of each individual flower are developed at the same time, but at the time of maturity mechanical contrivances exist which prevent the pollen falling on the stigma of the same flower; it must be carried by insects to other flowers.

In other cases, as Hildebrand has shown in the case of Corydalis cava ${ }^{2}$, the pollen does actually fall on the stigma of the same flower, but is there impotent, having the power of fertilising only when it falls on the stigma of a different flower, and only perfectly when carried to the flower of a different individual of the same species. Such a plant is therefore only morphologically hermaphrodite; it is

${ }^{1}$ K. C. Sprengel (Das neu entdeckte Geheimniss der Natur im Bau und in der Befruchtung der Blumen, Berlin, I 793, p 43) first gave expression to the pregnant idea, 'Since a large number of flowers are diclinous and probably at least as many hermaphrodite flowers are dichogamous, Nature appears to have designed that no flower shall be fertilised by its own pollen.' Darwin (On the Various Contrivances by which Orchids are Fertilised, London 1862, p. 359) says, 'Nature tells us in the most emphatic manner that she abhors perpetual self-fertilisation;' and again, 'No hermaphrodite fertilises itself for a perpetuity of generations.' [This last observation was first made by Andrew Knight in I 799 (Phil. Trans. p. 202). - See Darwin, The Effects of Cross* and Self-Fertilisation in the Vegetable Kingdom, 18;6.]

${ }^{2}$ [Ueber die Befruchtung von Corydalis cava, Jahıb. fuir wiss. Bot. I 866.] 
physiologically diœcious. J. Scott states that Oncidium microchilum exhibits the same phenomena, the pollen not being potent on the stigma of the same flower while cross-pollination ensures fertilisation ${ }^{1}$; the pollen and stigma are therefore without function except to the stigma and pollen of a different flower. Similar phenomena have been described by Gärtner in the case of Lobelia fulgens and Verbascum nigrum, and in species of Begonia by Fritz Müller ${ }^{2}$.

No less remarkable is another contrivance for the mutual fertilisation of different individuals of plants with hermaphrodite flowers,-Dimorphism ${ }^{3}$ (or Heterostylism), consisting in a difference between different individuals of the same species with reference to their reproductive organs. In one individual the flowers all have a long style and short filaments, while in another individual all the flowers have' a short style and long filaments, as in Linum perenne, Primula sinensis, and other species of Primula. It sometimes happens also, as in Lythrum Salicaria and many species of Oxalis $^{4}$, that the reproductive organs in the flowers of different specimens of the same species exhibit three different relative lengths (Trimorphism), there being an intermediate length of style between the long-styled and the short-styled forms. In these cases of dimorphism and trimorphism Darwin and Hildebrand have shown that fertilisation is possible only (in the case of Linum perenne) or at least has the best result when the pollen of the long-styled flower is carried to the short-styled stigma of another plant, and vice vers $\hat{a}^{\mathbf{b}}$. Where there are three different lengths of style, fertilisation succeeds best when the pollen is carried to the stigma which stands at the same height in another flower as the anthers from which the pollen came. It will be seen that this is but an expansion of the same rule.

While in the very numerous diclinous, dichogamous, dimorphic, and trimorphic flowers, insects carry pollen from one flower to another, it is comparatively rare for cross-pollination to take place without the help of insects. This occurs in some Urticaceæ, as Pilea and Broussonetia, where the anthers emerge suddenly from the bud and scatter their light pollen in the air like a fine cloud of dust, which is then blown to the female organs of other flowers. In the Rye the arrangement is still simpler; the flowers open separately, usually in the morning; the filaments elongate rapidly and push the ripe anthers out of the pales; the anthers then hang down at the end of the long filaments, open, and allow the heavy pollen to fall down, thus reaching the stigmas of other flowers lower down in the same spike or in neighbouring spikes, being assisted in this by the oscillations of the haulm under the influence of the wind ${ }^{6}$.

1 According to Fritz Müller (Bot. Zeit. I868, p. I I4), in some species of Oncidium the pollenmasses and stigmas of the same individual have a positively poisonous effect on one another.

${ }^{2}$ Fritz Müller, Bot. Zeit. I 864, p. 629.

${ }^{3}$ [Darwin, On the Two Forms, or Dimorphic Condition, in the Species of Primula, Journ. Proc. Linn. Soc. Bot. 1862, p. 77 ; ditto, On the Existence of Two Forms, \&c. of the Genus Linum, ibid., I863, p. 69 ; ditto, On Trimorphism in Lythrum Salicaria, ibid., I864, p. I69; ditto, On the Character and Hybrid-iike Nature of the Offspring from the Illegitimate Unions of Dimorphic and Trimorphic Plants, Journ. Linn. Soc. 1868, p. 393; ditto, The Different Forms of Flowers on Plants of the same Species, 1877.$]$

${ }^{4}$ Hildebrand, Bot. Zeit. 187 r, Nos. $25,26$.

${ }^{5}$ [Darwin has given the name of legitimate to the union of two distinct forms, illegitimate to the fertilisation of long- or short-styled plants by pollen from flowers of their own form.]

${ }^{6}$ [For a detailed account of the very remarkable phenomena connected with the pollination of 
In connection with the tendency so clearly evidenced even among Cryptogams, and still more among Phanerogams, to prevent self-fertilisation within the same hermaphrodite group of sexual organs, it is a very remarkable fact that there are a number of plants among Angiosperms which form two kinds of hermaphrodite flowers, viz. large flowers which can generally be fertilised by the pollen of other flowers, and small, more or less depauperated flowers, sometimes underground, which never open [Cleistogamous Flowers], the pollen emitting its tubes immediately from the anthers and thus fertilising the ovules. There occur therefore in these cases different kinds of flowers on the same individual, one kind being adapted for cross-, the other kind exclusively for self-fertilisation ${ }^{1}$. This occurs, for example, in Oxalis Acetosella, where the small flowers are formed close to the ground when the larger flowers have already ripened their fruit; in Impatiens Noli-me-tangere, Lamium amplexicaule, Specularia perfoliata, many species of Viola, as $V$. odorata, elatior, canina, mirabilis, \&c., Ruellia clandestina, many Papilionaceæ, as Amphicarpaa, and Voandzeia, Commelyna bengalensis, \&c. When in these cases the large typically developed flowers are fertile, cross-fertilisation with other flowers of the same species must happen occasionally in the course of generations, and the small depauperated self-fertilised flowers then seem to be a subsidiary contrivance whose purpose is altogether unknown. It is however remarkable, and apparently in contradiction to the general rule, that the large normal flowers sometimes exhibit a tendency to infertility (as in species of Viola) or are altogether unfruitful (as in Voandzeia), so that reproduction depends in such cases mainly or entirely on the cleistogamous selffertilised flowers. But since there are many questions in connection with this subject which find their solution in the foregoing facts, these rare exceptions cannot overthrow the general law ${ }^{2}$.

In other cases, as in most Fumariaceæ, Canna indica, Salvia hirta, Linum usitatissimum, Draba verna, Brassica Rapa, Oxalis micrantha and sensitiva, the pollen must also, according to Hildebrand, owing to the position of the sexual organs, fall on the stigma in the same flower, and is potent; but in such cases, since the flowers are visited by insects, an occasional crossing with other flowers is not impossible. Even among Orchideæ, where we find the most wonderful contrivances to prevent self-fertilisation, Darwin found an instance in Cephalanthera grandiflora in which the pollen-tubes are emitted from the pollen-grains on to the stigma while the former

Rye and other cereals, see Hildebrand in Gardener's Chronicle, March $\mathrm{I}_{5}$ and 22, and May 24, 1873; also A. S. Wilson, Trans. Bot. Soc. Edin. XI. 506 and XII. 84. Flowers the pollination of which is effected by the wind are termed anemophilous, in contradistinction to the entomophilous, or those pollinated by the agency of insects.]

1 H. v. Mohl, Einige Beobachtungen über dimorphe Blüthen, Bot. Zeit. 1863, Nos. 42, 43. [See also A. W. Bennett on the closed self-fertilised flowers of Impatiens in Journ. Linn. Soc. 1872, p. 147 ; ditto, Pop. Sci. Rev. 1873, p. 337. In Funcus bufonius the pollen-tubes are emitted while the pollen-grains are still enclosed in the anther, perforating the wall of the latter. Henslow, On the Self-fertilisation of Plants, Trans. Linn. Soc., Series II, vol. I, 1879.]

${ }^{2}$ [Herrmann Müller (Nature, vol. VIII. p. 433 et scq.) has pointed out the existence of another kind of dimorphism, in which a species presents two different forms of flowers, one adapted to selffertilisation, smaller and less brightly-coloured, growing in situations where there are but few insects, the other adapted to cross-fertilisation, larger and more brightly-coloured, growing where insects abound. These two forms have occasionally been described as distinct varieties or even species.] 
are still in the anthers; but according to Darwin's experiments the number of good seeds produced is smaller when the plant is allowed to fertilise itself than when pollination is effected by foreign pollen with the help of insects.

A clear comprehension of the phenomena of dichogamy, dimorphism, and the other contrivances for ensuring cross-fertilisation, can only be obtained by a careful study of numerous individual cases ${ }^{1}$.

It is more clearly seen in the fertilisation of flowers than almost anywhere else how exactly the development of the organs is adapted to the fulfilment of a perfectly definite purpose. Each plant has its own peculiar contrivance for the conveyance of the pollen to the stigma of another flower. It is not possible to make many general remarks on this subject; the following may suffice here.

It must be noted in the first place that insects ${ }^{2}$ carry pollen undesignedly while seeking the nectar of flowers which has been produced exclusively for their attraction. Flowers which are not visited by insects, and Cryptogams which do not require them, do not secrete any nectar. The position of the nectaries, usually concealed deep at the bottom of the flower, as well as the size, form, arrangement, and often also the movement of the parts of the flower during the time of pollination, are always of such a nature that the insect-sometimes of one particular species-must take up particular positions and make particular movements in obtaining the nectar, and thus cause the masses of pollen to become attached to its hairs, feet, or proboscis, and afterwards, when assuming similar positions, to be applied to the stigmas of other flowers. In dichogamous plants the movements of the stamens, styles, or branches of the stigmas assist this end, taking place frequently in such a way that at one time the open anthers occupy the same position in the flower that the receptive stigmas do at another time, so that the insect, when taking up the same position, touches the open anthers in one flower and the receptive stigmas in another flower with the same part of its body. The same result is also obtained in dimorphic flowers, the pollination being in these cases efficacious when anthers and stigmas which occupy the same position in different flowers are made mutually to act on one another. But there are besides many other contrivances, most variable in their nature and often perfectly astonishing, for effecting the conveyance of pollen by insects. A few examples may suffice.

1 See especially K. C. Sprengel, Das neu entdeckte Gelheimniss der Natur, \&c., Berlin 1793.Darwin, On the Fertilisation of Orchids, London 1862.-Hildebrand, Die Geschlechtervertheilung bei den Pflanzen, u. das Gesetz der vermiedenen $u$. unvortheilhaften stetigen Selbstbefruchtung, Leipzig 1867.-Strasburger in Jenaische Zeitschrift, vol. VI, 1870, and Jahrb. für wiss. Bot. vol. VII, where the mode of fertilisation of Gymnosperms, Marchantieæ, and Ferns is described. [The most complete account of the phenomena of the reciprocal adaptation of flowers and insects to cross-fertilisation is contained in Herrmann Müller's Befruchtung der Blumen durch Insecten u. die gegenseitigen Anpassungen beider, Leipzig, 1873, where also is a résumé of the literature of the subject. See also Kölreuter, Vorläufige Nachricht von einigen das Geschlecht betreffenden Versuchen, Leipzig I761.-Delpino, Ulteriori osservazioni sulla dicogamia, Milan I868-1870.-Axell, Om Anordningarna for fanerogama växternas befruktning, I 869.-Darwin, On the Agency of Bees in the Fertilisation of Papilionaceous Flowers, Ann. and Mag. Nat. Hist. 3rd series, vol. II. p. 46r. -Ogle in Pop. Sci. Rev. 1869, p. 26I, and I870, p. 45 (on Salvia).-Hildebrand in Leopoldina, I869 (Compositæ); ditto, in Monatsber. der Berlin. Akad. 1872 (Grasses).-Farrer in Ann. and Mag. Nat. Hist. 1868; Nature, vol. VI, 1872, p. 478 et seq. (Papilionaceæ).-A. W. Bennett, in Pop. Sci. Rev. I873, p. 337.-H. Müller, in Nature, vols. VIII, IX, and X.-Sir J. Lubbock, On British Wild Flowers considered in relation to Insects, London 1875.$]$

2 J. G. Kölreuter first recognised the necessity of insect help, and described special contrivances for pollination, in his Vorläufige Nachricht von einigen das Geschlecht der Pflanzen betreffenden Versuchen, i 761 . 
(I) Dichogamous Flowers ${ }^{1}$ are either protandrous or protogynous ${ }^{2}$. In the former the stamens are developed first, their anthers opening at a time when the stigmas are still undeveloped and not yet receptive; the stigmatic surface is only developed later, and usually not till the pollen has been carried away from the anthers by insects; they can then only be fertilised by the pollen of younger flowers. To this category belong the various species of Geranium, Pelargonium, Epilobium, Malva, Umbelliferæ, Compositæ, Campanulaceæ, Labiatæ, Digitalis, \&c. The phenomena referred to, especially the movements of the stamens and stigmas, are so readily observed in these cases, e.g. in Geranium and Altbrea, that no further description is necessary. In protogynous flowers

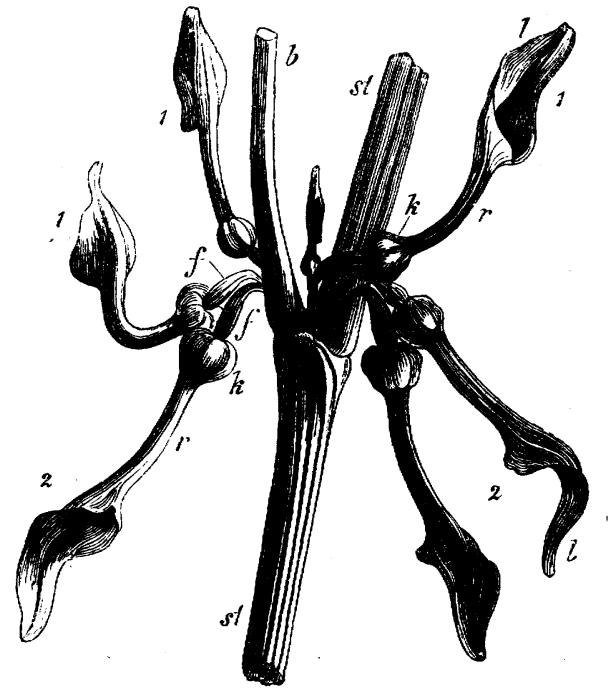

FIG. 488.-Aristolochia Clematitis: a piece of a stem st with petiole $b$; in the axil of this are flowers of different ages; $I, I$ young flowers not.yet fertilised; 2, 2 fertilised flowers, the pedicels bent downwards; $k$ swollen part of the tube of the perianth $r ; f$ the inferior ovary (natural size).

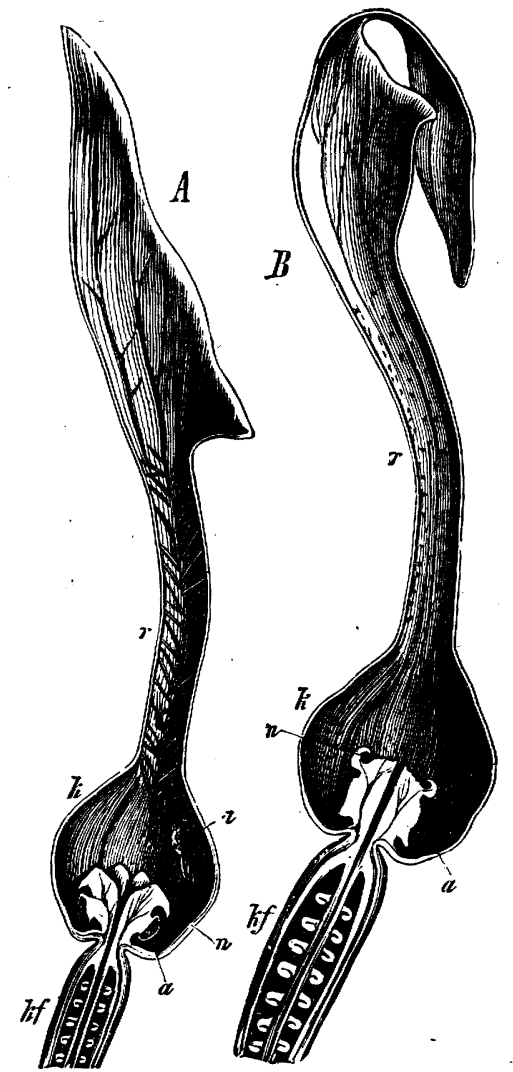

FIG. 489.-Aristolochia Clematitis: the perianth cut through longitudinally. $A$ before, $B$ after pollination (magnified).

the stigma is receptive before the anthers in the same flower are mature; when these subsequently open and allow the pollen to escape, the stigma has already been pollinated by foreign pollen or has even withered up and fallen off (as in Parietaria diffusa); and the pollen of these flowers can therefore only be applied to the fertilisation of younger

1 F. Delpino, Ulteriori osservazioni sulla dicogamia nel regno vegetabile, Atti della soc. Ital. di sci. nat. vol. XIII, I869, and Bot. Zeit. I871, No. 26 et seq.; ditto. in Bot. Zeit. I869, p. 792.

2 [For a list of British protandrous, protogynous, and 'synacmic' plants (or those in which the male and female organs are mature at nearly the same time), see A. W. Bennett in Journal of Potany, I87o, p. 315 , and 1873 , p. 329.$]$ 
flowers. To this class belong Scropbularia nodosa, Mandragora vernalis, Scopolia atropoides, Plantago media, Luzula pilosa, Antboxantbum odoratum, \&c. Among protogynous flowers Aristolocbia Clematitis is characterised by striking and peculiar contrivances.

In Fig. $489 A$ is shown a young flower cut through lengthwise; the stigmatic surface $n$ is already in a receptive condition, but the anthers are still closed; a small fly $i$, which has brought on its back a mass of pollen from an older flower, makes its way in through the narrow throat of the perianth, and runs about in the globular swelling $k$; as many as from six to ten flies are not unfrequently found in one flower. They are shut up and cannot escape, because the throat of the perianth $r$ is furnished with long hairs moving as on a hinge, which present no impediment to the entrance of the insect, but prevent its escape like a trap. While the insect is moving about in the cavity, its back laden with pollen comes into contact with the stigmatic surface and pollinates it, in consequence of which the lobes of the stigma curve upwards, as is shown in Fig. $489 B, n$. As soon as this has taken place, the anthers, previously closed, open; they are laid bare by the change in the position of the stigmas, and are rendered accessible by the withering up of the hairs at the bottom of the cavity of the flower, which has now become wider. The flies which have now carried their pollen on to the stigmatic surface can therefore creep down to the open anthers, where the pollen again becomes attached to them. By this time the throat of the perianth $r$ has again become passable, the net-work of hairs in it having died and withered away after the pollination of the stigma. The insect, laden with the pollen of this flower, can now escape, and again performs the same work in another flower. But while the changes which have been described are taking place inside the flower, its position has also altered. As long as the stigma is still receptive, the pedicel is erect and the perianth open outwards (Fig. $488 i i$ ), so that the visiting flies find a door hospitably open. But as soon as the pollination of the stigma has been effected, the pedicel bends sharply downwards just beneath the ovary, and when the flies, again laden with pollen, have flown out of the flower, the standard-like lobe of the perianth above the mouth of the tube (Fig. $489 \mathrm{~B}$ ) closes, preventing the entrance of the flies, whose visits would now be useless.

(2) Flowers in which the anthers and stigmas are mature at the same time, but selffertilisation is bindered or prevented by the position of the organs and by mechanical contrivances. The pollen is in these cases also usually carried to the stigma by insects, but generally in such a manner that the stigma can only be pollinated by the pollen from another flower, though sometimes, as in Asclepiadex, pollination from the same flower is not impossible in addition to cross-fertilisation. The contrivances for this purpose are astonishingly numerous, and sometimes so complicated that their purpose can only be detected by very careful investigation. To this category belong, for example, the various species of Iris, Crocus, and Pedicularis, many Labiatæ, Melastomaceæ, Passiforaceæ, and Papilionaceæ. Among the most interesting examples are the Asclepiadeæ, in which however the contrivances could be explained only by lengthy descriptions and a large number of illustrations'. In Salvia pratensis and some other species of this genus the mechanical contrivance for preventing self-fertilisation and for ensuring crossing ${ }^{2}$ is extremely beautiful and easy to understand. Fig. 490 represents a flower of S. pratensis seen from the side; at $n$ is the two-lipped stigma in a receptive condition; and indicated by a dotted line inside the upper lip of the corolla is the position of one of the two stamens. If a pin is inserted into the tube of the corolla in the direction of the arrow, the two stamens spring out, as indicated at $a$; if a humble-bee inserts its proboscis in order to obtain the honey, the open anthers strike the back of the insect, and some of the pollen adheres to a particular part; when the bee places itself in the same position in

1 For a fuller description, see R. Brown, Observations on the Organs and Mode of Fecundation in Orchideæ and Asclepiadeæ; Trans. Linn. Soc. I833, and Hildebrand in Bot. Zeit. 1867, No. 33.

${ }^{2}$ For further details, see Hildebrand, Jahrb. für wiss. Bot. vol. IV, I865, p. I. 
another flower, the pollen is rubbed off its back on to the stigma. The cause of the stamen springing out in this way is made sufficiently clear in Fig. $49 \circ \mathrm{B}$. This shows the short true filaments $f f$ which adhere by their bases to the sides of the corolla-tube, and bear at their upper end the long connective $c x$, which oscillates readily about its point of attachment. Only the upper longer and slender arm of each connective $c$ bears an anther-lobe $a$, the lower shorter $\operatorname{arm} x$ is without an anther, and is applied to that of the other stamen in such a manner that the two form together a kind of arm-chair. When the proboscis of the bee in search of honey penetrates the flower in the direction of
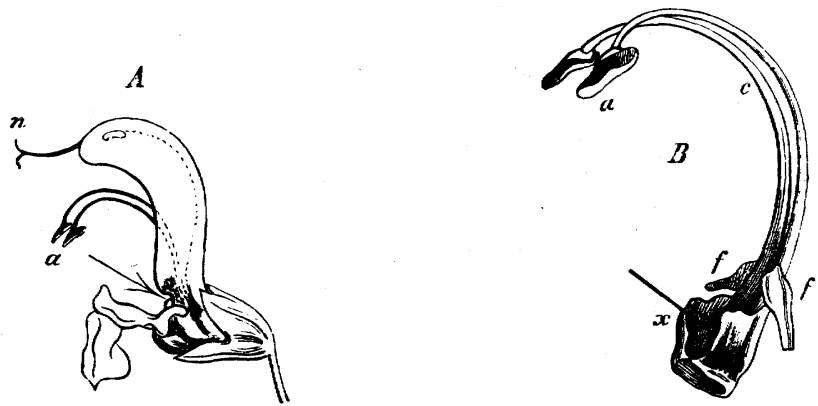

FIG. 490.-Salvia pratensis : a corolla with stigmas $n$ and fertile anther-lobes $a ; B$ stamens removed from corolla.

the arrow, the lower arm of the connective is pressed down, and the upper arm $c$ is made to move forward, and thus to strike the back of the insect.

In the Pansy (Viola tricolor) we have quite a different contrivance for preventing the possibility of self-fertilisation. In Fig. $49 \mathrm{I}, A$ and $B$, is shown the position and arrangement of the parts of the flower. The cavity of the flower enclosed by the petals is completely filled up by the anthers and ovary, with the exception of the tubular spur of the inferior petal in which the nectar collects, secreted by the appendages of the two inferior stamens. The only entrance to this nectary, which therefore lies behind the
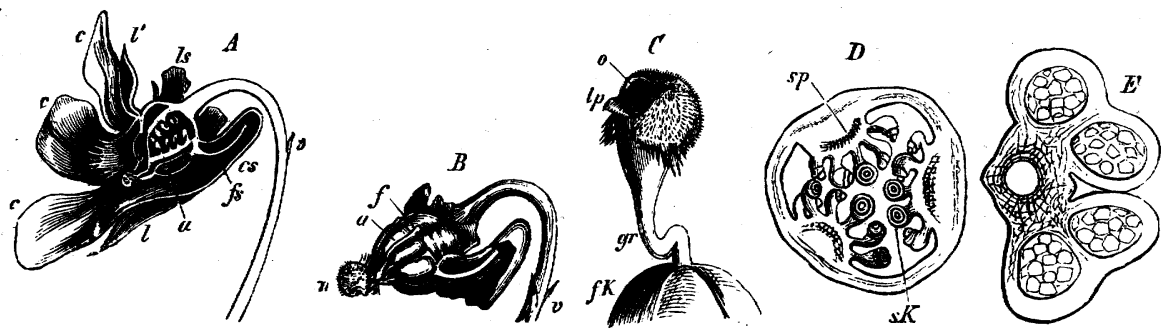

FIG. 491-Viola tricolor: $A$ longitudinal section through the flower (natural size); $B$ the ovary fertilised and swollen; the filaments have been ruptured and the anthers drawn up by the growth of the ovary; $C$ the stigma with its orifice $o$ and lip $l p$, on the style $g r$ (magnified); $l$ sepals, $l s$ prolonged base of the sepals, $c$ petals, $c s$ spur of the inferior petals or nectary; $f s$ appendages of the two inferior stamens projecting into the spur, which secrete the nectar, $a$ the anthers, $n$ stigma, $v$ bracts; $D$ horizontal section through the ovary with the three placentæ $s p$ and ovules $s K ; E$ horizontal section through an unripe anther.

reproductive organs, is through a deep channel in the inferior petal, lined with hairs. The upper and lateral petals incline towards one another in front of the ovary which is surrounded by the anthers, and above the channel in such a manner that the entrance to it is entirely filled up by the capitate stigma $B, n$. The stigma is seated on a flexible style $(C, g r)$, is hollow and opens by an orifice which faces the hairy channel of the lower petal; the lower and posterior margin of this orifice has a lip-like appendage: The anthers open of their own accord, and the pollen in the form of a yellow powder 
collects below and behind the stigma among the hairs of the channel. An insect which has already brought pollen on its proboscis from another flower inserts its proboscis beneath the stigma through the channel into the nectary. The foreign pollen, which is attached to the proboscis, is thus rubbed off on to the lip of the stigma, it is detained by the viscid secretion which fills up the hollow of the latter, and subsequently emits its poilen-tubes through the canal of the style. While the insect is sucking the nectar in the spur, the pollen of this flower, which lies in the channel behind the stigma, becomes attached to the proboscis; when the proboscis is again drawn out, this pollen does not come into contact with the viscid stigma, the lip being drawn forward by the motion of the proboscis, and the orifice of the stigma protected. The pollen that is removed from this flower is now carried, in the manner described, to the stigma of another flower. If the insect were to insert its proboscis again into the nectary of the same flower, the pollen would be detached into the cavity of its own

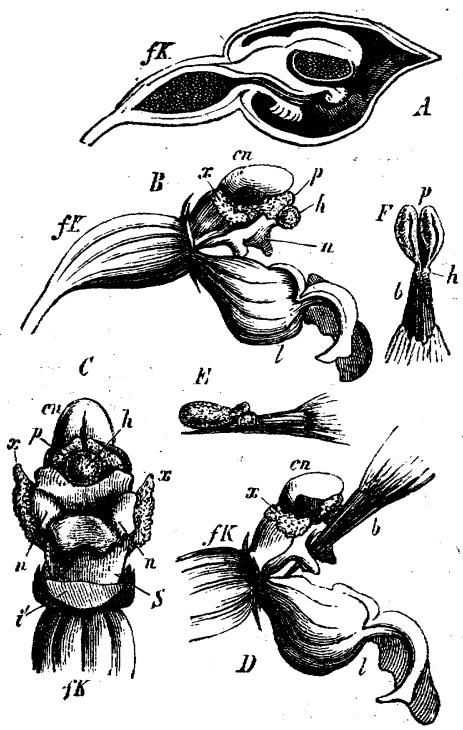

FIG. 492.-Epipactis latifolia: $A$ longitudinal section through a flower-bud; $B$ open flower after removal of the perianth with the exception of the labellum $l ; C$ the reproductive organs after removal of the perianth seen from below and in front; $D$ as $b$, the point of a lead-pencil $b$ inserted after the manner of the proboscis of an insect; $E$ and $F$ the lead-pencil with the pollinia attached; $f K$ ovary, $l$ labellum, its bag-like depression serving as a nectary, $n$ the broad stigma, $c n$ the connective of the single fertile anther, $\not p$ pollinia, $h$ the rostellum, $x \boldsymbol{x}$ the two lateral gland-like staminodes, $i$ place where the labellum has been cut off, $s$ the gynostemium.

stigma; but, as Hildebrand has remarked, insects do not usually do this, but suck up the nectar only once, and then visit another flower. The proceedings of the insect may be imitated by inserting a fine sharp pin beneath the stigma into the channel and again withdrawing it, and filling with the pollen thus removed the stigmatic cavity of another flower.

The contrivances for cross-pollination in Orchids, as numerous as they are complicated and ingenious, have been described in detail by Darwin in the work already named $^{1}$. One of the simpler cases, and the most frequent in its main features, may be briefly described in the case of Epipactis latifolia. At the time when the reproductive organs are mature, the flower stands, in consequence of a torsion of its pedicel, so that the true posterior leaf of the six that form the perianth (the labellum) hangs in front and

1 See also Wolff, Beiträge zur Entwickelungsgeschichte der Orchideen-blüthe, in Jahrb. für wiss. Bot. vol. IV, $186_{5}$. 
downward; it is hollowed out in its lower part, and is thus transformed into a receptacle for the nectar which it secretes (Fig. 492, $B, D, l$ ). The sexual organs, borne on the gynostemium $S$ (in $C$ ), project obliquely above this nectary; the stigma forms a disc with several lips hollowed out and viscid in the centre, the surface of which is inclined obliquely above the nectary. The two gland-like staminodes $x x$ stand right and left beside the stigma; above the stigma and covering it like a roof lies the single fertile anther, of considerable size, which is again on its part protected above by its cushion-like connective $c n$; the lateral walls of the two anther-lobes burst lengthwise right and left, so that their pollen-masses (pollinia) became partially exposed, the pollen-grains remaining attached to one another by a viscid substance. In front of the middle of the anther and above the stigmatic surface is the rostellum $b$, a peculiarly metamorphosed part of the stigma (see $A$ ); the tissue of the rostellum is transformed into a viscid substance covered only by a thin membrane. The flower of Epipactis is not fertilised if left to itself; the pollinia do not fall of their own accord out of the anther, and would even then not reach the stigmatic surface; they must be carried away by insects to the stigma of other flowers. The mode in which this is effected is explained by inserting the point of a black-lead pencil into the flower in a direction towards the bottom of the labellum and beneath the stigmatic surface; if it is then pressed slightly against the rostellum, and again withdrawn slowly in this position $(D)$, the viscid mass of the rostellum or adhesive disc of the pollinia to which the pollen-masses are attached remains sticking to the pencil. The pollinia are now completely removed from the two anther-lobes by the withdrawing of the pencil, as is shown in $E$ and $F$. If the pencil with its pollinia attached is now again inserted into another flower in the direction of the bottom of the labellum, the pollinia necessarily come into contact with the viscid stigmatic surface and adhere firmly to it; when the pencil is again withdrawn they are left behind, being partially or entirely torn from the pencil. In consequence of the form and position of the parts of the flower, an insect which settles on the anterior part of the labellum would in the same manner be able to creep into the bottom of the nectary without disturbing the rostellum; but when it again crept out after obtaining the nectar, it would strike against it and carry off the pollinia; and on crawling into a second flower, these would come into contact with the viscid stigma, and would remain attached to it. In some other Orchidex the contrivances are much more complicated.

(3) The ripe pollen has often to remain for a considerable time in the open anthers, in the case of flowers in which pollination is effected by insects, before it is carried away. During this time it might be blown away by the wind or wetted by rain or dew. In order to prevent this, numerous and very different contrivances exist which protect the pollen. For details see Kerner, Die Schutzmittel des Pollens (Innsbruck, 1873).

SECT. 34.-Hybridisation ${ }^{1}$. In the preceding paragraphs we have spoken only of the union of the reproductive cells of the same plant, or of two individuals of the same species. We learn however from experience that a fertile sexual union can take place between plants which are specifically distinct. A union of this kind is called Hybridisation, and its product a Hybrid. According as the union takes place between different varieties of one species, different species of one genus, or between

1 J. G. Kölreuter, Vorläufige Nachricht von einigen das Geschlecht der Pflanzen betreffenden Versuchen $\mathfrak{u}$. Beobachtungen, Leipzig $1_{7} 6_{1} ;$ Appendices in $176_{3}, 1764$, and 1766 .-W. Herbert, On Amaryllidaceæ, with a treatise on cross-bred vegetables; London, 1837.-Gärtner, Versuche u. Beobachtungen uiber die Bastarderzeugung im Pflanzenreich; Stuttgart, 1849. [See notice by Berkeley, Journ. Roy. Hort. Soc. vol. V, 1850 , p. 156.]-Wichura, Die Bastardbefruchtung im Pflanzenreich, erläutert an den Bastarden der Weiden (with two nature-printed plates); Breslau, 1865. [See abstract by Berkeley, Journ. Roy. Hort. Soc. new series, vol, I, 1850 , p. 57.-Focke, Pflanzen Mischlinge, I881.] 
two species belonging to different genera, the resulting hybrid may be termed a variety-hybrid, species-hybrid, or genus-hybrid.

Among Cryptogams only a few instances of hybridisation are known with certainty. Thuret (Ann. des sci. nat., I 855) obtained hybrid plants by bringing antherozoids of Fucus serratus into contact with oospheres of $F$. vesiculosus. In some other families of Cryptogams forms have been found which have been supposed, from their characters, to have a hybrid origin. Thus A. Braun (Verjüngung, p. 329) adduces instances of hybrids between Mosses ${ }^{1}$, Physcomitrium pyriforme and Funaria hygrometrica, and between Physcomitrium fasciculare and Funaria hygrometrica, and between the following species of Ferns-Gymnogramme chrysophylla and $G$. calomelana, G. chrysophylla and G. distans, and Aspidium Filix-mas and A. spinulosum ${ }^{2}$.

The most important observations from a scientific point of view, which have given us the clearest insight into the nature of the difference of sex, are however those made on hybrids between flowering plants, resulting from the artificial conveyance of pollen from one species to another. Nägeli has collected the results of many thousand experiments on hybridisation made by Kölreuter in the last century, and more recently by Knight, Gärtner, Herbert, Wichura, and other observers. The following facts are taken chiefly from Nägeli's resumé3 ${ }^{3}$.

I. Only those forms which are closely related genetically can produce hybrids. They are formed most easily between different varieties of the same species; with greater difficulty-but are still possible in a great number of cases-between two species of the same genus; of hybrids between species which belong to different genera only a very few instances are known, and it is probable that in these cases the species ought to be included in the same genus. The facility with which hybrids can be produced varies extremely in different orders, families, and genera of Angiosperms. The phenomenon is frequent among Liliaceæ, Irideæ, Nyctagineæ, Lobeliaceæ, Solanaceæ, Scrophulariaceæ, Gesneraceæ, Primulaceæ, Ericaceæ, Ranunculaceæ, Passifloraceæ, Cactaceæ, Caryophyllaceæ, Malvaceæ, Geraniaceæ, Enothereæ, Rosaceæ, and Salicineæ. It does not occur at all, or only very exceptionally, in Gramineæ, Urticaceæ, Labiatæ ‘, Convolvulaceæ, Polemoniaceæ, Grossulariaceæ, Papaveraceæ, Cruciferæ, Hypericineæ⿱ and Papilionaceæ. Even genera of the same order or family differ in this respect. Among Caryophyllaceæ, the species of Dianthus hybridise easily, those of Silene only with difficulty; among Solanaceæ, the species of Nicotiana and Datura have a tendency to produce hybrids, while those of Solanum, Physalis, and Nycandra have not; among Scrophulariaceæ, Verbascum $^{5}$ and Digitalis, but not Pentstemon, Linaria, or Antirrhinum; among Rosaceæ, Geum, but not Potentilla.

1 [See also H. Philibert, L'Hybridation dans les Mousses (Grimmia), Ann. des sci. nat. I873, vol. XVII. p. 225.]

${ }^{2}$ [See also T. Moore on Adiantum farleyense, Journ. Roy. Hort. Soc. new series, I. p. 83 ; Berkeley on Asplenium ebenoides, Scott, ibid. p. 137.]

${ }^{3}$ Nägeli, Sitzungsber. der k. bayer. Akad, der Wiss. in München, Dec. 23, 1865, and Jan. I3, I 866. Also Kerner, in Oesterreich. Bot. Zeitsch. Wien. XXI.

${ }^{4}$ [Stachys ambigua $\mathrm{Sm}$. is considered to be a hybrid between $S$. sylvatica and S.palustris.]

5 [On hybridity in the genus Verbascum, see Darwin, Journ. Linn. Soc. 1868, 'p. 437.] 
Hybridisation between species belonging to different genera has been observed between Lychnis and Silene, Rhododendron and Azalea, Rhododendron and Rhodora, Azalea and Rhodora, Rhododendron and Kalmia, Rhododendron and Menziesia ${ }^{1}$, Agilops and Triticum, and between Echinocaclus, Cereus, and Phyllocactus, to which must be added a few wild forms which appear to be genus-hybrids.

2, Besides the near genetic relationship, the possibility of the production of hybrids depends also on a certain relationship between the parent-plants, which is manifested only in the result of hybridisation, and which Nägeli calls 'Sexual Affinity.' This kind of affinity is not always concurrent with the external resemblance of the plants. Thus, for example, hybrids have never been obtained between the Apple and Pear ${ }^{2}$, Anagallis arvenis and carulea, Primula officinalis and elatior, or Nigella damascena and sativa, nor between many other pairs of species belonging to the same genus which are very nearly allied to one another; while in other cases very dissimilar forms unite, as Eilops ovata with Triticum vulgare, Lychnis diurna with L. Flos-cuculi, Cereus speciosissimus with Phyllocactus Phyllanthus, the Peach with the Almond. A still more striking proof of the difference between sexual and genetic affinity is afforded by the fact that varieties of the same species will sometimes be partially or altogether infertile with one another, as e.g. Silene inflata var. alpina with var. angustifolia, var. latifolia with var. littoralis, \&c.

3. When a sexual union is possible between two species A and B, A can usually produce hybrids when fertilised by the pollen of $B$, and $B$ when fertilised by the pollen of A (reciprocal hybridisation). But there are cases in which $\mathrm{A}$ can only be the male and $\mathrm{B}$ only the female parent plant, the pollination of $\mathrm{A}$ by $\mathrm{B}$ yielding no result. Thus Thuret found, as has already been mentioned, that Fucus vesiculosus produces hybrids with the antherozoids of $F$. serratus, while the oospheres of the latter species could not be fertilised by the antherozoids of the former. Gärtner states that Nicotiana paniculata produces hybrid seeds when acted on by the pollen of $N$. Langsdorfii, while the latter does not under the influence of the pollen of the former. Kölreuter easily obtained seeds of Mirabilis Jalapa with the pollen of $M$. longiflora, while more than two hundred experiments on pollinating the latter by the former species extending over eight years produced no result.

4. Sexual affinity presents a great variety of gradations. At one extreme we have complete infertility under the influence of the pollen of another variety or species, the pollen-tubes not even entering the stigma, and the pollinated flower behaving precisely as if no pollen had reached it; the other extreme is shown in the production of numerous hybrids, which not only grow vigorously, but are themselves fertile. The lowest degree of the action of pollen of a different kind consists in various changes taking place in the parts of the flower of the mother-plant, the ovary or even the ovules also growing, without any embryo being produced. A higher degree is manifested in the production of ripe normal fruits and seeds

1 [The history of the plant which is here intended is given in the Botanical Gazette, vol. III. p. 82. It was raised from seed of Bryanthus (Menziesia) empetriformis, supposed to be fertilised by the pollen of Rhodothamnus (Rhododendron) Chamacistus. It is figured under the name of Bryanthus erectus in Paxton's Flower Garden, vol. I. t. 19; but it agrees well with specimens of its female parent from the Rocky Mountains, and is probably therefore not a hybrid at all.]

${ }^{2}$ [An instance to the contrary is recorded in the Proc. Acad. Philadelphia, 187 I, vol. I. p. 10.] 
containing embryos, but these embryos having no power of germination. Further steps are indicated by the number of embryos which have the power of germination that are produced in the ovary $\mathbf{1}$.

5. When pollen from different species is applied simultaneously to the same stigma, only one kind is potent, viz. that from the species which has the greatest sexual affinity to the one that is pollinated. And since, as a general law, pollen is most efficacious on a different flower of the same species-in other words, the highest degree of sexual affinity occurs between different individuals of the same specieswhen a stigma is pollinated at the same time with pollen of the same and of another species, the first only is potent. But since, on the other hand, hybrids are sometimes more easily produced between varieties than between individuals of the same variety, in this case the foreign pollen may be prepotent over that of the same kind. When the pollen of different species reaches the stigma at the same time, and if that which reaches it later has a greater sexual affinity, it can only be potent when the first is not potent or acts injuriously. In Nicotiana the production of hybrids can no longer be prevented by its own pollen after two hours, in Malva and Hibiscus after three hours, in Dianthus after five or six hours.

6. The hybrid is possessed of external characters intermediate between those of its parent-forms, usually nearly half way between; less often it resembles one of the parent-forms more nearly than the other, and this is more often the case with varietyhybrids than with species-hybrids. It follows that in reciprocal hybrids from the species $\mathrm{A}$ and $\mathrm{B}$, the hybrid $\mathrm{A} \mathrm{B}$ is generally similar externally to the hybrid $\mathrm{B} A$, though the two forms may differ somewhat internally. Thus, according to Gärtner, the hybrid Nicotiana paniculato-rustica is more fertile than the reciprocal hybrid Nicotiana rustico-paniculata ${ }^{2}$. An internal difference between reciprocal hybrids is also shown by the fact that one is more variable than the other; thus, according to Gärtner, the progeny of Digitalis purpureo-lutea is more variable than that of D. luteopurpurea, the progeny of Dianthus pulchello-arenarius more variable than that of D. arenario-pulchellus.

When two species $\mathrm{A}$ and $\mathrm{B}$ hybridise, and the one species $\mathrm{A}$ exercises a greater influence on the form and properties of the hybrid than the other species $\mathrm{B}$, the hybrid or its descendants, if fertilised by $\mathrm{A}$, will revert more quickly to the parent-form A than it will to the parent-form B if fertilised by it. Thus Gärtner states that the hybrid of Dianthus chinensis and D. Caryophyllus reverts to the latter form after three or four generations if repeatedly fertilised by it, while it requires fertilisation for five or six generations by $D$. chinensis in order to revert to that form.

7. The characteristics of the parent-forms are as a rule so transmitted to the hybrid that the influence of both is manifested in all its characters, producing a fusion of the different peculiarities. This is more evident in the species- than in the variety-hybrids; in the latter some of the non-essential characters of the parents sometimes present themselves in the offspring uncombined side by side; e.g. various

1 See Hildebrand, Bastardirungsversuche an Orchideen, Bot. Zeit. 1865, No. 3 r.

${ }^{2}$ In this mode of designating hybrids, the name of the male parent-plant stands first; thus Nicotiana rustico-paniculata is the product of the fertilisation of $N$. paniculata by the pollen of N. rustica. 
kinds of streaks and blotches instead of a mixing of the colours of the flowers. Thus a hybrid which Sageret obtained from Cucumis Chate (female) with C. Melo Cantalupus (which had a reticulated rind) had a yellow flesh, a reticulate marking of the rind and moderately prominent ribs like the male parent, but white seeds and an acid flavour like the female parent. Another hybrid from the same species had, on the contrary, the sweet flavour and yellow flesh of the male, with the white seeds and smooth rind of the female parent. To this category belongs also, the hybrid of Cytisus Laburnum and purpureus [known as Cytisus Adami], some of the branches of which partially or entirely resembled one and some of them the other parent-form. I have found what seemed to be a hybrid Antirrhinum majus, in which the inflorescence bore on one side of the axis only dark-red, on the other side only yellow flowers, while between the two halves stood a single flower which was half red and half yellow.

8. In addition to its inherited properties, the hybrid usually possesses characters of its own by which it is distinguished from both its parent-forms. One of these new characters, which occurs especially with variety-hybrids, is the tendency to vary more strongly than its parent-forms. Species-hybrids are usually weak in their sexual properties; those derived from nearly related parent-species are, on the other hand, more vigorous in their growth than their parent-forms, while hybrids resulting from the union of species less nearly related are generally feebler in their development. The luxuriant growth of the hybrids from nearly allied species is displayed in their more numerous and larger leaves, in their taller and stouter stems, more copious root-system, and larger number of shoots (stolons, scions, \&c.). Hybrids have also a tendency to a longer duration of life; those of annual or biennial parentforms often live a number of years, probably in consequence of their producing a smaller number of seeds. Hybrids are also characterised by commencing to flower earlier, and continuing to do so longer and more abundantly, than the parentforms; sometimes they produce an extraordinary number of flowers, which are also larger, more enduring, and of brighter colour and stronger odour. They have also a tendency to become double, their staminal and carpellary leaves to increase in number and develope into petals. Along with this luxuriant vegetative growth, the sexual organs are usually weak, and this in every possible degree. 'The stamens,' says Nägeli, 'are, it is true, in some cases perfect externally, but partially or altogether infertile, the pollen-grains not attaining their proper development; while in others the stamens are altogether abortive and reduced to rudiments. The pistils (gynæceum) of hybrids are in most cases not distinguishable externally from those of the parent species, but their ovules have no power, or only to a slight degree, of becoming fertilised; either no oospheres are formed, or the embryos which begin to be developed from the oospheres perish sooner or later. Under favourable circumstances, when fertile seeds are produced, their number is smaller, and they manifest a certain degree of feebleness in their slow germination and the short duration of this capacity.' The feebleness of the sexual function is in some variety-hybrids scarcely perceptible, in others but small; in general it is the more marked the more distant the genetic and sexual affinity of the parent-forms. When species-hybrids have the power of producing seeds by self-pollination, and this is repeated in the progeny, their fertility generally diminishes from generation to 
generation; though this phenomenon probably depends less on the sexual feebleness of hybrids than on the circumstance that their flowers have probably been generally fertilised with their own pollen, instead of being pollinated from other flowers or other individuals of the same hybrid. Nägeli's rule holds true in the general way, that the male organs of species-hybrids are functionally weak to a higher degree than the female organs, although the rule is not without exceptions.

9. 'Hybrids usually vary less in the first generation, the less the degree of affinity between their parent-forms; species-hybrids therefore less than varietyhybrids; the former are often characterised by a great uniformity, the latter by a great variability. When hybrids are self-fertilised, the variability increases in the second and succeeding generations the more completely it was absent from the first; and three different varieties arise more certainly the less the affinity between the parent-forms; viz. one corresponding to the original (hybrid) type, the two others bearing a greater resemblance to the two parent-forms. But these varieties show but little constancy, passing easily into one another, at least in the earlier generations. An actual reversion to one of the two parent-forms (with pure breeding-in) takes place especially when the parent-forms are very nearly related, as in varietyhybrids and those from species that approximate to varieties. When this reversion occurs in other species-hybrids, it appears to be limited to those cases where one of the parent-species exercised a preponderating influence in the hybridisation.' (Nägeli, l.c.)

Io. When a hybrid is made to unite with one of its parent-forms, or with another parent-form, or with a hybrid of different origin, the product is termed a 'derivative hybrid;' and this may again on its part unite with one of the parentforms or with a hybrid of different origin. When a union is effected between a hybrid and one of its own parent-forms, and the hybrid thus obtained unites again with the same parent-form, and so on through several generations, the derived progeny approach more and more nearly in their characters to those of this parentform, until they come to resemble it in all respects. According as one or the other of the parent-forms is taken, a larger or smaller number of generations are required to effect the perfect reversion; and this behaviour has been reduced by Nägeli to a numerical expression (formula of heredity), which indicates in numbers the amount of influence exercised by a species in reference to the hereditary transmission of its qualities in hybridisation. In proportion as the derivative hybrid approaches one or the other of its parent-forms, its hybrid nature gradually decreases, and its fertility at the same time increases.

When a hybrid unites with a new parent-form or with a hybrid of another species, a derivative hybrid results in which three, four, or more species (or varieties) are combined; Wichura has united as many as six different species of Willow in one such derivative hybrid. Hybrids of this kind, which may conveniently be termed ' combined hybrids,' usually follow the same rules with reference to their form and other characters as hold good in the case of simple hybrids. Combined hybrids becoine less fertile the larger the number of different parent-forms that are united in them; and they are usually very variable. Wichura showed, from his own observations and those of Gärtner, that hybrid pollen produces a greater variety of forms in its progeny than does the pollen of true species. 
The results of hybridisation are important with respect to the theory of sexuality, because there is no boundary-line or essential distinction between the self-fertilisation of pure species or varieties and their fertilisation by other species or varieties; and because in the latter case-in other words in hybridisation-certain peculiarities of sexual differentiation and union are rendered more evident. The two extremes of the conditions under which a fertile union of sexual cells is possible lie at a great distance from one another, but are connected by very numerous transitions. One extreme is presented in the genus Rbyncbonema, where a fertile sexual union of sister-cells takes place regularly; the other extreme is furnished in genus-hybrids, where the uniting cells belong to very different forms of plants whose descent from a common ancestor dates back to a remote antiquity. But the great majority of phenomena in the vegetable kingdom show that sexual union is usually most productive when the cells stand neither in too close nor in too remote an affinity to one another; self-fertilisation is in the vast majority of cases as carefully avoided as the hybridisation of different species or genera. The phenomena may be comprised in the statement that the original form of sexual differentiation was probably the simultaneous formation of male and female organs in close juxtaposition on the same plant, but that sexual union is more potent and more favourable for the maintenance of the race when the closely contiguous cells do not unite, but those of different descent, a certain mean amount of difference of descent being established as the most favourable. This mean of the difference of descent associated with a maximum of sexual potency is obtained when the sexual cells belong to different individuals of the same species ${ }^{1}$. The arrangements described in the preceding paragraphs which are manifested in polygamy, diclinism, dichogamy, dimorphism, the impotence of pollen on the stigma of the same flower (as in Corydalis and Oncidium), the mechanical contrivances for rendering self-fertilisation impossible (as in Aristolocbia Clematitis, many Orchidex, \&c.), are different means for promoting the cross-fertilisation of individuals belonging to the same species or for rendering it alone possible.

CHA P T E R VII.

\section{THE ORIGIN OF SPECIES.}

SECr. 35.-Origin of Varieties. The characters of plants are transmitted to their descendants, or, in other words, are hereditary. But, in addition to the inherited properties, new characters may arise in a smaller or larger number of the descendants of a plant which were not possessed by the parent-plants. Thus, for example, Descemet obtained in $1803^{2}$, among the seedlings from Robinia Pseud-acacia, an individual without spines; Duchesne, in $176 \mathbf{1}^{3}$, among seedlings of the Strawberry, one with simple instead of trifoliolate leaves; and Godron ${ }^{4}$, among seedlings of Datura Tatula, one with smooth instead of spiny capsules.

1 [See Darwin, Variation of Animals and Plants under Domestication, vol. II. chap. xvii, where several illustrations of the law are given.]!

2 See Chevreul, Ann. des sci. nat. 1346, vol. VI. p. I57. [Journ. Roy. Hort. Soc. vol. VI, I $85 \mathrm{I}$, p. 6r.]

3 For further details, See Usteri, Annalen der Botanik, vol. V. p. 40.

* See Naudin, Compt. rend. 1867, vol. LXIV. p. 929. 
The characters which arise in single descendants are often only individual, i.e. they are not again transmitted to their descendants. Thus the seeds of the unarmed Robinia produce again spiny plants resembling, not their immediate ancestor, but more remote ones; while in other cases the new character is hereditary, though at first perhaps only partially so, the new form making its appearance only in a certain proportion of the descendants, while the others revert to the original form, as in Duchesne's unifoliolate Strawberry.

When a new character is transmitted by inheritance to new generations, the number of individuals that revert to the primitive form often decreases from generation to generation, or the hereditary permanence of the new character increases; they become more and more constant, and sometimes even as much so as those of the primitive form. Such new constant forms are termed Varieties ${ }^{1}$.

The same parent-form may produce a smaller or larger number either simultaneously or in succession, sometimes even hundreds, of new forms; and this is especially the case with cultivated plants. The enormous number of varieties of the Dahlia, differing in the colour, size, and form of the flowers and in their mode of growth, now cultivated in our gardens, have been derived since 1802 from the simple yellow-blossomed primitive form of Dahlia variabilis. The great variety of Pansies, distinguished chiefly by the colour of their flowers, have resulted since 1687 from the cultivation of the Viola tricolor of our fields with small flowers almost uniform in colour $^{2}$. Still more numerous are the varieties of Cucurbita Pepo, differing not only in the form of their fruit but also in all other characters; and the same is the case with the Cabbage (Brassica oleracea) and a vast number of other cultivated plants.

Some płants have a special tendency to variation; among native species, for example, the fruticose Rubi, and those of Rosa and Hieracium; others, on the contrary, are distinguished by great constancy in their characters, as for example Rye, which has as yet produced no hereditary varieties, notwithstanding long cultivation; while the nearly related species of Wheat (especially Triticum vulgare, amyleum and Spelta) are distinguished by a number of old varieties and an ever-increasing number of new ones.

By far the greater number of hereditary varieties are the product of sexual reproduction; thus among Phanerogams the new characters appear suddenly in individual seedlings which differ at once from the parent-plant in these respects. Sometimes however it happens that particular buds develope differently from the other shoots of the same stock; and of this Bud-variation ${ }^{3}$ two different cases must be carefully distinguished, since their significance is altogether different. In the one case the abnormal shoot of a stock which itself belongs to a variety resembles or reverts to the primitive form; and this therefore is an instance not of the production but of the cessation of a new form. In the Botanic Garden at Munich there is, for example, a Beech-tree with divided leaves, itself a variety, a single branch of which bears the

1 For examples, see Hofmeister, Allgemeine Morphologie, p. 565 .

2 Darwin, The Variation of Animals and Plants under Domestication, vol. I. p. 368 et seq.

3 [T. Meehan adduces a number of remarkable instances of bud-variation in which hybiidisation could not have taken any part;-in Rubus which rarely produces seeds in the wild state, Convolvulus Batatas, which seldom flowers in America, \&c. See Proceedings of the Philadelphia Acad. of Nat. Sci. Nov. 29, 1870.] 
ordinary undivided entire leaves, or has reverted to the primitive form. In the second case new characters not previously displayed arise on particular shoots of a stock. Thus, for instance, single shoots of the Myrtle are sometimes found with leaves in alternating whorls of threes, instead of in pairs; but these shoots again produce from the axils of their leaves the ordinary branches with decussate leaves. Knight (see Darwin, l.c. vol. I. p. 375) observed a Cherry (the May Duke) with one branch bearing fruit of a longer shape which always ripened later. The common 'Moss-Rose' is considered by Darwin (l.c. p. 379) to have probably arisen by 'bud-variation' from $R$. centifolia; the white and striped Moss-Roses made their appearance in $\mathbf{I} 788$ from a bud of the common red Moss-Rose; Rivers states that the seeds of the simple red Moss-Rose almost always again produce Moss-Roses ${ }^{1}$.

Those changes which are produced in a plant by the nature of its food and other external conditions must not be confounded with variation. Specimens of the same plant often differ conspicuously in the size and number of their leaves, shoots, flowers, and fruits, according as their supply of food has been abundant or deficient; deep shade frequently occasions the most striking changes in the habit of plants that usually grow in sunshine; but these changes are not hereditary; the descendants of such individuals revert, under normal conditions of light and nutrition, to the original characters of the species.

Those characters, on the contrary, which may become hereditary or form the groundwork of varieties, arise independently of the direct influence of soil, locality, climate, or other external influences; they appear seemingly without any cause. We must therefore assume either that external impulses which are altogether imperceptible first cause an imperceptible deviation in the process of development, which is always extremely complicated, and that this variation gradually increases until it becomes perceptible, or that the processes in the interior of the plant itself react upon one another in such a manner as to cause sooner or later an external change.

The fact that wild plants, when cultivated, usually begin to produce hereditary varieties, shows that the change in the external conditions of life disturbs to a certain extent the ordinary process of development; but it does not show that particular external influences produce particular hereditary varieties corresponding to them; for under the same conditions of cultivation the most different varieties arise simultaneously or successively from the same parent-form. The same is the case also in nature with wild plants; in the same locality under precisely the same vital conditions a number of varieties are often found by the side of their parent-form, and the same variety is often found in the most diverse localities ${ }^{2}$. It is for this very reason-because varieties are to so great an extent independent of external influences - that they are hereditary. A change produced in a plant by moisture, shade, or any similar cause, is not hereditary, because its descendants, when placed under other vital conditions, acquire again other non-permanent characters. That hereditary characters, or those which may become so, are not produced by external influences,

1 [See also M. J. Masters, On a pink sport of the Gloire de Dijon Rose, Journ. Roy. Hort. Soc. new series, vol. IV. p. I53--Braun, Abhand. d. Berl. Akad. I859, p. 2 I 9.

${ }^{2}$ Further details on this important subject are given by Nägeli in the Sitzungsberichte der kön. bayer. Akad, der Wiss. Dec. I5, 1865 . 
is proved most conclusively by the fact that seeds from the same fruit produce different varieties, either exclusively or together with the hereditary parent-form.

Although the production of varieties and the form they assume are not the direct results of external influences, yet the continuance of the existence of a variety may be determined by these influences. When a variety is produced, the question arises whether it will thrive best in damp or in dry ground, in sunny or shady places, and so forth; whether it can reproduce itself under these circumstances, or whether it will die out. The conclusion follows that hereditary varieties arise independently of direct external influences, but that the continuance of their existence depends on external causes. A variety which occurs only in a particular locality is not produced by the conditions of this particular locality; but it alone furnishes the peculiar conditions of life which this particular variety requires, while other varieties which have arisen at the same place disappear.

It has already been shown in Sect. 34 that hybrids show in general a tendency to the production of varieties. Two different sets of hereditary characters are combined in a hybrid, and there is hence a strong tendency towards the formation of new characters which may be more or less hereditary. Hybridisation is therefore one of the most important means at the command of the horticulturist for disturbing the constancy of inherited characters and producing a number of varieties from two distinct ancestral forms ${ }^{1}$. But even the ordinary sexual union of two individuals of a species, as in dicecious, dichogamous, or dimorphic plants, may be considered as a kind of hybridisation; in these cases also the individuals which unite must certainly be different, since otherwise their cross-fertilisation would be no more productive than self-fertilisation. In these cases therefore two sets of characters which differ, though it may be but slightly, also unite in the descendants; and if a hybrid from two different species exhibits a strong tendency to variation, the cross-fertilisation of two different individuals of one and the same species may at least give rise to a slight tendency in the same direction. It is therefore probable that in the cross-fertilisation of different individuals-towards which there is always a tendency in nature even in hermaphrodite flowers -we have a perpetual cause of variation in plants. But this is by no means the only cause of variation, as is shown by the existence of bud-variation, and by the reflection that the difference between individuals which produce a variable progeny is itself due to slight variation.

A great number of facts point to the conclusion that almost every plant has a tendency to vary continually and in different directions, while every new character which is not produced directly by external agencies tends at the same time to become hereditary. If notwithstanding this many wild plants and some cultivated ones are very constant and produce no varieties which can be distinguished externally, this is mainly the result of the fact that the newly produced varieties are unable to exist in the conditions by which they are surrounded, or at least soon disappear, a point to which I shall recur more in detail. The hereditary transmissibility of acquired characters exhibits itself in a most peculiar way when it does not affect the whole of the parent-plant, but only a particular branch. A still more remarkable case was observed by Bridgman. He noticed that the spores from the lower inner part of the lamina of the leaves of the varieties Scolopendrium

${ }^{1}$ See also Naudin, Compt. rend. I864, vol. LIX. p. 837. [Journ. Roy. Hort. Soc. new series, vol. I. p. I.] 
vulgare laceratum and $S$. vulgare Crista-galli, which was of the normal form, uniformly produced plants of the normal parent-form, while those produced on the outer abnormal part of the leaf reproduced the special varieties ${ }^{1}$.

SECT. 36. - Accumulation of new characters in the reproduction of varieties. The difference between a variety and its parent-form, or between the varieties of a common parent-form, is usually at first small and affects only a few characters. But the descendants of the variety may again vary, the new characters may thus become intensified, and other new characters of a different kind may be added to them. The amount of difference between parent-form and variety and between the various varieties of the same parent-form thus becomes greater; and if the tendency of the characters to become hereditary increases with the increase of their difference, the variety comes at length to differ so greatly from the parent-form that their genetic connection can only be proved historically or by the existence of transitional forms. This is the case with many of our cultivated plants, as $e . g$. the Pear, which varies much even in the wild state, but under cultivation has altered its mode of growth, form of leaf, flower, and especially its fruit, to such an extent that it would be impossible to suppose the finest sorts of Pears to be descendants of the wild Pyrus communis, if Decaisne had not proved their genetic connection by the study of the transitional forms (Darwin, l.c. vol. I. p. 350). In the same manner it scarcely admits of a doubt that all the cultivated kinds of Gooseberry are descended from the wild Ribes Grossularia of Central and Northern Europe; and Darwin brings forward historical evidence to show that the size of the fruit has been continually increased by cultivation since 1786 , so that in $185^{2}$ it had attained the weight of 895 grs. Darwin found that a small apple $6 \frac{1}{2}$ inches in circumference weighed as much (l.c.p. 356). The different varieties of Cabbage are all descended from one parent-species, or, according to Alph. de Candolle, from two or three closely related ones still growing in the neighbourhood of the Mediterranean. In this case hybridisation has also cooperated; the varieties are for the most part hereditary but without any great constancy. The extent of the variation which has taken place under cultivation is shown by the existence on the one hand of shrubby forms with branching woody stems, Io to 12 or even 16 feet high, on the other hand of the round Cabbage with a short stem and a spherical, pointed, or broad head consisting of leaves closely packed one over another; and again of the Savoy with its curled blistered leaves, the Kohl-Rabi with its stem swollen below, the Cauliflower with its crowded monstrous flowers, \&c. ${ }^{2}$

In the case of many cultivated plants the original wild form is unknown. It is possible that in a few cases it may have disappeared; but it is more probable that the varieties which have arisen under cultivation have gradually acquired such a number of new characters that their resemblance to the wild parent-form can no longer be traced. This is probably the case with the cultivated Cucurbitaceæ, Gourds, Bottle-Gourds, Melons, Water-Melons, \&c., the hundreds of varieties of which

1 Ann. and Mag. Nat. Hist. third series, vol. VIII, I86r, p. 490 ; Darwin, l. c. vol. II. p. 379. Also Nägeli, Sitzungsberichte d. k. bayer. Akad. d. Wiss. I866, p. 274.

${ }^{2}$ See Metzger, Landwirthschaftliche Pflanzenkunde, Frankfurt a. M. 1851, p. 1000; and Darwin, $l . c$. vol. I. p. 323 . 
have been traced back by Naudin to three primitive forms, Cucurbita Pepo, maxima, and moschata, neither of which however is known in the wild state. These original forms have been as it were evolved from the resemblances and differences of the numberless varieties, and have only an ideal existence; it is doubtful whether either of them ever actually existed, or whether these ideal parent-forms do not merely correspond to three principal varieties which arose from a single primitive form which possibly still exists, or from the hybridisation of several. The characters of many of these varieties are perfectly hereditary, and all the organs show the greatest degree of variation; how great and various these differences are is seen from the fact that Naudin has divided the group of forms which he includes under the name Cucurbita Pepo into seven sections, each of which again includes a number of subordinate varieties ${ }^{1}$. The fruit of one variety exceeds that of another variety more than two thousand fold in size; the original form of the fruit is probably ovoid, but in some varieties it is elongated into a cylinder, in others abbreviated into a flat plate; the colour of the rind varies almost infinitely in the different varieties; in some it is hard, in others soft; some have a sweet, others a bitter flesh; the seeds vary in length from 5 or 7 to $25 \mathrm{~mm}$; ; in some the tendrils are of enormous size, in others they are altogether wanting; in one variety they are transformed into branches which bear leaves, flowers, and fruits. Even characters which are normally constant throughout entire natural orders become extremely variable in the Gourds ; thus Naudin (Compt. rend. r867, vol. LXIV. p. 929) describes a Chinese variety of Cucurbita maxima which has a perfectly free or superior ovary, whereas it is inferior elsewhere in the Cucurbitaceæ and in the nearly allied orders ${ }^{2}$. The varieties of Melon (Cucumis Melo) Naudin divides into ten sections, which differ also not only in their fruit, but also in their leaves and their entire habit or mode of growth. Some Melons are no larger than small plums, others weigh as much as 66 lbs.; one variety has a scarlet fruit; another is only $\mathrm{I}$ inch in diameter but 3 feet long, and is coiled in a serpentine manner in all directions, the other organs being also greatly elongated. The fruits of one variety can scarcely be distinguished externally or internally from Cucumbers; one Algerian variety suddenly splits up into sections when ripe (Darwin, l.c. vol. I. p. 357).

The behaviour of the genus $Z e a$ is similar to that of Cucurbita. The cultivated varieties of Maize are probably descended from a single primitive wild form which has been cultivated in America for a very long period; but it seems doubtful whether the native Brazilian species, the only one known in the wild state, with long glumes enveloping the grains, is the primitive form; if it is not, then no plant is now known which can be considered as the ancestral form of our numerous and extremely diverse varieties of Maize. In this case also continued cultivation has increased the amount of difference between the different varieties, as well as to a prodigious extent that between them and the primitive form; and the separate varieties are distinguished from one another by a number of different characters. Some are only $\mathbf{I} \frac{1}{2}$ feet high, others as much as $I 5$ to 18 feet; the grains stand on the rachis in rows varying from

1 See Metzger, Landwirthschaftliche Pflanzenkunde, p. 692, and Darwin, l. c. vol. I. p. $35^{8}$.

2 Hooker states that a specimen of Begonia frigida at Kew produced, in addition to male and female flowers with inferior ovary, also hermaphrodite flowers with superior ovary. This variation was the product of seeds from a normal flower. (Darwin, l.c. p. $3^{6} 5$.) 
six to twenty in number; they may be white, yellow, red, orange, violet, streaked with black, blue, or copper-red; their weight varies sevenfold; their form also varies extremely; there are varieties with three kinds of fruit of different form and colour on one rachis; and a great number of other differences also occur ${ }^{1}$. These instances may suffice to show to what an extent the amount of deviation of the varieties of a primitive form may increase under cultivation ${ }^{2}$.

It is much more difficult, and to a great extent impossible, to prove directly to what extent the variation of wild forms can increase without cultivation, because historical evidence is in this case generally impossible, or can only be obtained indirectly or conjecturally. But since the laws of variation are unquestionably the same in the case of wild as of cultivated plants-although they operate in the two cases under different conditions-we may for the present at least assume as probable that plants vary as greatly in the wild as in the cultivated state. We shall however in the sequel have to examine a number of weighty considerations which lead to the conclusion that variation has produced.infinitely greater effects in originating the various wild forms of plants than those which we perceive in cultivated varieties ${ }^{3}$.

The variation of cultivated plants shows that there is only one cause for the internal and for the external hereditary resemblance between different plants, and that this cause is the common origin of similar forms from one and the same ancestral form. When we meet with corresponding phenomena in wild forms, and when we find that with them dissimilar forms are connected by a series of intermediate forms, just as we find to be the case between the primitive forms of cultivated plants and their most abnormal varieties, we are forced to the conclusion that in wild plants also a similar affinity is the only cause of resemblance. The extraordinarily numerous forms, for example, of the widely distributed genus Hieracium present phenomena similar in many respects to those of cultivated Gourds, Cabbages, \&c. In addition to a number of forms which are considered to be species, there are a still greater number of intermediate forms, some of which only are hybrids, the greater part perfectly fertile varieties. Nägeli ${ }^{4}$, who has made this genus the subject of close study, says:- 'If an attempt is made to unite into a single species all the types which are connected by perfectly fertile transitional forms, we should find only three species of native Hieracia, which have been erected by some authors into distinct genera:-Pilosella (Piloselloideæ), Hieracium (Archieracium), and Chlorocrepis (H. staticifolium). Between these three groups we have, at least in Europe, no transitional forms; hybrids between Piloselloideæ and Archieracium

1 See Darwin, $l$. c. vol. I. p. $3^{6} 5$, and Metzger, $l$. c. p. 207 . No great value with reference to variation and the constancy of varieties must be set on the result of experiments on cultivated plants, since the possibility of hybridisation was not excluded. Some varieties of Maize appear to hybridise with difficulty.

${ }^{2}$ Further material will be found collected in Darwin's and Metzger's works already quoted, and in De Candolle, Géographie botanique, Paris 1855 .

3 [H. Hoffmann gives in the Bot. Zeit. for April 27 and May 1,1874 , an account of an interesting series of experiments on the extent to which the characters which distinguish the allied species Papaver Rhoeas and dubium and Phaseolus vulgaris and multiflorus can be made to vary by cultivation, and on the tendency of the cultivated varieties to revert to the parent-form. See also his 'Ruickblick auf meine Variations-Versuche von 1855 -188o,' Bot. Zeitg. I 881.]

${ }^{4}$ Sitzungsberichte der kön. bayer. Akad. der Wiss. March Io, I 866. 
have been erroneously stated to occur, but the alleged hybrids are either true Piloselloideæ or true Archieracia. ... According to the present state of our knowledge, no other hypothesis is possible but that all the various species of Hieracium have sprung from the transmutation (descent with variation) of forms which have either disappeared or are still in existence; and a large number of the intermediate forms still occur which must have had their share in producing several new species by the splitting up of one original species, or which would have occurred in the transformation of a still living species into one derived from it. In the case of Hieracium the species have not become so completely separated by the suppression of the intermediate forms as is the case in most other genera.'

By the term Species is meant the aggregate of all the individual plants which have the same constant characters, these characters being different from those of other somewhat similar forms. It is clear from what has already been said that the only distinction between varieties of a known primitive form which have become constant, and the wild species comprised within a genus, is that in one case their descent is known, in the other it is not. The various cultivated varieties of a primitive form which have become constant are linked together by intermediate forms in which the progressive accumulation of new varietal characters may be perceived; but these intermediate forms may disappear, and then there is a more or less wide chasm between the various varieties themselves, as well as between them and the primitive form. Both of these cases occur also in wild plants. In some genera, like Hieracium, species the extreme forms of which differ greatly are connected together by a number of intermediate forms which occur along with them. The analogy of cultivated plants justifies us in considering these intermediate forms (so far as they are not hybrids) as varieties in a progressive state of development, some particular descendants of which have advanced furthest in the accumulation of new properties. But usually the intermediate forms, the connecting links so to speak between the ancestral and the derived forms, have disappeared; and the species of the same genus are then completely separated from one another, and their characters are at once distinguishable. The different species of the same genus agree among one another in a number of inherited characters, and are distinguished by only a few constant characters; the amount of resemblance is much greater than the amount of difference. The same relationship therefore exists, but in a greater degree, between the various species of one genus as between different varieties of the same primitive form. Since no other explanation is known of this relationship than common descent with variation and the heredity of the new characters, we are entitled to consider the species of a genus as varieties of a common ancestral form which have developed further and become constant,- the original form having possibly actually disappeared or being no longer recognisable as such. There is therefore no natural boundary-line between variety and species; they differ only in the amount of divergence of the characters and in the degree of their constancy. Just as a number of varieties are included in the idea of a species-the varietal characters being neglected in the diagnosis of the species-so several species are united into a genus by including in the diagnosis of the genus a maximum of their common characters. But since it is impossible either to determine by measure or by weight the most important characters of a plant, it is difficult and to a certain extent impossible to define, i.e. to determine by convention what amount of differentiation is necessary in order to classify two different but similar forms as species rather than varieties. In the same manner it is left to a great degree to personal judgment to decide whether two different but similar groups of forms should be regarded as two species each including several varieties, or as two distinct genera each including several species. The only object actually presented to the eye is the individual (and even this not always as a whole); the ideas Variety, Species, Genus are abstract ideas, and indicate a progressive scale of the differences between individuals 
which is small in the variety, larger in the species, and still larger in the genus. But in all three cases the points of difference are accompanied by a much greater amount of resemblance; and since in the phenomena of variation we learn that from forms which are similar others are derived which are constantly becoming more different by the continual accumulation of differences, we assume that the higher degree of variation of similar forms which we express by the terms Species and Genus has resulted from the accumulation of new characters in the variation from one ancestral form.

SECT. 37.-Causes of the progressive development of varieties. The characters of the cultivated varieties of one parent-form show, as Darwin was the first to point out, a constant striking and remarkable relation to the purpose for which the plant was cultivated by man. The varieties of Wheat differ from one another only slightly in the form of the haulm or leaves, which are of but small importance to mankind; but they show a great variety and extent of difference in the form and size of the grains, and the quantity of starch and proteid contained in them, i.e. in the characters of that part of the plant for the sake of which Wheat is cultivated, and in those properties of this part which under various circumstances are especially useful to mankind. The varieties of the Cabbage, on the other hand, scarcely differ at all in their seeds or even in their seed-vessels or flowers, the external properties of which are useless to man, and the internal properties only of value because the seed has to reproduce the variety; the varieties of Cabbage differ exclusively in the development of those parts which are used as vegetables, and to which therefore cultivation is directed. The object of cultivation is therefore, retaining the taste and value as food for man, sometimes to increase the succulence of the tissues, sometimes to attain as large a size as possible, sometimes to alter the time of the year at which the vegetable can be used. These and a number of other properties are furnished by the different varieties. The varieties of Beet differ only slightly in their flowers, more in their leaves, according as they are grown in the garden as ornamental foliage-plants or as agricultural crops ; the varieties in the latter case differ from one another in the size and shape of the roots and the amount of sugar they contain, properties which make the plant valuable on the one hand as food for cattle, on the other hand for the manufacture of sugar. Fruit-trees of the same kind differ but little in general in their roots, leaves, flowers, or stems, but to an extraordinary extent in the size, shape, colour, smell, taste, period of maturity, and keeping-properties of the fruit, according to the special purpose or prevalent mode in which it is employed. In garden-flowers it is generally the flowers and especially the corolla and inflorescence that differ in the varieties of a species, because the greater number are cultivated only for the shape, size, colour, or odour of the flowers.

This relation of cultivated varieties to the requirements of man is explained if we suppose that only those varieties were cultivated, at first undesignedly afterwards designedly, in which some character useful to man was more strongly manifested than in the others; those individuals were selected which best answered to a definite requirement; they alone were further cultivated; the particular character was again strongly displayed in some of their descendants, and only these individuals were again selected for reproduction; and the desired character was thus conţinually increased in strength. Other characters of the plant also varied at the same time, but they were disregarded, and the individuals in which they occurred were not preserved 
for reproduction, and no increase of these characters consequently took place from generation to generation.

The greatest service which Darwin has rendered to science is to have shown that wild plants are also subject to conditions of life the effect of which consists in this, that only some of the varieties of one primitive form maintain themselves and increase their peculiarities, while others perish. The relationship of the varying wild plant to its environment in the broadest sense of the word is however different from that of the cultivated plant to man; man protects his charges in order to preserve them; he places them under favourable conditions in order that those properties which are useful to him may become freely developed. Wild plants, on the contrary, have to protect themselves against all injury from without; their existence is continually threatened by other plants or animals or by the hostility of the elements; and in this Struggle for Existence, as Darwin has appropriately termed it, only those individuals are able to maintain themselves which are best able to resist the prejudicial influences to which they are exposed; and only those varieties which happen to be the best endowed in these respects will reproduce themselves and further develope their special properties. Hence the characters of wild plants, as far as they are not of a purely morphological nature, always show a perfectly definite relationship to the conditions in which they are placed; the form and other characters of the organs have essentially for their object to secure the existence of the plant under the local conditions of its habitat; varieties and species which are not endowed with qualities to endure the struggle for existence perish. The struggle for existence acts therefore in a certain sense similarly to the selection of the breeder; as the breeder developes only that which is suited to his own purposes, so in the struggle for existence only those varieties survive and reproduce their kind which are better adapted, through some property which they possess, to endure the struggle. Thus, finally, through imperceptible variation, through the destruction of those characters which are not beneficial, and through the further development of the useful ones-in one word, through what may be termed metaphorically Natural Selection by means of the struggle for existence,- - forms are produced which are as well or even better adapted for the purpose of self-preservation than cultivated plants are for the purposes of man. By the undesigned reciprocal influences of plants and of their living and physical environments, specialities of organisation finally arise which could scarcely be better adapted for the preservation of the plant under its special local conditions, and which give the impression of being the result of the greatest ingenuity and foresight.

'On the other hand, certain specialities of organisation which are essential in the struggle for existence may disappear in consequence of continued cultivation. Hildebrand points out ${ }^{1}$ that Peas, Beans, Lentils, Cereals, Buckwheat, all develope under cultivation large heavy seeds which cannot be self-sown, so that these plants when left to themselves do not become wild, but disappear in consequence of having lost the specialities of organisation which effect the dispersion of their seeds and which protect them from animals. The same is the case with cultivated plants the fruits of which have been modified for the use of man, and have become useless in

1 Hildebrand, Die Verbreitungsmittel der Pflanzen, Leipzig 1873 . 
the struggle for existence in the wild condition; such are cucurbitaceous plants, fruit-trees, \&c. Cultivation may so far affect edible fruits that they cease to develope seeds, as in the better kinds of Pears, in Grapes, Figs, Oranges, Dates, Bread-fruit, and Bananas; thus the first and most important element in maintaining the struggle for existence, namely, reproduction by means of seeds, is destroyed.

In order to understand clearly how the struggle for existence has caused the existing wild forms of plants to be so admirably adapted to their specific conditions of life, it must be borne in mind that all plants are continually varying to a very slight extent, and that the variation affects all their organs and all their characters, although usually to an imperceptible amount. On the other hand, the struggle for existence in plants (as well as in animals) is a perpetual and never-ceasing one, in which the smallest advantage that the plant has obtained through variation in any one direction may be of the utmost importance for its perpetuation.

The struggle which the plant carries on by means of its capacity for variation has two different aspects. On the one hand its tendency is to adapt its organisation completely to the conditions of food and growth afforded by the climate and the soil. It is evident that the organisation of a submerged water-plant must be different from that of a land-plant; that the assimilating organs of a plant that grows in the deep shade of a wood must be differently constructed from those of a plant exposed daily to bright sunshine, and so forth. The conditions of life of all plants growing at a great elevation and in Arctic countries must be different from those growing in the lowlands of the Tropic and Temperate zones. If we had to do only with these general conditions of plant-life, the struggle for existence would be a comparatively simple process. It would be easy to imagine how, among the varieties of a primitive form which grew in water, there would be some which would be occasionally subjected to a subsidence of the water, and how these would give birth to descendants which would gradually assume the character of marsh- and finally of land-plants, as is well illustrated in the case of Nasturtium amphibium, Polygonum amphibium, \&c. ${ }^{1}$ It may also be supposed that some of the descendants of a plant exhibit a somewhat greater power of resisting frost, that this property increases in the course of generations, and that thus a form which can at first only bear a temperate climate gradually produces varieties which can endure a more and more severe climate; and so forth. Even these comparatively simple conditions would necessarily lead to a great diversity in the varieties descended from one ancestral form; for each adaptation to new conditions of climate or locality would act in different ways; i.e. varieties of different descriptions would take up and carry out in different ways the struggle against the influences of the elements.

But the struggle for existence and the changes occasioned by it in the organisation of plants are greatly complicated by the fact that every plant, while struggling to adapt itself to its special conditions of life, has also to protect itself at the same time against a number of other plants and against the attacks of animals; or, what is more to the point, its capacity for variation enables it to make use of particular favourable conditions which are offered to it by other plants and animals in order to take ad-

1 A special interest attaches in this connection to Hildebrand's observation on Marsilia in Bot. Zeit. 1870, No. I, and Askenasy's on Ranunculus aquatilis and divaricatus in Bot. Zeit. 1870, p. 193 et seq. 
vantage of them ; as parasites of their hosts, dichogamous and other flowering plants of the visits of insects, \&c. These relationships are endless in their diversity, and can only be illustrated by examples.

We must here call special attention to a remark of Darwin's; that the individuals of the same species or variety are competitors for position, food, light, \&c. The fact that plants of the same species have the same requirements itself gives rise to a struggle for existence among them ; and the same is the case, though to a somewhat smaller but still to a great extent between the different varieties of the same primitive form, to a less extent between different species and genera. The result of these relationships is seen on the one hand in the fact that, in the case of plants which live socially, only the most vigorous seedlings arrive at full maturity, while the weaker ones are smothered, as may be seen in any young plantation; on the other hand, that species and genera which differ greatly from one another can thrive side by side, because their requirements are different and the competition between them is less.

From the fact that plants whose organisation differs can thrive better side by side on the same soil in consequence of the diminished competition between them, Darwin drew the important and pregnant conclusion that in the propagation of the varieties of one primitive form those new forms must be the best able to maintain themselves in the wild state which differ most from the primitive form and from one another, whereas the intermediate forms must be gradually dispossessed. This is the reason why the connecting forms between the different species of a genus are so often wanting, although the conclusion cannot be avoided that the species arose by variation from a single ancestral form, and by the propagation of varieties.

In its broader features (and on that account more conspicuously) the struggle for existence between the various forms of plants, the competition for space, food, and light, is manifested in the luxuriant growth of what we term weeds in our gardens and fields. Our cultivated plants are able to bear our climate, and the soil supplies what they require for their vigorous growth. But a number of wild plants are still better adapted to the climate; and they grow still more vigorously, rapidly, and luxuriantly on cultivated soil, and their seeds or rhizomes are everywhere present in enormous quantities. If the cultivated plants are not carefully protected from the weeds, the latter soon dispossess them of the ground which was set apart for them. Every country and every soil has its own peculiar weeds; i.e. under any particular external conditions there are always certain forms of plants which thrive best and drive out the cultivated plants. To a certain extent we have a measure of the amount of advantage which weeds have over cultivated plants in the amount of labour bestowed by man on their destruction in order to preserve and maintain his nurselings. The primitive forms of our cultivated plants are mostly natives of other countries, where they are not only sufficiently adapted for the climate, but are able to sustain competition with their neighbours.

The number of species or of individuals of any species which we find in a meadow, a marsh, \&c. is not a matter of chance; it does not depend merely on the number of seeds of one or another species produced or brought to the locality ; every one of these species would, if it alone existed there or were protected by cultivation, of itself cover the space of ground in a short time; but there is a definite relationship between the numbers of individuals of the different species when left to themselves, a relationship which depends on the specific power of each particular species to maintain itself in the struggle with the rest ${ }^{1}$.

1 [How the relationship subsisting between the species in permanent pastures may be disturbed by the application of different manures, may be seen in Lawes and Gilbert's paper on this subject in Journ. Roy. Agric. Soc. vol. XXIV, 1863.] 
How complicated may be this relationship in the cases of only two nearly related forms of plants in their struggle for existence in particular localities has been described as exhaustively as clearly by Nägeli in the case of various Alpine plants. 'The internecine war,' he says ${ }^{1}$, 'is obviously most severe between the species and races that are most nearly related, because they require the same conditions of existence. Achillea moscbata drives out $A$. atrata, or is driven out by it; they are seldom found side by side; while each grows along with $A$. Millefolium. It is clear that Acbillea moschata and atrata, being extremely similar to one another externally, make similar demands on their environment, while $A$. Millefolium, which is less nearly allied to both, does not properly compete with them, because it requires other conditions of existence. Still less do plants of different genera or orders compete with one another..... In the BerninaHeuthal (Upper Engadin) Acbillea moschata, atrata, and Millefolium occur in profusion, A. moschata and Millefolium on slate, $A$. atrata and Millefolium on limestone; where the slate ends and limestone begins, $A$. moschata always ceases and $A$. atrata takes its place. Both species are therefore here strictly circumscribed as to soil, and this I have found to be the case also at various spots in Bündten, where both species occur together. But where one species is absent the other is widely distributed. A. atrata is then found indiscriminately on slate or limestone: and although $A$. moschata does not apparently grow so readily on limestone as $A$. atrata does on slate, yet in the neighbourhood of the primary rocks it is found on a distinctly calcareous formation along with the vegetation characteristic of it. In the Bernina-Heuthal I found in the midst of the slate which was thickly covered with $A$. moschata a large erratic block of limestone covered with a crust of soil scarcely an inch thick, upon which a patch of $A$. moschata had established itself, because it did not here meet with any competition from $A$. atrata... . A similar relationship was observed in certain districts between Rbododendron birsutum and ferrugineum, Saussurea alpina and discolor, and between species of the genera Gentiana, Veronica, Erigeron, Hieracium, \&c.' The obvious objection, that there cannot possibly be any struggle between two forms of plants as long as there is space for both in the area in question, rests on an incorrect basis, and is disposed of by Nägeli as follows:- ' Upon a slate slope are a million plants of $A$. moschata; they obviously do not occupy the whole space, for a hundred millions or more could find room there; but the rest of the space is occupied by other plants. There is here a condition of equilibrium, which has been produced in reference to the nature of the soil and the preceding climatic influences. The number one million gives us also the proportion which $A$. moschata is able to maintain in relation to the rest of the vegetation; and the objection that there would still be plenty of room for $A$. atrata is an untenable one. If the space were accessible to species of Acbillea generally, it would be occupied by the species which is already present, and which in any case has the advantage, $A$. moschata. If we now imagine that the two species happened for once to be intermixed on the slate slope, perhaps in consequence of artificial transplanting, in equal quantities, say 500,000 plants of each, A. moschata would thrive the better of the two, as the soil contains but little lime; $A$. atrata would become weaker and its tissue less matured, and would in consequence have less power to withstand external prejudicial influences, as summer frosts, long-continued rainy weather, or persistent drought, \&c. If we suppose, for example, that every twentieth or fiftieth year a severe frost occurs at the time of flowering which destroys half the plants of $A$. atrata, while the more vigorous $A$. moschata resists it, the voids are again filled up by the dispersion of the seeds; but more plants of A. moschata spring up than of $A$. atrata, because the number of individuals of the latter was reduced by the frost to 250,000 , while that of the former remains at 500,000 . The million plants of Acbillea on the slope will in consequence be composed of say 670,000

1 Sitzungsber. der kön. bayer. Akad. der Wiss. Dec. I5, 1865. The manner in which the arrangements for the protection of the pollen with reference to certain insects on the one hand, and to climatic influences on the other, determine the distribution of certain plants, is clearly indicated by Kerner (Schutzmittel des Pollens, Innsbruck I873). 
$A$. moschata and $330,000 \mathrm{~A}$. atrata. After a second frost, which again destroys one half of the individuals of $A$. atrata, we should have about 800,000 of $A$. moschata to 200,000 of $A$. atrata. In this manner the number of the latter would decrease with every unusual summer frost, until at length it entirely disappeared, a nearly-allied hardier species becoming distributed over the locality in its place.' In conclusion, the following remark by the same author may be added :- ' From such a course of reasoning the conclusion might perhaps be drawn that this result would always take place, and that one of two plants would always be crowded out, because the two could hardly be precisely equally hardy. But this conclusion would be unsound, because it would hold good only for plants whose conditions of existence were as nearly as possible alike. We can imagine another case in which the two species suffer injury from altogether dissimilar external influences (one, e.g., from spring frost, the other from dry heat), so that sometimes the number of individuals of one species, sometimes that of the other species diminishes, and where moreover the production and the germination of the seeds are affected by altogether dissimilar external influences, so that sometimes the one sometimes the other species increases more rapidly and occupies the vacant spots. The numerical proportion of the two species must in this case be variable, but neither is able to expel the other.'

Just as the struggle between two species is the result of their thriving more or less vigorously on a soil of a particular chemical nature, so also the need for more or less water, light, heat, \&c. can determine also the nature of the struggle for existence. Nägeli gives some examples of the first case. When Primula officinalis and elatior occur together in a district, they are sometimes sharply separated from one another, $P$. officinalis preferring the dry, $P$. elatior the damp spots. Each is most vigorous in its own locality, and may expel the other. But when only one species occurs, it is not so particular; $P$. officinalis will choose damper, $P$. elatior drier situations, than if they were in company. Prunella vulgaris and grandifora behave in the same manner in reference to poorer and more fertile soils; as also do Rbinantbus Alectorolophus and minor, Hieracium Pilosella and boppeanum.

These examples may suffice to show what is meant by the Struggle for Existence. It must however be borne in mind that such a struggle must arise in reference to every vital phenomenon of a plant, and to each of its relationships to the external world, especially to the animal kingdom; and that its course must vary for the same plant in different localities. An understanding of the Theory of Descent, and especially an insight into the causes of the perfect adaptations of the organisation of the plant to its vital conditions which are often extremely local, depend essentially on a clear comprehension of the struggle for existence.

SECT. 38. - Relationship of the morphological nature of the organ to its adaptation to the conditions of plant-life. Every plant is very accurately adapted (though not absolutely so) to the conditions and circumstances under which it grows and is reproduced; its organs have the shape, size, mode of development, power of movement, chemical properties, \&c. needful for this purpose. If this were not the case, the plant would inevitably perish in the struggle for existence. But the vital conditions are extremely various, and undergo, in the course of time, endless changes. The diversity in the characters of plants corresponds to this infinite variety in the conditions of life; and yet even in the more highly differentiated classes there are only three or four morphologically distinct forms of structure, axis (caulomes), leaves (phyllomes), roots, and trichomes, which suffice for these conditions, while maintaining a constant morphological character through numberless variations in their physiological properties. This relationship has already been described in chap. iii of Book I as the metamorphosis of the morphological members of a plant, 
understanding by metamorphosis the adaptation to various physiological purposes of morphologically equivalent members. The diversity in the physiological development is related to the conditions of life of the plant ; and to this extent Metamorphosis is synonymous with what we have here termed Adaptation, and which has also been described as Accommodation. When we speak of Purpose in the structure of a plant, we mean in fact nothing more than that the form and the other characters of the organ are adapted to the conditions of its life, which may be at once inferred from the very fact of the survival of the plant in the struggle for existence. The terms Purpose, Adaptation, and Metamorphosis express therefore the same thing, and may be used as synonymous, as we have already repeatedly done ${ }^{1}$.

For the discussion of the questions to be treated of in the following paragraphs it is important to have as clear a conception as possible of the relationship of adaptation to the morphological nature of the organs, and of the great constancy of morphological characters and the infinite diversity of metamorphosis; for this relationship can be explained by no other theory than that of descent.

In its most general features the relationship of adaptation to the morphological nature of organs is manifested in the fact that all the various morphological members perform the most different functions and in an infinite variety of ways; in other words, that the morphological nature of the parts of a plant is not directly determined by their function, nor is the function of an organ determined directly by its morphological nature. Thus, for example, trichomes sometimes take the form of a protective envelope (mostly in buds), sometimes of glands, sometimes of absorptive organs (as root-hairs), sometimes of asexual organs of reproduction (as the sporangia of Ferns), \&c. The leaves again are usually organs of assimilation containing chlorophyll; but they may also be employed as protective envelopes to winter-buds (in most of our native woody plants), as reservoirs for reserve food-materials (in the seedlings of flowering plants and in bulbs). In the majority of plants the leaves bear the sporangia: in flowering plants the flowers are composed of peculiarly metamorphosed leaves. In many slender-stemmed Angiosperms the leaves are transformed into tendrils, in order to raise up the slender stem and fix it to neighbouring supports; the leaves of Nepenthes produce at their apex an appendage which forms a pitcher provided with a moveable lid and filled with the fluid which it itself secretes; some of the leaves contained in flowers are developed into nectaries and then perform the function of glands; not unfrequently they are transformed into hard woody spines; in other cases they are sensitive to irritation, contractile, and so forth. The parts of the axis are scarcely less varied in their development; sometimes they cling round upright supports; sometimes they are woody and able to retain themselves in an erect position; sometimes they are slender swaying branches, or thick fleshy succulent masses (Cactus), or round tubers filled

1 Many recent writers seem to be almost too anxious to avoid the use of the word 'purpose,' because it seems to suggest antiquated teleological views. The word 'useful' which they would substitute for 'purposive' has also a teleological significance in human affairs. If every word which has once been used to express an incorrect theory is to be discarded, the resulting diminution of the vocabulary would soon produce an evident impoverishment of the language. The mission of science is not to explain and alter words, but the ideas to which they correspond. Ought we to give up the use of the word 'root' in Botany, because it formerly bore a very different meaning from that which is now attached to it? 
with food-materials (Arum, Potato), or they become tendrils (the Vine), or spines (Gleditschia); sometimes they assume the form of foliage-leaves (Ruscus, Xylophyllum, \&c.). The adaptations of roots are less numerous; usually filiform, slender, cylindrical, and provided with root-hairs for absorbing water and dissolved mineral substances, they become tuberous reservoirs for reserve food-materials in the Dahlia; their tissue is loose and contains air and they resemble swimming bladders in Jussieua; in the Ivy, Ficus repens, \&c., they are simple organs of attachment for the stem ; in Vanilla aromatica they play the part of tendrils; but they never produce sporangia or sexual organs.

According to the definition already given of Purpose in the vegetable organisation, its relationship to the morphological nature of the organ can also be illustrated by keeping in view the end to be served, $i$.e. the condition of the plant which is most favourable in the struggle for existence, and then observing the means employed for attaining this end, i.e. what members of the plant become adapted, and what metamorphosis they undergo. A few examples will explain this ${ }^{1}$.

It is obviously useful for the greater number of flowering plants - in other words advantageous in the struggle for existence - that their stem should grow rapidly to a certain height, because the conditions of assimilation (light and warmth) are thus most perfectly fulfilled, and because-which is perhaps of greater importance - the flowers are more easily detected by insects on the wing, and the pollen transferred by them from one flower to another. Even where (as in many Coniferæ, \&c.) the light pollen is carried by the wind to the female flowers, this is accomplished better when the flowers are at a considerable height from the ground; and finally by this means the dissemination of the seeds by the wind or by frugivorous birds is promoted, or their scattering by the bursting of the fruits. That these arrangements for propagation are especially promoted by the upright growth of the stem is evident from the fact that in the large number of plants which develope their leaves in a rosette close to the ground or on a stem that creeps along it, a rapidly ascending flower-stem is formed only just before the unfolding of the flower-buds. Still more strikingly is this the case in parasites and saprophytes (Orobanche, Neottia, \&c.), which vegetate below and blossom above ground. If we recognise these and other special purposes of upright growth, it is of interest to see in what various ways this one purpose is attained in different species of plants. In many shrubs the growing stem is endowed with sufficient firmness and elasticity to support in an upright position the weight of the leaves, flowers, and fruits; if it happen to be broken down, or if it must raise itself from a previously creeping position, advantage is taken of the property of geotropism. But the slender haulms of Grasses are not themselves endowed with this power; and in their case the basal portion of each leaf-sheath forms a thick ring the tissue of which retains for a long time its power of growth ; and when the haulm is bent by the wind, or is in its early stage prostrate on the ground, the elevation into an erect position is brought about by the surface of the node which faces the ground

1 In these examples I am compelled to confine myself to the most important points. Most of the adaptations are so complicated that a detailed description of them in even a single plant would require a great deal of space. What was said in the fourth chapter of this Book on climbing plants and in the sixth on the adaptation of the foliar organs of a flower to the purpose of crossfertilisation may be consulted. 
growing rapidly and strongly; a knee-shaped bend is thus formed by which the upper part of the haulm is raised up. If, on the contrary, the stem is perennial, and has to bear a great weight of branches, leaves, and fruits, contrivances of this kind are not sufficient, and then the tissue becomes woody; if the weight of the crown increases year by year, the stem also becomes thicker each year, as in dicotyledonous trees and Conifers; if the weight of the foliage does not increase, as in Palms, the stem only retains the same thickness. In such cases a considerable quantity of assimilated food-material is necessary in order to produce the massive solid stem, while in many other cases the elevation is attained at the expense of a very small amount of organic substance, as in climbing and twining plants, such as are found in the most widely separated families of Angiosperms. Plants with a twining stem like the Hop presuppose in general the existence and proximity of other plants which are able themselves to grow upright and round which they twine; and in order that such a neighbouring support may be more easily and certainly taken hold of, the slender stem of climbing plants is endowed with a power of revolution by which the apex is carried round in a circle and is enabled to come into contact with the stem of an upright plant, up which it then climbs.

The greater number of plants provided with tendrils are also dependent on the proximity of erect plants round which they can climb; they are characterised by an extreme parsimony in the employment of organic substances for the purpose of an erect growth. Sometimes (as in the Grape-Vine) the tendrils are axial structures furnished with minute leaves and branching from the axils of these; but much more commonly (as in Clematis or Tropacolum) the petioles, or (as in Fumaria) the branched narrowly-divided lamina, or most often the metamorphosed apical parts of the foliage-leaves (Cobca scandens, the Pea and other Papilionaceæ) are developed in a filiform manner and perform the function of tendrils. The morphological significance of the tendrils of Cucurbitaceæ is not yet perfectly determined; but they are probably metamorphosed branches. Tendrils occur only in those plants whose stem is not able to bear in an erect position the weight of the foliage, flowers, and fruits; in the genus Vicia, for example, all the slender-stemmed species have leaftendrils; but in the thick-stemmed erect $V$. Faba they are rudimentary. The office of tendrils is to twine round the slender branches and the leaves of other neighbouring plants, and thus to fix the apex of the stem as with cords on various sides while it is growing upwards. The adaptation of tendrils, i.e. their endowment with useful properties corresponding to their purpose, is, as Darwin has shown, not only extremely diverse, but exhibits also very different grades of perfection, like that of climbing stems. Some tendrils are only of slight use; sometimes (as in some species of Bignonia) they are merely helps to an imperfectly climbing stem; but where they are perfectly adapted to their function, a variety of properties concur in a remarkable way to increase to a maximum this kind of adaptation to the use of the plant. The tendrils radiate in different directions from the growing apex of the shoot, which makes movements of revolving nutation by which the tendrils are brought into the greatest variety of positions, they themselves also revolving at the same time, so that within a certain area, often not a very small one, they assume an infinite number of positions, by which they must almost inevitably be brought into contact with some support, such as a branch or leaf, lying within this area. The 
supports are, so to speak, sought out in the most industrious manner; when one is touched by a tendril, the tendril bends in consequence of the stimulation and twines firmly round it; and when several tendrils do the same in different direction from the stem, it hangs suspended between the points of support. If this were all, the attachment would be a very weak one, and the elevation of the stem would only take place slowly; but the whole contrivance is perfected in the most ingenious way. When the tendrils have fixed themselves by their extremities, they draw the stem towards the support by twisting themselves spirally. When several tendrils do this in different directions, the stem which is suspended between them is tightly stretched, and the tenacity of the tendrils is at the same time considerably increased by the twisting. Many tendrils, while very tender at the time when they are sensitive, become afterwards hard and woody, and some become much thicker; this is strikingly the case in Clematis glandulosa and Solanum jasminoides. But the most perfect adaptation is shown in the tendrils of the Virginian Creeper, Bignonia capreolata, and some other plants : it is most perfect in Ampelopsis hederacea. As in the Grape-Vine, the tendrils are here branched axial structures, and are to a much greater extent negatively heliotropic; their power of twining round slender supports is but slightly developed, but when, in consequence of their negative heliotropism, they come into contact with a wall, or in the wild state with a rock, trunk of a tree, \&c., there is formed in the course of a few days on each branch of the tendril which touches the support with its curved and hooked apex, a cushionlike swelling which afterwards expands into a red flat disc, and becomes firmly attached by its surface to the support. The adhesion of this organ of attachment is probably at first occasioned by an exudation of viscid sap; but the attachment to the support is caused mainly by this organ forcing itself into all the depressions in the surface of the support and growing over the slight elevations. After this has taken place the whole tendril becomes thicker; it contracts spirally, the stem to which it belongs being thus drawn towards the wall, rock, \&c.; then it becomes woody, and the firmness of its tissue and the power of retention of the disc are so considerable that, according to Darwin ${ }^{1}$, a tendril ten years old and furnished with five of these discs can support a weight of rolbs. without giving way and without the disc becoming detached from the wall. Since a shoot which is growing upwards forms a number of tendrils, this attachment to the flat support is a very effectual one, and enables the plant to endure the annually increasing weight of the stem which is gradually becoming thicker and more woody; and in this way it climbs over the walls and roofs of buildings more than 100 feet high. The fact is very interesting that those tendrils of the Virginian Creeper which do not come into contact with the wall or rock die after some time, and wither up into slender threads which then fall off, no adhesive disc having been formed on them. But in order that these peculiar tendrils may more readily come into contact with the support, the upright shoot is scarcely at all positively heliotropic, since this property would cause it and its tendrils to move further away from the supports; while the young shoots which exhibit very slight heliotropism become erect under the influence of gravitation; otherwise the whole of the contrivances connected with the tendrils would be purposeless.

1. [Movements and Habits of Climbing Plants, 1875.$]$ 
If looked at merely superficially, the mode in which the Virginian Creeper climbs up rocks, walls, and thick trees, presents a certain resemblance to the climbing of the Ivy; but in fact the adaptations of the two are altogether different. It has already been shown how negative heliotropism causes the leafy branches of the Ivy to become closely pressed to the support, and how the summit of the branch at first exhibits slight positive heliotropism, so that the slight convexity is in contact with the support. At this point of pressure rows of aërial roots afterwards arise (not in consequence of pressure, for they make their appearance also on branches which hang free) which apply themselves to the inequalities of the bark of the tree or of the rock which serves as a support, and thus fix the Ivy-stem to it. Other weakstemmed plants attain the same object (that of elevating their assimilating and flowering shoots) by apparently much simpler means, as the Bramble, Rose, and some climbing Palms like Calamus, \&c., whose long shoots spread over neighbouring plants and are supported by them, their hooked prickles and other similar contrivances assisting in this.

It is of service to many plants in the struggle for existence that they should keep firm possession of the piece of ground they have once occupied, without forming for this purpose large woody masses, like trees and shrubs. The underground parts of such plants are perennial, and they send up separate shoots in each vegetative period to be exposed to the light and air where they will be able to assimilate, to produce flowers, and to scatter their seeds. This persistence of the underground parts has the advantage that the plant, although it assimilates and grows only at particular times of the year, is not compelled to seek each year, like annual plants, a new locality in which its seeds may germinate. The collection of reserve food-materials underground gives strength to the plant; it developes its buds beneath the soil to such an extent that at the right time they can grow up quickly at the expense of the rich supply of material. Every year very strong shoots are put forth, while in annual plants a number of feeble seedlings perish annually before some of them attain sufficient strength to protect themselves from the shade and humidity to which their neighbours subject them. Plants whose underground parts are perennial have in particular the power of resisting long and severe frost and the greatest variations of temperature, because these only penetrate slowly beneath the soil. It is for this reason that so large a number of Alpine and Arctic plants belong to this class. They are also able to grow in localities which are much too dry for the germination of the seeds of annual plants, because moisture is retained at a great depth for a longer period than near the surface. Numerous other advantages might also be mentioned which are compensated for in annual plants by other adaptations ${ }^{1}$.

This permanence of the underground parts is attained in the greatest variety of ways. Sometimes the plant possesses slender creeping underground shoots in which the reserve food-materials are collected and which themselves rise above the surface at a particular time, as in many Grasses; or sometimes the leafy stems are developed from lateral buds, as in Equisetum; or there are thick stout stems from which shoots

1 [This subject-and especially the relation of peculiar habits of life to the power of resisting great cold-is very fully discussed in Kerner's treatise Die Abhängigkeit der Pflanzengestalt von Klima und Boden, Innsbruck 1869.] 
appear each year at the same place. In some cases the whole plant is annually renewed; all the parts which existed the previous year die off, and a complete rejuvenescence of the individual is accomplished underground. In the Potato and Artichoke only the apical parts of the underground lateral shoots swollen into tubers remain over till the next year, the whole of the rest of the plant having perished. In many of our native Orchids the rejuvenescence takes place in a similar way (see p. 219 and fig. I58); and one of the most interesting cases of annual rejuvenescence occurs in Colchicum autumnale (see fig. 422). In these cases, with the exception of the Orchids, the reserve food materials accumulate in underground parts of the axis; in other cases this takes place in the swollen roots, which remain in connection with the underground part of the stem that bears the new buds, as in the Hop, Dahlia, and Bryony. In bulbs again the reserve accumulates in the leaves (bulb-scales) which surround the bud that developes into the new plant. The reserve often collects in cataphyllary leaves of peculiar development; in Allium Cepa in the lower part of the leaf-sheaths, which persist through the winter, while the upper parts of the leaves die off.

We have already in the last chapter spoken of the immense variety of the contrivances which have for their object the partial or entire prevention of the self-fertilisation of plants, in order to produce a stronger and more numerous offspring by the sexual union of different individuals; and only a few examples need now be mentioned. Just as the form, size, colour, position and movements of the parts of the flower are almost invariably adapted to facilitate the conveyance of pollen from one flower to another, generally by insects, and often also to render self-fertilisation impossible; and as a great diversity even of those forms of flowers which are constructed on the same morphological type results from this, so the properties of ripe seeds and fruits are no less adapted ${ }^{1}$ to bring about the dissemination of the seeds. Fruits which are very similar from a morphological point of view may nevertheless assume physiological properties which are altogether different, and fruits which are very different morphologically may become extremely similar in consequence of their adaptation to the purposes of dissemination. The service rendered by insects in the fertilisation of diclinous, dichogamous, dimorphic, and many other flowers, is performed by birds in the dissemination of a number of seeds which are concealed beneath fleshy edible pericarps ; in some cases, as the Mistletoe, it is scarcely possible to imagine any other mode of dissemination than the eating of the berries by birds. Dry fruits or the seeds which are shed by dry fruits are often provided with an apparatus adapted for transport by the wind, the morphological value of which is as various as possible. The wings on the seeds of the species of Abies are outgrowths of a superficial layer of the tissue of the scale, those on the seed of Bignonia muricata originate from the integument of the ovule; the wings of the indehiscent fruits (samaræ) of Acer, Ulmus, \&c. are outgrowths of the pericarp; the crown of hairs on the seed of Asclepias syriaca evidently performs the same service as the pappus of many Compositæ which is a metamorphosed calyx. In these cases it is obvious that the wind carries the seeds or fruits; in other cases

${ }^{1}$ It is scarcely needful to mention again that this mode of expression has only a metaphorical meaning from the stand-point here assumed, and is only used for the sake of convenience, 
animals of considerable size perform this office involuntarily, the hooked or rough fruits becoming attached to them and afterwards falling off ${ }^{1}$.

In most of these adaptations, both their purpose and the mechanical contrivances for its attainment are easily recognised; but not unfrequently the latter require a closer examination and some reflection in order to understand them. Among many other cases of this kind one only may be mentioned here which any one can easily observe for himself. The fruit of Erodium gruinum and other Geraniace $^{2}$ splits up into five mericarps each of which has the form of a cone with the apex pointing downwards, containing the seed and bearing above a long awn. When moist this awn is stretched out straight, but if it becomes dry while lying on the ground the outer side of the awn contracts strongly, causing the upper end to describe a sickle-shaped curve, which brings its point against the ground, the cone being thus placed with its apex downwards. The lower part of the awn now begins to contract into narrow spiral coils, causing the cone to turn on its axis and to penetrate the ground, and the erect hairs on it which point upwards retain it there like grappling-hooks. After the cone has penetrated the ground, the twisted part of the awn does the same, driving the part which contains the seed further and further into the soil. If the mericarp now becomes moistened, the coiled part attempts to straighten itself, but its coils are held by the hairs which stand on the convex surface; and thus this movement also contributes to drive the cone deeper into the soil. Whether therefore the moisture is greater or less, the mechanical contrivance produces the same effect, namely, to drive the part of the mericarp which contains the seed into the soil.

Some of the contrivances found in plants are extremely striking, from the concurrence of the most different properties for the attainment of a perfectly definite purpose corresponding only to certain specific conditions of life, as the adaptation of the Virginian Creeper to climbing up vertical walls, the contrivance to prevent self-fertilisation in the flowers of Aristolocbia Clematitis, the bursting of the fruit of Momordica Elaterium, and a thousand similar cases. The most remarkable instances are generally connected with the ordinary arrangements, or even with other extreme cases, by a number of the most diverse intermediate or transitional forms. These transitional forms have been described in detail by Darwin in the case of climbing and twining plants, and the fertilisation of Orchids, in his works already mentioned, and by Hildebrand in the case of the fertilisation of Salvia ${ }^{3}$, and of the dissemination of seeds.

SECT. 39.-The Theory of Descent. The facts and conclusions which have been indicated rather than described are the foundation of the Theory of Descent. This theory consists in the hypothesis that the most unlike forms of plants have a relationship to one another of the same kind as that which the varieties gradually developed from one ancestral form bear to it and to one another. It supposes that the different species of a genus are varieties derived from one progenitor which have undergone further development; and that in the same manner the various genera of an order owe their common characters to their descent from one and the

${ }^{1}$ [A remarkable instance of this is recorded by Dr. Shaw (Journ. Linn. Soc. vol. XIV, 1874, p. 202), in the introduction into South Africa and enormously rapid distribution of a European plant, Xanthium spinosum, by the spiny achenes clinging to the wool of the Merino sheep.] See also Hildebrand, Die Verbreitungsmittel der Pflanzen, Leipzig 1873 .

${ }^{2}$ See Hanstein, Sitzungsber. der niederrheinischen Ges. in Bonn, 1868.

${ }^{3}$ Jahrbuch für wiss. Bot. vol. IV, I865. Also Verbreitungsmittel der Pflanzen. 
same older ancestral form, and their differences to variation and to the accumulation of new characters in successive individuals through a long series of generations: The theory of descent goes still further, and assumes the same mutual relationship between the various orders of a class, between the various classes of a group, and finally between the various groups. It considers variation in the course of reproduction to be the cause of all the differences among plants, and the heredity of the varietal characters to be the cause of the agreement which subsists even between the most diverse forms of plants. What we call the common law of growth of a class, or in other words its Type, is the result of all the plants of this class being descended from one ancestral form or Archetype, as Darwin terms it. That which was long since termed in a merely metaphorical sense the affinity between different forms of plants is, according to the theory of descent, an actual affinity or bloodrelationship in various degrees. The differences have arisen in the course of a long: series of generations, by the descendants of the same archetype continuing to vary, by the variation of the different individuals in different ways, and by the continual and necessary increase of the differences between them under diverse conditions of climate, especially under the conditions imposed by the struggle for existence, in order that they may still be capable of maintaining themselves. At the same time numberless varieties, species, and genera have gradually disappeared, because they were not sufficiently adapted for the struggle for existence under the new conditions caused by geological changes, and in consequence of the appearance of other forms which were better adapted to resist them.

The scientific basis for the theory of descent rests in the fact that it alone is able to explain in a simple manner all the mutual relationships of plants to one another, to the animal kingdom, and to the facts of geology and palæontology, their distribution at different times over the surface of the earth, \&c.; for this no other hypothesis is necessary than descent with variation and the continued struggle for existence which permits those forms only to persist that are sufficiently endowed with useful properties, the others perishing sooner or later. Moreover both these hypotheses are supported by an infinite number of facts. The theory of descent involves only one hypothesis that is not directly demonstrated by facts, namely that the amount of variation may increase to any given extent in a sufficiently long time. But since the theory which involves this hypothesis is sufficient to explain the facts of morphology and adaptation, and since these are explained by no other scientific theory, we are justified in making this assumption.

The theory of descent explains intelligibly how plants have obtained their extraordinarily perfect adaptations for supporting the struggle for existence; this struggle has itself been the means of their obtaining them by the 'Survival of the Fittest,' that is, by permitting the existence and propagation of those newly-formed varieties alone which are endowed with the various characters that render them best fitted to the climate and to resist the rivalry of competitors, the attacks of animals, \&c. In this manner adaptations are gradually developed from a slight and imperceptible beginning by the accumulation of useful characters which have the appearance of being the result of the most careful and far-sighted calculation and deliberation, or sometimes even of the most cruel caprice (as in the fertilisation of Apocynum androscamifolium by flies which are tortured to death in the process). 
The fact that members which are morphologically similar are adapted for the most various functions is explicable when we consider that the morphological features in the structure of plants are those which are most certainly transmitted unchanged to posterity, either because they have no direct relation to the struggle for existence, or because they have proved useful in the various relations of life; as for example the differentiation into stem, root, leaves, \&c., and into the different tissue-systems, by which the division of physiological labour and the acquisition of the most various properties useful for the struggle for existence are facilitated. The structure of the Thallophytes and of the Hepaticæ shows that these morphological differentiations do not exist in the first or lowest forms of plants, but that they come gradually into existence; but when once fully developed they are preserved in the course of further variations, because they are never prejudicial, but often on the contrary advantageous for the purposes of adaptation.

The perfect heredity of morphological characters gives rise to a very remarkable phenomenon, the production of functionless members. It is obvious that hereditary peculiarities may have lost their use under the new conditions of life of the descendants, because the physiological requirements of the plant are supplied by other means, by fresh adaptations. Of this nature are, for example, the minute leaves on the root-like shoots of Psilotum, the formation of endosperm in the embryo-sac of many Dicotyledons whose embryo afterwards grows so vigorously as to supplant the endosperm, while it becomes itself filled with reserve food-materials which in other cases are stored up in the endosperm for the seedling. The most striking illustration however is the behaviour of parasites and saprophytes destitute of chlorophyll, which are found in various orders of plants, and the near allies of which form large green leaves containing chlorophyll, while these produce leaves similar in a morphological sense, but which are neither large nor green, and sometimes degenerated so as to have become obsolete. The explanation of this phenomenon is at once afforded by the theory of descent, viz. that the parasites and saprophytes which contain no chlorophyll are the transformed descendants of leafy ancestors which did form chlorophyll, but which have gradually become accustomed to take up the assimilated food-materials of other plants or the available products of their decomposition; and the more they did this the less needful did it become for the plants themselves to assimilate. The green leaves therefore became meaningless and ceased to form chlorophyll; but without chlorophyll the leaves were of little or no service to the new form, and therefore as little substance as possible was employed in their development, and they gradually degenerated.

Looked at from the point of view of the theory of descent, the natural system of the classification of plants represents their blood-relationship to one another. A species consists of all the varieties which are descended from a common ancestral form; a genus of all the species produced from an older progenitor, and which have become in the course of time further differentiated; an order includes all the genera which have been derived by variation from a still older ancestral form; and the first primitive form of all the orders comprised in a group belongs to a still older past ; finally there must have been a time when a primordial plant originated the whole series of development; and this must have produced in its varying descendants the primitive types of all the later forms. The relationships of the various 
classes and groups described at length in Book II might be represented by lines, which should express their actual affinity to one another; and the system of diverging lines which would thus be obtained might be compared to an irregular system of branching.

It has frequently been attempted to draw up genealogical trees for the whole Vegetable Kingdom and for the various groups of it, but the attempts made hitherto are by no means satisfactory, for the incompleteness of our knowledge as to the real relationships leaves too much room for the play of the imagination and of subjective impressions. I will merely suggest here, as I did repeatedly in Book II, that in endeavouring to form such a genealogical tree, the simplest forms of the various types or classes must be especially considered, for it is in them that the evidences of descent from common ancestral forms will most easily be detected. With each of these simple forms, which do not differ widely from each other, is connected a branching developmental series in which variation is taking place independently of other series ; in consequence of this variation the difference between the members of one series and those of another is increasing, so that the most perfect forms of different types are those which are the most widely separated.

The theory of descent requires that the various forms of plants must have arisen at different times, that the primitive forms of the separate classes and groups existed at an earlier period than the derived ones; and palæontological research, although at present it has but a very small amount of material at its disposal, supports this view.

In the same manner it is a necessary consequence of the theory that each plantform must have originated at a definite spot, that it must have spread gradually more widely from that spot, that its change of locality in the course of generations must have depended on climatic conditions, the competition of rivals, \&c., and that its distribution must have been impeded by hindrances or assisted by means of transport ${ }^{1}$. The geographical distribution of plants has already determined in the case of many forms the spots on the surface of the earth or centres of distribution from which they have gradually spread; it has shown how the distribution has been hindered sometimes by climate, sometimes by chains of mountains, sometimes by seas; how more recently formed islands have been peopled by the plants from the neighbouring continents which have become the ancestors of new species ${ }^{2}$; how some species when transported to a new soil (as European plants in America and vice versa ) have sometimes carried on a successful struggle for existence with the native plants and have increased enormously. In the distribution of plants at present existing, as for instance Alpine plants, it is possible to recognise the influences of the last great geological changes, of the entrance and disappearance of the glacial epoch and of earlier periods.

When we reflect what a number of generations our cultivated plants must have passed through before any considerable amount of new properties were manifested in

1 Kerner has given an illustration of what can be accomplished in this direction in the relationships, geographical distribution, and history of the species of Cytisus from the primitive form Tubocytisus, in his pamphlet Die Abhängigkeit der Pflanzengestalt von Klima und Boden, Innsbruck 1869 .

${ }^{2}$ See Dr. Hooker, On Insular Floras, Gardener's Chronicle, Jan. 1867; Ann. des sci. nat. $5^{\text {th }}$ series, vol. IV. p. 266. [Wallace, Island Life, I880.] 
their varieties, and how long it takes for these new properties to become hereditary, and further how enormous is the diversity of hereditary properties, we are forced to the conclusion that an inconceivably long period must have elapsed since the appearance of the first plants on the earth. But geology and the physical nature of the globe require as great a space of time for the explanation of other facts; and a few millions of years more or less is a matter of but little consequence in the explanation of facts which require lapse of time in order to reach a given magnitude.

The first rudiments of the Theory of Descent, which holds good for the animal as for the vegetable kingdom, may be traced to Lamarck, at the commencement of the century, in his Zoologie Philosophique (Paris, I80I); it was afterwards advocated by Geoffroy St. Hilaire; but it is only since the publication of Darwin's work 'On the Origin of Species by means of Natural Selection' (London, 1859) that it has become an integral part of science. Darwin's great service to science is to have established as a fact the struggle for existence which all living beings have to fight, and to have proved its action in the maintenance or destruction of new forms. It is only by means of the struggle for existence that the motive principle is recognised, and that the theory of descent is enabled to solve the great problem why parts which are morphologically similar are adapted for such different functions; and conversely also to show how purpose in organisation, and at the same time the relations of affinity among plants, can be explained. Darwin considers the Natural Selection which the struggle for existence brings about as the sole cause of the increasing differentiation of plants which are undergoing variation; he starts with the hypothesis that every plant varies in all directions without any definite tendency to become further developed in any one particular direction. He attributes to the struggle for existence alone the power of securing the perpetuation of one or more varieties among the countless numbers which are produced, and is convinced that in this way not only is a perfect adaptation of the new forms effected, but morphological differentiation is also carried further. Nägeli ${ }^{1}$ assumes, on the contrary, that each plant has in itself a tendency to vary in a definite direction, to increase the morphological differentiation, or, as it is commonly expressed, to perfect itself. The great differences of a purely morphological nature between the classes and smaller divisions of the vegetable kingdom may then owe their existence to this internal tendency towards a higher and more varied differentiation; while the struggle for existence brings about the adaptation of the separate forms. The great services of the theory of descent remain in either case.

In all future research it will be of primary importance to distinguish clearly between those peculiarities of plants which have no referen $?$ to the external world, which are, that is, purely morphological, and those without which it would be impossible for the plant to continue to exist under certain external conditions. It appears to be certain that the latter are only produced by adaptation in the struggle for existence ${ }^{2}$.

The first and simplest plants had no ancestors; they arose by spontaneous generation. Whether this took place only once; whether only one or a number of primitive plants were produced simultaneously, giving origin in the latter case to different developmental series, or whether, as Nägeli supposes, spontaneous generation has taken place at all times, and is now taking place, giving rise to new developmental series, are questions which still await solution, and which we cannot follow out further here.

1 Nägeli, Entstehung und Begriff der naturhistorischen Art, Munich 1865 .

${ }^{2}$ Pringsheim has recently drawn attention to certain phenomena in the Sphacelariæ which show that a continued development with increasing morphological differentiation may take place independently of the struggle for existence (Abh. Berl. Acad., 1873). Cultivated plants show, on the other hand, that a considerable accumulation of new physiological properties may take place unaccompanied by important morphological changes. 


\section{APPENDIX.}

\section{BOOK I.}

Page 8. Cell-formation by Rejuvenescence. In most cases the cell produced contains only one nucleus, but several may be present, as in the zoogonidium of Vaucheria and in the oosphere of those Saprolegnieæ in which there is only one oosphere in the oogonium.

The whole of the protoplasm of the cell is not necessarily involved in this process, as is shown by the development of the antherozoid in many cases.

Page 8. In the process of conjugation in Spirogyra the nuclei of the conjugating cells were observed by Schmitz (Sitzber. d. niederrhein. Ges. in Bonn, 1879) to coalesce.

Page 10. From the researches of Schmitz on the Myxomycetes (Sitzber. d. niederrhein. Ges. in Bonn, I879), it appears that the nuclei of the cells which coalesce to form the plasmodium do not fuse but remain distinct: this case of coalescence of cells cannot, therefore, be any longer regarded as an instance of cell-formation by conjugation.

Free cell-formation. From the account of this process given in the text, it is evident that the expression 'free cell-formation' is now used in a sense different from that which it originally possessed. In its original sense it implied the development de novo of fresh nuclei around which the protoplasm became aggregated so as to form cells: it is now applied to those cases in which many nuclear divisions take place before any corresponding cell-divisions occur. Taken in this sense, free cell-formation differs only in degree from cell-division, and it is not possible to distinguish sharply between them: for example, the development of the pollen-grains of Dicotyledons is usually regarded as coming under the head of cell-division, but it may equally well be considered to be a case of very limited free-cell formation in which only four nuclei are produced by division before any cell-division takes place.

It must not be assumed that there is no such thing as a formation of nuclei de novo. Strasburger (Bau u. Wachsth. d. Zellhäute) has pointed out that the appearance of the male pronucleus in the oosphere during fertilisation (see p. ${ }^{8}{ }_{4}$ ) is an instance of it, inasmuch as the nuclei of the pollen-grain do not pass directly into the oosphere, but break up and become diffused either in the pollen-grain or in the pollen-tube. Johow has observed the breaking-up and the reconstitution of nuclei in Chara (Bot. Zeitg. 1881).

Page 12. For minute details as to the development of spores and of pollen-grains, see Strasburger, Bau und Wachsthum der Zellhäute, I882.

Page 15. Cell-formation by Budding and Abstriction. Further instances of this are afforded in the formation of the spores of Pellia epipbylla and some other Liverworts. The mother-cell of the spores grows out into four protuberances, each of which becomes shut off by a septum in the narrow pedicle and forms a spore (Strasburger, Zelltheilung und Zellbildung, I 880).

Page 16. Vegetative Cell-formation. In the case of naked (primordial) cells division takes place by the gradual constriction of the protoplasm: this has been observed by Schmitz (Mittheil. aus der zool. Stat. zu Neapel, I, I 878) in the formation of zoogonidia of Halosphara viridis; by de Bary in the amœboid zoogonidia of Myxomycetes; by Pringsheim in the oospore of $E$ dogonium; and by Kirchner in the oospore of Volvox minor.

Page 17. The Behaviour of the Nucleus during Division. The accounts of the structure of the nucleus and of its behaviour during division given by Flemming and by Strasburger do not agree in all points. The following are the principal differences between them: (I) Flemming holds that the chromatin only exists in the form of fibrillæ; (2) he does not agree with Strasburger that, in the splitting of the equatorial plate, any division of 
the chromatin fibrillæ takes place, but he considers that two groups of fibrillæ are formed which travel to the poles of the spindle; (3) he is of opinion that the equatorial plate consists of chromatin and the rest of the spindle of achromatin, whereas Strasburger states that the equatorial plate consists of the whole of the nuclear substance (both chromatin and achromatin) and that the spindle consists of cell-protoplasm; (4) Flemming does not admit that the fibrillæ, derived from the equatorial plate, which travel to each pole, undergo fusion to form a new nucleus. (See Quart. Journ. Micros. Sci. 1882.)

The illustrative cases of cell-division which have been given in the text refer only to cells which contain a single nucleus. In cells which are or are about to become multinuclear,-in cases, that is, in which nuclear division is not followed by cell division,the process of nuclear division is usually simpler. In the older internodal cells of the Characeæ, in older parenchymatous cells of Lycopodium and of some Phanerogams (Taraxacum, Glyceria, Sempervivum, Cereus, Solanum, etc.), and occasionally in Valonia (in all of which cases the cells become multinuclear), the nucleus simply divides by constriction, the chromatin granules being shared equally between the two new nuclei without any indications of karyokinesis. Division may begin again in the two new nuclei even before they are separated from each other. This process of nuclear division has been termed fragmentation. In Valonia and in Codium a rudimentary form of karyokinesis has been observed by Schmitz and by Berthold (Mittheil. der zool. Stat. zu Neapel, II, 1880), which appears to occur commonly among Thallophytes. In such a case the nucleus becomes elongated; its ends enlarge, whereas the middle part remains narrow; the ground-substance of the nucleus now presents a faint longitudinal striation, and the chromatin-granules either simply travel to the two ends of the nucleus, or become rodshaped and aggregate in the equatorial plane to form a rudimentary nuclear disc, which splits in the ordinary way, each half travelling to one end of the nucleus. The narrow middle portion now undergoes absorption, and the two ends round themselves off to constitute two new nuclei. Treub (Sur des cellules végétales à plusieurs noyaux, Arch. Neerland., XV, 1880) has observed the division of the nucleus in the multinuclear bastfibres and laticiferous cells of various Phanerogams, and finds that it takes place in the manner described in the text for uninuclear cells, except that no cell-plate is formed. See also Johow, Die Zellkerne von Chara, Bot. Zeitg. 188r.

An illustration of the independence of cell-division with regard to nuclear division is afforded by Cladophora. The cells of this plant are multinuclear, and Strasburger has found that the division of the nuclei takes place in the manner observed by Treub in bast-fibres and laticiferous cells: the division of the cells bears no relation, either in time or space, to that of the nuclei; the new cell-wall is formed in much the same manner as in Spirogyra.

Page 19. Schmitz has come to the conclusion (loc. cit. 1880) that the cell-wall is formed by the actual conversion of a peripheral layer of the protoplasm into cellulose. $\mathrm{He}$ is also of opinion that stratified cell-walls are formed by the deposition, one within the other, of successive layers, and not by intussusceptive growth with subsequent differentiation. These views are also held by Strasburger; Bau und Wachsthum der Zellhäute, 1882 (see p. 960).

Both Schmitz and Strasburger hold that the surface-growth of cell-walls is not due to the intercalation of new solid particles (intussusception), but is simply the expression of the stretching of the cell-wall by the cell-contents.

Frommann (Protoplasma, 1880) believes that he has been able to trace a connexion of the protoplasm through the walls of adjacent cells. That protoplasm can pass through closed cell-walls is beyond doubt. See Strasburger, loc. cit. p. 247.

Page 23. The formation of bordered pits. A very different account from that in the text is given by Mikosch (Unters. üb. Entstehung und Bau der Hoftüpfel, Sitzber. d. k. k. Akad. in Wien, LXXXIV, I88r). Sachs has found (Ueb. die Porosität des Holzes, Arb. d. bot. Inst. in Würzburg, II, 1879) that in the spring-wood at least the bordered pits are closed by a membrane. See also Strasburger, Bau und Wachsth. d. Zellhäute, i 882. 
Page 29. With regard to the growth of the cell-wall by intussusception, see note above referring to page $\mathbf{~} 9$.

Page 30. With regard to the growth in thickness of the cell-wall, see the note referring to page 19 .

Page 36, r6th line from the top: for 'molecules' read 'micellæ.'

Page 37. Protoplasm. According to Schmitz (Unters. ueb. die Struktur des Protoplasmas und der Zellkerne, Sitzber. d. niederrhein. Ges. in Bonn, I880), a net-work of fine fibres exists in protoplasm, the meshes of which are occupied by a homogeneous fluid : the net-work readily stains with hæmatoxylin, but the fluid remains colourless. In the endoplasm, more particularly, a greater or smaller number of fine granules, termed by Hanstein microsomata, which stain deeply with hæmatoxylin, are present. (See also Frommann, Beob. üb. Structur und Bewegungserscheinungen des Protoplasma, Jena, 1880. -Hanstein, Das Protoplasma, Heidelberg, i 880 : id., Einige Züge aus der Biologie des Protoplasmas, Botanische Abhandlungen, IV. 2, r880.-Strasburger, Zellhäute, 1882.-On the Chemistry of Protoplasm, see Reinke, in Unters. aus dem Bot. Lab. der Univers. Göttingen, II, I88 r.)

Page 44. The Nucleus. Schmitz has detected one or more nuclei in a number of Thallophytes (Batrachospermum moniliforme, Codium, Vaucheria, Gaulerpa, Conferva, Gongrosira, Scbizogonium, Monostroma, Chlamydomonas, Chroolepus, Saprolegnia, Mucor, Saccharomyces, Mycoderma, Oidium, Exoascus, Peziza, Morchella, Ascobolus, Myxomycetes, etc. ; (Sitzber. d. niederrhein. Ges. in Bonn, 1879 and 1880 ) : he believes that the Phycochromaceæ and the Schizomycetes are the only plants in which no nucleus is present.

With regard to the function of the nucleus, it does not appear that it regulates the process of cell-division. Strasburger is of opinion (Bau und Wachsthum der Zellhäute, 1882, p. $24 \mathrm{r}$ ) that it is of importance in the nutrition of the cell, more especially as regards the formation of proteids.

With regard to the structure of the nucleus, see page 18 .

Chemically the nucleus consists of a substance termed nuclein, to which Miescher has given the formula $\mathrm{C}_{29} \mathrm{H}_{49} \mathrm{~N}_{9} \mathrm{P}_{3} \mathrm{O}_{22}$. (See Hoppe-Seyler, Physiologische Chemie; also Zacharias, Die chemische Beschaffenheit des Zellkerns, Bot. Zeitg. I 88r.)

Page 48. On the development of chlorophyll-granules, see Mikosch, Unters. üb. die Entstehung der Chlorophyllkörner, Sitzber. d. k. k. Akad. in Wien, LXXVIII, 1878.

Page 49. Structure of chlorophyll-granules. Pringsheim has observed (Ueb. Lichtwirkung und Chlorophyllfunction in der Pflanze, Jahrb. f. wiss. Bot., XII, I88I) that when green parts of plants are treated with dilute acids or with warm water the colouringmatter exudes in viscid drops, the colourless basis remains behind and presents a trabecular structure in the interstices of which the colouring-matter was previously contained.

Page 49-51. Crystalloids and Aleurone-grains. On Rhodospermin, see note on page 289.-Schimper, Unters. üb. die Proteinkrystalloide der Pflanzen, Strassburg, r879: id., Ueb. die Krystallisation der eiweissartigen Substanzen, Zeitschr. für Krystallographie, 1880.- Vines, On the Proteid Substances contained in the Seeds of Plants, Journal of Physiology, III, 1881.-Julius Klein, Die Krystalloide der Meeresalgen, and Die ZellkernKrystalloide von Pinguicula und Utricularia, Jahrb. f. wiss. Bot. XIII, I881.

An aleurone-grain consists of a mixture of proteids, some of which are soluble in water, and others in either saturated or dilute solution of common salt; the former belong to the chemical group of peptones, the latter to the globulins. In some cases the grain does not dissolve in salt solution; this is probably due to a chemical alteration of the globulins into albuminates. The crystalloids consist chemically of globulins or of altered globulins (albuminates).

Page 57 et seq. In the account of the growth of starch-grains the word ' micella' is to be substituted for 'molecule' (see Bk. III): it is also preferable to use the word ' hilum' instead of ' nucleus.'

With reference to the mode of growth of starch-grains, it appears from Schimper's observations (Unters. üb. das Wachsthum der Stärkekörner, Bot. Zeitg. I88I) that they 
grow by apposition and not by intussusception. He is led to the conclusion that a starchgrain is a sphero-crystal, built up of prismatic crystalloids. (By a 'crystalloid' is meant here and elsewhere a crystal which is capable of swelling-up.) This conclusion is supported by Meyer (ibid.), and by Strasburger (Bau und Wachsthum der Zellhäute, 1882), but is severely criticised by Nägeli (Unters. üb. das Wachsthum der Stärkekörner, Bot. Zeitg. I 88I).

It is known that when starch-grains are formed in parts of plants exposed to light, they arise in connexion with the chlorophyll-granules. Schimper has made the interesting observation (Bot. Zeitg. 1880; also Researches upon the Development of Starch-grains, Quart. Journ. Micr. Sci., I88I) that when they are formed in parts of plants not exposed to light they arise in connexion with small masses of protoplasm which he terms 'starchforming corpuscles' (Siärkebildner). That they are closely related to the chlorophyllgranules is shown by the fact that if cells containing starch-forming corpuscles are exposed to light, the corpuscles turn green and become in fact chlorophyll-granules. Errera suggests the name 'amidoplasts' for these bodies.

Schimper observed that the point of attachment of the starch-grain to the chlorophyllgranule or starch-forming corpuscle lies in the line of the long axis of the grain,- - that is, in the line of most rapid growth,-and at its broader end; the hilum is near the free narrow end of the grain. These facts afford a strong argument in favour of the growth of the grain by apposition.

Page 64, line 9 from the top. For 'erecta' read 'evecta.'

Page 65. On the distribution of calcium carbonate, see Molisch, Ueber die Ablagerung von kohlensaurem Kalk im Stamme dicotyler Holzgewächse, Sitzber. d. k. k. Akad. in Wien, LXXXIV, 1881 .

Page 68. Cystoliths. See also Richter, Beitr. zur genauern Kenntniss der Cystolithen, etc., Sitzber. d. k. k. Akad. in Wien, LXXVI, 1877, and Melnikoff, Unters. üb. das Vorkommen des Kohlensauren Kalkes in Pflanzen, Diss. Inaug., Bonn, 1877.

Page 70. The statement made here with reference to the formation of the endosperm in the embryo-sac of Phanerogams is not quite accurate: compare p. 585 .

For a more complete account of the Morphology of the Tissues, see de Bary, Vergleichende Anatomie der Vegetationsorgane der Phanerogamen und der Farne, I877.

Page 77. Leitgeb has found (Die Athemöffnungen der Marchantiaceen (Sitzber. d.k. k. Akad. in Wien, LXXXI, I880) that the hypodermal chambers of these plants is not formed, as described in the text, by the separation of the epidermal cells from the subjacent tissue: these chambers make their first appearance as pits which become overgrown by the epidermal cells which form their limits, and the communication between the cavity of the pit and the external air may be continuous from the beginning, or the pits may become completely closed in by the overgrowth of the surrounding epidermal cells, the communication being restored on the development of the stoma. In both cases the cells forming the stoma are not derived from a single mother cell; in the former case the stomatal cells are formed by the cutting off of contiguous segments from the cells bounding the opening; in the latter, by the cutting off of segments in a similar manner from the cells lying over the centre of the chamber, and by the subsequent separation of these segments so as to form an opening between them. The stomatal cells may then divide so as to form a series of superposed cells, and thus the complex stoma of Marchantia, for example, is produced.

Page 86. On the development of laticiferous vessels, see Schmalhausen, Beitr. z. Kenntniss der Milchsaftbehälter der Pflanzen, Mém. de l'Acad. imp. de St. Pétersbourg, XXIV, I877; also Scott, The Development of Articulated Laticiferous Vessels, Quart. Journ. Micr. Sci., 1882, and Schmidt. Bot. Zeitg., I 882.

Page 88, line 2 from the bottom. The statement in the text that sieve-tubes occur only in the fibro-vascular bundles is not correct. Scattered bundles of them occur in the stems of many Dicotyledons and Monocotyledons; in the periphery of the pith in Solanum tuberosum, Dulcamara, species of Nicotiana, Datura, and Cestrum, in many Campanulacex, and among Composites in Gundelia Tournefortii, and in the genera Lactuca, Scorzonera, 
Sonchus, Tragopogon, and Hieracium; in the cortex of thick-stemmed Cucurbitacex, such as Cucurbita, Lagenaria, Cucumis, Ecbalium, and in many species of Potamogeton (P. natans, lucens, pectinatus).

In Strycbnos no sieve-tubes are formed in the secondary phloëm, but they occur in the xylem (de Bary, Vergleichende Anatomie, p. 594).

Page 90. For a full account of the structure of sieve-tubes, and of their transverse connexion, see Wilhelm, Beitr. z. Kenntniss des Siebröhrenapparates Dicotyler Pflanzen, Leipzig, I880: also, Janczewski, Études comparées sur les tubes cribreux, Cherbourg, I88 $\mathrm{I}$.

Page 93. On the development of resin-ducts, see Kreuz, Beitr. zür Entwickelungsgeschichte der Harzgänge einiger Coniferen, Sitzber. der k. k. Akad. in Wien, LXXVI, 1877 .

Page 98. Stöhr (Ueb. Vorkommen von Chlorophyll in der Epidermis der Phanerogamen-Laubblätter, Sitzber. d. k. k. Akad. in Wien, LXXIX, I879) has found chlorophyllgranules in the epidermal cells of the leaves of a large number of plants.

Page 102. Mention should be made, under the head of Stomata, of the waterstomata or water-pores which occur on the leaves of those plants (such as Alchemilla, Zea, many Aroids, Saxifragaceæ, and Crassulacex) which excrete drops of water. In some of these an ordinary stoma serves as a water-stoma ; in others the water-stoma is larger than the ordinary stoma, and its guard-cells are incapable of opening and closing the aperture. In the Saxifragaceæ and Crassulaceæ the mesophyll-cells beneath the water-stoma are differentiated so as to form a more or less well-defined mass of tissue, the water-gland, which appears to effect the excretion of saline substances (principally calcic carbonate) in solution in the excreted water. In each such gland a fibro-vascular bundle terminates. See Gardiner, Quart. Journ. Micr. Sci., I88I ; also de Bary, Vergleichende Anatomie, pp. 55, I I 3,389 .

Page 105. On the development of the stomata of Marcbantia, see the note above which refers to p. 77 .

Page 106. On Cork, see von Höhnel, Ueber den Kork und verkorkte Gewebe überhaupt, Sitzber. d. k. k. Akad. in Wien, LXXVI, 1877. When cork is developed in roots it is formed by the division of the cells of the pericambium, the primary cortex being gradually thrown off.

Page 108. On Lenticels, see Haberlandt, Beiträge zur Kenntniss der Lenticellen, Sitzber. d. k. k. Akad. in Wien, LXXII, 1875, and Kreuz, ibid., Entwickelung der Lenticellen an beschatteten $Z_{\text {weigen von }}$ Ampelopsis bederacea.

Page 112. In addition to collateral and concentric bundles the following may also be distinguished:-

I. Bicollateral bundles, in which (as mentioned on page $\mathrm{I} \mathrm{I}$ ) there is a layer of phloëm on the inner as well as on the outer side of the xylem; Cucurbitacex; Melastomaceæ, Cichoriaceæ, Solanaceæ, Asclepiadaceæ, Apocyneæ, Strychnos, Dapbne, Eucalyptus Globulus, and probably also Metrosideros, Callistemon, Melaleuca, Myrtus, and the other species of Eucalyptus.

2. Radial Bundles, in which the xylem and the phloëm strands lie on different radii. This arrangement obtains almost universally in roots, exceptions being found in those of Dioscorea Batatas, of Ophrydex, and perhaps in those of Sedum Telepbium and its allies, in which the bundles are coilateral. The bundles in the stems of Lycopodiacex are rather to be regarded as radial than as concentric as is done in the text. (De Bary, Vergl. Anat.)

Page 114. The Fibro-vascular System of Roots. On the transition from the fibrovascular system of the stem to that of the root, see Sophie Goldsmith, Beitr. z. Entwickelungsgeschichte der Fibrovasalmassen im Stengel und in der Hauptwurzel der Dicotyledonen, Diss. Inaug. Zürich, 1876; Gérard, Passage de la Racine à la Tige, Ann. Sci. Nat. ser. 6, t. XI, I $88 \mathrm{I}$.

Page 116. The 'vessels' (trachex) in most plants are really tracheïdes: this is the 
case with the peripheral ends of fibro-vascular bundles in all plants; with the vascular elements of the secondary xylem of Conifers and Cycads, and with most of those of the secondary xylem of woody Dicotyledons and of many Monocotyledons; with the vascular elements of Ferns and their allies (true vessels are known only in Pteris aquilina and in the root of Atbyrium Filix fomina). See de Bary, Vergleichende Anatomie, p. 172.

The definition given in the text is not satisfactory: a tracheide is a closed vascular cell ; true vessels are formed by the fusion-that is, by the absorption of the intervening septa—of tracheïdes.

Page 118. De Bary (Vergleichende Anatomie) distinguishes the following kinds of prosenchymatous xylem-elements in Dicotyledons (see also p. $6_{5} \mathrm{I}$ ):-

(I) True Woody Fibres: differing from tracheides in the absence of the internal spirally-fibrous layer of their wall, and in that the pits are narrow, elongated transversely, and oblique; they contain a small residue of protoplasm.

(2) Fibres (secondary wood): septate or unseptate, of two kinds,

a. resembling the true woody fibres, but differing from them in that they almost always contain starch, and sometimes chlorophyll or tannin;

b. shorter fibres, tending to parenchymatous form (hence termed ersatzfasern or 'intermediate cells').

Page 130, line 13 from the top. Dele the word 'cortex.'

" line 16 from the top. For 'phloëm $(R)$ ' read ' cortex $(R)$.'

Page 131, line 20 from the top. For 'secondary cortex' read 'secondary phloëm.'

Page 132, line 25 from the top. Dele the word 'phloëm.'

Page 135. The cambium which is formed outside the primary xylem-bundles arises in the pericambium.

Page 136, section (c). The following is to be substituted for the last two paragraphs of this section:-

I. The successive rings of bundles originate in the primary cortex: Menispermex, Cycadeæ, Avicennieæ.

2. They originate in the primary phloëm: Pbytolacca.

3. They originate in the secondary phloëm: Wistaria, Baubinia, Rhynchosia (Leguminosæ); Securidaca (Polygalaceæ); Gnetum; Doliocarpus (Dilleniaceæ); Pbytocrene (Olacineæ). (See de Bary, Vergleichende Anatomie, 1877.)

Section (d). The account of the mode of growth of the stems of Bignoniacex given by de Bary (loc. cit.) differs somewhat from that in the text. According to him four symmetrically placed fibro-vascular bundles of the ring of primary bundles in the stem are from the first larger than the others, all the bundles being connected by a normal cambium-ring. When growth in thickness begins it proceeds normally at all points except in the four above-mentioned bundles; in these the formation of xylem eiements is very much smaller than in the adjoining bundles, whereas the formation of bast is much more considerable. The cambium layer consequently loses its originally circular outline and becomes deeply infolded where it extends inwards to each of these four bundles. Where it lies radially with respect to the primary bundles, that is, where it is parallel to the circumference, the cambium-layer gives rise to xylem and phloëm elements in the normal manner: where the infoldings take place, that is, where it lies upon the sides of the bundles in which the wood has been normally developed, it forms only parenchyma, so that considerable masses of cortical tissue are formed between the normally developed bundles, corresponding in position to the four abnormally developed bundles.

Page 137, line 21 from the bottom. None of the scattered bundles in the pith of Piperacex, Amarantaceæ (Amarantus, Euxolus), or Nyctagineæ are cauline: they are leaftraces (de Bary, Vergl. Anat.). The structure of these abnormal forms will be rendered more intelligible by the following considerations. The primary bundles in the dicotyledonous stem are in some cases all leaf-traces (e. g. Ricinus, Cucurbita), but more commonly other primary bundles which are cauline are formed between the primary leaf-traces. 
When all the bundles lie in a ring, the characteristic structure of a young dicotyledonous stem is seen in a transverse section; when they do not lie in a ring, the appearance presented by a section is that of an abnormal stem. In the instances mentioned above the latter is the case; but a more marked instance of this is afforded by Podophyllum, for example, in which, owing to the irregular distribution of the primary bundles (which are here all leaf-traces), a transverse section of the stem somewhat resembles that of a monocotyledonous stem.

The account given in the text of the formation of the cambium-ring in Cbavica (Piperacex) will be correct if for 'cauline bundles' 'inner leaf-trace bundles' be substituted: that given of the Begoniacex, in which the internal bundles are cauline, is correct.

Section 19. For an account of the laws according to which cell-divisions take place in growing organs, see Sachs' important paper Ueb. die Anordnung der Zellen in jüngsten Pflanzentheilen, Arb. d. bot. Inst. in Würzburg, II. I, I878. The following are the more important points to which he draws attention:-

r. The walls are formed at right angles to those which they intersect.

2. The planes of the walls in a growing-point are classified thus :

a. Periclinal, those which are curved in the same direction as the surface (seen in longitudinal section).

b. Anticlinal, those which intersect the surface and the periclinal walls at right angles; they thus constitute a system of orthogonal trajectories for the periclinal walls.

c. Radial, those which pass through the axis of growth and intersect the surface at right angles.

d. Transverse, those which intersect both the axis of growth and the surface at right angles.

The relation of the periclinal and anticlinal planes are illustrated by the following cases:-

a. If the outline (in longitudinal section) of the growing-point is a parabola, the periclinals will constitute a system of confocal parabolas of different parameter, the focus of the system being at the point of intersection of two lines of which one is the direction of the axis and the other of the parameter. In this case the anticlinals being the orthogonal trajectories of the periclinals, constitute a system of confocal parabolas the axis and focus of which coincide with those of the periclinals.

b. If the outline of the growing-point is a hyperbola, the periclinals will be confocal hyperbolas with the same axis but different parameter; the anticlinals will be confocal ellipses, with the same focus and axis as the periclinals.

c. If the outline of the growing-point is an ellipse, the periclinals will be confocal ellipses; the anticlinals will be confocal hyperbolas.

It must not be supposed that the outline of a growing-point is necessarily one or other of these well-known geometrical forms; they are selected merely because they serve to illustrate clearly the rectangular intersection of the cell-walls as they are formed, and because, when the nature of the periclinal and anticlinal curves is unknown, their relations may be inferred by analogy.

An interesting deviation is found in those roots (such as those of Papilionaceæ) in which the apex of the plerome are open, i. e. are directly continuous with the tissue of the root-cap; this is to be ascribed to the fact that the periclinals at the end of the root become parallel or even tend to diverge upwards.

3. The arrangement of the cell-walls in these planes is most perfectly seen in the growing-points of plants which do not possess a single apical cell but in which there is a small-celled meristem. A large apical cell interrupts the 
continuity of the planes which are evident in the divisions which take place in the segments cut off from the apical cell.

4. It is probable, contrary to the generally received view, that, in confocal growing-points, the apical cell represents the most slowly growing portion, whereas in non-confocal meristem-protuberances the most active growth may take place at the apex. Westermaier however has come to the conclusion (Ueb. die Wachsthumsintensität der Scheitelzelle, Jahrb. f. wiss. Bot., XII, r88I) that the maximum of increase in volume within the apical region is exhibited either by the apical cell itself or by the youngest segments.

Page 140. On the apical growth of Metzgeria furcata, see Goebel, Ueb. das Wachsthum von Metzgeria furcata und Aneura, in Arb. d. bot. des Bot. Inst. in Würzburg, II. 2, 1879 .

When the segments are cut off from the apical cell by oblique walls these are parts of anticlinals which are completed by the secondary divisions in the adjacent segments (see fig. $15 \circ A)$.

Page 146. On growing-points of stems without an apical cell, see Schmitz, Beob. ueb. die Entwickelung der Sprossspitze der Phanerogamen, Halle, 1874.

Page 147, line 3 from the bottom. It is only in certain cases that the root-cap of Phanerogams is derived from the dermatogen.

On the structure of the growing-points of roots, see Janczewski, Recherches sur le développement des racines dans les Phanérogames, Ann. d. Sci. Nat., sér. 5. t. XX, I874: - Treub, Le Méristème primitif de la Racine dans les Monocotylédones, Leyden, 1876:Eriksson, Ueb. das Urmeristem der Dikotylen-Wurzeln, Jahrb. f. wiss. Bot. XI, i 878 :Flahault, Recherches sur l'Accroissement terminal de la Racine chez les Phanérogames, Ann. d. Sci. Nat., sér. VI. t. 6, I878:-Olivier, Rech. sur l'appareil tegumentaire des Racines, Ann. d. Sci. Nat., sér. 6, t. XI, I88r. A good account is also given in de Bary's Vergleichende Anatomie.

From Janczewski's researches, of which the following is a brief abstract, it appears that in many cases there is a distinct meristematic layer, which he terms the calyptrogen, from which the root-cap is derived :-

Type I. The meristem consists of four distinct layers, Plerome, Periblem, Dermatogen, and Calyptrogen : Hydrocharis Morsus Ranc, Pistia Stratiotes.

Type 2. A distinct Plerome and Calyptrogen; the Periblem and the Dermatogen have common initial-cells : many Monocotyledons (Juncaceæ, Hæmodoraceæ, Cannaceæ, Zingiberaceæ, Typha, Cyperaceæ, Gramineæ, Commelineæ, Potameæ, Juncagineæ, Sagittaria, Limnocbaris, Stratiotes).

Type 3. A distinct Plerome; the Calyptrogen, Periblem, and the Dermatogen have common initial cells (Treub): many Monocotyledons (Liliaceæ, Astelieæ, Xerotideæ, Aspidistreæ, Ophicpogoneæ, Amaryllideæ, Hypoxide , Dioscoreæ, Taccaceæ, Bromeliaceæ, Musaceæ, Orchideæ, Palmæ, Pandaneæ, Cyclantheæ, Aroideæ (except Pistia), Irideæ, Pontederieæ, Sparganium, Butomus, Alisma (?).

Type 4. A distinct Plerome and Periblem; the Dermatogen and the Calyptrogen have common initial cells: most Dicotyledons (Heliantbus annuus, Fagopyrum, Raphanus sativus, Myriopbyllum, Salix, Casuarina stricta, Linum usitatissimum, Primulaceæ).

Type 5. A group of initial cells common to all four layers: some Dicotyledons (Cucurbita, Pisum, Pbaseolus, Cicer).

Type 6. A distinct Plerome and Periblem only; hence there is no true epidermis or root-cap, these being formed simply by the outer layers of the periblem (cortex): Gymnosperms.

Cryptogams. According to the observations of Strasburger (Coniferen und Gnetaceen) and of Bruchmann (Ueb. Wurzeln von Lycopodium und Isoëtes, 
Jena, 1874) the structure of the growing-point of the root of Lycopodium is the same as that described above under Type $\mathbf{r}$.

According to Bruchmann the structure of the growing-point of the root of Isoëtes agrees with that described under Type 4 .

At the growing-point of the root in the Marattiacex there is a group of several large polygonal cells. From these segments are cut off parallel to the base to form the root-cap, and internally segments are cut off to increase the Plerome. The plerome-segments are further divided by longitudinal walls, and the more external of the cells thus formed constitute the cortex, which is differentiated at a lower level into Dermatogen and Periblem. These roots are thus intermediate in their structure between those which have a single apical cell and those which have a small-celled meristem. In the case of stems, those of certain Selaginella (arborescens, Pervillei, Wallicbii, Lyalli) occupy an analogous position.

Page 149. For a discussion of the physiological causes of the morphological differentiation of Plants, see Sachs, Stoff und Form der Pflanzenorgane, Arb. d. bot. Inst. in Würzburg, II. 3, I880, and 4, I882: see also Vöchting, Ueb. Organbildung im Pflanzenreich, 1878, and F. Darwin, The Theory of the Growth of Cuttings, Journ. Linn. Soc., XVIII, $\mathbf{r} 88 \mathrm{r}$.

Page 153. On the Anatomy and Morphology of the Leaf, see J. Chatin, De la Feuille, Paris, I874; and Goebel, Beitr. zur Morphologie und Physiologie des Blattes, Bot. Zeitg. I 880 and 1882 .

Page 163, fig. 122. The root represented here affords a good illustration of the structure of the apex described above under Type 2 ; it is obvious that the original interpretation was the right one, namely, that the root-cap is not developed from the dermatogen.

Page 166. On the development of the lateral roots, see Janczewski, Recherches sur le Développement des Radicelles dans les Phanérogames, Ann. d. Sci. Nat., sér. 5, XX, 1874 .

In the Equisetacex the plerome- (bundle-) sheath consists of two layers (Van Tieghem), and it is from cells of the inner layer that the lateral roots are developed.

Line 5 from the bottom. For 'vascular' read 'xylem.'

Page 168, paragraph (e). For another case of the conversion of a root into a stem, see Goebel, Ueb. Wurzelsprosse von Anthurium longifolium, Bot. Zeitg. 1878.

Page 170. Welwitschia prodúces two leaves in addition to the cotyledons (see Bower, Q. J. M. S. I88I).

Page 171. It has been found (see p. 400) that the shoots of Equisetacex are not endogenous.

Page 172, paragraph (a). See Heinricher, Ueb. Adventivknospen an der Wedelspreite einiger Farne, and, Die jüngsten Stadien der Adventivknospen an der Wedelspreite von Asplenium bulbiferum, Sitzber. d. k. k. Wien. Akad. LXXVIII, 1878, and LXXXIV, $\mathrm{r} 88 \mathrm{r}$. He finds that in Asplenium the adventitious bud is developed from a single superficial cell.

Page 173, paragraphs (b) and (c). On the adventitious development of organs, see Hansen, Vergl. Unters. ueb. Adventivbildungen bei den Pflanzen, Frankfurt, I88 r.

Paragraph (e). It has been shown by Janczewski and by Famintzin that the lateral buds of Equisetaceæ are not of endogenous origin.

Page 184, paragraph (d). It is pointed out in Book II that the branching of the stem of the Lycopodiaceæ is not dichotomous in all cases.

Page 187. Phyllotaxis. On this subject see Schwendener, Mechanische Theorie der Blattstellungen, Leipzig, 1878. According to him the relative positions of lateral members depends upon ( $\mathrm{r}$ ) the relative size of the lateral members (when they are developed close together), and (2) the increase in length and in thickness of the axis bearing them. 
Page 204, paragraph (4). Sachs points out (Ueb. orthotrope und plagiotrope Pflanzentheile, Arb. d. bot. 'Inst. in Würzburg, II. 2, 1879) that most monosymmetrical or bilaterally symmetrical organs present not only two symmetrical (right and left) halves, but also dorsal and ventral halves which are of different internal structure; such organs he describes by the term dorsiventral. When this is the case the two halves react differently to external forces (light, gravity, etc.), and the organ is, according to his terminology, plagiotropic. Some bilateral organs are therefore plagiotropic, but this peculiarity is not confined to them, for some polysymmetrical organs are plagiotropic also (see Book III. p. 854 ; also Goebel, Ueb. die Verzweigung dorsiventraler Sprosse, Arb. d. bot. Inst. in Wüzburg, II. 3, 1880). The term 'actinomorphic' is often used as synonymous with 'polysymmetrical.'

Page 209. On the morphology of Begonia, see Eichler, Ueb. Wuchsverhältnisse der Begonien, Sitzber. d. Ges. naturfor. Freunde, Berlin, r 880.

Page 224, line 16 from bottom: dele 'germinal vesicle.'

For an account of sexual reproduction more in accordance with our present knowledge, see Book III. p. 896, et seq.

Page 226. Alternation of Generations in Thallophytes. It is extremely doubtful if any real alternation of generations can be detected in the life-history of Thallophytes, more particularly in the one (Penicillium) given as an example in the text. The sexual and the asexual modes of reproduction both occur, but their relation in time and space is not so definite as to warrant the comparison of the life-history of one of these plants with that of a Moss or a Fern. In some, such as the Coleochæteæ, sexual individuals occur periodically with a number of intervening asexual generations; the same might be said of some of the Æcidiomycetes (such as Ec. Berberidis) if only the existence of a sexual act were proved. These remarks are also applicable to the accounts of the life-histories of various plants given in the Introduction to the Thallophytes in Book II. (See Pringsheim, Jahrb. f. wiss. Bot. XI, 1878 ; and Vines, Journal of Botany, I879.)

\section{BOOK II.}

Page 246. Cyanophyceæ (or Phycochromacex, or Schizophyceæ). Goebel (Bot. Zeitg. 1880, p. 490 ) has observed the formation of zoogonidia in Merismopedia.

Janczewski, Observ. sur la Réprod. de quelques Nostocacées, Ann. Sci. Nat. sér. V, i 9.

The segments into which the filamentous forms of this group break up have been termed by Thuret Hormogonia.

Page 248. Rostafinski, Quelques mots sur l'Hæmatococcus lacustris, Mém. de la Soc. Nat. des Sc. Nat. de Cherbourg, 1875 ; Dyer, Sexual Reproduction of Thallophytes, Q.J. M. S. 1875.

For 'Clorophyll' read 'Chlorophyll.'

Schizomycetes. The formation of gonidia in these plants was first observed by Cohn in Bacillus (Beitr. z. Biol. d. Pfinzn. I). The filaments elongate very rapidly and become diffluent, and then rows of highly refractive gonidia make their appearance in them.

The following additional references will suffice as an introduction to the very extensive recent literature. Dallinger and Drysdale, On the Existence of Flagella (cilia) in Bacterium Termo, Monthly Microscop. Journ. XIX, 1875:-Warming, On new forms of Bacteria, Vidensk. Meddel., Copenhagen, I875:-Suringar, On Sarcina, Amsterdam, 1866 : - Papers by Cohn, Koch, and others, in Cohn's Beitr. z. Biol. d. Pflnzn. vol. 2, and vol. 3. I, 1877-79:-Geddes and Cossar Ewart, Proc. Roy. Soc. 1878 (Spirillum):-Reports in Q.J. M. S., vols. XVIII and XX, 1878-80):-Kuehn, Ein Beitrag zur Biologie der Bacterien, Dorpat, I 879 :-Nencki, Beiträge zur Biologie der Spaltpilze, Leipzig, 1880 :-Lister, 
On the Relation of Micro-organisms to Disease, Q. J. M. S. I88 I :-Prazmowski, Unters. üb. Entwick. und Fermentwirkung einiger Bacterien-Asten, 1880:-Papers in Nageli's Unters. ueb. niedere Pilze, Munich, I882 ; also Die niederen Pilze, I877 (sanitary).

Page 249. Saccharomycetes. Nägeli, Ueb. die chemische Zusammensetzung der Hefe, in Sitzungsber. d. Akad. d. Wiss. zu München, 1878; also, Theorie der Gährung, Munich, r879:-Hansen, Recherches sur la Physiologie et la Morphologie des Ferments alcooliques, Copenhagen, 1881:-Reess, Ueb. den Soorpilz (Ö̈dium albicans, Robin), Erlangen, 1877 .

Page 250, note 3 : for 'Lebrbuch' read 'Lehrbuch.'

Page 258. Note 2 : see also Book I. p. 16.

Page 260. Diatomacex. For a full account of this group, see Pfitzer, Die Bacillariaceen (Diatomaceen), in vol. II of Schenk's Handbuch der Botanik, part of the Scientific Encyclopedia published by Trewendt, Breslau, $\mathbf{1} 882$.

Page 261. Myxomycetes. See de Bary, Morph. und Physiol. der Pilze, Flechten und Myxomyceten :-Cooke, Myxomycetes of Great Britain, 1877 .

It has already been pointed out that the nuclei of the myxoamœbæ which coalesce to form the plasmodium remain distinct, p. 945 ; hence the plasmodium can no longer be regarded as the equivalent of a zygospore, and the position of the Myxomycetes among the Zygomycetes is untenable.

Page 264. Zygomycetes. Van Tieghem, Nouvelles Recherches sur les Mucorinées, Ann. d. Sci. Nat., sér. 6, t. I :-Brefeld, Ueb. copulirende Pilze, Sitzber. d. Ges. naturforsch. Freunde, Berlin, 1875, and Weitere Unters. (Mortierella), ibid. 1876. In Mortierella the zygospore becomes enclosed in a capsule of pseudoparenchyma formed by a felt of hyphæ. Brefeld classifies the Zygomycetes as follows :-

I. Mucorini (incl. Chætocladiaceæ) with simple zygospores; conidia formed by free cell-formation or by abstriction.

2. Mortierellex; zygospore enclosed in a capsule; conidia formed by free cellformation.

3. Piptocephalidex; zygospore possessing a temporary growing point and undergoing division to form three cells, one of which is the functional zygospore; conidia formed by division with subsequent rounding-off.

There is some ground for believing that the Entomophthoreæ belong to the group of Zygomycetes (see note on p. 277).

Page 271. Siphonex. Since conjugation of zoogonidia has been observed to take place in Botrydium and in Acetabularia these plants ought to be included among the Zygosporeæ in accordance with the classification followed in this work. See infra, note on the Fucoidex.

Page 275. Parthenogenesis of Saprolegnieæ. De Bary concludes from his observations (Beitr. z. Morphol. u. Physiol. d. Pilze, IV, I88I) that, in Saprolegnia, Acblya, and Aphanomyces, even when antheridia are formed and come into contact with oogonia, no act of fertilisation takes place, that is, no part of the contents of the antheridium enters the oogonium: hence the oospore is in all cases parthenogenetically produced. Fungi :-

The following is a brief résumé of the results of his observations on the oosporous

I. Pytbium; most of the protoplasm of the antheridium passes into the oosphere.

2. Phytophthora; a small quantity of the antheridial protoplasm enters the oosphere.

3. Peronospora; probably the same process as in the preceding genus.

4. Saprolegnia, Acblya, Aphanomyces; the antheridial tube does not open into the - oosphere, and no passage of substance can be observed.

5. Saprolegnia (S. torulosa, asterophora); the antheridia are closely applied to the oogonia, but no antheridial tubes, or only rudimentary ones, are developed.

6. No antheridia developed.

Page 278, line 9 from bottom; for 'Bulbocrate' read 'Bulbocbate.'

Page 281. Fucoidex. This group, like the Siphoneæ (see supra), includes forms 
in which conjugation and others in which fertilisation takes place. To be consistent with the classification followed in this work, it ought to be divided into two sub-groups, one belonging to the Zygosporeæ, the other to the Oosporex. Such a subdivision of the group, and this holds equally with regard to the Siphoneæ, would be obviously unnatural: the most satisfactory mode of meeting the difficulty would be to combine the $Z$ ygosporeæ and the Oosporex into one group. The following are the principal Orders of Fucoideæ or Melanophyceæ (Falkenberg, Die Algen, Schenk's Handbuch, vol. II) :-

Order I. Fucaceæ: reproduction by fertilisation; no zoogonidia.

„ 2. Cutleriacex : sexual cells both motile, the female being the larger; asexual reproduction by zoogonidia.

3. Phæosporeæ: sexual cells both motile at first, but the female cell comes to rest before fertilisation; asexual reproduction by zoogonidia. (Fertilisation observed by Berthold in Scytosiphon lomentarius and Ectocarpus siliculosus.)

a. Sphacelarieæ.

b. Ectocarpeæ; Mesoglœaceæ; Desmarestieæ.

c. Pbyllitis ; Scytosipbon; Colpomenia; Asperococcus; Punctaria.

" 4. Tilopteridex.

d. Laminariex.

" 5 (?) Dictyotacex.

It is doubtful if the Dictyotaceæ ought to be regarded as an Order of Fucoidex, for they differ from the other members of this group in that they produce tetraspores, and in that their antherozoids are not motile; in these respects they approach the Floridex. Probably the Dictyotaceæ constitute a group of Algæ intermediate between the Fucoideæ and the Florideæ.

In an interesting paper on Hydrurus (Akad. d. Wiss. Krakau, I88I) Rostafinski groups together the brown Algæ as follows:-

Phæoideæ.

I. Diatomaceæ.

2. Syngeneticæ (Chromopbyton, Hydrurus); no sexual reproduction (agamic).

3. Phæosporeæ;

(a) agamæ,

(b) isogamæ,

(c) oogamæ.

4. Cutlerieæ.

5. Fucacex.

6. Dictyotex.

Page 330. Lichens. Further evidence in support of the composite nature of Lichens is afforded by the discovery that the fungal-element of the Lichen-genus Cora, Fries, is a basidiomycetous Fungus. (Contribuzioni allo studio del genere Cora, Fries, del Dottore Oreste Mattirolo, Nuov. giorn. bot. ital. I881 ; also Bot. Zeitg. I881, p. 865.)

AEcidiomycetes. On Hemileia vastatrix, a fungus which most probably is to be referred to this group and which attacks Coffee plantations, see Marshall Ward, Q.J. M.S., 1882.

Page 335. Ustilagineæ. Woronin, Beitrag zur Kenntniss der Ustilagineen; being No. 5 of de Bary and Woronin's Beiträge zur Morph. und Physiol. d. Pilze, Frankfurt, 1882 .

Page 341. Relationships of the groups of Fungi. A short account may be given here of de Bary's views respecting the affinities of the groups of the higher Fungi as expressed in No. 4 of his Beiträge (188I). He considers that the Ascomycetes are connected with the Peronosporeæ through the Erysipheæ: the Uredineæ form one of the more highly developed groups of the Ascomycetous series. Among Basidiomycetes the Tremellineæ are closely connected with those Uredineæ which have no Ecidium-form (e. g. Chrysomyxa Abietis), their basidia being regarded by de Bary as homologous with the 
teleutospores of the Uredinex; it appears probable that all the other Basidiomycetes have sprung from the Tremellinex.

The Ustilaginex form a group of which it is difficult to trace the affinities. De Bary concludes, from a consideration of the simpler forms, such as Entyloma and Protomyces, that they are connected with the Chytridiaceæ through Nowakowski's Cladochytrieæ. (Cohn, Beiträge, II.)

Page 342. For a full account of the Muscineæ, see Goebel in Schenk's Handbuch, vol. II, 1882 .

Page 351. According to Goebel the embryo does not in all cases undergo the successive divisions which produce the octants: in some cases (Sphrocarpus, Targionia Micbelii) it is spindle-shaped, and undergoes at first only transverse divisions, four octant-cells being subsequently formed at the upper end by longitudinal divisions.

The relative differentiation of the sporogonium in the Hepaticæ is tabulated by Leitgeb as follows :-

I. The sporogonium consists of a parietal layer enclosing a mass of sporogenous cells: Riccia, Oxymitra.

2. Of the internal cells some are sporogenous, whereas others are sterile and act as deposits of nutriment : Corsinia, Riella, Notothylas.

3. The sterile cells develope into elaters: most Hepaticæ.

4. The axis of the capsule is occupied by a mass of sterile cells, the columella, which is covered above by the sporogenous layer: Anthocerotex (some species of Notothylas?).

Page 355, note I. On the development of the stomata of Marchantia, see Appendix, p. 948.

Page 386, note I. From the researches of Schmitz (Sitzber. d. niederrhein. Ges. zu Bonn, I880) and of Zacharias (Bot. Zeitg. I88 I, Ueb. die Spermatozoiden) it appears that the old view held by Hofmeister and Schacht is the correct one, that, namely, the nucleus of the mother-cell does not disappear, but becomes actually converted into the antherozoid, forming the greater portion of it, the remainder being derived from the protoplasm of the mother-cell.

Line 24 and 27 from top: for 'neck-cell' read 'canal-cell of the neck.'

Page 438. On the development of the spores, see p. I 3 .

Page 444. Rhizocarpex. The Female Prothallium. From the researches of Berggren (Om Azollas prothallium och embryo, Lunds Univ. Arsskrift, t. XVI; also Bot. Zeitg. r88I, and Nature, vol. 25. p. 327), it appears that the prothallium of Azolla caroliniana resembles that of Salvinia. On germination the endospore of the macrospore ruptures along its three edges, and the prothallium projects as a convex disc which is only one cell thick at its margin. Shortly after this a single archegonium is developed near the centre, consisting of four cells forming the ventral and of four forming the neck portion. When mature the prothallium is nearly hemispherical, and its cells contain chlorophyll. No mention is made of any canal-cell in the archegonium.

After fertilisation the oospore is divided by a transverse (basal) wall, and then by a median and a transverse wall, so that it consists of octants. Each octant is then divided by a wall parallel to the first (basal) and thus the embryo comes to consist of sixteen cells. The four uppermost cells (nearest to the neck of the archegonium) give rise to the foot; of the four lowermost cells, one becomes the apical cell of the stem, the second developes into a leaf-like organ, the third and fourth produce the scutiform leaf. It appears that these two organs can scarcely be regarded as true leaves; they seem to have the same morphological value as the stem.

Page 461-485. The alternate pages should be headed 'Dichotomeæ' instead of ' Filicineæ.'

Page 464. Apical growth of Psilotum. It is only the subterranean shoots of Psilotum which have a single apical cell; the subaerial shoots have a group of dividing cells at their apices (Strasburger, Bot. Zeitg. I 873). 
Page 478. An interesting abnormality has been observed by Goebel (Bot. Zeitg. 1879) in Isoëtes lacustris and ecbinospora. In a number of specimens no sporangia had been developed on the leaves, but in the place of each sporangium a. young plant had been produced by budding.

Page 481, seventh line from top. For ' delevopment' read 'development.'

Page 495, line 18 ; for 'Cocus' read 'Cocos.'

Page 504. Cycadeæ. On the development of the pollen sacs and pollen of Zamia muricata, see Treub, Ann. d. Sci. Nat., sér. 6, t. XV, 1882.

Page 506. Treub (loc. cit.) makes the following statements with regard to the development of the ovule of Ceratozamia longifolia.

Each scale of the female cone bears two sporangiferous lobes, in each of which a macrosporangium is developed.

The macrosporangium is visible in the lobe before any external differentiation can be detected.

The macrosporangium subsequently consists of a group of sporogenous cells (archesporium ?), surrounded by an external parietal layer and by an internal parietal layer consisting of several rows of cells.

Only one of the sporogenous cells gives rise to a macrospore. This cell undergoes no further division, but constitutes the single macrospore in the manner in which the embryo-sac is generally formed.

Shortly after the differentiation of the macrosporangium in the interior of the sporangiferous lobe, two new bodies are formed on the lobe superficially to the macrosporangium; these are the integument, and a mass of tissue immediately over the macrosporangium which Treub terms the nucellus.

The macrosporangium is, according to Treub, perfectly homologous with a sporangium of Ophioglossum; the nucellus and the integument are therefore new formations which have no equivalents in Cryptogams.

Page 519, line 6 from top. For 'are' read ' is.'

On the Morphology of the female flower in the Coniferæ, see Eichler, Ueb. die weiblichen Blüthen der Coniferen, in Monatsber. d. k. Akad. d. Wiss., Berlin, $188 \mathrm{r}$. As the result of comparative investigation of the female flowers in the various families of Coniferæ, he concludes that in the Araucarieæ, Abietineæ, and Taxodineæ each cone is a single flower, and that the scales of the cone are simple leaves (carpels) bearing ventral outgrowths (seminiferous scales) on which the ovules are borne; in the Cupressineæ the ovules are borne in the axils of the carpellary leaves; in the Taxineæ (incl. Podocarpex) the ovules are borne on the carpellary leaves (Microcachys, Dacrydium, Podocarpus), in Pbyllocladus they are axillary, in Taxus and Torreya they are terminal on lateral shoots, no carpels being present. In these two genera each ovule represents a single female flower, whereas in all the other genera and families the female flower consists of an aggregate of carpels bearing ovules either directly or in their axils. Eichler considers that Taxus and Torreya lead from the Coniferæ to the Gnetacex.

Eichler finds that it is no longer possible to hold Celakovsky's view that the ovule is either a modified leaf or a bud. (See note, p. 574, on the morphological significance of the placenta and of the ovule.)

Page 526, top line; for 'Juniperus' read 'Thuja.'

Page 530. Gnetacex. On the Embryology of the Gnetacex, see the paper by Bower, Q.J.M.S., I882, of which the following is a brief abstract:-

It appears to be the rule in Gnetum Gnemon (though not in all species of the genus) that the embryo is not developed until the seed begins to germinate: long tubular suspensors are however found in the endosperm of the ripe seed. On germination, embryos are formed at the apices of these suspensors, the mode of their development being similar to that which obtains in other Gymnosperms. It has been observed that the suspensors branch and that an embryo is developed at the extremity of each branch, a curious form of polyembryony : only one of the numerous embryos persists. 
After the two cotyledons of the developing embryo have made their appearance, an outgrowth, similar to that in Welwitschia, is produced at the base of the hypocotyledonary stem; but in Gnetum its position relatively to the planes of the cotyledons is not fixed, as in Welwitscbia, the point at which it is formed being determined by the action of gravity, so that it always developes on the under side of the hypocotyledonary stem. Further, the organ is larger in Gnetum, and the pith and vascular tissue take part in its formation, whereas in Welwitscbia it is derived only from the cortex and epidermis of the hypocotyledonary stem.

In the three genera of Gnetaceæ this organ is developed for the transfer of nutritive material from the seed to the seedling, the size of the organ being proportional to that of the seed and to the quantity of reserve material.

Page 534, line 2 from bottom (note). For 'Eilcher' read 'Eichler.'

Page 574. The morphological significance of the ovule. See above, note on the female flowers of Coniferæ.

Page 576. On the development of the ovule of the Loranthacex, see Treub, Observations sur les Loranthacées, Ann. d. Jardin botanique de Buitenzorg, vols. 2 and 3, Leyden, 1882 .

In Lorantbus spharocarpus there is, according to Treub, a central placenta which bears three or four free lateral segments; these Treub regards as rudimentary ovules. In each of these several embryo-sac-mother-cells (archesporial cells) are formed, but only one embryo-sac becomes fully developed: it is developed from the uppermost cell of the row formed by the division of the mother-cell.

In the Viscum album and articulatum there is no placenta and no ovule, but the embryo-sacs are developed in the tissue of the carpels; in $V$. album there is a relation between the number and position of the embryo-sacs and the carpels, but in $V$. articulatum this is not the case. In spite of the degradation of these plants, the mother-cells of the embryo-sacs are nevertheless of hypodermal origin as in the other Angiosperms.

Page 578, line 8 from top. For ' maculatam' read ' maculatum.'

In addition to the instances here given of deviation from what may be regarded as the typical mode of development of the embryo-sac, it may be added that in some cases it is the uppermost of the cells of the row formed by the division of the archesporial cell which developes into the embryo-sac, e.g. Lorantbus spbxrocarpus (Treub), Pyretbrum balsaminatum (Marshall Ward), Agraphis patula (Treub and Mellink). For the most recent researches on the embryo-sac of Angiosperms, see Guignard, Ann. d. Sci. Nat., sér. 6, t. XIII, r882.

Page 580. The Synergidæ. Strasburger (Bau und Wachsthum der Zellhäute, r 882) considers that the longitudinal striation (Filiform Apparatus of Schacht) mentioned in the text is due to the presence of delicate canals which are filled with protoplasm; the body of the Filiform Apparatus is probably not protoplasmic.

Page 588. Development of the Embryo. See also Rech. embryol. sur l'Orcbis maculata, Monteverde in Mélanges Biologiques de l'Acad. Imp. de St. Petersbourg, I880; Guignard, Rech. d'embryogénie végétale comparée, Ann. d. Sci. Nat., sér. 6, t. XII ; and Treub, Notes sur l'Embryon, le Sac embryonnaire, et l'Ovule, in Ann. d. Jardin bot. de Buitenzorg, III, I882.

Treub's researches refer to Peristylus grandis and to Avicennia officinalis. With regard to the former he finds that the embryo remains at first rudimentary, whereas the suspensor grows rapidly and until it projects through the micropyle; it then branches, and the branches become closely applied to the placenta. At this time the embryo begins to develope, and there is no doubt that it does so in consequence of the supply of nutritive material which is absorbed from the placenta and transmitted by the suspensor. These observations confirm Treub's previous conclusions as to the function of the suspensor in Orchids.

With regard to Avicennia, Treub's observations complete our knowledge of this curious 'viviparous' plant long ago described by Griffith (On the development of the 
ovulum in Avicennia, Trans. Linn. Soc. XX, I846). In the first place Treub points out that the ovule is not naked, as Griffith states, but that it has an integument developed from the dermatogen in the same way as that described for Thesium ebracteatum by Warming (Ann. Sci. Nat., sér. 6, t. V, 1878). One of the hypodermal cells enlarges and becomes the archesporial cell : this is divided transversely into two, the lower becoming the embryo-sac, the upper dividing transversely into two superposed tapetal cells. These tapetal cells are peculiar in that they persist for a considerable time, whereas in most plants they are absorbed before fertilisation. The embryo-sac enlarges, pushes aside the tapetal cells, and absorbs the epidermis at the micropylar end of the ovule. After fertilisation the embryo is soon to be seen surrounded by endosperm cells, and at one side of this group of cells there is a large cell, termed by Treub the 'cotyloid' cell, which elongates towards the apex of the embryo-sac. The endosperm now grows and projects through the micropyle, until finally it is quite external to the ovule. It still encloses the embryo, but as the embryo grows it ruptures the endosperm and the cotyledons project, the radicular end remaining inserted in the endosperm. During this time the cotyloid cell has enlarged, branching posteriorly in the ovule and penetrating anteriorly into the placenta. It acts as an absorptive organ, taking up nutritive substances from the ovule and the placenta, and transmitting them to the endosperm and thus also to the embryo. The radicular end of the embryo is peculiar in that it is destitute of a root-cap ; but previously to the dehiscence of the fruit adventitious roots, generally four in number, provided with root-caps, spring from it close to its attachment to the suspensor.

Page 603. On the flowers of Orchids, see Gérard, Sur l'Homologie et le Diagramme des Orchidées, Ann. d. Sci. Nat., sér. 6, t. VII.

Page 611. On the symmetry of the flower, see also Eichler, Ueb. einige zygomorphe Blüthen, in Sitzber. d. Ges. naturf. Freunde, Berlin, 1880.

Page 629. On abnormal fibro-vascular bundles in Monocotyledons, see Kny, Ueb. einige Abweichungen im Bau des Leitbündels der Monokotyledonen, Sitz. d. Bot. Ver. d. Prov. Brandenburg, $188 \mathrm{I}$.

Page 650, lines 5 and 9 from bottom ; for 'secondary cortex' read 'secondary phloëm.'

Page 653. On abnormal modes of thickening of the stem in Dicotyledons, see Appendix, p. $95^{\circ}$.

\section{BOOK III.}

Page 663. The Condition of Aggregation of organised structures. In his work on the structure and growth of the cell-wall (Ueb. Bau und Wachsthum der Zellhäute, I 882), Strasburger dissents entirely from Nägeli's theory of the structure of organised bodies which is given in the text. A short account of his views may be given here.

Strasburger comes to the conclusion that the forces which hold together the solid particles of organised bodies are of a chemical, as opposed to a physical, nature. The chemical molecules are not grouped together into micellæ by cohesion, and the micellæ are not connected into organised substance by attraction, as Nägeli would have it, but the molecules are linked together by chemical affinity, probably by means of multivalent atoms, into networks. Further, the water present is retained, according to Strasburger, in the intermolecular meshes by capillarity. The phenomenon of 'swelling-up' is therefore one of intermolecular capillarity, and depends upon the mobility of the molecules about their position of equilibrium.

Nägeli's micellar theory received considerable support from his observations upon the appearances presented by organised bodies (starch-grains, cell-walls) when examined in polarised light. He found that they were doubly refractive, and further that their double refraction was not affected by tension, strain, etc. It was from these facts that he inferred 
the crystalline form of the micellæ. Strasburger argues that cell-walls and starch-grains consist of numerous lamellæ which are in different states of tension and are at the same time very firmly adherent; it is to these tensions that the optical phenomena in question are due, and it is not probable that any mechanical force applied could so far modify these tensions as to produce an alteration in the optical phenomena.

Strasburger's views may be summarised as follows:-

I. Organised bodies consist of molecules of solid substance united by chemical affinity; the water which they contàin is retained by intermolecular capillarity. tensions.

2. The doubly refractive properties of starch-grains and cell-walls depend upon

3. The directions of swelling-up are determined by a certain anatomical structure.

4. The increase in surface of organised bodies depends upon stretching and swellingup (see also p. 946).

5. Increase in thickness or in bulk depends upon apposition.

Page 671. On the growth of artificial cells, see Traube, Experimente zur physikalischen Erklärung der Bildung der Zellhaut, ihres Wachsthum durch Intussusception, und des Aufwärtswachsens der Pflanzen, Bot. Zeitg. 1875 , p. $5^{6}$; also Reinke, ibid., p. 425, Bemerkungen über das Wachsthum anorganischer Zellen; further, Sachs and Traube, Bot. Zeitg. 1878 .

Page 678. On Transpiration, see also Wiesner, Untersuch. ueb. den Einfluss des Lichtes und der strahlenden Wärme auf die Transspiration der Pflanze, Sitzber. d. k. Akad. d. Wiss., Bd. LXXIV, Wien, 1876 (also Ann. d. Sci. Nat., sér. 6, t. IV, I876).

In note 4 , for ' 1836 ' read ' 1856 '

Page 688. On the absorption of water by roots, see Vesque, De l'influence des matières salines sur l'absorption de l'eau par les racines, Ann. d. Sci. nat., sér. 6, t. IX, I 880 .

Page 701. Absorption of substances by roots. See Phillips, On the Absorption of metallic oxides by plants, American Journal of Science, 1882 .

Page 703. On the chemistry of Assimilation, see Reinke, Theoretisches zur Assimilationsproblem, Bot. Zeitg. 1882 : also Strasburger, Bau und Wachsthum der Zellhäute, p. 237.

Page 707. It has been recently ascertained that glycogen occurs as a reservematerial in many plants: see Errera, L'Epiplasme des Ascomycètes et le Glycogène des Végétaux, Brussels, i 882 .

Page 712. From observations made on Euphorbia trigona, Treub comes to the conclusion that the laticiferous cells serve as channels for the transmission of amylaceous substances, and that the starch-grains which they contain are transitory (Ann. du Jardin bot. de Buitenzorg, III, I882).

Page 734, paragraph 3. See also Holzner, Beob. ueb. die Schütte der Kiefer oder Föhre und die Winterfärbung immergrüner Gewächse, Freising, 1877.

Page 737. On the action of light, see also Pauchon, Rech. sur le rôle de la lumière dans la germination, Ann. d. Sci. Nat., sér. 6, t. X, r88I.

Siemens, On the Influence of Electric Light upon Vegetation, Proc. Roy. Soc., I 880; also, On some Applications of Electric Energy to Horticulture and Agriculture, London, I88r.

Page 739 (2). See also Famintzin, De l'influence de l'intensité de la lumière sur la décomposition de l'acide carbonique par les plantes, Ann. d. Sci. Nat., sér. 6, t. X, I880.

Page $744(b)$. Famintzin, La décomposition de l'acide carbonique par les plantes exposées à la lumière artificielle, Ann. d. Sci. Nat., sér. 6, t. X; Dehérain et Maquenne, Sur la décomposition de l'acide carbonique par les feuilles éclairées par des lumières artificielles, Ann. d. Sci. Nat., sér. 6, t. IX (1880).

Page 761. On the function of chlorophyll, see further, Bonnier, Du rôle physiologique de la chlorophylle, Ann. d. Sci. Nat., sér. 6, t. X, I88I ; and Hansen, Geschichte der Assimilation und Chlorophyllfunction, Arb. d. bot. Inst. in Würzburg, II, I882. 
Page 768. On the action of electrical currents on growing roots, see Elfving, Ueb. eine Wirkung des galvanischen Stromes auf wachsende Wurzeln, Bot. Zeitg., 1882.

$\mathrm{He}$ finds that when a root is placed vertically between two electrodes, it curves towards the positive electrode; the curvature is evidently connected with the growth of the root, the current effecting a retardation. Continued exposure to the action of the current causes death.

When the current is parallel to the long axis of the root it appears to retard growth when it runs in opposition to the direction of growth.

Experiments with negatively heliotropic roots (Brassica oleracea, Lepidium sativum, Sinapis alba), the direction of the current being transverse, showed that they curved towards the negative electrode.

Page 769. For further researches on Dionaa, see Burdon-Sanderson, On the Electromotive Properties of the leaf of Dionxa, Phil. Trans. 1882.

Page 787. Turgidity does not necessarily cause an elongation of cells; it may also cause them to become shorter and thicker. See de Vries, Ueb. Verkürzung pflanzlicher Zellen durch Aufnahme von Wasser, Bot. Zeitg., 1879.

Page 812. Light has some influence on the development of root-hairs on the gemmæ of Marchantia; see Zimmermann, Ueb. die Einwirkung des Lichtes auf den Marchantienthallus, Arb. d. bot. Inst. in Würzburg, II, 1882.

Page 815. On the growth in length of stems, see further, Wiesner, Die undulirende Nutation der Internodien, Sitzber. d. k. k. Akad. d. Wiss. in Wien, LXXVII, I878.

Page 839. Geotropism. See F. Darwin, On the Connexion between Geotropism and Growth, Journ. Linn. Soc., vol. XIX, I882.

Page 854, note 2. Further, F. Darwin, On the power possessed by leaves of placing themselves at right angles to the direction of incident light, Journ. Linn. Soc., XVIII, $188 \mathrm{r}$.

Page 862. On Climbing Plants, see Schwendener, Ueb. das Winden der Schlingpflanzen, Monatsber. d. Berl. Akad., ı88 I; Sachs, Notiz über Schlingpflanzen, Arb. d. bot. Inst. in Würzburg, II, 1882 . 1880 .

Page 865. See de Vries, Over de Bewegingen der Ranken van Sicyos, Amsterdam,

Treub has recently drawn attention to a new group of climbing plants, those namely which climb by means of irritable hooks, the effect of irritation being an increase in the thickness of the hook. Such are Uncaria (Rubiacex), Ancistrocladus (Dipterocarpex), Artabotrys (Anonaceæ), Luvunga (Aurantiaceæ), Olax (Olacineæ), Hugonia (Linaceæ), Strychnos (Loganiacex). This group is not to be confounded with Darwin's 'hookclimbers,' the hooks of which are not irritable. (Treub, Sur une nouvelle catégorie de plantes grimpantes, Ann. du Jardin botanique de Buitenzorg, III, I882.)

Page 871, Sect. 26. Vöchting, Die Bewegungen der Blüthen und Früchte, Bonn, I882.

Page 920, Sect. 35. For some interesting observations as to the relation between plants and external conditions, made on the Flora of Scandinavia, see Bonnier et Flahault, Observations sur les Modifications des Végétaux suivant les conditions physiques du milieu, Ann. Sci. Nat. ser. 6, t. VII, 1879, and Flahault, ibid. t. IX, I880. 


\section{N D EX.}

Abies, pp. 514, 518, 939 (Figs. 350, 353).

Abietinex, 518, 527, 958.

Abortion, $219,602$.

Absorption, 700, 96 1 .

Absorption of assimilated substances, 720 .

Acaciex, 557.

Acalypheæ, $66 \mathrm{r}$.

Acanthace $, 65,68,658$.

Acanthus, 648.

Acarospora, 325.

Accumulation of characters, 924.

Acer, 535, 61 5, 617, 651, 940.

Acerineæ, 6 ro.

Acetabularia, 65, 272, 955.

Achenium, 6 I5.

Achillæa, 932.

Achlya, 12, 273, 902, 955 (Figs. 8, 9, 178, 179).

Achromatin, 18, 946 .

Aconitum, 495, 538, 607, 608 .

Acorn, 6 I 4 .

Acorus, I 15 (Fig. 96).

Acrocarpous Mosses, 370.

Acropetal order of development, 170.

Acrosticheæ, 44I.

Acrostichum, 435, 436, 44 I.

Actinomorphic, 954 .

Actinostrobex, 527 .

Acyclic, 600, 641 .

Adaptation, 934 .

Adhesion, 219, 546 .

Adiantum, 182, 424, 425,427, 442, 915 (Figs. 293, 295297).

Adventitious formations, 17 I, I73, 433, 639, 953 .

Ecidiomycetes, 244, 330, 956.

Eccidium, 330.

Esculineæ, 660.

Esculus, 644 (Fig. 455).

Atthalium, 262, 84 I.

Agapanthus, 22.

Agaricus, 336 (Figs. 225, 226, 227).
Agave, 556, 594, 621 .

Aggregatæ, 658.

Agrimonia, 578.

Agrostemma, 579.

Ailanthus, $65^{2}$ (Fig. 97).

Akebia, 534 (Fig. 357).

Albumen, 492.

Albuminoids, 706.

Albuminous, 587 .

Alchemilla, 568, 677 .

Aldrovanda, 649 .

Alepyrum, 542 .

Aletris, I 27.

Aleurone, 5 I, 947.

Algæ, 23I, 241.

Alisma, 589, 626, 952.

Alismaceæ, 626, 631 (Fig. 43I).

Alliaria, 574 .

Allionia, $58 \mathrm{r}$.

Allium, $17,88,581,623,624$, 629, 939 (Figs. I $5,73,424$, 425).

Almond, 637 .

Alnus, 555, 652 .

Aloë, I 27, 193, 629 (Fig. 152).

Aloinex, I36, 496.

Alopecurus, 596.

Alpinia, 625 (Fig. 429).

Alsineæ, 66r.

Alternate arrangement, I89, 601.

Alternation of generations, $222,225,233,239,342$, $385,486,488,899,903$, 954.

Althæa, 546, 552, 553 (Figs. $42,83,360,377,378$, $38 \mathrm{I})$.

Aluminium, 695 .

Alyssum, 544.

Amaranthaceæ, $137,645,66 \mathrm{I}$.

Amaranthus, 653, 950 .

Amentiferæ, 656 .

Amidoplasts, 948 .

Ammonia, 698 .

Amœboid-movement of protoplasm, 39, 263.

Amorpha, 599 .
Amorphophallus, 183,624 (Fig. 141).

Ampelidex, 644, 66o, 866.

Ampelopsis, $217,865,866$.

Amphigastria, 356 .

Amphithecium, 375 .

Amygdalex, 651, 662.

Amyridex, 660 .

Anacardiaceæ, 644, 660 (Fig. $\left.45^{2}\right)$.

Anagallis, 565, 616 (Fig. 392).

Ananasineæ, 632 .

Anaptychia, 323, 325 (Figs. 218, 219).

Anatropous, 492, 570.

Anchusa, 598.

Ancistrocladus, 962.

Andrexa, 373, 374 (Fig. 255).

Andreæace , $36 \mathrm{r}, 380$.

Andrœcium, 490, $54 \mathrm{I}$.

Androgonidium, 279.

Aneimia, 105, 42I, 423, $44{ }^{\circ}$

(Fig. 106).

Anemone, 640.

Anemophilous, 494, 908 .

Aneura, 346, 356, 358, 952 .

Angiocarpous, 306 .

Angiocarpous Lichens, 323.

Angiopteris, 93, 416 (Figs. 291, 292).

Angiosperms, 497, 534 .

Angle of divergence, 188,201 .

Anisocarpæ, 655, 658 .

Anisostemonous, $64 \mathrm{r}$.

Annual ring, $\mathrm{I}_{32}, 652,813$.

Annular vessels, 22, 23.

Annulus, 338, 381, 435 .

Anona, 557.

Anonaceæ, 657, 962 .

Anterior, 600 .

Anthela, 596.

Anthemis, 599.

Anther, 49 I, 54 I (Figs. 360, 382 ).

Anthericum, 577.

Antheridium, 234, 268, 290, 299, 343, 349, 387, 394, 4II, 423, 442, 469, 487, 515, 899 (Figs. 177, 179, 
I 81, I 83, I 85, I 87, I 89, 190, 198, 200, 201, 235, 237, 239, 241, 253, 254, $262,275,287,293,294$, $309,329,33 \mathrm{r}$ ).

Antherozoid, 224, 234, 268, 27 I $278,279,282,287$, 290, 298, 371, 386, 394, $424,443,446,469,524$, 897 (Figs. 2, I 77, I 83,185 , 187, 188, 198, 241, 254, 262, 275, 293, 309, 310, 3. 4, 329).

Anthoceros, 346, $35^{2}$ (Figs. 237,238 ).

Anthoceroteæ, 352.

Anthophore, 539 (Fig. 36 I).

Anthurium, 586, 953 .

Antipetalous, 60 I.

Antipodal cells, 486, 580 .

Antirrhinum, 616 .

Antisepalous, 6or.

Apetalous, $64 \mathrm{I}$.

Apex, 158, 175, 202.

Aphanocyclæ, 655,657 .

Aphanomyces, $273,955$.

Apheliotropic, 756 .

Apical cell, 137, 173, 289, 293, 347, 397, 401, 413, $429,449,450,463,464$, 476 (Figs. 108-II2, II 5, I 16, I 20, I 2 I, I 38, I42, $192,244,279,283,284$, $315 a, 33^{6}$ ).

Apical growth, 158 .

Apocynaceæ, 86, 658, 949.

Apogamy, 425.

Apopetalous, 539.

Apophyllous, 539.

Apophysis, 385 (Fig. 273).

Aposepalous, 539.

Apostasiaceæ, 633 .

Apostrophe, 750 .

Apothecium, 308, 323 (Figs. $7,205,218,219)$.

Apple, 6I4, 662.

Aqueous Tissue, 98, I 23.

Aquifoliacex, 660 .

Aquilegia, 570, 643 (Figs. 396,45 I).

Aralia, 654 .

Araliaceæ, 66r.

Araucaria, 507, 509, 510,513, 5 I 8,527 .

Araucarieæ, 507, 527, 958.

Arbutus, 543 (Fig. 527).

Arc-indicator, 826 (Fig. 480 ).

Arceuthos, 5 I 7.

Archegonium, 343, 349, 353 , $355,357,359,372,386$, 394,4 II $423,425,445$, $446,462,47 I, 487,498$, $506,521,529,582,899$ (Figs. 237, 240, 243, 256,
$26_{3}, 276,287,294,295$, 312, 330, 33 I, 354, 355).

Archesporium, 375, 388, 437, 491, 577 (Figs. 305, 337).

Archetype, 94I.

Archidium, 374, 380 (Figs. 264, 265).

Archieracium, 926.

Arcyria, 262.

Areca-Palm, 587.

Aril, 495, 513, 570, 6 I 8 (Fig. 348 ).

Aristolochia, 862, 9 ro, 940 (Figs. 488, 489).

Aristolochiaceæ, $6_{5} 6$.

Aroideæ, 65, 84, 93, I 20, 540, 631,952 .

Artabotrys, 962 .

Arthrotaxis, 5 I 8, 527.

Artocarpeæ, 656 .

Arum, 583, 585, 933 .

Asarineæ, 656 .

Asarum, 535 (Fig. 358).

Ascent of water, 685 .

Asclepiadeæ, 85, 658, 906, 9 I I, 949.

Ascobolus, 238, 309 ' (Fig. 204).

Ascogenous hyphæ, 308, 3 ro, 315,324 (Fig. 204).

Ascogonium, 309, 329, 898 (Figs. 204, 206, 207, 208). Ascomycetes, 244, 308, 899 . Ascospore, 238, 308 (Figs. 7, 205, 207, 208, 209, 2 I 9).

Ascus, 226, 238, 308, 310 (Figs. 7, 205, 207, 208, 209, $219)$.

Asexual generation, 225, 227 , $239,343,35$ I $387,395,4$ I 2, 4 I $6,425,447,462,472,488$, 899 .

Asexual propagation, 348 .

Asexual reproduction, 223.

Ashes, 36, 695.

Asparagin, 718.

Aspergillus, 3 I 2 (Fig. 208).

Asperifolieæ, 599.

Asphodelus, .552 .

Aspicilia; 323 :

Aspidieæ, $44^{2}$

Aspidium, 426,$427 ; 429,432$, $433,435,436 ; 437,438,439$, $44^{2}$, 915 (Figs. 302, 303, 304,306 ).

Asplenieæ, 422.

Asplenium, I 44, I $72,437,442$, 915,953 (Figs. I I2, 127 , 305).

Assimilation, 703, 729, 737, $96 \mathrm{r}$.

Asterophyllites, 408.

Astragalus, 56r, 6 I 5 .

Astrapæa, 555.
Astrocarpus, 609.

Atelanthera, 605 .

Atherurus, 622 .

Atom, 664.

Atrichum, 367, 368 .

Atriplex, 653 .

Aurantiaceæ, 646, 660, 962 .

Automatic periodic movements, 880,895 .

Autumnal wood, $8 \mathrm{I} 3$.

Auxanometer, 827 (Fig.481).

Auxospore, $26 \mathrm{r}$.

Avicennia, 652,959 .

Avicennieæ, 950.

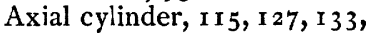
468.

Axial longitudinal section, 203.

Axial placentation, $56_{3}, 5^{6} 4$.

Axil, I 75, 489 .

Axillary placentation, 574 .

Axillary shoots, I 75, 489 .

Axis of growth, I59, 203, 206.

Azolla, 448, 450, 452, 454, 460,957 .

Bacillarieæ, 260.

Bacillus, 246, 249, 954

Bacteria, 249, 954 (Fig. 166).

Bactrospora, 325.

Bæomyces, 324.

Balanophora, 634 .

Balanophoreæ, 241 , 576, 648, 649,662 .

Balsamineæ, 660 .

Bambusa, 603 (Fig. 409).

Bangiaceæ, 289.

Barbula, 364, 368, 385 (Fig. $250)$.

Bark, ı06, 108.

Bartramia, 362, 365 .

Basal growth, 159.

Basal wall, $35 \mathrm{I}$.

Base, I77, 202.

Basidiomycetes, 240, 244, $305,335,956$.

Basidiospore, I 5, 336 (Fig. 227).

Basidium, $15,330,333,336$, 338 (Fig. 227 ).

Bassorin, 705.

Bast, I I I.

Batrachospermum, 289.

Bauhinia, 653, 950.

Beech, 587 .

Beggiatoa, 249.

Begonia, 204, 209, 639, 907, 925,954 .

Begoniaceæ, I 37, 662 .

Benthamia, $6 \mathrm{r} 4$.

Berberideæ, 646,657 .

Berberis, 332, 595, 647, 880, 883,894 . 
Berry, 525, 6 I6.

Betula, $6{ }_{52}$.

Betulaceæ, 656 .

Bicollateral bundles, 949 .

Bicornes, 659 .

Bifurcation, 177 .

Bignonia, ז 37, 6 × 7, 652, 866, 936, 939 .

Bignoniaceæ, 1 36, 658, 950.

Bilateral structure, 204, 854 954 .

Bilirubin, 758 .

Biota, 51 4, 527.

Biscutella, 570 (Fig. 396)

Bisexual, 490.

Bixaceæ, 659 .

Blackberry, 6 I 4 .

Blasia, 347, 348, 352, 357 .

Blastocolla, гог.

Bleeding of wood, 677 .

Bloom on plants, 99 .

Boletus, 96, 336 (Fig. 79).

Boragineæ, 597, 658 .

Borago, 598.

Bordered pits, 23, 25, $53 \mathrm{I}$, 533, 946.

Bostrychoid cyme, I 80 (Fig. I 36).

Bostrychoid dichotomy, i 78 (Fig. 134).

Bostryx, I80, 597.

Botrychium, 410 (Figs. 287, 289).

Botrydium, 27 I, 955.

Botrytis, 3 I 1 .

Bract, 214 , 540, 595 .

Bracteole, 297, 304, 49 I, 595.

Branch system, I 76 .

Branching, I 69.

Branching of leaves, 182 .

Branching of roots, $18 \mathrm{I}$.

Branching of stem, 184 .

Brasenia, 575 .

Brassica, 599, 605, 921 .

Brizula, 542.

Bromeliaceæ, 632.

Bromine, 695.

Broussonetia, 907.

Bryaceæ, 377, 381 .

Bryineæ, 362.

Bryonia, 867, 939 (Fig. 487).

Bryophyllum, 173,640 .

Bryopsis, 272.

Bryum, 96, 365, 375, 377 (Figs. 80, 249).

Bud, 156.

Bud-rudiment, 297.

Bud-variation, $92 \mathrm{I}$.

Bulb, 2 I 6, 620, 623, 704, 707, 715 (Figs. 421, 424, 470).

Bulbil, I 72, 223, 228, 295, 368, 622 (Fig. 250).

Bulbocapnos, 634 .

Bulbochæte, 27.8.
Bundle-sheath, I 12 , I 1 3, I 15 , I 23, I 24, 167, 407, 434, 468,483 .

Burmanniaceæ, 633 .

Burseraceæ, 660.

Butomaceæ, 601.

Butomus, 558, 568, 606, 626 (Fig. 382).

Buxbaumia, 366, 369.

Buxineæ, 66r.

Byttneriaceæ, 66r.

Cabombeæ, 657.

Cactaceæ, 638, 662 .

Cactus, 2 I 6, 935 .

Groma, 331 .

Cæsalpineæ, 633, 662 .

Cæsalpinia, 133 .

Caladium, 87 .

Calamites, 404, 405, 407 .

Calamodendron, $407,408$.

Calamostachys, 408 .

Calamus, 629, 938.

Calanthe, $5^{67}$ (Fig. 394).

Calcium, 699 .

Calcium carbonate, 65, 948 .

Calcium oxalate, $52,65,88$, 699.

Calcium sulphate, 699 .

Calendula, 876 .

Callistemon, 65 r, 949.

Callithamnion, $5 \mathrm{I}$.

Callitrichaceæ, 662

Callitriche, 570.

Callitris, 517, 527 (Fig. 352 ).

Callus, 8 ro.

Calodracon, I I 4, I 29.

Calothamnus, 544 (Fig. $3^{6}$ ).

Calycanthace $x, 662$.

Calycanthus, 651 .

Calycereæ, 659 .

Calycifloræ, 655, 662 .

Calycium, 324 .

Calyculus, 540.

Calypogeia, 359.

Calyptra, 344, 350, 351, 355, 374 (Figs. 23:6, $240, .246$, $257,263-266)$.

Calyptrogen, 489, 500, 952 .

Calyx, 538.

Cambiform tissue, i 19 .

Cambium, 82, I 1 0, I I 2, I 29, 810,813 .

Cambium-ring, $127,130,133$, 531,650 .

Camellia, 20, 65I (Fig. I6).

Campanula, $64^{2}, 8$ I $_{2}$ (Figs. 442, 479).

Campanulaceæ, 86, 642, 658 .

Campylotropous, 492, 570 .

Canal of the style, 568 (Fig. 395).

Canal-cell, 350, 374, 386, $395 ; 4$ I $2,424,44^{6}, 472$,
499, $521,522,582,957$

(Figs. 236, 256, 294, 312 , 331 ).

Candollea, 646 (Fig. $46 \mathrm{r}$ ).

Canna, 6r, 625, 586 (Fig.625).

Cannabineæ, 535,656 .

Cannabis, ror.

Cannaceæ, 99, 628, 632, 952 (Fig. 430).

Caoutchouc, $7 \mathbf{I} 2$.

Cap-cell of root, I45 (Figs. I I 2, I 20, I 2 I, I 38 ).

Capillary attraction, 684 .

Capillitium, 262 (Fig. 172).

Capitulum, 596 (Fig. I 26).

Capparideæ, 604, 657 (Fig. 4 I2).

Caprifoliaceæ, $6_{42}, 658$ (Fig. 440).

Capsella, 590, 591 (Fig. 403).

Capsule, 6I6.

Capsule, of Muscineæ, 344, 374,375 (Figs. 236-240, $246,263-268$ ).

Caragana, 652 .

Carbo-hydrates, 715 .

Carbon, 695, 696.

Carbon dioxide, 696, 703, 72 I , 737, 744 .

Carcerulus, 6 I 4 .

Carex, 577, 600 .

Carica, 87, 708.

Carpel, 2 I 4, 490, 5 I6, 518 , $526,536,559,567,574$.

Carpellary leaf, 493, 517 .

Carpinus, 555, $65 \mathrm{I}$.

Carpogonium, 236, 285, 286 , $287,290,297,301,307$, $309,312,313,314,329$, 897 (Figs. I64, I 88, I 89 , 190, 197, 199, 202, 204, 207, 208).

Carpophore, 6 I5.

Carpospore, 236,305 .

Carposporex, 236, 244, 284 .

Caruncle, 6 r8.

Caryolopha, 598.

Caryophylleæ, 563, 570, 66r, 9 I 5.

Caryophyllineæ, 645, 66 I.

Caryopsis, 6 I $5_{5}, 617$.

Cassia, 56 I , 6 I 5 .

Castanea, $65 \mathrm{I}$.

Casuarina, I I 6, 49 I, 54 r, $65 \mathrm{I}$.

Casuarineæ, 662 .

Catalpa, 588, 648 .

Cataphyllary leaves, 186,213 , $509,622,623,624,640$.

Catharinea, $199,217$.

Caulerpa, I 58, r6 r, 272, 947.

Cauline bundles, I37, I 56 , $440,468,482,483,630$, 649,950 .

Caulome, I50, 151 , $15 \%$. 
Caulotretus, 136 .

Cedreleæ, 660.

Cedrus, 509, 527.

Celastrineæ, 645,660 .

Celastrus, 602,65 I (Fig.407).

Cell, Primordial, 5 .

Cell, Structure of, $\mathbf{r}$.

Cell-division, 8, 12, 16, 275, 762.

Cell-families, 81, 246.

Cell-multiplication, 8 .

Cell-nucleus, 2, 17, 37, 44, $945,947$.

Cell-plate, 13, 18.

Cell-sap, 2, 62.

Cell-wall, I, 19, 946.

Cells, Bundle of, $8 \mathbf{I}$.

Cells, Filament of, 80 .

Cells, Formation of, 7, 945 .

Cells, Formation of the common wall of, 72 .

Cells, Forms of, 5,809 .

Cells, Group of, $8 \mathrm{r}$.

Cells, Layers of, 80.

Cellular tissue, $\mathrm{I}$.

Cellulose, 2, 19, 708.

Cielosia, 566, 645 (Fig. 460).

Celtideæ, 656 .

Centaurea, 883, 892 .

Centradenia, 543, 548, $65 \mathrm{I}$, (Fig. 364).

Central cell, 350, 353, 374, $386,395,4 \mathrm{II}, 424,446$, 472, 506, $52 \mathrm{I}$ (Figs. 294, 312,331 ).

Centranthus, 643 (Fig. 443).

Centrifugal force, action of, 772.

Centrifugal infloresence, 596 .

Centripetal infloresence,595.

Centrolepidace, 542.

Centrolepis, 542.

Centrospermæ, 630, 655, 66 I .

Cephalanthera, 908 .

Cephalotaxus, 66, $518,527$.

Cephalotus, 640 .

Ceramiex, 72, 290.

Ceramium, $5 \mathrm{I}$.

Cerastium, $56_{3}, 5_{64}, 616$.

Ceratonia, 20, 35, 36, 73, 652 (Fig. 39).

Ceratophyllacex, 662 .

Ceratophyllum, 49, 98, 586, 648,649 .

Ceratopteris, $16_{4}, 422,430$, $433,436$.

Ceratozamia, $501,5 \circ 3,506$, 958 (Fig. 345).

Cercis, I 85, 208 (Fig. I55).

Cereus, 99, 203, $2 \mathbf{1} 6$.

Cerinthe, 598 .

Ceropegia, 89 .

Cerorchideæ, 557.

Ceroxylon, 99 .
Chrtocladium, 267, 955 .

Chætomorpha, $28 \mathrm{I}$.

Chætophora, $28 \mathrm{I}$.

Chalaza, 492, $57 \mathrm{I}$.

Chamæcyparis, 527 .

Chantransia, 290.

Chara, 152, 293, 902 (Figs. I I 5, I 9 I-I 97, 203).

Characeæ, 18, 39, 174, 237, 238, 24 I, 244, 292, 897, $898,899,900,905$.

Characteristic torms of leaves and shoots, 2 I r.

Chavica, I 37, $95 \mathrm{I}$.

Cheiranthus, 578,652 .

Chelidonium, 87, 618, 647 (Fig. 464).

Chemical processes, 695.

Chenopodiaceæ, $574,66 \mathrm{r}$.

Chenopodium, 536, 577,578 , 871 (Fig. 359).

Chimonanthus, 634(Fig. 434).

Chlænaceæ, 660.

Chlamydococcus, 253.

Chlamydomonas, 246, 253, 947.

Chloranthex, 656 .

Chlorine, 695, 699 .

Chlorofucine, 765 .

Chlorophyll, 45, 58, 24I, 244, 260, 282, 289, 691, $697,703,729,737,743$, $757,96 \mathrm{r}$.

Chlorophyll band, I0, 16 .

Chlorophyll-bodies, $6,45,48$, 257 (Figs. 3, 5, 43, 170, I7I).

Chlorophyll-granules, 6, 9, I0, $17,46,47,747,75^{\circ}$, 947 (Figs. 5, 44, 45).

Chlorophyll spectrum, 759 (Fig. 476).

Chlorophyllan, 758 .

Chlorophytum, 756, 833, 838 .

Chordarieæ, 283.

Chorisis, 605 .

Chromatin, 18, 945.

Chroococcaceæ, 8 I, 246, 3 I 8, 328.

Chroolepus, 329, 947.

Chrysobalaneæ, 662.

Chrysodium, 433.

Chrysomyxa, 33I, 956.

Chrysosplenium, 60I.

Chrysotannin, 767 .

Chytridinex, 25 I, 264, 957.

Cibotium, 428, 435, $44 \mathrm{I}$.

Cichoriaceæ, 86, 93, 883, 949.

Cichorium, 23, 883 (Fig. 20).

Cicinal dichotomy, 178 .

Cicinus, I80, 597 .

Cilia, 4, 39, 234 .

Cilia in Mosses, $38 \mathrm{r}, 383$.
Cinnamomum,643 (Fig.450).

Circæa, 646 .

Circulation of protoplasm, 39 .

Circumnutation, 855 .

Cirsium, 93, 640 .

Cissus, 653,866 .

Cistineæ, 659 .

Citrus, 9 I, 593, 616, 646 (Fig. 462 ).

Gladonia, 319, 327 (Fig. 222).

Cladophora, I $5,28 \mathrm{I}, 946$.

Clathrate cells, 89 .

Claviceps, 316, 317 .

Claw, 539.

Cleistogamous flowers, 908 .

Clematis, 174, 540, 554, 936 (Fig. I 29).

Cleome, 605.

Climbing plants, 862, 936, 962.

Climbing stems, 217,862 .

Clinostat, 773 .

Closed bundles, I10, 439, 629.

Closing-cells, 446.

Closterium, 260.

Clusiaceæ, 660 .

Coalescence of cells, 75 .

Cobæa, 569, 936.

Cobalt, 695.

Cocconema, 26 r.

Cocculus, I 35, 653 .

Cocoa-nut, 585 .

Cocos, 495 .

Coefficients of Heat Expansion, 727.

Cœlastrum, 70.

Colebogyne, 593, 902.

Cœeloblastæ, 244, 269.

Cœnobium, 252, 278 (Figs. I67-I69).

Cœnogonium, 323, 329 .

Coffea, ror, 586.

Coffee-berry, 587.

Cohesion, 222, 538, 6or.

Colchicum, 584, 622, 627, 939 (Fig. 422).

Coleochæte, 236, 237, 240, 288, 897, 900 (Figs. 186, I 87 ).

Coleochæteæ, 237, 24 I, 244, 286, 290, 306, 329, 904 .

Coleorhiza, I65, 588, 619 (Figs. I 23, I 24).

Coleosporium, 33 I.

Coleus, 652 .

Collateral bundles, I I 2.

Collateral chorisis, 605 .

Collema, 319, 328, 329 (Fig. 2I2).

Collemacex, 318.

Collenchyma, 24, 95, 98, 123 , 654 (Fig. 21).

Colleter, Ior. 
Colloids, 670 .

Colouring-matters, 764 .

Colours of leaves in autumn, 734 .

Columella, 345, 353, 354, $375,379,380,38 \mathrm{I}, 44 \mathrm{I}$ (Figs. 238, 266, 273).

Columnea, 222, 548,6 I I (Fig. 4 I6).

Columniferæ, 66r.

Colymbea, 507 .

Combined hybrids, 9 9 .

Combretaceæ, 662 .

Comesperma, 653 .

Commelynaceæ, 88, 632 (Fig. 86).

Common bundles, 155,156 , $407,496,530,629,649$.

Compositæ, $93,176,194,538$, $54 \circ, 555,57$ I $, 572,575$, $587,596,609,61_{3}, 61_{5}$, 6І7 $639,643,659,87$, 872 , 910 (Fig. 445).

Compound glands, 86, 9 I (Figs. 76, 77).

Concentric bundles, I 2.

Conceptacle, 28 I (Fig. I84).

Concussion, Irritability to, $88 \mathrm{I}$.

Condition of aggregation of organised structures, $66_{3}$, 960.

Conducting tissue for the assimilated food-materials, 711 .

Conducting tissue of style, 568.

Conduction of heat, 725 .

Cone of growth, 138 .

Confervaceæ, $28 \mathrm{I}, 329$.

Conidia, $223,239$.

Coniferæ, 93, 104, III, II3, I30, I 58, I $74,18 \mathrm{I}, 185$, 204, 2 г3,486, 490, 497, $507,531,743,958$.

Conjugatæ, 9, 244, 252, 257, 897 .

Conjugation, 8, 9, 224, 234, $253,257,26$ I, 264, 897.

Connective, 49I, 54I (Figs. $364,490)$.

Contortæ, 658 .

Contractile organs, 756,888 .

Convallaria, 579, 624 .

Convolvulaceæ, 88, 555, 658, 915.

Convolvulus, $217,862$.

Copper, 695.

Corallina, 65.

Corallineæ, 289.

Corallorhiza, $164,214,24 \mathrm{I}$, $620,697$.

Cordyline, 29.

Coriaria, I 87 (Fig. 144).
Cork, 20, 33, 95, 106, 415, 949 (Fig. 90).

Cork-cambium, 82, ro7 (Fig. 90).

Corm, 622 (Figs. 422, 423).

Cormophytes, $15 \mathrm{I}$.

Cornus, 540.

Cornaceæ, 66 r.

Corolla, 538.

Corollifloræ, $632,655,662$.

Corona, 213, 539 (Fig. 361).

Corpusculum, $486,498,52 \mathrm{I}$.

Corrosion by roots, 702 .

Cortex, 96, 108, 120, 289, 293.

Cortical sheath, $65^{\circ}$.

Coryanthes, 677 .

Corydalis, 535, 604, 61 8 , 906 .

Cosmarium, 259 (Fig. I7 I).

Costus, 623,625 .

Cotyledon, $214,426,447$, 448, 473, 499, 501, 507, $587,618,634,704,715$.

Crambe, 544 .

Crassulacex, 104, 194, 645, $652,661,949$.

Cratægus, I08, 65 I.

Craterospermum, 257.

Cremocarp, 6 I 5 .

Crest, 6 I 8.

Crinum, 98, 586, 618, 628 .

Critenchyma, I 23.

Crocus, 568, 580, 581, 583, 872,875 (Fig. 423 ).

Crown, 299.

Crozophora, 644 (Fig. 453).

Crucibulum, 7 I, 339 (Figs. $55,228,229,230$ ).

Cruciferæ, 104, 494, 573, $574,578,595,599,604$, $607,616,633,634,637$, 64I, 647, 657 (Fig. 4I3).

Crucifloræ, 646, 655, 657 .

Crustaceous Lichens, 319.

Cryptomeria, 527.

Crystalloids, 49, 55, 289, $672,947,948$ (Fig. 48).

Crystals, 53, 64, 84 (Fig. 52 ).

Cucumis, 569,925 .

Cucurbita, 22, 29, 32, 36, $43,58,89,495,545,554$, $634,635,637,868,925$, 952 (Figs. 34, 35, 74, 98, $99,368,379,444)$.

Cucurbitacex, I I I, 2 I 7, 555, $570,587,643,654,658$, $865,924,936,949$.

Cunninghamia, $518,527,533$.

Cunninghamieæ, 527 .

Cunonia, I01, I02.

Cunoniaceæ, $66 \mathrm{r}$.

Cuphea, 579.

Cupressineæ, 209, 493, 507, 526.
Cupressus, 509, 5 16, 517, 527, 532.

Cupule, 348, 369, 540 (Figs. 233,25 I).

Cupuliferæ, 535, 587, 633, 656.

Curvature of concussion, 787 .

Cuscuta, 21 7, 24I, 634, 637, $649,721,865$.

Cuscuteæ, 658 .

Cuticle, $34,98$.

Cuticularisation of the cellwall, 20, 33 (Fig. 37).

Cutleriex, 956.

Eyanophyceæ, 244, 246, 954.

Cyatheaceæ, 391, 435, 438, $44 \mathrm{I}$.

Cyathium, 647 .

Cycadeæ, 93, 486, 488, 489, 490, 491, 496, 497, 498, 500, 50I, 515, 519, 331, $573,950,958$.

Cycas, 493, 499, 503, $53 \mathrm{I}$ (Fig. 343).

Cyclantheæ, 63 I, 952.

Cyclanthera, 54I.

Cyclic, 600, 608, 64I, 657 .

Cyclomyces, 336 .

Cydonia, 578.

Cylindrocystis, 258 .

Cyme, $179,597$.

Cymose infloresence, 596.

Cymose umbel, $179,596$.

Cynanchum, 598.

Cynara, 733, 883, 892 (Fig. 473).

Cynaraceæ, 93, 881, 883 , 886.

Cynarex, 887, 89 1, 894 .

Cynoglossum, 54, 598.

Cyperaceæ, 538, 627, 631, 952.

Cyperus, 596 (Fig. 372).

Cypripedium, 548, 557,603.

Cystocarp, 292.

Cystococcus, 249, 329.

Cystocoleus, 329 .

Cystolith, 36, 65, 68, 948 (Fig. 53).

Cystopteris, 430.

Cystopus, 235, 275, 277 (Figs. I80, I 81 ).

Cystoseira, 282.

Cytineæ, $6 \mathbf{5 6}$.

Cytisus, 599, 943.

Dacrydium, 527.

Dahlia, 26, 63, I 19, I66, 713 , 921 , 939 (Figs. 25, 26, 29, $5 \mathrm{I}, 74)$.

Dally periodicity of growth, 823.

Dammara, 5 I I , 5 I 3, 51 ), 527 .

Danæa, 4 I 6. 
Datura, I6I, 6 I6.

Daucus, 493.

Davallieæ, 442.

Decussate, I89, I 98 .

Dédoublement, 549,605, 645 .

Definite inflorescence, 596 .

Degradation of chlorophyll, 47.

Degradation-products, 705 .

Dehiscent fruits, 6 I5.

Delesseria, 289 .

Delphinium, 578, 579, 608.

Dentaria, 640 .

Deposits in the cell-wall, 36 .

Derivative hybrid, 9 I 9 .

Dermatogen, 147, 500, 590, 59 I, 592, 952 (Figs. I I 4, I 22, 125 ).

Descent, Theory of, 940 .

Desmanthus, 883 .

Desmidiex, 252, 258, 260.

Desmodium, 757, 88r, 884 .

Deutzia, 36 .

Development of Sexuality, 901 .

Development of the members of a branch-system, I 76 .

Diageotropism, 854 .

Diagonal plane, 600 .

Diagram of Shoot, 188, I89, I $91,192,193,194,196$ (Figs. I 46 , I 48 , I 49, I50, I 5 I, I 52 , I 53 ).

Diagrams, Floral, 6or (Figs. I 47, 406-4I4).

Development of Varieties, 928.

Diaheliotropism, 854 .

Dialypetalæ, 659 .

Diandræ, $6{ }_{5} 8$.

Dianthus, 540, 917 .

Diastase, 708.

Diatomaceæ, 47, 244, 252, $260,956$.

Diatomine, 260 .

Diatoms, 36, 260.

Dicentra, 604.

Dichasium, 179, 597 (Fig. I 35).

Dichogamy, 906.

Dichotomex, 391, 460 .

Dichotomy, 169, 177, I78, 460 (Figs. I $33,134,137$, I38).

Diclinous, 490, 500, 907.

Dicotyledons, I3, I4, 129 I $33,486,498,555,590$, 633 .

Dictamnus, $92,175,563,601$, $605,85_{6}$ (Figs. $76,77,1_{3} \mathrm{I}$, $388,389,4$ I 4,485$)$.

Dictyostelium, 262 .

Dictyota, I 77 (Fig. I33).
Dictyoteæ, $283,956$.

Dicyclic, 6or.

Didymium, 262 (Fig. I72).

Differentiation of cell-wall, 32.

Differentiation of tissues, 138 .

Digitalis, 599, 9 r7.

Dilleniaceæ, I $36,646,653$, 657,950 .

Dimorphism, 907.

Diœcious, 490, 500.

Diœcism, 905 .

Dion, 502 .

Dionæa, 769, 894, .962.

Dioscoreæ, 632, 952 .

Diosmex, 660.

Diospyrineæ, 659 .

Diplostemonous, 600.

Dipsacaceæ, $6{ }_{5} 8$.

Dipterocarpeæ, 660 .

Directions of growth, 202, 206.

Discomycetes, 308.

Discophoræ, $66 \mathrm{r}$.

Displacement, 2 I 9.

Diurnal and nocturnal positions of organs, $88 \mathrm{I}$.

Divergence, Angle of, 188 .

Dorsiventral, 854, 954 .

Dorstenia, 22 r, 656 .

Doubling of the flower, 542 .

Draba, 605.

Dracæna, 1 27, I 28, 621,629 (Fig. 104).

Draparnaldia, $28 \mathrm{I}$.

Dried substance of plants, 695.

Drimys, $65 \mathrm{r}$.

Drosera, r6 1, 597, 894 .

Drupe, 6 I 6.

Dryadeæ, 22 I, 662.

Dudresnaya, 292.

Dwarf males, 279, 280, 899

(Figs. $16_{3}, 183$ ).

Ebenaceæ, 659 .

Echeveria, I 97, 597 .

Echium, 598, 6 I 4 .

Ectocarpeæ, 283.

Ectoplasm, 40.

Egg-apparatus, 580 .

Elæagnaceæ, 662 .

Elæagnus, 559 (Fig. 384).

Elaëis, 54 .

Elaphomyces, 3 I 5 .

Elasticity, 779. 784 .

Elater, 23, 344, 345, 350, $352,403,404$ (Figs. I8, $236,238,240$ bis, 246 , 286).

Elatineæ, 662.

Electricity, 768, 962 .

Elementary constituents of the food of plants, 695 .
Eleutheropetalæ, 655, 659 .

Eleutheropetalous, 539 .

Eleutherophyllous, 539.

Eleutherosepalous, 539 .

Elodea, 750.

Embryo of-

Dicotyledons, 634 .

Monocotyledons, 6 I 8 .

Embryo-sac, 486, 492, 498, $506,520,529,576,900$, 959 (Figs. 397-401).

Embryology of -

Angiosperms, 587, 959 .

Coniferæ, 525 (Figs. 354, 355).

Cycadeæ, 506.

Dicotyledons, 590 (Figs. 403-405).

Equisetum, 395 (Fig. 277).

Ferns, 425 (Figs. 296298.).

Gnetaceæ, 958.

Hepaticæ, 35 I, 353, 355, 360, 957 (Figs. 237 , $238,240,246$ ).

Isoëtes, 472 .

Monocotyledons, $\quad 589$ (Figs. 402, 404).

Mosses, 375, 957 (Figs. 257, 264).

Ophioglosseæ, 4 I 2.

Rhizocarpeæ, 447 (Figs. 3 I I, 3 I 3-3 I 5).

Selaginella, 47 I (Figs. 331,332 ).

Embryonal tubes, 529.

Emergences, I6r.

Empetraceæ, 662.

Empetrum, 570.

Empirical diagram, 602 .

Empusa, 277.

Emulsin, 708 .

Enantioblastæ, 625, 632 .

Encephalartos, 50 I.

Endocarp, 594, 6 I 5 .

Endocarpon, 329.

Endogenous formations, 162 , I 70, 359, 400.

Endophyllum, 330, 331 .

Endoplasm, 40.

Endosmotic force, 672 .

Endosperm, 472, 486, 492, $495,497,498,506,521$, $529,585,618,633,7$ 15, 900 (Figs. 1 24, 331, 346, $348,354,355,400,401$, 4 I9, 434, 435).

Endospore, 32, 344, 404, 42 I, 437.

Endostome, 570 .

Endothecium, 375, 556.

Energy of growth, 821 .

Entomophilous, 494, 908. 
Entomophthorex, 277

Epacrideæ, 644, 659 454).

Epen, I 2 I.

Epenchyma, $12 \mathbf{r}$.

Ephebe, 2 I (Fig. 216).

Ephedra, 528, 530, 65I.

Epibasal cell, 35 I, 395, 426, $447,473$.

Epicalyx, 540.

Epicarp, 594, 6 I 5.

Epidermal tissue, 79, 94.

Epidermis, 97 (Figs. 37, 7989).

Epigynæ, 658 .

Epigynous, 559 .

Epilobium, 601 (Fig. 380).

Epimedium, 570, 646 (Fig. 396).

Epinasty, 857.

Epipactis, 91 3 (Figs. 396, 492 ).

Epiphragm, 341, 383 (Fig. 273).

Epiphyllum, 538.

Epipogium, 164, 620, 633, 697,721 .

Epispore, 31 1444, 446, 458, 556 (Figs. 33, 310, 311, $314,315,324)$.

Epistrophe, 750.

Equisetaceæ, 142, 225, 385, $390,392$.

Equisetineæ, 390.

Equisetum, I 3, 36, 143, 173, 393 (Figs. IO, III, I 28 , 274-286).

Eranthis, 540.

Ergot, 3 I 6 (Fig. 209).

Ericaceæ, 601, 644, 659 (Fig. 454).

Eriocaulonex, 632.

Erodium, 644, 940.

Eryngium, 559 (Fig. 383 ).

Erysiphe, 3 I I (Fig. 207).

Erythrophyll, 767 .

Erythroxylaceæ, 660 .

Escallonia, 570.

Escallonieæ, 66r.

Etiolation, 754.

Etiolin, 743.

Eucalyptus, 640, 949 .

Eucyclæ, 655, 659 .

Eucyclic, 6оr.

Eudorina, 278.

Eugenia, 652.

Euonymus, 570, $65 \mathrm{I}$.

Euphorbia, 85, 86, I79, I 89, 597, 618, 647 (Figs. 74, I48).

Euphorbiacex, 86, 644, 66I.

Euphorbiex, 66r.

Eupodium, 419.

Eurotiaæ, 312.
Eurotium, 31 2 (Fig. 208).

Eusporangiata, 388 .

Evernia, 329.

Exalbuminous, 587. Excipulum, 323, 324 (Fig.
2 1 8 ).

Exobasidium, 336.

Exogenous formations, I54, I 70.

Exospore, 32, 344, 404, $42 \mathrm{I}$, 438.

Exostome, 570.

Extensibility, 779, 784 .

External sheath, 420.

Extine, 32, 505, 514, 553, 555 (Figs. 35, 36, 350, $35 \mathrm{I}$, $38 \mathrm{I})$.

Extra-axillary branching, I 75, 639 .

Extrorse, 557.

Fagus, 584 .

False dichotomy, I79 (Fig. I35).

False tissue, $7 \mathbf{r}$.

Fascicular cambium, 130 .

Fascicular tissue, 79 .

Fascicular xylem, 130.

Female prothallium, 444, 470.

Female reproduetive cell, $224,897$.

Ferments, organised, 248 , 249.

Ferments, unorganised, 60 , 708.

Ferns, $42 \mathrm{I}$.

Fertilisation, 224, 233, 267, $495,523,582,897$.

Festuca, 542.

Fibrovascular bundle of-

Dicotyledons, 649 (Figs. 93, 95).

Equisetum, 407.

Ferns, 439 (Figs. 94, 308).

Gymnosperms, $53^{\circ}$.

Ligulatæ, ${ }_{482}$.

Lycopodiaceæ, 468 .

Marattiacex, 420 .

Monocotyledons, (Fig. 92).

Ophioglossex, $4 \mathrm{r} 5$.

Phanerogams, 496.

Rhizocarpeæ, 459.

Fibrovascular bundles, 79, I08, 387, 949.

Ficaria, 577 .

Ficus, $36,68,85,98,220$ (Figs. 53, 159).

Fig, 220, 594, 6r4.

Filament, 49I, 54I.

Filices, 229, 39I, 42 I.

Filicineæ, 390, 409 .
Filiform apparatus, 580,582 , 959.

Filobacteria, 249.

Fissidens, 1 98, 364, 366 .

Flexibility, 779 .

Flexibility of internodes, 785.

Float, 395 .

Floral diagram, 6or.

Floral formulæ, 606, 625, 642 .

Floridex, 5 I, 238, 244, 288, 898.

Flower, 490, 538, 599, 625, $64 \mathrm{I}$.

Flowers of $\tan , \mathbf{2 6 2}$.

Fluorescence of chlorophyll, 760.

Fluorine, 695 .

Foliaceous Lichens, 319 (Fig. 2 I I).

Foliage-leaves, 214.

Foliose Hepaticæ, 347 .

Follicle, 6I 5.

Fontinalis, 153, 195, I99, 372 (Figs. I 16, 267).

Food-materials, 696 .

Foot, 344, 35 I, 387, 395, 426, $448,472,473$ (Figs. 238 , $26_{4}, 265,296,3$ I $\left.3,3 I_{4}\right)$.

Foramen, 492 .

Formative materials, 705 .

Fossil Equisetacex, 407.

Fossil Lycopodiaceæ, 484.

Fourcroya, 557, 568.

Four-fold pollen-grains, 557 .

Fovea, 475 (Fig. 334).

Foveola, 475.

Fovilla, 556 .

Fragaria, 568, 578 .

Fragmentation, 946 .

Francoacex, 66 .

Frangulineæ, 660,

Frankeniaceæ, 659 .

Fraxinus, 652 .

Free cell-formation, I0, 945 .

Freezing, effects of, $73 \mathbf{I}$.

Frenela, 517, 527 .

Fritillaria, 192, 621, 677 (Figs. I $5 \mathrm{I}, 42 \mathrm{I}$ ),

629 Fruit, 495, 593, 614.

Frullania, 347, 359, 362 .

Fruticose lichens, 3 I 9 .

Fucaceæ, 3, 28I, 956.

Fuchsia, 572.

Fucoideæ; 244, 28 I, 955.

Fucoxanthine, 766 .

Fucus, 3, 235, 281, 897, 905, 915 (Figs. 2, I84, I85).

Fumaria, $61_{3}$.

Fumariaceæ, 604, 646, 657 (Fig. 4II).

Funaria, 47, 97, 362, 367, $368,370,37$ I $, 373,381,915$, 
936 (Figs. 45, 82, 247, 253, $254,256,257,266,268-$ 272 .

Fundamental tissue, 79, I20.

Fungi, 227, 24 1, 697, 956.

Funiculus, 492, 570 .

Funkia, I 5, 23, 55 I, 572, 581, 593 (Figs. 1 2, I 9, 375, 376, 398, 399).

Gagea, 495, 598 .

Gamopetalæ, 655, 658 .

Gamopetalous, 22 I, 539.

Gamophyllous, 539.

Gamosepalous, 22 I, 539.

Garidella, 608 .

Gases, movements of, $69 \mathrm{I}$.

Gasteromycetes, 7 1, 307, 338 .

Gelatinous lichens, 320.

Gelidium, 289.

Gemmæ, I $72,223,342,348$, 369,423 (Figs. 234,25 r).

General conditions of Plant Life, 725.

Generating tissue, 82 .

Generations, Alternation of, $222,233,342,385,486$, 488,899 .

Genetic spiral, r 90.

Gentianaceæ, 658 .

Genus, 927.

Genus-hybrid, 9 5 .

Geoglossum, 3 I I.

Geographical distribution of plants, 943 .

Geotropism, negative, 839 . positive, 839 (Figs. 482 , 484). transverse, 854 .

Geraniaceæ, 660 .

Germination, 7 I 5, 728, 73 I.

Germination of seeds, 501 , 507, 619, 635 (Figs. 346, $\left.419,435,43^{6}\right)$.

Germination of spores, 258 , $259,262,268,293,333$, $346,36 \mathrm{I}, 393,4 \mathrm{I} 6,42 \mathrm{I}$, $44^{2}, 46 \mathrm{I}, 469,5 \mathrm{I} 5,52 \mathrm{I}$, 580,582 .

Gesneraceæ, 658.

Geum, 22 I, 579 (Fig. I6r).

Ginkgo, 520, 578 .

Glabrous, 99.

Gladiolus, 555.

Glands, compound, 9 I (Fig. 76). simple, 84 .

Glandular hairs, IOI, I6I (Figs. 77, 359).

Glans, 6 I 5 .

Glaucium, 588.

Gieba, 307, 34I (Fig. 23I).

Gleditschia, $218,639,652$, 935 .
Gleichenia, 43 I.

Gleicheniaceæ, 39r, 44r.

Globoids, 52 (Fig. 48).

Globularia, 648 .

Globulariaceæ, 658 .

Globule, 298 (Figs. r97, r98, 200, 201).

Glœocapsa, 246 (Fig. I65).

Glœocystis, 248.

Glœothece, 247 .

Gloxinia, 584 .

Glucose, 7 I 2.

Glume, 63 .

Glumifloræ, 63 I.

Glyptostrobus, 527 .

Gnetaceæ, 497, 498, 500, 527, $95^{8}$.

Gnetum, 527, 578, 959 .

Gomphrena, 580 .

Gonidium, 223, 239, 3 I 8.

Gonium, 253.

Goodeniaceæ, 659 .

Gramineæ, 631 (Fig. 409).

Grand period of growth, 817.

Granulose, 56, 60.

Graphideæ, 329.

Graphis, 319.

Grass, flower of, 603 .

Grasses, embryo of, 6 I 8 .

Grateloupia, 292.

Gravitation, action of, 770 , 839 (Fig. 482).

Grossulariaceæ, 66r.

Growing point, I 38 (Figs. I 08 - I I 4).

Growth-

action of electricity on, 962 .

action of gravitation on, 770, 839 (Figs. 477, $482)$.

action of light on, 752, 832,962 .

action of temperature on, 730,828 .

causes of, 775 .

directions of, 202 .

in length, 815 .

in length of the root and stem, 137.

in thickness of the cellwall, $19,32$.

in thickness of the root, $127,133$.

in thickness of the stem and root, 125.

in thickness of the stem of monocotyledons, I 27 .

mechanics of, 773 .

of starch-grains, 57 .

periodicity of, 823 .

Gruinales, 660 .
Guard-cells of stomata, 98 , I03 (Figs. 6I-65, 84-89).

Gum, 36, 93, 705.

Gum-passages, 93 (Fig. 66).

Guttiferæ, 660.

Gymnadenia, 578 .

Gymnoascus, 307, 308 .

Gymnocarpous, 306 .

Gymnocarpous lichens, 323.

Gymnogramme, 42 I.

Gymnosperms, 497, 498, 534, 897.

Gymnosporangia, 33 I, 335 .

Gymnostachys, 626 (Fig. $433)$.

Gymnostomous, 38 r .

Gymnostomum, 383 .

Gynæceum, 490, 557, 627, 64 I (Figs. 386, 387 ).

Gynandræ, 633.

Gynobasic style, 563, 568 (Figs. 389, 390).

Gynophore, 547, 563 (Figs. $37 \mathrm{I}, 388)$.

Gynostemium, 547, 627, 633 (Figs. 372, 492).

Hæmatococcus, 954.

Hæmodoraceæ, 632 .

Hairs, 99, I 50, 160 (Figs. $42,83)$.

Hakea, 124,652 .

Halidrys, 282 .

Haloragideæ, 662.

Halymenia, 2 y2.

Haustoria, 275, 8 I 2 (Figs. I 75, 276).

Heat-

action of, 731,884 . conduction of, 725 . production of, 723 . radiation of, 725 .

Heat-expansion, Coëfficients of, 727 .

Heating apparatus for the microscope, 736 (Fig. 474).

Hebenstreitia, 648 .

Hedera, $65 \mathrm{I}$.

Hedychium, 625 (Fig. 429).

Helianthemum, 577, 597.

Helianthus, 567 (Fig. 393).

Helicoid cyme, I 80, 597 (Fig. I36).

Helicoid dichotomy, 178 (Fig. 134 ).

Heliotropism, 832 . negative, 756,833 . positive, 756,833 . transverse, 854 .

Heliotropium, 598.

Helleborus, 495.

Helobiæ, 630 .

Helvella, $3 \mathbf{1}$. 
Hemerocallis, 597, 598 .

Hemicyclic, 600, $64 \mathrm{I}$.

Hemileia, 956.

Hepaticæ, 345, 346.

Heppia, 328.

Heracleum, 610 (Fig. 4I5).

Hermaphrodite, 490.

Herminium, 2 I 8 (Fig. 158).

Herpothamnion, 29I (Fig. 189).

Hesperidex, 66o.

Hesperidium, 6 I 6 .

Heterocyst, 247.

Heterocism, 332.

Heteromerous, 60I.

Heteromerous lichens, 320 .

Heterosporeæ, 389 .

Heterostylism, 907 .

Hibiscus, 6 I 6 .

Hieracium, 926.

Hildenbrandtia, 289.

Hilum, 492, 570, 618 .

Himanthalia, 282.

Hippocastaneæ, 644, 660 (Fig. 455).

Hippocrateacex, 660 .

Hippurideæ, 662 .

Hippuris, 1 55, 537, 649 (Figs. I19, 360).

Histology of -

Dicotyledons, 649 .

Filices, 438 .

Gymnosperms, 530 .

Ligulatæ, 48r.

Lycopodiacex, 467 .

Marattiacex, 420.

Monocotyledons, 629.

Ophioglossacex, 4 I 5 .

Phanerogams, 496.

Rhizocarpeæ, 459.

Holargidium, 605 .

Holly, 34, 35 (Fig. 37).

Hollyhock, 43, IOI (Figs. 42,83 ).

Homoiomerous lichens, 320 .

Hook-climbers, 962.

Hop, 862, 939.

Hormogonia, 954 .

Hoya, 29, 85, 668 (Figs. 30, $466)$.

Humiriaceæ, 660.

Hyacinthus, 77 (Figs. 6I-64).

Hybrid, 9 I 4.

Hybridisation, 91 4 .

Hydnora, 576 .

Hydnoreæ, 656 .

Hydnum, 336 .

Hydrangea, 652 .

Hydrangex, 66r.

Hydrilla, 630 .

Hydrille ${ }^{2}, 63 \mathrm{r}$.

Hydrocharidex, 625, 63I.

Hydrocharis, 624,626 .

Hydrodictyeæ, 244, 255.
Hydrodictyon, 256.

Hydrogen, 695, 697.

Hydropeltidineæ, 655,657 .

Hydrophyllace, 658 .

Hydropleon, 664.

Hydrophylleæ, 539

(Fig. 483 ).

Hydrotropism, 845.

Hymenium, 306 (Figs. 205, 218, 219, 227).

Hymenomycetes, 336 .

Hymenophyllaceæ, $42 \mathrm{I}, 44 \mathrm{I}$.

Hymenophyllum, 422 .

Hyoscyamus, 598 .

Hypecoum, 604 .

Hypericinex, 544, 660.

Hypericum, 545, 602 (Figs. $367,408)$.

Hyphæ, 84, $3 \circ 7$ (Figs. 55, 205, 214-21 7, 225, 229).

Hyphal tissue, 84 .

Hypobasal cell, 35r, 395, $426,447,472$.

Hypochlorin, $7 \mathrm{I}$.

Hypocotyledonary axis, 507, 635.

Hypoderma, 95, 98, I 23 (Fig. IO2).

Hypodermix, 335 .

Hypoglossum, 289.

Hypogynæ, 658.

Hypogynous, 559.

Hyponasty, 857 .

Hypophysis, 589 (Figs. 402405).

Hypothallus, 323.

Hypothecium, 324 .

Hypsophyllary leaves, 214 , 595.

Iberis, 649 .

Ice, Formation of, 73 I (Fig. 473).

Idioblast, $84,9 \mathrm{r}$.

Illicium, 6 I 5 .

Imbibition, 790 .

Impatiens, 555,637 .

Incombustible deposits in cell-wall, 36 .

Indefinite inflorescence, 595 .

Indehiscent fruits, $61_{5}, 6$ I 6 .

Indusium, 419, 435, 455, 575 (Figs. 303, 304).

Inferior ovary, 566 (Figs. 393, 394).

Inflorescence, 490, 495, 594 .

Innovation, 185,342 .

Insect-agency in pollination, 494, 906 (Figs. 488-492).

Insertion of leaves, 155 .

Integument, 492, 498, 570, 575 (Figs. 397, 398).

Intercalary growth of cellwall, 22.
Intercalary vegetative zone, 536, 816 .

Intercellular spaces, $7.5,76$, 93, 98 (Figs. 58-66, 78).

Intercellular substance, $\mathbf{7 2}$, 76, I 19 .

Interfascicular cambium, $\mathrm{r} 30$.

Interfascicular phloem, $\mathrm{I} 30$.

Interfascicular xylem, 130 .

Intermediate cells, I 19, 65 r.

Intermediate tissue, 123.

Internodes, $15^{6}$.

Interposed members, $60 \mathrm{r}$.

Intine, 32, 505, 514, 553, 555 (Figs. 345, 35 I, 380, $38 \mathrm{I}$ ).

Intrapetiolar buds, 639 .

Introrse, 557.

Intussusception, 29, 58, 666 .

Inulin, 62, 707 (Fig. $5 \mathrm{I}$ ).

Involucel, 596 .

Involucre, 540, 596 .

Iodine, 695 .

Ipomæa, 599.

Irideæ, 625,632 (Fig. 427).

Iron, 696, 699 .

Irritability, 865,878 .

Isatis, I 75 (Fig. I 32 ).

Isoëtex, $389,392,460$.

Isoëtes, 173, 182, 215,388 , 468, 958 (Figs. I 38,329 , $330,333,334)$.

Isocarpeæ, 655, 659 .

Isomerous, 60I.

Isosporex, 389 .

Isostemonous, 600 .

Ivy, 78, 837, 935, 938 (Fig. $66)$.

Jasminiaceæ, 658 .

Jasminum, $65 \mathrm{r}$.

Jerusalem artichoke, 42, 63.

Juglandeæ, 662 .

Juglans, 633 .

Julifloræ, 655 .

Juncace ${ }^{6} 632$.

Juncagineæ, 626, 63I (Fig. 432).

Jungermannia, 347, 356(Figs. 244, 246).

Jungermanniex, 343, 349, $35 \mathrm{I}, 356$.

Juniperineæ, 527 .

Juniperus, 509, $5 \mathrm{Ir}, 5 \mathrm{I} 3$, 523 (Figs. 349, 355).

Jussieua, 935 .

Justicia, 555 .

Kalmia, 6 I 6.

Karyokinesis, I7.

Kaulfussia, $4 \mathbf{1} 6$.

Kenenchyma, 83 .

Kerria, 651. 
Kitaibelia, 540.

Klugia, 598.

Knight's experiments on the influence of gravitation, 853.

L.abellum, 567, 913 (Figs. $394,492)$.

Labiatæ, 548, 579, 585, 6 I 3 , $633,648,658$.

Labiatilloræ, 658 .

Labium, 475 (Fig. 334).

Laburnum, 613.

Lamina of Leaf, 2 I $2,640$.

Laminarieæ, 283.

Lamium, 549, 578, 640 (Fig. $373)$.

Larix, 509, $5 \mathrm{I}$.

Lateral budding, I69 (Figs. I 28-I 32 ).

Lateral plane, 600 .

Lateral roots, I 66 (Fig. I 25 ).

Lateral shoots, 173.

Lathræa, 50, 648 .

Laticiferous cells, 85, 96 I (Figs. 69, 7 I).

Laticiferous vessels, 86, 705, 7 I 2, 948 (Fig. 72).

Latticed cells, 89 .

Lauraceæ, 643 .

Laurineæ, 657 .

Leaf, I 50, 1 53, I 57, I87.

Leaf, development of, in Ferns, 430.

Leaf-bearing axes, 153 .

Leaf-blade, 2 I $2,640$.

Leat-branching, I 82 .

Leaf-forms, 2 I 1.

Leaf-sheath, 396.

Leaf-spines, 2 I 5.

Leaf-stalk, 2 I $2,640$.

Leaf-tendrils, 2 I $4_{4}, 86_{5}$.

Leaf-trace, I 55, 496 .

Leaf-veins, 213 .

Leaflet, 2 I 2.

Legume, 6I.5.

Legumin, 7 I 9 .

Leguminosæ, 633, 7 I 8.

Lejolisia, I 81, 29 I (Fig. 190).

Lemaneaceæ, 289 .

Lemna, I64, 621, 626, 628 .

Lemnaceæ, 630 .

Lempholemma, 328 .

Lentibulariaceæ, 659 .

Lenticels, 108.

Lepidium, 604.

Lepidodendron, 484 .

Lepidostrobus, 485 .

Leptogium, 328 (Fig. 2 I 5).

Leptosporangiata, 388 .

Lessonia, 283.

Leucin, 7 I 8.

Leucojum, 628.

Levisticum, 183 (Fig. I40).
Leycesteria, 642 (Fig. 440).

Libocedrus, 509.

Libriform Fibres, 35, I 18 (Fig. 97).

Lichens, 23I, 24 I, 318.

Lichina, 328.

Lichnoërythrine, 766 .

Lichnoxanthine, 766 .

Light-

action of, 737 .

chemical action of, 743 . mechanical action of, 749,832 .

Lignification of the cell-wall, $20,34,705$.

Ligulatæ, 392, 46r, 468 .

Ligule, 212 , 392, 475, 539, 624 (Figs. $36 \mathrm{I}, 425$ ).

Liliaceæ, 584, 602, 606, 628, 632 (Fig. 406).

Liliifloræ, 625, 632 .

Lilium, $223,623$.

Limnanthaceæ, 660.

Linaceæ, 660.

Linaria, 640.

Lingula, 475 (Fig. 334).

Linnea, 579 .

Linum, 563 .

Liriodendron, 652 .

Lithium, 695.

Lithocysts, 84 .

Lithospermum, 598.

Loasaceæ, 659.

Lobelia, 642 (Fig. 442).

Lobeliaceæ, 86, 6 I $3,658$.

Loculicidal dehiscence, 6 I 6 .

Lodicule, 538, 627 .

Loganiaceæ, $6{ }_{5} 8$.

Lomentum, 6 I 5 .

Lonicera, 640, 642, (Fig. 440).

Loranthaceæ, $576,585,599$, $662,959$.

Loranthus, 588 .

Lunularia, 348,355 .

Lupinus, 52, 718 (Fig. 47).

Luzula, 577 .

Lychnis, 536, 539 (Fig. 361).

Lycogala, 262 .

Lycopodiaceæ, 184, 385, 392, $46 \mathrm{I}, 484,953$.

Lycopodieæ, I 84, 389, 39.2, 460.

Lycopodineæ, 460.

Lycopodium, 72, 388, 389, ${ }_{4}^{6}$ I, 466, 5 I 5 (Figs. 326$328)$.

Lygodium, 21 7, 432, 435, 862.

Lysimachia, 579, 638 .

Lythrarieæ, 662 .

Macrocystis, 283 .

Macrosporangium, 453, 459,
476, 480, 49I (Figs. 319$321,325,335$ ).

Macrospore, 31, 386, 389, $44^{2}, 455,457,458,470$, 491, 497 (Figs. 33, 310, $311,314,330,331,338$ ).

Macrozamia, 503.

Macrozoogonidia, $28 \mathrm{r}$.

Magnesium, 52, 696, 699 .

Magnolia, 549, 612, 652 .

Magnoliaceæ, 600, 608, 64 I, 657.

Mahernia, 579.

Mahonia, 543,880 (Fig. 362).

Maianthemum, 606.

Maize (Figs. I 8 bis, 4I, 50, 58,92 , I I 7, I.22-I 24 ).

Malachium, 563 .

Malaxis, 622 .

Male Prothallium, 442.

Male reproductive cells, 224 , 897.

Malope, 540.

Malpighiaceæ, 660.

Malvaceæ, 66 I.

Manglesia, 547 (Fig. 370).

Manubrium, 298 (Fig. I98).

Marattia, 64, 93, 4I6.

Marattiaceæ, 39I, 4I6, 882.

Marchantia, 23, 78, 348, 350, 356,962 (Figs. 18, 65, 89, $233-236,24$ I-243).

Marchantia, stomata of, 75, 106, 948 (Figs. 65, 89, 233 ).

Marchantiex, 343, 352, 355 .

Marcgraviaceæ, 660 .

Marsilia, I62, I84, 443, 446, $447,45 \mathrm{l}, 456,459,882$ (Figs. 120, 12 I, 142, $3 \mathrm{IO}$, 3 I 4,3 I $5,317,321,325)$.

Marsiliaceæ, $39 \mathrm{I}, 460$.

Mazus, 648 .

Mechanics of growth, 773 .

Mechanism ofheliotropism, 837 . geotropism, 853 (Fig. 482 .

movements, 887 .

Median plane, I 88, 600 .

Median wall, 426 .

Medullary rays, I20, I29, I 31, 531, 649 (Fig. I05).

Medullary sheath, I 30,53 I, 650 (Fig. 105).

Megacarpæa, 605.

Megaclinium, 88 I.

Megalospora, 325.

Melaleuca, $65 \mathrm{I}$.

Melampsora, $33 \mathrm{r}$.

Melampyrum, 648 .

Melastomaceæ, 662 .

Meliaceæ, 660 .

Melilotus, 493.

Melobesia, 65. 
Melobesiaceæ, 290.

Members, I 49 .

Menispermaceæ, 643,653 , 657 (Fig. 449).

Mentha, 638 .

Menyanthes, I6I, 595 .

Mericarp, 6 4 $_{4}, 6$ I $5_{5}$.

Merismopedia, 247, 954 .

Meristem, 80, 137 .

Mertensia, 43I.

Mesembryanthemex, 653, 662.

Mesocarp, 594, 6r5.

Mesocarpeæ, 257.

Mesocarpus, 257.

Mesophyll, 2 I 3.

Mesotænium, 258.

Metamorphosischemical, 708 . of organs, I 49, I 5 I, 934 .

Metaplasm, 37, 40 .

Metastasis, 703.

Metzgeria, r 40, r 8 I, 347, 356, 952 (Figs. 109, 110,137 , 232).

Micella, r 9, $66_{4}$.

Micellar aggregate, $66_{4}$.

Michauxia, 642,646 .

Micranthæ, $63 \mathrm{I}$.

Microcachrys, 527.

Micropyle, 492, 570 (Figs. $352,397,400$ ).

Microsomata, I 8, 947.

Microsporangium, 453,455 , $459,475,476,479$ (Figs. 309, 319-32I, 325, 334, $335)$.

Microspore, 386, 389, 442, 469, 49I, 497 (Figs. 309, $310,329,331$ ).

Microzoogonidia, 256, $28 \mathrm{I}$.

Mid-rib, 2 I 3.

Middle (central) lamella, 25 , 34, 72 (Figs. 24, 32, 38, 57).

Mignonette, I87 (Fig. I45).

Mimosa, 882, 888 .

Mimoseæ, 550, 557, 662, 879 .

Mirabilis, 538, 653 .

Mistletoe, 634,837 .

Mnium, 342, 363 .

Molecular forces, $66_{3}$.

Momordica, 940.

Monocarpellary, 560.

Monocarpic, 594.

Monocarpous, 560 .

Monochlamydeæ, 655,656 .

Monoclex, 352, 354 .

Monocotyledons, 498, 6 I 8.

Monocyclic, 601.

Monœcious, 490, 900.

Monœcism, 905 .

Monopodial inflorescence, 595.
Monopodium, I77 (Figs. I 19, I 23,132 ).

Monosymmetrical, 204,6 I 1 .

Monotropa, 214, 241, 577, $578,584,634,649,697$, $72 \mathrm{I}$

Monotropeæ, 659 .

Monsonia, 644 .

Monsterineæ, 84 (Fig. 70).

Moreæ, 656.

Morchella, $3 \mathbf{I} \mathbf{r}$.

Morel, 3 I I.

Mosses, 342 .

Mosses (true), $38 \mathrm{r}$.

Mougeotia, 258.

Movement-action of light on, 756 , 885 .

ciliary, 4, 232, 240, $75 \mathrm{I}$. dependent on oxygen, 722,886 .

influence of temperature on, 729,884 .

of chlorophyll granules, 750.

of protoplasm, 38,40 , $26 \mathrm{I}, 730,749,769$.

of water, 674 .

Movements-

induced, 878 .

of gases, $69 \mathrm{I}$.

of nutation, 855,857 , 862,866 .

of nutation, influence of light and temperature on, $87 \mathrm{r}$.

- periodic, 878 . periodic, influence of light on, 882 .

Mucilage, conversion of the cell-wall into, 20, 33,35,705.

Mucor, 265,266 .

Mucorini, 265, 267.

Mühlenbeckia, 65I.

Mulberry, 594, 6 I 4 .

Multilateral structure, 205.

Musa, 555, 621.

Musaceæ,625,632(Fig. 428).

Muscari, I74 (Fig. I 30).

Musci, 345, 36r.

Muscineæ, 342.

Mushroom, 307, 336 (Figs. $225-227$ ).

Mycelium, 226, 305, 307 (Figs. $174,175,180,181$, 204, 208, 225).

Myosotis, 598.

Myosurus, 56r, 578 .

Myricaceæ, 657 .

Myriophyllum, 49, I7 I.

Myristica, 492, 570, 587 .

Myristicaceæ, 657 .

Myrosin, 708.

Myrsinaceæ, 570, 659 .
Myrtaceæ, $66 z$.

Myrtifloræ, 662.

Myxoamæbæ, 10, 39, 252,261, 955 (Fig. 173).

Myxomycetes, 10, 4r, 244, 26 I, 945 .

Naiadex, 573, 6r8, 630 .

Naias, 49 I, 54I, 557, 565, 566, $573,575,627$.

Nardus, 603 (Fig. 409).

Nasturtium, 930.

Natural Selection, 929.

Natural System, 942.

Nectar, 494, 569 .

Nectary, 494, 569.

Negative geotropism, 839 .

Negative heliotropism, 756 , 833.

Nelumbiaceæ, 64I, 657 .

Nemalieæ, 289, 290.

Nemalion, 238 (Figs. I64, r88).

Neottia, I68, 214, 697, 721, 935.

Nepentheæ, 656 .

Nepenthes, 640, 677, 688, 934 .

Nephrolepis, 428, 43 I, 433 .

Nerium, 539, 652.

Nickel, 695 .

Nicotiana, 494 .

Nidularieæ, 339 .

Nigella, 542 .

Niphobolus, 429, 430.

Nitella, r 7, 49, 298, 300, 302, 905 (Figs. I 4, I98-202).

Nitophyllum, 290.

Nitric acid, 698.

Nitrogen, 691, 696, 698.

Node, 156.

Nostoc, 247.

Nostocaceæ, 247, 328 .

Nothoscordum, 593.

Nucellus, 487,497 (Figs. 348, $349,352,354,355,383$, 391, 397, 398).

Nuclear disc, I8, (Fig. 10).

Nucleïn, 947.

Nucleoli, 38, 44.

Nucleus, 2, 38, 44, 947 . division of, 17 .

Nucule, 299.

Nuphar, 575, 586, 6I 7, 648 (Fig. 68).

Nut, 6 I 5 .

Nutation, 855 (Fig. 485).

Nutmeg, 587 .

Nutrition, 775 .

Nyctagineæ, 137, 653, 66r, 950.

Nyctitropic, 873 .

Nymphæa, 538, 549, $5 \varepsilon 6$, 6 I $2,6+8$. 
Nymphæaceæ, 536, 586, 600, $633,641,654,657$.

Oak, 6 r 4 .

Obdiplostemonous, 60I.

Ochnacex, 660.

Ochrolechia, 326.

Octamerous, 642.

Octants, $35 \mathrm{I}$.

Edogonieæ, 244, 278.

Edogonium, 9, 22, 235, 279, 897, 945 (Figs. 17, 163, I82, I83).

Enotherex, 662.

Oidium, 3 I I.

Oil, 52, 9I , 706, 7 I 5 .

Oleacex, 643,658 (Fig. 448).

Olfersia, 436 .

Omphalaria, 328.

Omphalodes, 598.

Onagrarieæ, 555 .

Onopordon, 640.

Oocardium, 248.

Oogonium, 3, 234, 267, 27 I, 273, 279, 281 (Figs. 16 3 , I $77,179,181,183,185)$.

Oophore, 225, 342, 385, 421 , 488.

Oosphere, 224, 234, 267, 350, $355,373,425,446,486$, 523, 580, 897 (Figs. I63, I77, 179, 181, 183, 185, $236,240,256,295,3$ I 4 , 33 I, 354, 355, 399).

Oospore, 224, 234, 343, 47 I, 585.

Oosporeæ, 244, 267.

Opegrapha, 329 .

Open bundles, 650 .

Opening and closing of flowers, 872 .

Operculum, 345, 38I (Fig. 266).

Ophioglosseæ, 395,39 I, 4 I 0 .

Ophioglossum, 4 IO, 4 I 2, 414 , 958 (Figs. 288, 290).

Ophrydeæ, $557,603,628$.

Opuntia, 538, 566 .

Orchideæ, 557, 576, 579, $583,586,593,603,6$ I 8 , $627,633,908,960$ (Fig. 4 IO).

Orchis, 57 I, 578 (Figs. 397, 4I8).

Order of succession of the parts of the flower, 608 .

Organic centre, 203.

Organs of plants, 149.

Origin of species, 920 .

Ornithogalum, 580, 588 .

Orobanche, 214, 24I, 634, 649,935 .

Orobanchex, 585,658 .

Orthostichy, I88 (Fig. I54).
Orthotropic, 854 .

Orthotropous, 492,570 .

Oscillatorieæ, $246,247$.

Osmunda, 423, 435 .

Osmundaceæ, 391, 410, 437, 440.

Ouvirandra, 625 .

Ovary, 493, 497, 558 (Figs. 382-395).

Ovule, 487, 491, 497, 498, 570 (Figs. 343, 347$349,397,398,400$ ).

development of, in Angiosperms, 576 .

development of, in Coniferæ, 520 .

development of, in Gnetaceæ, 529.

Oxalic acid, 699 .

Oxalideæ, 660, 879 .

Oxalis, 882, 89I.

Oxygen, 69I, 696, 698.

Oxy-salts, 698.

Ozothallia, 282.

Pæonia, 6r 4 .

Paleæ, I50, 435, 602, 63 I.

Paliurus, 599.

Pallisade - parenchyma, 84, 533, 735 .

Palmaceæ, 624, 631 .

Palmella, 248.

Palmellaceæ, 46, 244, 248, 329.

Palms, 620, 623, 629, 938.

Pandanaceæ, $63 \mathrm{I}$.

Pandorina, 234, 254 (Figs. I62, I67).

Pandorineæ, 244, 253.

Panicle, 596.

Panicled inflorescences, 596.

Pannaria, 328.

Papaïn, 708.

Papaver, 87, 616, 641, 647, 654 (Fig. 464).

Papaveraceæ, 87, 647, 657 .

Papayaceæ, 87, 659 .

Papilionaceæ, 607, 609, 613.

Pappus, 538, 617, 940 .

Paraphyses, 309, 310, 325, $336,338,343,370,+35$, 44I (Figs. 205, 219, 227, 253).

-Parasites, 2 I 4, 242, 649, 720 , 942.

Parastichy, 194.

Paratonic action of light, 757.

Parenchyma, 83 (Fig. I).

Parietales, 659 .

Paris, I 88, 606 (Fig. I 47).

Parmelia, 327.

Parnassia, 642, 856 (Fig. 44I).

Paronychieæ, 645, 66 $\mathrm{I}$.
Parthenogenesis, 275, 593, 902.

Passiflora, 536, 865, 870.

Passiflorace, 659.

Pastinaca, I83 (Fig. I40).

Paullinia, 653 .

Pectinaceoussubstances, 706 .

Pediastrum, 70, 256 (Figs. 54, I69).

Pedicularis, 588, 648 .

Peduncle, $49 \mathrm{I}$.

Peganum, 6ro, 644 .

Peissomelia, 289.

Pellia, 352, 360, 395, 422, 945.

Peltigera, 3 I 9.

Pelvetia, 282.

Penicillium, 226, 306, 314.

Pentamerous, $64 \mathrm{I}$.

Peperomia, 579.

Perianth, $490,528,538$.

Periblem, I 47, I63, 500, 952 (Fig. I I 4).

Pericambium, I15, I34, I67 (Figs. 96, 106, I1 4, 125).

Pericarp, 594, 6 I 5.

Perichætium, 343, 37 I.

Periderm, 95, 106.

Peridium, 306, 333, 340 (Fig. 23 I).

Perigynæ, 66r.

Perigynium, 343, 350 (Fig. 236).

Perigynous, 559.

Periodic movements of organs, 878 .

Periodicity of growth in length, $8 \mathrm{I}_{7}, 823$.

Periodicity of tension, 808 .

Perisperm, 492, 586.

Peristome, 38I (Figs. 266, $267,273)$.

Perithecium, 308, 312, 313, 317,323 (Figs. 207, 208, 209).

Permanent tissue, 82.

Peronosporeæ, 244, 275, 955.

Persea, 555.

Pertusaria, 319, 326 (Fig. 220 ).

Petal, 538.

Petiole, 2 I 2, 640.

Peziza, II, 310 (Figs. 205, 206).

Phæosporeæ, $283,956$.

Phalloideæ, $34 \mathrm{I}$.

Phallus, 340 (Fig. 23I).

Phascaceæ, 377, 380.

Phascum, 375.

Phaseolus, 24, I 34, I 35, I 47, $56 \mathrm{I}, 6{ }_{3} 6$ (Figs. 2I, 106, I07, II $3,385,437$ ).

Phegopteris, 430, $44 \mathrm{I}$.

Phelloderm, 107 (Fig. 90). 
Phellogen, 82, 107 (Fig. 90).

Philadelpheæ, 66r.

Philodendron, 93.

Phloëm, I I I, I 19.

Phloëm-ray, I I I, I 3 I.

Phloëm-sheath, 440,468 .

Phlomis, 564 (Fig. 390).

Phœnix, 61 8 (Fig. 419).

Phosphorescence, 723.

Phosphorus, 696, 699.

Phototactic, 752 .

Phototonus, 757, 885 .

Phragmidium, 332.

Phycocyanine, 246, 766 .

Phycoërythrine, 289, 766 .

Phycomyces, 265, 267 (Fig. I 74 ).

Phycophæine, 282.

Phycoxanthine, 260, 766.

Phyllactidium, 329.

Phyllanthaceæ, 66r.

Phyllanthex, 66r.

Phylliscium, 328.

Phylloclade, 2 I 7.

Phyllocladus, I 58, 2 I 7, 509, $510,527$.

Phyllade, 476.

Phyllode, 222.

Phylloglossum, 462, 463 .

Phyllome, I 50, i 57.

Phyllophoraceæ, 289.

Phyllophyte, I 5 I.

Phyllopode, 484.

Phyllotaxis, 187, 194, 953 (Figs. $146-154$ ).

Phyllotaxis of Ligulatæ, 476 .

Physalis, 638 .

Physarum (Fig. I73).

Physcia, 327 (Fig. 222).

Physma, 327 (Fig. 222).

Phytelephas, 587.

Phytocrene, 653, 950.

Phytolacca, 645, 653 (Fig. 459).

Phytolaccaceæ, I36, 645, $66 \mathrm{r}$.

Phytophthora, 275.

Picea, 5 I 7.

Pileus, 336, 337 .

Pilobolus, 267.

Pilularia, 3 I, 45 I, 455, 457, 458,460 (Figs. 33, 318 , $320,322-324)$.

Pine-apple, $6 \mathrm{I}_{4}$.

Pinus, 25, 30, 72, 74, 94, 105, I 24, 508, 527, 533 (Figs. $23,24,32,60,78,88,102$, I03, 346, 356).

Piperaceæ, I37, 492, 496, $564,573,575,633,654$, $656,95 \mathrm{I}$.

Piperineæ, 655 .

Piptocephalidæ, 267, 955 (Fig. I75).
Pistia, 585,628 .

Pisum, 52 (Fig. ${ }^{6} 6$ ).

Pitcher-like organs, 677.

Pith, I 20.

Pitted vessels, 24, I I6 (Figs. 25-27, 97).

Pittosporeæ, 93, 660 .

Placenta, 452, 492, 574.

Placentation, 561,563 .

Plagiotropic, 854,954 .

Plane of insertion, I 88.

Plane of symmetry, 204.

Plantagineæ, 643, 658 (Fig. 447).

Plasmodium, ro, 39, 252, 262, $841,955$.

Platanaceæ, 656 .

Platanus, 639.

Platycerium, I 70, 432.

Pleomorphy, 232.

Pleon, $66_{4}$.

Pleospora, 3 I 6.

Plerome, I 1 5, I47, I63, 500, 952 (Fig. I 4 ).

Plerome sheath, II5, I24, 4I 5 (Fig. 96).

Pleurocarpous Mosses, 370.

Pleurococcus, 246, $253,329$.

Plocamium, 289.

Plumbagineæ, 659 .

Plumule, 499, 593.

Podocarpeæ, 527.

Podocarpus, 500, 5 ro, 527.

Podophyllum, 647 .

Podosphæra, 238, 3 I 2.

Podostemonex, 662.

Point of insertion, 188 .

Polanisia, 605 (Fig. 4 I2).

Polar nuclei, 580.

Polarised light, $66_{5}$.

Polemoniaceæ, 658 .

Pollen, Development of, I 2, I $4,15,32,505,55 \mathrm{I}, 55^{2}$ (Figs. I 2, 34, 374, 375, 378,379 ).

Pollen-grain, 23, 34, 224, $487,49 \mathrm{r}, 498,506,5 \mathrm{I} 4$, 553, 555, 900 (Figs. 36, $345,350,351,378,380$, $38 \mathrm{I}$ ).

Pollen-sac, 49I, 505, 512, $54^{2}, 55 \mathrm{I}, 55^{2}, 55^{6}$ (Figs. $374,377)$.

Pollen-tube, 33, 515, 522, 554, 568 (Figs. 35, 345, $354,355,376,395)$.

Poilination, 494, 583 .

Pollinium, 557, 9 I 3.

Pollinodium, $236,307,309$, 312, 313, 898 (Figs. 204, 207).

Polyblastia, 329.

Polycarpæ, 631, 657 .

Polycarpellary, $56 \mathrm{I}$.
Polycarpic, 594.

Polycarpous, 560.

Polychidium, 328.

Polycyclic, 601.

Polyembryony, 526, 593.

Polygala, I 36, 6 I 2 (Fig. 4 I 7 ).

Polygalaceæ, 1 36, 653,66o.

Polygamous, 535, 905 .

Polygamy, 905.

Polygonaceæ, 493, 565, 662.

Polygonatum, I 86, 6r9 (Figs. 1 43, 420).

Polygonum, 580, 638, 930.

Polypodiacex, 39 I, 42 I, 44 I.

Polypodiea, 44I.

Polysymmetrical, 204, 6 I r.

Polytrichum, 365, 366, 368, 384 (Fig. 273).

Pomeæ, 662.

Pomegranate, 616.

Pontederiaceæ, 632 .

Populus, 637,640 .

Pore-capsule, 6 I 6.

Porlieria, $65 \mathrm{r}$.

Porocyphus, 328 .

Portulacaceæ, 66r.

Posterior, 600.

Potamogeton, 625, 630 .

Potamogetoneæ, 586, 63 I.

Potassium, 696, 699.

Potato, 50, 59.

Potentilla, 609, 65I.

Pottia, 36r.

Pressure, Effect of, on growth, 809 .

Prickle, roo.

Primary bast, $65^{\circ}$.

Primary cortex, 1 27, 1 30, 532, 649.

Primary endosperm, 582.

Primary fibro - vascular bundle, I 29, 649 .

Primary meristem, 80, 82, I 37.

Primary root, I64, r66.

Primary tissue, 80 .

Primary wood, 53 1, 650.

Primine, 57 I (Figs. 397, 398).

Primordia, 609.

Primordial cell, 5.

Primordial epidermis, 147.

Primordial utricle, 42.

Primulace, 493, 565, 574, 644,659 (Fig. 456).

Primulineæ, 659 .

Procambium, I 10.

Products-

of degradation, 705 .

of degradation of chlorophyll, 47 .

Pro-embryo, 293 (Fig. I9r).

Pro-embryonic branch, 296.

Prolification, 490, 503.

Promycelium, 330 (Fig. 224). 
Pro-nucleus, 495.

Prosenchyma, 83, 950.

Protandrous, 9 I 0.

Proteaceæ, 662.

Proteids, $5 \mathrm{I}$.

Proten, I 2 I.

Protenchyma, I 2 I.

Prothallium, 225, 385, 393,

- 394, 427, 444, 447, 47 I, $486,498,521,522,582$.

Protococcus, 248,329 .

Protogynous, 9 ro.

Protomyces, 335, 957.

Protonema, 226, 342, 346 , $361,362,363,376$ (Figs. $247,284,250,259$ ).

Protophyta, 244, 245.

Protoplasm, 2, 37, 947.

Prototaxites, 272.

Pseudaxis, $178,180$.

Pseudocarp, 22 I, 594, 6 I 4 .

Pseudo-parenchyma, 84, 307.

Pseudopodiumof Mosses, 369 . of Sphagnum, 379 (Fig. 263).

Pseudotsuga, 533.

Psiloteæ, 392.

Psilotum, 164, 460, 464, 942, 957.

Psoralea, 81 (Fig. 69).

Pteris, 24, 27, 28, 30, 35, 109, I I 3, I 23, I 44, 2 I 6, 424, $426,428,429,431,439$ (Figs. 22, 27, 28, $3 \mathrm{I}, 3^{8}$, 84,9 I , 95, I I I, I I 2, I 56, 294, 296, 299-301, 307, 308).

Puccinia, 33 r, 333, 334 (Figs. $223,224)$.

Pulvinus, 880, 882, 889 .

Punctum vegetationis, 138 .

Punica, 65 I.

Purpose, 934.

Puschkinia, 495.

Putamen, 6r6.

Pycnidium, 308, 316, 326.

Pycnophycus, 282.

Pyrenomycetes, 3 I 6.

Pyrola, 562, 634 (Fig. 387).

Pyrolaceæ, 659 .

Pyrus, 559.

Pythium, 273.

Pyxidium, 616.

Quercus, 593, 636 (Figs. $43^{8}$, 439).

Raceme, 595.

Racemose branching, I79.

Racemose inflorescence, 595.

Racoblenna, 328.

Radial bundles, 949 .

Radiation of heat, 725 .
Radicle, 593, 635 .

Radula, 343, 346, 356, 362 (Fig. 245).

Rafflesiaceæ, 634,656 .

Ramalina, 327.

Ramenta, 428, 435.

Ramondieæ, $6{ }_{5} 8$.

Ranunculaceæ, 634, 657 .

Raphe, 492, 570.

Raphides, 65, 84, 88 .

Reaumuriaceæ, 660.

Receptacle-

of Flower, 22 I , 490, 559, 6 I 4 (Figs. I 59-I6I). of Mosses, 370 (Figs. $253,256)$.

Receptacles for secretions, 93.

Receptive spot, 27 I, 280,425 .

Reciprocal hybrids, 9 I 6.

Regular flowers, 6 I r.

Rejuvenescence of the cell, 8,945 .

Reproductive cells, 223,896 .

Reseda, 608 (Fig. I 45).

Resedaceæ, 608, 659 .

Reserve-materials, 704 .

Reservoir of reserve-materials, 704 .

Resin, 705 .

Resin-passages, 93, 94, 532, 949 (Fig. 78).

Respiration, 72 I.

Restiaceæ, 632 .

Resupination, 604 .

Retardation of growth by light, 755, 832.

Revolving nutation, 855 .

Rhamnaceæ, 660 .

Rheum, 565 (Fig. 39I).

Rhinanthus, 579,648 .

Rhizantheæ, 656 .

Rhizine, 3 I 9 (Fig. 2 I $_{4}$ ).

Rhizocarpeæ, 385, 391, 442, 957.

Rhizocarpon, 323.

Rhizoclonium, 28 r.

Rhizoid, 231, 293, 296, 367 (Figs. 80, I9I, 248).

Rhizome, 216.

Rhizophore, I68, 477.

Rhododendron, 557.

Rhodoraceæ, 601, 659 .

Rhodospermine, 50, 51, 298.

Rhus, 644 (Fig. 452).

Rhynchonema, 904, 920.

Ribes, ro7 (Fig. 90).

Riccia, 344, 35I, 354 (Figs. 239, 240).

Ricciex, 352, 354 .

Ricinus, 53, I I 2, I I 4, I 82 , $544,635,705,716$ (Figs. $48,93,94$, I $39,366,435$, $47 \mathrm{I})$.
Rivularia, 248.

Rivularieæ, 247, 328 .

Robinia, 584, $65_{2}$.

Roccella, 329.

Roestella, 33 r, 335 .

Root, I 50, I62.

Root-cap, I 44, I62, 952

(Figs. I I 2, I I 4, I 20-I 22 ).

Root-hairs, 162.

Root-pressure, 676, 685, 688 (Figs. 467, 469).

Root-sheath, 165 (Figs. I23, 124 ).

Root-system, 164 .

Roots-

branching of, $164,18 \mathrm{I}$ (Figs. I 25, 138 ).

fibrovascular system of, I I 4, 949 (Figs. 96, I06).

growth in thickness of, I 33 (Figs. 106, 107).

Rosa, 220 (Fig. I6o).

Rosaceæ, 662.

Rose-hip, 22 I, 6 I4.

Rosette, $52 \mathrm{I}$.

Rosifloræ, 662 .

Rostellum, 9I 3 (Fig. 492).

Rotation of protoplasm, 39 , 43.

Rubiaceæ, 643，658 (Fig. 446).

Rubus, 578 .

Rudbeckia, 599.

Ruscus, 2 I 7, 935.

Rutaceæ, 660.

Ruteæ, 66 。

Sabal, I 9 r (Fig. I 49).

Sabina, 516, 527 .

Sabulina, 578 .

Saccharomyces, $244,249$.

Saccharomycetes, 244, 249, 955.

Sagittaria, 74, 624 (Fig. 59). Salicineæ, 660 .

Salisburia, 512, 527 (Fig. 347).

Salix, 599.

Salvia, 9 I 2, 940 (Fig. 498).

Salvinia, I9 I, 39 I, 443, 444, $445,449,450,453$ (Figs. I 50, 309, 3 I I-3I3, 3 I6, 39).

Salviniaceæ, 391, 459.

Samara, 6 I 5.

Sambucus, 650 (Fig. $46_{5}$ ).

Samolus, 566, 599 .

Samydaceæ, 659 .

Sanguisorba, 578 .

Sanguisorbeæ, 662 .

Santalaceæ, 576, 662 .

Santalum, 579, 580, 58r.

Sap-vesicles, 42 . 
Sapindaceæ, 653, 660.

Sapindeæ, 660.

Saponaria, 535 .

Sapotaceæ, 659.

Saprolegnia, 897, 955 (Fig. 163).

Saprolegniex, 235, 244, 272, 955.

Saprophytes, 214, 242, 620, 649, 697, 721 , 942 .

Sarcina, 249, 954 (Fig. I66).

Sarcocarp, 6 I 5 .

Sarcogyne, 325 .

Sarracenia, 640 .

Sauromatum, I83, 624 .

Saurureæ, 656.

Saxifraga, $5^{62}$ (Fig. 386).

Saxifragaceæ, 642 , 661, 949 .

Saxifragineæ, $66 \mathrm{r}$.

Scabiosa, 593.

Scalariform vessels, 25, 27, I I 4,439 (Figs. 27, 94, 308).

Scale-leaves, 186,2 I 4 .

Scattered arrangement, I 88.

Schizæa, 432 .

Schizæaceæ, 39 I, $44^{\circ}$.

Schizandreæ, 657.

Schizocarp, 6I 4, 6I 5 .

Schizomycetes, 244, 248, 954 (Fig. 166).

Schultz's solution, 69.

Sciadopitys, 519, 527 .

Scilla, 597 .

Scirpus, 625 (Fig. 426).

Scitamineæ, 632.

Sclerantheæ, $66 \mathrm{t}$.

Scleranthus, 645 (Fig. 458).

Sclerenchyma, 35, 84, I 22, 125 (Fig. 28).

Scleroblasts, 83,84 .

Sclerotium, 306, 315, 317.

Scolecite, 3 I 0.

Scorpioid cyme, I80, 597 (Fig. 136).

Scorpioid dichotomy, 178 (Fig. 134).

Scorzonera, 87 (Fig. 72).

Scrophularia, 90 (Fig. 75).

Scrophulariacex, $65^{8}$.

Scutellum, r66, 6 I8 (Figs. $123,124)$.

Scutiform leaf, 444, 447 (Fig. 3II).

Scytonema, 248.

Scytonemeæ, 248, 328 .

Scytosiphon, 956 .

Secondary-

bundles, $136,653,950$. endosperm, 582 .

meristem, 82 .

phloëm (bast), I 27, I 3 I, 650.

products of metastasis, 705 .
Secondary-

roots, 164 .

wood (xylem), I 27, I 3 I,

$53 \mathrm{I}, 650,65 \mathrm{I}$.

Secretion-canals, 93 .

Secundine, $57 \mathrm{I}$.

Securidaca, ז $36,653,950$.

Sedum, 597 (Fig. 85).

Seed, $486,593,618,633$.

Segmentation of the apical cell, I 39, 95 I (Figs. 108I I 2).

Selaginex, 658.

Selaginella, 47,80 , I22, $47 \mathrm{r}$, $473,476,477,479,480$, $48 \mathrm{r}, 482,483,486$ (Figs. $44,67,100,33 \mathrm{I}, 335-34 \mathrm{I}$ ).

Selaginellex, 392, $48 \mathrm{r}$.

Senebiera, 604.

Senecio, 578.

Sepal, 2 I $4_{4}, 538$.

Septate Fibres, 118, 65I (Fig. 97).

Septicidal dehiscence, 6 I6.

Septifragal dehiscence, 616 .

Sequoia, 509, 527 .

Serjania, 653 .

Serpentarieæ, 656 .

Seta-

of Cyperaceæ, 538 .

of Muscinex, 344, 35 I,

Sexual -374 374 (Figs. 266, 273).

affinity, 916.

generation, 225.

reproduction, 223,896 .

reproductive cells, 224 , 897.

Sexuality, development of, 901 .

Sheath-teeth, 400 (Figs. 278, 282).

Shells, formation of, in the cell-wall, 32 (Figs. $35-38$ ).

Shield, 298 (Figs. 198, 200).

Shoot, $158,215$.

Sieve-cells, 22.

Sieve-plates, 22, 89, 118 (Figs. 98, 99).

Sieve-pores, 89.

Sieve-tubes, 88, I I 8, 948 (Figs. 74, 98, 99).

Sigillaria, 485.

Silenex, $66 \mathrm{r}$.

Silicon, 696, 700 .

Siliqua, 6 16.

Silphium, 598, 640 .

Silver grain, $130,65 \mathrm{i}$.

Simarubex, 660.

Simple glands, 84 .

Simultaneous whorls, 187 .

Sinningia, 580 .

Siphoneæ, 271, 955.

Sirogonium, 258.
Sirosiphon, 248, 328.

Sisymbrium, 599.

Sisyrinchium, 578 .

Skeleton of cell-wall, 36 .

Skeleton of starch-grain, 60 .

Sleep of plants, 873 .

Sodium, 696, 699 .

Soft bast, I 19 .

Solanaceæ, III , 219, 597, 658.

Solanum, $55^{6}$.

Solorina, 326.

Solubility of starch-grains, $6 \mathrm{r}$.

Sorby's researches on chlorophyll, 764 .

Sordaria, 316 .

Soredial branch, $32 \mathrm{I}$ (Fig. 217).

Soredium, 326 (Fig. 22 r).

Sorus, 435 (Figs. 292, 304, 32 I).

Spadicifloræ; 63 I, 884 .

Spadix, 595.

Sparmannia, 65 I, 884.

Spathe, 540, 624.

Spathularia, 3 I I.

Special mother-cells, 15, 32, 554 (Figs. 34, 378, 379).

Species, 927 .

Species, Origin of, 920.

Species-hybrid, 91 5.

Spectrum of chlorophyll,759.

Spermatia, 308, 326, 329, 333,898 .

Spermogonium,308, 316,326, 330, 333 (Fig. 223).

Spermothamniex, 290.

Sphacelariex, 283, 944, 965.

Sphacelia, 3 I 7.

Sphæria, 3 I 6.

Sphærobacteria, 249.

Sphærococcus, 289.

Sphæromphale, 329.

Sphæroplea, 235, 269.

Sphærotheca, 3 I I.

Sphagnaceæ, 377.

Sphagnum, 376, 377, 378, 379 (Figs. 81, 258-263).

Sphenophyllum, 408.

Sphere-crystals, $63,64,420$ (Fig. 5I).

Spicate inflorescences, 595.

Spicular cells, 66, 84 (Fig. $52)$.

Spike, 595.

Spilonema, 328.

Spine, 2 I8.

Spiræa, $65 \mathrm{I}$.

Spiræeæ, 662.

Spiral arrangement, 190.

Spiral flowers, 600 .

Spiral theory of phyllotaxis, 201. 
Spiral vessels, 22, 90, 114 , I 6 (Figs. 75, 95).

Spirillum, 249, 954 (Fig. I66).

Spirobacteria, 249.

Spirochæte, 249.

Spirogyra, 10, 16, 46, 234, 258, 946 (Figs. 5, 6, I3, I70).

Spirulina, 249.

Splitting of the cell-wall, 73 (Figs. 58, 59).

Spongy parenchyma, 84 .

Spontaneous periodic movements, $880,88 \mathrm{I}, 895$.

Sporangium, 388, 402, 4I4, $436,453,455,456,459$, $466,475,476,479,480$ (Figs. 285, 290, 292, 304, $305,319-322,325,327$, $334,335,337,338)$.

Sporastatia, 323 .

Spore, 223, 228, 382, 404, $43^{8}$.

Spores, Mode of formation of, 12 (Figs. 7, 10, 188, 205, 223, 227, 268-270, $286,305,306,322,323$, 337):

Sporidia, 330, 334 (Fig. 224 ). Sporocarp-

of Carposporex, 236 , 284 (Figs. I64, I87I90, I99, 204, 207, 208, 218 ).

of Rhizocarpeæ, $45 \mathrm{I}$, 452, 453, 455, 456, 459 (Figs. 317-321, 325).

Sporogonium, 226, 342, 350, $354,355,360,374,379$, 380,381 (Figs. 238, 240 , $246,257,263-266)$.

Sporophore, 225, 343, 387 .

Spur, 570 (Figs. 396, 491).

Squamarieæ, 292.

Stamen, 490, 505, 514, 529, $54 \mathrm{I}, 543,544,545,546$ (Figs. 344, 347-350, 362366,490 ).

Staminal leaves, 541 .

Staminode, 548 .

Staphisagria, 608.

Staphylea, 65 I.

Staphyleaceæ, 660.

Starch, 6, 56, 707, 747 .

Starch-forming corpuscles, 948.

Starch grains, 46, 56, 6I (Figs. 45, 49).

Staurospermum, 257.

Stem, I 50, 157.

Stem-tendrils, 2 I $7,865$.

Stephanosphæra, 255 (Fig. I68).
Sterculia, 547 (Fig. 37 I).

Sterculiaceæ, 66 I .

Stereocaulon, 319.

Sterigma, 326 .

'Stichococcus, 329.

Sticta, 320 (Fig. 2 I4).

Stigeoclonium, 4, 8 (Fig. 3).

Stigma, 493, 558, 568 (Figs. $382,385-387,390-392$, $395,491,492)$.

Stigma, of Muscineæ, 343, 373 .

Stigmaria, 485 .

Stigmatic cells, $35 \mathrm{I}$.

Stinging hairs, roo.

Stipulatæ, 390, 4 ro.

Stipule, 2 I 2,2 I $5,640$.

Stipules of Chara, 295.

Stolon, 217.

Stomata, 77, 102, 103, 104, I 05, 106, 678, 949 (Figs. $84-89$ ).

Stone, I $22, \mathrm{I} 25$.

Stone-cells, 84 .

Stone-fruit, 6 I 6.

Stratification of the cellwall, 19, 27, $94^{6}$.

Stratioteæ, 631 .

Stratiotes, 952.

Strawberry, 22 I, 594, 6I 4 .

Strelitzia, 555 .

Striation of the cell-wall, 19, 27 (Figs. 28, 30, 32).

Stroma, 3 I 6 (Fig. 209).

Strontium, 695.

Strophiole, 6r 8.

Struggle for existence, 929 .

Struthiopteris, 428, 433 , 435.

Strychnaceæ, 658 .

Strychnos, 587, 949.

Style, 558, 567 (Figs. 383 , $386,388-390,392,393$, $395,49 \mathrm{I})$.

Stylidieæ, 659.

Stylogonidium, 239.

Stypocaulon, 139 (Fig. 108).

Styracaceæ, 659 .

Suberous change of cell-wall, 20.

Subhymenial layer, 310,323 , 325, 333, $33^{8}$ (Figs. 7, 205, $218,219,227$ ).

Successive whorls, I 87 .

Succulent tissue, 83 .

Sugar, 708.

Sulphur, 696,698 .

Sulphuric acid, 698 .

Sunflower, 7 I, I 54, I 7 I (Figs. I 18, i 26).

Superior gynæceum, 560 .

Superposed, I 89, 601, 645 .

Surface-growth of the cellwall, 2 I, 946 .
Survival of the Fittest, $94 \mathrm{r}$.

Suspensor, 472, 499, 523, 588 (Figs. 332, 354, 35j, $399,400,402-405)$.

Swarm-spore, 4, I 2, 240.

Swartzieæ, 662 .

Swelling - up, 35, 6r, 668, 778 .

Swimming of swarm-spores, 38.

Symmetry, 204, 537, 610, 960.

Sympetalæ, 658 .

Sympetalous, 22 I, 539.

Symphoricarpus, 642 (Fig. 440).

Symphyllous, 539.

Symphytum, 577, 598.

Sympodial inflorescence, 597 .

Sympodium, 178, r80.

Synacmic, 910.

Synalissia, 327.

Synandræ, 658 .

Syncarp, 6 I 4 .

Synchitrium, 252

Synergidæ, 580, 959 (Fig. 399).

Synsepalous, $221,539$.

Syntagma, $66_{4}$.

Syringa, 652 .

System, Natural, 942.

Taccaceæ, 632 .

Tagma, $66_{4}$.

Tamariscineæ, 660 .

Tannin, 706.

Tap-root, 637

Tapetum, 388, 437, 49 I (Figs. $305,322,337,374,377)$.

Targioneæ, 356 .

Tasmannia, $65 \mathrm{I}$.

Taxineæ, 527, 958 .

Taxodineæ, 527, 958 .

Taxodium, 510, 517,527 .

Taxus, 513, 522, 527 (Figs. $348,354)$.

Tecoma, I 37,654 .

Teeth, $38 \mathrm{r}, 383$.

Teleutospore, 330 (Figs. 223 , 224 ).

Temperature, Influence of, 725.

Tendril, 2 I 4, 2 I $7,865,934$, 936 (Fig. 486).

Tension-

in plants, 787 . of tissues, 794 .

Terebinthace $, 93,660$.

Terebinthineæ, 660 .

Terminal branching, I 76 .

Ternströmiaceæ, 660 .

Testa, 486, 492, 593 (Figs. $\left.346,435,436,43^{8}\right)$.

Tetracyclæ, 655,657 . 
Tetragonidia, 289, $95^{6}$ (Fig. 190).

Tetragonieæ, 662 .

Tetramerous, $64 \mathrm{I}$.

Tetraphis, 369 (Figs. 25 , 252).

Tetrapoma, 605 .

Tetraspora, 248 .

Thalloid Hepaticæ, 346.

Thallome, I 5 I, ${ }_{5} 8$.

Thallophytes, I 5 I, 23 I, 244.

Thallus, $15 \mathrm{I}$.

Thamnidium, 267.

Theca, 344, 374 (Figs. 266, $267,271-273)$.

Theobroma, 635 .

Theoretical diagram, 602.

Theory of descent, 940 .

Thesium, 599, 648 .

Thickening-ring, I 29, I47, 653.

Thread-indicator, 827 .

Thuja, 5I4, 527 (Fig. 35r).

Thujopsidæ, 527.

Thunbergia, 34 (Fig. 36).

Thymelæaceæ, 662 .

Thymelæineæ, 662 .

Thyrsopteris, $4 \mathrm{I} 9$.

Tilia, $65^{2}$ (Fig. $4_{3}$ ).

Tiliaceæ, 646, 661.

Tissues, Forms and Systems of, 79 .

Tissues, Morphology of, 70, 948.

Tmesipteris, 462 .

Torenia, $58 \mathrm{I}$.

Torreya, 519, 527, 958 .

Torsion, 859 .

Torus, 490, 559 .

Trabeculæ, 479 (Fig. 334).

Tracheides, 75, I $16,949$.

Traction, Action of, on growth, 809 .

Tradescantia, 554, 577 .

Trama, 338 (Fig. 227 ).

Transfusion-tissue, 533 .

Transpiration, $678,96 \mathrm{I}$.

Transpiration current, 682.

Transport of assimilated substances, 7 I I

Transverse wall, 426 .

Trapa, 586, 592, 633 (Fig. $125)$.

Traube's artificial cells, 67 I, 961 .

Tree-ferns, 2 I 5, 433.

Tremellineæ, 336, $95^{6}$.

Trichoblast, 85 (Figs. 70 , 466).

Trichogyne, 238, 288, 290 329 (Figs.' I 64, I 88-190).

Trichomanes, $42 \mathrm{I}, 44 \mathrm{I}$.

Trichome, 150,160 .

Trichomes, of Ferns, 435 .
Trichophore, 238, 288, 292 .

Tricoccæ, 66r.

Tricyclic, 60I.

Trifolium, 599.

Triglochin, 577, 626 .

Trimorphism, 907 .

Triphragmium, 332.

Tritonia, 577 .

Trochodendron, $65 \mathrm{I}$.

Tropæolaceæ, 660 .

Tropæolum, 14, 586, 640, 865,936 (Fig. 486 ).

Truffle, 3 I 4 .

Tsuga, 527, 533 .

Tuber, 21 5, 2 I6, 226, 704, 935.

Tuber (truffle), 308, 315 .

Tuberaceæ, 3 I 4 .

Tubifloræ, 658 .

Tulipa, 7 I 5 (Fig. 470).

Tüllen, 24,8 ro.

Turgidity, $78 \mathrm{I}, 788,962$.

Turneraceæ, 659 .

Twining of climbing plants, 862.

Twining of tendrils, 865 .

Twining stems, 217,862 .

Tyloses, 24, 8 ro.

Type, $94 \mathrm{r}$.

Typha, 491, 54 I, 542, 564 .

Typhaceæ, 573, 631 .

Tyrosine, 7 I 8 .

Udotea, 272.

Ulex, 652 .

Ulmaceæ, 656 .

Ulmus, 584,940 .

Ulothricaceæ, 257.

Ulothrix, 252, 253, 257, 901, 902 .

Ulvaceæ, $28 \mathrm{I}$.

Umbel, 596 .

Umbel, Cymose, I85, 596.

Umbellicaria, 325 .

Umbelliferæ, 66r.

Umbellifloræ, 66 I.

Uncaria, 962 .

Unequal growth, 854 .

Unguis, 539.

Unicellular plants, 79, 232, 692.

Unilateral cicinal cyme, 597 .

Unilateral helicoid cyme, 597.

Uredineæ, $330,956$.

Uredo, 330.

Uredospore, 330, 333 (Fig. 223 ).

Urn, 344, 374 .

Uromyces, 332 .

Uropedium, 603.

Urticaceæ, I о0, 656 .

Urticeæ, 656.

Urticineæ, 656 .
Usnea, 320, $32 \mathrm{I}, 327$ (Figs.

$213,217,221$ ).

Ustilagineæ, $335,956$.

Utricularvessels, 88 (Fig. 73).

Utricularia, 639 .

Vaccinieæ, 659 .

Vaccinium, 648.

Vacuole, 3, 38, 4 o.

Vaginula, 344, 374 .

Valeriana, 643 (Fig. 443).

Valerianaceæ, 643,658 .

Vallisneria, 43, 769 .

Vallisnerieæ, $63 \mathrm{I}$.

Valonia, 946.

Vanilla, 935 .

Variation, 777 .

Variation of hybrids, 918.

Varieties, Origin of, 920 .

Variety, $92 \mathrm{I}$.

Variety-hybrid, 9 I 5.

Vascular Cryptogams, 385.

Vaucheria, 4 I, 244, 269, 270, 27 I, 897 (Figs. 40, 176, I77).

Vegetable ivory, $5^{87}$.

Vegetative cone, 138 .

Veins, 122.

Velamen, 98 .

Velum, 337, 475 (Fig. 225).

Venation, $213,624,64 \mathrm{I}$.

Ventral canal-cell, 350,386 , $395,425,446$ (Figs. 256, 295).

Veratrum, 596.

Verbascum, 577.

Verbenaceæ, 658 .

Veronica, 599.

Verrucarieæ, 329.

Versatile anther, 543.

Verticillate flowers, 600.

Vessels, 80, I I6, 949.

Vibratile cilia, 4, 240.

Vicia, 635, 882, 936 (Figs. $436,477)$.

Victoria regia, 640 .

Vine, $837,935,936$.

Viola, 568, 586, 9 I 2 (Figs. $395,400,40 \mathrm{I}, 49 \mathrm{I})$.

Violaceæ, 659 .

Virginian creeper, 837, 937 .

Viscum, 578 .

Vitis, 639, 865 (Fig. 457).

Volkmannia, 408.

Volvocineæ, 244, 278.

Volvox, 278, 945 .

Water, Ascent of, from the root, 685 .

Water, Currents of, in the wood, 679 .

Water, Exudation of, 676 .

Water, Movements of, 674 .

Water of crystallisation, $3 \mathrm{I}$. 
Water of organisation, 31,62. Xanthoxylaceæ, 66o.

Water-pores, 949.

Watsonia, 584 .

Wax, 99.

Wellingtonia, 509 .

Welwitschia, 66, 170, 528 , $53 \mathrm{I}, 953$.

Wendungszellen, 299, 302 (Fig. 202).

Whorl, i70, 187 .

Whorl, Spurious, I 70, 187.

Widdringtonia, 527 .

Wistaria, 653, 950 .

Wood, Irir.

Woodwardia, 433.

Wrangeliex, 290.

Xanthium, 215, 940.

Xanthophyll, 765 .
Xylaria, 3 I 6.

Xylem, I I I.

Xylem-portion of fibrovascular bundle, i 16 .

X ylem ray, I I I, I3I.

Xylophylla, 2 17, 935 .

Xyrideæ, 632.

Yeast-fungi, 249.

Yew, 5 I 2.

Yucca, 127, 629.

Zamia, 50r, 505, $95^{8}$ (Figs. $342,344)$.

Zanichellia, 618 .

Zea, 580, 620, 925 .

Zinc, 695 .

Zingiberaceæ, 625, 632 (Fig. 429).
Zoogonidia, 12,240 (Figs. 3 , 4, 9, I62, I67, I68, I69, $173,176,178,180,182$ ).

Zoospore, 234, 254 (Figs. I67, I68, I8I, I83).

Zoosporeæ, 253.

Zostera, 557.

Zygnema, 46, 258 .

Zygnemex, 257 .

Zygogonium, 258.

Zygomorphic, 204, 610, 6I r, $6 \mathrm{I}_{2}, 6 \mathrm{r} 3$.

Zygomycetes, 9, 244, 955.

$Z$ ygophyllaceæ, 660 .

Zygospore, 9, 224, 234, 254, 258, 265, 897 (Figs. I62, I67, 168, I70, I7 I, I74, I75).

Zygosporeæ, 244, 250. 


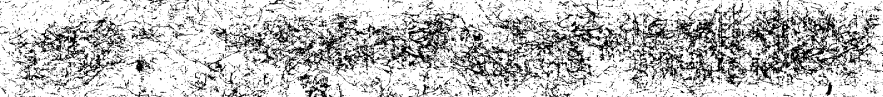

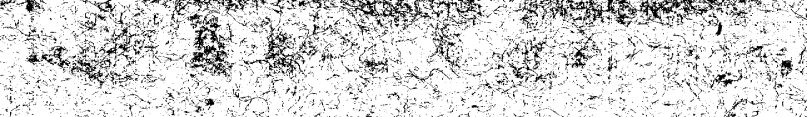

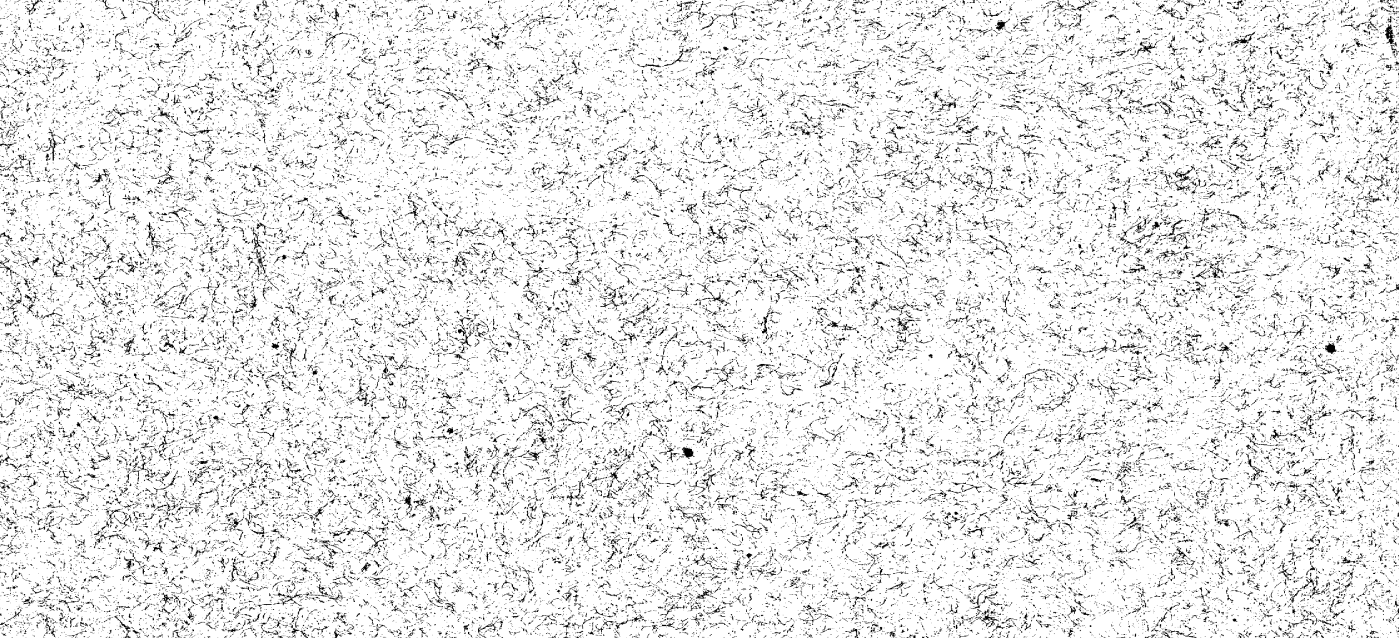


THE UNIVERSITY OF MICHIGAN

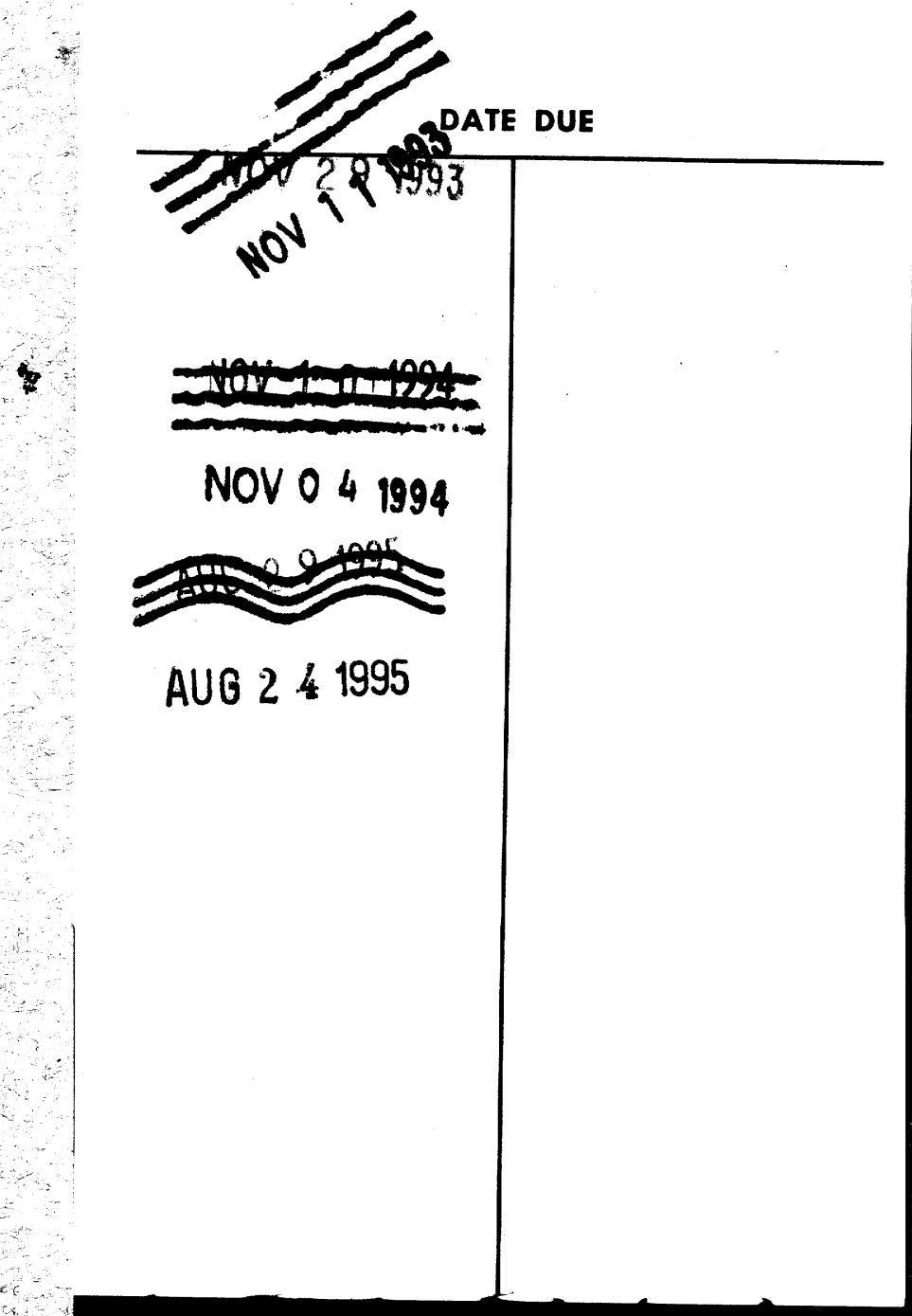





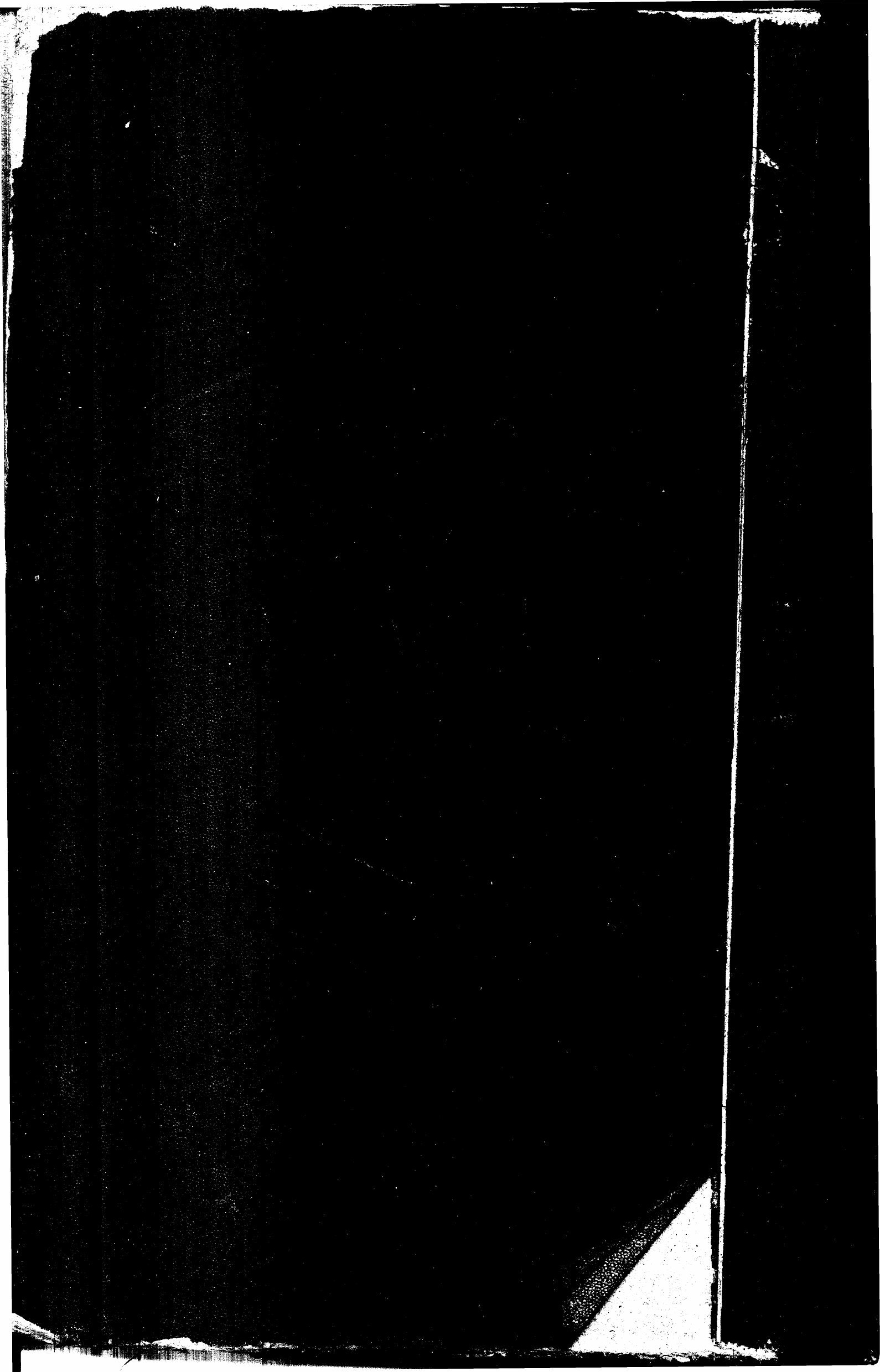

